\title{
1Q/2Q00 M-Area and Metallurgical Laboratory Hazardous Waste Management Facilities Groundwater Monitoring and Corrective-Action Report - First and Second Quarters 2000 - Volumes I, II, and II
}

by

J. Chase

Westinghouse Savannah River Company

Savannah River Site

Aiken, South Carolina 29808

This paper was prepared in connection with work done under the above contract number with the U.S. Department of Energy. By acceptance of this paper, the publisher and/or recipient acknowledges the U.S. Government's right to retain a nonexclusive, royalty-free license in and to any copyright covering this paper, along with the right to reproduce and to authorize others to reproduce all or part of the copyrighted paper. 
$y+x_{4}+\cdots+\cdots+\cdots$ 
WSRC-TR-2000-00242

Unclassified

\section{Q/2Q00 M-AREA AND METALLURGICAL LABORATORY HAZARDOUS WASTE MANAGEMENT FACILITIES GROUNDWATER MONITORING AND CORRECTIVE-ACTION REPORT (U)}

FIRST AND SECOND QUARTERS 2000

VOLUME I and II

Publication Date: September 2000

Authorized Derivative Classifier and Reviewing Official:

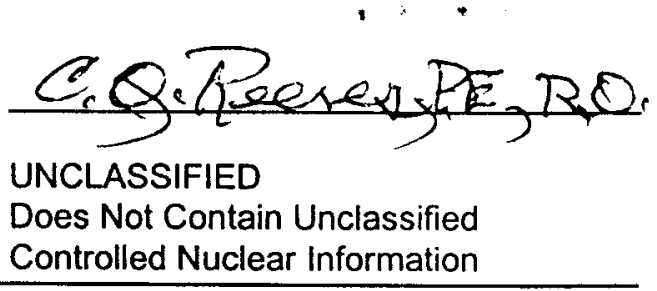

Westinghouse Savannah River Company Savannah River Site

Aiken, SC 29808 



\section{DISCLAIMER}

This report was prepared as an account of work sponsored by an agency of the United States Government. Neither the United States Government nor any agency thereof, nor any of their employees, make any warranty, express or implied, or assumes any legal liability or responsibility for the accuracy, completeness, or usefulness of any information, apparatus, product, or process disclosed, or represents that its use would not infringe privately owned rights. Reference herein to any specific commercial product, process, or service by trade name, trademark, manufacturer, or otherwise does not necessarily constitute or imply its endorsement, recommendation, or favoring by the United States Government or any agency thereof. The views and opinions of authors expressed herein do not necessarily state or reflect those of the United States Government or any agency thereof. 


\section{DISCLAIMER}

Portions of this document may be illegible in electronic image products. Images are produced from the best available original document. 
NOV $O 32000$

\section{OSTI}

\section{Q/2Q00 M-AREA AND METALLURGICAL LABORATORY HAZARDOUS WASTE MANAGEMENT FACILITIES GROUNDWATER MONITORING AND CORRECTIVE-ACTION REPORT (U)}

\section{FIRST AND SECOND QUARTERS 2000}

VOLUME I and II

Publication Date: September 2000

Authorized Derivative Classifier and Reviewing Official:

UNCLASSIFIED

Does Not Contain Unclassified

Controlled Nuclear Information

Westinghouse Savannah River Company

Savannah River Site

Aiken, SC 29808 


\section{DISCLAIMER}

This report was prepared as an account of work sponsored by an agency of the United States Government. Neither the United States Government nor any agency thereof, nor any of their employees, makes any warranty, express or implied, or assumes any legal liability or responsibility for the accuracy, completeness, or usefulness of any information, apparatus, product or process disclosed, or represents that its use would not infringe privately owned rights. Reference herein to any specific commercial product, process or service by trade name, trademark, manufacturer, or otherwise does not necessarily constitute or imply its endorsement, recommendation, or favoring by the United States Government or any agency thereof. The views and opinions of authors expressed herein do not necessarily state or reflect those of the United States Government or any agency thereof.

This report has been reproduced directly from the best available copy.

Available for sale to the public, in paper, from: U.S. Department of Commerce, National Technical Information Service, 5285 Port Royal Road, Springfield, VA 22161, phone: (800) 553-6847

fax: (703) 605-6900

email: orders@ntis.fedworld.gov

online ordering: http://www.ntis.gov/ordering.htm

Available electronically at http://www.doe.gov/bridge

Available for a processing fee to U.S. Department of Energy and its contractors, in paper, from: U.S. Department of Energy, Office of Scientific and Technical Information, P.O. Box 62, Oak Ridge, TN 37831-0062, phone: (865) 576-8401

fax: (865) 576-5728

email: reports@adonis.osti.gov 


\section{Contents}

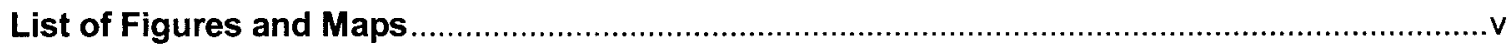

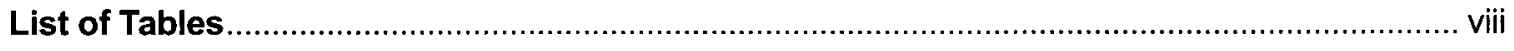

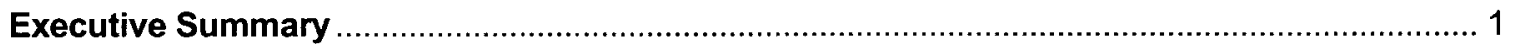

Introduction

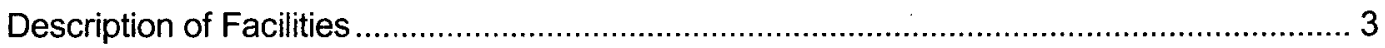

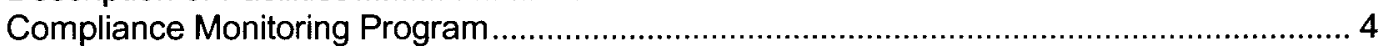

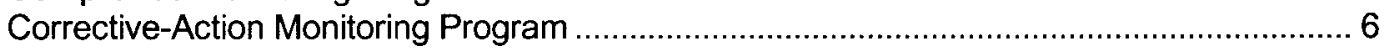

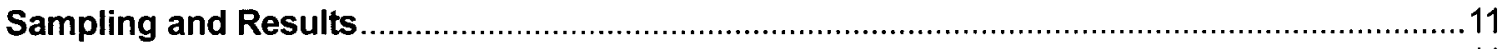

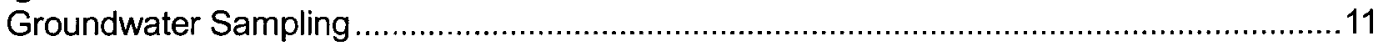

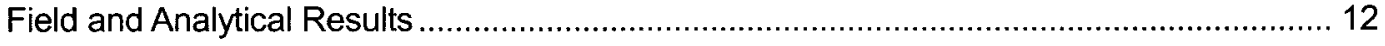

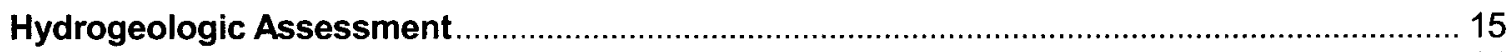

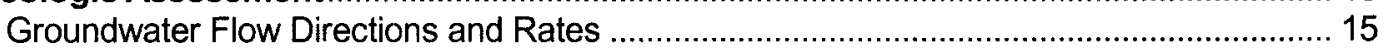

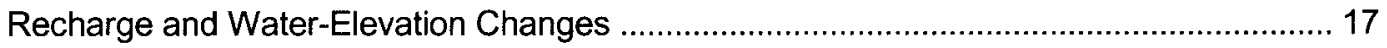

Effectiveness of the Corrective-Action Program ................................................................... 19

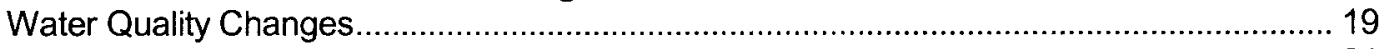

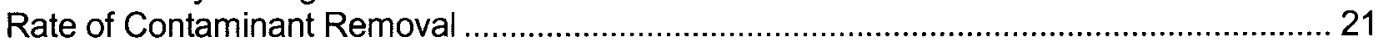

Corrective-Action System Operation and Performance ........................................................ 23

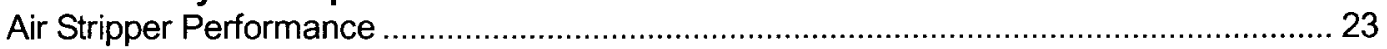

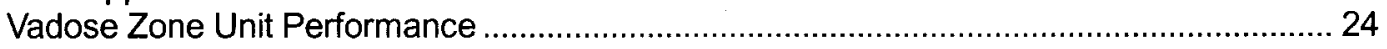

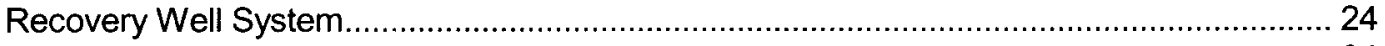

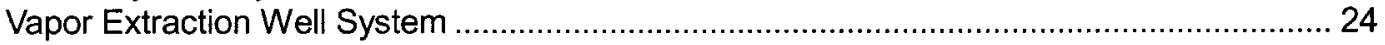

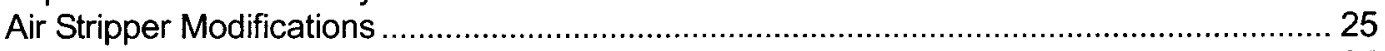

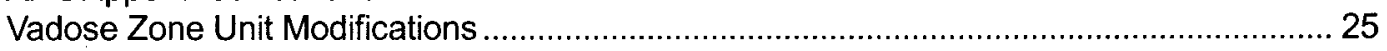

Monitoring and Corrective-Action Program Assessment............................................... 27

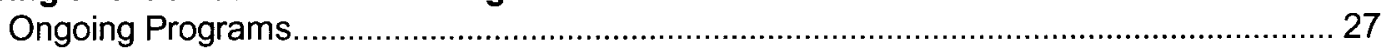

Proposed Monitoring and Corrective-Action Program Modifications .................................... 31

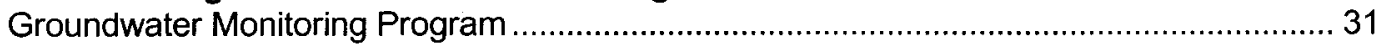

A/M-Areas Vadose Zone Remediation Program .................................................................. 31

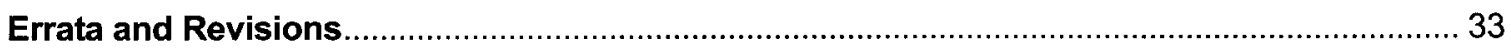

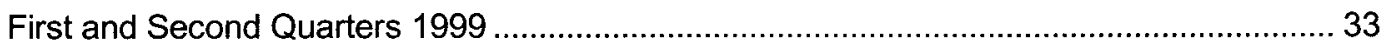

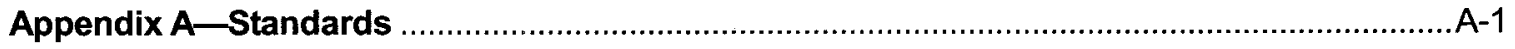

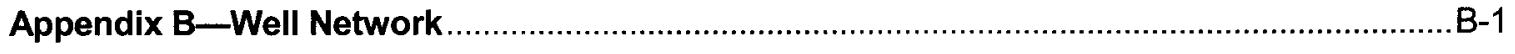

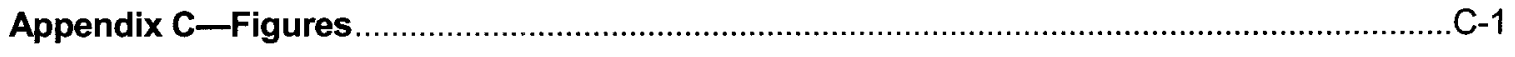

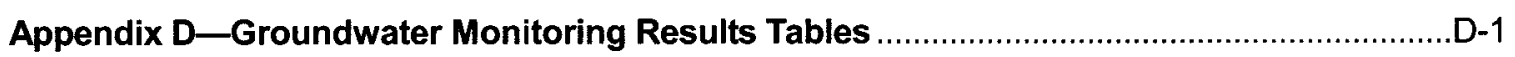


Volume II

Page

Appendix E-Data Quality/Usability Assessment

E-1

Appendix F-Time Series Plots $\mathrm{F}-1$

Appendix G-Hydrographs

G-1

Volume III

Page

Appendix $\mathrm{H}-$ Water-Elevation and Isoconcentration Contour Maps

. $\mathrm{H}-1$ 


\section{List of Figures and Maps}

Appendix C-Figures

Page

1. Location of the M-Area and Metallurgical Laboratory HWMFs at the Savannah River Site .....C-3

2. Location of Point-of-Compliance Wells at the M-Area HWMF .................................................

3. Location of Point-of-Compliance Wells at the Metallurgical Laboratory HWMF ........................-5

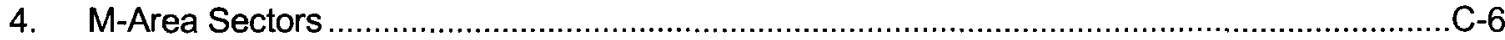

5. Location of Plume Definition and Background Wells at the M-Area HWMF …........................

6. Location of Plume Definition and Background Wells at the Metallurgical Laboratory HWMF ..C-8

7. Location of Recovery Wells at the M-Area HWMF

..C-9

Appendix H-Maps Page

1. Potentiometric Surface Map of the M-Area Aquifer Zone at the M-Area and Met Lab HWMFs,

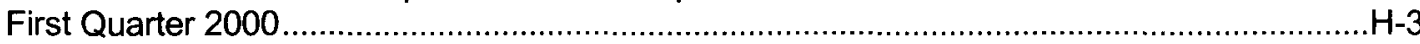

2. Potentiometric Surface Map of the Upper Lost Lake Aquifer Zone at the M-Area and Met Lab HWMFs, First Quarter 2000.

3. Potentiometric Surface Map of the Lower Lost Lake Aquifer Zone at the M-Area and Met Lab HWMFs, First Quarter 2000.

4. Potentiometric Surface Map of the Middle Sand of the Crouch Branch Confining Unit at the MArea and Met Lab HWMFs, First Quarter 2000

5. Potentiometric Surface Map of the Crouch Branch Aquifer Zone at the M-Area and Met Lab HWMFs, First Quarter 2000

6. Potentiometric Surface Map of the M-Area Aquifer Zone at the M-Area and Met Lab HWMFs, Second Quarter 2000

7. Potentiometric Surface Map of the Upper Lost Lake Aquifer Zone at the M-Area and Met Lab HWMFs, Second Quarter 2000.

8. Potentiometric Surface Map of the Lower Lost Lake Aquifer Zone at the M-Area and Met Lab HWMFs, Second Quarter 2000.

9. Potentiometric Surface Map of the Middle Sand of the Crouch Branch Confining Unit at the MArea and Met Lab HWMFs, Second Quarter 2000

10. Potentiometric Surface Map of the Crouch Branch Aquifer Zone at the M-Area and Met Lab HWMFs, Second Quarter 2000.

11. Zone of Capture and Tetrachloroethylene Isoconcentration Contour Map of the M-Area Aquifer Zone at the M-Area and Met Lab HWMFs, First Quarter 2000

12. Zone of Capture and Trichloroethylene Isoconcentration Contour Map at the M-Area and Met Lab HWMFs, First Quarter 2000.

13. Zone of Capture and Tetrachloroethylene Isoconcentration Contour Map of the Upper Lost Lake Aquifer at the M-Area and Met Lab HWMFs, First Quarter 2000

14. Zone of Capture and Trichloroethylene Isoconcentration Contour Map of the Upper Lost Lake Aquifer at the M-Area and Met Lab HWMFs, First Quarter 2000 
15. Zone of Capture and Tetrachloroethylene Isoconcentration Contour Map of the Lower Lost Lake Aquifer Zone at the M-Area and Met Lab HWMFs, First Quarter 2000

16. Zone of Capture and Trichloroethylene Isoconcentration Contour Map of the Lower Lost Lake Aquifer at the M-Area and Met Lab HWMFs, First Quarter 2000

17. Tetrachloroethylene Isoconcentration Contour Map of the Middle Sand of the Crouch Branch Confining Unit at the M-Area and Met Lab HWMFs, First Quarter 2000.

18. Trichloroethylene Isoconcentration Contour Map of the Middle Sand of the Crouch Branch Confining Unit at the M-Area HWMF, First Quarter 2000

19. Zone of Capture and Tetrachloroethylene Isoconcentration Contour Map of the Crouch Branch Aquifer Zone at the M-Area and Met Lab HWMFs, First Quarter 2000

20. Zone of Capture and Trichloroethylene Isoconcentration Contour Map of the Crouch Branch Aquifer Zone at the M-Area and Met Lab HWMFs, First Quarter 2000 


\section{List of Tables}

In-text

Page

1. A/M-Areas Precipitation Data for First and Second Quarters 2000

Appendix A

A-1. Standards

B-1. Well Network.

D-1. Synchronous Water Elevations

D-2. Groundwater Monitoring Results for Point-of-Compliance Wells, M-Area HWMF

D-3. Groundwater Monitoring Results for Background Wells, M-Area HWMF

D-4. Groundwater Monitoring Results for Plume Definition Wells, M-Area HWMF

D-5a. Groundwater Monitoring Results for Recovery Wells, January-April 1999 D5a-1

D-5b. Groundwater Monitoring Results for Recovery Wells, May-August 1999 D5b-1

D-5c. Groundwater Monitoring Results for Recovery Wells, September-December 1999 D5c-1

D-6. Groundwater Monitoring Results for Point-of-Compliance Wells, Met Lab HWMF. D6-1

D-7. Groundwater Monitoring Results for Background Wells, Met Lab HWMF D7-1

D-8. Groundwater Monitoring Results for Plume Definition Wells, Met Lab HWMF D8-1

D-9a. Groundwater Monitoring Results for QA Wells, Met Lab HWMF D9a-1

D-9b. Groundwater Monitoring Results for All Wells (Lab Duplicates), Met Lab HWMF D9b-1

D-10. Constituents Exceeding Groundwater Protection Standard or Monitoring Constituent Standard in Point-of-Compliance Wells, M-Area HWMF.

D-11. Constituents Exceeding Groundwater Protection Standard or Monitoring Constituent Standard in Point-of-Compliance Wells, Met Lab HWMF

D-12. Summary of Operation of the M-1 Air Stripper, First and Second Quarters 2000 D12-1

D-13. Summary of Operation of the A-2 Air Stripper, First and Second Quarters 2000 D13-1

D-14. Summary of Operation of the SVEU Vadose Zone Unit 782-3M, First and Second Quarters 2000

D-15. Summary of Operation of the SVEU Vadose Zone Unit 782-4M, First and Second Quarters 2000 D15-1

D-16. Summary of Operation of the SVEU Vadose Zone Unit 782-5M, First and Second Quarters 2000 D16-1

D-17. Summary of Operation of the SVEU Vadose Zone Unit 782-6M, First and Second Quarters 2000 D17-1

D-18. Summary of Operation of the SVEU Vadose Zone Unit 782-7M, First and Second Quarters 2000 D18-1 
D-19. Summary of Operation of the SVEU Vadose Zone Unit 782-8M, First and Second Quarters 2000 D19-1

D-20. Summary of Volume Pumped from Recovery Wells, First and Second Quarters 2000 . D20-1 D-21. Summary of Average Tetrachloroethylene and Trichloroethylene Concentrations in Recovery Wells, First and Second Quarters 2000 D21-1

D-22. Horizontal Gradients and Flow Rates in the Five Aquifers D22-1 


\section{Executive Summary}

This report describes the groundwater monitoring and corrective-action program at the M-Area Hazardous Waste Management Facility (HWMF) and the Metallurgical Laboratory (Met Lab) HWMF at the Savannah River Site (SRS) during first and second quarters of 2000. This program is required by South Carolina Hazardous Waste Permit SC1-890-008-989 and Section $264.100(\mathrm{~g})$ of the South Carolina Hazardous Waste Management Regulations. Report requirements are described in the 1995 RCRA Renewal Permit, effective October 5, 1995, Section IIIB.H.11.b for the M-Area HWMF and Section IIIG.H.11.b for the Met Lab HWMF. Program activities are summarized below.

- Sampling was conducted to determine compliance with the Groundwater Protection Standard (GWPS) and demonstrate the effectiveness of the corrective action program. Samples were collected and analyzed during first quarter of 2000 at M-Area HWMF and Met Lab point-ofcompliance (POC), background, and plume definition wells. M-Area HWMF recovery wells were sampled monthly. Semiannual monitoring is for $\mathrm{pH}$, specific conductance, temperature, and GWPS constituents (except polychlorinated biphenyls [PCBs]); only POC wells MSB 1D, 2D, 4D, and 59D are monitored for PCBs. The Monitoring Constituents and Appendix IX analyses are only conducted annually, during the third quarter of the calendar year.

- $\quad$ All 37 POC wells at the M-Area HWMF were sampled for semi-annual constituents listed in Appendix IIIB-A of the 1995 RCRA Renewal Permit. Approval was granted by SCDHEC (letter dated March 15, 1999; Frasier to Odum) to refine the list of inorganic Monitoring Constituents listed in Section II, Appendix IIIB-A of the permit (SCDHEC, 1995). The Monitoring Constituents SCDHEC approved for deletion from monitoring at the POC wells were: chromium, mercury, uranium, fluoride, cobalt (with the exception of the Annual Appendix IX analysis at $20 \%$ of POC wells), chloride, copper, zinc, manganese, sulfate, and total phosphates.

Samples from POC wells in the M-Area Aquifer Zone exceeded the Appendix IIIB-A Groundwater Protection Standard (GWPS) for tetrachloroethylene (PCE) (MSB 8A, 62D) and trichloroethylene (TCE) (MSB 62D). POC wells screened in the Upper and Lower Lost Lake Aquifer Zones also had TCE (MSB 6C, 7C, 8C) and PCE (MSB 7C, 8C, 63B) above the GWPS. One well in the Upper Lost Lake Aquifer Zone (MSB $8 C$ ) had 1,1-dichlorethylene above the standard, and one well in the Lower Lost Lake Aquifer Zone (MSB 63B) had chlorobenzene above the standard. (See the Field and Analytical Results subsection and Tables D-2 and D-10, Appendix D, Volume I.)

- All 6 background wells at the M-Area HWMF were sampled during first quarter 2000 . In the M-Area Aquifer Zone, MSB 29A had gross alpha and total radium above the GWPS, and iron above the Monitoring Constituent Standard (MCS). MSB 43D, screened in the M-Area Aquifer Zone and partially in the Green Clay Confining Zone, exceeded the GWPS (Appendix A) for lead. (See the Field and Analytical Results subsection and Table D-4, Appendix D, Volume I.)

- PCE, or TCE exceeded the GWPS in one or more of the plume definition wells at the M-Area HWMF during first quarter 2000 as follows: MSB 31C, 88B (M-Area Aquifer Zone); ABP 8C, MCB 5C, 6C, 7C, MSB 47C, 76C, 79C (Upper Lost Lake Aquifer Zone); MSB 21B, 31B, 33B, 36B, 47B, 66C, 68C, 69C, 74B, 89B (Lower Lost Lake Aquifer Zone); AMB 17A, AMB 18A, MSB 10A, MSB 33A, MSB 66B (Middle Sand Aquifer Zone-Crouch Branch Confining Unit); and MSB 42TA, 47TA (Crouch Branch Aquifer Unit). Concentrations of PCE and TCE and their distributions are provided in isoconcentration maps for each aquifer zone monitored. (See the Field and Analytical Results subsection and Table D-4, Appendix D, Volume I.)

- Monthly sampling events were scheduled for each recovery well during the first half of 2000 . The majority of recovery wells show a continued decreasing trend in TCE and PCE 
concentrations. (See the Field and Analytical Results subsection and Tables D-5a, b, and c, Appendix D, Volume I.)

- $\quad$ All 14 of the POC wells at the Met Lab HWMF were sampled for constituents listed in Appendix IIIG-A of the 1995 RCRA Renewal Permit. In the M-Area Aquifer Zone POC wells, no analyte exceeded the Appendix IIIG-A GWPS, and only sodium and sulfate exceeded the MCS (AMB 6, 8D, 9D) (Appendix A). In the Upper Lost Lake Aquifer Zone, lead (AMB 18C, 19C) exceeded the GWPS, and sodium and sulfate exceeded the MCS (AMB 19C), and in the Lower Lost Lake Aquifer Zone, only sodium exceeded the MCS (AMB 10B). However, one or more of the POC wells screened in the Middle Sand Aquifer Zone of the Crouch Branch Confining Unit had TCE (AMB 17A, 18A) and PCE (AMB 17A) above the GWPS, and total organic halogens (AMB 17A) above the MCS. (See the Field and Analytical Results subsection and Tables D-6 and D-10, Appendix D, Volume I.)

- All 8 background wells at the Met Lab HWMF were sampled during first quarter 2000. One of background wells in the M-Area Aquifer Zone (MSB 29D) contained gross alpha, total radium above the GWPS and iron above the MCS, and another (MSB 43D) exceeded the GWPS for lead. There were no analytes above standards in the background wells screened in the deeper aquifers. (See Field and Analytical Results subsection and Table D-7, Appendix D, Volume I.)

- All 8 of the plume definition wells at the Met Lab HWMF were sampled during first quarter 2000. In the M-Area Aquifer Zone, no analytes exceeded the GWPS but iron, sodium or sulfate exceeded the MCS in one or more of the wells. There were no analytes above the GWPS or MCS in the wells in the Upper or Lower Lost Lake Aquifer Zones. (See the Field and Analytical Results subsection and Table D-8, Appendix D, Volume I.)

- Statistical analysis of concentration trends of TCE and PCE are not performed on first quarter 2000 data. This evaluation is provided in the annual reports.

- Groundwater flow rates and directions are not significantly different from previous reports. Precipitation was below the historical average.

- During the first half of 2000 , the M-1 air stripper removed $5443.29 \mathrm{lbs}$. of solvent from the groundwater beneath the M-Area HWMF. (See the Air Stripper Performance subsection and Table D-15, Appendix D, Volume I.)

- During the first half of 2000 , the A-2 air stripper removed $759.3 \mathrm{lbs}$. of solvent from the groundwater beneath the M-Area HWMF. (See the Air Stripper Performance subsection and Table D-16, Appendix D, Volume I.)

- During the first half of 2000 , the SVEU Vadose Zone Unit 782-3M removed $7294.43 \mathrm{lbs}$. of solvent. (See the Vadose Zone Unit Performance subsection and Table D-17, Appendix D, Volume I.)

- During the first half of 2000, the SVEU Vadose Zone Unit 782-4M removed $9664 \mathrm{lbs}$. of solvent. (See the Vadose Zone Unit Performance subsection and Table D-18, Appendix D, Volume I.)

- During 1999, the SVEU Vadose Zone Units 782-5M and 782-8M are believed to have achieved their goals and have ceased operations. (See the Vadose Zone Unit Performance subsection and Table D-23, Appendix D, Volume 1.)

- During the first half of 2000 , the SVEU Vadose Zone Unit 782-6M removed $4996.93 \mathrm{lbs}$. of solvent. (See the Vadose Zone Unit Performance subsection and Table D-20, Appendix D, Volume 1.)

- During the first half of 2000 , the SVEU Vadose Zone Unit (formerly the IDU) $782-7 \mathrm{M}$ removed $1173.51 \mathrm{lbs}$. of solvent. (See the Vadose Zone Unit Performance subsection and Table D-22, Appendix D, Volume I.) 


\section{Introduction}

As directed by the 1995 RCRA Renewal Permit (SCDHEC, 1995), this report includes information on groundwater monitoring and corrective action at both the M-Area Hazardous Waste Management Facility (HWMF) and the Metallurgical Laboratory Hazardous Waste Management Facility (Met Lab HWMF). Requirements specified in Module III, Section B, M-Area Hazardous Waste Management Facility of the permit (SCDHEC, 1995) were approved for modification by SCDHEC (letter dated March 15, 1999; Frasier to Odum) in response to proposals for reducing monitoring requirements submitted to support SRS's permit modification request (Revision 12 to Volume III of the 1992 RCRA Part B Permit Renewal Application). SCDHEC reviewed the proposals, Technical Basis Report for Efficient Groundwater Monitoring at the A/M Area Network (WSRC-TR-98-00044) and Refinement of the List of Constituents for Groundwater Monitoring at M-Area (WSRC-TR00347) and outlined approval of specific portions of the SRS proposals in their response (letter dated March 15, 1999; Frasier to Odum).

\section{Description of Facilities}

\section{M-Area HWMF}

The M-Area HWMF, located in the northwest portion of the Savannah River Site (SRS) (Figure 1, Appendix C, Volume I), consists of the M-Area Settling Basin; a seepage area, overflow ditch, and inlet process sewer line; and Lost Lake, a shallow upland depression.

The unlined settling basin operated from 1958 until 1985, receiving wastewater that contained volatile organic solvents used for metal degreasing as well as chemical constituents and depleted uranium from fuel fabrication processes in M Area. The underground process sewer line transported M-Area process wastewater to the basin. Water periodically overflowed from the basin through the ditch to the seepage area adjacent to the ditch and to Lost Lake.

Because of the hazardous nature of the constituents released to the environment during operation of the basin, the M-Area HWMF is subject to the requirements of the Resource Conservation and Recovery Act (RCRA). Currently, the facility is operating under the 1995 RCRA Renewal Permit (South Carolina Hazardous Waste Permit SC1-890-008-989) (SCDHEC, 1995). A brief history of the M-Area HWMF compliance and post-closure programs is located in the Monitoring and Corrective-Action Program Assessment section of this report.

The AM-Areas corrective-action program addresses four sectors of the contaminant plume that are based on geography, subsurface conditions, and ongoing actions.

- The central sector includes the solvent storage and handling areas, the M-Area process sewer line, the M-Area Settling Basin, and the A-014 outfall area. Contamination came from direct release to the subsurface of large quantities of solvent with migration through the vadose zone and into the saturated zone. A separate DNAPL phase is present in both the saturated and unsaturated zones resulting in high aqueous concentrations typically above $1000 \mathrm{ppb}$ of TCE. The remediation strategy is to identify and remove DNAPL in the subsurface while continuing mass removal from the dissolved plume using the $\mathrm{M}-1$ air stripper recovery system. The majority of the concentrated contaminant plume and the primary remediation efforts are in this sector.

- The northern sector is outside the influence of the M-1 air stripper recovery system. This sector, which includes the Savannah River Laboratory Seepage Basins, the A-001 outfall, and part of the Savannah River Technology Center, is remediated by the A-2 air stripper recovery system.

- The western sector is west of the central sector and outside the M-1 air stripper zone of influence. Dense nonaqueous phase liquid (DNAPL) may have migrated and dispersed into 
this area. Although no remediation system is currently in place within the western sector, a proposal has been developed to perform remediation of DNAPL in the vicinity of the closed MBasin, which is believed to be the source of the Western Sector Plume. In addition a system of airlift wells is proposed for the distal portion of the plume. The proposed remedial actions were submitted to SCHDEC March 31, 2000

- The southern sector encompasses the contaminant plume south of the central sector outside the M-1 air stripper zone of influence. The contamination plume in the area is a result of advective transport processes from source zones in the Central Sector prior to installation of the $M-1$ pump and treat system. The plume is relatively large and has low concentration values. Groundwater flows south from this sector towards Upper Three Runs Creek. Remediation in the Lost Lake Aquifer Zone is currently addressed by a system of 12 vertical airlift recirculation wells at the distal portion of the plume. Other remediation technologies may be implemented as necessary.

\section{Met Lab HWMF}

The Met Lab HWMF is located in the eastern portion of the central sector of A/M Areas (Figure 1, Appendix C). The facility consists of the unlined Metallurgical Laboratory Basin, the abandoned portion of the influent process sewer line, an associated Carolina bay, and a drainage outfall to the bay.

Because of the hazardous nature of the constituents released to the environment during operation of the facility, the Met Lab HWMF is subject to the requirements of RCRA. Currently, the facility is operating under the 1995 RCRA Renewal Permit (South Carolina Hazardous Waste Permit SC1-890-008-989) (SCDHEC, 1995).

\section{Compliance Monitoring Program}

\section{M-Area HWMF}

\section{Requirements and Standards}

- Point-of-compliance (POC) wells (Figure 2, Appendix C, Volume I) monitor groundwater quality at the M-Area HWMF as mandated by RCRA and associated South Carolina regulations. Appendix IIIB-C in the 1995 RCRA Renewal Permit (SCDHEC, 1995) specifies the required monitoring regimen. This monitoring regimen was modified by SCDHEC (letter dated March 15, 1999; Frasier to Odum) in response to proposals for reducing monitoring requirements submitted to support SRS's permit modification request (Revision 12 to Volume III of the 1992 RCRA Part B Permit Renewal Application). SCDHEC reviewed the proposals, Technical Basis Report for Efficient Groundwater Monitoring at the AVM Area Network (WSRCTR-98-00044) and Refinement of the List of Constituents for Groundwater Monitoring at MArea (WSRC-TR-00347) and outlined approval of specific portions of the SRS proposals in their response (letter dated March 15, 1999; Frasier to Odum). quarterly monitoring for synchronous water elevations

- quarterly monitoring of new wells for one year for Groundwater Protection Standard (GWPS) constituents, inorganic monitoring constituents, and field parameters listed in Appendix IIIB-A of the permit

- semiannual monitoring for $\mathrm{pH}$, specific conductance, temperature, and GWPS constituents (except polychlorinated biphenyls [PCBs]); only POC wells MSB 1D, 2D, 4D, and 59D are monitored for PCBs. MSB 57D, 58D and 60D previously received monitoring for PCBs, but were approved for deletion from the groundwater monitoring well network (letter dated March 15, 1999; Frasier to Odum).

- annual monitoring for gross alpha, nonvolatile beta, total alpha-emitting radium, and the inorganic monitoring constituents listed in Section II, Appendix IIIB-A, of the permit 
- monitoring during the third quarter of each year of at least 20 percent of the POC wells for Appendix IX constituents (SCDHEC, 1994)

- Approval was granted by SCDHEC (letter dated March 15, 1999; Frasier to Odum) to refine the list of inorganic Monitoring Constituents listed in Section II, Appendix IIIB-A of the permit (SCDHEC, 1995). The Monitoring Constituents SCDHEC approved for deletion from monitoring at the POC wells were: chromium, mercury, uranium, fluoride, cobalt (with the exception of the Annual Appendix IX analysis at $20 \%$ of POC wells), chloride, copper, zinc, manganese, sulfate, and total phosphates.

Analytical results for the hazardous constituents listed in Section I, Appendix IIIB-A of the permit (SCDHEC, 1995) are compared to the GWPS as defined in Appendix IIIB-A of the permit (SCDHEC, 1995). The Monitoring Constituents listed in Section II, Appendix IIIB-A are compared to the Monitoring Constituents Standard (MCS), which is derived from the former GWPS as defined in WSRC (1994), M-Area HWMF background concentrations as defined in the 1987 Part B permit (WSRC, 1987), or drinking water standards (EPA, 1993a, 1993b). The GWPS and the MCS used for comparison are provided in Appendix A, Volume I. M-Area HWMF groundwater background concentrations were determined during the assessment monitoring program, using groundwater samples from background wells MSB 29B, 29C, 29D, 43A, 43B, and 43D (WSRC, 1987, 1994).

The SRS Environmental Restoration Department (ERD) reports the monitoring results to the South Carolina Department of Health and Environmental Control (SCDHEC) as required by the 1995 RCRA Renewal Permit (SCDHEC, 1995) and Section 264.100(g) of the South Carolina Hazardous Waste Management Regulations (SCDHEC, 1994).

\section{Point-of-Compliance Well Network}

Appendix $B$, Volume I, lists the wells in the groundwater monitoring network, and the units they monitor. Currently, the M-Area HWMF POC well network is composed of 37 wells: MSB 1B, 1C, 1D, 2B, 2C, 2D, 3B, 3C, 3D, 4B, 4C, 4D, 5A, 5B, 5C, 6A, 6B, 6C, 7A, 7B, 7C, 8A, 8B, 8C, 13A, 13CC, 13D, 39A, 59D, 62B, 62C, 62D, 63B, 63C, 63D, 64C, and 64D.

This network developed as follows:

- Wells MSB 1A, 2A, 3A, 4A, 5A, 6A, 7A, 8A, 13A, 13B, and 13C, installed in the early 1980s, were approved by SCDHEC as the original POC wells.

- Well MSB 22 was approved by SCDHEC for interim sampling to replace well MSB 3A.

- Sampling at wells MSB 1A, 2A, 3A, 4A, and 13C was stopped in 1990 due to declining water levels at the M-Area HWMF resulting from basin closure and the recovery system operation.

- Sampling of well MSB 22 as a POC well was stopped in 1991.

- Wells MSB 1B, 1C, 1CC, 1D, 2B, 2C, 2D, 3B, 3C, 3D, 4B, 4C, 4D, 5B, 5C, 6B, 6C, 7B, 7C, $8 \mathrm{~B}, 8 \mathrm{C}, 13 \mathrm{CC}, 13 \mathrm{D}, 57 \mathrm{D}, 58 \mathrm{D}, 59 \mathrm{D}, 60 \mathrm{D}, 62 \mathrm{~B}, 62 \mathrm{C}, 62 \mathrm{D}, 63 \mathrm{~B}, 63 \mathrm{C}, 63 \mathrm{D}, 64 \mathrm{~B}, 64 \mathrm{C}$, and 64D were installed in 1990 (MSB 1D, 2D, 3D, 4D, and 13D replaced wells MSB 1A, 2A, 3A, 4A, and 13C).

- Well MSB 13B was removed from the POC well network beginning third quarter 1994.

- Well MSB 39A was designated a POC well, and well MSB 64B, formerly a POC well, was designated a plume definition well in the new permit (SCDHEC, 1995).

- Wells MSB 1CC, 57D, 58D, and 60D were proposed for removal from the groundwater monitoring network in a permit modification request submitted to SCDHEC (Technical Basis Report for Efficient Groundwater Monitoring at the AVM Area Network dated June, 1998) to reduce the sampling requirements for the M-Area HWMF. SCDHEC granted approval to remove these wells from the groundwater monitoring program in response to the modification request (letter of March 15, 1999; Frasier to Odum). 


\section{Met Lab HWMF}

\section{Requirements and Standards}

POC wells (Figure 2, Appendix C, Volume I) monitor the groundwater quality at the Met Lab HWMF as mandated by RCRA and associated South Carolina regulations. Appendix IIIG-C of the 1995 RCRA Renewal Permit (SCDHEC, 1995) specifies the following monitoring regimen:

- quarterly monitoring for synchronous water elevations at all POC wells listed in Appendix IIIG$B$ of the permit

- quarterly monitoring of new POC wells for one year for GWPS constituents, inorganic monitoring constituents, and field parameters listed in Appendix IIIG-A of the permit

- semiannual monitoring for inorganic monitoring constituents, $\mathrm{pH}$, specific conductance, temperature, and GWPS constituents at all POC wells listed in Appendix IIIG-B of the permit

- annual monitoring for gross alpha, nonvolatile beta, and total alpha-emitting radium at all POC wells listed in Appendix IIIG-B of the permit

- monitoring during the first quarter of each year of at least 40 percent of the POC wells for Appendix IX constituents (SCDHEC, 1994)

Analytical results for hazardous constituents are compared to the GWPS as defined in Section I, Appendix IIIG-A of the permit (SCDHEC, 1995). Monitoring Constituents as defined in Section II, Appendix IIIG-A of the permit (SCDHEC, 1995) are compared to a Monitoring Constituent Standard which is derived from the former GWPS as defined in WSRC (1994), M-Area HWMF background concentrations as defined in the 1987 Part B permit (WSRC, 1987), or drinking water standards (EPA, 1993a, 1993b). The GWPS and the MCS used for comparison are provided in Appendix A, Volume I. M-Area HWMF groundwater background concentrations were determined during the assessment monitoring program, using groundwater samples from background wells MSB 29B, 29C, 29D, 43A, 43B, and 43D (WSRC, 1987, 1994). Data from background wells AMB 11D, and 12D were not included in development of the background standard (WSRC, 1987, 1994). Therefore the comparison is to groundwater background quality not impacted by contamination from the M-Area HWMF. ERD reports the monitoring results to SCDHEC to meet the requirements of the renewal permit (SCDHEC, 1995) and the South Carolina Hazardous Waste Management Regulations (SCDHEC, 1994).

\section{Point-of-Compliance Well Network}

Appendix $B$, Volume I, lists the wells in the groundwater monitoring network, and the units they monitor. Currently, the Met Lab HWMF POC well network is composed of 14 wells:

- 7 M-Area Aquifer Zone wells: AMB 4D, 5, 6, 8D, 9D, 10D, 16D

- 2 Upper Lost Lake Aquifer Zone wells: AMB 18C, 19C

- 1 Lower Lost Lake Aquifer Zone well: AMB 10B

- 4 Middle Sand Aquifer Zone of the Crouch Branch Confining Unit (CBCU) wells: AMB 4A, 10A, 17A, 18A

\section{Corrective-Action Monitoring Program}

\section{M-Area HWMF}

\section{Requirements and Standards}

Data from background, plume definition, and recovery wells (Figures 4 and 5 , Appendix $C$, Volume 1) are used to assess the effectiveness of the corrective-action program for the M-Area HWMF. Background and plume definition wells monitor the horizontal and vertical extent of groundwater contamination, groundwater flow rates and directions, and groundwater quality. Recovery wells 
pump contaminated groundwater to air strippers, which remove volatile organic contaminants from the water before it is returned to the ground.

The 1995 RCRA Renewal Permit (SCDHEC, 1995) specifies the monitoring regimen for these wells to meet RCRA requirements. This monitoring regimen was modified by SCDHEC (letter dated March 15, 1999; Frasier to Odum) in response to proposals for reducing monitoring requirements submitted to support SRS's permit modification request (Revision 12 to Volume III of the 1992 RCRA Part B Permit Renewal Application). SCDHEC reviewed the proposals, Technical Basis Report for Efficient Groundwater Monitoring at the AVM Area Network (WSRC-TR-98-00044) and Refinement of the List of Constituents for Groundwater Monitoring at M-Area (WSRC-TR00347) and outlined approval of specific portions of the SRS proposals in their response (letter dated March 15, 1999; Frasier to Odum).

\section{Background wells}

- quarterly monitoring for synchronous water elevations

- quarterly monitoring of new wells for one year for GWPS constituents, inorganic monitoring constituents, and field parameters listed in Appendix IIIB-A of the permit

- semiannual monitoring for $\mathrm{pH}$, specific conductance, temperature, and GWPS constituents (except PCBs)

- annual monitoring for gross alpha, nonvolatile beta, total alpha-emitting radium, and the inorganic monitoring constituents listed in Section II, Appendix IIIB-A, of the permit

Plume definition wells

- quarterly monitoring for synchronous water elevations

- quarterly monitoring of new wells for one year for GWPS constituents, inorganic monitoring constituents, and field parameters listed in Appendix IIIB-A of the permit

- semiannual monitoring for $\mathrm{pH}$, specific conductance, temperature, and GWPS constituents (except PCBs); selected plume definition wells are monitored semiannually for PCBs

- annual monitoring for gross alpha, nonvolatile beta, total alpha-emitting radium, and the inorganic monitoring constituents listed in Section II, Appendix IIIB-A, of the permit

- Approval was granted by SCDHEC (letter dated March 15, 1999; Fraiser to Odum) to refine the list of inorganic Monitoring Constituents listed in Section II, Appendix IIIB-A of the permit (SCDHEC, 1995). The SCDHEC approved deletion of chromium, mercury, uranium, fluoride, cobalt, chloride, copper, zinc, manganese, and total phosphates from the monitoring requirements for plume definition wells. In addition, SCDHEC (letter dated March 15, 1999; Fraiser to Odum) approved deletion of monitoring radionuclides (gross alpha, gross beta, and total radium) at most plume definition wells, continuing analyses only on samples from the wells in the MSB 17, MSB 18 and MSB 39 clusters.

\section{Recovery wells}

- monthly monitoring for water elevations

- monthly monitoring for $\mathrm{pH}$, specific conductance, temperature, and the organic GWPS constituents (except PCBs)

- semiannual monitoring for PCBs at recovery wells RWM 1, 6, and 10

\section{Piezometers}

- quarterly monitoring for synchronous water elevations

Analytical results for background, plume definition, and recovery wells are compared to standards and reported to SCDHEC as described for the POC wells. 


\section{Corrective-Action Well Network}

Wells were proposed for removal from the groundwater monitoring network in permit modification requests submitted to SCDHEC (Technical Basis for Proposed Modification to the Groundwater Monitoring Program, September 1996; Technical Basis Report for Efficient Groundwater Monitoring at the A/M Area Network dated June, 1998) to reduce the sampling requirements for the M-Area HWMF. In response, SCDHEC granted approval to remove some of the proposed piezometer wells (letter of March 4, 1997; Fraiser to Wells). SCDHEC granted approval to remove some of the proposed wells from the groundwater monitoring program in response to the modification request (letter of March 15, 1999; Fraiser to Odum). Appendix B, Volume I, lists the wells in the groundwater monitoring network, and the units they monitor. The network of background, plume definition, and recovery wells and piezometers currently consists of the following:

\section{Background wells ( 6 total)}

- 2 M-Area Aquifer Zone wells: MSB 29D, 43D

- 2 Upper Lost Lake Aquifer Zone wells: MSB 29C, 43B

- 2 Lower Lost Lake Aquifer Zone wells: MSB 29B, 43A

Plume definition wells (210 total)

1 well screened in a perched zone above the water table, 49 wells in the M-Area Aquifer Zone, 4 wells screened in the M-Area Aquifer Zone/Green Clay Confining Zone, 1 wells screened in the Green Clay Confining Unit, $\mathbf{4 3}$ wells screened in the Upper Lost Lake Aquifer Zone, 57 wells screened in the Lower Lost Lake Aquifer Zone, 1 well screened in the Upper Clay Confining ZoneCrouch Branch Confining Unit, 32 wells screened in the Middle Sand Aquifer Zone-Crouch Branch Confining Unit, and 23 wells screened in the Crouch Branch Aquifer Unit.

Recovery wells (17 total)

RWM 1, 2, 3, 4, 5, 6, 7, 8, 9, 10, 11, 12, 13B, 13C, 14B, 14C, 15B

Piezometers (57 total)

1 piezometer screened in the vadose zone; 26 piezometers screened in the M-Area Aquifer; 5 piezometers screened across the Green Clay Confining Unit such that the screen zone is in the M-Area Aquifer Zone/Green Clay Confining Zone/Upper Lost Lake Aquifer Zone; 6 screened in the Upper Lost Lake Aquifer, 9 screened in the Lower Lost Lake Aquifer, 3 screened in the Middle Sand Aquifer Zone-Crouch Branch Confining Unit, and 8 screened in the Crouch Branch Aquifer Unit.

\section{Met Lab HWMF}

\section{Requirements and Standards}

Data from background and plume definition wells (Figure 4, Appendix C, Volume I) are used to assess the effectiveness of the corrective-action program for the Met Lab HWMF. Background and plume definition wells monitor the horizontal and vertical extent of groundwater contamination, groundwater flow rates and directions, and groundwater quality.

Appendix IIIG-C of the 1995 RCRA Renewal Permit (SCDHEC, 1995) specifies the following monitoring regimen:

- quarterly monitoring for synchronous water elevations at all background and plume definition wells listed in Appendix IIIG-B of the permit

- quarterly monitoring of new background and plume definition wells for one year for GWPS constituents, inorganic monitoring constituents, and field parameters listed in Appendix IIIG-A of the permit 
- semiannual monitoring for inorganic monitoring constituents, $\mathrm{pH}$, specific conductance, temperature, and GWPS constituents at all background and plume definition wells listed in Appendix IIIG-B of the permit

- annual monitoring for gross alpha, nonvolatile beta, and total alpha-emitting radium at all background and plume definition wells listed in Appendix IIIG-B of the permit

Analytical results for background and plume definition wells are compared to standards and reported to SCDHEC as described for POC wells.

\section{Corrective-Action Well Network}

Appendix B, Volume I, lists the wells in the groundwater monitoring network, and the units they monitor. The network of background and plume definition wells currently consists of the following wells:

Background wells (8 total)

- 4 M-Area Aquifer Zone wells: AMB 11D, 12D; MSB 29D, 43D

- 2 Upper Lost Lake Aquifer Zone wells: MSB 29C, 43B

- 2 Lower Lost Lake Aquifer Zone wells: MSB 29B, 43A

Plume definition wells (8 total)

- 3 M-Area Aquifer Zone wells: AMB 7, 14D, 15D

- 1 Upper Lost Lake Aquifer Zone well: AMB 11B

- 2 Lower Lost Lake Aquifer Zone wells: AMB 4B, 7B

- 2 Middle Sand Aquifer Zone of the CBCU wells: AMB 7A, 13AR 
THIS PAGE LEFT BLANK INTENTIONALLY 


\section{Sampling and Results}

\section{Groundwater Sampling}

During first and second quarters 2000 , synchronous water elevations were measured on March $20-29,2000$, and June 24-29, 2000, respectively. Synchronous water levels for the first half of 2000 are presented in Table D-1 (Appendix D, Volume I).

The sampling procedure for wells with pumps (WSRC, 1992) requires evacuation of a minimum of two well volumes and stabilization of $\mathrm{pH}$, specific conductance, and turbidity prior to sample collection. Stability is established when a minimum of three successive measurements, taken at 10-minute intervals, are within 10 percent of each other.

If a well pumps dry before two well volumes are purged or before stabilization is achieved, it must be revisited within 24 hours for the data to be considered from a single sampling event. On the second visit within 24 hours, samples are taken without purging or stability measurements; thus, these samples may not be representative of the groundwater quality. Tables D-2 through D-8 (Appendix $D$, Volume I) show the number of well volumes purged for each well. The well volumes purged for some wells may be less than 2 because of rounding down in the calculations.

Variable-speed pumps are installed in wells that have histories of elevated metals and in some new wells. Samples from these wells are collected at a slower rate to minimize turbidity, which has been associated with elevated metals levels. Currently, variable-speed pumps are installed in wells AMB 14D, 15D, 16D, 18C, and 19C; MCB 5C; MSB 32B, 32C, 78DR, 87B, 87C, 88B, 88C, and $89 \mathrm{~B}$. Samples from wells MSB 15C, 16C, 42D, and $46 \mathrm{C}$ are collected using an open-bucket bailer. The remaining wells, except the recovery wells, have single-speed centrifugal downhole pumps. The recovery wells have pumps that run continuously. Pump types for all wells are indicated in Tables D-2 through D-8 (Appendix D, Volume I).

Samples were collected by RCS Corporation of Aiken, South Carolina, and analyzed by Environmental Science \& Engineering of General Engineering Laboratories of Charleston, South Carolina (South Carolina certification number 10120). Samples are treated as specified in the 1995 RCRA Renewal Permit (SCDHEC, 1995) and the Westinghouse Savannah River Company Quality Assurance Plan (WSRC, 1990).

A record of well installations, replacements, and abandonments is found in the EPD/EMS well inventory (EPD/EMS, 1997). The aquifer zones monitored by the POC well networks, and identified in this report in the Key to Reading the Tables section of Appendix D (Volume I), are defined in Ehrke et al. (1995).

Analytical results that exceeded the GWPS (Appendix A, Volume I) during first quarter 2000 are presented in this report (Tables D-2 through $D-10$, Appendix D). Previous sampling events in first and third quarters 1999 and third quarter 1998 also are included to fulfill permit requirement IIIB.H.11.b(i) for M Area and IIIG.H.11.b(i) for Met Lab. For simplicity, results that either equaled or exceeded standards are described as elevated, exceeding standards, or above standards.

\section{M-Area HWMF}

Groundwater sampling to determine water quality was conducted first quarter 2000 at POC, background, and plume definition wells and monthly at recovery wells. 


\section{Sampling Problems}

Tables D-2 through D-5 (Appendix D, Volume I) provide sampling codes (defined in the Key to Reading the Tables section of Appendix D, Volume I) that describe unusual sampling events for the M-Area wells.

Well MSB 3D contains DNAPL with dissolved PCBs in sediments trapped in the well sump (memorandum SWE-ERG-93-0966, M. A. Ebra, SRS, to J. W. Cook and J. V. Odum, SRS, December 7,1993$)$. This well is no longer sampled regularly for groundwater quality because of waste disposal issues.

Maintenance or access problems during first quarter 2000 for the remaining wells are summarized as follows:

- Well MSB 46C was scheduled but not sampled due to mechanical problems.

- Wells MSB $16 \mathrm{C}$ and $87 \mathrm{C}$ were not sampled during first quarter 2000 because there was no water to the surface in these wells.

- Well MSB 19A was abandoned in place prior to the first quarter 2000 sampling period.

- Well MSB 35A was not sampled due to a scheduling error. MSB 35A will be scheduled for sampling during third quarter 2000 .

- Well MSB 66D was not sampled because the well produces too much sand despite efforts at redevelopment.

- Well MSB 67D was dry.

- Well MSB 9C was dry.

\section{Met Lab HWMF}

Groundwater sampling to determine water quality was conducted during first quarter 2000 at POC, background, and plume definition wells.

\section{Sampling Problems}

Tables D-6 through D-8 provide sampling codes (defined in Key to Reading the Tables, Appendix D) that describe unusual sampling events for the Met Lab wells.

Unusual sampling events during first quarter 2000 are summarized as follows:

- Well AMB 14D was scheduled for sampling but was not sampled because it was dry.

\section{Field and Analytical Results}

The Key to Reading the Tables section of Appendix D (Volume I) defines abbreviations used in the data tables and provides discussions of holding times, data rounding, and data qualification. The section includes a description of the result modifiers used in Appendix D. The modifiers help define analytical accuracy and precision. Appendix $E$ (Volume I) provides a general assessment of the quality and usability of the data.

\section{M-Area HWMF}

Tables D-2 through D-5 (Appendix D) present the field and analytical results for samples collected from M-Area POC, background, plume definition, and recovery wells, respectively, for first quarter 2000. The tables identify the hazardous constituents that exceeded the Groundwater Protection Standard (GWPS) in Appendix IIIB-A of the 1995 RCRA Renewal Permit (SCDHEC, 1995). the Monitoring Constituents that exceeded the Monitoring Constituent Standard (MCS) (Appendix A, Volume I). Table D-10 summarizes the constituents that exceeded GWPS in M-Area POC wells during first quarter 2000. 
Constituent results in Tables D-2 through D-5 that appear to equal the standards in Appendix A but are not marked in the ST (exceeded standard) column are below the standards in the database. Database results, the results that are compared to the standards, have more significant digits than the results given in this report. Apparent discrepancies are due to the rounding of reported results.

Tables D-2 through D-5 also show the analytical laboratories that conducted the analyses, the dilution factors used in the analyses, the analyses that received modifiers or that exceeded the EPA-approved holding times during first quarter 2000, and information concerning the wells.

\section{Met Lab HWMF}

Tables D-6 through D-8 (Appendix D) present the field and analytical results for samples collected from Met Lab POC, background, and plume definition wells, respectively, for first quarter 2000. Water elevations were determined using the elevation of the top of casing as the water level measurement reference point. The tables identify the hazardous constituents that exceeded the GWPS in Appendix IIIG-A of the 1995 RCRA Renewal Permit (SCDHEC, 1995), or the MCS (Appendix A, Volume I). Table D-11 summarizes the constituents that exceeded GWPS in Met Lab HWMF POC wells during first quarter 2000.

Constituent results in Tables D-6 through D-8 that appear to equal the standards in Appendix A but are not marked in the ST (exceeded standard) column are below the standards in the database. Database results, the results that are compared to the standards, have more significant digits than the results given in this report. Apparent discrepancies are due to the rounding of reported results.

Tables D-6 through D-8 also show the analytical laboratories that conducted the analyses, the dilution factors used in the analyses, the analyses that received modifiers or that exceeded the EPA-approved holding times during first quarter 2000, and information concerning the wells. 
WSRC-TR-2000-00242

Unclassified

THIS PAGE LEFT BLANK INTENTIONALLY 


\section{Hydrogeologic Assessment}

The hydrogeologic system in AM Areas is evaluated using piezometric and potentiometric maps and cross sections (Maps 1 through 16, Appendix $\mathrm{H}$, Volume III) to determine groundwater flow rates and directions and precipitation data, hydrographs (Appendix $G$, Volume II), and zone of capture maps (Appendix H, Maps 17 through 20) to determine the effects of the recovery well system.

The piezometric and potentiometric maps for this report were constructed for first and second quarters 2000 using synchronous water elevations. Water elevations were determined using the elevation of the top of standpipe as the water measurement reference point, or the top of casing in the cases where a standpipe was not present. Maps were constructed for each of the aquifer zones described in Ehrke et al. (1995). Appendix B, Volume I, lists the wells in the groundwater monitoring network, and the units they monitor. Aquifer zones are identified in this report in the Key to Reading the Tables section of Appendix D (Volume I). A significant number of monitoring well screen zones were re-evaluated in the process of preparing the isoconcentration and potentiometric surface maps to resolve data anomalies. As a result of this re-evaluation, many of the wells were re-designated to the appropriate monitoring zone corresponding to the screened interval. The aquifer zones monitored by each well are provided in Appendix B, Volume I, M-Area HWMF and Met Lab HWMF Monitoring and Recovery Well Network. Map directions are oriented to SRS site north.

\section{Groundwater Flow Directions and Rates}

\section{Horizontal Flow Directions}

- Horizontal gradients in the M-Area Aquifer Zone (Maps 1 and 6, Appendix H, Volume III) are variable. The natural stratigraphy, variability in recharge, and the removal of water by wells RWM 3, 6, 9, 10, and 11 produce mounds and depressions found on the piezometric surface. South of the M-Area HWMF, near the Miscellaneous Chemical Basin, a water-table divide appears to divert flow naturally to the southwest to southeast.

- Potentiometric contours for the Upper Lost Lake Aquifer Zone (Maps 2 and 7, Appendix H, Volume III) indicate a flow pattern, with the highest water elevations located north of $A / M$ Areas, and flow away from this area to the southwest to southeast. Near the M-Area HWMF and A-014 outfall, flow is directed to the south. A mound in the potentiometric surface at the M-Area HWMF may result from local recharge from the M-Area Aquifer Zone at this location, possibly enhanced by recovery system water-level drawdown to the north. Another mound in the potentiometric surface located north of the Metallurgical Laboratory Seepage Basin may be the result of pumping by recovery wells RWM 12 to the northeast and RWM 9 and 11 to the southwest.

- Flow beneath the Lower Lost Lake Aquifer Zone (Maps 3 and 8, Appendix H, Volume III) is toward the south, southeast. The most prominent feature is a generally north-south oriented trough beneath the M-Area HWMF. The potentiometric depression that occurs near the recovery wells surrounding the M-Area HWMF may result from the recovery well system.

- The flow direction in the Middle Sand Aquifer Zone of the Crouch Branch Confining Unit (CBCU) (Maps 4 and 9, Appendix $\mathrm{H}$, Volume III) is toward the south, southeast. The effects of pumping in the overlying Upper and Lower Lost Lake Aquifer Zones cause a slight depression beneath the M-Area HWMF.

- Flow in the Crouch Branch Aquifer Unit (Maps 5 and 10, Appendix H, Volume III) is to the west-southwest, especially in the northern vicinity of A/M Area, apparently controlled by regional discharge to the Savannah River. 


\section{Horizontal Flow Rates}

Horizontal groundwater flow rates are calculated using the following equation:

$$
\text { Flow }(\mathrm{ft} / \text { day })=\frac{\text { HydraulicConductivity }(\mathrm{ft} / \text { day })}{\text { Porosity }(\text { unitless })} \times \frac{\mathrm{dh}(\mathrm{ft})}{\mathrm{dl}(\mathrm{ft})}
$$

Hydraulic conductivity constants estimated for the M-Area Aquifer Zone, the Upper Lost Lake Aquifer Zone, the Lower Lost Lake Aquifer Zone, the Middle Sand Aquifer Zone of the CBCU, and the Crouch Branch Aquifer Unit are 27, 45, 45, 45, and $40 \mathrm{ft} /$ day, respectively; the effective porosity value for each is 0.20 (Aadland et al, $1995 \& 1997$ ). The value $d h$ is the difference in head, and $d /$ is the length of the flow path shown on the map. The ratio $d h / d /$ is the horizontal gradient. Gradient, flow rate per day, and flow rate per year were each determined to two significant digits in the calculations.

Flow-rate estimates vary depending on the vertical gradient between wells, the size of the area under consideration, the number of data points, and the length and location of the flow path.

Because flow rates are based on inferred or estimated parameters, rate estimates should be considered accurate to an order of magnitude only.

The estimated groundwater flow rates for first and second quarters 2000 (Table D-22, Appendix D, Volume I) were as follows:

\section{First Quarter 2000}

- M-Area Aquifer Zone: 128 to $361 \mathrm{ft} /$ year

- Upper Lost Lake Aquifer Zone: 197 to $314 \mathrm{ft} /$ year

- Lower Lost Lake Aquifer Zone: 222 to $337 \mathrm{ft} /$ year

- Middle Sand Aquifer Zone of the CBCU: 140 to $320 \mathrm{ft} /$ year

- Crouch Branch Aquifer Unit: $248 \mathrm{ft} /$ year

Second Quarter 2000

- M-Area Aquifer Zone: 123 to $330 \mathrm{ft} /$ year

- Upper Lost Lake Aquifer Zone: 213 to $238 \mathrm{ft} /$ year

- Lower Lost Lake Aquifer Zone: 172 to $205 \mathrm{ft} /$ year

- Middle Sand Aquifer Zone of the CBCU: 123 to $329 \mathrm{ft} /$ year

- Crouch Branch Aquifer Unit: $226 \mathrm{ft} /$ year

These rates are within the same order of magnitude as the flow rates for previous quarters.

\section{Recharge and Water-Elevation Changes}

Precipitation measurements for the A/M Areas during first and second quarters 2000 are provided in Table 1 below. For comparison, the historical averages are also provided. 
Table 1. A/M-Areas Precipitation Data for First and Second Quarters 2000

\begin{tabular}{|ccc|}
\hline & Monthly Precipitation & Historical Average \\
(in.) & $1952-1999$ (in.) \\
January & 6.53 & 4.43 \\
February & 0.61 & 4.38 \\
March & 3.58 & 4.82 \\
April & 1.43 & 3.29 \\
May & 0.63 & 3.68 \\
June & 4.86 & 4.55 \\
July & --- & --- \\
August & --- & --- \\
September & --- & --- \\
October & --- & --- \\
November & --- & --- \\
\hline December & --- & Average total:25.15 \\
\hline Semi-Annual- total & 17.64 & \\
\hline
\end{tabular}

As in 1998 and 1999, precipitation in the first half of 2000 was several inches below the historical average. Concurrently, recharge rates would also be expected to be below the historical average. A reduction in recharge rates may be reflected in a decreasing trend in water elevations measured in shallow aquifers. Hydrographs showing water-elevation changes for M-Area HWMF and Met Lab HWMF monitoring wells from 1992 to present are provided in Appendix G (Volume II). Water elevations portrayed by the hydrographs were determined using the elevation of the top of standpipe as the water measurement reference point except for wells without standpipes, where top of casing was used as the reference point. Monitoring wells generally reflect a water-level decline since 1996 caused by the decrease in precipitation over the last several years. Water elevations in monitoring wells away from the recovery wells system show variations related to rainfall, proximity to streams and other physical features. 
THIS PAGE LEFT BLANK INTENTIONALLY 


\section{Effectiveness of the Corrective-Action Program}

The effectiveness of the corrective-action program is evaluated by assessing water quality changes and rate of contaminant removal. The duration of the cleanup program is assessed by tracking the time it takes to remove contaminants from the groundwater.

\section{Water Quality Changes}

\section{Extent of Contamination}

The discussion of the horizontal and vertical extent of contamination addresses the distribution of tetrachloroethylene and trichloroethylene contamination (Maps 11 through 20, Appendix H, Volume III). Tetrachloroethylene, although geometrically similar in distribution to trichloroethylene, is usually present in lower concentrations, and the plume is less extensive. The $5 \mu \mathrm{g} / \mathrm{L}$ contour lines on the isoconcentration maps encompass the areas where the constituent exceeded the GWPS during first quarter 2000 .

Isoconcentration maps of nitrate-nitrite as nitrogen, sodium, and sulfate are provided in the annual reports as indicators of contamination emanating from discrete sources in the A/M area, even though these constituents are not part of the list of hazardous constituents. Nitric acid and sodium hydroxide were used to clean aluminum parts in the A/M Area during the manufacture of fuel targets for reactors. Isoconcentration maps of sodium and nitrate may be useful in identifying the zone of contamination that could be attributed to the M-Area Basin. A separate zone of elevated sodium concentrations in the Northern Sector may be indicative of another release from the SRL Seepage Basins. A distinct plume of sulfate contamination was observed within the northern sector at the SRL Seepage Basins when isoconcentration maps were plotted using first quarter 1995 and third quarter 1996 data for the M-Area and Upper Lost lake aquifers. Therefore, isoconcentration maps of sulfate may be indicative as general tracers of the contamination zone attributed to the SRL Seepage Basins.

The results of a special program conducted to determine the extent of inorganic contamination near the M-Area HWMF were presented in the 1987 annual report (DuPont, 1988). The results indicate that a plume of mobile inorganic constituents is present south-southeast of the M-Area HWMF. The plume is evident in most of the hydrostratigraphic units composing the M-Area Aquifer Zone and appears to have a pattern similar to the trichloroethylene and tetrachloroethylene plumes. Plume maps for the inorganics also were shown in the revised RCRA Part B post-closure care permit application submitted May 31, 1988; they are not presented in this report. Because these constituents are within the more extensive chlorinated solvent plumes, they are addressed by the current corrective-action system or by other measures.

Contour lines on the isoconcentration maps are solid when the horizontal extent of the constituent was confidently interpreted and dashed when concentration boundaries were uncertain. Isoconcentration maps, showing the extent of contamination, are interpreted and plotted for the recorded specific concentration value of a monitoring well within a specific aquifer for that specific time frame. The plume geometry and apparent extent of contamination may differ between sampling periods. Differences may in part be attributed to the distribution of data points available for each sampling period being contoured. If a particular well at a controlling location could not be sampled due to access or mechanical problems, historical data from the well was reviewed to determine what the expected value would have been from the well, and efforts were made to honor the historical data while contouring. A similar review process was used in the case of data returned from the lab with the qualifiers " $\mathrm{J}$ " or " $\mathrm{L}$ " that were posted on the maps as "non decision data" (NDD) based on guidance received from SCDHEC (letter, Taylor to Cook, April 21, 1998). Thus the extent of the plume may not be representative of past extents of the depicted analyte. 


\section{M-Area Aquifer Zone}

Maps 11 -15 (Appendix $\mathrm{H}$, Volume III) show constituent distribution in the M-Area Aquifer Zone. The plume generally is limited to areas of known or suspected solvent disposal sites, which include the M-Area HWMF, the Solvent Storage Facility, the A-014 outfall, the Metallurgical Laboratory Seepage Basin, the A-Area Metals Burning Pit and Burning/Rubble Pit, and the Miscellaneous Chemicals Basin.

\section{Upper Lost Lake Aquifer Zone}

Maps 16 through 20 (Appendix $\mathrm{H}$, Volume III) show the extent of contamination in the Upper Lost Lake Aquifer Zone. The pattern of contamination in this zone in relation to known point sources is similar to, but more extensive than, the pattern in the M-Area Aquifer Zone. In general, the extent of the plume in this zone is greater in the downgradient direction than in the M-Area Aquifer Zone due to greater horizontal flow rates in both the Upper and Lower Lost Lake Aquifer Zones.

\section{Lower Lost Lake Aquifer Zone}

Constituent concentrations in the Lower Lost Lake Aquifer Zone are shown on Maps 21 though 25 (Appendix $\mathrm{H}$, Volume III). The lateral extent of the plume as defined by the $5 \mu \mathrm{g} / \mathrm{L}$ contour is greater in the downgradient directions than in the upper units because the Lower Lost Lake Aquifer Zone has a higher potential for horizontal flow. The $1,000 \mu \mathrm{g} / \mathrm{L}$ contour generally is below suspected sources of contamination.

\section{Middle Sand Aquifer Zone of the CBCU}

The distribution of constituents in the Middle Sand Aquifer Zone of the CBCU is shown on Maps 26 through 30 (Appendix H, Volume III). Monitoring is limited in the Middle Sand Aquifer Zone of the CBCU. Constituent concentrations in this zone, as in the overlying zones, are highest near known sources. Contamination in the Middle Sand Aquifer Zone of the CBCU is less extensive beneath the western part of M Area than in overlying zones. Beneath the eastern part of M Area, contamination patterns are similar to patterns in the Lower Lost Lake Aquifer Zone, but contamination is less extensive in the lower zone. A localized detection of PCE (7.7 ppb) has been recorded at well MSB 55B in the northern sector of AMM Area. However, the detection may be spurious because there were no surrounding detections in the area. A follow-up on the detection did not confirm the existence of PCE contamination.

\section{Crouch Branch Aquifer Unit}

The distribution of constituents in the Crouch Branch Aquifer Unit is shown on Maps 31 through 35 (Appendix H, Volume III). Contaminant concentrations in the Crouch Branch Aquifer Unit are primarily limited to the area beneath SRTC and an area extending from the SRTC north to well MSB 55TA, which is generally upgradient of these facilities. Downward vertical hydraulic gradients from the M-Area Aquifer Zone to the Crouch Branch Aquifer Unit characterize the SRTC area. The presence of a zone of increased transmissivity in the Middle Sand Aquifer Zone, which allows downward flow into the Crouch Branch Aquifer Unit, is indicated by constituent concentrations measured in wells monitoring that portion of the Crouch Branch Aquifer Unit.

\section{Concentration Changes over Time}

Changes in tetrachloroethylene and trichloroethylene concentrations during the past several years in samples from POC and plume definition wells are illustrated in time series plots in Appendix $F$ (Volume II). The plots use a fixed, logarithmic scale providing a consistent frame of reference for evaluating changes in concentration over time. Since the vertical scale begins at $1 \mathrm{ppb}$, occasionally a few values that were less than $1 \mathrm{ppb}$ drop below the $\mathrm{x}$ axis.

Statistical evaluations using the CUSUM test were previously performed as described in the 1987 Annual Monitoring and Corrective-Action Report [DuPont, 1988] and as modified in third quarter 1998. The CUSUM statistical evaluation program was rewritten in 1999 to be consistent with new EPA guidance for using CUSUM. 
The RCRA Part B Permit requires annual reports on the effectiveness of the corrective action program that are to include a statistical evaluation of water quality data for significant changes. The purpose of the statistical evaluation is to identify remediation system impacts to the groundwater quality in various wells. Identifying impacts requires a statistical evaluation since normal concentration fluctuations and trends make a non-statistical approach ineffective. The statistical evaluation included in the annual report, rather than this semi-annual submittal.

\section{Rate of Contaminant Removal}

SRS determines the rate of contaminant removal using mass balance calculations that subtract the concentration of VOCs in the effluent from the concentration of VOCs in the influent. This amount is then multiplied by the number of gallons of water or cubic feet of air that passes through the stripper column or SVEU during a given period. The results of this technique are described in the Corrective-Action System Operation and Performance section. 


\section{THIS PAGE LEFT BLANK INTENTIONALLY}




\section{Corrective-Action System Operation and Performance}

Corrective action is accomplished in A/M Areas by pumping contaminated groundwater to two air strippers where volatile organic compounds are removed and by pumping contaminated air, using vacuum extraction, from the vadose zone to six catalytic-oxidation units where the volatile organic compounds are destroyed.

The M-1 air stripper in M Area, Central Sector, is fed by 11 recovery wells (RWM series), began operating in April 1985 and has been operating full scale since September 1985. The A-2 air stripper is fed by 6 recovery wells in the northern sector. The production catalytic-oxidation unit at the Integrated Demonstration Site (782-7M), fed by a single vapor extraction well, began experimental operation in 1990 and has been in full-scale operation since August 1994. Four soil vacuum-extraction units (SVEU) vadose zone units, located in A/M Areas, have been operating since May 1995. These units are fed by 12 soil vapor extraction wells.

Twelve vertical, airlift recirculation wells (ARWs) are screened in the Upper and Lower Lost Lake Aquifer Zones in the Southern Sector of A/M Area. The ARW remediation system was initiated with the installation of the first SSR well in 1996. Currently 12 wells (SSR well series) are placed approximately 255 feet apart within the vicinity of the $500 \mathrm{ppb}$ isoconcentration contour of trichloroethylene (based on 1994 data). The SSR wells form a line diagonal to the groundwater flow path. Computer modeling predicts the SSR wells form a vertical capture zone extending through the majority of the Lost Lake Aquifer Zone (Aleman and Hamm, 1999). Eleven wells of this Southern Sector remediation system became operational during 1999.

A description of the systems that compose the corrective-action program follows.

\section{Air Stripper Performance}

\section{M-1 Air Stripper}

A summary of first and second quarter of 2000 operating and performance data for the $\mathrm{M}-1$ air stripper is presented in Table D-12 (Appendix D, Volume I). During the first half of the year, the stripper removed $5443.29 \mathrm{lbs}$. of degreaser solvent. The average total influent VOC concentration was $6,362 \mu \mathrm{g} / \mathrm{L}$. The average annual effluent total was $0 \mu \mathrm{g} / \mathrm{L}$.

The M-1 air stripper has removed a cumulative total of $362,055 \mathrm{lbs}$ of VOCs. When combined with the contaminated water treated by the pilot air stripper, the prototype air stripper, and the start-up of the full-scale M-1 air stripper system $(16,200,15,900$, and 2,100 lbs, respectively, for a total of $34,200 \mathrm{lbs}$ ), the total weight of tetrachloroethylene and trichloroethylene removed by the M-1 air stripper is $396,255 \mathrm{lbs}$.

The water feed rate to the M-1 air stripper has averaged $425 \mathrm{gpm}$ since startup. Total inlet solvent concentrations have decreased from roughly $47,100 \mu \mathrm{g} / \mathrm{L}$ in April 1985 to less than $5,730 \mu \mathrm{g} / \mathrm{L}$ in June 2000 . Stripping efficiency has always produced effluent concentrations substantially less than the permit limits.

\section{A-2 Air Stripper}

A summary of first and second quarter of 2000 operating and performance data for the A-2 air stripper is presented in Table D-13 (Appendix D, Volume I). During the first half of the year, the stripper removed $759.3 \mathrm{lbs}$. of degreaser solvent from RWM 12, RWM 13B, RWM 13C, RWM 14B, RWM 14C, and RWM 15. The average semi-annual influent concentration was $1,266 \mu \mathrm{g} / \mathrm{L}$, and the average semi-annual effluent VOC concentration was $1 \mu \mathrm{g} / \mathrm{L}$. 


\section{Vadose Zone Unit Performance}

\section{SVEU Vadose Zone Unit 782-3M}

A summary of first and second quarter of 2000 operating and performance data for the $782-3 \mathrm{M}$ is presented in Table D-14 (Appendix D, Volume I). During the first half of the year, the 782-3M removed a total of $7,294.43 \mathrm{lbs}$. of degreaser solvent. The average annual VOC influent concentration was 124.04 ppmv.

\section{SVEU Vadose Zone Unit 782-4M}

A summary of first and second quarter of 2000 operating and performance data for the $782-4 \mathrm{M}$ is presented in Table D-15 (Appendix D, Volume I). During the first half of the year, the 782-4M removed a total of $9664.00 \mathrm{lbs}$. of degreaser solvent. The average annual influent VOC concentration was $239.75 \mathrm{ppmv}$.

\section{SVEU Vadose Zone Unit 782-5M}

A summary of first and second quarter of 2000 operating and performance data for the $782-5 \mathrm{M}$ is presented in Table D-16 (Appendix D, Volume I). During 1999, the 782-5M achieved its goal and was taken out of operation.

\section{SVEU Vadose Zone Unit 782-6M}

A summary of first and second quarter of 2000 operating and performance data for the $782-6 \mathrm{M}$ is presented in Table D-17 (Appendix D, Volume I). During the first half of the year, the 782-6M removed a total of $4996.93 \mathrm{lbs}$. of degreaser solvent. The average annual influent VOC concentration was $323.34 \mathrm{ppmv}$.

\section{SVEU Vadose Zone Unit 782-7M (formerly the Integrated Demonstration Unit)}

A summary of first and second quarter of 2000 operating and performance data for the $782-7 \mathrm{M}$ (formerly the IDU) is presented in Table D-18 (Appendix D, Volume I). During the first half of the year, the $782-7 \mathrm{M}$ removed $1173.51 \mathrm{lbs}$. of degreaser solvent. The average annual influent VOC concentration was $35.37 \mathrm{ppmv}$ during this time.

\section{SVEU Vadose Zone Unit 782-8M}

A summary of first and second quarter of 2000 operating and performance data for the $782-8 \mathrm{M}$ is presented in Table D-19 (Appendix D, Volume I). During 1999, the 782-8M achieved its goal and was removed from service.

\section{Recovery Well System}

Monthly and cumulative volumes of groundwater pumped from the recovery well system and average flow rates for recovery wells RWM 1 through 15B are presented in Table D-20 (Appendix D, Volume I). Monthly concentrations ( $\mu \mathrm{g} / \mathrm{L}$ ) of tetrachloroethylene and trichloroethylene in each well are presented in Table D-21 (Appendix D, Volume I). Volume pumped for RWM 11 was unavailable due to equipment malfunction. Therefore, the average concentrations were estimated.

\section{Vapor Extraction Well System}

Vapor extraction well AMH 2 pumps contaminated air to the SVEU Vadose Zone Unit 782-7M (formerly the IDU). Table D-22 (Appendix D, Volume I) presents a summary of the first half of 2000 activity at the IDU.

Soil vapor extraction wells MVE 4, MVE 9, and MVE 10 pump contaminated air to the SVEU Vadose Zone Unit 782-3M, located in the A-014 outfall. Table D-14 (Appendix D, Volume I) presents a summary of first half of 2000 activity at the $782-3 \mathrm{M}$. 
Soil vapor extraction wells AMH 6 and AMH 7 pump contaminated air to the SVEU Vadose Zone Unit 782-4M, located in the M-Area Settling Basin. Table D-15 (Appendix D, Volume I) presents a summary of first half of 2000 activity at the $782-4 \mathrm{M}$.

Soil vapor extraction wells MVE 5, MVE 6, MVE 7, and MVE 8 pump contaminated air to the SVEU Vadose Zone Unit 782-5M, located in the M-Area HWMF process sewer. Table D-16 (Appendix D, Volume I) presents a summary of first half of 2000 activity at the $782-5 \mathrm{M}$.

Soil vapor extraction wells MVE 1, MVE 2 , and MVE 3 pump contaminated air to the SVEU Vadose Zone Unit 782-6M, located in the 321-M Solvent Storage Area. Table D-17 (Appendix D, Volume I) presents a summary of first half of 2000 activity at the $782-6 \mathrm{M}$.

Soil vapor extraction wells AMH 4 and AMH 5 pump contaminated air to the SVEU Vadose Zone Unit 782-8M, located near the IDU. Table D-18 (Appendix D, Volume I) presents the summary of activity for first half of 2000 at the $782-8 \mathrm{M}$. During the first half of $2000,782-8 \mathrm{M}$ was removed from service.

\section{Air Stripper Modifications}

Modifications were made to M1 and A2 Air Stripper operations during 1999. SCDHEC approved a modification to the Air Quality Control Permit to allow the M1 Air Stripper to operate without treating the PCE and TCE emissions from this unit. The operation of the M1 catalyst oxidation unit ceased in 1999.

SCDHEC approved a modification to the Air Quality Control Permit to allow the A2 Air Stripper to operate without treating the PCE and TCE emissions from this unit. The operation of the A2 catalyst oxidation unit ceased in 1999. A stack was added to the top of the air stripper per AQC permit modification.

Construction was completed on the connection from the Met Lab wells to the M1 Air Stripper In First and Second Quarter of 2000. Operation of RWM-17B commenced on June 12, 2000. RWM17D was dry on June 12, 2000 probably due to the recent draught.

\section{Vadose Zone Unit Modifications}

A modification was made in 1999 to Soil Vapor Extraction Units 782-3M, 4M, 5M, 6M, and 7M. SCDHEC approved a modification to the Air Quality Control Permit to allow these units to operate without treating the PCE and TCE emissions. The catalysts were removed and the heaters are no longer used to treat the emissions per AQC permit modification.

Soil Vapor Extraction Units 782-5M and 782-8M appear to have achieved their goal and ceased operations in 1999.

As stated in the M-Area Part B Permit Modification, Rev. 4, final shutdown of the soil vapor extraction units will be supported by an assessment of the areas under remediation. SRS plans to assess the areas using depth discrete soil cores and soil gas sampling. Sampling efforts are planned to take place by November, 2000. 


\section{Monitoring and Corrective-Action Program Assessment}

\section{Ongoing Programs}

\section{M-Area HWMF}

\section{Compliance and Post-Closure Programs}

- In 1980, the U.S. Department of Energy (DOE) submitted a notification of the SRS hazardous waste activities to SCDHEC; DOE then submitted a RCRA Part A hazardous waste permit application.

- SRS notified SCDHEC of volatile organic solvent contamination when it was first detected in the groundwater beneath the settling basin in June 1981. A network of monitoring wells was initiated to define the extent, concentration, distribution, and migration rate of the contaminant plume.

- Interim status monitoring began in 1983 and continued through September 1987; assessment monitoring was initiated second quarter 1986 and completed fourth quarter 1987.

- In February 1983, SRS voluntarily began a pilot remediation system to treat contaminated groundwater.

- A groundwater protection plan was submitted to Congress in June 1984 to meet the requirements of Public Law 98-181.

- In compliance with the requirements of Public Law 98-181, a closure plan for the settling basin was submitted to SCDHEC in 1984, with revisions in 1985 and 1987.

- A full-scale corrective-action program was initiated in 1985 to remove and treat hazardous constituents from the groundwater beneath the M-Area HWMF.

- The M-Area HWMF RCRA Part B post-closure care permit application was approved by SCDHEC in 1987.

- Closure was approved by SCDHEC on July 10,1987; a closure cap was completed during 1989 and 1990; and closure was certified by SCDHEC on April 26, 1991.

- The 1992 M-Area HWMF RCRA Part B permit renewal application was submitted to SCDHEC in April 1992 as specified in SCHWMR R.61-79.270.30(b) and 270.50(a).

- Revision 1 of the 1992 M-Area HWMF RCRA Part B permit renewal application was submitted to SCDHEC in March 1993. The revision included proposals to reduce the sampling, analysis, and reporting requirements for the groundwater monitoring program in AVM Areas.

- Modifications to the 1992 M-Area HWMF RCRA Part B permit renewal application (Revision 2) and the 1987 Part B permit application (Revision 4) were submitted to SCDHEC December 20,1993 . The modifications included a proposal to include PCB monitoring at specified $\mathrm{A} / \mathrm{M}$-Areas groundwater monitoring wells.

- To expedite SCDHEC's approval of the program to reduce sampling, analysis, and reporting, Revision 5 of the 1987 Part B permit application was submitted in February 1994. SRS received a Notice of Deficiency (NOD) on Revision 5 in June 1994.

- Revision 6 of the 1987 Part B permit application, submitted to SCDHEC in July 1994, was approved by SCDHEC. The revision addresses the NOD on Revision 5 , with modifications to the proposed reductions in sampling, analysis, and reporting. 
- Also in July 1994, Revision 3 of the 1992 Part B permit renewal application was submitted to SCDHEC in response to their NOD. Revision 3 modifications included updating the hydrostratigraphic nomenclature; updating information on the purged water disposal station, closure and corrective-action activities, DNAPL investigations, and proposed demonstration programs; adding information on new investigations in the western and southern sectors of AM Areas; and revising text on contingency plans, personnel training, and procedures to prevent hazards. The renewal document adheres to the format specified in the EPA Regulatory Completeness Checklist for Post-Closure Permit Applications (Revision 4, February 4, 1986). SCDHEC reviewed the application and held a comment and response period in March 1995.

- The final report updating the hydrostratigraphy of the A/M Areas and the Metallurgical Laboratory HWMF was submitted to SCDHEC in February 1995.

- The 1995 RCRA Renewal Permit (SCDHEC, 1995) for the M-Area HWMF and the Metallurgical Laboratory HWMF became effective October 5, 1995.

\begin{abstract}
A/M-Areas Vadose Zone Remediation
Although the groundwater corrective-action system in A/M Areas has proven effective in removing and treating contaminated groundwater, it does not remove contaminants from the vadose zone, which is the unsaturated layer of soil between the ground surface and the water table. Vadose zone remediation decreases the migration of residual contaminants into the groundwater and reduces the time required for groundwater remediation.
\end{abstract}

The following four sites, which have elevated levels of tetrachloroethylene and trichloroethylene in the vadose zone, have been chosen for vadose zone remediation using vacuum extraction: the abandoned process sewer line, the M-Area Settling Basin, the A-014 outfall, and the 321-M former solvent storage area. Vacuum extraction was selected after a successful pilot demonstration removed more than 1,500 lbs. of solvents during a 21-day test in 1987.

Four soil vacuum extraction (SVE) units were installed in A/M Areas during 1994. Design of the SVE units was completed March 11, 1994; construction began March 28, 1994, and was completed in April 1995. On March 25, 1994, SRS requested temporary authorization to initiate vadose zone vacuum extraction at the four locations. SCDHEC granted temporary authorization in June 1994 (letter, J. Litton to W. Wierzbicki, June 16, 1994) and again in June 1995. The SVE units have been operating since May 1995.

The soil vacuum extraction systems operate under air quality control permits 0080-0041-M-CA, 0080-0041-M-CB, 0080-0041-M-CC, and 0080-0041-A-CA issued by SCDHEC in January 1993.

Process modifications have been placed on the four SVE units to allow remote control operation of the systems. This system allows automatic processing changes while the units are operating. This dynamic process eliminates the manual manipulations necessary to operate the units while still meeting the air quality control permit requirements.

Two additional SVE units, located near the M-Area Settling Basin and the process sewer line, were on line in 1997.

Additional characterization of the vadose zone contamination plume was completed in 1997 and early 1998. These results were compiled for submittal and further action strategies.

The Vadose Zone Monitoring Plan was submitted under separate cover in March 1998.

\title{
Purge Water Management
}

During third quarter 1993, construction of a facility to receive, store, and transfer purged water from the M-Area monitoring wells to the $\mathrm{M}-1$ air stripper was completed. This Purged Water Disposal Station, $782-2 \mathrm{M}$, houses a 10,000-gal, double-wall tank. This facility has been used continuously for containment of purged water since January 1994. 


\section{Investigation Derived Waste Minimization}

In 1999, SRS initiated use of an alternate monitoring well design, the Westbay Multi-port Sampling System. The Westbay Multi-port Sampling System allows groundwater sampling from multiple screened intervals in the same well bore. The advantage in utilizing this technology is that only a single well bore need to be drilled for multiple completions. Also, because the sampling device is connected directly to the aquifer via a depressible "O-ring", the only Investigation Derived Waste (IDW) that is generated is from the unused portion of the groundwater sample brought to the surface in the sampling chambers. The best application of this technology is for VOC contaminated groundwater in thick (i.e., 40-60 feet) aquifers which exhibit clean sand formations with very thin clay laminae. It is especially useful when volatile contamination is present due to the low sample volume needed for analysis. The sampler is limited to approximately one-half liter per run in the hole. This is the major limiting factor. As many as four canisters can be strung together to produce one liter of sample, however then a 20 -foot sump below the sampling port is required.

The first Westbay system was installed in the spring of 1999, MSB-91TB. This pilot Westbay system was completed in the Crouch Branch Aquifer Unit with two screened intervals. Installation of this Westbay system provided dual benefits by reducing IDW generation, and also reducing installation costs since conventional well installation would have required using surface casing for two wells, instead of just one. Following this successful application of the Westbay system, the technology was applied to eight Effectiveness Monitoring wells (SSM $10-17$ ), designed to monitor the effectiveness of the recirculation well (SSR 1 -12) VOC remediation in the Southern Sector of AMM Area. These eight Westbay completions in the Lost Lake Aquifer Zone have two screened intervals each, one interval screened in the Upper Lost Lake Aquifer Zone, and one in the Lower Lost Lake Aquifer Zone. The southern sector wells were installed in two phases of four wells each. The first was in October/November 1999 time frame and the last four were installed in January 2000.

Planned activities are for installation of the Westbay Multi-port Sampling System in five Effectiveness Monitoring well installations at the Miscellaneous Chemical Basin (MCB). This remediation activity is for VOC contamination in the Lost Lake Aquifer Zone also. For the MCB installation two screened intervals will have the Westbay system installed. Although the MCB is not part of the M-Area or Met Lab HWMFs, monitoring activities are included in this report due to its proximity to the HWMFs, and its inclusion on area maps. Other planned activities are for the installation of as many as eight Westbay systems in the Crouch Branch Aquifer Unit, with up to three screened zones each. To date, all the screen zones in a given well bore have been in the same aquifer zone. That is to say, none of the Westbay systems have yet positioned screen zones above and below a confining unit in the same well bore.

\section{Central Sector Remediation}

The remediation strategy in the Central Sector has been to identify and remove DNAPL in the subsurface, while continuing mass removal from the dissolved plume using the M-1 Air Stripper and recovery well system. Remediation is complemented with Soil Vacuum Extraction operations in the vadose zone.

\section{Western Sector Characterization}

Soil head space was analyzed on eight borings in the western sector. The drilling report has recently been released and shows a refinement of the boundary of the trichloroethylene/tetrachloroethylene plume. The findings suggest that the primary source of trichloroethylene/tetrachloroethylene is from the M-Area Seepage Basin with the contamination spreading into the western sector from upgradient sources. In addition, fate and transport modeling is being developed to predict mobility of the DNAPL pool that is located near the M-Area Settling Basin and Lost Lake. Characterization was initiated and completed in the Western Sector during 1998 that further defined the plume and lithology in the area. 


\section{Southern Sector Remediation}

The southern sector plume has an estimated aerial extent of 800 acres, ranging from relatively dilute concentrations of volatile organic compounds to concentrations in the $1000 \mathrm{ppb}$ range, and is outside the zone of influence of the M-1 and A-2 Air Stripper groundwater recovery system. SRS investigated the southern sector to determine the boundary of solvent contamination and to define the geology and hydrology of the area. Based on the results of the assessments, in 1996 SRS installed two vertical recirculation wells in the Lost Lake Aquifer Zone to control downgradient migration of the $>500 \mathrm{ppb}$ boundary of the VOC plume. Ten additional wells were also installed in the Lost Lake Aquifer Zone and start-up of 11 of the 12 wells in the system began in 1999.

Monthly status reports have continued on the progress of the remedial system. A multi-stage, inwell aerator, developed by Davis Environmental, was installed into SSR-012.

An "effectiveness monitoring plan" was submitted to SCDHEC and was approved in 1999. The plan proposed installation of 8 wells, in addition to using 4 existing wells to monitor effectiveness of the ARW treatment system. Reports will be submitted annually to SCDHEC. The first report is due for submittal May 15, 2000.

\section{Northern Sector Characterization and Remediation}

Characterization of the northern sector was initiated in 1998 by drilling three soil borings. Initial results of the borings showed a positive VOC result in the Lost Lake and Crouch Branch Aquifers. . Follow-up testing has failed to confirm the VOC results in these borings. However, additional monitoring wells were installed in 1999 for continued monitoring of the Lost Lake and Crouch Branch Aquifers.

Groundwater contamination in the northern sector extends over approximately 174 acres. Controlling contamination in the uppermost aquifer zone will prevent further migration downward into the deeper Crouch Branch Aquifer Unit. The A-2 air stripper, with a feed rate of $300 \mathrm{gpm}$, is fed by six recovery wells: RWM 13B, 13C, 14B, 14C, 15B and RWM 12 (previously from the A-1 stripper unit). This unit began operating in fiscal year 1996.

Although not designed for remediation purposes, production wells 905-20A and 905-53A provide remediation benefits in the Crouch Branch Aquifer in the Northern Sector. The annual operating frequency of the production wells is expected to remove approximately $700 \mathrm{lbs}$. of solvent every year (Jackson et al., 1997).

\section{Met Lab HWMF}

Vadose zone remediation was initiated in 1998 with the installation and operation of 20 BaroBalls within the Met Lab area. Two additional recovery wells, RWM 17 and 18, began feeding groundwater into the M-1 Air Stripper as of July 2000.

A proposal for background wells is currently under review for acceptance. The set of proposed background wells will allow an accurate statistical assessment of the contamination found in and around the Met Lab area. 


\section{Proposed Monitoring and Corrective-Action Program Modifications}

The progress of proposed modifications is reported here after changes are approved by SCDHEC in other reports, e.g., status reports, monitoring-change proposals, and modifications.

\section{Groundwater Monitoring Program}

A modification to the current sampling program was submitted in 1998 to reduce the sampling requirements (analytes and wells) for the M-Area HWMF. This modification received partial approval in March 1999.

\section{A/M-Areas Vadose Zone Remediation Program}

During 1998, SRS prepared and delivered an air permit modification to SCDHEC for eliminating offgas treatment at the vadose zone units. This draft permit was presented for public comment in early 1999 and was approved. 
THIS PAGE LEFT BLANK INTENTIONALLY. 


\section{Errata and Revisions}

Result values for earlier quarters presented in this report may differ from the values for those same quarters presented in previous reports because some re-analyses may have occurred after the quarterly reports were printed.

Third and Fourth Quarters 1999

No errata have been reported. 
THIS PAGE LEFT BLANK INTENTIONALLY. 


\section{Appendix A}

Standards 
THIS PAGE LEFT BLANK INTENTIONALLY. 
Table A-1. Standards for M-Area HWMF

Constituent

Standard

$\underline{\text { Unit }}$

Source

Groundwater Protection Standard

261 Appendix VIII / 264 Appendix IX Hazardous Constituents

Inorganics

Barium

Cyanide

Lead

Nickel

Selenium

$2,000 \quad \mu g / L$

$\mu g / L$

$\mu g / L$

$\mu \mathrm{g} / \mathrm{L}$

$\mu \mathrm{g} / \mathrm{L}$

SCDHEC (1995)

$40^{\mathrm{b}}$

15

100

SCDHEC (1995)

SCDHEC (1995)

50

SCDHEC (1995)

Organics

Chlorobenzene

1,1-Dichloroethane

5

1,1-Dichloroethylene

7

trans-1,2-Dichloroethylene

PCB 1016

PCB 1221

PCB 1232

PCB 1242

PCB 1248

PCB 1254

PCB 1260

1,1,2,2-Tetrachloroethane

Tetrachloroethylene

1,1,1-Trichloroethane

Trichloroethylene

$\mu g / L$

SCDHEC (1995)

\section{Monitoring Constituents Standard}

Inorganics
Aluminum
Chloride
Chromium
Cobalt
Copper
Fluoride
Manganese
Mercury
Nitrate-nitrite as nitrogen
Sodium
Sulfate
Total phosphates (as P)

100
4,200
100
4
1,300
4,000
50
2
2,400
4,600
3,000
300

$\mu \mathrm{g} / \mathrm{L}$

WSRC (1987)

$\mu g / L$

$\mu g / L$

SCDHEC (1995)

SCDHEC (1995)

$\mu \mathrm{g} / \mathrm{L}$

SCDHEC (1995)

$\mu g / L$

SCDHEC (1995)

$\mu \mathrm{g} / \mathrm{L}$

SCDHEC (1995)

$\mu g / L$

SCDHEC (1995)

$\mu \mathrm{g} / \mathrm{L}$

SCDHEC (1995)

$\mu g / L$

SCDHEC (1995)

$\mu g / L$

SCDHEC (1995)

$\mu \mathrm{g} / \mathrm{L}$

SCDHEC (1995)

SCDHEC (1995)

$\mu g / L$

SCDHEC (1995)

$\mu g / L$

SCDHEC (1995)

$\mu \mathrm{g} / \mathrm{L}$

SCDHEC (1995)

$\mu g / L$

SCDHEC (1995)

Total phosphates (as $\mathrm{P}$ )

300

$\mu \mathrm{g} / \mathrm{L}$

$\mu g / L$

$\mu g / L$

$\mu g / L$

$\mu \mathrm{g} / \mathrm{L}$

$\mu g / L$

$\mu g / L$

$\mu g / L$

$\mu \mathrm{g} / \mathrm{L}$

$\mu \mathrm{g} / \mathrm{L}$

$\mu g / L$
WSRC (1987)

EPA (1993a) ${ }^{e}$

WSRC (1994)

WSRC (1994)

EPA (1993a)

$\operatorname{EPA}(1993 b)^{9}$

EPA (1993a)

WSRC (1987)

WSRC (1987)

WSRC (1987)

WSRC (1987) 


\section{Constituent}

Uranium

Zinc

\section{Radionuclides}

Gross alpha

Nonvolatile beta

Radium, total alpha-emitting ${ }^{h}$

\section{Standard}

100

5,000

15

50

5 $\underline{\text { Unit }}$

$\mu g / L$

$\mu g / L$

$\mathrm{pCi} / \mathrm{L}$

$\mathrm{pCi} / \mathrm{L}$

$\mathrm{pCi} / \mathrm{L}$
Source

WSRC (1987)

EPA (1993b)

EPA (1993a)

EPA (1993a)

EPA (1993a)

a Concentrations with SCDHEC (1995) sources are groundwater protection standards in the 1995 RCRA Renewal Permit.

b Concentration is the practical quantitation limit (PQL) for as defined in R.61-79.264 Appendix IX.

c Concentration is the PQL for EPA Method 8240 as defined in SCDHEC (1994). This PQL is the contractrequired $P Q L$ for $G E$ and $W A$.

d Concentrations with WSRC (1987) sources are background concentrations.

e Concentrations and activities with EPA (1993a) sources are primary drinking water standards.

f Concentrations with WSRC (1994) sources were groundwater protection standards under the previous permit.

g Concentrations with EPA (1993b) sources are secondary drinking water standards.

$\mathrm{h}$ The standard used for total alpha-emitting radium is the primary drinking water standard for total radium.

\section{Table A-2. Standards for Met Lab HWMF}

Constituent $\underline{\text { Standard Unit } \quad \underline{\text { Source }}}$

Groundwater Protection Standard

261 Appendix VIII / 264 Appendix IX Hazardous Constituents

\section{Inorganics}

Arsenic

Barium

Chromium

Lead

Mercury

Nickel

Silver

\section{Organics}

Acetone

Carbon tetrachloride

Chloroethene

Chloroform

1,1-Dichloroethane

1,2-Dichloroethane

1,1-Dichloroethylene

cis-1,2-Dichloroethylene

trans-1,2-Dichloroethylene

2,4-Dichlorophenoxyacetic acid

Lindane

Methyl methacrylate
50

2,000

100

15

2

100

50

$10^{\mathrm{b}}$

5

2

100

$5^{\mathrm{b}}$

5

7

70

100

$10^{c}$

0.2

$2^{b}$ $\mu \mathrm{g} / \mathrm{L}$

$\mu g / L$

$\mu g / L$

$\mu \mathrm{g} / \mathrm{L}$

$\mu g / L$

$\mu \mathrm{g} / \mathrm{L}$

$\mu g / L$

$\mu \mathrm{g} / \mathrm{L}$

$\mu g / L$

$\mu \mathrm{g} / \mathrm{L}$

$\mu g / L$

$\mu g / L$

$\mu g / L$

$\mu \mathrm{g} / \mathrm{L}$

$\mu g / L$

$\mu g / L$

$\mu g / L$

$\mu \mathrm{g} / \mathrm{L}$

$\mu \mathrm{g} / \mathrm{L}$
SCDHEC (1995) ${ }^{a}$

SCDHEC (1995)

SCDHEC (1995)

SCDHEC (1995)

SCDHEC (1995)

SCDHEC (1995)

SCDHEC (1995)

SCDHEC (1995)

SCDHEC (1995)

SCDHEC (1995)

SCDHEC (1995)

SCDHEC (1995)

SCDHEC (1995)

SCDHEC (1995)

SCDHEC (1995)

SCDHEC (1995)

SCDHEC (1995)

SCDHEC (1995)

SCDHEC (1995) 


\begin{tabular}{llll} 
Constituent & Standard & $\underline{\text { Unit }}$ & Source \\
\cline { 2 - 4 } Phenol & $10^{\mathrm{d}}$ & $\mu \mathrm{g} / \mathrm{L}$ & SCDHEC (1995) \\
Tetrachloroethylene & 5 & $\mu \mathrm{g} / \mathrm{L}$ & SCDHEC (1995) \\
$1,1,1$-Trichloroethane & 200 & $\mu \mathrm{g} / \mathrm{L}$ & SCDHEC (1995) \\
Trichloroethylene & 5 & $\mu \mathrm{g} / \mathrm{L}$ & SCDHEC (1995)
\end{tabular}

Monitoring Constituents Standard

\begin{tabular}{|c|c|c|c|}
\hline Chloride & 4,200 & $\mu \mathrm{g} / \mathrm{L}$ & WSRC $(1987)^{e}$ \\
\hline Cyanide & $40^{f}$ & $\mu \mathrm{g} / \mathrm{L}$ & SCDHEC (1995) \\
\hline Fluoride & 4,000 & $\mu g / L$ & EPA $(1993 a)^{g}$ \\
\hline Iron & 300 & $\mu g / L$ & EPA $(1993 b)^{h}$ \\
\hline Manganese & 50 & $\mu \mathrm{g} / \mathrm{L}$ & EPA (1993b) \\
\hline Nitrate-nitrite as nitrogen & 2,400 & $\mu g / L$ & WSRC (1987) \\
\hline Selenium & 50 & $\mu \mathrm{g} / \mathrm{L}$ & EPA (1993a) \\
\hline Sodium & 4,600 & $\mu g / L$ & WSRC (1987) \\
\hline Sulfate & 3,000 & $\mu \mathrm{g} / \mathrm{L}$ & WSRC (1987) \\
\hline Total organic carbon & $10,000^{i}$ & $\mu \mathrm{g} / \mathrm{L}$ & SCDHEC (1995) \\
\hline Total organic halogens & $50^{\mathrm{j}}$ & $\mu g / L$ & SCDHEC (1995) \\
\hline Total phosphates (as P) & 300 & $\mu g / L$ & WSRC (1987) \\
\hline \multicolumn{4}{|l|}{ Radionuclides } \\
\hline Gross alpha & 15 & $\mathrm{pCi} / \mathrm{L}$ & EPA (1993a) \\
\hline Nonvolatile beta & 50 & $\mathrm{pCi} / \mathrm{L}$ & EPA (1993a) \\
\hline Radium, total alpha-emitting ${ }^{k}$ & 5 & $\mathrm{pCi} / \mathrm{L}$ & EPA (1993a) \\
\hline \multicolumn{4}{|c|}{$\begin{array}{l}\text { Concentrations with SCDHEC (1995) sources are groundwater protection standards in the } 1995 \text { RCRA } \\
\text { Renewal Permit. }\end{array}$} \\
\hline \multirow{2}{*}{\multicolumn{4}{|c|}{$\begin{array}{l}\text { Concentration is the practical quanitation limit (PQL) as defined in R.61-79.264 Appendix IX. } \\
\text { Concentration is the PQL for EPA Method } 8150 \text { as defined in SCDHEC (1994). This PQL is the contract- } \\
\text { required PQL for GE and WA. }\end{array}$}} \\
\hline & & & \\
\hline \multicolumn{4}{|c|}{$\begin{array}{l}\text { Concentration is the PQL for EPA Method } 8270 \text { as listed in SCDHEC (1994). This PQL is the contract- } \\
\text { required PQL for GE and WA. }\end{array}$} \\
\hline \multicolumn{4}{|c|}{ Concentrations with WSRC (1987) sources are background concentrations. } \\
\hline \multicolumn{4}{|c|}{ Concentration is the PQL as defined in R.61-79.264 Appendix IX. } \\
\hline \multicolumn{4}{|l|}{$\begin{array}{l}\text { g } \text { Concentrations and activities } \\
\text { h } \text { Concentrations with EPA (19 }\end{array}$} \\
\hline \multicolumn{4}{|l|}{$\begin{array}{l}\text { Concentration is the PQL fo } \\
\text { Concentration is the PQL fo }\end{array}$} \\
\hline Concentration is the PQL fo & 020. & & W \\
\hline
\end{tabular}


THIS PAGE LEFT BLANK INTENTIONALLY. 
WSRC-TR-2000-00242

Unclassified

Appendix B

Well Network 
WSRC-TR-2000-00242

Unclassified

THIS PAGE LEFT BLANK INTENTIONALLY. 


\begin{tabular}{|c|c|c|}
\hline Well Name & Met Lab Well Use & Aquifer Name \\
\hline MSB 29B & Background Well & Lower Lost Lake Aquifer Zone \\
\hline MSB 43A & Background Well & Lower Lost Lake Aquifer Zone \\
\hline AMB 11D & Background Well & M-Area Aquifer Zone \\
\hline AMB 12D & Background Well & M-Area Aquifer Zone \\
\hline MSB 29D & Background Well & M-Area Aquifer Zone \\
\hline MSB 43D & Background Well & M-Area Aquifer Zone / Green Clay Confining Zone \\
\hline MSB 29C & Background Well & Upper Lost Lake Aquifer Zone \\
\hline MSB 43B & Background Well & Upper Lost Lake Aquifer Zone \\
\hline $\mathrm{AMB} 4 \mathrm{~B}$ & Plume Definition Well & Lower Lost Lake Aquifer Zone \\
\hline AMB 7B & Plume Definition Well & Lower Lost Lake Aquifer Zone \\
\hline AMB 7 & Plume Definition Well & M-Area Aquifer Zone \\
\hline AMB 14D & Plume Definition Well & M-Area Aquifer Zone \\
\hline AMB 15D & Plume Definition Well & M-Area Aquifer Zone \\
\hline AMB 7A & Plume Definition Well & Middle Sand Aquifer Zone-Crouch Branch Confining Unit \\
\hline AMB 13AR & Plume Definition Well & Middle Sand Aquifer Zone-Crouch Branch Confining Unit \\
\hline AMB 11B & Plume Definition Well & Upper Lost Lake Aquifer Zone \\
\hline AMB 10B & Point of Compliance Well & Lower Lost Lake Aquifer Zone \\
\hline AMB 4D & Point of Compliance Well & M-Area Aquifer Zone \\
\hline AMB 5 & Point of Compliance Well & M-Area Aquifer Zone \\
\hline AMB 6 & Point of Compliance Well & M-Area Aquifer Zone \\
\hline AMB 8D & Point of Compliance Well & M-Area Aquifer Zone \\
\hline AMB 9D & Point of Compliance Well & M-Area Aquifer Zone \\
\hline AMB 10D & Point of Compliance Well & M-Area Aquifer Zone \\
\hline AMB 16D & Point of Compliance Well & M-Area Aquifer Zone \\
\hline AMB $4 \mathrm{~A}$ & Point of Compliance Well & Middle Sand Aquifer Zone-Crouch Branch Confining Unit \\
\hline AMB 10A & Point of Compliance Well & Middle Sand Aquifer Zone-Crouch Branch Confining Unit \\
\hline AMB 17A & Point of Compliance Well & Middle Sand Aquifer Zone-Crouch Branch Confining Unit \\
\hline AMB 18A & Point of Compliance Well & Middle Sand Aquifer Zone-Crouch Branch Confining Unit \\
\hline AMB 18C & Point of Compliance Well & Upper Lost Lake Aquifer Zone \\
\hline AMB 19C & Point of Compliance Well & Upper Lost Lake Aquifer Zone \\
\hline
\end{tabular}




\begin{tabular}{|c|c|c|}
\hline Well Name & M Area Well Use & Aquifer Name \\
\hline MSB 29B & Background Well & Lower Lost Lake Aquifer Zone \\
\hline MSB 43A & Background Well & Lower Lost Lake Aquifer Zone \\
\hline MSB 29D & Background Well & M-Area Aquifer Zone \\
\hline MSB 43D & Background Well & M-Area Aquifer Zone / Green Clay Confining Zone \\
\hline MSB 29C & Background Well & Upper Lost Lake Aquifer Zone \\
\hline MSB 43B & Background Well & Upper Lost Lake Aquifer Zone \\
\hline MSB 21TA & Piezometer Well & Crouch Branch Aquifer Unit \\
\hline MSB 30A & Piezometer Well & Crouch Branch Aquifer Unit \\
\hline MSB 31A & Piezometer Well & Crouch Branch Aquifer Unit \\
\hline MSB 33TA & Piezometer Well & Crouch Branch Aquifer Unit \\
\hline MSB 37TA & Piezometer Well & Crouch Branch Aquifer Unit \\
\hline MSB 38TA & Piezometer Well & Crouch Branch Aquifer Unit \\
\hline MSB 40TA & Piezometer Well & Crouch Branch Aquifer Unit \\
\hline SRW 12A & Piezometer Well & Crouch Branch Confining Unit \\
\hline$A C \quad 2 A$ & Piezometer Well & Lower Lost Lake Aquifer Zone \\
\hline MSB 11A & Piezometer Well & Lower Lost Lake Aquifer Zone \\
\hline MSB 12A & Piezometer Well & Lower Lost Lake Aquifer Zone \\
\hline MSB 41C & Piezometer Well & Lower Lost Lake Aquifer Zone \\
\hline MSB 50B & Piezometer Well & Lower Lost Lake Aquifer Zone \\
\hline SRW 3BB & Piezometer Well & Lower Lost Lake Aquifer Zone \\
\hline SRW $9 A$ & Piezometer Well & Lower Lost Lake Aquifer Zone \\
\hline SRW 11BB & Piezometer Well & Lower Lost Lake Aquifer Zone \\
\hline SRW 13A & Piezometer Well & Lower Lost Lake Aquifer Zone \\
\hline$A C \quad 2 B$ & Piezometer Well & M-Area Aquifer Zone \\
\hline$A C \quad 3 B$ & Piezometer Well & M-Area Aquifer Zone \\
\hline$A C B 2 A$ & Piezometer Well & M-Area Aquifer Zone \\
\hline ASB 9 & Piezometer Well & M-Area Aquifer Zone \\
\hline MCB 2 & Piezometer Well & M-Area Aquifer Zone \\
\hline MCB 9D & Piezometer Well & M-Area Aquifer Zone \\
\hline MSB 25 & Piezometer Well & M-Area Aquifer Zone \\
\hline MSB 35D & Piezometer Well & M-Area Aquifer Zone \\
\hline MSB 37D & Piezometer Well & M-Area Aquifer Zone \\
\hline MSB 40D & Piezometer Well & M-Area Aquifer Zone \\
\hline MSB 41D & Piezometer Well & M-Area Aquifer Zone \\
\hline MSB 45C & Piezometer Well & M-Area Aquifer Zone \\
\hline MSB 51D & Piezometer Well & M-Area Aquifer Zone \\
\hline MSB 52D & Piezometer Well & M-Area Aquifer Zone \\
\hline MSB 53D & Piezometer Well & M-Area Aquifer Zone \\
\hline MSB 56D & Piezometer Well & M-Area Aquifer Zone \\
\hline MSB 77D & Piezometer Well & M-Area Aquifer Zone \\
\hline MSB 89C & Piezometer Well & M-Area Aquifer Zone \\
\hline
\end{tabular}




\begin{tabular}{|c|c|c|}
\hline Well Name & M Area Well Use & Aquifer Name \\
\hline SRW 3A & Piezometer Well & M-Area Aquifer Zone \\
\hline SRW 6 & Piezometer Well & M-Area Aquifer Zone \\
\hline SRW 9 & Piezometer Well & M-Area Aquifer Zone \\
\hline SRW 12C & Piezometer Well & M-Area Aquifer Zone \\
\hline SRW 13C & Piezometer Well & M-Area Aquifer Zone \\
\hline SRW 15C & Piezometer Well & M-Area Aquifer Zone \\
\hline SRW 16C & Piezometer Well & M-Area Aquifer Zone \\
\hline SRW 17DR & Piezometer Well & M-Area Aquifer Zone \\
\hline ARP $1 \mathrm{~A}$ & Piezometer Well & $\begin{array}{l}\text { M-Area Aquifer Zone / Green Clay Confining Zone / Upper } \\
\text { Lost Lake Aquifer Zone }\end{array}$ \\
\hline ARP 2 & Piezometer Well & $\begin{array}{l}\text { M-Area Aquifer Zone / Green Clay Confining Zone / Upper } \\
\text { Lost Lake Aquifer Zone }\end{array}$ \\
\hline ARP 3 & Piezometer Well & $\begin{array}{l}\text { M-Area Aquifer Zone / Green Clay Confining Zone / Upper } \\
\text { Lost Lake Aquifer Zone }\end{array}$ \\
\hline ARP 4 & Piezometer Well & $\begin{array}{l}\text { M-Area Aquifer Zone / Green Clay Confining Zone / Upper } \\
\text { Lost Lake Aquifer Zone }\end{array}$ \\
\hline ARP 5D & Piezometer Well & $\begin{array}{l}\text { M-Area Aquifer Zone / Green Clay Confining Zone / Upper } \\
\text { Lost Lake Aquifer Zone }\end{array}$ \\
\hline MSB 42A & Piezometer Well & Middle Sand Aquifer Zone-Crouch Branch Confining Unit \\
\hline MSB 44A & Piezometer Well & Middle Sand Aquifer Zone-Crouch Branch Confining Unit \\
\hline SRW 15A & Piezometer Well & Middle Sand Aquifer Zone-Crouch Branch Confining Unit \\
\hline$A C \quad 1 A$ & Piezometer Well & Upper Lost Lake Aquifer Zone \\
\hline MSB 24A & Piezometer Well & Upper Lost Lake Aquifer Zone \\
\hline MSB 46B & Piezometer Well & Upper Lost Lake Aquifer Zone \\
\hline MSB 50D & Piezometer Well & Upper Lost Lake Aquifer Zone \\
\hline SRW 13B & Piezometer Well & Upper Lost Lake Aquifer Zone \\
\hline SRW 15B & Piezometer Well & Upper Lost Lake Aquifer Zone \\
\hline ASB $6 T A$ & Plume Definition Well & Crouch Branch Aquifer Unit \\
\hline ASB 8TA & Plume Definition Well & Crouch Branch Aquifer Unit \\
\hline MSB 12TA & Plume Definition Well & Crouch Branch Aquifer Unit \\
\hline MSB 23TA & Plume Definition Well & Crouch Branch Aquifer Unit \\
\hline MSB 27TA & Plume Definition Well & Crouch Branch Aquifer Unit \\
\hline MSB 29TA & Plume Definition Well & Crouch Branch Aquifer Unit \\
\hline MSB 34TA & Plume Definition Well & Crouch Branch Aquifer Unit \\
\hline MSB 35TA & Plume Definition Well & Crouch Branch Aquifer Unit \\
\hline MSB 36TA & Plume Definition Well & Crouch Branch Aquifer Unit \\
\hline MSB 37A & Plume Definition Well & Crouch Branch Aquifer Unit \\
\hline MSB 39TA & Plume Definition Well & Crouch Branch Aquifer Unit \\
\hline MSB 41TA & Plume Definition Well & Crouch Branch Aquifer Unit \\
\hline MSB 42TA & Plume Definition Well & Crouch Branch Aquifer Unit \\
\hline MSB 43TA & Plume Definition Well & Crouch Branch Aquifer Unit \\
\hline MSB 47TA & Plume Definition Well & Crouch Branch Aquifer Unit \\
\hline MSB 48TA & Plume Definition Well & Crouch Branch Aquifer Unit \\
\hline MSB 54TA & Plume Definition Well & Crouch Branch Aquifer Unit \\
\hline MSB 55TA & Plume Definition Well & Crouch Branch Aquifer Unit \\
\hline MSB 66TA & Plume Definition Well & Crouch Branch Aquifer Unit \\
\hline
\end{tabular}




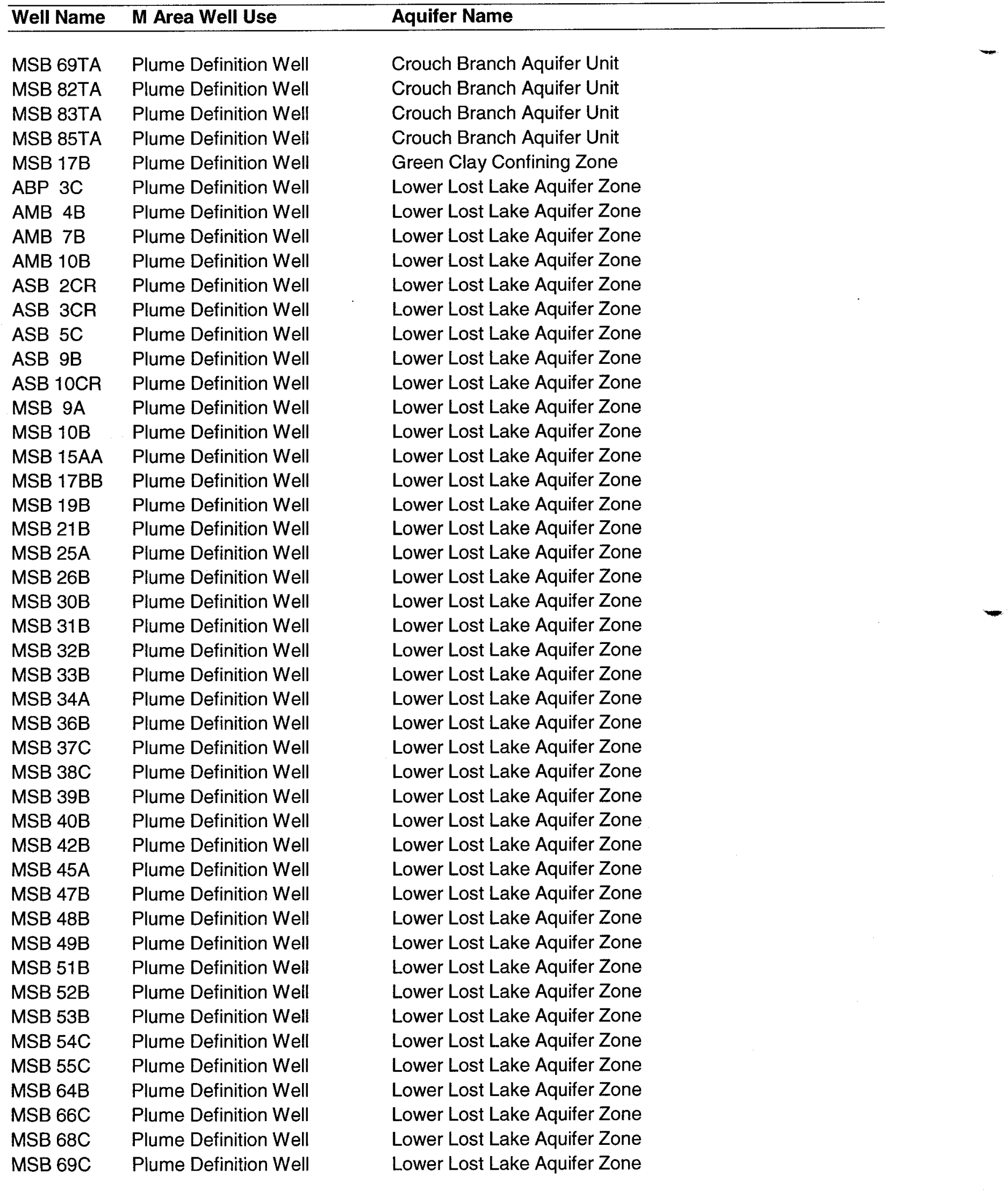




\begin{tabular}{|c|c|c|}
\hline Well Name & M Area Well Use & Aquifer Name \\
\hline MSB 71B & Plume Definition Well & Lower Lost Lake Aquifer Zone \\
\hline MSB 72B & Plume Definition Well & Lower Lost Lake Aquifer Zone \\
\hline MSB 73B & Plume Definition Well & Lower Lost Lake Aquifer Zone \\
\hline MSB 74B & Plume Definition Well & Lower Lost Lake Aquifer Zone \\
\hline MSB 75B & Plume Definition Well & Lower Lost Lake Aquifer Zone \\
\hline MSB 77B & Plume Definition Well & Lower Lost Lake Aquifer Zone \\
\hline MSB 79B & Plume Definition Well & Lower Lost Lake Aquifer Zone \\
\hline MSB 81B & Plume Definition Well & Lower Lost Lake Aquifer Zone \\
\hline MSB $82 C$ & Plume Definition Well & Lower Lost Lake Aquifer Zone \\
\hline MSB $83 C$ & Plume Definition Well & Lower Lost Lake Aquifer Zone \\
\hline MSB 84C & Plume Definition Well & Lower Lost Lake Aquifer Zone \\
\hline MSB 85C & Plume Definition Well & Lower Lost Lake Aquifer Zone \\
\hline MSB 86C & Plume Definition Well & Lower Lost Lake Aquifer Zone \\
\hline MSB $88 C$ & Plume Definition Well & Lower Lost Lake Aquifer Zone \\
\hline MSB 89B & Plume Definition Well & Lower Lost Lake Aquifer Zone \\
\hline SRW 16A & Plume Definition Well & Lower Lost Lake Aquifer Zone \\
\hline ABP $2 A$ & Plume Definition Well & M-Area Aquifer Zone \\
\hline AMB 4D & Plume Definition Well & M-Area Aquifer Zone \\
\hline AMB 7 & Plume Definition Well & M-Area Aquifer Zone \\
\hline AMB 9D & Plume Definition Well & M-Area Aquifer Zone \\
\hline AMB 12D & Plume Definition Well & M-Area Aquifer Zone \\
\hline AMB 15D & Plume Definition Well & M-Area Aquifer Zone \\
\hline AMB 16D & Plume Definition Well & M-Area Aquifer Zone \\
\hline $\mathrm{AOB} 1$ & Plume Definition Well & M-Area Aquifer Zone \\
\hline $\mathrm{AOB} 2$ & Plume Definition Well & M-Area Aquifer Zone \\
\hline ASB $2 A R$ & Plume Definition Well & M-Area Aquifer Zone \\
\hline ASB 3AR & Plume Definition Well & M-Area Aquifer Zone \\
\hline ASB 4 & Plume Definition Well & M-Area Aquifer Zone \\
\hline ASB 5 AR & Plume Definition Well & M-Area Aquifer Zone \\
\hline ASB $6 A$ & Plume Definition Well & M-Area Aquifer Zone \\
\hline ASB 8 & Plume Definition Well & M-Area Aquifer Zone \\
\hline MSB $9 \mathrm{C}$ & Plume Definition Well & M-Area Aquifer Zone \\
\hline MSB 11F & Plume Definition Well & M-Area Aquifer Zone \\
\hline MSB 15D & Plume Definition Well & M-Area Aquifer Zone \\
\hline MSB 16C & Plume Definition Well & M-Area Aquifer Zone \\
\hline MSB 18B & Plume Definition Well & M-Area Aquifer Zone \\
\hline MSB 19C & Plume Definition Well & M-Area Aquifer Zone \\
\hline MSB 20C & Plume Definition Well & M-Area Aquifer Zone \\
\hline MSB 21C & Plume Definition Well & M-Area Aquifer Zone \\
\hline MSB 24 & Plume Definition Well & M-Area Aquifer Zone \\
\hline MSB 26 & Plume Definition Well & M-Area Aquifer Zone \\
\hline MSB 27 & Plume Definition Well & M-Area Aquifer Zone \\
\hline MSB 28 & Plume Definition Well & M-Area Aquifer Zone \\
\hline MSB 31C & Plume Definition Well & M-Area Aquifer Zone \\
\hline MSB 32 & Plume Definition Well & M-Area Aquifer Zone \\
\hline
\end{tabular}




\begin{tabular}{|c|c|c|}
\hline Well Name & M Area Well Use & Aquifer Name \\
\hline MSB 33 & Plume Definition Well & M-Area Aquifer Zone \\
\hline MSB 34C & Plume Definition Well & M-Area Aquifer Zone \\
\hline MSB 36D & Plume Definition Well & M-Area Aquifer Zone \\
\hline MSB 39D & Plume Definition Well & M-Area Aquifer Zone \\
\hline MSB 42D & Plume Definition Well & M-Area Aquifer Zone \\
\hline MSB 46C & Plume Definition Well & M-Area Aquifer Zone \\
\hline MSB 47D & Plume Definition Well & M-Area Aquifer Zone \\
\hline MSB 48D & Plume Definition Well & M-Area Aquifer Zone \\
\hline MSB 49D & Plume Definition Well & M-Area Aquifer Zone \\
\hline MSB 65D & Plume Definition Well & M-Area Aquifer Zone \\
\hline MSB 66D & Plume Definition Well & M-Area Aquifer Zone \\
\hline MSB 67D & Plume Definition Well & M-Area Aquifer Zone \\
\hline MSB 69D & Plume Definition Well & M-Area Aquifer Zone \\
\hline MSB 70D & Plume Definition Well & M-Area Aquifer Zone \\
\hline MSB 74D & Plume Definition Well & M-Area Aquifer Zone \\
\hline MSB 82D & Plume Definition Well & M-Area Aquifer Zone \\
\hline MSB 85D & Plume Definition Well & M-Area Aquifer Zone \\
\hline MSB 87C & Plume Definition Well & M-Area Aquifer Zone \\
\hline MSB 88B & Plume Definition Well & M-Area Aquifer Zone \\
\hline SRW 16B & Plume Definition Well & M-Area Aquifer Zone \\
\hline SRW 18 & Plume Definition Well & M-Area Aquifer Zone \\
\hline ASB $1 A$ & Plume Definition Well & M-Area Aquifer Zone / Green Clay Confining Zone \\
\hline MSB 54D & Plume Definition Well & M-Area Aquifer Zone / Green Clay Confining Zone \\
\hline MSB 55D & Plume Definition Well & M-Area Aquifer Zone / Green Clay Confining Zone \\
\hline MSB 78DR & Plume Definition Well & M-Area Aquifer Zone / Green Clay Confining Zone \\
\hline AMB 4A & Plume Definition Well & Middle Sand Aquifer Zone-Crouch Branch Confining Unit \\
\hline $\mathrm{AMB} 7 \mathrm{~A}$ & Plume Definition Well & Middle Sand Aquifer Zone-Crouch Branch Confining Unit \\
\hline AMB 10A & Plume Definition Well & Middle Sand Aquifer Zone-Crouch Branch Confining Unit \\
\hline AMB 13AR & Plume Definition Well & Middle Sand Aquifer Zone-Crouch Branch Confining Unit \\
\hline AMB 17A & Plume Definition Well & Middle Sand Aquifer Zone-Crouch Branch Confining Unit \\
\hline$A M B 18 A$ & Plume Definition Well & Middle Sand Aquifer Zone-Crouch Branch Confining Unit \\
\hline ASB $6 A A$ & Plume Definition Well & Middle Sand Aquifer Zone-Crouch Branch Confining Unit \\
\hline ASB 8B & Plume Definition Well & Middle Sand Aquifer Zone-Crouch Branch Confining Unit \\
\hline MSB 10A & Plume Definition Well & Middle Sand Aquifer Zone-Crouch Branch Confining Unit \\
\hline MSB 19A & Plume Definition Well & Middle Sand Aquifer Zone-Crouch Branch Confining Unit \\
\hline MSB 29A & Plume Definition Well & Middle Sand Aquifer Zone-Crouch Branch Confining Unit \\
\hline MSB 30AA & Plume Definition Well & Middle Sand Aquifer Zone-Crouch Branch Confining Unit \\
\hline MSB 33A & Plume Definition Well & Middle Sand Aquifer Zone-Crouch Branch Confining Unit \\
\hline MSB 35A & Plume Definition Well & Middle Sand Aquifer Zone-Crouch Branch Confining Unit \\
\hline MSB 36A & Plume Definition Well & Middle Sand Aquifer Zone-Crouch Branch Confining Unit \\
\hline MSB 37B & Plume Definition Well & Middle Sand Aquifer Zone-Crouch Branch Confining Unit \\
\hline MSB 40A & Plume Definition Well & Middle Sand Aquifer Zone-Crouch Branch Confining Unit \\
\hline MSB 41B & Plume Definition Well & Middle Sand Aquifer Zone-Crouch Branch Confining Unit \\
\hline MSB 46A & Plume Definition Well & Middle Sand Aquifer Zone-Crouch Branch Confining Unit \\
\hline MSB 48A & Plume Definition Well & Middle Sand Aquifer Zone-Crouch Branch Confining Unit \\
\hline MSB 49A & Plume Definition Well & Middle Sand Aquifer Zone-Crouch Branch Confining Unit \\
\hline
\end{tabular}




\begin{tabular}{|c|c|c|}
\hline Well Name & M Area Well Use & Aquifer Name \\
\hline MSB 54B & Plume Definition Well & Middle Sand Aquifer Zone-Crouch Branch Confining Unit \\
\hline MSB 55B & Plume Definition Well & Middle Sand Aquifer Zone-Crouch Branch Confining Unit \\
\hline MSB 66B & Plume Definition Well & Middle Sand Aquifer Zone-Crouch Branch Confining Unit \\
\hline MSB 68B & Plume Definition Well & Middle Sand Aquifer Zone-Crouch Branch Confining Unit \\
\hline MSB 69B & Plume Definition Well & Middle Sand Aquifer Zone-Crouch Branch Confining Unit \\
\hline MSB $82 A$ & Plume Definition Well & Middle Sand Aquifer Zone-Crouch Branch Confining Unit \\
\hline MSB 83B & Plume Definition Well & Middle Sand Aquifer Zone-Crouch Branch Confining Unit \\
\hline MSB 84A & Plume Definition Well & Middle Sand Aquifer Zone-Crouch Branch Confining Unit \\
\hline MSB 85B & Plume Definition Well & Middle Sand Aquifer Zone-Crouch Branch Confining Unit \\
\hline SRW $2 A$ & Plume Definition Well & Middle Sand Aquifer Zone-Crouch Branch Confining Unit \\
\hline SRW 14A & Plume Definition Well & Middle Sand Aquifer Zone-Crouch Branch Confining Unit \\
\hline SRW 19 & Plume Definition Well & Perched Unit Above M-Area Aquifer Zone \\
\hline MSB 82B & Plume Definition Well & Upper Clay Confining Zone - Crouch Branch Confining Unit \\
\hline ABP $8 \mathrm{C}$ & Plume Definition Well & Upper Lost Lake Aquifer Zone \\
\hline$A C \quad 3 A$ & Plume Definition Well & Upper Lost Lake Aquifer Zone \\
\hline AMB 18C & Plume Definition Well & Upper Lost Lake Aquifer Zone \\
\hline AMB 19C & Plume Definition Well & Upper Lost Lake Aquifer Zone \\
\hline ASB $6 \mathrm{C}$ & Plume Definition Well & Upper Lost Lake Aquifer Zone \\
\hline ASB $8 \mathrm{C}$ & Plume Definition Well & Upper Lost Lake Aquifer Zone \\
\hline ASB $9 \mathrm{C}$ & Plume Definition Well & Upper Lost Lake Aquifer Zone \\
\hline MCB 5C & Plume Definition Well & Upper Lost Lake Aquifer Zone \\
\hline MCB $6 \mathrm{C}$ & Plume Definition Well & Upper Lost Lake Aquifer Zone \\
\hline MCB 7C & Plume Definition Well & Upper Lost Lake Aquifer Zone \\
\hline MSB 11C & Plume Definition Well & Upper Lost Lake Aquifer Zone \\
\hline MSB 12B & Plume Definition Well & Upper Lost Lake Aquifer Zone \\
\hline MSB 15A & Plume Definition Well & Upper Lost Lake Aquifer Zone \\
\hline MSB 16A & Plume Definition Well & Upper Lost Lake Aquifer Zone \\
\hline MSB 18A & Plume Definition Well & Upper Lost Lake Aquifer Zone \\
\hline MSB 20A & Plume Definition Well & Upper Lost Lake Aquifer Zone \\
\hline MSB 23B & Plume Definition Well & Upper Lost Lake Aquifer Zone \\
\hline MSB 27B & Plume Definition Well & Upper Lost Lake Aquifer Zone \\
\hline MSB 28A & Plume Definition Well & Upper Lost Lake Aquifer Zone \\
\hline MSB $30 C C$ & Plume Definition Well & Upper Lost Lake Aquifer Zone \\
\hline MSB $31 \mathrm{CC}$ & Plume Definition Well & Upper Lost Lake Aquifer Zone \\
\hline MSB 32C & Plume Definition Well & Upper Lost Lake Aquifer Zone \\
\hline MSB 33C & Plume Definition Well & Upper Lost Lake Aquifer Zone \\
\hline MSB 34B & Plume Definition Well & Upper Lost Lake Aquifer Zone \\
\hline MSB 35B & Plume Definition Well & Upper Lost Lake Aquifer Zone \\
\hline MSB 36C & Plume Definition Well & Upper Lost Lake Aquifer Zone \\
\hline MSB 39C & Plume Definition Well & Upper Lost Lake Aquifer Zone \\
\hline MSB 40C & Plume Definition Well & Upper Lost Lake Aquifer Zone \\
\hline MSB 42C & Plume Definition Well & Upper Lost Lake Aquifer Zone \\
\hline MSB 45B & Plume Definition Well & Upper Lost Lake Aquifer Zone \\
\hline MSB 47C & Plume Definition Well & Upper Lost Lake Aquifer Zone \\
\hline MSB 48C & Plume Definition Well & Upper Lost Lake Aquifer Zone \\
\hline
\end{tabular}




\begin{tabular}{|c|c|c|}
\hline Well Name & M Area Well Use & Aquifer Name \\
\hline MSB 53C & Plume Definition Well & Upper Lost Lake Aquifer Zone \\
\hline MSB $55 \mathrm{HC}$ & Plume Definition Well & Upper Lost Lake Aquifer Zone \\
\hline MSB 70C & Plume Definition Well & Upper Lost Lake Aquifer Zone \\
\hline MSB 74C & Plume Definition Well & Upper Lost Lake Aquifer Zone \\
\hline MSB 75C & Plume Definition Well & Upper Lost Lake Aquifer Zone \\
\hline MSB 76C & Plume Definition Well & Upper Lost Lake Aquifer Zone \\
\hline MSB 77C & Plume Definition Well & Upper Lost Lake Aquifer Zone \\
\hline MSB 79C & Plume Definition Well & Upper Lost Lake Aquifer Zone \\
\hline MSB 87B & Plume Definition Well & Upper Lost Lake Aquifer Zone \\
\hline SRW 2B & Plume Definition Well & Upper Lost Lake Aquifer Zone \\
\hline SRW 14B & Plume Definition Well & Upper Lost Lake Aquifer Zone \\
\hline MSB 1B & Point of Compliance Well & Lower Lost Lake Aquifer Zone \\
\hline MSB 2B & Point of Compliance Well & Lower Lost Lake Aquifer Zone \\
\hline MSB 3B & Point of Compliance Well & Lower Lost Lake Aquifer Zone \\
\hline MSB 4B & Point of Compliance Well & Lower Lost Lake Aquifer Zone \\
\hline MSB 5B & Point of Compliance Well & Lower Lost Lake Aquifer Zone \\
\hline MSB $6 B$ & Point of Compliance Well & Lower Lost Lake Aquifer Zone \\
\hline MSB 7B & Point of Compliance Well & Lower Lost Lake Aquifer Zone \\
\hline MSB 8B & Point of Compliance Well & Lower Lost Lake Aquifer Zone \\
\hline MSB 13A & Point of Compliance Well & Lower Lost Lake Aquifer Zone \\
\hline MSB 62B & Point of Compliance Well & Lower Lost Lake Aquifer Zone \\
\hline MSB 63B & Point of Compliance Well & Lower Lost Lake Aquifer Zone \\
\hline MSB 1D & Point of Compliance Well & M-Area Aquifer Zone \\
\hline MSB 2D & Point of Compliance Well & M-Area Aquifer Zone \\
\hline MSB 3D & Point of Compliance Well & M-Area Aquifer Zone \\
\hline MSB 4D & Point of Compliance Well & M-Area Aquifer Zone \\
\hline MSB $5 \mathrm{~A}$ & Point of Compliance Well & M-Area Aquifer Zone \\
\hline MSB $6 A$ & Point of Compliance Well & M-Area Aquifer Zone \\
\hline MSB 7A & Point of Compliance Well & M-Area Aquifer Zone \\
\hline MSB 8A & Point of Compliance Well & M-Area Aquifer Zone \\
\hline MSB 13D & Point of Compliance Well & M-Area Aquifer Zone \\
\hline MSB 59D & Point of Compliance Well & M-Area Aquifer Zone \\
\hline MSB 62D & Point of Compliance Well & M-Area Aquifer Zone \\
\hline MSB 63D & Point of Compliance Well & M-Area Aquifer Zone \\
\hline MSB 64D & Point of Compliance Well & M-Area Aquifer Zone \\
\hline MSB 39A & Point of Compliance Well & Middle Sand Aquifer Zone-Crouch Branch Confining Unit \\
\hline MSB $1 \mathrm{C}$ & Point of Compliance Well & Upper Lost Lake Aquifer Zone \\
\hline MSB 2C & Point of Compliance Well & Upper Lost Lake Aquifer Zone \\
\hline MSB $3 \mathrm{C}$ & Point of Compliance Well & Upper Lost Lake Aquifer Zone \\
\hline MSB 4C & Point of Compliance Well & Upper Lost Lake Aquifer Zone \\
\hline MSB 5C & Point of Compliance Well & Upper Lost Lake Aquifer Zone \\
\hline MSB $6 \mathrm{C}$ & Point of Compliance Well & Upper Lost Lake Aquifer Zone \\
\hline MSB $7 \mathrm{C}$ & Point of Compliance Well & Upper Lost Lake Aquifer Zone \\
\hline MSB $8 \mathrm{C}$ & Point of Compliance Well & Upper Lost Lake Aquifer Zone \\
\hline MSB 13CC & Point of Compliance Well & Upper Lost Lake Aquifer Zone \\
\hline
\end{tabular}




\begin{tabular}{|c|c|c|}
\hline Well Name & M Area Well Use & Aquifer Name \\
\hline MSB $62 C$ & Point of Compliance Well & Upper Lost Lake Aquifer Zone \\
\hline MSB 63C & Point of Compliance Well & Upper Lost Lake Aquifer Zone \\
\hline MSB 64C & Point of Compliance Well & Upper Lost Lake Aquifer Zone \\
\hline SSR 10 & Recirculation Well & $\begin{array}{l}\text { Upper Lost Lake Aquifer Zone / Lower Lost Lake Aquifer } \\
\text { Zone }\end{array}$ \\
\hline SSR 1 & Recirculation Well & $\begin{array}{l}\text { Upper Lost Lake Aquifer Zone / Lower Lost Lake Aquifer } \\
\text { Zone }\end{array}$ \\
\hline SSR 2 & Recirculation Well & $\begin{array}{l}\text { Upper Lost Lake Aquifer Zone / Lower Lost Lake Aquifer } \\
\text { Zone }\end{array}$ \\
\hline SSR 3 & Recirculation Well & $\begin{array}{l}\text { Upper Lost Lake Aquifer Zone / Lower Lost Lake Aquifer } \\
\text { Zone }\end{array}$ \\
\hline SSR 4 & Recirculation Well & $\begin{array}{l}\text { Upper Lost Lake Aquifer Żone / Lower Lost Lake Aquifer } \\
\text { Zone }\end{array}$ \\
\hline SSR 5 & Recirculation Well & $\begin{array}{l}\text { Upper Lost Lake Aquifer Zone / Lower Lost Lake Aquifer } \\
\text { Zone }\end{array}$ \\
\hline SSR 6 & Recirculation Well & $\begin{array}{l}\text { Upper Lost Lake Aquifer Zone / Lower Lost Lake Aquifer } \\
\text { Zone }\end{array}$ \\
\hline SSR 7 & Recirculation Well & $\begin{array}{l}\text { Upper Lost Lake Aquifer Zone / Lower Lost Lake Aquifer } \\
\text { Zone }\end{array}$ \\
\hline SSR 8 & Recirculation Well & $\begin{array}{l}\text { Upper Lost Lake Aquifer Zone / Lower Lost Lake Aquifer } \\
\text { Zone }\end{array}$ \\
\hline SSR 9 & Recirculation Well & $\begin{array}{l}\text { Upper Lost Lake Aquifer Zone / Lower Lost Lake Aquifer } \\
\text { Zone }\end{array}$ \\
\hline SSR 11 & Recirculation Well & $\begin{array}{l}\text { Upper Lost Lake Aquifer Zone / Lower Lost Lake Aquifer } \\
\text { Zone }\end{array}$ \\
\hline SSR 12 & Recirculation Well & $\begin{array}{l}\text { Upper Lost Lake Aquifer Zone / Lower Lost Lake Aquifer } \\
\text { Zone }\end{array}$ \\
\hline RWM 8 & Recovery Well & $\begin{array}{l}\text { Green Clay Confining Zone / Upper Lost Lake Aquifer } \\
\text { Zone / Lower Lost Lake Aquifer Zone }\end{array}$ \\
\hline RWM 13B & Recovery Well & Lower Lost Lake Aquifer Zone \\
\hline RWM 14B & Recovery Well & Lower Lost Lake Aquifer Zone \\
\hline RWM 15B & Recovery Well & Lower Lost Lake Aquifer Zone \\
\hline RWM 9 & Recovery Well & M-Area Aquifer Zone \\
\hline RWM 10 & Recovery Well & M-Area Aquifer Zone \\
\hline RWM 4 & Recovery Well & $\begin{array}{l}\text { M-Area Aquifer Zone / Green Clay Confining Zone / Upper } \\
\text { Lost Lake Aquifer Zone / Lower Lost Lake Aquifer Zone }\end{array}$ \\
\hline RWM 5 & Recovery Well & $\begin{array}{l}\text { M-Area Aquifer Zone / Green Clay Confining Zone / Upper } \\
\text { Lost Lake Aquifer Zone / Lower Lost Lake Aquifer Zone }\end{array}$ \\
\hline RWM 6 & Recovery Well & $\begin{array}{l}\text { M-Area Aquifer Zone / Green Clay Confining Zone / Upper } \\
\text { Lost Lake Aquifer Zone / Lower Lost Lake Aquifer Zone }\end{array}$ \\
\hline RWM 7 & Recovery Well & $\begin{array}{l}\text { M-Area Aquifer Zone / Green Clay Confining Zone / Upper } \\
\text { Lost Lake Aquifer Zone / Lower Lost Lake Aquifer Zone }\end{array}$ \\
\hline RWM 1 & Recovery Well & Unknown \\
\hline RWM 2 & Recovery Well & Unknown \\
\hline RWM 3 & Recovery Well & Unknown \\
\hline RWM 11 & Recovery Well & Unknown \\
\hline RWM 13C & Recovery Well & Upper Lost Lake Aquifer Zone \\
\hline RWM 14C & Recovery Well & Upper Lost Lake Aquifer Zone \\
\hline RWM 12 & Recovery Well & $\begin{array}{l}\text { Upper Lost Lake Aquifer Zone / Lower Lost Lake Aquifer } \\
\text { Zone }\end{array}$ \\
\hline
\end{tabular}


WSRC-TR-2000-00242

Unclassified

THIS PAGE INTENTIONALLY LEFT BLANK. 


\section{Appendix C}

Figures and Maps 


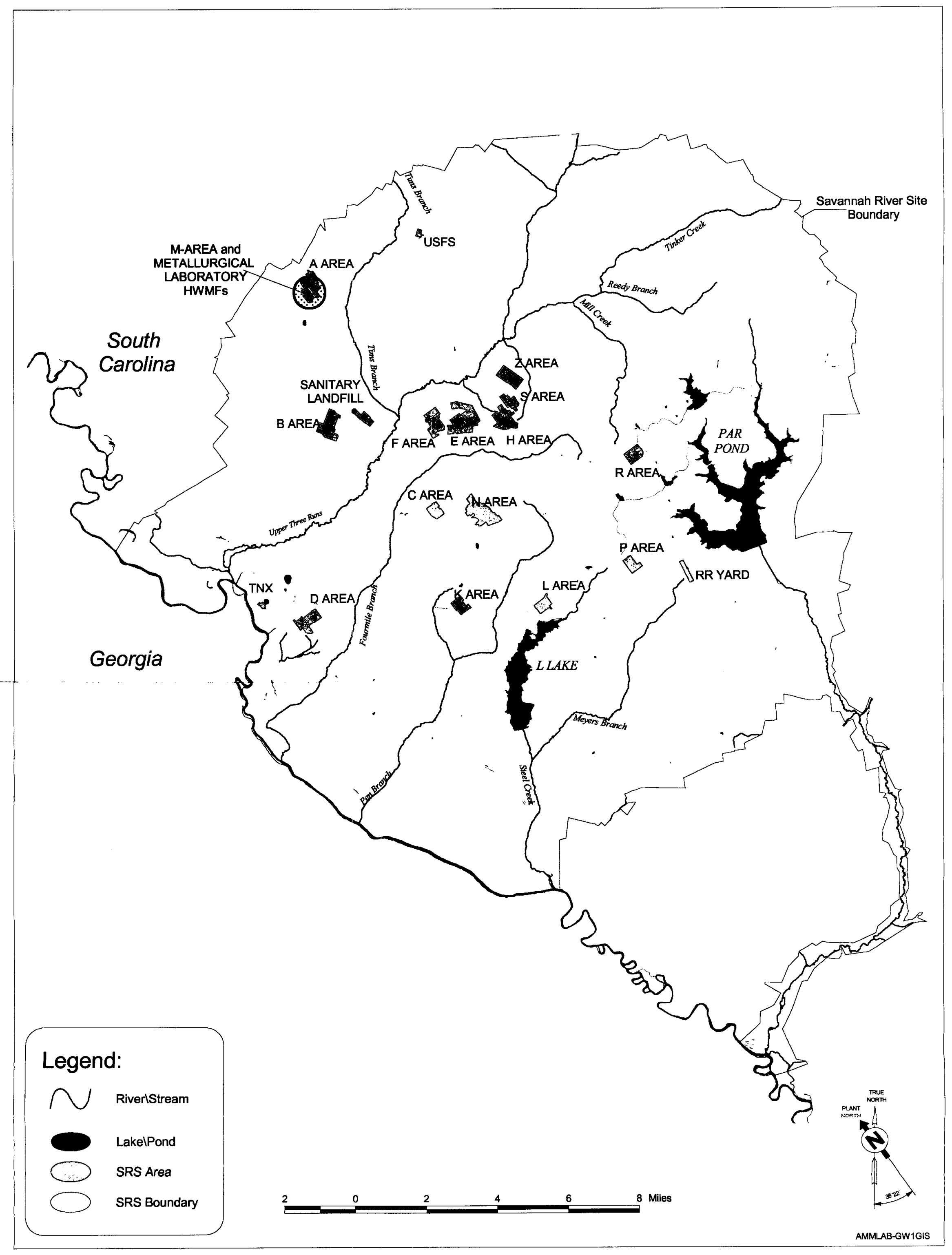

Figure 1. Location of the M-Area and Metallurgical Laboratory HWMFs at the Savannah River Site 


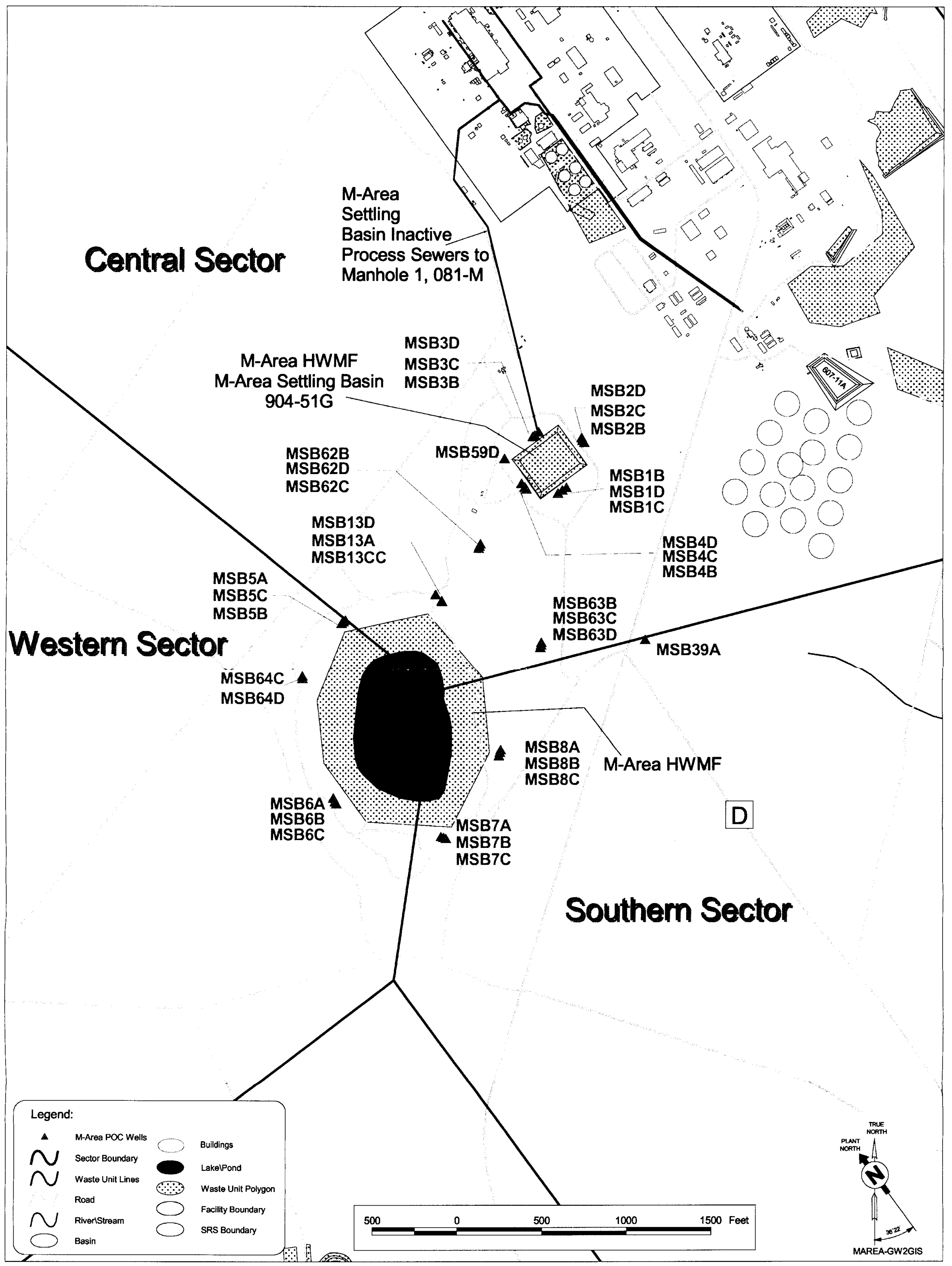

Figure 2. Location of Point-of-Compliance Wells at the M-Area HWMF 


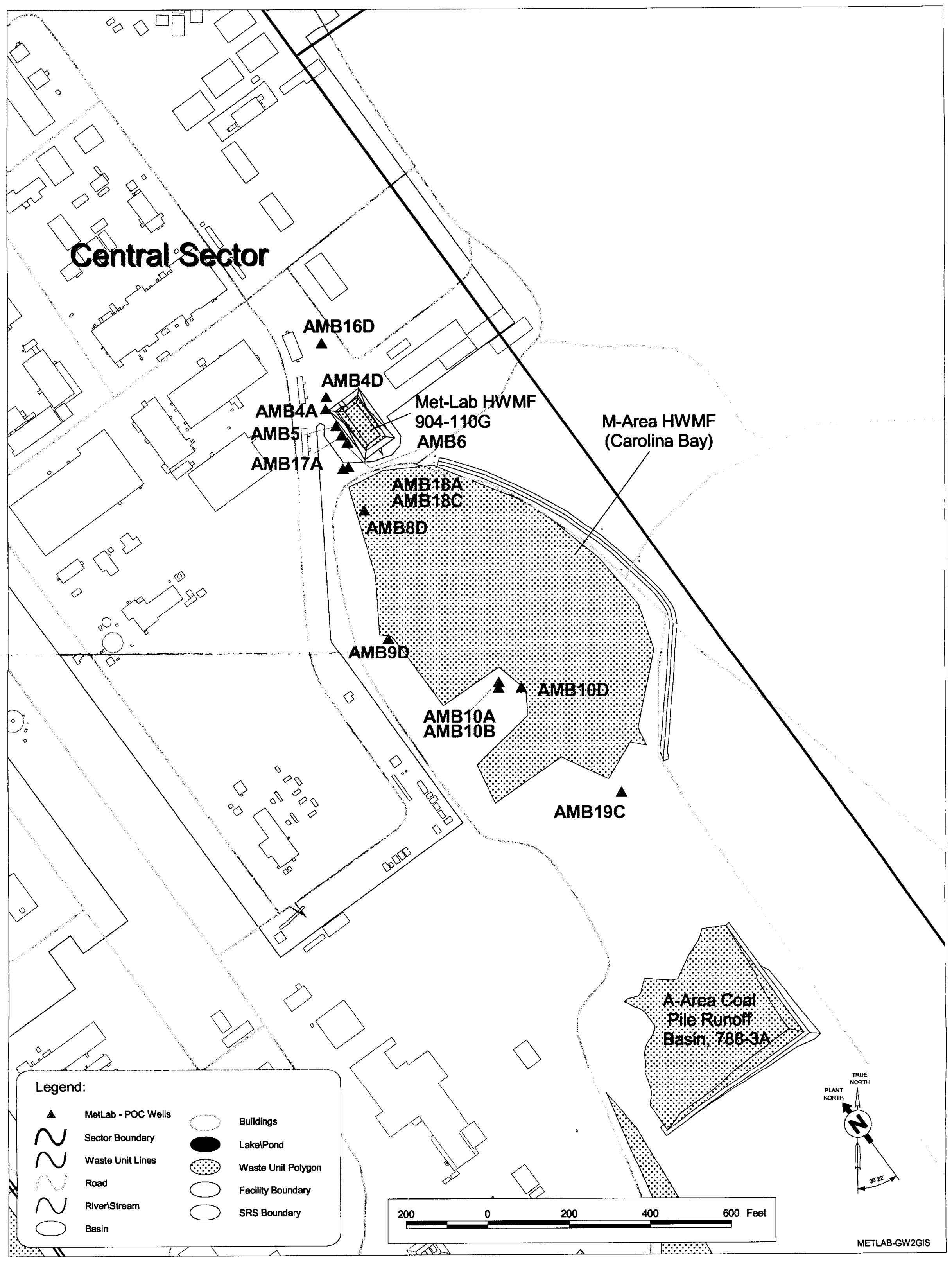

Figure 3. Location of Point-of-Compliance Wells at the Metallurgical Laboratory HWMF 


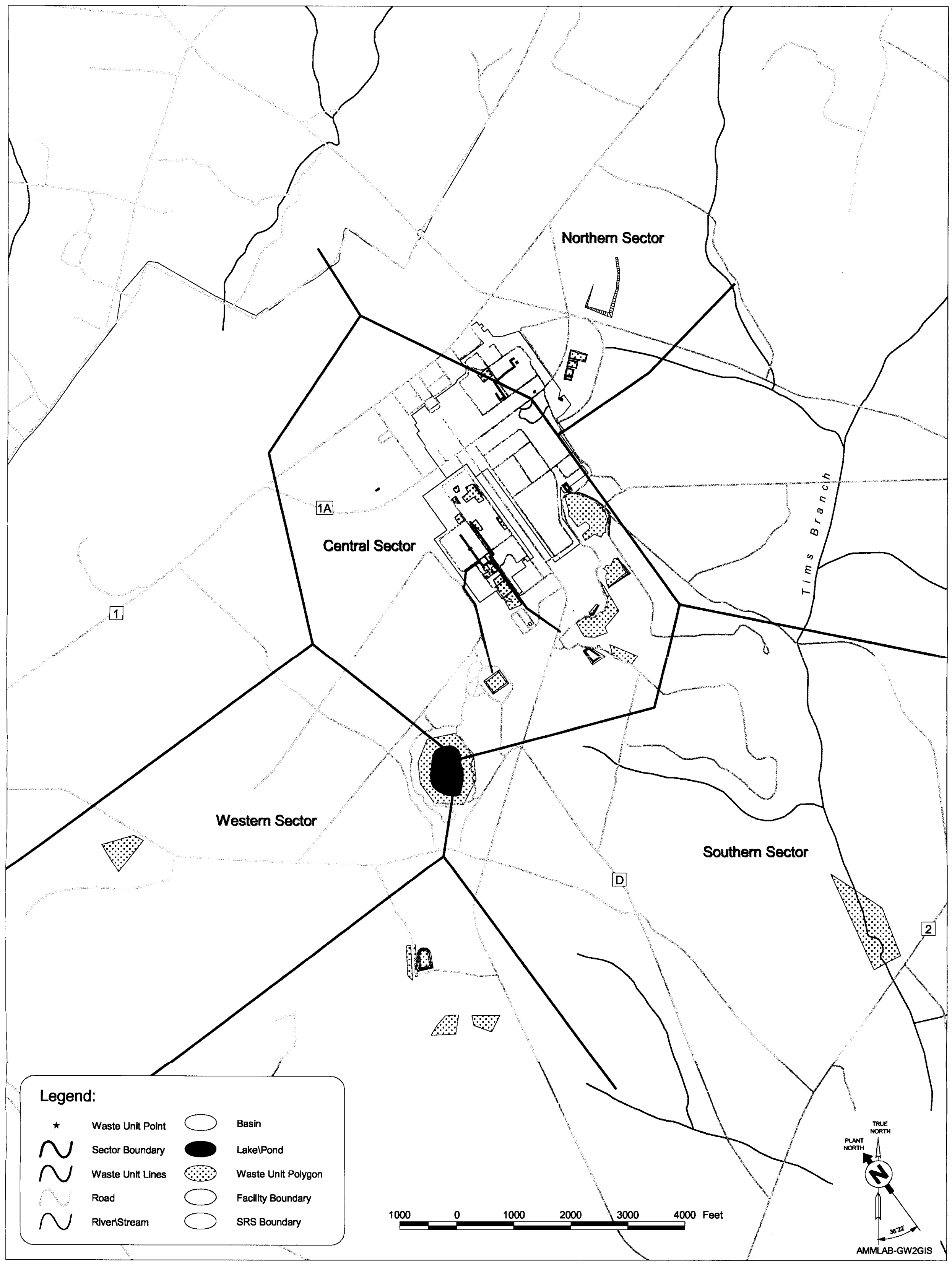

Figure 4. M-Area Sectors 


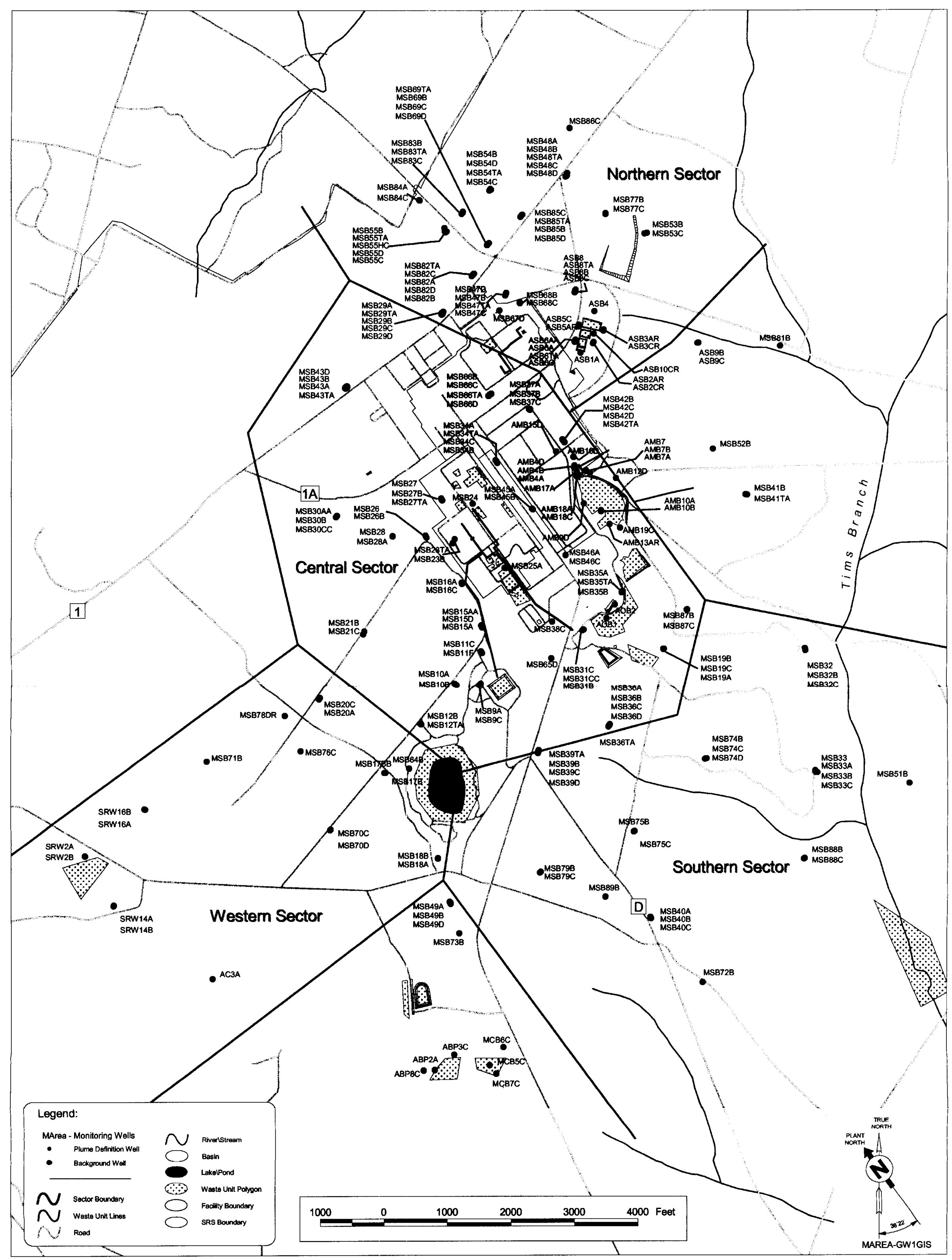

Figure 5. Location of Plume Definition and Background Wells at the M-Area HWMF 


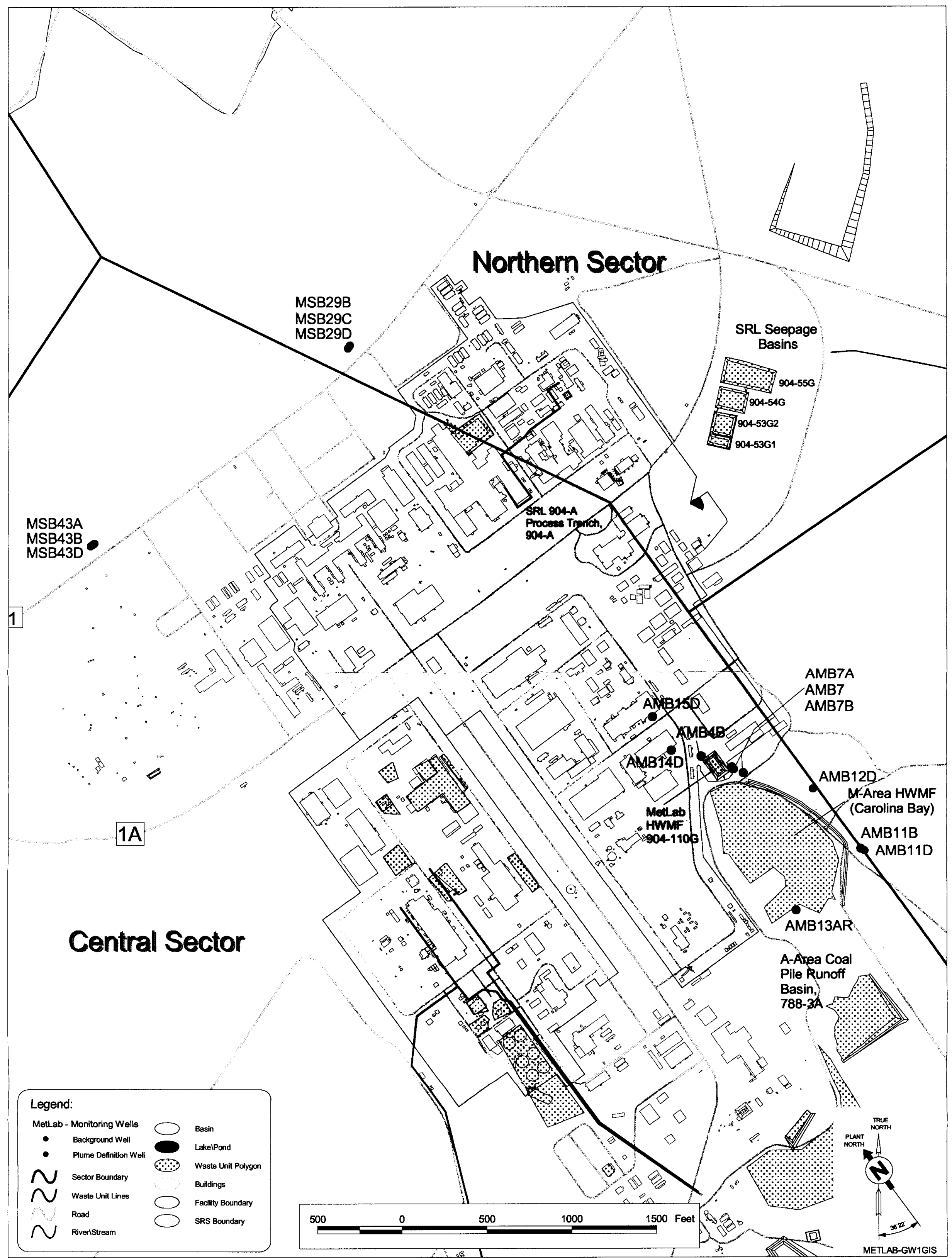

Figure 6. Location of Plume Definition and Background Wells at the Metallurgical Laboratory HWMF 


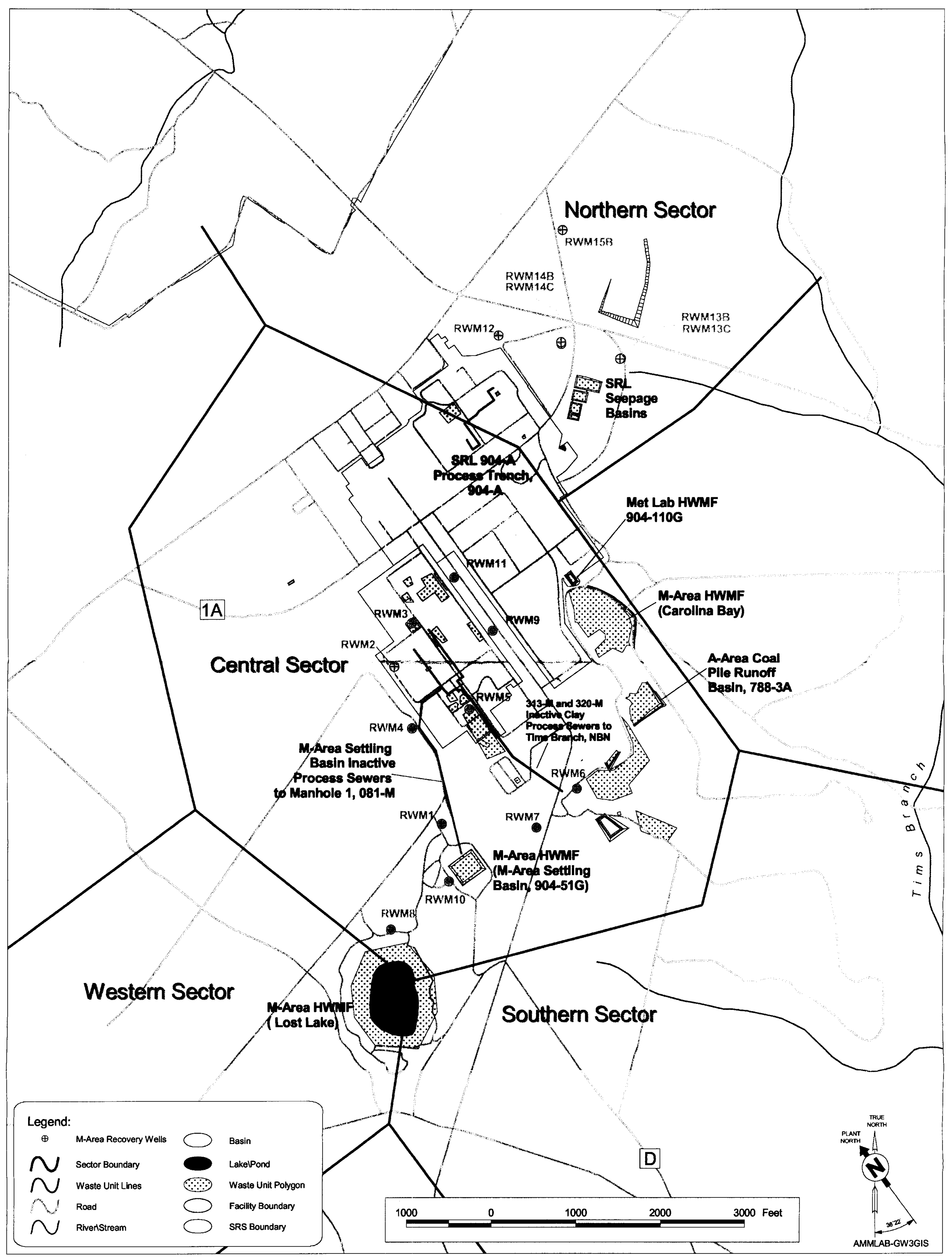

Figure 7. Location of Recovery Wells at the M-Area HWMF 
THIS PAGE LEFT BLANK INTENTIONALLY. 


\section{Appendix D}

\section{Groundwater Monitoring Results Tables}




\section{Key to Reading the Tables}

The following abbreviations may appear in the data tables:

\section{Constituents}

1,2,3,4,6,7,8-HPCDD
1,2,3,4,6,7,8-HPCDF
1,2,3,4,7,8-HXCDD
1,2,3,4,7,8-HXCDF
Lindane
PCB
1,2,3,7,8-PCDD
1,2,3,7,8-PCDF
Sp. conductance
TCDD
TCDF

\section{Laboratories}

$\mathrm{CN}$

EM

GE and GP

$\mathrm{SC}$

$\mathrm{SP}$

TM

WA and WS 1,2,3,4,6,7,8-heptachlorodibenzo-p-dioxin

$1,2,3,4,6,7,8$-heptachlorodibenzo-p-furan

$1,2,3,4,7,8$-hexachlorodibenzo-p-dioxin

1,2,3,4,7,8-hexachlorodibenzo-p-furan

gamma-benzene hexachloride

polychlorinated biphenyl

1,2,3,7,8-pentachlorodibenzo-p-dioxin

1,2,3,7,8-pentachlorodibenzo-p-furan

specific conductance

tetrachlorodibenzo-p-dioxin

tetrachlorodibenzo-p-furan

\section{Nomenclature}

$\begin{array}{lll}\text { AZ } & - & \text { Aquifer Zone } \\ \text { CBAU } & \text { CBA } & \text { Crouch Branch Aquifer Unit (previously Black Creek) } \\ \text { CBCU } & \text { CBC } & \text { Crouch Branch Confining Unit } \\ \text { GAU } & \text { GAU } & \text { Gordon Aquifer Unit } \\ \text { GCCZ } & \text { GC } & \text { Green Clay Confining Zone } \\ \text { LAZ_UTRA } & \text { LAZ } & \text { Lower Aquifer Zone-Upper Three Runs Aquifer Unit } \\ \text { LLAZ } & \text { LLAZ } & \text { Lost Lake Aquifer Zone } \\ \text { LLLAZ } & \text { LL } & \text { Lower Lost Lake Aquifer Zone (previously Lower Congaree) } \\ \text { MAAZ } & \text { M } & \text { M-Area Aquifer Zone (previously Water Table) } \\ \text { MSAZ_CBCU } & \text { MCBC } & \text { Middle Sand Aquifer Zone-CBCU (previously Ellenton Sand) } \\ \text { PZ } & \text { PZ } & \text { Perched Zone } \\ \text { UAZ_UTRA } & \text { UAZ } & \text { Upper Aquifer Zone-Upper Three Runs Aquifer Unit } \\ \text { ULLAZ } & \text { UL } & \text { Upper Lost Lake Aquifer Zone (previously Upper Congaree) } \\ \text { UNKNOWN } & \text { UNK } & \text { Unknown }\end{array}$




\section{Sampling Codes}

B

C

D

E

I

L

N

$P$

$\mathrm{S}$

$\mathrm{T}$

W

$x$

\section{Sampling Methods}

\section{B \\ O \\ $P$ \\ $S$ \\ V}

blank sample was collected

well was pumping continuously

well was dry

equipment blank was collected

well went dry during sampling; insufficient water to collect all samples

well went dry before sampling began; only depth to water can be determined

well was not stabilized before sampling began

inaccessibility or mechanical failure prevented sample collection and field analysis of the water

no water in standpipe

samples were collected, but some samples were not sent to the laboratory due to high turbidity

unable to sample well because of stabilization or sampling

equipment failure; water-level measurements were obtained

well went dry during purging; samples collected after well

recovered

\section{Units}

$\mathrm{mg} / \mathrm{L}$

msl

MSL

NTU

$\mathrm{pCi} / \mathrm{L}$

$\mathrm{pCi} / \mathrm{mL}$

$\mathrm{pH}$

$\mu g / L$

$\mu \mathrm{S} / \mathrm{cm}$

sample collected using an open-bucket bailer

sample collected by method other than bailer or pump

sample collected using a bladder pump

sample collected using a single-speed centrifugal downhole pump sample collected using a variable-speed pump

milligrams per liter

mean sea level

million structures per liter

nephelometric turbidity unit

picocuries per liter

picocuries per milliliter

pH unit

micrograms per liter

microsiemens per centimeter 


\section{Other Codes}

$\begin{array}{ll}\text { CLP } & \text { EPA Functional Guideline Codes } \\ \text { CS } & \text { carbon steel } \\ \text { DF } & \text { dilution factor column in data tables } \\ \text { E } & \text { exponential notation }\left(e . g ., 1.1 \mathrm{E}-09=1.1 \times 10^{-9}=0.0000000011\right) \\ \text { STORET } & \text { EPASTORET result qualifiers } \\ \text { Filt. } & \text { Data results after application of the Data Usability filter } \\ \text { GWPS } & \text { groundwater protection standard } \\ \text { MCS } & \text { monitoring constituents standard } \\ \text { Mod } & \text { modifier column in data tables } \\ \text { NDD } & \text { "not decision data" } \\ \text { PDWS } & \text { primary drinking water standard } \\ \text { PVC } & \text { polyvinyl chloride } \\ \text { ST } & \text { exceeded the GWPS or MCS column in data tables } \\ \text { TOC } & \text { top of casing } \\ \text { CEQL } & \text { less sample-specific estimated quantitation limit }\end{array}$

\section{Results Below Detection}

For radiological analyses, the analytical result field contains the result recorded on the analytical instrument and reported by the laboratory, even if it is negative. For nonradiological analyses, if the analyte is not detected, the sample-specific estimated quantitation limit (EQL) is entered into the result field and is reported with a less than $[<]$ sign. The EQL is defined as the lowest concentration that can be achieved reliably within specified limits of precision and accuracy during routine laboratory operating conditions. The sample-specific EQL is modified for sample concentration or dilution or unusual aliquot size that affects analytical sensitivity.

\section{Uncertainty and Data Usability}

In April 1998, SCDHEC accepted guidance proposed by SRS to apply a method for minimizing uncertainty in compliance decisions potentially affecting long-term monitoring or remediation (letter, Taylor to Cook; April 21, 1998). The method is applied by processing or "filtering" the data, using the EPA Functional Guideline Codes applied by the laboratories to qualify the analytical results. By removing all data with a result qualifier of " $L$ ", "R", " $U$ ", and " $J$ " from consideration, groundwater data users can ensure that only quantified numerical results are applied to the decision process. The output of the filtering process populates the "Filt" column as follows:

1) "Null" or "blank" - Data not remarked. The analytical result is acceptable for use as reported, and the result is not greater than an associated concentration limit for the analyte. If a concentration limit exists for the analyte, and the result is greater than the concentration limit, then the "Filt." Column will contain the applicable limit, and the "greater than" symbol. For example, in the case of trichloroethylene, " $>5$ " would indicate the result exceeded a concentration limit of 5 ug/L.

Rationale: The best result would be one without qualifiers, so the preferred choice would be the maximum result that did not have any qualifiers.

2) "J", "L", "N", "NJ", or "JL" - "J" identifies that the analyte was positively identified; the associated numerical value is an estimated concentration of the analyte in the sample. "L" Indicates the sample result is off scale high. "JL" Indicates an estimated quantity of a sample that is off scale high. " $N$ " is used for all TIC (tentatively identified compounds) and indicates the presence of an analyte for which there is presumptive evidence to make a tentative identification. "NJ" means the presence of an analyte that has been tentatively identified and the associated numerical value represents its approximate concentration. 
Rationale: an estimate can still provide useful information. Although there may be a range of uncertainty around the actual value, the value itself may still grossly exceed a regulatory standard. However, a estimated value is less certain than an unqualified result. Therefore, this would be labeled as "NDD" (not decision data).

3) "U" - material analyzed for, but not detected. The analyte concentration is less than the sample specific Estimated Quantitation Limit and labeled "<EQL".

Rationale: a result above the detection limit would be chosen before a result below detection so that the process is not biased toward false negatives.

4) "UJ" - result is not above the reported sample quantitation limit, but the reported quantitation limit itself is approximate, and may not represent the actual limit of quantitation necessary to accurately and precisely measure the analyte in the sample.

Rationale: the additional qualifiers make this result less reliable for use than the " $U$ " without qualifiers. These results would be labeled " $\angle E Q L$ ".

5) "Rejected" - The sample results are rejected due to serious deficiencies in the ability to analyze the sample and meet quality control criteria. The presence or absence of the analyte cannot be verified.

Rationale: the only value in providing this result in the report is to indicate that the lab attempted to analyze the sample. If there are any other results available, the result with the "R" qualifier should not be reported. If it is reported, it is definitely "NDD" (not decision data).

\section{Holding Times}

Standard analytical methods include a limit, called holding time, on the maximum elapsed time between sample collection and extraction or analysis by the laboratory. In the data tables, the result qualifier $Q$ in the "EPA" column indicates that holding time was exceeded. Analyses performed beyond holding times may not yield valid results.

The South Carolina Department of Health and Environmental Control (SCDHEC) allows only 15 minutes to elapse between sampling and analysis for $\mathrm{pH}$. Thus, only field $\mathrm{pH}$ measurements can meet the holding time criterion; laboratory $\mathrm{pH}$ analyses always will exceed it.

The laboratory procedure used for the determination of specific conductance allows one day to elapse between sampling and analysis. Thus, laboratory specific conductance measurements may exceed the holding time criterion.

\section{Data Qualification}

The contract laboratories submit sample- or batch-specific quality assurance/quality control information either at the same time as analytical results or in a quarterly summary. Properly defined and used, data qualifiers can be a key component in assessing data usability. The EPA Functional Guideline Codes used by the analytical laboratories are shown in the CLP result qualifier column are defined below. These modifiers appear in the data tables under the column CLP. EPA STORET codes appear in the data tables under the column labeled EPA. 


\section{"CLP" Qualifiers - EPA Functional Guideline Codes}

(Blank) Data not remarked. The analytical result is acceptable for use as reported.

$J$

$N$

$R$

$U$

NJ
The analyte was positively identified; the associated numerical value is an estimated concentration of the analyte in the sample.

The analysis indicates the presence of an analyte for which there is presumptive evidence to make a tentative identification. Used for all TIC results.

The sample results are rejected due to serious deficiencies in the ability to analyze the sample and meet quality control criteria. The presence of absence of the analyte cannot be verified. Assignment of $R$ requires approval by the appropriate WSRC data validation coordinator.

Material analyzed for but not detected. Analytical result reported is less than the sample quantitation limit.

The analysis indicates the presence of an analyte that has been tentatively identified and the associated numerical value represents its approximate concentration.

Note: These are only some of the qualifiers present in the database. All modifiers associated with the data are published in the result tables of EPD/EMS' quarterly groundwater monitoring reports, the official repository of the data.

"EPA" Qualifiers - EPA STORET Codes

$\begin{array}{ll}\text { (Blank) } & \text { Data not remarked } \\ C & \text { The result is calculated. } \\ I & \text { The result is less than the sSEQL, but equal to or greater than the MDL. Always } \\ K & \text { reported with an associated EPA functional Guideline Code qualifier of } U . \\ L & \text { The actual concentration is known to be less than the reported result. } \\ O & \text { The actual concentration is know to be less than the reported result. } \\ Q & \text { Sample received by laboratory, but the analysis was lost or not performed. } \\ & \text { Sample was held beyond normal holding time prior to analysis. }\end{array}$

Note: These are only some of the qualifiers present in the database. All modifiers associated with the data are published in the result tables of EPD/EMS' quarterly groundwater monitoring reports, the official repository of the data. 


\title{
TABLE D-1
}

\section{Synchronous Water Elevations}

\author{
Water Level Measurement Periods \\ $1 Q 00 \quad 03 / 20 / 00$ to $03 / 29 / 00$ \\ $2 Q 00 \quad 06 / 24 / 00$ to $06 / 29 / 00$
}




\begin{tabular}{|c|c|c|c|}
\hline $\begin{array}{l}\text { Well } \\
\text { Name }\end{array}$ & $1 Q 00$ & $2 \mathrm{QOOO}$ & Aquifer Name \\
\hline SRW 19 & NA & NA & Perched Unit Above M-Area Aquifer Zone \\
\hline $\mathrm{ABP} 2 \mathrm{~A}$ & 222.2 & 221.0 & M-Area Aquifer Zone \\
\hline $\mathrm{AC} \quad 2 \mathrm{~B}$ & 225.5 & 225.2 & M-Area Aquifer Zone \\
\hline$A C \quad 3 B$ & 211.2 & 210.6 & M-Area Aquifer Zone \\
\hline$A C B 2 A$ & 232.1 & 231.5 & M-Area Aquifer Zone \\
\hline AMB 4D & 229.6 & 228.9 & M-Area Aquifer Zone \\
\hline AMB 5 & 230.1 & 229.5 & M-Area Aquifer Zone \\
\hline AMB 6 & 230.1 & 229.4 & M-Area Aquifer Zone \\
\hline AMB 7 & 230.7 & 229.5 & M-Area Aquifer Zone \\
\hline$A M B$ 8D & 230.1 & 229.3 & M-Area Aquifer Zone \\
\hline AMB 9D & 229.9 & 229.2 & M-Area Aquifer Zone \\
\hline AMB 10D & 230.9 & 229.9 & M-Area Aquifer Zone \\
\hline AMB 11D & 231.2 & 230.5 & M-Area Aquifer Zone \\
\hline AMB 12D & 230.7 & 230.0 & M-Area Aquifer Zone \\
\hline AMB 14D & 228.5 & NA & M-Area Aquifer Zone \\
\hline AMB 15D & 230.1 & 238.7 & M-Area Aquifer Zone \\
\hline AMB 16D & 230.0 & 218.1 & M-Area Aquifer Zone \\
\hline AOB 1 & NA & NA & M-Area Aquifer Zone \\
\hline $\mathrm{AOB} 2$ & 231.2 & NA & M-Area Aquifer Zone \\
\hline ASB 2AR & 235.7 & 235.3 & M-Area Aquifer Zone \\
\hline ASB 3AR & 236.1 & 233.7 & M-Area Aquifer Zone \\
\hline ASB 4 & 236.1 & 233.8 & M-Area Aquifer Zone \\
\hline ASB 5AR & 233.5 & 230.7 & M-Area Aquifer Zone \\
\hline ASB $6 A$ & 228.8 & 209.1 & M-Area Aquifer Zone \\
\hline ASB 8 & 232.0 & 231.1 & M-Area Aquifer Zone \\
\hline ASB 9 & 239.4 & 238.7 & M-Area Aquifer Zone \\
\hline MCB 2 & 223.1 & 222.0 & M-Area Aquifer Zone \\
\hline MCB 9D & 222.2 & 221.1 & M-Area Aquifer Zone \\
\hline MSB 1D & 228.5 & 227.8 & M-Area Aquifer Zone \\
\hline MSB 2D & 229.0 & 228.4 & M-Area Aquifer Zone \\
\hline MSB 3D & NA & NA & M-Area Aquifer Zone \\
\hline MSB 4D & 227.7 & 227.0 & M-Area Aquifer Zone \\
\hline MSB 5A & 226.2 & NA & M-Area Aquifer Zone \\
\hline MSB $6 \mathrm{~A}$ & 226.3 & 224.9 & M-Area Aquifer Zone \\
\hline MSB 7A & 227.3 & 226.2 & M-Area Aquifer Zone \\
\hline MSB 8A & 168.0 & NA & M-Area Aquifer Zone \\
\hline MSB $9 \mathrm{C}$ & NA & NA & M-Area Aquifer Zone \\
\hline MSB 11F & 228.3 & 227.6 & M-Area Aquifer Zone \\
\hline MSB 13D & 226.6 & 225.9 & M-Area Aquifer Zone \\
\hline MSB 15D & NA & 237.5 & M-Area Aquifer Zone \\
\hline
\end{tabular}




\section{M-Area Aquifer Zone (Cont.)}

\begin{tabular}{|c|c|c|c|}
\hline $\begin{array}{l}\text { Well } \\
\text { Name }\end{array}$ & $1 Q 00$ & $2 Q 00$ & Aquifer Name \\
\hline MSB 16C & 228.5 & 227.5 & M-Area Aquifer Zone \\
\hline MSB 18B & 220.5 & 219.9 & M-Area Aquifer Zone \\
\hline MSB 19C & 233.3 & 232.6 & M-Area Aquifer Zone \\
\hline MSB 20C & 225.6 & 225.1 & M-Area Aquifer Zone \\
\hline MSB 21C & 228.1 & 227.1 & M-Area Aquifer Zone \\
\hline MSB 24 . & 233.9 & 233.5 & M-Area Aquifer Zone \\
\hline MSB 25 & NA & NA & M-Area Aquifer Zone \\
\hline MSB 26 & 229.4 & 228.6 & M-Area Aquifer Zone \\
\hline MSB 27 & NA & 226.5 & M-Area Aquifer Zone \\
\hline MSB 28 & 228.4 & 227.7 & M-Area Aquifer Zone \\
\hline MSB 29D & 230.4 & 229.9 & M-Area Aquifer Zone \\
\hline MSB 31C & 231.1 & 230.3 & M-Area Aquifer Zone \\
\hline MSB 32 & 223.0 & 221.9 & M-Area Aquifer Zone \\
\hline MSB 33 & 216.1 & 216.0 & M-Area Aquifer Zone \\
\hline MSB 34C & 228.4 & NA & M-Area Aquifer Zone \\
\hline MSB 35D & NA & 238.5 & M-Area Aquifer Zone \\
\hline MSB 36D & 232.5 & 231.8 & M-Area Aquifer Zone \\
\hline MSB 37D & 229.3 & 228.4 & M-Area Aquifer Zone \\
\hline MSB 39D & 230.3 & 229.3 & M-Area Aquifer Zone \\
\hline MSB 4OD & 226.5 & 225.8 & M-Area Aquifer Zone \\
\hline MSB 41D & 241.0 & 241.1 & M-Area Aquifer Zone \\
\hline MSB 42D & 229.7 & 230.5 & M-Area Aquifer Zone \\
\hline MSB 45C & NA & NA & M-Area Aquifer Zone \\
\hline MSB 46C & NA & NA & M-Area Aquifer Zone \\
\hline MSB 47D & 231.9 & 230.9 & M-Area Aquifer Zone \\
\hline MSB 48D & 231.4 & 230.5 & M-Area Aquifer Zone \\
\hline MSB 49D & 228.1 & 226.4 & M-Area Aquifer Zone \\
\hline MSB 51D & 209.5 & 209.0 & M-Area Aquifer Zone \\
\hline MSB 52D & 235.4 & 234.9 & M-Area Aquifer Zone \\
\hline MSB 53D & 231.4 & 220.1 & M-Area Aquifer Zone \\
\hline MSB 56D & 219.3 & 219.7 & M-Area Aquifer Zone \\
\hline MSB 59D & 228.1 & 227.4 & M-Area Aquifer Zone \\
\hline MSB 62D & 227.5 & 226.7 & M-Area Aquifer Zone \\
\hline MSB 63D & 227.8 & 227.2 & M-Area Aquifer Zone \\
\hline MSB 64D & 225.3 & 224.9 & M-Area Aquifer Zone \\
\hline MSB 65D & 230.1 & NA & M-Area Aquifer Zone \\
\hline MSB 66D & 267.2 & 267.1 & M-Area Aquifer Zone \\
\hline MSB 67D & NA & NA & M-Area Aquifer Zone \\
\hline MSB 69D & 231.8 & 231.0 & M-Area Aquifer Zone \\
\hline MSB 70D & 220.2 & 215.9 & M-Area Aquifer Zone \\
\hline
\end{tabular}




\section{M-Area Aquifer Zone (Cont.)}

\begin{tabular}{|c|c|c|c|}
\hline $\begin{array}{l}\text { Well } \\
\text { Name }\end{array}$ & $1 Q 00$ & $2 Q 00$ & Aquifer Name \\
\hline MSB 74D & 230.0 & 229.4 & M-Area Aquifer Zone \\
\hline MSB 77D & 232.0 & 231.4 & M-Area Aquifer Zone \\
\hline MSB 82D & 231.6 & 230.6 & M-Area Aquifer Zone \\
\hline MSB 85D & 231.5 & 230.5 & M-Area Aquifer Zone \\
\hline MSB $87 \mathrm{C}$ & NA & NA & M-Area Aquifer Zone \\
\hline MSB 88B & 200.4 & 200.1 & M-Area Aquifer Zone \\
\hline MSB $89 \mathrm{C}$ & 228.1 & 227.4 & M-Area Aquifer Zone \\
\hline SRW $3 A$ & 213.9 & 211.1 & M-Area Aquifer Zone \\
\hline SRW 6 & NA & NA & M-Area Aquifer Zone \\
\hline SRW 9 & 200.2 & 201.3 & M-Area Aquifer Zone \\
\hline SRW 12C & 192.0 & 191.4 & M-Area Aquifer Zone \\
\hline SRW 13C & 207.8 & 207.2 & M-Area Aquifer Zone \\
\hline SRW 15C & 210.8 & 210.0 & M-Area Aquifer Zone \\
\hline SRW 16B & 213.1 & 212.0 & M-Area Aquifer Zone \\
\hline SRW 16C & 213.4 & 212.6 & M-Area Aquifer Zone \\
\hline SRW 17DR & NA & NA & M-Area Aquifer Zone \\
\hline SRW 18 & NA & NA & M-Area Aquifer Zone \\
\hline
\end{tabular}




\section{Green Clay Confining Zone}

\begin{tabular}{|c|c|c|c|}
\hline $\begin{array}{l}\text { Well } \\
\text { Name }\end{array}$ & $1 Q 00$ & $2 Q 00$ & Aquifer Name \\
\hline ASB $1 \mathrm{~A}$ & 235.9 & NA & M-Area Aquifer Zone / Green Clay Confining Zone \\
\hline MSB 43D & 229.9 & 229.0 & M-Area Aquifer Zone / Green Clay Confining Zone \\
\hline MSB 54D & 231.8 & 230.9 & M-Area Aquifer Zone / Green Clay Confining Zone \\
\hline MSB 55D & 233.0 & NA & M-Area Aquifer Zone / Green Clay Confining Zone \\
\hline MSB 78DR & 221.6 & 221.2 & M-Area Aquifer Zone / Green Clay Confining Zone \\
\hline
\end{tabular}




\section{M-Area Aquifer Zone / Green Clay Confining Zone / Upper Lost Lake Aquifer Zone}

\begin{tabular}{|c|c|c|c|}
\hline $\begin{array}{l}\text { Well } \\
\text { Name }\end{array}$ & $1 Q 00$ & $2 Q 00$ & Aquifer Name \\
\hline ARP $1 \mathrm{~A}$ & NA & NA & $\begin{array}{l}\text { M-Area Aquifer Zone / Green Clay Confining Zone / } \\
\text { Upper Lost Lake Aquifer Zone }\end{array}$ \\
\hline ARP 2 & 218.0 & 216.7 & $\begin{array}{l}\text { M-Area Aquifer Zone / Green Clay Confining Zone / } \\
\text { Upper Lost Lake Aquifer Zone }\end{array}$ \\
\hline ARP 3 & 220.5 & 212.3 & $\begin{array}{l}\text { M-Area Aquifer Zone / Green Clay Confining Zone / } \\
\text { Upper Lost Lake Aquifer Zone }\end{array}$ \\
\hline ARP 4 & 218.0 & 216.8 & $\begin{array}{l}\text { M-Area Aquifer Zone / Green Clay Confining Zone / } \\
\text { Upper Lost Lake Aquifer Zone }\end{array}$ \\
\hline ARP 5D & NA & NA & $\begin{array}{l}\text { M-Area Aquifer Zone / Green Clay Confining Zone / } \\
\text { Upper Lost Lake Aquifer Zone }\end{array}$ \\
\hline MSB 17B & 224.4 & 223.6 & Green Clay Confining Zone \\
\hline
\end{tabular}




\section{Upper Lost Lake Aquifer Zone}

\begin{tabular}{|c|c|c|c|}
\hline $\begin{array}{l}\text { Well } \\
\text { Name }\end{array}$ & $1 Q 00$ & $2 Q 00$ & Aquifer Name \\
\hline $\mathrm{ABP} 8 \mathrm{C}$ & 195.9 & 194.4 & Upper Lost Lake Aquifer Zone \\
\hline$A C \quad 1 A$ & NA & NA & Upper Lost Lake Aquifer Zone \\
\hline$A C \quad 3 A$ & 209.5 & 208.5 & Upper Lost Lake Aquifer Zone \\
\hline$A M B 11 B$ & 220.7 & 220.8 & Upper Lost Lake Aquifer Zone \\
\hline AMB $18 \mathrm{C}$ & 229.7 & 229.1 & Upper Lost Lake Aquifer Zone \\
\hline AMB $19 \mathrm{C}$ & 327.3 & 226.4 & Upper Lost Lake Aquifer Zone \\
\hline ASB $6 C$ & 221.7 & 220.5 & Upper Lost Lake Aquifer Zone \\
\hline ASB $8 C$ & 218.5 & 217.4 & Upper Lost Lake Aquifer Zone \\
\hline ASB $9 \mathrm{C}$ & 219.8 & 218.5 & Upper Lost Lake Aquifer Zone \\
\hline MCB $5 \mathrm{C}$ & 194.3 & 192.8 & Upper Lost Lake Aquifer Zone \\
\hline MCB $6 \mathrm{C}$ & 194.9 & 193.5 & Upper Lost Lake Aquifer Zone \\
\hline MCB $7 \mathrm{C}$ & 193.4 & 191.9 & Upper Lost Lake Aquifer Zone \\
\hline MSB $1 \mathrm{C}$ & 214.4 & 214.2 & Upper Lost Lake Aquifer Zone \\
\hline MSB 2C & 215.5 & 215.3 & Upper Lost Lake Aquifer Zone \\
\hline MSB $3 C$ & 217.2 & 216.7 & Upper Lost Lake Aquifer Zone \\
\hline MSB $4 \mathrm{C}$ & 213.0 & 212.9 & Upper Lost Lake Aquifer Zone \\
\hline MSB $5 \mathrm{C}$ & 221.6 & 220.6 & Upper Lost Lake Aquifer Zone \\
\hline MSB $6 \mathrm{C}$ & 222.2 & 221.3 & Upper Lost Lake Aquifer Zone \\
\hline MSB $7 \mathrm{C}$ & 221.3 & 220.3 & Upper Lost Lake Aquifer Zone \\
\hline MSB $8 C$ & 218.7 & 211.7 & Upper Lost Lake Aquifer Zone \\
\hline MSB 11C & 217.7 & 217.0 & Upper Lost Lake Aquifer Zone \\
\hline MSB 12B & 216.5 & 215.6 & Upper Lost Lake Aquifer Zone \\
\hline MSB $13 C C$ & 223.4 & 222.2 & Upper Lost Lake Aquifer Zone \\
\hline MSB 15A & 217.8 & 217.1 & Upper Lost Lake Aquifer Zone \\
\hline MSB 16A & 217.0 & 215.9 & Upper Lost Lake Aquifer Zone \\
\hline MSB 18A & 211.1 & 210.2 & Upper Lost Lake Aquifer Zone \\
\hline MSB 20A & NA & 216.2 & Upper Lost Lake Aquifer Zone \\
\hline MSB 23B & 220.4 & NA & Upper Lost Lake Aquifer Zone \\
\hline MSB 24A & 222.7 & 221.7 & Upper Lost Lake Aquifer Zone \\
\hline MSB 27B & 224.7 & 222.1 & Upper Lost Lake Aquifer Zone \\
\hline MSB 28A & 222.1 & 221.0 & Upper Lost Lake Aquifer Zone \\
\hline MSB 29C & 228.4 & 227.6 & Upper Lost Lake Aquifer Zone \\
\hline MSB $30 \mathrm{CC}$ & 223.4 & 222.5 & Upper Lost Lake Aquifer Zone \\
\hline MSB 31CC & 212.4 & 210.3 & Upper Lost Lake Aquifer Zone \\
\hline MSB 32C & 215.5 & 214.8 & Upper Lost Lake Aquifer Zone \\
\hline MSB 33C & 210.2 & 209.7 & Upper Lost Lake Aquifer Zone \\
\hline MSB 34B & 226.5 & 223.6 & Upper Lost Lake Aquifer Zone \\
\hline MSB 35B & 217.6 & 216.5 & Upper Lost Lake Aquifer Zone \\
\hline MSB 36C & 213.3 & 212.0 & Upper Lost Lake Aquifer Zone \\
\hline MSB 39C & 213.6 & 213.1 & Upper Lost Lake Aquifer Zone \\
\hline MSB $40 C$ & 203.4 & 203.5 & Upper Lost Lake Aquifer Zone \\
\hline MSB 42C & 228.5 & 229.5 & Upper Lost Lake Aquifer Zone \\
\hline MSB 43B & 227.8 & 226.9 & Upper Lost Lake Aquifer Zone \\
\hline
\end{tabular}




\section{Upper Lost Lake Aquifer Zone (cont.)}

$\begin{array}{llll}\text { Well } & \text { 1Q00 } & \text { 2Q00 } & \text { Aquifer Name } \\ \text { Name } & & & \\ \text { MSB 45B } & 223.0 & 221.9 & \text { Upper Lost Lake Aquifer Zone } \\ \text { MSB 46B } & 226.1 & 225.9 & \text { Upper Lost Lake Aquifer Zone } \\ \text { MSB 47C } & 230.7 & 229.7 & \text { Upper Lost Lake Aquifer Zone } \\ \text { MSB 48C } & 223.0 & 222.1 & \text { Upper Lost Lake Aquifer Zone } \\ \text { MSB 50D } & \text { NA } & \text { NA } & \text { Upper Lost Lake Aquifer Zone } \\ \text { MSB 53C } & 221.3 & 231.4 & \text { Upper Lost Lake Aquifer Zone } \\ \text { MSB 55HC } & 231.5 & 230.6 & \text { Upper Lost Lake Aquifer Zone } \\ \text { MSB 62C } & 222.8 & 220.8 & \text { Upper Lost Lake Aquifer Zone } \\ \text { MSB 63C } & 219.0 & 218.3 & \text { Upper Lost Lake Aquifer Zone } \\ \text { MSB 64C } & 192.3 & 220.4 & \text { Upper Lost Lake Aquifer Zone } \\ \text { MSB 70C } & 216.7 & 218.8 & \text { Upper Lost Lake Aquifer Zone } \\ \text { MSB 74C } & 210.0 & 209.0 & \text { Upper Lost Lake Aquifer Zone } \\ \text { MSB 75C } & 208.6 & 207.8 & \text { Upper Lost Lake Aquifer Zone } \\ \text { MSB 76C } & 219.2 & 218.7 & \text { Upper Lost Lake Aquifer Zone } \\ \text { MSB 77C } & 222.0 & 221.3 & \text { Upper Lost Lake Aquifer Zone } \\ \text { MSB 79C } & 208.5 & 207.7 & \text { Upper Lost Lake Aquifer Zone } \\ \text { MSB 87B } & 316.9 & 210.4 & \text { Upper Lost Lake Aquifer Zone } \\ \text { SRW 2B } & 205.8 & \text { NA } & \text { Upper Lost Lake Aquifer Zone } \\ \text { SRW 13B } & 201.4 & 200.4 & \text { Upper Lost Lake Aquifer Zone } \\ \text { SRW 14B } & 203.6 & 202.3 & \text { Upper Lost Lake Aquifer Zone } \\ \text { SRW 15B } & 207.7 & 206.6 & \text { Upper Lost Lake Aquifer Zone } \\ & & & \end{array}$




\section{Lower Lost Lake Aquifer Zone}

\begin{tabular}{|c|c|c|c|}
\hline $\begin{array}{l}\text { Well } \\
\text { Name }\end{array}$ & $1 Q 00$ & $2 Q 00$ & Aquifer Name \\
\hline$A B P 3 C$ & 196.5 & 195.1 & Lower Lost Lake Aquifer Zone \\
\hline$A C \quad 2 A$ & 219.1 & 218.2 & Lower Lost Lake Aquifer Zone \\
\hline $\mathrm{AMB} 4 \mathrm{~B}$ & 222.6 & 221.2 & Lower Lost Lake Aquifer Zone \\
\hline $\mathrm{AMB} 7 \mathrm{~B}$ & 222.4 & 221.2 & Lower Lost Lake Aquifer Zone \\
\hline AMB $10 B$ & 221.3 & 220.0 & Lower Lost Lake Aquifer Zone \\
\hline ASB 2CR & 221.5 & 219.5 & Lower Lost Lake Aquifer Zone \\
\hline ASB $3 C R$ & 221.3 & 218.9 & Lower Lost Lake Aquifer Zone \\
\hline ASB $5 \mathrm{C}$ & 220.3 & 219.3 & Lower Lost Lake Aquifer Zone \\
\hline ASB $9 B$ & 219.4 & 218.1 & Lower Lost Lake Aquifer Zone \\
\hline ASB $10 \mathrm{CR}$ & 221.4 & 219.2 & Lower Lost Lake Aquifer Zone \\
\hline MSB $1 B$ & 208.1 & 207.3 & Lower Lost Lake Aquifer Zone \\
\hline MSB 2B & 209.3 & 209.1 & Lower Lost Lake Aquifer Zone \\
\hline MSB 3B & NA & NA & Lower Lost Lake Aquifer Zone \\
\hline MSB 4B & 206.6 & 206.1 & Lower Lost Lake Aquifer Zone \\
\hline MSB 5B & 206.4 & 205.3 & Lower Lost Lake Aquifer Zone \\
\hline MSB 6B & 205.1 & 204.3 & Lower Lost Lake Aquifer Zone \\
\hline MSB 7B & 205.7 & 204.8 & Lower Lost Lake Aquifer Zone \\
\hline MSB 8B & 207.1 & 209.1 & Lower Lost Lake Aquifer Zone \\
\hline MSB 9A & 208.7 & 207.9 & Lower Lost Lake Aquifer Zone \\
\hline MSB 10B & 209.9 & 210.7 & Lower Lost Lake Aquifer Zone \\
\hline MSB 11A & 210.7 & 209.6 & Lower Lost Lake Aquifer Zone \\
\hline MSB $12 \mathrm{~A}$ & 207.9 & 205.8 & Lower Lost Lake Aquifer Zone \\
\hline MSB 13A & 211.0 & 205.1 & Lower Lost Lake Aquifer Zone \\
\hline MSB 15AA & 211.5 & 210.5 & Lower Lost Lake Aquifer Zone \\
\hline MSB 17BB & 211.0 & 210.2 & Lower Lost Lake Aquifer Zone \\
\hline MSB 19B & 215.1 & 214.2 & Lower Lost Lake Aquifer Zone \\
\hline MSB 21B & 218.5 & 217.6 & Lower Lost Lake Aquifer Zone \\
\hline MSB 25A & 213.8 & 212.4 & Lower Lost Lake Aquifer Zone \\
\hline MSB 26B & 216.8 & 215.6 & Lower Lost Lake Aquifer Zone \\
\hline MSB 29B & 222.4 & 221.4 & Lower Lost Lake Aquifer Zone \\
\hline MSB 30B & 223.4 & 222.6 & Lower Lost Lake Aquifer Zone \\
\hline MSB 31B & 212.4 & 210.0 & Lower Lost Lake Aquifer Zone \\
\hline MSB 32B & 210.7 & 210.0 & Lower Lost Lake Aquifer Zone \\
\hline MSB 33B & 206.9 & 206.3 & Lower Lost Lake Aquifer Zone \\
\hline MSB 34A & 217.1 & 215.3 & Lower Lost Lake Aquifer Zone \\
\hline MSB 36B & 213.2 & 212.0 & Lower Lost Lake Aquifer Zone \\
\hline MSB 37C & 226.2 & 224.9 & Lower Lost Lake Aquifer Zone \\
\hline MSB 38C & 220.0 & 215.5 & Lower Lost Lake Aquifer Zone \\
\hline MSB 39B & 212.4 & 209.5 & Lower Lost Lake Aquifer Zone \\
\hline MSB 40B & 203.5 & 202.7 & Lower Lost Lake Aquifer Zone \\
\hline
\end{tabular}


Lower Lost Lake Aquifer Zone (Cont.)

\begin{tabular}{|c|c|c|c|}
\hline $\begin{array}{l}\text { Well } \\
\text { Name }\end{array}$ & $1 Q 00$ & $2 Q 00$ & Aquifer Name \\
\hline MSB 41C & 216.4 & 215.7 & Lower Lost Lake Aquifer Zone \\
\hline MSB 42B & 223.3 & 224.0 & Lower Lost Lake Aquifer Zone \\
\hline MSB 43A & 227.6 & 226.7 & Lower Lost Lake Aquifer Zone \\
\hline MSB 45A & 216.2 & 212.8 & Lower Lost Lake Aquifer Zone \\
\hline MSB 47B & 223.1 & 222.0 & Lower Lost Lake Aquifer Zone \\
\hline MSB 48B & 222.5 & 221.6 & Lower Lost Lake Aquifer Zone \\
\hline MSB 49B & 202.5 & 201.3 & Lower Lost Lake Aquifer Zone \\
\hline MSB 50B & 202.1 & 201.4 & Lower Lost Lake Aquifer Zone \\
\hline MSB 51B & 204.4 & 203.7 & Lower Lost Lake Aquifer Zone \\
\hline MSB 52B & 218.4 & 217.0 & Lower Lost Lake Aquifer Zone \\
\hline MSB 53B & 220.6 & 219.8 & Lower Lost Lake Aquifer Zone \\
\hline MSB 54C & 225.2 & 224.2 & Lower Lost Lake Aquifer Zone \\
\hline MSB 55C & 227.9 & 226.9 & Lower Lost Lake Aquifer Zone \\
\hline MSB 62B & 212.8 & 206.6 & Lower Lost Lake Aquifer Zone \\
\hline MSB 63B & 207.4 & 206.8 & Lower Lost Lake Aquifer Zone \\
\hline MSB 64B & 206.7 & 205.6 & Lower Lost Lake Aquifer Zone \\
\hline MSB 66C & 226.8 & 225.7 & Lower Lost Lake Aquifer Zone \\
\hline MSB $68 \mathrm{C}$ & 222.9 & 221.8 & Lower Lost Lake Aquifer Zone \\
\hline MSB $69 \mathrm{C}$ & 225.9 & 224.9 & Lower Lost Lake Aquifer Zone \\
\hline MSB 71B & 215.8 & 215.0 & Lower Lost Lake Aquifer Zone \\
\hline MSB 72B & 199.1 & 198.5 & Lower Lost Lake Aquifer Zone \\
\hline MSB 73B & 200.5 & 194.8 & Lower Lost Lake Aquifer Zone \\
\hline MSB 74B & 210.1 & 209.3 & Lower Lost Lake Aquifer Zone \\
\hline MSB 75B & 208.8 & 207.8 & Lower Lost Lake Aquifer Zone \\
\hline MSB 77B & 220.0 & 219.0 & Lower Lost Lake Aquifer Zone \\
\hline MSB 79B & 206.3 & 205.3 & Lower Lost Lake Aquifer Zone \\
\hline MSB 81B & 219.1 & 218.2 & Lower Lost Lake Aquifer Zone \\
\hline MSB $82 C$ & 226.3 & 225.2 & Lower Lost Lake Aquifer Zone \\
\hline MSB 83C & 226.9 & 225.8 & Lower Lost Lake Aquifer Zone \\
\hline MSB 84C & 228.6 & 227.6 & Lower Lost Lake Aquifer Zone \\
\hline MSB 85C & 223.0 & 222.1 & Lower Lost Lake Aquifer Zone \\
\hline MSB 86C & 223.9 & 223.2 & Lower Lost Lake Aquifer Zone \\
\hline MSB $88 \mathrm{C}$ & 202.9 & 202.6 & Lower Lost Lake Aquifer Zone \\
\hline MSB 89B & 206.2 & 205.5 & Lower Lost Lake Aquifer Zone \\
\hline SRW 3BB & 206.8 & 205.7 & Lower Lost Lake Aquifer Zone \\
\hline SRW 9A & 196.8 & 195.7 & Lower Lost Lake Aquifer Zone \\
\hline SRW 11BB & 203.7 & 202.7 & Lower Lost Lake Aquifer Zone \\
\hline SRW 13A & 199.6 & 198.5 & Lower Lost Lake Aquifer Zone \\
\hline SRW 16A & 212.5 & 211.2 & Lower Lost Lake Aquifer Zone \\
\hline
\end{tabular}




\section{Middle Sand Aquifer Zone-Crouch Branch Confining Unit}

$\begin{array}{lll}\text { Well } & 1 \mathbf{1 0 0} & \mathbf{2 Q 0 0} \\ \text { Name } & & \\ & & \\ \text { AMB 4A } & 217.2 & 216.2 \\ \text { AMB 7A } & 217.1 & 216.0 \\ \text { AMB 10A } & 216.7 & 215.3 \\ \text { AMB 13AR } & 217.0 & 225.1 \\ \text { AMB 17A } & 216.8 & 216.1 \\ \text { AMB 18A } & 216.8 & 215.6 \\ \text { ASB 6AA } & 216.7 & 216.5 \\ \text { ASB 8B } & 211.0 & 210.0 \\ \text { MSB 10A } & 209.0 & 208.1 \\ \text { MSB 19A } & \text { NA } & \text { NA } \\ \text { MSB 29A } & 218.0 & 217.1 \\ \text { MSB 30AA } & 222.5 & 221.6 \\ \text { MSB 33A } & 204.1 & 203.7 \\ \text { MSB 35A } & 215.0 & 214.1 \\ \text { MSB 36A } & 209.4 & 207.9 \\ \text { MSB 37B } & 217.9 & 216.1 \\ \text { MSB 39A } & 207.8 & 206.7 \\ \text { MSB 40A } & 202.1 & 201.2 \\ \text { MSB 41B } & 216.1 & 215.3 \\ \text { MSB 42A } & 217.2 & 217.7 \\ \text { MSB 44A } & 215.9 & 215.0 \\ \text { MSB 46A } & 214.2 & 212.7 \\ \text { MSB 48A } & 221.1 & 220.1 \\ \text { MSB 49A } & 196.5 & 195.4 \\ \text { MSB 54B } & 220.9 & 219.8 \\ \text { MSB 55B } & 220.5 & 219.5 \\ \text { MSB 66B } & 217.3 & 216.2 \\ \text { MSB 68B } & 217.2 & 216.2 \\ \text { MSB 69B } & 219.2 & 218.2 \\ \text { MSB 82A } & 213.1 & 218.4 \\ \text { MSB 83B } & 220.5 & 219.6 \\ \text { MSB 84A } & 197.0 & 217.9 \\ \text { MSB 85B } & 219.9 & 218.9 \\ \text { SRW 2A } & 204.6 & 203.6 \\ \text { SRW 14A } & 201.6 & 200.2 \\ \text { SRW 15A } & 207.4 & 206.4\end{array}$

\section{Aquifer Name}

Middle Sand Aquifer Zone-Crouch Branch Confining Unit Middle Sand Aquifer Zone-Crouch Branch Confining Unit Middle Sand Aquifer Zone-Crouch Branch Confining Unit Middle Sand Aquifer Zone-Crouch Branch Confining Unit Middle Sand Aquifer Zone-Crouch Branch Confining Unit Middle Sand Aquifer Zone-Crouch Branch Confining Unit Middle Sand Aquifer Zone-Crouch Branch Confining Unit Middle Sand Aquifer Zone-Crouch Branch Confining Unit Middle Sand Aquifer Zone-Crouch Branch Confining Unit Middle Sand Aquifer Zone-Crouch Branch Confining Unit Middle Sand Aquifer Zone-Crouch Branch Confining Unit Middle Sand Aquifer Zone-Crouch Branch Confining Unit Middle Sand Aquifer Zone-Crouch Branch Confining Unit Middle Sand Aquifer Zone-Crouch Branch Confining Unit Middle Sand Aquifer Zone-Crouch Branch Confining Unit Middle Sand Aquifer Zone-Crouch Branch Confining Unit Middle Sand Aquifer Zone-Crouch Branch Confining Unit Middle Sand Aquifer Zone-Crouch Branch Confining Unit Middle Sand Aquifer Zone-Crouch Branch Confining Unit Middle Sand Aquifer Zone-Crouch Branch Confining Unit Middle Sand Aquifer Zone-Crouch Branch Confining Unit Middle Sand Aquifer Zone-Crouch Branch Confining Unit Middle Sand Aquifer Zone-Crouch Branch Confining Unit Middle Sand Aquifer Zone-Crouch Branch Confining Unit Middle Sand Aquifer Zone-Crouch Branch Confining Unit Middle Sand Aquifer Zone-Crouch Branch Confining Unit Middle Sand Aquifer Zone-Crouch Branch Confining Unit Middle Sand Aquifer Zone-Crouch Branch Confining Unit Middle Sand Aquifer Zone-Crouch Branch Confining Unit Middle Sand Aquifer Zone-Crouch Branch Confining Unit Middle Sand Aquifer Zone-Crouch Branch Confining Unit Middle Sand Aquifer Zone-Crouch Branch Confining Unit Middle Sand Aquifer Zone-Crouch Branch Confining Unit Middle Sand Aquifer Zone-Crouch Branch Confining Unit Middle Sand Aquifer Zone-Crouch Branch Confining Unit Middle Sand Aquifer Zone-Crouch Branch Confining Unit 


\section{Upper Clay Confining Zone - Crouch Branch Confining Unit}

$\begin{array}{llll}\begin{array}{l}\text { Well } \\ \text { Name }\end{array} & 1 \mathrm{Q} 00 & 2 \mathrm{Q} 00 & \begin{array}{l}\text { Aquifer Name } \\ \text { MSB 82B }\end{array} \\ 218.4 & 217.3 & \begin{array}{l}\text { Upper Clay Confining Zone - Crouch Branch Confining } \\ \text { Unit }\end{array}\end{array}$




\section{Crouch Branch Aquifer Unit}

\begin{tabular}{|c|c|c|c|}
\hline $\begin{array}{l}\text { Well } \\
\text { Name }\end{array}$ & $1 Q 00$ & $2 Q 00$ & Aquifer Name \\
\hline ASB 6TA & 212.5 & 211.7 & Crouch Branch Aquifer Unit \\
\hline ASB 8TA & 214.3 & 213.3 & Crouch Branch Aquifer Unit \\
\hline MSB 12TA & 191.8 & 190.4 & Crouch Branch Aquifer Unit \\
\hline MSB 21TA & 193.3 & 192.0 & Crouch Branch Aquifer Unit \\
\hline MSB 23TA & 199.8 & 198.8 & Crouch Branch Aquifer Unit \\
\hline MSB 27TA & 199.5 & 198.5 & Crouch Branch Aquifer Unit \\
\hline MSB 29TA & 210.9 & 209.9 & Crouch Branch Aquifer Unit \\
\hline MSB 30A & 197.1 & 196.2 & Crouch Branch Aquifer Unit \\
\hline MSB $31 \mathrm{~A}$ & 197.4 & 195.7 & Crouch Branch Aquifer Unit \\
\hline MSB 33TA & 195.2 & 193.7 & Crouch Branch Aquifer Unit \\
\hline MSB 34TA & 200.9 & 199.7 & Crouch Branch Aquifer Unit \\
\hline MSB 35TA & 198.7 & 197.5 & Crouch Branch Aquifer Unit \\
\hline MSB $36 T A$ & 193.9 & 193.0 & Crouch Branch Aquifer Unit \\
\hline MSB 37A & NA & 204.7 & Crouch Branch Aquifer Unit \\
\hline MSB 37TA & 205.6 & 204.6 & Crouch Branch Aquifer Unit \\
\hline MSB 38TA & 197.4 & 195.6 & Crouch Branch Aquifer Unit \\
\hline MSB 39TA & 191.9 & 190.8 & Crouch Branch Aquifer Unit \\
\hline MSB 40TA & 189.5 & 188.1 & Crouch Branch Aquifer Unit \\
\hline MSB 41TA & 206.0 & 204.7 & Crouch Branch Aquifer Unit \\
\hline MSB 42TA & 203.7 & 205.0 & Crouch Branch Aquifer Unit \\
\hline MSB 43TA & 201.5 & 200.9 & Crouch Branch Aquifer Unit \\
\hline MSB 47TA & 214.7 & 213.8 & Crouch Branch Aquifer Unit \\
\hline MSB 48TA & 221.0 & 220.0 & Crouch Branch Aquifer Unit \\
\hline $\begin{array}{l}\text { MSB 54TA } \\
\text { MSB 55TA }\end{array}$ & $\begin{array}{l}218.4 \\
213.0\end{array}$ & $\begin{array}{l}217.4 \\
212.1\end{array}$ & $\begin{array}{l}\text { Crouch Branch Aquifer Unit } \\
\text { Crouch Branch Aquifer Unit }\end{array}$ \\
\hline MSB 66TA & 204.2 & 204.2 & Crouch Branch Aquifer Unit \\
\hline MSB 69TA & 214.0 & 213.2 & Crouch Branch Aquifer Unit \\
\hline MSB 82TA & 213.0 & 212.1 & Crouch Branch Aquifer Unit \\
\hline MSB 83TA & 214.5 & 213.5 & Crouch Branch Aquifer Unit \\
\hline MSB 85TA & 219.4 & 218.3 & Crouch Branch Aquifer Unit \\
\hline SRW 12A & 192.0 & 190.5 & Crouch Branch Confining Unit \\
\hline
\end{tabular}


WSRC-TR-2000-00242

Unclassified

THIS PAGE INTENTIONALLY LEFT BLANK. 
Table D-2. Groundwater Monitoring Results for Point-of-Compliance Wells, M-Area HWMF WELL MSB 1B

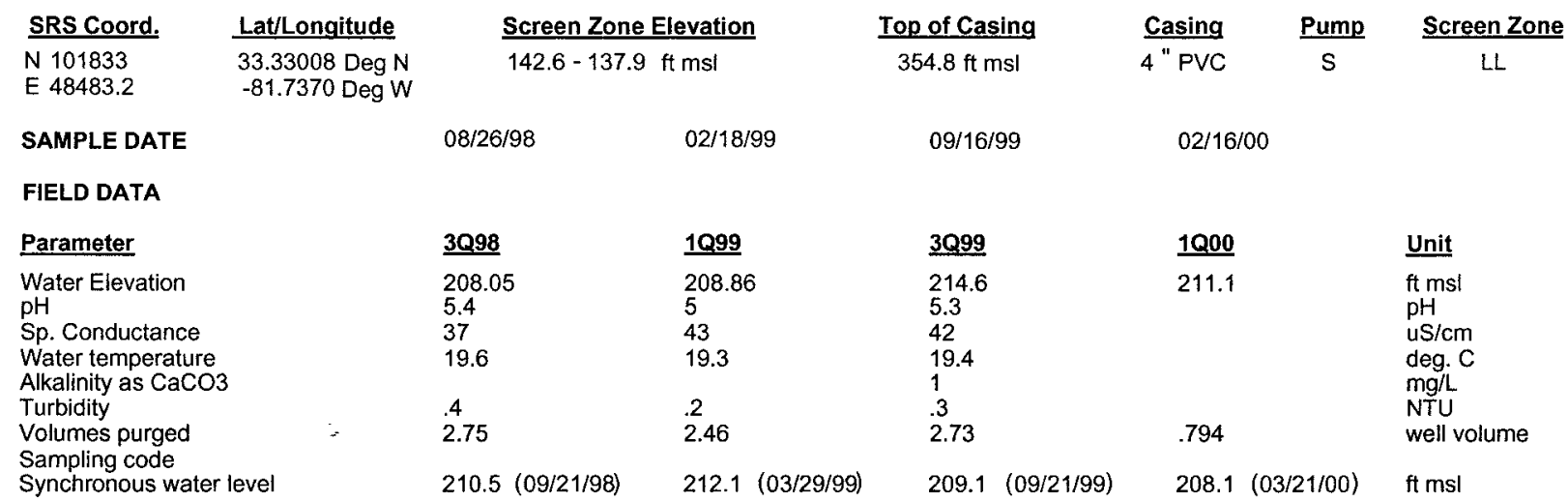

\section{ANALYTICAL DATA}

I. Groundwater Protection Standard

261 Appendix VIII/264 Appendix IX Hazardous Constituents

\begin{tabular}{|c|c|c|c|c|c|c|c|c|c|c|c|c|c|}
\hline ST Parameter & $\underline{3 Q 98}$ & CLPEPA & $\underline{1 Q 99}$ & CLP EPA & $\underline{3099}$ & CLPEPA & 1000 & CLP & EPA & Filt. & $\underline{\text { DF }}$ & Unit & $\underline{\text { Lab }}$ \\
\hline \multicolumn{14}{|l|}{ Inorganics } \\
\hline Barium, total recoverable & 15.8 & & $<10$ & $\mathrm{U}$ & 14.4 & & 46 & & & $<2000$ & 1 & $\mathrm{ug} / \mathrm{L}$ & ML \\
\hline Cyanide & $<10$ & $\mathrm{U}$ & $<10$ & $\mathrm{U}$ & $<10$ & JU $Q$ & & & & & & $\mathrm{ug} / \mathrm{L}$ & \\
\hline Lead, total recoverable & $<100$ & $\mathbf{U}$ & $<100$ & $\mathrm{U}$ & $<10$ & $\mathrm{U}$ & 10.5 & $\mathbf{J}$ & I & NDD & 1 & $\mathrm{ug} / \mathrm{L}$ & ML \\
\hline Nickel, total recoverable & 11.2 & J & $<50$ & $\mathrm{U}$ & $<50$ & $U$ & $<60$ & $\mathrm{U}$ & & $<$ EQL & 1 & $u g / L$ & ML \\
\hline Selenium, total recoverable & $<200$ & $\mathrm{U}$ & $<200$ & $\mathrm{U}$ & $<10$ & $\mathrm{U}$ & $<40$ & $\mathrm{U}$ & & $<$ EQL & 1 & $\mathrm{ug} / \mathrm{L}$ & ML \\
\hline \multicolumn{14}{|l|}{ Organics } \\
\hline Chlorobenzene & $<50$ & UJ & $<50$ & $\mathrm{U}$ & $<250$ & U & $<10$ & U & & $<E Q L$ & 10 & $\mathrm{ug} / \mathrm{L}$ & ML. \\
\hline 1,1-Dichloroethane & $<50$ & UJ & $<50$ & $U$ & $<250$ & U & $<10$ & $\mathrm{U}$ & & $<E Q L$ & 10 & $\mathrm{ug} / \mathrm{L}$ & ML \\
\hline 1,1-Dichloroethylene & $<50$ & US & $<50$ & $\mathrm{U}$ & $<250$ & $\mathrm{U}$ & $<10$ & $U$ & & $<E Q L$ & 10 & $\mathrm{ug} / \mathrm{L}$ & ML \\
\hline trans-1,2-Dichloroethylene & $<50$ & UJ & $<50$ & $\mathrm{U}$ & $<250$ & $\mathrm{U}$ & & & & & & $\mathrm{ug} / \mathrm{L}$ & \\
\hline PCB 1016 & & & & & $<1.9$ & $\mathrm{U}$ & & & & & & $u g / L$ & \\
\hline PCB 1221 & & & & & $<1.9$ & $U$ & & & & & & $\mathrm{ug} / \mathrm{L}$ & \\
\hline PCB 1232 & & & & & $<.94$ & $U$ & & & & & & $u g / L$ & \\
\hline PCB 1242 & & & & & $<.94$ & $\mathrm{U}$ & & & & & & $\mathrm{ug} / \mathrm{L}$ & \\
\hline РCB 1248 & & & & & $<.94$ & $\mathrm{U}$ & & & & & & $u g / L$ & \\
\hline PCB 1254 & & & & & $<.94$ & $\mathrm{U}$ & & & & & & $\mathrm{ug} / \mathrm{L}$ & \\
\hline PCB 1260 & & & & & $<.94$ & $\mathrm{U}$ & & & & & & $u g / L$ & \\
\hline 1,1,2,2-Tetrachloroethane & $<50$ & UJ & $<50$ & U & $<250$ & $\mathrm{U}$ & $<10$ & $\mathrm{U}$ & & $<E Q L$ & 10 & $\mathrm{ug} / \mathrm{L}$ & ML \\
\hline Tetrachloroethylene & $<50$ & UJ & $<50$ & $U$ & $<250$ & $\mathrm{U}$ & 10.3 & $\mathrm{~J}$ & $\mathrm{~K}$ & NDD & 10 & $\mathrm{ug} / \mathrm{L}$ & ML \\
\hline $1,1,1$-Trichloroethane & $<50$ & UJ & $<50$ & $U$ & $<250$ & $\mathrm{U}$ & $<10$ & U & & $<E Q L$ & 10 & $\mathrm{ug} / \mathrm{L}$ & ML \\
\hline Trichloroethylene & 1240 & $J$ & 1200 & & 1700 & & 1690 & $J$ & K & NDD & 10 & $\mathrm{ug} / \mathrm{L}$ & ML \\
\hline \multicolumn{14}{|l|}{ II. Monitoring Constituents } \\
\hline Parameter & $\underline{3096}$ & CLPEPA & $\underline{3097}$ & CLPEEPA & $\underline{3098}$ & CLPEPA & $\underline{3099}$ & $\underline{\text { CLP }}$ & EPA & Filt. & $\underline{\text { DF }}$ & Unit & Lab \\
\hline \multicolumn{14}{|l|}{ Inorganics } \\
\hline Aluminum, total recoverable & 272 & & $<20$ & $\mathrm{U}$ & $<200$ & $\mathbf{U}$ & $<200$ & $\mathrm{U}$ & & $<E Q L$ & 1 & ug/L & EX \\
\hline Nitrate-nitrite as nitrogen & 1860 & & 2420 & & 2240 & & 3120 & & & $>2400$ & 5 & $\mathrm{ug} / \mathrm{L}$ & $\mathrm{EX}$ \\
\hline Sodium, total recoverable & 2480 & & 2620 & & 2640 & & 2890 & & & $<4600$ & 1 & $\mathrm{ug} / \mathrm{L}$ & EX \\
\hline Sulfate & 264 & $J$ & 384 & $\mathbf{J}$ & 315 & $J$ & 243 & & & $<3000$ & 1 & ug/L & EX \\
\hline \multicolumn{14}{|l|}{ Radionuclides } \\
\hline Gross alpha & .985 & & -.35 & $\mathrm{U}$ & 2.02 & & 1.36 & $\mathrm{~J}$ & 1 & NDD & 1 & $\mathrm{pCi} / \mathrm{L}$ & GP \\
\hline Nonvolatile beta & 1.17 & & -1.04 & UIJ & 1.91 & & 1.26 & $\mathrm{U}$ & & $<$ EQL & 1 & $\mathrm{pCi} / \mathrm{L}$ & GP \\
\hline Radium, total alpha-emitting & .5 & UI & .88 & UIJ & .41 & & .2 & $\mathrm{U}$ & & $<\mathrm{EQL}$ & 1 & $\mathrm{pCi} / \mathrm{L}$ & GP \\
\hline
\end{tabular}

Notes: Concentrations in bold italics exceed the groundwater protection or monitoring constituent standards listed in Appendix A. Synchronous water levels are measured over a 3-5 day period or less. Dilution factors, Laboratory, and Filtered Data are for Groundwater Protection Standards First Quarter 2000 and Monitoring Constituents Third Quarter 1999 data only.

+ = exceeded the Groundwater Protection Standards (First Quarter 2000) or Monitoring Constituents (Third Quarter 1999) listed in Appendix A. 
Table D-2. Groundwater Monitoring Results for Point-of-Compliance Wells, M-Area HWMF (Cont.) WELL MSB 1C

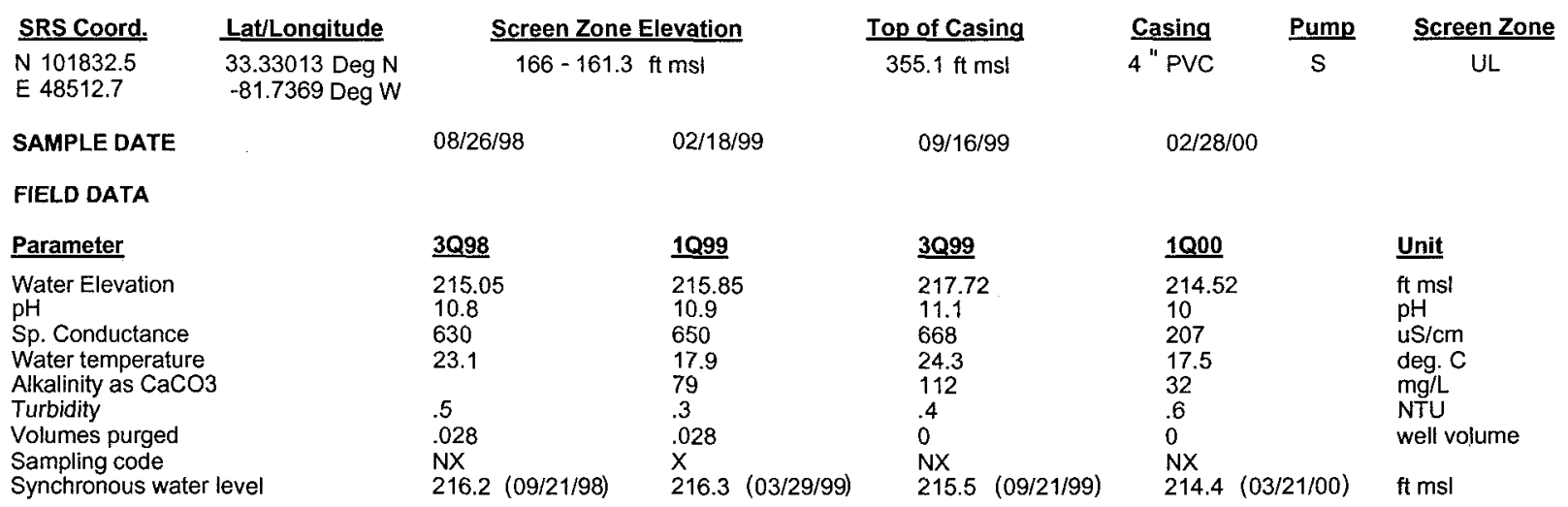

ANALYTICAL DATA

I. Groundwater Protection Standard

261 Appendix VIII/264 Appendix IX Hazardous Constituents

ST Parameter

Inorganics

Barium, total recoverable

Cyanide

Lead, total recoverable

Nickel, total recoverable

Selenium, total recoverable

Organics

Chlorobenzene

1,1-Dichloroethane

1,1-Dichloroethylene

trans-1,2-Dichloroethylene

PCB 1016

PCB 1221

PCB 1232

PCB 1242

PCB 1248

PCB 1254

PCB 1260

1,1,2,2-Tetrachloroethane

Tetrachloroethylerie

1,1,1-Trichloroethane

Trichloroethylene

II. Monitoring Constituents

ST Parameter

Inorganics

$+\quad$ Aluminum, total recoverable

$+\quad$ Nitrate-nitrite as nitrogen

+ Sodium, total recoverable Sulfate

Radionuclides

Gross alpha

Nonvolatile beta

Radium, total alpha-emitting
3Q98 CLPEPA

1099

\section{CLP EPA}

\section{$\underline{3099}$}

\section{CLPEPA}

1000 CLPEPA Filt. DF Unit Lab

55.9
$<10$
$<100$
14.9
$<200$

$\begin{array}{ll}<10 & U \\ <10 & U \\ <100 & U \\ <50 & U \\ <200 & U\end{array}$

$\begin{array}{ll} & 44.5 \\ <10 & U \\ <10 & U \\ <50 & U \\ <10 & U\end{array}$

$\begin{aligned} & 5.13 \\ & <20 \\ < & 60 \\ < & 40\end{aligned}$

J I NDD 1

$\mathrm{ug} / \mathrm{L} \quad \mathrm{ML}$

$U$
$U$

$\mathrm{U}$

$<25$ UJ

$<25$ UJ

$<25$ UJ

$<25$

UJ

$<25$
$<25$
$<25$

$<25$

$<25$

$\begin{array}{ll}<1 & U \\ <1 & U \\ 3.97 & \end{array}$

$<1$

U

$\begin{array}{lllll}<2 & U & & <E Q L & 2 \\ <2 & U & & <E Q L & 2 \\ 3.76 & J & K & \text { NDD } & 2\end{array}$

ug/L ML

$\mathrm{ug} / \mathrm{L}$

$<25$
436
$<25$
215

$\begin{array}{ll}<25 & U \\ 377 & \\ <25 & U \\ 161 & \end{array}$

$\begin{array}{lll}<1 & \mathrm{U} & \\ 349 & & \\ 69 & \mathrm{~J} & \mathrm{I} \\ 136 & & \end{array}$

$<2$
304
$<2$
152

$U$
$U$

< EQL 2

$u g / L \quad M L$

ug/L $M L$

$u g / L \quad M L$

\section{CLPEPA}

3097 CLPEPA

3098 CLPEPA

309

CLPEPA Filt. DF Unit Lab

\begin{tabular}{|c|c|c|}
\hline $\begin{array}{l}560 \\
14900\end{array}$ & $\begin{array}{l}73.6 \\
17100\end{array}$ & $\begin{array}{l}1770 \\
1170\end{array}$ \\
\hline 24800 & 19200 & 22600 \\
\hline 3630 & 2260 & 2750 \\
\hline 1.65 & 1.24 & 2.91 \\
\hline 5.59 & 4.09 & 10.9 \\
\hline .5 & 2.16 & .72 \\
\hline
\end{tabular}

\begin{tabular}{|c|c|c|c|c|}
\hline 1820 & & $>100$ & 1 & $\mathrm{ug} / \mathrm{L}$ \\
\hline 9410 & & $>2400$ & 5 & $\mathrm{ug} / \mathrm{L}$ \\
\hline 18700 & & $>4600$ & 1 & $\mathrm{ug} / \mathrm{L}$ \\
\hline 2870 & & $<3000$ & 1 & $u g / L$ \\
\hline .939 & $\mathrm{U}$ & $<E Q L$ & 1 & $\mathrm{pCi} / \mathrm{L}$ \\
\hline 5.97 & $\mathrm{~J} \quad \mathrm{~K}$ & NDD & 1 & $\mathrm{pCi} / \mathrm{L}$ \\
\hline .3 & $\mathrm{U}$ & $<\mathrm{EQL}$ & 1 & pCi/l \\
\hline
\end{tabular}

Notes: Concentrations in bold italics exceed the groundwater protection or monitoring constituent standards listed in Appendix A. Synchronous water levels are measured over a 3-5 day period or less. Dilution factors, Laboratory, and Filtered Data are for Groundwater Protection Standards First Quarter 2000 and Monitoring Constituents Third Quarter 1999 data only.

$+=$ exceeded the Groundwater Protection Standards (First Quarter 2000) or Monitoring Constituents (Third Quarter 1999) listed in Appendix A. 
Table D-2. Groundwater Monitoring Results for Point-of-Compliance Wells, M-Area HWMF (Cont.) WELL MSB 1D

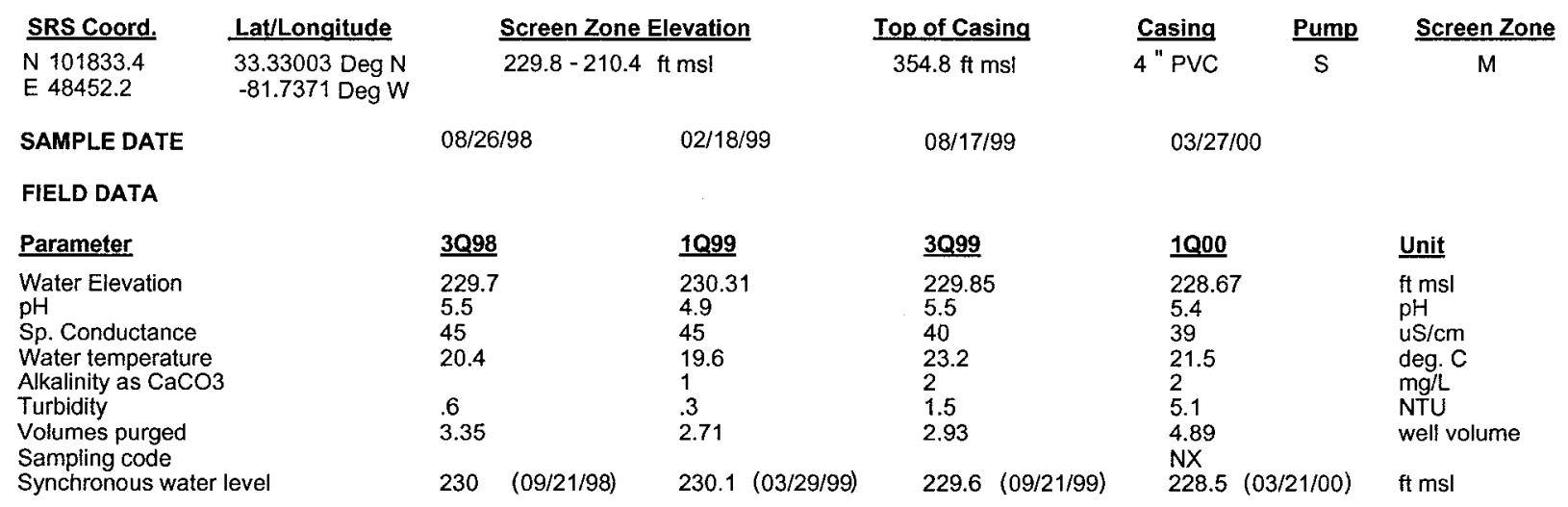

ANALYTICAL DATA

I. Groundwater Protection Standard

261 Appendix VIII/264 Appendix IX Hazardous Constituents

\begin{tabular}{|c|c|c|c|c|c|c|c|c|c|c|c|c|c|c|}
\hline$\underline{\text { ST }}$ & Parameter & $\underline{3 Q 98}$ & CLPEPA & 1099 & CLP EPA & $\underline{3099}$ & CLPEPA & 1000 & CLP & EPA & Filt. & $\underline{D F}$ & Unit & $\underline{\text { Lab }}$ \\
\hline Inor & ganics & & & & & & & & & & & & & \\
\hline & Barium, total recoverable & 14.3 & & $<10$ & $U$ & 14 & & 13.8 & $\jmath$ & 1 & NDD & 1 & ug/L & $M L$ \\
\hline & Cyanide & $<10$ & $U$ & $<10$ & $U$ & $<10$ & U & $<5.2$ & $\mathrm{U}$ & V & $<E Q L$ & 1 & $\mathrm{ug} / \mathrm{L}$ & $M L$ \\
\hline & Lead, total recoverable & $<100$ & $\mathrm{U}$ & $<100$ & $\mathrm{U}$ & $<100$ & $\mathrm{U}$ & $<5.83$ & JU & V & $<E Q L$ & 1 & $\mathrm{ug} / \mathrm{L}$ & ML \\
\hline & Nickel, total recoverable & 10.9 & $\mathrm{~J}$ & $<50$ & $\mathrm{U}$ & $<50$ & $\mathrm{U}$ & $<60$ & $\mathrm{U}$ & & $<E Q L$ & 1 & $\mathrm{ug} / \mathrm{L}$ & ML \\
\hline & Selenium, total recoverable & $<200$ & U & $<200$ & $\mathrm{U}$ & $<200$ & $\mathrm{U}$ & $<40$ & $\mathbf{U}$ & & $<E Q L$ & 1 & $\mathrm{ug} / \mathrm{L}$ & ML \\
\hline Org: & anics & & & & & & & & & & & & & \\
\hline & Chlorobenzene & $<5$ & US & $<5$ & $\mathrm{U}$ & $<5$ & $\mathrm{U}$ & $<5$ & $U$ & & $<E Q L$ & 5 & $\mathrm{ug} / \mathrm{L}$ & $\mathrm{ML}$ \\
\hline & 1,1-Dichloroethane & $<5$ & UJ & $<5$ & $U$ & $<5$ & $U$ & $<5$ & U & & $<\mathrm{EQL}$ & 5 & $\mathrm{ug} / \mathrm{L}$ & ML \\
\hline & 1,1-Dichloroethylene & $<5$ & UJ & $<5$ & $U$ & $<5$ & $U$ & $<5$ & $U$ & & $<\mathrm{EQL}$ & 5 & $\mathrm{ug} / \mathrm{L}$ & $M L$ \\
\hline & trans-1,2-Dichloroethylene & $<5$ & UJ & $<5$ & $\mathrm{U}$ & $<5$ & $U$ & $<5$ & $\mathrm{U}$ & & $<E Q L$ & 5 & $\mathrm{ug} / \mathrm{L}$ & $M L$ \\
\hline & PCB 1016 & & & $<2$ & $\mathrm{U}$ & $<2$ & $U$ & $<.971$ & $\mathrm{JU}$ & $Q$ & $<\mathrm{EQL}$ & 1 & $\mathrm{ug} / \mathrm{L}$ & $M L$ \\
\hline & PCB 1221 & & & $<2$ & $\mathrm{U}$ & $<2$ & $\mathrm{U}$ & $<.971$ & JU & $Q$ & $<\mathrm{EQL}$ & 1 & $\mathrm{ug} / \mathrm{L}$ & ML \\
\hline & РСВ 1232 & & & $<1$ & $U$ & $<.98$ & $\mathrm{U}$ & $<.971$ & JU & $Q$ & $<E Q L$ & 1 & $\mathrm{ug} / \mathrm{L}$ & ML \\
\hline & РCB 1242 & & & $<1$ & $\mathrm{U}$ & $<.98$ & U & $<.971$ & JU & $Q$ & $<\mathrm{EQL}$ & 1 & $\mathrm{ug} / \mathrm{L}$ & $M L$ \\
\hline & PCB 1248 & & & $<1$ & $U$ & $<.98$ & U & $<.971$ & JU & $Q$ & $<\mathrm{EQL}$ & 1 & $\mathrm{ug} / \mathrm{L}$ & $M L$ \\
\hline & PCB 1254 & & & $<1$ & U & $<.98$ & $U$ & $<.971$ & JU & $Q$ & $<\mathrm{EQL}$ & 1 & $\mathrm{ug} / \mathrm{L}$ & ML \\
\hline & PCB 1260 & & & $<1$ & $U$ & $<.98$ & $U$ & $<.971$ & JU & $Q$ & $<\mathrm{EQL}$ & 1 & $\mathrm{ug} / \mathrm{L}$ & $\mathrm{ML}$ \\
\hline & $1,1,2,2$-Tetrachloroethane & $<5$ & UJ & $<5$ & $\mathrm{U}$ & $<5$ & $U$ & $<5$ & $U$ & & $<\mathrm{EQL}$ & 5 & ug/L & ML \\
\hline+ & Tetrachloroethylene & 17.6 & $J$ & 11.9 & & 6 & & 17.8 & & & $>5$ & 5 & $\mathrm{ug} / \mathrm{L}$ & ML \\
\hline & 1,1,1-Trichloroethane & $<5$ & UJ & $<5$ & $\mathrm{U}$ & $<5$ & $U$ & $<5$ & $U$ & & $<\mathrm{EQL}$ & 5 & $\mathrm{ug} / \mathrm{L}$ & $\mathrm{ML}$ \\
\hline+ & Trichloroethylene & 5.9 & $J$ & 3.09 & $\mathrm{~J} \quad \mathrm{I}$ & 3.8 & $\mathbf{J} \quad \mathrm{I}$ & 17.3 & & & $>5$ & 5 & $\mathrm{ug} / \mathrm{L}$ & ML \\
\hline II. Mc & onitoring Constituents & & & & & & & & & & & & & \\
\hline ST & Parameter & $\underline{3096}$ & CLPEPA & $\underline{3097}$ & CLPE EPA & $\underline{3098}$ & CLPEPA & $\underline{3099}$ & CLP & EPA & Filt. & $\underline{D F}$ & Unit & $=\mathbf{a b}$ \\
\hline Inorg & ganics & & & & & & & & & & & & & \\
\hline & Aluminum, total recoverable & 48 & & 13.1 & $\mathrm{~J}$ & $<200$ & $\mathrm{U}$ & $<200$ & $\mathrm{U}$ & & $<E Q L$ & 1 & $u g / L$ & EX \\
\hline+ & Nitrate-nitrite as nitrogen & 2620 & & 2550 & & 2290 & & 2490 & & & $>2400$ & 1 & $\mathrm{ug} / \mathrm{L}$ & EX \\
\hline & Sodium, total recoverable & 4920 & & 4470 & & 5320 & & 4100 & & & $<4600$ & 1 & $\mathrm{ug} / \mathrm{L}$ & EX \\
\hline & Sulfate & 760 & $J$ & $<5000$ & $\mathrm{U}$ & 192 & $J$ & 278 & & & $<3000$ & 1 & $u g / L$ & EX \\
\hline Radi & ionuclides & & & & & & & & & & & & & \\
\hline & Gross alpha & 2.38 & & .7 & UI & 11 & & 5.84 & & & $<15$ & 1 & $\mathrm{pCi} / \mathrm{L}$ & GP \\
\hline & Nonvolatile beta & 1.98 & & 22.8 & $\mathbf{J}$ & 7.29 & & 2.63 & & & $<50$ & 1 & $\mathrm{pCi} / \mathrm{L}$ & GP \\
\hline & Radium, total alpha-emitting & 1.1 & UI & .95 & UI & 2.41 & & 1.4 & & & $<5$ & 1 & $\mathrm{pCi} / \mathrm{L}$ & \\
\hline
\end{tabular}

\footnotetext{
Notes: Concentrations in bold italics exceed the groundwater protection or monitoring constituent standards listed in Appendix A. Synchronous water levels are measured over a 3-5 day period or less. Dilution factors, Laboratory, and Filtered Data are for Groundwater Protection Standards First Quarter 2000 and Monitoring Constituents Third Quarter 1999 data only.

$+=$ exceeded the Groundwater Protection Standards (First Quarter 2000) or Monitoring Constituents (Third Quarter 1999) listed in Appendix A.
} 
Table D-2. Groundwater Monitoring Results for Point-of-Compliance Wells, M-Area HWMF (Cont.) WELL MSB 2B

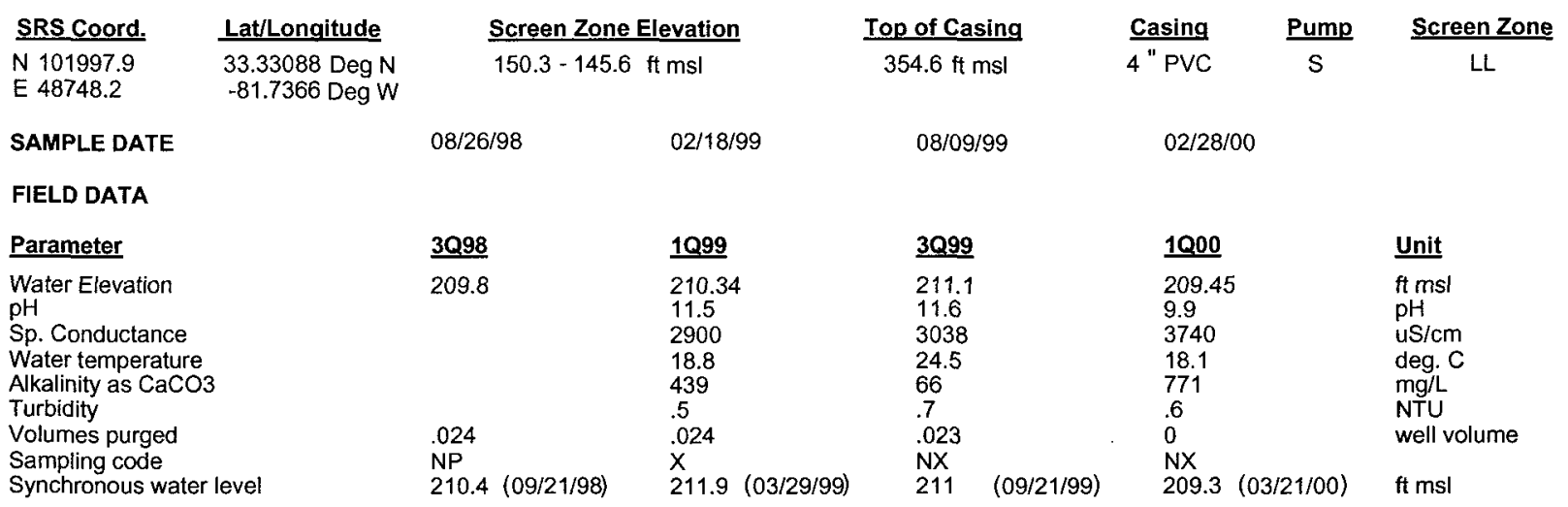

ANALYTICAL DATA

I. Groundwater Protection Standard

261 Appendix VIII/264 Appendix IX Hazardous Constituents

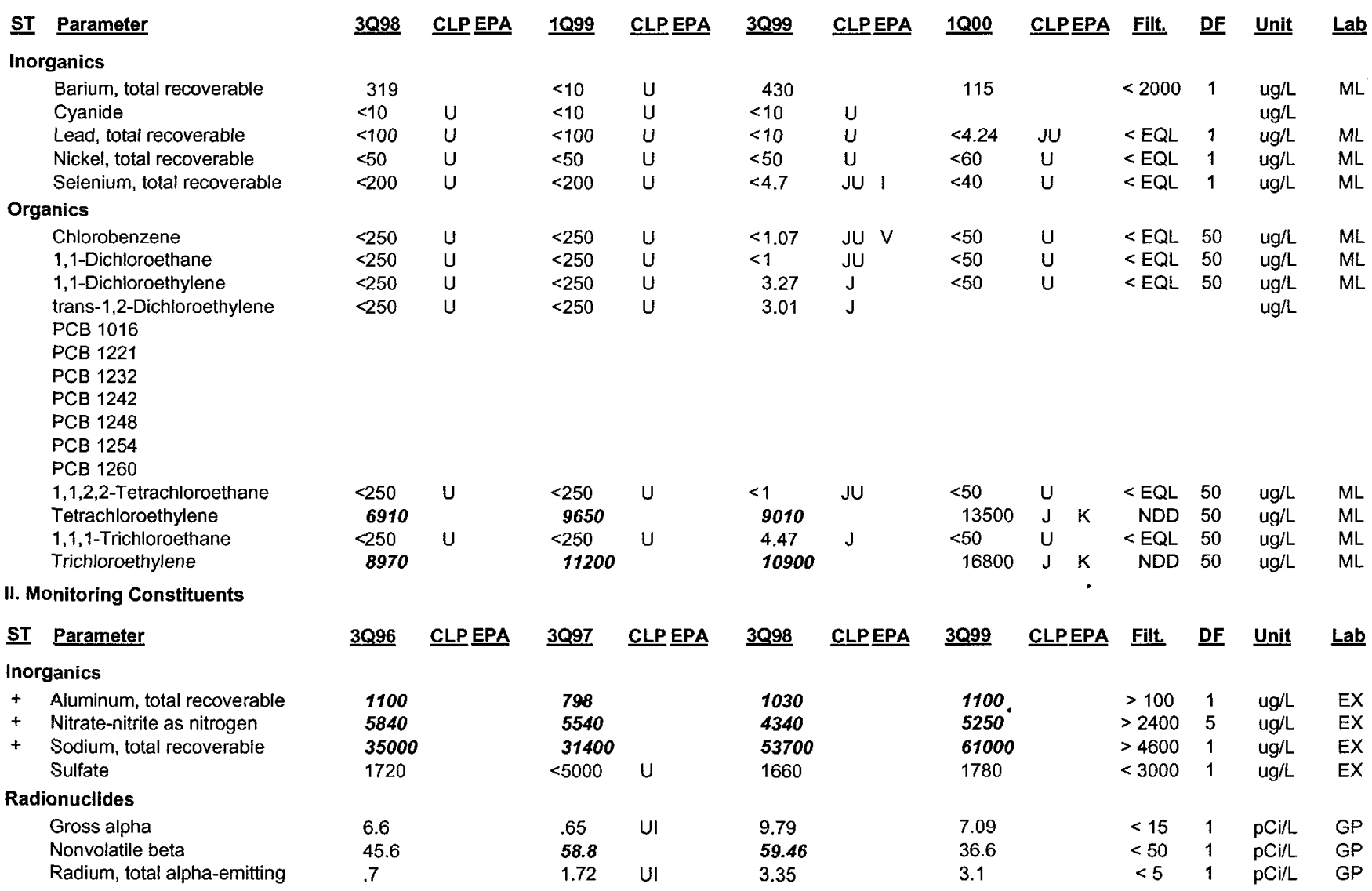

Notes: Concentrations in bold italics exceed the groundwater protection or monitoring constituent standards listed in Appendix A. Synchronous water levels are measured over a 3-5 day period or less. Dilution factors, Laboratory, and Filtered Data are for Groundwater Protection Standards First Quarter 2000 and Monitoring Constituents Third Quarter 1999 data only.

+ = exceeded the Groundwater Protection Standards (First Quarter 2000) or Monitoring Constituents (Third Quarter 1999) listed in Appendix A. 
Table D-2. Groundwater Monitoring Results for Point-of-Compliance Wells, M-Area HWMF (Cont.) WELL MSB 2C

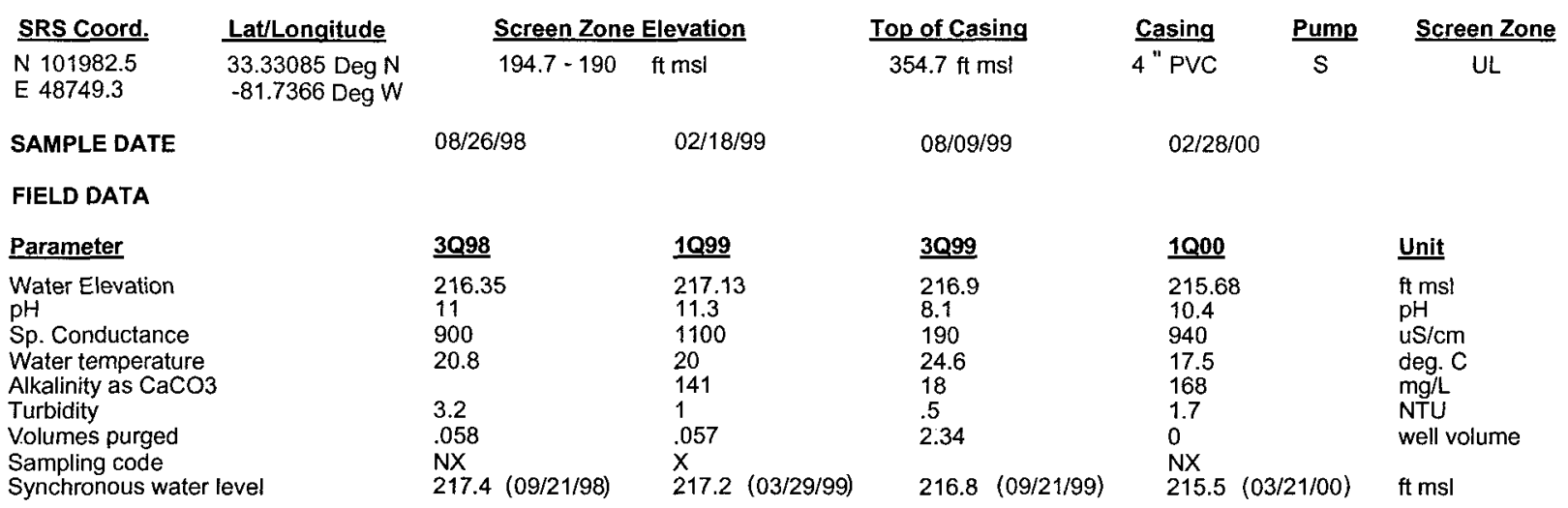

ANALYTICAL DATA

I. Groundwater Protection Standard

261 Appendix VIII/264 Appendix IX Hazardous Constituents

\begin{tabular}{|c|c|c|c|c|c|c|c|c|c|c|c|c|c|}
\hline$\underline{\mathbf{S T}}$ & Parameter & $\underline{3 Q 98}$ & CLPEPA & $\underline{1099}$ & $\underline{\text { CLP EPA }}$ & $\underline{3 Q 99}$ & CLPEPA & $\underline{1 Q 00}$ & CLPEPA & Filt. & $\underline{\text { DF }}$ & $\underline{\text { Unit }}$ & $\underline{\text { Lab }}$ \\
\hline \multicolumn{14}{|c|}{ Inorganics } \\
\hline & Barium, total recoverable & 99.3 & & $<10$ & $U$ & 62 & & 20.8 & & $<2000$ & 1 & $u g / L$ & ML \\
\hline & Cyanide & $<10$ & $\mathrm{U}$ & $<10$ & U & $<10$ & U & & & & & $u g / L$ & \\
\hline & Lead, total recoverable & $<100$ & $U$ & $<100$ & U & $<10$ & U & $<20$ & $U$ & $<E Q L$ & 1 & ug/L & ML. \\
\hline & Nickel, total recoverable & 9.66 & J & $<50$ & $\mathrm{U}$ & $<50$ & $U$ & $<60$ & $\mathrm{U}$ & $<\mathrm{EQL}$ & 1 & ug/L & ML \\
\hline & Selenium, total recoverable & $<200$ & $U$ & $<200$ & $\mathrm{U}$ & $<10$ & $U$ & $<40$ & $\mathrm{U}$ & $<E Q L$ & 1 & $\mathrm{ug} / \mathrm{L}$ & ML \\
\hline \multicolumn{14}{|c|}{ Organics } \\
\hline & Chlorobenzene & $<1250$ & UJ & $<1250$ & $\mathrm{u}$ & 4.68 & $\mathrm{~K}$ & $<100$ & $U$ & $<\mathrm{EQL}$ & 100 & $u g / t$ & ML \\
\hline & 1,1-Dichloroethane & $<1250$ & UJ & $<1250$ & $U$ & $<1$ & $\mathrm{U}$ & $<100$ & $U$ & $<E Q L$ & 100 & $\mathrm{ug} / \mathrm{L}$ & ML \\
\hline & 1,1-Dichloroethylene & $<1250$ & UJ & $<1250$ & $\mathrm{U}$ & .92 & J IK & $<100$ & $\mathrm{U}$ & $<E Q L$ & 100 & $\mathrm{ug} / \mathrm{L}$ & ML \\
\hline & $\begin{array}{l}\text { trans-1,2-Dichloroethylene } \\
\text { PCB } 1016\end{array}$ & $<1250$ & UJ & $<1250$ & $\mathrm{U}$ & $<1$ & $U$ & & & & & $u g / L$ & \\
\hline & PCB 1221 & & & & & & & & & & & & \\
\hline & PCB 1232 & & & & & & & & & & & & \\
\hline & PCB 1242 & & & & & & & & & & & & \\
\hline & PCB 1248 & & & & & & & & & & & & \\
\hline & PCB 1254 & & & & & & & & & & & & \\
\hline & PCB 1260 & & & & & & & & & & & & \\
\hline & $1,1,2,2$-Tetrachloroethane & $<1250$ & UJ & $<1250$ & $\mathrm{U}$ & 12.6 & $\mathrm{~K}$ & $<100$ & $U$ & $<E Q L$ & 100 & ug/L & ML \\
\hline & Tetrachloroethylene & 26900 & $\mathrm{~J}$ & 24700 & & 57400 & & 22200 & J & NDD & 100 & $u g / L$ & ML \\
\hline & $1,1,1$-Trichloroethane & $<1250$ & UJ & $<1250$ & $U$ & $<1$ & U & $<100$ & U & $<E Q L$ & 100 & $\mathrm{ug} / \mathrm{L}$ & ML \\
\hline & Trichloroethylene & 25500 & $\mathrm{~J}$ & 18300 & & 44500 & & 14200 & $\mathrm{~J} K$ & NDD & 100 & $\mathrm{ug} / \mathrm{L}$ & $M L$ \\
\hline \multicolumn{14}{|c|}{ II. Monitoring Constituents } \\
\hline$\underline{\mathbf{S T}}$ & Parameter & $\underline{3 Q 96}$ & CLPEPA & $\underline{3097}$ & CLP EPA & $\underline{3 Q 98}$ & CLPEPA & $\underline{3099}$ & CLPEPA & Filt. & $\underline{D F}$ & Unit & Lab \\
\hline \multicolumn{14}{|c|}{ Inorganics } \\
\hline & Aluminum, total recoverable & 494 & & 194 & & 727 & & $<200$ & $U$ & $<E Q L$ & 1 & $\mathrm{ug} / \mathrm{L}$ & $V$ \\
\hline+ & Nitrate-nitrite as nitrogen & 10800 & & 11000 & & 7830 & & 15300 & & $>2400$ & 5 & $\mathrm{ug} / \mathrm{L}$ & \\
\hline+ & Sodium, total recoverable & 20900 & & 13000 & & 20300 & & 13000 & & $>4600$ & 1 & $\mathrm{ug} / \mathrm{L}$ & EX \\
\hline & Sulfate & 1290 & & $<5000$ & $U$ & 811 & & 337 & & $<3000$ & 1 & $\mathrm{ug} / \mathrm{L}$ & - \\
\hline \multicolumn{14}{|c|}{ Radionuclides } \\
\hline & Gross alpha & 5.06 & & .9 & UI & 6.05 & & 3.31 & & $<15$ & 1 & $\mathrm{pCi} / \mathrm{L}$ & \\
\hline & Nonvolatile beta & 13.2 & & 4.01 & & 11.74 & & 4.37 & & $<50$ & 1 & $\mathrm{pCl} / \mathrm{L}$ & \\
\hline & Radium, total alpha-emitting & 1.8 & & 4.66 & J & 1.65 & & 3.7 & & $<5$ & 1 & $\mathrm{pCi} / \mathrm{L}$ & GP \\
\hline
\end{tabular}

Notes: Concentrations in bold italics exceed the groundwater protection or monitoring constituent standards listed in Appendix A. Synchronous water levels are measured over a 3-5 day period or less. Dilution factors, Laboratory, and Filtered Data are for Groundwater Protection Standards First Quarter 2000 and Monitoring Constituents Third Quarter 1999 data only.

$+=$ exceeded the Groundwater Protection Standards (First Quarter 2000) or Monitoring Constituents (Third Quarter 1999) listed in Appendix A. 
Table D-2. Groundwater Monitoring Results for Point-of-Compliance Wells, M-Area HWMF (Cont.) WELL MSB 2D

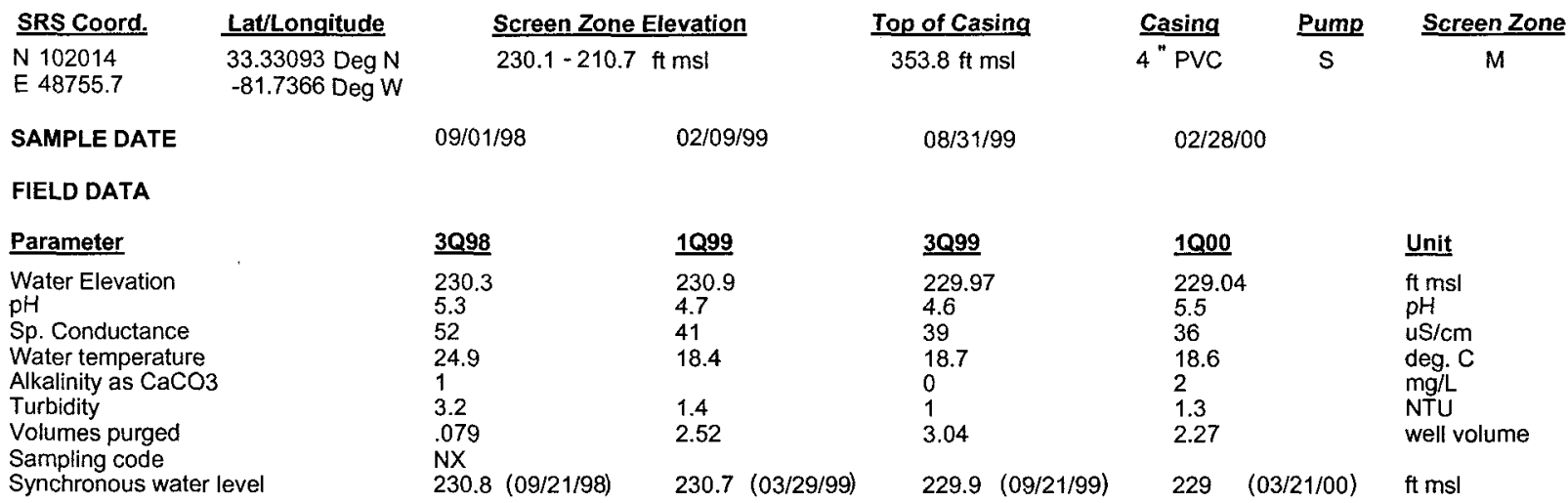

ANALYTICAL DATA

I. Groundwater Protection Standard

261 Appendix VIII/264 Appendix IX Hazardous Constituents

\begin{tabular}{|c|c|c|c|c|c|c|c|c|c|c|c|c|c|c|c|}
\hline$\underline{\mathbf{S T}}$ & Parameter & $\underline{3098}$ & CLPEPA & 1099 & CLPEPA & $\underline{3 Q 99}$ & $\underline{\text { CLPP }}$ & EPA & $1 \mathrm{Q00}$ & CLPP & EPA & Filt. & $\underline{D F}$ & Unit & $\underline{\text { Lab }}$ \\
\hline Ino & ganics & & & & & & & & & & & & & & \\
\hline & Barium, total recoverable & 10.5 & & $<10$ & $\mathrm{U}$ & 14 & & & 12 & J & 1 & NDD & 1 & ug/L & ML \\
\hline & Cyanide & $<10$ & $\mathrm{U}$ & $<10$ & $\mathrm{U}$ & $<10$ & $U$ & & $<20$ & U & & $<\mathrm{EQL}$ & 1 & ug/L & $M L$ \\
\hline & Lead, total recoverable & $<100$ & $\mathrm{U}$ & $<100$ & $\mathrm{U}$ & $<10$ & U & & $<20$ & $U$ & & $<\mathrm{EQL}$ & 1 & ug/L & ML \\
\hline & Nickel, total recoverable & $<50$ & $\mathrm{U}$ & $<50$ & $\mathrm{U}$ & $<50$ & $U$ & & $<60$ & $U$ & & $<E Q L$ & 1 & $\mathrm{ug} / \mathrm{L}$ & $M L$ \\
\hline & Selenium, total recoverable & $<3.33$ & UJ & $<200$ & $U$ & $<10$ & U & & $<40$ & $U$ & & $<E Q L$ & 1 & $\mathrm{ug} / \mathrm{h}$ & $M L$ \\
\hline $\mathrm{rg}$ & anics & & & & & & & & & & & & & & \\
\hline & Chlorobenzene & $<5$ & $\mathrm{u}$ & $<125$ & $U$ & $<1$ & $U$ & & $<2$ & $\mathrm{U}$ & & $<\mathrm{EQL}$ & 2 & $\mathrm{ug} / \mathrm{L}$ & ML \\
\hline & 1,1-Dichloroethane & $<5$ & $\mathrm{U}$ & $<125$ & $\mathrm{U}$ & $<1$ & $\mathrm{U}$ & & $<2$ & $\mathrm{U}$ & & $<\mathrm{EQL}$ & 2 & $\mathrm{ug} / \mathrm{L}$ & ML \\
\hline & 1,1-Dichloroethylene & $<5$ & $\mathrm{U}$ & $<125$ & $\mathrm{U}$ & 1.94 & $J$ & $\mathrm{~K}$ & 1.34 & $\mathrm{~J}$ & IK & NDD & 2 & $\mathrm{ug} / \mathrm{L}$ & ML \\
\hline & trans-1,2-Dichloroethylene & $<5$ & $\mathrm{U}$ & $<125$ & $\mathrm{U}$ & $<1$ & $\mathrm{U}$ & & & & & & & $\mathrm{ug} / \mathrm{L}$ & \\
\hline & PCB 1016 & $<2$ & $\mathrm{U}$ & $<2$ & $\mathrm{U}$ & $<2$ & $\mathrm{U}$ & & $<1$ & JU & & $<E Q L$ & 1 & $\mathrm{ug} / \mathrm{L}$ & $M L$ \\
\hline & PCB 1221 & $<2$ & $\mathrm{U}$ & $<2$ & $\mathrm{U}$ & $<2$ & $\mathrm{U}$ & & $<1$ & JU & & $<\mathrm{EQL}$ & 1 & ug/L & $M L$ \\
\hline & PC8 1232 & $<1$ & U & $<1$ & $\mathrm{U}$ & $<.99$ & $\mathrm{U}$ & & $<1$ & JU & & $<E Q L$ & 1 & $\mathrm{ug} / \mathrm{L}$ & $\mathrm{ML}$ \\
\hline & PCB 1242 & $<1$ & $U$ & $<1$ & $\mathrm{U}$ & $<.99$ & $\mathrm{U}$ & & $<1$ & JU & & $<E Q L$ & 1 & ug/L & $\mathrm{ML}$ \\
\hline & PCB 1248 & $<1$ & U & $<1$ & $U$ & $<.99$ & $\mathrm{U}$ & & $<1$ & $J U$ & & $<$ EQL & 1 & ug/L & $\mathrm{ML}$ \\
\hline & PCB 1254 & $<1$ & U & $<1$ & $U$ & $<.99$ & $\mathrm{U}$ & & $<1$ & JU & & $<E Q L$ & 1 & ug/L & ML \\
\hline & РСB 1260 & $<1$ & $U$ & $<1$ & $\mathrm{U}$ & $<.99$ & $\mathrm{U}$ & & $<1$ & JU & & $<$ EQL & 1 & $\mathrm{ug} / \mathrm{L}$ & $\mathrm{ML}$ \\
\hline & 1,1,2,2-Tetrachloroethane & $<5$ & $\mathrm{U}$ & $<125$ & $\mathrm{U}$ & $<1$ & $\mathrm{U}$ & & $<2$ & $\mathrm{U}$ & & $<E Q L$ & 2 & $\mathrm{ug} / \mathrm{L}$ & ML \\
\hline & Tetrachloroethylene & 137 & & 272 & & 507 & & & 599 & $J$ & $\mathrm{~K}$ & NDD & 2 & $\omega g / L$ & $\mathrm{ML}$ \\
\hline & 1,1,1-Trichloroethane & $<5$ & $\mathrm{U}$ & $<125$ & U & 14.5 & $J$ & $\mathrm{~K}$ & 20.6 & 」 & $\mathrm{K}$ & NDD & 2 & $\mathrm{ug} / \mathrm{L}$ & $M L$ \\
\hline & Trichloroethylene & 69.7 & & 98.9 & J 1 & 99.8 & $J$ & $\mathrm{~K}$ & 84.8 & J & K & NDD & 2 & ug/L & ML \\
\hline $\mathbf{N}$ & onitoring Constituents & & & & & & & & & & & & & & \\
\hline$\underline{\text { ST }}$ & Parameter & $\underline{3096}$ & CLPEPA & $\underline{3 Q 97}$ & CLP EPA & $\underline{3098}$ & CLP & EPA & $\underline{3099}$ & CLP & EPA & Filt. & $\underline{\text { DF }}$ & Unit & $\underline{\mathrm{Lab}}$ \\
\hline 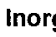 & ganics & & & & & & & & & & & & & & \\
\hline & Aluminum, total recoverable & 285 & & 224 & & 710 & & & 115 & J & I & NDD & 1 & $u g / L$ & WA \\
\hline+ & Nitrate-nitrite as nitrogen & 4120 & & 3370 & & 1800 & & & 2450 & & & $>2400$ & 5 & $\mathrm{ug} / \mathrm{L}$ & EX \\
\hline & Sodium, total recoverable & 2500 & & 2050 & & 2480 & & & 2100 & & & $<4600$ & 1 & $u g / L$ & EX \\
\hline & Sulfate & 118 & J & $<5000$ & $\mathrm{U}$ & 246 & $\mathrm{~J}$ & & $<400$ & $\mathrm{U}$ & & $<\mathrm{EQL}$ & 2 & $\mathrm{ug} / \mathrm{L}$ & EX \\
\hline Radi & onuclides & & & & & & & & & & & & & & \\
\hline & Gross alpha & 13.5 & & .48 & UI & 14.6 & & & 7.49 & & & $<15$ & 1 & $\mathrm{pCi} / \mathrm{L}$ & GP \\
\hline & Nonvolatile beta & 5.81 & & -1.26 & UI & 15.36 & & & 2.83 & J & 1 & NDD & 1 & $\mathrm{pCi} / \mathrm{L}$ & GP \\
\hline & Radium, total alpha-emitting & 8.9 & & 3.95 & UI & 3.14 & & & 4.9 & & & $<5$ & 1 & $\mathrm{pCi} / \mathrm{L}$ & GP \\
\hline
\end{tabular}

Notes: Concentrations in bold italics exceed the groundwater protection or monitoring constituent standards listed in Appendix A. Synchronous water levels are measured over a 3-5 day period or less. Dilution factors, Laboratory, and Filtered Data are for Groundwater Protection Standards First Quarter 2000 and Monitoring Constituents Third Quarter 1999 data only.

$+=$ exceeded the Groundwater Protection Standards (First Quarter 2000) or Monitoring Constituents (Third Quarter 1999) listed in Appendix A. 
Table D-2. Groundwater Monitoring Results for Point-of-Compliance Wells, M-Area HWMF (Cont.) WELL MSB 3B

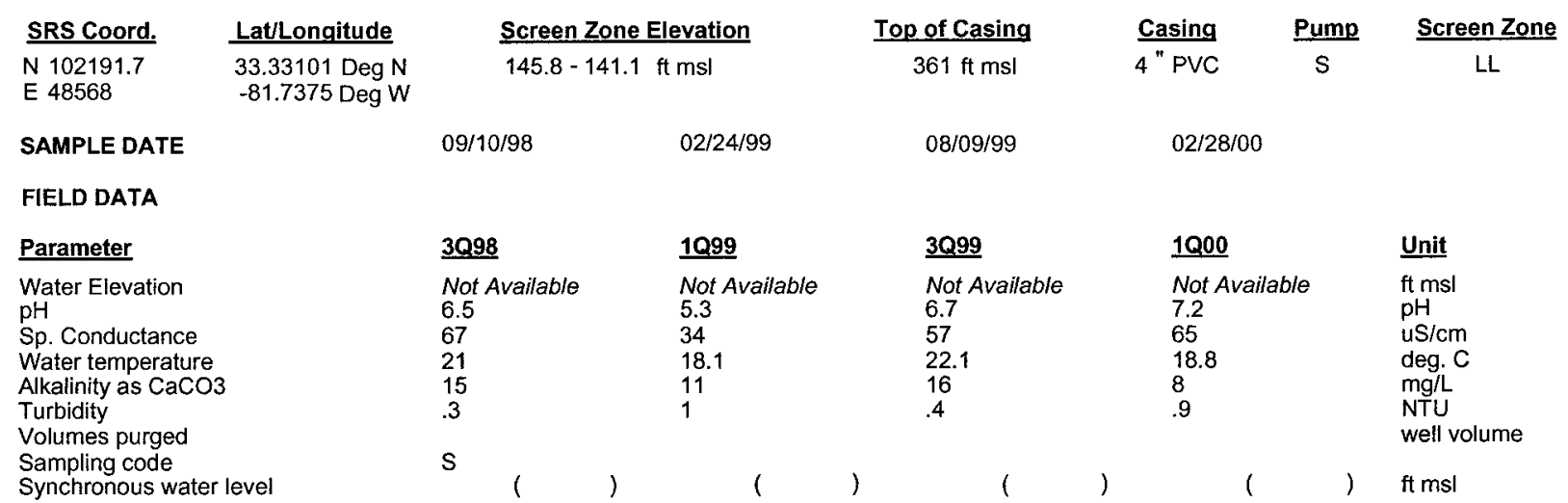

\section{ANALYTICAL DATA}

I. Groundwater Protection Standard

261 Appendix VIII/264 Appendix IX Hazardous Constituents

\begin{tabular}{|c|c|c|c|c|c|c|c|c|c|c|c|c|c|c|}
\hline \multirow{2}{*}{\multicolumn{2}{|c|}{$\frac{\text { ST }}{\text { Parameter }}$}} & \multirow[t]{2}{*}{$\underline{3 Q 98}$} & \multirow[t]{2}{*}{ CLPEPA } & \multirow[t]{2}{*}{1099} & \multirow[t]{2}{*}{ CLP EPA } & \multirow[t]{2}{*}{$\underline{3 Q 99}$} & \multirow[t]{2}{*}{ CLPEPA } & \multirow[t]{2}{*}{$\underline{1 Q 00}$} & \multicolumn{2}{|c|}{ CLPEPA } & \multirow[t]{2}{*}{ Filt. } & \multirow[t]{2}{*}{$\underline{\text { DF }}$} & \multirow[t]{2}{*}{$\underline{\text { Unit }}$} & \multirow[t]{2}{*}{$\underline{L a b}$} \\
\hline & & & & & & & & & & & & & & \\
\hline & Barium, total recoverable & 20.6 & & 17.2 & & 20 & & $<15$ & $U$ & & $<\mathrm{EQL}$ & 1 & $u g / L$ & $\mathrm{ML}$ \\
\hline & Cyanide & $<10$ & $\mathrm{U}$ & $<10$ & $\mathrm{U}$ & $<10$ & $U$ & & & & & & $u g / L$ & \\
\hline & Lead, total recoverable & $<100$ & $\mathrm{U}$ & $<100$ & $\mathrm{U}$ & $<10$ & U & $<5.8$ & JU & & $<E Q L$ & 1 & $u g / L$ & ML \\
\hline & Nickel, total recoverable & $<50$ & $u$ & $<50$ & $\mathrm{U}$ & $<8.7$ & JU I & $<60$ & $U$ & & $<\mathrm{EQL}$ & 1 & $u g / L$ & ML \\
\hline & Selenium, total recoverable & $<200$ & $U$ & $<200$ & U & $<3.7$ & JU I & $<40$ & $U$ & & $<E Q L$ & 1 & $u g / L$ & ML \\
\hline \multicolumn{15}{|c|}{ Organics } \\
\hline & Chlorobenzene & $<50$ & $\mathrm{U}$ & $<125$ & $\mathrm{U}$ & $<1$ & $\mathrm{U}$ & $<5$ & $\mathrm{U}$ & & $<E Q L$ & 5 & $\mathrm{ug} / \mathrm{L}$ & ML \\
\hline & 1,1-Dichioroethane & $<50$ & U & $<125$ & $\mathrm{U}$ & $<1$ & $\mathrm{U}$ & $<5$ & $\mathrm{U}$ & & $<\mathrm{EQL}$ & 5 & $u g / L$ & ML \\
\hline & 1,1-Dichloroethylene & $<50$ & $\mathrm{U}$ & $<125$ & $\mathbf{U}$ & $<1$ & $U$ & $<5$ & $U$ & & $<E Q L$ & 5 & $\mathrm{ug} / \mathrm{t}$ & ML \\
\hline & trans-1,2-Dichloroethylene & $<50$ & $\mathrm{U}$ & $<125$ & $\mathbf{U}$ & $<1$ & $U$ & & & & & & $\mathrm{ug} / \mathrm{L}$ & \\
\hline & PCB 1016 & $<2$ & $\mathrm{U}$ & & & & & & & & & & $u g / L$ & \\
\hline & PCB 1221 & $<2$ & $\mathrm{U}$ & & & & & & & & & & $u g / L$ & \\
\hline & PCB 1232 & $<1$ & $\mathrm{U}$ & & & & & & & & & & $u g / L$ & \\
\hline & PCB 1242 & $<1$ & $\mathrm{U}$ & & & & & & & & & & $u g / L$ & \\
\hline & PCB 1248 & $<1$ & U & & & & & & & & & & $u g / L$ & \\
\hline & PCB 1254 & $<1$ & $\mathrm{U}$ & & & & & & & & & & $u g / L$ & \\
\hline & PCB 1260 & $<1$ & $U$ & & & & & & & & & & $u g / L$ & \\
\hline & $1,1,2,2$-Tetrachloroethane & $<50$ & $U$ & $<125$ & U & $<1$ & $\mathrm{U}$ & $<5$ & $\mathrm{U}$ & & $<E Q L$ & 5 & $\mathrm{ug} / \mathrm{L}$ & ML \\
\hline & Tetrachloroethylene & 414 & & 894 & & 376 & & 551 & $J$ & $\mathrm{~K}$ & NDD & 5 & $u g / L$ & ML \\
\hline & 1,1,1-Trichloroethane & $<50$ & $U$ & $<125$ & $U$ & $<1$ & $\mathrm{U}$ & $<5$ & $U$ & & $<\mathrm{EQL}$ & 5 & $u g / L$ & ML \\
\hline & Trichloroethylene & 1770 & & 3260 & & 1290 & & 1730 & $J$ & $\mathrm{~K}$ & NDD & 5 & $\mathrm{ug} / \mathrm{L}$ & ML \\
\hline \multicolumn{15}{|c|}{ II. Monitoring Constituents } \\
\hline 21 & Parameter & $\underline{3096}$ & CLP EPA & $\underline{3097}$ & CLP EPA & $\underline{3 Q 98}$ & CLPEEA & $\underline{3099}$ & $\underline{\text { CLP }}$ & EPA & Filt. & $\underline{\text { DF }}$ & $\underline{\text { Unit }}$ & $\underline{\text { Lab }}$ \\
\hline \multicolumn{15}{|c|}{ Inorganics } \\
\hline & Aluminum, total recoverable & & & $<20$ & $\mathrm{U}$ & $<200$ & $\mathrm{U}$ & $<200$ & U & & $<E Q L$ & 1 & $u g / L$ & EX \\
\hline & Nitrate-nitrite as nitrogen & & & 1370 & & 1340 & & 1130 & & & $<2400$ & 5 & $u g / L$ & EX \\
\hline & Sodium, total recoverable & & & 2240 & & 2760 & & 2600 & & & $<4600$ & 1 & $\mathrm{ug} / \mathrm{L}$ & EX \\
\hline & Sulfate & & & 779 & $\mathrm{~J}$ & 706 & & 443 & & & $<3000$ & 1 & $u g / L$ & EX \\
\hline \multicolumn{15}{|c|}{ Radionuclides } \\
\hline & Gross alpha & & & .12 & $\mathrm{U}$ & 1.21 & & 6 & $\mathrm{~J}$ & 1 & NDD & 1 & $\mathrm{pCi} / \mathrm{L}$ & GP \\
\hline & Nonvolatile beta & & & 2.49 & $J$ & 1.42 & & 1.39 & $\mathrm{~J}$ & 1 & NDD & 1 & $\mathrm{pCi} / \mathrm{L}$ & GP \\
\hline & Radium, total alpha-emitting & & & .93 & UIJ & .25 & UI & .4 & $\mathrm{U}$ & & $<$ EQL & 1 & $\mathrm{pCi} / \mathrm{L}$ & GP \\
\hline
\end{tabular}

Notes: Concentrations in bold italics exceed the groundwater protection or monitoring constituent standards listed in Appendix A. Synchronous water levels are measured over a 3-5 day period or less. Dilution factors, Laboratory, and Filtered Data are for Groundwater Protection Standards First Quarter 2000 and Monitoring Constituents Third Quarter 1999 data only.

+ = exceeded the Groundwater Protection Standards (First Quarter 2000) or Monitoring Constituents (Third Quarter 1999) listed in Appendix A. 
Table D-2. Groundwater Monitoring Results for Point-of-Compliance Wells, M-Area HWMF (Cont.) WELL MSB 3C

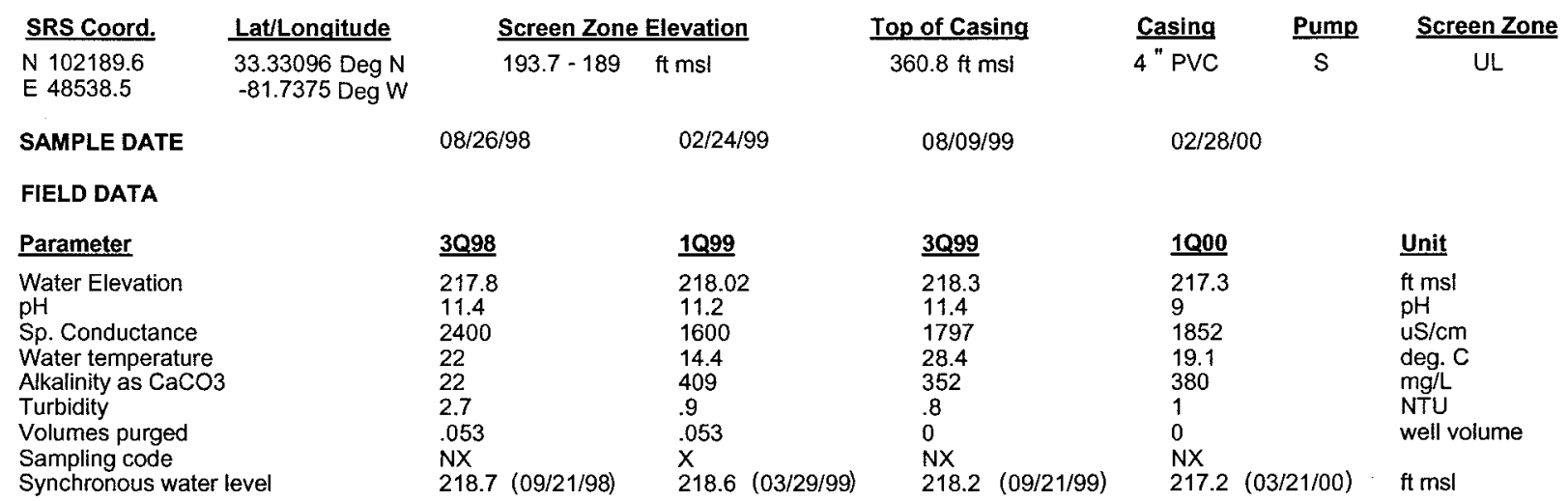

\section{ANALYTICAL DATA}

I. Groundwater Protection Standard

261 Appendix VIII/264 Appendix IX Hazardous Constituents

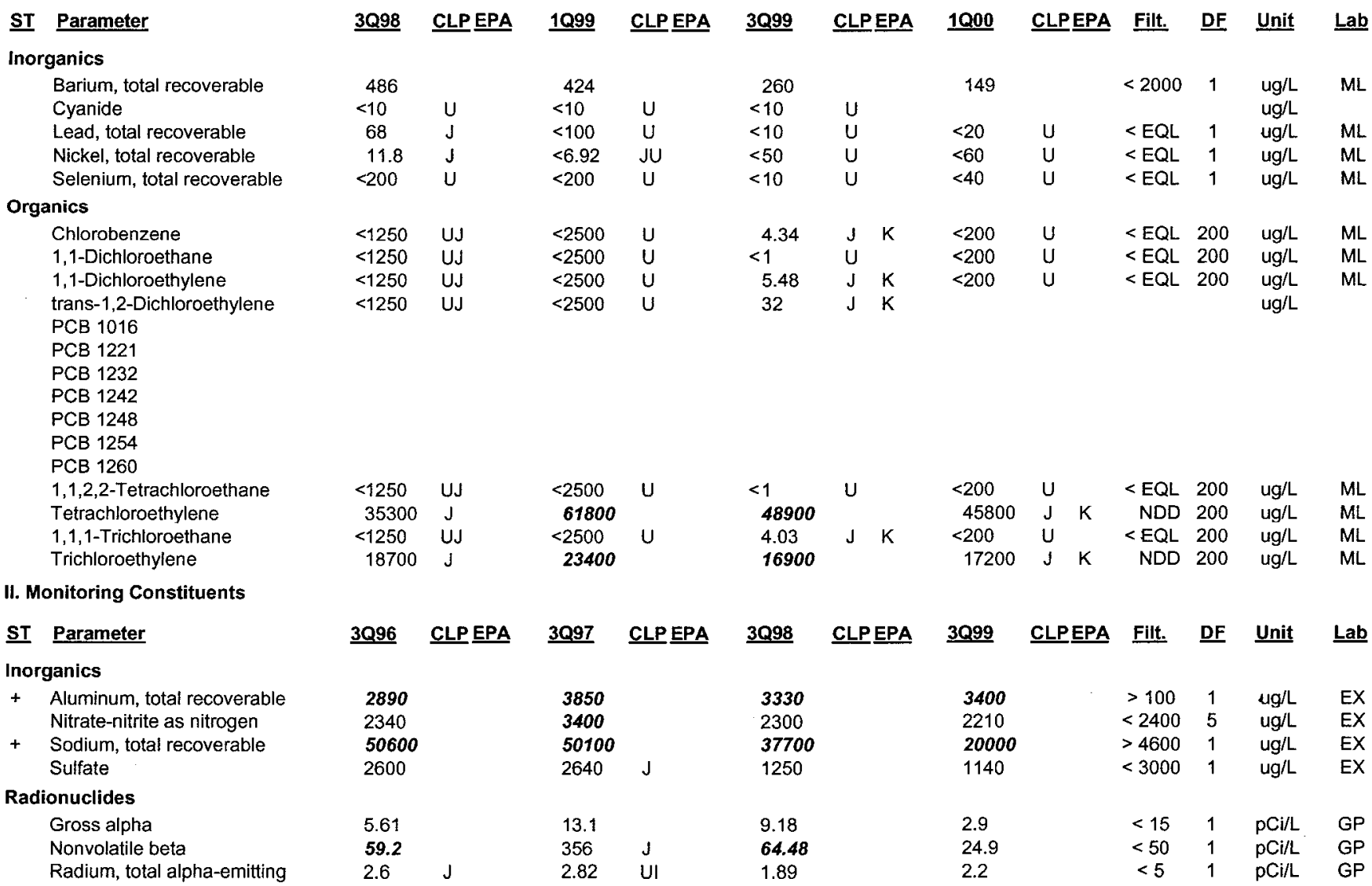

Notes: Concentrations in bold italics exceed the groundwater protection or monitoring constituent standards listed in Appendix A. Synchronous water levels are measured over a 3-5 day period or less. Dilution factors, Laboratory, and Filtered Data are for Groundwater Protection Standards First Quarter 2000 and Monitoring Constituents Third Quarter 1999 data only.

+= exceeded the Groundwater Protection Standards (First Quarter 2000) or Monitoring Constituents (Third Quarter 1999) listed in Appendix A. 
Table D-2. Groundwater Monitoring Results for Point-of-Compliance Wells, M-Area HWMF (Cont.) WELL MSB 4B

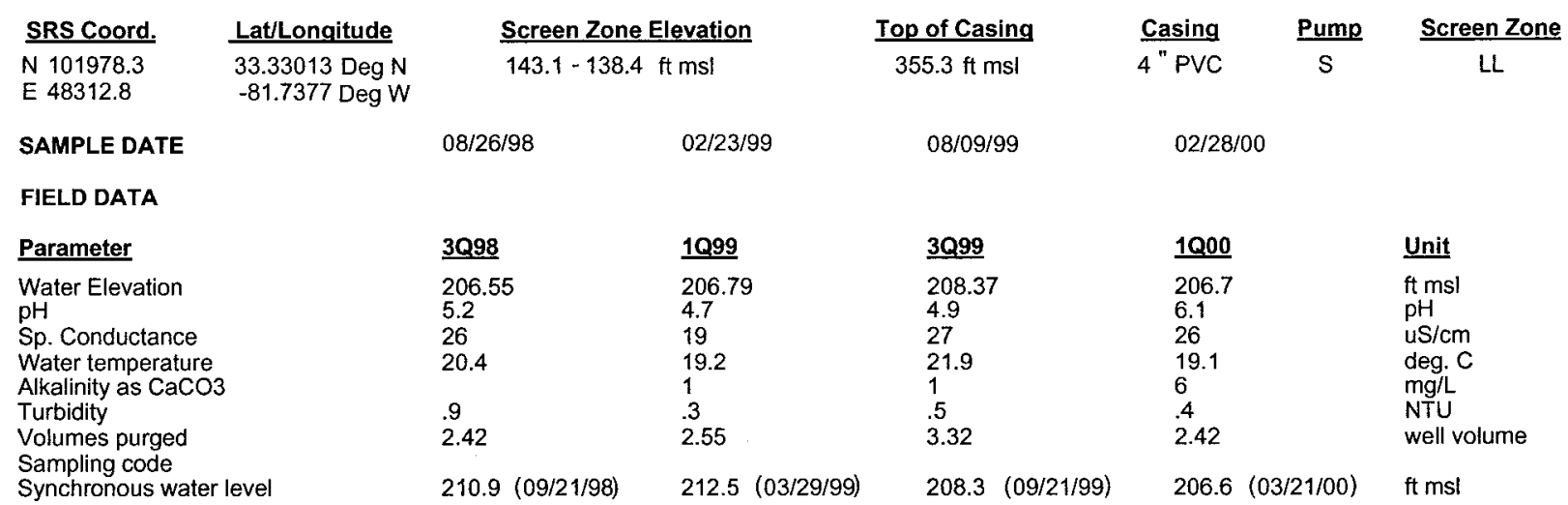

\section{ANALYTICAL DATA}

I. Groundwater Protection Standard

261 Appendix VIII/264 Appendix IX Hazardous Constituents

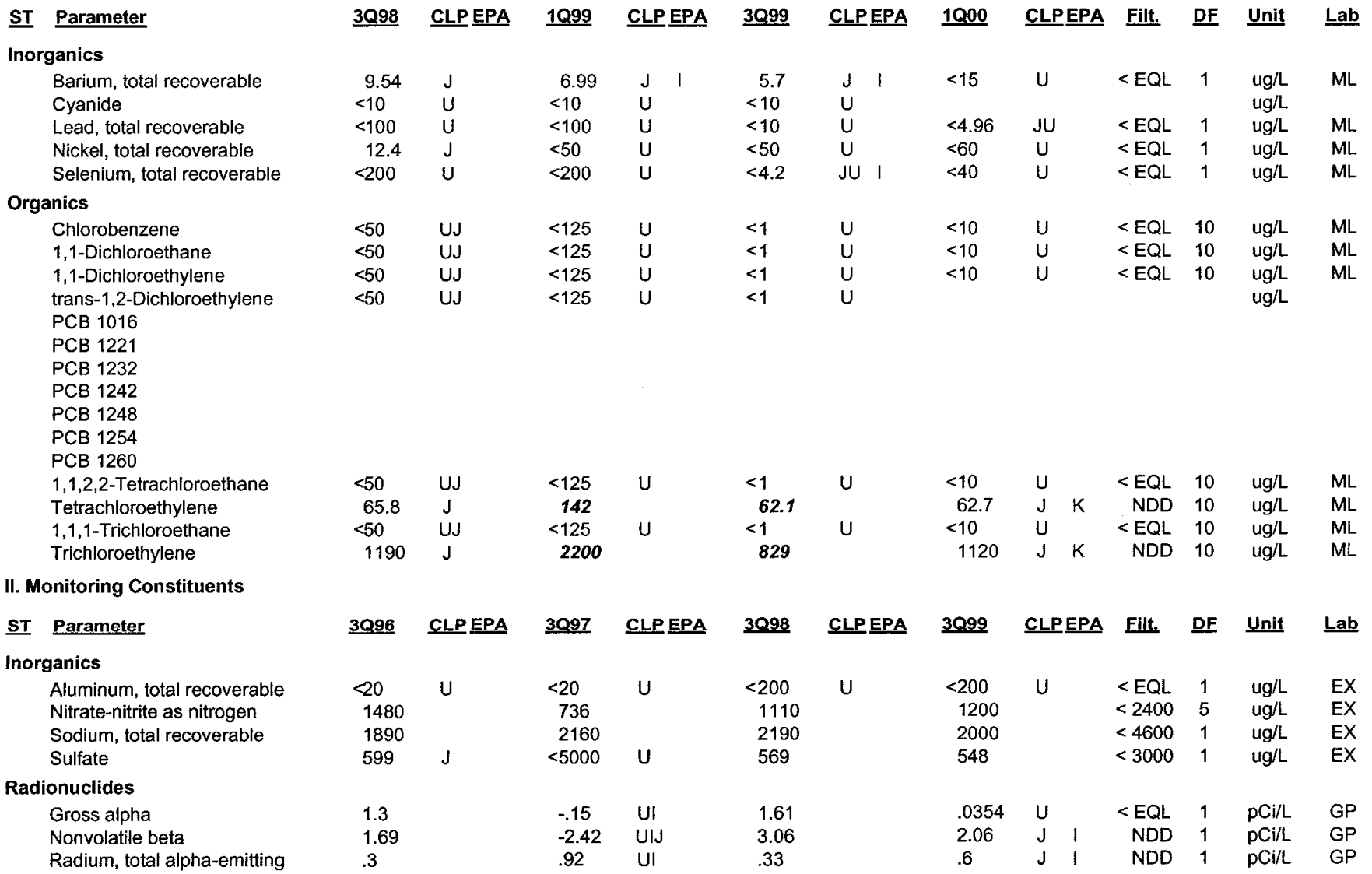

Notes: Concentrations in bold italics exceed the groundwater protection or monitoring constituent standards listed in Appendix A. Synchronous water levels are measured over a 3-5 day period or less. Dilution factors, Laboratory, and Filtered Data are for Groundwater Protection Standards First Quarter 2000 and Monitoring Constituents Third Quarter 1999 data only.

$+=$ exceeded the Groundwater Protection Standards (First Quarter 2000) or Monitoring Constituents (Third Quarter 1999) listed in Appendix A. 
Table D-2. Groundwater Monitoring Results for Point-of-Compliance Wells, M-Area HWMF (Cont.) WELL MSB 4C

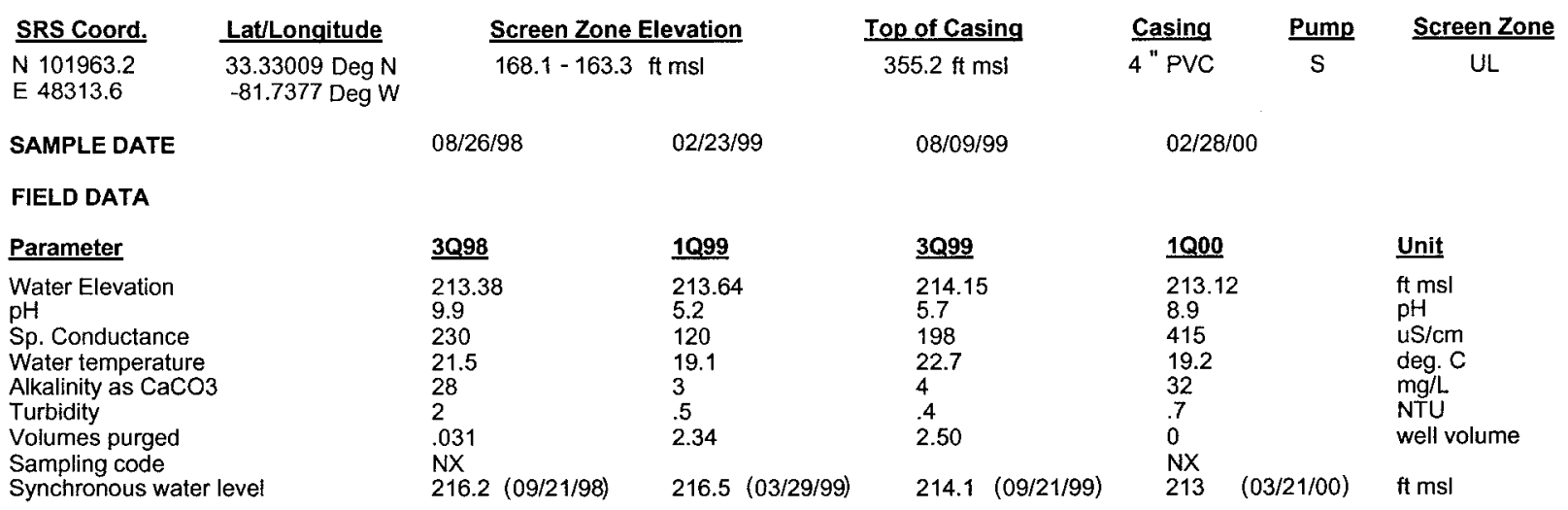

\section{ANALYTICAL DATA}

I. Groundwater Protection Standard

261 Appendix VIII/264 Appendix IX Hazardous Constituents

\begin{tabular}{|c|c|c|c|c|c|c|c|c|c|c|c|c|c|c|c|}
\hline \multirow{2}{*}{\multicolumn{2}{|c|}{$\frac{\text { ST }}{\text { Parameter }}$}} & \multirow[t]{2}{*}{$\underline{3 Q 98}$} & \multirow[t]{2}{*}{ CLP EPA } & \multirow[t]{2}{*}{$\underline{1 Q 99}$} & \multirow[t]{2}{*}{ CLP EPA } & \multirow[t]{2}{*}{$\underline{3 Q 99}$} & \multicolumn{2}{|c|}{ CLPEPA } & \multirow[t]{2}{*}{1000} & \multicolumn{2}{|c|}{ CLPEPA } & \multirow[t]{2}{*}{ Filt. } & \multirow[t]{2}{*}{$\underline{\text { DF }}$} & \multirow[t]{2}{*}{ Unit } & \multirow[t]{2}{*}{ Lab } \\
\hline & & & & & & & & & & & & & & & \\
\hline & Barium, total recoverable & 119 & & 72.3 & & 65 & & & 142 & & & $<2000$ & 1 & ug/L & ML \\
\hline & Cyanide & $<10$ & $U$ & $<10$ & $U$ & $<10$ & $U$ & & & & & & & ug/l. & \\
\hline & Lead, total recoverable & $<100$ & $\mathrm{U}$ & $<100$ & $\mathrm{U}$ & $<10$ & $\mathrm{U}$ & & $<20$ & U & & $<\mathrm{EQL}$ & 1 & $\mathrm{ug} / \mathrm{L}$ & ML \\
\hline & Nickel, total recoverable & 13.3 & J & $<50$ & $\mathrm{U}$ & $<50$ & U & & $<60$ & $\mathrm{U}$ & & $<E Q L$ & 1 & $\mathrm{ug} / \mathrm{L}$ & ML \\
\hline & Selenium, total recoverable & $<200$ & $U$ & $<200$ & $\mathrm{U}$ & $<10$ & $U$ & & $<40$ & $\mathrm{U}$ & & $<E Q L$ & 1 & $\mathrm{ug} / \mathrm{L}$ & ML \\
\hline \multicolumn{16}{|c|}{ Organics } \\
\hline & Chlorobenzene & $<500$ & UJ & $<500$ & $\mathrm{U}$ & $<1.06$ & JU & KV & $<50$ & $\mathrm{U}$ & & $<\mathrm{EQL}$ & 50 & $u g / L$ & $M L$ \\
\hline & 1,1-Dichloroethane & $<500$ & UJ & $<500$ & $\mathrm{U}$ & $<1$ & $\mathrm{U}$ & & $<50$ & $U$ & & $<\mathrm{EQL}$ & 50 & $\mathrm{ug} / \mathrm{L}$ & $M L$ \\
\hline & 1,1-Dichloroethylene & $<500$ & UJ & $<500$ & $\mathrm{U}$ & 6.79 & $\mathrm{~J}$ & K & $<50$ & $U$ & & $<E Q L$ & 50 & $\mathrm{ug} / \mathrm{L}$ & ML \\
\hline & trans-1,2-Dichloroethylene & $<500$ & U. & $<500$ & $\mathrm{U}$ & $<1$ & $U$ & & & & & & & $\mathrm{ug} / \mathrm{L}$ & \\
\hline & PCB 1016 & & & & & & & & & & & & & & \\
\hline & PCB 1221 & & & & & & & & & & & & & & \\
\hline & PCB 1232 & & & & & & & & & & & & & & \\
\hline & PCB 1242 & & & & & & & & & & & $\cdot$ & & & \\
\hline & PCB 1248 & & & & & & & & & & & & & & \\
\hline & PCB 1254 & & & & & & & & & & & & & & \\
\hline & PCB 1260 & & & & & & & & & & & & & & \\
\hline & 1,1,2,2-Tetrachloroethane & $<500$ & UJ & $<500$ & U & 1.54 & J & $\mathrm{K}$ & $<50$ & $U$ & & $<E Q L$ & 50 & $\mathrm{ug} / \mathrm{L}$ & ML \\
\hline & Tetrachloroethylene & 8240 & $J$ & 9280 & & 9590 & & & 7050 & $\sqrt{ }$ & K & NDD & 50 & $\mathrm{ug} / \mathrm{L}$ & ML \\
\hline & $1,1,1$-Trichloroethane & $<500$ & UJ & $<500$ & $\mathrm{u}$ & .7 & $J$ & IK & $<50$ & $U$ & & $<E Q L$ & 50 & $\mathrm{ug} / \mathrm{L}$ & $\mathrm{ML}$ \\
\hline & Trichloroethylene & 10600 & $J$ & 10600 & & 11300 & & & 10300 & $J$ & $\mathrm{~K}$ & NDD & 50 & ug/L & $\mathrm{ML}$ \\
\hline \multicolumn{16}{|c|}{ II. Monitoring Constituents } \\
\hline ST & Parameter & $\underline{3096}$ & CLP EPA & $\underline{3097}$ & CLP EPA & $\underline{3098}$ & CLP & EPA & $\underline{3099}$ & $\underline{C L P}$ & EPA & Filt. & $\underline{D F}$ & Unit & $\underline{\text { Lab }}$ \\
\hline \multicolumn{16}{|c|}{ Inorganics } \\
\hline & Aluminum, total recoverable & 17.9 & $J$ & 45.9 & & $<200$ & $u$ & & $<200$ & $\mathrm{u}$ & & $<\mathrm{EQL}$ & 1 & $\mathrm{ug} / \mathrm{L}$ & EX \\
\hline & Nitrate-nitrite as nitrogen & 19400 & & 29700 & & 17700 & & & 18800 & & & $>2400$ & 5 & $\mathrm{ug} / \mathrm{L}$ & EX \\
\hline+ & Sodium, total recoverable & 12800 & & 12900 & & 16800 & & & 15000 & & & $>4600$ & 1 & $u g / L$ & EX \\
\hline & Sulfate & 91 & $\mathrm{~J}$ & $<5000$ & $U$ & 292 & J & & 239 & & & $<3000$ & 1 & ug/L & EX \\
\hline \multicolumn{16}{|c|}{ Radionuclides } \\
\hline & Gross alpha & 9.06 & & .37 & UI & 10.78 & & & 2.93 & & & $<15$ & 1 & $\mathrm{pCi} / \mathrm{L}$ & GP \\
\hline & Nonvolatile beta & 8.65 & & 2.02 & UI & 25.95 & & & 7.03 & & & $<50$ & 1 & $\mathrm{pCi} / \mathrm{L}$ & GP \\
\hline & Radium, total alpha-emitting & 3.9 & & 3.46 & UI & 2.1 & & & 2 & & & $<5$ & 1 & $\mathrm{pCi} / \mathrm{L}$ & GP \\
\hline
\end{tabular}

Notes: Concentrations in bold italics exceed the groundwater protection or monitoring constituent standards listed in Appendix A. Synchronous water levels are measured over a 3-5 day period or less. Dilution factors, Laboratory, and Filtered Data are for Groundwater Protection Standards First Quarter 2000 and Monitoring Constituents Third Quarter 1999 data only.

$+=$ exceeded the Groundwater Protection Standards (First Quarter 2000) or Monitoring Constituents (Third Quarter 1999) listed in Appendix A. 
Table D-2. Groundwater Monitoring Results for Point-of-Compliance Wells, M-Area HWMF (Cont.) WELL MSB 4D

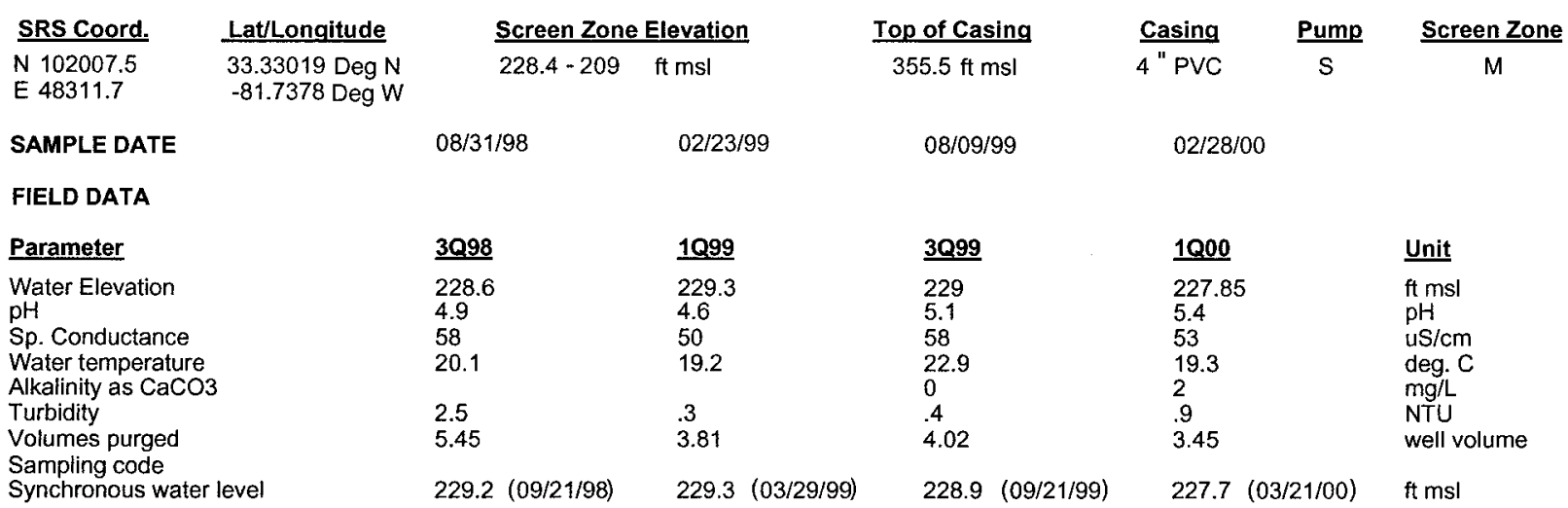

ANALYTICAL DATA

I. Groundwater Protection Standard

261 Appendix VIII/264 Appendix IX Hazardous Constituents

\begin{tabular}{|c|c|c|c|c|c|c|c|c|c|c|c|c|c|c|}
\hline \multirow{2}{*}{$\frac{\text { ST }}{\text { Inorg }}$} & \multirow{2}{*}{$\begin{array}{l}\text { Parameter } \\
\text { ganics }\end{array}$} & \multirow[t]{2}{*}{$\underline{3098}$} & \multirow[t]{2}{*}{ CLP EPA } & \multirow[t]{2}{*}{1099} & \multirow[t]{2}{*}{ CLP EPA } & \multirow[t]{2}{*}{$\underline{3 Q 99}$} & \multirow[t]{2}{*}{ CLPEPA } & \multirow[t]{2}{*}{$\underline{1000}$} & \multicolumn{2}{|c|}{ CLPEPA } & \multirow[t]{2}{*}{ Filt. } & \multirow[t]{2}{*}{$\underline{\text { DF }}$} & \multirow[t]{2}{*}{ Unit } & \multirow[t]{2}{*}{$\underline{\text { Lab }}$} \\
\hline & & & & & & & & & & & & & & \\
\hline & Barium, total recoverable & 10.7 & & 13.9 & & 17 & & 5.55 & $J$ & I & NDD & 1 & $\mathrm{ug} / \mathrm{L}$ & ML \\
\hline & Cyanide & $<10$ & $\mathrm{U}$ & $<10$ & $\mathrm{U}$ & $<10$ & $\mathrm{U}$ & 8.2 & $J$ & IL & NDD & 1 & $\mathrm{ug} / \mathrm{L}$ & ML \\
\hline & Lead, total recoverable & $<100$ & $\mathrm{U}$ & $<100$ & $u$ & $<10$ & U & $<20$ & $U$ & & $<E Q L$ & 1 & $\mathrm{ug} / \mathrm{L}$ & ML \\
\hline & Nickel, total recoverable & $<50$ & $\mathrm{U}$ & $<50$ & $\mathrm{U}$ & $<50$ & $\mathrm{U}$ & $<60$ & $\mathrm{U}$ & & $<E Q L$ & 1 & $u g / L$ & ML \\
\hline & Selenium, total recoverable & $<10$ & $U$ & $<200$ & $\mathrm{U}$ & $<10$ & $\mathrm{U}$ & $<40$ & $\mathrm{U}$ & & $<\mathrm{EQL}$ & 1 & $\mathrm{ug} / \mathrm{L}$ & ML \\
\hline \multicolumn{15}{|c|}{ Organics } \\
\hline & Chlorobenzene & $<25$ & $\mathrm{U}$ & $<25$ & $\mathrm{U}$ & $<1$ & 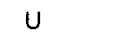 & $<1$ & $\mathrm{U}$ & & $<E Q L$ & 1 & ug/L & ML \\
\hline & 1,1-Dichloroethane & $<25$ & $\mathrm{U}$ & $<25$ & $\mathrm{U}$ & $<1$ & $\mathrm{U}$ & $<1$ & $\mathrm{U}$ & & $<E Q L$ & 1 & $\mathrm{ug} / \mathrm{L}$ & ML \\
\hline & 1,1-Dichloroethylene & $<25$ & $\mathrm{U}$ & $<25$ & $\mathrm{U}$ & $<1$ & $\mathrm{U}$ & $<1$ & $\mathrm{U}$ & & $<E Q L$ & 1 & $u g / L$ & ML \\
\hline & trans-1,2-Dichloroethylene & $<25$ & $\mathrm{U}$ & $<25$ & $U$ & $<1$ & $\mathrm{U}$ & & & & & & $u g / L$ & \\
\hline & PCB 1016 & $<2$ & $U$ & $<2$ & $\mathrm{U}$ & $<2$ & $\mathbf{U}$ & $<1$ & JU & & $<E Q L$ & 1 & ug/L & $\mathrm{ML}$ \\
\hline & PCB 1221 & $<2$ & $\mathrm{U}$ & $<2$ & $\mathrm{U}$ & $<2$ & $\mathrm{U}$ & $<1$ & JU & & $<\mathrm{EQL}$ & 1 & $\mathrm{ug} / \mathrm{L}$ & $M L$ \\
\hline & PCB 1232 & $<1$ & $\mathrm{U}$ & $<1$ & $\mathrm{U}$ & $<1$ & $\mathrm{U}$ & $<1$ & JU & & $<E Q L$ & 1 & $\mathrm{ug} / \mathrm{L}$ & $M L$ \\
\hline & PCB 1242 & $<1$ & $U$ & $<1$ & $\mathrm{U}$ & $<1$ & U & $<1$ & JU & & $<$ EQL & 1 & $\mathrm{ug} / \mathrm{L}$ & $M L$ \\
\hline & PCB 1248 & $<1$ & $U$ & $<1$ & $U$ & $<1$ & $\mathrm{U}$ & $<1$ & JU & & $<\mathrm{EQL}$ & 1 & $\mathrm{ug} / \mathrm{L}$ & $M L$ \\
\hline & РCB 1254 & $<1$ & $\mathrm{U}$ & $<1$ & $U$ & $<1$ & $U$ & $<1$ & JU & & $<E Q L$ & 1 & $\mathrm{ug} / \mathrm{L}$ & $M L$ \\
\hline & PCB 1260 & $<1$ & U & $<1$ & U & $<1$ & $\mathrm{U}$ & $<1$ & JU & & $<E Q L$ & 1 & $\mathrm{ug} / \mathrm{L}$ & $M L$ \\
\hline & 1,1,2,2-Tetrachloroethane & $<25$ & $U$ & $<25$ & $U$ & $<1$ & $\mathrm{U}$ & $<1$ & $\mathrm{U}$ & & $<\mathrm{EQL}$ & 1 & $\mathrm{ug} / \mathrm{L}$ & ML \\
\hline & Tetrachloroethylene & 79.3 & & 86.1 & & 68.7 & & 53.5 & $J$ & K & NDD & 1 & $\mathrm{ug} / \mathrm{L}$ & ML \\
\hline & 1,1,1-Trichloroethane & $<25$ & $\mathrm{U}$ & $<25$ & $\mathrm{U}$ & $<1$ & $U$ & $<1$ & $\mathrm{U}$ & & $<E Q L$ & 1 & $u g / \mathrm{L}$ & ML \\
\hline & Trichloroethylene & 80.5 & & 107 & & 104 & & 85.6 & $J$ & $\mathrm{~K}$ & NDD & 1 & $\mathrm{ug} / \mathrm{L}$ & ML \\
\hline \multicolumn{15}{|c|}{ II. Monitoring Constituents } \\
\hline$\underline{\text { ST }}$ & Parameter & $\underline{3096}$ & CLPEPA & $\underline{3097}$ & CLP EPA & $\underline{3098}$ & CLPEPA & $\underline{3099}$ & CLP & EPA & Filt. & $\underline{\text { DF }}$ & Unit & $\underline{\text { Lab }}$ \\
\hline \multicolumn{15}{|c|}{ Inorganics } \\
\hline & Aluminum, total recoverable & 159 & & 153 & & 111 & $J$ & 130 & $J$ & 1 & NDD & 1 & $\mathrm{ug} / \mathrm{L}$ & EX \\
\hline+ & Nitrate-nitrite as nitrogen & 4280 & & 2060 & & 2570 & & 4520 & & & $>2400$ & 5 & ug/L & EX \\
\hline & Sodium, total recoverable & 4480 & & 3830 & & 3730 & & 4400 & & & $<4600$ & 1 & $\mathrm{ug} / \mathrm{L}$ & EX \\
\hline & Sulfate & 1440 & & $<5000$ & $\mathrm{U}$ & 1480 & $J$ & 1540 & & & $<3000$ & 1 & $\mathrm{ug} / \mathrm{L}$ & EX \\
\hline \multicolumn{15}{|c|}{ Radionuclides } \\
\hline & Gross alpha & 8.91 & & 1.42 & & 10.82 & & 4.79 & & & $<15$ & 1 & $\mathrm{pCi} / \mathrm{L}$ & GP \\
\hline & Nonvolatile beta & 4.67 & & .04 & UI & 10.94 & $J$ & 4.46 & & & $<50$ & 1 & $\mathrm{pCi} / \mathrm{L}$ & GP \\
\hline & Radium, total alpha-emitting & 5.5 & & 4.74 & UI & 1.41 & & 4.4 & & & $<5$ & 1 & $\mathrm{pCi} / \mathrm{L}$ & GP \\
\hline
\end{tabular}

Notes: Concentrations in bold italics exceed the groundwater protection or monitoring constituent standards listed in Appendix A. Synchronous water levels are measured over a 3-5 day period or less. Dilution factors, Laboratory, and Filtered Data are for Groundwater Protection Standards First Quarter 2000 and Monitoring Constituents Third Quarter 1999 data only.

+= exceeded the Groundwater Protection Standards (First Quarter 2000) or Monitoring Constituents (Third Quarter 1999) listed in Appendix A. 
Table D-2. Groundwater Monitoring Results for Point-of-Compliance Wells, M-Area HWMF (Cont.) WELL MSB 5A

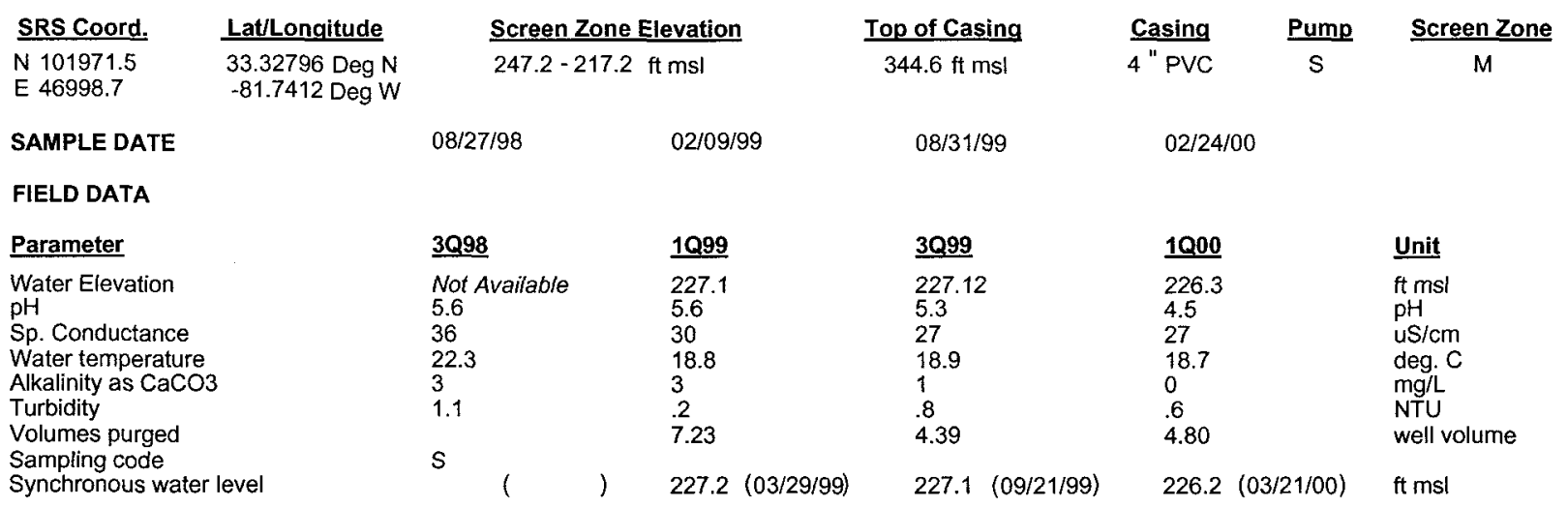

\section{ANALYTICAL DATA}

I. Groundwater Protection Standard

261 Appendix VIII/264 Appendix IX Hazardous Constituents

\begin{tabular}{|c|c|c|c|c|c|c|c|c|c|c|c|c|c|c|}
\hline$\underline{\mathrm{ST}}$ & Parameter & $\underline{3 Q 98}$ & $\underline{\text { CLP EPA }}$ & 1099 & CLP EPA & 3Q99 & CLPEPA & $\underline{1000}$ & $\underline{\text { CLP }}$ & EPA & Filt. & $\underline{D F}$ & Unit & $\underline{\text { Lab }}$ \\
\hline Inor & ganics & & & & & & & & & & & & & \\
\hline & Barium, total recoverable & 5.33 & $\mathrm{~J}$ & $<10$ & $u$ & $<10$ & $\mathrm{u}$ & $<15$ & $U$ & & $<E Q L$ & 1 & $u g / L$ & ML \\
\hline & Cyanide & $<10$ & $\mathrm{u}$ & $<10$ & $\cup$ & $<10$ & $\mathrm{U}$ & $<20$ & $U$ & & $<E Q L$ & 1 & $u g / L$ & ML \\
\hline & Lead, total recoverable & $<100$ & $U$ & $<100$ & $U$ & $<10$ & $U$ & $<9.84$ & JU & IV & $<E Q L$ & 1 & $u g / L$ & $M L$ \\
\hline & Nickel, total recoverable & 26.2 & $J$ & $<50$ & $\mathrm{U}$ & $<50$ & $\mathrm{U}$ & $<60$ & $U$ & & $<$ EQL & 1 & $\mathrm{ug} / \mathrm{L}$ & ML \\
\hline & Selenium, total recoverable & $<200$ & $U$ & $<200$ & $\mathrm{U}$ & $<10$ & $\mathrm{U}$ & $<40$ & $U$ & & $<E Q L$ & 1 & $\mathrm{ug} / \mathrm{L}$ & ML \\
\hline Org & anics & & & & & & & & & & & & & \\
\hline & Chlorobenzene & $<5$ & UJ & $<5$ & $\mathrm{U}$ & $<1$ & $\mathrm{U}$ & $<1$ & U & & $<\mathrm{EQL}$ & 1 & $u g / L$ & ML \\
\hline & 1,1-Dichloroethane & $<5$ & UJ & $<5$ & $\mathrm{U}$ & $<1$ & $\mathrm{U}$ & $<1$ & $U$ & & $<E Q L$ & 1 & $\mathrm{ug} / \mathrm{L}$ & ML \\
\hline & 1,1-Dichloroethylene & $<5$ & UJ & $<5$ & $\mathrm{U}$ & $<1$ & $U$ & $<1$ & $U$ & & $<E Q L$ & 1 & $\mathrm{ug} / \mathrm{L}$ & $M L$ \\
\hline & trans-1,2-Dichloroethylene & $<5$ & UJ & $<5$ & $\mathrm{U}$ & $<1$ & $U$ & $<1$ & $U$ & & $<\mathrm{EQL}$ & 1 & ug/L & ML \\
\hline & PCB 1016 & & & & & & & & & & & & & \\
\hline & PCB 1221 & & & & & & & & & & & & & \\
\hline & PCB 1232 & & & & & & & & & & & & & \\
\hline & $\begin{array}{l}\text { PCB } 1242 \\
\text { PCB } 1248\end{array}$ & & & & & & & & & & & & & \\
\hline - & PCB 1254 & & & & & & & & & & & & & \\
\hline & PCB 1260 & & & & & & & & & & & & & \\
\hline & 1,1,2,2-Tetrachloroethane & $<5$ & UJ & $<5$ & $U$ & $<1$ & $U$ & $<1$ & $\mathrm{U}$ & & $<E Q L$ & 1 & $\mathrm{ug} / \mathrm{L}$ & ML \\
\hline & Tetrachioroethylene & 11.1 & $J$ & $<5$ & $\mathrm{U}$ & .69 & IK & $<1$ & $\mathrm{U}$ & & $<\mathrm{EQL}$ & 1 & $\mathrm{ug} / \mathrm{L}$ & ML \\
\hline & 1,1,1-Trichloroethane & $<5$ & UJ & $<5$ & $U$ & $<1$ & $\mathrm{U}$ & $<1$ & $\mathrm{U}$ & & $<E Q L$ & 1 & $\mathrm{ug} / \mathrm{L}$ & ML. \\
\hline & Trichloroethylene & 21.7 & J & 3.12 & J IL & .73 & $\mathrm{~J} \quad \mathrm{IK}$ & $<1$ & $\mathrm{U}$ & & $<\mathrm{EQL}$ & 1 & $\mathrm{ug} / \mathrm{L}$ & ML \\
\hline $\mathbf{M}$ & onitoring Constituents & & & & & & & & & & & & & \\
\hline$\underline{\text { ST }}$ & Parameter & $\underline{3096}$ & CLPEPA & $\underline{3 Q 97}$ & CLP EPA & $\underline{3 Q 98}$ & CLPEPA & $\underline{3 Q 99}$ & $\underline{\text { CLP }}$ & EPA & Filt. & DF & $\underline{\text { Unit }}$ & Lab \\
\hline Inor & ganics & & & & & & & & & & & & & \\
\hline & Aluminum, total recoverable & $<20$ & U & 9 & $\mathrm{~J}$ & 99.9 & $J$ & $<200$ & $\mathrm{U}$ & & $<E Q L$ & 1 & ug/L & EX \\
\hline & Nitrate-nitrite as nitrogen & 2220 & & 2930 & & 1530 & & 1290 & & & $<2400$ & 5 & ug/L & EX \\
\hline & Sodium, total recoverable & 4420 & & 4670 & & 5550 & & 3700 & & & $<4600$ & 1 & ug/L & EX \\
\hline & Sulfate & 358 & $J$ & $<5000$ & $\mathrm{U}$ & 822 & & 701 & & & $<3000$ & 2 & ug/L & EX \\
\hline Rad & ionuclides & & & & & & & & & & & & & \\
\hline & Gross alpha & 2.81 & & 1.46 & & 1.47 & & 1.29 & $U$ & $\mathrm{v}$ & $<E Q L$ & 1 & $\mathrm{pCi} / \mathrm{L}$ & GP \\
\hline & Nonvolatile beta & 1.41 & & .003 & UI & 1.04 & UI & 1.08 & $U$ & & $<E Q L$ & 1 & $\mathrm{pCi} / \mathrm{L}$ & GP \\
\hline & Radium, total alpha-emitting & .8 & UI & 1.35 & UI & 1.26 & & .2 & U & & $<$ EQL & 1 & $\mathrm{pCi} / \mathrm{L}$ & GP \\
\hline
\end{tabular}

Notes: Concentrations in bold italics exceed the groundwater protection or monitoring constituent standards listed in Appendix A. Synchronous water levels are measured over a 3-5 day period or less. Dilution factors, Laboratory, and Filtered Data are for Groundwater Protection Standards First Quarter 2000 and Monitoring Constituents Third Quarter 1999 data only.

$+=$ exceeded the Groundwater Protection Standards (First Quarter 2000) or Monitoring Constituents (Third Quarter 1999) listed in Appendix A. 
Table D-2. Groundwater Monitoring Results for Point-of-Compliance Wells, M-Area HWMF (Cont.) WELL MSB 5B

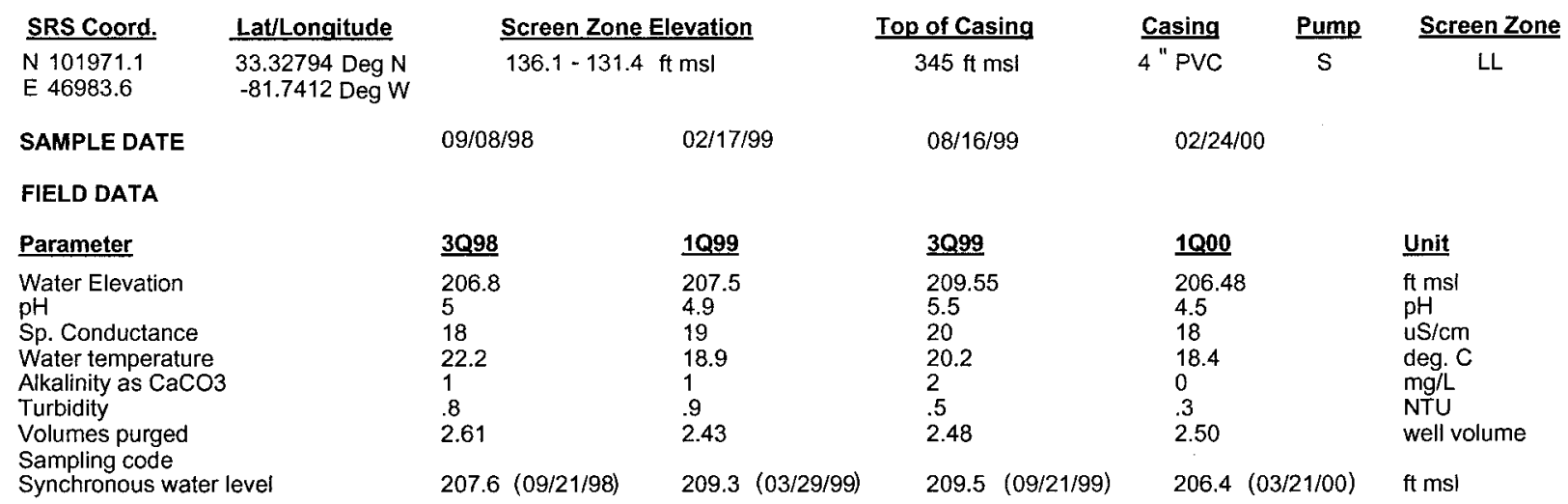

ANALYTICAL DATA

I. Groundwater Protection Standard

261 Appendix VIII/264 Appendix IX Hazardous Constituents

\begin{tabular}{|c|c|c|c|c|c|c|c|c|c|c|c|c|c|c|c|}
\hline$\underline{\mathrm{ST}}$ & Parameter & $\underline{3 Q 98}$ & CLP EPA & 1099 & CLP EPA & $\underline{3099}$ & $\underline{\text { CLP }}$ & EPA & 1000 & $\underline{\text { CLP }}$ & EPA & Filt. & DF & Unit & $\underline{\text { Lab }}$ \\
\hline Inor & ganics & & & & & & & & & & & & & & \\
\hline & Barium, total recoverable & 5.39 & $J$ & $<10$ & U & 6.4 & $J$ & I & 4.79 & $J$ & I & NDD & 1 & $\mathrm{ug} / \mathrm{L}$ & $\mathrm{ML}$ \\
\hline & Cyanide & $<10$ & $U$ & $<10$ & $\mathrm{U}$ & $<10$ & $U$ & & & & & & & $\mathrm{ug} / \mathrm{L}$ & \\
\hline & Lead, total recoverable & $<100$ & $U$ & $<100$ & $\mathrm{U}$ & $<1.96$ & U & V & $<5.36$ & JU & IV & $<\mathrm{EQL}$ & 1 & $\mathrm{ug} / \mathrm{L}$ & $\mathrm{ML}$ \\
\hline & Nickel, total recoverable & $<50$ & $\mathrm{U}$ & $<50$ & $\mathrm{U}$ & $<50$ & $U$ & & $<60$ & $U$ & & $<E Q L$ & 1 & $\mathrm{ug} / \mathrm{L}$ & ML \\
\hline & Selenium, total recoverable & $<10$ & $\mathrm{U}$ & $<200$ & $\mathrm{U}$ & $<5$ & $U$ & & $<40$ & $U$ & & $<\mathrm{EQL}$ & 1 & $\mathrm{ug} / \mathrm{L}$ & ML \\
\hline Org & anics & & & & & & & & & & & & & & \\
\hline & Chlorobenzene & $<5$ & UJ & $<5$ & U & $<1$ & $U$ & & $<1$ & $\cup$ & & $<E Q L$ & 1 & $\mathrm{ug} / \mathrm{L}$ & $M L$ \\
\hline & 1,1-Dichloroethane & $<5$ & UJ & $<5$ & $\mathrm{U}$ & $<1$ & $U$ & & $<1$ & $U$ & & $<E Q L$ & 1 & $\mathrm{ug} / \mathrm{L}$ & $M L$ \\
\hline & 1,1-Dichloroethylene & $<5$ & UJ & $<5$ & U & $<1$ & $U$ & & $<1$ & $U$ & & $<\mathrm{EQL}$ & 1 & $\mathrm{ug} / \mathrm{L}$ & ML \\
\hline & trans-1,2-Dichloroethylene & $<5$ & UJ & $<5$ & $\mathrm{U}$ & $<1$ & $U$ & & $<1$ & $U$ & & $<\mathrm{EQL}$ & 1 & $\mathrm{ug} / \mathrm{L}$ & ML \\
\hline & PCB 1016 & & & & & & & & & & & & & & \\
\hline & PCB 1221 & & & & & & & & & & & & & & \\
\hline & PCB 1232 & & & & & & & & & & & & & & \\
\hline & PCB 1242 & & & & & & & & & & & & & & \\
\hline & РСB 1248 & & & & & & & & & & & & & & \\
\hline & PCB 1254 & & & & & & & & & & & & & & \\
\hline & PCB 1260 & & & & & & & & & & & & & & \\
\hline & 1,1,2,2-Tetrachloroethane & $<5$ & UJ & $<5$ & $U$ & $<1$ & U & & $<1$ & U & & $<E Q L$ & 1 & ug/L & ML \\
\hline & Tetrachloroethylene & $<5$ & UJ & $<5$ & $U$ & $<1$ & $U$ & & $<1$ & U & & $<E Q L$ & 1 & $\mathrm{ug} / \mathrm{L}$ & ML \\
\hline & 1,1,1-Trichloroethane & $<5$ & UJ & $<5$ & $\mathrm{U}$ & $<1$ & U & & $<1$ & U & & $<\mathrm{EQL}$ & 1 & $\mathrm{ug} / \mathrm{L}$ & $\mathrm{ML}$ \\
\hline & Trichloroethylene & 7.25 & J & 29 & $J \quad L$ & 5 & $J$ & K & 10.9 & $J$ & $\mathrm{~K}$ & NDD & 1 & $\mathrm{ug} / \mathrm{L}$ & ML \\
\hline I. $\mathbf{M}$ & onitoring Constituents & & & & & & & & & & & & & & \\
\hline$\underline{\mathbf{S T}}$ & Parameter & $\underline{3 Q 96}$ & CLPEPA & $\underline{3097}$ & CLPP EPA & $\underline{3098}$ & CLP & EPA & $\underline{3099}$ & CLP & EPA & Filt. & $\underline{D F}$ & Unit & Lảab \\
\hline or & ganics & & & & & & & & & & & & & & \\
\hline & Aluminum, total recoverable & $<20$ & $U$ & $<20$ & $\mathrm{U}$ & $<200$ & $u$ & & $<200$ & $\mathrm{U}$ & & $<E Q L$ & 1 & $\mathrm{ug} / \mathrm{L}$ & EX \\
\hline & Nitrate-nitrite as nitrogen & 580 & & 342 & & 253 & $\mathrm{~J}$ & & 246 & J & $\mathrm{I}$ & NDD & 5 & $\mathrm{ug} / \mathrm{L}$ & EX \\
\hline & Sodium, total recoverable & 1470 & & 1550 & & 1600 & & & 1700 & & & $<4600$ & 1 & $\mathrm{ug} / \mathrm{L}$ & EX \\
\hline & Sulfate & 554 & $J$ & $<5000$ & $U$ & 568 & & & 701 & & & $<3000$ & 1 & $\mathrm{ug} / \mathrm{L}$ & EX \\
\hline Rad & onuclides & & & & & & & & & & & & & & \\
\hline & Gross alpha & .465 & UI & .11 & UI & 17.57 & $J$ & & .254 & JU & $\mathrm{L}$ & $<$ EQL & 1 & $\mathrm{pCi} / \mathrm{L}$ & GP \\
\hline & Nonvolatile beta & 1.1 & & -1.2 & UI & 41.7 & $\mathrm{~J}$ & & 1.37 & $U$ & & $<E Q L$ & 1 & $\mathrm{pCi} / \mathrm{L}$ & GP \\
\hline & Radium, total alpha-emitting & .3 & UI & .17 & UI & .18 & UI & & .1 & U & & $<$ EQL & 1 & $\mathrm{pCi} / \mathrm{L}$ & GP \\
\hline
\end{tabular}


Table D-2. Groundwater Monitoring Results for Point-of-Compliance Wells, M-Area HWMF (Cont.) WELL MSB 5C

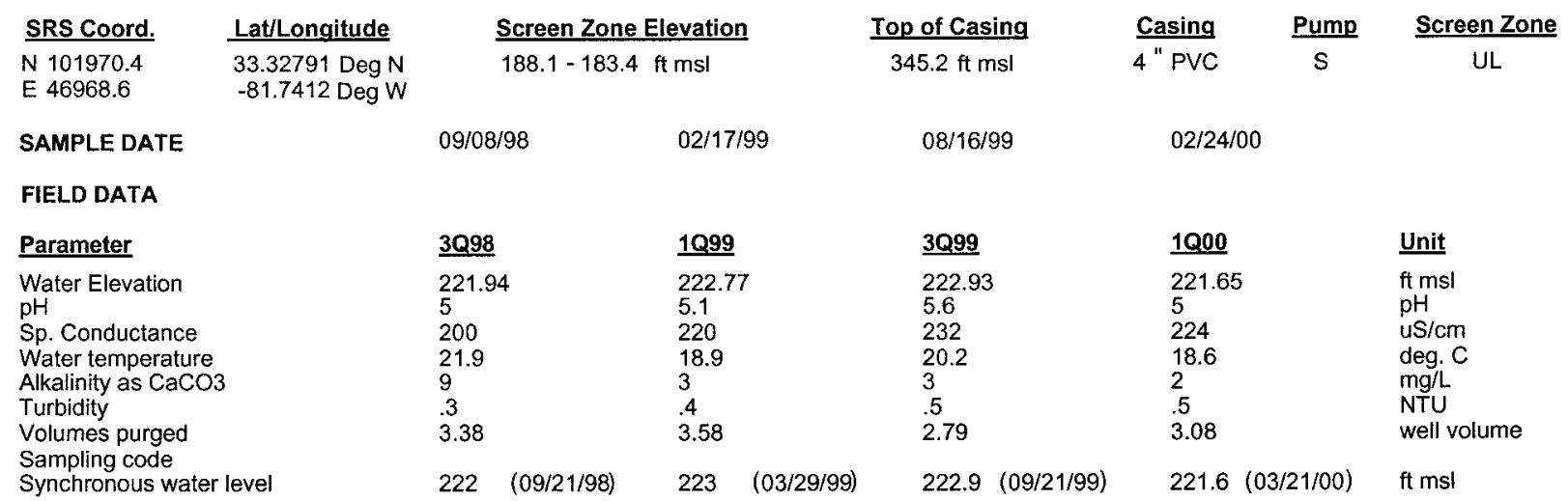

\section{ANALYTICAL DATA}

I. Groundwater Protection Standard

261 Appendix VIII/264 Appendix IX Hazardous Constituents

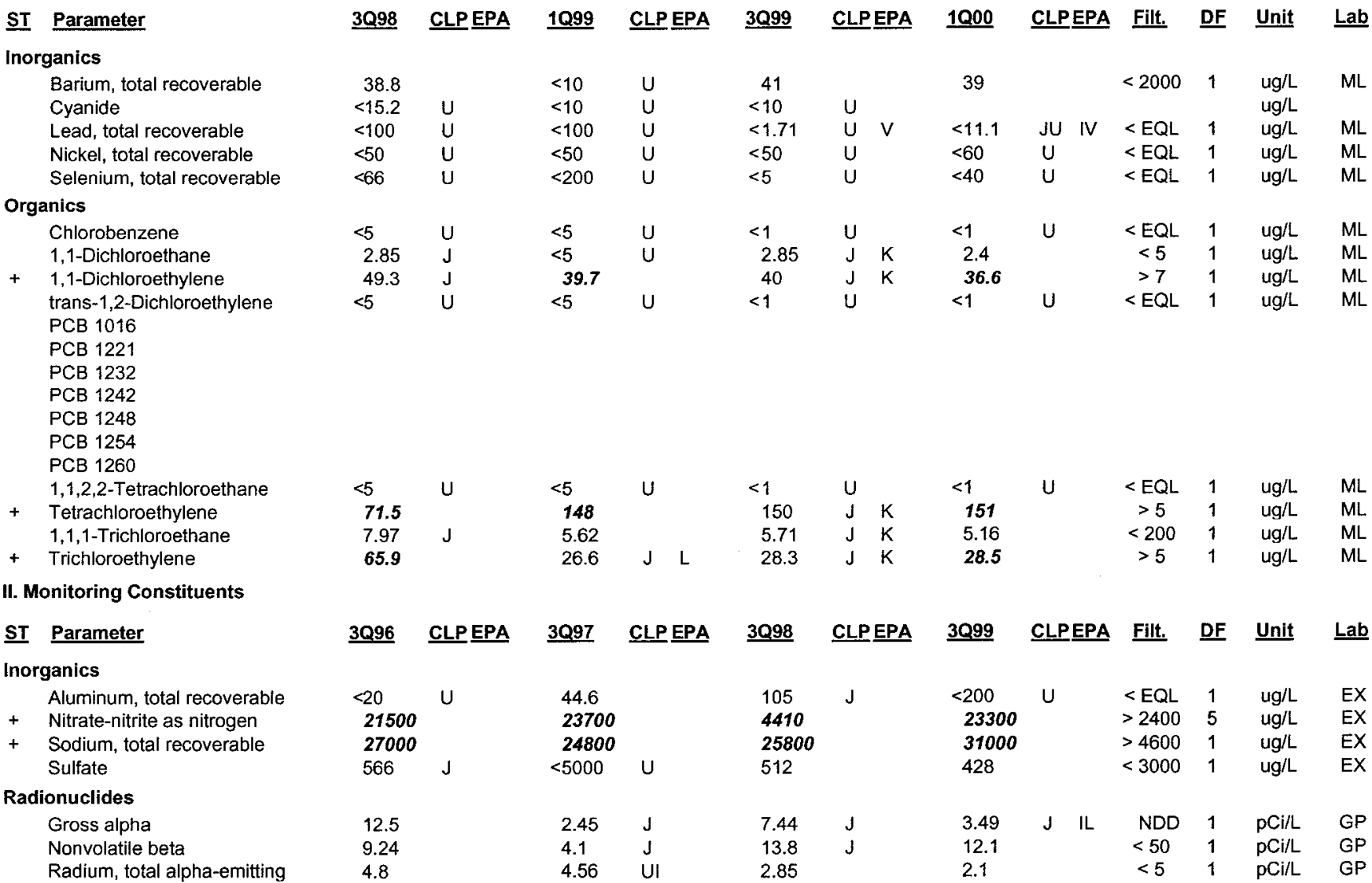

\footnotetext{
Notes: Concentrations in bold italics exceed the groundwater protection or monitoring constituent standards listed in Appendix A. Synchronous water levels are measured over a 3-5 day period or less. Dilution factors, Laboratory, and Filtered Data are for Groundwater Protection Standards First Quarter 2000 and Monitoring Constituents Third Quarter 1999 data only.
}

+ = exceeded the Groundwater Protection Standards (First Quarter 2000) or Monitoring Constituents (Third Quarter 1999) listed In Appendix A. 
Table D-2. Groundwater Monitoring Results for Point-of-Compliance Wells, M-Area HWMF (Cont.) WELL MSB 6 A

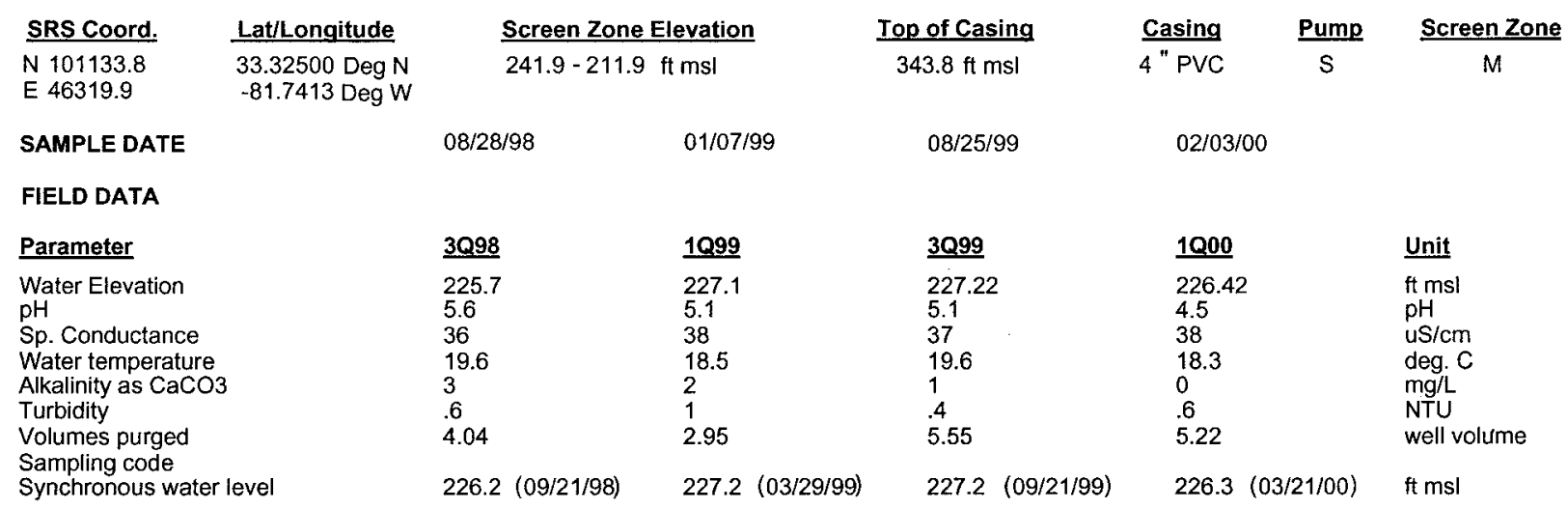

\section{ANALYTICAL DATA}

1. Groundwater Protection Standard

261 Appendix VIII/264 Appendix IX Hazardous Constituents

\begin{tabular}{|c|c|c|c|c|c|c|c|c|c|c|c|c|c|c|}
\hline \multirow{2}{*}{\multicolumn{2}{|c|}{$\begin{array}{l}\text { ST } \\
\text { Inorganics }\end{array}$}} & \multirow[t]{2}{*}{$\underline{3 Q 98}$} & \multirow[t]{2}{*}{ CLP EPA } & \multirow[t]{2}{*}{1099} & CLPEPA & \multirow[t]{2}{*}{$\underline{3 Q 99}$} & CLPEPA & \multirow[t]{2}{*}{1000} & \multicolumn{2}{|c|}{ CLPEPA } & \multirow[t]{2}{*}{ Filt. } & \multirow[t]{2}{*}{$\underline{\text { DF }}$} & \multirow[t]{2}{*}{ Unit } & \multirow[t]{2}{*}{$\underline{\text { Lab }}$} \\
\hline & & & & & & & & & & & & & & \\
\hline & Barium, total recoverable & 4.87 & $J$ & 6.46 & J I & 6.1 & $\mathrm{~J} \quad 1$ & 6.24 & $J$ & 1 & NDD & 1 & $\mathrm{ug} / \mathrm{L}$ & ML \\
\hline & Cyanide & $<10$ & $U$ & $<10$ & $U$ & $<10$ & $U$ & $<15.1$ & $\mathrm{U}$ & V & $<\mathrm{EQL}$ & 1 & $\mathrm{ug} / \mathrm{L}$ & ML \\
\hline & Lead, total recoverable & $<100$ & $U$ & $<100$ & $U$ & $<10$ & $\mathrm{U}$ & $<20$ & $\mathrm{U}$ & & $<E Q L$ & 1 & $\mathrm{ug} / \mathrm{L}$ & ML \\
\hline & Nickel, total recoverable & $<50$ & $U$ & $<50$ & $U$ & $<50$ & $U$ & $<60$ & $\mathrm{U}$ & & $<\mathrm{EQL}$ & 1 & $\mathrm{ug} / \mathrm{L}$ & $M L$ \\
\hline & Selenium, total recoverable & $<3.26$ & $U$ & $<10$ & $\mathrm{U}$ & $<10$ & $\mathrm{U}$ & $<40$ & $\mathrm{U}$ & & $<E Q L$ & 1 & $\mathrm{ug} / \mathrm{L}$ & ML \\
\hline \multicolumn{15}{|c|}{ Organics } \\
\hline & Chlorobenzene & $<5$ & UJ & $<5$ & $\mathrm{U}$ & $<1$ & $U$ & $<1$ & $\mathrm{U}$ & & $<$ EQL & 1 & ug/L & ML \\
\hline & 1,1-Dichloroethane & $<5$ & UJ & $<5$ & $U$ & $<1$ & $\mathrm{U}$ & $<1$ & $\mathrm{U}$ & & $<\mathrm{EQL}$ & 1 & $u g / L$ & $M L$ \\
\hline & 1,1-Dichloroethylene & $<5$ & UJ & $<5$ & $U$ & $<1$ & $\mathrm{U}$ & $<1$ & $\mathrm{U}$ & & $<\mathrm{EQL}$ & 1 & ug/L & ML \\
\hline & trans-1,2-Dichloroethylene & $<5$ & UJ & $<5$ & $\mathrm{U}$ & $<1$ & $U$ & $<1$ & $\mathrm{U}$ & & $<\mathrm{EQL}$ & 1 & $\mathrm{ug} / \mathrm{h}$ & ML \\
\hline & PCB 1016 & & & & & & & & & & & & & \\
\hline & РСB 1221 & & & & & & & & & & & & & \\
\hline & РCB 1232 & & & & & & & & & & & & & \\
\hline & PCB 1242 & & & & & & & & & & & & & \\
\hline & PCB 1248 & & & & & & & & & & & & & \\
\hline & PCB 1254 & & & & & & & & & & & & & \\
\hline & PCB 1260 & & & & & & & & & & & & & \\
\hline & $1,1,2,2$-Tetrachloroethane & $<5$ & UJ & $<5$ & $U$ & $<1$ & $U$ & $<1$ & $\mathrm{U}$ & & $<\mathrm{EQL}$ & 1 & $\mathrm{ug} / \mathrm{L}$ & ML \\
\hline & Tetrachloroethylene & $<5$ & UJ & $<5$ & $\mathrm{U}$ & $<1$ & $U$ & $<1$ & U & & $<\mathrm{EQL}$ & 1 & $u g / L$ & ML \\
\hline & $1,1,1$-Trichloroethane & $<5$ & UJ & $<5$ & $U$ & $<1$ & $\mathrm{U}$ & $<1$ & $U$ & & $<\mathrm{EQL}$ & 1 & $u g / L$ & $\mathrm{ML}$ \\
\hline & Trichloroethylene & $<5$ & UJ & $<5$ & U & $<1$ & $U$ & $<1$ & $\mathrm{U}$ & & $<E Q L$ & 1 & $\mathrm{ug} / \mathrm{L}$ & $\mathrm{ML}$ \\
\hline \multicolumn{15}{|c|}{ II. Monitoring Constituents } \\
\hline$\underline{\text { ST }}$ & Parameter & $\underline{3 Q 96}$ & CLPEPA & $\underline{3097}$ & CLP EPA & $\underline{3098}$ & CLPEPA & $\underline{3099}$ & CLP & EPA & Filt. & $\underline{\text { DE }}$ & Unit & $\underline{\text { Lab }}$ \\
\hline \multicolumn{15}{|c|}{ Inorganics } \\
\hline & Aluminum, total recoverable & $<94.5$ & U & $<20$ & $\mathrm{U}$ & $<200$ & $U$ & $<200$ & $U$ & & $<E Q L$ & 1 & $\mathrm{ug} / \mathrm{L}$ & EX \\
\hline & Nitrate-nitrite as nitrogen & 730 & & 336 & & 390 & $J$ & 384 & $\mathrm{~J}$ & 1 & NDD & 5 & $\mathrm{ug} / \mathrm{L}$ & EX \\
\hline+ & Sodium, total recoverable & 5140 & & 5750 & & 5250 & & 6300 & & & $>4600$ & 1 & $u g / L$ & EX \\
\hline & Sulfate & 299 & 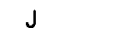 & 324 & J & 711 & & 350 & $\mathrm{~J}$ & 1 & NDD & 2 & $\mathrm{ug} / \mathrm{L}$ & EX \\
\hline \multicolumn{15}{|c|}{ Radionuclides } \\
\hline & Gross alpha & .637 & UI & -.58 & U & 3.13 & & 1.46 & $\mathrm{~J}$ & 1 & NDD & 1 & $\mathrm{pCi} / \mathrm{L}$ & GP \\
\hline & Nonvolatile beta & .907 & UI & -.28 & UIJ & 1.99 & & 1.02 & $J$ & I & NDD & 1 & $\mathrm{pCi} / \mathrm{L}$ & GP \\
\hline & Radium, total alpha-emitting & 1.7 & & 3.29 & $J$ & 1.1 & & .8 & $\mathrm{~J}$ & I & NDD & 1 & $\mathrm{pCi} / \mathrm{L}$ & GP \\
\hline
\end{tabular}


Table D-2. Groundwater Monitoring Results for Point-of-Compliance Wells, M-Area HWMF (Cont.) WELL MSB 6B

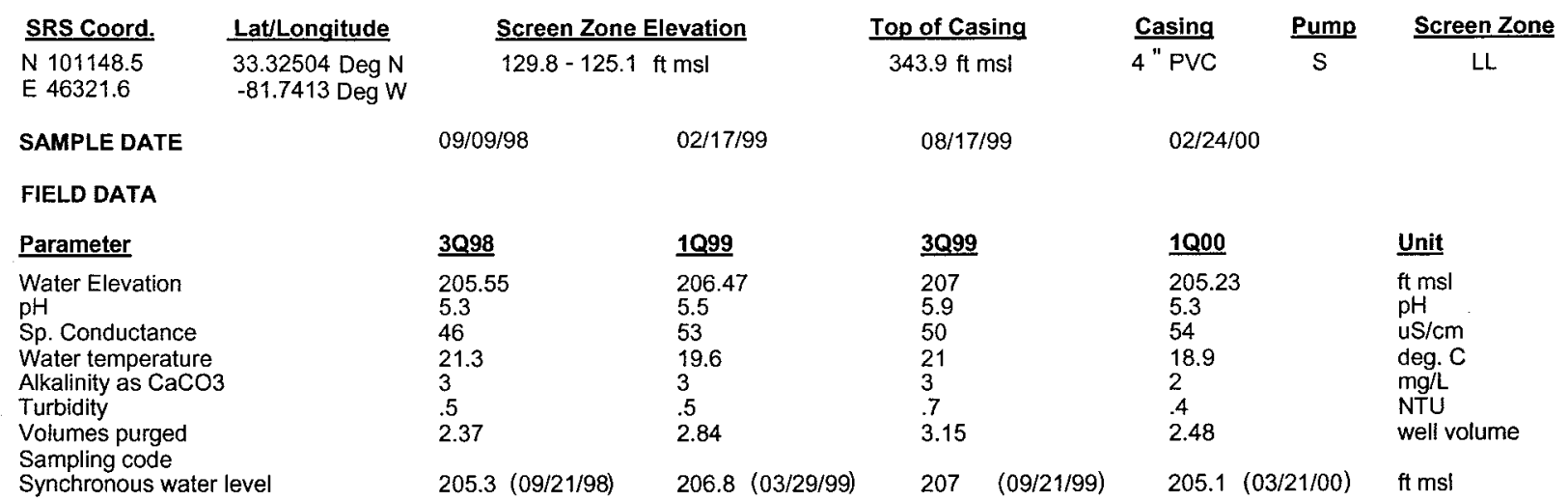

\section{ANALYTICAL DATA}

I. Groundwater Protection Standard

261 Appendix VIII/264 Appendix IX Hazardous Constituents

\begin{tabular}{|c|c|c|c|c|c|c|c|c|c|c|c|c|c|}
\hline \multirow{2}{*}{$\begin{array}{l}\text { ST Parameter } \\
\text { Inorganics }\end{array}$} & \multirow[t]{2}{*}{3098} & \multirow[t]{2}{*}{ CLPEPA } & \multirow[t]{2}{*}{$\underline{1099}$} & \multirow[t]{2}{*}{ CLPEPA } & \multirow[t]{2}{*}{$\underline{3099}$} & CLPEPA & \multirow[t]{2}{*}{1000} & \multicolumn{2}{|c|}{ CLPEPA } & \multirow[t]{2}{*}{ Fill. } & \multirow[t]{2}{*}{$\underline{\text { DF }}$} & \multirow[t]{2}{*}{$\underline{\text { Unit }}$} & \multirow[t]{2}{*}{$\underline{\text { Lab }}$} \\
\hline & & & & & & & & & & & & & \\
\hline Barium, total recoverable & 19.1 & & $<10$ & $\mathrm{u}$ & 18 & & 18.5 & & & $<2000$ & 1 & $\mathrm{ug} / \mathrm{L}$ & $\mathrm{ML}$ \\
\hline Cyanide & $<10$ & $\mathrm{u}$ & $<10$ & u & $<10$ & $\mathrm{u}$ & & & & & & $\mathrm{ug} / \mathrm{L}$ & \\
\hline Lead, total recoverable & $<100$ & u & $<100$ & $\mathrm{u}$ & $<10$ & $\mathrm{u}$ & $<5.41$ & ju & IV & $<E Q L$ & 1 & $\mathrm{ug} / \mathrm{L}$ & ML \\
\hline Nickel, total recoverable & $<50$ & u & $<50$ & $\mathrm{U}$ & $<50$ & $\mathrm{u}$ & $<60$ & $u$ & & $<E Q L$ & 1 & $\mathrm{ug} / \mathrm{L}$ & ML \\
\hline Selenium, total recoverable & $<200$ & $\mathrm{u}$ & $<200$ & $\mathrm{U}$ & $<10$ & $u$ & $<40$ & $\mathrm{u}$ & & $<E Q L$ & 1 & ug/L & ML \\
\hline \multicolumn{14}{|l|}{ Organics } \\
\hline Chiorobenzene & $<250$ & UJ & $<125$ & $\mathrm{u}$ & $<1$ & $\mathrm{u}$ & $<10$ & $\mathrm{u}$ & & $<E Q L$ & 10 & $u g / L$ & ML \\
\hline 1,1-Dichloroethane & $<250$ & UJ & $<125$ & U & $<1$ & $\mathrm{u}$ & $<10$ & U & & $<E Q L$ & 10 & $\mathrm{ug} / \mathrm{L}$ & ML \\
\hline 1,1-Dichloroethylene & $<250$ & UJ & $<125$ & u & $<1$ & u & $<10$ & $\mathrm{u}$ & & $<\mathrm{EQL}$ & 10 & $\mathrm{ug} / \mathrm{L}$ & ML \\
\hline trans-1,2-Dichloroethylene & $<250$ & UJ & $<125$ & $u$ & .94 & $\mathrm{~J} \quad \mathrm{IK}$ & $<10$ & $\mathrm{U}$ & & $<E Q L$ & 10 & $\mathrm{ug} / \mathrm{L}$ & ML \\
\hline PCB 1016 & & & & & & & & & & & & & \\
\hline PCB 1221 & & & & & & & & & & & & & \\
\hline PCB 1232 & & & & & & & & & & & & & \\
\hline PCB 1242 & & & & & & & & & & & & & \\
\hline PCB 1248 & & & & & & & & & & & & & \\
\hline PCB 1254 & & & & & & & & & & & & & \\
\hline РСВ 1260 & & & & & & & & & & & & & \\
\hline 1,1,2,2-Tetrachloroethane & $<250$ & UJ & $<125$ & $\mathrm{u}$ & $<1$ & $\mathrm{u}$ & $<10$ & $u$ & & $<E Q L$ & 10 & $\mathrm{ug} / \mathrm{L}$ & ML \\
\hline+ Tetrachloroethylene & 77.5 & $\mathrm{~J}$ & 94.3 & J I & 73.5 & $\mathrm{~J} K$ & 91.1 & & & $>5$ & 10 & $\mathrm{ug} / \mathrm{L}$ & ML \\
\hline 1,1,1-Trichloroethane & $<250$ & UJ & $<125$ & $\mathrm{U}$ & $<1$ & $\mathrm{u}$ & $<10$ & $\mathrm{u}$ & & $<E Q L$ & 10 & $\mathrm{ug} / \mathrm{L}$ & ML \\
\hline+ Trichloroethylene & 3010 & $\mathrm{~J}$ & 2930 & J L & 2260 & & 2880 & & & $>5$ & 10 & $\mathrm{ug} / \mathrm{L}$ & ML \\
\hline \multicolumn{14}{|l|}{ II. Monitoring Constituents } \\
\hline ST Parameter & $\underline{3096}$ & CLPEPA & $\underline{3 Q 97}$ & CLP EPA & $\underline{3 Q 98}$ & CLPEPA & $\underline{3099}$ & $\underline{\text { CLP }}$ & EPA & Filt. & $\underline{D F}$ & $\underline{\text { Unit }}$ & Lab \\
\hline \multicolumn{14}{|l|}{ Inorganics } \\
\hline Aluminum, total recoverable & 157 & & $<20$ & $u$ & $<200$ & $u$ & $<200$ & $U$ & & $<E Q L$ & 1 & $u g / L$ & EX \\
\hline$+\quad$ Nitrate-nitrite as nitrogen & 3250 & & 4190 & & 2990 & & 3730 & & & $>2400$ & 5 & ug/L & \\
\hline Sodium, total recoverable & 3040 & & 3020 & & 2980 & & 3400 & & & $<4600$ & 1 & $\mathrm{ug} / \mathrm{L}$ & EX \\
\hline Sulfate & 161 & $J$ & $<5000$ & U & 436 & & 219 & J & 1 & NDD & 2 & $\mathrm{ug} / \mathrm{L}$ & EX \\
\hline \multicolumn{14}{|l|}{ Radionuclides } \\
\hline Gross alpha & 5.28 & & .36 & UI & 2.51 & & 1.74 & J & 1 & NDD & 1 & $\mathrm{pCi} / \mathrm{L}$ & GP \\
\hline Nonvolatile beta & 4.89 & & .78 & UI & 4.13 & & 3.95 & & & $<50$ & 1 & $\mathrm{pCi} / \mathrm{L}$ & GP \\
\hline Radium, total alpha-emitting & 1.1 & UI & 2.65 & UI & .93 & & .9 & $\mathrm{~J}$ & 1 & NDD & 1 & $\mathrm{pCi} / \mathrm{L}$ & $P$ \\
\hline
\end{tabular}

Notes: Concentrations in bold italics exceed the groundwater protection or monitoring constituent standards listed in Appendix A. Synchronous water levels are measured over a 3-5 day period or less. Dilution factors, Laboratory, and Filtered Data are for Groundwater Protection Standards First Quarter 2000 and Monitoring Constituents Third Quarter 1999 data only.

$+=$ exceeded the Groundwater Protection Standards (First Quarter 2000) or Monitoring Constituents (Third Quarter 1999) listed in Appendix A. 
Table D-2. Groundwater Monitoring Results for Point-of-Compliance Wells, M-Area HWMF (Cont.) WELL MSB 6C

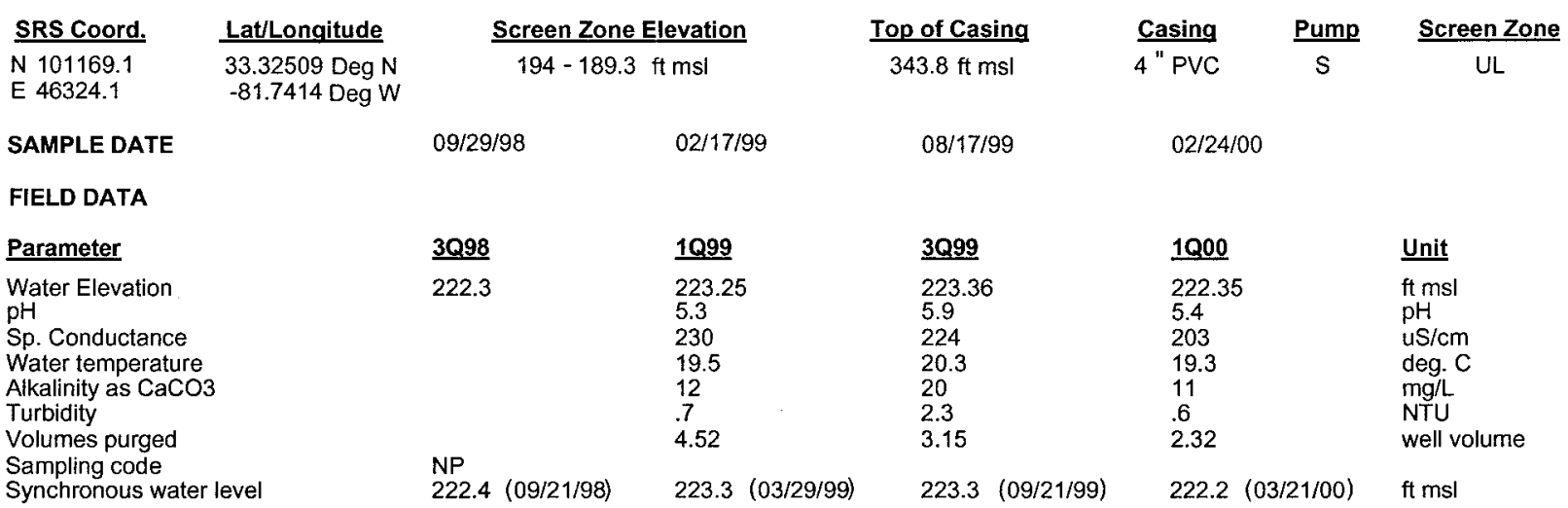

ANALYTICAL DATA

I. Groundwater Protection Standard

261 Appendix VIII/264 Appendix IX Hazardous Constituents

\begin{tabular}{|c|c|c|c|c|c|c|c|c|c|c|c|c|c|c|c|}
\hline \multirow{2}{*}{$\underline{\text { ST }}$} & Parameter & \multirow[t]{2}{*}{$\underline{3 Q 98}$} & \multirow[t]{2}{*}{$\underline{\text { CLP EPA }}$} & \multirow[t]{2}{*}{$\underline{1 \mathrm{Q} 99}$} & \multirow[t]{2}{*}{$\underline{\text { CLP EPA }}$} & \multirow[t]{2}{*}{$\underline{3 Q 99}$} & \multicolumn{2}{|c|}{ CLPEPA } & \multirow[t]{2}{*}{$\underline{1 Q 00}$} & \multicolumn{2}{|c|}{$\underline{\text { CLPEPA }}$} & \multirow[t]{2}{*}{ Filt. } & \multirow[t]{2}{*}{$\underline{\text { DF }}$} & \multirow[t]{2}{*}{ Unit } & \multirow[t]{2}{*}{ Lab } \\
\hline & ganics & & & & & & & & & & & & & & \\
\hline & Barium, total recoverable & & & $<10$ & $u$ & 4.7 & $J$ & 1 & 4.59 & J & 1 & NDD & 1 & $\mathrm{ug} / \mathrm{L}$ & ML \\
\hline & Cyanide & & & $<10$ & $\mathrm{U}$ & $<10$ & $\mathrm{U}$ & & & & & & & $\mathrm{ug} / \mathrm{L}$ & \\
\hline & Lead, total recoverable & & & $<100$ & $\mathrm{U}$ & $<10$ & $U$ & & $<6.13$ & JU & IV & $<$ EQL & 1 & $\mathrm{ug} / \mathrm{L}$ & ML \\
\hline & Nickel, total recoverable & & & $<50$ & $\mathrm{U}$ & $<50$ & U & & $<60$ & $U$ & & $<E Q L$ & 1 & ug/L & ML \\
\hline & Selenium, total recoverable & & & $<200$ & $\mathrm{U}$ & $<4.2$ & JU & 1 & $<40$ & $\mathrm{U}$ & & $<E Q L$ & 1 & $\mathrm{ug} / \mathrm{L}$ & ML \\
\hline \multicolumn{16}{|c|}{ Organics } \\
\hline & Chlorobenzene & & & $<5$ & $u$ & $<1$ & $\mathrm{U}$ & & $<1$ & $U$ & & $<E Q L$ & 1 & $\mathrm{ug} / \mathrm{L}$ & ML \\
\hline & 1,1-Dichloroethane & & & $<5$ & $\mathrm{U}$ & $<1$ & $\mathrm{U}$ & & $<1$ & $U$ & & $<E Q L$ & 1 & $u g / L$ & ML \\
\hline & 1,1-Dichloroethylene & & & $<5$ & $U$ & .62 & $J$ & IK & $<1$ & $\mathrm{U}$ & & $<E Q L$ & 1 & $u g / L$ & ML \\
\hline & trans-1,2-Dichloroethylene & & & $<5$ & U & $<1$ & U & & $<1$ & $\mathrm{U}$ & & $<E Q L$ & 1 & $\mathrm{ug} / \mathrm{L}$ & $M L$ \\
\hline & PCB 1016 & & & & & & & & & & & & & & \\
\hline & PCB 1221 & & & & & & & & & & & & & & \\
\hline & $\begin{array}{l}\text { PCB } 1232 \\
\text { PCB } 1242\end{array}$ & & & & & & & & & & & & & & \\
\hline & PCB 1248 & & & & & & & & & & & & & & \\
\hline & PCB 1254 & & & & & & & & & & & & & & \\
\hline & PCB 1260 & & & & & & & & & & & & & & \\
\hline & 1,1,2,2-Tetrachloroethane & & & $<5$ & $U$ & $<1$ & $u$ & & $<1$ & $\mathrm{U}$ & & $<E Q L$ & 1 & $u g / L$ & ML \\
\hline+ & Tetrachloroethylene & & & 58.3 & & 57.8 & 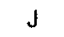 & $\mathrm{K}$ & 61.5 & & & $>5$ & 1 & ug/L & ML \\
\hline & 1,1,1-Trichloroethane & & & $<5$ & $\mathrm{u}$ & & $\mathrm{U}$ & & $<1$ & $\mathrm{U}$ & & $<$ EQL & 1 & $\mathrm{ug} / \mathrm{L}$ & ML \\
\hline+ & Trichioroethylene & & & 11.9 & J L & 10.9 & $J$ & $\mathrm{~K}$ & 11.3 & & & $>5$ & 1 & $u g / L$ & ML \\
\hline \multicolumn{16}{|c|}{ II. Monitoring Constituents } \\
\hline$\underline{\text { ST }}$ & Parameter & $\underline{3096}$ & CLPEEPA & $\underline{3097}$ & CLP EPA & $\underline{3098}$ & $\underline{\text { CLP }}$ & EPA & $\underline{3 Q 99}$ & CLP & EPA & Filt. & $\underline{D F}$ & $\underline{\text { Unit }}$ & $\underline{\text { Lab }}$ \\
\hline \multicolumn{16}{|c|}{ Inorganics } \\
\hline & Aluminum, total recoverable & $<20$ & U & & & & & & $<200$ & $\mathrm{U}$ & & $<E Q L$ & 1 & $\mathrm{ug} / \mathrm{L}$ & EX \\
\hline+ & Nitrate-nitrite as nitrogen & 40500 & & & & & & & 14900 & & & $>2400$ & 5 & $\mathrm{ug} / \mathrm{L}$ & EX \\
\hline+ & Sodium, total recoverable & 78200 & & & & & & & 40000 & & & $>4600$ & 1 & $\mathrm{ug} / \mathrm{L}$ & EX \\
\hline+ & Sulfate & 5960 & & & & & & & 16300 & & & $>3000$ & 1 & $\mathrm{ug} / \mathrm{L}$ & EX \\
\hline \multicolumn{16}{|c|}{ Radionuclides } \\
\hline & Gross alpha & 5.07 & & & & & & & .668 & $\mathrm{U}$ & & $<E Q L$ & 1 & $\mathrm{pCi} / \mathrm{L}$ & GP \\
\hline & Nonvolatile beta & 13.6 & & & & & & & 6.66 & & & $<50$ & 1 & $\mathrm{pCi} / \mathrm{L}$ & GP \\
\hline & Radium, total alpha-emitting & 1.1 & Ul & & & & & & .5 & J & 1 & NDD & 1 & $\mathrm{pCi} / \mathrm{L}$ & GP \\
\hline
\end{tabular}

Notes: Concentrations in bold italics exceed the groundwater protection or monitoring constituent standards listed in Appendix A. Synchronous water levels are measured over a 3-5 day period or less. Dilution factors, Laboratory, and Filtered Data are for Groundwater Protection Standards First Quarter 2000 and Monitoring Constituents Third Quarter 1999 data only.

$+=$ exceeded the Groundwater Protection Standards (First Quarter 2000) or Monitoring Constituents (Third Quarter 1999) listed in Appendix A. 
Table D-2. Groundwater Monitoring Results for Point-of-Compliance Wells, M-Area HWMF (Cont.) WELL MSB 7A

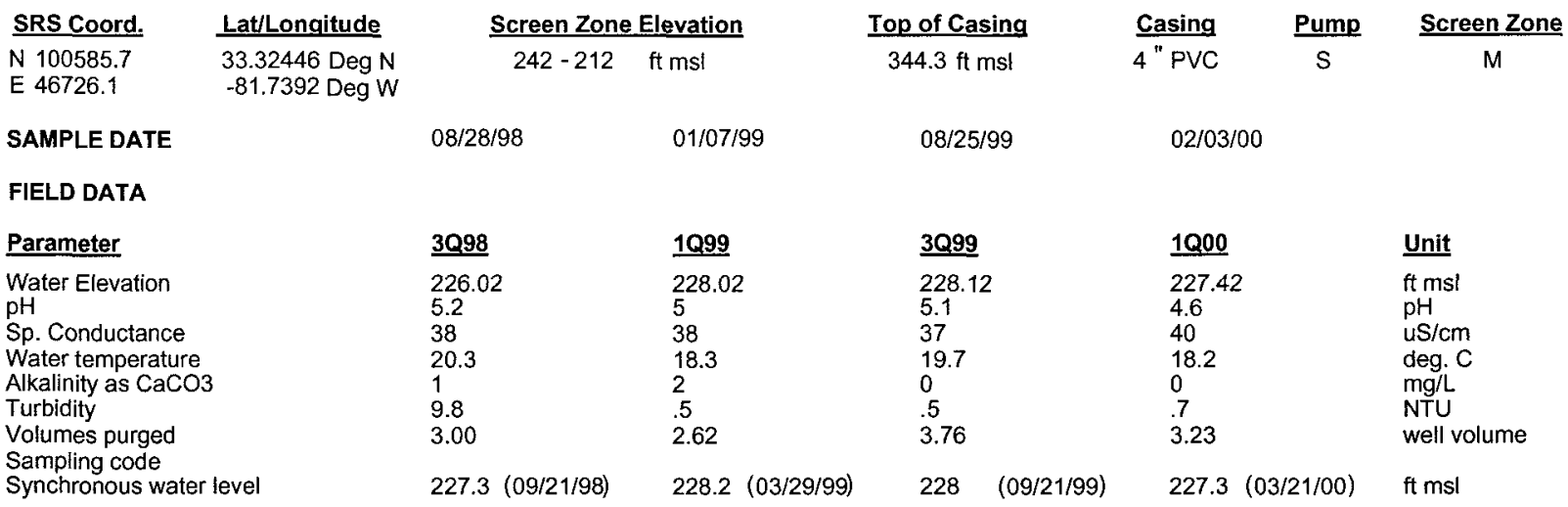

ANALYTICAL DATA

I. Groundwater Protection Standard

261 Appendix VIII/264 Appendix IX Hazardous Constituents

ST Parameter

Inorganics

Barium, total recoverable

Cyanide

Lead, total recoverable

Nickel, total recoverable

Selenium, total recoverable Organics

Chlorobenzene

1,1-Dichloroethane

1,1-Dichloroethylene

trans-1,2-Dichloroethylene

PCB 1016

PCB 1221

PCB 1232

PCB 1242

PCB 1248

PCB 1254

PCB 1260

1,1,2,2-Tetrachloroethane

Tetrachloroethylene

1,1,1-Trichloroethane

Trichloroethylene

II. Monitoring Constituents

ST Parameter

Inorganics

Aluminum, total recoverable Nitrate-nitrite as nitrogen

+ Sodium, total recoverable Sulfate

Radionuclides

Gross aipha

Nonvolatile beta

Radium, total alpha-emitting
3Q98 CLPEPA

1099

CLPEPA

$\underline{3 Q 99}$

CLPEPA

1000

CLPEPA

Filt. DF Unit

$\underline{\text { Lab }}$

$\begin{array}{ll}8.41 & \mathrm{~J} \\ <10 & \mathrm{U} \\ <100 & U \\ <50 & U\end{array}$

8.89
$<10$
$<100$
52.3
$<10$

$$
\begin{array}{ll}
\mathrm{U} & \mathrm{U} \\
\mathrm{U} & \mathrm{I}
\end{array}
$$

U

10
$<10$

$\begin{aligned} & 8.54 \\ < & 14.7 \\ < & 20 \\ < & 60 \\ < & <0\end{aligned}$

$\begin{array}{ll}J & I \\ U & V \\ U & \\ U & \\ U & \end{array}$

NDD 1

$u g / L \quad M L$

$<$ EQL $1 \quad$ ug/L $\quad M L$

$<$ EQL $1 \mathrm{ug} / \mathrm{L} \quad \mathrm{ML}$

< EQL 1 ug/L ML

$<5 \quad U$

$\begin{array}{ll}<5 & U \\ <5 & U \\ <5 & U\end{array}$

$\begin{array}{ll}<5 & \mathrm{U} \\ <5 & \mathrm{U} \\ <5 & \mathrm{U} \\ <5 & \mathrm{U}\end{array}$

$\begin{array}{ll}<1 & U \\ <1 & U \\ <1 & U \\ <1 & U\end{array}$

$<1$
$<1$
$<1$
$<1$

$<$ EQL

ug/L

$<$ EQL 1

$u g / L \quad M L$

$\begin{array}{lllll}\mathrm{U} & <\mathrm{EQL} & 1 & \mathrm{ug} / \mathrm{L} & \mathrm{ML} \\ \mathrm{U} & <\mathrm{EQL} & 1 & \mathrm{ug} / \mathrm{L} & \mathrm{ML} \\ \mathrm{U} & <\mathrm{EQL} & 1 & \mathrm{ug} / \mathrm{L} & \mathrm{ML} \\ \mathrm{U} & <\mathrm{EQL} & 1 & \mathrm{ug} / \mathrm{L} & \mathrm{ML}\end{array}$

$<5$
$<5$
$<5$
$<5$

$\mathrm{U}$
$\mathrm{U}$
$\mathrm{U}$

$\begin{array}{ll}<1 & U \\ <1 & U \\ <1 & U \\ <1 & U\end{array}$

$<1$

$<1$

$<1$

$<1$

3096 CLPEPA

3097

CLP EPA

3098

CLPEPA

3099

\begin{tabular}{|c|c|c|c|c|}
\hline$<38$ & $\mathrm{U}$ & $<20$ & U & $<200$ \\
\hline 1920 & & 1730 & & 1610 \\
\hline 5080 & & 5320 & & 6010 \\
\hline$<1000$ & $\mathrm{U}$ & $<1000$ & $\mathrm{U}$ & 405 \\
\hline 3.61 & & 1.15 & U & 4.78 \\
\hline 1.35 & U: & -.22 & UIJ & 3.13 \\
\hline 2.1 & & 3.09 & UIJ & 1.12 \\
\hline
\end{tabular}

$\begin{array}{lrrrrrr}<200 & \mathrm{U} & & <\mathrm{EQL} & 1 & \mathrm{ug} / \mathrm{L} & \mathrm{EX} \\ 1550 & & & <2400 & 5 & \mathrm{ug} / \mathrm{L} & \mathrm{EX} \\ 5900 & & & \begin{array}{c}>4600 \\ 1\end{array} & \mathrm{ug} / \mathrm{L} & \mathrm{EX} \\ 237 & \mathrm{~J} & \mathrm{I} & \mathrm{NDD} & 2 & \mathrm{ug} / \mathrm{L} & \mathrm{EX} \\ & & & & & & \\ 1.83 & \mathrm{~J} & \mathrm{I} & \mathrm{NDD} & 1 & \mathrm{pCi} / \mathrm{L} & \mathrm{GP} \\ .489 & \mathrm{U} & & <\mathrm{EQL} & 1 & \mathrm{pCi} / \mathrm{L} & \mathrm{GP} \\ 1.7 & & & <5 & 1 & \mathrm{pCi} / \mathrm{L} & \mathrm{GP}\end{array}$

Notes: Concentrations in bold italics exceed the groundwater protection or monitoring constituent standards listed in Appendix A. Synchronous water levels are measured over a 3-5 day period or less. Dilution factors, Laboratory, and Filtered Data are for Groundwater Protection Standards First Quarter 2000 and Monitoring Constituents Third Quarter 1999 data only.

$+=$ exceeded the Groundwater Protection Standards (First Quarter 2000) or Monitoring Constituents (Third Quarter 1999) listed in Appendix A. 
Table D-2. Groundwater Monitoring Results for Point-of-Compliance Wells, M-Area HWMF (Cont.) WELL MSB 7B

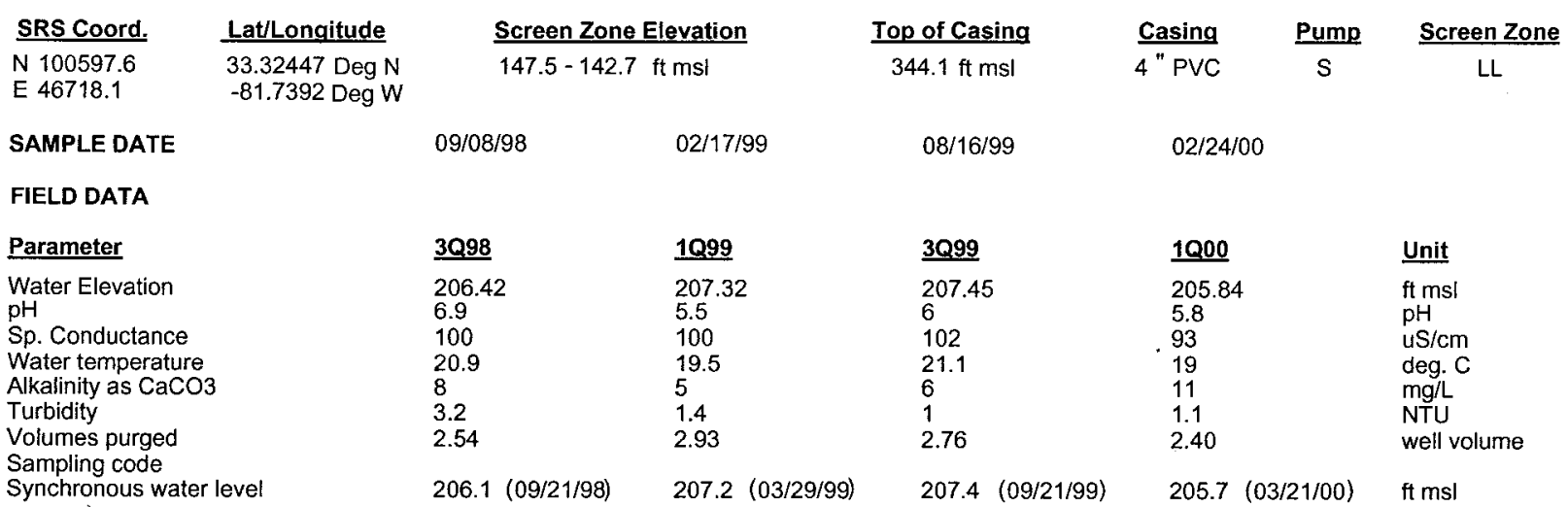

\section{ANALYTICAL DATA}

I. Groundwater Protection Standard

261 Appendix VIII/264 Appendix IX Hazardous Constituents

\begin{tabular}{|c|c|c|c|c|c|c|c|c|c|c|c|c|c|c|c|}
\hline \multirow{2}{*}{$\frac{\mathrm{ST}}{\text { Inorg }}$} & Parameter & \multirow[t]{2}{*}{$\underline{3 Q 98}$} & \multirow[t]{2}{*}{ CLP EPA } & \multirow[t]{2}{*}{1099} & \multirow[t]{2}{*}{ CLP EPA } & \multirow[t]{2}{*}{$\underline{3099}$} & \multicolumn{2}{|c|}{ CLPEPA } & \multirow[t]{2}{*}{$\underline{1000}$} & \multicolumn{2}{|c|}{ CLPEPA } & \multirow[t]{2}{*}{ Filt. } & \multirow[t]{2}{*}{ DF } & \multirow[t]{2}{*}{$\underline{\text { Unit }}$} & \multirow[t]{2}{*}{$\underline{\text { Lab }}$} \\
\hline & ganics & & & & & & & & & & & & & & \\
\hline & Barium, total recoverable & 50.4 & & $<10$ & $U$ & 53 & & & 104 & & & $<2000$ & 1 & ug/L & $M L$ \\
\hline & Cyanide & $<10$ & $U$ & $<10$ & $\mathrm{U}$ & $<10$ & $u$ & & & & & & & ug/L & \\
\hline & Lead, total recoverable & $<100$ & $\mathrm{U}$ & $<100$ & $\mathrm{U}$ & $<1.6$ & U & V & $<5.39$ & JU & IV & $<\mathrm{EQL}$ & 1 & $\mathrm{ug} / \mathrm{L}$ & $M L$ \\
\hline & Nickel, total recoverable & $<50$ & $\mathrm{U}$ & $<50$ & $\mathrm{U}$ & $<50$ & $\mathrm{U}$ & & $<60$ & $\mathrm{U}$ & & $<E Q L$ & 1 & $\mathrm{ug} / \mathrm{L}$ & ML \\
\hline & Selenium, total recoverable & $<10$ & $\mathrm{U}$ & $<200$ & $\mathrm{U}$ & $<5$ & $\mathrm{U}$ & & $<40$ & U & & $<\mathrm{EQL}$ & 1 & $u g / L$ & ML \\
\hline \multicolumn{16}{|c|}{ Organics } \\
\hline & Chlorobenzene & $<5$ & US & $<5$ & U & $<1$ & $u$ & & $<1$ & $U$ & & $<E Q L$ & 1 & $u g / L$ & ML \\
\hline & 1,1-Dichloroethane & $<5$ & UJ & $<5$ & $U$ & $<1$ & $u$ & & $<1$ & $U$ & & $<E Q L$ & 1 & $\mathrm{ug} / \mathrm{L}$ & ML \\
\hline & 1,1-Dichloroethylene & 6.28 & $\jmath$ & 6.77 & & 6.63 & $\mathrm{~J}$ & $\mathrm{~K}$ & 6.48 & & & $<7$ & 1 & $\mathrm{ug} / \mathrm{L}$ & ML \\
\hline & $\begin{array}{l}\text { trans-1,2-Dichloroethylene } \\
\text { PCB } 1016\end{array}$ & $<5$ & UJ & $<5$ & $\mathrm{u}$ & $<1$ & $U$ & & $<1$ & $\mathrm{U}$ & & $<\mathrm{EQL}$ & 1 & $\mathrm{ug} / \mathrm{L}$ & ML \\
\hline & $\begin{array}{l}\text { PCB } 1016 \\
\text { PCB } 1221\end{array}$ & & & & & & & & & & & & & & \\
\hline & PCB 1232 & & & & & & & & & & & & & & \\
\hline & PCB 1242 & & & & & & & & & & & & & & \\
\hline & PCB 1248 & & & & & & & & & & & & & & \\
\hline & PCB 1254 & & & & & & & & & & & & & & \\
\hline & PCB 1260 & & & & & & & & & & & & & & \\
\hline & $1,1,2,2$-Tetrachloroethane & $<5$ & UJ & $<5$ & $\mathrm{U}$ & $<1$ & $\mathrm{U}$ & & $<1$ & U & & $<$ EQL & 1 & $\mathrm{ug} / \mathrm{L}$ & $M L$ \\
\hline+ & Tetrachloroethylene & 86.6 & $\mathrm{~J}$ & 77.8 & & 68.9 & $\mathrm{~J}$ & $\mathrm{~K}$ & 66.1 & & & $>5$ & 1 & $\mathrm{ug} / \mathrm{L}$ & ML \\
\hline & 1,1,1-Trichioroethane & $<5$ & UJ & $<5$ & $\mathrm{U}$ & $<1$ & $\mathrm{U}$ & & 1.02 & & & $<200$ & 1 & $\mathrm{ug} / \mathrm{L}$ & ML \\
\hline+ & Trichloroethylene & 66.3 & $J$ & 56.1 & $J \quad L$ & 56.2 & J & K & 50.4 & & & $>5$ & 1 & $\mathrm{ug} / \mathrm{L}$ & ML \\
\hline \multicolumn{16}{|c|}{ II. Monitoring Constituents } \\
\hline$\underline{\text { ST }}$ & Parameter & 3Q996 & CLPEEPA & $\underline{3097}$ & CLPEPA & $\underline{3098}$ & CLP & EPA & $\underline{3099}$ & $\underline{\text { CLP }}$ & EPA & Filt. & $\underline{\text { DF }}$ & Unit & Lab \\
\hline \multicolumn{16}{|c|}{ Inorganics } \\
\hline & Aluminum, total recoverable & 28.7 & & 41.5 & & $<200$ & $\mathrm{U}$ & & $<200$ & U & & $<\mathrm{EQL}$ & 1 & $\mathrm{ug} / \mathrm{L}$ & EX \\
\hline+ & Nitrate-nitrite as nitrogen & 7900 & & 8720 & & 5460 & & & 7740 & & & $>2400$ & 5 & $\mathrm{ug} / \mathrm{L}$ & EX \\
\hline+ & Sodium, total recoverable & 6890 & & 8080 & & 7750 & & & 9200 & & & $>4600$ & 1 & $\mathrm{ug} / \mathrm{L}$ & EX \\
\hline+ & Sulfate & 2520 & & $<5000$ & $\mathrm{U}$ & 3290 & & & 3140 & & & $>3000$ & 1 & $\mathrm{ug} / \mathrm{L}$ & EX \\
\hline \multicolumn{16}{|c|}{ Radionuclides } \\
\hline & Gross alpha & 2.33 & & 1.09 & UI & 1.71 & $\mathrm{~J}$ & & .89 & JU & $L$ & $<\mathrm{EQL}$ & 1 & $\mathrm{pCi} / \mathrm{L}$ & GP \\
\hline & Nonvolatile beta & 2.76 & & 1.22 & UI & 1.88 & $J$ & & 2.46 & $J$ & 1 & NDD & 1 & $\mathrm{pCi} / \mathrm{L}$ & GP \\
\hline & Radium, total alpha-emitting & 1.4 & Ui & 2.13 & $J$ & 1.63 & & & 6 & $J$ & 1 & NDD & 1 & $\mathrm{pCi} / \mathrm{L}$ & GP \\
\hline
\end{tabular}

Notes: Concentrations in bold italics exceed the groundwater protection or monitoring constituent standards listed in Appendix A. Synchronous water levels are measured over a 3-5 day period or less. Dilution factors, Laboratory, and Filtered Data are for Groundwater Protection Standards First Quarter 2000 and Monitoring Constituents Third Quarter 1999 data only.

+ = exceeded the Groundwater Protection Standards (First Quarter 2000) or Monitoring Constituents (Third Quarter 1999) listed in Appendix A. 
Table D-2. Groundwater Monitoring Results for Point-of-Compliance Wells, M-Area HWMF (Cont.) WELL MSB 7C

\begin{tabular}{|c|c|c|c|c|c|c|c|}
\hline SRS Coord. & Lat/Longitude & \multicolumn{2}{|c|}{ Screen Zone Elevation } & Top of Casing & Casing & Pump & Screen Zone \\
\hline $\begin{array}{l}\text { N } 100609.2 \\
\text { E 46709.1 }\end{array}$ & $\begin{array}{l}\text { 33.32448 Deg } N \\
\text {-81.7393 Deg } W\end{array}$ & \multicolumn{2}{|c|}{$200.1-195.4 \mathrm{ft} \mathrm{msl}$} & $344.5 \mathrm{ft} \mathrm{msl}$ & 4 " PVC & $s$ & $\mathrm{UL}$ \\
\hline SAMPLE DATE & & $09 / 08 / 98$ & $02 / 17 / 99$ & $08 / 16 / 99$ & $02 / 18 / 0$ & & \\
\hline \multicolumn{8}{|l|}{ FIELD DATA } \\
\hline Parameter & & $\underline{3098}$ & 1099 & $\underline{3 Q 99}$ & \multicolumn{2}{|l|}{1000} & Unit \\
\hline $\begin{array}{l}\text { Water Elevation } \\
\text { pH } \\
\text { Sp. Conductance } \\
\text { Water temperature } \\
\text { Alkalinity as } \mathrm{CaCO} 3 \\
\text { Turbidity } \\
\text { Volumes purged }\end{array}$ & & $\begin{array}{l}221.87 \\
6.7 \\
240 \\
24.5 \\
26 \\
2.6\end{array}$ & $\begin{array}{l}222.8 \\
4.9 \\
200 \\
19.5 \\
1 \\
.9 \\
2.75\end{array}$ & $\begin{array}{l}222.45 \\
5.4 \\
200 \\
20.8 \\
1 \\
.7 \\
2.95\end{array}$ & \multicolumn{2}{|l|}{$\begin{array}{l}221.4 \\
5.3 \\
179 \\
19.7 \\
2 \\
1.9 \\
2.90\end{array}$} & $\begin{array}{l}\mathrm{ftmsl} \\
\mathrm{pH} \\
\text { uS/cm } \\
\text { deg. C } \\
\mathrm{mg} / \mathrm{L} \\
\text { NTU } \\
\text { well volume }\end{array}$ \\
\hline $\begin{array}{l}\text { Sampling code } \\
\text { Synchronous water I }\end{array}$ & & $\begin{array}{l}\text { NX } \\
221.8 \quad(09 / 21 / 98)\end{array}$ & $222.7(03 / 29 / 99)$ & $222.4(09 / 21 / 99)$ & \multicolumn{2}{|c|}{$221.3(03 / 21 / 00)$} & $\mathrm{ft} \mathrm{msl}$ \\
\hline
\end{tabular}

ANALYTICAL DATA

I. Groundwater Protection Standard

261 Appendix VIII/264 Appendix IX Hazardous Constituents

\begin{tabular}{|c|c|c|c|c|c|c|c|c|c|c|c|c|}
\hline ST Parameter & $\underline{3098}$ & CLP EPA & 1099 & CLP EPA & $\underline{3 Q 99}$ & CLPEPA & 1000 & CLPEEPA & Filt. & $\underline{\mathrm{DF}}$ & Unit & $\underline{\text { Lab }}$ \\
\hline \multicolumn{13}{|l|}{ Inorganics } \\
\hline Barium, total recoverable & 84 & & $<10$ & $\mathrm{u}$ & 30 & & 28.1 & & $<2000$ & 1 & $u g / L$ & ML \\
\hline Cyanide & $<10$ & $u$ & $<10$ & $\mathrm{u}$ & $<10$ & $u$ & & & & & $\mathrm{ug} / \mathrm{L}$ & \\
\hline Lead, total recoverable & $<100$ & $u$ & $<100$ & $\mathrm{u}$ & $<2.14$ & $u v$ & $<4.44$ & $\mathrm{JU}$ & $<E Q L$ & 1 & $\mathrm{ug} / \mathrm{L}$ & $\mathrm{ML}$ \\
\hline Nickel, total recoverable & $<50$ & $u$ & $<50$ & $\mathrm{u}$ & $<7.4$ & $\mathrm{JU} I$ & $<60$ & $u$ & $<E Q L$ & 1 & $\operatorname{ug} / \mathrm{L}$ & ML \\
\hline Selenium, total recoverable & $<10$ & $u$ & $<200$ & $u$ & $<5$ & $\mathrm{U}$ & $<40$ & $\mathrm{u}$ & $<E Q L$ & 1 & ug/L & ML \\
\hline \multicolumn{13}{|l|}{ Organics } \\
\hline Chlorobenzene & $<5$ & UJ & $<5$ & $\mathrm{u}$ & $<1$ & $\mathrm{u}$ & $<1$ & $\mathrm{u}$ & $<E Q L$ & 1 & $u g / L$ & ML \\
\hline 1,1-Dichloroethane & $<5$ & UJ & $<5$ & $\mathrm{u}$ & $<1$ & $\mathrm{u}$ & $<1$ & $\mathrm{u}$ & $<E Q L$ & 1 & $\mathrm{ug} / \mathrm{L}$ & ML \\
\hline 1,1-Dichioroethylene & $<5$ & UJ & $<5$ & $u$ & 1.68 & $\mathrm{~J} \mathrm{~K}$ & 1.88 & & $<7$ & 1 & $u g / L$ & ML \\
\hline trans-1,2-Dichloroethylene & $<5$ & UJ & $<5$ & $u$ & $<1$ & $u$ & $<1$ & $u$ & $<E Q L$ & 1 & $\mathrm{ug} / \mathrm{L}$ & ML \\
\hline $\begin{array}{l}\text { РCB } 1016 \\
\text { PCB } 1221\end{array}$ & & & & & & & & & & & & \\
\hline $\begin{array}{l}\text { PCB } 1221 \\
\text { PCB } 1232\end{array}$ & & & & & & & & & & & & \\
\hline PCB 1242 & & & & & & & & & & & & \\
\hline PCB 1248 & & & & & & & & & & & & \\
\hline PCB 1254 & & & & & & & & & & & & \\
\hline PCB 1260 & & & & & & & & & & & & \\
\hline 1,1,2,2-Tetrachloroethane & $<5$ & UJ & $<5$ & $u$ & $<1$ & $u$ & $<1$ & $u$ & $<E Q L$ & 1 & $\mathrm{ug} / \mathrm{L}$ & ML \\
\hline + Tetrachloroethylene & 58.6 & $\mathrm{~J}$ & 56.2 & & 56.9 & $\mathrm{~J} K$ & 62.1 & & $>5$ & 1 & $\mathrm{ug} / \mathrm{L}$ & ML \\
\hline 1,1,1-Trichloroethane & $<5$ & UJ & $<5$ & $u$ & $<1$ & $u$ & $<1$ & $\mathrm{u}$ & $<E Q L$ & 1 & $\mathrm{ug} / \mathrm{L}$ & $\mathrm{ML}$ \\
\hline+ Trichloroethylene & 19 & $\mathrm{~J}$ & 18.4 & J L & 20 & $\mathrm{~J} \mathrm{~K}$ & 20.9 & & $>5$ & 1 & $\mathrm{ug} / \mathrm{L}$ & $M L$ \\
\hline \multicolumn{13}{|l|}{ II. Monitoring Constituents } \\
\hline$\underline{\text { ST Parameter }}$ & $\underline{3096}$ & CLPEPA & $\underline{3097}$ & CLP EPA & $\underline{3098}$ & CLPEPA & $\underline{3099}$ & CLPEPA & Filt. & $\underline{\mathrm{DF}}$ & Unit & Lab \\
\hline \multicolumn{13}{|l|}{ Inorganics } \\
\hline Aluminum, total recoverable & 82.8 & & 125 & & 167 & $\mathrm{~J}$ & $<200$ & $u$ & $<E Q L$ & 1 & $\mathrm{ug} / \mathrm{L}$ & EX \\
\hline$+\quad$ Nitrate-nitrite as nitrogen & 30000 & & 26700 & & 13600 & & 20200 & & $>2400$ & 5 & $\mathrm{ug} / \mathrm{L}$ & EX \\
\hline+ Sodium, total recoverable & 41500 & & 31000 & & 27500 & & 29000 & & $>4600$ & 1 & $\mathrm{ug} / \mathrm{L}$ & EX \\
\hline Sulfate & 111 & $J$ & $<5000$ & $U$ & 364 & $\mathrm{~J}$ & $<200$ & $u$ & $<E Q L$ & 1 & ug/L & EX \\
\hline \multicolumn{13}{|l|}{ Radionuclides } \\
\hline Gross alpha & 14.8 & & 7.79 & UI & 7.8 & $\mathrm{~J}$ & 3.07 & $J \quad L$ & NDD & 1 & $\mathrm{pCi} / \mathrm{L}$ & GP \\
\hline Nonvolatile beta & 45.4 & & 67.4 & & 53.21 & $\mathrm{~J}$ & 30.6 & & $<50$ & 1 & $\mathrm{pCi} / \mathrm{L}$ & GP \\
\hline Radium, total alpha-emitting & 5.1 & & 7.3 & UIJ & 3.42 & & 1.8 & & $<5$ & 1 & $\mathrm{pCi} / \mathrm{L}$ & GP \\
\hline
\end{tabular}

Notes: Concentrations in bold italics exceed the groundwater protection or monitoring constituent standards listed in Appendix A. Synchronous water levels are measured over a 3-5 day period or less. Dilution factors, Laboratory, and Filtered Data are for Groundwater Protection Standards First Quarter 2000 and Monitoring Constituents Third Quarter 1999 data only.

+ = exceeded the Groundwater Protection Standards (First Quarter 2000) or Monitoring Constituents (Third Quarter 1999) listed in Appendix A. 
Table D-2. Groundwater Monitoring Results for Point-of-Compliance Wells, M-Area HWMF (Cont.) WELL MSB 8A

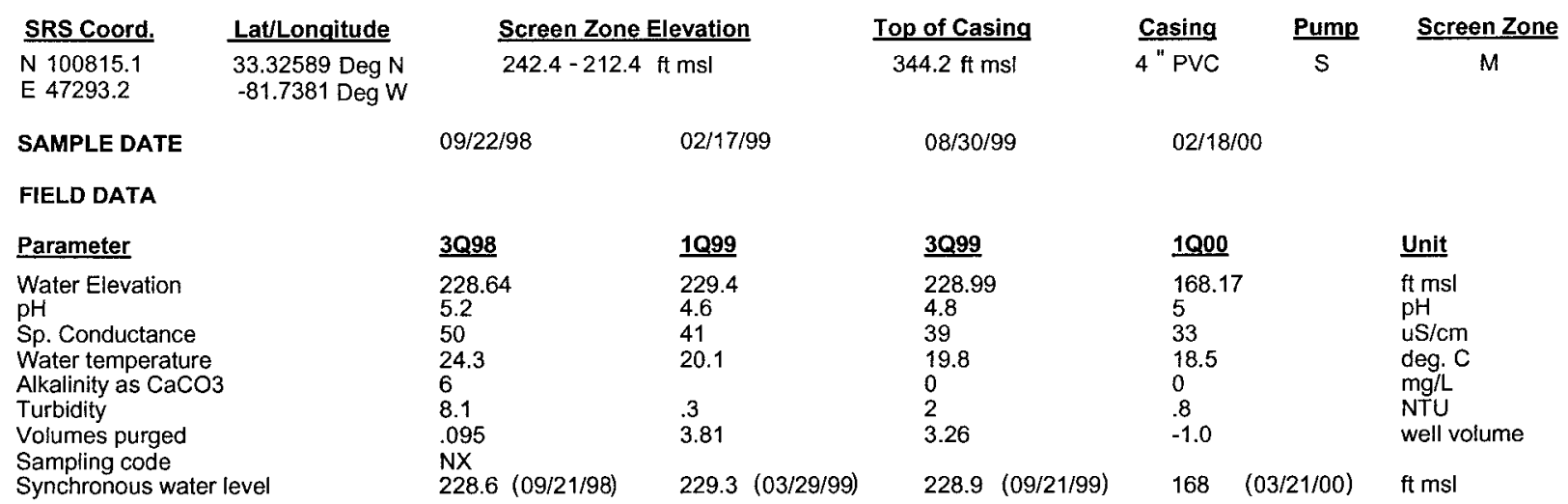

\section{ANALYTICAL DATA}

I. Groundwater Protection Standard

261 Appendix VIII/264 Appendix IX Hazardous Constituents

\begin{tabular}{|c|c|c|c|c|c|c|c|c|c|c|c|c|c|c|}
\hline$\underline{\text { ST }}$ & Parameter & $\underline{3 Q 98}$ & CLP EPA & $\underline{1 Q 99}$ & CLP EPA & $\underline{3 Q 99}$ & CLPEPA & $\underline{1000}$ & $\underline{\text { CLP }}$ & EPA & Filt. & $\underline{\mathrm{DF}}$ & $\underline{\text { Unit }}$ & $\underline{\text { Lab }}$ \\
\hline \multicolumn{15}{|c|}{ Inorganics } \\
\hline & Barium, total recoverable & 18.1 & & $<10$ & $\mathrm{U}$ & 6.5 & J 1 & 6.18 & $J$ & 1 & NDD & 1 & $u g / L$ & ML \\
\hline & Cyanide & $<10$ & $\mathrm{U}$ & $<10$ & $U$ & $<10$ & $\mathrm{U}$ & $<20$ & $\mathrm{U}$ & & $<$ EQL & 1 & $u g / L$ & ML \\
\hline & Lead, total recoverable & $<100$ & $\mathrm{U}$ & $<100$ & U & $<10$ & U & $<4.64$ & JU & & $<E Q L$ & 1 & $u g / L$ & ML \\
\hline & Nickel, total recoverable & 112 & & $<50$ & $U$ & $<50$ & $\mathrm{U}$ & $<60$ & $\mathrm{U}$ & & $<E Q L$ & 1 & $\mathrm{ug} / \mathrm{L}$ & ML. \\
\hline & Selenium, total recoverable & $<200$ & $\mathrm{U}$ & $<200$ & $U$ & $<3.8$ & JU 1 & $<40$ & $u$ & & $<E Q L$ & 1 & $\mathrm{ug} / \mathrm{L}$ & ML \\
\hline \multicolumn{15}{|c|}{ Organics } \\
\hline & Chlorobenzene & $<5$ & $\mathrm{U}$ & $<5$ & U & $<1$ & $\mathrm{U}$ & $<1$ & $\mathrm{U}$ & & $<E Q L$ & 1 & $u g / L$ & $\mathrm{ML}$ \\
\hline & 1,1-Dichloroethane & $<5$ & $\mathrm{U}$ & $<5$ & $U$ & $<1$ & U & $<1$ & $\mathrm{U}$ & & $<E Q L$ & 1 & $\mathrm{ug} / \mathrm{L}$ & IL \\
\hline & 1,1-Dichloroethylene & $<5$ & $U$ & $<5$ & $U$ & .59 & $\mathrm{~J} \quad \mathrm{IK}$ & $<1$ & $\mathrm{U}$ & & $<E Q L$ & 1 & $u g / L$ & IL \\
\hline & trans-1,2-Dichloroethylene & $<5$ & $\mathrm{U}$ & $<5$ & $U$ & $<1$ & U & $<1$ & $\mathrm{U}$ & & $<E Q L$ & 1 & $\mathrm{ug} / \mathrm{L}$ & ML \\
\hline & PCB 1016 & $<2$ & U & & & & & & & & & & ug $/ \mathrm{L}$ & \\
\hline & PCB 1221 & $<2$ & $U$ & & & & & & & & & & $u g / L$ & \\
\hline & РCB 1232 & $<1$ & $U$ & & & & & & & & & & $\mathrm{ug} / \mathrm{L}$ & \\
\hline & РCB 1242 & $<1$ & $\mathrm{U}$ & & & & & & & & & & $\mathrm{ug} / \mathrm{L}$ & \\
\hline & РСВ 1248 & $<1$ & $\mathrm{U}$ & & & & & & & & & & $\mathrm{ug} / \mathrm{L}$ & \\
\hline & PCB 1254 & $<1$ & $\mathrm{U}$ & & & & & & & & & & $u g / L$ & \\
\hline & PCB 1260 & $<1$ & $\mathrm{U}$ & & & & & & & & & & $u g / L$ & \\
\hline & 1,1,2,2-Tetrachloroethane & $<5$ & $U$ & $<5$ & U & $<1$ & $\mathrm{U}$ & $<1$ & $\mathrm{U}$ & & $<$ EQL & 1 & $u g / L$ & ML \\
\hline+ & Tetrachloroethylene & 22.9 & & 60.9 & & 53.9 & $\mathrm{~J} K$ & 36.7 & & & $>5$ & 1 & $\mathrm{ug} / \mathrm{L}$ & I \\
\hline & 1,1,1-Trichloroethane & $<5$ & $\mathrm{U}$ & $<5$ & U & & $\mathrm{U}$ & $<1$ & $\mathrm{U}$ & & $<E Q L$ & 1 & $\mathrm{ug} / \mathrm{L}$ & $M L$ \\
\hline+ & Trichloroethylene & 5.72 & & 19 & $J \quad L$ & 16.2 & $\mathrm{~J} K$ & 5.96 & & & $>5$ & 1 & $u g / L$ & \\
\hline \multicolumn{15}{|c|}{ II. Monitoring Constituents } \\
\hline$\underline{\text { ST }}$ & Parameter & $\underline{3096}$ & CLPEPA & $\underline{3097}$ & CLPEPA & $\underline{3098}$ & CLPPEPA & $\underline{3099}$ & $\underline{\text { CLF }}$ & EPA & Filt. & $\underline{\text { DF }}$ & Unit & \\
\hline \multicolumn{15}{|c|}{ Inorganics } \\
\hline & Aluminum, total recoverable & $<20$ & $U$ & 19.6 & $J$ & 544 & & $<200$ & $\mathrm{U}$ & & $<\mathrm{EQL}$ & 1 & $\mathrm{ug} / \mathrm{L}$ & EX \\
\hline+ & Nitrate-nitrite as nitrogen & 3960 & & 2510 & & 1290 & & 2790 & & & $>2400$ & 5 & ug/L & \\
\hline & Sodium, total recoverable & 3430 & & 3080 & & 2780 & & 3500 & & & $<4600$ & 1 & $\mathrm{ug} / \mathrm{L}$ & \\
\hline & Sulfate & 288 & $J$ & $<5000$ & $\mathrm{U}$ & 621 & & 569 & & & $<3000$ & 2 & $\mathrm{ug} / \mathrm{L}$ & \\
\hline \multicolumn{14}{|c|}{ Radionuclides } & \\
\hline & Gross alpha & 5.56 & & 3.31 & & 10.1 & & 3.17 & & & $<15$ & 1 & $\mathrm{pCi} / \mathrm{L}$ & GP \\
\hline & Nonvolatile beta & 3.79 & & -1.9 & UI & 9.71 & & 2.11 & $J$ & 1 & NDD & 1 & $\mathrm{pCi} / \mathrm{L}$ & GP \\
\hline & Radium, total alpha-emitting & 3 & & 2.61 & Ul & 1.7 & & 2.4 & U & V & $<E Q L$ & 1 & $\mathrm{pCi} / \mathrm{L}$ & \\
\hline
\end{tabular}

Notes: Concentrations in bold italics exceed the groundwater protection or monitoring constituent standards listed in Appendix A. Synchronous water levels are measured over a 3-5 day period or less. Dilution factors, Laboratory, and Filtered Data are for Groundwater Protection Standards First Quarter 2000 and Monitoring Constituents Third Quarter 1999 data only.

$+=$ exceeded the Groundwater Protection Standards (First Quarter 2000) or Monitoring Constituents (Third Quarter 1999) listed in Appendix A. 
Table D-2. Groundwater Monitoring Results for Point-of-Compliance Wells, M-Area HWMF (Cont.) WELL MSB 8B

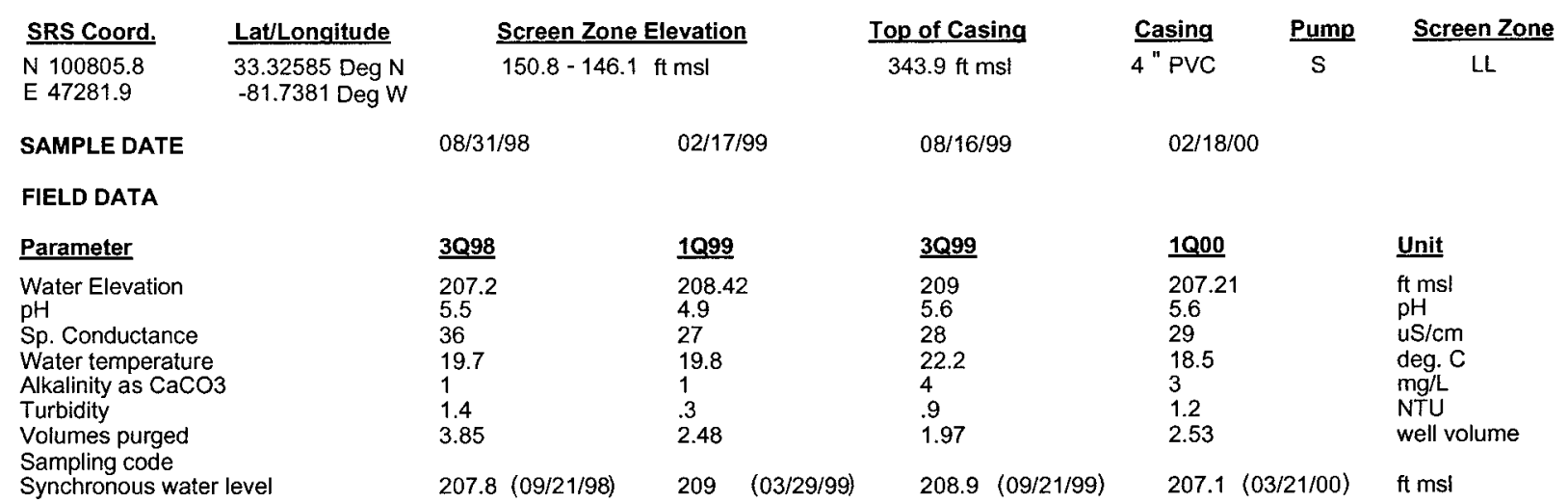

ANALYTICAL DATA

I. Groundwater Protection Standard

261 Appendix VIII/264 Appendix IX Hazardous Constituents

\begin{tabular}{|c|c|c|c|c|c|c|c|c|c|c|c|c|c|c|}
\hline \multirow{2}{*}{\multicolumn{2}{|c|}{$\begin{array}{l}\underline{\text { ST }} \text { Parameter } \\
\text { Inorganics }\end{array}$}} & \multirow[t]{2}{*}{$\underline{3098}$} & \multirow[t]{2}{*}{ CLPEPA } & \multirow[t]{2}{*}{$\underline{1 Q 99}$} & \multirow[t]{2}{*}{ CLPEPA } & \multirow[t]{2}{*}{$\underline{3 Q 99}$} & \multicolumn{2}{|c|}{ CLPEPA } & \multirow[t]{2}{*}{$\underline{1 Q 00}$} & \multirow[t]{2}{*}{ CLPEPA } & \multirow[t]{2}{*}{ Filt. } & \multirow[t]{2}{*}{$\underline{\mathrm{DF}}$} & \multirow[t]{2}{*}{$\underline{\text { Unit }}$} & \multirow[t]{2}{*}{$\underline{\underline{L a b}}$} \\
\hline & & & & & & & & & & & & & & \\
\hline & Barium, total recoverable & 13.2 & & $<10$ & $u$ & 17 & & & 26.4 & & $<2000$ & 1 & ug/L & ML \\
\hline & Cyanide & $<10$ & $\mathrm{u}$ & $<10$ & $\mathrm{u}$ & $<10$ & $u$ & & & & & & ug/L & \\
\hline & Lead, total recoverable & $<10$ & $u$ & $<100$ & $\mathrm{U}$ & $<1.92$ & $\mathrm{U}$ & $\mathrm{v}$ & $<20$ & $U$ & $<E Q L$ & 1 & ug/L & ML \\
\hline & Nickel, total recoverable & $<50$ & $u$ & $<50$ & $u$ & $<7.8$ & JU & $\mathrm{I}$ & $<60$ & U & $<E Q L$ & 1 & $\mathrm{ug} / \mathrm{L}$ & $\mathrm{ML}$ \\
\hline & Selenium, total recoverable & 4.01 & $\mathrm{~J}$ & $<200$ & $u$ & $<5$ & u & & $<40$ & $u$ & $<E Q L$ & 1 & ug/L & ML \\
\hline \multicolumn{15}{|c|}{ Organics } \\
\hline & Chlorobenzene & $<5$ & UJ & $<5$ & $u$ & $<1$ & $u$ & & $<1$ & $U$ & $<E Q L$ & 1 & $\mathrm{ug} / \mathrm{L}$ & ML \\
\hline & 1,1-Dichloroethane & $<5$ & UJ & $<5$ & $u$ & $<1$ & $u$ & & $<1$ & U & $<\mathrm{EQL}$ & 1 & $\mathrm{ug} / \mathrm{L}$ & ML \\
\hline & 1,1-Dichloroethylene & $<5$ & UJ & $<5$ & $u$ & $<1$ & $u$ & & $<1$ & $u$ & $<E Q L$ & 1 & $\mathrm{ug} / \mathrm{L}$ & ML \\
\hline & trans-1,2-Dichloroethylene & $<5$ & UJ & $<5$ & u & $<1$ & $u$ & & $<1$ & $\mathrm{u}$ & $<E Q L$ & 1 & ug/L & $\mathrm{ML}$ \\
\hline & PCB 1016 & $<.2$ & $u$ & & & & & & & & & & ug/L & \\
\hline & PCB 1221 & $<.2$ & u & & & & & & & & & & $\mathrm{ug} / \mathrm{L}$ & \\
\hline & РCB 1232 & $<.2$ & $\mathrm{u}$ & & & & & & & & & & $\mathrm{ug} / \mathrm{L}$ & \\
\hline & PCB 1242 & $<.1$ & $u$ & & & & & & & & & & $\mathrm{ug} / \mathrm{L}$ & \\
\hline & PCB 1248 & $<.1$ & u & & & & & & & & & & $\mathrm{ug} / \mathrm{L}$ & \\
\hline & PCB 1254 & $<.1$ & $u$ & & & & & & & & & & $\mathrm{ug} / \mathrm{L}$ & \\
\hline & PCB 1260 & $<.1$ & $u$ & & & & & & & & & & $\mathrm{ug} / \mathrm{h}$ & \\
\hline & 1,1,2,2-Tetrachloroethane & $<5$ & UJ & $<5$ & $\mathrm{u}$ & $<1$ & $\mathrm{u}$ & & $<1$ & u & $<E Q L$ & 1 & ug/L & ML \\
\hline+ & Tetrachloroethylene & 4.46 & $\mathrm{~J}$ & 4.74 & J 1 & 4.72 & $\mathrm{~J}$ & $\mathrm{~K}$ & 5.38 & & $>5$ & 1 & $\mathrm{ug} / \mathrm{L}$ & $\mathrm{ML}$ \\
\hline & $1,1,1$-Trichloroethane & $<5$ & UJ & $<5$ & u & $<1$ & $u$ & & $<1$ & $\mathrm{u}$ & $<E Q L$ & 1 & $\mathrm{ug} / \mathrm{L}$ & $\mathrm{ML}$ \\
\hline+ & Trichloroethylene & 44.9 & J & 45.6 & $J \quad L$ & 46.8 & J & $\mathrm{K}$ & 47.5 & & $>5$ & 1 & $\mathrm{ug} / \mathrm{L}$ & $\mathrm{ML}$ \\
\hline \multicolumn{15}{|c|}{ II. Monitoring Constituents } \\
\hline$\underline{\text { ST }}$ & Parameter & $\underline{3096}$ & CLPEPA & $\underline{3097}$ & CLPEPA & $\underline{3098}$ & $\underline{\text { CLP }}$ & EPA & $\underline{3099}$ & CLPEPA & Filt. & DF & $\underline{\text { Unit }}$ & Lab \\
\hline \multicolumn{15}{|c|}{ Inorganics } \\
\hline & Aluminum, total recoverable & $<20$ & $u$ & $<20$ & $u$ & $<200$ & $u$ & & $<200$ & $u$ & $<E Q L$ & 1 & $\mathrm{ug} / \mathrm{L}$ & EX \\
\hline & Nitrate-nitrite as nitrogen & 1860 & & 1030 & & 1070 & & & 1140 & & $<2400$ & 5 & $\mathrm{ug} / \mathrm{L}$ & EX \\
\hline & Sodium, total recoverable & 2110 & & 1920 & & 2310 & & & 2600 & & $<4600$ & 1 & $\mathrm{ug} / \mathrm{L}$ & EX \\
\hline & Sulfate & 415 & $\mathrm{~J}$ & $<5000$ & $u$ & 654 & J & & 426 & & $<3000$ & 1 & $\mathrm{ug} / \mathrm{L}$ & EX \\
\hline \multicolumn{15}{|c|}{ Radionuclides } \\
\hline & Gross alpha & .454 & Ul & -.29 & UI & 1.41 & & & .521 & JU L & $<E Q L$ & 1 & $\mathrm{pCi} / \mathrm{L}$ & GP \\
\hline & Nonvolatile beta & 1.79 & & .21 & $\mathrm{UI}$ & 3.17 & J & & 1.01 & U & $<\mathrm{EQL}$ & 1 & $\mathrm{pCi} / \mathrm{L}$ & GP \\
\hline & Radium, total alpha-emitting & -.2 & UI & 1.11 & & .34 & 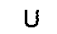 & & 0 & $U$ & $<E Q L$ & 1 & $\mathrm{pCi} / \mathrm{L}$ & GP \\
\hline
\end{tabular}

Notes: Concentrations in bold italics exceed the groundwater protection or monitoring constituent standards listed in Appendix A. Synchronous water levels are measured over a 3-5 day period or less. Dilution factors, Laboratory, and Filtered Data are for Groundwater Protection Standards First Quarter 2000 and Monitoring Constituents Third Quarter 1999 data only.

$+=$ exceeded the Groundwater Protection Standards (First Quarter 2000) or Monitoring Constituents (Third Quarter 1999) listed in Appendix A. 
Table D-2. Groundwater Monitoring Results for Point-of-Compliance Wells, M-Area HWMF (Cont.) WELL MSB 8C

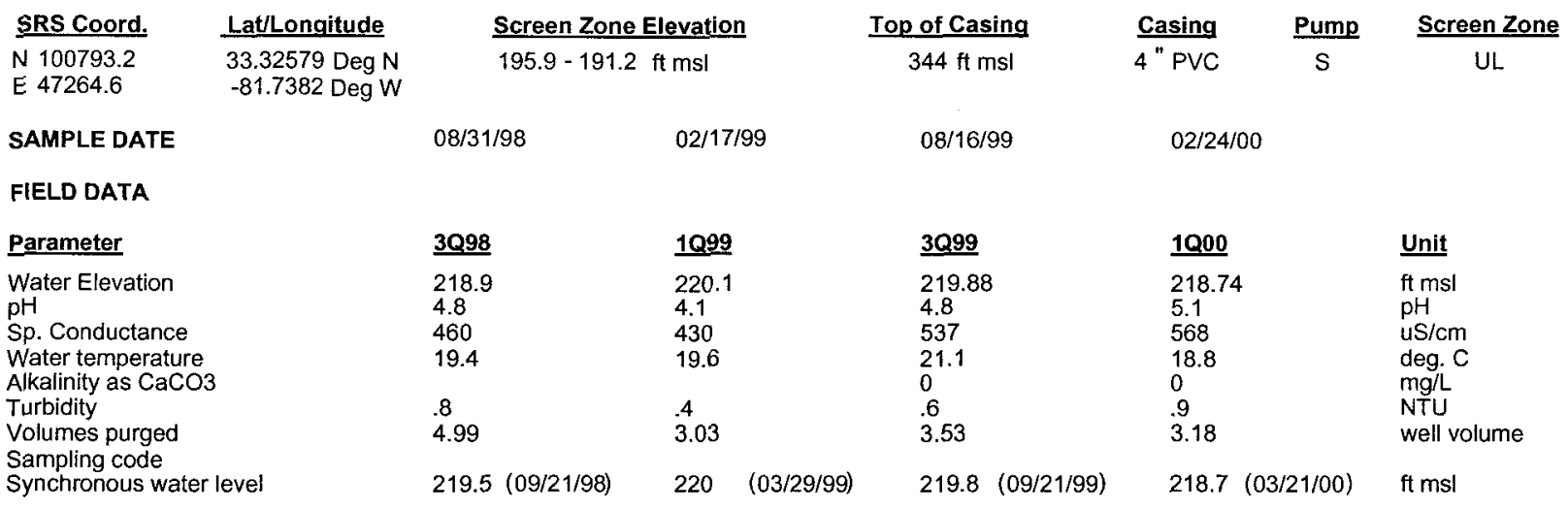

ANALYTICAL DATA

I. Groundwater Protection Standard

261 Appendix VIII/264 Appendix IX Hazardous Constituents

\begin{tabular}{|c|c|c|c|c|c|c|c|c|c|c|c|c|c|c|}
\hline$\underline{\mathbf{S T}}$ & Parameter & 3098 & CLP EPA & $1 \mathrm{Q99}$ & CLP EPA & 3Q999 & CLPEPA & $1 Q 00$ & CLF & EPA & Filt. & $\underline{\text { DF }}$ & $\underline{\text { Unit }}$ & $\underline{\text { Lab }}$ \\
\hline or & ganics & & & & & & & & & & & & & \\
\hline & Barium, total recoverable & 102 & & $<10$ & U & 130 & & 137 & & & $<2000$ & 1 & ug/L & ML \\
\hline & Cyanide & $<10$ & $U$ & $<10$ & $U$ & $<10$ & $U$ & & & & & & $\mathrm{ug} / \mathrm{L}$ & \\
\hline & Lead, total recoverable & $<10$ & $\mathrm{U}$ & $<100$ & $\mathrm{U}$ & $<100$ & $\mathrm{U}$ & $<5.91$ & JU & IV & $<\mathrm{EQL}$ & 1 & ug/L & ML \\
\hline & Nickel, total recoverable & $<50$ & $U$ & $<50$ & JU & $<50$ & $U$ & $<60$ & $\mathrm{U}$ & & $<E Q L$ & 1 & $\mathrm{ug} / \mathrm{L}$ & ML \\
\hline & Selenium, total recoverable & $<10$ & $U$ & $<200$ & $\mathrm{U}$ & $<200$ & $U$ & $<40$ & $U$ & & $<\mathrm{EQL}$ & 1 & $\mathrm{ug} / \mathrm{L}$ & ML \\
\hline $\operatorname{rg}$ & anics & & & & & & & & & & & & & \\
\hline & Chlorobenzene & $<5$ & UJ & $<5$ & $\mathrm{U}$ & $<5$ & $U$ & $<1$ & $\mathrm{U}$ & & $<\mathrm{EQL}$ & 1 & $\mathrm{ug} / \mathrm{L}$ & $\mathrm{ML}$ \\
\hline & 1,1-Dichloroethane & $<5$ & UJ & $<5$ & $\mathrm{U}$ & $<5$ & $U$ & .49 & J & 1 & NDD & 1 & ug/L & ML \\
\hline+ & 1,1-Dichloroethylene & 11.2 & $\mathrm{~J}$ & 8.56 & & $<5$ & U & 7.96 & & & $>7$ & 1 & $\mathrm{ug} / \mathrm{L}$ & ML \\
\hline & trans-1,2-Dichloroethylene & $<5$ & UJ & $<5$ & $u$ & $<5$ & $U$ & $<1$ & $u$ & & $<\mathrm{EQL}$ & 1 & $\mathrm{ug} / \mathrm{L}$ & $\mathrm{ML}$ \\
\hline & PCB 1016 & $<.2$ & $u$ & & & $<1.9$ & $U$ & & & & & & $\mathrm{ug} / \mathrm{L}$ & \\
\hline & PCB 1221 & $<.2$ & $\mathrm{U}$ & & & $<1.9$ & $U$ & & & & & & ugh $/ \mathrm{L}$ & \\
\hline & PCB 1232 & $<.2$ & $\mathrm{U}$ & & & $<.96$ & $u$ & & & & & & $\mathrm{ug} / \mathrm{h}$ & \\
\hline & PCB 1242 & $<.1$ & $\mathrm{u}$ & & & $<.96$ & U & & & & & & $\mathrm{ug} / \mathrm{L}$ & \\
\hline & PCB 1248 & $<.1$ & $\mathrm{U}$ & & & $<.96$ & $\mathrm{U}$ & & & & & & $\mathrm{ug} / \mathrm{L}$ & \\
\hline & PCB 1254 & $<.1$ & $\mathrm{U}$ & & & $<.96$ & $U$ & & & & & & $\mathrm{ug} / \mathrm{L}$ & \\
\hline & PCB 1260 & $<.1$ & $U$ & & & $<.96$ & $U$ & & & & & & $\mathrm{ug} / \mathrm{L}$ & \\
\hline & $1,1,2,2$-Tetrachloroethane & $<5$ & UJ & $<5$ & $U$ & $<5$ & $U$ & $<1$ & $\mathrm{U}$ & & $<\mathrm{EQL}$ & 1 & ug/L & ML \\
\hline+ & Tetrachloroethylene & 84.2 & $J$ & 76.1 & & 69 & & 68.1 & & & $>5$ & 1 & $\mathrm{ug} / \mathrm{L}$ & ML \\
\hline & 1,1,1-Trichloroethane & $<5$ & UJ & $<5$ & U & 1.7 & J I & 1.62 & & & $<200$ & 1 & $u g / L$ & ML \\
\hline+ & Trichloroethylene & 51.7 & $j$ & 48.5 & J L & 46 & & 43.4 & & & $>5$ & 1 & $u g / L$ & $\mathrm{ML}$ \\
\hline II & onitoring Constituents & & & & & & & & & & & & & \\
\hline$\underline{\text { ST }}$ & Parameter & $\underline{3096}$ & CLP EPA & $\underline{3097}$ & CLP EPA & $\underline{3098}$ & CLPPEPA & 3099 & $\underline{\text { CLP }}$ & EPA & Filt. & $\underline{\text { DF }}$ & Unit & Lab \\
\hline or & ganics & & & & & & & & & & & & & \\
\hline+ & Aluminum, total recoverable & 114 & & 143 & & 180 & 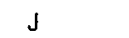 & 260 & & & $>100$ & 1 & $\mathrm{ug} / \mathrm{L}$ & EX \\
\hline+ & Nitrate-nitrite as nitrogen & 14500 & & 39800 & & 45000 & & 59000 & & & $>2400$ & 20 & $\mathrm{ug} / \mathrm{L}$ & EX \\
\hline+ & Sodium, total recoverable & 23700 & & 31300 & & 34600 & & 51000 & & & $>4600$ & 1 & $\mathrm{ug} / \mathrm{L}$ & EX \\
\hline & Sulfate & 372 & $J$ & $<5000$ & $\mathrm{U}$ & 404 & $J$ & 343 & $J$ & I & NDD & 2 & $u g / L$ & EX \\
\hline Radi & ionuclides & & & & & & & & & & & & & \\
\hline & Gross alpha & 6.16 & & 4.59 & & 20.82 & & 6.61 & $J$ & L & NDD & 1 & $\mathrm{pCi} / \mathrm{L}$ & GP \\
\hline+ & Nonvolatile beta & 35.9 & & 44.9 & & 120.46 & J & 84 & & & $>50$ & 1 & $\mathrm{pCi} / \mathrm{L}$ & GP \\
\hline & Radium, total alpha-emitting & 3.6 & & 3.69 & & 2.07 & & 4.2 & & & $<5$ & 1 & $\mathrm{pCi} / \mathrm{L}$ & GP \\
\hline
\end{tabular}

Notes: Concentrations in bold italics exceed the groundwater protection or monitoring constituent standards listed in Appendix A. Synchronous water levels are measured over a 3-5 day period or less. Dilution factors, Laboratory, and Filtered Data are for Groundwater Protection Standards First Quarter 2000 and Monitoring Constituents Third Quarter 1999 data only.

+= exceeded the Groundwater Protection Standards (First Quarter 2000) or Monitoring Constituents (Third Quarter 1999) listed in Appendix A. 
Table D-2. Groundwater Monitoring Results for Point-of-Compliance Wells, M-Area HWMF (Cont.) WELL MSB 13A

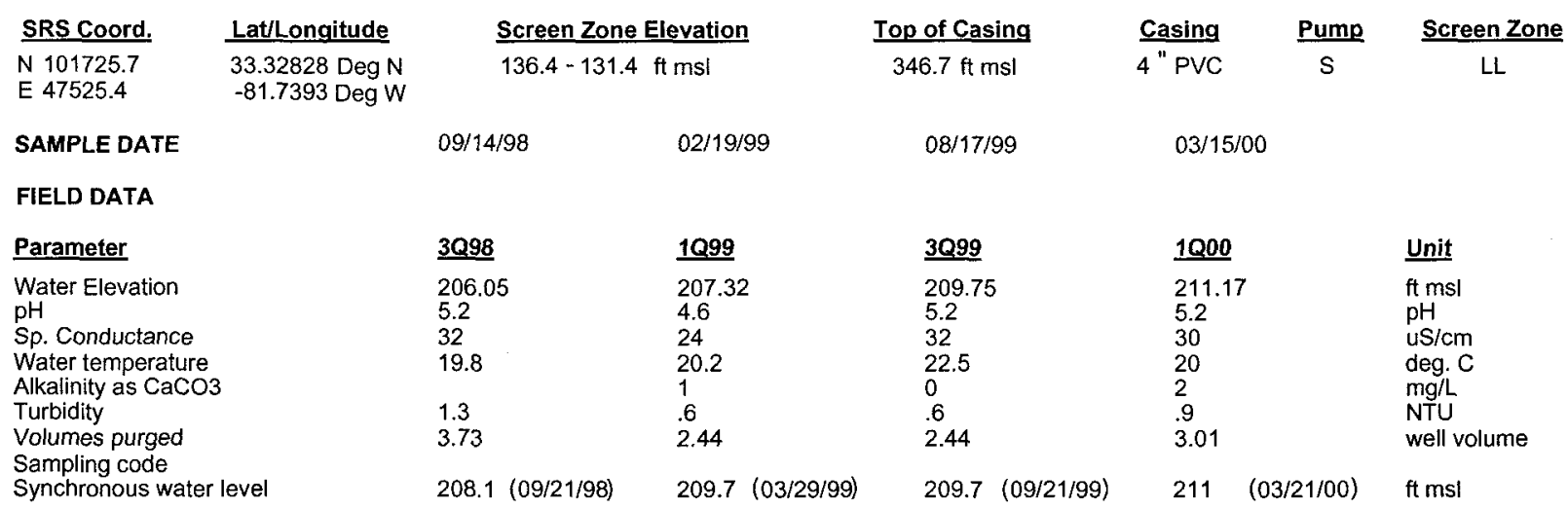

ANALYTICAL DATA

I. Groundwater Protection Standard

261 Appendix VIII/264 Appendix IX Hazardous Constituents

\begin{tabular}{|c|c|c|c|c|c|c|c|c|c|c|c|c|c|}
\hline$\underline{\text { ST }}$ & Parameter & 3Q98 & CLPEPA & 1099 & CLP EPA & 3099 & CLPEPA & 1000 & CLPEPA & Filt. & $\underline{\text { DF }}$ & Unit & $\underline{\text { Lab }}$ \\
\hline \multicolumn{14}{|c|}{ Inorganics } \\
\hline & Barium, total recoverable & 7.41 & $J$ & 8.52 & J 1 & 7.9 & $\jmath \quad 1$ & 7.37 & J I & NDD & 1 & $u g / L$ & ML \\
\hline & Cyanide & $<10$ & $\mathrm{U}$ & $<10$ & $J U L$ & $<10$ & $\mathrm{U}$ & & & & & $\mathrm{ug} / \mathrm{L}$ & \\
\hline & Lead, total recoverable & $<100$ & $\mathrm{U}$ & $<100$ & $\mathrm{U}$ & $<100$ & $\mathrm{U}$ & $<20$ & $\mathrm{U}$ & $<E Q L$ & 1 & $\mathrm{ug} / \mathrm{L}$ & ML \\
\hline & Nickel, total recoverable & $<50$ & $\mathrm{U}$ & $<50$ & $\mathrm{U}$ & $<50$ & U & $<60$ & $U$ & $<E Q L$ & 1 & $\mathrm{ug} / \mathrm{L}$ & $M L$ \\
\hline & Selenium, total recoverable & 81.6 & J & $<10$ & $U$ & $<200$ & $U$ & $<40$ & $\mathrm{U}$ & $<\mathrm{EQL}$ & 1 & $\mathrm{ug} / \mathrm{L}$ & ML \\
\hline \multicolumn{14}{|c|}{ Organics } \\
\hline+ & Chlorobenzene & $<25$ & $\mathrm{u}$ & $<25$ & $\mathrm{U}$ & $<50$ & $u$ & 11.3 & & $>5$ & 5 & $u g / L$ & $M L$ \\
\hline & 1,1-Dichloroethane & $<25$ & $\mathrm{U}$ & $<25$ & $\mathrm{U}$ & $<50$ & $\mathrm{U}$ & $<5$ & $\mathrm{U}$ & $<\mathrm{EQL}$ & 5 & $\mathrm{ug} / \mathrm{L}$ & ML \\
\hline & 1,1-Dichloroethylene & $<25$ & $U$ & $<25$ & $\mathrm{U}$ & $<50$ & $\mathrm{U}$ & $<5$ & U & $<E Q L$ & 5 & $\mathrm{ug} / \mathrm{L}$ & $\mathrm{ML}$ \\
\hline & trans-1,2-Dichloroethylene & $<25$ & $\mathrm{U}$ & $<25$ & $u$ & $<50$ & $\mathrm{U}$ & $<5$ & $\mathrm{U}$ & $<\overline{E Q L}$ & 5 & $\mathrm{ug} / \mathrm{L}$ & ML \\
\hline & PCB 1016 & & & & & $<1.9$ & $\mathrm{U}$ & & & & & $\mathrm{ug} / \mathrm{L}$ & \\
\hline & PCB 1221 & & & & & $<1.9$ & $\mathrm{U}$ & & & & & $\mathrm{ug} / \mathrm{L}$ & \\
\hline & PCB 1232 & & & & & $<.96$ & $U$ & & & & & $\mathrm{ug} / \mathrm{L}$ & \\
\hline & PCB 1242 & & & & & $<.96$ & U & & & & & $\mathrm{ug} / \mathrm{L}$ & \\
\hline & PCB 1248 & & & & & $<.96$ & $U$ & & & & & $\mathrm{ug} / \mathrm{L}$ & \\
\hline & PCB 1254 & & & & & $<.96$ & $\mathrm{U}$ & & & & & $\mathrm{ug} / \mathrm{L}$ & \\
\hline & PCB 1260 & & & & & $<.96$ & $\cup$ & & & & & ug/L & \\
\hline & 1,1,2,2-Tetrachloroethane & $<25$ & $\mathrm{U}$ & $<25$ & $U$ & $<50$ & U & $<5$ & $\mathrm{U}$ & $<E Q L$ & 5 & $\mathrm{ug} / \mathrm{L}$ & ML \\
\hline+ & Tetrachloroethylene & 39.1 & & 41.1 & & 29 & J 1 & 15.8 & & $>5$ & 5 & $u g / L$ & ML \\
\hline & 1,1,1-Trichloroethane & $<25$ & $\mathrm{U}$ & $<25$ & $\mathrm{U}$ & $<50$ & $\mathrm{U}$ & $<5$ & $\mathrm{U}$ & $<E Q L$ & 5 & $\mathrm{ug} / \mathrm{L}$ & ML \\
\hline+ & Trichloroethylene & 878 & & 904 & & 1000 & & 1210 & & $>5$ & 5 & $u g / L$ & ML \\
\hline \multicolumn{14}{|c|}{ II. Monitoring Constituents } \\
\hline$\underline{\mathbf{S T}}$ & Parameter & $\underline{3096}$ & CLPEPA & $\underline{3097}$ & CLP EPA & $\underline{3 Q 98}$ & CLPEPA & $\underline{3099}$ & CLPEPA & Filt. & DF & Unit & $\underline{L a b}$ \\
\hline \multicolumn{14}{|c|}{ Inorganics } \\
\hline & Aluminum, total recoverable & $<20$ & $\mathrm{U}$ & $<20$ & $U$ & $<200$ & $\mathrm{U}$ & $<200$ & $\mathrm{U}$ & $<E Q L$ & 1 & ug/L & EX \\
\hline & Nitrate-nitrite as nitrogen & 2400 & & 1840 & & 1860 & & 1830 & & $<2400$ & 5 & $\mathrm{ug} / \mathrm{L}$ & EX \\
\hline & Sodium, total recoverable & 2170 & & 2090 & & 2400 & & 2300 & & $<4600$ & 1 & ug/L & EX \\
\hline & Sulfate & 554 & J & $<5000$ & $\mathrm{U}$ & 587 & & 551 & & $<3000$ & 2 & ug/L & EX \\
\hline \multicolumn{14}{|c|}{ Radionuclides } \\
\hline & Gross alpha & 1.52 & & -.19 & UI & 2.09 & & .386 & $\mathrm{U}$ & $<\mathrm{EQL}$ & 1 & $\mathrm{pCi} / \mathrm{L}$ & GP \\
\hline & Nonvolatile beta & 1.84 & & -1.4 & UI & 2.92 & & 1.2 & $\mathrm{U}$ & $<E Q L$ & 1 & $\mathrm{pCi} / \mathrm{L}$ & GP \\
\hline & Radium, total alpha-emitting & .4 & & 1.26 & & .09 & UI & .4 & $U$ & $<E Q L$ & 1 & pCi/l & GP \\
\hline
\end{tabular}

Notes: Concentrations in bold italics exceed the groundwater protection or monitoring constituent standards listed in Appendix A. Synchronous water levels are measured over a 3-5 day period or less. Dilution factors, Laboratory, and Filtered Data are for Groundwater Protection Standards First Quarter 2000 and Monitoring Constituents Third Quarter 1999 data only.

$+=$ exceeded the Groundwater Protection Standards (First Quarter 2000) or Monitoring Constituents (Third Quarter 1999) listed in Appendix A. 
Table D-2. Groundwater Monitoring Results for Point-of-Compliance Wells, M-Area HWMF (Cont.) WELL MSB 13CC

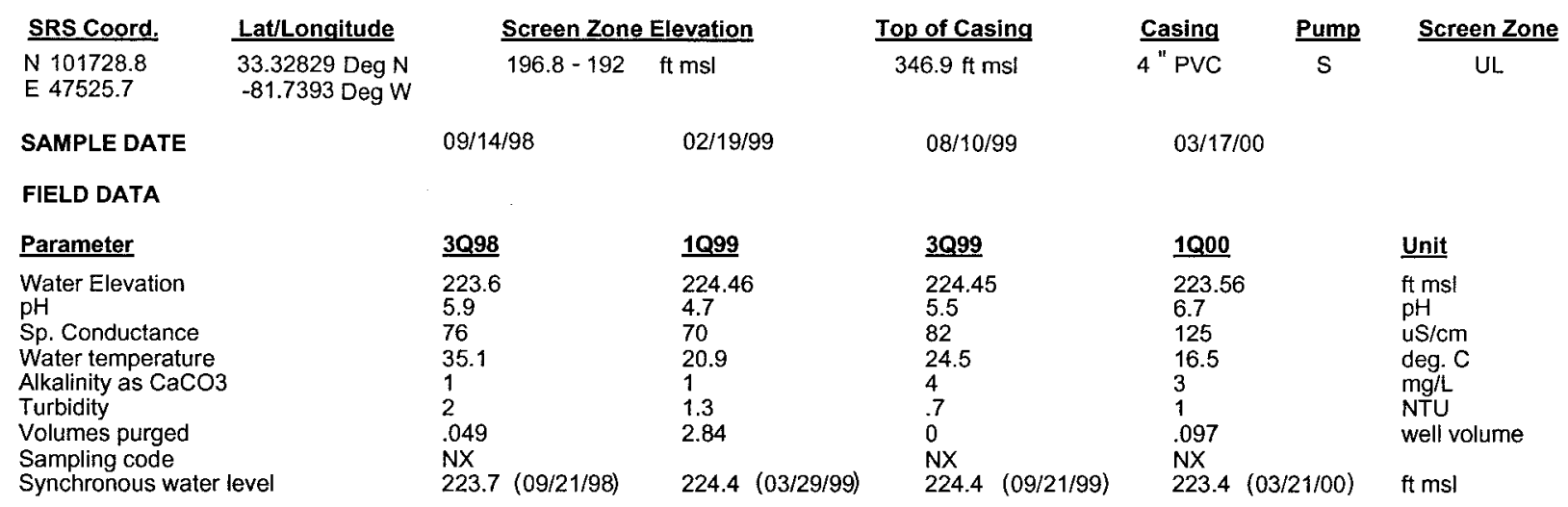

ANALYTICAL DATA

I. Groundwater Protection Standard

261 Appendix VIII/264 Appendix IX Hazardous Constituents

\begin{tabular}{|c|c|c|c|c|c|c|c|c|c|c|c|c|c|c|}
\hline$\underline{\mathrm{ST}}$ & Parameter & $\underline{3 Q 98}$ & $\underline{\text { CLP EPA }}$ & 1099 & $\underline{\text { CLP EPA }}$ & $\underline{3 Q 99}$ & CLPEPA & 1000 & $\underline{\text { CLP }}$ & EPA & Filt. & $\underline{\text { DF }}$ & Unit & $\underline{L a b}$ \\
\hline \multicolumn{15}{|c|}{ Inorganics } \\
\hline & Barium, total recoverable & $<10$ & $U$ & $<10$ & $\mathrm{U}$ & $<10$ & $\mathrm{U}$ & $<15$ & $U$ & & $<E Q L$ & 1 & $\mathrm{ug} / \mathrm{L}$ & ML \\
\hline & Cyanide & $<10$ & $\mathrm{U}$ & $<10$ & JU L & $<10$ & $\mathrm{U}$ & & & & & & $\mathrm{ug} / \mathrm{L}$ & \\
\hline & Lead, total recoverable & $<100$ & $U$ & $<100$ & $u$ & 10 & & 18.1 & $\mathrm{~J}$ & I & NDD & 1 & $\mathrm{ug} / \mathrm{L}$ & ML \\
\hline & Nickel, total recoverable & $<50$ & $U$ & $<50$ & U & $<50$ & $U$ & $<60$ & $\mathrm{U}$ & & $<E Q L$ & 1 & $\mathrm{ug} / \mathrm{L}$ & \\
\hline & Selenium, total recoverable & $<200$ & $\mathrm{U}$ & $<10$ & $U$ & $<10$ & $\mathrm{U}$ & $<40$ & $\mathrm{U}$ & & $<\mathrm{EQL}$ & 1 & ug/L & \& \\
\hline \multicolumn{15}{|c|}{ Organics } \\
\hline & Chlorobenzene & $<5$ & $\mathrm{U}$ & $<5$ & $U$ & $<1$ & $\mathrm{U}$ & $<1$ & $U$ & & $<E Q L$ & 1 & $\mathrm{ug} / \mathrm{L}$ & \\
\hline & 1,1-Dichloroethane & $<5$ & U & $<5$ & U & $<1$ & $\mathrm{U}$ & $<1$ & $\mathrm{u}$ & & $<\mathrm{EQL}$ & 1 & $\mathrm{ug} / \mathrm{L}$ & \\
\hline & 1,1-Dichloroethylene & $<5$ & $\mathrm{U}$ & $<5$ & U & 2.09 & & 2.86 & & & $<7$ & 1 & $\mathrm{ug} / \mathrm{L}$ & \\
\hline & trans-1,2-Dichloroethylene & $<5$ & $U$ & $<5$ & U & $<1$ & $\mathrm{U}$ & $<1$ & $\mathrm{U}$ & & $<E Q L$ & 1 & $\mathrm{ug} / \mathrm{L}$ & \\
\hline & $\begin{array}{l}\text { PCB } 1016 \\
\text { PCB } 1221\end{array}$ & & & & & & & & & & & & & \\
\hline & PCB 1232 & & & & & & & & & & & & & \\
\hline & PCB 1242 & & & & & & & & & & & & & \\
\hline & PCB 1248 & & & & & & & & & & & & & \\
\hline & РCB 1254 & & & & & & & & & & & & & \\
\hline & PCB 1260 & & & & & & & & & & & & & \\
\hline & $1,1,2,2$-Tetrachloroethane & $<5$ & $\mathrm{U}$ & $<5$ & $\mathrm{U}$ & $<1$ & $U$ & $<1$ & $u$ & & $<\mathrm{EQL}$ & 1 & $\mathrm{ug} / \mathrm{L}$ & \\
\hline+ & Tetrachloroethylene & 14.3 & & 12 & & 12.9 & & 14.9 & & & $>5$ & 1 & $\mathrm{ug} / \mathrm{L}$ & \\
\hline & $1,1,1$-Trichloroethane & $<5$ & $\mathrm{U}$ & $<5$ & $\mathrm{U}$ & 1.91 & & 2.31 & & & $<200$ & 1 & $\mathrm{ug} / \mathrm{L}$ & $1 \mathrm{~V}$ \\
\hline+ & Trichloroethylene & 13.4 & & 11.6 & & 10.8 & & 9.88 & & & $>5$ & 1 & ug/L & \\
\hline \multicolumn{15}{|c|}{ II. Monitoring Constituents } \\
\hline$\underline{\mathbf{S T}}$ & Parameter & $\underline{3 Q 96}$ & CLPEPA & $\underline{3097}$ & CLPP EPA & $\underline{3 Q 98}$ & CLPEPA & $\underline{3099}$ & $\underline{\text { CLP }}$ & EPA & Filt. & $\underline{\text { DF }}$ & $\underline{\text { Unit }}$ & \\
\hline \multicolumn{15}{|c|}{ Inorganics } \\
\hline+ & Aluminum, total recoverable & $<20$ & $\mathrm{U}$ & 114 & & 181 & $\mathrm{~J}$ & 98 & $J$ & 1 & NDD & $\begin{array}{l}1 \\
5\end{array}$ & $\mathrm{ug} / \mathrm{L}$ & \\
\hline+ & Sodium, total recoverable & 19600 & & 15800 & & 15600 & & 19000 & & & $>4600$ & $\begin{array}{l}3 \\
1\end{array}$ & $u g / L$ & \\
\hline+ & Sulfate & 6010 & & 5420 & & 4450 & & 4130 & & & $>3000$ & 1 & $\mathrm{ug} / \mathrm{L}$ & \\
\hline \multicolumn{15}{|c|}{ Radionuclides } \\
\hline & Gross alpha & .198 & UI & -.79 & UI & 1.56 & & 2.35 & & & $<15$ & 1 & $\mathrm{pCi} / \mathrm{L}$ & \\
\hline & Nonvolatile beta & 1.69 & & 2.72 & & 5 & & 2.07 & $J$ & I & NDD & 1 & $\mathrm{pCi} / \mathrm{L}$ & \\
\hline & Radium, total alpha-emitting & 0 & UI & .48 & & .14 & UI & 0 & $U$ & & $<E Q L$ & 1 & $\mathrm{pCi} / \mathrm{L}$ & \\
\hline
\end{tabular}

Notes: Concentrations in bold italics exceed the groundwater protection or monitoring constituent standards listed in Appendix A. Synchronous water levels are measured over a 3-5 day period or less. Dilution factors, Laboratory, and Filtered Data are for Groundwater Protection Standards First Quarter 2000 and Monitoring Constituents Third Quarter 1999 data only.

+ = exceeded the Groundwater Protection Standards (First Quarter 2000) or Monitoring Constituents (Third Quarter 1999) listed in Appendix A. 
Table D-2. Groundwater Monitoring Results for Point-of-Compliance Wells, M-Area HWMF (Cont.) WELL MSB 13D

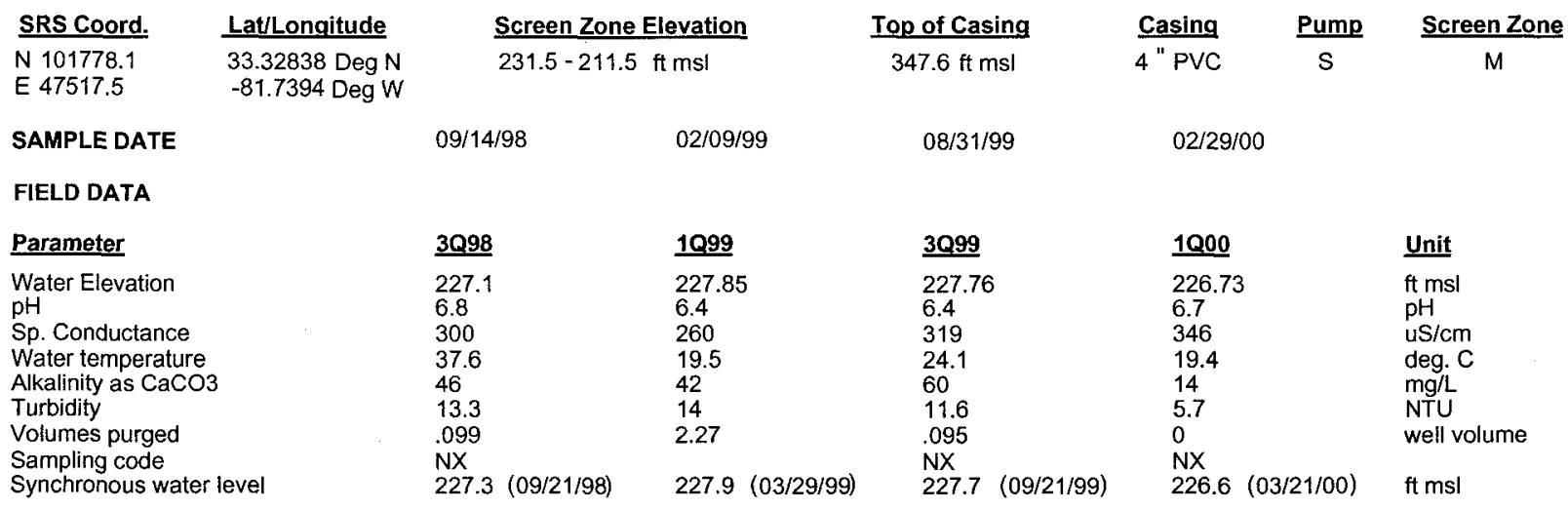

\section{ANALYTICAL DATA}

I. Groundwater Protection Standard

261 Appendix VIII/264 Appendix IX Hazardous Constituents

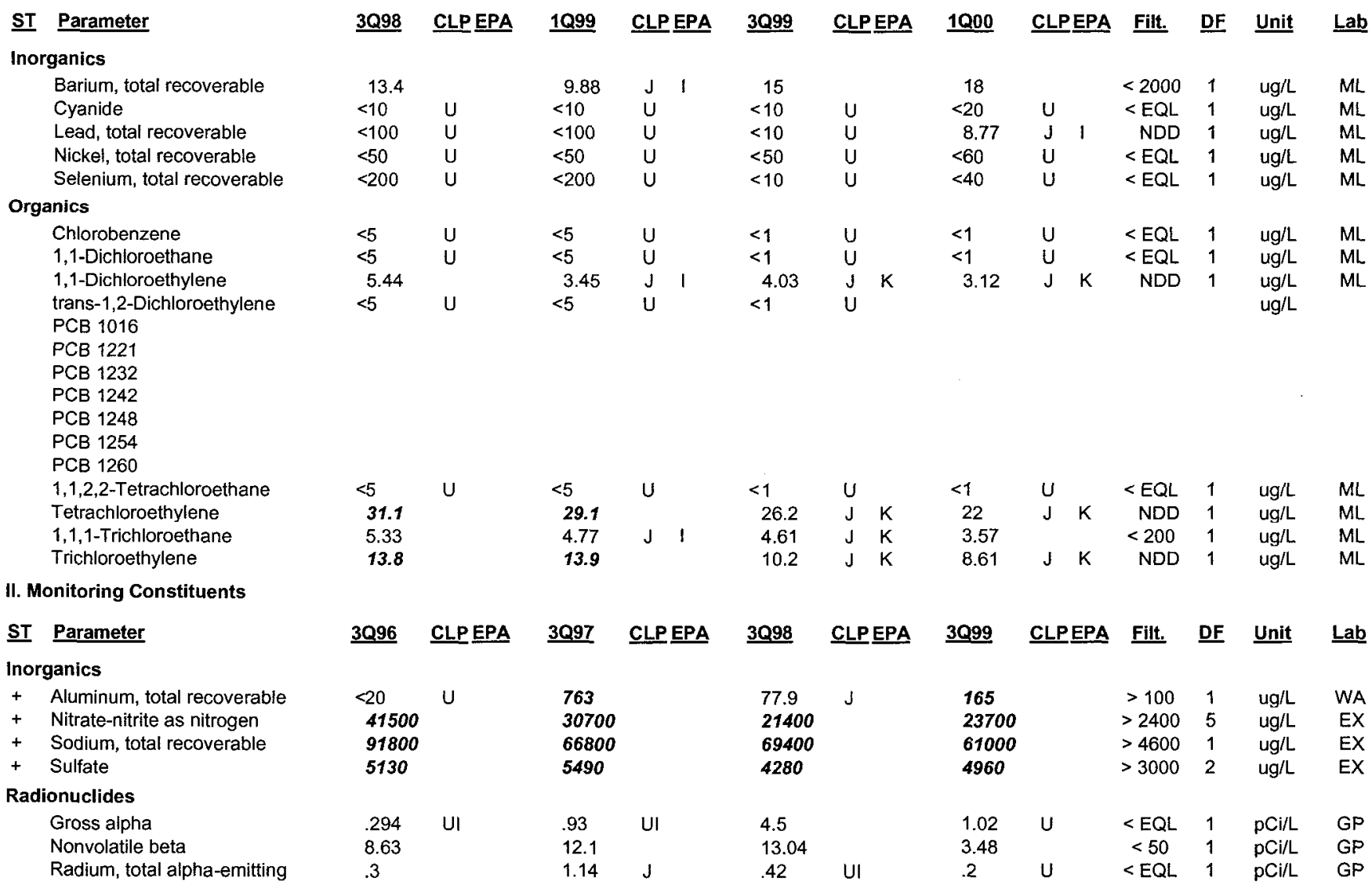

\footnotetext{
Notes: Concentrations in bold italics exceed the groundwater protection or monitoring constituent standards listed in Appendix A. Synchronous water levels are measured over a 3-5 day period or less. Dilution factors, Laboratory, and Filtered Data are for Groundwater Protection Standards First Quarter 2000 and Monitoring Constituents Third Quarter 1999 data only.

$+=$ exceeded the Groundwater Protection Standards (First Quarter 2000) or Monitoring Constituents (Third Quarter 1999) listed in Appendix A.
} 
Table D-2. Groundwater Monitoring Results for Point-of-Compliance Wells, M-Area HWMF (Cont.) WELL MSB 39A

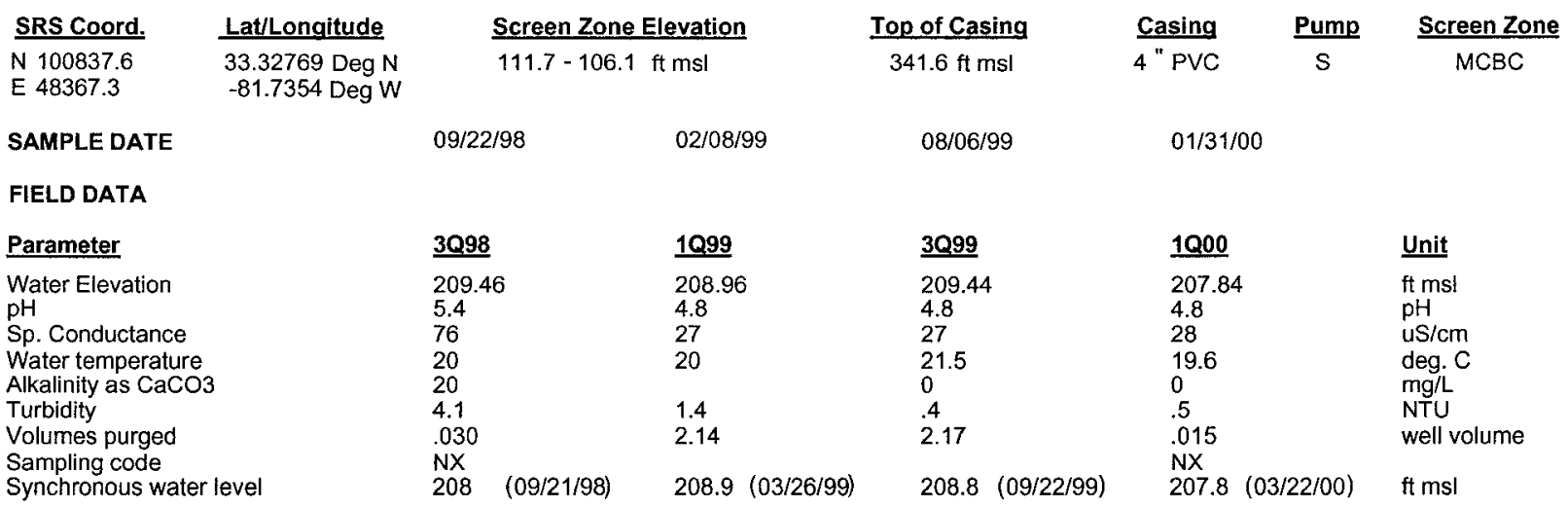

ANALYTICAL DATA

I. Groundwater Protection Standard

261 Appendix VIII/264 Appendix IX Hazardous Constituents

\begin{tabular}{|c|c|c|c|c|c|c|c|c|c|c|c|c|c|}
\hline \multirow{2}{*}{$\frac{\underline{\text { ST }}}{\text { Inorganics }}$} & \multirow[t]{2}{*}{$\underline{3 Q 98}$} & \multirow[t]{2}{*}{ CLPEPA } & \multirow[t]{2}{*}{$\underline{1099}$} & \multirow[t]{2}{*}{ CLP EPA } & \multirow[t]{2}{*}{$\underline{3 Q 99}$} & CLPEPA & \multirow[t]{2}{*}{1000} & \multicolumn{2}{|c|}{ CLPEPA } & \multirow[t]{2}{*}{ Filt. } & \multirow[t]{2}{*}{$\underline{\mathrm{DF}}$} & \multirow[t]{2}{*}{$\underline{\text { Unit }}$} & \multirow[t]{2}{*}{ Lab } \\
\hline & & & & & & & & & & & & & \\
\hline Barium, total recoverable & 13.2 & & $<10$ & $u$ & 6.6 & $\mathrm{~J} \quad \mathrm{I}$ & & & & & & $\mathrm{ug} / \mathrm{L}$ & \\
\hline Cyanide & $<10$ & $u$ & $<10$ & $\mathrm{u}$ & $<10$ & $\mathrm{u}$ & & & & & & $\mathrm{ug} / \mathrm{L}$ & \\
\hline Lead, total recoverable & $<100$ & $\mathrm{u}$ & $<100$ & $u$ & $<100$ & $\mathrm{U}$ & & & & & & $\mathrm{ug} / \mathrm{L}$ & \\
\hline Nickel, total recoverable & 21.1 & $\mathrm{~J}$ & $<50$ & u & $<50$ & $\mathrm{u}$ & & & & & & $\mathrm{ug} / \mathrm{L}$ & \\
\hline Selenium, total recoverable & $<200$ & $\mathrm{u}$ & $<200$ & $\mathrm{u}$ & $<200$ & $u$ & & & & & & $u g / L$ & \\
\hline \multicolumn{14}{|l|}{ Organics } \\
\hline Chlorobenzene & $<5$ & $u$ & $<5$ & $u$ & $<5$ & $u$ & $<1$ & JU & L & $<E Q L$ & 1 & $\mathrm{ug} / \mathrm{L}$ & ML \\
\hline 1,1-Dichloroethane & $<5$ & $u$ & $<5$ & $\mathrm{u}$ & $<5$ & $u$ & $<1$ & Ju & $\bar{L}$ & $<$ EQL & 1 & $\mathrm{ug} / \mathrm{L}$ & ML \\
\hline 1,1-Dichloroethylene & $<5$ & $\mathrm{U}$ & $<5$ & $\mathrm{u}$ & $<5$ & $u$ & $<1$ & su & L & $<E Q L$ & 1 & $\mathrm{ug} / \mathrm{L}$ & ML \\
\hline trans-1,2-Dichloroethylene & $<5$ & $\mathrm{u}$ & $<5$ & $\mathrm{u}$ & $<5$ & $\mathrm{u}$ & & & & & & $\mathrm{ug} / \mathrm{L}$ & \\
\hline PCB 1016 & & & & & $<2$ & $\mathrm{u}$ & & & & & & $\mathrm{ug} / \mathrm{L}$ & \\
\hline PCB 1221 & & & & & $<2$ & $\mathrm{u}$ & & & & & & $u g / L$ & \\
\hline PCB 1232 & & & & & $<1$ & $\mathrm{u}$ & & & & & & $\mathrm{ug} / \mathrm{L}$ & \\
\hline PCB 1242 & & & & & $<1$ & $\mathrm{u}$ & & & & & & $\mathrm{ug} / \mathrm{L}$ & \\
\hline PCB 1248 & & & & & $<1$ & $\mathrm{u}$ & & & & & & $u g / L$ & \\
\hline PCB 1254 & & & & & $<1$ & $u$ & & & & & & $\mathrm{ug} / \mathrm{L}$ & \\
\hline PCB 1260 & & & & & $<1$ & $u$ & & & & & & $\mathrm{ug} / \mathrm{L}$ & \\
\hline $1,1,2,2$-Tetrachloroethane & $<5$ & $u$ & $<5$ & $\mathrm{u}$ & $<5$ & $u$ & $<1$ & Ju & L & $<E Q L$ & 1 & $\mathrm{ug} / \mathrm{L}$ & ML \\
\hline Tetrachloroethylene & $<5$ & $u$ & $<5$ & $u$ & $<5$ & $u$ & $<1$ & JU & L & $<E Q L$ & 1 & $\mathrm{ug} / \mathrm{L}$ & $M L$ \\
\hline 1,1,1-Trichloroethane & $<5$ & $u$ & $<5$ & $u$ & $<5$ & $u$ & $<1$ & Ju & L & $<E Q L$ & 1 & $\mathrm{ug} / \mathrm{L}$ & $\mathrm{ML}$ \\
\hline Trichloroethylene & 4.65 & J & 3.35 & J 1 & 3.1 & $\mathrm{~J} 1$ & 3.63 & $J$ & $\mathrm{~L}$ & NDD & 1 & $\mathrm{ug} / \mathrm{L}$ & ML \\
\hline \multicolumn{14}{|l|}{ II. Monitoring Constituents } \\
\hline ST Parameter & $\underline{3096}$ & CLPEPA & $\underline{3097}$ & CLPEPA & 3098 & CLPEEPA & $\underline{3099}$ & $\underline{\text { CLP }}$ & EPA & Fill. & DF & Unit & $\underline{\text { Lab }}$ \\
\hline \multicolumn{14}{|l|}{ Inorganics } \\
\hline Aluminum, total recoverable & 354 & & 32.4 & & 695 & & $<200$ & $\mathrm{U}$ & & $<E Q L$ & 1 & $\mathrm{ug} / \mathrm{L}$ & EX \\
\hline Nitrate-nitrite as nitrogen & 2740 & & 163 & & 735 & & 74.8 & J & I & NDD & 5 & $\mathrm{ug} / \mathrm{L}$ & EX \\
\hline Sodium, total recoverable & 4720 & & 1720 & & 4070 & & 1600 & & & $<4600$ & 1 & $\mathrm{ug} / \mathrm{L}$ & EX \\
\hline$+\quad$ Sulfate & 3950 & & 4850 & $\mathrm{~J}$ & 3930 & & 4350 & & & $>3000$ & 2 & ug/L & EX \\
\hline \multicolumn{14}{|l|}{ Radionuclides } \\
\hline Gross alpha & 1.01 & & .04 & UI & 2.93 & & 1.81 & $J$ & 1 & NDD & 1 & $\mathrm{pCi} / \mathrm{L}$ & GP \\
\hline Nonvolatile beta & 2.32 & & -.27 & UI & 6.04 & & 3.28 & & & $<50$ & 1 & $\mathrm{pCi} / \mathrm{L}$ & GP \\
\hline Radium, total alpha-emitting & 0 & UI & 9.12 & UIJ & 1.04 & & 2.8 & & & $<5$ & 1 & $\mathrm{pCi} / \mathrm{L}$ & GP \\
\hline
\end{tabular}

Notes: Concentrations in bold italics exceed the groundwater protection or monitoring constituent standards listed in Appendix A. Synchronous water levels are measured over a 3-5 day period or less. Dilution factors, Laboratory, and Filtered Data are for Groundwater Protection Standards First Quarter 2000 and Monitoring Constituents Third Quarter 1999 data only.

$+=$ exceeded the Groundwater Protection Standards (First Quarter 2000) or Monitoring Constituents (Third Quarter 1999) listed in Appendix A. 
Table D-2. Groundwater Monitoring Results for Point-of-Compliance Wells, M-Area HWMF (Cont.) WELL MSB 59D

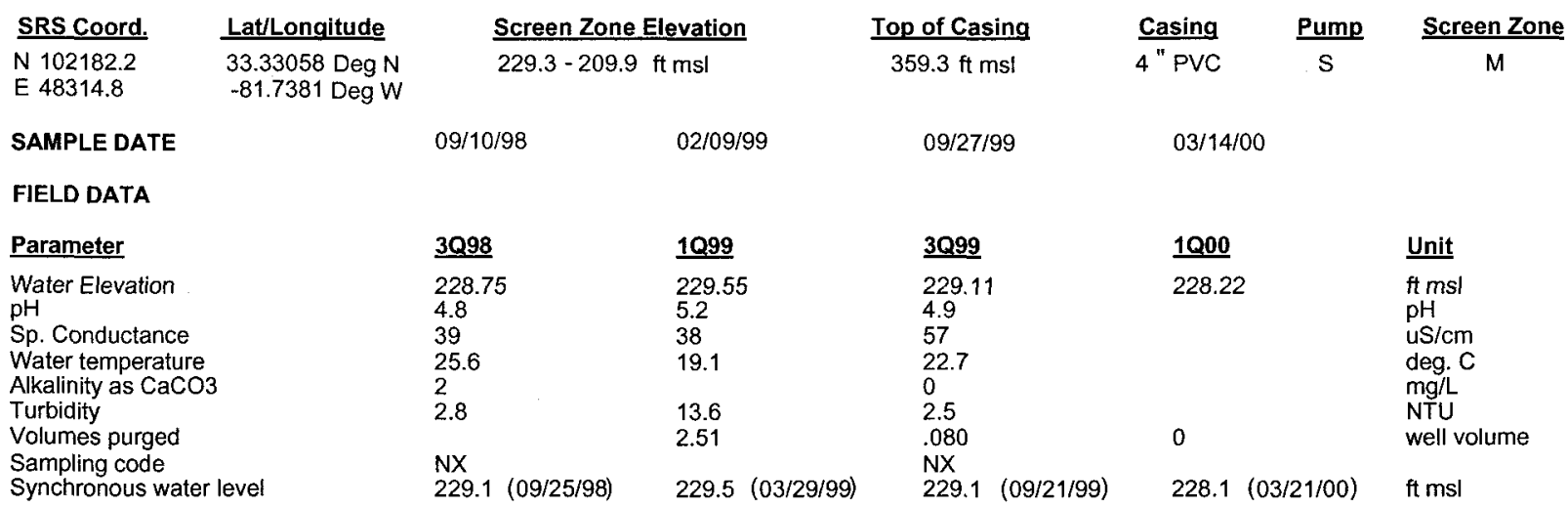

ANALYTICAL DATA

I. Groundwater Protection Standard

261 Appendix VIII/264 Appendix IX Hazardous Constituents

\begin{tabular}{|c|c|c|c|c|c|c|c|c|c|c|c|c|c|c|}
\hline$\underline{\text { ST }}$ & Parameter & 3Q98 & CLPEPA & 10.99 & CLP EPA & 3Q999 & CLPEP & $\underline{1 Q 00}$ & CLP & EPA & Filt. & DF & Unit & Lab \\
\hline Inor & ganics & & & & & & & & & & & & & \\
\hline & Barium, total recoverable & 22.2 & & 14 & & 31.4 & & 13.2 & $J$ & 1 & NDD & 1 & $u g / L$ & ML \\
\hline & Cyanide & $<10$ & $\mathrm{U}$ & $<10$ & $U$ & $<10$ & $J U Q$ & $<35.1$ & JU & LV & $<$ EQL & 1 & $\mathrm{ug} / \mathrm{L}$ & ML \\
\hline & Lead, total recoverable & $<100$ & $U$ & $<100$ & $U$ & $<10$ & $U$ & 10.4 & $J$ & 1 & NDD & 1 & $u g / L$ & $\mathrm{ML}$ \\
\hline & Nickel, total recoverable & 7.76 & 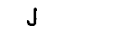 & $<50$ & $\mathrm{U}$ & $<50$ & $\mathrm{U}$ & $<60$ & $U$ & & $<E Q L$ & 1 & $\mathrm{ug} / \mathrm{L}$ & ML \\
\hline & Selenium, total recoverable & $<200$ & $U$ & $<200$ & $\mathrm{U}$ & $<10$ & $\mathbf{U}$ & $<40$ & $U$ & & $<\mathrm{EQL}$ & 1 & $\mathrm{ug} / \mathrm{L}$ & ML \\
\hline Org & anics & & & & & & & & & & & & & \\
\hline & Chlorobenzene & $<2500$ & UJ & $<2500$ & $\mathrm{U}$ & 3.93 & & $<200$ & $\mathrm{U}$ & & $<E Q L$ & 200 & $\mathrm{ug} / \mathrm{L}$ & $M L$ \\
\hline & 1,1-Dichloroethane & $<2500$ & UJ & $<2500$ & U & $<1$ & $\mathrm{U}$ & $<200$ & U & & $<\mathrm{EQL}$ & 200 & $u g / L$ & ML \\
\hline & 1,1-Dichloroethylene & $<2500$ & UJ & $<2500$ & $U$ & 3.43 & & $<200$ & $U$ & & $<$ EQL & 200 & $u g / L$ & ML \\
\hline & trans-1,2-Dichloroethylene & $<2500$ & UJ & $<2500$ & $\mathrm{U}$ & $<1$ & $\mathrm{U}$ & $<200$ & U & & $<E Q L$ & 200 & $\mathrm{ug} / \mathrm{L}$ & $M L$ \\
\hline & РСВ 1016 & $<2$ & $\mathrm{U}$ & $<2$ & $\mathrm{U}$ & $<2$ & $\mathrm{U}$ & $<.99$ & $U$ & & < EQL & 1 & $\mathrm{ug} / \mathrm{L}$ & ML \\
\hline & PCB 1221 & $<2$ & $U$ & $<2$ & $U$ & $<2$ & $U$ & $<.99$ & $U$ & & $<E Q L$ & 1 & $u g / L$ & ML \\
\hline & PCB 1232 & $<1$ & $U$ & $<1$ & $U$ & $<.99$ & $U$ & $<.99$ & U & & $<E Q L$ & 1 & $u g / L$ & ML \\
\hline & РСB 1242 & $<1$ & U & $<1$ & U & $<.99$ & $\mathrm{U}$ & $<.99$ & U & & $<\mathrm{EQL}$ & 1 & $\mathrm{ug} / \mathrm{L}$ & $M L$ \\
\hline & РСВ 1248 & $<1$ & $\mathrm{U}$ & $<1$ & U & $<.99$ & $\mathrm{U}$ & $<.99$ & $\mathrm{U}$ & & $<E Q L$ & 1 & $\mathrm{ug} / \mathrm{L}$ & $M L$ \\
\hline & РСВ 1254 & $<1$ & U & $<1$ & $\mathrm{U}$ & $<.99$ & U & $<.99$ & $\mathrm{U}$ & & $<E Q L$ & 1 & $\mathrm{ug} / \mathrm{L}$ & $M L$ \\
\hline & РСB 1260 & $<1$ & $U$ & $<1$ & $\mathrm{U}$ & $<.99$ & $\mathrm{U}$ & $<.99$ & $\mathrm{U}$ & & $<E Q L$ & 1 & $\mathrm{ug} / \mathrm{L}$ & ML \\
\hline & $1,1,2,2$-Tetrachloroethane & $<2500$ & UJ & $<2500$ & $\mathrm{U}$ & 9.28 & & $<200$ & $\mathrm{U}$ & & $<E Q L$ & 200 & $\mathrm{ug} / \mathrm{L}$ & ML \\
\hline & Tetrachloroethylene & 58000 & 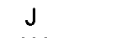 & 66700 & & 57300 & & 127000 & $J$ & $\mathrm{~K}$ & NDD & 200 & $\mathrm{ug} / \mathrm{L}$ & ML \\
\hline & $1,1,1-$ Trichloroethane & $<2500$ & UJ & $<2500$ & $U$ & 4.12 & & $<200$ & $U$ & & $<E Q L$ & 200 & $\mathrm{ug} / \mathrm{L}$ & ML \\
\hline & Trichloroethylene & 14600 & $J$ & 7550 & & 11000 & & 18000 & $J$ & $\mathrm{~K}$ & NDD & 200 & $u g / L$ & ML \\
\hline II. $M$ & onitoring Constituents & & & & & & & & & & & & & \\
\hline$\underline{\text { ST }}$ & Parameter & $\underline{3096}$ & $\underline{\text { CLP EPA }}$ & $\underline{3 Q 97}$ & CLPP EPA & $\underline{3 Q 98}$ & CLP EPA & $\underline{3099}$ & CLㄹ & EPA & Filt. & $\underline{\text { DF }}$ & Unit & $\underline{\text { Lab }}$ \\
\hline Inor & ganics & & & & & & & & & & & & & \\
\hline & Aluminum, total recoverable & 242 & & 265 & & 162 & $J$ & 130 & J & IK & NDD & 1 & $\mathrm{ug} / \mathrm{L}$ & WA \\
\hline+ & Nitrate-nitrite as nitrogen & 3000 & & 2420 & & 2030 & & 4360 & & & $>2400$ & 5 & ug/L & EX \\
\hline & Sodium, total recoverable & 2960 & $J$ & 2740 & & 2980 & & 4010 & & & $<4600$ & 1 & $\mathrm{ug} / \mathrm{L}$ & $\mathrm{EX}$ \\
\hline & Sulfate & 147 & $J$ & $<5000$ & U & 215 & $J$ & 629 & & & $<3000$ & 2 & $\mathrm{ug} / \mathrm{L}$ & EX \\
\hline Rad & onuclides & & & & & & & & & & & & & \\
\hline & Gross alpha & 3.7 & & 5.14 & & 5.98 & & 6.59 & & & $<15$ & 1 & $\mathrm{pCi} / \mathrm{L}$ & GP \\
\hline & Nonvolatile beta & 2.4 & & 1.94 & UI & 5.62 & & 2.98 & & & $<50$ & 1 & $\mathrm{pCi} / \mathrm{L}$ & GP \\
\hline & Radium, total alpha-emitting & 1.4 & & 2.44 & บIJ & 1.67 & & 1 & $\mathrm{~J}$ & I & NDD & 1 & $\mathrm{pCi} / \mathrm{L}$ & GP \\
\hline
\end{tabular}

Notes: Concentrations in bold italics exceed the groundwater protection or monitoring constituent standards listed in Appendix A. Synchronous water levels are measured over a 3-5 day period or less. Dilution factors, Laboratory, and Filtered Data are for Groundwater Protection Standards First Quarter 2000 and Monitoring Constituents Third Quarter 1999 data only.

$+=$ exceeded the Groundwater Protection Standards (First Quarter 2000) or Monitoring Constituents (Third Quarter 1999) listed in Appendix A. 
Table D-2. Groundwater Monitoring Results for Point-of-Compliance Wells, M-Area HWMF (Cont.) WELL MSB 62B

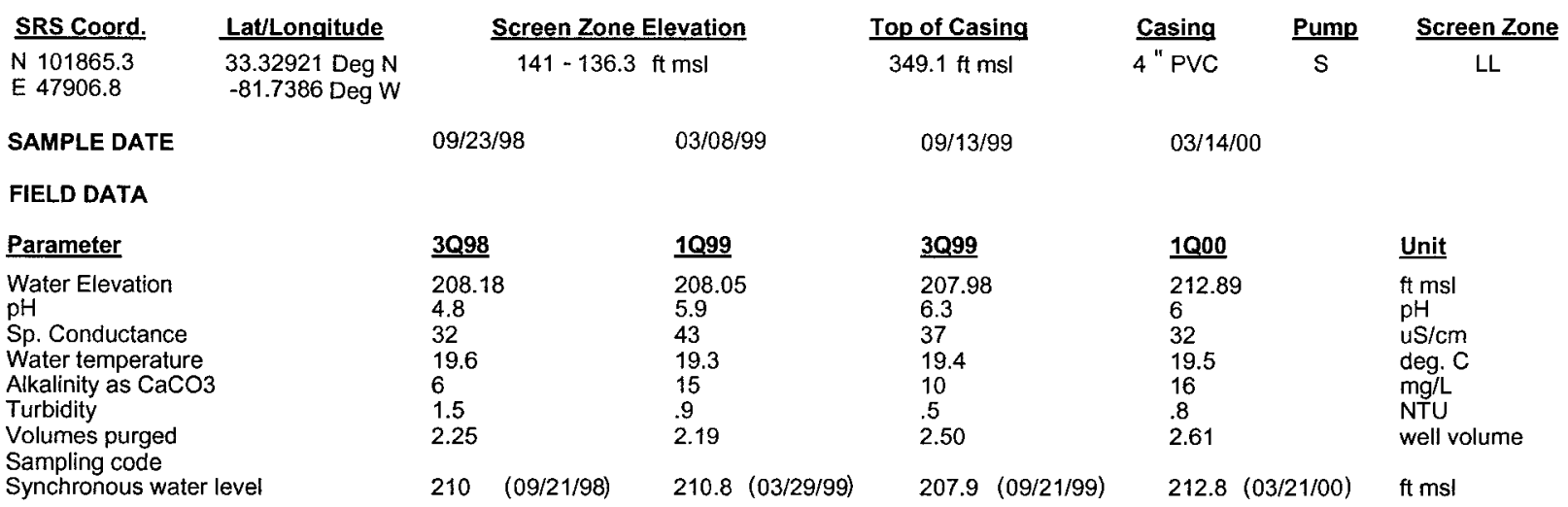

ANALYTICAL DATA

I. Groundwater Protection Standard

261 Appendix VIII/264 Appendix IX Hazardous Constituents

\begin{tabular}{|c|c|c|c|c|c|c|c|c|c|c|c|c|c|c|}
\hline$\underline{\text { ST }}$ & Parameter & $\underline{3 Q 98}$ & CLP EPA & 1099 & CLPEPA & $\underline{3 Q 99}$ & CLPEPA & 1000 & CLF & EPA & Filt. & $\underline{\mathrm{DF}}$ & Unit & $\underline{\text { Lab }}$ \\
\hline & ganics & & & & & & & & & & & & & \\
\hline & Barium, total recoverable & 19.5 & & 23.9 & & 18.7 & & 16 & & & $<2000$ & 1 & $u g / L$ & $\mathrm{ML}$ \\
\hline & Cyanide & $<10^{\circ}$ & $\mathrm{U}$ & $<15.2$ & $\mathrm{U}$ & $<10$ & $\mathrm{U}$ & & & & & & $\mathrm{ug} / \mathrm{L}$ & \\
\hline & Lead, total recoverable & $<100$ & $\mathrm{U}$ & $<100$ & $\mathrm{U}$ & $<10$ & $U$ & $<20$ & $U$ & & $<E Q L$ & 1 & ug/L & ML \\
\hline & Nickel, total recoverable & $<50$ & $U$ & $<50$ & $\mathrm{U}$ & $<50$ & $U$ & $<60$ & $\mathrm{U}$ & & $<E Q L$ & 1 & ug/L & ML \\
\hline & Selenium, total recoverable & $<200$ & $U$ & $<200$ & $U$ & $<10$ & $\mathrm{U}$ & $<40$ & $\mathbf{U}$ & & $<E Q L$ & 1 & $\mathrm{ug} / \mathrm{L}$ & ML \\
\hline Org & anics & & & & & & & & & & & & & \\
\hline & Chlorobenzene & $<5$ & $\mathrm{U}$ & $<5$ & $U$ & $<.35$ & U V & $<1$ & $\mathrm{U}$ & & $<$ EQL & 1 & $u g / L$ & ML \\
\hline & 1,1-Dichloroethane & $<5$ & U & $<5$ & $U$ & $<1$ & $\mathrm{U}$ & $<1$ & $\mathrm{U}$ & & $<E Q L$ & 1 & $\mathrm{ug} / \mathrm{L}$ & ML \\
\hline & 1,1-Dichloroethylene & $<5$ & $U$ & $<5$ & $U$ & $<1$ & $\mathrm{U}$ & $<1$ & $\mathrm{U}$ & & $<E Q L$ & 1 & $\mathrm{ug} / \mathrm{L}$ & ML \\
\hline & trans-1,2-Dichloroethylene & $<5$ & $U$ & $<5$ & $U$ & $<1$ & $\mathrm{U}$ & $<1$ & U & & $<E Q L$ & 1 & $\mathrm{ug} / \mathrm{L}$ & ML \\
\hline & $\begin{array}{l}\text { PCB } 1016 \\
\text { PCB } 1221\end{array}$ & & & & & & & & & & & & & \\
\hline & PCB 1232 & & & & & & & & & & & & & \\
\hline & PCB 1242 & & & & & & & & & & & & & \\
\hline & PCB 1248 & & & & & & & & & & & & & \\
\hline & PCB 1254 & & & & & & & & & & & & & \\
\hline & PCB 1260 & & & & & & & & & & & & & \\
\hline & $1,1,2,2$-Tetrachloroethane & $<5$ & $U$ & $<5$ & $\mathrm{U}$ & $<1$ & $U$ & $<1$ & $\mathrm{u}$ & & $<\mathrm{EQL}$ & 1 & $\mathrm{ug} / \mathrm{L}$ & ML \\
\hline & Tetrachloroethylene & 26.3 & & 17,4 & & 18.8 & & 14.6 & J & $\mathrm{K}$ & NDD & 1 & $\mathrm{ug} / \mathrm{L}$ & ML \\
\hline & 1,1,1-Trichloroethane & $<5$ & $\mathrm{U}$ & $<5$ & $U$ & $<1$ & $\mathrm{U}$ & $<1$ & $\mathrm{U}$ & & $<E Q L$ & 1 & $\mathrm{ug} / \mathrm{L}$ & ML \\
\hline & Trichloroethylene & 44.8 & & 41.2 & & 49.5 & & 38.2 & $\mathrm{~J}$ & $\mathrm{~K}$ & NDD & 1 & $\mathrm{ug} / \mathrm{L}$ & ML \\
\hline II. $\mathbf{M}$ & onitoring Constituents & & & & & & & & & & & & & \\
\hline$\underline{\mathbf{S T}}$ & Parameter & $\underline{3 Q 96}$ & CLPEPA & $\underline{3097}$ & CLP EPA & $\underline{3098}$ & CLPEPA & 3099 & CLP & EPA & Filt. & $\underline{\text { DF }}$ & Unit & $\underline{\text { Lab }}$ \\
\hline Ino & ganics & & & & & & & & & & & & & \\
\hline & Aluminum, total recoverable & $<20$ & U & 45.3 & & $<200$ & $U$ & $<200$ & $\mathrm{U}$ & & $<E Q L$ & 1 & $\mathrm{ug} / \mathrm{L}$ & EX \\
\hline & Nitrate-nitrite as nitrogen & 1230 & & 845 & & 1550 & & 570 & & & $<2400$ & 5 & $\mathrm{ug} / \mathrm{L}$ & EX \\
\hline & Sodium, total recoverable & 3590 & & 1970 & & 2470 & & 2400 & & & $<4600$ & 1 & ug $/ \mathrm{L}$ & EX \\
\hline & Sulfate & 415 & J & $<5000$ & $\mathbf{u}$ & 507 & & 560 & & & $<3000$ & 1 & $\mathrm{ug} / \mathrm{L}$ & EX \\
\hline Rad & ionuclides & & & & & & & & & & & & & \\
\hline+ & Gross alpha & .614 & & .2 & Ul & .9 & & 27 & & & $>15$ & 1 & $\mathrm{pCi} / \mathrm{L}$ & GP \\
\hline & Nonvolatile beta & 1.04 & Ul & .34 & UI & 1.97 & J & 6.33 & $J$ & $\mathrm{~K}$ & NDD & 1 & $\mathrm{pCi} / \mathrm{L}$ & GP \\
\hline & Radium, total alpha-emitting & 0 & UIJ & 8.72 & $\mathrm{~J}$ & .65 & & .2 & $U$ & & $<E Q L$ & 1 & $\mathrm{pCi} / \mathrm{L}$ & GP \\
\hline
\end{tabular}

Notes: Concentrations in bold italics exceed the groundwater protection or monitoring constituent standards listed in Appendix A. Synchronous water levels are measured over a 3-5 day period or less. Dilution factors, Laboratory, and Filtered Data are for Groundwater Protection Standards First Quarter 2000 and Monitoring Constituents Third Quarter 1999 data only.

$+=$ exceeded the Groundwater Protection Standards (First Quarter 2000) or Monitoring Constituents (Third Quarter 1999) listed in Appendix A. 
Table D-2. Groundwater Monitoring Results for Point-of-Compliance Wells, M-Area HWMF (Cont.) WELL MSB 62C

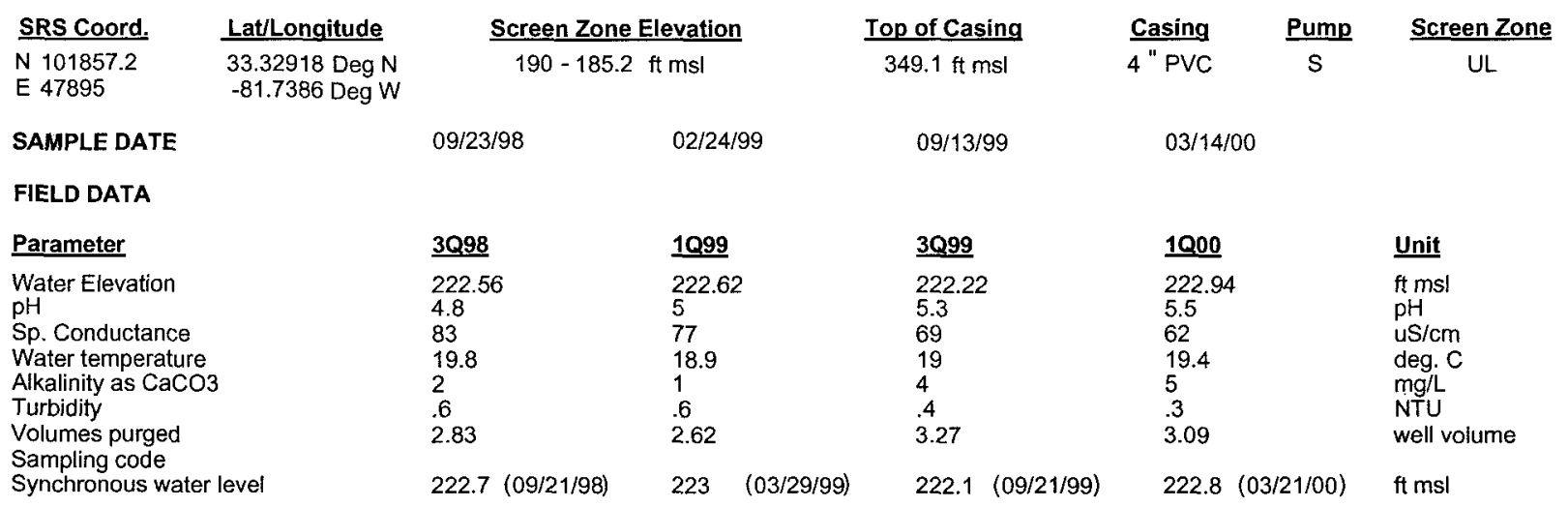

ANALYTICAL DATA

I. Groundwater Protection Standard

261 Appendix VIII/264 Appendix IX Hazardous Constituents

\begin{tabular}{|c|c|c|c|c|c|c|c|c|c|c|c|c|c|c|c|}
\hline$\underline{\text { ST }}$ & Parameter & $\underline{3 Q 98}$ & CLPPEPA & $\underline{1 Q 99}$ & CLPEPA & 3Q999 & $\underline{\text { CLP }}$ & EPA & 1000 & $\underline{\mathrm{CLF}}$ & EPA & Filt. & $\underline{\text { DF }}$ & Unit & Lab \\
\hline Inor & ganics & & & & & & & & & & & & & & \\
\hline & Barium, total recoverable & 6.09 & $\mathrm{~J}$ & 4.79 & J I & 4.2 & $J$ & 1 & 3.74 & $\mathrm{~J}$ & 1 & NDD & 1 & $u g / L$ & ML \\
\hline & Cyanide & $<10$ & $U$ & $<10$ & $U$ & $<10$ & $\mathrm{U}$ & & & & & & & $\mathrm{ug} / \mathrm{L}$ & \\
\hline & Lead, total recoverable & $<100$ & $U$ & $<100$ & $U$ & $<10$ & $U$ & & $<20$ & $\mathrm{U}$ & & $<E Q L$ & 1 & $\mathrm{ug} / \mathrm{L}$ & ML \\
\hline & Nickel, total recoverable & $<50$ & $U$ & $<50$ & $U$ & $<50$ & $\mathrm{U}$ & & $<60$ & $\mathrm{U}$ & & $<E Q L$ & 1 & ug/L & $M L$ \\
\hline & Selenium, total recoverable & $<200$ & $\mathrm{U}$ & $<10$ & $\mathrm{U}$ & $<10$ & $U$ & & $<40$ & $\mathrm{U}$ & & $<E Q L$ & 1 & $\mathrm{ug} / \mathrm{L}$ & $M L$ \\
\hline Org & anics & & & & & & & & & & & & & & \\
\hline & Chlorobenzene & $<5$ & $u$ & $<5$ & u & $<.42$ & $\mathrm{U}$ & V & $<1$ & $\mathrm{U}$ & & $<$ EQL & 1 & $\mathrm{ug} / \mathrm{L}$ & $\mathrm{ML}$ \\
\hline & 1,1-Dichloroethane & $<5$ & $U$ & $<5$ & $\mathrm{U}$ & $<1$ & U & & $<1$ & $\mathrm{U}$ & & $<E Q L$ & 1 & $u g / L$ & $\mathrm{ML}$ \\
\hline & 1,1-Dichloroethylene & 2.19 & J & $<5$ & $\mathrm{U}$ & .93 & J & I & .81 & $\mathrm{~J}$ & I & NDD & 1 & $\mathrm{ug} / \mathrm{L}$ & $M L$ \\
\hline & trans-1,2-Dichloroethylene & $<5$ & U & $<5$ & $\mathrm{U}$ & $<1$ & $\mathrm{U}$ & & $<1$ & $\mathrm{U}$ & & $<\mathrm{EQL}$ & 1 & $\mathrm{ug} / \mathrm{L}$ & $\mathrm{ML}$ \\
\hline & PCB 1016 & & & & & & & & & & & & & & \\
\hline & $\begin{array}{l}\text { PCB } 1221 \\
\text { PCB } 1232\end{array}$ & & & & & & & & & & & & & & \\
\hline & $\begin{array}{l}\text { PCB } 1232 \\
\text { PCB } 1242\end{array}$ & & & & & & & & & & & & & & \\
\hline & $\begin{array}{l}\text { PCB } 1242 \\
\text { PCB } 1248\end{array}$ & & & & & & & & & & & & & & \\
\hline & $\begin{array}{l}\text { PCB } 1248 \\
\text { PCB } 1254\end{array}$ & & & & & & & & & & & & & & \\
\hline & PCB 1254 & & & & & & & & & & & & & & \\
\hline & PCB 1260 & & & & & & & & & & & & & & \\
\hline & 1,1,2,2-Tetrachloroethane & $<5$ & $\mathrm{U}$ & $<5$ & $U$ & $<1$ & $U$ & & $<1$ & $\mathrm{U}$ & & $<E Q L$ & 1 & $\mathrm{ug} / \mathrm{L}$ & ML \\
\hline+ & Tetrachloroethylene & 39.7 & & 31.2 & & 21.6 & & & 17.6 & & & $>5$ & 1 & $\mathrm{ug} / \mathrm{L}$ & ML \\
\hline & 1,1,1-Trichloroethane & $<5$ & U & $<5$ & $u$ & $<1$ & $\mathrm{U}$ & & $<1$ & $\mathrm{U}$ & & $<\mathrm{EQL}$ & 1 & ug/L & ML \\
\hline+ & Trichloroethylene & 33.3 & & 29.4 & & 25.3 & & & 20.8 & & & $>5$ & 1 & $\mathrm{ug} / \mathrm{L}$ & ML \\
\hline II. $\mathbf{M}$ & onitoring Constituents & & & & & & & & & & & & & & \\
\hline$\underline{\text { ST }}$ & Parameter & $\underline{3096}$ & CLP EPA & $\underline{3097}$ & CLP EPA & $\underline{3098}$ & $\underline{\text { CLP }}$ & EPA & $\underline{3099}$ & $\underline{\text { CLF }}$ & EPA & Filt. & $\underline{\mathrm{DF}}$ & Unit & $\underline{\text { Lab }}$ \\
\hline Inor & ganics & & & & & & & & & & & & & & \\
\hline & Aluminum, total recoverable & $<20$ & U & 48.9 & & $<200$ & U & & $<200$ & $\mathrm{U}$ & & $<E Q L$ & 1 & $\mathrm{ug} / \mathrm{L}$ & EX \\
\hline+ & Nitrate-nitrite as nitrogen & 12700 & & 684 & & 496 & $J$ & & 4400 & & & $>2400$ & 5 & $\mathrm{ug} / \mathrm{L}$ & EX \\
\hline+ & Sodium, total recoverable & 19400 & $J$ & 15200 & & 14200 & & & 11700 & & & $>4600$ & 1 & $\mathrm{ug} / \mathrm{L}$ & EX \\
\hline+ & Sulfate & 1250 & & 4220 & $\mathrm{~J}$ & 5910 & & & 5000 & & & $>3000$ & 1 & ug/L & EX \\
\hline Radi & ionuclides & & & & & & & & & & & & & & \\
\hline+ & Gross alpha & .962 & & .63 & UI & .8 & & & 17.9 & & & $>15$ & 1 & $\mathrm{pCi} / \mathrm{L}$ & GP \\
\hline & Nonvolatile beta & 2.34 & & 2.62 & & .33 & UIJ & & 4.33 & $\mathrm{~J}$ & $\mathrm{~K}$ & NDD & 1 & $\mathrm{pCi} / \mathrm{L}$ & GP \\
\hline & Radium, total alpha-emitting & .4 & $J$ & 5.44 & $\mathrm{~J}$ & .44 & UI & & -.3 & $\bigcup$ & & $<\mathrm{EQL}$ & 1 & $\mathrm{pCi} / \mathrm{L}$ & GP \\
\hline
\end{tabular}

Notes: Concentrations in bold italics exceed the groundwater protection or monitoring constituent standards listed in Appendix A. Synchronous water levels are measured over a 3-5 day period or less. Dilution factors, Laboratory, and Filtered Data are for Groundwater Protection Standards First Quarter 2000 and Monitoring Constituents Third Quarter 1999 data only.

$+=$ exceeded the Groundwater Protection Standards (First Quarter 2000) or Monitoring Constituents (Third Quarter 1999) listed in Appendix A. 
Table D-2. Groundwater Monitoring Results for Point-of-Compliance Wells, M-Area HWMF (Cont.) WELL MSB 62D

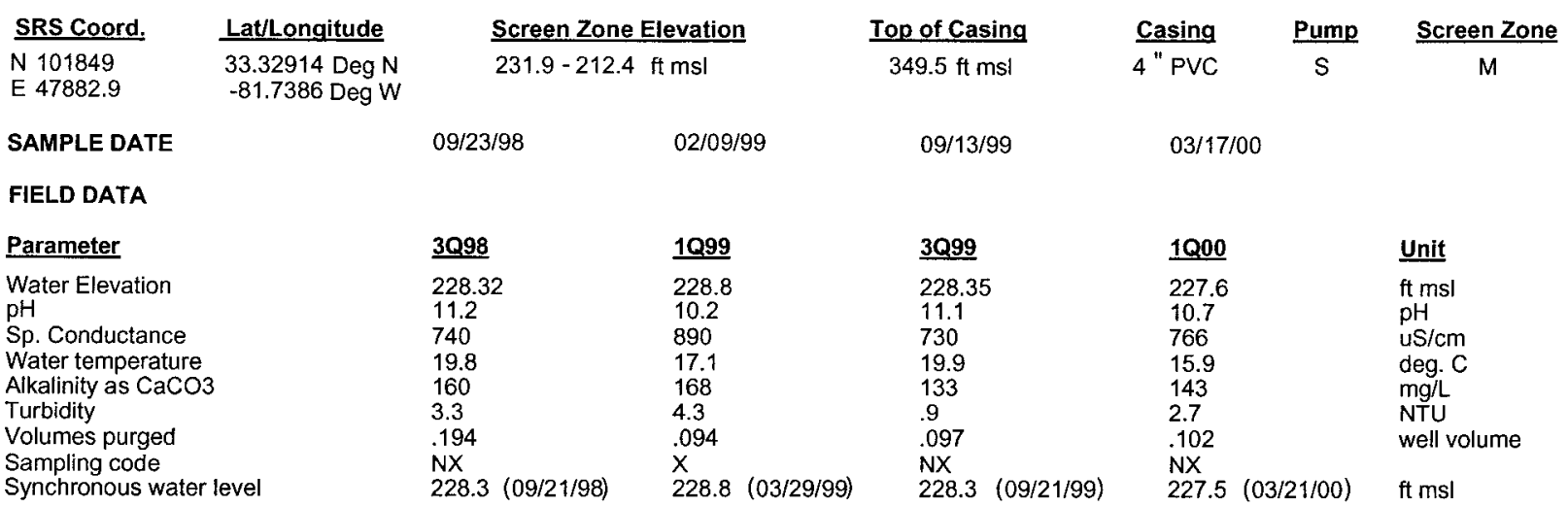

ANALYTICAL DATA

I. Groundwater Protection Standard

261 Appendix VIII/264 Appendix IX Hazardous Constituents

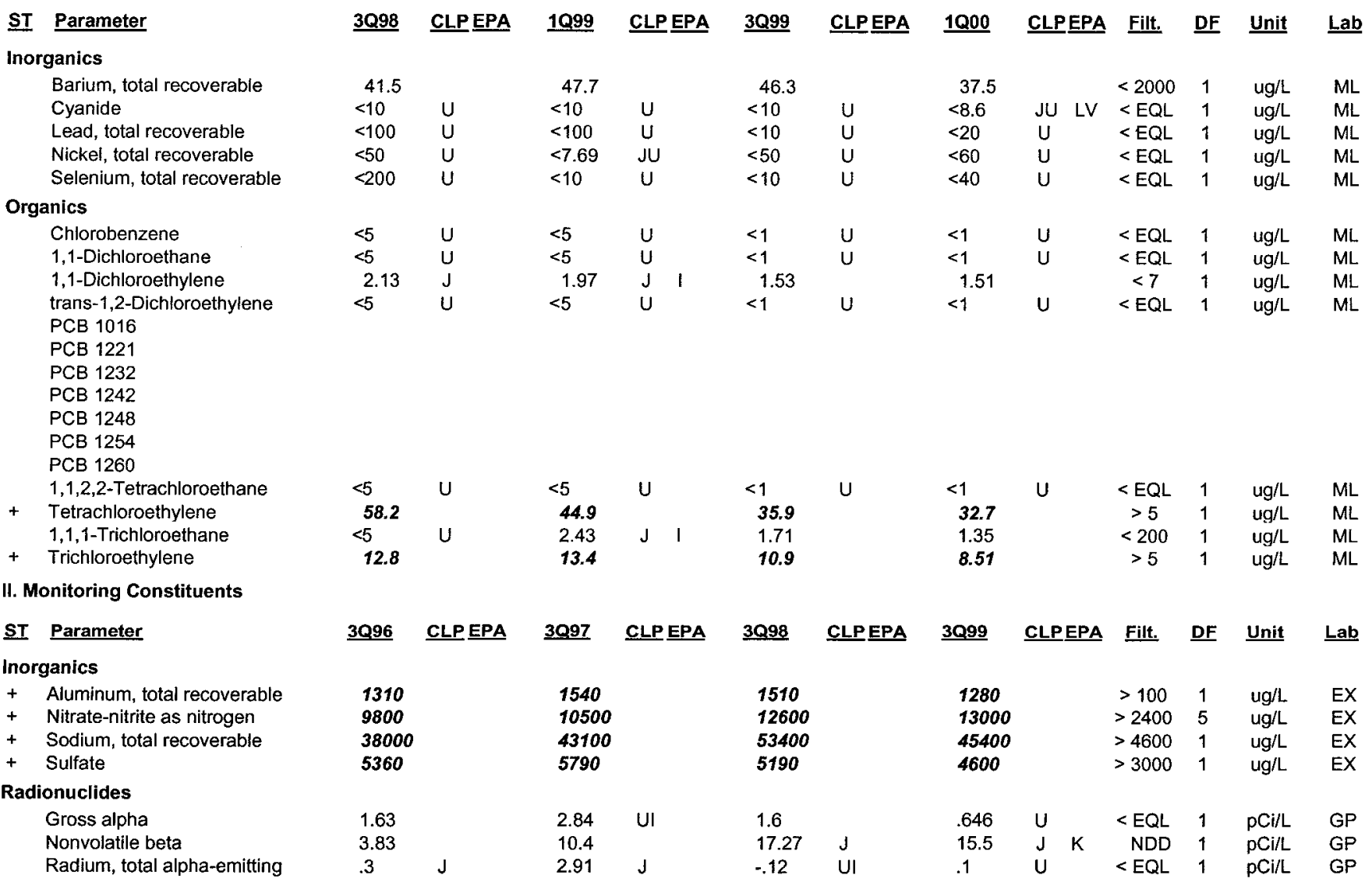

Notes: Concentrations in bold italics exceed the groundwater protection or monitoring constituent standards listed in Appendix A. Synchronous water levels are measured over a 3-5 day period or less. Dilution factors, Laboratory, and Filtered Data are for Groundwater Protection Standards First Quarter 2000 and Monitoring Constituents Third Quarter 1999 data only.

$+=$ exceeded the Groundwater Protection Standards (First Quarter 2000) or Monitoring Constituents (Third Quarter 1999) listed in Appendix A. 
Table D-2. Groundwater Monitoring Results for Point-of-Compliance Wells, M-Area HWMF (Cont.) WELL MSB 63B

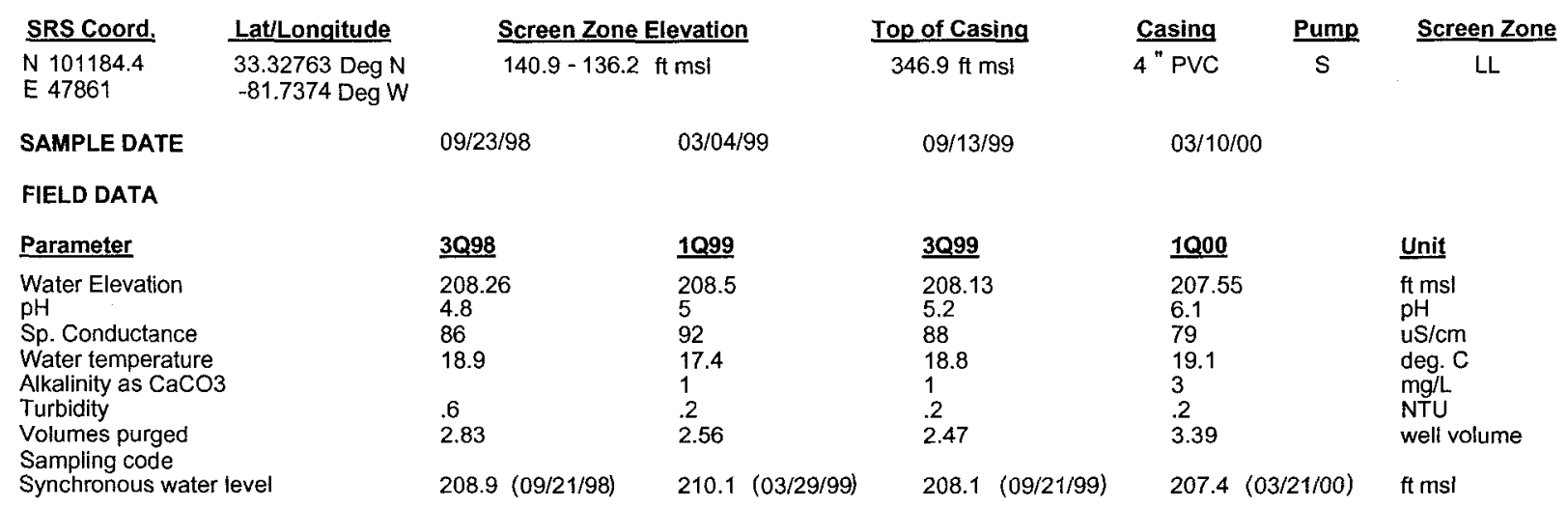

\section{ANALYTICAL DATA}

I. Groundwater Protection Standard

261 Appendix VIII/264 Appendix IX Hazardous Constituents

\begin{tabular}{|c|c|c|c|c|c|c|c|c|c|c|c|c|c|}
\hline \multirow{2}{*}{$\begin{array}{l}\text { ST Parameter } \\
\text { Inorganics }\end{array}$} & \multirow[t]{2}{*}{$\underline{3098}$} & \multirow[t]{2}{*}{ CLPEPA } & \multirow[t]{2}{*}{$\underline{1099}$} & \multirow[t]{2}{*}{ CLPEPA } & \multirow[t]{2}{*}{$\underline{3099}$} & CLPEPA & \multirow[t]{2}{*}{$\underline{1000}$} & \multicolumn{2}{|c|}{ CLPEPA } & \multirow[t]{2}{*}{ Filt. } & \multirow[t]{2}{*}{$\underline{\mathrm{DF}}$} & \multirow[t]{2}{*}{$\underline{\text { Unit }}$} & \multirow[t]{2}{*}{$\underline{\text { Lab }}$} \\
\hline & & & & & & & & & & & & & \\
\hline Barium, total recoverable & 20.5 & & 24.3 & & 24.2 & & & & & & & $\mathrm{ug} / \mathrm{L}$ & \\
\hline Cyanide & $<10$ & $\mathrm{u}$ & $<10$ & $u$ & $<10$ & $\mathrm{u}$ & & & & & & $u g / L$ & \\
\hline Lead, total recoverable & $<100$ & $\mathrm{U}$ & $<100$ & $\mathrm{u}$ & $<10$ & $u$ & & & & & & $\mathrm{ug} / \mathrm{L}$ & \\
\hline Nickel, total recoverable & $<50$ & $u$ & $<50$ & $u$ & $<50$ & $u$ & & & & & & ug/ & \\
\hline Selenium, total recoverable & $<200$ & $U$ & $<10$ & $u$ & $<10$ & $\mathrm{U}$ & & & & & & $\mathrm{ug} / \mathrm{L}$ & \\
\hline \multicolumn{13}{|l|}{ Organics } & \\
\hline+ Chlorobenzene & $<25$ & $\mathrm{u}$ & $<5$ & $\mathrm{u}$ & $<1$ & u & 6.5 & & & $>5$ & 5 & $u g / L$ & \\
\hline 1,1-Dichloroethane & $<25$ & $u$ & $<5$ & u & $<1$ & $u$ & $<5$ & $u$ & & $<E Q L$ & 5 & $\mathrm{ug} / \mathrm{L}$ & \\
\hline 1,1-Dichloroethylene & $<25$ & $u$ & $<5$ & $u$ & 2.61 & $\mathrm{~J} K$ & $<5$ & $u$ & & $<E Q L$ & 5 & $\mathrm{ug} / \mathrm{L}$ & \\
\hline trans-1,2-Dichloroethylene & $<25$ & $\mathrm{U}$ & $<5$ & $u$ & $<1$ & u & & & & & & $u g / L$ & \\
\hline PCB 1016 & & & & & & & & & & & & & \\
\hline РСB 1221 & & & & & & & & & & & & & \\
\hline PCB 1232 & & & & & & & & & & & & & \\
\hline PCB 1242 & & & & & & & & & & & & & \\
\hline PCB 1248 & & & & & & & & & & & & & \\
\hline PCB 1254 & & & & & & & & & & & & & \\
\hline PCB 1260 & & & & & & & & & & & & & \\
\hline 1,1,2,2-Tetrachloroethane & $<25$ & u & $<5$ & $\mathrm{U}$ & $<1$ & $\mathrm{u}$ & $<5$ & $\mathrm{u}$ & & $<E Q L$ & 5 & $\mathrm{ug} / \mathrm{L}$ & \\
\hline + Tetrachloroethylene & 149 & & 167 & & 170 & $\mathrm{~J} K$ & 125 & & & $>5$ & 5 & $\mathrm{ug} / \mathrm{L}$ & \\
\hline 1,1,1-Trichloroethane & $<25$ & $\mathrm{u}$ & $<5$ & $\mathrm{u}$ & $<1$ & $\mathrm{U}$ & $<5$ & $u$ & & $<E Q L$ & 5 & $\mathrm{ug} / \mathrm{L}$ & \\
\hline+ Trichloroethylene & 490 & & 426 & & 545 & & 684 & & & $>5$ & 5 & $\mathrm{ug} / \mathrm{L}$ & \\
\hline \multicolumn{13}{|l|}{ II. Monitoring Constituents } & \\
\hline ST Parameter & $\underline{3096}$ & CLPEPA & $\underline{3 Q 97}$ & CLPEPA & $\underline{3 Q 98}$ & CLPEPA & $\underline{3 Q 99}$ & $\underline{\text { CLP }}$ & DEPA & Filt. & $\underline{\mathrm{DF}}$ & $\underline{\text { Unit }}$ & \\
\hline \multicolumn{13}{|l|}{ Inorganics } & \\
\hline Aluminum, total recoverable & $<20$ & $u$ & $<20$ & $\mathrm{u}$ & $<200$ & $u$ & $<200$ & $u$ & & $<E Q L$ & 1 & $\mathrm{ug} / \mathrm{L}$ & \\
\hline$+\quad$ Nitrate-nitrite as nitrogen & 2760 & & 5170 & & 6990 & & 7800 & & & $>2400$ & 5 & $\mathrm{ug} / \mathrm{L}$ & \\
\hline+ Sodium, total recoverable & 2220 & $\mathrm{~J}$ & 3870 & & 5950 & & 6660 & & & $>4600$ & 1 & $u g / L$ & \\
\hline Sulfate & 139 & J & $<5000$ & $u$ & 359 & J & 120 & J & 1 & NDD & 1 & $u g / L$ & \\
\hline \multicolumn{13}{|l|}{ Radionuclides } & \\
\hline Gross alpha & .456 & $\mathrm{UI}$ & 1.16 & $\mathrm{UI}$ & 3.36 & & 1.84 & & & $<15$ & 1 & $\mathrm{pCi} / \mathrm{L}$ & \\
\hline Nonvolatile beta & .797 & $\mathrm{UI}$ & 1.27 & Ul & 7.29 & $\mathrm{~J}$ & 3.91 & $\mathrm{~J}$ & $\mathrm{k}$ & NDD & 1 & $\mathrm{pCi} / \mathrm{L}$ & G \\
\hline Radium, total alpha-emitting & .2 & $\mathrm{Ul}$ & 1.46 & UIJ & .93 & & .9 & $J$ & 1 & NDD & 1 & $\mathrm{pCi} / \mathrm{L}$ & \\
\hline
\end{tabular}

Notes: Concentrations in bold italics exceed the groundwater protection or monitoring constituent standards listed in Appendix A. Synchronous water levels are measured over a 3-5 day period or less. Dilution factors, Laboratory, and Filtered Data are for Groundwater Protection Standards First Quarter 2000 and Monitoring Constituents Third Quarter 1999 data only.

+ = exceeded the Groundwater Protection Standards (First Quarter 2000) or Monitoring Constituents (Third Quarter 1999) listed in Appendix A. 
Table D-2. Groundwater Monitoring Results for Point-of-Compliance Wells, M-Area HWMF (Cont.) WELL MSB 63C

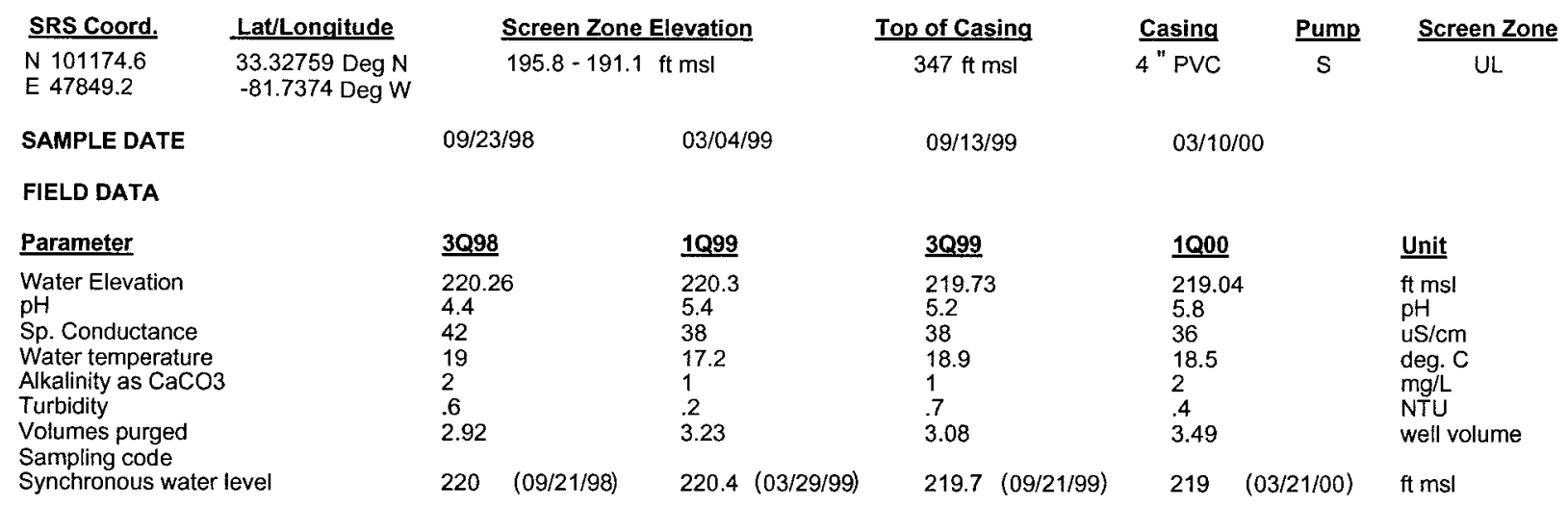

\section{ANALYTICAL DATA}

I. Groundwater Protection Standard

261 Appendix VIII/264 Appendix IX Hazardous Constituents

\begin{tabular}{|c|c|c|c|c|c|c|c|c|c|c|c|c|c|}
\hline ST Parameter & $\underline{3 Q 98}$ & CLPEPA & 1099 & CLP EPA & $\underline{3099}$ & $\underline{\text { CLPEPA }}$ & 1Q00 & CLF & EPA & Filt. & DF & $\underline{\text { Unit }}$ & $\underline{\text { Lab }}$ \\
\hline \multicolumn{14}{|l|}{ Inorganics } \\
\hline Barium, total recoverable & 6.74 & $J$ & 6.14 & J I & 7.13 & J I & 6.18 & $\mathrm{~J}$ & 1 & NDD & 1 & ug/L & ML \\
\hline Cyanide & $<10$ & $U$ & $<10$ & $\mathrm{U}$ & $<10$ & $\mathrm{U}$ & & & & & & $\mathrm{ug} / \mathrm{L}$ & \\
\hline Lead, total recoverable & $<100$ & $U$ & $<100$ & $\mathrm{U}$ & $<10$ & U & $<20$ & $U$ & & $<E Q L$ & 1 & $\mathrm{ug} / \mathrm{L}$ & ML \\
\hline Nickel, total recoverable & $<50$ & $\mathrm{U}$ & $<50$ & $\mathrm{U}$ & $<50$ & $U$ & $<60$ & $\mathrm{U}$ & & $<\overline{E Q L}$ & 1 & $\mathrm{ug} / \mathrm{L}$ & ML \\
\hline Selenium, total recoverable & $<200$ & $U$ & $<10$ & $U$ & $<10$ & $\mathrm{U}$ & $<40$ & $U$ & & $<E Q L$ & 1 & ug/L & ML \\
\hline \multicolumn{14}{|l|}{ Organics } \\
\hline Chlorobenzene & $<25$ & $\mathrm{U}$ & $<10$ & $U$ & $<1$ & $\mathrm{U}$ & $<1$ & $U$ & & $<\mathrm{EQL}$ & 1 & ug $/ \mathrm{L}$ & $M L$ \\
\hline 1,1-Dichloroethane & $<25$ & $\mathrm{U}$ & $<10$ & $\mathrm{U}$ & $<1$ & $U$ & $<1$ & $\mathrm{U}$ & & $<\mathrm{EQL}$ & 1 & $\mathrm{ug} / \mathrm{L}$ & $\mathrm{ML}$ \\
\hline 1,1-Dichloroethylene & $<25$ & $\mathrm{U}$ & $<10$ & $\mathrm{U}$ & $<1$ & $\mathrm{U}$ & .6 & $J$ & $\mathrm{IK}$ & NDD & 1 & $u g / L$ & $M L$ \\
\hline trans-1,2-Dichloroethylene & $<25$ & $\mathrm{U}$ & $<10$ & $\mathrm{U}$ & $<1$ & $\mathrm{U}$ & & & & & & $\mathrm{ug} / \mathrm{L}$ & \\
\hline \multicolumn{14}{|l|}{ PCB 1016} \\
\hline \multicolumn{14}{|l|}{ PCB 1221} \\
\hline \multicolumn{14}{|l|}{$\begin{array}{l}\text { PCB } 1232 \\
\text { PCB } 1242\end{array}$} \\
\hline \multicolumn{14}{|l|}{ PCB 1248} \\
\hline \multicolumn{14}{|l|}{ PCB 1254} \\
\hline \multicolumn{14}{|l|}{ PCB 1260} \\
\hline $1,1,2,2$-Tetrachloroethane & $<25$ & $\mathrm{U}$ & $<10$ & $U$ & $<1$ & $\mathrm{U}$ & $<1$ & $\mathrm{u}$ & & $<\mathrm{EQL}$ & 1 & $\mathrm{ug} / \mathrm{L}$ & ML \\
\hline Tetrachloroethylene & 167 & & 168 & & 128 & & 155 & j & K & NDD & 1 & $u g / L$ & ML \\
\hline 1,1,1-Trichloroethane & $<25$ & $U$ & $<10$ & $U$ & $<1$ & $u$ & $<1$ & $\mathrm{U}$ & & $<E Q L$ & 1 & $\mathrm{ug} / \mathrm{L}$ & ML \\
\hline Trichloroethylene & 25 & & 30.3 & & 22.4 & & 22.6 & $\mathrm{~J}$ & K & NDD & 1 & $\mathrm{ug} / \mathrm{L}$ & ML \\
\hline \multicolumn{14}{|l|}{ II. Monitoring Constituents } \\
\hline Parameter & $\underline{3 Q 96}$ & CLP EPA & $\underline{3097}$ & CLP EPA & $\underline{3 Q 98}$ & CLPEPA & $\underline{3 Q 99}$ & CLP & EPA & Filt. & $\underline{\text { DF }}$ & Unit & $\underline{\mathrm{Lab}}$ \\
\hline \multicolumn{14}{|l|}{ Inorganics } \\
\hline Aluminum, total recoverable & $<20$ & $\mathrm{U}$ & 11.1 & $\mathrm{~J}$ & $<200$ & $U$ & $<200$ & $U$ & & $<\mathrm{EQL}$ & 1 & $\mathrm{ug} / \mathrm{L}$ & EX \\
\hline Nitrate-nitrite as nitrogen & 2520 & & 2640 & & 1900 & & 2400 & & & $<2400$ & 5 & $\mathrm{ug} / \mathrm{L}$ & EX \\
\hline Sodium, total recoverable & 2930 & $J$ & 3060 & & 3230 & & 2950 & & & $<4600$ & 1 & $\mathrm{ug} / \mathrm{L}$ & EX \\
\hline Sulfate & 168 & $J$ & $<5000$ & $\mathrm{U}$ & 427 & & 250 & & & $<3000$ & 1 & $\mathrm{ug} / \mathrm{L}$ & EX \\
\hline \multicolumn{14}{|l|}{ Radionuclides } \\
\hline Gross alpha & 1.71 & & 1.44 & & 2.87 & & 1.05 & $J$ & 1 & NDD & 1 & $\mathrm{pCi} / \mathrm{L}$ & GP \\
\hline Nonvolatile beta & 1.93 & & -.65 & UI & 2.57 & J & 1.78 & $J$ & IK & NDD & 1 & $\mathrm{pCi} / \mathrm{L}$ & GP \\
\hline Radium, total alpha-emitting & .4 & $\mathrm{~J}$ & 1.92 & UIJ & .5 & UI & .5 & $\mathrm{U}$ & & $<\mathrm{EQL}$ & 1 & $\mathrm{pCi} / \mathrm{L}$ & GP \\
\hline
\end{tabular}

Notes: Concentrations in bold italics exceed the groundwater protection or monitoring constituent standards listed in Appendix A. Synchronous water levels are measured over a 3-5 day period or less. Dilution factors, Laboratory, and Filtered Data are for Groundwater Protection Standards First Quarter 2000 and Monitoring Constituents Third Quarter 1999 data only.

$+=$ exceeded the Groundwater Protection Standards (First Quarter 2000) or Monitoring Constituents (Third Quarter 1999) listed in Appendix A. 
Table D-2. Groundwater Monitoring Results for Point-of-Compliance Wells, M-Area HWMF (Cont.) WELL MSB 63D

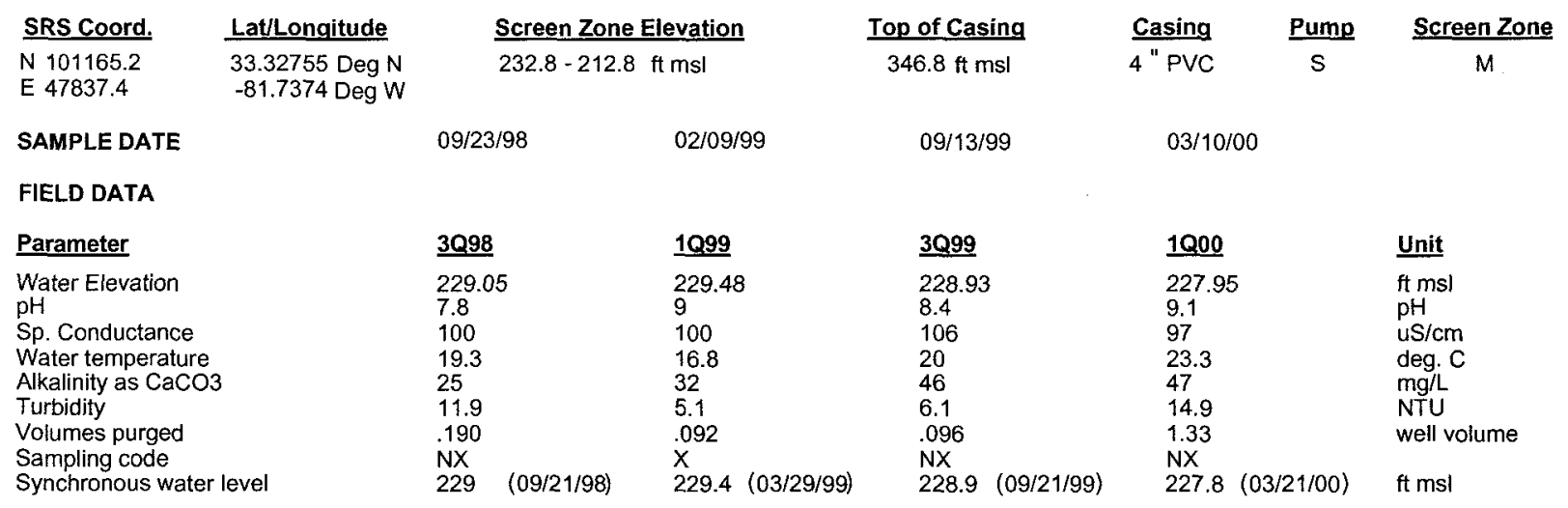

\section{ANALYTICAL DATA}

I. Groundwater Protection Standard

261 Appendix VIII/264 Appendix IX Hazardous Constituents

\begin{tabular}{|c|c|c|c|c|c|c|c|c|c|c|c|c|c|}
\hline \multirow{2}{*}{$\frac{\text { ST }}{\text { Inorganics }}$} & \multirow[t]{2}{*}{$\underline{3098}$} & \multirow[t]{2}{*}{ CLPEPA } & \multirow[t]{2}{*}{1099} & \multirow[t]{2}{*}{$\underline{\text { CLPEPA }}$} & \multirow[t]{2}{*}{$\underline{3099}$} & \multirow[t]{2}{*}{ CLPEPA } & \multirow[t]{2}{*}{$\underline{1000}$} & \multicolumn{2}{|c|}{ CLPEPA } & \multirow[t]{2}{*}{ Filt. } & \multirow[t]{2}{*}{$\underline{\text { DF }}$} & \multirow[t]{2}{*}{ Unit } & \multirow[t]{2}{*}{ Lab } \\
\hline & & & & & & & & & & & & & \\
\hline Barium, total recoverable & 8.13 & J & 11.3 & & 8.1 & J 1 & 12 & $\mathrm{~J}$ & 1 & NDD & 1 & $u g / L$ & ML \\
\hline Cyanide & $<10$ & $u$ & $<10$ & u & $<10$ & $\mathrm{u}$ & $<8.6$ & JU & ILV & $<E Q L$ & 1 & $\mathrm{ug} / \mathrm{L}$ & ML \\
\hline Lead, total recoverable & $<100$ & $u$ & $<100$ & $u$ & $<10$ & $U$ & $<7.48$ & JU & IV & $<\mathrm{EQL}$ & 1 & $\mathrm{ug} / \mathrm{L}$ & ML \\
\hline Nickel, total recoverable & $<50$ & $\mathrm{u}$ & $<50$ & $\mathrm{u}$ & $<50$ & $\mathrm{u}$ & $<60$ & u & & $<E Q L$ & 1 & $\mathrm{ug} / \mathrm{L}$ & $\mathrm{ML}$ \\
\hline Selenium, total recoverabte & $<200$ & $\mathrm{u}$ & $<10$ & $\mathrm{u}$ & $<10$ & $\mathrm{u}$ & $<40$ & $\mathrm{u}$ & & $<E Q L$ & 1 & $\mathrm{ug} / \mathrm{L}$ & ML \\
\hline \multicolumn{14}{|l|}{ Organics } \\
\hline Chlorobenzene & $<25$ & $\mathrm{u}$ & $<25$ & $u$ & $<1$ & $u$ & $<1$ & $\mathrm{u}$ & & $<E Q L$ & 1 & $\mathrm{ug} / \mathrm{L}$ & ML \\
\hline 1,1-Dichloroethane & $<25$ & $\mathrm{u}$ & $<25$ & $u$ & $<1$ & $u$ & $<1$ & $u$ & & $<E Q L$ & 1 & $\mathrm{ug} / \mathrm{L}$ & ML \\
\hline 1,1-Dichloroethylene & $<25$ & $\mathrm{u}$ & $<25$ & $u$ & $<1$ & $\mathrm{u}$ & $<1$ & $u$ & & $<E Q L$ & 1 & ug/L & $M$ \\
\hline trans-1,2-Dichloroethylene & $<25$ & $u$ & $<25$ & $u$ & $<1$ & $\mathrm{u}$ & & & & & & $\mathrm{ug} / \mathrm{L}$ & \\
\hline PCB 1016 & & & & & & & & & & & & & \\
\hline PCB 1221 & & & & & & & & & & & & & \\
\hline PCB 1232 & & & & & & & & & & & & & \\
\hline PCB 1242 & & & & & & & & & & & & & \\
\hline PCB 1248 & & & & & & & & & & & & & \\
\hline PCB 1254 & & & & & & & & & & & & & \\
\hline PCB 1260 & & & & & & & & & & & & & \\
\hline 1,1,2,2-Tetrachloroethane & $<25$ & $\mathrm{u}$ & $<25$ & $\mathrm{u}$ & $<1$ & $u$ & $<1$ & $\mathrm{u}$ & & $<E Q L$ & 1 & $u g / L$ & $\Lambda \mathrm{L}$ \\
\hline + Tetrachloroethylene & 257 & & 283 & & 250 & & 220 & & & $>5$ & 1 & $\mathrm{ug} / \mathrm{L}$ & L \\
\hline 1,1,1-Trichloroethane & $<25$ & $\mathrm{u}$ & $<25$ & $\mathrm{u}$ & $<1$ & $u$ & $<1$ & $\mathrm{u}$ & & $<E Q L$ & 1 & $\mathrm{ug} / \mathrm{L}$ & M \\
\hline+ Trichloroethylene & 30.2 & & 40.8 & & 25.9 & & 20.2 & & & $>5$ & 1 & ug/L & \\
\hline \multicolumn{14}{|l|}{ II. Monitoring Constituents } \\
\hline ST Parameter & $\underline{3096}$ & CLPEPA & $\underline{3 Q 97}$ & CLPEPA & $\underline{3098}$ & CLPEPA & $\underline{3099}$ & CLP. & EPA & Fillt. & $\underline{\mathrm{DF}}$ & $\underline{\text { Unit }}$ & Lab \\
\hline \multicolumn{14}{|l|}{ Inorganics } \\
\hline$+\quad$ Aluminum, total recoverable & 1100 & & 1200 & & 1020 & & 688 & & & $>100$ & 1 & ug/L & EX \\
\hline$+\quad$ Nitrate-nitrite as nitrogen & 3380 & & 4050 & & 2770 & & 2500 & & & $>2400$ & 5 & ug/L & EX \\
\hline Sodium, total recoverable & 3960 & & 4690 & & 4070 & & 2950 & & & $<4600$ & 1 & $\mathrm{ug} / \mathrm{L}$ & EX \\
\hline Sulfate & 770 & J & $<5000$ & $\mathrm{u}$ & 1440 & & 730 & & & $<3000$ & 1 & ug/L & EX \\
\hline \multicolumn{14}{|l|}{ Radionuclides } \\
\hline Gross alpha & 1.48 & & .12 & $\mathrm{UI}$ & 2.72 & & .634 & $u$ & & $<E Q L$ & 1 & $\mathrm{pCi} / \mathrm{L}$ & GP \\
\hline Nonvolatile beta & 2.45 & & .31 & $\mathrm{Ul}$ & 12.99 & $\mathrm{~J}$ & 1.4 & $\mathrm{~J}$ & $\mathrm{IK}$ & NDD & 1 & $\mathrm{pCi} / \mathrm{L}$ & GP \\
\hline Radium, total alpha-emitting & .3 & $\mathrm{~J}$ & 1.39 & UIJ & .53 & & .2 & $u$ & & $<E Q L$ & 1 & $\mathrm{pCi} / \mathrm{L}$ & GP \\
\hline
\end{tabular}

Notes: Concentrations in bold italics exceed the groundwater protection or monitoring constituent standards listed in Appendix A. Synchronous water levels are measured over a 3-5 day period or less. Dilution factors, Laboratory, and Filtered Data are for Groundwater Protection Standards First Quarter 2000 and Monitoring Constituents Third Quarter 1999 data only.

$+=$ exceeded the Groundwater Protection Standards (First Quarter 2000) or Monitoring Constituents (Third Quarter 1999) listed in Appendix A. 
Table D-2. Groundwater Monitoring Results for Point-of-Compliance Wells, M-Area HWMF (Cont.) WELL MSB 64C

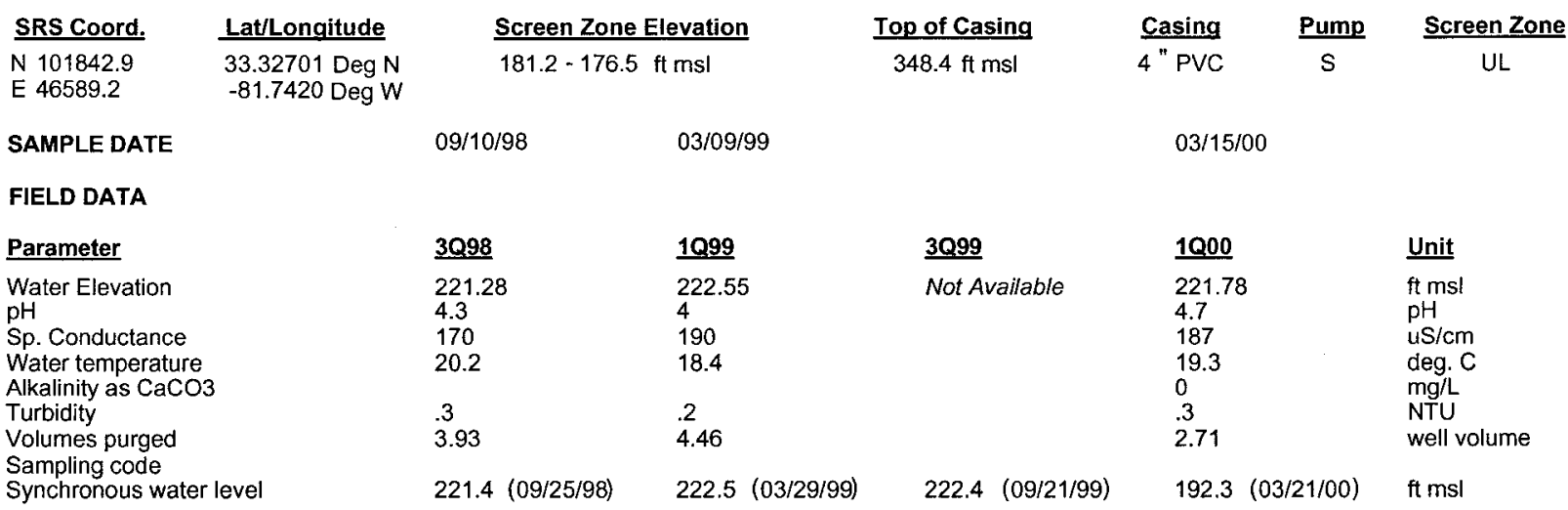

\section{ANALYTICAL DATA}

I. Groundwater Protection Standard

261 Appendix VIII/264 Appendix IX Hazardous Constituents

\begin{tabular}{|c|c|c|c|c|c|c|c|c|c|c|c|}
\hline ST Parameter & 3Q98 & CLPEPA & 1099 & CLP EPA & $\underline{3 Q 99}$ & CLPEPA & 1000 & CLPEPA & Filt. & $\underline{\mathrm{DF}}$ & Unit \\
\hline \multicolumn{12}{|l|}{ Inorganics } \\
\hline Barium, total recoverable & 32.1 & & 30.1 & & & & 29.6 & & $<2000$ & 1 & $\mathrm{ug} / \mathrm{L}$ \\
\hline Cyanide & $<10$ & $U$ & $<10$ & U & & & & & & & . \\
\hline Lead, total recoverable & $<100$ & $U$ & $<100$ & U & & & $<8.29$ & JU IV & $<E Q L$ & 1 & $u g / L$ \\
\hline Nickel, total recoverable & 14.8 & $\mathrm{~J}$ & $<50$ & $U$ & & & $<60$ & $\mathrm{U}$ & $<E Q L$ & 1 & $u g / h$ \\
\hline Selenium, total recoverable & $<200$ & $U$ & $<200$ & $\mathrm{U}$ & & & $<40$ & $U$ & $<E Q L$ & 1 & $\mathrm{ug} / \mathrm{L}$ \\
\hline \multicolumn{12}{|l|}{ Organics } \\
\hline Chlorobenzene & $<50$ & $U$ & $<50$ & $\mathrm{U}$ & & & $<1$ & JU & $<E Q L$ & 1 & $\mathrm{ug} / \mathrm{L}$ \\
\hline 1,1-Dichloroethane & $<50$ & $U$ & $<50$ & $U$ & & & .93 & J 1 & NDD & 1 & ug \\
\hline 1,1-Dichloroethylene & $<50$ & U & $<50$ & 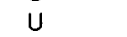 & & & 26 & $\mathrm{~J}$ & NDD & 1 & $\mathrm{ug} / \mathrm{L}$ \\
\hline trans-1,2-Dichloroethylene & $<50$ & $\bigcup$ & $<50$ & U & & & $<1$ & JU & $<E Q L$ & 1 & $\mathrm{ug} / \mathrm{L}$ \\
\hline PCB 1016 & $<2$ & $U$ & & & & & & & & & ug \\
\hline PCB 1221 & $<2$ & $\mathrm{U}$ & & & & & & & & & ug \\
\hline PCB 1232 & $<1$ & U & & & & & & & & & $\mathrm{ug} / \mathrm{L}$ \\
\hline PCB 1242 & $<1$ & U & & & & & & & & & ug \\
\hline PCB 1248 & $<1$ & $\mathrm{U}$ & & & & & & & & & ug \\
\hline PCB 1254 & $<1$ & $\mathrm{U}$ & & . & & & & & & & ug \\
\hline PCB 1260 & $<1$ & U & & & & & & & & & ug \\
\hline $1,1,2,2$-Tetrachloroethane & $<50$ & $U$ & $<50$ & $\mathrm{U}$ & & & $<1$ & JU & $<\mathrm{EQL}$ & 1 & $\mathrm{ug} / \mathrm{L}$ \\
\hline Tetrachloroethylene & 307 & J & 259 & & & & 217 & $\mathrm{~J}$ & NDD & 1 & $\mathrm{ug} / \mathrm{L}$ \\
\hline $1,1,1$-Trichloroethane & $<50$ & $\mathrm{U}$ & $<50$ & $U$ & & & 2.8 & $\mathrm{~J}$ & NDD & 1 & $\mathrm{ug} / \mathrm{L}$ \\
\hline Trichloroethylene & 39.3 & $\mathrm{~J}$ & 34.9 & J I & & & 27.1 & $J$ & NDD & 1 & $u g / L$ \\
\hline \multicolumn{12}{|l|}{ II. Monitoring Constituents } \\
\hline ST Parameter & $\underline{3096}$ & CLPEPA & $\underline{3097}$ & CLPEPA & $\underline{3098}$ & CLPEPA & $\underline{3099}$ & CLPEPA & Filt. & $\underline{\mathrm{DF}}$ & Unit \\
\hline \multicolumn{12}{|l|}{ Inorganics } \\
\hline Aluminum, total recoverable & 167 & & 206 & & 230 & & & & & & $\mathrm{ug} / \mathrm{L}$ \\
\hline Nitrate-nitrite as nitrogen & 15600 & & 20800 & & 16800 & & & & & & ug/L. \\
\hline Sodium, total recoverable & 12900 & & 13000 & & 14400 & & & & & & ug \\
\hline Sulfate & 130 & J & $<5000$ & $\mathrm{U}$ & $<400$ & U & & & & & $\mathrm{ug} / \mathrm{L}$ \\
\hline \multicolumn{12}{|l|}{ Radionuclides } \\
\hline Gross alpha & 9.34 & & 5.47 & & 11.05 & & & & & & $\mathrm{pCi} / \mathrm{L}$ \\
\hline Nonvolatile beta & 8.45 & & 8.53 & & 15.86 & & & & & & $\mathrm{pC}$ \\
\hline Radium, total alpha-emitting & 2.9 & & 5.98 & $\mathrm{~J}$ & 1.96 & & & & & & \\
\hline
\end{tabular}

Notes: Concentrations in bold italics exceed the groundwater protection or monitoring constituent standards listed in Appendix A. Synchronous water levels are measured over a 3-5 day period or less. Dilution factors, Laboratory, and Filtered Data are for Groundwater Protection Standards First Quarter 2000 and Monitoring Constituents Third Quarter 1999 data only.

$+=$ exceeded the Groundwater Protection Standards (First Quarter 2000) or Monitoring Constituents (Third Quarter 1999) listed in Appendix A. 
Table D-2. Groundwater Monitoring Results for Point-of-Compliance Wells, M-Area HWMF (Cont.) WELL MSB 64D

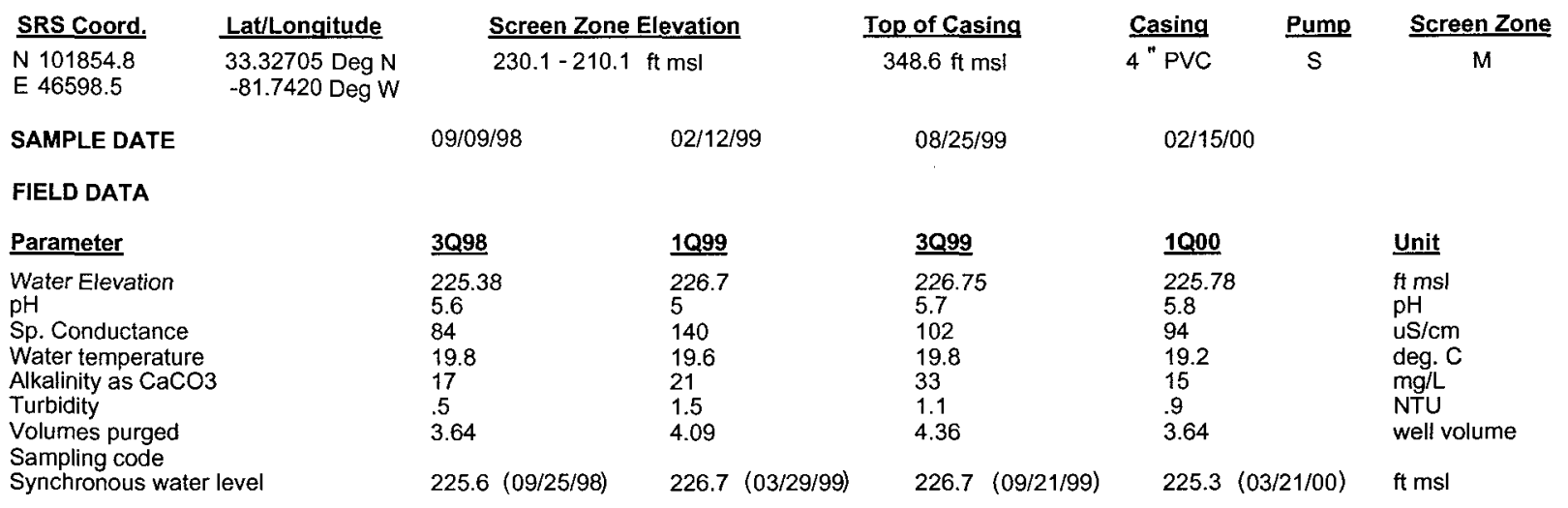

ANALYTICAL DATA

I. Groundwater Protection Standard

261 Appendix VIII/264 Appendix IX Hazardous Constituents

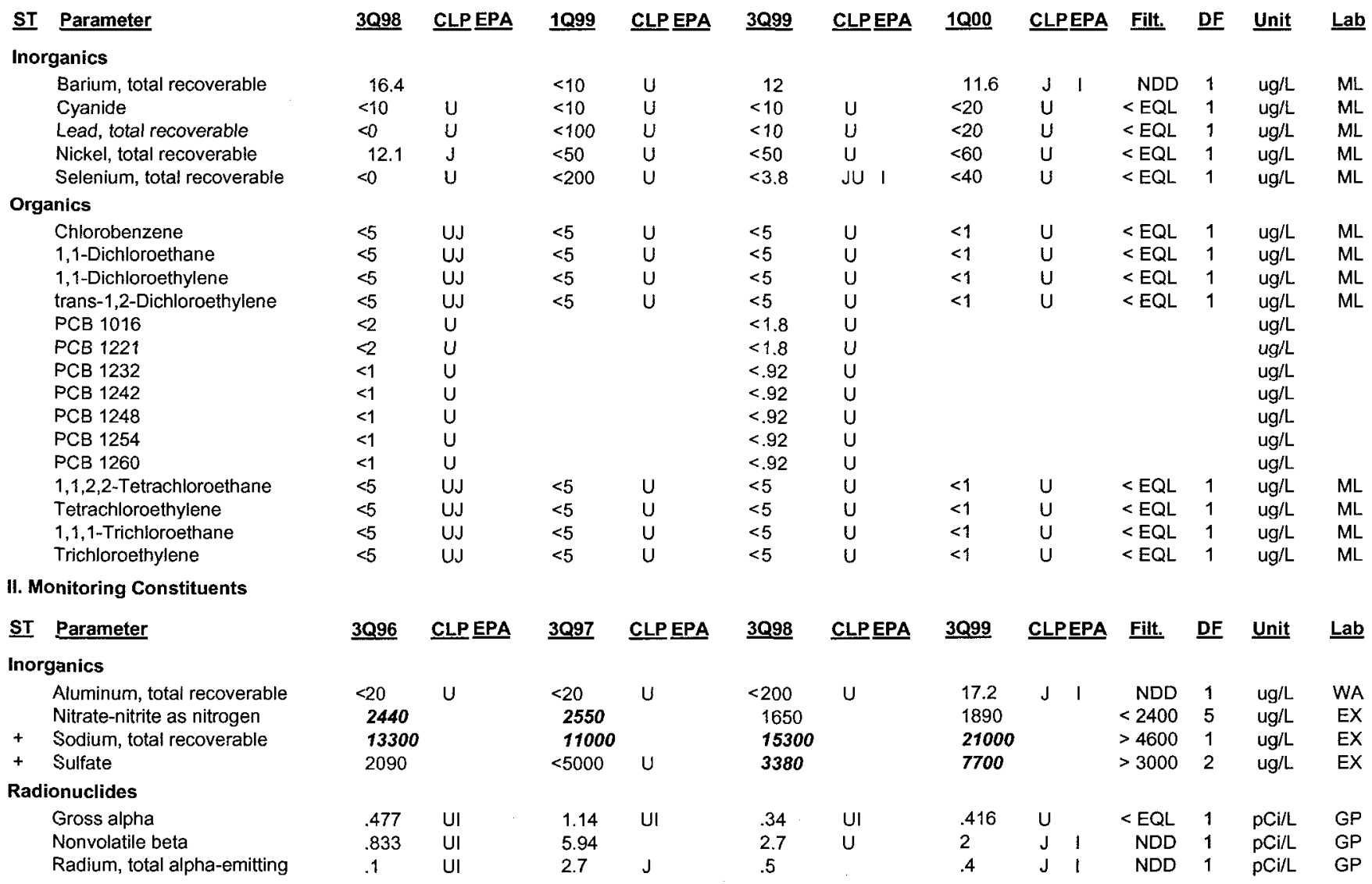

Notes: Concentrations in bold italics exceed the groundwater protection or monitoring constituent standards listed in Appendix A. Synchronous water levels are measured over a 3-5 day period or less. Dilution factors, Laboratory, and Filtered Data are for Groundwater Protection Standards First Quarter 2000 and Monitoring Constituents Third Quarter 1999 data only.

$+=$ exceeded the Groundwater Protection Standards (First Quarter 2000) or Monitoring Constituents (Third Quarter 1999) listed in Appendix A. 
Table D-3. Groundwater Monitoring Results for Background Wells, M-Area HWMF WELL MSB 29B

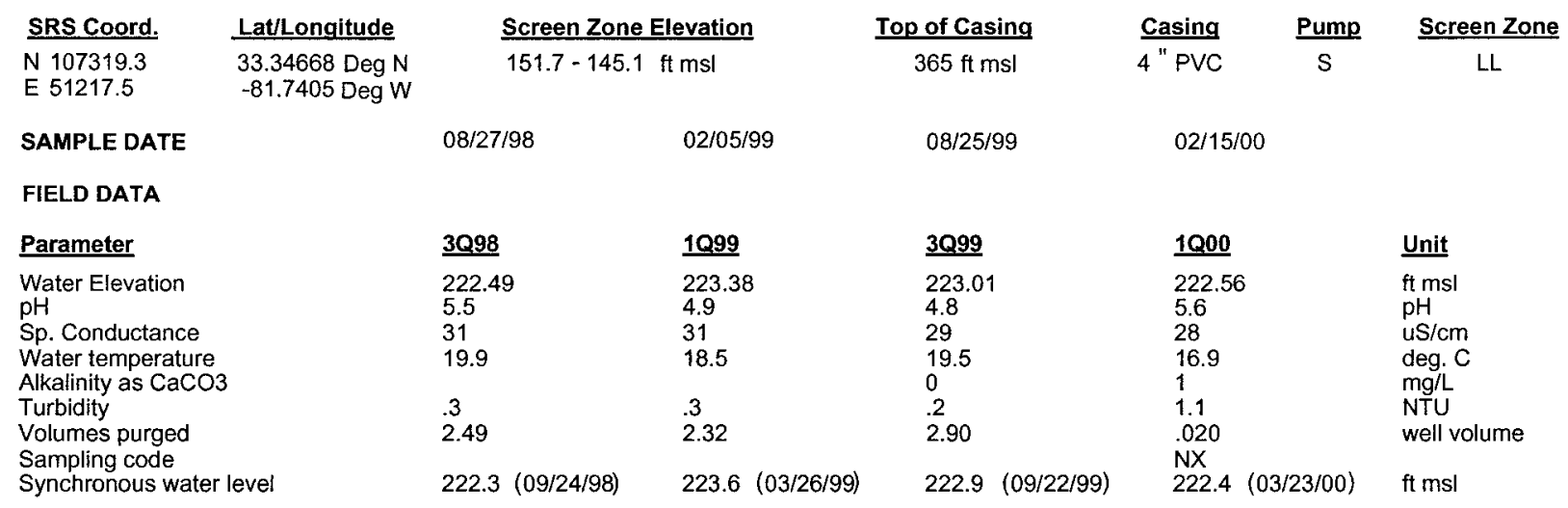

ANALYTICAL DATA

1. Groundwater Protection Standard

261 Appendix VIII/264 Appendix IX Hazardous Constituents

ST Parameter

Inorganics

Barium, total recoverable

Cyanide

Lead, total recoverable

Nickel, total recoverable

Selenium, total recoverable

Organics

Chlorobenzene

1,1-Dichloroethane

1,1-Dichloroethylene

trans-1,2-Dichloroethylene

PCB 1016

PCB 1221

PCB 1232

PCB 1242

PCB 1248

PCB 1254

PCB 1260

1,1,2,2-Tetrachloroethane

Tetrachloroethylene

1,1,1-Trichloroethane

Trichloroethylene

II. Monitoring Constituents

ST Parameter

Inorganics

Aluminum, total recoverable

Chloride

Chromium, total recoverable

Cobalt, total recoverable

Copper, total recoverable

Fluoride

Manganese, total recoverable

Mercury, total recoverable

Nitrate-nitrite as nitrogen

Sodium, total recoverable

Sulfate

Total phosphates (as P)

Uranium, total recoverable

Zinc, total recoverable

Radionuclides

Gross alpha

Nonvolatile beta

Radium, total alpha-emitting
3 Q98 CLPEPA

1099

CLPEPA

$\underline{3099}$

$<10$
$<10$
$<100$
$<50$

$\begin{array}{lll}\begin{array}{l}7.5 \\ <10\end{array} & \mathrm{U} & \text { I } \\ <10 & U \\ <50 & U \\ <10 & \mathrm{U} & \end{array}$

$\quad 7.35$
$<10$
$<10$
$<50$
$<10$

$<200 \quad \mathrm{U}$

$\begin{array}{ll}<5 & U \\ <5 & U \\ <5 & U \\ <5 & U\end{array}$

$\begin{array}{ll}<5 & \mathrm{U} \\ <5 & \mathrm{U} \\ <5 & \mathrm{U} \\ <5 & \mathrm{U}\end{array}$

$<5$
$<5$
$<5$
$<5$

$U$
$U$
$U$

$\begin{array}{ll}<5 & U \\ <5 & U \\ <5 & U \\ <5 & U\end{array}$

$\begin{array}{ll}<5 & U \\ <5 & U \\ <5 & U \\ <5 & U\end{array}$

$\underline{3096}$

CLPPEPA

\section{CLP EPA}

$\underline{3098}$

\section{CLPEPA}

$\begin{array}{ll}<200 & \mathrm{U} \\ 2770 & \\ <10 & \mathrm{U} \\ <20 & \mathrm{U} \\ <20 & \mathrm{U} \\ <200 & \mathrm{U} \\ 1.77 & \mathrm{~J} \\ <.5 & \mathrm{U} \\ 1360 & \\ 2440 & \\ 469 & \\ <2500 & \mathrm{U} \\ & \\ <20 & \mathrm{U}\end{array}$

$<5$
$<5$
$<5$
$<5$

$\begin{array}{ll}<5 & U \\ <5 & U \\ <5 & U \\ <5 & U\end{array}$

$\begin{array}{ll}<5 & U \\ <5 & U \\ <5 & U \\ <5 & U\end{array}$

$\begin{array}{lll}<E Q L & 1 & u g / L \\ <E Q L & 1 & u g / L \\ <E Q L & 1 & u g / L \\ <E Q L & 1 & u g / L\end{array}$

EX

EX

EX
EX

$\begin{array}{ll}41 & \\ 2310 & \\ <4 & \mathrm{U} \\ .25 & \mathrm{~J} \\ 1.24 & \mathrm{~J} \\ <100 & \mathrm{U} \\ 1.37 & \mathrm{~J} \\ .023 & \mathrm{~J} \\ 1800 & \\ 2280 & \\ 133 & \mathrm{~J} \\ <50 & \mathrm{UJ} \\ & \\ <5 & \mathrm{U}\end{array}$

$\begin{array}{ll}88.8 & \\ 2820 & \\ <5 & U \\ <5 & U \\ <5 & U \\ <100 & U \\ 2 & J \\ <.2 & U \\ 1510 & \\ 2490 & \\ <5000 & U \\ <50 & U \\ & \\ 9.8 & J\end{array}$

\section{3}

3.06

1.4

2.02
.33

1.63

.33
.72

UI

3 Q99 CLPEPA Filt. DF Unit Lab

\begin{tabular}{|c|c|c|c|c|c|}
\hline $\begin{array}{l}<200 \\
2540\end{array}$ & U & & $\begin{array}{l}<E Q L \\
<4200\end{array}$ & $\begin{array}{l}1 \\
2\end{array}$ & $\begin{array}{l}u g / L \\
u g / L\end{array}$ \\
\hline$<10$ & U & & $<E Q L$ & 1 & $\mathrm{ug} / \mathrm{L}$ \\
\hline$<20$ & $U$ & & $<E Q L$ & 1 & $\mathrm{ug} / \mathrm{L}$ \\
\hline$<20$ & U & & $<E Q L$ & 1 & $\mathrm{ug} / \mathrm{L}$ \\
\hline$<200$ & $U$ & & $<E Q L$ & 2 & $u g / L$ \\
\hline$<1.4$ & $U$ & V & $<E Q L$ & 1 & $\mathrm{ug} / \mathrm{L}$ \\
\hline$<.5$ & U & & $<E Q L$ & 1 & ug/L \\
\hline 1460 & & & $<2400$ & 5 & $u g / L$ \\
\hline 2600 & & & $<4600$ & 1 & $\mathrm{ug} / \mathrm{L}$ \\
\hline$<400$ & U & & $<E Q L$ & 2 & ug/L \\
\hline$<2500$ & U & & $<\mathrm{EQL}$ & 5 & ug/L \\
\hline$<20$ & $\mathrm{U}$ & & $<E Q L$ & 1 & ug/L. \\
\hline .898 & U & & $<E Q L$ & 1 & $\mathrm{pCi} / \mathrm{L}$ \\
\hline 1.28 & U & & $<E Q L$ & 1 & $\mathrm{pCi} / \mathrm{L}$ \\
\hline 1.5 & & 1 & NDD & 1 & $\mathrm{pCi} / \mathrm{L}$ \\
\hline
\end{tabular}

Notes: Concentrations in bold italics exceed the groundwater protection or monitoring constituent standards listed in Appendix A. Synchronous water levels are measured over a 3-5 day period or less. Dilution factors, Laboratory, and Filtered Data are for Groundwater Protection Standards First Quarter 2000 and Monitoring Constituents Third Quarter 1999 data only.

$+=$ exceeded the Groundwater Protection Standards (First Quarter 2000) or Monitoring Constituents (Third Quarter 1999) listed in Appendix A. 
Table D-3. Groundwater Monitoring Results for Background Wells, M-Area HWMF (Cont.) WELL MSB 29C

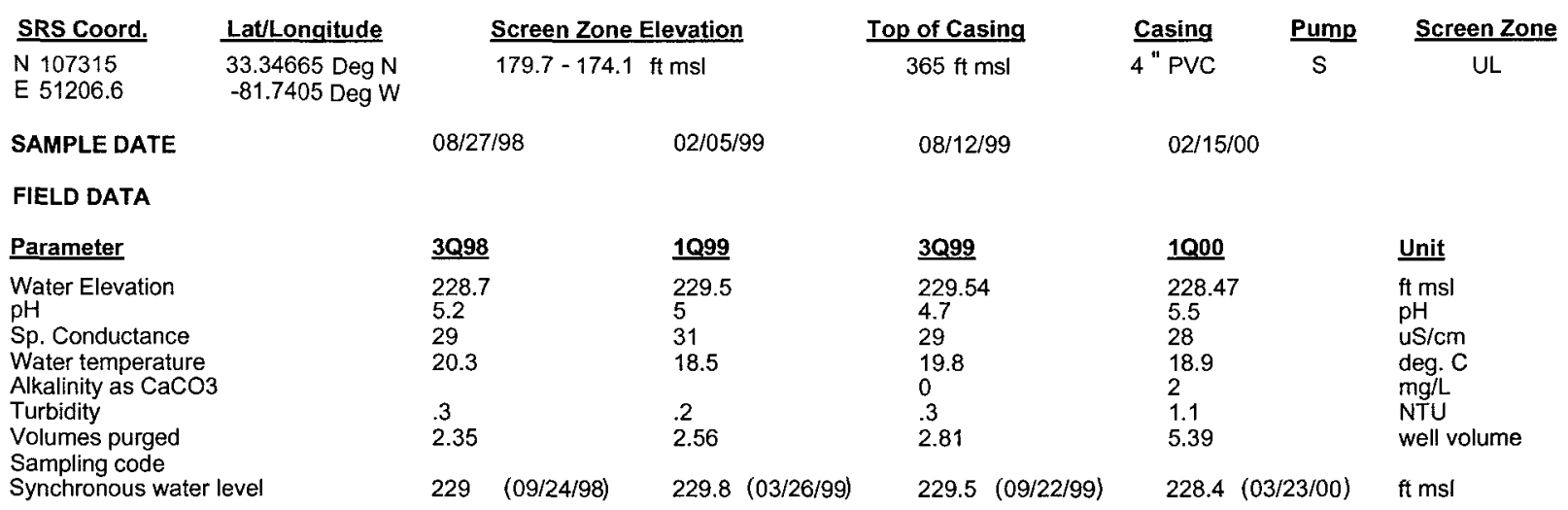

ANALYTICAL DATA

I. Groundwater Protection Standard

261 Appendix VIII/264 Appendix IX Hazardous Constituents

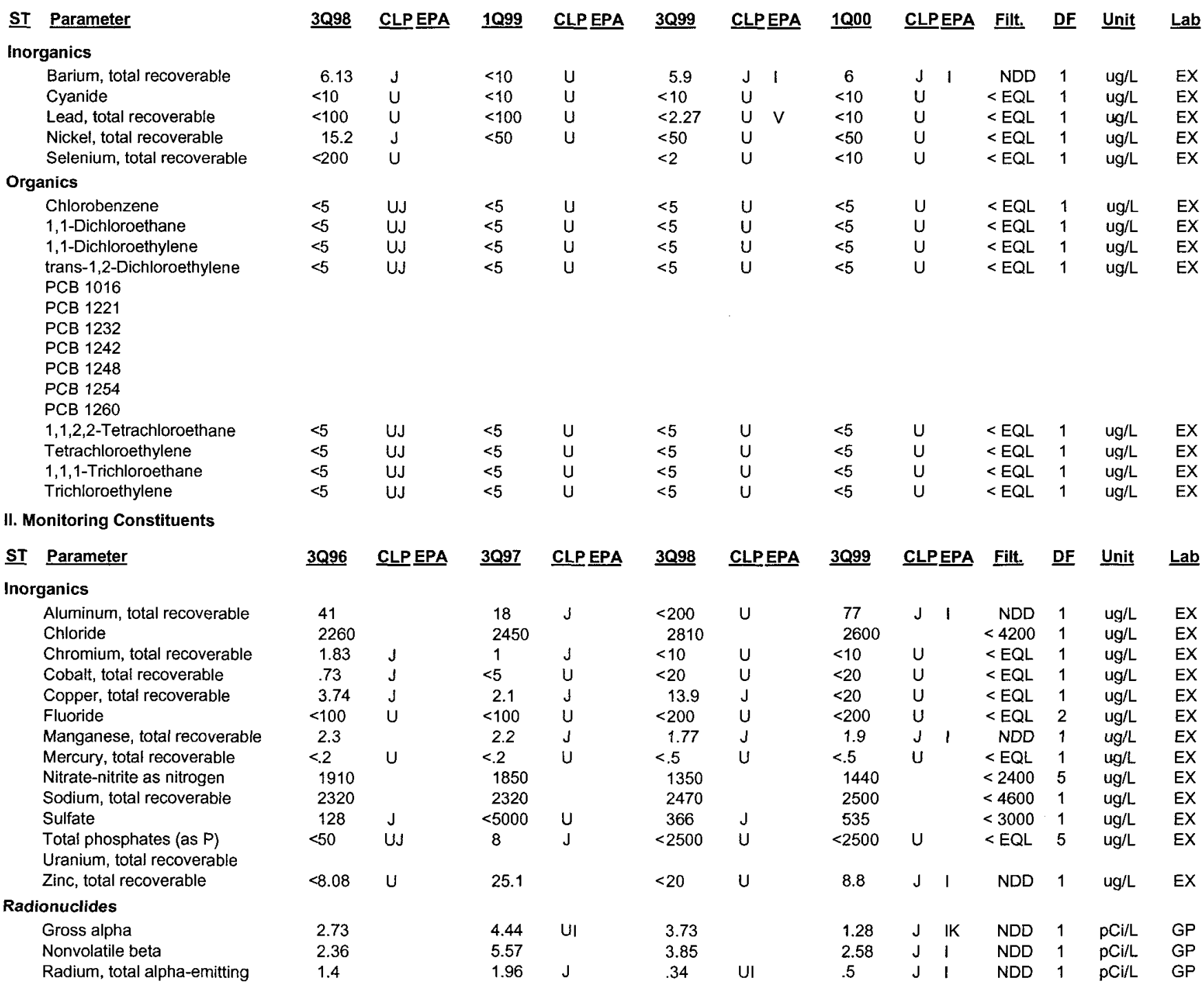

Notes: Concentrations in bold italics exceed the groundwater protection or monitoring constituent standards listed in Appendix A. Synchronous water levels are measured over a 3-5 day period or less. Dilution factors, Laboratory, and Filtered Data are for Groundwater Protection Standards First Quarter 2000 and Monitoring Constituents Third Quarter 1999 data only.

$+=$ exceeded the Groundwater Protection Standards (First Quarter 2000) or Monitoring Constituents (Third Quarter 1999) listed in Appendix A. 
Table D-3. Groundwater Monitoring Results for Background Wells, M-Area HWMF (Cont.) WELL MSB 29D

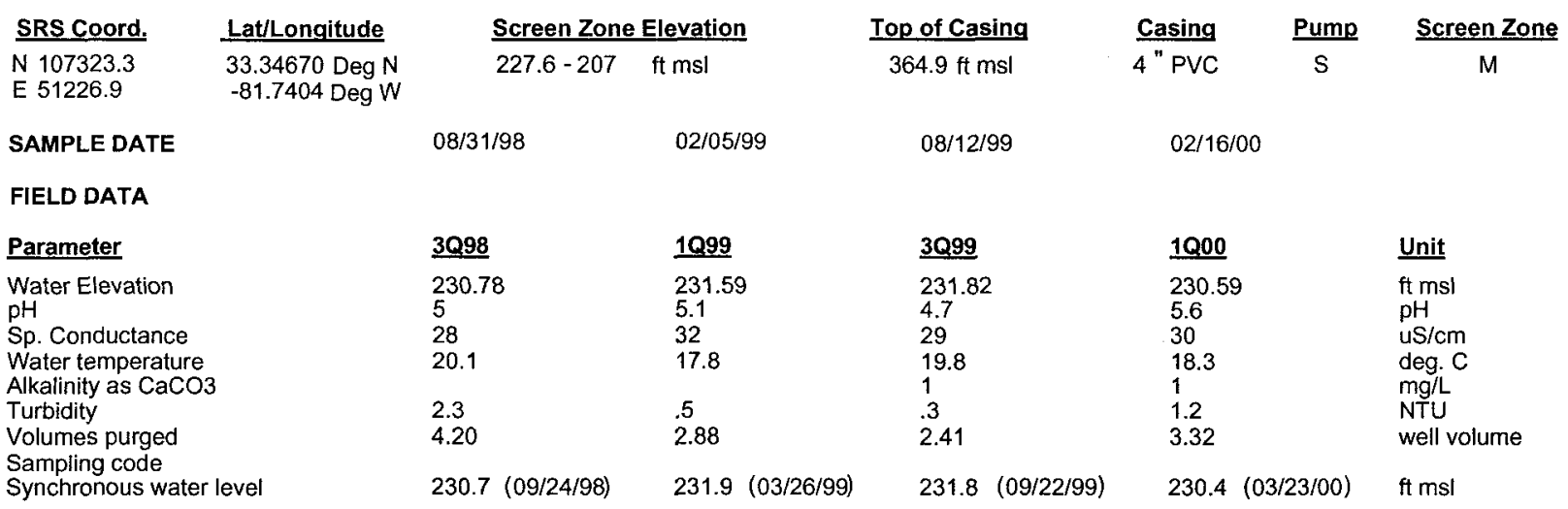

ANALYTICAL DATA

I. Groundwater Protection Standard

261 Appendix VIII/264 Appendix IX Hazardous Constituents

\begin{tabular}{|c|c|c|c|c|c|c|c|c|c|c|c|c|c|c|}
\hline$\underline{\text { ST }}$ & Parameter & $\underline{3 Q 98}$ & CLPEPA & $\underline{1 Q 99}$ & CLP EPA & $\underline{3 Q 99}$ & CLPEPA & 1000 & CLP & EPA & Filt. & $\underline{\text { DF }}$ & Unit & $\underline{\mathrm{Lab}}$ \\
\hline or & ganics & & & & & & & & & & & & & \\
\hline & Barium, total recoverable & 8.84 & J & $<10$ & $\mathrm{U}$ & 9.1 & J I & $<8.64$ & $U$ & V & $<E Q L$ & 1 & $\mathrm{ug} / \mathrm{L}$ & EX \\
\hline & Cyanide & $<10$ & $\mathrm{U}$ & $<10$ & $\mathrm{U}$ & $<10$ & $\mathrm{U}$ & $<10$ & $U$ & & $<E Q L$ & 1 & ug/L & EX \\
\hline & Lead, total recoverable & $<100$ & U & $<100$ & $U$ & $<4.14$ & U V & 11.2 & & & $<15$ & 1 & ugh & EX \\
\hline & Nickel, total recoverable & $<50$ & $U$ & $<50$ & $U$ & $<9.5$ & JU 1 & $<10.9$ & JU & V & $<E Q L$ & 1 & $\mathrm{ug} / \mathrm{L}$ & EX \\
\hline & Selenium, total recoverable & $<3.38$ & UJ & & & $<2$ & $\mathrm{U}$ & $<10$ & $U$ & & $<E Q L$ & 1 & ug/L & EX \\
\hline Org & anics & & & & & & & & & & & & & \\
\hline & Chlorobenzene & $<5$ & UJ & $<5$ & $U$ & $<5$ & $U$ & $<5$ & $U$ & & $<E Q L$ & 1 & $\mathrm{ug} / \mathrm{L}$ & EX \\
\hline & 1,1-Dichloroethane & $<5$ & UJ & $<5$ & U & $<5$ & U & $<5$ & $U$ & & $<E Q L$ & 1 & $\mathrm{ug} / \mathrm{L}$ & EX \\
\hline & 1,1-Dichloroethylene & $<5$ & UJ & $<5$ & $\mathrm{U}$ & $<5$ & $\mathrm{U}$ & $<5$ & $U$ & & $<\mathrm{EQL}$ & 1 & ug/L & EX \\
\hline & $\begin{array}{l}\text { trans-1,2-Dichloroethylene } \\
\text { PCB } 1016\end{array}$ & $<5$ & UJ & $<5$ & $\mathrm{U}$ & $<5$ & $U$ & $<5$ & $U$ & & $<E Q L$ & 1 & $\mathrm{ug} / \mathrm{L}$ & EX \\
\hline & PCB 1221 & & & & & & & & & & & & & \\
\hline & PCB 1232 & & & & & & & & & & & & & \\
\hline & PCB 1242 & & & & & & & & & & & & & \\
\hline & PCB 1248 & & & & & & & & & & & & & \\
\hline & PCB 1254 & & & & & & & & & & & & & \\
\hline & PCB 1260 & & & & & & & & & & & & & \\
\hline & $1,1,2,2$-Tetrachloroethane & $<5$ & UJ & $<5$ & $U$ & $<5$ & $\mathrm{U}$ & $<5$ & $U$ & & $<E Q L$ & 1 & $u g / L$ & EX \\
\hline & Tetrachloroethylene & $<5$ & UJ & $<5$ & $\mathrm{U}$ & $<5$ & $\mathrm{U}$ & $<5$ & $\mathrm{U}$ & & $<E Q L$ & 1 & ug/L & EX \\
\hline & 1,1,1-Trichloroethane & $<5$ & UJ & $<5$ & $\mathrm{U}$ & $<5$ & $\mathrm{U}$ & $<5$ & U & & $<E Q L$ & 1 & ug/L & EX \\
\hline & Trichloroethylene & $<5$ & UJ & $<5$ & $U$ & $<5$ & $U$ & $<5$ & $U$ & & $<E Q L$ & 1 & ug/L & EX \\
\hline $\mathbf{N}$ & anitoring Constituents & & & & & & & & & & & & & \\
\hline$\underline{\text { ST }}$ & Parameter & $\underline{3 Q 96}$ & CLPPEPA & $\underline{3097}$ & CLPEPA & $\underline{3098}$ & CLPEPA & $\underline{3099}$ & CLP & EPA & Filt. & $\underline{\text { DF }}$ & Unit & Lab \\
\hline$O$ & ganics & & & & & & & & & & & & & \\
\hline & Aluminum, total recoverable & 47.8 & & 16.9 & $\mathrm{~J}$ & $<200$ & $U$ & $<200$ & $U$ & & $<\mathrm{EQL}$ & 1 & $u g / L$ & EX \\
\hline & Chloride & 3850 & & 3610 & & 3450 & $J$ & 3340 & & & $<4200$ & 1 & $\mathrm{ug} / \mathrm{L}$ & $\mathrm{EX}$ \\
\hline & Chromium, total recoverable & $<4$ & $U$ & $<3$ & $U$ & 6.18 & J & $<10$ & $\mathrm{U}$ & & $<E Q L$ & 1 & $u g / L$ & EX \\
\hline & Cobalt, total recoverable & .219 & $J$ & $<5$ & $\mathrm{U}$ & $<20$ & $\mathrm{U}$ & $<20$ & $U$ & & $<\mathrm{EQL}$ & 1 & $\mathrm{ug} / \mathrm{L}$ & EX \\
\hline & Copper, total recoverable & 17.1 & & 7.9 & & 17.4 & $J$ & 19 & $J$ & $\mathbf{I}$ & NDD & 1 & ug/L & EX \\
\hline & Fluoride & $<100$ & $\mathrm{U}$ & $<100$ & $\cup$ & 188 & $J$ & $<200$ & $U$ & & $<\mathrm{EQL}$ & 2 & $\mathrm{ug} / \mathrm{L}$ & EX \\
\hline & Manganese, total recoverable & 4.78 & & 5.1 & & 8.76 & $J$ & 14 & & & $<50$ & 1 & $u g / L$ & EX \\
\hline & Mercury, total recoverable & .022 & $J$ & .33 & & $<.5$ & $\mathbf{U}$ & $<.5$ & $\mathrm{U}$ & & $<\mathrm{EQL}$ & 1 & $u g / L$ & EX \\
\hline & Nitrate-nitrite as nitrogen & 1270 & & 1020 & & 867 & & 1400 & & & $<2400$ & 5 & $\mathrm{ug} / \mathrm{L}$ & $\mathrm{EX}$ \\
\hline & Sodium, total recoverable & 2440 & & 2260 & & 2700 & & 3400 & & & $<4600$ & 1 & $\mathrm{ug} / \mathrm{L}$ & $\mathrm{EX}$ \\
\hline & Sulfate & 757 & $J$ & 562 & $J$ & 356 & $J$ & 647 & & & $<3000$ & 1 & $\mathrm{ug} / \mathrm{L}$ & EX \\
\hline & Total phosphates (as P) & $<50$ & UJ & $<50$ & U & 2500 & $\mathrm{~J}$ & $<2500$ & $U$ & & $<\mathrm{EQL}$ & 5 & $\mathrm{ug} / \mathrm{L}$ & EX \\
\hline & Uranium, total recoverable & & & & & & & & & & & & & \\
\hline & Zinc, total recoverable & $<3.11$ & $\mathrm{U}$ & 14.3 & & 7.82 & $J$ & 7.5 & $\mathrm{~J}$ & 1 & NDD & 1 & $u g / L$ & EX \\
\hline & ionuclides & & & & & & & & & & & & & \\
\hline & Gross alpha & 10.2 & & 5.06 & & 28.5 & & 12.4 & $J$ & K & NDD & 1 & $\mathrm{pCi} / \mathrm{L}$ & GP \\
\hline & Nonvolatile beta & 3.53 & & 1.37 & $\mathrm{U}$ & 19.3 & $J$ & 4.93 & & & $<50$ & 1 & $\mathrm{pCi} / \mathrm{L}$ & GP \\
\hline & Radium, total alpha-emitting & 6.6 & & 6.88 & & 3.33 & & 3.7 & & & $<5$ & 1 & $\mathrm{pCi} / \mathrm{L}$ & GP \\
\hline
\end{tabular}

Notes: Concentrations in bold italics exceed the groundwater protection or monitoring constituent standards listed in Appendix A. Synchronous water levels are measured over a 3-5 day period or less. Dilution factors, Laboratory, and Filtered Data are for Groundwater Protection Standards First Quarter 2000 and Monitoring Constituents Third Quarter 1999 data only.

+ = exceeded the Groundwater Protection Standards (First Quarter 2000) or Monitoring Constituents (Third Quarter 1999) listed in Appendix A. 
Table D-3. Groundwater Monitoring Results for Background Wells, M-Area HWMF (Cont.) WELL MSB 43A

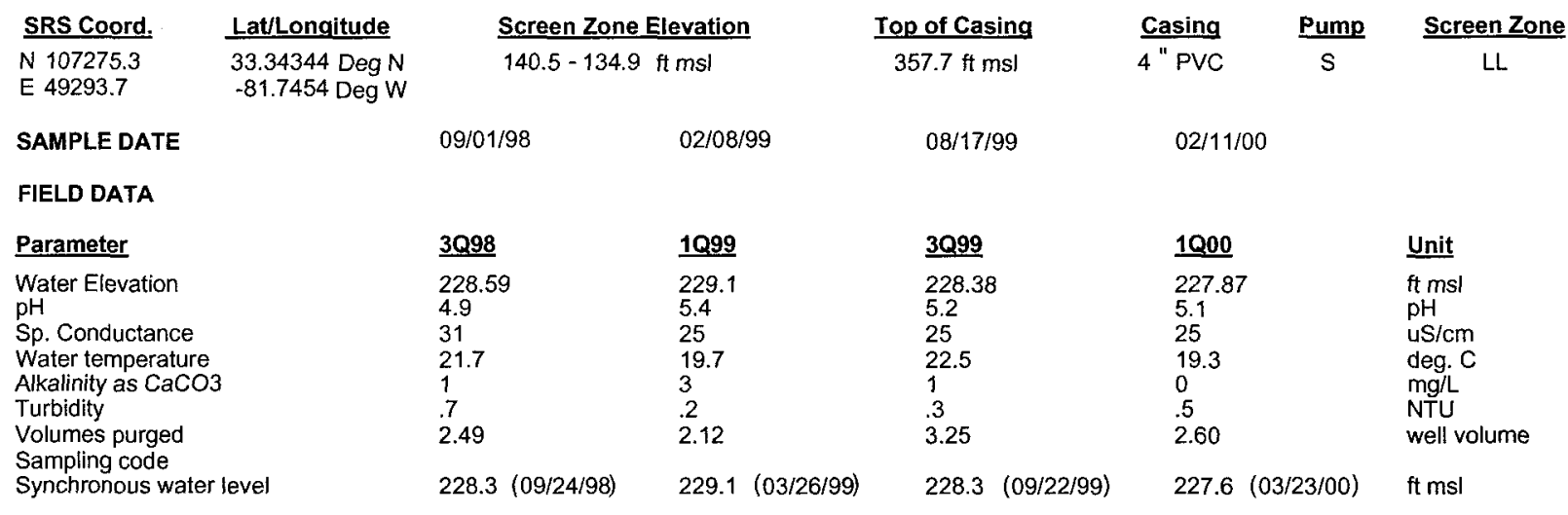

ANALYTICAL DATA

I. Groundwater Protection Standard

261 Appendix VIII/264 Appendix IX Hazardous Constituents

\begin{tabular}{|c|c|c|c|c|c|c|c|c|c|c|c|c|c|c|}
\hline$\underline{\mathrm{ST}}$ & Parameter & $\underline{3 Q 98}$ & CLP EPA & $\underline{1099}$ & CLP EPA & $\underline{3099}$ & CLPEPA & 1000 & CLF & EPA & Filt. & $\underline{\mathrm{DF}}$ & Unit & Lab \\
\hline \multicolumn{15}{|c|}{ Inorganics } \\
\hline & Barium, total recoverable & 14.1 & & 7.77 & $\mathrm{~J} \quad \mathrm{I}$ & 9.8 & J I & 9.54 & $J$ & 1 & NDD & 1 & ug/L & EX \\
\hline & Cyanide & $<10$ & $U$ & $<10$ & $\mathrm{U}$ & $<10$ & U & $<10$ & $\mathrm{U}$ & & $<\mathrm{EQL}$ & 1 & $\mathrm{ug} / \mathrm{L}$ & EX \\
\hline & Lead, total recoverable & $<100$ & $\mathrm{U}$ & $<100$ & $\mathrm{U}$ & $<10$ & $\mathrm{U}$ & $<1.93$ & JU & & $<E Q L$ & 1 & $u g / L$ & EX \\
\hline & Nickel, total recoverable & $<50$ & $\mathrm{U}$ & $<50$ & $\mathrm{U}$ & $<50$ & $\mathrm{U}$ & $<50$ & $U$ & & $<\mathrm{EQL}$ & 1 & ug/L & EX \\
\hline & Selenium, total recoverable & $<10$ & $U$ & $<200$ & $\mathrm{U}$ & $<10$ & U & $<10$ & $\mathrm{U}$ & & $<\mathrm{EQL}$ & 1 & $\mathrm{ug} / \mathrm{L}$ & EX \\
\hline \multicolumn{15}{|c|}{ Organics } \\
\hline & Chlorobenzene & $<5$ & UJ & $<5$ & $\mathrm{U}$ & $<5$ & $u$ & $<5$ & $\mathrm{U}$ & & $<\mathrm{EQL}$ & 1 & $\mathrm{ug} / \mathrm{L}$ & EX \\
\hline & 1,1-Dichloroethane & $<5$ & UJ & $<5$ & $\mathrm{U}$ & $<5$ & $\mathrm{U}$ & $<5$ & $\mathrm{U}$ & & $<\overline{E Q L}$ & 1 & $\mathrm{ug} / \mathrm{L}$ & $\mathrm{EX}$ \\
\hline & 1,1-Dichloroethylene & $<5$ & UJ & $<5$ & $\mathrm{U}$ & $<5$ & $U$ & $<5$ & U & & $<E Q L$ & 1 & $\mathrm{ug} / \mathrm{L}$ & $\mathrm{EX}$ \\
\hline & trans-1,2-Dichloroethylene & $<5$ & UJ & $<5$ & $U$ & $<5$ & $U$ & $<5$ & $\mathrm{U}$ & & $<\mathrm{EQL}$ & 1 & $\mathrm{ug} / \mathrm{L}$ & EX \\
\hline & PCB 1016 & & & & & & & & & & & & & \\
\hline & PCB 1221 & & & & & & & & & & & & & \\
\hline & PCB 1232 & & & & & & & & & & & & & \\
\hline & PCB 1242 & & & & & & & & & & & & & \\
\hline & PCB 1248 & & & & & & & & & & & & & \\
\hline & РСВ 1254 & & & & & & & & & & & & & \\
\hline & PCB 1260 & & & & & & & & & & & & & \\
\hline & $1,1,2,2$-Tetrachloroethane & $<5$ & UJ & $<5$ & $\mathrm{u}$ & $<5$ & $\mathrm{U}$ & $<5$ & $\mathrm{U}$ & & $<E Q L$ & 1 & $\mathrm{ug} / \mathrm{L}$ & EX \\
\hline & Tetrachloroethylene & $<5$ & UJ & $<5$ & $U$ & $<5$ & $\mathrm{U}$ & $<5$ & $\mathrm{U}$ & & $<E Q L$ & 1 & ug/L & EX \\
\hline & 1,1,1-Trichloroethane & $<5$ & UJ & $<5$ & U & $<5$ & $U$ & $<5$ & $\mathrm{U}$ & & $<E Q L$ & 1 & $\mathrm{ug} / \mathrm{L}$ & EX \\
\hline & Trichloroethylene & $<5$ & UJ & $<5$ & $\mathrm{U}$ & $<5$ & $\mathrm{U}$ & $<5$ & $\mathrm{U}$ & & $<\overline{\mathrm{EQL}}$ & 1 & $\mathrm{ug} / \mathrm{L}$ & EX \\
\hline \multicolumn{15}{|c|}{ II. Monitoring Constituents } \\
\hline$\underline{\mathbf{S T}}$ & Parameter & $\underline{3096}$ & CLPEPA & $\underline{3 Q 97}$ & CLP EPA & 3Q98 & CLPEPA & $\underline{3 Q 99}$ & $\underline{C L F}$ & EPA & Filt. & $\underline{D F}$ & Unit & Lab \\
\hline \multicolumn{15}{|c|}{ Inorganics } \\
\hline & Aluminum, total recoverable & $<68.5$ & $\mathrm{u}$ & $<20$ & $U$ & $<200$ & $U$ & $<200$ & $\mathrm{U}$ & & $<E Q L$ & 1 & $\mathrm{ug} / \mathrm{L}$ & EX \\
\hline & Chloride & 1190 & & 1360 & & 1530 & & 1620 & & & $<4200$ & 2 & $\mathrm{ug} / \mathrm{L}$ & EX \\
\hline & Chromium, total recoverable & $<4$ & $\mathrm{U}$ & .7 & $\mathrm{~J}$ & $<10$ & $U$ & $<10$ & U & & $<E Q L$ & 1 & $u g / L$ & EX \\
\hline & Cobalt, total recoverable & .371 & $J$ & $<5$ & $\mathrm{U}$ & $<20$ & $\mathrm{U}$ & $<20$ & U & & $<E Q L$ & 1 & $\mathrm{ug} / \mathrm{L}$ & EX \\
\hline & Copper, total recoverable & 17.5 & & $<3$ & $\mathrm{U}$ & $<20$ & $\mathrm{U}$ & $<20$ & $\mathrm{U}$ & & $<E Q L$ & 1 & $u g / L$ & EX \\
\hline & Fluoride & $<100$ & $\mathrm{U}$ & $<100$ & $\mathrm{U}$ & 391 & & 153 & $\mathrm{~J}$ & 1 & NDD & 2 & $\mathrm{ug} / \mathrm{L}$ & EX \\
\hline & Manganese, total recoverable & 2.27 & & 2.9 & $\mathrm{~J}$ & 2.51 & $\mathrm{~J}$ & 2.3 & $\mathrm{~J}$ & 1 & NDD & 1 & $\mathrm{ug} / \mathrm{L}$ & EX \\
\hline & Mercury, total recoverable & $<.2$ & $U$ & $<.2$ & $\mathrm{U}$ & $<.5$ & $U$ & $<.5$ & U & & $<E Q L$ & 1 & $u g / L$ & EX \\
\hline & Nitrate-nitrite as nitrogen & 1560 & & 1700 & & 1470 & & 1620 & & & $<2400$ & 5 & $u g / L$ & $\mathrm{EX}$ \\
\hline & Sodium, total recoverable & 1750 & & 1740 & & 1700 & & 2000 & & & $<4600$ & 1 & $u g / L$ & EX \\
\hline & Sulfate & 102 & $J$ & $<5000$ & $\mathrm{U}$ & 284 & J & 252 & $J$ & 1 & NDD & 2 & $u g / L$ & EX \\
\hline & Total phosphates (as P) & 140 & & $<10$ & $U$ & $<2500$ & $U$ & $<2500$ & $\mathrm{U}$ & & $<E Q L$ & 5 & $\mathrm{ug} / \mathrm{L}$ & EX \\
\hline & Uranium, total recoverable & & & & & & & & & & & & & \\
\hline & Zinc, total recoverable & 13.9 & $\mathrm{~J}$ & 15.6 & & 4.38 & J & 6.3 & J & 1 & NDD & 1 & $\mathrm{ug} / \mathrm{L}$ & EX \\
\hline \multicolumn{15}{|c|}{ Radionuclides } \\
\hline & Gross alpha & 1.49 & & -.1 & UI & .76 & UI & .531 & $\mathrm{U}$ & & $<E Q L$ & 1 & $\mathrm{pCi} / \mathrm{L}$ & GP \\
\hline & Nonvolatile beta & 1.35 & & -.19 & U! & 2.16 & & 4.32 & & & $<50$ & 1 & $\mathrm{pCi} / \mathrm{L}$ & GP \\
\hline & Radium, total alpha-emilting & .4 & UI & .15 & U: & .72 & $\mathrm{U}$ & .1 & $\mathrm{U}$ & & $<\mathrm{EQL}$ & 1 & $\mathrm{pCi} / \mathrm{L}$ & GP \\
\hline
\end{tabular}

Notes: Concentrations in bold italics exceed the groundwater protection or monitoring constituent standards listed in Appendix A. Synchronous water levels are measured over a 3-5 day period or less. Dilution factors, Laboratory, and Filtered Data are for Groundwater Protection Standards First Quarter 2000 and Monitoring Constituents Third Quarter 1999 data only.

+ = exceeded the Groundwater Protection Standards (First Quarter 2000) or Monitoring Constituents (Third Quarter 1999) listed in Appendix A. 
Table D-3. Groundwater Monitoring Results for Background Wells, M-Area HWMF (Cont.) WELL MSB 43B

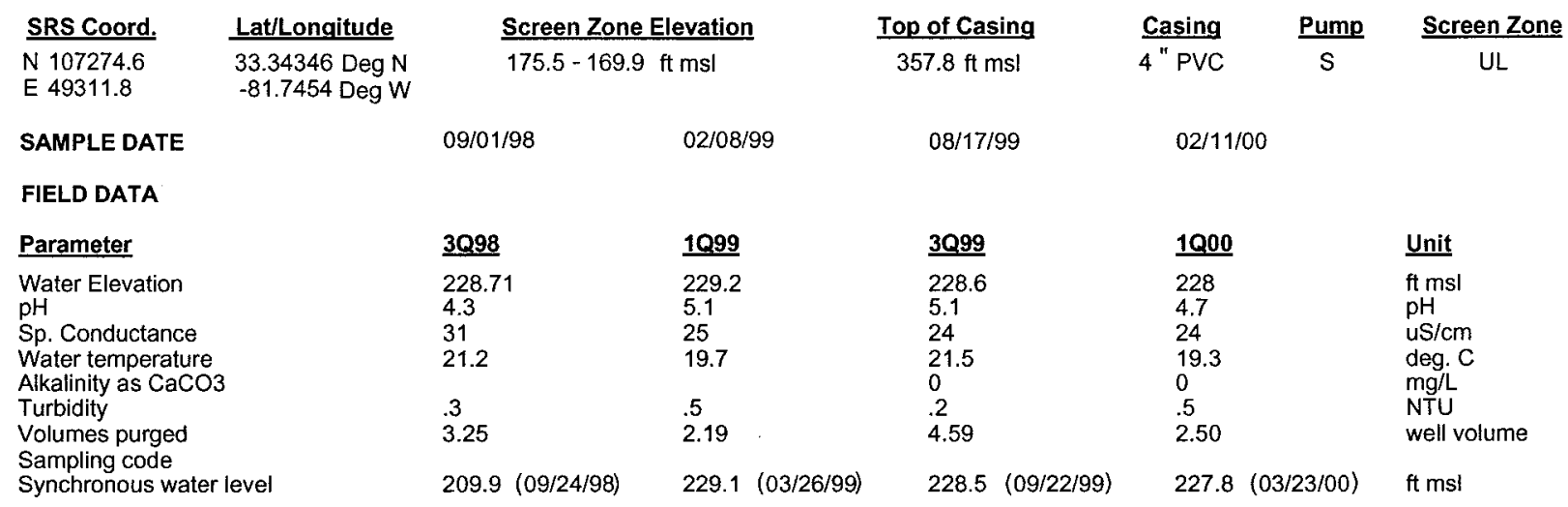

ANALYTICAL DATA

I. Groundwater Protection Standard

261 Appendix VIII/264 Appendix IX Hazardous Constituents

\begin{tabular}{|c|c|c|c|c|c|c|c|c|c|c|c|c|c|}
\hline ST Parameter & 3Q98 & CLPEPA & 1099 & CLP EPA & $\underline{3099}$ & CLPEPA & $\underline{1000}$ & CLP & EPA & Filt. & $\underline{D F}$ & Unit & $\underline{\text { Lab }}$ \\
\hline \multicolumn{14}{|l|}{ Inorganics } \\
\hline Barium, total recoverable & 2.89 & $\mathrm{~J}$ & 2.43 & J 1 & 3.1 & $\mathrm{~J} \quad \mathrm{I}$ & $<3.22$ & $\mathrm{U}$ & V & $<\mathrm{EQL}$ & 1 & $u g / L$ & EX \\
\hline Cyanide & $<10$ & $\mathrm{U}$ & $<10$ & $\mathrm{U}$ & $<10$ & $\mathrm{U}$ & $<10$ & $\mathrm{U}$ & & $<E Q L$ & 1 & $\mathrm{ug} / \mathrm{L}$ & EX \\
\hline Lead, total recoverable & $<100$ & $\mathrm{U}$ & $<100$ & $\mathrm{U}$ & $<10$ & U & $<10$ & $\mathrm{U}$ & & $<E Q L$ & 1 & $\mathrm{ug} / \mathrm{L}$ & EX \\
\hline Nickel, total recoverable & $<50$ & $\mathrm{U}$ & $<50$ & $\mathrm{U}$ & $<6.7$ & JU 1 & $<50$ & $\mathrm{U}$ & & $<E Q L$ & 1 & $u g / L$ & EX \\
\hline Selenium, total recoverable & $<10$ & $\mathrm{U}$ & $<200$ & $U$ & $<10$ & $\mathrm{U}$ & $<10$ & $\mathrm{U}$ & & $<\mathrm{EQL}$ & 1 & $\mathrm{ug} / \mathrm{L}$ & EX \\
\hline \multicolumn{14}{|l|}{ Organics } \\
\hline Chlorobenzene & $<5$ & UJ & $<5$ & $\mathrm{u}$ & $<5$ & $\mathrm{U}$ & $<5$ & $\mathrm{U}$ & & $<$ EQL & 1 & $\mathrm{ug} / \mathrm{L}$ & EX \\
\hline 1,1-Dichloroethane & $<5$ & UJ & $<5$ & $\mathrm{U}$ & $<5$ & $\mathrm{U}$ & $<5$ & $\mathrm{U}$ & & $<E Q L$ & 1 & $u g / L$ & $\mathrm{EX}$ \\
\hline 1,1-Dichloroethylene & $<5$ & Us & $<5$ & $\mathrm{U}$ & $<5$ & $U$ & $<5$ & U & & $<E Q L$ & 1 & $\mathrm{ug} / \mathrm{L}$ & EX \\
\hline trans-1,2-Dichloroethylene & $<5$ & UJ & $<5$ & $\mathrm{U}$ & $<5$ & $\mathrm{u}$ & $<5$ & $\mathrm{U}$ & & $<E Q L$ & 1 & $\mathrm{ug} / \mathrm{L}$ & EX \\
\hline \multicolumn{14}{|l|}{ PCB 1016} \\
\hline \multicolumn{14}{|l|}{ PCB 1221} \\
\hline \multicolumn{14}{|l|}{$\begin{array}{l}\text { PCB } 1232 \\
\text { PCB } 1242\end{array}$} \\
\hline \multicolumn{14}{|l|}{ PCB 1248} \\
\hline \multicolumn{14}{|l|}{ PCB 1254} \\
\hline \multicolumn{14}{|l|}{ PCB 1260} \\
\hline $1,1,2,2-$ Tetrachloroethane & $<5$ & UJ & $<5$ & $\cup$ & $<5$ & $\mathrm{U}$ & $<5$ & $\mathrm{U}$ & & $<$ EQL & 1 & $u g / L$ & EX \\
\hline Tetrachloroethylene & $<5$ & UJ & $<5$ & 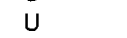 & $<5$ & $\mathrm{U}$ & $<5$ & $\mathrm{U}$ & & $<E Q L$ & 1 & $\mathrm{ug} / \mathrm{L}$ & EX \\
\hline 1,1,1-Trichloroethane & $<5$ & UJ & $<5$ & $\mathrm{U}$ & $<5$ & $U$ & $<5$ & $\mathrm{U}$ & & $<E Q L$ & 1 & ug $/ \mathrm{L}$ & EX \\
\hline Trichloroethylene & $<5$ & UJ & $<5$ & $U$ & $<5$ & U & $<5$ & $U$ & & $<$ EQL & 1 & $\mathrm{ug} / \mathrm{L}$ & $\mathrm{EX}$ \\
\hline \multicolumn{14}{|l|}{ II. Monitoring Constituents } \\
\hline Parameter & $\underline{3096}$ & CLP EPA & $\underline{3 Q 97}$ & CLP EPA & $\underline{3098}$ & CLPEPA & $\underline{3099}$ & $\underline{\text { CLP }}$ & EPA & Filt. & $\underline{D F}$ & Unit & Lab \\
\hline \multicolumn{14}{|l|}{ Inorganics } \\
\hline Aluminum, total recoverable & 13.2 & $J$ & 12.6 & 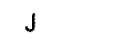 & $<200$ & $\mathrm{U}$ & $<200$ & $\mathrm{U}$ & & $<E Q L$ & 1 & $\operatorname{ug} / \mathrm{L}$ & EX \\
\hline Chloride & 1800 & & 1860 & & 1950 & & 1900 & & & $<4200$ & 2 & ug/L & EX \\
\hline Chromium, total recoverable & $<4$ & $\mathrm{U}$ & 1 & $J$ & $<10$ & $\mathrm{U}$ & $<10$ & U & & $<\mathrm{EQL}$ & 1 & $\mathrm{ug} / \mathrm{L}$ & EX \\
\hline Cobalt, total recoverable & $<.686$ & U & $<5$ & U & $<20$ & $\mathrm{U}$ & $<20$ & $\mathrm{U}$ & & $<E Q L$ & 1 & $\mathrm{ug} / \mathrm{L}$ & EX \\
\hline Copper, total recoverable & 6.93 & & $<3$ & U & $<20$ & $\mathrm{U}$ & $<4.6$ & JU & 1 & $<E Q L$ & 1 & ug/L & EX \\
\hline Fluoride & $<100$ & $U$ & $<100$ & $U$ & 373 & & 159 & $J$ & $i$ & NDD & 2 & $\mathrm{ug} / \mathrm{L}$ & EX \\
\hline Manganese, total recoverable & 1.57 & $J$ & 1.7 & $\mathrm{~J}$ & 1.43 & J & 2.5 & $\mathrm{~J}$ & $i$ & NDD & 1 & $\mathrm{ug} / \mathrm{L}$ & EX \\
\hline Mercury, total recoverable & $<.2$ & $U$ & $<.2$ & U & $<.5$ & U & $<.5$ & $\mathrm{u}$ & & $<E Q L$ & 1 & $\mathrm{ug} / \mathrm{L}$ & EX \\
\hline Nitrate-nitrite as nitrogen & 1680 & & 1880 & & 1410 & & 1480 & & & $<2400$ & 5 & $\mathrm{ug} / \mathrm{L}$ & EX \\
\hline Sodium, total recoverable & 2250 & & 2250 & & 2330 & & 2400 & & & $<4600$ & 1 & $\mathrm{ug} / \mathrm{L}$ & EX \\
\hline Sulfate & 215 & $J$ & $<5000$ & $\mathrm{U}$ & 356 & $J$ & 374 & $J$ & 1 & NDD & 2 & $u g / L$ & EX \\
\hline $\begin{array}{l}\text { Total phosphates (as P) } \\
\text { Uranium, total recoverable }\end{array}$ & $<50$ & $\mathrm{U}$ & $<10$ & $\mathrm{U}$ & $<2500$ & $U$ & $<2500$ & $\mathbf{U}$ & & $<E Q L$ & 5 & ug/L & $\mathrm{EX}$ \\
\hline Zinc, total recoverable & 8.25 & & 17 & & 10.3 & $J$ & 12 & $\mathrm{~J}$ & 1 & NDD & 1 & $\mathrm{ug} / \mathrm{L}$ & EX \\
\hline \multicolumn{14}{|l|}{ Radionuclides } \\
\hline Gross alpha & 2.69 & & 3.84 & UI & 3.76 & & 1.44 & $\mathrm{~J}$ & 1 & NDD & 1 & $\mathrm{pCi} / \mathrm{L}$ & GP \\
\hline Nonvolatile beta & 1.74 & & 3.32 & & 4.24 & & 1.48 & $\mathrm{U}$ & & $<\mathrm{EQL}$ & 1 & $\mathrm{pCi} / \mathrm{L}$ & GP \\
\hline Radium, total alpha-emitting & .8 & & 2.08 & J & .99 & $u$ & .5 & $\mathrm{U}$ & v & $<E Q L$ & 1 & $\mathrm{pCi} / \mathrm{L}$ & GP \\
\hline
\end{tabular}

Notes: Concentrations in bold italics exceed the groundwater protection or monitoring constituent standards listed in Appendix A. Synchronous water levels are measured over a 3-5 day period or less. Dilution factors, Laboratory, and Filtered Data are for Groundwater Protection Standards First Quarter 2000 and Monitoring Constituents Third Quarter 1999 data only.

$+=$ exceeded the Groundwater Protection Standards (First Quarter 2000) or Monitoring Constituents (Third Quarter 1999) listed in Appendix A. 
Table D-3. Groundwater Monitoring Results for Background Wells, M-Area HWMF (Cont.) WELL MSB 43D

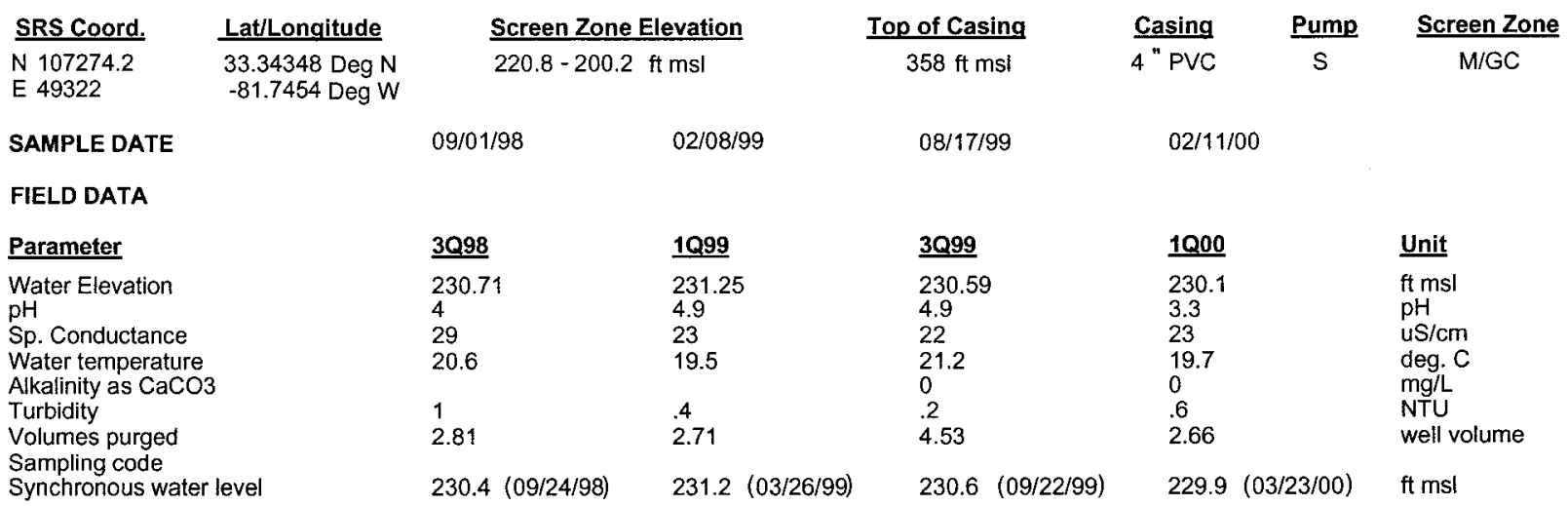

ANALYTICAL DATA

I. Groundwater Protection Standard

261 Appendix VIII/264 Appendix IX Hazardous Constituents

\begin{tabular}{|c|c|c|c|c|c|c|c|c|c|c|c|c|c|}
\hline \multirow{2}{*}{$\begin{array}{l}\underline{\text { ST }} \text { Parameter } \\
\text { Inorganics }\end{array}$} & \multirow[t]{2}{*}{$\underline{3 Q 98}$} & \multirow[t]{2}{*}{ CLPEPA } & \multirow[t]{2}{*}{$\underline{1 Q 99}$} & CLPEPA & \multirow[t]{2}{*}{$\underline{3099}$} & CLPEPA & \multirow[t]{2}{*}{$\underline{1000}$} & \multicolumn{2}{|c|}{ CLPEPA } & \multirow[t]{2}{*}{ Fillt. } & \multirow[t]{2}{*}{$\underline{\text { DF }}$} & \multirow[t]{2}{*}{$\underline{\text { Unit }}$} & \\
\hline & & & & & & & & & & & & & \\
\hline Barium, total recoverable & 3.12 & $\mathrm{~J}$ & 3.37 & J 1 & 3.6 & J 1 & $<3.61$ & $\mathrm{u}$ & $\mathrm{v}$ & $<E Q L$ & 1 & $\mathrm{ug} / \mathrm{L}$ & \\
\hline Cyanide & $<10$ & $u$ & $<10$ & $u$ & $<10$ & u & $<10$ & $u$ & & $<E Q L$ & 1 & $\mathrm{ug} / \mathrm{L}$ & \\
\hline$+\quad$ Lead, total recoverable & $<100$ & $\mathrm{u}$ & $<100$ & $u$ & 7.5 & J 1 & 25.8 & & & $>15$ & 1 & $\mathrm{ug} / \mathrm{L}$ & \\
\hline Nickel, total recoverable & $<50$ & $u$ & $<50$ & $u$ & $<50$ & $u$ & $<50$ & $\mathrm{u}$ & & $<E Q L$ & 1 & $\mathrm{ug} / \mathrm{L}$ & \\
\hline Selenium, total recoverable & $<10$ & $u$ & $<200$ & $u$ & $<3.8$ & JU 1 & $<10$ & $\mathrm{u}$ & & $<E Q L$ & 1 & $\mathrm{ug} / \mathrm{L}$ & \\
\hline \multicolumn{13}{|l|}{ Organics } & \\
\hline Chlorobenzene & $<5$ & UJ & $<5$ & $u$ & $<5$ & $\mathrm{u}$ & $<5$ & $\mathrm{U}$ & & $<\mathrm{EQL}$ & 1 & $\mathrm{ug} / \mathrm{L}$ & \\
\hline 1,1-Dichloroethane & $<5$ & UJ & $<5$ & $u$ & $<5$ & $u$ & $<5$ & $\mathrm{U}$ & & $<E Q L$ & 1 & $u g / L$ & \\
\hline 1,1-Dichloroethylene & $<5$ & UJ & $<5$ & $u$ & $<5$ & $\mathrm{u}$ & $<5$ & u & & $<E Q L$ & 1 & $\mathrm{ug} / \mathrm{L}$ & \\
\hline trans-1,2-Dichloroethylene & $<5$ & UJ & $<5$ & $\mathrm{u}$ & $<5$ & $\mathrm{U}$ & $<5$ & $u$ & & $<E Q L$ & 1 & ug/L & \\
\hline PCB 1016 & & & & - & & & & & & & & & \\
\hline PCB 1221 & & & & & & & & & & & & & \\
\hline PCB 1232 & & & & & & & & & & & & & \\
\hline PCB 1242 & & & & & & & & & & & & & \\
\hline PCB 1248 & & & & & & & & & & & & & \\
\hline PCB 1254 & & & & & & & & & & & & & \\
\hline PCB 1260 & & & & & & & & & & & & & \\
\hline 1,1,2,2-Tetrachloroethane & $<5$ & UJ & $<5$ & $\mathrm{u}$ & $<5$ & $u$ & $<5$ & $\mathrm{u}$ & & $<E Q L$ & 1 & $\mathrm{ug} / \mathrm{L}$ & \\
\hline Tetrachloroethylene & $<5$ & UJ & $<5$ & $\mathrm{u}$ & $<5$ & $u$ & $<5$ & $\mathrm{u}$ & & $<E Q L$ & 1 & $\mathrm{ug} / \mathrm{L}$ & \\
\hline 1,1,1-Trichloroethane & $<5$ & UJ & $<5$ & u & $<5$ & $\mathrm{u}$ & $<5$ & $\mathrm{u}$ & & $<E Q L$ & 1 & $\mathrm{ug} / \mathrm{L}$ & \\
\hline Trichioroethylene & $<5$ & UJ & $<5$ & $u$ & $<5$ & $u$ & $<5$ & $\mathrm{U}$ & & $<E Q L$ & 1 & $\mathrm{ug} / \mathrm{L}$ & \\
\hline \multicolumn{13}{|l|}{ II. Monitoring Constituents } & \\
\hline ST Parameter & $\underline{3 Q 96}$ & CLPEPA & $\underline{3 Q 97}$ & CLPEPA & $\underline{3098}$ & CLPEPA & $\underline{3099}$ & $\underline{\text { CLP }}$ & EPA & Filt. & $\underline{\text { DF }}$ & $\underline{\text { Unit }}$ & \\
\hline \multicolumn{13}{|l|}{ Inorganics } & \\
\hline Aluminum, total recoverable & 62.9 & & 73.1 & & 84.9 & $\mathrm{~J}$ & $<200$ & $U$ & & $<E Q L$ & 1 & $u g / L$ & \\
\hline Chloride & 1660 & & 1840 & & 1920 & & 1710 & & & $<4200$ & 2 & $\mathrm{ug} / \mathrm{L}$ & \\
\hline Chromium, total recoverable & $<4$ & $u$ & 5.8 & & 4.2 & $\mathrm{~J}$ & $<10$ & $u$ & & $<E Q L$ & 1 & $\mathrm{ug} / \mathrm{L}$ & \\
\hline Cobalt, total recoverable & $<.917$ & $\mathrm{u}$ & $<5$ & $\mathrm{u}$ & 4.66 & $\mathrm{~J}$ & $<20$ & $u$ & & $<E Q L$ & 1 & $\mathrm{ug} / \mathrm{L}$ & \\
\hline Copper, total recoverable & 16.4 & & 28.5 & & 119 & & 37 & & & $<1300$ & 1 & $\mathrm{ug} / \mathrm{L}$ & \\
\hline Fluoride & $<100$ & $\mathrm{u}$ & $<100$ & $\mathrm{U}$ & 309 & & $<200$ & $\mathrm{U}$ & & $<E Q L$ & 2 & $\mathrm{ug} / \mathrm{L}$ & \\
\hline Manganese, total recoverable & 6.66 & & 5.3 & & 4.83 & $\mathrm{~J}$ & 4.8 & J & 1 & NDD & 1 & $\mathrm{ug} / \mathrm{L}$ & \\
\hline Mercury, total recoverable & $<.2$ & $\mathrm{u}$ & $<.2$ & $\mathrm{u}$ & $<.5$ & $u$ & $<.5$ & $\mathrm{u}$ & & $<E Q L$ & 1 & $u g / L$ & \\
\hline Nitrate-nitrite as nitrogen & 1680 & & 1150 & & 953 & & 1050 & & & $<2400$ & 5 & $\mathrm{ug} / \mathrm{L}$ & \\
\hline Sodium, total recoverable & 1410 & & 1370 & & 1510 & & 1500 & & & $<4600$ & 1 & $\mathrm{ug} / \mathrm{L}$ & \\
\hline Sulfate & 398 & $\mathrm{~J}$ & $<5000$ & $u$ & 508 & & 308 & J & 1 & NDD & 2 & $\mathrm{ug} / \mathrm{L}$ & \\
\hline $\begin{array}{l}\text { Total phosphates (as } \mathrm{P} \text { ) } \\
\text { Uranium, total recoverable }\end{array}$ & $<50$ & $u$ & $<10$ & $u$ & $<2500$ & U & $<2500$ & $u$ & & $<E Q L$ & 5 & $\mathrm{ug} / \mathrm{L}$ & \\
\hline Zinc, total recoverable & 6.15 & & 29.3 & & 18.4 & J & 13 & $j$ & I & NDD & 1 & $\mathrm{ug} / \mathrm{L}$ & \\
\hline \multicolumn{13}{|l|}{ Radionuclides } & \\
\hline Gross alpha & 1.51 & & 5.37 & UI & 1.31 & & .495 & $u$ & & $<E Q L$ & 1 & $\mathrm{pCi} / \mathrm{L}$ & \\
\hline Nonvolatile beta & 1.01 & $\mathrm{UI}$ & 6.42 & & 1.39 & Ui & 2.12 & J & 1 & NDD & 1 & $\mathrm{pCi} / \mathrm{L}$ & \\
\hline Radium, total alpha-emitting & 1 & & .95 & $\mathrm{~J}$ & .45 & $\mathrm{u}$ & .3 & $\mathrm{u}$ & & $<E Q L$ & 1 & $\mathrm{pCi} / \mathrm{L}$ & \\
\hline
\end{tabular}

Notes: Concentrations in bold italics exceed the groundwater protection or monitoring constituent standards listed in Appendix A. Synchronous water levels are measured over a 3-5 day period or less. Dilution factors, Laboratory, and Filtered Data are for Groundwater Protection Standards First Quarter 2000 and Monitoring Constituents Third Quarter 1999 data only.

$+=$ exceeded the Groundwater Protection Standards (First Quarter 2000) or Monitoring Constituents (Third Quarter 1999) listed in Appendix A. 
Table D-4. Groundwater Monitoring Results for Plume Definition Wells, M-Area HWMF WELL ABP 2A

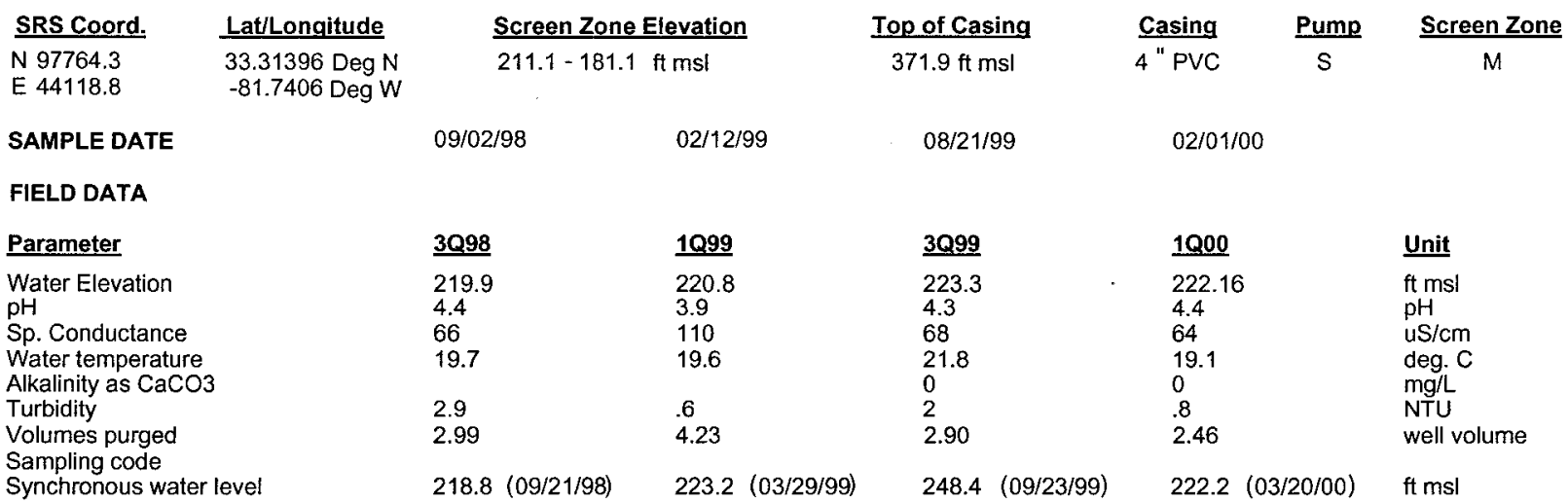

ANALYTICAL DATA

I. Groundwater Protection Standard

261 Appendix VIII/264 Appendix IX Hazardous Constituents

\begin{tabular}{|c|c|c|c|c|c|c|c|c|c|c|c|c|}
\hline ST Parameter & $\underline{3 Q 98}$ & CLPEPA & $\underline{1099}$ & CLP EPA & $\underline{3 Q 99}$ & CLPEPA & 1000 & $\underline{\text { CLPEPA }}$ & Filt. & $\underline{\text { DF }}$ & $\underline{\text { Unit }}$ & $\underline{\text { Lab }}$ \\
\hline \multicolumn{13}{|l|}{ Inorganics } \\
\hline Barium, total recoverable & 24.8 & & 27.6 & & 29 & & & & & & $u g / L$ & \\
\hline Cyanide & $<15.2$ & $U$ & 2.39 & $\mathrm{~J} \quad \mathrm{I}$ & $<10$ & $\mathrm{U}$ & & & & & ug/L & \\
\hline Lead, total recoverable & $<47$ & $U$ & $<100$ & U & $<10$ & U & & & & & $\mathrm{ug} / \mathrm{L}$ & \\
\hline Nickel, total recoverable & 2.6 & $J$ & $<50$ & U & $<50$ & $U$ & & & & & ug/L & \\
\hline Selenium, total recoverable & $<66$ & $U$ & $<200$ & $U$ & $<10$ & $U$ & & & & & ug/L & \\
\hline \multicolumn{13}{|l|}{ Organics } \\
\hline Chlorobenzene & $<5$ & $\mathrm{U}$ & $<5$ & $\mathrm{U}$ & $<5$ & $\mathrm{U}$ & $<1$ & JU & $<\mathrm{EQL}$ & 1 & ug/L & ML \\
\hline 1,1-Dichloroethane & $<5$ & $\mathrm{U}$ & $<5$ & $\mathrm{U}$ & $<5$ & U & $<1$ & JU & $<E Q$ & 1 & $\mathrm{ug} / \mathrm{L}$ & $\mathrm{ML}$ \\
\hline 1,1-Dichloroethylene & $<5$ & $\mathrm{U}$ & $<5$ & $\mathrm{U}$ & $<5$ & $U$ & $<1$ & JU & $<E Q L$ & 1 & $u g / L$ & ML \\
\hline trans-1,2-Dichloroethylene & & & $<5$ & $\mathrm{U}$ & $<5$ & $\mathrm{U}$ & $<1$ & JU & $<E Q L$ & 1 & $\mathrm{ug} / \mathrm{L}$ & ML \\
\hline PCB 1016 & & & & & & & & & & & & \\
\hline PCB 1221 & & & & & & & & & & & & \\
\hline PCB 1232 & & & & & & & & & & & & \\
\hline PCB 1242 & & & & & & & & & & & & \\
\hline PCB 1248 & & & & & & & & & & & & \\
\hline PCB 1254 & & & & & & & & & & & & \\
\hline PCB 1260 & & & & & & & & & & & & \\
\hline $1,1,2,2$-Tetrachloroethane & $<5$ & U & $<5$ & $U$ & $<5$ & $U$ & $<1$ & JU & $<E Q L$ & 1 & $u g / L$ & ML \\
\hline Tetrachloroethylene & $<5$ & $\mathrm{U}$ & $<5$ & $\mathrm{U}$ & $<5$ & $\mathrm{U}$ & $<1$ & JU & $<E Q L$ & 1 & $\mathrm{ug} / \mathrm{L}$ & ML \\
\hline $1,1,1$-Trichloroethane & $<5$ & $\mathrm{U}$ & $<5$ & $\mathrm{U}$ & $<5$ & $\mathrm{U}$ & $<1$ & $\mathrm{JU}$ & $<E Q L$ & 1 & $\mathrm{ug} / \mathrm{L}$ & ML \\
\hline Trichloroethylene & 1.51 & $J$ & 1.21 & J I & $<5$ & $\mathrm{U}$ & .99 & J 1 & NDD & 1 & $\mathrm{ug} / \mathrm{L}$ & ML \\
\hline \multicolumn{13}{|l|}{ II. Monitoring Constituents } \\
\hline ST Parameter & $\underline{3 Q 96}$ & CLPEPA & $\underline{3097}$ & CLP EPA & $\underline{3 Q 98}$ & CLPEPA & $\underline{3099}$ & CLPEPA & Filt. & DF & $\underline{\text { Unit }}$ & $\underline{\text { Lab }}$ \\
\hline \multicolumn{13}{|l|}{ Inorganics } \\
\hline Aluminum, total recoverable & 181 & & 744 & & 114 & $\mathrm{~J}$ & 120 & J I & NDD & 1 & $u g / L$ & EX \\
\hline Nitrate-nitrite as nitrogen & 7600 & & 7600 & & 5640 & & 6220 & & $>2400$ & 5 & $\mathrm{ug} / \mathrm{L}$ & $\mathrm{EX}$ \\
\hline Sodium, total recoverable & 2140 & & 2040 & & 1820 & & 2200 & & $<4600$ & 1 & $\mathrm{ug} / \mathrm{L}$ & EX \\
\hline Sulfate & $<1000$ & $U$ & $<1000$ & $U$ & 190 & $J$ & 276 & & $<3000$ & 1 & $\mathrm{ug} / \mathrm{L}$ & EX \\
\hline \multicolumn{13}{|l|}{ Radionuclides } \\
\hline Gross alpha & 14.9 & & 5.47 & & 9.7 & J & 6.22 & & $<15$ & 1 & $\mathrm{pCi} / \mathrm{L}$ & GP \\
\hline Nonvolatile beta & 7.15 & & .84 & UIJ & 5.04 & UIJ & 3.55 & & $<50$ & 1 & $\mathrm{pCi} / \mathrm{L}$ & GP \\
\hline Radium, total alpha-emitting & 7.4 & & 11.6 & $\mathrm{~J}$ & 3.24 & & 3.5 & & $<5$ & 1 & $\mathrm{pCi} / \mathrm{L}$ & GP \\
\hline
\end{tabular}

Notes: Concentrations in bold italics exceed the groundwater protection or monitoring constituent standards listed in Appendix A. Synchronous water levels are measured over a 3-5 day period or less. Dilution factors, Laboratory, and Filtered Data are for Groundwater Protection Standards First Quarter 2000 and Monitoring Constituents Third Quarter 1999 data only.

+ = exceeded the Groundwater Protection Standards (First Quarter 2000) or Monitoring Constituents (Third Quarter 1999) listed in Appendix A. 
Table D-4. Groundwater Monitoring Results for Plume Definition Wells, M-Area HWMF (Cont.) WELL ABP 3C

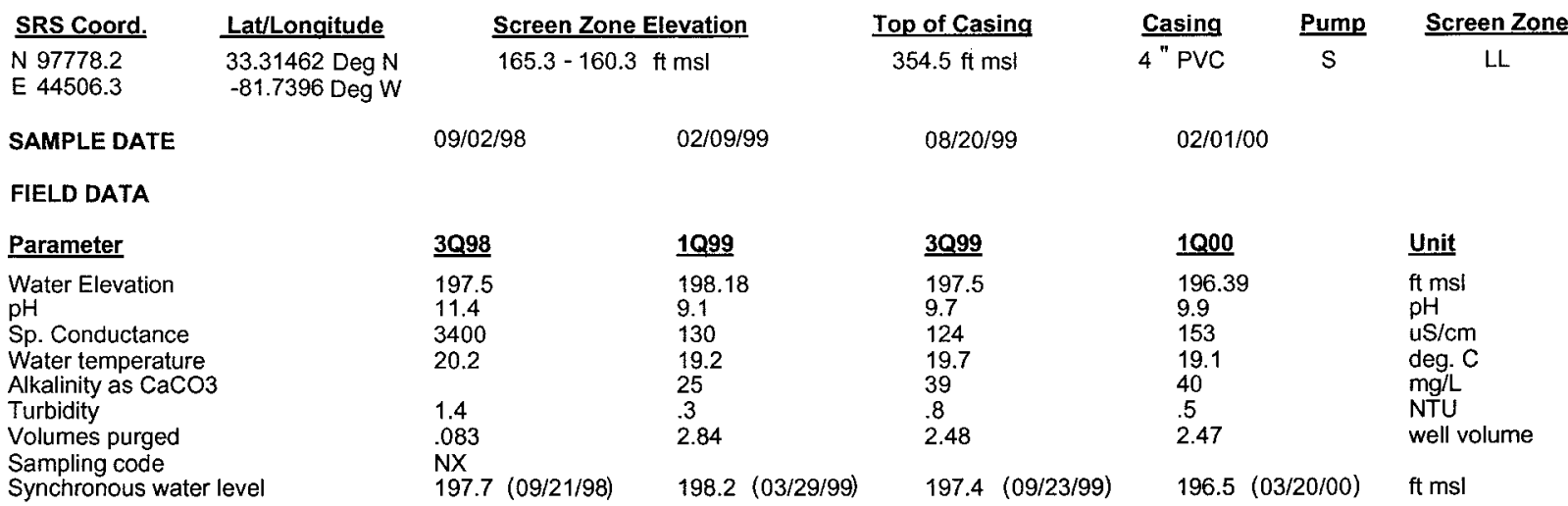

ANALYTICAL DATA

I. Groundwater Protection Standard

261 Appendix VIII/264 Appendix IX Hazardous Constituents

\begin{tabular}{|c|c|c|c|c|c|c|c|c|c|c|c|c|c|c|}
\hline$\underline{\mathrm{ST}}$ & Parameter & $\underline{3 Q 98}$ & CLPEPA & 1099 & $\underline{\text { CLP }}$ & EPA & $\underline{3 Q 99}$ & $\underline{\text { CLPEPA }}$ & 1000 & CLPEPA & Filt. & DF & Unit & Lab \\
\hline \multicolumn{15}{|c|}{ Inorganics } \\
\hline & Barium, total recoverable & 83.1 & & 28.3 & & & 32 & & & & & & $\mathrm{ug} / \mathrm{L}$ & \\
\hline & Cyanide & $<15.2$ & U & $<15.2$ & $\mathrm{U}$ & & $<10$ & $\mathrm{U}$ & & & & & $u g / L$ & \\
\hline & Lead, total recoverable & $<47$ & U & $<47$ & $\mathrm{u}$ & & $<10$ & $\mathrm{U}$ & & & & & $\mathrm{ug} / \mathrm{L}$ & \\
\hline & Nickel, total recoverable & $<26$ & $\mathrm{U}$ & $<26$ & $\mathrm{u}$ & & $<50$ & $\mathrm{U}$ & & & & & $u g / L$ & \\
\hline & Selenium, total recoverable & $<66$ & $\mathrm{U}$ & $<66$ & U & & $<10$ & $\mathrm{U}$ & & & & & $\mathrm{ug} / \mathrm{L}$ & \\
\hline \multicolumn{15}{|c|}{ Organics } \\
\hline & Chlorobenzene & $<5$ & $\mathrm{u}$ & $<5$ & JU & $Q$ & $<5$ & $\mathrm{U}$ & $<1$ & JU & $<E Q L$ & 1 & ug/L & ML \\
\hline & 1,1-Dichloroethane & $<5$ & U & $<5$ & JU & $\mathrm{Q}$ & $<5$ & $\mathrm{U}$ & $<1$ & JU & $<E Q L$ & 1 & ug/L & ML \\
\hline & 1,1-Dichloroethylene & 2.31 & $J$ & 1.02 & $J$ & $\mathrm{IKQ}$ & $<5$ & U & 1.3 & $J$ & NDD & 1 & ug/L & ML \\
\hline & trans-1,2-Dichloroethylene & & & $<5$ & JU & $\mathrm{Q}$ & $<5$ & $\mathrm{U}$ & $<1$ & JU & $<E Q L$ & 1 & $\mathrm{ug} / \mathrm{L}$ & ML \\
\hline & PCB 1016 & & & & & & & & & & & & & \\
\hline & PCB 1221 & & & & & & & & & & & & & \\
\hline & PCB 1232 & & & & & & & & & & & & & \\
\hline & PCB 1242 & & & & & & & & & & & & & \\
\hline & PCB 1248 & & & & & & & & & & & & & \\
\hline & PCB 1254 & & & & & & & & & & & & & \\
\hline & PCB 1260 & & & & & & & & & & & & & \\
\hline & 1,1,2,2-Tetrachloroethane & $<5$ & $\mathrm{U}$ & $<5$ & JU & $Q$ & $<5$ & $\mathrm{U}$ & $<1$ & JU & $<E Q L$ & 1 & $\mathrm{ug} / \mathrm{L}$ & ML \\
\hline & Tetrachloroethylene & 36.7 & & 30.9 & & $\mathrm{KQ}$ & 31 & & 29.9 & $\mathrm{~J}$ & NDD & 1 & $\mathrm{ug} / \mathrm{L}$ & ML \\
\hline & 1,1,1-Trichloroethane & $<5$ & $\mathrm{U}$ & $<5$ & JU & $Q$ & $<5$ & U & $<1$ & JU & $<E Q L$ & 1 & $\mathrm{ug} / \mathrm{L}$ & ML \\
\hline & Trichloroethylene & 39.9 & & 32.4 & $\mathrm{j}$ & KQ & 33 & & 32.9 & $J$ & NDD & 1 & ug/L & ML \\
\hline \multicolumn{15}{|c|}{ II. Monitoring Constituents } \\
\hline ST & Parameter & $\underline{3096}$ & CLP EPA & $\underline{3097}$ & CLP. & EPA & $\underline{3098}$ & CLPEPA & $\underline{3 Q 99}$ & CLPEPA & Filt. & DF & Unit & $\underline{\text { Lab }}$ \\
\hline \multicolumn{15}{|c|}{ Inorganics } \\
\hline+ & Aluminum, total recoverable & 149 & & 372 & & & 359 & & 290 & & $>100$ & 1 & $u g / L$ & EX \\
\hline+ & Nitrate-nitrite as nitrogen & 4240 & & 3740 & & & 3790 & & 3600 & & $>2400$ & 5 & $\mathrm{ug} / \mathrm{L}$ & EX \\
\hline & Sodium, total recoverable & 3570 & & 3880 & & & 4160 & & 4200 & & $<4600$ & 1 & $\mathrm{ug} / \mathrm{L}$ & EX \\
\hline & Sulfate & 349 & & 345 & $J$ & & 509 & & 222 & & $<3000$ & 1 & $u g / L$ & EX \\
\hline \multicolumn{15}{|c|}{ Radionuclides } \\
\hline & Gross alpha & 1.66 & & .83 & $\mathrm{U}$ & & 4.54 & $\jmath$ & .996 & $\mathrm{U}$ & $<\mathrm{EQL}$ & 1 & $\mathrm{pCi} / \mathrm{L}$ & GP \\
\hline & Nonvolatile beta & 4.14 & & -.95 & UIJ & & 3.32 & UIJ & 1.25 & $\mathrm{U}$ & $<E Q L$ & 1 & $\mathrm{pCi} / \mathrm{L}$ & GP \\
\hline & Radium, total alpha-emitting & 1.4 & & 2.48 & & & 1.18 & & .3 & $\mathrm{U}$ & $<E Q L$ & 1 & $\mathrm{pCi} / \mathrm{L}$ & \\
\hline
\end{tabular}

Notes: Concentrations in bold italics exceed the groundwater protection or monitoring constituent standards listed in Appendix A. Synchronous water levels are measured over a 3-5 day period or less. Dilution factors, Laboratory, and Filtered Data are for Groundwater Protection Standards First Quarter 2000 and Monitoring Constituents Third Quarter 1999 data only.

$+=$ exceeded the Groundwater Protection Standards (First Quarter 2000) or Monitoring Constituents (Third Quarter 1999) listed in Appendix A. 
Table D-4. Groundwater Monitoring Results for Plume Definition Wells, M-Area HWMF (Cont.) WELL ABP 8C

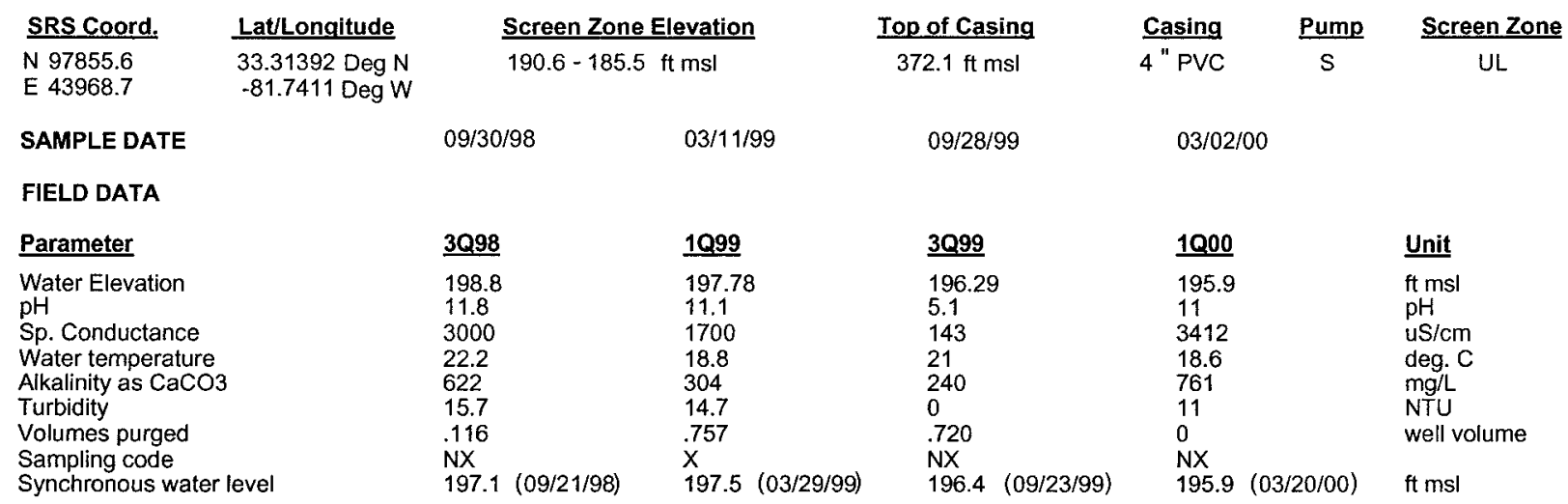

\section{ANALYTICAL DATA}

I. Groundwater Protection Standard

261 Appendix VIII/264 Appendix IX Hazardous Constituents

\begin{tabular}{|c|c|c|c|c|c|c|c|c|c|c|c|c|c|}
\hline$\underline{\mathbf{S T}}$ & Parameter & 3098 & CLP EPA & 10,99 & CLPEPA & $\underline{3 Q 99}$ & CLPEPA & 1000 & CLP트A & Filt. & DF & Unit & Lab \\
\hline \multicolumn{14}{|c|}{ Inorganics } \\
\hline & Barium, total recoverable & 455 & & 316 & & 302 & & & & & & $u g / L$ & \\
\hline & Cyanide & $<15.2$ & U & $<15.2$ & $U$ & $<10$ & JU Q & & & & & ug/L & \\
\hline & Lead, total recoverable & 213 & & 142 & & $<10$ & $\mathrm{U}$ & & & & & ug/L & \\
\hline & Nickel, total recoverable & 5.3 & $J$ & 6.8 & $J \quad I$ & $<8.56$ & JU 1 & & & & & $\mathrm{ug} / \mathrm{L}$ & \\
\hline & Selenium, totai recoverable & $<66$ & $U$ & $<66$ & U & $<10$ & $\mathrm{U}$ & & & & & $u g / L$ & \\
\hline \multicolumn{14}{|c|}{ Organics } \\
\hline & Chlorobenzene & $<5$ & $U$ & $<5$ & $U$ & $<5$ & $U$ & $<1$ & $\mathrm{U}$ & $<E Q L$ & 1 & $\mathrm{ug} / \mathrm{L}$ & ML \\
\hline & 1,1-Dichloroethane & $<5$ & $\mathrm{U}$ & $<5$ & $\mathrm{U}$ & $<5$ & $U$ & $<1$ & $\mathrm{U}$ & $<E Q L$ & 1 & ug/L & $\mathrm{ML}$ \\
\hline & 1,1-Dichloroethylene & $<5$ & U & $<5$ & $\mathrm{U}$ & $<5$ & $\mathrm{U}$ & $<1$ & $\mathrm{U}$ & $<\mathrm{EQL}$ & 1 & $\mathrm{ug} / \mathrm{L}$ & $M L$ \\
\hline & trans-1,2-Dichloroethylene & $<5$ & $U$ & $<5$ & $\mathrm{U}$ & $<5$ & $\mathrm{U}$ & & & & & ug/L & \\
\hline & PCB 1016 & & & & & & & & & & & & \\
\hline & PCB 1221 & & & & & & & & & & & & \\
\hline & PCB 1232 & & & & & & & & & & & & \\
\hline & PCB 1242 & & & & & & & & & & & & \\
\hline & PCB 1248 & & & & & & & & & & & & \\
\hline & PCB 1254 & & & & & & & & & & & & \\
\hline & PCB 1260 & & & & & & & & & & & & \\
\hline & $1,1,2,2$-Tetrachloroethane & $<5$ & $\mathrm{U}$ & $<5$ & $U$ & $<5$ & $U$ & $<1$ & $\mathrm{U}$ & $<E Q L$ & 1 & ug/L & $M L$ \\
\hline & Tetrachloroethylene & 6.92 & & $<5$ & $\mathrm{U}$ & $<5$ & U & $<1$ & $U$ & $<\mathrm{EQL}$ & 1 & $\mathrm{ug} / \mathrm{L}$ & ML \\
\hline & 1,1,1-Trichloroethane & $<5$ & $\mathrm{U}$ & $<5$ & $U$ & $<5$ & $\mathrm{U}$ & $<1$ & $U$ & $<E Q L$ & 1 & $u g / L$ & $M L$ \\
\hline+ & Trichloroethylene & 9.34 & & 10.2 & & 10 & & 5.02 & & $>5$ & 1 & ug/L & ML \\
\hline \multicolumn{14}{|c|}{ II. Monitoring Constituents } \\
\hline$\underline{\text { ST }}$ & Parameter & $\underline{3096}$ & CLPEPA & 3097 & CLPP EPA & 3098 & CLPPEPA & 3099 & CLPEPA & Filt. & $\underline{\text { DF }}$ & $\underline{\text { Unit }}$ & $\underline{\text { Lab }}$ \\
\hline \multicolumn{14}{|c|}{ Inorganics } \\
\hline+ & Aluminum, total recoverable & & & & & 3740 & & 3090 & & $>100$ & 1 & ug/L & EX \\
\hline & Nitrate-nitrite as nitrogen & & & & & 159 & & 458 & J I & NDD & 5 & $\mathrm{ug} / \mathrm{L}$ & EX \\
\hline+ & Sodium, total recoverable & & & & & 29000 & & 18700 & & $>4600$ & 1 & ug/L & EX \\
\hline & Sulfate & & & & & 4430 & $J$ & 2960 & & $<3000$ & 2 & $u g / L$ & EX \\
\hline \multicolumn{14}{|c|}{ Radionuclides } \\
\hline & Gross alpha & & & & & 10.86 & & 3.09 & & $<15$ & 1 & $\mathrm{pCi} / \mathrm{L}$ & GP \\
\hline & Nonvolatile beta & & & & & 37.95 & & 13.6 & & $<50$ & 1 & $\mathrm{pCi} / \mathrm{L}$ & GP \\
\hline & Radium, total alpha-emitting & & & & & 3.5 & & 1.4 & J 1 & NDD & 1 & $\mathrm{pCi} / \mathrm{L}$ & GP \\
\hline
\end{tabular}

Notes: Concentrations in bold italics exceed the groundwater protection or monitoring constituent standards listed in Appendix A. Synchronous water levels are measured over a 3-5 day period or less. Dilution factors, Laboratory, and Filtered Data are for Groundwater Protection Standards First Quarter 2000 and Monitoring Constituents Third Quarter 1999 data only.

+= exceeded the Groundwater Protection Standards (First Quarter 2000) or Monitoring Constituents (Third Quarter 1999) listed in Appendix A. 
Table D-4. Groundwater Monitoring Results for Plume Definition Wells, M-Area HWMF (Cont.) WELL AC 3 A

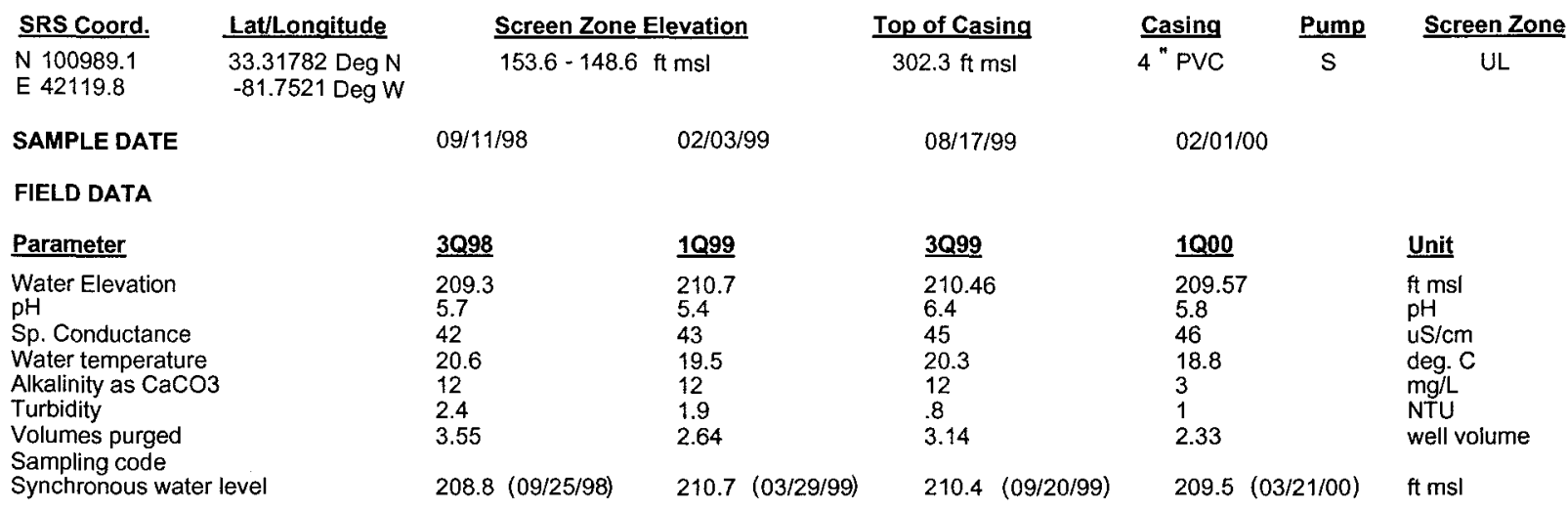

ANALYTICAL DATA

I. Groundwater Protection Standard

261 Appendix VIII/264 Appendix IX Hazardous Constituents

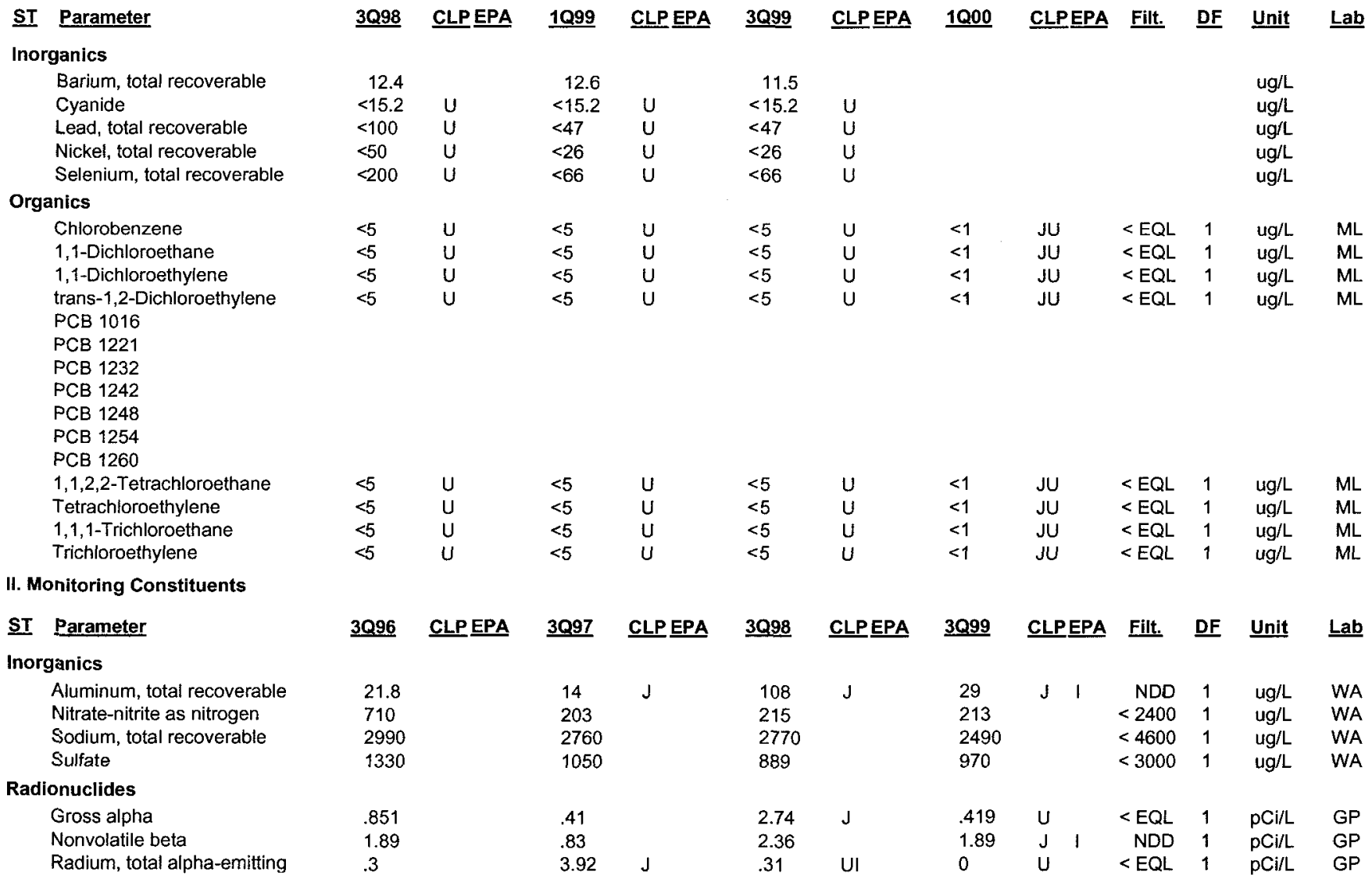

Notes: Concentrations in bold italics exceed the groundwater protection or monitoring constituent standards listed in Appendix A. Synchronous water levels are measured over a 3-5 day period or less. Dilution factors, Laboratory, and Filtered Data are for Groundwater Protection Standards First Quarter 2000 and Monitoring Constituents Third Quarter 1999 data only.

$+=$ exceeded the Groundwater Protection Standards (First Quarter 2000) or Monitoring Constituents (Third Quarter 1999) listed in Appendix A. 
Table D-4. Groundwater Monitoring Results for Plume Definition Wells, M-Area HWMF (Cont.) WELL AMB 4A

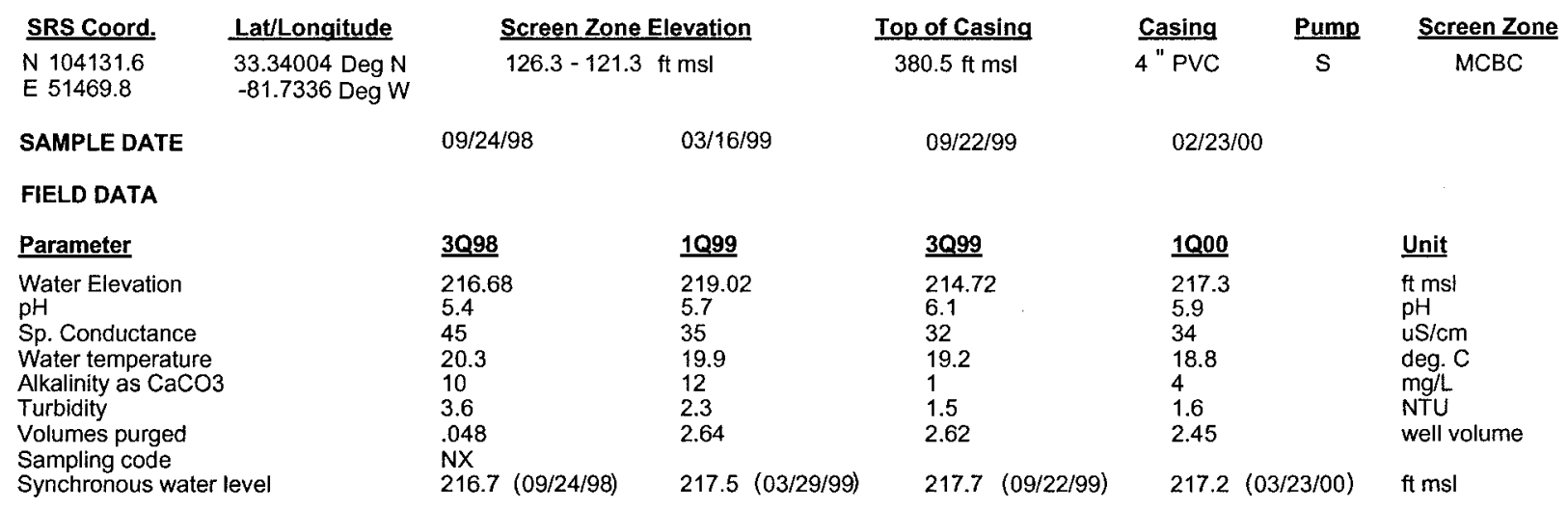

ANALYTICAL DATA

I. Groundwater Protection Standard

261 Appendix VIII/264 Appendix IX Hazardous Constituents

\begin{tabular}{|c|c|c|c|c|c|c|c|c|c|c|c|c|c|c|}
\hline$\underline{\mathrm{ST}}$ & Parameter & 3098 & CLP EPA & 1099 & CLPEPA & 3099 & CLPEPA & 1000 & CLF & EPA & Filt. & DF & Unit & Lab \\
\hline Inor & ganics & & & & & & & & & & & & & \\
\hline & Barium, total recoverable & 4.7 & & 5.9 & & 5.4 & & $<6.16$ & $\mathrm{U}$ & V & $<E Q L$ & 1 & ug/L & EX \\
\hline & Cyanide & $<15.2$ & $\mathrm{U}$ & $<15.2$ & $U$ & $<15.2$ & $\mathrm{U}$ & $<10$ & $U$ & & $<E Q L$ & 1 & $\mathrm{ug} / \mathrm{L}$ & EX \\
\hline & Lead, total recoverable & $<47$ & $\mathrm{U}$ & $<47$ & U & $<47$ & $\mathrm{U}$ & $<10$ & $U$ & & $<\mathrm{EQL}$ & 1 & ugh & EX \\
\hline & Nickel, total recoverable & $<26$ & $\mathrm{U}$ & $<26$ & $\mathrm{U}$ & $<26$ & $\mathrm{U}$ & $<6.24$ & JU & V & $<\mathrm{EQL}$ & 1 & ug/L & EX \\
\hline & Selenium, total recoverable & $<66$ & $U$ & $<66$ & U & $<66$ & $\mathrm{U}$ & $<10$ & $\mathrm{U}$ & & $<\mathrm{EQL}$ & 1 & $u g / L$ & EX \\
\hline Org & anics & & & & & & & & & & & & & \\
\hline & Chlorobenzene & $<25$ & $U$ & $<25$ & $\mathrm{U}$ & $<25$ & $u$ & $<25$ & $U$ & & $<\mathrm{EQL}$ & 5 & $u g / L$ & EX \\
\hline & 1,1-Dichloroethane & $<25$ & $U$ & $<25$ & $\mathrm{U}$ & $<25$ & $\mathrm{U}$ & $<25$ & $U$ & & $<\mathrm{EQL}$ & 5 & ug/L & EX \\
\hline & 1,1-Dichloroethylene & $<25$ & $\mathrm{U}$ & $<25$ & $U$ & $<25$ & $\mathrm{U}$ & $<25$ & $\mathrm{U}$ & & $<\mathrm{EQL}$ & 5 & ug/L & EX \\
\hline & $\begin{array}{l}\text { trans-1,2-Dichloroethylene } \\
\text { PCB } 1016\end{array}$ & $<25$ & $U$ & $<25$ & $U$ & $<25$ & U & $<25$ & $\mathrm{U}$ & & $<\mathrm{EQL}$ & 5 & ug/L & EX \\
\hline & PCB 1221 & & & & & & & & & & & & & \\
\hline & PCB 1232 & & & & & & & & & & & & & \\
\hline & PCB 1242 & & & & & & & & & & & & & \\
\hline & РСВ 1248 & & & & & & & & & & & & & \\
\hline & PCB 1254 & & & & & & & & & & & & & \\
\hline & PCB 1260 & & & & & & & & & & & & & \\
\hline & $1,1,2,2$-Tetrachloroethane & $<25$ & U & $<25$ & $\mathrm{U}$ & $<25$ & U & $<25$ & $\mathrm{U}$ & & $<E Q L$ & 5 & $\mathrm{ug} / \mathrm{L}$ & EX \\
\hline+ & Tetrachloroethylene & 64.6 & & 73.1 & & 58.5 & & 62 & & & $>5$ & 5 & ug/L & EX \\
\hline & 1,1,1-Trichloroethane & $<25$ & $U$ & $<25$ & $\mathrm{U}$ & $<25$ & $u$ & $<25$ & $\mathrm{U}$ & & $<\mathrm{EQL}$ & 5 & $\mathrm{ug} / \mathrm{L}$ & EX \\
\hline+ & Trichloroethylene & 439 & & 526 & & 429 & & 380 & & & $>5$ & 5 & $u g / L$ & EX \\
\hline II. $M$ & onitoring Constituents & & & & & & & & & & & & & \\
\hline$\underline{\text { ST }}$ & Parameter & $\underline{3096}$ & CLPEPA & 3097 & CLP EPA & $\underline{3 Q 98}$ & CLPEPA & 3099 & $\underline{C L P}$ & EPA & Filt. & DF & Unit & $\underline{L a b}$ \\
\hline Inor & ganics & & & & & & & & & & & & & \\
\hline & Aluminum, total recoverable & 152 & & $<20$ & $\mathrm{U}$ & 55.7 & $J$ & 17.8 & $J$ & $\mathbf{I}$ & NDD & 1 & ugiL & WA \\
\hline & Nitrate-nitrite as nitrogen & 962 & & 1150 & & 953 & & 899 & & & $<2400$ & 1 & $u g / L$ & WA \\
\hline & Sodium, total recoverable & 10900 & & 2360 & & 2320 & & 2030 & & & $<4600$ & 1 & ug/L & WA \\
\hline & Sulfate & 3260 & & $<5000$ & $U$ & 784 & $J$ & 616 & & & $<3000$ & 1 & $u g / L$ & WA \\
\hline Rad & Ionuclides & & & & & & & & & & & & & \\
\hline & Gross alpha & 1.09 & & -.61 & UI & 1.05 & & .0121 & $\mathrm{U}$ & & $<\mathrm{EQL}$ & 1 & $\mathrm{pCi} / \mathrm{L}$ & GP \\
\hline & Nonvolatile beta & 4.54 & & -.75 & UI & 6.6 & $J$ & .357 & $\mathrm{U}$ & & $<E Q L$ & 1 & $\mathrm{pCi} / \mathrm{L}$ & GP \\
\hline & Radium, total alpha-emitting & .26 & J & 1.2 & $\mathrm{~J}$ & .13 & UI & 0 & $\mathrm{U}$ & & $<E Q L$ & 1 & $\mathrm{pCi} / \mathrm{L}$ & GP \\
\hline
\end{tabular}

Notes: Concentrations in bold italics exceed the groundwater protection or monitoring constituent standards listed in Appendix A. Synchronous water levels are measured over a 3-5 day period or less. Dilution factors, Laboratory, and Filtered Data are for Groundwater Protection Standards First Quarter 2000 and Monitoring Constituents Third Quarter 1999 data only.

$+=$ exceeded the Groundwater Protection Standards (First Quarter 2000) or Monitoring Constituents (Third Quarter 1999) listed in Appendix A. 
Table D-4. Groundwater Monitoring Results for Plume Definition Wells, M-Area HWMF (Cont.) WELL AMB 4B

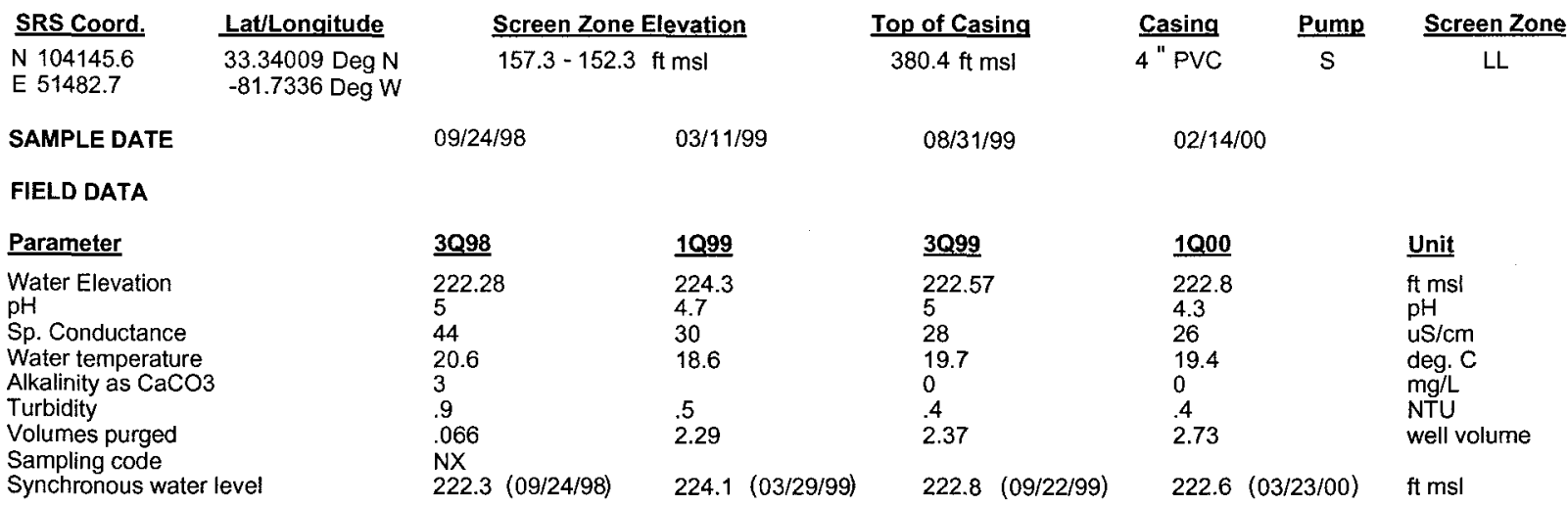

ANALYTICAL DATA

I. Groundwater Protection Standard

261 Appendix VIII/264 Appendix IX Hazardous Constituents

\begin{tabular}{|c|c|c|c|c|c|c|c|c|c|c|c|c|c|c|}
\hline \multirow{2}{*}{\multicolumn{2}{|c|}{$\begin{array}{l}\text { ST Parameter } \\
\text { Inorganics }\end{array}$}} & \multirow[t]{2}{*}{$\underline{3 Q 98}$} & \multirow[t]{2}{*}{ CLPEPA } & \multirow[t]{2}{*}{1099} & \multirow[t]{2}{*}{$\underline{\text { CLP EPA }}$} & \multirow[t]{2}{*}{ 3Q99 } & \multirow[t]{2}{*}{$\underline{\text { CLPEPA }}$} & \multirow[t]{2}{*}{$\underline{1000}$} & \multicolumn{2}{|c|}{ CLPEPA } & \multirow[t]{2}{*}{ Filt. } & \multirow[t]{2}{*}{$\underline{\text { DF }}$} & \multirow[t]{2}{*}{$\underline{\text { Unit }}$} & \multirow[t]{2}{*}{$\underline{L a b}$} \\
\hline & & & & & & & & & & & & & & \\
\hline & Barium, total recoverable & 9.6 & J & 5.1 & & 6.1 & & 5.16 & $\mathrm{~J}$ & 1 & NDD & 1 & $\mathrm{ug} / \mathrm{L}$ & EX \\
\hline & Cyanide & $<15.2$ & $\mathrm{U}$ & $<15.2$ & $\mathrm{U}$ & $<15.2$ & $\mathrm{U}$ & $<10$ & U & & $<E Q L$ & 1 & $\mathrm{ug} / \mathrm{L}$ & EX \\
\hline & Lead, total recoverable & $<100$ & $\mathrm{U}$ & $<47$ & $U$ & $<47$ & $\mathrm{U}$ & $<10$ & $\mathrm{U}$ & & $<E Q L$ & 1 & $\mathrm{ug} / \mathrm{L}$ & EX \\
\hline & Nickel, total recoverable & $<26$ & $\mathrm{U}$ & $<26$ & $U$ & $<26$ & $\mathrm{U}$ & $<50$ & $\mathrm{U}$ & & < EQL & 1 & $\mathrm{ug} / \mathrm{L}$ & EX \\
\hline & Selenium, total recoverable & $<200$ & $\mathrm{U}$ & $<66$ & $U$ & $<66$ & $\mathrm{U}$ & $<10$ & $U$ & & $<E Q L$ & 1 & $\mathrm{ug} / \mathrm{L}$ & EX \\
\hline \multicolumn{15}{|c|}{ Organics } \\
\hline & Chlorobenzene & $<5$ & $\mathrm{U}$ & $<5$ & $U$ & $<5$ & $\mathrm{u}$ & $<5$ & $\mathrm{U}$ & & $<\mathrm{EQL}$ & 1 & $u g / L$ & EX \\
\hline & 1,1-Dichloroethane & $<5$ & $\mathrm{U}$ & $<5$ & $U$ & $<5$ & $\mathrm{U}$ & $<5$ & $\mathrm{U}$ & & $<E Q L$ & 1 & $\mathrm{ug} / \mathrm{L}$ & EX \\
\hline & 1,1-Dichloroethylene & $<5$ & $\mathrm{U}$ & $<5$ & $\mathrm{U}$ & $<5$ & $\mathrm{U}$ & $<5$ & U & & $<E Q L$ & 1 & ug/L & EX \\
\hline & trans-1,2-Dichloroethylene & $<5$ & $\mathrm{U}$ & $<5$ & $\mathrm{U}$ & $<5$ & $\mathrm{U}$ & $<5$ & $\mathrm{U}$ & & $<\mathrm{EQL}$ & 1 & $\mathrm{ug} / \mathrm{L}$ & EX \\
\hline & PCB 1016 & & & & & & & & & & & & & \\
\hline & PCB 1221 & & & & & & & & & & & & & \\
\hline & РCB 1232 & & & & & & & & & & & & & \\
\hline & PCB 1242 & & & & & & & & & & & & & \\
\hline & PCB 1248 & & & & & & & & & & & & & \\
\hline & PCB 1254 & & & & & & & & & & & & & \\
\hline & PCB 1260 & & & & & & & & & & & & & \\
\hline & $1,1,2,2$-Tetrachloroethane & $<5$ & U & $<5$ & $\mathrm{U}$ & $<5$ & $\mathrm{U}$ & $<5$ & $\mathrm{U}$ & & $<E Q L$ & 1 & $\mathrm{ug} / \mathrm{L}$ & EX \\
\hline & Tetrachloroethylene & $<5$ & $U$ & $<5$ & $\mathrm{U}$ & $<5$ & $\mathrm{U}$ & $<5$ & $U$ & & $<E Q L$ & 1 & $\mathrm{ug} / \mathrm{L}$ & $\mathrm{EX}$ \\
\hline & 1,1,1-Trichloroethane & $<5$ & $\mathrm{U}$ & $<5$ & $U$ & $<5$ & $\mathrm{U}$ & $<5$ & $\mathrm{U}$ & & $<\mathrm{EQL}$ & 1 & $\mathrm{ug} / \mathrm{L}$ & EX \\
\hline & Trichtoroethylene & 3.56 & $J$ & 2.82 & $\sqrt{1}$ & 3.26 & J 1 & 4.5 & 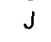 & 1 & NDD & 1 & $\mathrm{ug} / \mathrm{L}$ & EX \\
\hline \multicolumn{15}{|c|}{ II. Monitoring Constituents } \\
\hline$\underline{\text { ST }}$ & Parameter & $\underline{3 Q 96}$ & CLP EPA & $\underline{3097}$ & CLP EPA & $\underline{3098}$ & CLPEPA & $\underline{3099}$ & CLP & EPA & Filt. & DF & $\underline{\text { Unit }}$ & $\underline{\text { Lab }}$ \\
\hline \multicolumn{15}{|c|}{ Inorganics } \\
\hline & Aluminum, total recoverable & $<37$ & $U$ & 40.3 & & 292 & & 29 & $J$ & 1 & NDD & 1 & ug/L & WA \\
\hline & Nitrate-nitrite as nitrogen & 950 & & 646 & & 440 & $\mathrm{~J}$ & 444 & & & $<2400$ & 1 & $\mathrm{ug} / \mathrm{L}$ & WA \\
\hline & Sodium, total recoverable & 2980 & & 2990 & & 3210 & & 2960 & & & $<4600$ & 1 & $\mathrm{ug} / \mathrm{L}$ & WA \\
\hline & Sulfate & 208 & $J$ & $<5000$ & 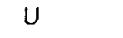 & 1180 & & 402 & & & $<3000$ & 1 & ug/L & WA \\
\hline \multicolumn{15}{|c|}{ Radionuclides } \\
\hline & Gross alpha & 1.57 & & 4.66 & UI & 1.85 & & .636 & $u$ & & $<E Q L$ & 1 & $\mathrm{pCi} / \mathrm{L}$ & GP \\
\hline & Nonvolatile beta & 1.35 & UI & 4.81 & UI & 8.38 & UI & .463 & U & & $<E Q L$ & 1 & $\mathrm{pCi} / \mathrm{L}$ & GP \\
\hline & Radium, total alpha-emitting & .7 & & 1.72 & $J$ & .74 & & .3 & U & & $<E Q L$ & 1 & $\mathrm{pCi} / \mathrm{L}$ & GP \\
\hline
\end{tabular}

Notes: Concentrations in bold italics exceed the groundwater protection or monitoring constituent standards listed in Appendix A. Synchronous water Jevels are measured over a 3-5 day period or less. Dilution factors, Laboratory, and Filtered Data are for Groundwater Protection Standards First Quarter 2000 and Monitoring Constituents Third Quarter 1999 data only.

+ = exceeded the Groundwater Protection Standards (First Quarter 2000) or Monitoring Constituents (Third Quarter 1999) listed in Appendix A. 
Table D-4. Groundwater Monitoring Results for Plume Definition Wells, M-Area HWMF (Cont.) WELL AMB 4D

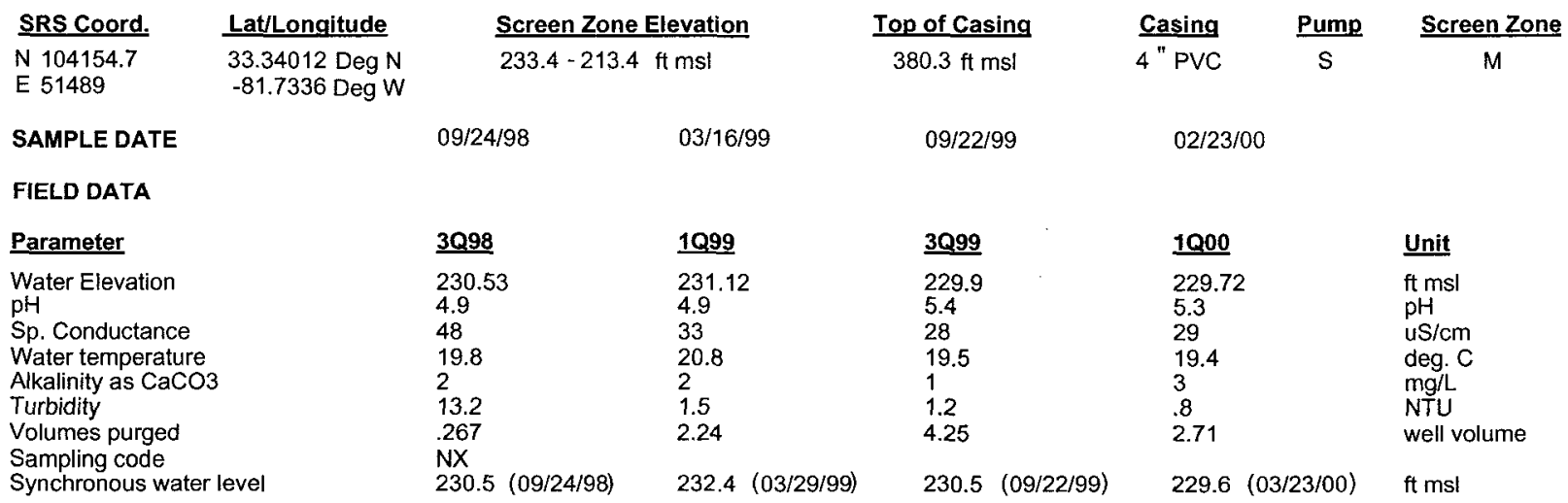

ANALYTICAL DATA

I. Groundwater Protection Standard

261 Appendix VIII/264 Appendix IX Hazardous Constituents

\begin{tabular}{|c|c|c|c|c|c|c|c|c|c|c|c|c|c|c|}
\hline$\underline{\mathbf{S T}}$ & Parameter & 3098 & CLPEPA & 1099 & CLP EPA & $\underline{3099}$ & CLPEPA & $\underline{1000}$ & $\underline{\text { CLP }}$ & EPA & Filt. & $\underline{\mathrm{DF}}$ & Unit & $\underline{\operatorname{Lab}}$ \\
\hline \multicolumn{15}{|c|}{ Inorganics } \\
\hline & Barium, total recoverable & 8.7 & & 6.5 & & 4.9 & & $<4.42$ & $U$ & V & $<E Q L$ & 1 & $u g / L$ & EX \\
\hline & Cyanide & $<15.2$ & $\mathrm{U}$ & 2.16 & J $\quad 1$ & $<15.2$ & $\mathrm{U}$ & $<10$ & $\mathrm{U}$ & & $<E Q L$ & 1 & $u g / L$ & EX \\
\hline & Lead, total recoverable & 6 & $J$ & $<47$ & $\mathrm{U}$ & $<47$ & U & $<10$ & $\mathrm{U}$ & & $<E Q L$ & 1 & $\mathrm{ug} / \mathrm{L}$ & EX \\
\hline & Nickel, total recoverable & 2.8 & J & $<26$ & $U$ & $<26$ & $\mathrm{U}$ & $<50$ & $\mathrm{U}$ & & $<E Q L$ & 1 & $\mathrm{ug} / \mathrm{L}$ & EX \\
\hline & Selenium, total recoverable & $<66$ & $U$ & $<66$ & $U$ & $<66$ & $\mathrm{U}$ & $<10$ & $\mathrm{U}$ & & $<E Q L$ & 1 & $u g / L$ & EX \\
\hline \multicolumn{15}{|c|}{ Organics } \\
\hline & Chlorobenzene & $<5$ & $U$ & $<5$ & $U$ & $<5$ & $\mathrm{U}$ & $<5$ & JU & $\mathrm{L}$ & $<E Q L$ & 1 & $u g / L$ & EX \\
\hline & 1,1-Dichloroethane & $<5$ & $\mathrm{U}$ & $<5$ & $\mathrm{U}$ & $<5$ & $\mathbf{U}$ & $<5$ & JU & L. & $<$ EQL & 1 & $u g / L$ & EX \\
\hline & 1,1-Dichloroethylene & $<5$ & $\mathrm{U}$ & $<5$ & $U$ & $<5$ & $\mathrm{U}$ & $<5$ & JU & $\mathrm{L}$ & $<$ EQL & 1 & $u g / L$ & EX \\
\hline & trans-1,2-Dichloroethylene & $<5$ & $\mathrm{U}$ & $<5$ & $\mathrm{U}$ & $<5$ & $\mathrm{U}$ & $<5$ & JU & $\mathrm{L}$ & $<E Q L$ & 1 & $u g / L$ & EX \\
\hline & PCB 1016 & & & & & & & $<.97$ & $\mathrm{U}$ & & $<E Q L$ & .970 & $u g / L$ & EX \\
\hline & PCB 1221 & & & & & & & $<.97$ & U & & $<E Q L$ & .970 & ug/L & EX \\
\hline & PCB 1232 & & & & & & & $<.97$ & $\mathrm{U}$ & & $<E Q L$ & .970 & $\mathrm{ug} / \mathrm{L}$ & $\mathrm{EX}$ \\
\hline & PCB 1242 & & & & & & & $<1.9$ & $\mathrm{U}$ & & $<E Q L$ & .970 & ug/L & EX \\
\hline & PCB 1248 & & & & & & & $<.97$ & $U$ & & $<\mathrm{EQL}$ & .970 & ug/L & EX \\
\hline & PCB 1254 & & & & & & & $<.97$ & $U$ & & $<E Q L$ & .970 & $\mathrm{ug} / \mathrm{L}$ & EX \\
\hline & PCB 1260 & & & & & & & $<.97$ & $U$ & & $<E Q L$ & .970 & ug/L & EX \\
\hline & $1,1,2,2$-Tetrachloroethane & $<5$ & $U$ & $<5$ & $U$ & $<5$ & $U$ & $<5$ & JU & $\mathrm{L}$ & $<E Q L$ & 1 & ug/L & EX \\
\hline & Tetrachloroethylene & 1.89 & $\mathrm{~J}$ & $<5$ & $U$ & $<5$ & $\mathrm{U}$ & $<5$ & JU & $\mathrm{L}$ & $<E Q L$ & 1 & $\mathrm{ug} / \mathrm{L}$ & EX \\
\hline & $1,1,1$-Trichloroethane & $<5$ & $U$ & $<5$ & $U$ & $<5$ & $\mathrm{U}$ & $<5$ & JU & $\bar{L}$ & $<E Q L$ & 1 & $\mathrm{ug} / \mathrm{L}$ & EX \\
\hline & Trichloroethylene & 64 & & 24.9 & & 7.37 & & 7.6 & J & L & NDD & 1 & ug/L & EX \\
\hline \multicolumn{15}{|c|}{ II. Monitoring Constituents } \\
\hline ST & Parameter & 3096 & $\underline{\text { CLPP EPA }}$ & 3097 & CLPEPA & $3 Q 998$ & CLPEPA & 3099 & $\underline{\text { CLP }}$ & EPA & Filt. & $\underline{\text { DF }}$ & Unit & $\underline{\text { Lab }}$ \\
\hline \multicolumn{15}{|c|}{ Inorganics } \\
\hline & Aluminum, total recoverable & 43.3 & & 332 & & 84.4 & $J$ & 26.8 & $J$ & IK & NDD & 1 & $\mathrm{ug} / \mathrm{L}$ & WA \\
\hline & Nitrate-nitrite as nitrogen & 1370 & & 907 & & 2380 & & 1390 & & & $<2400$ & 5 & $\mathrm{ug} / \mathrm{L}$ & WA \\
\hline & Sodium, total recoverable & 7390 & & 6770 & & 5620 & & 4090 & & & $<4600$ & 1 & ug/L & WA \\
\hline & Sulfate & 11100 & & 3830 & $J$ & 1170 & & 2560 & & & $<3000$ & 1 & $\mathrm{ug} / \mathrm{L}$ & WA \\
\hline \multicolumn{15}{|c|}{ Radionuclides } \\
\hline & Gross alpha & 1.09 & & 6.83 & & 17.2 & & 1.99 & $J$ & I & NDD & 1 & $\mathrm{pCi} / \mathrm{L}$ & GP \\
\hline & Nonvolatile beta & 69.8 & & .74 & UI & 12.17 & $\mathrm{~J}$ & 1.16 & $\mathrm{U}$ & & $<E Q L$ & 1 & $\mathrm{pCi} / \mathrm{L}$ & GP \\
\hline & Radium, total alpha-emitting & -.8 & U! & 4.65 & UI & 4.04 & & 2.2 & & & $<5$ & 1 & $\mathrm{pC} / \mathrm{L}$ & GP \\
\hline
\end{tabular}

Notes: Concentrations in bold italics exceed the groundwater protection or monitoring constituent standards listed in Appendix A. Synchronous water levels are measured over a 3-5 day period or less. Dilution factors, Laboratory, and Filtered Data are for Groundwater Protection Standards First Quarter 2000 and Monitoring Constituents Third Quarter 1999 data only.

$+=$ exceeded the Groundwater Protection Standards (First Quarter 2000) or Monitoring Constituents (Third Quarter 1999) listed in Appendix A. 
WSRC-TR- 2000-00242

Unclassified

Table D-4. Groundwater Monitoring Results for Plume Definition Wells, M-Area HWMF (Cont.) WELL AMB 7

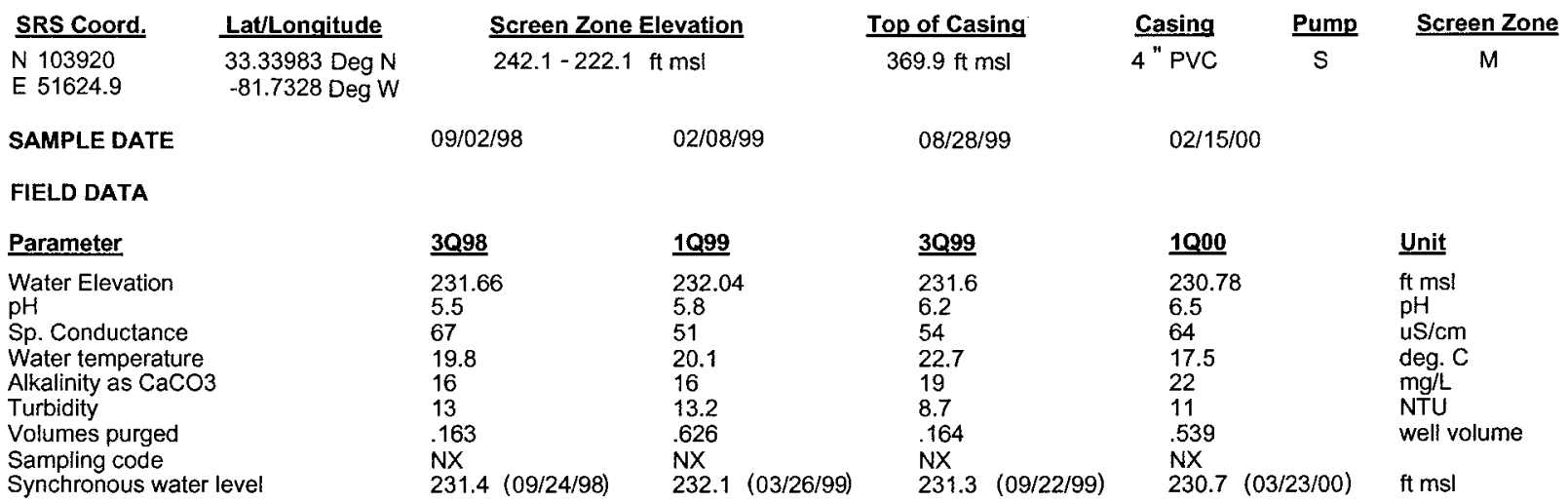

ANALYTICAL DATA

I. Groundwater Protection Standard

261 Appendix VIII/264 Appendix IX Hazardous Constituents

\begin{tabular}{|c|c|c|c|c|c|c|c|c|c|c|c|c|c|}
\hline \multirow{2}{*}{$\begin{array}{l}\text { ST Parameter } \\
\text { Inorganics }\end{array}$} & \multirow[t]{2}{*}{$\underline{3 Q 98}$} & \multirow[t]{2}{*}{ CLPEPA } & \multirow[t]{2}{*}{$\underline{1099}$} & CLPEPA & \multirow[t]{2}{*}{$\underline{3 Q 99}$} & CLPEPA & \multirow[t]{2}{*}{1000} & \multicolumn{2}{|c|}{ CLPEPA } & \multirow[t]{2}{*}{ Filt. } & \multirow[t]{2}{*}{$\underline{\mathrm{DF}}$} & \multirow[t]{2}{*}{$\underline{\text { Unit }}$} & \multirow[t]{2}{*}{ Lab } \\
\hline & & & & & & & & & & & & & \\
\hline Barium, total recoverable & 9.9 & & 6.8 & & 10.1 & & 10.1 & & & $<2000$ & 1 & $u g / L$ & EX \\
\hline Cyanide & $<15.2$ & $u$ & 2.41 & J 1 & $<15.2$ & $u$ & $<10$ & $\mathrm{u}$ & & $<E Q L$ & 1 & $\mathrm{ug} / \mathrm{L}$ & EX \\
\hline Lead, total recoverable & 5.5 & $\mathrm{~J}$ & 9.4 & J i & 6 & J 1 & 7.2 & $\mathrm{~J}$ & I & NDD & 1 & $\mathrm{ug} / \mathrm{L}$ & EX \\
\hline Nickel, total recoverable & 4.4 & J & 3.8 & J 1 & 3.3 & J 1 & $<11.3$ & $u$ & $\mathrm{v}$ & $<E Q L$ & 1 & $\mathrm{ug} / \mathrm{L}$ & EX \\
\hline Selenium, total recoverable & $<66$ & $u$ & $<66$ & $\mathrm{u}$ & $<66$ & $\mathrm{u}$ & $<10$ & $\mathrm{u}$ & & $<E Q L$ & 1 & $\mathrm{ug} / \mathrm{L}$ & EX \\
\hline \multicolumn{14}{|l|}{ Organics } \\
\hline Chlorobenzene & $<5$ & $\mathrm{u}$ & $<5$ & JU $Q$ & $<5$ & $\mathrm{u}$ & $<5$ & $\mathrm{u}$ & & $<E Q L$ & 1 & $u g / L$ & EX \\
\hline 1,1-Dichloroethane & $<5$ & $\mathrm{u}$ & $<5$ & JU Q & $<5$ & $\mathrm{u}$ & $<5$ & $\mathrm{U}$ & & $<E Q L$ & 1 & $\mathrm{ug} / \mathrm{L}$ & $E x$ \\
\hline 1,1-Dichloroethylene & $<5$ & $u$ & $<5$ & $J U Q$ & $<5$ & $\mathrm{u}$ & $<5$ & $\mathrm{u}$ & & $<E Q L$ & 1 & $\mathrm{ug} / \mathrm{L}$ & $E x$ \\
\hline trans-1,2-Dichloroethylene & $<5$ & $\mathrm{u}$ & $<5$ & $J \cup Q$ & $<5$ & $\mathrm{u}$ & $<5$ & $\mathrm{u}$ & & $<E Q L$ & 1 & $\mathrm{ug} / \mathrm{L}$ & -7 \\
\hline PCB 1016 & & & & & & & & & & & & & \\
\hline РСВ 1221 & & & & & & & & & & & & & \\
\hline PCB 1232 & & & & & & & & & & & & & \\
\hline PCB 1242 & & & & & & & & & & & & & \\
\hline PCB 1248 & & & & & & & & & & & & & \\
\hline PCB 1254 & & & & & & & & & & & & & \\
\hline PCB 1260 & & & & & & & & & & & & & \\
\hline 1,1,2,2-Tetrachloroethane & $<5$ & $\mathrm{u}$ & $<5$ & $J U Q$ & $<5$ & $u$ & $<5$ & $\mathrm{u}$ & & $<E Q L$ & 1 & $u g / L$ & 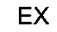 \\
\hline Tetrachloroethylene & $<5$ & $u$ & $<5$ & $J \cup Q$ & $<5$ & $\mathrm{u}$ & $<5$ & $\mathrm{u}$ & & $<E Q L$ & 1 & $\mathrm{ug} / \mathrm{L}$ & 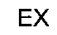 \\
\hline 1,1,1-Trichloroethane & $<5$ & $\mathrm{u}$ & $<5$ & JU $Q$ & $<5$ & $\mathrm{u}$ & $<5$ & $u$ & & $<E Q L$ & 1 & $u g / L$ & \\
\hline Trichloroethylene & 1.04 & J & $<5$ & $J U Q$ & $<5$ & u & $<5$ & $\mathrm{U}$ & & $<E Q L$ & 1 & $\mathrm{ug} / \mathrm{L}$ & x \\
\hline \multicolumn{14}{|l|}{ II. Monitoring Constituents } \\
\hline ST Parameter & $\underline{3096}$ & CLPEPA & $\underline{3097}$ & CLPEPA & $\underline{3 Q 98}$ & CLPEPA & $\underline{3099}$ & $\underline{\text { CLP }}$ & EPA & Filt. & $\underline{\text { DF }}$ & $\underline{\text { Unit }}$ & \\
\hline \multicolumn{14}{|l|}{ Inorganics } \\
\hline Aluminum, total recoverable & 163 & & 331 & & 334 & & 342 & & & $>100$ & 1 & ug/L & A \\
\hline Nitrate-nitrite as nitrogen & 470 & & 184 & & 285 & & 575 & & & $<2400$ & 1 & $\mathrm{ug} / \mathrm{L}$ & WA \\
\hline$+\quad$ Sodium, total recoverable & 19300 & & 12000 & & 10800 & & 8140 & & & $>4600$ & 1 & $\mathrm{ug} / \mathrm{L}$ & WA \\
\hline+ Sulfate & 1920 & & 3040 & J & 3970 & & 4010 & & & $>3000$ & 1 & $\mathrm{ug} / \mathrm{L}$ & WA \\
\hline \multicolumn{13}{|l|}{ Radionuclides } & \\
\hline Gross alpha & 1.55 & & 1.12 & UI & 1.75 & $\mathrm{~J}$ & .55 & $\mathrm{~J}$ & IK & NDD & 1 & $\mathrm{pCi} / \mathrm{L}$ & \\
\hline Nonvolatile beta & 2.15 & & 3.14 & & 1.82 & UIJ & .93 & $\mathrm{U}$ & & $<\mathrm{EQL}$ & 1 & $\mathrm{pCi} / \mathrm{L}$ & \\
\hline Radium, total alpha-emitting & 6 & & 1.4 & UIJ & .43 & & .5 & $\mathrm{~J}$ & 1 & NDD & 1 & $\mathrm{pCi} / \mathrm{L}$ & b \\
\hline
\end{tabular}

Notes: Concentrations in bold italics exceed the groundwater protection or monitoring constituent standards listed in Appendix A. Synchronous water levels are measured over a 3-5 day period or less. Dilution factors, Laboratory, and Filtered Data are for Groundwater Protection Standards First Quarter 2000 and Monitoring Constituents Third Quarter 1999 data only.

$+=$ exceeded the Groundwater Protection Standards (First Quarter 2000) or Monitoring Constituents (Third Quarter 1999) listed in Appendix A. 
Table D-4. Groundwater Monitoring Results for Plume Definition Wells, M-Area HWMF (Cont.) WELL AMB 7A

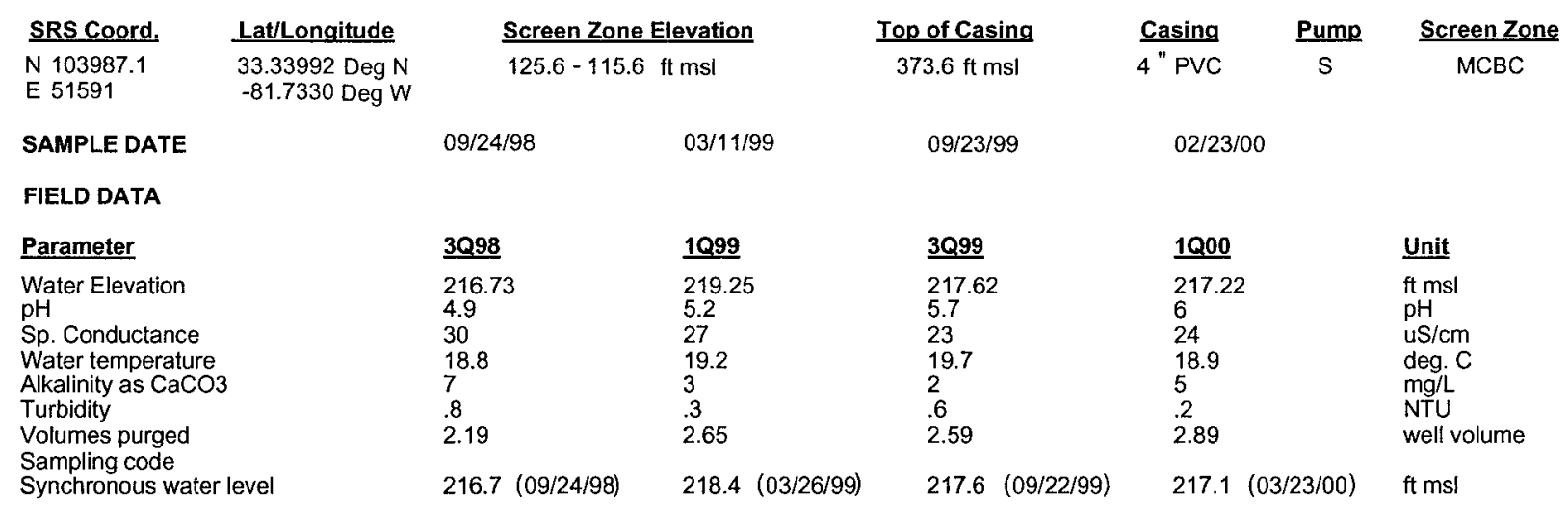

ANALYTICAL DATA

I. Groundwater Protection Standard

261 Appendix VIII/264 Appendix IX Hazardous Constituents

\begin{tabular}{|c|c|c|c|c|c|c|c|c|c|c|c|c|c|c|}
\hline \multirow{2}{*}{$\frac{\mathbf{S T}}{\text { Inorg }}$} & Parameter & \multirow[t]{2}{*}{$\underline{3 Q 98}$} & \multirow[t]{2}{*}{ CLPEPA } & \multirow[t]{2}{*}{1099} & \multirow[t]{2}{*}{$\underline{\text { CLP EPA }}$} & \multirow[t]{2}{*}{$\underline{3 Q 99}$} & \multirow[t]{2}{*}{ CLPEPA } & \multirow[t]{2}{*}{$\underline{1 Q 00}$} & \multicolumn{2}{|c|}{$\underline{\text { CLPEPA }}$} & \multirow[t]{2}{*}{ Filt. } & \multirow[t]{2}{*}{ DF } & \multirow[t]{2}{*}{ Unit } & \multirow[t]{2}{*}{$\underline{\text { Lab }}$} \\
\hline & ganics & & & & & & & & & & & & & \\
\hline & Barium, total recoverable & 6.1 & & 4.6 & & 4.5 & & $<5.23$ & U & V & $<E Q L$ & 1 & $\mathrm{ug} / \mathrm{L}$ & EX \\
\hline & Cyanide & $<15.2$ & $\mathrm{U}$ & $<15.2$ & $\mathrm{U}$ & $<15.2$ & $U$ & $<10$ & $U$ & & $<\mathrm{EQL}$ & 1 & $\mathrm{ug} / \mathrm{L}$ & EX \\
\hline & Lead, total recoverable & 4.9 & J & $<47$ & $u$ & $<47$ & $U$ & $<10$ & U & & $<$ EQL & 1 & $\mathrm{ug} / \mathrm{L}$ & EX \\
\hline & Nickel, total recoverable & 9.81 & $J$ & $<26$ & $U$ & $<26$ & $\mathrm{U}$ & $<12.3$ & JU & V & $<\mathrm{EQL}$ & 1 & ug/L & EX \\
\hline & Selenium, total recoverable & $<200$ & $U$ & $<66$ & $U$ & $<66$ & $U$ & $<10$ & $\mathrm{U}$ & & $<\mathrm{EQL}$ & 1 & ug/L. & EX \\
\hline \multicolumn{15}{|c|}{ Organics } \\
\hline & Chlorobenzene & $<5$ & $U$ & $<5$ & U & $<5$ & $\mathrm{U}$ & $<5$ & $u$ & & $<\mathrm{EQL}$ & 1 & ug/L & EX \\
\hline & 1,1-Dichloroethane & $<5$ & $\mathrm{U}$ & $<5$ & U & $<5$ & $\mathrm{U}$ & $<5$ & $u$ & & $<E Q L$ & 1 & $\mathrm{ug} / \mathrm{L}$ & EX \\
\hline & 1,1-Dichloroethylene & $<5$ & $U$ & $<5$ & $\mathrm{U}$ & $<5$ & $\mathrm{U}$ & $<5$ & $U$ & & $<\mathrm{EQL}$ & 1 & $\mathrm{ug} / \mathrm{L}$ & EX \\
\hline & trans-1,2-Dichloroethylene & $<5$ & $\mathrm{U}$ & $<5$ & $\mathrm{U}$ & $<5$ & $\mathrm{U}$ & $<5$ & $\mathrm{U}$ & & $<E Q L$ & 1 & $\mathrm{ug} / \mathrm{L}$ & EX \\
\hline & PCB 1016 & & & & & & & & & & & & & \\
\hline & РСВ 1221 & & & & & & & & & & & & & \\
\hline & PCB 1232 & & & & & & & & & & & & & \\
\hline & PCB 1242 & & & & & & & & & & & & & \\
\hline & PCB 1248 & & & & & & & & & & & & & \\
\hline & РCB 1254 & & & & & & & & & & & & & \\
\hline & $\mathrm{PCB} 1260$ & & & & & & & & & & & & & \\
\hline & $1,1,2,2$-Tetrachloroethane & $<5$ & $\mathrm{U}$ & $<5$ & $\mathrm{U}$ & $<5$ & $\mathrm{U}$ & $<5$ & $\mathrm{U}$ & & $<\mathrm{EQL}$ & 1 & $\mathrm{ug} / \mathrm{L}$ & EX \\
\hline+ & Tetrachloroethylene & 40.4 & & 34.5 & & 34.8 & & 41 & & & $>5$ & 1 & $\mathrm{ug} / \mathrm{L}$ & EX \\
\hline & 1,1,1-Trichloroethane & $<5$ & $\mathrm{U}$ & $<5$ & $\mathrm{U}$ & $<5$ & $\mathrm{U}$ & $<5$ & $U$ & & $<\mathrm{EQL}$ & 1 & $\mathrm{ug} / \mathrm{L}$ & EX \\
\hline+ & Trichloroethylene & 127 & & 102 & & 90.3 & & 73 & & & $>5$ & 1 & ug/L & EX \\
\hline \multicolumn{15}{|c|}{ II. Monitoring Constituents } \\
\hline$\underline{\mathbf{S T}}$ & Parameter & $\underline{3 Q 96}$ & CLPEPA & $\underline{3097}$ & CLP EPA & $\underline{3098}$ & CLPEPA & $\underline{3099}$ & $\underline{\text { CLP }}$ & EPA & Filt. & $\underline{\text { DF }}$ & $\underline{\text { Unit }}$ & $\underline{\text { Lab }}$ \\
\hline \multicolumn{15}{|c|}{ Inorganics } \\
\hline & Aluminum, total recoverable & 30.4 & & $<20$ & U & 43.7 & $\mathrm{~J}$ & $<146$ & $U$ & & $<\mathrm{EQL}$ & 1 & $u g / L$ & WA \\
\hline & Nitrate-nitrite as nitrogen & 1100 & & 966 & & 837 & & 935 & & & $<2400$ & 1 & $\mathrm{ug} / \mathrm{L}$ & WA \\
\hline & Sodium, total recoverable & 1790 & & 1760 & & 2290 & & 1620 & & & $<4600$ & 1 & $\mathrm{ug} / \mathrm{L}$ & WA \\
\hline & Sulfate & 444 & $\mathrm{~J}$ & $<5000$ & $U$ & 789 & & 495 & & & $<3000$ & 1 & $u g / L$ & WA \\
\hline \multicolumn{15}{|c|}{ Radionuclides } \\
\hline & Gross alpha & .283 & UI & .49 & UI & .85 & & .689 & $J$ & I & NDD & 1 & $\mathrm{pCi} / \mathrm{L}$ & GP \\
\hline & Nonvolatile beta & 1.02 & UI & .9 & UI & 1.41 & & .8 & $U$ & & $<\mathrm{EQL}$ & 1 & $\mathrm{pCi} / \mathrm{L}$ & GP \\
\hline & Radium, total alpha-emitting & -.7 & UI & 1.15 & $\mathrm{~J}$ & .1 & UI & .2 & $\mathrm{U}$ & & $<E Q L$ & 1 & $\mathrm{pCi} / \mathrm{L}$ & GP \\
\hline
\end{tabular}

Notes: Concentrations in bold italics exceed the groundwater protection or monitoring constituent standards listed in Appendix A. Synchronous water levels are measured over a 3-5 day period or less. Dilution factors, Laboratory, and Filtered Data are for Groundwater Protection Standards First Quarter 2000 and Monitoring Constituents Third Quarter 1999 data only.

+ = exceeded the Groundwater Protection Standards (First Quarter 2000) or Monitoring Constituents (Third Quarter 1999) listed in Appendix A. 
Table D-4. Groundwater Monitoring Results for Plume Definition Wells, M-Area HWMF (Cont.) WELL AMB 7B

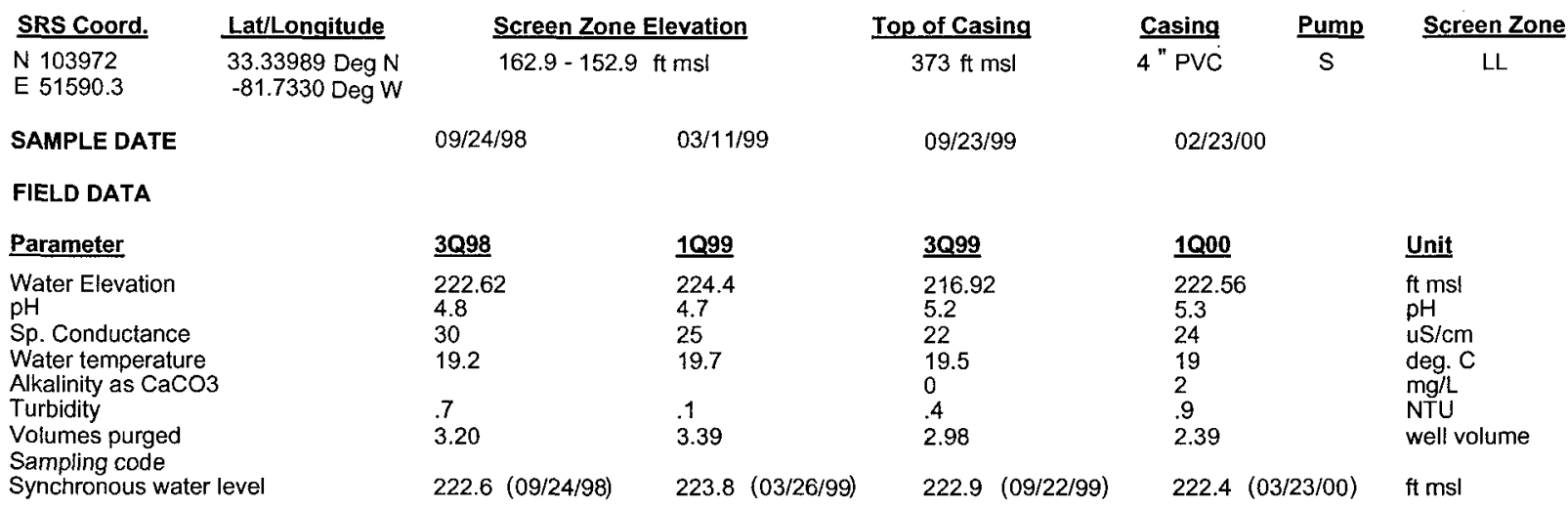

ANALYTICAL DATA

I. Groundwater Protection Standard

261 Appendix VIII/264 Appendix IX Hazardous Constituents

\begin{tabular}{|c|c|c|c|c|c|c|c|c|c|c|c|c|c|c|}
\hline$\underline{\mathbf{S T}}$ & Parameter & $\underline{3098}$ & CLPP EPA & 1099 & $\underline{\text { CLPEPA }}$ & $\underline{3 Q 99}$ & CLPEPA & 1000 & $\underline{\text { CLP }}$ & EPA & Filt. & DF & Unit & $\underline{\underline{L a b}}$ \\
\hline \multicolumn{15}{|c|}{ Inorganics } \\
\hline & Barium, total recoverable & 2.5 & & 2.5 & & 2.8 & & $<2.88$ & u & V & $<E Q L$ & 1 & $\mathrm{ug} / \mathrm{L}$ & EX \\
\hline & Cyanide & $<15.2$ & $\mathrm{U}$ & $<15.2$ & $\mathrm{u}$ & $<15.2$ & $\mathrm{U}$ & $<10$ & $U$ & & $<E Q L$ & 1 & $\mathrm{ug} / \mathrm{L}$ & EX \\
\hline & Lead, total recoverable & 18.6 & J & $<100$ & $\mathrm{U}$ & $<47$ & $\mathrm{U}$ & $<10$ & $\mathrm{U}$ & & $<E Q L$ & 1 & $\mathrm{ug} / \mathrm{L}$ & EX \\
\hline & Nickel, total recoverable & $<26$ & $\mathrm{U}$ & $<50$ & $\mathrm{U}$ & $<26$ & U & $<5.95$ & JU & V & $<E Q L$ & 1 & $\mathrm{ug} / \mathrm{L}$ & EX \\
\hline & Selenium, total recoverable & $<66$ & $\mathrm{U}$ & $<200$ & $\mathrm{U}$ & $<66$ & U & $<10$ & $\mathrm{U}$ & & $<E Q L$ & 1 & $\mathrm{ug} / \mathrm{L}$ & EX \\
\hline \multicolumn{15}{|c|}{ Organics } \\
\hline & Chlorobenzene & $<5$ & $u$ & $<5$ & $u$ & $<5$ & $U$ & $<5$ & U & & $<E Q L$ & 1 & $u g / L$ & EX \\
\hline & 1,1-Dichloroethane & $<5$ & $\mathrm{U}$ & $<5$ & 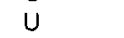 & $<5$ & U & $<5$ & U & & $<E Q L$ & 1 & $\mathrm{ug} / \mathrm{L}$ & EX \\
\hline & 1,1-Dichloroethylene & $<5$ & $\mathrm{U}$ & $<5$ & $\mathrm{U}$ & $<5$ & $\mathrm{U}$ & $<5$ & $\mathrm{U}$ & & $<E Q L$ & 1 & ug $/ \mathrm{L}$ & EX \\
\hline & trans-1,2-Dichloroethylene & $<5$ & $\mathrm{U}$ & $<5$ & $\mathrm{U}$ & $<5$ & $\mathrm{U}$ & $<5$ & $U$ & & $<E Q L$ & 1 & $\mathrm{ug} / \mathrm{L}$ & EX \\
\hline & PCB 1016 & & & & & & & & & & & & & \\
\hline & PCB 1221 & & & & & & & & & & & & & \\
\hline & PCB 1232 & & & & & & & & & & & & & \\
\hline & PCB 1242 & & & & & & & & & & & & & \\
\hline & PCB 1248 & & & & & & & & & & & & & \\
\hline & PCB 1254 & & & & & & & & & & & & & \\
\hline & PCB 1260 & & & & & & & & & & & & & \\
\hline & $1,1,2,2$-Tetrachloroethane & $<5$ & $U$ & $<5$ & $U$ & $<5$ & U & $<5$ & $U$ & & $<$ EQL & 1 & $\mathrm{ug} / \mathrm{L}$ & EX \\
\hline & Tetrachloroethylene & $<5$ & $\mathrm{U}$ & $<5$ & $U$ & $<5$ & U & $<5$ & $\mathrm{U}$ & & $<E Q L$ & 1 & Uğ/L & EX \\
\hline & 1,1,1-Trichloroethane & $<5$ & $\mathrm{U}$ & $<5$ & $\mathrm{U}$ & $<5$ & $\mathrm{U}$ & $<5$ & $\mathrm{U}$ & & $<E Q L$ & 1 & ug/L & EX \\
\hline+ & Trichloroethylene & 6.06 & & 6.25 & & 5.26 & & 5.9 & & & $>5$ & 1 & $\mathrm{ug} / \mathrm{L}$ & EX \\
\hline \multicolumn{15}{|c|}{ II. Monitoring Constituents } \\
\hline$\underline{\mathbf{S T}}$ & Parameter & $\underline{3 Q 96}$ & CLP EPA & $\underline{3097}$ & CLPEPA & $\underline{3 Q 98}$ & CLPEEA & $\underline{3099}$ & $\underline{\text { CLP }}$ & EPA & Filt. & $\underline{\mathrm{DF}}$ & $\underline{\text { Unit }}$ & $\underline{\text { Lab }}$ \\
\hline \multicolumn{15}{|c|}{ Inorganics } \\
\hline & Aluminum, total recoverable & $<40.2$ & $\mathrm{U}$ & 9.3 & J & 23.3 & $\mathrm{~J}$ & $<146$ & $\mathrm{U}$ & & $<E Q L$ & 1 & $u g / L$ & WA \\
\hline & Nitrate-nitrite as nitrogen & 800 & & 659 & & 593 & & 636 & & & $<2400$ & 1 & $\mathrm{ug} / \mathrm{L}$ & WA \\
\hline & Sodium, total recoverable & 3840 & & 3620 & & 3300 & & 3130 & & & $<4600$ & 1 & $\mathrm{ug} / \mathrm{L}$ & WA \\
\hline & Sulfate & 863 & $J$ & 1030 & & 836 & & 821 & & & $<3000$ & 1 & $\mathrm{ug} / \mathrm{L}$ & WA \\
\hline \multicolumn{15}{|c|}{ Radionuclides } \\
\hline & Gross alpha & .583 & Ui & -.66 & $\mathrm{U}$ & .7 & U1 & .611 & $\mathrm{U}$ & & $<\mathrm{EQL}$ & 1 & $\mathrm{pCi} / \mathrm{L}$ & GP \\
\hline & Nonvolatile beta & 1.19 & UI & -.87 & UIJ & .4 & UiJ & .778 & $U$ & & $<E Q L$ & 1 & $\mathrm{pCi} / \mathrm{L}$ & GP \\
\hline & Radium, total alpha-emitting & 0 & UI & 1.72 & $\mathrm{j}$ & .09 & UI & .2 & $\mathrm{U}$ & & $<\overline{E Q L}$ & 1 & $\mathrm{pCi} / \mathrm{L}$ & GP \\
\hline
\end{tabular}

Notes: Concentrations in bold italics exceed the groundwater protection or monitoring constituent standards listed in Appendix A. Synchronous water levels are measured over a 3-5 day period or less. Dilution factors, Laboratory, and Filtered Data are for Groundwater Protection Standards First Quarter 2000 and Monitoring Constituents Third Quarter 1999 data only.

$+=$ exceeded the Groundwater Protection Standards (First Quarter 2000) or Monitoring Constituents (Third Quarter 1999) listed in Appendix A. 
Table D-4. Groundwater Monitoring Results for Plume Definition Wells, M-Area HWMF (Cont.) WELL AMB 9D

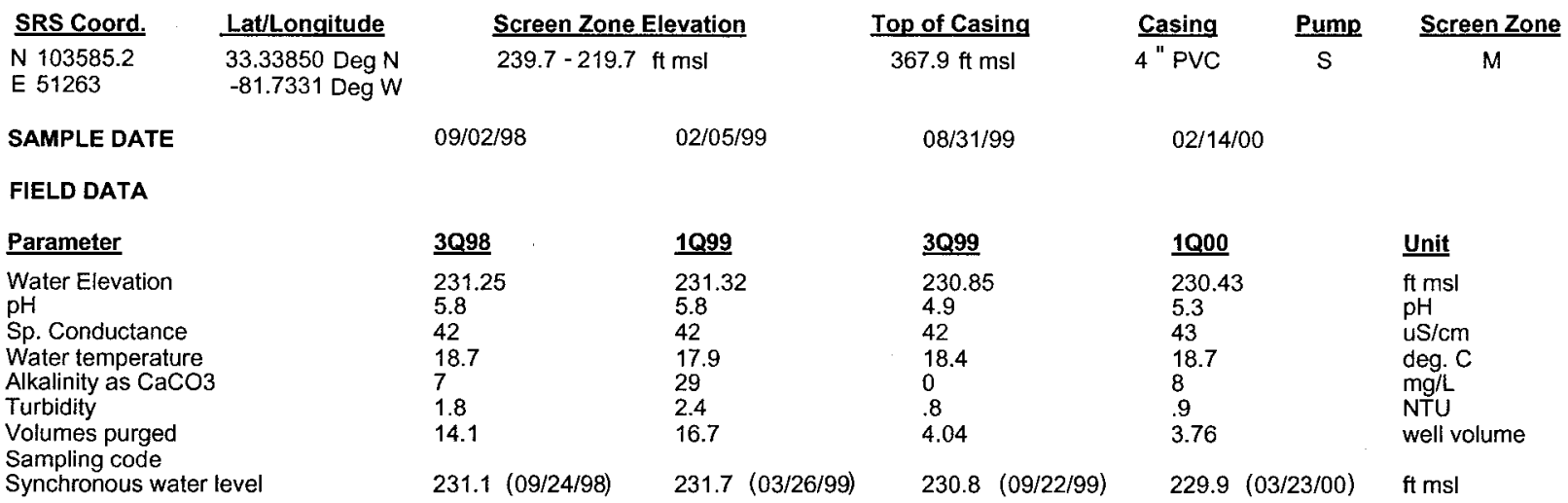

ANALYTICAL DATA

I. Groundwater Protection Standard

261 Appendix VIII/264 Appendix IX Hazardous Constituents

\begin{tabular}{|c|c|c|c|c|c|c|c|c|c|c|c|c|c|c|}
\hline \multirow{2}{*}{\multicolumn{2}{|c|}{$\frac{\text { ST }}{\text { Inorganics }}$}} & \multirow[t]{2}{*}{$\underline{3 Q 98}$} & \multirow[t]{2}{*}{ CLP EPA } & \multirow[t]{2}{*}{1099} & CLPEPA & \multirow[t]{2}{*}{$\underline{3099}$} & CLP EPA & \multirow[t]{2}{*}{$\underline{1000}$} & \multicolumn{2}{|c|}{ CLPEPA } & \multirow[t]{2}{*}{ Filt. } & \multirow[t]{2}{*}{ DF } & \multirow[t]{2}{*}{$\underline{\text { Unit }}$} & \multirow[t]{2}{*}{$\underline{L a b}$} \\
\hline & & & & & & & & & & & & & & \\
\hline & Barium, total recoverable & 3.4 & & 2.6 & & 2.8 & & $<2.77$ & $\mathrm{u}$ & V & $<E Q L$ & 1 & ug/L & EX \\
\hline & Cyanide & $<15.2$ & $U$ & $<15.2$ & $U$ & $<15.2$ & $U$ & $<10$ & $\mathrm{U}$ & & $<E Q L$ & 1 & ug/L & EX \\
\hline & Lead, total recoverable & $<47$ & $\mathrm{U}$ & $<47$ & U & $<47$ & $\mathrm{U}$ & $<10$ & $\mathrm{U}$ & & $<E Q L$ & 1 & $\mathrm{ug} / \mathrm{L}$ & EX \\
\hline & Nickel, total recoverable & $<26$ & $\mathrm{U}$ & $<26$ & U & $<26$ & $\mathrm{U}$ & $<50$ & $\mathrm{U}$ & & $<E Q L$ & 1 & ug $/ \mathrm{L}$ & EX \\
\hline & Selenium, total recoverable & $<66$ & $\mathrm{U}$ & $<66$ & $\mathrm{U}$ & $<66$ & $U$ & $<10$ & $\mathrm{U}$ & & $<\mathrm{EQL}$ & 1 & $\mathrm{ug} / \mathrm{L}$ & EX \\
\hline \multicolumn{15}{|c|}{ Organics } \\
\hline & Chlorobenzene & $<5$ & $U$ & $<5$ & $\mathrm{U}$ & $<5$ & $J U Q$ & $<5$ & $\mathbf{u}$ & & $<E Q L$ & 1 & ug/L & EX \\
\hline & 1,1-Dichloroethane & $<5$ & $\mathrm{U}$ & $<5$ & $\mathrm{U}$ & $<5$ & JU $Q$ & $<5$ & $u$ & & $<E Q L$ & 1 & ug/L & EX \\
\hline & 1,1-Dichloroethylene & $<5$ & U & $<5$ & $U$ & $<5$ & JU $Q$ & $<5$ & $\mathrm{U}$ & & $<E Q L$ & 1 & $u g / L$ & EX \\
\hline & trans-1,2-Dichloroethylene & $<5$ & $U$ & $<5$ & U & $<5$ & JU $Q$ & $<5$ & $\mathrm{U}$ & & $<E Q L$ & 1 & $\mathrm{ug} / \mathrm{L}$ & EX \\
\hline & PCB 1016 & & & $<1.02$ & $J U Q$ & & & & & & & & ug/L & \\
\hline & PCB 1221 & & & $<2.04$ & $J U Q$ & & & & & & & & $u g / L$ & \\
\hline & PCB 1232 & & & $<1.02$ & $J U Q$ & & & & & & & & $\mathrm{ug} / \mathrm{L}$ & \\
\hline & PCB 1242 & & & $<1.02$ & $J U \bar{Q}$ & & & & & & & & ug/L & \\
\hline & РCB 1248 & & & $<1.02$ & $J U Q$ & & & & & & & & $u g / L$ & \\
\hline & РCB 1254 & & & $<1.02$ & $J U Q$ & & & & & & & & ug/L & \\
\hline & PCB 1260 & & & $<1.02$ & JU $Q$ & & & & & & & & $\mathrm{ug} / \mathrm{L}$ & \\
\hline & $1,1,2,2$-Tetrachloroethane & $<5$ & $U$ & $<5$ & $\mathrm{U}$ & $<5$ & $\mathrm{JU} Q$ & $<5$ & $\mathrm{U}$ & & $<\mathrm{EQL}$ & 1 & ug/L & EX \\
\hline & Tetrachloroethylene & $<5$ & $U$ & $<5$ & $\mathrm{U}$ & $<5$ & JU $Q$ & $<5$ & $\mathrm{U}$ & & $<\mathrm{EQL}$ & 1 & ug/L & EX \\
\hline & 1,1,1-Trichloroethane & $<5$ & $\mathrm{U}$ & $<5$ & $\mathrm{U}$ & $<5$ & JU Q & $<5$ & $\mathrm{U}$ & & $<\mathrm{EQL}$ & 1 & $\mathrm{ug} / \mathrm{L}$ & EX \\
\hline & Trichloroethylene & $<5$ & $\mathrm{u}$ & $<5$ & $\mathrm{U}$ & $<5$ & $J U Q$ & $<5$ & $U$ & & $<\mathrm{EQL}$ & 1 & $\mathrm{ug} / \mathrm{L}$ & EX \\
\hline \multicolumn{15}{|c|}{ II. Monitoring Constituents } \\
\hline$\underline{\text { ST }}$ & Parameter & $\underline{3096}$ & CLPEPA & $\underline{3 Q 997}$ & CLP EPA & $\underline{3098}$ & CLPEPA & $\underline{3099}$ & $\underline{\text { CLP }}$ & EPA & Filt. & DF & $\underline{\text { Unit }}$ & $\underline{\text { Lab }}$ \\
\hline \multicolumn{15}{|c|}{ Inorganics } \\
\hline & Aluminum, total recoverable & $<22.2$ & $\mathrm{U}$ & 19.5 & $J$ & 953 & & $<146$ & $U$ & & $<$ EQL & 1 & $u g / L$ & WA \\
\hline & Nitrate-nitrite as nitrogen & 410 & & 174 & & 173 & & 117 & & & $<2400$ & 1 & $\mathrm{ug} / \mathrm{L}$ & WA \\
\hline+ & Sodium, total recoverable & 8560 & & 8240 & & 8830 & & 7710 & & & $>4600$ & 1 & $\mathrm{ug} / \mathrm{L}$ & WA \\
\hline+ & Sulfate & 4800 & & 5040 & & 4900 & & 5380 & & & $>3000$ & 1 & $\mathrm{ug} / \mathrm{L}$ & WA \\
\hline \multicolumn{15}{|c|}{ Radionuclides } \\
\hline & Gross alpha & 1.03 & & 2.9 & & 2.35 & $J$ & 1.1 & $\mathrm{U}$ & $\mathrm{V}$ & $<\mathrm{EQL}$ & 1 & $\mathrm{pCi} / \mathrm{L}$ & GP \\
\hline & Nonvolatile beta & .301 & UI & .87 & $\mathrm{U}$ & 1.59 & UIJ & .573 & $U$ & & $<E Q L$ & 1 & $\mathrm{pCi} / \mathrm{L}$ & GP \\
\hline & Radium, total alpha-emitting & .7 & & 2.88 & & 1 & & .9 & $\mathrm{~J}$ & I & NDD & 1 & $\mathrm{pCi} / \mathrm{L}$ & GP \\
\hline
\end{tabular}

Notes: Concentrations in bold italics exceed the groundwater protection or monitoring constituent standards listed in Appendix A. Synchronous water levels are measured over a 3-5 day period or less. Dilution factors, Laboratory, and Filtered Data are for Groundwater Protection Standards First Quarter 2000 and Monitoring Constituents Third Quarter 1999 data only.

$+=$ exceeded the Groundwater Protection Standards (First Quarter 2000) or Monitoring Constituents (Third Quarter 1999) listed in Appendix A. 
Table D-4. Groundwater Monitoring Results for Plume Definition Wells, M-Area HWMF (Cont.) WELL AMB 10A

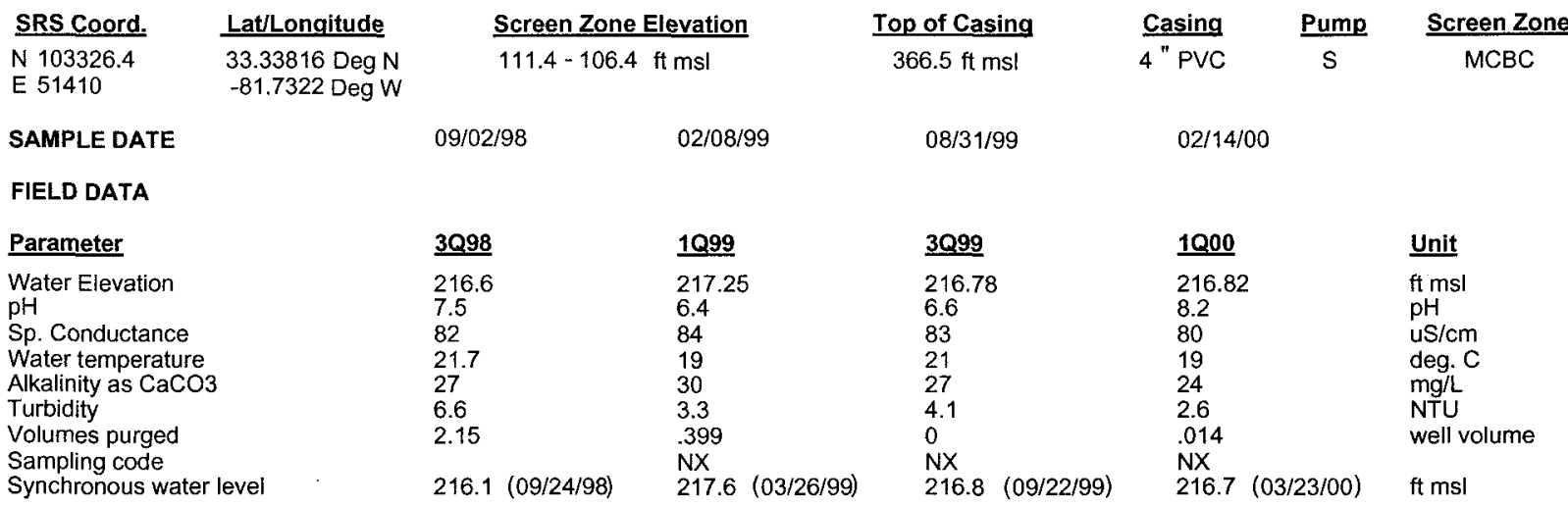

ANALYTICAL DATA

I. Groundwater Protection Standard

261 Appendix VIII/264 Appendix IX Hazardous Constituents

\begin{tabular}{|c|c|c|c|c|c|c|c|c|c|c|c|c|c|c|}
\hline$\underline{\text { ST }}$ & Parameter & $\underline{3 Q 98}$ & CLPEEPA & 1099 & $\underline{\text { CLP }}$ & EPA & $\underline{3099}$ & CLPEPA & 1000 & CLPEPA & Filt. & DF & Unit & Lab \\
\hline Inc & ganics & & & & & & & & & & & & & \\
\hline & Barium, total recoverable & 11.9 & & 12.9 & & & 10.9 & & 12.1 & & $<2000$ & 1 & ug $/ \mathrm{L}$ & EX \\
\hline & Cyanide & $<15.2$ & $\mathrm{U}$ & 1.54 & J & 1 & $<15.2$ & $\mathrm{U}$ & $<10$ & $\mathrm{U}$ & $<\mathrm{EQL}$ & 1 & $\mathrm{ug} / \mathrm{L}$ & EX \\
\hline & Lead, total recoverable & $<47$ & $\mathrm{U}$ & $<47$ & U & & $<47$ & $\mathrm{U}$ & $<10$ & $\mathrm{U}$ & $<E Q L$ & 1 & $\mathrm{ug} / \mathrm{L}$ & EX \\
\hline & Nickel, total recoverable & $<26$ & U & $<26$ & $\mathrm{U}$ & & $<26$ & $U$ & $<50$ & $U$ & $<\overline{E Q L}$ & 1 & $\mathrm{ug} / \mathrm{L}$ & EX \\
\hline & Selenium, total recoverable & $<66$ & $\mathrm{U}$ & $<66$ & $\mathrm{U}$ & & $<66$ & $\mathrm{U}$ & $<10$ & $U$ & $<E Q L$ & 1 & $\mathrm{ug} / \mathrm{L}$ & EX \\
\hline Org & anics & & & & & & & & & & & & & \\
\hline & Chlorobenzene & $<5$ & $\mathrm{U}$ & $<5$ & JU & $Q$ & $<5$ & $\mathrm{U}$ & $<5$ & $\mathrm{U}$ & $<\mathrm{EQL}$ & 1 & $\mathrm{ug} / \mathrm{L}$ & EX \\
\hline & 1,1-Dichloroethane & $<5$ & U & $<5$ & JU & $\vec{Q}$ & $<5$ & U & $<5$ & U & $<\mathrm{EQL}$ & 1 & $\mathrm{ug} / \mathrm{L}$ & EX \\
\hline & 1,1-Dichloroethylene & $<5$ & $u$ & $<5$ & jU & $\widehat{Q}$ & $<5$ & $\mathrm{U}$ & $<5$ & $u$ & $<\mathrm{EQL}$ & 1 & $\mathrm{ug} / \mathrm{L}$ & EX \\
\hline & trans-1,2-Dichloroethylene & $<5$ & U & $<5$ & JU & $\bar{Q}$ & $<5$ & $\mathrm{U}$ & $<5$ & $U$ & $<\mathrm{EQL}$ & 1 & $\mathrm{ug} / \mathrm{L}$ & EX \\
\hline & РСВ 1016 & & & & & & & & & & & & & \\
\hline & РCB 1221 & & & & & & & & & & & & & \\
\hline & PCB 1232 & & & & & & & & & & & & & \\
\hline & PCB 1242 & & & & & & & & & & & & & \\
\hline & PCB 1248 & & & & & & & & & & & & & \\
\hline & PCB 1254 & & & & & & & & & & & & & \\
\hline & PCB 1260 & & & & & & & & & & & & & \\
\hline & $1,1,2,2$-Tetrachloroethane & $<5$ & $\mathrm{U}$ & $<5$ & JU & $Q$ & $<5$ & $U$ & $<5$ & $\mathrm{U}$ & $<$ EQL & 1 & ug/L. & EX \\
\hline & Tetrachloroethylene & $<5$ & $\mathrm{U}$ & $<5$ & JU & $\widehat{Q}$ & $<5$ & $U$ & $<5$ & $U$ & $<E Q L$ & 1 & $\mathrm{ug} / \mathrm{L}$ & EX \\
\hline & $1,1,1$-Trichloroethane & $<5$ & $\mathrm{U}$ & $<5$ & JU & $\bar{Q}$ & $<5$ & $\mathrm{U}$ & $<5$ & $\mathrm{U}$ & $<E Q L$ & 1 & ug/L & EX \\
\hline & Trichloroethylene & $<5$ & $U$ & $<5$ & JU & $Q$ & $<5$ & $u$ & $<5$ & $U$ & $<E Q L$ & 1 & $u g / L$ & EX \\
\hline II. $\mathrm{M}$ & onitoring Constituents & & & & & & & & & & & & & \\
\hline$\underline{\mathbf{S T}}$ & Parameter & $\underline{3096}$ & CLP EPA & 3Q997 & CLP & EPA & $\underline{3098}$ & CLPEPA & $\underline{3099}$ & CLPEPA & Filt. & DF & Unit & Lab \\
\hline Inor & ganics & & & & & & & & & & & & & \\
\hline & Aluminum, total recoverable & 881 & & 938 & & & 156 & & 66 & J 1 & NDD & 1 & $\mathrm{ug} / \mathrm{L}$ & WA \\
\hline & Nitrate-nitrite as nitrogen & 400 & & 283 & & & 263 & & 270 & & $<2400$ & 1 & $\mathrm{ug} / \mathrm{L}$ & WA \\
\hline+ & Sodium, total recoverable & 13700 & & 10500 & & & 5540 & & 4910 & & $>4600$ & 1 & $\mathrm{ug} / \mathrm{L}$ & WA \\
\hline+ & Sulfate & 5160 & & 4330 & $J$ & & 3610 & & 3700 & & $>3000$ & 1 & $\mathrm{ug} / \mathrm{L}$ & WA \\
\hline Radi & onuclides & & & & & & & & & & & & & \\
\hline & Gross alpha & 1.09 & & .73 & Uf & & .09 & UIJ & .0302 & $U$ & < EQL. & 1 & $\mathrm{pCi} / \mathrm{L}$ & GP \\
\hline & Nonvolatile beta & 9.37 & & 3.62 & & & 1.81 & UIJ & 1.08 & $\mathrm{U}$ & $<\mathrm{EQL}$ & 1 & $\mathrm{pCi} / \mathrm{L}$ & GP \\
\hline & Radium, total alpha-emitting & .7 & & 2.66 & & & -.11 & UI & .2 & $u$ & $<E Q L$ & 1 & $\mathrm{pCi} / \mathrm{L}$ & GP \\
\hline
\end{tabular}

Notes: Concentrations in bold italics exceed the groundwater protection or monitoring constituent standards listed in Appendix A. Synchronous water levels are measured over a 3-5 day period or less. Dilution factors, Laboratory, and Filtered Data are for Groundwater Protection Standards First Quarter 2000 and Monitoring Constituents Third Quarter 1999 data only.

$+=$ exceeded the Groundwater Protection Standards (First Quarter 2000) or Monitoring Constituents (Third Quarter 1999) listed in Appendix A. 
Table D-4. Groundwater Monitoring Results for Plume Definition Wells, M-Area HWMF (Cont.) WELL AMB 10B

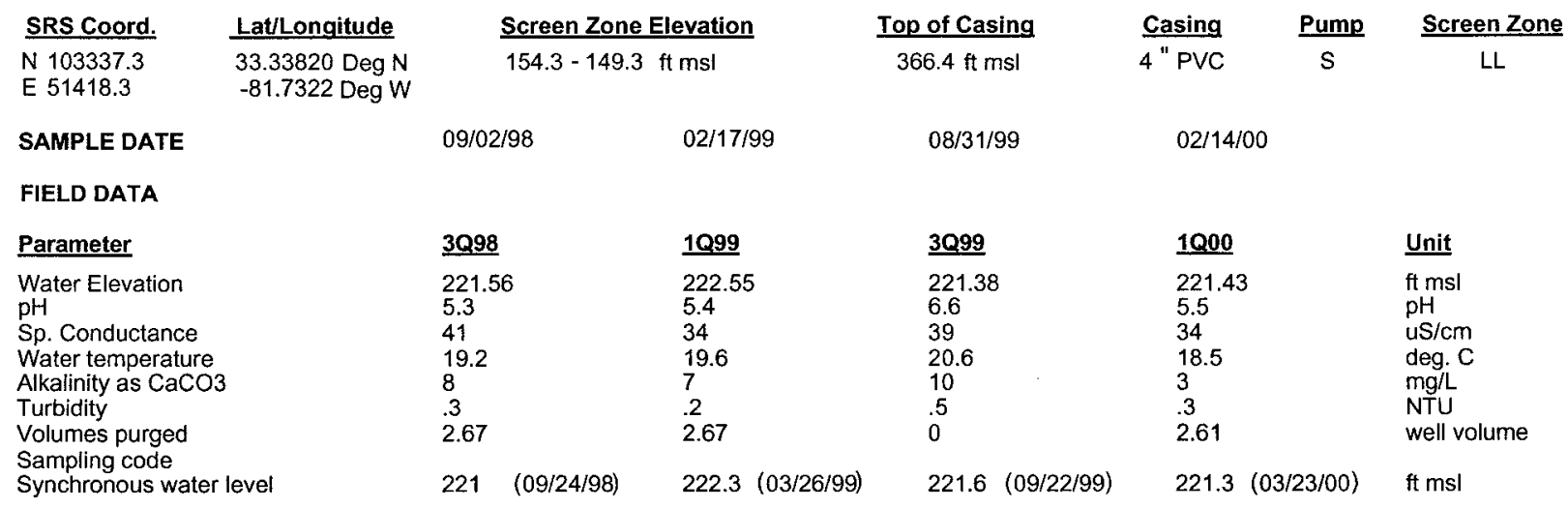

ANALYTICAL DATA

I. Groundwater Protection Standard

261 Appendix VIII/264 Appendix IX Hazardous Constituents

\begin{tabular}{|c|c|c|c|c|c|c|c|c|c|c|c|c|}
\hline ST Parameter & $\underline{3098}$ & CLPEPA & $\underline{1 Q 99}$ & CLPEPA & $\underline{3099}$ & CLPEPA & $\underline{1000}$ & CLPEPA & Filt. & $\underline{\text { DF }}$ & Unit & $\underline{\text { Lab }}$ \\
\hline \multicolumn{13}{|l|}{ Inorganics } \\
\hline Barium, total recoverable & 6.3 & & 9.47 & & 9.9 & & 8.51 & J I & NDD & 1 & $\mathrm{ug} / \mathrm{h}$ & EX \\
\hline Cyanide & $<15.2$ & $u$ & $<15.2$ & $\mathrm{u}$ & $<15.2$ & $\mathrm{u}$ & $<10$ & $\mathrm{u}$ & $<E Q L$ & 1 & $\mathrm{ug} / \mathrm{L}$ & EX \\
\hline Lead, total recoverable & $<47$ & $u$ & $<47$ & $u$ & $<47$ & $\mathrm{u}$ & $<10$ & $\mathrm{U}$ & $<E Q L$ & 1 & $\mathrm{ug} / \mathrm{L}$ & EX \\
\hline Nickel, total recoverable & $<26$ & $u$ & $<26$ & $u$ & $<26$ & $u$ & $<5.71$ & $u \mathrm{~V}$ & $<E Q L$ & 1 & $\mathrm{ug} / \mathrm{L}$ & EX \\
\hline Selenium, total recoverable & $<66$ & $u$ & $<66$ & $U$ & $<66$ & $u$ & $<10$ & $u$ & $<E Q L$ & 1 & ug/L & EX \\
\hline \multicolumn{13}{|l|}{ Organics } \\
\hline Chlorobenzene & $<5$ & $\mathrm{u}$ & $<5$ & $u$ & $<5$ & $\mathrm{u}$ & $<5$ & $\mathrm{u}$ & $<\mathrm{EQL}$ & 1 & $\mathrm{ug} / \mathrm{L}$ & EX \\
\hline 1,1-Dichloroethane & $<5$ & $u$ & $<5$ & $\mathrm{U}$ & $<5$ & $u$ & $<5$ & $u$ & $<E Q L$ & 1 & $\mathrm{ug} / \mathrm{L}$ & EX \\
\hline 1,1-Dichloroethylene & $<5$ & $\mathrm{u}$ & $<5$ & U & $<5$ & $\mathrm{U}$ & $<5$ & $u$ & $<E Q L$ & 1 & $\mathrm{ug} / \mathrm{L}$ & EX \\
\hline trans-1,2-Dichloroethylene & $<5$ & $u$ & $<5$ & $u$ & $<5$ & $u$ & $<5$ & $u$ & $<E Q L$ & 1 & $\mathrm{ug} / \mathrm{L}$ & EX \\
\hline РСВ 1016 & & & $<1.02$ & $u$ & & & $<.96$ & $\mathrm{u}$ & $<E Q L$ & .960 & ug/L & EX \\
\hline PCB 1221 & & & $<2.04$ & U & & & $<.96$ & $\mathrm{U}$ & $<E Q L$ & .960 & $\mathrm{ug} / \mathrm{L}$ & EX \\
\hline РCB 1232 & & & $<1.02$ & $\mathrm{u}$ & & & $<.96$ & $\mathrm{u}$ & $<E Q L$ & .960 & $\mathrm{ug} / \mathrm{L}$ & EX \\
\hline РСВ 1242 & & & $<1.02$ & $u$ & & & $<1.9$ & $\mathrm{U}$ & $<\mathrm{EQL}$ & .960 & $\mathrm{ug} / \mathrm{L}$ & EX \\
\hline РСB 1248 & & & $<1.02$ & u & & & $<.96$ & $u$ & $<E Q L$ & .960 & ug/L & EX \\
\hline PCB 1254 & & & $<1.02$ & $u$ & & & $<.96$ & $u$ & $<$ EQL & .960 & $\mathrm{ug} / \mathrm{L}$ & EX \\
\hline PCB 1260 & & & $<1.02$ & $u$ & & & $<.96$ & $\mathrm{u}$ & $<E Q L$ & .960 & $\mathrm{ug} / \mathrm{L}$ & EX \\
\hline 1,1,2,2-Tetrachloroethane & $<5$ & $u$ & $<5$ & $u$ & $<5$ & $\mathrm{U}$ & $<5$ & $\mathrm{U}$ & $<\mathrm{EQL}$ & 1 & $\mathrm{ug} / \mathrm{L}$ & EX \\
\hline Tetrachloroethylene & $<5$ & $\mathrm{u}$ & $<5$ & u & $<5$ & $\mathrm{U}$ & $<5$ & $\mathrm{u}$ & $<E Q L$ & 1 & $\mathrm{ug} / \mathrm{L}$ & EX \\
\hline 1,1,1-Trichloroethane & $<5$ & $\mathrm{U}$ & $<5$ & $\mathrm{u}$ & $<5$ & $\mathrm{u}$ & $<5$ & $\mathrm{u}$ & $<E Q L$ & 1 & $\mathrm{ug} / \mathrm{L}$ & EX \\
\hline Trichloroethylene & $<5$ & $u$ & $<5$ & $u$ & $<5$ & $u$ & $<5$ & U & $<E Q L$ & 1 & ug/L & EX \\
\hline \multicolumn{13}{|l|}{ II. Monitoring Constituents } \\
\hline ST Parameter & 3096 & CLPEPA & $\underline{3097}$ & CLPEPA & 3098 & ELPEPA & $\underline{3099}$ & CLPEPA & Filt. & $\underline{\text { DF }}$ & $\underline{\text { Unit }}$ & Lab \\
\hline \multicolumn{13}{|l|}{ Inorganics } \\
\hline Aluminum, total recoverable & $<13$ & $u$ & $<20$ & $u$ & $<146$ & $u$ & $<146$ & $U$ & $<\mathrm{EQL}$ & 1 & $\mathrm{ug} / \mathrm{L}$ & WA \\
\hline Nitrate-nitrite as nitrogen & 410 & & 192 & & 206 & & 239 & & $<2400$ & 1 & $\mathrm{ug} / \mathrm{L}$ & WA \\
\hline Sodium, total recoverable & 3770 & & 4170 & & 4260 & & 4400 & & $<4600$ & 1 & $\mathrm{ug} / \mathrm{L}$ & WA \\
\hline Sulfate & 625 & $J$ & $<5000$ & U & 865 & & 1060 & & $<3000$ & 1 & $\mathrm{ug} / \mathrm{L}$ & WA \\
\hline \multicolumn{13}{|l|}{ Radionuclides } \\
\hline Gross alpha & .267 & UI & 1.47 & & .36 & UIJ & .013 & $\mathrm{u}$ & $<E Q L$ & 1 & $\mathrm{pCi} / \mathrm{L}$ & GP \\
\hline Nonvolatile beta & .387 & $\mathrm{UI}$ & .74 & $\mathrm{UI}$ & 6.68 & $\mathrm{~J}$ & .542 & $\mathrm{U}$ & $<E Q L$ & 1 & $\mathrm{pCi} / \mathrm{L}$ & GP \\
\hline Radium, total alpha-emitting & .6 & & 1.86 & UIJ & .18 & UI & .1 & U & $<E Q L$ & 1 & $\mathrm{pCi} / \mathrm{L}$ & GP \\
\hline
\end{tabular}

Notes: Concentrations in bold italics exceed the groundwater protection or monitoring constituent standards listed in Appendix A. Synchronous water levels are measured over a 3-5 day period or less. Dilution factors, Laboratory, and Filtered Data are for Groundwater Protection Standards First Quarter 2000 and Monitoring Constituents Third Quarter 1999 data only.

$+=$ exceeded the Groundwater Protection Standards (First Quarter 2000) or Monitoring Constituents (Third Quarter 1999) listed in Appendix A. 
Table D-4. Groundwater Monitoring Results for Plume Definition Wells, M-Area HWMF (Cont.) WELL AMB 12D

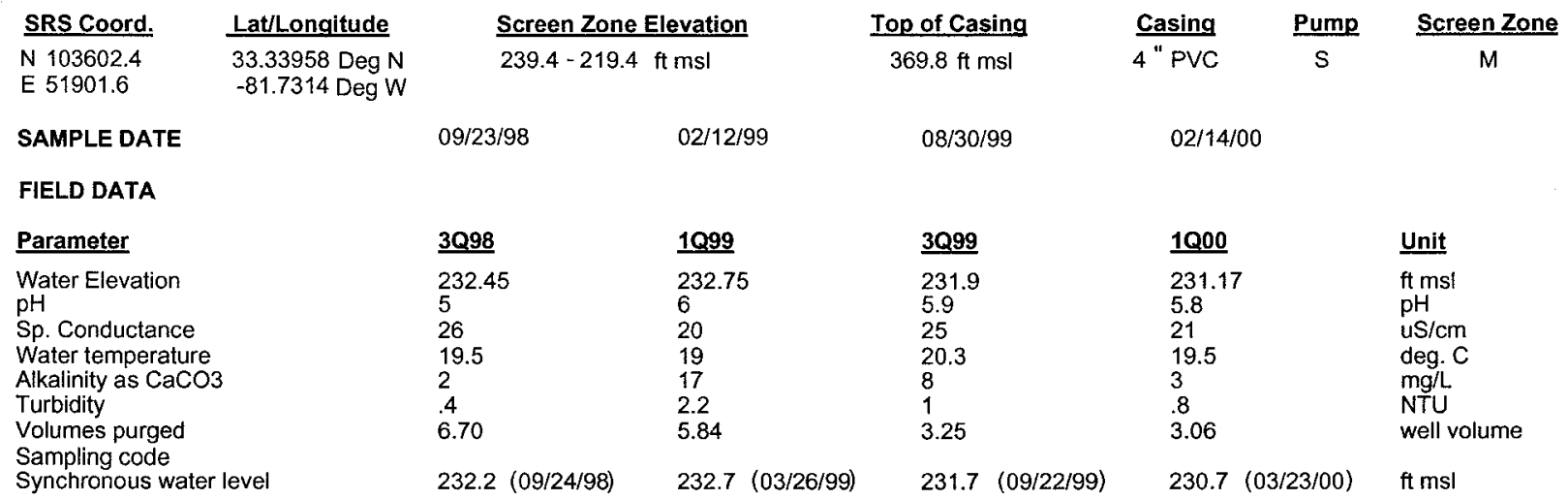

ANALYTICAL DATA

I. Groundwater Protection Standard

261 Appendix VIII/264 Appendix IX Hazardous Constituents

\begin{tabular}{|c|c|c|c|c|c|c|c|c|c|c|c|c|c|c|}
\hline$\underline{\text { ST }}$ & Parameter & $\underline{3 Q 998}$ & CLP EPA & 1099 & CLP EPA & 3Q99 & CLPEEA & $1 Q 00$ & $\underline{\text { CLF }}$ & EPA & Filt. & $\underline{\text { DF }}$ & Unit & $\underline{L a b}$ \\
\hline Inor & ganics & & & & & & & & & & & & & \\
\hline & Barium, total recoverable & 6.4 & & 6.7 & & 7.3 & & 7.61 & $J$ & 1 & NDD & 1 & $\mathrm{ug} / \mathrm{L}$ & EX \\
\hline & Cyanide & $<15.2$ & $U$ & $<15.2$ & $U$ & $<15.2$ & U & $<10$ & $\mathrm{U}$ & & $<\mathrm{EQL}$ & 1 & $\mathrm{ug} / \mathrm{L}$ & EX \\
\hline & Lead, total recoverable & $<47$ & $\mathrm{U}$ & $<47$ & $U$ & $<47$ & $\cup$ & $<10$ & $\mathrm{U}$ & & $<E Q L$ & 1 & $\mathrm{ug} / \mathrm{L}$ & EX \\
\hline & Nickel, total recoverable & $<26$ & $\mathrm{U}$ & $<26$ & $U$ & $<26$ & $U$ & $<50$ & $\mathrm{U}$ & & $<$ EQL & 1 & $\mathrm{ug} / \mathrm{L}$ & Ex \\
\hline & Selenium, total recoverable & $<66$ & U & $<66$ & $\mathrm{U}$ & $<66$ & U & $<10$ & $\mathrm{u}$ & & $<\mathrm{EQL}$ & 1 & ug/L & Ex \\
\hline $\operatorname{rg}$ & anics & & & & & & & & & & & & & \\
\hline & Chlorobenzene & $<5$ & $u$ & $<5$ & $U$ & $<5$ & $u$ & $<5$ & U & & $<\mathrm{EQL}$ & 1 & ug/L & $x$ \\
\hline & 1,1-Dichloroethane & $<5$ & $U$ & $<5$ & $\mathrm{U}$ & $<5$ & $\mathrm{U}$ & $<5$ & $\mathrm{U}$ & & $<E Q L$ & 1 & $\mathrm{ug} / \mathrm{L}$ & $x$ \\
\hline & 1,1-Dichloroethylene & $<5$ & 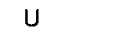 & $<5$ & $\mathrm{U}$ & $<5$ & $\mathrm{U}$ & $<5$ & $\mathbf{U}$ & & $<\mathrm{EQL}$ & 1 & $\mathrm{ug} / \mathrm{L}$ & $x$ \\
\hline & $\begin{array}{l}\text { trans-1,2-Dichloroethylene } \\
\text { PCB } 1016\end{array}$ & $<5$ & 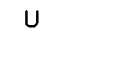 & $<5$ & 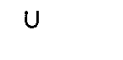 & $<5$ & $\mathrm{U}$ & $<5$ & $\mathrm{U}$ & & $<\mathrm{EQL}$ & 1 & $\mathrm{ug} / \mathrm{L}$ & \\
\hline & PCB 1221 & & & & & & & & & & & & & \\
\hline & PCB 1232 & & & & & & & & & & & & & \\
\hline & PCB 1242 & & & & & & & & & & & & & \\
\hline & PCB 1248 & & & & & & & & & & & & & \\
\hline & PCB 1254 & & & & & & & & & & & & & \\
\hline & PCB 1260 & & & & & & & & & & & & & \\
\hline & $1,1,2,2-$-Tetrachloroethane & $<5$ & $\cup$ & $<5$ & $U$ & $<5$ & $U$ & $<5$ & $\mathrm{U}$ & & $<\mathrm{EQL}$ & 1 & $\mathrm{ug} / \mathrm{L}$ & EX \\
\hline & Tetrachloroethylene & 3.54 & $J$ & $<5$ & $\mathrm{U}$ & $<5$ & $\mathrm{U}$ & $<5$ & $\mathrm{U}$ & & $<\mathrm{EQL}$ & 1 & $\mathrm{ug} / \mathrm{L}$ & 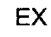 \\
\hline & 1,1,1-Trichloroethane & $<5$ & U & $<5$ & $\mathrm{U}$ & $<5$ & $\mathrm{U}$ & $<5$ & $\mathrm{U}$ & & $<\mathrm{EQL}$ & 1 & $\mathrm{ug} / \mathrm{L}$ & K \\
\hline & Trichloroethylene & 10.2 & & $<5$ & $\mathrm{U}$ & $<5$ & U & $<5$ & $\mathrm{U}$ & & $<E Q L$ & 1 & ug/L & $E$ \\
\hline II. $\mathrm{M}$ & onitoring Constituents & & & & & & & & & & & & & \\
\hline$\underline{\text { ST }}$ & Parameter & $\underline{3096}$ & CLP EPA & $\underline{3097}$ & $\underline{\text { CLP EPA }}$ & $\underline{3098}$ & CLPEPA & $\underline{3099}$ & $\underline{C L P}$ & EPA & Filt. & $\underline{\text { DF }}$ & Unit & $\underline{\mathrm{g}}$ \\
\hline Ino & ganics & & & & & & & & & & & & & \\
\hline & Aluminum, total recoverable & $<35.8$ & $\mathrm{U}$ & 293 & & $<146$ & $\mathrm{U}$ & $<146$ & $\mathrm{U}$ & & $<E Q L$ & 1 & ug/L & WA \\
\hline & Nitrate-nitrite as nitrogen & 1290 & & 255 & & 841 & & 814 & & & $<2400$ & 1 & ug/L & WA \\
\hline & Sodium, total recoverable & 2050 & & 1970 & & 1530 & & 1660 & & & $<4600$ & 1 & $\mathrm{ug} / \mathrm{L}$ & WA \\
\hline & Sulfate & 421 & $J$ & $<5000$ & $\mathrm{U}$ & & & 601 & & & $<3000$ & 1 & $\mathrm{ug} / \mathrm{L}$ & WA \\
\hline Radi & ionuclides & & & & & & & & & & & & & \\
\hline & Gross alpha & .776 & & .09 & UI & 2.51 & & 1.08 & $J$ & 1 & NDD & 1 & $\mathrm{pCi} / \mathrm{L}$ & P \\
\hline & Nonvolatile beta & 3.14 & & -.15 & UI & 8.37 & $J$ & 1.42 & $J$ & 1 & NDD & 1 & $\mathrm{pCi} / \mathrm{L}$ & GP \\
\hline & Radium, total alpha-emitting & .3 & & 1.56 & UI & .35 & UI & .1 & $U$ & & $<\mathrm{EQL}$ & 1 & $\mathrm{pCi} / \mathrm{L}$ & GP \\
\hline
\end{tabular}

Notes: Concentrations in bold italics exceed the groundwater protection or monitoring constituent standards listed in Appendix A. Synchronous water levels are measured over a 3-5 day period or less. Dilution factors, Laboratory, and Filtered Data are for Groundwater Protection Standards First Quarter 2000 and Monitoring Constituents Third Quarter 1999 data only.

+ = exceeded the Groundwater Protection Standards (First Quarter 2000) or Monitoring Constituents (Third Quarter 1999) listed in Appendix A. 
Table D-4. Groundwater Monitoring Results for Plume Definition Wells, M-Area HWMF (Cont.) WELL AMB 13AR

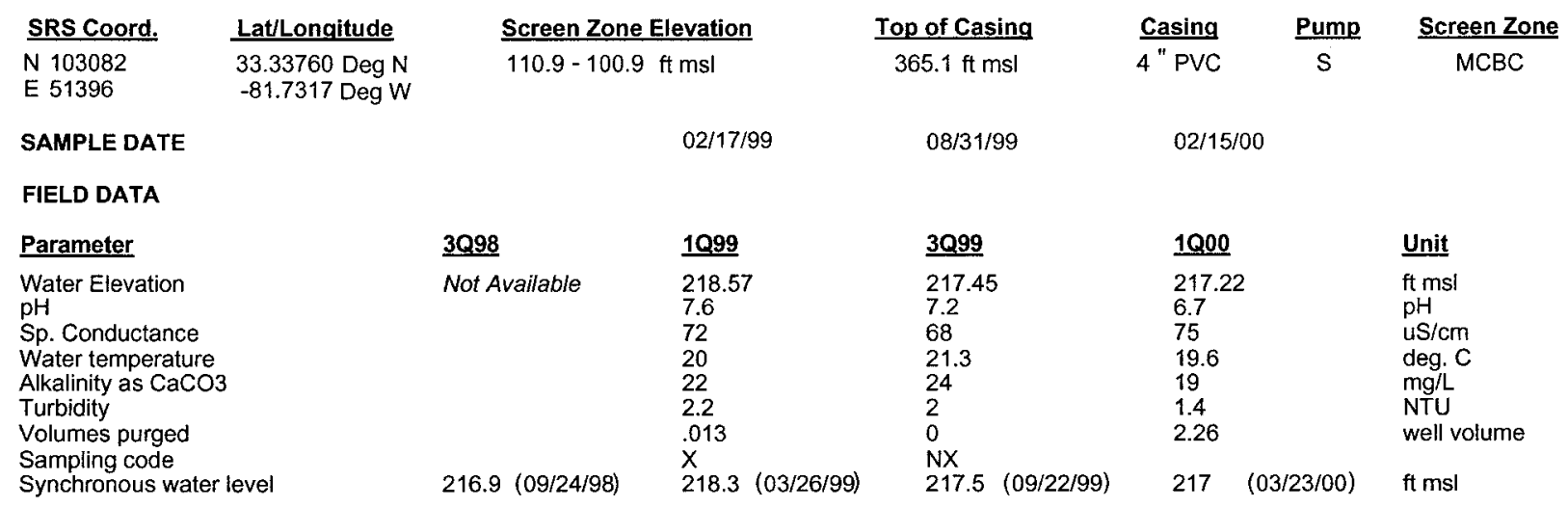

ANALYTICAL DATA

I. Groundwater Protection Standard

261 Appendix VIII/264 Appendix IX Hazardous Constituents

\begin{tabular}{|c|c|c|c|c|c|c|c|c|c|c|c|c|c|c|}
\hline ST & Parameter & $\underline{3098}$ & CLP EPA & 1099 & CLP EPA & $\underline{3099}$ & CLPEPA & $\underline{1000}$ & CL다 & EPA & Filt. & $\underline{\text { DF }}$ & Unit & $\underline{L a b}$ \\
\hline Inor & ganics & & & & & & & & & & & & & \\
\hline & Barium, total recoverable & & & 28 & & 19.3 & & 22.2 & & & $<2000$ & 1 & $\mathrm{ug} / \mathrm{L}$ & EX \\
\hline & Cyanide & & & $<15.2$ & $\mathrm{U}$ & 5.21 & J 1 & $<10$ & $\mathrm{U}$ & & $<E Q L$ & 1 & $\mathrm{ug} / \mathrm{L}$ & EX \\
\hline & Lead, total recoverable & & & $<47$ & U & $<47$ & $U$ & $<10$ & $\mathrm{U}$ & & $<\mathrm{EQL}$ & 1 & $\mathrm{ug} / \mathrm{L}$ & EX \\
\hline & Nickel, total recoverable & & & $<26$ & $\mathrm{U}$ & $<26$ & $\mathrm{U}$ & $<5.09$ & $\mathrm{U}$ & V & $<\mathrm{EQL}$ & 1 & $\mathrm{ug} / \mathrm{L}$ & EX \\
\hline & Selenium, total recoverable & & & $<66$ & $\mathrm{U}$ & $<66$ & $U$ & $<10$ & $U$ & & $<E Q L$ & 1 & $\mathrm{ug} / \mathrm{L}$ & EX \\
\hline Org & anics & & & & & & & & & & & & & \\
\hline & Chlorobenzene & & & $<5$ & $\mathrm{U}$ & $<5$ & $\mathrm{U}$ & $<5$ & $U$ & & $<\mathrm{EQL}$ & 1 & $\mathrm{ug} / \mathrm{L}$ & EX \\
\hline & 1,1-Dichloroethane & & & $<5$ & $\mathrm{U}$ & $<5$ & U & $<5$ & $\mathrm{U}$ & & $<\mathrm{EQL}$ & 1 & $\mathrm{ug} / \mathrm{L}$ & EX \\
\hline & 1,1-Dichloroethylene & & & $<5$ & $\mathrm{U}$ & $<5$ & U & $<5$ & $U$ & & $<E Q L$ & 1 & $\mathrm{ug} / \mathrm{L}$ & EX \\
\hline & trans-1,2-Dichloroethylene & & & $<5$ & $\mathrm{U}$ & $<5$ & $\mathrm{U}$ & $<5$ & $U$ & & $<\mathrm{EQL}$ & 1 & ug/L & EX \\
\hline & PCB 1016 & & & & & & & & & & & & & \\
\hline & PCB 1221 & & & & & & & & & & & & & \\
\hline & PCB 1232 & & & & & & & & & & & & & \\
\hline & PCB 1242 & & & & & & & & & & & & & \\
\hline & РCB 1248 & & & & & & & & & & & & & \\
\hline & PCB 1254 & & & & & & & & & & & & & \\
\hline & PCB 1260 & & & & & & & & & & & & & \\
\hline & $1,1,2,2$-Tetrachloroethane & & & $<5$ & $U$ & $<5$ & $U$ & $<5$ & $U$ & & $<\mathrm{EQL}$ & 1 & ug/L & EX \\
\hline & Tetrachioroethylene & & & $<5$ & U & $<5$ & $U$ & $<5$ & U & & $<\mathrm{EQL}$ & 1 & $\mathrm{ug} / \mathrm{L}$ & EX \\
\hline & 1,1,1-Trichloroethane & & & $<5$ & $\mathrm{U}$ & $<5$ & U & $<5$ & $U$ & & $<E Q L$ & 1 & $\mathrm{ug} / \mathrm{L}$ & EX \\
\hline & Trichloroethylene & & & $<5$ & $\mathrm{U}$ & $<5$ & $U$ & $<5$ & $\mathrm{U}$ & & $<E Q L$ & 1 & $\mathrm{ug} / \mathrm{L}$ & EX \\
\hline II. $\mathbf{M}$ & onitoring Constituents & & & & & & & & & & & & & \\
\hline$\underline{\text { ST }}$ & Parameter & $\underline{3996}$ & CLPPEPA & $\underline{3097}$ & CLPEPA & $\underline{3098}$ & CLPEEPA & $\underline{3 Q 99}$ & $\underline{C L F}$ & EPA & Filt. & DF & $\underline{\text { Unit }}$ & $L a b$ \\
\hline Inor & ganics & & & & & & & & & & & & & \\
\hline+ & Aluminum, total recoverable & 360 & & 100 & & & & 226 & & & $>100$ & 1 & $\mathrm{ug} / \mathrm{L}$ & WA \\
\hline & Nitrate-nitrite as nitrogen & 1560 & . & 1080 & & & & 978 & & & $<2400$ & 1 & ug/L & WA \\
\hline & Sodium, total recoverable & 5550 & & 3350 & & & & 3380 & & & $<4600$ & 1 & $u g / L$ & WA \\
\hline & Sulfate & 1380 & & $<5000$ & $\mathrm{U}$ & & & 1510 & & & $<3000$ & 1 & $\mathrm{ug} / \mathrm{L}$ & WA \\
\hline $\operatorname{Rad}$ & ionuclides & & & & & & r. & & & & & & & \\
\hline & Gross alpha & 1.68 & & .92 & UI & & & .384 & $\mathrm{U}$ & & $<E Q L$ & 1 & $\mathrm{pCi} / \mathrm{L}$ & GP \\
\hline & Nonvolatile beta & 3.53 & & 2.12 & & & & 2 & $\mathrm{~J}$ & 1 & NDD & 1 & $\mathrm{pCi} / \mathrm{L}$ & GP \\
\hline & Radium, total alpha-emitting & .7 & & 3.91 & UIJ . & & & .5 & $J$ & 1 & NDD & 1 & $\mathrm{pCi} / \mathrm{L}$ & GP \\
\hline
\end{tabular}

Notes: Concentrations in bold italics exceed the groundwater protection or monitoring constituent standards listed in Appendix A. Synchronous water levels are measured over a 3-5 day period or less. Dilution factors, Laboratory, and Filtered Data are for Groundwater Protection Standards First Quarter 2000 and Monitoring Constituents Third Quarter 1999 data only.

$+=$ exceeded the Groundwater Protection Standards (First Quarter 2000) or Monitoring Constituents (Third Quarter 1999) listed in Appendix A. 
Table D-4. Groundwater Monitoring Results for Plume Definition Wells, M-Area HWMF (Cont.) WELL AMB 15D

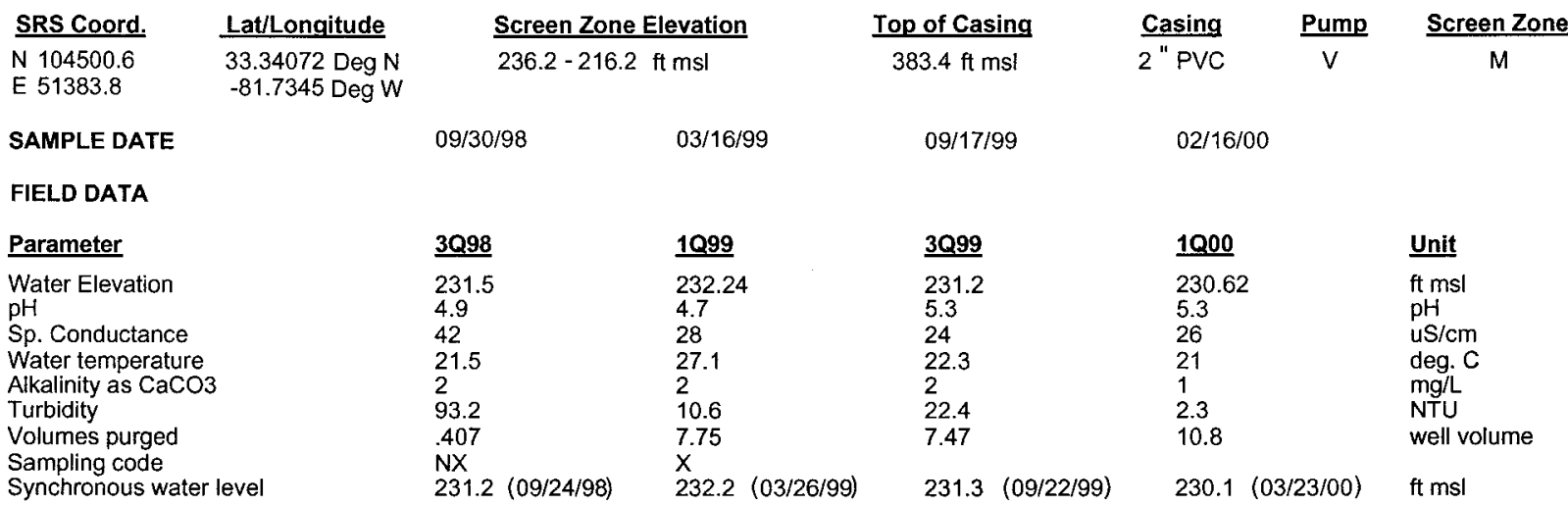

ANALYTICAL DATA

I. Groundwater Protection Standard

261 Appendix VIII/264 Appendix IX Hazardous Constituents

\begin{tabular}{|c|c|c|c|c|c|c|c|c|c|c|c|c|c|c|}
\hline$\underline{\mathrm{ST}}$ & Parameter & $\underline{3 Q 98}$ & $\underline{\text { CLP EPA }}$ & 1099 & CLP EPA & 3Q999 & CLPEPA & 1000 & CLP & EPA & Filt. & DF & Unit & Lab \\
\hline \multicolumn{15}{|c|}{ Inorganics } \\
\hline & Barium, total recoverable & 11.4 & & 7.3 & & 8.8 & & $<4.35$ & $\mathrm{U}$ & V & $<E Q L$ & 1 & ug/L & EX \\
\hline & Cyanide & $<15.2$ & $\mathrm{U}$ & $<15.2$ & $U$ & $<15.2$ & $J U Q$ & $<10$ & $\mathrm{U}$ & & $<\mathrm{EQL}$ & 1 & $\mathrm{ug} / \mathrm{L}$ & EX \\
\hline & Lead, total recoverable & $<47$ & $U$ & $<47$ & $\mathrm{U}$ & $<47$ & $\mathrm{U}$ & $<10$ & $\mathrm{U}$ & & $<\mathrm{EQL}$ & 1 & ug/L & EX \\
\hline & Nickel, total recoverable & 6.5 & $\mathrm{~J}$ & 5.3 & J 1 & 3.4 & J I & $<8.41$ & JU & V & $<E Q L$ & 1 & $\mathrm{ug} / \mathrm{L}$ & EX \\
\hline & Selenium, total recoverable & $<66$ & $\mathrm{U}$ & $<66$ & $U$ & $<66$ & $\mathrm{U}$ & $<10$ & $\mathrm{U}$ & & $<\mathrm{EQL}$ & 1 & ug/L & EX \\
\hline \multicolumn{15}{|c|}{ Organics } \\
\hline & Chlorobenzene & $<5$ & $\mathrm{U}$ & $<5$ & $U$ & $<5$ & $U$ & $<5$ & $U$ & & $<$ EQL & 1 & $\mathrm{ug} / \mathrm{L}$ & EX \\
\hline & 1,1-Dichloroethane & $<5$ & $U$ & $<5$ & $U$ & $<5$ & $\mathrm{U}$ & $<5$ & $\mathrm{U}$ & & $<E Q L$ & 1 & $\mathrm{ug} / \mathrm{L}$ & EX \\
\hline & 1,1-Dichloroethylene & $<5$ & $U$ & $<5$ & $U$ & $<5$ & $\mathrm{U}$ & $<5$ & $\mathrm{U}$ & & $<E Q L$ & 1 & $\mathrm{ug} / \mathrm{L}$ & EX \\
\hline & trans-1,2-Dichloroethylene & $<5$ & $\mathrm{U}$ & $<5$ & $U$ & $<5$ & $\mathrm{U}$ & $<5$ & $\mathrm{U}$ & & $<E Q L$ & 1 & ug/L & $\mathrm{EX}$ \\
\hline & PCB 1016 & & & & & & & & & & & & & \\
\hline & РСB 1221 & & & & & & & & & & & & & \\
\hline & PCB 1232 & & & & & & & & & & & & & \\
\hline & PCB 1242 & & & & & & & & & & & & & \\
\hline & РСB 1248 & & & & & & & & & & & & & \\
\hline & РСB 1254 & & & & & & & & & & & & & \\
\hline & РCB 1260 & & & & & & & & & & & & & \\
\hline & 1,1,2,2-Tetrachloroethane & $<5$ & $U$ & $<5$ & U & $<5$ & $\mathrm{U}$ & $<5$ & $\mathrm{U}$ & & $<\mathrm{EQL}$ & 1 & $\mathrm{ug} / \mathrm{L}$ & EX \\
\hline & Tetrachloroethylene & $<5$ & $\mathrm{U}$ & $<5$ & U & $<5$ & $\mathrm{U}$ & $<5$ & $\mathrm{U}$ & & $<$ EQL & 1 & $\mathrm{ug} / \mathrm{L}$ & EX \\
\hline & 1,1,1-Trichloroethane & $<5$ & $\mathrm{U}$ & $<5$ & $U$ & $<5$ & $\mathrm{U}$ & $<5$ & $\mathrm{U}$ & & $<$ EQL. & 1 & $\mathrm{ug} / \mathrm{L}$ & EX \\
\hline & Trichloroethylene & 6.16 & & $<5$ & $\mathrm{U}$ & $<5$ & $\mathrm{U}$ & $<5$ & $\mathrm{U}$ & & $<$ EQL & 1 & $\mathrm{ug} / \mathrm{L}$ & EX \\
\hline \multicolumn{15}{|c|}{ II. Monitoring Constituents } \\
\hline$\underline{\text { ST }}$ & Parameter & $\underline{3096}$ & $\underline{\text { CLP EPA }}$ & $\underline{3097}$ & CLP EPA & $\underline{3098}$ & CLP ExPA & $\underline{3099}$ & $\underline{\text { CLP }}$ & EPA & Filt. & $\underline{\text { DF }}$ & Unit & Lab \\
\hline \multicolumn{15}{|c|}{ Inorganics } \\
\hline+ & Aluminum, total recoverable & 872 & J & 1730 & & 890 & & 637 & & & $>100$ & 1 & $\mathrm{ug} / \mathrm{L}$ & WA \\
\hline & Nitrate-nitrite as nitrogen & 900 & & 857 & & 717 & , & 1160 & & & $<2400$ & 5 & $\mathrm{ug} / \mathrm{L}$ & WA \\
\hline & Sodium, total recoverable & 6110 & & 5430 & & 4000 & & 2800 & & & $<4600$ & 1 & ug/L & WA \\
\hline & Sulfate & 2240 & & 3600 & $\mathrm{~J}$ & 371 & $\mathrm{~J}$ & 1620 & & & $<3000$ & 1 & ug/L & WA \\
\hline \multicolumn{15}{|c|}{ Radionuclides } \\
\hline & Gross alpha & 1.39 & & 4.24 & U! & 11.07 & & 1.03 & $\mathrm{~J}$ & I & NDD & 1 & $\mathrm{pCi} / \mathrm{L}$ & GP \\
\hline & Nonvolatile beta & 1.96 & & 2.92 & Ut & 6.66 & & .8 & U & & $<\mathrm{EQL}$ & 1 & $\mathrm{pCi} / \mathrm{L}$ & GP \\
\hline & Radium, total alpha-emitting & 0 & UI & 1.57 & $\mathrm{~J}$ & 1.09 & & .9 & $\mathrm{~J}$ & I & NDD & 1 & $\mathrm{pCi} / \mathrm{L}$ & GP \\
\hline
\end{tabular}

Notes: Concentrations in bold italics exceed the groundwater protection or monitoring constituent standards listed in Appendix A. Synchronous water levels are measured over a 3-5 day period or less. Dilution factors, Laboratory, and Filtered Data are for Groundwater Protection Standards First Quarter 2000 and Monitoring Constituents Third Quarter 1999 data only.

$+=$ exceeded the Groundwater Protection Standards (First Quarter 2000) or Monitoring Constituents (Third Quarter 1999) listed in Appendix A. 
Table D-4. Groundwater Monitoring Results for Plume Definition Wells, M-Area HWMF (Cont.) WELL AMB 16D

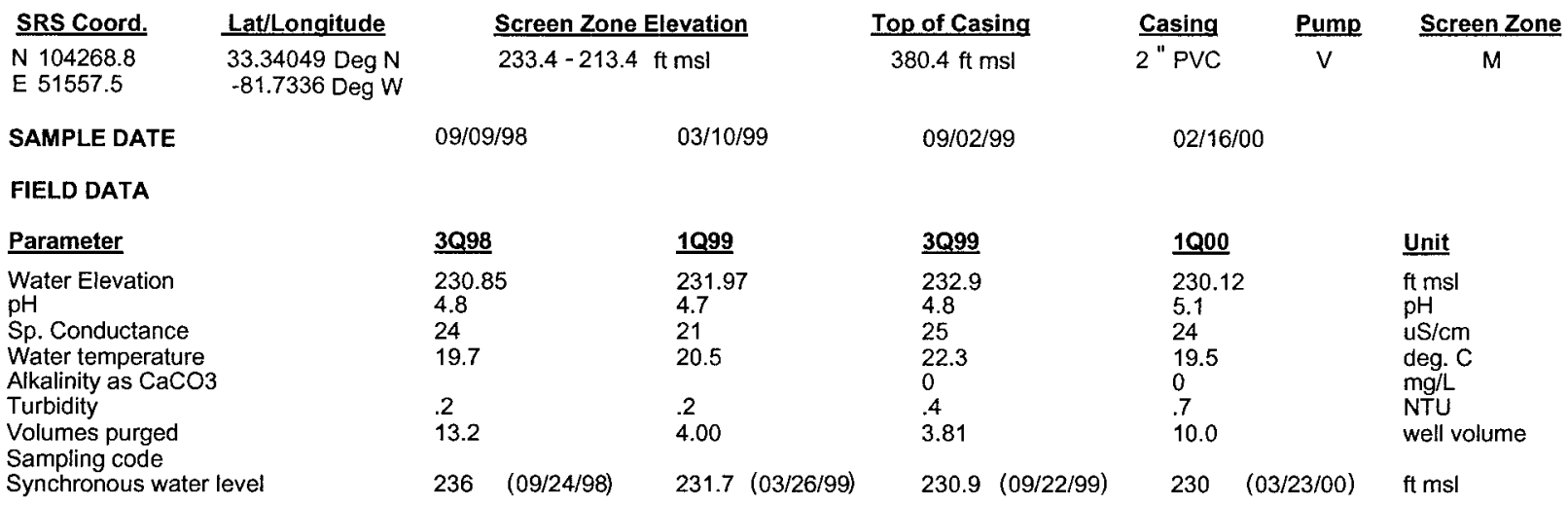

ANALYTICAL DATA

I. Groundwater Protection Standard

261 Appendix VIII/264 Appendix IX Hazardous Constituents

\begin{tabular}{|c|c|c|c|c|c|c|c|c|c|c|c|c|c|c|}
\hline \multirow{2}{*}{$\begin{array}{l}\text { ST Parameter } \\
\text { Inorganics }\end{array}$} & \multirow[t]{2}{*}{$\underline{3 Q 98}$} & \multirow[t]{2}{*}{ CLPEPA } & \multirow[t]{2}{*}{$\underline{1099}$} & \multirow[t]{2}{*}{ CLP EPA } & \multirow[t]{2}{*}{$\underline{3099}$} & \multicolumn{2}{|c|}{ CLPEPA } & \multirow[t]{2}{*}{1000} & \multicolumn{2}{|c|}{ CLPEPA } & \multirow[t]{2}{*}{ Filt. } & \multirow[t]{2}{*}{ DF } & \multirow[t]{2}{*}{ Unit } & \multirow[t]{2}{*}{$\underline{\text { Lab }}$} \\
\hline & & & & & & & & & & & & & & \\
\hline Barium, total recoverable & 4.2 & & 4.6 & & 4 & & & $<4.35$ & $\mathrm{u}$ & $\mathrm{v}$ & $<E Q L$ & 1 & $u g / L$ & EX \\
\hline Cyanide & $<15.2$ & $u$ & $<15.2$ & $u$ & $<15.2$ & $u$ & & $<10$ & $u$ & & $<E Q L$ & 1 & ug/L & EX \\
\hline Lead, total recoverable & $<47$ & $u$ & $<47$ & $\mathrm{u}$ & 27.8 & J & I & $<10$ & $u$ & & $<E Q L$ & 1 & $\mathrm{ug} / \mathrm{L}$ & EX \\
\hline Nickel, total recoverable & $<26$ & $u$ & $<26$ & $u$ & $<26$ & $\mathrm{U}$ & & $<7.83$ & JU & $\mathrm{v}$ & $<E Q L$ & 1 & $\mathrm{ug} / \mathrm{L}$ & EX \\
\hline Selenium, total recoverable & $<66$ & $\mathrm{u}$ & $<66$ & $u$ & $<66$ & $\mathrm{u}$ & & $<10$ & $\mathrm{u}$ & & $<E Q L$ & 1 & $\mathrm{ug} / \mathrm{L}$ & EX \\
\hline \multicolumn{15}{|l|}{ Organics } \\
\hline Chlorobenzene & $<5$ & $\mathrm{u}$ & $<5$ & $u$ & $<5$ & JU & $\mathrm{Q}$ & $<5$ & $\mathrm{u}$ & & $<\mathrm{EQL}$ & 1 & $u g / L$ & EX \\
\hline 1,1-Dichloroethane & $<5$ & $u$ & $<5$ & $\mathrm{u}$ & $<5$ & $J U$ & $\mathrm{Q}$ & $<5$ & $u$ & & $<\mathrm{EQL}$ & 1 & ug/L & EX \\
\hline 1,1-Dichloroethylene & $<5$ & $u$ & $<5$ & $\mathrm{u}$ & $<5$ & JU & $\mathrm{Q}$ & $<5$ & $\mathrm{u}$ & & $<\mathrm{EQL}$ & 1 & $\mathrm{ug} / \mathrm{L}$ & EX \\
\hline trans-1,2-Dichloroethylene & $<5$ & $\mathrm{U}$ & $<5$ & $\mathrm{u}$ & $<5$ & JU & $\mathrm{Q}$ & $<5$ & $\mathrm{u}$ & & $<E Q L$ & 1 & ug/L & EX \\
\hline PCB 1016 & & & & & & & & $<.94$ & $\mathrm{u}$ & & $<\mathrm{EQL}$ & .940 & $\mathrm{ug} / \mathrm{L}$ & EX \\
\hline РСВ 1221 & & & & & & & & $<.94$ & $\mathrm{U}$ & & $<E Q L$ & .940 & $\mathrm{ug} / \mathrm{L}$ & EX \\
\hline РСВ 1232 & & & & & & & & $<.94$ & $\mathrm{U}$ & & $<\mathrm{EQL}$ & 940 & $\mathrm{ug} / \mathrm{L}$ & EX \\
\hline PCB 1242 & & & & & & & & $<1.9$ & $\mathrm{u}$ & & $<E Q L$ & .940 & $\mathrm{ug} / \mathrm{L}$ & EX \\
\hline РCB 1248 & & & & & & & & $<.94$ & $U$ & & $<E Q L$ & .940 & $\mathrm{ug} / \mathrm{L}$ & EX \\
\hline PCB 1254 & & & & & & & & $<.94$ & $U$ & & $<E Q L$ & .940 & $\mathrm{ug} / \mathrm{L}$ & EX \\
\hline РСВ 1260 & & & & & & & & $<.94$ & $u$ & & $<E Q L$ & .940 & ug/L & EX \\
\hline 1,1,2,2-Tetrachloroethane & $<5$ & u & $<5$ & $u$ & $<5$ & JU & $Q$ & $<5$ & $\mathrm{U}$ & & $<E Q L$ & 1 & $\mathrm{ug} / \mathrm{L}$ & EX \\
\hline Tetrachloroethylene & $<5$ & $u$ & $<5$ & $\mathrm{u}$ & $<5$ & JU & $Q$ & $<5$ & $\mathrm{U}$ & & $<E Q L$ & 1 & $\mathrm{ug} / \mathrm{L}$ & EX \\
\hline 1,1,1-Trichloroethane & $<5$ & $\mathrm{U}$ & $<5$ & u & $<5$ & JU & Q & $<5$ & $\mathrm{U}$ & & $<E Q L$ & 1 & $\mathrm{ug} / \mathrm{L}$ & EX \\
\hline Trichloroethylene & 4.13 & $J$ & 1.54 & J 1 & 2.13 & $J$ & IQ & 3.1 & $J$ & 1 & NDD & 1 & $u g / L$ & EX \\
\hline \multicolumn{15}{|l|}{ II. Monitoring Constituents } \\
\hline Parameter & $\underline{3 Q 96}$ & CLPPEPA & $\underline{3097}$ & CLPP EPA & $\underline{3098}$ & $\underline{\text { CLP }}$ & EPA & $\underline{3099}$ & CLP & EPA & Filt. & $\underline{\text { DF }}$ & $\underline{\text { Unit }}$ & $\underline{\text { Lab }}$ \\
\hline \multicolumn{15}{|l|}{ Inorganics } \\
\hline Aluminum, total recoverable & $<20$ & UJ & $<20$ & $u$ & $<146$ & $U$ & & 59.8 & J & I & NDD & 1 & $\mathrm{ug} / \mathrm{L}$ & WA \\
\hline Nitrate-nitrite as nitrogen & 890 & & 7120 & & 1030 & & & 1400 & & & $<2400$ & 5 & $\mathrm{ug} / \mathrm{L}$ & WA \\
\hline Sodium, total recoverable & 4710 & & 1500 & & 1910 & & & 2920 & & & $<4600$ & 1 & $u g / L$ & WA \\
\hline Sulfate & 2300 & & $<5000$ & $U$ & 352 & & & 414 & & & $<3000$ & 1 & $\mathrm{ug} / \mathrm{L}$ & WA \\
\hline \multicolumn{15}{|l|}{ Radionuclides } \\
\hline Gross alpha & 1.17 & & 4.04 & & 7.09 & & & 4.02 & & & $<15$ & 1 & $\mathrm{pCi} / \mathrm{L}$ & GP \\
\hline Nonvolatile beta & .926 & & .25 & UI & 3.31 & u & & 2.41 & & & $<50$ & 1 & $\mathrm{pCi} / \mathrm{L}$ & GP \\
\hline Radium, total alpha-emitting & .6 & & 4.27 & $\mathrm{~J}$ & 1.26 & & & 1.8 & $\mathrm{~J}$ & I & NDD & 1 & $\mathrm{pCi} / \mathrm{L}$ & GP \\
\hline
\end{tabular}

Notes: Concentrations in bold italics exceed the groundwater protection or monitoring constituent standards listed in Appendix A. Synchronous water levels are measured over a 3-5 day period or less. Dilution factors, Laboratory, and Filtered Data are for Groundwater Protection Standards First Quarter 2000 and Monitoring Constituents Third Quarter 1999 data only.

$+=$ exceeded the Groundwater Protection Standards (First Quarter 2000) or Monitoring Constituents (Third Quarter 1999) listed in Appendix A. 
Table D-4. Groundwater Monitoring Results for Plume Definition Wells, M-Area HWMF (Cont.) WELL AMB 17A

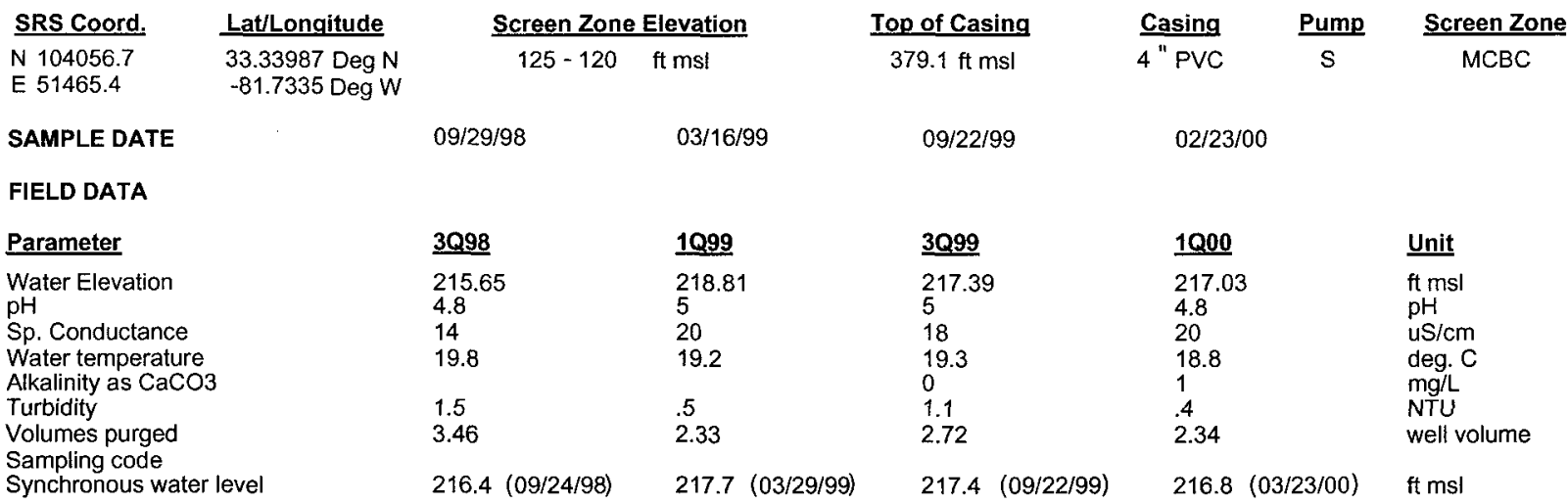

ANALYTICAL DATA

I. Groundwater Protection Standard

261 Appendix VIII/264 Appendix IX Hazardous Constituents

\begin{tabular}{|c|c|c|c|c|c|c|c|c|c|c|c|c|c|}
\hline$\underline{\text { ST }}$ & Parameter & $\underline{3098}$ & CLPEPA & $\underline{1099}$ & $\underline{\text { CLP EPA }}$ & 3099 & $\underline{\text { CLPPEPA }}$ & $\underline{1000}$ & CLPEPA & Filt. & $\underline{\mathrm{DF}}$ & Unit & $\underline{\text { Lab }}$ \\
\hline \multicolumn{14}{|c|}{ Inorganics } \\
\hline & Barium, total recoverable & 2.8 & & 3.2 & & 2.7 & & $<3.28$ & U V & $<E Q L$ & 1 & $u g / L$ & EX \\
\hline & Cyanide & $<15.2$ & $U$ & $<15.2$ & $\mathrm{u}$ & $<15.2$ & $U$ & $<10$ & $\mathrm{U}$ & $<\mathrm{EQL}$ & 1 & $\mathrm{ug} / \mathrm{L}$ & EX \\
\hline & Lead, total recoverable & 40.3 & J & $<47$ & $U$ & $<47$ & $\mathrm{U}$ & $<10$ & $U$ & $<$ EQL & 1 & $\mathrm{ug} / \mathrm{L}$ & EX \\
\hline & Nickel, total recoverable & 27.3 & $J$ & $<26$ & $\mathrm{U}$ & $<26$ & $U$ & $<50$ & $U$ & $<$ EQL & 1 & $u g / L$ & EX \\
\hline & Selenium, total recoverable & $<200$ & $U$ & $<66$ & $U$ & $<66$ & $\mathrm{U}$ & $<10$ & U & $<E Q L$ & 1 & $\mathrm{ug} / \mathrm{L}$ & EX \\
\hline \multicolumn{14}{|c|}{ Organics } \\
\hline & Chlorobenzene & $<25$ & $u$ & $<10$ & $u$ & $<10$ & $\mathbf{u}$ & $<5$ & U & $<E Q L$ & 1 & $\mathrm{ug} / \mathrm{L}$ & EX \\
\hline & 1,1-Dichloroethane & $<25$ & $\mathrm{U}$ & $<10$ & $\mathrm{U}$ & $<10$ & $\mathrm{U}$ & $<5$ & $U$ & $<E Q L$ & 1 & $\mathrm{ug} / \mathrm{L}$ & $\mathrm{EX}$ \\
\hline & 1,1-Dichloroethylene & $<25$ & U & $<10$ & $\mathrm{U}$ & $<10$ & $\mathrm{U}$ & $<5$ & $U$ & $<E Q L$ & 1 & $\mathrm{ug} / \mathrm{L}$ & $\mathrm{EX}$ \\
\hline & trans-1,2-Dichloroethylene & $<25$ & $\mathrm{U}$ & $<10$ & $\mathrm{U}$ & $<10$ & $\mathrm{U}$ & $<5$ & $U$ & $<\mathrm{EQL}$ & 1 & $\mathrm{ug} / \mathrm{L}$ & EX \\
\hline & PCB 1016 & & & $<1.02$ & $J U Q$ & & & $<.98$ & $U$ & $<E Q L$ & .980 & $\mathrm{ug} / \mathrm{L}$ & EX \\
\hline & PCB 1221 & & & $<2.04$ & JU $Q$ & & & $<.98$ & $\mathrm{U}$ & $<E Q L$ & .980 & $\mathrm{ug} / \mathrm{L}$ & EX \\
\hline & PCB 1232 & & & $<1.02$ & $J \cup Q$ & & & $<.98$ & U & $<E Q L$ & .980 & $\mathrm{ug} / \mathrm{L}$ & EX \\
\hline & РCB 1242 & & & $<1.02$ & JU $Q$ & & & $<2$ & $U$ & $<\mathrm{EQL}$ & .980 & $\mathrm{ug} / \mathrm{L}$ & EX \\
\hline & PCB 1248 & & & $<1.02$ & $J \cup Q$ & & & $<.98$ & U & $<E Q L$ & .980 & $\mathrm{ug} / \mathrm{L}$ & EX \\
\hline & PCB 1254 & & & $<1.02$ & JU $\bar{Q}$ & & & $<.98$ & $\mathrm{U}$ & $<E Q L$ & .980 & $\mathrm{ug} / \mathrm{L}$ & EX \\
\hline & PCB 1260 & & & $<1.02$ & JU $Q$ & & & $<.98$ & $\mathrm{U}$ & $<\overline{E Q L}$ & .980 & $\mathrm{ug} / \mathrm{L}$ & $\mathrm{EX}$ \\
\hline & 1,1,2,2-Tetrachloroethane & $<25$ & $U$ & $<10$ & $\mathrm{U}$ & $<10$ & $\mathrm{U}$ & $<5$ & $U$ & $<\mathrm{EQL}$ & 1 & $\mathrm{ug} / \mathrm{L}$ & EX \\
\hline+ & Tetrachloroethylene & 22.8 & & 26 & & 26.3 & & 25 & & $>5$ & 1 & $\mathrm{ug} / \mathrm{L}$ & EX \\
\hline & 1,1,1-Trichloroethane & $<25$ & $U$ & $<10$ & U & $<10$ & $\mathrm{U}$ & $<5$ & $U$ & $<E Q L$ & 1 & $\mathrm{ug} / \mathrm{L}$ & EX \\
\hline+ & Trichloroethylene & 241 & & 208 & & 176 & & 170 & & $>5$ & 1 & $\mathrm{ug} / \mathrm{L}$ & EX \\
\hline \multicolumn{14}{|c|}{ II. Monitoring Constituents } \\
\hline$\underline{\text { ST }}$ & Parameter & $\underline{3096}$ & CLP EPA & $\underline{3097}$ & CLP EPA & $\underline{3 Q 98}$ & $\underline{\text { CLP EPA }}$ & $\underline{\mathbf{3 Q 9 9}}$ & CLPEPA & Filt. & $\underline{\text { DF }}$ & $\underline{\text { Unit }}$ & $\underline{\text { Lab }}$ \\
\hline \multicolumn{14}{|c|}{ Inorganics } \\
\hline & Aluminum, total recoverable & 60.1 & & $<20$ & $U$ & $<200$ & $u$ & $<146$ & $\mathrm{U}$ & $<E Q L$ & 1 & $u g / L$ & WA \\
\hline & Nitrate-nitrite as nitrogen & 1250 & & 1130 & & 868 & & 926 & & $<2400$ & 1 & $\mathrm{ug} / \mathrm{L}$ & WA \\
\hline & Sodium, total recoverable & 1920 & & 2010 & & 2040 & & 1680 & & $<4600$ & 1 & $\mathrm{ug} / \mathrm{L}$ & WA \\
\hline & Sulfate & 478 & & $<5000$ & $\mathrm{U}$ & 514 & & 452 & & $<3000$ & 1 & $\mathrm{ug} / \mathrm{L}$ & WA \\
\hline \multicolumn{14}{|c|}{ Radionuclides } \\
\hline & Gross alpha & .79 & UI & -.13 & UI & 1.21 & & .122 & U & $<\mathrm{EQL}$ & 1 & $\mathrm{pCi} / \mathrm{L}$ & GP \\
\hline & Nonvolatile beta & 1.48 & UI & -1.24 & UI & 10.04 & & .497 & U & $<\mathrm{EQL}$ & 1 & $\mathrm{pCi} / \mathrm{L}$ & GP \\
\hline & Radium, total alpha-emitting & 8.29 & & .81 & Ul & .32 & UI & -.1 & $\mathrm{U}$ & $<\mathrm{EQL}$ & 1 & $\mathrm{pCi} / \mathrm{L}$ & GP \\
\hline
\end{tabular}

Notes: Concentrations in bold italics exceed the groundwater protection or monitoring constituent standards listed in Appendix A. Synchronous water levels are measured over a 3-5 day period or less. Dilution factors, Laboratory, and Filtered Data are for Groundwater Protection Standards First Quarter 2000 and Monitoring Constituents Third Quarter 1999 data only.

+ = exceeded the Groundwater Protection Standards (First Quarter 2000) or Monitoring Constituents (Third Quarter 1999) listed in Appendix A. 
Table D-4. Groundwater Monitoring Results for Plume Definition Wells, M-Area HWMF (Cont.) WELL AMB 18A

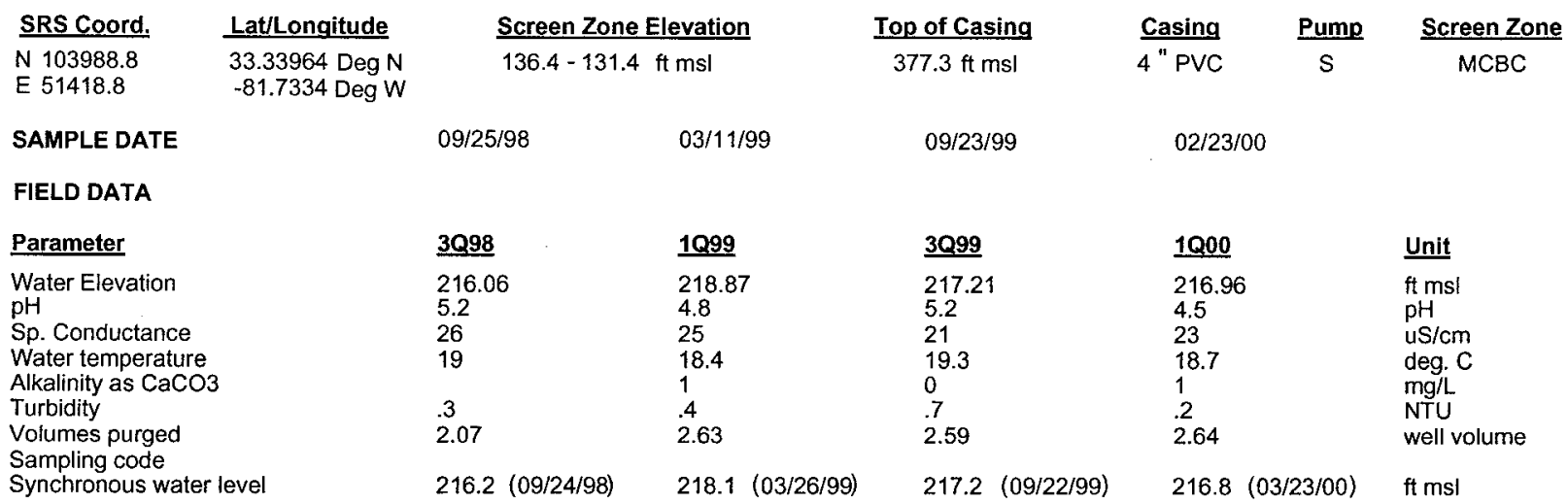

ANALYTICAL DATA

I. Groundwater Protection Standard

261 Appendix VIII/264 Appendix IX Hazardous Constituents

\begin{tabular}{|c|c|c|c|c|c|c|c|c|c|c|c|c|c|}
\hline ST & Parameter & $\underline{3098}$ & $\underline{\text { CLP EPA }}$ & 1099 & CLP EPA & $\underline{3 Q 99}$ & $\underline{\text { CLPEPA }}$ & $1 Q 00$ & CLPEPA & Filt. & $\underline{\text { DF }}$ & $\underline{\text { Unit }}$ & $\underline{\mathrm{Lab}}$ \\
\hline \multicolumn{14}{|c|}{ Inorganics } \\
\hline & Barium, total recoverable & 6.1 & & 5.7 & & 5.4 & & 44.7 & & $<2000$ & 1 & $\mathrm{ug} / \mathrm{L}$ & EX \\
\hline & Cyanide & $<15.2$ & UJ & $<15.2$ & $\mathrm{U}$ & $<15.2$ & 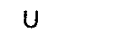 & $<10$ & $U$ & $<E Q L$ & 1 & $\mathrm{ug} / \mathrm{L}$ & EX \\
\hline & Lead, total recoverable & $<47$ & $U$ & $<47$ & U & $<47$ & $\mathrm{U}$ & $<10$ & $U$ & $<E Q L$ & 1 & $\mathrm{ug} / \mathrm{L}$ & EX \\
\hline & Nickel, total recoverable & $<26$ & $\mathrm{U}$ & $<26$ & $\mathrm{U}$ & $<26$ & $\mathrm{U}$ & $<6.24$ & JU V & $<E Q L$ & 1 & $\mathrm{ug} / \mathrm{L}$ & EX \\
\hline & Selenium, total recoverable & $<66$ & $U$ & $<66$ & $\mathrm{U}$ & $<66$ & $\mathrm{U}$ & $<10$ & $\mathrm{U}$ & $<$ EQL & 1 & $\mathrm{ug} / \mathrm{L}$ & EX \\
\hline \multicolumn{14}{|c|}{ Organics } \\
\hline & Chlorobenzene & $<5$ & $\mathrm{U}$ & $<5$ & $\mathrm{U}$ & $<5$ & $\mathrm{U}$ & $<5$ & U & $<\mathrm{EQL}$ & 1 & $\mathrm{ug} / \mathrm{L}$ & EX \\
\hline & 1,1-Dichloroethane & $<5$ & U & $<5$ & $\mathrm{U}$ & $<5$ & $\mathrm{U}$ & $<5$ & $\mathrm{U}$ & $<\mathrm{EQL}$ & 1 & $\mathrm{ug} / \mathrm{L}$ & EX \\
\hline & 1,1-Dichloroethylene & $<5$ & $\mathrm{U}$ & $<5$ & $\mathrm{U}$ & $<5$ & $\mathrm{U}$ & $<5$ & $U$ & $<E Q L$ & 1 & $\mathrm{ug} / \mathrm{L}$ & EX \\
\hline & trans-1,2-Dichloroethylene & $<5$ & U & $<5$ & U & $<5$ & $\mathrm{U}$ & $<5$ & U & $<E Q L$ & 1 & $\mathrm{ug} / \mathrm{L}$ & EX \\
\hline & PCB 1016 & & & $<1.02$ & $J U Q$ & & & $<.96$ & U & $<E Q$ & .960 & $\mathrm{ug} / \mathrm{L}$ & EX \\
\hline & PCB 1221 & & & $<2.04$ & JU $Q$ & & & $<.96$ & $\mathrm{U}$ & $<E Q L$ & .960 & ug/L & EX \\
\hline & PCB 1232 & & & $<1.02$ & JU $Q$ & & & $<.96$ & $\mathrm{U}$ & $<\mathrm{EQL}$ & .960 & ug/L & EX \\
\hline & PCB 1242 & & & $<1.02$ & JU $Q$ & & & $<1.9$ & U & $<E Q L$ & .960 & $\mathrm{ug} / \mathrm{L}$ & EX \\
\hline & PCB 1248 & & & $<1.02$ & JU $Q$ & & & $<.96$ & $\mathrm{U}$ & $<\mathrm{EQL}$ & .960 & $\mathrm{ug} / \mathrm{L}$ & EX \\
\hline & PCB 1254 & & & $<1.02$ & JU $Q$ & & & $<.96$ & $\mathrm{U}$ & $<\mathrm{EQL}$ & .960 & $\mathrm{ug} / \mathrm{L}$ & EX \\
\hline & PCB 1260 & & & $<1.02$ & JU $Q$ & & & $<.96$ & $\mathrm{U}$ & $<E Q L$ & .960 & $\mathrm{ug} / \mathrm{L}$ & EX \\
\hline & $1,1,2,2$-Tetrachloroethane & $<5$ & U & $<5$ & $\mathrm{U}$ & $<5$ & $\mathrm{U}$ & $<5$ & $\mathrm{U}$ & $<E Q L$ & 1 & $\mathrm{ug} / \mathrm{L}$ & EX \\
\hline & Tetrachloroethylene & 4.59 & $\mathrm{~J}$ & $<5$ & $U$ & 2.36 & J I & 4.6 & J 1 & NDD & 1 & $u g / L$ & EX \\
\hline & 1,1,1-Trichloroethane & $<5$ & $U$ & $<5$ & $U$ & & $U$ & $<5$ & $\mathrm{U}$ & $<\mathrm{EQL}$ & 1 & $\mathrm{ug} / \mathrm{L}$ & EX \\
\hline+ & Trichloroethylene & 36.8 & & 27.8 & & 19.3 & & 24 & & $>5$ & 1 & $\mathrm{ug} / \mathrm{L}$ & EX \\
\hline \multicolumn{14}{|c|}{ II. Monitoring Constituents } \\
\hline$\underline{\text { ST }}$ & Parameter & $\underline{3096}$ & CLPEPA & $\underline{3097}$ & CLPEPA & $\underline{3098}$ & CLP EPA & $\underline{3099}$ & CLPEPA & Filt. & $\underline{\text { DF }}$ & Unit & $\underline{\text { Lab }}$ \\
\hline \multicolumn{14}{|c|}{ Inorganics } \\
\hline & Aluminum, total recoverable & $<20$ & UJ & 21.6 & & $<55.5$ & 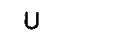 & $<146$ & $\mathrm{U}$ & $<\mathrm{EQL}$ & 1 & ug/L & WA \\
\hline & Nitrate-nitrite as nitrogen & 1050 & & 1110 & & 813 & & 795 & & $<2400$ & 1 & $\mathrm{ug} / \mathrm{L}$ & WA \\
\hline & Sodium, total recoverable & 2100 & & 2270 & & 2180 & & 1900 & & $<4600$ & 1 & $\mathrm{ug} / \mathrm{L}$ & WA \\
\hline & Sulfate & $<336$ & $U$ & $<5000$ & $\mathrm{U}$ & 471 & $J$ & 432 & & $<3000$ & 1 & $\mathrm{ug} / \mathrm{L}$ & WA \\
\hline \multicolumn{14}{|c|}{ Radionuclides } \\
\hline & Gross alpha & .089 & UI & .86 & UI & .28 & UIJ & .106 & $\mathrm{U}$ & $<E Q L$ & 1 & $\mathrm{pCi} / \mathrm{L}$ & GP \\
\hline & Nonvolatile beta & .088 & UI & .211 & UI & 1.64 & UI & .482 & $\mathrm{U}$ & $<E Q L$ & 1 & $\mathrm{pCi} / \mathrm{L}$ & $\mathrm{GP}$ \\
\hline & Radium, total alpha-emitting & -.1 & UI & 6 & & .35 & UI & 0 & $\mathrm{U}$ & $<\mathrm{EQL}$ & 1 & $\mathrm{pCi} / \mathrm{L}$ & GP \\
\hline
\end{tabular}

Notes: Concentrations in bold italics exceed the groundwater protection or monitoring constituent standards listed in Appendix A. Synchronous water levels are measured over a 3-5 day period or less. Dilution factors, Laboratory, and Filtered Data are for Groundwater Protection Standards First Quarter 2000 and Monitoring Constituents Third Quarter 1999 data only.

$+=$ exceeded the Groundwater Protection Standards (First Quarter 2000) or Monitoring Constituents (Third Quarter 1999) listed in Appendix A. 
Table D-4. Groundwater Monitoring Results for Plume Definition Wells, M-Area HWMF (Cont.) WELL AMB $18 \mathrm{C}$

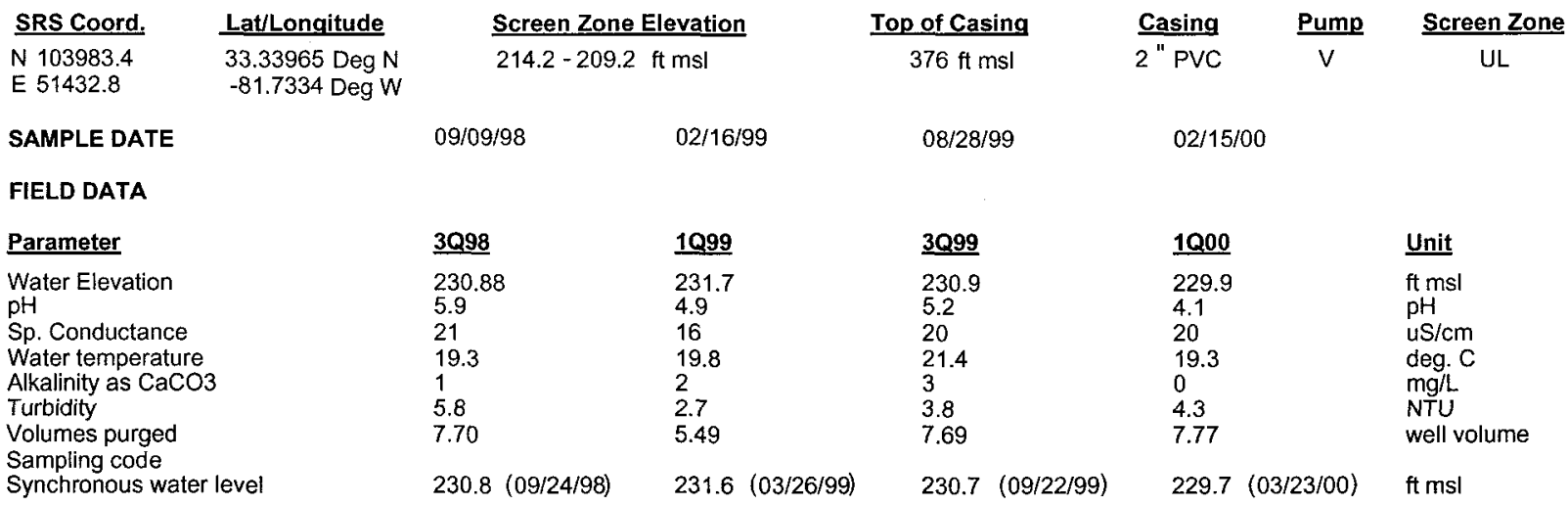

ANALYTICAL DATA

I. Groundwater Protection Standard

261 Appendix VIII/264 Appendix IX Hazardous Constituents

\begin{tabular}{|c|c|c|c|c|c|c|c|c|c|c|c|c|c|c|c|}
\hline \multirow{2}{*}{\multicolumn{2}{|c|}{$\begin{array}{l}\text { ST Parameter } \\
\text { Inorganics }\end{array}$}} & \multirow[t]{2}{*}{$\underline{3 Q 98}$} & \multirow[t]{2}{*}{$\underline{\text { CLP EPA }}$} & \multirow[t]{2}{*}{$\underline{1 Q 99}$} & \multirow[t]{2}{*}{ CLP EPA } & \multirow[t]{2}{*}{$\underline{3099}$} & \multicolumn{2}{|c|}{ CLPEPA } & \multirow[t]{2}{*}{$\underline{1000}$} & \multicolumn{2}{|c|}{ CLPEPA } & \multirow[t]{2}{*}{ Filt. } & \multirow[t]{2}{*}{$\underline{\mathrm{DF}}$} & \multirow[t]{2}{*}{ Unit } & \multirow[t]{2}{*}{ Lab } \\
\hline & & & & & & & & & & & & & & & \\
\hline & Barium, total recoverable & 3.9 & & 3.7 & & $<4.1$ & $U$ & V & 4.25 & J & 1 & NDD & 1 & ug/L & EX \\
\hline & Cyanide & $<15.2$ & $\mathrm{U}$ & $<15.2$ & $\mathrm{U}$ & $<15.2$ & $U$ & & $<10$ & $U$ & & $<E Q L$ & 1 & $\mathrm{ug} / \mathrm{L}$ & EX \\
\hline+ & Lead, total recoverable & 52.4 & & 6.9 & $\jmath 1$ & $<47$ & $U$ & & 15.7 & & & $>15$ & 1 & $u g / L$ & EX \\
\hline & Nickel, total recoverable & $<26$ & $U$ & $<26$ & $\mathbf{U}$ & $<26$ & $U$ & & $<6.96$ & $U$ & V & $<E Q L$ & 1 & $\mathrm{ug} / \mathrm{L}$ & EX \\
\hline & Selenium, total recoverable & $<66$ & $U$ & $<66$ & $\mathrm{U}$ & $<66$ & $U$ & & $<10$ & $U$ & & $<\mathrm{EQL}$ & 1 & $u g / L$ & EX \\
\hline \multicolumn{16}{|c|}{ Organics } \\
\hline & Chlorobenzene & $<5$ & $u$ & $<5$ & $\mathrm{u}$ & $<5$ & JU & $Q$ & $<5$ & $\mathrm{U}$ & & $<E Q L$ & 1 & $\mathrm{ug} / \mathrm{L}$ & EX \\
\hline & 1,1-Dichloroethane & $<5$ & $\mathrm{U}$ & $<5$ & $\mathrm{U}$ & $<5$ & JU & $Q$ & $<5$ & $U$ & & $<E Q L$ & 1 & $\mathrm{ug} / \mathrm{L}$ & EX \\
\hline & 1,1-Dichloroethylene & $<5$ & $\mathrm{U}$ & $<5$ & $\mathrm{U}$ & $<5$ & JU & $\vec{Q}$ & $<5$ & U & & $<$ EQL & 1 & $\mathrm{ug} / \mathrm{L}$ & EX \\
\hline & trans-1,2-Dichloroethylene & $<5$ & $\mathrm{U}$ & $<5$ & $\mathrm{U}$ & $<5$ & JU & $\mathrm{Q}$ & $<5$ & $U$ & & $<E Q L$ & 1 & $\mathrm{ug} / \mathrm{L}$ & EX \\
\hline & PCB 1016 & & & $<1.01$ & $\mathrm{U}$ & & & & $<.97$ & $U$ & & $<E Q L$ & .970 & $u g / L$ & EX \\
\hline & PCB 1221 & & & $<2.02$ & $\mathrm{U}$ & & & & $<.97$ & $U$ & & $<E Q L$ & .970 & ug/L & EX \\
\hline & PCB 1232 & & & $<1.01$ & $\mathrm{U}$ & & & & $<.97$ & U & & $<E Q L$ & .970 & $\mathrm{ug} / \mathrm{L}$ & EX \\
\hline & PCB 1242 & & & $<1.01$ & $\mathrm{U}$ & & & & $<1.9$ & U & & $<$ EQL & .970 & $u g / L$ & EX \\
\hline & PCB 1248 & & & $<1.01$ & U & & & & $<.97$ & $U$ & & $<E Q L$ & .970 & $u g / L$ & EX \\
\hline & PCB 1254 & & & $<1.01$ & U & & & & $<.97$ & U & & $<E Q L$ & .970 & $\mathrm{ug} / \mathrm{L}$ & EX \\
\hline & PCB 1260 & & & $<1.01$ & $\mathrm{U}$ & & & & $<.97$ & $\mathrm{U}$ & & $<\mathrm{EQL}$ & .970 & $\mathrm{ug} / \mathrm{L}$ & EX \\
\hline & 1,1,2,2-Tetrachloroethane & $<5$ & $\mathrm{U}$ & $<5$ & $\mathrm{U}$ & $<5$ & JU & $Q$ & $<5$ & U & & $<\mathrm{EQL}$ & 1 & $\mathrm{ug} / \mathrm{L}$ & EX \\
\hline & Tetrachloroethylene & $<5$ & $\mathrm{U}$ & $<5$ & $\mathrm{U}$ & $<5$ & JU & $Q$ & $<5$ & $\mathrm{U}$ & & $<E Q L$ & 1 & $u g / L$ & EX \\
\hline & 1,1,1-Trichloroethane & $<5$ & $U$ & $<5$ & $U$ & $<5$ & JU & $Q$ & $<5$ & $\mathrm{U}$ & & $<E Q L$ & 1 & $\mathrm{ug} / \mathrm{L}$ & EX \\
\hline & Trichloroethylene & $<5$ & $\mathrm{U}$ & $<5$ & U & $<5$ & JU & $Q$ & $<5$ & $\mathrm{U}$ & & $<\mathrm{EQL}$ & 1 & $\mathrm{ug} / \mathrm{L}$ & EX \\
\hline \multicolumn{16}{|c|}{ II. Monitoring Constituents } \\
\hline$\underline{\text { ST }}$ & Parameter & $\underline{3 Q 96}$ & CLPEPA & $\underline{3097}$ & CLP EPA & $\underline{3098}$ & $\underline{\text { CLP }}$ & EPA & $\underline{3 Q 99}$ & $\underline{\text { CLF }}$ & EPA & Filt. & $\underline{\text { DF }}$ & Unit & Lab \\
\hline \multicolumn{16}{|c|}{ Inorganics } \\
\hline & Aluminum, total recoverable & 73.2 & & 202 & & 87.1 & $J$ & & 63.8 & $J$ & J & NDD & 1 & $u g / L$ & WA \\
\hline & Nitrate-nitrite as nitrogen & 740 & & 1030 & & 884 & & & 763 & & & $<2400$ & 1 & $\mathrm{ug} / \mathrm{L}$ & WA \\
\hline & Sodium, total recoverable & 4700 & $J$ & 3700 & & 1960 & & & 1960 & & & $<4600$ & 1 & $\mathrm{ug} / \mathrm{L}$ & WA \\
\hline & Sulfate & $<1120$ & $U$ & $<5000$ & $\mathrm{U}$ & 708 & & & 629 & & & $<3000$ & 1 & $\mathrm{ug} / \mathrm{L}$ & WA \\
\hline \multicolumn{16}{|c|}{ Radionuclides } \\
\hline & Gross alpha & .079 & UI & .92 & & 1.07 & & & 1.39 & $J$ & IK & NDD & 1 & $\mathrm{pCi} / \mathrm{L}$ & GP \\
\hline & Nonvolatile beta & .893 & UI & -.92 & UI & .91 & UI & & .676 & U & & $<\mathrm{EQL}$ & 1 & $\mathrm{pCi} / \mathrm{L}$ & GP \\
\hline & Radium, total alpha-emitting & 0 & UI & 2.31 & UIJ & 1.07 & & & .3 & $J$ & 1 & NDD & 1 & $\mathrm{pCi} / \mathrm{L}$ & GP \\
\hline
\end{tabular}

Notes: Concentrations in bold italics exceed the groundwater protection or monitoring constituent standards listed in Appendix A. Synchronous water levels are measured over a 3-5 day period or less. Dilution factors, Laboratory, and Filtered Data are for Groundwater Protection Standards First Quarter 2000 and Monitoring Constituents Third Quarter 1999 data only.

$+=$ exceeded the Groundwater Protection Standards (First Quarter 2000) or Monitoring Constituents (Third Quarter 1999) listed in Appendix A. 
Table D-4. Groundwater Monitoring Results for Plume Definition Wells, M-Area HWMF (Cont.) WELL AMB 19C

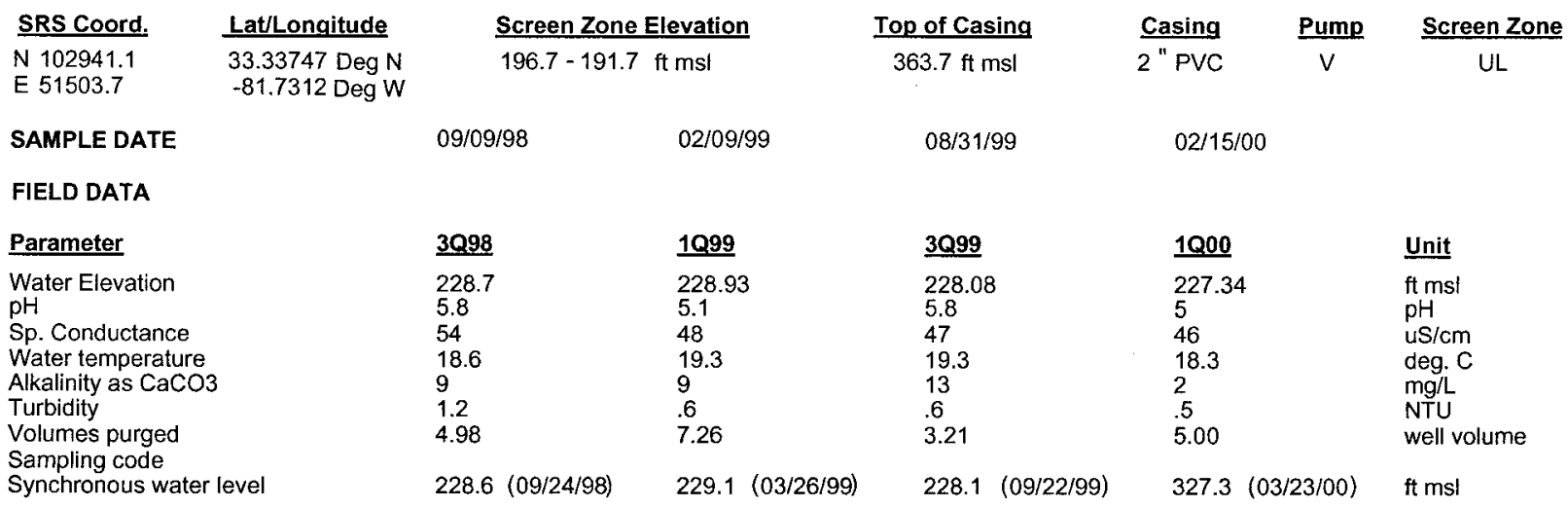

ANALYTICAL DATA

1. Groundwater Protection Standard

261 Appendix VIII/264 Appendix IX Hazardous Constituents

\begin{tabular}{|c|c|c|c|c|c|c|c|c|c|c|c|c|c|}
\hline$\underline{\text { ST }}$ & Parameter & $\underline{3 Q 98}$ & CLP EPA & 1099 & CLP EPA & $\underline{3099}$ & CLPEPA & $1 Q 00$ & CLPEPA & Filt. & $\underline{\text { DF }}$ & Unit & $\underline{\text { Lab }}$ \\
\hline \multicolumn{14}{|c|}{ Inorganics } \\
\hline & Barium, total recoverable & 1.2 & $J$ & $<1.4$ & V & 2.2 & & $<1.8$ & V & $<E Q L$ & 1 & $u g / L$ & EX \\
\hline & Cyanide & $<15.2$ & $U$ & $<15.2$ & $U$ & 2.5 & J 1 & $<10$ & $U$ & $<E Q L$ & 1 & $\mathrm{ug} / \mathrm{L}$ & EX \\
\hline+ & Lead, total recoverable & $<47$ & $U$ & $<47$ & $\mathrm{U}$ & $<47$ & $\mathrm{U}$ & 98.8 & & $>15$ & 1 & $\mathrm{ug} / \mathrm{L}$ & EX \\
\hline & Nickel, total recoverable & $<26$ & $\mathrm{U}$ & $<26$ & $\mathrm{U}$ & $<26$ & $\mathrm{U}$ & $<50$ & $U$ & $<E Q L$ & 1 & $\mathrm{ug} / \mathrm{L}$ & EX \\
\hline & Selenium, total recoverable & $<66$ & $\mathrm{U}$ & $<66$ & $U$ & $<66$ & $\mathrm{U}$ & $<10$ & $U$ & $<E Q L$ & 1 & $\mathrm{ug} / \mathrm{L}$ & EX \\
\hline \multicolumn{14}{|c|}{ Organics } \\
\hline & Chlorobenzene & $<5$ & U & $<5$ & $U$ & $<5$ & $U$ & $<5$ & U & $<E Q L$ & 1 & $\mathrm{ug} / \mathrm{L}$ & $\mathrm{EX}$ \\
\hline & 1,1-Dichloroethane & $<5$ & $U$ & $<5$ & $\mathrm{U}$ & $<5$ & $U$ & $<5$ & $\mathrm{U}$ & $<E Q L$ & 1 & $\mathrm{ug} / \mathrm{L}$ & EX \\
\hline & 1,1-Dichloroethylene & $<5$ & $U$ & $<5$ & $\mathrm{U}$ & $<5$ & $\mathrm{U}$ & $<5$ & $\mathrm{U}$ & $<E Q L$ & 1 & $\mathrm{ug} / \mathrm{L}$ & EX \\
\hline & trans-1,2-Dichloroethylene & $<5$ & $\mathrm{U}$ & $<5$ & $U$ & $<5$ & $\mathrm{U}$ & $<5$ & $\mathrm{U}$ & $<E Q L$ & 1 & $\mathrm{ug} / \mathrm{L}$ & EX \\
\hline & PCB 1016 & & & & & & & & & & & & \\
\hline & PCB 1221 & & & & & & & & & & & & \\
\hline & PCB 1232 & & & & & & & & & & & & \\
\hline & РСВ 1242 & & & & & & & & & & & & \\
\hline & PCB 1248 & & & & & & & & & & & & \\
\hline & PCB 1254 & & & & & & & & & & & & \\
\hline & PCB 1260 & & & & & & & & & & & & \\
\hline & 1,1,2,2-Tetrachloroethane & $<5$ & U & $<5$ & $U$ & $<5$ & $U$ & $<5$ & $\mathrm{U}$ & $<\mathrm{EQL}$ & 1 & $\mathrm{ug} / \mathrm{L}$ & EX \\
\hline & Tetrachloroethylene & $<5$ & $\mathrm{U}$ & $<5$ & $U$ & $<5$ & $U$ & $<5$ & U & $<\mathrm{EQL}$ & 1 & $\mathrm{ug} / \mathrm{L}$ & EX \\
\hline & 1,1,1-Trichloroethane & $<5$ & $U$ & $<5$ & $U$ & $<5$ & U & $<5$ & U & $<E Q L$ & 1 & $\mathrm{ug} / \mathrm{L}$ & EX \\
\hline & Trichloroethylene & $<5$ & $\mathrm{U}$ & $<5$ & $U$ & $<5$ & $\mathrm{U}$ & $<5$ & $\mathrm{U}$ & $<E Q L$ & 1 & $\mathrm{ug} / \mathrm{L}$ & EX \\
\hline \multicolumn{14}{|c|}{ II. Monitoring Constituents } \\
\hline$\underline{\text { ST }}$ & Parameter & 3096 & CLP EPA & 3097 & CLPEEPA & $\underline{3 Q 98}$ & CLPEPA & $\underline{3099}$ & CLPEPA & Filt. & DF & Unit & $\underline{\text { Lab }}$ \\
\hline \multicolumn{14}{|c|}{ Inorganics } \\
\hline & Aluminum, total recoverable & $<22.4$ & $\mathrm{U}$ & 74.7 & & $<146$ & U & $<146$ & U & $<\mathrm{EQL}$ & 1 & $\mathrm{ug} / \mathrm{L}$ & WA \\
\hline & Nitrate-nitrite as nitrogen & 420 & & 116 & & 68 & & 71 & & $<2400$ & 1 & $\mathrm{ug} / \mathrm{L}$ & WA \\
\hline+ & Sodium, total recoverable & 11000 & & 10000 & & 9440 & & 9160 & & $>4600$ & 1 & $\mathrm{ug} / \mathrm{L}$ & WA \\
\hline+ & Sulfate & 8760 & & 7700 & & 6540 & & 6160 & & $>3000$ & 1 & $\mathrm{ug} / \mathrm{L}$ & WA \\
\hline \multicolumn{14}{|c|}{ Radionuclides } \\
\hline & Gross alpha & .493 & UI & -.12 & UI & 1.43 & & .425 & $U$ & $<\mathrm{EQL}$ & 1 & $\mathrm{pCi} / \mathrm{L}$ & GP \\
\hline & Nonvolatile beta & .259 & UI & .04 & UI & 1.07 & UI & .756 & $U$ & $<E Q L$ & 1 & $\mathrm{pCi} / \mathrm{L}$ & GP \\
\hline & Radium, total alpha-emitting & -.1 & UI & 4.91 & UIJ & .14 & UI & -.1 & $\mathrm{U}$ & $<\mathrm{EQL}$ & 1 & $\mathrm{pCi} / \mathrm{L}$ & GP \\
\hline
\end{tabular}

Notes: Concentrations in bold italics exceed the groundwater protection or monitoring constituent standards listed in Appendix A. Synchronous water levels are measured over a 3-5 day period or less. Dilution factors, Laboratory, and Filtered Data are for Groundwater Protection Standards First Quarter 2000 and Monitoring Constituents Third Quarter 1999 data only.

$+=$ exceeded the Groundwater Protection Standards (First Quarter 2000) or Monitoring Constituents (Third Quarter 1999) listed in Appendix A. 
Table D-4. Groundwater Monitoring Results for Plume Definition Wells, M-Area HWMF (Cont.) WELL AOB 1

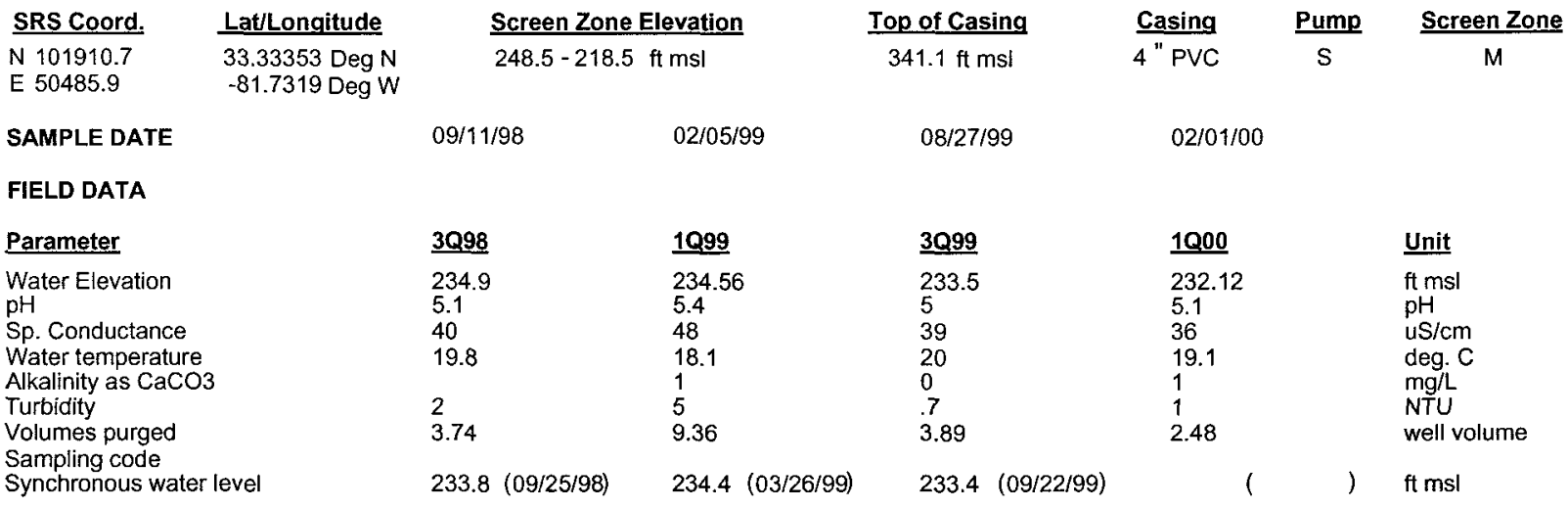

ANALYTICAL DATA

I. Groundwater Protection Standard

261 Appendix VIII/264 Appendix IX Hazardous Constituents

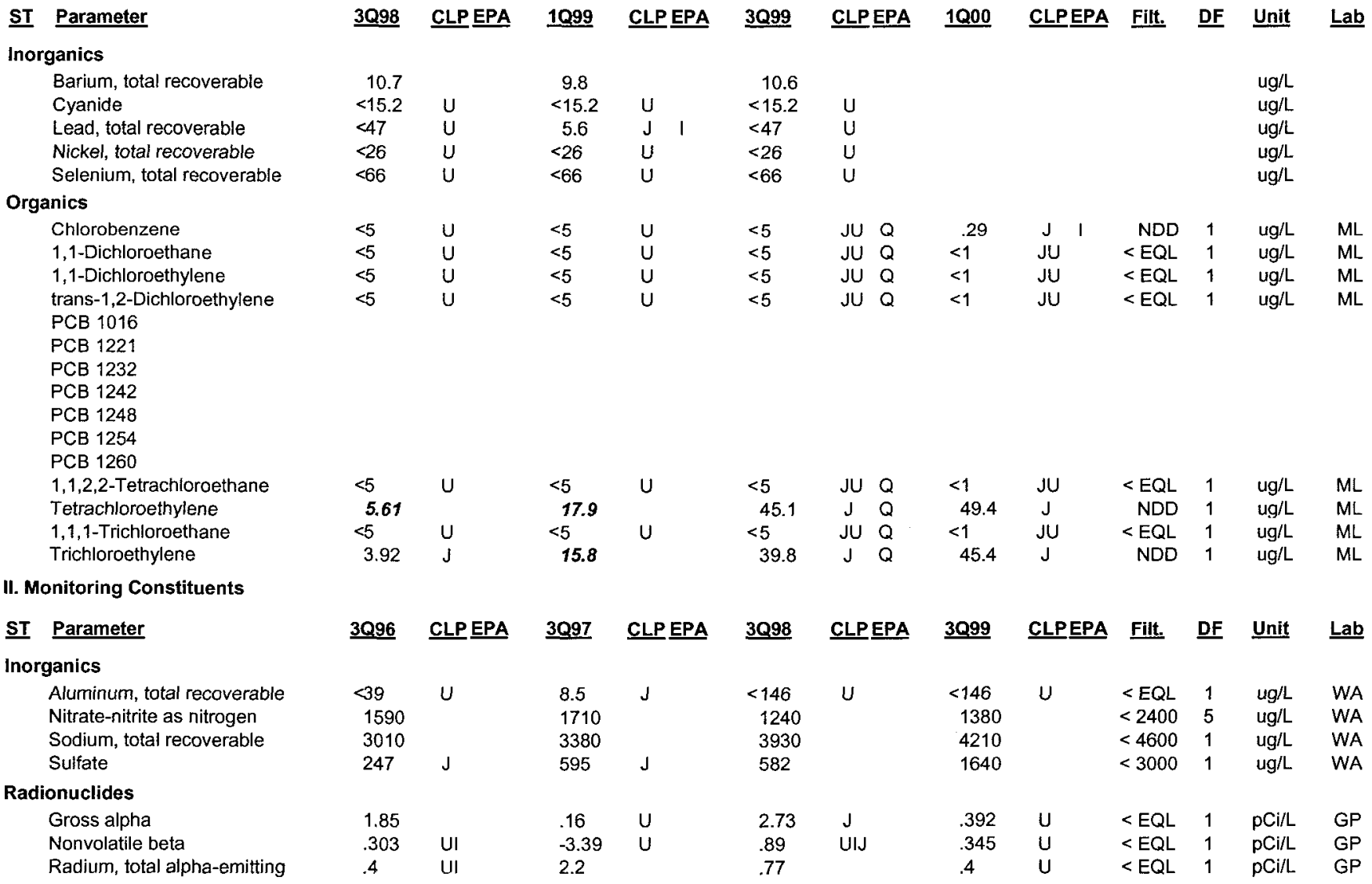

\footnotetext{
Notes: Concentrations in bold italics exceed the groundwater protection or monitoring constituent standards listed in Appendix A. Synchronous wate levels are measured over a 3-5 day period or less. Dilution factors, Laboratory, and Filtered Data are for Groundwater Protection Standards First Quarter 2000 and Monitoring Constituents Third Quarter 1999 data only.

$+=$ exceeded the Groundwater Protection Standards (First Quarter 2000) or Monitoring Constituents (Third Quarter 1999) listed in Appendix A.
} 
Table D-4. Groundwater Monitoring Results for Plume Definition Wells, M-Area HWMF (Cont.) WELL AOB 2

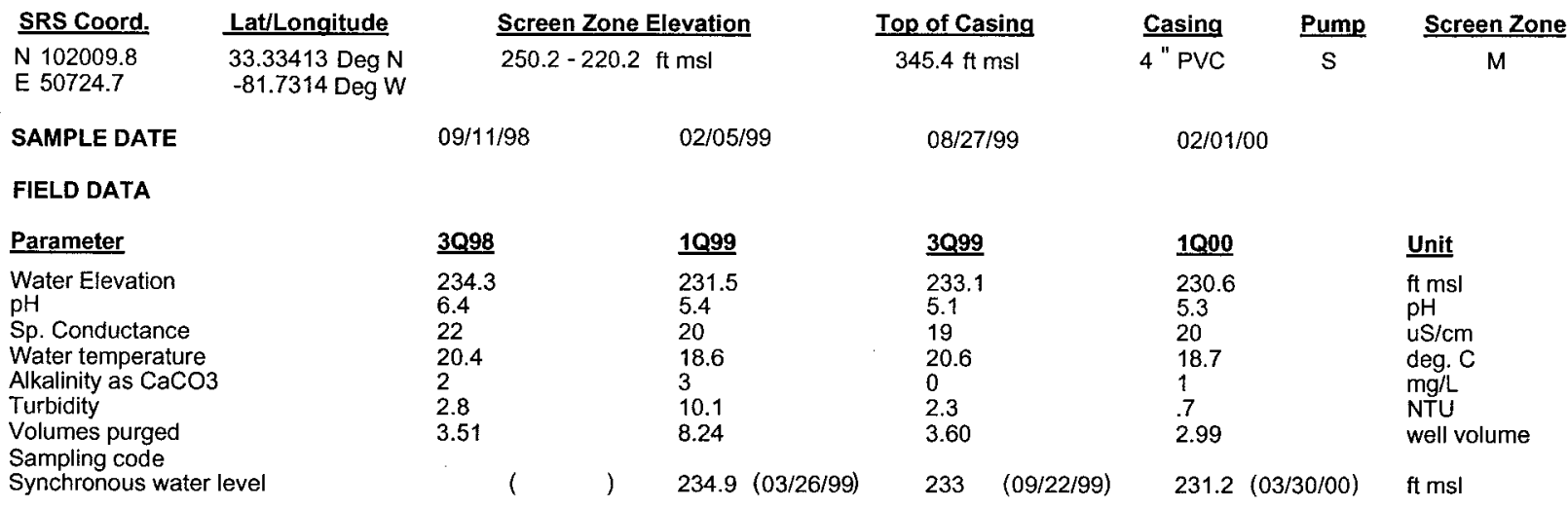

ANALYTICAL DATA

I. Groundwater Protection Standard

261 Appendix VIII/264 Appendix IX Hazardous Constituents

\begin{tabular}{|c|c|c|c|c|c|c|c|c|c|c|c|c|c|c|c|}
\hline \multirow{2}{*}{\multicolumn{2}{|c|}{$\begin{array}{l}\text { ST Parameter } \\
\text { Inorganics }\end{array}$}} & \multirow[t]{2}{*}{$\underline{3 Q 98}$} & \multirow[t]{2}{*}{ CLP EPA } & \multirow[t]{2}{*}{1099} & \multicolumn{2}{|c|}{$\underline{\text { CLP EPA }}$} & \multirow[t]{2}{*}{$\underline{3099}$} & \multicolumn{2}{|c|}{ CLPEPA } & \multirow[t]{2}{*}{$\underline{1000}$} & CLPEPA & \multirow[t]{2}{*}{ Filt. } & \multirow[t]{2}{*}{ DF } & \multirow[t]{2}{*}{ Unit } & \multirow[t]{2}{*}{$\underline{L a b}$} \\
\hline & & & & & & & & & & & & & & & \\
\hline & Barium, total recoverable & 3.9 & & 3.6 & & & $<3.3$ & $\mathrm{U}$ & V & & & & & $\mathrm{ug} / \mathrm{L}$ & \\
\hline & Cyanide & $<15.2$ & $\mathrm{U}$ & $<15.2$ & $\mathrm{U}$ & & $<15.2$ & $\mathrm{U}$ & & & & & & $\mathrm{ug} / \mathrm{L}$ & \\
\hline & Lead, total recoverable & $<47$ & $U$ & $<47$ & $U$ & & $<47$ & $\mathrm{U}$ & & & & & & $\mathrm{ug} / \mathrm{L}$ & \\
\hline & Nickel, total recoverable & $<26$ & $\mathrm{U}$ & 18.5 & $\mathbf{J}$ & I & 5.4 & J & I & & & & & $\mathrm{ug} / \mathrm{L}$ & \\
\hline & Selenium, total recoverable & $<66$ & $\mathrm{U}$ & $<66$ & $U$ & & $<66$ & $\mathrm{U}$ & & & & & & $\mathrm{ug} / \mathrm{L}$ & \\
\hline \multicolumn{16}{|c|}{ Organics } \\
\hline & Chlorobenzene & $<5$ & $U$ & $<5$ & JU & $Q$ & $<5$ & JU & $Q$ & $<1$ & JU & $<\mathrm{EQL}$ & 1 & $\mathrm{ug} / \mathrm{L}$ & ML \\
\hline & 1,1-Dichloroethane & $<5$ & U & $<5$ & JU & $Q$ & $<5$ & JU & $\mathrm{Q}$ & $<1$ & JU & $<\mathrm{EQL}$ & 1 & $\mathrm{ug} / \mathrm{L}$ & ML \\
\hline & 1,1-Dichloroethylene & $<5$ & $U$ & $<5$ & JU & $Q$ & $<5$ & JU & $Q$ & $<1$ & JU & $<$ EQL & 1 & $\mathrm{ug} / \mathrm{L}$ & $M L$ \\
\hline & $\begin{array}{l}\text { trans-1,2-Dichloroethylene } \\
\text { PCB } 1016\end{array}$ & $<5$ & $\mathrm{U}$ & $<5$ & JU & $Q$ & $<5$ & JU & $Q$ & $<1$ & JU & $<\mathrm{EQL}$ & 1 & $\mathrm{ug} / \mathrm{L}$ & $M L$ \\
\hline & PCB 1221 & & & & & & & & & & & & & & \\
\hline & PCB 1232 & & & & & & & & & & & & & & \\
\hline & PCB 1242 & & & & & & & & & & & & & & \\
\hline & PCB 1248 & & & & & & & & & & & & & & \\
\hline & PCB 1254 & & & & & & & & & & & & & & \\
\hline & PCB 1260 & & & & & & & & & & & & & & \\
\hline & $1,1,2,2-$ Tetrachloroethane & $<5$ & U & $<5$ & JU & $Q$ & $<5$ & JU & $Q$ & $<1$ & JU & $<E Q L$ & 1 & $\mathrm{ug} / \mathrm{L}$ & $M L$ \\
\hline & Tetrachloroethylene & $<5$ & $\mathrm{U}$ & $<5$ & JU & $Q$ & $<5$ & JU & $Q$ & $<1$ & JU & $<$ EQL & 1 & $\mathrm{ug} / \mathrm{L}$ & $\mathrm{ML}$ \\
\hline & 1,1,1-Trichloroethane & $<5$ & $\mathrm{U}$ & $<5$ & JU & $Q$ & $<5$ & JU & $Q$ & $<1$ & JU & $<E Q L$ & 1 & $\mathrm{ug} / \mathrm{L}$ & ML \\
\hline & Trichloroethylene & $<5$ & $\mathrm{U}$ & $<5$ & JU & $Q$ & $<5$ & $\mathrm{JU}$ & $Q$ & $<1$ & $\mathrm{JU}$ & $<E Q L$ & 1 & $\mathrm{ug} / \mathrm{L}$ & ML \\
\hline \multicolumn{16}{|c|}{ II. Monitoring Constituents } \\
\hline$\underline{\mathbf{S T}}$ & Parameter & $\underline{3096}$ & CLPEPA & $\underline{3097}$ & $\underline{\text { CLP }}$ & EPA & $\underline{3098}$ & CLP & EPA & $\underline{3099}$ & CLPEPA & Filt. & $\underline{\text { DF }}$ & Unit & Lab \\
\hline \multicolumn{16}{|c|}{ Inorganics } \\
\hline & Aluminum, total recoverable & $<39.8$ & $U$ & & & & $<146$ & $U$ & & 18.5 & J I & NDD & 1 & $u g / L$ & WA \\
\hline & Nitrate-nitrite as nitrogen & 1240 & & & & & 1220 & & & 922 & & $<2400$ & 1 & $u g / L$ & WA \\
\hline & Sodium, total recoverable & 2710 & & & & & 1840 & & & 2410 & & $<4600$ & 1 & $u g / L$ & WA \\
\hline & Sulfate & 439 & J & & & & 348 & & & 515 & & $<3000$ & 1 & $u g / L$ & WA \\
\hline \multicolumn{16}{|c|}{ Radionuclides } \\
\hline & Gross alpha & .842 & & & & & 2.21 & $J$ & & .554 & $\mathrm{U}$ & $<E Q L$ & 1 & $\mathrm{pCi} / \mathrm{L}$ & GP \\
\hline & Nonvolatile beta & 1.39 & UI & & & & 1.87 & $J$ & & .315 & $\mathrm{U}$ & $<$ EQL & 1 & $\mathrm{pCi} / \mathrm{L}$ & GP \\
\hline & Radium, total alpha-emitting & .5 & & & & & .71 & & & .3 & U & $<E Q L$ & 1 & $\mathrm{pCi} / \mathrm{L}$ & GP \\
\hline
\end{tabular}

Notes: Concentrations in bold italics exceed the groundwater protection or monitoring constituent standards listed in Appendix A. Synchronous water levels are measured over a 3-5 day period or less. Dilution factors, Laboratory, and Filtered Data are for Groundwater Protection Standards First Quarter 2000 and Monitoring Constituents Third Quarter 1999 data only.

+= exceeded the Groundwater Protection Standards (First Quarter 2000) or Monitoring Constituents (Third Quarter 1999) listed in Appendix A. 
Table D-4. Groundwater Monitoring Results for Plume Definition Wells, M-Area HWMF (Cont.) WELL ASB 1A

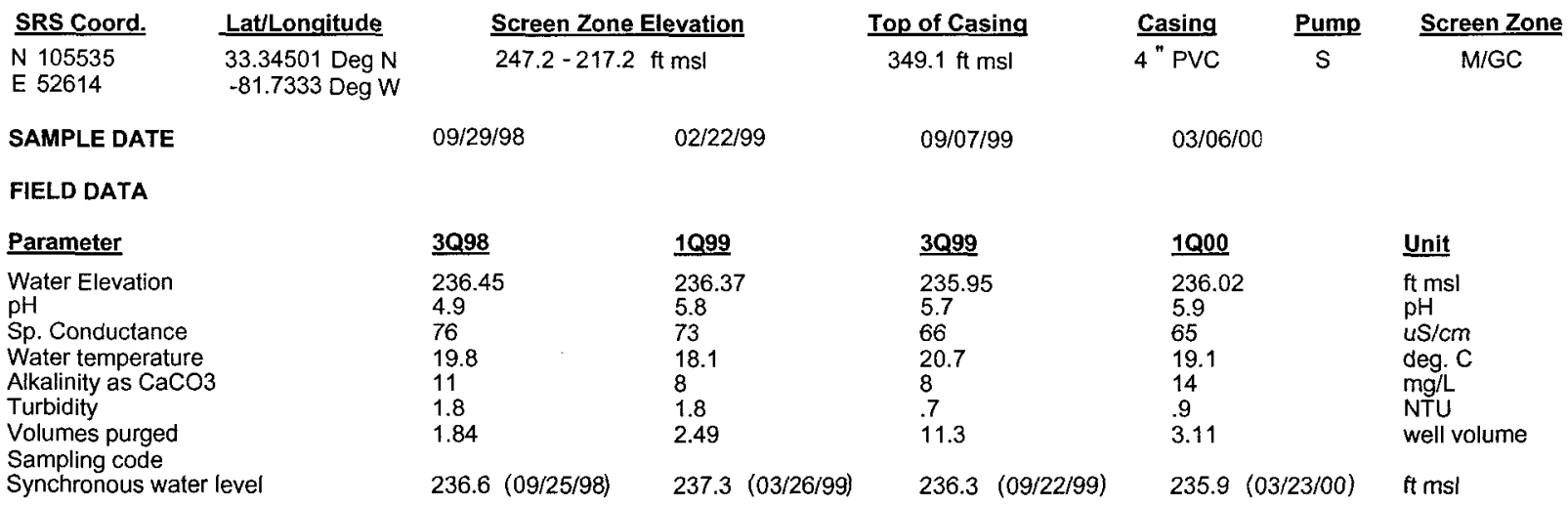

ANALYTICAL DATA

I. Groundwater Protection Standard

261 Appendix VIII/264 Appendix IX Hazardous Constituents

\begin{tabular}{|c|c|c|c|c|c|c|c|c|c|c|c|c|c|}
\hline$\underline{\text { ST }}$ & Parameter & $\underline{3 Q 98}$ & CLPPEPA & $\underline{1 Q 99}$ & CLPEPA & $\underline{3 Q 99}$ & CLPEPA & $\underline{1000}$ & CLPEEA & Filt. & DF & $\underline{\text { Unit }}$ & $\underline{L a b}$ \\
\hline \multicolumn{14}{|c|}{ Inorganics } \\
\hline & Barium, total recoverable & 6 & & 7.6 & & 4.7 & & & & & & $\mathrm{ug} / \mathrm{L}$ & \\
\hline & Cyanide & $<15.2$ & UJ & 72.3 & & $<15.2$ & $\mathrm{U}$ & & & & & ug/L & \\
\hline & Lead, total recoverable & $<47$ & $\mathrm{U}$ & $<47$ & $\mathrm{U}$ & $<47$ & $\mathrm{U}$ & & & & & $\mathrm{ug} / \mathrm{L}$ & \\
\hline & Nickel, total recoverable & $<26$ & $U$ & $<26$ & $\mathrm{U}$ & $<26$ & $\mathrm{U}$ & & & & & $\mathrm{ug} / \mathrm{L}$ & \\
\hline & Selenium, total recoverable & $<66$ & $U$ & $<66$ & $U$ & $<66$ & $\mathrm{U}$ & & & & & $\mathrm{ug} / \mathrm{L}$ & \\
\hline \multicolumn{14}{|c|}{ Organics } \\
\hline & Chlorobenzene & $<5$ & $u$ & $<5$ & $u$ & $<5$ & $u$ & $<1$ & JU & $<E Q L$ & 1 & $u g / L$ & ML \\
\hline & 1,1-Dichloroethane & $<5$ & U & $<5$ & $U$ & $<5$ & U & $<1$ & JU & $<$ EQL & 1 & $u g / L$ & ML \\
\hline & 1,1-Dichloroethylene & $<5$ & $\mathrm{U}$ & $<5$ & $\mathrm{U}$ & $<5$ & U & $<1$ & JU & $<E Q L$ & 1 & $\mathrm{ug} / \mathrm{L}$ & $M L$ \\
\hline & trans-1,2-Dichloroethylene & $<5$ & $\mathrm{U}$ & $<5$ & $\mathrm{U}$ & $<5$ & $\mathrm{U}$ & $<1$ & JU & $<E Q L$ & 1 & $\mathrm{ug} / \mathrm{L}$ & ML \\
\hline & PCB 1016 & & & & & & & & & & & & \\
\hline & PCB 1221 & & & & & & & & & & & & \\
\hline & PCB 1232 & & & & & & & & & & & & \\
\hline & PCB 1242 & & & & & & & & & & & & \\
\hline & РСВ 1248 & & & & & & & & & & & & \\
\hline & PCB 1254 & & & & & & & & & & & & \\
\hline & PCB 1260 & & & & & & & & & & & & \\
\hline & $1,1,2,2$-Tetrachloroethane & $<5$ & $\mathrm{U}$ & $<5$ & $\mathrm{U}$ & $<5$ & $\mathrm{U}$ & $<1$ & JU & $<E Q L$ & 1 & $u g / L$ & $M L$ \\
\hline & Tetrachloroethylene & 2.12 & $J$ & $<5$ & $U$ & 1.53 & $\mathrm{~J} \quad 1$ & 1.15 & $\mathrm{~J}$ & NDD & 1 & $\mathrm{ug} / \mathrm{L}$ & $M L$ \\
\hline & 1,1,1-Trichloroethane & $<5$ & $U$ & $<5$ & $\mathrm{U}$ & $<5$ & $\mathrm{U}$ & $<1$ & JU & $<\mathrm{EQL}$ & 1 & $\mathrm{ug} / \mathrm{L}$ & ML \\
\hline & Trichloroethylene & 1.21 & $\mathrm{~J}$ & $<5$ & U & 1.13 & J 1 & 1.43 & $J$ & NDD & 1 & ug/L & ML \\
\hline \multicolumn{14}{|c|}{ II. Monitoring Constituents } \\
\hline$\underline{\text { ST }}$ & Parameter & $\underline{3096}$ & CLP EPA & $\underline{3 Q 97}$ & CLP EPA & $\underline{3 Q 98}$ & CLPEPA & $\underline{3 Q 99}$ & CLPEPA & Filt. & $\underline{\text { DF }}$ & Unit & $\underline{\underline{L a b}}$ \\
\hline \multicolumn{14}{|c|}{ Inorganics } \\
\hline & Aluminum, total recoverable & $<34.9$ & $U$ & 28.4 & & $<146$ & $u$ & $<146$ & $U$ & $<$ EQL & 1 & $u g / L$ & WA \\
\hline & Nitrate-nitrite as nitrogen & 680 & & 645 & & 962 & & 1390 & & $<2400$ & 5 & $\mathrm{ug} / \mathrm{L}$ & WA \\
\hline+ & Sodium, total recoverable & 12800 & & 12100 & & 12000 & & 12000 & & $>4600$ & 1 & $\mathrm{ug} / \mathrm{L}$ & WA \\
\hline+ & Sulfate & 5970 & & 7490 & & 4960 & & 6800 & & $>3000$ & 1 & ug/L & WA \\
\hline \multicolumn{14}{|c|}{ Radionuclides } \\
\hline & Gross alpha & .679 & UI & -.55 & UIJ & 2.09 & & .643 & $u$ & $<E Q L$ & 1 & $\mathrm{pCi} / \mathrm{L}$ & GP \\
\hline & Nonvolatile beta & 2.88 & & -2.08 & UIJ & 4.3 & & 1.15 & J 1 & NDD & 1 & $\mathrm{pCi} / \mathrm{L}$ & GP \\
\hline & Radium, total alpha-emitting & 0 & UI & 1.01 & & 1.03 & & .5 & $\mathrm{~J} i$ & NDD & 1 & $\mathrm{pCi} / \mathrm{L}$ & GP \\
\hline
\end{tabular}

Notes: Concentrations in bold italics exceed the groundwater protection or monitoring constituent standards listed in Appendix A. Synchronous water levels are measured over a 3-5 day period or less. Dilution factors, Laboratory, and Filtered Data are for Groundwater Protection Standards First Quarter 2000 and Monitoring Constituents Third Quarter 1999 data only.

$+=$ exceeded the Groundwater Protection Standards (First Quarter 2000) or Monitoring Constituents (Third Quarter 1999) listed in Appendix A. 
Table D-4. Groundwater Monitoring Results for Plume Definition Wells, M-Area HWMF (Cont.) WELL ASB 2AR

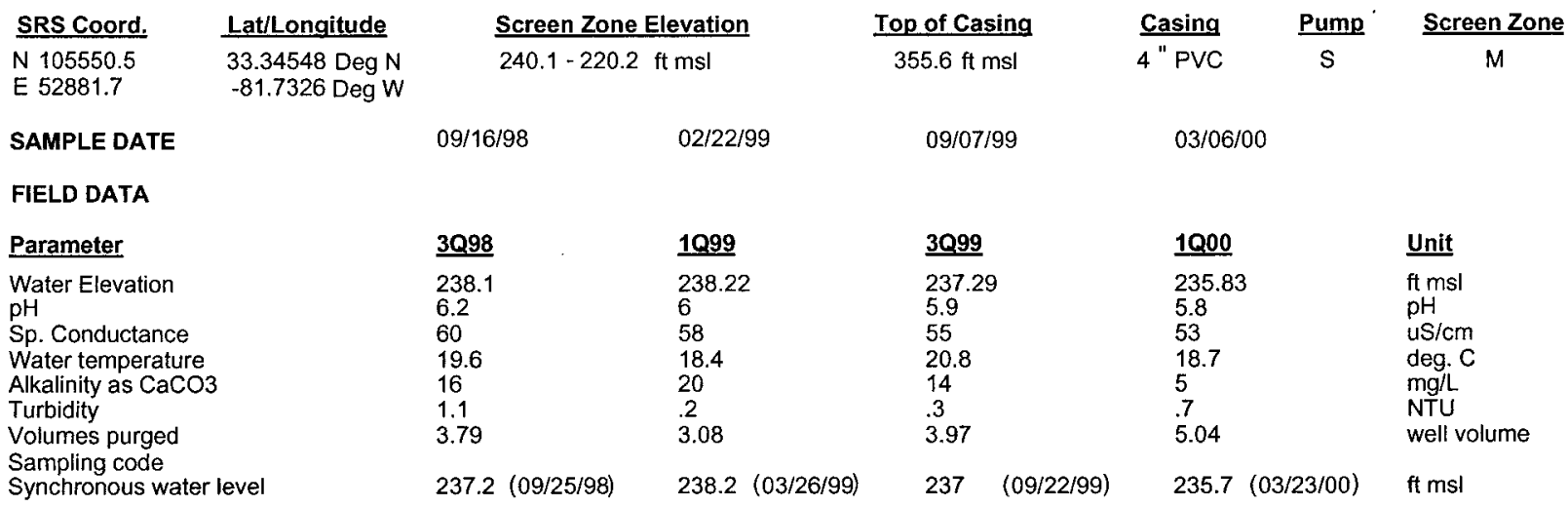

ANALYTICAL DATA

I. Groundwater Protection Standard

261 Appendix VIII/264 Appendix IX Hazardous Constituents

\begin{tabular}{|c|c|c|c|c|c|c|c|c|c|c|c|c|c|}
\hline$\underline{\text { ST }}$ & Parameter & $\underline{3 Q 98}$ & CLP EPA & 1099 & $\underline{\text { CLP EPA }}$ & $\underline{3 Q 99}$ & CLPEPA & 1000 & CLPEPA & Filt. & $\underline{\mathrm{DF}}$ & Unit & $\underline{\text { Lab }}$ \\
\hline \multicolumn{14}{|c|}{ Inorganics } \\
\hline & Barium, total recoverable & 7 & & 7 & & 7 & & & & & & $\mathrm{ug} / \mathrm{L}$ & \\
\hline & Cyanide & $<15.2$ & $\mathrm{U}$ & $<15.2$ & $\mathrm{U}$ & $<15.2$ & $\mathrm{U}$ & & & & & ug/L & \\
\hline & Lead, total recoverable & $<47$ & $\mathrm{U}$ & $<47$ & $\mathrm{U}$ & $<47$ & $\mathrm{U}$ & & & & & $\mathrm{ug} / \mathrm{L}$ & \\
\hline & Nickel, total recoverable & $<26$ & $\mathrm{u}$ & $<26$ & $\mathrm{U}$ & 2.7 & J 1 & & & & & $\mathrm{ug} / \mathrm{L}$ & \\
\hline & Selenium, total recoverable & $<66$ & $\mathrm{U}$ & $<66$ & $\mathrm{U}$ & $<66$ & $\mathrm{U}$ & & & & & $\mathrm{ug} / \mathrm{L}$ & \\
\hline \multicolumn{14}{|c|}{ Organics } \\
\hline & Chlorobenzene & $<5$ & U & $<5$ & $\mathrm{U}$ & $<5$ & $\mathrm{U}$ & $<1$ & JU & $<E Q L$ & 1 & ug/L & $M L$ \\
\hline & 1,1-Dichloroethane & $<5$ & $\mathrm{U}$ & $<5$ & $\mathrm{U}$ & $<5$ & $\mathrm{U}$ & $<1$ & JU & $<E Q L$ & 1 & $\mathrm{ug} / \mathrm{L}$ & $M L$ \\
\hline & 1,1-Dichloroethylene & $<5$ & $\mathrm{U}$ & $<5$ & $U$ & $<5$ & $\mathrm{U}$ & $<1$ & JU & $<\mathrm{EQL}$ & 1 & $\mathrm{ug} / \mathrm{L}$ & ML \\
\hline & $\begin{array}{l}\text { trans-1,2-Dichloroethylene } \\
\text { PCB } 1016\end{array}$ & $<5$ & $\mathrm{U}$ & $<5$ & $\mathrm{U}$ & $<5$ & $\mathrm{U}$ & $<1$ & JU & $<E Q L$ & 1 & ug/L & ML \\
\hline & PCB 1221 & & & & & & & & & & & & \\
\hline & PCB 1232 & & & & & & & & & & & & \\
\hline & PCB 1242 & & & & & & & & & & & & \\
\hline & PCB 1248 & & & & & & & & & & & & \\
\hline & PCB 1254 & & & & & & & & & & & & \\
\hline & PCB 1260 & & & & & & & & & & & & \\
\hline & 1,1,2,2-Tetrachloroethane & $<5$ & $U$ & $<5$ & $\mathrm{U}$ & $<5$ & $\mathrm{U}$ & $<1$ & JU & $<E Q L$ & 1 & $\mathrm{ug} / \mathrm{L}$ & ML \\
\hline & Tetrachloroethylene & $<5$ & $U$ & $<5$ & $\mathrm{U}$ & $<5$ & $\mathrm{U}$ & $<1$ & JU & $<E Q L$ & 1 & $\mathrm{ug} / \mathrm{L}$ & ML \\
\hline & $1,1,1$-Trichloroethane & $<5$ & $\mathrm{U}$ & $<5$ & $\mathrm{U}$ & $<5$ & $\mathrm{U}$ & $<1$ & JU & $<E Q L$ & 1 & $\mathrm{ug} / \mathrm{L}$ & ML \\
\hline & Trichloroethylene & $<5$ & $\mathrm{U}$ & 1.68 & $\mathrm{~J} \quad \mathrm{I}$ & $<5$ & $U$ & $<1$ & JU & $<\mathrm{EQL}$ & 1 & $\mathrm{ug} / \mathrm{L}$ & $M L$ \\
\hline \multicolumn{14}{|c|}{ II. Monitoring Constituents } \\
\hline$\underline{\mathbf{S T}}$ & Parameter & $\underline{3096}$ & CLP EPA & $\underline{3097}$ & CLP EPA & $\underline{3098}$ & CLPEPA & 3099 & CLPEPA & Filt. & $\underline{\text { DF }}$ & Unit & $\underline{\text { Lab }}$ \\
\hline \multicolumn{14}{|c|}{ Inorganics } \\
\hline & Aluminum, total recoverable & $<31.9$ & $U$ & 46.5 & & $<146$ & U & $<146$ & $U$ & $<\mathrm{EQL}$ & 1 & $u g / L$ & WA \\
\hline & Nitrate-nitrite as nitrogen & 460 & & 249 & & 400 & & 489 & & $<2400$ & 1 & $\mathrm{ug} / \mathrm{L}$ & WA \\
\hline+ & Sodium, total recoverable & 12300 & & 11600 & & 11300 & & 10100 & & $>4600$ & 1 & $\mathrm{ug} / \mathrm{L}$ & WA \\
\hline+ & Sulfate & 4870 & & 4980 & $J$ & 3840 & & 3280 & & $>3000$ & 1 & $\mathrm{ug} / \mathrm{L}$ & WA \\
\hline \multicolumn{14}{|c|}{ Radionuclides } \\
\hline & Gross alpha & 1.21 & & .81 & Ui & 3.9 & & 2.59 & J 1 & NDD & 1 & $\mathrm{pCi} / \mathrm{L}$ & GP \\
\hline & Nonvolatile beta & 3.04 & & -.25 & UI & 127.85 & $J$ & 1.36 & J 1 & NDD & 1 & $\mathrm{pCi} / \mathrm{L}$ & GP \\
\hline & Radium, total alpha-emitting & 1.1 & & 1.49 & & .91 & & .7 & J I & NDD & 1 & $\mathrm{pCi} / \mathrm{L}$ & GP \\
\hline
\end{tabular}

Notes: Concentrations in bold italics exceed the groundwater protection or monitoring constituent standards listed in Appendix A. Synchronous water levels are measured over a 3-5 day period or less. Dilution factors, Laboratory, and Filtered Data are for Groundwater Protection Standards First Quarter 2000 and Monitoring Constituents Third Quarter 1999 data only.

+ = exceeded the Groundwater Protection Standards (First Quarter 2000) or Monitoring Constituents (Third Quarter 1999) listed in Appendix A. 
Table D-4. Groundwater Monitoring Results for Plume Definition Wells, M-Area HWMF (Cont.) WELL ASB 2CR

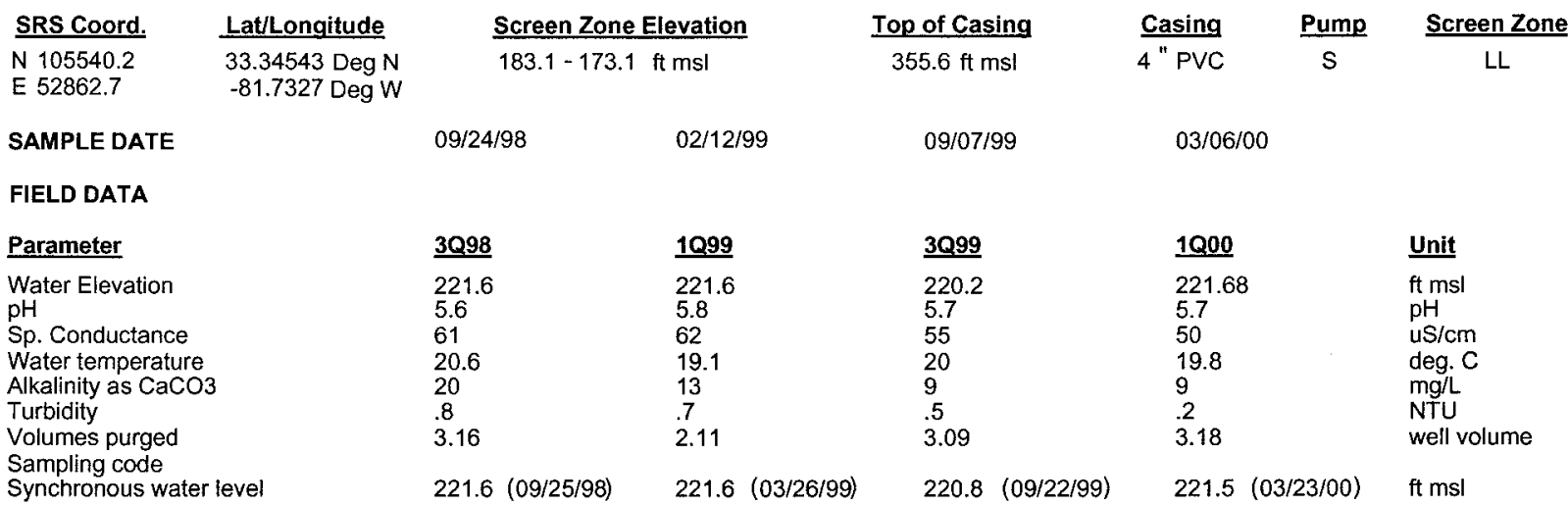

ANALYTICAL DATA

I. Groundwater Protection Standard

261 Appendix VIII/264 Appendix IX Hazardous Constituents

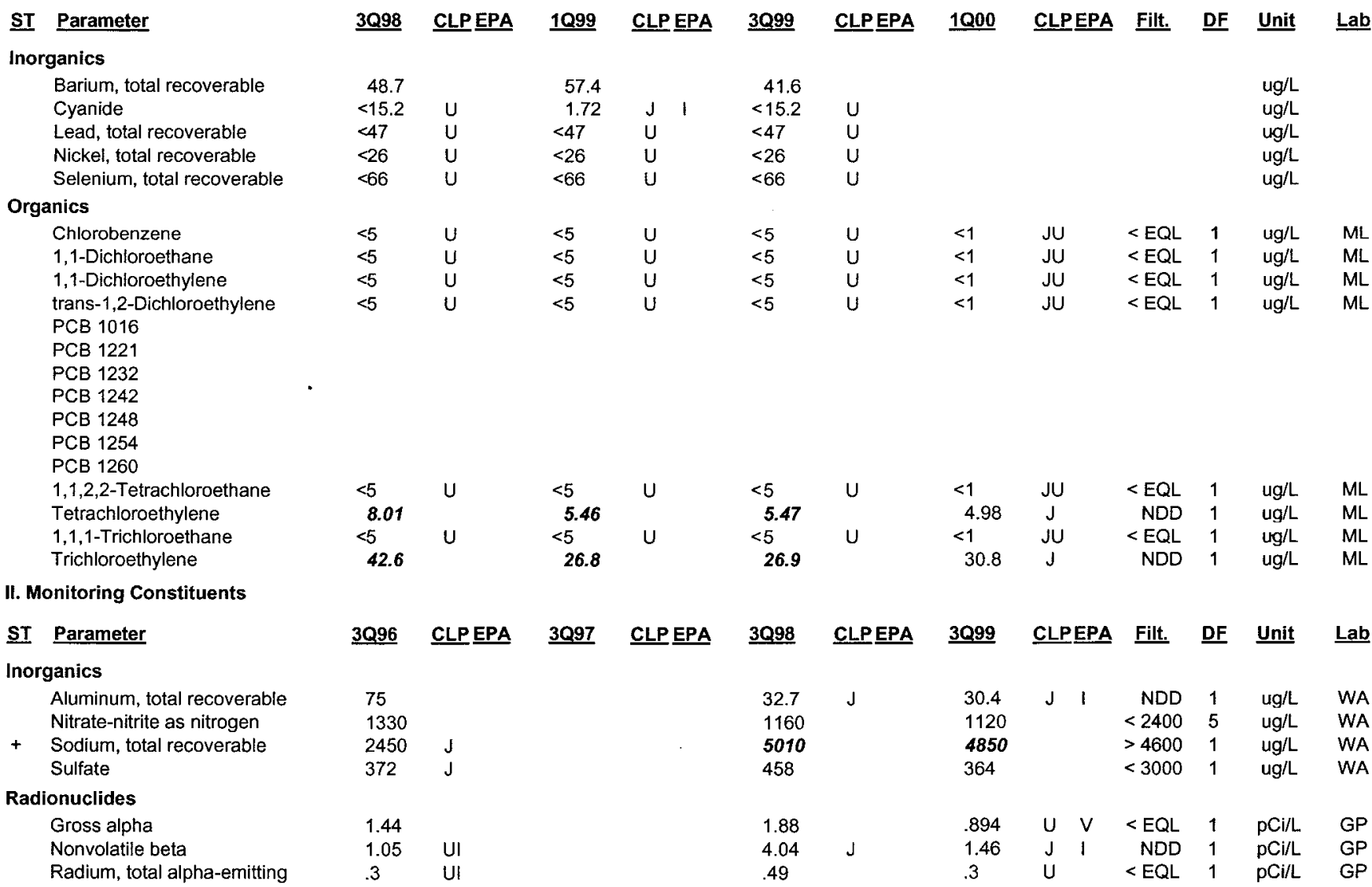

Notes: Concentrations in bold italics exceed the groundwater protection or monitoring constituent standards listed in Appendix A. Synchronous water levels are measured over a 3-5 day period or less. Dilution factors, Laboratory, and Filtered Data are for Groundwater Protection Standards First Quarter 2000 and Monitoring Constituents Third Quarter 1999 data only.

$+=$ exceeded the Groundwater Protection Standards (First Quarter 2000) or Monitoring Constituents (Third Quarter 1999) listed in Appendix A. 
Table D-4. Groundwater Monitoring Results for Plume Definition Wells, M-Area HWMF (Cont.) WELL ASB 3AR

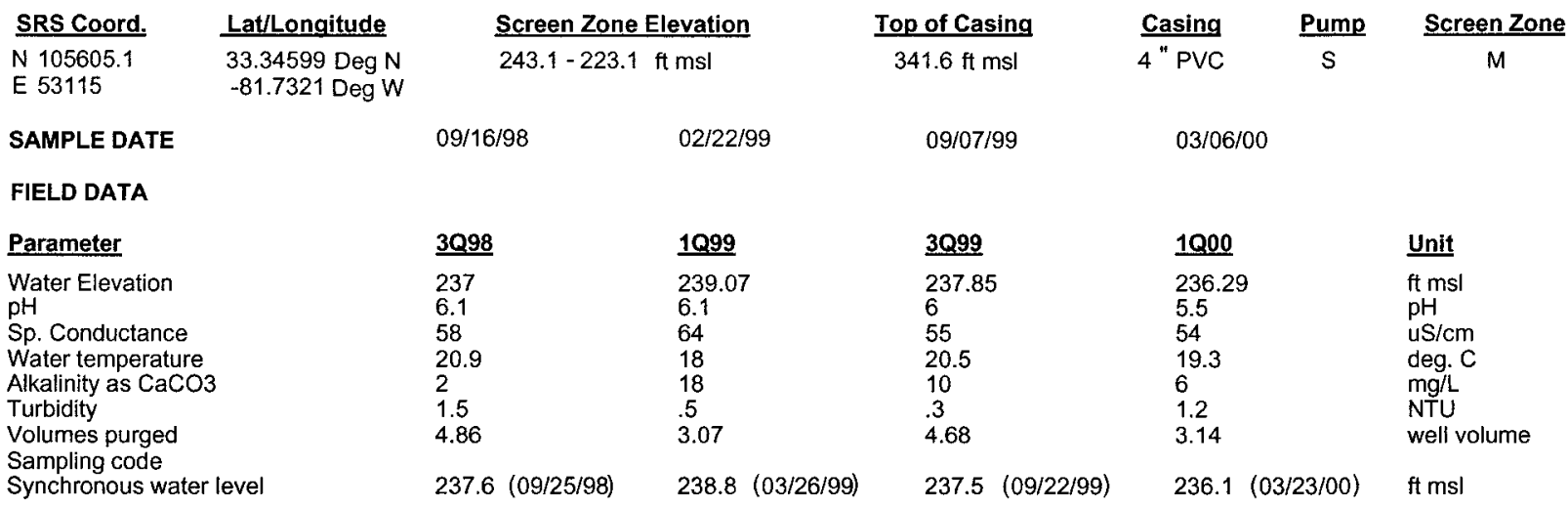

ANALYTICAL DATA

I. Groundwater Protection Standard

261 Appendix VIII/264 Appendix IX Hazardous Constituents

\begin{tabular}{|c|c|c|c|c|c|c|c|c|c|c|c|c|c|c|}
\hline$\underline{\text { ST }}$ & Parameter & $\underline{3 Q 98}$ & CLPEPA & 1099 & $\underline{\text { CLP EPA }}$ & $\underline{3099}$ & CLPEPA & 1000 & \multicolumn{2}{|c|}{ CLPEPA } & Filt. & DF & Unit & Lab \\
\hline \multicolumn{15}{|c|}{ Inorganics } \\
\hline & Barium, total recoverable & 7.1 & & 9.4 & & 9.9 & & & & & & & $\mathrm{ug} / \mathrm{L}$ & \\
\hline & Cyanide & $<15.2$ & $U$ & $<15.2$ & $\mathrm{U}$ & $<15.2$ & $U$ & & & & & & $\mathrm{ug} / \mathrm{L}$ & \\
\hline & Lead, total recoverable & $<47$ & $\mathrm{U}$ & $<47$ & $\mathrm{U}$ & $<47$ & $U$ & & & & & & $\mathrm{ug} / \mathrm{L}$ & \\
\hline & Nickel, total recoverable & $<26$ & $\mathrm{U}$ & $<26$ & $\mathrm{U}$ & $<26$ & $U$ & & & & & & $\mathrm{ug} / \mathrm{L}$ & \\
\hline & Selenium, total recoverable & $<66$ & $U$ & $<66$ & $\mathrm{U}$ & $<66$ & $\mathrm{U}$ & & & & & & $\mathrm{ug} / \mathrm{L}$ & \\
\hline \multicolumn{15}{|c|}{ Organics } \\
\hline & Chlorobenzene & $<5$ & $\mathrm{U}$ & $<5$ & $\mathrm{U}$ & $<5$ & $\mathrm{U}$ & $<1$ & JU & & $<E Q L$ & 1 & $\mathrm{ug} / \mathrm{L}$ & ML \\
\hline & 1,1-Dichloroethane & $<5$ & $\mathrm{U}$ & $<5$ & $\mathrm{U}$ & $<5$ & $\mathrm{U}$ & $<1$ & JU & & $<\mathrm{EQL}$ & 1 & $u g / L$ & ML \\
\hline & 1,1-Dichloroethylene & $<5$ & $\mathrm{U}$ & $<5$ & U & $<5$ & $\mathrm{U}$ & $<1$ & JU & & $<\mathrm{EQL}$ & 1 & ug/L & ML \\
\hline & trans-1,2-Dichloroethylene & $<5$ & $\mathrm{U}$ & $<5$ & $\mathrm{U}$ & $<5$ & $\mathrm{U}$ & $<1$ & JU & & $<\mathrm{EQL}$ & 1 & $u g / L$ & ML \\
\hline & PCB 1016 & & & & & & & & & & & & & \\
\hline & PCB 1221 & & & & & & & & & & & & & \\
\hline & $\begin{array}{l}\text { PCB } 1232 \\
\text { PCB } 1242\end{array}$ & & & & & & & & & & & & & \\
\hline & PCB 1248 & & & & & & & & & & & & & \\
\hline & PCB 1254 & & & & & & & & & & & & & \\
\hline & РCB 1260 & & & & & & & & & & & & & \\
\hline & 1,1,2,2-Tetrachloroethane & $<5$ & $\mathrm{U}$ & $<5$ & $U$ & $<5$ & $\mathrm{U}$ & $<1$ & JU & & $<\mathrm{EQL}$ & 1 & $\mathrm{ug} / \mathrm{L}$ & ML \\
\hline & Tetrachloroethylene & $<5$ & $\mathrm{U}$ & $<5$ & $\mathrm{U}$ & $<5$ & $\mathrm{U}$ & $<1$ & JU & & $<E Q L$ & 1 & $\mathrm{ug} / \mathrm{L}$ & ML \\
\hline & 1,1,1-Trichloroethane & $<5$ & $\mathrm{U}$ & $<5$ & $\mathrm{U}$ & $<5$ & $\mathrm{U}$ & $<1$ & JU & & $<E Q L$ & 1 & $u g / L$ & ML \\
\hline & Trichloroethylene & $<5$ & $\mathrm{U}$ & $<5$ & $\mathrm{U}$ & $<5$ & $\mathbf{U}$ & $<1$ & JU & & $<E Q L$ & 1 & $\mathrm{ug} / \mathrm{L}$ & ML \\
\hline \multicolumn{15}{|c|}{ II. Monitoring Constituents } \\
\hline$\underline{\text { ST }}$ & Parameter & $\underline{3 Q 96}$ & CLP EPA & $\underline{3097}$ & CLPEPA & $\underline{3 Q 98}$ & CLPEPA & 3099 & CLP & EPA & Filt. & $\underline{\text { DF }}$ & Unit & $\underline{\text { Lab }}$ \\
\hline \multicolumn{15}{|c|}{ Inorganics } \\
\hline & Aluminum, total recoverable & $<35$ & $\mathrm{U}$ & 20.4 & & 35.7 & J & 106 & $\mathrm{~J}$ & 1 & NDD & 1 & $\mathrm{ug} / \mathrm{L}$ & WA \\
\hline & Nitrate-nitrite as nitrogen & 460 & & 581 & & 569 & & 894 & & & $<2400$ & 1 & $\mathrm{ug} / \mathrm{L}$ & WA \\
\hline+ & Sodium, total, recoverable & 11300 & & 10900 & & 11400 & & 10700 & & & $>4600$ & 1 & $u g / L$ & WA \\
\hline+ & Sulfate & 5200 & & 4870 & $J$ & 4900 & & 4290 & & & $>3000$ & 1 & $\mathrm{ug} / \mathrm{L}$ & WA \\
\hline \multicolumn{15}{|c|}{ Radionuclides } \\
\hline & Gross alpha & -.107 & UI & .32 & UI & 1.45 & & 1.24 & $\mathrm{U}$ & V & $<\mathrm{EQL}$ & 1 & $\mathrm{pCi} / \mathrm{L}$ & GP \\
\hline & Nonvolatile beta & .889 & UI & -1.13 & UI & 3.78 & 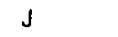 & 1.76 & $\mathrm{~J}$ & I & NDD & 1 & $\mathrm{pCi} / \mathrm{L}$ & GP \\
\hline & Radium, total alpha-emitting & .1 & UI & .9 & & .38 & UI & .5 & $\mathrm{~J}$ & 1 & NDD & 1 & $\mathrm{pCi} / \mathrm{L}$ & GP \\
\hline
\end{tabular}

Notes: Concentrations in bold italics exceed the groundwater protection or monitoring constituent standards listed in Appendix A. Synchronous water levels are measured over a 3-5 day period or less. Dilution factors, Laboratory, and Filtered Data are for Groundwater Protection Standards First Quarter 2000 and Monitoring Constituents Third Quarter 1999 data only.

+ = exceeded the Groundwater Protection Standards (First Quarter 2000) or Monitoring Constituents (Third Quarter 1999) listed in Appendix A. 
Table D-4. Groundwater Monitoring Results for Plume Definition Wells, M-Area HWMF (Cont.) WELL ASB 3CR

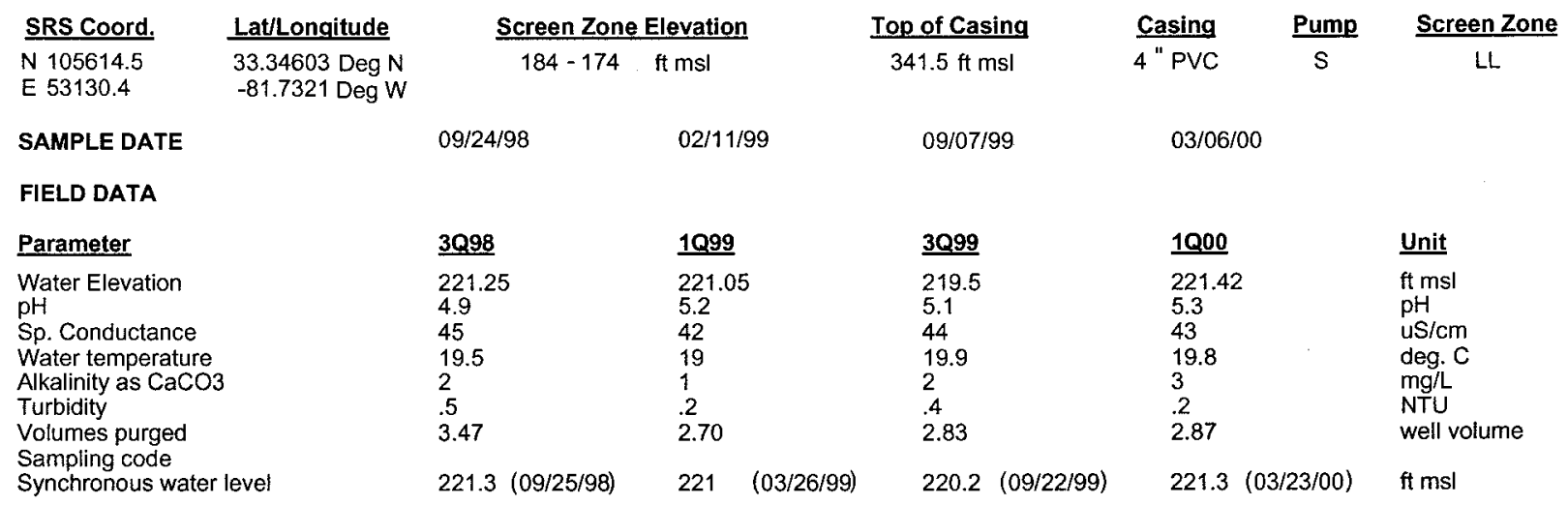

ANALYTICAL DATA

I. Groundwater Protection Standard

261 Appendix VIII/264 Appendix IX Hazardous Constituents

\begin{tabular}{|c|c|c|c|c|c|c|c|c|c|c|c|c|c|}
\hline$\underline{\mathrm{ST}}$ & Parameter & $\underline{3 Q 98}$ & CLP EPA & $\underline{1099}$ & CLP EPA & 3Q99 & CLPEPA & 1000 & CLPEPA & Filt. & DF & Unit & $\underline{\text { Lab }}$ \\
\hline \multicolumn{14}{|c|}{ Inorganics } \\
\hline & Barium, total recoverable & 503 & & 472 & & 419 & & & & & & ug/L & \\
\hline & Cyanide & $<15.2$ & $\mathrm{U}$ & $<15.2$ & $U$ & $<15.2$ & $\mathrm{U}$ & & & & & ug/L & \\
\hline & Lead, total recoverable & $<47$ & $\mathrm{U}$ & $<47$ & $U$ & $<47$ & $U$ & & & & & $\mathrm{ug} / \mathrm{L}$ & \\
\hline & Nickel, total recoverable & $<26$ & $\mathrm{U}$ & $<26$ & $U$ & $<26$ & $\mathrm{U}$ & & & & & ug/L & \\
\hline & Selenium, total recoverable & $<66$ & $\mathrm{U}$ & $<66$ & $\mathrm{U}$ & $<66$ & $\mathrm{U}$ & & & & & $\mathrm{ug} / \mathrm{L}$ & \\
\hline \multicolumn{14}{|c|}{ Organics } \\
\hline & Chlorobenzene & $<5$ & U & $<5$ & U & $<5$ & $U$ & $<1$ & JU & $<\mathrm{EQL}$ & 1 & $\mathrm{ug} / \mathrm{L}$ & $M L$ \\
\hline & 1,1-Dichloroethane & $<5$ & $U$ & $<5$ & U & $<5$ & $U$ & $<1$ & JU & $<\mathrm{EQL}$ & 1 & $\mathrm{ug} / \mathrm{L}$ & $M L$ \\
\hline & 1,1-Dichloroethylene & $<5$ & $U$ & $<5$ & $U$ & $<5$ & $\mathrm{U}$ & $<1$ & JU & $<\mathrm{EQL}$ & 1 & ug/L & $M L$ \\
\hline & trans-1,2-Dichloroethylene & $<5$ & $\mathrm{U}$ & $<5$ & $U$ & $<5$ & $U$ & $<1$ & JU & $<\mathrm{EQL}$ & 1 & ug/L & $M L$ \\
\hline & PCB 1221 & & & & & & & & & & & & \\
\hline & PCB 1232 & & & & & & & & & & & & \\
\hline & РCB 1242 & & & & & & & & & & & & \\
\hline & PCB 1248 & & & & & & & & & & & & \\
\hline & PCB 1254 & & & & & & & & & & & & \\
\hline & PCB 1260 & & & & & & & & & & & & \\
\hline & $1,1,2,2$-Tetrachloroethane & $<5$ & U & $<5$ & U & $<5$ & $U$ & $<1$ & JU & $<\mathrm{EQL}$ & 1 & ug $/ \mathrm{L}$ & ML \\
\hline & Tetrachloroethylene & 6.51 & & $<5$ & U & 3.99 & J 1 & 3.79 & $J$ & NDD & 1 & $\mathrm{ug} / \mathrm{L}$ & ML \\
\hline & $1,1,1-$ Trichloroethane & $<5$ & $u$ & $<5$ & U & $<5$ & $\mathrm{U}$ & $<1$ & JU & $<\mathrm{EQL}$ & 1 & $\mathrm{ug} / \mathrm{L}$ & $M L$ \\
\hline & Trichloroethylene & 20.6 & & 17.4 & & 20.7 & & 15.5 & $J$ & NDD & 1 & $\mathrm{ug} / \mathrm{L}$ & $\mathrm{ML}$ \\
\hline \multicolumn{14}{|c|}{ II. Monitoring Constituents } \\
\hline$\underline{\text { ST }}$ & Parameter & $\underline{3 Q 96}$ & $\underline{\text { CLP EPA }}$ & $\underline{3097}$ & CLP EPA & $\underline{3 Q 98}$ & CLPEPA & $\underline{3099}$ & CLPEPA & Filt. & $\underline{\mathrm{DF}}$ & $\underline{\text { Unit }}$ & Lab \\
\hline \multicolumn{14}{|c|}{ Inorganics } \\
\hline & Aluminum, total recoverable & $<20$ & U & & & 55.6 & $J$ & $<146$ & $U$ & $<\mathrm{EQL}$ & 1 & $\mathrm{ug} / \mathrm{L}$ & WA \\
\hline & Nitrate-nitrite as nitrogen & 740 & & & & 1060 & & 1220 & & $<2400$ & 5 & ug/L & WA \\
\hline & Sodium, total recoverable & 4220 & $\mathrm{~J}$ & & & 4110 & & 4500 & & $<4600$ & 1 & $u g / L$ & WA \\
\hline & Sulfate & 587 & $\mathrm{~J}$ & & & 670 & & 600 & & $<3000$ & 1 & $u g / L$ & WA \\
\hline \multicolumn{14}{|c|}{ Radionuclides } \\
\hline & Gross alpha & .971 & & & & 1.9 & & .95 & $\mathrm{~J} \quad 1$ & NDD & 1 & $\mathrm{pCi} / \mathrm{L}$ & GP \\
\hline & Nonvolatile beta & 1.2 & UI & & & 3.41 & $J$ & 1.79 & J 1 & NDD & 1 & $\mathrm{pCi} / \mathrm{L}$ & GP \\
\hline & Radium, total alpha-emitting & .3 & UI & & & .86 & & .4 & J 1 & NDD & 1 & $\mathrm{pCi} / \mathrm{L}$ & GP \\
\hline
\end{tabular}

Notes: Concentrations in bold italics exceed the groundwater protection or monitoring constituent standards listed in Appendix A. Synchronous water levels are measured over a 3-5 day period or less. Dilution factors, Laboratory, and Filtered Data are for Groundwater Protection Standards First Quarter 2000 and Monitoring Constituents Third Quarter 1999 data only.

$+=$ exceeded the Groundwater Protection Standards (First Quarter 2000) or Monitoring Constituents (Third Quarter 1999) listed in Appendix A. 
Table D-4. Groundwater Monitoring Results for Plume Definition Wells, M-Area HWMF (Cont.) WELL ASB 4

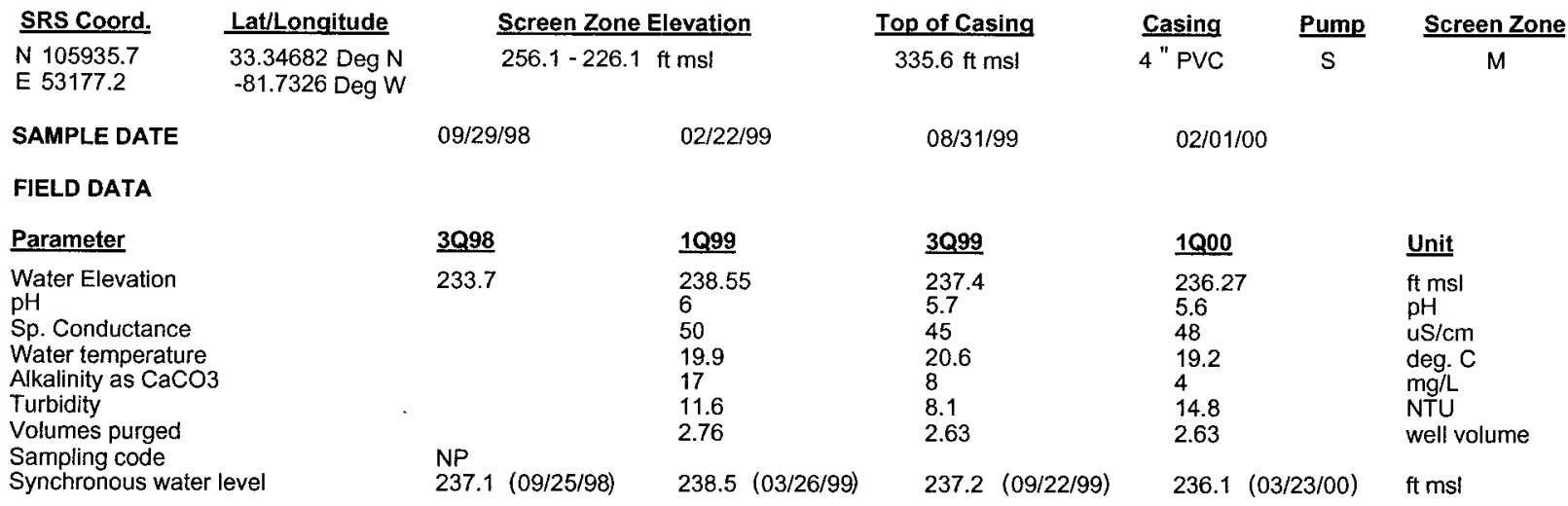

ANALYTICAL DATA

I. Groundwater Protection Standard

261 Appendix VIII/264 Appendix IX Hazardous Constituents

\begin{tabular}{|c|c|c|c|c|c|c|c|c|c|c|c|c|c|c|}
\hline \multirow{2}{*}{\multicolumn{2}{|c|}{$\begin{array}{l}\text { ST Parameter } \\
\text { Inorganics }\end{array}$}} & \multirow[t]{2}{*}{$\underline{3098}$} & \multirow[t]{2}{*}{ CLP EPA } & \multirow[t]{2}{*}{1099} & $\underline{\text { CLP EPA }}$ & \multirow[t]{2}{*}{$\underline{3 Q 99}$} & \multirow[t]{2}{*}{ CLPEPA } & \multirow[t]{2}{*}{10,00} & \multicolumn{2}{|c|}{ CLPEPA } & \multirow[t]{2}{*}{ Filt. } & \multirow[t]{2}{*}{$\underline{\text { DF }}$} & \multirow[t]{2}{*}{$\underline{\text { Unit }}$} & \multirow[t]{2}{*}{$\underline{\text { Lab }}$} \\
\hline & & & & & & & & & & & & & & \\
\hline & Barium, total recoverable & & & 8.3 & & 8.5 & & & & & & & $u g / L$ & \\
\hline & Cyanide & & & $<15.2$ & $U$ & $<15.2$ & $\mathrm{U}$ & & & & & & $\mathrm{ug} / \mathrm{L}$ & \\
\hline & Lead, total recoverable & & & $<47$ & $U$ & $<47$ & $\mathrm{U}$ & & & & & & $\mathrm{ug} / \mathrm{L}$ & \\
\hline & Nickel, total recoverable & & & 2.8 & $\mathrm{~J} \quad \mathrm{I}$ & $<26$ & U & & & & & & $\mathrm{ug} / \mathrm{L}$ & \\
\hline & Selenium, total recoverable & & & $<66$ & $U$ & $<66$ & $\mathrm{U}$ & & & & & & ug/L & \\
\hline \multicolumn{15}{|c|}{ Organics } \\
\hline & Chlorobenzene & & & $<5$ & $U$ & $<5$ & $J U Q$ & $<1$ & JU & & $<\mathrm{EQL}$ & 1 & $\mathrm{ug} / \mathrm{L}$ & $M L$ \\
\hline & 1,1-Dichloroethane & & & $<5$ & $u$ & $<5$ & $J \cup Q$ & $<1$ & JU & & $<\mathrm{EQL}$ & 1 & $\mathrm{ug} / \mathrm{L}$ & ML \\
\hline & 1,1-Dichloroethylene & & & $<5$ & $\mathrm{U}$ & $<5$ & $J \cup Q$ & $<1$ & JU & & $<\mathrm{EQL}$ & 1 & $\mathrm{ug} / \mathrm{L}$ & $M L$ \\
\hline & trans-1,2-Dichloroethylene & & & $<5$ & $\mathrm{U}$ & $<5$ & JU Q & $<1$ & JU & & $<\mathrm{EQL}$ & 1 & $\mathrm{ug} / \mathrm{L}$ & $\mathrm{ML}$ \\
\hline & PCB 1016 & & & & & & & & & & & & & \\
\hline & РСВ 1221 & & & & & & & & & & & & & \\
\hline & PCB 1232 & & & & & & & & & & & & & \\
\hline & PCB 1242 & & & & & & & & & & & & & \\
\hline & РСB 1248 & & & & & & & & & & & & & \\
\hline & PCB 1254 & & & & & & & & & & & & & \\
\hline & PCB 1260 & & & & & & & & & & & & & \\
\hline & 1,1,2,2-Tetrachloroethane & & & $<5$ & $\mathrm{U}$ & $<5$ & $J U Q$ & $<1$ & JU & & $<\mathrm{EQL}$ & 1 & $\mathrm{ug} / \mathrm{L}$ & ML \\
\hline & Tetrachloroethylene & & & $<5$ & $\mathrm{U}$ & $<5$ & $\mathrm{JU} Q$ & $<1$ & JU & & $<\mathrm{EQL}$ & 1 & $\mathrm{ug} / \mathrm{L}$ & ML \\
\hline & $1,1,1$-Trichloroethane & & & $<5$ & U & $<5$ & $\mathrm{JU} Q$ & $<1$ & JU & & $<E Q L$ & 1 & $\mathrm{ug} / \mathrm{L}$ & ML \\
\hline & Trichloroethylene & & & $<5$ & $U$ & $<5$ & $\mathrm{JU} Q$ & .73 & $\mathrm{~J}$ & 1 & NDD & 1 & $u g / L$ & ML \\
\hline \multicolumn{15}{|c|}{ II. Monitoring Constituents } \\
\hline$\underline{\mathbf{S T}}$ & Parameter & $\underline{3096}$ & $\underline{\text { CLP EPA }}$ & $\underline{3 Q 97}$ & CLPPEPA & $\underline{3098}$ & CLPEPA & $\underline{3099}$ & $\underline{\text { CLP }}$ & EPA & Filt. & $\underline{\mathrm{DF}}$ & Unit & Lab \\
\hline \multicolumn{15}{|c|}{ Inorganics } \\
\hline+ & Aluminum, total recoverable & 636 & $J$ & 57.2 & & & & 150 & & & $>100$ & 1 & $u g / L$ & WA \\
\hline & Nitrate-nitrite as nitrogen & 990 & & 900 & & & & 898 & & & $<2400$ & 1 & $\mathrm{ug} / \mathrm{L}$ & WA \\
\hline+ & Sodium, total recoverable & 9180 & & 11200 & & & & 6950 & & & $>4600$ & 1 & $\mathrm{ug} / \mathrm{L}$ & WA \\
\hline+ & Sulfate & 4550 & & 5100 & & & & 3230 & & & $>3000$ & 1 & $\mathrm{ug} / \mathrm{L}$ & WA \\
\hline \multicolumn{15}{|c|}{ Radionuclides } \\
\hline & Gross alpha & 19.2 & & .41 & UI & & & 1.06 & $\mathrm{U}$ & V & $<E Q L$ & 1 & $\mathrm{pCi} / \mathrm{L}$ & GP \\
\hline & Nonvolatile beta & 539 & & .3 & UI & & & .657 & $\mathrm{U}$ & & $<E Q L$ & 1 & $\mathrm{pCi} / \mathrm{L}$ & GP \\
\hline & Radium, total alpha-emitting & 5.3 & & 1.14 & & & & .7 & $J$ & 1 & NDD & 1 & $\mathrm{pCi} / \mathrm{L}$ & GP \\
\hline
\end{tabular}

Notes: Concentrations in bold italics exceed the groundwater protection or monitoring constituent standards listed in Appendix A. Synchronous water levels are measured over a 3-5 day period or less. Dilution factors, Laboratory, and Filtered Data are for Groundwater Protection Standards First Quarter 2000 and Monitoring Constituents Third Quarter 1999 data only.

$+=$ exceeded the Groundwater Protection Standards (First Quarter 2000) or Monitoring Constituents (Third Quarter 1999) listed in Appendix A. 
Table D-4. Groundwater Monitoring Results for Plume Definition Wells, M-Area HWMF (Cont.) WELL ASB 5AR

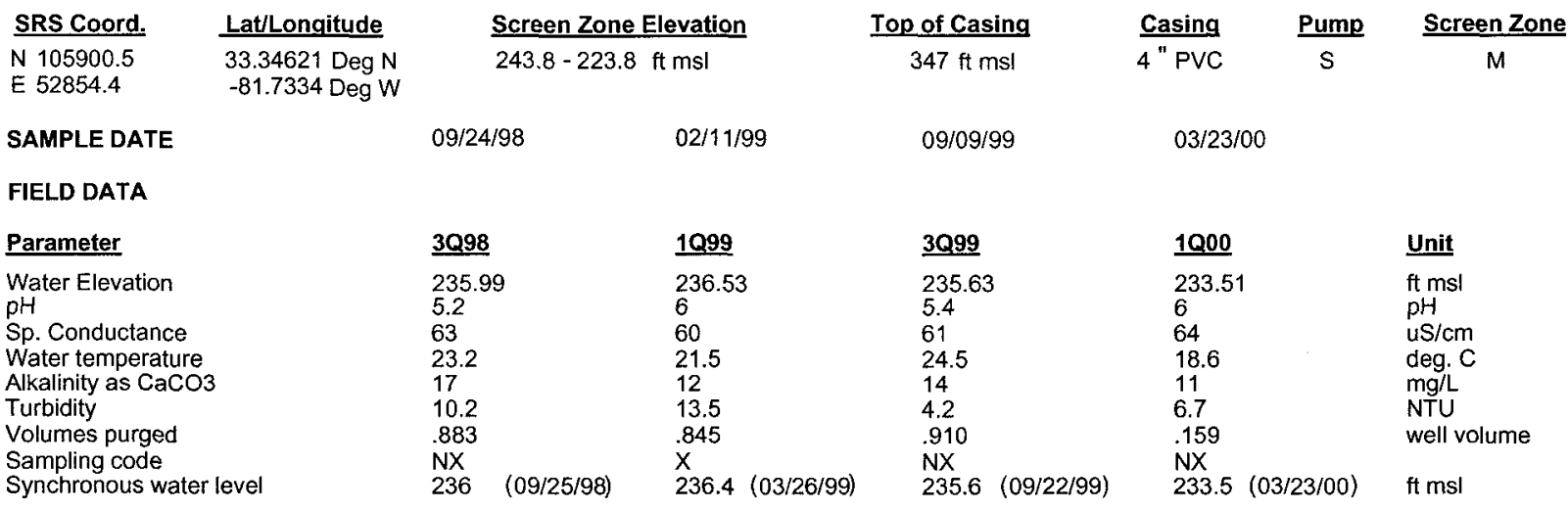

ANALYTICAL DATA

I. Groundwater Protection Standard

261 Appendix VIII/264 Appendix IX Hazardous Constituents

\begin{tabular}{|c|c|c|c|c|c|c|c|c|c|c|c|c|c|}
\hline \multirow{2}{*}{$\begin{array}{l}\text { ST Parameter } \\
\text { Inorganics }\end{array}$} & \multirow[t]{2}{*}{$\underline{3 Q 98}$} & \multirow[t]{2}{*}{ CLPEPA } & \multirow[t]{2}{*}{$\underline{1 Q 99}$} & CLPEPA & \multirow[t]{2}{*}{$\underline{3 Q 99}$} & CLPEPA & \multirow[t]{2}{*}{$\underline{1 Q 00}$} & \multicolumn{2}{|c|}{ CLPEPA } & \multirow[t]{2}{*}{ Filt. } & \multirow[t]{2}{*}{$\underline{\mathrm{DF}}$} & \multirow[t]{2}{*}{ Unit } & \multirow[t]{2}{*}{$\underline{\text { Lab }}$} \\
\hline & & & & & & & & & & & & & \\
\hline Barium, total recoverable & 4 & & 3.1 & & 2.5 & & & & & & & $u g / L$ & \\
\hline Cyanide & $<15.2$ & $\mathrm{u}$ & $<15.2$ & $\mathrm{u}$ & $<15.2$ & $\mathrm{u}$ & & & & & & $u g / L$ & \\
\hline Lead, total recoverable & $<47$ & $u$ & $<47$ & $u$ & 4.7 & J 1 & & & & & & $u g / L$ & \\
\hline Nickel, total recoverable & $<26$ & $\mathrm{u}$ & 2.8 & J 1 & $<26$ & $\mathrm{u}$ & & & & & & $u g / L$ & \\
\hline Selenium, total recoverable & $<66$ & $u$ & $<66$ & $\mathrm{u}$ & $<66$ & $u$ & & & & & & $\mathrm{ug} / \mathrm{L}$ & \\
\hline \multicolumn{14}{|l|}{ Organics } \\
\hline Chlorobenzene & $<5$ & $\mathrm{u}$ & $<5$ & $u$ & $<5$ & $u$ & $<1$ & $\mathrm{U}$ & & $<E Q L$ & 1 & $u g / L$ & ML \\
\hline 1,1-Dichloroethane & $<5$ & $u$ & $<5$ & u & $<5$ & $\mathrm{u}$ & $<1$ & $u$ & & $<E Q L$ & 1 & ug/L & 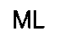 \\
\hline 1,1-Dichloroethylene & $<5$ & u & $<5$ & u & $<5$ & $\mathrm{u}$ & $<1$ & $u$ & & $<E Q L$ & 1 & ug/L & \\
\hline trans-1,2-Dichloroethylene & $<5$ & $u$ & $<5$ & $u$ & $<5$ & $\mathrm{u}$ & $<1$ & $u$ & & $<E Q L$ & 1 & $\mathrm{ug} / \mathrm{L}$ & ML \\
\hline PCB 1016 & & & & & & & & & - & & & & \\
\hline PCB 1221 & & & & & & & & & & & & & \\
\hline PCB 1232 & & & & & & & & & & & & & \\
\hline PCB 1242 & & & & & & & & & & & & & \\
\hline PCB 1248 & & & & & & & & & & & & & \\
\hline PCB 1254 & & & & & & & & & & & & & \\
\hline PCB 1260 & & & & & & & & & & & & & \\
\hline 1,1,2,2-Tetrachloroethane & $<5$ & $\mathrm{u}$ & $<5$ & $u$ & $<5$ & $u$ & $<1$ & $u$ & & $<E Q L$ & 1 & $\mathrm{ug} / \mathrm{L}$ & ML \\
\hline Tetrachloroethylene & $<5$ & u & $<5$ & $u$ & $<5$ & $u$ & 1.89 & $\mathrm{~J}$ & $\mathrm{~K}$ & NDD & 1 & $\mathrm{ug} / \mathrm{L}$ & \\
\hline 1,1,1-Trichloroethane & $<5$ & u & $<5$ & $u$ & $<5$ & $u$ & $<1$ & $u$ & & $<E Q L$ & 1 & $\mathrm{ug} / \mathrm{L}$ & 1L \\
\hline Trichloroethylene & 103 & & 79.9 & & 39.4 & & 17.8 & $J$ & K & NDD & 1 & $\mathrm{ug} / \mathrm{L}$ & ML \\
\hline \multicolumn{13}{|l|}{ II. Monitoring Constituents } & \\
\hline ST Parameter & $\underline{3 Q 96}$ & CLPEPA & $\underline{3097}$ & CLP EPA & $\underline{3098}$ & CLPEPA & $\underline{3099}$ & CLP & EPA & Fill. & $\underline{\text { DF }}$ & $\underline{\text { Unit }}$ & \\
\hline \multicolumn{13}{|l|}{ Inorganics } & \\
\hline Aluminum, total recoverable & $<44.3$ & $u$ & 38.4 & & 126 & $\mathrm{~J}$ & 41.6 & $J$ & I & NDD & 1 & ug/L & WA \\
\hline Nitrate-nitrite as nitrogen & 520 & & 458 & & 530 & & 626 & & & $<2400$ & 1 & $\mathrm{ug} / \mathrm{L}$ & \\
\hline+ Sodium, total recoverable & 10700 & & 11200 & & 10900 & & 10400 & & & $>4600$ & 1 & $\mathrm{ug} / \mathrm{L}$ & w \\
\hline$+\quad$ Sulfate & 6060 & & 8090 & & 5900 & & 7300 & & & $>3000$ & 1 & ug/L & WA \\
\hline \multicolumn{13}{|l|}{ Radionuclides } & \\
\hline Gross alpha & .667 & UI & .4 & UI & .85 & Ut & 4.01 & & & $<15$ & 1 & $\mathrm{pCi} / \mathrm{L}$ & GP \\
\hline Nonvolatile beta & .571 & Ul & -1.2 & Ui & .7 & UIJ & 3.14 & $\mathrm{~J}$ & I & NDD & 1 & $\mathrm{pCi} / \mathrm{L}$ & GP \\
\hline Radium, total alpha-emitting & .1 & UI & 1.51 & J & .25 & UI & 6 & $\mathrm{~J}$ & I & NDD & 1 & $\mathrm{pCi} / \mathrm{L}$ & GP \\
\hline
\end{tabular}

Notes: Concentrations in bold italics exceed the groundwater protection or monitoring constituent standards listed in Appendix A. Synchronous water levels are measured over a 3-5 day period or less. Dilution factors, Laboratory, and Filtered Data are for Groundwater Protection Standards First Quarter 2000 and Monitoring Constituents Third Quarter 1999 data only.

$+=$ exceeded the Groundwater Protection Standards (First Quarter 2000) or Monitoring Constituents (Third Quarter 1999) listed in Appendix A. 
Table D-4. Groundwater Monitoring Results for Plume Definition Wells, M-Area HWMF (Cont.) WELL ASB 5C

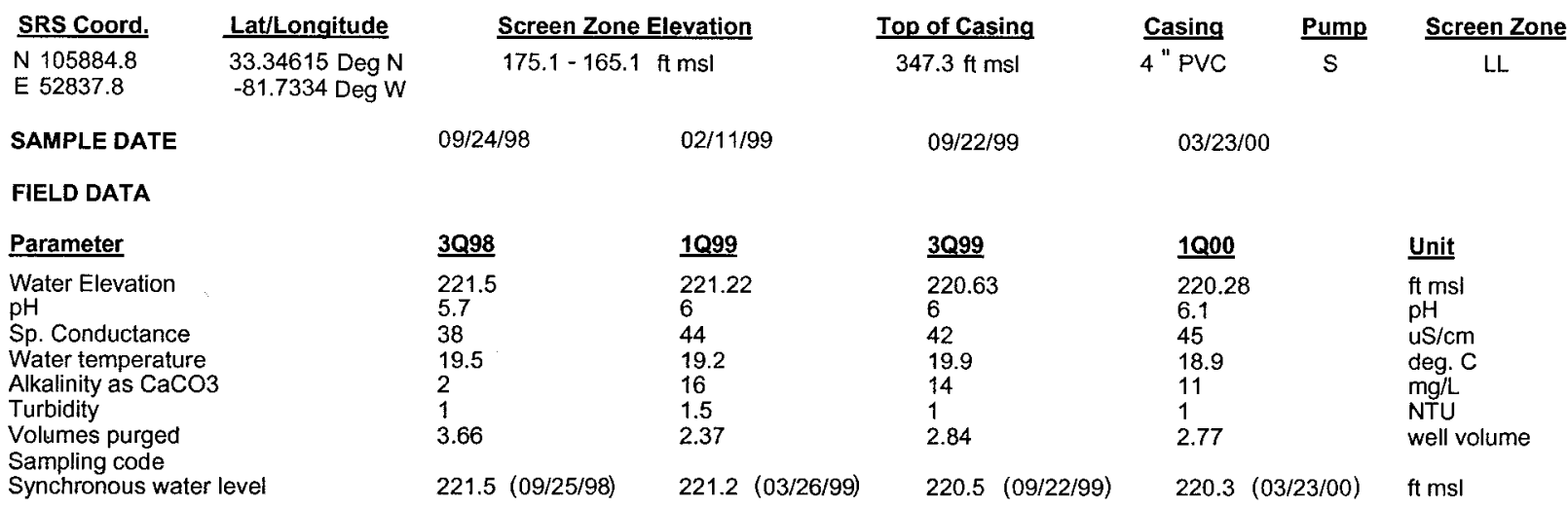

ANALYTICAL DATA

I. Groundwater Protection Standard

261 Appendix VIII/264 Appendix IX Hazardous Constituents

\begin{tabular}{|c|c|c|c|c|c|c|c|c|c|c|c|c|c|c|}
\hline$\underline{\text { ST }}$ & Parameter & $\underline{3 Q 98}$ & CLP EPA & 1099 & CLP EPA & $\underline{3 Q 99}$ & CLPEPA & $\underline{1 Q 00}$ & \multicolumn{2}{|c|}{ CLPEPA } & Filt. & $\underline{D F}$ & Unit & $\underline{L a b}$ \\
\hline \multicolumn{15}{|c|}{ Inorganics } \\
\hline & Barium, total recoverable & 9.2 & & 10.1 & & 10.1 & & & & & & & $u g / L$ & \\
\hline & Cyanide & $<15.2$ & $\mathrm{u}$ & $<15.2$ & $\mathrm{U}$ & $<15.2$ & $\mathrm{U}$ & & & & & & $\mathrm{ug} / \mathrm{L}$ & \\
\hline & Lead, total recoverable & $<47$ & $U$ & $<47$ & $\mathrm{U}$ & $<47$ & $\mathrm{U}$ & & & & & & $u g / L$ & \\
\hline & Nickel, total recoverable & $<26$ & $U$ & $<26$ & U & $<26$ & $\mathrm{U}$ & & & & & & $u g / L$ & \\
\hline & Selenium, total recoverable & $<66$ & $U$ & $<66$ & U & $<66$ & $\mathrm{U}$ & & & & & & $\mathrm{ug} / \mathrm{L}$ & \\
\hline \multicolumn{15}{|c|}{ Organics } \\
\hline & Chlorobenzene & $<5$ & $\mathrm{U}$ & $<5$ & $\mathrm{U}$ & $<5$ & $u$ & $<1$ & U & & $<E Q L$ & 1 & $\mathrm{ug} / \mathrm{L}$ & $\mathrm{ML}$ \\
\hline & 1,1-Dichloroethane & $<5$ & U & $<5$ & $\mathrm{U}$ & $<5$ & $U$ & $<1$ & $\mathrm{U}$ & & $<E Q L$ & 1 & ug/L & ML \\
\hline & 1,1-Dichloroethylene & $<5$ & $\mathrm{U}$ & $<5$ & $U$ & $<5$ & $U$ & $<1$ & $U$ & & $<E Q L$ & 1 & ug/L & ML \\
\hline & $\begin{array}{l}\text { trans-1,2-Dichloroethylene } \\
\text { РСВ } 1016\end{array}$ & $<5$ & $U$ & $<5$ & $U$ & $<5$ & $U$ & $<1$ & $\mathrm{U}$ & & $<E Q L$ & 1 & $\mathrm{ug} / \mathrm{L}$ & ML \\
\hline & PCB 1221 & & & & & & & & & & & & & \\
\hline & PCB 1232 & & & & & & & & & & & & & \\
\hline & PCB 1242 & & & & & & & & & & & & & \\
\hline & PCB 1248 & & & & & & & & & & & & & \\
\hline & PCB 1254 & & & & & & & & & & & & & \\
\hline & PCB 1260 & & & & & & & & & & & & & \\
\hline & $1,1,2,2$-Tetrachloroethane & $<5$ & $\mathrm{U}$ & $<5$ & $\mathrm{U}$ & $<5$ & $U$ & $<1$ & $\mathrm{U}$ & & $<\mathrm{EQL}$ & 1 & ug/L & ML \\
\hline & Tetrachloroethylene & 89.5 & & 79.3 & & 60 & & 54.7 & $\mathrm{~J}$ & K & NDD & 1 & $\mathrm{ug} / \mathrm{L}$ & ML \\
\hline & 1,1,1-Trichloroethane & $<5$ & $\mathrm{U}$ & $<5$ & $U$ & $<5$ & U & $<1$ & $\mathrm{U}$ & & $<E Q L$ & 1 & $\mathrm{ug} / \mathrm{h}$ & $M L$ \\
\hline & Trichloroethylene & 177 & & 167 & & 143 & & 186 & $\mathrm{~J}$ & K & NDD & 1 & $\mathrm{ug} / \mathrm{L}$ & $M L$ \\
\hline \multicolumn{15}{|c|}{ II. Monitoring Constituents } \\
\hline$\underline{\mathbf{S T}}$ & Parameter & 3096 & CLPEPA & 3097 & CLPEPA & $\underline{3 Q 98}$ & CLPEPA & 3099 & $\underline{\text { CLF }}$ & EPA & Filt. & $\underline{\text { DF }}$ & Unit & $\underline{\text { Lab }}$ \\
\hline \multicolumn{15}{|c|}{ Inorganics } \\
\hline & Aluminum, total recoverable & $<20$ & $U$ & 7.2 & J & $<146$ & $U$ & 41.3 & $J$ & 1 & NDD & 1 & $u g / L$ & WA \\
\hline & Nitrate-nitrite as nitrogen & 1160 & & 1470 & & 1090 & & 1060 & & & $<2400$ & 5 & ug/L & WA \\
\hline & Sodium, total recoverable & 2420 & J & 2180 & & 1920 & & 2280 & & & $<4600$ & 1 & $\mathrm{ug} / \mathrm{L}$ & WA \\
\hline & Sulfate & $<241$ & U & $<5000$ & $\mathrm{U}$ & 392 & & 339 & $J$ & 1 & NDD & 1 & $\mathrm{ug} / \mathrm{L}$ & WA \\
\hline \multicolumn{15}{|c|}{ Radionuclides } \\
\hline & Gross alpha & .436 & IU & -.13 & UI & .33 & UI & .664 & $\mathrm{U}$ & & $<E Q L$ & 1 & $\mathrm{pCi} / \mathrm{L}$ & GP \\
\hline & Nonvolatile beta & 1.16 & & 1 & UI & .24 & UIJ & .703 & $\mathrm{U}$ & & $<\mathrm{EQL}$ & 1 & $\mathrm{pCi} / \mathrm{L}$ & GP \\
\hline & Radium, total alpha-emitting & .3 & UI & 2.87 & UIJ & .34 & & 0 & $U$ & & $<E Q L$ & 1 & $\mathrm{pCi} / \mathrm{L}$ & GP \\
\hline
\end{tabular}

Notes: Concentrations in bold italics exceed the groundwater protection or monitoring constituent standards listed in Appendix A. Synchronous water levels are measured over a 3-5 day period or less. Dilution factors, Laboratory, and Filtered Data are for Groundwater Protection Standards First Quarter 2000 and Monitoring Constituents Third Quarter 1999 data only.

+ = exceeded the Groundwater Protection Standards (First Quarter 2000) or Monitoring Constituents (Third Quarter 1999) listed in Appendix A. 
Table D-4. Groundwater Monitoring Results for Plume Definition Wells, M-Area HWMF (Cont.) WELL ASB 6A

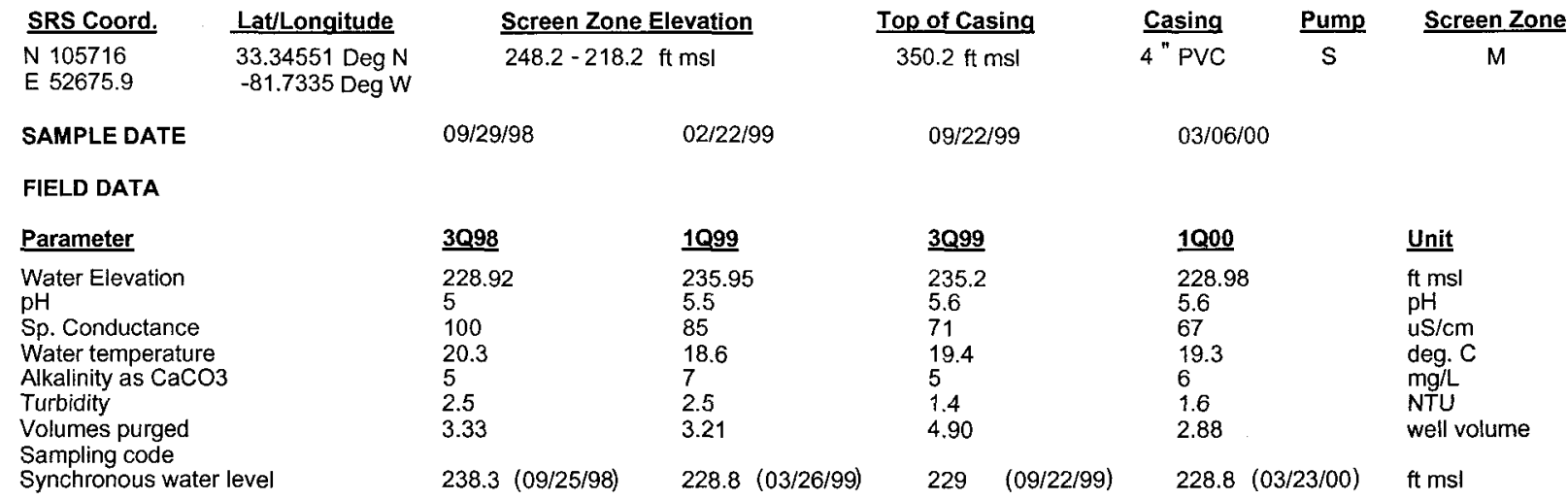

\section{ANALYTICAL DATA}

I. Groundwater Protection Standard

261 Appendix VIII/264 Appendix IX Hazardous Constituents

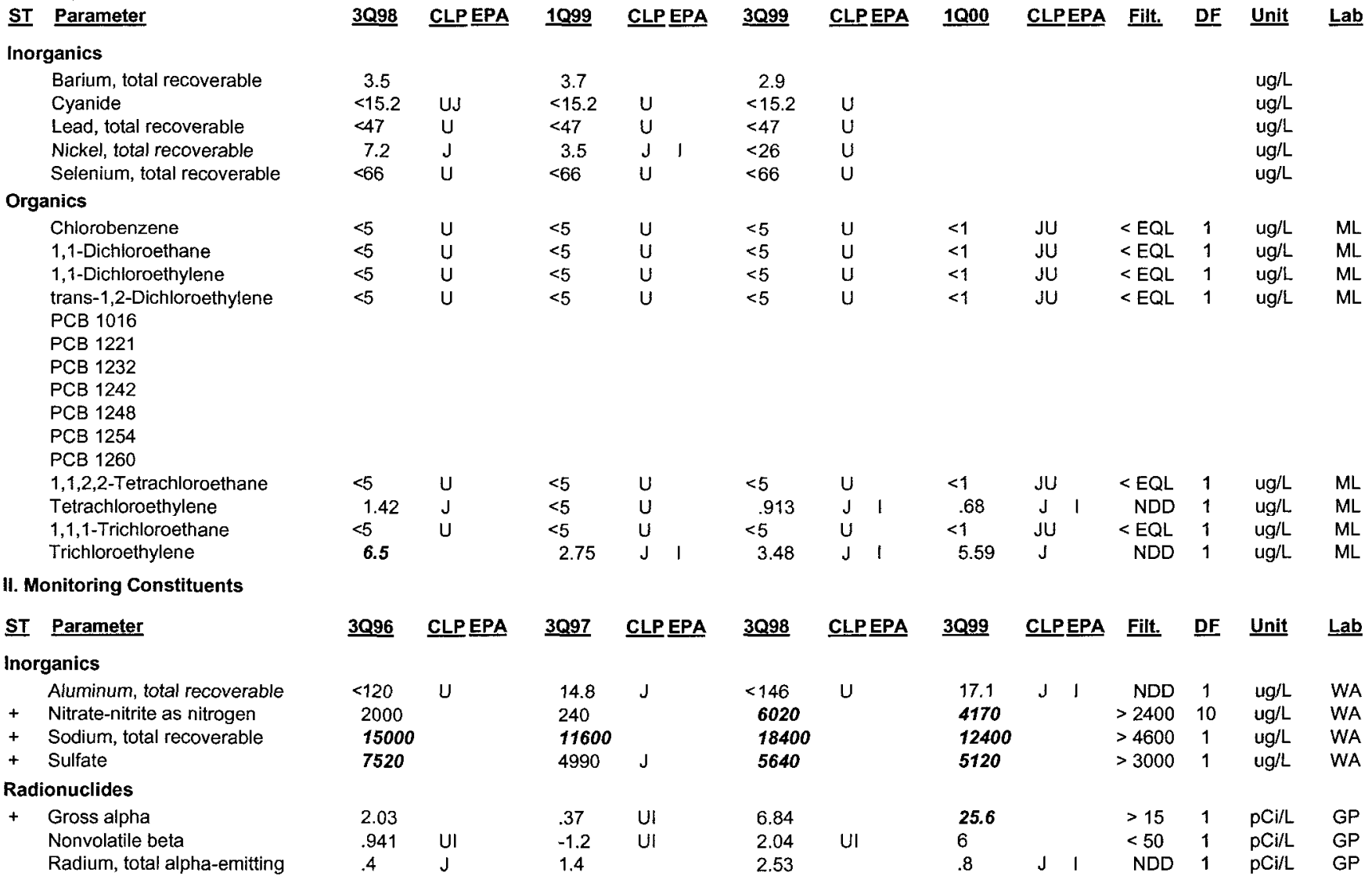

Notes: Concentrations in bold italics exceed the groundwater protection or monitoring constituent standards listed in Appendix A. Synchronous water levels are measured over a 3-5 day period or less. Dilution factors, Laboratory, and Filtered Data are for Groundwater Protection Standards First Quarter 2000 and Monitoring Constituents Third Quarter 1999 data only.

+ = exceeded the Groundwater Protection Standards (First Quarter 2000) or Monitoring Constituents (Third Quarter 1999) listed in Appendix A. 
Table D-4. Groundwater Monitoring Results for Plume Definition Wells, M-Area HWMF (Cont.) WELL ASB 6AA

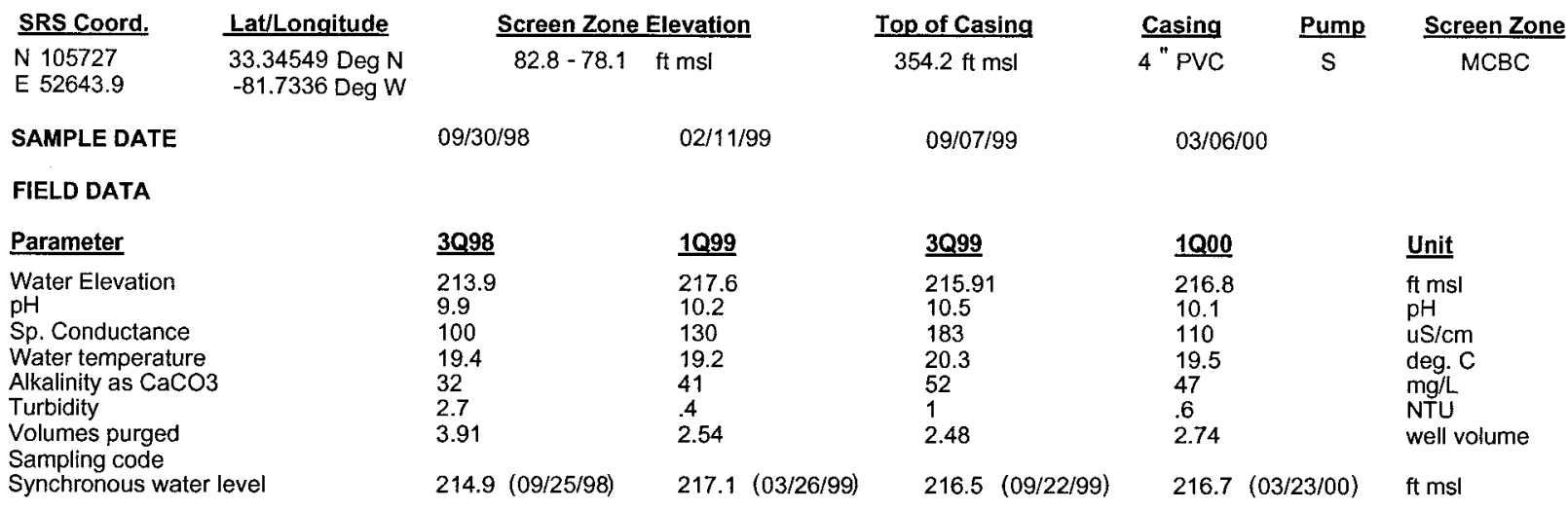

ANALYTICAL DATA

I. Groundwater Protection Standard

261 Appendix VIII/264 Appendix IX Hazardous Constituents

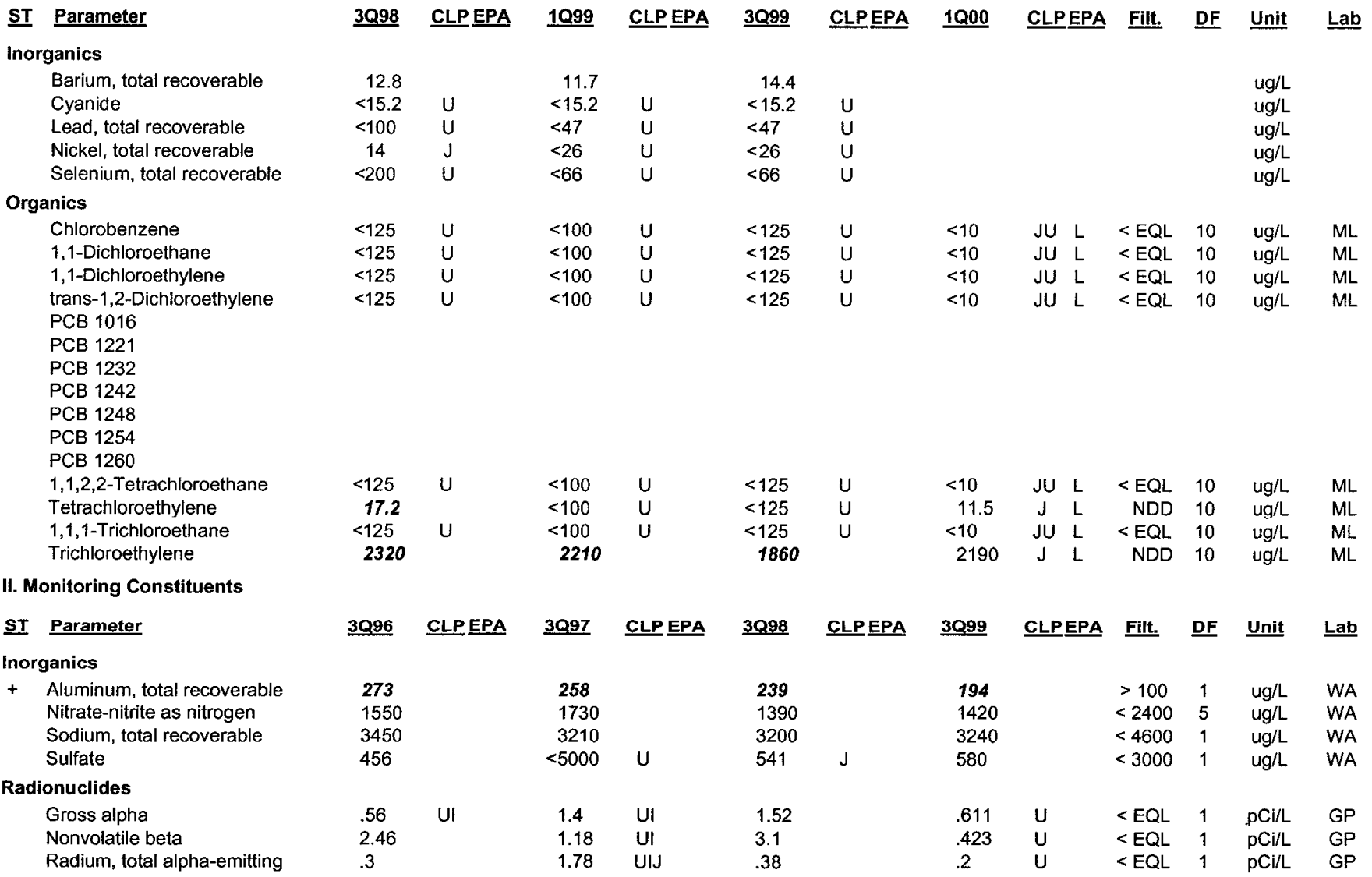

Notes: Concentrations in bold italics exceed the groundwater protection or monitoring constituent standards listed in Appendix A. Synchronous water levels are measured over a 3-5 day period or less. Dilution factors, Laboratory, and Filtered Data are for Groundwater Protection Standards First Quarter 2000 and Monitoring Constituents Third Quarter 1999 data only.

+ = exceeded the Groundwater Protection Standards (First Quarter 2000) or Monitoring Constituents (Third Quarter 1999) listed in Appendix A. 
Table D-4. Groundwater Monitoring Results for Plume Definition Wells, M-Area HWMF (Cont.) WELL ASB 6C

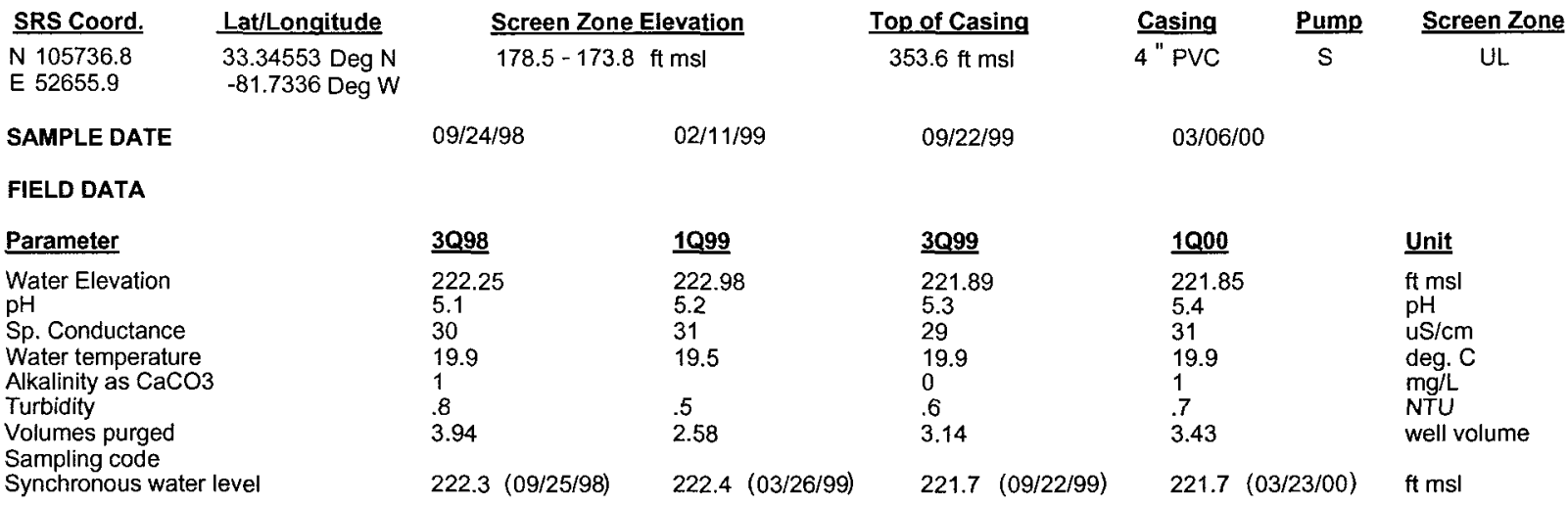

ANALYTICAL DATA

I. Groundwater Protection Standard

261 Appendix VIII/264 Appendix IX Hazardous Constituents

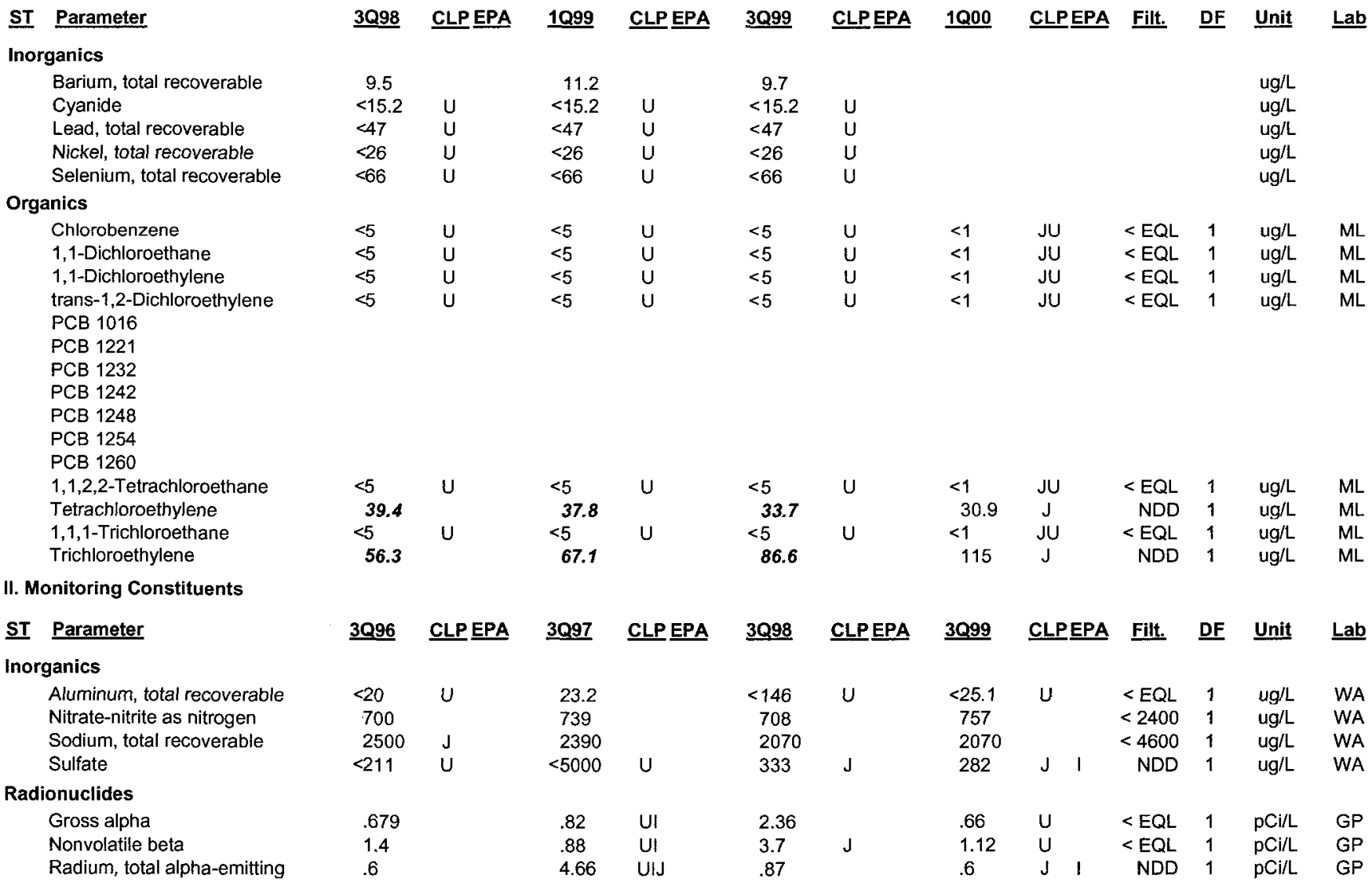

Notes: Concentrations in bold italics exceed the groundwater protection or monitoring constituent standards listed in Appendix A. Synchronous water levels are measured over a 3-5 day period or less. Dilution factors, Laboratory, and Filtered Data are for Groundwater Protection Standards First Quarter 2000 and Monitoring Constituents Third Quarter 1999 data only.

+ = exceeded the Groundwater Protection Standards (First Quarter 2000) or Monitoring Constituents (Third Quarter 1999) listed in Appendix A. 
Table D-4. Groundwater Monitoring Results for Plume Definition Wells, M-Area HWMF (Cont.) WELL ASB 6TA

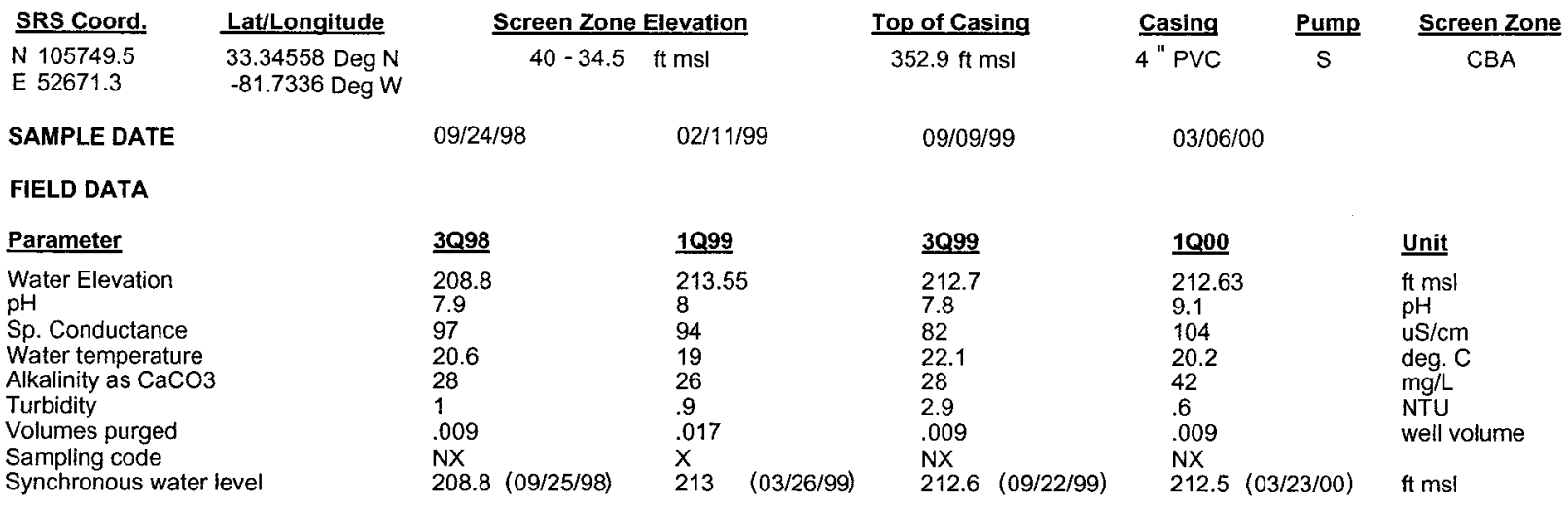

ANALYTICAL DATA

I. Groundwater Protection Standard

261 Appendix VIII/264 Appendix IX Hazardous Constituents

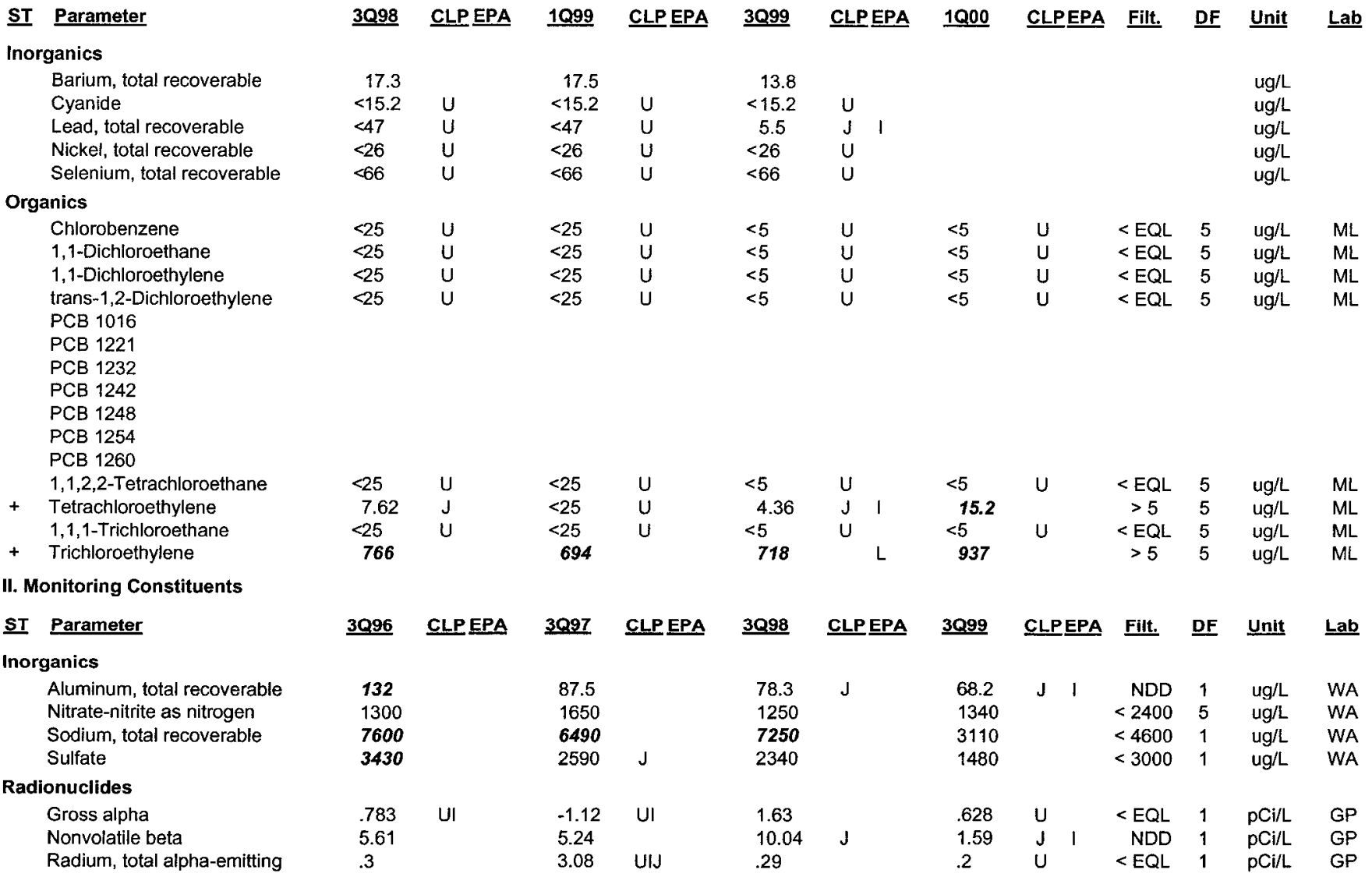

Notes: Concentrations in bold italics exceed the groundwater protection or monitoring constituent standards listed in Appendix A. Synchronous water levels are measured over a 3-5 day period or less. Dilution factors, Laboratory, and Filtered Data are for Groundwater Protection Standards First Quarter 2000 and Monitoring Constituents Third Quarter 1999 data only.

+ = exceeded the Groundwater Protection Standards (First Quarter 2000) or Monitoring Constituents (Third Quarter 1999) listed in Appendix A. 
Table D-4. Groundwater Monitoring Results for Plume Definition Wells, M-Area HWMF (Cont.) WELL ASB 8

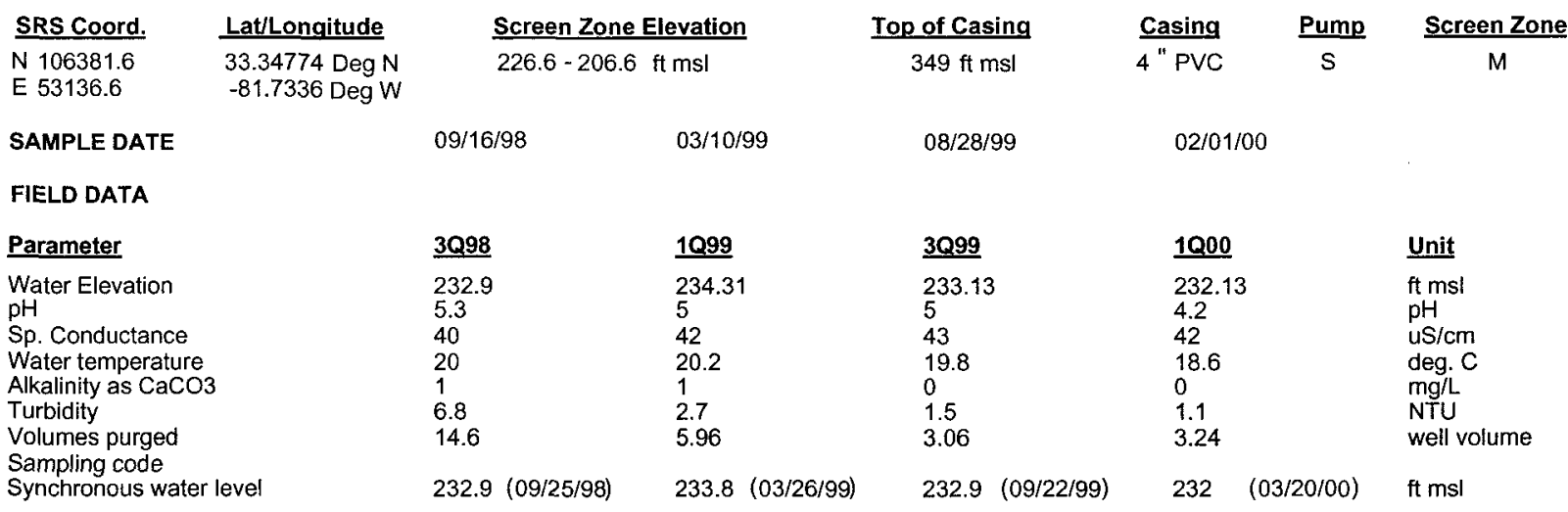

ANALYTICAL DATA

I. Groundwater Protection Standard

261 Appendix VIII/264 Appendix IX Hazardous Constituents

\begin{tabular}{|c|c|c|c|c|c|c|c|c|c|c|c|c|c|}
\hline ST & Parameter & $\underline{3 Q 98}$ & CLP EPA & $\underline{1 Q 99}$ & CLPEPA & $\underline{3099}$ & CLP EPA & $\underline{1000}$ & CLPEPA & Filt. & $\underline{\text { DF }}$ & Unit & $\underline{L a b}$ \\
\hline \multicolumn{14}{|c|}{ Inorganics } \\
\hline & Barium, total recoverable & 1.4 & $J$ & $<1.5$ & U V & $<2.1$ & U V & & & & & $u g / L$ & \\
\hline & Cyanide & $<15.2$ & $\mathrm{U}$ & $<15.2$ & $\mathrm{U}$ & $<15.2$ & $\mathbf{u}$ & & & & & $u g / L$ & \\
\hline & Lead, total recoverable & $<47$ & $\mathrm{U}$ & $<47$ & $\mathrm{U}$ & $<47$ & $U$ & & & & & $\mathrm{ug} / \mathrm{L}$ & \\
\hline & Nickel, total recoverable & $<26$ & $\mathrm{U}$ & $<26$ & $\mathrm{u}$ & $<26$ & U & & & & & $\mathrm{ug} / \mathrm{L}$ & \\
\hline & Selenium, total recoverable & $<66$ & $\mathrm{U}$ & $<66$ & $\mathrm{U}$ & $<66$ & $\mathrm{U}$ & & & & & $\mathrm{ug} / \mathrm{L}$ & \\
\hline \multicolumn{14}{|c|}{ Organics } \\
\hline & Chlorobenzene & $<5$ & $u$ & $<5$ & $u$ & $<5$ & U & $<1$ & JU & $<E Q L$ & 1 & ug/L & ML \\
\hline & 1,1-Dichloroethane & $<5$ & U & $<5$ & $\mathrm{U}$ & $<5$ & $\mathrm{U}$ & $<1$ & JU & $<E Q L$ & 1 & $\mathrm{ug} / \mathrm{L}$ & ML \\
\hline & 1,1-Dichioroethylene & $<5$ & U & $<5$ & $\mathrm{U}$ & $<5$ & $\mathrm{U}$ & $<1$ & JU & $<\mathrm{EQL}$ & 1 & $\mathrm{ug} / \mathrm{L}$ & ML \\
\hline & trans-1,2-Dichloroethylene & $<5$ & $U$ & $<5$ & $U$ & $<5$ & $\mathrm{U}$ & $<1$ & JU & $<E Q L$ & 1 & $\mathrm{ug} / \mathrm{L}$ & $M L$ \\
\hline & PCB 1016 & & & & & & & & & & & & \\
\hline & PCB 1221 & & & & & & & & & & & & \\
\hline & PCB 1232 & & & & & & & & & & & & \\
\hline & PCB 1242 & & & & & & & & & & & & \\
\hline & PCB 1248 & & & & & & & & & & & & \\
\hline & PCB 1254 & & & & & & & & & & & & \\
\hline & РСB 1260 & & & & & & & & & & & & \\
\hline & 1,1,2,2-Tetrachloroethane & $<5$ & $u$ & $<5$ & $\mathrm{U}$ & $<5$ & $\mathrm{U}$ & $<1$ & JU & $<E Q L$ & 1 & $u g / L$ & ML \\
\hline & Tetrachloroethylene & $<5$ & $U$ & $<5$ & $\mathrm{U}$ & $<5$ & $\mathrm{U}$ & $<1$ & JU & $<\mathrm{EQL}$ & 1 & $\mathrm{ug} / \mathrm{L}$ & ML \\
\hline & $1,1,1-$ Trichloroethane & $<5$ & $U$ & $<5$ & $\mathrm{U}$ & $<5$ & $U$ & $<1$ & JU & $<E Q L$ & 1 & $\mathrm{ug} / \mathrm{L}$ & $\mathrm{ML}$ \\
\hline & Trichloroethylene & 3.37 & $J$ & 1.7 & J 1 & .99 & J I & 1.82 & $J$ & NDD & 1 & $\mathrm{ug} / \mathrm{L}$ & $\mathrm{ML}$ \\
\hline \multicolumn{14}{|c|}{ II. Monitoring Constituents } \\
\hline$\underline{\text { ST }}$ & Parameter & $\underline{3 Q 96}$ & CLPPEPA & $\underline{3097}$ & CLPEPA & $\underline{3 Q 98}$ & CLPEPA & $\underline{3099}$ & CLPEPA & Filt. & $\underline{D F}$ & Unit & $\underline{L a b}$ \\
\hline \multicolumn{14}{|c|}{ Inorganics } \\
\hline & Aluminum, total recoverable & $<49.7$ & $U$ & $<20$ & $U$ & 27.8 & $\mathrm{~J}$ & $<146$ & $\mathrm{U}$ & $<E Q L$ & 1 & $\mathrm{ug} / \mathrm{L}$ & WA \\
\hline & Nitrate-nitrite as nitrogen & 810 & & 1370 & & 627 & & 842 & & $<2400$ & 1 & $\mathrm{ug} / \mathrm{L}$ & WA \\
\hline+ & Sodium, total recoverable & 6100 & & 1430 & & 7240 & & 6990 & & $>4600$ & 1 & $\mathrm{ug} / \mathrm{L}$ & WA \\
\hline+ & Sulfate & 4550 & & $<5000$ & $\mathrm{U}$ & 6730 & & 5440 & & $>3000$ & 1 & $\mathrm{ug} / \mathrm{L}$ & WA \\
\hline \multicolumn{14}{|c|}{ Radionuclides } \\
\hline & Gross alpha & 1.91 & & 1.83 & & .57 & UI & .539 & $\mathrm{U}$ & $<E Q L$ & 1 & $\mathrm{pCi} / \mathrm{L}$ & GP \\
\hline & Nonvolatile beta & 2.12 & & 2.93 & $J$ & 4.7 & $J$ & 1.37 & $\mathrm{U}$ & $<E Q L$ & 1 & $\mathrm{pCi} / \mathrm{L}$ & GP \\
\hline & Radium, total alpha-emitting & .6 & & 3.19 & UIJ & 1.2 & & .1 & $\mathrm{U}$ & $<\mathrm{EQL}$ & 1 & $\mathrm{pCi} / \mathrm{L}$ & GP \\
\hline
\end{tabular}

Notes: Concentrations in bold italics exceed the groundwater protection or monitoring constituent standards listed in Appendix A. Synchronous water levels are measured over a 3-5 day period or less. Dilution factors, Laboratory, and Filtered Data are for Groundwater Protection Standards First Quarter 2000 and Monitoring Constituents Third Quarter 1999 data only.

$+=$ exceeded the Groundwater Protection Standards (First Quarter 2000) or Monitoring Constituents (Third Quarter 1999) listed in Appendix A. 
Table D-4. Groundwater Monitoring Results for Plume Definition Wells, M-Area HWMF (Cont.) WELL ASB 8B

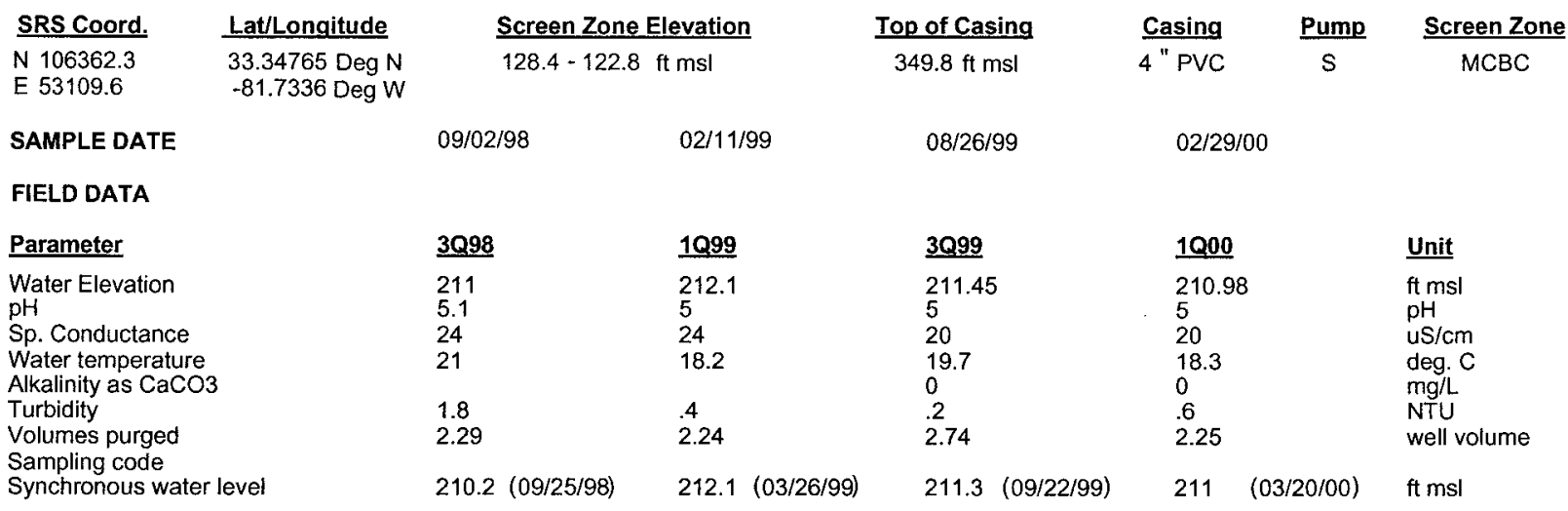

ANALYTICAL DATA

I. Groundwater Protection Standard

261 Appendix VIII/264 Appendix IX Hazardous Constituents

\begin{tabular}{|c|c|c|c|c|c|c|c|c|c|c|c|c|c|c|c|}
\hline \multirow{2}{*}{\multicolumn{2}{|c|}{$\begin{array}{l}\text { ST } \\
\text { Inorgameter }\end{array}$}} & \multirow[t]{2}{*}{$\underline{3098}$} & \multirow[t]{2}{*}{ CLP EPA } & \multirow[t]{2}{*}{1099} & \multirow[t]{2}{*}{ CLP EPA } & \multirow[t]{2}{*}{$\underline{3099}$} & \multicolumn{2}{|c|}{ CLPEPA } & \multirow[t]{2}{*}{1000} & \multicolumn{2}{|c|}{ CLPEPA } & \multirow[t]{2}{*}{ Filt. } & \multirow[t]{2}{*}{$\underline{\text { DF }}$} & \multirow[t]{2}{*}{ Unit } & \multirow[t]{2}{*}{$\underline{L a b}$} \\
\hline & & & & & & & & & & & & & & & \\
\hline & Barium, total recoverable & 4.6 & & 3.6 & & $<4.3$ & U & V & & & & & & $\mathrm{ug} / \mathrm{L}$ & \\
\hline & Cyanide & $<15.2$ & $\mathrm{U}$ & $<15.2$ & $U$ & $<15.2$ & $U$ & & & & & & & ug/L & \\
\hline & Lead, total recoverable & $<47$ & $\mathrm{U}$ & $<100$ & $\mathrm{U}$ & $<47$ & $\mathrm{U}$ & & & & & & & $\mathrm{ug} / \mathrm{L}$ & \\
\hline & Nickel, total recoverable & $<26$ & $\mathrm{U}$ & $<50$ & $\mathrm{U}$ & $<26$ & $\mathrm{u}$ & & & & & & & $\mathrm{ug} / \mathrm{L}$ & \\
\hline & Selenium, total recoverable & $<66$ & $\mathrm{U}$ & $<66$ & $\mathrm{U}$ & $<66$ & $u$ & & & & & & & ug/L & \\
\hline \multicolumn{16}{|c|}{ Organics } \\
\hline & Chlorobenzene & $<5$ & $\mathrm{U}$ & $<25$ & $U$ & $<5$ & JU & $Q$ & $<1$ & $\mathrm{U}$ & & $<E Q L$ & 1 & ug/L & ML \\
\hline & 1,1-Dichloroethane & $<5$ & $\mathrm{U}$ & $<25$ & $U$ & $<5$ & JU & $\mathrm{Q}$ & $<1$ & $\mathrm{U}$ & & $<\mathrm{EQL}$ & 1 & $\mathrm{ug} / \mathrm{L}$ & ML \\
\hline & 1,1-Dichloroethylene & $<5$ & $\mathrm{U}$ & $<25$ & $\mathrm{U}$ & $<5$ & JU & $Q$ & $<1$ & $\mathrm{U}$ & & $<E Q L$ & 1 & $\mathrm{ug} / \mathrm{L}$ & ML \\
\hline & trans-1,2-Dichloroethylene & & & $<25$ & $U$ & $<5$ & JU & $Q$ & & & & & & ug/L & \\
\hline & РСB 1016 & & & & & & & & & & & & & & \\
\hline & PCB 1221 & & & & & & & & & & & & & & \\
\hline & $\begin{array}{l}\text { PCB } 1232 \\
\text { PCB } 1242\end{array}$ & & & & & & & & & & & & & & \\
\hline & PCB 1248 & & & & & & & & & & & & & & \\
\hline & PCB 1254 & & & & & & & & & & & & & & \\
\hline & PCB 1260 & & & & & & & & & & & & & & \\
\hline & 1,1,2,2-Tetrachloroethane & $<5$ & U & $<25$ & $\mathrm{U}$ & $<5$ & JU & $Q$ & $<1$ & $\mathrm{U}$ & & $<\mathrm{EQL}$ & 1 & $\mathrm{ug} / \mathrm{L}$ & ML \\
\hline & Tetrachloroethylene & 1.44 & J & $<25$ & $U$ & $<5$ & JU & $Q$ & 1.76 & $J$ & $K$ & NDD & 1 & ug/L & $M L$ \\
\hline & 1,1,1-Trichloroethane & $<5$ & $U$ & $<25$ & $\mathrm{U}$ & $<5$ & JU & $Q$ & $<1$ & $\mathrm{U}$ & & $<\mathrm{EQL}$ & 1 & $\mathrm{ug} / \mathrm{L}$ & $M L$ \\
\hline & Trichloroethylene & & $L$ & 341 & & 192 & J & $Q$ & 107 & $\mathrm{~J}$ & $\mathrm{~K}$ & NDD & 1 & $\mathrm{ug} / \mathrm{L}$ & ML \\
\hline \multicolumn{16}{|c|}{ Il. Monitoring Constituents } \\
\hline$\underline{\mathbf{S T}}$ & Parameter & $\underline{3096}$ & CLPEPA & $\underline{3097}$ & CLPEPA & 3098 & $\underline{\text { CLP }}$ & EPA & $\underline{3 Q 99}$ & $\underline{\text { CLP }}$ & EPA & Filt. & $\underline{\text { DF }}$ & Unit & $\underline{L a b}$ \\
\hline \multicolumn{16}{|c|}{ Inorganics } \\
\hline & Aluminum, total recoverable & 27.6 & $J$ & $<20$ & $\mathrm{U}$ & 22.6 & $J$ & & $<146$ & $u$ & & $<E Q L$ & 1 & ug/L & WA \\
\hline & Nitrate-nitrite as nitrogen & 1100 & & 2200 & & 1280 & & & 775 & & & $<2400$ & 1 & ug/L & WA \\
\hline & Sodium, total recoverable & 2460 & & 2510 & & 2110 & & & 1680 & & & $<4600$ & 1 & ug/L & WA \\
\hline & Sulfate & 162 & $J$ & $<5000$ & U & 287 & $\mathrm{~J}$ & & 384 & & & $<3000$ & 1 & ug/L & WA \\
\hline \multicolumn{16}{|c|}{ Radionuclides } \\
\hline & Gross alpha & 1.23 & & 2.32 & & 3.79 & $J$ & & .48 & $\mathrm{U}$ & & $<\mathrm{EQL}$ & 1 & $\mathrm{pCi} / \mathrm{L}$ & GP \\
\hline & Nonvolatile beta & 1.47 & & 1.44 & Ui & 8.25 & $\mathrm{~J}$ & & 1.41 & $\mathrm{~J}$ & I & NDD & 1 & pCi/L & GP \\
\hline & Radium, total alpha-emitting & .8 & & 2.36 & UIJ & .47 & & & .1 & $\mathrm{U}$ & & $<E Q L$ & 1 & $\mathrm{pCi} / \mathrm{L}$ & GP \\
\hline
\end{tabular}

Notes: Concentrations in bold italics exceed the groundwater protection or monitoring constituent standards listed in Appendix A. Synchronous water levels are measured over a 3-5 day period or less. Dilution factors, Laboratory, and Filtered Data are for Groundwater Protection Standards First Quarter 2000 and Monitoring Constituents Third Quarter 1999 data only.

+口 exceeded the Groundwater Protection Standards (First Quarter 2000) or Monitoring Constituents (Third Quarter 1999) listed in Appendix A. 
Table D-4. Groundwater Monitoring Results for Plume Definition Wells, M-Area HWMF (Cont.) WELL ASB 8C

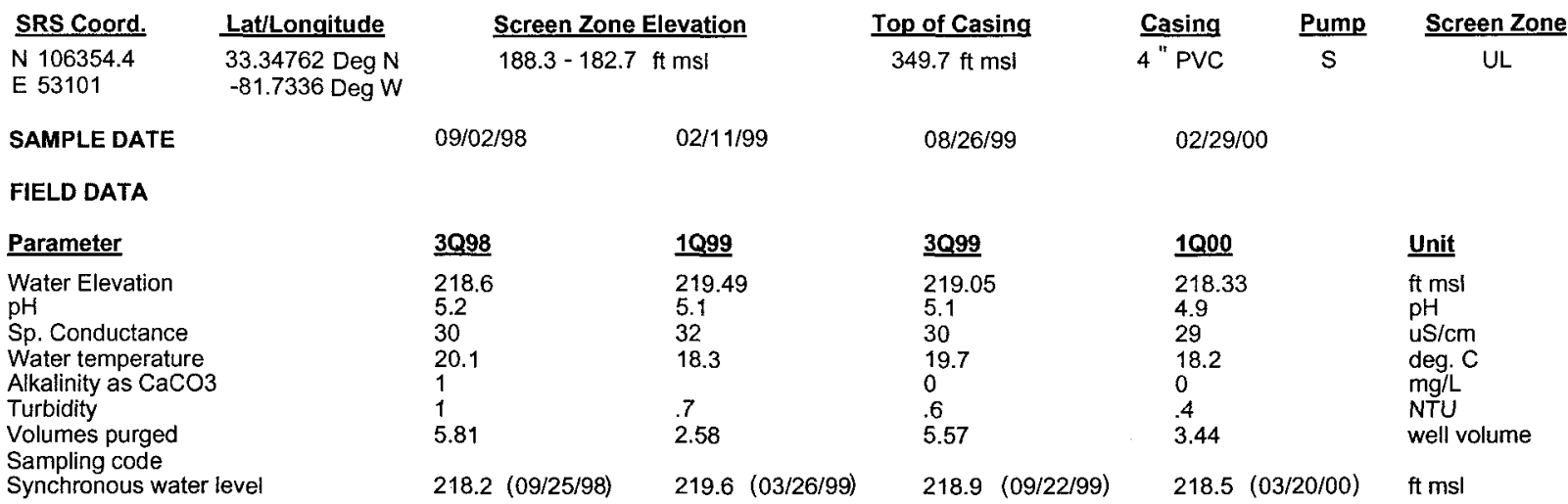

ANALYTICAL DATA

1. Groundwater Protection Standard

261 Appendix VIII/264 Appendix IX Hazardous Constituents

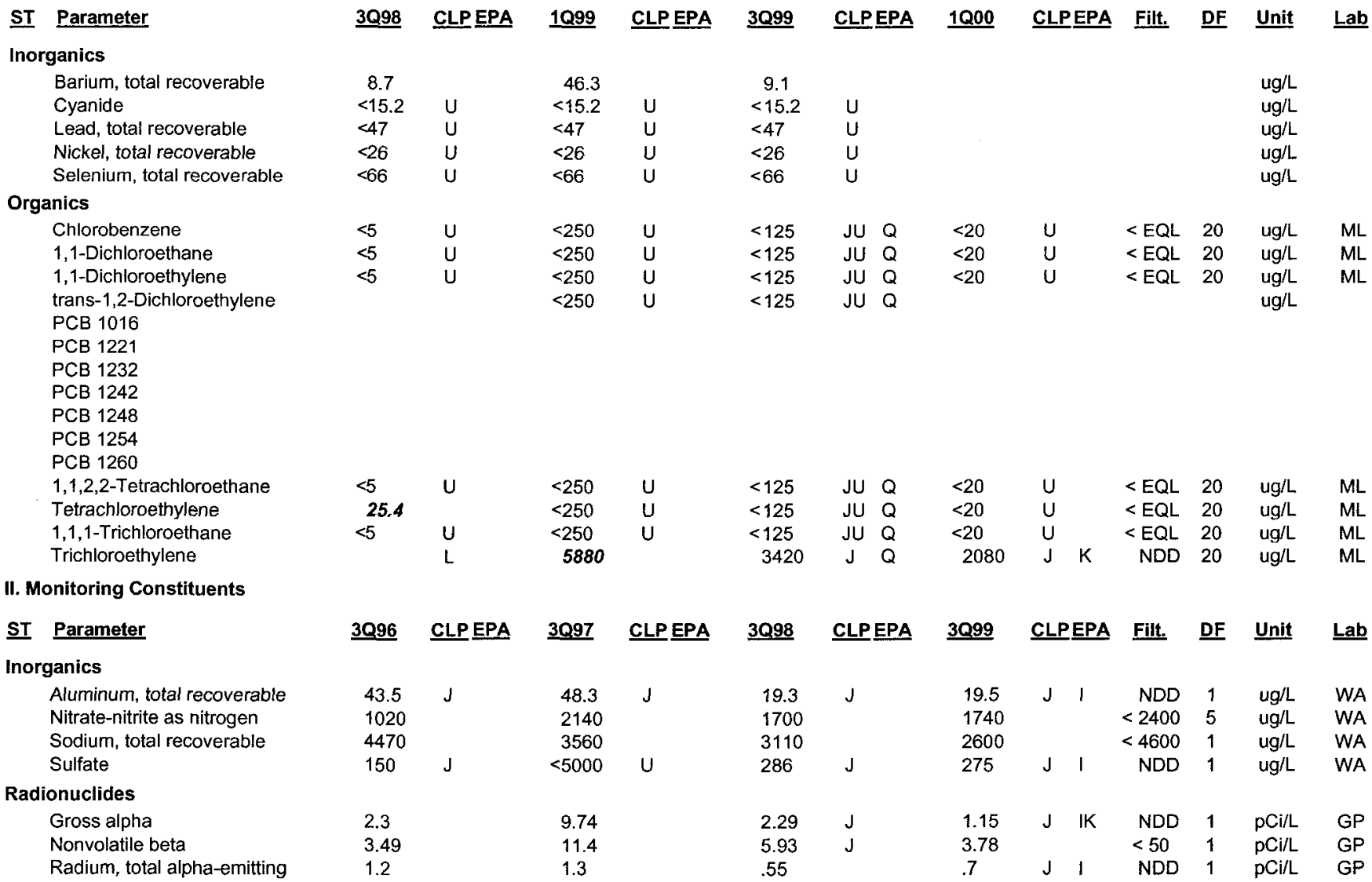

Notes: Concentrations in bold italics exceed the groundwater protection or monitoring constituent standards listed in Appendix A. Synchronous water Jevels are measured over a 3-5 day period or less. Dilution factors, Laboratory, and Filtered Data are for Groundwater Protection Standards First Quarter 2000 and Monitoring Constituents Third Quarter 1999 data only.

$+=$ exceeded the Groundwater Protection Standards (First Quarter 2000) or Monitoring Constituents (Third Quarter 1999) listed in Appendix A. 
Table D-4. Groundwater Monitoring Results for Plume Definition Wells, M-Area HWMF (Cont.) WELL ASB 8TA

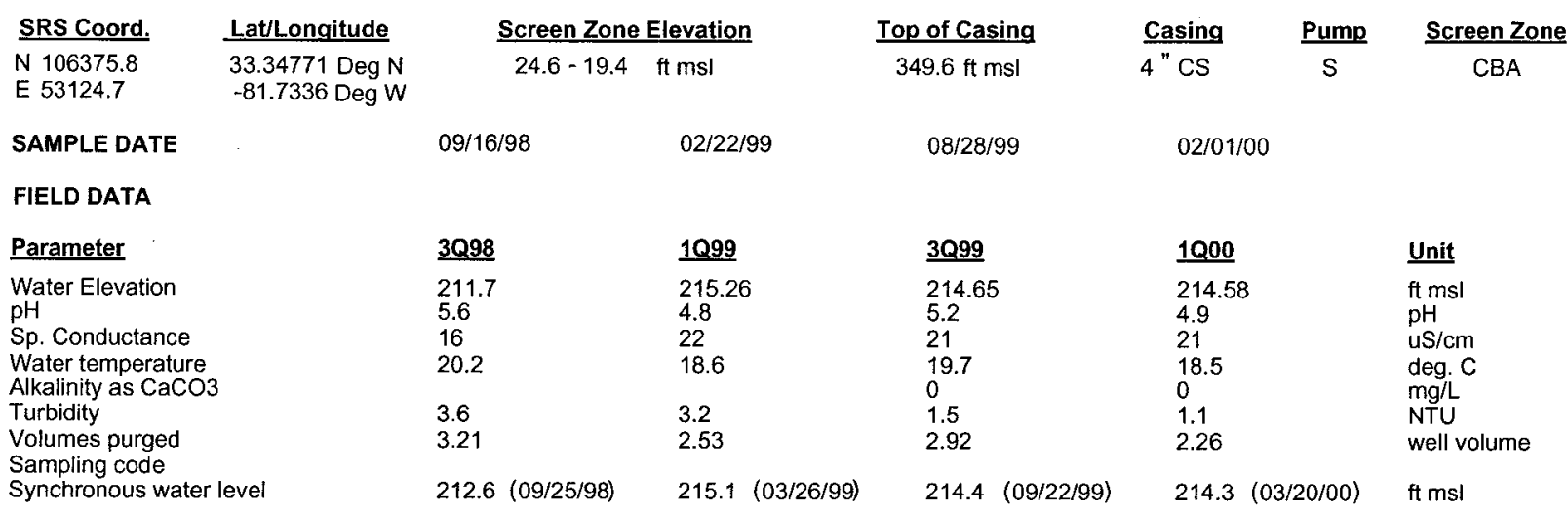

ANALYTICAL DATA

I. Groundwater Protection Standard

261 Appendix VIII/264 Appendix IX Hazardous Constituents

\begin{tabular}{|c|c|c|c|c|c|c|c|c|c|c|c|c|c|}
\hline$\underline{\text { ST }}$ & Parameter & $\underline{3 Q 98}$ & CLP EPA & $\underline{1099}$ & $\underline{\text { CLP EPA }}$ & 3099 & $\underline{\text { CLPEPA }}$ & 1000 & CLPEPA & Filt. & DF & $\underline{\text { Unit }}$ & $\underline{\text { Lab }}$ \\
\hline \multicolumn{14}{|c|}{ Inorganics } \\
\hline & Barium, total recoverable & 9.9 & & 8.3 & & 8.8 & & & & & & $\mathrm{ug} / \mathrm{L}$ & \\
\hline & Cyanide & $<15.2$ & $\mathrm{U}$ & $<15.2$ & $\mathrm{U}$ & $<15.2$ & $U$ & & & & & ug/L & \\
\hline & Lead, total recoverable & 9.9 & J & 14 & $\mathrm{~J} \quad \mathrm{I}$ & 12.8 & J I & & & & & $u g / L$ & \\
\hline & Nickel, total recoverable & $<26$ & $\mathrm{U}$ & $<50$ & $\mathrm{U}$ & $<26$ & $\mathrm{U}$ & & & & & $\mathrm{ug} / \mathrm{L}$ & \\
\hline & Selenium, total recoverable & $<66$ & $\mathrm{U}$ & $<200$ & $\mathrm{U}$ & $<66$ & $\mathrm{U}$ & & & & & $\mathrm{ug} / \mathrm{L}$ & \\
\hline \multicolumn{14}{|c|}{ Organics } \\
\hline & Chlorobenzene & $<5$ & $u$ & $<5$ & $\mathrm{U}$ & $<5$ & $U$ & $<1$ & JU & $<\mathrm{EQL}$ & 1 & $u g / L$ & $\mathrm{ML}$ \\
\hline & 1,1-Dichloroethane & $<5$ & $\mathrm{U}$ & $<5$ & $\mathrm{U}$ & $<5$ & $\mathrm{U}$ & $<1$ & JU & $<E Q L$ & 1 & $\mathrm{ug} / \mathrm{L}$ & ML \\
\hline & 1,1-Dichloroethylene & $<5$ & $\mathrm{U}$ & $<5$ & $\mathrm{U}$ & $<5$ & $\mathrm{U}$ & $<1$ & JU & $<E Q L$ & 1 & $u g / L$ & ML \\
\hline & $\begin{array}{l}\text { trans- } 1,2 \text {-Dichloroethylene } \\
\text { PCB } 1016\end{array}$ & $<5$ & $\mathrm{U}$ & $<5$ & $\mathrm{U}$ & $<5$ & U & $<1$ & JU & $<E Q L$ & 1 & $\mathrm{ug} / \mathrm{L}$ & ML \\
\hline & PCB 1221 & & & & & & & & & & & & \\
\hline & РСВ 1232 & & & & & & & & & & & & \\
\hline & PCB 1242 & & & & & & & & & & & & \\
\hline & PCB 1248 & & & & & & & & & & & & \\
\hline & PCB 1254 & & & & & & & & & & & & \\
\hline & PCB 1260 & & & & & & & & & & & & \\
\hline & 1,1,2,2-Tetrachloroethane & $<5$ & $U$ & $<5$ & $\mathrm{U}$ & $<5$ & $\mathrm{U}$ & $<1$ & JU & $<\mathrm{EQL}$ & 1 & ug/L & $M L$ \\
\hline & Tetrachloroethylene & $<5$ & $\mathrm{U}$ & $<5$ & $\mathrm{U}$ & $<5$ & $\mathrm{U}$ & $<1$ & JU & $<E Q L$ & 1 & $\mathrm{ug} / \mathrm{L}$ & $M L$ \\
\hline & 1,1,1-Trichloroethane & $<5$ & $U$ & $<5$ & $\mathrm{U}$ & $<5$ & $\mathrm{U}$ & $<1$ & JU & $<E Q L$ & 1 & $\mathrm{ug} / \mathrm{L}$ & ML \\
\hline & Trichloroethylene & $<5$ & $u$. & $<5$ & U & $<5$ & $\mathrm{U}$ & $<1$ & JU & $<\mathrm{EQL}$ & 1 & $\mathrm{ug} / \mathrm{L}$ & ML \\
\hline \multicolumn{14}{|c|}{ II. Monitoring Constituents } \\
\hline$\underline{\mathbf{S T}}$ & Parameter & $\underline{3096}$ & CLP EPA & $\underline{3097}$ & CLP EPA & $\underline{3 Q 98}$ & CLPEPA & $\underline{3 Q 99}$ & CLPEPA & Filt. & $\underline{\mathrm{DF}}$ & Unit & $\underline{\text { Lab }}$ \\
\hline \multicolumn{14}{|c|}{ Inorganics } \\
\hline & Aluminum, total recoverable & $<146$ & $\mathrm{U}$ & $<20$ & U & $<146$ & $\mathrm{U}$ & $<146$ & $\mathbf{u}$ & $<E Q L$ & 1 & ug/L & WA \\
\hline & Nitrate-nitrite as nitrogen & 1440 & & 1300 & & 1060 & & 1100 & & $<2400$ & 5 & ug/L & WA \\
\hline & Sodium, total recoverable & 1630 & & 1480 & & 1390 & & 1380 & & $<4600$ & 1 & $u g / L$ & WA \\
\hline & Sulfate & 318 & $J$ & $<5000$ & $\mathrm{U}$ & 288 & $\mathrm{~J}$ & 231 & $\mathrm{~J} \quad \mathrm{I}$ & NDD & 1 & $\mathrm{ug} / \mathrm{L}$ & WA \\
\hline \multicolumn{14}{|c|}{ Radionuclides } \\
\hline & Gross alpha & 1.79 & & 1.33 & & 1.37 & & .336 & $\mathbf{U}$ & $<\mathrm{EQL}$ & 1 & $\mathrm{pCi} / \mathrm{L}$ & GP \\
\hline & Nonvolatile beta & 1.8 & & .5 & U! & 2 & 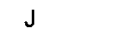 & 1.57 & J 1 & NDD & 1 & $\mathrm{pCi} / \mathrm{L}$ & GP \\
\hline & Radium, total alpha-emitting & 1.33 & & 2.24 & UIJ & .27 & UI & .3 & $\mathrm{U}$ & $<\mathrm{EQL}$ & 1 & $\mathrm{pCi} / \mathrm{L}$ & GP \\
\hline
\end{tabular}

Notes: Concentrations in bold italics exceed the groundwater protection or monitoring constituent standards listed in Appendix A. Synchronous water levels are measured over a 3-5 day period or less. Dilution factors, Laboratory, and Filtered Data are for Groundwater Protection Standards First Quarter 2000 and Monitoring Constituents Third Quarter 1999 data only.

+= exceeded the Groundwater Protection Standards (First Quarter 2000) or Monitoring Constituents (Third Quarter 1999) listed in Appendix A. 
Table D-4. Groundwater Monitoring Results for Plume Definition Wells, M-Area HWMF (Cont.) WELL ASB 9B

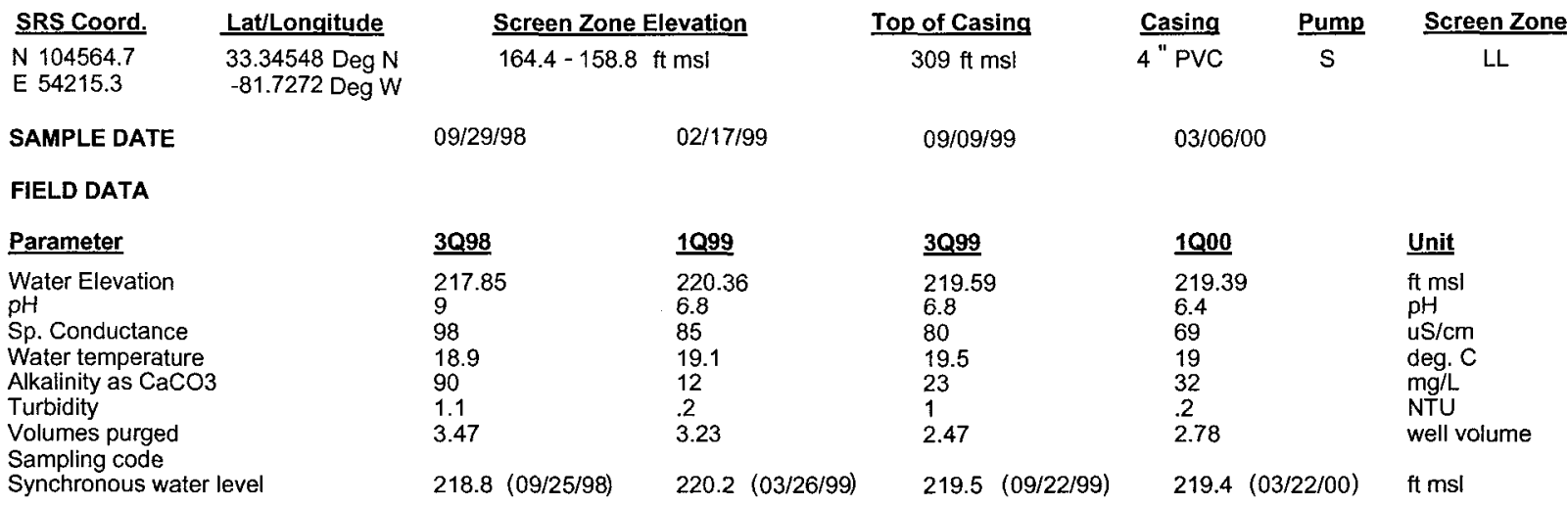

ANALYTICAL DATA

I. Groundwater Protection Standard

261 Appendix VIII/264 Appendix IX Hazardous Constituents

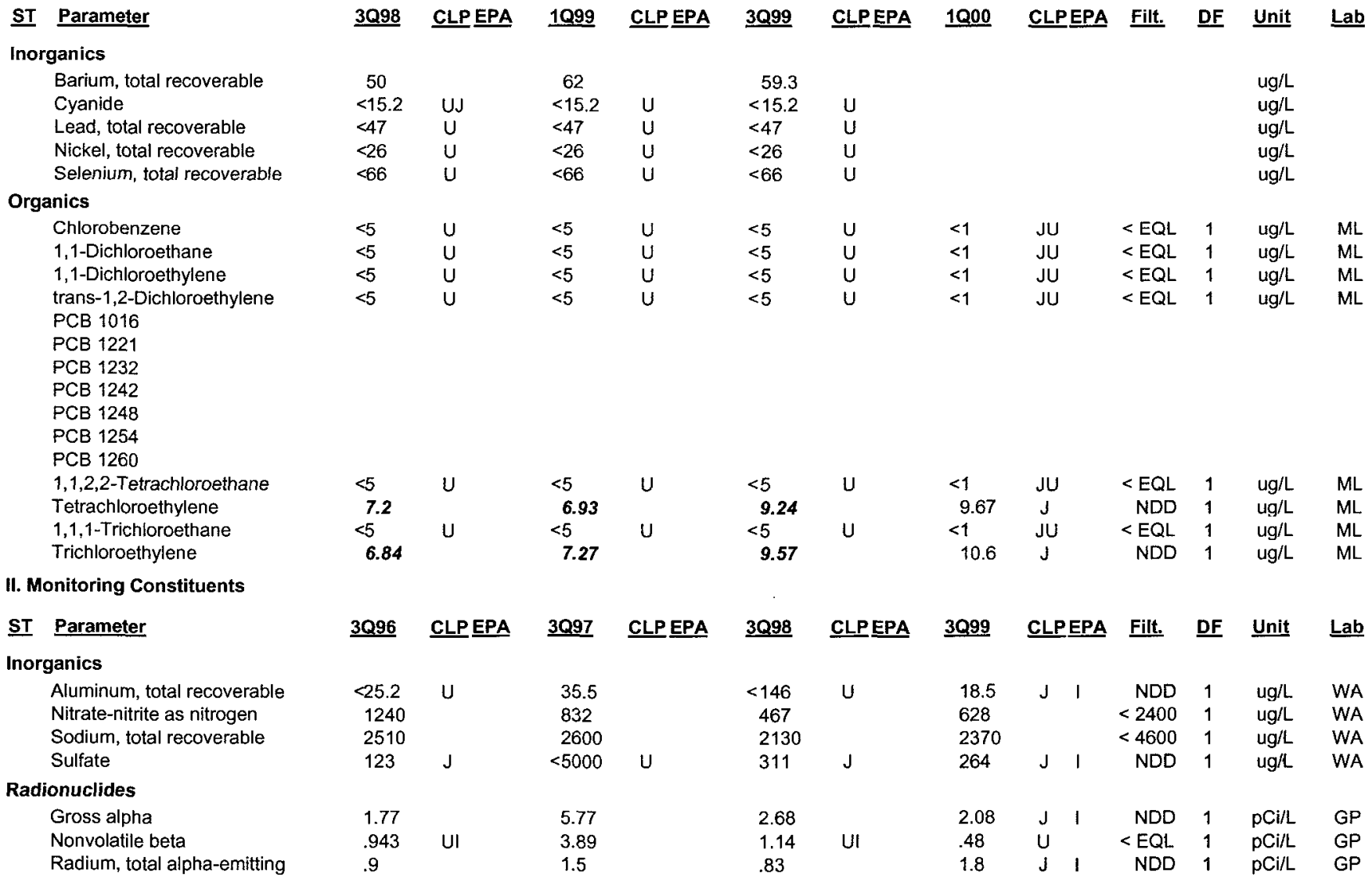

Notes: Concentrations in bold italics exceed the groundwater protection or monitoring constituent standards listed in Appendix A. Synchronous water levels are measured over a 3-5 day period or less. Dilution factors, Laboratory, and Filtered Data are for Groundwater Protection Standards First Quarter 2000 and Monitoring Constituents Third Quarter 1999 data only.

$+=$ exceeded the Groundwater Protection Standards (First Quarter 2000) or Monitoring Constituents (Third Quarter 1999) listed in Appendix A. 
Table D-4. Groundwater Monitoring Results for Plume Definition Wells, M-Area HWMF (Cont.) WELL ASB 9C

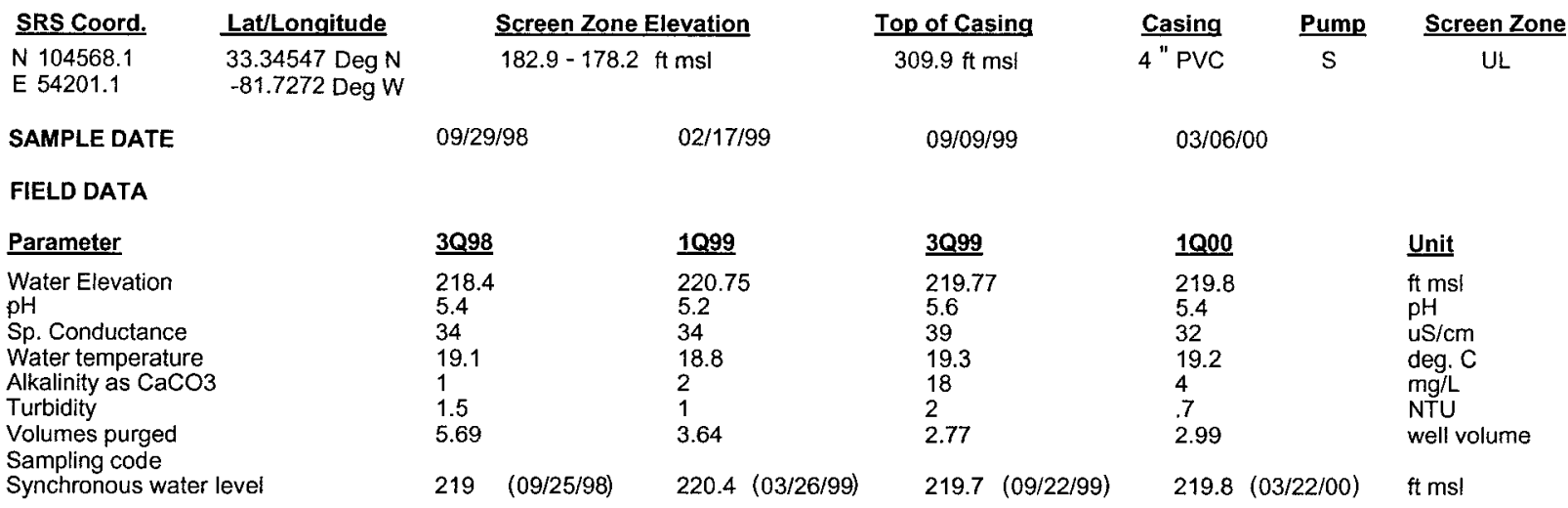

ANALYTICAL DATA

I. Groundwater Protection Standard

261 Appendix VIII/264 Appendix IX Hazardous Constituents

\begin{tabular}{|c|c|c|c|c|c|c|c|c|c|c|c|c|c|c|}
\hline \multirow{2}{*}{$\underline{\text { ST }}$} & \multirow{2}{*}{$\begin{array}{l}\text { Parameter } \\
\text { ganics }\end{array}$} & \multirow[t]{2}{*}{$\underline{3098}$} & \multirow[t]{2}{*}{ CLP EPA } & \multirow[t]{2}{*}{1099} & \multirow[t]{2}{*}{ CLP EPA } & \multirow[t]{2}{*}{$\underline{3 Q 99}$} & CLPEPA & \multirow[t]{2}{*}{1000} & \multicolumn{2}{|c|}{ CLPEPA } & \multirow[t]{2}{*}{ Filt. } & \multirow[t]{2}{*}{ DF } & \multirow[t]{2}{*}{ Unit } & $\underline{\text { Lab }}$ \\
\hline & & & & & & & & & & & & & & \\
\hline & Barium, total recoverable & 20.4 & & 19.4 & & 18.3 & & & & & & & $\mathrm{ug} / \mathrm{L}$ & \\
\hline & Cyanide & $<15.2$ & $\mathrm{U}$ & $<15.2$ & $\mathrm{U}$ & $<15.2$ & $\mathrm{U}$ & & & & & & ug $/ \mathrm{L}$ & \\
\hline & Lead, total recoverable & $<47$ & $\mathrm{U}$ & $<47$ & $\mathrm{U}$ & 7.1 & J 1 & & & & & & $u g / L$ & \\
\hline & Nickel, total recoverable & $<26$ & $U$ & $<26$ & $\mathrm{U}$ & $<26$ & $\mathrm{U}$ & & & & & & $\mathrm{ug} / \mathrm{L}$ & \\
\hline & Selenium, total recoverable & $<66$ & $\mathrm{U}$ & $<66$ & $\mathrm{U}$ & $<66$ & $\mathrm{U}$ & & & & & & $u g / L$ & \\
\hline \multicolumn{15}{|c|}{ Organics } \\
\hline & Chlorobenzene & $<5$ & $\mathrm{U}$ & $<5$ & $\mathrm{U}$ & $<5$ & $\mathrm{U}$ & $<1$ & JU & & $<$ EQL & 1 & ug/L & $M L$ \\
\hline & 1,1-Dichloroethane & $<5$ & $\mathrm{U}$ & $<5$ & $\mathrm{U}$ & $<5$ & $\mathrm{U}$ & $<1$ & $\mathrm{JU}$ & & $<E Q L$ & 1 & $\mathrm{ug} / \mathrm{L}$ & ML. \\
\hline & 1,1-Dichloroethylene & $<5$ & $\mathrm{U}$ & $<5$ & $\mathrm{U}$ & $<5$ & $\mathrm{U}$ & $<1$ & JU & & $<E Q L$ & 1 & $u g / L$ & ML \\
\hline & trans-1,2-Dichloroethylene & $<5$ & $\mathrm{U}$ & $<5$ & $\mathrm{U}$ & $<5$ & $\mathrm{U}$ & $<1$ & JU & & $<E Q L$ & 1 & $u g / L$ & ML \\
\hline & PCB 1016 & & & & & & & & & & & & & \\
\hline & PCB 1221 & & & & & & & & & & & & & \\
\hline & РCB 1232 & & & & & & & & & & & & & \\
\hline & PCB 1242 & & & & & & & & & & & & & \\
\hline & PCB 1248 & & & & & & & & & & & & & \\
\hline & РСВ 1254 & & & & & & & & & & & & & \\
\hline & РСВ 1260 & & & & & & & & & & & & & \\
\hline & 1,1,2,2-Tetrachloroethane & $<5$ & $\mathrm{U}$ & $<5$ & $\mathrm{U}$ & $<5$ & $\mathrm{U}$ & $<1$ & JU & & $<\mathrm{EQL}$ & 1 & $\mathrm{ug} / \mathrm{L}$ & ML \\
\hline & Tetrachloroethylene & 15.3 & & 11.8 & & 10.9 & & 11.6 & $\mathrm{~J}$ & & NDD & 1 & $\mathrm{ug} / \mathrm{L}$ & ML \\
\hline & 1,1,1-Trichloroethane & $<5$ & $\mathrm{U}$ & $<5$ & $\mathrm{U}$ & $<5$ & $\mathrm{U}$ & $<1$ & JU & & $<E Q L$ & 1 & $\mathrm{ug} / \mathrm{L}$ & ML \\
\hline & Trichloroethylene & 16.1 & & 11.8 & & 9.98 & & 11.4 & $J$ & & NDD & 1 & $\mathrm{ug} / \mathrm{L}$ & $M L$ \\
\hline \multicolumn{15}{|c|}{ II. Monitoring Constituents } \\
\hline$\underline{\text { ST }}$ & Parameter & $\underline{3096}$ & CLPEEPA & $\underline{3097}$ & CLP EPA & $\underline{3098}$ & CLPEEPA & $\underline{3099}$ & $\underline{\text { CLP }}$ & EPA & Filt. & $\underline{\mathrm{DF}}$ & Unit & $\underline{\text { Lab }}$ \\
\hline \multicolumn{15}{|c|}{ Inorganics } \\
\hline & Aluminum, total recoverable & $<20$ & $\mathrm{U}$ & 13.9 & $J$ & $<146$ & $\mathrm{U}$ & 22.7 & $J$ & 1 & NDD & 1 & ug/L & WA \\
\hline & Nitrate-nitrite as nitrogen & 900 & & 1010 & & 729 & & 773 & & & $<2400$ & 1 & ug/L & WA \\
\hline & Sodium, total recoverable & 2830 & $\mathrm{~J}$ & 2710 & & 2610 & & 2570 & & & $<4600$ & 1 & ug/L & WA \\
\hline & Sulfate & 137 & $J$ & $<5000$ & U & 323 & $J$ & 272 & $\mathrm{~J}$ & 1 & NDD & 1 & $\mathrm{ug} / \mathrm{L}$ & WA \\
\hline \multicolumn{15}{|c|}{ Radionuclides } \\
\hline & Gross alpha & 1.64 & & 1.08 & & 2.57 & & .799 & $J$ & 1 & NDD & 1 & $\mathrm{pCi} / \mathrm{L}$ & GP \\
\hline & Nonvolatile beta & 1.51 & UI & -.09 & UI & 1.25 & UI & .964 & $\mathrm{U}$ & & $<\mathrm{EQL}$ & 1 & $\mathrm{pCi} / \mathrm{L}$ & GP \\
\hline & Radium, total alpha-emitting & .7 & & 1.38 & UIJ & .44 & UI & .2 & $\mathrm{U}$ & & $<\mathrm{EQL}$ & 1 & $\mathrm{pCi} / \mathrm{L}$ & GP \\
\hline
\end{tabular}

Notes: Concentrations in bold italics exceed the groundwater protection or monitoring constituent standards listed in Appendix A. Synchronous water levels are measured over a 3-5 day period or less. Dilution factors, Laboratory, and Filtered Data are for Groundwater Protection Standards First Quarter 2000 and Monitoring Constituents Third Quarter 1999 data only.

$+=$ exceeded the Groundwater Protection Standards (First Quarter 2000) or Monitoring Constituents (Third Quarter 1999) listed in Appendix A. 
Table D-4. Groundwater Monitoring Results for Plume Definition Wells, M-Area HWMF (Cont.) WELL ASB 10CR

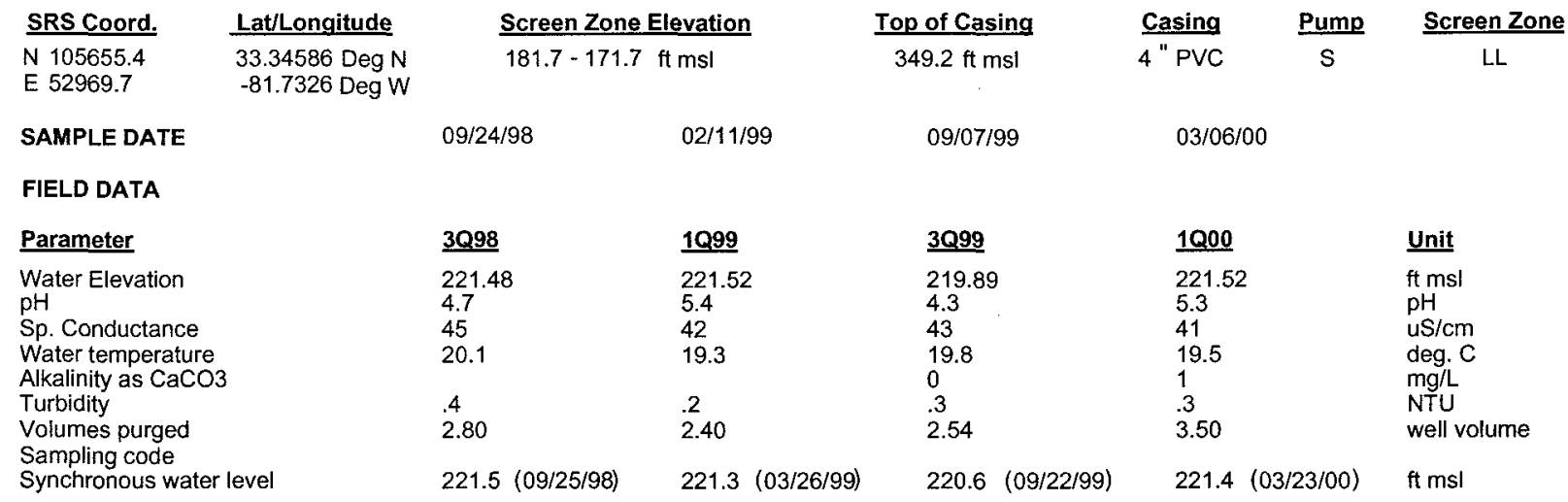

ANALYTICAL DATA

I. Groundwater Protection Standard

261 Appendix VIII/264 Appendix IX Hazardous Constituents

\begin{tabular}{|c|c|c|c|c|c|c|c|c|c|c|c|c|c|}
\hline$\underline{\mathrm{ST}}$ & Parameter & $\underline{3 Q 98}$ & $\underline{\text { CLP EPA }}$ & $\underline{1 Q 99}$ & CLP EPA & 3Q99 & CLPPEPA & $\underline{1 Q 00}$ & CLPEPA & Filt. & $\underline{\mathrm{DF}}$ & $\underline{\text { Unit }}$ & $\underline{\text { Lab }}$ \\
\hline \multicolumn{14}{|c|}{ Inorganics } \\
\hline & Barium, total recoverable & 13 & & 8.6 & & 8.6 & & & & & & ug/L & \\
\hline & Cyanide & $<15.2$ & $\mathrm{U}$ & $<15.2$ & $\mathrm{U}$ & $<15.2$ & $u$ & & & & & ug/L & \\
\hline & Lead, total recoverable & $<4$ & U & $<47$ & U & $<47$ & U & & & & & $u g / L$ & \\
\hline & Nickel, total recoverable & $<26$ & U & $<26$ & $\mathrm{U}$ & $<26$ & $U$ & & & & & $\mathrm{ug} / \mathrm{L}$ & \\
\hline & Selenium, total recoverable & $<66$ & $\mathrm{U}$ & $<66$ & $\mathrm{U}$ & $<66$ & U & & & & & $\mathrm{ug} / \mathrm{L}$ & \\
\hline \multicolumn{14}{|c|}{ Organics } \\
\hline & Chlorobenzene & $<10$ & $U$ & $<5$ & $U$ & $<5$ & $\mathrm{U}$ & $<1$ & $\mathrm{JU}$ & $<E Q L$ & 1 & $\mathrm{ug} / \mathrm{L}$ & ML \\
\hline & 1,1-Dichloroethane & $<10$ & $U$ & $<5$ & $\mathrm{U}$ & $<5$ & $U$ & $<1$ & JU & $<E Q L$ & 1 & $\mathrm{ug} / \mathrm{L}$ & ML \\
\hline & 1,1-Dichloroethylene & $<10$ & U & $<5$ & $U$ & $<5$ & $U$ & $<1$ & JU & $<E Q L$ & 1 & ug/L. & $M L$ \\
\hline & trans-1,2-Dichloroethylene & $<10$ & $\mathrm{U}$ & $<5$ & $\mathrm{U}$ & $<5$ & $\mathrm{U}$ & $<1$ & JU & $<E Q L$ & 1 & $u g / L$ & $M L$ \\
\hline & PCB 1016 & & & & & & & & & & & & \\
\hline & PCB 1221 & & & & & & & & & & & & \\
\hline & PCB 1232 & & & & & & & & & & & & \\
\hline & PCB 1242 & & & & & & & & & & & & \\
\hline & РСB 1248 & & & & & & & & & & & & \\
\hline & PCB 1254 & & & & & & & & & & & & \\
\hline & PCB 1260 & & & & & & & & & & & & \\
\hline & 1,1,2,2-Tetrachloroethane & $<10$ & U & $<5$ & $U$ & $<5$ & U & $<1$ & JU & $<$ EQL & 1 & $\mathrm{ug} / \mathrm{L}$ & $\mathrm{ML}$ \\
\hline & Tetrachloroethylene & 6.71 & J & $<5$ & $U$ & 3.86 & J I & 3.35 & $J$ & NDD & 1 & $\mathrm{ug} / \mathrm{L}$ & $M L$ \\
\hline & 1,1,1-Trichloroethane & $<10$ & $\mathrm{U}$ & $<5$ & $U$ & $<5$ & $\mathrm{U}$ & $<1$ & JU & $<E Q L$ & 1 & $u g / L$ & $M L$ \\
\hline & Trichloroethylene & 223 & & 192 & & 179 & & 179 & $J$ & NDD & 1 & $u g / L$ & ML \\
\hline \multicolumn{14}{|c|}{ II. Monitoring Constituents } \\
\hline$\underline{\text { ST }}$ & Parameter & $\underline{3096}$ & CLP EPA & $\underline{3097}$ & CLP EPA & $\underline{3 Q 98}$ & CLPEPA & $\underline{3099}$ & CLPEPA & Filt. & $\underline{\mathrm{DF}}$ & Unit & Lab \\
\hline \multicolumn{14}{|c|}{ Inorganics } \\
\hline & Aluminum, total recoverable & $<20$ & $U$ & & & 16.8 & J & $<146$ & $U$ & $<E Q L$ & 1 & ug/L & WA \\
\hline & Nitrate-nitrite as nitrogen & 1370 & & & & 1260 & & 1260 & & $<2400$ & 5 & $\mathrm{ug} / \mathrm{L}$ & WA \\
\hline+ & Sodium, total recoverable & 38500 & $\mathrm{~J}$ & & & 5080 & & 4910 & & $>4600$ & 1 & ug/L & WA \\
\hline & Sulfate & 11700 & & & & 400 & & 399 & & $<3000$ & 1 & $u g / L$ & WA \\
\hline \multicolumn{14}{|c|}{ Radionuclides } \\
\hline & Gross alpha & 1.04 & & & & 2.21 & & .827 & $\mathrm{~J} \quad \mathrm{I}$ & NDD & 1 & $\mathrm{pCi} / \mathrm{L}$ & GP \\
\hline & Nonvolatile beta & 1.16 & UI & & & 13.23 & $J$ & 2.5 & & $<50$ & 1 & $\mathrm{pCi} / \mathrm{L}$ & GP \\
\hline & Radium, total alpha-emitting & .9 & & & & .41 & & .3 & $\mathrm{U}$ & $<E Q L$ & 1 & $\mathrm{pCi} / \mathrm{L}$ & GP \\
\hline
\end{tabular}

Notes: Concentrations in bold italics exceed the groundwater protection or monitoring constituent standards listed in Appendix A. Synchronous water levels are measured over a 3-5 day period or less. Dilution factors, Laboratory, and Filtered Data are for Groundwater Protection Standards First Quarter 2000 and Monitoring Constituents Third Quarter 1999 data only.

+ = exceeded the Groundwater Protection Standards (First Quarter 2000) or Monitoring Constituents (Third Quarter 1999) listed in Appendix A. 
Table D-4. Groundwater Monitoring Results for Plume Definition Wells, M-Area HWMF (Cont.) WELL MCB 5C

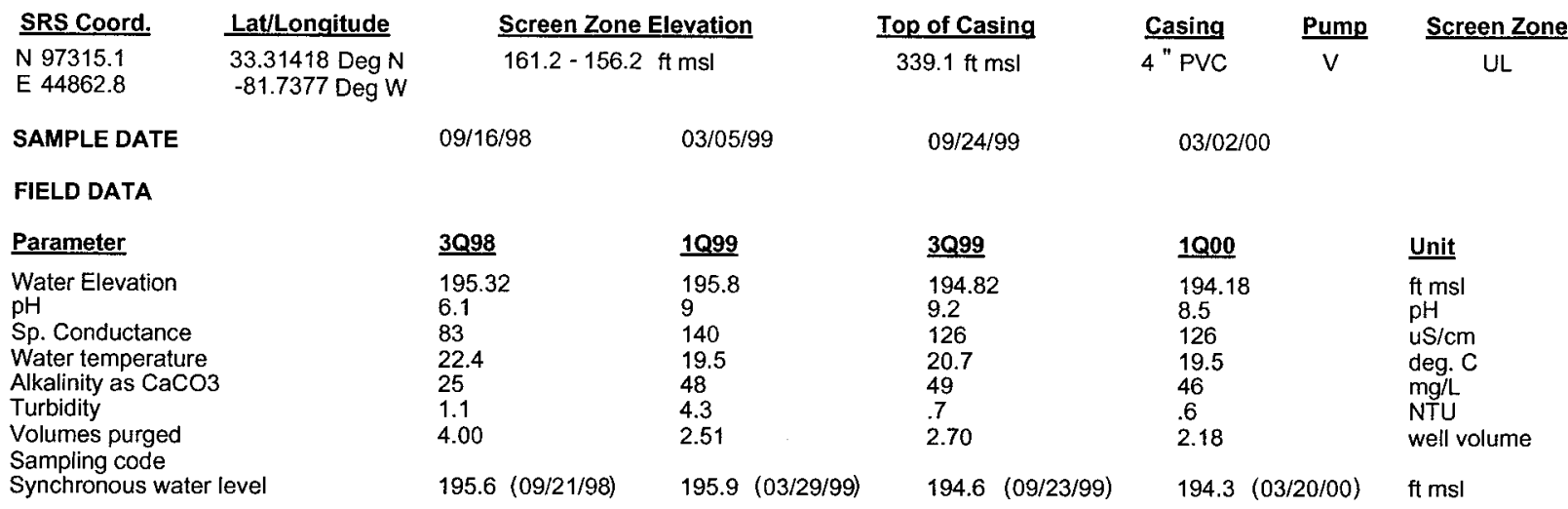

ANALYTICAL DATA

I. Groundwater Protection Standard

261 Appendix VIII/264 Appendix IX Hazardous Constituents

\begin{tabular}{|c|c|c|c|c|c|c|c|c|c|c|c|c|c|c|}
\hline$\underline{\text { ST }}$ & Parameter & $\underline{3098}$ & CLPP EPA & 1099 & CLPEPA & $\underline{3099}$ & CLPEPA & $1 \mathrm{Q00}$ & $\underline{\text { CLF }}$ & EPA & Filt. & $\underline{\mathrm{DF}}$ & Unit & $\underline{\text { Lab }}$ \\
\hline \multicolumn{15}{|c|}{ Inorganics } \\
\hline & Barium, total recoverable & & & 62.3 & & 77.8 & & & & & & & $u g / L$ & \\
\hline & Cyanide & & & $<15.2$ & $\mathrm{U}$ & $<10$ & U & & & & & & $\mathrm{ug} / \mathrm{L}$ & \\
\hline & Lead, total recoverable & & & $<47$ & $\mathrm{U}$ & $<10$ & U & & & & & & ug/L & \\
\hline & Nickel, total recoverable & & & $<26$ & $\mathrm{U}$ & $<50$ & $\mathrm{U}$ & & & & & & $\mathrm{ug} / \mathrm{L}$ & \\
\hline & Selenium, total recoverable & & & $<66$ & $\mathrm{U}$ & $<10$ & $\mathrm{U}$ & & & & & & $\mathrm{ug} / \mathrm{L}$ & \\
\hline \multicolumn{15}{|c|}{ Organics } \\
\hline & Chlorobenzene & & & $<5$ & $\mathrm{U}$ & $<5$ & $\mathrm{U}$ & $<1$ & $\mathrm{U}$ & & $<E Q L$ & 1 & $\mathrm{ug} / \mathrm{L}$ & $M L$ \\
\hline & 1,1-Dichloroethane & & & $<5$ & $U$ & $<5$ & $\mathrm{U}$ & $<1$ & $\mathrm{U}$ & & $<\mathrm{EQL}$ & 1 & $\mathrm{ug} / \mathrm{L}$ & $M L$ \\
\hline & 1,1-Dichloroethylene & & & $<5$ & $U$ & $<5$ & $\mathrm{U}$ & .97 & $\mathrm{~J}$ & 1 & NDD & 1 & $\mathrm{ug} / \mathrm{L}$ & $M L$ \\
\hline & trans-1,2-Dichloroethylene & & & $<5$ & $\mathrm{U}$ & $<5$ & $\mathrm{U}$ & & & & & & ug/L & \\
\hline & РСВ 1016 & & & & & & & & & & & & & \\
\hline & PCB 1221 & & & & & & & & & & & & & \\
\hline & РСВ 1232 & & & & & & & & & & & & & \\
\hline & PCB 1242 & & & & & & & & & & & & & \\
\hline & PCB 1248 & & & & & & & & & & & & & \\
\hline & PCB 1254 & & & & & & & & & & & & & \\
\hline & РСВ 1260 & & & & & & & & & & & & & \\
\hline & 1,1,2,2-Tetrachloroethane & & & $<5$ & U & $<5$ & $\mathrm{U}$ & $<1$ & $\mathrm{u}$ & & $<\mathrm{EQL}$ & 1 & ug/L & $M L$ \\
\hline+ & Tetrachloroethylene & & & 69.8 & & 70 & & 74.8 & & & $>5$ & 1 & $\mathrm{ug} / \mathrm{L}$ & $\mathrm{ML}$ \\
\hline & 1,1,1-Trichloroethane & & & $<5$ & $\mathrm{U}$ & $<5$ & $U$ & $<1$ & $U$ & & $<\mathrm{EQL}$ & 1 & $\mathrm{ug} / \mathrm{L}$ & $M L$ \\
\hline+ & Trichloroethylene & & & 64 & & 61 & & 59 & & & $>5$ & 1 & $\mathrm{ug} / \mathrm{L}$ & $M L$ \\
\hline \multicolumn{15}{|c|}{ II. Monitoring Constituents } \\
\hline$\underline{\mathrm{ST}}$ & Parameter & $\underline{3096}$ & CLP틀 & $\underline{3 Q 97}$ & CLP EPA & $\underline{3098}$ & CLPEPA & $\underline{3099}$ & $\underline{\text { CLP }}$ & EPA & Filt. & $\underline{\text { DF }}$ & Unit & $\underline{\text { Lab }}$ \\
\hline \multicolumn{15}{|c|}{ Inorganics } \\
\hline & Aluminum, total recoverable & 128 & $J$ & 67 & & & & 173 & $\mathrm{~J}$ & 1 & NDD & 1 & ug/L & EX \\
\hline+ & Nitrate-nitrite as nitrogen & 4200 & & 4320 & & & & 3790 & & & $>2400$ & 5 & $\mathrm{ug} / \mathrm{L}$ & $\mathrm{EX}$ \\
\hline+ & Sodium, total recoverable & 6210 & & 5140 & & & & 5610 & & & $>4600$ & 1 & $\mathrm{ug} / \mathrm{L}$ & EX \\
\hline & Sulfate & 881 & $J$ & $<5000$ & U & & & 960 & & & $<3000$ & 2 & $u g / L$ & EX \\
\hline \multicolumn{15}{|c|}{ Radionuclides } \\
\hline & Gross alpha & 1.57 & $J$ & 3.53 & & 3.04 & & 1.64 & $J$ & 1 & NDD & 1 & $\mathrm{pCi} / \mathrm{L}$ & GP \\
\hline & Nonvolatile beta & 5.09 & & 4.26 & & 5.68 & $\mathrm{~J}$ & 5.07 & & & $<50$ & 1 & $\mathrm{pCi} / \mathrm{L}$ & GP \\
\hline & Radium, total alpha-emitting & .2 & UI & 2.22 & UIJ & .5 & UI & 1.2 & $J$ & I & NDD & 1 & $\mathrm{pCi} / \mathrm{L}$ & $\mathrm{GP}$ \\
\hline
\end{tabular}

Notes: Concentrations in bold italics exceed the groundwater protection or monitoring constituent standards listed in Appendix A. Synchronous water levels are measured over a 3-5 day period or less. Dilution factors, Laboratory, and Filtered Data are for Groundwater Protection Standards First Quarter 2000 and Monitoring Constituents Third Quarter 1999 data only.

+= exceeded the Groundwater Protection Standards (First Quarter 2000) or Monitoring Constituents (Third Quarter 1999) listed in Appendix A. 
Table D-4. Groundwater Monitoring Results for Plume Definition Wells, M-Area HWMF (Cont.) WELL MCB 6C

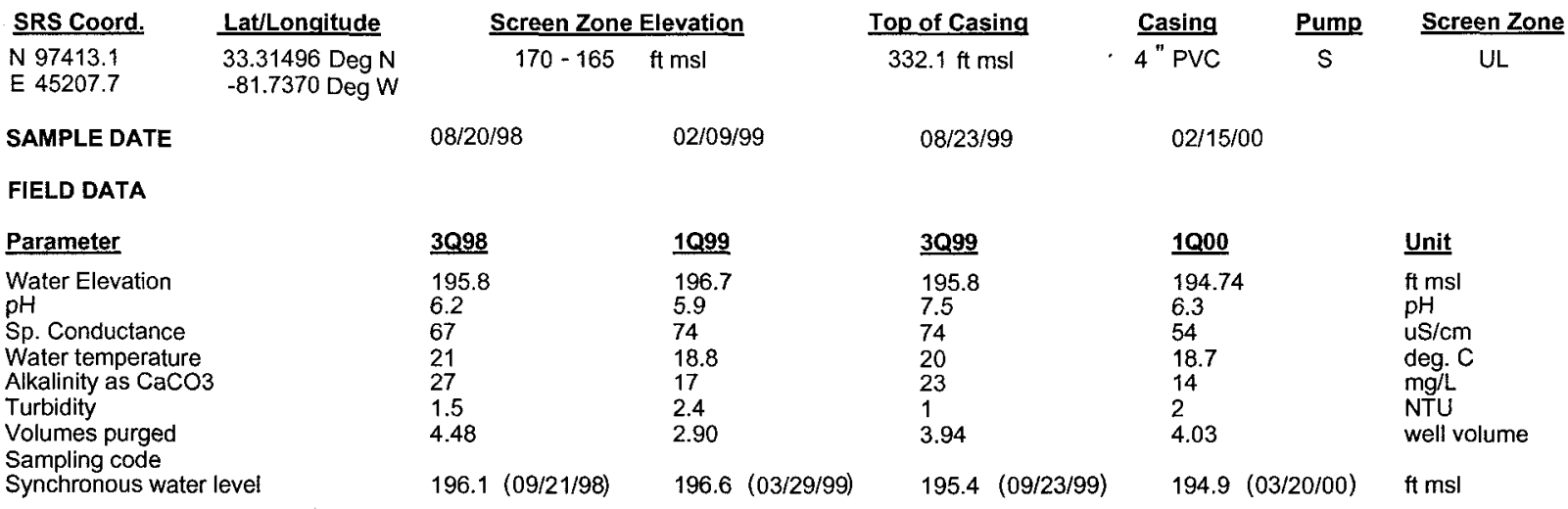

ANALYTICAL DATA

1. Groundwater Protection Standard

261 Appendix VIII/264 Appendix IX Hazardous Constituents

\begin{tabular}{|c|c|c|c|c|c|c|c|c|c|c|c|c|c|c|}
\hline$\underline{\text { ST }}$ & Parameter & 3Q98 & CLPEPA & $1 Q 99$ & CLP. & EPA & 3Q99 & CLPEPA & 1000 & CLPEPA & Filt. & DF & Unit & $\underline{\text { Lab }}$ \\
\hline Inor & ganics & & & & & & & & & & & & & \\
\hline & Barium, total recoverable & 36.2 & & 35 & & & 40 & & & & & & ug/L & \\
\hline & Cyanide & $<15.2$ & $\mathrm{U}$ & $<15.2$ & U & & $<10$ & $\mathrm{U}$ & & & & & ug/L & \\
\hline & Lead, total recoverable & $<47$ & $\mathrm{U}$ & $<47$ & $U$ & & $<10$ & $U$ & & & & & $\mathrm{ug} / \mathrm{L}$ & \\
\hline & Nickel, total recoverable & $<26$ & $U$ & $<26$ & U & & $<50$ & $U$ & & & & & $\mathrm{ug} / \mathrm{L}$ & \\
\hline & Selenium, total recoverable & $<66$ & U & $<66$ & $U$ & & $<10$ & $U$ & & & & & ug/L & \\
\hline Org & anics & & & & & & & & & & & & & \\
\hline & Chlorobenzene & $<5$ & $U$ & $<5$ & JU & $Q$ & $<5$ & $U$ & $<1$ & $U$ & $<\mathrm{EQL}$ & 1 & $\mathrm{ug} / \mathrm{L}$ & ML \\
\hline & 1,1-Dichloroethane & $<5$ & $U$ & $<5$ & JU & $Q$ & $<5$ & $U$ & $<1$ & $U$ & $<\mathrm{EQL}$ & 1 & $\mathrm{ug} / \mathrm{L}$ & ML \\
\hline & 1,1-Dichloroethylene & $<5$ & U & $<5$ & JU & $Q$ & $<5$ & $\mathrm{U}$ & $<1$ & $\mathrm{U}$ & $<\mathrm{EQL}$ & 1 & ug/L & $M L$ \\
\hline & trans-1,2-Dichloroethylene & $<5$ & $\mathrm{U}$ & $<5$ & JU & $Q$ & $<5$ & $U$ & $<1$ & $\mathrm{U}$ & $<\mathrm{EQL}$ & 1 & ug/L. & ML \\
\hline & PCB 1016 & & & & & & & & & & & & & \\
\hline & PCB 1221 & & & & & & & & & & & & & \\
\hline & PCB 1232 & & & & & & & & & & & & & \\
\hline & PCB 1242 & & & & & & & & & & & & & \\
\hline & PCB 1248 & & & & & & & & & & & & & \\
\hline & PCB 1254 & & & & & & & & & & & & & \\
\hline & PCB 1260 & & & & & & & & & & & & & \\
\hline & 1,1,2,2-Tetrachloroethane & $<5$ & $U$ & $<5$ & $J U$ & $Q$ & $<5$ & $\mathrm{U}$ & $<1$ & $U$ & $<\mathrm{EQL}$ & 1 & ug/L & $\mathrm{ML}$ \\
\hline & Tetrachloroethylene & $<5$ & $\mathrm{U}$ & $<5$ & JU & $Q$ & $<5$ & $\mathrm{U}$ & 1.38 & & $<5$ & 1 & ug/L & ML \\
\hline & 1,1,1-Trichloroethane & $<5$ & U & $<5$ & JU & $Q$ & $<5$ & $\mathrm{U}$ & $<1$ & $\mathrm{U}$ & $<\mathrm{EQL}$ & 1 & ug/L & $\mathrm{ML}$ \\
\hline+ & Trichloroethylene & 4.99 & $\mathrm{~J}$ & 4.23 & $\jmath$ & $1 Q$ & 8.7 & & 10.8 & & $>5$ & 1 & $\mathrm{ug} / \mathrm{L}$ & $\mathrm{ML}$ \\
\hline II. M & onitoring Constituents & & & & & & & & & & & & & \\
\hline$\underline{\text { ST }}$ & Parameter & $\underline{3096}$ & CLP EPA & 3Q97 & CLP & EPA & $\underline{3098}$ & CLPEPA & $\underline{3099}$ & CLPEPA & Filt. & $\underline{\text { DF }}$ & Unit & $\underline{\mathrm{Lab}}$ \\
\hline Inor & ganics & & & & & & & & & & & & & \\
\hline & Aluminum, total recoverable & 34.6 & & 63.6 & & & 34.7 & J & $<200$ & $U$ & $<$ EQL & 1 & $u g / L$ & EX \\
\hline & Nitrate-nitrite as nitrogen & 1050 & & 962 & & & 770 & & 915 & & $<2400$ & 5 & $u g / L$ & EX \\
\hline & Sodium, total recoverable & 2720 & & 3090 & & & 2350 & & 2800 & & $<4600$ & 1 & ug/L & EX \\
\hline & Sulfate & 2570 & & 2500 & & & 2210 & & 2260 & & $<3000$ & 1 & ug/L & EX \\
\hline Rad & ionuclides & & & & & & & & & & & & & \\
\hline & Gross alpha & 2.07 & J & .05 & $U$ & & .63 & & .0701 & $\mathrm{U}$ & $<E Q L$ & 1 & $\mathrm{pCl} / \mathrm{L}$ & GP \\
\hline & Nonvolatile beta & 3.33 & & .22 & UIJ & & 2.1 & & 2.46 & $\mathrm{~J} \quad \mathrm{I}$ & NDD & 1 & $\mathrm{pCi} / \mathrm{L}$ & GP \\
\hline & Radium, total alpha-emitting & .45 & J & .54 & UIJ & & .06 & UI & -.1 & U & < EQL & 1 & $\mathrm{pCi} / \mathrm{L}$ & $P$ \\
\hline
\end{tabular}

\footnotetext{
Notes: Concentrations in bold italics exceed the groundwater protection or monitoring constituent standards listed in Appendix A. Synchronous wate levels are measured over a 3-5 day period or less. Dilution factors, Laboratory, and Filtered Data are for Groundwater Protection Standards First Quarter 2000 and Monitoring Constituents Third Quarter 1999 data only.

$+=$ exceeded the Groundwater Protection Standards (First Quarter 2000) or Monitoring Constituents (Third Quarter 1999) listed in Appendix A.
} 
Table D-4. Groundwater Monitoring Results for Plume Definition Wells, M-Area HWMF (Cont.) WELL MCB 7C

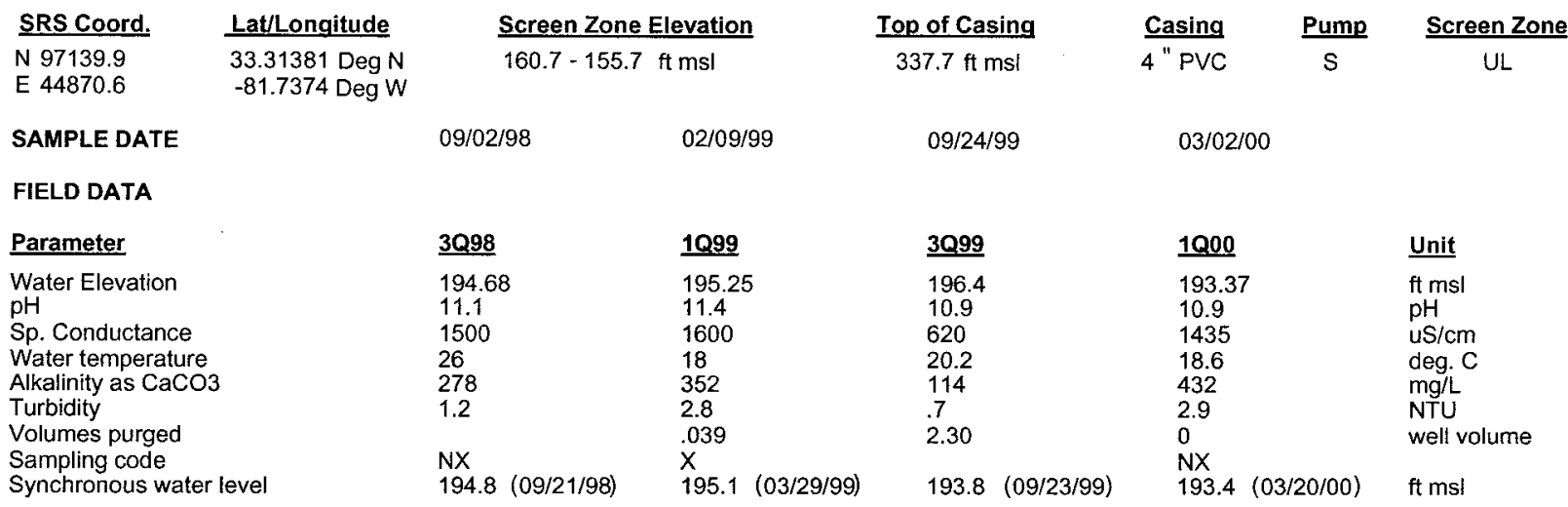

ANALYTICAL DATA

I. Groundwater Protection Standard

261 Appendix VIII/264 Appendix IX Hazardous Constituents

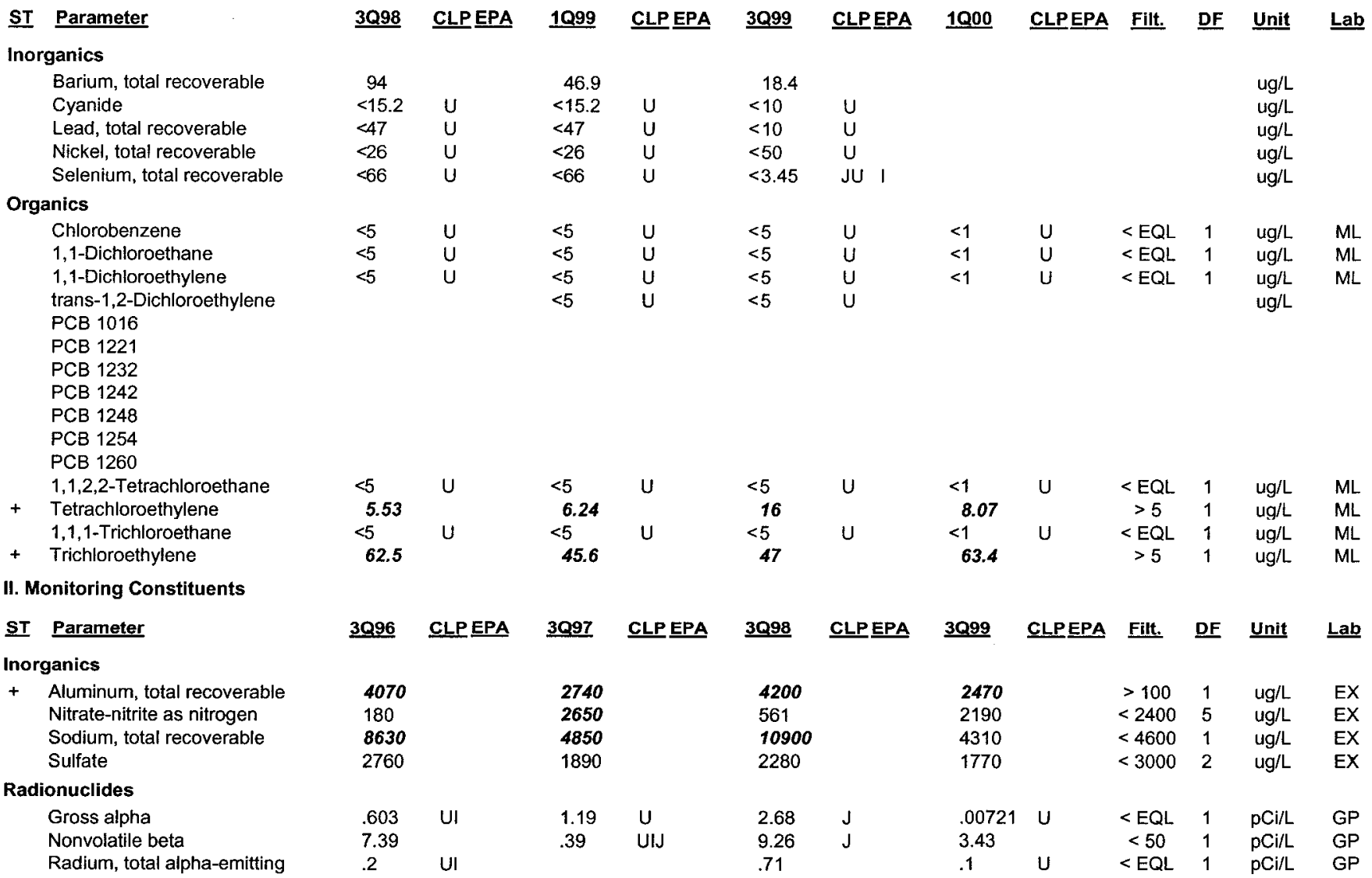

Notes: Concentrations in bold italics exceed the groundwater protection or monitoring constituent standards listed in Appendix A. Synchronous water levels are measured over a 3-5 day period or less. Dilution factors, Laboratory, and Filtered Data are for Groundwater Protection Standards First Quarter 2000 and Monitoring Constituents Third Quarter 1999 data only.

+ = exceeded the Groundwater Protection Standards (First Quarter 2000) or Monitoring Constituents (Third Quarter 1999) listed in Appendix A. 
Table D-4. Groundwater Monitoring Results for Plume Definition Wells, M-Area HWMF (Cont.) WELL MSB 9A

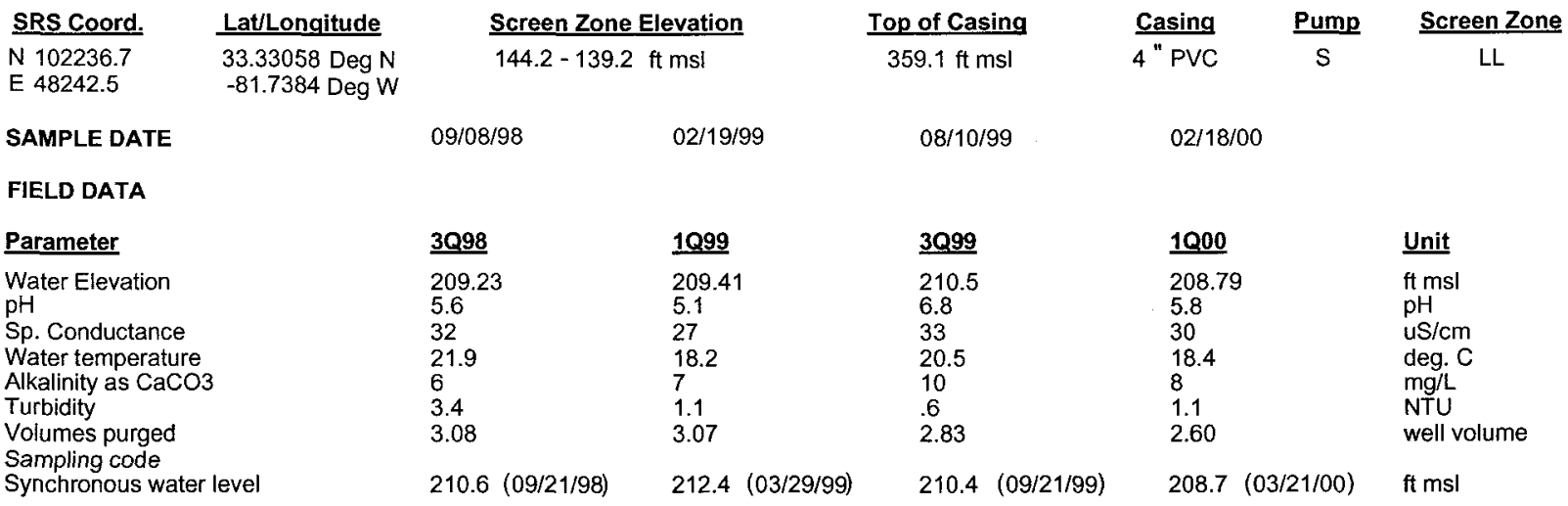

ANALYTICAL DATA

I. Groundwater Protection Standard

261 Appendix VIII/264 Appendix IX Hazardous Constituents

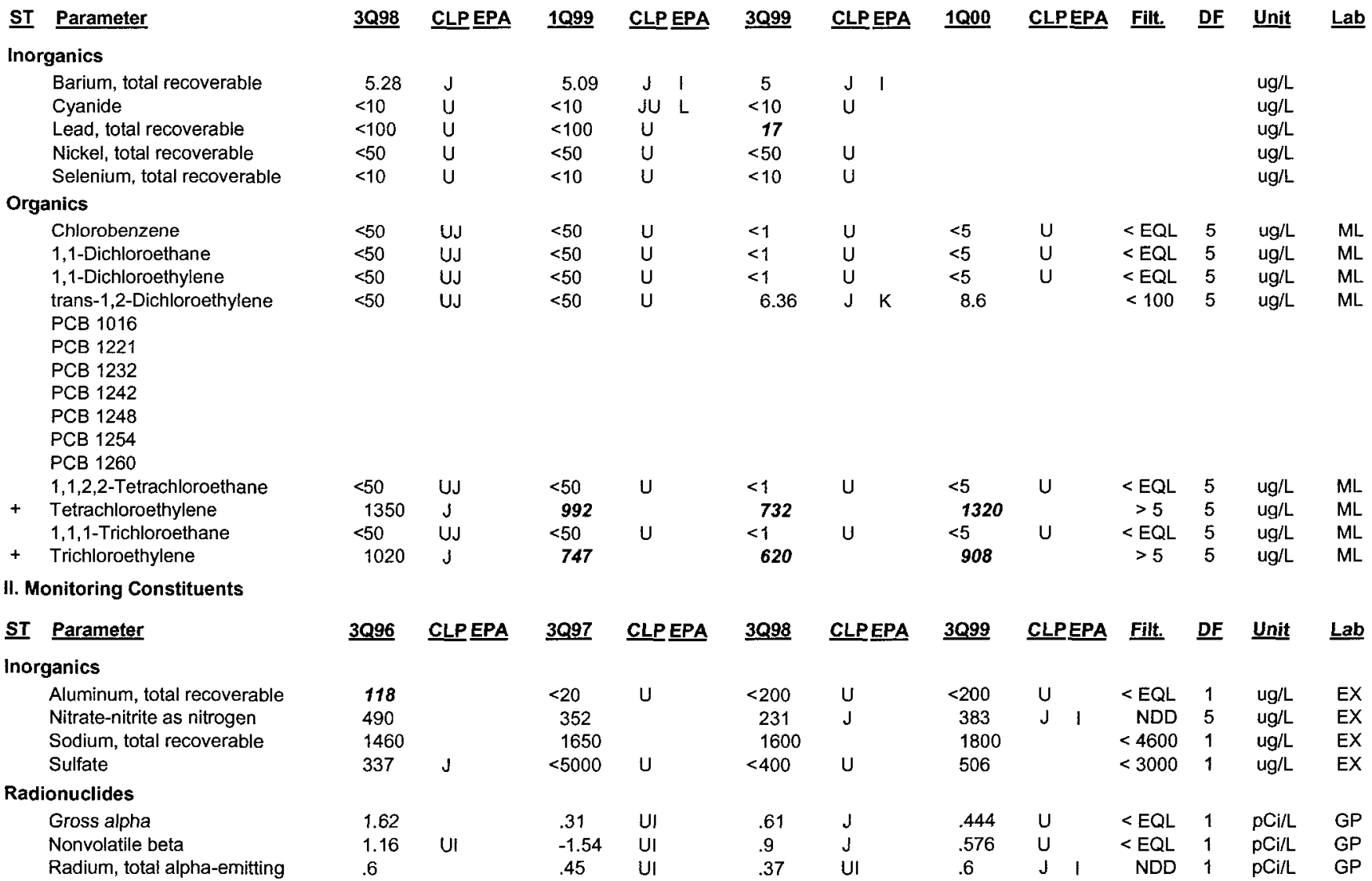

Notes: Concentrations in bold italics exceed the groundwater protection or monitoring constituent standards listed in Appendix A. Synchronous water levels are measured over a 3-5 day period or less. Dilution factors, Laboratory, and Filtered Data are for Groundwater Protection Standards First Quarter 2000 and Monitoring Constituents Third Quarter 1999 data only.

$+=$ exceeded the Groundwater Protection Standards (First Quarter 2000) or Monitoring Constituents (Third Quarter 1999) listed in Appendix A. 
Table D-4. Groundwater Monitoring Results for Plume Definition Wells, M-Area HWMF (Cont.) WELL MSB 10A

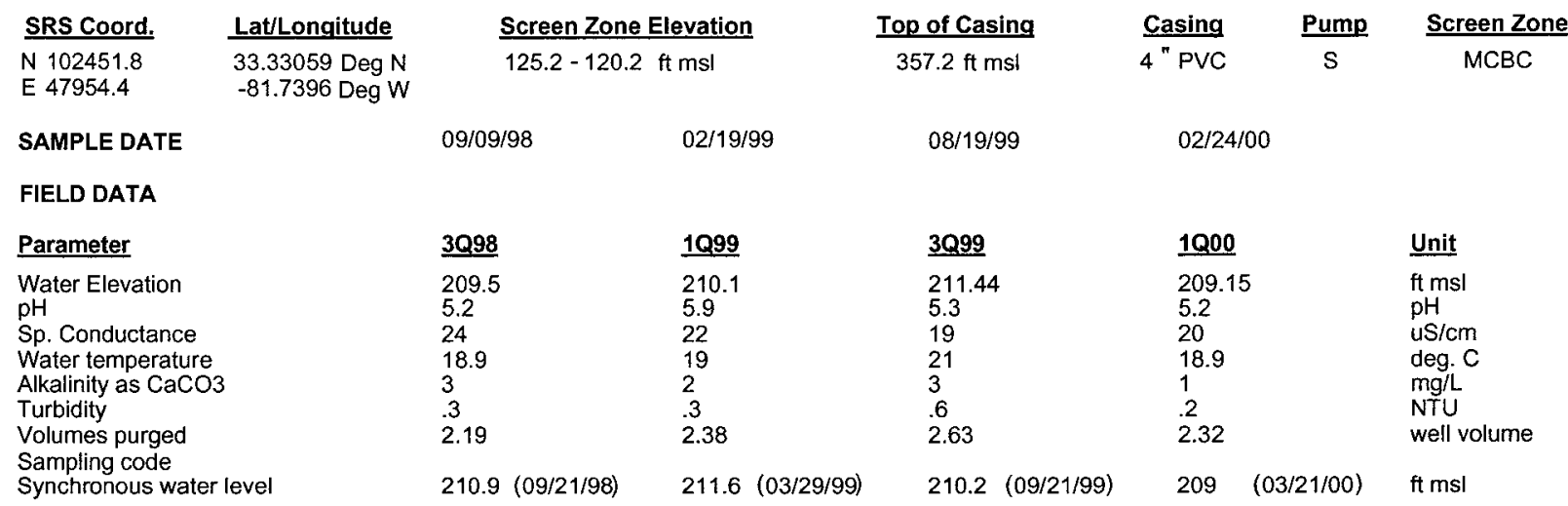

ANALYTICAL DATA

I. Groundwater Protection Standard

261 Appendix VIII/264 Appendix IX Hazardous Constituents

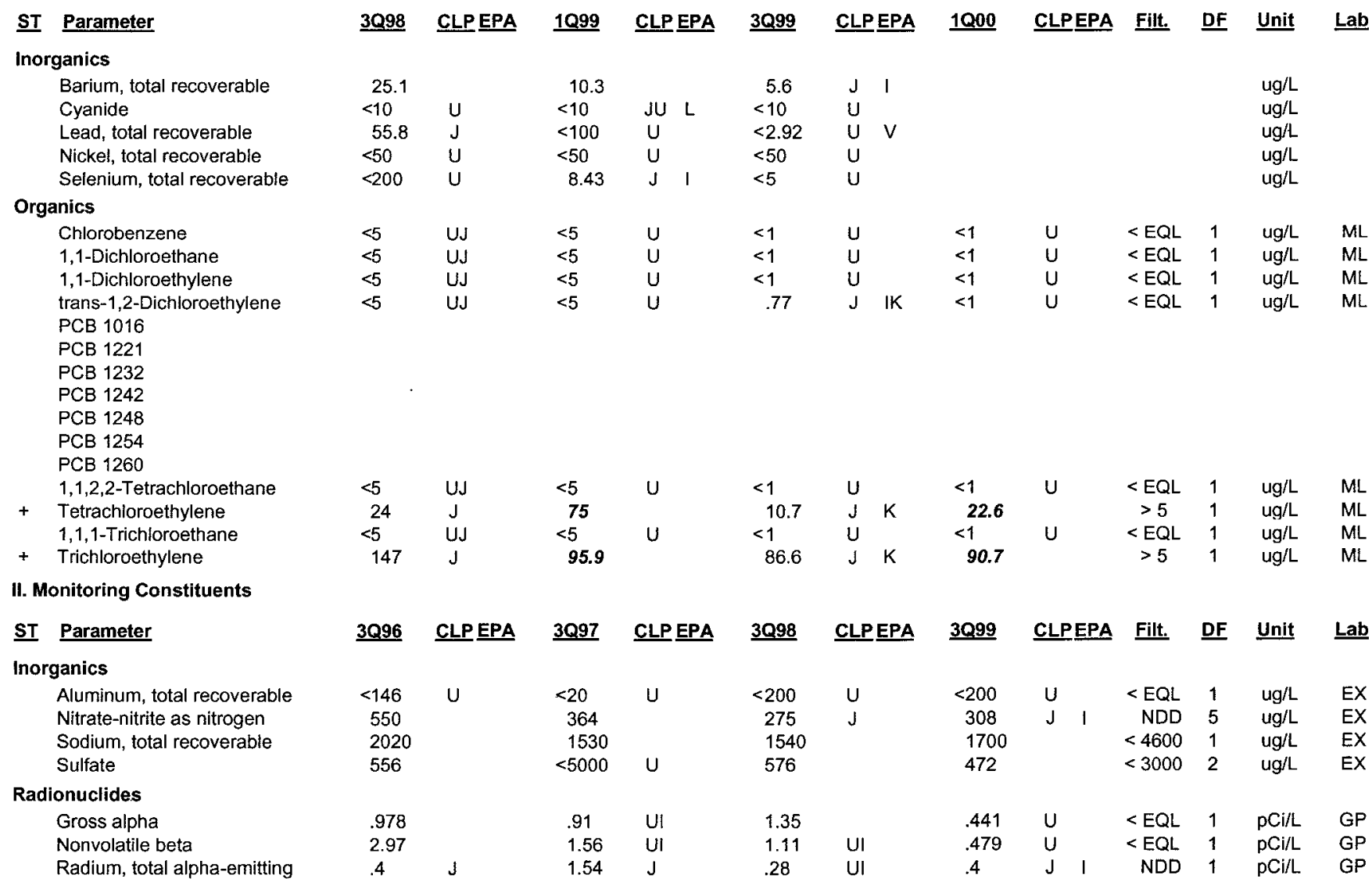

Notes: Concentrations in bold italics exceed the groundwater protection or monitoring constituent standards listed in Appendix A. Synchronous water levels are measured over a 3-5 day period or less. Dilution factors, Laboratory, and Filtered Data are for Groundwater Protection Standards First Quarter 2000 and Monitoring Constituents Third Quarter 1999 data only.

$+=$ exceeded the Groundwater Protection Standards (First Quarter 2000) or Monitoring Constituents (Third Quarter 1999) listed in Appendix A. 
Table D-4. Groundwater Monitoring Results for Plume Definition Wells, M-Area HWMF (Cont.) WELL MSB 10B

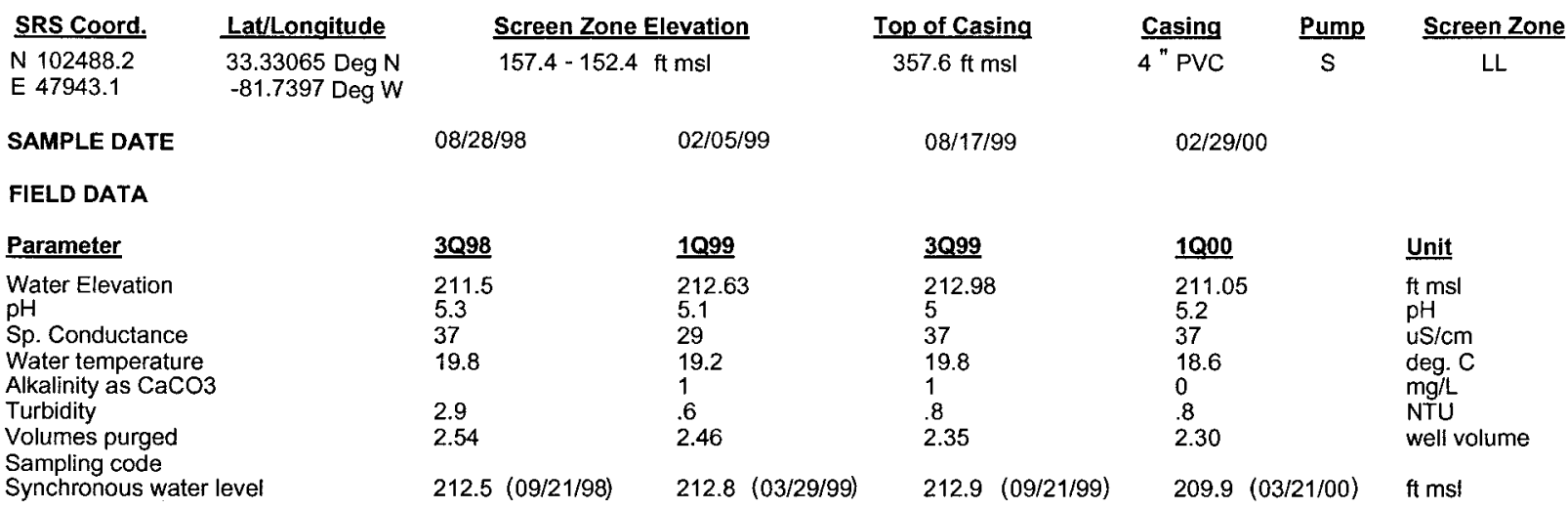

ANALYTICAL DATA

I. Groundwater Protection Standard

261 Appendix VIII/264 Appendix IX Hazardous Constituents

\begin{tabular}{|c|c|c|c|c|c|c|c|c|c|c|c|c|c|c|}
\hline$\underline{\text { ST }}$ & Parameter & $\underline{3 Q 98}$ & CLPEPA & 1099 & $\underline{\text { CLPEPA }}$ & $\underline{3 Q 99}$ & $\underline{\text { CLPEPA }}$ & $\underline{1 Q 00}$ & $\underline{\text { CLF }}$ & EPA & Filt. & $\underline{\text { DF }}$ & Unit & $\underline{\text { Lab }}$ \\
\hline In & ganics & & & & & & & & & & & & & \\
\hline & Barium, total recoverable & 12.7 & & 12 & & 14 & & & & & & & $u g / L$ & \\
\hline & Cyanide & $<10$ & $\mathrm{U}$ & $<10$ & $\mathrm{U}$ & $<10$ & U & & & & & & $\mathrm{ug} / \mathrm{L}$ & \\
\hline & Lead, total recoverable & $<100$ & $\mathrm{U}$ & $<100$ & $\mathrm{U}$ & $<10$ & U & & & & & & ug/L & \\
\hline & Nickel, total recoverable & $<50$ & $\mathrm{U}$ & $<50$ & $\mathrm{U}$ & $<9.3$ & JU I & & & & & & $\mathrm{ug} / \mathrm{L}$ & \\
\hline & Selenium, total recoverable & $<10$ & $\mathrm{U}$ & $<10$ & $\mathrm{U}$ & $<10$ & $\mathrm{U}$ & & & & & & $\mathrm{ug} / \mathrm{L}$ & \\
\hline rg & anics & & & & & & & & & & & & & \\
\hline & Chlorobenzene & $<5$ & $\mathrm{U}$ & $<5$ & $\mathrm{U}$ & $<1$ & U & $<1$ & $U$ & & $<E Q L$ & 1 & ug/L & $M L$ \\
\hline & 1,1-Dichloroethane & $<5$ & $U$ & $<5$ & $U$ & $<1$ & $\mathrm{U}$ & $<1$ & $U$ & & $<\mathrm{EQL}$ & 1 & ug/L & $M L$ \\
\hline & 1,1-Dichloroethylene & $<5$ & $\mathrm{U}$ & $<5$ & $U$ & $<1$ & $\mathrm{U}$ & $<1$ & $\mathrm{U}$ & & $<\mathrm{EQL}$ & 1 & ug/L & $\mathrm{ML}$ \\
\hline & trans-1,2-Dichloroethylene & $<5$ & $U$ & $<5$ & $\mathrm{U}$ & $<1$ & $\mathrm{U}$ & & & & & & $\mathrm{ug} / \mathrm{L}$ & \\
\hline & PCB 1016 & & & & & & & & & & & & & \\
\hline & PCB 1221 & & & & & & & & & & & & & \\
\hline & PCB 1232 & & & & & & & & & & & & & \\
\hline & PCB 1242 & & & & & & & & & & & & & \\
\hline & PCB 1248 & & & & & & & & & & & & & \\
\hline & PCB 1254 & & & & & & & & & & & & & \\
\hline & PCB 1260 & & & & & & & & & & & & & \\
\hline & 1,1,2,2-Tetrachioroethane & $<5$ & $U$ & $<5$ & $\mathrm{U}$ & $<1$ & $\mathrm{U}$ & $<1$ & U & & $<\mathrm{EQL}$ & 1 & ug/L & ML \\
\hline & Tetrachloroethylene & $<5$ & $U$ & 2.75 & J I & 4.35 & $\mathrm{~J} \quad \mathrm{~K}$ & 15.6 & $J$ & $\mathrm{~K}$ & NDD & 1 & $\mathrm{ug} / \mathrm{L}$ & $\mathrm{ML}$ \\
\hline & 1,1,1-Trichloroethane & $<5$ & $U$ & $<5$ & U & $<1$ & U & $<1$ & $\mathrm{U}$ & & $<E Q L$ & 1 & $\mathrm{ug} / \mathrm{L}$ & $M L$ \\
\hline & Trichloroethylene & 4.04 & $J$ & 6.9 & & 9.03 & $\mathrm{~J} \quad \mathrm{~K}$ & 19.4 & J & K & NDD & 1 & ug/L & ML \\
\hline $\mathbf{M}$ & onitoring Constituents & & & & & & & & & & & & & \\
\hline ST & Parameter & 3996 & CLPEPA & $\underline{3097}$ & CLPEPA & $3 Q 98$ & CLPEPA & $\underline{3099}$ & CLP & EPA & Filt. & $\underline{\text { DF }}$ & Unit & Lab \\
\hline Inor & ganics & & & & & & & & & & & & & \\
\hline & Aluminum, total recoverable & 38.2 & & $<20$ & $U$ & $<200$ & $\mathrm{U}$ & $<200$ & $\mathrm{U}$ & & $<E Q L$ & 1 & $\mathrm{ug} / \mathrm{L}$ & EX \\
\hline & Nitrate-nitrite as nitrogen & 160 & & 70 & & 96 & $J$ & 80.1 & $J$ & 1 & NDD & 5 & ug/L & EX \\
\hline & Sodium, total recoverable & 2320 & & 2460 & & 2290 & & 2800 & & & $<4600$ & 1 & ug/L & EX \\
\hline+ & Sulfate & 7760 & & 8160 & & 8090 & & 7980 & & & $>3000$ & 1 & ug/L & EX \\
\hline Rad & ionuclides & & & & & & & & & & & & & \\
\hline & Gross alpha & .015 & U1 & -.17 & UI & 1 & UI & -.0344 & $\mathrm{U}$ & & $<\mathrm{EQL}$ & 1 & $\mathrm{pCi} / \mathrm{L}$ & GP \\
\hline & Nonvolatile beta & .805 & UI & -1.49 & UI & 1.57 & UI & .687 & $\mathrm{U}$ & & $<\mathrm{EQL}$ & 1 & $\mathrm{pCi} / \mathrm{L}$ & GP \\
\hline & Radium, total alpha-emitting & 0 & Ul & .92 & & .37 & & 0 & $\mathrm{U}$ & & $<\mathrm{EQL}$ & 1 & $\mathrm{pCi} / \mathrm{L}$ & GP \\
\hline
\end{tabular}

Notes: Concentrations in bold italics exceed the groundwater protection or monitoring constituent standards listed in Appendix A. Synchronous water levels are measured over a 3-5 day period or less. Dilution factors, Laboratory, and Filtered Data are for Groundwater Protection Standards First Quarter 2000 and Monitoring Constituents Third Quarter 1999 data only.

$+=$ exceeded the Groundwater Protection Standards (First Quarter 2000) or Monitoring Constituents (Third Quarter 1999) listed in Appendix A. 
Table D-4. Groundwater Monitoring Results for Plume Definition Wells, M-Area HWMF (Cont.) WELL MSB 11C

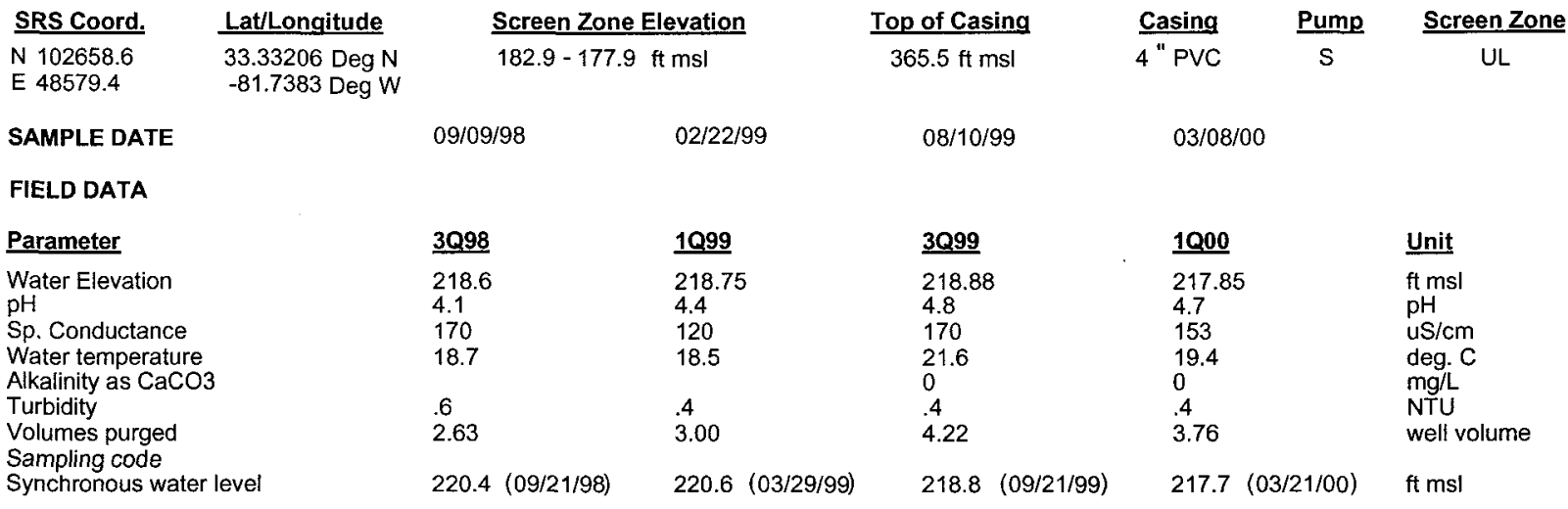

ANALYTICAL DATA

I. Groundwater Protection Standard

261 Appendix VIII/264 Appendix IX Hazardous Constituents

\begin{tabular}{|c|c|c|c|c|c|c|c|c|c|c|c|c|c|}
\hline \multirow{2}{*}{$\begin{array}{l}\text { ST Parameter } \\
\text { Inorganics }\end{array}$} & \multirow[t]{2}{*}{$\underline{3098}$} & \multirow[t]{2}{*}{ CLPEPA } & \multirow[t]{2}{*}{1099} & \multirow[t]{2}{*}{ CLPEPA } & \multirow[t]{2}{*}{$\underline{3 Q 99}$} & CLPEPA & \multirow[t]{2}{*}{$\underline{1000}$} & \multicolumn{2}{|c|}{ CLPEPA } & \multirow[t]{2}{*}{ Filt. } & \multirow[t]{2}{*}{$\underline{\mathrm{DF}}$} & \multirow[t]{2}{*}{$\underline{\text { Unit }}$} & \multirow[t]{2}{*}{$\underline{L a b}$} \\
\hline & & & & & & & & & & & & & \\
\hline Barium, total recoverable & 55.5 & & 47.1 & & 56 & & 48.7 & & & $<2000$ & 1 & ug/L & $M L$ \\
\hline Cyanide & $<10$ & $\mathrm{u}$ & $<10$ & $u$ & $<10$ & $\mathrm{u}$ & & & & & & ug/L & \\
\hline Lead, total recoverable & $<100$ & $\mathrm{u}$ & $<100$ & $u$ & $<10$ & $\mathrm{u}$ & $<20$ & $\mathrm{u}$ & & $<E Q L$ & 1 & $\mathrm{ug} / \mathrm{L}$ & ML \\
\hline Nickel, total recoverable & $<50$ & $\mathrm{U}$ & $<7.65$ & JU & $<50$ & $\mathrm{u}$ & $<60$ & $\mathrm{u}$ & & $<E Q L$ & 1 & $\mathrm{ug} / \mathrm{L}$ & ML \\
\hline Selenium, total recoverable & $<200$ & $\mathrm{u}$ & $<200$ & $\mathrm{u}$ & $<10$ & $\mathrm{u}$ & $<40$ & $\mathrm{u}$ & & $<\overline{E Q L}$ & 1 & $u g / L$ & ML \\
\hline \multicolumn{14}{|l|}{ Organics } \\
\hline Chlorobenzene & $<2500$ & us & $<2500$ & $\mathrm{u}$ & $<1$ & $\mathrm{u}$ & 112 & $\mathrm{~J}$ & IK & NDD & 200 & $\mathrm{ug} / \mathrm{L}$ & \\
\hline 1,1-Dichloroethane & $<2500$ & us & $<2500$ & $\mathrm{u}$ & $<1$ & $u$ & $<200$ & $u$ & & $<E Q L$ & 200 & $u g / L$ & $\mathrm{ML}$ \\
\hline 1,1-Dichloroethylene & $<2500$ & UJ & $<2500$ & u & $<1$ & $u$ & $<200$ & $u$ & & $<E Q L$ & 200 & $u g / L$ & $\mathbf{n}$ \\
\hline trans-1,2-Dichloroethylene & $<2500$ & UJ & $<2500$ & $u$ & $<1$ & U & $<200$ & $u$ & & $<E Q L$ & 200 & $\mathrm{ug} / \mathrm{L}$ & \\
\hline РСВ 1016 & & & & & & & & & & & & & \\
\hline $\begin{array}{l}\text { PCB } 1221 \\
\text { PCB } 1232\end{array}$ & & & & & & & & & & & & & \\
\hline $\begin{array}{l}\text { PCB } 1232 \\
\text { PCB } 1242\end{array}$ & & & & & & & & & & & & & \\
\hline PCB 1248 & & & & & & & & & & & & & \\
\hline PCB 1254 & & & & & & & & & & & & & \\
\hline РCB 1260 & & & & & & & & & & & & & \\
\hline 1,1,2,2-Tetrachloroethane & $<2500$ & UJ & $<2500$ & $\mathrm{u}$ & $<1$ & $\mathrm{u}$ & $<200$ & $\mathrm{u}$ & & $<E Q L$ & 200 & $u g / L$ & ML \\
\hline Tetrachloroethylene & $<2500$ & UJ & $<2500$ & u & 93.1 & $\mathrm{~J} \quad \mathrm{~K}$ & $<200$ & JU & $\mathrm{K}$ & $<E Q L$ & 200 & $\mathrm{ug} / \mathrm{L}$ & \\
\hline 1,1,1-Trichloroethane & $<2500$ & UJ & $<2500$ & U & $<1$ & $\mathrm{U}$ & $<200$ & $\mathrm{u}$ & & $<\mathrm{EQL}$ & 200 & $\mathrm{ug} / \mathrm{L}$ & $I L$ \\
\hline Trichloroethylene & 44700 & $\mathrm{~J}$ & 42900 & & 40300 & & 40300 & j & K & NDD & 200 & $\mathrm{ug} / \mathrm{L}$ & ML \\
\hline \multicolumn{14}{|l|}{ II. Monitoring Constituents } \\
\hline ST Parameter & $\underline{3096}$ & CLPEPA & $\underline{3097}$ & CLPEPA & $\underline{3098}$ & CLPPEPA & $\underline{3099}$ & $\underline{\text { CLP }}$ & EPA & Filt. & $\underline{\mathrm{DF}}$ & Unit & La \\
\hline \multicolumn{14}{|l|}{ Inorganics } \\
\hline Aluminum, total recoverable & 88.1 & & 101 & & 122 & $\mathrm{~J}$ & 160 & $\mathrm{~J}$ & 1 & NDD & 1 & $\mathrm{ug} / \mathrm{L}$ & $E X$ \\
\hline$+\quad$ Nitrate-nitrite as nitrogen & 17300 & & 16700 & & 15500 & & 16900 & & & $>2400$ & 5 & $\mathrm{ug} / \mathrm{L}$ & \\
\hline+ Sodium, total recoverable & 8060 & & 7150 & & 5870 & & 5700 & & & $>4600$ & 1 & ug/L & \\
\hline Sulfate & 263 & $\mathrm{~J}$ & $<5000$ & $u$ & 252 & J & 165 & $J$ & 1 & NDD & 1 & $\mathrm{ug} / \mathrm{L}$ & \\
\hline \multicolumn{13}{|l|}{ Radionuclides } & \\
\hline Gross alpha & 6.44 & & 2.23 & & 9.66 & & 5.1 & & & $<15$ & 1 & $\mathrm{pCi} / \mathrm{L}$ & \\
\hline Nonvolatile beta & 11.39 & & 1.12 & UI & 9.48 & & 4.23 & & & $<50$ & 1 & $\mathrm{pCi} / \mathrm{L}$ & \\
\hline Radium, total alpha-emitting & 4.44 & & .23 & $\mathrm{UI}$ & 3.19 & & 2.9 & & & $<5$ & 1 & $\mathrm{pCi} / \mathrm{L}$ & \\
\hline
\end{tabular}

Notes: Concentrations in bold italics exceed the groundwater protection or monitoring constituent standards listed in Appendix A. Synchronous water levels are measured over a 3-5 day period or less. Dilution factors, Laboratory, and Filtered Data are for Groundwater Protection Standards First Quarter 2000 and Monitoring Constituents Third Quarter 1999 data only.

+ = exceeded the Groundwater Protection Standards (First Quarter 2000) or Monitoring Constituents (Third Quarter 1999) listed in Appendix A. 
Table.D-4. Groundwater Monitoring Results for Plume Definition Wells, M-Area HWMF (Cont.) WELL MSB 11F

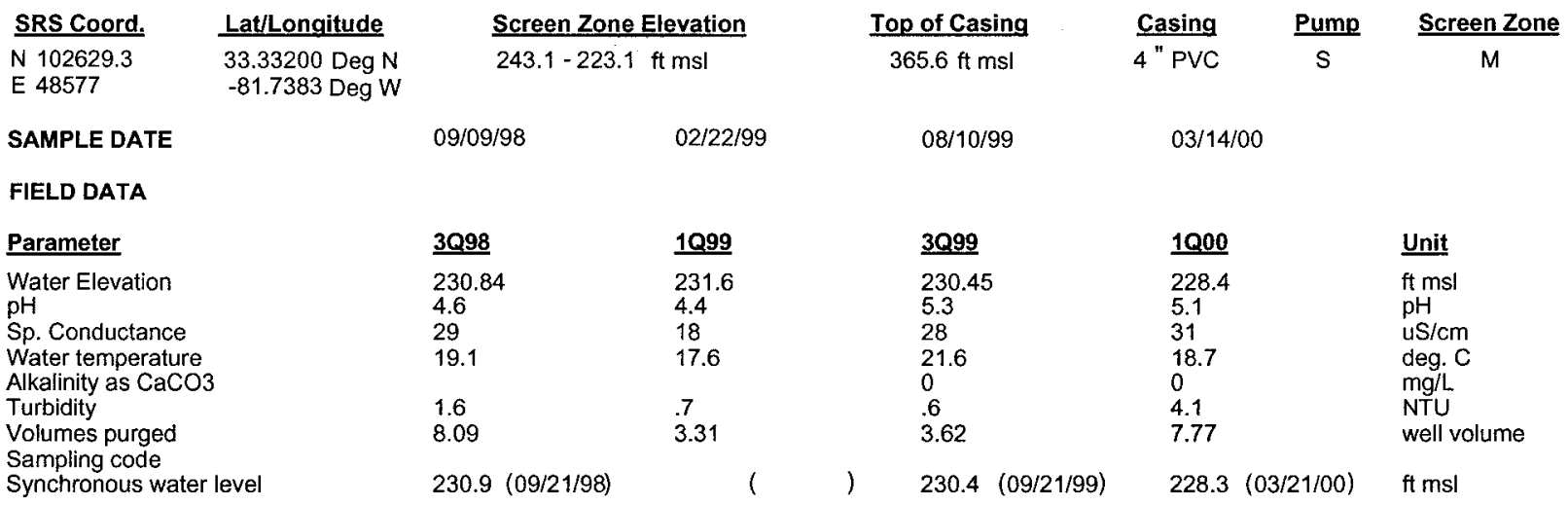

ANALYTICAL DATA

I. Groundwater Protection Standard

261 Appendix VIII/264 Appendix IX Hazardous Constituents

\begin{tabular}{|c|c|c|c|c|c|c|c|c|c|c|c|c|c|c|c|}
\hline \multirow{2}{*}{\multicolumn{2}{|c|}{$\begin{array}{l}\text { ST } \\
\text { Inorganics }\end{array}$}} & \multirow[t]{2}{*}{$\underline{3098}$} & \multirow[t]{2}{*}{ CLP EPA } & \multirow[t]{2}{*}{1099} & \multirow[t]{2}{*}{ CLP EPA } & \multirow[t]{2}{*}{$\underline{3099}$} & \multicolumn{2}{|c|}{ CLPEPA } & \multirow[t]{2}{*}{1000} & \multicolumn{2}{|c|}{ CLPEPA } & \multirow[t]{2}{*}{ Filt. } & \multirow[t]{2}{*}{ DF } & \multirow[t]{2}{*}{ Unit } & \multirow[t]{2}{*}{ Lab } \\
\hline & & & & & & & & & & & & & & & \\
\hline & Barium, total recoverable & 3.96 & $J$ & $<10$ & $U$ & 4.4 & $J$ & I & 4.5 & $J$ & 1 & NDD & 1 & $\mathrm{ug} / \mathrm{L}$ & ML \\
\hline & Cyanide & $<10$ & $U$ & $<10$ & $\mathrm{U}$ & $<10$ & $\mathrm{U}$ & & & & & & & $\mathrm{ug} / \mathrm{L}$ & \\
\hline & Lead, total recoverable & $<100$ & $\mathrm{U}$ & $<100$ & $\mathrm{U}$ & $<10$ & $\bar{U}$ & & $<6.72$ & JU & IV & $<\mathrm{EQL}$ & 1 & $\mathrm{ug} / \mathrm{L}$ & ML \\
\hline & Nickel, total recoverable & 7.28 & $J$ & $<50$ & $\mathrm{U}$ & $<50$ & $U$ & & $<60$ & $\mathbf{U}$ & & $<\mathrm{EQL}$ & 1 & ug/t & ML \\
\hline & Selenium, total recoverable & $<200$ & $U$ & $<200$ & $\mathrm{U}$ & $<10$ & $\mathrm{U}$ & & $<40$ & $\mathrm{U}$ & & $<\mathrm{EQL}$ & 1 & $\mathrm{ug} / \mathrm{L}$ & ML \\
\hline \multicolumn{16}{|c|}{ Organics } \\
\hline & Chlorobenzene & $<500$ & $U$ & $<500$ & U & .41 & $J$ & $\mathrm{IK}$ & 1.4 & $J$ & $\mathbb{K}$ & NDD & 5 & $\mathrm{ug} / \mathrm{L}$ & $M L$ \\
\hline & 1,1-Dichloroethane & $<500$ & $U$ & $<500$ & $U$ & $<1$ & U & & $<5$ & $\mathrm{U}$ & & $<\mathrm{EQL}$ & 5 & ug/L & $M L$ \\
\hline & 1,1-Dichloroethylene & $<500$ & $U$ & $<500$ & $U$ & 1.94 & $\mathrm{~J}$ & $\mathrm{~K}$ & $<5$ & $\mathrm{U}$ & & $<\mathrm{EQL}$ & 5 & ug/L & ML \\
\hline & trans-1,2-Dichloroethylene & $<500$ & $\mathrm{U}$ & $<500$ & $\mathrm{U}$ & $<1$ & $\mathrm{U}$ & & $<5$ & $\mathrm{u}$ & & $<\mathrm{EQL}$ & 5 & $\mathrm{ug} / \mathrm{L}$ & $M L$ \\
\hline & PCB 1016 & & & & & & & & & & & & & & \\
\hline & PCB 1221 & & & & & & & & & & & & & & \\
\hline & РСB 1232 & & & & & & & & & & & & & & \\
\hline & PCB 1242 & & & & & & & & & & & & & & \\
\hline & PCB 1248 & & & & & & & & & & & & & & \\
\hline & PCB 1254 & & & & & & & & & & & & & & \\
\hline & PCB 1260 & & & & & & & & & & & & & & \\
\hline & 1,1,2,2-Tetrachloroethane & $<500$ & $U$ & $<500$ & $U$ & $<1$ & $U$ & & $<5$ & U & & $<\mathrm{EQL}$ & 5 & ug/L & ML \\
\hline & Tetrachloroethylene & 4180 & & 1170 & & 1230 & & & 1600 & $J$ & $\mathrm{~K}$ & NDD & 5 & ug/L & ML \\
\hline & 1,1,1-Trichloroethane & 20.5 & $J$ & $<500$ & $\mathrm{U}$ & 3.81 & $J$ & $\mathrm{~K}$ & 4.55 & J & IK & NDD & 5 & ug/L & ML \\
\hline & Trichloroethylene & 1750 & & 563 & & 523 & & & 843 & $J$ & $\mathrm{~K}$ & NDO & 5 & ug/L & $\mathrm{ML}$ \\
\hline \multicolumn{16}{|c|}{ II. Monitoring Constituents } \\
\hline$\underline{\mathbf{S T}}$ & Parameter & $\underline{3096}$ & CLPEPA & $3 Q 97$ & CLPP EPA & $\underline{3 Q 98}$ & CLLF & EPA & 3Q999 & CLP & EPA & Filt. & $\underline{\text { DF }}$ & Unit & $\underline{\text { Lab }}$ \\
\hline \multicolumn{16}{|c|}{ Inorganics } \\
\hline & Aluminum, total recoverable & 57.4 & & & & 191 & $J$ & & 160 & $J$ & 1 & NDD & 1 & $\mathrm{ug} / \mathrm{L}$ & EX \\
\hline & Nitrate-nitrite as nitrogen & 820 & & 1210 & & 755 & & & 824 & & & $<2400$ & 5 & ug/L & EX \\
\hline & Sodium, total recoverable & 2340 & & & & 2890 & & & 3800 & & & $<4600$ & 1 & ug/L & EX \\
\hline & Sulfate & 835 & J & $<5000$ & $U$ & 886 & & & 961 & & & $<3000$ & 1 & ug/L & EX \\
\hline \multicolumn{16}{|c|}{ Radionuclides } \\
\hline & Gross alpha & 1.51 & & .26 & UI & 6.27 & & & 1.77 & & & $<15$ & 1 & $\mathrm{pCi} / \mathrm{L}$ & GP \\
\hline & Nonvolatile beta & 2.36 & & -.62 & UI & 1.8 & $\mathrm{U}$ & & 1.13 & $\mathrm{U}$ & & $<\mathrm{EQL}$ & 1 & $\mathrm{pCi} / \mathrm{L}$ & GP \\
\hline & Radium, total alpha-emitting & 1.1 & & 2.36 & & 1.66 & & & .8 & $\mathrm{~J}$ & 1 & NDD & 1 & $\mathrm{pCi} / \mathrm{L}$ & GP \\
\hline
\end{tabular}

Notes: Concentrations in bold italics exceed the groundwater protection or monitoring constituent standards listed in Appendix A. Synchronous water levels are measured over a 3-5 day period or less. Dilution factors, Laboratory, and Filtered Data are for Groundwater Protection Standards First Quarter 2000 and Monitoring Constituents Third Quarter 1999 data only.

$+=$ exceeded the Groundwater Protection Standards (First Quarter 2000) or Monitoring Constituents (Third Quarter 1999) listed in Appendix A. 
Table D-4. Groundwater Monitoring Results for Plume Definition Wells, M-Area HWMF (Cont.) WELL MSB 12B

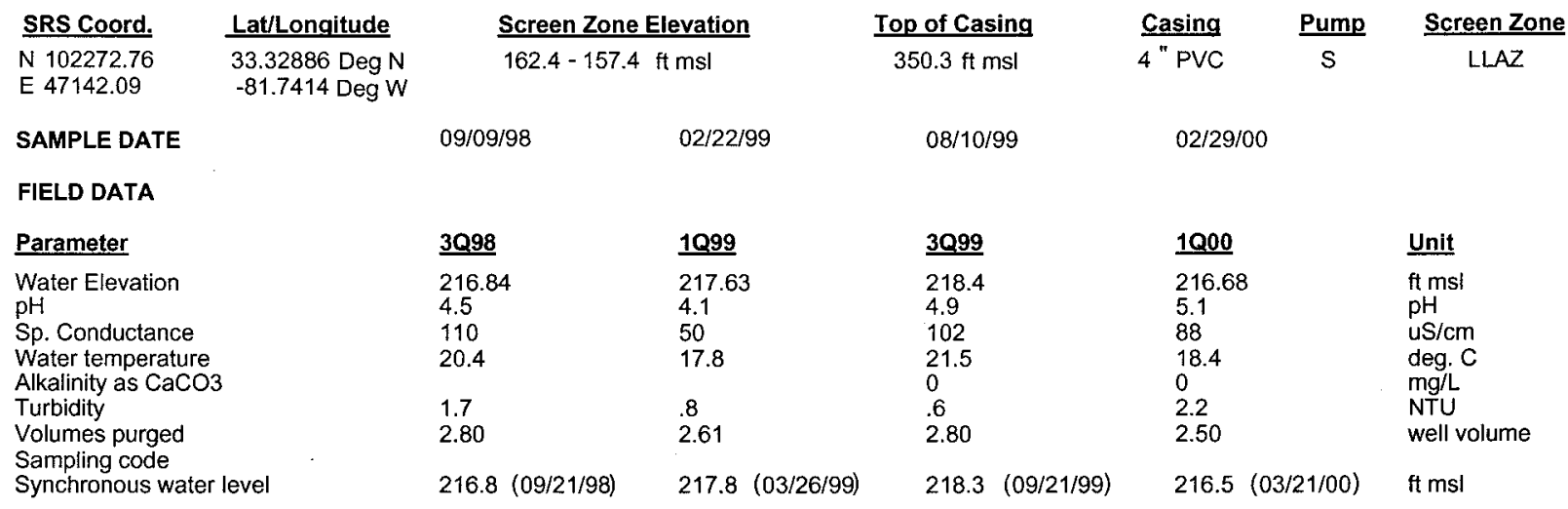

ANALYTICAL DATA

I. Groundwater Protection Standard

261 Appendix VIII/264 Appendix IX Hazardous Constituents

\begin{tabular}{|c|c|c|c|c|c|c|c|c|c|c|c|c|c|c|c|}
\hline$\underline{\text { ST }}$ & Parameter & 3Q98 & CLP EPA & 1099 & CLP EPA & $\underline{3 Q 99}$ & $\underline{\text { CLP }}$ & EPA & $\underline{1 Q 00}$ & $\underline{\text { CLP }}$ & EPA & Filt. & $\underline{\text { DF }}$ & Unit & $\underline{\text { Lab }}$ \\
\hline 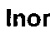 & ganics & & & & & & & & & & & & & & \\
\hline & Barium, total recoverable & 25 & & - 23.6 & & 25 & & & & & & & & $u g / L$ & \\
\hline & Cyanide & $<10$ & U & $<10$ & $\mathrm{U}$ & $<10$ & $\mathrm{U}$ & & & & & & & $\mathrm{ug} / \mathrm{L}$ & \\
\hline & Lead, total recoverable & $<100$ & $U$ & $<100$ & U & $<10$ & $\mathrm{U}$ & & & & & & & $u g / L$ & \\
\hline & Nickel, total recoverable & $<50$ & $U$ & $<50$ & $\mathrm{U}$ & $<50$ & U & & & & & & & $\mathrm{ug} / \mathrm{L}$ & \\
\hline & Selenium, total recoverable & $<200$ & $U$ & $<200$ & $\mathrm{U}$ & $<10$ & $U$ & & & & & & & $\mathrm{ug} / \mathrm{L}$ & \\
\hline Org & anics & & & & & & & & & & & & & & \\
\hline & Chlorobenzene & $<1000$ & $u$ & $<1250$ & $\mathrm{U}$ & $<1.39$ & JU & $\mathrm{KV}$ & $<50$ & $\mathrm{u}$ & & $<\mathrm{EQL}$ & 50 & ug/L & ML \\
\hline & 1,1-Dichloroethane & $<1000$ & U & $<1250$ & U & $<1$ & $U$ & & $<50$ & $U$ & & $<$ EQL & 50 & $\mathrm{ug} / \mathrm{L}$ & $M L$ \\
\hline & 1,1-Dichloroethylene & $<1000$ & $U$ & $<1250$ & U & 7.53 & $J$ & K & $<50$ & $U$ & & $<$ EQL & 50 & $u g / L$ & $M L$ \\
\hline & trans-1,2-Dichloroethylene & $<1000$ & U & $<1250$ & $U$ & $<1$ & U & & & & & & & $u g / L$ & \\
\hline & PCB 1016 & & & & & & & & & & & & & & \\
\hline & PCB 1221 & & & & & & & & & & & & & & \\
\hline & PCB 1232 & & & & & & & & & & & & & & \\
\hline & PCB 1242 & & & & & & & & & & & & & & \\
\hline & РCB 1248 & & & & & & & & & & & & & & \\
\hline & PCB 1254 & & & & & & & & & & & & & & \\
\hline & PCB 1260 & & & & & & & & & & & & & & \\
\hline & $1,1,2,2$-Tetrachloroethane & $<1000$ & $\mathrm{U}$ & $<1250$ & $\mathrm{U}$ & 3.35 & $J$ & $\mathrm{~K}$ & $<50$ & $U$ & & $<\mathrm{EQL}$ & 50 & $u g / L$ & $M L$ \\
\hline & Tetrachloroethylene & 24000 & & 18300 & & 13500 & & & 18200 & $\mathrm{~J}$ & $\mathrm{~K}$ & NDD & 50 & $\mathrm{ug} / \mathrm{L}$ & $M L$ \\
\hline & $1,1,1$-Trichloroethane & $<1000$ & $U$ & $<1250$ & $\mathrm{U}$ & 1.47 & $\mathrm{~J}$ & $\mathrm{~K}$ & $<50$ & $U$ & & $<E Q L$ & 50 & $\mathrm{ug} / \mathrm{L}$ & ML \\
\hline & Trichloroethylene & 23800 & & 15600 & & 15400 & & & 20000 & $J$ & $\mathrm{~K}$ & NDD & 50 & $\mathrm{ug} / \mathrm{L}$ & $\mathrm{ML}$ \\
\hline II. $\mathbf{M}$ & onitoring Constituents & & & & & & & & & & & & & & \\
\hline$\underline{\text { ST }}$ & Parameter & $\underline{3096}$ & CLP EPA & $\underline{3 Q 97}$ & CLP EPA & $\underline{3 Q 98}$ & $\underline{\text { CLP }}$ & EPA & $\underline{3099}$ & $\underline{\text { CLP }}$ & EPA & Filt. & $\underline{\mathrm{DF}}$ & Unit & La \\
\hline Inor & ganics & & & & & & & & & & & & & & \\
\hline & Aluminum, total recoverable & 95.4 & J & 39.9 & & $<200$ & $u$ & & 160 & $J$ & $\mathbf{i}$ & NDD & 1 & $\mathrm{ug} / \mathrm{L}$ & EX \\
\hline+ & Nitrate-nitrite as nitrogen & 13100 & & 12000 & & 9950 & & & 9870 & & & $>2400$ & 5 & $\mathrm{ug} / \mathrm{L}$ & EX \\
\hline+ & Sodium, total recoverable & 13900 & $J$ & 10600 & & 9450 & & & 12000 & & & $>4600$ & 1 & $\mathrm{ug} / \mathrm{L}$ & EX \\
\hline & Sulfate & 249 & J & $<5000$ & $\mathrm{U}$ & 325 & 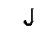 & & 306 & & & $<3000$ & 1 & $\mathrm{ug} / \mathrm{L}$ & EX \\
\hline Rac & onuclides & & & & & & & & & & & & & & \\
\hline & Gross alpha & 6.1 & & 5.51 & & 8.89 & & & 4.39 & & & $<15$ & 1 & $\mathrm{pCi} / \mathrm{L}$ & GP \\
\hline & Nonvolatile beta & 7.61 & & 9.53 & & 10.89 & & & 6 & & & $<50$ & 1 & $\mathrm{pCi} / \mathrm{L}$ & GP \\
\hline & Radium, total alpha-emitting & 3 & & 4.86 & UIJ & 2.61 & & & 1.4 & $J$ & 1 & NDD & 1 & $\mathrm{pCi} / \mathrm{L}$ & GP \\
\hline
\end{tabular}

Notes: Concentrations in bold italics exceed the groundwater protection or monitoring constituent standards listed in Appendix A. Synchronous water levels are measured over a 3-5 day period or less. Dilution factors, Laboratory, and Filtered Data are for Groundwater Protection Standards First Quarter 2000 and Monitoring Constituents Third Quarter 1999 data only.

+ = exceeded the Groundwater Protection Standards (First Quarter 2000) or Monitoring Constituents (Third Quarter 1999) listed in Appendix A. 
Table D-4. Groundwater Monitoring Results for Plume Definition Wells, M-Area HWMF (Cont.) WELL MSB 12TA

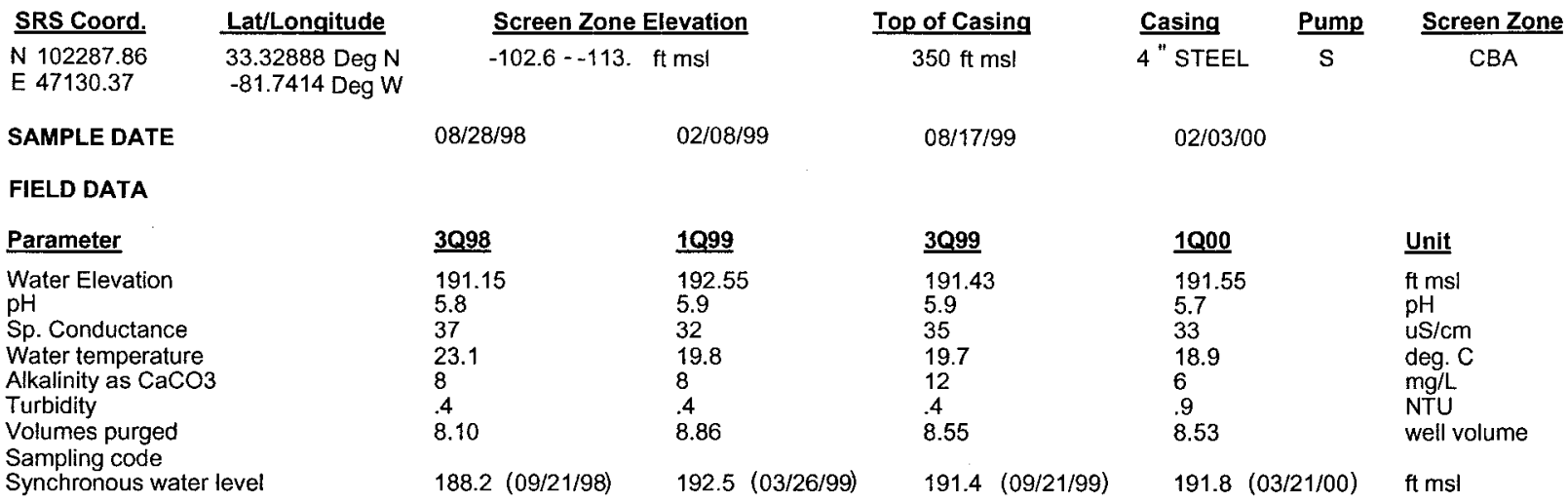

\section{ANALYTICAL DATA}

I. Groundwater Protection Standard

261 Appendix VIII/264 Appendix IX Hazardous Constituents

\begin{tabular}{|c|c|c|c|c|c|c|c|c|c|c|c|c|}
\hline ST Parameter & $\underline{3 Q 98}$ & CLPPEPA & 1099 & $\underline{\text { CLP EPA }}$ & $\underline{3099}$ & $\underline{\text { CLP EPA }}$ & 1000 & CLPPEPA & Filt. & $\underline{\text { DF }}$ & Unit & $\underline{\text { Lab }}$ \\
\hline \multicolumn{13}{|l|}{ Inorganics } \\
\hline Barium, total recoverable & 13.7 & & $<10$ & $\mathrm{U}$ & 12 & & & & & & $u g / L$ & \\
\hline Cyanide & $<10$ & $\mathrm{U}$ & $<10$ & $\mathrm{U}$ & $<10$ & $U$ & & & & & ug/L & \\
\hline Lead, total recoverable & $<100$ & $\mathrm{U}$ & $<100$ & $\mathrm{U}$ & $<10$ & $\mathrm{U}$ & & & & & $u g / L$ & \\
\hline Nickel, total recoverable & 198 & & $<50$ & U & $<50$ & $U$ & & & & & $\mathrm{ug} / \mathrm{L}$ & \\
\hline Selenium, total recoverable & $<10$ & $U$ & $<200$ & $U$ & $<10$ & $U$ & & & & & ug/L & \\
\hline \multicolumn{13}{|l|}{ Organics } \\
\hline Chlorobenzene & $<5$ & UJ & $<5$ & $U$ & $<1$ & $\mathrm{U}$ & $<1$ & U & $<\mathrm{EQL}$ & 1 & $\mathrm{ug} / \mathrm{L}$ & $M L$ \\
\hline 1,1-Dichloroethane & $<5$ & US & $<5$ & $\mathrm{U}$ & $<1$ & U & $<1$ & $\mathrm{U}$ & $<\mathrm{EQL}$ & 1 & $\mathrm{ug} / \mathrm{L}$ & ML \\
\hline 1,1-Dichloroethylene & $<5$ & UJ & $<5$ & $\mathrm{U}$ & $<1$ & U & $<1$ & $\mathrm{U}$ & $<\mathrm{EQL}$ & 1 & $\mathrm{ug} / \mathrm{L}$ & $M L$ \\
\hline trans-1,2-Dichloroethylene & $<5$ & UJ & $<5$ & $\mathrm{U}$ & $<1$ & $U$ & $<1$ & $U$ & $<\mathrm{EQL}$ & 1 & $\mathrm{ug} / \mathrm{L}$ & $M L$ \\
\hline РCB 1016 & & & & & & & & & & & & \\
\hline PCB 1221 & & & & & & & & & & & & \\
\hline РСВ 1232 & & & & & & & & & & & & \\
\hline PCB 1242 & & & & & & & & & & & & \\
\hline PCB 1248 & & & & & & & & & & & & \\
\hline PCB 1254 & & & & & & & & & & & & \\
\hline PCB 1260 & & & & & & & & & & & & \\
\hline $1,1,2,2-$ Tetrachloroethane & $<5$ & UJ & $<5$ & $\mathrm{U}$ & $<1$ & $U$ & $<1$ & $\mathrm{U}$ & $<\mathrm{EQL}$ & 1 & $\mathrm{ug} / \mathrm{L}$ & $M L$ \\
\hline Tetrachloroethylene & $<5$ & UJ & $<5$ & $\mathrm{U}$ & $<1$ & $\mathrm{U}$ & $<1$ & $\mathrm{U}$ & $<E Q L$ & 1 & $\mathrm{ug} / \mathrm{L}$ & $M L$ \\
\hline 1,1,1-Trichloroethane & $<5$ & UJ & $<5$ & $\mathrm{U}$ & $<1$ & $\mathrm{U}$ & $<1$ & $U$ & $<E Q L$ & 1 & $\mathrm{ug} / \mathrm{L}$ & $M L$ \\
\hline Trichloroethylene & $<5$ & UJ & $<5$ & $U$ & $<1$ & $\mathrm{U}$ & $<1$ & $U$ & $<$ EQL & 1 & $u g / L$ & $\mathrm{ML}$ \\
\hline \multicolumn{13}{|l|}{ II. Monitoring Constituents } \\
\hline ST Parameter & $\underline{3 Q 96}$ & CLPEPA & $\underline{3097}$ & CLPEPA & 3098 & CLPEPA & 3099 & CLPEPA & Filt. & $\underline{\mathrm{DF}}$ & Unit & $\underline{\text { Lab }}$ \\
\hline \multicolumn{13}{|l|}{ Inorganics } \\
\hline Aluminum, total recoverable & $<146$ & $U$ & $<20$ & $U$ & 74.4 & $\mathrm{~J}$ & $<200$ & $\mathrm{U}$ & $<E Q L$ & 1 & $\mathrm{ug} / \mathrm{L}$ & EX \\
\hline Nitrate-nitrite as nitrogen & 370 & & 35 & & $<500$ & $\mathrm{U}$ & $<500$ & $\mathrm{U}$ & $<E Q L$ & 5 & $u g / L$ & $\mathrm{EX}$ \\
\hline Sodium, total recoverable & 1300 & & 1240 & & 1280 & & 1400 & & $<4600$ & 1 & $u g / L$ & $\mathrm{EX}$ \\
\hline Sulfate & 880 & & $<5000$ & $U$ & 827 & & 579 & & $<3000$ & 1 & $\mathrm{ug} / \mathrm{L}$ & $\mathrm{EX}$ \\
\hline \multicolumn{13}{|l|}{ Radionuclides } \\
\hline Gross alpha & 9.03 & & .17 & UI & 2.29 & & 1.16 & $J \quad I$ & NDD & 1 & $\mathrm{pCi} / \mathrm{L}$ & GP \\
\hline Nonvolatile beta & 4.86 & & .54 & UI & 1.05 & UI & 1.11 & $\mathrm{U}$ & $<E Q L$ & 1 & $\mathrm{pCi} / \mathrm{L}$ & GP \\
\hline Radium, total alpha-emitting & 1.2 & & .94 & & .62 & & .4 & $\mathbf{J} \quad \mathbf{I}$ & NDD & 1 & $\mathrm{pCi} / \mathrm{L}$ & GP \\
\hline
\end{tabular}

Notes: Concentrations in bold italics exceed the groundwater protection or monitoring constituent standards listed in Appendix A. Synchronous water levels are measured over a 3-5 day period or less. Dilution factors, Laboratory, and Filtered Data are for Groundwater Protection Standards First Quarter 2000 and Monitoring Constituents Third Quarter 1999 data only.

+ = exceeded the Groundwater Protection Standards (First Quarter 2000) or Monitoring Constituents (Third Quarter 1999) listed in Appendix A. 
Table D-4. Groundwater Monitoring Results for Plume Definition Wells, M-Area HWMF (Cont.) WELL MSB 15A

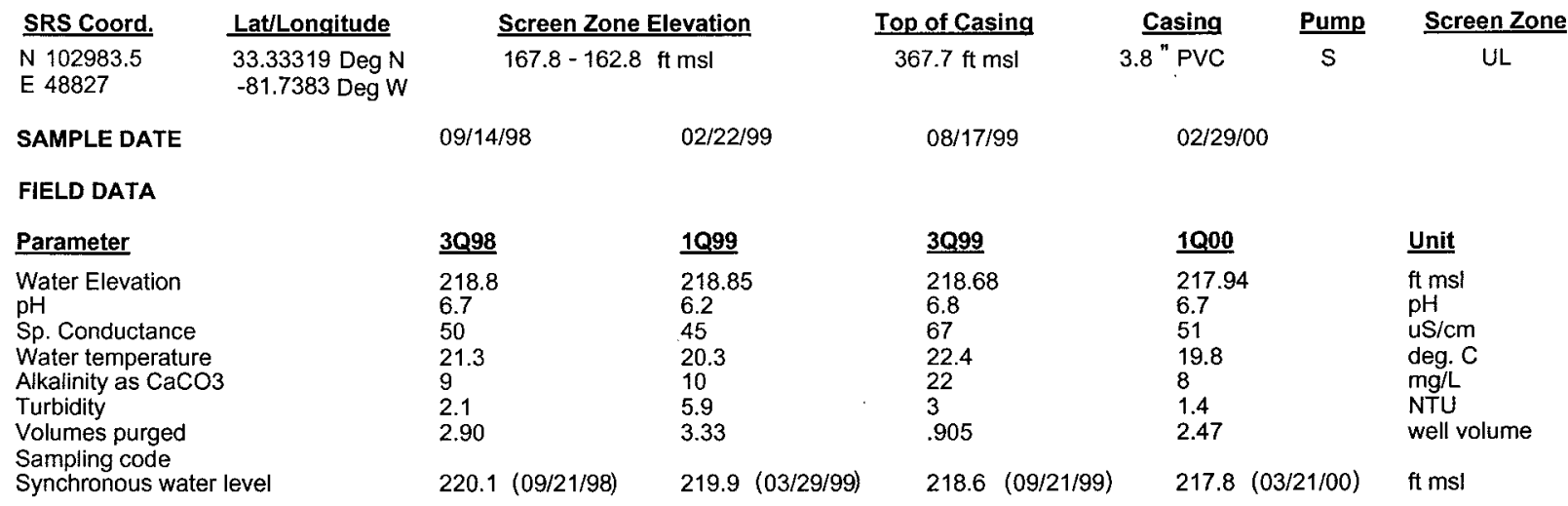

ANALYTICAL DATA

I. Groundwater Protection Standard

261 Appendix VIII/264 Appendix IX Hazardous Constituents

\begin{tabular}{|c|c|c|c|c|c|c|c|c|c|c|c|c|c|}
\hline ST & Parameter & $\underline{3 Q 98}$ & CLP EPA & 1099 & CLP EPA & $\underline{3 Q 99}$ & CLPEPA & $\underline{1 Q 00}$ & CLPEPA & Filt. & $\underline{D F}$ & $\underline{\text { Unit }}$ & $\underline{\text { Lab }}$ \\
\hline Inor & ganics & & & & & & & & & & & & \\
\hline & Barium, total recoverable & 16.8 & & 12 & & 11 & & & & & & ug/L & \\
\hline & Cyanide & $<10$ & $u$ & $<10$ & $U$ & $<10$ & $\mathrm{U}$ & & & & & ug/L & \\
\hline & Lead, total recoverable & $<100$ & $\mathrm{U}$ & $<100$ & $U$ & 3.7 & J 1 & & & & & $\mathrm{ug} / \mathrm{L}$ & \\
\hline & Nickel, total recoverable & $<50$ & U & $<50$ & $U$ & $<9.5$ & JU 1 & & & & & $\mathrm{ug} / \mathrm{L}$ & \\
\hline & Selenium, total recoverable & $<200$ & $\mathrm{U}$ & $<200$ & $U$ & $<10$ & $\mathrm{U}$ & & & & & $\mathrm{ug} / \mathrm{L}$ & \\
\hline Org & anics & & & & & & & & & & & & \\
\hline & Chlorobenzene & $<250$ & $u$ & $<500$ & $u$ & .47 & IK & $<50$ & $\mathrm{U}$ & $<\mathrm{EQL}$ & 50 & ug/L & $M L$ \\
\hline & 1,1-Dichloroethane & $<250$ & $\mathrm{U}$ & $<500$ & $U$ & $<1$ & U & $<50$ & $\mathrm{U}$ & $<E Q L$ & 50 & $\mathrm{ug} / \mathrm{L}$ & ML \\
\hline & 1,1-Dichloroethylene & $<250$ & $\mathrm{U}$ & $<500$ & $U$ & $<1$ & $U$ & $<50$ & $\mathrm{U}$ & $<\mathrm{EQL}$ & 50 & $u g / L$ & ML \\
\hline & trans-1,2-Dichloroethylene & $<250$ & $U$ & $<500$ & $U$ & $<1$ & 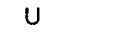 & & & & & $\mathrm{ug} / \mathrm{L}$ & \\
\hline & PCB 1016 & & & & & & & & & & & & \\
\hline & PCB 1221 & & & & & & & & & & & & \\
\hline & PCB 1232 & & & & & & & & & & & & \\
\hline & $\begin{array}{l}\text { PCB } 1242 \\
\text { PCB } 1248\end{array}$ & & & & & & & & & & & & \\
\hline & PCB 1248 & & & & & & & & & & & & \\
\hline & PCB 1254 & & & & & & & & & & & & \\
\hline & PCB 1260 & & & & & & & & & & & & \\
\hline & 1,1,2,2-Tetrachloroethane & $<250$ & $U$ & $<500$ & $U$ & $<1$ & $\mathrm{U}$ & $<50$ & $U$ & $<\mathrm{EQL}$ & 50 & $\mathrm{ug} / \mathrm{L}$ & $M L$ \\
\hline & Tetrachloroethylene & 1240 & & 1660 & & 1240 & & 2020 & $\mathrm{~J} K$ & NDD & 50 & $\mathrm{ug} / \mathrm{L}$ & $M L$ \\
\hline & 1,1,1-Trichloroethane & $<250$ & U & $<500$ & U & $<1$ & $U$ & $<50$ & $\mathrm{U}$ & $<\mathrm{EQL}$ & 50 & $\mathrm{ug} / \mathrm{L}$ & $M L$ \\
\hline & Trichloroethylene & 8310 & & 8740 & & 7990 & & 12100 & $\mathrm{~J} K$ & NDD & 50 & $\mathrm{ug} / \mathrm{L}$ & $M L$ \\
\hline $\mathbf{M}$ & onitoring Constituents & & & & & & & & & & & & \\
\hline$\underline{\mathbf{S T}}$ & Parameter & $\underline{3 Q 96}$ & CLPEPA & $\underline{3097}$ & CLP EPA & $\underline{3 Q 98}$ & CLPEPA & $\underline{3099}$ & CLPEPA & Filt. & $\underline{D F}$ & Unit & b \\
\hline Inor & ganics & & & & & & & & & & & & \\
\hline & Aluminum, total recoverable & $<29.6$ & $U$ & $<20$ & U & $<200$ & $\mathrm{U}$ & $<200$ & $\mathrm{U}$ & $<\mathrm{EQL}$ & 1 & $\mathrm{ug} / \mathrm{L}$ & EX \\
\hline & Nitrate-nitrite as nitrogen & 820 & & 779 & & 550 & & 590 & & $<2400$ & 5 & ug/L & 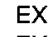 \\
\hline & Sodium, total recoverable & 1850 & & 2100 & & 2210 & & 2000 & & $<4600$ & 1 & $\mathrm{ug} / \mathrm{L}$ & $x$ \\
\hline & Sulfate & 348 & $J$ & $<5000$ & $\mathrm{U}$ & 361 & $J$ & 307 & & $<3000$ & 1 & $\mathrm{ug} / \mathrm{L}$ & $E X$ \\
\hline ad & Ionuclides & & & & & & & & & & & & \\
\hline & Gross alpha & .807 & & .51 & UI & 1.56 & & .491 & $U$ & $<\mathrm{EQL}$ & 1 & $\mathrm{pCi} / \mathrm{L}$ & GP \\
\hline & Nonvolatile beta & 1.08 & Ul & -1.07 & UI & 2.48 & & 1.3 & $U$ & $<E Q L$ & 1 & $\mathrm{pCi} / \mathrm{L}$ & GP \\
\hline & Radium, total alpha-emitting & .5 & & 1.2 & & .36 & UI & .2 & $U$ & $<\mathrm{EQL}$ & 1 & $\mathrm{pCi} / \mathrm{L}$ & GP \\
\hline
\end{tabular}

Notes: Concentrations in bold italics exceed the groundwater protection or monitoring constituent standards listed in Appendix A. Synchronous water levels are measured over a 3-5 day period or less. Dilution factors, Laboratory, and Filtered Data are for Groundwater Protection Standards First Quarter 2000 and Monitoring Constituents Third Quarter 1999 data only.

$+=$ exceeded the Groundwater Protection Standards (First Quarter 2000) or Monitoring Constituents (Third Quarter 1999) listed in Appendix A. 
Table D-4. Groundwater Monitoring Results for Plume Definition Wells, M-Area HWMF (Cont.) WELL MSB 15AA

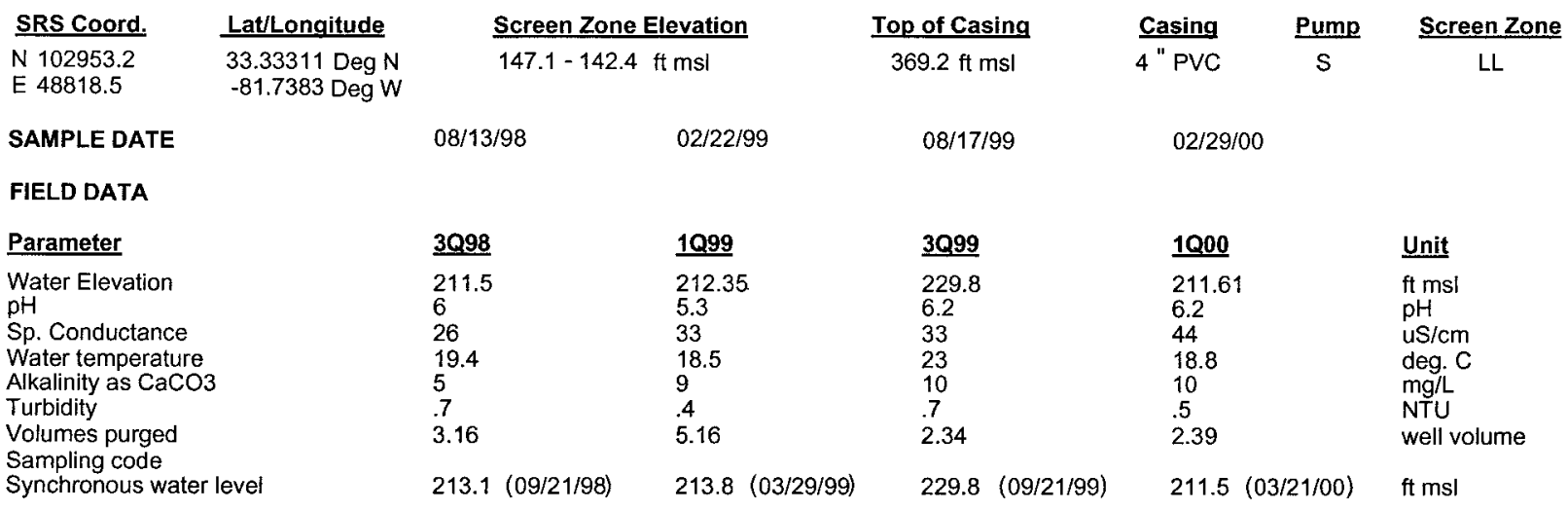

ANALYTICAL DATA

I. Groundwater Protection Standard

261 Appendix VIII/264 Appendix IX Hazardous Constituents

\begin{tabular}{|c|c|c|c|c|c|c|c|c|c|c|c|c|c|}
\hline \multirow{2}{*}{$\begin{array}{l}\text { ST Parameter } \\
\text { Inorganics }\end{array}$} & \multirow[t]{2}{*}{$\underline{3 Q 98}$} & \multirow[t]{2}{*}{ CLP EPA } & \multirow[t]{2}{*}{$\underline{1 Q 99}$} & \multirow[t]{2}{*}{ CLPEPA } & \multirow[t]{2}{*}{$\underline{3 Q 99}$} & \multirow[t]{2}{*}{ CLPEPA } & \multirow[t]{2}{*}{1000} & \multirow{2}{*}{\multicolumn{2}{|c|}{ CLPEPA }} & \multirow[t]{2}{*}{ Filt. } & \multirow[t]{2}{*}{ DF } & \multirow[t]{2}{*}{$\underline{\text { Unit }}$} & \multirow[t]{2}{*}{$\underline{\text { Lab }}$} \\
\hline & & & & & & & & & & & & & \\
\hline Barium, total recoverable & 10.9 & & 15.7 & & 13 & & 16.9 & & & $<2000$ & 1 & ug/L & ML \\
\hline Cyanide & $<10$ & $u$ & $<10$ & $u$ & $<10$ & $u$ & & & & & & $\mathrm{ug} / \mathrm{L}$ & \\
\hline Lead, total recoverable & $<10$ & $u$ & $<100$ & $u$ & $<10$ & $\mathrm{u}$ & $<20$ & $\mathrm{u}$ & & $<E Q L$ & 1 & $\mathrm{ug} / \mathrm{L}$ & ML \\
\hline Nickel, total recoverable & $<50$ & $u$ & $<50$ & $u$ & $<9.6$ & JU I & $<60$ & $u$ & & $<E Q L$ & 1 & $\mathrm{ug} / \mathrm{L}$ & ML \\
\hline Selenium, total recoverable & $<10$ & $u$ & $<200$ & $\mathrm{u}$ & $<4.1$ & JU I & $<40$ & $u$ & & $<E Q L$ & 1 & $\mathrm{ug} / \mathrm{L}$ & ML \\
\hline \multicolumn{14}{|l|}{ Organics } \\
\hline Chlorobenzene & $<25$ & $u$ & $<25$ & u & $<1$ & $\mathrm{u}$ & $<1$ & $u$ & & $<E Q L$ & 1 & ug/L & ML \\
\hline 1,1-Dichloroethane & $<25$ & $u$ & $<25$ & $u$ & $<1$ & $\mathrm{u}$ & $<1$ & U & & $<E Q L$ & 1 & ug/L & $\mathrm{ML}$ \\
\hline 1,1-Dichloroethylene & $<25$ & $u$ & $<25$ & $\mathrm{u}$ & $<1$ & $u$ & $<1$ & $u$ & & $<\mathrm{EQL}$ & 1 & ug/L & ML \\
\hline trans-1,2-Dichloroethylene & $<25$ & $u$ & $<25$ & $u$ & $<1$ & $u$ & & & & & & ug/L & \\
\hline PCB 1016 & & & & & & & & & & & & & \\
\hline PCB 1221 & & & & & & & & & & & & & \\
\hline PCB 1232 & & & & & & & & & & & & & \\
\hline PCB 1242 & & & & & & & & & & & & & \\
\hline PCB 1248 & & & & & & & & & & & & & \\
\hline PCB 1254 & & & & & & & & & & & & & \\
\hline PCB 1260 & & & & & & & & & & & & & \\
\hline 1,1,2,2-Tetrachloroethane & $<25$ & $u$ & $<25$ & $u$ & $<1$ & $u$ & $<1$ & $\mathrm{U}$ & & $<E Q L$ & 1 & $\mathrm{ug} / \mathrm{L}$ & ML \\
\hline Tetrachloroethylene & 10.5 & J & 14.1 & J 1 & 11 & $\mathrm{~J} \mathrm{~K}$ & 13.6 & $\mathrm{~J}$ & $\mathrm{~K}$ & NDD & 1 & $\mathrm{ug} / \mathrm{L}$ & ML \\
\hline 1,1,1-Trichloroethane & $<25$ & $\mathrm{u}$ & $<25$ & $U$ & $<1$ & U & $<1$ & $\mathrm{u}$ & & $<E Q L$ & 1 & $\mathrm{ug} / \mathrm{L}$ & ML \\
\hline Trichloroethylene & 149 & & 298 & & 275 & $\mathrm{~J} \mathrm{~K}$ & 263 & $\mathrm{~J}$ & K & NDD & 1 & $\mathrm{ug} / \mathrm{L}$ & ML \\
\hline \multicolumn{14}{|l|}{ II. Monitoring Constituents } \\
\hline Parameter & $\underline{3096}$ & CLPEPA & $\underline{3097}$ & CLPEPA & $\underline{3098}$ & CLPEPA & $\underline{3099}$ & CLP & EPA & Filt. & DE & $\underline{\text { Unit }}$ & $\underline{\text { Lab }}$ \\
\hline \multicolumn{14}{|l|}{ Inorganics } \\
\hline Aluminum, total recoverable & $<20$ & $u$ & $<20$ & $u$ & $<200$ & $u$ & $<200$ & $u$ & & $<E Q L$ & 1 & $u g / L$ & EX \\
\hline Nitrate-nitrite as nitrogen & 450 & & 120 & & $<500$ & $u$ & 153 & J & 1 & NDD & 5 & $\mathrm{ug} / \mathrm{L}$ & EX \\
\hline Sodium, total recoverable & 1800 & & 1580 & & 1530 & & 1700 & & & $<4600$ & 1 & $\mathrm{ug} / \mathrm{L}$ & EX \\
\hline Sulfate & 386 & $\mathrm{~J}$ & $<5000$ & $u$ & $<200$ & $\mathrm{u}$ & 381 & & & $<3000$ & 1 & $\mathrm{ug} / \mathrm{L}$ & EX \\
\hline \multicolumn{14}{|l|}{ Radionuclides } \\
\hline Gross alpha & .156 & UI & 2.35 & U1 & .24 & UI & -.0638 & $u$ & & $<E Q L$ & 1 & $\mathrm{pCi} / \mathrm{L}$ & GP \\
\hline Nonvolatile beta & 1.03 & & 3.81 & & -.6 & UI & .473 & $u$ & & $<E Q L$ & 1 & $\mathrm{pCi} / \mathrm{L}$ & GP \\
\hline Radium, total alpha-emitting & .1 & UI & -.25 & UI & .12 & $u$ & .1 & $u$ & & $<E Q L$ & 1 & $\mathrm{pCi} / \mathrm{L}$ & GP \\
\hline
\end{tabular}

Notes: Concentrations in bold italics exceed the groundwater protection or monitoring constituent standards listed in Appendix A. Synchronous water levels are measured over a 3-5 day period or less. Dilution factors, Laboratory, and Filtered Data are for Groundwater Protection Standards First Quarter 2000 and Monitoring Constituents Third Quarter 1999 data only.

$+=$ exceeded the Groundwater Protection Standards (First Quarter 2000) or Monitoring Constituents (Third Quarter 1999) listed in Appendix A. 
Table D-4. Groundwater Monitoring Results for Plume Definition Wells, M-Area HWMF (Cont.) WELL MSB 15D

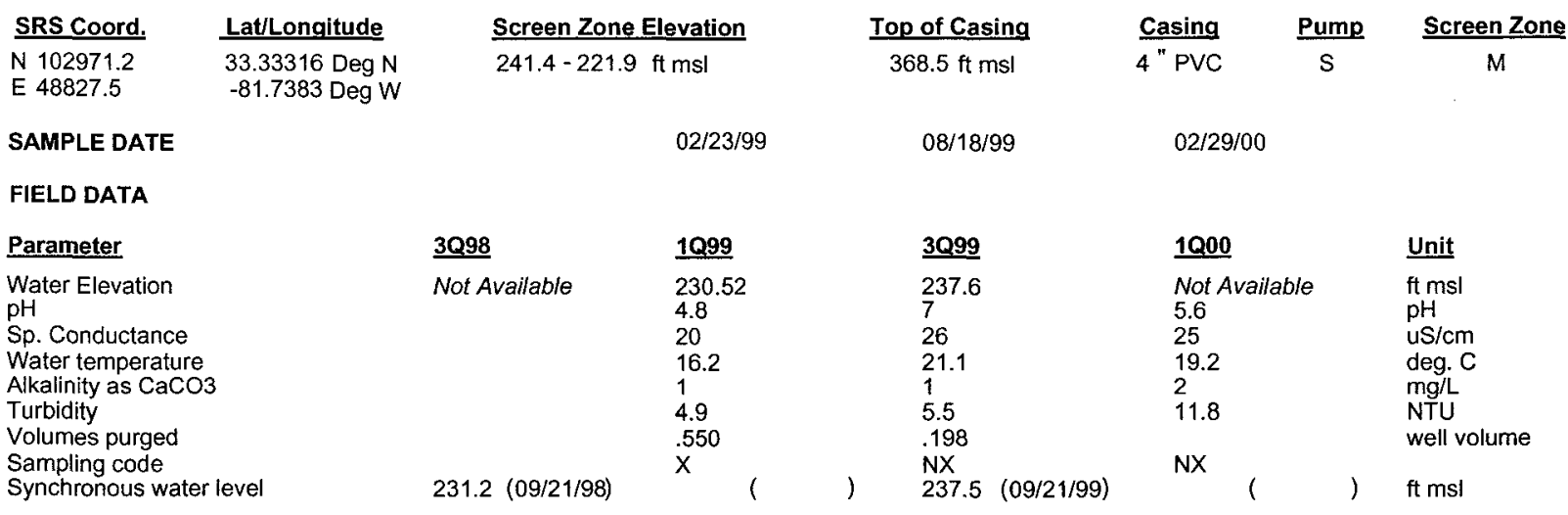

ANALYTICAL DATA

1. Groundwater Protection Standard

261 Appendix VIII/264 Appendix IX Hazardous Constituents

\begin{tabular}{|c|c|c|c|c|c|c|c|c|c|c|c|c|c|}
\hline \multirow{2}{*}{$\begin{array}{l}\text { ST Parameter } \\
\text { Inorganics }\end{array}$} & \multirow[t]{2}{*}{$\underline{3098}$} & \multirow[t]{2}{*}{ CLPEPA } & \multirow[t]{2}{*}{1099} & \multirow[t]{2}{*}{ CLPEPA } & \multirow[t]{2}{*}{$\underline{3099}$} & CLPEPA & \multirow[t]{2}{*}{1000} & \multicolumn{2}{|c|}{ CLPEPA } & \multirow[t]{2}{*}{ Filt. } & \multirow[t]{2}{*}{ DF } & \multirow[t]{2}{*}{$\underline{\text { Unit }}$} & \multirow[t]{2}{*}{$\underline{\text { Lab }}$} \\
\hline & & & & & & & & & & & & & \\
\hline Barium, total recoverable & & & 3.63 & J 1 & 2.9 & J 1 & & & & & & $\mathrm{ug} / \mathrm{L}$ & \\
\hline Cyanide & & & $<10$ & $\mathrm{u}$ & $<10$ & $\mathrm{u}$ & & & & & & $\mathrm{ug} / \mathrm{L}$ & \\
\hline Lead, total recoverable & & & $<100$ & $\mathrm{u}$ & 5.75 & & & & & & & $\mathrm{ug} / \mathrm{L}$ & \\
\hline Nickel, total recoverable & & & $<50$ & $\mathrm{u}$ & $<13$ & JU 1 & & & & & & $\mathrm{ug} / \mathrm{L}$ & \\
\hline Selenium, total recoverable & & & $<200$ & $u$ & $<5$ & $\mathrm{u}$ & & & & & & $\mathrm{ug} / \mathrm{L}$ & \\
\hline \multicolumn{14}{|l|}{ Organics } \\
\hline Chlorobenzene & & & $<250$ & $\mathrm{u}$ & $<1$ & $\mathrm{u}$ & $<2$ & u & & $<E Q L$ & 2 & $\mathrm{ug} / \mathrm{L}$ & ML \\
\hline 1,1-Dichloroethane & & & $<250$ & u & $<1$ & $\mathrm{u}$ & $<2$ & u & & $<E Q L$ & 2 & $\mathrm{ug} / \mathrm{h}$ & ML \\
\hline i,1-Dichloroethylene & & & $<250$ & u & $<1$ & u & $<2$ & u & & $<E Q L$ & 2 & $\mathrm{ug} / \mathrm{L}$ & ML \\
\hline trans-1,2-Dichloroethylene & & & $<250$ & $u$ & $<1$ & $u$ & & & & & & $\mathrm{ug} / \mathrm{L}$ & \\
\hline PCB 1016 & & & & & & & & & & & & & \\
\hline PCB 1221 & & & & & & & & & & & & & \\
\hline РCB 1232 & & & & & & & & & & & & & \\
\hline PCB 1242 & & & & & & & & & & & & & \\
\hline PCB 1248 & & & & & & & & & & & & & \\
\hline PCB 1254 & & & & & & & & & & & & & \\
\hline PCB 1260 & & & & & & & & & & & & & \\
\hline 1,1,2,2-Tetrachloroethane & & & $<250$ & $u$ & $<1$ & $\mathrm{u}$ & $<2$ & $\mathrm{u}$ & & $<E Q L$ & 2 & $\mathrm{ug} / \mathrm{L}$ & ML \\
\hline Tetrachloroethylene & & & 326 & & 176 & $J \quad K$ & 95.4 & J & $\mathrm{K}$ & NDD & 2 & $\mathrm{ug} / \mathrm{L}$ & ML \\
\hline 1,1,1-Trichloroethane & & & $<250$ & $\mathrm{u}$ & .75 & J IK & $<2$ & u & & $<E Q L$ & 2 & ug/L & $\mathrm{ML}$ \\
\hline Trichloroethylene & & & 2670 & & 1580 & & 595 & J & K & NDD & 2 & $\mathrm{ug} / \mathrm{L}$ & $M L$ \\
\hline \multicolumn{14}{|l|}{ II. Monitoring Constituents } \\
\hline ST Parameter & $\underline{3 Q 96}$ & CLPEPA & $\underline{3097}$ & CLP EPA & $\underline{3098}$ & CLPEPA & $\underline{3099}$ & $\underline{\text { CLP }}$ & EPA & Filt. & $\underline{\mathrm{DF}}$ & $\underline{\text { Unit }}$ & $\underline{\mathrm{Lab}}$ \\
\hline \multicolumn{14}{|l|}{ Inorganics } \\
\hline Aluminum, total recoverable & 51.3 & $J$ & & & & & $<200$ & $\mathrm{u}$ & & $<E Q L$ & 1 & $\mathrm{ug} / \mathrm{L}$ & EX \\
\hline Nitrate-nitrite as nitrogen & 800 & & & & & & 1280 & & & $<2400$ & 5 & $u g / L$ & EX \\
\hline Sodium, total recoverable & 3050 & & & & & & 3500 & & & $<4600$ & 1 & ug/L & EX \\
\hline Sulfate & 380 & $J$ & & & & & 345 & & & $<3000$ & 1 & ug/L & $E X$ \\
\hline \multicolumn{14}{|l|}{ Radionuclides } \\
\hline Gross alpha & 3.52 & & & & & & .88 & J & 1 & NDD & 1 & $\mathrm{pCi} / \mathrm{L}$ & GP \\
\hline Nonvolatile beta & 4.28 & & & & & & .858 & $u$ & & $<E Q L$ & 1 & $\mathrm{pCi} / \mathrm{L}$ & GP \\
\hline Radium, total alpha-emitting & 6 & & & & & & .1 & $u$ & & $<E Q L$ & 1 & $\mathrm{pCi} / \mathrm{L}$ & GP \\
\hline
\end{tabular}

\footnotetext{
Notes: Concentrations in bold italics exceed the groundwater protection or monitoring constituent standards listed in Appendix A. Synchronous water levels are measured over a 3-5 day period or less. Dilution factors, Laboratory, and Filtered Data are for Groundwater Protection Standards First Quarter 2000 and Monitoring Constituents Third Quarter 1999 data only.
}

+ = exceeded the Groundwater Protection Standards (First Quarter 2000) or Monitoring Constituents (Third Quarter 1999) listed in Appendix A. 
Table D-4. Groundwater Monitoring Results for Plume Definition Wells, M-Area HWMF (Cont.) WELL MSB 16A

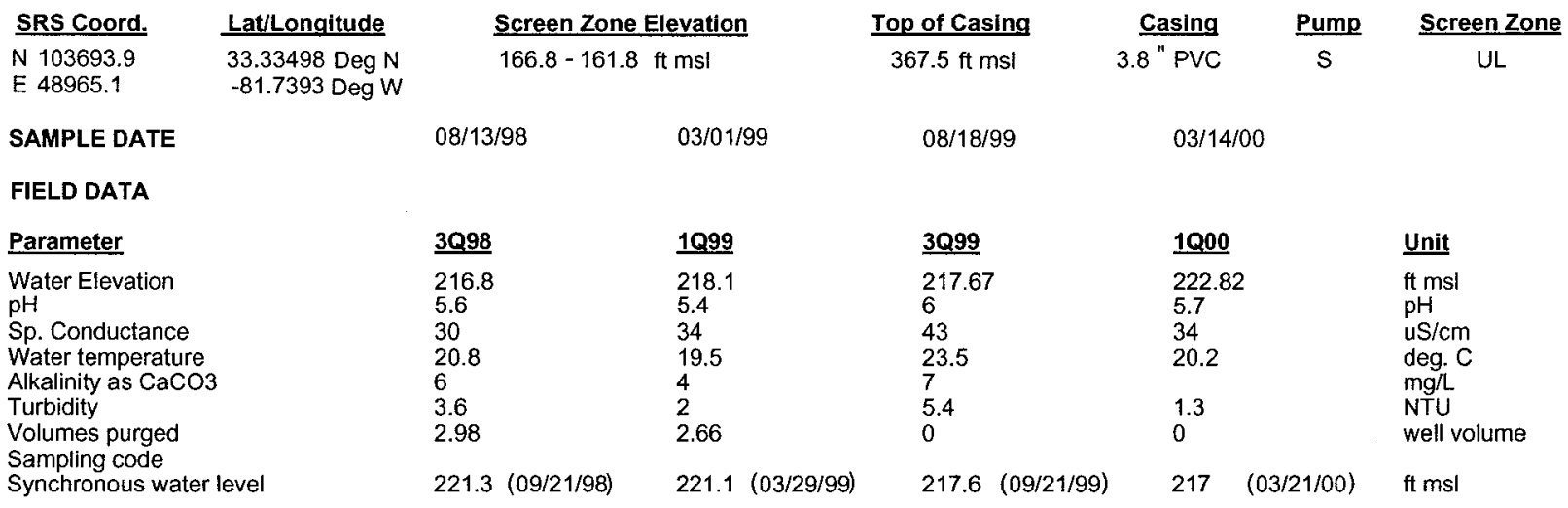

ANALYTICAL DATA

I. Groundwater Protection Standard

261 Appendix VIII/264 Appendix IX Hazardous Constituents

\begin{tabular}{|c|c|c|c|c|c|c|c|c|c|c|c|c|c|c|c|}
\hline \multirow{2}{*}{$\frac{\mathrm{ST}}{\text { Inorg }}$} & Parameter & \multirow[t]{2}{*}{$\underline{3 Q 98}$} & \multirow[t]{2}{*}{$\underline{\text { CLP EPA }}$} & \multirow[t]{2}{*}{$\underline{1099}$} & $\underline{\text { CLP EPA }}$ & \multirow[t]{2}{*}{$\underline{3099}$} & \multicolumn{2}{|c|}{ CLPEPA } & \multirow[t]{2}{*}{$\underline{1000}$} & \multicolumn{2}{|c|}{ CLPEPA } & \multirow[t]{2}{*}{ Filt. } & \multirow[t]{2}{*}{$\underline{\mathrm{DF}}$} & \multirow[t]{2}{*}{ Unit } & \multirow[t]{2}{*}{$\underline{L a b}$} \\
\hline & ganics & & & & & & & & & & & & & & \\
\hline & Barium, total recoverable & 6.81 & $J$ & 8.74 & $\mathrm{~J} \quad \mathrm{I}$ & 6.2 & $J$ & 1 & 10.7 & $J$ & I & NDD & 1 & ug/L & ML \\
\hline & Cyanide & $<10$ & $\mathrm{U}$ & $<10$ & $\mathrm{U}$ & $<10$ & $U$ & & & & & & & ug/L & \\
\hline & Lead, total recoverable & 4.7 & $\mathrm{~J}$ & $<100$ & $\mathrm{U}$ & 14.8 & & & 14.8 & J & IV & NDD & 1 & $\mathrm{ug} / \mathrm{L}$ & ML \\
\hline & Nickel, total recoverable & $<50$ & $\mathrm{U}$ & $<50$ & $\mathrm{U}$ & 23 & $\mathrm{~J}$ & I & $<60$ & $\mathrm{U}$ & & $<E Q L$ & 1 & $\mathrm{ug} / \mathrm{L}$ & ML \\
\hline & Selenium, total recoverable & $<10$ & $U$ & $<200$ & $U$ & $<5$ & $U$ & & $<40$ & $U$ & & $<\mathrm{EQL}$ & 1 & $\mathrm{ug} / \mathrm{L}$ & ML \\
\hline \multicolumn{16}{|c|}{ Organics } \\
\hline & Chlorobenzene & $<500$ & $\mathrm{U}$ & $<1250$ & $\mathrm{U}$ & $<1$ & $\mathrm{u}$ & & 23 & $J$ & 1 & NDD & 50 & $\mathrm{ug} / \mathrm{L}$ & $M L$ \\
\hline & 1,1-Dichloroethane & $<500$ & $\mathrm{U}$ & $<1250$ & $U$ & $<1$ & $\mathrm{U}$ & & $<50$ & U & & $<\mathrm{EQL}$ & 50 & $\mathrm{ug} / \mathrm{L}$ & $M L$ \\
\hline & 1,1-Dichloroethylene & $<500$ & $U$ & $<1250$ & $U$ & 3.31 & J & $\mathrm{K}$ & $<50$ & $\mathrm{U}$ & & $<\mathrm{EQL}$ & 50 & $\mathrm{ug} / \mathrm{L}$ & ML \\
\hline & trans-1,2-Dichloroethylene & $<500$ & $\mathrm{U}$ & $<1250$ & $U$ & $<1$ & $\mathrm{U}$ & & $<50$ & $\mathrm{U}$ & & $<E Q L$ & 50 & $\mathrm{ug} / \mathrm{L}$ & $M L$ \\
\hline & PCB 1016 & & & & & & & & & & & & & & \\
\hline & PCB 1221 & & & & & & & & & & & & & & \\
\hline & PCB 1232 & & & & & & & & & & & & & & \\
\hline & PCB 1242 & & & & & & & & & & & & & & \\
\hline & РCB 1248 & & & & & & & & & & & & & & \\
\hline & PCB 1254 & & & & & & & & & & & & & & \\
\hline & PCB 1260 & & & & & & & & & & & & & & \\
\hline & $1,1,2,2$-Tetrachloroethane & $<500$ & $U$ & $<1250$ & $\mathrm{U}$ & $<1$ & $U$ & & $<50$ & $\mathrm{U}$ & & $<\mathrm{EQL}$ & 50 & $\mathrm{ug} / \mathrm{L}$ & ML \\
\hline+ & Tetrachloroethylene & 1370 & & 1400 & & 1600 & & & 1480 & & & $>5$ & 50 & $\mathrm{ug} / \mathrm{L}$ & ML \\
\hline & 1,1,1-Trichloroethane & $<500$ & $\mathrm{U}$ & $<1250$ & $\mathrm{U}$ & & J & IK & $<50$ & $\mathrm{U}$ & & $<$ EQL & 50 & $\mathrm{ug} / \mathrm{L}$ & ML \\
\hline+ & Trichloroethylene & 9890 & & 9130 & & 10600 & & & 12700 & & & $>5$ & 50 & $\mathrm{ug} / \mathrm{L}$ & ML \\
\hline \multicolumn{16}{|c|}{ II. Monitoring Constituents } \\
\hline$\underline{\mathbf{S T}}$ & Parameter & $\underline{3 Q 96}$ & CLPEPA & $\underline{3097}$ & CLPEPA & $\underline{3 Q 98}$ & CLP & EPA & $\underline{3099}$ & CLP & EPA & Filt. & DF & Unit & $\underline{\text { Lab }}$ \\
\hline \multicolumn{16}{|c|}{ Inorganics } \\
\hline & Aluminum, total recoverable & $<22.9$ & $U$ & $<20$ & $\mathrm{U}$ & $<200$ & $\mathrm{U}$ & & $<200$ & $u$ & & $<E Q L$ & 1 & $\mathrm{ug} / \mathrm{L}$ & EX \\
\hline & Nitrate-nitrite as nitrogen & 1280 & J & 1420 & & 1490 & & & 1800 & & & $<2400$ & 5 & $\mathrm{ug} / \mathrm{L}$ & EX \\
\hline & Sodium, total recoverable & 2000 & & 2370 & & 2340 & & & 2800 & & & $<4600$ & 1 & $\mathrm{ug} / \mathrm{L}$ & EX \\
\hline & Sulfate & 194 & $J$ & $<5000$ & $\mathrm{U}$ & $<200$ & $\mathrm{U}$ & & 111 & $\mathrm{~J}$ & 1 & NDD & 1 & $\mathrm{ug} / \mathrm{L}$ & EX \\
\hline \multicolumn{16}{|c|}{ Radionuclides } \\
\hline & Gross alpha & .589 & UI & 1.12 & UI & 1.32 & & & .754 & J & 1 & NDD & 1 & $\mathrm{pCi} / \mathrm{L}$ & GP \\
\hline & Nonvolatile beta & 1.85 & & -.14 & $\mathrm{UI}$ & .76 & $\mathrm{UI}$ & & 1.82 & J & 1 & NDD & 1 & $\mathrm{pCi} / \mathrm{L}$ & GP \\
\hline & Radium, total alpha-emitting & .3 & & .6 & $\mathrm{~J}$ & .93 & & & 2.2 & $\mathrm{U}$ & V & $<\mathrm{EQL}$ & 1 & $\mathrm{pCi} / \mathrm{L}$ & GP \\
\hline
\end{tabular}

Notes: Concentrations in bold italics exceed the groundwater protection or monitoring constituent standards listed in Appendix A. Synchronous water levels are measured over a 3-5 day period or less. Dilution factors, Laboratory, and Filtered Data are for Groundwater Protection Standards First Quarter 2000 and Monitoring Constituents Third Quarter 1999 data only.

$+=$ exceeded the Groundwater Protection Standards (First Quarter 2000) or Monitoring Constituents (Third Quarter 1999) listed in Appendix A. 
Table D-4. Groundwater Monitoring Results for Plume Definition Wells, M-Area HWMF (Cont.) WELL MSB 16C

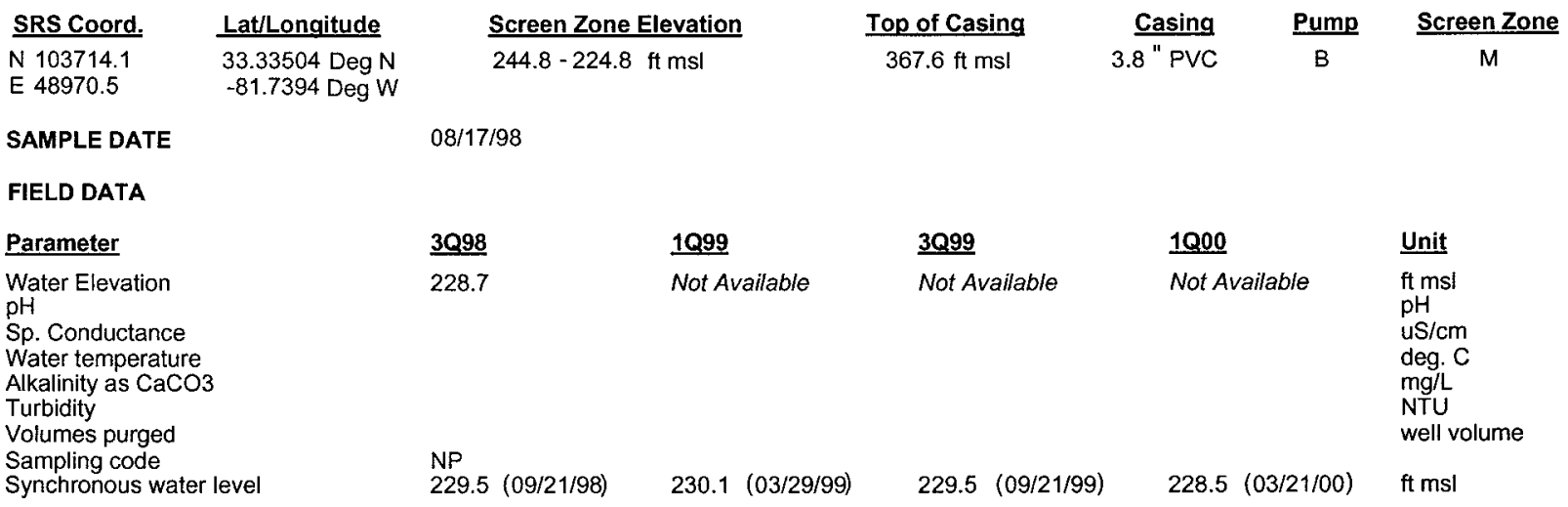

ANALYTICAL DATA

I. Groundwater Protection Standard

261 Appendix VIII/264 Appendix IX Hazardous Constituents

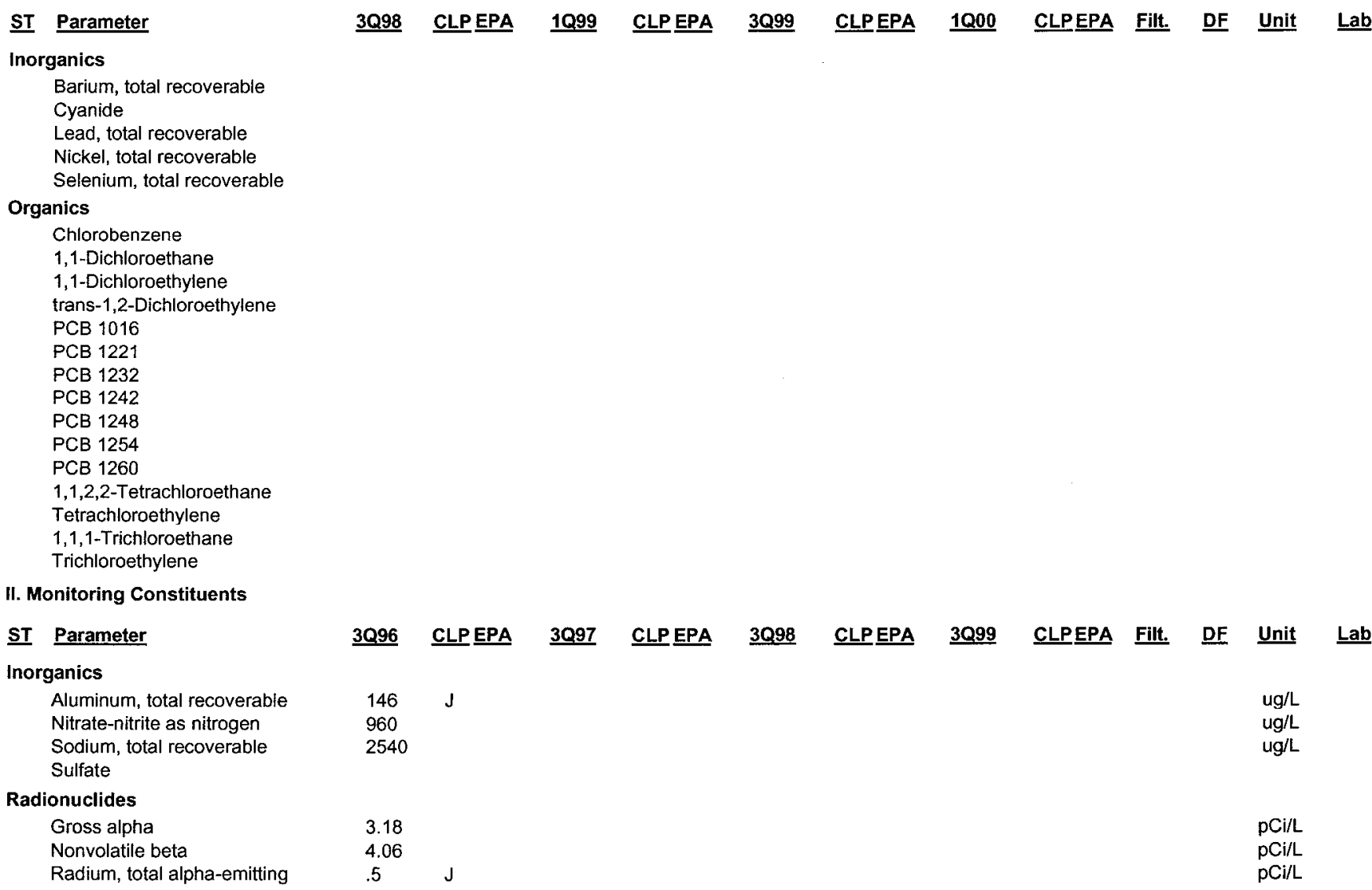

Notes: Concentrations in bold italics exceed the groundwater protection or monitoring constituent standards listed in Appendix A. Synchronous water levels are measured over a 3-5 day period or less. Dilution factors, Laboratory, and Filtered Data are for Groundwater Protection Standards First Quarter 2000 and Monitoring Constituents Third Quarter 1999 data only.

$+=$ exceeded the Groundwater Protection Standards (First Quarter 2000) or Monitoring Constituents (Third Quarter 1999) listed in Appendix A. 
Table D-4. Groundwater Monitoring Results for Plume Definition Wells, M-Area HWMF (Cont.) WELL MSB 17B

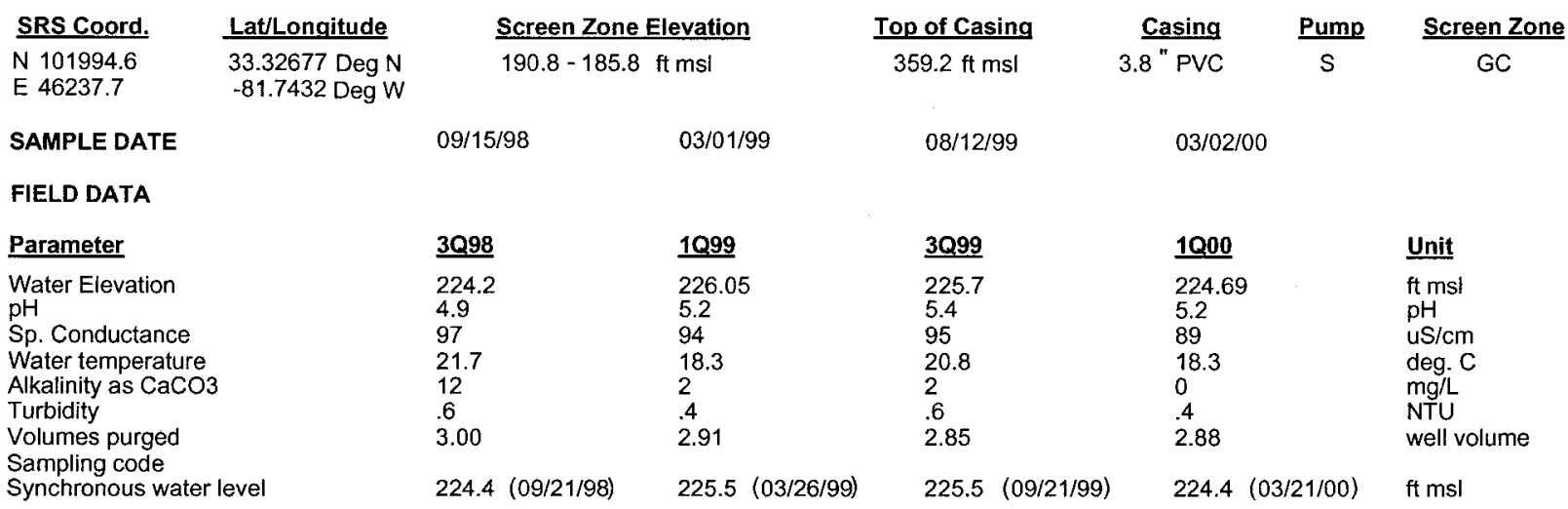

ANALYTICAL DATA

I. Groundwater Protection Standard

261 Appendix VIII/264 Appendix IX Hazardous Constituents

ST Parameter

Inorganics

Barium, total recoverable

Cyanide

Lead, total recoverable

Nickel, total recoverable

Selenium, total recoverable

Organics

$\begin{array}{llll}\text { Chlorobenzene } & <250 & U & <1250 \\ 1,1 \text {-Dichloroethane } & <250 & U & <1250 \\ 1,1 \text {-Dichloroethylene } & <250 & U & <1250 \\ \text { trans } 1 \text { - } & <50 & U & <1250\end{array}$

trans-1,2-Dichloroethylene

PCB 1016

PCB 1221

PCB 1232

PCB 1242

PCB 1248

PCB 1254

PCB 1260

1,1,2,2-Tetrachloroethane

Tetrachloroethylene

1,1,1-Trichloroethane

Trichloroethylene

II. Monitoring Constituents

ST Parameter

Aluminum total recoverable

$+\quad$ Nitrate-nitrite as nitrogen

+ Sodium, total recoverable Sulfate

Radionuclides

Gross alpha

Nonvolatile beta

Radium, total alpha-emitting
3 Q98 CLPEPA

1 Q99 CLPEPA

3 Q99 CLPEPA

1000

CLPEPA

Filt. DF Unit

44
$<10 \quad U$

5.7

$\begin{array}{cc}8.9 & \text { J } \\ <5 & U\end{array}$

$\begin{array}{lll}4 & \mathrm{~J} & 1 \mathrm{~K} \\ <1 & \mathrm{U} & \\ 12.6 & \mathrm{~J} & \mathrm{~K} \\ <1 & \mathrm{U} & \end{array}$

$<50$
$<50$
$<50$

$J U L<E Q L \quad 50$

$J U L<E Q L 50$

JU $L<E Q L 50$

$\mathrm{ug} / \mathrm{L}$

$\mathrm{ug} / \mathrm{L}$

$\mathrm{ug} / \mathrm{L}$

$\mathrm{ug} / \mathrm{L}$

$\mathrm{ug} / \mathrm{L}$

$\mathrm{ug} / \mathrm{L} \quad \mathrm{ML}$

ug/L ML

$u g / L \quad M L$

\begin{tabular}{|c|c|c|c|c|c|c|c|c|c|c|c|c|}
\hline $\begin{array}{l}<250 \\
1070\end{array}$ & $U$ & $\begin{array}{r}<1250 \\
1680\end{array}$ & $U$ & $\begin{array}{l}<1 \\
\quad 1720\end{array}$ & $\mathrm{U}$ & & $\begin{array}{l}<50 \\
1980\end{array}$ & $\begin{array}{l}\mathrm{JU} \\
\mathrm{J}\end{array}$ & $\begin{array}{l}\mathrm{L} \\
\mathrm{L}\end{array}$ & $\begin{array}{r}<\mathrm{EQL} \\
\mathrm{NDD}\end{array}$ & $\begin{array}{l}50 \\
50\end{array}$ & $\begin{array}{l}\mathrm{ug} / \mathrm{L} \\
\mathrm{ug} / \mathrm{L}\end{array}$ \\
\hline $\begin{array}{l}<250 \\
7140\end{array}$ & $U$ & $\begin{array}{r}<1250 \\
12100\end{array}$ & $\mathrm{U}$ & $\begin{array}{l}2 \\
11200\end{array}$ & $\mathbf{J}$ & $\mathrm{K}$ & $\begin{array}{l}<50 \\
\quad 11300\end{array}$ & $\begin{array}{l}\mathrm{JU} \\
\mathrm{J}\end{array}$ & $L$ & $\begin{array}{r}<\mathrm{EQL} \\
\mathrm{NDD}\end{array}$ & $\begin{array}{l}50 \\
50\end{array}$ & $\begin{array}{l}\mathrm{ug} / \mathrm{L} \\
\mathrm{ug} / \mathrm{L}\end{array}$ \\
\hline
\end{tabular}

\begin{tabular}{|c|c|c|c|c|c|c|c|c|c|c|c|c|}
\hline 3096 & CLPEPA & 3097 & CLP EPA & 3098 & CLPEPA & 3099 & CL & EPA & Filt. & DF & Unit & Lab \\
\hline 71.6 & & 71.6 & & 91.6 & J & 97 & $J$ & I & NDD & 1 & $\mathrm{ug} / \mathrm{L}$ & EX \\
\hline 10300 & & 9150 & & 8340 & & 8800 & & & $>2400$ & 5 & $\mathrm{ug} / \mathrm{L}$ & EX \\
\hline 12000 & & 10100 & & 11200 & & 10000 & & & $>4600$ & 1 & $\mathrm{ug} / \mathrm{L}$ & EX \\
\hline$<1000$ & $\mathrm{U}$ & $<5000$ & $\mathrm{U}$ & $<400$ & U & $<400$ & U & & $<E Q L$ & 2 & $\mathrm{ug} / \mathrm{L}$ & EX \\
\hline 9.97 & & 4.83 & & 13.93 & & 6.9 & $J$ & K & NDD & 1 & $\mathrm{pCi} / \mathrm{L}$ & GP \\
\hline 6.49 & & 3.9 & U1 & 11.84 & & 5.51 & & & $<50$ & 1 & $\mathrm{pCi} / \mathrm{L}$ & $\mathrm{GP}$ \\
\hline 5 & & 4.75 & $J$ & 4.1 & $J$ & 3.7 & & & $<5$ & 1 & $\mathrm{pCi} / \mathrm{L}$ & GP \\
\hline
\end{tabular}

Notes: Concentrations in bold italics exceed the groundwater protection or monitoring constituent standards listed in Appendix A. Synchronous water levels are measured over a 3-5 day period or less. Dilution factors, Laboratory, and Filtered Data are for Groundwater Protection Standards First Quarter 2000 and Monitoring Constituents Third Quarter 1999 data only.

$+=$ exceeded the Groundwater Protection Standards (First Quarter 2000) or Monitoring Constituents (Third Quarter 1999) listed in Appendix A. 
Table D-4. Groundwater Monitoring Results for Plume Definition Wells, M-Area HWMF (Cont.) WELL MSB 17BB

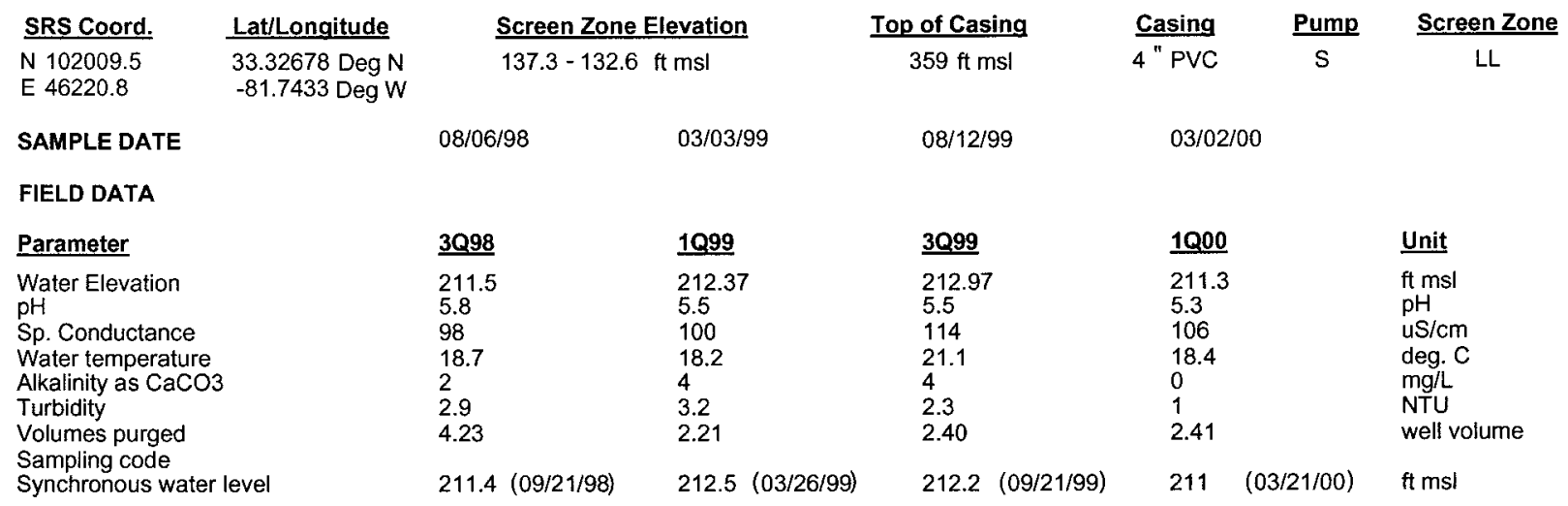

ANALYTICAL DATA

I. Groundwater Protection Standard

261 Appendix VIII/264 Appendix IX Hazardous Constituents

\begin{tabular}{|c|c|c|c|c|c|c|c|c|c|c|c|c|c|c|c|}
\hline$\underline{\text { ST }}$ & Parameter & $\underline{3 Q 98}$ & CLPEPA & 1099 & CLP EPA & $\underline{3 Q 99}$ & $\underline{\text { CLP }}$ & EPA & 1000 & $\underline{\text { CLP }}$ & EPA & Filt. & $\underline{\text { DF }}$ & $\underline{\text { Unit }}$ & Lab \\
\hline & ganics & & & & & & & & & & & & & & \\
\hline & Barium, total recoverable & 30.8 & & 35 & & 35 & & & & & & & & $\mathrm{ug} / \mathrm{L}$ & \\
\hline & Cyanide & $<10$ & $\mathrm{U}$ & $<15.2$ & $U$ & $<10$ & $\mathbf{U}$ & & & & & & & $u g / L$ & \\
\hline & Lead, total recoverable & $<10$ & $\mathrm{U}$ & $<100$ & $\mathrm{U}$ & $<1.8$ & $\mathrm{U}$ & V & & & & & & ug/L & \\
\hline & Nickel, total recoverable & $<50$ & $U$ & $<50$ & U & $<8.1$ & JU & 1 & & & & & & $u g / L$ & \\
\hline & Selenium, total recoverable & $<10$ & $\mathrm{U}$ & $<200$ & U & $<5$ & $U$ & & & & & & & $\mathrm{ug} / \mathrm{L}$ & \\
\hline Org & anics & & & & & & & & & & & & & & \\
\hline & Chlorobenzene & $<50$ & $\mathrm{U}$ & $<125$ & U & $<1$ & $\mathrm{U}$ & & $<10$ & JU & $\mathbf{L}$ & $<E Q L$ & 10 & $u g / L$ & ML \\
\hline & 1,1-Dichloroethane & $<50$ & $\mathrm{U}$ & $<125$ & U & $<1$ & $\mathrm{U}$ & & $<10$ & JU & $\bar{L}$ & $<E Q L$ & 10 & $u g / L$ & $M L$ \\
\hline & 1,1-Dichloroethylene & $<50$ & $\mathrm{U}$ & $<125$ & U & .89 & $\mathrm{~J}$ & IK & $<10$ & JU & $\mathrm{L}$ & $<E Q L$ & 10 & $u g /\llcorner$ & $\mathrm{ML}$ \\
\hline & trans-1,2-Dichloroethylene & $<50$ & $\mathrm{U}$ & $<125$ & $U$ & 1.62 & $\mathrm{~J}$ & $\mathrm{~K}$ & & & & & & $u g / L$ & \\
\hline & PCB 1016 & & & & & & & & & & & & & & \\
\hline & PCB 1221 & & & & & & & & & & & & & & \\
\hline & PCB 1232 & & & & & & & & & & & & & & \\
\hline & PCB 1242 & & & & & & & & & & & & & & \\
\hline & PCB 1248 & & & & & & & & & & & & & & \\
\hline & PCB 1254 & & & & & & & & & & & & & & \\
\hline & PCB 1260 & & & & & & & & . & & & & & & \\
\hline & $1,1,2,2$-Tetrachloroethane & $<50$ & $\mathrm{U}$ & $<125$ & $\mathrm{U}$ & $<1$ & $\mathrm{U}$ & & $<10$ & JU & $L$ & $<\mathrm{EQL}$ & 10 & $u g / L$ & $M L$ \\
\hline & Tetrachloroethylene & 387 & & 626 & & 487 & & & 619 & $\mathrm{~J}$ & $\bar{L}$ & NDD & 10 & $u g / L$ & ML \\
\hline & $1,1,1$-Trichloroethane & $<50$ & $U$ & $<125$ & U & $<1$ & $\mathrm{U}$ & & $<10$ & $\mathrm{JU}$ & $\mathrm{L}$ & $<E Q L$ & 10 & $u g / L$ & $M L$ \\
\hline & Trichloroethylene & 1470 & & 2620 & & 1950 & & & 2430 & $J$ & $\mathrm{~L}$ & NDD & 10 & $u g / L$ & $M L$ \\
\hline$N$ & onitoring Constituents & & & & & & & & & & & & & & \\
\hline$\underline{\text { ST }}$ & Parameter & $3 Q 96$ & CLP EPA & $\underline{3 Q 97}$ & CLP EPA & $\underline{3 Q 98}$ & CLP & EPA & $\underline{3 Q 99}$ & $\underline{\text { CLP }}$ & EPA & Filt. & $\underline{\mathrm{DF}}$ & Unit & $\underline{\text { Lab }}$ \\
\hline In & ganics & & & & & & & & & & & & & & \\
\hline & Aluminum, total recoverable & 58.3 & & 38.1 & & 73.1 & J & & 86 & J & 1 & NDD & 1 & $u g / L$ & EX \\
\hline+ & Nitrate-nitrite as nitrogen & 11600 & & 8550 & & 10500 & & & 10700 & & & $>2400$ & 5 & $u g / L$ & EX \\
\hline+ & Sodium, total recoverable & 7300 & & 5670 & & 6780 & & & 7600 & & & $>4600$ & 1 & $u g / L$ & EX \\
\hline & Sulfate & 357 & & $<5000$ & $U$ & $<200$ & $\mathrm{U}$ & & 405 & & & $<3000$ & 1 & $\mathrm{ug} / \mathrm{L}$ & EX \\
\hline & ionuclides & & & & & & & & & & & & & & \\
\hline & Gross alpha & 7.4 & & 6.79 & UI & 3.27 & & & 1.22 & $\mathrm{~J}$ & IK & NDD & 1 & $\mathrm{pCi} / \mathrm{L}$ & GP \\
\hline & Nonvolatile beta & 7.64 & & 6.5 & & 5.8 & UJ & & 3.64 & & & $<50$ & 1 & $\mathrm{pCi} / \mathrm{L}$ & GP \\
\hline & Radium, total alpha-emitting & 1.5 & & 1.74 & $J$ & .82 & & & .3 & $\mathrm{U}$ & & $<\mathrm{EQL}$ & 1 & $\mathrm{pCi} / \mathrm{L}$ & $\mathrm{GP}$ \\
\hline
\end{tabular}

Notes: Concentrations in bold italics exceed the groundwater protection or monitoring constituent standards listed in Appendix A. Synchronous water levels are measured over a 3-5 day period or less. Dilution factors, Laboratory, and Filtered Data are for Groundwater Protection Standards First Quarter 2000 and Monitoring Constituents Third Quarter 1999 data only.

$+=$ exceeded the Groundwater Protection Standards (First Quarter 2000) or Monitoring Constituents (Third Quarter 1999) listed in Appendix A. 
Table D-4. Groundwater Monitoring Results for Plume Definition Wells, M-Area HWMF (Cont.) WELL MSB 18A

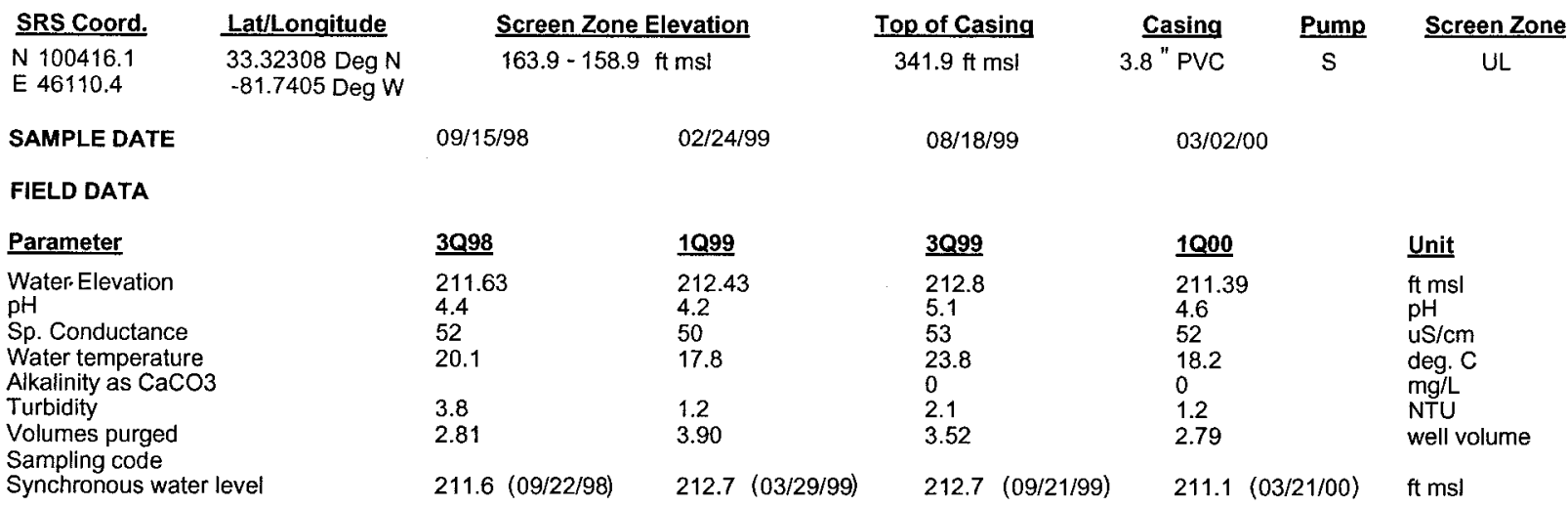

ANALYTICAL DATA

I. Groundwater Protection Standard

261 Appendix VIII/264 Appendix IX Hazardous Constituents

\begin{tabular}{|c|c|c|c|c|c|c|c|c|c|c|c|c|c|}
\hline$\underline{\text { ST }}$ & Parameter & 3Q98 & CLPEPA & 1099 & CLPEPA & $\underline{3099}$ & CLPEPA & 1000 & CLPEPA & Filt. & DF & Unit & Lab \\
\hline \multicolumn{14}{|c|}{ Inorganics } \\
\hline & Barium, total recoverable & 18.7 & & 20.4 & & 17 & & & & & & $\mathrm{ug} / \mathrm{L}$ & \\
\hline & Cyanide & $<10$ & $\mathrm{U}$ & $<10$ & $\mathrm{U}$ & $<10$ & $U$ & & & & & $\mathrm{ug} / \mathrm{L}$ & \\
\hline & Lead, total recoverable & $<100$ & $\mathrm{U}$ & $<100$ & $\mathrm{U}$ & 4.12 & $\mathrm{~J} \quad 1$ & & & & & $\mathrm{ug} / \mathrm{L}$ & \\
\hline & Nickel, total recoverable & 8.37 & J & $<12.6$ & JU & 20 & J I & & & & & $\mathrm{ug} / \mathrm{L}$ & \\
\hline & Selenium, total recoverable & $<200$ & $\mathrm{U}$ & $<200$ & $\mathrm{U}$ & $<5$ & $U$ & & & & & $\mathrm{ug} / \mathrm{L}$ & \\
\hline \multicolumn{14}{|c|}{ Organics } \\
\hline & Chlorobenzene & $<5$ & $\mathrm{U}$ & $<5$ & $\mathrm{U}$ & $<1$ & $\mathrm{U}$ & $<1$ & $\mathrm{U}$ & $<E Q L$ & 1 & ug/L. & ML \\
\hline & 1,1-Dichloroethane & $<5$ & $\mathrm{U}$ & $<5$ & $U$ & $<1$ & $\mathrm{U}$ & $<1$ & $\mathrm{U}$ & $<\mathrm{EQL}$ & 1 & $\mathrm{ug} / \mathrm{L}$ & ML \\
\hline & 1,1-Dichloroethylene & $<5$ & $\mathrm{U}$ & $<5$ & U & 1.09 & $\mathrm{~J} K$ & 1.06 & & $<7$ & 1 & ug/L & $M L$ \\
\hline & trans-1,2-Dichloroethylene & $<5$ & $\mathrm{U}$ & $<5$ & $U$ & $<1$ & $U$ & & & & & $\mathrm{ug} / \mathrm{L}$ & \\
\hline & $\begin{array}{l}\text { PCB } 1016 \\
\text { PCB } 1221\end{array}$ & & & & & & & & & & & & \\
\hline & PCB 1221 & & & & & & & & & & & & \\
\hline & PCB 1232 & & & & & & & & & & & & \\
\hline & PCB 1242 & & & & & & & & & & & & \\
\hline & PCB 1248 & & & & & & & & & & & & \\
\hline & PCB 1254 & & & & & & & & & & & & \\
\hline & PCB 1260 & & & & & & & & & & & & \\
\hline & $1,1,2,2$-Tetrachloroethane & $<5$ & $\mathrm{U}$ & $<5$ & $\mathrm{U}$ & $<1$ & $U$ & $<1$ & $\mathrm{U}$ & $<E Q L$ & 1 & $\mathrm{ug} / \mathrm{L}$ & $M L$ \\
\hline+ & Tetrachloroethylene & 11.6 & & 12 & & 10.8 & $\mathrm{~J}$ & 13.3 & & $>5$ & 1 & $\mathrm{ug} / \mathrm{L}$ & $M L$ \\
\hline & 1,1,1-Trichloroethane & $<5$ & U & $<5$ & $\mathrm{U}$ & $<1$ & $\mathrm{U}$ & $<1$ & $\mathrm{U}$ & $<\mathrm{EQL}$ & 1 & $\mathrm{ug} / \mathrm{L}$ & $M L$ \\
\hline+ & Trichloroethylene & 30 & & 30.3 & & 30.4 & $J K$ & 35.2 & & $>5$ & 1 & $\mathrm{ug} / \mathrm{L}$ & $M L$ \\
\hline \multicolumn{14}{|c|}{ II. Monitoring Constituents } \\
\hline$\underline{\mathbf{S T}}$ & Parameter & $\underline{3 Q 96}$ & CLPEPA & 3097 & CLPEPA & $\underline{3 Q 98}$ & GLPEPA & 3099 & CLPEPA & Filt. & $\underline{\mathrm{DF}}$ & $\underline{\text { Unit }}$ & $\underline{\text { Lab }}$ \\
\hline \multicolumn{14}{|c|}{ Inorganics } \\
\hline & $\begin{array}{l}\text { Aluminum, total recoverable } \\
\text { Nitrate-nitrite as nitrogen }\end{array}$ & $\begin{array}{l}<20 \\
3360\end{array}$ & $\mathbf{U}$ & $\begin{array}{l}<20 \\
4460\end{array}$ & $\mathrm{U}$ & $\begin{array}{l}<200 \\
3540\end{array}$ & $\mathrm{U}$ & $\begin{array}{l}<200 \\
3840\end{array}$ & $\mathrm{U}$ & $\begin{array}{l}<\mathrm{EQL} \\
>2400\end{array}$ & $\begin{array}{l}1 \\
5\end{array}$ & $\begin{array}{l}u g / L \\
u g / L\end{array}$ & $\begin{array}{l}\text { EX } \\
\text { EX }\end{array}$ \\
\hline & Sodium, total recoverable & 2840 & & 3030 & & 3360 & & 3500 & & $<4600$ & 1 & ug/L & EX \\
\hline & Sulfate & 391 & J & $<5000$ & $\mathbf{U}$ & 491 & & 307 & & $<3000$ & 1 & $u g / L$ & EX \\
\hline \multicolumn{14}{|c|}{ Radionuclides } \\
\hline & Gross alpha & .95 & & 3.21 & UI & .39 & UI & .359 & U & $<\mathrm{EQL}$ & 1 & $\mathrm{pCi} / \mathrm{L}$ & GP \\
\hline & Nonvolatile beta & 1.86 & & 3.7 & & 4.21 & & 2.69 & & $<50$ & 1 & $\mathrm{pCi} / \mathrm{L}$ & GP \\
\hline & Radium, total alpha-emitting & .4 & UI & .73 & $J$ & .64 & UI & .2 & U & $<\mathrm{EQL}$ & 1 & $\mathrm{pCi} / \mathrm{L}$ & GP \\
\hline
\end{tabular}

Notes: Concentrations in bold italics exceed the groundwater protection or monitoring constituent standards listed in Appendix A. Synchronous water levels are measured over a 3-5 day period or less. Dilution factors, Laboratory, and Filtered Data are for Groundwater Protection Standards First Quarter 2000 and Monitoring Constituents Third Quarter 1999 data only.

$+=$ exceeded the Groundwater Protection Standards (First Quarter 2000) or Monitoring Constituents (Third Quarter 1999) listed in Appendix A. 
Table D-4. Groundwater Monitoring Results for Plume Definition Wells, M-Area HWMF (Cont.) WELL MSB 18B

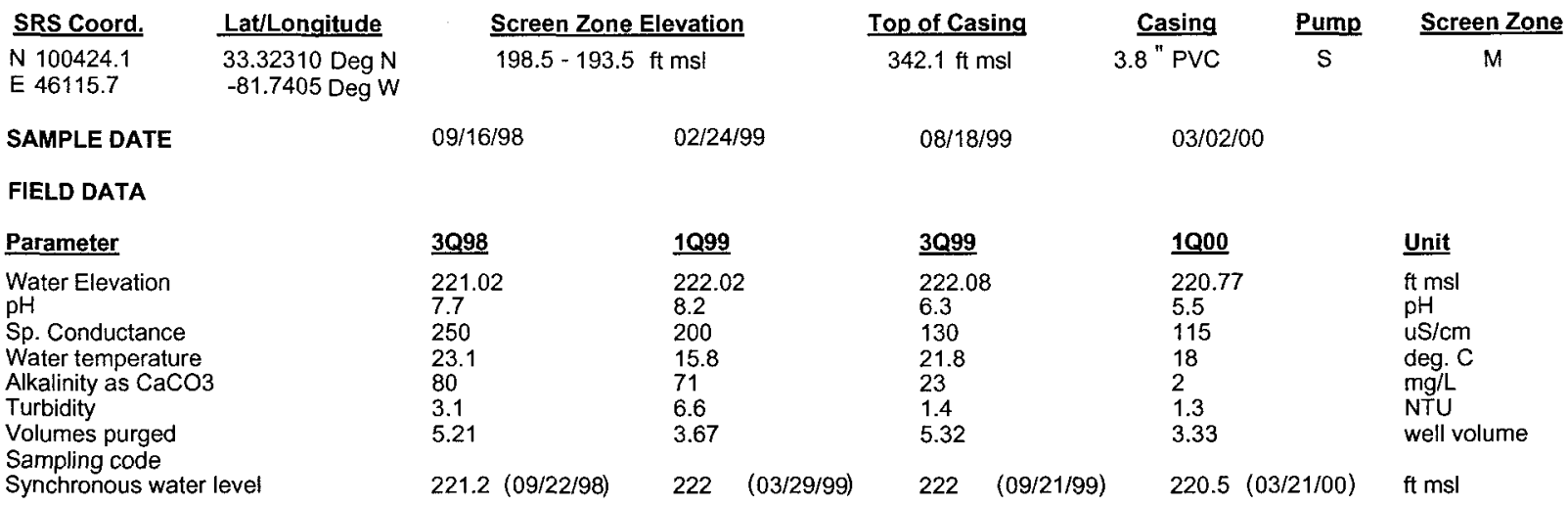

\section{ANALYTICAL DATA}

I. Groundwater Protection Standard

261 Appendix VIII/264 Appendix IX Hazardous Constituents

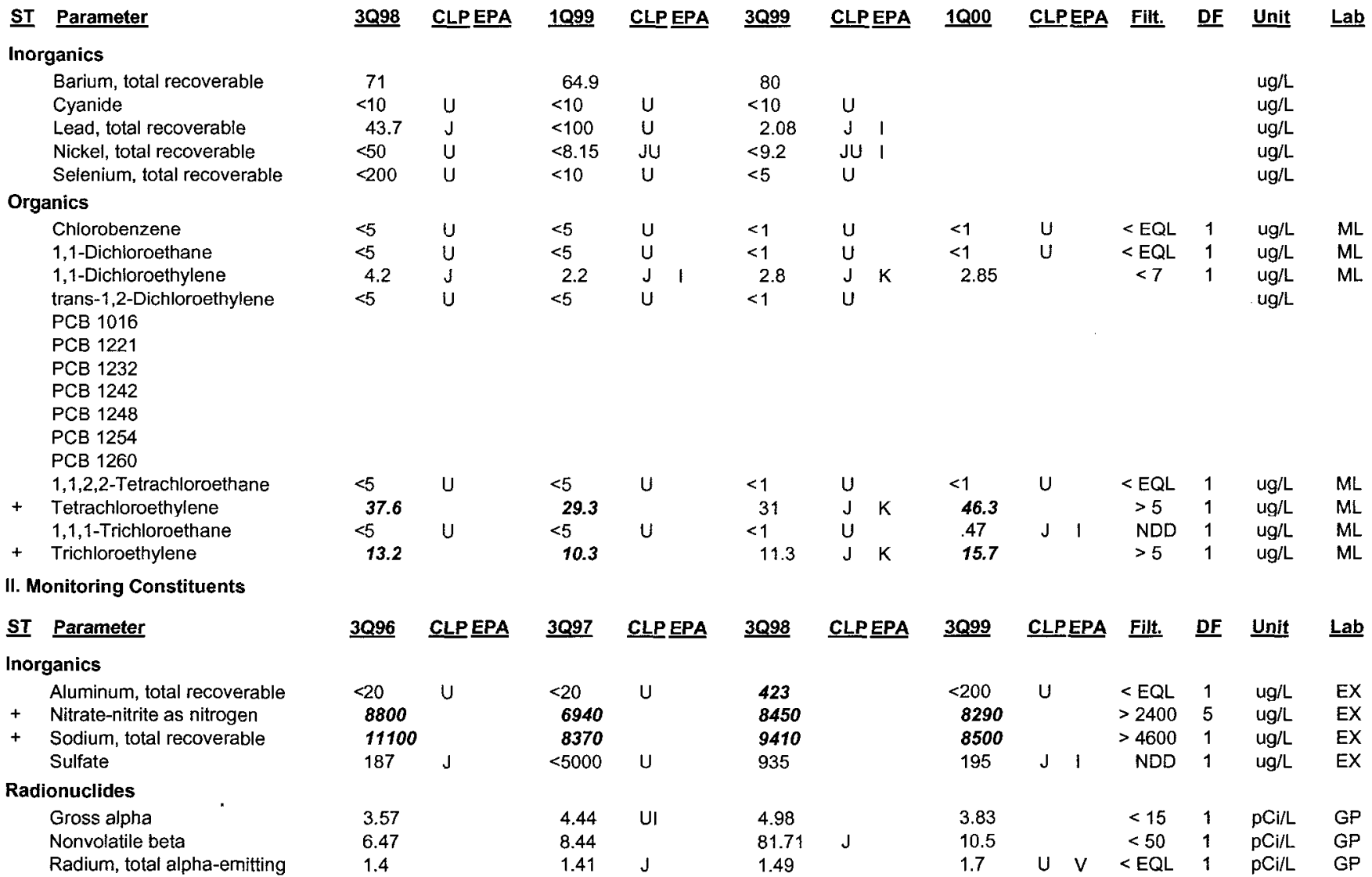

Notes: Concentrations in bold italics exceed the groundwater protection or monitoring constituent standards listed in Appendix A. Synchronous water levels are measured over a 3-5 day period or less. Dilution factors, Laboratory, and Filtered Data are for Groundwater Protection Standards First Quarter 2000 and Monitoring Constituents Third Quarter 1999 data only.

$+=$ exceeded the Groundwater Protection Standards (First Quarter 2000) or Monitoring Constituents (Third Quarter 1999) listed in Appendix A. 
Table D-4. Groundwater Monitoring Results for Plume Definition Wells, M-Area HWMF (Cont.) WELL MSB 19B

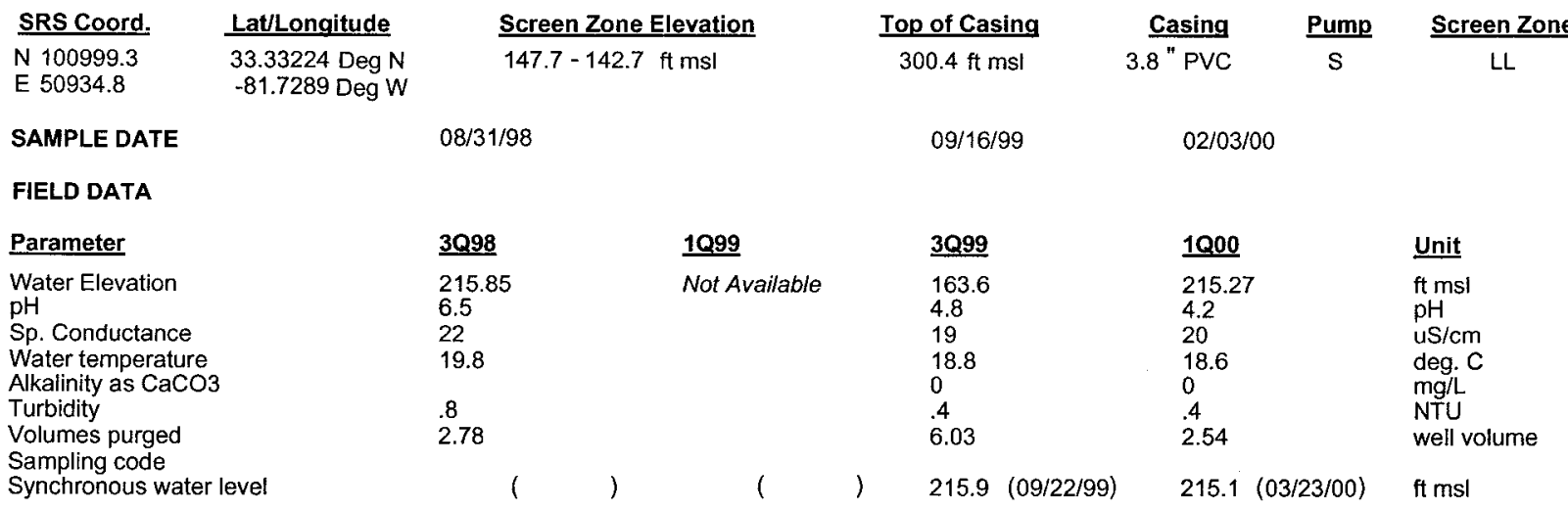

ANALYTICAL DATA

I. Groundwater Protection Standard

261 Appendix VIII/264 Appendix IX Hazardous Constituents

\begin{tabular}{|c|c|c|c|c|c|c|c|c|c|c|c|c|c|}
\hline$\underline{\mathbf{S T}}$ & Parameter & $\underline{3098}$ & CLP EPA & $\underline{1 Q 99}$ & CLP EPA & $\underline{3 Q 99}$ & CLPPEPA & 1000 & CLPEPA & Filt. & $\underline{\text { DF }}$ & Unit & $\underline{\text { Lab }}$ \\
\hline \multicolumn{14}{|c|}{ Inorganics } \\
\hline & Barium, total recoverable & 4.53 & $\mathrm{~J}$ & & & 3.72 & J 1 & & & & & $u g / L$ & \\
\hline & Cyanide & $<10$ & $\mathrm{u}$ & & & $<10$ & $\mathrm{U}$ & & & & & $\mathrm{ug} / \mathrm{L}$ & \\
\hline & Lead, total recoverable & $<100$ & U & & & $<10$ & $\mathrm{U}$ & & & & & $\mathrm{ug} / \mathrm{L}$ & \\
\hline & Nickel, total recoverable & $<50$ & $U$ & & & $<50$ & $\mathrm{U}$ & & & & & ug/L & \\
\hline & Selenium, total recoverable & $<10$ & $\mathrm{U}$ & & & $<10$ & $\mathrm{U}$ & & & & & $\mathrm{ug} / \mathrm{L}$ & \\
\hline \multicolumn{14}{|c|}{ Organics } \\
\hline & Chlorobenzene & $<5$ & UJ & & & $<1$ & $U$ & $<1$ & $U$ & $<E Q L$ & 1 & $u g / L$ & ML \\
\hline & 1,1-Dichloroethane & $<5$ & UJ & & & $<1$ & $\mathrm{U}$ & $<1$ & $\mathrm{U}$ & $<\mathrm{EQL}$ & 1 & $\mathrm{ug} / \mathrm{L}$ & ML \\
\hline & 1,1-Dichloroethylene & $<5$ & UJ & & & $<1$ & $\mathrm{U}$ & $<1$ & $\mathbf{U}$ & $<\mathrm{EQL}$ & 1 & $\mathrm{ug} / \mathrm{L}$ & $M L$ \\
\hline & $\begin{array}{l}\text { trans-1,2-Dichloroethylene } \\
\text { PCB } 1016\end{array}$ & $<5$ & UJ & & & $<1$ & $\mathrm{U}$ & $<1$ & $\mathrm{U}$ & $<\mathrm{EQL}$ & 1 & $\mathrm{ug} / \mathrm{h}$ & $M L$ \\
\hline & PCB 1221 & & & & & & & & & & & & \\
\hline & PCB 1232 & & & & & & & & & & & & \\
\hline & PCB 1242 & & & & & & & & & & & & \\
\hline & PCB 1248 & & & & & & & & & & & & \\
\hline & PCB 1254 & & & & & & & & & & & & \\
\hline & PCB 1260 & & & & & & & & & & & & \\
\hline & 1,1,2,2-Tetrachloroethane & $<5$ & UJ & & & $<1$ & $\mathrm{U}$ & $<1$ & $\mathrm{U}$ & $<\mathrm{EQL}$ & 1 & ug/L & ML \\
\hline & Tetrachloroethylene & $<5$ & UJ & & & $<1$ & $U$ & $<1$ & $\mathrm{U}$ & $<E Q L$ & 1 & $\mathrm{ug} / \mathrm{L}$ & ML \\
\hline & 1,1,1-Trichloroethane & $<5$ & UJ & & & $<1$ & $\mathrm{U}$ & $<1$ & $U$ & $<E Q L$ & 1 & $\mathrm{ug} / \mathrm{L}$ & ML \\
\hline & Trichloroethylene & $<5$ & UJ & & & $<1$ & $\mathrm{U}$ & $<1$ & $U$ & $<\mathrm{EQL}$ & 1 & $\mathrm{ug} / \mathrm{L}$ & $M L$ \\
\hline \multicolumn{14}{|c|}{ II. Monitoring Constituents } \\
\hline$\underline{\mathbf{S T}}$ & Parameter & 3096 & CLP EPA & $\underline{3 Q 97}$ & CLP EPA & 3098 & CLPEPA & $\underline{3099}$ & CLPEPA & Filt. & $\underline{\text { DF }}$ & Unit & Lab \\
\hline \multicolumn{14}{|c|}{ Inorganics } \\
\hline & Aluminum, total recoverable & 102 & & $<20$ & $\mathrm{U}$ & $<200$ & $\mathrm{U}$ & $<200$ & $\mathbf{U}$ & $<\mathrm{EQL}$ & 1 & ug/L & EX \\
\hline+ & Nitrate-nitrite as nitrogen & 1310 & & 980 & & 501 & & 2760 & & $>2400$ & 5 & ug/L & EX \\
\hline & Sodium, total recoverable & 1570 & & 1490 & & 1600 & & 1550 & & $<4600$ & 1 & $u g / L$ & EX \\
\hline & Sulfate & 245 & J & $<5000$ & $\mathrm{U}$ & 2260 & $J$ & 285 & & $<3000$ & 1 & ug/L & EX \\
\hline \multicolumn{14}{|c|}{ Radionuclides } \\
\hline & Gross alpha & 1.47 & & .23 & UI & 3.25 & & .844 & J 1 & NDD & 1 & $\mathrm{pCi} / \mathrm{L}$ & GP \\
\hline & Nonvolatile beta & 1.18 & Ui & -.79 & U! & 4.12 & $\mathrm{~J}$ & .58 & $U$ & $<\mathrm{EQL}$ & 1 & $\mathrm{pCi} / \mathrm{L}$ & GP \\
\hline & Radium, total alpha-emitting & .3 & & .003 & UI & .28 & 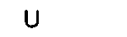 & .5 & U & $<$ EQL & 1 & $\mathrm{pCi} / \mathrm{L}$ & GP \\
\hline
\end{tabular}

Notes: Concentrations in bold italics exceed the groundwater protection or monitoring constituent standards listed in Appendix A. Synchronous water levels are measured over a 3-5 day period or less. Dilution factors, Laboratory, and Filtered Data are for Groundwater Protection Standards First Quarter 2000 and Monitoring Constituents Third Quarter 1999 data only.

+= exceeded the Groundwater Protection Standards (First Quarter 2000) or Monitoring Constituents (Third Quarter 1999) listed in Appendix A. 
Table D-4. Groundwater Monitoring Results for Plume Definition Wells, M-Area HWMF (Cont.) WELL MSB 19C

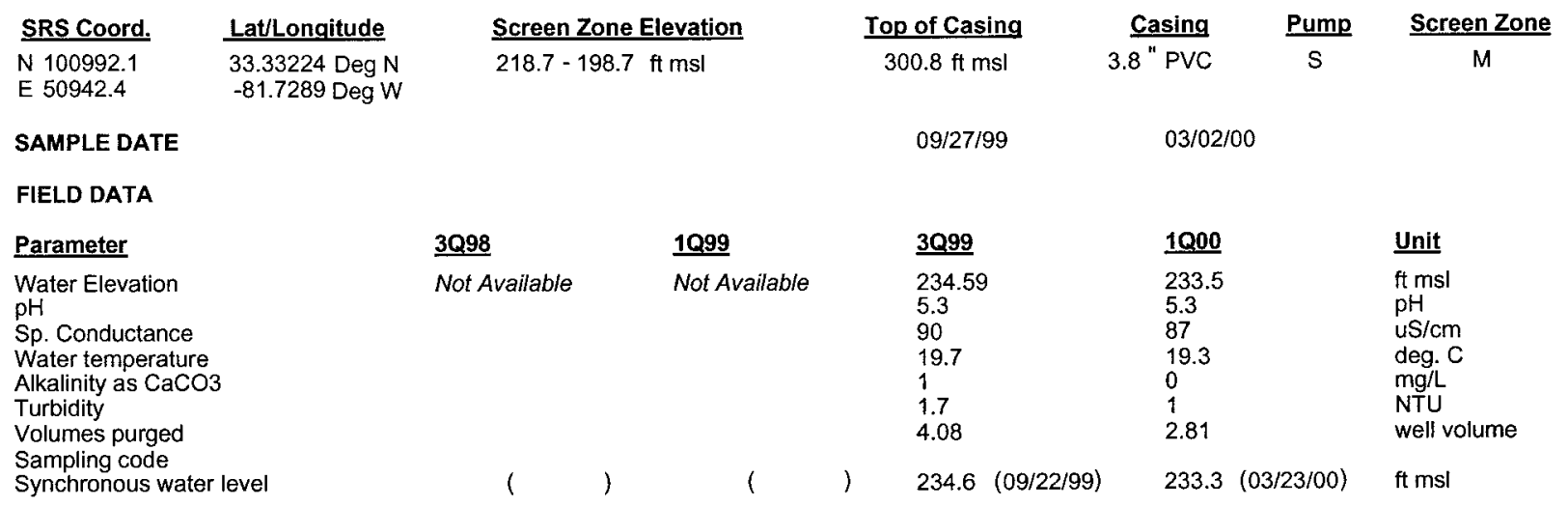

ANALYTICAL DATA

I. Groundwater Protection Standard

261 Appendix VIII/264 Appendix IX Hazardous Constituents

\begin{tabular}{|c|c|c|c|c|c|c|c|c|c|c|c|c|c|c|}
\hline$\underline{\mathrm{ST}}$ & Parameter & $\underline{3 Q 98}$ & $\underline{\text { CLP EPA }}$ & $1 \mathrm{Q99}$ & CLP EPA & $\underline{3 Q 99}$ & $\underline{\text { CLP }}$ & EPA & $\underline{1000}$ & CLPEPA & Filt. & $\underline{\mathrm{DF}}$ & $\underline{\text { Unit }}$ & $\underline{\text { Lab }}$ \\
\hline \multicolumn{15}{|c|}{ Inorganics } \\
\hline & Barium, total recoverable & & & & & 4.99 & $J$ & I & & & & & $u g / L$ & \\
\hline & Cyanide & & & & & $<10$ & JU & $Q$ & & & & & $\mathrm{ug} / \mathrm{L}$ & \\
\hline & Lead, total recoverable & & & & & 4.15 & $J$ & I & & & & & $u g / L$ & \\
\hline & Nickel, total recoverable & & & & & $<50$ & $U$ & & & & & & $\mathrm{ug} / \mathrm{L}$ & \\
\hline & Selenium, total recoverable & & & & & $<10$ & U & & & & & & $\mathrm{ug} / \mathrm{L}$ & \\
\hline \multicolumn{15}{|c|}{ Organics } \\
\hline & Chlorobenzene & & & & & $<.48$ & U & v & $<1$ & $u$ & $<E Q L$ & 1 & $u g / L$ & ML \\
\hline & 1,1-Dichloroethane & & & & & $<1$ & U & & $<1$ & $\mathrm{U}$ & $<E Q L$ & 1 & $\mathrm{ug} / \mathrm{L}$ & ML \\
\hline & 1,1-Dichloroethylene & & & & & $<1$ & $\mathrm{U}$ & & $<1$ & $\mathrm{U}$ & $<E Q L$ & 1 & ug/L & ML \\
\hline & trans-1,2-Dichloroethylene & & & & & $<1$ & $\mathrm{U}$ & & & & & & ug/L & \\
\hline & PCB 1016 & & & & & & & & & & & & & \\
\hline & PCB 1221 & & & & & & & & & & & & & \\
\hline & PCB 1232 & & & & & & & & & & & & & \\
\hline & PCB 1242 & & & & & & & & & & & & & \\
\hline & PCB 1248 & & & & & & & & & & & & & \\
\hline & PCB 1254 & & & & & & & & & & & & & \\
\hline & PCB 1260 & & & & & & & & & & & & & \\
\hline & $1,1,2,2$-Tetrachloroethane & & & & & $<1$ & $u$ & & $<1$ & $\mathrm{U}$ & $<E Q L$ & 1 & ug/L & ML \\
\hline & Tetrachloroethylene & & & & & 3.81 & $\mathrm{~J}$ & K & 3.77 & & $<5$ & 1 & $u g / L$ & ML \\
\hline & $1,1,1$-Trichloroethane & & & & & $<1$ & U & & $<1$ & $\mathrm{U}$ & $<\mathrm{EQL}$ & 1 & ug/L & ML \\
\hline+ & Trichloroethylene & & & & & 26.4 & $J$ & K & 20.3 & & $>5$ & 1 & $\mathrm{ug} / \mathrm{L}$ & ML \\
\hline \multicolumn{15}{|c|}{ II. Monitoring Constituents } \\
\hline ST & Parameter & $\underline{3 Q 96}$ & $\underline{\text { CLPEPA }}$ & $\underline{3097}$ & CLPEPA & $\underline{3098}$ & CLLP & EPA & $\underline{3 Q 99}$ & CLPEPA & Filt. & DF & $\underline{\text { Unit }}$ & La \\
\hline \multicolumn{15}{|c|}{ Inorganics } \\
\hline & Aluminum, total recoverable & $<20$ & $U$ & $<20$ & $\mathrm{U}$ & & & & $<200$ & $\mathrm{U}$ & $<\mathrm{EQL}$ & 1 & $u g / L$ & $E X$ \\
\hline+ & Nitrate-nitrite as nitrogen & 8900 & & 9830 & & & & & 4400 & & $>2400$ & 5 & ug/L & $E x$ \\
\hline+ & Sodium, total recoverable & 15000 & & 16100 & & & & & 16000 & & $>4600$ & 1 & $\mathrm{ug} / \mathrm{L}$ & EX \\
\hline+ & Sulfate & 5100 & & 4580 & $\jmath$ & & & & 13400 & & $>3000$ & 2 & ug/L & $E x$ \\
\hline \multicolumn{15}{|c|}{ Radionuclides } \\
\hline & Gross alpha & 2.12 & & 1.39 & UI & & & & .767 & $\mathrm{U}$ & $<\mathrm{EQL}$ & 1 & $\mathrm{pCi} / \mathrm{L}$ & GP \\
\hline & Nonvolatile beta & 1.96 & & 1.53 & UI & & & & 1.34 & J I & NDD & 1 & $\mathrm{pCi} / \mathrm{L}$ & GP \\
\hline & Radium, total alpha-emitting & 1.1 & & 3.54 & UI & & & & .4 & $\mathrm{U}$ & $<E Q L$ & 1 & $\mathrm{pCi} / \mathrm{L}$ & GP \\
\hline
\end{tabular}

Notes: Concentrations in bold italics exceed the groundwater protection or monitoring constituent standards listed in Appendix A. Synchronous water levels are measured over a 3-5 day period or less. Dilution factors, Laboratory, and Filtered Data are for Groundwater Protection Standards First Quarter 2000 and Monitoring Constituents Third Quarter 1999 data only.

$+=$ exceeded the Groundwater Protection Standards (First Quarter 2000) or Monitoring Constituents (Third Quarter 1999) listed in Appendix A. 
Table D-4. Groundwater Monitoring Results for Plume Definition Wells, M-Area HWMF (Cont.) WELL MSB 20A

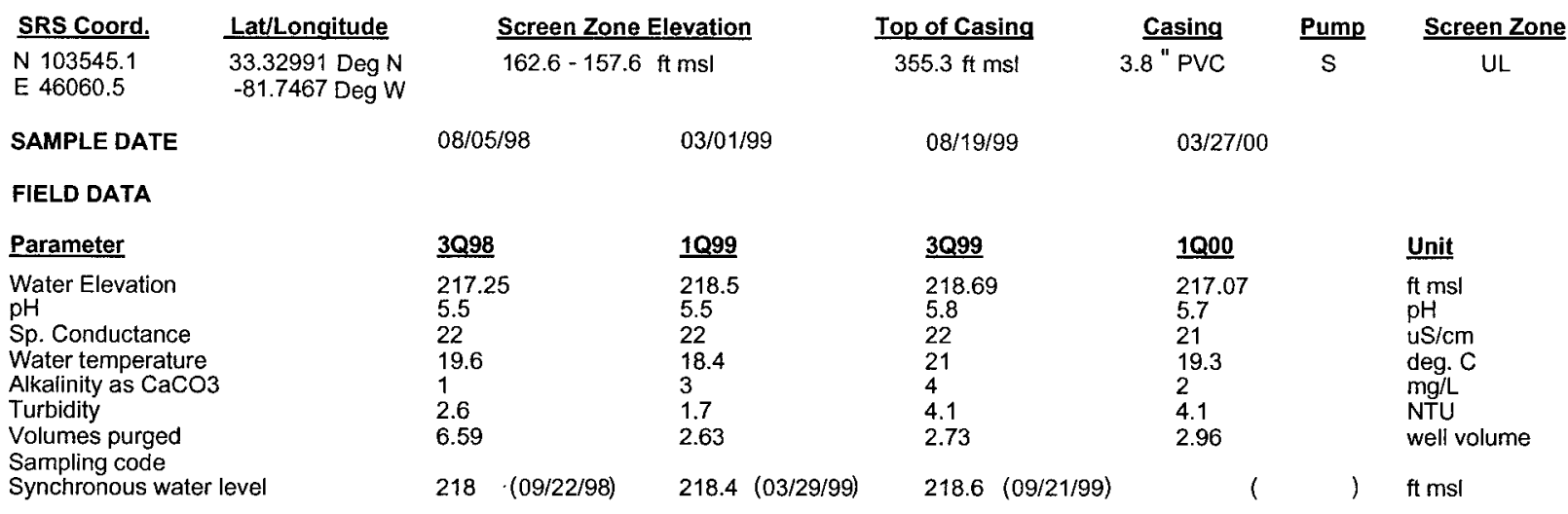

ANALYTICAL DATA

I. Groundwater Protection Standard

261 Appendix VIII/264 Appendix IX Hazardous Constituents

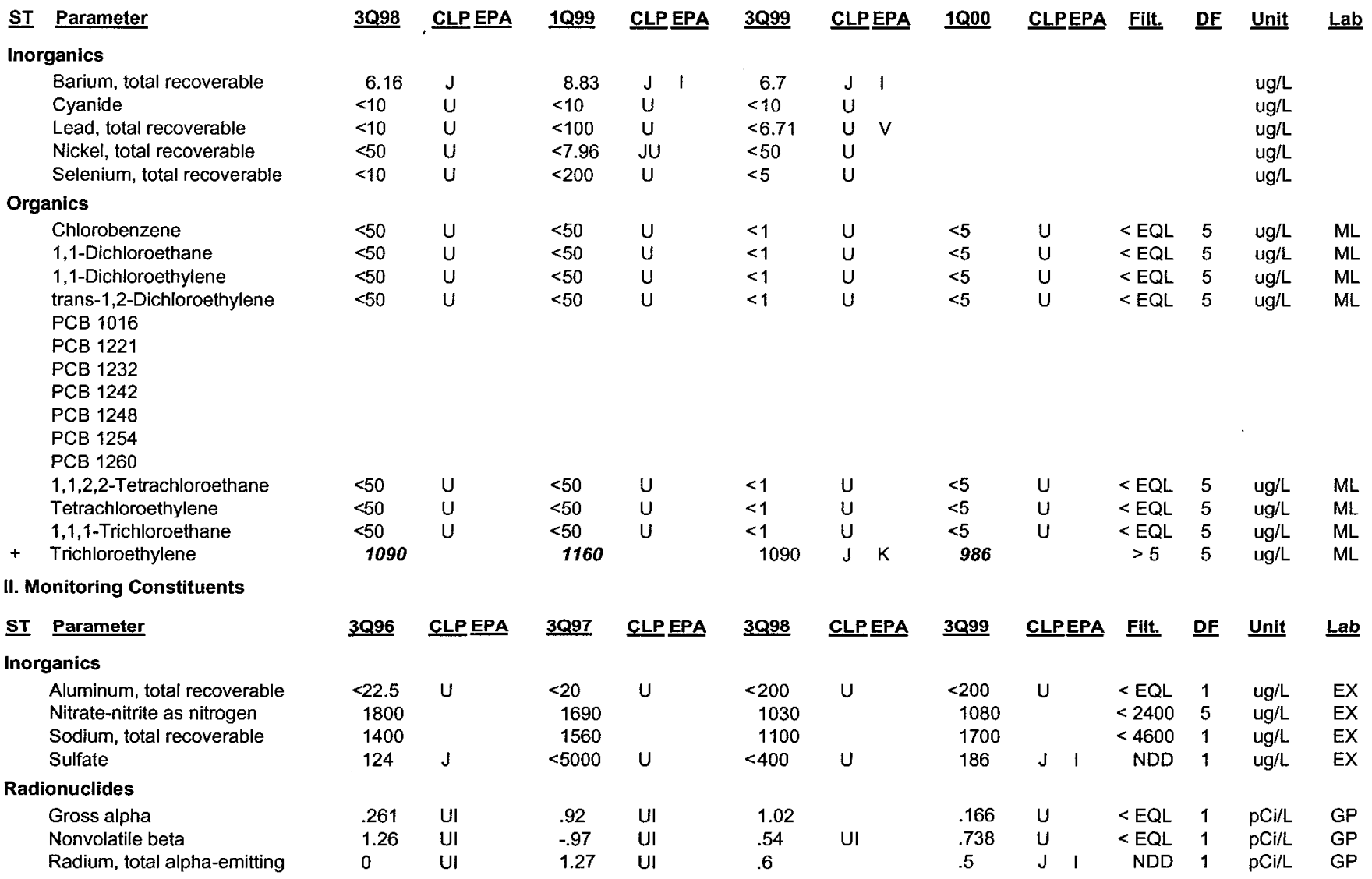

Notes: Concentrations in bold italics exceed the groundwater protection or monitoring constituent standards listed in Appendix A. Synchronous water levels are measured over a 3-5 day period or less. Dilution factors, Laboratory, and Filtered Data are for Groundwater Protection Standards First Quarter 2000 and Monitoring Constituents Third Quarter 1999 data only.

+= exceeded the Groundwater Protection Standards (First Quarter 2000) or Monitoring Constituents (Third Quarter 1999) listed in Appendix A. 
Table D-4. Groundwater Monitoring Results for Plume Definition Wells, M-Area HWMF (Cont.)

WELL MSB 20C

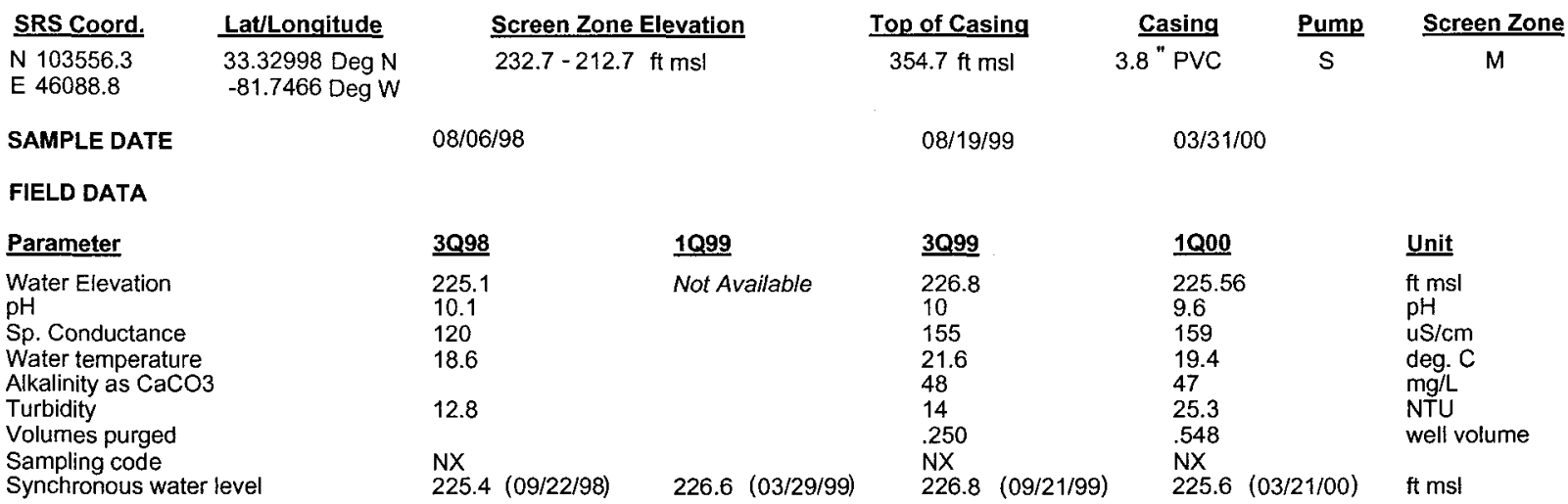

ANALYTICAL DATA

I. Groundwater Protection Standard

261 Appendix VIII/264 Appendix IX Hazardous Constituents

ST Inorganics

Barium, total recoverable

Cyanide

Lead, total recoverable

Nickel, total recoverable

Selenium, total recoverable

Organics

1,1-Dichloroethane

1,1-Dichloroethylene

trans-1,2-Dichloroethylene

PCB 1016

PCB 1221

PCB 1232

PCB 1242

PCB 1248

PCB 1254

PCB 1260

1,1,2,2-Tetrachloroethane

Tetrachloroethylene

1,1,1-Trichloroethane

Trichloroethylene

II. Monitoring Constituents

ST Parameter

Inorganics

+ Aluminum, total recoverable

Nitrate-nitrite as nitrogen

$+\quad$ Sodium, total recoverable

+ Sulfate

Radionuclides

Gross alpha

Nonvolatile beta

Radium, total alpha-emitting
3 Q98 CLPEPA

1 Q99 CLPEPA

3Q99

CLPEPA

1000

CLPEPA

Filt. DF Unit

$\underline{\text { Lab }}$

$\begin{array}{cc}4.57 & \mathrm{~J} \\ <10 & \mathrm{U} \\ 78 & \\ <50 & \mathrm{U} \\ <10 & \mathrm{U}\end{array}$

$\begin{array}{lll} & 5.51 & \text { J I } \\ <10 & \text { U } \\ <100 & \text { U } \\ <9.99 & \text { JU } \\ <200 & \text { U }\end{array}$

\begin{tabular}{|c|c|}
\hline 4 & $\mathrm{~J}$ \\
\hline$<10$ & $\mathrm{U}$ \\
\hline$<8.73$ & $\mathrm{U}$ \\
\hline$<17$ & JU \\
\hline$<5$ & $U$ \\
\hline
\end{tabular}

$\mathrm{ug} / \mathrm{L}$

ug/L

ug/L

ug/L

ug/L

$\begin{array}{lll}<5 & U \\ <5 & U \\ <5 & U \\ <5 & U\end{array}$

$\begin{array}{ll}<5 & U \\ <5 & U \\ <5 & U \\ <5 & U\end{array}$

$\begin{array}{ll}<1 & U \\ <1 & U \\ <1 & U \\ <1 & U\end{array}$

$<1$
$<1$
$<1$

$<E Q L$

$\mathrm{ug} / \mathrm{L} \quad \mathrm{ML}$

$<E Q L \quad 1 \quad u g / L \quad M L$

$<$ EQL $1 \mathrm{ug} / \mathrm{L} \quad \mathrm{ML}$

$\begin{array}{lll}<\text { EQL } & 1 \quad \mathrm{ug} / \mathrm{L} \quad \mathrm{ML}\end{array}$

\begin{tabular}{|c|c|c|}
\hline$<5$ & $U$ & $<1$ \\
\hline$<5$ & $U$ & $<1$ \\
\hline$<5$ & $U$ & $<1$ \\
\hline 3.51 & J 1 & $<1$ \\
\hline
\end{tabular}

$\underline{3096}$

CLPEPA

566

2140

6670

6330

$.548 \quad$ UI

1.34

$-2$

UI

$\begin{array}{lll} & \mathbf{5 9 7} & \\ & 2390 & \\ & 4470 & \\ & \mathbf{7 7 6 0} & \\ & & \\ & & \\ & 7.66 & \text { UI } \\ & 8.73 & \\ & 1.05 & \mathrm{~J}\end{array}$

$$
\begin{aligned}
& \mathbf{5 0 2} \\
& 1720 \\
& 3240 \\
& \mathbf{1 2 2 0 0}
\end{aligned}
$$

$U$
$U$
$U$

$<1$
$<1$
$<1$
$<1$

$<\mathrm{EQL}$
$<\mathrm{EQL}$
$<\mathrm{EQL}$
$<\mathrm{EQL}$

ug/L

$\mathrm{ug} / \mathrm{L}$

$\mathrm{ug} / \mathrm{L}$

ML

$M L$

ug/L $\quad M L$ 
Table D-4. Groundwater Monitoring Results for Plume Definition Wells, M-Area HWMF (Cont.) WELL MSB 21B

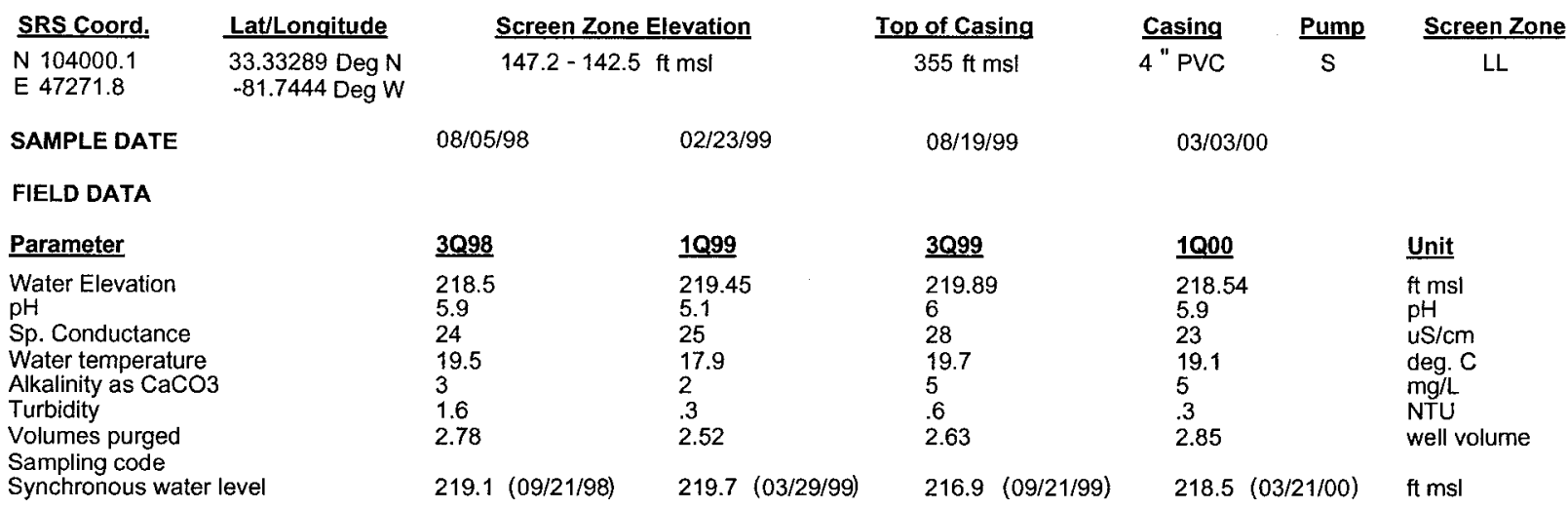

ANALYTICAL DATA

I. Groundwater Protection Standard

261 Appendix VIII/264 Appendix IX Hazardous Constituents

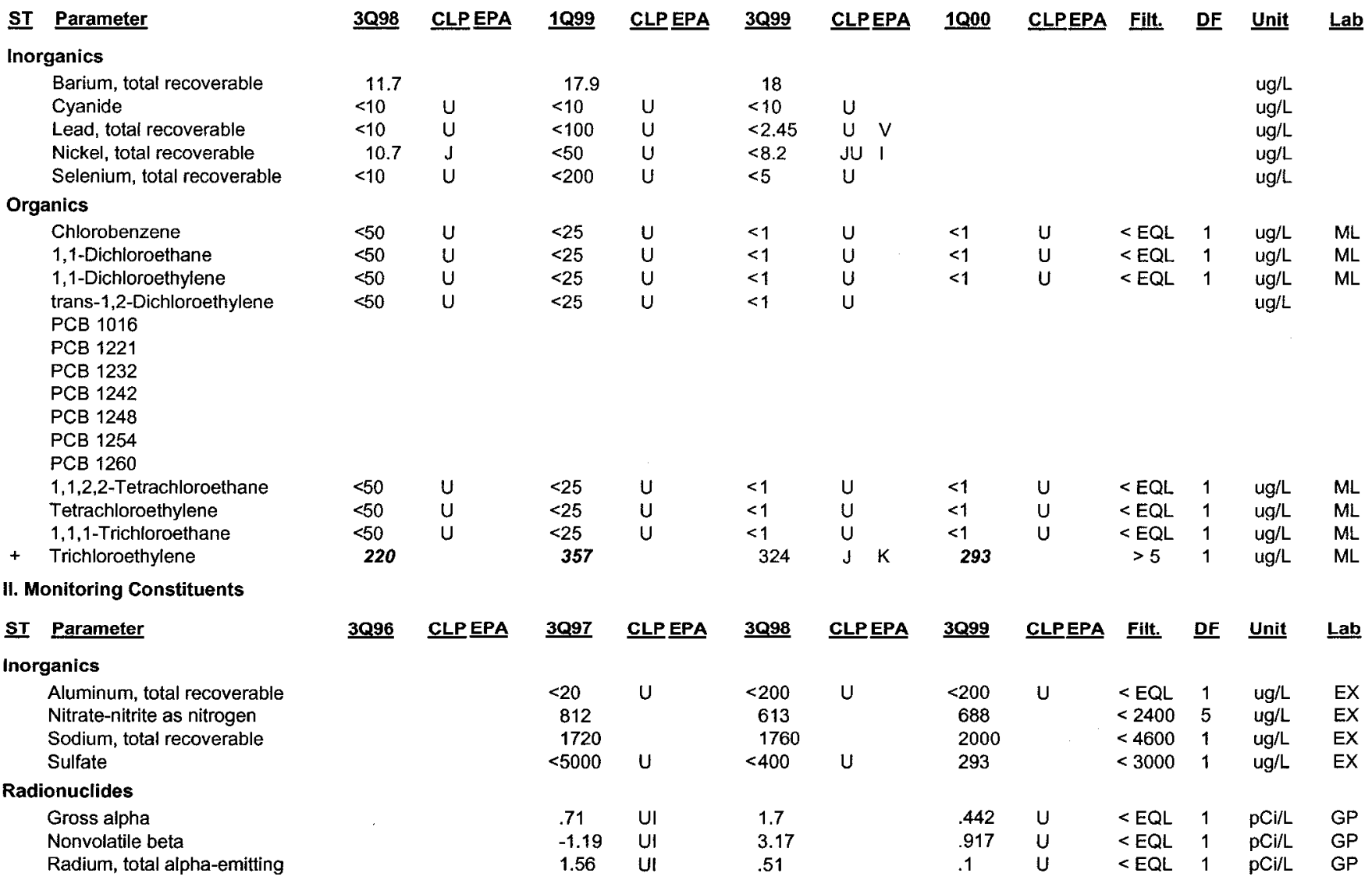

Notes: Concentrations in bold italics exceed the groundwater protection or monitoring constituent standards listed in Appendix A. Synchronous water levels are measured over a 3-5 day period or less. Dilution factors, Laboratory, and Filtered Data are for Groundwater Protection Standards First Quarter 2000 and Monitoring Constituents Third Quarter 1999 data only.

$+=$ exceeded the Groundwater Protection Standards (First Quarter 2000) or Monitoring Constituents (Third Quarter 1999) listed in Appendix A. 
Table D-4. Groundwater Monitoring Results for Plume Definition Wells, M-Area HWMF (Cont.) WELL MSB 21C

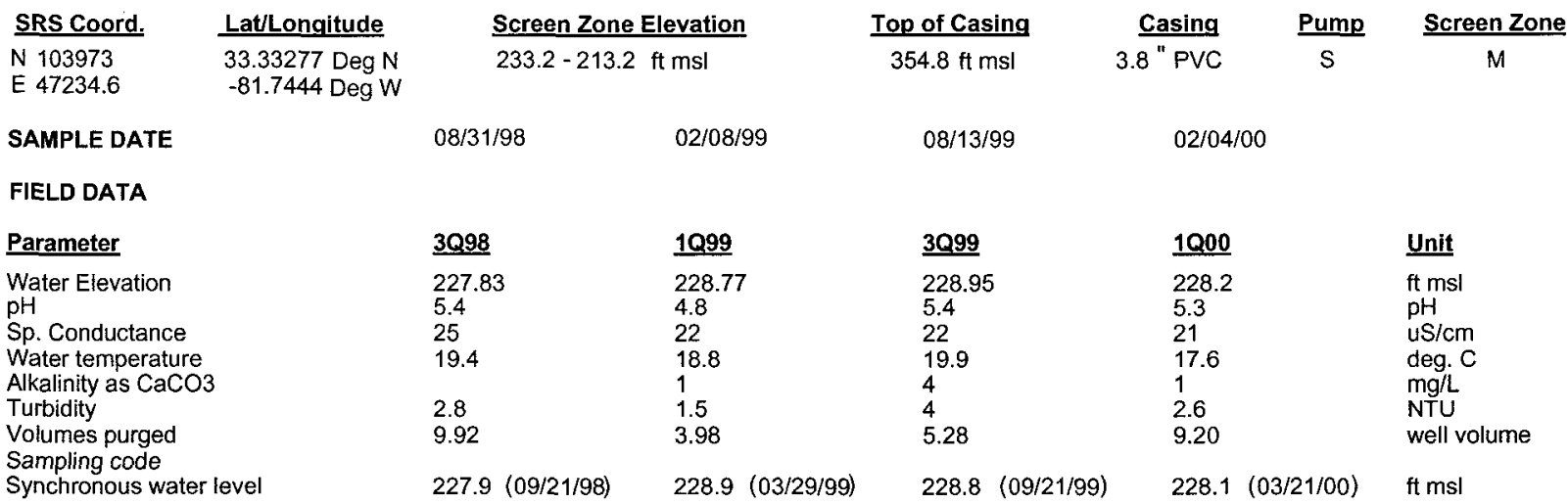

ANALYTICAL DATA

I. Groundwater Protection Standard

261 Appendix VIII/264 Appendix IX Hazardous Constituents

\begin{tabular}{|c|c|c|c|c|c|c|c|c|c|c|c|c|c|}
\hline$\underline{\text { ST }}$ & Parameter & $\underline{3 Q 98}$ & CLP EPA & $\underline{1099}$ & CLP EPA & $\underline{3099}$ & CLPEPA & $\underline{1 Q 00}$ & CLPEPA & Filt. & DF & $\underline{\text { Unit }}$ & $\underline{\text { Lab }}$ \\
\hline \multicolumn{14}{|c|}{ Inorganics } \\
\hline & Barium, total recoverable & 6.9 & J & $<10$ & $\mathrm{U}$ & 7.9 & J I & & & & & $\mathrm{ug} / \mathrm{L}$ & \\
\hline & Cyanide & $<10$ & U & $<10$ & $\mathrm{U}$ & $<10$ & $\mathrm{U}$ & & & & & $\mathrm{ug} / \mathrm{L}$ & \\
\hline & Lead, total recoverable & $<100$ & U & $<100$ & $\mathrm{U}$ & 8.99 & & & & & & ug/L & \\
\hline & Nickel, total recoverable & 9.5 & J & $<50$ & $\mathrm{U}$ & $<11$ & JU I & & & & & $\mathrm{ug} / \mathrm{L}$ & \\
\hline & Selenium, total recoverable & $<10$ & $\mathrm{U}$ & $<200$ & $\mathrm{U}$ & $<5$ & $\mathrm{U}$ & & & & & $\mathrm{ug} / \mathrm{L}$ & \\
\hline \multicolumn{14}{|c|}{ Organics } \\
\hline & Chiorobenzene & $<5$ & UJ & $<5$ & U & $<1$ & U & $<1$ & JU & $<E Q L$ & 1 & $u g / L$ & ML \\
\hline & 1,1-Dichloroethane & $<5$ & UJ & $<5$ & $U$ & $<1$ & $\mathrm{U}$ & $<1$ & JU & $<E Q L$ & 1 & $\mathrm{ug} / \mathrm{L}$ & ML \\
\hline & 1,1-Dichloroethylene & $<5$ & UJ & $<5$ & 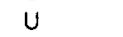 & $<1$ & $\mathrm{U}$ & $<1$ & JU & $<E Q L$ & 1 & $\mathrm{ug} / \mathrm{L}$ & ML \\
\hline & $\begin{array}{l}\text { trans-1,2-Dichioroethylene } \\
\text { PCB } 1016\end{array}$ & $<5$ & UJ & $<5$ & $\mathrm{U}$ & $<1$ & $u$ & $<1$ & JU & $<E Q L$ & 1 & $\mathrm{ug} / \mathrm{L}$ & $\mathrm{ML}$ \\
\hline & PCB 1221 & & & & & & & & & & & & \\
\hline & PCB 1232 & & & & & & & & & & & & \\
\hline & PCB 1242 & & & & & & & & & & & & \\
\hline & PCB 1248 & & & & & & & & & & & & \\
\hline & PCB 1254 & & & & & & & & & & & & \\
\hline & PCB 1260 & & & & & & & & & & & & \\
\hline & $1,1,2,2$-Tetrachloroethane & $<5$ & UJ & $<5$ & $\mathrm{U}$ & $<1$ & $\mathrm{U}$ & $<1$ & jU & $<\mathrm{EQL}$ & 1 & ug/L. & $M L$ \\
\hline & Tetrachloroethylene & $<5$ & UJ & $<5$ & $\mathrm{U}$ & $<1$ & $\mathrm{U}$ & $<1$ & JU & $<E Q L$ & 1 & ug/L & $M L$ \\
\hline & $1,1,1$-Trichloroethane & $<5$ & UJ & $<5$ & $\mathrm{U}$ & $<1$ & $\mathrm{U}$ & $<1$ & JU & $<E Q L$ & 1 & $\mathrm{ug} / \mathrm{L}$ & $M L$ \\
\hline & Trichloroethylene & $<5$ & UJ & $<5$ & $U$ & $<1$ & $\mathrm{U}$ & $<1$ & JU & $<\mathrm{EQL}$ & 1 & $\mathrm{ug} / \mathrm{L}$ & $M L$ \\
\hline \multicolumn{14}{|c|}{ II. Monitoring Constituents } \\
\hline$\underline{\text { ST }}$ & Parameter & $\underline{3096}$ & CLPEPA & $\underline{3 Q 97}$ & CLPEPA & $\underline{3098}$ & CLPEPA & $\underline{3 Q 99}$ & CLPEPA & Filt. & $\underline{D F}$ & Unit & $\underline{L a b}$ \\
\hline \multicolumn{14}{|c|}{ Inorganics } \\
\hline & Aluminum, total recoverable & 61.5 & & $<50$ & $u$ & $<200$ & U & $<200$ & $\mathrm{U}$ & $<\mathrm{EQL}$ & 1 & $\mathrm{ug} / \mathrm{L}$ & EX \\
\hline & Nitrate-nitrite as nitrogen & 1320 & & 1280 & & 824 & & 996 & & $<2400$ & 5 & $\mathrm{ug} / \mathrm{L}$ & EX \\
\hline & Sodium, total recoverable & 1540 & & 1580 & & 1940 & & 2200 & & $<4600$ & 1 & $\mathrm{ug} / \mathrm{L}$ & EX \\
\hline & Sulfate & 224 & J & $<5000$ & $\mathrm{U}$ & 2170 & $J$ & 472 & & $<3000$ & 1 & $\mathrm{ug} / \mathrm{L}$ & EX \\
\hline \multicolumn{14}{|c|}{ Radionuclides } \\
\hline & Gross alpha & 2.67 & & 2.61 & & 8.12 & & 2.98 & $\mathrm{~K}$ & NDD & 1 & $\mathrm{pCi} / \mathrm{L}$ & GP \\
\hline & Nonvolatile beta & 2.02 & & 1.94 & & 7.26 & $\mathrm{~J}$ & 2.35 & & $<50$ & 1 & $\mathrm{pCi} / \mathrm{L}$ & GP \\
\hline & Radium, total alpha-emitting & 1.6 & & 2.39 & & 3.13 & & 1.2 & & $<5$ & 1 & $\mathrm{pCi} / \mathrm{L}$ & GP \\
\hline
\end{tabular}

Notes: Concentrations in bold italics exceed the groundwater protection or monitoring constituent standards listed in Appendix A. Synchronous water levels are measured over a 3-5 day period or less. Dilution factors, Laboratory, and Filtered Data are for Groundwater Protection Standards First Quarter 2000 and Monitoring Constituents Third Quarter 1999 data only.

+ = exceeded the Groundwater Protection Standards (First Quarter 2000) or Monitoring Constituents (Third Quarter 1999) listed in Appendix A. 
Table D-4. Groundwater Monitoring Results for Plume Definition Wells, M-Area HWMF (Cont.) WELL MSB 23B

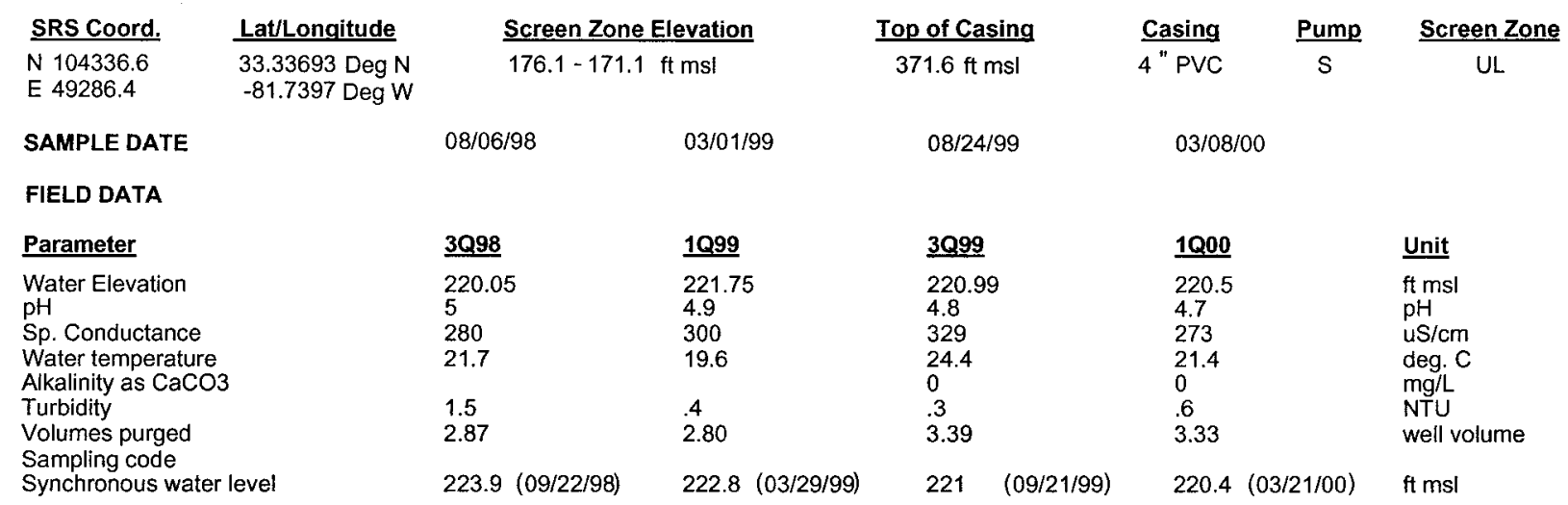

\section{ANALYTICAL DATA}

I. Groundwater Protection Standard

261 Appendix VIII/264 Appendix IX Hazardous Constituents

\begin{tabular}{|c|c|c|c|c|c|c|c|c|c|c|c|c|c|c|c|}
\hline \multirow{2}{*}{$\frac{\mathbf{S T}}{\text { Inor }}$} & Parameter & \multirow[t]{2}{*}{$\underline{3 Q 98}$} & \multirow[t]{2}{*}{ CLPEPA } & \multirow[t]{2}{*}{$\underline{1 Q 99}$} & \multirow[t]{2}{*}{ CLP EPA } & \multirow[t]{2}{*}{$\underline{\mathbf{3 Q 9 9}}$} & \multicolumn{2}{|c|}{ CLPEPA } & \multirow[t]{2}{*}{1000} & \multicolumn{2}{|c|}{ CLPEPA } & \multirow[t]{2}{*}{ Filt. } & \multirow[t]{2}{*}{$\underline{\text { DF }}$} & \multirow[t]{2}{*}{$\underline{\text { Unit }}$} & \multirow[t]{2}{*}{ Lab } \\
\hline & rganics & & & & & & & & & & & & & & \\
\hline & Barium, total recoverable & 143 & & 129 & & 120 & & & 116 & & & $<2000$ & 1 & $\mathrm{ug} / \mathrm{L}$ & $M L$ \\
\hline & Cyanide & $<10$ & $U$ & $<10$ & $U$ & $<10$ & $\mathrm{U}$ & & & & & & & $\mathrm{ug} / \mathrm{L}$ & \\
\hline & Lead, total recoverable & $<10$ & $U$ & $<100$ & $\mathrm{U}$ & $<2.93$ & $\mathrm{U}$ & V & $<6.58$ & JU & & $<\mathrm{EQL}$ & 1 & $\mathrm{ug} / \mathrm{L}$ & $\mathrm{ML}$ \\
\hline & Nickel, total recoverable & 12.2 & $\mathrm{~J}$ & $<11.6$ & JU & $<8.9$ & JU & I & $<60$ & $\mathrm{U}$ & & $<\mathrm{EQL}$ & 1 & $\mathrm{ug} / \mathrm{L}$ & ML \\
\hline & Selenium, total recoverable & $<10$ & $U$ & $<200$ & $\mathrm{U}$ & $<5$ & $\mathrm{U}$ & & $<40$ & $U$ & & $<E Q L$ & 1 & $\mathrm{ug} / \mathrm{L}$ & $\mathrm{ML}$ \\
\hline \multicolumn{16}{|c|}{ Organics } \\
\hline & Chlorobenzene & $<1000$ & $\mathrm{U}$ & $<1250$ & $\mathrm{U}$ & 1.7 & $J$ & K & 150 & J & IK & NDD & 200 & $\mathrm{ug} / \mathrm{L}$ & $\mathrm{ML}$ \\
\hline & 1,1-Dichloroethane & $<1000$ & U & $<1250$ & $\mathrm{U}$ & $<1$ & $\mathrm{U}$ & & $<200$ & $\mathrm{U}$ & & $<\mathrm{EQL}$ & 200 & $\mathrm{ug} / \mathrm{L}$ & $\mathrm{ML}$ \\
\hline & 1,1-Dichloroethylene & $<1000$ & U & $<1250$ & $U$ & 89.6 & $\mathrm{~J}$ & $\mathrm{~K}$ & $<200$ & $\mathrm{U}$ & & $<E Q L$ & 200 & $\mathrm{ug} / \mathrm{L}$ & $\mathrm{ML}$ \\
\hline & trans-1,2-Dichloroethylene & $<1000$ & $\mathrm{U}$ & $<1250$ & $\mathrm{U}$ & $<1$ & $\mathrm{U}$ & & $<200$ & $\mathrm{U}$ & & $<E Q L$ & 200 & ug/L & $\mathrm{ML}$ \\
\hline & РСВ 1016 & & & & & & & & & & & & & & \\
\hline & PCB 1221 & & & & & & & & & & & & & & \\
\hline & PCB 1232 & & & & & & & & & & & & & & \\
\hline & PCB 1242 & & & & & & & & & & & & & & \\
\hline & PCB 1248 & & & & & & & & & & & & & & \\
\hline & PCB 1254 & & & & & & & & & & & & & & \\
\hline & PCB 1260 & & & & & & & & & & & & & & \\
\hline & 1,1,2,2-Tetrachloroethane & $<1000$ & $\mathrm{U}$ & $<1250$ & $\mathrm{U}$ & $<1$ & $U$ & & $<200$ & $U$ & & $<E Q L$ & 200 & $u g / L$ & $\mathrm{ML}$ \\
\hline & Tetrachloroethylene & 38000 & & 34500 & & 30800 & & & 33100 & $\mathrm{~J}$ & K & NDD & 200 & ug/L & $\mathrm{ML}$ \\
\hline & 1,1,1-Trichloroethane & $<1000$ & $\mathrm{U}$ & $<1250$ & $\mathrm{U}$ & 13.9 & $J$ & $\mathrm{~K}$ & $<200$ & $\mathrm{U}$ & & $<E Q L$ & 200 & $\mathrm{ug} / \mathrm{L}$ & $\mathrm{ML}$ \\
\hline & Trichloroethylene & 30100 & & 29800 & & 23600 & & & 26800 & $\mathrm{~J}$ & $\mathrm{~K}$ & NDD & 200 & $\mathrm{ug} / \mathrm{L}$ & $\mathrm{ML}$ \\
\hline \multicolumn{16}{|c|}{ II. Monitoring Constituents } \\
\hline$\underline{\mathbf{S T}}$ & Parameter & $\underline{3096}$ & CLP EPA & $\underline{3097}$ & CLP EPA & $\underline{3098}$ & $\underline{\text { CLP }}$ & EPA & $\underline{3099}$ & $\underline{\text { CLP }}$ & EPA & Filt. & DF & $\underline{\text { Unit }}$ & $\underline{\text { Lab }}$ \\
\hline \multicolumn{16}{|c|}{ Inorganics } \\
\hline+ & Aluminum, total recoverable & 692 & $\mathrm{~J}$ & 711 & & 761 & & & 730 & & & $>100$ & 1 & ug/L. & EX \\
\hline & Nitrate-nitrite as nitrogen & 48000 & & 47200 & & 39300 & & & 38400 & & & $>2400$ & 10 & $\mathrm{ug} / \mathrm{L}$ & EX \\
\hline+ & Sodium, total recoverable & 14900 & $J$ & 13100 & & 11600 & & & 10000 & & & $>4600$ & 1 & $u g / L$ & EX \\
\hline & Sulfate & 206 & $\mathrm{~J}$ & $<5000$ & $\mathrm{U}$ & $<200$ & $\mathrm{U}$ & & 347 & $J$ & I & NDD & 2 & $\mathrm{ug} / \mathrm{L}$ & EX \\
\hline \multicolumn{16}{|c|}{ Radionuclides } \\
\hline+ & Gross alpha & 23.7 & & 31.2 & & 54.07 & & & 32.7 & & & $>15$ & 1 & $\mathrm{pCi} / \mathrm{L}$ & GP \\
\hline & Nonvolatile beta & 19.4 & & 16.2 & & 40.87 & $\mathrm{~J}$ & & 17.1 & & & $<50$ & 1 & $\mathrm{pCi} / \mathrm{L}$ & GP \\
\hline+ & Radium, total alpha-emitting & 14.4 & & 19.4 & & 12.94 & & & 11.6 & & & $>5$ & 1 & $\mathrm{pCi} / \mathrm{L}$ & GP \\
\hline
\end{tabular}

Notes: Concentrations in bold italics exceed the groundwater protection or monitoring constituent standards listed in Appendix A. Synchronous water levels are measured over a 3-5 day period or less. Dilution factors, Laboratory, and Filtered Data are for Groundwater Protection Standards First Quarter 2000 and Monitoring Constituents Third Quarter 1999 data only.

+ = exceeded the Groundwater Protection Standards (First Quarter 2000) or Monitoring Constituents (Third Quarter 1999) listed in Appendix A. 
Table D-4. Groundwater Monitoring Results for Plume Definition Wells, M-Area HWMF (Cont.) WELL MSB 23TA

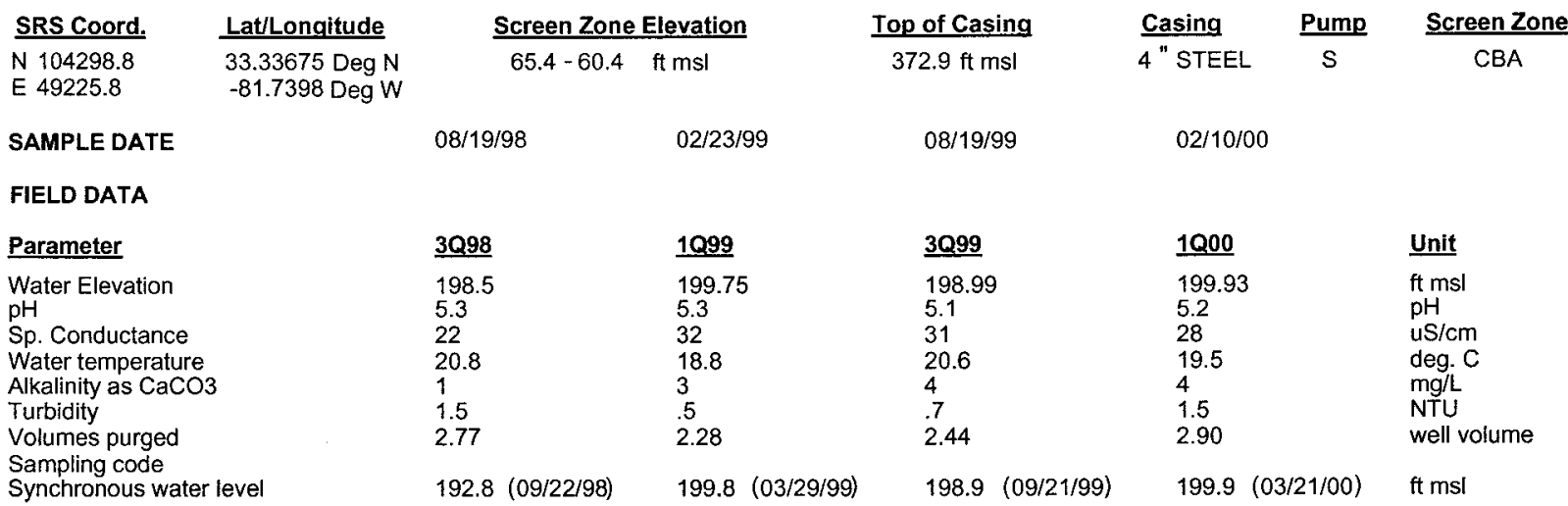

ANALYTICAL DATA

I. Groundwater Protection Standard

261 Appendix VIII/264 Appendix IX Hazardous Constituents

\begin{tabular}{|c|c|c|c|c|c|c|c|c|c|c|c|c|c|}
\hline$\underline{\text { ST }}$ & Parameter & $\underline{3 Q 98}$ & CLP EPA & 1099 & $\underline{\text { CLP EPA }}$ & $\underline{3 Q 99}$ & $\underline{\text { CLPEPA }}$ & 1000 & CLPEPA & Filt. & DF & Unit & Lab \\
\hline $\ln$ & ganics & & & & & & & & & & & & \\
\hline & Barium, total recoverable & 8.57 & J & 8.72 & $J$ & 9 & $\mathrm{~J}$ & & & & & $u g / L$ & \\
\hline & Cyanide & $<10$ & U & $<10$ & $\mathrm{U}$ & $<10$ & U & & & & & ug/L & \\
\hline & Lead, total recoverable & $<10$ & U & $<100$ & U & $<2.54$ & U V & & & & & $\mathrm{ug} / \mathrm{L}$ & \\
\hline & Nickel, total recoverable & $<18.9$ & U & $<50$ & $U$ & $<50$ & $\mathrm{U}$ & & & & & $\mathrm{ug} / \mathrm{L}$ & \\
\hline & Selenium, total recoverable & $<10$ & U & $<200$ & $U$ & $<5$ & $\mathrm{U}$ & & & & & $u g / L$ & \\
\hline Org & anics & & & & & & & & & & & & \\
\hline & Chlorobenzene & $<5$ & $\mathrm{U}$ & $<5$ & $\mathrm{U}$ & $<1$ & $\mathrm{U}$ & $<1$ & $u$ & $<\mathrm{EQL}$ & 1 & $u g / L$ & ML \\
\hline & 1,1-Dichloroethane & $<5$ & $\mathrm{U}$ & $<5$ & $\mathrm{U}$ & $<1$ & $\mathrm{U}$ & $<1$ & $\mathrm{U}$ & $<E Q L$ & 1 & $\mathrm{ug} / \mathrm{L}$ & $M L$ \\
\hline & 1,1-Dichloroethylene & $<5$ & $\mathrm{U}$ & $<5$ & $\mathrm{U}$ & $<1$ & $\mathrm{U}$ & $<1$ & U & $<E Q L$ & 1 & $\mathrm{ug} / \mathrm{L}$ & ML \\
\hline & trans-1,2-Dichloroethylene & $<5$ & $\mathrm{U}$ & $<5$ & $\mathrm{U}$ & $<1$ & $\mathrm{U}$ & & & & & $u g / L$ & \\
\hline & PCB 1016 & & & & & & & & & & & & \\
\hline & PCB 1221 & & & & & & & & & & & & \\
\hline & $\begin{array}{l}\text { PCB } 1232 \\
\text { PCB } 1242\end{array}$ & & & & & & & & & & & & \\
\hline & PCB 1248 & & & & & & & & & & & & \\
\hline & PCB 1254 & & & & & & & & & & & & \\
\hline & PCB 1260 & & & & & & & & & & & & \\
\hline & 1,1,2,2-Tetrachloroethane & $<5$ & $\mathrm{U}$ & $<5$ & $\mathrm{U}$ & $<1$ & $\mathrm{U}$ & $<1$ & $\mathrm{U}$ & $<E Q L$ & 1 & $\mathrm{ug} / \mathrm{L}$ & $M L$ \\
\hline & Tetrachloroethylene & $<5$ & $\mathrm{U}$ & $<5$ & $\mathrm{U}$ & $<1$ & $\mathrm{U}$ & $<1$ & $\mathrm{U}$ & $<E Q L$ & 1 & $u g / L$ & $M L$ \\
\hline & $1,1,1$-Trichloroethane & $<5$ & $\mathrm{U}$ & $<5$ & $\mathrm{U}$ & $<1$ & $\mathrm{U}$ & $<1$ & U & $<\mathrm{EQL}$ & 1 & $\mathrm{ug} / \mathrm{L}$ & ML \\
\hline & Trichloroethylene & $<5$ & $\mathrm{U}$ & $<5$ & $\mathrm{U}$ & $<1$ & $\mathrm{U}$ & $<1$ & $\mathrm{U}$ & $<E Q L$ & 1 & $\mathrm{ug} / \mathrm{L}$ & $M L$ \\
\hline II. $M$ & onitoring Constituents & & & & & & & & & & & & \\
\hline$\underline{\text { ST }}$ & Parameter & $\underline{3096}$ & CLP EPA & $\underline{3097}$ & CLP EPA & $\underline{30,98}$ & CLPEPA & $\underline{3099}$ & CLPEPA & Filt. & $\underline{\mathrm{DF}}$ & Unit & $\underline{\text { Lab }}$ \\
\hline Inor & ganics & & & & & & & & & & & & \\
\hline & Aluminum, total recoverable & $<42.4$ & $\mathrm{U}$ & 16.5 & $J$ & $<200$ & $\mathrm{u}$ & $<200$ & $\mathrm{U}$ & $<E Q L$ & 1 & ug/L & EX \\
\hline & Nitrate-nitrite as nitrogen & 270 & & 5 & $\mathrm{j}$ & $<100$ & $\mathrm{U}$ & $<500$ & U & $<E Q L$ & 5 & $\mathrm{ug} / \mathrm{L}$ & EX \\
\hline & Sodium, total recoverable & 2160 & & 1920 & & 2250 & & 2300 & & $<4600$ & 1 & $\mathrm{ug} / \mathrm{L}$ & EX \\
\hline+ & Sulfate & 4590 & & 4400 & $J$ & 4400 & & 4300 & & $>3000$ & 1 & $\mathrm{ug} / \mathrm{L}$ & EX \\
\hline Rac & onuclides & & & & & & & & & & & & \\
\hline & Gross alpha & 1.07 & & -.19 & UI & 2.65 & $J$ & .564 & $\mathrm{U}$ & $<E Q L$ & 1 & $\mathrm{pCi} / \mathrm{L}$ & GP \\
\hline & Nonvolatile beta & 1.55 & & -.75 & UI & & & 1.94 & $\mathrm{~J} \quad \mathrm{I}$ & NDD & 1 & $\mathrm{pCi} / \mathrm{L}$ & GP \\
\hline & Radium, total alpha-emitting & .5 & & .79 & UI & .53 & & .5 & $\mathrm{~J} \quad \mathrm{I}$ & NDD & 1 & $\mathrm{pCi} / \mathrm{h}$ & GP \\
\hline
\end{tabular}

Notes: Concentrations in bold italics exceed the groundwater protection or monitoring constituent standards listed in Appendix A. Synchronous water levels are measured over a 3-5 day period or less. Dilution factors, Laboratory, and Filtered Data are for Groundwater Protection Standards First Quarter 2000 and Monitoring Constituents Third Quarter 1999 data only.

+ = exceeded the Groundwater Protection Standards (First Quarter 2000) or Monitoring Constituents (Third Quarter 1999) listed in Appendix A. 
Table D-4. Groundwater Monitoring Results for Plume Definition Wells, M-Area HWMF (Cont.) WELL MSB 24

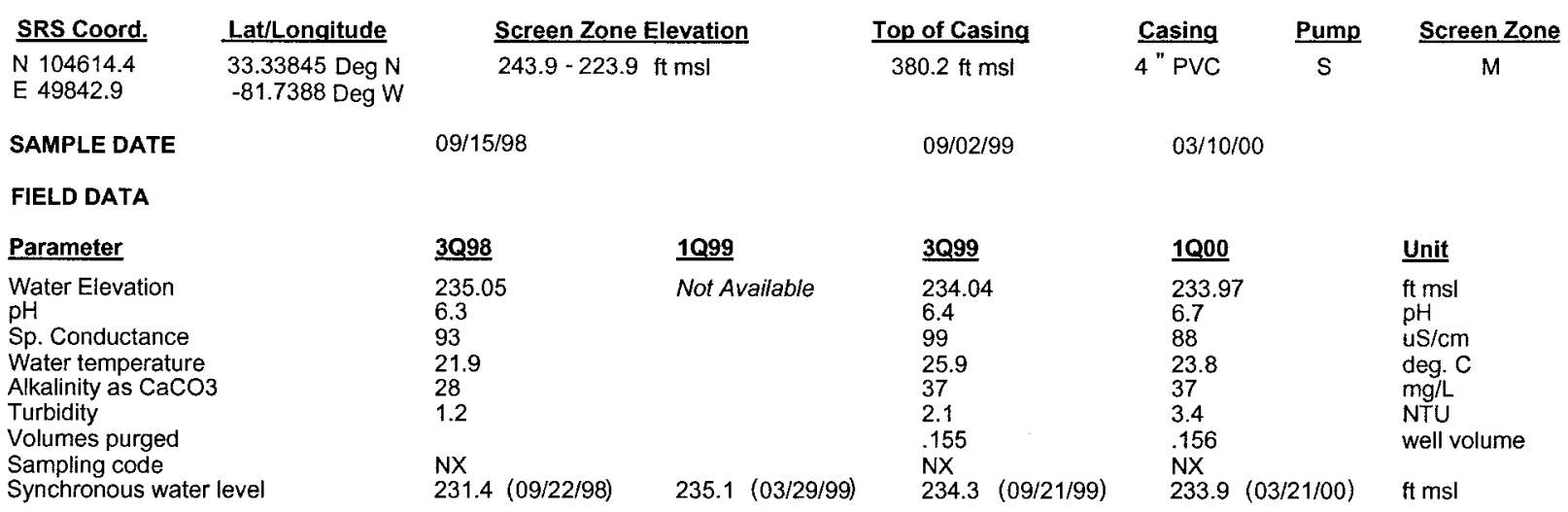

ANALYTICAL DATA

I. Groundwater Protection Standard

261 Appendix VIII/264 Appendix IX Hazardous Constituents

\begin{tabular}{|c|c|c|c|c|c|c|c|c|c|c|c|c|c|}
\hline$\underline{\text { ST }}$ & Parameter & $\underline{3 Q 98}$ & $\underline{\text { CLP EPA }}$ & $\underline{1 Q 99}$ & CLP EPA & $\underline{3099}$ & CLPEPA & $1 Q 00$ & CLPEPA & Filt. & $\underline{D F}$ & $\underline{\text { Unit }}$ & $\underline{\text { Lab }}$ \\
\hline \multicolumn{14}{|c|}{ Inorganics } \\
\hline & Barium, total recoverable & 8.71 & $\mathrm{~J}$ & 12.2 & & 7.6 & $\mathrm{~J} \quad \mathrm{I}$ & & & & & $u g / L$ & \\
\hline & Cyanide & $<10$ & $\mathrm{U}$ & $<10$ & $U$ & $<10$ & U & & & & & $\mathrm{ug} / \mathrm{L}$ & \\
\hline & Lead, total recoverable & $<100$ & $U$ & $<100$ & $U$ & $<10$ & $\mathrm{U}$ & & & & & $\mathrm{ug} / \mathrm{L}$ & \\
\hline & Nickel, total recoverable & $<50$ & $U$ & $<50$ & $U$ & $<50$ & $U$ & & & & & $\mathrm{ug} / \mathrm{L}$ & \\
\hline & Selenium, total recoverable & $<200$ & $U$ & $<200$ & $U$ & $<10$ & $\mathrm{U}$ & & & & & ug/L & \\
\hline \multicolumn{14}{|c|}{ Organics } \\
\hline & Chlorobenzene & $<250$ & $\mathrm{U}$ & $<250$ & $U$ & $<1$ & $\cup$ & $<10$ & JU & $<\mathrm{EQL}$ & 10 & $\mathrm{ug} / \mathrm{L}$ & $M L$ \\
\hline & 1,1-Dichloroethane & $<250$ & $U$ & $<250$ & $U$ & $<1$ & $\mathrm{U}$ & $<10$ & JU & $<$ EQL & 10 & $\mathrm{ug} / \mathrm{L}$ & $M L$ \\
\hline & 1,1-Dichloroethylene & $<250$ & $U$ & $<250$ & U & 2.63 & $J \quad K$ & $<10$ & JU & $<\mathrm{EQL}$ & 10 & $\mathrm{ug} / \mathrm{L}$ & ML \\
\hline & $\begin{array}{l}\text { trans-1,2-Dichloroethylene } \\
\text { PCB } 1016\end{array}$ & $<250$ & $U$ & $<250$ & $U$ & $<1$ & $U$ & & & & & $\mathrm{ug} / \mathrm{L}$ & \\
\hline & PCB 1221 & & & & & & & & & & & & \\
\hline & PCB 1232 & & & & & & & & & & & & \\
\hline & PCB 1242 & & & & & & & & & & & & \\
\hline & PCB 1248 & & & & & & & & & & & & \\
\hline & PCB 1254 & & & & & & & & & & & & \\
\hline & PCB 1260 & & & & & & & & & & & & \\
\hline & $1,1,2,2-$ Tetrachloroethane & $<250$ & $\mathrm{U}$ & $<250$ & $\mathrm{U}$ & $<1$ & $\mathrm{U}$ & $<10$ & JU & $<$ EQL & 10 & $\mathrm{ug} / \mathrm{L}$ & $\mathrm{ML}$ \\
\hline & Tetrachloroethylene & 1650 & $J$ & 1780 & & 957 & & 618 & $J$ & NDD & 10 & $\mathrm{ug} / \mathrm{L}$ & $M L$ \\
\hline & 1,1,1-Trichloroethane & $<250$ & $U$ & $<250$ & $U$ & $<1$ & $U$ & $<10$ & JU & $<\mathrm{EQL}$ & 10 & ug/L & $M L$ \\
\hline & Trichloroethylene & 4740 & $J$ & 5020 & & 2880 & & 2240 & $\mathrm{~J}$ & NDD & 10 & ug/L. & ML \\
\hline \multicolumn{14}{|c|}{ If. Monitoring Constituents } \\
\hline$\underline{\mathbf{S T}}$ & Parameter & $\underline{3096}$ & CLPEPA & 3097 & CLP EPA & $\underline{3098}$ & CLPEPA & 3099 & CLPEPA & Filt. & $\underline{\text { DF }}$ & $\underline{\text { Unit }}$ & $\underline{L a b}$ \\
\hline \multicolumn{14}{|c|}{ Inorganics } \\
\hline & Aluminum, total recoverable & 129 & $J$ & 135 & & 207 & & 160 & J 1 & NDD & 1 & ug/L & EX \\
\hline & Nitrate-nitrite as nitrogen & 2360 & & 2590 & & 2000 & & 2150 & & $<2400$ & 5 & $\mathrm{ug} / \mathrm{L}$ & EX \\
\hline & Sodium, total recoverable & 3870 & J & 3860 & & 4320 & & 4000 & & $<4600$ & 1 & $\mathrm{ug} / \mathrm{L}$ & EX \\
\hline & Sulfate & 3290 & & 3420 & $J$ & 2320 & & 1960 & & $<3000$ & 2 & $\mathrm{ug} / \mathrm{L}$ & $\mathrm{EX}$ \\
\hline \multicolumn{14}{|c|}{ Radionuclides } \\
\hline & Gross alpha & 1.56 & & .69 & UI & 2.29 & & 1.45 & U V & $<E Q L$ & 1 & $\mathrm{pCi} / \mathrm{L}$ & GP \\
\hline & Nonvolatile beta & 1.69 & & -.58 & UI & 16.13 & & .971 & $U$ & $<\mathrm{EQL}$ & 1 & $\mathrm{pCi} / \mathrm{L}$ & GP \\
\hline & Radium, total alpha-emitting & .2 & UI & 2.06 & UI & .38 & UI & .3 & $\mathrm{U}$ & $<\mathrm{EQL}$ & 1 & $\mathrm{pCi} / \mathrm{L}$ & GP \\
\hline
\end{tabular}

Notes: Concentrations in bold italics exceed the groundwater protection or monitoring constituent standards listed in Appendix A. Synchronous water levels are measured over a 3-5 day period or less. Dilution factors, Laboratory, and Filtered Data are for Groundwater Protection Standards First Quarter 2000 and Monitoring Constituents Third Quarter 1999 data only.

$+=$ exceeded the Groundwater Protection Standards (First Quarter 2000) or Monitoring Constituents (Third Quarter 1999) listed in Appendix A. 
Table D-4. Groundwater Monitoring Results for Plume Definition Wells, M-Area HWMF (Cont.) WELL MSB 25A

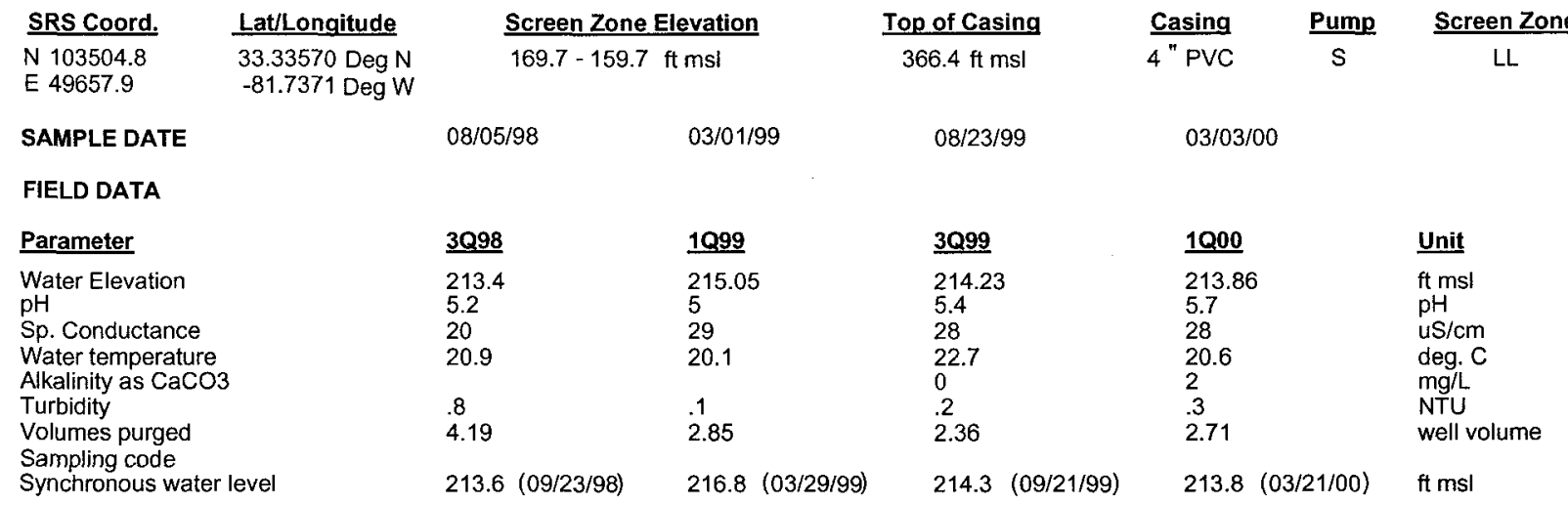

\section{ANALYTICAL DATA}

I. Groundwater Protection Standard

261 Appendix VIII/264 Appendix IX Hazardous Constituents

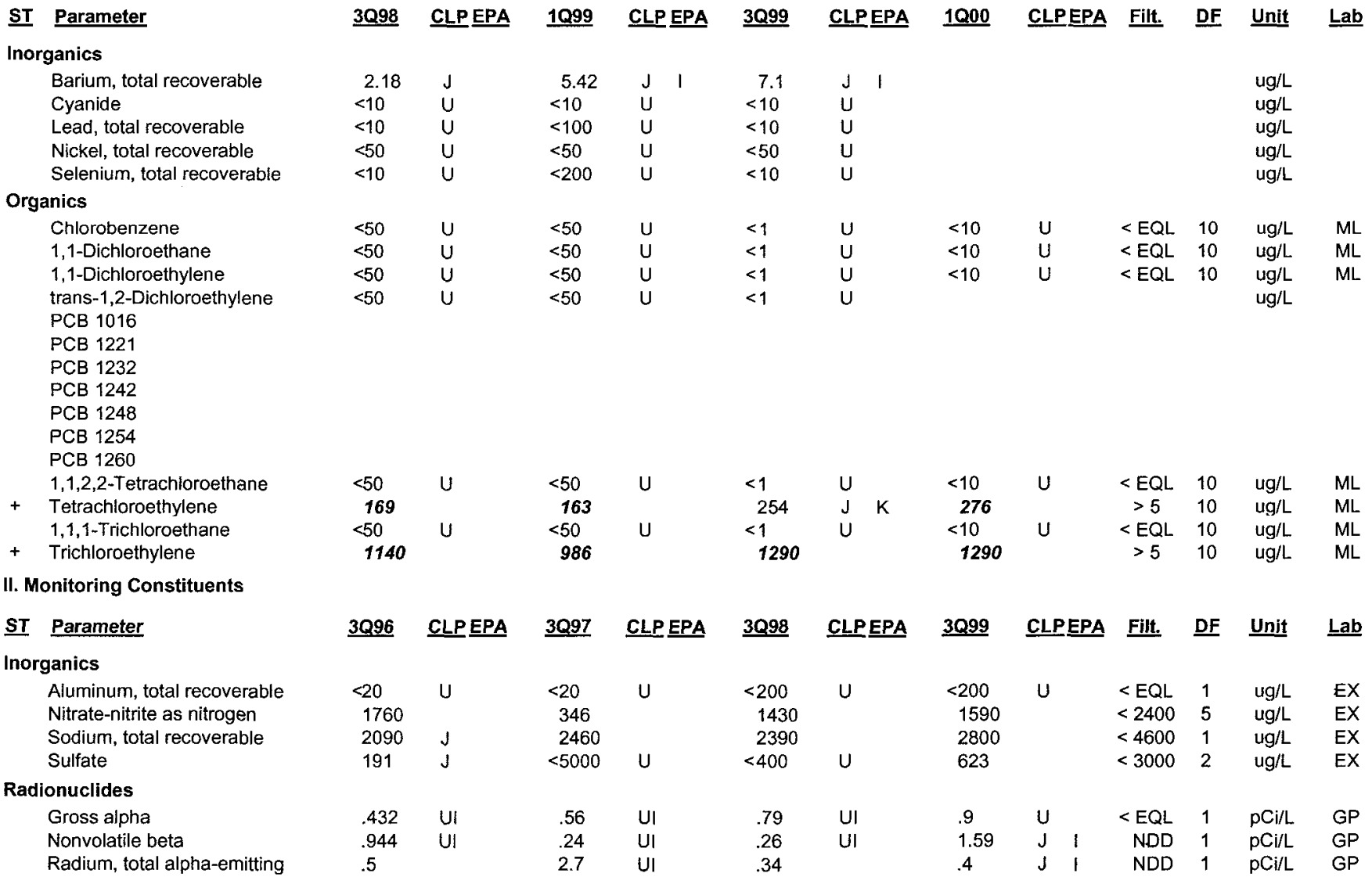

Notes: Concentrations in bold italics exceed the groundwater protection or monitoring constituent standards listed in Appendix A. Synchronous water levels are measured over a 3-5 day period or less. Dilution factors, Laboratory, and Filtered Data are for Groundwater Protection Standards First Quarter 2000 and Monitoring Constituents Third Quarter 1999 data only.

$+=$ exceeded the Groundwater Protection Standards (First Quarter 2000) or Monitoring Constituents (Third Quarter 1999) listed in Appendix A. 
Table D-4. Groundwater Monitoring Results for Plume Definition Wells, M-Area HWMF (Cont.) WELL MSB 26

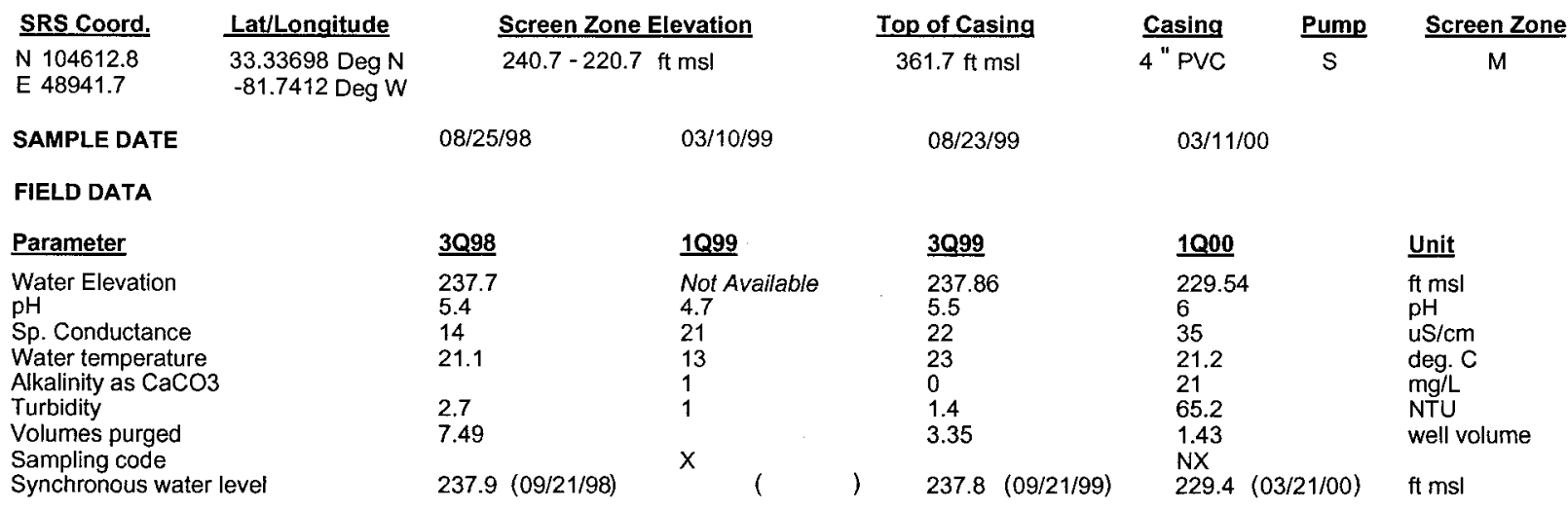

ANALYTICAL DATA

I. Groundwater Protection Standard

261 Appendix VIII/264 Appendix IX Hazardous Constituents

\begin{tabular}{|c|c|c|c|c|c|c|c|c|c|c|c|c|c|c|}
\hline \multirow{2}{*}{\multicolumn{2}{|c|}{$\begin{array}{l}\text { ST } \\
\text { Inorganics }\end{array}$}} & \multirow[t]{2}{*}{$\underline{3 Q 98}$} & \multirow[t]{2}{*}{ CLP EPA } & \multirow[t]{2}{*}{$\underline{1099}$} & CLP EPA & \multirow[t]{2}{*}{$\underline{3 Q 99}$} & CLPEPA & \multirow[t]{2}{*}{$\underline{1000}$} & \multicolumn{2}{|c|}{ CLPEPA } & \multirow[t]{2}{*}{ Filt. } & \multirow[t]{2}{*}{$\underline{\mathrm{DF}}$} & \multirow[t]{2}{*}{ Unit } & \multirow[t]{2}{*}{$\underline{L a b}$} \\
\hline & & & & & & & & & & & & & & \\
\hline & Barium, total recoverable & 2.95 & $J$ & 2.42 & $\mathrm{~J} \quad 1$ & 3.4 & J 1 & & & & & & $\mathrm{ug} / \mathrm{L}$ & \\
\hline & Cyanide & $<10$ & $\mathrm{U}$ & $<10$ & JU L & $<10$ & $\mathrm{U}$ & & & & & & $\mathrm{ug} / \mathrm{L}$ & \\
\hline & Lead, total recoverable & $<100$ & U & $<100$ & $\mathrm{U}$ & $<10$ & $\mathrm{U}$ & & & & & & $\mathrm{ug} / \mathrm{L}$ & \\
\hline & Nickel, total recoverable & 18.7 & $J$ & $<9.89$ & JU & $<50$ & $\mathrm{U}$ & & & & & & $\mathrm{ug} / \mathrm{L}$ & \\
\hline & Selenium, total recoverable & $<200$ & $\mathrm{U}$ & $<200$ & $U$ & $<10$ & $\mathrm{U}$ & & & & & & $\mathrm{ug} / \mathrm{L}$ & \\
\hline \multicolumn{15}{|c|}{ Organics } \\
\hline & Chlorobenzene & $<10$ & UJ & $<25$ & $\mathrm{U}$ & $<1$ & $\mathrm{U}$ & $<1$ & $\mathrm{U}$ & & $<$ EQL & 1 & ug/L & ML \\
\hline & 1,1-Dichloroethane & $<10$ & UJ & $<25$ & $\mathrm{U}$ & $<1$ & $\mathrm{u}$ & $<1$ & $U$ & & $<E Q L$ & 1 & $\mathrm{ug} / \mathrm{L}$ & $M L$ \\
\hline & 1,1-Dichloroethylene & $<10$ & UJ & $<25$ & $U$ & $<1$ & $\mathbf{U}$ & $<1$ & $\mathrm{U}$ & & $<E Q L$ & 1 & $\mathrm{ug} / \mathrm{L}$ & ML \\
\hline & trans-1,2-Dichloroethylene & $<10$ & UJ & $<25$ & $\mathrm{U}$ & $<1$ & $\mathrm{U}$ & & & & & & $\mathrm{ug} / \mathrm{L}$ & \\
\hline & PCB 1016 & & & & & & & & & & & & & \\
\hline & РCB 1221 & & & & & & & & & & & & & \\
\hline & РCB 1232 & & & & & & & & & & & & & \\
\hline & PCB 1242 & & & & & & & & & & & & & \\
\hline & PCB 1248 & & & & & & & & & & & & & \\
\hline & PCB 1254 & & & & & & & & & & & & & \\
\hline & PCB 1260 & & & & & & & & & & & & & \\
\hline & 1,1,2,2-Tetrachloroethane & $<10$ & UJ & $<25$ & $\mathrm{U}$ & $<1$ & $\mathrm{U}$ & $<1$ & $U$ & & $<E Q L$ & 1 & ug/L & ML \\
\hline & Tetrachloroethylene & 46 & $\mathrm{~J}$ & 45.3 & & 69.9 & $\mathrm{~J} \quad \mathrm{~K}$ & 45.4 & $\mathrm{~J}$ & $\mathrm{~K}$ & NDD & 1 & $\mathrm{ug} / \mathrm{L}$ & $M L$ \\
\hline & 1,1,1-Trichloroethane & $<10$ & UJ & $<25$ & $\mathrm{U}$ & $<1$ & $\mathrm{U}$ & $<1$ & $\mathrm{U}$ & & $<E Q L$ & 1 & $\mathrm{ug} / \mathrm{L}$ & $M L$ \\
\hline & Trichloroethylene & 238 & $J$ & 226 & & 300 & $\mathrm{~J} K$ & 186 & 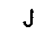 & K & NDD & 1 & $\mathrm{ug} / \mathrm{L}$ & $M L$ \\
\hline \multicolumn{15}{|c|}{ II. Monitoring Constituents } \\
\hline$\underline{\mathbf{S T}}$ & Parameter & $\underline{3 Q 96}$ & CLPEPA & $\underline{3097}$ & CLPEPA & $\underline{3 Q 98}$ & CLPEPA & $\underline{3099}$ & CLP & EPA & Filt. & DF & $\underline{\text { Unit }}$ & $\underline{\text { Lab }}$ \\
\hline \multicolumn{15}{|c|}{ Inorganics } \\
\hline & Aluminum, total recoverable & $<40.3$ & $\mathrm{U}$ & $<20$ & U & $<200$ & $U$ & $<200$ & $\mathrm{U}$ & & $<\mathrm{EQL}$ & 1 & ug/L & EX \\
\hline & Nitrate-nitrite as nitrogen & 580 & & 336 & & 235 & $J$ & 262 & $\checkmark$ & 1 & NDD & 5 & ug/L & EX \\
\hline & Sodium, total recoverable & 2180 & & 2610 & & 2440 & & 2800 & & & $<4600$ & 1 & $\mathrm{ug} / \mathrm{L}$ & EX \\
\hline & Sulfate & 813 & $\mathrm{~J}$ & $<5000$ & $\mathrm{U}$ & 1660 & & 923 & & & $<3000$ & 2 & $\mathrm{ug} / \mathrm{L}$ & EX \\
\hline \multicolumn{15}{|c|}{ Radionuclides } \\
\hline & Gross alpha & .439 & Ul & .58 & UI & 4.54 & & .558 & $\mathbf{U}$ & & $<E Q L$ & 1 & $\mathrm{pCi} / \mathrm{L}$ & GP \\
\hline & Nonvolatile beta & .38 & UI & .15 & UI & 13.14 & $J$ & .861 & $\mathrm{U}$ & & $<\mathrm{EQL}$ & 1 & $\mathrm{pCi} / \mathrm{L}$ & GP \\
\hline & Radium, total alpha-emitting & .2 & & 1.98 & UI & .23 & UI & .3 & $\mathrm{U}$ & & $<E Q L$ & 1 & $\mathrm{pCi} / \mathrm{L}$ & GP \\
\hline
\end{tabular}

Notes: Concentrations in bold italics exceed the groundwater protection or monitoring constituent standards listed in Appendix A. Synchronous water levels are measured over a 3-5 day period or less. Dilution factors, Laboratory, and Filtered Data are for Groundwater Protection Standards First Quarter 2000 and Monitoring Constituents Third Quarter 1999 data only.

+= exceeded the Groundwater Protection Standards (First Quarter 2000) or Monitoring Constituents (Third Quarter 1999) listed in Appendix A. 
Table D-4. Groundwater Monitoring Results for Plume Definition Wells, M-Area HWMF (Cont.) WELL MSB 26B

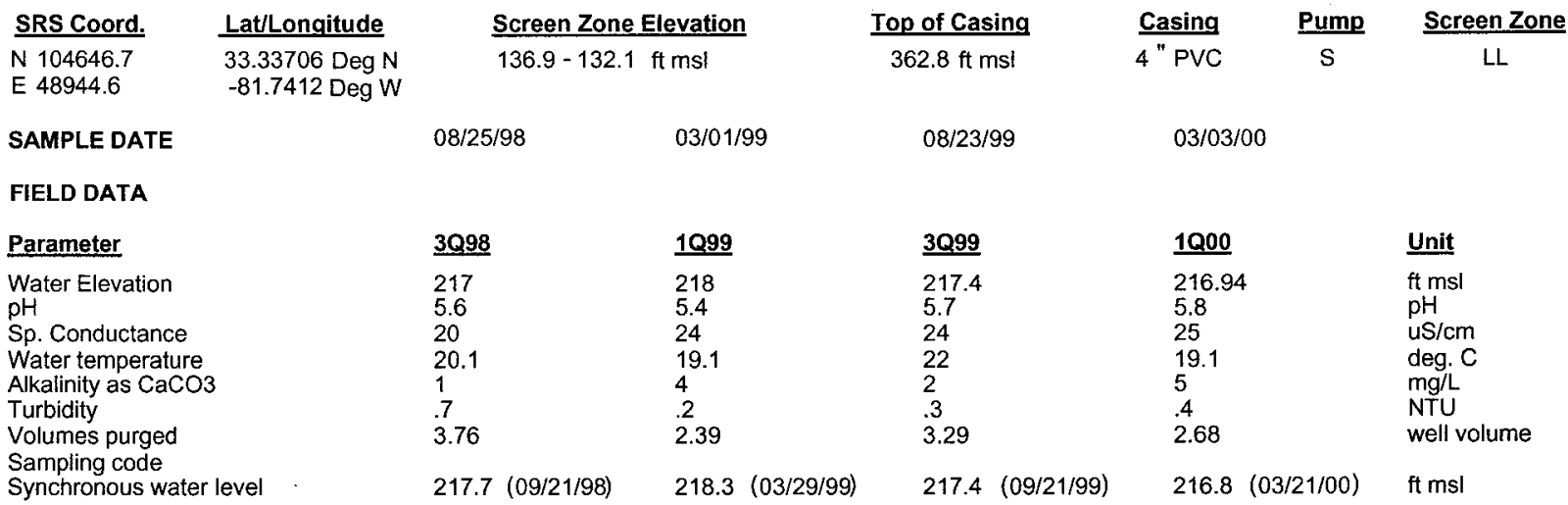

ANALYTICAL DATA

I. Groundwater Protection Standard

261 Appendix VIII/264 Appendix IX Hazardous Constituents

\begin{tabular}{|c|c|c|c|c|c|c|c|c|c|c|c|c|c|c|}
\hline \multirow{2}{*}{$\frac{\text { ST }}{\text { Inorg }}$} & Parameter & \multirow[t]{2}{*}{$\underline{3 Q 98}$} & \multirow[t]{2}{*}{ CLP EPA } & \multirow[t]{2}{*}{$1 Q 99$} & \multirow[t]{2}{*}{ CLPEPA } & \multirow[t]{2}{*}{$\underline{3 Q 99}$} & CLPEPA & \multirow[t]{2}{*}{1000} & \multicolumn{2}{|c|}{$\underline{\text { CLPEPA }}$} & \multirow[t]{2}{*}{ Filt. } & \multirow[t]{2}{*}{$\underline{D F}$} & \multirow[t]{2}{*}{ Unit } & \multirow[t]{2}{*}{$\underline{\text { Lab }}$} \\
\hline & ganics & & & & & & & & & & & & & \\
\hline & Barium, total recoverable & 7.83 & $J$ & $\$ 1.3$ & & 7.8 & $\mathrm{~J} \quad \mathrm{I}$ & & & & & & $\mathrm{ug} / \mathrm{L}$ & \\
\hline & Cyanide & $<10$ & $\mathrm{U}$ & $<10$ & $U$ & $<10$ & $\mathrm{U}$ & & & & & & $\mathrm{ug} / \mathrm{L}$ & \\
\hline & Lead, total recoverable & $<100$ & $\mathrm{U}$ & $<100$ & $U$ & $<10$ & $\mathrm{U}$ & & & & & & $\mathrm{ug} / \mathrm{L}$ & \\
\hline & Nickel, total recoverable & 8.44 & $\mathrm{~J}$ & $<50$ & $\mathrm{U}$ & $<50$ & $\mathrm{U}$ & & & & & & $u g / L$ & \\
\hline & Selenium, total recoverable & $<200$ & $\mathrm{U}$ & $<200$ & $\mathrm{U}$ & $<10$ & $\mathrm{U}$ & & & & & & $\mathrm{ug} / \mathrm{L}$ & \\
\hline \multicolumn{15}{|c|}{ Organics } \\
\hline & Chlorobenzene & $<25$ & UJ & $<25$ & $u$ & $<1$ & $U$ & $<10$ & JU & $\mathrm{L}$ & $<E Q L$ & 10 & $\mathrm{ug} / \mathrm{L}$ & $M L$ \\
\hline & 1,1-Dichloroethane & $<25$ & UJ & $<25$ & $U$ & $<1$ & $\mathrm{U}$ & $<10$ & JU & $L$ & $<E Q L$ & 10 & $\mathrm{ug} / \mathrm{L}$ & $M L$ \\
\hline & 1,1-Dichloroethylene & $<25$ & UJ & $<25$ & $U$ & $<1$ & $\mathrm{U}$ & $<10$ & JU & $L$ & $<E Q L$ & 10 & $\mathrm{ug} / \mathrm{L}$ & ML \\
\hline & trans-1,2-Dichloroethylene & $<25$ & UJ & $<25$ & $\mathrm{U}$ & $<1$ & $\mathrm{U}$ & & & & & & $u g / L$ & \\
\hline & PCB 1016 & & & & & & & & & & & & & \\
\hline & PCB 1221 & & & & & & & & & & & & & \\
\hline & PCB 1232 & & & & & & & & & & & & & \\
\hline & PCB 1242 & & & & & & & & & & & & & \\
\hline & PCB 1248 & & & & & & & & & & & & & \\
\hline & PCB 1254 & & & & & & & & & & & & & \\
\hline & PCB 1260 & & & & & & & & & & & & & \\
\hline & $1,1,2,2$-Tetrachloroethane & $<25$ & UJ & $<25$ & $\mathrm{U}$ & $<1$ & $\mathrm{U}$ & $<10$ & JU & $\mathrm{L}$ & $<E Q L$ & 10 & $\mathrm{ug} / \mathrm{L}$ & ML \\
\hline & Tetrachloroethylene & 18 & $J$ & 112 & & 24.1 & $\mathrm{~J} K$ & 55.4 & $J$ & $\mathrm{~L}$ & NDD & 10 & $\mathrm{ug} / \mathrm{L}$ & ML \\
\hline & $1,1,1$-Trichloroethane & $<25$ & UJ & $<25$ & $U$ & $<1$ & U & $<10$ & JU & $\mathrm{L}$ & $<E Q L$ & 10 & $\mathrm{ug} / \mathrm{L}$ & ML \\
\hline & Trichloroethylene & 331 & $J$ & 749 & & 1480 & & 2180 & $J$ & $\mathrm{~L}$ & NDD & 10 & $\mathrm{ug} / \mathrm{L}$ & ML \\
\hline \multicolumn{15}{|c|}{ II. Monitoring Constituents } \\
\hline$\underline{\mathrm{ST}}$ & Parameter & $\underline{3096}$ & $\underline{\text { CLP EPA }}$ & $\underline{3097}$ & CLPEPA & $\underline{3098}$ & CLPEPA & $\underline{3099}$ & $\underline{\text { CLP }}$ & EPA & Filt. & $\underline{\text { DF }}$ & Unit & $\underline{\text { Lab }}$ \\
\hline \multicolumn{15}{|c|}{ Inorganics } \\
\hline & Aluminum, total recoverable & $<20$ & $\mathrm{U}$ & $<20$ & $U$ & $<200$ & U & $<200$ & $\mathrm{U}$ & & $<E Q L$ & 1 & $\mathrm{ug} / \mathrm{L}$ & EX \\
\hline & Nitrate-nitrite as nitrogen & 550 & & 668 & & 598 & & 631 & & & $<2400$ & 5 & ug/L & EX \\
\hline & Sodium, total recoverable & 1860 & & 2010 & & 1870 & & 2200 & & & $<4600$ & 1 & $\mathrm{ug} / \mathrm{L}$ & EX \\
\hline & Sulfate & 327 & $J$ & $<5000$ & $\mathrm{U}$ & 309 & & 426 & & & $<3000$ & 2 & $\mathrm{ug} / \mathrm{L}$ & EX \\
\hline \multicolumn{15}{|c|}{ Radionuclides } \\
\hline & Gross alpha & -.322 & UI & 1.11 & UI & .34 & UI & .462 & U & & $<E Q L$ & 1 & $\mathrm{pCi} / \mathrm{L}$ & GP \\
\hline & Nonvolatile beta & .909 & UI & .63 & UI & .45 & UIJ & .663 & U & & $<E Q L$ & 1 & $\mathrm{pCi} / \mathrm{L}$ & GP \\
\hline & Radium, total alpha-emitting & -.1 & UI & 1.81 & UIJ & .23 & UI & -.1 & $\mathrm{U}$ & & $<E Q L$ & 1 & $\mathrm{pCi} / \mathrm{L}$ & GP \\
\hline
\end{tabular}

Notes: Concentrations in bold italics exceed the groundwater protection or monitoring constituent standards listed in Appendix A. Synchronous water levels are measured over a 3-5 day period or less. Dilution factors, Laboratory, and Filtered Data are for Groundwater Protection Standards First Quarter 2000 and Monitoring Constituents Third Quarter 1999 data only.

$+=$ exceeded the Groundwater Protection Standards (First Quarter 2000) or Monitoring Constituents (Third Quarter 1999) listed in Appendix A. 
Table D-4. Groundwater Monitoring Results for Plume Definition Wells, M-Area HWMF (Cont.) WELL MSB 27

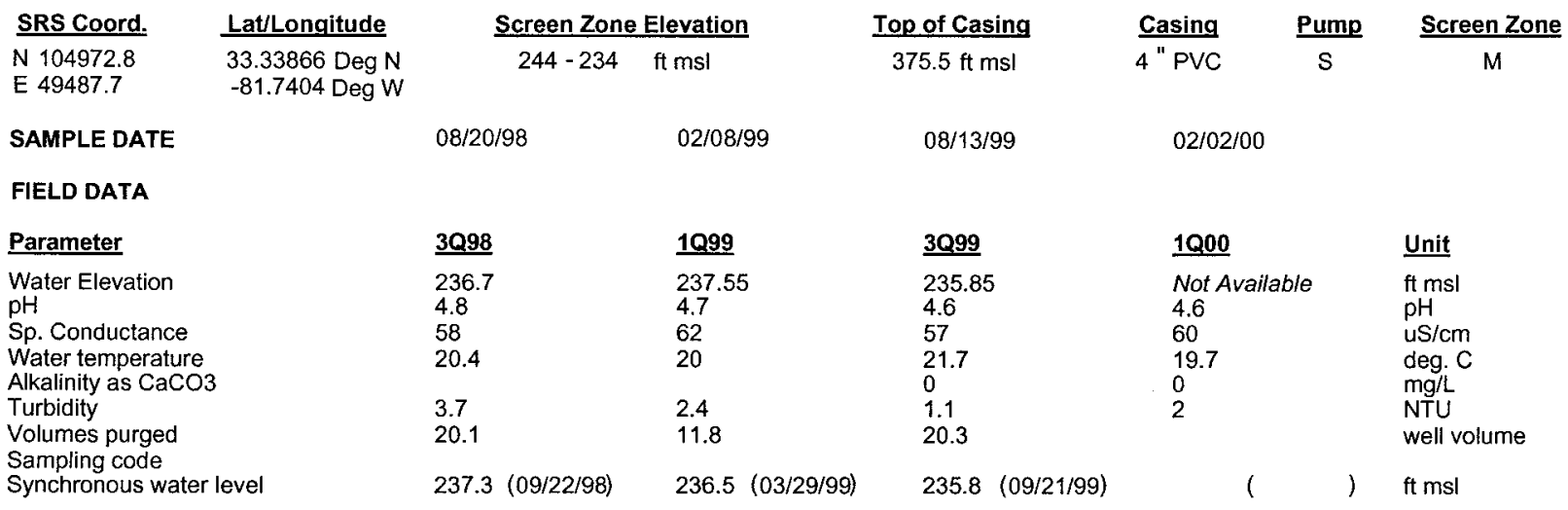

ANALYTICAL DATA

I. Groundwater Protection Standard

261 Appendix VIII/264 Appendix IX Hazardous Constituents

\begin{tabular}{|c|c|c|c|c|c|c|c|c|c|c|c|c|c|c|c|}
\hline \multirow{2}{*}{$\frac{\text { ST }}{\text { Inorg }}$} & Parameter & \multirow[t]{2}{*}{$\underline{3098}$} & \multirow[t]{2}{*}{ CLP EPA } & \multirow[t]{2}{*}{1099} & \multirow[t]{2}{*}{ CLP EPA } & \multirow[t]{2}{*}{$\underline{3 Q 99}$} & \multicolumn{2}{|c|}{ CLPEPA } & \multirow[t]{2}{*}{1000} & \multicolumn{2}{|c|}{ CLPEPA } & \multirow[t]{2}{*}{ Filt. } & \multirow[t]{2}{*}{$\underline{\text { DF }}$} & \multirow[t]{2}{*}{ Unit } & \multirow[t]{2}{*}{$\underline{L a b}$} \\
\hline & ganics & & & & & & & & & & & & & & \\
\hline & Barium, total recoverable & 20.4 & & $<10$ & $\mathrm{U}$ & 16 & & & 16.6 & & & $<2000$ & 1 & $u g / L$ & ML \\
\hline & Cyanide & $<10$ & $\mathrm{U}$ & $<10$ & $U$ & $<10$ & $U$ & & & & & & & $\mathrm{ug} / \mathrm{L}$ & \\
\hline & Lead, total recoverable & $<10$ & U & $<100$ & $\mathrm{U}$ & $<2.2$ & $\mathrm{U}$ & V & $<20$ & $\mathrm{U}$ & & $<E Q L$ & 1 & $\mathrm{ug} / \mathrm{L}$ & ML \\
\hline & Nickel, total recoverable & 14.9 & $\mathrm{~J}$ & $<50$ & $U$ & $<50$ & $U$ & & $<60$ & $\mathrm{U}$ & & $<$ EQL & 1 & $\mathrm{ug} / \mathrm{L}$ & ML \\
\hline & Selenium, total recoverable & $<10$ & U & $<200$ & $U$ & $<5$ & $\mathrm{U}$ & & $<40$ & $U$ & & $<\mathrm{EQL}$ & 1 & ug/L & ML \\
\hline \multicolumn{16}{|c|}{ Organics } \\
\hline & Chlorobenzene & $<5$ & $\mathrm{U}$ & $<5$ & U & $<1$ & $u$ & & $<1$ & JU & $L$ & $<\mathrm{EQL}$ & 1 & $u g / L$ & $M L$ \\
\hline & 1,1-Dichloroethane & $<5$ & $\mathrm{U}$ & $<5$ & $\mathrm{U}$ & $<1$ & $\mathrm{U}$ & & $<1$ & JU & $L$ & $<\mathrm{EQL}$ & 1 & ug/t & $M L$ \\
\hline & 1,1-Dichloroethylene & $<5$ & $U$ & $<5$ & $\mathrm{U}$ & $<1$ & $\mathrm{U}$ & & $<1$ & JU & L & $<\mathrm{EQL}$ & 1 & ug/L & $M L$ \\
\hline & $\begin{array}{l}\text { trans-1,2-Dichloroethylene } \\
\text { PCB } 1016\end{array}$ & $<5$ & $U$ & $<5$ & $\mathrm{U}$ & $<1$ & $\mathrm{U}$ & & $<1$ & JU & $\mathrm{L}$ & $<$ EQL & 1 & $\mathrm{ug} / \mathrm{L}$ & ML \\
\hline & PCB 1221 & & & & & & & & & & & & & & \\
\hline & PCB 1232 & & & & & & & & & & & & & & \\
\hline & PCB 1242 & & & & & & & & & & & & & & \\
\hline & PCB 1248 & & & & & & & & & & & & & & \\
\hline & PCB 1254 & & & & & & & & & & & & & & \\
\hline & PCB 1260 & & & & & & & & & & & & & & \\
\hline & $1,1,2,2-$ Tetrachloroethane & $<5$ & $\mathrm{U}$ & $<5$ & $U$ & $<1$ & $\cup$ & & $<1$ & JU & $L$ & $<\mathrm{EQL}$ & 1 & ug/L & ML \\
\hline & Tetrachloroethylene & $<5$ & $\mathrm{U}$ & $<5$ & $U$ & $<1.05$ & $U$ & V & .57 & $J$ & IL & NDD & 1 & $\mathrm{ug} / \mathrm{L}$ & ML \\
\hline & 1,1,1-Trichloroethane & $<5$ & $\mathrm{U}$ & $<5$ & $\mathrm{U}$ & $<1$ & $U$ & & $<1$ & JU & $\mathrm{L}$ & $<E Q L$ & 1 & $\mathrm{ug} / \mathrm{L}$ & ML \\
\hline & Trichloroethylene & $<5$ & U & $<5$ & $\mathrm{U}$ & $<1.21$ & $U$ & V & $<1$ & JU & $\mathrm{L}$ & $<E Q L$ & 1 & $\mathrm{ug} / \mathrm{L}$ & $\mathrm{ML}$ \\
\hline \multicolumn{16}{|c|}{ II. Monitoring Constituents } \\
\hline$\underline{\text { ST }}$ & Parameter & $\underline{3 Q 996}$ & CLPEPA & $\underline{3097}$ & CLP EPA & $\underline{3098}$ & CLP & EPA & $\underline{3099}$ & $\underline{\text { CLP }}$ & EPA & Filt. & $\underline{\text { DF }}$ & Unit & Lab \\
\hline \multicolumn{16}{|c|}{ Inorganics } \\
\hline & Aluminum, total recoverable & 153 & & 240 & & 142 & $\mathrm{~J}$ & & 120 & $\mathrm{~J}$ & 1 & NDD & 1 & $\mathrm{ug} / \mathrm{L}$ & EX \\
\hline+ & Nitrate-nitrite as nitrogen & 4080 & & 4890 & & 3760 & & & 3270 & & & $>2400$ & 5 & ug/L & $\mathrm{EX}$ \\
\hline+ & Sodium, total recoverable & 4620 & & 4810 & & 5920 & & & 4900 & & & $>4600$ & 1 & $u g / L$ & EX \\
\hline & Sulfate & $<1000$ & $\mathrm{U}$ & $<5000$ & $\mathrm{U}$ & $<400$ & $U$ & & 291 & & & $<3000$ & 1 & $u g / L$ & EX \\
\hline \multicolumn{16}{|c|}{ Radionuclides } \\
\hline & Gross alpha & 1.88 & & .31 & U! & 4.21 & & & 2.06 & $\mathrm{~J}$ & K & NDD & 1 & $\mathrm{pCi} / \mathrm{L}$ & GP \\
\hline & Nonvolatile beta & 1.91 & & .39 & UI & 3.4 & & & 1.19 & $\mathrm{~J}$ & 1 & NDD & 1 & $\mathrm{pCi} / \mathrm{L}$ & GP \\
\hline & Radium, total alpha-emitting & .5 & & 2.79 & UIJ & .78 & & & .7 & $J$ & I & NDD & 1 & $\mathrm{pCi} / \mathrm{L}$ & GP \\
\hline
\end{tabular}

Notes: Concentrations in bold italics exceed the groundwater protection or monitoring constituent standards listed in Appendix A. Synchronous water levels are measured over a 3-5 day period or less. Dilution factors, Laboratory, and Filtered Data are for Groundwater Protection Standards First Quarter 2000 and Monitoring Constituents Third Quarter 1999 data only.

+ = exceeded the Groundwater Protection Standards (First Quarter 2000) or Monitoring Constituents (Third Quarter 1999) listed in Appendix A. 
Table D-4. Groundwater Monitoring Results for Plume Definition Wells, M-Area HWMF (Cont.) WELL MSB 27B

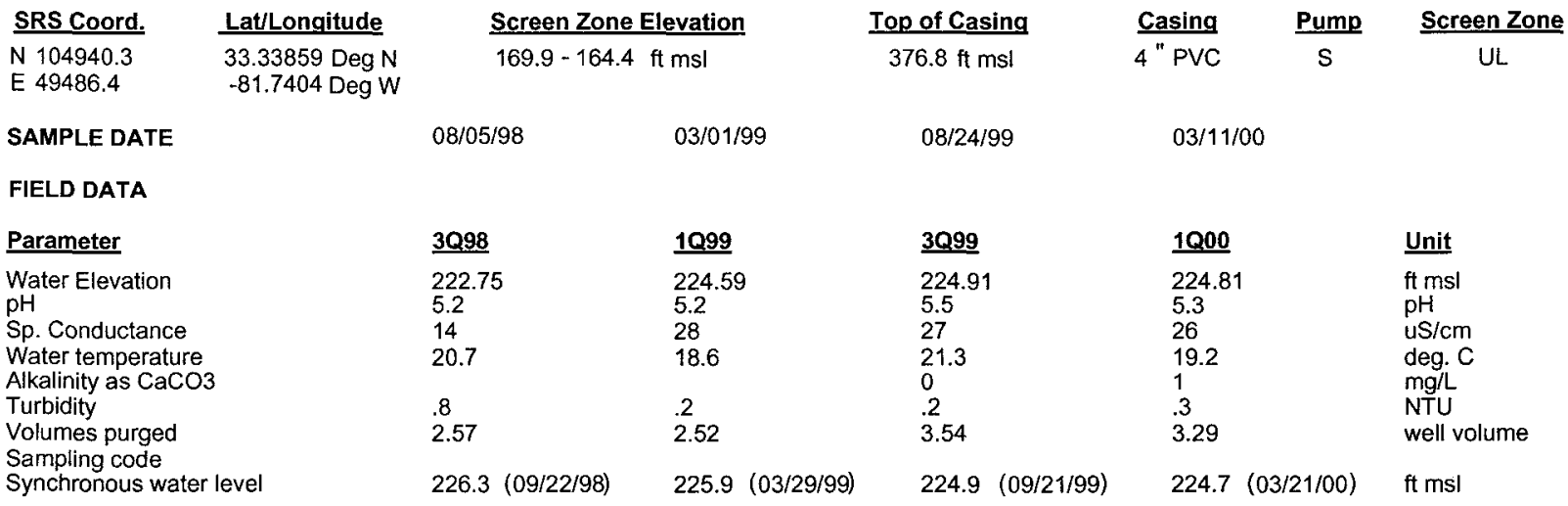

ANALYTICAL DATA

I. Groundwater Protection Standard

261 Appendix VIII/264 Appendix IX Hazardous Constituents

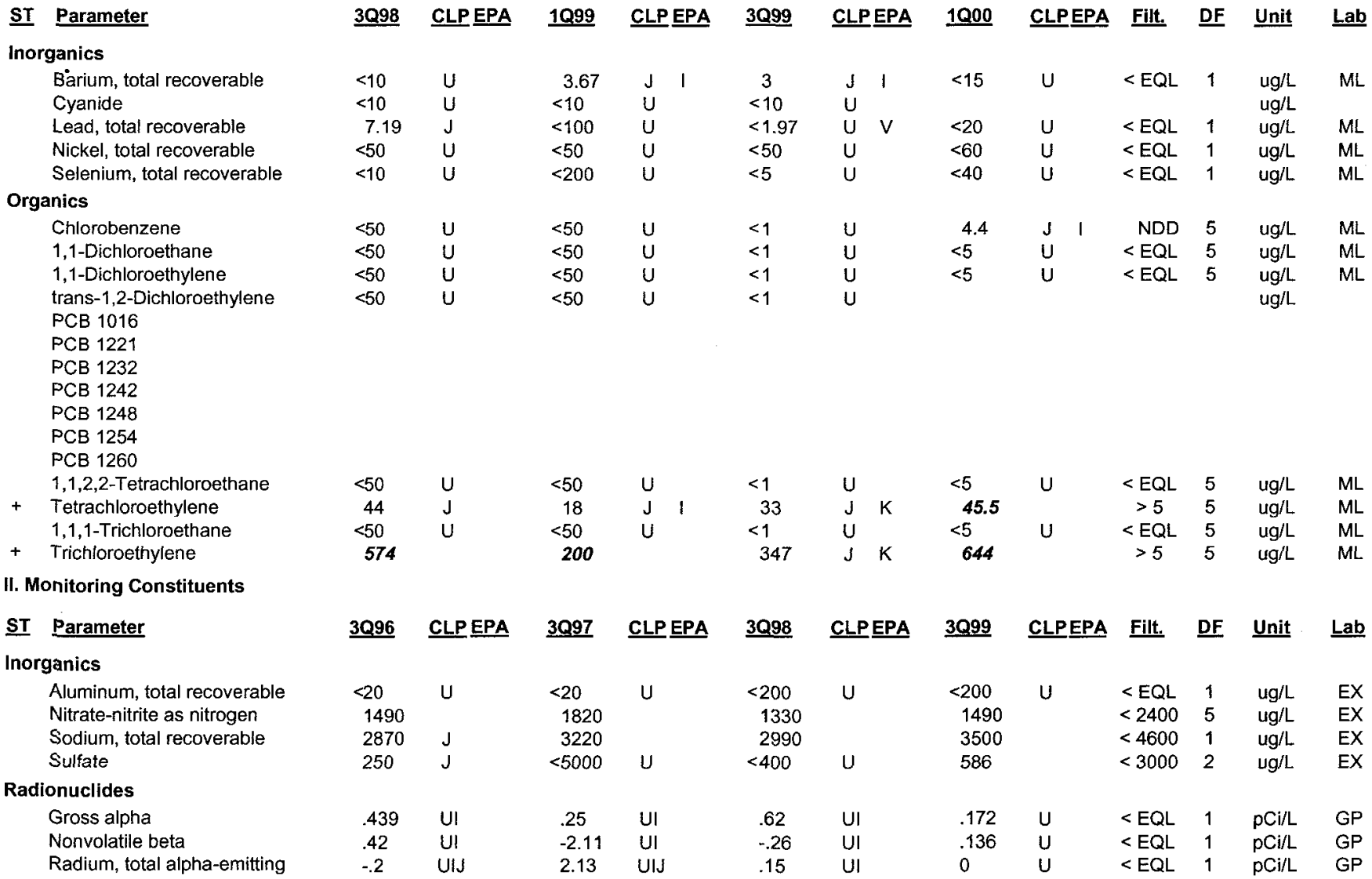

Notes: Concentrations in bold italics exceed the groundwater protection or monitoring constituent standards listed in Appendix A. Synchronous water levels are measured over a 3-5 day period or less. Dilution factors, Laboratory, and Filtered Data are for Groundwater Protection Standards First Quarter 2000 and Monitoring Constituents Third Quarter 1999 data only.

$+=$ exceeded the Groundwater Protection Standards (First Quarter 2000) or Monitoring Constituents (Third Quarter 1999) listed in Appendix A. 
Table D-4. Groundwater Monitoring Results for Plume Definition Wells, M-Area HWMF (Cont.) WELL MSB 27TA

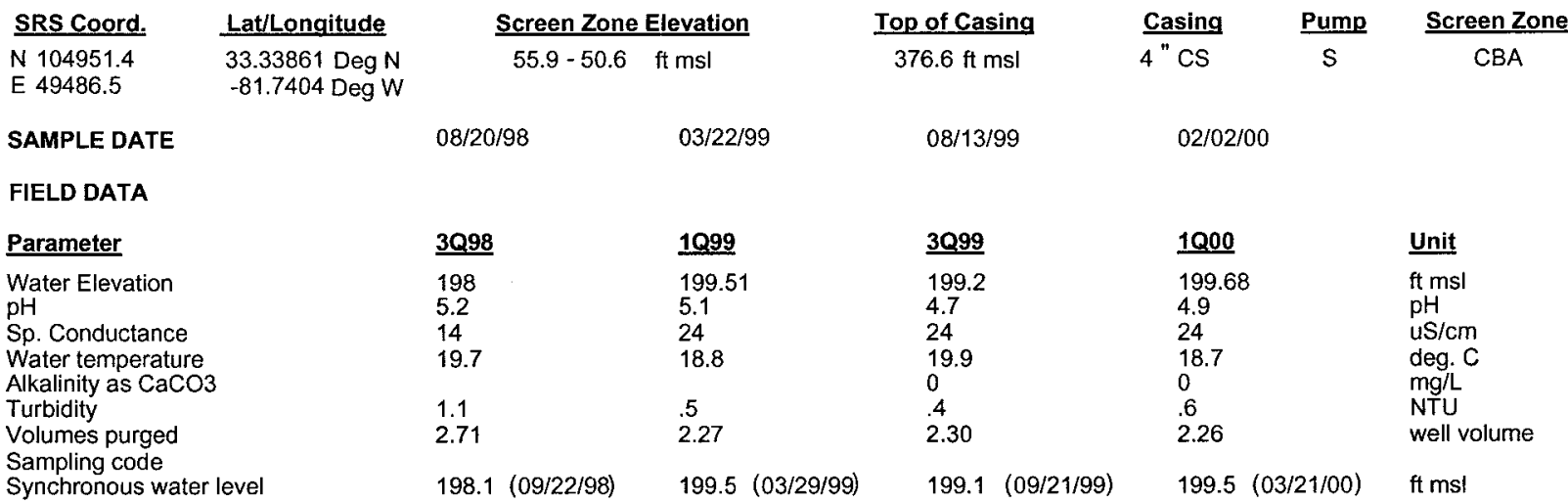

ANALYTICAL DATA

1. Groundwater Protection Standard

261 Appendix VIII/264 Appendix IX Hazardous Constituents

\begin{tabular}{|c|c|c|c|c|c|c|c|c|c|c|c|c|c|}
\hline$\underline{\text { ST }}$ & Parameter & $\underline{3 Q 98}$ & CLP EPA & 1099 & $\underline{\text { CLP EPA }}$ & $\underline{3 Q 99}$ & CLP EPA & 1000 & CLPEPA & Filt. & DF & Unit & $\underline{\text { Lab }}$ \\
\hline \multicolumn{14}{|c|}{ Inorganics } \\
\hline & Barium, total recoverable & 5.24 & J & 5.58 & $\mathrm{~J}$ & 6.4 & J 1 & & & & & $u g / L$ & \\
\hline & Cyanide & $<10$ & $U$ & $<10$ & U & $<10$ & U & & & & & $\mathrm{ug} / \mathrm{L}$ & \\
\hline & Lead, total recoverable & $<10$ & $\mathrm{U}$ & $<100$ & $u$ & $<3.41$ & U V & & & & & $\mathrm{ug} / \mathrm{L}$ & \\
\hline & Nickel, total recoverable & 12.6 & $\mathrm{~J}$ & $<50$ & U & $<50$ & $U$ & & & & & $\mathrm{ug} / \mathrm{L}$ & \\
\hline & Selenium, total recoverable & $<10$ & U & $<10$ & $U$ & $<5$ & $\mathrm{U}$ & & & & & $\mathrm{ug} / \mathrm{L}$ & \\
\hline \multicolumn{14}{|c|}{ Organics } \\
\hline & Chlorobenzene & $<5$ & $\mathrm{U}$ & $<5$ & $\mathrm{U}$ & $<1$ & $\mathrm{U}$ & $<1$ & u & $<E Q L$ & 1 & $u g / L$ & ML \\
\hline & 1,1-Dichloroethane & $<5$ & $\mathrm{U}$ & $<5$ & $\mathrm{U}$ & $<1$ & U & $<1$ & $U$ & $<E Q L$ & 1 & $\mathrm{ug} / \mathrm{L}$ & ML \\
\hline & 1,1-Dichloroethylene & $<5$ & $\mathrm{U}$ & $<5$ & $\mathrm{U}$ & $<1$ & $U$ & $<1$ & $U$ & $<E Q L$ & 1 & $\mathrm{ug} / \mathrm{L}$ & $M L$ \\
\hline & trans-1,2-Dichloroethylene & $<5$ & $U$ & $<5$ & $\mathrm{U}$ & $<1$ & U & $<1$ & $U$ & $<\mathrm{EQL}$ & 1 & $u g / L$ & ML \\
\hline & PCB 1016 & & & & & & & & & & & & \\
\hline & PCB 1221 & & & & & & & & & & & & \\
\hline & PCB 1232 & & & & & & & & & & & & \\
\hline & PCB 1242 & & & & & & & & & & & & \\
\hline & РCB 1248 & & & & & & & & & & & & \\
\hline & PCB 1254 & & & & & & & & & & & & \\
\hline & PCB 1260 & & & & & & & & & & & & \\
\hline & $1,1,2,2$-Tetrachloroethane & $<5$ & $\mathrm{U}$ & $<5$ & $U$ & $<1$ & $u$ & $<1$ & $U$ & $<E Q L$ & 1 & $\mathrm{ug} / \mathrm{L}$ & ML \\
\hline & Tetrachloroethylene & $<5$ & $\mathrm{U}$ & $<5$ & $\mathrm{U}$ & $<1$ & $\mathrm{U}$ & $<1$ & $\mathrm{U}$ & $<$ EQL & 1 & $u g / L$ & $M L$ \\
\hline & 1,1,1-Trichloroethane & $<5$ & $\mathrm{u}$ & $<5$ & $\mathrm{U}$ & $<1$ & $U$ & $<1$ & $\mathrm{U}$ & $<E Q L$ & 1 & ug/L & ML \\
\hline & Trichloroethylene & $<5$ & $\mathrm{U}$ & $<5$ & $\mathrm{U}$ & $<1$ & $U$ & $<1$ & $\mathrm{U}$ & $<\overline{E Q L}$ & 1 & $\mathrm{ug} / \mathrm{L}$ & ML \\
\hline \multicolumn{14}{|c|}{ II. Monitoring Constituents } \\
\hline$\underline{\mathbf{S T}}$ & Parameter & $\underline{3096}$ & CLP EPA & 3097 & CLPEPA & 3Q998 & CLPEPA & $\underline{3099}$ & CLPEPA & Filt. & $\underline{D F}$ & Unit & $\underline{\text { Lab }}$ \\
\hline \multicolumn{14}{|c|}{ Inorganics } \\
\hline & Aluminum, total recoverable & 19.8 & J & 31.3 & & $<200$ & $U$ & $<200$ & $\mathrm{U}$ & $<E Q L$ & 1 & $\mathrm{ug} / \mathrm{L}$ & EX \\
\hline & Nitrate-nitrite as nitrogen & 360 & & $<19$ & $U$ & $<200$ & U & $<500$ & $U$ & $<E Q L$ & 5 & $\mathrm{ug} / \mathrm{L}$ & EX \\
\hline & Sodium, total recoverable & 1530 & & 1530 & & $<1660$ & U & 1600 & & $<4600$ & 1 & $\mathrm{ug} / \mathrm{L}$ & EX \\
\hline+ & Sulfate & 3380 & & 3720 & J & 3170 & & 3520 & & $>3000$ & 1 & $\mathrm{ug} / \mathrm{L}$ & EX \\
\hline \multicolumn{14}{|c|}{ Radionuclides } \\
\hline & Gross alpha & .919 & & .32 & UI & .79 & & .826 & $\mathrm{~J} \quad \mathbb{I K}$ & NDD & 1 & $\mathrm{pCi} / \mathrm{L}$ & GP \\
\hline & Nonvolatile beta & .724 & UI & -.61 & UI & .57 & UI & .99 & $U$ & $<E Q L$ & 1 & $\mathrm{pCi} / \mathrm{L}$ & GP \\
\hline & Radium, total alpha-emitting & .5 & & 2.24 & UIJ & .37 & UI & 0 & U & $<E Q L$ & 1 & $\mathrm{pCi} / \mathrm{L}$ & GP \\
\hline
\end{tabular}

Notes: Concentrations in bold italics exceed the groundwater protection or monitoring constituent standards listed in Appendix A. Synchronous water levels are measured over a 3-5 day period or less. Dilution factors, Laboratory, and Filtered Data are for Groundwater Protection Standards First Quarter 2000 and Monitoring Constituents Third Quarter 1999 data only.

$+=$ exceeded the Groundwater Protection Standards (First Quarter 2000) or Monitoring Constituents (Third Quarter 1999) listed in Appendix A. 
Table D-4. Groundwater Monitoring Results for Plume Definition Wells, M-Area HWMF (Cont.) WELL MSB 28

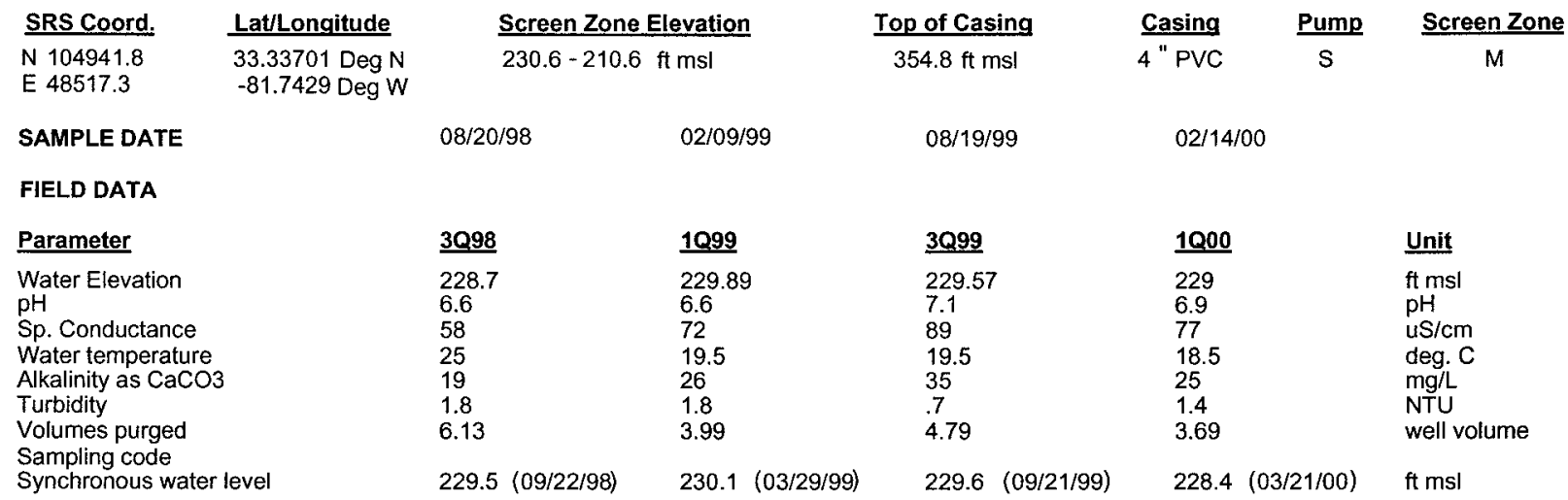

ANALYTICAL. DATA

I. Groundwater Protection Standard

261 Appendix VIII/264 Appendix IX Hazardous Constituents

\begin{tabular}{|c|c|c|c|c|c|c|c|c|c|c|c|c|c|c|}
\hline \multirow{2}{*}{\multicolumn{2}{|c|}{$\frac{\text { ST }}{\text { Inorganics }}$}} & \multirow[t]{2}{*}{$\underline{3 Q 98}$} & \multirow[t]{2}{*}{ CLPEPA } & \multirow[t]{2}{*}{$1 \mathrm{Q99}$} & \multicolumn{2}{|c|}{ CLP EPA } & \multirow[t]{2}{*}{$\underline{3 Q 99}$} & CLPEPA & \multirow[t]{2}{*}{$1 \mathrm{Q00}$} & CLPEPA & \multirow[t]{2}{*}{ Filt. } & \multirow[t]{2}{*}{$\underline{\text { DF }}$} & \multirow[t]{2}{*}{ Unit } & \multirow[t]{2}{*}{ Lab } \\
\hline & & & & & & & & & & & & & & \\
\hline & Barium, total recoverable & 4.54 & $J$ & 5.49 & $J$ & I & 5.3 & J I & & & & & $\mathrm{ug} / \mathrm{L}$ & \\
\hline & Cyanide & $<10$ & $U$ & $<10$ & $U$ & & $<10$ & $\mathrm{U}$ & & & & & $u g / L$ & \\
\hline & Lead, total recoverable & $<10$ & $U$ & $<100$ & $\mathrm{U}$ & & $<2.4$ & U V & & & & & $\mathrm{ug} / \mathrm{L}$ & \\
\hline & Nickel, total recoverable & $<50$ & $U$ & $<50$ & $U$ & & $<50$ & $\mathrm{U}$ & & & & & $\mathrm{ug} / \mathrm{L}$ & \\
\hline & Selenium, total recoverable & $<10$ & U & $<200$ & U & & $<5$ & $u$ & & & & & $\mathrm{ug} / \mathrm{L}$ & \\
\hline \multicolumn{15}{|c|}{ Organics } \\
\hline & Chlorobenzene & $<5$ & $u$ & $<5$ & JU & $Q$ & $<1$ & $u$ & $<1$ & $u$ & $<\mathrm{EQL}$ & 1 & $\mathrm{ug} / \mathrm{L}$ & $M L$ \\
\hline & 1,1-Dichloroethane & $<5$ & $\cup$ & $<5$ & JU & $\widehat{Q}$ & $<1$ & $\mathrm{U}$ & $<1$ & $U$ & $<\mathrm{EQL}$ & 1 & $\mathrm{ug} / \mathrm{L}$ & ML \\
\hline & 1,1-Dichloroethylene & $<5$ & $\cup$ & $<5$ & JU & $\bar{Q}$ & $<1$ & $u$ & $<1$ & $\cup$ & $<$ EQL & 1 & $\mathrm{ug} / \mathrm{L}$ & ML \\
\hline & trans-1,2-Dichloroethylene & $<5$ & $\cup$ & $<5$ & JU & $Q$ & $<1$ & $U$ & $<1$ & $U$ & $<\mathrm{EQL}$ & 1 & $\mathrm{ug} / \mathrm{L}$ & ML \\
\hline & & & & & & & & & & & & & & \\
\hline & $\begin{array}{l}\text { PCB } 1221 \\
\text { PCB } 1232\end{array}$ & & & & & & & & & & & & & \\
\hline & $\begin{array}{l}\text { PCB } 1232 \\
\text { PCB } 1242\end{array}$ & & & & & & & & & & & & & \\
\hline & PCB 1248 & & & & & & & & & & & & & \\
\hline & PCB 1254 & & & & & & & & & & & & & \\
\hline & PCB 1260 & & & & & & & & & & & & & \\
\hline & 1,1,2,2-Tetrachloroethane & $<5$ & $\mathrm{U}$ & $<5$ & JU & $Q$ & $<1$ & $U$ & $<1$ & $U$ & $<E Q L$ & 1 & $\mathrm{ug} / \mathrm{L}$ & ML \\
\hline & Tetrachloroethylene & $<5$ & U & $<5$ & JU & $\bar{Q}$ & $<1$ & $U$ & $<1$ & $U$ & $<E Q L$ & 1 & $\mathrm{ug} / \mathrm{L}$ & ML \\
\hline & $1,1,1$-Trichloroethane & $<5$ & $\mathrm{U}$ & $<5$ & JU & $Q$ & $<1$ & $U$ & $<1$ & $\mathrm{U}$ & $<\mathrm{EQL}$ & 1 & $\mathrm{ug} / \mathrm{L}$ & ML \\
\hline & Trichloroethylene & $<5$ & $\mathrm{U}$ & $<5$ & JU & $\mathbf{Q}$ & $<1$ & U & $<1$ & $\mathrm{U}$ & $<E Q L$ & 1 & $\mathrm{ug} / \mathrm{L}$ & $\mathrm{ML}$ \\
\hline \multicolumn{15}{|c|}{ II. Monitoring Constituents } \\
\hline$\underline{\mathbf{S T}}$ & Parameter & $\underline{3096}$ & CLP EPA & 3097 & CLP & EPA & $\underline{3 Q 98}$ & CLPEPA & $\underline{3099}$ & CLPEPA & Filt. & DF & $\underline{\text { Unit }}$ & La \\
\hline \multicolumn{15}{|c|}{ Inorganics } \\
\hline & Aluminum, total recoverable & $<37.7$ & $U$ & 31.6 & & & $<200$ & $\mathrm{U}$ & $<200$ & $\mathrm{U}$ & $<E Q L$ & 1 & $\mathrm{ug} / \mathrm{L}$ & EX \\
\hline & Nitrate-nitrite as nitrogen & 560 & & 190 & & & 204 & & 198 & J 1 & NDD & 5 & $\mathrm{ug} / \mathrm{L}$ & EX \\
\hline & Sodium, total recoverable & 1570 & & 1510 & & & $<1630$ & $\mathrm{U}$ & 1700 & & $<4600$ & 1 & $\mathrm{ug} / \mathrm{L}$ & EX \\
\hline & Sulfate & 1120 & & 1150 & & & 838 & & 2340 & & $<3000$ & 1 & $\mathrm{ug} / \mathrm{L}$ & EX \\
\hline \multicolumn{15}{|c|}{ Radionuclides } \\
\hline & Gross alpha & .579 & UI & -.3 & $\mathrm{U}$ & & 1.43 & & .732 & $U$ & $<\mathrm{EQL}$ & 1 & $\mathrm{pCi} / \mathrm{L}$ & GP \\
\hline & Nonvolatile beta & .712 & $\mathrm{UI}$ & -.49 & UIJ & & .63 & UI & .393 & $\mathrm{U}$ & $<E Q L$ & 1 & $\mathrm{pCi} / \mathrm{L}$ & GP \\
\hline & Radium, total alpha-emitting & -.1 & UI & -.62 & UI & & .03 & UI & 0 & $\mathrm{U}$ & $<\overline{E Q L}$ & 1 & $\mathrm{pCi} / \mathrm{L}$ & GP \\
\hline
\end{tabular}

Notes: Concentrations in bold italics exceed the groundwater protection or monitoring constituent standards listed in Appendix A. Synchronous water levels are measured over a 3-5 day period or less. Dilution factors, Laboratory, and Filtered Data are for Groundwater Protection Standards First Quarter 2000 and Monitoring Constituents Third Quarter 1999 data only.

$+=$ exceeded the Groundwater Protection Standards (First Quarter 2000) or Monitoring Constituents (Third Quarter 1999) listed in Appendix A. 
Table D-4. Groundwater Monitoring Results for Plume Definition Wells, M-Area HWMF (Cont.) WELL MSB 28A

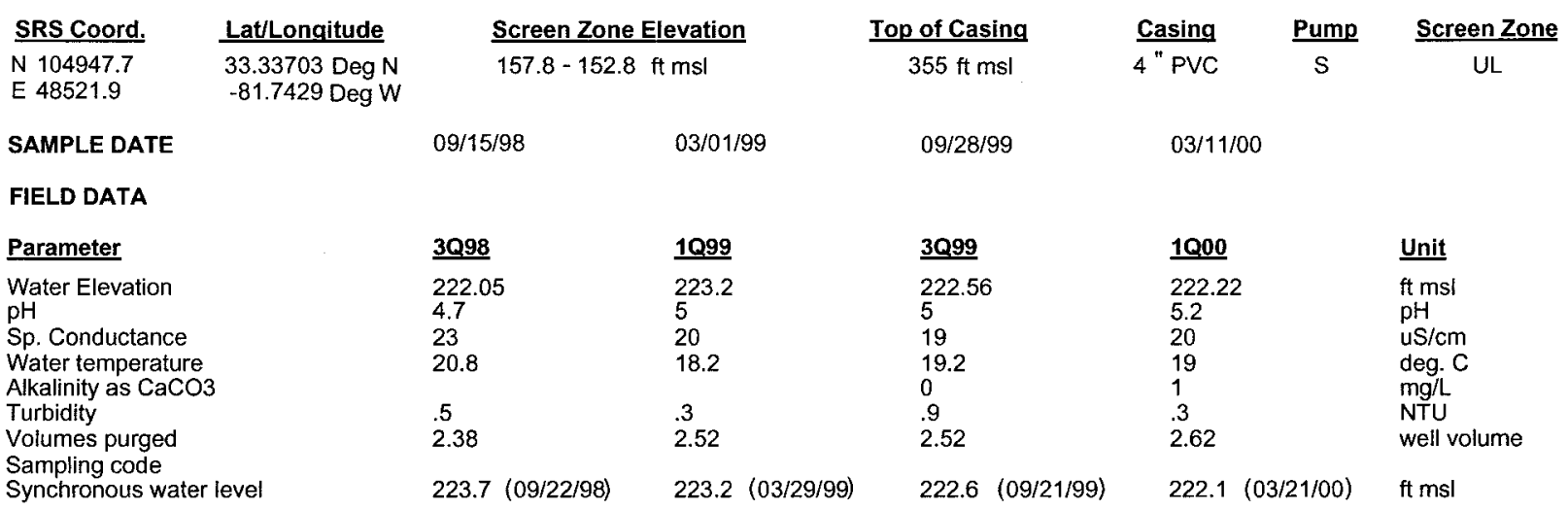

ANALYTICAL DATA

I. Groundwater Protection Standard

261 Appendix VIII/264 Appendix IX Hazardous Constituents

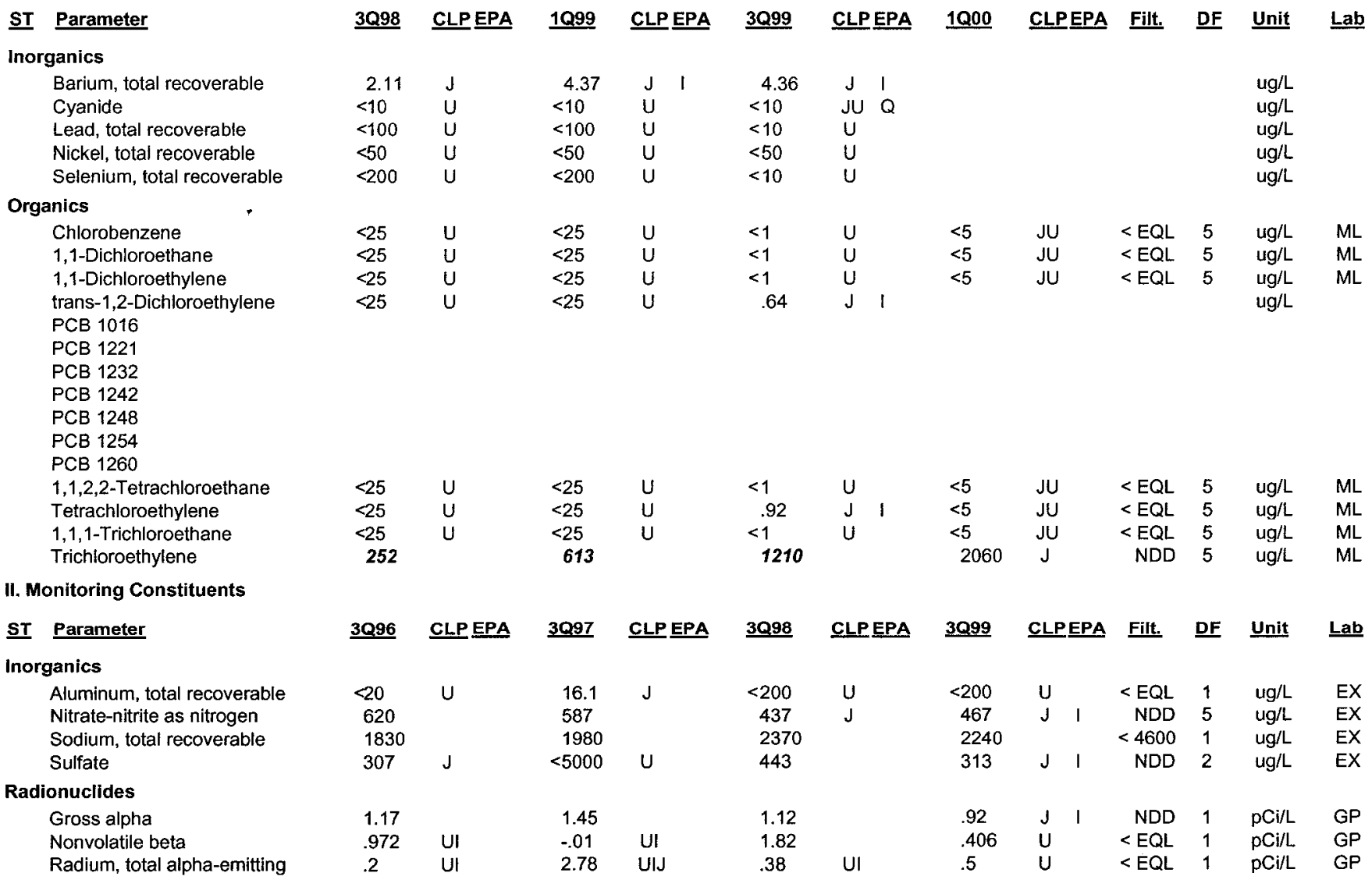

Notes: Concentrations in bold italics exceed the groundwater protection or monitoring constituent standards listed in Appendix A. Synchronous water levels are measured over a 3-5 day period or less. Dilution factors, Laboratory, and Filtered Data are for Groundwater Protection Standards First Quarter 2000 and Monitoring Constituents Third Quarter 1999 data only.

$+=$ exceeded the Groundwater Protection Standards (First Quarter 2000) or Monitoring Constituents (Third Quarter 1999) listed in Appendix A. 
Table D-4. Groundwater Monitoring Results for Plume Definition Wells, M-Area HWMF (Cont.) WELL MSB 29A

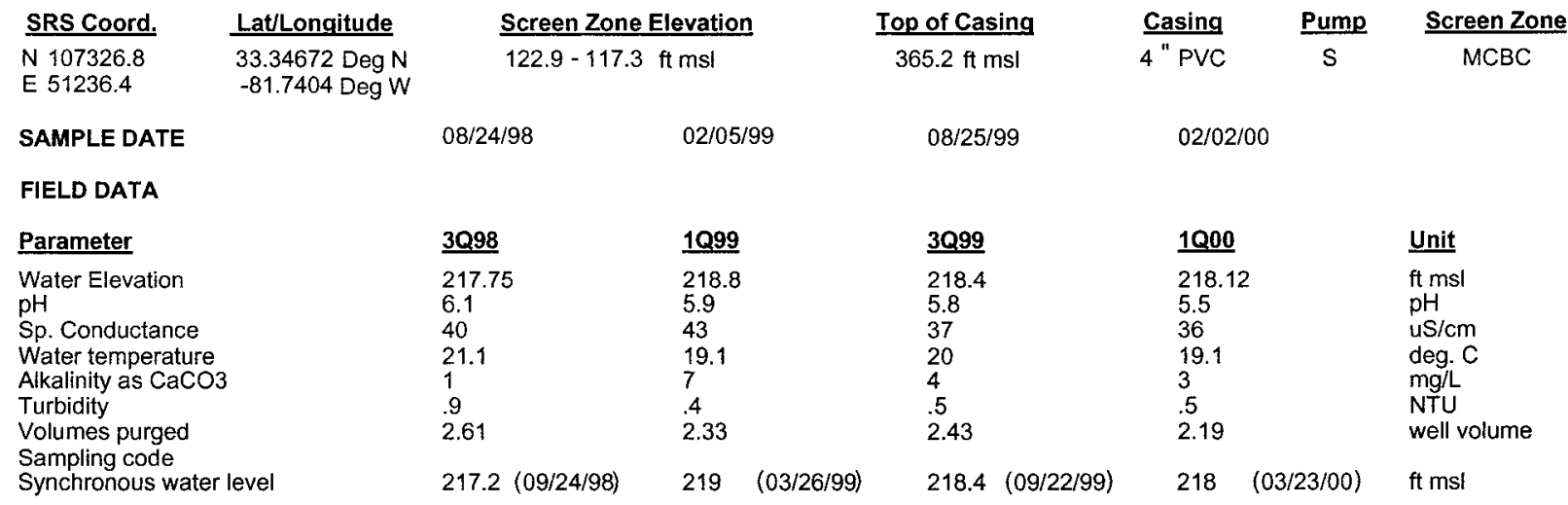

ANALYTICAL DATA

I. Groundwater Protection Standard

261 Appendix VIII/264 Appendix IX Hazardous Constituents

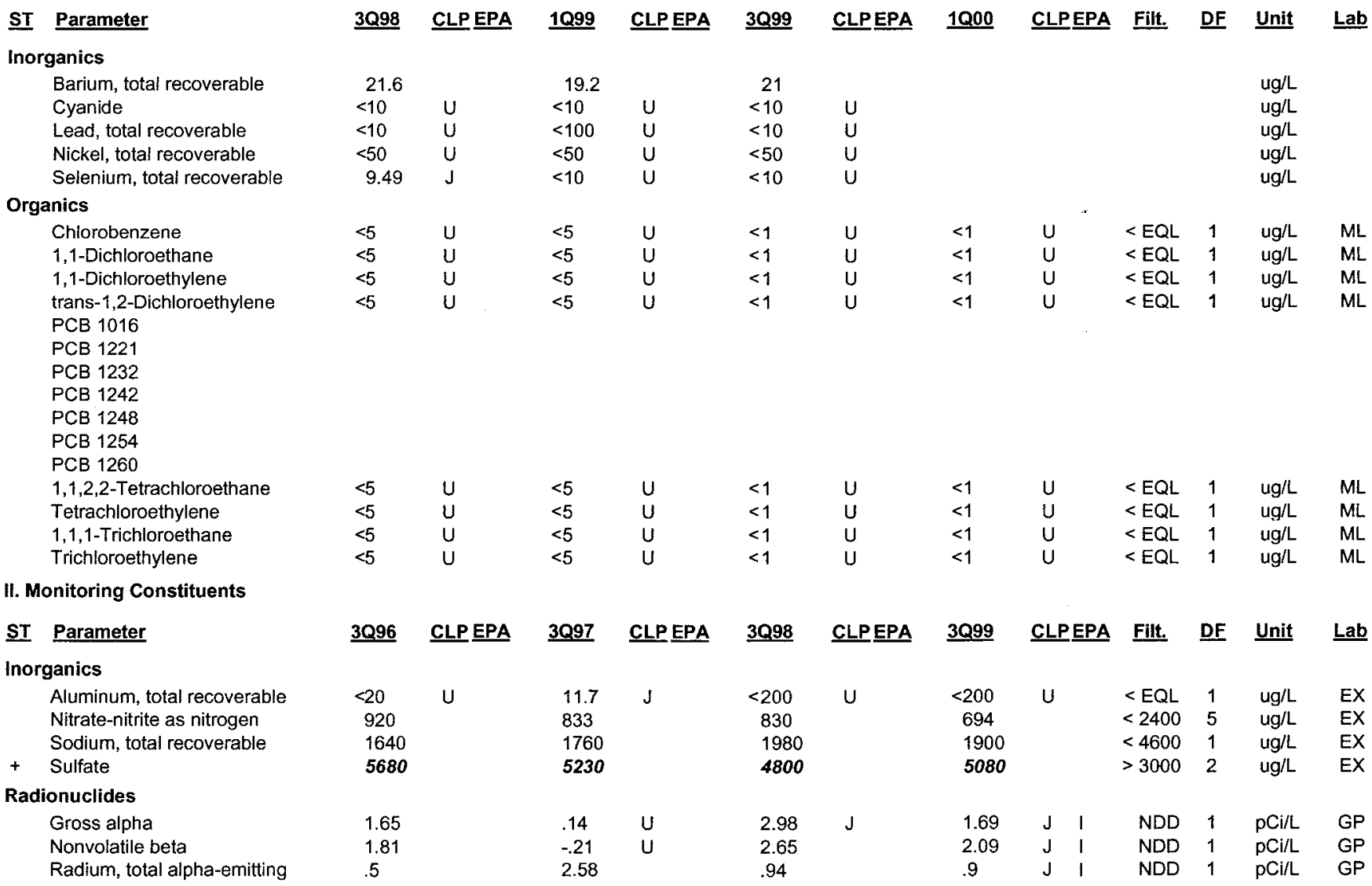

Notes: Concentrations in bold italics exceed the groundwater protection or monitoring constituent standards listed in Appendix A. Synchronous water levels are measured over a 3-5 day period or less. Dilution factors, Laboratory, and Filtered Data are for Groundwater Protection Standards First Quarter 2000 and Monitoring Constituents Third Quarter 1999 data only.

$+=$ exceeded the Groundwater Protection Standards (First Quarter 2000) or Monitoring Constituents (Third Quarter 1999) listed in Appendix A. 
Table D-4. Groundwater Monitoring Results for Plume Definition Wells, M-Area HWMF (Cont.) WELL MSB 29TA

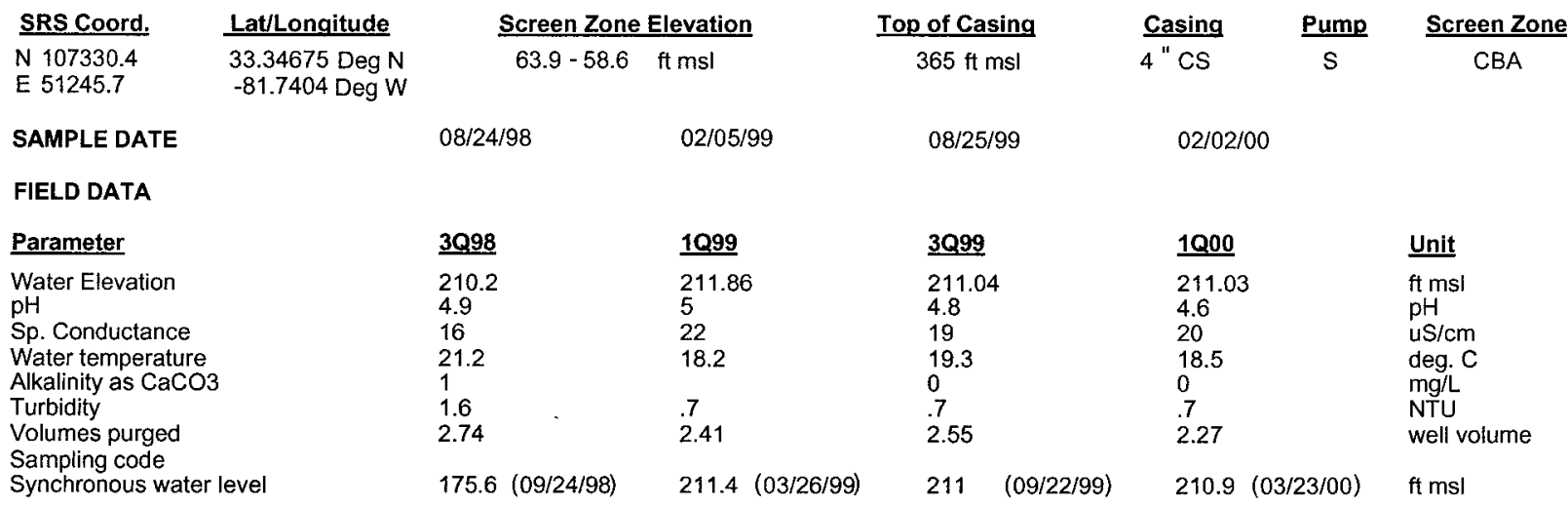

ANALYTICAL DATA

I. Groundwater Protection Standard

261 Appendix VIII/264 Appendix IX Hazardous Constituents

\begin{tabular}{|c|c|c|c|c|c|c|c|c|c|c|c|c|c|}
\hline ST & Parameter & $\underline{3 Q 98}$ & CLP EPA & 1099 & CLP EPA & $\underline{3 Q 99}$ & $\underline{\text { CLPEPA }}$ & 1000 & $\underline{\text { CLPEPA }}$ & Filt. & $\underline{\text { DF }}$ & $\underline{\text { Unit }}$ & Lab \\
\hline \multicolumn{14}{|c|}{ Inorganics } \\
\hline & Barium, total recoverable & 3.67 & $\mathrm{~J}$ & 3.2 & & 3.4 & J 1 & & & & & $\mathrm{ug} / \mathrm{L}$ & \\
\hline & Cyanide & $<10$ & U & $<15.2$ & $\mathrm{U}$ & $<10$ & $\mathrm{U}$ & & & & & $\mathrm{ug} / \mathrm{L}$ & \\
\hline & Lead, total recoverable & $<10$ & $\mathrm{U}$ & $<100$ & $\mathrm{U}$ & 4.9 & $\mathrm{~J} \quad \mathrm{I}$ & & & & & $\mathrm{ug} / \mathrm{L}$ & \\
\hline & Nickel, total recoverable & $<50$ & $\mathrm{U}$ & $<50$ & $\mathrm{U}$ & $<50$ & U & & & & & $\mathrm{ug} / \mathrm{L}$ & \\
\hline & Selenium, total recoverable & 4.02 & $\downarrow$ & $<66$ & U & $<10$ & U & & & & & $\mathrm{ug} / \mathrm{L}$ & \\
\hline \multicolumn{14}{|c|}{ Organics } \\
\hline & Chlorobenzene & $<5$ & $\mathrm{U}$ & $<5$ & $\mathrm{U}$ & $<1$ & $\mathrm{U}$ & $<1$ & U & $<E Q L$ & 1 & $u g / L$ & ML \\
\hline & 1,1-Dichloroethane & $<5$ & $u$ & $<5$ & $\mathrm{U}$ & $<1$ & $\mathrm{U}$ & $<1$ & $u$ & $<$ EQL & 1 & $\mathrm{ug} / \mathrm{L}$ & ML \\
\hline & 1,1-Dichloroethylene & $<5$ & $\mathrm{U}$ & $<5$ & $\mathrm{U}$ & $<1$ & $\mathrm{U}$ & $<1$ & $\mathrm{U}$ & $<E Q L$ & 1 & $\mathrm{ug} / \mathrm{L}$ & ML \\
\hline & trans-1,2-Dichloroethylene & $<5$ & $U$ & $<5$ & $U$ & $<1$ & $U$ & $<1$ & $U$ & $<E Q L$ & 1 & $\mathrm{ug} / \mathrm{L}$ & ML \\
\hline & PCB 1016 & & & & & & & & & & & & \\
\hline & PCB 1221 & & & & & & & & & & & & \\
\hline & PCB 1232 & & & & & & & & & & & & \\
\hline & PCB 1242 & & & & & & & & & & & & \\
\hline & PCB 1248 & & & & & & & & & & & & \\
\hline & РСВ 1254 & & & & & & & & & & & & \\
\hline & PCB 1260 & & & & & & & & & & & & \\
\hline & $1,1,2,2$-Tetrachloroethane & $<5$ & $\mathrm{U}$ & $<5$ & U & $<1$ & $\mathrm{U}$ & $<1$ & $u$ & $<\mathrm{EQL}$ & 1 & $\mathrm{ug} / \mathrm{L}$ & ML \\
\hline & Tetrachloroethylene & $<5$ & $\mathrm{U}$ & $<5$ & $\mathrm{U}$ & $<.61$ & $\mathrm{U} V$ & $<1$ & U & $<$ EQL & 1 & $\mathrm{ug} / \mathrm{L}$ & ML \\
\hline & 1,1,1-Trichloroethane & $<5$ & $\mathrm{U}$ & $<5$ & $U$ & $<1$ & $U$ & $<1$ & $\mathrm{U}$ & $<E Q L$ & 1 & $\mathrm{ug} / \mathrm{L}$ & ML \\
\hline & Trichloroethylene & 3.59 & $J$ & 4.15 & $\mathrm{~J} \quad \mathrm{I}$ & 7.2 & $\mathrm{~J} K$ & 9.06 & $\mathrm{~J} K$ & NDD & 1 & $\operatorname{lig} / \mathrm{L}$ & $\mathrm{ML}$ \\
\hline \multicolumn{14}{|c|}{ II. Monitoring Constituents } \\
\hline$\underline{\mathbf{S T}}$ & Parameter & $\underline{3096}$ & CLPEPA & $\underline{3097}$ & CLP EPA & $\underline{3098}$ & CLPEPA & $\underline{3099}$ & CLPEPA & Filt. & $\underline{D F}$ & Unit & $\underline{\text { Lab }}$ \\
\hline \multicolumn{14}{|c|}{ Inorganics } \\
\hline & Aluminum, total recoverable & $<20$ & $\mathrm{U}$ & $<20$ & U & $<200$ & $\mathrm{U}$ & $<200$ & $\mathrm{U}$ & $<E Q L$ & 1 & $u g / L$ & EX \\
\hline & Nitrate-nitrite as nitrogen & 1050 & & 742 & & 618 & & 680 & & $<2400$ & 5 & $\mathrm{ug} / \mathrm{L}$ & EX \\
\hline & Sodium, total recoverable & 1480 & & 1660 & & 1930 & & 1500 & & $<4600$ & 1 & $\mathrm{ug} / \mathrm{L}$ & EX \\
\hline & Sulfate & 789 & $\mathrm{~J}$ & 892 & $\mathrm{~J}$ & 931 & & 785 & & $<3000$ & 2 & ug/L. & EX \\
\hline \multicolumn{14}{|c|}{ Radionuclides } \\
\hline & Gross alpha & .396 & UI & -.26 & $\mathrm{U}$ & .01 & UIJ & .428 & $\mathrm{u}$ & $<E Q L$ & 1 & $\mathrm{pCi} / \mathrm{L}$ & $G P$ \\
\hline & Nonvolatile beta & .892 & UI & -3 & $\mathrm{U}$ & -.36 & Ui & .273 & $\mathrm{U}$ & $<\mathrm{EQL}$ & 1 & $\mathrm{pCi} / \mathrm{L}$ & GP \\
\hline & Radium, total alpha-emitting & .3 & & 1.59 & & .44 & & .1 & $\mathrm{U}$ & $<\mathrm{EQL}$ & 1 & $\mathrm{pCi} / \mathrm{L}$ & GP \\
\hline
\end{tabular}

Notes: Concentrations in bold italics exceed the groundwater protection or monitoring constituent standards Iisted in Appendix A. Synchronous water levels are measured over a 3-5 day period or less. Dilution factors, Laboratory, and Filtered Data are for Groundwater Protection Standards First Quarter 2000 and Monitoring Constituents Third Quarter 1999 data only.

+ = exceeded the Groundwater Protection Standards (First Quarter 2000) or Monitoring Constituents (Third Quarter 1999) listed in Appendix A. 
Table D-4. Groundwater Monitoring Results for Plume Definition Wells, M-Area HWMF (Cont.) WELL MSB 30AA

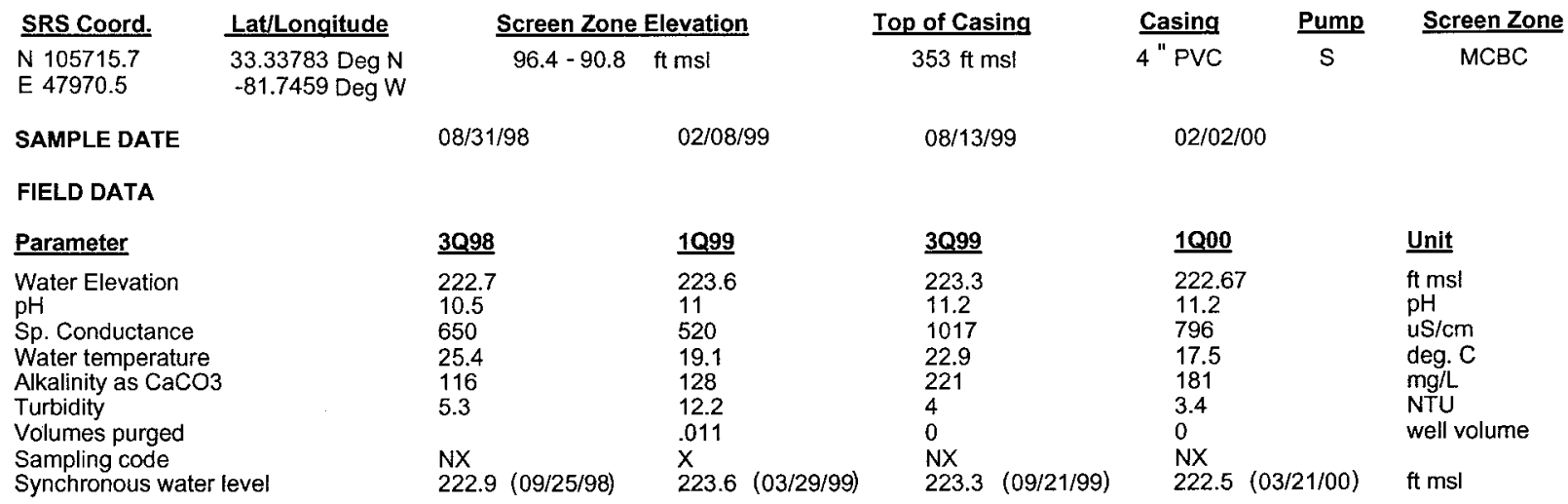

ANALYTICAL DATA

I. Groundwater Protection Standard

261 Appendix VIII/264 Appendix IX Hazardous Constituents

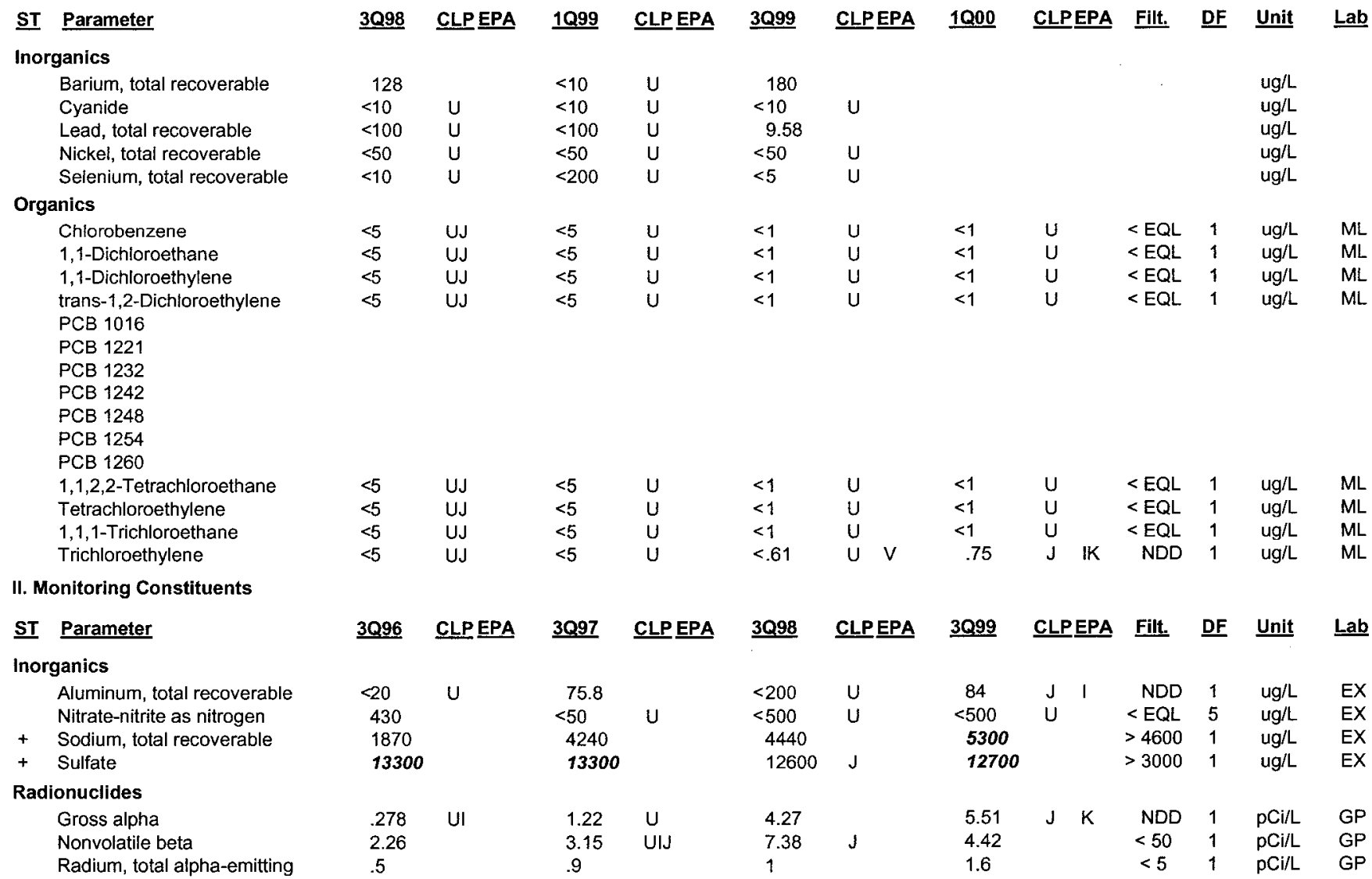

Notes: Concentrations in bold italics exceed the groundwater protection or monitoring constituent standards listed in Appendix A. Synchronous water levels are measured over a 3-5 day period or less. Dilution factors, Laboratory, and Filtered Data are for Groundwater Protection Standards First Quarter 2000 and Monitoring Constituents Third Quarter 1999 data only.

+= exceeded the Groundwater Protection Standards (First Quarter 2000) or Monitoring Constituents (Third Quarter 1999) listed in Appendix A. 
Table D-4. Groundwater Monitoring Results for Plume Definition Wells, M-Area HWMF (Cont.) WELL MSB 30B

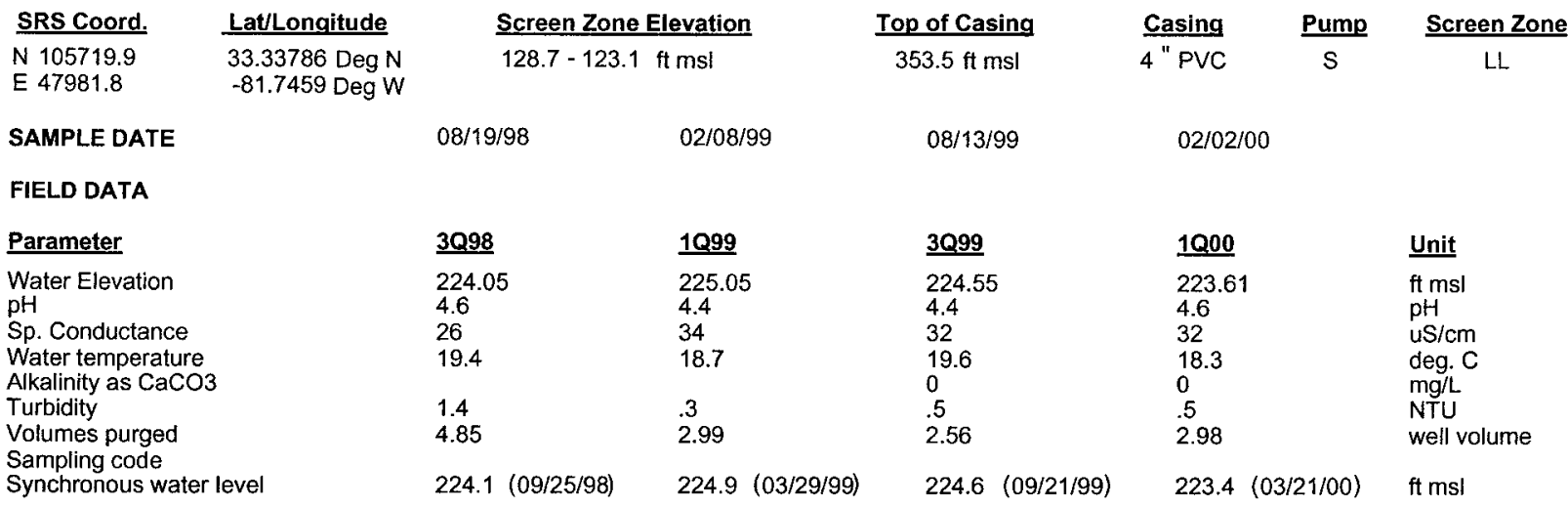

ANALYTICAL DATA

I. Groundwater Protection Standard

261 Appendix VIII/264 Appendix IX Hazardous Constituents

\begin{tabular}{|c|c|c|c|c|c|c|c|c|c|c|c|c|c|}
\hline ST & Parameter & $\underline{3 Q 98}$ & CLPEPA & 1099 & CLP EPA & $\underline{3099}$ & CLPEPA & 1000 & CLPEPA & Filt. & $\underline{\text { DF }}$ & Unit & Lab \\
\hline \multicolumn{14}{|c|}{ Inorganics } \\
\hline & Barium, total recoverable & 9.25 & $J$ & $<10$ & $U$ & 8.2 & J 1 & & & & & $\mathrm{ug} / \mathrm{L}$ & \\
\hline & Cyanide & $<10$ & $\mathrm{u}$ & $<10$ & $\mathrm{U}$ & $<10$ & $\mathrm{U}$ & & & & & $\mathrm{ug} / \mathrm{L}$ & \\
\hline & Lead, total recoverable & $<10$ & $\mathrm{U}$ & $<100$ & $\mathrm{U}$ & $<2.95$ & U V & & & & & $\mathrm{ug} / \mathrm{L}$ & \\
\hline & Nickel, total recoverable & $<11.5$ & $\mathrm{U}$ & $<50$ & $\mathrm{U}$ & $<50$ & $\mathrm{U}$ & & & & & $\mathrm{ug} / \mathrm{L}$ & \\
\hline & Selenium, total recoverable & $<10$ & $\mathrm{U}$ & $<200$ & $\mathrm{U}$ & $<5$ & $\mathrm{U}$ & & & & & $\mathrm{ug} / \mathrm{L}$ & \\
\hline \multicolumn{14}{|c|}{ Organics } \\
\hline & Chlorobenzene & $<5$ & $\mathrm{U}$ & $<5$ & $\mathrm{U}$ & $<1$ & $\mathrm{U}$ & $<1$ & $U$ & $<\mathrm{EQL}$ & 1 & $\mathrm{ug} / \mathrm{L}$ & $M L$ \\
\hline & 1,1-Dichloroethane & $<5$ & $\mathrm{u}$ & $<5$ & $\mathrm{U}$ & $<1$ & $\mathrm{U}$ & $<1$ & $\mathrm{U}$ & $<E Q L$ & 1 & $\mathrm{ug} / \mathrm{L}$ & $M L$ \\
\hline & 1,1-Dichloroethylene & $<5$ & $\mathrm{U}$ & $<5$ & $\mathbf{U}$ & $<1$ & $\mathrm{U}$ & $<1$ & $\mathrm{U}$ & $<\mathrm{EQL}$ & 1 & $\mathrm{ug} / \mathrm{L}$ & $M L$ \\
\hline & $\begin{array}{l}\text { trans-1,2-Dichloroethylene } \\
\text { PCB } 1016\end{array}$ & $<5$ & $\mathrm{U}$ & $<5$ & $\mathrm{U}$ & $<1$ & $u$ & $<1$ & $U$ & $<$ EQL & 1 & $\mathrm{ug} / \mathrm{L}$ & $M L$ \\
\hline & PCB 1221 & & & & & & & & & & & & \\
\hline & PCB 1232 & & & & & & & & & & & & \\
\hline & PCB 1242 & & & & & & & & & & & & \\
\hline & PCB 1248 & & & & & & & & & & & & \\
\hline & PCB 1254 & & & & & & & & & & & & \\
\hline & $\mathrm{PCB} 1260$ & & & & & & & & & & & & \\
\hline & $1,1,2,2$-Tetrachloroethane & $<5$ & $\mathrm{U}$ & $<5$ & $U$ & $<1$ & $u$ & $<1$ & $U$ & $<E Q L$ & 1 & ug/L & ML \\
\hline & Tetrachloroethylene & $<5$ & $U$ & $<5$ & $\mathrm{U}$ & $<1$ & $\mathrm{U}$ & $<1$ & $\mathrm{U}$ & $<\mathrm{EQL}$ & 1 & $u g / L$ & ML \\
\hline & 1,1,1-Trichloroethane & $<5$ & $\mathrm{U}$ & $<5$ & U & $<1$ & $\mathrm{U}$ & $<1$ & $\mathrm{U}$ & $<$ EQL & 1 & $\mathrm{ug} / \mathrm{L}$ & $M L$ \\
\hline & Trichloroethylene & $<5$ & $\mathrm{u}$ & $<5$ & $\mathrm{U}$ & $<1$ & $\mathrm{U}$ & $<1$ & $u$ & $<E Q L$ & 1 & $\mathrm{ug} / \mathrm{L}$ & ML \\
\hline \multicolumn{14}{|c|}{ II. Monitoring Constituents } \\
\hline$\underline{\mathbf{S T}}$ & Parameter & 3Q996 & CLPEPA & 3097 & CLPEPA & $\underline{3098}$ & CLPPEPA & $\underline{3099}$ & CLPEPA & Filt. & $\underline{\text { DF }}$ & $\underline{\text { Unit }}$ & $\underline{\text { Lab }}$ \\
\hline \multicolumn{14}{|c|}{ Inorganics } \\
\hline & Aluminum, total recoverable & 42.5 & & 74.5 & & $<200$ & $U$ & $<200$ & $\mathrm{U}$ & $<E Q L$ & 1 & $\mathrm{ug} / \mathrm{L}$ & EX \\
\hline & Nitrate-nitrite as nitrogen & 530 & & 86 & & $<100$ & $U$ & $<500$ & $U$ & $<\mathrm{EQL}$ & 5 & $\mathrm{ug} / \mathrm{L}$ & EX \\
\hline & Sodium, total recoverable & 1490 & & 1530 & & 1770 & & 1600 & & $<4600$ & 1 & $\mathrm{ug} / \mathrm{L}$ & $\mathrm{EX}$ \\
\hline+ & Sulfate & 4790 & & 4910 & J & 5280 & & 5710 & & $>3000$ & 1 & $\mathrm{ug} / \mathrm{L}$ & EX \\
\hline \multicolumn{14}{|c|}{ Radionuclides } \\
\hline & Gross alpha & .805 & & .26 & UI & .38 & UIJ & .198 & $\mathbf{U}$ & $<E Q L$ & 1 & $\mathrm{pCi} / \mathrm{L}$ & GP \\
\hline & Nonvolatile beta & 1.55 & & -.09 & UI & & & .609 & $\mathbf{U}$ & $<\mathrm{EQL}$ & 1 & $\mathrm{pCi} / \mathrm{L}$ & GP \\
\hline & Radium, total alpha-emitting & .9 & & .86 & UI & .46 & & 1.1 & J $\quad I$ & NDD & 1 & $\mathrm{pCi} / \mathrm{L}$ & GP \\
\hline
\end{tabular}

Notes: Concentrations in bold italics exceed the groundwater protection or monitoring constituent standards listed in Appendix A. Synchronous water levels are measured over a 3-5 day period or less. Dilution factors, Laboratory, and Filtered Data are for Groundwater Protection Standards First Quarter 2000 and Monitoring Constituents Third Quarter 1999 data only.

+= exceeded the Groundwater Protection Standards (First Quarter 2000) or Monitoring Constituents (Third Quarter 1999) listed in Appendix A. 
Table D-4. Groundwater Monitoring Results for Plume Definition Wells, M-Area HWMF (Cont.) WELL MSB 30CC

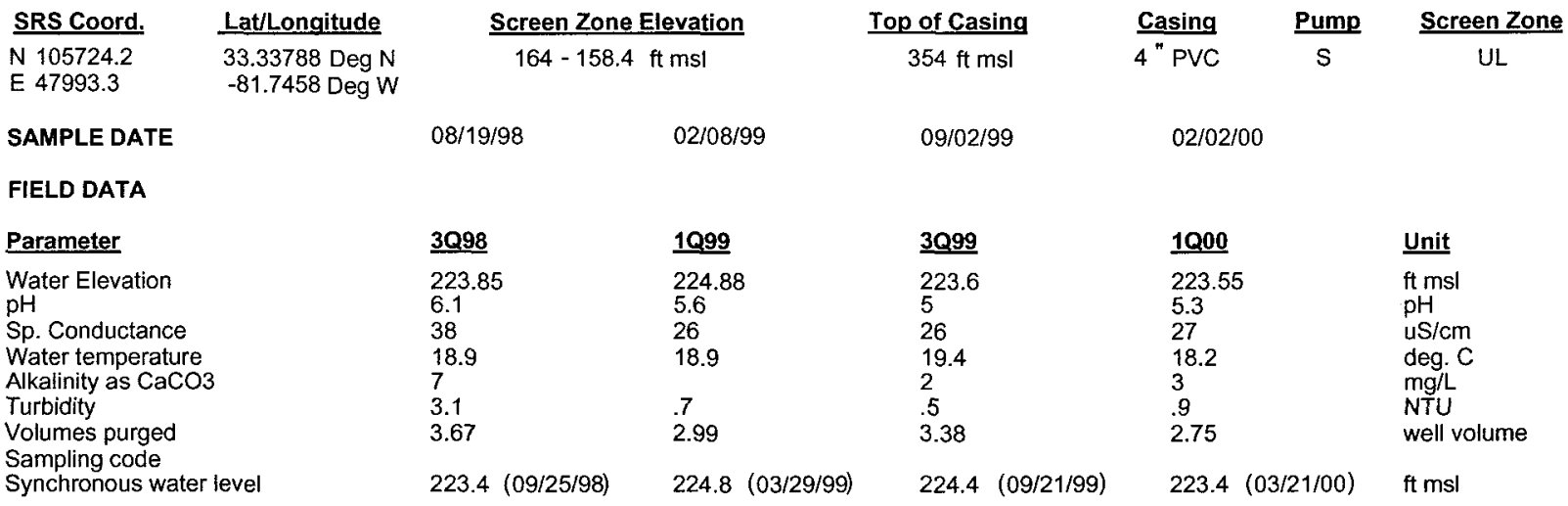

ANALYTICAL DATA

I. Groundwater Protection Standard

261 Appendix VIII/264 Appendix IX Hazardous Constituents

\begin{tabular}{|c|c|c|c|c|c|c|c|c|c|c|c|c|c|}
\hline$\underline{\text { ST }}$ & Parameter & 3098 & CLPEEPA & 1099 & CLP EPA & 3099 & CLP EPA & 1000 & CLPEPA & Filt. & DF & Unit & Lab \\
\hline Inor & ganics & & & & & & & & & & & & \\
\hline & Barium, total recoverable & 27.6 & & $<10$ & $U$ & 15 & & & & & & $\mathrm{ug} / \mathrm{L}$ & \\
\hline & Cyanide & $<10$ & $U$ & $<10$ & $\mathrm{U}$ & $<10$ & $U$ & & & & & $\mathrm{ug} / \mathrm{L}$ & \\
\hline & Lead, total recoverable & $<10$ & $\mathrm{U}$ & $<100$ & $\mathrm{U}$ & $<1.47$ & U V & & & & & $\mathrm{ug} / \mathrm{L}$ & \\
\hline & Nickel, total recoverable & $<10.5$ & $\mathrm{U}$ & $<50$ & $\mathrm{U}$ & $<11$ & JU 1 & & & & & ug/L & \\
\hline & Selenium, total recoverable & $<10$ & $U$ & $<200$ & $\mathrm{U}$ & $<5$ & $\mathrm{U}$ & & & & & $\mathrm{ug} / \mathrm{L}$ & \\
\hline Org & anics & & & & & & & & & & & & \\
\hline & Chlorobenzene & $<5$ & $\mathrm{U}$ & $<5$ & U & $<1$ & $\mathrm{U}$ & $<1$ & JU L & $<\mathrm{EQL}$ & 1 & $\mathrm{ug} / \mathrm{L}$ & ML \\
\hline & 1,1-Dichloroethane & $<5$ & $U$ & $<5$ & U & $<1$ & $U$ & $<1$ & JU L & $<E Q L$ & 1 & $\mathrm{ug} / \mathrm{L}$ & $M L$ \\
\hline & 1,1-Dichloroethylene & $<5$ & $\mathrm{U}$ & $<5$ & U & $<1$ & $U$ & $<1$ & JU L & $<\mathrm{EQL}$ & 1 & $\mathrm{ug} / \mathrm{L}$ & $\mathrm{ML}$ \\
\hline & trans-1,2-Dichloroethylene & $<5$ & $U$ & $<5$ & U & $<1$ & U & $<1$ & JU L & $<\mathrm{EQL}$ & 1 & $\mathrm{ug} / \mathrm{L}$ & ML \\
\hline & PCB 1016 & & & & & & & & & & & & \\
\hline & PCB 1221 & & & & & & & & & & & & \\
\hline & PCB 1232 & & & & & & & & & & & & \\
\hline & PCB 1242 & & & & & & & & & & & & \\
\hline & PCB 1248 & & & & & & & & & & & & \\
\hline & PCB 1254 & & & & & & & & & & & & \\
\hline & PCB 1260 & & & & & & & & & & & & \\
\hline & 1,1,2,2-Tetrachloroethane & $<5$ & $u$ & $<5$ & $\mathrm{U}$ & $<1$ & U & $<1$ & JU $\mathbf{L}$ & $<$ EQL & 1 & $\mathrm{ug} / \mathrm{L}$ & $\mathrm{ML}$ \\
\hline & Tetrachloroethylene & $<5$ & $U$ & $<5$ & $\mathrm{U}$ & $<1$ & $U$ & $<1$ & JU L & $<E Q L$ & 1 & $\mathrm{ug} / \mathrm{L}$ & $M L$ \\
\hline & $1,1,1$-Trichloroethane & $<5$ & U & $<5$ & $\mathrm{U}$ & $<1$ & $\mathrm{U}$ & $<1$ & JU L & $<E Q L$ & 1 & $\mathrm{ug} / \mathrm{L}$ & ML. \\
\hline & Trichloroethylene & $<5$ & $U$ & $<5$ & $\mathrm{U}$ & $<1$ & $\mathrm{U}$ & $<1$ & JU L & $<E Q L$ & 1 & ug/L & ML \\
\hline II. $\mathrm{M}$ & onitoring Constituents & & & & & & & & & & & & \\
\hline$\underline{\mathbf{S T}}$ & Parameter & $\underline{3 Q 96}$ & $\underline{\text { CLP EPA }}$ & $\underline{3 Q 97}$ & CLP EPA & $\underline{3 Q 98}$ & CLPEPA & $\underline{3099}$ & CLPEPA & Filt. & $\underline{\text { DF }}$ & $\underline{\text { Unit }}$ & Lab \\
\hline Inor & ganics & & & & & & & & & & & & \\
\hline & Aluminum, total recoverable & $<20$ & $U$ & $<20$ & U & $<200$ & $U$ & $<200$ & $U$ & $<E Q L$ & 1 & ug/L & EX \\
\hline & Nitrate-nitrite as nitrogen & 1400 & & 1290 & & 1050 & & 1030 & & $<2400$ & 5 & ug/L & EX \\
\hline & Sodium, total recoverable & 1670 & & 1830 & & 1910 & & 1900 & & $<4600$ & 1 & $\mathrm{ug} / \mathrm{L}$ & EX \\
\hline & Sulfate & 941 & J & $<5000$ & $U$ & $<200$ & $\mathrm{U}$ & 949 & & $<3000$ & 1 & $u g / L$ & EX \\
\hline Rad & ionuclides & & & & & & & & & & & & \\
\hline & Gross a!pha & .672 & UI & -.03 & UIJ & .93 & UIJ & .763 & $U$ & $<E Q L$ & 1 & $\mathrm{pCi} / \mathrm{L}$ & GP \\
\hline & Nonvolatile beta & .455 & UI & -2.41 & UIJ & & & .186 & $\mathrm{U}$ & $<E Q L$ & 1 & $\mathrm{pCi} / \mathrm{L}$ & GP \\
\hline & Radium, total alpha-emitting & 0 & U! & .6 & UI & .27 & UI & .2 & $U$ & $<\mathrm{EQL}$ & 1 & $\mathrm{pCi} / \mathrm{L}$ & $\mathrm{GP}$ \\
\hline
\end{tabular}

Notes: Concentrations in bold italics exceed the groundwater protection or monitoring constituent standards listed in Appendix A. Synchronous water levels are measured over a 3-5 day period or less. Dilution factors, Laboratory, and Filtered Data are for Groundwater Protection Standards First Quarter 2000 and Monitoring Constituents Third Quarter 1999 data only.

+ = exceeded the Groundwater Protection Standards (First Quarter 2000) or Monitoring Constituents (Third Quarter 1999) listed in Appendix A. 
Table D-4. Groundwater Monitoring Results for Plume Definition Wells, M-Area HWMF (Cont.) WELL MSB 31B

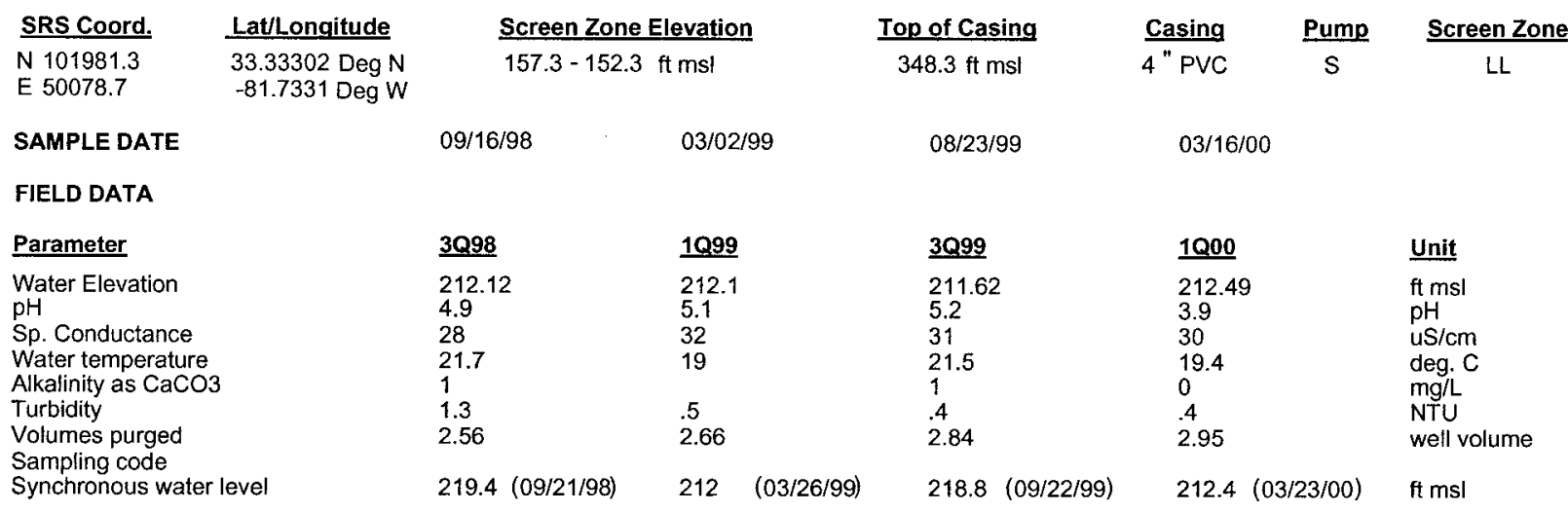

ANALYTICAL DATA

I. Groundwater Protection Standard

261 Appendix VIII/264 Appendix IX Hazardous Constituents

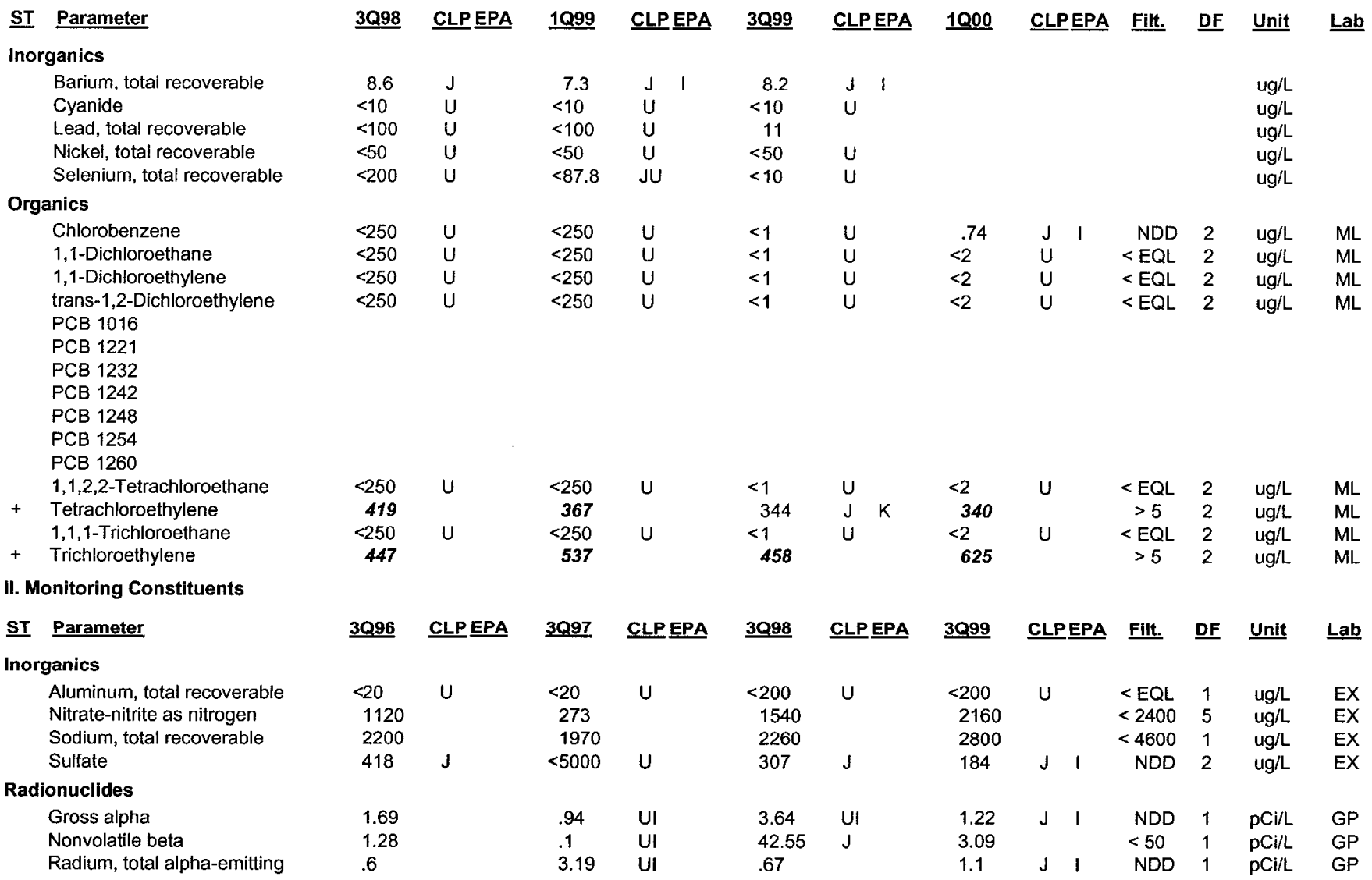

Notes: Concentrations in bold italics exceed the groundwater protection or monitoring constituent standards listed in Appendix A. Synchronous water levels are measured over a 3-5 day period or less. Dilution factors, Laboratory, and Filtered Data are for Groundwater Protection Standards First Quarter 2000 and Monitoring Constituents Third Quarter 1999 data only.

+ = exceeded the Groundwater Protection Standards (First Quarter 2000) or Monitoring Constituents (Third Quarter 1999) listed in Appendix A. 
Table D-4. Groundwater Monitoring Results for Plume Definition Wells, M-Area HWMF (Cont.) WELL MSB 31C

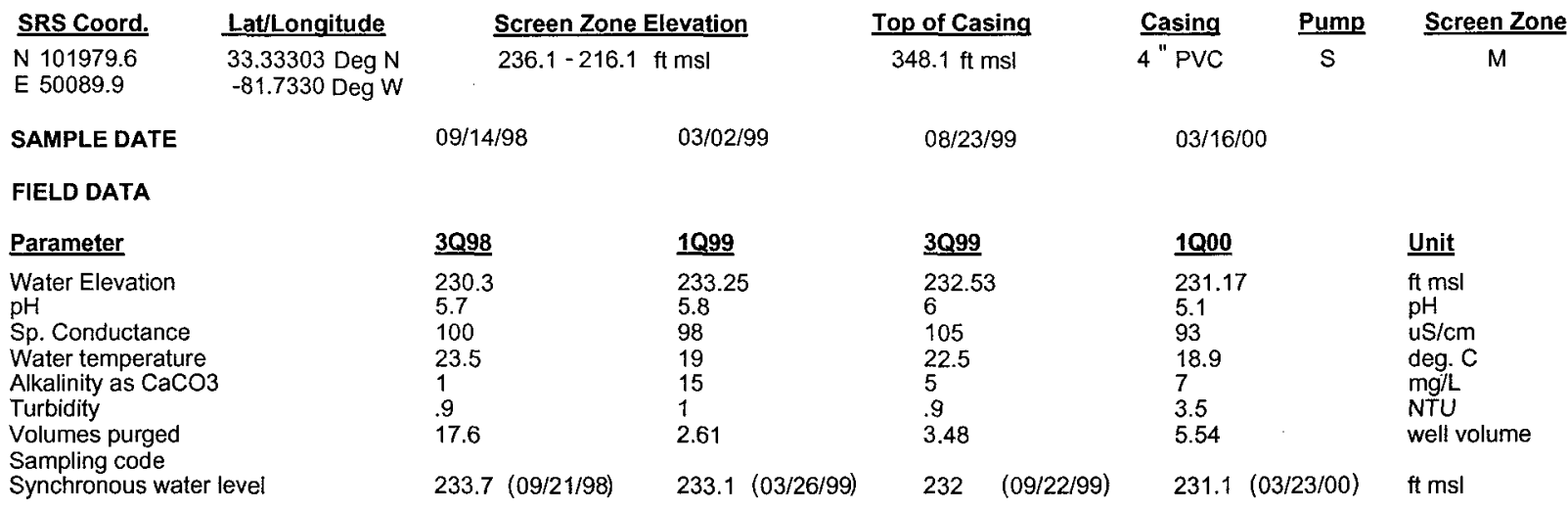

ANALYTICAL DATA

I. Groundwater Protection Standard

261 Appendix VIII/264 Appendix IX Hazardous Constituents

\begin{tabular}{|c|c|c|c|c|c|c|c|c|c|c|c|c|}
\hline$\underline{\text { ST }}$ Parameter & $\underline{3 Q 98}$ & CLPEPA & 1099 & CLP EPA & $\underline{3 Q 99}$ & CLPEPA & $\underline{1000}$ & CLPEPA & Filt. & $\underline{\mathrm{DF}}$ & Unit & $\underline{\text { Lab }}$ \\
\hline \multicolumn{13}{|l|}{ Inorganics } \\
\hline Barium, total recoverable & 13.3 & & 16.1 & & & & & & & & $\mathrm{ug} / \mathrm{L}$ & \\
\hline Cyanide & $<10$ & $u$ & $<15.2$ & U & & & & & & & ug/L & \\
\hline Lead, total recoverable & $<100$ & $u$ & $<100$ & $u$ & & & & & & & $\mathrm{ug} / \mathrm{L}$ & \\
\hline Nickel, total recoverable & $<50$ & $u$ & $<50$ & $\mathrm{U}$ & & & & & & & $u g / L$ & \\
\hline Selenium, total recoverable & $<200$ & $u$ & $<200$ & $u$ & & & & & & & $\mathrm{ug} / \mathrm{L}$ & \\
\hline \multicolumn{13}{|l|}{ Organics } \\
\hline Chlorobenzene & $<500$ & $u$ & $<1250$ & $u$ & $<1$ & $\mathrm{u}$ & $<200$ & $u$ & $<\mathrm{EQL}$ & 200 & $u g / L$ & AL \\
\hline 1,1-Dichloroethane & $<500$ & $\mathrm{U}$ & $<1250$ & u & $<1$ & $\mathrm{U}$ & $<200$ & $\mathrm{u}$ & $<E Q L$ & 200 & $\mathrm{ug} / \mathrm{L}$ & UL \\
\hline 1,1-Dichloroethylene & $<500$ & u & $<1250$ & $u$ & $<1$ & U & $<200$ & $\mathrm{u}$ & $<E Q L$ & 200 & $u g / L$ & IL \\
\hline trans-1,2-Dichloroethylene & $<500$ & $\mathrm{u}$ & $<1250$ & $u$ & $<1$ & $\mathrm{u}$ & $<200$ & $\mathrm{u}$ & $<E Q L$ & 200 & $u g / L$ & \\
\hline РСВ 1016 & $<2$ & $\mathrm{U}$ & $<2$ & $\mathrm{u}$ & & & $<1$ & $u$ & $<E Q L$ & 1 & $u g / L$ & UL \\
\hline PCB 1221 & $<2$ & U & $<2.04$ & $u$ & & & $<1$ & $\mathrm{u}$ & $<E Q L$ & 1 & $\mathrm{ug} / \mathrm{L}$ & IL \\
\hline PCB 1232 & $<1$ & $u$ & $<1.02$ & $u$ & & & $<1$ & $\mathrm{u}$ & $<E Q L$ & 1 & $\mathrm{ug} / \mathrm{L}$ & \\
\hline PCB 1242 & $<1$ & u & $<1.02$ & $u$ & & & $<1$ & $\mathrm{u}$ & $<E Q L$ & 1 & $\mathrm{ug} / \mathrm{L}$ & \\
\hline РCB 1248 & $<1$ & $u$ & $<1.02$ & $u$ & & & $<1$ & $\mathrm{u}$ & $<E Q L$ & 1 & $\mathrm{ug} / \mathrm{L}$ & TVIL \\
\hline PCB 1254 & $<1$ & $u$ & $<1.02$ & $u$ & & & $<1$ & $\mathrm{u}$ & $<E Q L$ & 1 & $\mathrm{ug} / \mathrm{L}$ & \\
\hline PCB 1260 & $<1$ & u & $<1.02$ & $\mathrm{u}$ & & & $<1$ & $\mathrm{u}$ & $<\mathrm{EQL}$ & 1 & $\mathrm{ug} / \mathrm{L}$ & \\
\hline 1,1,2,2-Tetrachloroethane & $<500$ & $u$ & $<1250$ & $u$ & $<1$ & $\mathrm{u}$ & $<200$ & $\mathrm{u}$ & $<E Q L$ & 200 & $\mathrm{ug} / \mathrm{L}$ & ML \\
\hline Tetrachloroethylene & 11000 & & 22600 & & 34600 & & 22500 & & $>5$ & 200 & $\mathrm{ug} / \mathrm{L}$ & \\
\hline 1,1,1-Trichloroethane & $<500$ & $u$ & $<1250$ & $\mathrm{u}$ & .76 & J IK & $<200$ & $\mathrm{u}$ & $<E Q L$ & 200 & $\mathrm{ug} / \mathrm{L}$ & \\
\hline+ Trichloroethylene & 2330 & & 3670 & & 2590 & & 1210 & & $>5$ & 200 & $\mathrm{ug} / \mathrm{L}$ & \\
\hline \multicolumn{12}{|l|}{ II. Monitoring Constituents } & \\
\hline ST Parameter & $\underline{3 Q 96}$ & CLPEPA & $\underline{3097}$ & CLP EPA & $\underline{3098}$ & CLPEPA & $\underline{3099}$ & CLPEPA & Filt. & $\underline{D F}$ & $\underline{\text { Unit }}$ & Lab \\
\hline \multicolumn{12}{|l|}{ Inorganics } & \\
\hline Aluminum, total recoverable & 22.5 & & 9.7 & J & $<200$ & $u$ & & & & & $\mathrm{ug} / \mathrm{L}$ & \\
\hline Nitrate-nitrite as nitrogen & 5680 & & 8 & $\mathrm{~J}$ & 5500 & & & & & & $\mathrm{ug} / \mathrm{L}$ & \\
\hline Sodium, total recoverable & 10600 & & 9120 & & 12600 & & & & & & $\mathrm{ug} / \mathrm{L}$ & \\
\hline Sulfate & 8310 & & 7260 & & 9270 & & & & & & ug/L & \\
\hline \multicolumn{12}{|l|}{ Radionuclides } & \\
\hline Gross alpha & 6.62 & & 4.09 & & 7.36 & & 3.79 & & $<15$ & 1 & $\mathrm{pCi} / \mathrm{L}$ & GP \\
\hline Nonvolatile beta & 5.43 & & 4.27 & & 9.5 & & 3.54 & & $<50$ & 1 & $\mathrm{pCi} / \mathrm{L}$ & \\
\hline Radium, total alpha-emitting & 3.5 & & 6.46 & & 3.11 & $\mathrm{~J}$ & 2.1 & & $<5$ & 1 & $\mathrm{pCi} / \mathrm{L}$ & or \\
\hline
\end{tabular}

\footnotetext{
Notes: Concentrations in bold italics exceed the groundwater protection or monitoring constituent standards listed in Appendix A. Synchronous water levels are measured over a 3-5 day period or less. Dilution factors, Laboratory, and Filtered Data are for Groundwater Protection Standards First Quarter 2000 and Monitoring Constituents Third Quarter 1999 data only.
}

+ = exceeded the Groundwater Protection Standards (First Quarter 2000) or Monitoring Constituents (Third Quarter 1999) listed in Appendix A. 
Table D-4. Groundwater Monitoring Results for Plume Definition Wells, M-Area HWMF (Cont.) WELL MSB 31CC

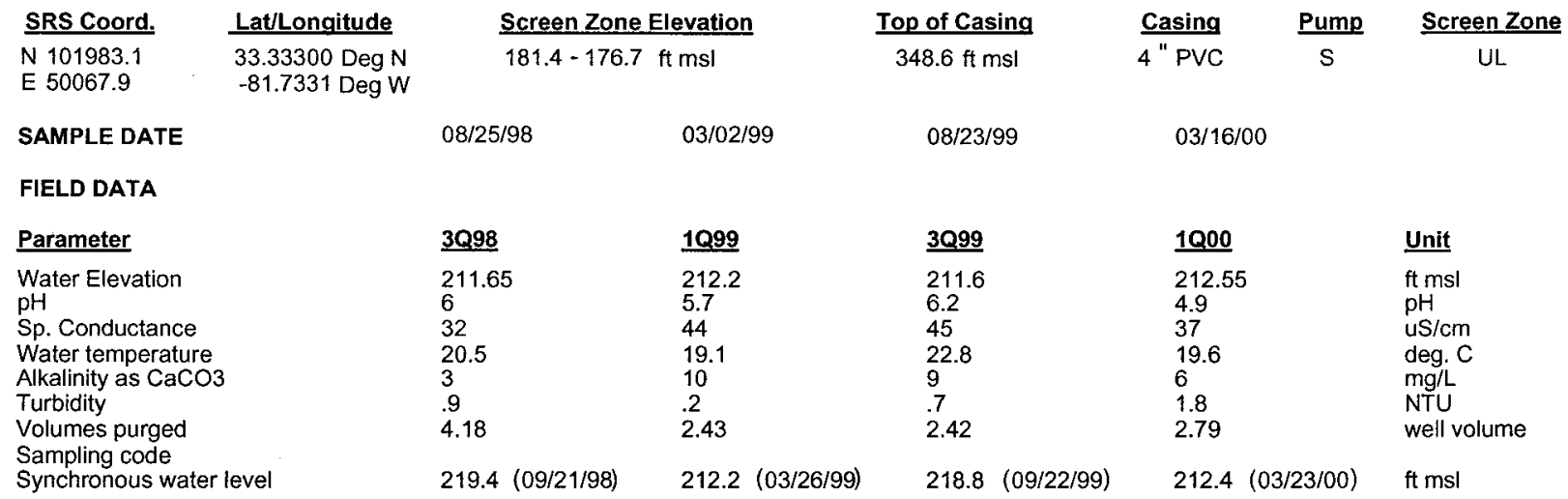

\section{ANALYTICAL DATA}

I. Groundwater Protection Standard

261 Appendix VIII/264 Appendix IX Hazardous Constituents

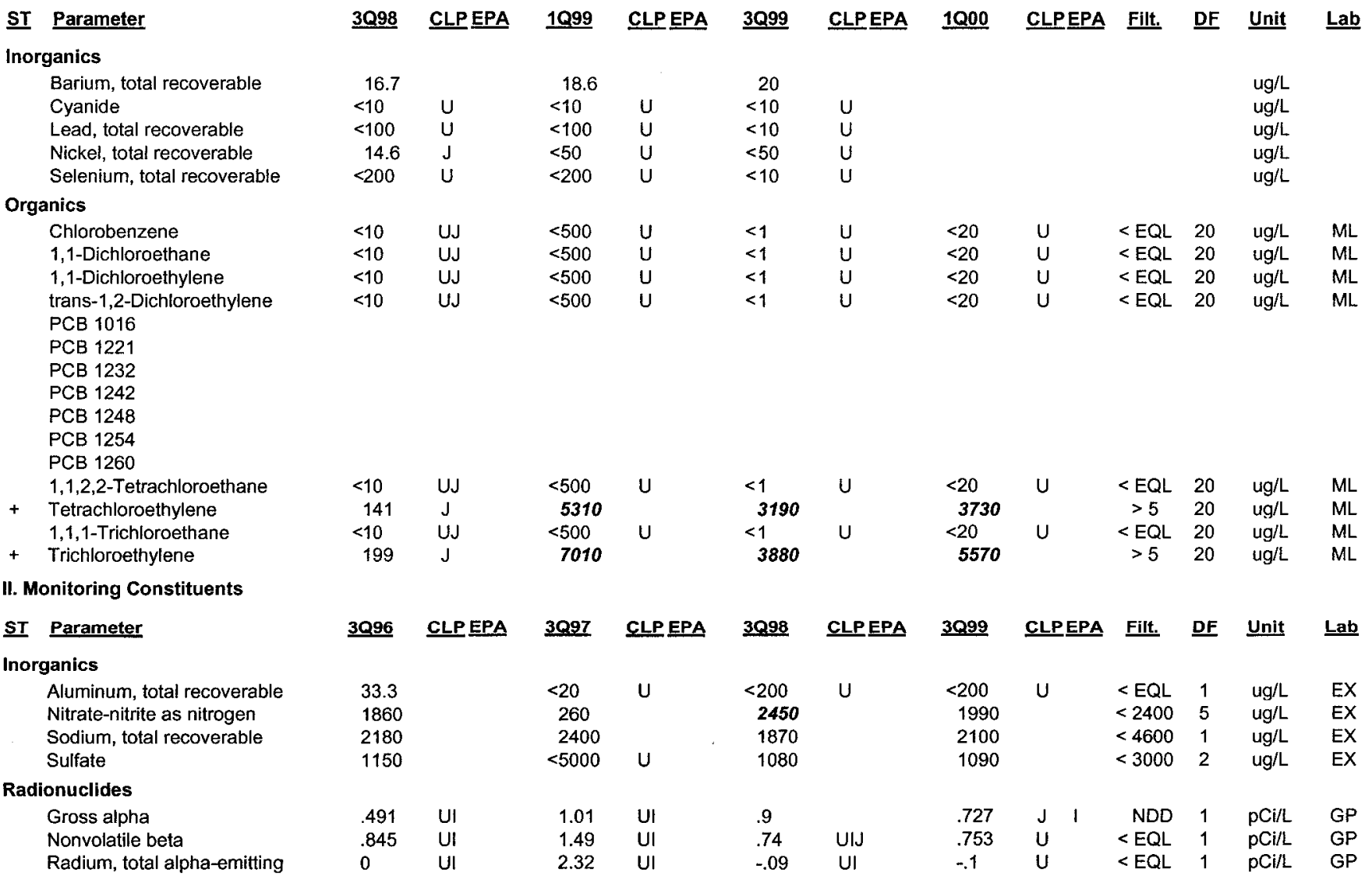

Notes: Concentrations in bold italics exceed the groundwater protection or monitoring constituent standards listed in Appendix A. Synchronous water levels are measured over a 3-5 day period or less. Dilution factors, Laboratory, and Filtered Data are for Groundwater Protection Standards First Quarter 2000 and Monitoring Constituents Third Quarter 1999 data only.

$+=$ exceeded the Groundwater Protection Standards (First Quarter 2000) or Monitoring Constituents (Third Quarter 1999) listed in Appendix A. 
Table D-4. Groundwater Monitoring Results for Plume Definition Wells, M-Area HWMF (Cont.) WELL MSB 32

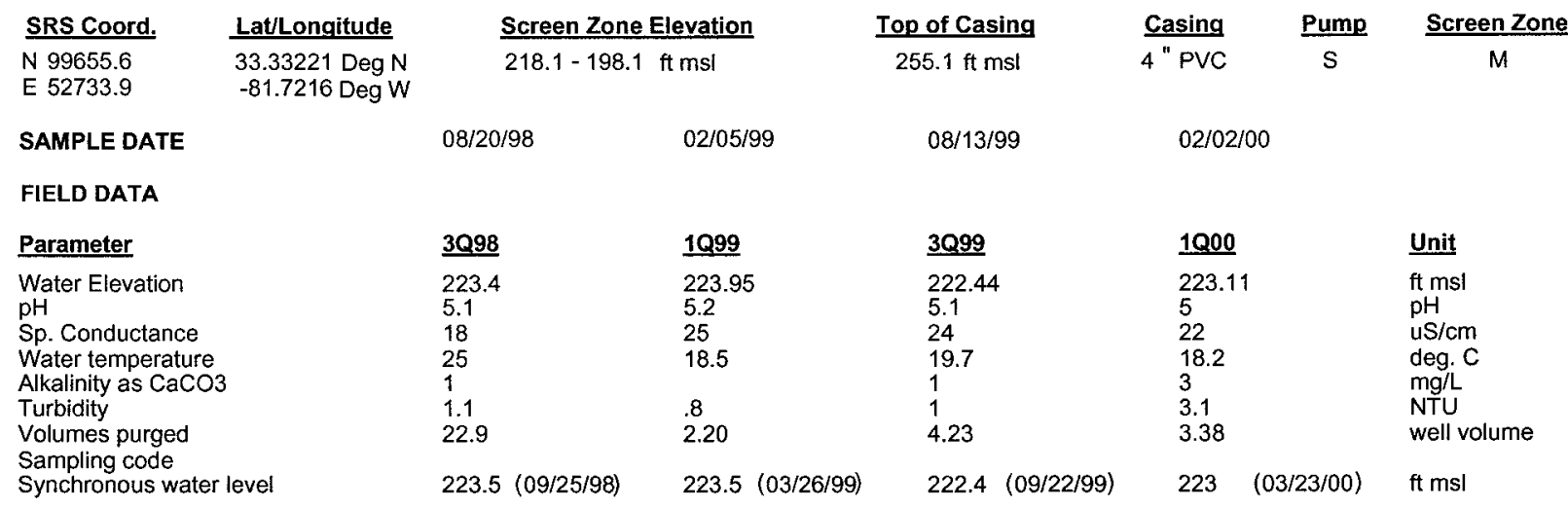

ANALYTICAL DATA

I. Groundwater Protection Standard

261 Appendix VIII/264 Appendix IX Hazardous Constituents

\begin{tabular}{|c|c|c|c|c|c|c|c|c|c|c|c|c|c|}
\hline$\underline{\text { ST }}$ & Parameter & 3Q98 & CLPPEPA & 1099 & CLP EPA & 3Q99 & CLPEPA & $1 Q 00$ & CLPEPA & Filt. & DF & Unit & $\underline{\text { Lab }}$ \\
\hline \multicolumn{14}{|c|}{ Inorganics ${ }^{-}$} \\
\hline & Barium, total recoverable & $<10$ & U & $<10$ & U & $<10$ & $u$ & & & & & $u g / h$ & \\
\hline & Cyanide & $<10$ & $\mathrm{u}$ & $<10$ & $U$ & $<10$ & $\mathrm{U}$ & & & & & $\mathrm{ug} / \mathrm{L}$ & \\
\hline & Lead, total recoverable & $<10$ & $\mathrm{U}$ & $<100$ & $U$ & $<1.48$ & $\mathrm{U} V$ & & & & & ug/L & \\
\hline & Nickel, total recoverable & 9.65 & $J$ & $<50$ & $U$ & $<50$ & $\mathrm{U}$ & & & & & ug/L & \\
\hline & Selenium, total recoverable & $<10$ & $\mathrm{U}$ & $<10$ & U & $<5$ & $U$ & & & & & ug/L & \\
\hline \multicolumn{14}{|c|}{ Organics } \\
\hline & Chlorobenzene & $<5$ & $U$ & $<5$ & $u$ & $<1$ & $u$ & $<1$ & JU L & $<\mathrm{EQL}$ & 1 & ug $/ \mathrm{L}$ & $M L$ \\
\hline & 1,1-Dichloroethane & $<5$ & $U$ & $<5$ & $U$ & $<1$ & U & $<1$ & JU L & $<E Q L$ & 1 & $\mathrm{ug} / \mathrm{L}$ & ML \\
\hline & 1,1-Dichloroethylene & $<5$ & $\mathrm{U}$ & $<5$ & $\mathrm{U}$ & $<1$ & U & $<1$ & JU L & $<E Q L$ & 1 & ug/L & ML \\
\hline & $\begin{array}{l}\text { trans-1,2-Dichloroethylene } \\
\text { PCB } 1016\end{array}$ & $<5$ & $U$ & $<5$ & $\mathrm{U}$ & $<1$ & $\mathrm{U}$ & $<1$ & JU L & $<\mathrm{EQL}$ & 1 & $\mathrm{ug} / \mathrm{L}$ & $M L$ \\
\hline & PCB 1221 & & & & & & & & & & & & \\
\hline & PCB 1232 & & & & & & & & & & & & \\
\hline & PCB 1242 & & & & & & & & & & & & \\
\hline & PCB 1248 & & & & & & & & & & & & \\
\hline & PCB 1254 & & & & & & & & & & & & \\
\hline & PCB 1260 & & & & & & & & & & & & \\
\hline & $1,1,2,2$-Tetrachloroethane & $<5$ & $U$ & $<5$ & U & $<1$ & $\mathrm{U}$ & $<1$ & JU L & $<\mathrm{EQL}$ & 1 & $u g / L$ & ML \\
\hline & Tetrachloroethylene & $<5$ & U & $<5$ & $U$ & $<1$ & U & $<1$ & JU L & $<\mathrm{EQL}$ & 1 & $\mathrm{ug} / \mathrm{L}$ & ML \\
\hline & $1,1,1$-Trichloroethane & $<5$ & $\mathrm{U}$ & $<5$ & 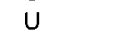 & $<1$ & U & $<1$ & JU L & $<\mathrm{EQL}$ & 1 & $\mathrm{ug} / \mathrm{L}$ & ML \\
\hline & Trichloroethylene & $<5$ & $U$ & $<5$ & $\mathrm{U}$ & $<1$ & $U$ & $<1$ & JU L & $<\mathrm{EQL}$ & 1 & $\mathrm{ug} / \mathrm{L}$ & ML \\
\hline \multicolumn{14}{|c|}{ II. Monitoring Constituents } \\
\hline$\underline{\mathbf{S T}}$ & Parameter & $\underline{3 Q 96}$ & $\underline{\text { CLP EPA }}$ & $\underline{3 Q 97}$ & $\underline{\text { CLP EPA }}$ & $\underline{3 Q 98}$ & CLPEPA & $\underline{3099}$ & CLPEPA & Filt. & $\underline{\text { DF }}$ & Unit & Lab \\
\hline \multicolumn{14}{|c|}{ Inorganics } \\
\hline & Aluminum, total recoverable & 12.6 & $J$ & 31 & & $<200$ & $\mathrm{U}$ & $<200$ & $\mathrm{U}$ & $<\mathrm{EQL}$ & 1 & $\mathrm{ug} / \mathrm{L}$ & EX \\
\hline & Nitrate-nitrite as nitrogen & 1170 & & 903 & & 776 & & 856 & & $<2400$ & 5 & $\mathrm{ug} / \mathrm{L}$ & EX \\
\hline & Sodium, total recoverable & 3280 & & 2760 & & 2760 & & 3700 & & $<4600$ & 1 & $\mathrm{ug} / \mathrm{L}$ & EX \\
\hline & Sulfate & 982 & $J$ & $<5000$ & U & 696 & & 1180 & & $<3000$ & 1 & $\mathrm{ug} / \mathrm{L}$ & EX \\
\hline \multicolumn{13}{|c|}{ Radionuclides } & \\
\hline & Gross alpha & .11 & UI & 0 & UIJ & .9 & & .32 & $U$ & $<E Q L$ & 1 & $\mathrm{pCi} / \mathrm{L}$ & GP \\
\hline & Nonvolatile beta & .82 & UI & -1.15 & UI & .71 & UI & 1.15 & $U$ & $<E Q L$ & 1 & $\mathrm{pCi} / \mathrm{L}$ & GP \\
\hline & Radium, total alpha-emitting & .2 & UI & 2.44 & $J$ & .06 & UI & 0 & $U$ & $<E Q L$ & 1 & $\mathrm{pCi} / \mathrm{L}$ & \\
\hline
\end{tabular}

Notes: Concentrations in bold italics exceed the groundwater protection or monitoring constituent standards listed in Appendix A. Synchronous water levels are measured over a 3-5 day period or less. Dilution factors, Laboratory, and Filtered Data are for Groundwater Protection Standards First Quarter 2000 and Monitoring Constituents Third Quarter 1999 data only.

$+=$ exceeded the Groundwater Protection Standards (First Quarter 2000) or Monitoring Constituents (Third Quarter 1999) listed in Appendix A. 
Table D-4. Groundwater Monitoring Results for Plume Definition Wells, M-Area HWMF (Cont.) WELL MSB 32B

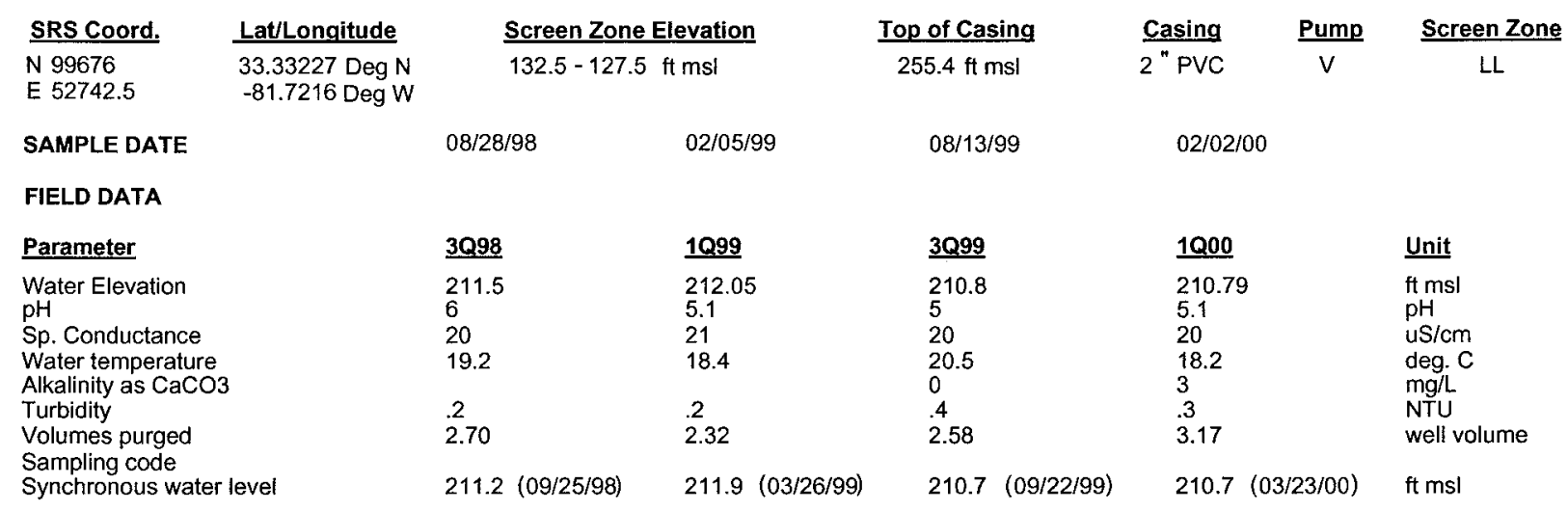

\section{ANALYTICAL DATA}

I. Groundwater Protection Standard

261 Appendix VIII/264 Appendix IX Hazardous Constituents

\begin{tabular}{|c|c|c|c|c|c|c|c|c|c|c|c|c|c|}
\hline$\underline{\text { ST }}$ & Parameter & $\underline{3 Q 98}$ & CLP EPA & 1099 & CLP EPA & $\underline{3 Q 99}$ & $\underline{\text { CLPEPA }}$ & $\underline{1000}$ & CLPEPA & Filt. & $\underline{\text { DF }}$ & $\underline{\text { Unit }}$ & $\underline{\text { Lab }}$ \\
\hline Inor & ganics & & & & & & & & & & & & \\
\hline & Barium, total recoverable & 4.09 & $J$ & 3.74 & $\mathrm{~J} \quad \mathrm{I}$ & 3.5 & J $\quad$ I & & & & & $u g / L$ & \\
\hline & Cyanide & $<10$ & $U$ & $<10$ & $\mathrm{U}$ & $<10$ & U & & & & & ug/L & \\
\hline & Lead, total recoverable & $<100$ & U & $<100$ & $U$ & $<1.55$ & U V & & & & & ug/L & \\
\hline & Nickel, total recoverable & $<50$ & $U$ & $<50$ & $U$ & $<50$ & $U$ & & & & & ug/L & \\
\hline & Selenium, total recoverable & $<10$ & $U$ & $<10$ & U & $<5$ & $\mathrm{U}$ & & & & & ug/L & \\
\hline Org & anics & & & & & & & & & & & & \\
\hline & Chlorobenzene & $<5$ & $\mathrm{U}$ & $<5$ & $\mathrm{U}$ & $<1$ & $\mathrm{U}$ & $<1$ & $\mathrm{U}$ & $<E Q L$ & 1 & $\mathrm{ug} / \mathrm{L}$ & ML \\
\hline & 1,1-Dichloroethane & $<5$ & $U$ & $<5$ & $\mathrm{U}$ & $<1$ & $\mathrm{U}$ & $<1$ & $\mathrm{U}$ & $<E Q L$ & 1 & ug/L & ML \\
\hline & 1,1-Dichloroethylene & $<5$ & U & $<5$ & $U$ & $<1$ & $U$ & $<1$ & $U$ & $<\mathrm{EQL}$ & 1 & $u g / L$ & ML \\
\hline & trans-1,2-Dichloroethylene & $<5$ & $\mathrm{U}$ & $<5$ & $U$ & $<1$ & U & $<1$ & $U$ & $<\mathrm{EQL}$ & 1 & ug/L & ML \\
\hline & PCB 1016 & & & & & & & & & & & & \\
\hline & PCB 1221 & & & & & & & & & & & & \\
\hline & PCB 1232 & & & & & & & & & & & & \\
\hline & PCB 1242 & & & & & & & & & & & & \\
\hline & РCB 1248 & & & & & & & & & & & & \\
\hline & РCB 1254 & & & & & & & & & & & & \\
\hline & PCB 1260 & & & & & & & & & & & & \\
\hline & $1,1,2,2$-Tetrachloroethane & $<5$ & $\mathrm{U}$ & $<5$ & $\mathrm{U}$ & $<1$ & $U$ & $<1$ & $\mathrm{U}$ & $<\mathrm{EQL}$ & 1 & ug/L & ML \\
\hline & Tetrachloroethylene & $<5$ & $\mathrm{U}$ & $<5$ & $\mathrm{U}$ & $<1$ & $U$ & $<1$ & $U$ & $<\mathrm{EQL}$ & 1 & $\mathrm{ug} / \mathrm{L}$ & $\mathrm{ML}$ \\
\hline & $1,1,1$-Trichloroethane & $<5$ & $\mathrm{U}$ & $<5$ & U & $<1$ & U & $<1$ & $U$ & $<\mathrm{EQL}$ & 1 & $\mathrm{ug} / \mathrm{L}$ & ML \\
\hline & Trichloroethylene & $<5$ & $\mathrm{U}$ & $<5$ & $\mathrm{U}$ & $<1$ & $U$ & $<1$ & $U$ & $<E Q L$ & 1 & $\mathrm{ug} / \mathrm{L}$ & ML \\
\hline II. $\mathrm{M}$ & onitoring Constituents & & & & & & & & & & & & \\
\hline$\underline{\text { ST }}$ & Parameter & $\underline{3 Q 96}$ & CLPEPA & 3097 & CLP EPA & $\underline{3098}$ & CLPEPA & $\underline{3099}$ & CLPEPA & Filt. & $\underline{\text { DF }}$ & Unit & $\underline{\text { Lab }}$ \\
\hline Inor & ganics & & & & & & & & & & & & \\
\hline & Aluminum, total recoverable & $<26.1$ & $\mathrm{U}$ & 23.6 & & $<200$ & U & $<200$ & $\mathrm{U}$ & $<E Q L$ & 1 & $\mathrm{ug} / \mathrm{L}$ & EX \\
\hline & Nitrate-nitrite as nitrogen & 1330 & & 1130 & & $<500$ & $\mathrm{U}$ & 896 & & $<2400$ & 5 & $\mathrm{ug} / \mathrm{L}$ & EX \\
\hline & Sodium, total recoverable & 1350 & & 1450 & & 1360 & & 1600 & & $<4600$ & 1 & $\mathrm{ug} / \mathrm{L}$ & EX \\
\hline & Sulfate & 283 & $J$ & $<5000$ & $U$ & 424 & & 308 & & $<3000$ & 1 & $\mathrm{ug} / \mathrm{L}$ & EX \\
\hline Rad & ionuclides & & & & & & & & & & & & \\
\hline & Gross alpha & .435 & UI & .04 & UI & .78 & UI & -.049 & JU L & $<E Q L$ & 1 & $\mathrm{pCi} / \mathrm{L}$ & GP \\
\hline & Nonvolatile beta & 1.34 & Ui & -.05 & UI & 1.48 & UI & 1.48 & $\mathrm{~J}$ & NDD & 1 & $\mathrm{pCi} / \mathrm{L}$ & GP \\
\hline & Radium, total alpha-emitting & .2 & & 1.54 & UIJ & .31 & UI & 0 & $U$ & $<E Q L$ & 1 & $\mathrm{pCi} / \mathrm{L}$ & GP \\
\hline
\end{tabular}

Notes: Concentrations in bold italics exceed the groundwater protection or monitoring constituent standards listed in Appendix A. Synchronous water levels are measured over a 3-5 day period or less. Dilution factors, Laboratory, and Filtered Data are for Groundwater Protection Standards First Quarter 2000 and Monitoring Constituents Third Quarter 1999 data only.

$+=$ exceeded the Groundwater Protection Standards (First Quarter 2000) or Monitoring Constituents (Third Quarter 1999) listed in Appendix A. 
Table D-4. Groundwater Monitoring Results for Plume Definition Wells, M-Area HWMF (Cont.) WELL MSB 32C

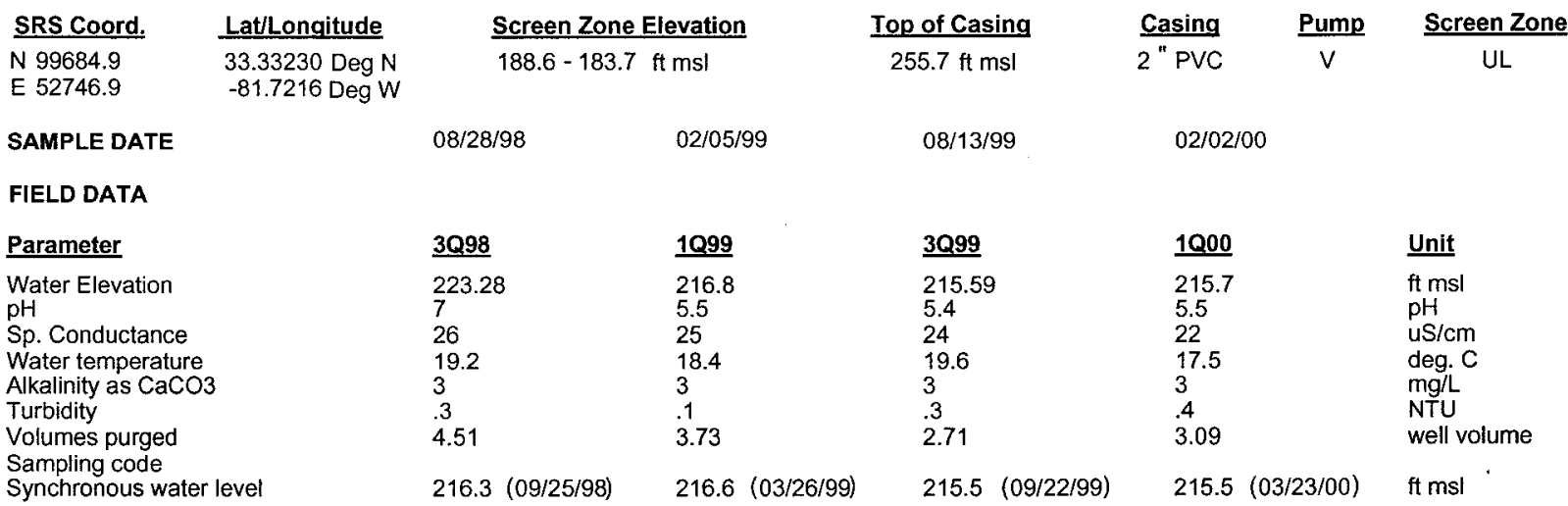

ANALYTICAL DATA

I. Groundwater Protection Standard

261 Appendix VIII/264 Appendix IX Hazardous Constituents

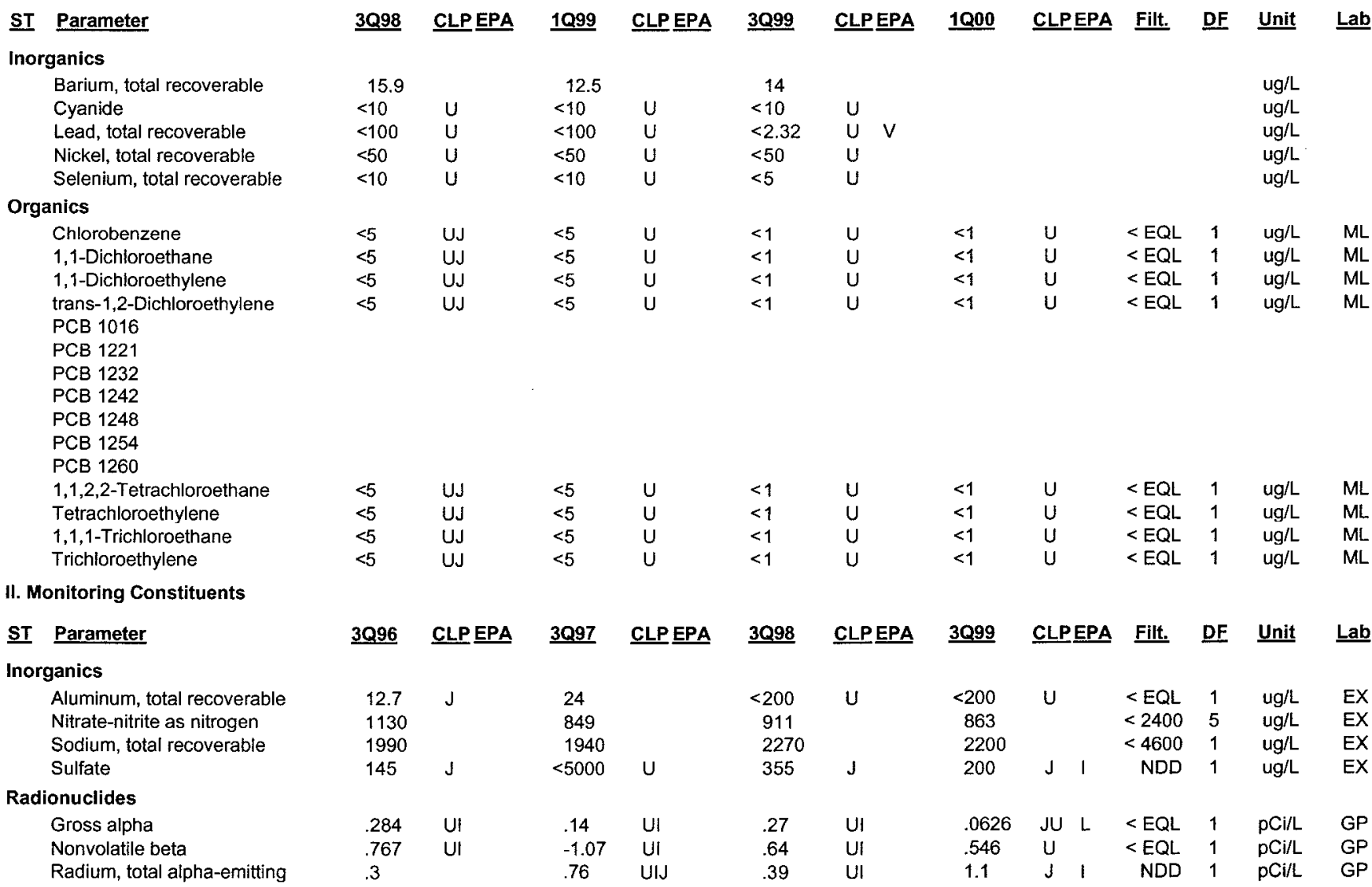

Notes: Concentrations in bold italics exceed the groundwater protection or monitoring constituent standards listed in Appendix A. Synchronous water levels are measured over a 3-5 day period or less. Dilution factors, Laboratory, and Filtered Data are for Groundwater Protection Standards First Quarter 2000 and Monitoring Constituents Third Quarter 1999 data only.

$+=$ exceeded the Groundwater Protection Standards (First Quarter 2000) or Monitoring Constituents (Third Quarter 1999) listed in Appendix A. 
Table D-4. Groundwater Monitoring Results for Plume Definition Wells, M-Area HWMF (Cont.) WELL MSB 33

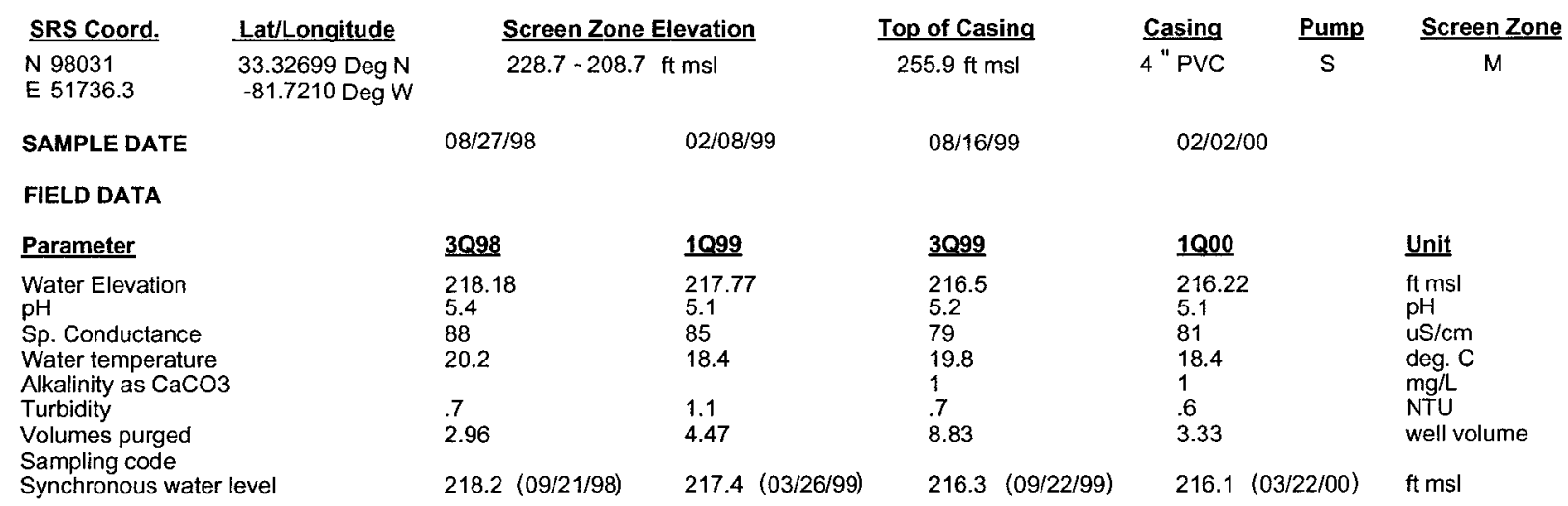

\section{ANALYTICAL DATA}

I. Groundwater Protection Standard

261 Appendix VIII/264 Appendix IX Hazardous Constituents

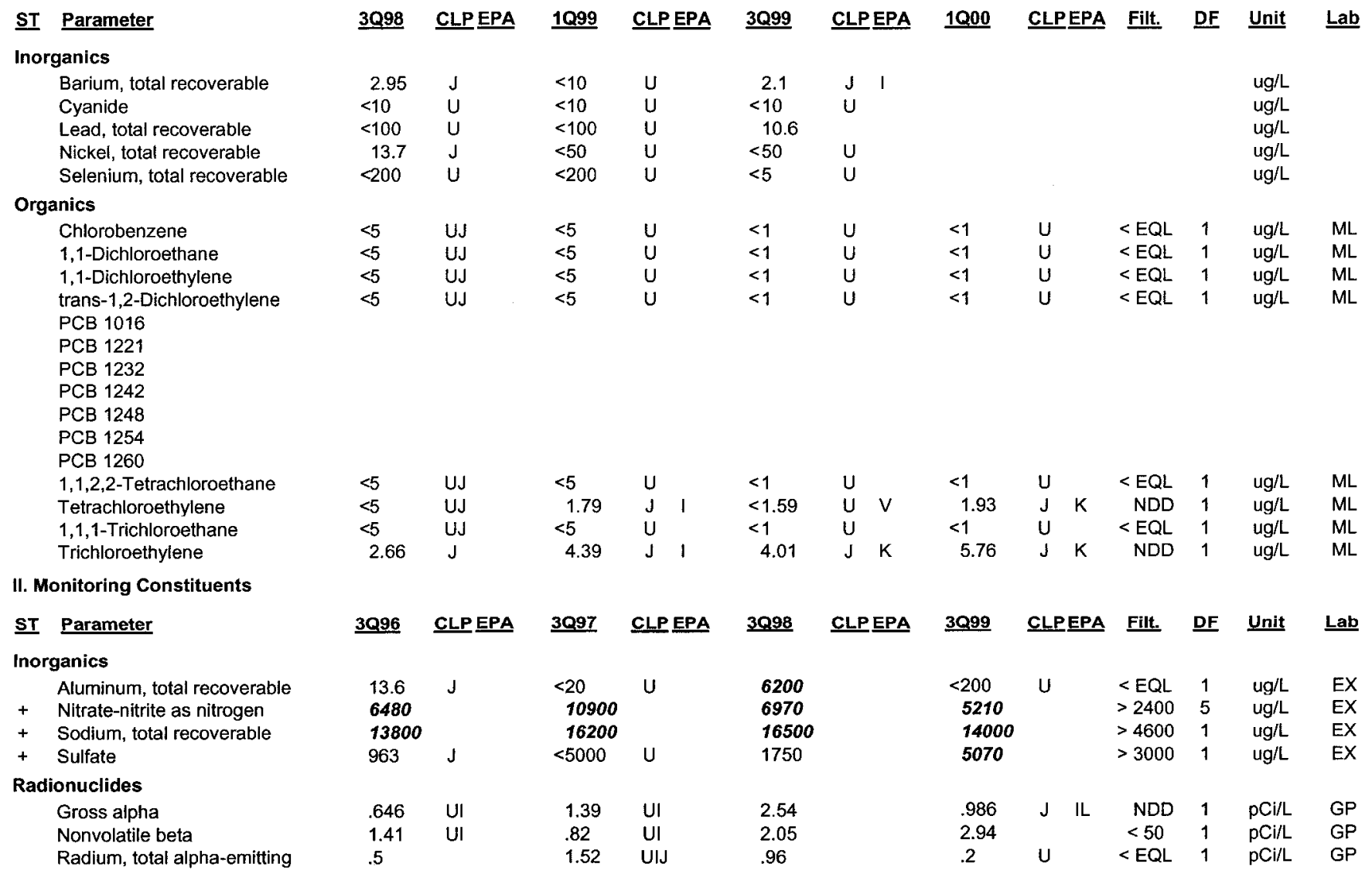

Notes: Concentrations in bold italics exceed the groundwater protection or monitoring constituent standards listed in Appendix A. Synchronous water levels are measured over a 3-5 day period or less. Dilution factors, Laboratory, and Filtered Data are for Groundwater Protection Standards First Quarter 2000 and Monitoring Constituents Third Quarter 1999 data only.

$+=$ exceeded the Groundwater Protection Standards (First Quarter 2000) or Monitoring Constituents (Third Quarter 1999) listed in Appendix A. 
Table D-4. Groundwater Monitoring Results for Plume Definition Wells, M-Area HWMF (Cont.) WELL MSB 33A

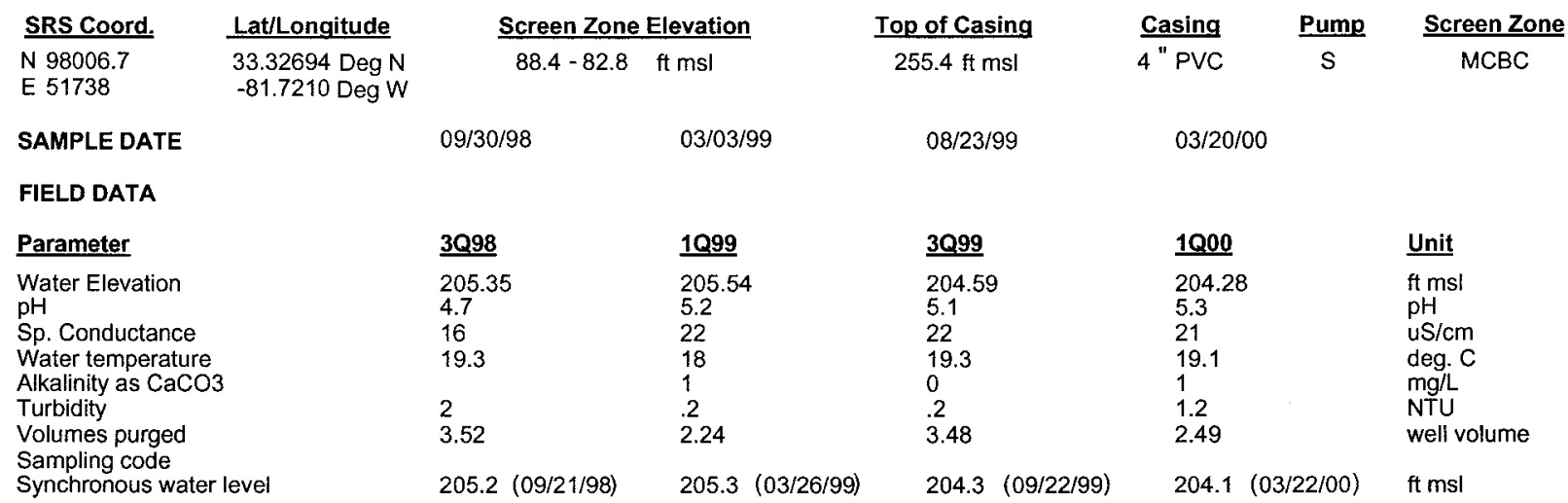

\section{ANALYTICAL DATA}

1. Groundwater Protection Standard

261 Appendix VIII/264 Appendix IX Hazardous Constituents

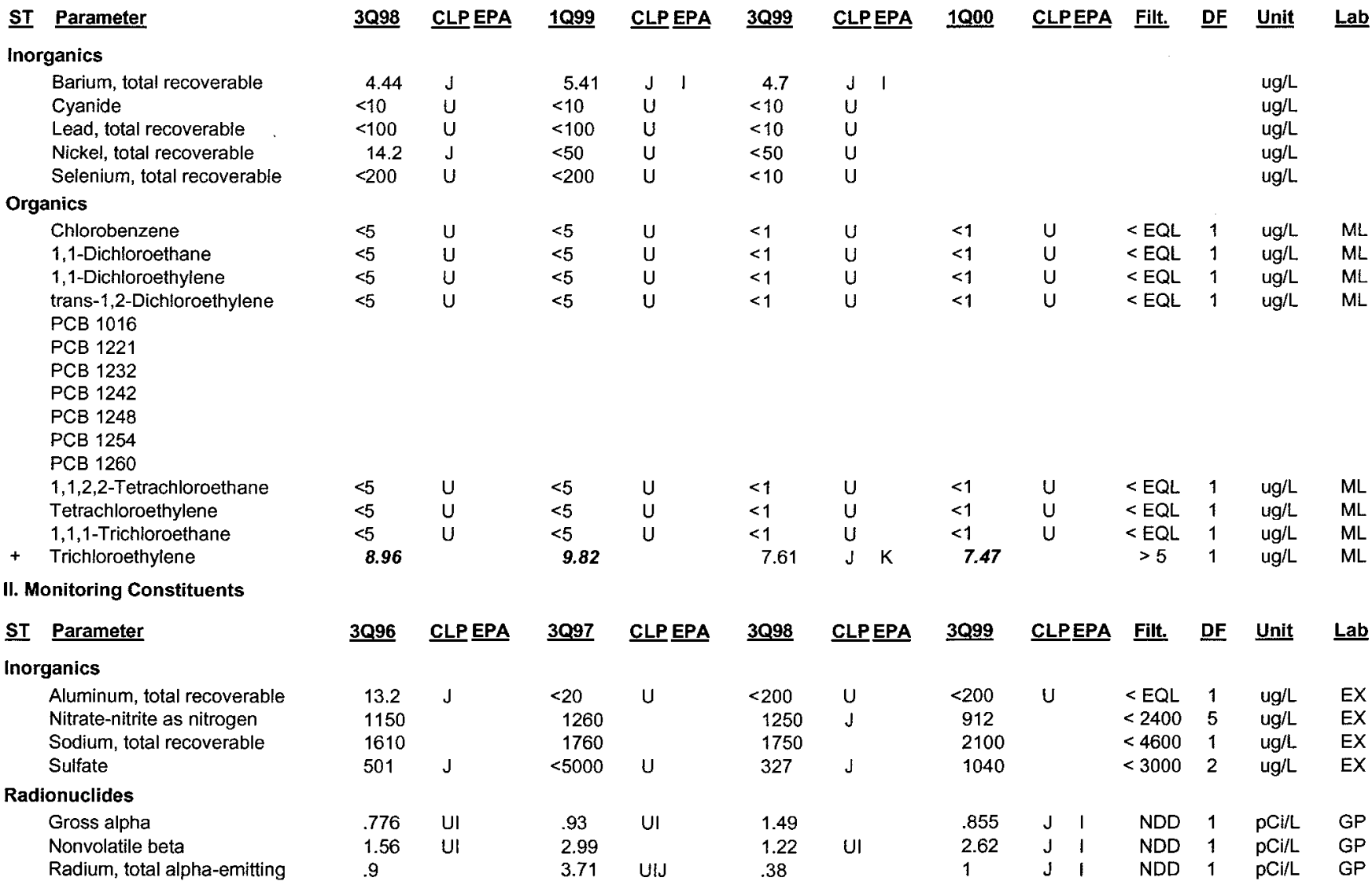

Notes: Concentrations in bold italics exceed the groundwater protection or monitoring constituent standards listed in Appendix A. Synchronous water levels are measured over a 3-5 day period or less. Dilution factors, Laboratory, and Filtered Data are for Groundwater Protection Standards First Quarter 2000 and Monitoring Constituents Third Quarter 1999 data only.

+ = exceeded the Groundwater Protection Standards (First Quarter 2000) or Monitoring Constituents (Third Quarter 1999) listed in Appendix A. 
Table D-4. Groundwater Monitoring Results for Plume Definition Wells, M-Area HWMF (Cont.) WELL MSB 33B

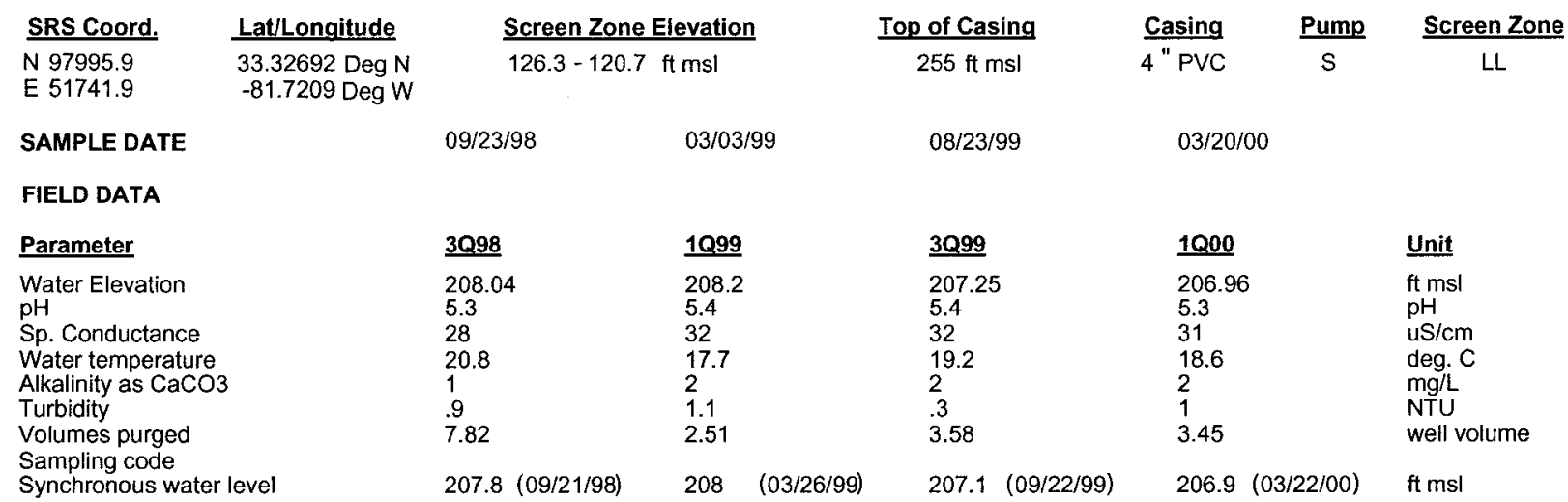

\section{ANALYTICAL DATA}

I. Groundwater Protection Standard

261 Appendix VIII/264 Appendix IX Hazardous Constituents

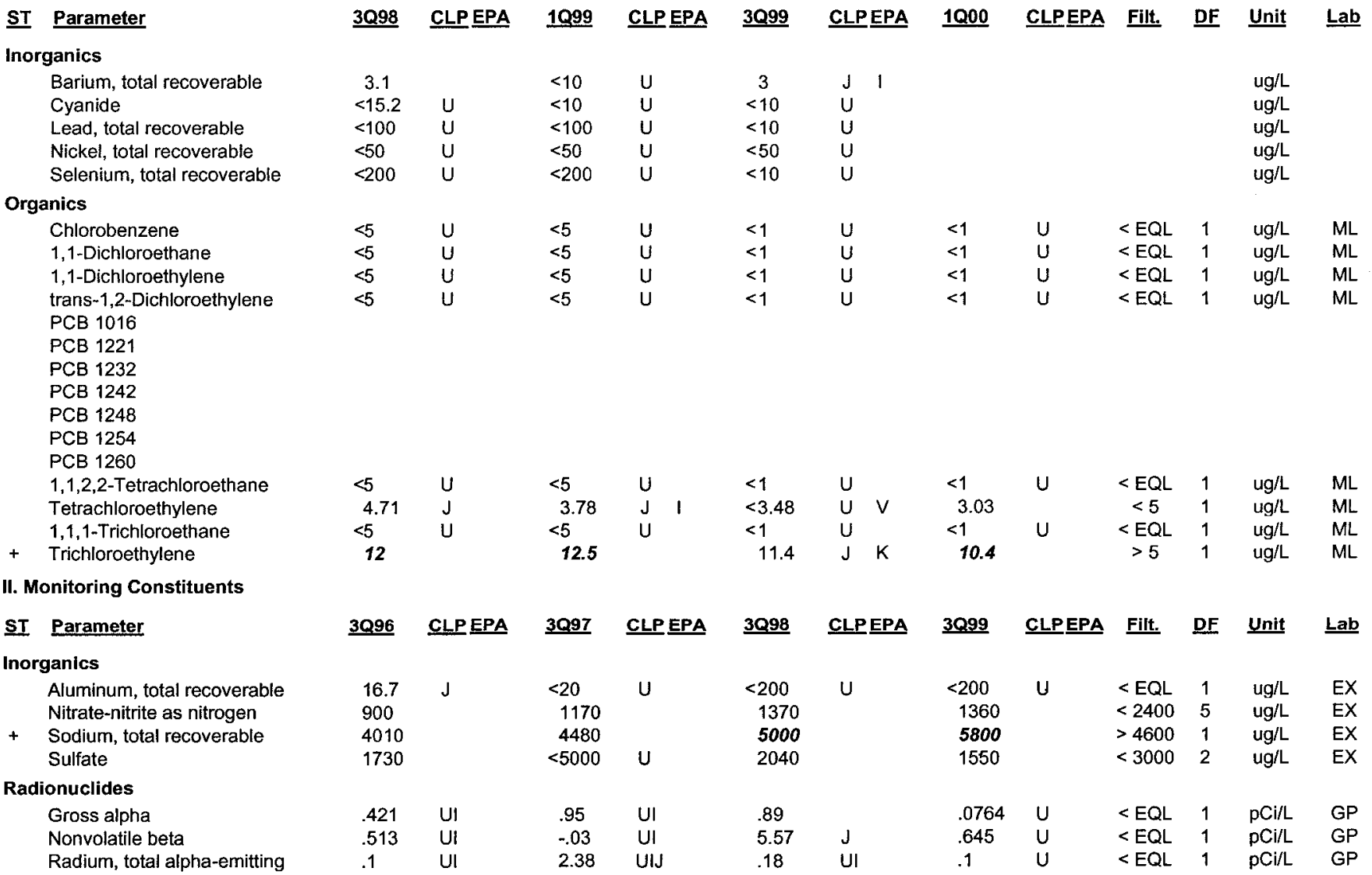

Notes: Concentrations in bold italics exceed the groundwater protection or monitoring constituent standards listed in Appendix A. Synchronous water levels are measured over a 3-5 day period or less. Dilution factors, Laboratory, and Filtered Data are for Groundwater Protection Standards First Quarter 2000 and Monitoring Constituents Third Quarter 1999 data only.

$+=$ exceeded the Groundwater Protection Standards (First Quarter 2000) or Monitoring Constituents (Third Quarter 1999) listed in Appendix A. 
Table D-4. Groundwater Monitoring Results for Plume Definition Wells, M-Area HWMF (Cont.) WELL MSB 33C

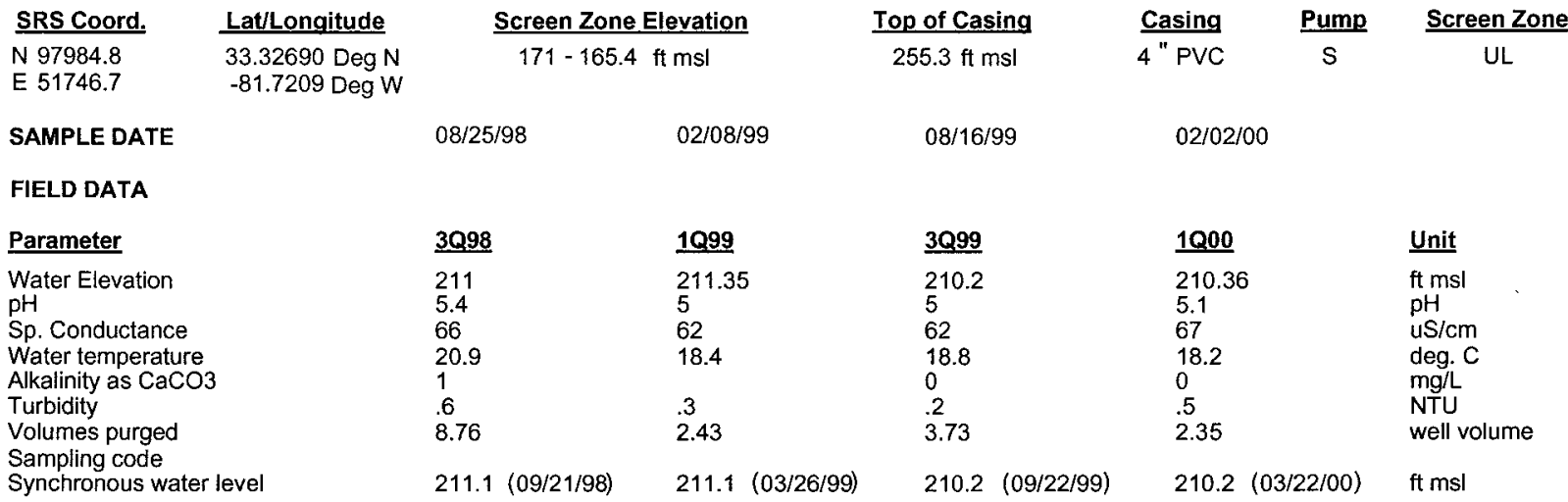

ANALYTICAL DATA

I. Groundwater Protection Standard

261 Appendix VIII/264 Appendix IX Hazardous Constituents

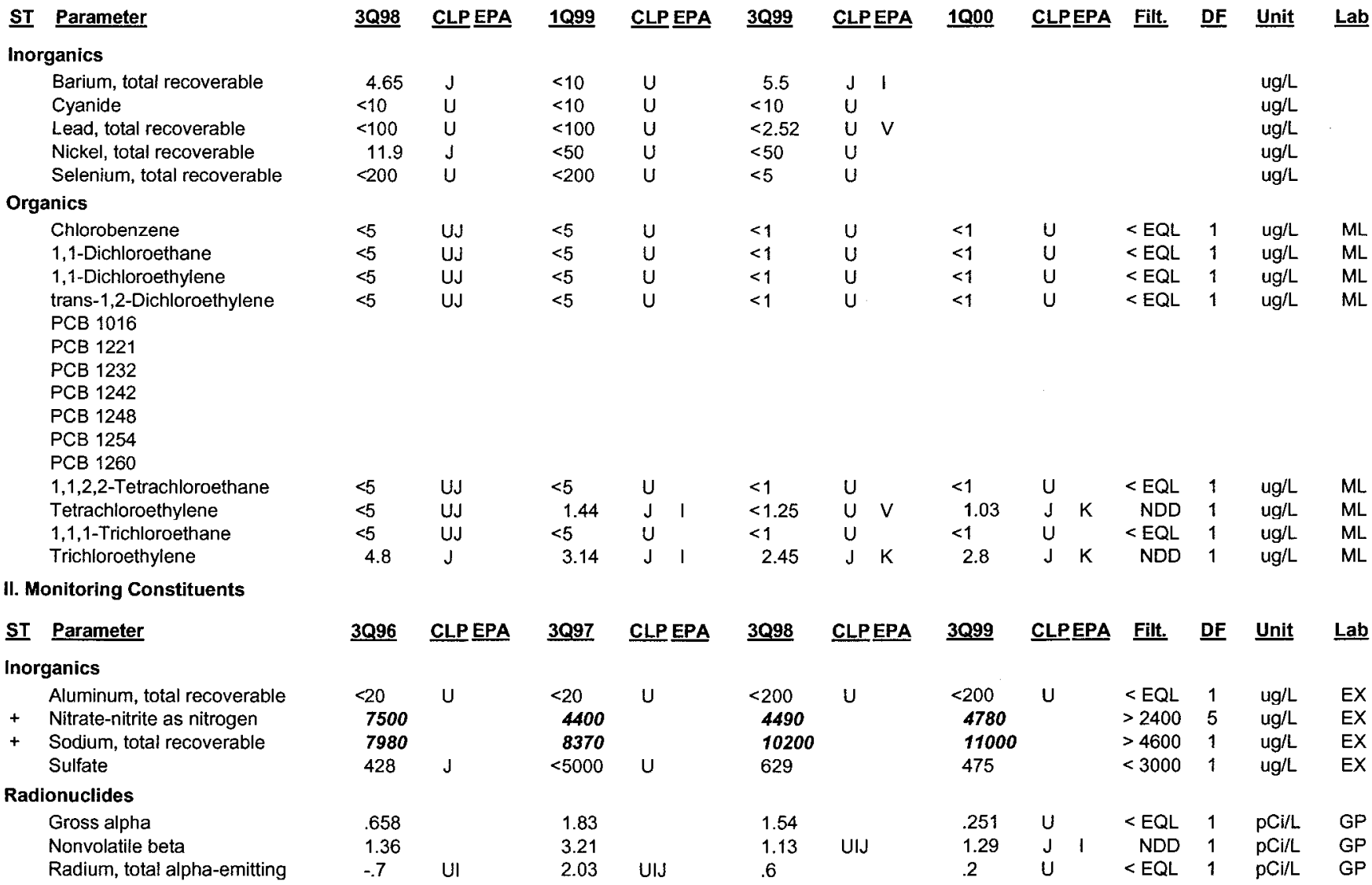

Notes: Concentrations in bold italics exceed the groundwater protection or monitoring constituent standards listed in Appendix A. Synchronous water levels are measured over a 3-5 day period or less. Dilution factors, Laboratory, and Filtered Data are for Groundwater Protection Standards First Quarter 2000 and Monitoring Constituents Third Quarter 1999 data only.

+= exceeded the Groundwater Protection Standards (First Quarter 2000) or Monitoring Constituents (Third Quarter 1999) listed in Appendix A. 
Table D-4. Groundwater Monitoring Results for Plume Definition Wells, M-Area HWMF (Cont.) WELL MSB 34A

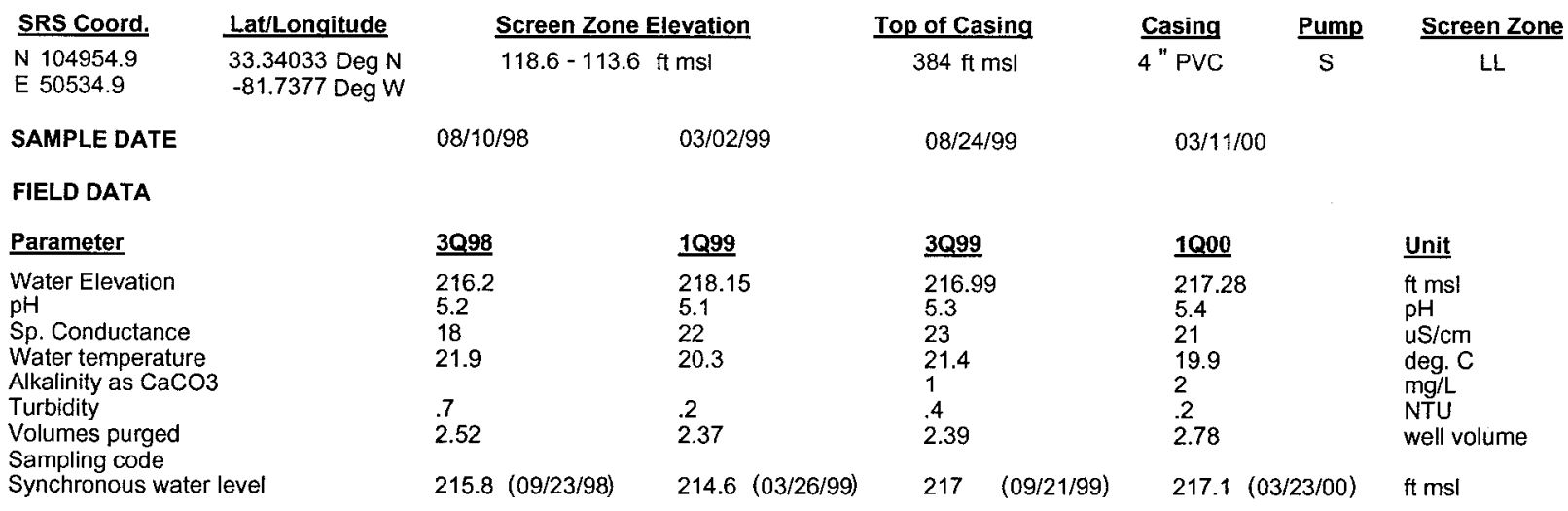

ANALYTICAL DATA

I. Groundwater Protection Standard

261 Appendix VIII/264 Appendix IX Hazardous Constituents

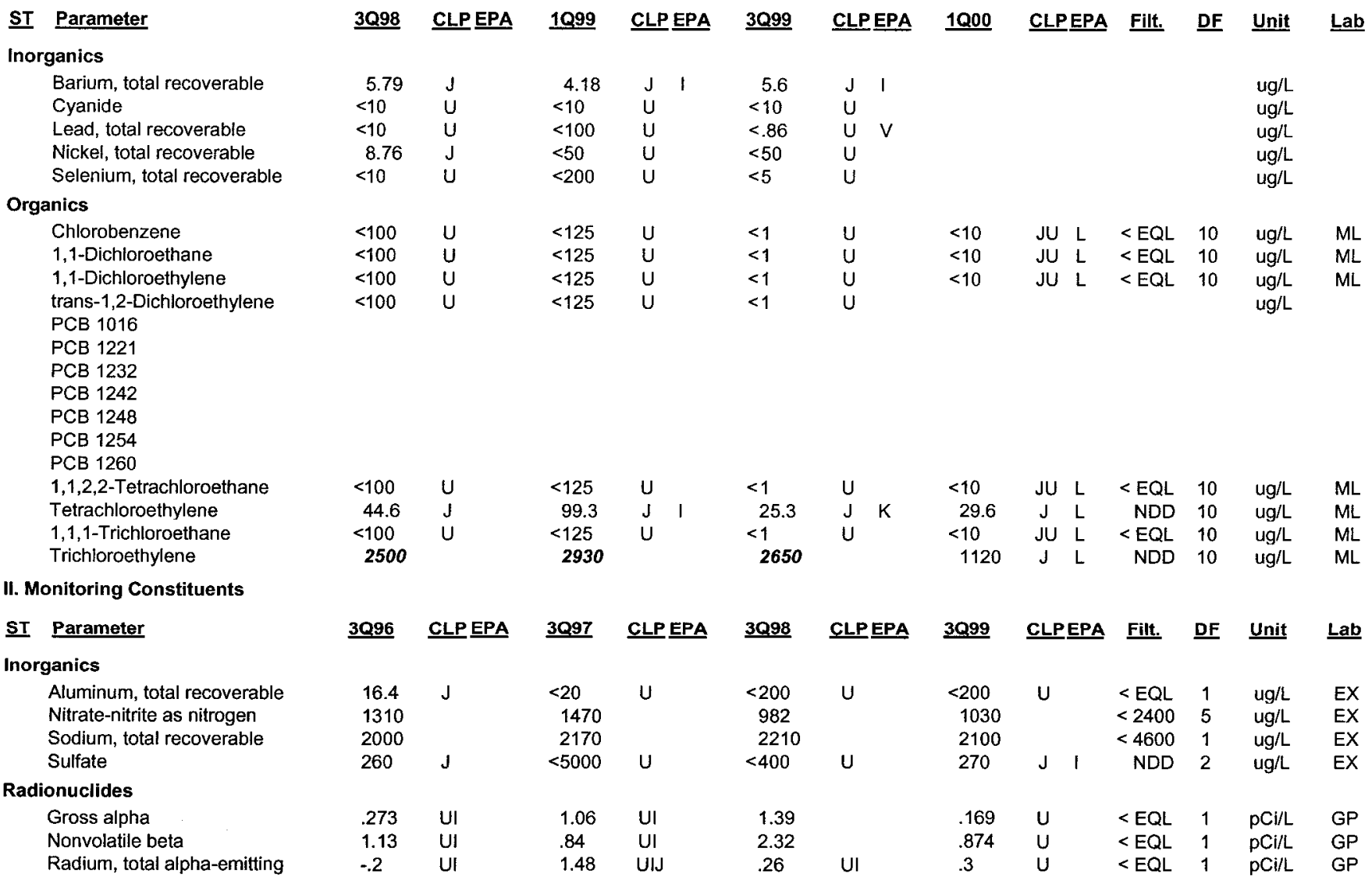

Notes: Concentrations in bold italics exceed the groundwater protection or monitoring constituent standards listed in Appendix A. Synchronous water levels are measured over a 3-5 day period or less. Dilution factors, Laboratory, and Filtered Data are for Groundwater Protection Standards First Quarter 2000 and Monitoring Constituents Third Quarter 1999 data only.

$+=$ exceeded the Groundwater Protection Standards (First Quarter 2000) or Monitoring Constituents (Third Quarter 1999) listed in Appendix A. 
Table D-4. Groundwater Monitoring Results for Plume Definition Wells, M-Area HWMF (Cont.) WELL MSB 34B

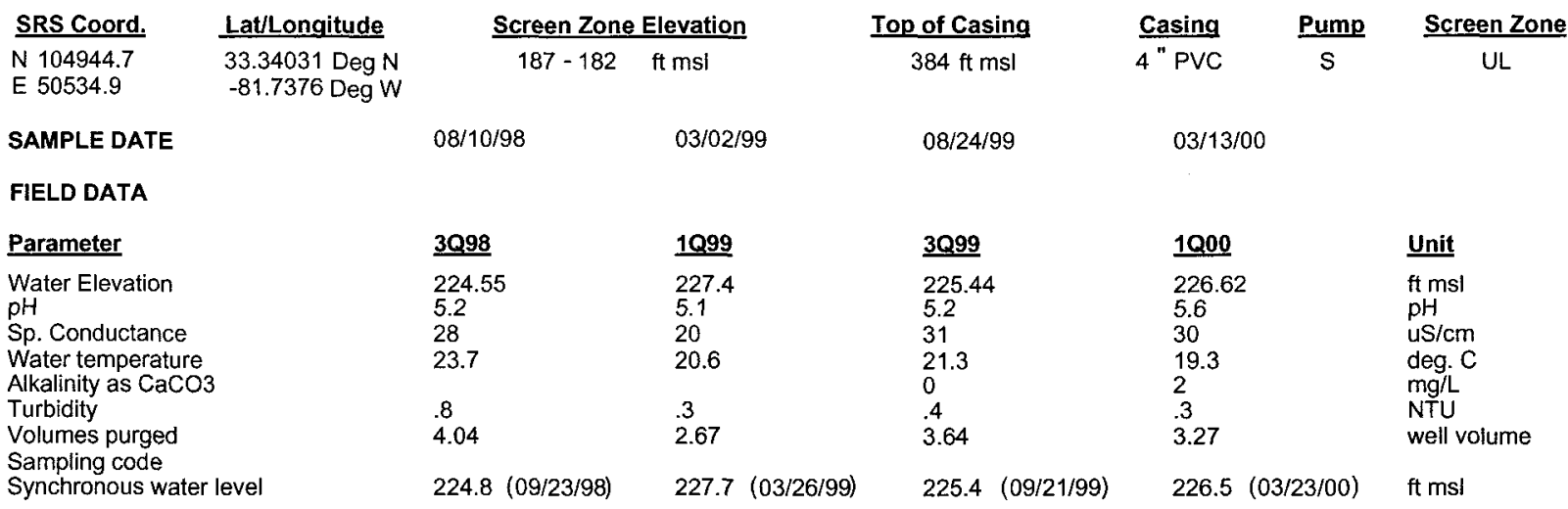

ANALYTICAL DATA

I. Groundwater Protection Standard

\begin{tabular}{|c|c|c|c|c|c|c|c|c|c|c|c|c|c|c|c|}
\hline \multirow{2}{*}{ ST } & Parameter & \multirow[t]{2}{*}{$\underline{3 Q 98}$} & \multirow{2}{*}{ CLP EPA } & \multirow[t]{2}{*}{1099.} & \multirow{2}{*}{ CLP EPA } & \multirow[t]{2}{*}{$\underline{3 Q 99}$} & \multicolumn{2}{|c|}{ CLPEPA } & \multirow{2}{*}{$1 \mathrm{Q00}$} & \multicolumn{2}{|c|}{ CLPEPA } & \multirow[t]{2}{*}{ Filt. } & \multirow[t]{2}{*}{$\underline{\text { DF }}$} & \multirow[t]{2}{*}{ Unit } & \multirow[t]{2}{*}{ Lab } \\
\hline & ganics & & & & & & & & & & & & & & \\
\hline & Barium, total recoverable & 6.88 & $\mathrm{~J}$ & 7.57 & J I & 6.4 & $J$ & I & & & & & & ug/L & \\
\hline & Cyanide & $<10$ & U & $<10$ & $\mathrm{U}$ & $<10$ & $\mathrm{U}$ & & & & & & & $u g / L$ & \\
\hline & Lead, total recoverable & 4.46 & $\mathrm{~J}$ & $<100$ & $\mathrm{U}$ & $<2.88$ & $\mathrm{U}$ & V & & & & & & $u g / L$ & \\
\hline & Nickel, total recoverable & $<50$ & U & $<50$ & $\mathrm{U}$ & $<50$ & $\mathrm{U}$ & & & & & & & $u g / L$ & \\
\hline & Selenium, total recoverable & $<10$ & $\mathrm{U}$ & $<200$ & $\mathrm{U}$ & $<5$ & $\mathrm{U}$ & & & & & & & $\mathrm{ug} / \mathrm{L}$ & \\
\hline \multicolumn{16}{|c|}{ Organics } \\
\hline & Chlorobenzene & $<5$ & $\mathrm{U}$ & $<5$ & $\mathrm{U}$ & $<1$ & $u$ & & 3.28 & $\mathrm{~J}$ & $\mathrm{~L}$ & NDD & 1 & ug/L & $\mathrm{ML}$ \\
\hline & 1,1-Dichloroethane & $<5$ & $\mathrm{U}$ & $<5$ & $\mathrm{U}$ & $<1$ & $\mathrm{U}$ & & $<1$ & JU & $\mathrm{L}$ & $<E Q L$ & 1 & $\mathrm{ug} / \mathrm{L}$ & $\mathrm{ML}$ \\
\hline & 1,1-Dichloroethylene & $<5$ & $\mathrm{U}$ & $<5$ & $\mathrm{U}$ & $<1$ & $\mathrm{U}$ & & $<1$ & JU & $L$ & $<E Q L$ & 1 & $u g / L$ & ML \\
\hline & trans-1,2-Dichloroethylene & $<5$ & $\mathrm{U}$ & $<5$ & $\mathrm{u}$ & $<1$ & $\mathrm{u}$ & & $<1$ & JU & $\mathrm{L}$ & $<$ EQL & 1 & ug/L & $M L$ \\
\hline & PCB 1016 & & & & & & & & & & & & & & \\
\hline & PCB 1221 & & & & & & & & & & & & & & \\
\hline & PCB 1232 & & & & & & & & & & & & & & \\
\hline & PCB 1242 & & & & & & & & & & & & & & \\
\hline & PCB 1248 & & & & & & & & & & & & & & \\
\hline & PCB 1254 & & & & & & & & & & & & & & \\
\hline & PCB 1260 & & & & & & & & & & & & & & \\
\hline & 1,1,2,2-Tetrachloroethane & $<5$ & $U$ & $<5$ & $\mathrm{U}$ & $<1$ & $\mathrm{U}$ & & $<1$ & JU & $L$ & $<$ EQL & 1 & $\mathrm{ug} / \mathrm{L}$ & $M L$ \\
\hline & Tetrachloroethylene & 16.9 & & 19.4 & & 21.3 & $\mathrm{~J}$ & $\mathrm{~K}$ & 19.6 & $J$ & $\mathrm{~L}$ & NDD & 1 & $\mathrm{ug} / \mathrm{L}$ & $\mathrm{ML}$ \\
\hline & 1,1,1-Trichloroethane & $<5$ & $U$ & $<5$ & $\cup$ & $<1$ & $\mathrm{U}$ & & $<1$ & JU & $L$ & $<$ EQL & 1 & $\mathrm{ug} / \mathrm{L}$ & $M L$ \\
\hline & Trichloroethylene & 26.4 & & 21.6 & & 25.9 & $J$ & K & 50.7 & $J$ & $L$ & NDD & 1 & $\mathrm{ug} / \mathrm{L}$ & $\mathrm{ML}$ \\
\hline \multicolumn{16}{|c|}{ II. Monitoring Constituents } \\
\hline$\underline{\mathbf{S T}}$ & Parameter & $\underline{3 Q 996}$ & CLP EPA & $\underline{3097}$ & CLP EPA & $\underline{3 Q 98}$ & CLP & EPA & $\underline{3099}$ & CLP. & EPA & Filt. & $\underline{\text { DF }}$ & $\underline{\text { Unit }}$ & $\underline{\text { Lab }}$ \\
\hline \multicolumn{16}{|c|}{ Inorganics } \\
\hline & Aluminum, total recoverable & 30.7 & & 24.4 & & $<200$ & $U$ & & $<200$ & $\mathrm{U}$ & & $<\mathrm{EQL}$ & 1 & $\mathrm{ug} / \mathrm{L}$ & EX \\
\hline & Nitrate-nitrite as nitrogen & 1260 & & 1070 & & 1130 & & & 881 & & & $<2400$ & 5 & $\mathrm{ug} / \mathrm{L}$ & EX \\
\hline & Sodium, total recoverable & 2240 & & 2300 & & 2700 & & & 3000 & & & $<4600$ & 1 & ug/L & EX \\
\hline & Sulfate & 162 & $J$ & $<5000$ & $\mathrm{U}$ & $<400$ & $\mathrm{U}$ & & 577 & & & $<3000$ & 2 & ug/L & EX \\
\hline \multicolumn{16}{|c|}{ Radionuclides } \\
\hline & Gross alpha & 1.39 & & 3.86 & & 3.88 & & & 1.8 & & & $<15$ & 1 & $\mathrm{pCi} / \mathrm{L}$ & GP \\
\hline & Nonvolatile beta & 1.69 & & 3.03 & & 4.79 & & & 1.93 & $\mathrm{~J}$ & 1 & NDD & 1 & $\mathrm{pCi} / \mathrm{L}$ & GP \\
\hline & Radium, total alpha-emitting & 1.3 & & 6.28 & $\mathrm{~J}$ & .91 & & & 1.3 & $\mathrm{~J}$ & I & NDD & 1 & $\mathrm{pCi} / \mathrm{L}$ & GP \\
\hline
\end{tabular}

Notes: Concentrations in bold italics exceed the groundwater protection or monitoring constituent standards listed in Appendix A. Synchronous water levels are measured over a 3-5 day period or less. Dilution factors, Laboratory, and Filtered Data are for Groundwater Protection Standards First Quarter 2000 and Monitoring Constituents Third Quarter 1999 data only.

+ = exceeded the Groundwater Protection Standards (First Quarter 2000) or Monitoring Constituents (Third Quarter 1999) listed in Appendix A. 
Table D-4. Groundwater Monitoring Results for Plume Definition Wells, M-Area HWMF (Cont.) WELL MSB 34C

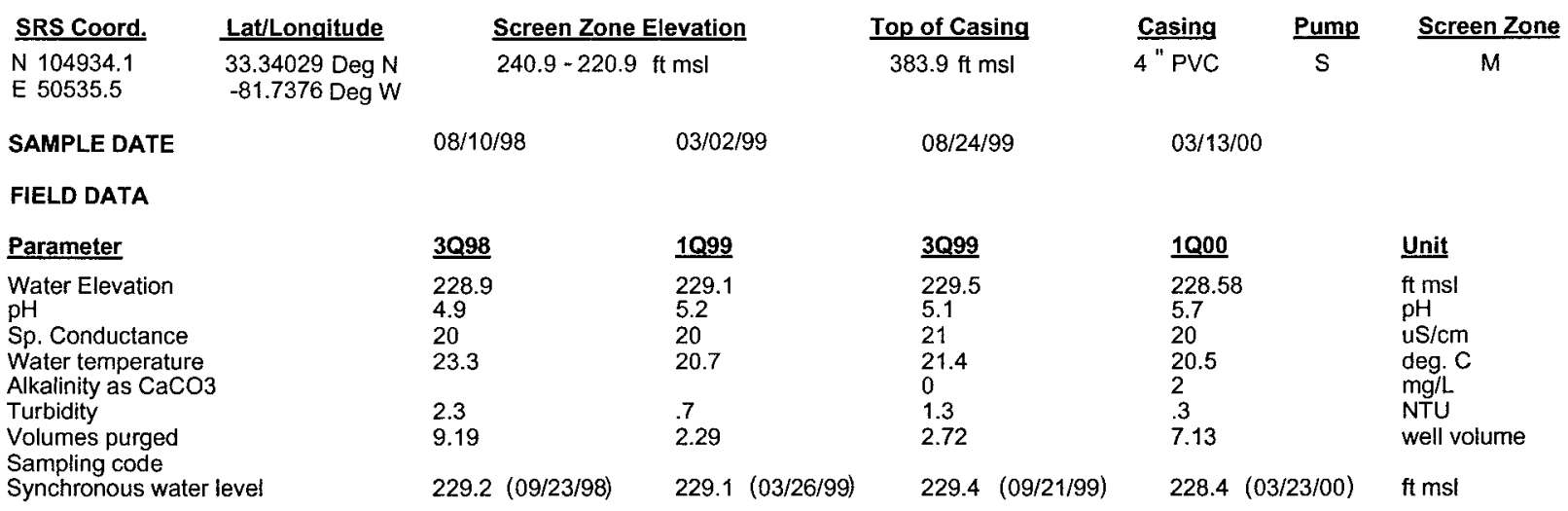

ANALYTICAL DATA

I. Groundwater Protection Standard

261 Appendix VIII/264 Appendix IX Hazardous Constituents

\begin{tabular}{|c|c|c|c|c|c|c|c|c|c|c|c|c|c|c|}
\hline \multirow{2}{*}{$\frac{\text { ST }}{\text { Inors }}$} & Parameter & \multirow[t]{2}{*}{$\underline{3 Q 98}$} & \multirow[t]{2}{*}{ CLP EPA } & \multirow[t]{2}{*}{1099} & CLP EPA & \multirow[t]{2}{*}{$\underline{3 Q 99}$} & CLPEPA & \multirow[t]{2}{*}{$\underline{1 Q 00}$} & \multicolumn{2}{|c|}{ CLPEPA } & \multirow[t]{2}{*}{ Filt. } & \multirow[t]{2}{*}{$\underline{\text { DF }}$} & \multirow[t]{2}{*}{$\underline{\text { Unit }}$} & \multirow[t]{2}{*}{$\underline{\text { Lab }}$} \\
\hline & ganics & & & & & & & & & & & & & \\
\hline & Barium, total recoverable & 4.08 & J & 3.2 & J I & 3.7 & J I & & & & & & ug̊/L & \\
\hline & Cyanide & $<10$ & $\mathrm{U}$ & $<10$ & $U$ & $<10$ & $\mathrm{U}$ & & & & & & $\mathrm{ug} / \mathrm{L}$ & \\
\hline & Lead, total recoverable & 12.3 & & $<100$ & U & 15.2 & & & & & & & $\mathrm{ug} / \mathrm{L}$ & \\
\hline & Nickel, total recoverable & 8.06 & $\mathrm{~J}$ & $<50$ & U & $<50$ & $\mathrm{U}$ & & & & & & $\mathrm{ug} / \mathrm{L}$ & \\
\hline & Selenium, total recoverable & $<10$ & $\mathrm{U}$ & $<200$ & U & $<5$ & U & & & & & & ug/L & \\
\hline \multicolumn{15}{|c|}{ Organics } \\
\hline & Chlorobenzene & $<25$ & $\mathrm{U}$ & $<5$ & $\mathrm{U}$ & $<1$ & $\mathrm{U}$ & 4.66 & $J$ & $\mathrm{~L}$ & NDD & 1 & $\mathrm{ug} / \mathrm{L}$ & ML \\
\hline & 1,1-Dichloroethane & $<25$ & $\mathrm{U}$ & $<5$ & U & $<1$ & $\mathrm{U}$ & $<1$ & JU & $\bar{L}$ & $<E Q L$ & 1 & $\mathrm{ug} / \mathrm{L}$ & $M L$ \\
\hline & 1,1-Dichloroethylene & $<25$ & $\mathrm{U}$ & $<5$ & $U$ & $<1$ & U & $<1$ & JU & $L$ & $<\mathrm{EQL}$ & 1 & $\mathrm{ug} / \mathrm{L}$ & ML \\
\hline & trans-1,2-Dichloroethylene & $<25$ & $\mathrm{u}$ & $<5$ & $u$ & $<1$ & $U$ & $<1$ & JU & $\bar{L}$ & $<E Q L$ & 1 & $\mathrm{ug} / \mathrm{L}$ & $\mathrm{ML}$ \\
\hline & PCB 1016 & & & & & & & & & & & & & \\
\hline & PCB 1221 & & & & & & & & & & & & & \\
\hline & PCB 1232 & & & & & & & & & & & & & \\
\hline & РСВ 1242 & & & & & & & & & & & & & \\
\hline & РСВ 1248 & & & & & & & & & & & & & \\
\hline & PCB 1254 & & & & & & & & & & & & & \\
\hline & PCB 1260 & & & & & & & & & & & & & \\
\hline & 1,1,2,2-Tetrachloroethane & $<25$ & $U$ & $<5$ & $u$ & $<1$ & $\mathrm{U}$ & $<1$ & JU & $\mathrm{L}$ & $<$ EQL & 1 & $\mathrm{ug} / \mathrm{L}$ & $M L$ \\
\hline & Tetrachloroethylene & 5.43 & & 4.27 & $J$ & $<3.18$ & U V & 6.5 & $\mathrm{~J}$ & $\mathrm{~L}$ & NDD & 1 & $\mathrm{ug} / \mathrm{L}$ & $M L$ \\
\hline & $1,1,1$-Trichloroethane & $<25$ & $u$ & $<5$ & $\mathrm{U}$ & $<1$ & $U$ & $<1$ & JU & $\mathrm{L}$ & $<E Q L$ & 1 & $\mathrm{ug} / \mathrm{L}$ & $M L$ \\
\hline & Trichloroethylene & 12.8 & & 7.58 & & 5.65 & $\mathrm{~J} K$ & 11.3 & $J$ & $\bar{L}$ & NDD & 1 & $\mathrm{ug} / \mathrm{L}$ & $\mathrm{ML}$ \\
\hline \multicolumn{15}{|c|}{ II. Monitoring Constituents } \\
\hline$\underline{\mathrm{ST}}$ & Parameter & $\underline{3096}$ & CLP EPA & 3097 & CLP EPA & $\underline{3 Q 98}$ & CLPEPA & $\underline{3099}$ & $\underline{\text { CLF }}$ & EPA & Filt. & $\underline{\text { DF }}$ & Unit & $\underline{L a b}$ \\
\hline \multicolumn{15}{|c|}{ Inorganics } \\
\hline & Aluminum, total recoverable & 47.6 & & 38.8 & & 72.6 & $J$ & $<200$ & $\mathrm{U}$ & & $<\mathrm{EQL}$ & 1 & ug/L & EX \\
\hline & Nitrate-nitrite as nitrogen & 1760 & & 1250 & & 1350 & & 1250 & & & $<2400$ & 5 & ug/L & EX \\
\hline & Sodium, total recoverable & 1550 & & 1610 & & 2120 & & 2100 & & & $<4600$ & 1 & $\mathrm{ug} / \mathrm{L}$ & EX \\
\hline & Sulfate & 238 & $J$ & $<5000$ & U & $<400$ & $\mathrm{U}$ & 755 & & & $<3000$ & 2 & $\mathrm{ug} / \mathrm{L}$ & EX \\
\hline \multicolumn{15}{|c|}{ Radionuclides } \\
\hline & Gross alpha & 2.93 & & 3.08 & & 4.77 & & 2.32 & & & $<15$ & 1 & $\mathrm{pCi} / \mathrm{L}$ & GP \\
\hline & Nonvolatile beta & 2.02 & & 2.41 & & 5.33 & & 3 & & & $<50$ & 1 & $\mathrm{pCi} / \mathrm{L}$ & GP \\
\hline & Radium, total alpha-emitting & .9 & & 3.08 & $\mathrm{~J}$ & 1.72 & & 1.4 & $\mathrm{~J}$ & 1 & NDD & 1 & $\mathrm{pCi} / \mathrm{L}$ & GP \\
\hline
\end{tabular}

Notes: Concentrations in bold italics exceed the groundwater protection or monitoring constituent standards listed in Appendix A. Synchronous water levels are measured over a 3-5 day period or less. Dilution factors, Laboratory, and Filtered Data are for Groundwater Protection Standards First Quarter 2000 and Monitoring Constituents Third Quarter 1999 data only.

+ = exceeded the Groundwater Protection Standards (First Quarter 2000) or Monitoring Constituents (Third Quarter 1999) listed in Appendix A. 
Table D-4. Groundwater Monitoring Results for Plume Definition Wells, M-Area HWMF (Cont.) WELL MSB 34TA

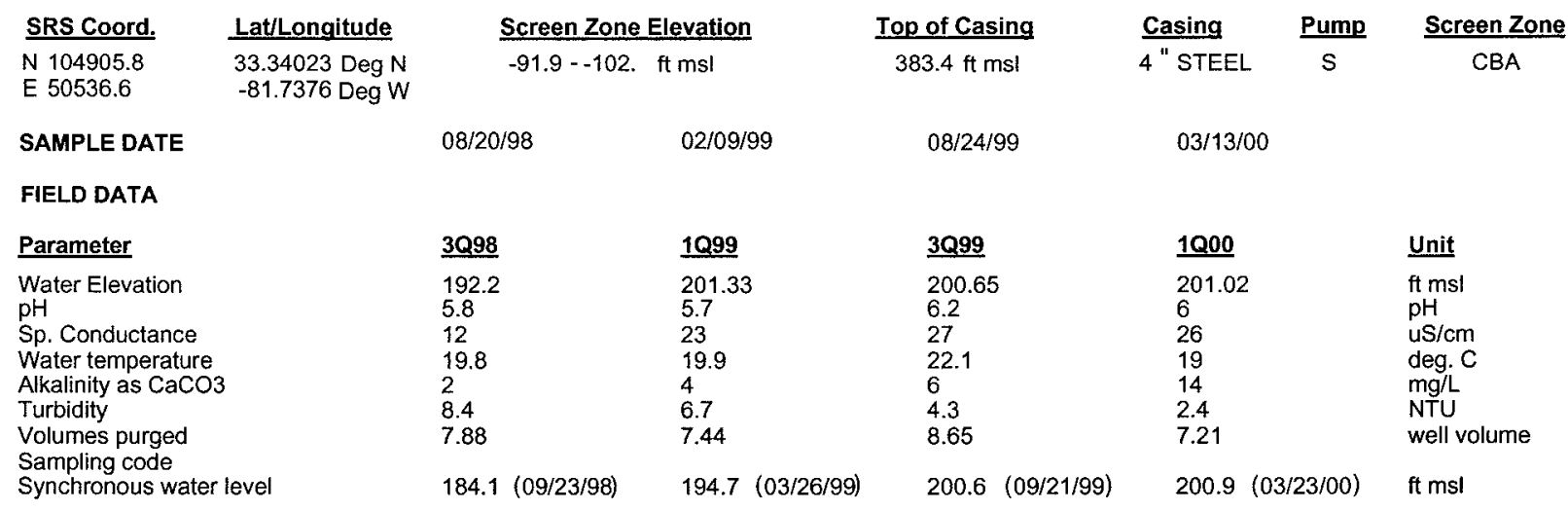

ANALYTICAL DATA

I. Groundwater Protection Standard

261 Appendix VIII/264 Appendix IX Hazardous Constituents

\begin{tabular}{|c|c|c|c|c|c|c|c|c|c|c|c|c|c|c|c|c|}
\hline \multirow{2}{*}{\multicolumn{2}{|c|}{$\frac{\text { ST }}{\text { Parameter }}$}} & \multirow[t]{2}{*}{$\underline{3 Q 98}$} & \multirow[t]{2}{*}{ CLPEPA } & \multirow[t]{2}{*}{1099} & \multicolumn{2}{|c|}{ CLP EPA } & \multirow[t]{2}{*}{$\underline{3 Q 99}$} & \multicolumn{2}{|c|}{ CLPEPA } & \multirow[t]{2}{*}{$\underline{1 Q 00}$} & \multicolumn{2}{|c|}{ CLPEPA } & \multirow[t]{2}{*}{ Filt. } & \multirow[t]{2}{*}{$\underline{\mathrm{DF}}$} & \multirow[t]{2}{*}{ Unit } & \multirow[t]{2}{*}{$\underline{L a b}$} \\
\hline & & & & & & & & & & & & & & & & \\
\hline & Barium, total recoverable & 2.97 & $J$ & 2.27 & $J$ & 1 & 2.5 & $J$ & 1 & & & & & & ug/L & \\
\hline & Cyanide & $<10$ & $\mathrm{U}$ & $<10$ & $U$ & & $<10$ & $\mathrm{U}$ & & & & & & & $\mathrm{ug} / \mathrm{L}$ & \\
\hline & Lead, total recoverable & $<10$ & $U$ & $<100$ & $U$ & & $<.91$ & $\mathrm{U}$ & V & & & & & & $\mathrm{ug} / \mathrm{L}$ & \\
\hline & Nickel, total recoverable & $<50$ & $U$ & $<50$ & $U$ & & $<50$ & $\mathrm{U}$ & & & & & & & $\mathrm{ug} / \mathrm{L}$ & \\
\hline & Selenium, total recoverable & $<10$ & $\mathrm{U}$ & $<200$ & $U$ & & $<1.26$ & $\mathrm{U}$ & V & & & & & & ug/L & \\
\hline \multicolumn{17}{|c|}{ Organics } \\
\hline & Chlorobenzene & $<5$ & $\mathrm{U}$ & $<5$ & JU & $Q$ & $<1$ & $\mathrm{u}$ & & 1.75 & $J$ & $L$ & NDD & 1 & $\mathrm{ug} / \mathrm{L}$ & ML \\
\hline & 1,1-Dichloroethane & $<5$ & $\mathrm{U}$ & $<5$ & JU & $Q$ & $<1$ & $\mathrm{U}$ & & $<1$ & JU & $L$ & $<\mathrm{EQL}$ & 1 & $\mathrm{ug} / \mathrm{L}$ & $M L$ \\
\hline & 1,1-Dichloroethylene & $<5$ & U & $<5$ & JU & $Q$ & $<1$ & $\mathrm{U}$ & & $<1$ & JU & $\mathrm{L}$ & $<\mathrm{EQL}$ & 1 & $\mathrm{ug} / \mathrm{L}$ & $M L$ \\
\hline & trans-1,2-Dichloroethylene & $<5$ & $\mathrm{U}$ & $<5$ & JU & $Q$ & $<1$ & $\mathrm{U}$ & & $<1$ & JU & L & $<\mathrm{EQL}$ & 1 & $u g / L$ & $\mathrm{ML}$ \\
\hline & PCB 1016 & & & & & & & & & & & & & & & \\
\hline & PCB 1221 & & & & & & & & & & & & & & & \\
\hline & РCB 1232 & & & & & & & & & & & & & & & \\
\hline & PCB 1242 & & & & & & & & & & & & & & & \\
\hline & PCB 1248 & & & & & & & & & & & & & & & \\
\hline & PCB 1254 & & & & & & & & & & & & & & & \\
\hline & PCB 1260 & & & & & & & & & & & & & & & \\
\hline & 1,1,2,2-Tetrachloroethane & $<5$ & $U$ & $<5$ & JU & $Q$ & $<1$ & $\mathrm{U}$ & & $<1$ & JU & $L$ & $<\mathrm{EQL}$ & 1 & $\mathrm{ug} / \mathrm{L}$ & ML \\
\hline & Tetrachloroethylene & $<5$ & $U$ & $<5$ & JU & $Q$ & $<1$ & $\mathrm{U}$ & & $<1$ & JU & $\mathrm{L}$ & $<\mathrm{EQL}$ & 1 & $\mathrm{ug} / \mathrm{L}$ & $M L$ \\
\hline & 1,1,1-Trichloroethane & $<5$ & $\mathrm{U}$ & $<5$ & JU & $Q$ & $<1$ & $\mathrm{U}$ & & $<1$ & JU & L & $<\mathrm{EQL}$ & 1 & $\mathrm{ug} / \mathrm{L}$ & ML \\
\hline & Trichloroethylene & 14 & & 12.8 & $\mathrm{~J}$ & $Q$ & 13.1 & $J$ & K & 12.1 & $\mathrm{~J}$ & L & NDD & 1 & $\mathrm{ug} / \mathrm{L}$ & $\mathrm{ML}$ \\
\hline \multicolumn{17}{|c|}{ II. Monitoring Constituents } \\
\hline$\underline{\text { ST }}$ & Parameter & $\underline{3 Q 96}$ & CLPEPA & $\underline{3 Q 97}$ & $\underline{\text { CLP }}$ & EPA & $\underline{3098}$ & CLP & EPA & $\underline{3099}$ & CLP & EPA & Filt. & DF & Unit & 느 \\
\hline \multicolumn{17}{|c|}{ Inorganics } \\
\hline & Aluminum, total recoverable & $<20$ & $U$ & 17.3 & J & & $<200$ & $\mathrm{U}$ & & $<200$ & $\mathrm{U}$ & & $<\mathrm{EQL}$ & 1 & $\mathrm{ug} / \mathrm{L}$ & EX \\
\hline & Nitrate-nitrite as nitrogen & 990 & & 694 & & & 718 & & & 699 & & & $<2400$ & 5 & $\mathrm{ug} / \mathrm{L}$ & EX \\
\hline & Sodium, total recoverable & 1690 & & 1780 & & & $<1670$ & $\mathrm{U}$ & & 1900 & & & $<4600$ & 1 & $u g / L$ & EX \\
\hline & Sulfate & 429 & $J$ & $<5000$ & $\mathrm{U}$ & & 971 & & & 369 & $J$ & 1 & NDD & 2 & ug/L & EX \\
\hline \multicolumn{17}{|c|}{ Radionuclides } \\
\hline & Gross alpha & .293 & UI & 2.69 & UI & & .87 & & & .603 & $U$ & & < EQL & 1 & $\mathrm{pCi} / \mathrm{L}$ & $\mathrm{GP}$ \\
\hline & Nonvolatile beta & 1.19 & UI & 2.23 & UI & & 1.34 & UI & & 1.4 & $\mathrm{~J}$ & I & NDD & 1 & $\mathrm{pCi} / \mathrm{L}$ & GP \\
\hline & Radium, total alpha-emitting & .1 & UI & .92 & $\mathrm{~J}$ & & .62 & & & .3 & $\mathrm{U}$ & & $<E Q L$ & 1 & $\mathrm{pCi} / \mathrm{L}$ & GP \\
\hline
\end{tabular}

Notes: Concentrations in bold italics exceed the groundwater protection or monitoring constituent standards listed in Appendix A. Synchronous water levels are measured over a 3-5 day period or less. Dilution factors, Laboratory, and Filtered Data are for Groundwater Protection Standards First Quarter 2000 and Monitoring Constituents Third Quarter 1999 data only.

+ = exceeded the Groundwater Protection Standards (First Quarter 2000) or Monitoring Constituents (Third Quarter 1999) listed in Appendix A. 
Table. D-4. Groundwater Monitoring Results for Plume Definition Wells, M-Area HWMF (Cont.) WELL MSB 35A

\begin{tabular}{|c|c|c|c|c|c|c|}
\hline SRS Coord. & Lat/Longitude & Screen Zone Elevation & Top of Casing & Casing & Pump & Screen Zone \\
\hline $\begin{array}{l}\text { N } 102098 \\
E ~ 50945.2\end{array}$ & $\begin{array}{l}\text { 33.33469 Deg N } \\
-81.7310 \mathrm{Deg} W\end{array}$ & $128.8-123.2 \mathrm{ft} \mathrm{msl}$ & $350.9 \mathrm{ft} \mathrm{msl}$ & $4 " \mathrm{PVC}$ & $S$ & MCBC \\
\hline
\end{tabular}

SAMPLE DATE

FIELD DATA

Parameter

Water Elevation

$\mathrm{pH}$

Sp. Conductance

Water temperature

Alkalinity as $\mathrm{CaCO} 3$

Turbidity

Volumes purged

Sampling code

Synchronous water level

ANALYTICAL DATA

I. Groundwater Protection Standard

\begin{tabular}{|c|c|c|c|c|}
\hline 3098 & 1099 & 3099 & 1000 & Unit \\
\hline Not Available & Not Available & Not Available & Not Available & $\begin{array}{l}\mathrm{ft} \mathrm{msl} \\
\mathrm{pH} \\
\mathrm{uS} / \mathrm{cm} \\
\mathrm{deg} . \mathrm{C} \\
\mathrm{mg} / \mathrm{L} \\
\mathrm{NTU} \\
\text { well volume }\end{array}$ \\
\hline $216.1(09 / 21 / 98)$ & $216.3(03 / 26 / 99)$ & $216 \quad(09 / 22 / 99)$ & $215 \quad(03 / 23 / 00)$ & ft msl \\
\hline
\end{tabular}

Notes: Concentrations in bold italics exceed the groundwater protection or monitoring constituent standards listed in Appendix A. Synchronous water levels are measured over a 3-5 day period or less. Dilution factors, Laboratory, and Filtered Data are for Groundwater Protection Standards First Quarter 2000 and Monitoring Constituents Third Quarter 1999 data only.

$+=$ exceeded the Groundwater Protection Standards (First Quarter 2000) or Monitoring Constituents (Third Quarter 1999) listed in Appendix A. 
Table D-4. Groundwater Monitoring Results for Plume Definition Wells, M-Area HWMF (Cont.) WELL MSB 35B

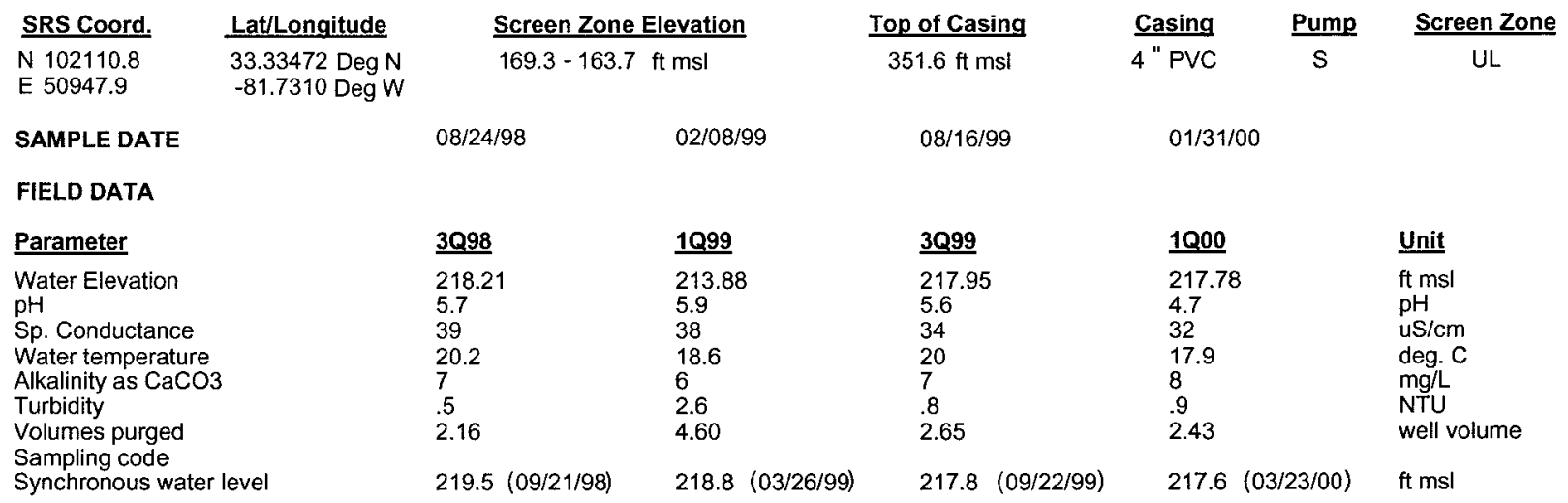

ANALYTICAL DATA

I. Groundwater Protection Standard

261 Appendix VIII/264 Appendix IX Hazardous Constituents

\begin{tabular}{|c|c|c|c|c|c|c|c|c|c|c|c|c|c|c|}
\hline$\underline{\text { ST }}$ & Parameter & $\underline{3 Q 98}$ & CLP EPA & $\underline{1 Q 99}$ & CLPPEPA & $\underline{3 Q 99}$ & CLPEPA & 10,00 & $\underline{\text { CLP }}$ & EPA & Filt. & $\underline{\text { DF }}$ & Unit & Lab \\
\hline Inor & ganics & & & & & & & & & & & & & \\
\hline & Barium, total recoverable & 4.78 & $J$ & $<10$ & $U$ & 3.8 & J I & & & & & & ug/L & \\
\hline & Cyanide & $<10$ & U & $<10$ & $U$ & $<10$ & U & & & & & & $\mathrm{ug} / \mathrm{L}$ & \\
\hline & Lead, total recoverable & $<10$ & $U$ & $<100$ & $U$ & $<2.56$ & $\mathrm{U} V$ & & & & & & $\mathrm{ug} / \mathrm{L}$ & \\
\hline & Nickel, total recoverable & $<50$ & $\mathrm{U}$ & $<50$ & $U$ & $<50$ & $\cup$ & & & & & & ug/L & \\
\hline & Selenium, total recoverable & 6.16 & $J$ & $<200$ & $\mathrm{U}$ & $<5$ & $U$ & & & & & & $\mathrm{ug} / \mathrm{L}$ & \\
\hline Org & anics & & & & & & & & & & & & & \\
\hline & Chlorobenzene & $<5$ & $u$ & $<5$ & $u$ & $<1$ & $U$ & $<1$ & JU & $\mathrm{L}$ & $<$ EQL & 1 & $\mathrm{ug} / \mathrm{L}$ & $\mathrm{ML}$ \\
\hline & 1,1-Dichloroethane & $<5$ & $\mathrm{U}$ & $<5$ & $U$ & $<1$ & $\cup$ & $<1$ & JU & $\mathrm{L}$ & $<\mathrm{EQL}$ & 1 & $\mathrm{ug} / \mathrm{L}$ & ML \\
\hline & 1,1-Dichloroethylene & $<5$ & U & $<5$ & $\mathrm{U}$ & $<1$ & $\mathrm{U}$ & $<1$ & JU & $\mathrm{L}$ & $<\mathrm{EQL}$ & 1 & $\mathrm{ug} / \mathrm{L}$ & $M L$ \\
\hline & trans-1,2-Dichloroethylene & $<5$ & $U$ & $<5$ & U & $<1$ & $\mathrm{U}$ & & & & & & $\mathrm{ug} / \mathrm{L}$ & \\
\hline & РCB 1016 & & & & & & & & & & & & & \\
\hline & PCB 1221 & & & & & & & & & & & & & \\
\hline & PCB 1232 & & & & & & & & & & & & & \\
\hline & PCB 1242 & & & & & & & & & & & & & \\
\hline & PCB 1248 & & & & & & & & & & & & & \\
\hline & PCB 1254 & & & & & & & & & & & & & \\
\hline & PCB 1260 & & & & & & & & & & & & & \\
\hline & $1,1,2,2$-Tetrachloroethane & $<5$ & $\mathrm{U}$ & $<5$ & $\mathrm{U}$ & $<1$ & U & $<1$ & JU & $\mathrm{L}$ & $<$ EQL & 1 & $\mathrm{ug} / \mathrm{L}$ & ML \\
\hline & Tetrachloroethylene & $<5$ & $\mathrm{U}$ & $<5$ & $\mathrm{U}$ & $<1$ & $U$ & $<1$ & $J U$ & $\mathrm{~L}$ & $<\mathrm{EQL}$ & 1 & $u g / L$ & ML \\
\hline & $1,1,1$-Trichloroethane & $<5$ & U & $<5$ & $\mathrm{U}$ & $<1$ & $\mathrm{U}$ & $<1$ & JU & $L$ & $<\mathrm{EQL}$ & 1 & $u g / L$ & ML \\
\hline & Trichloroethylene & $<5$ & U & $<5$ & $\mathrm{U}$ & $<1$ & $\mathrm{U}$ & $<1$ & JU & $L$ & $<\mathrm{EQL}$ & 1 & ug/L & ML \\
\hline II. $\mathbf{M}$ & onitoring Constituents & & & & & & & & & & & & & \\
\hline$\underline{\text { ST }}$ & Parameter & $\underline{3096}$ & CLP EPA & $\underline{3097}$ & CLP EPA & $\underline{3 Q 98}$ & CLPEPA & $\underline{3099}$ & $\underline{\text { CLP }}$ & EPA & Fill. & $\underline{D F}$ & Unit & Lab \\
\hline Inor & ganics & & & & & & & & & & & & & \\
\hline & Aluminum, total recoverable & 34.7 & J & $<20$ & $U$ & $<200$ & $\mathrm{U}$ & $<200$ & $\mathrm{U}$ & & $<E Q L$ & 1 & $\mathrm{ug} / \mathrm{L}$ & EX \\
\hline & Nitrate-nitrite as nitrogen & 320 & & 205 & & 216 & J & 215 & $J$ & 1 & NDD & 5 & $\mathrm{ug} / \mathrm{L}$ & EX \\
\hline & Sodium, total recoverable & 4210 & J & 4470 & & 4650 & & 4400 & & & $<4600$ & 1 & $u g / L$ & EX \\
\hline & Sulfate & 1610 & & $<5000$ & $\mathrm{U}$ & 1410 & & 1370 & & & $<3000$ & 1 & $\mathrm{ug} / \mathrm{L}$ & EX \\
\hline Rac & onuclides & & & & & & & & & & & & & \\
\hline & Gross alpha & .749 & Ui & .58 & U: & .83 & UIJ & .0953 & U & & $<E Q L$ & 1 & $\mathrm{pCi} / \mathrm{L}$ & GP \\
\hline & Nonvolatile beta & .246 & $\mathrm{UI}$ & -1.08 & UI & -.1 & UI & 1.21 & $\mathrm{U}$ & & $<$ EQL & 1 & $\mathrm{pCi} / \mathrm{L}$ & GP \\
\hline & Radium, total alpha-emitting & -.1 & UI & 2.06 & UIJ & .02 & UI & -.1 & $\mathrm{U}$ & & $<$ EQL & 1 & $\mathrm{pCi} / \mathrm{L}$ & \\
\hline
\end{tabular}

Notes: Concentrations in bold italics exceed the groundwater protection or monitoring constituent standards listed in Appendix A. Synchronous water levels are measured over a 3-5 day period or less. Dilution factors, Laboratory, and Filtered Data are for Groundwater Protection Standards First Quarter 2000 and Monitoring Constituents Third Quarter 1999 data only.

+ = exceeded the Groundwater Protection Standards (First Quarter 2000) or Monitoring Constituents (Third Quarter 1999) listed in Appendix A. 
Table D-4. Groundwater Monitoring Results for Plume Definition Wells, M-Area HWMF (Cont.) WELL MSB 35TA

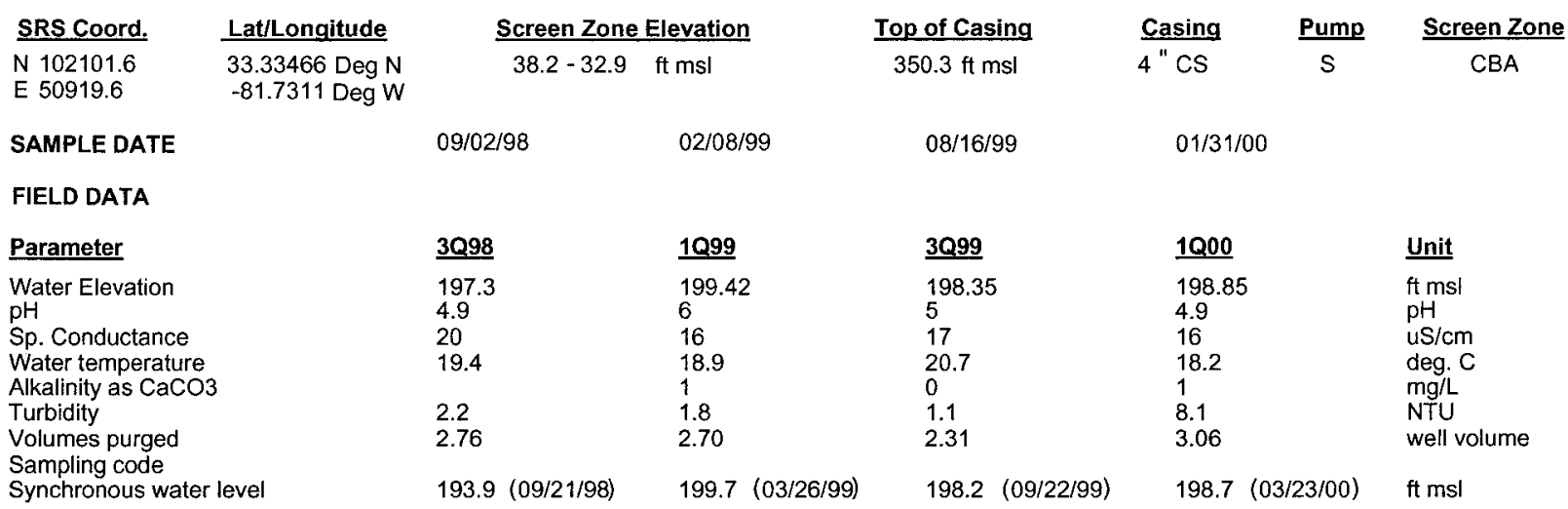

ANALYTICAL DATA

I. Groundwater Protection Standard

261 Appendix VIII/264 Appendix IX Hazardous Constituents

\begin{tabular}{|c|c|c|c|c|c|c|c|c|c|c|c|c|c|c|}
\hline \multirow{2}{*}{$\underline{\mathbf{S T}}$} & Parameter & \multirow[t]{2}{*}{$\underline{3098}$} & \multirow[t]{2}{*}{ CLP EPA } & \multirow[t]{2}{*}{1099} & \multirow[t]{2}{*}{$\underline{\text { CLP EPA }}$} & \multirow[t]{2}{*}{$\underline{3 Q 99}$} & CLPEPA & \multirow[t]{2}{*}{$\underline{1 Q 00}$} & \multicolumn{2}{|c|}{ CLPEPA } & \multirow[t]{2}{*}{ Filt. } & \multirow[t]{2}{*}{$\underline{\mathrm{DF}}$} & $\underline{\text { Unit }}$ & \multirow[t]{2}{*}{$\underline{\text { Lab }}$} \\
\hline & ganics & & & & & & & & & & & & & \\
\hline & Barium, total recoverable & 3.4 & & $<10$ & U & 2.6 & J 1 & & & & & & $\mathrm{ug} / \mathrm{L}$ & \\
\hline & Cyanide & $<15.2$ & $\mathrm{U}$ & $<10$ & $\mathrm{U}$ & $<10$ & $\mathrm{U}$ & & & & & & ug/L & \\
\hline & Lead, total recoverable & 6.6 & $J$ & $<100$ & $\mathrm{U}$ & 5.96 & & & & & & & $\mathrm{ug} / \mathrm{L}$ & \\
\hline & Nickel, total recoverable & $<50$ & $\mathrm{U}$ & $<50$ & $\mathrm{U}$ & $<50$ & $\mathrm{U}$ & & & & & & ug/L & \\
\hline & Selenium, total recoverable & $<66$ & $U$ & $<200$ & $U$ & $<5$ & $U$ & & & & & & $\mathrm{ug} / \mathrm{L}$ & \\
\hline \multicolumn{15}{|c|}{ Organics } \\
\hline & Chlorobenzene & $<5$ & $\mathrm{U}$ & $<5$ & $\mathrm{U}$ & $<1$ & $\mathrm{U}$ & $<1$ & JU & $\mathrm{L}$ & $<\mathrm{EQL}$ & 1 & ug/L & ML \\
\hline & 1,1-Dichloroethane & $<5$ & $\mathrm{U}$ & $<5$ & $\mathrm{U}$ & $<1$ & $\mathrm{U}$ & $<1$ & JU & $\mathrm{L}$ & $<\mathrm{EQL}$ & 1 & $\mathrm{ug} / \mathrm{L}$ & ML \\
\hline & 1,1-Dichloroethylene & $<5$ & $\mathrm{U}$ & $<5$ & $U$ & $<1$ & $U$ & $<1$ & JU & $L$ & $<E Q L$ & 1 & $\operatorname{ug} / \mathrm{L}$ & ML \\
\hline & trans-1,2-Dichloroethylene & $<5$ & $U$ & $<5$ & $\mathrm{U}$ & $<1$ & U & & & & & & $\mathrm{ug} / \mathrm{L}$ & \\
\hline & PCB 1016 & & & & & & & & & & & & & \\
\hline & PCB 1221 & & & & & & & & & & & & & \\
\hline & PCB 1232 & & & & & & & & & & & & & \\
\hline & PCB 1242 & & & & & & & & & & & & & \\
\hline & PCB 1248 & & & & & & & & & & & & & \\
\hline & РCB 1254 & & & & & & & & & & & & & \\
\hline & PCB 1260 & & & & & & & & & & & & & \\
\hline & 1,1,2,2-Tetrachloroethane & $<5$ & $\mathrm{U}$ & $<5$ & $U$ & $<1$ & $\mathrm{U}$ & $<1$ & JU & $\mathrm{L}$ & $<E Q L$ & 1 & $\mathrm{ug} / \mathrm{L}$ & ML \\
\hline & Tetrachloroethylene & $<5$ & $U$ & $<5$ & 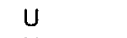 & $<1$ & $\mathrm{U}$ & $<1$ & JU & $\mathrm{L}$ & $<E Q L$ & 1 & $\mathrm{ug} / \mathrm{L}$ & ML \\
\hline & 1,1,1-Trichloroethane & $<5$ & $U$ & $<5$ & $\mathrm{U}$ & $<1$ & $\mathrm{U}$ & $<1$ & JU & $\mathrm{L}$ & $<\mathrm{EQL}$ & 1 & $\mathrm{ug} / \mathrm{L}$ & ML \\
\hline & Trichloroethylene & $<5$ & $\mathrm{U}$ & $<5$ & $\mathrm{U}$ & $<1$ & $U$ & $<1$ & JU & $L$ & $<E Q L$ & 1 & $\mathrm{ug} / \mathrm{L}$ & ML \\
\hline \multicolumn{15}{|c|}{ II. Monitoring Constituents } \\
\hline$\underline{\text { ST }}$ & Parameter & $\underline{3 Q 96}$ & CLPEPA & $\underline{3097}$ & CLP EPA & $3 Q 998$ & CLPPEPA & $\underline{3 Q 99}$ & $\underline{\text { CLP }}$ & EPA & Filt. & DF & Unit & $\underline{\text { Lab }}$ \\
\hline \multicolumn{15}{|c|}{ Inorganics } \\
\hline & Aluminum, total recoverable & 49.2 & $J$ & $<20$ & $U$ & $<200$ & $\mathrm{U}$ & $<200$ & $U$ & & $<E Q L$ & 1 & $\mathrm{ug} / \mathrm{L}$ & EX \\
\hline & Nitrate-nitrite as nitrogen & 790 & & 368 & & 9630 & & 349 & $\mathrm{~J}$ & 1 & NDD & 5 & $\mathrm{ug} / \mathrm{L}$ & EX \\
\hline & Sodium, total recoverable & 1420 & $J$ & 1480 & & 1510 & & 1500 & & & $<4600$ & 1 & $\mathrm{ug} / \mathrm{L}$ & EX \\
\hline & Sulfate & 197 & $J$ & $<5000$ & $U$ & 673 & & 693 & & & $<3000$ & 1 & $\mathrm{ug} / \mathrm{L}$ & EX \\
\hline \multicolumn{15}{|c|}{ Radionuclides } \\
\hline & Gross alpha & .363 & UI & .46 & UI & .661 & & .306 & U & & $<E Q L$ & 1 & $\mathrm{pCi} / \mathrm{L}$ & GP \\
\hline & Nonvolatile beta & .307 & UI & -1.94 & UI & .533 & UI & .729 & $U$ & & $<E Q L$ & 1 & $\mathrm{pCl} / \mathrm{L}$ & GP \\
\hline & Radium, total alpha-emitting & -.2 & UI & .96 & UIJ & .67 & & -.1 & $U$ & & $<\mathrm{EQL}$ & 1 & $\mathrm{pCi} / \mathrm{L}$ & GP \\
\hline
\end{tabular}

Notes: Concentrations in bold italics exceed the groundwater protection or monitoring constituent standards listed in Appendix A. Synchronous water levels are measured over a 3-5 day period or less. Dilution factors, Laboratory, and Filtered Data are for Groundwater Protection Standards First Quarter 2000 and Monitoring Constituents Third Quarter 1999 data only.

+ = exceeded the Groundwater Protection Standards (First Quarter 2000) or Monitoring Constituents (Third Quarter 1999) listed in Appendix A. 
Table D-4. Groundwater Monitoring Results for Plume Definition Wells, M-Area HWMF (Cont.) WELL MSB 36A

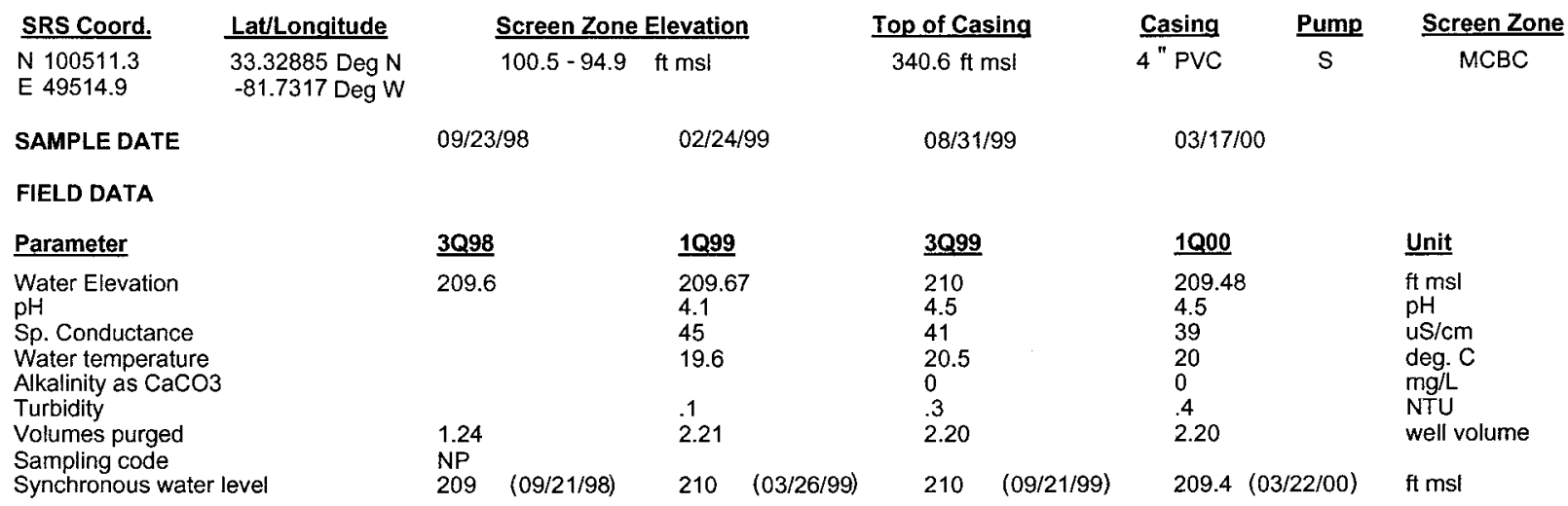

ANALYTICAL DATA

I. Groundwater Protection Standard

261 Appendix VIII/264 Appendix IX Hazardous Constituents

\begin{tabular}{|c|c|c|c|c|c|c|c|c|c|c|c|c|c|c|}
\hline \multirow{2}{*}{$\underline{\underline{\mathrm{ST}}}$} & Parameter & \multirow[t]{2}{*}{$\underline{3098}$} & \multirow[t]{2}{*}{$\underline{\text { CLPP EPA }}$} & \multirow[t]{2}{*}{$\underline{1099}$} & \multirow[t]{2}{*}{ CLPEEA } & \multirow[t]{2}{*}{$\underline{3 Q 99}$} & CLPEPA & \multirow[t]{2}{*}{1000} & \multicolumn{2}{|c|}{ CLPEPA } & \multirow[t]{2}{*}{ Filt. } & \multirow[t]{2}{*}{$\underline{\text { DF }}$} & \multirow[t]{2}{*}{ Unit } & \multirow[t]{2}{*}{$\underline{\text { Lab }}$} \\
\hline & ganics & & & & & & & & & & & & & \\
\hline & Barium, total recoverable & & & 12.2 & & 12 & & & & & & & $\mathrm{ug} / \mathrm{L}$ & \\
\hline & Cyanide & & & $<10$ & $\mathrm{U}$ & $<10$ & $U$ & & & & & & ug/L & \\
\hline & Lead, total recoverable & & & $<100$ & $\mathrm{U}$ & 5 & J I & & & & & & $\mathrm{ug} / \mathrm{L}$ & \\
\hline & Nickel, total recoverable & & & $<8.92$ & $J U$ & $<50$ & $\mathrm{U}$ & & & & & & $u g / L$ & \\
\hline & Selenium, total recoverable & & & $<10$ & $\mathrm{U}$ & $<10$ & $\mathrm{U}$ & & & & & & $\mathrm{ug} / \mathrm{L}$ & \\
\hline \multicolumn{15}{|c|}{ Organics } \\
\hline & Chlorobenzene & & & $<125$ & $u$ & $<1$ & $U$ & $<2$ & $\mathrm{JU}$ & & $<E Q L$ & 2 & $u g / L$ & ML \\
\hline & 1,1-Dichloroethane & & & $<125$ & $\mathrm{U}$ & $<1$ & $U$ & $<2$ & JU & & $<E Q L$ & 2 & $\mathrm{ug} / \mathrm{L}$ & $M L$ \\
\hline & 1,1-Dichloroethylene & & & $<125$ & $\mathrm{U}$ & $<1$ & U & $<2$ & JU & & $<\mathrm{EQL}$ & 2 & ug/L & ML \\
\hline & trans-1,2-Dichloroethylene & & & $<125$ & $\mathrm{U}$ & $<1$ & $U$ & $<2$ & JU & & $<E Q L$ & 2 & ug/L & $\mathrm{ML}$ \\
\hline & PCB 1016 & & & & & & & & & & & & & \\
\hline & PCB 1221 & & & & & & & & & & & & & \\
\hline & PCB 1232 & & & & & & & & & & & & & \\
\hline & PCB 1242 & & & & & & & & & & & & & \\
\hline & PCB 1248 & & & & & & & & & & & & & \\
\hline & PCB 1254 & & & & & & & & & & & & & \\
\hline & PCB 1260 & & & & & & & & & & & & & \\
\hline & $1,1,2,2$-Tetrachloroethane & & & $<125$ & U & $<1$ & $\mathrm{U}$ & $<2$ & JU & & $<E Q L$ & 2 & $u g / L$ & ML \\
\hline & Tetrachloroethylene & & & 76.2 & J 1 & 24.6 & $J K$ & 15.4 & $J$ & & NDD & 2 & $\mathrm{ug} / \mathrm{L}$ & ML \\
\hline & 1,1,1-Trichloroethane & & & $<125$ & $\mathrm{U}$ & $<1$ & $\mathrm{U}$ & $<2$ & JU & & $<E Q L$ & 2 & $\mathrm{ug} / \mathrm{L}$ & ML \\
\hline & Trichloroethylene & & & 1410 & & 546 & & 542 & J & & NDD & 2 & $\mathrm{ug} / \mathrm{L}$ & ML \\
\hline \multicolumn{15}{|c|}{ II. Monitoring Constituents } \\
\hline$\underline{\mathbf{S T}}$ & Parameter & $\underline{3096}$ & CLPEPA & $\underline{3097}$ & CLPEPA & $\underline{3098}$ & CLPEPA & $\underline{3 Q 99}$ & $\underline{\text { CLP }}$ & EPA & Filt. & $\underline{\mathrm{DF}}$ & Unit & $\underline{\text { Lab }}$ \\
\hline \multicolumn{15}{|c|}{ Inorganics } \\
\hline & Aluminum, total recoverable & 68.1 & & 46.6 & & & & 73 & $\mathrm{~J}$ & 1 & NDD & 1 & $u g / L$ & EX \\
\hline & Nitrate-nitrite as nitrogen & 740 & $J$ & 516 & & & & 392 & $\mathrm{~J}$ & 1 & NDD & 5 & $u g / L$ & EX \\
\hline & Sodium, total recoverable & 1780 & & 1820 & & & & 1900 & & & $<4600$ & 1 & ug/L & EX \\
\hline+ & Sulfate & 6490 & & 6650 & & & & 6610 & & & $>3000$ & 2 & $\mathrm{ug} / \mathrm{L}$ & EX \\
\hline \multicolumn{15}{|c|}{ Radionuclides } \\
\hline & Gross alpha & 1.87 & & 2.03 & & & & 1.64 & $\mathrm{U}$ & V & $<\mathrm{EQL}$ & 1 & $\mathrm{pCi} / \mathrm{L}$ & GP \\
\hline & Nonvolatile beta & 2.39 & & 1.24 & UI & & & 2.08 & $J$ & I & NDD & 1 & $\mathrm{pCi} / \mathrm{L}$ & GP \\
\hline & Radium, total alpha-emitting & .8 & & 4.49 & UIJ & & & .4 & 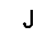 & 1 & NDD & 1 & $\mathrm{pCi} / \mathrm{L}$ & GP \\
\hline
\end{tabular}

Notes: Concentrations in bold italics exceed the groundwater protection or monitoring constituent standards listed in Appendix A. Synchronous water levels are measured over a 3-5 day period or less. Dilution factors, Laboratory, and Filtered Data are for Groundwater Protection Standards First Quarter 2000 and Monitoring Constituents Third Quarter 1999 data only.

$+=$ exceeded the Groundwater Protection Standards (First Quarter 2000) or Monitoring Constituents (Third Quarter 1999) listed in Appendix A. 
Table D-4. Groundwater Monitoring Results for Plume Definition Wells, M-Area HWMF (Cont.) WELL MSB 36B

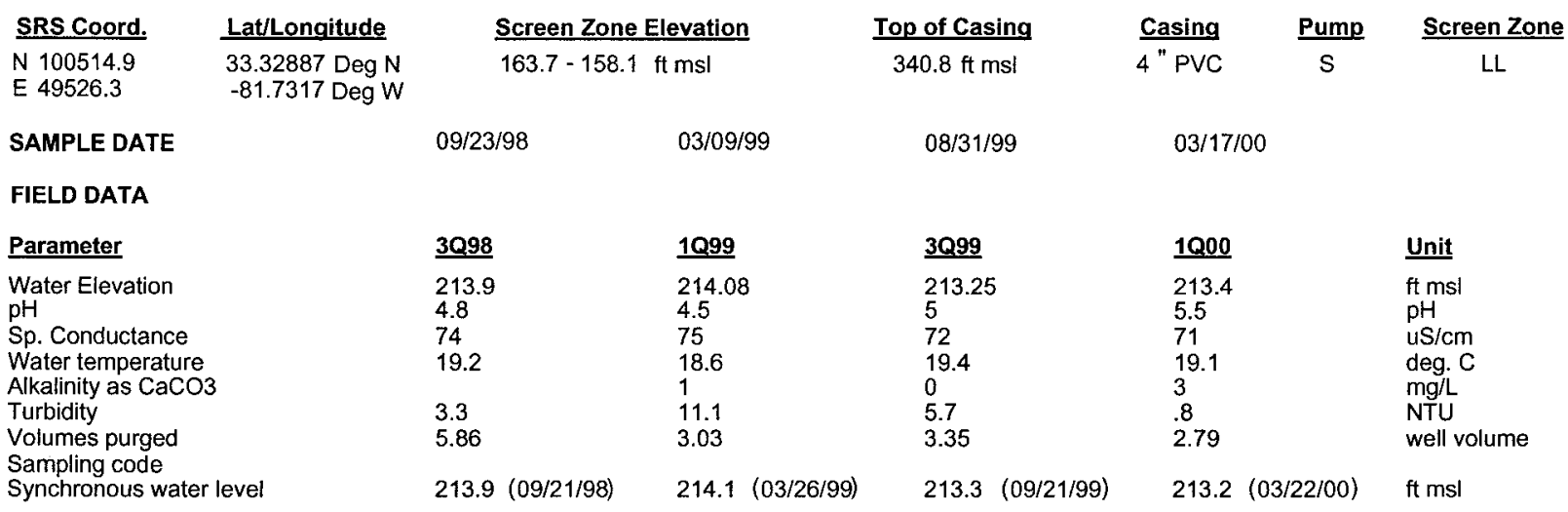

ANALYTICAL DATA

I. Groundwater Protection Standard

261 Appendix VIII/264 Appendix IX Hazardous Constituents

\begin{tabular}{|c|c|c|c|c|c|c|c|c|c|c|c|c|c|}
\hline ST Parameter & $\underline{3098}$ & CLPPEPA & 1099 & CLPEPA & $\underline{3 Q 99}$ & CLPEPA & $\underline{1000}$ & \multicolumn{2}{|c|}{ CLPEPA } & Filt. & DF & $\underline{\text { Unit }}$ & $\underline{\text { Lab }}$ \\
\hline \multicolumn{14}{|l|}{ Inorganics } \\
\hline Barium, total recoverable & 23.7 & & 23.1 & & 21 & & & & & & & $\mathrm{ug} / \mathrm{L}$ & \\
\hline Cyanide & $<15.2$ & $\mathrm{U}$ & $<10$ & $\mathrm{U}$ & $<10$ & $U$ & & & & & & $\mathrm{ug} / \mathrm{L}$ & \\
\hline Lead, total recoverable & $<100$ & $\mathrm{U}$ & $<100$ & $\mathrm{U}$ & $<10$ & $U$ & & & & & & $\mathrm{ug} / \mathrm{L}$ & \\
\hline Nickel, total recoverable & 9.69 & $J$ & $<50$ & $U$ & $<50$ & $\mathrm{U}$ & & & & & & $u g / L$ & \\
\hline Selenium, total recoverable & $<200$ & $U$ & $<200$ & $U$ & $<10$ & $U$ & & & & & & $\mathrm{ug} / \mathrm{L}$ & \\
\hline \multicolumn{14}{|l|}{ Organics } \\
\hline Chlorobenzene & $<100$ & $\mathrm{U}$ & $<125$ & $\mathrm{U}$ & $<1$ & $\mathrm{U}$ & $<5$ & $\mathrm{U}$ & & $<\mathrm{EQL}$ & 5 & $u g / L$ & ML \\
\hline 1,1-Dichloroethane & $<100$ & $\mathrm{U}$ & $<125$ & $\mathrm{U}$ & $<1$ & $\mathrm{U}$ & $<5$ & $\mathrm{U}$ & & $<E Q L$ & 5 & $\mathrm{ug} / \mathrm{L}$ & ML \\
\hline 1,1-Dichloroethylene & $<100$ & U & $<125$ & $\mathrm{U}$ & $<1$ & $U$ & $<5$ & $\mathrm{U}$ & & $<E Q L$ & 5 & ug/L & $M L$ \\
\hline trans-1,2-Dichloroethylene & $<100$ & $U$ & $<125$ & $U$ & $<1$ & $\mathrm{U}$ & $<5$ & $\mathrm{U}$ & & $<E Q L$ & 5 & $\mathrm{ug} / \mathrm{L}$ & ML \\
\hline РСВ 1016 & & & & & & & & & & & & & \\
\hline PCB 1221 & & & & & & & & & & & & & \\
\hline PCB 1232 & & & & & & & & & & & & & \\
\hline PCB 1242 & & & & & & & & & & & & & \\
\hline PCB 1248 & & & & & & & & & & & & & \\
\hline PCB 1254 & & & & & & & & & & & & & \\
\hline PCB 1260 & & & & & & & & & & & & & \\
\hline 1,1,2,2-Tetrachloroethane & $<100$ & $\mathrm{U}$ & $<125$ & $\mathrm{U}$ & $<1$ & $\mathrm{U}$ & $<5$ & U & & $<$ EQL & 5 & ug/L & $M L$ \\
\hline Tetrachloroethylene & $<100$ & $U$ & $<125$ & $\mathrm{U}$ & 1.8 & $J K$ & 2.3 & $J$ & 1 & NDD & 5 & $u g / L$ & ML \\
\hline $1,1,1$-Trichloroethane & $<100$ & $U$ & $<125$ & $U$ & $<1$ & $\mathrm{U}$ & $<5$ & $\mathrm{U}$ & & $<\mathrm{EQL}$ & 5 & $\mathrm{ug} / \mathrm{L}$ & $\mathrm{ML}$ \\
\hline+ Trichloroethylene & 1140 & & 1930 & & 1330 & & 1180 & & & $>5$ & 5 & $\mathrm{ug} / \mathrm{L}$ & $\mathrm{ML}$ \\
\hline \multicolumn{14}{|l|}{ II. Monitoring Constituents } \\
\hline ST Parameter & $\underline{3096}$ & CLPEPA & $\underline{3 Q 97}$ & CLPEPA & 3098 & CLPEPA & $\underline{3099}$ & $\underline{\text { CLP }}$ & EPA & Filt. & $\underline{\text { DF }}$ & Unit & Lab \\
\hline \multicolumn{14}{|l|}{ Inorganics } \\
\hline Aluminum, total recoverable & 106 & & 3610 & & 235 & & 190 & $J$ & 1 & NDD & 1 & $\mathrm{ug} / \mathrm{L}$ & EX \\
\hline Nitrate-nitrite as nitrogen & 15200 & $J$ & 11300 & & 7520 & & 6690 & & & $>2400$ & 5 & $\mathrm{ug} / \mathrm{L}$ & EX \\
\hline Sodium, total recoverable & 5250 & & 4410 & & 4620 & & 4400 & & & $<4600$ & 1 & $\mathrm{ug} / \mathrm{L}$ & EX \\
\hline Sulfate & 411 & $J$ & $<5000$ & $\mathrm{U}$ & 400 & & 397 & $J$ & 1 & NDD & 2 & $u g / L$ & EX \\
\hline \multicolumn{14}{|l|}{ Radionuclides } \\
\hline Gross alpha & 5.94 & & 20.9 & & 6.28 & & 2.55 & $U$ & V & $<\mathrm{EQL}$ & 1 & $\mathrm{pCl} / \mathrm{L}$ & GP \\
\hline Nonvolatile beta & 5.02 & & 8.4 & & 5.04 & & 2.02 & $J$ & I & $\cdot \mathrm{NDD}$ & 1 & $\mathrm{pCl} / \mathrm{L}$ & GP \\
\hline Radium, total alpha-emitting & 2.1 & & 5 & UIJ & 3.95 & & .8 & $J$ & I & NDD & 1 & $\mathrm{pCi} / \mathrm{L}$ & GP \\
\hline
\end{tabular}

Notes: Concentrations in bold italics exceed the groundwater protection or monitoring constituent standards listed in Appendix A. Synchronous water levels are measured over a 3-5 day period or less. Dilution factors, Laboratory, and Filtered Data are for Groundwater Protection Standards First Quarter 2000 and Monitoring Constituents Third Quarter 1999 data only.

+= exceeded the Groundwater Protection Standards (First Quarter 2000) or Monitoring Constituents (Third Quarter 1999) listed in Appendix A. 
Table D-4. Groundwater Monitoring Results for Plume Definition Wells, M-Area HWMF (Cont.) WELL MSB 36C

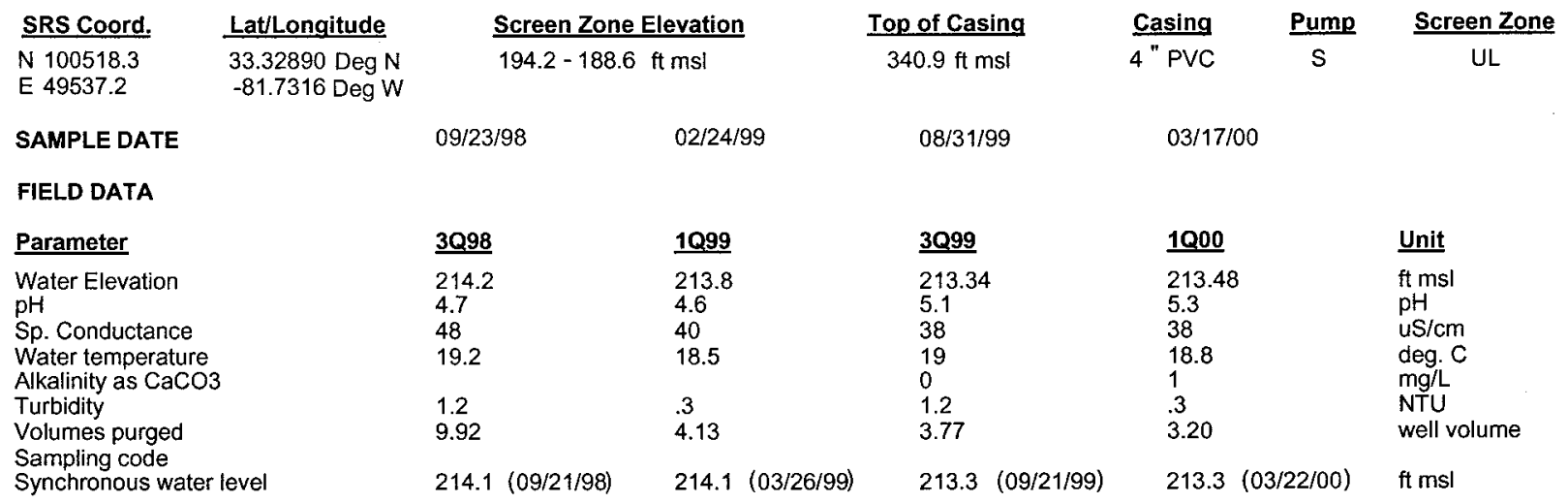

ANALYTICAL DATA

I. Groundwater Protection Standard

261 Appendix VIII/264 Appendix IX Hazardous Constituents

\begin{tabular}{|c|c|c|c|c|c|c|c|c|c|c|c|c|c|c|}
\hline \multirow{2}{*}{$\frac{\mathbf{S T}}{\text { Inorg }}$} & Parameter & \multirow[t]{2}{*}{$\underline{3 Q 98}$} & \multirow[t]{2}{*}{$\underline{\text { CLPEPA }}$} & \multirow[t]{2}{*}{$1 \mathrm{Q99}$} & \multirow[t]{2}{*}{ CLP EPA } & \multirow[t]{2}{*}{$\underline{3 Q 99}$} & CLPEPA & \multirow[t]{2}{*}{1000} & \multicolumn{2}{|c|}{$\underline{\text { CLPPEPA }}$} & \multirow[t]{2}{*}{ Filt. } & \multirow[t]{2}{*}{$\underline{\text { DF }}$} & \multirow[t]{2}{*}{ Unit } & \multirow[t]{2}{*}{$\underline{L a b}$} \\
\hline & ganics & & & & & & & & & & & & & \\
\hline & Barium, total recoverable & 14.2 & & 11.1 & & 9.6 & J 1 & & & & & & $\mathrm{ug} / \mathrm{L}$ & \\
\hline & Cyanide & $<10$ & $\mathrm{U}$ & $<10$ & $U$ & $<10$ & $U$ & & & & & & $\mathrm{ug} / \mathrm{L}$ & \\
\hline & Lead, total recoverable & $<100$ & $\mathrm{U}$ & $<100$ & $U$ & $<10$ & $U$ & & & & & & $\mathrm{ug} / \mathrm{L}$ & \\
\hline & Nickel, total recoverable & $<50$ & $\mathrm{U}$ & $<50$ & U & $<50$ & $\mathrm{U}$ & & & & & & $\mathrm{ug} / \mathrm{L}$ & \\
\hline & Selenium, total recoverable & $<200$ & $U$ & $<10$ & $\mathrm{U}$ & $<10$ & $U$ & & & & & & $u g / L$ & \\
\hline \multicolumn{15}{|c|}{ Organics } \\
\hline & Chlorobenzene & $<5$ & $u$ & $<5$ & $u$ & $<1$ & $U$ & $<1$ & $U$ & & $<E Q L$ & 1 & ug/L & ML \\
\hline & 1,1-Dichloroethane & $<5$ & $U$ & $<5$ & $\mathrm{U}$ & $<1$ & $U$ & $<1$ & U & & $<\mathrm{EQL}$ & 1 & $\mathrm{ug} / \mathrm{L}$ & ML \\
\hline & 1,1-Dichloroethylene & $<5$ & $\cup$ & $<5$ & $\mathrm{U}$ & $<1$ & $U$ & $<1$ & $u$ & & $<$ EQL & 1 & $\mathrm{ug} / \mathrm{L}$ & ML \\
\hline & trans-1,2-Dichloroethylene & $<5$ & $\cup$ & $<5$ & $u$ & $<1$ & $U$ & $<1$ & $u$ & & $<E Q L$ & 1 & ug/L & ML \\
\hline & PCB 1016 & & & & & & & & & & & & & \\
\hline & PCB 1221 & & & & & & & & & & & & & \\
\hline & PCB 1232 & & & & & & & & & & & & & \\
\hline & PCB 1242 & & & & & & & & & & & & & \\
\hline & PCB 1248 & & & & & & & & & & & & & \\
\hline & PCB 1254 & & & & & & & & & & & & & \\
\hline & PCB 1260 & & & & & & & & & & & & & \\
\hline & 1,1,2,2-Tetrachloroethane & $<5$ & $U$ & $<5$ & $\mathrm{U}$ & $<1$ & $U$ & $<1$ & $\mathrm{U}$ & & $<\mathrm{EQL}$ & 1 & $\mathrm{ug} / \mathrm{L}$ & ML \\
\hline & Tetrachloroethylene & 5.59 & & 1.22 & $\mathrm{~J} \quad \mathrm{I}$ & .95 & IK & .77 & $\mathbf{J}$ & 1 & NDD & 1 & $\mathrm{ug} / \mathrm{L}$ & ML \\
\hline & 1,1,1-Trichloroethane & $<5$ & $\mathrm{U}$ & $<5$ & $U$ & $<1$ & U & $<1$ & $\mathrm{U}$ & & $<\mathrm{EQL}$ & 1 & $\mathrm{ug} / \mathrm{L}$ & ML \\
\hline+ & Trichloroethylene & 53 & & 13.2 & & 10.1 & K & 8.48 & & & $>5$ & 1 & ug/L & ML \\
\hline \multicolumn{15}{|c|}{ II. Monitoring Constituents } \\
\hline$\underline{\text { ST }}$ & Parameter & $\underline{3 Q 96}$ & CLPEPA & $\underline{3097}$ & $\underline{\text { CLP EPA }}$ & 3098 & $\underline{\text { CLPEPA }}$ & $\underline{3 Q 99}$ & $\underline{\text { CLF }}$ & EPA & Filt. & $\underline{\text { DF }}$ & Unit & $\underline{\text { Lab }}$ \\
\hline \multicolumn{15}{|c|}{ Inorganics } \\
\hline & Aluminum, total recoverable & 49.8 & & 38.2 & & 131 & J & $<200$ & $\mathrm{U}$ & & $<\mathrm{EQL}$ & 1 & $\mathrm{ug} / \mathrm{L}$ & EX \\
\hline+ & Nitrate-nitrite as nitrogen & 3000 & & 3050 & & $<500$ & $U$ & 2430 & & & $>2400$ & 5 & $u g / L$ & EX \\
\hline & Sodium, total recoverable & 4040 & & 4060 & & 5310 & & 3500 & & & $<4600$ & 1 & $\mathrm{ug} / \mathrm{L}$ & EX \\
\hline & Sulfate & 154 & J & $<5000$ & $U$ & $<400$ & $U$ & $<400$ & $U$ & & $<E Q L$ & 2 & ug/L & EX \\
\hline \multicolumn{15}{|c|}{ Radionuclides } \\
\hline & Gross alpha & 2.39 & & 3.87 & & 5.73 & & 1.67 & $U$ & V & $<\mathrm{EQL}$ & 1 & $\mathrm{pCi} / \mathrm{L}$ & GP \\
\hline & Nonvolatile beta & 1.75 & & 3.12 & & 3.16 & $J$ & .9 & $U$ & & $<E Q L$ & 1 & $\mathrm{pCi} / \mathrm{L}$ & GP \\
\hline & Radium, total alpha-emitting & .9 & & 7.4 & $\mathrm{~J}$ & 1.22 & & .4 & $\mathrm{~J}$ & 1 & NDD & 1 & $\mathrm{pCi} / \mathrm{L}$ & GP \\
\hline
\end{tabular}

Notes: Concentrations in bold italics exceed the groundwater protection or monitoring constituent standards listed in Appendix A. Synchronous water levels are measured over a 3-5 day period or less. Dilution factors, Laboratory, and Filtered Data are for Groundwater Protection Standards First Quarter 2000 and Monitoring Constituents Third Quarter 1999 data only.

+= exceeded the Groundwater Protection Standards (First Quarter 2000) or Monitoring Constituents (Third Quarter 1999) listed in Appendix A. 
Table D-4. Groundwater Monitoring Results for Plume Definition Wells, M-Area HWMF (Cont.) WELL MSB 36D

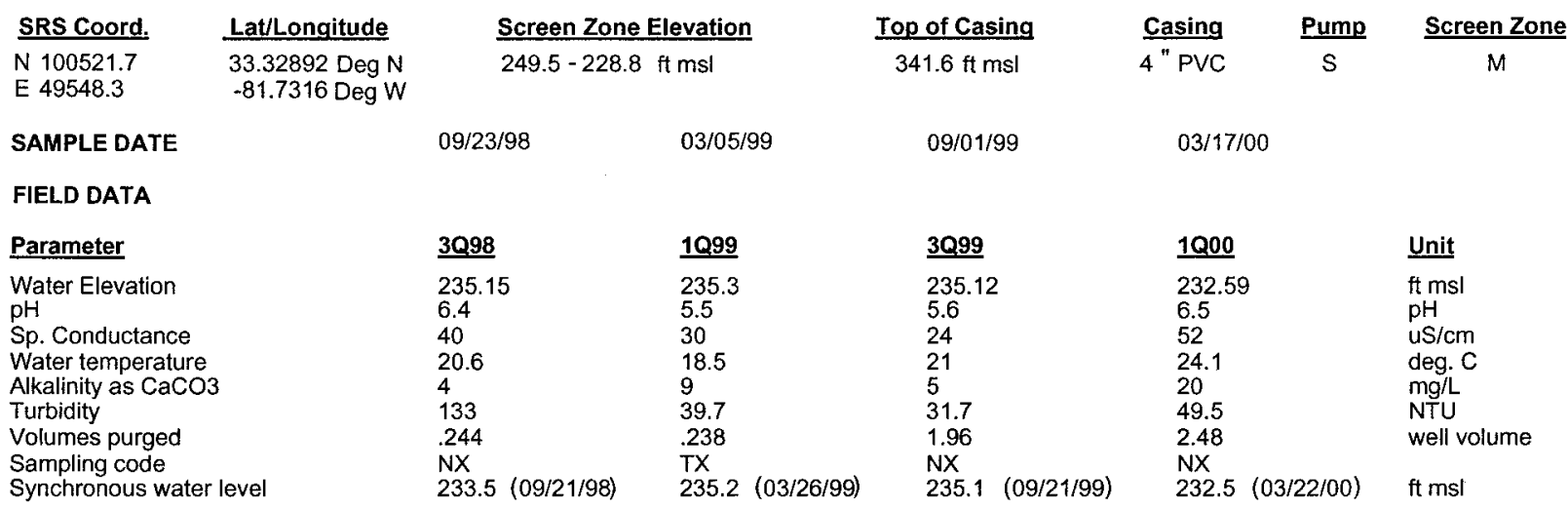

ANALYTICAL DATA

I. Groundwater Protection Standard

261 Appendix VIII/264 Appendix IX Hazardous Constituents

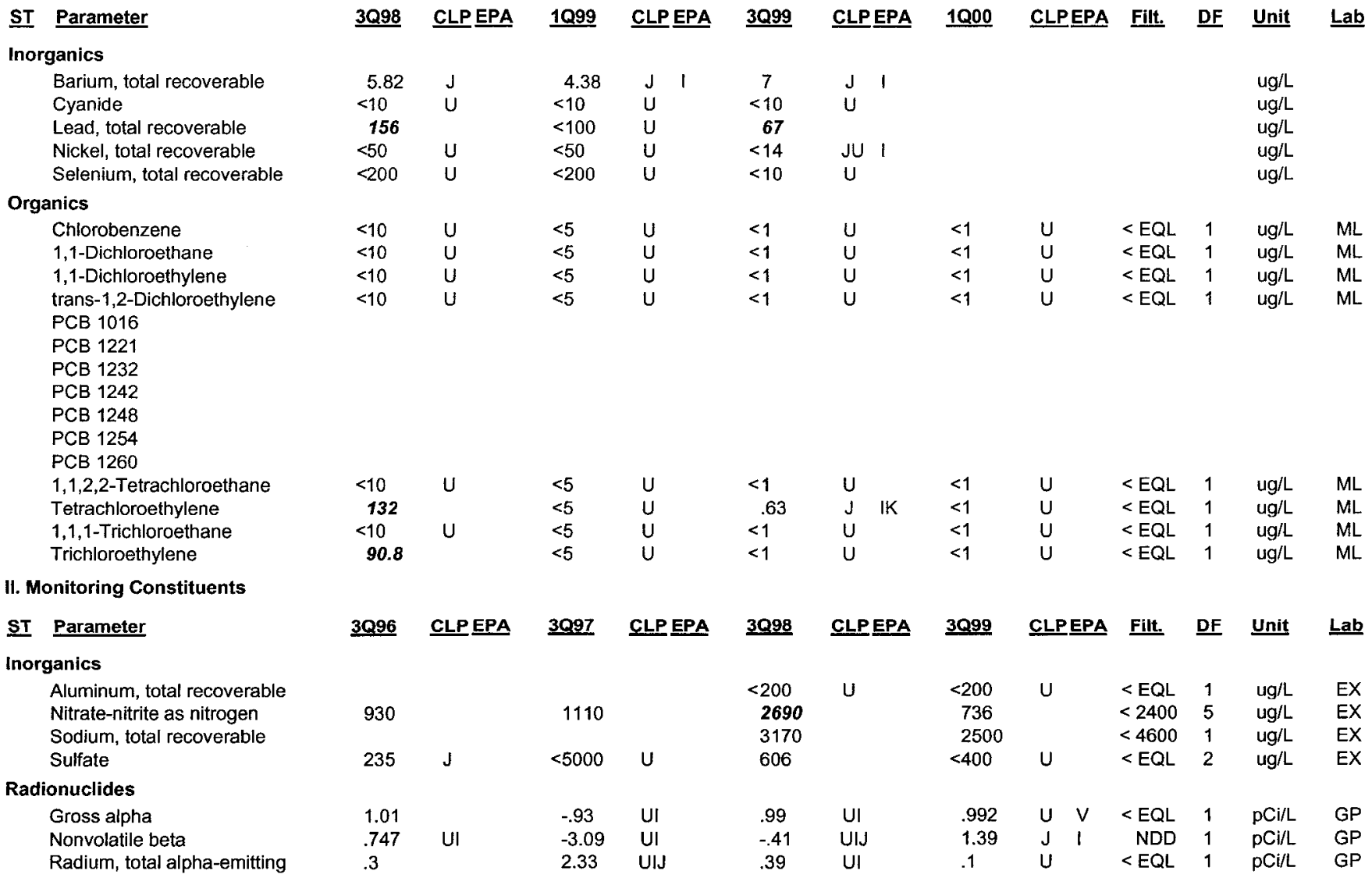

Notes: Concentrations in bold italics exceed the groundwater protection or monitoring constituent standards listed in Appendix A. Synchronous water levels are measured over a 3-5 day period or less. Dilution factors, Laboratory, and Filtered Data are for Groundwater Protection Standards First Quarter 2000 and Monitoring Constituents Third Quarter 1999 data only.

$+=$ exceeded the Groundwater Protection Standards (First Quarter 2000) or Monitoring Constituents (Third Quarter 1999) listed in Appendix A. 
Table D-4. Groundwater Monitoring Results for Plume Definition Wells, M-Area HWMF (Cont.) WELL MSB 36TA

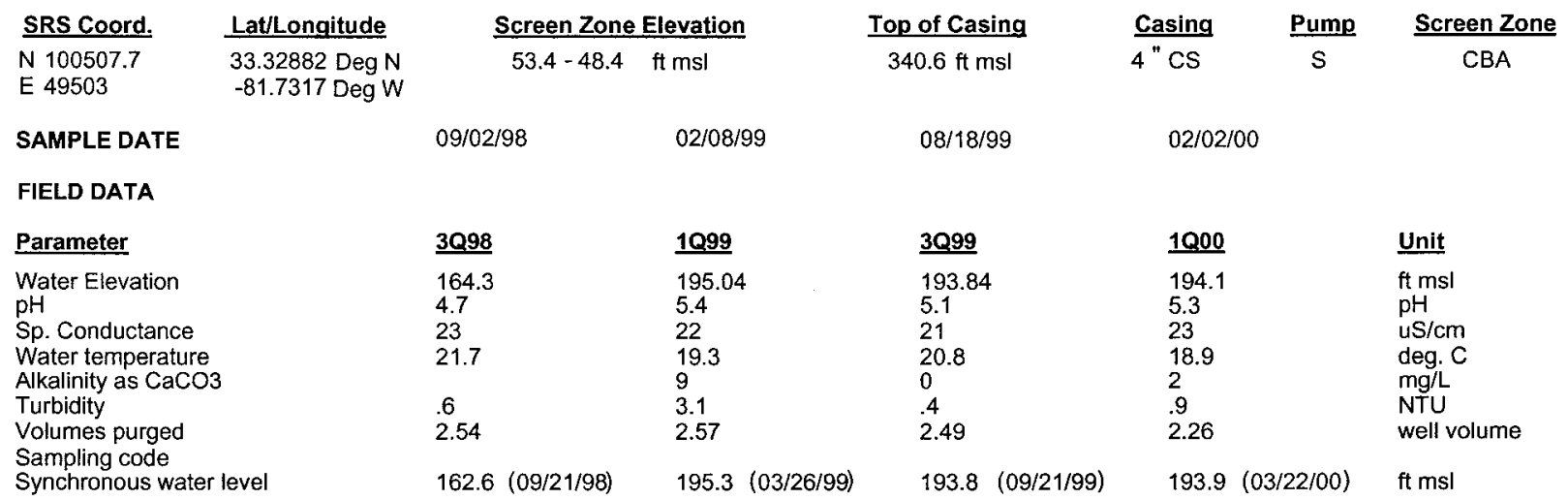

ANALYTICAL DATA

I. Groundwater Protection Standard

261 Appendix VIII/264 Appendix IX Hazardous Constituents

\begin{tabular}{|c|c|c|c|c|c|c|c|c|c|c|c|c|c|}
\hline$\underline{\text { ST }}$ & Parameter & $\underline{3 Q 98}$ & CLP EPA & $1 \mathrm{Q99}$ & CLP EPA & $\underline{3 Q 99}$ & CLP EPA & 1000 & CLPEPA & Filt. & DF & Unit & Lab \\
\hline \multicolumn{14}{|c|}{ Inorganics } \\
\hline & Barium, total recoverable & 5.64 & $\mathbf{J}$ & $<10$ & $U$ & 5 & J 1 & & & & & ug/L & \\
\hline & Cyanide & $<10$ & $\mathrm{U}$ & $<10$ & $\mathrm{U}$ & $<10$ & $\mathrm{U}$ & & & & & ug/L & \\
\hline & Lead, total recoverable & $<100$ & $U$ & $<100$ & $U$ & 7.09 & & & & & & ug/L & \\
\hline & Nickel, total recoverable & $<50$ & $\mathrm{U}$ & $<50$ & $\mathrm{U}$ & $<12$ & JU I & & & & & $\mathrm{ug} / \mathrm{h}$ & \\
\hline & Selenium, total recoverable & $<10$ & $U$ & $<200$ & $\mathrm{U}$ & $<5$ & $U$ & & & & & $\mathrm{ug} / \mathrm{L}$ & \\
\hline \multicolumn{14}{|c|}{ Organics } \\
\hline & Chlorobenzene & $<5$ & $\mathrm{U}$ & $<5$ & U & $<1$ & $U$ & $<1$ & $\mathrm{U}$ & $<\mathrm{EQL}$ & 1 & $\mathrm{ug} / \mathrm{L}$ & $M L$ \\
\hline & 1,1-Dichloroethane & $<5$ & $U$ & $<5$ & U & $<1$ & $U$ & $<1$ & $\mathrm{U}$ & $<E Q L$ & 1 & $\mathrm{ug} / \mathrm{L}$ & ML \\
\hline & 1,1-Dichloroethylene & $<5$ & $U$ & $<5$ & $\mathrm{U}$ & $<1$ & $\mathrm{U}$ & $<1$ & $\mathrm{U}$ & $<\mathrm{EQL}$ & 1 & ug/L & ML \\
\hline & trans-1,2-Dichloroethylene & $<5$ & $\mathrm{U}$ & $<5$ & $\mathrm{u}$ & $<1$ & $\mathrm{U}$ & $<1$ & $\mathrm{U}$ & $<\mathrm{EQL}$ & 1 & $\mathrm{ug} / \mathrm{L}$ & $\mathrm{ML}$ \\
\hline & PCB 1016 & & & & & & & & & & & & \\
\hline & PCB 1221 & & & & & & & & & & & & \\
\hline & $\begin{array}{l}\text { PCB } 1232 \\
\text { PCB } 1242\end{array}$ & & & & & & & & & & & & \\
\hline & PCB 1248 & & & & & & & & & & & & \\
\hline & PCB 1254 & & & & & & & & & & & & \\
\hline & PCB 1260 & & & & & & & & & & & & \\
\hline & 1,1,2,2-Tetrachloroethane & $<5$ & U & $<5$ & $\mathrm{U}$ & $<1$ & $U$ & $<1$ & $\mathrm{U}$ & $<\mathrm{EQL}$ & 1 & $\mathrm{ug} / \mathrm{L}$ & ML \\
\hline & Tetrachloroethylene & $<5$ & U & $<5$ & $\mathrm{U}$ & $<1$ & $U$ & $<1$ & $U$ & $<\mathrm{EQL}$ & 1 & ug/L & $M L$ \\
\hline & $1,1,1$-Trichloroethane & $<5$ & $\mathrm{U}$ & $<5$ & $\mathrm{U}$ & $<1$ & $U$ & $<1$ & $\mathrm{U}$ & $<E Q L$ & 1 & $\mathrm{ug} / \mathrm{L}$ & ML \\
\hline & Trichloroethylene & $<5$ & $\mathrm{U}$ & $<5$ & $\mathrm{U}$ & $<1$ & $\mathrm{U}$ & $<1$ & $\mathrm{U}$ & $<E Q L$ & 1 & ug/L. & ML \\
\hline \multicolumn{14}{|c|}{ II. Monitoring Constituents } \\
\hline$\underline{\text { ST }}$ & Parameter & $\underline{3096}$ & $\underline{\text { CLP EPA }}$ & $\underline{3097}$ & $\underline{\text { CLP EPA }}$ & $\underline{3 Q 98}$ & CLPEPA & $\underline{3099}$ & $\underline{\text { CLPEPA }}$ & Filt. & DF & Unit & $\underline{\mathrm{Le}}$ \\
\hline \multicolumn{14}{|c|}{ Inorganics } \\
\hline & Aluminum, total recoverable & $<20$ & $\mathrm{U}$ & 79.4 & & $<200$ & U & $<200$ & $\mathrm{U}$ & $<\mathrm{EQL}$ & 1 & $\mathrm{ug} / \mathrm{L}$ & EX \\
\hline & Nitrate-nitrite as nitrogen & 330 & & $<59$ & $U$ & 721 & & $<500$ & $U$ & $<E Q L$ & 5 & $\mathrm{ug} / \mathrm{L}$ & EX \\
\hline & Sodium, total recoverable & 1480 & & 1520 & & 1490 & & 1700 & & $<4600$ & 1 & $u g / L$ & EX \\
\hline & Sulfate & 2300 & & $<5000$ & $\mathrm{U}$ & 2050 & & 2350 & & $<3000$ & 1 & $u g / L$ & EX \\
\hline \multicolumn{14}{|c|}{ Radionuclides } \\
\hline & Gross alpha & .628 & UI & .73 & U! & 1.42 & $\downarrow$ & .306 & $\mathrm{U}$ & $<E Q L$ & 1 & $\mathrm{pCi} / \mathrm{L}$ & GP \\
\hline & Nonvolatile beta & .674 & UI & 3.43 & & 3.37 & J & .121 & $\mathrm{U}$ & $<$ EQL & 1 & $\mathrm{pCi} / \mathrm{L}$ & GP \\
\hline & Radium, total alpha-emitting & .3 & & 1.27 & & .14 & UI & -.1 & $\mathrm{U}$ & $<E Q L$ & 1 & $\mathrm{pCi} / \mathrm{L}$ & GP \\
\hline
\end{tabular}

Notes: Concentrations in bold italics exceed the groundwater protection or monitoring constituent standards listed in Appendix A. Synchronous water levels are measured over a 3-5 day period or less. Dilution factors, Laboratory, and Filtered Data are for Groundwater Protection Standards First Quarter 2000 and Monitoring Constituents Third Quarter 1999 data only.

+ = exceeded the Groundwater Protection Standards (First Quarter 2000) or Monitoring Constituents (Third Quarter 1999) listed in Appendix A. 
Table D-4. Groundwater Monitoring Results for Plume Definition Wells, M-Area HWMF (Cont.) WELL MSB 37A

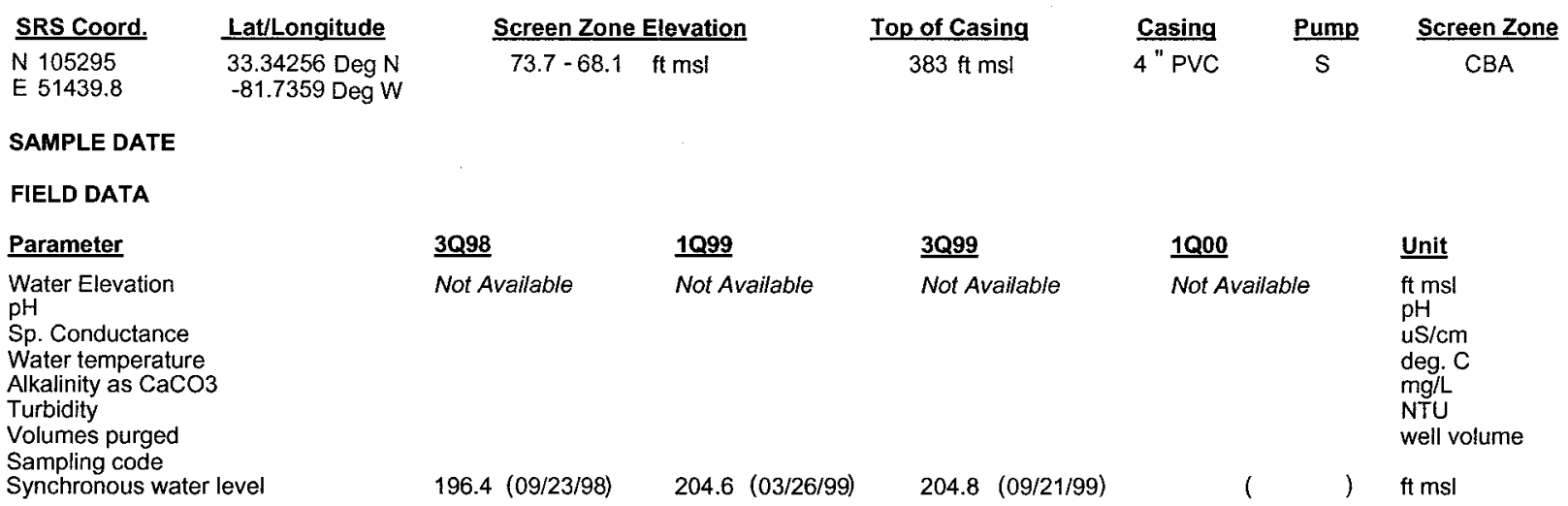

\section{ANALYTICAL DATA}

I. Groundwater Protection Standard

Notes: Concentrations in bold italics exceed the groundwater protection or monitoring constituent standards listed in Appendix A. Synchronous water levels are measured over a 3-5 day period or less. Dilution factors, Laboratory, and Filtered Data are for Groundwater Protection Standards First Quarter 2000 and Monitoring Constituents Third Quarter 1999 data only.

+ = exceeded the Groundwater Protection Standards (First Quarter 2000) or Monitoring Constituents (Third Quarter 1999) listed in Appendix A. 
Table D-4. Groundwater Monitoring Results for Plume Definition Wells, M-Area HWMF (Cont.) WELL MSB 37B

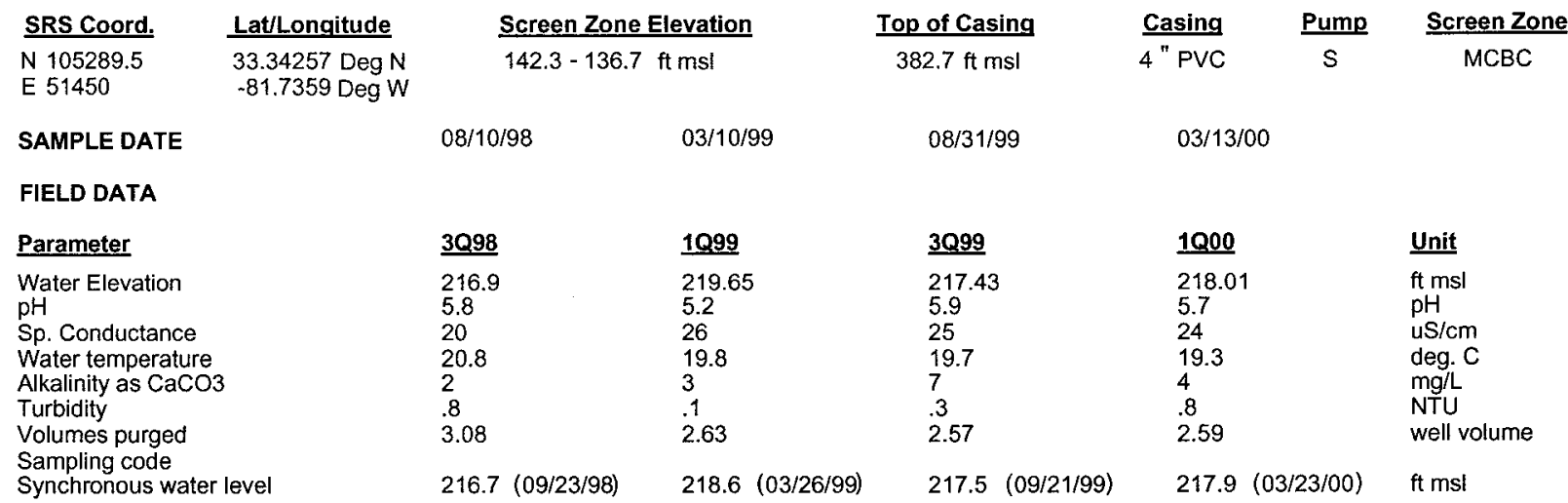

ANALYTICAL DATA

I. Groundwater Protection Standard

261 Appendix VIII/264 Appendix IX Hazardous Constituents

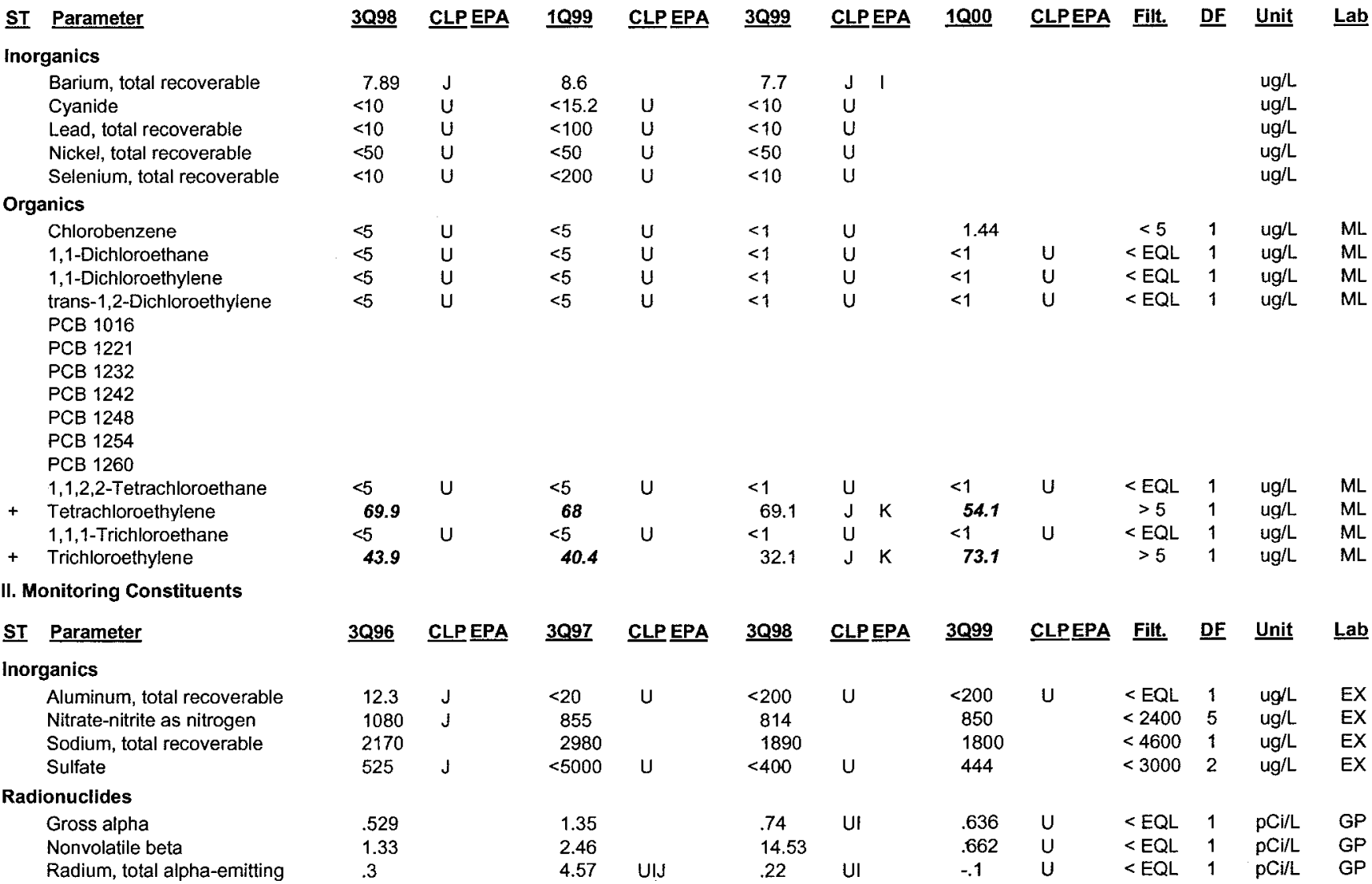

Notes: Concentrations in bold italics exceed the groundwater protection or monitoring constituent standards listed in Appendix A. Synchronous water levels are measured over a 3-5 day period or less. Dilution factors, Laboratory, and Filtered Data are for Groundwater Protection Standards First Quarter 2000 and Monitoring Constituents Third Quarter 1999 data only.

$+=$ exceeded the Groundwater Protection Standards (First Quarter 2000) or Monitoring Constituents (Third Quarter 1999) listed in Appendix A. 
Table D-4. Groundwater Monitoring Results for Plume Definition Wells, M-Area HWMF (Cont.) WELL MSB 37C

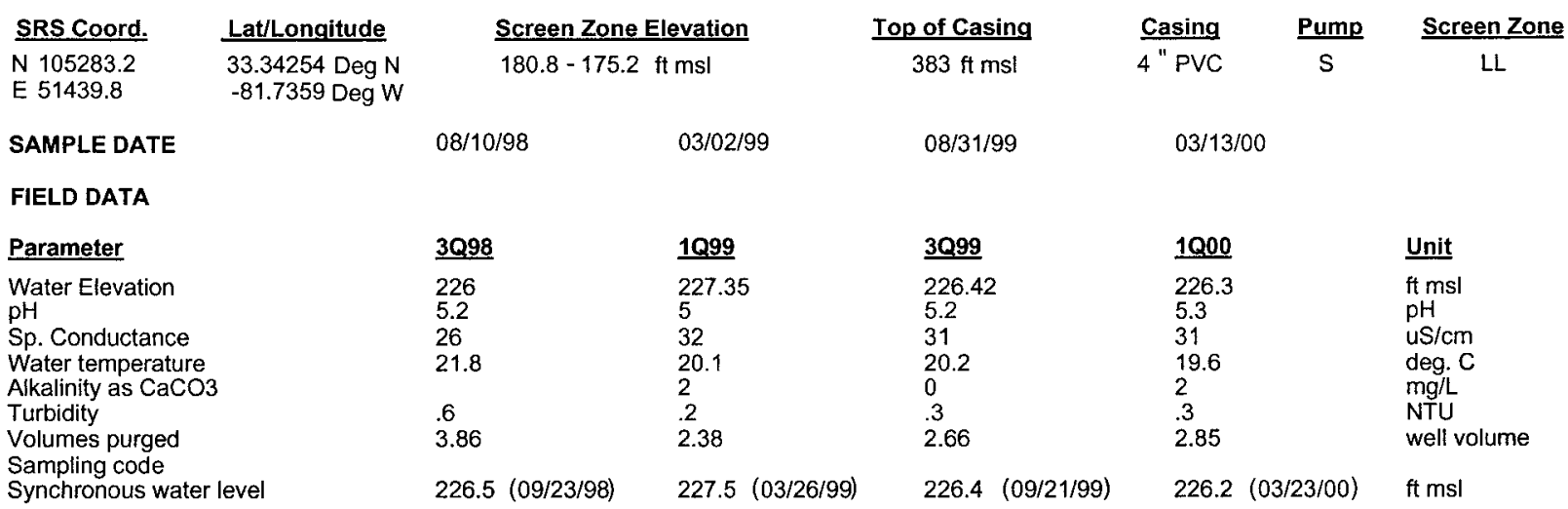

ANALYTICAL DATA

I. Groundwater Protection Standard

261 Appendix VIII/264 Appendix IX Hazardous Constituents

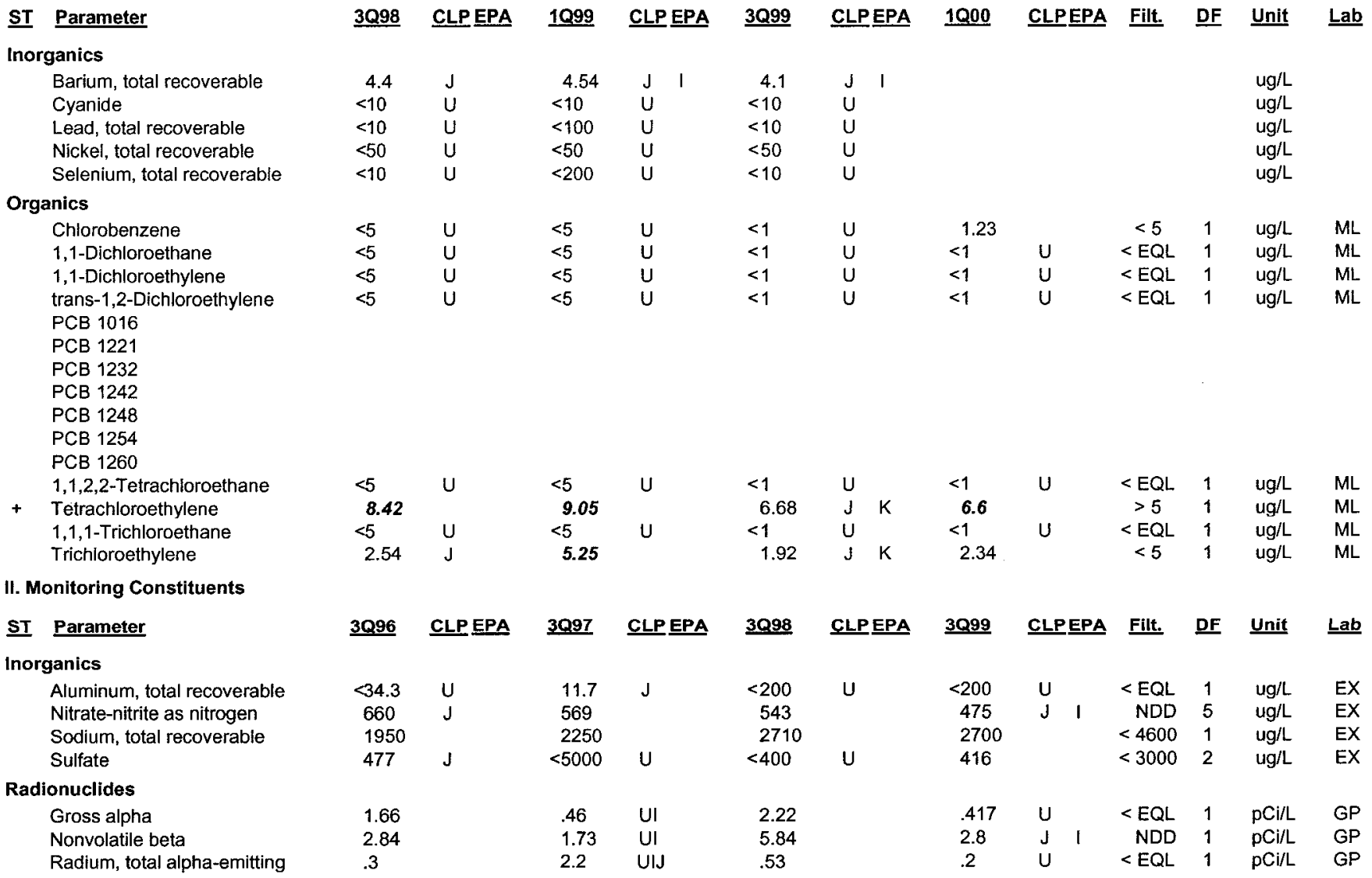

Notes: Concentrations in bold italics exceed the groundwater protection or monitoring constituent standards listed in Appendix A. Synchronous water levels are measured over a 3-5 day period or less. Dilution factors, Laboratory, and Filtered Data are for Groundwater Protection Standards First Quarter 2000 and Monitoring Constituents Third Quarter 1999 data only.

$+=$ exceeded the Groundwater Protection Standards (First Quarter 2000) or Monitoring Constituents (Third Quarter 1999) listed in Appendix A. 
Table D-4. Groundwater Monitoring Results for Plume Definition Wells, M-Area HWMF (Cont.) WELL MSB 38C

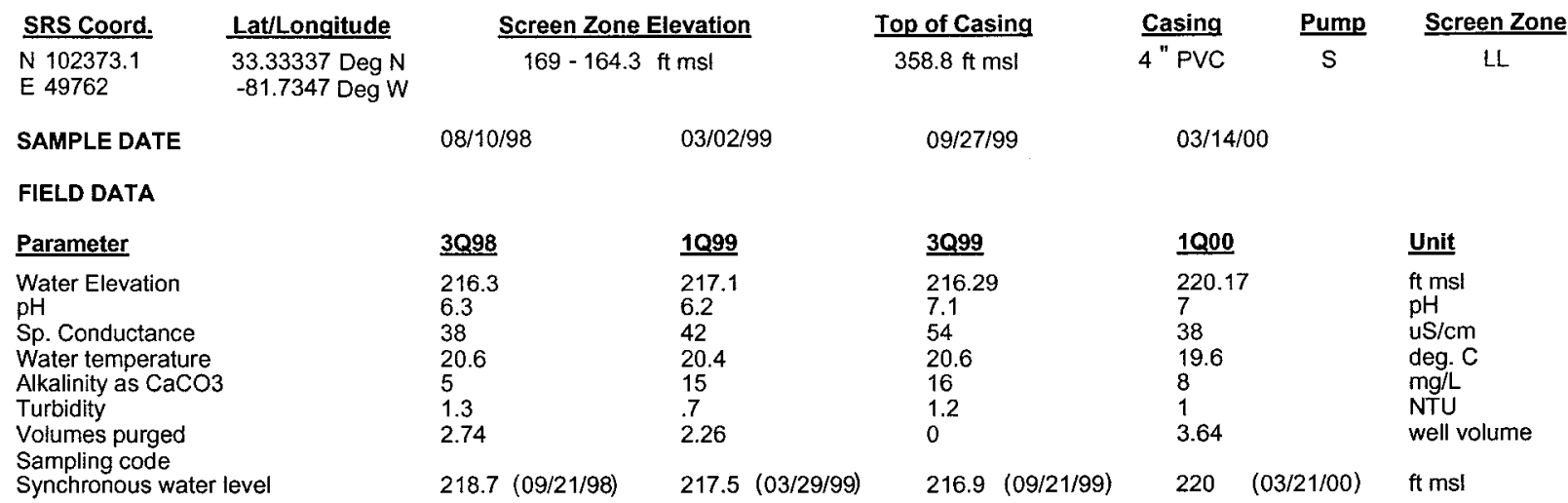

ANALYTICAL DATA

I. Groundwater Protection Standard

261 Appendix VIII/264 Appendix IX Hazardous Constituents

\begin{tabular}{|c|c|c|c|c|c|c|c|c|c|c|c|c|c|c|}
\hline ST & Parameter & $\underline{3 Q 98}$ & CLP EPA & 1099 & $\underline{\text { CLPPEPA }}$ & $\underline{3 Q 99}$ & CLPEPA & 1000 & $\underline{C L F}$ & EPA & Filt. & $\underline{D F}$ & Unit & Lab \\
\hline $\ln O$ & ganics & & & & & & & & & & & & & \\
\hline & Barium, total recoverable & 24.4 & & 38.1 & & 36.9 & & & & & & & $\mathrm{ug} / \mathrm{L}$ & \\
\hline & Cyanide & $<10$ & $\mathrm{U}$ & $<10$ & $\mathrm{U}$ & $<10$ & $\mathrm{U}$ & & & & & & $\mathrm{ug} / \mathrm{L}$ & \\
\hline & Lead, total recoverable & $<10$ & U & $<100$ & $\mathrm{U}$ & $<10$ & $\mathrm{U}$ & & & & & & $\mathrm{ug} / \mathrm{L}$ & \\
\hline & Nickel, total recoverable & $<50$ & $U$ & $<50$ & $\mathrm{U}$ & $<50$ & $\mathrm{U}$ & & & & & & $\mathrm{ug} / \mathrm{L}$ & \\
\hline & Selenium, total recoverable & $<10$ & $\mathrm{U}$ & $<200$ & $\mathrm{U}$ & $<10$ & $\mathrm{U}$ & & & & & & ug/L & \\
\hline Org & anics & & & & & & & & & & & & & \\
\hline & Chlorobenzene & $<250$ & $u$ & $<500$ & $U$ & $<.52$ & U V & $<50$ & $\mathrm{U}$ & & $<\mathrm{EQL}$ & 50 & $\mathrm{ug} / \mathrm{L}$ & ML \\
\hline & 1,1-Dichloroethane & $<250$ & U & $<500$ & $\mathrm{U}$ & $<1$ & $\mathrm{U}$ & $<50$ & $\mathrm{U}$ & & $<E Q L$ & 50 & $u g / L$ & ML \\
\hline & 1,1-Dichloroethylene & $<250$ & $\mathrm{U}$ & $<500$ & $\mathrm{U}$ & $<1$ & $\mathrm{U}$ & $<50$ & $\mathrm{U}$ & & $<E Q L$ & 50 & $\mathrm{ug} / \mathrm{L}$ & ML \\
\hline & trans-1,2-Dichloroethylene & $<250$ & $U$ & $<500$ & $\mathrm{U}$ & $<1$ & $\mathrm{U}$ & $<50$ & U & & $<E Q L$ & 50 & $\mathrm{ug} / \mathrm{L}$ & $\mathrm{ML}$ \\
\hline & PCB 1016 & & & & & & & & & & & & & \\
\hline & PCB 1221 & & & & & & & & & & & & & \\
\hline & PCB 1232 & & & & & & & & & & & & & \\
\hline & PCB 1242 & & & & & & & & & & & & & \\
\hline & PCB 1248 & & & & & & & & & & & & & \\
\hline & PCB 1254 & & & & & & & & & & & & & \\
\hline & PCB 1260 & & & & & & & & & & & & & \\
\hline & 1,1,2,2-Tetrachloroethane & $<250$ & $\mathrm{U}$ & $<500$ & $\mathrm{U}$ & $<1$ & $\mathrm{U}$ & $<50$ & $U$ & & $<E Q L$ & 50 & $u g / L$ & $M L$ \\
\hline & Tetrachloroethylene & 6630 & & 18600 & & 8120 & & 7100 & $\mathrm{~J}$ & $\mathrm{~K}$ & NDD & 50 & $\mathrm{ug} / \mathrm{L}$ & $\mathrm{ML}$ \\
\hline & 1,1,1-Trichloroethane & $<250$ & $\mathrm{U}$ & $<500$ & U & $<1$ & $\mathrm{U}$ & $<50$ & $U$ & & $<E Q L$ & 50 & $u g / L$ & $\mathrm{ML}$ \\
\hline & Trichloroethylene & 6880 & & 18700 & & 9500 & & 8760 & $\mathrm{~J}$ & K & NDD & 50 & $u g / L$ & $M L$ \\
\hline II. $\mathbf{M}$ & onitoring Constituents & & & & & & & & & & & & & \\
\hline$\underline{\text { ST }}$ & Parameter & 3096 & CLP EPA & 3097 & $\underline{\text { CLP EPA }}$ & $\underline{3098}$ & CLPEPA & $\underline{3099}$ & $\underline{\text { CLF }}$ & EPA & Filt. & $\underline{\text { DF }}$ & $\underline{\text { Unit }}$ & $\underline{\text { Lab }}$ \\
\hline Inor & ganics & & & & & & & & & & & & & \\
\hline & Aluminum, total recoverable & $<56.5$ & $\mathrm{U}$ & 43.7 & & $<200$ & U & $<200$ & $\mathrm{U}$ & & $<E Q L$ & 1 & $u g / L$ & EX \\
\hline & Nitrate-nitrite as nitrogen & 1860 & & 2190 & & 1460 & & 1440 & & & $<2400$ & 5 & $\mathrm{ug} / \mathrm{L}$ & EX \\
\hline & Sodium, total recoverable & 2930 & $J$ & 1970 & & 2170 & & 2270 & & & $<4600$ & 1 & $\mathrm{ug} / \mathrm{L}$ & EX \\
\hline & Sulfate & 243 & $J$ & $<5000$ & $u$ & $<400$ & $\mathrm{U}$ & 178 & $\mathrm{~J}$ & 1 & NDD & 2 & $\mathrm{ug} / \mathrm{L}$ & EX \\
\hline Rad & ionuclides & & & & & & & & & & & & & \\
\hline & Gross alpha & 1.34 & Ut & .02 & UI & 1.23 & & 1.2 & $\mathrm{~J}$ & I & NDD & 1 & $\mathrm{pCi} / \mathrm{L}$ & GP \\
\hline & Nonvolatile beta & 1.92 & & .3 & UI & 2.38 & & .472 & $u$ & & $<E Q L$ & 1 & $\mathrm{pCi} / \mathrm{L}$ & GP \\
\hline & Radium, total alpha-emitting & 0 & UI & .89 & UIJ & .22 & UI & .6 & $\mathrm{~J}$ & 1 & NDD & 1 & $\mathrm{pCi} / \mathrm{L}$ & GP \\
\hline
\end{tabular}

Notes: Concentrations in bold italics exceed the groundwater protection or monitoring constituent standards listed in Appendix A. Synchronous water levels are measured over a 3-5 day period or less. Dilution factors, Laboratory, and Filtered Data are for Groundwater Protection Standards First Quarter 2000 and Monitoring Constituents Third Quarter 1999 data only.

+ = exceeded the Groundwater Protection Standards (First Quarter 2000) or Monitoring Constituents (Third Quarter 1999) listed in Appendix A. 
Table D-4. Groundwater Monitoring Results for Plume Definition Wells, M-Area HWMF (Cont.) WELL MSB 39B

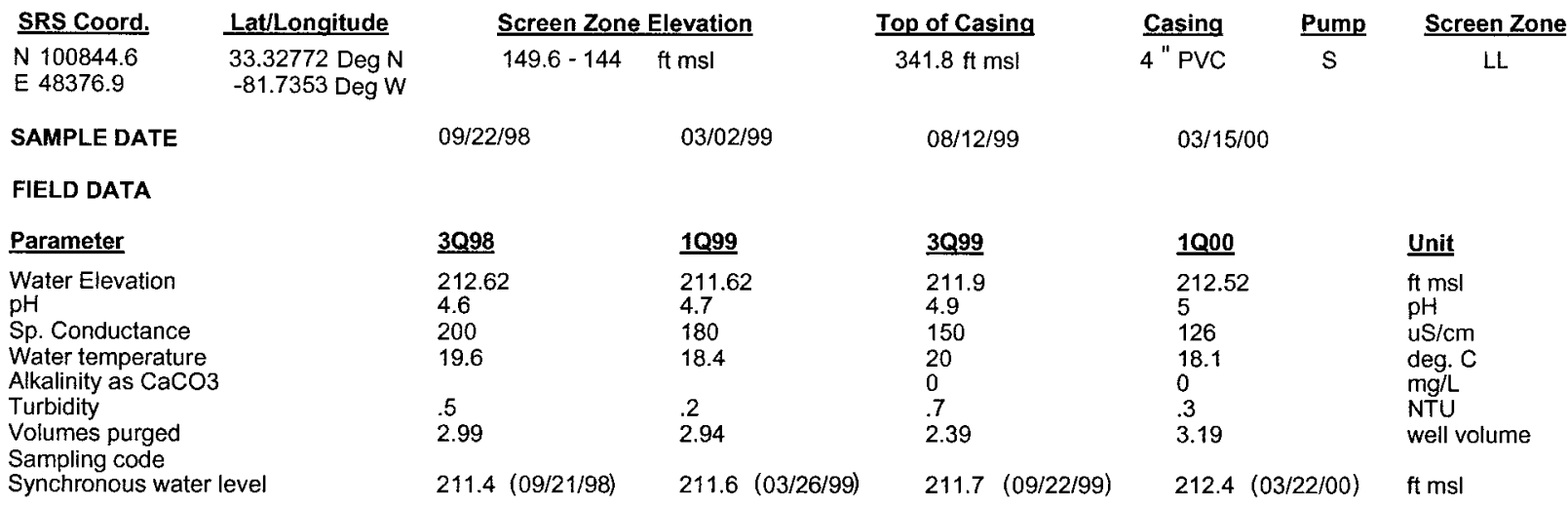

ANALYTICAL DATA

I. Groundwater Protection Standard

261 Appendix VIII/264 Appendix IX Hazardous Constituents

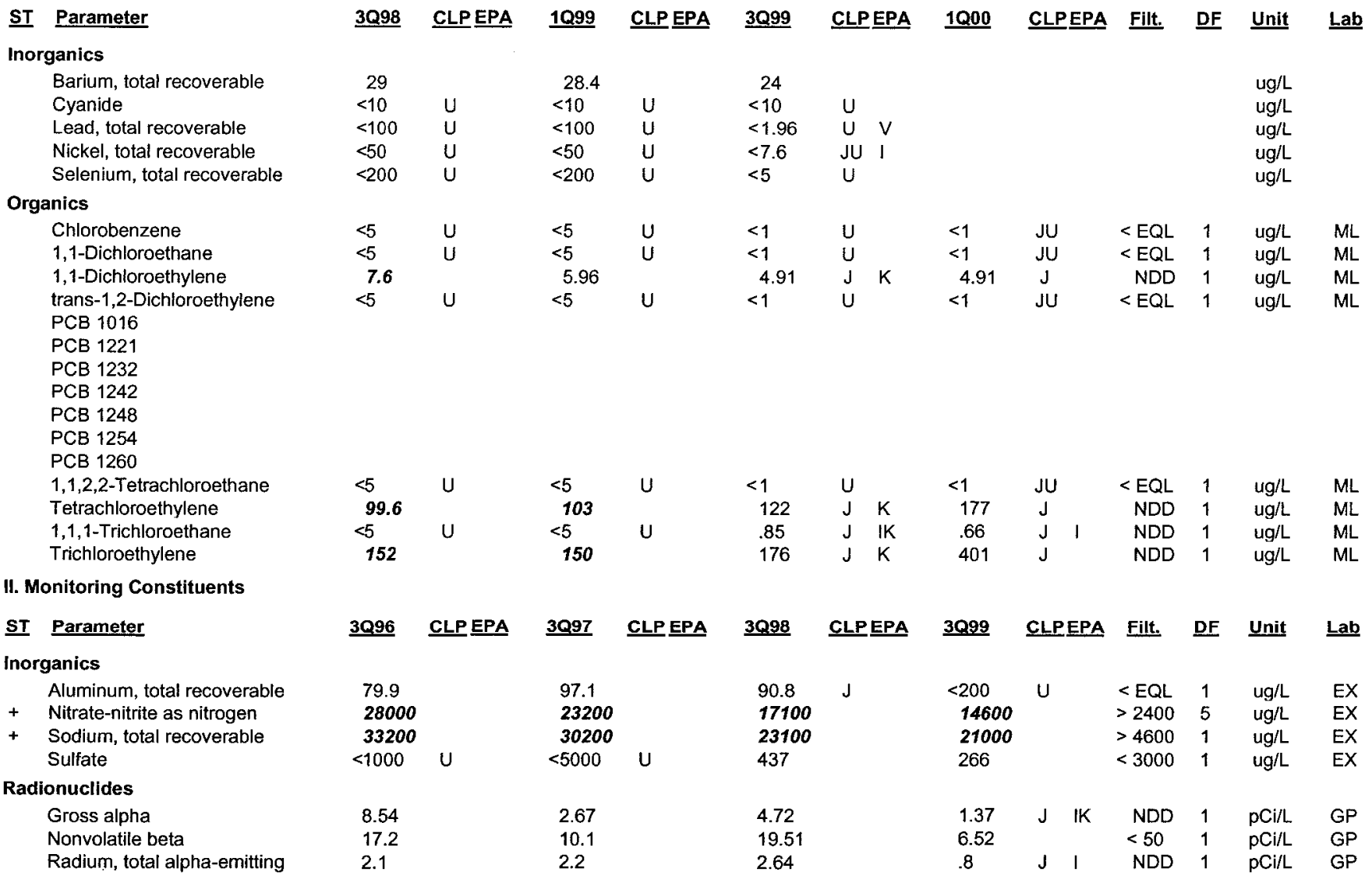

Notes: Concentrations in bold italics exceed the groundwater protection or monitoring constituent standards listed in Appendix A. Synchronous water levels are measured over a 3-5 day period or less. Dilution factors, Laboratory, and Filtered Data are for Groundwater Protection Standards First Quarter 2000 and Monitoring Constituents Third Quarter 1999 data only.

$+=$ exceeded the Groundwater Protection Standards (First Quarter 2000) or Monitoring Constituents (Third Quarter 1999) listed in Appendix A. 
Table D-4. Groundwater Monitoring Results for Plume Definition Wells, M-Area HWMF (Cont.) WELL MSB 39C

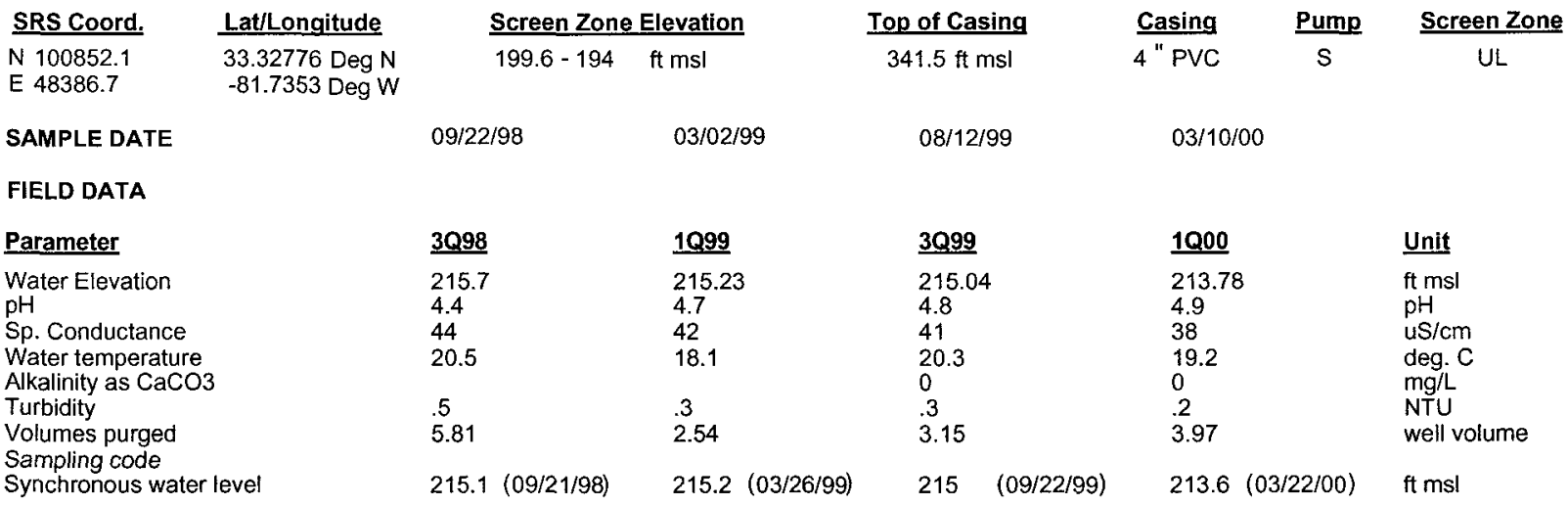

ANALYTICAL DATA

I. Groundwater Protection Standard

261 Appendix VIII/264 Appendix IX Hazardous Constituents

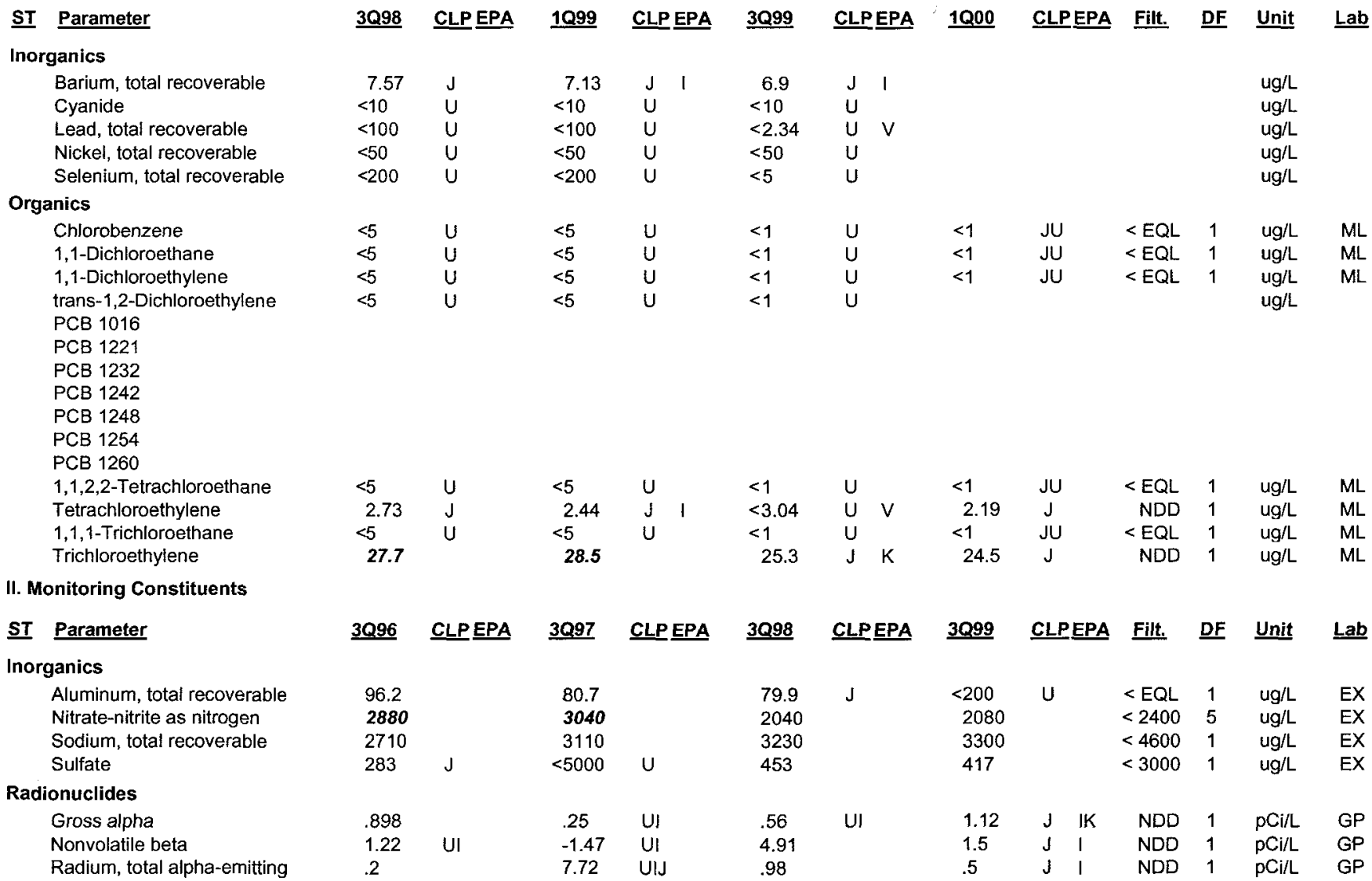

Notes: Concentrations in bold italics exceed the groundwater protection or monitoring constituent standards listed in Appendix A. Synchronous water levels are measured over a 3-5 day period or less. Dilution factors, Laboratory, and Filtered Data are for Groundwater Protection Standards First Quarter 2000 and Monitoring Constituents Third Quarter 1999 data only.

$+=$ exceeded the Groundwater Protection Standards (First Quarter 2000) or Monitoring Constituents (Third Quarter 1999) listed in Appendix A. 
Table D-4. Groundwater Monitoring Results for Plume Definition Wells, M-Area HWMF (Cont.) WELL MSB 39D

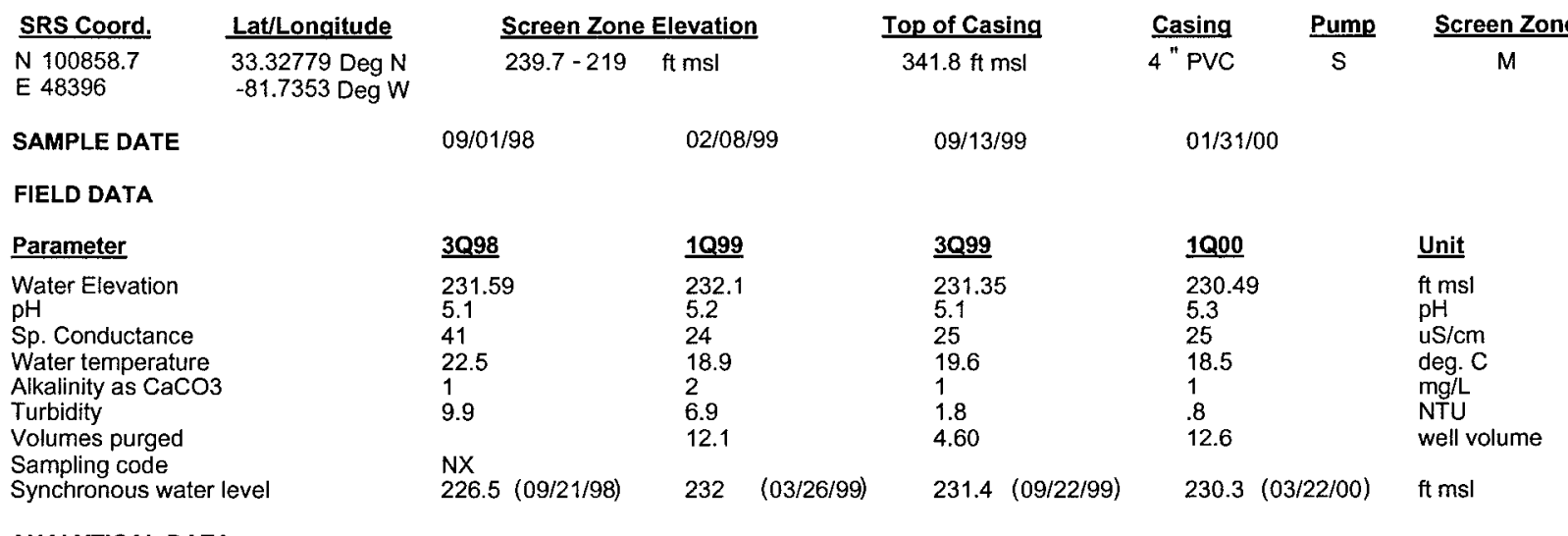

ANALYTICAL DATA

I. Groundwater Protection Standard

261 Appendix VIII/264 Appendix IX Hazardous Constituents

\begin{tabular}{|c|c|c|c|c|c|c|c|c|c|c|c|c|c|c|c|}
\hline \multirow{2}{*}{\multicolumn{2}{|c|}{$\begin{array}{l}\text { ST } \\
\text { Inorganics }\end{array}$}} & \multirow[t]{2}{*}{$\underline{3 Q 98}$} & \multirow[t]{2}{*}{ CLP EPA } & \multirow[t]{2}{*}{1099} & \multirow[t]{2}{*}{ CLP EPA } & \multirow[t]{2}{*}{$\underline{3099}$} & \multicolumn{2}{|c|}{ CLPEPA } & \multirow[t]{2}{*}{$\underline{1 Q 00}$} & \multicolumn{2}{|c|}{ CLPEPA } & \multirow[t]{2}{*}{ Filt. } & \multirow[t]{2}{*}{$\underline{\text { DF }}$} & \multirow[t]{2}{*}{ Unit } & \multirow[t]{2}{*}{$\underline{\text { Lab }}$} \\
\hline & & & & & & & & & & & & & & & \\
\hline & Barium, total recoverable & 6.48 & $J$ & $<10$ & $\mathrm{U}$ & 4.81 & $J$ & I & & & & & & $\mathrm{ug} / \mathrm{L}$ & \\
\hline & Cyanide & $<10$ & $U$ & $<10$ & $\mathrm{U}$ & $<10$ & $\mathrm{U}$ & & & & & & & ug/L & \\
\hline & Lead, total recoverable & $<100$ & U & $<100$ & $U$ & 3.64 & $J$ & 1 & & & & & & $\mathrm{ug} / \mathrm{L}$ & \\
\hline & Nickel, total recoverable & $<10.9$ & $\mathrm{U}$ & $<50$ & $\mathrm{U}$ & 26.6 & $J$ & 1 & & & & & & $\mathrm{ug} / \mathrm{L}$ & \\
\hline & Selenium, total recoverable & $<10$ & $\mathrm{U}$ & $<200$ & $\mathrm{U}$ & $<10$ & $U$ & & & & & & & $\mathrm{ug} / \mathrm{L}$ & \\
\hline \multicolumn{16}{|c|}{ Organics } \\
\hline & Chlorobenzene & $<5$ & US & $<5$ & $\mathrm{U}$ & $<1$ & $U$ & & $<1$ & JU & $\mathbf{L}$ & $<\mathrm{EQL}$ & 1 & $\mathrm{ug} / \mathrm{L}$ & ML \\
\hline & 1,1-Dichloroethane & $<5$ & UJ & $<5$ & $\mathrm{U}$ & $<1$ & $U$ & & $<1$ & JU & L & $<E Q L$ & 1 & $\mathrm{ug} / \mathrm{L}$ & ML \\
\hline & 1,1-Dichloroethylene & $<5$ & UJ & $<5$ & $\mathrm{U}$ & $<1$ & $U$ & & $<1$ & JU & L & $<\mathrm{EQL}$ & 1 & ug/L & ML \\
\hline & trans-1,2-Dichloroethylene & $<5$ & UJ & $<5$ & $\mathrm{U}$ & $<1$ & $U$ & & & & & & & $\mathrm{ug} / \mathrm{L}$ & \\
\hline & PCB 1016 & & & & & & & & & & & & & & \\
\hline & PCB 1221 & & & & & & & & & & & & & & \\
\hline & PCB 1232 & & & & & & & & & & & & & & \\
\hline & РCB 1242 & & & & & & & & & & & & & & \\
\hline & PCB 1248 & & & & & & & & & & & & & & \\
\hline & PCB 1254 & & & & & & & & & & & & & & \\
\hline & PCB 1260 & & & & & & & & & & & & & & \\
\hline & 1,1,2,2-Tetrachloroethane & $<5$ & UJ & $<5$ & $\mathrm{U}$ & $<1$ & $U$ & & $<1$ & JU & $\mathbf{L}$ & $<E Q L$ & 1 & $\mathrm{ug} / \mathrm{L}$ & ML \\
\hline & Tetrachloroethylene & $<5$ & UJ & $<5$ & $\mathrm{U}$ & $<1$ & $U$ & & $<1$ & JU & $\mathbf{L}$ & $<E Q L$ & 1 & $\mathrm{ug} / \mathrm{L}$ & ML \\
\hline & 1,1,1-Trichloroethane & $<5$ & UJ & $<5$ & $\mathrm{U}$ & $<1$ & $\mathrm{U}$ & & $<1$ & JU & $L$ & $<E Q L$ & 1 & $\mathrm{ug} / \mathrm{L}$ & ML \\
\hline & Trichloroethylene & 3.41 & $J$ & 1.49 & J 1 & .67 & $\mathbf{J}$ & 1 & $<1$ & JU & $\mathbf{L}$ & $<E Q L$ & 1 & $\mathrm{ug} / \mathrm{L}$ & ML \\
\hline \multicolumn{16}{|c|}{ II. Monitoring Constituents } \\
\hline$\underline{\text { ST }}$ & Parameter & $\underline{3096}$ & CLPEPA & $\underline{3097}$ & CLPEPA & $\underline{3098}$ & $\underline{\text { CLP }}$ & EPA & $\underline{3 Q 99}$ & GLP & EPA & Filt. & $\underline{\text { DF }}$ & Unit & $\underline{\text { Lab }}$ \\
\hline \multicolumn{16}{|c|}{ Inorganics } \\
\hline & Aluminum, total recoverable & 40.1 & & 53.3 & & $<200$ & $U$ & & $<200$ & U & & $<E Q L$ & 1 & $u g / L$ & EX \\
\hline & Nitrate-nitrite as nitrogen & 1500 & & 851 & & 99 & $J$ & & 860 & & & $<2400$ & 5 & ug/L & EX \\
\hline & Sodium, total recoverable & 2370 & & 2450 & & 2900 & & & 1980 & & & $<4600$ & 1 & $\mathrm{ug} / \mathrm{L}$ & EX \\
\hline & Sulfate & 204 & $J$ & $<5000$ & $\mathrm{U}$ & 405 & & & 490 & & & $<3000$ & 2 & $u g / L$ & EX \\
\hline \multicolumn{16}{|c|}{ Radionuclides } \\
\hline & Gross alpha & 5.54 & & 1.68 & & 4 & & & 2.15 & & & $<15$ & 1 & $\mathrm{pCi} / \mathrm{L}$ & GP \\
\hline & Nonvolatile beta & 2.61 & & .32 & UI & 2.74 & $\mathrm{U}$ & & 1.35 & $J$ & IK & NDD & 1 & $\mathrm{pCi} / \mathrm{L}$ & GP \\
\hline & Radium, total alpha-emitting & 2.1 & & 9.14 & $J$ & .73 & $U$ & & 1.2 & $\mathrm{~J}$ & 1 & NDD & 1 & $\mathrm{pCi} / \mathrm{L}$ & GP \\
\hline
\end{tabular}

Notes: Concentrations in bold italics exceed the groundwater protection or monitoring constituent standards listed in Appendix A. Synchronous water levels are measured over a 3-5 day period or less. Dilution factors, Laboratory, and Filtered Data are for Groundwater Protection Standards First Quarter 2000 and Monitoring Constituents Third Quarter 1999 data only.

$+=$ exceeded the Groundwater Protection Standards (First Quarter 2000) or Monitoring Constituents (Third Quarter 1999) listed in Appendix A. 
Table D-4. Groundwater Monitoring Results for Plume Definition Wells, M-Area HWMF (Cont.) WELL MSB 39TA

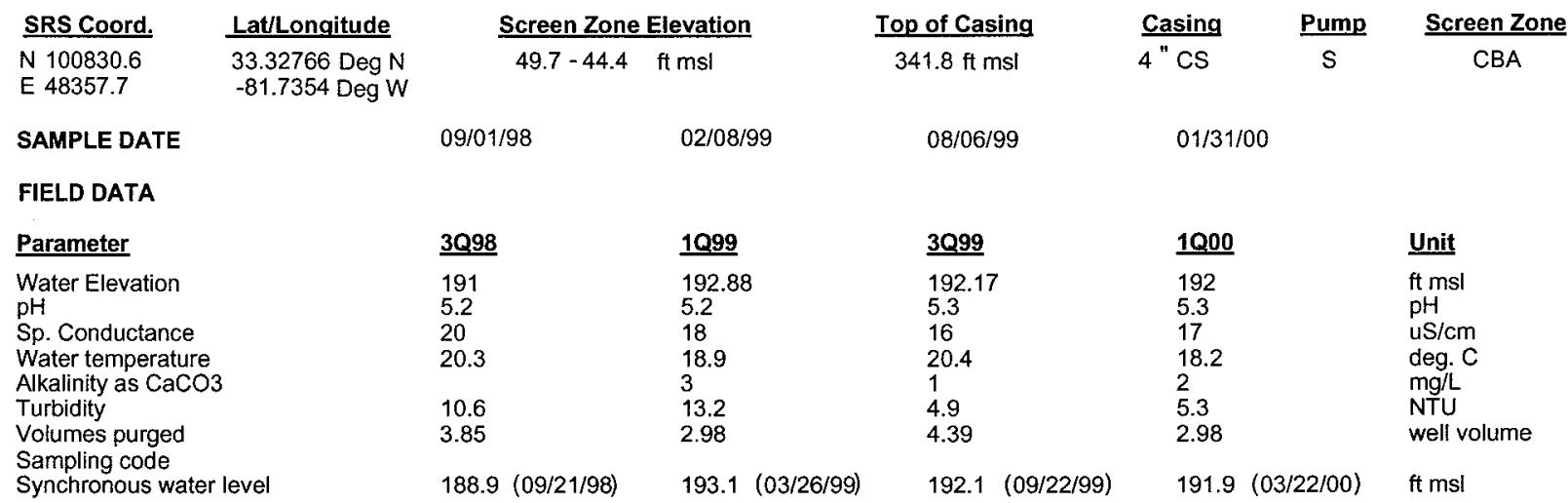

ANALYTICAL DATA

I. Groundwater Protection Standard

261 Appendix VIII/264 Appendix IX Hazardous Constituents

\begin{tabular}{|c|c|c|c|c|c|c|c|c|c|c|c|c|c|c|}
\hline$\underline{\text { ST }}$ & Parameter & $\underline{3 Q 98}$ & CLP EPA & 1099 & $\underline{\text { CLP EPA }}$ & $\underline{3 Q 99}$ & CLPEPA & 1000 & \multicolumn{2}{|c|}{ CLPEPA } & Filt. & $\underline{\text { DF }}$ & Unit & $\underline{L a b}$ \\
\hline \multicolumn{15}{|c|}{ Inorganics } \\
\hline & Barium, total recoverable & 3.4 & & $<10$ & $\mathrm{U}$ & 2.6 & J 1 & & & & & & $\mathrm{ug} / \mathrm{L}$ & \\
\hline & Cyanide & 2.77 & $\mathrm{~J}$ & $<10$ & $\mathrm{U}$ & $<10$ & $\mathrm{U}$ & & & & & & $u g / L$ & \\
\hline & Lead, total recoverable & 5.6 & $\mathrm{~J}$ & $<100$ & $\mathrm{U}$ & $<10$ & $\mathrm{U}$ & & & & & & ug/L & \\
\hline & Nickel, total recoverable & $<50$ & $\mathrm{U}$ & $<50$ & U & $<50$ & $\mathrm{U}$ & & & & & & $u g / L$ & \\
\hline & Selenium, total recoverable & $<66$ & $\mathrm{U}$ & $<200$ & $\mathrm{U}$ & $<10$ & $\mathrm{U}$ & & & & & & $\mathrm{ug} / \mathrm{L}$ & \\
\hline \multicolumn{15}{|c|}{ Organics } \\
\hline & Chlorobenzene & $<5$ & U & $<5$ & $u$ & $<1$ & $u$ & $<1$ & JU & $\mathrm{L}$ & $<E Q L$ & 1 & $u g / L$ & $\mathrm{ML}$ \\
\hline & 1,1-Dichloroethane & $<5$ & $U$ & $<5$ & $\mathrm{U}$ & $<1$ & $\mathrm{U}$ & $<1$ & JU & $\mathrm{L}$ & $<E Q L$ & 1 & ug/L & ML \\
\hline & 1,1-Dichloroethylene & $<5$ & $\mathrm{U}$ & $<5$ & $\mathrm{U}$ & $<1$ & $\mathrm{U}$ & $<1$ & JU & $L$ & $<E Q L$ & 1 & ug/L & ML \\
\hline & trans-1,2-Dichloroethylene & $<5$ & $\mathrm{U}$ & $<5$ & $\mathrm{U}$ & $<1$ & $u$ & & & & & & ug/L & \\
\hline & PCB 1016 & & & & & & & & & & & & & \\
\hline & PCB 1221 & & & & & & & & & & & & & \\
\hline & PCB 1232 & & & & & & & & & & & & & \\
\hline & PCB 1242 & & & & & & & & & & & & & \\
\hline & PCB 1248 & & & & & & & & & & & & & \\
\hline & PCB 1254 & & & & & & & & & & & & & \\
\hline & PCB 1260 & & & & & & & & & & & & & \\
\hline & 1,1,2,2-Tetrachloroethane & $<5$ & $\mathrm{U}$ & $<5$ & $\mathrm{U}$ & $<1$ & $U$ & $<1$ & JU & $\mathrm{L}$ & $<$ EQL & 1 & $\mathrm{ug} / \mathrm{L}$ & ML \\
\hline & Tetrachloroethylene & $<5$ & $\mathrm{U}$ & $<5$ & $\mathrm{U}$ & $<1$ & $\mathrm{U}$ & $<1$ & JU & $\mathrm{L}$ & $<E Q L$ & 1 & $u g / L$ & $\mathrm{ML}$ \\
\hline & 1,1,1-Trichloroethane & $<5$ & $\mathrm{U}$ & $<5$ & $\mathrm{U}$ & $<1$ & $\mathrm{U}$ & $<1$ & JU & $\mathrm{L}$ & $<E Q L$ & 1 & $u g / L$ & ML \\
\hline & Trichloroethylene & $<5$ & $\mathrm{U}$ & $<5$ & $\mathrm{U}$ & $<1$ & U & $<1$ & JU & $\mathrm{L}$ & $<E Q L$ & 1 & ug/L & ML \\
\hline \multicolumn{15}{|c|}{ II. Monitoring Constituents } \\
\hline$\underline{\text { ST }}$ & Parameter & $\underline{3 Q 96}$ & CLP EPA & $\underline{3 Q 97}$ & CLPEPA & $\underline{3 Q 98}$ & CLPEPA & $\underline{3099}$ & CLP & EPA & Filt. & DF & $\underline{\text { Unit }}$ & $\underline{L a b}$ \\
\hline \multicolumn{15}{|c|}{ Inorganics } \\
\hline & Aluminum, total recoverable & 33.6 & & 24.8 & & $<200$ & 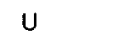 & $<200$ & $\mathrm{U}$ & & $<$ EQL & 1 & $\operatorname{ug} / \mathrm{L}$ & EX \\
\hline & Nitrate-nitrite as nitrogen & 210 & & $<10$ & $U$ & 4 & $\mathrm{~J}$ & $<500$ & $\mathrm{U}$ & & $<E Q L$ & 5 & ug/L & EX \\
\hline & Sodium, total recoverable & 1420 & & 1410 & & 1510 & & 1400 & & & $<4600$ & 1 & $\mathrm{ug} / \mathrm{L}$ & EX \\
\hline & Sulfate & 1120 & & $<5000$ & $\mathrm{U}$ & $* * * * * * *$ & & 1430 & & & $<3000$ & 1 & ug/L & EX \\
\hline \multicolumn{15}{|c|}{ Radionuclides } \\
\hline & Gross alpha & .225 & Ul & -.67 & UI & .509 & & 1.13 & $J$ & 1 & NDD & 1 & $\mathrm{pCi} / \mathrm{L}$ & GP \\
\hline & Nonvolatile beta & 1.43 & Ui & -1.12 & UI & 3.15 & $U$ & 3.47 & & & $<50$ & 1 & $\mathrm{pCi} / \mathrm{L}$ & GP \\
\hline & Radium, total alpha-emitting & .3 & & 9.94 & $\mathrm{~J}$ & .5 & & 1.3 & $J$ & 1 & NDD & 1 & $\mathrm{pCi} / \mathrm{L}$ & GP \\
\hline
\end{tabular}

Notes: Concentrations in bold italics exceed the groundwater protection or monitoring constituent standards listed in Appendix A. Synchronous water levels are measured over a 3-5 day period or less. Dilution factors, Laboratory, and Filtered Data are for Groundwater Protection Standards First Quarter 2000 and Monitoring Constituents Third Quarter 1999 data only.

+= exceeded the Groundwater Protection Standards (First Quarter 2000) or Monitoring Constituents (Third Quarter 1999) listed in Appendix A. 
Table D-4. Groundwater Monitoring Results for Plume Definition Wells, M-Area HWMF (Cont.) WELL MSB 40A

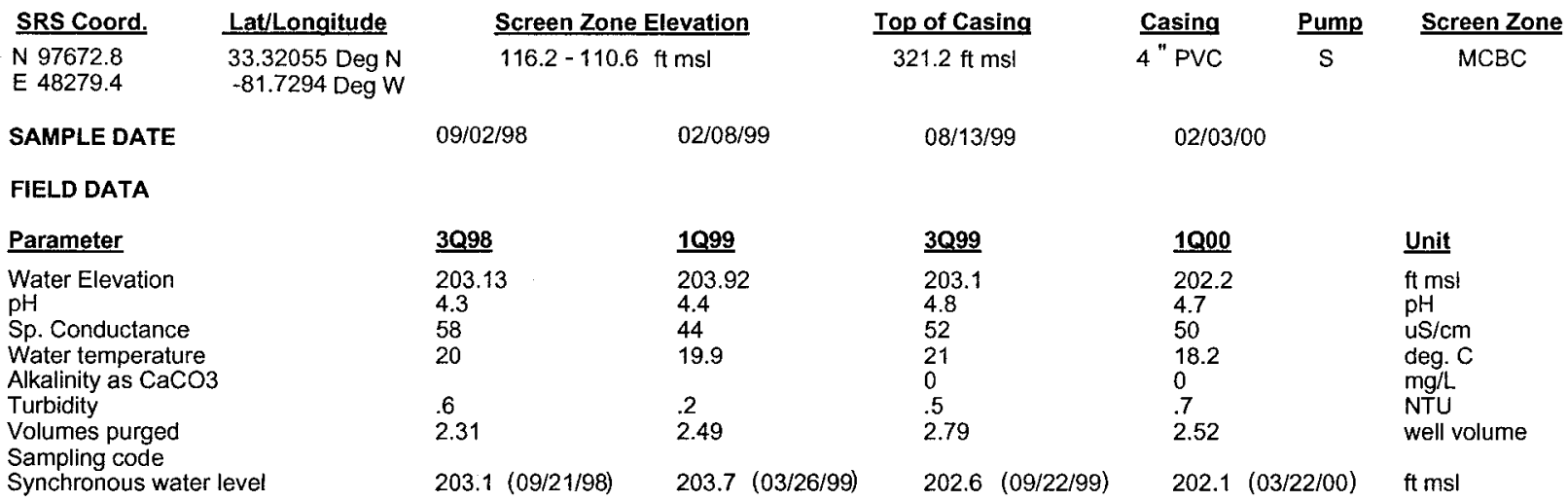

ANALYTICAL DATA

I. Groundwater Protection Standard

261 Appendix VIII/264 Appendix IX Hazardous Constituents

\begin{tabular}{|c|c|c|c|c|c|c|c|c|c|c|c|c|c|}
\hline$\underline{\mathbf{S T}}$ & Parameter & $\underline{3098}$ & CLP EPA & 1099 & $\underline{\text { CLP EPA }}$ & $\underline{3 Q 99}$ & CLPEPA & 1000 & $\underline{\text { CLPEPAA }}$ & Filt. & $\underline{\mathrm{DF}}$ & $\underline{\text { Unit }}$ & Lab \\
\hline \multicolumn{14}{|c|}{ Inorganics } \\
\hline & Barium, total recoverable & 17.6 & & $<10$ & $u$ & 18 & & & & & & $u g / L$ & \\
\hline & Cyanide & $<15.2$ & $\mathrm{U}$ & $<10$ & $\mathrm{U}$ & $<10$ & U & & & & & $u g / L$ & \\
\hline & Lead, total recoverable & $<100$ & $\mathrm{U}$ & $<100$ & $\mathrm{U}$ & $<3.73$ & U V & & & & & $\mathrm{ug} / \mathrm{L}$ & \\
\hline & Nickel, total recoverable & 5.3 & $J$ & $<50$ & $\mathrm{U}$ & $<50$ & $U$ & & & & & $\mathrm{ug} / \mathrm{L}$ & \\
\hline & Selenium, total recoverable & $<66$ & $U$ & $<200$ & $\mathrm{U}$ & $<5$ & $\mathrm{U}$ & & & & & ug/L & \\
\hline \multicolumn{14}{|c|}{ Organics } \\
\hline & Chlorobenzene & $<5$ & $\mathrm{U}$ & $<5$ & $u$ & $<1$ & $\mathrm{U}$ & $<1$ & $\mathrm{U}$ & $<E Q L$ & 1 & $\mathrm{ug} / \mathrm{L}$ & $M L$ \\
\hline & 1,1-Dichloroethane & $<5$ & $\mathrm{U}$ & $<5$ & $U$ & $<1$ & $\mathrm{U}$ & $<1$ & $\mathrm{U}$ & $<\mathrm{EQL}$ & 1 & $u g / L$ & $M L$ \\
\hline & 1,1-Dichloroethylene & $<5$ & $\mathrm{U}$ & $<5$ & $\mathrm{U}$ & $<1$ & $\mathrm{U}$ & $<1$ & $\mathrm{U}$ & $<E Q L$ & 1 & ug/L & ML \\
\hline & trans-1,2-Dichloroethylene & $<5$ & $\mathrm{U}$ & $<5$ & $\mathrm{U}$ & $<1$ & $\mathrm{U}$ & $<1$ & $U$ & $<\mathrm{EQL}$ & 1 & $\mathrm{ug} / \mathrm{L}$ & $M L$ \\
\hline & PCB 1016 & & & & & & & & & & & & \\
\hline & PCB 1221 & & & & & & & & & & & & \\
\hline & PCB 1232 & & & & & & & & & & & & \\
\hline & $\begin{array}{l}\text { PCB } 1242 \\
\text { PCB } 1248\end{array}$ & & & & & & & & & & & & \\
\hline & & & & & & & & & & & & & \\
\hline & PCB 1254 & & & & & & & & & & & & \\
\hline & PCB 1260 & & & & & & & & & & & & \\
\hline & 1,1,2,2-Tetrachloroethane & $<5$ & $U$ & $<5$ & $U$ & $<1$ & $\mathrm{U}$ & $<1$ & $\mathrm{U}$ & $<\mathrm{EQL}$ & 1 & ug/L & $M L$ \\
\hline & Tetrachloroethylene & $<5$ & U & $<5$ & $U$ & $<1$ & $\mathrm{U}$ & $<1$ & $\mathrm{U}$ & $<E Q L$ & 1 & $\mathrm{ug} / \mathrm{L}$ & $M L$ \\
\hline & 1,1,1-Trichloroethane & $<5$ & $U$ & $<5$ & $U$ & $<1$ & $\mathrm{U}$ & $<1$ & $\mathrm{U}$ & $<E Q L$ & 1 & $\mathrm{ug} / \mathrm{L}$ & $M L$ \\
\hline & Trichloroethylene & 3.86 & $J$ & $<5$ & $\mathrm{U}$ & $<1$ & $\mathrm{U}$ & $<1$ & $\mathrm{U}$ & $<E Q L$ & 1 & $\mathrm{ug} / \mathrm{L}$ & $M L$ \\
\hline \multicolumn{14}{|c|}{ II. Monitoring Constituents } \\
\hline$\underline{\mathbf{S T}}$ & Parameter & 3096 & CLPEPA & $\underline{3097}$ & CLPEPA & $3 Q 98$ & CLPEPA & $\underline{3099}$ & CLPEPA & Filt. & $\underline{\text { DF }}$ & $\underline{\text { Unit }}$ & $\underline{\text { Lab }}$ \\
\hline \multicolumn{14}{|c|}{ Inorganics } \\
\hline & Aluminum, total recoverable & 75.9 & & 60.3 & & 96.1 & J & $<200$ & $\mathrm{U}$ & $<\mathrm{EQL}$ & 1 & $\mathrm{ug} / \mathrm{L}$ & EX \\
\hline & Nitrate-nitrite as nitrogen & 350 & & $<10$ & $U$ & 403 & J & $<500$ & U & $<E Q L$ & 5 & $\mathrm{ug} / \mathrm{L}$ & EX \\
\hline & Sodium, total recoverable & 1960 & & 2050 & & 2080 & & 2500 & & $<4600$ & 1 & ug/L & EX \\
\hline+ & Sulfate & 12200 & & 11800 & & 11800 & & 13300 & & $>3000$ & 1 & ug/L & EX \\
\hline \multicolumn{14}{|c|}{ Radionuclides } \\
\hline & Gross alpha & 1.33 & & 1.31 & & 1.71 & $J$ & .608 & JU $L$ & $<\mathrm{EQL}$ & 1 & $\mathrm{pCi} / \mathrm{L}$ & GP \\
\hline & Nonvolatile beta & 1.03 & UI & 1.76 & UI & 4.64 & $J$ & 1.96 & J 1 & NDD & 1 & $\mathrm{pCi} / \mathrm{L}$ & GP \\
\hline & Radium, total alpha-emitting & .9 & & 3.99 & UI & .4 & Ui & 0 & $\mathrm{U}$ & $<\mathrm{EQL}$ & 1 & $\mathrm{pCi} / \mathrm{L}$ & GP \\
\hline
\end{tabular}

Notes: Concentrations in bold italics exceed the groundwater protection or monitoring constituent standards listed in Appendix A. Synchronous water levels are measured over a 3-5 day period or less. Dilution factors, Laboratory, and Filtered Data are for Groundwater Protection Standards First Quarter 2000 and Monitoring Constituents Third Quarter 1999 data only.

+= exceeded the Groundwater Protection Standards (First Quarter 2000) or Monitoring Constituents (Third Quarter 1999) listed in Appendix A. 
Table D-4. Groundwater Monitoring Results for Plume Definition Wells, M-Area HWMF (Cont.) WELL MSB 40B

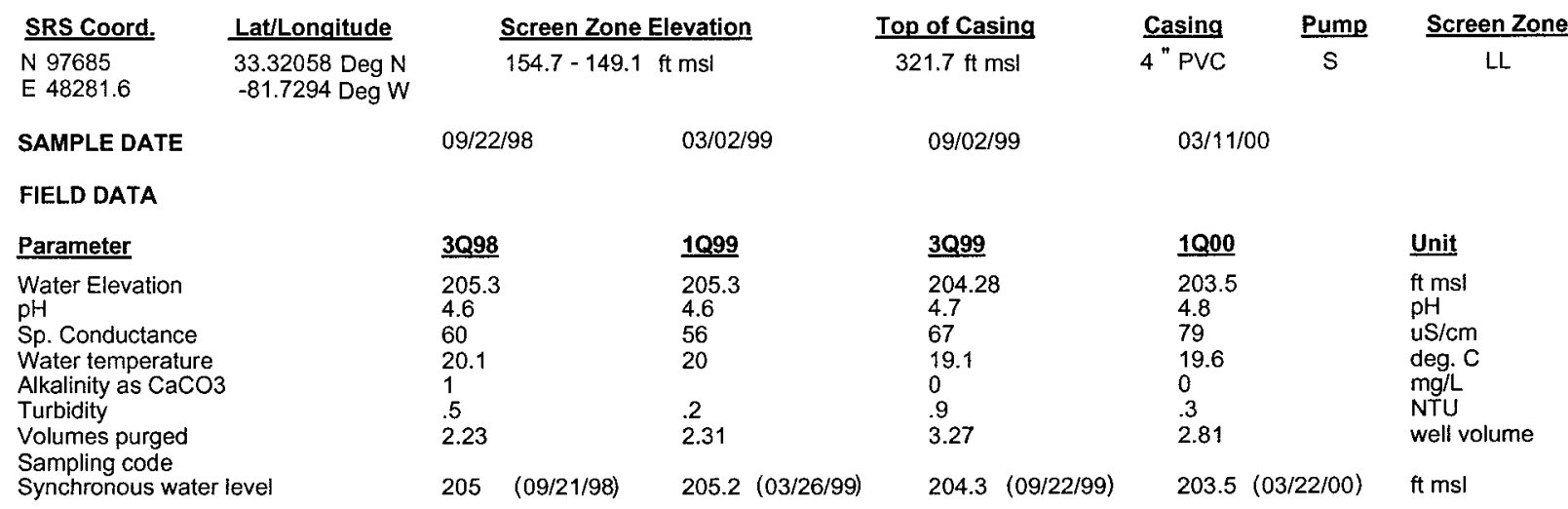

ANALYTICAL DATA

I. Groundwater Protection Standard

261 Appendix VIII/264 Appendix IX Hazardous Constituents

\begin{tabular}{|c|c|c|c|c|c|c|c|c|c|c|c|c|c|c|}
\hline$\underline{\text { ST }}$ & Parameter & $\underline{3 Q 98}$ & CLP EPA & $\underline{1 Q 99}$ & CLP EPA & $\underline{3 Q 99}$ & $\underline{\text { CLPEPA }}$ & 1000 & \multicolumn{2}{|c|}{ CLPEPA } & Filt. & $\underline{\text { DF }}$ & Unit & $\underline{\text { Lab }}$ \\
\hline \multicolumn{15}{|c|}{ Inorganics } \\
\hline & Barium, total recoverable & 14.4 & & 14.3 & & 18 & & & & & & & $\mathrm{ug} / \mathrm{L}$ & \\
\hline & Cyanide & $<10$ & $u$ & $<10$ & $\mathrm{U}$ & $<10$ & $\mathrm{U}$ & & & & & & $\mathrm{ug} / \mathrm{L}$ & \\
\hline & Lead, total recoverable & $<100$ & $\mathrm{U}$ & $<100$ & $\mathrm{U}$ & $<10$ & $U$ & & & & & & $\mathrm{ug} / \mathrm{L}$ & \\
\hline & Nickel, total recoverable & $<50$ & $U$ & $<50$ & $\mathrm{U}$ & $<50$ & $U$ & & & & & & $u g / L$ & \\
\hline & Selenium, total recoverable & $<200$ & $U$ & $<200$ & $\mathrm{U}$ & $<10$ & $\mathrm{U}$ & & & & & & $\mathrm{ug} / \mathrm{L}$ & \\
\hline \multicolumn{15}{|c|}{ Organics } \\
\hline & Chlorobenzene & $<50$ & $u$ & $<125$ & $U$ & $<1$ & U & $<10$ & $\mathrm{U}$ & & $<E Q L$ & 10 & $\mathrm{ug} / \mathrm{L}$ & ML \\
\hline & 1,1-Dichloroethane & $<50$ & $U$ & $<125$ & $\mathrm{U}$ & $<1$ & $U$ & $<10$ & $\mathrm{U}$ & & $<\mathrm{EQL}$ & 10 & $\mathrm{ug} / \mathrm{L}$ & ML \\
\hline & 1,1-Dichloroethylene & $<50$ & $\mathrm{U}$ & $<125$ & $\mathrm{U}$ & $<1$ & $\mathrm{U}$ & $<10$ & $\mathrm{U}$ & & $<\mathrm{EQL}$ & 10 & $\mathrm{ug} / \mathrm{L}$ & ML \\
\hline & $\begin{array}{l}\text { trans-1,2-Dichloroethylene } \\
\text { PCB } 1016\end{array}$ & $<50$ & $\mathrm{U}$ & $<125$ & $\mathrm{U}$ & $<1$ & $U$ & & & & & & $\mathrm{ug} / \mathrm{L}$ & \\
\hline & PCB 1221 & & & & & & & & & & & & & \\
\hline & PCB 1232 & & & & & & & & & & & & & \\
\hline & PCB 1242 & & & & & & & & & & & & & \\
\hline & РCB 1248 & & & & & & & & & & & & & \\
\hline & PCB 1254 & & & & & & & & & & & & & \\
\hline & PCB 1260 & & & & & & & & & & & & & \\
\hline & $1,1,2,2$-Tetrachloroethane & $<50$ & $U$ & $<125$ & $U$ & $<1$ & $U$ & $<10$ & $U$ & & $<\mathrm{EQL}$ & 10 & $u g / L$ & ML \\
\hline & Tetrachloroethylene & $<50$ & $\cup$ & $<125$ & $\mathrm{U}$ & .89 & J IK & $<10$ & $U$ & & $<E Q L$ & 10 & $\mathrm{ug} / \mathrm{L}$ & ML \\
\hline & 1,1,1-Trichloroethane & $<50$ & $U$ & $<125$ & U & $<1$ & $U$ & $<10$ & $\mathrm{U}$ & & $<E Q L$ & 10 & $u g / L$ & ML \\
\hline & Trichloroethylene & 1380 & & 2430 & & 1720 & & 1810 & $J$ & $\mathrm{~K}$ & NDD & 10 & $\mathrm{ug} / \mathrm{L}$ & ML \\
\hline \multicolumn{15}{|c|}{ Il. Monitoring Constituents } \\
\hline$\underline{\text { ST }}$ & Parameter & $\underline{3 Q 96}$ & CLP EPA & $\underline{3097}$ & CLPEPA & $\underline{3098}$ & CLPEPA & $\underline{3099}$ & $\underline{\text { CLP }}$ & EPA & Filt. & DF & Unit & $\underline{\text { Lab }}$ \\
\hline \multicolumn{15}{|c|}{ Inorganics } \\
\hline & Aluminum, total recoverable & $<51.6$ & $\mathrm{U}$ & 30.5 & & 184 & $J$ & $<200$ & $\mathrm{U}$ & & $<\mathrm{EQL}$ & 1 & $\mathrm{ug} / \mathrm{L}$ & EX \\
\hline+ & Nitrate-nitrite as nitrogen & 4800 & & 3930 & & 3760 & & 5720 & & & $>2400$ & 5 & $\mathrm{ug} / \mathrm{L}$ & EX \\
\hline & Sodium, total recoverable & 3640 & & 3590 & & 4070 & & 4300 & & & $<4600$ & 1 & $\mathrm{ug} / \mathrm{L}$ & EX \\
\hline & Sulfate & 1260 & & $<5000$ & $\mathrm{U}$ & 1240 & & 1140 & & & $<3000$ & 2 & $\mathrm{ug} / \mathrm{L}$ & EX \\
\hline \multicolumn{15}{|c|}{ Radionuclides } \\
\hline & Gross alpha & 3.45 & & 1.74 & & 2.8 & & 2.07 & $U$ & V & $<E Q L$ & 1 & $\mathrm{pCi} / \mathrm{L}$ & GP \\
\hline & Nonvolatile beta & 3.96 & & 1.53 & UI & 4.35 & & 4.72 & & & $<50$ & 1 & $\mathrm{pCi} / \mathrm{L}$ & GP \\
\hline & Radium, total alpha-emitting & .9 & & 4.41 & UIJ & 1.66 & & 1.5 & $\mathrm{~J}$ & I & NDD & 1 & $\mathrm{pCi} / \mathrm{L}$ & GP \\
\hline
\end{tabular}

Notes: Concentrations in bold italics exceed the groundwater protection or monitoring constituent standards listed in Appendix A. Synchronous water levels are measured over a 3-5 day period or less. Dilution factors, Laboratory, and Filtered Data are for Groundwater Protection Standards First Quarter 2000 and Monitoring Constituents Third Quarter 1999 data only.

+= exceeded the Groundwater Protection Standards (First Quarter 2000) or Monitoring Constituents (Third Quarter 1999) listed in Appendix A. 
Table D-4. Groundwater Monitoring Results for Plume Definition Wells, M-Area HWMF (Cont.) WELL MSB 40C

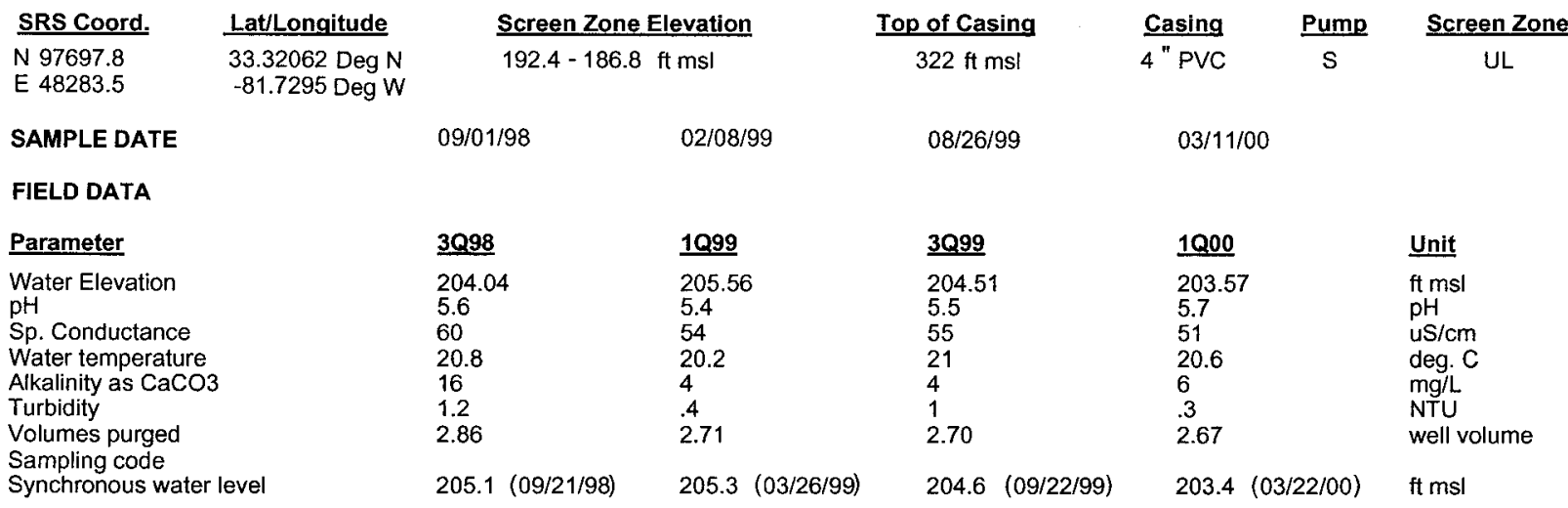

ANALYTICAL DATA

I. Groundwater Protection Standard

261 Appendix VIII/264 Appendix IX Hazardous Constituents

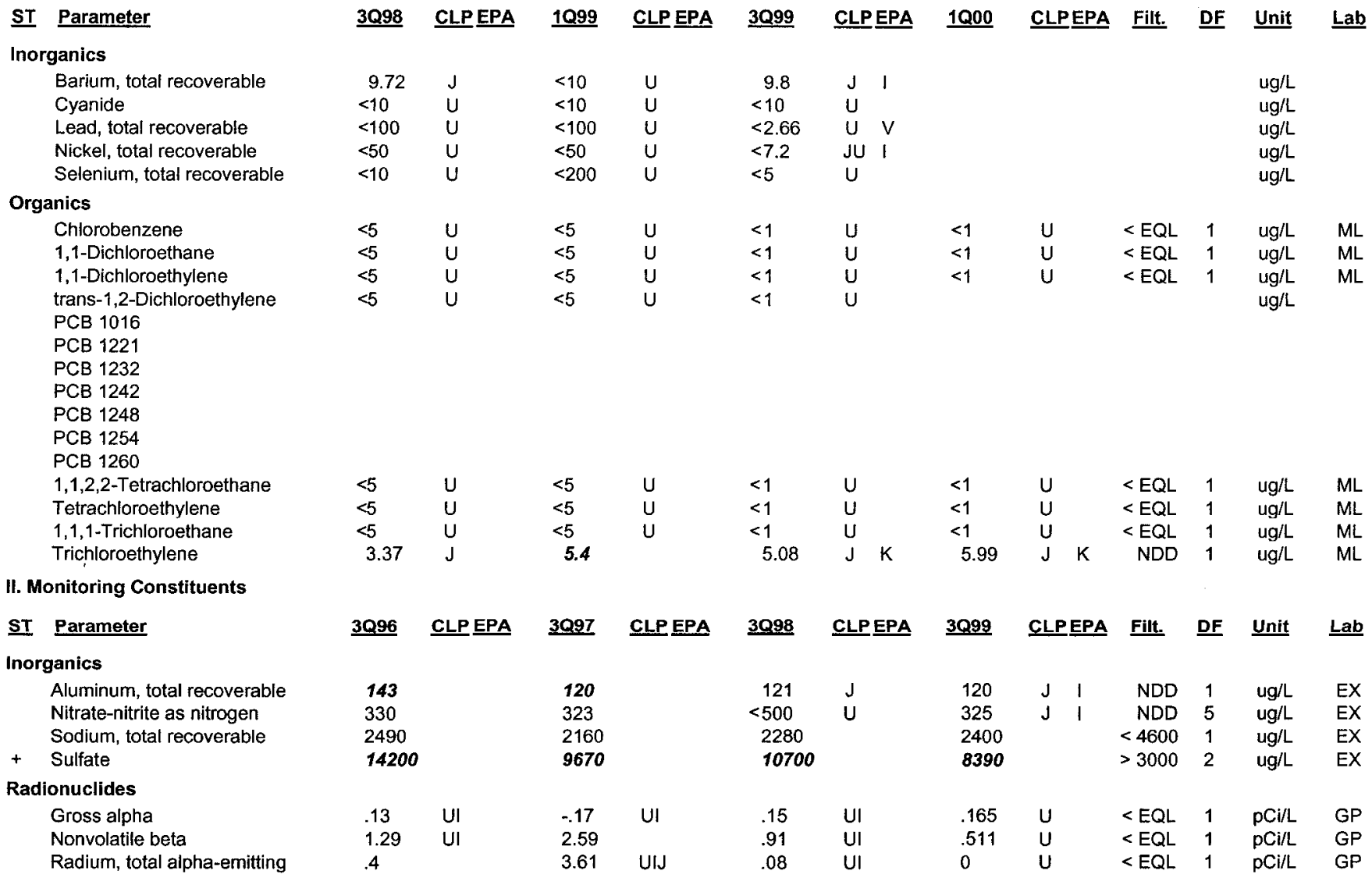

Notes: Concentrations in bold italics exceed the groundwater protection or monitoring constituent standards listed in Appendix A. Synchronous water levels are measured over a 3-5 day period or less. Dilution factors, Laboratory, and Filtered Data are for Groundwater Protection Standards First Quarter 2000 and Monitoring Constituents Third Quarter 1999 data only.

+ = exceeded the Groundwater Protection Standards (First Quarter 2000) or Monitoring Constituents (Third Quarter 1999) listed in Appendix A. 
Table D-4. Groundwater Monitoring Results for Plume Definition Wells, M-Area HWMF (Cont.) WELL MSB 41B

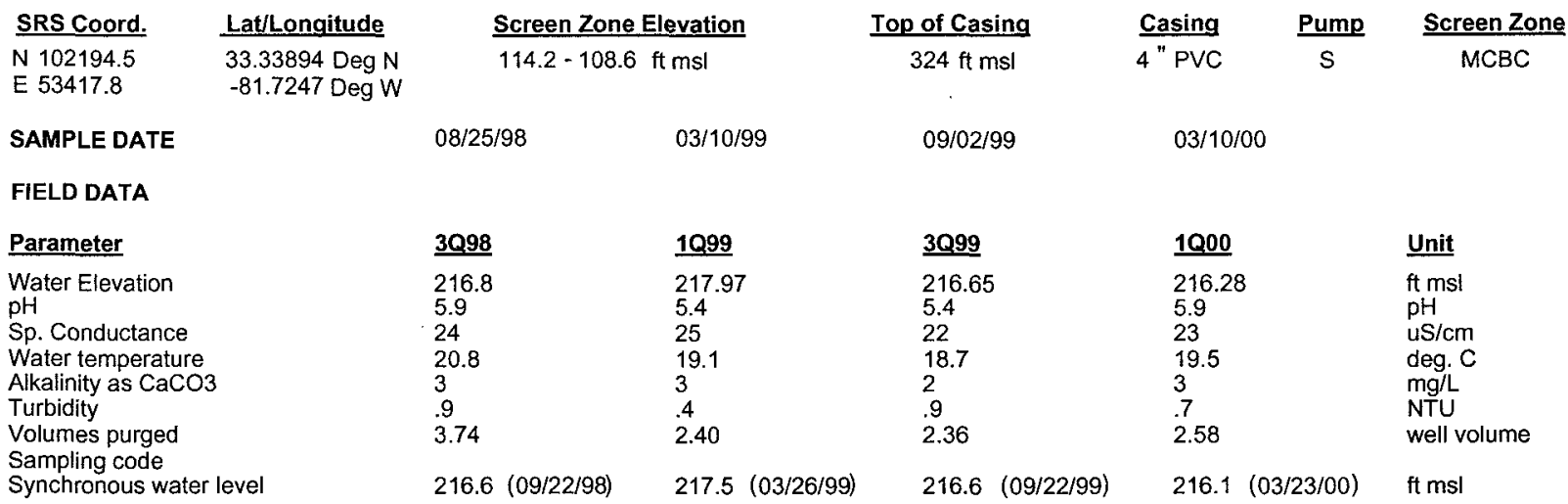

ANALYTICAL DATA

I. Groundwater Protection Standard

261 Appendix VIII/264 Appendix IX Hazardous Constituents

\begin{tabular}{|c|c|c|c|c|c|c|c|c|c|c|c|c|c|c|}
\hline \multirow{2}{*}{\multicolumn{2}{|c|}{$\underline{\text { ST }} \underset{\text { Parameter }}{\text { Inorganics }}$}} & \multirow[t]{2}{*}{$\underline{3 Q 98}$} & \multirow[t]{2}{*}{ CLPEPA } & \multirow[t]{2}{*}{$\underline{1 Q 99}$} & \multirow[t]{2}{*}{ CLPEPA } & \multirow[t]{2}{*}{ 3Q99 } & CLPEPA & \multirow[t]{2}{*}{$1 \mathrm{Q00}$} & \multicolumn{2}{|c|}{ CLPEPA } & \multirow[t]{2}{*}{ Filt. } & \multirow[t]{2}{*}{$\underline{D F}$} & \multirow[t]{2}{*}{$\underline{\text { Unit }}$} & \multirow[t]{2}{*}{$\underline{\text { Lab }}$} \\
\hline & & & & & & & & & & & & & & \\
\hline & Barium, total recoverable & 9.88 & $\mathrm{~J}$ & 11.5 & & 11 & & & & & & & $\mathrm{ug} / \mathrm{L}$ & \\
\hline & Cyanide & $<10$ & $\mathrm{U}$ & $<10$ & JU L & $<10$ & $\mathrm{U}$ & & & & & & ug/L & \\
\hline & Lead, total recoverable & $<100$ & $U$ & $<100$ & $\mathrm{U}$ & $<10$ & $U$ & & & & & & ug/L & \\
\hline & Nickel, total recoverable & 13.1 & $\mathrm{~J}$ & $<50$ & $\mathrm{U}$ & $<50$ & $\mathrm{U}$ & & & & & & ug/L & \\
\hline & Selenium, total recoverable & $<200$ & $U$ & $<200$ & $\mathrm{U}$ & $<10$ & $\mathrm{U}$ & & & & & & $u g / L$ & \\
\hline \multicolumn{15}{|c|}{ Organics } \\
\hline & Chlorobenzene & $<5$ & UJ & $<5$ & $\mathrm{U}$ & $<1$ & $\mathrm{U}$ & $<1$ & $\mathrm{u}$ & & $<\mathrm{EQL}$ & 1 & $\mathrm{ug} / \mathrm{L}$ & ML \\
\hline & 1,1-Dichloroethane & $<5$ & UJ & $<5$ & $\mathrm{U}$ & $<1$ & $\mathrm{U}$ & $<1$ & $\mathrm{U}$ & & $<\mathrm{EQL}$ & 1 & $\mathrm{ug} / \mathrm{L}$ & ML \\
\hline & 1,1-Dichloroethylene & $<5$ & UJ & $<5$ & $\mathrm{U}$ & $<1$ & $U$ & $<1$ & $\mathrm{U}$ & & $<\mathrm{EQL}$ & 1 & $\mathrm{ug} / \mathrm{L}$ & $M L$ \\
\hline & trans-1,2-Dichloroethylene & $<5$ & UJ & $<5$ & $\mathrm{U}$ & $<1$ & $\mathrm{U}$ & & & & & & $\mathrm{ug} / \mathrm{L}$ & \\
\hline & PCB 1016 & & & & & & & & & & & & & \\
\hline & РCB 1221 & & & & & & & & & & & & & \\
\hline & PCB 1232 & & & & & & & & & & & & & \\
\hline & PCB 1242 & & & & & & & & & & & & & \\
\hline & PCB 1248 & & & & & & & & & & & & & \\
\hline & PCB 1254 & & & & & & & & & & & & & \\
\hline & PCB 1260 & & & & & & & & & & & & & \\
\hline & $1,1,2,2$-Tetrachloroethane & $<5$ & US & $<5$ & $\mathrm{U}$ & $<1$ & $\mathrm{U}$ & $<1$ & $U$ & & $<\mathrm{EQL}$ & 1 & ug/L & ML \\
\hline & Tetrachioroethylene & 5.48 & $J$ & 7.22 & & 7.53 & $J \mathrm{~K}$ & 6.17 & $\mathrm{~J}$ & $\mathrm{~K}$ & NDD & 1 & ug/L & $\mathrm{ML}$ \\
\hline & 1,1,1-Trichloroethane & $<5$ & US & $<5$ & U & $<1$ & U & $<1$ & $U$ & & $<E Q L$ & 1 & $\mathrm{ug} / \mathrm{h}$ & ML \\
\hline & Trichloroethylene & $<5$ & UJ & 3.09 & $J \quad 1$ & 3.2 & $J \quad K$ & 2.94 & $J$ & K & NDD & 1 & $u g / L$ & ML \\
\hline \multicolumn{15}{|c|}{ II. Monitoring Constituents } \\
\hline$\underline{\text { ST }}$ & Parameter & $\underline{3096}$ & $\underline{\text { CLP EPA }}$ & $\underline{3097}$ & CLP EPA & 3098 & $\underline{\text { CLPEPA }}$ & $\underline{3099}$ & $\underline{\text { CLP }}$ & EPA & Filt. & DF & $\underline{\text { Unit }}$ & $\underline{\text { Lab }}$ \\
\hline \multicolumn{15}{|c|}{ Inorganics } \\
\hline & Aluminum, total recoverable & & & $<20$ & $U$ & $<200$ & $\mathrm{U}$ & $<200$ & 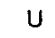 & & $<E Q L$ & 1 & $u g / L$ & $\mathrm{EX}$ \\
\hline & Nitrate-nitrite as nitrogen & & & 720 & & 1070 & & 1050 & & & $<2400$ & 5 & $\mathrm{ug} / \mathrm{L}$ & EX \\
\hline & Sodium, total recoverable & & & 1410 & & 1430 & & 1400 & & & $<4600$ & 1 & $u g / L$ & EX \\
\hline & Sulfate & & & $<5000$ & $U$ & 423 & & 193 & $J$ & 1 & NDD & 2 & $u g / L$ & EX \\
\hline \multicolumn{15}{|c|}{ Radionuclides } \\
\hline & Gross alpha & & & 1.01 & UI & 3.16 & & 1.93 & $U$ & V & $<E Q L$ & 1 & $\mathrm{pCi} / \mathrm{L}$ & GP \\
\hline & Nonvolatile beta & & & 1.03 & UI & 2.75 & $J$ & 2.61 & $J$ & 1 & NDD & 1 & $\mathrm{pCi} / \mathrm{L}$ & GP \\
\hline & Radium, total alpha-emitting & & & 2.97 & UIJ & .64 & & .8 & $\jmath$ & 1 & NDD & 1 & $\mathrm{pCi} / \mathrm{L}$ & GP \\
\hline
\end{tabular}

Notes: Concentrations in bold italics exceed the groundwater protection or monitoring constituent standards listed in Appendix A. Synchronous water levels are measured over a 3-5 day period or less. Dilution factors, Laboratory, and Filtered Data are for Groundwater Protection Standards First Quarter 2000 and Monitoring Constituents Third Quarter 1999 data only.

$+=$ exceeded the Groundwater Protection Standards (First Quarter 2000) or Monitoring Constituents (Third Quarter 1999) listed in Appendix A. 
Table D-4. Groundwater Monitoring Results for Plume Definition Wells, M-Area HWMF (Cont.) WELL MSB 41TA

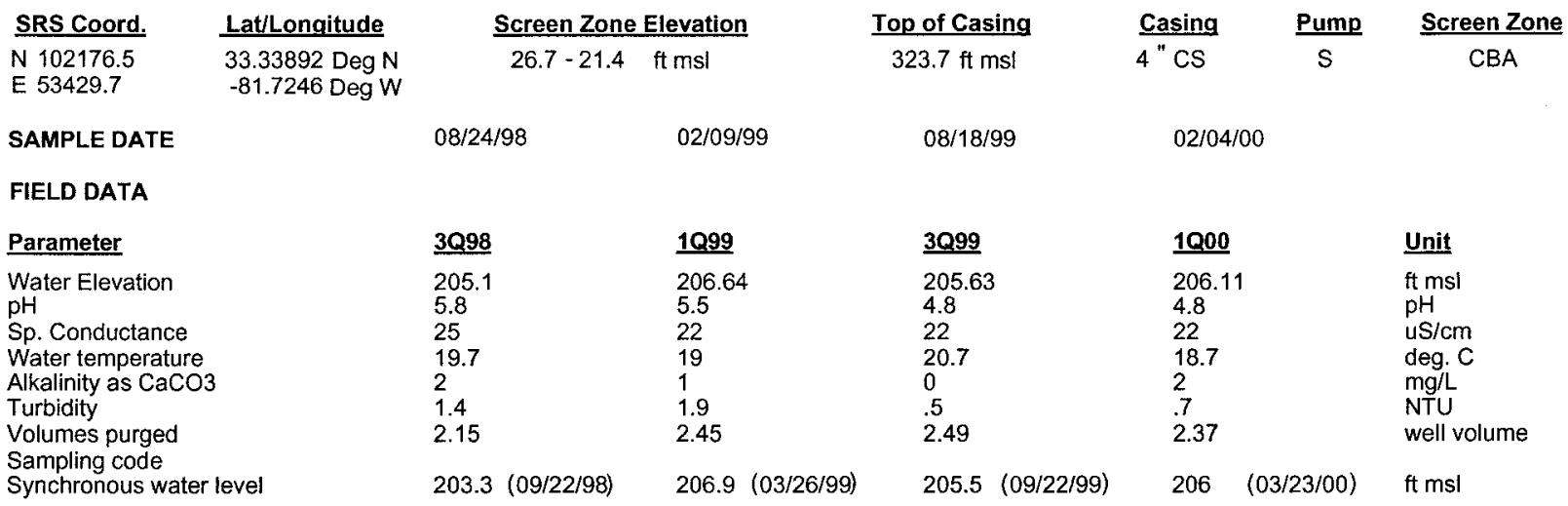

ANALYTICAL DATA

I. Groundwater Protection Standard

261 Appendix VIII/264 Appendix IX Hazardous Constituents

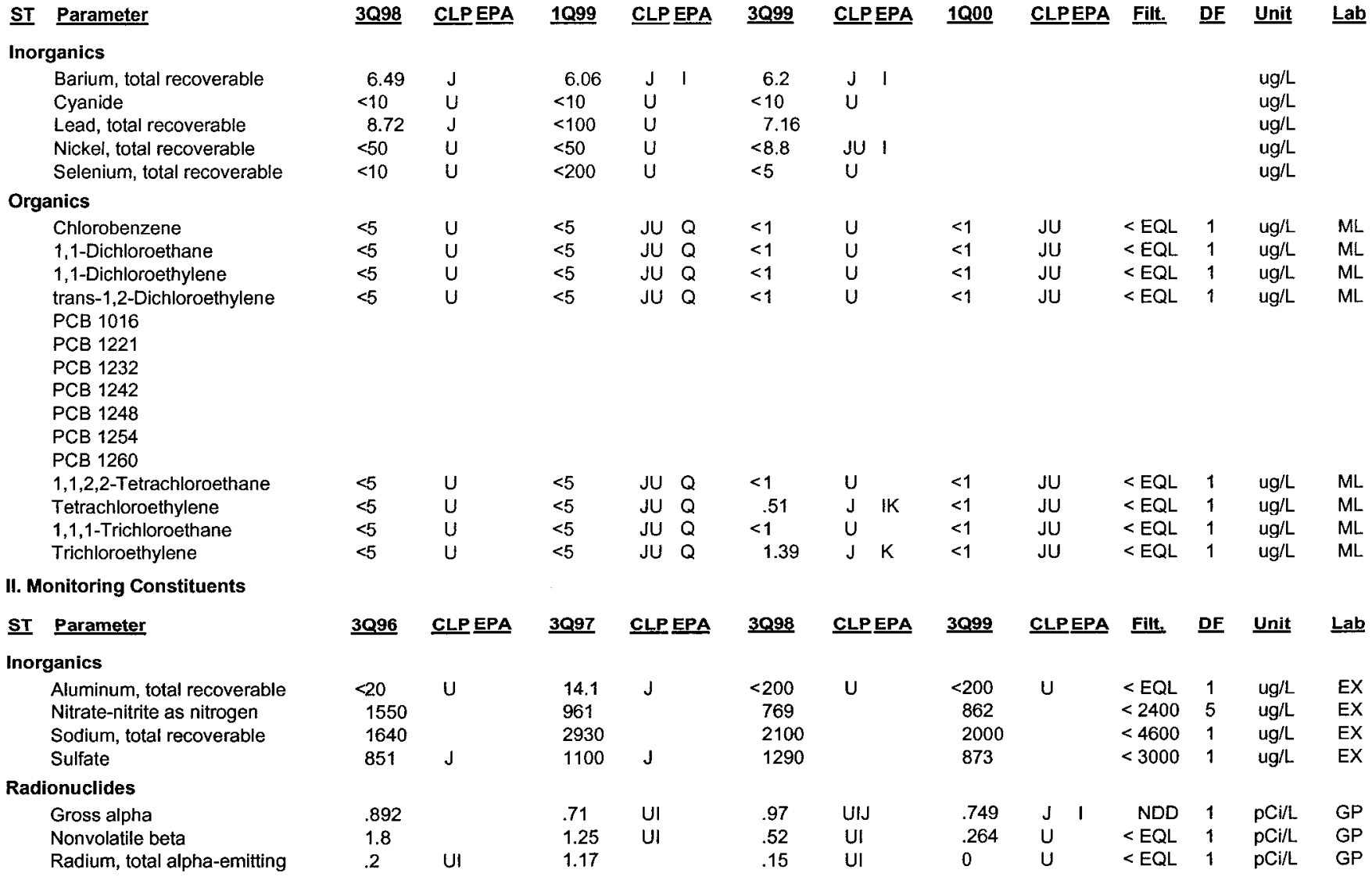

Notes: Concentrations in bold italics exceed the groundwater protection or monitoring constituent standards listed in Appendix A. Synchronous water levels are measured over a 3-5 day period or less. Dilution factors, Laboratory, and Filtered Data are for Groundwater Protection Standards First Quarter 2000 and Monitoring Constituents Third Quarter 1999 data only.

+ = exceeded the Groundwater Protection Standards (First Quarter 2000) or Monitoring Constituents (Third Quarter 1999) listed in Appendix A. 
Table D-4. Groundwater Monitoring Results for Plume Definition Wells, M-Area HWMF (Cont.) WELL MSB 42B

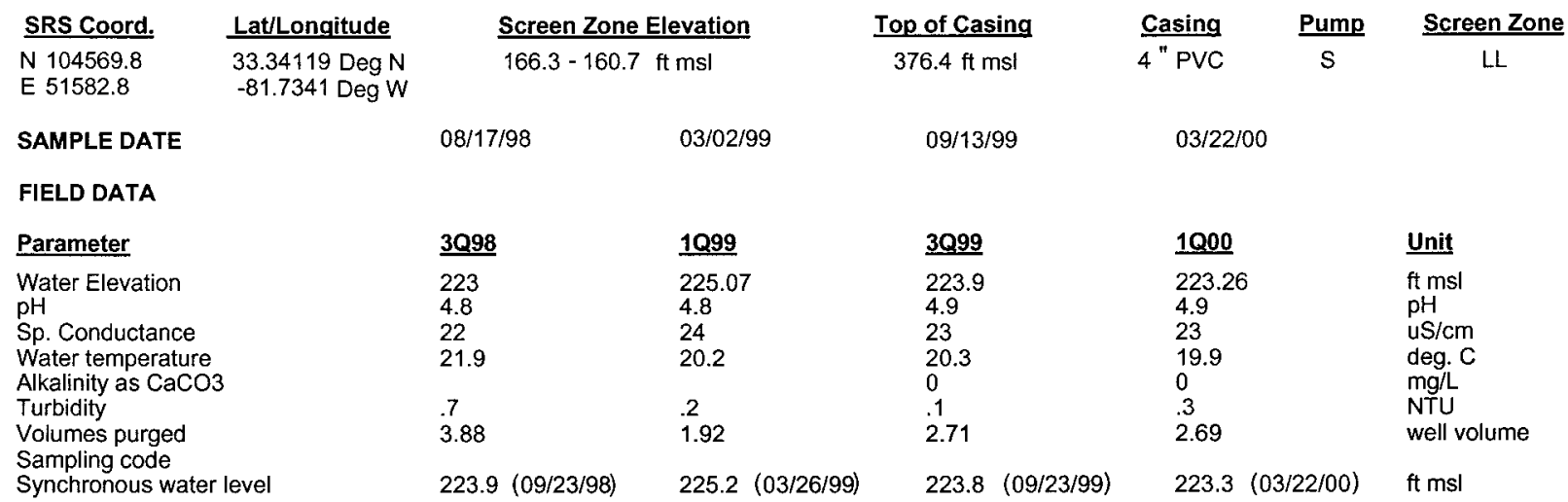

ANALYTICAL DATA

I. Groundwater Protection Standard

261 Appendix VIII/264 Appendix IX Hazardous Constituents

\begin{tabular}{|c|c|c|c|c|c|c|c|c|c|c|c|c|c|c|}
\hline \multirow{2}{*}{\multicolumn{2}{|c|}{$\frac{\text { ST Parameter }}{\text { Inorganics }}$}} & \multirow[t]{2}{*}{$\underline{3 Q 98}$} & \multirow[t]{2}{*}{$\underline{\text { CLP EPA }}$} & \multirow[t]{2}{*}{$\underline{1 Q 99}$} & \multirow[t]{2}{*}{ CLP EPA } & \multirow[t]{2}{*}{$\underline{3099}$} & CLPEPA & \multirow[t]{2}{*}{1000} & \multicolumn{2}{|c|}{ CLPEPA } & \multirow[t]{2}{*}{ Filt. } & \multirow[t]{2}{*}{$\underline{\text { DF }}$} & \multirow[t]{2}{*}{ Unit } & \multirow[t]{2}{*}{ Lab } \\
\hline & & & & & & & & & & & & & & \\
\hline & Barium, total recoverable & 3.66 & $J$ & 42.9 & & 3.65 & J I & & & & & & $u g / L$ & \\
\hline & Cyanide & $<10$ & $U$ & 1.52 & J 1 & $<10$ & $\mathrm{U}$ & & & & & & $\mathrm{ug} / \mathrm{L}$ & \\
\hline & Lead, total recoverable & $<10$ & $U$ & 57.3 & & $<10$ & $\bigcup$ & & & & & & $\mathrm{ug} / \mathrm{L}$ & \\
\hline & Nickel, total recoverable & $<50$ & $U$ & $<50$ & $\mathrm{U}$ & $<50$ & U & & & & & & $\mathrm{ug} / \mathrm{L}$ & \\
\hline & Selenium, total recoverable & $<10$ & $\cup$ & $<200$ & $\mathrm{U}$ & $<10$ & $U$ & & & & & & $\mathrm{ug} / \mathrm{L}$ & \\
\hline \multicolumn{15}{|c|}{ Organics } \\
\hline & Chlorobenzene & $<5$ & $u$ & $<5$ & $\mathrm{U}$ & $<1$ & U & $<1$ & $u$ & & $<\mathrm{EQL}$ & 1 & $\mathrm{ug} / \mathrm{L}$ & ML \\
\hline & 1,1-Dichloroethane & $<5$ & $\mathrm{U}$ & $<5$ & $\mathrm{U}$ & $<1$ & $U$ & $<1$ & $\mathrm{U}$ & & $<\mathrm{EQL}$ & 1 & $\mathrm{ug} / \mathrm{L}$ & ML \\
\hline & 1,1-Dichloroethylene & $<5$ & U & $<5$ & $\mathrm{U}$ & $<1$ & $\cup$ & $<1$ & $u$ & & $<\mathrm{EQL}$ & 1 & $\mathrm{ug} / \mathrm{L}$ & ML \\
\hline & trans-1,2-Dichloroethylene & $<5$ & U & $<5$ & $\mathrm{U}$ & $<1$ & $\mathrm{U}$ & $<1$ & U & & $<E Q L$ & 1 & $\mathrm{ug} / \mathrm{L}$ & ML \\
\hline & PCB 1016 & & & & & & & & & & & & & \\
\hline & PCB 1221 & & & & & & & & & & & & & \\
\hline & PCB 1232 & & & & & & & & & & & & & \\
\hline & PCB 1242 & & & & & & & & & & & & & \\
\hline & PCB 1248 & & & & & & & & & & & & & \\
\hline & РCB 1254 & & & & & & & & & & & & & \\
\hline & PCB 1260 & & & & & & & & & & & & & \\
\hline & $1,1,2,2$-Tetrachloroethane & $<5$ & $U$ & $<5$ & $U$ & $<1$ & U & $<1$ & $\mathrm{U}$ & & $<$ EQL & 1 & $\mathrm{ug} / \mathrm{L}$ & ML \\
\hline & Tetrachloroethylene & 1.39 & $J$ & $<5$ & $U$ & .95 & J I & $<1$ & U & & $<\mathrm{EQL}$ & 1 & $\mathrm{ug} / \mathrm{L}$ & ML \\
\hline & 1,1,1-Trichloroethane & $<5$ & $\mathrm{U}$ & $<5$ & $u$ & $<1$ & $\mathrm{U}$ & $<1$ & $U$ & & $<\mathrm{EQL}$ & 1 & $\mathrm{ug} / \mathrm{L}$ & ML \\
\hline & Trichloroethylene & 13.2 & & 14.6 & & 9.53 & & 5.8 & $J$ & $\mathrm{~K}$ & NDD & 1 & ug/L & ML \\
\hline \multicolumn{15}{|c|}{ II. Monitoring Constituents } \\
\hline$\underline{\text { ST }}$ & Parameter & $\underline{3096}$ & CLPEPA & $\underline{3097}$ & CLPEPA & $\underline{3098}$ & CLPEPA & $\underline{3099}$ & $\underline{\text { CLP }}$ & EPA & Filt. & $\underline{\text { DF }}$ & Unit & $\underline{\text { Lab }}$ \\
\hline \multicolumn{15}{|c|}{ Inorganics } \\
\hline & Aluminum, total recoverable & $<20$ & $\mathrm{U}$ & $<20$ & $\mathrm{u}$ & $<200$ & $\mathrm{u}$ & $<200$ & $\mathrm{U}$ & & $<E Q L$ & 1 & $u g / L$ & EX \\
\hline & Nitrate-nitrite as nitrogen & 1540 & & 1180 & & 1100 & & 950 & & & $<2400$ & 5 & $\mathrm{ug} / \mathrm{L}$ & EX \\
\hline & Sodium, total recoverable & 2170 & & 2040 & & 2290 & & 2160 & & & $<4600$ & 1 & $\mathrm{ug} / \mathrm{L}$ & EX \\
\hline & Sulfate & 241 & $J$ & $<5000$ & $\mathrm{U}$ & $<200$ & $\mathrm{U}$ & 480 & & & $<3000$ & 1 & ug/L & EX \\
\hline \multicolumn{15}{|c|}{ Radionuclides } \\
\hline & Gross alpha & 1.88 & Ul & .97 & UI & 1.84 & & .728 & $\mathrm{~J}$ & 1 & NDD & 1 & $\mathrm{pCi} / \mathrm{L}$ & GP \\
\hline & Nonvolatile beta & 8.75 & & .16 & UI & 3.88 & & 1.01 & $J$ & IK & NDD & 1 & $\mathrm{pCi} / \mathrm{L}$ & GP \\
\hline & Radium, total alpha-emitting & .5 & & 3.42 & UIJ & .42 & $\mathrm{U}$ & .3 & $u$ & & $<E Q L$ & 1 & $\mathrm{pCi} / \mathrm{L}$ & GP \\
\hline
\end{tabular}

Notes: Concentrations in bold italics exceed the groundwater protection or monitoring constituent standards listed in Appendix A. Synchronous water levels are measured over a 3-5 day period or less. Dilution factors, Laboratory, and Filtered Data are for Groundwater Protection Standards First Quarter 2000 and Monitoring Constituents Third Quarter 1999 data only.

$+=$ exceeded the Groundwater Protection Standards (First Quarter 2000) or Monitoring Constituents (Third Quarter 1999) listed in Appendix A. 
Table D-4. Groundwater Monitoring Results for Plume Definition Wells, M-Area HWMF (Cont.) WELL MSB 42C

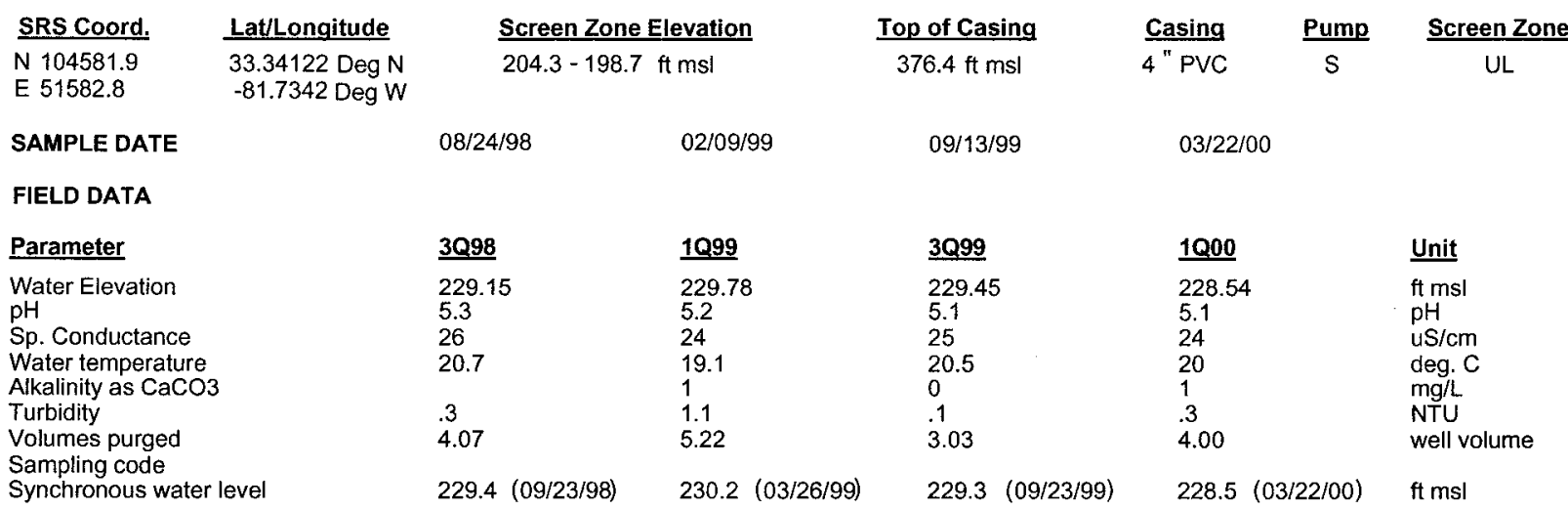

ANALYTICAL DATA

I. Groundwater Protection Standard

261 Appendix VIII/264 Appendix IX Hazardous Constituents

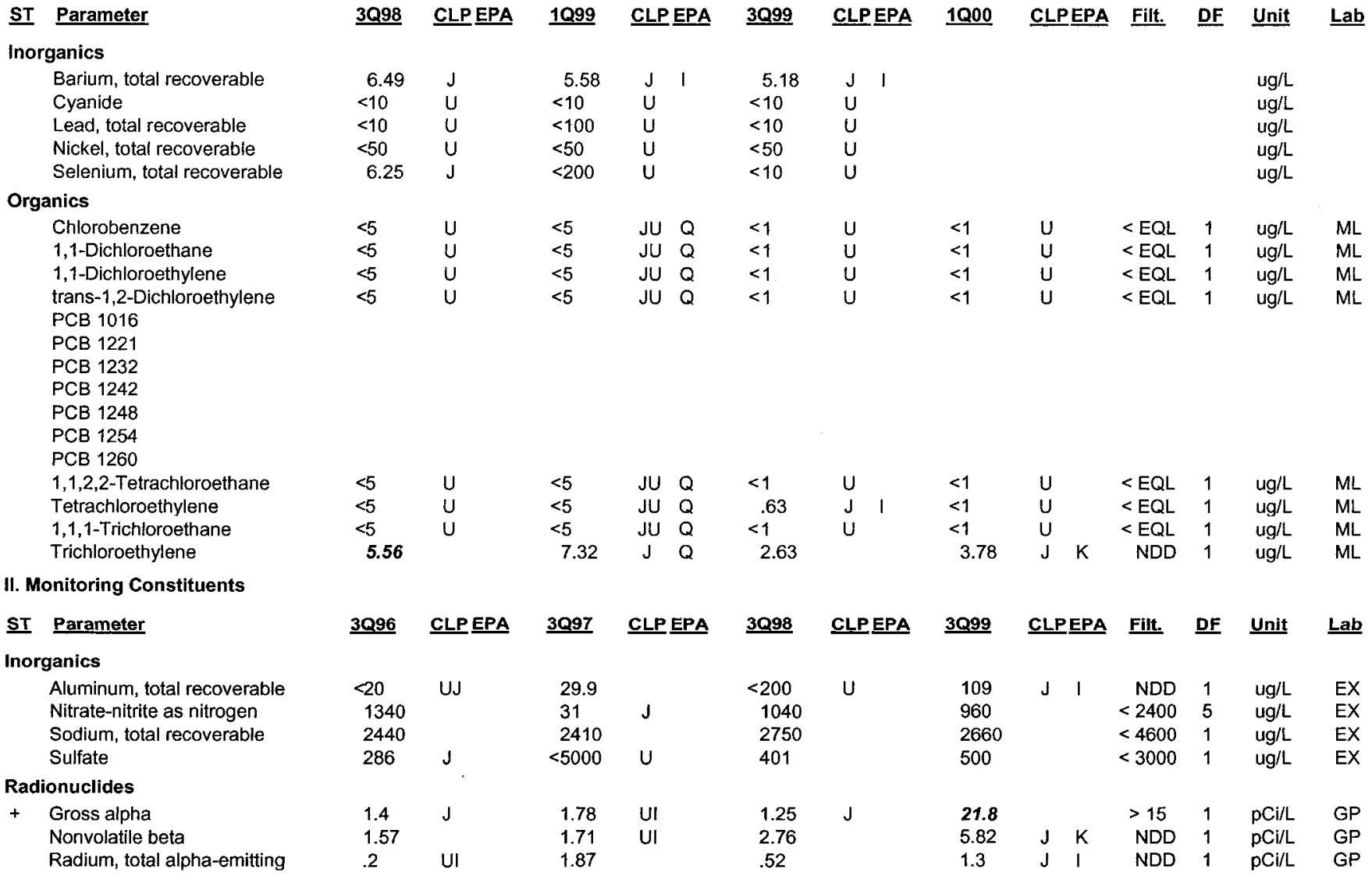

Notes: Concentrations in bold italics exceed the groundwater protection or monitoring constituent standards listed in Appendix A. Synchronous water levels are measured over a 3-5 day period or less. Dilution factors, Laboratory, and Filtered Data are for Groundwater Protection Standards First Quarter 2000 and Monitoring Constituents Third Quarter 1999 data only.

+= exceeded the Groundwater Protection Standards (First Quarter 2000) or Monitoring Constituents (Third Quarter 1999) listed in Appendix A. 
Table D-4. Groundwater Monitoring Results for Plume Definition Wells, M-Area HWMF (Cont.) WELL MSB 42D

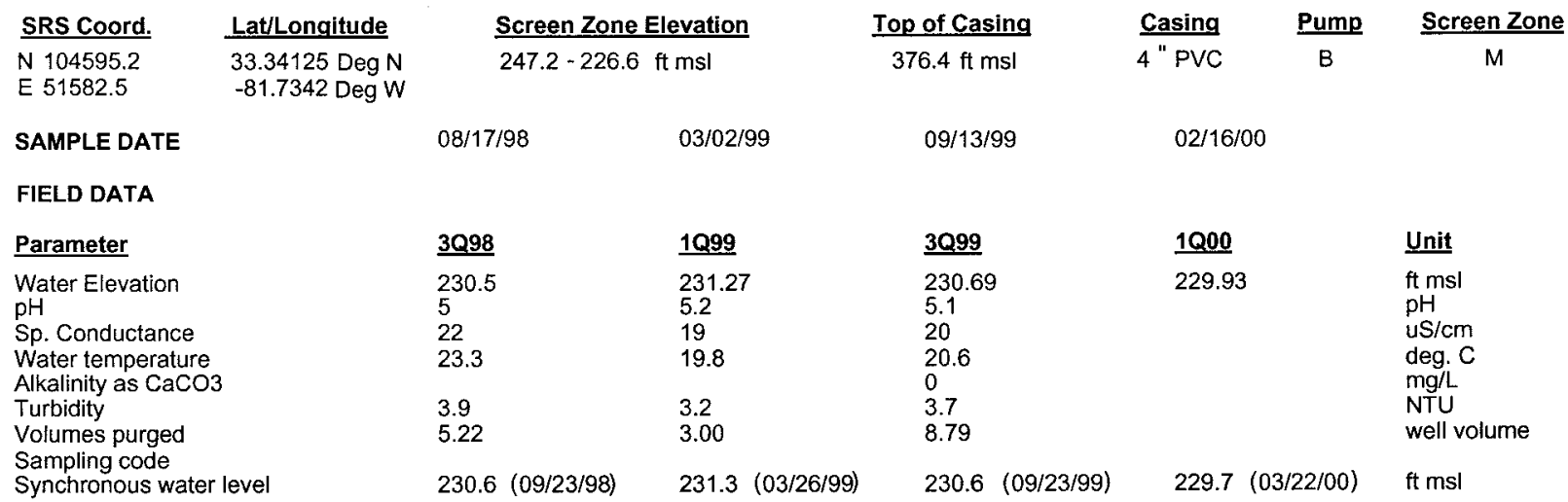

ANALYTICAL DATA

I. Groundwater Protection Standard

261 Appendix VIII/264 Appendix IX Hazardous Constituents

\begin{tabular}{|c|c|c|c|c|c|c|c|c|c|c|c|c|c|}
\hline$\underline{\mathbf{S T}}$ & Parameter & $\underline{3 Q 98}$ & CLP EPA & $\underline{1099}$ & CLPEPA & $\underline{3099}$ & CLPEPA & 1000 & CLP토A & Filt. & $\underline{\text { DF }}$ & Unit & $\underline{\mathrm{Lab}}$ \\
\hline \multicolumn{14}{|c|}{ Inorganics } \\
\hline & Barium, total recoverable & 9.21 & $J$ & 6.5 & $\mathrm{~J} 1$ & 5.42 & J 1 & & & & & $u g / L$ & \\
\hline & Cyanide & $<10$ & U & $<10$ & $\mathrm{U}$ & $<10$ & $\mathrm{U}$ & & & & & $\mathrm{ug} / \mathrm{L}$ & \\
\hline & Lead, total recoverable & $<10$ & $U$ & $<100$ & $\mathrm{U}$ & $<10$ & $\mathrm{U}$ & & & & & $\mathrm{ug} / \mathrm{L}$ & \\
\hline & Nickel, total recoverable & 8.55 & $J$ & $<50$ & $\mathrm{U}$ & $<50$ & $\mathrm{u}$ & & & & & $\mathrm{ug} / \mathrm{L}$ & \\
\hline & Selenium, total recoverable & $<10$ & $\mathrm{U}$ & $<200$ & $\mathrm{U}$ & $<10$ & $\mathrm{U}$ & & & & & $\mathrm{ug} / \mathrm{L}$ & \\
\hline \multicolumn{14}{|c|}{ Organics } \\
\hline & Chlorobenzene & $<5$ & $\mathrm{U}$ & $<5$ & U & $<1$ & $u$ & $<1$ & $\mathrm{u}$ & $<$ EQL & 1 & $u g / L$ & ML \\
\hline & 1,1-Dichloroethane & $<5$ & $\mathrm{U}$ & $<5$ & U & $<1$ & $\mathrm{U}$ & $<1$ & $\mathrm{U}$ & $<E Q L$ & 1 & $\mathrm{ug} / \mathrm{L}$ & $\mathrm{ML}$ \\
\hline & 1,1-Dichioroethylene & $<5$ & $\mathrm{U}$ & $<5$ & $\mathrm{U}$ & $<1$ & $\mathrm{U}$ & $<1$ & U & $<E Q L$ & 1 & $u g / L$ & $\mathrm{ML}$ \\
\hline & trans-1,2-Dichloroethylene & $<5$ & $\mathrm{u}$ & $<5$ & U & $<1$ & $\mathrm{U}$ & $<1$ & $\mathrm{U}$ & $<E Q L$ & 1 & $u g / L$ & ML \\
\hline & PCB 1016 & & & & & & & & & & & & \\
\hline & PCB 1221 & & & & & & & & & & & & \\
\hline & PCB 1232 & & & & & & & & & & & & \\
\hline & РCB 1242 & & & & & & & & & & & & \\
\hline & PCB 1248 & & & & & & & & & & & & \\
\hline & PCB 1254 & & & & & & & & & & & & \\
\hline & PCB 1260 & & & & & & & & & & & & \\
\hline & $1,1,2,2$-Tetrachloroethane & $<5$ & $\mathrm{U}$ & $<5$ & U & $<1$ & $\mathrm{U}$ & $<1$ & $\mathrm{U}$ & $<$ EQL & 1 & $u g / L$ & $M L$ \\
\hline & Tetrachloroethylene & $<5$ & $\mathrm{U}$ & $<5$ & $\mathrm{U}$ & $<1$ & $\mathrm{U}$ & $<1$ & U & $<E Q L$ & 1 & ug/L & ML \\
\hline & $1,1,1$-Trichloroethane & $<5$ & $\mathrm{U}$ & $<5$ & $\mathrm{U}$ & $<1$ & $U$ & $<1$ & $U$ & $<$ EQL & 1 & $u g / L$ & ML \\
\hline & Trichloroethylene & 3.6 & $J$ & 3.95 & J 1 & 3.55 & & 4.2 & & $<5$ & 1 & $\mathrm{ug} / \mathrm{L}$ & ML \\
\hline \multicolumn{14}{|c|}{ II. Monitoring Constituents } \\
\hline$\underline{\mathbf{S T}}$ & Parameter & $\underline{3 Q 96}$ & CLP EPA & $\underline{3 Q 97}$ & CLP EPA & $\underline{3098}$ & CLPEEA & $\underline{3 Q 99}$ & CLPEPA & Filt. & DF & Unit & $\underline{\text { Lab }}$ \\
\hline \multicolumn{14}{|c|}{ Inorganics } \\
\hline & Aluminum, total recoverable & 182 & $J$ & $<20$ & $\mathrm{U}$ & $<200$ & $\mathrm{U}$ & $<200$ & $U$ & $<E Q L$ & 1 & $u g / L$ & EX \\
\hline & Nitrate-nitrite as nitrogen & 1190 & & 1030 & & 780 & & 660 & & $<2400$ & 5 & $\mathrm{ug} / \mathrm{L}$ & EX \\
\hline & Sodium, total recoverable & 2030 & & 1630 & & $<1760$ & $\mathrm{U}$ & 1640 & & $<4600$ & 1 & ug/L & EX \\
\hline & Sulfate & 272 & $\mathrm{~J}$ & $<5000$ & $u$ & $<400$ & $\mathrm{U}$ & 770 & & $<3000$ & 2 & $\mathrm{ug} / \mathrm{L}$ & EX \\
\hline \multicolumn{14}{|c|}{ Radionuclides } \\
\hline & Gross alpha & 3.73 & & 10.5 & & 8.19 & & 2.19 & & $<15$ & 1 & $\mathrm{pCi} / \mathrm{L}$ & GP \\
\hline & Nonvolatile beta & 2.73 & & 12.6 & & 18.68 & & 1.53 & $\mathrm{~J} \quad \mathrm{~K}$ & NDD & 1 & $\mathrm{pCi} / \mathrm{L}$ & GP \\
\hline & Radium, total alpha-emitting & 1.3 & & 3.46 & UIJ & 2.02 & & 1.5 & J 1 & NDD & 1 & $\mathrm{pCi} / \mathrm{L}$ & GP \\
\hline
\end{tabular}

Notes: Concentrations in bold italics exceed the groundwater protection or monitoring constituent standards listed in Appendix A. Synchronous water levels are measured over a 3-5 day period or less. Dilution factors, Laboratory, and Filtered Data are for Groundwater Protection Standards First Quarter 2000 and Monitoring Constituents Third Quarter 1999 data only.

+= exceeded the Groundwater Protection Standards (First Quarter 2000) or Monitoring Constituents (Third Quarter 1999) listed in Appendix A. 
Table D-4. Groundwater Monitoring Results for Plume Definition Wells, M-Area HWMF (Cont.) WELL MSB 42TA

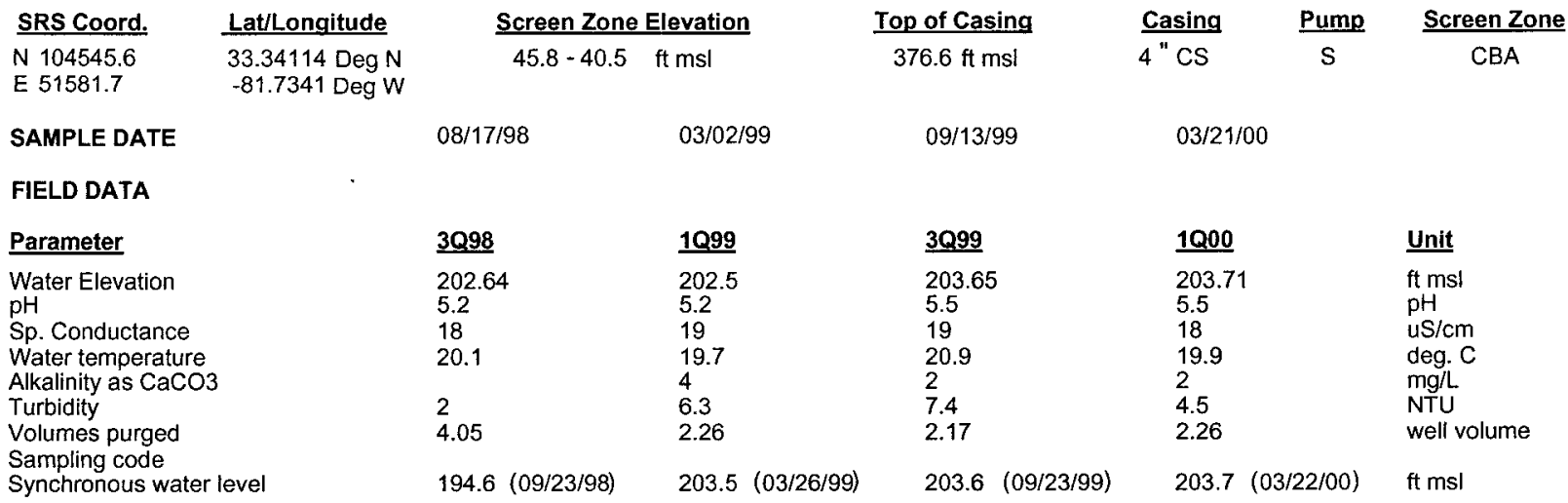

ANALYTICAL DATA

I. Groundwater Protection Standard

261 Appendix VIII/264 Appendix IX Hazardous Constituents

\begin{tabular}{|c|c|c|c|c|c|c|c|c|c|c|c|c|c|}
\hline$\underline{\text { ST }}$ & Parameter & $\underline{3098}$ & CLP EPA & $\underline{1 Q 99}$ & CLP EPA & $\underline{3 Q 99}$ & CLPEPA & $\underline{1 Q 00}$ & CLPEPA & Filt. & $\underline{\text { DF }}$ & Unit & Lab \\
\hline \multicolumn{14}{|c|}{ Inorganics } \\
\hline & Barium, total recoverable & 3.1 & $\mathrm{~J}$ & 3.29 & J 1 & 3.41 & J 1 & & & & & ug/L & \\
\hline & Cyanide & $<10$ & $U$ & $<10$ & $\mathrm{U}$ & $<10$ & $\mathrm{U}$ & & & & & $\mathrm{ug} / \mathrm{L}$ & \\
\hline & Lead, total recoverable & 8.07 & J & $<100$ & $\mathrm{U}$ & 13 & & & & & & $u g / L$ & \\
\hline & Nickel, total recoverable & $<50$ & $\mathrm{U}$ & $<50$ & $\mathbf{U}$ & $<50$ & $\mathrm{U}$ & & & & & $u g / L$ & \\
\hline & Selenium, total recoverable & $<10$ & $\mathrm{U}$ & $<200$ & $\mathrm{U}$ & $<10$ & $U$ & & & & & $\mathrm{ug} / \mathrm{L}$ & \\
\hline \multicolumn{14}{|c|}{ Organics } \\
\hline & Chlorobenzene & $<5$ & UJ & $<5$ & $\mathrm{U}$ & $<1$ & $\mathrm{U}$ & $<1$ & $U$ & $<E Q L$ & 1 & $u g / L$ & ML \\
\hline & 1,1-Dichloroethane & $<5$ & UJ & $<5$ & $\mathrm{U}$ & $<1$ & U & $<1$ & $U$ & $<E Q L$ & 1 & $u g / L$ & ML \\
\hline & 1,1-Dichloroethylene & $<5$ & UJ & $<5$ & $\mathrm{U}$ & $<1$ & $U$ & $<1$ & $U$ & $<E Q L$ & 1 & ug/L & ML \\
\hline & trans-1,2-Dichloroethylene & $<5$ & UJ & $<5$ & U & $<1$ & U & $<1$ & $\mathrm{U}$ & $<E Q L$ & 1 & $\mathrm{ug} / \mathrm{L}$ & ML \\
\hline & PCB 1016 & & & & & & & & & & & & \\
\hline & PCB 1221 & & & & & & & & & & & & \\
\hline & PCB 1232 & & & & & & & & & & & & \\
\hline & PCB 1242 & & & & & & & & & & & & \\
\hline & PCB 1248 & & & & & & & & & & & & \\
\hline & PCB 1254 & & & & & & & & & & & & \\
\hline & PCB 1260 & & & & & & & & & & & & \\
\hline & $1,1,2,2$-Tetrachloroethane & $<5$ & UJ & $<5$ & $\mathrm{U}$ & $<1$ & $\mathrm{U}$ & $<1$ & $U$ & $<E Q L$ & 1 & $\mathrm{ug} / \mathrm{L}$ & $M L$ \\
\hline+ & Tetrachloroethylene & 32.8 & $\mathrm{~J}$ & 41.6 & & 40.1 & & 34.7 & & $>5$ & 1 & $u g / L$ & $\mathrm{ML}$ \\
\hline & 1,1,1-Trichloroethane & & UJ & $<5$ & $\mathrm{U}$ & $<1$ & $\mathrm{U}$ & $<1$ & $\mathrm{U}$ & $<E Q L$ & 1 & $u g / t$ & ML \\
\hline+ & Trichloroethylene & 68.7 & $J$ & 87.3 & & 88.7 & & 73.5 & & $>5$ & 1 & $\mathrm{ug} / \mathrm{L}$ & ML \\
\hline \multicolumn{14}{|c|}{ II. Monitoring Constituents } \\
\hline$\underline{\mathrm{ST}}$ & Parameter & $\underline{3096}$ & CLPPEPA & $\underline{3097}$ & CLP EPA & 3098 & CLPEPA & 3099 & CLPEPA & Filit. & $\underline{\mathrm{DF}}$ & $\underline{\text { Unit }}$ & Lab \\
\hline \multicolumn{14}{|c|}{ Inorganics } \\
\hline & Aluminum, total recoverable & $<20$ & $\mathrm{U}$ & 13.3 & $\mathrm{~J}$ & $<200$ & $\mathrm{U}$ & $<200$ & $U$ & $<\mathrm{EQL}$ & 1 & $\mathrm{ug} / \mathrm{L}$ & EX \\
\hline & Nitrate-nitrite as nitrogen & 1020 & & 816 & & 640 & & 620 & & $<2400$ & 5 & $\mathrm{ug} / \mathrm{L}$ & EX \\
\hline & Sodium, total recoverable & 1490 & & 1450 & & $<1720$ & $\mathrm{U}$ & 1590 & & $<4600$ & 1 & $\mathrm{ug} / \mathrm{L}$ & EX \\
\hline & Sulfate & 274 & $J$ & $<5000$ & 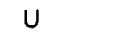 & $<400$ & $\mathrm{U}$ & 360 & & $<3000$ & 1 & $\mathrm{ug} / \mathrm{L}$ & EX \\
\hline \multicolumn{14}{|c|}{ Radionuclides } \\
\hline+ & Gross alpha & 1.48 & UI & .23 & Ui & 1.39 & & 19.6 & & $>15$ & 1 & $\mathrm{pCi} / \mathrm{L}$ & GP \\
\hline & Nonvolatile beta & 1.13 & UI & .65 & UI & 7.24 & & 4.39 & $\mathrm{~J} \mathrm{~K}$ & NDD & 1 & $\mathrm{pCi} / \mathrm{L}$ & GP \\
\hline+ & Radium, total alpha-emitting & .1 & UI & 7.81 & UIJ & .37 & $\mathbf{U}$ & 6.7 & & $>5$ & 1 & $\mathrm{pCi} / \mathrm{L}$ & GP \\
\hline
\end{tabular}

Notes: Concentrations in bold italics exceed the groundwater protection or monitoring constituent standards listed in Appendix A. Synchronous water levels are measured over a 3-5 day period or less. Dilution factors, Laboratory, and Filtered Data are for Groundwater Protection Standards First Quarter 2000 and Monitoring Constituents Third Quarter 1999 data only.

$+=$ exceeded the Groundwater Protection Standards (First Quarter 2000) or Monitoring Constituents (Third Quarter 1999) listed in Appendix A. 
Table D-4. Groundwater Monitoring Results for Plume Definition Wells, M-Area HWMF (Cont.) WELL MSB 43TA

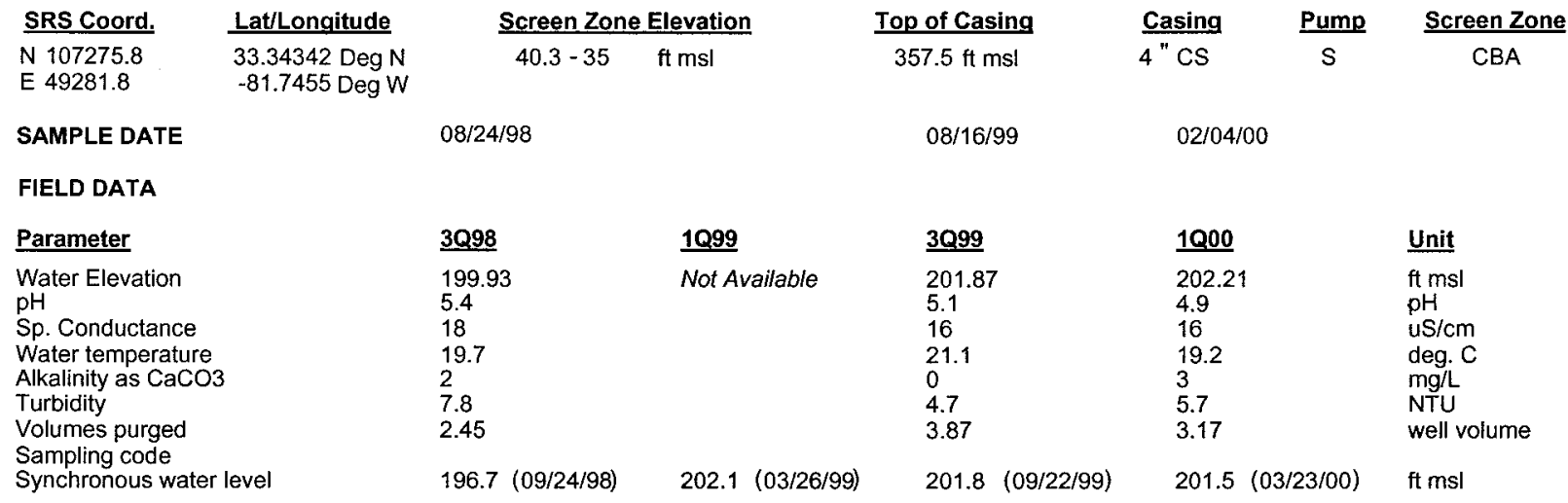

ANALYTICAL DATA

I. Groundwater Protection Standard

261 Appendix VIII/264 Appendix IX Hazardous Constituents

\begin{tabular}{|c|c|c|c|c|c|c|c|c|c|c|c|c|c|}
\hline$\underline{\mathbf{S T}}$ & Parameter & $\underline{3 Q 98}$ & CLP EPA & $\underline{1 Q 99}$ & CLP EPA & $\underline{3 Q 99}$ & CLPEPA & $\underline{1 Q 00}$ & CLPEPA & Filt. & $\underline{\mathrm{DF}}$ & Unit & Lab \\
\hline \multicolumn{14}{|c|}{ Inorganics } \\
\hline & Barium, total recoverable & $<10$ & $\mathrm{U}$ & $<10$ & $\mathrm{U}$ & $<10$ & $\mathrm{U}$ & & & & & $\mathrm{ug} / \mathrm{L}$ & \\
\hline & Cyanide & $<10$ & $\mathrm{U}$ & $<10$ & $\mathrm{U}$ & $<10$ & $\mathrm{U}$ & & & & & $\mathrm{ug} / \mathrm{L}$ & \\
\hline & Lead, total recoverable & $<10$ & $\mathrm{U}$ & $<100$ & $\mathrm{U}$ & $<3.68$ & U V & & & & & $\mathrm{ug} / \mathrm{L}$ & \\
\hline & Nickel, total recoverable & $<50$ & $\mathrm{U}$ & $<50$ & $\mathrm{U}$ & $<8.4$ & JU 1 & & & & & $\mathrm{ug} / \mathrm{L}$ & \\
\hline & Selenium, total recoverable & $<10$ & $\mathrm{U}$ & $<200$ & $\mathrm{U}$ & $<5$ & $\mathrm{U}$ & & & & & $\mathrm{ug} / \mathrm{L}$ & \\
\hline \multicolumn{14}{|c|}{ Organics } \\
\hline & Chlorobenzene & $<5$ & $\mathrm{u}$ & $<5$ & $U$ & $<1$ & U & $<1$ & JU & $<$ EQL & 1 & $\mathrm{ug} / \mathrm{L}$ & $M L$ \\
\hline & 1,1-Dichloroethane & $<5$ & $\mathrm{U}$ & $<5$ & 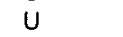 & $<1$ & $\mathrm{U}$ & $<1$ & JU & $<E Q L$ & 1 & $\mathrm{ug} / \mathrm{L}$ & $M L$ \\
\hline & 1,1-Dichloroethylene & $<5$ & $\mathrm{U}$ & $<5$ & $\mathrm{U}$ & $<1$ & $\mathrm{U}$ & $<1$ & JU & $<\mathrm{EQL}$ & 1 & ug/L & ML \\
\hline & trans-1,2-Dichloroethylene & $<5$ & $\mathrm{U}$ & $<5$ & $U$ & $<1$ & $\mathrm{U}$ & $<1$ & JU & $<\mathrm{EQL}$ & 1 & $\mathrm{ug} / \mathrm{L}$ & ML \\
\hline & PCB 1016 & & & & & & & & & & & & \\
\hline & PCB 1221 & & & & & & & & & & & & \\
\hline & PCB 1232 & & & & & & & & & & & & \\
\hline & $\begin{array}{l}\text { PCB } 1242 \\
\text { PCB } 1248\end{array}$ & & & & & & & & & & & & \\
\hline & PCB 1254 & & & & & & & & & & & & \\
\hline & PCB 1260 & & & & & & & & & & & & \\
\hline & 1,1,2,2-Tetrachloroethane & $<5$ & $U$ & $<5$ & $U$ & $<1$ & $\mathrm{U}$ & $<1$ & JU & $<E Q L$ & 1 & $\mathrm{ug} / \mathrm{L}$ & $M L$ \\
\hline & Tetrachloroethylene & $<5$ & $U$ & $<5$ & $U$ & $<1$ & $U$ & $<1$ & JU & $<\mathrm{EQL}$ & 1 & $\mathrm{ug} / \mathrm{L}$ & ML \\
\hline & 1,1,1-Trichloroethane & $<5$ & $U$ & $<5$ & $U$ & $<1$ & $U$ & $<1$ & JU & $<\mathrm{EQL}$ & 1 & $\mathrm{ug} / \mathrm{L}$ & $\mathrm{ML}$ \\
\hline & Trichloroethylene & $<5$ & $\mathrm{U}$ & $<5$ & $U$ & $<1$ & $U$ & $<1$ & JU & $<\mathrm{EQL}$ & 1 & $\mathrm{ug} / \mathrm{L}$ & $M L$ \\
\hline \multicolumn{14}{|c|}{ II. Monitoring Constituents } \\
\hline$\underline{\text { ST }}$ & Parameter & $\underline{3 Q 96}$ & CLP EPA & $\underline{3097}$ & $\underline{\text { CLP EPA }}$ & $\underline{3 Q 98}$ & CLPEPA & $\underline{3099}$ & CLPEPA & Filt. & $\underline{\text { DF }}$ & Unit & Lab \\
\hline \multicolumn{14}{|c|}{ Inorganics } \\
\hline & Aluminum, total recoverable & 44.4 & & 14.2 & $\mathrm{~J}$ & $<200$ & U & $<200$ & $U$ & $<\mathrm{EQL}$ & 1 & $\mathrm{ug} / \mathrm{L}$ & EX \\
\hline & Nitrate-nitrite as nitrogen & 500 & & 97 & & 106 & $J$ & 103 & J 1 & NDD & 5 & ug/L & EX \\
\hline & Sodium, total recoverable & 1400 & & 1400 & & 1610 & & 1500 & & $<4600$ & 1 & $\mathrm{ug} / \mathrm{L}$ & EX \\
\hline & Sulfate & 680 & $J$ & $<5000$ & $\mathrm{U}$ & 758 & & 839 & & $<3000$ & 1 & $\mathrm{ug} / \mathrm{l}$ & EX \\
\hline \multicolumn{14}{|c|}{ Radionuclides } \\
\hline & Gross alpha & .242 & UI & .06 & $\mathrm{Ul}$ & .24 & UIJ & .366 & $U$ & $<E Q L$ & 1 & $\mathrm{pCi} / \mathrm{L}$ & GP \\
\hline & Nonvolatile beta & .705 & UI & -.14 & UI & -.32 & UI & .671 & $U$ & $<E Q L$ & 1 & $\mathrm{pCi} / \mathrm{L}$ & GP \\
\hline & Radium, total alpha-emitting & .1 & UI & .57 & UIJ & .02 & UI & -.2 & $U$ & $<\mathrm{EQL}$ & 1 & $\mathrm{pCi} / \mathrm{L}$ & GP \\
\hline
\end{tabular}

Notes: Concentrations in bold italics exceed the groundwater protection or monitoring constituent standards listed in Appendix A. Synchronous water levels are measured over a 3-5 day period or less. Dilution factors, Laboratory, and Filtered Data are for Groundwater Protection Standards First Quarter 2000 and Monitoring Constituents Third Quarter 1999 data only.

$+=$ exceeded the Groundwater Protection Standards (First Quarter 2000) or Monitoring Constituents (Third Quarter 1999) listed in Appendix A. 
Table D-4. Groundwater Monitoring Results for Plume Definition Wells, M-Area HWMF (Cont.) WELL MSB 45A

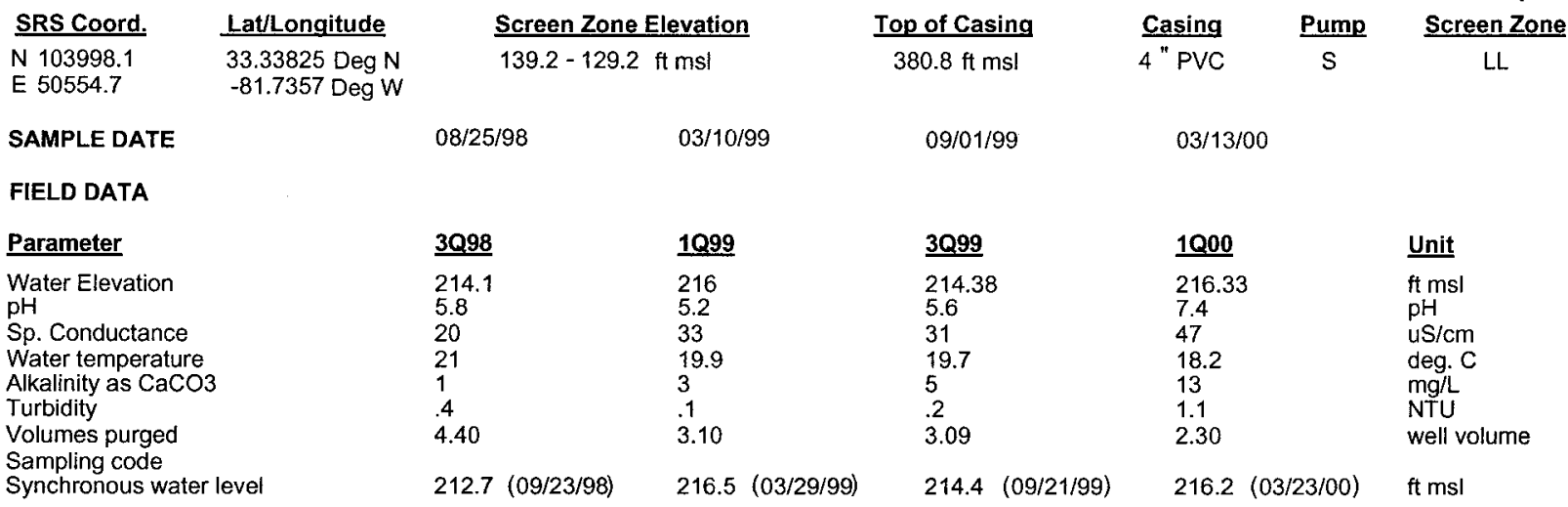

\section{ANALYTICAL DATA}

I. Groundwater Protection Standard

261 Appendix VIII/264 Appendix IX Hazardous Constituents

\begin{tabular}{|c|c|c|c|c|c|c|c|c|c|c|c|c|c|c|}
\hline \multirow{2}{*}{\multicolumn{2}{|c|}{$\begin{array}{l}\text { ST Parameter } \\
\text { Inorganics }\end{array}$}} & \multirow[t]{2}{*}{$\underline{3 Q 98}$} & \multirow[t]{2}{*}{$\underline{\text { CLPP EPA }}$} & \multirow[t]{2}{*}{1099} & \multirow[t]{2}{*}{ CLP EPA } & \multirow[t]{2}{*}{$\underline{3 Q 99}$} & CLPEPA & \multirow[t]{2}{*}{$\underline{1 Q 00}$} & \multicolumn{2}{|c|}{ CLPEPA } & \multirow[t]{2}{*}{ Filt. } & \multirow[t]{2}{*}{$\underline{\text { DF }}$} & \multirow[t]{2}{*}{$\underline{\text { Unit }}$} & \multirow[t]{2}{*}{$\underline{\mathrm{Lab}}$} \\
\hline & & & & & & & & & & & & & & \\
\hline & Barium, total recoverable & 10.4 & & 24.5 & & 17 & & & & & & & $u g / L$ & \\
\hline & Cyanide & $<10$ & $\mathrm{U}$ & $<10$ & $J U L$ & $<10$ & $\mathrm{U}$ & & & & & & $\mathrm{ug} / \mathrm{L}$ & \\
\hline & Lead, total recoverable & $<100$ & $U$ & $<100$ & $\mathrm{U}$ & $<10$ & $U$ & & & & & & ug/L & \\
\hline & Nickel, total recoverable & 14.4 & $\mathrm{~J}$ & $<50$ & $\mathrm{U}$ & $<50$ & $U$ & & & & & & $\mathrm{ug} / \mathrm{L}$ & \\
\hline & Selenium, total recoverable & $<200$ & $\mathrm{U}$ & $<200$ & $\mathrm{U}$ & $<10$ & $U$ & & & & & & $\mathrm{ug} / \mathrm{L}$ & \\
\hline \multicolumn{15}{|c|}{ Organics } \\
\hline & Chlorobenzene & $<25$ & UJ & $<50$ & $\mathrm{u}$ & $<1$ & U & $<5$ & JU & & $<$ EQL & 5 & $\mathrm{ug} / \mathrm{L}$ & ML \\
\hline & 1,1-Dichloroethane & $<25$ & UJ & $<50$ & $\mathrm{U}$ & $<1$ & U & $<5$ & JU & & $<E Q L$ & 5 & $\mathrm{ug} / \mathrm{L}$ & $\mathrm{ML}$ \\
\hline & 1,1-Dichtoroethylene & $<25$ & UJ & $<50$ & U & $<1$ & U & $<5$ & JU & & $<E Q L$ & 5 & $\mathrm{ug} / \mathrm{L}$ & $\mathrm{ML}$ \\
\hline & $\begin{array}{l}\text { trans-1,2-Dichloroethylene } \\
\text { PCB } 1016\end{array}$ & $<25$ & UJ & $<50$ & $\mathrm{U}$ & $<1$ & $\mathrm{U}$ & $<5$ & JU & & $<E Q L$ & 5 & $\mathrm{ug} / \mathrm{L}$ & $\mathrm{ML}$ \\
\hline & PCB 1221 & & & & & & & & & & & & & \\
\hline & PCB 1232 & & & & & & & & & & & & & \\
\hline & PCB 1242 & & & & & & & & & & & & & \\
\hline & PCB 1248 & & & & & & & & & & & & & \\
\hline & PCB 1254 & & & & & & & & & & & & & \\
\hline & PCB 1260 & & & & & & & & & & & & & \\
\hline & $1,1,2,2$-Tetrachloroethane & $<25$ & UJ & $<50$ & $\mathrm{U}$ & $<1$ & $\mathrm{U}$ & $<5$ & JU & & $<$ EQL & 5 & ug/L & $M L$ \\
\hline & Tetrachloroethylene & 142 & $J$ & 116 & & 120 & $\mathrm{~J} \quad \mathrm{~K}$ & 100 & J & & NDD & 5 & $\mathrm{ug} / \mathrm{L}$ & $M L$ \\
\hline & $1,1,1$-Trichloroethane & $<25$ & UJ & $<50$ & $u$ & $<1$ & U & $<5$ & JU & & $<$ EQL & 5 & $\mathrm{ug} / \mathrm{L}$ & $M L$ \\
\hline & Trichloroethylene & 806 & $\mathrm{~J}$ & 706 & & 725 & & 776 & $J$ & & NDD & 5 & $\mathrm{ug} / \mathrm{L}$ & ML \\
\hline \multicolumn{15}{|c|}{ II. Monitoring Constituents } \\
\hline$\underline{\text { ST }}$ & Parameter & $\underline{3096}$ & CLPEPA & $\underline{3097}$ & CLPPEPA & $\underline{3098}$ & CLPEPA & $\underline{3099}$ & CLP & EPA & Filt. & $\underline{\text { DF }}$ & $\underline{\text { Unit }}$ & Lab \\
\hline \multicolumn{15}{|c|}{ Inorganics } \\
\hline & Aluminum, total recoverable & $<20$ & U & & & $<200$ & $u$ & $<200$ & $\mathrm{U}$ & & $<E Q L$ & 1 & $\mathrm{ug} / \mathrm{L}$ & EX \\
\hline & Nitrate-nitrite as nitrogen & 1340 & & & & 993 & & 962 & & & $<2400$ & 5 & $\mathrm{ug} / \mathrm{L}$ & $\mathrm{EX}$ \\
\hline & Sodium, total recoverable & 2850 & & & & 2580 & & 2600 & & & $<4600$ & 1 & $\mathrm{ug} / \mathrm{L}$ & EX \\
\hline & Sulfate & 353 & $J$ & & & 582 & & 372 & $J$ & 1 & NDD & 2 & $\mathrm{ug} / \mathrm{L}$ & EX \\
\hline \multicolumn{15}{|c|}{ Radionuclides } \\
\hline & Gross alpha & 1.68 & UI & & & 2.58 & & .96 & $\mathrm{u}$ & V & $<\mathrm{EQL}$ & 1 & $\mathrm{pCi} / \mathrm{L}$ & GP \\
\hline & Nonvolatile beta & 1.65 & & & & 3.86 & J & 1.47 & $J$ & 1 & NDD & 1 & $\mathrm{pCi} / \mathrm{L}$ & GP \\
\hline & Radium, total alpha-emitting & -.1 & UI & & & .55 & & .1 & U & & $<E Q L$ & 1 & $\mathrm{pCi} / \mathrm{L}$ & GP \\
\hline
\end{tabular}

Notes: Concentrations in bold italics exceed the groundwater protection or monitoring constituent standards listed in Appendix A. Synchronous water levels are measured over a 3-5 day period or less. Dilution factors, Laboratory, and Filtered Data are for Groundwater Protection Standards First Quarter 2000 and Monitoring Constituents Third Quarter 1999 data only.

+ = exceeded the Groundwater Protection Standards (First Quarter 2000) or Monitoring Constituents (Third Quarter 1999) listed in Appendix A. 
Table D-4. Groundwater Monitoring Results for Plume Definition Wells, M-Area HWMF (Cont.) WELL MSB 45B

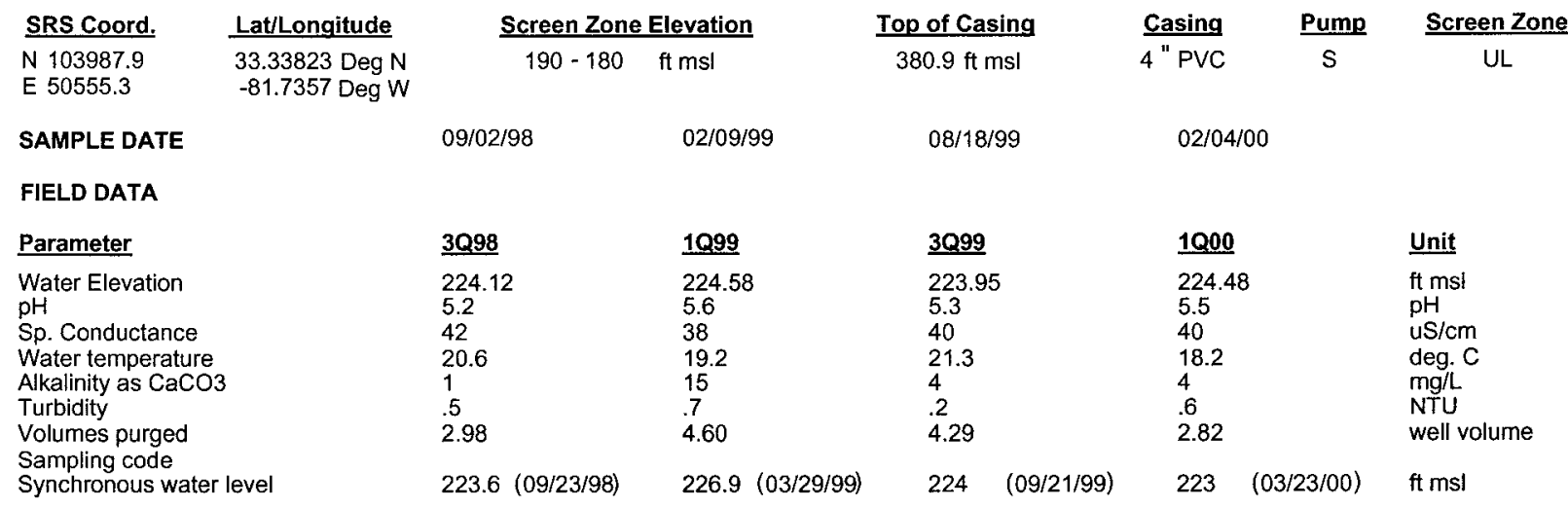

ANALYTICAL DATA

I. Groundwater Protection Standard

261 Appendix VIII/264 Appendix IX Hazardous Constituents

\begin{tabular}{|c|c|c|c|c|c|c|c|c|c|c|c|c|c|c|c|}
\hline$\underline{\text { ST }}$ & Parameter & 3098 & CLP EPA & 1099 & CLP & EPA & 3Q99 & CLPEPA & 1000 & $\underline{\text { CLPP }}$ & EPA & Filt. & $\underline{\text { DF }}$ & Unit & $\underline{\text { Lab }}$ \\
\hline Inor & ganics & & & & & & & & & & & & & & \\
\hline & Barium, total recoverable & $<10$ & $\mathrm{U}$ & $<10$ & $\mathrm{U}$ & & $<10$ & $u$ & & & & & & ug/L & \\
\hline & Cyanide & $<10$ & $\mathrm{U}$ & $<10$ & $\mathrm{U}$ & & $<10$ & $\mathrm{U}$ & & & & & & $\mathrm{ug} / \mathrm{L}$ & \\
\hline & Lead, total recoverable & $<100$ & U & $<100$ & $\mathrm{U}$ & & 1.89 & J 1 & & & & & & $\mathrm{ug} / \mathrm{L}$ & \\
\hline & Nickel, total recoverable & $<50$ & U & $<50$ & $\mathrm{U}$ & & $<11$ & JU I & & & & & & $\mathrm{ug} / \mathrm{L}$ & \\
\hline & Selenium, total recoverable & $<10$ & $\mathrm{U}$ & $<200$ & $\mathrm{U}$ & & $<5$ & $\mathrm{U}$ & & & & & & ug/L & \\
\hline Orge & anics & & & & & & & & & & & & & & \\
\hline & Chlorobenzene & $<5$ & $\mathrm{U}$ & $<5$ & JU & $Q$ & $<1$ & $u$ & $<1$ & JU & $\mathbf{L}$ & $<\mathrm{EQL}$ & 1 & $u g / L$ & ML \\
\hline & 1,1-Dichloroethane & $<5$ & U & $<5$ & JU & $Q$ & $<1$ & $\mathrm{U}$ & $<1$ & JU & $\mathrm{L}$ & $<E Q L$ & 1 & $\mathrm{ug} / \mathrm{L}$ & $M L$ \\
\hline & 1,1-Dichloroethylene & $<5$ & $U$ & $<5$ & JU & $Q$ & $<1$ & $\mathrm{U}$ & $<1$ & JU & $\mathbf{L}$ & $<\mathrm{EQL}$ & 1 & $\mathrm{ug} / \mathrm{L}$ & $\mathrm{ML}$ \\
\hline & trans-1,2-Dichloroethylene & $<5$ & $U$ & $<5$ & JU & $Q$ & $<1$ & $U$ & $<1$ & JU & $\mathbf{L}$ & $<E Q L$ & 1 & $\mathrm{ug} / \mathrm{L}$ & $\mathrm{ML}$ \\
\hline & PCB 1016 & & & & & & & & & & & & & & \\
\hline & PCB 1221 & & & & & & & & & & & & & & \\
\hline & PCB 1232 & & & & & & & & & & & & & & \\
\hline & PCB 1242 & & & & & & & & & & & & & & \\
\hline & PCB 1248 & & & & & & & & & & & & & & \\
\hline & PCB 1254 & & & & & & & & & & & & & & \\
\hline & PCB 1260 & & & & & & & & & & & & & & \\
\hline & $1,1,2,2-$ Tetrachloroethane & $<5$ & $U$ & $<5$ & $\jmath U$ & $Q$ & $<1$ & $U$ & $<1$ & JU & $L$ & $<E Q L$ & 1 & $u g / L$ & ML \\
\hline & Tetrachloroethylene & $<5$ & U & $<5$ & JU & $\bar{Q}$ & $<1$ & $U$ & $<1$ & JU & $\mathbf{L}$ & $<\mathrm{EQL}$ & 1 & $\mathrm{ug} / \mathrm{L}$ & ML \\
\hline & $1,1,1$-Trichloroethane & $<5$ & U & $<5$ & JU & $\vec{Q}$ & $<1$ & $\mathrm{U}$ & $<1$ & JU & $\mathbf{L}$ & $<\mathrm{EQL}$ & 1 & $\mathrm{ug} / \mathrm{L}$ & ML \\
\hline & Trichloroethylene & $<5$ & $U$ & $<5$ & JU & $Q$ & $<1$ & $U$ & $<1$ & JU & L & $<\mathrm{EQL}$ & 1 & $\mathrm{ug} / \mathrm{L}$ & ML \\
\hline $\mathbf{M}$ & nitoring Constituents & & & & & & & & & & & & & & \\
\hline$\underline{\mathbf{S T}}$ & Parameter & $\underline{3 Q 96}$ & CLPEPA & $\underline{3 Q 97}$ & $\underline{\text { CLP }}$ & EPA & $\underline{3098}$ & CLPEPA & $\underline{3099}$ & CLP & EPA & Filt. & DF & Unit & Lab \\
\hline Inor & ganics & & & & & & & & & & & & & & \\
\hline & Aluminum, total recoverable & $<20$ & $\mathrm{U}$ & 20.8 & & & $<200$ & $\mathrm{U}$ & $<200$ & $U$ & & $<E Q L$ & 1 & $\mathrm{ug} / \mathrm{L}$ & EX \\
\hline & Nitrate-nitrite as nitrogen & 530 & & 1200 & & & 595 & & 255 & J & 1 & NDD & 5 & $\mathrm{ug} / \mathrm{L}$ & EX \\
\hline+ & Sodium, total recoverable & 6480 & & 7310 & & & 7480 & & 8100 & & & $>4600$ & 1 & $\mathrm{ug} / \mathrm{L}$ & EX \\
\hline+ & Sulfate & 5870 & & 6470 & & & 4720 & & 5640 & & & $>3000$ & 1 & $\mathrm{ug} / \mathrm{L}$ & EX \\
\hline Rad & onuclides & & & & & & & & & & & & & & \\
\hline & Gross alpha & .083 & UI & .88 & UI & & .43 & UIJ & .635 & $\mathrm{U}$ & & $<E Q L$ & 1 & $\mathrm{pCi} / \mathrm{L}$ & GP \\
\hline & Nonvolatile beta & .64 & UI & 2.02 & & & .21 & UIJ & .774 & U & & $<E Q L$ & 1 & $\mathrm{pCi} / \mathrm{L}$ & GP \\
\hline & Radium, total alpha-emitting & .1 & Ut & 1.4 & $\mathrm{~J}$ & & .22 & UI & .2 & $U$ & & $<E Q L$ & 1 & $\mathrm{pCi} / \mathrm{L}$ & GP \\
\hline
\end{tabular}

Notes: Concentrations in bold italics exceed the groundwater protection or monitoring constituent standards listed in Appendix A. Synchronous water levels are measured over a 3-5 day period or less. Dilution factors, Laboratory, and Filtered Data are for Groundwater Protection Standards First Quarter 2000 and Monitoring Constituents Third Quarter 1999 data only.

$+=$ exceeded the Groundwater Protection Standards (First Quarter 2000) or Monitoring Constituents (Third Quarter 1999) listed in Appendix A. 
Table D-4. Groundwater Monitoring Results for Plume Definition Wells, M-Area HWMF (Cont.) WELL MSB 46A

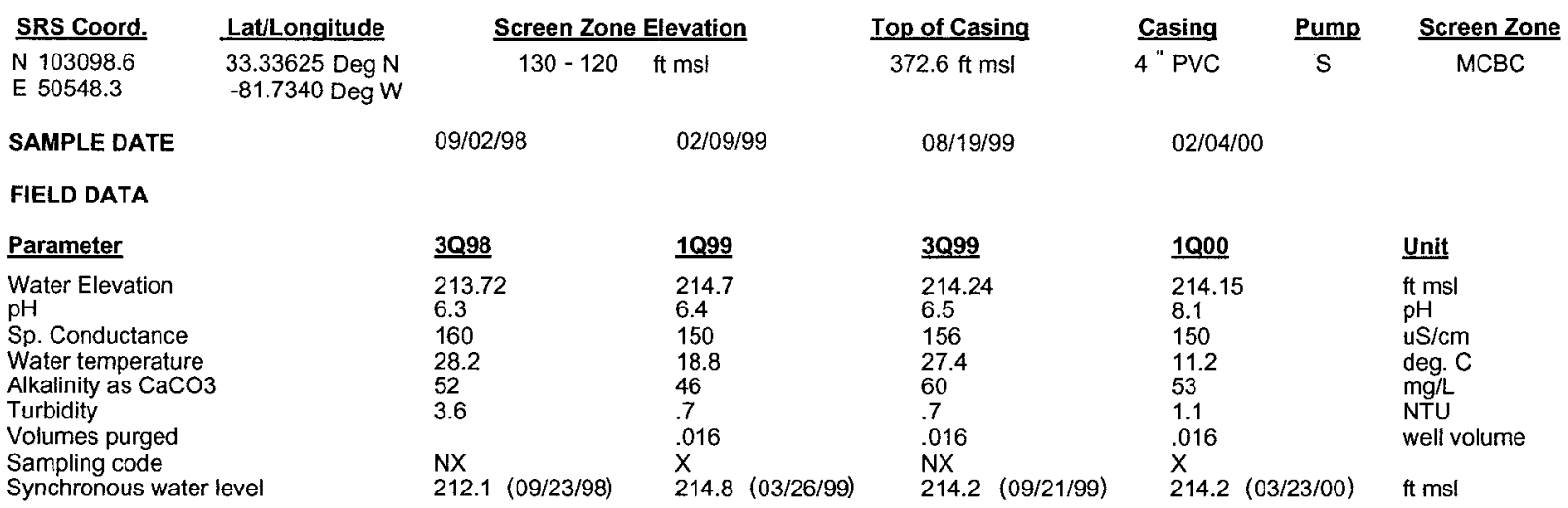

ANALYTICAL DATA

I. Groundwater Protection Standard

261 Appendix VIII/264 Appendix IX Hazardous Constituents

\begin{tabular}{|c|c|c|c|c|c|c|c|c|c|c|c|c|c|}
\hline$\underline{\text { ST }}$ & Parameter & $\underline{3 Q 98}$ & CLPPEPA & 1099 & $\underline{\text { CLP EPA }}$ & $\underline{3 Q 99}$ & $\underline{\text { CLPEPA }}$ & 1000 & CLPEPA & Filt. & $\underline{\text { DF }}$ & Unit & $\underline{\text { Lab }}$ \\
\hline \multicolumn{14}{|c|}{ Inorganics } \\
\hline & Barium, total recoverable & 26.7 & & 20.2 & & 22 & & & & & & $\mathrm{ug} / \mathrm{L}$ & \\
\hline & Cyanide & $<10$ & $\mathrm{U}$ & $<10$ & $U$ & $<10$ & $\mathrm{U}$ & & & & & $\mathrm{ug} / \mathrm{L}$ & \\
\hline & Lead, total recoverable & $<100$ & $\mathrm{U}$ & $<100$ & $U$ & $<4.02$ & U V & & & & & $\mathrm{ug} / \mathrm{L}$ & \\
\hline & Nickel, total recoverable & $<50$ & U & $<50$ & $U$ & $<50$ & $\mathrm{U}$ & & & & & $\mathrm{ug} / \mathrm{L}$ & \\
\hline & Selenium, total recoverable & $<10$ & $\mathrm{U}$ & $<200$ & $U$ & $<5$ & U & & & & & $\mathrm{ug} / \mathrm{L}$ & \\
\hline \multicolumn{14}{|c|}{ Organics } \\
\hline & Chlorobenzene & $<5$ & US & $<5$ & JU $Q$ & $<1$ & $\mathrm{U}$ & $<1$ & JU & $<E Q L$ & 1 & $\mathrm{ug} / \mathrm{L}$ & ML \\
\hline & 1,1-Dichloroethane & $<5$ & UJ & $<5$ & JU Q & $<1$ & $\mathrm{U}$ & $<1$ & JU & $<$ EQL. & 1 & $\mathrm{ug} / \mathrm{L}$ & $M L$ \\
\hline & 1,1-Dichloroethylene & $<5$ & UJ & $<5$ & JU $Q$ & $<1$ & $\mathrm{U}$ & $<1$ & JU & $<\mathrm{EQL}$ & 1 & ug/L & $\mathrm{ML}$ \\
\hline & trans-1,2-Dichloroethylene & $<5$ & UJ & $<5$ & JU $\bar{Q}$ & $<1$ & $\mathrm{U}$ & $<1$ & JU & $<E Q L$ & 1 & $\mathrm{ug} / \mathrm{L}$ & $\mathrm{ML}$ \\
\hline & PCB 1016 & & & & & & & & & & & & \\
\hline & РСВ 1221 & & & & & & & & & & & & \\
\hline & PCB 1232 & & & & & & & & & & & & \\
\hline & РСВ 1242 & & & & & & & & & & & & \\
\hline & PCB 1248 & & & & & & & & & & & & \\
\hline & PCB 1254 & & & & & & & & & & & & \\
\hline & PCB 1260 & & & & & & & & & & & & \\
\hline & 1,1,2,2-Tetrachloroethane & $<5$ & UJ & $<5$ & JU $Q$ & $<1$ & $U$ & $<1$ & JU & $<E Q L$ & 1 & $\mathrm{ug} / \mathrm{L}$ & ML \\
\hline & Tetrachloroethylene & $<5$ & UJ & $<5$ & JU $Q$ & $<1$ & $\mathrm{U}$ & $<1$ & JU & $<$ EQL & 1 & $\mathrm{ug} / \mathrm{L}$ & $\mathrm{ML}$ \\
\hline & 1,1,1-Trichloroethane & $<5$ & UJ & $<5$ & $J U Q$ & $<1$ & $U$ & $<1$ & JU & $<\mathrm{EQL}$ & 1 & $\mathrm{ug} / \mathrm{L}$ & ML \\
\hline & Trichloroethylene & $<5$ & UJ & $<5$ & $J U Q$ & $<1$ & $U$ & $<1$ & $\mathrm{JU}$ & $<$ EQL & 1 & ug/L & $\mathrm{ML}$ \\
\hline \multicolumn{14}{|c|}{ II. Monitoring Constituents } \\
\hline$\underline{\mathbf{S T}}$ & Parameter & $\underline{3 Q 96}$ & CLPEPA & $\underline{3097}$ & CLP EPA & 3098 & CLPEPA & $\underline{3099}$ & CLPEPA & Filt. & $\underline{\text { DF }}$ & Unit & Lab \\
\hline \multicolumn{14}{|c|}{ Inorganics } \\
\hline & Aluminum, total recoverable & 623 & J & 38.4 & & $<200$ & U & $<200$ & U & $<E Q L$ & 1 & $\mathrm{ug} / \mathrm{L}$ & EX \\
\hline & Nitrate-nitrite as nitrogen & 2500 & $J$ & 25 & & 365 & $J$ & $<100$ & $U$ & $<\mathrm{EQL}$ & 1 & $\mathrm{ug} / \mathrm{L}$ & EX \\
\hline+ & Sodium, total recoverable & 6850 & & 5160 & & 4520 & & 5000 & & $>4600$ & 1 & $\mathrm{ug} / \mathrm{L}$ & EX \\
\hline+ & Sulfate & 4990 & & 11200 & & 8040 & & 10900 & & $>3000$ & 1 & $\mathrm{ug} / \mathrm{L}$ & EX \\
\hline \multicolumn{14}{|c|}{ Radionuclides } \\
\hline & Gross alpha & 6.08 & $J$ & .34 & UI & -.36 & UIJ & .555 & $\mathrm{U}$ & $<\mathrm{EQL}$ & 1 & $\mathrm{pCi} / \mathrm{L}$ & GP \\
\hline & Nonvolatile beta & 4.07 & $J$ & 4.63 & & 4.24 & $J$ & 5.56 & & $<50$ & 1 & $\mathrm{pCi} / \mathrm{L}$ & GP \\
\hline & Radium, total alpha-emitting & 1.2 & & 3.78 & UIJ & .14 & UI & 0 & $\mathrm{U}$ & $<\mathrm{EQL}$ & 1 & $\mathrm{pCi} / \mathrm{L}$ & $G P$ \\
\hline
\end{tabular}

Notes: Concentrations in bold italics exceed the groundwater protection or monitoring constituent standards listed in Appendix A. Synchronous water levels are measured over a 3-5 day period or less. Dilution factors, Laboratory, and Filtered Data are for Groundwater Protection Standards First Quarter 2000 and Monitoring Constituents Third Quarter 1999 data only.

$+=$ exceeded the Groundwater Protection Standards (First Quarter 2000) or Monitoring Constituents (Third Quarter 1999) listed in Appendix A. 
Table D-4. Groundwater Monitoring Results for Plume Definition Wells, M-Area HWMF (Cont.) WELL MSB 46C

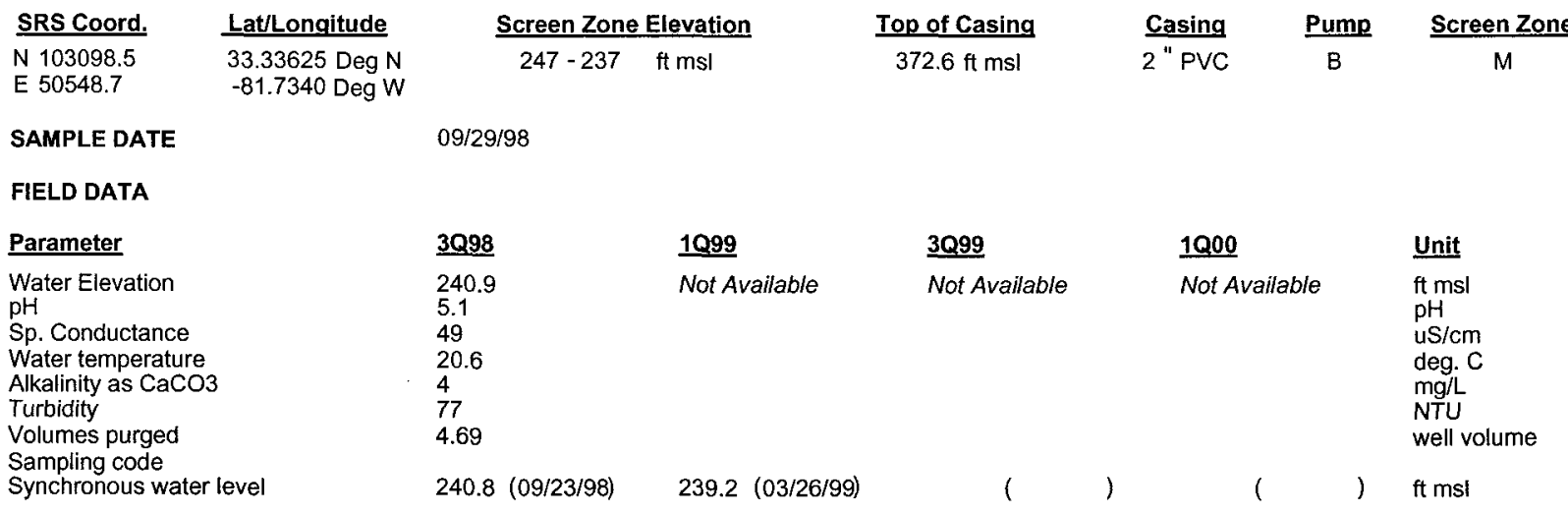

ANALYTICAL DATA

I. Groundwater Protection Standard

261 Appendix VIII/264 Appendix IX Hazardous Constituent

\begin{tabular}{|c|c|c|c|c|c|c|c|c|c|c|c|c|}
\hline$\underline{\text { ST }}$ & Parameter & $\underline{3 Q 98}$ & $\underline{\text { CLPEPA }}$ & $1 \mathrm{Q99}$ & CLPEPA & $\underline{3099}$ & CLPEPA & 1000 & CLPEPA & Filt. & $\underline{\text { DF }}$ & $\underline{\text { Unit }}$ \\
\hline \multicolumn{13}{|c|}{ Inorganics } \\
\hline & Barium, total recoverable & 10.7 & & & & & & & & & & ug \\
\hline & Cyanide & $<10$ & $u$ & & & & & & & & & \\
\hline & Lead, total recoverable & 42.4 & $\mathrm{~J}$ & & & & & & & & & ug \\
\hline & Nickel, total recoverable & 27.3 & $J$ & & & & & & & & & ug \\
\hline & Selenium, total recoverable & $<200$ & U & & & & & & & & & \\
\hline \multicolumn{13}{|c|}{ Organics } \\
\hline & Chlorobenzene & $<5$ & $\mathrm{U}$ & & & & & & & & & ug \\
\hline & 1,1-Dichloroethane & $<5$ & $\mathrm{U}$ & & & & & & & & & ug \\
\hline & 1,1-Dichloroethylene & $<5$ & $\mathrm{U}$ & & & & & & & & & ug \\
\hline & trans-1,2-Dichloroethylene & $<5$ & $\mathrm{U}$ & & & & & & & & & \\
\hline & PCB 1016 & & & & & & & & & & & \\
\hline & PCB 1221 & & & & & & & & & & & \\
\hline & PCB 1232 & & & & & & & & & & & \\
\hline & PCB 1242 & & & & & & & & & & & \\
\hline & PCB 1248 & & & & & & & & & & & \\
\hline & PCB 1254 & & & & & & & & & & & \\
\hline & PCB 1260 & & & & & & & & & & & \\
\hline & $1,1,2,2$-Tetrachloroethane & $<5$ & $\mathrm{U}$ & & & & & & & & & ug \\
\hline & Tetrachloroethylene & $<5$ & $\mathrm{U}$ & & & & & & & & & ug \\
\hline & 1,1,1-Trichloroethane & $<5$ & $\mathrm{U}$ & & & & & & & & & ug \\
\hline & Trichloroethylene & $<5$ & $\mathrm{U}$ & & & & & & & & & ug \\
\hline \multicolumn{13}{|c|}{ II. Monitoring Constituents } \\
\hline$\underline{\mathbf{S T}}$ & Parameter & $\underline{3096}$ & CLP EPA & $\underline{3097}$ & CLP EPA & $\underline{3 Q 98}$ & CLPEPA & $\underline{3 Q 99}$ & CLPEPA & Filt. & $\underline{\mathbf{D F}}$ & $\underline{\text { Un }}$ \\
\hline \multicolumn{13}{|c|}{ Inorganics } \\
\hline & Aluminum, total recoverable & & & & & 936 & & & & & & ug \\
\hline & Nitrate-nitrite as nitrogen & & & & & 2790 & & & & & & ug \\
\hline & Sodium, total recoverable & & & & & 2140 & & & & & & \\
\hline & Sulfate & & & & & 576 & & & & & & \\
\hline \multicolumn{13}{|c|}{ Radionuclides } \\
\hline & Gross alpha & & & & & 12.1 & & & & & & \\
\hline & Nonvolatile beta & & & & & 20.57 & & & & & & \\
\hline & Radium, total alpha-emitting & & & & & 1.82 & & & & & & \\
\hline
\end{tabular}

Notes: Concentrations in bold italics exceed the groundwater protection or monitoring constituent standards listed in Appendix A. Synchronous water levels are measured over a 3-5 day period or less. Dilution factors, Laboratory, and Filtered Data are for Groundwater Protection Standards First Quarter 2000 and Monitoring Constituents Third Quarter 1999 data only.

+ = exceeded the Groundwater Protection Standards (First Quarter 2000) or Monitoring Constituents (Third Quarter 1999) listed in Appendix A. 
Table D-4. Groundwater Monitoring Results for Plume Definition Wells, M-Area HWMF (Cont.) WELL MSB 47B

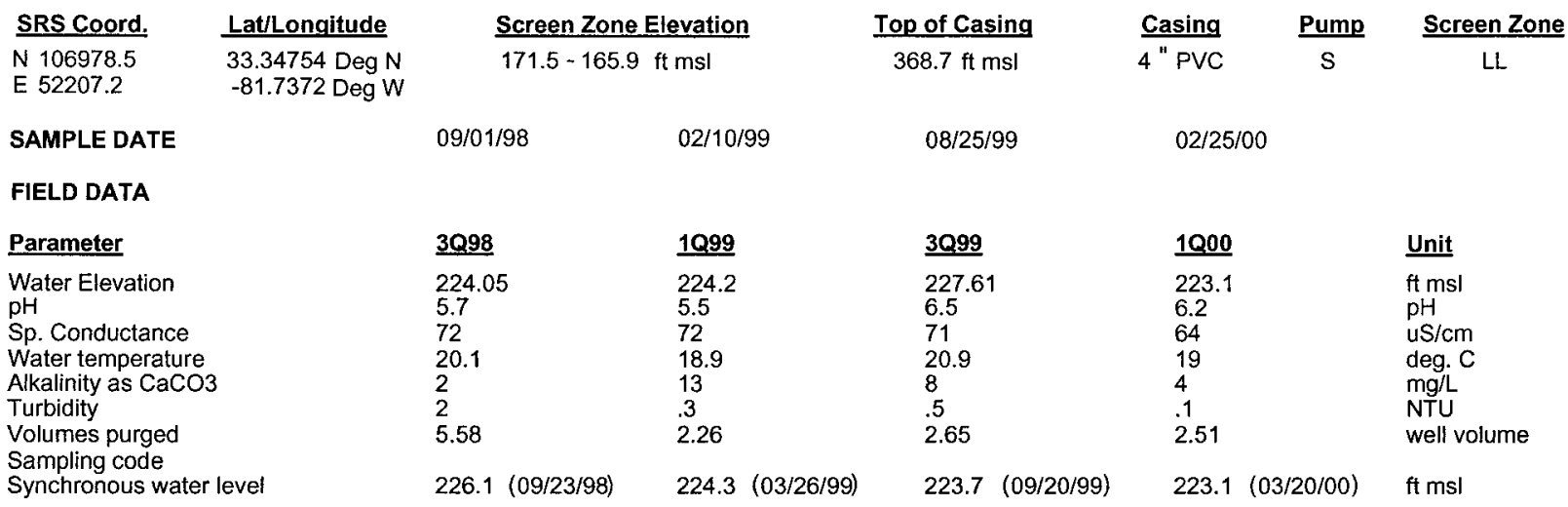

ANALYTICAL DATA

I. Groundwater Protection Standard

261 Appendix VIII/264 Appendix IX Hazardous Constituents

\begin{tabular}{|c|c|c|c|c|c|c|c|c|c|c|c|c|c|}
\hline$\underline{\mathbf{S T}}$ & Parameter & $\underline{3 Q 98}$ & CLP EPA & 1099 & CLP EPA & 3099 & CLPEPA & 1000 & CLPEPA & Filt. & $\underline{\text { DF }}$ & $\underline{\text { Unit }}$ & $\underline{\text { Lab }}$ \\
\hline \multicolumn{14}{|c|}{ Inorganics } \\
\hline & Barium, total recoverable & 43.3 & & 27 & & 34 & & & & & & $\mathrm{ug} / \mathrm{L}$ & \\
\hline & Cyanide & $<10$ & $U$ & $<10$ & $\mathrm{U}$ & $<10$ & $\mathrm{U}$ & & & & & $\mathrm{ug} / \mathrm{L}$ & \\
\hline & Lead, total recoverable & $<100$ & $\mathrm{U}$ & $<100$ & $U$ & $<10$ & $U$ & & & & & $\mathrm{ug} / \mathrm{L}$ & \\
\hline & Nickel, total recoverable & $<50$ & U & $<50$ & $U$ & $<50$ & $\mathrm{U}$ & & & & & $\mathrm{ug} / \mathrm{L}$ & \\
\hline & Selenium, total recoverable & $<10$ & $\mathrm{U}$ & $<10$ & $U$ & $<10$ & $U$ & & & & & $\mathrm{ug} / \mathrm{L}$ & \\
\hline \multicolumn{14}{|c|}{ Organics } \\
\hline & Chlorobenzene & $<125$ & UJ & $<125$ & $U$ & $<1$ & $U$ & $<5$ & $\mathrm{U}$ & $<\mathrm{EQL}$ & 5 & $u g / L$ & $\mathrm{ML}$ \\
\hline & 1,1-Dichloroethane & $<125$ & UJ & $<125$ & $\mathrm{U}$ & $<1$ & $\mathrm{U}$ & $<5$ & $\mathrm{U}$ & $<\mathrm{EQL}$ & 5 & $\mathrm{ug} / \mathrm{L}$ & ML. \\
\hline & 1,1-Dichloroethylene & $<125$ & UJ & $<125$ & U & $<1$ & $\mathrm{U}$ & $<5$ & $\mathrm{u}$ & $<\mathrm{EQL}$ & 5 & $\mathrm{ug} / \mathrm{L}$ & ML \\
\hline & trans-1,2-Dichloroethylene & $<125$ & UJ & $<125$ & $\mathrm{U}$ & $<1$ & $\mathrm{U}$ & $<5$ & $U$ & $<E Q L$ & 5 & $\mathrm{ug} / \mathrm{L}$ & $M L$ \\
\hline & PCB 1016 & & & & & & & & & & & & \\
\hline & PCB 1221 & & & & & & & & & & & & \\
\hline & PCB 1232 & & & & & & & & & & & & \\
\hline & РСВ 1242 & & & & & & & & & & & & \\
\hline & РСB 1248 & & & & & & & & & & & & \\
\hline & PCB 1254 & & & & & & & & & & & & \\
\hline & PCB 1260 & & & & & & & & & & & & \\
\hline & 1,1,2,2-Tetrachloroethane & $<125$ & UJ & $<125$ & $U$ & $<1$ & $U$ & $<5$ & $\mathrm{U}$ & $<\mathrm{EQL}$ & 5 & ug/L & ML \\
\hline & Tetrachloroethylene & $<125$ & UJ & $<125$ & $\mathrm{U}$ & $<1.56$ & U V & $<5$ & $U$ & $<\mathrm{EQL}$ & 5 & $\mathrm{ug} / \mathrm{L}$ & ML \\
\hline & 1,1,1-Trichloroethane & $<125$ & UJ & $<125$ & $U$ & $<1$ & $\mathrm{U}$ & $<5$ & $U$ & $<E Q L$ & 5 & $\mathrm{ug} / \mathrm{L}$ & $M L$ \\
\hline+ & Trichloroethylene & 2000 & $J$ & 1150 & & 1400 & & 1180 & & $>5$ & 5 & ug/L & $\mathrm{ML}$ \\
\hline \multicolumn{14}{|c|}{ II. Monitoring Constituents } \\
\hline$\underline{\text { ST }}$ & Parameter & 3096 & CLPEEPA & 3097 & CLP EPA & $\underline{3098}$ & CLPPEPA & $\underline{3099}$ & CLPEPA & Filt. & $\underline{\text { DF }}$ & Unit & Lab \\
\hline \multicolumn{14}{|c|}{ Inorganics } \\
\hline & Aluminum, total recoverable & 63.3 & & 60.7 & & $<200$ & $\mathrm{U}$ & $<200$ & U & $<E Q L$ & 1 & $u g / L$ & EX \\
\hline+ & Nitrate-nitrite as nitrogen & 4320 & & 3830 & & 3580 & & 3510 & & $>2400$ & 5 & $\mathrm{ug} / \mathrm{L}$ & EX \\
\hline+ & Sodium, total recoverable & 6330 & & 6160 & & 6370 & & 6800 & & $>4600$ & 1 & $u g / L$ & EX \\
\hline & Sulfate & 183 & J & $<5000$ & U & 356 & $J$ & 183 & J 1 & NDD & 2 & ug/L & EX \\
\hline \multicolumn{14}{|c|}{ Radionuclides } \\
\hline & Gross alpha & 3.56 & & 3.33 & & 7.98 & & 4.07 & & $<15$ & 1 & $\mathrm{pCi} / \mathrm{L}$ & GP \\
\hline & Nonvolatile beta & 4.1 & & 6.04 & & 10.24 & & 5.62 & & $<50$ & 1 & $\mathrm{pCi} / \mathrm{L}$ & GP \\
\hline & Radium, total alpha-emitting & 2.2 & & 10.2 & J & 1.57 & & 2.2 & & $<5$ & 1 & $\mathrm{pCi} / \mathrm{L}$ & GP \\
\hline
\end{tabular}

Notes: Concentrations in bold italics exceed the groundwater protection or monitoring constituent standards listed in Appendix A. Synchronous water levels are measured over a 3-5 day period or less. Dilution factors, Laboratory, and Filtered Data are for Groundwater Protection Standards First Quarter 2000 and Monitoring Constituents Third Quarter 1999 data only.

+ = exceeded the Groundwater Protection Standards (First Quarter 2000) or Monitoring Constituents (Third Quarter 1999) listed in Appendix A. 
Table D-4. Groundwater Monitoring Results for Plume Definition Wells, M-Area HWMF (Cont.) WELL MSB 47C

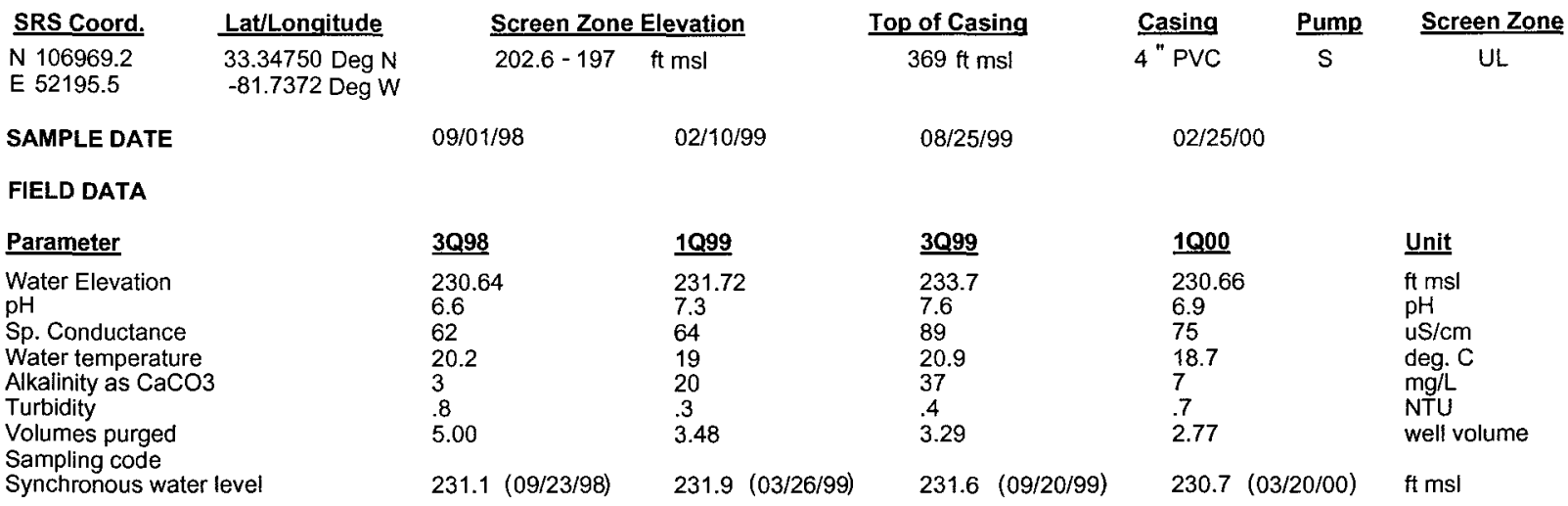

ANALYTICAL DATA

I. Groundwater Protection Standard

261 Appendix VIII/264 Appendix IX Hazardous Constituents

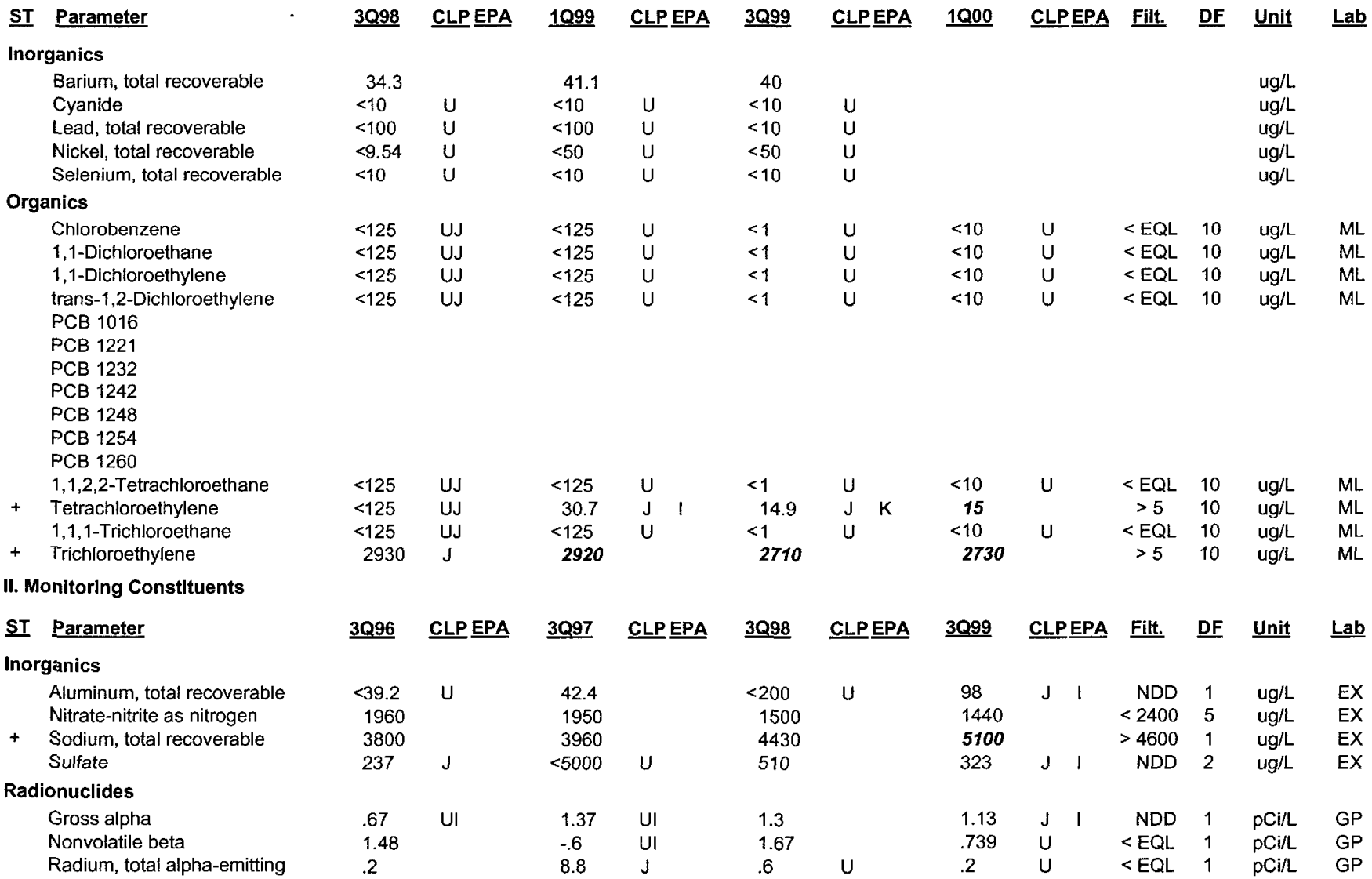

Notes: Concentrations in bold italics exceed the groundwater protection or monitoring constituent standards listed in Appendix A. Synchronous water levels are measured over a 3-5 day period or less. Dilution factors, Laboratory, and Filtered Data are for Groundwater Protection Standards First Quarter 2000 and Monitoring Constituents Third Quarter 1999 data only.

+= exceeded the Groundwater Protection Standards (First Quarter 2000) or Monitoring Constituents (Third Quarter 1999) listed in Appendix A. 
Table D-4. Groundwater Monitoring Results for Plume Definition Wells, M-Area HWMF (Cont.) WELL MSB 47D

\begin{tabular}{|c|c|c|c|c|c|c|c|}
\hline SRS Coord. & Lat/Longitude & \multicolumn{2}{|c|}{ Screen Zone Elevation } & Top of Casing & Casing & Pump & Screen Zone \\
\hline $\begin{array}{l}\text { N } 106960.1 \\
\text { E } 52184\end{array}$ & $\begin{array}{l}\text { 33.34746 Deg N } \\
-81.7372 \text { Deg } W\end{array}$ & $246.1-226.5$ & $\mathrm{ft} \mathrm{msl}$ & $368.8 \mathrm{ft} \mathrm{msl}$ & 4 "PVC & $S$ & M \\
\hline SAMPLE DATE & & $08 / 20 / 98$ & $02 / 09 / 99$ & & $03 / 08 /$ & & \\
\hline \multicolumn{8}{|l|}{ FIELD DATA } \\
\hline Parameter & & $\underline{3 Q 98}$ & 1099 & 3Q999 & 1000 & & $\underline{\text { Unit }}$ \\
\hline $\begin{array}{l}\text { Water Elevation } \\
\text { pH } \\
\text { Sp. Conductance } \\
\text { Water temperature } \\
\text { Alkalinity as CaCO3 } \\
\text { Turbidity } \\
\text { Volumes purged }\end{array}$ & & $\begin{array}{l}231.5 \\
5.1 \\
54 \\
25 \\
1 \\
1.2 \\
21.0\end{array}$ & $\begin{array}{l}232.88 \\
4.8 \\
48 \\
18.7 \\
1 \\
1.5 \\
31.8\end{array}$ & Not Available & $\begin{array}{l}231.78 \\
6 \\
84 \\
20.5 \\
12 \\
8.5 \\
12.6\end{array}$ & & $\begin{array}{l}\text { ft msl } \\
\mathrm{pH} \\
\text { uS/cm } \\
\text { deg. C } \\
\mathrm{mg} / \mathrm{L} \\
\text { NTU } \\
\text { well volume }\end{array}$ \\
\hline $\begin{array}{l}\text { Sampling code } \\
\text { Synchronous water }\end{array}$ & level & $232.1(09 / 23 / 98)$ & $233.1 \quad(03 / 26 / 99)$ & $(09 / 20 / 99)$ & 231.9 & $3 / 20 / 00)$ & $\mathrm{ft} \mathrm{msl}$ \\
\hline
\end{tabular}

ANALYTICAL DATA

I. Groundwater Protection Standard

261 Appendix VIII/264 Appendix IX Hazardous Constituents

\begin{tabular}{|c|c|c|c|c|c|c|c|c|c|c|c|c|c|}
\hline$\underline{\text { ST }}$ & Parameter & $\underline{3 Q 98}$ & CLP EPA & 1099 & $\underline{\text { CLP EPA }}$ & $\underline{3 Q 99}$ & $\underline{\text { CLPEPA }}$ & $\underline{1 Q 00}$ & CLPEPA & Filt. & $\underline{\text { DF }}$ & $\underline{\text { Unit }}$ & $\underline{\text { Lab }}$ \\
\hline \multicolumn{14}{|c|}{ Inorganics } \\
\hline & Barium, total recoverable & 28.3 & & 29 & & & & & & & & $u g / L$ & \\
\hline & Cyanide & $<10$ & $\mathrm{U}$ & $<10$ & $\mathrm{U}$ & & & & & & & $\mathrm{ug} / \mathrm{L}$ & \\
\hline & Lead, total recoverable & $<10$ & $\mathrm{U}$ & $<100$ & $\mathrm{U}$ & & & & & & & $\mathrm{ug} / \mathrm{L}$ & \\
\hline & Nickel, total recoverable & 9.94 & $\mathrm{~J}$ & $<50$ & $\mathrm{U}$ & & & & & & & $\mathrm{ug} / \mathrm{L}$ & \\
\hline & Selenium, total recoverable & $<10$ & $\mathrm{U}$ & $<200$ & $\mathrm{U}$ & & & & & & & $\mathrm{ug} / \mathrm{L}$ & \\
\hline \multicolumn{14}{|c|}{ Organics } \\
\hline & Chiorobenzene & $<5$ & $\mathrm{U}$ & $<5$ & $\mathrm{u}$ & & & $<.61$ & U & $<E Q L$ & 1 & $u g / L$ & ML \\
\hline & 1,1-Dichloroethane & $<5$ & $\mathrm{U}$ & $<5$ & $\mathrm{U}$ & & & $<1$ & U & $<E Q L$ & 1 & $\mathrm{ug} / \mathrm{L}$ & ML. \\
\hline & 1,1-Dichloroethylene & $<5$ & $\mathrm{U}$ & $<5$ & $\mathrm{U}$ & & & $<1$ & $U$ & $<E Q L$ & 1 & $u g / L$ & ML \\
\hline & trans-1,2-Dichloroethylene & $<5$ & $\mathrm{U}$ & $<5$ & $U$ & & & $<1$ & U & $<E Q L$ & 1 & $\mathrm{ug} / \mathrm{L}$ & $M L$ \\
\hline & PCB 1016 & & & & & & & & & & & & \\
\hline & PCB 1221 & & & & & & & & & & & & \\
\hline & PCB 1232 & & & & & & & & & & & & \\
\hline & PCB 1242 & & & & & & & & & & & & \\
\hline & PCB 1248 & & & & & & & & & & & & \\
\hline & PCB 1254 & & & & & & & & & & & & \\
\hline & PCB 1260 & & & & & & & & & & & & \\
\hline & $1,1,2,2$-Tetrachloroethane & $<5$ & U & $<5$ & $\mathrm{U}$ & & & $<1$ & $\mathrm{u}$ & $<E Q L$ & 1 & $u g / L$ & ML. \\
\hline & Tetrachloroethylene & $<5$ & $U$ & $<5$ & $\mathrm{U}$ & & & $<1$ & $U$ & $<E Q L$ & 1 & $\mathrm{ug} / \mathrm{L}$ & $M L$ \\
\hline & $1,1,1$-Trichloroethane & $<5$ & U & $<5$ & U & & & $<1$ & $\mathrm{U}$ & $<E Q L$ & 1 & $\mathrm{ug} / \mathrm{L}$ & ML \\
\hline & Trichloroethylene & 1.52 & $\jmath$ & 1.73 & J 1 & & & .85 & $\mathrm{~J} \quad \mathrm{IK}$ & NDD & 1 & $\mathrm{ug} / \mathrm{L}$ & ML \\
\hline \multicolumn{14}{|c|}{ II. Monitoring Constituents } \\
\hline$\underline{\mathbf{S T}}$ & Parameter & $\underline{3096}$ & CLPEPA & $\underline{3097}$ & CLPEPA & $\underline{3098}$ & CLPEPA & $\underline{3099}$ & CLPEPA & Filt. & DF & $\underline{\text { Unit }}$ & $\underline{\text { Lab }}$ \\
\hline \multicolumn{13}{|c|}{ Inorganics } & \\
\hline & Aluminum, total recoverable & $<20$ & $\mathrm{U}$ & 29.4 & & $<200$ & $\mathrm{U}$ & & & & & $\mathrm{ug} / \mathrm{L}$ & \\
\hline & Nitrate-nitrite as nitrogen & 5600 & & 4320 & & 3070 & & & & & & $u g / L$ & \\
\hline & Sodium, total recoverable & 11400 & & 8950 & & 5830 & & & & & & $\mathrm{ug} / \mathrm{L}$ & \\
\hline & Sulfate & 233 & $J$ & $<5000$ & $\mathrm{U}$ & 346 & J & & & & & $u g / L$ & \\
\hline \multicolumn{13}{|c|}{ Radionuclides } & \\
\hline & Gross alpha & 11.3 & & 4.4 & UI & 18.46 & & & & & & $\mathrm{pCi} / \mathrm{L}$ & \\
\hline & Nonvolatile beta & 6.04 & & 3.1 & UI & 12.25 & & & & & & $\mathrm{pCi} / \mathrm{L}$ & \\
\hline & Radium, total alpha-emitting & 4 & & 3.87 & $\mathrm{~J}$ & 8.38 & & & & & & $\mathrm{pCi} / \mathrm{L}$ & \\
\hline
\end{tabular}

Notes: Concentrations in bold italics exceed the groundwater protection or monitoring constituent standards listed in Appendix A. Synchronous water levels are measured over a 3-5 day period or less. Dilution factors, Laboratory, and Filtered Data are for Groundwater Protection Standards First Quarter 2000 and Monitoring Constituents Third Quarter 1999 data only.

+ = exceeded the Groundwater Protection Standards (First Quarter 2000) or Monitoring Constituents (Third Quarter 1999) listed in Appendix A. 
Table D-4. Groundwater Monitoring Results for Plume Definition Wells, M-Area HWMF (Cont.) WELL MSB 47TA

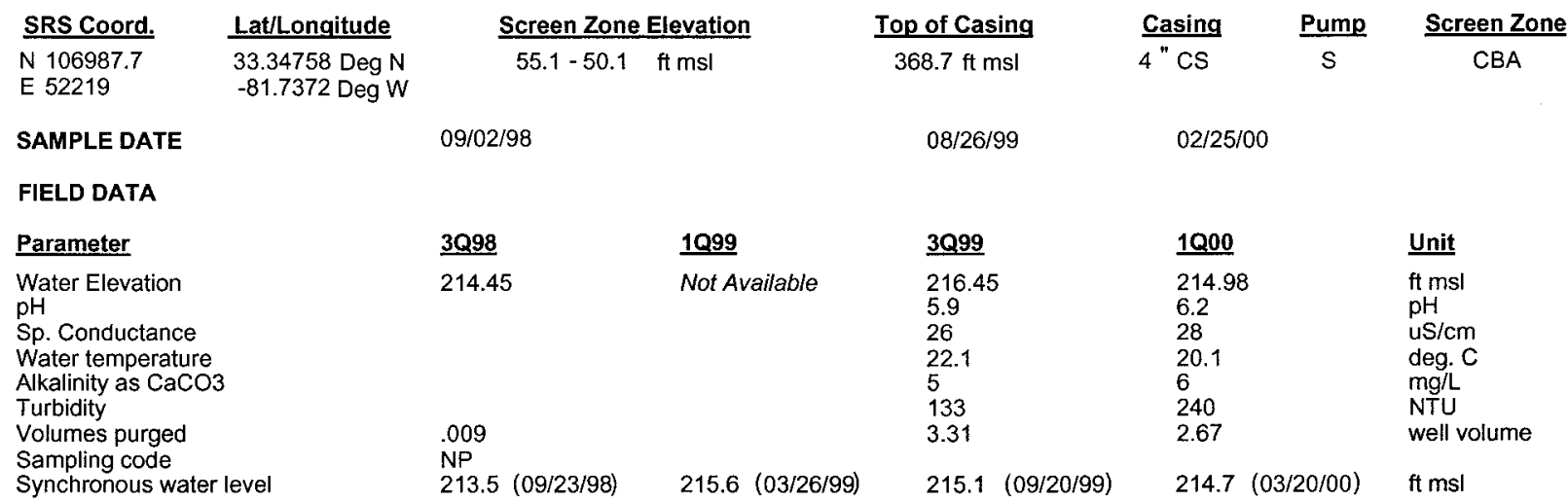

ANALYTICAL DATA

I. Groundwater Protection Standard

261 Appendix VIII/264 Appendix IX Hazardous Constituents

\begin{tabular}{|c|c|c|c|c|c|c|c|c|c|c|c|c|c|c|}
\hline$\underline{\mathbf{S T}}$ & Parameter & $3 Q 98$ & CLPEPA & 1099 & CLPEPA & $\underline{3 Q 99}$ & CLPEPA & \multirow[t]{2}{*}{$\underline{1 Q 00}$} & \multicolumn{2}{|c|}{ CLPEPA } & \multirow[t]{2}{*}{ Filt. } & \multirow[t]{2}{*}{ DF } & \multirow[t]{2}{*}{$\underline{\text { Unit }}$} & \multirow[t]{2}{*}{$\underline{\text { Lab }}$} \\
\hline \multicolumn{10}{|c|}{ Inorganics } & & & & & \\
\hline & Barium, total recoverable & & & $<10$ & $\mathrm{U}$ & 12 & & & & & & & $\mathrm{ug} / \mathrm{L}$ & \\
\hline & Cyanide & & & $<10$ & $U$ & $<10$ & $U$ & & & & & & $u g / L$ & \\
\hline & Lead, total recoverable & & & $<100$ & $\mathrm{U}$ & $<1.13$ & U V & & & & & & $\mathrm{ug} / \mathrm{L}$ & \\
\hline & Nickel, total recoverable & & & $<50$ & JU & $<7.4$ & JU I & & & & & & $\mathrm{ug} / \mathrm{L}$ & \\
\hline & Selenium, total recoverable & & & $<200$ & U & $<5$ & $\mathrm{U}$ & & & & & & $\mathrm{ug} / \mathrm{L}$ & \\
\hline \multicolumn{15}{|c|}{ Organics } \\
\hline & Chlorobenzene & & & $<25$ & $\mathrm{U}$ & $<1$ & $u$ & $<2$ & $\mathrm{U}$ & & $<$ EQL & 2 & $\mathrm{ug} / \mathrm{L}$ & ML \\
\hline & 1,1-Dichloroethane & & & $<25$ & $\mathrm{U}$ & $<1$ & $\mathrm{U}$ & $<2$ & $\mathrm{U}$ & & $<\mathrm{EQL}$ & 2 & $\mathrm{ug} / \mathrm{L}$ & ML \\
\hline & 1,1-Dichloroethylene & & & $<25$ & $\mathrm{U}$ & $<1$ & $\mathrm{U}$ & $<2$ & $\mathrm{U}$ & & $<\mathrm{EQL}$ & 2 & $\mathrm{ug} / \mathrm{L}$ & ML \\
\hline & trans-1,2-Dichloroethylene & & & $<25$ & $u$ & $<1$ & $\mathrm{U}$ & $<2$ & $U$ & & $<\mathrm{EQL}$ & 2 & $\mathrm{ug} / \mathrm{L}$ & ML \\
\hline & PCB 1016 & & & & & & & & & & & & & \\
\hline & PCB 1221 & & & & & & & & & & & & & \\
\hline & PCB 1232 & & & & & & & & & & & & & \\
\hline & PCB 1242 & & & & & & & & & & & & & \\
\hline & PCB 1248 & & & & & & & & & & & & & \\
\hline & PCB 1254 & & & & & & & & & & & & & \\
\hline & PCB 1260 & & & & & & & & & & & & & \\
\hline & 1,1,2,2-Tetrachloroethane & & & $<25$ & $\mathrm{U}$ & $<1$ & $\mathrm{U}$ & $<2$ & $U$ & & $<E Q L$ & 2 & $\mathrm{ug} / \mathrm{L}$ & ML \\
\hline & Tetrachloroethylene & & & $<25$ & $\mathrm{U}$ & 1.77 & $\downarrow K$ & 1.42 & $J$ & 1 & NDD & 2 & $\mathrm{ug} / \mathrm{L}$ & ML \\
\hline & 1,1,1-Trichloroethane & & & $<25$ & $\mathrm{U}$ & $<1$ & $\mathrm{u}$ & $<2$ & $\mathrm{u}$ & & $<E Q L$ & 2 & $\mathrm{ug} / \mathrm{L}$ & ML \\
\hline+ & Trichloroethylene & & & 884 & & 751 & & 589 & & & $>5$ & 2 & $\mathrm{ug} / \mathrm{L}$ & ML \\
\hline \multicolumn{15}{|c|}{ II. Monitoring Constituents } \\
\hline$\underline{\mathbf{S T}}$ & Parameter & $\underline{3 Q 96}$ & CLPEEPA & $\underline{3 Q 97}$ & CLPEPA & $\underline{3 Q 98}$ & CLPEPA & $\underline{3099}$ & $\underline{C L F}$ & EPA & Filt. & $\underline{D F}$ & Unit & Lab \\
\hline \multicolumn{15}{|c|}{ Inorganics } \\
\hline & Aluminum, total recoverable & $<20$ & $\mathrm{U}$ & 17.9 & $\mathrm{~J}$ & & & $<200$ & $\mathrm{U}$ & & $<E Q L$ & 1 & $\mathrm{ug} / \mathrm{L}$ & EX \\
\hline & Nitrate-nitrite as nitrogen & 1510 & & 1320 & & & & 1170 & & & $<2400$ & 5 & ug/L & EX \\
\hline & Sodium, total recoverable & 2040 & & 2440 & & & & 2100 & & & $<4600$ & 1 & $\mathrm{ug} / \mathrm{L}$ & EX \\
\hline & Sulfate & 129 & $J$ & $<5000$ & $\mathrm{U}$ & & & 1050 & & & $<3000$ & 2 & $\mathrm{ug} / \mathrm{L}$ & EX \\
\hline \multicolumn{15}{|c|}{ Radionuclides } \\
\hline & Gross alpha & 1.18 & & 2.49 & & & & 1.89 & $J$ & IK & NDD & 1 & $\mathrm{pCi} / \mathrm{L}$ & GP \\
\hline & Nonvolatile beta & 1.44 & UI & 1.12 & UI & & & 1.5 & $\mathrm{U}$ & & $<E Q L$ & 1 & $\mathrm{pCi} / \mathrm{L}$ & GP \\
\hline & Radium, total alpha-emitting & 3 & UI & 1.47 & UI & & & .7 & $J$ & 1 & NDD & 1 & $\mathrm{pCi} / \mathrm{L}$ & GP \\
\hline
\end{tabular}

Notes: Concentrations in bold italics exceed the groundwater protection or monitoring constituent standards listed in Appendix A. Synchronous water levels are measured over a 3-5 day period or less. Dilution factors, Laboratory, and Filtered Data are for Groundwater Protection Standards First Quarter 2000 and Monitoring Constituents Third Quarter 1999 data only.

+ = exceeded the Groundwater Protection Standards (First Quarter 2000) or Monitoring Constituents (Third Quarter 1999) listed in Appendix A. 
Table D-4. Groundwater Monitoring Results for Plume Definition Wells, M-Area HWMF (Cont.) WELL MSB 48A

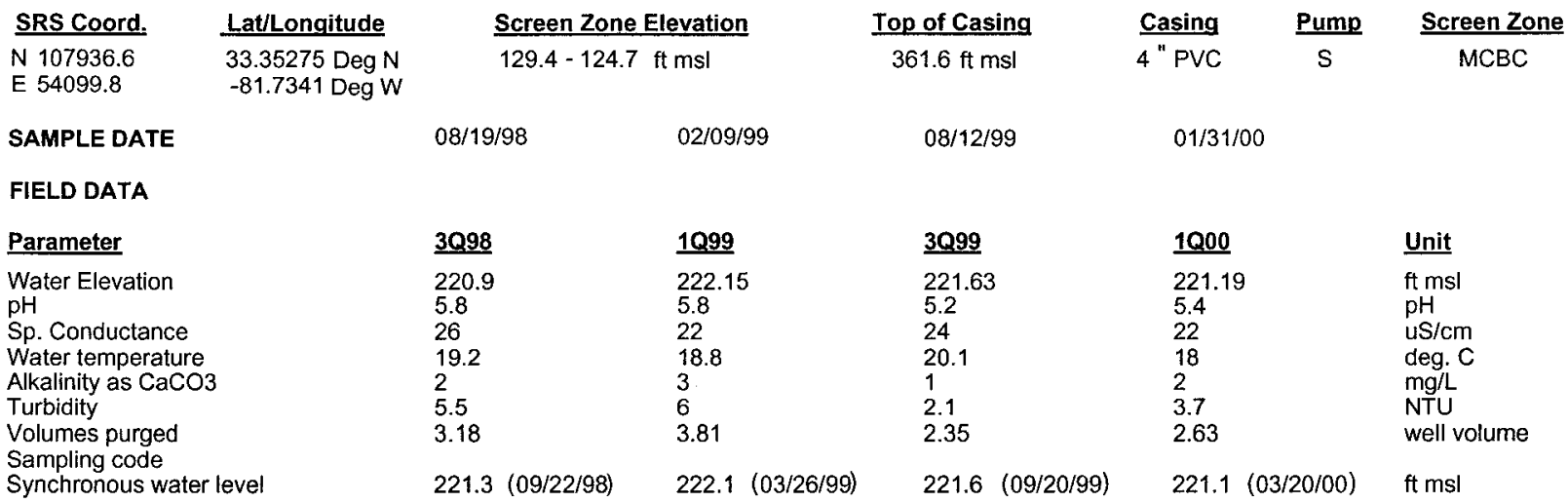

ANALYTICAL DATA

I. Groundwater Protection Standard

261 Appendix VIII/264 Appendix IX Hazardous Constituents

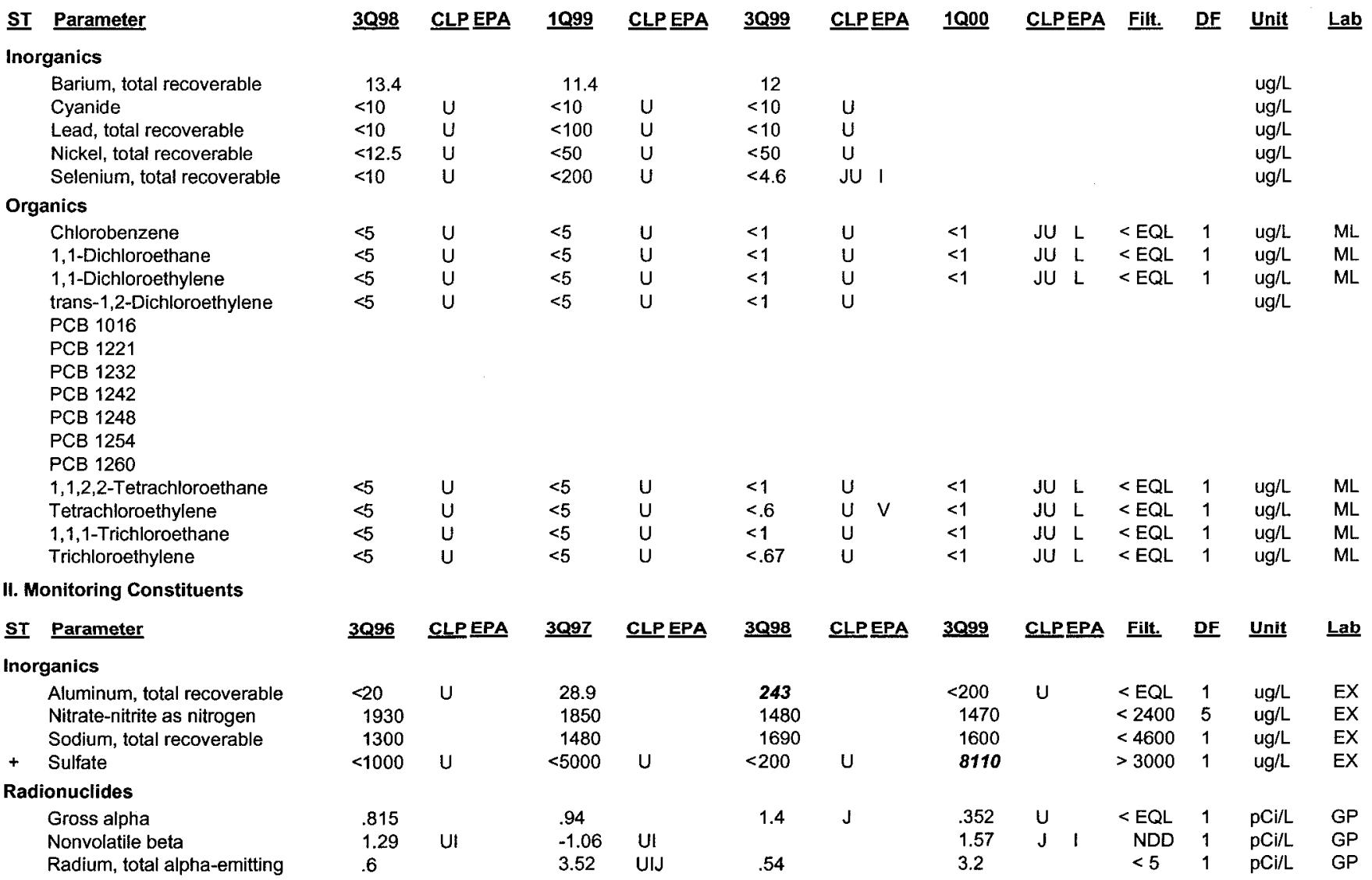

Notes: Concentrations in bold italics exceed the groundwater protection or monitoring constituent standards listed in Appendix A. Synchronous water levels are measured over a 3-5 day period or less. Dilution factors, Laboratory, and Filtered Data are for Groundwater Protection Standards First Quarter 2000 and Monitoring Constituents Third Quarter 1999 data only.

$+=$ exceeded the Groundwater Protection Standards (First Quarter 2000) or Monitoring Constituents (Third Quarter 1999) listed in Appendix A. 
Table D-4. Groundwater Monitoring Results for Plume Definition Wells, M-Area HWMF (Cont.) WELL MSB 48B

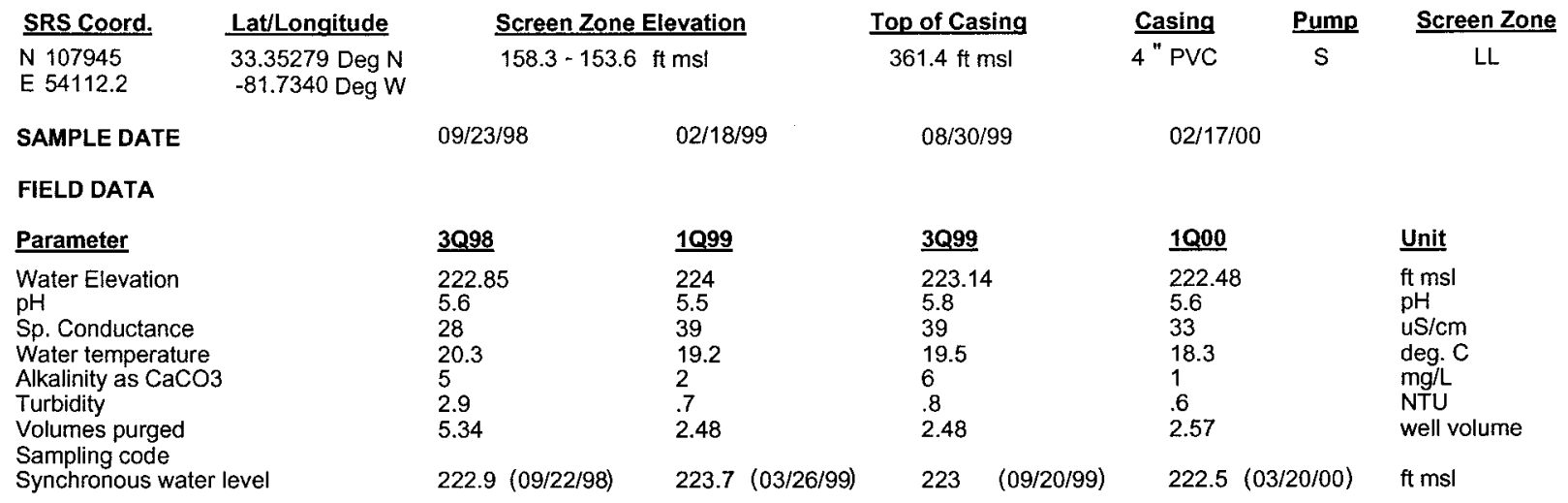

ANALYTICAL DATA

I. Groundwater Protection Standard

261 Appendix VIII/264 Appendix IX Hazardous Constituents

\begin{tabular}{|c|c|c|c|c|c|c|c|c|c|c|c|c|c|c|}
\hline$\underline{\mathbf{S T}}$ & Parameter & $\underline{3 Q 98}$ & CLPEPA & 1099 & CLPEPA & $\underline{3 Q 99}$ & CLP EPA & 1000 & CLP & EPA & Filt. & $\underline{\text { DF }}$ & Unit & $\underline{\text { Lab }}$ \\
\hline nor & ganics & & & & & & & & & & & & & \\
\hline & Barium, total recoverable & 12.1 & & $<10$ & $\mathrm{U}$ & 14 & & & & & & & $u g / L$ & \\
\hline & Cyanide & $<10$ & $\mathrm{U}$ & $<10$ & $U$ & $<10$ & $\mathrm{U}$ & & & & & & $\mathrm{ug} / \mathrm{L}$ & \\
\hline & Lead, total recoverable & $<100$ & $U$ & $<100$ & $U$ & $<10$ & $\mathrm{U}$ & & & & & & $\mathrm{ug} / \mathrm{L}$ & \\
\hline & Nickel, total recoverable & $<50$ & U & $<50$ & $\mathrm{U}$ & $<50$ & $\mathrm{U}$ & & & & & & $\mathrm{ug} / \mathrm{L}$ & \\
\hline & Selenium, total recoverable & $<200$ & $\mathrm{U}$ & $<200$ & $U$ & $<10$ & $\mathrm{U}$ & & & & & & $\mathrm{ug} / \mathrm{L}$ & \\
\hline rg & anics & & & & & & & & & & & & & \\
\hline & Chtorobenzene & $<5$ & $u$ & $<5$ & $u$ & $<1$ & $\mathrm{U}$ & $<1$ & JU & L & $<\mathrm{EQL}$ & 1 & $u g / L$ & ML \\
\hline & 1,1-Dichloroethane & $<5$ & $U$ & $<5$ & $U$ & $<1$ & $\mathrm{U}$ & $<1$ & JU & $\mathrm{L}$ & $<\mathrm{EQL}$ & 1 & $\mathrm{ug} / \mathrm{L}$ & $M L$ \\
\hline & 1,1-Dichloroethylene & $<5$ & $U$ & $<5$ & $U$ & $<1$ & $\mathrm{U}$ & $<1$ & JU & $L$ & $<E Q L$ & 1 & $\mathrm{ug} / \mathrm{L}$ & ML \\
\hline & trans-1,2-Dichloroethylene & $<5$ & $U$ & $<5$ & $\mathrm{U}$ & $<1$ & $\mathrm{U}$ & $<1$ & JU & $L$ & $<\mathrm{EQL}$ & 1 & $\mathrm{ug} / \mathrm{L}$ & ML \\
\hline & PCB 1016 & & & & & & & & & & & & & \\
\hline & PCB 1221 & & & & & & & & & & & & & \\
\hline & PCB 1232 & & & & & & & & & & & & & \\
\hline & PCB 1242 & & & & & & & & & & & & & \\
\hline & PCB 1248 & & & & & & & & & & & & & \\
\hline & PCB 1254 & & & & & & & & & & & & & \\
\hline & PCB 1260 & & & & & & & & & & & & & \\
\hline & $1,1,2,2$-Tetrachloroethane & $<5$ & $U$ & $<5$ & $U$ & $<1$ & $\mathrm{U}$ & $<1$ & JU & $L$ & $<\mathrm{EQL}$ & 1 & $\mathrm{ug} / \mathrm{L}$ & ML \\
\hline & Tetrachloroethylene & $<5$ & $u$ & $<5$ & $\mathrm{U}$ & $<1$ & $\mathrm{U}$ & $<1$ & JU & $\mathrm{L}$ & $<E Q L$ & 1 & $\mathrm{ug} / \mathrm{L}$ & $M L$ \\
\hline & 1,1,1-Trichloroethane & $<5$ & U & $<5$ & $U$ & $<1$ & $\mathrm{U}$ & $<1$ & JU & $\mathrm{L}$ & $<\mathrm{EQL}$ & 1 & $\mathrm{ug} / \mathrm{L}$ & $M L$ \\
\hline & Trichloroethylene & 23 & & 18.4 & & 15.5 & $\mathrm{~J} K$ & 10.8 & J & & NDD & 1 & ug/L & $M L$ \\
\hline II. $\mathbf{M}$ & onitoring Constituents & & & & & & & & & & & & & \\
\hline$\underline{\mathrm{ST}}$ & Parameter & $\underline{3 Q 96}$ & CLPEPA & $\underline{3097}$ & $\underline{\text { CLP EPA }}$ & $\underline{3 Q 98}$ & CLPEPA & $\underline{3099}$ & CLP & EPA & Filt. & $\underline{\text { DF }}$ & Unit & Lab \\
\hline Inor & ganics & & & & & & & & & & & & & \\
\hline & Aluminum, total recoverable & 88.3 & & 52.5 & & $<200$ & U & $<200$ & $\mathrm{U}$ & & $<\mathrm{EQL}$ & 1 & $\mathrm{ug} / \mathrm{L}$ & EX \\
\hline & Nitrate-nitrite as nitrogen & 1400 & $\mathrm{~J}$ & 1130 & & 339 & $\mathrm{~J}$ & 2180 & & & $<2400$ & 5 & $\mathrm{ug} / \mathrm{L}$ & EX \\
\hline & Sodium, total recoverable & 1750 & & 1720 & & 2000 & & 2000 & & & $<4600$ & 1 & $\mathrm{ug} / \mathrm{L}$ & EX \\
\hline & Sulfate & 156 & $\mathrm{~J}$ & $<5000$ & $U$ & 331 & $J$ & $<400$ & $u$ & & $<\mathrm{EQL}$ & 2 & $\mathrm{ug} / \mathrm{L}$ & EX \\
\hline Rad & ionuclides & & & & & & & & & & & & & \\
\hline & Gross alpha & 1.35 & & .28 & UI & 2.57 & & 1.67 & $J$ & 1 & NDD & 1 & $\mathrm{pCi} / \mathrm{L}$ & GP \\
\hline & Nonvolatile beta & 1.38 & UI & 1.73 & UI & 4.11 & $J$ & 2.08 & J & I & NDD & 1 & $\mathrm{pCi} / \mathrm{L}$ & GP \\
\hline & Radium, total alpha-emitting & .4 & & 1.18 & UIJ & .7 & & .3 & $U$ & & $<\mathrm{EQL}$ & 1 & $\mathrm{pCi} / \mathrm{L}$ & GP \\
\hline
\end{tabular}

Notes: Concentrations in bold italics exceed the groundwater protection or monitoring constituent standards listed in Appendix A. Synchronous water levels are measured over a 3-5 day period or less. Dilution factors, Laboratory, and Filtered Data are for Groundwater Protection Standards First Quarter 2000 and Monitoring Constituents Third Quarter 1999 data only.

$+=$ exceeded the Groundwater Protection Standards (First Quarter 2000) or Monitoring Constituents (Third Quarter 1999) listed in Appendix A. 
Table D-4. Groundwater Monitoring Results for Plume Definition Wells, M-Area HWMF (Cont.) WELL MSB 48C

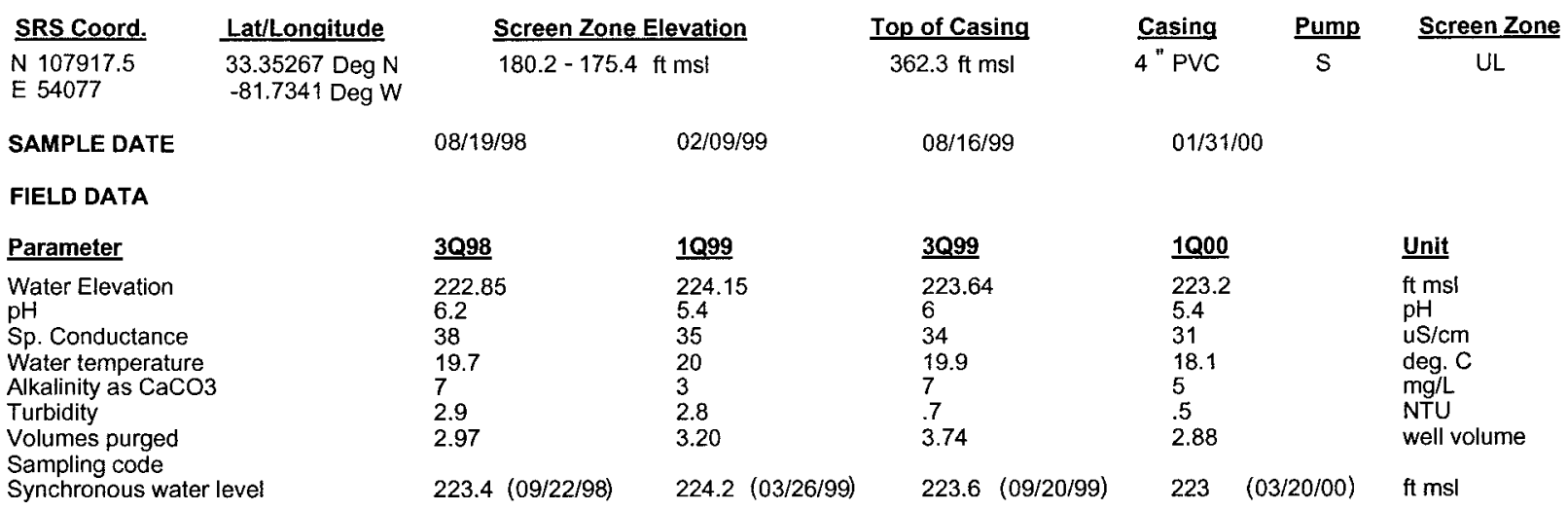

ANALYTICAL DATA

I. Groundwater Protection Standard

261 Appendix VIII/264 Appendix IX Hazardous Constituents

\begin{tabular}{|c|c|c|c|c|c|c|c|c|c|c|c|c|c|}
\hline \multirow{2}{*}{$\begin{array}{l}\text { ST Parameter } \\
\text { Inorganics }\end{array}$} & \multirow[t]{2}{*}{$\underline{3098}$} & \multirow[t]{2}{*}{ CLPEPA } & \multirow[t]{2}{*}{1099} & CLPEPA & \multirow[t]{2}{*}{$\underline{3 Q 99}$} & CLPEPA & \multirow[t]{2}{*}{$\underline{1000}$} & \multicolumn{2}{|c|}{ CLPEPA } & \multirow[t]{2}{*}{ Filt. } & \multirow[t]{2}{*}{$\underline{\text { DF }}$} & \multirow[t]{2}{*}{ Unit } & \multirow[t]{2}{*}{$\underline{\text { Lab }}$} \\
\hline & & & & & & & & & & & & & \\
\hline Barium, total recoverable & 12 & & 9.28 & J I & 8.9 & J I & & & & & & $u g / L$ & \\
\hline Cyanide & $<10$ & $\mathrm{u}$ & $<10$ & $u$ & $<10$ & $u$ & & & & & & $\mathrm{ug} / \mathrm{L}$ & \\
\hline Lead, total recoverable & $<10$ & $\mathrm{u}$ & $<100$ & $u$ & $<1.03$ & $u v$ & & & & & & $\mathrm{ug} / \mathrm{L}$ & \\
\hline Nickel, total recoverable & $<14.6$ & $\mathrm{u}$ & $<50$ & $\mathrm{U}$ & $<50$ & $u$ & & & & & & $\mathrm{ug} / \mathrm{L}$ & \\
\hline Selenium, total recoverable & $<10$ & $u$ & $<200$ & $U$ & $<5$ & $u$ & & & & & & $\mathrm{ug} / \mathrm{L}$ & \\
\hline \multicolumn{14}{|l|}{ Organics } \\
\hline Chlorobenzene & $<5$ & $\mathrm{u}$ & $<5$ & $u$ & $<1$ & $\mathrm{u}$ & $<1$ & JU & $\mathrm{L}$ & $<E Q L$ & 1 & $u g / L$ & ML \\
\hline 1,1-Dichloroethane & $<5$ & $u$ & $<5$ & u & $<1$ & $\mathrm{u}$ & $<1$ & ju & L & $<E Q L$ & 1 & $\mathrm{ug} / \mathrm{L}$ & ML \\
\hline 1,1-Dichloroethylene & $<5$ & $u$ & $<5$ & $\mathrm{u}$ & $<1$ & $\mathrm{u}$ & $<1$ & JU & L & $<E Q L$ & 1 & $\mathrm{ug} / \mathrm{L}$ & ML \\
\hline trans-1,2-Dichloroethylene & $<5$ & $\mathrm{u}$ & $<5$ & $u$ & $<1$ & $\mathrm{u}$ & & & & & & $\mathrm{ug} / \mathrm{L}$ & \\
\hline PCB 1016 & & & & & & & & & & & & & \\
\hline РCB 1221 & & & & & & & & & & & & & \\
\hline PCB 1232 & & & & & & & & & & & & & \\
\hline PCB 1242 & & & & & & & & & & & & & \\
\hline РCB 1248 & & & & & & & & & & & & & \\
\hline PCB 1254 & & & & & & & & & & & & & \\
\hline PCB 1260 & & & & & & & & & & & & & \\
\hline 1,1,2,2-Tetrachloroethane & $<5$ & $u$ & $<5$ & U & $<1$ & $u$ & $<1$ & JU & L & $<E Q L$ & 1 & $u g / L$ & $\mathrm{ML}$ \\
\hline Tetrachloroethylene & $<5$ & $u$ & $<5$ & U & $<1$ & $\mathrm{u}$ & $<1$ & JU & L & $<E Q L$ & 1 & ug/L & $\mathrm{ML}$ \\
\hline $1,1,1$-Trichloroethane & $<5$ & u & $<5$ & $u$ & $<1$ & $\mathrm{u}$ & $<1$ & JU & $\mathrm{L}$ & $<E Q L$ & 1 & ug/L & ML \\
\hline Trichloroethylene & $<5$ & $u$ & $<5$ & $u$ & $<1$ & $\mathrm{u}$ & $<1$ & JU & $\mathrm{L}$ & $<E Q L$ & 1 & $\mathrm{ug} / \mathrm{L}$ & ML \\
\hline \multicolumn{14}{|l|}{ II. Monitoring Constituents } \\
\hline SI Parameter & $\underline{3096}$ & CLPPEPA & $\underline{3097}$ & CLPEEPA & $\underline{3 Q 98}$ & CLPEPA & $\underline{3099}$ & $\underline{\text { CLP }}$ & EPA & Fill. & $\underline{\text { DF }}$ & $\underline{\text { Unit }}$ & $\underline{\text { Lab }}$ \\
\hline \multicolumn{14}{|l|}{ Inorganics } \\
\hline Aluminum, total recoverable & 110 & & 76.8 & & 74 & J & $<200$ & $\mathrm{U}$ & & $<E Q L$ & 1 & $u g / L$ & $\mathrm{EX}$ \\
\hline Nitrate-nitrite as nitrogen & 1750 & $\mathrm{~J}$ & 1730 & & 1370 & & 1510 & & & $<2400$ & 5 & $\mathrm{ug} / \mathrm{L}$ & EX \\
\hline Sodium, total recoverable & 2920 & & 2500 & & 2310 & & 2200 & & & $<4600$ & 1 & $\mathrm{ug} / \mathrm{L}$ & EX \\
\hline Sulfate & 902 & J & $<5000$ & $u$ & $<200$ & U & 423 & & & $<3000$ & 1 & $\mathrm{ug} / \mathrm{L}$ & EX \\
\hline \multicolumn{14}{|l|}{ Radionuclides } \\
\hline Gross alpha & .909 & & 6 & UI & 1.35 & UIJ & .576 & $u$ & & $<E Q L$ & 1 & $\mathrm{pCi} / \mathrm{L}$ & GP \\
\hline Nonvolatile beta & 3.42 & & 2.6 & & & & 2.35 & $\mathrm{~J}$ & I & NDD & 1 & $\mathrm{pCl} / \mathrm{L}$ & GP \\
\hline Radium, total alpha-emitting & .2 & & 1.14 & UIJ & .58 & & .6 & $\mathrm{~J}$ & I & NDD & 1 & $\mathrm{pCi} / \mathrm{L}$ & GP \\
\hline
\end{tabular}

Notes: Concentrations in bold italics exceed the groundwater protection or monitoring constituent standards listed in Appendix A. Synchronous water levels are measured over a 3-5 day period or less. Dilution factors, Laboratory, and Filtered Data are for Groundwater Protection Standards First Quarter 2000 and Monitoring Constituents Third Quarter 1999 data only.

$+=$ exceeded the Groundwater Protection Standards (First Quarter 2000) or Monitoring Constituents (Third Quarter 1999) listed in Appendix A. 
Table D-4. Groundwater Monitoring Results for Plume Definition Wells, M-Area HWMF (Cont.) WELL MSB 48D

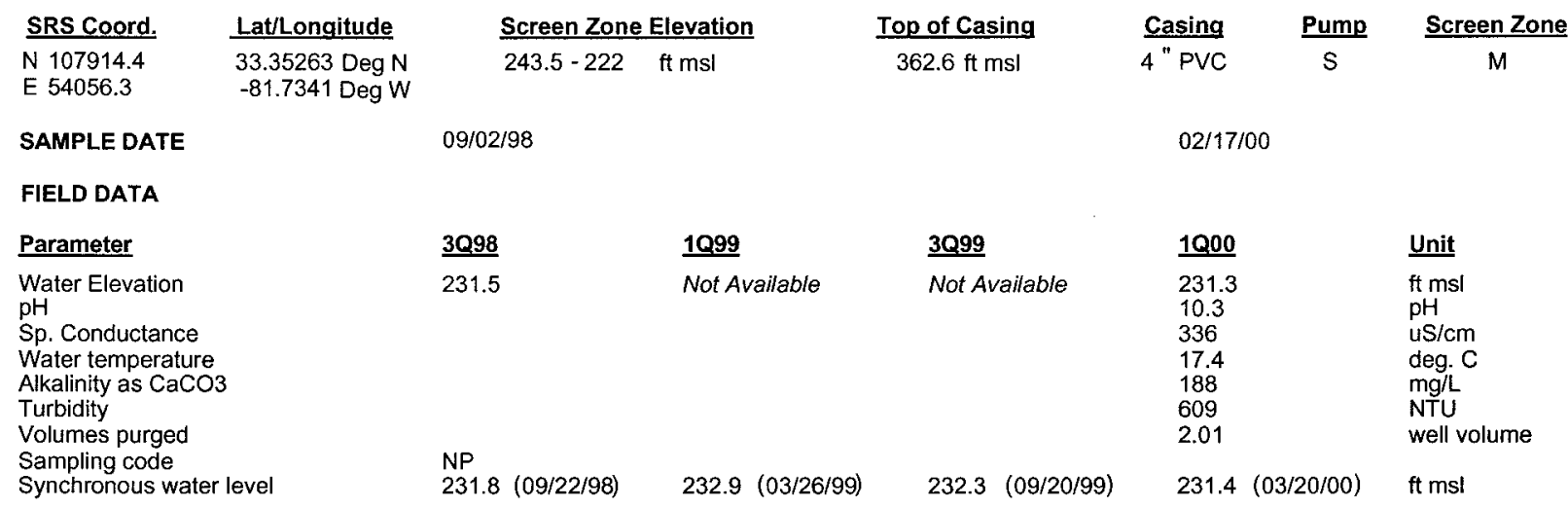

ANALYTICAL DATA

I. Groundwater Protection Standard

261 Appendix VIII/264 Appendix IX Hazardous Constituents

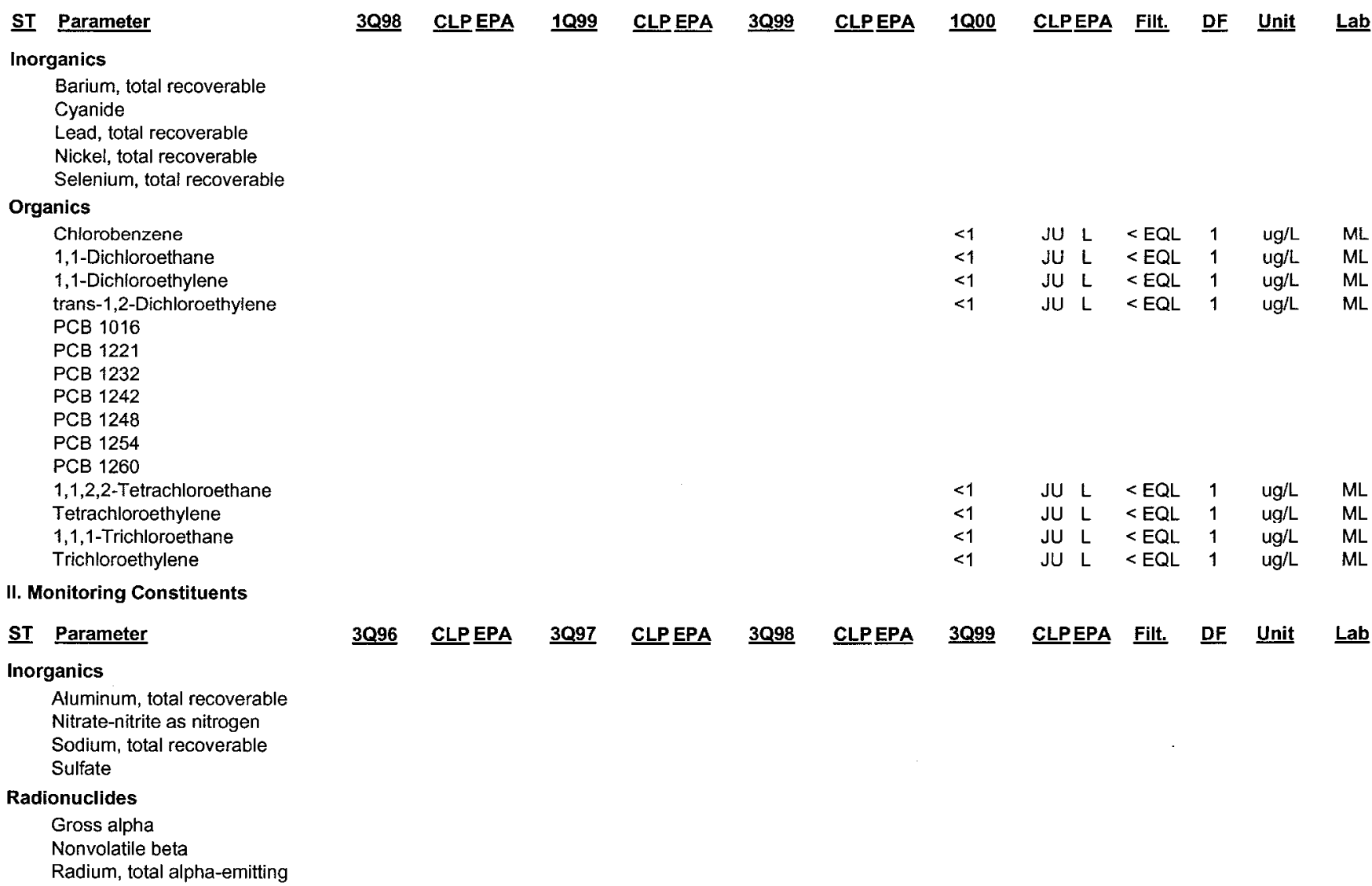

Notes: Concentrations in bold italics exceed the groundwater protection or monitoring constituent standards listed in Appendix A. Synchronous water levels are measured over a 3-5 day period or less. Dilution factors, Laboratory, and Filtered Data are for Groundwater Protection Standards First Quarter 2000 and Monitoring Constituents Third Quarter 1999 data only.

+ = exceeded the Groundwater Protection Standards (First Quarter 2000) or Monitoring Constituents (Third Quarter 1999) listed in Appendix A. 
Table D-4. Groundwater Monitoring Results for Plume Definition Wells, M-Area HWMF (Cont.) WELL MSB 48TA

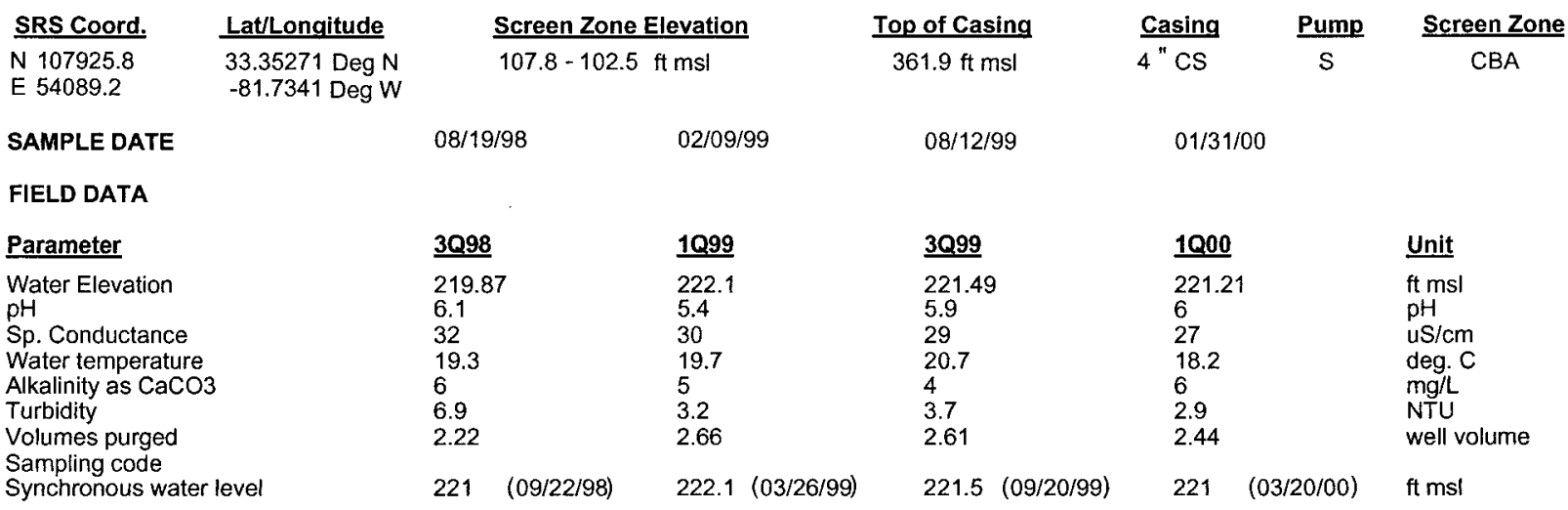

ANALYTICAL DATA

I. Groundwater Protection Standard

261 Appendix VIII/264 Appendix IX Hazardous Constituents

\begin{tabular}{|c|c|c|c|c|c|c|c|c|c|c|c|c|c|}
\hline$\underline{\text { ST }}$ & Parameter & $\underline{3098}$ & CLPEPA & 1099 & CLP EPA & 3Q999 & CLPEPA & 1000 & CLPEPA & Filt. & DF & Unit & Lab \\
\hline \multicolumn{14}{|c|}{ Inorganics } \\
\hline & Barium, total recoverable & 6.62 & J & 5.68 & $J$ & 6.4 & J 1 & & & & & $\mathrm{ug} / \mathrm{L}$ & \\
\hline & Cyanide & $<10$ & $\mathrm{U}$ & $<10$ & $\mathrm{U}$ & $<10$ & $\mathrm{U}$ & & & & & $\mathrm{ug} / \mathrm{L}$ & \\
\hline & Lead, total recoverable & $<10$ & $\mathrm{U}$ & $<100$ & $\mathrm{U}$ & $<10$ & $U$ & & & & & $u g / L$ & \\
\hline & Nickel, total recoverable & $<9.45$ & $\mathrm{U}$ & $<50$ & $\mathrm{U}$ & $<50$ & $\mathrm{U}$ & & & & & $u g / L$ & \\
\hline & Selenium, total recoverable & $<10$ & U & $<200$ & $\mathrm{U}$ & $<10$ & $\mathrm{U}$ & & & & & $\mathrm{ug} / \mathrm{L}$ & \\
\hline \multicolumn{14}{|c|}{ Organics } \\
\hline & Chlorobenzene & $<5$ & $\mathrm{U}$ & $<5$ & $\mathrm{U}$ & $<1$ & $U$ & $<1$ & JU L & $<E Q L$ & 1 & ug/L & ML \\
\hline & 1,1-Dichloroethane & $<5$ & $U$ & $<5$ & $\mathrm{U}$ & $<1$ & $U$ & $<1$ & JU L & $<E Q L$ & 1 & $\mathrm{ug} / \mathrm{L}$ & $M L$ \\
\hline & 1,1-Dichloroethylene & $<5$ & $\mathrm{U}$ & $<5$ & $\mathrm{U}$ & $<1$ & $\mathrm{U}$ & $<1$ & JU L & $<E Q L$ & 1 & $\mathrm{ug} / \mathrm{L}$ & $M L$ \\
\hline & trans- 1,2 -Dichloroethylene & $<5$ & $U$ & $<5$ & $\mathrm{U}$ & $<1$ & $\mathrm{U}$ & & & & & $\mathrm{ug} / \mathrm{L}$ & \\
\hline & PCB 1016 & & & & & & & & & & & & \\
\hline & PCB 1221 & & & & & & & & & & & & \\
\hline & РCB 1232 & & & & & & & & & & & & \\
\hline & РCB 1242 & & & & & & & & & & & & \\
\hline & PCB 1248 & & & & & & & & & & & & \\
\hline & PCB 1254 & & & & & & & & & & & & \\
\hline & PCB 1260 & & & & & & & & & & & & \\
\hline & 1,1,2,2-Tetrachloroethane & $<5$ & U & $<5$ & $\mathrm{U}$ & $<1$ & $\mathrm{U}$ & $<1$ & $J U L$ & $<E Q L$ & 1 & $\mathrm{ug} / \mathrm{L}$ & ML \\
\hline & Tetrachloroethylene & $<5$ & $\mathrm{U}$ & $<5$ & $U$ & $<.43$ & U V & $<1$ & JU L & $<E Q L$ & 1 & $\mathrm{ug} / \mathrm{L}$ & ML \\
\hline & 1,1,1-Trichloroethane & $<5$ & U & $<5$ & U & $<1$ & $\mathrm{U}$ & $<1$ & JU L & $<E Q L$ & 1 & $\mathrm{ug} / \mathrm{L}$ & ML \\
\hline & Trichloroethylene & $<5$ & $U$ & $<5$ & $\mathrm{U}$ & $<1$ & U & $<1$ & JU L & $<$ EQL & 1 & ug/L & $M L$ \\
\hline \multicolumn{14}{|c|}{ II. Monitoring Constituents } \\
\hline$\underline{\mathbf{S T}}$ & Parameter & 3096 & CLP EPA & $\underline{3097}$ & CLPEEA & $\underline{3 Q 98}$ & CLPEPA & $\underline{3099}$ & CLPPEPA & Filt. & $\underline{\text { DF }}$ & $\underline{\text { Unit }}$ & $\underline{\text { Lab }}$ \\
\hline \multicolumn{14}{|c|}{ Inorganics } \\
\hline & Aluminum, total recoverable & $<20$ & $U$ & $<20$ & $U$ & 130 & $\mathrm{~J}$ & $<200$ & $\mathrm{U}$ & $<$ EQL & 1 & $\mathrm{ug} / \mathrm{L}$ & EX \\
\hline & Nitrate-nitrite as nitrogen & 1740 & & 1530 & & 1160 & & 1230 & & $<2400$ & 5 & $\mathrm{ug} / \mathrm{L}$ & EX \\
\hline & Sodium, total recoverable & 2090 & & 1670 & & 1680 & & 1700 & & $<4600$ & 1 & $\mathrm{ug} / \mathrm{L}$ & EX \\
\hline & Sulfate & 315 & $J$ & $<5000$ & $\mathrm{U}$ & $<200$ & $\mathrm{U}$ & 331 & & $<3000$ & 1 & $\mathrm{ug} / \mathrm{L}$ & EX \\
\hline \multicolumn{14}{|c|}{ Radionuclides } \\
\hline & Gross alpha & 1.38 & & 5.16 & UI & .87 & UIJ & .427 & $\mathrm{u}$ & $<\mathrm{EQL}$ & 1 & $\mathrm{pCi} / \mathrm{L}$ & GP \\
\hline & Nonvolatile beta & .887 & UI & 4.52 & & & & 1.37 & $\mathrm{U}$ & $<E Q L$ & 1 & $\mathrm{pCi} / \mathrm{L}$ & GP \\
\hline & Radium, total alpha-emitting & .5 & Ui & 1.59 & $J$ & .78 & & .2 & $\mathrm{U}$ & $<E Q L$ & 1 & $\mathrm{pCi} / \mathrm{L}$ & GP \\
\hline
\end{tabular}

Notes: Concentrations in bold italics exceed the groundwater protection or monitoring constituent standards listed in Appendix A. Synchronous water levels are measured over a 3-5 day period or less. Dilution factors, Laboratory, and Filtered Data are for Groundwater Protection Standards First Quarter 2000 and Monitoring Constituents Third Quarter 1999 data only.

$+=$ exceeded the Groundwater Protection Standards (First Quarter 2000) or Monitoring Constituents (Third Quarter 1999) listed in Appendix A. 
Table D-4. Groundwater Monitoring Results for Plume Definition Wells, M-Area HWMF (Cont.) WELL MSB 49A

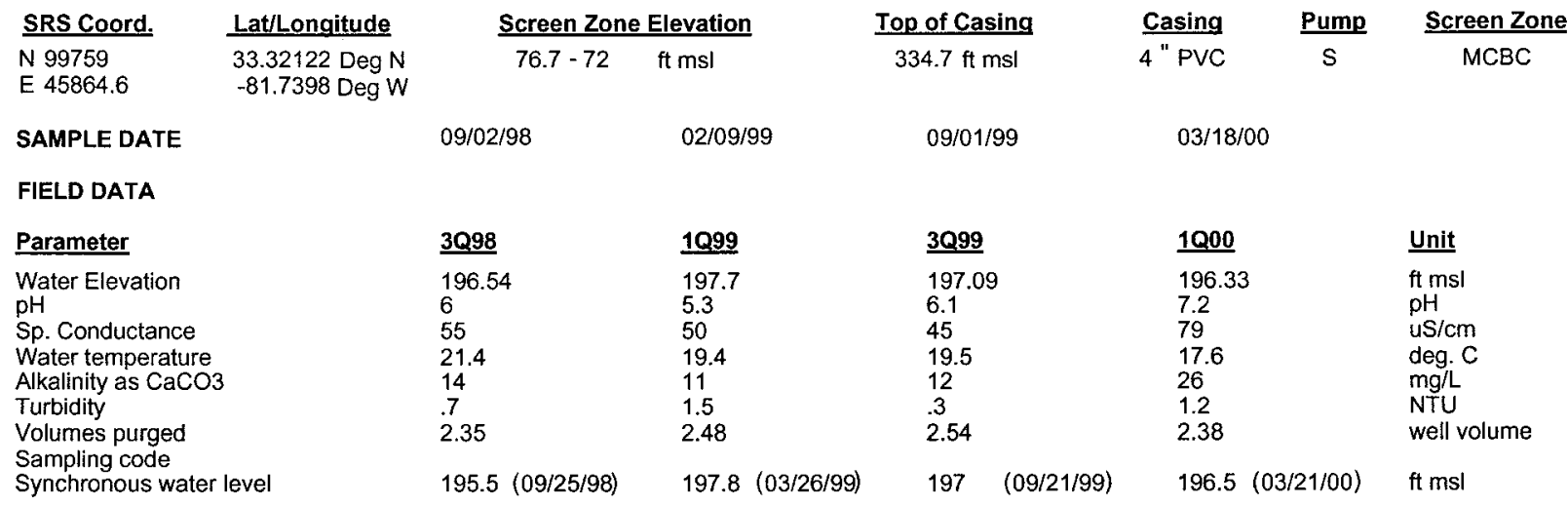

ANALYTICAL DATA

I. Groundwater Protection Standard

261 Appendix VIII/264 Appendix IX Hazardous Constituents

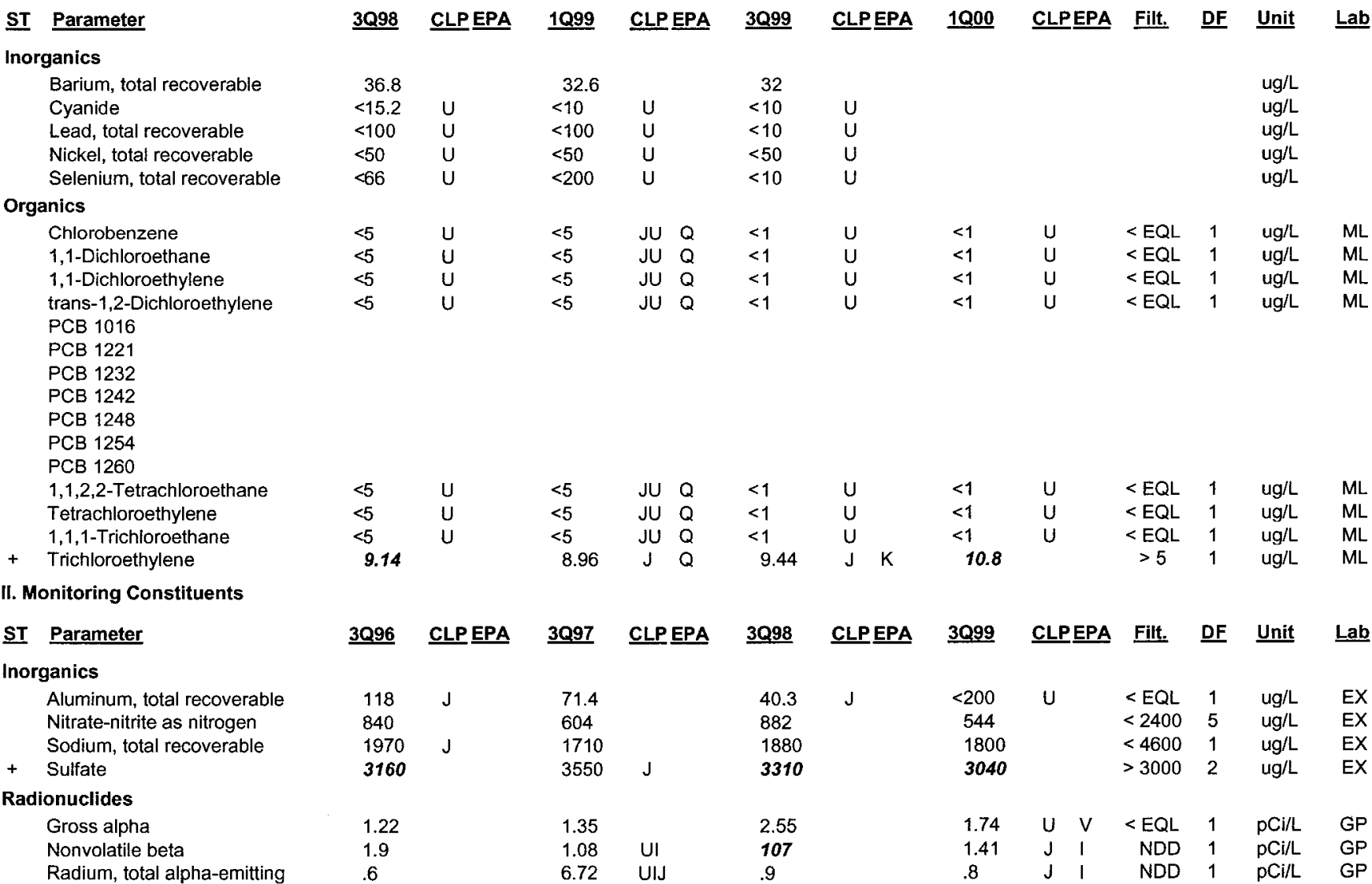

Notes: Concentrations in bold italics exceed the groundwater protection or monitoring constituent standards listed in Appendix A. Synchronous water levels are measured over a 3-5 day period or less. Dilution factors, Laboratory, and Filtered Data are for Groundwater Protection Standards First Quarter 2000 and Monitoring Constituents Third Quarter 1999 data only.

$+=$ exceeded the Groundwater Protection Standards (First Quarter 2000) or Monitoring Constituents (Third Quarter 1999) listed in Appendix A. 
Table D-4. Groundwater Monitoring Results for Plume Definition Wells, M-Area HWMF (Cont.) WELL MSB 49B

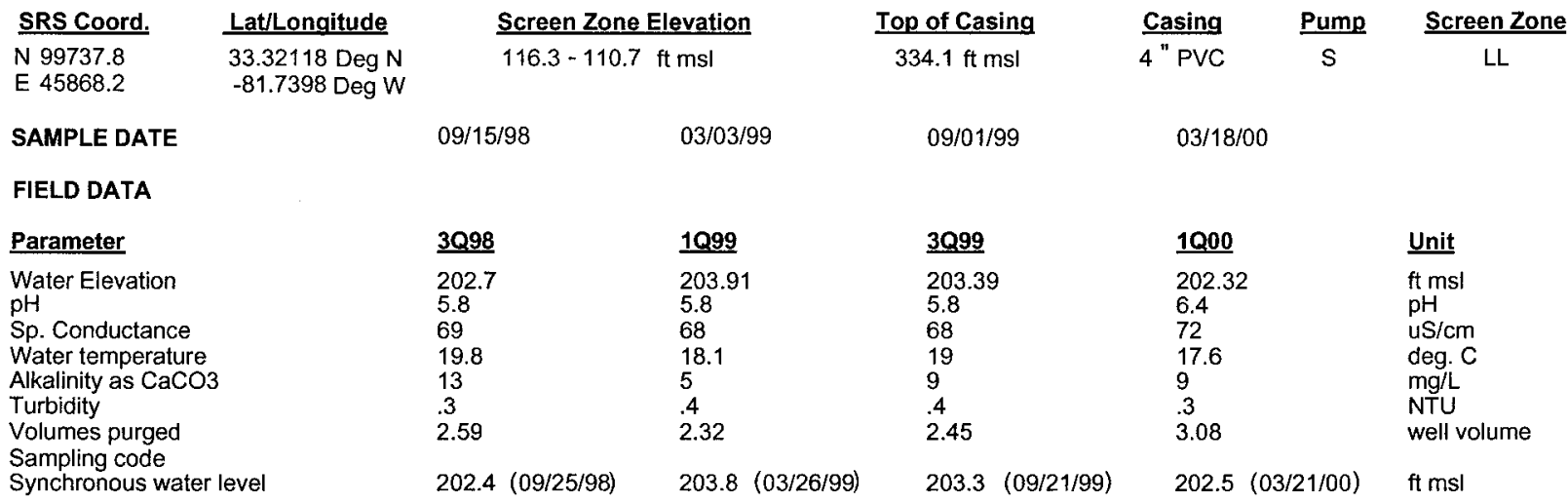

ANALYTICAL DATA

I. Groundwater Protection Standard

261 Appendix VIII/264 Appendix IX Hazardous Constituents

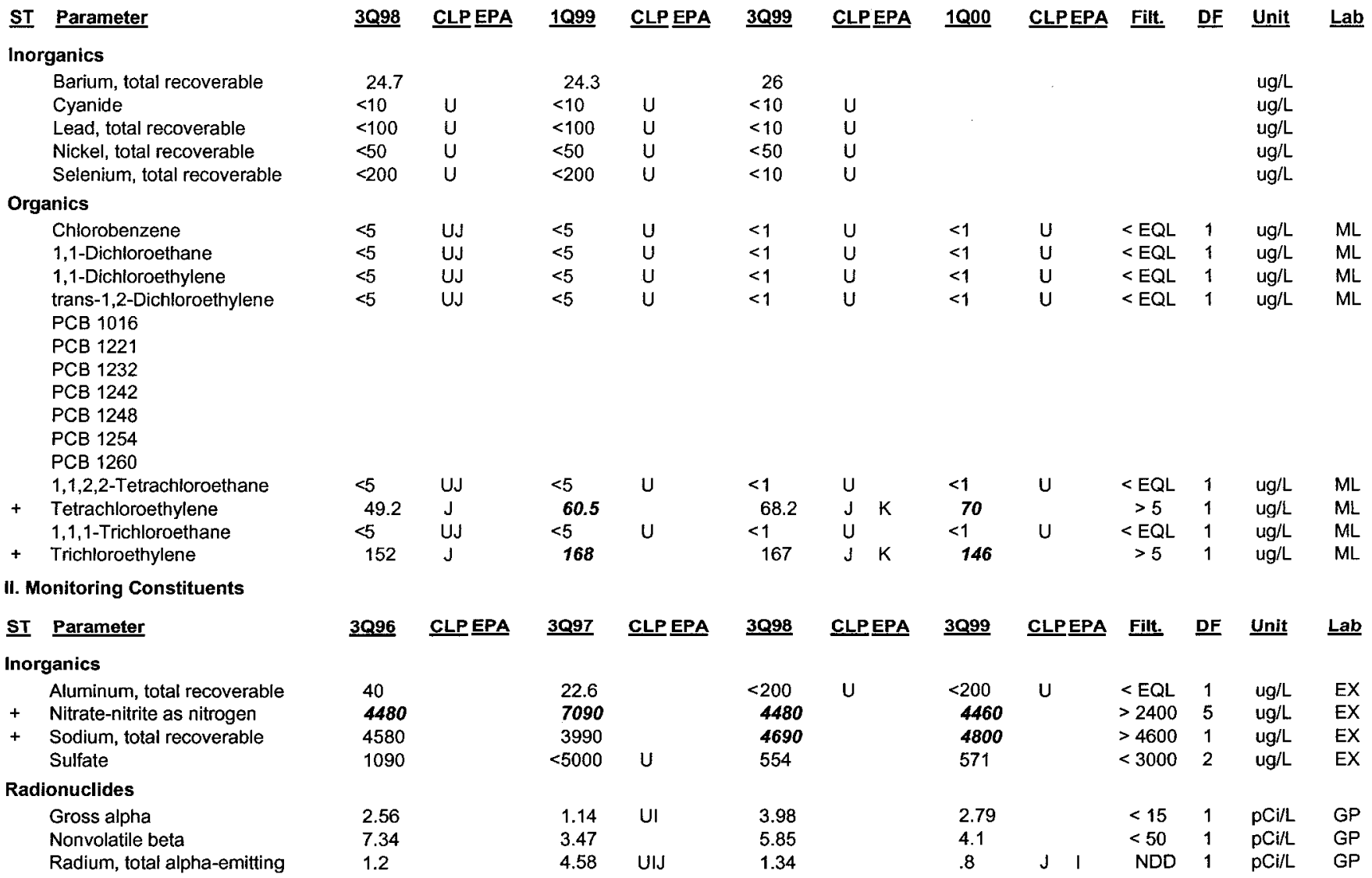

Notes: Concentrations in bold italics exceed the groundwater protection or monitoring constituent standards listed in Appendix A. Synchronous water levels are measured over a 3-5 day period or less. Dilution factors, Laboratory, and Filtered Data are for Groundwater Protection Standards First Quarter 2000 and Monitoring Constituents Third Quarter 1999 data only.

$+=$ exceeded the Groundwater Protection Standards (First Quarter 2000) or Monitoring Constituents (Third Quarter 1999) listed in Appendix A. 
Table D-4. Groundwater Monitoring Results for Plume Definition Wells, M-Area HWMF (Cont.) WELL MSB 49D

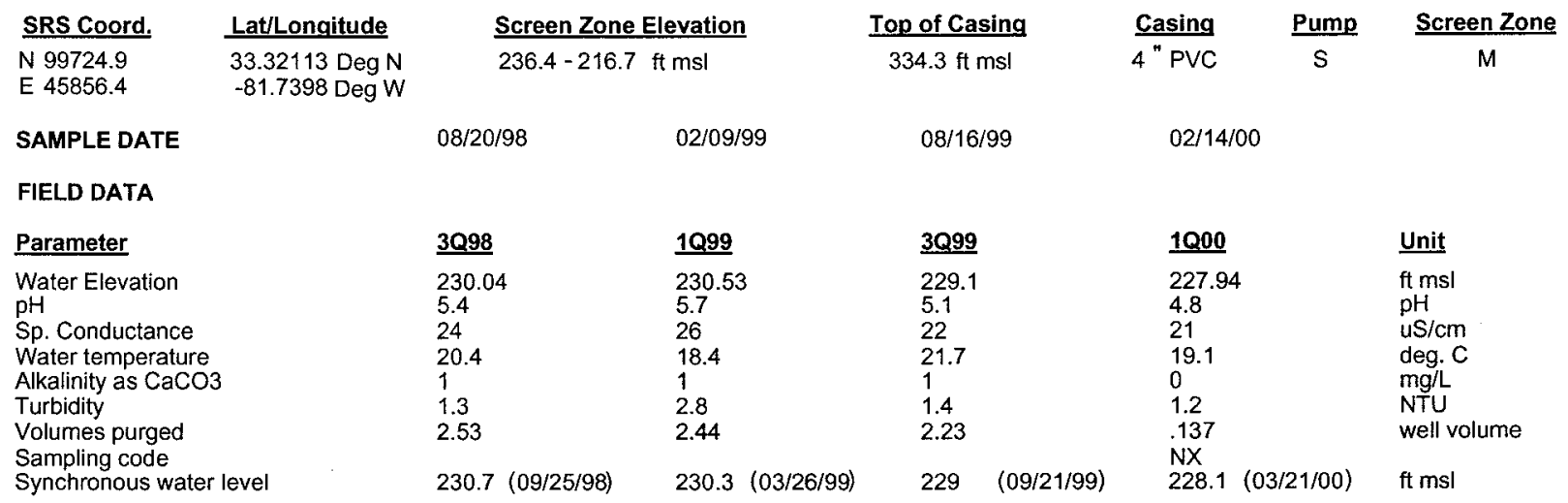

ANALYTICAL DATA

I. Groundwater Protection Standard

261 Appendix VIII/264 Appendix IX Hazardous Constituents

\begin{tabular}{|c|c|c|c|c|c|c|c|c|c|c|c|c|c|}
\hline$\underline{\mathrm{ST}}$ & Parameter & $\underline{3 Q 98}$ & CLPEPA & 1099 & $\underline{\text { CLP EPA }}$ & $\underline{3 Q 99}$ & CLPEPA & 1000 & CLPEPA & Filt. & $\underline{\text { DF }}$ & Unit & Lab \\
\hline \multicolumn{14}{|c|}{ Inorganics } \\
\hline & Barium, total recoverable & 8.91 & $\mathbf{J}$ & 11.4 & & 11 & & & & & & $u g / L$ & \\
\hline & Cyanide & $<10$ & $\mathrm{U}$ & $<10$ & $U$ & $<10$ & $U$ & & & & & ug/L & \\
\hline & Lead, total recoverable & $<10$ & $\mathrm{U}$ & $<100$ & $U$ & $<5.02$ & U V & & & & & $\mathrm{ug} / \mathrm{L}$ & \\
\hline & Nickel, total recoverable & 9.16 & $J$ & $<50$ & $\mathrm{U}$ & $<50$ & $\mathrm{U}$ & & & & & $\mathrm{ug} / \mathrm{L}$ & \\
\hline & Selenium, total recoverable & $<10$ & U & $<200$ & $U$ & $<5$ & $U$ & & & & & ug/L & \\
\hline \multicolumn{14}{|c|}{ Organics } \\
\hline & Chlorobenzene & $<5$ & $u$ & $<5$ & JU $Q$ & $<1$ & $u$ & $<1$ & $\mathrm{U}$ & $<\mathrm{EQL}$ & 1 & $u g / L$ & ML \\
\hline & 1,1-Dichloroethane & $<5$ & U & $<5$ & JU $Q$ & $<1$ & $U$ & $<1$ & U & $<\mathrm{EQL}$ & 1 & $\mathrm{ug} / \mathrm{L}$ & ML \\
\hline & 1,1-Dichloroethylene & $<5$ & $\cup$ & $<5$ & JU $Q$ & $<1$ & $\mathrm{U}$ & $<1$ & $\mathrm{U}$ & $<\mathrm{EQL}$ & 1 & $\mathrm{ug} / \mathrm{L}$ & ML \\
\hline & trans-1,2-Dichloroethylene & $<5$ & $U$ & $<5$ & $J U Q$ & $<1$ & $\mathrm{U}$ & $<1$ & $\mathrm{U}$ & $<\mathrm{EQL}$ & 1 & ug/L & $M L$ \\
\hline & PCB 1016 & & & & & & & & & & & & \\
\hline & PCB 1221 & & & & & & & & & & & & \\
\hline & PCB 1232 & & & & & & & & & & & & \\
\hline & PCB 1242 & & & & & & & & & & & & \\
\hline & PCB 1248 & & & & & & & & & & & & \\
\hline & PCB 1254 & & & & & & & & & & & & \\
\hline & PCB 1260 & & & & & & & & & & & & \\
\hline & 1,1,2,2-Tetrachloroethane & $<5$ & $U$ & $<5$ & $J U Q$ & $<1$ & $\mathrm{U}$ & $<1$ & $\mathrm{u}$ & $<\mathrm{EQL}$ & 1 & $\mathrm{ug} / \mathrm{L}$ & $M L$ \\
\hline & Tetrachloroethylene & $<5$ & $\mathrm{U}$ & $<5$ & $J U Q$ & $<1$ & $U$ & $<1$ & $\mathrm{U}$ & $<$ EQL & 1 & $\mathrm{ug} / \mathrm{L}$ & ML \\
\hline & 1,1,1-Trichloroethane & $<5$ & $U$ & $<5$ & JU Q & $<1$ & $U$ & $<1$ & $\mathrm{U}$ & $<\mathrm{EQL}$ & 1 & $\mathrm{ug} / \mathrm{L}$ & M \\
\hline & Trichloroethylene & $<5$ & U & $<5$ & JU $Q$ & $<1$ & $U$ & $<1$ & $\mathrm{U}$ & $<\mathrm{EQL}$ & 1 & $\mathrm{ug} / \mathrm{L}$ & ML \\
\hline \multicolumn{14}{|c|}{ II. Monitoring Constituents } \\
\hline$\underline{\mathbf{S T}}$ & Parameter & $\underline{3 Q 96}$ & CLPEPA & $\underline{3 Q 97}$ & CLP EPA & $\underline{3 Q 98}$ & CLPEPA & 3099 & CLPEEA & Filt. & DF & Unit & \\
\hline \multicolumn{14}{|c|}{ Inorganics } \\
\hline & Aluminum, total recoverable & $<20$ & $\mathrm{U}$ & 41.2 & & $<200$ & U & $<200$ & U & $<\mathrm{EQL}$ & 1 & $\mathrm{ug} / \mathrm{L}$ & $E$ \\
\hline & Nitrate-nitrite as nitrogen & 1060 & & 862 & & 716 & & 838 & & $<2400$ & 5 & $\mathrm{ug} / \mathrm{L}$ & $\mathrm{E}$ \\
\hline & Sodium, total recoverable & 1230 & & 1290 & & 2340 & & 1700 & & $<4600$ & 1 & $\mathrm{ug} / \mathrm{L}$ & EX \\
\hline & Sulfate & 200 & $\mathrm{~J}$ & $<5000$ & $\mathrm{U}$ & 354 & $J$ & 192 & J 1 & NDD & 1 & $\mathrm{ug} / \mathrm{L}$ & EX \\
\hline \multicolumn{14}{|c|}{ Radionuclides } \\
\hline & Gross alpha & 3.47 & & 1.79 & & 8.06 & & 3.55 & & $<15$ & 1 & $\mathrm{pCi} / \mathrm{L}$ & G \\
\hline & Nonvolatile beta & 1.47 & UI & .78 & UI & 5.88 & & 2.54 & & $<50$ & 1 & $\mathrm{pCi} / \mathrm{L}$ & GP \\
\hline & Radium, total alpha-emitting & 1.7 & & 4.56 & UIJ & 1.18 & & 1.6 & & $<5$ & 1 & $\mathrm{pCi} / \mathrm{L}$ & \\
\hline
\end{tabular}

Notes: Concentrations in bold italics exceed the groundwater protection or monitoring constituent standards listed in Appendix A. Synchronous water levels are measured over a 3-5 day period or less. Dilution factors, Laboratory, and Filtered Data are for Groundwater Protection Standards First Quarter 2000 and Monitoring Constituents Third Quarter 1999 data only.

$+=$ exceeded the Groundwater Protection Standards (First Quarter 2000) or Monitoring Constituents (Third Quarter 1999) listed in Appendix A. 
Table D-4. Groundwater Monitoring Results for Plume Definition Wells, M-Area HWMF (Cont.) WELL MSB 51B

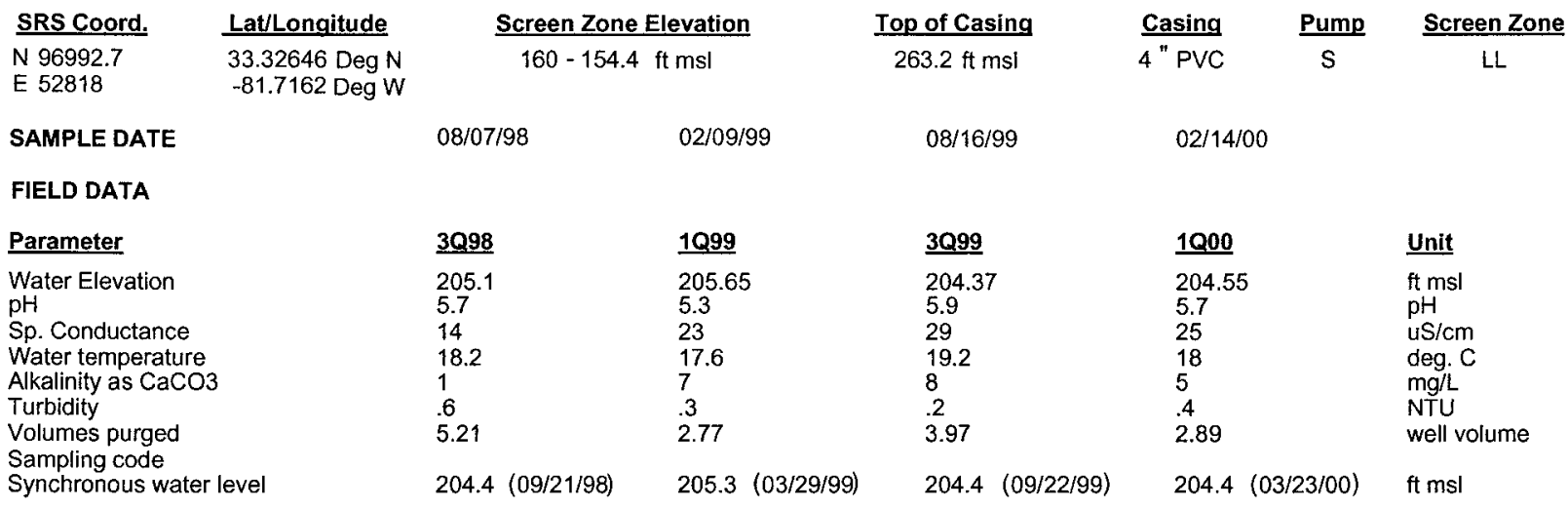

ANALYTICAL DATA

I. Groundwater Protection Standard

261 Appendix VIII/264 Appendix IX Hazardous Constituents

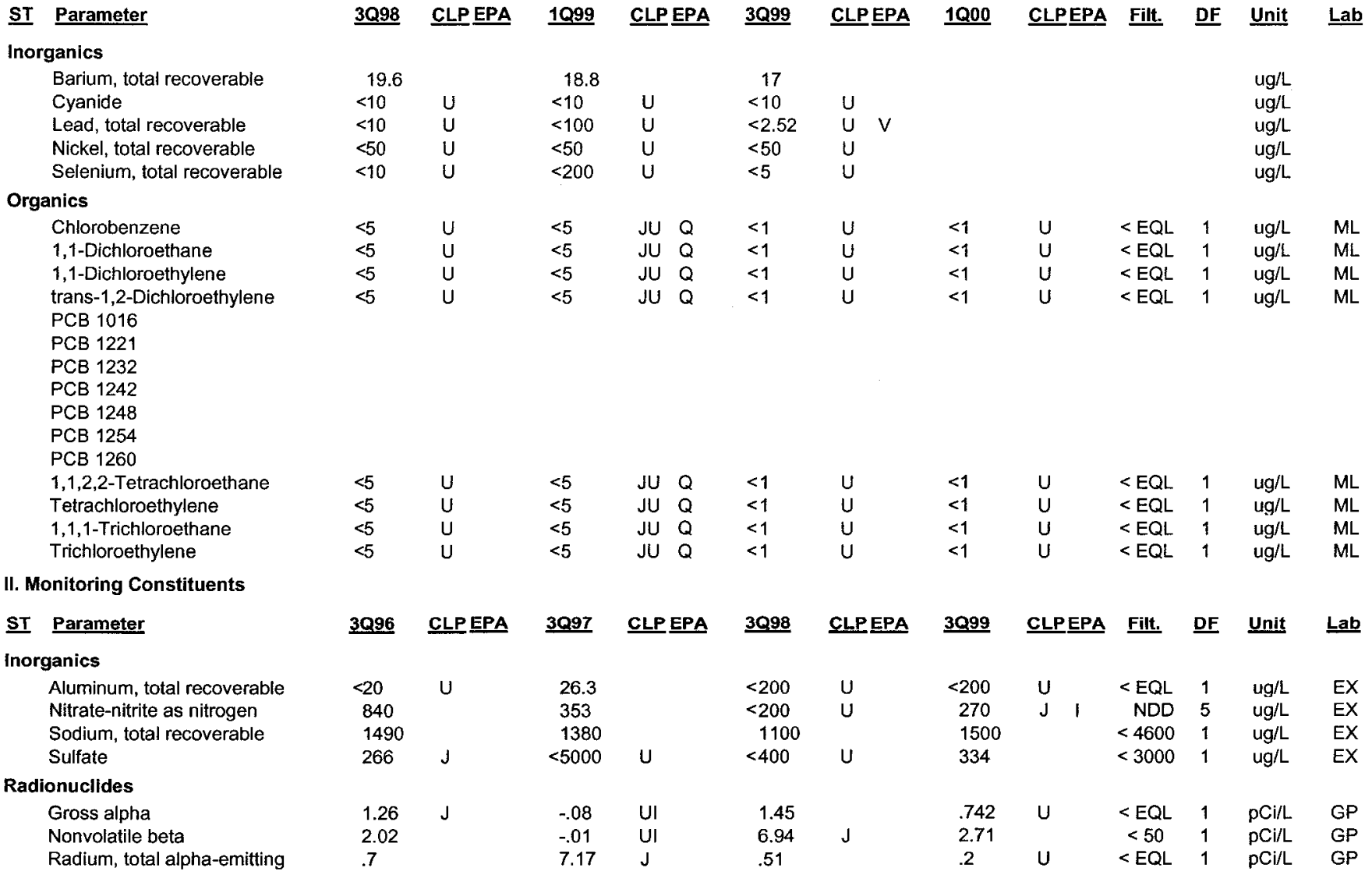

Notes: Concentrations in bold italics exceed the groundwater protection or monitoring constituent standards listed in Appendix A. Synchronous water levels are measured over a 3-5 day period or less. Dilution factors, Laboratory, and Filtered Data are for Groundwater Protection Standards First Quarter 2000 and Monitoring Constituents Third Quarter 1999 data only.

$+=$ exceeded the Groundwater Protection Standards (First Quarter 2000) or Monitoring Constituents (Third Quarter 1999) listed in Appendix A. 
Table D-4. Groundwater Monitoring Results for Plume Definition Wells, M-Area HWMF (Cont.) WELL MSB 52B

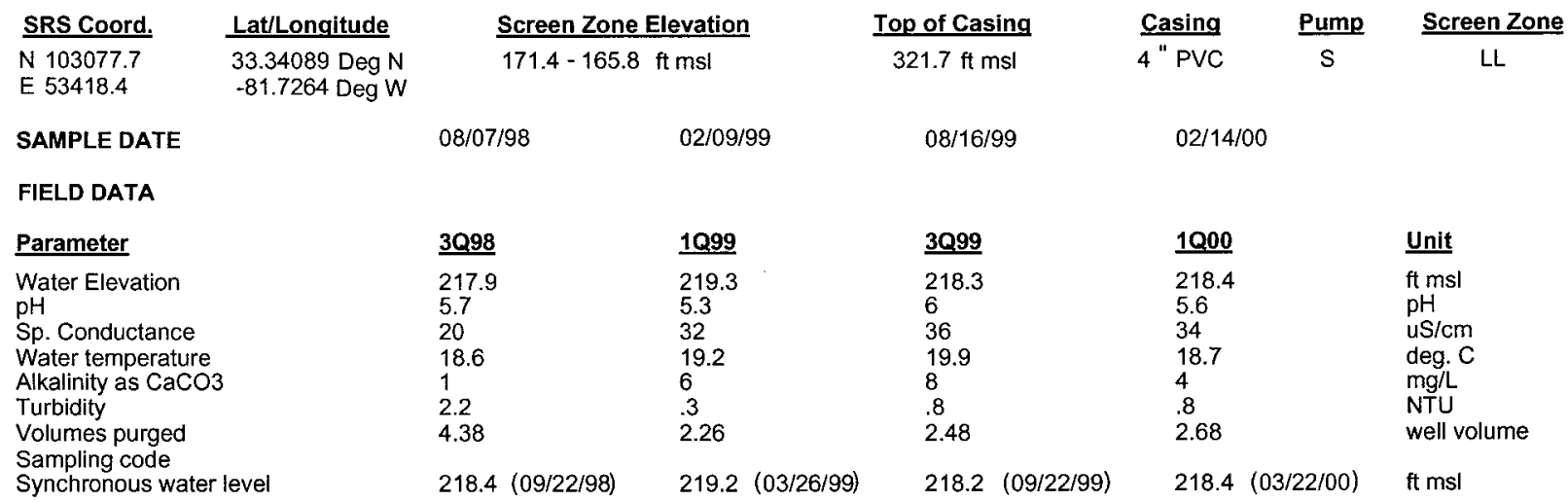

ANALYTICAL DATA

I. Groundwater Protection Standard

261 Appendix VIII/264 Appendix IX Hazardous Constituents

\begin{tabular}{|c|c|c|c|c|c|c|c|c|c|c|c|c|c|c|c|c|}
\hline \multirow{2}{*}{$\underbrace{S T}_{\text {Inorg }}$} & Parameter & \multirow[t]{2}{*}{$\underline{3 Q 98}$} & \multirow[t]{2}{*}{ CLPEPA } & \multirow[t]{2}{*}{1099} & \multicolumn{2}{|c|}{$\underline{\text { CLP EPA }}$} & \multirow[t]{2}{*}{$\underline{3 Q 99}$} & \multicolumn{2}{|c|}{ CLPEPA } & \multirow[t]{2}{*}{1000} & \multicolumn{2}{|c|}{ CLPEPA } & \multirow[t]{2}{*}{ Filt. } & \multirow[t]{2}{*}{$\underline{\text { DF }}$} & \multirow[t]{2}{*}{ Unit } & \multirow[t]{2}{*}{$\underline{\text { Lab }}$} \\
\hline & ganics & & & & & & & & & & & & & & & \\
\hline & Barium, total recoverable & 6.96 & $J$ & 9.84 & $\mathrm{~J}$ & I & 7.3 & $J$ & 1 & & & & & & ug/L & \\
\hline & Cyanide & $<10$ & $U$ & $<10$ & $\mathrm{U}$ & & $<10$ & $U$ & & & & & & & ug/L & \\
\hline & Lead, total recoverable & $<10$ & $U$ & $<100$ & $\mathrm{U}$ & & $<1.18$ & $\mathrm{U}$ & V & & & & & & ug/L & \\
\hline & Nickel, total recoverable & $<50$ & U & $<50$ & $\mathrm{U}$ & & $<50$ & $\mathrm{U}$ & & & & & & & $\mathrm{ug} / \mathrm{L}$ & \\
\hline & Selenium, total recoverable & $<10$ & U & $<200$ & $\mathrm{U}$ & & $<5$ & $U$ & & & & & & & $\mathrm{ug} / \mathrm{L}$ & \\
\hline \multicolumn{17}{|c|}{ Organics } \\
\hline & Chlorobenzene & $<5$ & $\mathrm{U}$ & $<5$ & JU & $Q$ & $<1$ & $\mathrm{U}$ & & $<1$ & $\mathrm{U}$ & & $<\mathrm{EQL}$ & 1 & ug/L & $\mathrm{ML}$ \\
\hline & 1,1-Dichloroethane & $<5$ & $\mathrm{U}$ & $<5$ & JU & $Q$ & $<1$ & $\mathrm{U}$ & & $<1$ & $\mathrm{U}$ & & $<\mathrm{EQL}$ & 1 & ug/L & ML \\
\hline & 1,1-Dichloroethylene & $<5$ & $\mathrm{U}$ & $<5$ & JU & $Q$ & $<1$ & $U$ & & $<1$ & $\mathrm{U}$ & & $<\mathrm{EQL}$ & 1 & ug/L & $M L$ \\
\hline & trans-1,2-Dichloroethylene & $<5$ & 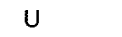 & $<5$ & JU & $Q$ & $<1$ & $\mathrm{U}$ & & $<1$ & $\mathrm{U}$ & & $<\mathrm{EQL}$ & 1 & $\mathrm{ug} / \mathrm{L}$ & ML \\
\hline & PCB 1016 & & & & & & & & & & & & & & & \\
\hline & PCB 1221 & & & & & & & & & & & & & & & \\
\hline & PCB 1232 & & & & & & & & & & & & & & & \\
\hline & PCB 1242 & & & & & & & & & & & & & & & \\
\hline & PCB 1248 & & & & & & & & & & & & & & & \\
\hline & PCB 1254 & & & & & & & & & & & & & & & \\
\hline & PCB 1260 & & & & & & & & & & & & & & & \\
\hline & $1,1,2,2$-Tetrachloroethane & $<5$ & $\mathrm{U}$ & $<5$ & JU & $Q$ & $<1$ & $\mathrm{U}$ & & $<1$ & $\mathrm{U}$ & & $<\mathrm{EQL}$ & 1 & ug/L & ML \\
\hline & Tetrachloroethylene & 3.26 & $\mathrm{~J}$ & $<5$ & JU & $Q$ & 2.67 & $J$ & K & 2.57 & $J$ & $\mathrm{~K}$ & NDD & 1 & ug/L & ML \\
\hline & $1,1,1$-Trichloroethane & $<5$ & $\mathrm{U}$ & $<5$ & JU & $Q$ & $<1$ & $U$ & & $<1$ & U & & $<\mathrm{EQL}$ & 1 & $\mathrm{ug} / \mathrm{L}$ & ML \\
\hline & Trichloroethylene & 2.15 & $\mathrm{~J}$ & 1.51 & $\mathrm{~J}$ & $1 Q$ & 1.82 & $\mathrm{~J}$ & $\mathrm{~K}$ & 1.77 & $\mathrm{~J}$ & $\mathrm{~K}$ & NDD & 1 & $\mathrm{ug} / \mathrm{L}$ & ML \\
\hline \multicolumn{17}{|c|}{ II. Monitoring Constituents } \\
\hline ST & Parameter & $\underline{3096}$ & CLPEPA & $\underline{3097}$ & $\underline{\text { CLP }}$. & EPA & 3098 & $\underline{\text { CLP }}$ & EPA & $\underline{3 Q 99}$ & $\underline{\text { CLF }}$ & EPA & Filt. & $\underline{\mathrm{DF}}$ & $\underline{\text { Unit }}$ & \\
\hline \multicolumn{17}{|c|}{ Inorganics } \\
\hline & Aluminum, total recoverable & $<20$ & $\mathrm{U}$ & 113 & & & $<200$ & $\mathrm{U}$ & & $<200$ & $\mathrm{U}$ & & $<E Q L$ & 1 & $u g / L$ & EX \\
\hline & Nitrate-nitrite as nitrogen & 1080 & & 896 & & & 610 & & & 574 & & & $<2400$ & 5 & $\mathrm{ug} / \mathrm{L}$ & EX \\
\hline & Sodium, total recoverable & 2220 & & 2230 & & & 2240 & & & 2500 & & & $<4600$ & 1 & $\mathrm{ug} / \mathrm{L}$ & EX \\
\hline & Sulfate & 230 & $J$ & $<5000$ & $\mathrm{U}$ & & $<400$ & $U$ & & 217 & & & $<3000$ & 1 & $\mathrm{ug} / \mathrm{L}$ & EX \\
\hline \multicolumn{16}{|c|}{ Radionuclides } & \\
\hline & Gross alpha & 1.69 & & 1.74 & & & 1.48 & & & .716 & $J$ & $\mathbf{I}$ & NDD & 1 & $\mathrm{pCi} / \mathrm{L}$ & GP \\
\hline & Nonvolatile beta & .757 & $\mathrm{UI}$ & 7.8 & & & 2.16 & UJ & & 3.68 & & & $<50$ & 1 & $p C i / L$ & GP \\
\hline & Radium, total alpha-emitting & 0 & $\mathrm{UI}$ & 1.97 & UIJ & & .37 & & & .4 & $\mathrm{U}$ & & $<E Q L$ & 1 & $\mathrm{pCi} / \mathrm{L}$ & \\
\hline
\end{tabular}

Notes: Concentrations in bold italics exceed the groundwater protection or monitoring constituent standards listed in Appendix A. Synchronous water levels are measured over a 3-5 day period or less. Dilution factors, Laboratory, and Filtered Data are for Groundwater Protection Standards First Quarter 2000 and Monitoring Constituents Third Quarter 1999 data only.

+ = exceeded the Groundwater Protection Standards (First Quarter 2000) or Monitoring Constituents (Third Quarter 1999) listed in Appendix A. 
Table D-4. Groundwater Monitoring Results for Plume Definition Wells, M-Area HWMF (Cont.) WELL MSB 53B

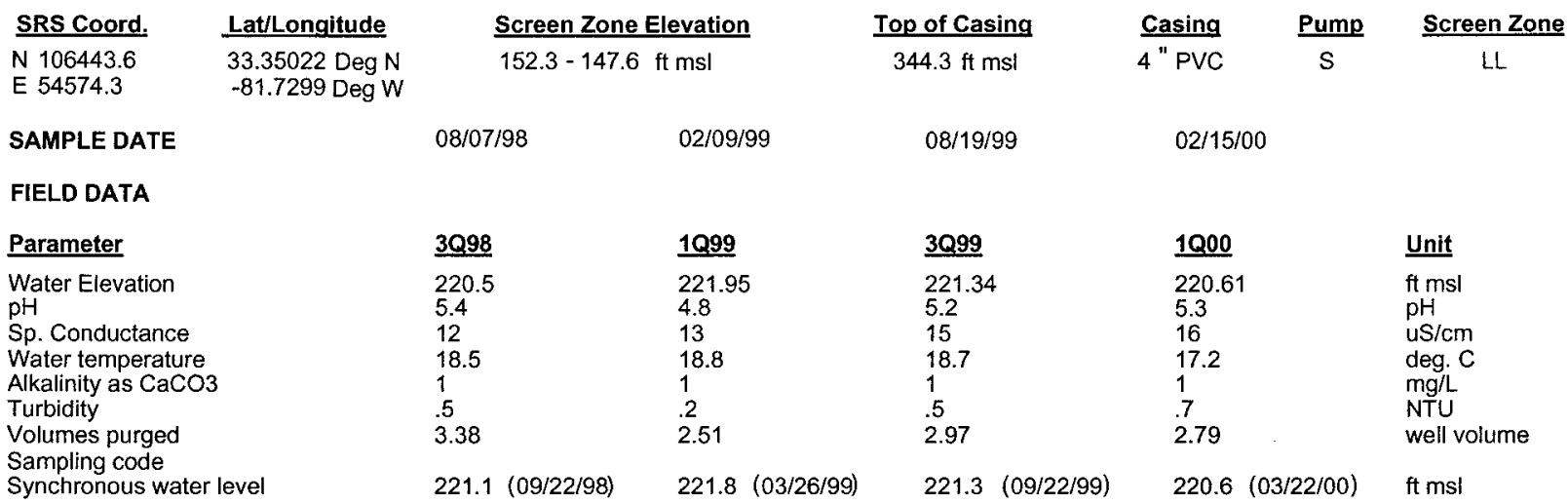

ANALYTICAL DATA

I. Groundwater Protection Standard

261 Appendix VIII/264 Appendix IX Hazardous Constituents

\begin{tabular}{|c|c|c|c|c|c|c|c|c|c|c|c|c|c|c|c|}
\hline \multirow{2}{*}{$\frac{\text { ST }}{\text { Inor }}$} & Parameter & \multirow[t]{2}{*}{$\underline{3098}$} & \multirow[t]{2}{*}{ CLP EPA } & \multirow[t]{2}{*}{$\underline{1 Q 99}$} & \multicolumn{2}{|c|}{ CLP EPA } & \multirow[t]{2}{*}{$\underline{3 Q 99}$} & CLPEPA & \multirow[t]{2}{*}{1000} & \multicolumn{2}{|c|}{ CLPEPA } & \multirow[t]{2}{*}{ Fitt. } & \multirow[t]{2}{*}{ DF } & \multirow[t]{2}{*}{ Unit } & \multirow[t]{2}{*}{ Lab } \\
\hline & ganics & & & & & & & & & & & & & & \\
\hline & Barium, total recoverable & 2.88 & $\mathrm{~J}$ & 5.11 & $J$ & 1 & 4.3 & J I & & & & & & $u g / L$ & \\
\hline & Cyanide & $<10$ & $U$ & $<10$ & $\mathrm{U}$ & & $<10$ & $U$ & & & & & & $\mathrm{ug} / \mathrm{L}$ & \\
\hline & Lead, total recoverable & $<10$ & $U$ & $<100$ & $U$ & & $<2.45$ & U V & & & & & & ug/L & \\
\hline & Nickel, total recoverable & $<50$ & $\mathrm{U}$ & $<50$ & $\mathrm{U}$ & & $<50$ & $\mathrm{U}$ & & & & & & ug/L & \\
\hline & Selenium, total recoverable & $<10$ & $\mathrm{U}$ & $<200$ & $U$ & & $<5$ & U & & & & & & ug/L & \\
\hline \multicolumn{16}{|c|}{ Organics } \\
\hline & Chlorobenzene & $<5$ & $U$ & $<5$ & JU & $Q$ & $<1$ & $U$ & $<1$ & $\mathrm{u}$ & & $<\mathrm{EQL}$ & 1 & $\mathrm{ug} / \mathrm{L}$ & ML. \\
\hline & 1,1-Dichloroethane & $<5$ & $U$ & $<5$ & JU & $Q$ & $<1$ & $\mathrm{U}$ & $<1$ & $\mathrm{u}$ & & $<E Q L$ & 1 & $\mathrm{ug} / \mathrm{L}$ & ML \\
\hline & 1,1-Dichloroethylene & $<5$ & $\mathrm{U}$ & $<5$ & JU & $Q$ & $<1$ & $\mathrm{U}$ & $<1$ & $\mathrm{U}$ & & $<\mathrm{EQL}$ & 1 & $\mathrm{ug} / \mathrm{L}$ & ML \\
\hline & trans-1,2-Dichloroethylene & $<5$ & $U$ & $<5$ & $\mathrm{JU}$ & $\mathrm{Q}$ & $<1$ & $U$ & $<1$ & $u$ & & $<\mathrm{EQL}$ & 1 & $\mathrm{ug} / \mathrm{L}$ & ML. \\
\hline & PCB 1016 & & & & & & & & & & & & & & \\
\hline & PCB 1221 & & & & & & & & & & & & & & \\
\hline & PCB 1232 & & & & & & & & & & & & & & \\
\hline & PCB 1242 & & & & & & & & & & & & & & \\
\hline & РCB 1248 & & & & & & & & & & & & & & \\
\hline & PCB 1254 & & & & & & & & & & & & & & \\
\hline & PCB 1260 & & & & & & & & & & & & & & \\
\hline & $1,1,2,2$-Tetrachloroethane & $<5$ & $U$ & $<5$ & JU & $Q$ & $<1$ & $U$ & $<1$ & $\mathrm{U}$ & & $<\mathrm{EQL}$ & 1 & $\mathrm{ug} / \mathrm{L}$ & ML \\
\hline & Tetrachloroethylene & $<5$ & $U$ & $<5$ & JU & $Q$ & $<1$ & $U$ & $<1$ & $\mathrm{U}$ & & $<\mathrm{EQL}$ & 1 & $\mathrm{ug} / \mathrm{L}$ & $M L$ \\
\hline & 1,1,1-Trichloroethane & $<5$ & $\mathrm{U}$ & $<5$ & JU & $Q$ & $<1$ & $U$ & $<1$ & $\mathrm{U}$ & & $<\mathrm{EQL}$ & 1 & $\mathrm{ug} / \mathrm{L}$ & $M L$ \\
\hline & Trichloroethylene & $<5$ & $\mathrm{U}$ & $<5$ & $\mathrm{JU}$ & $Q$ & $<1$ & U & $<1$ & $U$ & & $<\mathrm{EQL}$ & 1 & $\mathrm{ug} / \mathrm{L}$ & $M L$ \\
\hline \multicolumn{16}{|c|}{ II. Monitoring Constituents } \\
\hline$\underline{\text { ST }}$ & Parameter & $\underline{3096}$ & CLPEPA & $\underline{3097}$ & $\underline{\text { CLP }}$ & EPA & $\underline{3098}$ & CLPEPA & $\underline{3099}$ & CLP & EPA & Filt. & $\underline{\text { DF }}$ & $\underline{\text { Unit }}$ & $\underline{\text { Lab }}$ \\
\hline \multicolumn{16}{|c|}{ Inorganics } \\
\hline & Aluminum, total recoverable & $<20$ & U & 13.4 & $J$ & & $<200$ & $U$ & $<200$ & $\mathrm{U}$ & & $<\mathrm{EQL}$ & 1 & $\mathrm{ug} / \mathrm{L}$ & EX \\
\hline & Nitrate-nitrite as nitrogen & 920 & & 153 & & & $<200$ & $\mathrm{U}$ & 202 & $\mathrm{~J}$ & I & NDD & 5 & ug/L & EX \\
\hline & Sodium, total recoverable & 1900 & & 1550 & & & 1100 & & 1700 & & & $<4600$ & 1 & $\mathrm{ug} / \mathrm{L}$ & EX \\
\hline & Sulfate & 168 & $J$ & $<5000$ & $\mathrm{U}$ & & $<400$ & $U$ & 1350 & & & $<3000$ & 1 & $\mathrm{ug} / \mathrm{L}$ & EX \\
\hline \multicolumn{16}{|c|}{ Radionuclides } \\
\hline & Gross alpha & .991 & & -.43 & UI & & 1.47 & & .883 & $J$ & 1 & NDD & 1 & $\mathrm{pCi} / \mathrm{L}$ & GP \\
\hline & Nonvolatile beta & 1.78 & & 4.42 & & & 1.31 & UIJ & .737 & $U$ & & $<\mathrm{EQL}$ & 1 & $\mathrm{pCi} / \mathrm{L}$ & GP \\
\hline & Radium, total alpha-emitting & .4 & & 1.28 & UIJ & & .51 & & .3 & $\mathrm{U}$ & & $<E Q L$ & 1 & $\mathrm{pCi} / \mathrm{L}$ & GP \\
\hline
\end{tabular}

Notes: Concentrations in bold italics exceed the groundwater protection or monitoring constituent standards listed in Appendix A. Synchronous water levels are measured over a 3-5 day period or less. Dilution factors, Laboratory, and Filtered Data are for Groundwater Protection Standards First Quarter 2000 and Monitoring Constituents Third Quarter 1999 data only.

+ = exceeded the Groundwater Protection Standards (First Quarter 2000) or Monitoring Constituents (Third Quarter 1999) listed in Appendix A. 
Table D-4. Groundwater Monitoring Results for Plume Definition Wells, M-Area HWMF (Cont.) WELL MSB 53C

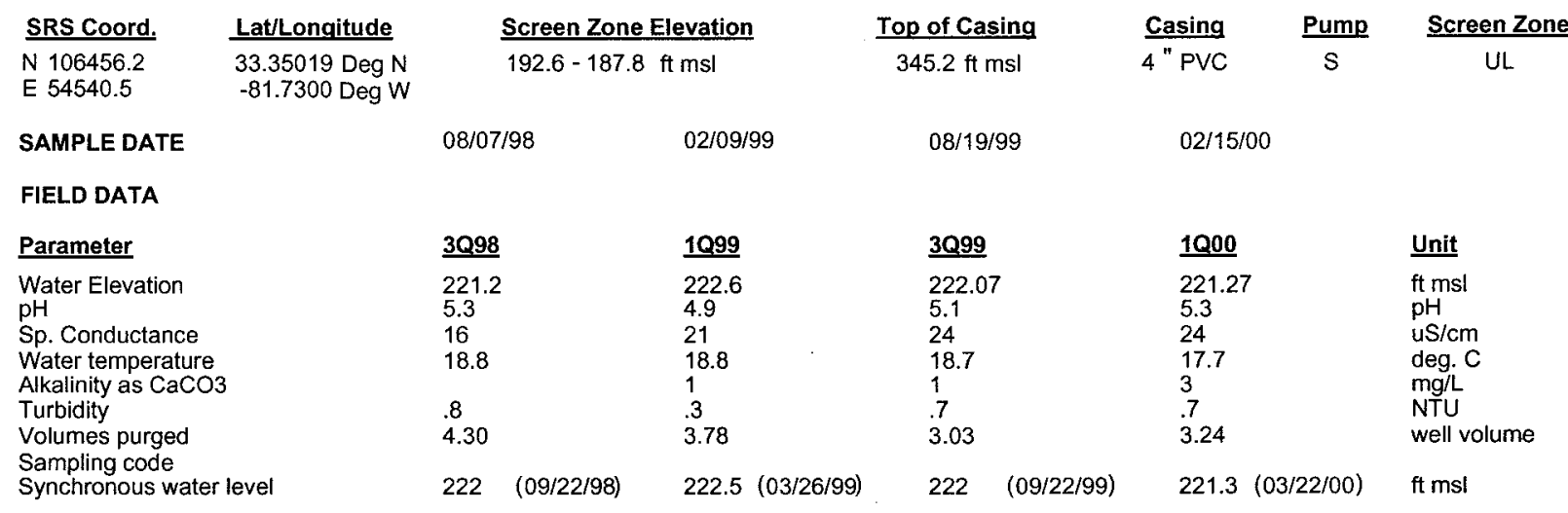

ANALYTICAL DATA

I. Groundwater Protection Standard

261 Appendix VIII/264 Appendix IX Hazardous Constituents

\begin{tabular}{|c|c|c|c|c|c|c|c|c|c|c|c|c|c|c|c|}
\hline$\underline{\mathbf{S T}}$ & Parameter & $\underline{3 Q 98}$ & CLP EPA & 1099 & CLP & EPA & $\underline{3 Q 99}$ & $\underline{\text { CLF }}$ & EPA & 1000 & CLPEPA & Filt. & $\underline{\text { DF }}$ & Unit & Lab \\
\hline or & ganics & & & & & & & & & & & & & & \\
\hline & Barium, total recoverable & 8.05 & $J$ & 7.19 & $J$ & 1 & 6.6 & J & 1 & & & & & $\mathrm{ug} / \mathrm{L}$ & \\
\hline & Cyanide & $<10$ & $\mathrm{U}$ & $<10$ & $\mathrm{U}$ & & $<10$ & $\mathrm{U}$ & & & & & & ug/L & \\
\hline & Lead, total recoverable & $<10$ & $\mathrm{U}$ & $<100$ & U & & $<3.71$ & $\mathrm{U}$ & V & & & & & $\mathrm{ug} / \mathrm{L}$ & \\
\hline & Nickel, total recoverable & $<50$ & $\mathrm{U}$ & $<50$ & U & & $<50$ & $\mathrm{U}$ & & & & & & ug/L & \\
\hline & Selenium, total recoverable & $<10$ & $U$ & $<200$ & $\mathrm{U}$ & & $<5$ & $\mathrm{U}$ & & & & & & $\mathrm{ug} / \mathrm{L}$ & \\
\hline Org & anics & & & & & & & & & & & & & & \\
\hline & Chlorobenzene & $<5$ & $u$ & $<5$ & JU & $Q$ & $<1$ & $\mathrm{U}$ & & $<1$ & $\mathrm{U}$ & $<$ EQL & 1 & $\mathrm{ug} / \mathrm{L}$ & ML \\
\hline & 1,1-Dichloroethane & $<5$ & $\mathrm{U}$ & $<5$ & $\mathrm{JU}$ & $Q$ & $<1$ & $\mathrm{U}$ & & $<1$ & U & $<E Q L$ & 1 & ug/L & ML \\
\hline & 1,1-Dichloroethylene & $<5$ & $\mathrm{U}$ & $<5$ & JU & $\vec{Q}$ & $<1$ & $\mathrm{U}$ & & $<1$ & $\mathrm{U}$ & $<\mathrm{EQL}$ & 1 & $\mathrm{ug} / \mathrm{L}$ & ML \\
\hline & $\begin{array}{l}\text { trans-1,2-Dichloroethylene } \\
\text { PCB } 1016\end{array}$ & $<5$ & $U$ & $<5$ & JU & $Q$ & $<1$ & $\mathrm{U}$ & & $<1$ & U & $<\mathrm{EQL}$ & 1 & ug/L & ML \\
\hline & PCB 1221 & & & & & & & & & & & & & & \\
\hline & PCB 1232 & & & & & & & & & & & & & & \\
\hline & PCB 1242 & & & & & & & & & & & & & & \\
\hline & PCB 1248 & & & & & & & & & & & & & & \\
\hline & PCB 1254 & & & & & & & & & & & & & & \\
\hline & PCB 1260 & & & & & & & & & & & & & & \\
\hline & $1,1,2,2$-Tetrachloroethane & $<5$ & $\mathrm{U}$ & $<5$ & JU & $Q$ & $<1$ & U & & $<1$ & $\mathrm{U}$ & $<E Q L$ & 1 & ug/L & ML \\
\hline & Tetrachloroethylene & $<5$ & $U$ & $<5$ & JU & $\bar{Q}$ & $<1$ & $U$ & & $<1$ & $\mathrm{U}$ & $<\mathrm{EQL}$ & 1 & $\mathrm{ug} / \mathrm{L}$ & ML \\
\hline & 1,1,1-Trichloroethane & $<5$ & $\mathrm{U}$ & $<5$ & JU & $\bar{Q}$ & $<1$ & $\mathrm{U}$ & & $<1$ & $\mathrm{U}$ & $<E Q L$ & 1 & $\mathrm{ug} / \mathrm{L}$ & ML \\
\hline & Trichloroethylene & $<5$ & $U$ & $<5$ & JU & $Q$ & $<1$ & $\mathrm{U}$ & & $<1$ & $U$ & $<E Q L$ & 1 & ug/L & ML \\
\hline II. $M$ & onitoring Constituents & & & & & & & & & & & & & & \\
\hline$\underline{\mathbf{S T}}$ & Parameter & $\underline{3 Q 96}$ & CLP EPA & $\underline{3097}$ & $\underline{\text { CLP }}$ & EPA & $\underline{3 Q 98}$ & $\underline{\text { CLF }}$ & EPA & $\underline{3 Q 99}$ & CLPEPA & Filt. & $\underline{\text { DF }}$ & $\underline{\text { Unit }}$ & $\underline{\operatorname{Lab}}$ \\
\hline Inor & ganics & & & & & & & & & & & & & & \\
\hline & Aluminum, total recoverable & $<20$ & U & 26.7 & & & $<200$ & $\mathrm{U}$ & & $<200$ & $U$ & $<\mathrm{EQL}$ & 1 & $\mathrm{ug} / \mathrm{L}$ & $x$ \\
\hline & Nitrate-nitrite as nitrogen & 240 & & 1610 & & & 1120 & & & 1170 & & $<2400$ & 5 & ug/L & EX \\
\hline & Sodium, total recoverable & 1490 & & 2440 & & & 2310 & & & 2700 & & $<4600$ & 1 & $\mathrm{ug} / \mathrm{L}$ & EX \\
\hline & Sulfate & 182 & $J$ & $<5000$ & $\mathrm{U}$ & & $<400$ & $U$ & & 224 & & $<3000$ & 1 & ug/L. & EX \\
\hline Rad & onuclides & & & & & & & & & & & & & & \\
\hline & Gross alpha & .724 & U! & .26 & UI & & 2.87 & & & 1.35 & $\mathrm{~J} \quad \mathrm{I}$ & NDD & 1 & $\mathrm{pCi} / \mathrm{L}$ & GP \\
\hline & Nonvolatile beta & 1.35 & Ui & .28 & UI & & 2.88 & $J$ & & 1.39 & $\mathrm{U}$ & $<E Q L$ & 1 & $\mathrm{pCi} / \mathrm{L}$ & GP \\
\hline & Radium, total alpha-emitting & .3 & & 1.44 & UIJ & & .49 & & & .5 & J I & NDD & 1 & $\mathrm{pCi} / \mathrm{L}$ & GP \\
\hline
\end{tabular}

Notes: Concentrations in bold italics exceed the groundwater protection or monitoring constituent standards listed in Appendix A. Synchronous water levels are measured over a 3-5 day period or less. Dilution factors, Laboratory, and Filtered Data are for Groundwater Protection Standards First Quarter 2000 and Monitoring Constituents Third Quarter 1999 data only.

$+=$ exceeded the Groundwater Protection Standards (First Quarter 2000) or Monitoring Constituents (Third Quarter 1999) listed in Appendix A. 
Table D-4. Groundwater Monitoring Results for Plume Definition Wells, M-Area HWMF (Cont.) WELL MSB 54B

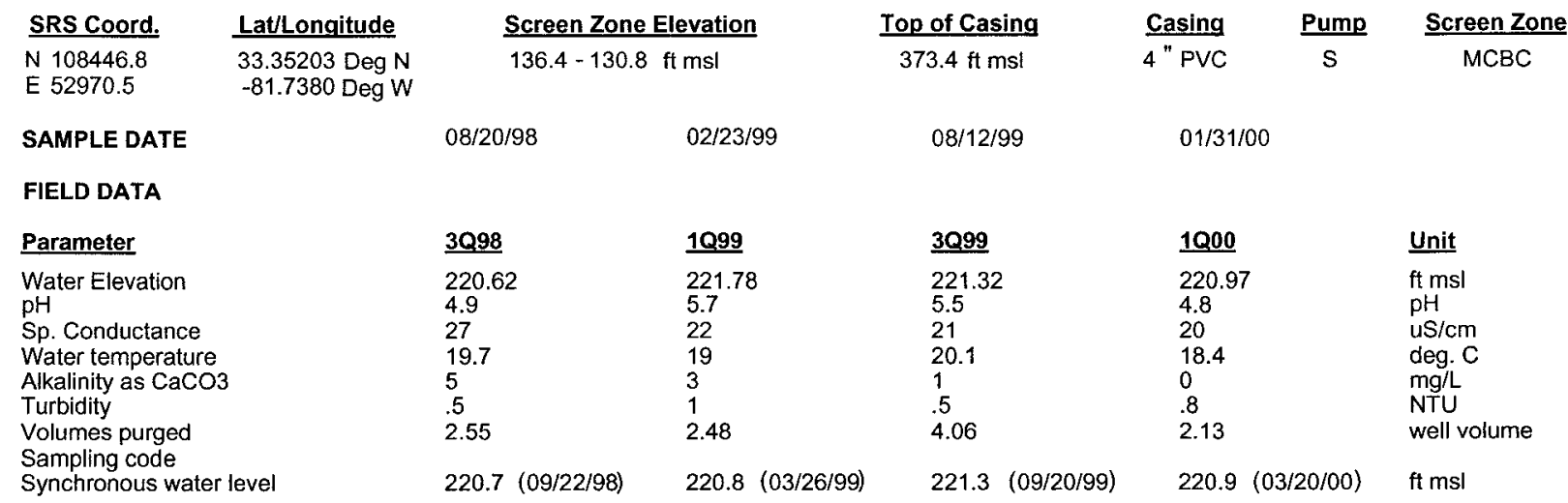

ANALYTICAL DATA

I. Groundwater Protection Standard

261 Appendix VIII/264 Appendix IX Hazardous Constituents

\begin{tabular}{|c|c|c|c|c|c|c|c|c|c|c|c|c|c|c|}
\hline \multirow{2}{*}{$\begin{array}{l}\underline{\text { ST }} \\
\text { Inoro }\end{array}$} & \multirow{2}{*}{ Parameter } & \multirow[t]{2}{*}{$\underline{3 Q 98}$} & \multirow[t]{2}{*}{ CLP EPA } & \multirow[t]{2}{*}{1099} & CLPEPA & \multirow[t]{2}{*}{$\underline{3 Q 99}$} & $\underline{\text { CLP EPA }}$ & \multirow[t]{2}{*}{1000} & \multicolumn{2}{|c|}{ CLPEPA } & \multirow[t]{2}{*}{ Filt. } & \multirow[t]{2}{*}{$\underline{\mathrm{DF}}$} & \multirow[t]{2}{*}{ Unit } & \multirow[t]{2}{*}{$\underline{\text { Lab }}$} \\
\hline & & & & & & & & & & & & & & \\
\hline & Barium, total recoverable & 6.9 & $\mathrm{~J}$ & 4.3 & & 4.8 & J 1 & & & & & & $\mathrm{ug} / \mathrm{L}$ & \\
\hline & Cyanide & $<10$ & $\mathrm{U}$ & 19.8 & & $<10$ & $\mathrm{U}$ & & & & & & $u g / L$ & \\
\hline & Lead, total recoverable & $<10$ & $\mathrm{U}$ & $<100$ & $\mathrm{U}$ & $<10$ & $\mathrm{U}$ & & & & & & ug/L & \\
\hline & Nickel, total recoverable & 7.38 & $\mathrm{~J}$ & 2.7 & J I & $<50$ & $\mathrm{U}$ & & & & & & $\mathrm{ug} / \mathrm{L}$ & \\
\hline & Selenium, total recoverable & $<10$ & U & $<200$ & $U$ & $<10$ & $\mathbf{U}$ & & & & & & $\mathrm{ug} / \mathrm{L}$ & \\
\hline \multicolumn{15}{|c|}{ Organics } \\
\hline & Chiorobenzene & $<5$ & $\mathrm{U}$ & $<5$ & $u$ & $<1$ & $\mathbf{u}$ & $<1$ & JU & $L$ & $<\mathrm{EQL}$ & 1 & $u g / L$ & ML \\
\hline & 1,1-Dichloroethane & $<5$ & $u$ & $<5$ & $\mathrm{U}$ & $<1$ & $\mathrm{U}$ & $<1$ & JU & $\mathrm{L}$ & $<E Q L$ & 1 & $\mathrm{ug} / \mathrm{L}$ & ML \\
\hline & 1,1-Dichloroethylene & $<5$ & $U$ & $<5$ & $U$ & $<1$ & $\mathrm{U}$ & $<1$ & JU & $L$ & $<\mathrm{EQL}$ & 1 & $\mathrm{ug} / \mathrm{L}$ & $M L$ \\
\hline & trans-1,2-Dichloroethylene & $<5$ & $U$ & $<5$ & $\mathrm{U}$ & $<1$ & $\mathrm{U}$ & & & & & & $\mathrm{ug} / \mathrm{L}$ & \\
\hline & PCB 1016 & & & & & & & & & & & & & \\
\hline & PCB 1221 & & & & & & & & & & & & & \\
\hline & PCB 1232 & & & & & & & & & & & & & \\
\hline & PCB 1242 & & & & & & & & & & & & & \\
\hline & PCB 1248 & & & & & & & & & & & & & \\
\hline & PCB 1254 & & & & & & & & & & & & & \\
\hline & PCB 1260 & & & & & & & & & & & & & \\
\hline & 1,1,2,2-Tetrachloroethane & $<5$ & $U$ & $<5$ & $\mathrm{U}$ & $<1$ & $\mathrm{U}$ & $<1$ & JU & $L$ & $<\mathrm{EQL}$ & 1 & ug/L & ML \\
\hline & Tetrachloroethylene & $<5$ & $\mathrm{U}$ & $<5$ & $U$ & $<.7$ & U V & $<1$ & JU & $L$ & $<E Q L$ & 1 & $\mathrm{ug} / \mathrm{L}$ & ML \\
\hline & 1,1,1-Trichloroethane & $<5$ & $U$ & $<5$ & $U$ & $<1$ & $\mathrm{U}$ & $<1$ & JU & $\mathrm{L}$ & $<E Q L$ & 1 & $\mathrm{ug} / \mathrm{L}$ & $M L$ \\
\hline & Trichloroethylene & $<5$ & $\mathrm{U}$ & $<5$ & $\mathrm{U}$ & $<.97$ & $U$ & $<1$ & $\mathrm{JU}$ & $\mathrm{L}$ & $<\mathrm{EQL}$ & 1 & $\mathrm{ug} / \mathrm{L}$ & ML \\
\hline \multicolumn{15}{|c|}{ II. Monitoring Constituents } \\
\hline$\underline{\mathbf{s T}}$ & Parameter & $\underline{3096}$ & CLPEPA & $\underline{3 Q 97}$ & CLPEPA & 3098 & CLPEPA & $\underline{3999}$ & $\underline{\text { CLP }}$ & EPA & Filt. & $\underline{\text { DF }}$ & $\underline{\text { Unit }}$ & $\underline{\text { Lab }}$ \\
\hline \multicolumn{15}{|c|}{ Inorganics } \\
\hline & Aluminum, total recoverable & 66.3 & $\mathrm{~J}$ & 8.3 & $\mathrm{~J}$ & $<200$ & $\mathbf{U}$ & $<200$ & U & & $<E Q L$ & 1 & $u g / L$ & EX \\
\hline & Nitrate-nitrite as nitrogen & 970 & & 1070 & & 904 & & 906 & & & $<2400$ & 5 & $\mathrm{ug} / \mathrm{L}$ & EX \\
\hline & Sodium, total recoverable & 1560 & & 1550 & & $<1670$ & U & 1800 & & & $<4600$ & 1 & ug/L & EX \\
\hline & Sulfate & 274 & $J$ & $<5000$ & $\mathrm{U}$ & 276 & $\mathrm{~J}$ & 340 & & & $<3000$ & 1 & $\mathrm{ug} / \mathrm{L}$ & EX \\
\hline \multicolumn{15}{|c|}{ Radionuclides } \\
\hline & Gross alpha & 1.8 & J & -.09 & UI & 2.46 & & .62 & J & IK & NDD & 1 & $\mathrm{pCi} / \mathrm{L}$ & GP \\
\hline & Nonvolatile beta & .691 & UI & .28 & UI & 2.38 & & 1.99 & $J$ & 1 & NDD & 1 & $\mathrm{pCi} / \mathrm{L}$ & GP \\
\hline & Radium, total alpha-emitting & .7 & & 2.15 & UIJ & .21 & UI & .4 & $\mathrm{~J}$ & I & NDD & 1 & $\mathrm{pCi} / \mathrm{L}$ & GP \\
\hline
\end{tabular}

Notes: Concentrations in bold italics exceed the groundwater protection or monitoring constituent standards listed in Appendix A. Synchronous water levels are measured over a 3-5 day period or less. Dilution factors, Laboratory, and Filtered Data are for Groundwater Protection Standards First Quarter 2000 and Monitoring Constituents Third Quarter 1999 data only.

$+=$ exceeded the Groundwater Protection Standards (First Quarter 2000) or Monitoring Constituents (Third Quarter 1999) listed in Appendix A. 
Table D-4. Groundwater Monitoring Results for Plume Definition Wells, M-Area HWMF (Cont.) WELL MSB 54C

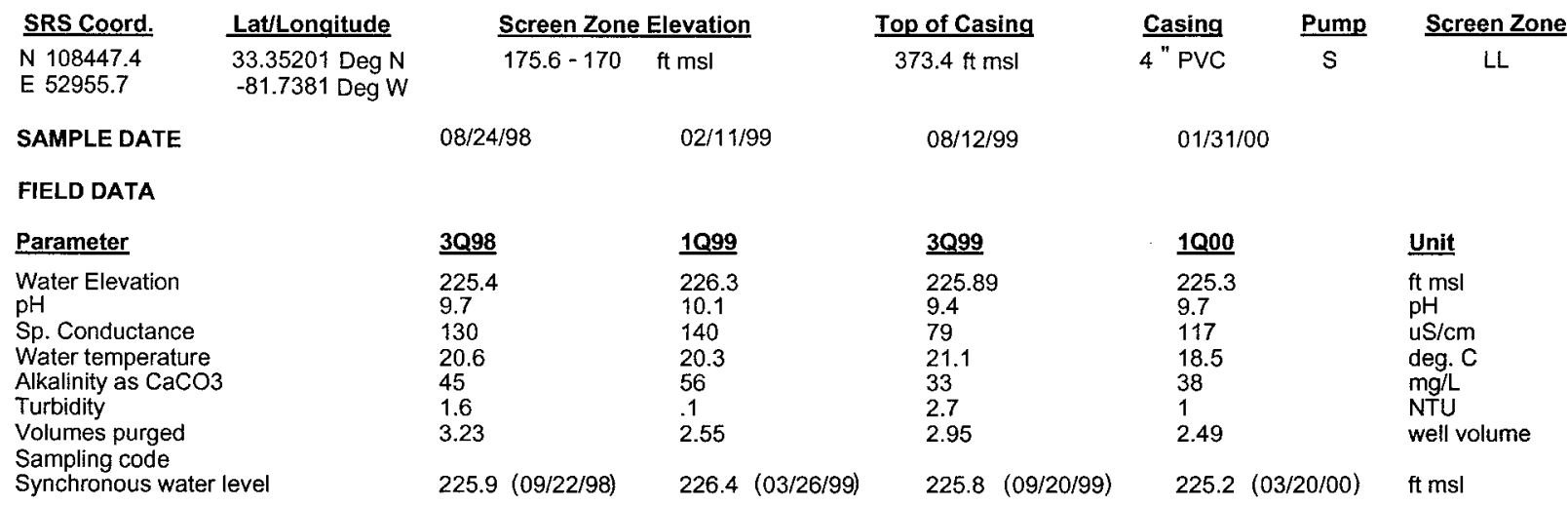

ANALYTICAL DATA

I. Groundwater Protection Standard

261 Appendix VIII/264 Appendix IX Hazardous Constituents

\begin{tabular}{|c|c|c|c|c|c|c|c|c|c|c|c|c|c|c|}
\hline$\underline{\text { ST }}$ & Parameter & 3Q98 & CLPEPA & 1 Q99 & CLP EPA & 3Q99 & CLPEPA & 1000 & \multicolumn{2}{|c|}{ CLPEPA } & Filt. & DF & $\underline{\text { Unit }}$ & $\underline{\text { Lab }}$ \\
\hline \multicolumn{15}{|c|}{ Inorganics } \\
\hline & Barium, total recoverable & 50.3 & & $<10$ & $u$ & 34 & & & & & & & $u g / L$ & \\
\hline & Cyanide & $<10$ & $U$ & $<10$ & $\mathrm{U}$ & $<10$ & U & & & & & & $\mathrm{ug} / \mathrm{L}$ & \\
\hline & Lead, total recoverable & $<10$ & $U$ & $<100$ & $\mathrm{U}$ & $<10$ & $U$ & & & & & & $\mathrm{ug} / \mathrm{L}$ & \\
\hline & Nickel, total recoverable & $<50$ & $U$ & $<50$ & $\mathrm{U}$ & $<50$ & $\mathrm{U}$ & & & & & & $u g / L$ & \\
\hline & Selenium, total recoverable & $<10$ & $U$ & & & $<10$ & $U$ & & & & & & ug $/ \mathrm{L}$ & \\
\hline \multicolumn{15}{|c|}{ Organics } \\
\hline & Chlorobenzene & $<5$ & $U$ & $<5$ & $\mathrm{U}$ & $<1$ & $\mathrm{U}$ & $<1$ & JU & $\mathrm{L}$ & $<\mathrm{EQL}$ & 1 & $u g / L$ & ML \\
\hline & 1,1-Dichloroethane & $<5$ & $U$ & $<5$ & $\mathrm{U}$ & $<1$ & $\mathrm{U}$ & $<1$ & JU & $\mathrm{L}$ & $<\mathrm{EQL}$ & 1 & $u g / L$ & ML \\
\hline & 1,1-Dichloroethylene & $<5$ & $\mathrm{U}$ & $<5$ & U & $<1$ & $U$ & $<1$ & JU & $\mathrm{L}$ & $<\mathrm{EQL}$ & 1 & $\mathrm{ug} / \mathrm{L}$ & $M L$ \\
\hline & trans-1,2-Dichloroethylene & $<5$ & $\mathrm{U}$ & $<5$ & $\mathrm{U}$ & $<1$ & $U$ & & & & & & ug/L & \\
\hline & PCB 1016 & & & & & & & & & & & & & \\
\hline & PCB 1221 & & & & & & & & & & & & & \\
\hline & PCB 1232 & & & & & & & & & & & & & \\
\hline & PCB 1242 & & & & & & & & & & & & & \\
\hline & PCB 1248 & & & & & & & & & & & & & \\
\hline & PCB 1254 & & & & & & & & & & & & & \\
\hline & PCB 1260 & & & & & & & & & & & & & \\
\hline & 1,1,2,2-Tetrachloroethane & $<5$ & $\mathrm{U}$ & $<5$ & $\mathrm{U}$ & $<1$ & $\mathrm{U}$ & $<1$ & JU & $\mathrm{L}$ & $<E Q L$ & 1 & ug/L & ML \\
\hline & Tetrachloroethylene & $<5$ & $\mathrm{U}$ & $<5$ & $\mathrm{U}$ & $<.69$ & U V & $<1$ & JU & $L$ & $<E Q L$ & 1 & $u g / L$ & ML \\
\hline & 1,1,1-Trichloroethane & $<5$ & $\mathrm{U}$ & $<5$ & $\mathrm{U}$ & $<1$ & $\mathrm{U}$ & $<1$ & JU & $\mathrm{L}$ & $<E Q L$ & 1 & $\mathrm{ug} / \mathrm{L}$ & ML. \\
\hline & Trichloroethylene & $<5$ & $\mathrm{U}$ & $<5$ & $\mathrm{U}$ & $<.77$ & $\mathrm{U}$ & $<1$ & $\mathrm{JU}$ & $\mathrm{L}$ & $<E Q L$ & 1 & $\mathrm{ug} / \mathrm{L}$ & ML \\
\hline \multicolumn{15}{|c|}{ II. Monitoring Constituents } \\
\hline$\underline{\text { ST }}$ & Parameter & $\underline{3 Q 96}$ & CLP EPA & $\underline{3097}$ & $\underline{\text { CLP EPA }}$ & $\underline{3 Q 98}$ & CLPEEPA & $\underline{3099}$ & CLP & EPA & Filt. & $\underline{\mathrm{DF}}$ & Unit & $\underline{L a b}$ \\
\hline \multicolumn{15}{|c|}{ Inorganics } \\
\hline & Aluminum, total recoverable & 360 & & 208 & & $<200$ & $U$ & 140 & $J$ & I & NDD & 1 & $\mathrm{ug} / \mathrm{L}$ & EX \\
\hline & Nitrate-nitrite as nitrogen & 1820 & & 1280 & & 1110 & & 1210 & & & $<2400$ & 5 & $\mathrm{ug} / \mathrm{L}$ & EX \\
\hline & Sodium, total recoverable & 2600 & & 2100 & & 2130 & & 2000 & & & $<4600$ & 1 & $\mathrm{ug} / \mathrm{L}$ & EX \\
\hline & Sulfate & 417 & $\mathrm{~J}$ & $<5000$ & $\mathrm{U}$ & 425 & & 319 & & & $<3000$ & 1 & $\mathrm{ug} / \mathrm{L}$ & EX \\
\hline \multicolumn{15}{|c|}{ Radionuclides } \\
\hline & Gross alpha & 3.68 & & 2.73 & & 2.55 & $\mathrm{~J}$ & .857 & J & IK & NDD & 1 & $\mathrm{pCi} / \mathrm{L}$ & GP \\
\hline & Nonvolatile beta & 2.41 & & .85 & UI & 2.93 & & 1.64 & $\mathrm{~J}$ & 1 & NDD & 1 & $\mathrm{pCi} / \mathrm{L}$ & GP \\
\hline & Radium, total alpha-emitting & 1.2 & & 3.45 & UIJ & .83 & & .9 & $J$ & 1 & NDD & 1 & $\mathrm{pCi} / \mathrm{L}$ & GP \\
\hline
\end{tabular}

Notes: Concentrations in bold italics exceed the groundwater protection or monitoring constituent standards listed in Appendix A. Synchronous water levels are measured over a 3-5 day period or less. Dilution factors, Laboratory, and Filtered Data are for Groundwater Protection Standards First Quarter 2000 and Monitoring Constituents Third Quarter 1999 data only.

$+=$ exceeded the Groundwater Protection Standards (First Quarter 2000) or Monitoring Constituents (Third Quarter 1999) listed in Appendix A. 
Table D-4. Groundwater Monitoring Results for Plume Definition Wells, M-Area HWMF (Cont.) WELL MSB 54D

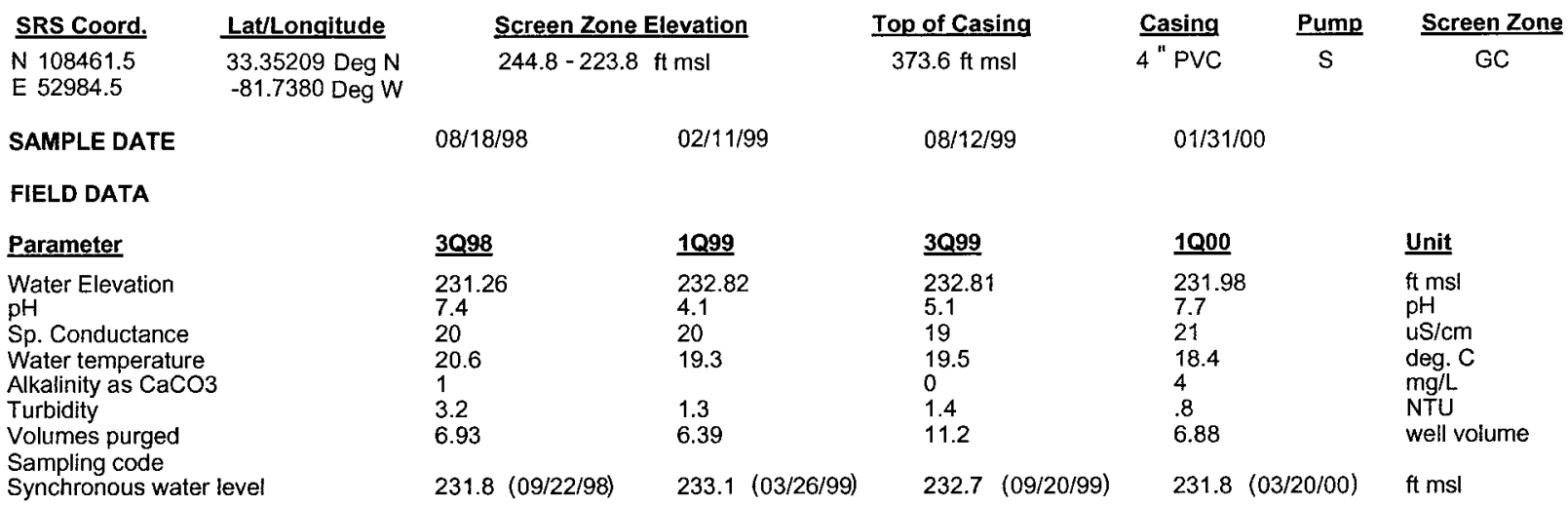

ANALYTICAL DATA

I. Groundwater Protection Standard

261 Appendix VIII/264 Appendix IX Hazardous Constituents

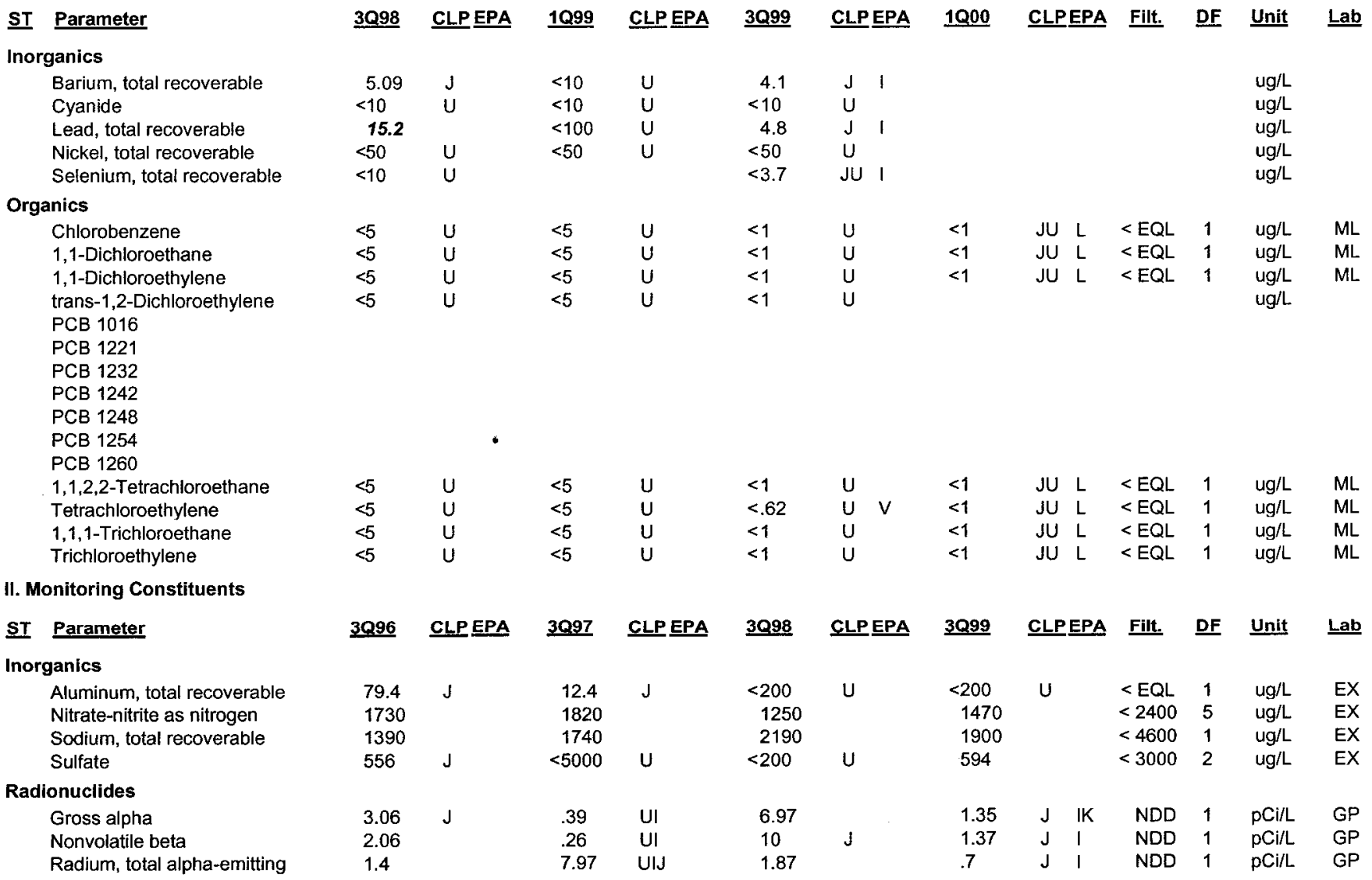

Notes: Concentrations in bold italics exceed the groundwater protection or monitoring constituent standards listed in Appendix A. Synchronous water levels are measured over a 3-5 day period or less. Dilution factors, Laboratory, and Filtered Data are for Groundwater Protection Standards First Quarter 2000 and Monitoring Constituents Third Quarter 1999 data only.

+= exceeded the Groundwater Protection Standards (First Quarter 2000) or Monitoring Constituents (Third Quarter 1999) listed in Appendix A. 
Table D-4. Groundwater Monitoring Results for Plume Definition Wells, M-Area HWMF (Cont.) WELL MSB 54TA

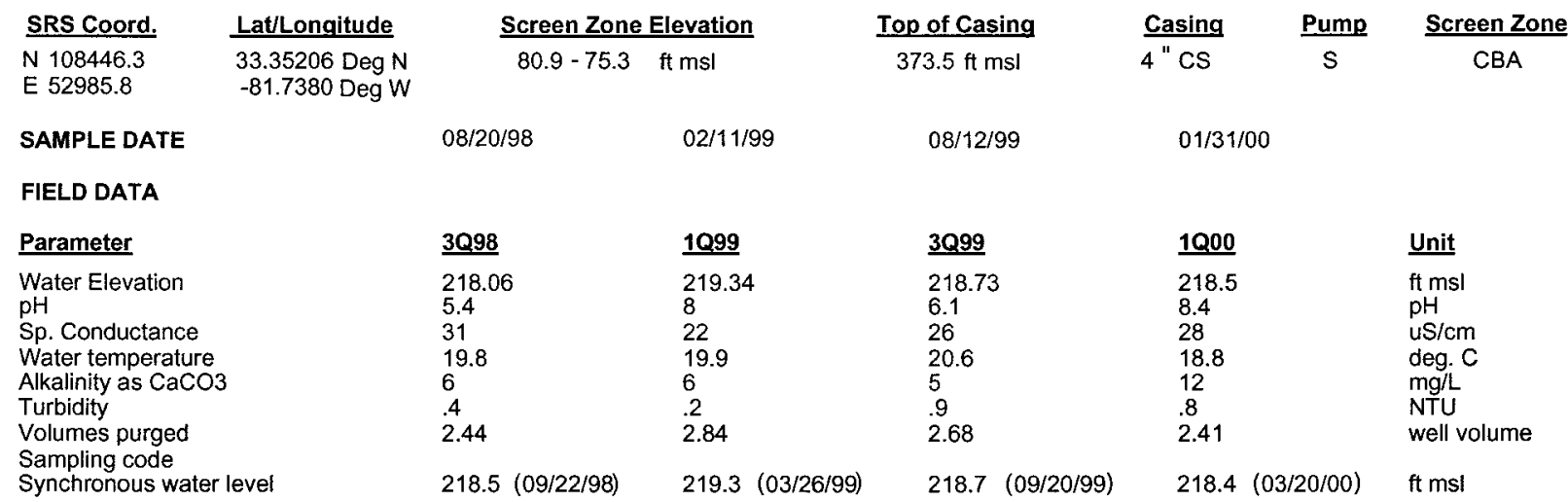

ANALYTICAL DATA

I. Groundwater Protection Standard

261 Appendix VIII/264 Appendix IX Hazardous Constituents

\begin{tabular}{|c|c|c|c|c|c|c|c|c|c|c|c|c|c|}
\hline ST Parameter & $\underline{\mathbf{3 Q 9 8}}$ & CLP EPA & 1099 & $\underline{\text { CLP EPA }}$ & $\underline{3 Q 99}$ & CLPEPA & 1000 & $\underline{\text { CLP }}$ & EPA & Filt. & $\underline{\text { DF }}$ & Unit & $\underline{\text { Lab }}$ \\
\hline \multicolumn{14}{|l|}{ Inorganics } \\
\hline Barium, total recoverable & 7.16 & $\mathrm{~J}$ & $<10$ & U & 8.5 & J I & & & & & & $\mathrm{ug} / \mathrm{L}$ & \\
\hline Cyanide & $<10$ & $\mathrm{U}$ & $<10$ & $U$ & $<10$ & U & & & & & & $\mathrm{ug} / \mathrm{L}$ & \\
\hline Lead, total recoverable & $<10$ & $\mathrm{U}$ & $<100$ & $\mathrm{U}$ & $<10$ & $\mathrm{U}$ & & & & & & $\mathrm{ug} / \mathrm{L}$ & \\
\hline Nickel, total recoverable & 8.16 & $\mathrm{~J}$ & $<50$ & U & $<8.8$ & JU 1 & & & & & & $\mathrm{ug} / \mathrm{L}$ & \\
\hline Selenium, total recoverable & $<10$ & $\mathrm{U}$ & & & $<10$ & $\mathrm{U}$ & & & & & & $\mathrm{ug} / \mathrm{L}$ & \\
\hline \multicolumn{14}{|l|}{ Organics } \\
\hline Chlorobenzene & $<5$ & $\mathrm{U}$ & $<5$ & $u$ & $<1$ & $\mathrm{U}$ & $<1$ & JU & $L$ & $<E Q L$ & 1 & $u g / L$ & $M L$ \\
\hline 1,1-Dichloroethane & $<5$ & $U$ & $<5$ & $U$ & $<1$ & $\mathrm{U}$ & $<1$ & JU & $L$ & $<\mathrm{EQL}$ & 1 & $\mathrm{ug} / \mathrm{L}$ & $M L$ \\
\hline 1,1-Dichloroethylene & $<5$ & $U$ & $<5$ & $U$ & $<1$ & $\mathrm{U}$ & $<1$ & JU & $L$ & $<\mathrm{EQL}$ & 1 & $u g / L$ & $M L$ \\
\hline trans-1,2-Dichloroethylene & $<5$ & $U$ & $<5$ & $U$ & $<1$ & $\mathrm{U}$ & & & & & & $\mathrm{ug} / \mathrm{L}$ & \\
\hline PCB 1016 & & & & & & & & & & & & & \\
\hline PCB 1221 & & & & & & & & & & & & & \\
\hline PCB 1232 & & & & & & & & & & & & & \\
\hline РСB 1242 & & & & & & & & & & & & & \\
\hline PCB 1248 & & & & & & & & & & & & & \\
\hline PCB 1254 & & & & & & & & & & & & & \\
\hline PCB 1260 & & & & & & & & & & & & & \\
\hline $1,1,2,2$-Tetrachloroethane & $<5$ & $U$ & $<5$ & $U$ & $<1$ & $U$ & $<1$ & JU & $\mathrm{L}$ & $<\mathrm{EQL}$ & 1 & $\mathrm{ug} / \mathrm{L}$ & $M L$ \\
\hline Tetrachloroethylene & $<5$ & $\cup$ & $<5$ & $U$ & $<.83$ & $\mathrm{U} V$ & $<1$ & JU & $\mathrm{L}$ & $<\mathrm{EQL}$ & 1 & $\mathrm{ug} / \mathrm{L}$ & ML \\
\hline 1,1,1-Trichloroethane & $<5$ & $\cup$ & $<5$ & $U$ & $<1$ & $\mathrm{U}$ & $<1$ & JU & $\mathrm{L}$ & $<\mathrm{EQL}$ & 1 & $\mathrm{ug} / \mathrm{L}$ & $M L$ \\
\hline Trichloroethylene & $<5$ & $\mathrm{U}$ & $<5$ & $\mathrm{U}$ & $<1.18$ & $\mathrm{U}$ & $<1$ & JU & $\mathrm{L}$ & $<\mathrm{EQL}$ & 1 & $\mathrm{ug} / \mathrm{L}$ & ML \\
\hline \multicolumn{14}{|l|}{ II. Monitoring Constituents } \\
\hline Parameter & $\underline{3 Q 96}$ & CLPEPA & $\underline{3097}$ & CLPEPA & $\underline{3098}$ & CLPEPA & $\underline{3099}$ & $\underline{\text { CLP }}$ & EPA & Filt, & $\underline{\mathrm{DF}}$ & Unit & \\
\hline \multicolumn{14}{|l|}{ Inorganics } \\
\hline Aluminum, total recoverable & 127 & J & 20.5 & & $<200$ & $U$ & 120 & $J$ & 1 & NDD & 1 & $\mathrm{ug} / \mathrm{L}$ & EX \\
\hline Nitrate-nitrite as nitrogen & 1180 & & 1000 & & 885 & & 894 & & & $<2400$ & 5 & $\mathrm{ug} / \mathrm{L}$ & EX \\
\hline Sodium, total recoverable & 1620 & & 1520 & & $<1320$ & $U$ & 1600 & & & $<4600$ & 1 & $\mathrm{ug} / \mathrm{L}$ & EX \\
\hline Sulfate & 322 & $J$ & $<5000$ & $\mathrm{U}$ & 237 & $J$ & 234 & & & $<3000$ & 1 & $\mathrm{ug} / \mathrm{L}$ & EX \\
\hline \multicolumn{14}{|l|}{ Radionuclides } \\
\hline Gross alpha & .464 & UIJ & .98 & UI & 1.67 & & 1.01 & $J$ & IK & NDD & 1 & $\mathrm{pCi} / \mathrm{L}$ & GP \\
\hline Nonvolatile beta & .953 & UI & .67 & UI & .71 & UI & 1.11 & J & 1 & NDD & 1 & $\mathrm{pCi} / \mathrm{L}$ & GP \\
\hline Radium, total alpha-emitting & .7 & & 6.03 & UIJ & .27 & UI & 6 & $J$ & 1 & NDD & 1 & $\mathrm{pCi} / \mathrm{L}$ & GP \\
\hline
\end{tabular}

Notes: Concentrations in bold italics exceed the groundwater protection or monitoring constituent standards listed in Appendix A. Synchronous water levels are measured over a 3-5 day period or less. Dilution factors, Laboratory, and Filtered Data are for Groundwater Protection Standards First Quarter 2000 and Monitoring Constituents Third Quarter 1999 data only.

+ = exceeded the Groundwater Protection Standards (First Quarter 2000) or Monitoring Constituents (Third Quarter 1999) listed in Appendix A. 
Table D-4. Groundwater Monitoring Results for Plume Definition Wells, M-Area HWMF (Cont.) WELL MSB 55B

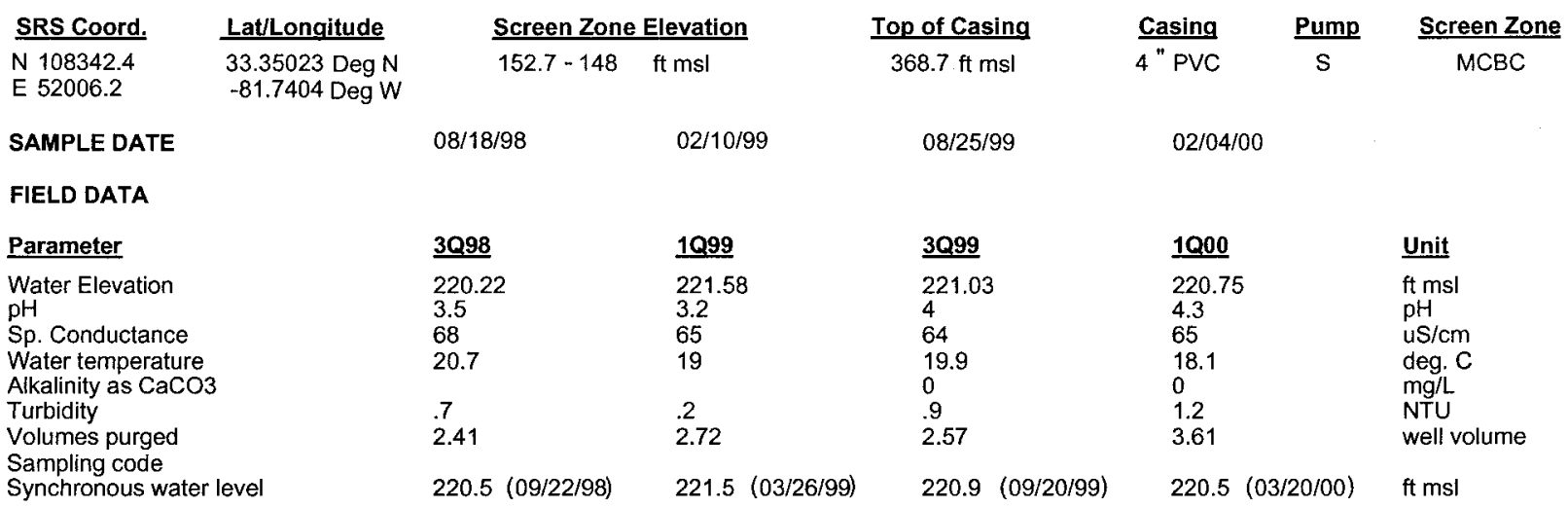

ANALYTICAL DATA

I. Groundwater Protection Standard

261 Appendix VIII/264 Appendix IX Hazardous Constituents

\begin{tabular}{|c|c|c|c|c|c|c|c|c|c|c|c|c|c|}
\hline$\underline{\mathbf{S T}}$ & Parameter & $\underline{3 Q 98}$ & CLP EPA & 1099 & CLP EPA & $\underline{3 Q 99}$ & CLPEPA & 1000 & CLPEPA & Filt. & $\underline{\mathrm{DF}}$ & Unit & $\underline{\text { Lab }}$ \\
\hline \multicolumn{14}{|c|}{ Inorganics } \\
\hline & Barium, total recoverable & 7.62 & $J$ & 8.31 & J I & 9.3 & $\mathrm{~J} \quad 1$ & & & & & $\mathrm{ug} / \mathrm{L}$ & \\
\hline & Cyanide & $<10$ & $U$ & $<10$ & $\mathrm{U}$ & $<10$ & $\mathrm{U}$ & & & & & $\mathrm{ug} / \mathrm{L}$ & \\
\hline & Lead, total recoverable & $<10$ & $\mathrm{U}$ & $<100$ & $U$ & $<10$ & $U$ & & & & & $u g / L$ & \\
\hline & Nickel, total recoverable & 12.6 & $J$ & $<50$ & $U$ & $<50$ & $\mathrm{U}$ & & & & & $\mathrm{ug} / \mathrm{L}$ & \\
\hline & Selenium, total recoverable & $<10$ & $u$ & $<10$ & $U$ & $<10$ & $\mathrm{U}$ & & & & & $\mathrm{ug} / \mathrm{L}$ & \\
\hline \multicolumn{14}{|c|}{ Organics } \\
\hline & Chlorobenzene & $<5$ & $\mathrm{U}$ & $<5$ & $U$ & $<1$ & $\mathrm{U}$ & $<1$ & JU & $<$ EQL & 1 & $u g / L$ & $M L$ \\
\hline & 1,1-Dichloroethane & $<5$ & $\mathrm{U}$ & $<5$ & U & $<1$ & $\mathrm{U}$ & $<1$ & JU & $<E Q L$ & 1 & $\mathrm{ug} / \mathrm{L}$ & ML \\
\hline & 1,1-Dichloroethylene & $<5$ & $\mathrm{U}$ & $<5$ & $U$ & $<1$ & $\mathrm{U}$ & $<1$ & JU & $<$ EQL & 1 & $\mathrm{ug} / \mathrm{L}$ & ML \\
\hline & trans-1,2-Dichloroethylene & $<5$ & $\mathrm{U}$ & $<5$ & $\mathrm{U}$ & $<1$ & $\mathrm{U}$ & $<1$ & JU & $<\mathrm{EQL}$ & 1 & $\mathrm{ug} / L$ & ML \\
\hline & PCB 1016 & & & & & & & & & & & & \\
\hline & РCB 1221 & & & & & & & & & & & & \\
\hline & РCB 1232 & & & & & & & & & & & & \\
\hline & РСВ 1242 & & & & & & & & & & & & \\
\hline & PCB 1248 & & & & & & & & & & & & \\
\hline & PCB 1254 & & & & & & & & & & & & \\
\hline & PCB 1260 & & & & & & & & & & & & \\
\hline & 1,1,2,2-Tetrachloroethane & $<5$ & $U$ & $<5$ & $U$ & $<1$ & $\mathrm{U}$ & $<1$ & JU & $<E Q L$ & 1 & ug/L & ML \\
\hline & Tetrachloroethylene & $<5$ & $U$ & $<5$ & $U$ & $<1$ & $U$ & $<1$ & JU & $<E Q L$ & 1 & $\mathrm{ug} / \mathrm{L}$ & ML \\
\hline & $1,1,1$-Trichloroethane & $<5$ & $U$ & $<5$ & $\mathrm{U}$ & $<1$ & $U$ & $<1$ & JU & $<E Q L$ & 1 & $\mathrm{ug} / \mathrm{L}$ & ML \\
\hline & Trichloroethylene & $<5$ & $u$ & $<5$ & $U$ & $<1$ & $\mathrm{U}$ & $<1$ & JU & $<\mathrm{EQL}$ & 1 & $\mathrm{ug} / \mathrm{L}$ & ML \\
\hline \multicolumn{14}{|c|}{ II. Monitoring Constituents } \\
\hline$\underline{\mathbf{S T}}$ & Parameter & $\underline{3096}$ & CLPEPA & $\underline{3097}$ & CLP EPA & $\underline{3098}$ & CLPEPA & $\underline{3099}$ & CLPEPA & Filt. & $\underline{D F}$ & Unit & $\underline{\text { Lab }}$ \\
\hline \multicolumn{14}{|c|}{ Inorganics } \\
\hline+ & Aluminum, total recoverable & 392 & $\mathrm{~J}$ & 375 & & 443 & & 510 & & $>100$ & 1 & $u g / L$ & EX \\
\hline & Nitrate-nitrite as nitrogen & 230 & & 13 & & $<500$ & $\mathrm{U}$ & $<500$ & $U$ & $<\mathrm{EQL}$ & 5 & $\mathrm{ug} / \mathrm{L}$ & EX \\
\hline & Sodium, total recoverable & 1690 & & 1730 & & $<2030$ & $\mathrm{u}$ & 2100 & & $<4600$ & 1 & $\mathrm{ug} / \mathrm{L}$ & EX \\
\hline+ & Sulfate & 10600 & & 11100 & & 13100 & & 10800 & & $>3000$ & 2 & ug/L & EX \\
\hline \multicolumn{14}{|c|}{ Radionuclides } \\
\hline & Gross alpha & 4.22 & & 8.34 & & 3.65 & & 1.75 & $\mathrm{~J} \quad \mathrm{I}$ & NDD & 1 & $\mathrm{pCi} / \mathrm{L}$ & GP \\
\hline & Nonvolatile beta & 5.1 & & 13.2 & & 4.12 & $J$ & 3.62 & & $<50$ & 1 & $\mathrm{pCi} / \mathrm{L}$ & GP \\
\hline & Radium, total alpha-emitting & 6.9 & & 8.32 & UIJ & 1.05 & & .6 & $\mathrm{U}$ & $<\mathrm{EQL}$ & 1 & $\mathrm{pCi} / \mathrm{L}$ & GP \\
\hline
\end{tabular}

Notes: Concentrations in bold italics exceed the groundwater protection or monitoring constituent standards listed in Appendix A. Synchronous water levels are measured over a 3-5 day period or less. Dilution factors, Laboratory, and Filtered Data are for Groundwater Protection Standards First Quarter 2000 and Monitoring Constituents Third Quarter 1999 data only.

+ = exceeded the Groundwater Protection Standards (First Quarter 2000) or Monitoring Constituents (Third Quarter 1999) listed in Appendix A. 
Table D-4. Groundwater Monitoring Results for Plume Definition Wells, M-Area HWMF (Cont.) WELL MSB 55C

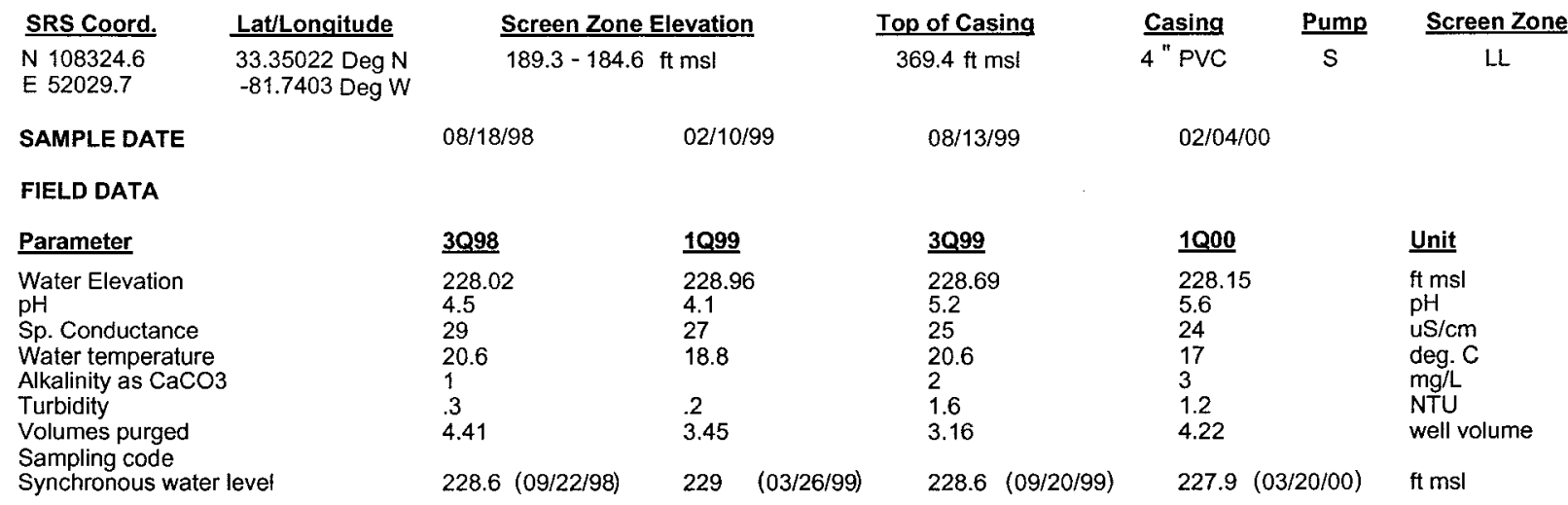

ANALYTICAL DATA

I. Groundwater Protection Standard

261 Appendix VIII/264 Appendix IX Hazardous Constituents

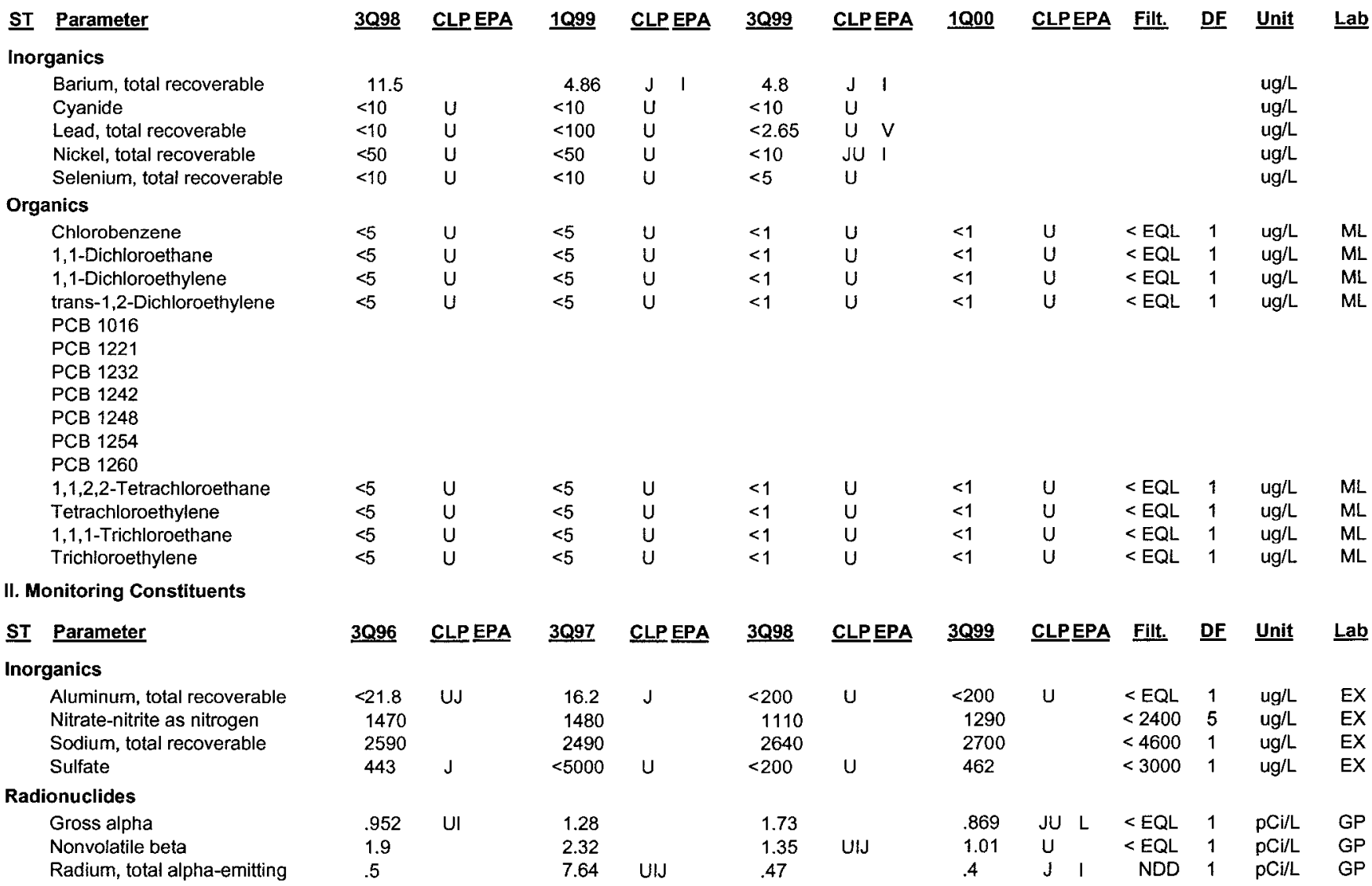

Notes: Concentrations in bold italics exceed the groundwater protection or monitoring constituent standards listed in Appendix A. Synchronous water levels are measured over a 3-5 day period or less. Dilution factors, Laboratory, and Filtered Data are for Groundwater Protection Standards First Quarter 2000 and Monitoring Constituents Third Quarter 1999 data only.

$+=$ exceeded the Groundwater Protection Standards (First Quarter 2000) or Monitoring Constituents (Third Quarter 1999) listed in Appendix A. 
Table D-4. Groundwater Monitoring Results for Plume Definition Wells, M-Area HWMF (Cont.) WELL MSB 55D

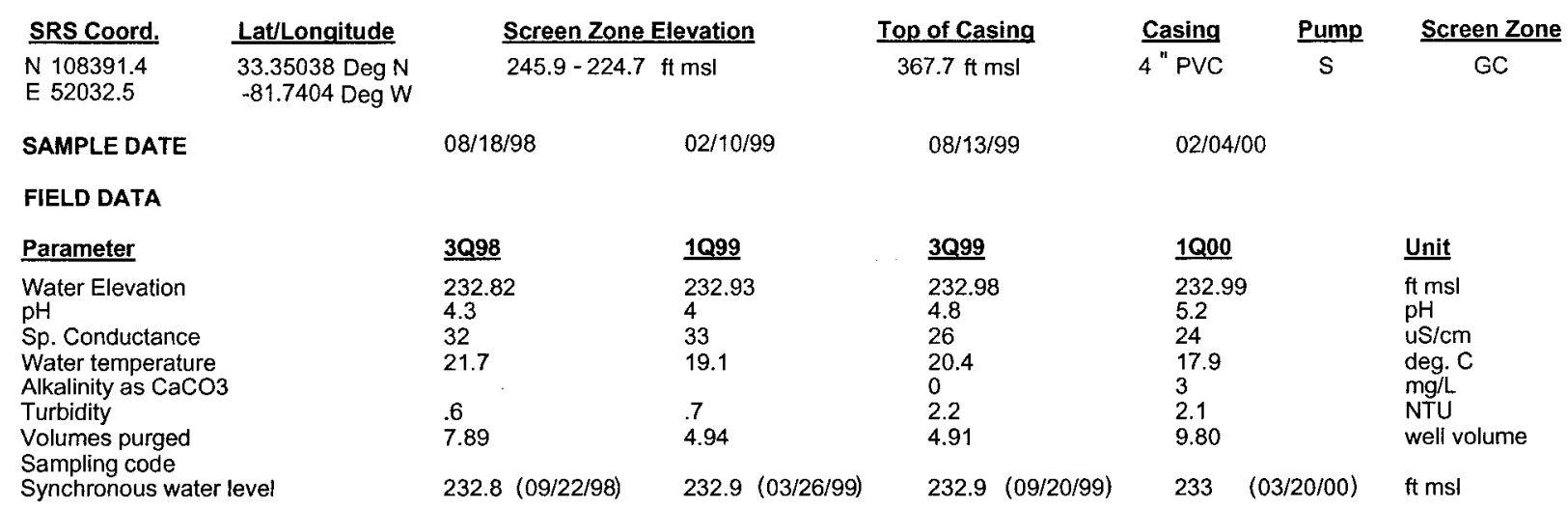

ANALYTICAL DATA

I. Groundwater Protection Standard

261 Appendix VIII/264 Appendix IX Hazardous Constituents

\begin{tabular}{|c|c|c|c|c|c|c|c|c|c|c|c|c|c|c|}
\hline \multirow{2}{*}{$\frac{\text { ST }}{\text { Inorg }}$} & \multirow{2}{*}{$\begin{array}{l}\text { Parameter } \\
\text { ganics }\end{array}$} & \multirow[t]{2}{*}{$\underline{3 Q 98}$} & \multirow[t]{2}{*}{ CLP EPA } & \multirow[t]{2}{*}{1099} & CLPEPA & \multirow[t]{2}{*}{$\underline{3 Q 99}$} & $\underline{\text { CLP EPA }}$ & \multirow[t]{2}{*}{$1 \mathrm{Q00}$} & \multicolumn{2}{|c|}{ CLPEPA } & \multirow[t]{2}{*}{ Filt. } & \multirow[t]{2}{*}{$\underline{\text { DF }}$} & \multirow[t]{2}{*}{ Unit } & \multirow[t]{2}{*}{ Lab } \\
\hline & & & & & & & & & & & & & & \\
\hline & Barium, total recoverable & 6.28 & 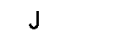 & 8.4 & J I & 8.4 & J 1 & & & & & & $\mathrm{ug} / \mathrm{L}$ & \\
\hline & Cyanide & $<10$ & $\mathrm{U}$ & $<10$ & U & $<10$ & $\mathrm{U}$ & & & & & & $\mathrm{ug} / \mathrm{L}$ & \\
\hline & Lead, total recoverable & 16.6 & & $<100$ & $U$ & 16.4 & & & & & & & $\mathrm{ug} / \mathrm{L}$ & \\
\hline & Nickel, total recoverable & $<50$ & $\mathrm{U}$ & $<50$ & $U$ & $<50$ & $\mathrm{U}$ & & & & & & $\mathrm{ug} / \mathrm{L}$ & \\
\hline & Selenium, total recoverable & $<10$ & $\mathrm{U}$ & $<10$ & $\cup$ & $<5$ & $\mathrm{U}$ & & & & & & $\mathrm{ug} / \mathrm{L}$ & \\
\hline \multicolumn{15}{|c|}{ Organics } \\
\hline & Chlorobenzene & $<5$ & $\mathrm{U}$ & $<5$ & U & $<1$ & $\mathrm{U}$ & $<1$ & JU & & $<E Q L$ & 1 & ug/L & ML \\
\hline & 1,1-Dichloroethane & $<5$ & $\mathrm{U}$ & $<5$ & $U$ & $<1$ & $\mathrm{U}$ & $<1$ & JU & & $<E Q L$ & 1 & $\mathrm{ug} / \mathrm{L}$ & $M L$ \\
\hline & 1,1-Dichloroethylene & $<5$ & $U$ & $<5$ & $U$ & $<1$ & $\mathrm{U}$ & $<1$ & JU & & $<E Q L$ & 1 & $\mathrm{ug} / \mathrm{L}$ & ML \\
\hline & trans-1,2-Dichloroethylene & $<5$ & $U$ & $<5$ & $\mathrm{U}$ & $<1$ & $\mathrm{U}$ & $<1$ & JU & & $<E Q L$ & 1 & $\mathrm{ug} / \mathrm{L}$ & ML \\
\hline & PCB 1016 & & & & & & & & & & & & & \\
\hline & PCB 1221 & & & & & & & & & & & & & \\
\hline & PCB 1232 & & & & & & & & & & & & & \\
\hline & PCB 1242 & & & & & & & & & & & & & \\
\hline & PCB 1248 & & & & & & & & & & & & & \\
\hline & PCB 1254 & & & & & & & & & & & & & \\
\hline & PCB 1260 & & & & & & & & & & & & & \\
\hline & 1,1,2,2-Tetrachloroethane & $<5$ & $\mathrm{U}$ & $<5$ & $\mathrm{U}$ & $<1$ & $\mathrm{U}$ & $<1$ & JU & & $<\mathrm{EQL}$ & 1 & $\mathrm{ug} / \mathrm{L}$ & ML \\
\hline & Tetrachloroethylene & $<5$ & $u$ & $<5$ & $U$ & $<1$ & $\mathrm{U}$ & $<1$ & JU & & $<\mathrm{EQL}$ & 1 & ug/L & $M L$ \\
\hline & $1,1,1$-Trichloroethane & $<5$ & $U$ & $<5$ & $\mathrm{U}$ & $<1$ & $\mathrm{U}$ & $<1$ & JU & & $<E Q L$ & 1 & ug/L & ML \\
\hline & Trichloroethylene & $<5$ & $U$ & $<5$ & $\mathrm{U}$ & $<1$ & $U$ & $<1$ & JU & & $<\mathrm{EQL}$ & 1 & $\mathrm{ug} / \mathrm{L}$ & $M L$ \\
\hline \multicolumn{15}{|c|}{ II. Monitoring Constituents } \\
\hline$\underline{\mathbf{S T}}$ & Parameter & $\underline{3096}$ & CLPEPA & 3097 & CLPEPA & $\underline{3 Q 98}$ & CLPEPA & $\underline{3099}$ & $\underline{\text { CLP }}$ & EPA & Filt. & $\underline{\text { DF }}$ & Unit & $\underline{\text { Lab }}$ \\
\hline \multicolumn{15}{|c|}{ Inorganics } \\
\hline & Aluminum, total recoverable & $<50.8$ & $\mathrm{U}$ & 6.9 & $J$ & $<200$ & $U$ & $<200$ & $\mathrm{U}$ & & $<\mathrm{EQL}$ & 1 & $\mathrm{ug} / \mathrm{L}$ & EX \\
\hline & Nitrate-nitrite as nitrogen & 1810 & & 1920 & & 1640 & & 1840 & & & $<2400$ & 5 & $\mathrm{ug} / \mathrm{L}$ & EX \\
\hline & Sodium, total recoverable & 1600 & & 1680 & & 2800 & & 1900 & & & $<4600$ & 1 & ug/L & EX \\
\hline & Sulfate & 179 & $J$ & $<5000$ & $\mathrm{U}$ & $<200$ & $\mathrm{U}$ & 192 & $J$ & 1 & NDD & 1 & $\mathrm{ug} / \mathrm{L}$ & EX \\
\hline \multicolumn{15}{|c|}{ Radionuclides } \\
\hline & Gross alpha & 6.19 & & 3.45 & & 7.87 & & 3.34 & $\mathrm{~J}$ & $\mathrm{~L}$ & NDD & 1 & $\mathrm{pCi} / \mathrm{L}$ & GP \\
\hline & Nonvolatile beta & 3.06 & & 1.8 & UI & 2.77 & $J$ & 1.79 & $\mathrm{~J}$ & 1 & NDD & 1 & $\mathrm{pCi} / \mathrm{L}$ & GP \\
\hline & Radium, total alpha-emitting & 3.8 & & 9.53 & J & 2.57 & & 2.5 & & & $<5$ & 1 & $\mathrm{pCi} / \mathrm{L}$ & GP \\
\hline
\end{tabular}

Notes: Concentrations in bold italics exceed the groundwater protection or monitoring constituent standards listed in Appendix A. Synchronous water levels are measured over a 3-5 day period or less. Dilution factors, Laboratory, and Filtered Data are for Groundwater Protection Standards First Quarter 2000 and Monitoring Constituents Third Quarter 1999 data only.

$+=$ exceeded the Groundwater Protection Standards (First Quarter 2000) or Monitoring Constituents (Third Quarter 1999) listed in Appendix A. 
Table D-4. Groundwater Monitoring Results for Plume Definition Wells, M-Area HWMF (Cont.) WELL MSB 55HC

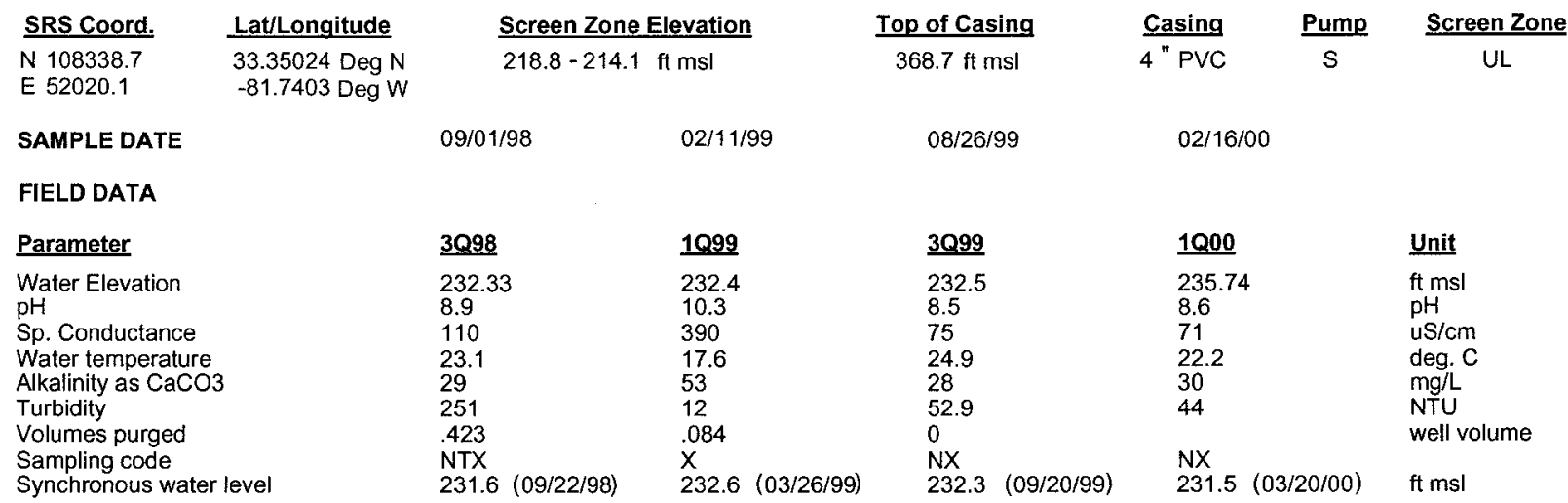

ANALYTICAL DATA

I. Groundwater Protection Standard

261 Appendix VIII/264 Appendix IX Hazardous Constituents

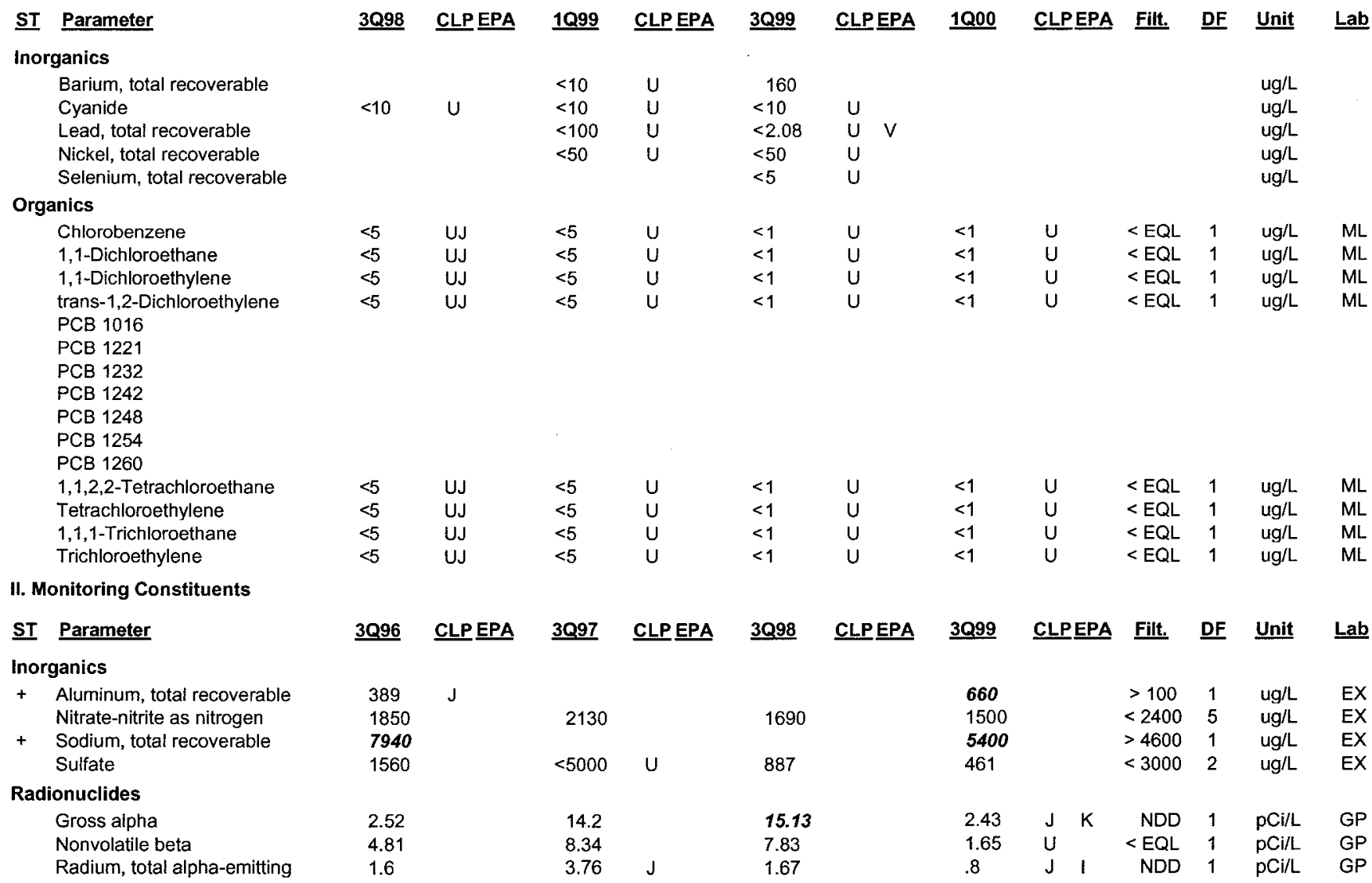

Notes: Concentrations in bold italics exceed the groundwater protection or monitoring constituent standards listed in Appendix A. Synchronous water levels are measured over a 3-5 day period or less. Dilution factors, Laboratory, and Filtered Data are for Groundwater Protection Standards First Quarter 2000 and Monitoring Constituents Third Quarter 1999 data only.

$+=$ exceeded the Groundwater Protection Standards (First Quarter 2000) or Monitoring Constituents (Third Quarter 1999) listed in Appendix A. 
Table D-4. Groundwater Monitoring Results for Plume Definition Wells, M-Area HWMF (Cont.) WELL MSB 55TA

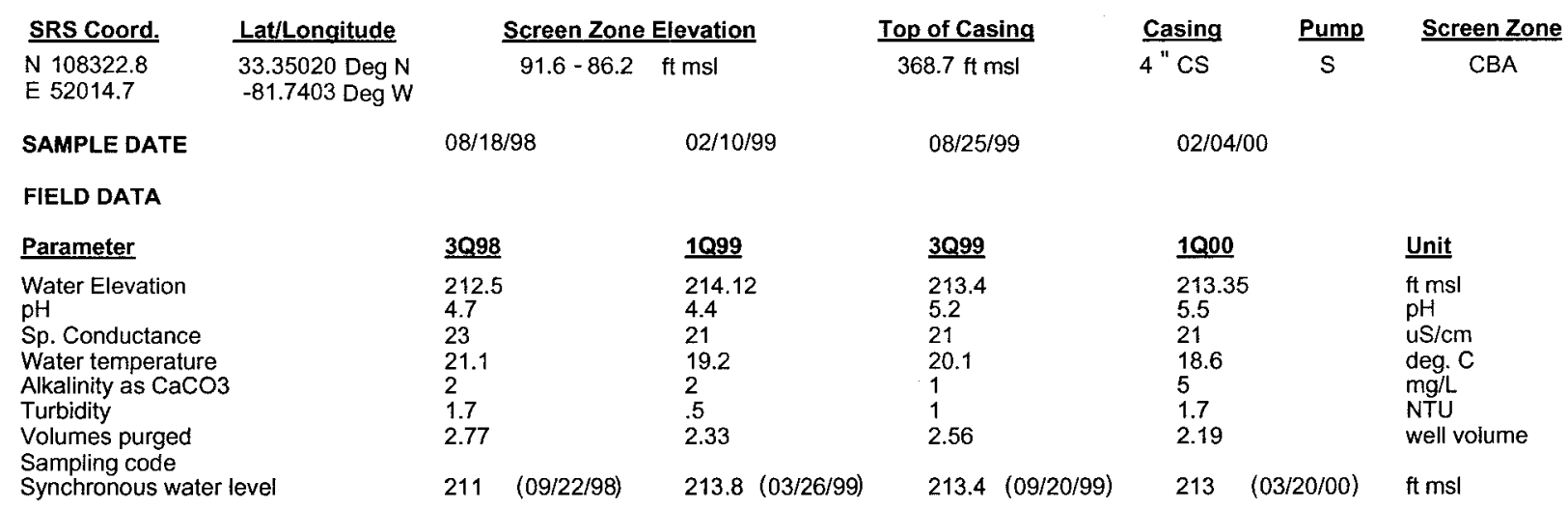

ANALYTICAL DATA

I. Groundwater Protection Standard

261 Appendix VIII/264 Appendix IX Hazardous Constituents

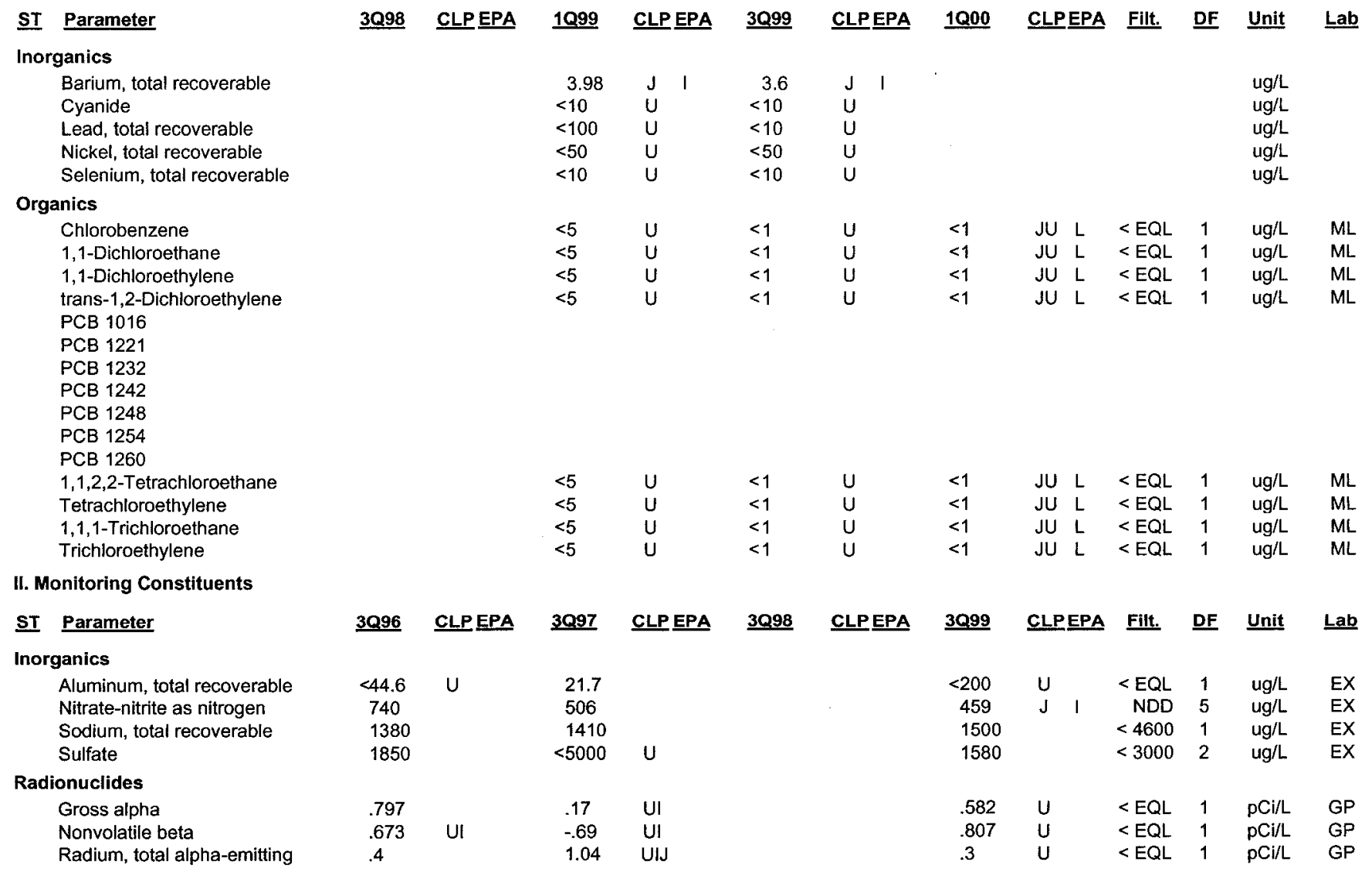

Notes: Concentrations in bold italics exceed the groundwater protection or monitoring constituent standards listed in Appendix A. Synchronous water levels are measured over a 3-5 day period or less. Dilution factors, Laboratory, and Filtered Data are for Groundwater Protection Standards First Quarter 2000 and Monitoring Constituents Third Quarter 1999 data only.

$+=$ exceeded the Groundwater Protection Standards (First Quarter 2000) or Monitoring Constituents (Third Quarter 1999) listed in Appendix A. 
Table D-4. Groundwater Monitoring Results for Plume Definition Wells, M-Area HWMF (Cont.) WELL MSB 64B

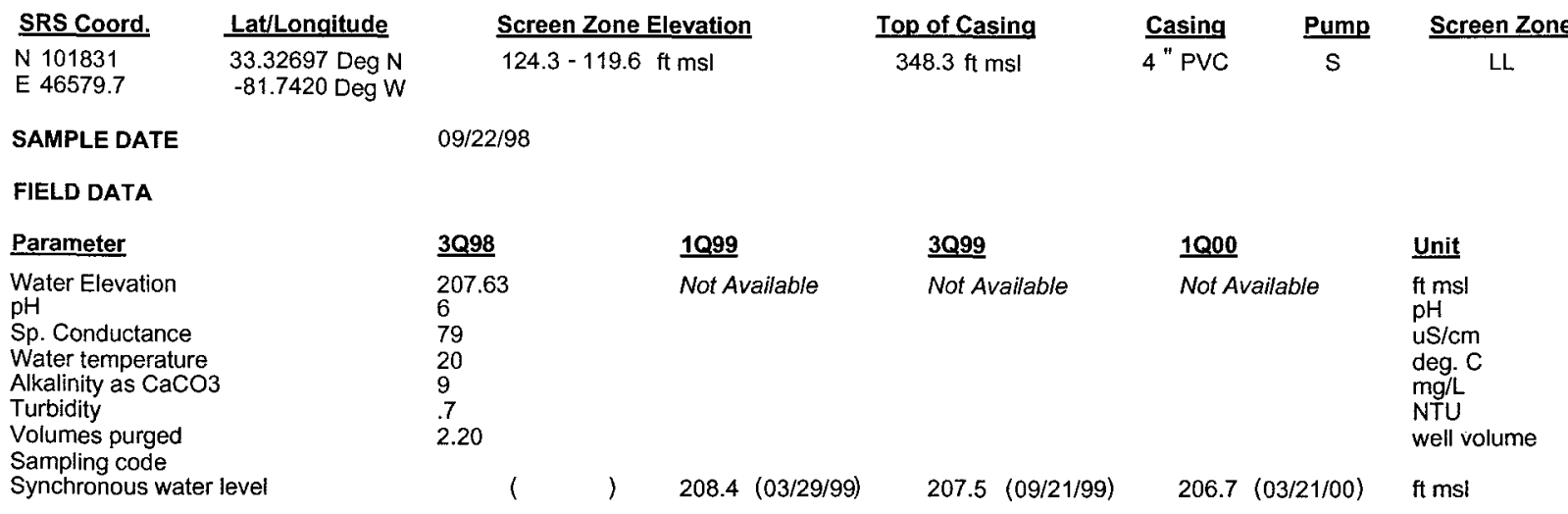

ANALYTICAL DATA

I. Groundwater Protection Standard

261 Appendix VIII/264 Appendix IX Hazardous Constituents

\begin{tabular}{|c|c|c|c|c|c|c|c|c|c|c|c|c|}
\hline$\underline{\text { ST }}$ & Parameter & $\underline{3 Q 98}$ & CLP EPA & 1099 & CLP EPA & $\underline{3 Q 99}$ & CLPEPA & 1000 & CLPEPA & Filt. & DF & Unit \\
\hline \multicolumn{13}{|c|}{ Inorganics } \\
\hline & Barium, total recoverable & 50.4 & & & & & & & & & & $\mathrm{ug} / \mathrm{L}$ \\
\hline & Cyanide & $<10$ & $u$ & & & & & & & & & ug/L \\
\hline & Lead, total recoverable & $<100$ & $U$ & & & & & & & & & $\mathrm{ug} / \mathrm{L}$ \\
\hline & Nickel, total recoverable & $<50$ & $U$ & & & & & & & & & $\mathrm{ug} / \mathrm{L}$ \\
\hline & Selenium, total recoverable & $<200$ & $\mathrm{U}$ & & & & & & & & & $\mathrm{ug} / \mathrm{L}$ \\
\hline \multicolumn{13}{|c|}{ Organics } \\
\hline & Chlorobenzene & $<125$ & $U$ & & & & & & & & & $\mathrm{ug} / \mathrm{L}$ \\
\hline & 1,1-Dichloroethane & $<125$ & $\mathrm{U}$ & & & & & & & & & ug/L \\
\hline & 1,1-Dichloroethylene & $<125$ & U & & & & & & & & & $\mathrm{ug} / \mathrm{L}$ \\
\hline & trans-1,2-Dichloroethylene & $<125$ & $U$ & & & & & & & & & $\mathrm{ug} / \mathrm{L}$ \\
\hline & PCB 1016 & $<2$ & $\mathrm{U}$ & & & & & & & & & $\mathrm{ug} / \mathrm{L}$ \\
\hline & PCB 1221 & $<2$ & $\mathrm{U}$ & & & & & & & & & $\mathrm{ug} / \mathrm{L}$ \\
\hline & PCB 1232 & $<1$ & U & & & & & & & & & $\mathrm{ug} / \mathrm{L}$ \\
\hline & PCB 1242 & $<1$ & $U$ & & & & & & & & & $\mathrm{ug} / \mathrm{L}$ \\
\hline & РCB 1248 & $<1$ & $\mathrm{U}$ & & & & & & & & & $\mathrm{ug} / \mathrm{L}$ \\
\hline & PCB 1254 & $<1$ & $U$ & & & & & & & & & $\mathrm{ug} / \mathrm{L}$ \\
\hline & PCB 1260 & $<1$ & $U$ & & & & & & & & & ug/L \\
\hline & 1,1,2,2-Tetrachloroethane & $<125$ & $\mathrm{U}$ & & & & & & & & & $\mathrm{ug} / \mathrm{L}$ \\
\hline & Tetrachloroethylene & 87.2 & $J$ & & & & & & & & & $\mathrm{ug} / \mathrm{L}$ \\
\hline & 1,1,1-Trichloroethane & $<125$ & $\mathrm{U}$ & & & & & & & & & $\mathrm{ug} / \mathrm{L}$ \\
\hline & Trichioroethylene & 3680 & & & & & & & & & & $u g / L$ \\
\hline \multicolumn{13}{|c|}{ II. Monitoring Constituents } \\
\hline$\underline{\mathbf{S T}}$ & Parameter & $\underline{3096}$ & CLPEPA & $\underline{3097}$ & CLP EPA & $\underline{3098}$ & CLPEPA & $\underline{3099}$ & CLPEPA & Filt. & $\underline{\text { DF }}$ & Unit \\
\hline \multicolumn{13}{|c|}{ Inorganics } \\
\hline & Aluminum, total recoverable & & & 43.1 & & $<200$ & U & & & & & $\mathrm{ug} / \mathrm{L}$ \\
\hline & Nitrate-nitrite as nitrogen & & & 4410 & & & & & & & & $\mathrm{ug} / \mathrm{L}$ \\
\hline & Sodium, total recoverable & & & 4060 & & 4070 & & & & & & $\mathrm{ug} / \mathrm{L}$ \\
\hline & Sulfate & & & $<5000$ & $U$ & & & & & & & $\mathrm{ug} / \mathrm{L}$ \\
\hline \multicolumn{13}{|c|}{ Radionuclides } \\
\hline & Gross alpha & & & .51 & UI & & & & & & & $\mathrm{pCi} / \mathrm{L}$ \\
\hline & Nonvolatile beta & & & .82 & UI & & & & & & & $\mathrm{pCi} / \mathrm{L}$ \\
\hline & Radium, total alpha-emitting & & & 3.69 & $\mathrm{~J}$ & & & & & & & \\
\hline
\end{tabular}

Notes: Concentrations in bold italics exceed the groundwater protection or monitoring constituent standards listed in Appendix A. Synchronous water levels are measured over a 3-5 day period or less. Dilution factors, Laboratory, and Filtered Data are for Groundwater Protection Standards First Quarter 2000 and Monitoring Constituents Third Quarter 1999 data only.

$+=$ exceeded the Groundwater Protection Standards (First Quarter 2000) or Monitoring Constituents (Third Quarter 1999) listed in Appendix A. 
Table D-4. Groundwater Monitoring Results for Plume Definition Wells, M-Area HWMF (Cont.) WELL MSB 65D

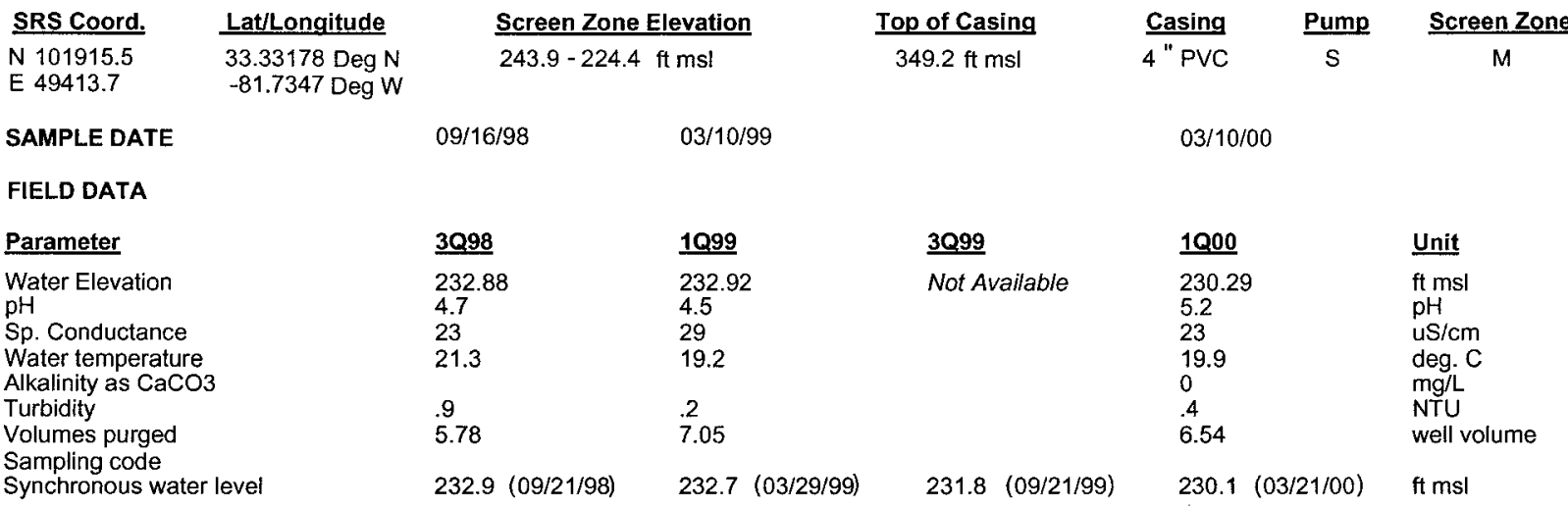

ANALYTICAL DATA

I. Groundwater Protection Standard

261 Appendix VIII/264 Appendix IX Hazardous Constituents

\begin{tabular}{|c|c|c|c|c|c|c|c|c|c|c|c|c|c|}
\hline$\underline{\text { ST }}$ & Parameter & $\underline{3098}$ & CLPEPA & 1099 & CLP EPA & $\underline{3099}$ & CLPEPA & 1000 & CLPEPA & Filt. & $\underline{\text { DF }}$ & $\underline{\text { Unit }}$ & Lab \\
\hline \multicolumn{14}{|c|}{ Inorganics } \\
\hline & Barium, total recoverable & 9.63 & $\mathrm{~J}$ & 8.37 & $\sqrt{ } 1$ & & & & & & & $u g / L$ & \\
\hline & Cyanide & $<10$ & $\mathrm{U}$ & $<10$ & JU L & & & & & & & $\mathrm{ug} / \mathrm{L}$ & \\
\hline & Lead, total recoverable & $<100$ & $\mathrm{U}$ & $<100$ & $U$ & & & & & & & $\mathrm{ug} / \mathrm{L}$ & \\
\hline & Nickel, total recoverable & $<50$ & $U$ & $<7.01$ & JU & & & & & & & $\mathrm{ug} / \mathrm{L}$ & \\
\hline & Selenium, total recoverable & $<200$ & $\mathrm{U}$ & $<200$ & $\mathrm{U}$ & & & & & & & $\mathrm{ug} / \mathrm{L}$ & \\
\hline \multicolumn{14}{|c|}{ Organics } \\
\hline & Chlorobenzene & $<5$ & $\mathrm{U}$ & $<5$ & $U$ & & & $<1$ & $U$ & $<E Q L$ & 1 & $\mathrm{ug} / \mathrm{L}$ & ML \\
\hline & 1,1-Dichloroethane & $<5$ & $\mathrm{U}$ & $<5$ & $U$ & & & $<1$ & $\mathrm{U}$ & $<\mathrm{EQL}$ & 1 & $\mathrm{ug} / \mathrm{L}$ & ML \\
\hline & 1,1-Dichloroethylene & $<5$ & $\mathrm{U}$ & $<5$ & $U$ & & & $<1$ & $\mathrm{U}$ & $<E Q L$ & 1 & $\mathrm{ug} / \mathrm{L}$ & ML \\
\hline & trans-1,2-Dichloroethylene & $<5$ & $\mathrm{U}$ & $<5$ & $\mathrm{U}$ & & & & & & & $\mathrm{ug} / \mathrm{L}$ & \\
\hline & PCB 1016 & & & & & & & & & & & & \\
\hline & PCB 1221 & & & & & & & & & & & & \\
\hline & PCB 1232 & & & & & & & & & & & & \\
\hline & PCB 1242 & & & & & & & & & & & & \\
\hline & PCB 1248 & & & & & & & & & & & & \\
\hline & PCB 1254 & & & & & & & & & & & & \\
\hline & РСВ 1260 & & & & & & & & & & & & \\
\hline & 1,1,2,2-Tetrachloroethane & $<5$ & $\mathrm{U}$ & $<5$ & $\mathrm{U}$ & & & $<1$ & $\mathrm{U}$ & $<E Q L$ & 1 & ug/L & ML \\
\hline & Tetrachloroethylene & 12.4 & & 6.99 & & & & 16.9 & $\mathrm{~J} K$ & NDD & 1 & $\mathrm{ug} / \mathrm{L}$ & ML \\
\hline & 1,1,1-Trichloroethane & $<5$ & $U$ & $<5$ & U & & & $<1$ & $\mathrm{U}$ & $<E Q L$ & 1 & $\mathrm{ug} / \mathrm{L}$ & ML \\
\hline & Trichloroethylene & 5.85 & & 4.53 & J I & & & 9.32 & $\mathrm{~J} \quad \mathrm{~K}$ & NDD & 1 & $\mathrm{ug} / \mathrm{L}$ & ML \\
\hline \multicolumn{14}{|c|}{ II. Monitoring Constituents } \\
\hline$\underline{\mathbf{S T}}$ & Parameter & $\underline{3096}$ & CLPEPA & $\underline{3097}$ & CLPEPA & $\underline{3098}$ & CLPEPA & 3099 & CLPEPA & Filt. & $\underline{\text { DF }}$ & Unit & Lab \\
\hline \multicolumn{13}{|c|}{ Inorganics } & \\
\hline & Aluminum, total recoverable & 59.4 & & 40.8 & & 108 & $J$ & & & & & $u g / L$ & \\
\hline & Nitrate-nitrite as nitrogen & 1550 & & 1300 & & 1090 & & & & & & ug/L & \\
\hline & Sodium, total recoverable & 1860 & & 1920 & & 1860 & & & & & & $\mathrm{ug} / \mathrm{L}$ & \\
\hline & Sulfate & 975 & $J$ & $<5000$ & $\mathrm{U}$ & 726 & & & & & & ug/L & \\
\hline \multicolumn{13}{|c|}{ Radionuclides } & \\
\hline & Gross alpha & 1.72 & & 1.77 & & 4.64 & & & & & & $\mathrm{pCi} / \mathrm{L}$ & \\
\hline & Nonvolatile beta & 2.05 & & 1.74 & UI & 2.18 & J & & & & & $\mathrm{pCi} / \mathrm{L}$ & \\
\hline & Radium, total alpha-emitting & .7 & $\mathrm{~J}$ & 1.81 & $\mathrm{UI}$ & 1.17 & & & & & & $\mathrm{pCi} / \mathrm{L}$ & \\
\hline
\end{tabular}

Notes: Concentrations in bold italics exceed the groundwater protection or monitoring constituent standards listed in Appendix A. Synchronous water levels are measured over a 3-5 day period or less. Dilution factors, Laboratory, and Filtered Data are for Groundwater Protection Standards First Quarter 2000 and Monitoring Constituents Third Quarter 1999 data only.

$+=$ exceeded the Groundwater Protection Standards (First Quarter 2000) or Monitoring Constituents (Third Quarter 1999) listed in Appendix A. 
Table D-4. Groundwater Monitoring Results for Plume Definition Wells, M-Area HWMF (Cont.) WELL MSB 66B

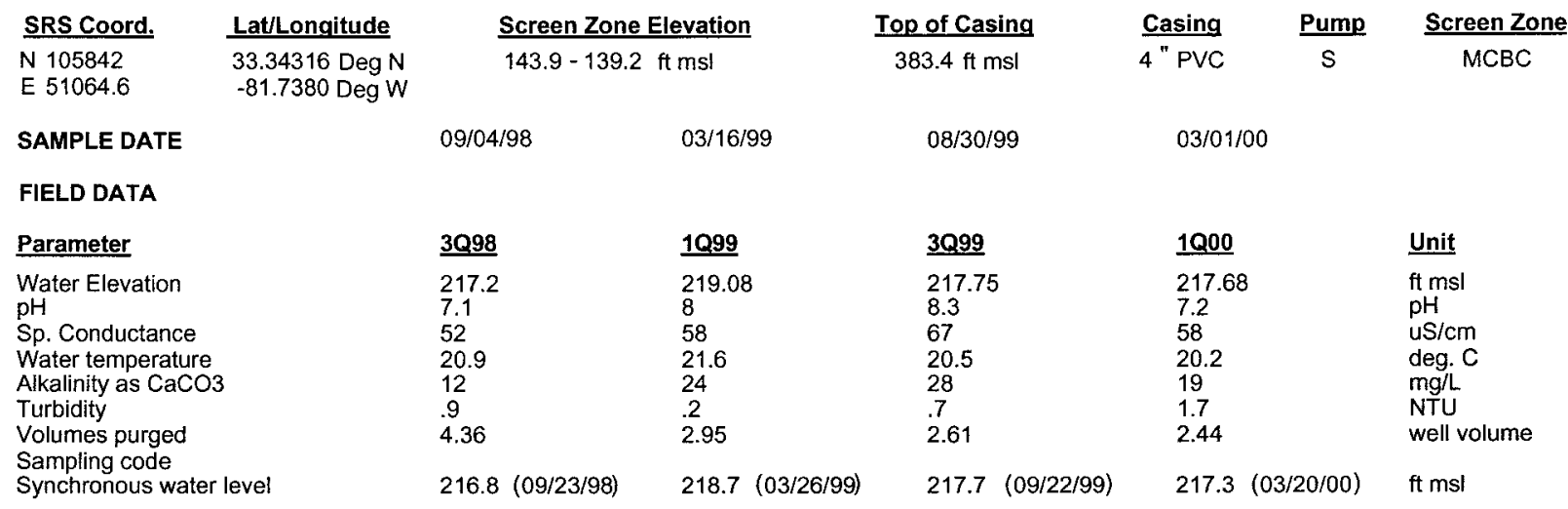

ANALYTICAL DATA

I. Groundwater Protection Standard

261 Appendix VIII/264 Appendix IX Hazardous Constituents

\begin{tabular}{|c|c|c|c|c|c|c|c|c|c|c|c|c|c|}
\hline ST & Parameter & $\underline{3 Q 98}$ & CLP EPA & $\underline{1099}$ & CLP EPA & $\underline{3 Q 99}$ & $\underline{\text { CLP EPA }}$ & $\underline{1 Q 00}$ & CLPEPA & Filt. & DF & $\underline{\text { Unit }}$ & $\underline{\text { Lab }}$ \\
\hline \multicolumn{14}{|c|}{ Inorganics } \\
\hline & Barium, total recoverable & 18.1 & & 21.6 & & 23 & & & & & & ug/L & \\
\hline & Cyanide & $<10$ & $\mathrm{U}$ & $<10$ & $\mathrm{U}$ & $<10$ & $U$ & & & & & $\mathrm{ug} / \mathrm{L}$ & \\
\hline & Lead, total recoverable & $<100$ & $\mathrm{U}$ & $<100$ & $\mathrm{U}$ & $<10$ & $\mathrm{U}$ & & & & & $\mathrm{ug} / \mathrm{L}$ & \\
\hline & Nickel, total recoverable & $<50$ & $\mathrm{U}$ & $<50$ & $U$ & $<50$ & $\mathrm{U}$ & & & & & ug/L & \\
\hline & Selenium, total recoverable & $<10$ & $\mathrm{U}$ & $<200$ & $\mathrm{U}$ & $<10$ & $\mathrm{U}$ & & & & & $\mathrm{ug} / \mathrm{L}$ & \\
\hline \multicolumn{14}{|c|}{ Organics } \\
\hline & Chlorobenzene & $<25$ & $u$ & $<25$ & $u$ & $<1$ & U & $<2$ & $\mathrm{U}$ & $<E Q L$ & 2 & $\mathrm{ug} / \mathrm{L}$ & ML \\
\hline & 1,1-Dichloroethane & $<25$ & $u$ & $<25$ & U & $<1$ & U & $<\overline{2}$ & $\mathrm{U}$ & $<E Q L$ & 2 & $\mathrm{ug} / \mathrm{L}$ & ML \\
\hline & 1,1-Dichloroethylene & $<25$ & $U$ & $<25$ & $\mathrm{U}$ & $<1$ & $\mathrm{U}$ & $<2$ & $\mathrm{U}$ & $<\mathrm{EQL}$ & 2 & $\mathrm{ug} / \mathrm{L}$ & ML \\
\hline & trans-1,2-Dichloroethylene & $<25$ & $U$ & $<25$ & $U$ & $<1$ & $\mathrm{U}$ & & & & & $\operatorname{ug} / \mathrm{L}$ & \\
\hline & $\begin{array}{l}\text { PCB } 1016 \\
\text { PCB } 1221\end{array}$ & & & & & & & & & & & & \\
\hline & $\begin{array}{l}\text { PCB } 1221 \\
\text { PCB } 1232\end{array}$ & & & & & & & & & & & & \\
\hline & $\begin{array}{l}\text { PCB } 1232 \\
\text { PCB } 1242\end{array}$ & & & & & & & & & & & & \\
\hline & PCB 1242 & & & & & & & & & & & & \\
\hline & PCB 1248 & & & & & & & & & & & & \\
\hline & PCB 1254 & & & & & & & & & & & & \\
\hline & РСВ 1260 & & & & & & & & & & & & \\
\hline & $1,1,2,2$-Tetrachloroethane & $<25$ & $\mathrm{U}$ & $<25$ & $U$ & $<1$ & $\mathrm{U}$ & $<2$ & $\mathrm{u}$ & $<E Q L$ & 2 & ug/L & $M L$ \\
\hline+ & Tetrachloroethylene & 7.26 & $J$ & $<25$ & $U$ & 9.33 & K & 11.8 & & $>5$ & 2 & $\mathrm{ug} / \mathrm{L}$ & ML \\
\hline & $1,1,1$-Trichloroethane & $<25$ & $U$ & $<25$ & $\mathrm{U}$ & $<1$ & $\mathrm{U}$ & $<2$ & $\mathrm{U}$ & $<E Q L$ & 2 & $u g / L$ & ML \\
\hline+ & Trichloroethylene & 292 & & 287 & & 383 & $\mathrm{~J} \quad \mathrm{~K}$ & 559 & & $>5$ & 2 & ug/L & $M L$ \\
\hline \multicolumn{14}{|c|}{ II. Monitoring Constituents } \\
\hline$\underline{\mathbf{S T}}$ & Parameter & $\underline{3 Q 96}$ & CLPEPA & $\underline{3 Q 97}$ & $\underline{\text { CLP EPA }}$ & $\underline{3 Q 98}$ & CLPEPA & $\underline{3 Q 99}$ & CLPEPA & Filt. & DF & Unit & $\underline{\text { Lab }}$ \\
\hline \multicolumn{14}{|c|}{ Inorganics } \\
\hline & Aluminum, total recoverable & 79.2 & & 61 & & $<200$ & $\mathrm{U}$ & $<200$ & $U$ & $<E Q L$ & 1 & ug/L & $\mathrm{EX}$ \\
\hline & Nitrate-nitrite as nitrogen & 1690 & & 1870 & & 1440 & & 1570 & & $<2400$ & 5 & $u g h$ & EX \\
\hline & Sodium, total recoverable & 2110 & & 2000 & & 2130 & & 2630 & & $<4600$ & 1 & $\mathrm{ug} / \mathrm{L}$ & EX \\
\hline & Sulfate & 170 & $J$ & $<5000$ & $U$ & 225 & $J$ & $<400$ & $\mathrm{U}$ & $<\mathrm{EQL}$ & 2 & $\mathrm{ug} / \mathrm{L}$ & EX \\
\hline \multicolumn{14}{|c|}{ Radionuclides } \\
\hline & Gross alpha & .754 & & 1.84 & Ul & 1.88 & $J$ & .35 & $\mathrm{U}$ & $<E Q L$ & 1 & $\mathrm{pCi} / \mathrm{L}$ & GP \\
\hline & Nonvolatile beta & 1.76 & & 1.15 & Ut & 5.69 & & 1.17 & $\mathrm{U}$ & $<E Q L$ & 1 & $\mathrm{pCi} / \mathrm{L}$ & GP \\
\hline & Radium, total alpha-emitting & -.1 & UIJ & 5.15 & $\mathrm{~J}$ & .89 & & .4 & $\mathrm{U}$ & $<\mathrm{EQL}$ & 1 & $\mathrm{pCi} / \mathrm{L}$ & GP \\
\hline
\end{tabular}

Notes: Concentrations in bold italics exceed the groundwater protection or monitoring constituent standards listed in Appendix A. Synchronous water levels are measured over a 3-5 day period or less. Dilution factors, Laboratory, and Filtered Data are for Groundwater Protection Standards First Quarter 2000 and Monitoring Constituents Third Quarter 1999 data only.

$+=$ exceeded the Groundwater Protection Standards (First Quarter 2000) or Monitoring Constituents (Third Quarter 1999) listed in Appendix A. 
Table D-4. Groundwater Monitoring Results for Plume Definition Wells, M-Area HWMF (Cont.) WELL MSB 66C

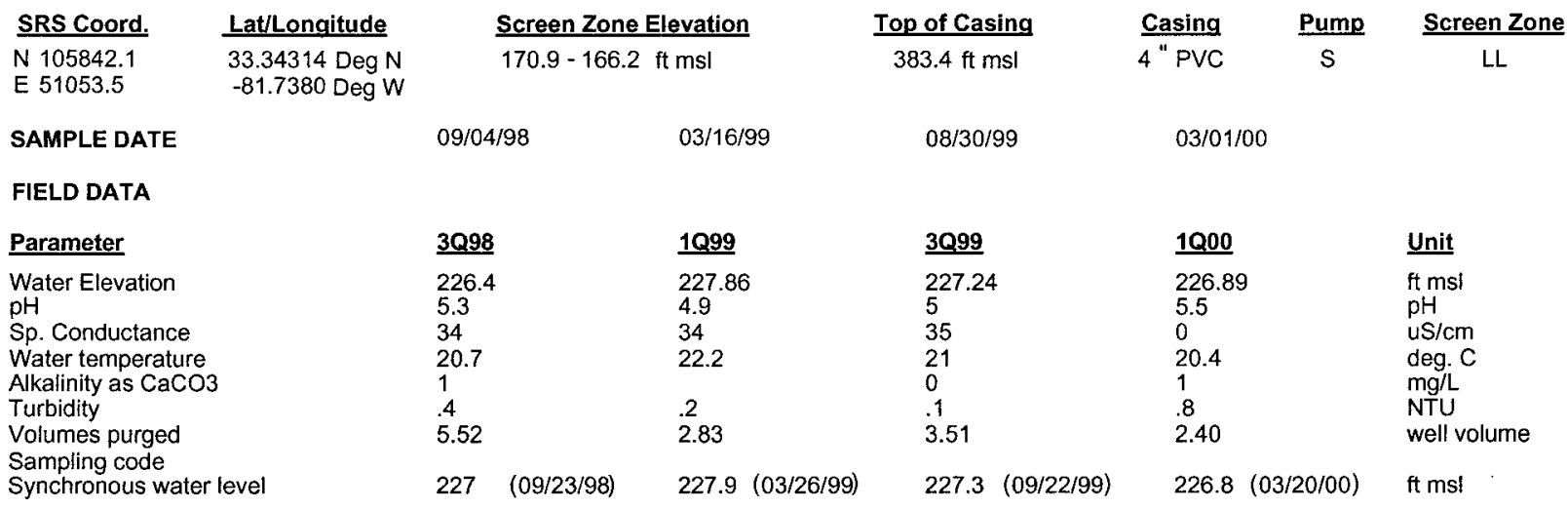

ANALYTICAL DATA

I. Groundwater Protection Standard

261 Appendix VIII/264 Appendix IX Hazardous Constituents

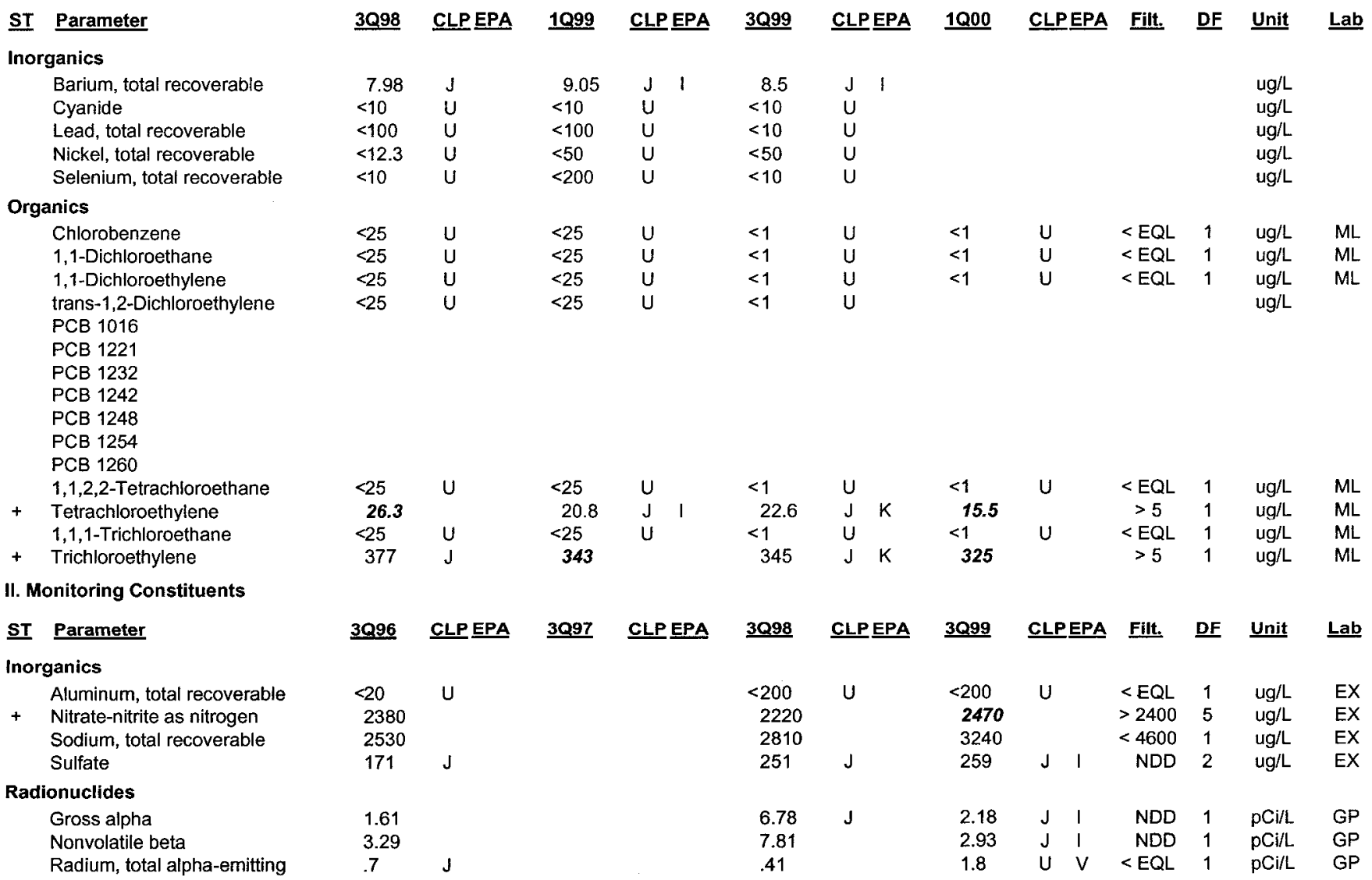

Notes: Concentrations in bold italics exceed the groundwater protection or monitoring constituent standards listed in Appendix A. Synchronous water levels are measured over a 3-5 day period or less. Dilution factors, Laboratory, and Filtered Data are for Groundwater Protection Standards First Quarter 2000 and Monitoring Constituents Third Quarter 1999 data only.

$+=$ exceeded the Groundwater Protection Standards (First Quarter 2000) or Monitoring Constituents (Third Quarter 1999) listed in Appendix A. 
Table D-4. Groundwater Monitoring Results for Plume Definition Wells, M-Area HWMF (Cont.) WELL MSB 66D

\begin{tabular}{|c|c|c|c|c|c|c|}
\hline SRS Coord. & Lat/Longitude & Screen Zone Elevation & Top of Casing & Casing & Pump & Screen Zone \\
\hline $\begin{array}{l}\text { N } 105841.8 \\
\text { E } 51044\end{array}$ & $\begin{array}{l}33.34313 \operatorname{Deg} N \\
-81.7380 \mathrm{Deg} W\end{array}$ & $239.5-220 \quad \mathrm{ft} \mathrm{msl}$ & $383.2 \mathrm{ft} \mathrm{msl}$ & 4 "PVC & & $M$ \\
\hline
\end{tabular}

SAMPLE DATE

FIELD DATA

Parameter

Water Elevation

$\mathrm{pH}$

Sp. Conductance

Water temperature

Alkalinity as $\mathrm{CaCO} 3$

Turbidity

Volumes purged

Sampling code

Synchronous water level

ANALYTICAL DATA

I. Groundwater Protection Standard

\begin{tabular}{|c|c|c|c|c|}
\hline$\underline{3098}$ & 1099 & $\underline{3099}$ & 1000 & Unit \\
\hline Not Available & Not Available & Not Available & Not Available & $\begin{array}{l}\text { ft msl } \\
\mathrm{pH} \\
\mathrm{uS} / \mathrm{cm} \\
\mathrm{deg} . \mathrm{C} \\
\mathrm{mg} / \mathrm{L} \\
\mathrm{NTU} \\
\text { well volume }\end{array}$ \\
\hline $267.1(09 / 23 / 98)$ & $231 \quad(03 / 26 / 99)$ & $230.5(09 / 22 / 99)$ & $267.2(03 / 20 / 00)$ & $\mathrm{ft} \mathrm{msl}$ \\
\hline
\end{tabular}

$267.1(09 / 23 / 98) \quad 231 \quad(03 / 26 / 99) \quad 230.5(09 / 22 / 99) \quad 267.2(03 / 20 / 00) \quad$ ft ms

Notes: Concentrations in bold italics exceed the groundwater protection or monitoring constituent standards listed in Appendix A. Synchronous water levels are measured over a 3-5 day period or less. Dilution factors, Laboratory, and Filtered Data are for Groundwater Protection Standards First Quarter 2000 and Monitoring Constituents Third Quarter 1999 data only.

$+=$ exceeded the Groundwater Protection Standards (First Quarter 2000) or Monitoring Constituents (Third Quarter 1999) listed in Appendix A. 
Table D-4. Groundwater Monitoring Results for Plume Definition Wells, M-Area HWMF (Cont.) WELL MSB 66TA

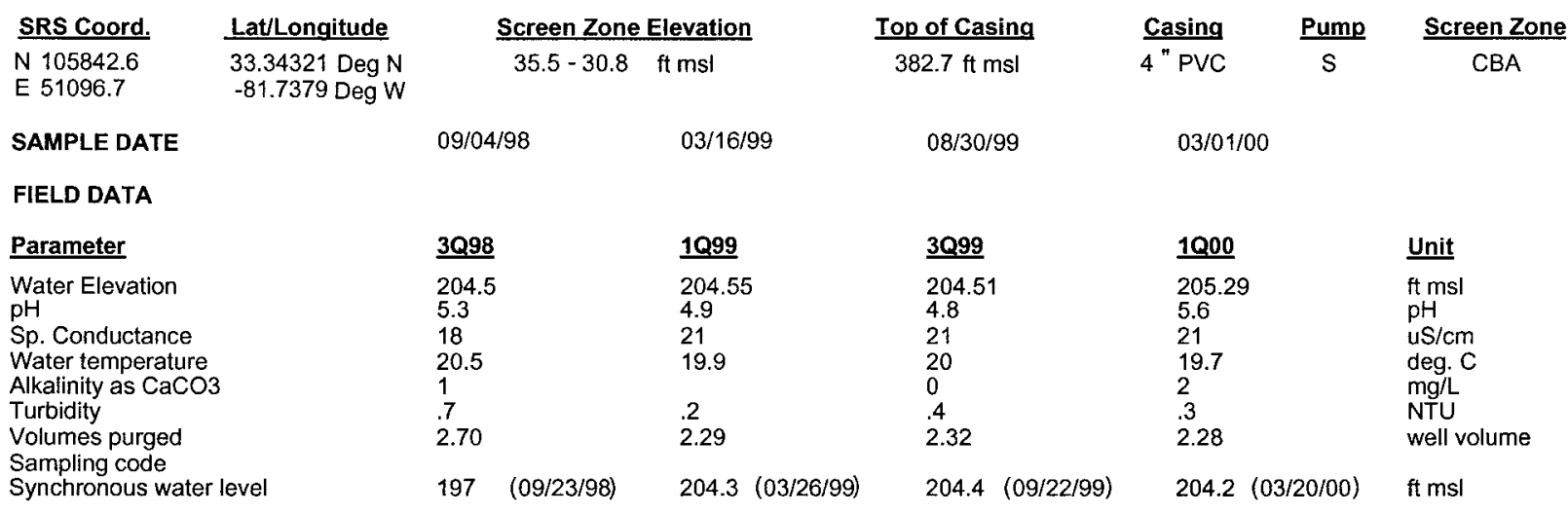

ANALYTICAL DATA

I. Groundwater Protection Standard

261 Appendix VIII/264 Appendix IX Hazardous Constituents

\begin{tabular}{|c|c|c|c|c|c|c|c|c|c|c|c|c|}
\hline ST Parameter & $\underline{3 Q 98}$ & CLPEPA & $\underline{1 Q 99}$ & CLPEPA & $\underline{3 Q 99}$ & CLPEPA & $\underline{1000}$ & CLPEPA & Fllt. & $\underline{D F}$ & $\underline{\text { Unit }}$ & $\underline{\text { Lab }}$ \\
\hline \multicolumn{13}{|l|}{ Inorganics } \\
\hline Barium, total recoverable & 3.01 & J & 3.38 & J 1 & 2.5 & J 1 & & & & & $u g / L$ & \\
\hline Cyanide & $<10$ & $\mathrm{u}$ & $<10$ & $\mathrm{u}$ & $<10$ & $\mathrm{U}$ & & & & & $\mathrm{ug} / \mathrm{L}$ & \\
\hline Lead, total recoverable & $<100$ & $\mathrm{u}$ & $<100$ & $\mathrm{u}$ & $<10$ & $u$ & & & & & $\mathrm{ug} / \mathrm{L}$ & \\
\hline Nickel, total recoverable & $<9.05$ & $\mathrm{u}$ & $<50$ & $\mathrm{u}$ & $<50$ & $\mathrm{u}$ & & & & & $\mathrm{ug} / \mathrm{L}$ & \\
\hline Selenium, total recoverable & $<10$ & $\mathrm{u}$ & $<200$ & $u$ & $<10$ & $u$ & & & & & $u g / L$ & \\
\hline \multicolumn{13}{|l|}{ Organics } \\
\hline Chlorobenzene & $<5$ & UJ & $<5$ & $u$ & $<1$ & $\mathrm{u}$ & $<1$ & $\mathrm{u}$ & $<E Q L$ & 1 & $u g / L$ & ML \\
\hline 1,1-Dichloroethane & $<5$ & UJ & $<5$ & $u$ & $<1$ & $\mathrm{u}$ & $<1$ & $\mathrm{u}$ & $<E Q L$ & 1 & $u g / L$ & $\mathrm{ML}$ \\
\hline 1,1-Dichloroethylene & $<5$ & UJ & $<5$ & $\mathrm{u}$ & $<1$ & $\mathrm{u}$ & $<1$ & $\mathrm{U}$ & $<E Q L$ & 1 & $u g / L$ & ML \\
\hline trans-1,2-Dichloroethylene & $<5$ & UJ & $<5$ & $\mathrm{u}$ & $<1$ & $\mathrm{u}$ & & & & & $u g / L$ & \\
\hline \multicolumn{13}{|l|}{ PCB 1016} \\
\hline \multirow{2}{*}{\multicolumn{13}{|c|}{ РCB 1221}} \\
\hline & & & & & & & & & & & & \\
\hline & \multicolumn{2}{|c|}{$\begin{array}{l}\text { PCB } 1232 \\
\text { PCB } 1242\end{array}$} & & & & & & & & & & \\
\hline \multicolumn{13}{|l|}{$\begin{array}{l}\text { РCB } 1242 \\
\text { РCB } 1248\end{array}$} \\
\hline \multicolumn{13}{|l|}{ PCB 1254} \\
\hline \multicolumn{13}{|l|}{ PCB 1260} \\
\hline 1,1,2,2-Tetrachloroethane & $<5$ & UJ & $<5$ & $\mathrm{U}$ & $<1$ & $\mathrm{u}$ & $<1$ & $\mathrm{U}$ & $<E Q L$ & 1 & $\mathrm{ug} / \mathrm{L}$ & ML \\
\hline Tetrachloroethylene & 7.05 & $\mathrm{~J}$ & 6.2 & & 6.8 & $J K$ & 6.74 & $J \quad K$ & NDD & 1 & $\mathrm{ug} / \mathrm{L}$ & ML \\
\hline 1,1,1-Trichloroethane & $<5$ & UJ & $<5$ & u & $<1$ & $\mathrm{u}$ & $<1$ & $\mathrm{u}$ & $<\mathrm{EQL}$ & 1 & $\mathrm{ug} / \mathrm{L}$ & $\mathrm{ML}$ \\
\hline Trichloroethylene & 5.16 & J & 5.01 & & 5.26 & $J \quad K$ & 5.56 & $J \quad K$ & NDD & 1 & $u g / L$ & ML \\
\hline \multicolumn{13}{|l|}{ II. Monitoring Constituents } \\
\hline ST Parameter & $\underline{3096}$ & CLPPEPA & $\underline{3097}$ & CLPPEPA & $\underline{3098}$ & CLPEPA & $\underline{3099}$ & CLPEPA & Filt. & $\underline{\text { DF }}$ & $\underline{\text { Unit }}$ & $\underline{\text { Lab }}$ \\
\hline \multicolumn{13}{|l|}{ Inorganics } \\
\hline Aluminum, total recoverable & 43.8 & $J$ & 57.7 & & $<200$ & u & $<200$ & u & $<E Q L$ & 1 & $\mathrm{ug} / \mathrm{L}$ & EX \\
\hline Nitrate-nitrite as nitrogen & 1010 & & 782 & & 682 & & 695 & & $<2400$ & 5 & $\mathrm{ug} / \mathrm{L}$ & EX \\
\hline Sodium, total recoverable & 1480 & $\mathrm{~J}$ & 1450 & & 1510 & & 1560 & & $<4600$ & 1 & $\mathrm{ug} / \mathrm{L}$ & EX \\
\hline Sulfate & 1500 & & $<5000$ & $U$ & 1300 & & 1380 & & $<3000$ & 2 & $\mathrm{ug} / \mathrm{L}$ & EX \\
\hline \multicolumn{13}{|l|}{ Radionuclides } \\
\hline Gross alpha & .743 & & 1.02 & UI & 1.15 & $\mathrm{~J}$ & .189 & $\mathrm{u}$ & $<E Q L$ & 1 & $\mathrm{pCi} / \mathrm{L}$ & GP \\
\hline Nonvolatile beta & .398 & UI & -.38 & UI & .67 & $\mathrm{UI}$ & .576 & $\mathrm{U}$ & $<\mathrm{EQL}$ & 1 & $\mathrm{pCi} / \mathrm{L}$ & GP \\
\hline Radium, total alpha-emitting & .7 & & 6.54 & $\mathrm{~J}$ & 1.08 & & .3 & u & $<E Q L$ & 1 & $\mathrm{pCi} / \mathrm{L}$ & GP \\
\hline
\end{tabular}

Notes: Concentrations in bold italics exceed the groundwater protection or monitoring constituent standards listed in Appendix A. Synchronous water levels are measured over a 3-5 day period or less. Dilution factors, Laboratory, and Filtered Data are for Groundwater Protection Standards First Quarter 2000 and Monitoring Constituents Third Quarter 1999 data only.

+ = exceeded the Groundwater Protection Standards (First Quarter 2000) or Monitoring Constituents (Third Quarter 1999) listed in Appendix A. 
Table D-4. Groundwater Monitoring Results for Plume Definition Wells, M-Area HWMF (Cont.) WELL MSB 67D

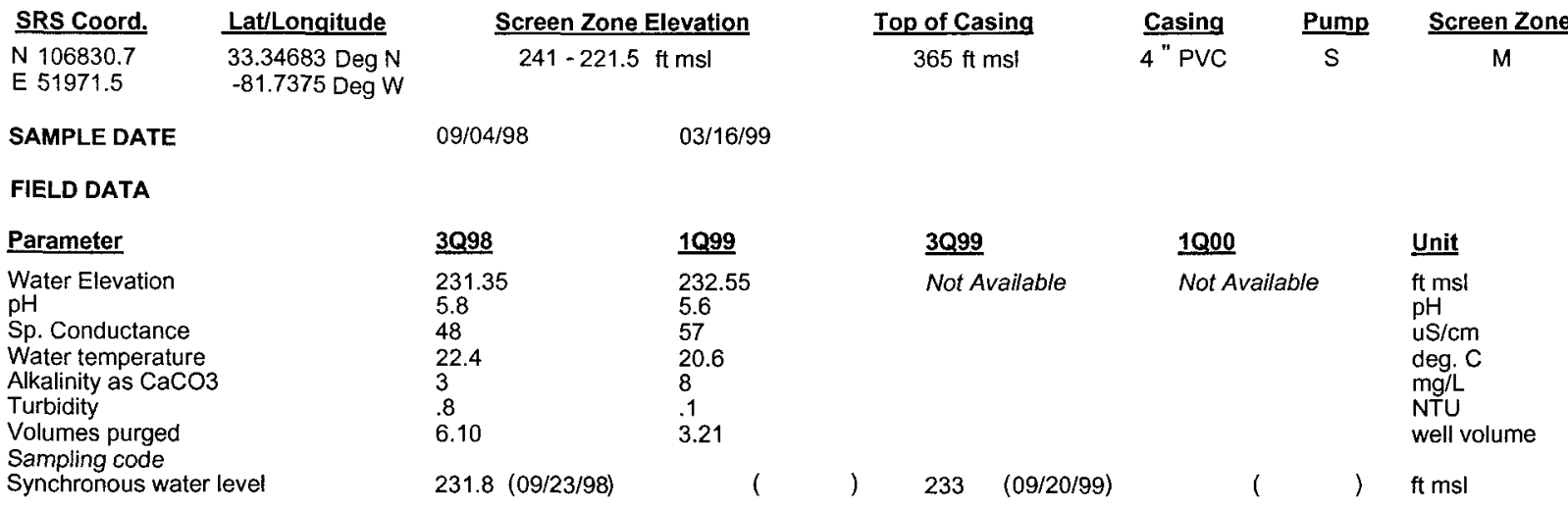

ANALYTICAL DATA

1. Groundwater Protection Standard

261 Appendix VIII/264 Appendix IX Hazardous Constituents

\begin{tabular}{|c|c|c|c|c|c|c|c|c|c|c|c|c|c|}
\hline$\underline{\text { ST }}$ & Parameter & $\underline{3 Q 98}$ & CLPEPA & 1099 & CLP EPA & 3Q99 & CLPEPA & $1 \mathrm{Q00}$ & CLPEPA & Filt. & DF & Unit & Lab \\
\hline \multicolumn{14}{|c|}{ Inorganics } \\
\hline & Barium, total recoverable & 33.4 & & 35.3 & & & & & & & & $\mathrm{ug} / \mathrm{L}$ & \\
\hline & Cyanide & $<10$ & $U$ & $<10$ & U & & & & & & & $\mathrm{ug} / \mathrm{L}$ & \\
\hline & Lead, total recoverable & $<100$ & $\mathrm{U}$ & $<100$ & $U$ & & & & & & & ug/L & \\
\hline & Nickel, total recoverable & $<12.7$ & $U$ & $<50$ & $\mathrm{U}$ & & & & & & & $\mathrm{ug} / \mathrm{L}$ & \\
\hline & Selenium, total recoverable & $<10$ & $U$ & $<200$ & $\mathrm{U}$ & & & & & & & $\mathrm{ug} / \mathrm{L}$ & \\
\hline \multicolumn{14}{|c|}{ Organics } \\
\hline & Chlorobenzene & $<50$ & $\mathrm{U}$ & $<125$ & $\mathrm{U}$ & & & & & & & ug/L & \\
\hline & 1,1-Dichloroethane & $<50$ & $\mathrm{U}$ & $<125$ & $\mathrm{U}$ & & & & & & & ug/L. & \\
\hline & 1,1-Dichloroethylene & $<50$ & U & $<125$ & $\mathrm{U}$ & & & & & & & $\mathrm{ug} / \mathrm{L}$ & \\
\hline & trans-1,2-Dichloroethylene & $<50$ & $\mathrm{U}$ & $<125$ & $U$ & & & & & & & $\mathrm{ug} / \mathrm{L}$ & \\
\hline & $\begin{array}{l}\text { PCB } 1016 \\
\text { PCB } 1221\end{array}$ & & & & & & & & & & & & \\
\hline & PCB 1232 & & & & & & & & & & & & \\
\hline & PCB 1242 & & & & & & & & & & & & \\
\hline & PCB 1248 & & & & & & & & & & & & \\
\hline & PCB 1254 & & & & & & & & & & & & \\
\hline & PCB 1260 & & & & & & & & & & & & \\
\hline & 1,1,2,2-Tetrachloroethane & $<50$ & $\mathrm{U}$ & $<125$ & $\mathrm{U}$ & & & & & & & $\mathrm{ug} / \mathrm{L}$ & \\
\hline & Tetrachloroethylene & $<50$ & $\mathrm{U}$ & $<125$ & $\mathrm{U}$ & & & & & & & $\mathrm{ug} / \mathrm{L}$ & \\
\hline & 1,1,1-Trichloroethane & $<50$ & $\mathrm{U}$ & $<125$ & $\mathrm{U}$ & & & & & & & $\mathrm{ug} / \mathrm{L}$ & \\
\hline & Trichloroethylene & 1370 & $J$ & 3030 & & & & & & & & ug/L & \\
\hline \multicolumn{14}{|c|}{ II. Monitoring Constituents } \\
\hline$\underline{\text { ST }}$ & Parameter & $\underline{3 Q 96}$ & CLP EPA & 3097 & $\underline{\text { CLP EPA }}$ & 3098 & CLPEPA & 30999 & CLPEEPA & Filt. & DF & $\underline{\text { Unit }}$ & $\underline{L a b}$ \\
\hline \multicolumn{13}{|c|}{ Inorganics } & \\
\hline & Aluminum, total recoverable & 18.8 & $J$ & 26.6 & & $<200$ & $\mathrm{U}$ & & & & & $u g / L$ & \\
\hline & Nitrate-nitrite as nitrogen & 2200 & & 1950 & & 2960 & & & & & & $u g / L$ & \\
\hline & Sodium, total recoverable & 2060 & & 3400 & & 3710 & & & & & & $u g / L$ & \\
\hline & Sulfate & 230 & $\mathrm{~J}$ & $<5000$ & $\mathrm{U}$ & 269 & $J$ & & & & & $u g / L$ & \\
\hline \multicolumn{13}{|c|}{ Radionuclides } & \\
\hline & Gross alpha & 1.28 & & 1.05 & UI & 5.83 & $\jmath$ & & & & & $\mathrm{pCi} / \mathrm{L}$ & \\
\hline & Nonvolatile beta & 1.45 & & -.35 & UI & 8.27 & & & & & & $\mathrm{pCi} / \mathrm{L}$ & \\
\hline & Radium, total alpha-emitting & .4 & $J$ & 5.91 & UIJ & 1.08 & UI & & & & & $\mathrm{pCi} / \mathrm{L}$ & \\
\hline
\end{tabular}

Notes: Concentrations in bold italics exceed the groundwater protection or monitoring constituent standards listed in Appendix A. Synchronous water levels are measured over a 3-5 day period or less. Dilution factors, Laboratory, and Filtered Data are for Groundwater Protection Standards First Quarter 2000 and Monitoring Constituents Third Quarter 1999 data only.

+ = exceeded the Groundwater Protection Standards (First Quarter 2000) or Monitoring Constituents (Third Quarter 1999) listed in Appendix A. 
Table D-4. Groundwater Monitoring Results for Plume Definition Wells, M-Area HWMF (Cont.) WELL MSB 68B

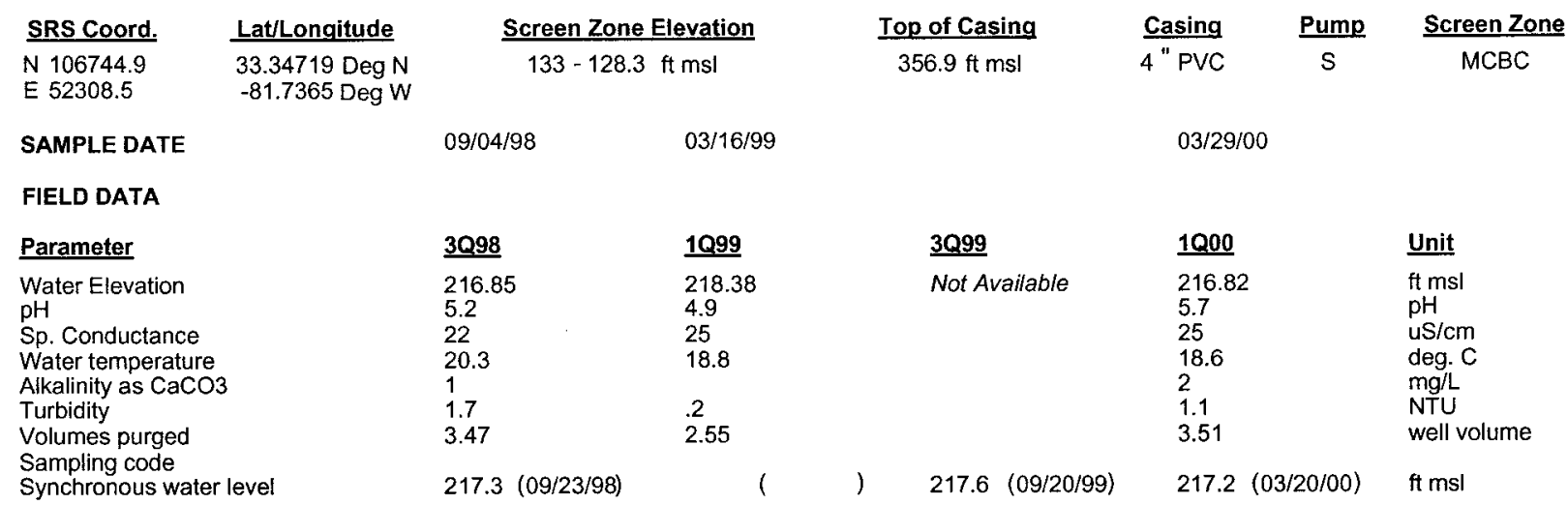

ANALYTICAL DATA

I. Groundwater Protection Standard

261 Appendix VIII/264 Appendix IX Hazardous Constituents

\begin{tabular}{|c|c|c|c|c|c|c|c|c|c|c|c|c|c|}
\hline$\underline{\text { ST }}$ & Parameter & $\underline{3 Q 98}$ & CLP EPA & 1099 & CLP EPA & 3Q99 & CLPEPA & $\underline{1 Q 00}$ & CLPEPA & Filt. & $\underline{\text { DF }}$ & Unit & $\underline{\text { Lab }}$ \\
\hline \multicolumn{14}{|c|}{ Inorganics } \\
\hline & Barium, total recoverable & 4.28 & $J$ & 5.07 & J I & & & & & & & $\mathrm{ug} / \mathrm{L}$ & \\
\hline & Cyanide & $<10$ & $\mathrm{U}$ & $<10$ & $U$ & & & & & & & $\mathrm{ug} / \mathrm{L}$ & \\
\hline & Lead, total recoverable & $<100$ & $\mathrm{U}$ & $<100$ & $U$ & & & & & & & ug/L & \\
\hline & Nickel, total recoverable & $<50$ & $\mathrm{U}$ & $<50$ & $\mathrm{U}$ & & & & & & & $\mathrm{ug} / \mathrm{L}$ & \\
\hline & Selenium, total recoverable & $<10$ & $\mathrm{U}$ & $<200$ & $U$ & & & & & & & $\mathrm{ug} / \mathrm{L}$ & \\
\hline \multicolumn{14}{|c|}{ Organics } \\
\hline & Chlorobenzene & $<25$ & $\mathrm{U}$ & $<25$ & $U$ & & & $<1$ & $U$ & $<\mathrm{EQL}$ & 1 & $\mathrm{ug} / \mathrm{L}$ & ML \\
\hline & 1,1-Dichloroethane & $<25$ & $\mathrm{U}$ & $<25$ & $\mathrm{U}$ & & . & $<1$ & $U$ & $<\mathrm{EQL}$ & 1 & $\mathrm{ug} / \mathrm{L}$ & $M L$ \\
\hline & 1,1-Dichloroethylene & $<25$ & $\mathrm{U}$ & $<25$ & U & & & $<1$ & $\mathrm{U}$ & $<E Q L$ & 1 & $\mathrm{ug} / \mathrm{L}$ & $M L$ \\
\hline & $\begin{array}{l}\text { trans-1,2-Dichloroethylene } \\
\text { PCB } 1016\end{array}$ & $<25$ & $\mathrm{U}$ & $<25$ & $U$ & & & $<1$ & $\mathrm{U}$ & $<$ EQL & 1 & $\mathrm{ug} / \mathrm{L}$ & ML \\
\hline & PCB 1221 & & & & & & & & & & & & \\
\hline & PCB 1232 & & & & & & & & & & & & \\
\hline & PCB 1242 & & & & & & & & & & & & \\
\hline & РCB 1248 & & & & & & & & & & & & \\
\hline & PCB 1254 & & & & & & & & & & & & \\
\hline & PCB 1260 & & & & & & & & & & & & \\
\hline & 1,1,2,2-Tetrachloroethane & $<25$ & $U$ & $<25$ & $\cup$ & & & $<1$ & $\mathrm{U}$ & $<\mathrm{EQL}$ & 1 & ug/L & $M L$ \\
\hline+ & Tetrachloroethylene & 29 & & 18.1 & J 1 & & & 13.3 & & $>5$ & 1 & $\mathrm{ug} / \mathrm{L}$ & $M L$ \\
\hline & 1,1,1-Trichloroethane & $<25$ & $U$ & $<25$ & $U$ & & & $<1$ & U & $<E Q L$ & 1 & $u g / L$ & $M L$ \\
\hline+ & Trichloroethylene & 346 & & 205 & & & & 211 & & $>5$ & 1 & $\mathrm{ug} / \mathrm{L}$ & $M L$ \\
\hline \multicolumn{14}{|c|}{ II. Monitoring Constituents } \\
\hline$\underline{\mathrm{ST}}$ & Parameter & $\underline{3096}$ & CLP EPA & $\underline{3097}$ & CLPPEPA & $\underline{3098}$ & CLPEPA & $\underline{3099}$ & CLPEPA & Filt. & $\underline{\text { DF }}$ & Unit & $\underline{\text { Lab }}$ \\
\hline \multicolumn{13}{|c|}{ Inorganics } & \\
\hline & Aluminum, total recoverable & 14.9 & $J$ & 32.4 & & $<200$ & $U$ & & & & & $\mathrm{ug} / \mathrm{L}$ & \\
\hline & Nitrate-nitrite as nitrogen & 1620 & & 1600 & & 1310 & & & & & & $\mathrm{ug} / \mathrm{L}$ & \\
\hline & Sodium, total recoverable & 2030 & & 2020 & & 2040 & & & & & & $\mathrm{ug} / \mathrm{L}$ & \\
\hline & Sulfate & 280 & $J$ & $<5000$ & $U$ & 334 & $J$ & & & & & $\mathrm{ug} / \mathrm{L}$ & \\
\hline \multicolumn{13}{|c|}{ Radionuclides } & \\
\hline & Gross alpha & .972 & & 1.27 & & 2.14 & J & & & & & $\mathrm{pCi} / \mathrm{L}$ & \\
\hline & Nonvolatife beta & 1.65 & & -.26 & UI & 4.23 & & & & & & $\mathrm{pCi} / \mathrm{L}$ & \\
\hline & Radium, total alpha-emitting & .2 & UIJ & 3.13 & UIJ & .21 & UI & & & & & $\mathrm{pCi} / \mathrm{L}$ & \\
\hline
\end{tabular}

Notes: Concentrations in bold italics exceed the groundwater protection or monitoring constituent standards listed in Appendix A. Synchronous water levels are measured over a 3-5 day period or less. Dilution factors, Laboratory, and Filtered Data are for Groundwater Protection Standards First Quarter 2000 and Monitoring Constituents Third Quarter 1999 data only.

$+=$ exceeded the Groundwater Protection Standards (First Quarter 2000) or Monitoring Constituents (Third Quarter 1999) listed in Appendix A. 
Table D-4. Groundwater Monitoring Results for Plume Definition Wells, M-Area HWMF (Cont.) WELL MSB 68C

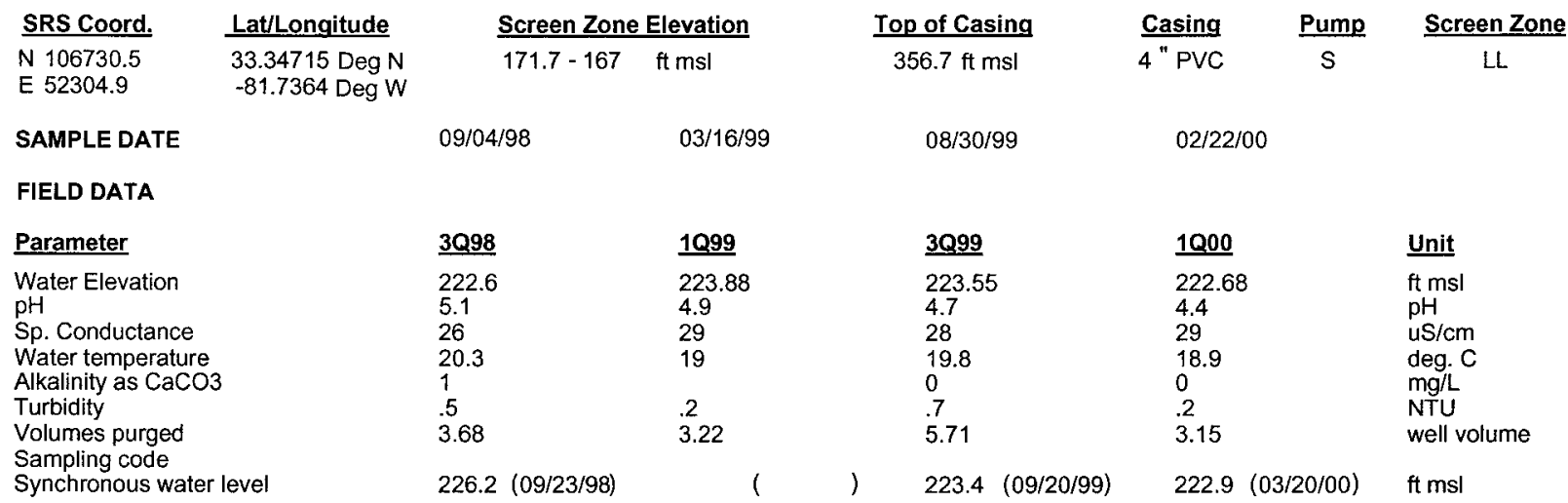

ANALYTICAL DATA

I. Groundwater Protection Standard

261 Appendix VIII/264 Appendix IX Hazardous Constituents

\begin{tabular}{|c|c|c|c|c|c|c|c|c|c|c|c|c|c|c|}
\hline$\underline{\mathrm{ST}}$ & Parameter & $\underline{3 Q 98}$ & $\underline{\text { CLP EPA }}$ & 1099 & CLPP EPA & $\underline{3 Q 99}$ & CLPEPA & 1000 & $\underline{\text { CLP }}$ & EPA & Filt. & $\underline{\text { DF }}$ & $\underline{\text { Unit }}$ & $\underline{\text { Lab }}$ \\
\hline Inor & ganics & & & & & & & & & & & & & \\
\hline & Barium, total recoverable & 4.51 & J & 6.4 & $\mathrm{~J}$ & 5.4 & J 1 & & & & & & $u g / L$ & \\
\hline & Cyanide & $<10$ & $U$ & $<10$ & $\mathrm{U}$ & $<10$ & U & & & & & & ug/L & \\
\hline & Lead, total recoverable & $<100$ & $U$ & $<100$ & $\mathrm{U}$ & $<10$ & $U$ & & & & & & $\mathrm{ug} / \mathrm{L}$ & \\
\hline & Nickel, total recoverable & $<50$ & U & $<50$ & $\mathrm{U}$ & $<50$ & $U$ & & & & & & $\mathrm{ug} / \mathrm{L}$ & \\
\hline & Selenium, total recoverable & $<10$ & $\mathrm{U}$ & $<200$ & $\mathrm{U}$ & $<10$ & $\mathrm{U}$ & & & & & & $\mathrm{ug} / \mathrm{L}$ & \\
\hline Orge & anics & & & & & & & & & & & & & \\
\hline & Chlorobenzene & $<50$ & $u$ & $<50$ & U & $<1$ & $\mathrm{U}$ & $<5$ & $\mathrm{u}$ & & $<\mathrm{EQL}$ & 5 & $u g / L$ & $M L$ \\
\hline & 1,1-Dichloroethane & $<50$ & $U$ & $<50$ & $\mathrm{U}$ & $<1$ & $U$ & $<5$ & $U$ & & $<E Q L$ & 5 & $\mathrm{ug} / \mathrm{h}$ & ML \\
\hline & 1,1-Dichloroethylene & $<50$ & $\mathrm{U}$ & $<50$ & $U$ & $<1$ & $\mathrm{U}$ & $<5$ & $\mathrm{U}$ & & $<\mathrm{EQL}$ & 5 & $u g / L$ & ML \\
\hline & $\begin{array}{l}\text { trans-1,2-Dichloroethylene } \\
\text { PCB } 1016\end{array}$ & $<50$ & $\mathrm{u}$ & $<50$ & $\mathrm{U}$ & $<1$ & $u$ & $<5$ & $\mathrm{u}$ & & $<E Q L$ & 5 & $u g / L$ & ML \\
\hline & PCB 1221 & & & & & & & & & & & & & \\
\hline & PCB 1232 & & & & & & & & & & & & & \\
\hline & PCB 1242 & & & & & & & & & & & & & \\
\hline & PCB 1248 & & & & & & & & & & & & & \\
\hline & PCB 1254 & & & & & & & & & & & & & \\
\hline & PCB 1260 & & & & & & & & & & & & & \\
\hline & 1,1,2,2-Tetrachloroethane & $<50$ & $\mathrm{U}$ & $<50$ & $U$ & $<1$ & U & $<5$ & $U$ & & $<E Q L$ & 5 & $\mathrm{ug} / \mathrm{L}$ & ML \\
\hline+ & Tetrachloroethylene & $<50$ & $\mathrm{U}$ & $<50$ & $\mathrm{U}$ & 6.13 & $\mathrm{~J} K$ & 8.6 & & & $>5$ & 5 & $\mathrm{ug} / \mathrm{L}$ & ML \\
\hline & 1,1,1-Trichloroethane & $<50$ & $\mathrm{U}$ & $<50$ & $\mathrm{U}$ & $<1$ & $\mathrm{U}$ & $<5$ & $\mathrm{U}$ & & $<\mathrm{EQL}$ & 5 & $\mathrm{ug} / \mathrm{L}$ & ML \\
\hline+ & Trichloroethylene & 702 & $\mathrm{~J}$ & 724 & & 835 & & 1070 & & & $>5$ & 5 & ug/L & ML \\
\hline II. Mc & onitoring Constituents & & & & & & & & & & & & & \\
\hline$\underline{\mathbf{S T}}$ & Parameter & $\underline{3 Q 96}$ & CLPEPA & $\underline{3097}$ & CLPEPA & $\underline{3 Q 98}$ & CLPEPA & $\underline{3099}$ & $\underline{\text { CLP }}$ & EPA & Filt. & $\underline{\text { DF }}$ & $\underline{\text { Unit }}$ & Lab \\
\hline Inors & ganics & & & & & & & & & & & & & \\
\hline & Aluminum, total recoverable & $<20$ & $\mathrm{U}$ & 30.7 & & $<200$ & $\mathrm{U}$ & $<200$ & $\mathrm{U}$ & & $<\mathrm{EQL}$ & 1 & $\mathrm{ug} / \mathrm{L}$ & EX \\
\hline & Nitrate-nitrite as nitrogen & 2000 & & 1090 & & 1780 & & 1660 & & & $<2400$ & 5 & $\mathrm{ug} / \mathrm{L}$ & EX \\
\hline & Sodium, total recoverable & 2230 & & 2240 & & 2390 & & 2820 & & & $<4600$ & 1 & $\mathrm{ug} / \mathrm{L}$ & EX \\
\hline & Sulfate & 175 & $J$ & $<5000$ & $\mathrm{U}$ & 2480 & & 263 & $J$ & 1 & NDD & 2 & $u g / L$ & EX \\
\hline Radi & ionuclides & & & & & & & & & & & & & \\
\hline & Gross alpha & 1.23 & & 1.51 & & 4.71 & J & 1.49 & J & I & NDD & 1 & $\mathrm{pCi} / \mathrm{L}$ & GP \\
\hline & Nonvolatile beta & 2.98 & & 1.1 & UI & 15.4 & & 3.22 & & & $<50$ & 1 & $\mathrm{pCi} / \mathrm{L}$ & GP \\
\hline & Radium, total alpha-emitting & .1 & UIJ & 2.82 & UIJ & 1.07 & & .8 & $\mathrm{U}$ & V & $<E Q L$ & 1 & $\mathrm{pCi} / \mathrm{L}$ & GP \\
\hline
\end{tabular}

Notes: Concentrations in bold italics exceed the groundwater protection or monitoring constituent standards listed in Appendix A. Synchronous water levels are measured over a 3-5 day period or less. Dilution factors, Laboratory, and Filtered Data are for Groundwater Protection Standards First Quarter 2000 and Monitoring Constituents Third Quarter 1999 data only.

$+=$ exceeded the Groundwater Protection Standards (First Quarter 2000) or Monitoring Constituents (Third Quarter 1999) listed in Appendix A. 
Table D-4. Groundwater Monitoring Results for Plume Definition Wells, M-Area HWMF (Cont.) WELL MSB 69B

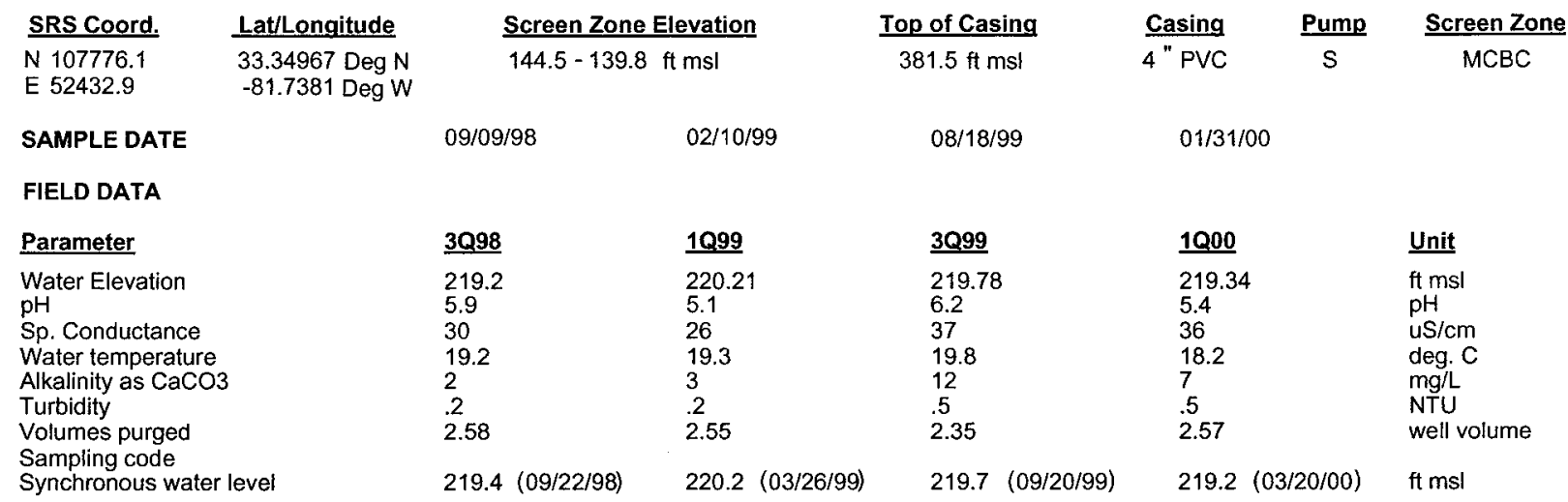

ANALYTICAL DATA

I. Groundwater Protection Standard

261 Appendix VIII/264 Appendix IX Hazardous Constituents

\begin{tabular}{|c|c|c|c|c|c|c|c|c|c|c|c|c|c|c|}
\hline \multirow{2}{*}{$\underline{\text { ST }}$} & \multirow{2}{*}{$\begin{array}{l}\text { Parameter } \\
\text { ganics }\end{array}$} & \multirow[t]{2}{*}{$\underline{3 Q 98}$} & \multirow[t]{2}{*}{ CLP EPA } & \multirow[t]{2}{*}{$\underline{1 Q 99}$} & CLP EPA & \multirow[t]{2}{*}{$\underline{3 Q 99}$} & CLPEPA & \multirow[t]{2}{*}{1000} & \multicolumn{2}{|c|}{ CLPEPA } & \multirow[t]{2}{*}{ Filt. } & \multirow[t]{2}{*}{$\underline{\text { DF }}$} & \multirow[t]{2}{*}{ Unit } & \multirow[t]{2}{*}{$\underline{\text { Lab }}$} \\
\hline & & & & & & & & & & & & & & \\
\hline & Barium, total recoverable & 14.6 & & 5.04 & J I & 7.6 & J I & & & & & & ug/L & \\
\hline & Cyanide & $<15.2$ & $U$ & $<10$ & U & $<10$ & $\mathrm{U}$ & & & & & & ug/L & \\
\hline & Lead, total recoverable & $<100$ & $\mathrm{U}$ & $<100$ & $\mathrm{U}$ & .59 & J I & & & & & & ug/L & \\
\hline & Nickel, total recoverable & $<50$ & $U$ & $<50$ & $\mathrm{U}$ & $<9.3$ & JU 1 & & & & & & $u g / L$ & \\
\hline & Selenium, total recoverable & $<200$ & $U$ & $<10$ & $U$ & $<5$ & $U$ & & & & & & $u g / L$ & \\
\hline \multicolumn{15}{|c|}{ Organics } \\
\hline & Chlorobenzene & $<5$ & $u$ & $<5$ & $\mathrm{U}$ & $<1$ & $\mathrm{U}$ & $<1$ & JU & $\mathrm{L}$ & $<E Q L$ & 1 & ug/L & $\mathrm{ML}$ \\
\hline & 1,1-Dichloroethane & $<5$ & U & $<5$ & $\mathrm{U}$ & $<1$ & $\mathrm{U}$ & $<1$ & JU & $L$ & $<\mathrm{EQL}$ & 1 & ug/L & $M L$ \\
\hline & 1,1-Dichloroethylene & $<5$ & $U$ & $<5$ & U & $<1$ & $\mathrm{U}$ & $<1$ & JU & $L$ & $<\mathrm{EQL}$ & 1 & ug/L & ML \\
\hline & trans-1,2-Dichloroethylene & $<5$ & $\mathrm{U}$ & $<5$ & U & $<1$ & $\mathrm{U}$ & & & & & & ug/L & \\
\hline & РСВ 1016 & & & & & & & & & & & & & \\
\hline & PCB 1221 & & & & & & & & & & & & & \\
\hline & РСB 1232 & & & & & & & & & & & & & \\
\hline & PCB 1242 & & & & & & & & & & & & & \\
\hline & PCB 1248 & & & & & & & & & & & & & \\
\hline & РСВ 1254 & & & & & & & & & & & & & \\
\hline & РCB 1260 & & & & & & & & & & & & & \\
\hline & 1,1,2,2-Tetrachloroethane & $<5$ & $\mathrm{U}$ & $<5$ & $\mathrm{U}$ & $<1$ & $\mathrm{U}$ & $<1$ & JU & $L$ & $<\mathrm{EQL}$ & 1 & ug/L & $M L$ \\
\hline & Tetrachloroethylene & $<5$ & $U$ & $<5$ & $\mathrm{U}$ & $<1$ & $\mathrm{U}$ & $<1$ & JU & $\mathrm{L}$ & $<\mathrm{EQL}$ & 1 & ug/L & $M L$ \\
\hline & $1,1,1$-Trichloroethane & $<5$ & $\mathrm{U}$ & $<5$ & $\mathrm{U}$ & $<1$ & $\mathrm{U}$ & $<1$ & JU & $L$ & $<\mathrm{EQL}$ & 1 & ug/L & $M L$ \\
\hline & Trichloroethylene & $<5$ & $U$ & $<5$ & U & $<1$ & $\mathrm{U}$ & $<1$ & JU & $L$ & $<E Q L$ & 1 & ug/L & $\mathrm{ML}$ \\
\hline \multicolumn{15}{|c|}{ II. Monitoring Constituents } \\
\hline$\underline{\mathbf{S T}}$ & Parameter & $\underline{3 Q 96}$ & CLP EPA & $\underline{3097}$ & CLPEPA & 3098 & CLPEPA & $\underline{3099}$ & $\underline{\text { CLP }}$ & EPA & Filt. & DF & $\underline{\text { Unit }}$ & Lab \\
\hline \multicolumn{15}{|c|}{ Inorganics } \\
\hline & Aluminum, total recoverable & $<20$ & U & 22 & & 39.5 & $\mathrm{~J}$ & $<200$ & $U$ & & $<E Q L$ & 1 & ug/L & EX \\
\hline & Nitrate-nitrite as nitrogen & 820 & & 768 & & 563 & & 550 & & & $<2400$ & 5 & $\mathrm{ug} / \mathrm{L}$ & $\mathrm{EX}$ \\
\hline & Sodium, total recoverable & 1550 & & 1530 & & 1980 & & 2000 & & & $<4600$ & 1 & ug/L & EX \\
\hline & Sulfate & 1160 & & $<5000$ & $\mathrm{U}$ & 1500 & & 1290 & & & $<3000$ & 1 & $\mathrm{ug} / \mathrm{L}$ & EX \\
\hline \multicolumn{15}{|c|}{ Radionuclides } \\
\hline & Gross alpha & 1.53 & $J$ & .25 & UI & 1.44 & & 1.14 & $J$ & I & NDD & 1 & $\mathrm{pCi} / \mathrm{L}$ & GP \\
\hline & Nonvolatile beta & 2.26 & & -1.07 & U! & 1.71 & & 2.07 & $\mathrm{~J}$ & 1 & NDD & 1 & $\mathrm{pCi} / \mathrm{L}$ & GP \\
\hline & Radium, total alpha-emitting & .5 & & 3.18 & UIJ & .7 & & .4 & $\mathrm{U}$ & V & $<\mathrm{EQL}$ & 1 & $\mathrm{pCi} / \mathrm{L}$ & GP \\
\hline
\end{tabular}

Notes: Concentrations in bold italics exceed the groundwater protection or monitoring constituent standards listed in Appendix A. Synchronous water levels are measured over a 3-5 day period or less. Dilution factors, Laboratory, and Filtered Data are for Groundwater Protection Standards First Quarter 2000 and Monitoring Constituents Third Quarter 1999 data only.

$+=$ exceeded the Groundwater Protection Standards (First Quarter 2000) or Monitoring Constituents (Third Quarter 1999) listed in Appendix A. 
Table D-4. Groundwater Monitoring Results for Plume Definition Wells, M-Area HWMF (Cont.) WELL MSB 69C

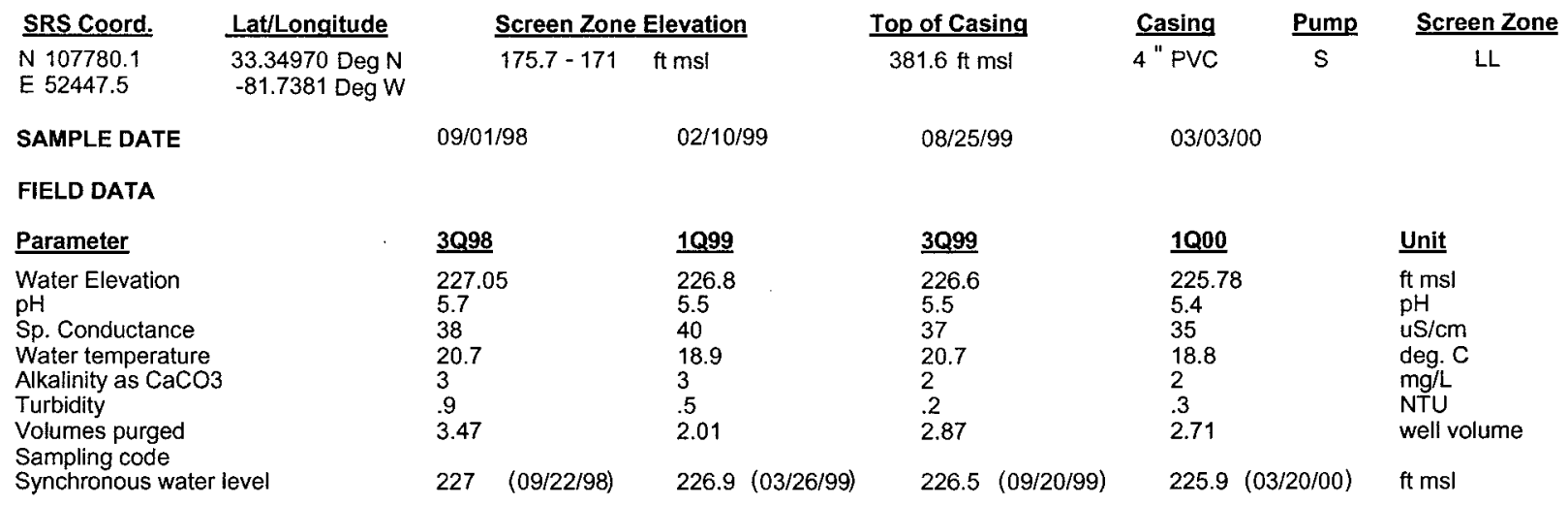

ANALYTICAL DATA

I. Groundwater Protection Standard

261 Appendix VIII/264 Appendix IX Hazardous Constituents

\begin{tabular}{|c|c|c|c|c|c|c|c|c|c|c|c|c|c|}
\hline$\underline{\mathbf{S T}}$ & Parameter & $\underline{3 Q 98}$ & CLP EPA & 1099 & CLP EPA & $\underline{3 Q 99}$ & $\underline{\text { CLPEPA }}$ & $\underline{1 Q 00}$ & CLPEPA & Filt. & $\underline{\mathrm{DF}}$ & Unit & $\underline{\mathrm{Lab}}$ \\
\hline \multicolumn{14}{|c|}{ Inorganics } \\
\hline & Barium, total recoverable & 8.33 & $\mathrm{~J}$ & 9.29 & $\mathrm{~J} \quad 1$ & 9.9 & $J$ & & & & & ug/L & \\
\hline & Cyanide & $<10$ & $\mathrm{U}$ & $<10$ & $U$ & $<10$ & $\mathrm{U}$ & & & & & $\mathrm{ug} / \mathrm{L}$ & \\
\hline & Lead, total recoverable & $<100$ & $\mathrm{U}$ & $<100$ & $U$ & $<10$ & $U$ & & & & & ug/L & \\
\hline & Nickel, total recoverable & $<50$ & $\mathrm{U}$ & $<50$ & $U$ & $<50$ & $\mathrm{u}$ & & & & & ug/L & \\
\hline & Selenium, total recoverable & $<10$ & $\mathrm{U}$ & $<10$ & $\mathrm{U}$ & $<3.6$ & JU I & & & & & $\mathrm{ug} / \mathrm{L}$ & \\
\hline \multicolumn{14}{|c|}{ Organics } \\
\hline & Chlorobenzene & $<5$ & UJ & $<5$ & $u$ & $<1$ & $\mathrm{U}$ & $<1$ & $U$ & $<\mathrm{EQL}$ & 1 & $\mathrm{ug} / \mathrm{L}$ & ML \\
\hline & 1,1-Dichloroethane & $<5$ & UJ & $<5$ & $U$ & $<1$ & $\mathrm{U}$ & $<1$ & $\mathrm{U}$ & $<\mathrm{EQL}$ & 1 & $\mathrm{ug} / \mathrm{L}$ & ML \\
\hline & 1,1-Dichloroethylene & $<5$ & UJ & $<5$ & $U$ & $<1$ & U & $<1$ & 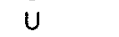 & $<\mathrm{EQL}$ & 1 & $\mathrm{ug} / \mathrm{L}$ & ML \\
\hline & trans-1,2-Dichloroethylene & $<5$ & UJ & $<5$ & $U$ & $<1$ & $u$ & & & & & ug/L & \\
\hline & РСB 1016 & & & & & & & & & & & & \\
\hline & PCB 1221 & & & & & & & & & & & & \\
\hline & PCB 1232 & & & & & & & & & & & & \\
\hline & PCB 1242 & & & & & & & & & & & & \\
\hline & PCB 1248 & & & & & & & & & & & & \\
\hline & PCB 1254 & & & & & & & & & & & & \\
\hline & PCB 1260 & & & & & & & & & & & & \\
\hline & $1,1,2,2$-Tetrachloroethane & $<5$ & UJ & $<5$ & $U$ & $<1$ & $U$ & $<1$ & $U$ & $<\mathrm{EQL}$ & 1 & ug/L & ML \\
\hline & Tetrachloroethylene & $<5$ & UJ & $<5$ & $\mathrm{U}$ & $<.58$ & U V & $<1$ & $\mathrm{U}$ & $<E Q L$ & 1 & ug/L & $M L$ \\
\hline & 1,1,1-Trichloroethane & $<5$ & UJ & $<5$ & $\mathrm{U}$ & $<1$ & $U$ & $<1$ & $\mathrm{U}$ & $<$ EQL & 1 & $\mathrm{ug} / \mathrm{L}$ & ML \\
\hline+ & Trichloroethylene & 37.5 & $J$ & 49.4 & & 45.1 & $\mathrm{~J} K$ & 39 & & $>5$ & 1 & ug/L & $M$ \\
\hline \multicolumn{14}{|c|}{ II. Monitoring Constituents } \\
\hline$\underline{\text { ST }}$ & Parameter & $\underline{3 Q 96}$ & CLPEPA & $\underline{3097}$ & CLP EPA & $\underline{3098}$ & CLPEPA & $\underline{3099}$ & CLPEPA & Filt. & $\underline{\text { DF }}$ & Unit & \\
\hline \multicolumn{14}{|c|}{ Inorganics } \\
\hline & Aluminum, total recoverable & $<20$ & UJ & 21.8 & & $<200$ & $\mathrm{U}$ & $<200$ & $\mathrm{U}$ & $<$ EQL & 1 & $\mathrm{ug} / \mathrm{L}$ & EX \\
\hline & Nitrate-nitrite as nitrogen & 2260 & $\mathrm{~J}$ & 2940 & & 2050 & & 2380 & & $<2400$ & 5 & $\mathrm{ug} / \mathrm{L}$ & \\
\hline & Sodium, total recoverable & 3760 & & 3220 & & 3640 & & 4000 & & $<4600$ & 1 & $\mathrm{ug} / \mathrm{L}$ & EX \\
\hline & Sulfate & & & $<5000$ & $\mathrm{U}$ & 309 & $J$ & $<400$ & $\mathrm{U}$ & $<\mathrm{EQL}$ & 2 & $\mathrm{ug} / \mathrm{L}$ & \\
\hline \multicolumn{14}{|c|}{ Radionuclides } \\
\hline & Gross alpha & 1.41 & & -.23 & UI & 3.54 & & .228 & $U$ & $<E Q L$ & 1 & $\mathrm{pCi} / \mathrm{L}$ & GP \\
\hline & Nonvolatile beta & 1.07 & & -1.79 & UI & 4.2 & & 1.45 & $\mathrm{~J} \quad \mathrm{I}$ & NDD & 1 & $\mathrm{pCi} / \mathrm{L}$ & GP \\
\hline & Radium, total alpha-emitting & .6 & $J$ & 3.77 & UIJ & 1.01 & $\mathrm{U}$ & .8 & J $\quad 1$ & NDD & 1 & $\mathrm{pCi} / \mathrm{L}$ & GP \\
\hline
\end{tabular}

Notes: Concentrations in bold italics exceed the groundwater protection or monitoring constituent standards listed in Appendix A. Synchronous water levels are measured over a 3-5 day period or less. Dilution factors, Laboratory, and Filtered Data are for Groundwater Protection Standards First Quarter 2000 and Monitoring Constituents Third Quarter 1999 data only.

+ = exceeded the Groundwater Protection Standards (First Quarter 2000) or Monitoring Constituents (Third Quarter 1999) listed in Appendix A. 
Table D-4. Groundwater Monitoring Results for Plume Definition Wells, M-Area HWMF (Cont.) WELL MSB 69D

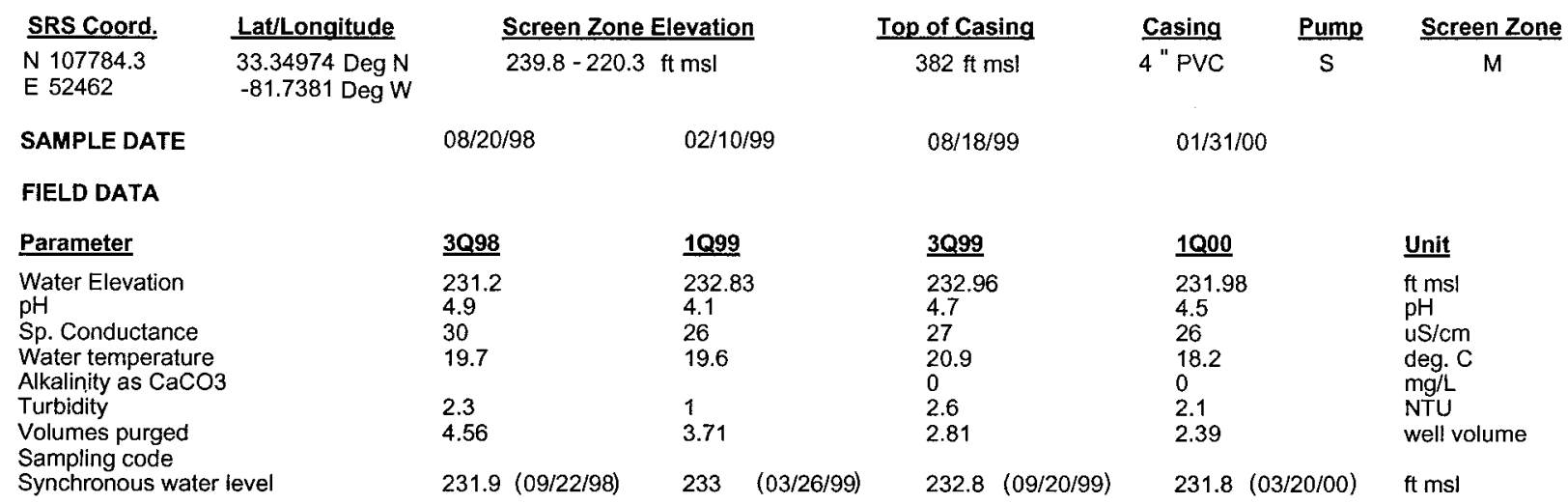

\section{ANALYTICAL DATA}

I. Groundwater Protection Standard

261 Appendix VIII/264 Appendix IX Hazardous Constituents

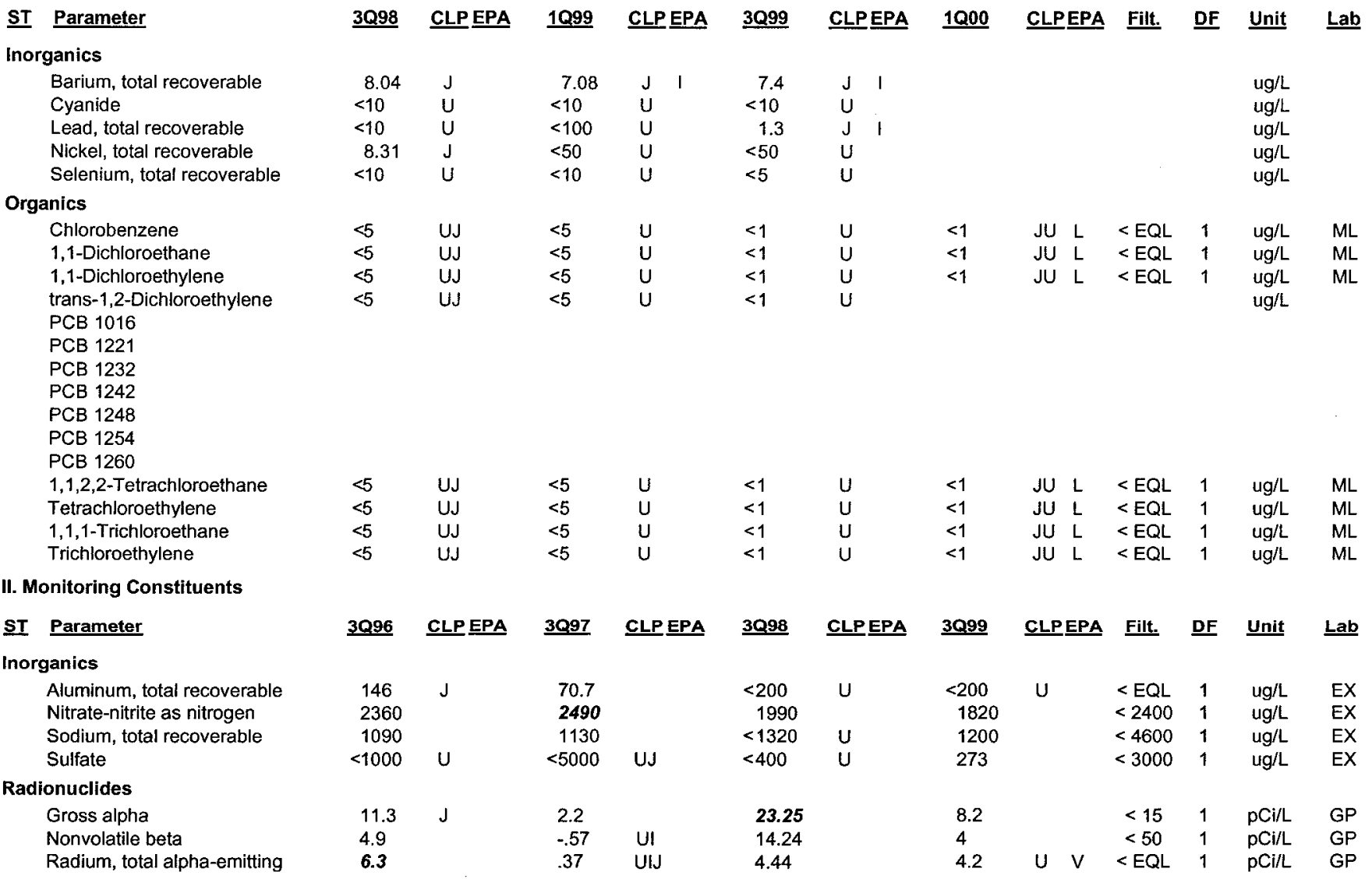

Notes: Concentrations in bold italics exceed the groundwater protection or monitoring constituent standards listed in Appendix A. Synchronous water levels are measured over a 3-5 day period or less. Dilution factors, Laboratory, and Filtered Data are for Groundwater Protection Standards First Quarter 2000 and Monitoring Constituents Third Quarter 1999 data only.

$+=$ exceeded the Groundwater Protection Standards (First Quarter 2000) or Monitoring Constituents (Third Quarter 1999) listed in Appendix A. 
Table D-4. Groundwater Monitoring Results for Plume Definition Wells, M-Area HWMF (Cont.) WELL MSB 69TA

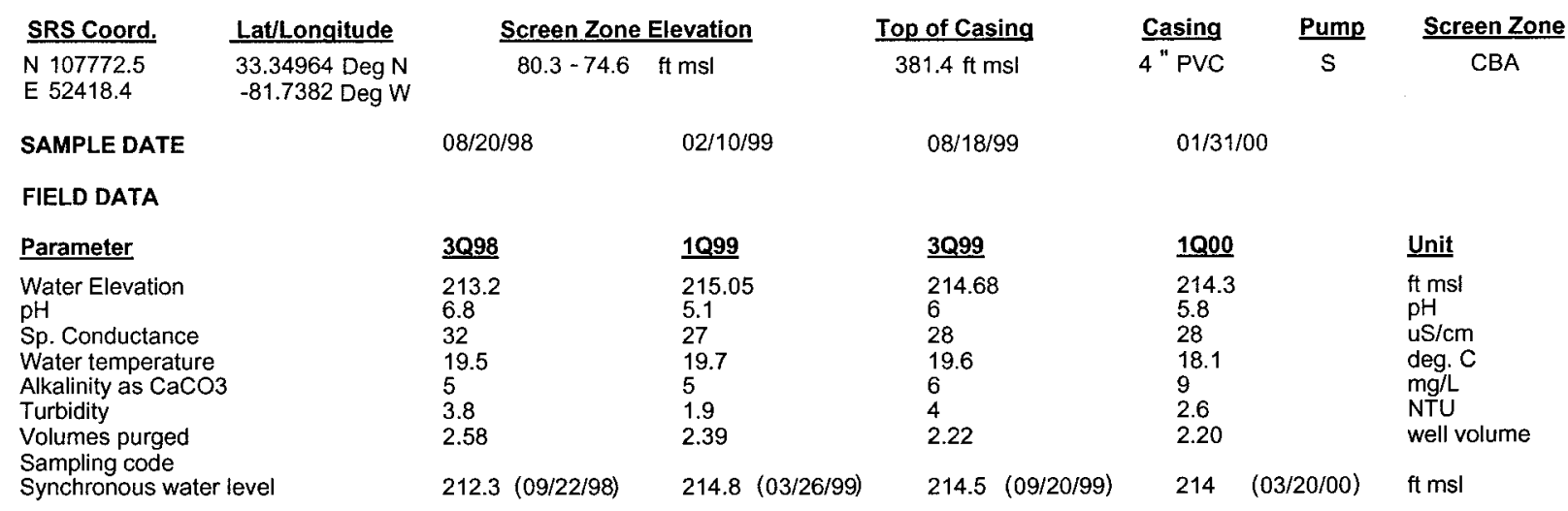

ANALYTICAL DATA

I. Groundwater Protection Standard

261 Appendix VIII/264 Appendix IX Hazardous Constituents

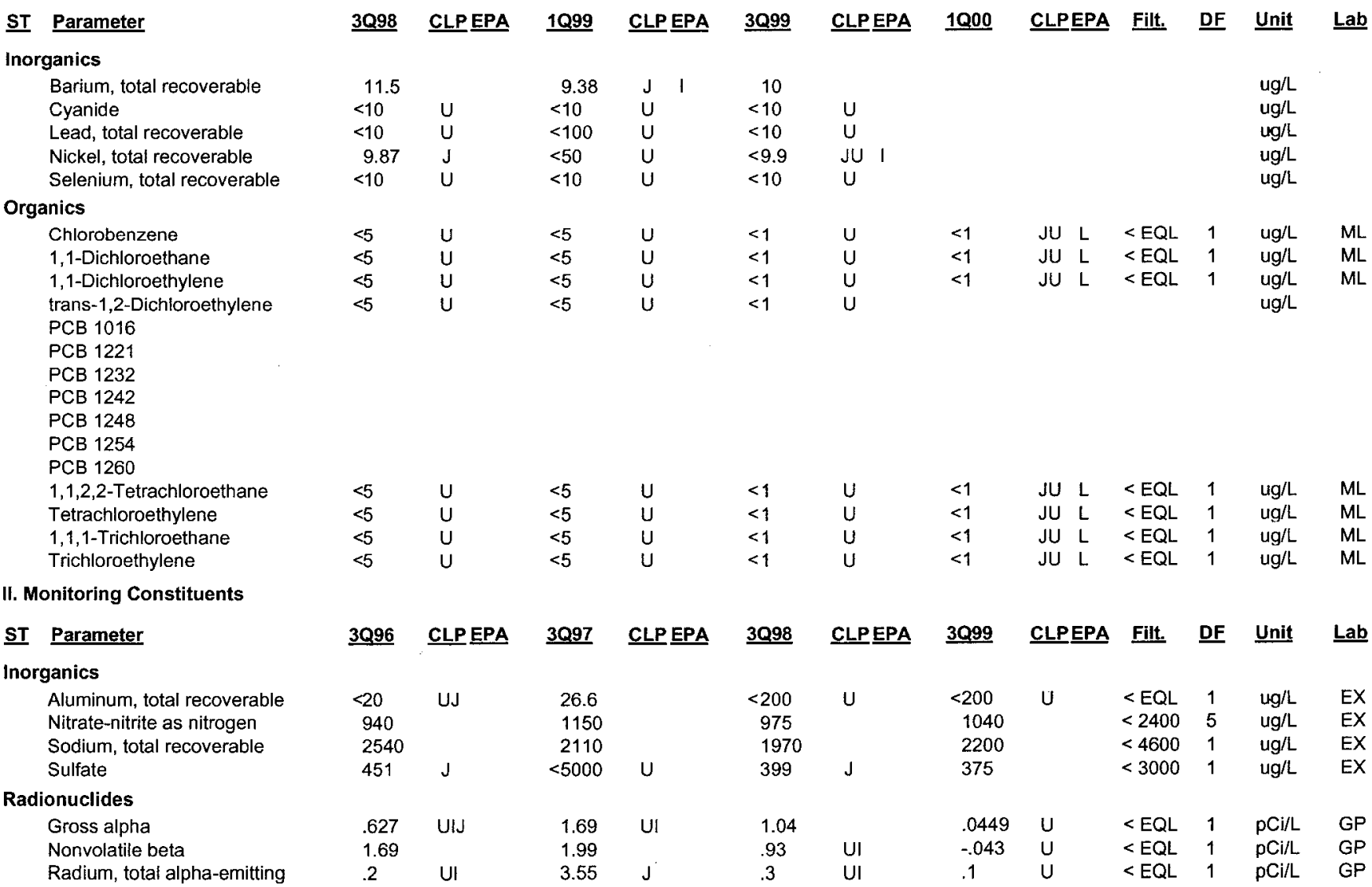

Notes: Concentrations in bold italics exceed the groundwater protection or monitoring constituent standards listed in Appendix A. Synchronous water leveis are measured over a 3-5 day period or less. Dilution factors, Laboratory, and Filtered Data are for Groundwater Protection Standards First Quarter 2000 and Monitoring Constituents Third Quarter 1999 data only.

$+=$ exceeded the Groundwater Protection Standards (First Quarter 2000) or Monitoring Constituents (Third Quarter 1999) listed in Appendix A. 
Table D-4. Groundwater Monitoring Results for Plume Definition Wells, M-Area HWMF (Cont.) WELL MSB 70C

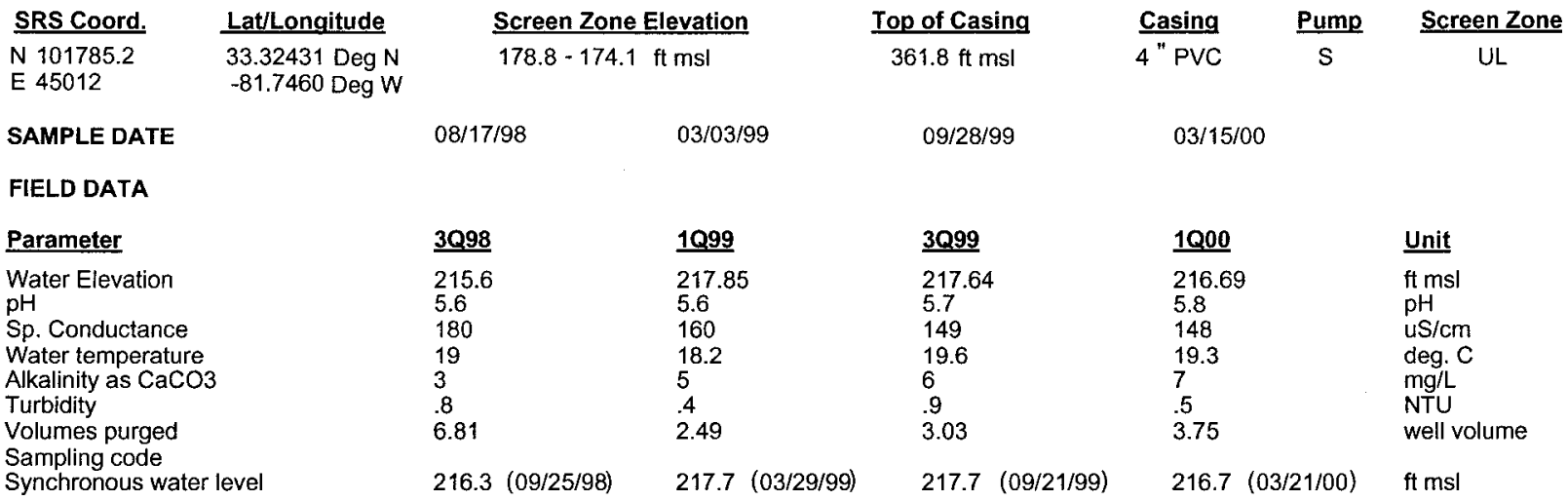

\section{ANALYTICAL DATA}

I. Groundwater Protection Standard

261 Appendix VIII/264 Appendix IX Hazardous Constituents

\begin{tabular}{|c|c|c|c|c|c|c|c|c|c|c|c|c|c|}
\hline \multirow{2}{*}{$\begin{array}{l}\text { SI Parameter } \\
\text { Inorganics }\end{array}$} & \multirow[t]{2}{*}{$\underline{3 Q 98}$} & \multirow[t]{2}{*}{ CLPEPA } & \multirow[t]{2}{*}{$\underline{1099}$} & CLPEPA & \multirow[t]{2}{*}{$\underline{3099}$} & \multirow[t]{2}{*}{ CLPEPA } & \multirow[t]{2}{*}{10,00} & \multicolumn{2}{|c|}{ CLPEPA } & \multirow[t]{2}{*}{ Filt. } & \multirow[t]{2}{*}{$\underline{\text { DF }}$} & \multirow[t]{2}{*}{$\underline{\text { Unit }}$} & \multirow[t]{2}{*}{$\underline{\mathrm{Lab}}$} \\
\hline & & & & & & & & & & & & & \\
\hline Barium, total recoverable & 37.1 & & 39.6 & & 43.1 & & & & & & & $\mathrm{ug} / \mathrm{L}$ & \\
\hline Cyanide & $<10$ & $u$ & $<10$ & $\mathrm{u}$ & $<10$ & JU $Q$ & & & & & & $\mathrm{ug} / \mathrm{L}$ & \\
\hline Lead, total recoverable & $<10$ & $u$ & $<100$ & $\mathrm{u}$ & $<10$ & U & & & & & & $\mathrm{ug} / \mathrm{L}$ & \\
\hline Nickel, total recoverable & $<50$ & $u$ & $<50$ & $\mathrm{u}$ & $<50$ & $\mathrm{u}$ & & & & & & $\mathrm{ug} / \mathrm{L}$ & \\
\hline Selenium, total recoverable & $<10$ & $u$ & $<200$ & $\mathrm{u}$ & $<10$ & $u$ & & & & & & $\mathrm{ug} / \mathrm{L}$ & \\
\hline \multicolumn{14}{|l|}{ Organics } \\
\hline Chlorobenzene & $<500$ & $\mathrm{u}$ & $<500$ & JU $Q$ & $<1$ & $u$ & 20.5 & $\mathrm{~J}$ & IK & NDD & 50 & $u g / L$ & ML \\
\hline 1,1-Dichloroethane & $<500$ & $u$ & $<500$ & $J U Q$ & $<1$ & $u$ & $<50$ & $U$ & & $<E Q L$ & 50 & ug/L & ML \\
\hline 1,1-Dichloroethylene & 41.1 & & $<500$ & $J U Q$ & 32.6 & & 35 & $J$ & IK & NDD & 50 & $\mathrm{ug} / \mathrm{L}$ & ML \\
\hline trans-1,2-Dichloroethylene & $<500$ & $\mathrm{u}$ & $<500$ & $\mathrm{JU} Q$ & $<1$ & $\mathrm{u}$ & $<50$ & U & & $<E Q L$ & 50 & $\mathrm{ug} / \mathrm{h}$ & ML. \\
\hline PCB 1016 & & & & & & & & & & & & & \\
\hline PCB 1221 & & & & & & & & & & & & & \\
\hline PCB 1232 & & & & & & & & & & & & & \\
\hline РСВ 1242 & & & & & & & & & & & & & \\
\hline PCB 1248 & & & & & & & & & & & & & \\
\hline PCB 1254 & & & & & & & & & & & & & \\
\hline PCB 1260 & & & & & & & & & & & & & \\
\hline 1,1,2,2-Tetrachloroethane & $<500$ & $\mathrm{u}$ & $<500$ & JU $Q$ & $<1$ & $u$ & $<50$ & $u$ & & $<E Q L$ & 50 & $\mathrm{ug} / \mathrm{L}$ & ML \\
\hline Tetrachloroethylene & 1030 & & 1360 & $J \quad Q$ & 1170 & & 1140 & $\mathrm{~J}$ & $\mathrm{~K}$ & NDD & 50 & $\mathrm{ug} / \mathrm{L}$ & ML \\
\hline 1,1,1-Trichloroethane & $<500$ & $u$ & $<500$ & $J \cup Q$ & 4.32 & & $<50$ & $u$ & & $<\mathrm{EQL}$ & 50 & $\mathrm{ug} / \mathrm{L}$ & ML \\
\hline Trichloroethylene & 3810 & & 5660 & $J \quad Q$ & 5990 & & 6490 & $J$ & K & NDD & 50 & $\mathrm{ug} / \mathrm{L}$ & ML \\
\hline \multicolumn{14}{|l|}{ II. Monitoring Constituents } \\
\hline ST Parameter & $\underline{3096}$ & CLPEPA & $\underline{3097}$ & CLPEPA & $\underline{3098}$ & CLPEPA & $\underline{3099}$ & CLP & EPA & Filt. & $\underline{\mathrm{DF}}$ & $\underline{\text { Unit }}$ & $\underline{\text { Lab }}$ \\
\hline \multicolumn{14}{|l|}{ Inorganics } \\
\hline $\begin{array}{l}\quad \text { Aluminum, total recoverable } \\
+\quad \text { Nitrate-nitrite as nitrogen }\end{array}$ & $\begin{array}{l}16.1 \\
19100\end{array}$ & J & $\begin{array}{l}12.6 \\
19200\end{array}$ & J & $\begin{array}{l}<200 \\
16400\end{array}$ & $u$ & $\begin{array}{l}<200 \\
14400\end{array}$ & $u$ & & $\begin{array}{l}<E Q L \\
>2400\end{array}$ & $\begin{array}{l}1 \\
5\end{array}$ & $\begin{array}{l}u g / L \\
u g / L\end{array}$ & $\begin{array}{l}\text { EX } \\
\text { EX }\end{array}$ \\
\hline + Sodium, total recoverable & 21300 & & 21000 & & 22100 & & 21800 & & & $>4600$ & 1 & $\mathrm{ug} / \mathrm{L}$ & EX \\
\hline Sulfate & 163 & $J$ & $<5000$ & $u$ & $<200$ & $u$ & 263 & $\mathrm{~J}$ & 1 & NDD & 2 & $\mathrm{ug} / \mathrm{L}$ & EX \\
\hline \multicolumn{14}{|l|}{ Radionuclides } \\
\hline Gross alpha & 3.75 & & 3.32 & & 4.8 & & 2.99 & & & $<15$ & 1 & $\mathrm{pCi} / \mathrm{L}$ & GP \\
\hline Nonvolatile beta & 4.02 & & 2.98 & & 5.28 & & 3.47 & $u$ & $\mathrm{v}$ & $<E Q L$ & 1 & $\mathrm{pCi} / \mathrm{L}$ & GP \\
\hline Radium, total alpha-emitting & 2.5 & $\mathrm{~J}$ & 11.6 & $\mathrm{~J}$ & 5.21 & & 2.2 & $\mathrm{~J}$ & 1 & NDD & 1 & $\mathrm{pCi} / \mathrm{L}$ & GP \\
\hline
\end{tabular}

Notes: Concentrations in bold italics exceed the groundwater protection or monitoring constituent standards listed in Appendix A. Synchronous water levels are measured over a 3-5 day period or less. Dilution factors, Laboratory, and Filtered Data are for Groundwater Protection Standards First Quarter 2000 and Monitoring Constituents Third Quarter 1999 data only.

$+=$ exceeded the Groundwater Protection Standards (First Quarter 2000) or Monitoring Constituents (Third Quarter 1999) listed in Appendix A. 
Table D-4. Groundwater Monitoring Results for Plume Definition Wells, M-Area HWMF (Cont.) WELL MSB 70D

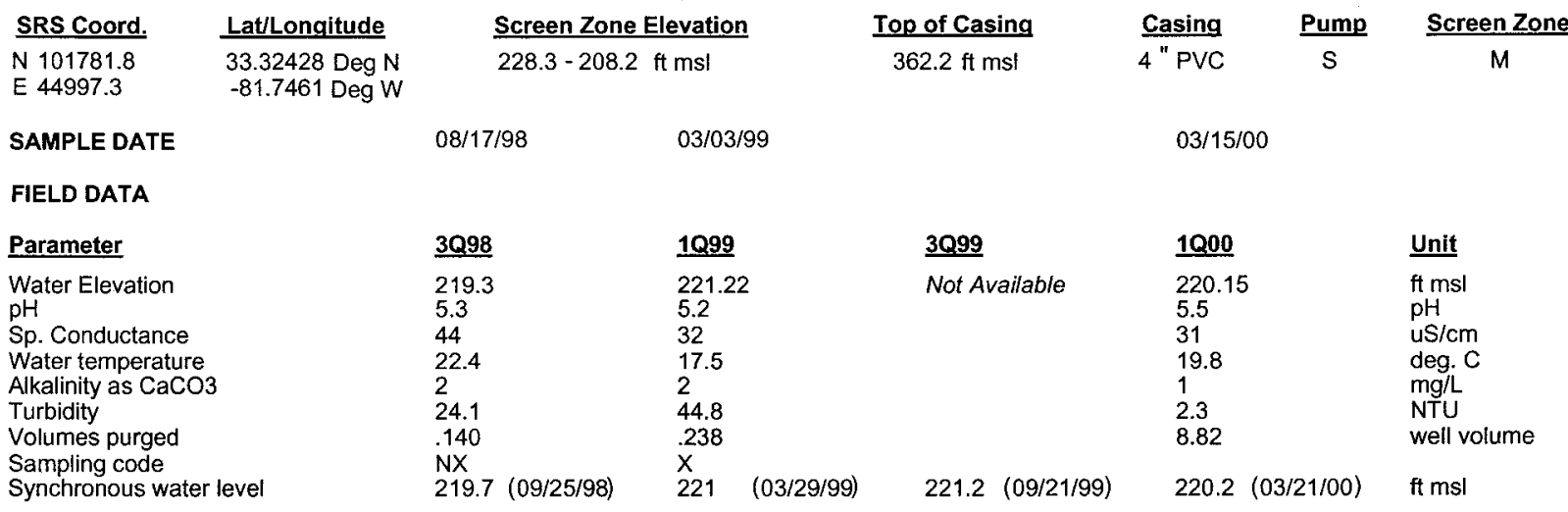

ANALYTICAL DATA

I. Groundwater Protection Standard

261 Appendix VIII/264 Appendix IX Hazardous Constituents

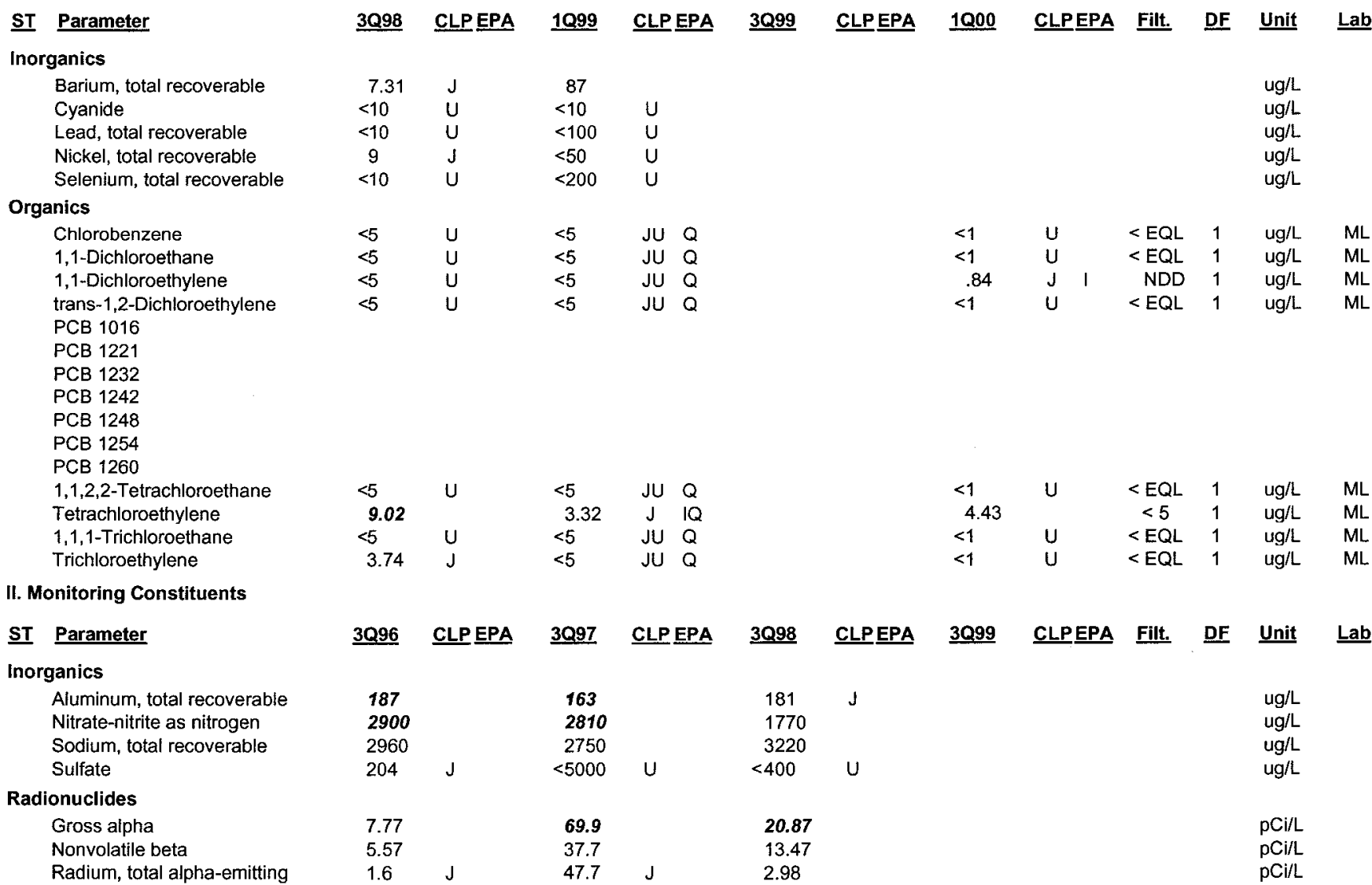

Notes: Concentrations in bold italics exceed the groundwater protection or monitoring constituent standards listed in Appendix A. Synchronous water levels are measured over a 3-5 day period or less. Dilution factors, Laboratory, and Filtered Data are for Groundwater Protection Standards First Quarter 2000 and Monitoring Constituents Third Quarter 1999 data only.

$+=$ exceeded the Groundwater Protection Standards (First Quarter 2000) or Monitoring Constituents (Third Quarter 1999) listed in Appendix A. 
Table D-4. Groundwater Monitoring Results for Plume Definition Wells, M-Area HWMF (Cont.) WELL MSB 71B

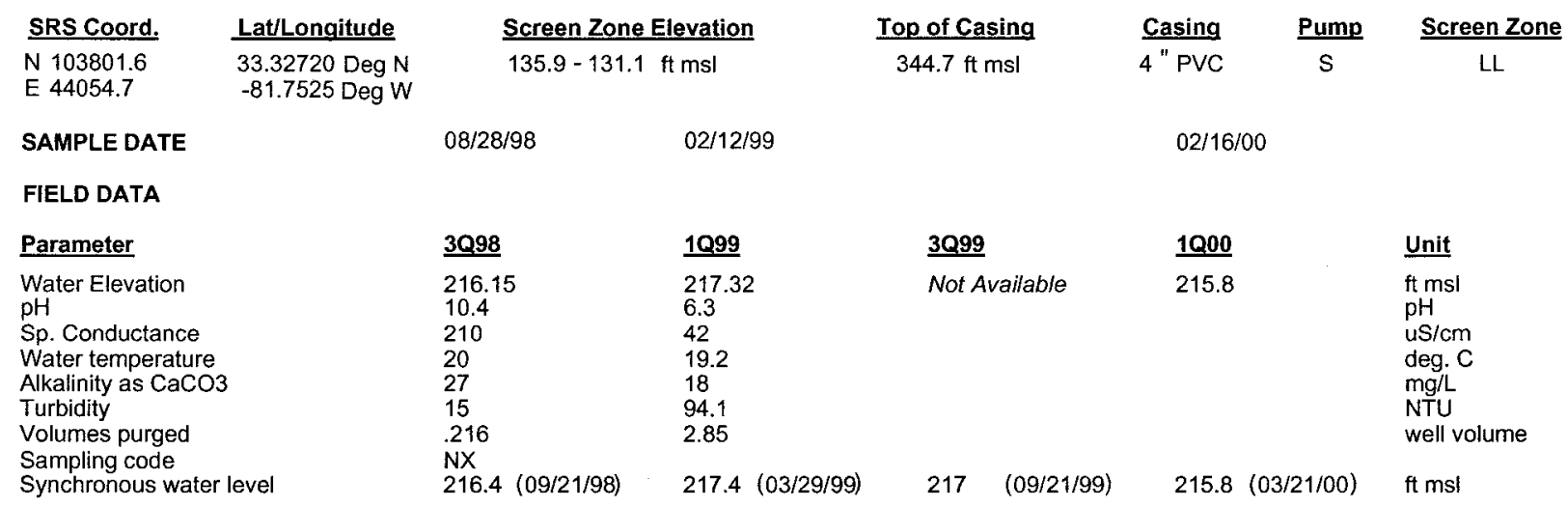

ANALYTICAL DATA

I. Groundwater Protection Standard

261 Appendix VIII/264 Appendix IX Hazardous Constituents

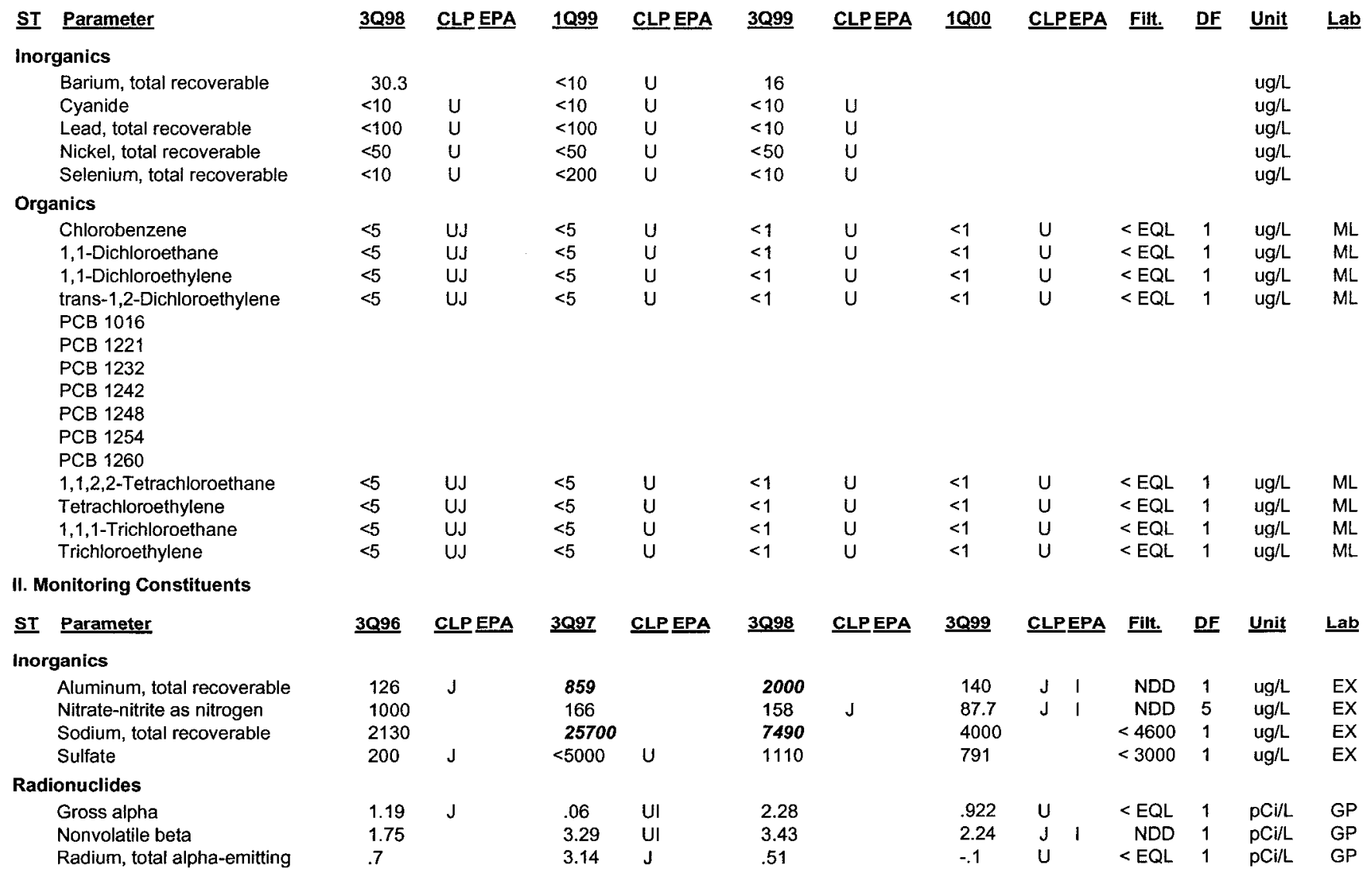

Notes: Concentrations in bold italics exceed the groundwater protection or monitoring constituent standards listed in Appendix A. Synchronous water levels are measured over a 3-5 day period or less. Dilution factors, Laboratory, and Filtered Data are for Groundwater Protection Standards First Quarter 2000 and Monitoring Constituents Third Quarter 1999 data only.

+= exceeded the Groundwater Protection Standards (First Quarter 2000) or Monitoring Constituents (Third Quarter 1999) listed in Appendix A. 
Table D-4. Groundwater Monitoring Results for Plume Definition Wells, M-Area HWMF (Cont.) WELL MSB 72B

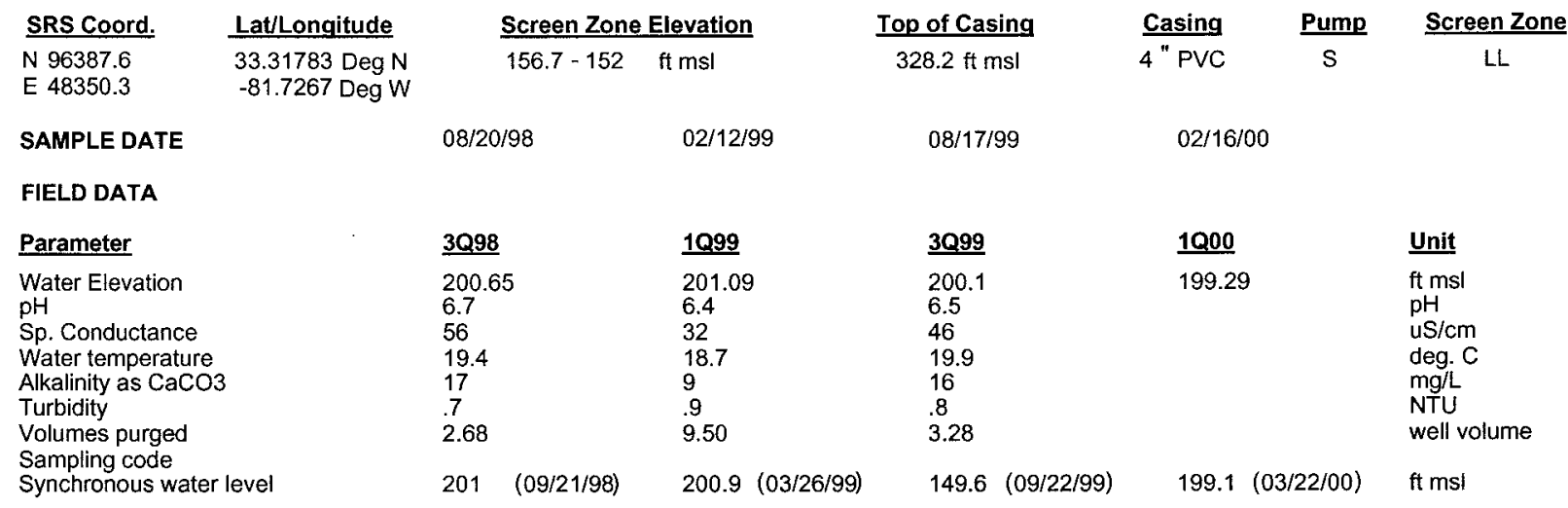

ANALYTICAL DATA

I. Groundwater Protection Standard

261 Appendix VIII/264 Appendix IX Hazardous Constituents

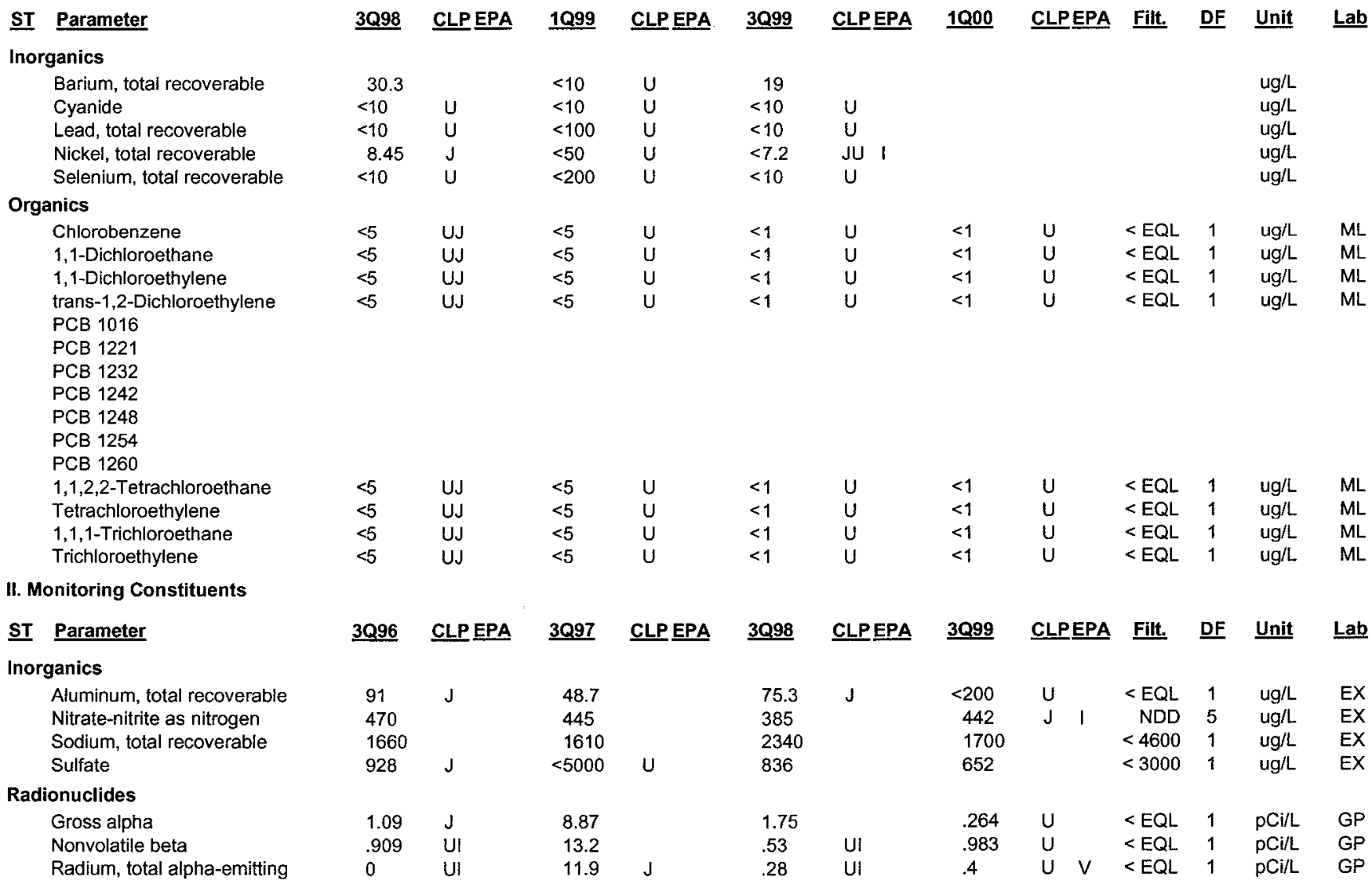

Notes: Concentrations in bold italics exceed the groundwater protection or monitoring constituent standards listed in Appendix A. Synchronous water levels are measured over a 3-5 day period or less. Dilution factors, Laboratory, and Filtered Data are for Groundwater Protection Standards First Quarter 2000 and Monitoring Constituents Third Quarter 1999 data only.

$+=$ exceeded the Groundwater Protection Standards (First Quarter 2000) or Monitoring Constituents (Third Quarter 1999) listed in Appendix A. 
Table D-4. Groundwater Monitoring Results for Plume Definition Wells, M-Area HWMF (Cont.) WELL MSB 73B

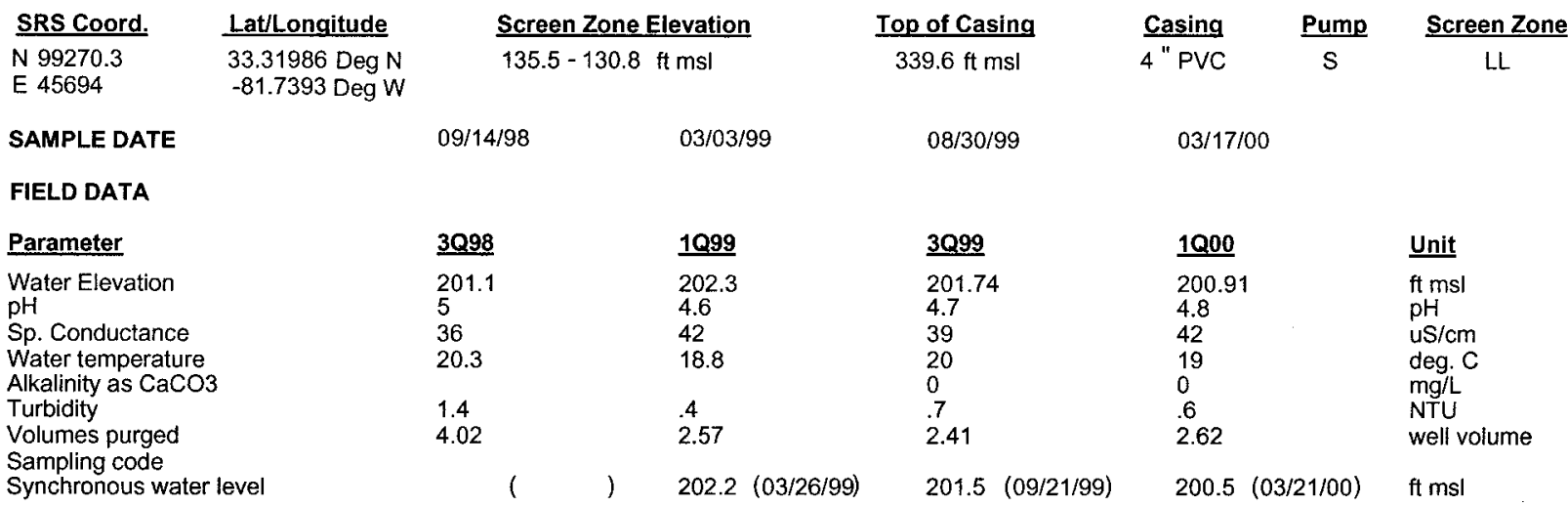

ANALYTICAL. DATA

I. Groundwater Protection Standard

261 Appendix VIII/264 Appendix IX Hazardous Constituents

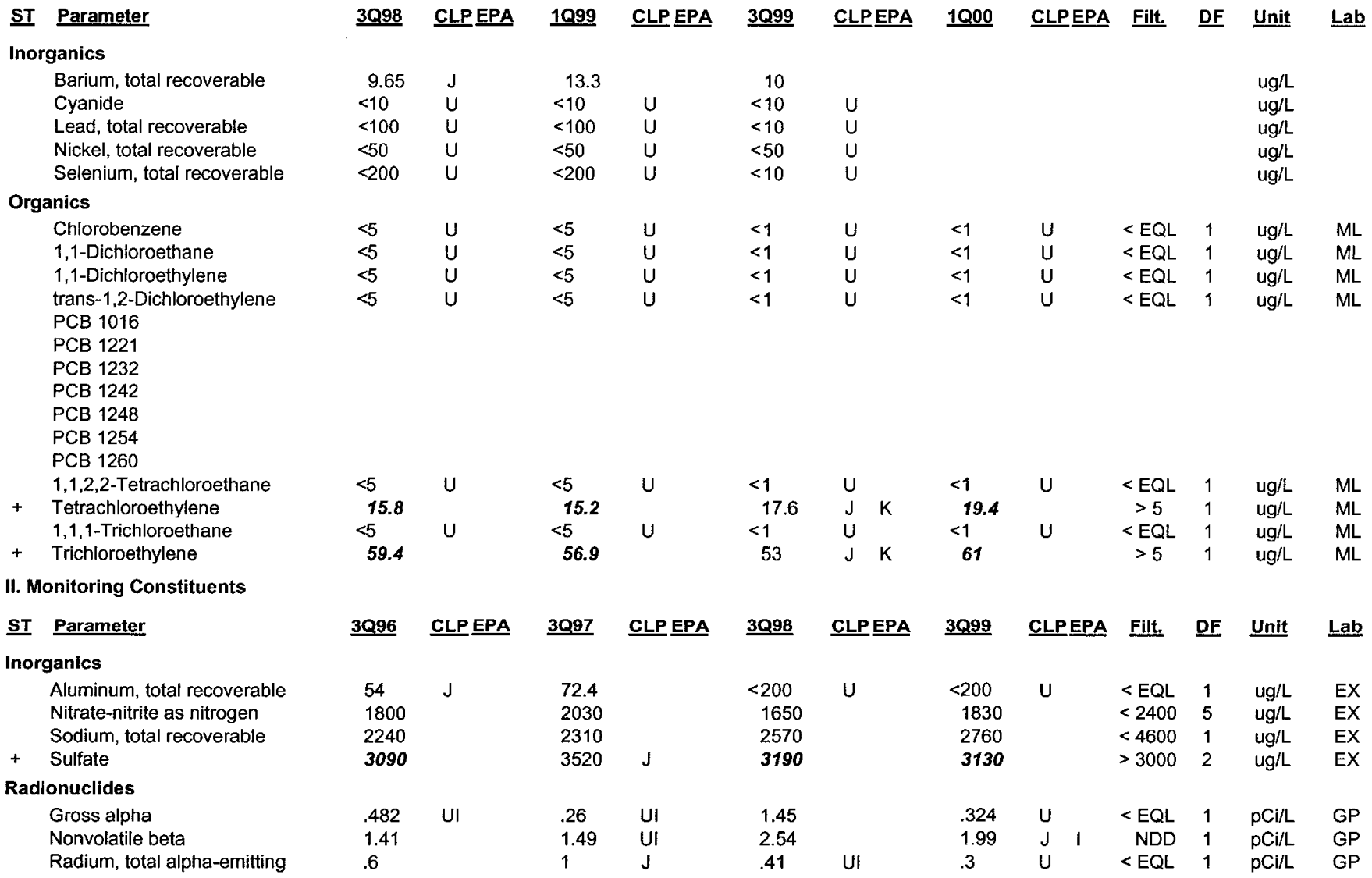

Notes: Concentrations in bold italics exceed the groundwater protection or monitoring constituent standards listed in Appendix A. Synchronous water levels are measured over a 3-5 day period or less. Dilution factors, Laboratory, and Filtered Data are for Groundwater Protection Standards First Quarter 2000 and Monitoring Constituents Third Quarter 1999 data only.

+ = exceeded the Groundwater Protection Standards (First Quarter 2000) or Monitoring Constituents (Third Quarter 1999) listed in Appendix A. 
Table D-4. Groundwater Monitoring Results for Plume Definition Wells, M-Area HWMF (Cont.) WELL MSB 74B

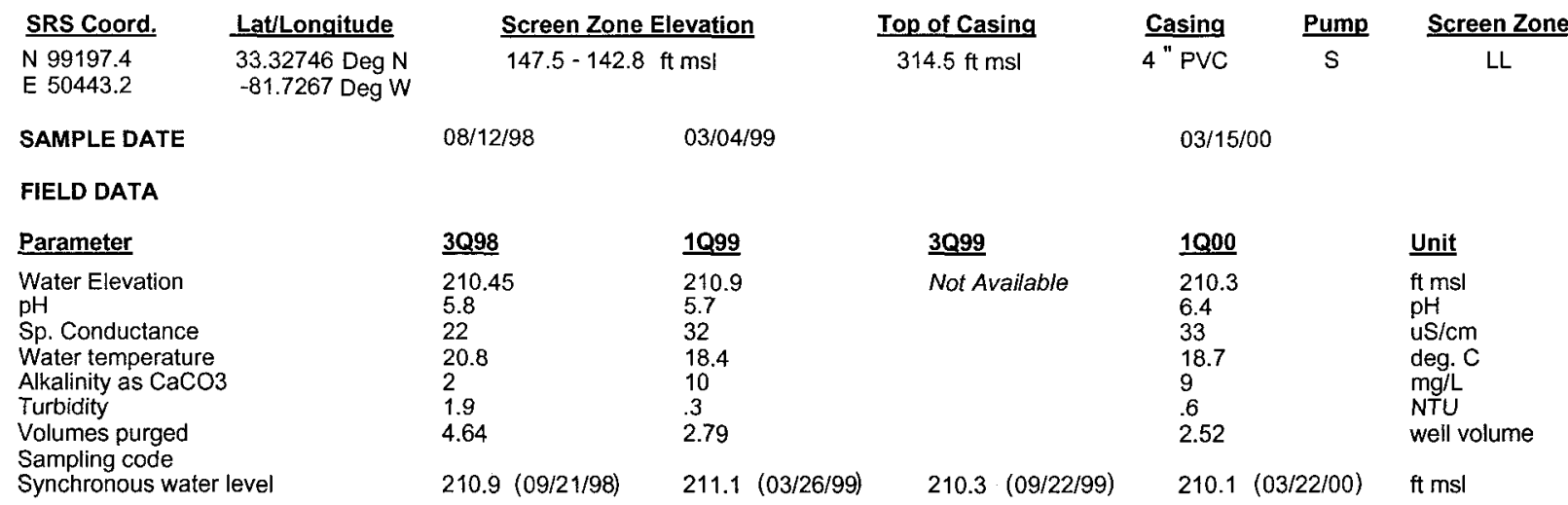

\section{ANALYTICAL DATA}

I. Groundwater Protection Standard

261 Appendix VIII/264 Appendix IX Hazardous Constituents

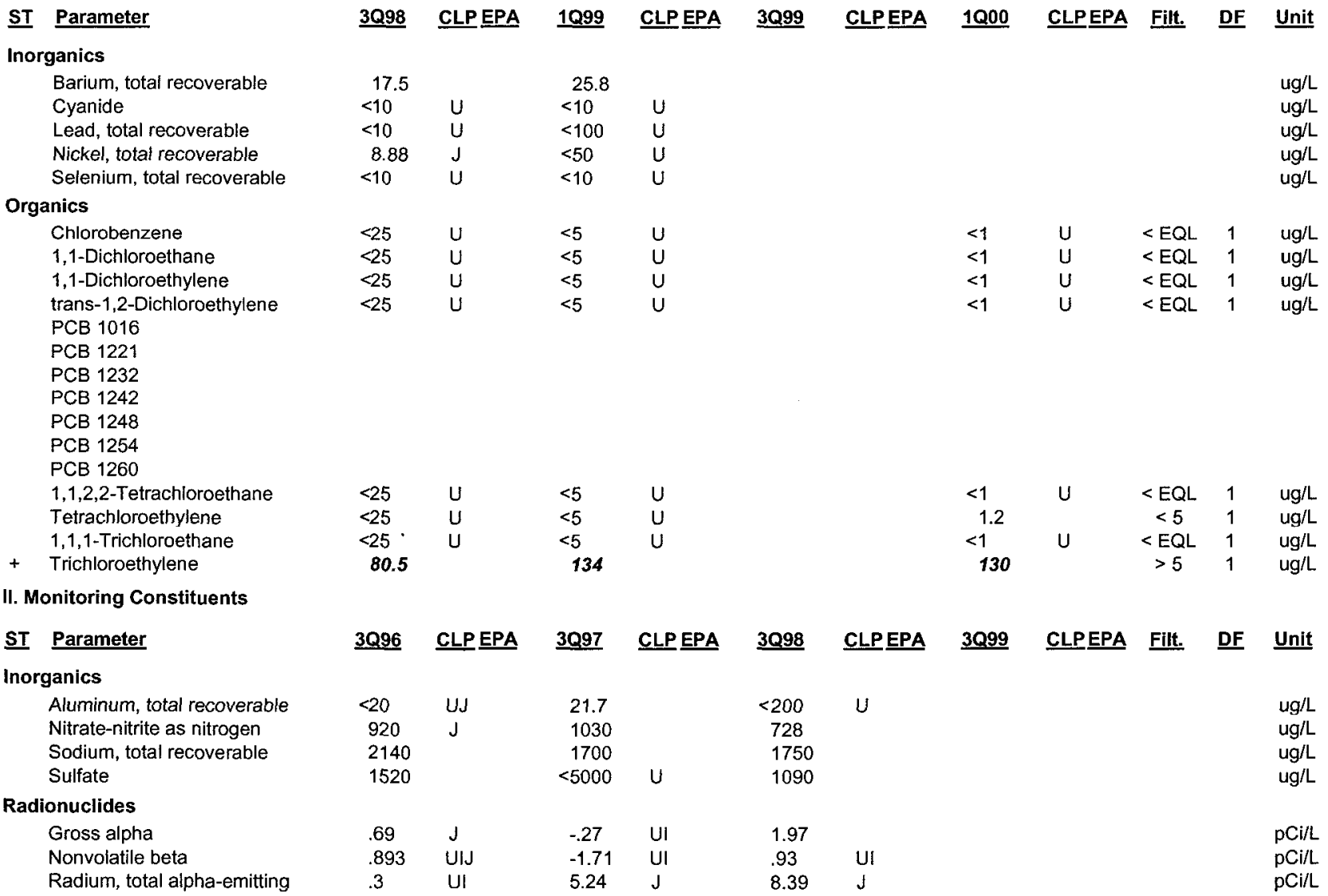

Notes: Concentrations in bold italics exceed the groundwater protection or monitoring constituent standards listed in Appendix A. Synchronous water levels are measured over a 3-5 day period or less. Dilution factors, Laboratory, and Filtered Data are for Groundwater Protection Standards First Quarter 2000 and Monitoring Constituents Third Quarter 1999 data only.

$+=$ exceeded the Groundwater Protection Standards (First Quarter 2000) or Monitoring Constituents (Third Quarter 1999) listed in Appendix A. 
Table D-4. Groundwater Monitoring Results for Plume Definition Wells, M-Area HWMF (Cont.) WELL MSB 74C

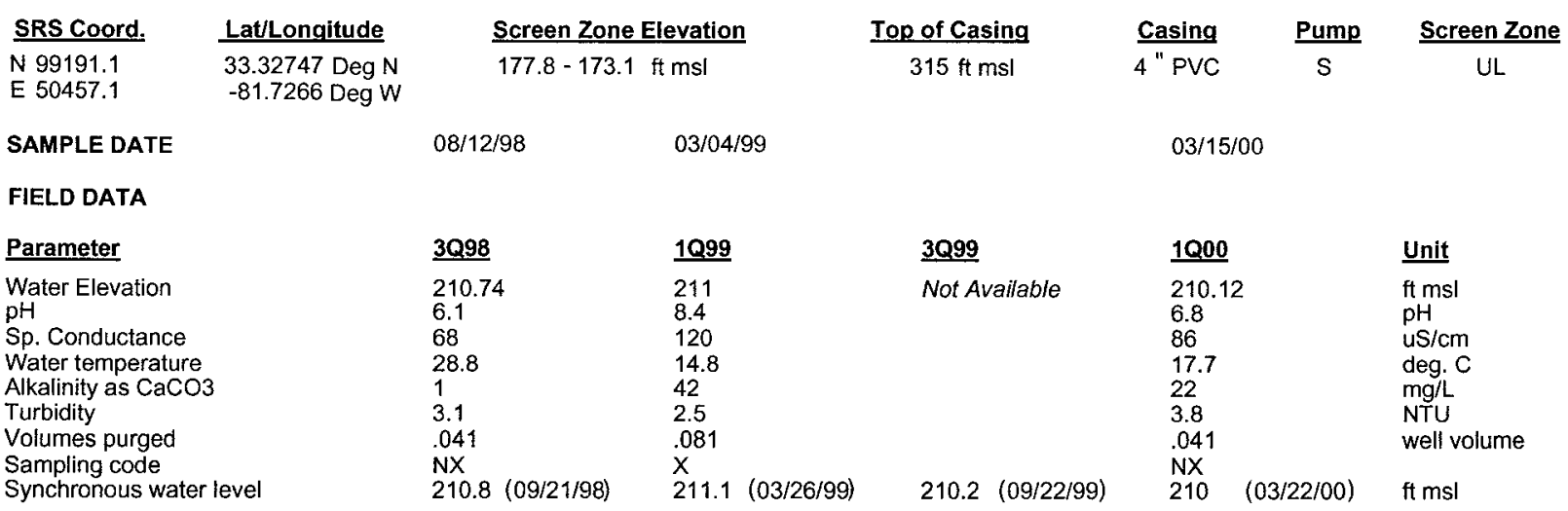

ANALYTICAL DATA

I. Groundwater Protection Standard

261 Appendix VIII/264 Appendix IX Hazardous Constituents

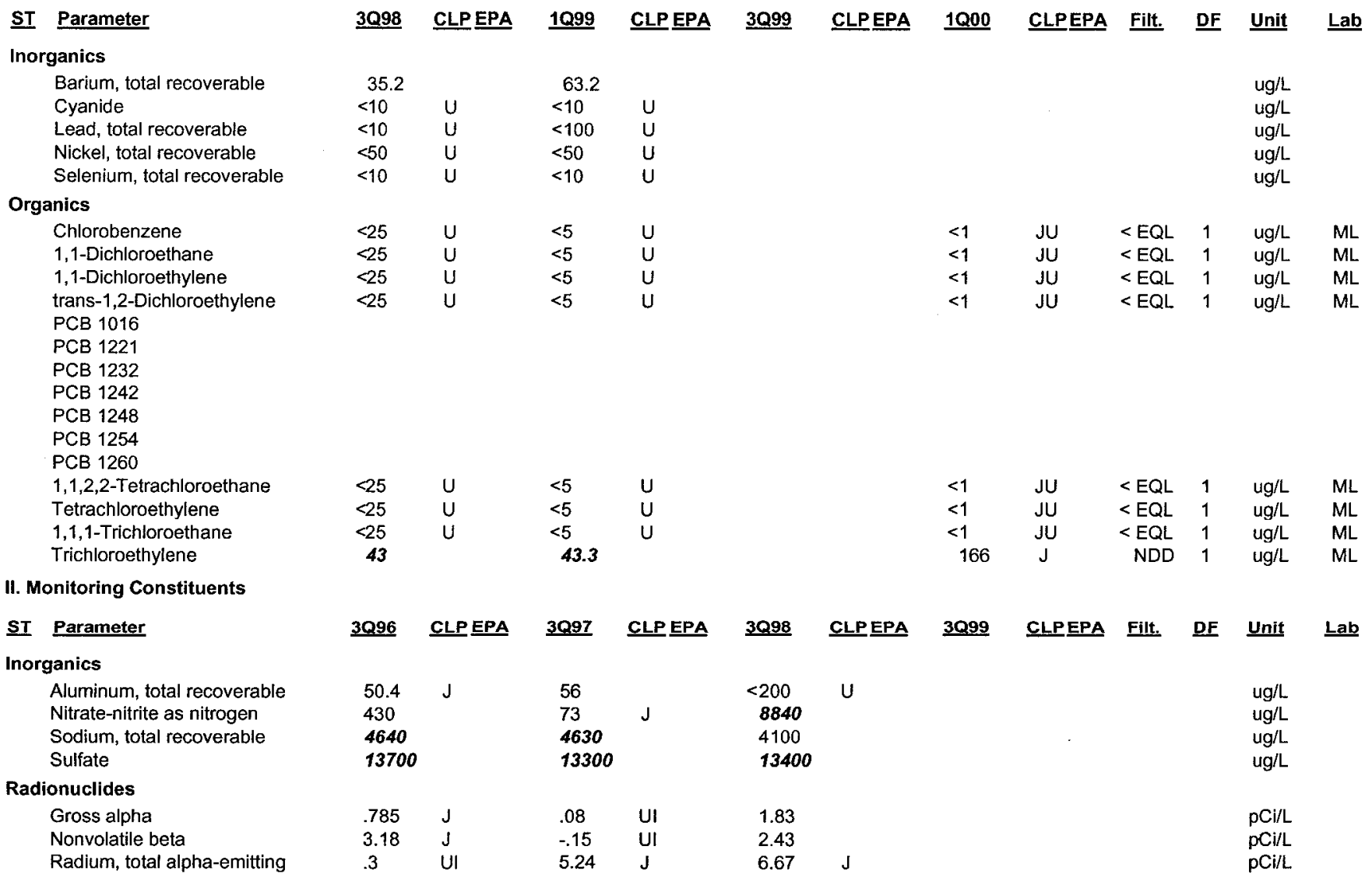

Notes: Concentrations in bold italics exceed the groundwater protection or monitoring constituent standards listed in Appendix A. Synchronous water levels are measured over a 3-5 day period or less. Dilution factors, Laboratory, and Filtered Data are for Groundwater Protection Standards First Quarter 2000 and Monitoring Constituents Third Quarter 1999 data only.

$+=$ exceeded the Groundwater Protection Standards (First Quarter 2000) or Monitoring Constituents (Third Quarter 1999) listed in Appendix A. 
Table D-4. Groundwater Monitoring Results for Plume Definition Wells, M-Area HWMF (Cont.) WELL MSB 74D

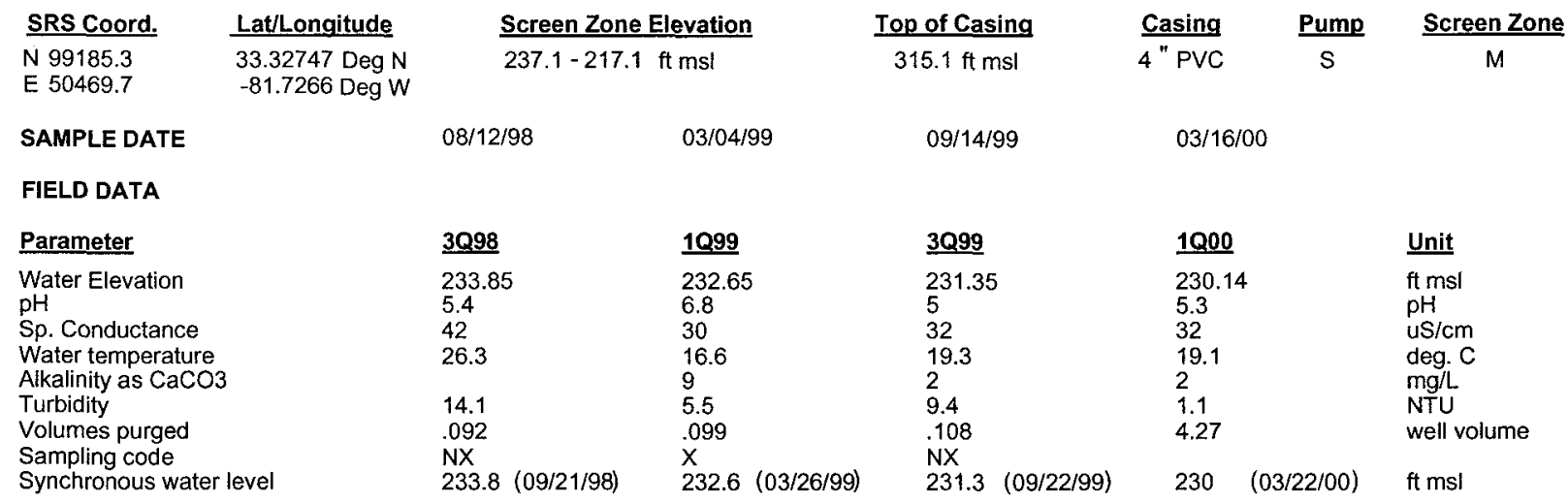

\section{ANALYTICAL DATA}

I. Groundwater Protection Standard

261 Appendix VIII/264 Appendix IX Hazardous Constituents

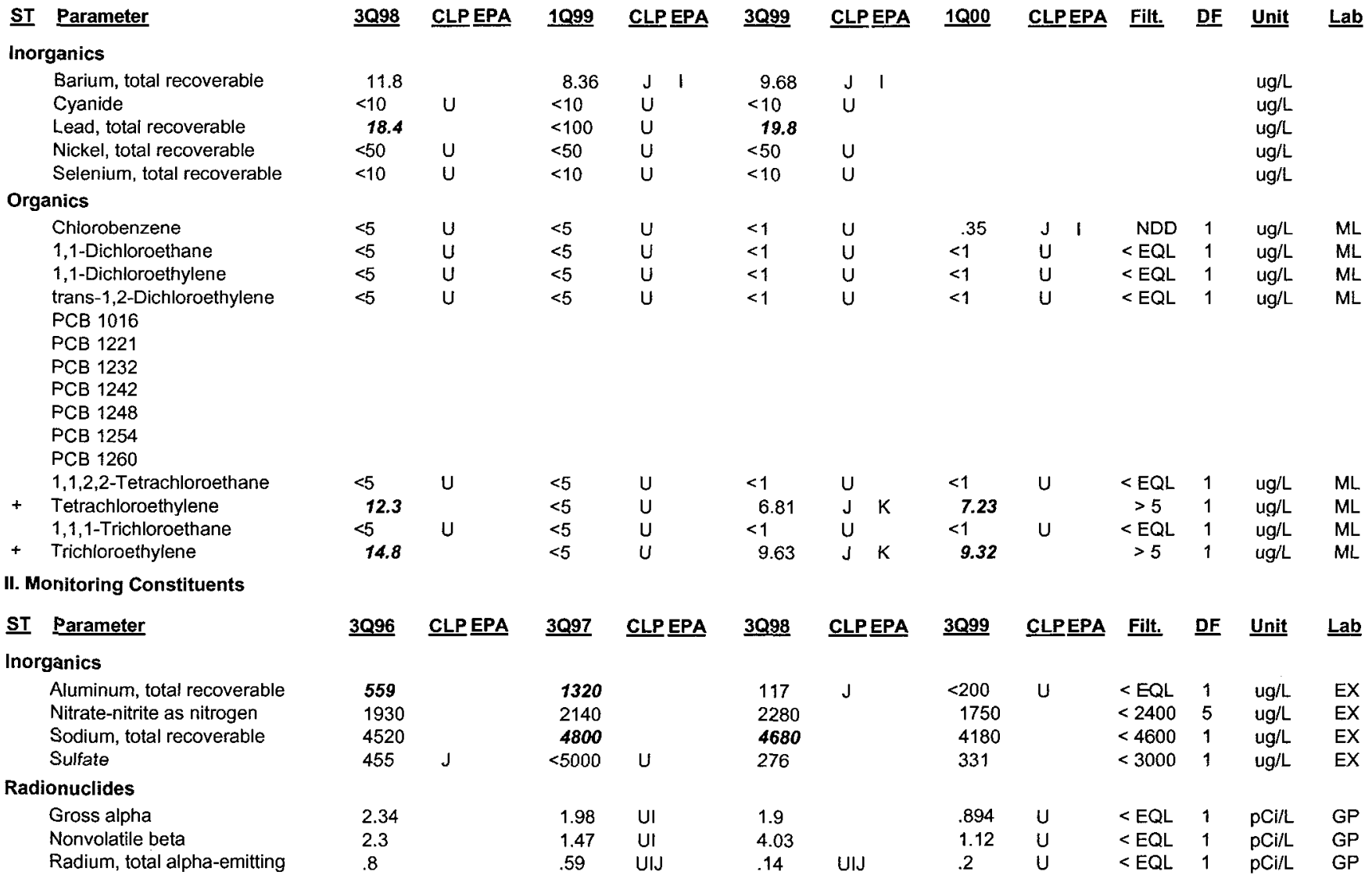

Notes: Concentrations in bold italics exceed the groundwater protection or monitoring constituent standards listed in Appendix A. Synchronous water levels are measured over a 3-5 day period or less. Dilution factors, Laboratory, and Filtered Data are for Groundwater Protection Standards First Quarter 2000 and Monitoring Constituents Third Quarter 1999 data only.

+ = exceeded the Groundwater Protection Standards (First Quarter 2000) or Monitoring Constituents (Third Quarter 1999) listed in Appendix A. 
Table D-4. Groundwater Monitoring Results for Plume Definition Wells, M-Area HWMF (Cont.) WELL MSB 75B

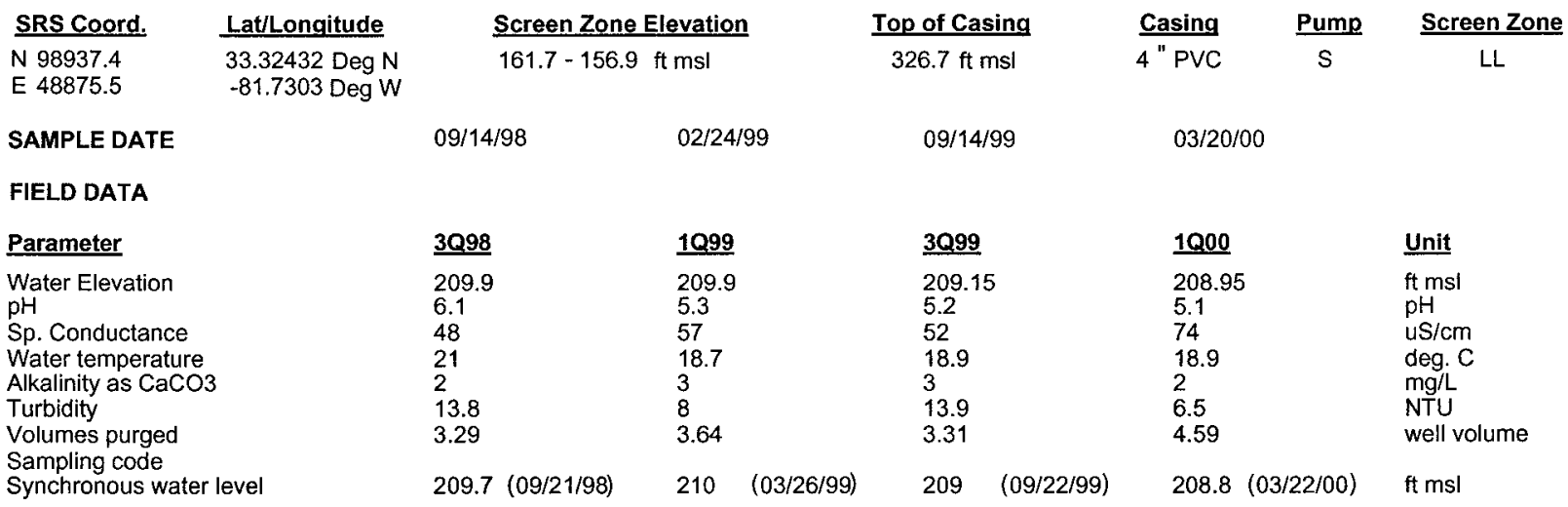

ANALYTICAL DATA

I. Groundwater Protection Standard

261 Appendix VIII/264 Appendix IX Hazardous Constituents

\begin{tabular}{|c|c|c|c|c|c|c|c|c|c|c|c|c|c|}
\hline$\underline{\mathrm{ST}}$ & Parameter & $\underline{3098}$ & CLP EPA & 1099 & CLP EPA & $\underline{3 Q 99}$ & CLPEPA & 1000 & CLPEPA & Filt. & $\underline{\mathrm{DF}}$ & Unit & $\underline{\text { Lab }}$ \\
\hline \multicolumn{14}{|c|}{ Inorganics } \\
\hline & Barium, total recoverable & 19.2 & & 25 & & 24.9 & & & & & & $\mathrm{ug} / \mathrm{L}$ & \\
\hline & Cyanide & $<10$ & $U$ & $<10$ & $U$ & $<10$ & $\mathrm{U}$ & & & & & $\mathrm{ug} / \mathrm{L}$ & \\
\hline & Lead, total recoverable & $<100$ & $U$ & $<100$ & U & $<10$ & $\mathrm{U}$ & & & & & $\mathrm{ug} / \mathrm{L}$ & \\
\hline & Nickel, total recoverable & $<50$ & $\mathrm{U}$ & $<50$ & $\mathrm{U}$ & $<50$ & $\mathrm{U}$ & & & & & ug/L & \\
\hline & Selenium, total recoverable & $<200$ & $\mathrm{U}$ & $<10$ & $U$ & $<10$ & $U$ & & & & & $\mathrm{ug} / \mathrm{L}$ & \\
\hline \multicolumn{14}{|c|}{ Organics } \\
\hline & Chlorobenzene & $<50$ & $U$ & $<125$ & $U$ & $<1$ & $u$ & $<10$ & $\mathrm{U}$ & $<\mathrm{EQL}$ & 10 & $\mathrm{ug} / \mathrm{L}$ & ML \\
\hline & 1,1-Dichloroethane & $<50$ & $\mathrm{U}$ & $<125$ & $\mathrm{U}$ & $<1$ & $\mathrm{U}$ & $<10$ & $U$ & $<\mathrm{EQL}$ & 10 & $\mathrm{ug} / \mathrm{L}$ & ML \\
\hline & 1,1-Dichloroethylene & $<50$ & $\mathrm{U}$ & $<125$ & $\mathrm{U}$ & $<1$ & $\mathrm{U}$ & $<10$ & $U$ & $<\mathrm{EQL}$ & 10 & $\mathrm{ug} / \mathrm{L}$ & $M L$ \\
\hline & trans-1,2-Dichloroethylene & $<50$ & $U$ & $<125$ & $\mathrm{U}$ & $<1$ & $\mathrm{U}$ & $<10$ & $\mathrm{U}$ & $<\mathrm{EQL}$ & 10 & ug/L & ML \\
\hline & PCB 1016 & & & & & & & & & & & & \\
\hline & PCB 1221 & & & & & & & & & & & & \\
\hline & PCB 1232 & & & & & & & & & & & & \\
\hline & PCB 1242 & & & & & & & & & & & & \\
\hline & PCB 1248 & & & & & & & & & & & & \\
\hline & PCB 1254 & & & & & & & & & & & & \\
\hline & PCB 1260 & & & & & & & & & & & & \\
\hline & $1,1,2,2$-Tetrachloroethane & $<50$ & $U$ & $<125$ & $\mathrm{U}$ & $<1$ & $U$ & $<10$ & $U$ & $<\mathrm{EQL}$ & 10 & $\mathrm{ug} / \mathrm{L}$ & ML. \\
\hline & Tetrachloroethylene & $<50$ & $\mathrm{U}$ & $<125$ & $\mathrm{U}$ & $<1$ & $\mathrm{U}$ & $<10$ & $\mathrm{U}$ & $<E Q L$ & 10 & $\mathrm{ug} / \mathrm{L}$ & ML \\
\hline & 1,1,1-Trichloroethane & $<50$ & $\mathrm{U}$ & $<125$ & $\mathrm{U}$ & $<1$ & $\mathrm{U}$ & $<10$ & $\mathrm{U}$ & $<$ EQL & 10 & $\mathrm{ug} / \mathrm{L}$ & $\mathrm{ML}$ \\
\hline+ & Trichloroethylene & 1880 & & 4080 & & 2820 & & 2650 & & $>5$ & 10 & $\mathrm{ug} / \mathrm{L}$ & ML \\
\hline \multicolumn{14}{|c|}{ II. Monitoring Constituents } \\
\hline$\underline{\text { ST }}$ & Parameter & $\underline{3096}$ & CLPEPA & $\underline{3 Q 97}$ & CLP EPA & $\underline{3098}$ & CLPEPA & $\underline{3099}$ & CLPEPA & Filt. & DF & Unit & Lab \\
\hline \multicolumn{14}{|c|}{ Inorganics } \\
\hline & Aluminum, total recoverable & 355 & J & 122 & & 94 & $J$ & $<200$ & U & $<E Q L$ & 1 & $\mathrm{ug} / \mathrm{L}$ & EX \\
\hline+ & Nitrate-nitrite as nitrogen & 3240 & J & 5540 & & 3000 & & 4710 & & $>2400$ & 5 & $\mathrm{ug} / \mathrm{L}$ & EX \\
\hline & Sodium, total recoverable & 2880 & & 2960 & & 3360 & & 3720 & & $<4600$ & 1 & ug/L & EX \\
\hline & Sulfate & 1540 & & $<5000$ & $\mathrm{U}$ & 1460 & & 1140 & & $<3000$ & 1 & ug/L & EX \\
\hline \multicolumn{14}{|c|}{ Radionuclides } \\
\hline & Gross alpha & 2.44 & $J$ & 1.55 & & 2.86 & & 2.37 & & $<15$ & 1 & $\mathrm{pCi} / \mathrm{L}$ & GP \\
\hline & Nonvolatile beta & 2.98 & $j$ & 1.83 & Ul & 4.67 & & 2.55 & J IK & NDD & 1 & $\mathrm{pCi} / \mathrm{L}$ & GP \\
\hline & Radium, total alpha-emitting & 1.3 & & 1.43 & $\mathrm{~J}$ & 1.38 & & .9 & J i & NDD & 1 & $\mathrm{pCi} / \mathrm{L}$ & GP \\
\hline
\end{tabular}

Notes: Concentrations in bold italics exceed the groundwater protection or monitoring constituent standards listed in Appendix A. Synchronous water levels are measured over a 3-5 day period or less. Dilution factors, Laboratory, and Filtered Data are for Groundwater Protection Standards First Quarter 2000 and Monitoring Constituents Third Quarter 1999 data only.

$+=$ exceeded the Groundwater Protection Standards (First Quarter 2000) or Monitoring Constituents (Third Quarter 1999) listed in Appendix A. 
Table D-4. Groundwater Monitoring Results for Plume Definition Wells, M-Area HWMF (Cont.) WELL MSB 75C

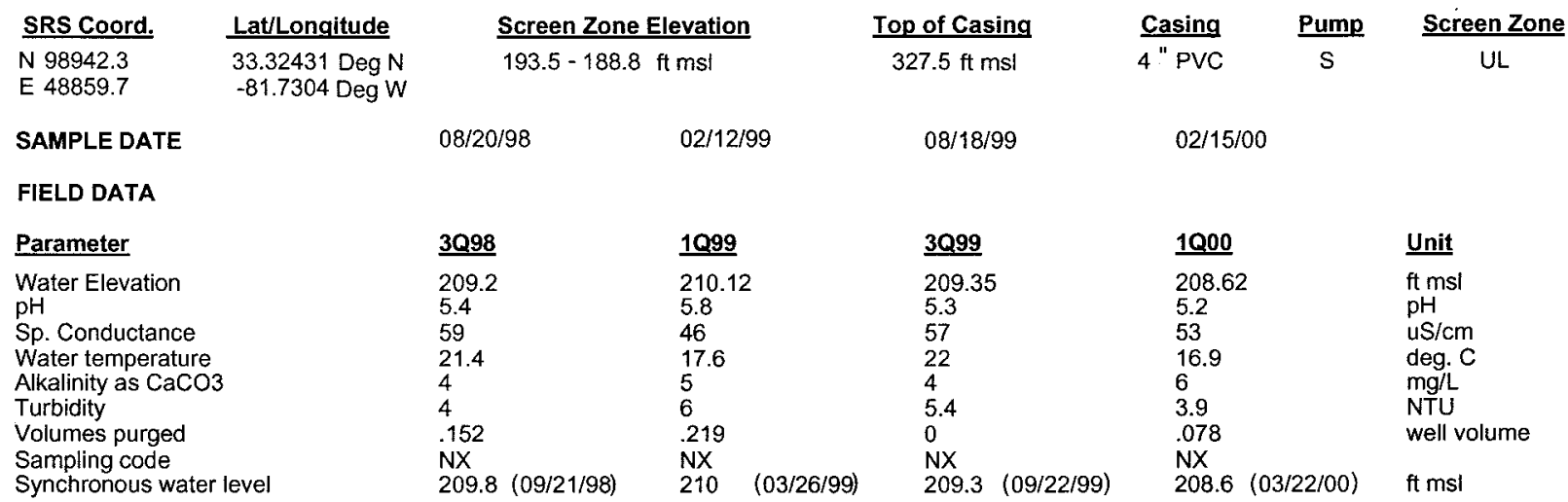

ANALYTICAL DATA

I. Groundwater Protection Standard

261 Appendix VIII/264 Appendix IX Hazardous Constituents

ST

Inorganics

Barium, total recoverable

Cyanide

Lead, total recoverable

Nickel, total recoverable

Selenium, total recoverable

Organics

Chlorobenzene

1,1-Dichloroethane

1,1-Dichloroethylene

trans-1,2-Dichloroethylene

PCB 1016

PCB 1221

PCB 1232

PCB 1242

PCB 1248

$P C B 1254$

PCB 1260

$1,1,2,2$-Tetrachloroethane

Tetrachloroethylene

1,1,1-Trichloroethane

Trichloroethylene

II. Monitoring Constituents

\section{ST Parameter \\ Inorganics}

Aluminum, total recoverable

Nitrate-nitrite as nitrogen

+ Sodium total recoverable

+ Sulfate

Radionuclides

Gross alpha

Nonvolatile beta

Radium, total alpha-emitting
$3 Q 98$ CLP EPA

1099

22.9

$<10 \quad U$

11.3
$<0$

$<10 \quad U$

$<5 \quad U$

$\begin{array}{ll}<5 & U \\ <5 & U\end{array}$

$<5 \quad U$

$\begin{array}{ll}<10 & U \\ <10 & U \\ <100 & U \\ <50 & U \\ <200 & U\end{array}$

20
$<10 \quad U$

12.4

$\begin{array}{ll}<7.6 & \text { JU } \\ <5 & \mathrm{U}\end{array}$

$\begin{array}{ll}<5 & \cup \\ <5 & U \\ <5 & U \\ <5 & U\end{array}$

$\begin{array}{ll}<1 & \mathrm{U} \\ <1 & \mathrm{U} \\ <1 & \mathrm{U} \\ <1 & \mathrm{U}\end{array}$

$\begin{array}{ll}<1 & U \\ <1 & U \\ <1 & U\end{array}$

< EQL 1

$<\mathrm{EQL} \quad 1$

$<E Q L$

$<\mathrm{EQL}$

$\mathrm{ug} / \mathrm{L}$

$u g / L$

$u g / L$

$\mathrm{ug} / \mathrm{L}$

$\mathrm{ug} / \mathrm{L}$

ug/L. ML

ug/L $\quad M L$

ug/ $\mathrm{L} \quad \mathrm{ML}$

$u g / L \quad M L$

$\begin{array}{lll}<5 & U & <5 \\ <5 & U & <5 \\ <5 & U & <5 \\ <5 & U & <5\end{array}$

$<5$
$<5$
$<5$

$\begin{array}{lll}U & <1 & U \\ U & <1 & U \\ U & <1 & U \\ U & <1 & U\end{array}$

$\begin{array}{ll}<1 & U \\ <1 & 1 \\ <1 & U\end{array}$

$<E Q L$
$<E Q L$
$<E Q L$
$<E Q L$

ug/L

ugl

U

ug/L

$M L$

3096 CLPEPA

3097 CLPEPA

3098 CLPEPA

3099 CLPEPA Filt. DF Unit Lab

\begin{tabular}{|c|c|c|c|c|c|c|c|c|c|c|c|}
\hline 102 & $\mathrm{~J}$ & 78.4 & & $<200$ & U & $<200$ & $\mathrm{U}$ & $<\mathrm{EQL}$ & 1 & $\mathrm{ug} / \mathrm{L}$ & EX \\
\hline 260 & & 316 & & $<200$ & U & $<500$ & $\mathrm{U}$ & $<\mathrm{EQL}$ & 5 & $\mathrm{ug} / \mathrm{L}$ & EX \\
\hline 5820 & $\mathrm{~J}$ & 5340 & & 6270 & & 5300 & & $>4600$ & 1 & $\mathrm{ug} / \mathrm{L}$ & EX \\
\hline 13400 & & 13400 & & 12700 & & 14500 & & $>3000$ & 1 & ug/L & EX \\
\hline 1.21 & & 1.88 & Ul & 1.78 & & .257 & $U$ & $<E Q L$ & 1 & $\mathrm{pCi} / \mathrm{L}$ & GP \\
\hline 3.23 & & 2.76 & UI & 3.3 & & 2.5 & $\mathrm{~J}$ & NDD & 1 & $\mathrm{pCi} / \mathrm{L}$ & GP \\
\hline 1.1 & & 1.21 & $\mathrm{~J}$ & .46 & & 1.3 & & $<5$ & 1 & $\mathrm{pCi} / \mathrm{L}$ & GP \\
\hline
\end{tabular}

Notes: Concentrations in bold italics exceed the groundwater protection or monitoring constituent standards listed in Appendix A. Synchronous water levels are measured over a 3-5 day period or less. Dilution factors, Laboratory, and Filtered Data are for Groundwater Protection Standards First Quarter 2000 and Monitoring Constituents Third Quarter 1999 data only.

$+=$ exceeded the Groundwater Protection Standards (First Quarter 2000) or Monitoring Constituents (Third Quarter 1999) listed in Appendix A. 
Table D-4. Groundwater Monitoring Results for Plume Definition Wells, M-Area HWMF (Cont.) WELL MSB 76C

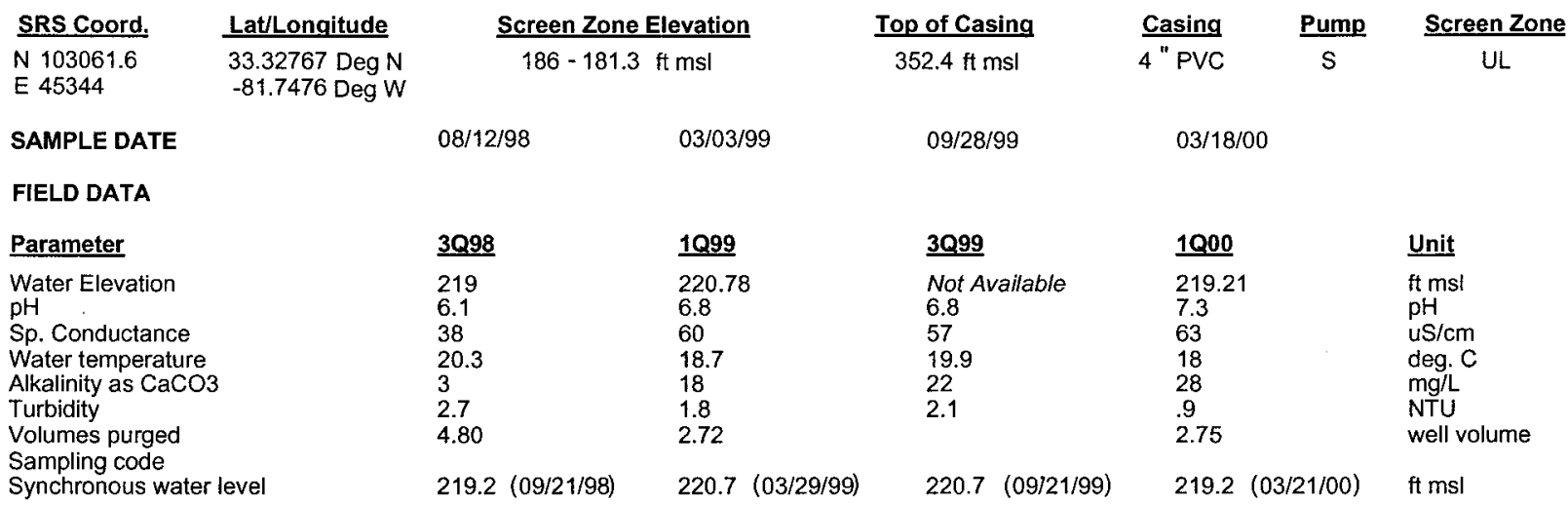

ANALYTICAL DATA

I. Groundwater Protection Standard

261 Appendix VIII/264 Appendix IX Hazardous Constituents

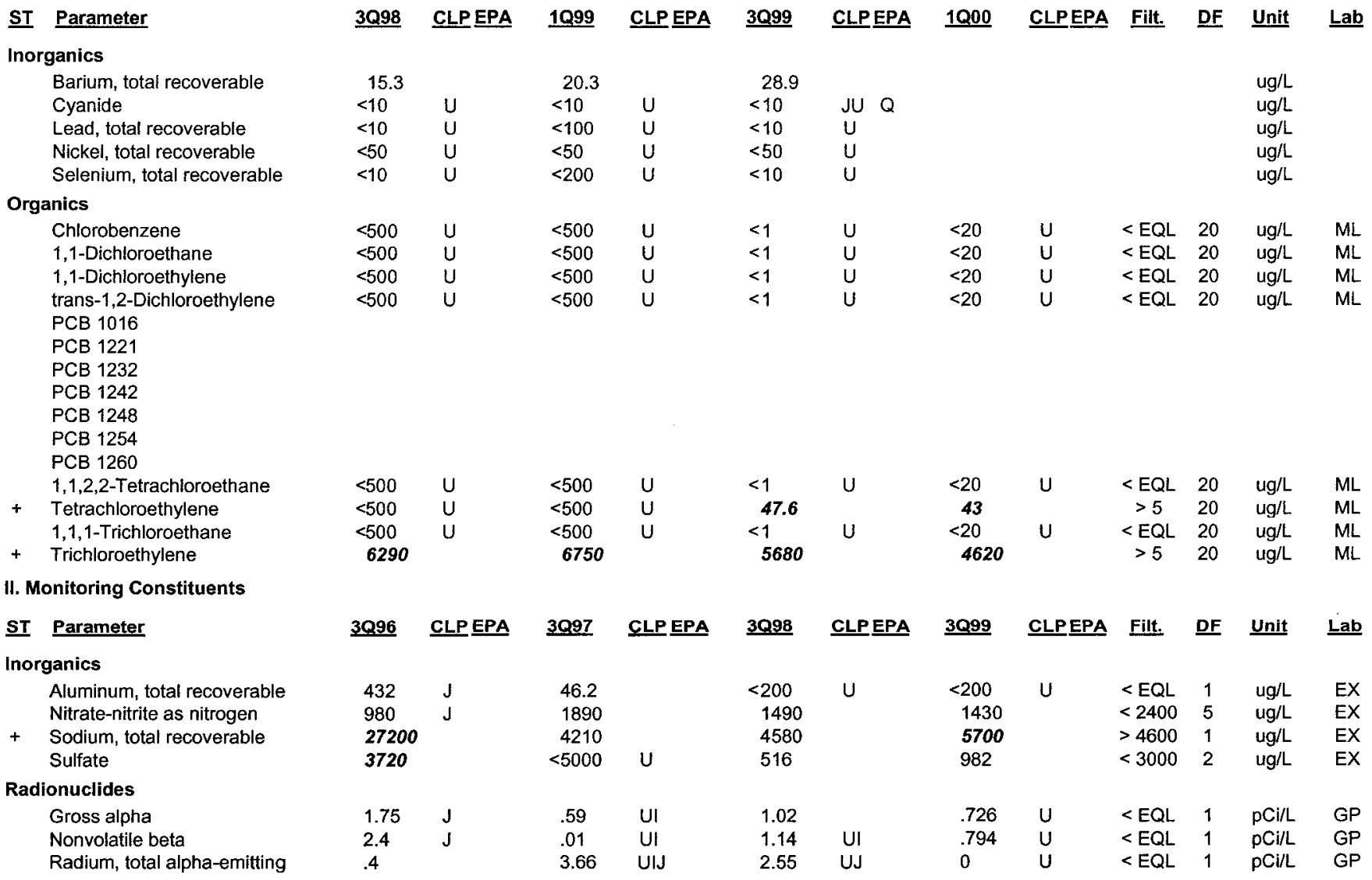

Notes: Concentrations in bold italics exceed the groundwater protection or monitoring constituent standards listed in Appendix A. Synchronous water levels are measured over a 3-5 day period or less. Dilution factors, Laboratory, and Filtered Data are for Groundwater Protection Standards First Quarter 2000 and Monitoring Constituents Third Quarter 1999 data only.

+ = exceeded the Groundwater Protection Standards (First Quarter 2000) or Monitoring Constituents (Third Quarter 1999) listed in Appendix A. 
Table D-4. Groundwater Monitoring Results for Plume Definition Wells, M-Area HWMF (Cont.) WELL MSB 77B

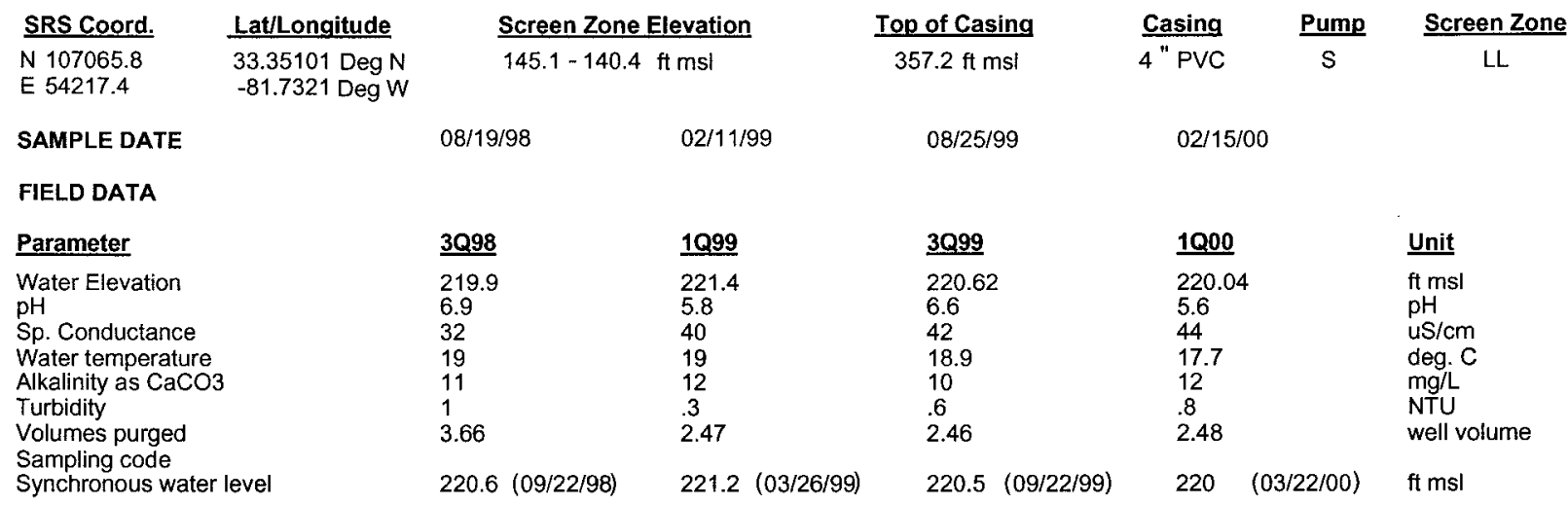

ANALYTICAL DATA

I. Groundwater Protection Standard

261 Appendix VIII/264 Appendix IX Hazardous Constituents

\begin{tabular}{|c|c|c|c|c|c|c|c|c|c|c|c|c|c|}
\hline$\underline{\mathbf{S T}}$ & Parameter & $\underline{3 Q 98}$ & $\underline{\text { CLPEPA }}$ & 1099 & $\underline{\text { CLP EPA }}$ & $\underline{3 Q 99}$ & CLPEPA & $\underline{1 Q 00}$ & $\underline{\text { CLPEPA }}$ & Filt. & $\underline{\mathrm{DF}}$ & $\underline{\text { Unit }}$ & Lab \\
\hline \multicolumn{14}{|c|}{ Inorganics } \\
\hline & Barium, total recoverable & 23.7 & & $<10$ & $\mathrm{U}$ & 34 & & & & & & $\mathrm{ug} / \mathrm{L}$ & \\
\hline & Cyanide & $<10$ & $\mathrm{U}$ & $<10$ & $\mathrm{U}$ & $<10$ & $\mathrm{U}$ & & & & & $\mathrm{ug} / \mathrm{L}$ & \\
\hline & Lead, total recoverable & $<10$ & $\mathrm{U}$ & $<100$ & $U$ & $<10$ & $\mathrm{U}$ & & & & & $\mathrm{ug} / \mathrm{L}$ & \\
\hline & Nickel, total recoverable & $<9.99$ & $\mathrm{U}$ & $<50$ & $\mathrm{U}$ & $<50$ & U & & & & & $\mathrm{ug} / \mathrm{L}$ & \\
\hline & Selenium, total recoverable & $<10$ & U & & & $<10$ & $\mathrm{U}$ & & & & & $\mathrm{ug} / \mathrm{L}$ & \\
\hline \multicolumn{14}{|c|}{ Organics } \\
\hline & Chlorobenzene & $<5$ & $U$ & $<5$ & $\mathrm{U}$ & $<1$ & $U$ & $<1$ & U & $<$ EQL & 1 & $\mathrm{ug} / \mathrm{L}$ & ML \\
\hline & 1,1-Dichloroethane & $<5$ & U & $<5$ & $\mathrm{U}$ & $<1$ & $\mathrm{U}$ & $<1$ & $\mathrm{U}$ & $<E Q L$ & 1 & $\mathrm{ug} / \mathrm{L}$ & ML \\
\hline & 1,1-Dichloroethylene & $<5$ & $\mathrm{U}$ & $<5$ & $\mathrm{U}$ & $<1$ & $\mathrm{U}$ & $<1$ & $\mathrm{U}$ & $<E Q L$ & 1 & $\mathrm{ug} / \mathrm{L}$ & $M L$ \\
\hline & trans-1,2-Dichloroethylene & $<5$ & $\mathrm{U}$ & $<5$ & $\mathrm{U}$ & $<1$ & $\mathrm{U}$ & $<1$ & $U$ & $<$ EQL & 1 & ug/L & $\mathrm{ML}$ \\
\hline & PCB 1016 & & & & & & & & & & & & \\
\hline & PCB 1221 & & & & & & & & & & & & \\
\hline & PCB 1232 & & & & & & & & & & & & \\
\hline & PCB 1242 & & & & & & & & & & & & \\
\hline & РСВ 1248 & & & & & & & & & & & & \\
\hline & PCB 1254 & & & & & & & & & & & & \\
\hline & РCB 1260 & & & & & & & & & & & & \\
\hline & 1,1,2,2-Tetrachloroethane & $<5$ & $U$ & $<5$ & $U$ & $<1$ & $\mathrm{U}$ & $<1$ & $\mathrm{U}$ & $<E Q L$ & 1 & $\mathrm{ug} / \mathrm{L}$ & ML \\
\hline & Tetrachloroethylene & $<5$ & U & $<5$ & $\mathrm{U}$ & $<1$ & $\mathrm{U}$ & $<1$ & $\mathrm{U}$ & $<E Q L$ & 1 & $\mathrm{ug} / \mathrm{L}$ & ML \\
\hline & 1,1,1-Trichtoroethane & $<5$ & $U$ & $<5$ & $\mathrm{U}$ & $<1$ & $\mathrm{U}$ & $<1$ & $\mathrm{U}$ & $<E Q L$ & 1 & $\mathrm{ug} / \mathrm{L}$ & ML \\
\hline & Trichloroethylene & $<5$ & $\mathrm{U}$ & $<5$ & $\mathrm{U}$ & $<1$ & $\mathrm{U}$ & $<1$ & $\mathrm{U}$ & $<E Q L$ & 1 & $\mathrm{ug} / \mathrm{L}$ & ML \\
\hline \multicolumn{14}{|c|}{ II. Monitoring Constituents } \\
\hline$\underline{\mathbf{S T}}$ & Parameter & 3096 & $\underline{\text { CLP EPA }}$ & $\underline{3 Q 97}$ & CLP EPA & $\underline{3098}$ & CLPEPA & $\underline{3099}$ & CLPEPA & Filt. & DF & Unit & $\underline{\mathrm{Lab}}$ \\
\hline \multicolumn{14}{|c|}{ Inorganics } \\
\hline & Aluminum, total recoverable & 553 & & 125 & & 104 & J & $<200$ & $\mathrm{U}$ & $<\mathrm{EQL}$ & 1 & $\mathrm{ug} / \mathrm{L}$ & EX \\
\hline & Nitrate-nitrite as nitrogen & 400 & & 129 & & 848 & & 873 & & $<2400$ & 5 & ug/L & EX \\
\hline & Sodium, total recoverable & 5410 & & 1730 & & 1910 & & 2000 & & $<4600$ & 1 & $\mathrm{ug} / \mathrm{L}$ & EX \\
\hline & Sulfate & 823 & $\mathrm{~J}$ & $<5000$ & $\mathrm{U}$ & $<200$ & $\mathrm{U}$ & $<400$ & $\mathrm{U}$ & $<E Q L$ & 2 & ug/L & EX \\
\hline \multicolumn{14}{|c|}{ Radionuclides } \\
\hline & Gross alpha & 6.3 & $\mathrm{~J}$ & .72 & UI & 2.35 & J & 1.58 & J 1 & NDD & 1 & $\mathrm{pCi} / \mathrm{L}$ & GP \\
\hline & Nonvolatile beta & 4.4 & & 1.78 & UI & 7.08 & $J$ & 1.19 & $\mathrm{U}$ & $<E Q L$ & 1 & $\mathrm{pCi} / \mathrm{L}$ & GP \\
\hline & Radium, total alpha-emitting & 1.1 & & 4.54 & $J$ & .35 & UI & .3 & J I & NDD & 1 & $\mathrm{pCi} / \mathrm{L}$ & GP \\
\hline
\end{tabular}

Notes: Concentrations in bold italics exceed the groundwater protection or monitoring constituent standards listed in Appendix A. Synchronous water levels are measured over a 3-5 day period or less. Dilution factors, Laboratory, and Filtered Data are for Groundwater Protection Standards First Quarter 2000 and Monitoring Constituents Third Quarter 1999 data only.

+= exceeded the Groundwater Protection Standards (First Quarter 2000) or Monitoring Constituents (Third Quarter 1999) listed in Appendix A. 
Table D-4. Groundwater Monitoring Results for Plume Definition Wells, M-Area HWMF (Cont.) WELL MSB 77C

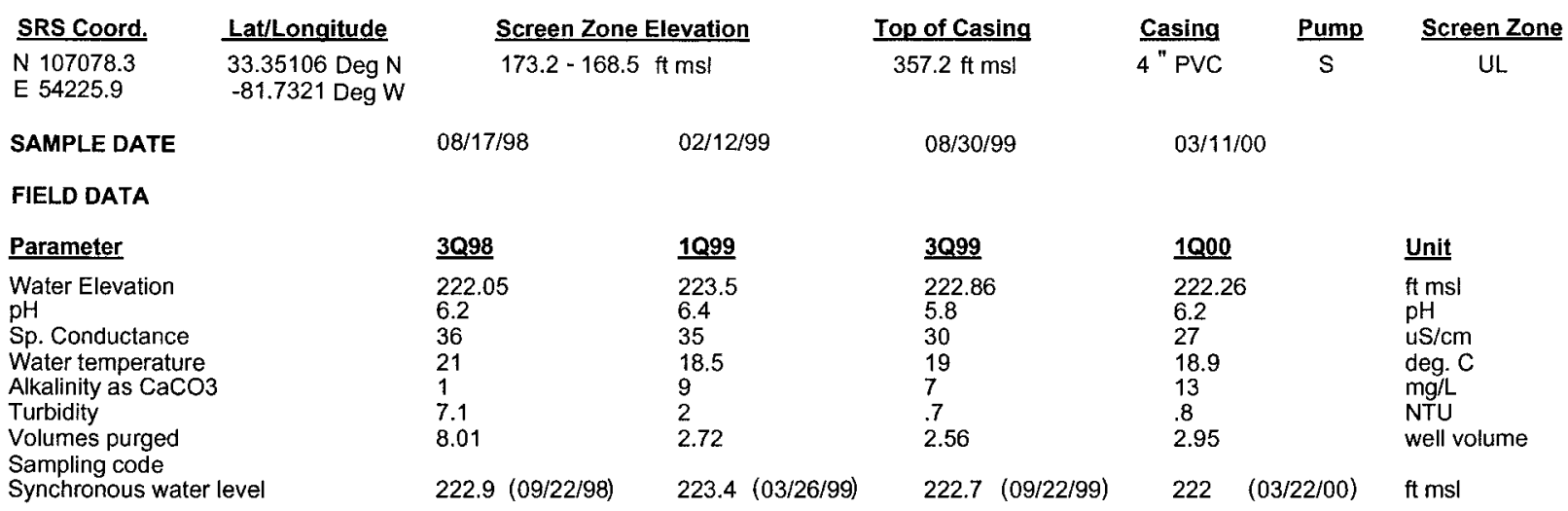

ANALYTICAL DATA

I. Groundwater Protection Standard

261 Appendix VIII/264 Appendix IX Hazardous Constituents

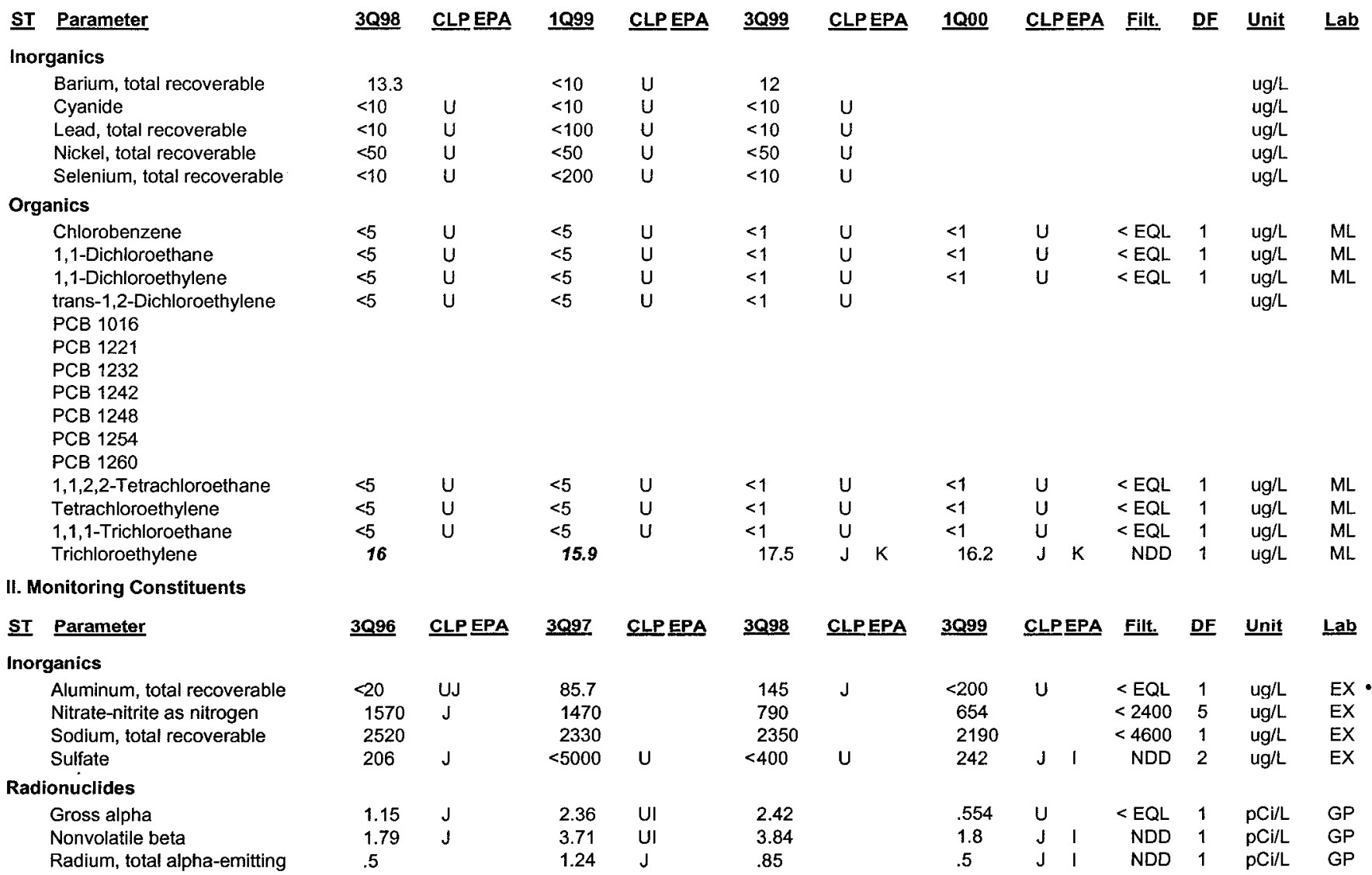

Notes: Concentrations in bold italics exceed the groundwater protection or monitoring constituent standards listed in Appendix A. Synchronous water levels are measured over a 3-5 day period or less. Dilution factors, Laboratory, and Filtered Data are for Groundwater Protection Standards First Quarter 2000 and Monitoring Constituents Third Quarter 1999 data only.

$+=$ exceeded the Groundwater Protection Standards (First Quarter 2000) or Monitoring Constituents (Third Quarter 1999) listed in Appendix A. 
Table D-4. Groundwater Monitoring Results for Plume Definition Wells, M-Area HWMF (Cont.) WELL MSB 78DR

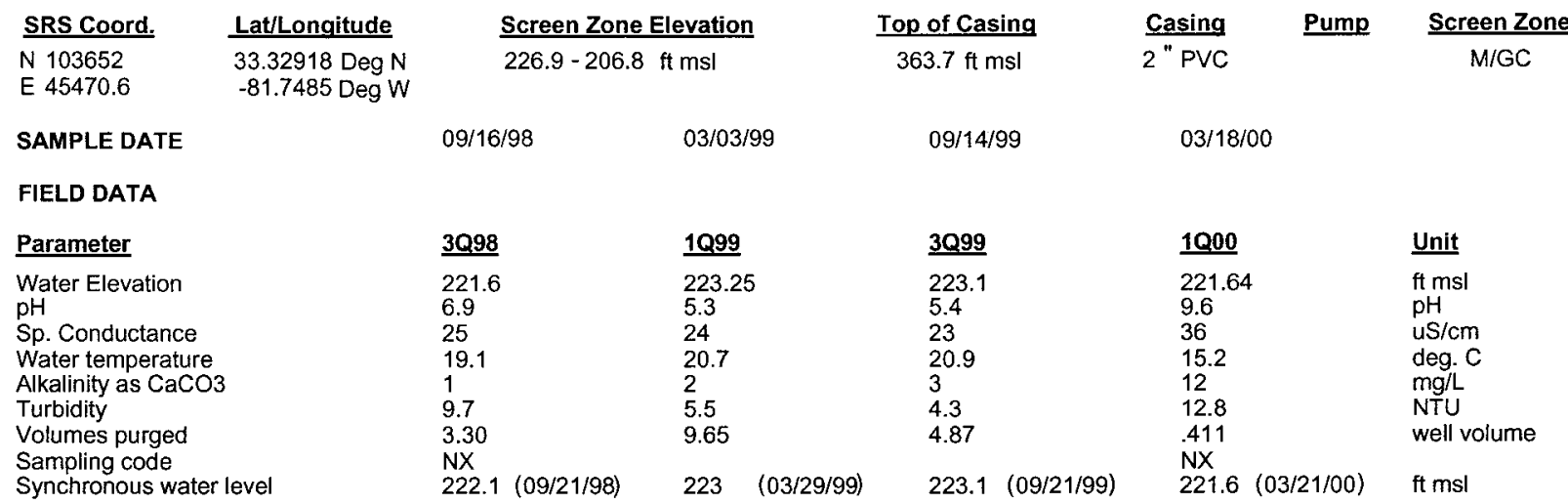

ANALYTICAL DATA

I. Groundwater Protection Standard

261 Appendix VIII/264 Appendix IX Hazardous Constituents

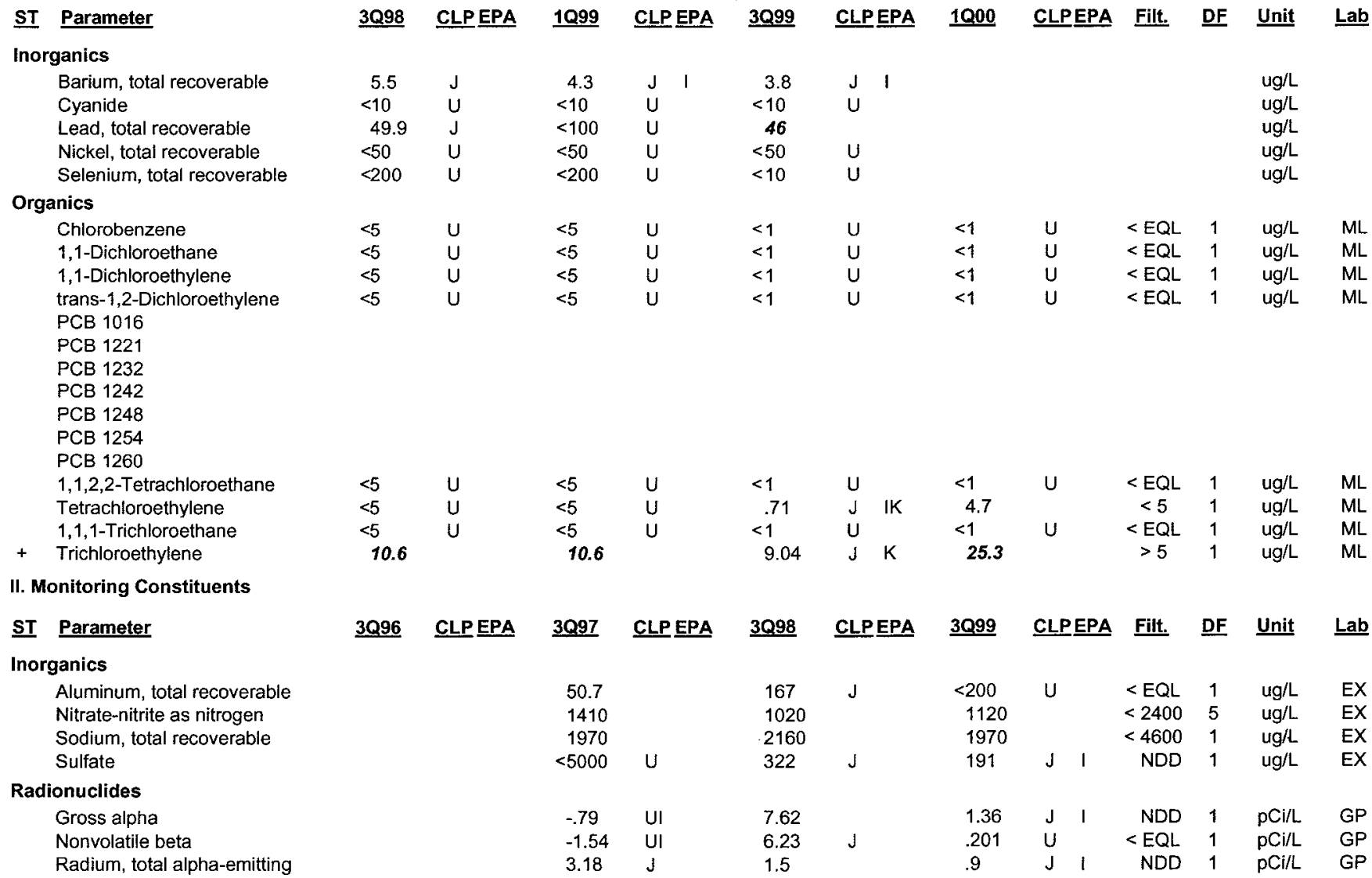

Notes: Concentrations in bold italics exceed the groundwater protection or monitoring constituent standards listed in Appendix A. Synchronous water levels are measured over a 3-5 day period or less. Dilution factors, Laboratory, and Filtered Data are for Groundwater Protection Standards First Quarter 2000 and Monitoring Constituents Third Quarter 1999 data only.

$+=$ exceeded the Groundwater Protection Standards (First Quarter 2000) or Monitoring Constituents (Third Quarter 1999) listed in Appendix A. 
Table D-4. Groundwater Monitoring Results for Plume Definition Wells, M-Area HWMF (Cont.) WELL MSB 79B

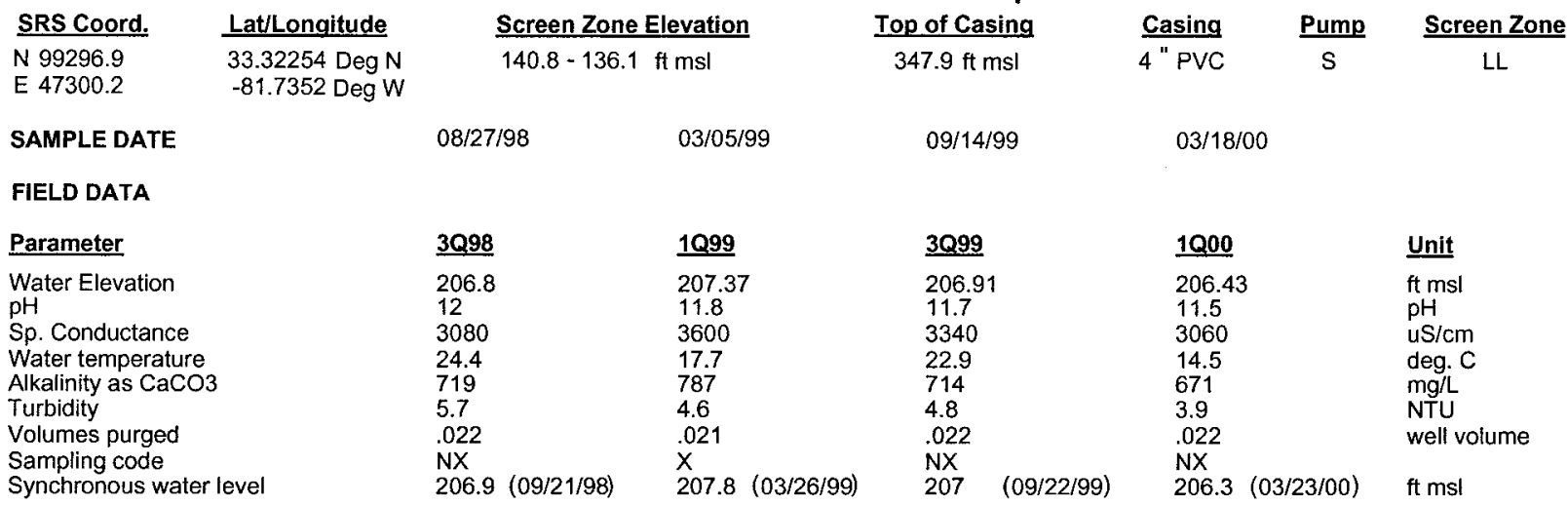

ANALYTICAL DATA

I. Groundwater Protection Standard

261 Appendix VIII/264 Appendix IX Hazardous Constituents

\begin{tabular}{|c|c|c|c|c|c|c|c|c|c|c|c|c|c|c|}
\hline$\underline{\text { ST }}$ & Parameter & $\underline{3098}$ & CLP EPA & $\underline{1099}$ & CLP EPA & $\underline{3099}$ & CLPEEA & 1000 & CLP & EPA & Filt. & DF & Unit & $\underline{\text { Lab }}$ \\
\hline \multicolumn{15}{|c|}{ Inorganics } \\
\hline & Barium, total recoverable & 819 & & 934 & & 790 & & & & & & & $\mathrm{ug} / \mathrm{L}$ & \\
\hline & Cyanide & $<10$ & U & $<10$ & $\mathrm{U}$ & $<10$ & $\mathrm{U}$ & & & & & & $\mathrm{ug} / \mathrm{L}$ & \\
\hline & Lead, total recoverable & 55.1 & $j$ & $<100$ & $U$ & $<10$ & $\mathrm{U}$ & & & & & & $\mathrm{ug} / \mathrm{L}$ & \\
\hline & Nickel, total recoverable & 19 & $J$ & $<50$ & $U$ & $<50$ & $\mathrm{U}$ & & & & & & ug/L & \\
\hline & Selenium, total recoverable & $<200$ & $U$ & $<200$ & $U$ & $<10$ & $U$ & & & & & & $\mathrm{ug} / \mathrm{L}$ & \\
\hline \multicolumn{15}{|c|}{ Organics } \\
\hline & Chlorobenzene & $<5$ & UJ & $<5$ & $U$ & $<1$ & $U$ & $<1$ & JU & $L$ & $<E Q L$ & 1 & $u g / L$ & $M L$ \\
\hline & 1,1-Dichioroethane & $<5$ & UJ & $<5$ & $\mathrm{U}$ & $<1$ & $\mathrm{U}$ & $<1$ & JU & $L$ & $<$ EQL & 1 & $\mathrm{ug} / \mathrm{L}$ & $M L$ \\
\hline & 1,1-Dichloroethylene & $<5$ & UJ & $<5$ & $\mathrm{U}$ & $<1$ & $\mathrm{U}$ & $<1$ & JU & $L$ & $<\mathrm{EQL}$ & 1 & $\mathrm{ug} / \mathrm{L}$ & ML \\
\hline & trans-1,2-Dichloroethylene & $<5$ & UJ & $<5$ & $U$ & $<1$ & $u$ & $<1$ & JU & $\mathrm{L}$ & $<E Q L$ & 1 & $\mathrm{ug} / \mathrm{L}$ & ML \\
\hline & $\begin{array}{l}\text { PCB } 1016 \\
\text { PCB } 1221\end{array}$ & & & & & & & & & & & & & \\
\hline & PCB 1232 & & & & & & & & & & & & & \\
\hline & PCB 1242 & & & & & & & & & & & & & \\
\hline & PCB 1248 & & & & & & & & & & & & & \\
\hline & PCB 1254 & & & & & & & & & & & & & \\
\hline & PCB 1260 & & & & & & & & & & & & & \\
\hline & 1,1,2,2-Tetrachloroethane & $<5$ & UJ & $<5$ & $U$ & $<1$ & $U$ & $<1$ & JU & $L$ & $<\mathrm{EQL}$ & 1 & $\mathrm{ug} / \mathrm{L}$ & ML \\
\hline & Tetrachloroethylene & 4.85 & J & 4.97 & $J \quad 1$ & 5.9 & & 5.92 & $J$ & $L$ & NDD & 1 & $\mathrm{ug} / \mathrm{L}$ & ML \\
\hline & 1,1,1-Trichloroethane & $<5$ & UJ & $<5$ & $\mathrm{U}$ & $<1$ & $U$ & $<1$ & JU & $\mathrm{L}$ & $<\mathrm{EQL}$ & 1 & $\mathrm{ug} / \mathrm{L}$ & ML \\
\hline & Trichloroethylene & 24.1 & $J$ & 26.9 & & 31.8 & & 28.5 & $J$ & $\mathrm{~L}$ & NDD & 1 & $\mathrm{ug} / \mathrm{L}$ & ML \\
\hline \multicolumn{15}{|c|}{ II. Monitoring Constituents } \\
\hline$\underline{\text { ST }}$ & Parameter & $\underline{3 Q 96}$ & CLPEPA & $\underline{3 Q 97}$ & CLPEPA & $\underline{3 Q 98}$ & CLPEPA & $\underline{3 Q 99}$ & $\underline{\text { CLP }}$ & EPA & Filt. & $\underline{\text { DF }}$ & $\underline{\text { Unit }}$ & $\underline{\mathrm{Lab}}$ \\
\hline \multicolumn{15}{|c|}{ Inorganics } \\
\hline+ & Aluminum, total recoverable & 2140 & J & 2190 & & 2190 & & 1720 & & & $>100$ & 1 & ug/L & EX \\
\hline & Nitrate-nitrite as nitrogen & 1420 & J & 1150 & & 880 & & 783 & & & $<2400$ & 5 & ug/L & EX \\
\hline+ & Sodium, total recoverable & 29300 & & 26400 & & 25100 & & 19300 & & & $>4600$ & 1 & $\mathrm{ug} / \mathrm{L}$ & EX \\
\hline+ & Sulfate & 3200 & & 3480 & $J$ & 3640 & & 3690 & & & $>3000$ & 1 & ug/L & EX \\
\hline \multicolumn{15}{|c|}{ Radionuclides } \\
\hline & Gross alpha & 7.04 & $J$ & 16.2 & UI & 22.05 & & 6.51 & & & $<15$ & 1 & $\mathrm{pCi} / \mathrm{L}$ & GP \\
\hline & Nonvolatile beta & 17.2 & $J$ & 7.44 & UI & 16.4 & & 17.6 & $J$ & $\mathrm{~K}$ & NDD & 1 & $\mathrm{pCi} / \mathrm{L}$ & GP \\
\hline & Radium, total alpha-emitting & 2.2 & & 12.7 & $J$ & 4.47 & & 2.9 & & & $<5$ & 1 & $\mathrm{pCi} / \mathrm{L}$ & GP \\
\hline
\end{tabular}

Notes: Concentrations in bold italics exceed the groundwater protection or monitoring constituent standards listed in Appendix A. Synchronous water levels are measured over a 3-5 day period or less. Dilution factors, Laboratory, and Filtered Data are for Groundwater Protection Standards First Quarter 2000 and Monitoring Constituents Third Quarter 1999 data only.

$+=$ exceeded the Groundwater Protection Standards (First Quarter 2000) or Monitoring Constituents (Third Quarter 1999) listed in Appendix A. 
Table D-4. Groundwater Monitoring Results for Plume Definition Wells, M-Area HWMF (Cont.) WELL MSB 79C

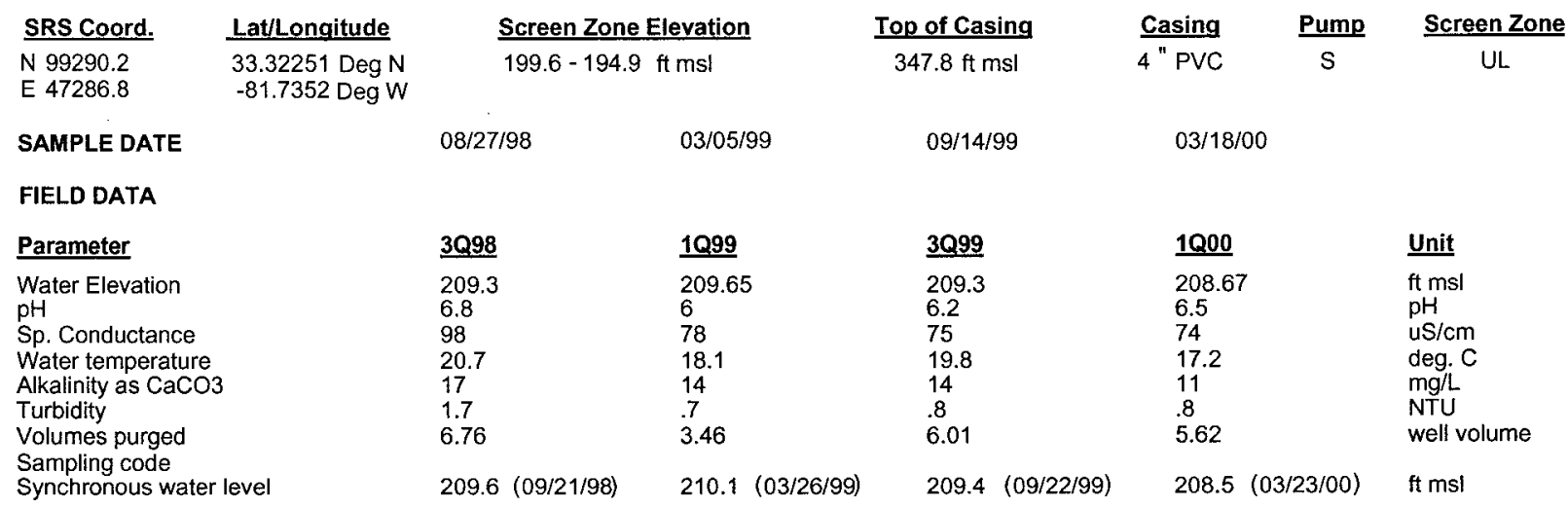

ANALYTICAL DATA

I. Groundwater Protection Standard

261 Appendix VIII/264 Appendix IX Hazardous Constituents

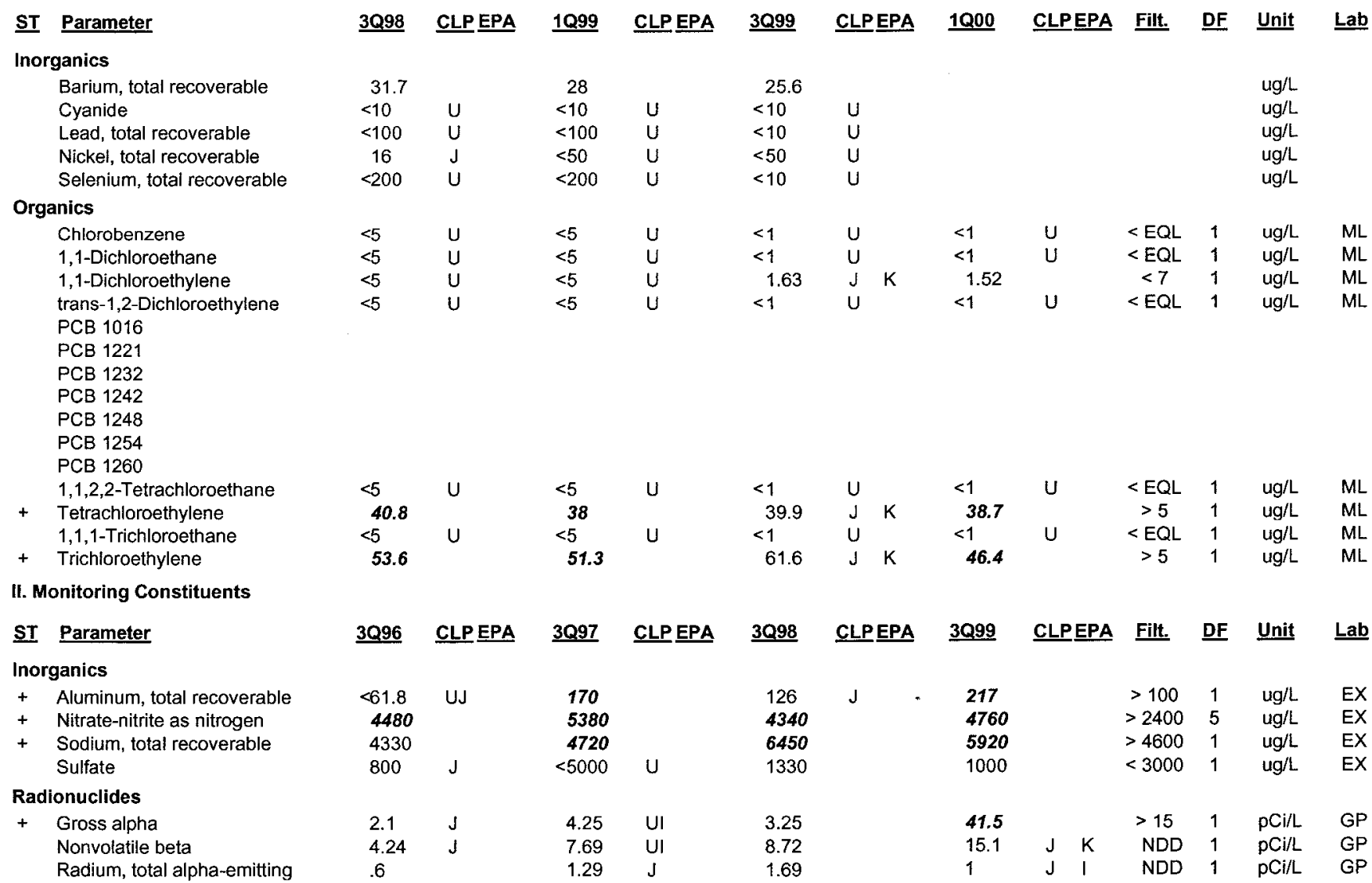

Notes: Concentrations in bold italics exceed the groundwater protection or monitoring constituent standards listed in Appendix A. Synchronous water levels are measured over a 3-5 day period or less. Dilution factors, Laboratory, and Filtered Data are for Groundwater Protection Standards First Quarter 2000 and Monitoring Constituents Third Quarter 1999 data only.

+= exceeded the Groundwater Protection Standards (First Quarter 2000) or Monitoring Constituents (Third Quarter 1999) listed in Appendix A. 
Table D-4. Groundwater Monitoring Results for Plume Definition Wells, M-Area HWMF (Cont.) WELL MSB 81B

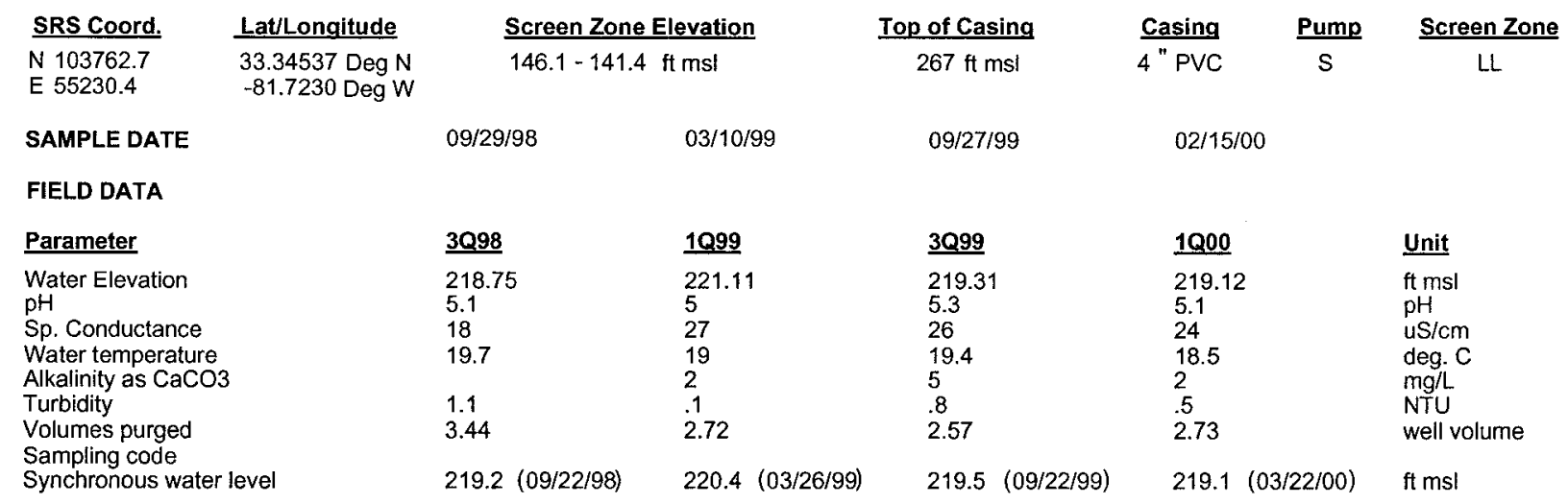

\section{ANALYTICAL DATA}

I. Groundwater Protection Standard

261 Appendix VIII/264 Appendix IX Hazardous Constituents

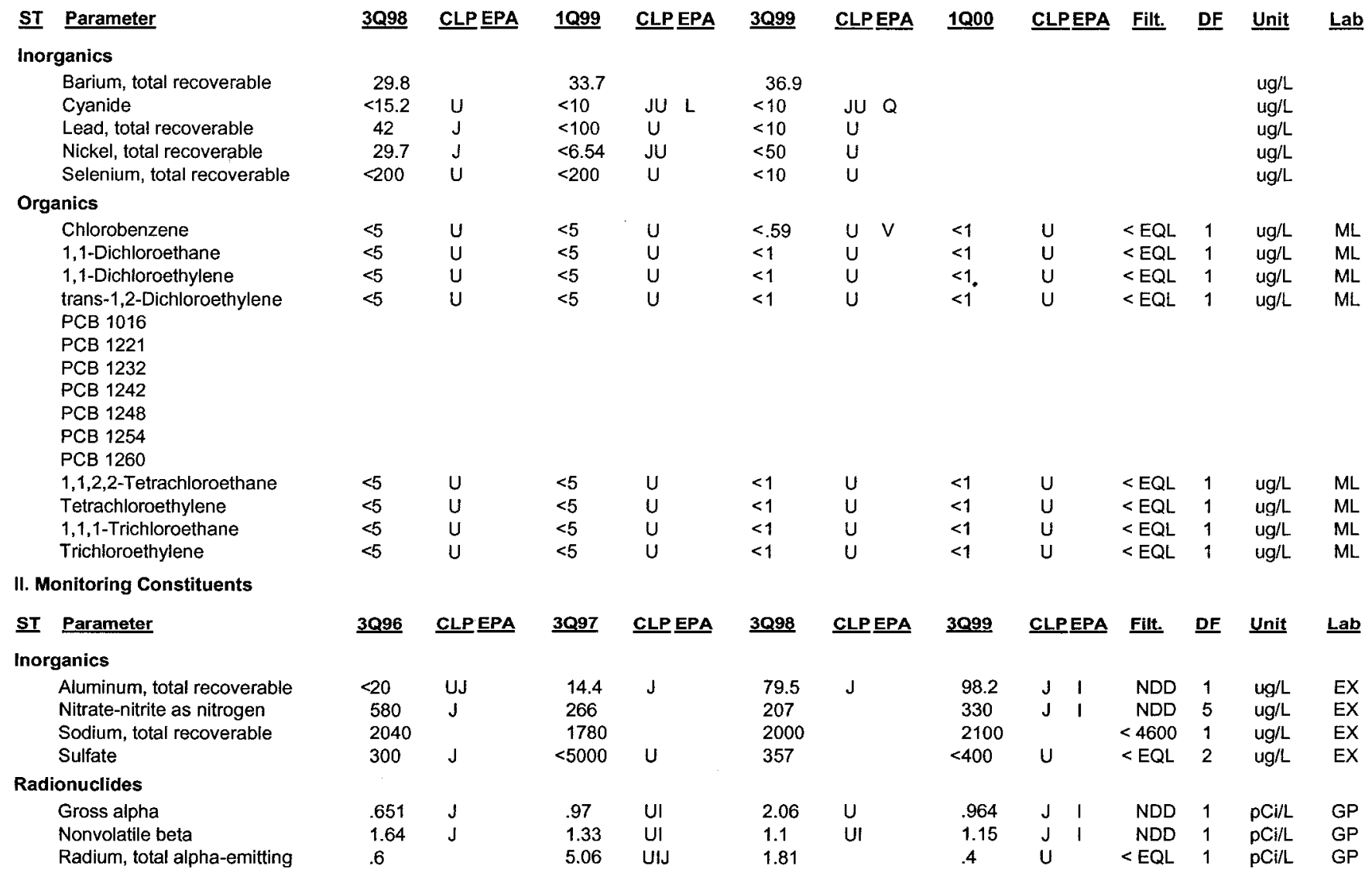

Notes: Concentrations in bold italics exceed the groundwater protection or monitoring constituent standards listed in Appendix A. Synchronous water levels are measured over a 3-5 day period or less. Dilution factors, Laboratory, and Filtered Data are for Groundwater Protection Standards First Quarter 2000 and Monitoring Constituents Third Quarter 1999 data only.

$+=$ exceeded the Groundwater Protection Standards (First Quarter 2000) or Monitoring Constituents (Third Quarter 1999) listed in Appendix A. 
Table D-4. Groundwater Monitoring Results for Plume Definition Wells, M-Area HWMF (Cont.) WELL MSB 82A

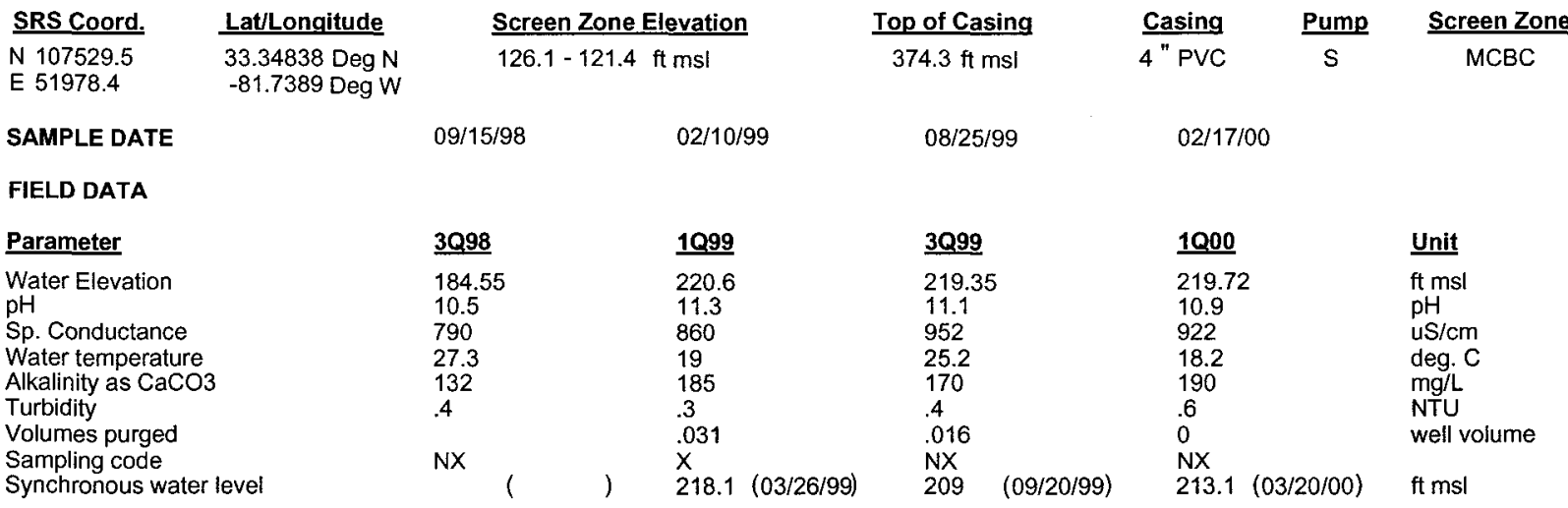

ANALYTICAL DATA

I. Groundwater Protection Standard

261 Appendix VIII/264 Appendix IX Hazardous Constituents

\begin{tabular}{|c|c|c|c|c|c|c|c|c|c|c|c|c|c|}
\hline$\underline{\mathrm{ST}}$ & Parameter & $\underline{3 Q 98}$ & $\underline{\text { CLP EPA }}$ & 1099 & $\underline{\text { CLPP EPA }}$ & $\underline{3 Q 99}$ & $\underline{\text { CLPEPA }}$ & 1000 & CLPEPA & Filt. & $\underline{\text { DF }}$ & $\underline{\text { Unit }}$ & Lab \\
\hline \multicolumn{14}{|c|}{ Inorganics } \\
\hline & Barium, total recoverable & 184 & & 139 & & 170 & & & & & & $u g / L$ & \\
\hline & Cyanide & $<10$ & $u$ & $<10$ & $U$ & $<10$ & U & & & & & $\mathrm{ug} / \mathrm{L}$ & \\
\hline & Lead, total recoverable & $<100$ & $\mathrm{U}$ & $<100$ & $U$ & $<10$ & $\mathrm{U}$ & & & & & $\mathrm{ug} / \mathrm{L}$ & \\
\hline & Nickel, total recoverable & $<50$ & $\mathrm{U}$ & $<50$ & $U$ & $<50$ & $\mathrm{U}$ & & & & & $\mathrm{ug} / \mathrm{L}$ & \\
\hline & Selenium, total recoverable & $<200$ & U & $<10$ & $\bigcup$ & $<4.4$ & JU 1 & & & & & $\mathrm{ug} / \mathrm{L}$ & \\
\hline \multicolumn{14}{|c|}{ Organics } \\
\hline & Chlorobenzene & $<25$ & $\mathrm{U}$ & $<5$ & $u$ & $<1$ & $\mathrm{U}$ & $<1$ & U & $<\mathrm{EQL}$ & 1 & $u g / L$ & ML \\
\hline & 1,1-Dichloroethane & $<25$ & $U$ & $<5$ & $\mathrm{U}$ & $<1$ & $\mathrm{U}$ & $<1$ & $\mathrm{U}$ & $<E Q L$ & 1 & $\mathrm{ug} / \mathrm{L}$ & ML \\
\hline & 1,1-Dichloroethylene & $<25$ & $\mathrm{U}$ & $<5$ & U & $<1$ & $\mathrm{U}$ & $<1$ & $\mathrm{U}$ & $<\mathrm{EQL}$ & 1 & $\mathrm{ug} / \mathrm{L}$ & ML. \\
\hline & trans-1,2-Dichloroethylene & $<25$ & $\mathrm{U}$ & $<5$ & U & $<1$ & $\mathrm{U}$ & $<1$ & U & $<\mathrm{EQL}$ & 1 & $\mathrm{ug} / \mathrm{L}$ & ML \\
\hline & PCB 1016 & & & & & & & & & & & & \\
\hline & PCB 1221 & & & & & & & & & & & & \\
\hline & PCB 1232 & & & & & & & & & & & & \\
\hline & $\begin{array}{l}\text { PCB } 1242 \\
\text { PCB } 1248\end{array}$ & & & & & & & & & & & & \\
\hline & PCB 1254 & & & & & & & & & & & & \\
\hline & PCB 1260 & & & & & & & & & & & & \\
\hline & $1,1,2,2$-Tetrachloroethane & $<25$ & $U$ & $<5$ & $U$ & $<1$ & $\mathrm{U}$ & $<1$ & $\mathrm{U}$ & $<\mathrm{EQL}$ & 1 & ug $/ \mathrm{L}$ & $\mathrm{ML}$ \\
\hline & Tetrachloroethylene & $<25$ & $U$ & $<5$ & $\mathrm{U}$ & $<1$ & $\mathrm{U}$ & $<1$ & U & $<\mathrm{EQL}$ & 1 & $\mathrm{ug} / \mathrm{L}$ & $M L$ \\
\hline & $1,1,1$-Trichloroethane & $<25$ & $\cup$ & $<5$ & U & $<1$ & $\mathrm{U}$ & $<1$ & $\mathrm{U}$ & $<E Q L$ & 1 & $\mathrm{ug} / \mathrm{L}$ & $\mathrm{ML}$ \\
\hline+ & Trichloroethylene & 131 & & 89.7 & & 77.8 & $\mathrm{~J} K$ & 65.8 & & $>5$ & 1 & $\mathrm{ug} / \mathrm{L}$ & ML \\
\hline \multicolumn{14}{|c|}{ II. Monitoring Constituents } \\
\hline$\underline{\text { ST }}$ & Parameter & $\underline{3 Q 96}$ & CLP EPA & $\underline{3 Q 97}$ & CLP EPA & $\underline{3098}$ & CLPEPA & $\underline{3099}$ & CLPEPA & Filt. & DF & Unit & Lab \\
\hline \multicolumn{14}{|c|}{ Inorganics } \\
\hline+ & Aluminum, total recoverable & 764 & J & 1100 & & 793 & & 850 & & $>100$ & 1 & ug/L & EX \\
\hline & Nitrate-nitrite as nitrogen & 570 & & 1020 & & 104 & $\mathrm{~J}$ & 87.5 & $\mathrm{~J} \quad \mathrm{I}$ & NDD & 5 & $\mathrm{ug} / \mathrm{L}$ & EX \\
\hline+ & Sodium, total recoverable & 27000 & & 20300 & & 23500 & & 20000 & & $>4600$ & 1 & ug/L & EX \\
\hline+ & Sulfate & 25300 & & 23300 & & 24000 & & 22400 & & $>3000$ & 2 & $\mathrm{ug} / \mathrm{L}$ & EX \\
\hline \multicolumn{14}{|c|}{ Radionuclides } \\
\hline & Gross alpha & 1.67 & & 5.61 & UI & 3.7 & & .89 & U & $<E Q L$ & 1 & $\mathrm{pCi} / \mathrm{L}$ & GP \\
\hline & Nonvolatile beta & 11.9 & & 9.36 & UI & 8.27 & & 6.49 & & $<50$ & 1 & $\mathrm{pCi} / \mathrm{L}$ & GP \\
\hline & Radium, total alpha-emitting & 6 & $\mathrm{~J}$ & 1.31 & $\mathrm{j}$ & .25 & UI & .7 & J 1 & NDD & 1 & $\mathrm{pCi} / \mathrm{L}$ & GP \\
\hline
\end{tabular}

Notes: Concentrations in bold italics exceed the groundwater protection or monitoring constituent standards listed in Appendix A. Synchronous water levels are measured over a 3-5 day period or less. Dilution factors, Laboratory, and Filtered Data are for Groundwater Protection Standards First Quarter 2000 and Monitoring Constituents Third Quarter 1999 data only.

$+=$ exceeded the Groundwater Protection Standards (First Quarter 2000) or Monitoring Constituents (Third Quarter 1999) listed in Appendix A. 
Table D-4. Groundwater Monitoring Results for Plume Definition Wells, M-Area HWMF (Cont.) WELL MSB 82B

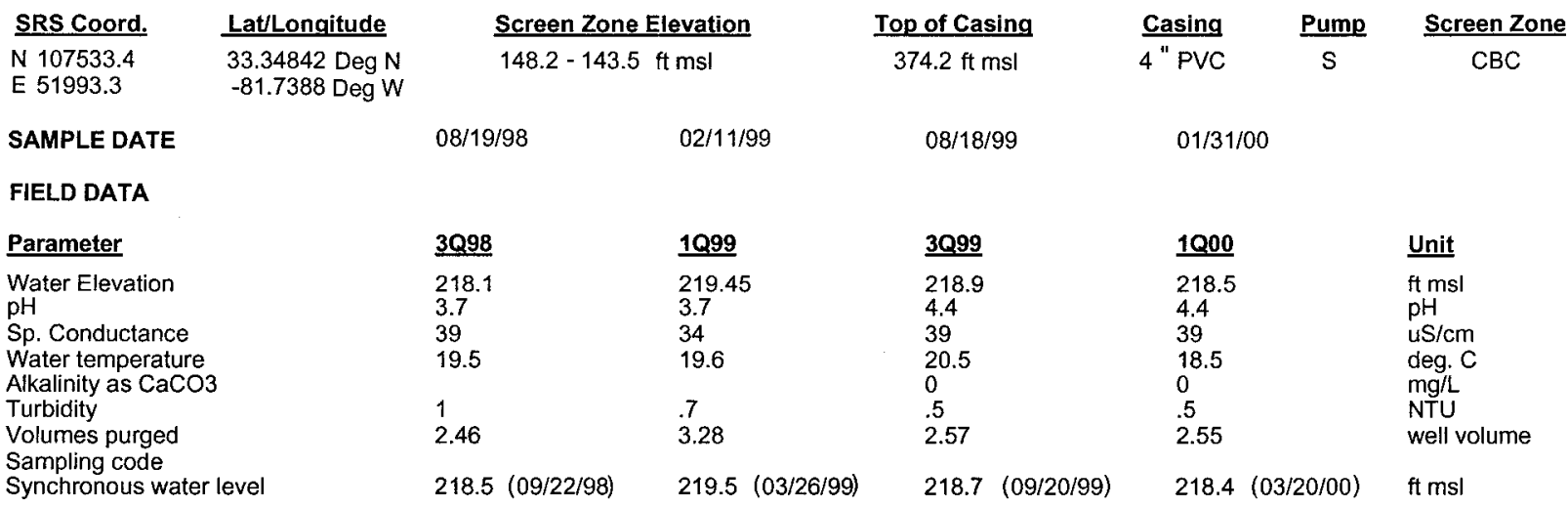

ANALYTICAL DATA

I. Groundwater Protection Standard

261 Appendix VIII/264 Appendix IX Hazardous Constituents

\begin{tabular}{|c|c|c|c|c|c|c|c|c|c|c|c|c|c|c|}
\hline \multirow{2}{*}{$\underline{\text { Inor }}$} & Parameter & \multirow[t]{2}{*}{$\underline{3 Q 98}$} & \multirow[t]{2}{*}{$\underline{\text { CLPEPA }}$} & \multirow[t]{2}{*}{1099} & \multirow[t]{2}{*}{$\underline{\text { CLP EPA }}$} & \multirow[t]{2}{*}{ 3099 } & \multirow[t]{2}{*}{ CLPEPA } & \multirow[t]{2}{*}{10,00} & \multicolumn{2}{|c|}{ CLPEPA } & \multirow[t]{2}{*}{ Filt. } & \multirow[t]{2}{*}{$\underline{\text { DF }}$} & \multirow[t]{2}{*}{ Unit } & \multirow[t]{2}{*}{ Lab } \\
\hline & ganics & & & & & & & & & & & & & \\
\hline & Barium, total recoverable & 10.3 & & $<10$ & $U$ & 11 & & & & & & & ug/L & \\
\hline & Cyanide & $<10$ & $U$ & $<10$ & $\mathrm{U}$ & $<10$ & $\mathrm{U}$ & & & & & & ug/L & \\
\hline & Lead, total recoverable & $<10$ & $U$ & $<100$ & $\mathrm{U}$ & $<10$ & U & & & & & & $u g / L$ & \\
\hline & Nickel, total recoverable & $<7.9$ & $\mathrm{U}$ & $<50$ & JU & $<16$ & JU I & & & & & & $\mathrm{ug} / \mathrm{L}$ & \\
\hline & Selenium, total recoverable & $<10$ & $\mathrm{U}$ & & & $<10$ & $U$ & & & & & & $\mathrm{ug} / \mathrm{L}$ & \\
\hline \multicolumn{15}{|c|}{ Organics } \\
\hline & Chlorobenzene & $<5$ & $\mathrm{U}$ & $<5$ & $\mathrm{U}$ & $<1$ & $U$ & $<1$ & JU & L & $<\mathrm{EQL}$ & 1 & $\mathrm{ug} / \mathrm{L}$ & ML \\
\hline & 1,1-Dichloroethane & $<5$ & $\mathrm{U}$ & $<5$ & $\mathrm{U}$ & $<1$ & $U$ & $<1$ & JU & $\bar{L}$ & $<\mathrm{EQL}$ & 1 & $\mathrm{ug} / \mathrm{L}$ & ML \\
\hline & 1,1-Dichloroethylene & $<5$ & $\mathrm{U}$ & $<5$ & $\mathbf{U}$ & $<1$ & $\mathrm{U}$ & $<1$ & JU & L & $<\mathrm{EQL}$ & 1 & $\mathrm{ug} / \mathrm{L}$ & ML \\
\hline & trans-1,2-Dichloroethylene & $<5$ & $u$ & $<5$ & $\mathrm{U}$ & $<1$ & $\mathrm{U}$ & & & & & & $\mathrm{ug} / \mathrm{L}$ & \\
\hline & PCB 1016 & & & & & & & & & & & & & \\
\hline & PCB 1221 & & & & & & & & & & & & & \\
\hline & РCB 1232 & & & & & & & & & & & & & \\
\hline & PCB 1242 & & & & & & & & & & & & & \\
\hline & PCB 1248 & & & & & & & & & & & & & \\
\hline & PCB 1254 & & & & & & & & & & & & & \\
\hline & PCB 1260 & & & & & & & & & & & & & \\
\hline & $1,1,2,2$-Tetrachloroethane & $<5$ & $\mathrm{U}$ & $<5$ & $\mathrm{U}$ & $<1$ & $\mathrm{U}$ & $<1$ & JU & L & $<\mathrm{EQL}$ & 1 & $u g / L$ & $M L$ \\
\hline & Tetrachloroethylene & $<5$ & $\mathrm{U}$ & $<5$ & $U$ & $<1$ & $\mathrm{U}$ & $<1$ & JU & $\mathbf{L}$ & $<E Q L$ & 1 & $\mathrm{ug} / \mathrm{L}$ & ML \\
\hline & 1,1,1-Trichloroethane & $<5$ & $\mathrm{U}$ & $<5$ & $\mathrm{U}$ & $<1$ & $\mathrm{U}$ & $<1$ & $\mathrm{JU}$ & L & $<$ EQL & 1 & $\mathrm{ug} / \mathrm{L}$ & ML \\
\hline & Trichloroethylene & $<5$ & U & $<5$ & $\mathrm{U}$ & $<1$ & $\mathrm{U}$ & $<1$ & JU & L & $<E Q L$ & 1 & $\mathrm{ug} / \mathrm{L}$ & ML \\
\hline \multicolumn{15}{|c|}{ II. Monitoring Constituents } \\
\hline$\underline{\text { ST }}$ & Parameter & $\underline{3096}$ & CLPEPA & $\underline{3097}$ & CLP EPA & 3ุQ98 & CLPEPA & $\underline{3099}$ & CLP & EPA & Filt. & $\underline{\mathrm{DF}}$ & $\underline{\text { Unit }}$ & Lab \\
\hline \multicolumn{15}{|c|}{ Inorganics } \\
\hline & Aluminum, total recoverable & $<131$ & 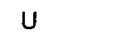 & 124 & & 101 & J & 76 & $\mathrm{~J}$ & $\mathbf{I}$ & NDD & 1 & $u g / L$ & EX \\
\hline & Nitrate-nitrite as nitrogen & 290 & & 89 & & $<100$ & $\mathrm{U}$ & 101 & J & 1 & NDD & 5 & ug/L & EX \\
\hline & Sodium, total recoverable & 1770 & & 1730 & & 1820 & & 2000 & & & $<4600$ & 1 & $\mathrm{ug} / \mathrm{L}$ & EX \\
\hline+ & Sulfate & 7040 & & 7370 & & 7910 & & 7590 & & & $>3000$ & 1 & $\mathrm{ug} / \mathrm{L}$ & EX \\
\hline \multicolumn{15}{|c|}{ Radionuclides } \\
\hline & Gross alpha & 11.1 & & 1.32 & & 2.67 & $J$ & 1.95 & 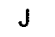 & 1 & NDD & 1 & $\mathrm{pCi} / \mathrm{L}$ & GP \\
\hline & Nonvolatile beta & 9.35 & & 2.79 & & 6.45 & $J$ & 4.79 & & & $<50$ & 1 & $\mathrm{pCi} / \mathrm{L}$ & GP \\
\hline & Radium, total alpha-emitting & 4.7 & & 7.42 & $J$ & 1.32 & & .8 & $\mathrm{U}$ & & $<E Q L$ & 1 & $\mathrm{pCi} / \mathrm{L}$ & GP \\
\hline
\end{tabular}

Notes: Concentrations in bold italics exceed the groundwater protection or monitoring constituent standards listed in Appendix A. Synchronous water levels are measured over a 3-5 day period or less. Dilution factors, Laboratory, and Filtered Data are for Groundwater Protection Standards First Quarter 2000 and Monitoring Constituents Third Quarter 1999 data only.

$+=$ exceeded the Groundwater Protection Standards (First Quarter 2000) or Monitoring Constituents (Third Quarter 1999) listed in Appendix A. 
Table D-4. Groundwater Monitoring Results for Plume Definition Wells, M-Area HWMF (Cont.) WELL MSB 82C

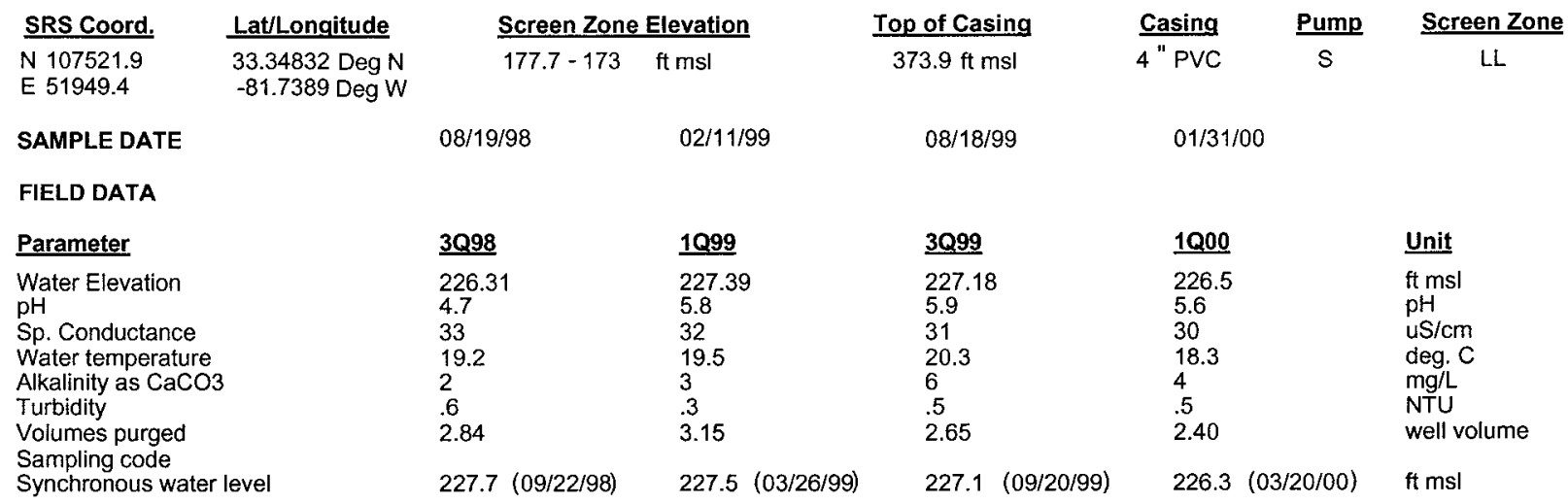

ANALYTICAL DATA

1. Groundwater Protection Standard

261 Appendix VIII/264 Appendix IX Hazardous Constituents

\begin{tabular}{|c|c|c|c|c|c|c|c|c|c|c|c|c|c|c|}
\hline$\underline{\text { ST }}$ & Parameter & $\underline{3 Q 98}$ & CLP EPA & 1099 & CLP EPA & $\underline{3 Q 99}$ & CLPEPA & $\underline{1000}$ & \multicolumn{2}{|c|}{ CLPEPA } & Filt. & DF & Unit & $\underline{\text { Lab }}$ \\
\hline \multicolumn{15}{|c|}{ Inorganics } \\
\hline & Barium, total recoverable & 19.7 & & $<10$ & $\mathrm{U}$ & 20 & & & & & & & $\mathrm{ug} / \mathrm{L}$ & \\
\hline & Cyanide & $<10$ & $\mathrm{U}$ & $<10$ & $\mathrm{U}$ & $<10$ & U & & & & & & $\mathrm{ug} / \mathrm{L}$ & \\
\hline & Lead, total recoverable & $<10$ & $U$ & $<100$ & $\mathrm{U}$ & .57 & J I & & & & & & $\mathrm{ug} / \mathrm{L}$ & \\
\hline & Nickel, total recoverable & $<11.8$ & $U$ & $<50$ & $\mathrm{U}$ & $<50$ & $\mathrm{U}$ & & & & & & $\mathrm{ug} / \mathrm{L}$ & \\
\hline & Selenium, total recoverable & $<10$ & $U$ & & & $<5$ & $U$ & & & & & & $\mathrm{ug} / \mathrm{L}$ & \\
\hline \multicolumn{15}{|c|}{ Organics } \\
\hline & Chlorobenzene & $<5$ & $U$ & $<5$ & $U$ & $<1$ & $\mathrm{U}$ & $<1$ & JU & $\mathbf{L}$ & $<\mathrm{EQL}$ & 1 & ug/L & ML \\
\hline & 1,1-Dichloroethane & $<5$ & $U$ & $<5$ & $\mathrm{U}$ & $<1$ & $U$ & $<1$ & JU & $\mathrm{L}$ & $<\mathrm{EQL}$ & 1 & $\mathrm{ug} / \mathrm{L}$ & $M L$ \\
\hline & 1,1-Dichloroethylene & $<5$ & $U$ & $<5$ & $U$ & $<1$ & 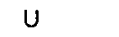 & $<1$ & JU & $\mathbf{L}$ & $<E Q L$ & 1 & ug/L & $M L$ \\
\hline & trans-1,2-Dichloroethylene & $<5$ & U & $<5$ & $\mathrm{U}$ & $<1$ & U & & & & & & ug/L & \\
\hline & PCB 1016 & & & & & & & & & & & & & \\
\hline & PCB 1221 & & & & & & & & & & & & & \\
\hline & PCB 1232 & & & & & & & & & & & & & \\
\hline & PCB 1242 & & & & & & & & & & & & & \\
\hline & PCB 1248 & & & & & & & & & & & & & \\
\hline & РCB 1254 & & & & & & & & & & & & & \\
\hline & PCB 1260 & & & & & & & & & & & & & \\
\hline & 1,1,2,2-Tetrachloroethane & $<5$ & $U$ & $<5$ & $\mathrm{U}$ & $<1$ & $U$ & $<1$ & JU & $\mathrm{L}$ & $<\mathrm{EQL}$ & 1 & $u g / L$ & ML \\
\hline & Tetrachloroethylene & $<5$ & $U$ & $<5$ & $\mathrm{U}$ & $<1$ & U & $<1$ & JU & $\mathrm{L}$ & $<\mathrm{EQL}$ & 1 & ug/L & ML \\
\hline & $1,1,1$-Trichloroethane & $<5$ & $\mathrm{U}$ & $<5$ & $\mathrm{U}$ & $<1$ & $U$ & $<1$ & JU & $\mathrm{L}$ & $<\mathrm{EQL}$ & 1 & $\mathrm{ug} / \mathrm{L}$ & $\mathrm{ML}$ \\
\hline & Trichloroethylene & $<5$ & $\mathrm{U}$ & $<5$ & $\mathrm{U}$ & $<1$ & $U$ & $<1$ & JU & $L$ & $<\mathrm{EQL}$ & 1 & ug/L & ML \\
\hline \multicolumn{15}{|c|}{ II. Monitoring Constituents } \\
\hline$\underline{\text { ST }}$ & Parameter & $\underline{3 Q 96}$ & CLP EPA & $\underline{3 Q 97}$ & CLPEPA & $\underline{3098}$ & CLPEPA & $\underline{3099}$ & $\underline{\text { CLP }}$ & EPA & Filt. & $\underline{\text { DF }}$ & Unit & Lab \\
\hline \multicolumn{15}{|c|}{ Inorganics } \\
\hline & Aluminum, total recoverable & $<39.7$ & U & $<50$ & $\mathrm{U}$ & 78.2 & $J$ & $<200$ & $\mathrm{U}$ & & $<E Q L$ & 1 & $\mathrm{ug} / \mathrm{L}$ & $E x$ \\
\hline & Nitrate-nitrite as nitrogen & 1850 & & 1640 & & 1520 & & 1640 & & & $<2400$ & 5 & $\mathrm{ug} / \mathrm{L}$ & EX \\
\hline & Sodium, total recoverable & 2720 & & 2880 & & 3000 & & 3300 & & & $<4600$ & 1 & $\mathrm{ug} / \mathrm{L}$ & EX \\
\hline & Sulfate & 169 & J & $<5000$ & $\mathrm{U}$ & $<200$ & $\mathrm{U}$ & 182 & $\mathrm{~J}$ & 1 & NDD & 1 & $\mathrm{ug} / \mathrm{L}$ & EX \\
\hline \multicolumn{14}{|c|}{ Radionuclides } & \\
\hline & Gross alpha & 1.17 & & 1.31 & & 2.31 & $J$ & 1.09 & J & I & NDD & 1 & $\mathrm{pCi} / \mathrm{L}$ & GP \\
\hline & Nonvolatile beta & .683 & Ul & 3.69 & UI & 1.61 & UIJ & 2.26 & J & 1 & NDD & 1 & $\mathrm{pCi} / \mathrm{L}$ & GP \\
\hline & Radium, total alpha-emitting & .4 & & .9 & & .46 & & .4 & $\mathrm{U}$ & & $<E Q L$ & 1 & $\mathrm{pCi} / \mathrm{L}$ & \\
\hline
\end{tabular}

Notes: Concentrations in bold italics exceed the groundwater protection or monitoring constituent standards listed in Appendix A. Synchronous water levels are measured over a 3-5 day period or less. Dilution factors, Laboratory, and Filtered Data are for Groundwater Protection Standards First Quarter 2000 and Monitoring Constituents Third Quarter 1999 data only.

$+=$ exceeded the Groundwater Protection Standards (First Quarter 2000) or Monitoring Constituents (Third Quarter 1999) listed in Appendix A. 
Table D-4. Groundwater Monitoring Results for Plume Definition Wells, M-Area HWMF (Cont.) WELL MSB 82D

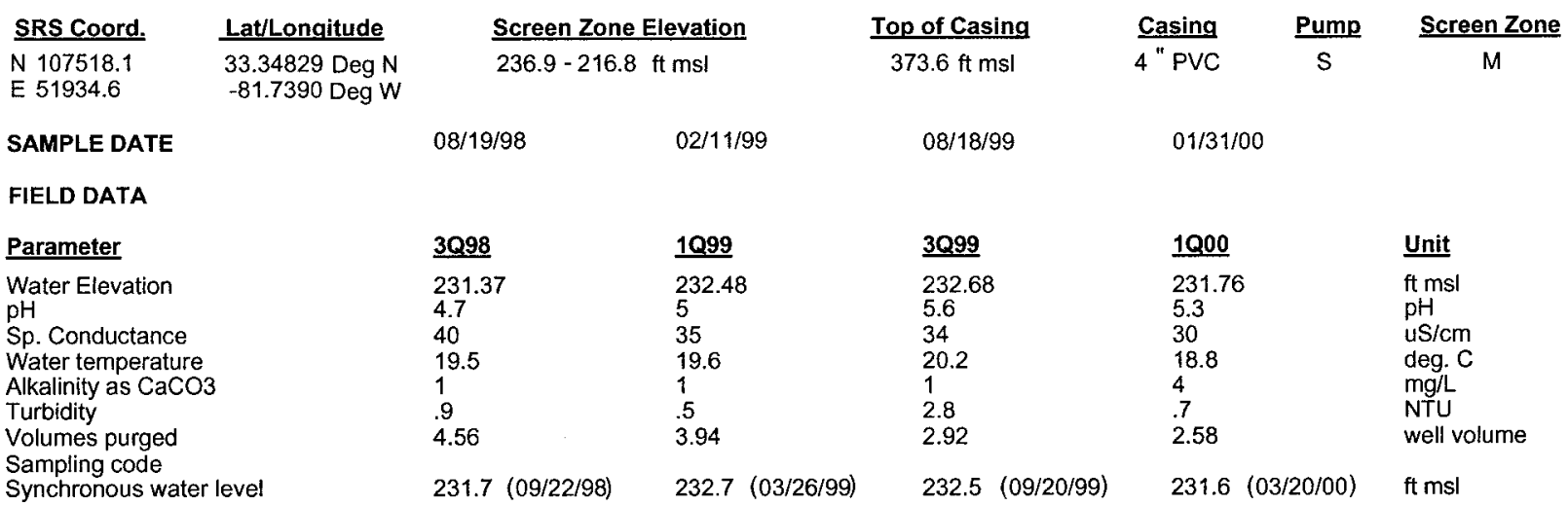

ANALYTICAL DATA

I. Groundwater Protection Standard

261 Appendix VIII/264 Appendix IX Hazardous Constituents

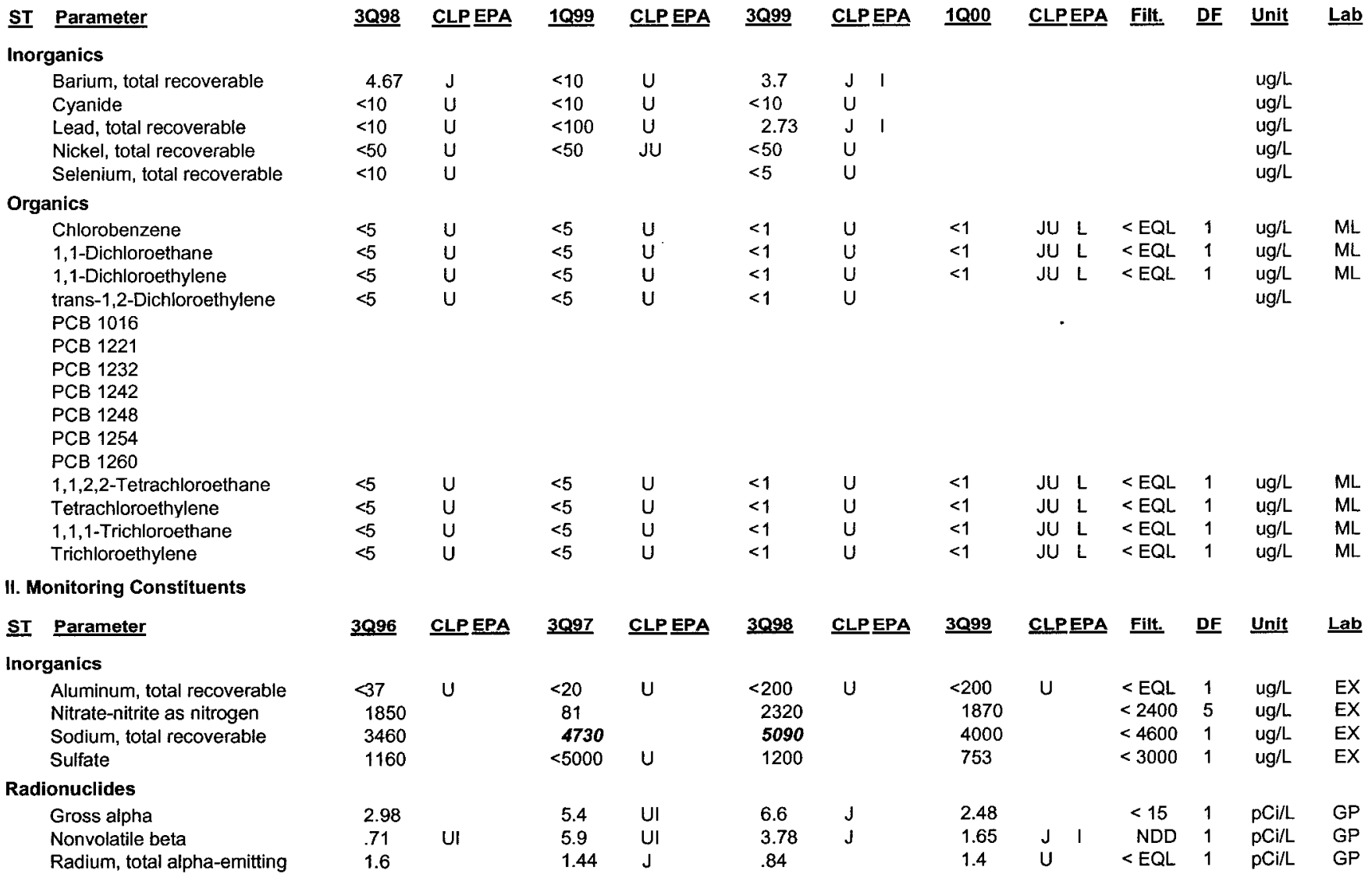

Notes: Concentrations in bold italics exceed the groundwater protection or monitoring constituent standards listed in Appendix A. Synchronous water levels are measured over a 3-5 day period or less. Dilution factors, Laboratory, and Filtered Data are for Groundwater Protection Standards First Quarter 2000 and Monitoring Constituents Third Quarter 1999 data only.

$+=$ exceeded the Groundwater Protection Standards (First Quarter 2000) or Monitoring Constituents (Third Quarter 1999) listed in Appendix A. 
Table D-4. Groundwater Monitoring Results for Plume Definition Wells, M-Area HWMF (Cont.) WELL MSB 82TA

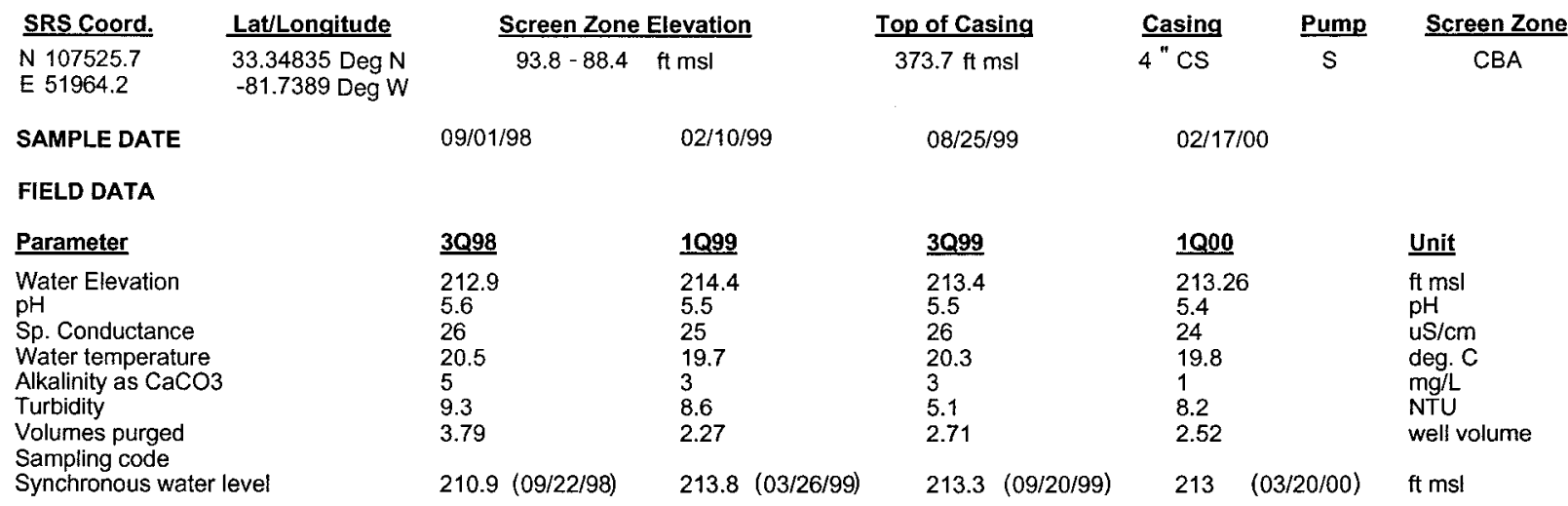

ANALYTICAL DATA

I. Groundwater Protection Standard

261 Appendix VIII/264 Appendix IX Hazardous Constituents

\begin{tabular}{|c|c|c|c|c|c|c|c|c|c|c|c|c|c|c|}
\hline$\underline{\mathbf{S T}}$ & Parameter & $\underline{3 Q 98}$ & CLP EPA & $\underline{1 Q 99}$ & $\underline{\text { CLP EPA }}$ & 3Q99 & CLPEPA & $\underline{1000}$ & $\underline{\text { CLP }}$ & EPA & Filt. & $\underline{\mathrm{DF}}$ & Unit & $\underline{\text { Lab }}$ \\
\hline Inor & ganics & & & & & & & & & & & & & \\
\hline & Barium, total recoverable & 3.01 & $\mathrm{~J}$ & 3.8 & J 1 & 4.3 & J I & & & & & & $\mathrm{ug} / \mathrm{L}$ & \\
\hline & Cyanide & $<10$ & $U$ & $<10$ & U & $<10$ & U & & & & & & $\mathrm{ug} / \mathrm{L}$ & \\
\hline & Lead, total recoverable & $<100$ & $U$ & $<100$ & $U$ & $<10$ & U & & & & & & $\mathrm{ug} / \mathrm{L}$ & \\
\hline & Nickel, total recoverable & $<50$ & $\mathrm{U}$ & $<50$ & $U$ & $<50$ & $U$ & & & & & & $\mathrm{ug} / \mathrm{L}$ & \\
\hline & Selenium, total recoverable & $<10$ & U & $<10$ & $U$ & $<10$ & $\mathrm{U}$ & & & & & & $\mathrm{ug} / \mathrm{L}$ & \\
\hline Org & anics & & & & & & & & & & & & & \\
\hline & Chlorobenzene & $<5$ & UJ & $<5$ & $\mathrm{U}$ & $<1$ & 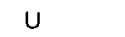 & $<1$ & JU & $\mathrm{L}$ & $<\mathrm{EQL}$ & 1 & $\mathrm{ug} / \mathrm{L}$ & $M L$ \\
\hline & 1,1-Dichloroethane & $<5$ & UJ & $<5$ & $\mathrm{U}$ & $<1$ & $\mathrm{U}$ & $<1$ & JU & $\mathrm{L}$ & $<\mathrm{EQL}$ & 1 & $\mathrm{ug} / \mathrm{L}$ & $M L$ \\
\hline & 1,1-Dichloroethylene & $<5$ & UJ & $<5$ & $\mathrm{u}$ & $<1$ & $\mathrm{U}$ & $<1$ & JU & $\mathrm{L}$ & $<$ EQL & 1 & $\mathrm{ug} / \mathrm{L}$ & $M L$ \\
\hline & trans-1,2-Dichloroethylene & $<5$ & UJ & $<5$ & $\mathrm{U}$ & $<1$ & $\mathrm{U}$ & $<1$ & JU & $\mathrm{L}$ & $<\mathrm{EQL}$ & 1 & $u g / L$ & ML \\
\hline & PCB 1016 & & & & & & & & & & & & & \\
\hline & РСВ 1221 & & & & & & & & & & & & & \\
\hline & PCB 1232 & & & & & & & & & & & & & \\
\hline & PCB 1242 & & & & & & & & & & & & & \\
\hline & PCB 1248 & & & & & & & & & & & & & \\
\hline & PCB 1254 & & & & & & & & & & & & & \\
\hline & PCB 1260 & & & & & & & & & & & & & \\
\hline & $1,1,2,2$-Tetrachloroethane & $<5$ & UJ & $<5$ & U & $<1$ & $\mathrm{U}$ & $<1$ & JU & $\mathrm{L}$ & $<\mathrm{EQL}$ & 1 & $u g / L$ & ML. \\
\hline & Tetrachloroethylene & $<5$ & UJ & $<5$ & $\mathrm{U}$ & $<1$ & $\mathrm{U}$ & $<1$ & JU & $\mathbf{L}$ & $<\mathrm{EQL}$ & 1 & $\mathrm{ug} / \mathrm{L}$ & $\mathrm{ML}$ \\
\hline & 1,1,1-Trichloroethane & $<5$ & UJ & $<5$ & U & $<1$ & $\mathrm{U}$ & $<1$ & JU & $\mathbf{L}$ & $<\mathrm{EQL}$ & 1 & $\mathrm{ug} / \mathrm{L}$ & $M L$ \\
\hline & Trichloroethylene & 85.9 & $J$ & 81.6 & & 96.8 & $j K$ & 123 & $\mathrm{~J}$ & $\mathbf{L}$ & NDD & 1 & $\mathrm{ug} / \mathrm{L}$ & ML. \\
\hline II. $M$ & onitoring Constituents & & & & & & & & & & & & & \\
\hline$\underline{\text { ST }}$ & Parameter & $\underline{3096}$ & CLP EPA & $\underline{3097}$ & CLP EPA & $\underline{3098}$ & CLPEPA & $\underline{3099}$ & CLP & EPA & Filt. & $\underline{\mathrm{DF}}$ & Unit & Lab \\
\hline Ino & ganics & & & & & & & & & & & & & \\
\hline & Aluminum, total recoverable & 30.5 & $J$ & 13.3 & $\mathrm{~J}$ & $<200$ & U & $<200$ & $\mathrm{U}$ & & $<\mathrm{EQL}$ & 1 & $u g / L$ & EX \\
\hline & Nitrate-nitrite as nitrogen & 820 & J & 1580 & & 543 & & 531 & & & $<2400$ & 5 & $u g / L$ & EX \\
\hline & Sodium, total recoverable & 1640 & & 1500 & & 1700 & & 2000 & & & $<4600$ & 1 & ug/L & EX \\
\hline & Sulfate & 1930 & & $<5000$ & $U$ & 2060 & & 1970 & & & $<3000$ & 2 & $\mathrm{ug} / \mathrm{L}$ & EX \\
\hline $\mathbf{R}$ & ionuclides & & & & & & & & & & & & & \\
\hline & Gross alpha & .947 & & .82 & U! & 1.6 & & .0863 & U & & $<E Q L$ & 1 & $\mathrm{pCi} / \mathrm{L}$ & GP \\
\hline & Nonvolatile beta & 1.42 & & -.42 & UI & 13.64 & & 1.39 & $\mathrm{~J}$ & 1 & NDD & 1 & $\mathrm{pCi} / \mathrm{L}$ & GP \\
\hline & Radium, total alpha-emitting & .3 & $\sqrt{ }$ & 2.24 & UIJ & 1.32 & & .3 & $\mathrm{~J}$ & 1 & NDD & 1 & $\mathrm{pCi} / \mathrm{L}$ & $\mathrm{GP}$ \\
\hline
\end{tabular}

Notes: Concentrations in bold italics exceed the groundwater protection or monitoring constituent standards listed in Appendix A. Synchronous water levels are measured over a 3-5 day period or less. Dilution factors, Laboratory, and Filtered Data are for Groundwater Protection Standards First Quarter 2000 and Monitoring Constituents Third Quarter 1999 data only.

+= exceeded the Groundwater Protection Standards (First Quarter 2000) or Monitoring Constituents (Third Quarter 1999) Iisted in Appendix A. 
Table D-4. Groundwater Monitoring Results for Plume Definition Wells, M-Area HWMF (Cont.) WELL MSB 83B

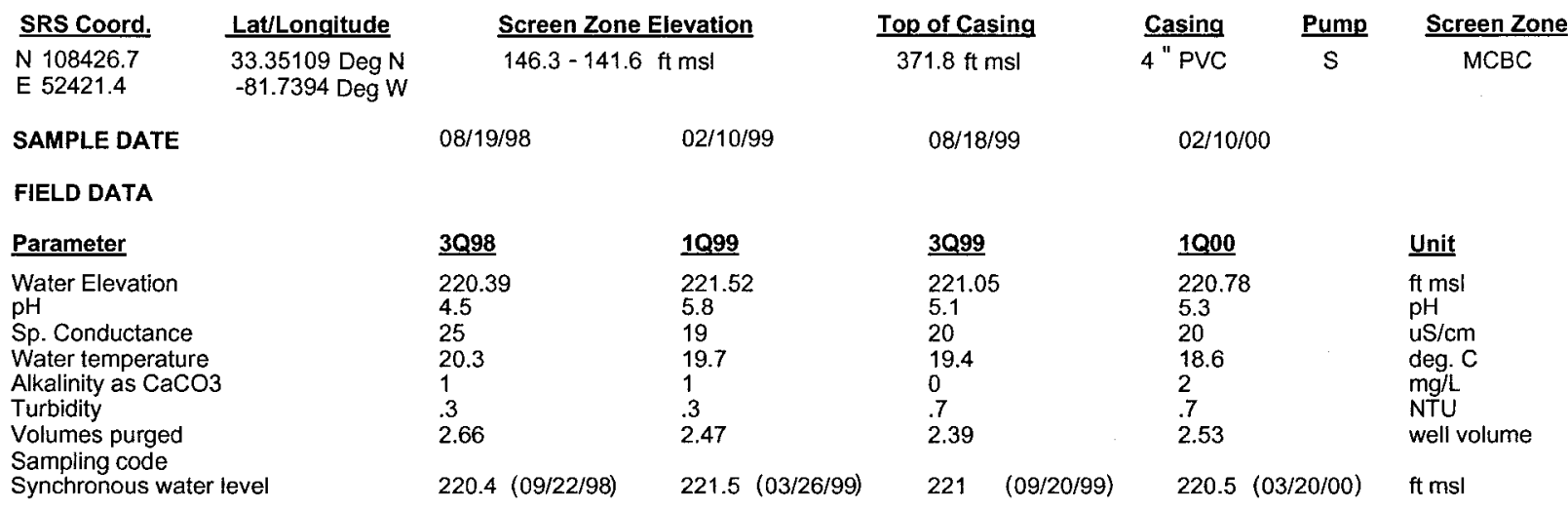

ANALYTICAL DATA

I. Groundwater Protection Standard

261 Appendix VIII/264 Appendix IX Hazardous Constituents

\begin{tabular}{|c|c|c|c|c|c|c|c|c|c|c|c|c|c|}
\hline \multirow{2}{*}{$\begin{array}{l}\text { ST Parameter } \\
\text { Inorganics }\end{array}$} & \multirow[t]{2}{*}{$\underline{3098}$} & \multirow[t]{2}{*}{ CLPEPA } & \multirow[t]{2}{*}{1099} & CLPEPA & \multirow[t]{2}{*}{$\underline{3 Q 99}$} & CLPEPA & \multirow[t]{2}{*}{$\underline{10,00}$} & \multicolumn{2}{|c|}{ CLPEPA } & \multirow[t]{2}{*}{ Fillt. } & \multirow[t]{2}{*}{ DF } & \multirow[t]{2}{*}{$\underline{\text { Unit }}$} & \multirow[t]{2}{*}{$\underline{\mathrm{Lab}}$} \\
\hline & & & & & & & & & & & & & \\
\hline Barium, total recoverable & 9.5 & $\mathrm{~J}$ & 6.81 & $\mathrm{~J} \quad \mathrm{I}$ & 6.5 & J 1 & & & & & & $u g / L$ & \\
\hline Cyanide & $<10$ & $u$ & $<10$ & U & $<10$ & $u$ & & & & & & $\mathrm{ug} / \mathrm{L}$ & \\
\hline Lead, total recoverable & $<10$ & $u$ & $<100$ & $\mathrm{u}$ & $<5$ & $u$ & & & & & & $u g / L$ & \\
\hline Nickel, total recoverable & $<10.9$ & $\mathrm{u}$ & $<50$ & $\mathrm{u}$ & $<50$ & $u$ & & & & & & ug/L & \\
\hline Selenium, total recoverable & $<10$ & $u$ & $<10$ & $\mathrm{u}$ & $<5$ & $\mathrm{u}$ & & & & & & ug/h & \\
\hline \multicolumn{14}{|l|}{ Organics } \\
\hline Chlorobenzene & $<5$ & $\mathrm{u}$ & $<5$ & $\mathrm{u}$ & $<1$ & $\mathrm{u}$ & $<1$ & $\mathrm{u}$ & & $<E Q L$ & 1 & $u g / \mathrm{L}$ & ML \\
\hline 1,1-Dichloroethane & $<5$ & $\mathrm{u}$ & $<5$ & $u$ & $<1$ & $u$ & $<1$ & $u$ & & $<\mathrm{EQL}$ & 1 & $\mathrm{ug} / \mathrm{L}$ & ML \\
\hline 1,1-Dichloroethylene & $<5$ & $\mathrm{u}$ & $<5$ & $\mathrm{U}$ & $<1$ & u & $<1$ & $\mathrm{u}$ & & $<E Q L$ & 1 & $\mathrm{ug} / \mathrm{h}$ & $\mathrm{ML}$ \\
\hline trans-1,2-Dichloroethylene & $<5$ & $u$ & $<5$ & $u$ & $<1$ & $u$ & & & & & & $\mathrm{ug} / \mathrm{L}$ & \\
\hline PCB 1016 & & & & & & & & & & & & & \\
\hline РCB 1221 & & & & & & & & & & & & & \\
\hline PCB 1232 & & & & & & & & & & & & & \\
\hline PCB 1242 & & & & & & & & & & & & & \\
\hline PCB 1248 & & & & & & & & & & & & & \\
\hline PCB 1254 & & & & & & & & & & & & & \\
\hline PCB 1260 & & & & & & & & & & & & & \\
\hline 1,1,2,2-Tetrachloroethane & $<5$ & 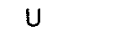 & $<5$ & $u$ & $<1$ & $\mathrm{U}$ & $<1$ & $u$ & & $<E Q L$ & 1 & $\mathrm{ug} / \mathrm{L}$ & $\mathrm{ML}$ \\
\hline Tetrachloroethylene & $<5$ & $\mathrm{U}$ & $<5$ & $\mathrm{U}$ & $<1$ & $U$ & $<1$ & $\mathrm{U}$ & & $<E Q L$ & 1 & $u g / L$ & $\mathrm{ML}$ \\
\hline 1,1,1-Trichloroethane & $<5$ & $\mathrm{u}$ & $<5$ & $\mathrm{u}$ & $<1$ & U & $<1$ & $u$ & & $<E Q L$ & 1 & $\mathrm{ug} / \mathrm{h}$ & ML \\
\hline Trichloroethylene & $<5$ & $u$ & $<5$ & $u$ & $<1$ & $u$ & $<1$ & $u$ & & $<E Q L$ & 1 & $\mathrm{ug} / \mathrm{L}$ & $\mathrm{ML}$ \\
\hline \multicolumn{14}{|l|}{ II. Monitoring Constituents } \\
\hline ST Parameter & $\underline{3 Q 96}$ & CLPEPA & $\underline{3097}$ & CLPEPA & $\underline{3098}$ & CLPEPA & 3099 & CLPP & EPA & Filt. & $\underline{\text { DF }}$ & $\underline{\text { Unit }}$ & $\underline{\text { Lab }}$ \\
\hline \multicolumn{14}{|l|}{ Inorganics } \\
\hline Aluminum, total recoverable & 19.6 & $J$ & 20.2 & & $<200$ & $u$ & $<200$ & $\mathrm{u}$ & & $<E Q L$ & 1 & $u g / L$ & EX \\
\hline Nitrate-nitrite as nitrogen & 700 & & 576 & & 427 & & 497 & $\mathrm{~J}$ & I & NDD & 5 & $u g / L$ & EX \\
\hline Sodium, total recoverable & 1600 & & 1640 & & 1860 & & 1800 & & & $<4600$ & 1 & $\mathrm{ug} / \mathrm{L}$ & EX \\
\hline Sulfate & 1390 & & $<5000$ & U & 1370 & & 1360 & & & $<3000$ & 1 & ug/L & EX \\
\hline \multicolumn{14}{|l|}{ Radionuclides } \\
\hline Gross alpha & 1.3 & & -.35 & UI & 2.39 & $\mathrm{~J}$ & 1.71 & $\mathrm{~J}$ & 1 & NDD & 1 & $\mathrm{pCi} / \mathrm{L}$ & GP \\
\hline Nonvolatile beta & 2.72 & & .04 & UI & 3.44 & $\mathrm{~J}$ & 3.18 & & & $<50$ & 1 & $\mathrm{pCi} / \mathrm{L}$ & GP \\
\hline Radium, total alpha-emitting & 1 & & 2.16 & UIJ & 1.33 & & .4 & $\mathrm{~J}$ & 1 & NDD & 1 & $\mathrm{pCl} / \mathrm{L}$ & GP \\
\hline
\end{tabular}

Notes: Concentrations in bold italics exceed the groundwater protection or monitoring constituent standards listed in Appendix A. Synchronous water levels are measured over a 3-5 day period or less. Dilution factors, Laboratory, and Filtered Data are for Groundwater Protection Standards First Quarter 2000 and Monitoring Constituents Third Quarter 1999 data only.

$+=$ exceeded the Groundwater Protection Standards (First Quarter 2000) or Monitoring Constituents (Third Quarter 1999) listed in Appendix A. 
Table D-4. Groundwater Monitoring Results for Plume Definition Wells, M-Area HWMF (Cont.) WELL MSB 83C

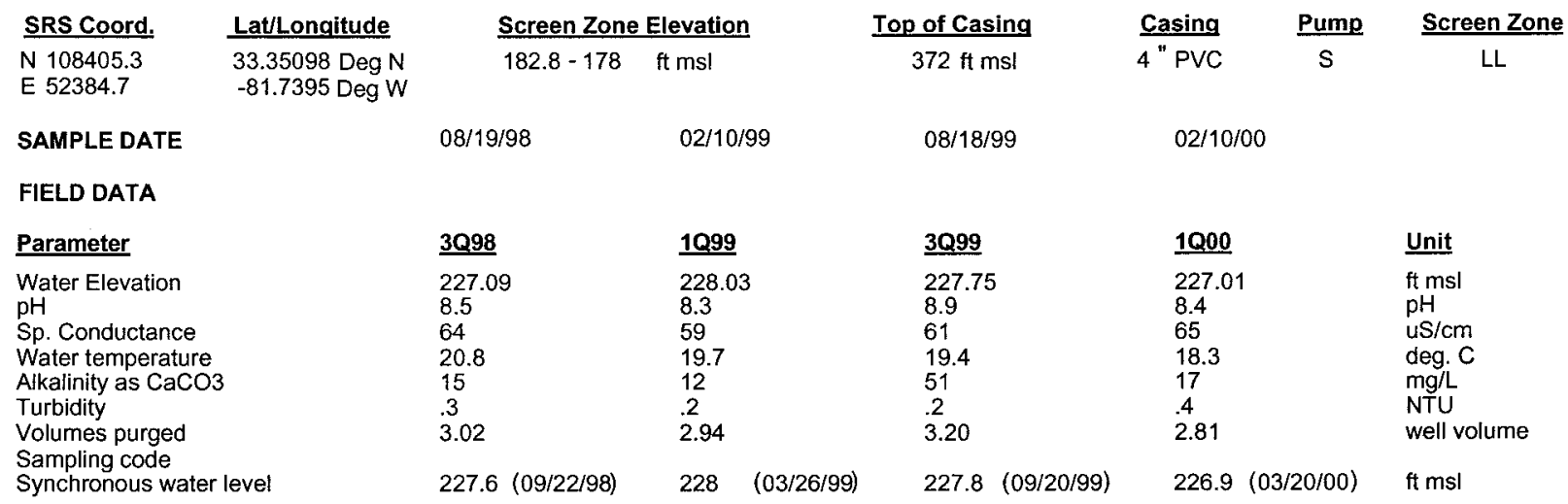

ANALYTICAL DATA

I. Groundwater Protection Standard

261 Appendix VIII/264 Appendix IX Hazardous Constituents

\begin{tabular}{|c|c|c|c|c|c|c|c|c|c|c|c|c|c|c|}
\hline$\underline{\mathrm{ST}}$ & Parameter & 3098 & CLP EPA & 1 Q99 & CLPPEPA & $\underline{3 Q 99}$ & CLPEPA & $\underline{1 Q 00}$ & \multicolumn{2}{|c|}{ CLPEPA } & Filt. & $\underline{\text { DF }}$ & Unit & $\underline{\text { Lab }}$ \\
\hline \multicolumn{15}{|c|}{ Inorganics } \\
\hline & Barium, total recoverable & 18.1 & & 16.4 & & 16 & & & & & & & $\mathrm{ug} / \mathrm{L}$ & \\
\hline & Cyanide & $<10$ & $U$ & $<10$ & $U$ & $<10$ & U & & & & & & $\mathrm{ug} / \mathrm{L}$ & \\
\hline & Lead, total recoverable & $<10$ & $U$ & $<100$ & $\mathrm{U}$ & $<5$ & $U$ & & & & & & $\mathrm{ug} / \mathrm{L}$ & \\
\hline & Nickel, total recoverable & $<18.2$ & $U$ & $<50$ & $\mathrm{U}$ & $<50$ & $U$ & & & & & & $\mathrm{ug} / \mathrm{L}$ & \\
\hline & Selenium, total recoverable & $<10$ & $U$ & $<10$ & $U$ & $<5$ & $U$ & & & & & & $\mathrm{ug} / \mathrm{L}$ & \\
\hline \multicolumn{15}{|c|}{ Organics } \\
\hline & Chlorobenzene & $<5$ & $u$ & $<5$ & $\mathrm{U}$ & $<1$ & $U$ & $<1$ & $\mathrm{U}$ & & $<$ EQL & 1 & ug/L & ML \\
\hline & 1,1-Dichloroethane & $<5$ & $U$ & $<5$ & $\mathrm{U}$ & $<1$ & $\mathrm{U}$ & $<1$ & $\cup$ & & $<\mathrm{EQL}$ & 1 & ug/L & ML. \\
\hline & 1,1-Dichloroethylene & $<5$ & $U$ & $<5$ & $\mathrm{U}$ & $<1$ & $U$ & $<1$ & U & & $<\mathrm{EQL}$ & 1 & ug/L & ML \\
\hline & trans-1,2-Dichloroethylene & $<5$ & $U$ & $<5$ & $\mathrm{U}$ & $<1$ & $\mathrm{U}$ & & & & & & $\mathrm{ug} / \mathrm{L}$ & \\
\hline & PCB 1016 & & & & & & & & & & & & & \\
\hline & PCB 1221 & & & & & & & & & & & & & \\
\hline & PCB 1232 & & & & & & & & & & & & & \\
\hline & РCB 1242 & & & & & & & & & & & & & \\
\hline & PCB 1248 & & & & & & & & & & & & & \\
\hline & РCB 1254 & & & & & & & & & & & & & \\
\hline & PCB 1260 & & & & & & & & & & & & & \\
\hline & 1,1,2,2-Tetrachloroethane & $<5$ & $U$ & $<5$ & $\mathrm{U}$ & $<1$ & U & $<1$ & $\mathrm{U}$ & & $<\mathrm{EQL}$ & 1 & $\mathrm{ug} / \mathrm{L}$ & ML \\
\hline & Tetrachloroethylene & $<5$ & $\mathrm{U}$ & $<5$ & $\mathrm{U}$ & $<1$ & $\mathrm{U}$ & $<1$ & $U$ & & $<\mathrm{EQL}$ & 1 & $\mathrm{ug} / \mathrm{L}$ & ML \\
\hline & $1,1,1$-Trichloroethane & $<5$ & $\mathrm{U}$ & $<5$ & $\mathrm{U}$ & $<1$ & $U$ & $<1$ & $\mathrm{U}$ & & $<E Q L$ & 1 & $u g / L$ & ML \\
\hline & Trichloroethylene & $<5$ & $U$ & $<5$ & $\mathrm{U}$ & $<1$ & $\cup$ & $<1$ & $U$ & & $<E Q L$ & 1 & ug/L & ML \\
\hline \multicolumn{15}{|c|}{ II. Monitoring Constituents } \\
\hline$\underline{\mathbf{S T}}$ & Parameter & $\underline{3 Q 96}$ & CLP EPA & $\underline{3 Q 97}$ & CLPEPA & $\underline{3098}$ & CLPEPA & $\underline{3099}$ & $\underline{\text { CLF }}$ & EPA & Filt. & $\underline{\mathrm{DF}}$ & Unit & La \\
\hline \multicolumn{15}{|c|}{ Inorganics } \\
\hline & Aluminum, total recoverable & $<106$ & $\mathrm{U}$ & 162 & & 197 & $J$ & $<200$ & $\mathrm{U}$ & & $<E Q L$ & 1 & $\mathrm{ug} / \mathrm{L}$ & EX \\
\hline & Nitrate-nitrite as nitrogen & 1910 & & 1900 & & 1360 & & 1410 & & & $<2400$ & 5 & ug/L & EX \\
\hline & Sodium, total recoverable & 2450 & & 2400 & & 2430 & & 2300 & & & $<4600$ & 1 & $u g / L$ & EX \\
\hline & Sulfate & 211 & $J$ & $<5000$ & $\mathrm{U}$ & $<200$ & $\mathrm{U}$ & 174 & $J$ & 1 & NDD & 1 & $u g / L$ & EX \\
\hline \multicolumn{15}{|c|}{ Radionuclides } \\
\hline & Gross alpha & 1.27 & & 0 & UI & 1.06 & UIJ & .479 & $\mathrm{U}$ & & $<E Q L$ & 1 & $\mathrm{pCi} / \mathrm{L}$ & GP \\
\hline & Nonvolatile beta & 1.46 & & -2.2 & Ul & 1.5 & UIJ & 1.36 & $J$ & 1 & NDD & 1 & $\mathrm{pCi} / \mathrm{L}$ & GP \\
\hline & Radium, total alpha-emitting & .2 & UI & 5.14 & UIJ & .43 & & .3 & $J$ & 1 & NDD & 1 & $\mathrm{pCi} / \mathrm{L}$ & ס \\
\hline
\end{tabular}

Notes: Concentrations in bold italics exceed the groundwater protection or monitoring constituent standards listed in Appendix A. Synchronous water levels are measured over a 3-5 day period or less. Dilution factors, Laboratory, and Filtered Data are for Groundwater Protection Standards First Quarter 2000 and Monitoring Constituents Third Quarter 1999 data only.

$+=$ exceeded the Groundwater Protection Standards (First Quarter 2000) or Monitoring Constituents (Third Quarter 1999) listed in Appendix A. 
Table D-4. Groundwater Monitoring Results for Plume Definition Wells, M-Area HWMF (Cont.) WELL MSB 83TA

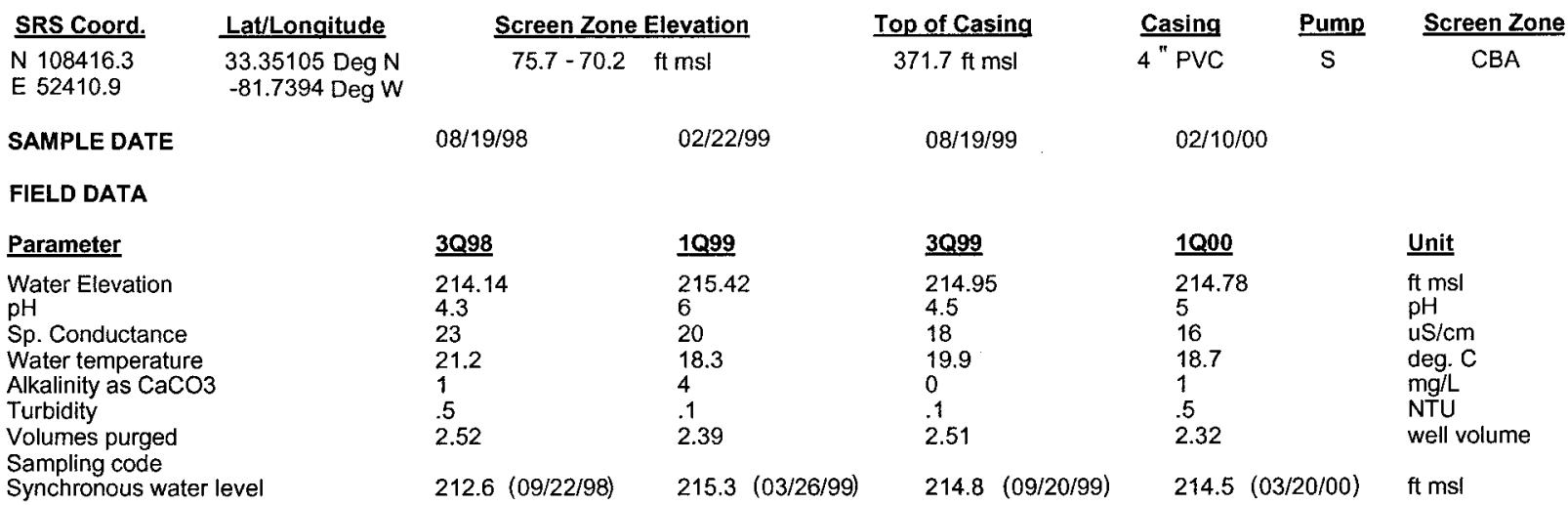

ANALYTICAL DATA

I. Groundwater Protection Standard

261 Appendix VIII/264 Appendix IX Hazardous Constituents

\begin{tabular}{|c|c|c|c|c|c|c|c|c|c|c|c|c|c|}
\hline$\underline{\mathbf{S T}}$ & Parameter & $\underline{3 Q 98}$ & $\underline{\text { CLP EPA }}$ & 1099 & $\underline{\text { CLP EPA }}$ & $\underline{3 Q 99}$ & CLPEPA & 1000 & CLPEPA & Filt. & DF & Unit & $\underline{\text { Lab }}$ \\
\hline \multicolumn{14}{|c|}{ Inorganics } \\
\hline & Barium, total recoverable & 4.08 & J & 4.3 & & 3.6 & J 1 & & & & & ug/L & \\
\hline & Cyanide & $<10$ & $\mathrm{U}$ & $<15.2$ & $\mathrm{U}$ & $<10$ & U & & & & & $\mathrm{ug} / \mathrm{L}$ & \\
\hline & Lead, total recoverable & $<10$ & $\mathrm{U}$ & $<100$ & $\mathrm{U}$ & $<2.88$ & U V & & & & & $\mathrm{ug} / \mathrm{L}$ & \\
\hline & Nickel, total recoverable & $<9.18$ & $\mathrm{U}$ & $<26$ & $U$ & $<50$ & $\mathrm{U}$ & & & & & ug/L & \\
\hline & Selenium, total recoverable & $<10$ & $U$ & $<200$ & $U$ & $<5$ & $\mathrm{U}$ & & & & & $\mathrm{ug} / \mathrm{L}$ & \\
\hline \multicolumn{14}{|c|}{ Organics } \\
\hline & Chlorobenzene & $<5$ & $\mathrm{U}$ & $<5$ & $U$ & $<1$ & $\mathrm{U}$ & $<1$ & $U$ & $<E Q L$ & 1 & $\mathrm{ug} / \mathrm{L}$ & ML \\
\hline & 1,1-Dichloroethane & $<5$ & $\mathrm{U}$ & $<5$ & $\mathrm{U}$ & $<1$ & $\mathrm{U}$ & $<1$ & $U$ & $<E Q L$ & 1 & $\mathrm{ug} / \mathrm{L}$ & $M L$ \\
\hline & 1,1-Dichloroethylene & $<5$ & $U$ & $<5$ & $U$ & $<1$ & $\mathrm{U}$ & $<1$ & $\mathrm{U}$ & $<\mathrm{EQL}$ & 1 & $\mathrm{ug} / \mathrm{L}$ & $M L$ \\
\hline & trans-1,2-Dichioroethylene & $<5$ & $\mathrm{U}$ & $<5$ & $\bigcup$ & $<1$ & $\mathrm{U}$ & & & & & $\mathrm{ug} / \mathrm{L}$ & \\
\hline & PCB 1016 & & & & & & & & & & & & \\
\hline & РCB 1221 & & & & & & & & & & & & \\
\hline & PCB 1232 & & & & & & & & & & & & \\
\hline & PCB 1242 & & & & & & & & & & & & \\
\hline & PCB 1248 & & & & & & & & & & & & \\
\hline & PCB 1254 & & & & & & & & & & & & \\
\hline & PCB 1260 & & & & & & & & & & & & \\
\hline & 1,1,2,2-Tetrachloroethane & $<5$ & $\mathrm{U}$ & $<5$ & $U$ & $<1$ & $U$ & $<1$ & $\mathrm{U}$ & $<E Q L$ & 1 & $\mathrm{ug} / \mathrm{L}$ & ML \\
\hline & Tetrachloroethylene & $<5$ & $\mathrm{U}$ & $<5$ & $U$ & $<1$ & $U$ & $<1$ & U & $<$ EQL & 1 & $\mathrm{ug} / \mathrm{L}$ & ML \\
\hline & $1,1,1$-Trichloroethane & $<5$ & U & $<5$ & $U$ & $<1$ & $U$ & $<1$ & $\mathrm{U}$ & $<E Q L$ & 1 & ug/L & ML \\
\hline & Trichloroethylene & $<5$ & $\mathrm{U}$ & 2 & $\mathbf{J} \quad 1$ & $<1$ & $\mathrm{U}$ & $<1$ & $\mathrm{U}$ & $<\mathrm{EQL}$ & 1 & $\mathrm{ug} / \mathrm{L}$ & $\mathrm{ML}$ \\
\hline \multicolumn{14}{|c|}{ II. Monitoring Constituents } \\
\hline$\underline{\mathbf{S T}}$ & Parameter & $\underline{3096}$ & CLPEPA & $\underline{3097}$ & CLPEPA & $\underline{3098}$ & CLPEPA & $\underline{3099}$ & CLPEPA & Filt. & $\underline{\mathrm{DF}}$ & $\underline{\text { Unit }}$ & $\underline{\text { Lab }}$ \\
\hline \multicolumn{14}{|c|}{ Inorganics } \\
\hline & Aluminum, total recoverable & $<29.1$ & $U$ & 11.6 & J & $<200$ & U & $<200$ & U & $<E Q L$ & 1 & ug/L & EX \\
\hline & Nitrate-nitrite as nitrogen & 900 & & 757 & & 730 & & 757 & & $<2400$ & 5 & $\mathrm{ug} / \mathrm{L}$ & EX \\
\hline & Sodium, total recoverable & 1340 & & 1320 & & 1270 & & 1600 & & $<4600$ & 1 & $\mathrm{ug} / \mathrm{L}$ & EX \\
\hline & Sulfate & 515 & $\mathrm{~J}$ & $<5000$ & $U$ & $<200$ & $U$ & 546 & & $<3000$ & 1 & ug/L & EX \\
\hline \multicolumn{14}{|c|}{ Radionuclides } \\
\hline & Gross alpha & .494 & UI & .29 & UI & 1.03 & $J$ & .665 & $U$ & $<\mathrm{EQL}$ & 1 & $\mathrm{pCi} / \mathrm{L}$ & GP \\
\hline & Nonvolatile beta & .302 & UI & -1.32 & Ul & -.51 & UIJ & .829 & $\mathrm{U}$ & $<E Q L$ & 1 & $\mathrm{pCi} / \mathrm{L}$ & GP \\
\hline & Radium, total alpha-emitting & .1 & UI & 3.37 & UIJ & .61 & & .3 & $\mathrm{U}$ & $<\mathrm{EQL}$ & 1 & $\mathrm{pCi} / \mathrm{L}$ & GP \\
\hline
\end{tabular}

Notes: Concentrations in bold italics exceed the groundwater protection or monitoring constituent standards listed in Appendix A. Synchronous water levels are measured over a 3-5 day period or less. Dilution factors, Laboratory, and Filtered Data are for Groundwater Protection Standards First Quarter 2000 and Monitoring Constituents Third Quarter 1999 data only.

+= exceeded the Groundwater Protection Standards (First Quarter 2000) or Monitoring Constituents (Third Quarter 1999) listed in Appendix A. 
Table D-4. Groundwater Monitoring Results for Plume Definition Wells, M-Area HWMF (Cont.) WELL MSB 84A

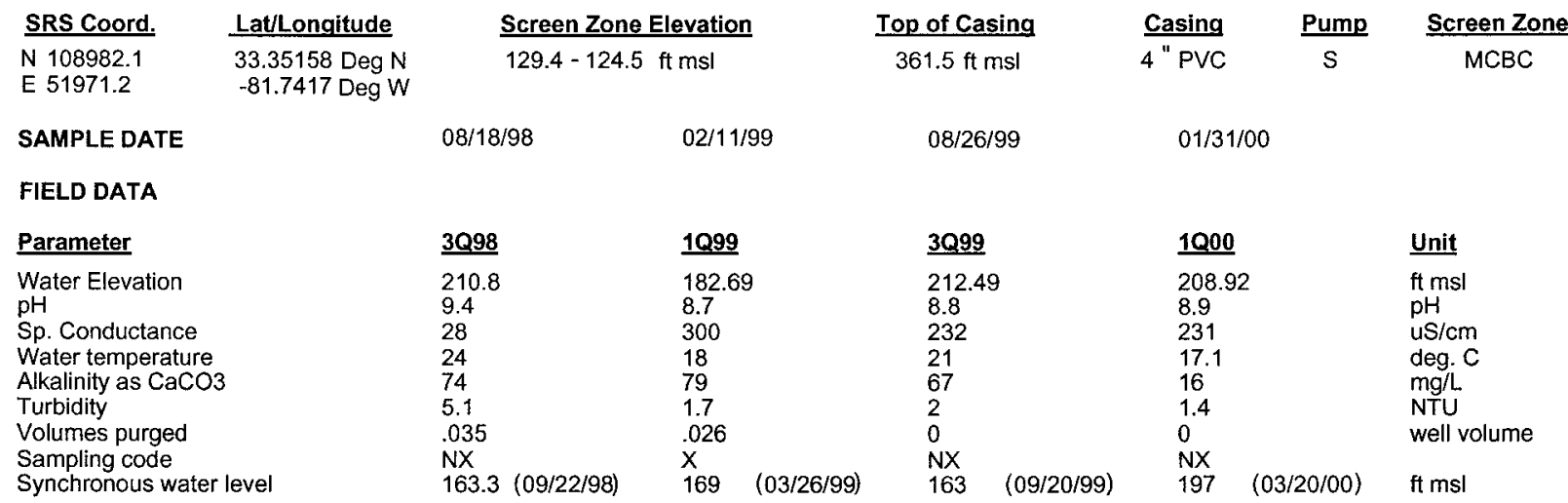

ANALYTICAL DATA

I. Groundwater Protection Standard

261 Appendix VIII/264 Appendix IX Hazardous Constituents

\begin{tabular}{|c|c|c|c|c|c|c|c|c|c|c|c|c|c|}
\hline$\underline{\text { ST }}$ & Parameter & $\underline{3098}$ & CLP EPA & 1099 & CLP EPA & $\underline{3 Q 99}$ & CLPEPA & 1000 & CLPEPA & Filt. & $\underline{\text { DF }}$ & $\underline{\text { Unit }}$ & $\underline{\text { Lab }}$ \\
\hline \multicolumn{14}{|c|}{ Inorganics } \\
\hline & Barium, total recoverable & 30 & & $<10$ & $U$ & 44 & & & & & & $\mathrm{ug} / \mathrm{L}$ & \\
\hline & Cyanide & $<10$ & $U$ & $<10$ & $\mathrm{U}$ & $<10$ & $U$ & & & & & $\mathrm{ug} / \mathrm{L}$ & \\
\hline & Lead, total recoverable & $<10$ & $\mathrm{U}$ & $<100$ & $\mathrm{U}$ & $<5$ & $\mathrm{U}$ & r & & & & $\mathrm{ug} / \mathrm{L}$ & \\
\hline & Nickel, total recoverable & $<50$ & $\mathrm{U}$ & $<50$ & $\mathrm{U}$ & $<50$ & $U$ & & & & & $\mathrm{ug} / \mathrm{L}$ & \\
\hline & Selenium, total recoverable & $<10$ & $\mathrm{U}$ & & & $<5$ & $U$ & & & & & $u g / L$ & \\
\hline \multicolumn{14}{|c|}{ Organics } \\
\hline & Chiorobenzene & $<5$ & $\mathrm{U}$ & $<5$ & $\mathrm{U}$ & $<1$ & $\mathrm{U}$ & $<1$ & JU L & $<\mathrm{EQL}$ & 1 & $\mathrm{ug} / \mathrm{L}$ & ML \\
\hline & 1,1-Dichloroethane & $<5$ & $\mathrm{U}$ & $<5$ & $\mathrm{U}$ & $<1$ & $\mathrm{U}$ & $<1$ & JU $L$ & $<E Q L$ & 1 & $\mathrm{ug} / \mathrm{L}$ & ML \\
\hline & 1,1-Dichloroethylene & $<5$ & U & $<5$ & $\mathrm{U}$ & $<1$ & $U$ & $<1$ & JU L & $<\mathrm{EQL}$ & 1 & $\mathrm{ug} / \mathrm{L}$ & ML \\
\hline & $\begin{array}{l}\text { trans-1,2-Dichloroethylene } \\
\text { PCB } 1016\end{array}$ & $<5$ & $\mathrm{U}$ & $<5$ & $\mathrm{U}$ & $<1$ & $\mathrm{U}$ & & & & & $\mathrm{ug} / \mathrm{L}$ & \\
\hline & PCB 1221 & & & & & & & & & & & & \\
\hline & PCB 1232 & & & & & & & & & & & & \\
\hline & PCB 1242 & & & & & & & & & & & & \\
\hline & PCB 1248 & & & & & & & & & & & & \\
\hline & PCB 1254 & & & & & & & & & & & & \\
\hline & PCB 1260 & & & & & & & & & & & & \\
\hline & 1,1,2,2-Tetrachloroethane & $<5$ & $\mathrm{U}$ & $<5$ & $\mathrm{U}$ & $<1$ & $\mathrm{U}$ & $<1$ & $J U L$ & $<E Q L$ & 1 & $\mathrm{ug} / \mathrm{L}$ & $M L$ \\
\hline & Tetrachloroethylene & $<5$ & $\mathrm{U}$ & $<5$ & $\mathrm{U}$ & $<1$ & $\mathrm{U}$ & $<1$ & $J U$ L & $<E Q L$ & 1 & $\mathrm{ug} / \mathrm{L}$ & $M L$ \\
\hline & 1,1,1-Trichloroethane & $<5$ & $\mathrm{U}$ & $<5$ & $u$ & $<1$ & $\mathrm{U}$ & $<1$ & JU L & $<E Q L$ & 1 & $\mathrm{ug} / \mathrm{L}$ & $M L$ \\
\hline & Trichloroethylene & $<5$ & $\mathrm{U}$ & $<5$ & $U$ & $<1$ & $\mathrm{U}$ & $<1$ & $J U L$ & $<E Q L$ & 1 & $\mathrm{ug} / \mathrm{L}$ & ML \\
\hline \multicolumn{14}{|c|}{ II. Monitoring Constituents } \\
\hline$\underline{\mathbf{S T}}$ & Parameter & $\underline{3 Q 96}$ & CLP EPA & $\underline{3097}$ & CLPEPA & $\underline{3098}$ & CLPEPA & $\underline{3099}$ & $\underline{\text { CLPEPA }}$ & Filt. & DF & Unit & $\underline{L a b}$ \\
\hline \multicolumn{14}{|c|}{ Inorganics } \\
\hline & Aluminum, total recoverable & 286 & & 112 & & 115 & $J$ & $<200$ & U & $<\mathrm{EQL}$ & 1 & ug/L & EX \\
\hline & Nitrate-nitrite as nitrogen & 230 & & $<10$ & $\mathrm{U}$ & $<500$ & $\mathrm{U}$ & $<500$ & $U$ & $<\mathrm{EQL}$ & 5 & ug/L & EX \\
\hline+ & Sodium, total recoverable & 88300 & & 51600 & & 40700 & & 30000 & & $>4600$ & 1 & $\mathrm{ug} / \mathrm{L}$ & EX \\
\hline+ & Sulfate & 45200 & & 37300 & & 12800 & & 25800 & & $>3000$ & 2 & ug/L & EX \\
\hline \multicolumn{14}{|c|}{ Radionuclides } \\
\hline & Gross alpha & -.061 & UI & .64 & UI & 3.04 & & .538 & $\mathrm{U}$ & $<E Q L$ & 1 & $\mathrm{pCi} / \mathrm{L}$ & GP \\
\hline & Nonvolatile beta & 14 & & 28.7 & & 25.88 & J & 13.4 & & $<50$ & 1 & $\mathrm{pCi} / \mathrm{L}$ & GP \\
\hline & Radium, total alpha-emitting & .2 & UI & 2.55 & $J$ & .39 & & .4 & J 1 & NDD & 1 & $\mathrm{pCi} / \mathrm{L}$ & GP \\
\hline
\end{tabular}

Notes: Concentrations in bold italics exceed the groundwater protection or monitoring constituent standards listed in Appendix A. Synchronous water levels are measured over a 3-5 day period or less. Dilution factors, Laboratory, and Filtered Data are for Groundwater Protection Standards First Quarter 2000 and Monitoring Constituents Third Quarter 1999 data only.

$+=$ exceeded the Groundwater Protection Standards (First Quarter 2000) or Monitoring Constituents (Third Quarter 1999) listed in Appendix A. 
Table D-4. Groundwater Monitoring Results for Plume Definition Wells, M-Area HWMF (Cont.) WELL MSB 84C

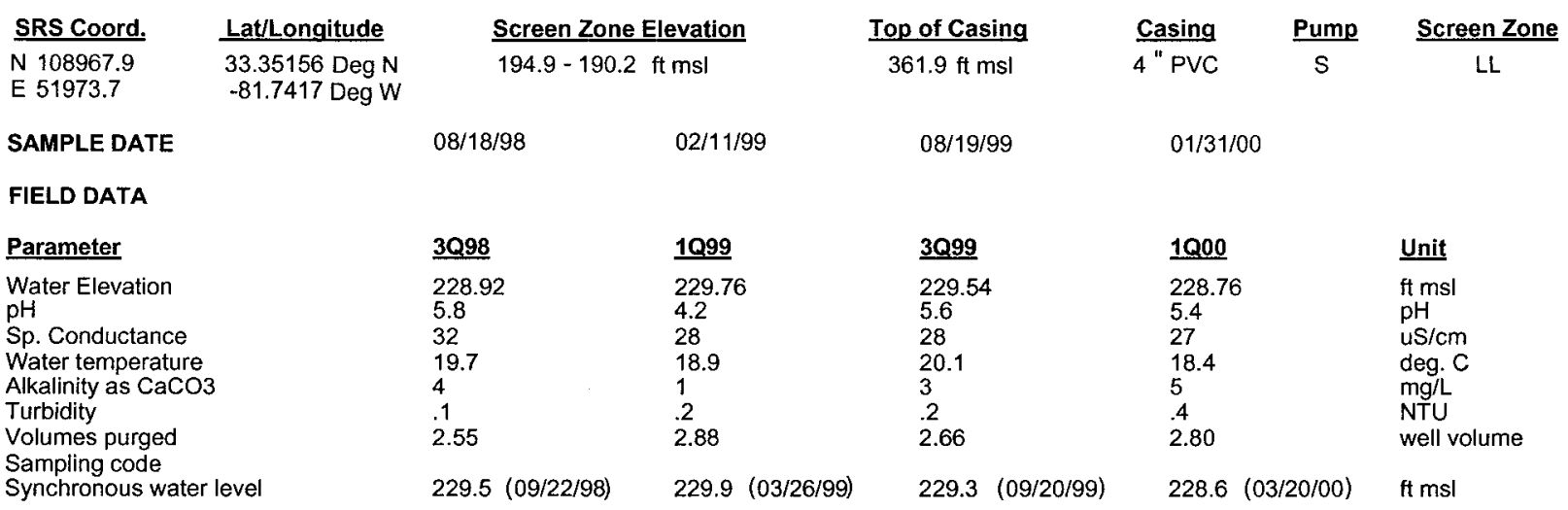

ANALYTICAL DATA

I. Groundwater Protection Standard

261 Appendix VIII/264 Appendix IX Hazardous Constituents

\begin{tabular}{|c|c|c|c|c|c|c|c|c|c|c|c|c|c|}
\hline$\underline{\mathbf{S T}}$ & Parameter & $\underline{3098}$ & CLPEPA & 1099 & $\underline{\text { CLP EPA }}$ & $\underline{3 Q 99}$ & CLPEPA & 1000 & CLPEPA & Filt. & $\underline{\text { DF }}$ & $\underline{\text { Unit }}$ & $\underline{\text { Lab }}$ \\
\hline \multicolumn{14}{|c|}{ Inorganics } \\
\hline & Barium, total recoverable & 8.34 & $J$ & $<10$ & U & 7.1 & $\mathrm{~J} \quad 1$ & & & & & $u g / L$ & \\
\hline & Cyanide & $<10$ & $U$ & $<10$ & U & $<10$ & $\mathrm{U}$ & & & & & $u g / L$ & \\
\hline & Lead, total recoverable & $<10$ & $U$ & $<100$ & $\mathrm{U}$ & $<3.24$ & U V & & & & & $\mathrm{ug} / \mathrm{L}$ & \\
\hline & Nickel, total recoverable & $<50$ & $\mathrm{U}$ & $<50$ & $U$ & $<50$ & $U$ & & & & & $u g / L$ & \\
\hline & Selenium, total recoverable & $<10$ & $\mathrm{U}$ & & & $<5$ & $\mathrm{U}$ & & & & & $\mathrm{ug} / \mathrm{L}$ & \\
\hline \multicolumn{14}{|c|}{ Organics } \\
\hline & Chlorobenzene & $<5$ & $\mathrm{U}$ & $<5$ & $U$ & $<1$ & U & $<1$ & JU L & $<E Q L$ & 1 & $u g / L$ & ML \\
\hline & 1,1-Dichloroethane & $<5$ & U & $<5$ & $U$ & $<1$ & $\mathrm{U}$ & $<1$ & JU L & $<E Q L$ & 1 & $\mathrm{ug} / \mathrm{L}$ & $M L$ \\
\hline & 1,1-Dichloroethylene & $<5$ & U & $<5$ & $U$ & $<1$ & $\mathrm{U}$ & $<1$ & $J U L$ & $<E Q L$ & 1 & $\mathrm{ug} / \mathrm{L}$ & $\mathrm{ML}$ \\
\hline & trans-1,2-Dichloroethylene & $<5$ & $\mathrm{U}$ & $<5$ & $\mathrm{U}$ & $<1$ & $\mathrm{U}$ & & & & & $u g / L$ & \\
\hline & PCB 1016 & & & & & & & & & & & & \\
\hline & PCB 1221 & & & & & & & & & & & & \\
\hline & PCB 1232 & & & & & & & & & & & & \\
\hline & PCB 1242 & & & & & & & & & & & & \\
\hline & PCB 1248 & & & & & & & & & & & & \\
\hline & PCB 1254 & & & & & & & & & & & & \\
\hline & PCB 1260 & & & & & & & & & & & & \\
\hline & $1,1,2,2$-Tetrachloroethane & $<5$ & $U$ & $<5$ & $\mathrm{U}$ & $<1$ & $\mathrm{U}$ & $<1$ & JU L & $<E Q L$ & 1 & $\mathrm{ug} / \mathrm{L}$ & $M L$ \\
\hline & Tetrachloroethylene & $<5$ & $\mathrm{U}$ & $<5$ & $\mathrm{U}$ & $<1$ & $\mathrm{U}$ & $<1$ & JU L & $<E Q L$ & 1 & ug/L & $M L$ \\
\hline & $1,1,1$-Trichloroethane & $<5$ & $\mathrm{U}$ & $<5$ & U & $<1$ & $\mathrm{U}$ & $<1$ & $J U L$ & $<E Q L$ & 1 & $\mathrm{ug} / \mathrm{L}$ & $M L$ \\
\hline & Trichloroethylene & $<5$ & $\mathrm{U}$ & $<5$ & $\mathrm{U}$ & $<1$ & $\mathrm{U}$ & $<1$ & JU L & $<E Q L$ & 1 & $\mathrm{ug} / \mathrm{L}$ & $M L$ \\
\hline \multicolumn{14}{|c|}{ II. Monitoring Constituents } \\
\hline$\underline{\mathbf{S T}}$ & Parameter & 3096 & CLP EPA & $\underline{3097}$ & CLP EPA & $\underline{3 Q 98}$ & CLPEPA & $\underline{3 Q 99}$ & CLPEPA & Filt. & $\underline{\text { DF }}$ & Unit & Lab \\
\hline \multicolumn{14}{|c|}{ Inorganics } \\
\hline & Aluminum, total recoverable & $<20$ & U & 42.4 & & $<200$ & $u$ & $<200$ & U & $<$ EQL & 1 & $\mathrm{ug} / \mathrm{L}$ & EX \\
\hline & Nitrate-nitrite as nitrogen & 1940 & & 2040 & & 1250 & & 1330 & & $<2400$ & 5 & ug/L & EX \\
\hline & Sodium, total recoverable & 2460 & & 2490 & & 2350 & & 2300 & & $<4600$ & 1 & $\mathrm{ug} / \mathrm{L}$ & EX \\
\hline & Sulfate & 204 & $J$ & $<5000$ & $\mathbf{U}$ & $<200$ & $\mathbf{U}$ & 234 & & $<3000$ & 1 & $\mathrm{ug} / \mathrm{L}$ & EX \\
\hline \multicolumn{14}{|c|}{ Radionuclides } \\
\hline & Gross alpha & .873 & & 1.11 & UI & 2.92 & & .553 & $\mathrm{U}$ & $<\mathrm{EQL}$ & 1 & $\mathrm{pCi} / \mathrm{L}$ & GP \\
\hline & Nonvolatile beta & 1.11 & UI & 2.21 & $\mathrm{UI}$ & 1.41 & UIJ & .799 & $\mathrm{U}$ & $<E Q L$ & 1 & $\mathrm{pCi} / \mathrm{L}$ & GP \\
\hline & Radium, total alpha-emitting & .4 & & 3.26 & UIJ & .53 & & .1 & $U$ & $<\mathrm{EQL}$ & 1 & $\mathrm{pCi} / \mathrm{L}$ & GP \\
\hline
\end{tabular}

Notes: Concentrations in bold italics exceed the groundwater protection or monitoring constituent standards listed in Appendix A. Synchronous water levels are measured over a 3-5 day period or less. Dilution factors, Laboratory, and Filtered Data are for Groundwater Protection Standards First Quarter 2000 and Monitoring Constituents Third Quarter 1999 data only.

+= exceeded the Groundwater Protection Standards (First Quarter 2000) or Monitoring Constituents (Third Quarter 1999) listed in Appendix A. 
Table D-4. Groundwater Monitoring Results for Plume Definition Wells, M-Area HWMF (Cont.) WELL MSB 85B

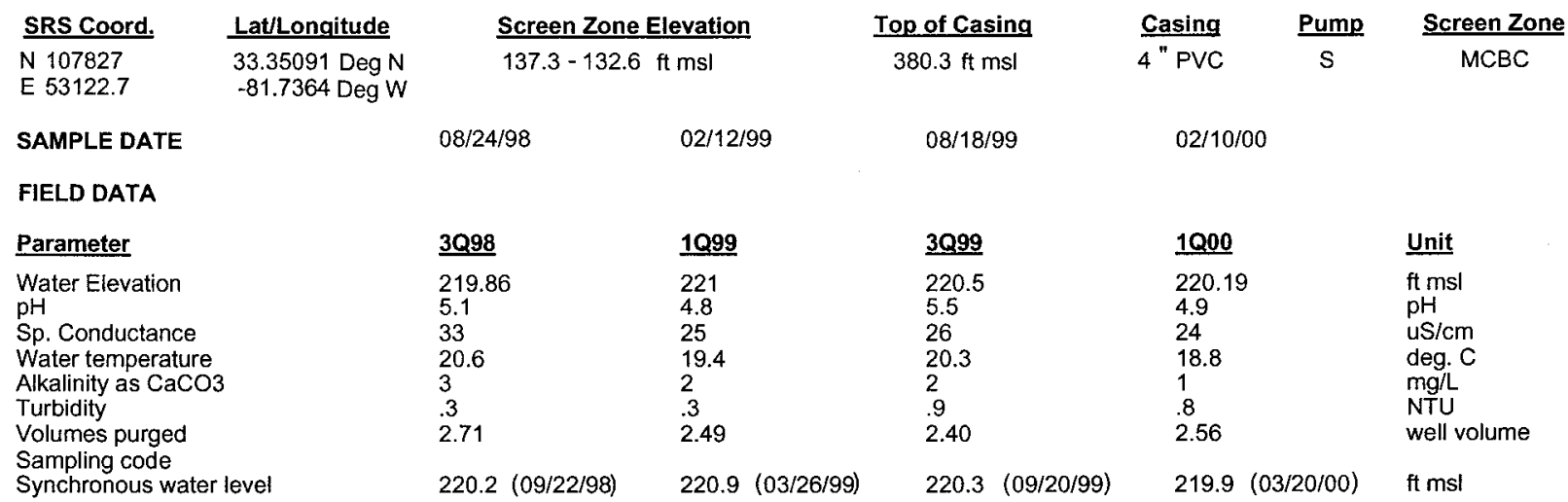

ANALYTICAL DATA

I. Groundwater Protection Standard

261 Appendix VIII/264 Appendix IX Hazardous Constituents

\begin{tabular}{|c|c|c|c|c|c|c|c|c|c|c|c|c|c|}
\hline$\underline{\mathrm{ST}}$ & Parameter & 3Q98 & CLPEPA & 1099 & CLP EPA & $\underline{3 Q 99}$ & CLPEEPA & 1000 & CLPEPA & Filt. & $\underline{\text { DF }}$ & $\underline{\text { Unit }}$ & Lab \\
\hline \multicolumn{14}{|c|}{ Inorganics } \\
\hline & Barium, total recoverable & 16 & & $<10$ & $U$ & 11 & & & & & & $\mathrm{ug} / \mathrm{L}$ & \\
\hline & Cyanide & $<10$ & u & $<10$ & $U$ & $<10$ & $\mathrm{U}$ & & & & & $\mathrm{ug} / \mathrm{L}$ & \\
\hline & Lead, total recoverable & $<10$ & U & $<100$ & $\mathrm{U}$ & $<5$ & U & & & & & $\mathrm{ug} / \mathrm{L}$ & \\
\hline & Nickel, total recoverable & $<50$ & U & $<50$ & $U$ & $<8.8$ & JU I & & & & & $\mathrm{ug} / \mathrm{L}$ & \\
\hline & Selenium, total recoverable & $<10$ & $U$ & $<200$ & $\mathrm{U}$ & $<5$ & $U$ & & & & & $\mathrm{ug} / \mathrm{L}$ & \\
\hline \multicolumn{14}{|c|}{ Organics } \\
\hline & Chlorobenzene & $<5$ & $u$ & $<5$ & U & $<1$ & $u$ & $<1$ & $\mathrm{U}$ & $<\mathrm{EQL}$ & 1 & ug/L & $M L$ \\
\hline & 1,1-Dichloroethane & $<5$ & $\mathrm{U}$ & $<5$ & $U$ & $<1$ & $U$ & $<1$ & $\mathrm{U}$ & $<E Q L$ & 1 & $\mathrm{ug} / \mathrm{L}$ & $\mathrm{ML}$ \\
\hline & 1,1-Dichloroethylene & $<5$ & $U$ & $<5$ & $\mathrm{U}$ & $<1$ & $U$ & $<1$ & $\mathrm{U}$ & $<E Q L$ & 1 & $\mathrm{ug} / \mathrm{L}$ & ML \\
\hline & trans-1,2-Dichloroethylene & $<5$ & $\mathrm{U}$ & $<5$ & $\mathrm{U}$ & $<1$ & $U$ & & & & & $\mathrm{ug} / \mathrm{L}$ & \\
\hline & РCB 1016 & & & & & & & & & & & & \\
\hline & РСВ 1221 & & & & & & & & & & & & \\
\hline & PCB 1232 & & & & & & & & & & & & \\
\hline & PCB 1242 & & & & & & & & & & & & \\
\hline & РСB 1248 & & & & & & & & & & & & \\
\hline & PCB 1254 & & & & & & & & & & & & \\
\hline & PCB 1260 & & & & & & & & & & & & \\
\hline & 1,1,2,2-Tetrachloroethane & $<5$ & $U$ & $<5$ & $\mathrm{U}$ & $<1$ & $U$ & $<1$ & $U$ & $<\mathrm{EQL}$ & 1 & $\mathrm{ug} / \mathrm{L}$ & $\mathrm{ML}$ \\
\hline & Tetrachloroethylene & $<5$ & $\mathrm{U}$ & $<5$ & $\mathrm{U}$ & $<1$ & $U$ & $<1$ & $\mathrm{U}$ & $<\mathrm{EQL}$ & 1 & $\mathrm{ug} / \mathrm{L}$ & ML \\
\hline & 1,1,1-Trichloroethane & $<5$ & $\mathrm{U}$ & $<5$ & $\mathrm{U}$ & $<1$ & $U$ & $<1$ & $\mathrm{U}$ & $<\mathrm{EQL}$ & 1 & $\mathrm{ug} / \mathrm{L}$ & $\mathrm{ML}$ \\
\hline & Trichloroethylene & $<5$ & $U$ & $<5$ & $\mathrm{U}$ & .61 & IK & $<1$ & $\mathrm{U}$ & $<$ EQL & 1 & ug/L & ML \\
\hline \multicolumn{14}{|c|}{ II. Monitoring Constituents } \\
\hline$\underline{\mathbf{S T}}$ & Parameter & $\underline{3 Q 96}$ & CLP EPA & $\underline{3 Q 97}$ & CLP EPA & $\underline{3 Q 98}$ & CLPEPA & 3099 & CLPEPA & Filt. & DF & Unit & Lab \\
\hline \multicolumn{14}{|c|}{ Inorganics } \\
\hline & Aluminum, total recoverable & $<45.2$ & $\mathrm{U}$ & 17.3 & $\jmath$ & $<200$ & $\mathrm{U}$ & $<200$ & $U$ & $<\mathrm{EQL}$ & 1 & $\mathrm{ug} / \mathrm{L}$ & EX \\
\hline & Nitrate-nitrite as nitrogen & 1700 & & 1710 & & 1340 & & 1460 & & $<2400$ & 5 & $\mathrm{ug} / \mathrm{L}$ & EX \\
\hline & Sodium, total recoverable & 2530 & & 2210 & & 2780 & & 2300 & & $<4600$ & 1 & $\mathrm{ug} / \mathrm{L}$ & EX \\
\hline & Sulfate & 283 & $J$ & $<5000$ & $U$ & 261 & $J$ & 198 & J 1 & NDD & 1 & $\mathrm{ug} / \mathrm{L}$ & EX \\
\hline \multicolumn{13}{|c|}{ Radionuclides } & \\
\hline & Gross alpha & .697 & & -.76 & UI & .94 & UIJ & .523 & $U$ & $<\mathrm{EQL}$ & 1 & $\mathrm{pCi} / \mathrm{L}$ & GP \\
\hline & Nonvolatile beta & 1.09 & UI & -1.2 & UI & .18 & UI & 1.4 & U & $<\mathrm{EQL}$ & 1 & $\mathrm{pCi} / \mathrm{L}$ & GP \\
\hline & Radium, total alpha-emitting & .1 & UI & 3.61 & & .1 & UI & .2 & $U$ & $<\mathrm{EQL}$ & 1 & $\mathrm{pCi} / \mathrm{L}$ & \\
\hline
\end{tabular}

Notes: Concentrations in bold italics exceed the groundwater protection or monitoring constituent standards listed in Appendix A. Synchronous water levels are measured over a 3-5 day period or less. Dilution factors, Laboratory, and Filtered Data are for Groundwater Protection Standards First Quarter 2000 and Monitoring Constituents Third Quarter 1999 data only.

+ = exceeded the Groundwater Protection Standards (First Quarter 2000) or Monitoring Constituents (Third Quarter 1999) listed in Appendix A. 
Table D-4. Groundwater Monitoring Results for Plume Definition Wells, M-Area HWMF (Cont.) WELL MSB 85C

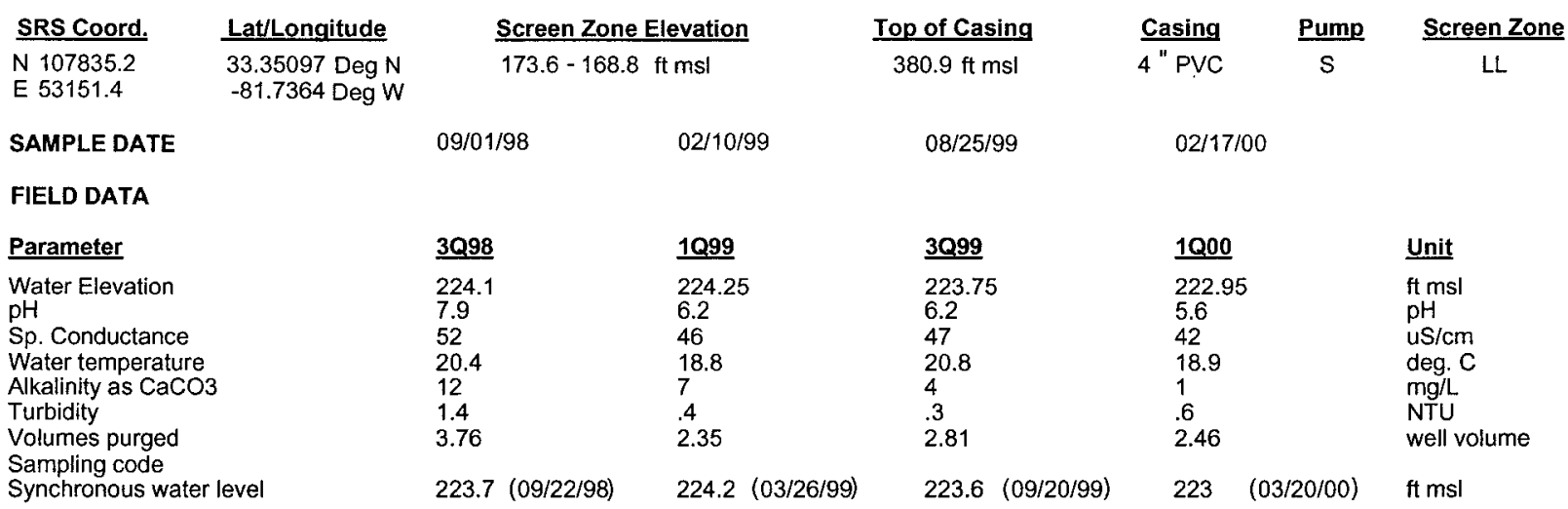

\section{ANALYTICAL DATA}

I. Groundwater Protection Standard

261 Appendix VIII/264 Appendix IX Hazardous Constituents

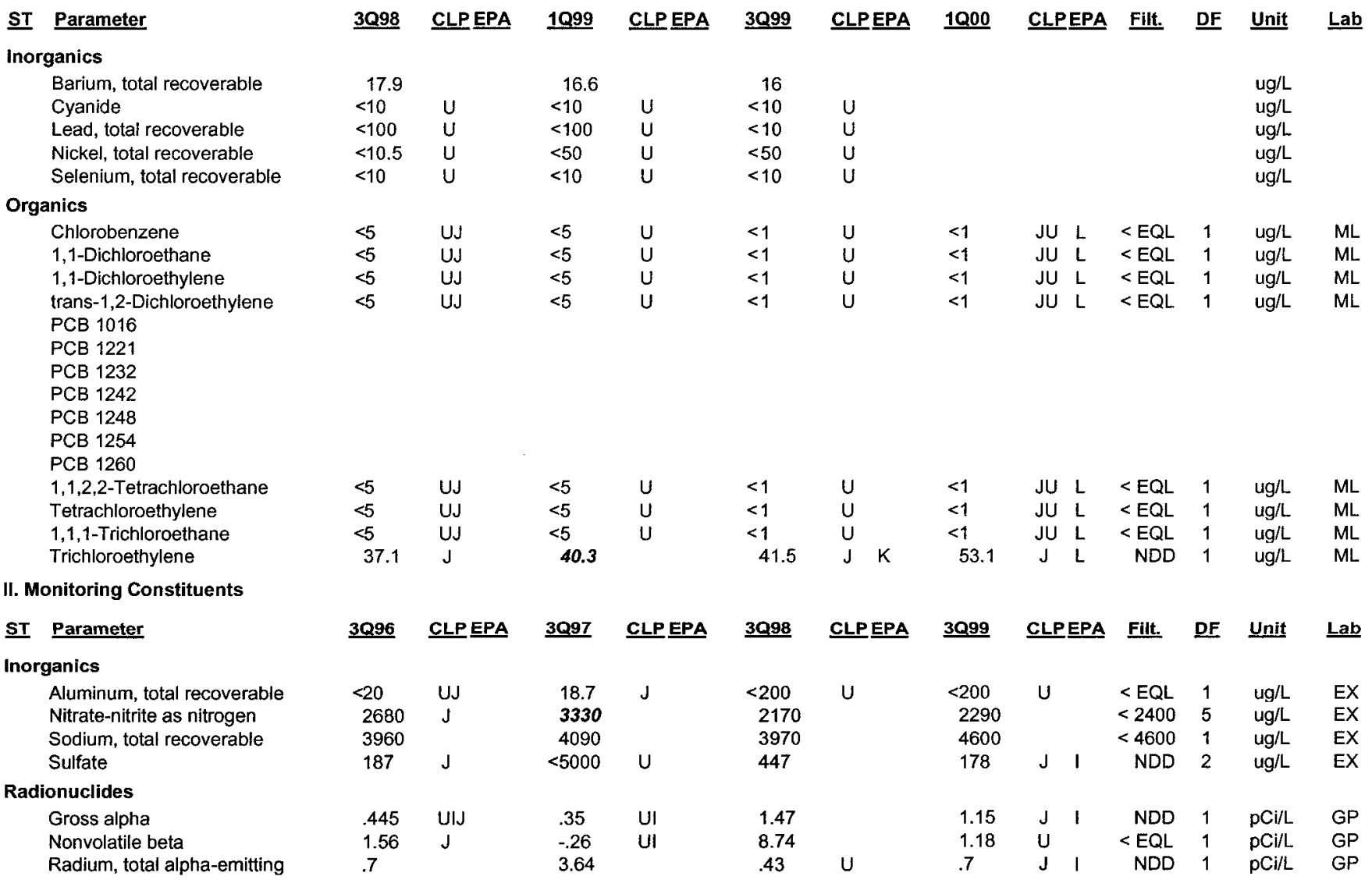

Notes: Concentrations in bold italics exceed the groundwater protection or monitoring constituent standards listed in Appendix A. Synchronous water levels are measured over a 3-5 day period or less. Dilution factors, Laboratory, and Filtered Data are for Groundwater Protection Standards First Quarter 2000 and Monitoring Constituents Third Quarter 1999 data only.

+= exceeded the Groundwater Protection Standards (First Quarter 2000) or Monitoring Constituents (Third Quarter 1999) listed in Appendix A. 
Table D-4. Groundwater Monitoring Results for Plume Definition Wells, M-Area HWMF (Cont.) WELL MSB 85D

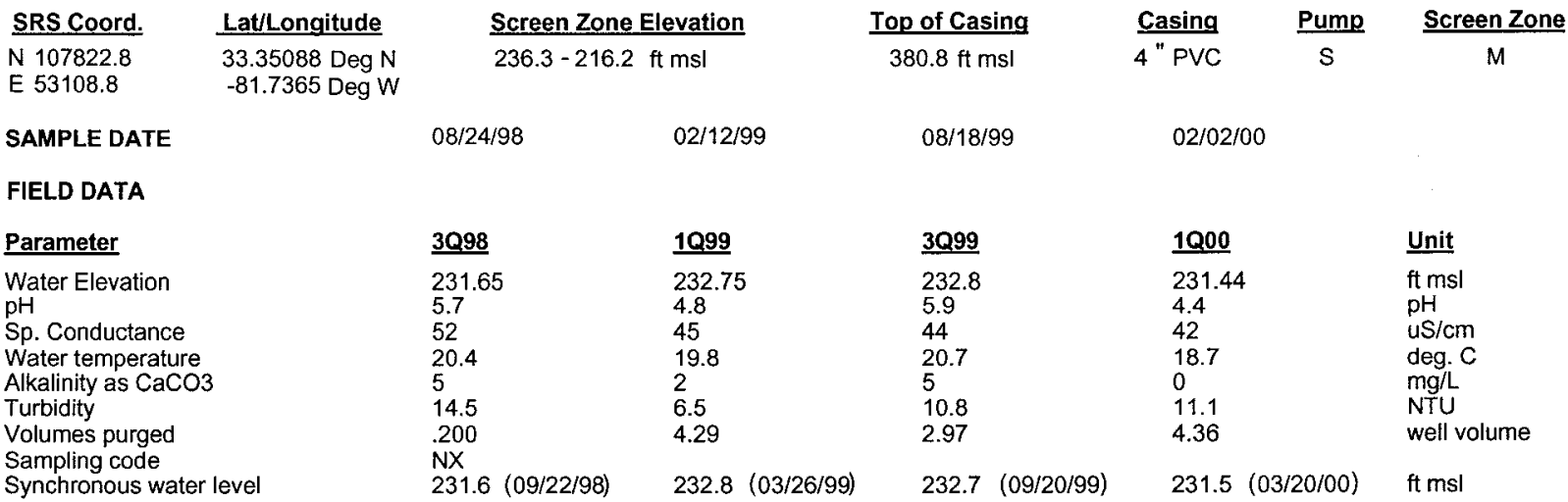

ANALYTICAL DATA

I. Groundwater Protection Standard

261 Appendix VIII/264 Appendix IX Hazardous Constituents

\begin{tabular}{|c|c|c|c|c|c|c|c|c|c|c|c|c|c|}
\hline$\underline{\text { ST }}$ & Parameter & $\underline{3 Q 98}$ & CLP EPA & 1099 & CLP EPA & $\underline{3 Q 99}$ & CLPEPA & 1000 & CLPEPA & Filt. & $\underline{\text { DF }}$ & Unit & $\underline{\text { Lab }}$ \\
\hline \multicolumn{14}{|c|}{ Inorganics } \\
\hline & Barium, total recoverable & 27.2 & & $<10$ & $\mathrm{U}$ & 20 & & & & & & $\mathrm{ug} / \mathrm{L}$ & \\
\hline & Cyanide & $<10$ & $U$ & $<10$ & $\mathrm{U}$ & $<10$ & $\mathrm{U}$ & & & & & $\mathrm{ug} / \mathrm{L}$ & \\
\hline & Lead, total recoverable & 4.62 & $J$ & $<100$ & $\cup$ & 1.13 & 51 & & & & & $u g / L$ & \\
\hline & Nickel, total recoverable & $<50$ & U & $<50$ & $U$ & $<11$ & JU 1 & & & & & $\mathrm{ug} / \mathrm{L}$ & \\
\hline & Selenium, total recoverable & 5.47 & J & $<200$ & $U$ & $<5$ & U & & & & & $\mathrm{ug} / \mathrm{L}$ & \\
\hline \multicolumn{14}{|c|}{ Organics } \\
\hline & Chlorobenzene & $<5$ & $u$ & $<5$ & $u$ & $<1$ & $u$ & $<1$ & $\mathrm{u}$ & $<\mathrm{EQL}$ & 1 & $\mathrm{ug} / \mathrm{L}$ & ML. \\
\hline & 1,1-Dichloroethane & $<5$ & U & $<5$ & $\cup$ & $<1$ & $\cup$ & $<1$ & $\mathrm{U}$ & $<E Q L$ & 1 & $\mathrm{ug} / \mathrm{L}$ & ML \\
\hline & 1,1-Dichloroethylene & $<5$ & $U$ & $<5$ & $U$ & $<1$ & $\mathrm{U}$ & $<1$ & $\mathrm{U}$ & $<\mathrm{EQL}$ & 1 & $\mathrm{ug} / \mathrm{h}$ & ML \\
\hline & trans-1,2-Dichloroethylene & $<5$ & $U$ & $<5$ & $U$ & $<1$ & $\mathrm{U}$ & $<1$ & $u$ & $<E Q L$ & 1 & $\mathrm{ug} / \mathrm{L}$ & ML \\
\hline & PCB 1016 & & & & & & & & & & & & \\
\hline & PCB 1221 & & & & & & & & & & & & \\
\hline & PCB 1232 & & & & & & & & & & & & \\
\hline & РCB 1242 & & & & & & & & & & & & \\
\hline & РCB 1248 & & & & & & & & & & & & \\
\hline & PCB 1254 & & & & & & & & & & & & \\
\hline & PCB 1260 & & & & & & & & & & & & \\
\hline & $1,1,2,2$-Tetrachloroethane & $<5$ & $U$ & $<5$ & $U$ & $<1$ & $U$ & $<1$ & $\mathrm{U}$ & $<\mathrm{EQL}$ & 1 & ug/L & $M L$ \\
\hline & Tetrachloroethylene & $<5$ & U & $<5$ & $U$ & $<1$ & U & $<1$ & $U$ & $<\mathrm{EQL}$ & 1 & $\mathrm{ug} / \mathrm{L}$ & ML \\
\hline & $1,1,1$-Trichloroethane & $<5$ & $\mathrm{U}$ & $<5$ & U & $<1$ & $U$ & $<1$ & U & $<E Q L$ & 1 & $\mathrm{ug} / \mathrm{L}$ & ML \\
\hline & Trichloroethylene & $<5$ & $U$ & $<5$ & $\mathrm{U}$ & $<1$ & $\mathrm{U}$ & .83 & J IK & NDD & 1 & $\mathrm{ug} / \mathrm{L}$ & ML \\
\hline \multicolumn{14}{|c|}{ II. Monitoring Constituents } \\
\hline$\underline{\mathbf{S T}}$ & Parameter & $\underline{3 Q 96}$ & CLPEPA & $\underline{3 Q 97}$ & CLP EPA & $\underline{3 Q 98}$ & CLPEPA & $\underline{3 Q 99}$ & CLPEPA & Filt. & DF & Unit & $\underline{\text { Lab }}$ \\
\hline \multicolumn{14}{|c|}{ Inorganics } \\
\hline & Aluminum, total recoverable & 680 & & 240 & & 96.9 & J & $<200$ & $\mathrm{U}$ & $<\mathrm{EQL}$ & 1 & $\mathrm{ug} / \mathrm{L}$ & EX \\
\hline+ & Nitrate-nitrite as nitrogen & 3440 & & 4230 & & 3260 & & 2830 & & $>2400$ & 5 & $\mathrm{ug} / \mathrm{L}$ & EX \\
\hline+ & Sodium, total recoverable & 5700 & & 2600 & & 4000 & & 4700 & & $>4600$ & 1 & $\mathrm{ug} / \mathrm{L}$ & EX \\
\hline & Sulfate & 877 & J & $<5000$ & $\mathrm{U}$ & 313 & J & 308 & & $<3000$ & 1 & ug/L & EX \\
\hline \multicolumn{14}{|c|}{ Radionuclides } \\
\hline & Gross alpha & 6.12 & & 5.35 & & 16.55 & J & 3.85 & & $<15$ & 1 & $\mathrm{pCi} / \mathrm{L}$ & GP \\
\hline & Nonvolatile beta & 4.66 & & 3.32 & & 11.65 & & 3.95 & & $<50$ & 1 & $\mathrm{pCi} / \mathrm{L}$ & GP \\
\hline & Radium, total alpha-emitting & 3.3 & & 4.62 & & 3.15 & & 2.4 & & $<5$ & 1 & $\mathrm{pCi} / \mathrm{L}$ & GP \\
\hline
\end{tabular}

Notes: Concentrations in bold italics exceed the groundwater protection or monitoring constituent standards listed in Appendix A. Synchronous water levels are measured over a 3-5 day period or less. Dilution factors, Laboratory, and Filtered Data are for Groundwater Protection Standards First Quarter 2000 and Monitoring Constituents Third Quarter 1999 data only.

$+=$ exceeded the Groundwater Protection Standards (First Quarter 2000) or Monitoring Constituents (Third Quarter 1999) listed in Appendix A. 
Table D-4. Groundwater Monitoring Results for Plume Definition Wells, M-Area HWMF (Cont.) WELL MSB 85TA

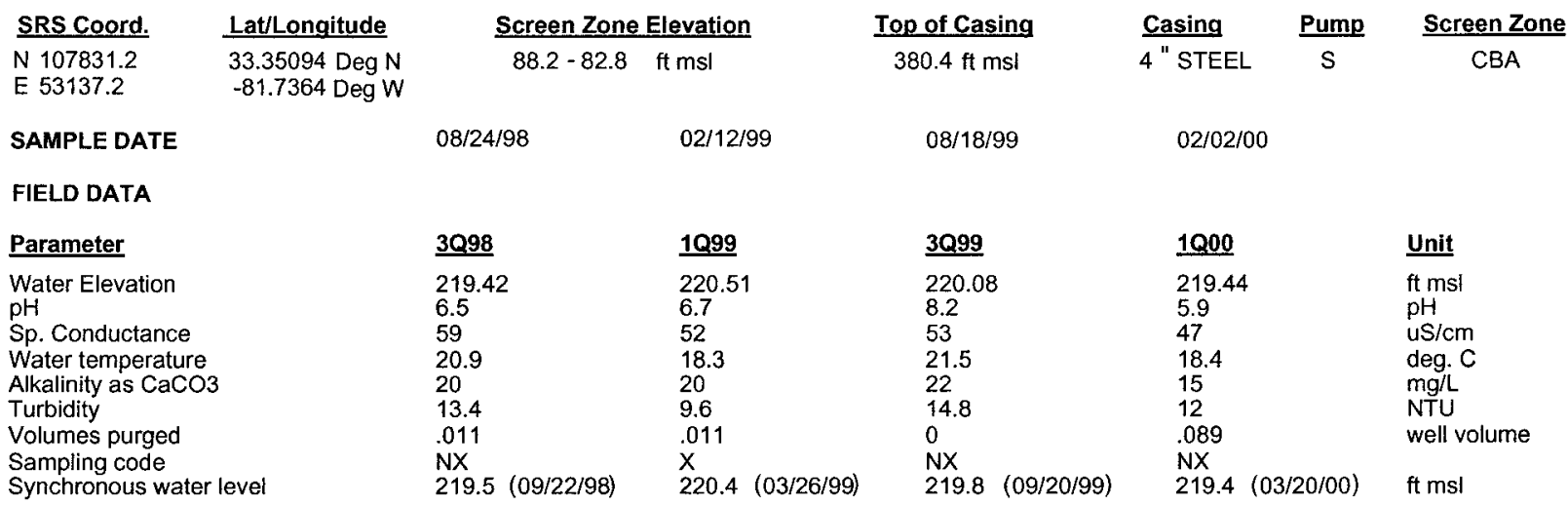

ANALYTICAL DATA

I. Groundwater Protection Standard

261 Appendix VIII/264 Appendix IX Hazardous Constituents

\begin{tabular}{|c|c|c|c|c|c|c|c|c|c|c|c|c|c|}
\hline ST & Parameter & $\underline{3 Q 98}$ & CLP EPA & $\underline{1099}$ & CLPP EPA & $\underline{3 Q 99}$ & CLPEPA & $\underline{1000}$ & CLPEPA & Filt. & $\underline{\text { DF }}$ & Unit & $\underline{\text { Lab }}$ \\
\hline \multicolumn{14}{|c|}{ Inorganics } \\
\hline & Barium, total recoverable & 18.8 & & $<10$ & $\mathrm{U}$ & 18 & & & & & & $\mathrm{ug} / \mathrm{L}$ & \\
\hline & Cyanide & $<10$ & U & $<10$ & $\mathrm{U}$ & $<10$ & $\mathrm{U}$ & & & & & $u g / L$ & \\
\hline & Lead, total recoverable & $<10$ & $\mathrm{U}$ & $<100$ & $\mathrm{U}$ & $<5$ & $U$ & & & & & $u g / L$ & \\
\hline & Nickel, total recoverable & $<50$ & $\mathrm{U}$ & $<50$ & $\mathrm{U}$ & $<12$ & JU I & & & & & $u g / L$ & \\
\hline & Selenium, total recoverable & $<10$ & $\mathrm{U}$ & $<200$ & $\mathrm{U}$ & $<5$ & U & & & & & $\mathrm{ug} / \mathrm{L}$ & \\
\hline \multicolumn{14}{|c|}{ Organics } \\
\hline & Chlorobenzene & $<5$ & $\mathrm{u}$ & $<5$ & $\mathrm{u}$ & $<1$ & $U$ & $<1$ & $\mathrm{U}$ & $<E Q L$ & 1 & $\mathrm{ug} / \mathrm{L}$ & ML \\
\hline & 1,1-Dichloroethane & $<5$ & $\mathrm{U}$ & $<5$ & U & $<1$ & U & $<1$ & U & $<E Q L$ & 1 & $\mathrm{ug} / \mathrm{L}$ & ML \\
\hline & 1,1-Dichloroethylene & $<5$ & $U$ & $<5$ & U & $<1$ & $\mathrm{U}$ & $<1$ & $\mathrm{U}$ & $<E Q L$ & 1 & $u g / L$ & ML \\
\hline & trans-1,2-Dichloroethylene & $<5$ & $\mathrm{U}$ & $<5$ & $\mathrm{U}$ & $<1$ & U & $<1$ & U & $<E Q L$ & 1 & $\mathrm{ug} / \mathrm{L}$ & ML \\
\hline & PCB 1016 & & & & & & & & & & & & \\
\hline & РCB 1221 & & & & & & & & & & & & \\
\hline & PCB 1232 & & & & & & & & & & & & \\
\hline & PCB 1242 & & & & & & & & & & & & \\
\hline & PCB 1248 & & & & & & & & & & & & \\
\hline & PCB 1254 & & & & & & & & & & & & \\
\hline & PCB 1260 & & & & & & & & & & & & \\
\hline & 1,1,2,2-Tetrachloroethane & $<5$ & $\mathrm{U}$ & $<5$ & $\mathrm{U}$ & $<1$ & $\mathrm{U}$ & $<1$ & $\mathrm{U}$ & $<E Q L$ & 1 & $u g / L$ & ML \\
\hline & Tetrachloroethylene & $<5$ & $\mathrm{U}$ & $<5$ & $U$ & $<1$ & $\mathrm{U}$ & $<1$ & $\mathrm{U}$ & $<E Q L$ & 1 & $\mathrm{ug} / \mathrm{L}$ & ML \\
\hline & $1,1,1$-Trichloroethane & $<5$ & $\mathrm{U}$ & $<5$ & $U$ & $<1$ & $\mathrm{U}$ & $<1$ & $\mathrm{U}$ & $<E Q L$ & 1 & $\mathrm{ug} / \mathrm{L}$ & $M L$ \\
\hline & Trichloroethylene & $<5$ & $\mathrm{U}$ & 4.12 & J 1 & 1.69 & $\mathrm{~J} \quad \mathrm{~K}$ & 1.56 & & $<5$ & 1 & $\mathrm{ug} / \mathrm{L}$ & $M L$ \\
\hline \multicolumn{14}{|c|}{ II. Monitoring Constituents } \\
\hline$\underline{\text { ST }}$ & Parameter & 3QQ96 & CLPEEA & 3097 & CLP EPA & $\underline{3098}$ & CLPEPA & $\underline{3099}$ & CLPEPA & Filt. & $\underline{\text { DF }}$ & Unit & $\underline{\text { Lab }}$ \\
\hline \multicolumn{14}{|c|}{ Inorganics } \\
\hline & Aluminum, total recoverable & 75.4 & $\mathrm{~J}$ & 344 & & $<200$ & U & $<200$ & $u$ & $<E Q L$ & 1 & ug/L & EX \\
\hline & Nitrate-nitrite as nitrogen & 1000 & $\mathrm{~J}$ & 2520 & & $<500$ & $U$ & 82.1 & J I & NDD & 5 & $\mathrm{ug} / \mathrm{L}$ & EX \\
\hline & Sodium, total recoverable & 5870 & & 3250 & & 5190 & & 4400 & & $<4600$ & 1 & $\mathrm{ug} / \mathrm{L}$ & EX \\
\hline & Sulfate & 271 & $\mathbf{J}$ & $<5000$ & $\cup$ & 217 & $J$ & $<200$ & U & $<E Q L$ & 1 & $\mathrm{ug} / \mathrm{L}$ & EX \\
\hline \multicolumn{14}{|c|}{ Radionuclides } \\
\hline & Gross alpha & .17 & UIJ & .93 & UI & .13 & UIJ & -.0774 & U & $<E Q L$ & 1 & $\mathrm{pCi} / \mathrm{L}$ & GP \\
\hline & Nonvolatile beta & 1.34 & $J$ & -.55 & UI & 2.23 & & .998 & $U$ & $<E Q L$ & 1 & $\mathrm{pCi} / \mathrm{L}$ & GP \\
\hline & Radium, total alpha-emitting & 0 & UI & 2.29 & UIJ & .31 & UI & 0 & $\mathrm{U}$ & $<E Q L$ & 1 & $\mathrm{pCi} / \mathrm{L}$ & GP \\
\hline
\end{tabular}

Notes: Concentrations in bold italics exceed the groundwater protection or monitoring constituent standards listed in Appendix A. Synchronous water levels are measured over a 3-5 day period or less. Dilution factors, Laboratory, and Filtered Data are for Groundwater Protection Standards First Quarter 2000 and Monitoring Constituents Third Quarter 1999 data only.

+ = exceeded the Groundwater Protection Standards (First Quarter 2000) or Monitoring Constituents (Third Quarter 1999) listed in Appendix A. 
Table D-4. Groundwater Monitoring Results for Plume Definition Wells, M-Area HWMF (Cont.) WELL MSB 86C

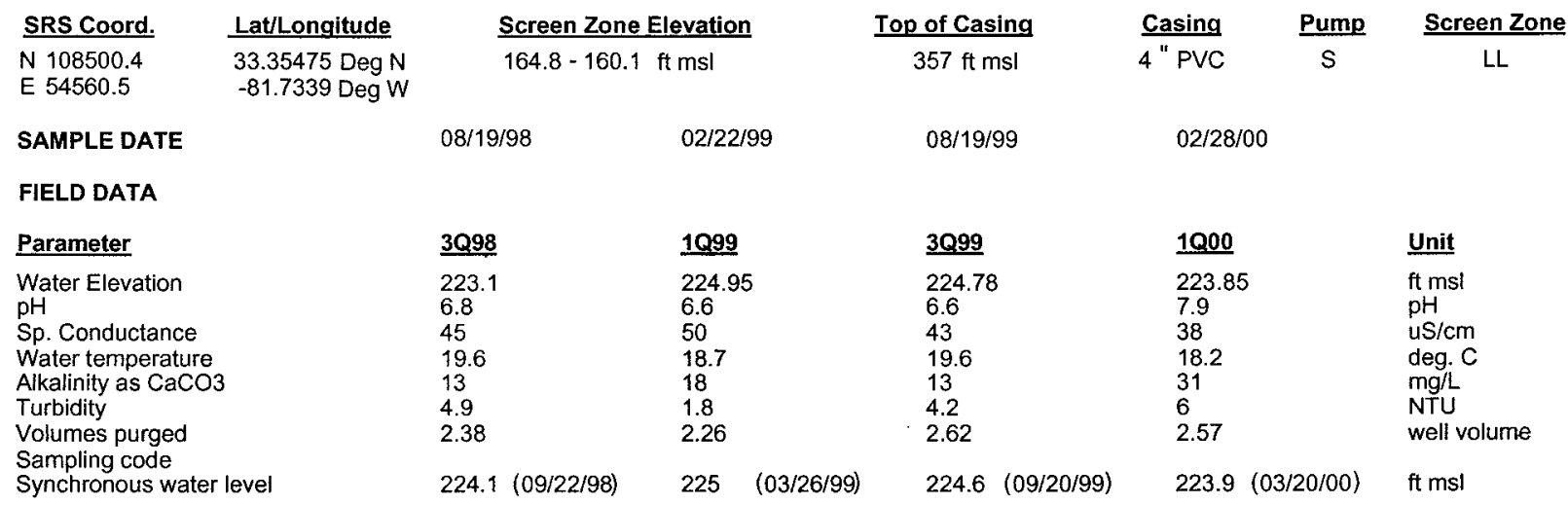

ANALYTICAL DATA

I. Groundwater Protection Standard

261 Appendix VIII/264 Appendix IX Hazardous Constituents

\begin{tabular}{|c|c|c|c|c|c|c|c|c|c|c|c|c|c|}
\hline$\underline{\mathbf{S T}}$ & Parameter & $\underline{3 Q 98}$ & CLP EPA & $1 Q 99$ & CLP EPA & 3Q99 & CLPEPA & 1000 & CLPEPA & Filt. & $\underline{\mathrm{DF}}$ & Unit & $\underline{\text { Lab }}$ \\
\hline \multicolumn{14}{|c|}{ Inorganics } \\
\hline & Barium, total recoverable & 17.8 & & 17.3 & & 17 & & & & & & $u g / L$ & \\
\hline & Cyanide & $<10$ & U & $<15.2$ & $\mathrm{U}$ & $<10$ & $U$ & & & & & $\mathrm{ug} / \mathrm{L}$ & \\
\hline & Lead, total recoverable & $<10$ & U & $<100$ & $\mathrm{U}$ & $<2.18$ & U V & & & & & $\mathrm{ug} / \mathrm{L}$ & \\
\hline & Nickel, total recoverable & $<13.9$ & $U$ & $<50$ & $\mathrm{U}$ & $<50$ & $\mathrm{U}$ & & & & & $\mathrm{ug} / \mathrm{L}$ & \\
\hline & Selenium, total recoverable & $<10$ & U & $<200$ & U & $<5$ & $\mathrm{U}$ & & & & & $\mathrm{ug} / \mathrm{L}$ & \\
\hline \multicolumn{14}{|c|}{ Organics } \\
\hline & Chlorobenzene & $<5$ & $U$ & $<5$ & $u$ & $<1$ & $U$ & $<1$ & $U$ & $<E Q L$ & 1 & $\mathrm{ug} / \mathrm{L}$ & ML \\
\hline & 1,1-Dichloroethane & $<5$ & $\mathrm{U}$ & $<5$ & $\mathrm{U}$ & $<1$ & U & $<1$ & $\mathrm{U}$ & $<E Q L$ & 1 & $\mathrm{ug} / \mathrm{L}$ & $\mathrm{ML}$ \\
\hline & 1,1-Dichloroethylene & $<5$ & $\mathrm{U}$ & $<5$ & $\mathrm{U}$ & $<1$ & $\mathrm{U}$ & $<1$ & $\mathrm{U}$ & $<E Q L$ & 1 & $u g / L$ & $M L$ \\
\hline & trans-1,2-Dichloroethylene & $<5$ & $\mathrm{U}$ & $<5$ & $\mathrm{U}$ & $<1$ & $\mathrm{U}$ & & & & & $\mathrm{ug} / \mathrm{L}$ & \\
\hline & PCB 1016 & & & & & & & & & & & & \\
\hline & PCB 1221 & & & & & & & & & & & & \\
\hline & PCB 1232 & & & & & & & & & & & & \\
\hline & PCB 1242 & & & & & & & & & & & & \\
\hline & PCB 1248 & & & & & & & & & & & & \\
\hline & PCB 1254 & & & & & & & & & & & & \\
\hline & PCB 1260 & & & & & & & & & & & & \\
\hline & 1,1,2,2-Tetrachloroethane & $<5$ & $U$ & $<5$ & $\mathrm{U}$ & $<1$ & $\mathrm{U}$ & $<1$ & U & $<\mathrm{EQL}$ & 1 & $\mathrm{ug} / \mathrm{L}$ & $\mathrm{ML}$ \\
\hline & Tetrachloroethylene & $<5$ & $\mathrm{U}$ & $<5$ & $\mathrm{U}$ & $<1$ & $\mathrm{U}$ & $<1$ & $\mathrm{U}$ & $<\mathrm{EQL}$ & 1 & $\mathrm{ug} / \mathrm{L}$ & ML \\
\hline & 1,1,1-Trichloroethane & $<5$ & $\mathrm{U}$ & $<5$ & $\mathrm{U}$ & $<1$ & $\mathrm{U}$ & $<1$ & $\mathrm{U}$ & $<E Q L$ & 1 & $\mathrm{ug} / \mathrm{L}$ & $\mathrm{ML}$ \\
\hline & Trichloroethylene & $<5$ & $\mathrm{U}$ & 1.03 & $\mathrm{~J} \quad \mathrm{I}$ & $<1$ & $\mathrm{U}$ & $<1$ & $\mathrm{U}$ & $<\mathrm{EQL}$ & 1 & $u g / L$ & $\mathrm{ML}$ \\
\hline \multicolumn{14}{|c|}{ II. Monitoring Constituents } \\
\hline$\underline{\mathbf{S T}}$ & Parameter & $\underline{3 Q 96}$ & CLPEPA & $\underline{3097}$ & CLP EPA & $\underline{3098}$ & CLPEPA & $\underline{30,99}$ & CLPEPA & Filt. & DF & Unit & Lab \\
\hline \multicolumn{14}{|c|}{ Inorganics } \\
\hline & Aluminum, total recoverable & 152 & & 170 & & 361 & & $<200$ & $\mathrm{U}$ & $<$ EQL & 1 & $u g / L$ & EX \\
\hline & Nitrate-nitrite as nitrogen & 1570 & & 1170 & & 1100 & & 1140 & & $<2400$ & 5 & $\mathrm{ug} / \mathrm{L}$ & EX \\
\hline & Sodium, total recoverable & 2550 & & 2590 & & 3120 & & 2300 & & $<4600$ & 1 & $\mathrm{ug} / \mathrm{L}$ & EX \\
\hline & Sulfate & 272 & $J$ & $<5000$ & $\mathrm{U}$ & $<200$ & $\mathrm{U}$ & 213 & & $<3000$ & 1 & $\mathrm{ug} / \mathrm{L}$ & EX \\
\hline \multicolumn{14}{|c|}{ Radionuclides } \\
\hline & Gross alpha & .643 & & -.51 & UI & 3.13 & $\checkmark$ & .889 & J 1 & NDD & 1 & $\mathrm{pCi} / \mathrm{L}$ & GP \\
\hline & Nonvolatile beta & .892 & Ul & -.8 & UI & 6.07 & J & 1.2 & $U$ & $<E Q L$ & 1 & $\mathrm{pCi} / \mathrm{L}$ & GP \\
\hline & Radium, total alpha-emitting & .4 & & 16.1 & $\mathrm{~J}$ & .19 & UI & .1 & $\mathrm{U}$ & $<E Q L$ & 1 & $\mathrm{pCi} / \mathrm{L}$ & GP \\
\hline
\end{tabular}

Notes: Concentrations in bold italics exceed the groundwater protection or monitoring constituent standards listed in Appendix A. Synchronous water levels are measured over a 3-5 day period or less. Dilution factors, Laboratory, and Filtered Data are for Groundwater Protection Standards First Quarter 2000 and Monitoring Constituents Third Quarter 1999 data only.

+ = exceeded the Groundwater Protection Standards (First Quarter 2000) or Monitoring Constituents (Third Quarter 1999) listed in Appendix A. 
Table D-4. Groundwater Monitoring Results for Plume Definition Wells, M-Area HWMF (Cont.) WELL MSB 87B

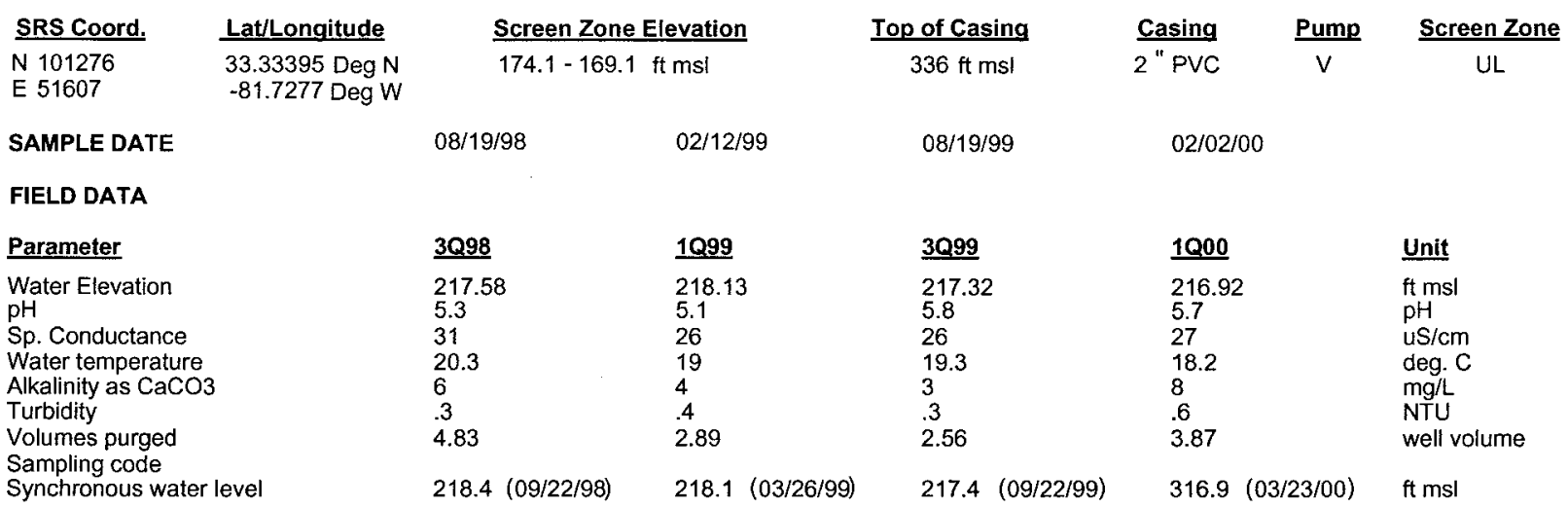

ANALYTICAL DATA

I. Groundwater Protection Standard

261 Appendix VIII/264 Appendix IX Hazardous Constituents

\begin{tabular}{|c|c|c|c|c|c|c|c|c|c|c|c|c|c|}
\hline$\underline{\text { ST }}$ & Parameter & $\underline{3 Q 98}$ & CLP EPA & $\underline{1 Q 99}$ & $\underline{\text { CLP EPA }}$ & $\underline{3 Q 99}$ & CLPEPA & 1000 & CLPEPA & Filt. & $\underline{\text { DF }}$ & $\underline{\text { Unit }}$ & Lab \\
\hline \multicolumn{14}{|c|}{ Inorganics } \\
\hline & Barium, total recoverable & 23.7 & & $<10$ & $u$ & 82 & & & & & & $\mathrm{ug} / \mathrm{L}$ & \\
\hline & Cyanide & $<10$ & $U$ & $<10$ & $\mathrm{U}$ & $<10$ & $\mathrm{U}$ & & & & & $\mathrm{ug} / \mathrm{L}$ & \\
\hline & Lead, total recoverable & $<10$ & U & $<100$ & $u$ & $<4.24$ & U V & & & & & $\mathrm{ug} / \mathrm{L}$ & \\
\hline & Nickel, total recoverable & $<12.5$ & U & $<50$ & $\mathrm{U}$ & $<50$ & $\mathrm{U}$ & & & & & $\mathrm{ug} / \mathrm{L}$ & \\
\hline & Selenium, total recoverable & $<10$ & $\mathrm{U}$ & $<200$ & U & $<5$ & $U$ & & & & & $\mathrm{ug} / \mathrm{L}$ & \\
\hline \multicolumn{14}{|c|}{ Organics } \\
\hline & Chlorobenzene & $<5$ & $U$ & $<5$ & $u$ & $<1$ & $\mathrm{U}$ & $<1$ & $\mathbf{U}$ & $<E Q L$ & 1 & $\mathrm{ug} / \mathrm{L}$ & $\mathrm{ML}$ \\
\hline & 1,1-Dichloroethane & $<5$ & $\mathrm{U}$ & $<5$ & $\mathrm{U}$ & $<1$ & $\mathrm{U}$ & $<1$ & $\mathrm{U}$ & $<E Q L$ & 1 & $\mathrm{ug} / \mathrm{L}$ & $M L$ \\
\hline & 1,1-Dichloroethylene & $<5$ & $\mathrm{U}$ & $<5$ & $U$ & $<1$ & U & $<1$ & $\mathrm{U}$ & $<E Q L$ & 1 & $\mathrm{ug} / \mathrm{L}$ & $\mathrm{ML}$ \\
\hline & trans-1,2-Dichloroethylene & $<5$ & U & $<5$ & $\mathrm{U}$ & $<1$ & $U$ & $<1$ & $U$ & $<E Q L$ & 1 & $\mathrm{ug} / \mathrm{L}$ & $M L$ \\
\hline & $\begin{array}{l}\text { PCB } 1016 \\
\text { PCB } 1221\end{array}$ & & & & & & & & & & & & \\
\hline & PCB 1232 & & & & & & & & & & & & \\
\hline & PCB 1242 & & & & & & & & & & & & \\
\hline & PCB 1248 & & & & & & & & & & & & \\
\hline & РCB 1254 & & & & & & & & & & & & \\
\hline & PCB 1260 & & & & & & & & & & & & \\
\hline & 1,1,2,2-Tetrachloroethane & $<5$ & $\mathrm{U}$ & $<5$ & $\mathrm{U}$ & $<1$ & $U$ & $<1$ & $\mathrm{U}$ & $<\mathrm{EQL}$ & 1 & $\mathrm{ug} / \mathrm{L}$ & ML \\
\hline & Tetrachloroethylene & $<5$ & $\mathrm{U}$ & $<5$ & $\mathrm{U}$ & $<1$ & $\mathrm{U}$ & $<1$ & $\mathrm{U}$ & $<E Q L$ & 1 & $\mathrm{ug} / \mathrm{L}$ & $\mathrm{ML}$ \\
\hline & 1,1,1-Trichloroethane & $<5$ & $\mathrm{U}$ & $<5$ & $\mathrm{U}$ & $<1$ & $U$ & $<1$ & $U$ & $<$ EQL & 1 & $\mathrm{ug} / \mathrm{L}$ & ML \\
\hline & Trichloroethylene & $<5$ & $\mathrm{U}$ & $<5$ & $\mathrm{U}$ & $<1$ & U & $<1$ & U & $<\mathrm{EQL}$ & 1 & ug/L & $\mathrm{ML}$ \\
\hline \multicolumn{14}{|c|}{ II. Monitoring Constituents } \\
\hline$\underline{\mathbf{S T}}$ & Parameter & $\underline{3 Q 96}$ & CLPEPA & $\underline{3097}$ & CLPEEA & $\underline{3 Q 98}$ & CLPEPA & $\underline{3099}$ & CLPEPA & Filt. & $\underline{\text { DF }}$ & Unit & $\underline{\text { Lab }}$ \\
\hline \multicolumn{14}{|c|}{ Inorganics } \\
\hline & Aluminum, total recoverable & $<41.3$ & $\mathrm{u}$ & 23.4 & & $<200$ & $\mathrm{U}$ & $<200$ & U & $<E Q L$ & 1 & $u g / L$ & EX \\
\hline & Nitrate-nitrite as nitrogen & 920 & & 785 & & 569 & & 598 & & $<2400$ & 5 & $\mathrm{ug} / \mathrm{L}$ & EX \\
\hline & Sodium, total recoverable & 1480 & & 1460 & & 1570 & & 1700 & & $<4600$ & 1 & $\mathrm{ug} / \mathrm{L}$ & EX \\
\hline & Sulfate & 1790 & & $<5000$ & U & 1270 & & 1280 & & $<3000$ & 1 & $\mathrm{ug} / \mathrm{L}$ & EX \\
\hline \multicolumn{14}{|c|}{ Radionuclides } \\
\hline & Gross alpha & .538 & UI & 0 & UI & .01 & UIJ & .498 & $U$ & $<E Q L$ & 1 & $\mathrm{pCi} / \mathrm{L}$ & $G P$ \\
\hline & Nonvolatile beta & .744 & UI & -1.62 & UI & 1.16 & UIJ & .505 & $\mathrm{U}$ & $<$ EQL & 1 & $\mathrm{pCi} / \mathrm{L}$ & GP \\
\hline & Radium, total alpha-emitting & .1 & UI & 4.04 & $\mathrm{~J}$ & .05 & UI & -.1 & $\mathrm{U}$ & $<E Q L$ & 1 & $\mathrm{pCi} / \mathrm{L}$ & GP \\
\hline
\end{tabular}

Notes: Concentrations in bold italics exceed the groundwater protection or monitoring constituent standards listed in Appendix A. Synchronous water levels are measured over a 3-5 day period or less. Dilution factors, Laboratory, and Filtered Data are for Groundwater Protection Standards First Quarter 2000 and Monitoring Constituents Third Quarter 1999 data only.

$+=$ exceeded the Groundwater Protection Standards (First Quarter 2000) or Monitoring Constituents (Third Quarter 1999) listed in Appendix A. 
Table D-4. Groundwater Monitoring Results for Plume Definition Wells, M-Area HWMF (Cont.) WELL MSB 87C

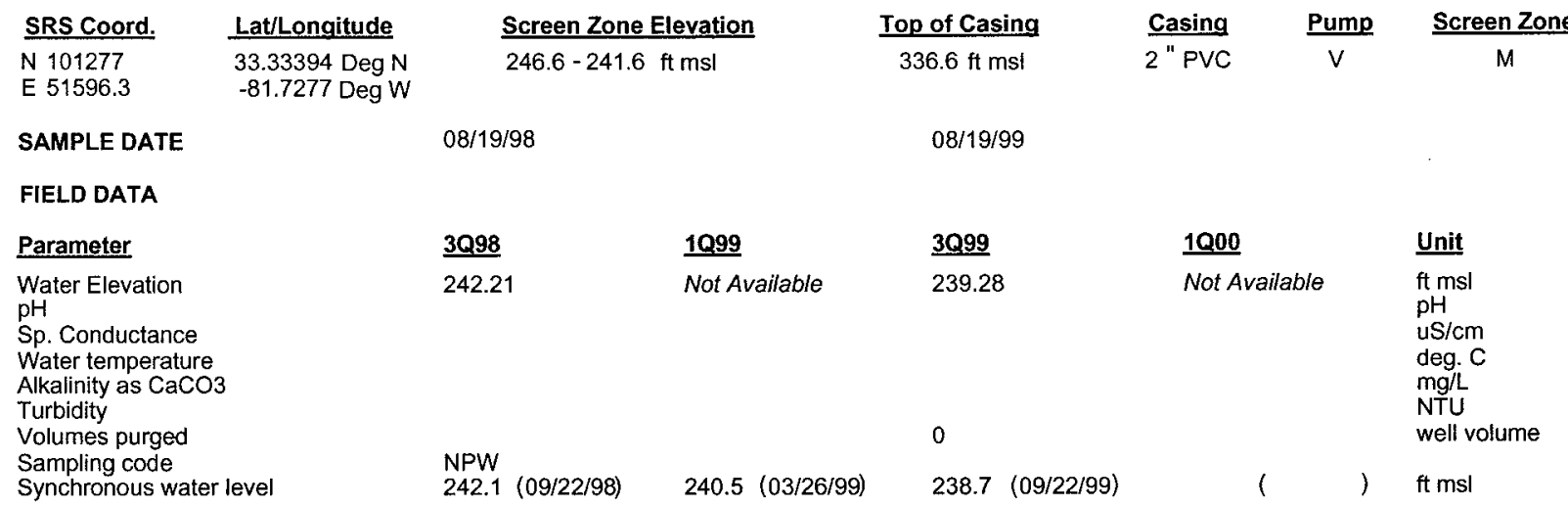

ANALYTICAL DATA

I. Groundwater Protection Standard

261 Appendix VIII/264 Appendix IX Hazardous Constituents

ST $3 \mathrm{Q}$

\section{CLPE}

$\underline{1099}$

CLPEPA

$\underline{3 Q 99}$

CLPEPA

1000

Barium, total recoverable

Cyanide

Lead, total recoverable

Nickel, total recoverable

Selenium, total recoverable

Organics

Chlorobenzene

1,1-Dichloroethane

1,1-Dichloroethylene

trans-1,2-Dichloroethylene

PCB 1016

PCB 1221

PCB 1232

PCB 1242

PCB 1248

PCB 1254

PCB 1260

1,1,2,2-Tetrachloroethane

Tetrachloroethylene

1,1,1-Trichloroethane

Trichloroethylene

II. Monitoring Constituents

\begin{tabular}{|c|c|c|c|c|c|c|c|c|c|c|c|c|}
\hline ST & Parameter & 3Q96 & CLPEPA & $\underline{3097}$ & CLP EPA & $\underline{3098}$ & CLP EPA & 3099 & CLPEPA & Filt. & $\underline{\text { DF }}$ & Unit \\
\hline \multicolumn{13}{|c|}{ Inorganics } \\
\hline & Aluminum, total recoverable & $<41.8$ & $\mathrm{U}$ & & & & & & & & & $\mathrm{ug} / \mathrm{L}$ \\
\hline & Nitrate-nitrite as nitrogen & 770 & & & & & & & & & & ug/L \\
\hline & Sodium, total recoverable & 3120 & & & & & & & & & & $\mathrm{ug} / \mathrm{L}$ \\
\hline & Sulfate & 458 & $J$ & & & & & & & & & ug/L \\
\hline \multicolumn{13}{|c|}{ Radionuclides } \\
\hline & Gross alpha & .102 & UI & & & & & & & & & $\mathrm{pCi} / \mathrm{L}$ \\
\hline & Nonvolatile beta & .361 & UI & & & & & & & & & $\mathrm{pCi} / \mathrm{L}$ \\
\hline & Radium, total alpha-emitting & -.1 & UI & & & & & & & & & $\mathrm{pCi} / \mathrm{L}$ \\
\hline
\end{tabular}

Notes: Concentrations in bold italics exceed the groundwater protection or monitoring constituent standards listed in Appendix A. Synchronous water levels are measured over a 3-5 day period or less. Dilution factors, Laboratory, and Filtered Data are for Groundwater Protection Standards First Quarter 2000 and Monitoring Constituents Third Quarter 1999 data only.

$+=$ exceeded the Groundwater Protection Standards (First Quarter 2000) or Monitoring Constituents (Third Quarter 1999) listed in Appendix A. 
Table D-4. Groundwater Monitoring Results for Plume Definition Wells, M-Area HWMF (Cont.) WELL MSB 88B

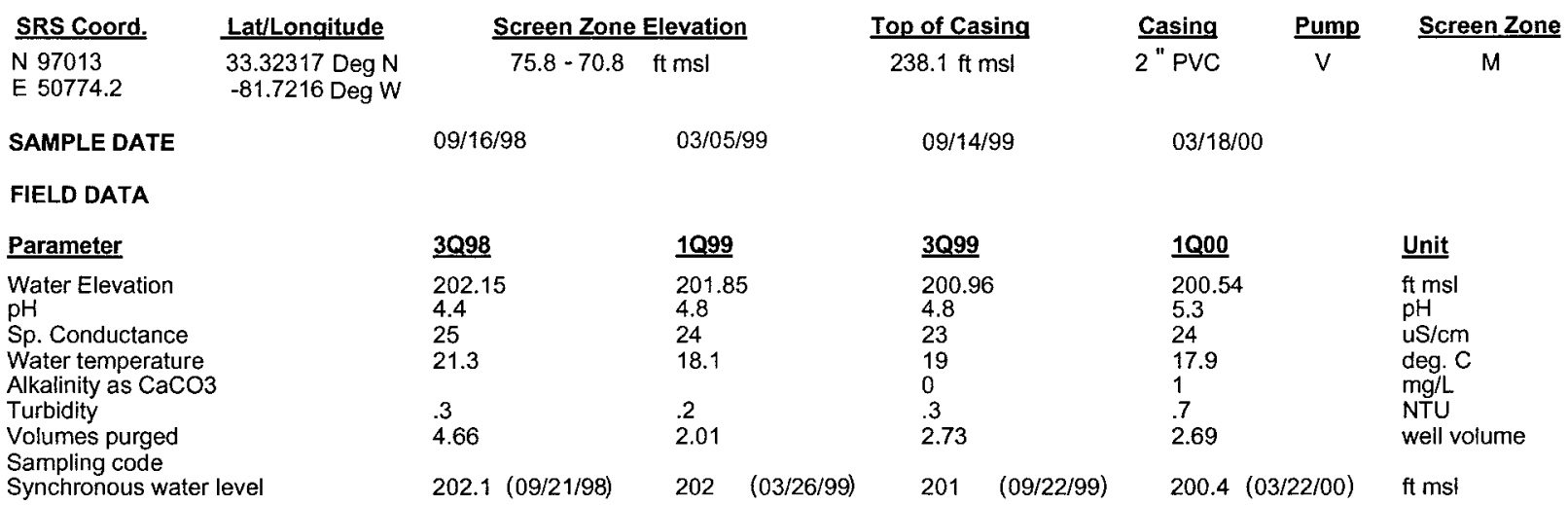

ANALYTICAL DATA

I. Groundwater Protection Standard

261 Appendix VIII/264 Appendix IX Hazardous Constituents

\begin{tabular}{|c|c|c|c|c|c|c|c|c|c|c|c|c|c|}
\hline$\underline{\mathrm{ST}}$ & Parameter & $\underline{3098}$ & CLP EPA & 1099 & CLPEPA & $\underline{3099}$ & CLPEEA & 1000 & CLPEPA & Filt. & DF & Unit & $\underline{\text { Lab }}$ \\
\hline \multicolumn{14}{|c|}{ Inorganics } \\
\hline & Barium, total recoverable & 7.11 & $\sqrt{ }$ & 4.98 & J I & 5.04 & J 1 & & & & & $u g / L$ & \\
\hline & Cyanide & $<10$ & $\mathrm{U}$ & $<10$ & $U$ & $<10$ & $\mathrm{U}$ & & & & & ug/L & \\
\hline & Lead, total recoverable & 46.4 & $\mathrm{~J}$ & $<100$ & $\mathrm{U}$ & $<10$ & $\mathrm{U}$ & & & & & ug/L & \\
\hline & Nickel, total recoverable & $<50$ & $\mathrm{U}$ & $<50$ & $U$ & $<50$ & $\mathrm{U}$ & & & & & ug/L & \\
\hline & Selenium, total recoverable & $<200$ & $\mathrm{U}$ & $<200$ & $U$ & $<10$ & $\mathbf{U}$ & & & & & ug/L & \\
\hline \multicolumn{14}{|c|}{ Organics } \\
\hline & Chlorobenzene & $<5$ & $\mathrm{U}$ & $<5$ & $U$ & $<1$ & U & $<1$ & $\mathrm{U}$ & $<\mathrm{EQL}$ & 1 & $u g / L$ & ML \\
\hline & 1,1-Dichloroethane & $<5$ & $U$ & $<5$ & U & $<1$ & $U$ & $<1$ & U & $<E Q L$ & 1 & ug/L & ML \\
\hline & 1,1-Dichloroethylene & $<5$ & $\mathrm{U}$ & $<5$ & $\mathrm{U}$ & $<1$ & $\mathrm{U}$ & $<1$ & $\mathrm{U}$ & $<\mathrm{EQL}$ & 1 & $\mathrm{ug} / \mathrm{L}$ & ML \\
\hline & trans-1,2-Dichloroethylene & $<5$ & $\mathrm{U}$ & $<5$ & $\mathrm{U}$ & $<1$ & $\mathrm{U}$ & $<1$ & $\mathrm{U}$ & $<\mathrm{EQL}$ & 1 & $\mathrm{ug} / \mathrm{L}$ & $\mathrm{ML}$ \\
\hline & PCB 1016 & & & & & & & & & & & & \\
\hline & PCB 1221 & & & & & & & & & & & & \\
\hline & PCB 1232 & & & & & & & & & & & & \\
\hline & PCB 1242 & & & & & & & & & & & & \\
\hline & PCB 1248 & & & & & & & & & & & & \\
\hline & PCB 1254 & & & & & & & & & & & & \\
\hline & PCB 1260 & & & & & & & & & & & & \\
\hline & 1,1,2,2-Tetrachloroethane & $<5$ & $U$ & $<5$ & $U$ & $<1$ & $U$ & $<1$ & $\mathrm{U}$ & $<\mathrm{EQL}$ & 1 & $\mathrm{ug} / \mathrm{L}$ & ML \\
\hline & Tetrachloroethylene & $<5$ & $U$ & $<5$ & $U$ & $<1$ & $U$ & $<1$ & $U$ & $<\mathrm{EQL}$ & 1 & $\mathrm{ug} / \mathrm{L}$ & ML \\
\hline & $1,1,1$-Trichloroethane & $<5$ & $U$ & $<5$ & $\mathrm{U}$ & $<1$ & $U$ & $<1$ & $U$ & $<\mathrm{EQL}$ & 1 & $\mathrm{ug} / \mathrm{L}$ & $M L$ \\
\hline+ & Trichloroethylene & 24.1 & & 25.5 & & 31.3 & $\mathrm{~J} \quad \mathrm{~K}$ & 27.3 & & $>5$ & 1 & $\mathrm{ug} / \mathrm{L}$ & ML \\
\hline \multicolumn{14}{|c|}{ II. Monitoring Constituents } \\
\hline$\underline{\text { ST }}$ & Parameter & $\underline{3096}$ & CLPEPA & $\underline{3097}$ & CLPPEPA & $\underline{3098}$ & CLPPEPA & 3099 & CLPEPA & Filt. & $\underline{\text { DF }}$ & Unit & $\underline{\text { Lab }}$ \\
\hline \multicolumn{14}{|c|}{ Inorganics } \\
\hline & Aluminum, total recoverable & $<20$ & $\mathrm{U}$ & 79.7 & & $<200$ & $\mathrm{U}$ & $<200$ & U & $<\mathrm{EQL}$ & 1 & $u g / L$ & EX \\
\hline & Nitrate-nitrite as nitrogen & 990 & & $<100$ & $U$ & 710 & & 800 & & $<2400$ & 5 & $\mathrm{ug} / \mathrm{L}$ & $\mathrm{EX}$ \\
\hline & Sodium, total recoverable & 1630 & & 1670 & & 1740 & & 1750 & & $<4600$ & 1 & ug/l & EX \\
\hline & Sulfate & 1900 & & $<5000$ & $\mathbf{U}$ & 1740 & & 1940 & & $<3000$ & 1 & $\mathrm{ug} / \mathrm{L}$ & $\mathrm{EX}$ \\
\hline \multicolumn{14}{|c|}{ Radionuclides } \\
\hline & Gross alpha & .238 & Ul & .71 & Ut & 1.24 & & .148 & $\mathrm{U}$ & $<$ EQL & 1 & $\mathrm{pCi} / \mathrm{L}$ & GP \\
\hline & Nonvolatile beta & .58 & UI & -.13 & UI & 1.31 & UI & 1.49 & $\mathrm{U}$ & $<\mathrm{EQL}$ & 1 & $\mathrm{pCi} / \mathrm{L}$ & GP \\
\hline & Radium, total alpha-emitting & -.5 & UI & 1.39 & UIJ & .17 & UIJ & 0 & $U$ & $<E Q L$ & 1 & $\mathrm{pCi} / \mathrm{L}$ & GP \\
\hline
\end{tabular}

Notes: Concentrations in bold italics exceed the groundwater protection or monitoring constituent standards listed in Appendix A. Synchronous water levels are measured over a 3-5 day period or less. Dilution factors, Laboratory, and Filtered Data are for Groundwater Protection Standards First Quarter 2000 and Monitoring Constituents Third Quarter 1999 data only.

$+=$ exceeded the Groundwater Protection Standards (First Quarter 2000) or Monitoring Constituents (Third Quarter 1999) listed in Appendix A. 
Table D-4. Groundwater Monitoring Results for Plume Definition Wells, M-Area HWMF (Cont.) WELL MSB 88C

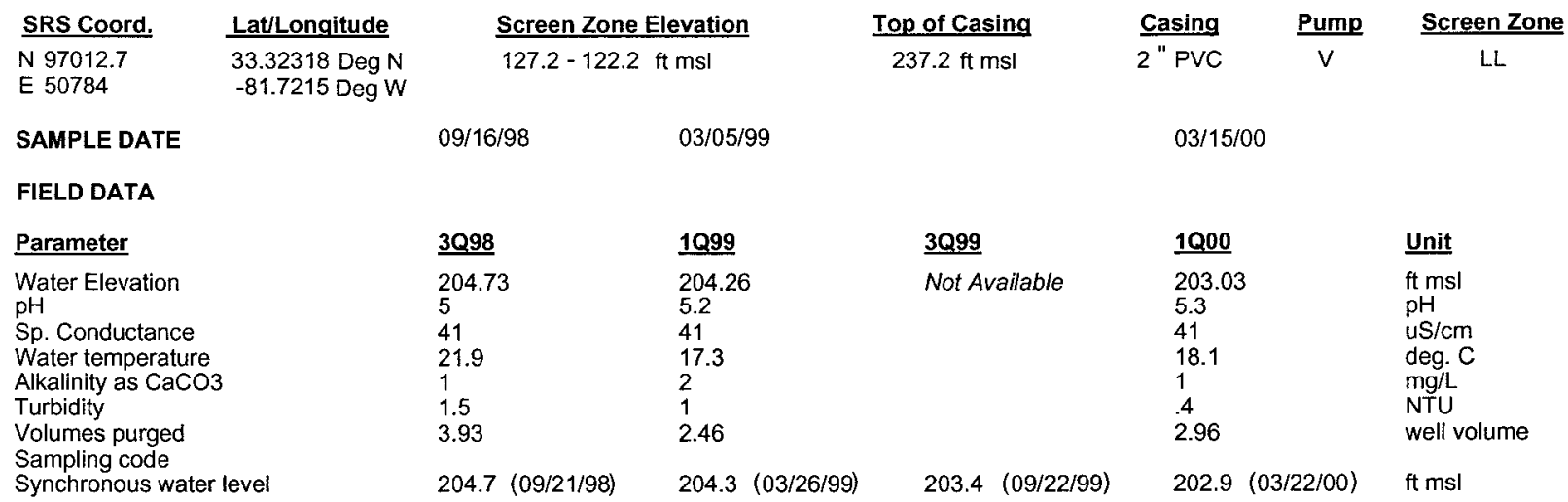

ANALYTICAL DATA

I. Groundwater Protection Standard

261 Appendix VIII/264 Appendix IX Hazardous Constituents

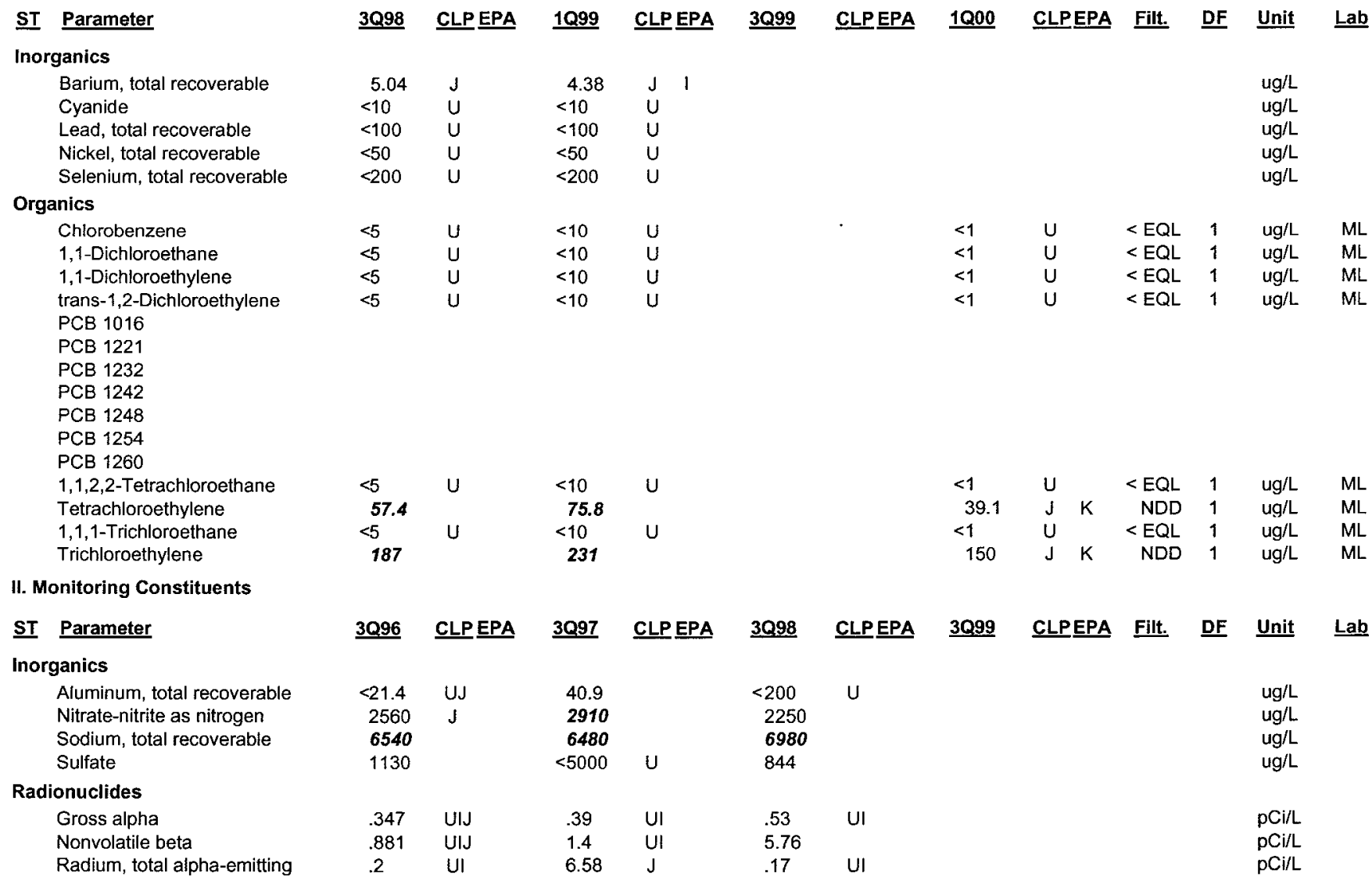

Notes: Concentrations in bold italics exceed the groundwater protection or monitoring constituent standards listed in Appendix A. Synchronous water levels are measured over a 3-5 day period or less. Dilution factors, Laboratory, and Filtered Data are for Groundwater Protection Standards First Quarter 2000 and Monitoring Constituents Third Quarter 1999 data only.

$+=$ exceeded the Groundwater Protection Standards (First Quarter 2000) or Monitoring Constituents (Third Quarter 1999) listed in Appendix A. 
Table D-4. Groundwater Monitoring Results for Plume Definition Wells, M-Area HWMF (Cont.) WELL MSB 89B

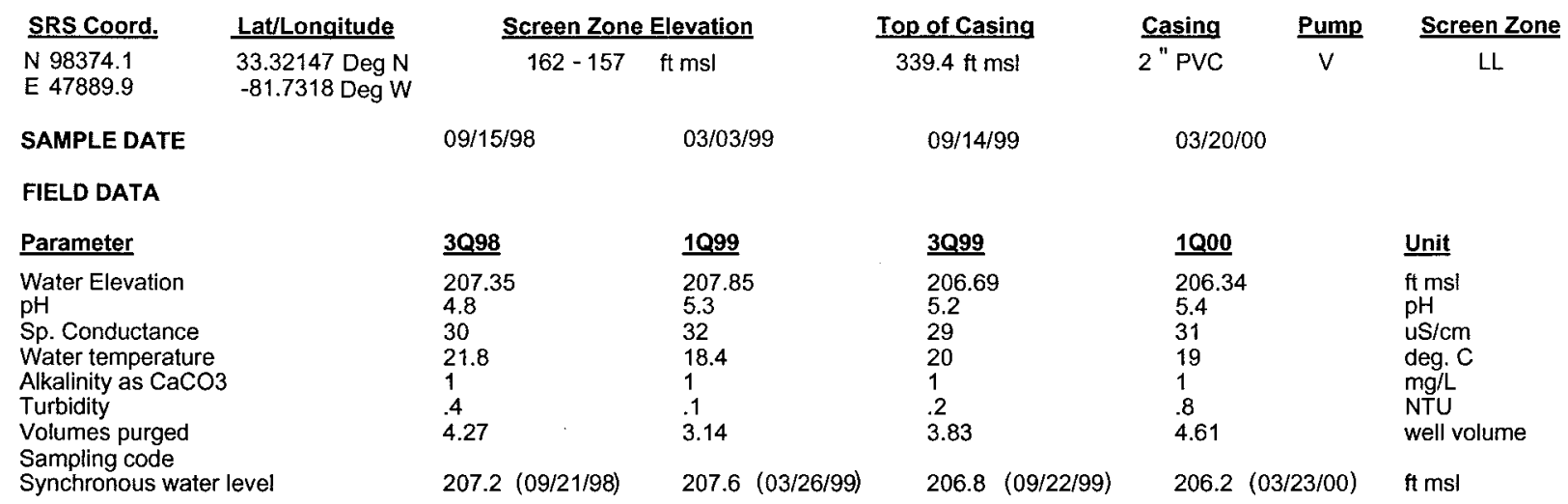

\section{ANALYTICAL DATA}

I. Groundwater Protection Standard

261 Appendix VIII/264 Appendix IX Hazardous Constituents

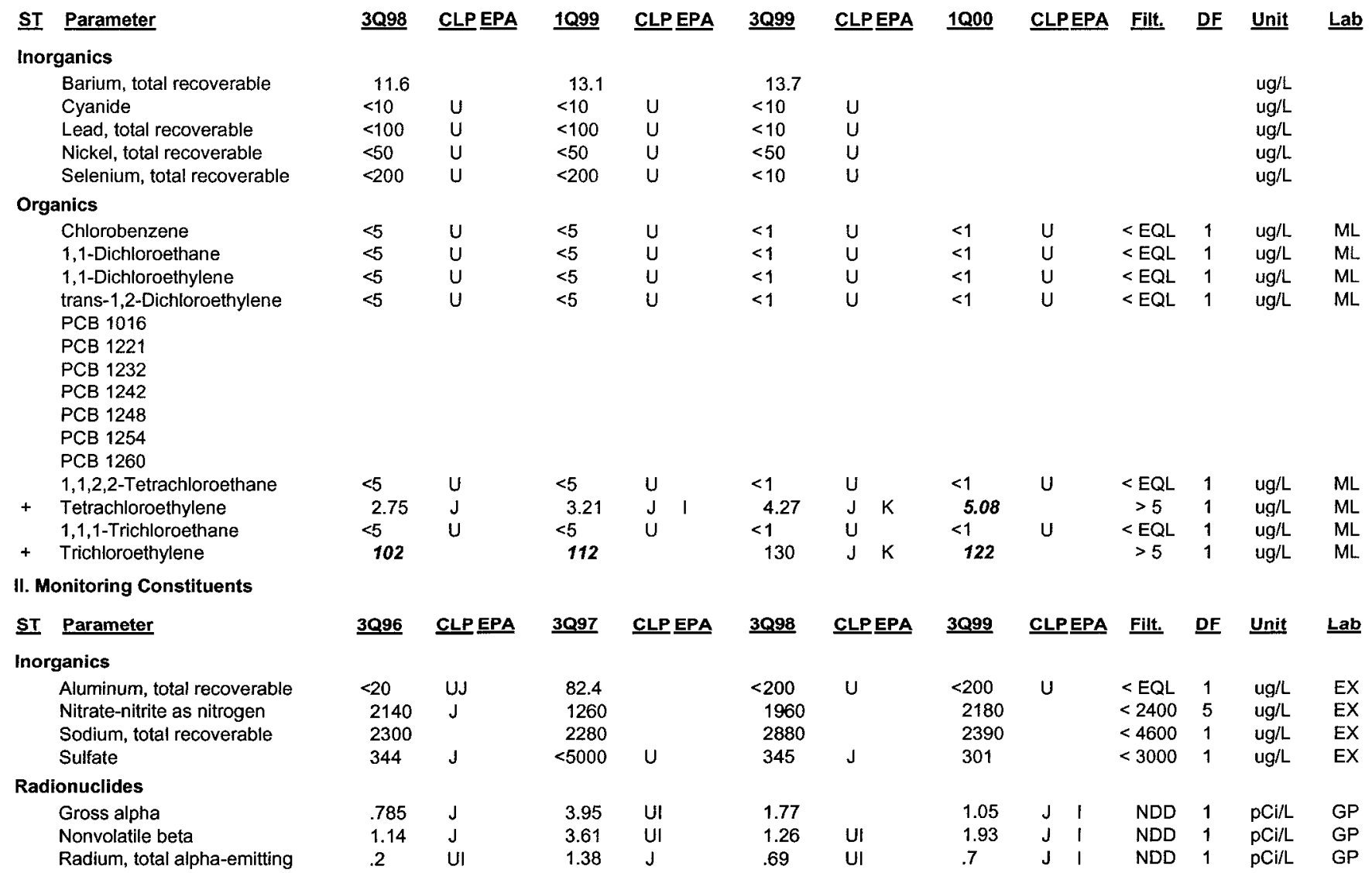

Notes: Concentrations in bold italics exceed the groundwater protection or monitoring constituent standards listed in Appendix A. Synchronous water levels are measured over a 3-5 day period or less. Dilution factors, Laboratory, and Filtered Data are for Groundwater Protection Standards First Quarter 2000 and Monitoring Constituents Third Quarter 1999 data only.

+= exceeded the Groundwater Protection Standards (First Quarter 2000) or Monitoring Constituents (Third Quarter 1999) listed in Appendix A. 
Table D-4. Groundwater Monitoring Results for Plume Definition Wells, M-Area HWMF (Cont.)

WELL SRW 2A

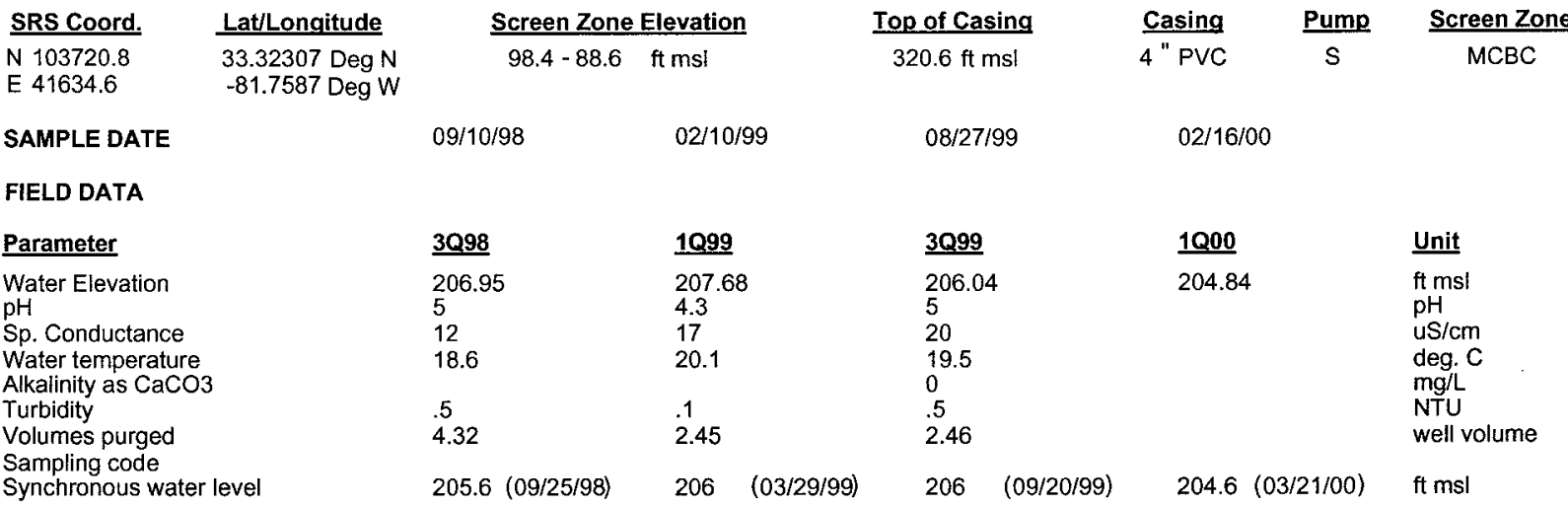

ANALYTICAL DATA

I. Groundwater Protection Standard

261 Appendix VIII/264 Appendix IX Hazardous Constituents

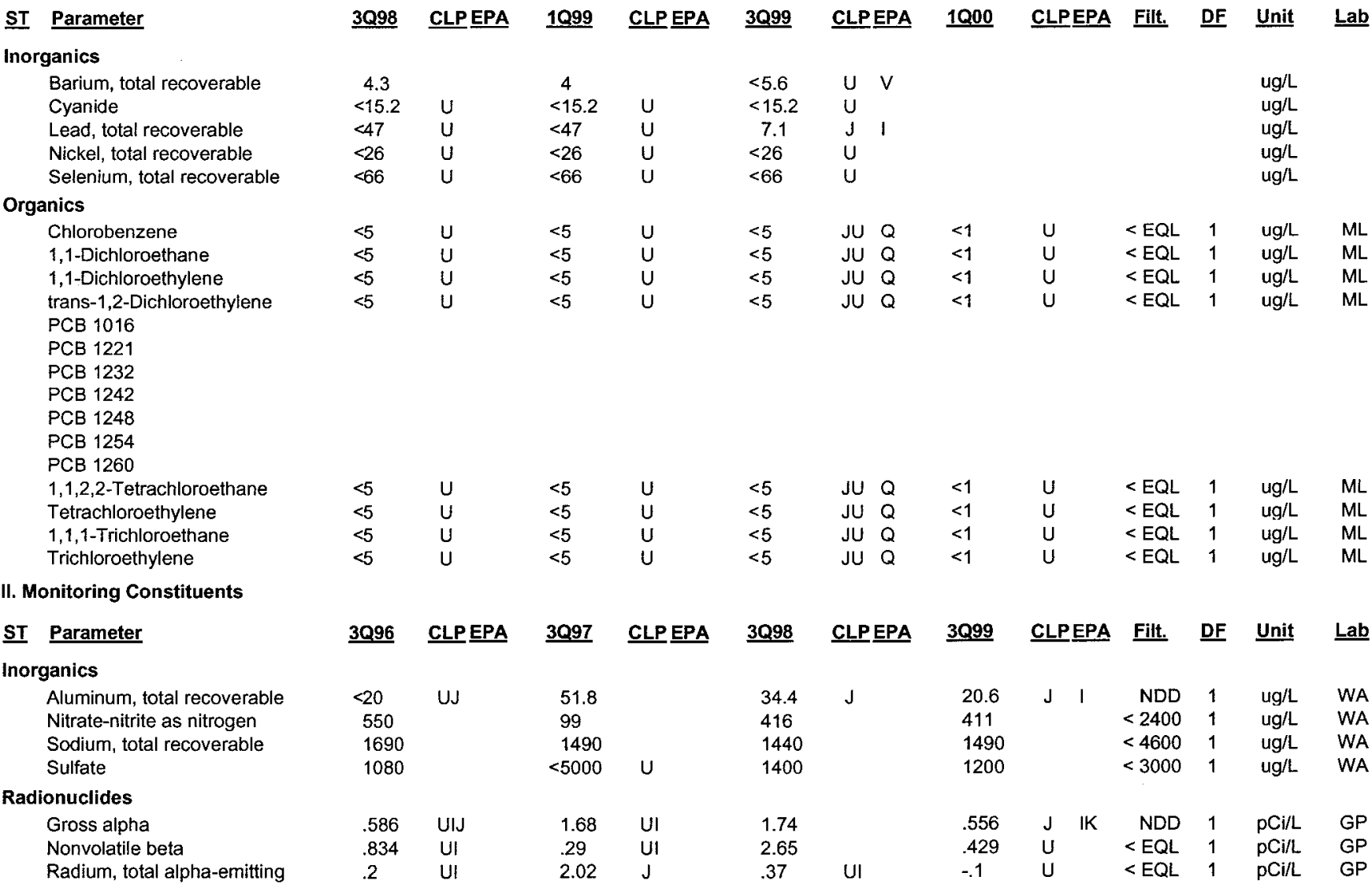

Notes: Concentrations in bold italics exceed the groundwater protection or monitoring constituent standards listed in Appendix A. Synchronous water levels are measured over a 3-5 day period or less. Dilution factors, Laboratory, and Filtered Data are for Groundwater Protection Standards First Quarter 2000 and Monitoring Constituents Third Quarter 1999 data only.

$+=$ exceeded the Groundwater Protection Standards (First Quarter 2000) or Monitoring Constituents (Third Quarter 1999) listed in Appendix A. 
Table D-4. Groundwater Monitoring Results for Plume Definition Wells, M-Area HWMF (Cont.) WELL SRW 2B

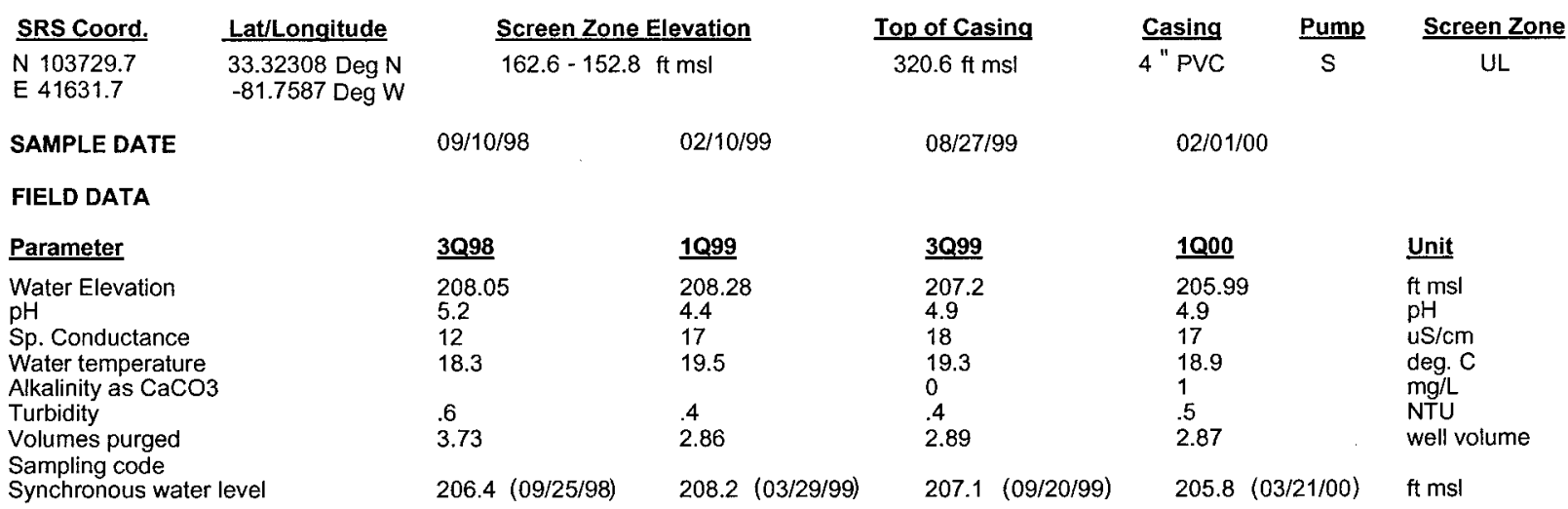

ANALYTICAL DATA

I. Groundwater Protection Standard

261 Appendix VIII/264 Appendix IX Hazardous Constituents

\begin{tabular}{|c|c|c|c|c|c|c|c|c|c|c|c|c|c|c|}
\hline$\underline{\text { ST }}$ & Parameter & $\underline{3 Q 98}$ & CLP EPA & $\underline{1099}$ & $\underline{\text { CLP EPA }}$ & $\underline{3 Q 99}$ & $\underline{\text { CLP }}$ & EPA & $1 \mathrm{Q00}$ & $\underline{\text { CLPEPA }}$ & Filt. & $\underline{D F}$ & Unit & $\underline{\text { Lab }}$ \\
\hline & ganics & & & & & & & & & & & & & \\
\hline & Barium, total recoverable & 6 & & 6.1 & & $<6.1$ & $\mathrm{u}$ & V & & & & & $u g / L$ & \\
\hline & Cyanide & $<15.2$ & U & $<15.2$ & $\mathrm{U}$ & $<15.2$ & $\mathrm{U}$ & & & & & & $u g / L$ & \\
\hline & Lead, total recoverable & 7.9 & $J$ & 6.6 & J 1 & 9.2 & $\mathrm{~J}$ & 1 & & & & & $u g / L$ & \\
\hline & Nickel, total recoverable & $<26$ & $\mathrm{U}$ & $<26$ & $\mathrm{U}$ & $<26$ & $\mathrm{U}$ & & & & & & $\mathrm{ug} / \mathrm{L}$ & \\
\hline & Selenium, total recoverable & $<66$ & U & $<66$ & $\mathrm{U}$ & $<66$ & $U$ & & & & & & $u g / L$ & \\
\hline Org & anics & & & & & & & & & & & & & \\
\hline & Chlorobenzene & $<5$ & $\mathrm{U}$ & $<5$ & $\mathrm{u}$ & $<5$ & JU & $Q$ & $<1$ & JU & $<E Q L$ & 1 & $u g / L$ & ML \\
\hline & 1,1-Dichloroethane & $<5$ & $\mathrm{U}$ & $<5$ & $\mathrm{U}$ & $<5$ & JU & $Q$ & $<1$ & JU & $<E Q L$ & 1 & $\mathrm{ug} / \mathrm{L}$ & ML \\
\hline & 1,1-Dichloroethylene & $<5$ & $\mathrm{U}$ & $<5$ & $\mathrm{U}$ & $<5$ & JU & $Q$ & $<1$ & JU & $<E Q L$ & 1 & $u g / L$ & ML \\
\hline & trans-1,2-Dichloroethylene & $<5$ & $\mathrm{U}$ & $<5$ & $U$ & $<5$ & JU & $Q$ & $<1$ & JU & $<E Q L$ & 1 & $u g / L$ & ML \\
\hline & PCB 1016 & & & & & & & & & & & & & \\
\hline & РCB 1221 & & & & & & & & & & & & & \\
\hline & PCB 1232 & & & & & & & & & & & & & \\
\hline & PCB 1242 & & & & & & & & & & & & & \\
\hline & РCB 1248 & & & & & & & & & & & & & \\
\hline & PCB 1254 & & & & & & & & & & & & & \\
\hline & PCB 1260 & & & & & & & & & & & & & \\
\hline & $1,1,2,2$-Tetrachloroethane & $<5$ & $\mathrm{U}$ & $<5$ & $\mathrm{U}$ & $<5$ & JU & $Q$ & $<1$ & JU & $<E Q L$ & 1 & $u g / L$ & ML \\
\hline & Tetrachloroethylene & $<5$ & $\mathrm{U}$ & $<5$ & $\mathrm{U}$ & $<5$ & JU & $Q$ & $<1$ & JU & $<E Q L$ & 1 & $u g / L$ & ML \\
\hline & 1,1,1-Trichloroethane & $<5$ & $\mathrm{U}$ & $<5$ & $\mathrm{U}$ & $<5$ & JU & $Q$ & $<1$ & JU & $<E Q L$ & 1 & $\mathrm{ug} / \mathrm{L}$ & ML \\
\hline & Trichloroethylene & 1.4 & $J$ & $<5$ & $\mathrm{U}$ & $<5$ & JU & $Q$ & $<1$ & JU & $<E Q L$ & 1 & ug/L & ML \\
\hline II. $\mathbf{M}$ & onitoring Constituents & & & & & & & & & & & & & \\
\hline$\underline{\text { ST }}$ & Parameter & $\underline{3 Q 96}$ & CLP EPA & $\underline{3097}$ & CLPEPA & $\underline{3098}$ & CLP & EPA & $\underline{3099}$ & CLPEPA & Filt. & $\underline{\text { DF }}$ & Unit & Lab \\
\hline Inor & ganics & & & & & & & & & & & & & \\
\hline+ & Aluminum, total recoverable & 29 & & 18.7 & $J$ & 28.2 & $\mathrm{~J}$ & & 1420 & & $>100$ & 1 & ug/L & WA \\
\hline & Nitrate-nitrite as nitrogen & 830 & & 154 & & 650 & & & 674 & & $<2400$ & 1 & $\mathrm{ug} / \mathrm{L}$ & WA \\
\hline & Sodium, total recoverable & 1430 & & 1400 & & 1440 & & & 1460 & & $<4600$ & 1 & $u g / L$ & WA \\
\hline & Sulfate & 446 & & $<5000$ & $U$ & 411 & & & 429 & & $<3000$ & 1 & ug/L & WA \\
\hline ad & onuclides & & & & & & & & & & & & & \\
\hline & Gross alpha & 1.98 & & 1.24 & UI & 3.52 & & & 1.42 & J IK & NDD & 1 & $\mathrm{pCi} / \mathrm{L}$ & GP \\
\hline & Nonvolatile beta & 1.91 & & 1.29 & UI & 12.55 & & & .709 & U & $<E Q L$ & 1 & $\mathrm{pCi} / \mathrm{L}$ & GP \\
\hline & Radium, total alpha-emitting & 1.2 & & 9.71 & J & .77 & & & 1.1 & U V & $<E Q L$ & 1 & $\mathrm{pCi} / \mathrm{L}$ & GP \\
\hline
\end{tabular}

Notes: Concentrations in bold italics exceed the groundwater protection or monitoring constituent standards listed in Appendix A. Synchronous water levels are measured over a 3-5 day period or less. Dilution factors, Laboratory, and Filtered Data are for Groundwater Protection Standards First Quarter 2000 and Monitoring Constituents Third Quarter 1999 data only.

$+=$ exceeded the Groundwater Protection Standards (First Quarter 2000) or Monitoring Constituents (Third Quarter 1999) listed in Appendix A. 
Table D-4. Groundwater Monitoring Results for Plume Definition Wells, M-Area HWMF (Cont.) WELL SRW 14A

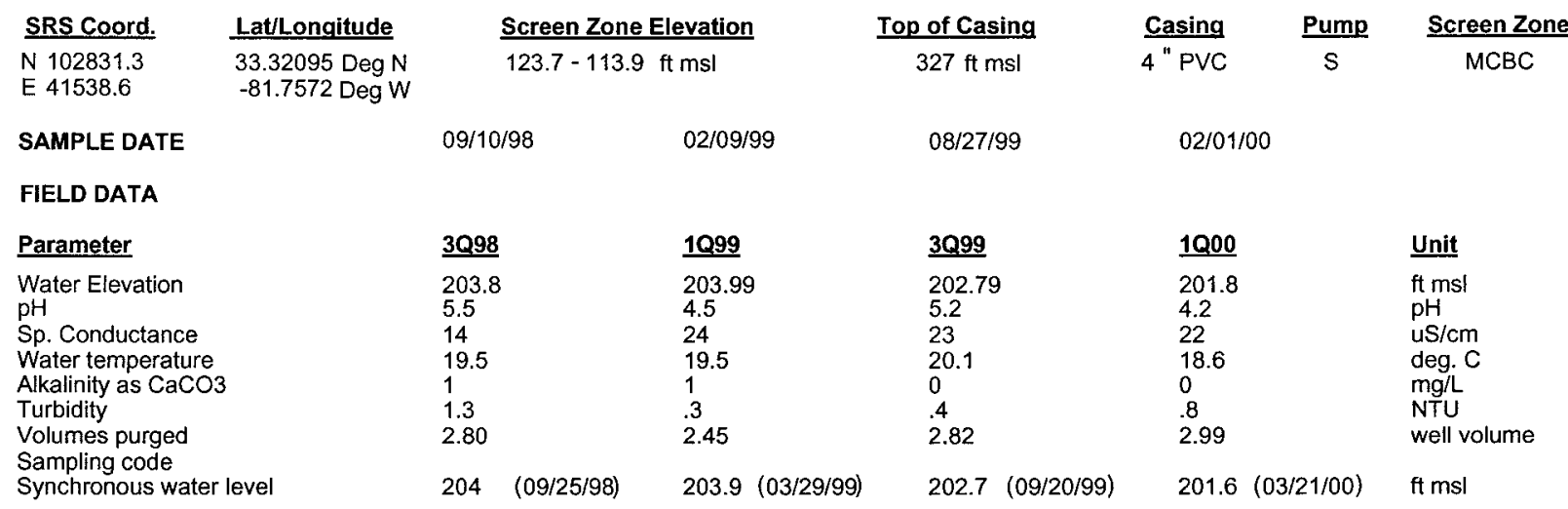

ANALYTICAL DATA

1. Groundwater Protection Standard

261 Appendix VIII/264 Appendix IX Hazardous Constituents

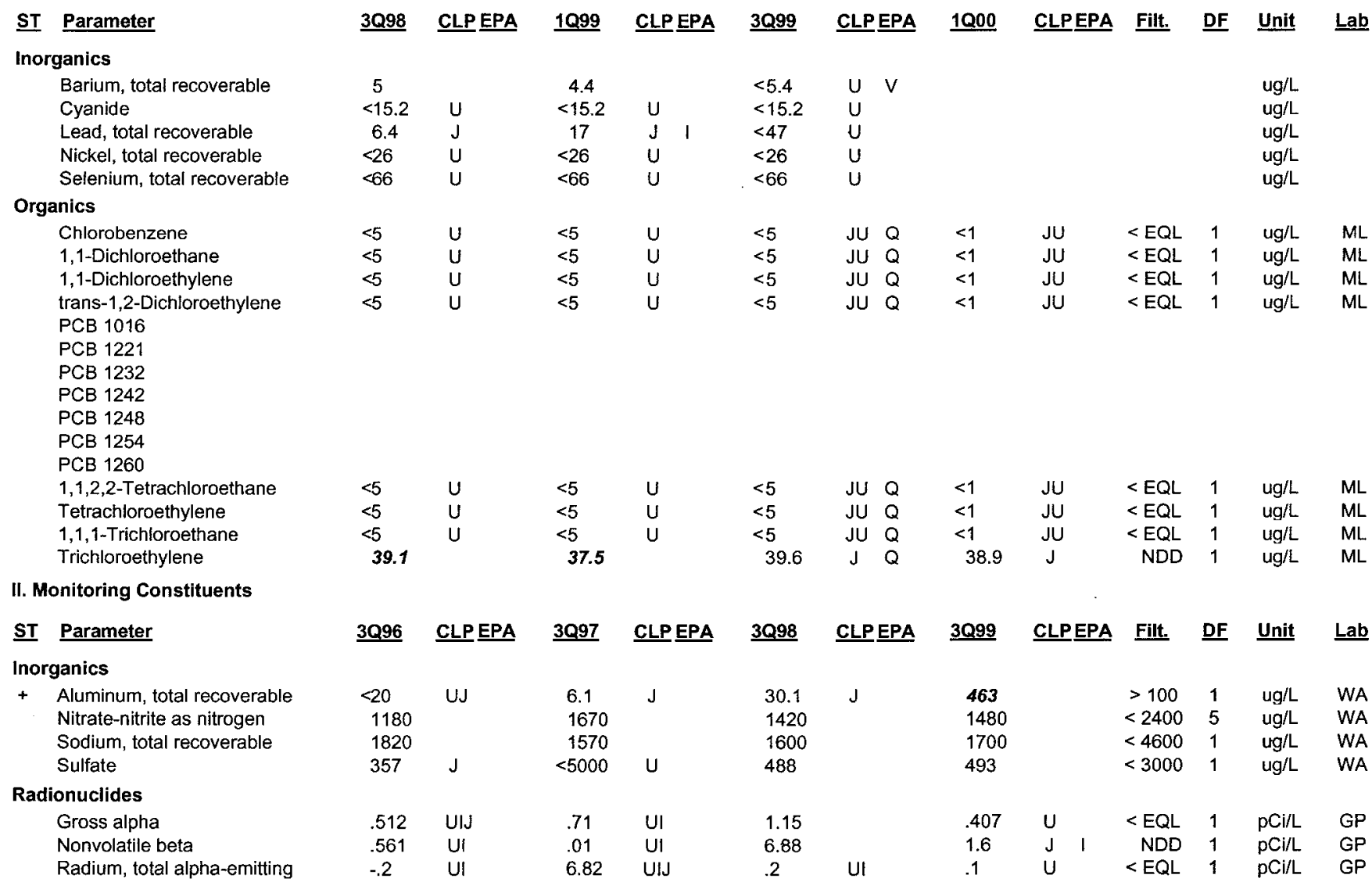

Notes: Concentrations in bold italics exceed the groundwater protection or monitoring constituent standards listed in Appendix A. Synchronous water levels are measured over a 3-5 day period or less. Dilution factors, Laboratory, and Filtered Data are for Groundwater Protection Standards First Quarter 2000 and Monitoring Constituents Third Quarter 1999 data only.

$+=$ exceeded the Groundwater Protection Standards (First Quarter 2000) or Monitoring Constituents (Third Quarter 1999) listed in Appendix A. 
Table D-4. Groundwater Monitoring Results for Plume Definition Wells, M-Area HWMF (Cont.) WELL SRW 14B

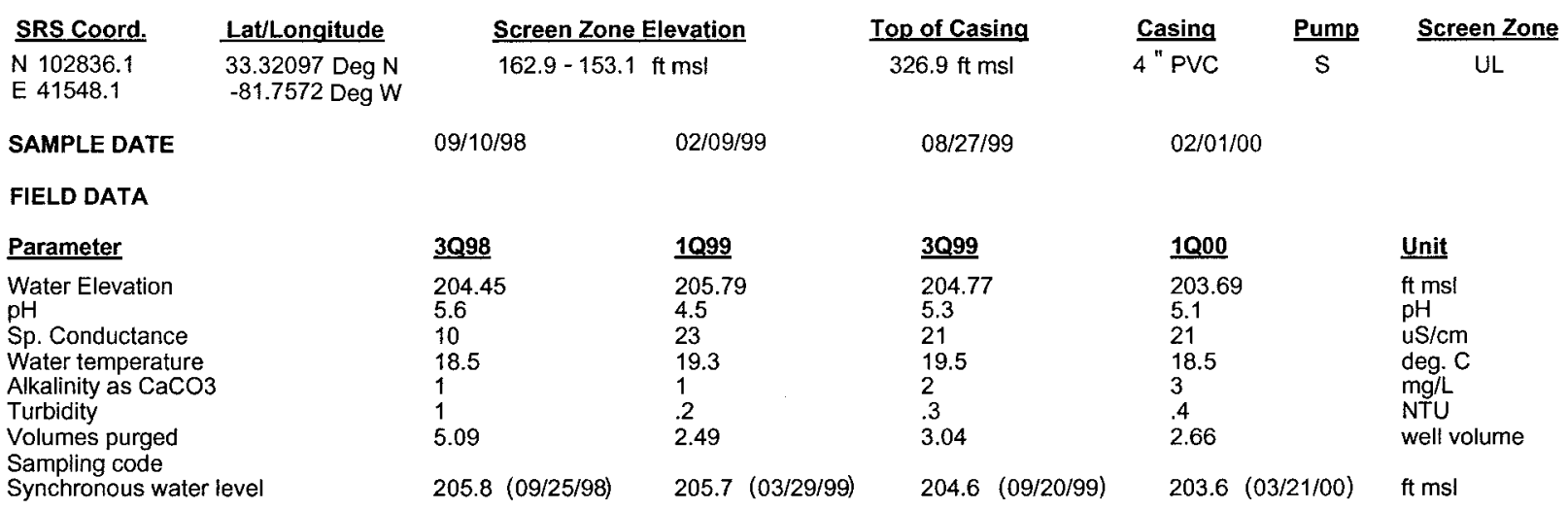

\section{ANALYTICAL DATA}

I. Groundwater Protection Standard

261 Appendix VIII/264 Appendix IX Hazardous Constituents

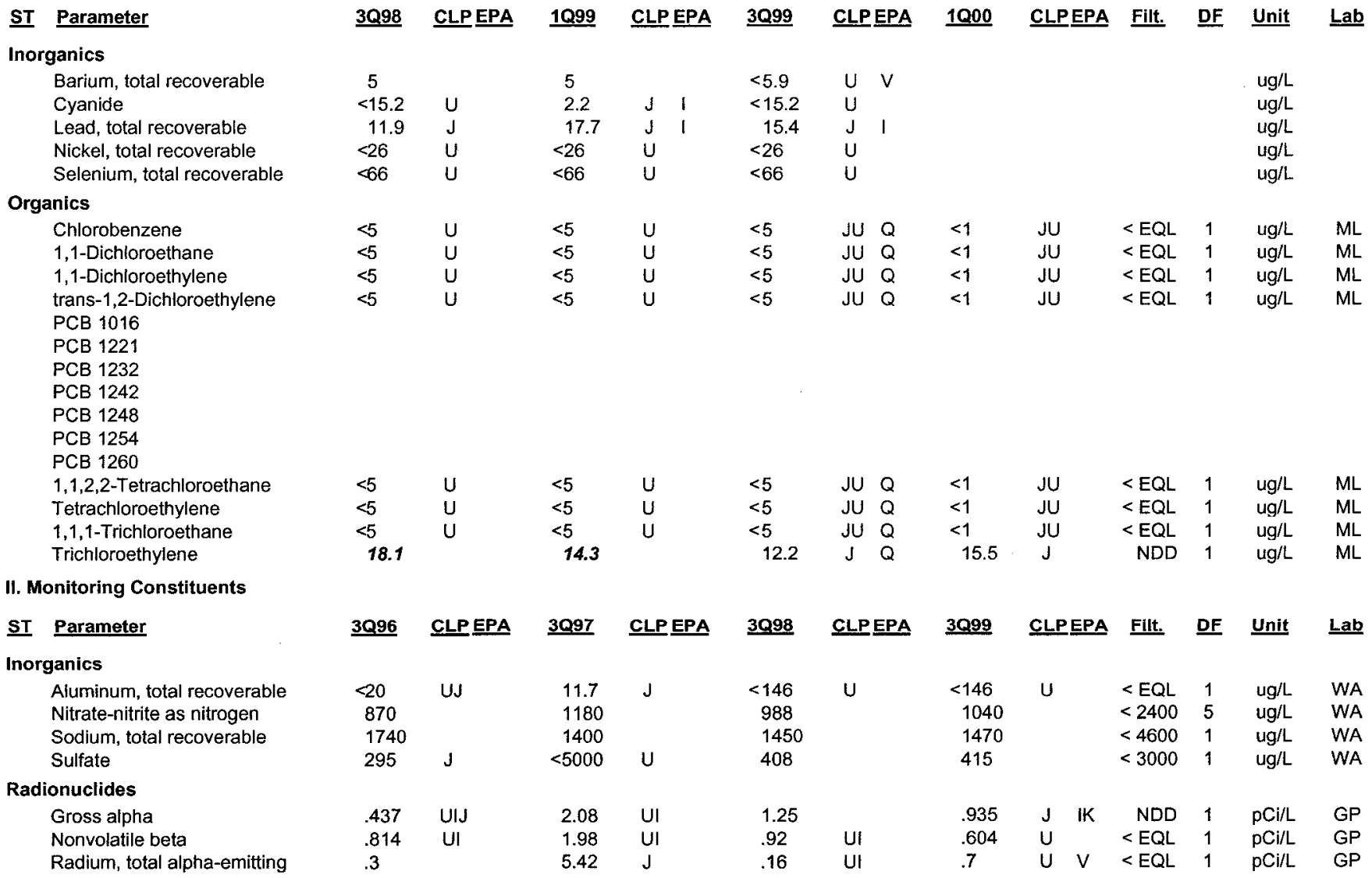

Notes: Concentrations in bold italics exceed the groundwater protection or monitoring constituent standards listed in Appendix A. Synchronous water levels are measured over a 3-5 day period or less. Dilution factors, Laboratory, and Filtered Data are for Groundwater Protection Standards First Quarter 2000 and Monitoring Constituents Third Quarter 1999 data only.

$+=$ exceeded the Groundwater Protection Standards (First Quarter 2000) or Monitoring Constituents (Third Quarter 1999) listed in Appendix A. 
Table D-4. Groundwater Monitoring Results for Plume Definition Wells, M-Area HWMF (Cont.) WELL SRW 16A

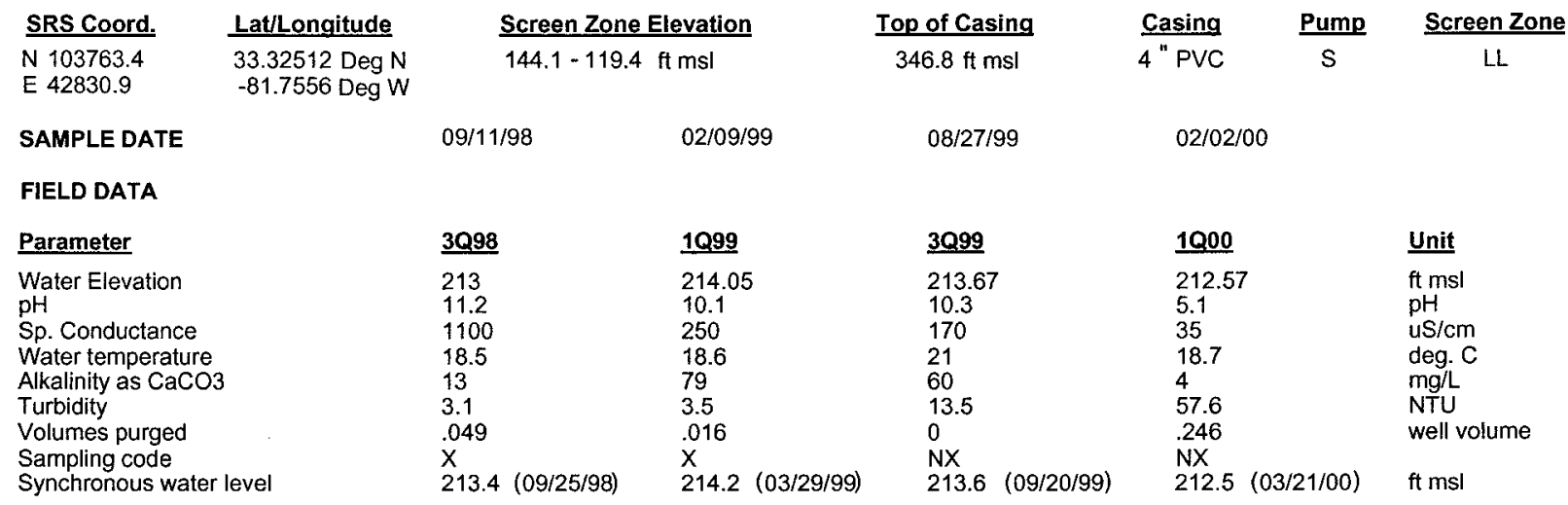

ANALYTICAL DATA

I. Groundwater Protection Standard

261 Appendix VIII/264 Appendix IX Hazardous Constituents

\begin{tabular}{|c|c|c|c|c|c|c|c|c|c|c|c|c|c|c|c|}
\hline \multirow{2}{*}{$\begin{array}{l}\text { ST } \\
\text { Inorg }\end{array}$} & \multirow{2}{*}{$\frac{\text { Parameter }}{\text { ganics }}$} & \multirow[t]{2}{*}{$\underline{3 Q 98}$} & \multirow[t]{2}{*}{ CLP EPA } & \multirow[t]{2}{*}{$1 \mathrm{Q99}$} & CLPEPA & \multirow[t]{2}{*}{$\underline{3 Q 99}$} & \multicolumn{2}{|c|}{ CLPEPA } & \multirow[t]{2}{*}{1000} & \multicolumn{2}{|c|}{ CLPEPA } & \multirow[t]{2}{*}{ Filt. } & \multirow[t]{2}{*}{$\underline{\mathrm{DF}}$} & \multirow[t]{2}{*}{ Unit } & Lab \\
\hline & & & & & & & & & & & & & & & \\
\hline & Barium, total recoverable & 101 & & 44.3 & & 38 & & & & & & & & $\mathrm{ug} / \mathrm{L}$ & \\
\hline & Cyanide & $<15.2$ & $\mathrm{U}$ & 1.54 & J I & $<15.2$ & $U$ & & & & & & & $\mathrm{ug} / \mathrm{L}$ & \\
\hline & Lead, total recoverable & 4.8 & $\mathrm{~J}$ & $<47$ & $U$ & $<47$ & $U$ & & & & & & & $\mathrm{ug} / \mathrm{L}$ & \\
\hline & Nickel, total recoverable & 6.6 & J & $<26$ & $\mathrm{U}$ & 3.7 & $\mathrm{~J}$ & 1 & & & & & & $\mathrm{ug} / \mathrm{L}$ & \\
\hline & Selenium, total recoverable & $<66$ & $\mathrm{U}$ & $<66$ & $\mathrm{U}$ & $<66$ & $\mathrm{U}$ & & & & & & & $\mathrm{ug} / \mathrm{L}$ & \\
\hline \multicolumn{16}{|c|}{ Organics } \\
\hline & Chlorobenzene & $<5$ & $U$ & $<5$ & $u$ & $<5$ & JU & $Q$ & $<1$ & $\mathrm{U}$ & & $<\mathrm{EQL}$ & 1 & $\mathrm{ug} / \mathrm{L}$ & ML \\
\hline & 1,1-Dichloroethane & $<5$ & $\mathrm{U}$ & $<5$ & $\mathrm{U}$ & $<5$ & JU & $\bar{Q}$ & $<1$ & $\mathrm{U}$ & & $<E Q L$ & 1 & $\mathrm{ug} / \mathrm{L}$ & ML \\
\hline & 1,1-Dichloroethylene & $<5$ & $\mathrm{U}$ & $<5$ & $U$ & $<5$ & JU & $Q$ & $<1$ & $\mathrm{U}$ & & $<$ EQL & 1 & $\mathrm{ug} / \mathrm{L}$ & ML \\
\hline & trans-1,2-Dichloroethylene & $<5$ & $\mathrm{U}$ & $<5$ & $U$ & $<5$ & JU & $Q$ & $<1$ & $\mathrm{U}$ & & $<\mathrm{EQL}$ & 1 & $\mathrm{ug} / \mathrm{L}$ & ML \\
\hline & PCB 1016 & & & & & & & & & & & & & & \\
\hline & PCB 1221 & & & & & & & & & & & & & & \\
\hline & PCB 1232 & & & & & & & & & & & & & & \\
\hline & PCB 1242 & & & & & & & & & & & & & & \\
\hline & PCB 1248 & & & & & & & & & & & & & & \\
\hline & PCB 1254 & & & & & & & & & & & & & & \\
\hline & PCB 1260 & & & & & & & & & & & & & & \\
\hline & $1,1,2,2$-Tetrachloroethane & $<5$ & $\cup$ & $<5$ & $\mathrm{U}$ & $<5$ & JU & $Q$ & $<1$ & $\mathrm{u}$ & & $<\mathrm{EQL}$ & 1 & $\mathrm{ug} / \mathrm{L}$ & ML \\
\hline & Tetrachloroethylene & $<5$ & $\mathrm{U}$ & $<5$ & $\mathrm{U}$ & $<5$ & JU & $Q$ & $<1$ & $\mathrm{U}$ & & $<\mathrm{EQL}$ & 1 & $\mathrm{ug} / \mathrm{L}$ & ML \\
\hline & 1,1,1-Trichloroethane & $<5$ & $\mathrm{U}$ & $<5$ & U & $<5$ & JU & $Q$ & $<1$ & $U$ & & $<\mathrm{EQL}$ & 1 & $\mathrm{ug} / \mathrm{L}$ & ML \\
\hline & Trichloroethylene & $<5$ & $\mathrm{U}$ & $<5$ & $\mathrm{U}$ & $<5$ & JU & $Q$ & 1.22 & & & $<5$ & 1 & ug/L & $\mathrm{ML}$ \\
\hline \multicolumn{16}{|c|}{ II. Monitoring Constituents } \\
\hline$\underline{\text { ST }}$ & Parameter & $\underline{3096}$ & CLPEPA & $\underline{3097}$ & CLP EPA & $\underline{3098}$ & $\underline{\text { CLP }}$ & EPA & $\underline{3099}$ & $\underline{\text { CLF }}$ & EPA & Filt. & $\underline{\text { DF }}$ & Unit & Le \\
\hline \multicolumn{16}{|c|}{ Inorganics } \\
\hline+ & Aluminum, total recoverable & 1340 & $J$ & 803 & . & 1050 & & & 608 & & & $>100$ & 1 & $\mathrm{ug} / \mathrm{L}$ & WA \\
\hline & Nitrate-nitrite as nitrogen & 340 & & 359 & & 220 & & & 473 & & & $<2400$ & 1 & $\mathrm{ug} / \mathrm{L}$ & WA \\
\hline & Sodium, total recoverable & 7180 & & 5120 & & 6080 & & & 3540 & & & $<4600$ & 1 & $\mathrm{ug} / \mathrm{L}$ & WA \\
\hline & Sulfate & 841 & 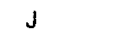 & $<5000$ & $\mathrm{U}$ & 867 & $J$ & & 633 & & & $<3000$ & 1 & ug/L & WA \\
\hline \multicolumn{16}{|c|}{ Radionuclides } \\
\hline & Gross alpha & 4.73 & $J$ & 2.98 & UI & 9.03 & $J$ & & 1.96 & $J$ & IK & NDD & 1 & $\mathrm{pCi} / \mathrm{L}$ & GP \\
\hline & Nonvolatile beta & 3.81 & & 1.16 & U: & 9.33 & $\mathrm{~J}$ & & 2.9 & & & $<50$ & 1 & $\mathrm{pCi} / \mathrm{L}$ & GP \\
\hline & Radium, total alpha-emitting & 1.6 & & 3.15 & $\mathrm{~J}$ & 1.3 & & & 1 & $\mathrm{U}$ & $\mathrm{V}$ & $<\mathrm{EQL}$ & 1 & $\mathrm{pCi} / \mathrm{L}$ & \\
\hline
\end{tabular}

Notes: Concentrations in bold italics exceed tbe groundwater protection or monitoring constituent standards listed in Appendix A. Synchronous water levels are measured over a 3-5 day period or less. Dilution factors, Laboratory, and Filtered Data are for Groundwater Protection Standards First Quarter 2000 and Monitoring Constituents Third Quarter 1999 data only.

+= exceeded the Groundwater Protection Standards (First Quarter 2000) or Monitoring Constituents (Third Quarter 1999) listed in Appendix A. 
Table D-4. Groundwater Monitoring Results for Plume Definition Wells, M-Area HWMF (Cont.) WELL SRW 16B

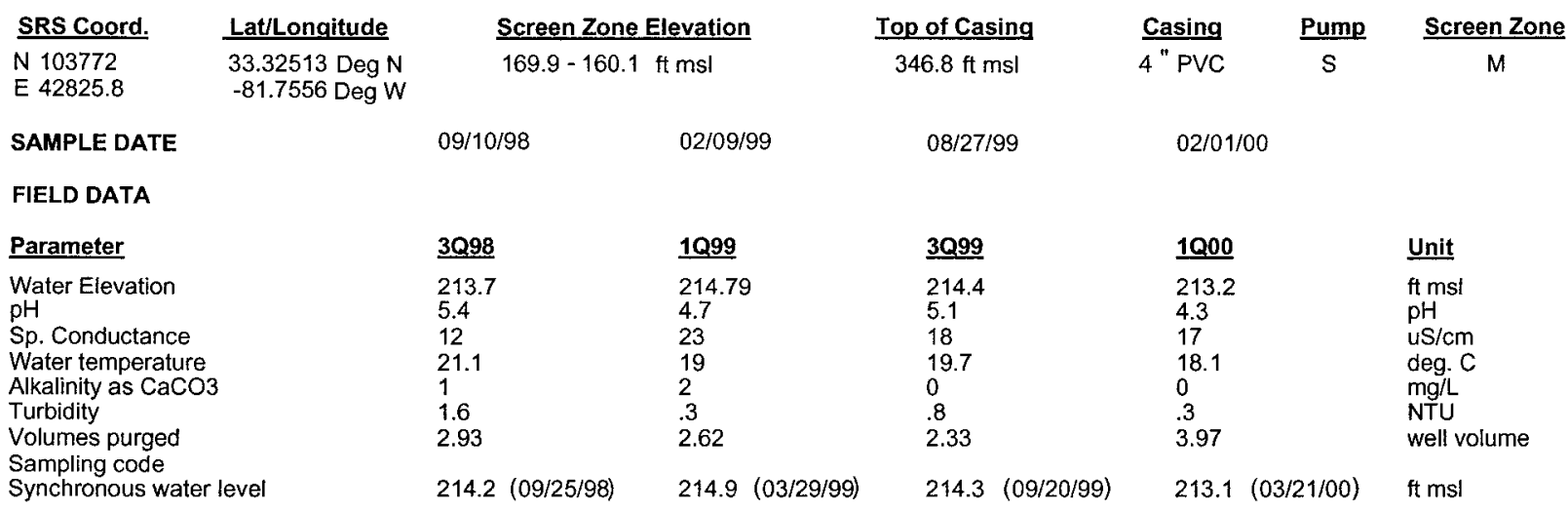

ANALYTICAL DATA

I. Groundwater Protection Standard

261 Appendix VIII/264 Appendix IX Hazardous Constituents

\begin{tabular}{|c|c|c|c|c|c|c|c|c|c|c|c|c|c|}
\hline$\underline{\mathbf{S T}}$ & Parameter & $\underline{3 Q 98}$ & $\underline{\text { CLP EPA }}$ & 1099 & CLP EPA & $\underline{3 Q 99}$ & $\underline{\text { CLP EPA }}$ & $\underline{1 Q 00}$ & CLPEPA & Filt. & $\underline{\mathrm{DF}}$ & $\underline{\text { Unit }}$ & $\underline{\text { Lab }}$ \\
\hline \multicolumn{14}{|c|}{ Inorganics } \\
\hline & Barium, total recoverable & 7.1 & & 6 & & 6.8 & & & & & & $\mathrm{ug} / \mathrm{L}$ & \\
\hline & Cyanide & $<15.2$ & $\mathrm{U}$ & 1.87 & J 1 & $<15.2$ & $\mathrm{U}$ & & & & & ug/L & \\
\hline & Lead, total recoverable & 15.7 & $\mathrm{~J}$ & 8 & J I & 28.9 & J I & & & & & $\mathrm{ug} / \mathrm{L}$ & \\
\hline & Nickel, total recoverable & $<26$ & $\mathrm{U}$ & 3.6 & $\mathrm{~J} \quad 1$ & $<26$ & $\mathrm{U}$ & & & & & $\mathrm{ug} / \mathrm{L}$ & \\
\hline & Selenium, total recoverable & $<66$ & $\mathrm{U}$ & $<66$ & $\mathrm{U}$ & $<66$ & $U$ & & & & & $\mathrm{ug} / \mathrm{L}$ & \\
\hline \multicolumn{14}{|c|}{ Organics } \\
\hline & Chlorobenzene & $<5$ & $\mathrm{U}$ & $<5$ & $\mathrm{U}$ & $<5$ & $J U Q$ & $<1$ & JU & $<E Q L$ & 1 & $\mathrm{ug} / \mathrm{L}$ & $M L$ \\
\hline & 1,1-Dichloroethane & $<5$ & $\mathrm{U}$ & $<5$ & $\mathrm{U}$ & $<5$ & $J U Q$ & $<1$ & JU & $<E Q L$ & 1 & $\mathrm{ug} / \mathrm{L}$ & ML \\
\hline & 1,1-Dichloroethylene & $<5$ & $\mathrm{U}$ & $<5$ & $\mathrm{U}$ & $<5$ & $J U Q$ & $<1$ & JU & $<E Q L$ & 1 & $\mathrm{ug} / \mathrm{L}$ & ML \\
\hline & trans-1,2-Dichloroethylene & $<5$ & U & $<5$ & $\mathrm{U}$ & $<5$ & $J U Q$ & $<1$ & JU & $<E Q L$ & 1 & $\mathrm{ug} / \mathrm{L}$ & ML \\
\hline & $\begin{array}{l}\text { PCB } 1016 \\
\text { PCB } 1221\end{array}$ & & & & & & & & & & & & \\
\hline & PCB 1232 & & & & & & & & & & & & \\
\hline & PCB 1242 & & & & & & & & & & & & \\
\hline & PCB 1248 & & & & & & & & & & & & \\
\hline & PCB 1254 & & & & & & & & & & & & \\
\hline & PCB 1260 & & & & & & & & & & & & \\
\hline & 1,1,2,2-Tetrachloroethane & $<5$ & $\mathrm{U}$ & $<5$ & $U$ & $<5$ & $J \cup Q$ & $<1$ & JU & $<E Q L$ & 1 & $\mathrm{ug} / \mathrm{L}$ & $M L$ \\
\hline & Tetrachloroethylene & $<5$ & $\mathrm{U}$ & $<5$ & $U$ & $<5$ & $J U Q$ & $<1$ & JU & $<E Q L$ & 1 & $\mathrm{ug} / \mathrm{L}$ & $M L$ \\
\hline & 1,1,1-Trichloroethane & $<5$ & $\mathrm{U}$ & $<5$ & $\mathrm{U}$ & $<5$ & JU $Q$ & $<1$ & JU & $<E Q L$ & 1 & $\mathrm{ug} / \mathrm{L}$ & ML \\
\hline & Trichloroethylene & $<5$ & $U$ & $<5$ & $\cup$ & $<5$ & $\mathrm{JU} Q$ & $<1$ & JU & $<\overrightarrow{E Q L}$ & 1 & $\mathrm{ug} / \mathrm{L}$ & $\mathrm{ML}$ \\
\hline \multicolumn{14}{|c|}{ II. Monitoring Constituents } \\
\hline$\underline{\mathbf{S T}}$ & Parameter & 3096 & $\underline{\text { CLPEPA }}$ & $\underline{3 Q 97}$ & CLP EPA & $\underline{3098}$ & CLPEPA & $\underline{3099}$ & CLPEPA & Filt. & $\underline{\text { DF }}$ & $\underline{\text { Unit }}$ & Lab \\
\hline \multicolumn{14}{|c|}{ Inorganics } \\
\hline & Aluminum, total recoverable & $<20$ & UJ & 53.3 & & $<146$ & U & $<146$ & U & $<E Q L$ & 1 & $u g / L$ & WA \\
\hline & Nitrate-nitrite as nitrogen & 990 & & 620 & & 731 & & 730 & & $<2400$ & 1 & $\mathrm{ug} / \mathrm{L}$ & WA \\
\hline & Sodium, total recoverable & 1870 & & 1490 & & 1330 & & 1340 & & $<4600$ & 1 & ug/L & WA \\
\hline & Sulfate & 254 & $\jmath$ & $<5000$ & $\mathrm{U}$ & 414 & & 409 & & $<3000$ & 1 & $\mathrm{ug} / \mathrm{L}$ & WA \\
\hline \multicolumn{14}{|c|}{ Radionuclides } \\
\hline & Gross alpha & 2.19 & J & 3.36 & UI & 7.35 & & 1.72 & J 1 & NDD & 1 & $\mathrm{pCi} / \mathrm{L}$ & GP \\
\hline & Nonvolatile beta & 1.66 & & 2.16 & & 7.18 & & 1.54 & $\mathrm{U}$ & $<E Q L$ & 1 & $\mathrm{pCi} / \mathrm{L}$ & GP \\
\hline & Radium, total alpha-emitting & .6 & & 2.1 & & .98 & & .5 & U V & $<E Q L$ & 1 & $\mathrm{pCi} / \mathrm{L}$ & GP \\
\hline
\end{tabular}

Notes: Concentrations in bold italics exceed the groundwater protection or monitoring constituent standards listed in Appendix A. Synchronous water levels are measured over a 3-5 day period or less. Dilution factors, Laboratory, and Filtered Data are for Groundwater Protection Standards First Quarter 2000 and Monitoring Constituents Third Quarter 1999 data only.

+= exceeded the Groundwater Protection Standards (First Quarter 2000) or Monitoring Constituents (Third Quarter 1999) listed in Appendix A. 
WSRC-TR-2000-00242

Unclassified

\section{THIS PAGE LEFT BLANK INTENTIONALLY}


Table D-5a. Groundwater Monitoring Results for Recovery Wells, July-October 1999 WELL RWM 1

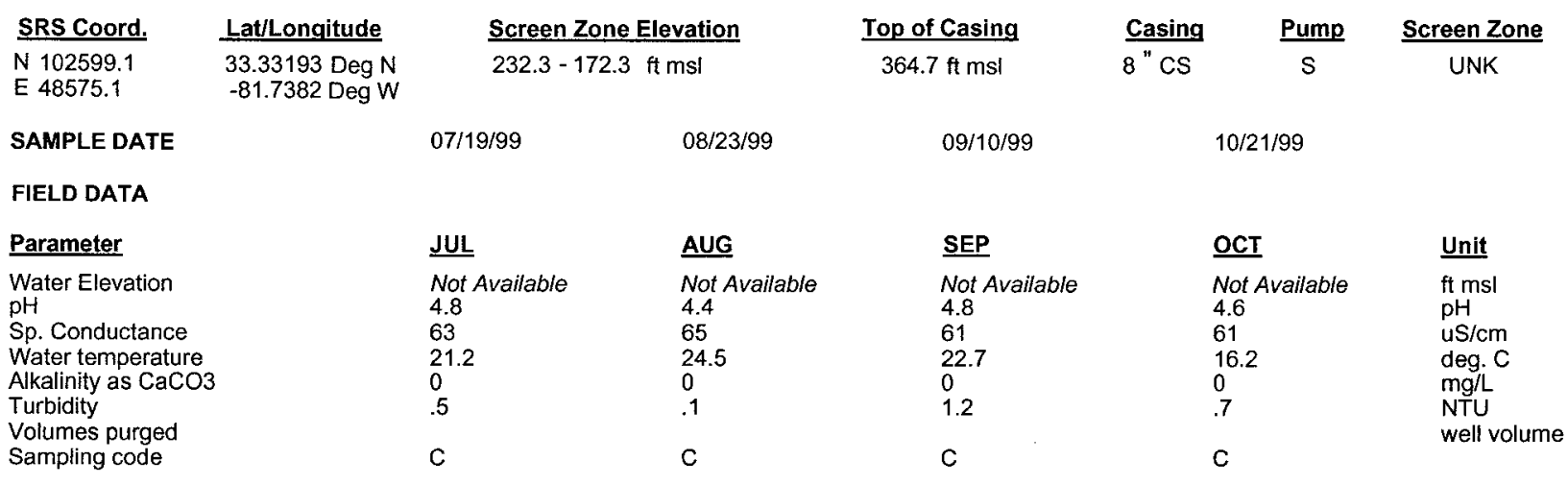

ANALYTICAL DATA

I. Groundwater Protection Standard

261 Appendix VIII/264 Appendix IX Hazardous Constituents

\begin{tabular}{|c|c|c|c|c|c|c|c|c|c|c|c|c|}
\hline$\underline{\mathbf{S T}}$ & Parameter & $\underline{\text { JUL }}$ & CLP EPA & $\underline{\text { AUG }}$ & CLPEPA & SEP & CLPEPA & $\underline{\text { OCT }}$ & CLPEPA & $\underline{\text { DF }}$ & Unit & $\underline{\text { Lab }}$ \\
\hline \multicolumn{13}{|c|}{ Inorganics } \\
\hline \multicolumn{13}{|c|}{ Barium, total recoverable } \\
\hline \multicolumn{13}{|c|}{ Cyanide } \\
\hline \multicolumn{13}{|c|}{ Lead, total recoverable } \\
\hline \multicolumn{13}{|c|}{ Nickel, total recoverable } \\
\hline \multicolumn{13}{|c|}{ Selenium, total recoverable } \\
\hline \multicolumn{13}{|c|}{ Organics } \\
\hline & Chlorobenzene & $<500$ & $u$ & $<500$ & $u$ & $<500$ & U & $<200$ & JU $Q$ & 200 & $\mathrm{ug} / \mathrm{L}$ & ML \\
\hline & 1,1-Dichloroethane & $<500$ & $U$ & $<500$ & $\mathrm{U}$ & $<500$ & $U$ & $<200$ & JU $Q$ & 200 & $\mathrm{ug} / \mathrm{L}$ & $M L$ \\
\hline & 1,1-Dichloroethylene & $<500$ & $\mathrm{U}$ & $<500$ & $\mathrm{U}$ & $<500$ & $U$ & $<200$ & JU $Q$ & 200 & $\mathrm{ug} / \mathrm{L}$ & $M L$ \\
\hline & trans-1,2-Dichloroethylene & $<500$ & $\mathrm{U}$ & $<500$ & $\mathrm{U}$ & $<500$ & $\mathrm{U}$ & & & & $\mathrm{ug} / \mathrm{L}$ & \\
\hline & РCB 1016 & $<1.05$ & $\mathrm{U}$ & & & & & & & & $\mathrm{ug} / \mathrm{L}$ & \\
\hline & PCB 1221 & $<2.1$ & $\mathrm{U}$ & & & & & & & & ug/L & \\
\hline & PCB 1232 & $<1.05$ & $U$ & & & & & & & & $\mathrm{ug} / \mathrm{L}$ & \\
\hline & PCB 1242 & $<1.05$ & $U$ & & & & & & & & ug/L & \\
\hline & PCB 1248 & $<1.05$ & $\mathrm{U}$ & & & & & & & & $\mathrm{ug} / \mathrm{L}$ & \\
\hline & РCB 1254 & $<1.05$ & U & & & & & & & & $\mathrm{ug} / \mathrm{L}$ & \\
\hline & PCB 1260 & $<1.05$ & $\mathrm{U}$ & & & & & & & & $\mathrm{ug} / \mathrm{L}$ & \\
\hline & $1,1,2,2$-Tetrachloroethane & $<500$ & $U$ & $<500$ & U & $<500$ & $\mathrm{U}$ & $<200$ & JU $Q$ & 200 & $\mathrm{ug} / \mathrm{L}$ & ML \\
\hline & Tetrachloroethylene & 13600 & & 12200 & & 13100 & & 11300 & $J \quad Q$ & 200 & $u g / L$ & ML \\
\hline & 1,1,1-Trichloroethane & $<500$ & $\mathrm{U}$ & $<500$ & $\mathrm{U}$ & $<500$ & $\mathrm{U}$ & $<200$ & JU Q & 200 & $u g / L$ & $\mathrm{ML}$ \\
\hline & Trichloroethylene & 20000 & & 1690 & & 19100 & & 15900 & $J \quad Q$ & 200 & ug/L & $\mathrm{ML}$ \\
\hline \multicolumn{13}{|c|}{ II. Monitoring Constituents } \\
\hline$\underline{\mathbf{S T}}$ & Parameter & $\underline{J U L}$ & CLPEPA & AUG & CLPEPA & SEP & $\underline{\text { CLPEPA }}$ & $\underline{\text { OCT }}$ & CLPEPA & $\underline{\mathrm{DF}}$ & $\underline{\text { Unit }}$ & $\underline{\text { Lab }}$ \\
\hline \multicolumn{13}{|c|}{ Inorganics } \\
\hline \multicolumn{13}{|c|}{ Aluminum, total recoverable } \\
\hline \multicolumn{13}{|c|}{ Nitrate-nitrite as nitrogen } \\
\hline \multicolumn{13}{|c|}{ Sodium, total recoverable } \\
\hline \multicolumn{12}{|c|}{ Sulfate } & \\
\hline Rad & ionuclides & & & & ' & & & & & & & \\
\hline \multicolumn{12}{|c|}{$\begin{array}{l}\text { Gross alpha } \\
\text { Nonvolatile beta } \\
\text { Radium total alpha-emitting }\end{array}$} & \\
\hline
\end{tabular}

Notes: Concentrations in bold italics exceed the groundwater protection or monitoring constituent standards listed in Appendix A. Synchronous water levels are measured over a 3-5 day. period or less. Dilution factors and Laboratory Data are October 1999 data only.

$+=$ exceeded the groundwater protection or monitoring constituent standards listed in Appendix A for October 1999. 
Table D-5a. Groundwater Monitoring Results for Recovery Wells, July-October 1999 (Cont.) WELL RWM 2

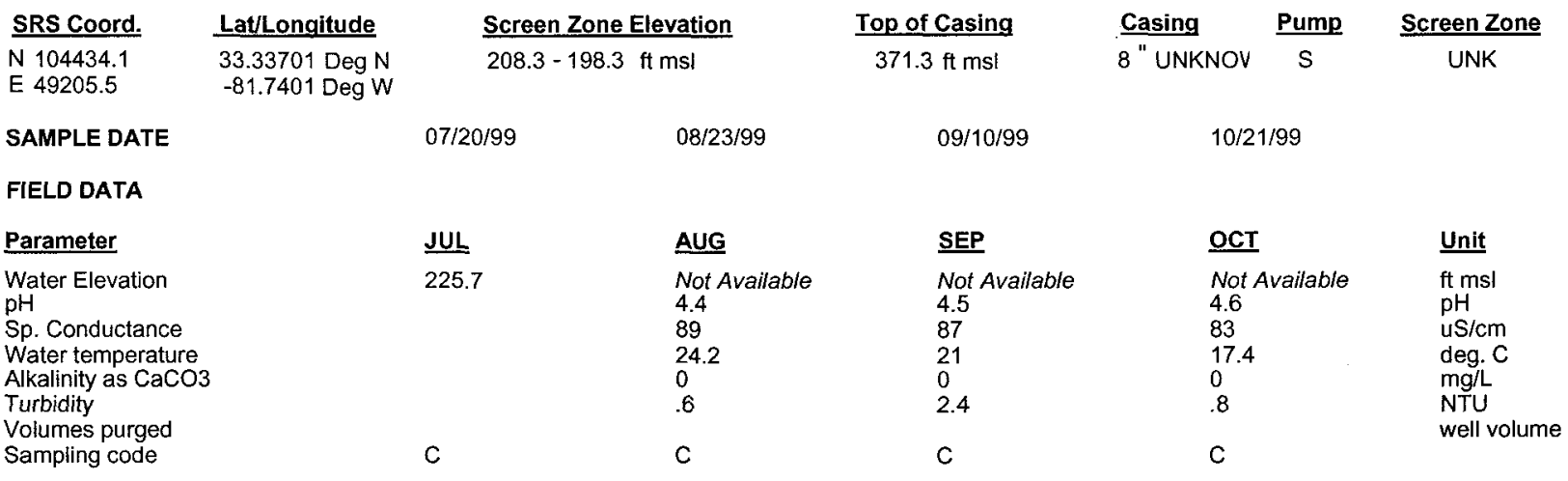

ANALYTICAL DATA

I. Groundwater Protection Standard

261 Appendix VIII/264 Appendix IX Hazardous Constituents

\begin{tabular}{|c|c|c|c|c|c|c|c|c|c|c|c|c|}
\hline$\underline{\mathbf{S T}}$ & Parameter & $\underline{\text { JUL }}$ & $\underline{\text { CLP EPA }}$ & $\underline{\text { AUG }}$ & CLP EPA & $\underline{\text { SEP }}$ & CLPEEA & $\underline{\text { OCT }}$ & CLPEPA & $\underline{\text { DF }}$ & Unit & $\underline{\underline{L a b}}$ \\
\hline \multicolumn{13}{|c|}{ Inorganics } \\
\hline \multicolumn{13}{|c|}{ Barium, total recoverable } \\
\hline \multicolumn{13}{|c|}{ Cyanide } \\
\hline \multicolumn{13}{|c|}{ Lead, total recoverable } \\
\hline \multicolumn{13}{|c|}{ Nickel, total recoverable } \\
\hline \multicolumn{13}{|c|}{ Selenium, total recoverable } \\
\hline \multicolumn{13}{|c|}{ Organics } \\
\hline \multirow{4}{*}{\multicolumn{2}{|c|}{$\begin{array}{l}\text { Chlorobenzene } \\
\text { 1,1-Dichloroethane } \\
\text { 1,1-Dichloroethylene } \\
\text { trans-1,2-Dichloroethylene }\end{array}$}} & & . & $<500$ & $U$ & $<500$ & $\mathrm{U}$ & $<50$ & $J U Q$ & 50 & $\mathrm{ug} / \mathrm{L}$ & $M L$ \\
\hline & & & & $<500$ & 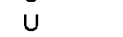 & $<500$ & $\mathrm{U}$ & $<50$ & JU Q & 50 & $\mathrm{ug} / \mathrm{L}$ & $\mathrm{ML}$ \\
\hline & & & & $<500$ & 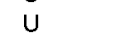 & $<500$ & $\mathrm{U}$ & $<50$ & $J U$ & 50 & $\mathrm{ug} / \mathrm{L}$ & $\mathrm{ML}$ \\
\hline & & & & $<500$ & U & $<500$ & U & & & & $u g / L$ & \\
\hline \multicolumn{13}{|c|}{ PCB 1016} \\
\hline \multicolumn{13}{|c|}{ PCB 1221} \\
\hline \multicolumn{13}{|c|}{ PCB 1232} \\
\hline \multicolumn{13}{|c|}{ PCB 1242} \\
\hline \multicolumn{13}{|c|}{ PCB 1248} \\
\hline \multicolumn{13}{|c|}{ PCB 1254} \\
\hline \multicolumn{13}{|c|}{ PCB 1260} \\
\hline & $1,1,2,2$-Tetrachloroethane & & & $<500$ & $U$ & $<500$ & $\mathrm{U}$ & $<50$ & $J U Q$ & 50 & $\mathrm{ug} / \mathrm{L}$ & $M L$ \\
\hline & Tetrachloroethylene & & & 9870 & & 9690 & & 12500 & $J \quad Q$ & 50 & $\mathrm{ug} / \mathrm{L}$ & $\mathrm{ML}$ \\
\hline & $1,1,1$-Trichloroethane & & & $<500$ & U & $<500$ & $\mathrm{U}$ & $<50$ & JU $Q$ & 50 & $\mathrm{ug} / \mathrm{L}$ & $\mathrm{ML}$ \\
\hline & Trichloroethylene & & & 9500 & & 10600 & & 13500 & $J \quad \bar{Q}$ & 50 & $\mathrm{ug} / \mathrm{L}$ & ML \\
\hline \multicolumn{13}{|c|}{ II. Monitoring Constituents } \\
\hline$\underline{\mathbf{S T}}$ & Parameter & $\underline{\mathrm{JUL}}$ & CLPEPA & AUG & CLP EPA & SEP & CLPEPA & $\underline{\text { OCT }}$ & CLPEPA & $\underline{\text { DF }}$ & Unit & $\underline{\text { Lab }}$ \\
\hline \multicolumn{13}{|c|}{ Inorganics } \\
\hline \multicolumn{12}{|c|}{ Aluminum, total recoverable } & \\
\hline \multicolumn{12}{|c|}{ Nitrate-nitrite as nitrogen } & \\
\hline \multicolumn{12}{|c|}{ Sodium, total recoverable } & \\
\hline \multicolumn{12}{|c|}{ Sulfate } & \\
\hline \multicolumn{13}{|c|}{ Radionuclides } \\
\hline \multicolumn{12}{|c|}{ Gross alpha } & \\
\hline & Nonvolatile beta & & & & & & & & & & & \\
\hline & Radium, total alpha-emitting & & & & & & & & & & & \\
\hline
\end{tabular}

\footnotetext{
Notes: Concentrations in bold italics exceed the groundwater protection or monitoring constituent standards listed in Appendix A. Synchronous water levels are measured over a 3-5 day period or less. Dilution factors and Laboratory Data are October 1999 data only.

$+=$ exceeded the groundwater protection or monitoring constituent standards listed in Appendix A for October 1999.
} 
Table D-5a. Groundwater Monitoring Results for Recovery Wells, July-October 1999 (Cont.) WELL RWM 3

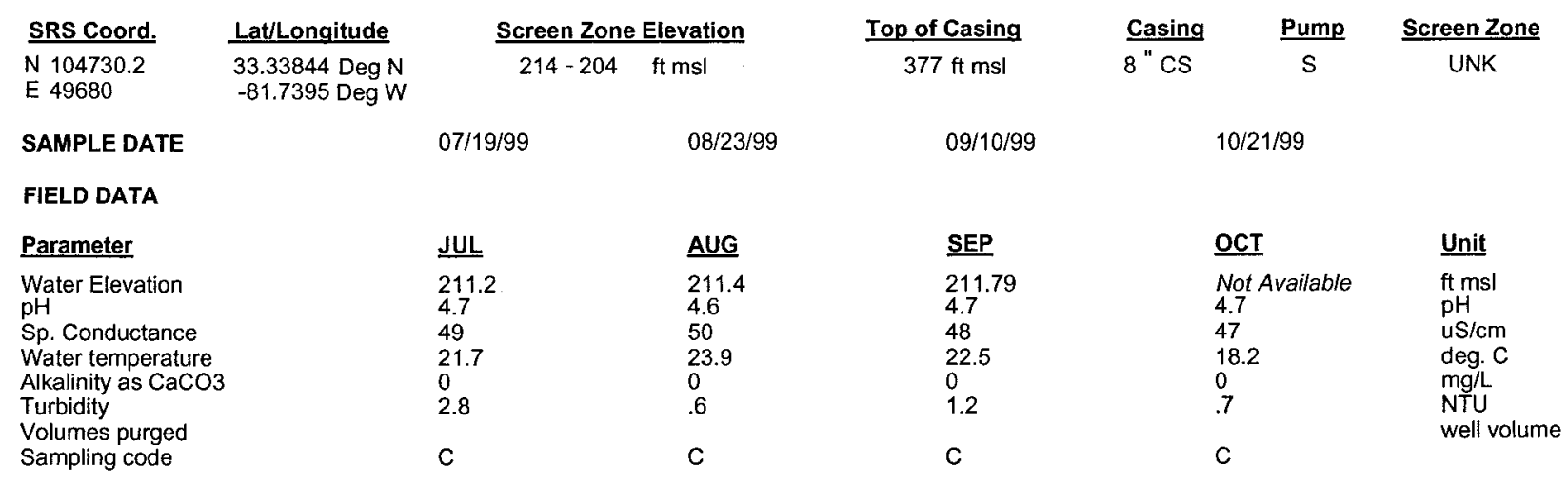

\section{ANALYTICAL DATA}

I. Groundwater Protection Standard

261 Appendix VIII/264 Appendix IX Hazardous Constituents

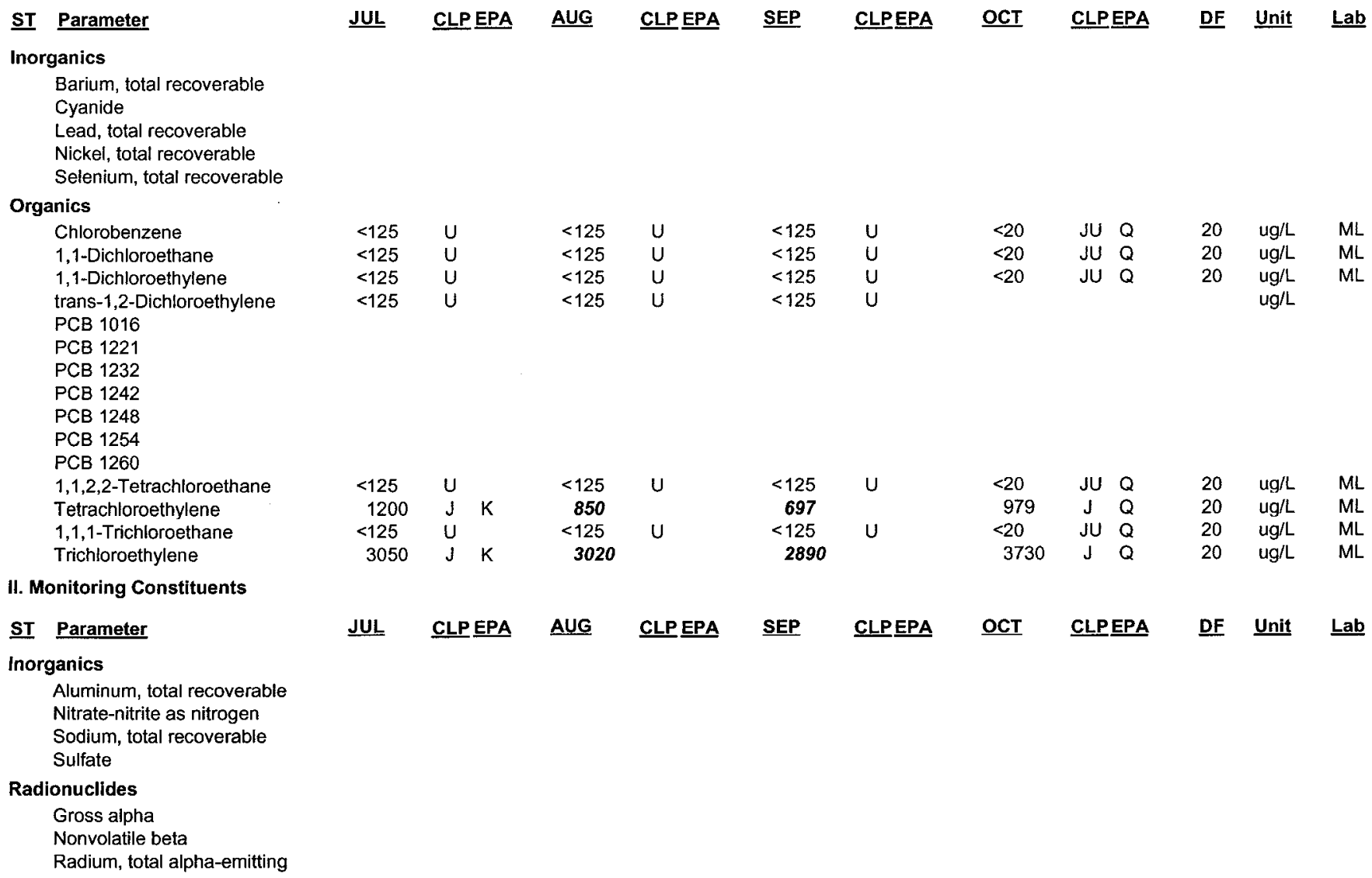

Notes: Concentrations in bold italics exceed the groundwater protection or monitoring constituent standards listed in Appendix A. Synchronous water levels are measured over a 3-5 day period or less. Dilution factors and Laboratory Data are October 1999 data only.

$+=$ exceeded the groundwater protection or monitoring constituent standards listed in Appendix A for October 1999. 
Table D-5a. Groundwater Monitoring Results for Recovery Wells, July-October 1999 (Cont.) WELL RWM 4

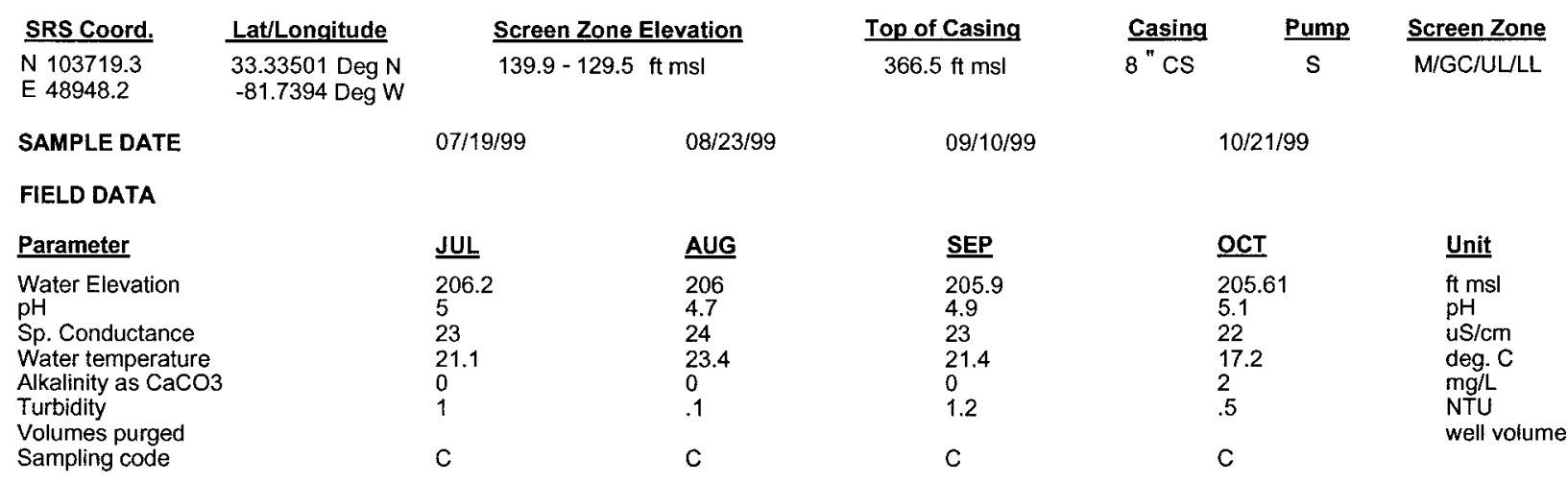

\section{ANALYTICAL DATA}

I. Groundwater Protection Standard

261 Appendix VIII/264 Appendix IX Hazardous Constituents

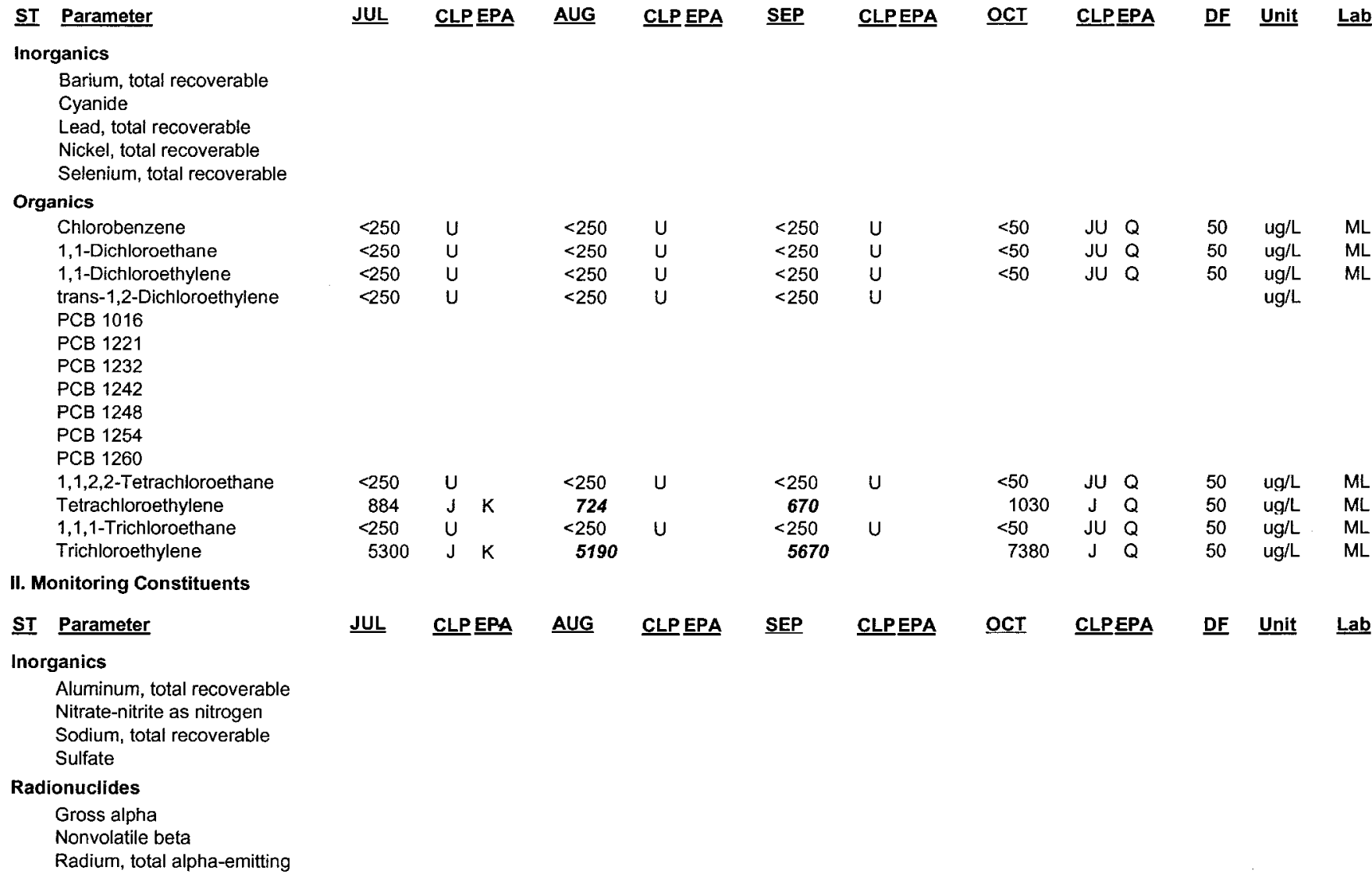

Notes: Concentrations in bold italics exceed the groundwater protection or monitoring constituent standards listed in Appendix A. Synchronous water levels are measured over a 3-5 day period or less. Dilution factors and Laboratory Data are October 1999 data only.

$+=$ exceeded the groundwater protection or monitoring constituent standards Iisted in Appendix A for October 1999. 
Table D-5a. Groundwater Monitoring Results for Recovery Wells, July-October 1999 (Cont.) WELL RWM 5

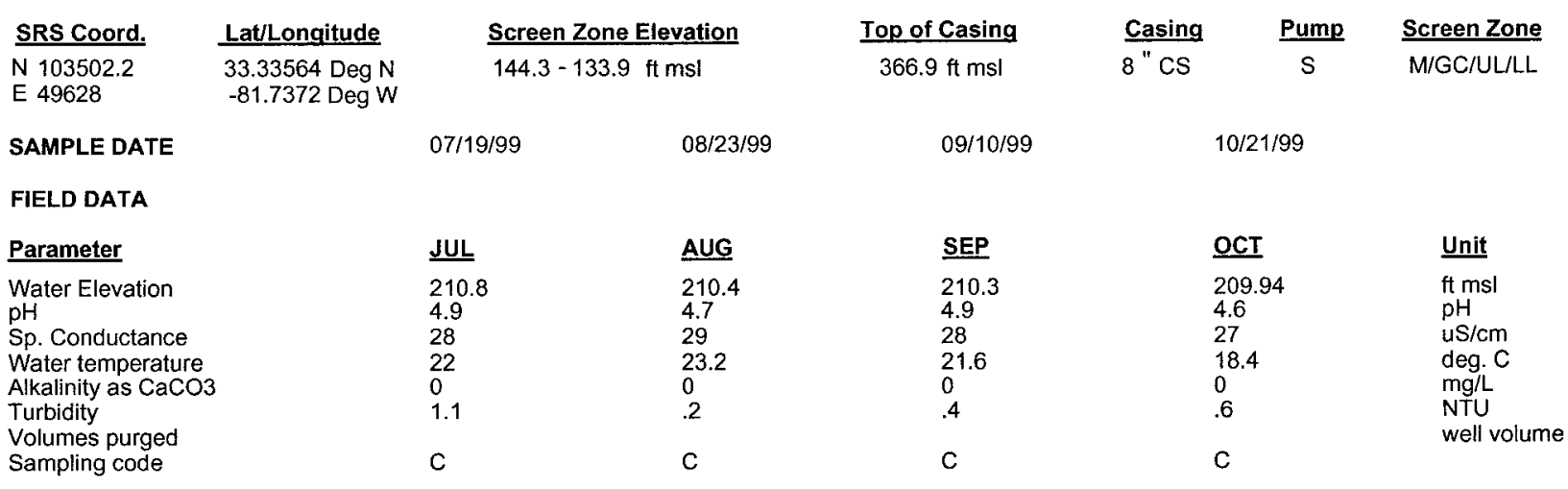

\section{ANALYTICAL DATA}

I. Groundwater Protection Standard

261 Appendix VIII/264 Appendix IX Hazardous Constituents

\begin{tabular}{|c|c|c|c|c|c|c|c|c|c|c|c|c|}
\hline ST & Parameter & $\underline{\text { JUL }}$ & CLPEPA & $\underline{\text { AUG }}$ & CLP EPA & $\underline{\text { SEP }}$ & CLPEPA & $\underline{\mathrm{OCT}}$ & CLPEPA & $\underline{\text { DF }}$ & Unit & Lab \\
\hline \multicolumn{13}{|c|}{ Inorganics } \\
\hline \multicolumn{13}{|c|}{ Barium, total recoverable } \\
\hline \multicolumn{13}{|c|}{ Cyanide } \\
\hline \multicolumn{13}{|c|}{ Lead, total recoverable } \\
\hline \multicolumn{13}{|c|}{ Nickel, total recoverable } \\
\hline \multicolumn{13}{|c|}{ Selenium, total recoverable } \\
\hline \multicolumn{13}{|c|}{ Organics } \\
\hline & Chlorobenzene & $<50$ & $U$ & $<50$ & $U$ & $<50$ & $U$ & $<10$ & $J U Q$ & 10 & ug/L & $M L$ \\
\hline & 1,1-Dichloroethane & $<50$ & $\mathrm{U}$ & $<50$ & $U$ & $<50$ & $\mathrm{U}$ & $<10$ & JU $Q$ & 10 & ug/L & ML \\
\hline & 1,1-Dichloroethylene & $<50$ & $\mathrm{U}$ & $<50$ & U & $<50$ & $\mathrm{U}$ & $<10$ & JU Q & 10 & $\mathrm{ug} / \mathrm{L}$ & ML \\
\hline & trans-1,2-Dichloroethylene & $<50$ & $\mathrm{u}$ & $<50$ & U & $<50$ & $U$ & & & & $\mathrm{ug} / \mathrm{L}$ & \\
\hline \multicolumn{13}{|c|}{ PCB 1016} \\
\hline \multicolumn{13}{|c|}{ PCB 1221} \\
\hline \multicolumn{13}{|c|}{ PCB 1232} \\
\hline \multicolumn{13}{|c|}{ PCB 1242} \\
\hline \multicolumn{13}{|c|}{ PCB 1248} \\
\hline \multicolumn{13}{|c|}{ PCB 1254} \\
\hline \multicolumn{13}{|c|}{ PCB 1260} \\
\hline & $1,1,2,2$-Tetrachloroethane & $<50$ & U & $<50$ & $U$ & $<50$ & U & $<10$ & JU $Q$ & 10 & $\mathrm{ug} / \mathrm{L}$ & $M L$ \\
\hline & Tetrachloroethylene & 498 & & 482 & & 497 & & 595 & $J \quad Q$ & 10 & $u g / L$ & $M L$ \\
\hline & $1,1,1$-Trichloroethane & $<50$ & $\mathrm{U}$ & $<50$ & $U$ & $<50$ & $U$ & $<10$ & JU $\bar{Q}$ & 10 & $\mathrm{ug} / \mathrm{L}$ & $\mathrm{ML}$ \\
\hline & Trichloroethylene & 1260 & & 1240 & & 1380 & & 1630 & $J \quad Q$ & 10 & $\mathrm{ug} / \mathrm{L}$ & $\mathrm{ML}$ \\
\hline \multicolumn{13}{|c|}{ II. Monitoring Constituents } \\
\hline$\underline{\text { ST }}$ & Parameter & JUL & CLPEPA & $\underline{A U G}$ & CLP EPA & SEP & CLPEPA & $\underline{\text { OCT }}$ & CLPEEPA & $\underline{D F}$ & Unit & Lab \\
\hline \multicolumn{13}{|c|}{ Inorganics } \\
\hline \multicolumn{12}{|c|}{ Aluminum, total recoverable } & \\
\hline \multicolumn{12}{|c|}{ Nitrate-nitrite as nitrogen } & \\
\hline \multicolumn{12}{|c|}{ Sodium, total recoverable } & \\
\hline \multicolumn{12}{|c|}{ Sulfate } & \\
\hline \multicolumn{13}{|c|}{ Radionuclides } \\
\hline \multirow{3}{*}{\multicolumn{12}{|c|}{$\begin{array}{l}\text { Gross alpha } \\
\text { Nonvolatile beta } \\
\text { Radium total alpha-emitting }\end{array}$}} & \\
\hline & & & & & & & & & & & & \\
\hline & & & & & & & & & & & & \\
\hline
\end{tabular}

\footnotetext{
Notes: Concentrations in bold italics exceed the groundwater protection or monitoring constituent standards listed in Appendix A. Synchronous water levels are measured over a 3-5 day period or less. Dilution factors and Laboratory Data are October 1999 data only.

$+=$ exceeded the groundwater protection or monitoring constituent standards listed in Appendix A for October 1999.
} 
Table D-5a. Groundwater Monitoring Results for Recovery Wells, July-October 1999 (Cont.) WELL RWM 6

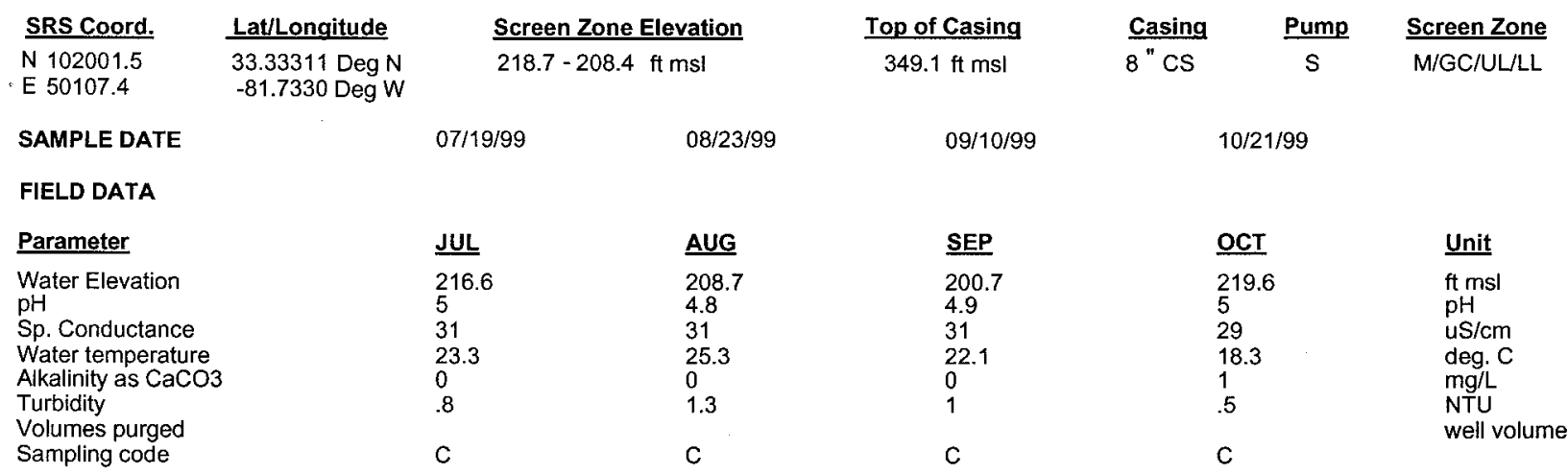

\section{ANALYTICAL DATA}

I. Groundwater Protection Standard

261 Appendix VIII/264 Appendix IX Hazardous Constituents

\begin{tabular}{|c|c|c|c|c|c|c|c|c|c|c|c|}
\hline$\underline{\mathbf{S T}}$ & Parameter & $\underline{\text { JUL }}$ & CLPEPA & $\underline{\text { AUG }}$ & CLP EPA & $\underline{\text { SEP }}$ & CLPEPA & $\underline{\text { OCT }}$ & CLPEPA & $\underline{\text { DF }}$ & Unit \\
\hline \multicolumn{12}{|c|}{ Inorganics } \\
\hline & $\begin{array}{l}\text { Barium, total recoverable } \\
\text { Cyanide } \\
\text { Lead, total recoverable } \\
\text { Nickel, total recoverable } \\
\text { Selenium, total recoverable }\end{array}$ & & & & & & & & & & \\
\hline \multicolumn{12}{|c|}{ Organics } \\
\hline & Chlorobenzene & $<125$ & $\mathrm{U}$ & $<100$ & $\mathrm{U}$ & $<125$ & $U$ & $<20$ & JU $Q$ & 20 & ug/L \\
\hline & 1,1-Dichloroethane & $<125$ & $\mathrm{U}$ & $<100$ & $\mathrm{U}$ & $<125$ & $U$ & $<20$ & JU $\bar{Q}$ & 20 & ug/L \\
\hline & 1,1-Dichloroethylene & $<125$ & $U$ & $<100$ & $u$ & $<125$ & $u$ & $<20$ & JU $Q$ & 20 & $\mathrm{ug} / \mathrm{L}$ \\
\hline & trans-1,2-Dichloroethylene & $<125$ & $\mathrm{U}$ & $<100$ & U & $<125$ & U & & & & $\mathrm{ug} / \mathrm{L}$ \\
\hline & PCB 1016 & $<1.05$ & $\mathrm{U}$ & & & & & & & & ug/L \\
\hline & PCB 1221 & $<2.1$ & $U$ & & & & & & & & $\mathrm{ug} / \mathrm{L}$ \\
\hline & PCB 1232 & $<1.05$ & $\mathrm{U}$ & & & & & & & & $\mathrm{ug} / \mathrm{L}$ \\
\hline & PCB 1242 & $<1.05$ & $\mathrm{U}$ & & & & & & & & $\mathrm{ug} / \mathrm{L}$ \\
\hline & PCB 1248 & $<1.05$ & $\mathrm{U}$ & & & & & & & & $\mathrm{ug} / \mathrm{L}$ \\
\hline & PCB 1254 & $<1.05$ & $\mathrm{U}$ & & & & & & & & ug/L \\
\hline & РСB 1260 & $<1.05$ & U & & & & & & & & $\mathrm{ug} / \mathrm{L}$ \\
\hline & 1,1,2,2-Tetrachloroethane & $<125$ & $\mathrm{U}$ & $<100$ & $\mathrm{U}$ & $<125$ & $\mathrm{U}$ & $<20$ & JU Q & 20 & $\mathrm{ug} / \mathrm{L}$ \\
\hline & Tetrachloroethylene & 2470 & & 2420 & & 2450 & & 2670 & $J \quad Q$ & 20 & $\mathrm{ug} / \mathrm{L}$ \\
\hline & 1,1,1-Trichloroethane & $<125$ & $U$ & $<100$ & $U$ & $<125$ & $\mathrm{U}$ & $<20$ & $\mathrm{JU} Q$ & 20 & $\mathrm{ug} / \mathrm{L}$ \\
\hline & Trichloroethylene & 1830 & & 1740 & & 1990 & & 2200 & $J \quad Q$ & 20 & $\mathrm{ug} / \mathrm{L}$ \\
\hline \multicolumn{12}{|c|}{ II. Monitoring Constituents } \\
\hline$\underline{\text { ST }}$ & Parameter & $\underline{\text { JUL }}$ & CLP EPA & $\underline{\text { AUG }}$ & CLP EPA & SEP & CLPEPA & $\underline{\text { OCT }}$ & CLPEPA & $\underline{\mathrm{DF}}$ & Unit \\
\hline \multicolumn{12}{|c|}{ Inorganics } \\
\hline \multicolumn{12}{|c|}{ Aluminum, total recoverable } \\
\hline \multicolumn{12}{|c|}{ Nitrate-nitrite as nitrogen } \\
\hline \multicolumn{12}{|c|}{ Sodium, total recoverable } \\
\hline \multicolumn{12}{|c|}{ Sulfate } \\
\hline \multicolumn{12}{|c|}{ Radionuclides } \\
\hline \multicolumn{12}{|c|}{$\begin{array}{l}\text { Gross alpha } \\
\text { Nonvolatile beta }\end{array}$} \\
\hline
\end{tabular}

Notes: Concentrations in bold italics exceed the groundwater protection or monitoring constituent standards listed in Appendix A. Synchronous water levels are measured over a 3-5 day period or less. Dilution factors and Laboratory Data are October 1999 data only.

$+=$ exceeded the groundwater protection or monitoring constituent standards listed in Appendix A for October 1999. 
Table D-5a. Groundwater Monitoring Results for Recovery Wells, July-October 1999 (Cont.) WELL RWM 7

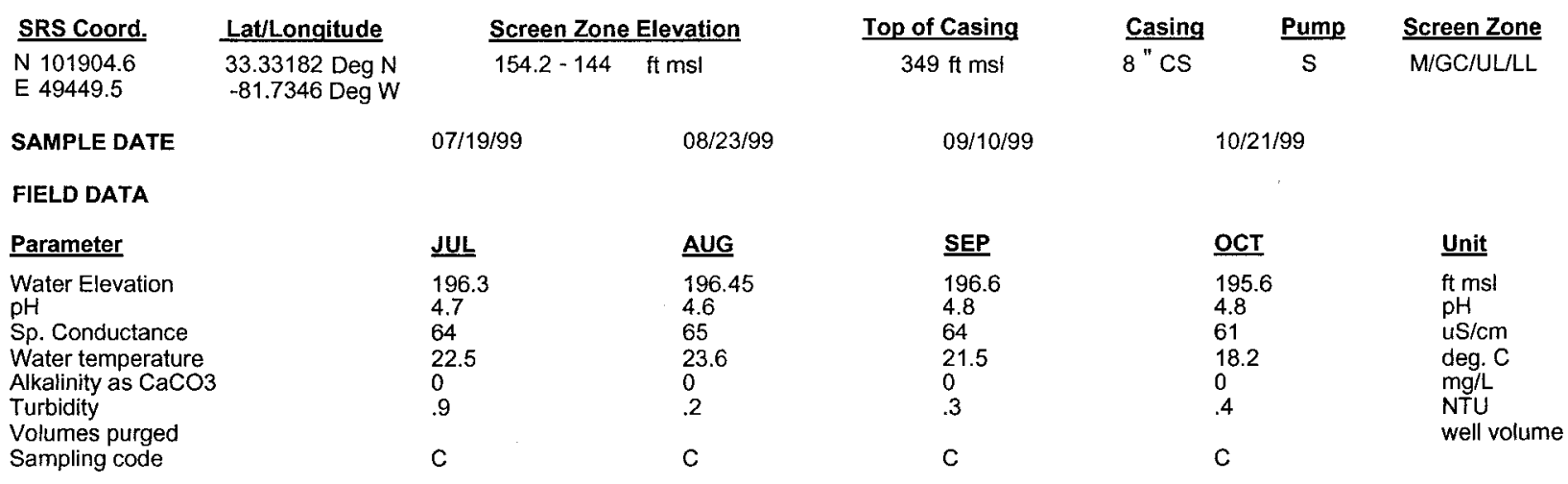

ANALYTICAL DATA

I. Groundwater Protection Standard

261 Appendix VIII/264 Appendix IX Hazardous Constituents

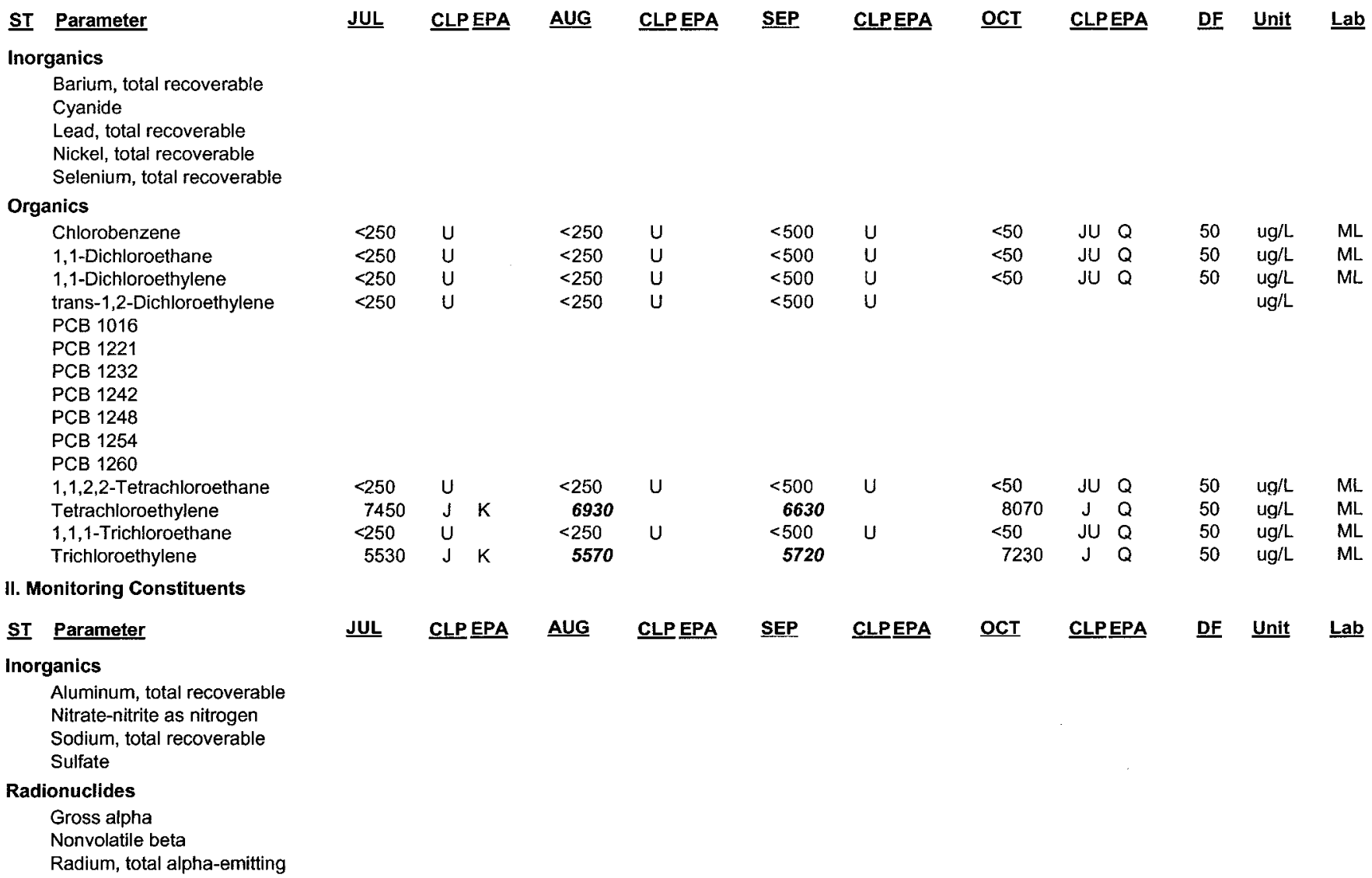


WSRC-TR- 2000-00242

Unclassified

Table D-5a. Groundwater Monitoring Results for Recovery Wells, July-October 1999 (Cont.) WELL RWM 8

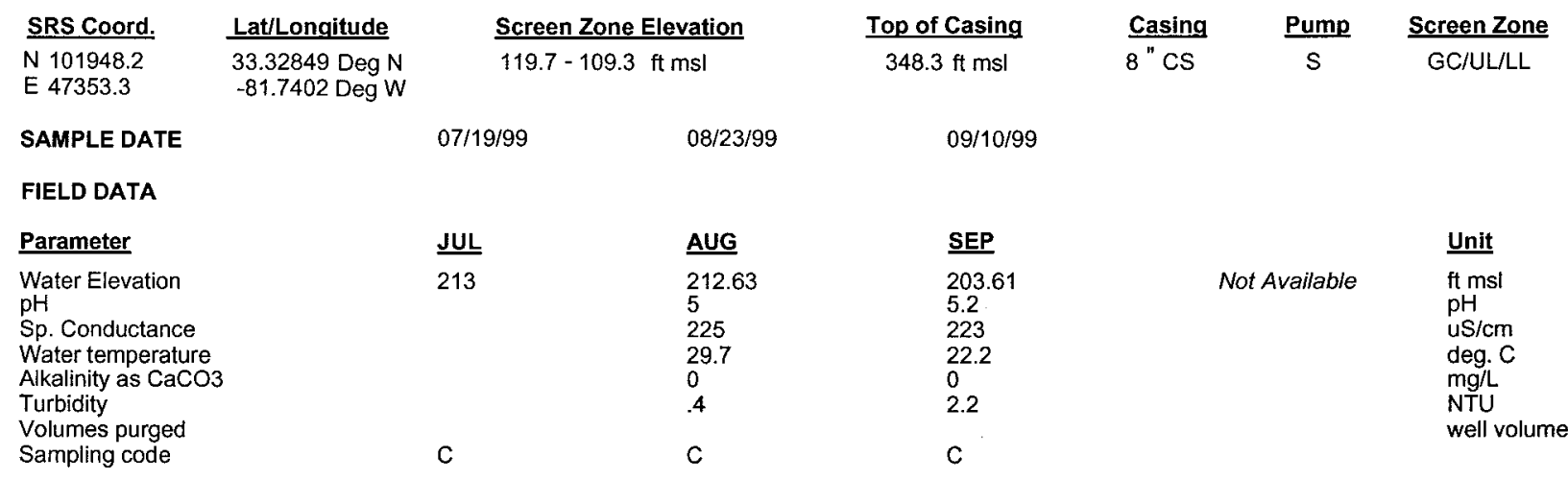

ANALYTICAL. DATA

I. Groundwater Protection Standard

261 Appendix VIII/264 Appendix IX Hazardous Constituents

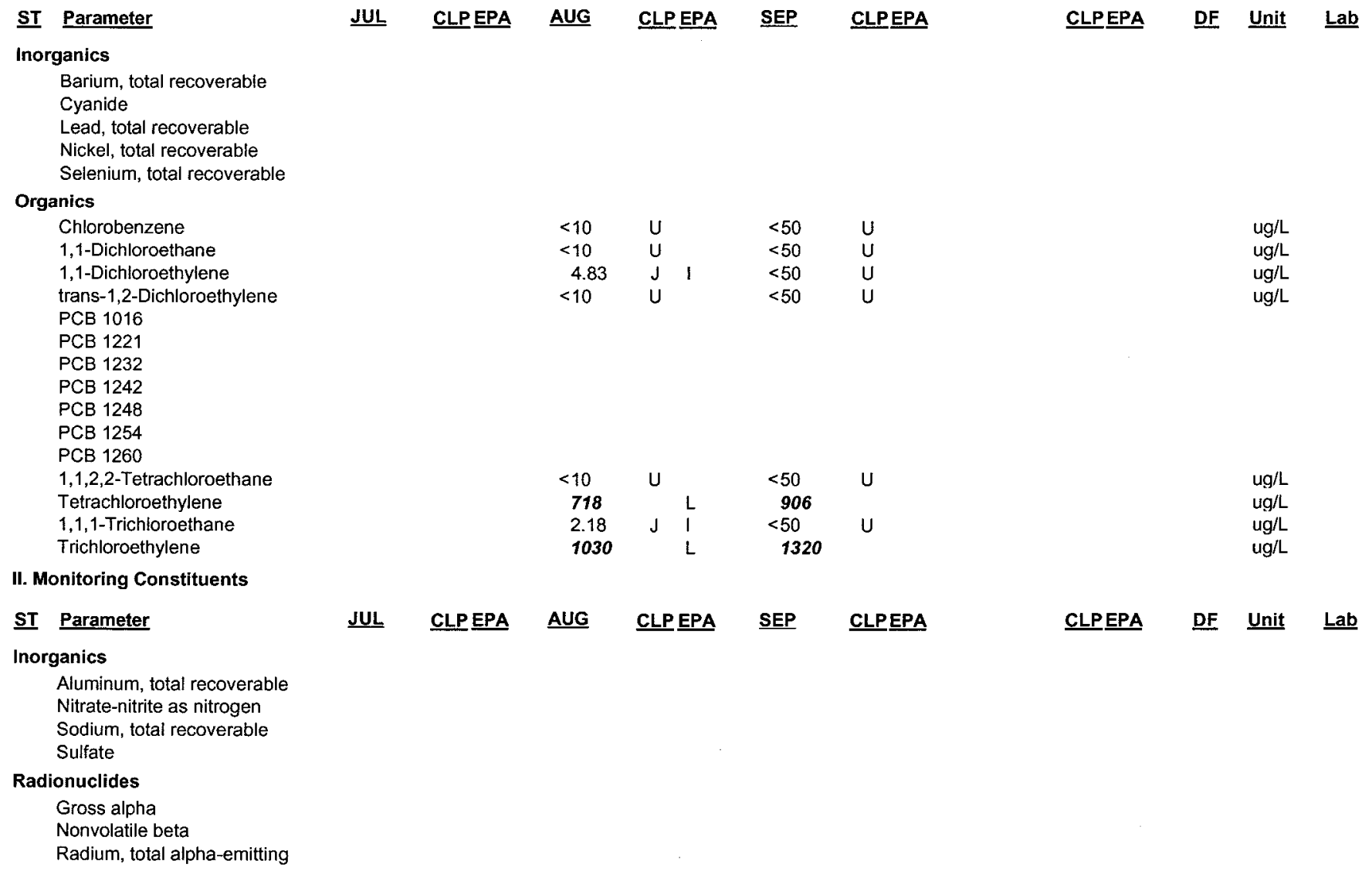

Notes: Concentrations in bold italics exceed the groundwater protection or monitoring constituent standards listed in Appendix A. Synchronous water levels are measured over a 3-5 day period or less. Dilution factors and Laboratory Data are October 1999 data only.

$+=$ exceeded the groundwater protection or monitoring constituent standards listed in Appendix A for October 1999. 
Table D-5a. Groundwater Monitoring Results for Recovery Wells, July-October 1999 (Cont.) WELL RWM 9

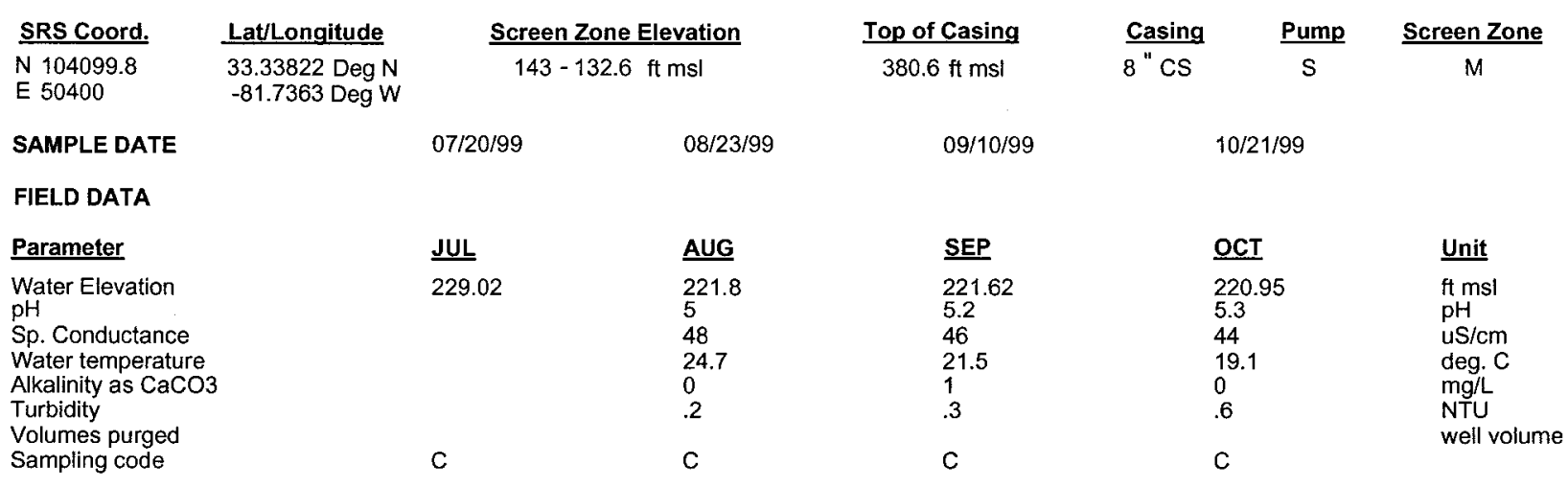

\section{ANALYTICAL DATA}

I. Groundwater Protection Standard

261 Appendix VIII/264 Appendix IX Hazardous Constituents

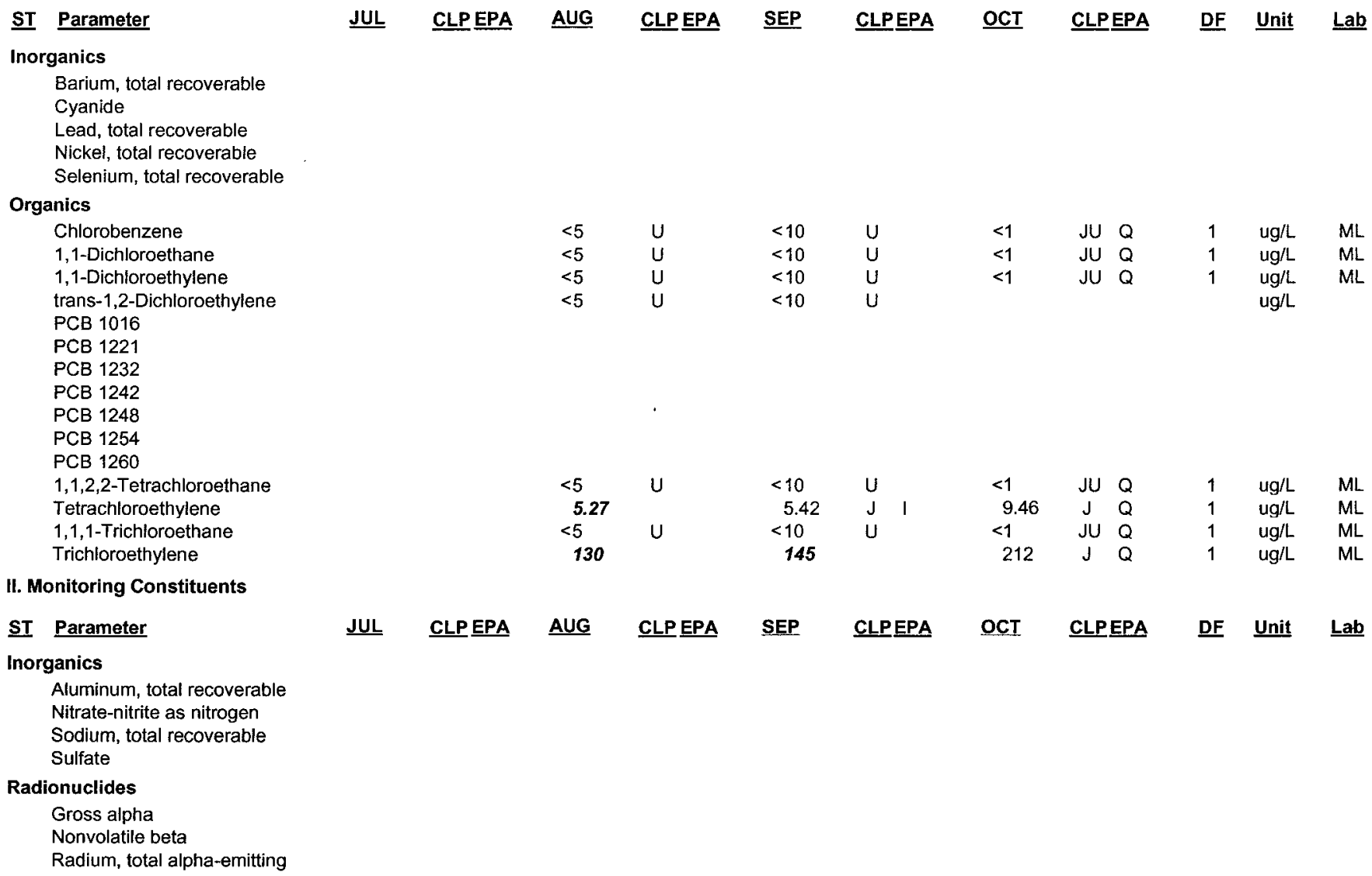

Notes: Concentrations in bold italics exceed the groundwater protection or monitoring constituent standards listed in Appendix A. Synchronous water levels are measured over a 3-5 day period or less. Dilution factors and Laboratory Data are October 1999 data only.

$+=$ exceeded the groundwater protection or monitoring constituent standards listed in Appendix A for October 1999. 
Table D-5a. Groundwater Monitoring Results for Recovery Wells, July-October 1999 (Cont.) WELL RWM 10

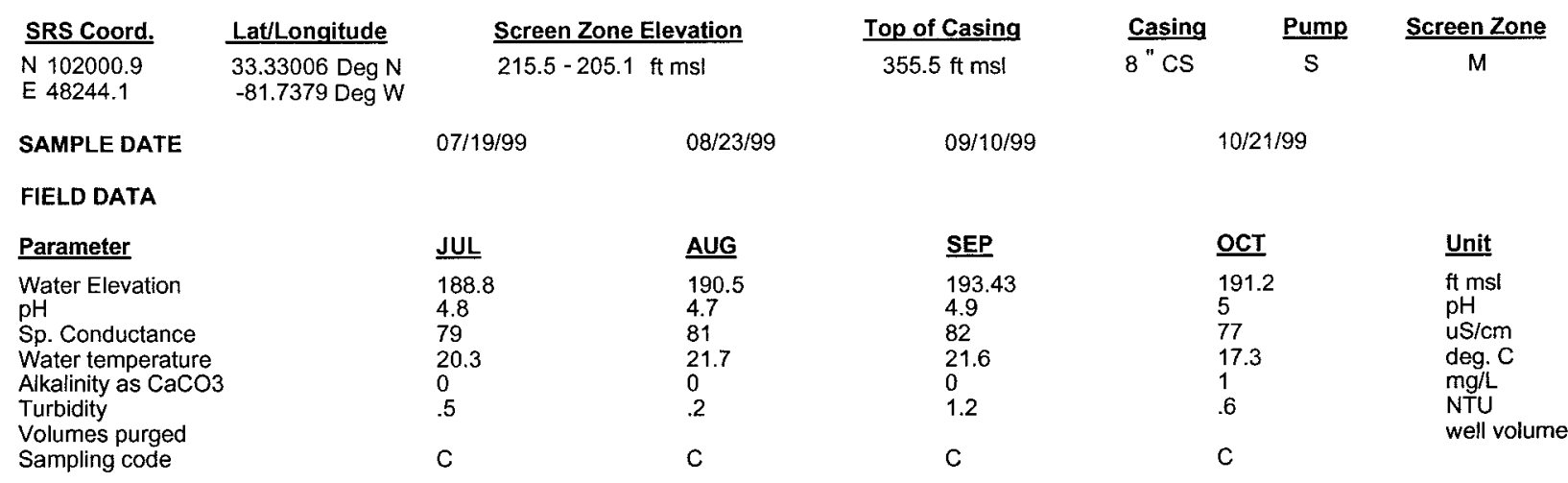

\section{ANALYTICAL DATA}

I. Groundwater Protection Standard

261 Appendix VIII/264 Appendix IX Hazardous Constituents

\begin{tabular}{|c|c|c|c|c|c|c|c|c|c|c|c|c|}
\hline$\underline{\mathbf{S T}}$ & Parameter & JUL & CLP EPA & $\underline{\text { AUG }}$ & CLPEPA & SEP & CLPEPA & $\underline{\text { OCT }}$ & CLPEPA & DF & Unit & $\underline{\mathrm{Lab}}$ \\
\hline \multicolumn{13}{|c|}{ Inorganics } \\
\hline \multicolumn{13}{|c|}{ Barium, total recoverable } \\
\hline \multicolumn{13}{|c|}{ Cyanide } \\
\hline \multicolumn{13}{|c|}{ Lead, total recoverable } \\
\hline \multicolumn{13}{|c|}{ Nickel, total recoverable } \\
\hline \multicolumn{13}{|c|}{ Selenium, total recoverable } \\
\hline \multicolumn{13}{|c|}{ Organics } \\
\hline & Chlorobenzene & $<250$ & $\mathrm{U}$ & $<250$ & $u$ & $<500$ & $\mathrm{U}$ & $<50$ & JU $Q$ & 50 & $\mathrm{ug} / \mathrm{L}$ & $M L$ \\
\hline & 1,1-Dichloroethane & $<250$ & $U$ & $<250$ & $\mathrm{U}$ & $<500$ & U & $<50$ & JU $Q$ & 50 & $\mathrm{ug} / \mathrm{L}$ & $M L$ \\
\hline & 1,1-Dichloroethylene & $<250$ & $U$ & $<250$ & $\mathrm{U}$ & $<500$ & $u$ & $<50$ & JU $Q$ & 50 & $\mathrm{ug} / \mathrm{L}$ & ML \\
\hline & trans-1,2-Dichloroethylene & $<250$ & $U$ & $<250$ & $\mathrm{U}$ & $<500$ & $\mathrm{U}$ & & & & $\mathrm{ug} / \mathrm{L}$ & \\
\hline & PCB 1016 & $<1.05$ & $\mathrm{U}$ & & & & & & & & $\mathrm{ug} / \mathrm{L}$ & \\
\hline & PCB 1221 & $<2.1$ & $U$ & & & & & & & & $u g / L$ & \\
\hline & PCB 1232 & $<1.05$ & $U$ & & & & & & & & $\mathrm{ug} / \mathrm{L}$ & \\
\hline & PCB 1242 & $<1.05$ & $\mathrm{U}$ & & & & & & & & $\mathrm{ug} / \mathrm{L}$ & \\
\hline & PCB 1248 & $<1.05$ & $\mathrm{U}$ & & & & & & & & $\mathrm{ug} / \mathrm{L}$ & \\
\hline & PCB 1254 & $<1.05$ & $u$ & & & & & & & & $u g / L$ & \\
\hline & PCB 1260 & $<1.05$ & $u$ & & & & & & & & $\mathrm{ug} / \mathrm{L}$ & \\
\hline & $1,1,2,2$-Tetrachloroethane & $<250$ & $\bigcup$ & $<250$ & $U$ & $<500$ & $\mathrm{u}$ & $<50$ & $J U Q$ & 50 & $\mathrm{ug} / \mathrm{L}$ & $M L$ \\
\hline & Tetrachloroethylene & 7180 & & 7610 & & 7140 & & 8950 & $\mathrm{~J} \quad \mathrm{KQ}$ & 50 & $u g / L$ & ML \\
\hline & $1,1,1$-Trichloroethane & $<250$ & $U$ & $<250$ & $U$ & $<500$ & $\mathrm{u}$ & $<50$ & JU $Q$ & 50 & $\mathrm{ug} / \mathrm{L}$ & ML \\
\hline & Trichloroethylene & 4560 & & 4600 & & 4150 & & 5140 & $\mathrm{~J} \quad \mathrm{KQ}$ & 50 & $u g / L$ & $\mathrm{ML}$ \\
\hline \multicolumn{13}{|c|}{ II. Monitoring Constituents } \\
\hline$\underline{\text { ST }}$ & Parameter & $\underline{\text { JUL }}$ & CLP EPA & $\underline{\text { AUG }}$ & CLP EPA & $\underline{\text { SEP }}$ & CL.PEPA & $\underline{\text { OCT }}$ & CLPEPA & DF & Unit & $\underline{L a b}$ \\
\hline \multicolumn{13}{|c|}{ Inorganics } \\
\hline \multicolumn{13}{|c|}{ Aluminum, total recoverable } \\
\hline \multicolumn{13}{|c|}{ Nitrate-nitrite as nitrogen } \\
\hline \multicolumn{13}{|c|}{ Sodium, total recoverable } \\
\hline \multicolumn{13}{|c|}{ Sulfate } \\
\hline \multicolumn{13}{|c|}{ Radionuclides } \\
\hline \multirow{2}{*}{\multicolumn{12}{|c|}{$\begin{array}{l}\text { Gross alpha } \\
\text { Nonvolatile beta }\end{array}$}} & \\
\hline & & & & & & & & & & & & \\
\hline & Radium, total alpha-emitting & & & & & & & & & & & \\
\hline
\end{tabular}

Notes: Concentrations in bold italics exceed the groundwater protection or monitoring constituent standards listed in Appendix A. Synchronous water levels are measured over a 3-5 day period or less. Dilution factors and Laboratory Data are October 1999 data only.

$+=$ exceeded the groundwater protection or monitoring constituent standards listed in Appendix A for October 1999. 
Table D-5a. Groundwater Monitoring Results for Recovery Wells, July-October 1999 (Cont.) WELL RWM 11

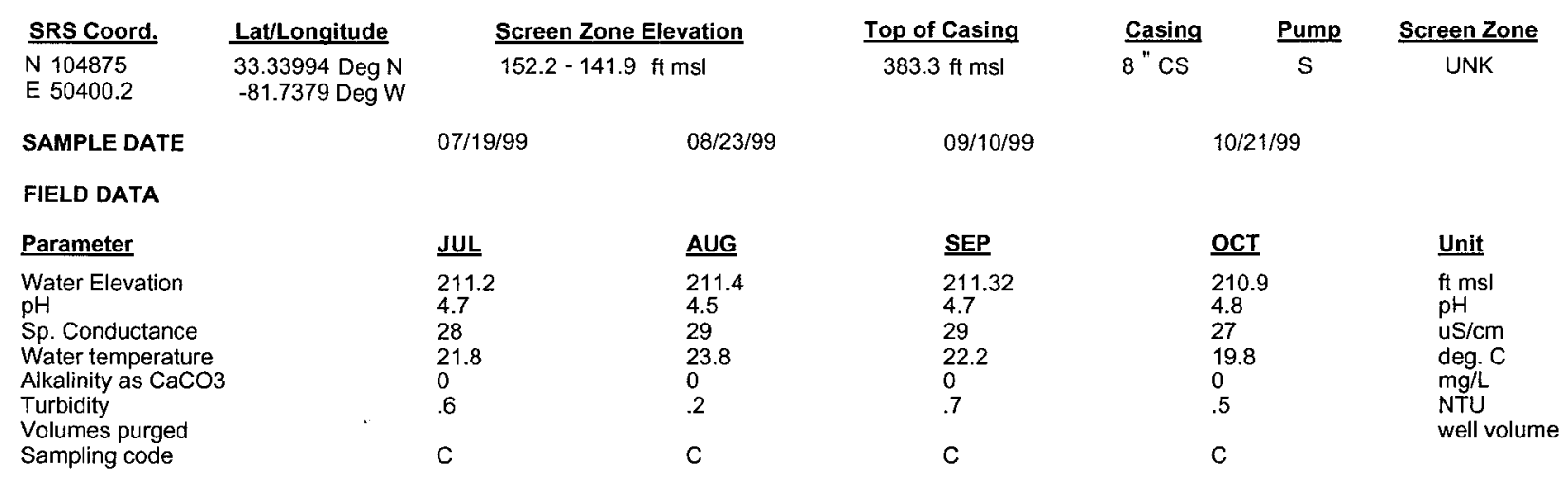

\section{ANALYTICAL DATA}

I. Groundwater Protection Standard

261 Appendix VIII/264 Appendix IX Hazardous Constituents

\begin{tabular}{|c|c|c|c|c|c|c|c|c|c|c|c|c|}
\hline$\underline{\text { ST }}$ & Parameter & $\underline{\text { JUL }}$ & CLP EPA & AUG & CLPEPA & SEP & CLPEPA & $\underline{\mathrm{OCT}}$ & CLPEPA & $\underline{\text { DF }}$ & Unit & $\underline{L a b}$ \\
\hline \multicolumn{13}{|c|}{ Inorganics } \\
\hline \multicolumn{13}{|c|}{ Barium, total recoverable } \\
\hline \multicolumn{13}{|c|}{ Cyanide } \\
\hline \multicolumn{13}{|c|}{ Lead, total recoverable } \\
\hline \multicolumn{13}{|c|}{ Nickel, total recoverable } \\
\hline & Selenium, total recoverable & \\
\hline \multicolumn{13}{|c|}{ Organics } \\
\hline & Chlorobenzene & $<50$ & $U$ & $<50$ & $U$ & $<50$ & $\mathrm{U}$ & $<5$ & $J U Q$ & 5 & $u g / L$ & ML \\
\hline & 1,1-Dichloroethane & $<50$ & $\mathrm{U}$ & $<50$ & U & $<50$ & U & $<5$ & $\mathrm{JU} \bar{Q}$ & 5 & $\mathrm{ug} / \mathrm{L}$ & $M L$ \\
\hline & 1,1-Dichloroethylene & $<50$ & $U$ & $<50$ & $\mathrm{U}$ & $<50$ & $U$ & $<5$ & $J U Q$ & 5 & $\mathrm{ug} / \mathrm{L}$ & $\mathrm{ML}$ \\
\hline & trans-1,2-Dichloroethylene & $<50$ & $\mathrm{U}$ & $<50$ & $U$ & $<50$ & $U$ & & & & $u g / L$ & \\
\hline \multicolumn{13}{|c|}{ PCB 1016} \\
\hline \multicolumn{13}{|c|}{ PCB 1221} \\
\hline \multicolumn{13}{|c|}{ PCB 1232} \\
\hline \multicolumn{13}{|c|}{ PCB 1242} \\
\hline \multicolumn{13}{|c|}{ PCB 1248} \\
\hline \multicolumn{13}{|c|}{ PCB 1254} \\
\hline \multicolumn{13}{|c|}{ PCB 1260} \\
\hline & $1,1,2,2-$-Tetrachloroethane & $<50$ & $U$ & $<50$ & $U$ & $<50$ & U & $<5$ & JU $Q$ & 5 & $\mathrm{ug} / \mathrm{L}$ & ML \\
\hline & Tetrachioroethylene & 112 & & 50.1 & & 43.6 & J I & 51.9 & $J \quad Q$ & 5 & $u g / L$ & ML \\
\hline & 1,1,1-Trichloroethane & $<50$ & $\mathrm{U}$ & $<50$ & $U$ & $<50$ & $\mathrm{U}$ & $<5$ & JU $\vec{Q}$ & 5 & ug/L & ML \\
\hline & Trichloroethylene & 749 & & 785 & & 736 & & 820 & $J \quad Q$ & 5 & $u g / L$ & ML \\
\hline \multicolumn{13}{|c|}{ II. Monitoring Constituents } \\
\hline ST & Parameter & JULL & CLPEPA & $\underline{\text { AUG }}$ & $\underline{\text { CLP EPA }}$ & $\underline{\text { SEP }}$ & CLP토A & OCT & CLPEPA & $\underline{\text { DF }}$ & $\underline{\text { Unit }}$ & $\underline{\text { Lab }}$ \\
\hline \multicolumn{13}{|c|}{ Inorganics } \\
\hline \multicolumn{12}{|c|}{ Aluminum, total recoverable } & \\
\hline \multicolumn{12}{|c|}{ Nitrate-nitrite as nitrogen } & \\
\hline \multicolumn{12}{|c|}{ Sodium, total recoverable } & \\
\hline \multicolumn{12}{|c|}{ Sulfate } & \\
\hline \multicolumn{13}{|c|}{ Radionuclides } \\
\hline \multirow{2}{*}{\multicolumn{12}{|c|}{$\begin{array}{l}\text { Gross alpha } \\
\text { Nonvolatile beta }\end{array}$}} & \\
\hline & & & & & & & & & & & & \\
\hline
\end{tabular}

Notes: Concentrations in bold italics exceed the groundwater protection or monitoring constituent standards listed in Appendix A. Synchronous water levels are measured over a 3-5 day period or less. Dilution factors and Laboratory Data are October 1999 data only.

$+=$ exceeded the groundwater protection or monitoring constituent standards listed in Appendix A for October 1999. 
Table D-5a. Groundwater Monitoring Results for Recovery Wells, July-October 1999 (Cont.) WELL RWM 12

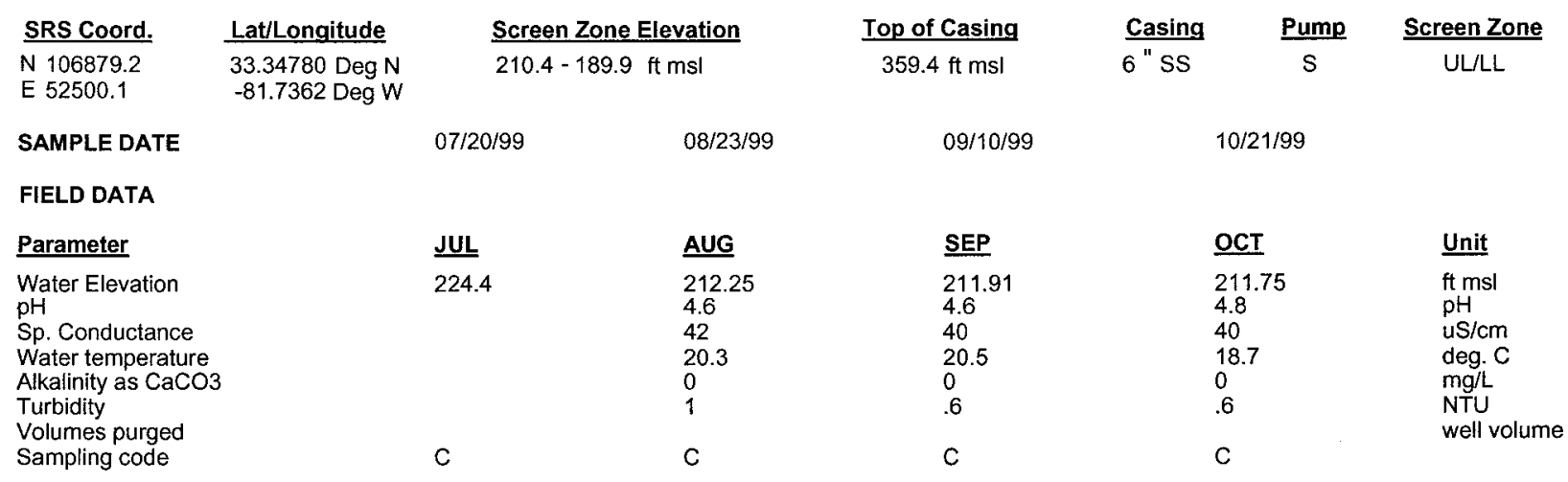

\section{ANALYTICAL DATA}

I. Groundwater Protection Standard

261 Appendix VIII/264 Appendix IX Hazardous Constituents

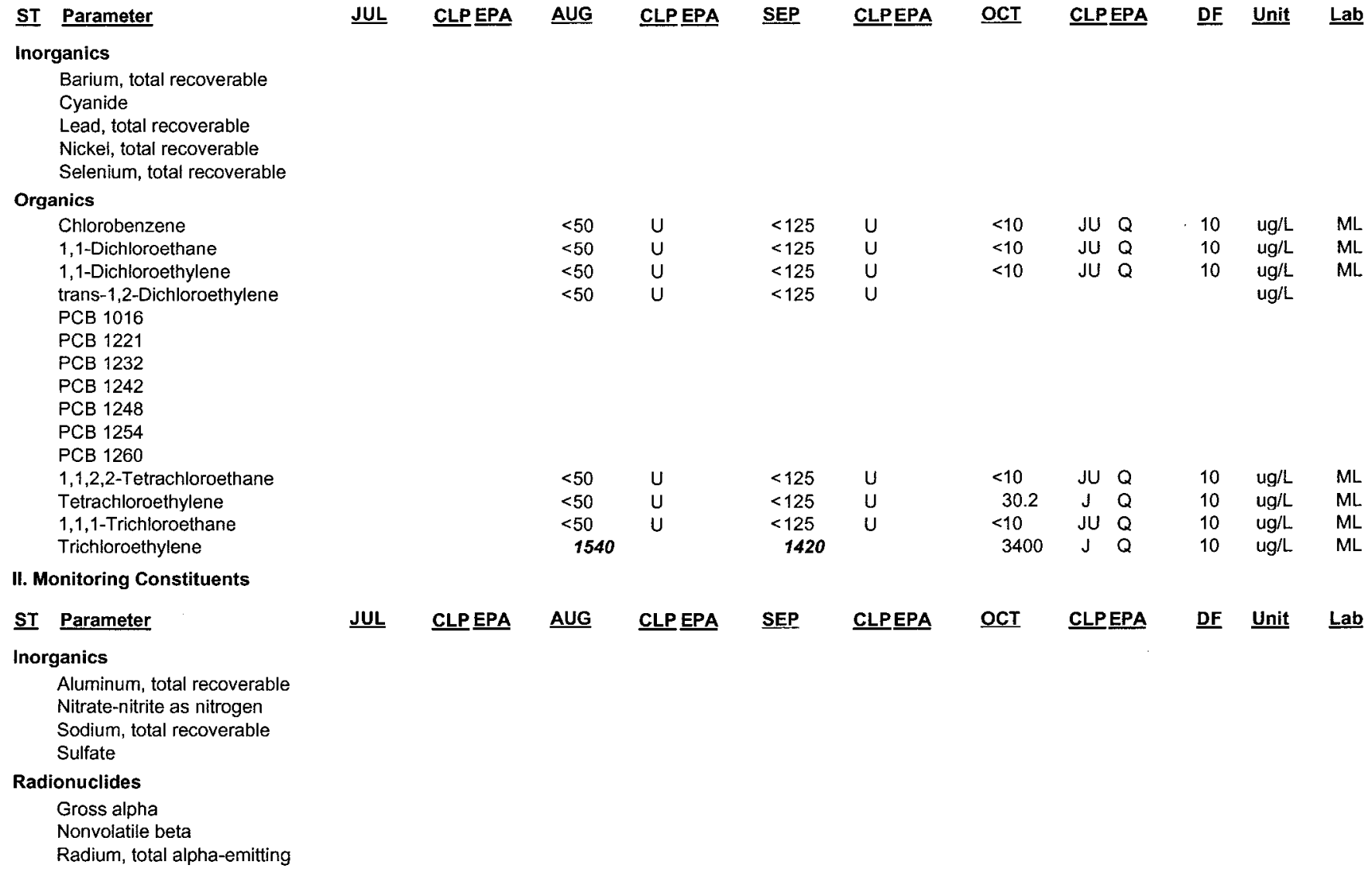

Notes: Concentrations in bold italics exceed the groundwater protection or monitoring constituent standards listed in Appendix A. Synchronous water levels are measured over a 3-5 day period or less. Dilution factors and Laboratory Data are October 1999 data only.

$+=$ exceeded the groundwater protection or monitoring constituent standards listed in Appendix A for October 1999. 
Table D-5a. Groundwater Monitoring Results for Recovery Wells, July-October 1999 (Cont.) WELL RWM 13B

\begin{tabular}{|c|c|c|c|c|c|c|}
\hline SRS Coord. & Lat/Longitude & \multicolumn{2}{|c|}{ Screen Zone Elevation } & Top of Casing & Casing & Screen Zone \\
\hline $\begin{array}{l}\text { N } 105803.3 \\
\text { E 53516.3 }\end{array}$ & $\begin{array}{l}\text { 33.34708 Deg } N \\
-81.7315 \operatorname{Deg} W\end{array}$ & $138.2-113$ & $\mathrm{ft} \mathrm{msl}$ & $336.2 \mathrm{ft} \mathrm{msl}$ & $6 " \mathrm{cs}$ & $\mathrm{LL}$ \\
\hline SAMPLE DATE & & $07 / 19 / 99$ & $08 / 23 / 99$ & $09 / 10 / 99$ & $10 / 21 / 99$ & \\
\hline FIELD DATA & & & & & & \\
\hline Parameter & & $\underline{\mathrm{JUL}}$ & AUG & SEP & $\underline{\mathrm{OCT}}$ & $\underline{\text { Unit }}$ \\
\hline $\begin{array}{l}\text { Water Elevation } \\
\text { pH } \\
\text { Sp. Conductance } \\
\text { Water temperature } \\
\text { Alkalinity as } \mathrm{CaCO} 3 \\
\text { Turbidity } \\
\text { Volumes purged } \\
\text { Sampling }\end{array}$ & & $\begin{array}{l}202.2 \\
5 \\
21 \\
21.8 \\
0 \\
1.8\end{array}$ & $\begin{array}{l}202.09 \\
4.8 \\
22 \\
21.3 \\
0 \\
.3\end{array}$ & $\begin{array}{l}201.69 \\
4.9 \\
21 \\
19.7 \\
0 \\
1.4\end{array}$ & $\begin{array}{l}202.7 \\
5 \\
20 \\
18.7 \\
1 \\
.3\end{array}$ & $\begin{array}{l}\mathrm{ft} \mathrm{msl} \\
\mathrm{pH} \\
\mathrm{uS} / \mathrm{cm} \\
\text { deg. C } \\
\mathrm{mg} / \mathrm{L} \\
\text { NTU } \\
\text { well volume }\end{array}$ \\
\hline Sampling code & & C & C & C & c & \\
\hline
\end{tabular}

\section{ANALYTICAL DATA}

I. Groundwater Protection Standard

261 Appendix VIII/264 Appendix IX Hazardous Constituents

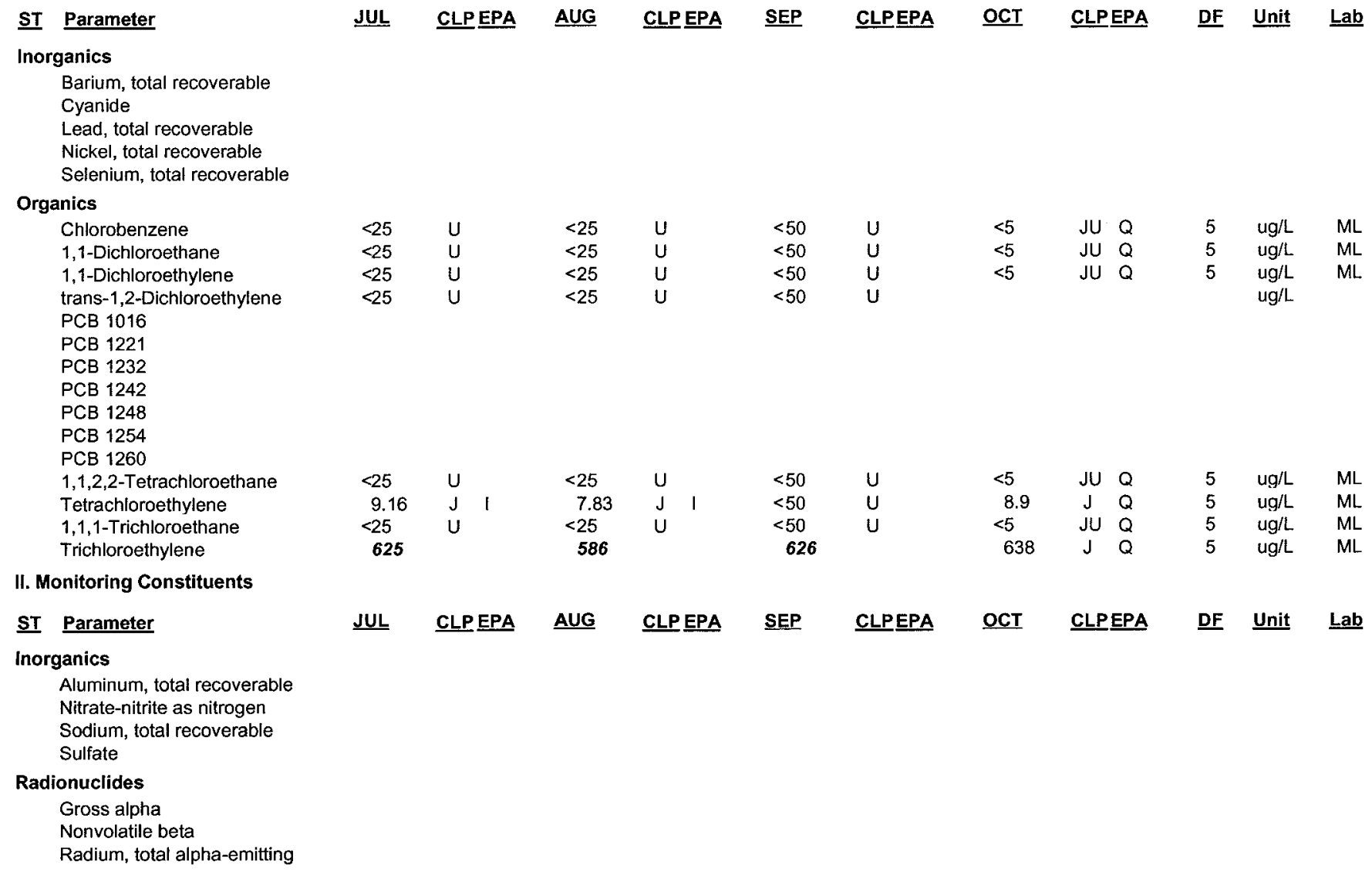

\footnotetext{
Notes: Concentrations in bold italics exceed the groundwater protection or monitoring constituent standards listed in Appendix A. Synchronous water levels are measured over a 3-5 day period or less. Dilution factors and Laboratory Data are October 1999 data only.

$+=$ exceeded the groundwater protection or monitoring constituent standards listed in Appendix A for October 1999.
} 
Table D-5a. Groundwater Monitoring Results for Recovery Wells, July-October 1999 (Cont.) WELL RWM 13C

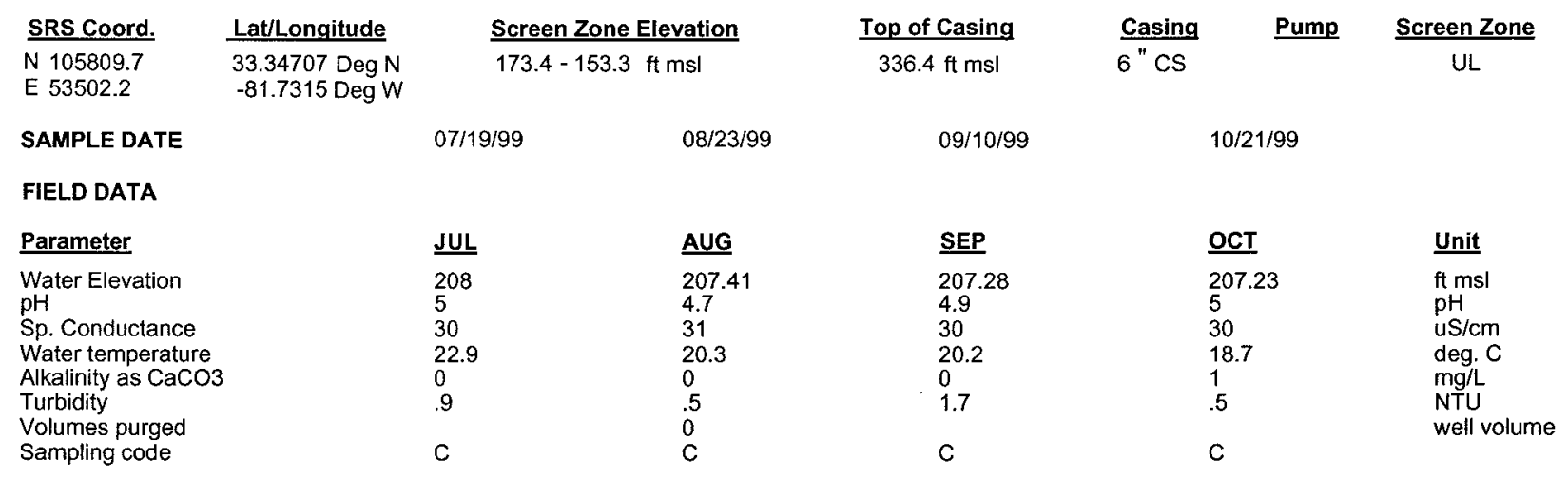

\section{ANALYTICAL DATA}

I. Groundwater Protection Standard

261 Appendix VIII/264 Appendix IX Hazardous Constituents

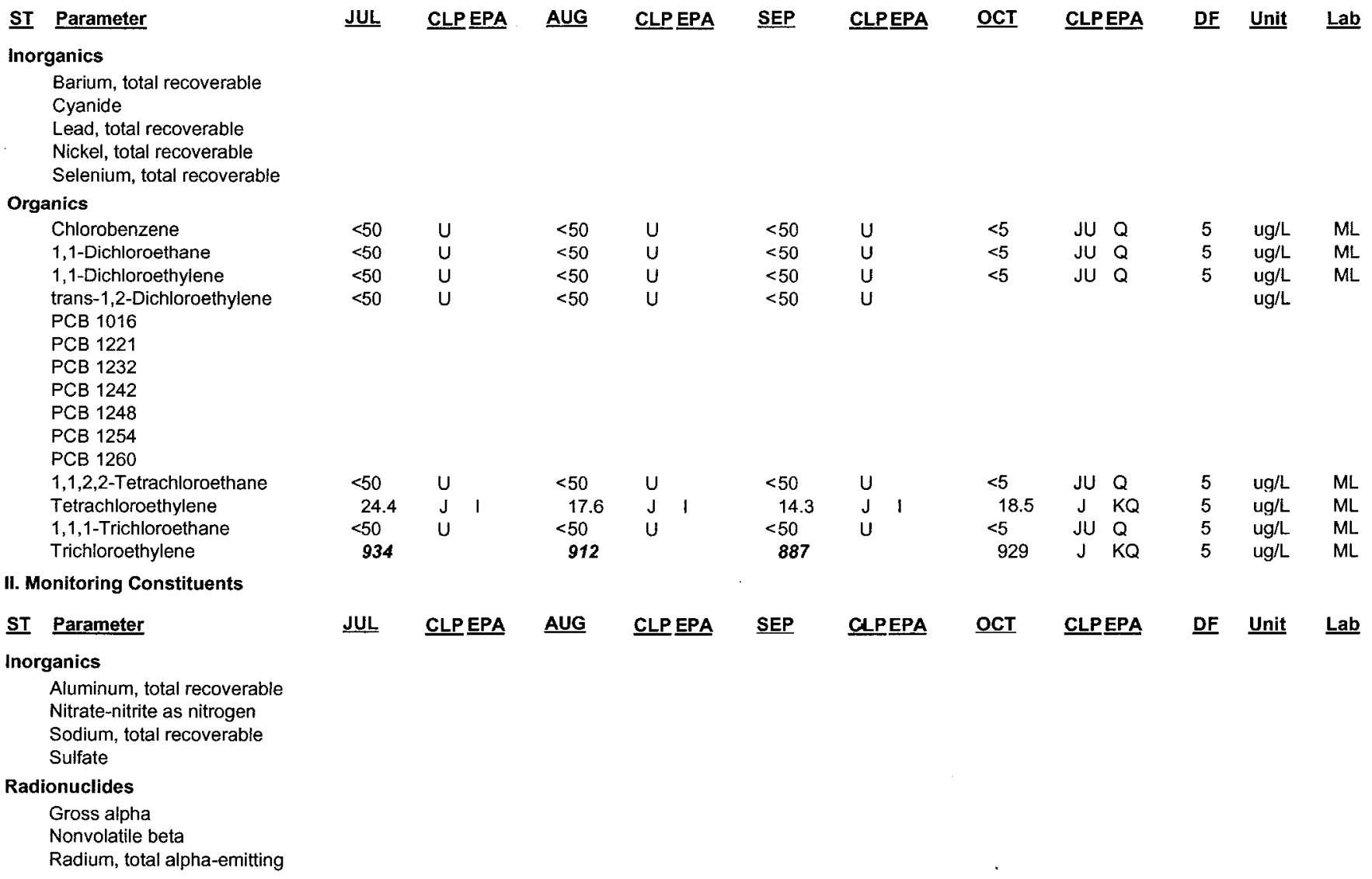

Notes: Concentrations in bold italics exceed the groundwater protection or monitoring constituent standards listed in Appendix A. Synchronous water levels are measured over a 3-5 day period or less. Dilution factors and Laboratory Data are October 1999 data only.

$+=$ exceeded the groundwater protection or monitoring constituent standards listed in Appendix A for October 1999. 
Table D-5a. Groundwater Monitoring Results for Recovery Wells, July-October 1999 (Cont.) WELL RWM 14B

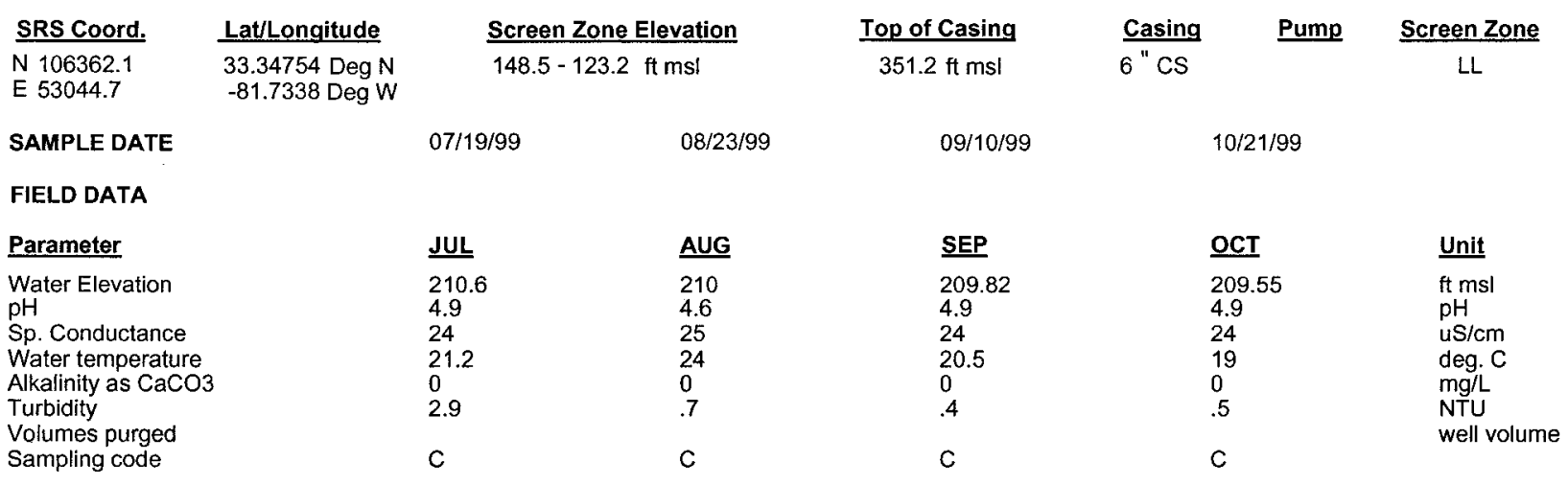

ANALYTICAL DATA

I. Groundwater Protection Standard

261 Appendix VIII/264 Appendix IX Hazardous Constituents

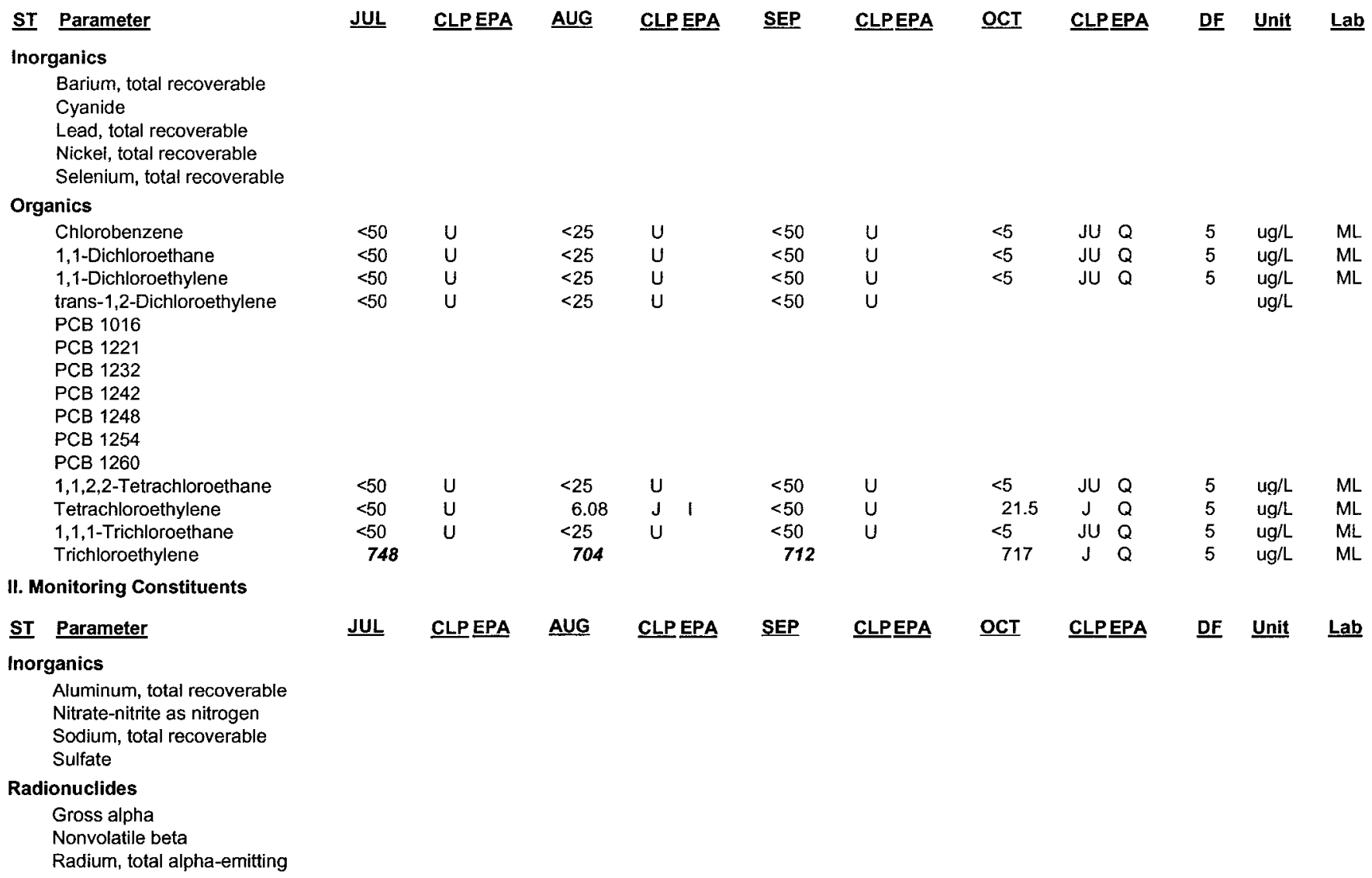

Notes: Concentrations in bold italics exceed the groundwater protection or monitoring constituent standards listed in Appendix A. Synchronous water levels are measured over a 3-5 day period or less. Dilution factors and Laboratory Data are October 1999 data only.

$+=$ exceeded the groundwater protection or monitoring constituent standards listed in Appendix A for October 1999. 
Table D-5a. Groundwater Monitoring Results for Recovery Wells, July-October 1999 (Cont.) WELL RWM 14C

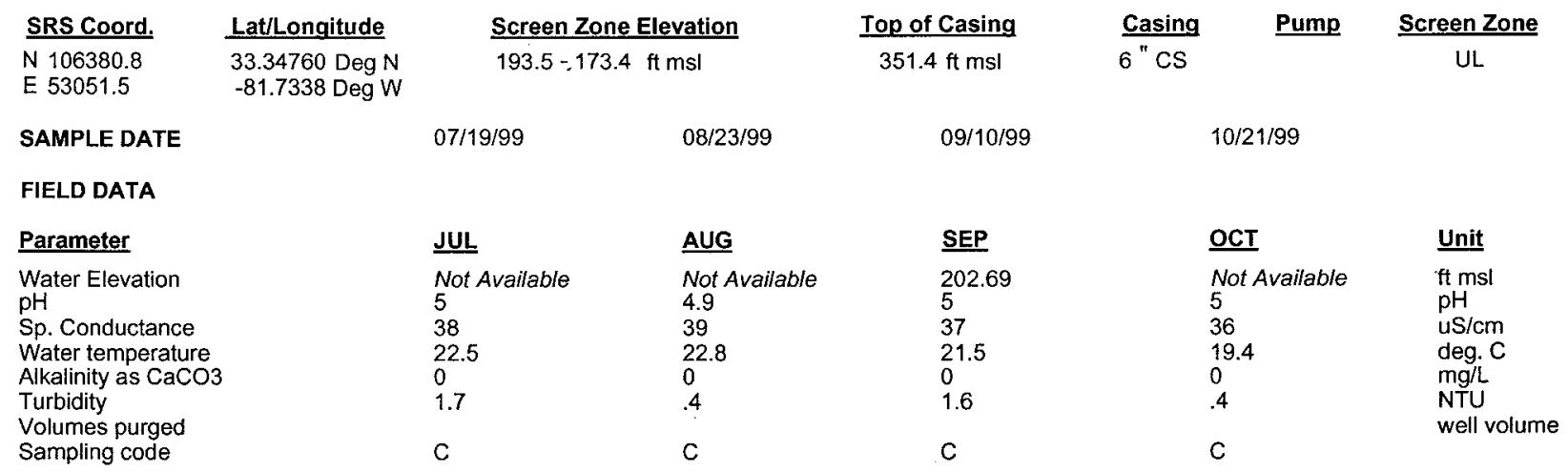

ANALYTICAL DATA

I. Groundwater Protection Standard

261 Appendix VIII/264 Appendix IX Hazardous Constituents

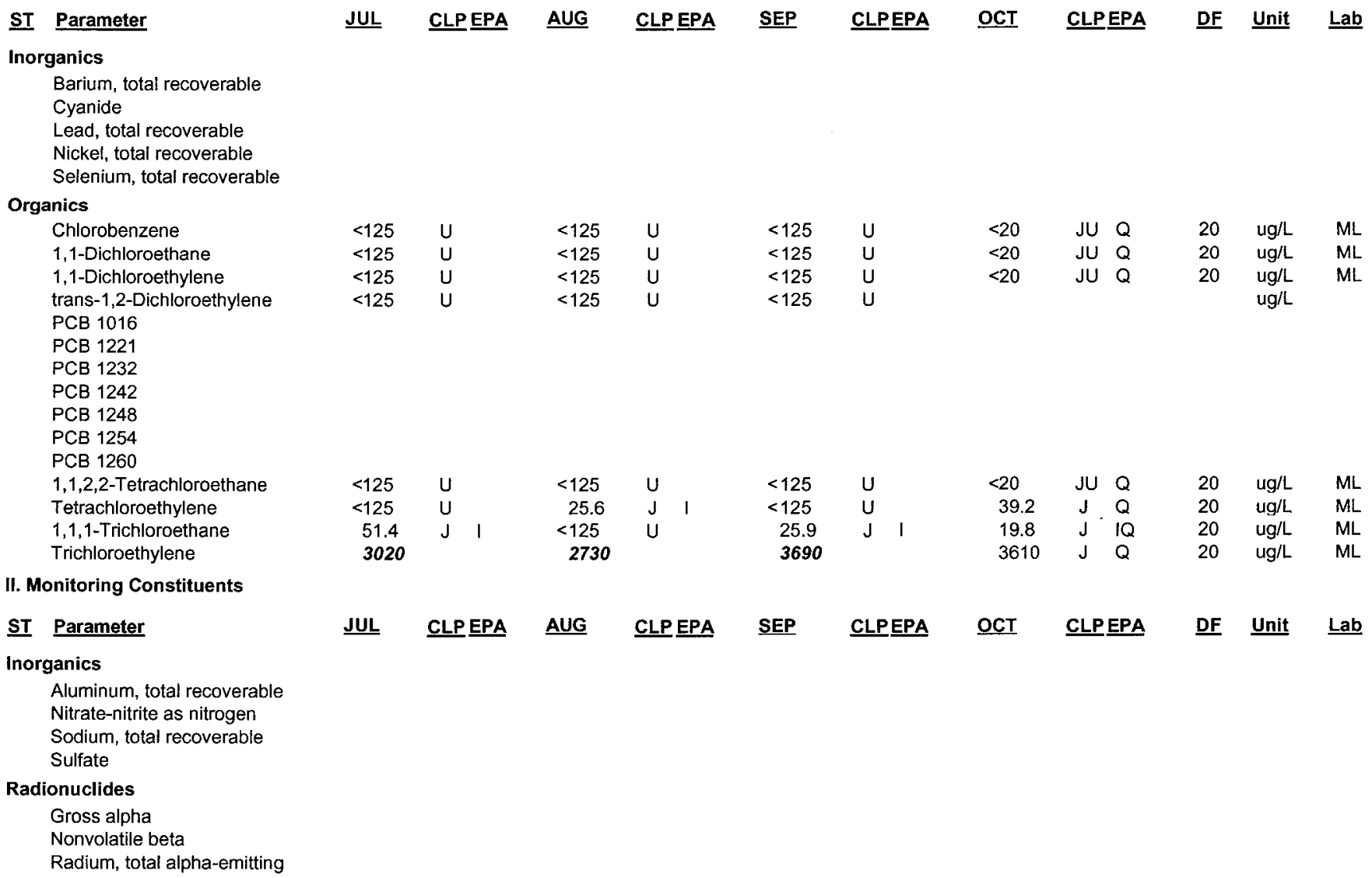


Table D-5a. Groundwater Monitoring Results for Recovery Wells, July-October 1999 (Cont.) WELL RWM 15B

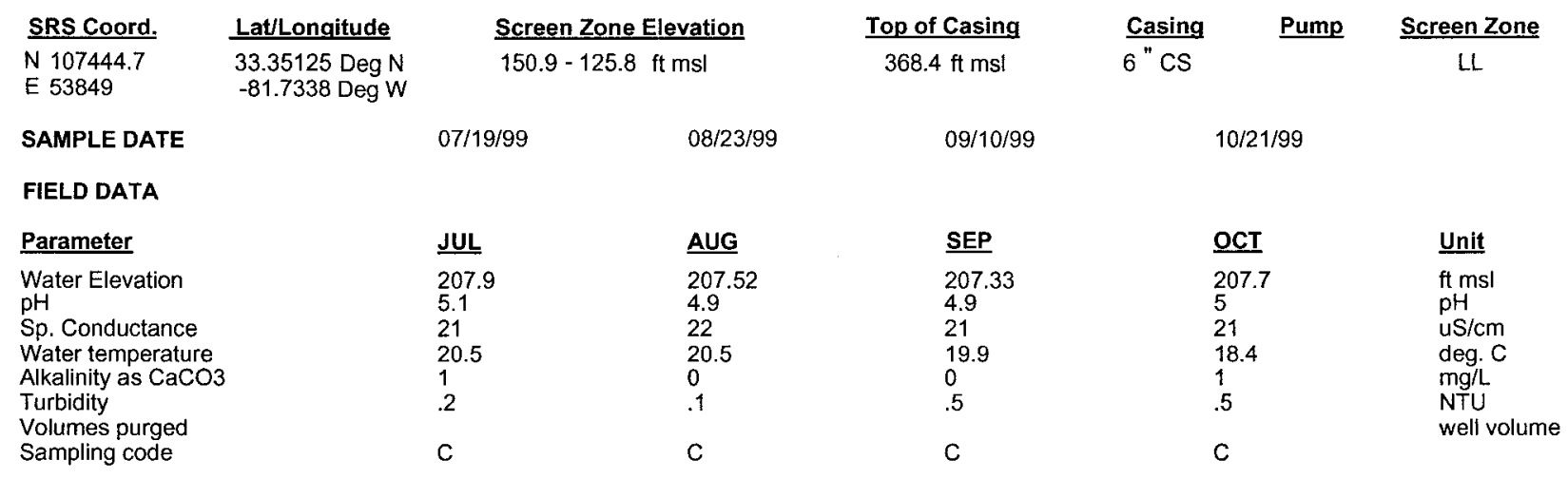

\section{ANALYTICAL DATA}

I. Groundwater Protection Standard

261 Appendix VIII/264 Appendix IX Hazardous Constituents

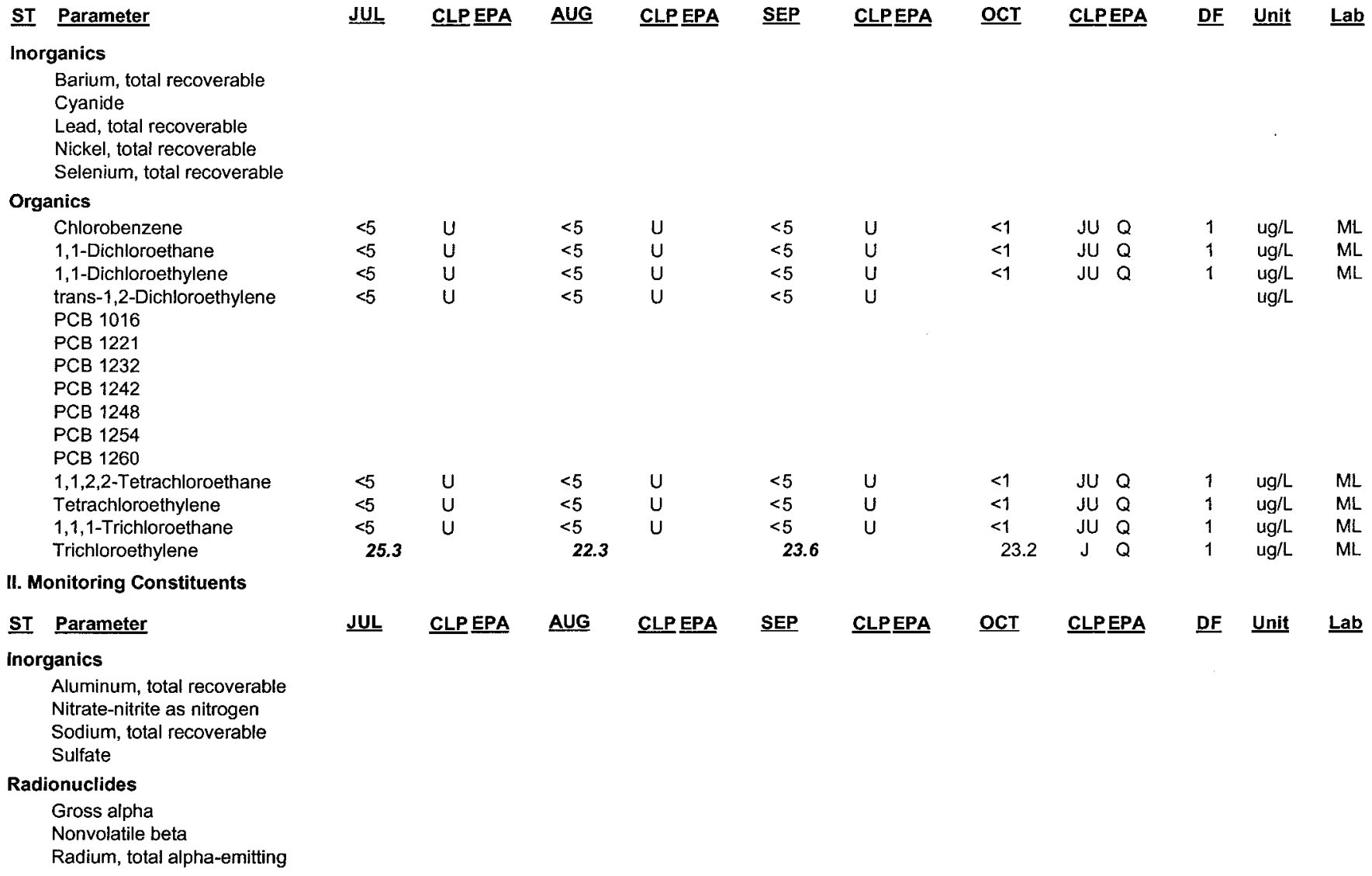

Notes: Concentrations in bold italics exceed the groundwater protection or monitoring constituent standards listed in Appendix A. Synchronous water levels are measured over a 3-5 day period or less. Dilution factors and Laboratory Data are October 1999 data only.

$+=$ exceeded the groundwater protection or monitoring constituent standards listed in Appendix A for October 1999. 
WSRC-TR-2000-00242

Unclassified

THIS PAGE LEFT BLANK INTENTIONALLY 
Table D-5b. Groundwater Monitoring Results for Recovery Wells, November 1999-February 2000 WELL RWM 1

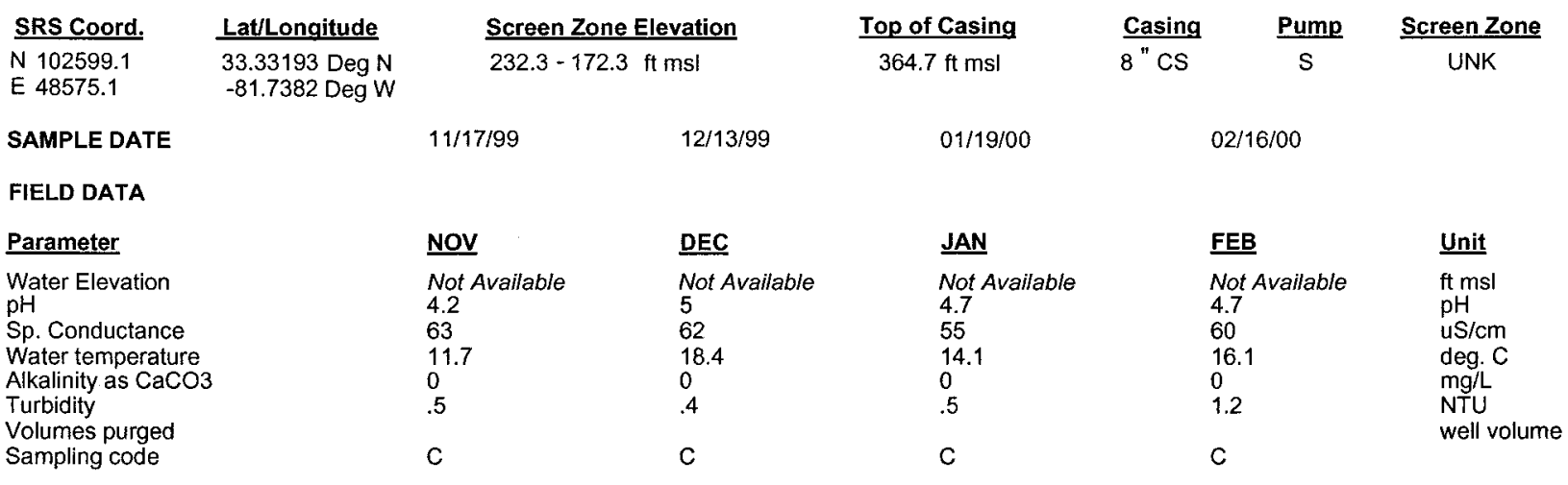

ANALYTICAL DATA

I. Groundwater Protection Standard

261 Appendix VIII/264 Appendix IX Hazardous Constituents

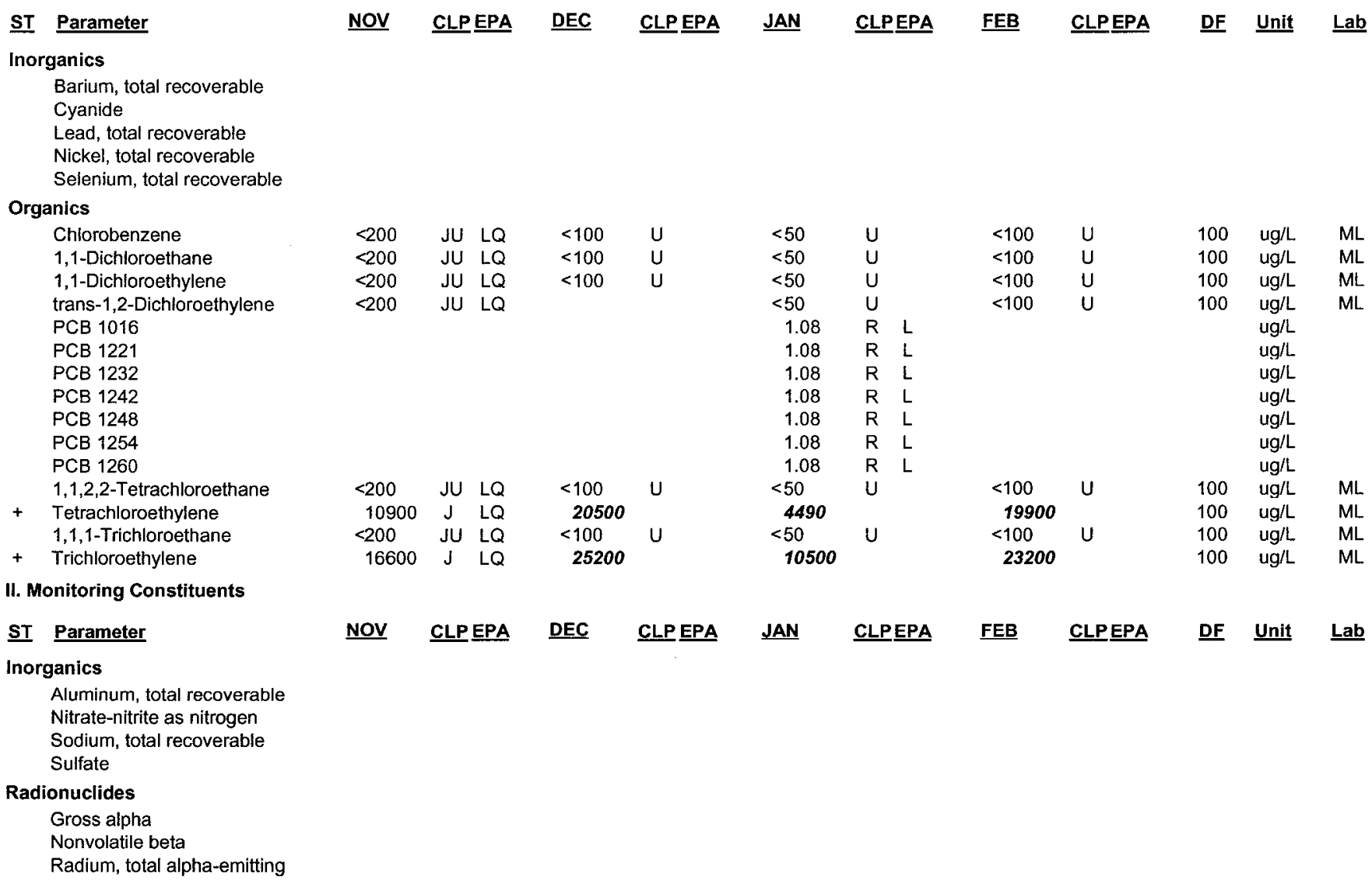

Notes: Concentrations in bold italics exceed the groundwater protection or monitoring constituent standards listed in Appendix A. Synchronous water levels are measured over a 3-5 day period or less. Dilution factors and Laboratory Data are February 2000 data only.

$+=$ exceeded the groundwater protection or monitoring constituent standards listed in Appendix A for February 2000. 
Table D-5b. Groundwater Monitoring Results for Recovery Wells, November 1999-February 2000 (Cont.) WELL RWM 2

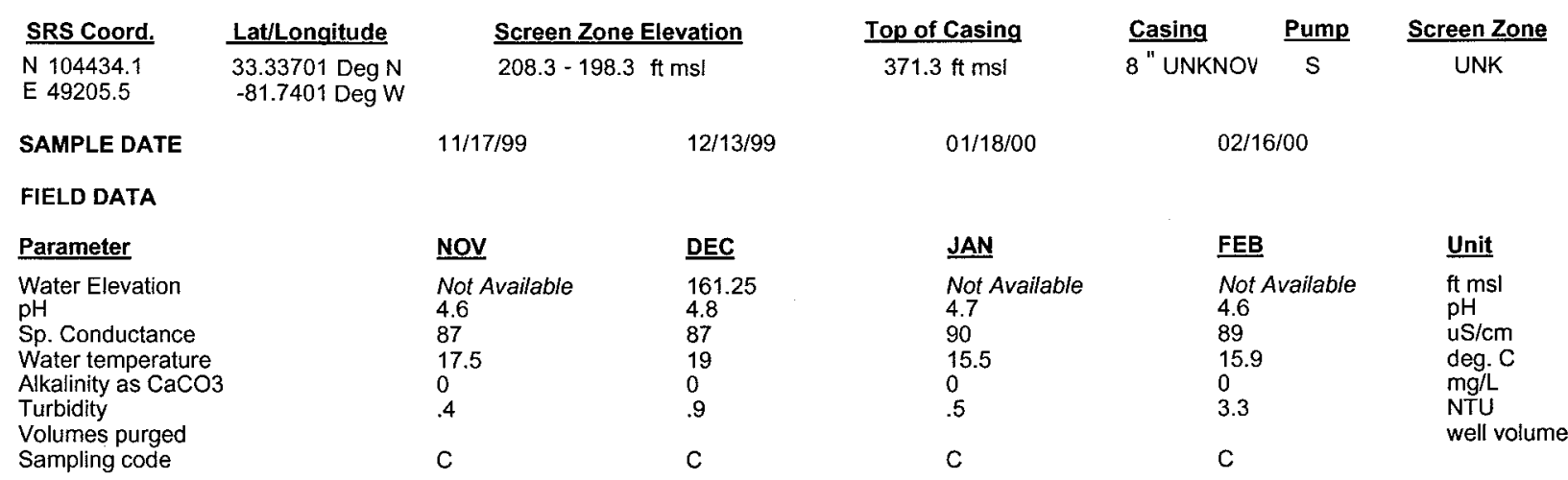

\section{ANALYTICAL DATA}

I. Groundwater Protection Standard

261 Appendix VIII/264 Appendix IX Hazardous Constituents

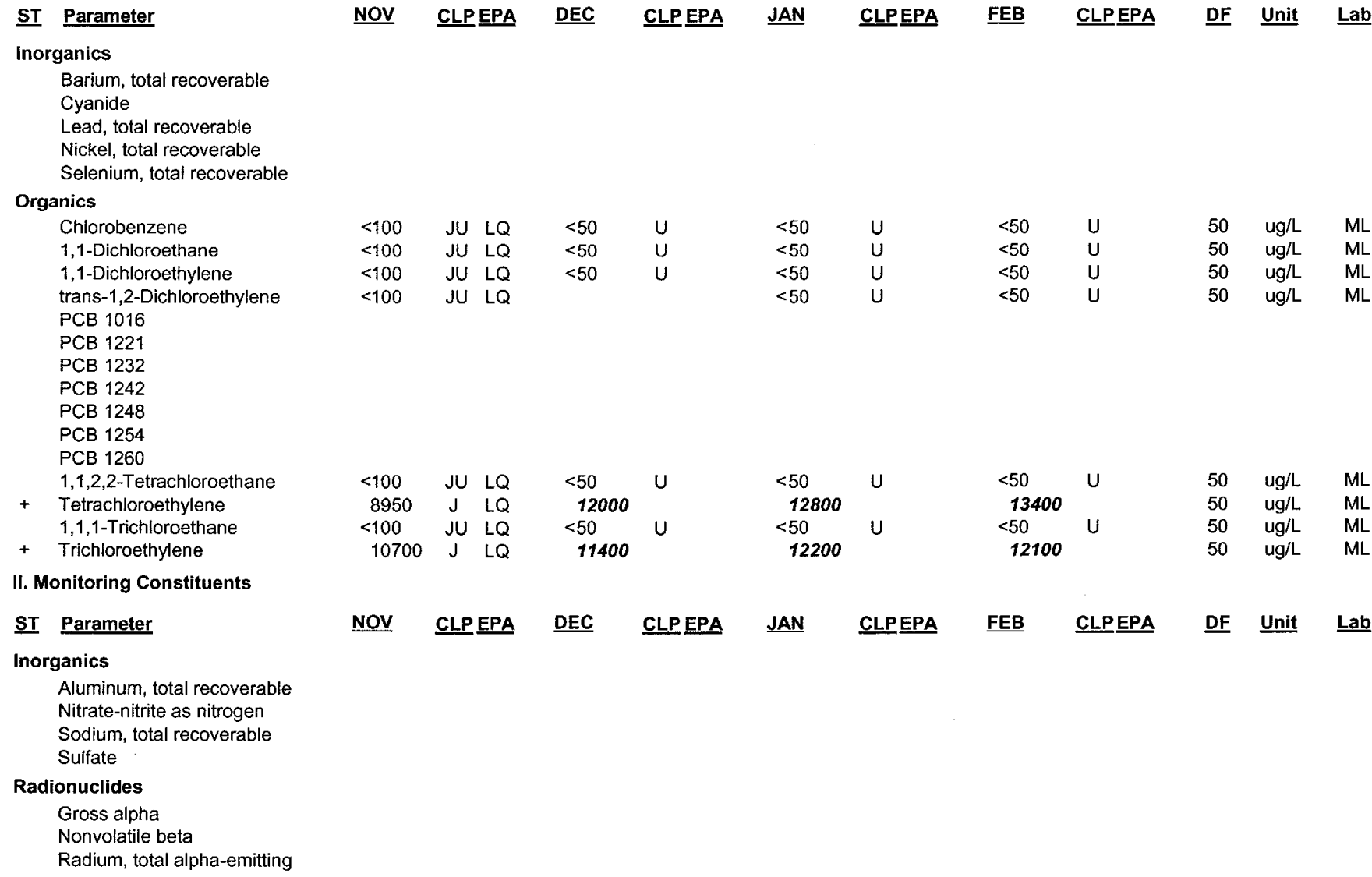

Notes: Concentrations in bold italics exceed the groundwater protection or monitoring constituent standards listed in Appendix A. Synchronous water levels are measured over a 3-5 day period or less. Dilution factors and Laboratory Data are February 2000 data only.

$+=$ exceeded the groundwater protection or monitoring constituent standards listed in Appendix A for February 2000. 
Table D-5b. Groundwater Monitoring Results for Recovery Wells, November 1999-February 2000 (Cont.) WELL RWM 3

\begin{tabular}{|c|c|c|c|c|c|c|c|}
\hline SRS Coord. & Lat/Longitude & \multicolumn{2}{|c|}{ Screen Zone Elevation } & Top of Casing & Casing & Pump & Screen Zone \\
\hline $\begin{array}{l}\text { N } 104730.2 \\
\text { E } 49680\end{array}$ & $\begin{array}{l}\text { 33.33844 Deg } N \\
-81.7395 \text { Deg } W\end{array}$ & \multicolumn{2}{|l|}{$214-204$} & $377 \mathrm{ft} \mathrm{msl}$ & $8 " \mathrm{CS}$ & $\mathrm{S}$ & \multirow[t]{3}{*}{ UNK } \\
\hline SAMPLE DATE & & $11 / 17 / 99$ & $12 / 13 / 99$ & $01 / 18 / 00$ & \multirow{2}{*}{\multicolumn{2}{|c|}{$02 / 16 / 00$}} & \\
\hline FIELD DATA & & & & & & & \\
\hline Parameter & & NOV & DEC & JAN & \multicolumn{2}{|r|}{ FEB } & \multirow{3}{*}{$\begin{array}{l}\text { Unit } \\
\mathrm{ft} \mathrm{msl} \\
\mathrm{pH} \\
\text { uS/cm } \\
\mathrm{deg} . \mathrm{C} \\
\mathrm{mg} / \mathrm{L} \\
\mathrm{NTU} \\
\text { well volume }\end{array}$} \\
\hline $\begin{array}{l}\text { Water Elevation } \\
\text { pH } \\
\text { Sp. Conductance } \\
\text { Water temperature } \\
\text { Alkalinity as CaCO3 } \\
\text { Turbidity } \\
\text { Volumes purged }\end{array}$ & . & $\begin{array}{l}\text { Not Available } \\
4.6 \\
49 \\
17 \\
0 \\
.5\end{array}$ & $\begin{array}{l}210.62 \\
4.9 \\
49 \\
19.2 \\
0 \\
1.9\end{array}$ & $\begin{array}{l}\text { Not Available } \\
4.8 \\
49 \\
13.3 \\
0 \\
.4\end{array}$ & \multicolumn{2}{|r|}{$\begin{array}{l}\text { Not Available } \\
4.1 \\
50 \\
18.9 \\
0 \\
.5\end{array}$} & \\
\hline Sampling code & & $\mathrm{C}$ & C & $\mathrm{C}$ & \multicolumn{2}{|r|}{$\mathrm{C}$} & \\
\hline
\end{tabular}

ANALYTICAL DATA

I. Groundwater Protection Standard

261 Appendix VIII/264 Appendix IX Hazardous Constituents

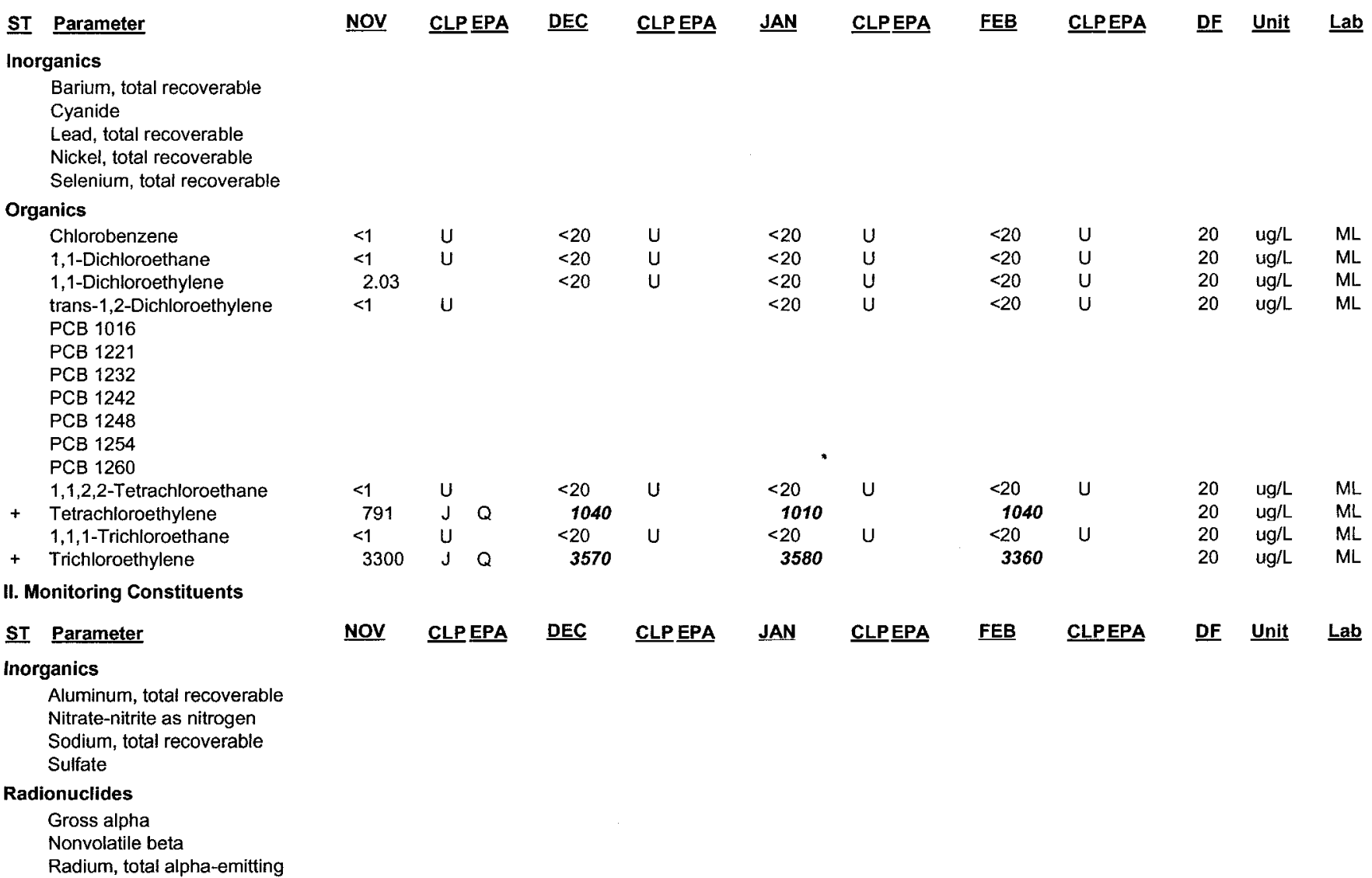


Table D-5b. Groundwater Monitoring Results for Recovery Wells, November 1999-February 2000 (Cont.) WELL RWM 4

\begin{tabular}{|c|c|c|c|c|c|c|c|}
\hline SRS Coord. & Lat/Longitude & \multicolumn{2}{|c|}{ Screen Zone Elevation } & Top of Casing & Casing & Pump & Screen Zone \\
\hline $\begin{array}{l}\text { N } 103719.3 \\
\text { E } 48948.2\end{array}$ & $\begin{array}{l}\text { 33.33501 Deg } N \\
-81.7394 \operatorname{Deg} W\end{array}$ & \multicolumn{2}{|c|}{$139.9-129.5 \mathrm{ft} \mathrm{msl}$} & $366.5 \mathrm{ft} \mathrm{msl}$ & $8 " \mathrm{Cs}$ & $S$ & M/GC/UL/LL \\
\hline SAMPLE DATE & & $11 / 17 / 99$ & $12 / 13 / 99$ & $01 / 18 / 00$ & & $02 / 16 / 00$ & \\
\hline \multicolumn{8}{|l|}{ FIELD DATA } \\
\hline Parameter & & NOV & DEC & JAN & & FEB & Unit \\
\hline $\begin{array}{l}\text { Water Elevation } \\
\text { pH } \\
\text { Sp. Conductance } \\
\text { Water temperature } \\
\text { Alkalinity as CaCO3 } \\
\text { Turbidity } \\
\text { Volumes purged } \\
\text { Sampling code }\end{array}$ & & $\begin{array}{l}205.5 \\
4.8 \\
23 \\
13.6 \\
0 \\
.6 \\
\text { C }\end{array}$ & $\begin{array}{l}205.6 \\
5.1 \\
23 \\
18.6 \\
1 \\
.4 \\
C\end{array}$ & $\begin{array}{l}207.25 \\
5.4 \\
24 \\
15.9 \\
1 \\
.4 \\
.015 \\
C\end{array}$ & & $\begin{array}{l}205.6 \\
5 \\
23 \\
16.4 \\
0 \\
.6 \\
\\
c\end{array}$ & $\begin{array}{l}\text { ft msl } \\
\mathrm{pH} \\
\text { uS/cm } \\
\text { deg. C } \\
\text { mg/L } \\
\text { NTU } \\
\text { well volume }\end{array}$ \\
\hline
\end{tabular}

\section{ANALYTICAL DATA}

I. Groundwater Protection Standard

261 Appendix VIII/264 Appendix IX Hazardous Constituent's

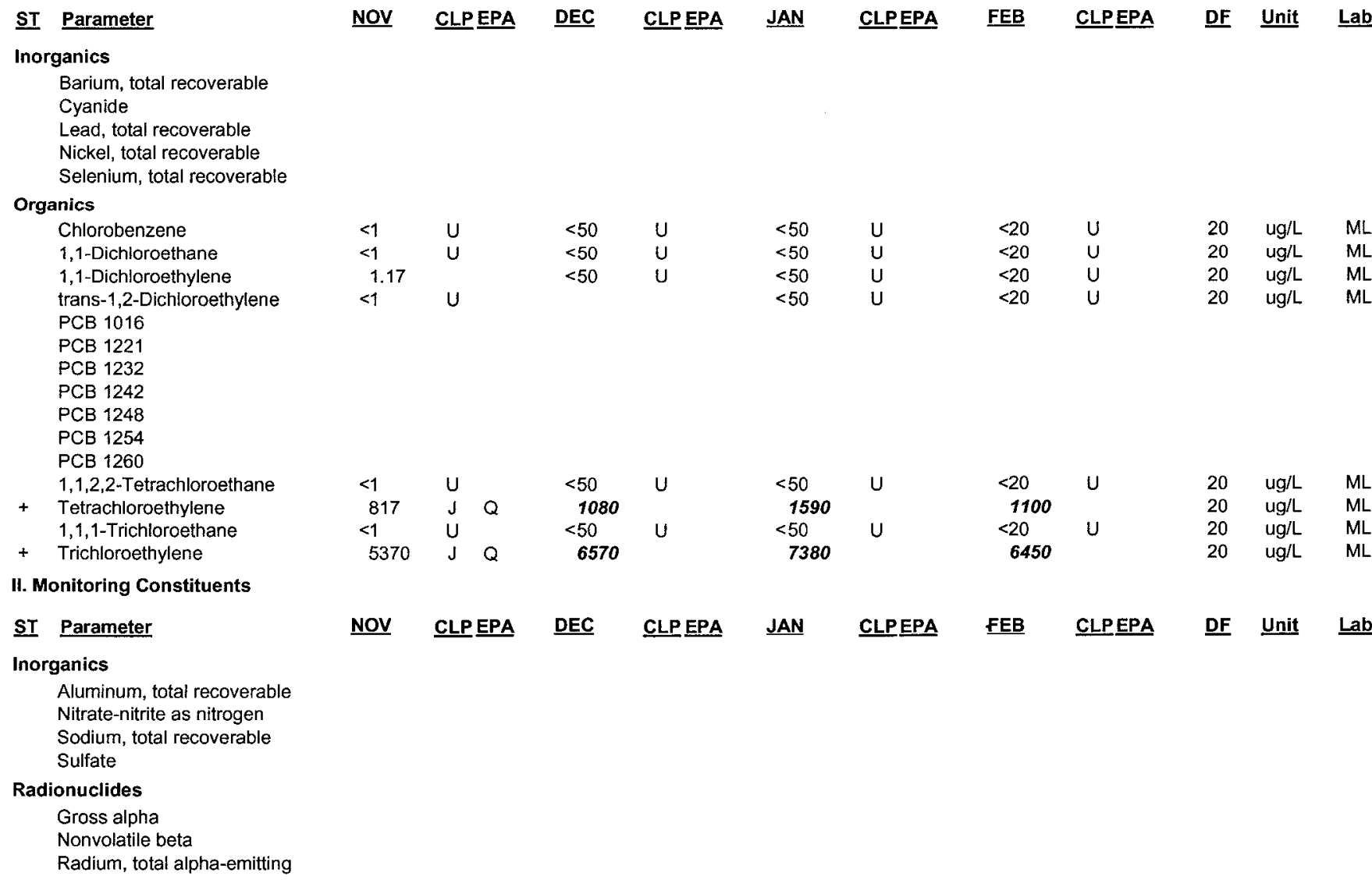

Notes: Concentrations in bold italics exceed the groundwater protection or monitoring constituent standards listed in Appendix A. Synchronous water levels are measured over a 3-5 day period or less. Dilution factors and Laboratory Data are February 2000 data only.

$+=$ exceeded the groundwater protection or monitoring constituent standards listed in Appendix A for February 2000. 
Table D-5b. Groundwater Monitoring Results for Recovery Wells, November 1999-February 2000 (Cont.) WELL RWM 5

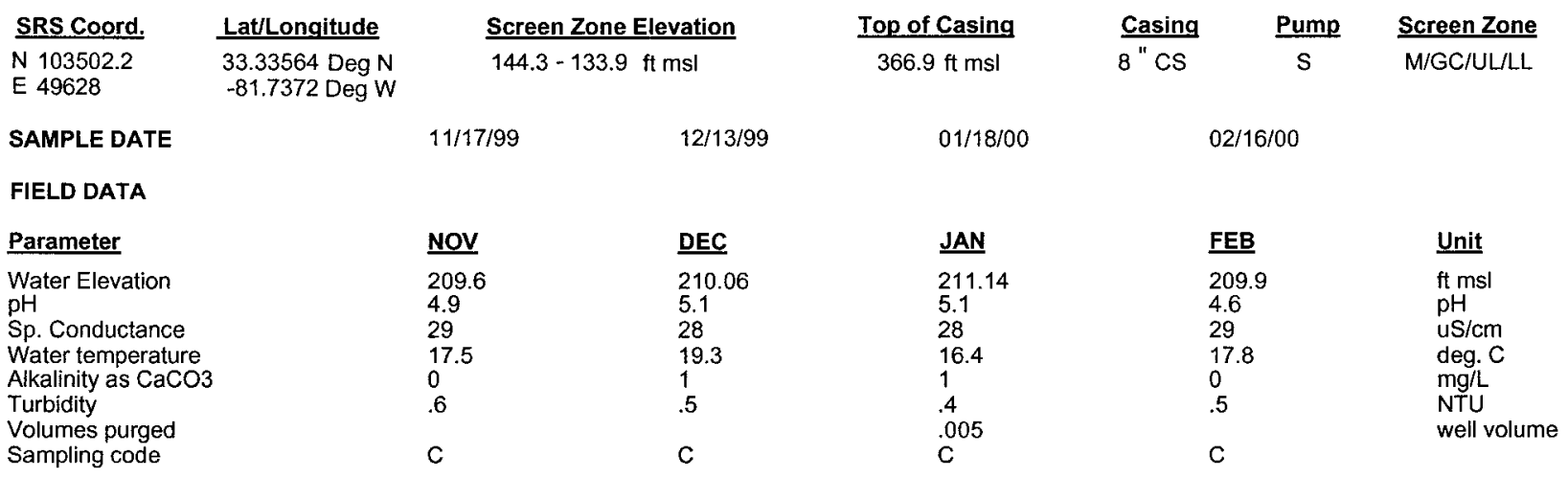

ANALYTICAL DATA

I. Groundwater Protection Standard

261 Appendix VIII/264 Appendix IX Hazardous Constituents

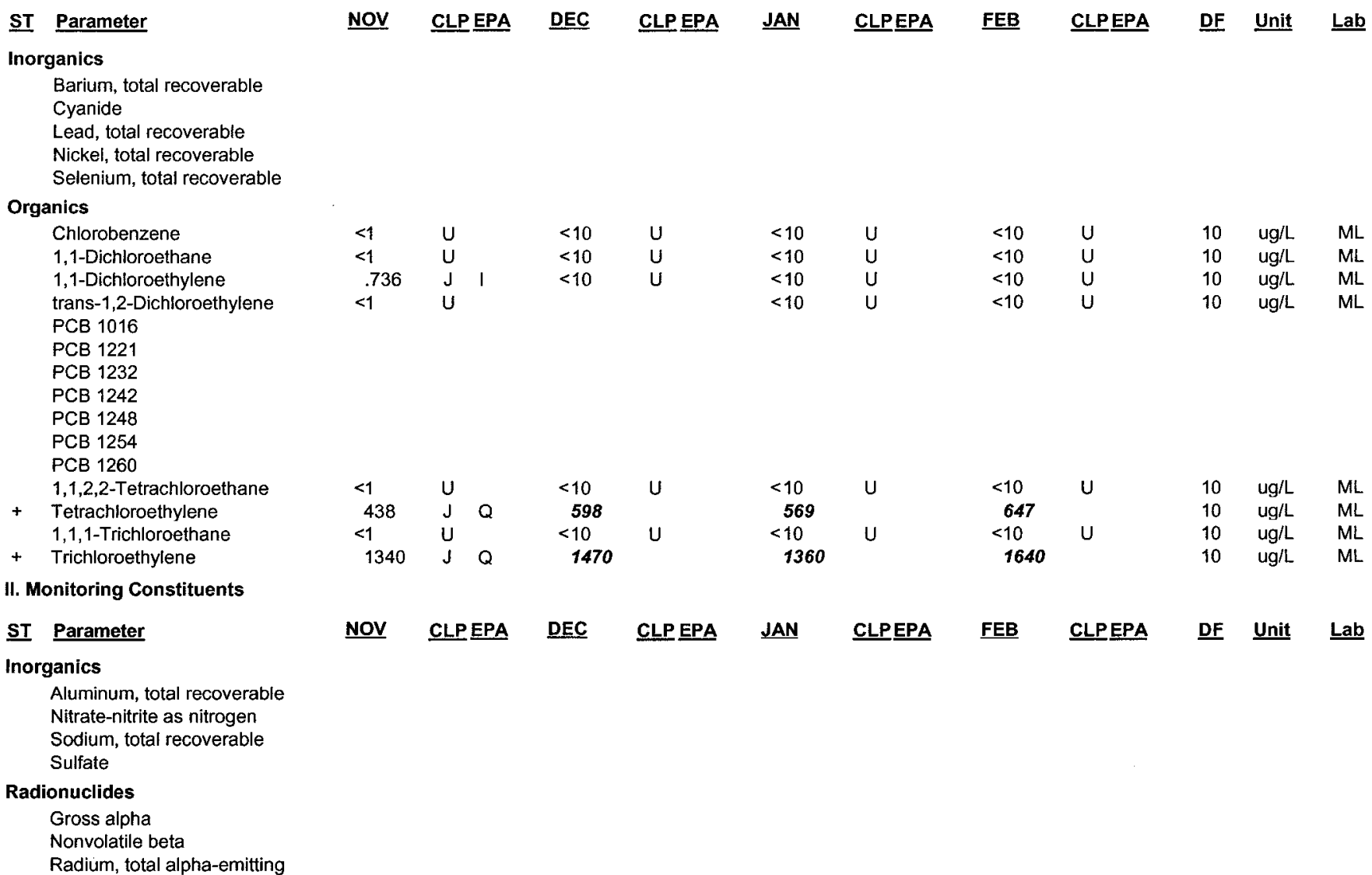

\footnotetext{
Notes: Concentrations in bold italics exceed the groundwater protection or monitoring constituent standards listed in Appendix A. Synchronous water levels are measured over a 3-5 day period or less. Dilution factors and Laboratory Data are February 2000 data only.

$+=$ exceeded the groundwater protection or monitoring constituent standards listed in Appendix A for February 2000.
} 
Table D-5b. Groundwater Monitoring Results for Recovery Wells, November 1999-February 2000 (Cont.) WELL RWM 6

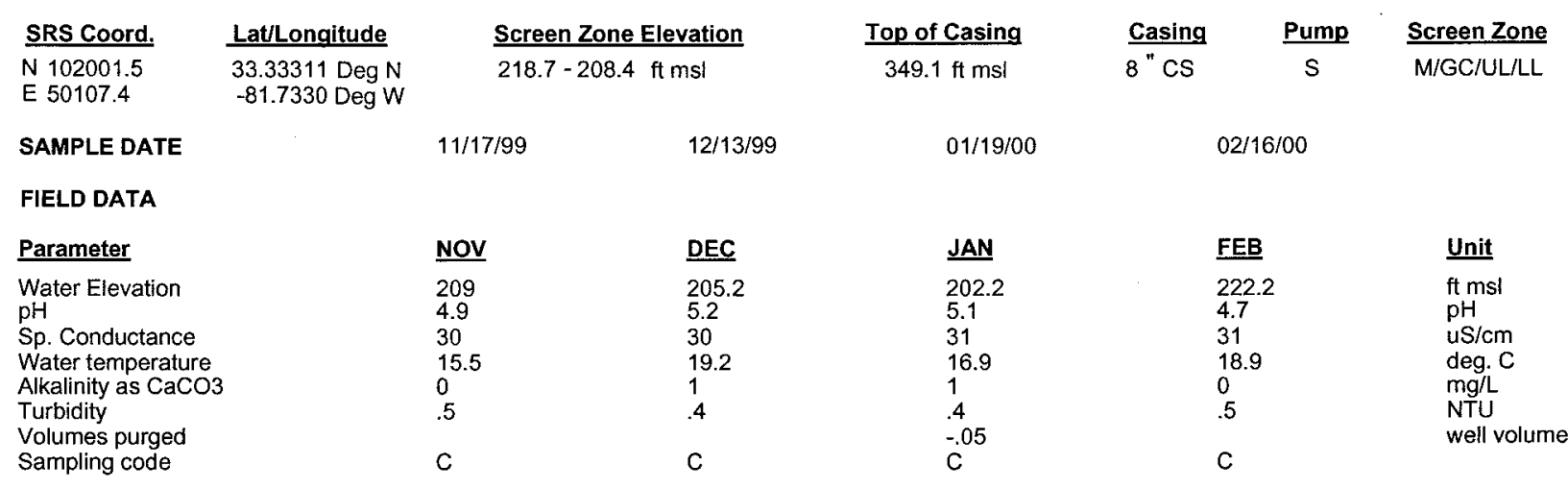

\section{ANALYTICAL DATA}

I. Groundwater Protection Standard

261 Appendix VIII/264 Appendix IX Hazardous Constituents

\begin{tabular}{|c|c|c|c|c|c|c|c|c|c|c|c|}
\hline$\underline{\text { ST }}$ & Parameter & $\underline{\text { NOV }}$ & CLP EPA & $\underline{\text { DEC }}$ & CLP EPA & JAN & CLPEPA & $\underline{\text { FEB }}$ & CLPEPA & $\underline{\mathrm{DF}}$ & Unit \\
\hline Ino & ganics & & & & & & & & & & \\
\hline & Barium, total recoverable & & & & & & & & & & \\
\hline & Cyanide & & & & & & & & & & \\
\hline & Lead, total recoverable & & & & & & & & & & \\
\hline & Nickel, total recoverable & & & & & & & & & & \\
\hline & Selenium, total recoverable & & & & & & & & & & \\
\hline Org & anics & & & & & & & & & & \\
\hline & Chlorobenzene & $<1$ & $U$ & $<20$ & $\mathrm{U}$ & $<20$ & $\mathrm{U}$ & $<20$ & U & 20 & $u g / L$ \\
\hline & 1,1-Dichloroethane & $<1$ & $u$ & $<20$ & $\mathrm{U}$ & $<20$ & U & $<20$ & U & 20 & $\mathrm{ug} / \mathrm{L}$ \\
\hline & 1,1-Dichloroethylene & $<1$ & $\mathrm{U}$ & $<20$ & $U$ & $<20$ & $U$ & $<20$ & U & 20 & $\mathrm{ug} / \mathrm{h}$ \\
\hline & trans-1,2-Dichloroethylene & $<1$ & $U$ & & & $<20$ & $\mathrm{U}$ & $<20$ & $U$ & 20 & $\mathrm{ug} / \mathrm{L}$ \\
\hline & PCB 1016 & & & & & 1.04 & $\mathrm{R} \quad \mathrm{L}$ & & & & $u g / L$ \\
\hline & PCB 1221 & & & & & 1.04 & $R \quad L$ & & & & $\mathrm{ug} / \mathrm{L}$ \\
\hline & PCB 1232 & & & & & 1.04 & R L & & & & $\mathrm{ug} / \mathrm{L}$ \\
\hline & PCB 1242 & & & & & 1.04 & R L L & & & & $u g / L$ \\
\hline & PCB 1248 & & & & & 1.04 & R L & & & & $\mathrm{ug} / \mathrm{L}$ \\
\hline & PCB 1254 & & & & & 1.04 & R L & & & & $u g / L$ \\
\hline & PCB 1260 & & & & & 1.04 & R L & & & & $u g / L$ \\
\hline & 1,1,2,2-Tetrachloroethane & $<1$ & $U$ & $<20$ & $u$ & $<20$ & $\mathrm{U}$ & $<20$ & U & 20 & $u g / L$ \\
\hline+ & Tetrachloroethylene & 2090 & $Q$ & 2780 & & 134 & & 2950 & & 20 & $\mathrm{ug} / \mathrm{L}$ \\
\hline & 1,1,1-Trichloroethane & $<1$ & $\mathrm{U}$ & $<20$ & U & $<20$ & $U$ & $<20$ & U & 20 & $\mathrm{ug} / \mathrm{L}$ \\
\hline+ & Trichloroethylene & 1840 & $J \quad Q$ & 2130 & & 2470 & & 2100 & & 20 & $\mathrm{ug} / \mathrm{L}$ \\
\hline II. $M$ & onitoring Constituents & & & & & & & & & & \\
\hline$\underline{\text { ST }}$ & Parameter & NOV & CLP EPA & $\underline{D E C}$ & CLP EPA & JAN & CLPEPA & FEB & CLPEPA & $\underline{\text { DF }}$ & Unit \\
\hline Inor & ganics & & & & & & & & & & \\
\hline & Aluminum, total recoverable & & & & & & & & & & \\
\hline & Nitrate-nitrite as nitrogen & & & & & & & & & & \\
\hline & Sodium, total recoverable & & & & & & & & & & \\
\hline & Sulfate & & & & & & & & & & \\
\hline $\operatorname{Rad}$ & onuclides & & & & & & & & & & \\
\hline & Gross alpha & & & & & & & & & & \\
\hline & Nonvolatile beta & & & & & & & & & & \\
\hline & Radium, total alpha-emitting & & & & & & & & & & \\
\hline
\end{tabular}

Notes: Concentrations in bold italics exceed the groundwater protection or monitoring constituent standards listed in Appendix A. Synchronous water levels are measured over a 3-5 day period or less. Dilution factors and Laboratory Data are February 2000 data only.

$+=$ exceeded the groundwater protection or monitoring constituent standards listed in Appendix A for February 2000. 
Table D-5b. Groundwater Monitoring Results for Recovery Wells, November 1999-February 2000 (Cont.) WELL RWM 7

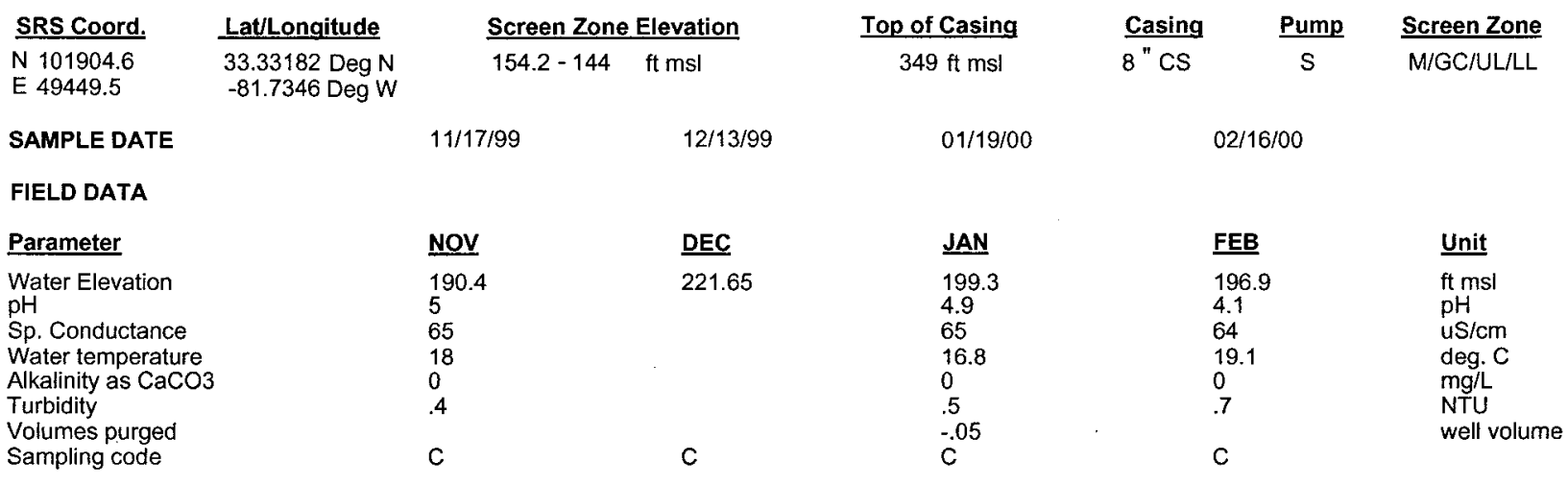

ANALYTICAL DATA

1. Groundwater Protection Standard

261 Appendix VIII/264 Appendix IX Hazardous Constituents

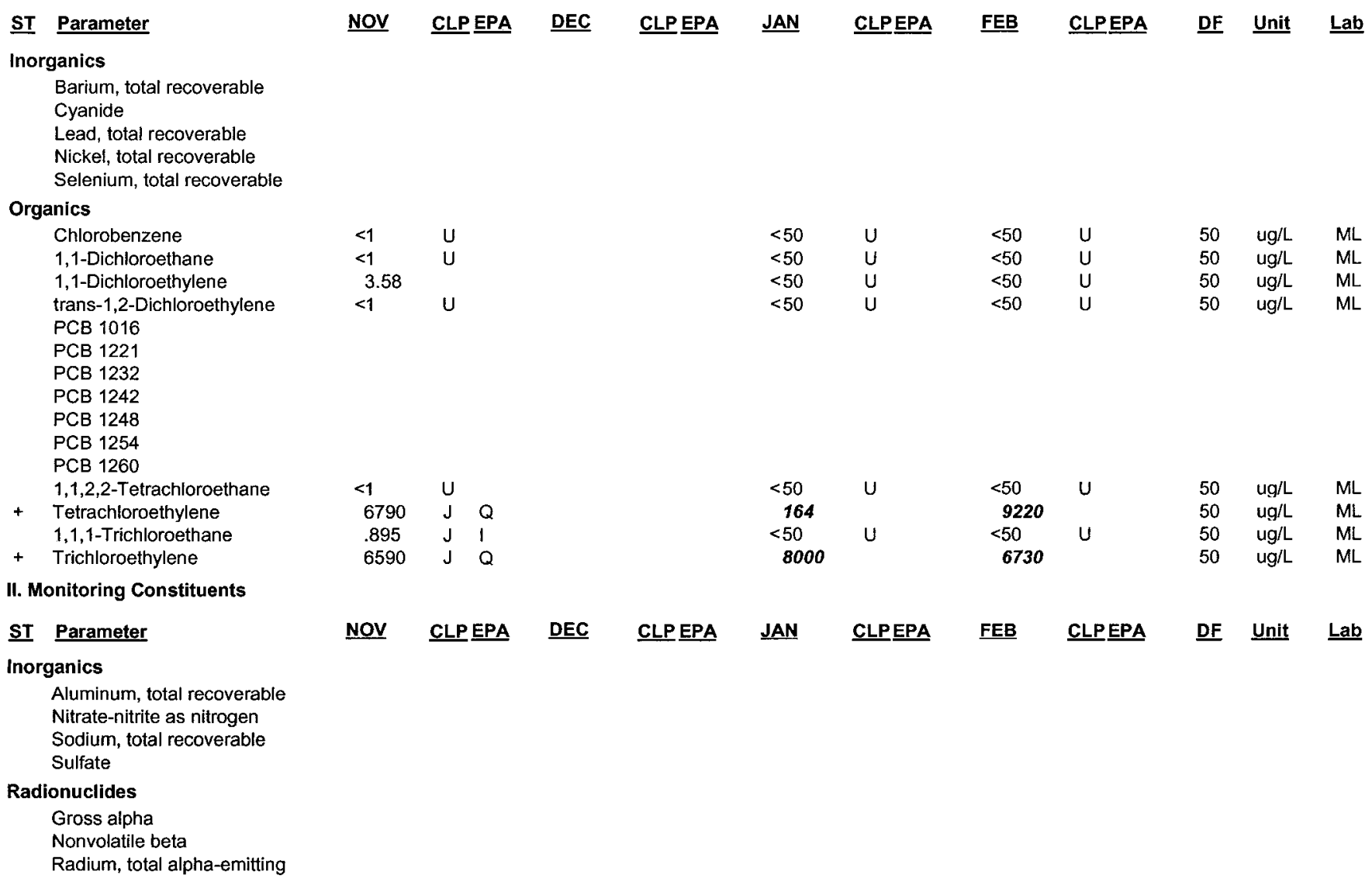


Table D-5b. Groundwater Monitoring Results for Recovery Wells, November 1999-February 2000 (Cont.) WELL RWM 8

$\begin{array}{ll}\text { SRS Coord. } & \text { Lat/Longitude } \\ \text { N } 101948.2 & \text { 33.32849 Deg N } \\ \text { E } 47353.3 & -81.7402 \text { Deg W }\end{array}$

SAMPLE DATE

FIELD DATA

Parameter

Water Elevation

$\mathrm{pH}$

Sp. Conductance

Water temperature

Alkalinity as $\mathrm{CaCO} 3$

Turbidity

Volumes purged

Sampling code

ANALYTICAL DATA

I. Groundwater Protection Standard

261 Appendix VIII/264 Appendix IX Hazardous Constituents

ST Paramete

Inorganics

Barium, total recoverable

Cyanide

Lead, total recoverable

Nickel, total recoverable

Selenium, total recoverable

Organics

Chlorobenzene

1,1-Dichloroethane

1,1-Dichloroethylene

trans-1,2-Dichloroethylene

PCB 1016

PCB 1221

PCB 1232

PCB 1242

PCB 1248

PCB 1254

PCB 1260

1,1,2,2-Tetrachloroethane

+ Tetrachloroethylene

1,1,1-Trichloroethane

+ Trichloroethylene

II. Monitoring Constituents

\section{ST Parameter}

Inorganics

Aluminum, total recoverable

Nitrate-nitrite as nitrogen

Sodium, total recoverable

Sulfate

Radionuclides

Gross alpha

Nonvolatile beta

Radium, total alpha-emitting
Screen Zone Elevation

$119.7-109.3 \mathrm{ft} \mathrm{msi}$

$12 / 13 / 99$

DEC

Not Available

212.49
DEC

CLP EPA

DEC

CLP EPA

\section{JAN}

CLPEPA

FEB

JAN

213.38

$\stackrel{0}{\mathrm{C}}$

$1 / 18 / 00$

Casing

Pump

Screen Zone

GC/UL/LL

$02 / 16 / 00$

$\begin{array}{ll}\text { FEB } & \text { Unit } \\ 202.85 & \text { ft msl } \\ 5.1 & \mathrm{pH} \\ 173 & \text { uS/cm } \\ 17.4 & \text { deg. C } \\ 0 & \text { mg/L } \\ .9 & \text { NTU } \\ \text { C } & \text { well volume }\end{array}$

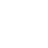


Table D-5b. Groundwater Monitoring Results for Recovery Wells, November 1999-February 2000 (Cont.) WELL RWM 9

\begin{tabular}{ll} 
SRS Coord. & Lat/Longitude \\
\cline { 2 - 2 } N 104099.8 & 33.33822 Deg N \\
E 50400 & $-81.7363 \operatorname{Deg} W$
\end{tabular}

SAMPLE DATE

FIELD DATA

Parameter

Water Elevation

$\mathrm{pH}$

$\mathrm{Sp}$. Conductance

Water temperature

Alkalinity as $\mathrm{CaCO} 3$

Turbidity

Volumes purged

Sampling code

ANALYTICAL DATA

I. Groundwater Protection Standard

261 Appendix VIII/264 Appendix IX Hazardous Constituents

$\frac{\text { Screen Zone Elevation }}{143-132.6 \mathrm{ft} \mathrm{msl}} \quad \frac{\text { Top of Casing }}{380.6 \mathrm{ft} \mathrm{msl}} \quad \frac{\text { Casing }}{8^{\prime \prime C S}} \quad \frac{\text { Pump }}{\mathrm{S}} \quad \frac{\text { Screen Zone }}{\mathrm{M}}$

$\begin{array}{lll}12 / 13 / 99 & 01 / 19 / 00 & 02 / 16 / 00\end{array}$

\begin{tabular}{lllll} 
Not Available & DEC & JAN & FEB & Unit \\
\cline { 2 - 4 } & Not Available & 222.05 & $\mathrm{ft} \mathrm{msl}$ \\
& & 5.3 & $\mathrm{pH}$ \\
& & 49 & US/cm \\
& & 18.2 & deg. C \\
& & .6 & $\mathrm{mg} / \mathrm{L}$ \\
& & $\mathrm{NTU}$ & well volume \\
& $\mathrm{C}$ & $\mathrm{C}$ & $\mathrm{C}$ &
\end{tabular}


Table D-5b. Groundwater Monitoring Results for Recovery Wells, November 1999-February 2000 (Cont.) WELL RWM 10

\begin{tabular}{|c|c|c|c|c|c|c|c|}
\hline SRS Coord. & Lat/Longitude & \multicolumn{2}{|c|}{ Screen Zone Elevation } & Top of Casing & Casing & Pump & Screen Zone \\
\hline $\begin{array}{l}\text { N } 102000.9 \\
\text { E } 48244.1\end{array}$ & $\begin{array}{l}\text { 33.33006 Deg } N \\
\text {-81.7379 Deg } W\end{array}$ & \multicolumn{2}{|c|}{$215.5-205.1 \mathrm{ft} \mathrm{msl}$} & $355.5 \mathrm{ft} \mathrm{msl}$ & $8 " \mathrm{CS}$ & $\mathrm{S}$ & $M$ \\
\hline SAMPLE DATE & & $11 / 17 / 99$ & $12 / 13 / 99$ & $01 / 18 / 00$ & & $6 / 00$ & \\
\hline \multicolumn{8}{|l|}{ FIELD DATA } \\
\hline Parameter & & NOV & DEC & $\underline{\text { JAN }}$ & \multicolumn{2}{|c|}{$\underline{\text { FEB }}$} & Unit \\
\hline $\begin{array}{l}\text { Water Elevation } \\
\text { pH } \\
\text { Sp. Conductance } \\
\text { Water temperature } \\
\text { Alkalinity as CaCO3 } \\
\text { Turbidity } \\
\text { Volumes purged } \\
\text { Sampling code }\end{array}$ & & $\begin{array}{l}190.1 \\
4.4 \\
81 \\
16.5 \\
0 \\
.6 \\
\\
c\end{array}$ & $\begin{array}{l}189.99 \\
5.5 \\
80 \\
18.5 \\
1 \\
.5 \\
\\
C\end{array}$ & $\begin{array}{l}195.9 \\
5.1 \\
124 \\
16.6 \\
2 \\
.5 \\
-.04 \\
\mathrm{C}\end{array}$ & \multicolumn{2}{|c|}{216.5} & $\begin{array}{l}\mathrm{ft} \mathrm{msl} \\
\mathrm{pH} \\
\mathrm{uS} / \mathrm{cm} \\
\mathrm{deg} . \mathrm{C} \\
\mathrm{mg} / \mathrm{L} \\
\mathrm{NTU} \\
\text { well volum }\end{array}$ \\
\hline
\end{tabular}

ANALYTICAL DATA

I. Groundwater Protection Standard

261 Appendix VIII/264 Appendix IX Hazardous Constituents

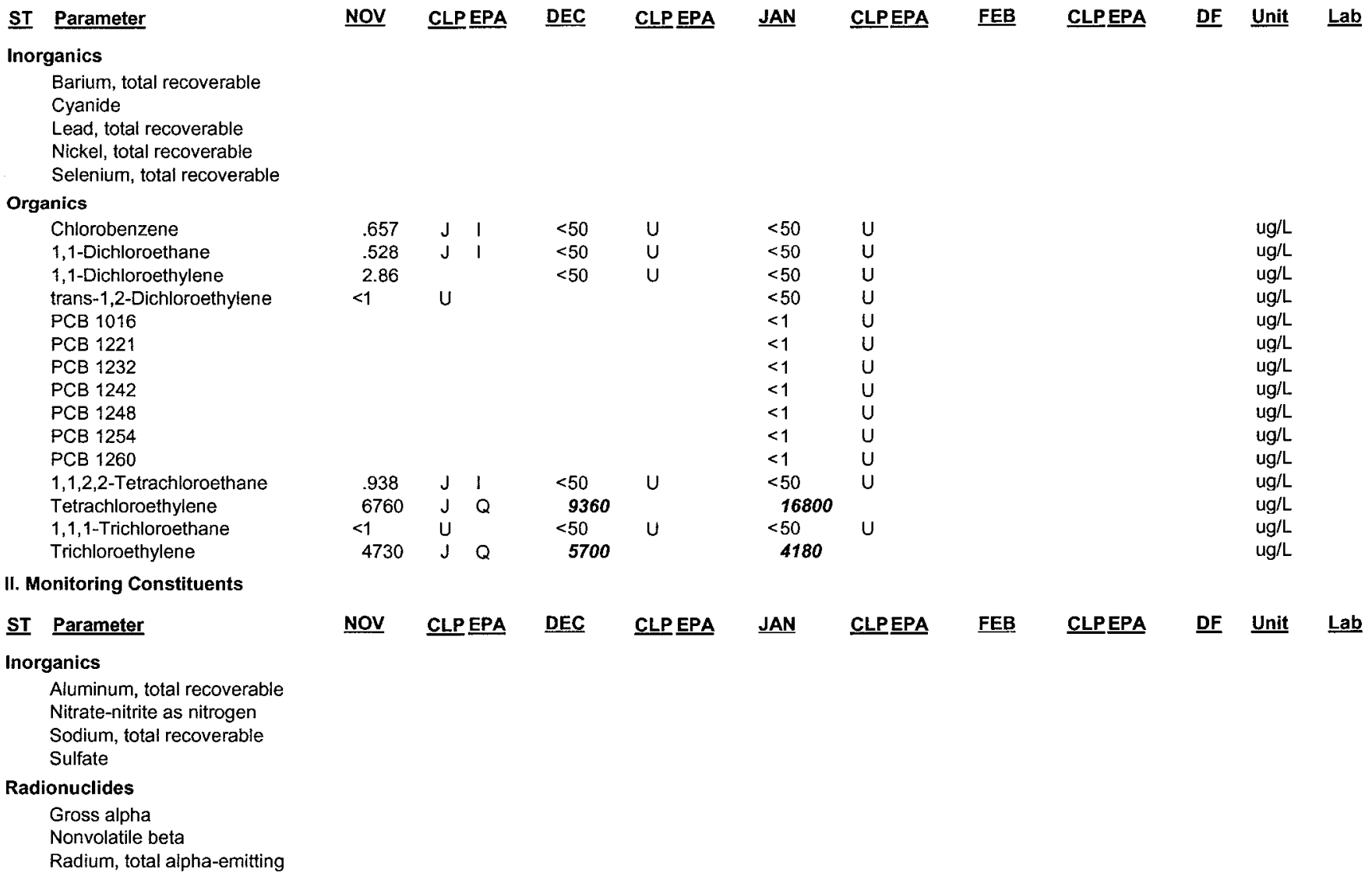

Notes: Concentrations in bold italics exceed the groundwater protection or monitoring constituent standards listed in Appendix A. Synchronous water levels are measured over a 3-5 day period or less. Dilution factors and Laboratory Data are February 2000 data only.

$+=$ exceeded the groundwater protection or monitoring constituent standards listed in Appendix A for February 2000. 
Table D-5b. Groundwater Monitoring Results for Recovery Wells, November 1999-February 2000 (Cont.) WELL RWM 11

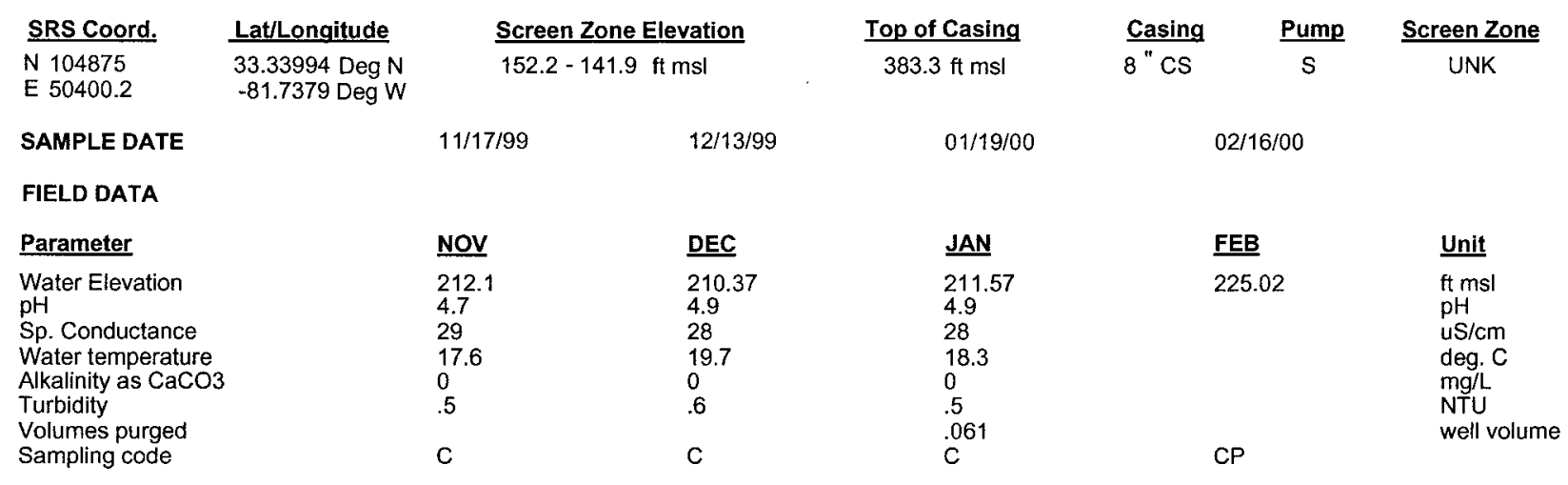

\section{ANALYTICAL DATA}

I. Groundwater Protection Standard

261 Appendix VIII/264 Appendix IX Hazardous Constituents

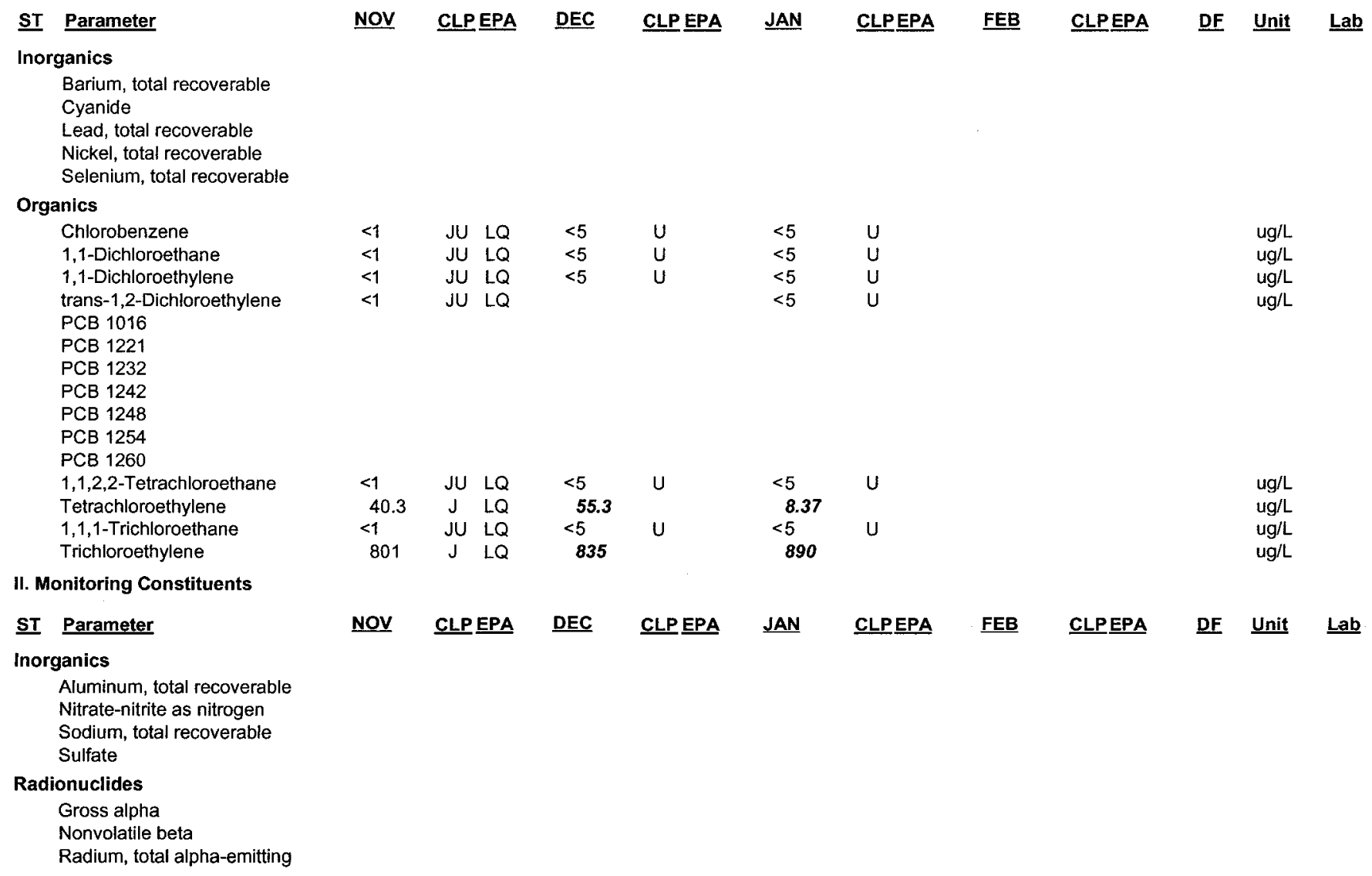

Notes: Concentrations in bold italics exceed the groundwater protection or monitoring constituent standards listed in Appendix A. Synchronous water levels are measured over a 3-5 day period or less. Dilution factors and Laboratory Data are February 2000 data only.

$+=$ exceeded the groundwater protection or monitoring constituent standards listed in Appendix A for February 2000. 
Table D-5b. Groundwater Monitoring Results for Recovery Wells, November 1999-February 2000 (Cont.) WELL RWM 12

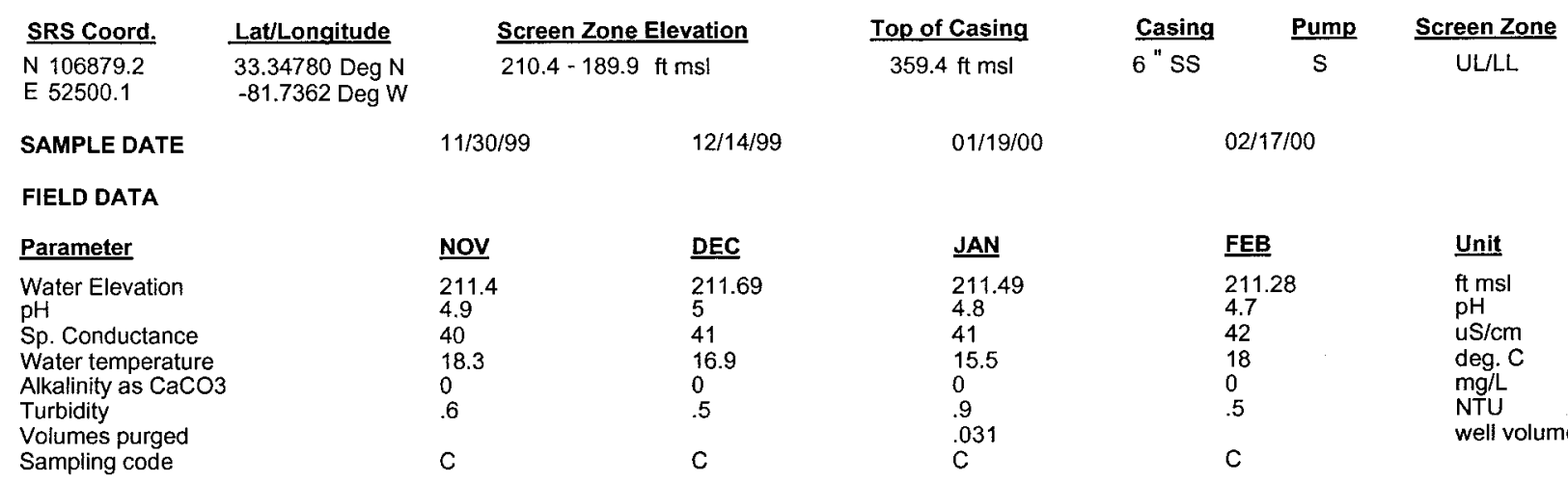

ANALYTICAL DATA

1. Groundwater Protection Standard

261 Appendix VIII/264 Appendix IX Hazardous Constituents

\begin{tabular}{|c|c|c|c|c|c|c|c|c|c|c|c|c|}
\hline$\underline{\mathbf{S T}}$ & Parameter & $\underline{\text { NOV }}$ & CLPEPA & $\underline{D E C}$ & CLP EPA & JAN & CLPEPA & FEB & CLPEPA & $\underline{\text { DF }}$ & Unit & $\underline{\text { Lab }}$ \\
\hline \multicolumn{13}{|c|}{ Inorganics } \\
\hline & $\begin{array}{l}\text { Barium, total recoverable } \\
\text { Cyanide } \\
\text { Lead, total recoverable } \\
\text { Nickel, total recoverable } \\
\text { Selenium, total recoverable }\end{array}$ & & & & & & & & & & & \\
\hline \multicolumn{13}{|c|}{ Organics } \\
\hline & Chlorobenzene & $<1$ & U & $<10$ & U & $<20$ & $\mathrm{U}$ & $<10$ & $\mathrm{U}$ & 10 & $u g / L$ & $M L$ \\
\hline & 1,1-Dichloroethane & $<1$ & $\mathrm{U}$ & $<10$ & U & $<20$ & $\mathrm{U}$ & $<10$ & U & 10 & $\mathrm{ug} / \mathrm{L}$ & ML \\
\hline & 1,1-Dichloroethylene & $<1$ & $u$ & $<10$ & U & $<20$ & $U$ & $<10$ & $\mathrm{U}$ & 10 & $\mathrm{ug} / \mathrm{L}$ & ML \\
\hline & trans-1,2-Dichloroethylene & $<1$ & $U$ & & & $<20$ & $U$ & $<10$ & $\mathrm{U}$ & 10 & $\mathrm{ug} / \mathrm{L}$ & $\mathrm{ML}$ \\
\hline \multicolumn{13}{|c|}{ PCB 1016} \\
\hline \multicolumn{13}{|c|}{ PCB 1221} \\
\hline \multicolumn{13}{|c|}{ PCB 1232} \\
\hline \multicolumn{13}{|c|}{ PCB 1242} \\
\hline \multicolumn{13}{|c|}{ PCB 1248} \\
\hline \multicolumn{13}{|c|}{ PCB 1254} \\
\hline \multicolumn{13}{|c|}{ PCB 1260} \\
\hline & 1,1,2,2-Tetrachloroethane & $<1$ & $\mathrm{U}$ & $<10$ & $\mathrm{U}$ & $<20$ & $\mathrm{U}$ & $<10$ & U & 10 & $\mathrm{ug} / \mathrm{L}$ & ML \\
\hline & Tetrachloroethylene & 9.52 & $\mathrm{~J} K$ & 16.3 & & $<20$ & $\mathrm{U}$ & 7.8 & $\mathrm{~J} \quad 1$ & 10 & $\mathrm{ug} / \mathrm{L}$ & $\mathrm{ML}$ \\
\hline & $1,1,1$-Trichloroethane & $<1$ & $\mathrm{U}$ & $<10$ & $\mathrm{U}$ & $<20$ & $U$ & $<10$ & $U$ & 10 & ug/L & $\mathrm{ML}$ \\
\hline+ & Trichtoroethylene & 1530 & $\mathrm{~J} K$ & 1880 & & 2000 & & 2280 & & 10 & $\mathrm{ug} / \mathrm{L}$ & $\mathrm{ML}$ \\
\hline \multicolumn{13}{|c|}{ II. Monitoring Constituents } \\
\hline$\underline{\text { ST }}$ & Parameter & NOV & CLPPEPA & $\underline{D E C}$ & CLPEPA & JAN & CLPEPA & FEB & CLPEPA & DF & Unit & $\underline{\text { Lab }}$ \\
\hline \multicolumn{13}{|c|}{ Inorganics } \\
\hline \multicolumn{12}{|c|}{ Aluminum, total recoverable } & \\
\hline \multicolumn{12}{|c|}{ Nitrate-nitrite as nitrogen } & \\
\hline \multicolumn{12}{|c|}{ Sodium, total recoverable } & \\
\hline \multicolumn{12}{|c|}{ Sulfate } & \\
\hline \multicolumn{12}{|c|}{ Radionuclides } & \\
\hline \multirow{3}{*}{\multicolumn{12}{|c|}{$\begin{array}{l}\text { Gross alpha } \\
\text { Nonvolatile beta } \\
\text { Radium, total alpha }\end{array}$}} & \\
\hline & & & & & & & & & & & & \\
\hline & & & & & & & & & & & & \\
\hline
\end{tabular}

Notes: Concentrations in bold italics exceed the groundwater protection or monitoring constituent standards listed in Appendix A. Synchronous water levels are measured over a 3-5 day period or less. Dilution factors and Laboratory Data are February 2000 data only.

$+=$ exceeded the groundwater protection or monitoring constituent standards listed in Appendix A for February 2000. 
Table D-5b. Groundwater Monitoring Results for Recovery Wells, November 1999-February 2000 (Cont.) WELL RWM 13B

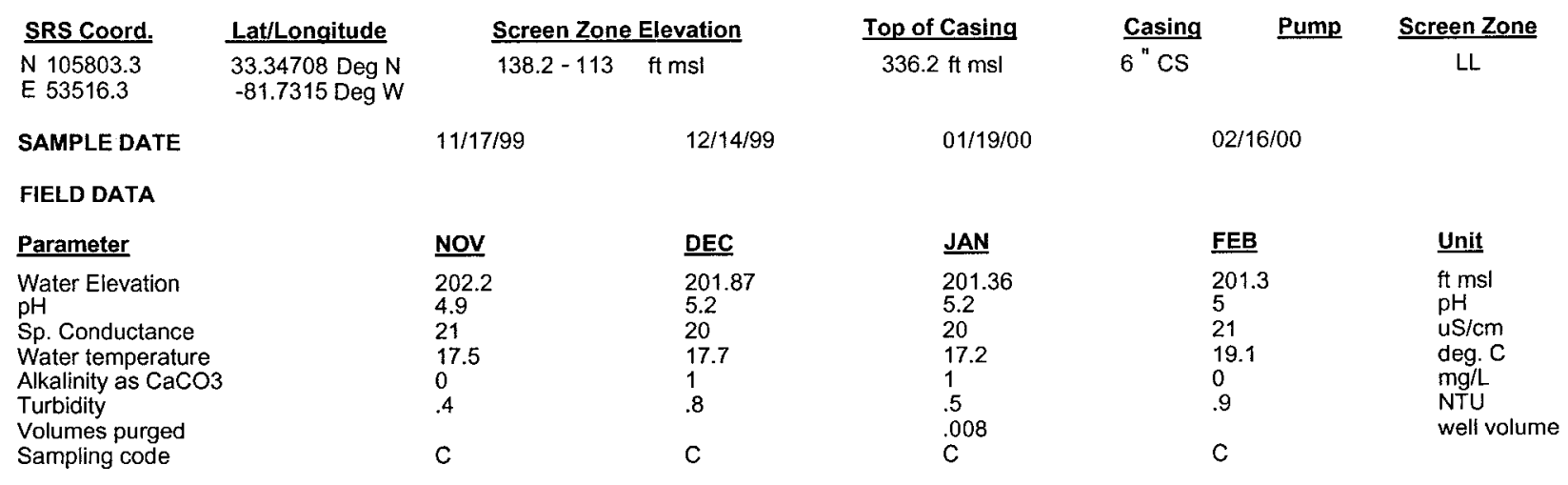

ANALYTICAL DATA

I. Groundwater Protection Standard

261 Appendix VIII/264 Appendix IX Hazardous Constituents

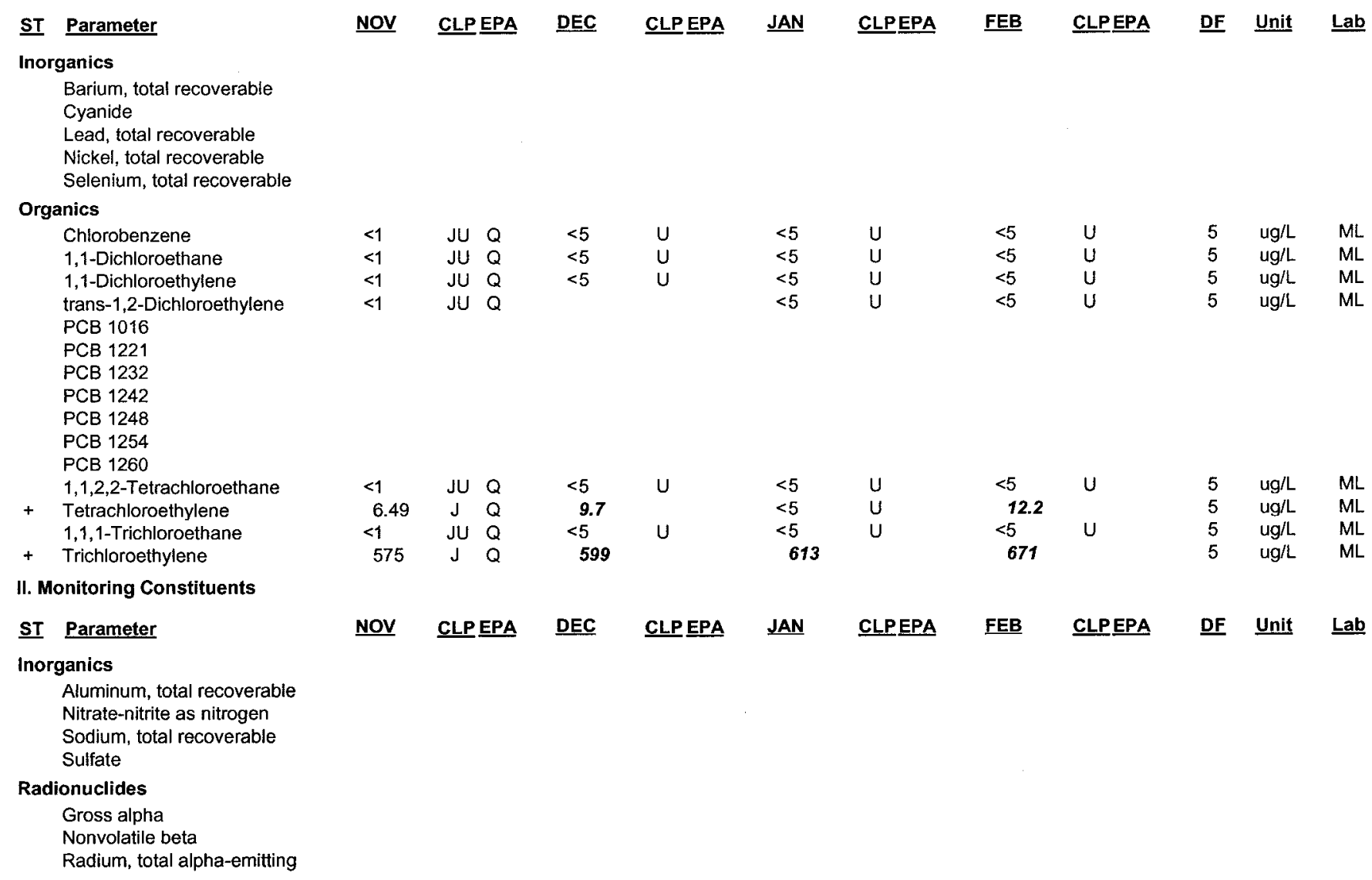

Notes: Concentrations in bold italics exceed the groundwater protection or monitoring constituent standards listed in Appendix A. Synchronous water levels are measured over a 3-5 day period or less. Dilution factors and Laboratory Data are February 2000 data only.

$+=$ exceeded the groundwater protection or monitoring constituent standards listed in Appendix A for February 2000. 
Table D-5b. Groundwater Monitoring Results for Recovery Wells, November 1999-February 2000 (Cont.) WELL RWM 13C

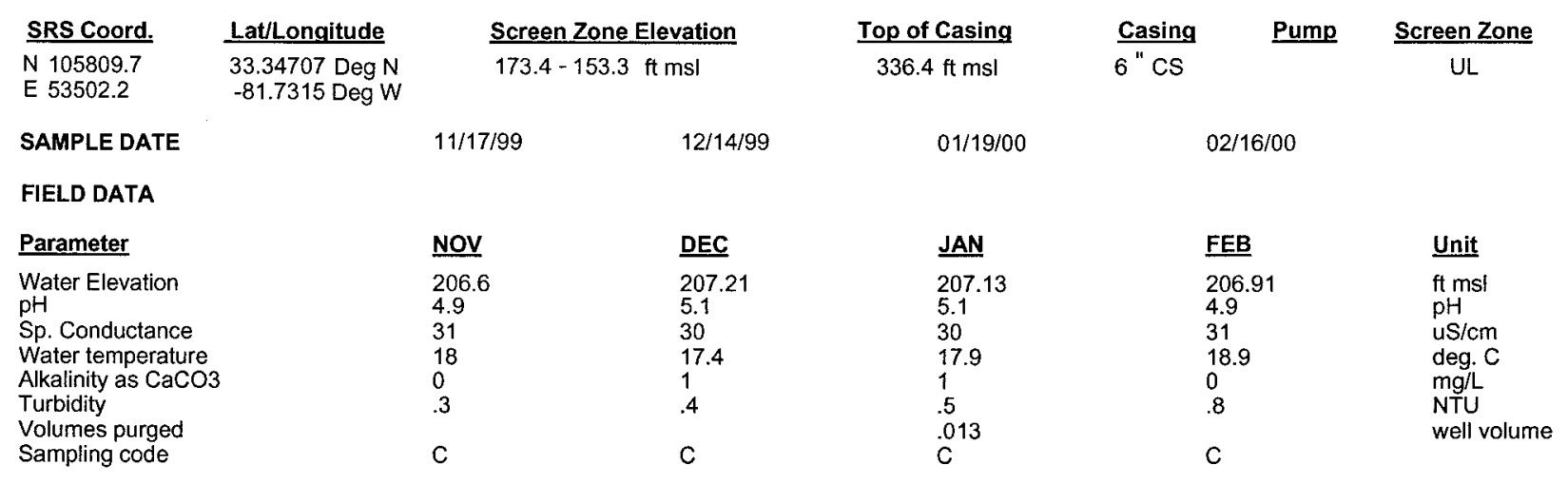

\section{ANALYTICAL DATA}

I. Groundwater Protection Standard

261 Appendix VIII/264 Appendix IX Hazardous Constituents

\begin{tabular}{|c|c|c|c|c|c|c|c|c|c|c|c|}
\hline$\underline{\text { sT }}$ & Parameter & NOV & CLP EPA & $\underline{\mathrm{DEC}}$ & CLP EPA & $\underline{\text { JAN }}$ & CLPEEPA & $\underline{\text { FEB }}$ & CLPEPA & $\underline{\text { DF }}$ & Unit \\
\hline Inor & $\begin{array}{l}\text { ganics } \\
\text { Barium, total recoverable } \\
\text { Cyanide } \\
\text { Lead, total recoverable } \\
\text { Nickel, total recoverable } \\
\text { Selenium, total recoverable }\end{array}$ & & & & & & & & & & \\
\hline \multicolumn{12}{|c|}{ Organics } \\
\hline & $\begin{array}{l}\text { Chlorobenzene } \\
\text { 1,1-Dichloroethane } \\
\text { 1,1-Dichloroethylene } \\
\text { trans-1,2-Dichloroethylene } \\
\text { PCB } 1016 \\
\text { PCB } 1221 \\
\text { PCB } 1232 \\
\text { PCB } 1242 \\
\text { PCB } 1248 \\
\text { PCB } 1254 \\
\text { PCB } 1260\end{array}$ & $\begin{array}{l}<50 \\
<50 \\
<50 \\
<50\end{array}$ & $\begin{array}{ll}J U & L Q \\
J U & L Q \\
J U & L Q \\
J U & L Q\end{array}$ & $\begin{array}{l}<5 \\
<5 \\
<5\end{array}$ & $\begin{array}{l}U \\
U \\
U\end{array}$ & $\begin{array}{l}<5 \\
<5 \\
<5 \\
<5\end{array}$ & $\begin{array}{l}U \\
U \\
U \\
U\end{array}$ & $\begin{array}{l}<5 \\
<5 \\
<5 \\
<5\end{array}$ & $\begin{array}{l}U \\
U \\
U \\
U\end{array}$ & $\begin{array}{l}5 \\
5 \\
5 \\
5\end{array}$ & $\begin{array}{l}\mathrm{ug} / \mathrm{L} \\
\mathrm{ug} / \mathrm{L} \\
\mathrm{ug} / \mathrm{L} \\
\mathrm{ug} / \mathrm{L}\end{array}$ \\
\hline $\begin{array}{l}+ \\
+\end{array}$ & $\begin{array}{l}\text { 1,1,2,2-Tetrachloroethane } \\
\text { Tetrachloroethylene } \\
\text { 1,1,1-Trichloroethane } \\
\text { Trichloroethylene }\end{array}$ & $\begin{array}{l}<50 \\
<50 \\
<50 \\
847\end{array}$ & $\begin{array}{ll}J U & L Q \\
J U & L Q \\
J U & L Q \\
J & L Q\end{array}$ & $\begin{array}{l}<5 \\
20.7 \\
<5 \\
882\end{array}$ & $\mathrm{U}$ & $\begin{array}{l}<5 \\
2.77 \\
<5 \\
950\end{array}$ & $\begin{array}{ll}\mathrm{U} & \\
\mathrm{J} & 1 \\
\mathrm{U} & \end{array}$ & $\begin{array}{l}<5 \\
23.3 \\
<5 \\
993\end{array}$ & u & $\begin{array}{l}5 \\
5 \\
5 \\
5\end{array}$ & $\begin{array}{l}\mathrm{ug} / \mathrm{L} \\
\mathrm{ug} / \mathrm{L} \\
\mathrm{ug} / \mathrm{L} \\
\mathrm{ug} / \mathrm{L}\end{array}$ \\
\hline \multicolumn{12}{|c|}{ II. Monitoring Constituents } \\
\hline$\underline{\mathbf{S T}}$ & Parameter & $\underline{\text { NOV }}$ & $\underline{\text { CLP EPA }}$ & $\underline{\mathrm{DEC}}$ & $\underline{\text { CLP EPA }}$ & $\underline{\text { JAN }}$ & CLPEPA & FEB & CLPEPA & $\underline{D F}$ & Unit \\
\hline Inors & $\begin{array}{l}\text { ganics } \\
\text { Aluminum, total recoverable } \\
\text { Nitrate-nitrite as nitrogen } \\
\text { Sodium, total recoverable } \\
\text { Sulfate }\end{array}$ & & & & & & & & & & \\
\hline Radi & $\begin{array}{l}\text { onuclides } \\
\text { Gross alpha } \\
\text { Nonvolatile beta } \\
\text { Radium, total alpha-emitting }\end{array}$ & & & & & & & & & & \\
\hline
\end{tabular}


Table D-5b. Groundwater Monitoring Results for Recovery Wells, November 1999-February 2000 (Cont.) WELL RWM 14B

\begin{tabular}{|c|c|c|c|c|c|c|c|}
\hline SRS Coord. & Lat/Longitude & \multicolumn{2}{|c|}{ Screen Zone Elevation } & Top of Casing & Casing & Pump & Screen Zone \\
\hline $\begin{array}{l}\text { N } 106362.1 \\
\text { E } 53044.7\end{array}$ & $\begin{array}{l}\text { 33.34754 Deg N } \\
-81.7338 \text { Deg } W\end{array}$ & \multicolumn{2}{|c|}{$148.5-123.2 \mathrm{ft} \mathrm{msl}$} & $351.2 \mathrm{ft} \mathrm{msl}$ & $6 " \mathrm{CS}$ & & $\mathrm{LL}$ \\
\hline SAMPLE DATE & & $11 / 30 / 99$ & $12 / 14 / 99$ & $01 / 19 / 00$ & & $02 / 17 / 00$ & \\
\hline \multicolumn{8}{|l|}{ FIELD DATA } \\
\hline Parameter & & Nov & $\underline{\mathrm{DEC}}$ & $\underline{\text { JAN }}$ & & FEB & $\underline{\text { Unit }}$ \\
\hline $\begin{array}{l}\text { Water Elevation } \\
\text { pH } \\
\text { Sp. Conductance } \\
\text { Water temperature } \\
\text { Alkalinity as CaCO3 } \\
\text { Turbidity } \\
\text { Volumes purged } \\
\text { Sampling code }\end{array}$ & & $\begin{array}{l}209.55 \\
5.1 \\
24 \\
16.7 \\
0 \\
.3 \\
C\end{array}$ & $\begin{array}{l}209.8 \\
5.1 \\
24 \\
16.3 \\
0 \\
.4 \\
C\end{array}$ & $\begin{array}{l}209.73 \\
4.5 \\
24 \\
15.8 \\
0 \\
.4 \\
.008 \\
C\end{array}$ & & $\begin{array}{l}209.57 \\
4.5 \\
25 \\
17.8 \\
0 \\
.4 \\
C\end{array}$ & $\begin{array}{l}\mathrm{ft} \mathrm{msl} \\
\mathrm{pH} \\
\mathrm{uS} / \mathrm{cm} \\
\text { deg. C } \\
\mathrm{mg} / \mathrm{L} \\
\text { NTU } \\
\text { well volume }\end{array}$ \\
\hline
\end{tabular}

\section{ANAL.YTICAL DATA}

I. Groundwater Protection Standard

261 Appendix VIII/264 Appendix IX Hazardous Constituents

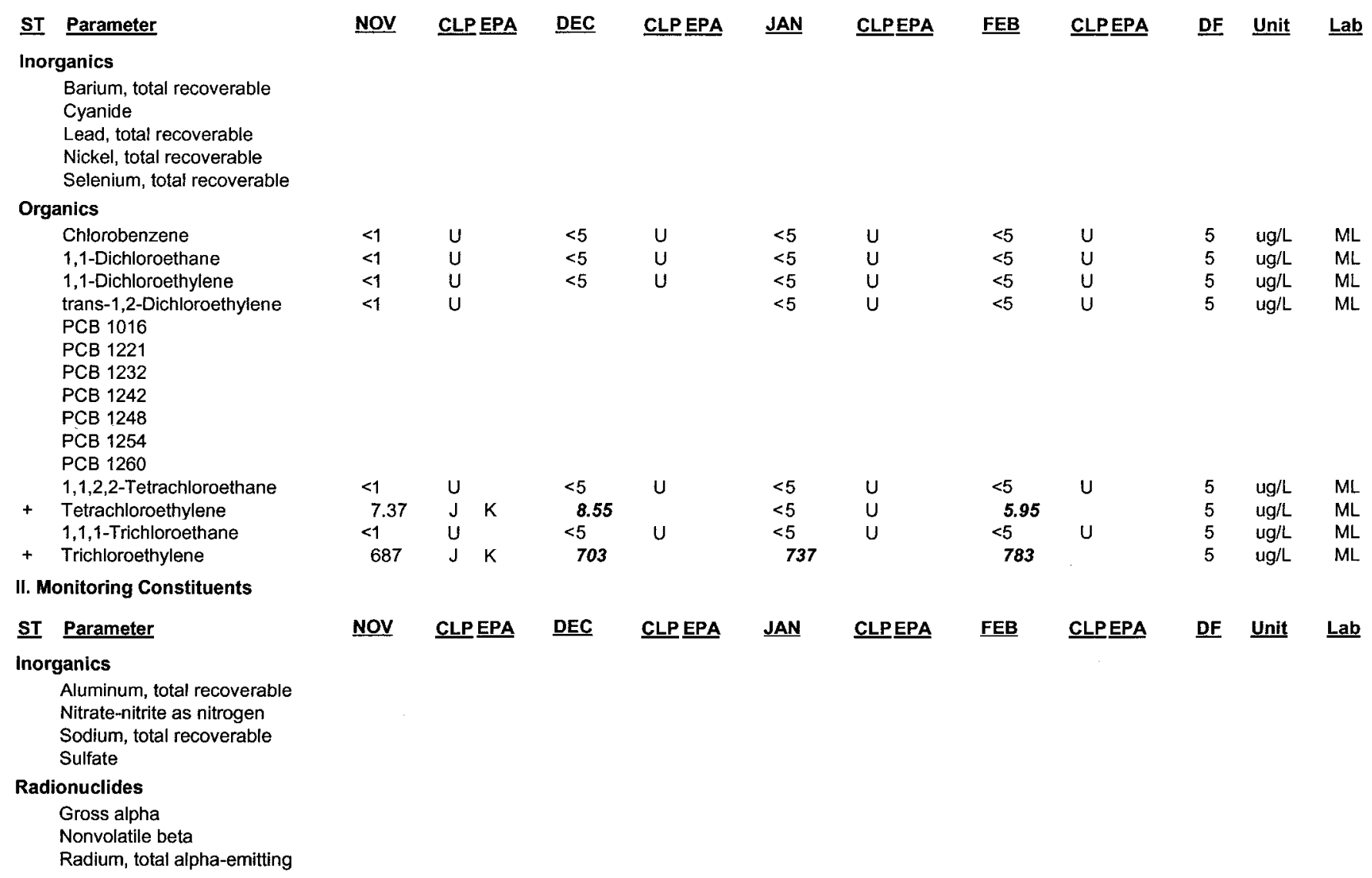


Table D-5b. Groundwater Monitoring Results for Recovery Wells, November 1999-February 2000 (Cont.) WELL RWM 14C

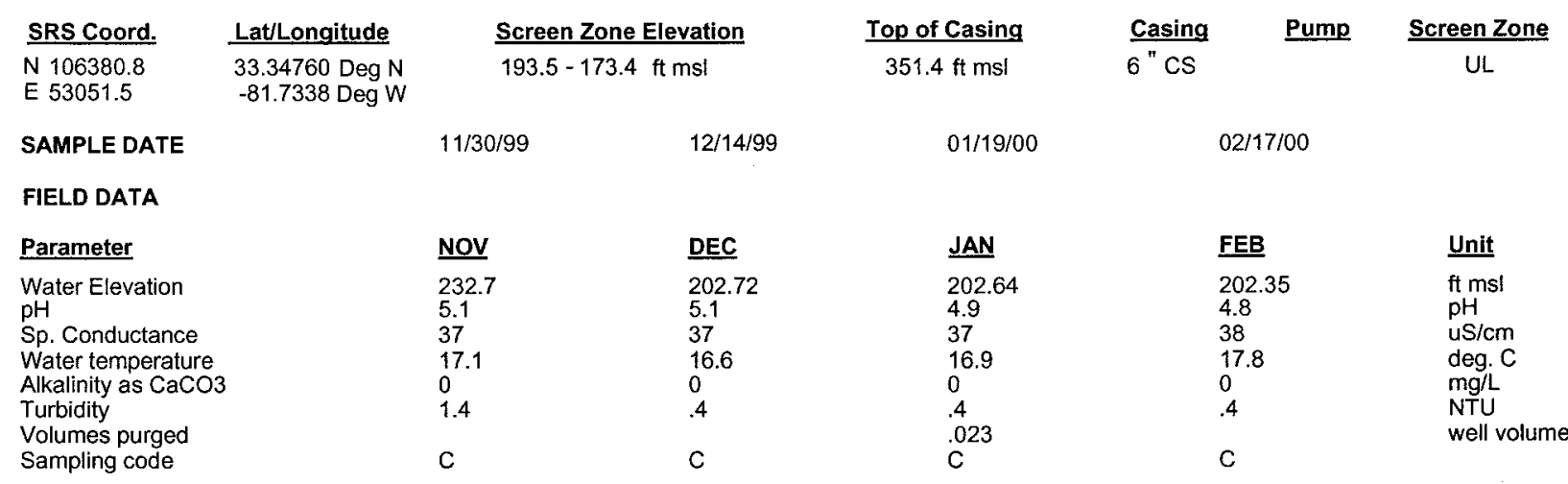

ANALYTICAL DATA

I. Groundwater Protection Standard

261 Appendix VIII/264 Appendix IX Hazardous Constituents

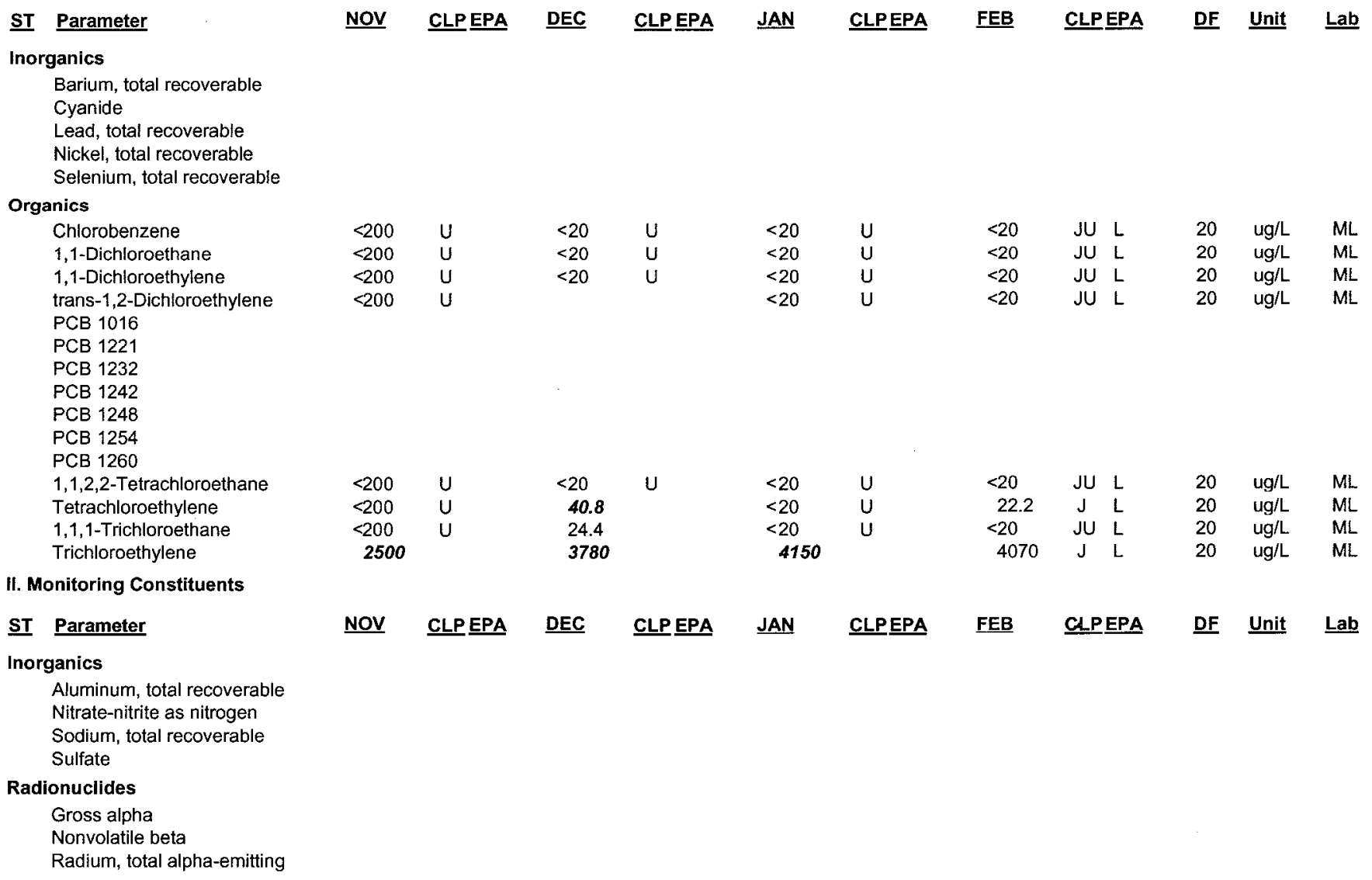

Notes: Concentrations in bold italics exceed the groundwater protection or monitoring constituent standards listed in Appendix A. Synchronous water levels are measured over a 3-5 day period or less. Dilution factors and Laboratory Data are February 2000 data only.

$+=$ exceeded the groundwater protection or monitoring constituent standards listed in Appendix A for February 2000. 
Table D-5b. Groundwater Monitoring Results for Recovery Wells, November 1999-February 2000 (Cont.) WELL RWM 15B

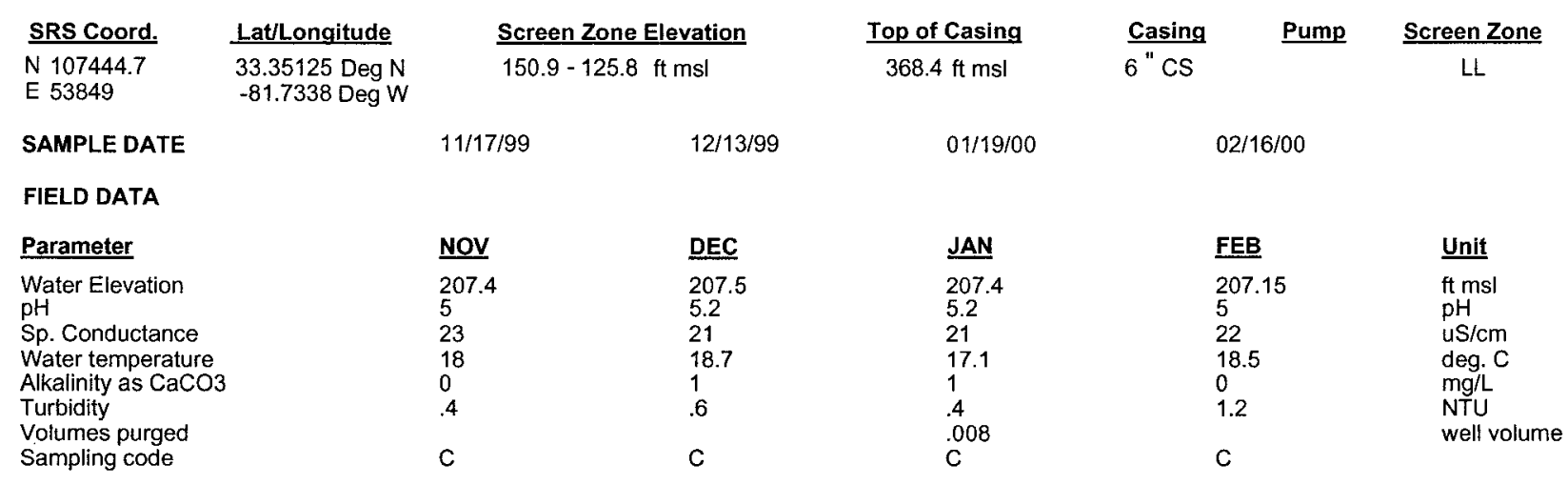

\section{ANALYTICAL DATA}

I. Groundwater Protection Standard

261 Appendix VIII/264 Appendix IX Hazardous Constituents

\begin{tabular}{|c|c|c|c|c|c|c|c|c|c|c|c|c|}
\hline$\underline{\mathrm{ST}}$ & Parameter & NOV & CLPEPA & DEC & CLP EPA & $\underline{\text { JAN }}$ & CLPEEPA & FEB & CLPEEPA & $\underline{\text { DF }}$ & $\underline{\text { Unit }}$ & Lab \\
\hline \multicolumn{13}{|c|}{ Inorganics } \\
\hline \multicolumn{13}{|c|}{ Barium, total recoverable } \\
\hline \multicolumn{13}{|c|}{ Cyanide } \\
\hline \multicolumn{13}{|c|}{ Lead, total recoverable } \\
\hline \multicolumn{13}{|c|}{ Nickel, total recoverable } \\
\hline \multicolumn{13}{|c|}{ Selenium, total recoverable } \\
\hline \multicolumn{13}{|c|}{ Organics } \\
\hline & Chlorobenzene & $<1$ & $J \cup Q$ & $<1$ & $u$ & $<1$ & $\mathrm{U}$ & $<1$ & $U$ & 1 & $\mathrm{ug} / \mathrm{L}$ & ML \\
\hline & 1,1-Dichloroethane & $<1$ & $\mathrm{JU} Q$ & $<1$ & $\mathrm{U}$ & $<1$ & $\mathrm{U}$ & $<1$ & $\mathrm{U}$ & 1 & $\mathrm{ug} / \mathrm{L}$ & ML. \\
\hline & 1,1-Dichloroethylene & $<1$ & JU $Q$ & $<1$ & $\mathrm{U}$ & $<1$ & $\mathrm{U}$ & $<1$ & $U$ & 1 & $\mathrm{ug} / \mathrm{L}$ & ML \\
\hline & trans-1,2-Dichloroethylene & $<1$ & JU $Q$ & & & $<1$ & $\mathrm{U}$ & $<1$ & $U$ & 1 & $\mathrm{ug} / \mathrm{L}$ & $M L$ \\
\hline \multicolumn{13}{|c|}{ PCB 1016} \\
\hline \multicolumn{13}{|c|}{ PCB 1221} \\
\hline \multicolumn{13}{|c|}{ PCB 1232} \\
\hline \multicolumn{13}{|c|}{ PCB 1242} \\
\hline \multicolumn{13}{|c|}{ РСВ 1248} \\
\hline \multicolumn{13}{|c|}{ PCB 1254} \\
\hline \multicolumn{13}{|c|}{ PCB 1260} \\
\hline & 1,1,2,2-Tetrachloroethane & $<1$ & JU $Q$ & $<1$ & U & $<1$ & $\mathrm{U}$ & $<1$ & $\mathrm{U}$ & 1 & $u g / L$ & $M L$ \\
\hline & Tetrachloroethylene & $<1$ & $J \cup Q$ & $<1$ & U & $<1$ & U & $<1$ & $\mathrm{U}$ & 1 & $\mathrm{ug} / \mathrm{L}$ & ML \\
\hline & $1,1,1$-Trichloroethane & $<1$ & $J \cup Q$ & $<1$ & $\mathrm{U}$ & $<1$ & $\mathrm{U}$ & $<1$ & $\mathrm{U}$ & 1 & $u g / L$ & ML \\
\hline+ & Trichloroethylene & 20.9 & $J \quad Q$ & 23.7 & & 25.5 & & 23.4 & & 1 & $u g / L$ & ML \\
\hline \multicolumn{13}{|c|}{ II. Monitoring Constituents } \\
\hline$\underline{\mathbf{S T}}$ & Parameter & NOV & CLPEPA & $\underline{\text { DEC }}$ & $\underline{\text { CLP EPA }}$ & $\underline{\text { JAN }}$ & CLPEPA & $\underline{\text { FEB }}$ & CLPEPA & $\underline{\mathrm{DF}}$ & $\underline{\text { Unit }}$ & $\underline{\text { Lab }}$ \\
\hline \multicolumn{13}{|c|}{ Inorganics } \\
\hline \multicolumn{12}{|c|}{ Aluminum, total recoverable } & \\
\hline \multicolumn{12}{|c|}{ Nitrate-nitrite as nitrogen } & \\
\hline \multicolumn{12}{|c|}{ Sodium, total recoverable } & \\
\hline \multicolumn{12}{|c|}{ Sulfate } & \\
\hline \multicolumn{13}{|c|}{ Radionuclides } \\
\hline \multirow{2}{*}{\multicolumn{12}{|c|}{$\begin{array}{l}\text { Gross alpha } \\
\text { Nonvolatile beta } \\
\text { Radium total alpha }\end{array}$}} & \\
\hline & & & & & & & & & & & & \\
\hline
\end{tabular}

Notes: Concentrations in bold italics exceed the groundwater protection or monitoring constituent standards listed in Appendix A. Synchronous water levels are measured over a 3-5 day period or less. Dilution factors and Laboratory Data are February 2000 data only.

$+=$ exceeded the groundwater protection or monitoring constituent standards listed in Appendix A for February 2000. 
WSRC-TR-2000-00242

Unclassified

\section{THIS PAGE LEFT BLANK INTENTIONALLY}


Table D-5c. Groundwater Monitoring Results for Recovery Wells, March-June 2000

WELL RWM 1

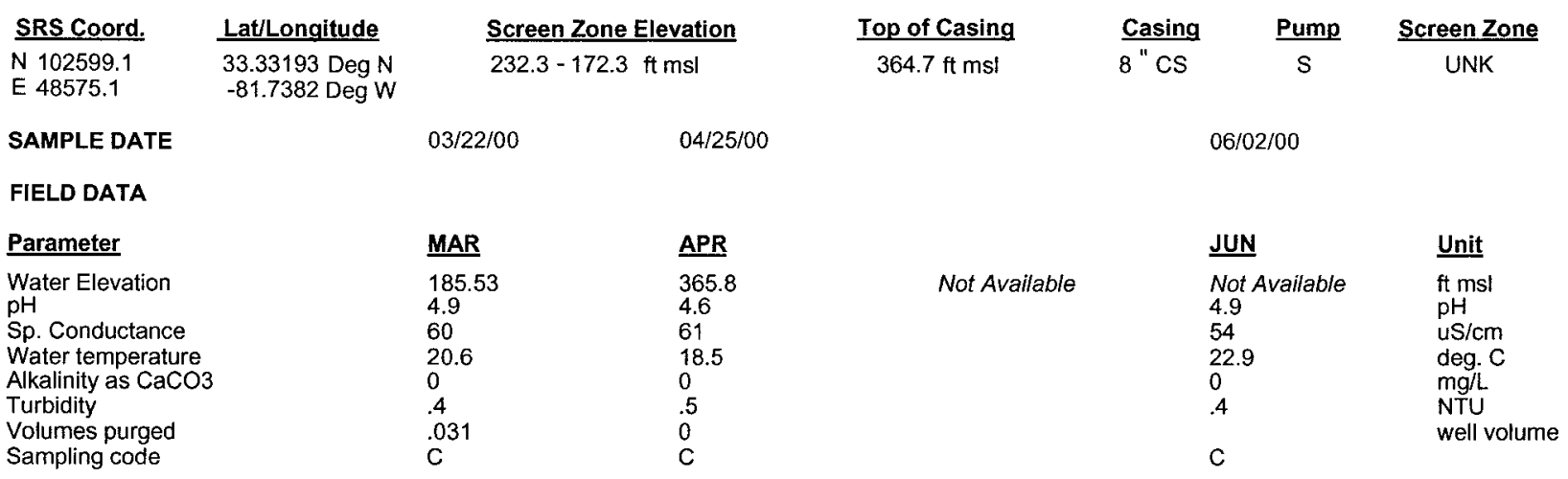

ANALYTICAL DATA

1. Groundwater Protection Standard

261 Appendix VIII/264 Appendix IX Hazardous Constituents

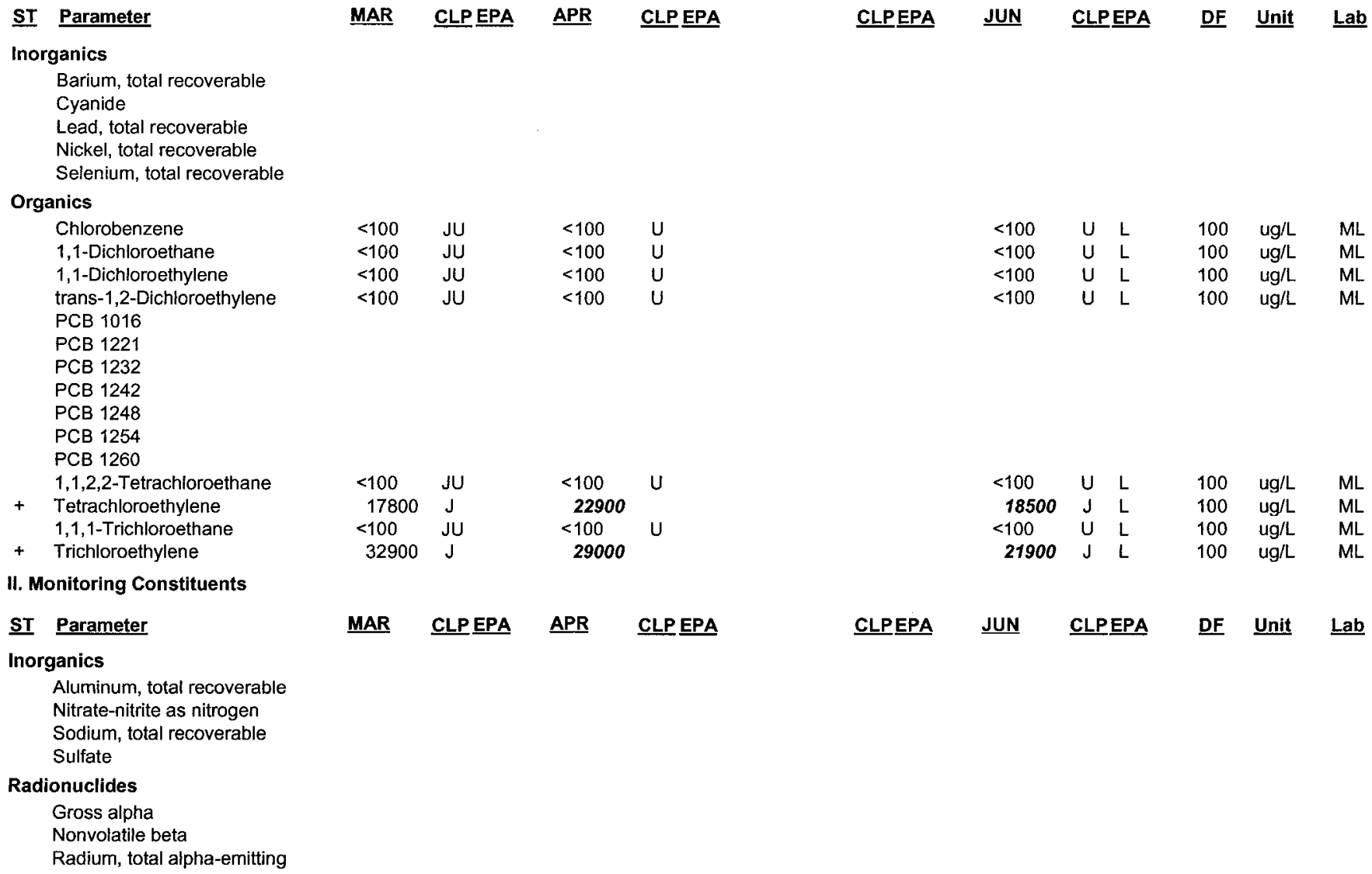
Notes: Concentrations in bold italics exceed the groundwater protection or monitoring constituent standards listed in Appendix A. Synchronous water
levels are measured over a 3-5 day period or less. Dilution factors and Laboratory Data are June 2000 data only.

$+=$ exceeded the groundwater protection or monitoring constituent standards listed in Appendix A for June 2000. 
Table D-5c. Groundwater Monitoring Results for Recovery Wells, March-June 2000 (Cont.)

WELL RWM 2

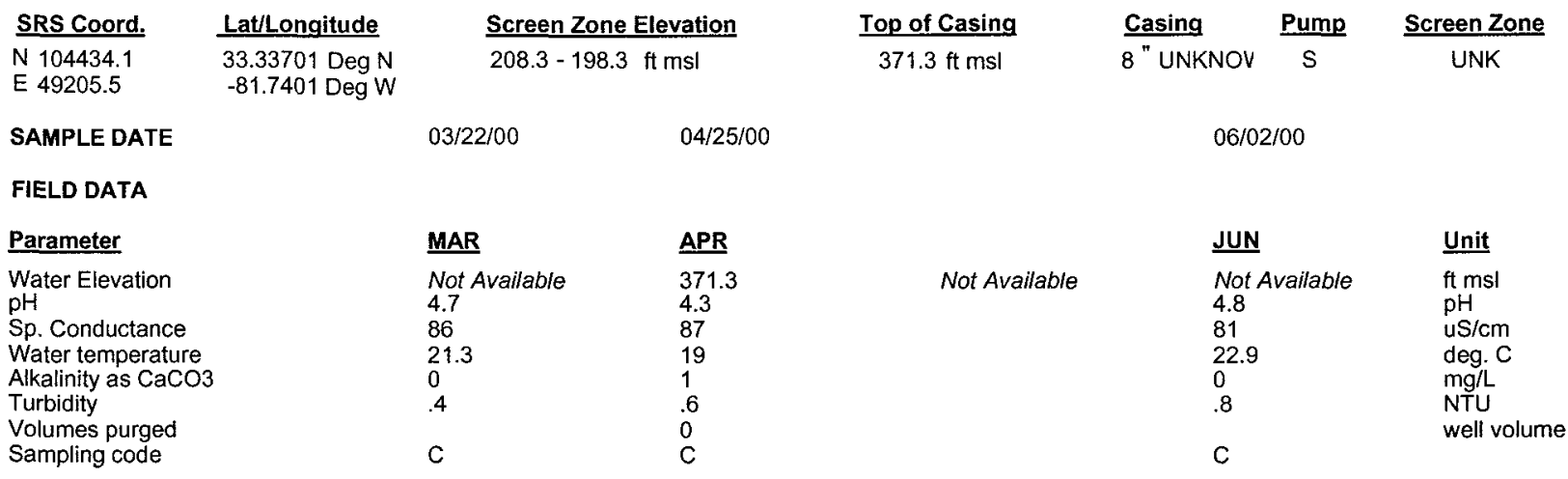

ANALYTICAL DATA

I. Groundwater Protection Standard

261 Appendix VIII/264 Appendix IX Hazardous Constituents

\begin{tabular}{|c|c|c|c|c|c|c|c|c|c|c|c|}
\hline ST & Parameter & MAR & CLP EPA & $\underline{A P R}$ & CLP EPA & CLPEPA & $\underline{\text { JUN }}$ & CLPEPA & $\underline{\text { DF }}$ & Unit & $\underline{\text { Lab }}$ \\
\hline \multicolumn{12}{|c|}{ Inorganics } \\
\hline \multicolumn{12}{|c|}{ Barium, total recoverable } \\
\hline \multicolumn{12}{|c|}{ Cyanide } \\
\hline \multicolumn{12}{|c|}{ Lead, total recoverable } \\
\hline \multicolumn{12}{|c|}{ Nickel, total recoverable } \\
\hline \multicolumn{12}{|c|}{ Selenium, total recoverable } \\
\hline \multicolumn{12}{|c|}{ Organics } \\
\hline & Chlorobenzene & $<50$ & JU & $<100$ & $U$ & & $<500$ & JU & 100 & $\mathrm{ug} / \mathrm{L}$ & EX \\
\hline & 1,1-Dichloroethane & $<50$ & $\mathrm{JU}$ & $<100$ & $u$ & & $<500$ & JU & 100 & $\mathrm{ug} / \mathrm{L}$ & EX \\
\hline & 1,1-Dichtoroethylene & $<50$ & JU & $<100$ & $\mathrm{U}$ & & $<500$ & JU & 100 & $\mathrm{ug} / \mathrm{L}$ & $\mathrm{EX}$ \\
\hline & trans-1,2-Dichloroethylene & $<50$ & JU & $<100$ & $\mathrm{U}$ & & $<500$ & JU & 100 & $\mathrm{ug} / \mathrm{L}$ & $\mathrm{EX}$ \\
\hline \multicolumn{12}{|c|}{ PCB 1016} \\
\hline \multicolumn{12}{|c|}{ PCB 1221} \\
\hline \multicolumn{12}{|c|}{ PCB 1232} \\
\hline \multicolumn{12}{|c|}{ PCB 1242} \\
\hline \multicolumn{12}{|c|}{ PCB 1248} \\
\hline \multicolumn{12}{|c|}{ PCB 1254} \\
\hline \multicolumn{12}{|c|}{ PCB 1260} \\
\hline & $1,1,2,2$-Tetrachloroethane & $<50$ & JU & $<100$ & U & & $<500$ & $\mathrm{JU}$ & 100 & $\mathrm{ug} / \mathrm{L}$ & EX \\
\hline & Tetrachloroethylene & 11500 & $J$ & 14200 & & & 12600 & $\mathrm{~J} \quad \mathrm{~L}$ & 50 & ug/L & $M L$ \\
\hline & $1,1,1-$ Trichloroethane & $<50$ & JU & $<100$ & U & & $<500$ & JU & 100 & $\mathrm{ug} / \mathrm{L}$ & EX \\
\hline & Trichloroethylene & 14200 & $\mathrm{~J}$ & 15400 & & & 8740 & $J \quad L$ & 50 & ug/L & $M L$ \\
\hline \multicolumn{12}{|c|}{ II. Monitoring Constituents } \\
\hline$\underline{\mathbf{S T}}$ & Parameter & MAR & CLPEEPA & $\underline{\text { APR }}$ & CLPP EPA & CLPEPA & JUN & CLPEPA & $\underline{\text { DF }}$ & Unit & $\underline{L a b}$ \\
\hline \multicolumn{12}{|c|}{ Inorganics } \\
\hline \multicolumn{11}{|c|}{ Aluminum, total recoverable } & \\
\hline \multicolumn{11}{|c|}{ Nitrate-nitrite as nitrogen } & \\
\hline \multicolumn{12}{|c|}{ Sodium, total recoverable } \\
\hline \multicolumn{11}{|c|}{ Sulfate } & \\
\hline \multicolumn{11}{|c|}{ Radionuclides } & \\
\hline & Gross alpha & & & & & & & & & & \\
\hline & $\begin{array}{l}\text { Nonvolatile beta } \\
\text { Radium, total alpha-emitting }\end{array}$ & & & & & & & & & & \\
\hline
\end{tabular}

Notes: Concentrations in bold italics exceed the groundwater protection or monitoring constituent standards listed in Appendix A. Synchronous water levels are measured over a 3-5 day period or less. Dilution factors and Laboratory Data are June 2000 data only.

$+=$ exceeded the groundwater protection or monitoring constituent standards listed in Appendix A for June 2000. 
Table D-5c. Groundwater Monitoring Results for Recovery Wells, March-June 2000 (Cont.) WELL RWM 3

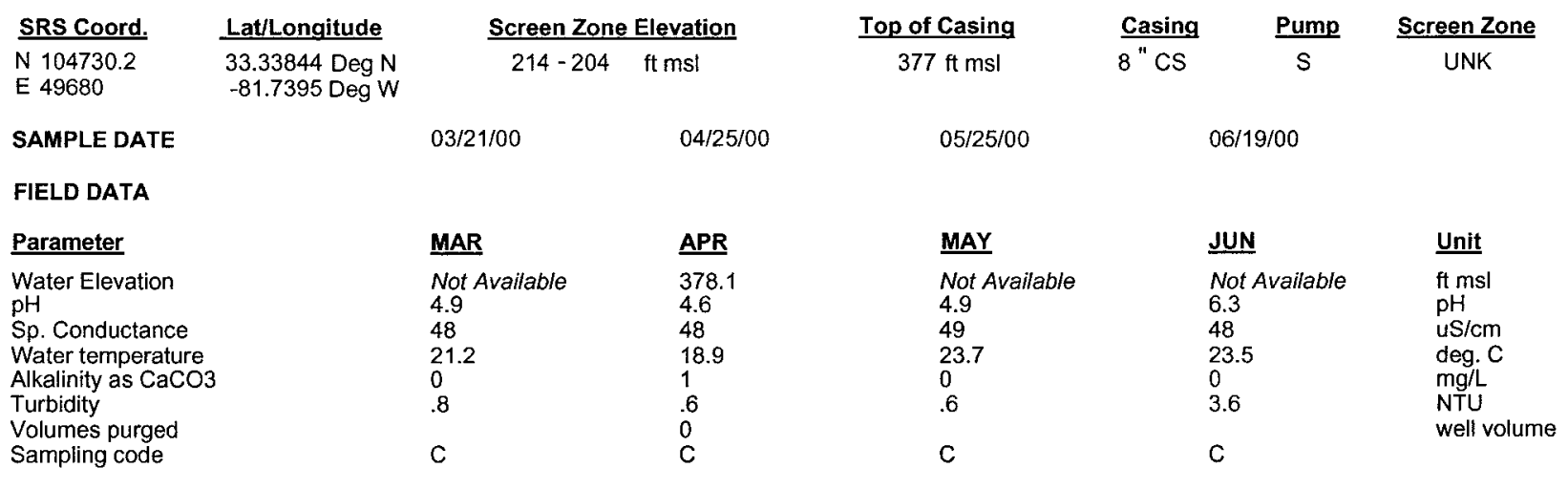

ANALYTICAL DATA

I. Groundwater Protection Standard

261 Appendix VIII/264 Appendix IX Hazardous Constituents

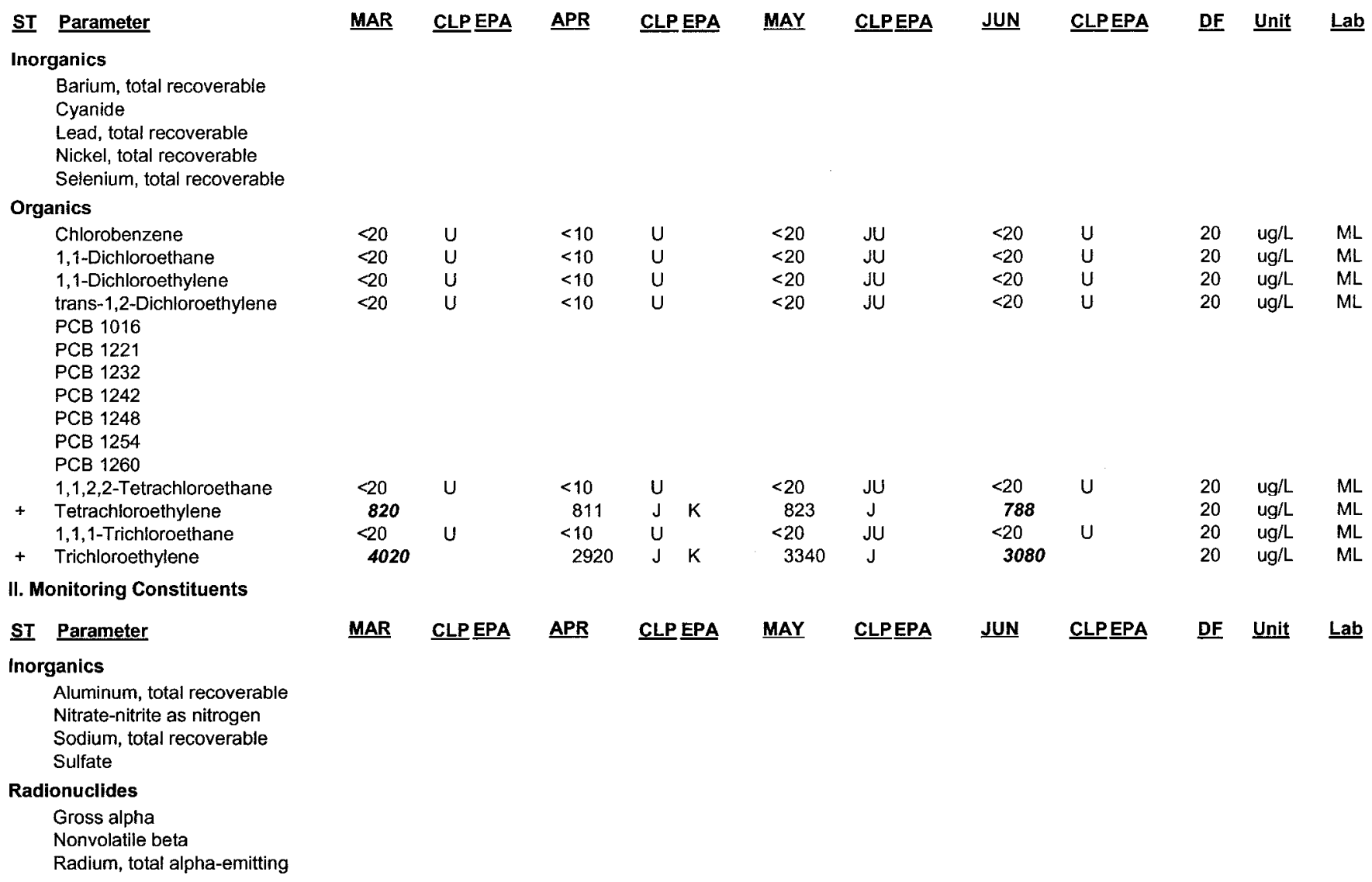

\footnotetext{
Notes: Concentrations in bold italics exceed the groundwater protection or monitoring constituent standards listed in Appendix A. Synchronous water levels are measured over a 3-5 day period or less. Dilution factors and Laboratory Data are June 2000 data only. 
Table D-5c. Groundwater Monitoring Results for Recovery Wells, March-June 2000 (Cont.) WELL RWM 4

\begin{tabular}{|c|c|c|c|c|c|c|c|}
\hline SRS Coord. & Lat/Longitude & \multicolumn{2}{|c|}{ Screen Zone Elevation } & Top of Casing & Casing & Pump & Screen Zone \\
\hline $\begin{array}{l}\text { N } 103719.3 \\
\text { E } 48948.2\end{array}$ & $\begin{array}{l}\text { 33.33501 Deg } N \\
-81.7394 \operatorname{Deg} W\end{array}$ & \multicolumn{2}{|c|}{$139.9-129.5 \mathrm{ft} \mathrm{msl}$} & $366.5 \mathrm{ft} \mathrm{mst}$ & $8 " \mathrm{CS}$ & $S$ & $\overline{M / G C / U L / L L}$ \\
\hline SAMPLE DATE & & $03 / 21 / 00$ & $04 / 25 / 00$ & $05 / 25 / 00$ & & $06 / 19 / 00$ & \\
\hline \multicolumn{8}{|l|}{ FIELD DATA } \\
\hline Parameter & & MAR & APR & MAY & & JUN & Unit \\
\hline $\begin{array}{l}\text { Water Elevation } \\
\text { pH } \\
\text { Sp. Conductance } \\
\text { Water temperature } \\
\text { Alkalinity as CaCO3 } \\
\text { Turbidity } \\
\text { Volumes purged } \\
\text { Sampling code }\end{array}$ & & $\begin{array}{l}207.14 \\
5.1 \\
23 \\
20.8 \\
2 \\
.6 \\
.005 \\
C\end{array}$ & $\begin{array}{l}205.4 \\
4.9 \\
23 \\
18.4 \\
1 \\
.4 \\
0 \\
C\end{array}$ & $\begin{array}{l}204.46 \\
5.1 \\
24 \\
23.2 \\
0 \\
.8 \\
.005 \\
C\end{array}$ & & $\begin{array}{l}\text { Not Available } \\
6.5 \\
22 \\
24 \\
3 \\
.7 \\
\text { C }\end{array}$ & $\begin{array}{l}\mathrm{ft} \mathrm{msl} \\
\mathrm{pH} \\
\mathrm{uS} / \mathrm{cm} \\
\text { deg. C } \\
\text { mg/l } \\
\text { NTU } \\
\text { well volume }\end{array}$ \\
\hline
\end{tabular}

\section{ANALYTICAL DATA}

I. Groundwater Protection Standard

261 Appendix VIII/264 Appendix IX Hazardous Constituents

\begin{tabular}{|c|c|c|c|c|c|c|c|c|c|c|c|}
\hline ST & Parameter & MAR & CLP EPA & APR & CLPEPA & MAY & CLPEPA & JUN & CLPEPA & DF & Unit \\
\hline \multicolumn{12}{|c|}{ Inorganics } \\
\hline & $\begin{array}{l}\text { Barium, total recoverable } \\
\text { Cyanide } \\
\text { Lead, total recoverable } \\
\text { Nickel, total recoverable } \\
\text { Selenium, total recoverable }\end{array}$ & & & & & & & & & & \\
\hline \multicolumn{12}{|c|}{ Organics } \\
\hline & Chlorobenzene & $<50$ & נU & $<20$ & $\mathrm{U}$ & $<50$ & JU & $<50$ & $\mathrm{U}$ & 50 & ug/L \\
\hline & 1,1-Dichloroethane & $<50$ & JU & $<20$ & $\mathrm{U}$ & $<50$ & JU & $<50$ & $\mathrm{U}$ & 50 & $\mathrm{ug} / \mathrm{L}$ \\
\hline & 1,1-Dichloroethylene & $<50$ & JU & $<20$ & $\mathrm{U}$ & $<50$ & JU & 1.3 & $J \quad I L$ & 1 & ug/L \\
\hline & trans-1,2-Dichloroethylene & $<50$ & JU & $<20$ & $\mathrm{u}$ & $<50$ & JU & $<50$ & $\mathrm{u}$ & 50 & $\mathrm{ug} / \mathrm{L}$ \\
\hline \multicolumn{12}{|c|}{ PCB 1016} \\
\hline & \multicolumn{11}{|l|}{ PCB 1221} \\
\hline & \multicolumn{11}{|l|}{ PCB 1232} \\
\hline & \multicolumn{11}{|l|}{ PCB 1242} \\
\hline & \multicolumn{11}{|l|}{ PCB 1248} \\
\hline & \multicolumn{11}{|l|}{ PCB 1254} \\
\hline & \multicolumn{11}{|l|}{ PCB 1260} \\
\hline \multirow{3}{*}{+} & 1,1,2,2-Tetrachloroethane & $<50$ & JU & $<20$ & $U$ & $<50$ & JU & $<50$ & $\mathrm{U}$ & 50 & $\mathrm{ug} / \mathrm{L}$ \\
\hline & Tetrachloroethylene & 1510 & $J$ & 1070 & & 1000 & $\mathrm{~J}$ & 1200 & & 50 & $\mathrm{ug} / \mathrm{L}$ \\
\hline & 1,1,1-Trichloroethane & $<50$ & JU & $<20$ & $\mathrm{U}$ & $<50$ & JU & $<50$ & $U$ & 50 & $\mathrm{ug} / \mathrm{L}$ \\
\hline+ & Trichloroethylene & 8700 & $J$ & 6090 & & 6690 & $\mathrm{~J}$ & 7100 & & 50 & $\mathrm{ug} / \mathrm{L}$ \\
\hline \multicolumn{12}{|c|}{ II. Monitoring Constituents } \\
\hline ST & Parameter & MAR & CLP EPA & APR & CLP EPA & $\underline{\text { MAY }}$ & CLPEPA & JUN & CLPEPA & DF & Unit \\
\hline \multicolumn{12}{|c|}{ Inorganics } \\
\hline \multicolumn{12}{|c|}{ Aluminum, total recoverable } \\
\hline \multicolumn{12}{|c|}{ Nitrate-nitrite as nitrogen } \\
\hline \multicolumn{12}{|c|}{ Sodium, total recoverable } \\
\hline \multicolumn{12}{|c|}{ Sulfate } \\
\hline \multicolumn{12}{|c|}{ Radionuclides } \\
\hline \multirow{3}{*}{\multicolumn{12}{|c|}{$\begin{array}{l}\text { Gross alpha } \\
\text { Nonvolatile beta }\end{array}$}} \\
\hline & & & & & & & & & & & \\
\hline & Radium, total alpha-emitting & & & & & & & & & & \\
\hline
\end{tabular}


Table D-5c. Groundwater Monitoring Results for Recovery Wells, March-June 2000 (Cont.) WELL RWM 5

\begin{tabular}{|c|c|c|c|c|c|c|c|}
\hline SRS Coord. & Lat/Longitude & \multicolumn{2}{|c|}{ Screen Zone Elevation } & Top of Casing & Casing & Pump & Screen Zone \\
\hline $\begin{array}{l}N 103502.2 \\
\text { E } 49628\end{array}$ & $\begin{array}{l}\text { 33.33564 Deg } N \\
-81.7372 \operatorname{Deg} W\end{array}$ & \multicolumn{2}{|c|}{$144.3-133.9 \mathrm{ft} \mathrm{ms}$} & $366.9 \mathrm{ft} \mathrm{msl}$ & $8^{\prime \prime} \mathrm{CS}$ & $\mathrm{S}$ & $\overline{M / G C / U L / L L}$ \\
\hline SAMPLE DATE & & $03 / 21 / 00$ & $04 / 25 / 00$ & $05 / 25 / 00$ & & $06 / 19 / 00$ & \\
\hline \multicolumn{8}{|l|}{ FIELD DATA } \\
\hline Parameter & & MAR & APR & MAY & & $\underline{\text { JUN }}$ & Unit \\
\hline $\begin{array}{l}\text { Water Elevation } \\
\text { pH } \\
\text { Sp. Conductance } \\
\text { Water temperature } \\
\text { Alkalinity as CaCO3 } \\
\text { Turbidity } \\
\text { Volumes purged } \\
\text { Sampling code }\end{array}$ & & $\begin{array}{l}209.76 \\
5.2 \\
28 \\
20.8 \\
0 \\
.5 \\
.176 \\
C\end{array}$ & $\begin{array}{l}209.45 \\
4.6 \\
33 \\
19 \\
0 \\
.6 \\
0 \\
C\end{array}$ & $\begin{array}{l}208.68 \\
5.2 \\
32 \\
23.8 \\
0 \\
.9 \\
.353 \\
\mathrm{C}\end{array}$ & & $\begin{array}{l}208.45 \\
6.6 \\
28 \\
23.3 \\
1 \\
.9 \\
\text { C }\end{array}$ & $\begin{array}{l}\mathrm{ft} \text { msl } \\
\mathrm{pH} \\
\mathrm{uS} / \mathrm{cm} \\
\mathrm{deg} . \mathrm{C} \\
\mathrm{mg} / \mathrm{L} \\
\mathrm{NTU} \\
\text { well volume }\end{array}$ \\
\hline
\end{tabular}

ANALYTICAL DATA

I. Groundwater Protection Standard

261 Appendix VIII/264 Appendix IX Hazardous Constituents

\begin{tabular}{|c|c|c|c|c|c|c|c|c|c|c|c|}
\hline$\underline{\text { ST }}$ & Parameter & MAR & CLPEPA & APR & CLP EPA & MAY & CLPEPA & $\underline{\text { JUN }}$ & CLPEPA & $\underline{\mathrm{DF}}$ & Unit \\
\hline \multicolumn{12}{|c|}{ Inorganics } \\
\hline \multicolumn{12}{|c|}{ Barium, total recoverable } \\
\hline \multicolumn{12}{|c|}{ Cyanide } \\
\hline \multicolumn{12}{|c|}{ Lead, total recoverable } \\
\hline \multicolumn{12}{|c|}{ Nickel, total recoverable } \\
\hline \multicolumn{12}{|c|}{ Selenium, total recoverable } \\
\hline \multicolumn{12}{|c|}{ Organics } \\
\hline & Chlorobenzene & $<5$ & $\mathrm{U}$ & $<10$ & $U$ & $<10$ & JU & $<10$ & $\mathrm{U}$ & 10 & $\mathrm{ug} / \mathrm{L}$ \\
\hline & 1,1-Dichloroethane & $<5$ & U & $<10$ & $\mathrm{U}$ & $<10$ & JU & $<10$ & $\mathrm{U}$ & 10 & $\mathrm{ug} / \mathrm{L}$ \\
\hline & 1,1-Dichloroethylene & $<5$ & $\mathrm{U}$ & $<10$ & $\mathrm{U}$ & $<10$ & $\mathrm{JU}$ & $<10$ & $U$ & 10 & $\mathrm{ug} / \mathrm{L}$ \\
\hline & trans-1,2-Dichloroethylene & $<5$ & $\mathrm{U}$ & $<10$ & $U$ & $<10$ & JU & $<10$ & $\mathrm{U}$ & 10 & $\mathrm{ug} / \mathrm{L}$ \\
\hline \multicolumn{12}{|c|}{ PCB 1016} \\
\hline \multicolumn{12}{|c|}{ PCB 1221} \\
\hline \multicolumn{12}{|c|}{ PCB 1232} \\
\hline \multicolumn{12}{|c|}{ PCB 1242} \\
\hline \multicolumn{12}{|c|}{ PCB 1248} \\
\hline \multicolumn{12}{|c|}{ PCB 1254} \\
\hline \multicolumn{12}{|c|}{ PCB 1260} \\
\hline & 1,1,2,2-Tetrachloroethane & $<5$ & U & $<10$ & $\mathrm{U}$ & $<10$ & JU & $<10$ & $U$ & 10 & $\mathrm{ug} / \mathrm{L}$ \\
\hline & Tetrachloroethylene & 524 & & 523 & & 574 & J & 585 & $\mathrm{~J} \quad \mathrm{~K}$ & 10 & $\mathrm{ug} / \mathrm{L}$ \\
\hline & 1,1,1-Trichloroethane & & $\mathrm{U}$ & $<10$ & $\mathrm{U}$ & $<10$ & JU & $<10$ & $\mathrm{U}$ & 10 & $\mathrm{ug} / \mathrm{L}$ \\
\hline & Trichloroethylene & 1680 & & 1530 & & 1690 & $J$ & 1550 & $J K$ & 10 & ug/L \\
\hline \multicolumn{12}{|c|}{ II. Monitoring Constituents } \\
\hline$\underline{\mathbf{S I}}$ & Parameter & $\underline{\text { MAR }}$ & CLP EPA & $\underline{\text { APR }}$ & CLP EPA & MAY & CLPEPA & $\underline{\text { JUN }}$ & CLPEPA & DF & Unit \\
\hline \multicolumn{12}{|c|}{ Inorganics } \\
\hline \multicolumn{12}{|c|}{ Aluminum, total recoverable } \\
\hline \multicolumn{12}{|c|}{ Nitrate-nitrite as nitrogen } \\
\hline \multicolumn{12}{|c|}{ Sodium, total recoverable } \\
\hline \multicolumn{12}{|c|}{ Sulfate } \\
\hline \multicolumn{12}{|c|}{ Radionuclides } \\
\hline \multirow{2}{*}{\multicolumn{12}{|c|}{$\begin{array}{l}\text { Gross alpha } \\
\text { Nonvolatile beta } \\
\text { Radium total aloha-emittina }\end{array}$}} \\
\hline & & & & & & & & & & & \\
\hline & Radium, total alpha-emitting & & & & & & & & & & \\
\hline
\end{tabular}

Notes: Concentrations in bold italics exceed the groundwater protection or monitoring constituent standards listed in Appendix A. Synchronous water levels are measured over a 3-5 day period or less. Dilution factors and Laboratory Data are June 2000 data only.

$+=$ exceeded the groundwater protection or monitoring constituent standards listed in Appendix A for June 2000. 
Table D-5c. Groundwater Monitoring Results for Recovery Wells, March-June 2000 (Cont.)

WELL RWM 7

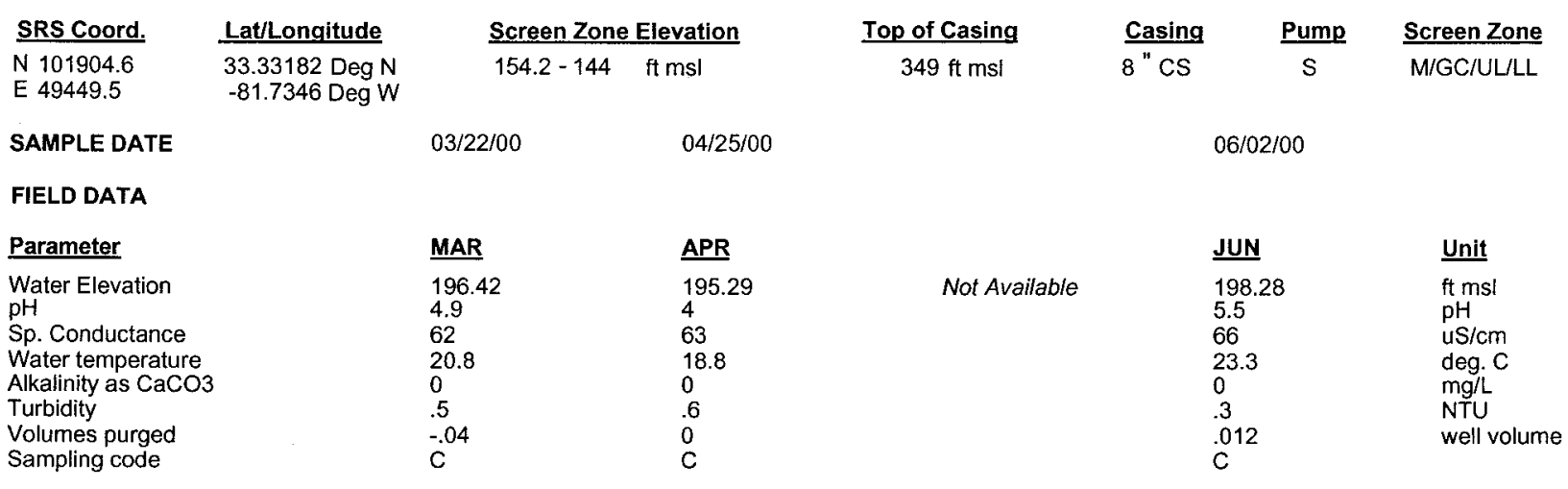

ANALYTICAL DATA

I. Groundwater Protection Standard

261 Appendix VIII/264 Appendix IX Hazardous Constituents

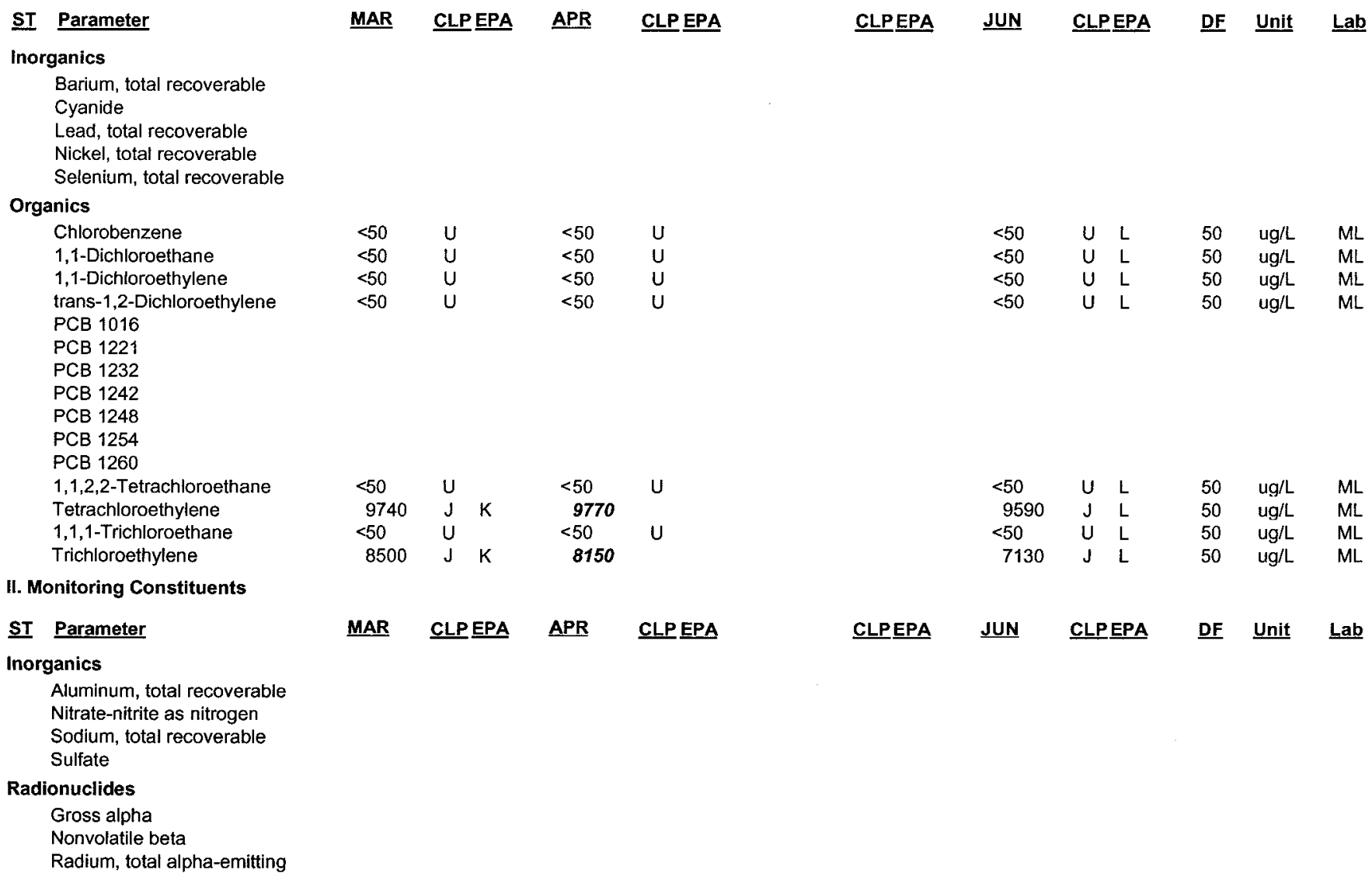

Notes: Concentrations in bold italics exceed the groundwater protection or monitoring constituent standards listed in Appendix A. Synchronous water levels are measured over a 3-5 day period or less. Dilution factors and Laboratory Data are June 2000 data only.

+ = exceeded the groundwater protection or monitoring constituent standards listed in Appendix A for June 2000. 
Table D-5c. Groundwater Monitoring Results for Recovery Wells, March-June 2000 (Cont.) WELL RWM 8

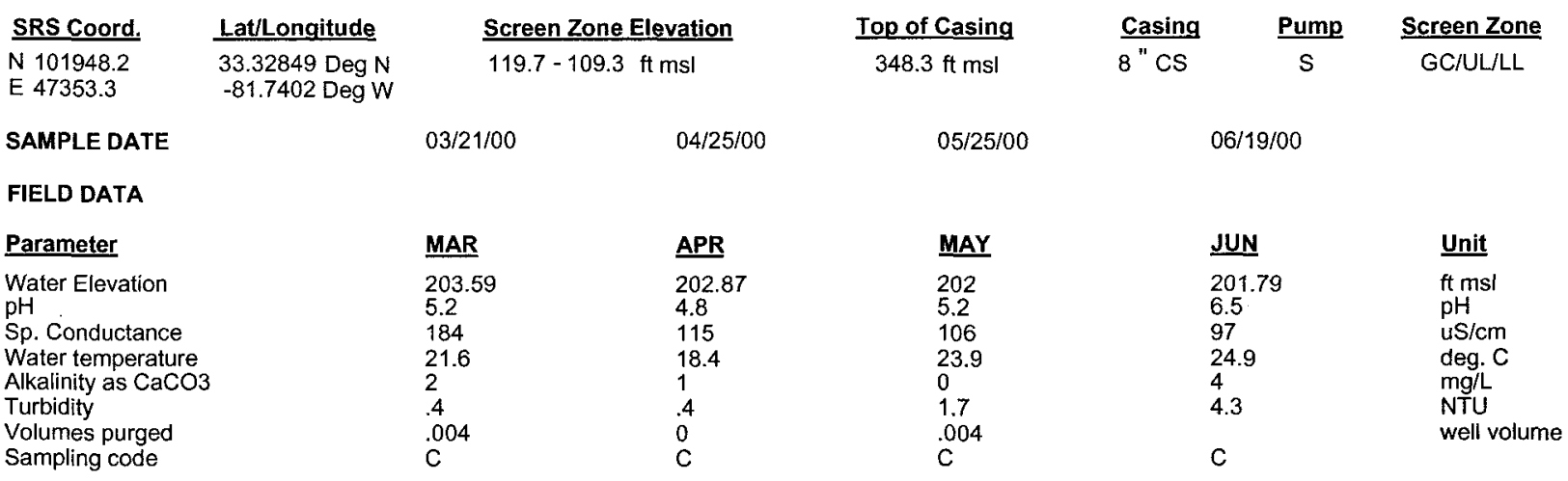

\section{ANALYTICAL DATA}

I. Groundwater Protection Standard

261 Appendix VIII/264 Appendix IX Hazardous Constituents

\begin{tabular}{|c|c|c|c|c|c|c|c|c|c|c|c|c|}
\hline$\underline{\text { ST }}$ & Parameter & MAR & CLPEPA & APR & CLP EPA & MAY & CLPEPA & JUN & CLPEPA & $\underline{\text { DF }}$ & $\underline{\text { Unit }}$ & Lab \\
\hline \multicolumn{13}{|c|}{ Inorganics } \\
\hline & $\begin{array}{l}\text { Barium, total recoverable } \\
\text { Cyanide } \\
\text { Lead, total recoverable } \\
\text { Nickel, total recoverable } \\
\text { Selenium, total recoverable }\end{array}$ & & & & & & & & & & & \\
\hline \multicolumn{13}{|c|}{ Organics } \\
\hline & Chlorobenzene & $<5$ & $\mathrm{U}$ & $<5$ & $U$ & $<10$ & $J U L$ & $<5$ & $U$ & 5 & $u g / L$ & $M L$ \\
\hline & 1,1-Dichloroethane & $<5$ & $U$ & $<5$ & $U$ & $<10$ & JU L & $<5$ & U & 5 & $u g / L$ & $M L$ \\
\hline & 1,1-Dichloroethylene & 4.85 & J $\quad 1$ & 6.85 & & $<10$ & JU L & $<5$ & U & 5 & $u g / L$ & ML \\
\hline & trans-1,2-Dichloroethylene & $<5$ & $\mathrm{U}$ & $<5$ & $U$ & $<10$ & $J U \mathrm{~L}$ & $<5$ & U & 5 & $\mathrm{ug} / \mathrm{L}$ & $\mathrm{ML}$ \\
\hline \multicolumn{13}{|c|}{ PCB 1016} \\
\hline \multicolumn{13}{|c|}{ PCB 1221} \\
\hline \multicolumn{13}{|c|}{ РCB 1232} \\
\hline \multicolumn{13}{|c|}{ PCB 1242} \\
\hline \multicolumn{13}{|c|}{ PCB 1248} \\
\hline \multicolumn{13}{|c|}{ PCB 1254} \\
\hline \multicolumn{13}{|c|}{ PCB 1260} \\
\hline & 1,1,2,2-Tetrachioroethane & $<5$ & $U$ & $<5$ & $U$ & $<10$ & JU L & $<5$ & $\mathrm{U}$ & 5 & $\mathrm{ug} / \mathrm{L}$ & ML \\
\hline & Tetrachloroethylene & 734 & & 1020 & & 466 & $\mathrm{~J} \quad \mathrm{~L}$ & 431 & $\mathrm{~J} K$ & 5 & ug/L & ML \\
\hline & 1,1,1-Trichloroethane & $<5$ & U & 2.35 & $\mathrm{~J} \quad 1$ & $<10$ & $J U L$ & $<5$ & $\mathrm{U}$ & 5 & ug/L & ML \\
\hline & Trichloroethylene & 1280 & & 1660 & & 786 & $J \quad L$ & 743 & $J \quad K$ & 5 & $u g / L$ & $\mathrm{ML}$ \\
\hline \multicolumn{13}{|c|}{ II. Monitoring Constituents } \\
\hline$\underline{\text { ST }}$ & Parameter & MAR & CLPEPA & APR & CLPEPA & MAY & CLPEPA & JUN & CLPEPA & DF & Unit & $\underline{\underline{L a b}}$ \\
\hline \multicolumn{13}{|c|}{ Inorganics } \\
\hline \multicolumn{12}{|c|}{ Aluminum, total recoverable } & \\
\hline \multicolumn{12}{|c|}{ Nitrate-nitrite as nitrogen } & \\
\hline \multicolumn{12}{|c|}{ Sodium, total recoverable } & \\
\hline \multicolumn{12}{|c|}{ Sulfate } & \\
\hline \multicolumn{12}{|c|}{ Radionuclides } & \\
\hline & Gross alpha & & & & & & & & & & & \\
\hline & $\begin{array}{l}\text { Nonvolatile beta } \\
\text { Radium, total alpha-emitting }\end{array}$ & & & & & & & & & & & \\
\hline
\end{tabular}

Notes: Concentrations in bold italics exceed the groundwater protection or monitoring constituent standards listed in Appendix A. Synchronous water levels are measured over a 3-5 day period or less. Dilution factors and Laboratory Data are June 2000 data only.

$+=$ exceeded the groundwater protection or monitoring constituent standards listed in Appendix A for June 2000. 
Table D-5c. Groundwater Monitoring Results for Recovery Wells, March-June 2000 (Cont.) WELL RWM 9

$\begin{array}{ll}\text { SRS Coord. } & \text { Lat/Longitude } \\ & \text { 33.33822 Deg N } \\ \text { E } 504099.8 & -81.7363 \text { Deg W }\end{array}$

SAMPLE DATE

FIELD DATA

\section{Screen Zone Elevation \\ $143-132.6 \mathrm{ft} \mathrm{msl}$}

$03 / 21 / 00$

$04 / 25 / 00$
Top of Casing

$380.6 \mathrm{ft} \mathrm{msl}$

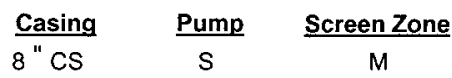

$06 / 19 / 00$

\begin{tabular}{lll} 
Parameter & MAR & APR \\
\hline Water Elevation & 220.74 & 219.45 \\
pH & 5.5 & 4.7 \\
Sp. Conductance & 46 & 46 \\
Water temperature & 20.9 & 18.6 \\
Alkalinity as CaCO3 & 3 & 2 \\
Turbidity & .5 & .5 \\
Volumes purged & .041 & 0 \\
Sampling code & $\mathrm{C}$ & $\mathrm{C}$
\end{tabular}

$\begin{array}{ll}\text { JUN } & \text { Unit } \\ 217.78 & \mathrm{ft} \mathrm{msl} \\ 6.7 & \mathrm{pH} \\ 44 & \text { uS/cm } \\ 22.8 & \mathrm{deg.} \mathrm{C} \\ 2 & \mathrm{mg} / \mathrm{L} \\ .5 & \text { NTU } \\ \mathrm{C} & \text { well volume }\end{array}$

\section{ANALYTICAL DATA}

I. Groundwater Protection Standard

261 Appendix VIII/264 Appendix IX Hazardous Constituents

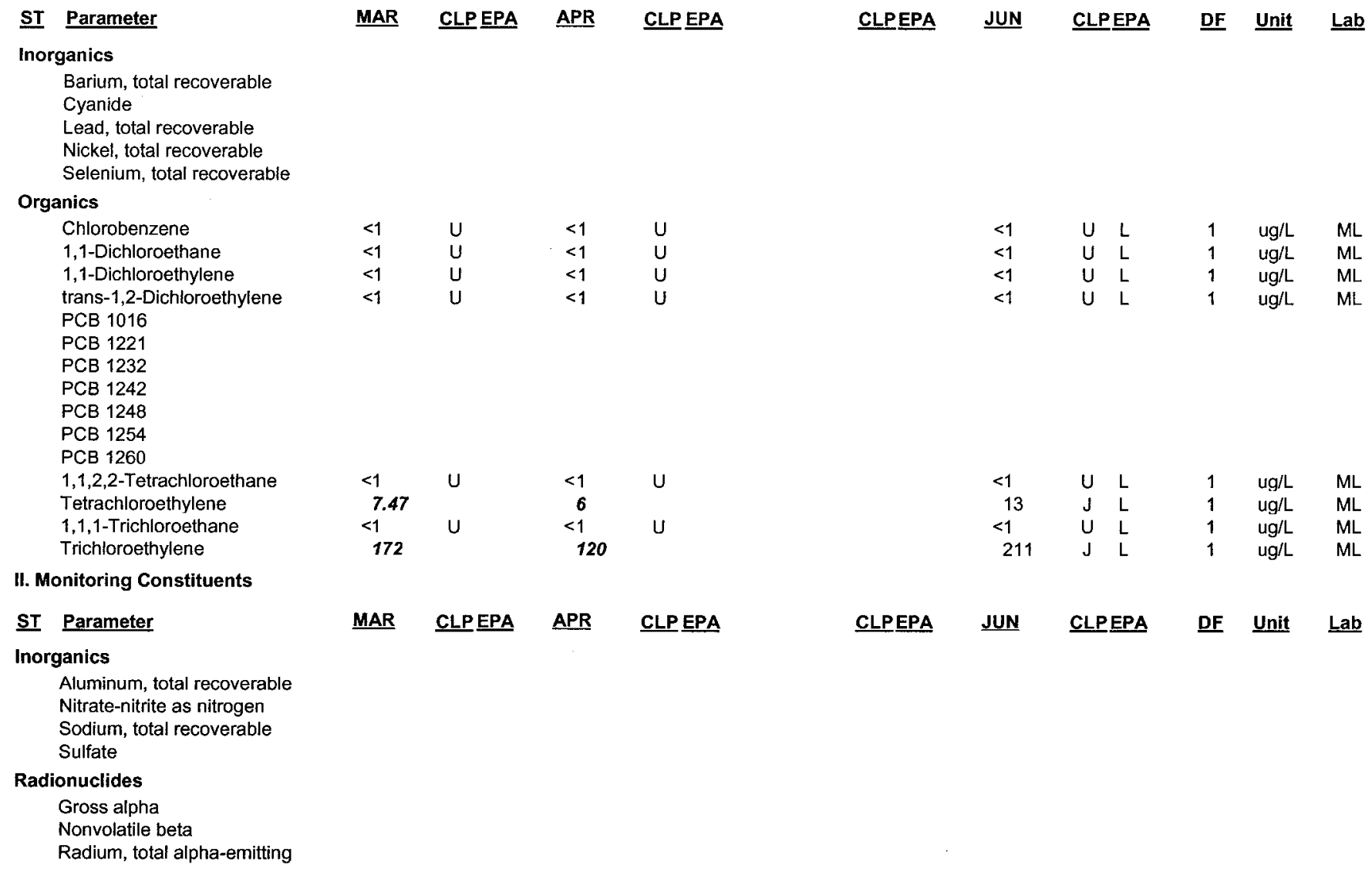

Notes: Concentrations in bold italics exceed the groundwater protection or monitoring constituent standards listed in Appendix A. Synchronous water levels are measured over a 3-5 day period or less. Dilution factors and Laboratory Data are June 2000 data only.

$+=$ exceeded the groundwater protection or monitoring constituent standards listed in Appendix A for June 2000. 
Table D-5c. Groundwater Monitoring Results for Recovery Wells, March-June 2000 (Cont.) WELL RWM 10

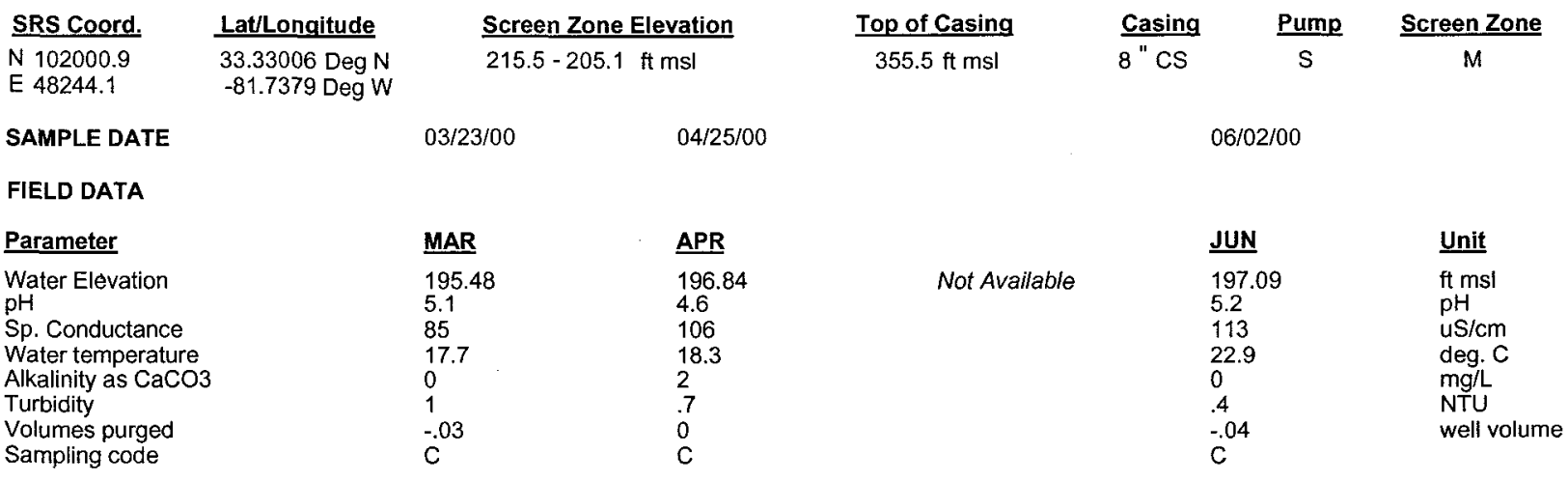

ANALYTICAL DATA

I. Groundwater Protection Standard

261 Appendix VIII/264 Appendix IX Hazardous Constituents

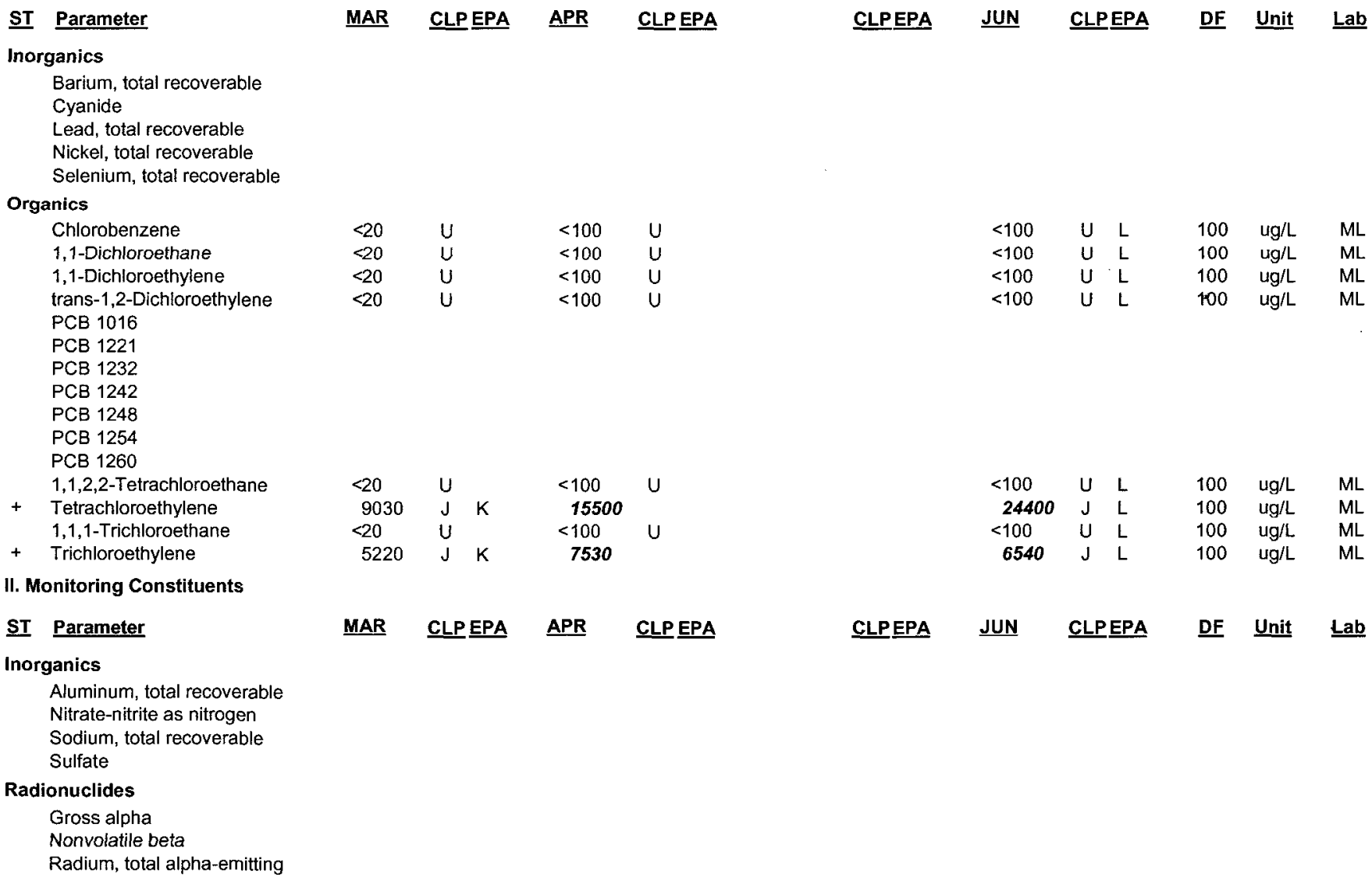

Notes: Concentrations in bold italics exceed the groundwater protection or monitoring constituent standards listed in Appendix A. Synchronous water levels are measured over a 3-5 day period or less. Dilution factors and Laboratory Data are June 2000 data only. $+=$ exceeded the groundwater protection or monitoring constituent standards listed in Appendix A for June 2000. 
Table D-5c. Groundwater Monitoring Results for Recovery Wells, March-June 2000 (Cont.) WELL RWM 11

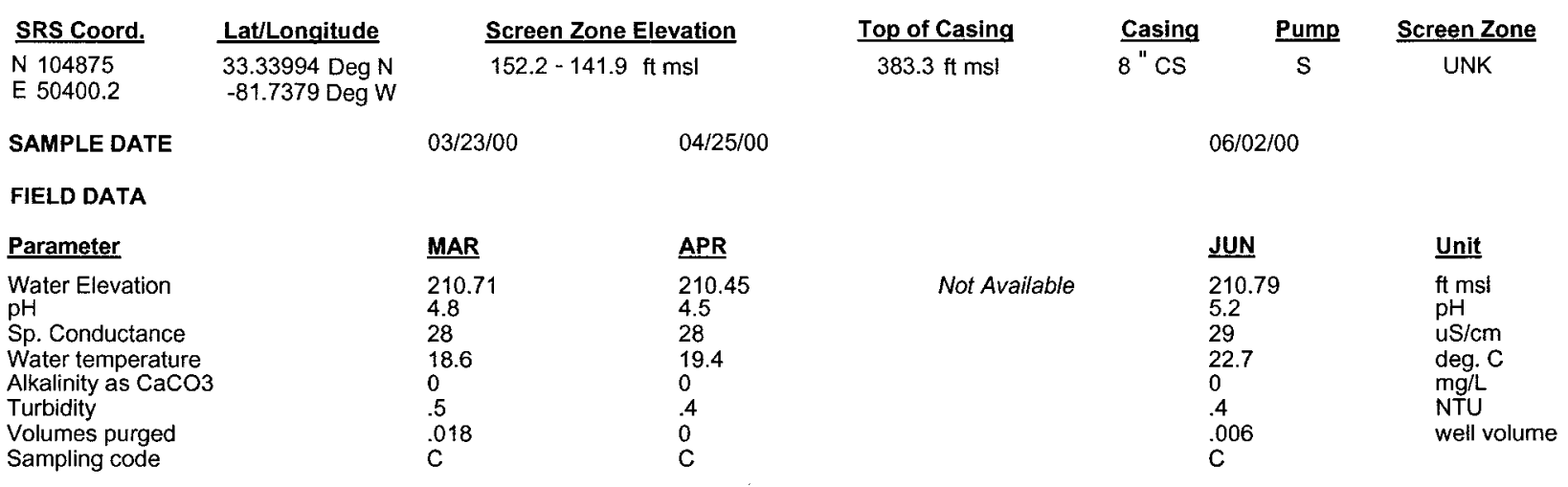

\section{ANALYTICAL DATA}

I. Groundwater Protection Standard

261 Appendix VIII/264 Appendix IX Hazardous Constituents

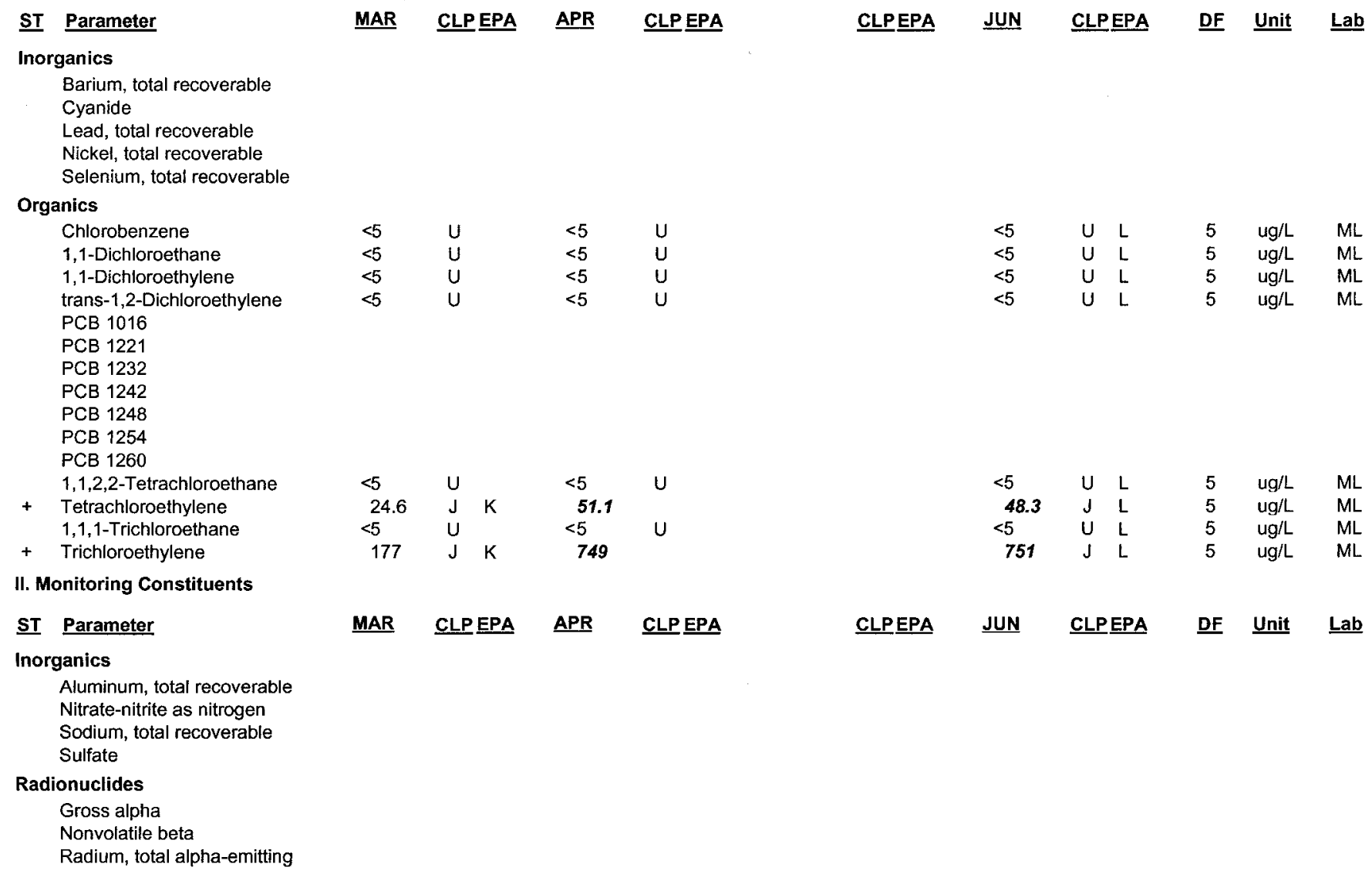

Notes: Concentrations in bold italics exceed the groundwater protection or monitoring constituent standards listed in Appendix A. Synchronous water levels are measured over a 3-5 day period or less. Dilution factors and Laboratory Data are June 2000 data only.

$+=$ exceeded the groundwater protection or monitoring constituent standards listed in Appendix A for June 2000. 
Table D-5c. Groundwater Monitoring Results for Recovery Wells, March-June 2000 (Cont.) WELL RWM 12

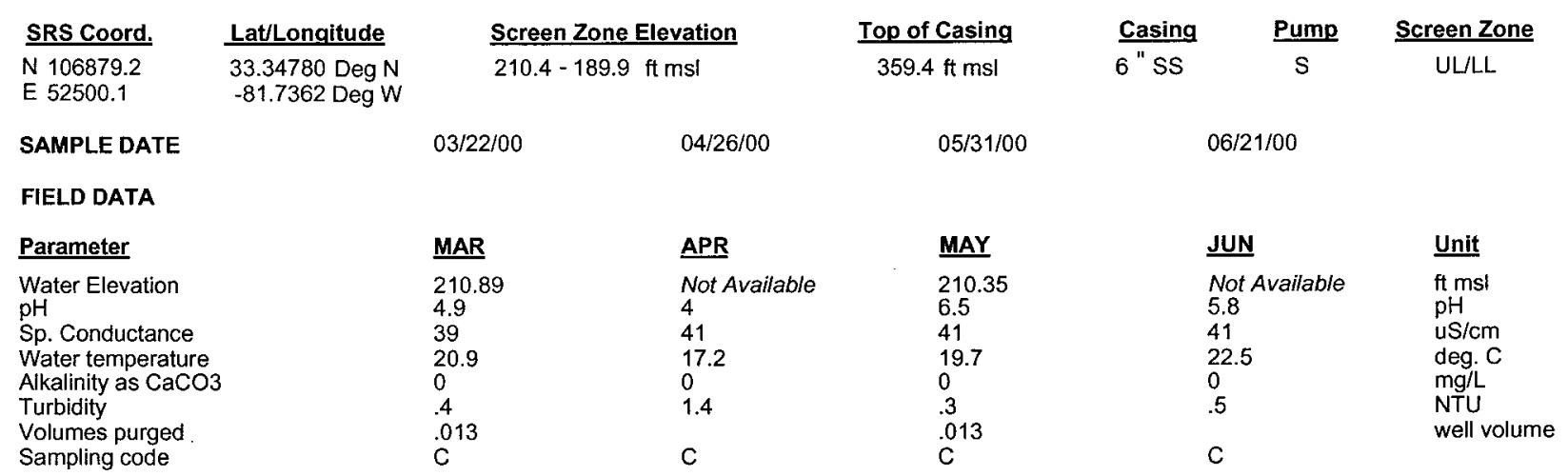

\section{ANALYTICAL DATA}

I. Groundwater Protection Standard

261 Appendix VIII/264 Appendix IX Hazardous Constituents

\begin{tabular}{|c|c|c|c|c|c|c|c|c|c|c|c|c|}
\hline$\underline{\mathbf{S T}}$ & Parameter & MAR & CLP EPA & $\underline{A P R}$ & CLP EPA & MAY & CLPEEA & JUN & CLPEPA & $\underline{\mathrm{DF}}$ & $\underline{\text { Unit }}$ & Lab \\
\hline \multicolumn{13}{|c|}{ Inorganics } \\
\hline & $\begin{array}{l}\text { Barium, total recoverable } \\
\text { Cyanide } \\
\text { Lead, total recoverable } \\
\text { Nickel, total recoverable } \\
\text { Selenium, total recoverable }\end{array}$ & & & & & & & & & & & \\
\hline \multicolumn{13}{|c|}{ Organics } \\
\hline & Chlorobenzene & $<5$ & JU & $<10$ & U & $<10$ & JU L & $<10$ & $\mathrm{U}$ & 10 & ug/L & ML \\
\hline & 1,1-Dichloroethane & $<5$ & JU & $<10$ & U & $<10$ & JU L & $<10$ & $\mathrm{U}$ & 10 & $\mathrm{ug} / \mathrm{L}$ & ML \\
\hline & 1,1-Dichloroethylene & $<5$ & JU & $<10$ & U & $<10$ & JU L & $<10$ & $\mathrm{U}$ & 10 & $u g / L$ & $\mathrm{ML}$ \\
\hline \\
\hline \multicolumn{13}{|c|}{ PCB 1221} \\
\hline \multicolumn{13}{|c|}{ PCB 1232} \\
\hline \multicolumn{13}{|c|}{ PCB 1242} \\
\hline \multicolumn{13}{|c|}{ РСB 1248} \\
\hline \multicolumn{13}{|c|}{ PCB 1254} \\
\hline \multicolumn{13}{|c|}{ PCB 1260} \\
\hline \multirow{3}{*}{+} & 1,1,2,2-Tetrachloroethane & $<5$ & JU & $<10$ & U & $<10$ & $J U$ L & $<10$ & $\mathrm{U}$ & 10 & ug/L & $\mathrm{ML}$ \\
\hline & Tetrachloroethylene & 9.35 & $J$ & 38.8 & $\mathrm{~J}$ & 12 & $J \quad L$ & 10.6 & & 10 & $u g / L$ & $M L$ \\
\hline & 1,1,1-Trichloroethane & & JU & $<10$ & $\mathrm{U}$ & $<10$ & JU L & $<10$ & U & 10 & $\mathrm{ug} / \mathrm{L}$ & $M L$ \\
\hline+ & Trichloroethylene & 2100 & $\mathrm{~J}$ & 1760 & $\mathrm{~J} K$ & 1800 & J L & 1860 & & 10 & ug/L & $M L$ \\
\hline \multicolumn{13}{|c|}{ II. Monitoring Constituents } \\
\hline$\underline{\text { ST }}$ & Parameter & $\underline{\text { MAR }}$ & $\underline{\text { CLPPEPA }}$ & $\underline{A P R}$ & CLP EPA & MAY & CLPEPA & JUN & CLPEEA & DF & $\underline{\text { Unit }}$ & Lab \\
\hline \multicolumn{13}{|c|}{ Inorganics } \\
\hline \multicolumn{13}{|c|}{ Aluminum, total recoverable } \\
\hline \multicolumn{12}{|c|}{ Nitrate-nitrite as nitrogen } & \\
\hline \multicolumn{13}{|c|}{ Sodium, total recoverable } \\
\hline \multicolumn{13}{|c|}{ Sulfate } \\
\hline \multicolumn{13}{|c|}{ Radionuclides } \\
\hline \multicolumn{12}{|c|}{$\begin{array}{l}\text { Gross alpha } \\
\text { Nonvolatile beta }\end{array}$} & \\
\hline
\end{tabular}

Notes: Concentrations in bold italics exceed the groundwater protection or monitoring constituent standards listed in Appendix A. Synchronous water levels are measured over a 3-5 day period or less. Dilution factors and Laboratory Data are June 2000 data only.

$+=$ exceeded the groundwater protection or monitoring constituent standards listed in Appendix A for June 2000. 
Table D-5c. Groundwater Monitoring Results for Recovery Wells, March-June 2000 (Cont.) WELL RWM 13B

\begin{tabular}{|c|c|c|c|c|c|c|}
\hline SRS Coord. & Lat/Longitude & \multicolumn{2}{|c|}{ Screen Zone Elevation } & \multirow{2}{*}{$\frac{\text { Top of Casing }}{336.2 \mathrm{ft} \mathrm{msl}}$} & \multirow{2}{*}{$\frac{\text { Casing }}{6 " C S}$} & \multirow{2}{*}{$\frac{\text { Screen Zone }}{L L}$} \\
\hline $\begin{array}{l}\text { N } 105803.3 \\
\text { E } 53516.3\end{array}$ & $\begin{array}{l}\text { 33.34708 Deg } N \\
-81.7315 \operatorname{Deg} W\end{array}$ & $138.2-113$ & $\mathrm{ft} \mathrm{msl}$ & & & \\
\hline SAMPLE DATE & & $03 / 23 / 00$ & $04 / 25 / 00$ & & $06 / 01 / 00$ & \\
\hline \multicolumn{7}{|l|}{ FIELD DATA } \\
\hline Parameter & & MAR & APR & & JUN & Unit \\
\hline $\begin{array}{l}\text { Water Elevation } \\
\text { pH } \\
\text { Sp. Conductance } \\
\text { Water temperature } \\
\text { Alkalinity as CaCO3 } \\
\text { Turbidity } \\
\text { Volumes purged } \\
\text { Sampling code }\end{array}$ & & $\begin{array}{l}200.42 \\
5.1 \\
22 \\
17.5 \\
1 \\
.4 \\
.008 \\
C\end{array}$ & $\begin{array}{l}200.05 \\
5 \\
20 \\
18.6 \\
1 \\
.6 \\
0 \\
C\end{array}$ & Not Available & $\begin{array}{l}199.97 \\
5.5 \\
20 \\
20.4 \\
0 \\
.4 \\
.008 \\
C\end{array}$ & $\begin{array}{l}\text { ft msl } \\
\mathrm{pH} \\
\mathrm{uS} / \mathrm{cm} \\
\mathrm{deg} . \mathrm{C} \\
\mathrm{mg} / \mathrm{L} \\
\mathrm{NTU} \\
\text { well volume }\end{array}$ \\
\hline
\end{tabular}

ANALYTICAL DATA

I. Groundwater Protection Standard

261 Appendix VIII/264 Appendix IX Hazardous Constituents

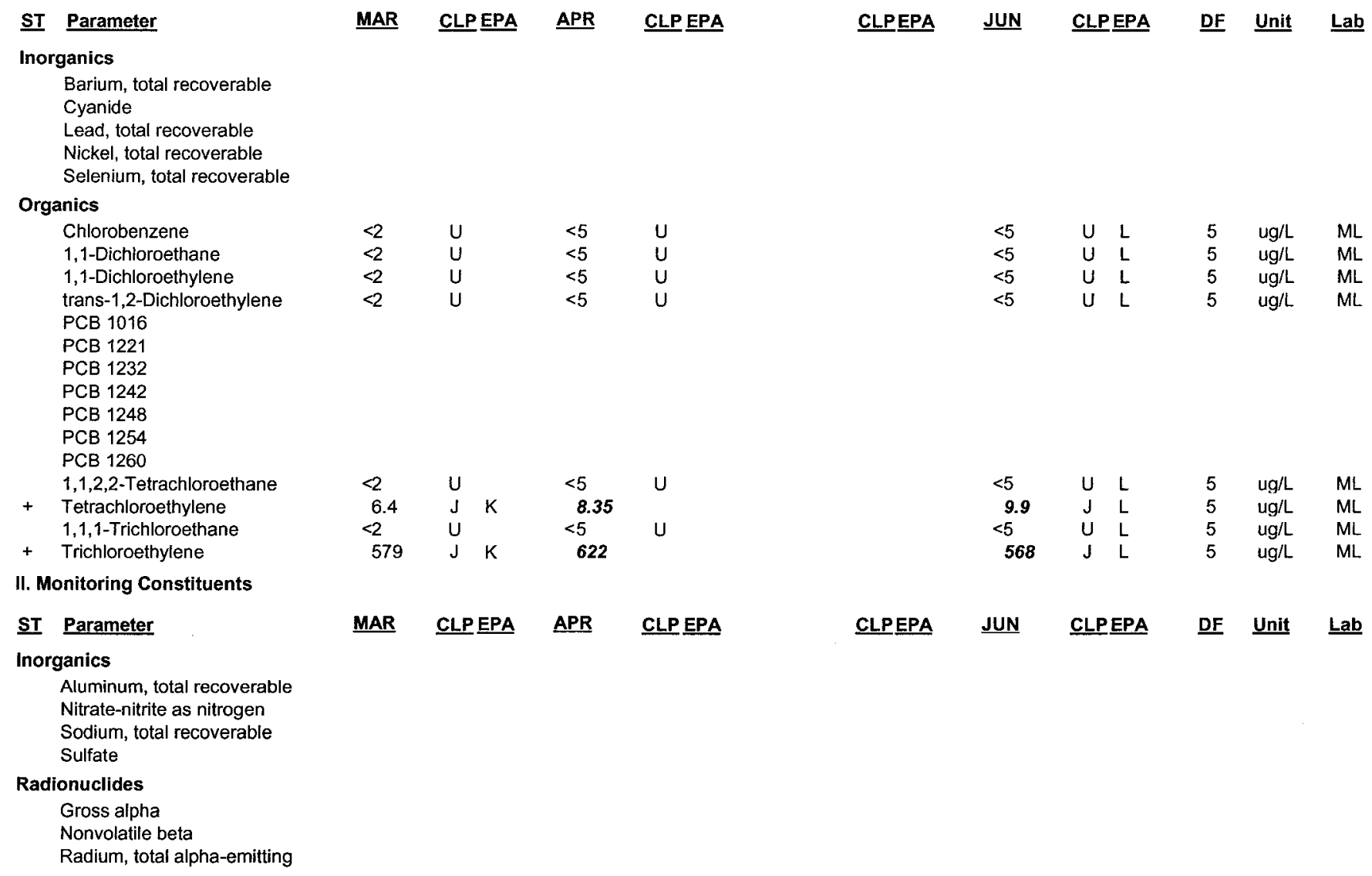

Notes: Concentrations in bold italics exceed the groundwater protection or monitoring constituent standards listed in Appendix A. Synchronous water levels are measured over a 3-5 day period or less. Dilution factors and Laboratory Data are June 2000 data only.

$+=$ exceeded the groundwater protection or monitoring constituent standards listed in Appendix A for June 2000. 
Table D-5c. Groundwater Monitoring Results for Recovery Wells, March-June 2000 (Cont.) WELL RWM 13C

\begin{tabular}{|c|c|c|c|c|c|c|c|}
\hline SRS Coord. & Lat/Longitude & \multicolumn{2}{|c|}{ Screen Zone Elevation } & Top of Casing & Casing & Pump & Screen Zone \\
\hline $\begin{array}{l}\text { N } 105809.7 \\
\text { E } 53502.2\end{array}$ & $\begin{array}{l}\text { 33.34707 Deg } N \\
-81.7315 \operatorname{Deg} W\end{array}$ & \multicolumn{2}{|c|}{$173.4-153.3 \mathrm{ft} \mathrm{mst}$} & $336.4 \mathrm{ft} \mathrm{msl}$ & $6 " \mathrm{CS}$ & & UL \\
\hline SAMPLE DATE & & $03 / 23 / 00$ & $04 / 25 / 00$ & & & $06 / 01 / 00$ & \\
\hline \multicolumn{8}{|l|}{ FIELD DATA } \\
\hline Parameter & & MAR & APR & & & $\underline{\text { JUN }}$ & Unit \\
\hline $\begin{array}{l}\text { Water Elevation } \\
\text { pH } \\
\text { Sp. Conductance } \\
\text { Water temperature } \\
\text { Alkalinity as CaCO3 } \\
\text { Turbidity } \\
\text { Volumes purged } \\
\text { Sampling code }\end{array}$ & & $\begin{array}{l}206.6 \\
5.4 \\
29 \\
17.3 \\
1 \\
.3 \\
.013 \\
C\end{array}$ & $\begin{array}{l}206.55 \\
4.9 \\
30 \\
18.9 \\
1 \\
.8 \\
0 \\
C\end{array}$ & Not Available & & $\begin{array}{l}205.99 \\
5.4 \\
30 \\
21.6 \\
0 \\
.4 \\
C\end{array}$ & $\begin{array}{l}\mathrm{ft} \mathrm{msl} \\
\mathrm{pH} \\
\mathrm{uS} / \mathrm{cm} \\
\text { deg. C } \\
\mathrm{mg} / \mathrm{L} \\
\text { NTU } \\
\text { well volume }\end{array}$ \\
\hline
\end{tabular}

ANALYTICAL DATA

I. Groundwater Protection Standard

261 Appendix VIII/264 Appendix IX Hazardous Constituents

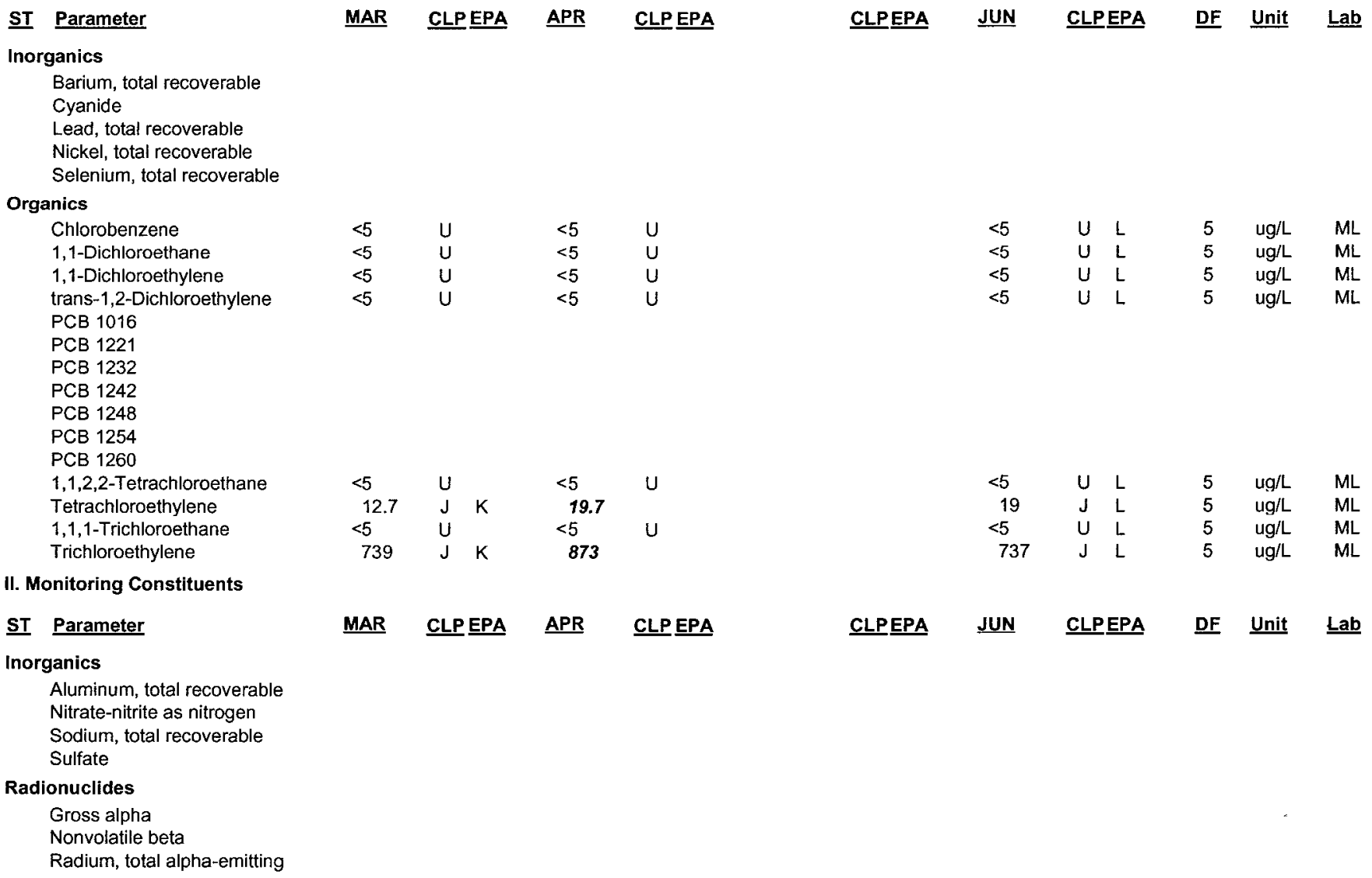

Notes: Concentrations in bold italics exceed the groundwater protection or monitoring constituent standards listed in Appendix A. Synchronous water levels are measured over a 3-5 day period or less. Dilution factors and Laboratory Data are June 2000 data only.

$+=$ exceeded the groundwater protection or monitoring constituent standards listed in Appendix A for June 2000. 
Table D-5c. Groundwater Monitoring Results for Recovery Wells, March-June 2000 (Cont.) WELL RWM 14B

\begin{tabular}{|c|c|c|c|c|c|c|c|}
\hline SRS Coord. & Lat/Longitude & \multicolumn{2}{|c|}{ Screen Zone Elevation } & Top of Casing & Casing & Pump & Screen Zone \\
\hline $\begin{array}{l}\text { N } 106362.1 \\
\text { E } 53044.7\end{array}$ & $\begin{array}{l}\text { 33.34754 Deg } N \\
-81.7338 \text { Deg } W\end{array}$ & \multicolumn{2}{|c|}{$148.5-123.2 \mathrm{ft} \mathrm{msl}$} & $351.2 \mathrm{ft} \mathrm{msl}$ & $6 " \mathrm{cs}$ & & LL \\
\hline SAMPLE DATE & & $03 / 22 / 00$ & $04 / 26 / 00$ & & & $06 / 01 / 00$ & \\
\hline \multicolumn{8}{|l|}{ FIELD DATA } \\
\hline Parameter & & MAR & APR & & & JUN & $\underline{\text { Unit }}$ \\
\hline $\begin{array}{l}\text { Water Elevation } \\
\text { pH } \\
\text { Sp. Conductance } \\
\text { Water temperature } \\
\text { Alkalinity as } \mathrm{CaCO} 3 \\
\text { Turbidity } \\
\text { Volumes purged } \\
\text { Sampling code }\end{array}$ & & $\begin{array}{l}209.2 \\
5.1 \\
23 \\
19.8 \\
1 \\
.3 \\
.008 \\
C\end{array}$ & $\begin{array}{l}155.24 \\
3.8 \\
23 \\
17 \\
0 \\
1.2 \\
0 \\
C\end{array}$ & Not Available & & $\begin{array}{l}208.46 \\
6.2 \\
25 \\
19.5 \\
0 \\
1 \\
.008 \\
\text { C }\end{array}$ & $\begin{array}{l}\mathrm{ft} \mathrm{msl} \\
\mathrm{pH} \\
\text { uS/cm } \\
\text { deg. C } \\
\mathrm{mg} / \mathrm{L} \\
\text { NTU } \\
\text { well volume }\end{array}$ \\
\hline
\end{tabular}

ANALYTICAL DATA

I. Groundwater Protection Standard

261 Appendix VIII/264 Appendix IX Hazardous Constituents

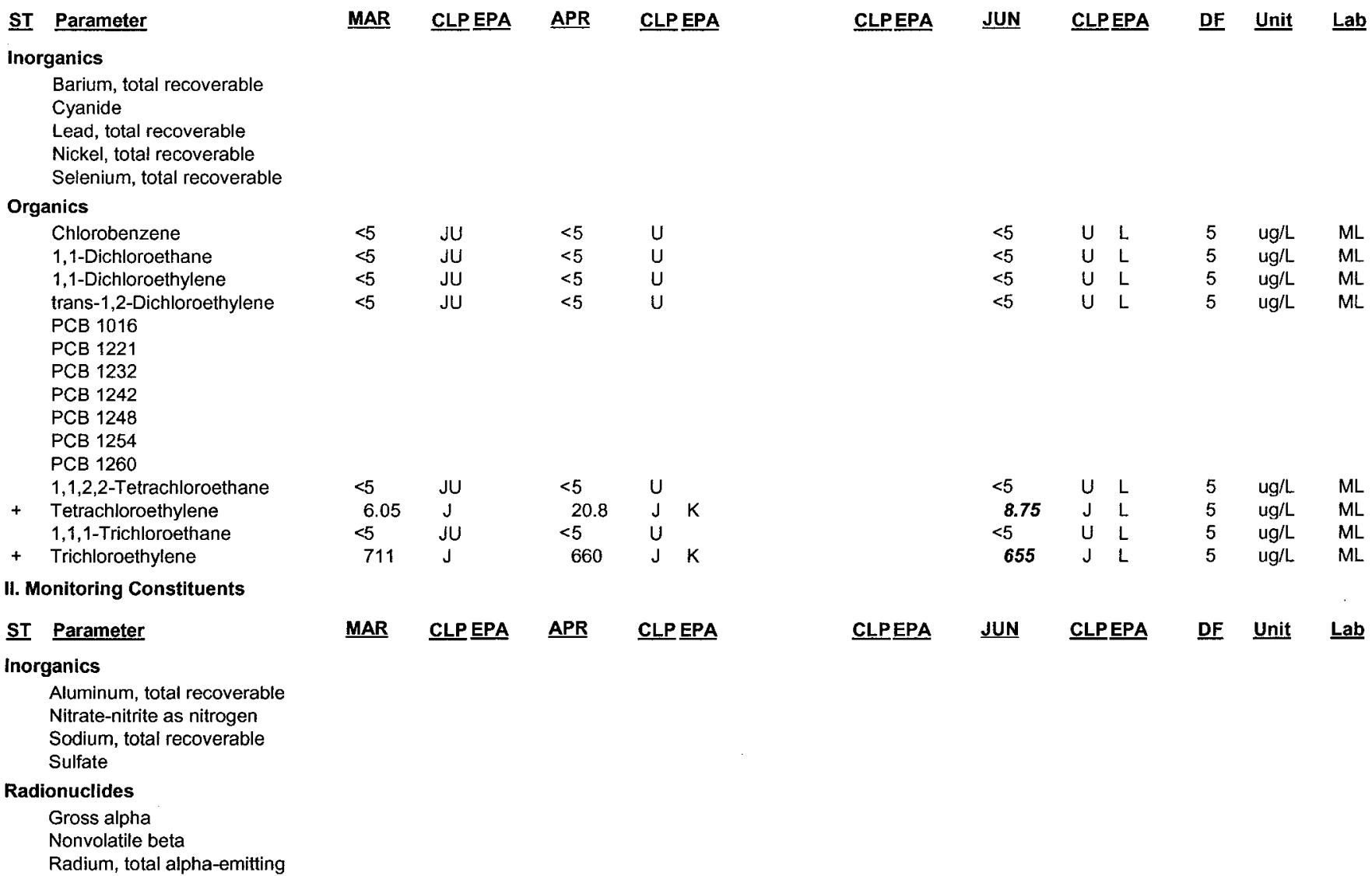

\footnotetext{
Notes: Concentrations in bold italics exceed the groundwater protection or monitoring constituent standards listed in Appendix A. Synchronous water levels are measured over a 3-5 day period or less. Dilution factors and Laboratory Data are June 2000 data only. 
Table D-5c. Groundwater Monitoring Results for Recovery Wells, March-June 2000 (Cont.) WELL RWM 14C

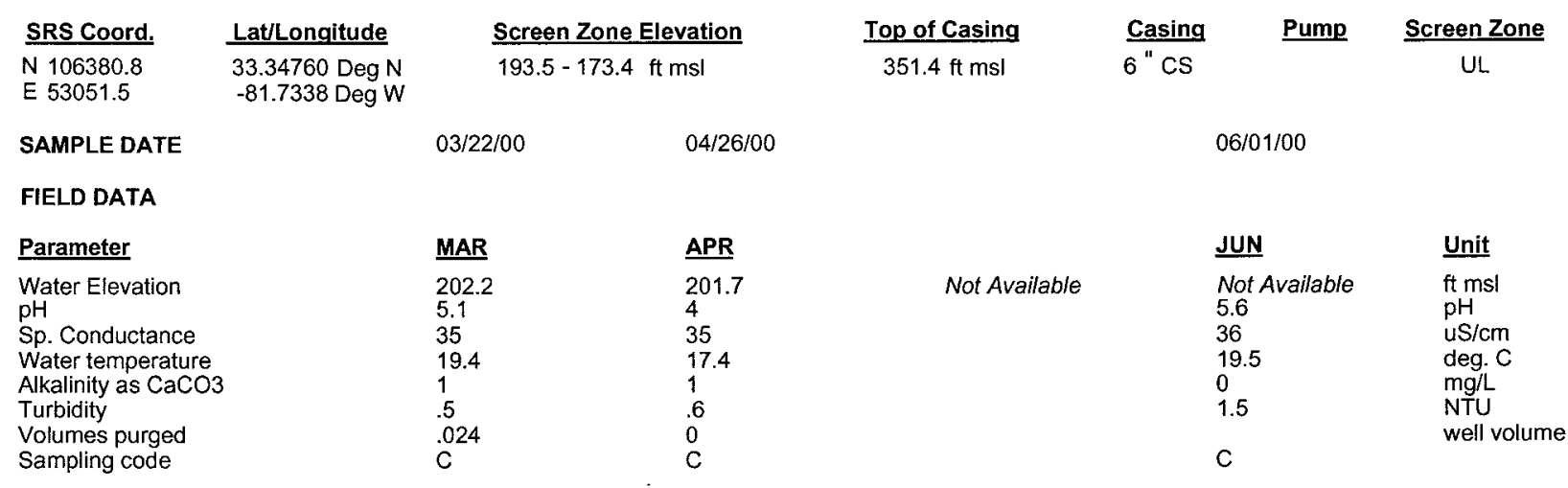

\section{ANALYTICAL DATA}

I. Groundwater Protection Standard

261 Appendix VIII/264 Appendix IX Hazardous Constituents

\begin{tabular}{|c|c|c|c|c|c|c|c|c|c|c|}
\hline$\underline{\text { ST }}$ & Parameter & $\underline{\text { MAR }}$ & CLPP EPA & $\underline{\text { APR }}$ & CLPEPA & CLPEPA & $\underline{\text { JUN }}$ & CLPEPA & $\underline{\text { DF }}$ & Unit \\
\hline 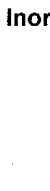 & $\begin{array}{l}\text { ganics } \\
\text { Barium, total recoverable } \\
\text { Cyanide } \\
\text { Lead, total recoverable } \\
\text { Nickel, total recoverable } \\
\text { Selenium, total recoverable }\end{array}$ & & & & & & & & & \\
\hline \multicolumn{11}{|c|}{ Organics } \\
\hline & $\begin{array}{l}\text { Chlorobenzene } \\
\text { 1,1-Dichloroethane } \\
\text { 1,1-Dichloroethylene } \\
\text { trans-1,2-Dichloroethylene } \\
\text { PCB } 1016 \\
\text { PCB } 1221 \\
\text { PCB } 1232 \\
\text { PCB } 1242 \\
\text { PCB } 1248 \\
\text { PCB } 1254 \\
\text { PCB } 1260\end{array}$ & $\begin{array}{l}<20 \\
<20 \\
<20 \\
<20\end{array}$ & $\begin{array}{l}J U \\
J U \\
J U \\
\text { JU }\end{array}$ & $\begin{array}{l}<20 \\
<20 \\
<20 \\
<20\end{array}$ & $\begin{array}{l}U \\
U \\
U \\
U\end{array}$ & & $\begin{array}{l}<20 \\
<20 \\
<20 \\
<20\end{array}$ & $\begin{array}{l}U \\
U \\
U \\
U\end{array}$ & $\begin{array}{l}20 \\
20 \\
20 \\
20\end{array}$ & $\begin{array}{l}u g / L \\
u g / L \\
u g / L \\
u g / L\end{array}$ \\
\hline $\begin{array}{l}+ \\
+\end{array}$ & $\begin{array}{l}\text { 1,1,2,2-Tetrachloroethane } \\
\text { Tetrachloroethylene } \\
\text { 1,1,1-Trichloroethane } \\
\text { Trichloroethylene }\end{array}$ & $\begin{array}{c}<20 \\
31 \\
<20 \\
5640\end{array}$ & $\begin{array}{l}\mathrm{J} \\
\mathrm{J} \\
\mathrm{J}\end{array}$ & $\begin{array}{l}<20 \\
81 \\
13.4 \\
3640\end{array}$ & $\begin{array}{ll}\mathrm{J} & \mathrm{I} \\
\mathrm{u} & \end{array}$ & & $\begin{array}{l}<20 \\
31.6 \\
<20 \\
3570\end{array}$ & $\begin{array}{l}\mathrm{U} \\
\mathrm{J} \\
\mathrm{J}\end{array}$ & $\begin{array}{l}20 \\
20 \\
20 \\
20\end{array}$ & $\begin{array}{l}\mathrm{ug} / \mathrm{L} \\
\mathrm{ug} / \mathrm{L} \\
\mathrm{ug} / \mathrm{L} \\
\mathrm{ug} / \mathrm{L}\end{array}$ \\
\hline \multicolumn{11}{|c|}{ II. Monitoring Constituents } \\
\hline$\underline{\text { ST }}$ & Parameter & MAR & CLP EPA & $\underline{A P R}$ & CLP EPA & CLPEPA & $\underline{\text { JUN }}$ & CLPEPA & $\underline{\text { DF }}$ & Unit \\
\hline Inor & $\begin{array}{l}\text { ganics } \\
\text { Aluminum, total recoverable } \\
\text { Nitrate-nitrite as nitrogen } \\
\text { Sodium, total recoverable } \\
\text { Sulfate }\end{array}$ & & & & & & & & & \\
\hline Rad & $\begin{array}{l}\text { Gonuclides } \\
\text { Gross alpha } \\
\text { Nonvolatile beta } \\
\text { Radium, total alpha-emitting }\end{array}$ & & & & & & & & & \\
\hline
\end{tabular}

Notes: Concentrations in bold italics exceed the groundwater protection or monitoring constituent standards listed in Appendix A. Synchronous water levels are measured over a 3-5 day period or less. Dilution factors and Laboratory Data are June 2000 data only.

$+=$ exceeded the groundwater protection or monitoring constituent standards listed in Appendix A for June 2000 
Table D-5c. Groundwater Monitoring Results for Recovery Wells, March-June 2000 (Cont.) WELL RWM 15B

\begin{tabular}{|c|c|c|c|c|c|c|c|}
\hline SRS Coord. & Lat/Longitude & \multicolumn{2}{|c|}{ Screen Zone Elevation } & Top of Casing & Casing & Pump & Screen Zone \\
\hline $\begin{array}{l}\text { N } 107444.7 \\
\text { E } 53849\end{array}$ & $\begin{array}{l}\text { 33.35125 Deg } N \\
-81.7338 \text { Deg } W\end{array}$ & \multicolumn{2}{|c|}{$150.9-125.8 \mathrm{ft} \mathrm{msl}$} & $368.4 \mathrm{ft} \mathrm{ms!}$ & $6 " \mathrm{Cs}$ & & $L L$ \\
\hline SAMPLE DATE & & $03 / 23 / 00$ & $04 / 25 / 00$ & & & $06 / 01 / 00$ & \\
\hline \multicolumn{8}{|l|}{ FIELD DATA } \\
\hline Parameter & & MAR & APR & & & JUN & Unit \\
\hline $\begin{array}{l}\text { Water Elevation } \\
\text { pH } \\
\text { Sp. Conductance } \\
\text { Water temperature } \\
\text { Alkalinity as CaCO3 } \\
\text { Turbidity } \\
\text { Volumes purged } \\
\text { Sampling code }\end{array}$ & & $\begin{array}{l}206.9 \\
5.2 \\
21 \\
16.6 \\
1 \\
.4 \\
.008 \\
c\end{array}$ & $\begin{array}{l}206.75 \\
5.1 \\
21 \\
18.6 \\
1 \\
.7 \\
0 \\
C\end{array}$ & Not Available & & $\begin{array}{l}206.44 \\
5.4 \\
21 \\
23 \\
0 \\
.4 \\
.008 \\
C\end{array}$ & $\begin{array}{l}\text { ft msl } \\
\text { pH } \\
\text { uS/cm } \\
\text { deg. C } \\
\text { mg/L } \\
\text { NTU } \\
\text { well volume }\end{array}$ \\
\hline
\end{tabular}

\section{ANALYTICAL DATA}

I. Groundwater Protection Standard

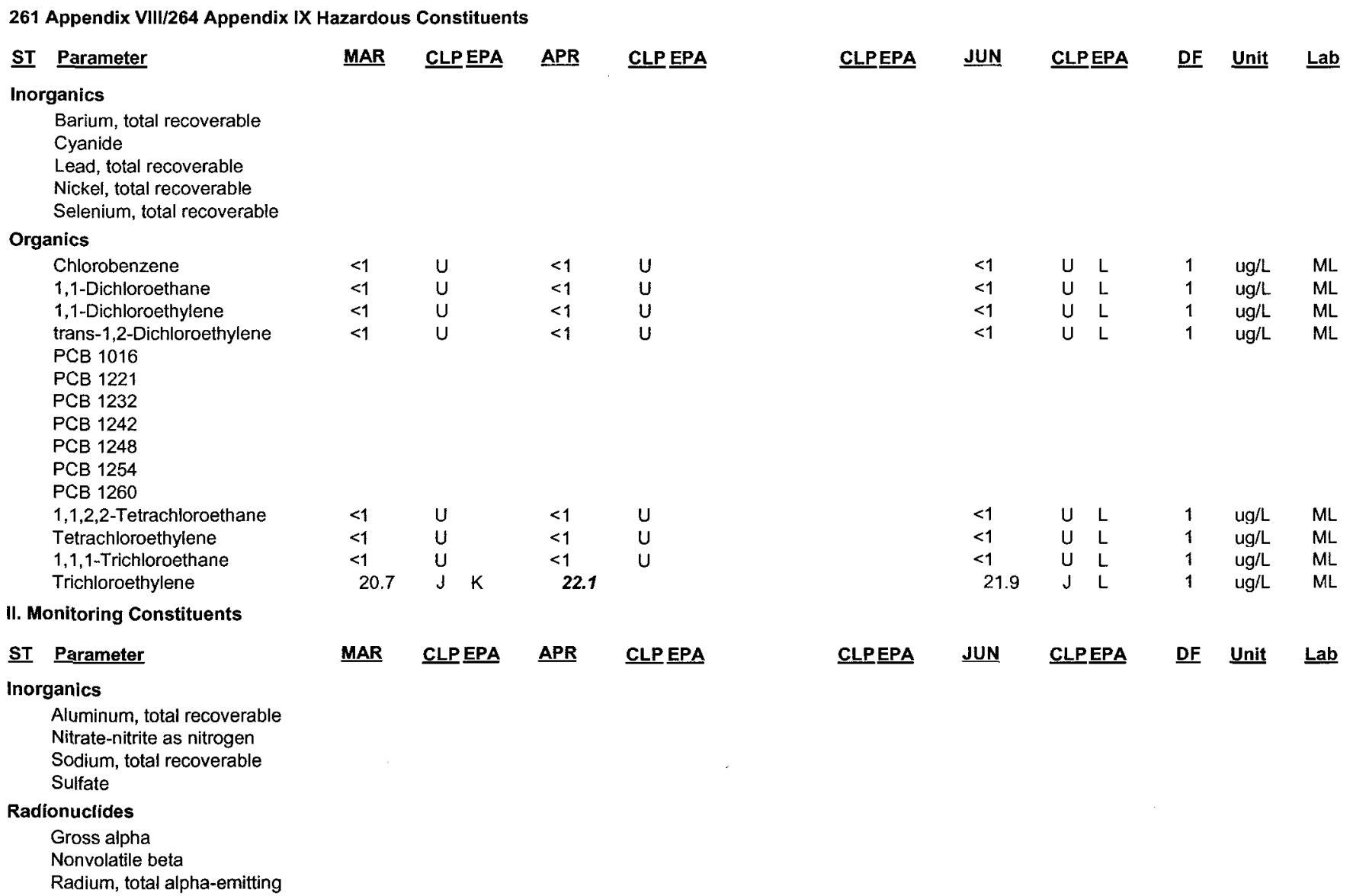




\section{THIS PAGE LEFT BLANK INTENTIONALLY}


Table D-6. Groundwater Monitoring Results for Point-of-Compliance Wells, Met Lab HWMF WELL AMB 4A

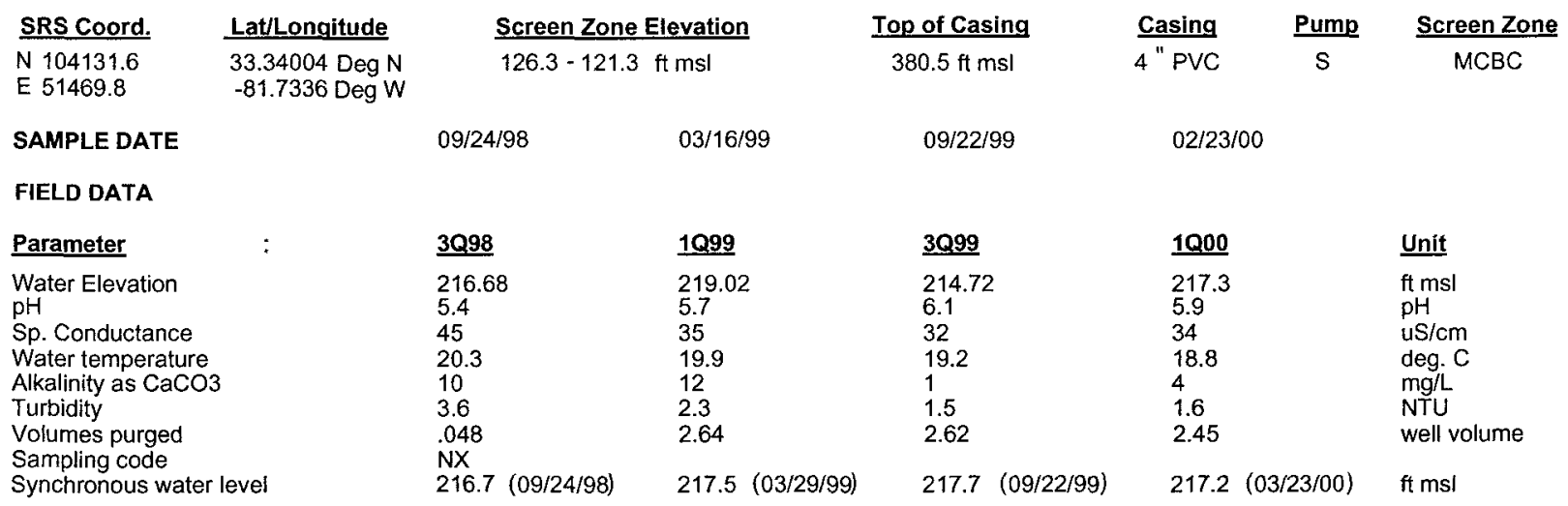

ANALYTICAL DATA

I. Groundwater Protection Standard

261 Appendix VIII/264 Appendix IX Hazardous Constituents

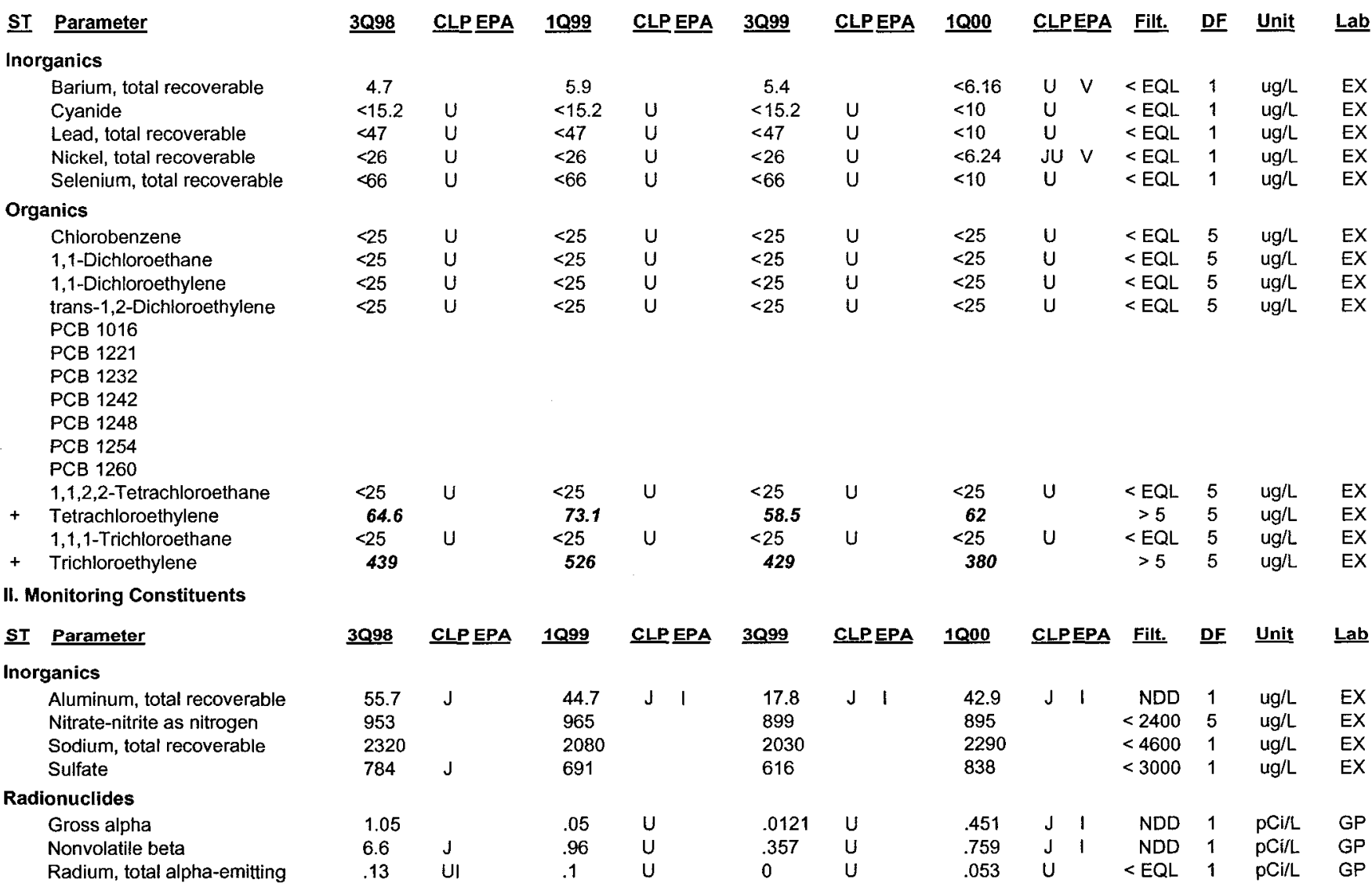

Notes: Concentrations in bold italics exceed the groundwater protection or monitoring constituent standards listed in Appendix A. Synchronous water levels are measured over a 3-5 day period or less. Dilution factors, Laboratory, and Filtered Data are for First Quarter 2000 data only.

$+=$ exceeded the Groundwater Protection Standards or Monitoring Constituents listed in Appendix A for First Quarter 2000. 
Table D-6. Groundwater Monitoring Results for Point-of-Compliance Wells, Met Lab HWMF (Cont.) WELL AMB 4D

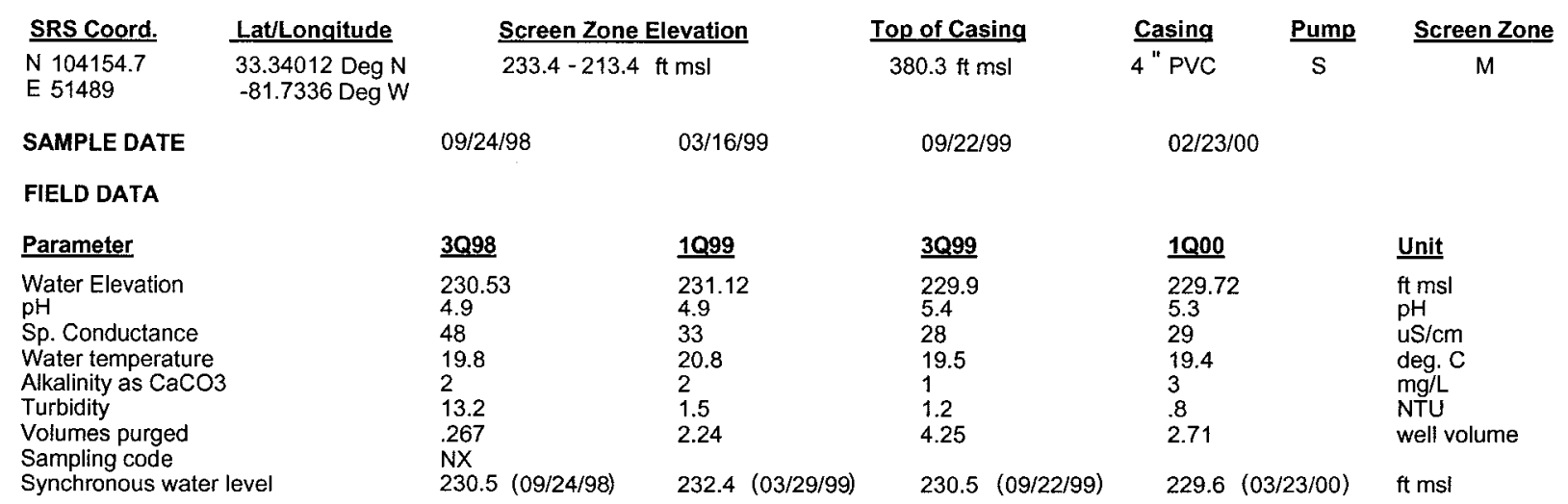

\section{ANALYTICAL DATA}

I. Groundwater Protection Standard

261 Appendix VIII/264 Appendix IX Hazardous Constituents

\begin{tabular}{|c|c|c|c|c|c|c|c|c|c|c|c|c|c|c|c|}
\hline \multirow{2}{*}{\multicolumn{2}{|c|}{$\begin{array}{l}\text { ST Parameter } \\
\text { Inorganics }\end{array}$}} & \multirow[t]{2}{*}{$\underline{3098}$} & \multirow[t]{2}{*}{$\underline{\text { CLP EPA }}$} & \multirow[t]{2}{*}{1099} & \multicolumn{2}{|c|}{$\underline{\text { CLP EPA }}$} & \multirow[t]{2}{*}{$\underline{3 Q 99}$} & CLPEPA & \multirow[t]{2}{*}{$1 \mathrm{Q} 00$} & \multicolumn{2}{|c|}{ CLPEPA } & \multirow[t]{2}{*}{ Filt. } & \multirow[t]{2}{*}{$\underline{\text { DF }}$} & \multirow[t]{2}{*}{ Unit } & \multirow[t]{2}{*}{$\underline{\text { Lab }}$} \\
\hline & & & & & & & & & & & & & & & \\
\hline & Barium, total recoverable & 8.7 & & 6.5 & & & 4.9 & & $<4.42$ & U & V & $<E Q L$ & 1 & ug/L & EX \\
\hline & Cyanide & $<15.2$ & $\mathrm{U}$ & 2.16 & J & 1 & $<15.2$ & $U$ & $<10$ & $\mathrm{U}$ & & $<E Q L$ & 1 & ug/L & EX \\
\hline & Lead, total recoverable & 6 & $\mathrm{~J}$ & $<47$ & $\cup$ & & $<47$ & $U$ & $<10$ & $\mathrm{U}$ & & $<\mathrm{EQL}$ & 1 & $\mathrm{ug} / \mathrm{L}$ & EX \\
\hline & Nickel, total recoverable & 2.8 & 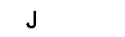 & $<26$ & $\cup$ & & $<26$ & $U$ & $<50$ & $U$ & & $<\mathrm{EQL}$ & 1 & $\mathrm{ug} / \mathrm{L}$ & EX \\
\hline & Selenium, total recoverable & $<66$ & $U$ & $<66$ & $U$ & & $<66$ & $u$ & $<10$ & $\mathrm{u}$ & & $<E Q L$ & 1 & $\mathrm{ug} / \mathrm{L}$ & EX \\
\hline \multicolumn{16}{|c|}{ Organics } \\
\hline & Chlorobenzene & $<5$ & $\mathrm{u}$ & $<5$ & $\mathrm{U}$ & & $<5$ & $u$ & $<5$ & JU & $\mathrm{L}$ & $<\mathrm{EQL}$ & 1 & $u g / L$ & EX \\
\hline & 1,1-Dichloroethane & $<5$ & U & $<5$ & $\mathrm{U}$ & & $<5$ & U & $<5$ & JU & $\mathrm{L}$ & $<E Q L$ & 1 & $\mathrm{ug} / \mathrm{L}$ & EX \\
\hline & 1,1-Dichloroethylene & $<5$ & U & $<5$ & $\mathrm{U}$ & & $<5$ & $\mathrm{U}$ & $<5$ & JU & $\mathrm{L}$ & $<E Q L$ & 1 & $\mathrm{ug} / \mathrm{L}$ & $E x$ \\
\hline & trans-1,2-Dichloroethylene & $<5$ & $u$ & $<5$ & $u$ & & $<5$ & U & $<5$ & JU & $\mathrm{L}$ & $<E Q L$ & 1 & $\mathrm{ug} / \mathrm{L}$ & $x$ \\
\hline & PCB 1016 & & & & & & & & $<.97$ & $\mathrm{U}$ & & $<E Q L$ & .970 & $\mathrm{ug} / \mathrm{L}$ & $x$ \\
\hline & PCB 1221 & & & & & & & & $<.97$ & $U$ & & $<E Q L$ & .970 & ug $/ \mathrm{L}$ & $x$ \\
\hline & PCB 1232 & & & & & & & & $<.97$ & U & & $<\mathrm{EQL}$ & .970 & $\mathrm{ug} / \mathrm{L}$ & EX \\
\hline & PCB 1242 & & & & & & & & $<1.9$ & $\mathrm{U}$ & & $<E Q L$ & .970 & ug/L. & Ex \\
\hline & PCB 1248 & & & & & & & & $<.97$ & $\mathrm{U}$ & & $<\mathrm{EQL}$ & .970 & $\mathrm{ug} / \mathrm{L}$ & $E x$ \\
\hline & РСB 1254 & & & & & & & & $<.97$ & $U$ & & $<\mathrm{EQL}$ & .970 & $\mathrm{ug} / \mathrm{L}$ & Ex \\
\hline & PCB 1260 & & & & & & & & $<.97$ & $\mathrm{U}$ & & $<\mathrm{EQL}$ & .970 & $\mathrm{ug} / \mathrm{L}$ & EX \\
\hline & 1,1,2,2-Tetrachloroethane & $<5$ & $\mathrm{U}$ & $<5$ & $\mathrm{U}$ & & $<5$ & $\mathrm{U}$ & $<5$ & JU & $\mathrm{L}$ & $<\overline{E Q L}$ & 1 & $\mathrm{ug} / \mathrm{L}$ & EX \\
\hline & Tetrachloroethylene & 1.89 & J & $<5$ & $u$ & & $<5$ & U & $<5$ & JU & $\bar{L}$ & $<E Q L$ & 1 & $\mathrm{ug} / \mathrm{L}$ & EX \\
\hline & 1,1,1-Trichloroethane & $<5$ & $\mathrm{U}$ & $<5$ & $\mathrm{U}$ & & $<5$ & $\mathrm{U}$ & $<5$ & JU & $L$ & $<E Q L$ & 1 & ug/L & EX \\
\hline & Trichloroethylene & 64 & & 24.9 & & & 7.37 & & 7.6 & $J$ & $\mathrm{~L}$ & NDD & 1 & $\mathrm{ug} / \mathrm{L}$ & EX \\
\hline \multicolumn{16}{|c|}{ II. Monitoring Constituents } \\
\hline$\underline{\mathbf{S T}}$ & Parameter & $\underline{3 Q 98}$ & CLPEPA & 1099 & $\underline{\text { CLP }}$ & EPA & $\underline{3 Q 99}$ & CLPEPA & $\underline{1 \mathrm{Q} 00}$ & $\underline{\text { CLP }}$ & EPA & Filt. & $\underline{\text { DF }}$ & $\underline{\text { Unit }}$ & $\underline{\text { La }}$ \\
\hline \multicolumn{16}{|c|}{ Inorganics } \\
\hline & Aluminum, total recoverable & 84.4 & J & 85 & J & । & 38.2 & J 1 & 41.2 & $J$ & 1 & NDD & 1 & $u g / L$ & EX \\
\hline & Nitrate-nitrite as nitrogen & 2380 & & 1630 & & & 1390 & & 1100 & & & $<2400$ & 5 & ug/L & EX \\
\hline & Sodium, total recoverable & 5620 & & 4310 & & & 4090 & & 4460 & & & $<4600$ & 1 & $\mathrm{ug} / \mathrm{L}$ & EX \\
\hline & Sulfate & 1170 & & 1400 & & & 2560 & & 1750 & & & $<3000$ & 1 & $\mathrm{ug} / \mathrm{L}$ & EX \\
\hline \multicolumn{16}{|c|}{ Radionuclides } \\
\hline & Gross alpha & 17.2 & & 7.28 & $J$ & $\mathrm{~K}$ & 1.99 & J 1 & 3.14 & & & $<15$ & 1 & $\mathrm{pCi} / \mathrm{L}$ & GP \\
\hline & Nonvolatile beta & 12.17 & $J$ & 2.6 & $J$ & I & 1.16 & $U$ & 1.69 & $J$ & 1 & NDD & 1 & $\mathrm{pCi} / \mathrm{L}$ & GP \\
\hline & Radium, total alpha-emitting & 4.04 & & 3.81 & & & 2.2 & & 1.81 & & & $<5$ & 1 & $\mathrm{pCi} / \mathrm{L}$ & GP \\
\hline
\end{tabular}

Notes: Concentrations in bold italics exceed the groundwater protection or monitoring constituent standards listed in Appendix A. Synchronous water levels are measured over a 3-5 day period or less. Dilution factors, Laboratory, and Filtered Data are for First Quarter 2000 data only.

$+=$ exceeded the Groundwater Protection Standards or Monitoring Constituents listed in Appendix A for First Quarter 2000. 
Table D-6. Groundwater Monitoring Results for Point-of-Compliance Wells, Met Lab HWMF (Cont.) WELL AMB 5

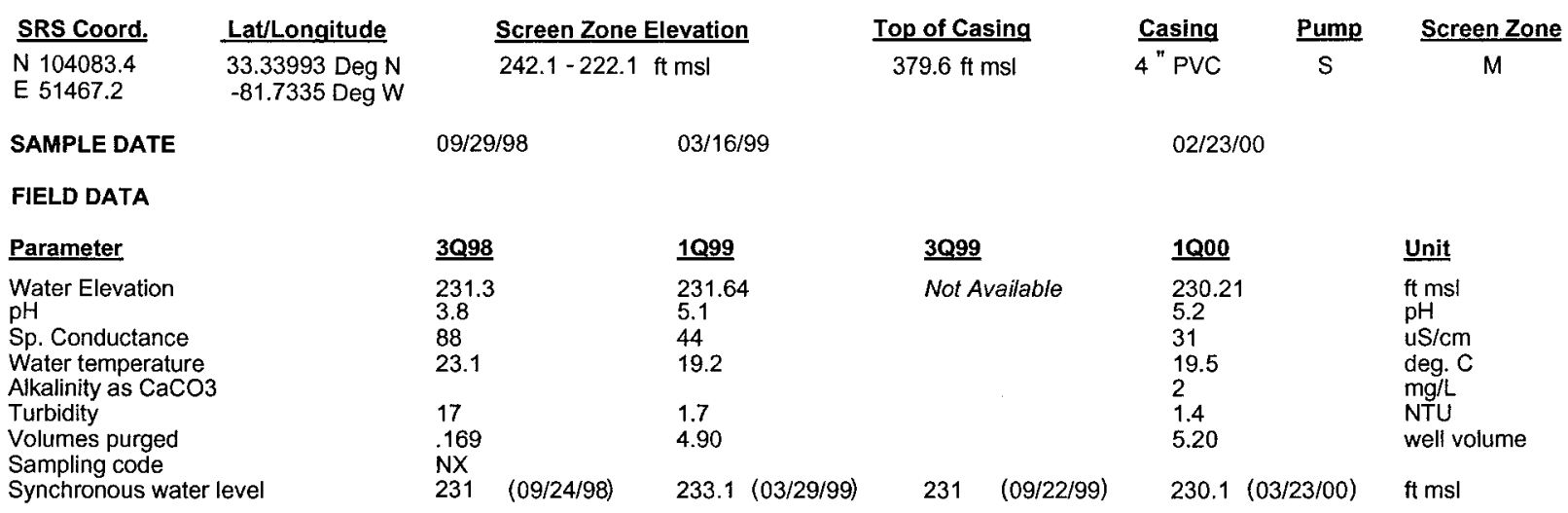

ANALYTICAL DATA

1. Groundwater Protection Standard

261 Appendix VIII/264 Appendix IX Hazardous Constituents

\begin{tabular}{|c|c|c|c|c|c|c|c|c|c|c|c|c|}
\hline ST Parameter & $\underline{3098}$ & CLPEPA & $\underline{1 \mathrm{Q} 99}$ & CLP EPA & $\underline{3 Q 99}$ & CLPEPA & 1000 & CLPEPA & Filt. & $\underline{D F}$ & $\underline{\text { Unit }}$ & $\underline{\text { Lab }}$ \\
\hline \multicolumn{13}{|l|}{ Inorganics } \\
\hline Barium, total recoverable & 23.2 & & 6.7 & & & & $<4.62$ & $U \mathrm{~V}$ & $<E Q L$ & 1 & $u g / L$ & EX \\
\hline Cyanide & $<15.2$ & UJ & $<15.2$ & $\mathrm{U}$ & & & $<10$ & 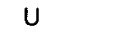 & $<E Q L$ & 1 & $u g / L$ & EX \\
\hline Lead, total recoverable & 11.3 & $\mathrm{~J}$ & $<47$ & $\mathrm{u}$ & & & $<10$ & $\mathrm{U}$ & $<E Q L$ & 1 & $\mathrm{ug} / \mathrm{L}$ & EX \\
\hline Nickel, total recoverable & 5.2 & J & $<26$ & $\mathrm{u}$ & & & $<9.29$ & JU V & $<\mathrm{EQL}$ & 1 & $u g / L$ & EX \\
\hline Selenium, total recoverable & $<66$ & u & $<66$ & $u$ & & & $<10$ & $\mathrm{U}$ & $<E Q L$ & 1 & $\mathrm{ug} / \mathrm{L}$ & EX \\
\hline \multicolumn{13}{|l|}{ Organics } \\
\hline Chlorobenzene & $<5$ & $u$ & $<5$ & $u$ & & & $<5$ & $u$ & $<E Q L$ & 1 & $\mathrm{ug} / \mathrm{L}$ & EX \\
\hline 1,1-Dichloroethane & $<5$ & $u$ & $<5$ & $\mathrm{u}$ & & & $<5$ & $u$ & $<E Q L$ & 1 & $u g / L$ & EX \\
\hline 1,1-Dichloroethylene & $<5$ & $\mathrm{u}$ & $<5$ & $u$ & & & $<5$ & $u$ & $<E Q L$ & 1 & $u g / L$ & EX \\
\hline trans-1,2-Dichloroethylene & $<5$ & $u$ & $<5$ & $u$ & & & $<5$ & $u$ & $<E Q L$ & 1 & $\mathrm{ug} / \mathrm{L}$ & EX \\
\hline $\begin{array}{l}\text { PCB } 1016 \\
\text { PCB } 1221\end{array}$ & & & & & & & & & & & & \\
\hline РCB 1232 & & & & & & & & & & & & \\
\hline PCB 1242 & & & & & & & & & & & & \\
\hline PCB 1248 & & & & & & & & & & & & \\
\hline PCB 1254 & & & & & & & & & & & & \\
\hline PCB 1260 & & & & & & & & & & & & \\
\hline 1,1,2,2-Tetrachloroethane & $<5$ & $u$ & $<5$ & 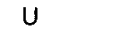 & & & $<5$ & $\mathrm{U}$ & $<E Q L$ & 1 & $u g / L$ & EX \\
\hline Tetrachloroethylene & $<5$ & $\mathrm{u}$ & $<5$ & $u$ & & & $<5$ & $\mathrm{u}$ & $<E Q L$ & 1 & $u g / L$ & EX \\
\hline 1,1,1-Trichloroethane & $<5$ & $u$ & $<5$ & $\mathrm{U}$ & & & $<5$ & $\mathrm{u}$ & $<E Q L$ & 1 & $\mathrm{ug} / \mathrm{L}$ & EX \\
\hline+ Trichloroethylene & 19.2 & & 18.2 & & & & 5.7 & & $>5$ & 1 & $\mathrm{ug} / \mathrm{L}$ & EX \\
\hline \multicolumn{13}{|l|}{ II. Monitoring Constituents } \\
\hline ST Parameter & $\underline{3098}$ & CLPEPA & 1099 & CLPEPA & 3099 & CLPPEPA & 1000 & CLPEPA & Filt. & $\underline{\text { DF }}$ & $\underline{\text { Unit }}$ & $\underline{\text { Lab }}$ \\
\hline \multicolumn{13}{|l|}{ Inorganics } \\
\hline Aluminum, total recoverable & 1320 & & 106 & J 1 & & & $<200$ & $\mathrm{u}$ & $<E Q L$ & 1 & $u g / L$ & EX \\
\hline Nitrate-nitrite as nitrogen & 3080 & & 1700 & & & & 1360 & & $<2400$ & 5 & $\mathrm{ug} / \mathrm{L}$ & EX \\
\hline+ Sodium, total recoverable & 5720 & & 7280 & & & & 5040 & & $>4600$ & 1 & ug/L & EX \\
\hline Sulfate & 846 & & 4570 & & & & 2190 & & $<3000$ & 1 & $\mathrm{ug} / \mathrm{h}$ & EX \\
\hline \multicolumn{13}{|l|}{ Radionuclides } \\
\hline Gross alpha & 16.74 & & 4.61 & $\mathrm{~J} \quad \mathrm{~K}$ & & & 3.46 & & $<15$ & 1 & $\mathrm{pCi} / \mathrm{L}$ & GP \\
\hline Nonvolatile beta & 10.29 & & 1.56 & J 1 & & & 2.52 & & $<50$ & 1 & $\mathrm{pCi} / \mathrm{L}$ & GP \\
\hline Radium, total alpha-emitting & 6.19 & & 2.15 & J 1 & & & 1.75 & & $<5$ & 1 & $\mathrm{pCi} / \mathrm{L}$ & GP \\
\hline
\end{tabular}

Notes: Concentrations in bold italics exceed the groundwater protection or monitoring constituent standards listed in Appendix A. Synchronous water levels are measured over a 3-5 day period or less. Dilution factors, Laboratory, and Filtered Data are for First Quarter 2000 data only.

$+=$ exceeded the Groundwater Protection Standards or Monitoring Constituents listed in Appendix A for First Quarter 2000. 
Table D-6. Groundwater Monitoring Results for Point-of-Compliance Wells, Met Lab HWMF (Cont.) WELL AMB 6

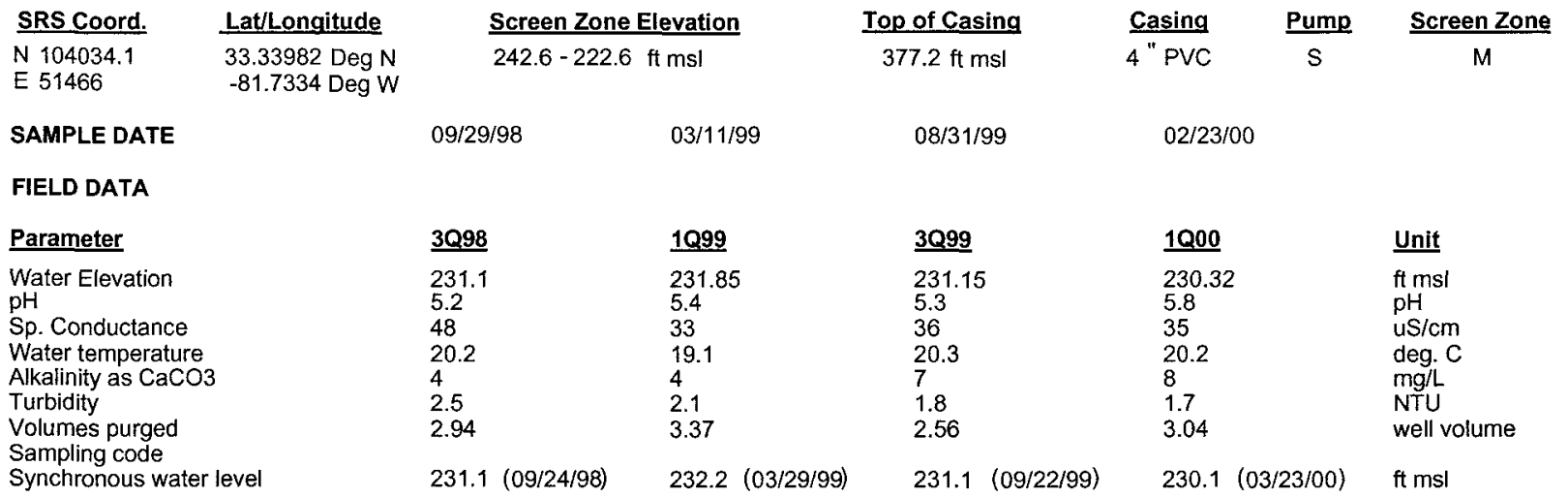

ANALYTICAL DATA

I. Groundwater Protection Standard

261 Appendix VIII/264 Appendix IX Hazardous Constituents

ST Parameter

Inorganics

Barium, total recoverable Cyanide

+ Lead, total recoverable Nickel, total recoverable

Selenium, total recoverable Organics

Chlorobenzene

1,1-Dichloroethane

1,1-Dichloroethylene

trans-1,2-Dichloroethylene

PCB 1016

PCB 1221

PCB 1232

PCB 1242

PCB 1248

PCB 1254

PCB 1260

1,1,2,2-Tetrachloroethane

Tetrachloroethylene

1,1,1-Trichloroethane

Trichloroethylene

II. Monitoring Constituents

ST Parameter

Inorganics

Nitrate-nitrite as nitrogen

+ Sodium, total recoverable

+ Sulfate

Radionuclides

Gross alpha

Nonvolatile beta

Radium, total alpha-emitting

\section{$\underline{309}$}

CLPEPA

1099

$\begin{array}{lll}2.2 & & \\ <15.2 & U J & \\ <47 & U & \\ <26 & U & \\ <66 & U & \\ & & \\ <5 & U & \\ <5 & U & \\ <5 & U & \\ <5 & U & <56\end{array}$

2.3
$<15.2$
17.8
$<26$
$<66$

$<5$
$<5$
$<5$
$<5$

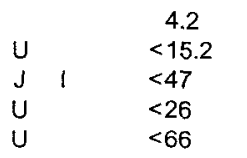

CLPEPA

1000

\begin{tabular}{|c|c|c|c|c|c|c|}
\hline$<2.14$ & U & V & $<\mathrm{EQL}$ & 1 & $u g / L$ & EX \\
\hline$<10$ & U & & $<\mathrm{EQL}$ & 1 & $\mathrm{ug} / \mathrm{L}$ & EX \\
\hline 15.1 & & & $>15$ & 1 & $\mathrm{ug} / \mathrm{L}$ & $E X$ \\
\hline$<9.72$ & JU & V & $<E Q L$ & 1 & $\mathrm{ug} / \mathrm{L}$ & EX \\
\hline$<10$ & U & & $<\mathrm{EQL}$ & 1 & $\mathrm{ug} / \mathrm{L}$ & EX \\
\hline$<5$ & $\mathrm{u}$ & & $<E O L$ & 1 & $u g / L$ & EX \\
\hline$<5$ & U & & $<\mathrm{EQL}$ & 1 & $\mathrm{ug} / \mathrm{L}$ & EX \\
\hline$<5$ & U & & $<\mathrm{EQL}$ & 1 & $\mathrm{ug} / \mathrm{L}$ & EX \\
\hline$<5$ & U & & $<\mathrm{EQL}$ & 1 & $\mathrm{ug} / \mathrm{L}$ & EX \\
\hline
\end{tabular}

$\begin{array}{lll}<5 & U & \\ <5 & U & \\ <5 & U & \\ 2.09 & \mathrm{~J} & <5\end{array}$

$<5$
$<5$
$<5$
$<5$

$\begin{array}{lll}U & <5 & U \\ U & <5 & U \\ U & <5 & U \\ U & <5 & U\end{array}$

$<5$
$<5$
$<5$
$<5$

$u$
$U$
$U$

$<E Q L$

$<$ EQL

ug/L

ug/L

$\underline{3 Q 98} \quad \underline{\text { CLPEPA }}$

1099

CLP EPA

3099

CLPEPA

1000

CLPEPA

Filt.

\begin{tabular}{|c|c|c|c|c|c|}
\hline$<884$ & U & & $<E Q L$ & 1 & $\mathrm{ug} / \mathrm{L}$ \\
\hline 820 & & & $<2400$ & 5 & $\mathrm{ug} / \mathrm{L}$ \\
\hline 7030 & & & $>4600$ & 1 & $\mathrm{ug} / \mathrm{L}$ \\
\hline 4010 & & & $>3000$ & 1 & $\mathrm{ug} / \mathrm{L}$ \\
\hline .687 & $J$ & 1 & NDD & 1 & $\mathrm{pCi} / \mathrm{L}$ \\
\hline 1.2 & $\mathrm{~J}$ & 1 & NDD & 1 & $\mathrm{pCi} / \mathrm{L}$ \\
\hline-.038 & $U$ & & $<E Q L$ & 1 & $\mathrm{pCi} / \mathrm{L}$ \\
\hline
\end{tabular}

Notes: Concentrations in bold italics exceed the groundwater protection or monitoring constituent standards listed in Appendix A. Synchronous water levels are measured over a 3-5 day period or less. Dilution factors, Laboratory, and Filtered Data are for First Quarter 2000 data only.

$+=$ exceeded the Groundwater Protection Standards or Monitoring Constituents listed in Appendix A for First Quarter 2000. 
Table D-6. Groundwater Monitoring Results for Point-of-Compliance Wells, Met Lab HWMF (Cont.) WELL AMB 8D

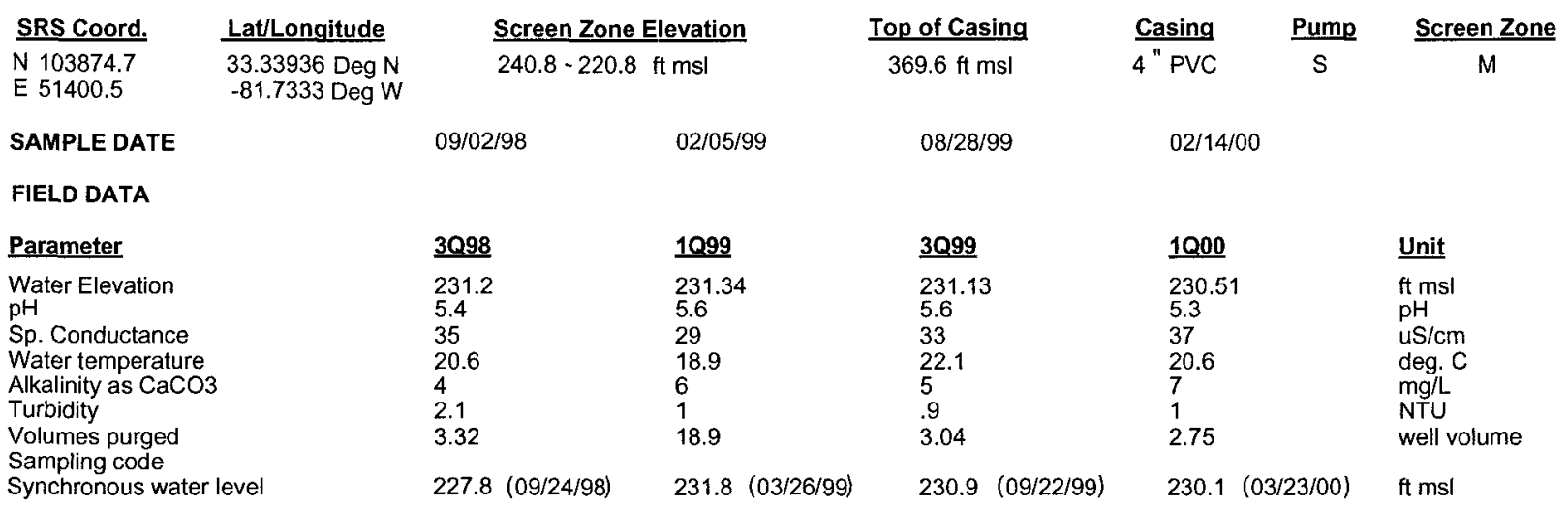

ANALYTICAL DATA

1. Groundwater Protection Standard

261 Appendix VIII/264 Appendix IX Hazardous Constituents

\begin{tabular}{|c|c|c|c|c|c|c|c|c|c|c|c|c|c|c|c|}
\hline \multirow{2}{*}{$\begin{array}{l}\text { ST Parameter } \\
\text { Inorganics }\end{array}$} & \multirow[t]{2}{*}{$\underline{3 Q 98}$} & \multirow[t]{2}{*}{ CLPEPA } & \multirow[t]{2}{*}{$\underline{1099}$} & \multicolumn{2}{|c|}{ CLPEPA } & \multirow[t]{2}{*}{$\underline{3 Q 99}$} & \multicolumn{2}{|c|}{ CLPEPA } & \multirow[t]{2}{*}{$\underline{1 Q 00}$} & \multicolumn{2}{|c|}{ CLPEPA } & \multirow[t]{2}{*}{ Filt. } & \multirow[t]{2}{*}{ DF } & \multirow[t]{2}{*}{$\underline{\text { Unit }}$} & \multirow{2}{*}{$\underline{\underline{L a b}}$} \\
\hline & & & & & & & & & & & & & & & \\
\hline Barium, total recoverable & 4.1 & & 3.6 & & & $<4.9$ & $\mathrm{u}$ & $\mathrm{v}$ & $<3.22$ & $u$ & $\mathrm{v}$ & $<E Q L$ & 1 & $\mathrm{ug} / \mathrm{L}$ & \\
\hline Cyanide & $<15.2$ & $u$ & 1.54 & $\mathrm{~J}$ & 1 & $<15.2$ & $\mathrm{U}$ & & $<10$ & $u$ & & $<E Q L$ & 1 & $u g / L$ & \\
\hline Lead, total recoverable & 5.2 & $\mathrm{~J}$ & $<47$ & $\mathrm{u}$ & & $<47$ & $\mathrm{U}$ & & $<10$ & $\mathrm{u}$ & & $<E Q L$ & 1 & $\mathrm{ug} / \mathrm{L}$ & \\
\hline Nickel, total recoverable & 2.9 & $\mathrm{~J}$ & $<26$ & $\mathrm{u}$ & & $<26$ & $\mathrm{u}$ & & $<50$ & $u$ & & $<\mathrm{EQL}$ & 1 & $\mathrm{ug} / \mathrm{L}$ & \\
\hline Selenium, total recoverable & $<66$ & $u$ & $<66$ & $u$ & & $<66$ & $U$ & & $<10$ & $\mathrm{U}$ & & $<\mathrm{EQL}$ & 1 & $\mathrm{ug} / \mathrm{L}$ & \\
\hline \multicolumn{15}{|l|}{ Organics } & \\
\hline Chlorobenzene & $<5$ & $u$ & $<5$ & $u$ & & $<5$ & JU & $\mathrm{Q}$ & $<5$ & $\mathrm{u}$ & & $<\mathrm{EQL}$ & 1 & $\mathrm{ug} / \mathrm{L}$ & \\
\hline 1,1-Dichloroethane & $<5$ & $u$ & $<5$ & $\mathrm{u}$ & & $<5$ & JU & $\mathrm{Q}$ & $<5$ & $\mathrm{u}$ & & $<E Q L$ & 1 & $\mathrm{ug} / \mathrm{L}$ & \\
\hline 1,1-Dichloroethylene & $<5$ & $\mathrm{u}$ & $<5$ & $u$ & & $<5$ & JU & $\mathrm{Q}$ & $<5$ & $\mathrm{u}$ & & $<\mathrm{EQL}$ & 1 & $\mathrm{ug} / \mathrm{h}$ & \\
\hline $\begin{array}{l}\text { trans-1,2-Dichloroethylene } \\
\text { PCB } 1016\end{array}$ & $<5$ & $u$ & $<5$ & $u$ & & $<5$ & JU & Q & $<5$ & $\mathrm{U}$ & & $<E Q L$ & 1 & $\mathrm{ug} / \mathrm{L}$ & \\
\hline РCB 1221 & & & & & & & & & & & & & & & \\
\hline PCB 1232 & & & & & & & & & & & & & & & \\
\hline PCB 1242 & & & & & & & & & & & & & & & \\
\hline PCB 1248 & & & & & & & & & & & & & & & \\
\hline PCB 1254 & & & & & & & & & & & & & & & \\
\hline $\begin{array}{l}\text { PCB } 1260 \\
1,1,2,2 \text {-Tetrachloroethane }\end{array}$ & $<5$ & $\mathrm{u}$ & $<5$ & u & & & 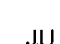 & 0 & & & & & & & \\
\hline Tetrachloroethylene & $<5$ & $\mathrm{u}$ & $<5$ & u & & $\begin{array}{l}<5 \\
<5\end{array}$ & JU & $a^{2}$ & $<5$ & (u) & & $<E Q L$ & 1 & $\mathrm{ug} / \mathrm{L}$ & \\
\hline 1,1,1-Trichloroethane & $<5$ & $\mathrm{u}$ & $<5$ & u & & $<5$ & JU & $Q$ & $<5$ & u & & $<E Q L$ & 1 & ug/L & \\
\hline Trichloroethylene & 1.65 & J & $<5$ & $u$ & & $<5$ & JU & $Q$ & 1.1 & $\mathrm{~J}$ & 1 & NDD & 1 & $\mathrm{ug} / \mathrm{L}$ & \\
\hline \multicolumn{15}{|l|}{ II. Monitoring Constituents } & \\
\hline ST Parameter & $\underline{3 Q 98}$ & CLLPEPA & 1099 & C나 & EPA & $\underline{3099}$ & CLP & EPA & 1000 & $\underline{\text { CLP }}$ & EPA & Filt. & $\underline{D F}$ & Unit & \\
\hline \multicolumn{15}{|l|}{ Inorganics } & \\
\hline Aluminum, total recoverable & 76.1 & J & 55 & $\mathrm{~J}$ & 1 & 18.6 & $\mathrm{~J}$ & I & $<55.3$ & u & $\mathrm{v}$ & $<E Q L$ & 1 & $\mathrm{ug} / \mathrm{L}$ & \\
\hline Nitrate-nitrite as nitrogen & 464 & & 700 & & & 627 & & & 508 & & & $<2400$ & 5 & $\mathrm{ug} / \mathrm{L}$ & \\
\hline Sodium, total recoverable & 5450 & & 4070 & & & 4960 & & & 6810 & & & $>4600$ & 1 & $\mathrm{ug} / \mathrm{L}$ & \\
\hline Sulfate & 1780 & & 1500 & & & 1910 & & & 2730 & & & $<3000$ & 2 & $\mathrm{ug} / \mathrm{L}$ & \\
\hline \multicolumn{15}{|l|}{ Radionuclides } & \\
\hline Gross alpha & 4.77 & $\mathrm{~J}$ & 4.54 & & & 2.34 & J & $\mathrm{K}$ & 4.36 & & & $<15$ & 1 & $\mathrm{pCi} / \mathrm{L}$ & \\
\hline Nonvolatile beta & 2.54 & UIJ & 4.03 & & & 1.25 & $u$ & & 2.15 & $\mathrm{~J}$ & 1 & NDD & 1 & $\mathrm{pCi} / \mathrm{L}$ & \\
\hline Radium, total alpha-emitting & .98 & & 1.66 & $\mathrm{~J}$ & 1 & .8 & J & I & .656 & J & i & NDD & 1 & $\mathrm{pCi} / \mathrm{L}$ & \\
\hline
\end{tabular}

Notes: Concentrations in bold italics exceed the groundwater protection or monitoring constituent standards listed in Appendix A. Synchronous water levels are measured over a 3-5 day period or less. Dilution factors, Laboratory, and Filtered Data are for First Quarter 2000 data only.

+ = exceeded the Groundwater Protection Standards or Monitoring Constituents listed in Appendix A for First Quarter 2000. 
Table D-6. Groundwater Monitoring Results for Point-of-Compliance Wells, Met Lab HWMF (Cont.) WELL AMB 9D

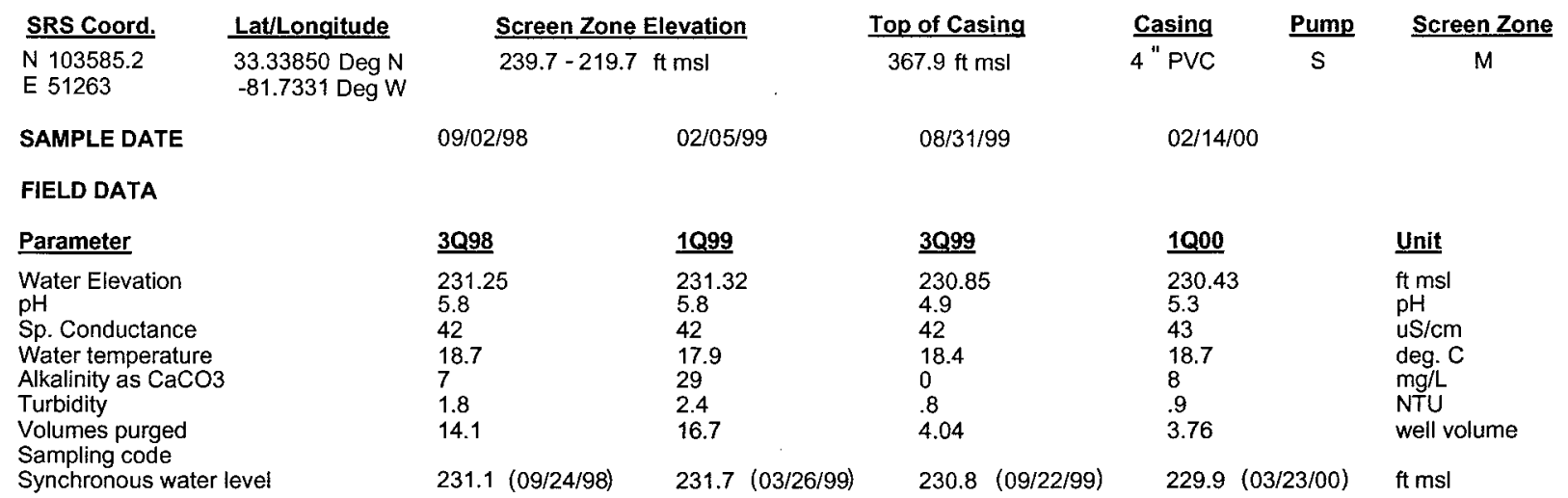

ANALYTICAL DATA

I. Groundwater Protection Standard

261 Appendix VIII/264 Appendix IX Hazardous Constituents

\begin{tabular}{|c|c|c|c|c|c|c|c|c|c|c|c|c|c|c|c|c|}
\hline \multirow{2}{*}{$\underline{\text { ST }}$} & Parameter & \multirow[t]{2}{*}{$\underline{3 Q 98}$} & \multirow[t]{2}{*}{ CLP EPA } & \multirow[t]{2}{*}{1099} & \multicolumn{2}{|c|}{ CLPEPA } & \multirow[t]{2}{*}{$\underline{3 Q 99}$} & \multicolumn{2}{|c|}{ CLPEPA } & \multirow[t]{2}{*}{$1 Q 00$} & \multicolumn{2}{|c|}{ CLPEPA } & \multirow[t]{2}{*}{ Filt. } & \multirow[t]{2}{*}{$\underline{\text { DF }}$} & \multirow[t]{2}{*}{ Unit } & \multirow[t]{2}{*}{$\underline{\text { Lab }}$} \\
\hline & ganics & & & & & & & & & & & & & & & \\
\hline & Barium, total recoverable & 3.4 & & 2.6 & & & 2.8 & & & $<2.77$ & U & V & $<E Q L$ & 1 & ug/L & EX \\
\hline & Cyanide & $<15.2$ & $U$ & $<15.2$ & $U$ & & $<15.2$ & $U$ & & $<10$ & $\mathrm{U}$ & & $<E Q L$ & 1 & ug/L & EX \\
\hline & Lead, total recoverable & $<47$ & $\mathrm{U}$ & $<47$ & U & & $<47$ & $\bigcup$ & & $<10$ & $\mathrm{U}$ & & $<$ EQL & 1 & $\mathrm{ug} / \mathrm{L}$ & EX \\
\hline & Nickel, total recoverable & $<26$ & $U$ & $<26$ & $\mathrm{U}$ & & $<26$ & $U$ & & $<50$ & $U$ & & $<E Q L$ & 1 & $\mathrm{ug} / \mathrm{L}$ & EX \\
\hline & Selenium, total recoverable & $<66$ & $\mathrm{U}$ & $<66$ & $\mathrm{U}$ & & $<66$ & $U$ & & $<10$ & U & & $<E Q L$ & 1 & $u g / L$ & EX \\
\hline \multicolumn{17}{|c|}{ Organics } \\
\hline & Chlorobenzene & $<5$ & $\mathrm{U}$ & $<5$ & $\mathrm{U}$ & & $<5$ & JU & $Q$ & $<5$ & U & & $<E Q L$ & 1 & $u g / L$ & EX \\
\hline & 1,1-Dichloroethane & $<5$ & $\mathrm{U}$ & $<5$ & $U$ & & $<5$ & JU & $Q$ & $<5$ & $\mathrm{U}$ & & $<$ EQL & 1 & $u g / L$ & EX \\
\hline & 1,1-Dichloroethylene & $<5$ & $\mathrm{U}$ & $<5$ & $U$ & & $<5$ & JU & $Q$ & $<5$ & $\mathrm{U}$ & & $<E Q L$ & 1 & $u g / L$ & EX \\
\hline & trans-1,2-Dichloroethyiene & $<5$ & $\mathrm{U}$ & $<5$ & $\mathrm{U}$ & & $<5$ & $\mathrm{JU}$ & $Q$ & $<5$ & U & & $<E Q L$ & 1 & ug/L & EX \\
\hline & PCB 1016 & & & $<1.02$ & JU & $Q$ & & & & & & & & & ug/L & \\
\hline & PCB 1221 & & & $<2.04$ & JU & $Q$ & & & & & & & & & ug/L & \\
\hline & PCB 1232 & & & $<1.02$ & JU & $\bar{Q}$ & & & & & & & & & ug/L & \\
\hline & РCB 1242 & & & $<1.02$ & JU & $Q$ & & & & & & & & & ug/L & \\
\hline & PCB 1248 & & & $<1.02$ & JU & $Q$ & & & & & & & & & ug/L & \\
\hline & PCB 1254 & & & $<1.02$ & JU & $Q$ & & & & & & & & & ug/L & \\
\hline & PCB 1260 & & & $<1.02$ & JU & $Q$ & & & & & & & & & $\mathrm{ug} / \mathrm{L}$ & \\
\hline & $1,1,2,2$-Tetrachloroethane & $<5$ & $\mathrm{U}$ & $<5$ & $\mathrm{U}$ & & $<5$ & JU & $Q$ & $<5$ & $U$ & & $<E Q L$ & 1 & $\mathrm{ug} / \mathrm{L}$ & EX \\
\hline & Tetrachloroethylene & $<5$ & $\mathrm{U}$ & $<5$ & $U$ & & $<5$ & JU & $Q$ & $<5$ & $\mathrm{U}$ & & $<E Q L$ & 1 & $\mathrm{ug} / \mathrm{L}$ & EX \\
\hline & 1,1,1-Trichloroethane & $<5$ & $\mathbf{U}$ & $<5$ & $U$ & & $<5$ & JU & $Q$ & $<5$ & $U$ & & $<E Q L$ & 1 & $\mathrm{ug} / \mathrm{L}$ & EX \\
\hline & Trichloroethylene & $<5$ & U & $<5$ & $\mathrm{U}$ & & $<5$ & JU & $Q$ & $<5$ & $\mathrm{U}$ & & $<E Q L$ & 1 & $\mathrm{ug} / \mathrm{L}$ & EX \\
\hline \multicolumn{17}{|c|}{ II. Monitoring Constituents } \\
\hline$\underline{\text { ST }}$ & Parameter & $\underline{3098}$ & CLPEPA & 1099 & $\underline{\text { CLP }}$ & EPA & $\underline{3099}$ & $\underline{\text { CLP }}$ & EPA & 1000 & $\underline{\text { CLP }}$ & EPA & Filt. & DF & Unit & $\underline{L a b}$ \\
\hline \multicolumn{17}{|c|}{ Inorganics } \\
\hline & Aluminum, total recoverable & 953 & & 30.8 & $\jmath$ & 1 & $<146$ & $\mathrm{U}$ & & $<46$ & $U$ & V & $<E Q L$ & 1 & $\mathrm{ug} / \mathrm{L}$ & EX \\
\hline & Nitrate-nitrite as nitrogen & 173 & & 143 & & & 117 & & & $<500$ & $\mathrm{U}$ & & $<E Q L$ & 5 & $\mathrm{ug} / \mathrm{L}$ & EX \\
\hline+ & Sodium, total recoverable & 8830 & & 7820 & & & 7710 & & & 9760 & & & $>4600$ & 1 & $\mathrm{ug} / \mathrm{L}$ & EX \\
\hline+ & Sulfate & 4900 & & 6490 & & & 5380 & & & 4800 & & & $>3000$ & 2 & $\mathrm{ug} / \mathrm{L}$ & EX \\
\hline \multicolumn{17}{|c|}{ Radionuclides } \\
\hline & Gross alpha & 2.35 & $J$ & 1.22 & $U$ & & 1.1 & $\mathrm{U}$ & V & .681 & $\mathrm{U}$ & & $<E Q L$ & 1 & $\mathrm{pCi} / \mathrm{L}$ & GP \\
\hline & Nonvolatile beta & 1.59 & UIJ & .51 & $U$ & & .573 & $\mathrm{U}$ & & .924 & $\mathrm{U}$ & & $<E Q L$ & 1 & $\mathrm{pCi} / \mathrm{L}$ & GP \\
\hline & Radium, total alpha-emitting & 1 & & 1.24 & $j$ & 1 & .9 & $\mathrm{~J}$ & 1 & .693 & $J$ & 1 & NDD & 1 & $\mathrm{pCi} / \mathrm{L}$ & GP \\
\hline
\end{tabular}

Notes: Concentrations in bold italics exceed the groundwater protection or monitoring constituent standards listed in Appendix A. Synchronous water levels are measured over a 3-5 day period or less. Dilution factors, Laboratory, and Filtered Data are for First Quarter 2000 data only.

$+=$ exceeded the Groundwater Protection Standards or Monitoring Constituents listed in Appendix A for First Quarter 2000. 
Table D-6. Groundwater Monitoring Results for Point-of-Compliance Wells, Met Lab HWMF (Cont.) WELL AMB 10A

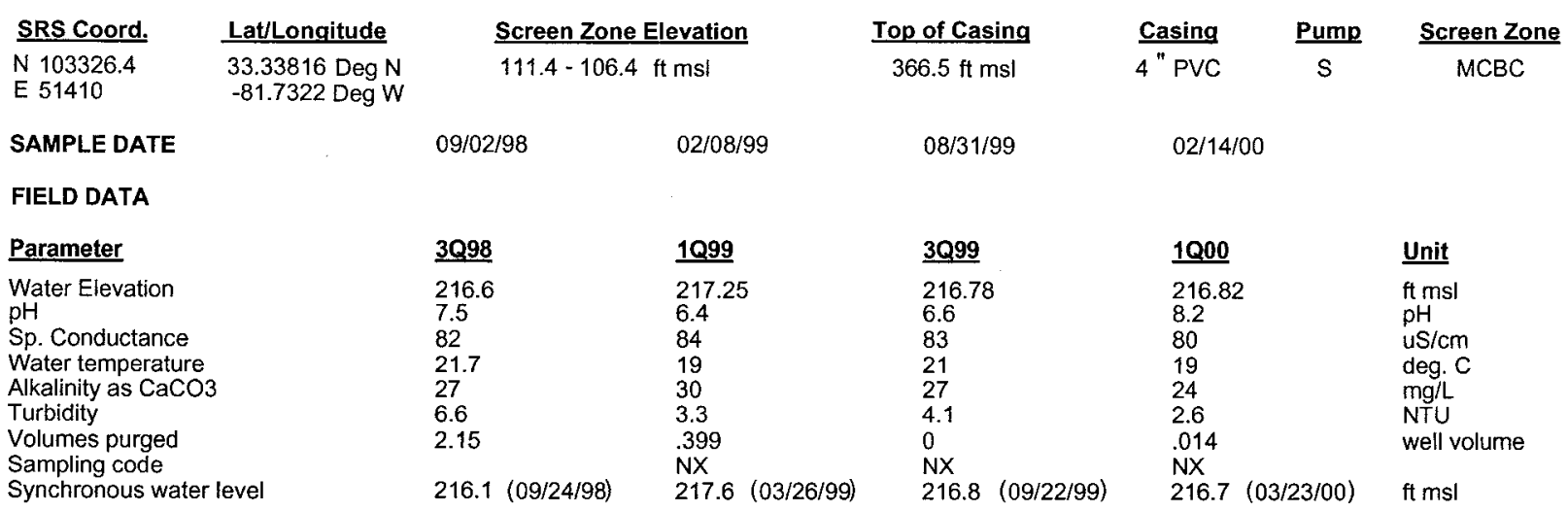

ANALYTICAL DATA

I. Groundwater Protection Standard

261 Appendix VIII/264 Appendix IX Hazardous Constituents

\begin{tabular}{|c|c|c|c|c|c|c|c|c|c|c|c|c|c|}
\hline \multirow{2}{*}{$\begin{array}{l}\text { ST Parameter } \\
\text { Inorganics }\end{array}$} & \multirow[t]{2}{*}{$\underline{30,98}$} & \multirow[t]{2}{*}{ CLPEPA } & \multirow[t]{2}{*}{$\underline{1099}$} & \multicolumn{2}{|c|}{ CLPPEPA } & \multirow[t]{2}{*}{$\underline{3099}$} & \multirow[t]{2}{*}{ CLPEPA } & \multirow[t]{2}{*}{1000} & CLPEPA & \multirow[t]{2}{*}{ Filt. } & \multirow[t]{2}{*}{$\underline{\text { DF }}$} & \multirow[t]{2}{*}{$\underline{\text { Unit }}$} & \multirow[t]{2}{*}{$\underline{\text { Lab }}$} \\
\hline & & & & & & & & & & & & & \\
\hline Barium, total recoverable & 11.9 & & 12.9 & & & 10.9 & & 12.1 & & $<2000$ & 1 & ug/L & EX \\
\hline Cyanide & $<15.2$ & $u$ & 1.54 & $\mathrm{~J}$ & 1 & $<15.2$ & $u$ & $<10$ & $u$ & $<\mathrm{EQL}$ & 1 & $\mathrm{ug} / \mathrm{L}$ & EX \\
\hline Lead, total recoverable & $<47$ & U & $<47$ & U & & $<47$ & $\mathrm{U}$ & $<10$ & $u$ & $<E Q L$ & 1 & $\mathrm{ug} / \mathrm{L}$ & EX \\
\hline Nickel, total recoverable & $<26$ & $u$ & $<26$ & $u$ & & $<26$ & $\mathrm{u}$ & $<50$ & $u$ & $<E Q L$ & 1 & $\mathrm{ug} / \mathrm{L}$ & EX \\
\hline Selenium, total recoverable & $<66$ & $u$ & $<66$ & $\mathrm{u}$ & & $<66$ & $\mathrm{u}$ & $<10$ & $u$ & $<E Q L$ & 1 & $\mathrm{ug} / \mathrm{L}$ & EX \\
\hline \multicolumn{14}{|l|}{ Organics } \\
\hline Chlorobenzene & $<5$ & $U$ & $<5$ & JU & $Q$ & $<5$ & $U$ & $<5$ & $\mathrm{U}$ & $<E Q L$ & 1 & $u g / L$ & EX \\
\hline 1,1-Dichloroethane & $<5$ & $u$ & $<5$ & JU & $Q$ & $<5$ & $u$ & $<5$ & $\mathrm{U}$ & $<E Q L$ & 1 & $\mathrm{ug} / \mathrm{L}$ & EX \\
\hline 1,1-Dichloroethylene & $<5$ & $u$ & $<5$ & JU & $Q$ & $<5$ & $u$ & $<5$ & $\mathrm{u}$ & $<E Q L$ & 1 & $\mathrm{ug} / \mathrm{L}$ & EX \\
\hline $\begin{array}{l}\text { trans-1,2-Dichloroethylene } \\
\text { PCB } 1016\end{array}$ & $<5$ & $u$ & $<5$ & JU & $Q$ & $<5$ & $u$ & $<5$ & $u$ & $<E Q L$ & 1 & $\mathrm{ug} / \mathrm{L}$ & EX \\
\hline PCB 1221 & & & & & & & & & & & & & \\
\hline РСB 1232 & & & & & & & & & & & & & \\
\hline РСВ 1242 & & & & & & & & & & & & & \\
\hline PCB 1248 & & & & & & & & & & & & & \\
\hline PCB 1254 & & & & & & & & & & & & & \\
\hline РCB 1260 & & & & & & & & & & & & & \\
\hline 1,1,2,2-Tetrachloroethane & $<5$ & $\mathrm{u}$ & $<5$ & JU & $Q$ & $<5$ & $u$ & $<5$ & $\mathrm{u}$ & $<E Q L$ & 1 & $\mathrm{ug} / \mathrm{L}$ & EX \\
\hline Tetrachloroethylene & $<5$ & U & $<5$ & JU & Q & $<5$ & U & $<5$ & U & $<E Q L$ & 1 & $\mathrm{ug} / \mathrm{L}$ & EX \\
\hline 1,1,1-Trichloroethane & $<5$ & U & $<5$ & JU & Q & $<5$ & u & $<5$ & U & $<E Q L$ & 1 & $\mathrm{ug} / \mathrm{L}$ & EX \\
\hline Trichloroethylene & $<5$ & $u$ & $<5$ & JU & Q & $<5$ & $u$ & $<5$ & $u$ & $<E Q L$ & 1 & $\mathrm{ug} / \mathrm{L}$ & EX \\
\hline \multicolumn{14}{|l|}{ II. Monitoring Constituents } \\
\hline ST Parameter & $\underline{3098}$ & CLPEPA & 1099 & CLP. & EPA & $\underline{3099}$ & CLPEEPA & 1000 & CLPEPA & Filt. & $\underline{\mathrm{DF}}$ & $\underline{\text { Unit }}$ & $\underline{\text { Lab }}$ \\
\hline \multicolumn{14}{|l|}{ Inorganics } \\
\hline Aluminum, total recoverable & 156 & & 66.7 & $J$ & 1 & 66 & J 1 & $<172$ & $\mathrm{U} V$ & $<E Q L$ & 1 & $u g / L$ & EX \\
\hline Nitrate-nitrite as nitrogen & 263 & & 268 & & & 270 & & $<500$ & $\mathrm{u}$ & $<E Q L$ & 5 & $\mathrm{ug} / \mathrm{L}$ & EX \\
\hline+ Sodium, total recoverable & 5540 & & 4760 & & & 4910 & & 7170 & & $>4600$ & 1 & $\mathrm{ug} / \mathrm{L}$ & EX \\
\hline+ Sulfate & 3610 & & 3500 & & & 3700 & & 5510 & & $>3000$ & 2 & $u g / L$ & EX \\
\hline \multicolumn{14}{|l|}{ Radionuclides } \\
\hline Gross alpha & .09 & UIJ & .44 & $\mathrm{u}$ & v & .0302 & $\mathrm{U}$ & .457 & $\mathrm{u}$ & $<E Q L$ & 1 & $\mathrm{pCi} / \mathrm{L}$ & GP \\
\hline Nonvolatile beta & 1.81 & UIJ & 2.76 & & & 1.08 & $\mathrm{u}$ & 2.18 & J 1 & NDD & 1 & $\mathrm{pCi} / \mathrm{L}$ & GP \\
\hline Radium, total alpha-emitting & -.11 & UI & 1.01 & $\mathrm{~J}$ & 1 & .2 & $u$ & .039 & U & $<E Q L$ & 1 & $\mathrm{pCi} / \mathrm{L}$ & GP \\
\hline
\end{tabular}

Notes: Concentrations in bold italics exceed the groundwater protection or monitoring constituent standards listed in Appendix A. Synchronous water levels are measured over a 3-5 day period or less. Dilution factors, Laboratory, and Filtered Data are for First Quarter 2000 data only.

$+=$ exceeded the Groundwater Protection Standards or Monitoring Constituents listed in Appendix A for First Quarter 2000. 
Table D-6. Groundwater Monitoring Results for Point-of-Compliance Wells, Met Lab HWMF (Cont.) WELL AMB 10B

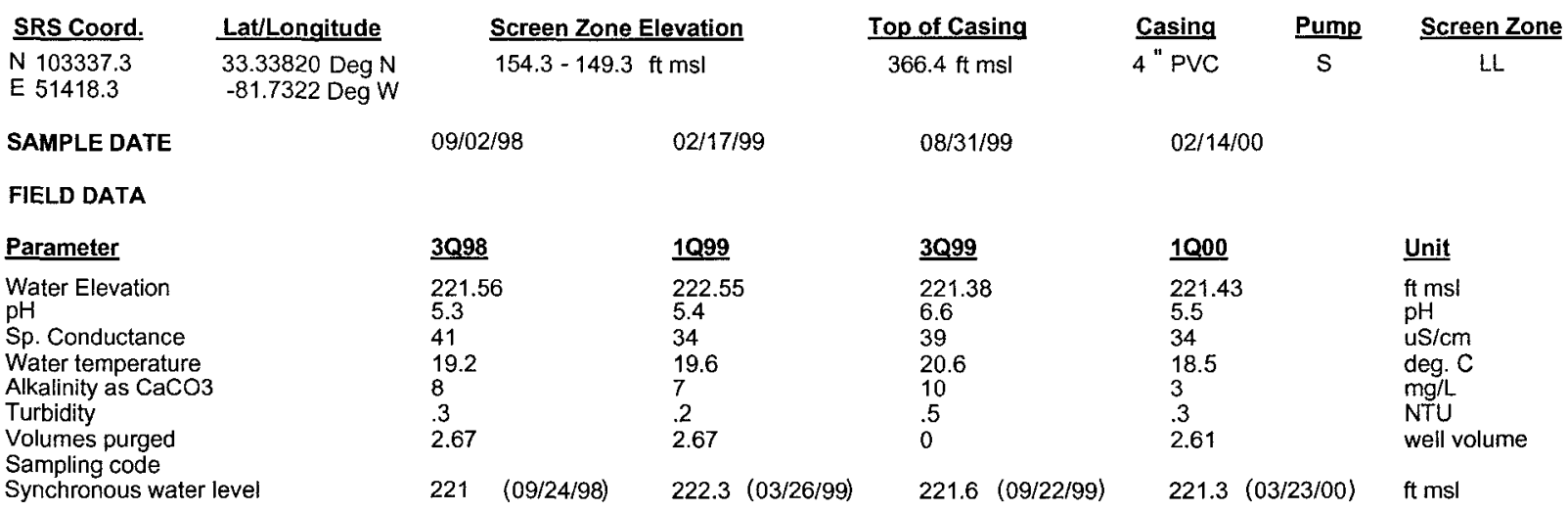

ANALYTICAL DATA

I. Groundwater Protection Standard

261 Appendix VIII/264 Appendix IX Hazardous Constituents

\begin{tabular}{|c|c|c|c|c|c|c|c|c|c|c|c|c|c|c|}
\hline$\underline{\text { ST }}$ & Parameter & $\underline{\mathbf{3 Q 9 8}}$ & CLPP EPA & 1099 & CLPEPA & 3Q99 & CLPEPA & 1000 & $\underline{\text { CLF }}$ & EPA & Filt. & $\underline{\text { DF }}$ & Unit & $\underline{\mathrm{Lab}}$ \\
\hline \multicolumn{15}{|c|}{ Inorganics } \\
\hline & Barium, total recoverable & 6.3 & & 9.47 & & 9.9 & & 8.51 & $J$ & 1 & NDD & 1 & $\mathrm{ug} / \mathrm{L}$ & EX \\
\hline & Cyanide & $<15.2$ & U & $<15.2$ & $\mathrm{U}$ & $<15.2$ & $\mathrm{U}$ & $<10$ & $\mathrm{U}$ & & $<\mathrm{EQL}$ & 1 & $\mathrm{ug} / \mathrm{L}$ & $\mathrm{EX}$ \\
\hline & Lead, total recoverable & $<47$ & $U$ & $<47$ & $U$ & $<47$ & $U$ & $<10$ & $U$ & & $<E Q L$ & 1 & ug/L & EX \\
\hline & Nickel, total recoverable & $<26$ & $U$ & $<26$ & $U$ & $<26$ & $U$ & $<5.71$ & $\mathrm{U}$ & V & $<E Q L$ & 1 & ug/L & EX \\
\hline & Selenium, total recoverable & $<66$ & $U$ & $<66$ & $U$ & $<66$ & $U$ & $<10$ & $U$ & & $<E Q L$ & 1 & $\mathrm{ug} / \mathrm{L}$ & EX \\
\hline \multicolumn{15}{|c|}{ Organics } \\
\hline & Chlorobenzene & $<5$ & $U$ & $<5$ & $\mathrm{U}$ & $<5$ & $\mathrm{U}$ & $<5$ & $\mathrm{U}$ & & $<E Q L$ & 1 & $\mathrm{ug} / \mathrm{L}$ & EX \\
\hline & 1,1-Dichloroethane & $<5$ & U & $<5$ & $\mathrm{U}$ & $<5$ & $U$ & $<5$ & $\mathrm{U}$ & & $<E Q L$ & 1 & $\mathrm{ug} / \mathrm{L}$ & EX \\
\hline & 1,1-Dichloroethylene & $<5$ & $\mathrm{U}$ & $<5$ & $\mathrm{U}$ & $<5$ & $U$ & $<5$ & $\mathrm{U}$ & & $<E Q L$ & 1 & $\mathrm{ug} / \mathrm{L}$ & EX \\
\hline & trans-1,2-Dichloroethylene & $<5$ & $\mathrm{U}$ & $<5$ & $\mathrm{U}$ & $<5$ & $\mathrm{U}$ & $<5$ & $\mathrm{U}$ & & $<\mathrm{EQL}$ & 1 & $u g / L$ & EX \\
\hline & PCB 1016 & & & $<1.02$ & $\mathrm{U}$ & & & $<.96$ & $\mathrm{U}$ & & $<\mathrm{EQL}$ & .960 & ug/L & EX \\
\hline & PCB 1221 & & & $<2.04$ & $\mathrm{U}$ & & & $<.96$ & $\mathrm{U}$ & & $<\mathrm{EQL}$ & .960 & $u g / L$ & EX \\
\hline & PCB 1232 & & & $<1.02$ & $\mathrm{U}$ & & & $<, 96$ & $U$ & & $<\mathrm{EQL}$ & .960 & ug/L & EX \\
\hline & PCB 1242 & & & $<1.02$ & U & & & $<1.9$ & $U$ & & $<\mathrm{EQL}$ & .960 & $\mathrm{ug} / \mathrm{L}$ & EX \\
\hline & РCB 1248 & & & $<1.02$ & U & & & $<.96$ & $\mathrm{U}$ & & $<E Q L$ & .960 & ug/L & EX \\
\hline & PCB 1254 & & & $<1.02$ & $U$ & & & $<.96$ & $U$ & & $<E Q L$ & .960 & ught & $E X$ \\
\hline & PCB 1260 & & & $<1.02$ & $\mathrm{U}$ & & & $<.96$ & $\mathrm{U}$ & & $<\mathrm{EQL}$ & .960 & $\mathrm{ug} / \mathrm{L}$ & EX \\
\hline & 1,1,2,2-Tetrachloroethane & $<5$ & $\mathrm{U}$ & $<5$ & $\mathrm{U}$ & $<5$ & U & $<5$ & $\mathrm{U}$ & & $<\mathrm{EQL}$ & 1 & $u g / L$ & EX \\
\hline & Tetrachloroethylene & $<5$ & $\mathrm{U}$ & $<5$ & $\mathrm{U}$ & $<5$ & $\mathrm{U}$ & $<5$ & $\mathrm{U}$ & & $<\mathrm{EQL}$ & 1 & $u g / L$ & EX \\
\hline & 1,1,1-Trichloroethane & $<5$ & $\mathrm{U}$ & $<5$ & $\mathrm{U}$ & $<5$ & $\mathrm{U}$ & $<5$ & $\mathrm{U}$ & & $<E Q L$ & 1 & $\mathrm{ug} / \mathrm{L}$ & EX \\
\hline & Trichloroethylene & $<5$ & $\mathrm{U}$ & $<5$ & $\mathrm{U}$ & $<5$ & $\mathrm{U}$ & $<5$ & $\mathrm{U}$ & & $<\mathrm{EQL}$ & 1 & ugg/L & EX \\
\hline \multicolumn{15}{|c|}{ II. Monitoring Constituents } \\
\hline ST & Parameter & $\underline{3098}$ & CLP EPA & 1099 & CLP EPA & $\underline{3099}$ & CLPEPA & 1000 & CLF & EPA & Filt. & $\underline{\text { DF }}$ & Unit & Lab \\
\hline \multicolumn{15}{|c|}{ Inorganics } \\
\hline & Aluminum, total recoverable & $<146$ & $\mathrm{U}$ & $<18.9$ & $\mathrm{U} V$ & $<146$ & $\mathrm{u}$ & $<200$ & $U$ & & $<E Q L$ & 1 & $\mathrm{ug} / \mathrm{L}$ & EX \\
\hline & Nitrate-nitrite as nitrogen & 206 & & 231 & & 239 & & $<500$ & $\mathrm{U}$ & & $<\mathrm{EQL}$ & 5 & $\mathrm{ug} / \mathrm{L}$ & EX \\
\hline+ & Sodium, total recoverable & 4260 & & 4250 & & 4400 & & 4950 & & & $>4600$ & 1 & ug/L & EX \\
\hline & Sulfate & 865 & & 1010 & & 1060 & & 1370 & & & $<3000$ & 2 & ug/L & EX \\
\hline \multicolumn{15}{|c|}{ Radionuclides } \\
\hline & Gross alpha & .36 & UIJ & .53 & J I & .013 & $U$ & .285 & $U$ & & $<E Q L$ & 1 & $\mathrm{pCi} / \mathrm{L}$ & GP \\
\hline & Nonvolatile beta & 6.68 & $J$ & -.09 & $\mathrm{U}$ & .542 & $U$ & .575 & $U$ & & $<E Q L$ & 1 & $\mathrm{pCi} / \mathrm{L}$ & GP \\
\hline & Radium, total alpha-emitting & .18 & UI & .23 & $U$ & .1 & $\mathrm{U}$ & .072 & $U$ & & $<E Q L$ & 1 & $\mathrm{pCi} / \mathrm{L}$ & $\mathrm{GP}$ \\
\hline
\end{tabular}

Notes: Concentrations in bold italics exceed the groundwater protection or monitoring constituent standards listed in Appendix A. Synchronous water levels are measured over a 3-5 day period or less. Dilution factors, Laboratory, and Filtered Data are for First Quarter 2000 data only.

$+=$ exceeded the Groundwater Protection Standards or Monitoring Constituents listed in Appendix A for First Quarter 2000. 
Table D-6. Groundwater Monitoring Results for Point-of-Compliance Wells, Met Lab HWMF (Cont.) WELL AMB 10D

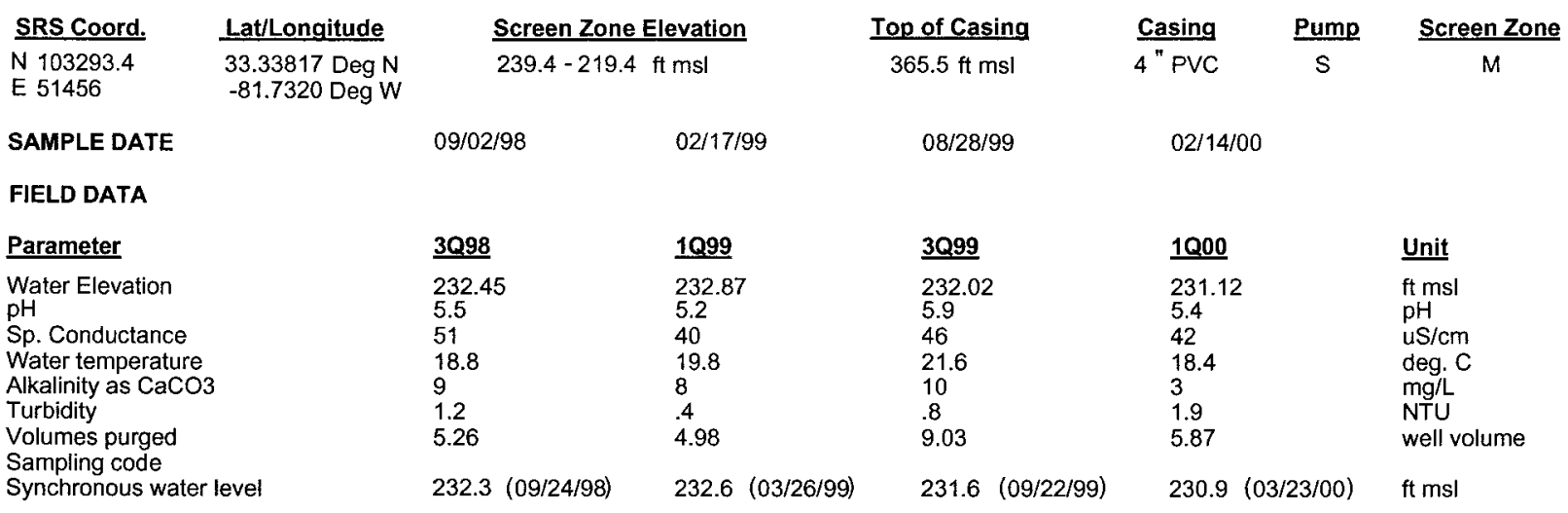

ANALYTICAL DATA

I. Groundwater Protection Standard

261 Appendix VIII/264 Appendix IX Hazardous Constituents

\begin{tabular}{|c|c|c|c|c|c|c|c|c|c|c|c|c|c|c|}
\hline \multirow{2}{*}{$\begin{array}{l}\text { ST Parameter } \\
\text { Inorganics }\end{array}$} & \multirow[t]{2}{*}{$\underline{3098}$} & \multirow[t]{2}{*}{ CLPEPA } & \multirow[t]{2}{*}{1099} & CLP EPA & \multirow[t]{2}{*}{$\underline{3099}$} & \multicolumn{2}{|c|}{ CLPEPA } & \multirow[t]{2}{*}{1000} & \multirow{2}{*}{\multicolumn{2}{|c|}{ CLPEPA }} & \multirow[t]{2}{*}{ Filt. } & \multirow[t]{2}{*}{ DF } & \multirow[t]{2}{*}{ Unit } & \multirow[t]{2}{*}{$\underline{L a b}$} \\
\hline & & & & & & & & & & & & & & \\
\hline Barium, total recoverable & 5 & & 5.99 & & $<4.8$ & $u$ & V & 14.4 & & & $<2000$ & 1 & ug/L & EX \\
\hline Cyanide & $<15.2$ & $u$ & $<15.2$ & $\mathrm{u}$ & $<15.2$ & $\mathrm{u}$ & & $<10$ & $\mathrm{u}$ & & $<E Q L$ & 1 & $\mathrm{ug} / \mathrm{L}$ & EX \\
\hline Lead, total recoverable & $<47$ & $u$ & $<47$ & $u$ & $<47$ & $u$ & & $<10$ & $\mathrm{u}$ & & $<\mathrm{EQL}$ & 1 & $\mathrm{ug} / \mathrm{L}$ & EX \\
\hline Nickel, total recoverable & $<26$ & $\mathrm{u}$ & $<26$ & $\mathrm{u}$ & $<26$ & $\mathrm{U}$ & & $<50$ & $\mathrm{u}$ & & $<E Q L$ & 1 & $\mathrm{ug} / \mathrm{L}$ & EX \\
\hline Selenium, total recoverable & $<66$ & $\mathrm{u}$ & $<66$ & $\mathrm{u}$ & $<66$ & $u$ & & $<10$ & $u$ & & $<\mathrm{EQL}$ & 1 & $\mathrm{ug} / \mathrm{L}$ & EX \\
\hline \multicolumn{15}{|l|}{ Organics } \\
\hline Chlorobenzene & $<5$ & $\mathrm{u}$ & $<5$ & $u$ & $<5$ & sU & $Q$ & $<5$ & $\mathrm{u}$ & & $<E Q L$ & 1 & $\mathrm{ug} / \mathrm{L}$ & EX \\
\hline 1,1-Dichloroethane & $<5$ & $u$ & $<5$ & $\mathrm{u}$ & $<5$ & JU & $\hat{Q}$ & $<5$ & $u$ & & $<E Q L$ & 1 & $\mathrm{ug} / \mathrm{L}$ & EX \\
\hline 1,1-Dichloroethylene & $<5$ & u & $<5$ & $\mathrm{u}$ & $<5$ & $\mathrm{JU}$ & $Q$ & $<5$ & $\mathrm{u}$ & & $<E Q L$ & 1 & $\mathrm{ug} / \mathrm{L}$ & EX \\
\hline trans-1,2-Dichloroethylene & $<5$ & $\mathrm{u}$ & $<5$ & $\mathrm{u}$ & $<5$ & $\mathrm{JU}$ & $Q$ & $<5$ & $\mathrm{u}$ & & $<E Q L$ & 1 & $\mathrm{ug} / \mathrm{L}$ & EX \\
\hline РСВ 1016 & & & $<1.02$ & $u$ & & & & & & & & & $\mathrm{ug} / \mathrm{L}$ & \\
\hline PCB 1221 & & & $<2.04$ & $u$ & & & & & & & & & $\mathrm{ug} / \mathrm{L}$ & \\
\hline PCB 1232 & & & $<1.02$ & $u$ & & & & & & & & & $\mathrm{ug} / \mathrm{L}$ & \\
\hline РCB 1242 & & & $<1.02$ & $u$ & & & & & & & & & $\mathrm{ug} / \mathrm{L}$ & \\
\hline PCB 1248 & & & $<1.02$ & $u$ & & & & & & & & & $\mathrm{ug} / \mathrm{L}$ & \\
\hline PCB 1254 & & & $<1.02$ & $u$ & & & & & & & & & $\mathrm{ug} / \mathrm{L}$ & \\
\hline PCB 1260 & & & $<1.02$ & $u$ & & & & & & & & & $\mathrm{ug} / \mathrm{L}$ & \\
\hline 1,1,2,2-Tetrachloroethane & $<5$ & $u$ & $<5$ & u & $<5$ & JU & $Q$ & $<5$ & $\mathrm{U}$ & & $<E Q L$ & 1 & $\mathrm{ug} / \mathrm{L}$ & EX \\
\hline Tetrachloroethylene & $<5$ & $u$ & $<5$ & $u$ & $<5$ & JU & $\mathbf{Q}$ & $<5$ & $\mathrm{U}$ & & $<E Q L$ & 1 & $\mathrm{ug} / \mathrm{L}$ & EX \\
\hline 1,1,1-Trichloroethane & $<5$ & $\mathrm{u}$ & $<5$ & $u$ & $<5$ & JU & $Q$ & $<5$ & $\mathrm{U}$ & & $<E Q L$ & 1 & $\mathrm{ug} / \mathrm{L}$ & EX \\
\hline Trichloroethylene & $<5$ & $u$ & $<5$ & $\mathrm{U}$ & $<5$ & jU & $\mathrm{Q}$ & $<5$ & $u$ & & $<E Q L$ & 1 & $\mathrm{ug} / \mathrm{L}$ & EX \\
\hline \multicolumn{15}{|l|}{ II. Monitoring Constituents } \\
\hline ST Parameter & $\underline{3098}$ & CLPPEPA & $\underline{1099}$ & CLPPEPA & $\underline{3099}$ & CLP & EPA & $\underline{10,00}$ & $\underline{\text { CLP }}$ & EPA & Filt. & $\underline{\text { DF }}$ & $\underline{\text { Unit }}$ & $\underline{\text { Lab }}$ \\
\hline \multicolumn{15}{|l|}{ Inorganics } \\
\hline Aluminum, total recoverable & 19.6 & $J$ & $<62.5$ & U V & 432 & & & $<176$ & $\mathbf{u}$ & $v$ & $<\mathrm{EQL}$ & 1 & $\mathrm{ug} / \mathrm{L}$ & EX \\
\hline Nitrate-nitrite as nitrogen & 60 & & 66 & & 80 & & & $<500$ & $\mathrm{u}$ & & $<E Q L$ & 5 & $\mathrm{ug} / \mathrm{L}$ & EX \\
\hline+ Sodium, total recoverable & 8050 & & 8060 & & 8440 & & & 8980 & & & $>4600$ & 1 & $\mathrm{ug} / \mathrm{L}$ & EX \\
\hline+ Sulfate & 3490 & & 4160 & & 4450 & & & 4270 & & & $>3000$ & 2 & $\mathrm{ug} / \mathrm{L}$ & EX \\
\hline \multicolumn{15}{|l|}{ Radionuclides } \\
\hline Gross alpha & 1.45 & 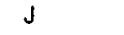 & .81 & J 1 & .0639 & $\mathrm{U}$ & & .978 & J & 1 & NDD & 1 & $\mathrm{pCi} / \mathrm{L}$ & GP \\
\hline Nonvolatile beta & 1.2 & UIJ & .74 & $\mathrm{u}$ & -.207 & $U$ & & .857 & $u$ & & $<E Q L$ & 1 & $\mathrm{pCi} / \mathrm{L}$ & GP \\
\hline Radium, total alpha-emitting & .25 & UI & .97 & $U \mathrm{~V}$ & .3 & $\mathrm{~J}$ & 1 & .25 & u & & $<\mathrm{EQL}$ & 1 & $\mathrm{pCi} / \mathrm{L}$ & GP \\
\hline
\end{tabular}

\footnotetext{
Notes: Concentrations in bold italics exceed the groundwater protection or monitoring constituent standards listed in Appendix A. Synchronous water levels are measured over a 3-5 day period or less. Dilution factors, Laboratory, and Filtered Data are for First Quarter 2000 data only.

$+=$ exceeded the Groundwater Protection Standards or Monitoring Constituents listed in Appendix A for First Quarter 2000.
} 
Table D-6. Groundwater Monitoring Results for Point-of-Compliance Wells, Met Lab HWMF (Cont.) WELL AMB 16D

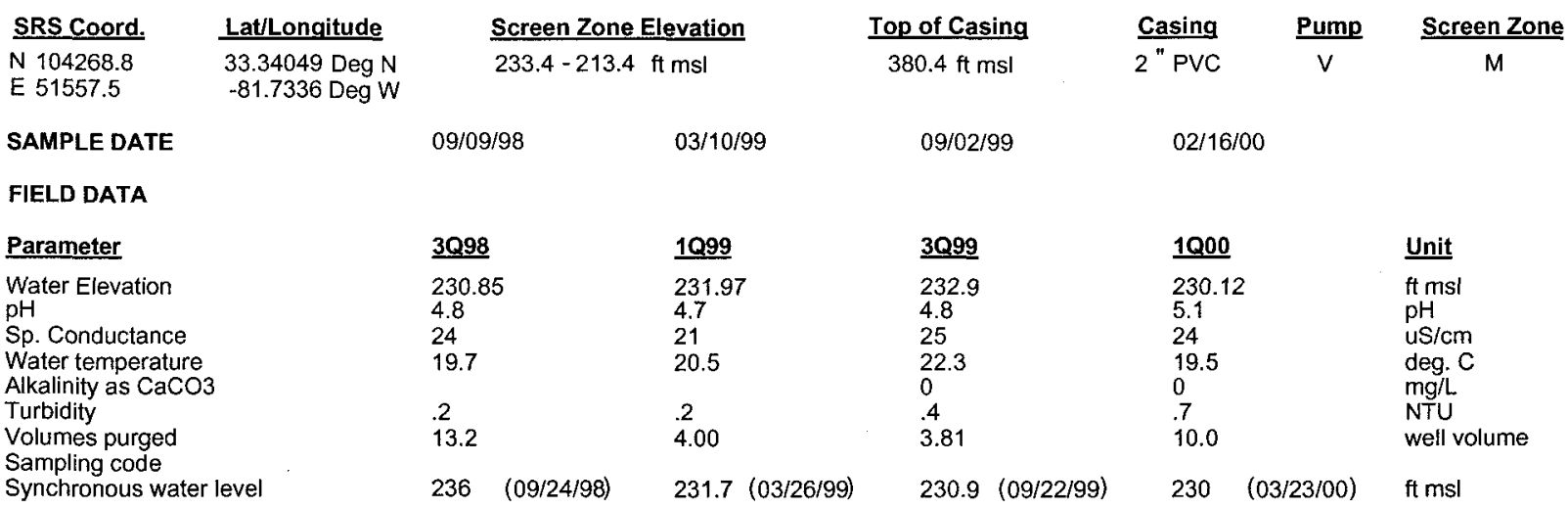

ANALYTICAL DATA

I. Groundwater Protection Standard

261 Appendix VIII/264 Appendix IX Hazardous Constituents

ST Parameter

Inorganics

Barium, total recoverable

Cyanide

Lead, total recoverable

Nickel, total recoverable

Selenium, total recoverable

Organics

Chlorobenzene

1,1-Dichloroethane

1,1-Dichloroethylene

trans-1,2-Dichloroethylene

PCB 1016

PCB 1221

PCB 1232

PCB 1242

PCB 1248

PCB 1254

PCB 1260

1,1,2,2-Tetrachloroethane

Tetrachloroethylene

1,1,1-Trichloroethane

Trichloroethylene

II. Monitoring Constituents

ST Parameter

Inorganics

Nitrate-nitrite as nitrogen

Sodium, total recoverable

Sulfate

Radionuclides

Gross alpha

Nonvolatile beta

Radium, total alpha-emitting

\section{9}

CLPEPA

1099

CLPP EPA

3Q99

CLPEPA

1000

$\begin{array}{ll} & 4.2 \\ <15.2 & U \\ <47 & U \\ <26 & U \\ <66 & U\end{array}$

$\begin{aligned} & 4.6 \\ &< 15.2 \\ &< 47 \\ &<<6 \\ &<66\end{aligned}$

$<5 \quad$

$\begin{array}{ll}< & U \\ <5 & U\end{array}$

$<5$

U

$<5$
$<5$
$<5$
$<5$

$U$
$U$
$U$

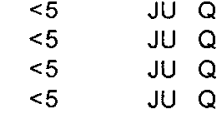

$\begin{array}{ll}<5 & U \\ <5 & U \\ <5 & U \\ 4.13 & J\end{array}$

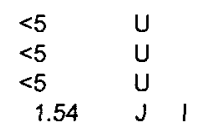

$<5$

$$
<5
$$$$
<5
$$

3098 CLPEPA

1099 CLPEPA

3Q99

$\begin{aligned} & 4 \\ &< 15.2 \\ & 27.8 \\ &< 26 \\ &< 66 \\ & \\ &< 5 \\ &< 5 \\ &<5 \\ &<5\end{aligned}$

$\begin{array}{ll}U \\ J \\ U & \\ U & \end{array}$

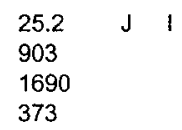

6.35

1.38
2.72

$\begin{array}{lll}7.09 & & 6.35 \\ 3.31 & \mathrm{U} & 1.38 \\ 1.26 & & 2.72\end{array}$

$\begin{array}{lllll}<4.35 & \mathrm{U} & \mathrm{V} & <\mathrm{EQL} & 1 \\ <10 & \mathrm{U} & & <\mathrm{EQL} & 1\end{array}$

$<10$ JU $V<$ EQL $1 \quad \mathrm{ug} / \mathrm{L}$

ug/L EX

$<5 \quad \mathrm{U} \quad<E Q L 1 \quad$ ug/L $\quad E X$

$<5 \quad \mathrm{U} \quad<\mathrm{EQL} 1 \mathrm{ug} / \mathrm{L} \quad \mathrm{EX}$

$<5 \quad U \quad<E Q L 11$ ug/L $\quad E X$

$<5 \quad U \quad<E Q L 11 \mathrm{ug} / \mathrm{L} \quad \mathrm{EX}$

$<.94 \quad \mathrm{U} \quad<$ EQL $.940 \mathrm{ug} / \mathrm{L} \quad \mathrm{EX}$

$<94 \quad U<E Q 1940 \quad$ ugl $\quad E X$

$<.94 \quad \mathrm{U} \quad<\mathrm{EQL} .940 \quad \mathrm{ug} / \mathrm{L}$

$<1.9 \quad \mathrm{U}<\mathrm{EQL} 940 \mathrm{ug} / \mathrm{L}$

$<.94 \quad \mathrm{U} \quad<$ EQL $.940 \quad \mathrm{ug} / \mathrm{L}$

$<.94 \quad \mathrm{U}<\mathrm{EQL} .940 \mathrm{ug} / \mathrm{L}$

$<.94 \quad \mathrm{U}<\mathrm{EQL} .940 \mathrm{ug} / \mathrm{h}$

$<\mathrm{EQL} 1 \mathrm{ug} / \mathrm{l}$

$<$ EQL $1 \mathrm{ug} / \mathrm{l}$

$<$ EQL 1 ug/

NDD 1 ug/L

3.1

J 1

(2)

1000 CLPEPA Filt. DF Unit Lab

$59.8 \quad J$
1400
2920
414

$\begin{array}{lllr}36.5 & \mathrm{~J} & \mathrm{I} & \text { NDD } \\ 1320 & & & <2400 \\ 3380 & & & <4600 \\ 768 & \mathrm{~J} & \mathrm{I} & \text { NDD }\end{array}$

ug/L EX

ug/L EX

$\mathrm{ug} / \mathrm{L} \quad \mathrm{EX}$

4.07

2.79

1.6 $\begin{array}{llll}<15 & 1 & \mathrm{pCi} / \mathrm{L} & \mathrm{GP} \\ <50 & 1 & \mathrm{pCi} / \mathrm{L} & \mathrm{GP} \\ <5 & 1 & \mathrm{pCi} / \mathrm{L} & \mathrm{GP}\end{array}$

Notes: Concentrations in bold italics exceed the groundwater protection or monitoring constituent standards listed in Appendix A. Synchronous water levels are measured over a 3-5 day period or less. Dilution factors, Laboratory, and Filtered Data are for First Quarter 2000 data only.

$+=$ exceeded the Groundwater Protection Standards or Monitoring Constituents listed in Appendix A for First Quarter 2000. 
Table D-6. Groundwater Monitoring Results for Point-of-Compliance Wells, Met Lab HWMF (Cont.) WELL AMB 17A

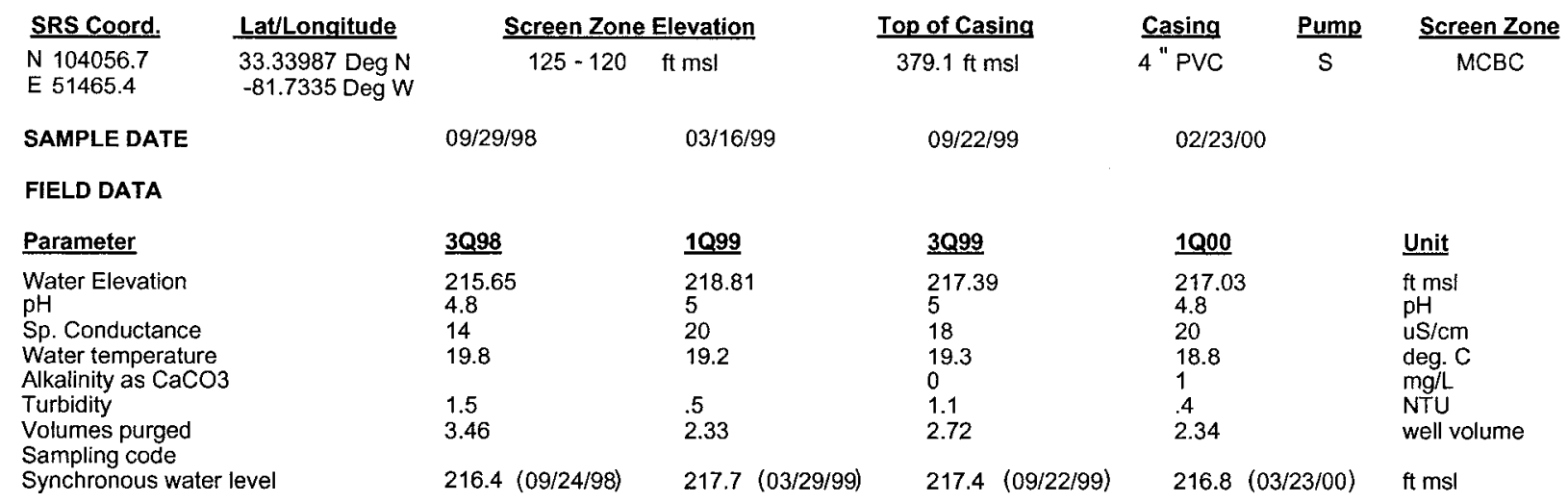

ANALYTICAL DATA

I. Groundwater Protection Standard

261 Appendix VIII/264 Appendix IX Hazardous Constituents

\begin{tabular}{|c|c|c|c|c|c|c|c|c|c|c|c|c|c|}
\hline \multirow{2}{*}{$\begin{array}{l}\text { ST Parameter } \\
\text { Inorganics }\end{array}$} & \multirow[t]{2}{*}{$\underline{3 Q 98}$} & \multirow[t]{2}{*}{ CLPEPA } & \multirow[t]{2}{*}{1099} & CLPEPA & \multirow[t]{2}{*}{$\underline{3 Q 99}$} & \multirow[t]{2}{*}{ CLPEPA } & \multirow[t]{2}{*}{$\underline{1000}$} & \multicolumn{2}{|c|}{ CLPEPA } & \multirow[t]{2}{*}{ Filt. } & \multirow[t]{2}{*}{$\underline{\text { DF }}$} & \multirow[t]{2}{*}{$\underline{\text { Unit }}$} & \multirow[t]{2}{*}{$\underline{\text { Lab }}$} \\
\hline & & & & & & & & & & & & & \\
\hline Barium, total recoverable & 2.8 & & 3.2 & & 2.7 & & $<3.28$ & $u$ & v & $<$ EQL & 1 & $\mathrm{ug} / \mathrm{L}$ & EX \\
\hline Cyanide & $<15.2$ & $u$ & $<15.2$ & $u$ & $<15.2$ & u & $<10$ & $\mathrm{u}$ & & $<\mathrm{EQL}$ & 1 & $\mathrm{ug} / \mathrm{L}$ & EX \\
\hline Lead, total recoverable & 40.3 & $\mathrm{~J}$ & $<47$ & $\mathrm{u}$ & $<47$ & $\mathrm{u}$ & $<10$ & $\mathrm{u}$ & & $<\mathrm{EQL}$ & 1 & $\mathrm{ug} / \mathrm{L}$ & EX \\
\hline Nickel, total recoverable & 27.3 & $\mathrm{~J}$ & $<26$ & $u$ & $<26$ & $\mathrm{u}$ & $<50$ & $\mathrm{u}$ & & $<E Q L$ & 1 & $\mathrm{ug} / \mathrm{L}$ & EX \\
\hline Selenium, total recoverable & $<200$ & $u$ & $<66$ & $\mathrm{u}$ & $<66$ & $u$ & $<10$ & $\mathrm{u}$ & & $<E Q L$ & 1 & $\mathrm{ug} / \mathrm{L}$ & EX \\
\hline \multicolumn{14}{|l|}{ Organics } \\
\hline Chlorobenzene & $<25$ & $u$ & $<10$ & $u$ & $<10$ & $\mathrm{u}$ & $<5$ & $u$ & & $<\mathrm{EQL}$ & 1 & $\mathrm{ug} / \mathrm{L}$ & EX \\
\hline 1,1-Dichloroethane & $<25$ & $u$ & $<10$ & $\mathrm{u}$ & $<10$ & $u$ & $<5$ & $u$ & & $<\mathrm{EQL}$ & 1 & $\mathrm{ug} / \mathrm{L}$ & EX \\
\hline 1,1-Dichloroethylene & $<25$ & $\mathrm{u}$ & $<10$ & $\mathrm{U}$ & $<10$ & $\mathrm{u}$ & $<5$ & $\mathrm{U}$ & & $<E Q L$ & 1 & $\mathrm{ug} / \mathrm{L}$ & EX \\
\hline trans-1,2-Dichloroethylene & $<25$ & $u$ & $<10$ & $\mathrm{u}$ & $<10$ & $\mathrm{u}$ & $<5$ & $\mathrm{U}$ & & $<\mathrm{EQL}$ & 1 & $\mathrm{ug} / \mathrm{L}$ & EX \\
\hline PCB 1016 & & & $<1.02$ & JU $Q$ & & & $<.98$ & $\mathrm{U}$ & & $<E Q L$ & .980 & $\mathrm{ug} / \mathrm{L}$ & EX \\
\hline PCB 1221 & & & $<2.04$ & JU $Q$ & & & $<.98$ & $\mathrm{u}$ & & $<\mathrm{EQL}$ & .980 & $\mathrm{ug} / \mathrm{L}$ & EX \\
\hline PCB 1232 & & & $<1.02$ & JU Q & & & $<.98$ & $U$ & & $<E Q L$ & .980 & $\mathrm{ug} / \mathrm{L}$ & EX \\
\hline РСВ 1242 & & & $<1.02$ & JU $Q$ & & & $<2$ & $u$ & & $<E Q L$ & .980 & $\mathrm{ug} / \mathrm{L}$ & EX \\
\hline РСВ 1248 & & & $<1.02$ & JU $Q$ & & & $<.98$ & $\mathrm{u}$ & & $<E Q L$ & .980 & $\mathrm{ug} / \mathrm{L}$ & EX \\
\hline РCB 1254 & & & $<1.02$ & $J U Q$ & & & $<, 98$ & $u$ & & $<E Q L$ & .980 & $\mathrm{ug} / \mathrm{L}$ & EX \\
\hline PCB 1260 & & & $<1.02$ & $J U Q$ & & & $<.98$ & $u$ & & $<E Q L$ & .980 & $\mathrm{ug} / \mathrm{h}$ & EX \\
\hline 1,1,2,2-Tetrachloroethane & $<25$ & $\mathrm{u}$ & $<10$ & $u$ & $<10$ & $u$ & $<5$ & U & & $<E Q L$ & 1 & $\mathrm{ug} / \mathrm{L}$ & EX \\
\hline Tetrachloroethylene & 22.8 & & 26 & & 26.3 & & 25 & & & $>5$ & 1 & ug/L & EX \\
\hline 1,1,1-Trichloroethane & $<25$ & $\mathrm{U}$ & $<10$ & $\mathrm{u}$ & $<10$ & $\mathrm{U}$ & $<5$ & $u$ & & $<E Q L$ & 1 & $\mathrm{ug} / \mathrm{L}$ & EX \\
\hline+ Trichloroethylene & 241 & & 208 & & 176 & & 170 & & & $>5$ & 1 & $\mathrm{ug} / \mathrm{L}$ & EX \\
\hline \multicolumn{14}{|l|}{ II. Monitoring Constituents } \\
\hline ST Parameter & $\underline{3098}$ & CLPEPA & 1099 & CLLP EPA & $\underline{3099}$ & CLPEPA & $\underline{1000}$ & CLP & EPA & Filt. & $\underline{D F}$ & $\underline{\text { Unit }}$ & $\underline{\text { Lab }}$ \\
\hline \multicolumn{14}{|l|}{ Inorganics } \\
\hline Aluminum, total recoverable & $<200$ & $u$ & $<146$ & $u$ & $<146$ & $u$ & 39.8 & J & 1 & NDD & 1 & $u g / L$ & EX \\
\hline Nitrate-nitrite as nitrogen & 868 & & 857 & & 926 & & 838 & & & $<2400$ & 5 & $\mathrm{ug} / \mathrm{L}$ & EX \\
\hline Sodium, total recoverable & 2040 & & 1700 & & 1680 & & 1870 & & & $<4600$ & 1 & $u g / L$ & EX \\
\hline Sulfate & 514 & & 487 & & 452 & & $<636$ & U & & $<E Q L$ & 1 & $\mathrm{ug} / \mathrm{L}$ & EX \\
\hline \multicolumn{14}{|l|}{ Radionuclides } \\
\hline Gross alpha & 1.21 & & .38 & $\mathrm{u}$ & .122 & $\mathrm{u}$ & .0614 & $\mathrm{u}$ & & $<E Q L$ & 1 & $\mathrm{pCi} / \mathrm{L}$ & GP \\
\hline Nonvolatile beta & 10.04 & & .46 & u & .497 & $\mathrm{u}$ & .217 & $u$ & & $<E Q L$ & 1 & $\mathrm{pCi} / \mathrm{L}$ & GP \\
\hline Radium, total alpha-emitting & .32 & UI & .48 & $u$ & -.1 & $u$ & .055 & $u$ & & $<E Q L$ & 1 & $\mathrm{pCi} / \mathrm{L}$ & GP \\
\hline
\end{tabular}

Notes: Concentrations in bold italics exceed the groundwater protection or monitoring constituent standards listed in Appendix A. Synchronous water levels are measured over a 3-5 day period or less. Dilution factors, Laboratory, and Filtered Data are for First Quarter 2000 data only.

$+=$ exceeded the Groundwater Protection Standards or Monitoring Constituents listed in Appendix A for First Quarter 2000. 
Table D-6. Groundwater Monitoring Results for Point-of-Compliance Wells, Met Lab HWMF (Cont.) WELL AMB 18A

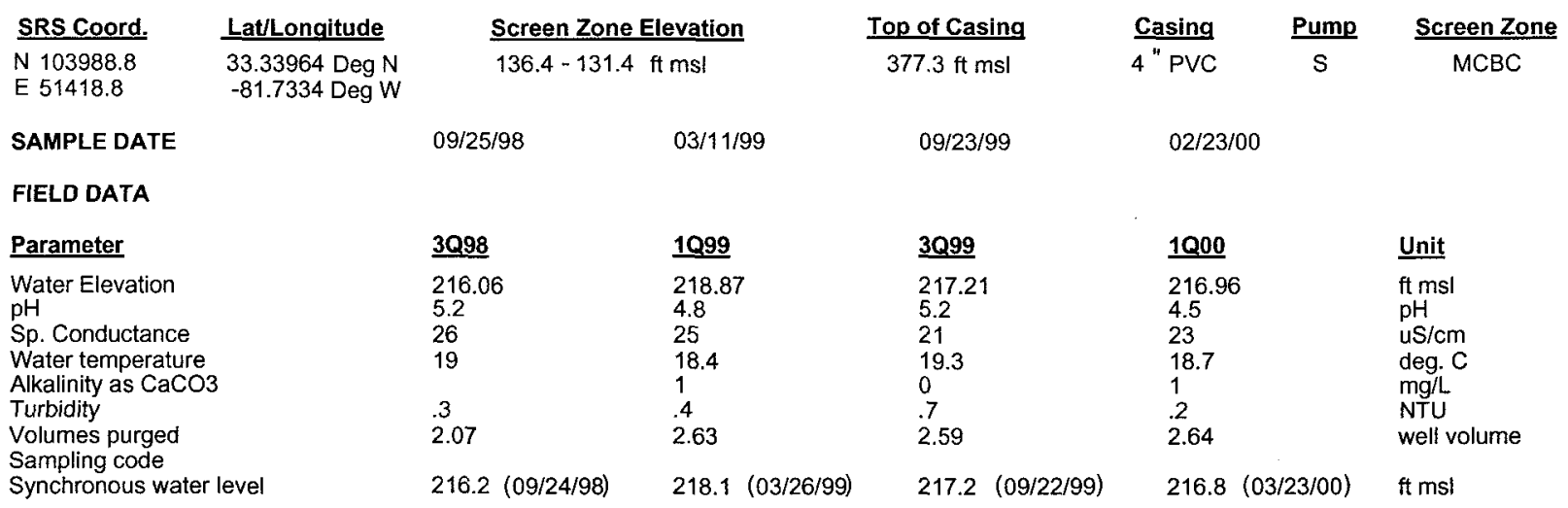

ANALYTICAL DATA

I. Groundwater Protection Standard

261 Appendix VIII/264 Appendix IX Hazardous Constituents

ST Parameter

Inorganics

Barium, total recoverable

Cyanide

Lead, total recoverable

Nickel, total recoverable

Selenium, total recoverable

Organics

Chlorobenzene

1,1-Dichloroethane

1,1-Dichloroethylene

trans-1,2-Dichloroethylene

PCB 1016

PCB 1221

PCB 1232

PCB 1242

PCB 1248

PCB 1254

PCB 1260

1,1,2,2-Tetrachloroethane

Tetrachloroethylene

1,1,1-Trichloroethane

+ Trichloroethylene

II. Monitoring Constituents

ST Parameter

Inorganics

Aluminum, total recoverable

Nitrate-nitrite as nitrogen

Sodium, total recoverable

Sulfate

Radionuclides

Gross alpha

Nonvolatile beta

Radium, total alpha-emitting

\section{$\underline{3 Q 98}$}

$\begin{array}{ll}6.1 & \\ <15.2 & U J \\ <47 & U \\ <26 & U \\ <66 & U\end{array}$

$<5 \quad$ U

$<5 \quad \mathrm{U}$

$<5$

$\begin{array}{ll} & \\ & \\ & \\ 4 & \\ 4.59 & \\ <5 & \\ 36.8\end{array}$

3098 CLPEPA

1099

$<55.5 \quad U$
813
2180

$<146$
790

471 J

1920
442

$\begin{array}{ll}.28 & \text { UIJ } \\ 1.64 & \text { UI } \\ .35 & \text { UI }\end{array}$

$$
\begin{aligned}
& \quad 5.7 \\
& <15.2 \\
& <47 \\
& <26 \\
& <66
\end{aligned}
$$

CLP EPA

3Q99

\section{CLPEPA}

\section{0}

CLPEPA

$\begin{array}{ll} & 5.4 \\ <15.2 & U \\ <47 & U \\ <26 & U \\ <66 & U\end{array}$

$\begin{array}{lll} & & \\ <14.7 & \mathrm{U} & <2000 \\ <10 & \mathrm{E} & <\mathrm{EQL} \\ <10 & \mathrm{U} & <\mathrm{EQL} \\ <6.24 & \mathrm{JU} V & <\mathrm{EQL} \\ <10 & \mathrm{U} & <\mathrm{EQL}\end{array}$

ug/L

ug/L

ug/L

ug/L

$\mathrm{ug} / \mathrm{L}$

$\begin{array}{llll}<5 & U & <5 & U \\ <5 & U & <5 & U \\ <5 & U & <5 & U \\ <5 & U & <5 & U\end{array}$

$<5$
$<5$
$<5$

$<5$

$<.96$

$<2.04$ JU $Q$

$<1.02$ JU $Q$

$<1.02$ JU $Q$

$<1.02$ JU $Q$

$<1.02$ JU $Q$

$<1.02$ JU Q

$\begin{array}{ll}<5 & \mathrm{U} \\ <5 & \mathrm{U} \\ <5 & \mathrm{U} \\ 27.8 & \end{array}$

$\begin{array}{lll}<5 & U & \\ 2.36 & \mathrm{~J} & \text { I } \\ <5 & \mathrm{U} & \\ 19.3 & & \end{array}$

$<.96$
$<1.9$

$<.96$

$<.96$

$<.96 \quad u$

$\begin{array}{ccc}<5 & \mathrm{U} & \\ 4.6 & \mathrm{~J} & \text { । } \\ <5 & \mathrm{U} & \end{array}$

$<\mathrm{EQL}$

ug/L EX

$<$ EQL 1 ug/L EX

$\mathrm{U}<\mathrm{EQL} 1 \mathrm{ug} / \mathrm{L} \quad \mathrm{EX}$

$<$ EQL 11 ug/L EX

U < EQL $.960 \quad \mathrm{ug} / \mathrm{L} \quad \mathrm{EX}$

$<E Q L .960$ ug/t

$\mathrm{U} \quad<\mathrm{EQL} .960 \mathrm{ug} / \mathrm{L}$

U $<E Q L .960$ ug/

$<E Q L .960$ ug/L

$<$ EQL $.960 \mathrm{ug} / \mathrm{h}$

$<$ EQL $.960 \mathrm{ug} / \mathrm{L}$

$<$ EQL 1 ug/l

NDD 1 ug/L

$<\mathrm{EQL} \quad 1 \quad \mathrm{ug} / \mathrm{L}$

$\begin{array}{lll}>5 & 1 & \mathrm{ug} / \mathrm{L}\end{array}$

EX

3099 CLPEPA 1000 CLPEPA Filt. DF Unit Lab

\section{CLPEPA}

$<146 \quad U$
795
1900
432

\begin{tabular}{|c|c|c|}
\hline$<200$ & u & $<E Q L$ \\
\hline 734 & & $<2400$ \\
\hline 2310 & & $<4600$ \\
\hline$<555$ & U & $<E Q L$ \\
\hline
\end{tabular}

$\mathrm{ug} / \mathrm{L} \quad \mathrm{EX}$

$u g / L \quad E X$

ug/L EX

$\begin{array}{lll}.72 & \mathrm{U} & \\ -.45 & \mathrm{U} & \\ 1.01 & \mathrm{~J} & \mathrm{I}\end{array}$

$\begin{array}{lllllll}.838 & \mathrm{~J} & \mathrm{I} & \mathrm{NDD} & 1 & \mathrm{pCi} / \mathrm{L} & \mathrm{GP} \\ .827 & \mathrm{U} & & <\mathrm{EQL} & 1 & \mathrm{pCi} / \mathrm{L} & \mathrm{GP} \\ -.081 & \mathrm{U} & & <\mathrm{EQL} & 1 & \mathrm{pCi} / \mathrm{L} & \mathrm{GP}\end{array}$

Notes: Concentrations in bold italics exceed the groundwater protection or monitoring constituent standards listed in Appendix A. Synchronous water levels are measured over a 3-5 day period or less. Dilution factors, Laboratory, and Filtered Data are for First Quarter 2000 data only.

$+=$ exceeded the Groundwater Protection Standards or Monitoring Constituents listed in Appendix A for First Quarter 2000. 
Table D-6. Groundwater Monitoring Results for Point-of-Compliance Wells, Met Lab HWMF (Cont.) WELL AMB $18 \mathrm{C}$

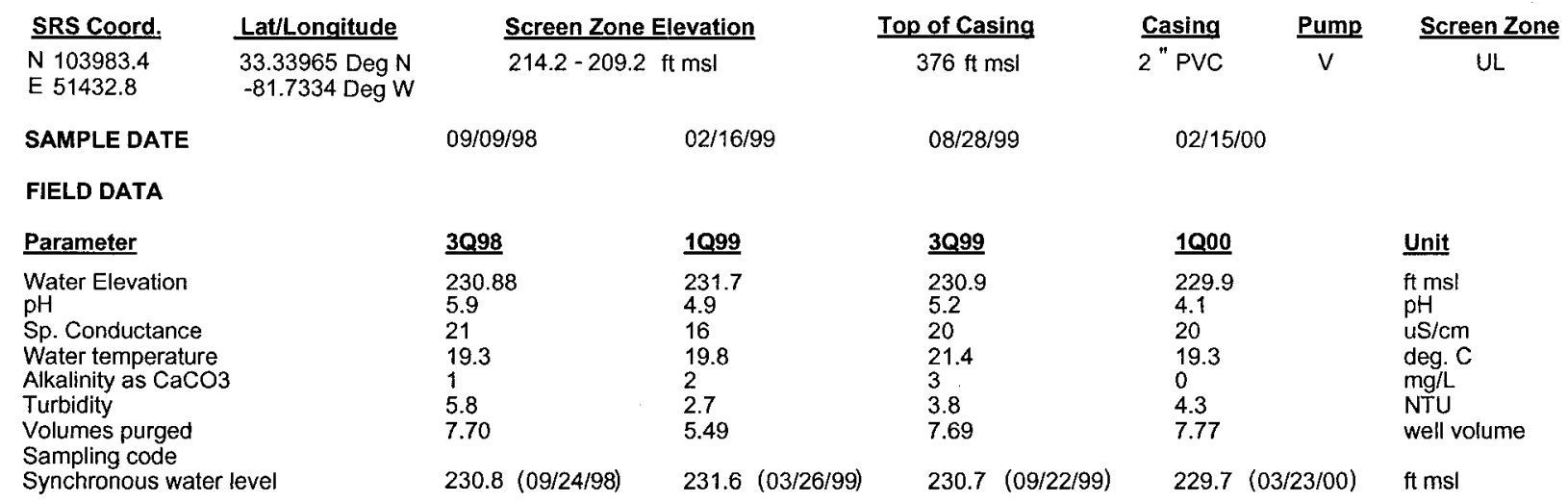

ANALYTICAL DATA

I. Groundwater Protection Standard

261 Appendix VIII/264 Appendix IX Hazardous Constituents

\begin{tabular}{|c|c|c|c|c|c|c|c|c|c|c|c|c|c|c|c|}
\hline \multirow{2}{*}{$\begin{array}{l}\text { ST Parameter } \\
\text { Inorganics }\end{array}$} & \multirow[t]{2}{*}{$\underline{3 Q 98}$} & \multirow[t]{2}{*}{ CLPEPA } & \multirow[t]{2}{*}{$\underline{1099}$} & \multicolumn{2}{|c|}{ CLPEPA } & \multirow[t]{2}{*}{$\underline{3099}$} & \multicolumn{2}{|c|}{ CLPEPA } & \multirow[t]{2}{*}{$\underline{1000}$} & \multicolumn{2}{|c|}{ CLPEPA } & \multirow[t]{2}{*}{ Filt. } & \multirow[t]{2}{*}{$\underline{\mathrm{DF}}$} & \multirow[t]{2}{*}{$\underline{\text { Unit }}$} & \multirow[t]{2}{*}{$\underline{\text { Lab }}$} \\
\hline & & & & & & & & & & & & & & & \\
\hline Barium, total recoverable & 3.9 & & 3.7 & & & $<4.1$ & $\mathrm{u}$ & $\mathrm{v}$ & 4.25 & $\mathrm{~J}$ & 1 & NDD & 1 & ug/L & EX \\
\hline Cyanide & $<15.2$ & $\mathrm{u}$ & $<15.2$ & $\mathrm{u}$ & & $<15.2$ & $\mathrm{u}$ & & $<10$ & $\mathrm{U}$ & & $<E Q L$ & 1 & ug/L & EX \\
\hline + Lead, total recoverable & 52.4 & & 6.9 & $\mathrm{~J}$ & 1 & $<47$ & $\mathrm{u}$ & & 15.7 & & & $>15$ & 1 & $\mathrm{ug} / \mathrm{L}$ & EX \\
\hline Nickel, total recoverable & $<26$ & $\mathrm{u}$ & $<26$ & $u$ & & $<26$ & $\mathrm{u}$ & & $<6.96$ & $\mathrm{u}$ & $\mathrm{v}$ & $<E Q L$ & 1 & $\mathrm{ug} / \mathrm{L}$ & EX \\
\hline Selenium, total recoverable & $<66$ & $u$ & $<66$ & $\mathrm{u}$ & & $<66$ & $\mathrm{u}$ & & $<10$ & $\mathrm{u}$ & & $<E Q L$ & 1 & ug/L & EX \\
\hline \multicolumn{16}{|l|}{ Organics } \\
\hline Chlorobenzene & $<5$ & $\mathrm{u}$ & $<5$ & $u$ & & $<5$ & JU & $Q$ & $<5$ & $\mathrm{u}$ & & $<E Q L$ & 1 & $\mathrm{ug} / \mathrm{L}$ & EX \\
\hline 1,1-Dichloroethane & $<5$ & $\mathrm{u}$ & $<5$ & U & & $<5$ & JU & $\mathrm{Q}$ & $<5$ & u & & $<\mathrm{EQL}$ & 1 & $\mathrm{ug} / \mathrm{L}$ & EX \\
\hline 1,1-Dichloroethylene & $<5$ & $u$ & $<5$ & U & & $<5$ & JU & $\mathrm{Q}$ & $<5$ & $u$ & & $<\mathrm{EQL}$ & 1 & $\mathrm{ug} / \mathrm{L}$ & EX \\
\hline trans-1,2-Dichloroethylene & $<5$ & $u$ & $<5$ & $u$ & & $<5$ & JU & $Q$ & $<5$ & $\mathrm{u}$ & & $<E Q L$ & 1 & $\mathrm{ug} / \mathrm{L}$ & EX \\
\hline PCB 1016 & & & $<1.01$ & u & & & & & $<.97$ & $\mathrm{u}$ & & $<\mathrm{EQL}$ & .970 & ug/L & EX \\
\hline PCB 1221 & & & $<2.02$ & $u$ & & & & & $<.97$ & $\mathrm{u}$ & & $<E Q L$ & .970 & ug/h & EX \\
\hline PCB 1232 & & & $<1.01$ & $u$ & & & & & $<.97$ & $u$ & & $<E Q L$ & .970 & $\mathrm{ug} / \mathrm{L}$ & EX \\
\hline PCB 1242 & & & $<1.01$ & $u$ & & & & & $<1.9$ & $u$ & & $<E Q L$ & .970 & ug/L & EX \\
\hline PCB 1248 & & & $<1.01$ & $u$ & & & & & $<.97$ & $\mathrm{u}$ & & $<\mathrm{EQL}$ & .970 & ug/L & EX \\
\hline PCB 1254 & & & $<1.01$ & $u$ & & & & & $<.97$ & $u$ & & $<E Q L$ & .970 & $\mathrm{ug} / \mathrm{L}$ & EX \\
\hline РСВ 1260 & & & $<1.01$ & $u$ & & & & & $<.97$ & $u$ & & $<E Q L$ & .970 & $\mathrm{ug} / \mathrm{L}$ & EX \\
\hline 1,1,2,2-Tetrachloroethane & $<5$ & $u$ & $<5$ & $u$ & & $<5$ & JU & $Q$ & $<5$ & $\mathrm{U}$ & & $<E Q L$ & 1 & $\mathrm{ug} / \mathrm{L}$ & EX \\
\hline Tetrachloroethylene & $<5$ & $u$ & $<5$ & $u$ & & $<5$ & JU & $Q$ & $<5$ & $\mathrm{u}$ & & $<E Q L$ & 1 & $\mathrm{ug} / \mathrm{L}$ & EX \\
\hline 1,1,1-Trichloroethane & $<5$ & $u$ & $<5$ & $u$ & & $<5$ & JU & $Q$ & $<5$ & $\mathrm{u}$ & & $<E Q L$ & 1 & ug/L & EX \\
\hline Trichloroethylene & $<5$ & $u$ & $<5$ & $u$ & & $<5$ & JU & $\vec{Q}$ & $<5$ & $\mathrm{U}$ & & $<E Q L$ & 1 & $\mathrm{ug} / \mathrm{L}$ & EX \\
\hline \multicolumn{16}{|l|}{ II. Monitoring Constituents } \\
\hline ST Parameter & $\underline{3098}$ & CLPEPA & 1099 & CLP & EPA & $\underline{3099}$ & CLLP & EPA & 1000 & CLP & EPA & Filt. & $\underline{D F}$ & Unit & $\underline{\text { Lab }}$ \\
\hline \multicolumn{16}{|l|}{ Inorganics } \\
\hline Aluminum, total recoverable & 87.1 & $\mathrm{~J}$ & 97.7 & $\mathrm{~J}$ & 1 & 63.8 & $\mathrm{~J}$ & 1 & $<173$ & $\mathrm{u}$ & $\mathrm{v}$ & $<\mathrm{EQL}$ & 1 & $\mathrm{ug} / \mathrm{L}$ & EX \\
\hline Nitrate-nitrite as nitrogen & 884 & & 678 & & & 763 & & & 702 & & & $<2400$ & 5 & $\mathrm{ug} / \mathrm{L}$ & EX \\
\hline Sodium, total recoverable & 1960 & & 1970 & & & 1960 & & & 2370 & & & $<4600$ & 1 & $\mathrm{ug} / \mathrm{L}$ & EX \\
\hline Sulfate & 708 & & 1170 & & & 629 & & & 969 & $\mathrm{~J}$ & 1 & NDD & 2 & $\mathrm{ug} / \mathrm{L}$ & EX \\
\hline \multicolumn{16}{|l|}{ Radionuclides } \\
\hline Gross alpha & 1.07 & & .83 & J & IK & 1.39 & $\mathrm{~J}$ & IK & 2.32 & Ј & 1 & NDD & 1 & $\mathrm{pCi} / \mathrm{L}$ & GP \\
\hline Nonvolatile beta & .91 & UI & .85 & $\mathrm{u}$ & & .676 & u & & .709 & $u$ & & $<E Q L$ & 1 & $\mathrm{pCi} / \mathrm{L}$ & GP \\
\hline Radium, total alpha-emitting & 1.07 & & 1.73 & $\mathrm{~J}$ & 1 & .3 & $\mathrm{~J}$ & I & .402 & $\mathrm{~J}$ & 1 & NDD & 1 & $\mathrm{pCi} / \mathrm{L}$ & GP \\
\hline
\end{tabular}


Table D-6. Groundwater Monitoring Results for Point-of-Compliance Wells, Met Lab HWMF (Cont.) WELL AMB 19C

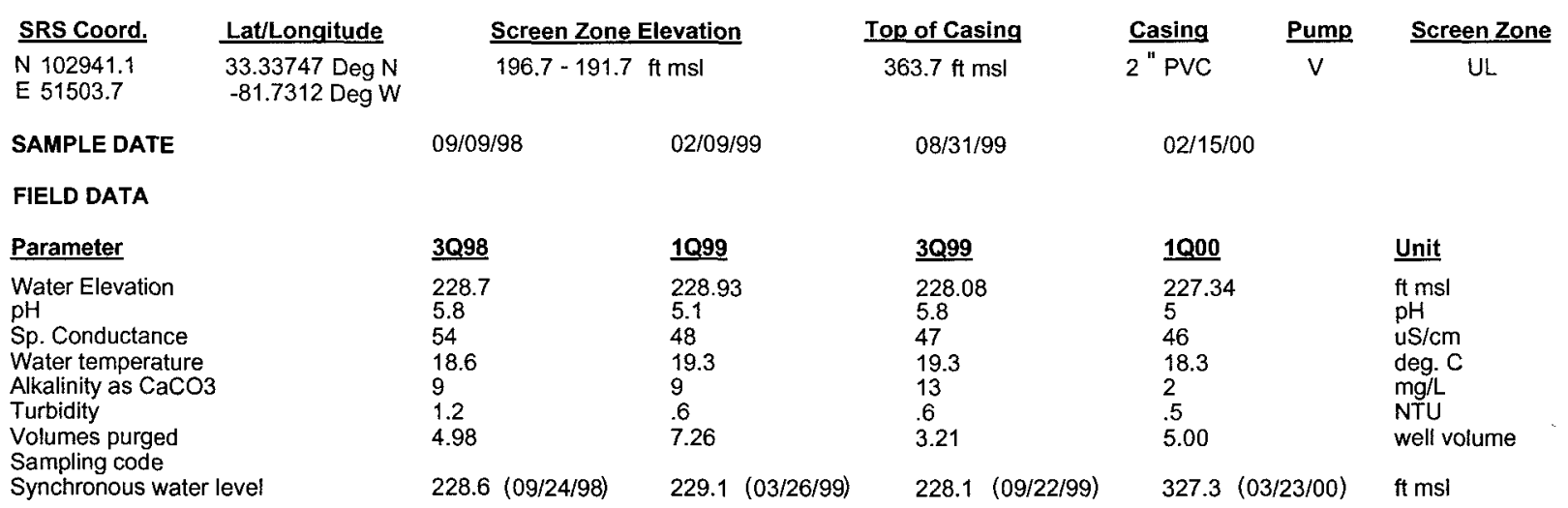

ANALYTICAL DATA

I. Groundwater Protection Standard

261 Appendix VIII/264 Appendix IX Hazardous Constituents

ST Parameter

Inorganics

Barium, total recoverable Cyanide

$+\quad$ Lead, total recoverable

Nickel, total recoverable

Selenium, total recoverable

$\begin{array}{ll}1.2 & J \\ <15.2 & U \\ <47 & U \\ <26 & U \\ <66 & U\end{array}$

$1 \mathrm{Q99}$

CLPEPA $\quad \underline{3 Q 99}$

CLPEPA 1000

CLPEPA Filt. DF Unit Lab Organics

Chlorobenzene

1,1-Dichloroethane

1,1-Dichloroethylene

trans-1,2-Dichloroethylene

PCB 1016

$\begin{array}{ll}<1.4 & U \\ <15.2 & U \\ <47 & U \\ <26 & U \\ <66 & U\end{array}$

2.2
2.5
$<47$
$<26$
$<66$

$\begin{array}{ll}J & 1 \\ U & \end{array}$

$<1.8$
$<10$
$\quad 98.8$
$<50$
$<10$

$<5$
$<5$
$<5$
$<5$

PCB 1221

PCB 1232

PCB 1242

PCB 1248

PCB 1254

PCB 1260

1,1,2,2-Tetrachloroethane

Tetrachloroethylene

1,1,1-Trichloroethane

Trichloroethylene

$\begin{array}{ll}<5 & U \\ <5 & U \\ <5 & U\end{array}$

$\begin{array}{ll}<5 & \cup \\ <5 & U \\ <5 & U \\ <5 & \mathrm{U}\end{array}$

$<5$
$<5$
$<5$
$<5$

$\mathrm{U}$
$\mathrm{U}$
$\mathrm{U}$

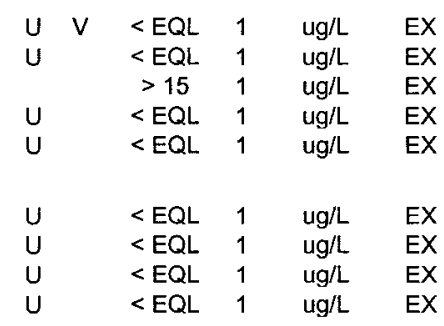

II. Monitoring Constituents

ST Parameter

Inorganics

Aluminum, total recoverable

$\begin{array}{ll}<5 & U \\ <5 & \\ <5 & U\end{array}$

$<5$
$<5$
$<5$
$<5$

$\begin{array}{ll}U & <5 \\ U & <5 \\ U & <5 \\ U & <5\end{array}$

$U$
$U$
$U$

$<5$
$<5$
$<5$

$\begin{array}{lll}\mathrm{U} & <E Q L & 1 \\ \mathrm{U} & <\mathrm{EQL} & 1 \\ \mathrm{U} & <\mathrm{EQL} & 1 \\ \mathrm{U} & <\mathrm{EQL} & 1\end{array}$

ug/L EX

ug/L EX

ug/h EX

3098 CLPEPA 1

\section{CLP EPA 3 3Q99}

CLPEPA

1000 CLPEPA

Filt. DF Unit Lab

Nitrate-nitrite as nitrogen

$+\quad$ Sodium, total recoverable

+ Sulfate

$<146 \quad 4$

68
9440

U

9440

Radionuclides

Gross alpha

Nonvolatile beta

0540

$\begin{array}{lllll}44.5 & \mathrm{~J} & \mathrm{I} & <146 & \mathrm{U} \\ 68 & & & 71 & \\ 8970 & & & 9160 & \\ 6750 & & & 6160 & \\ & & & & \\ -.24 & \mathrm{U} & .425 & \mathrm{U} \\ .5 & \mathrm{U} & .756 & \mathrm{U} \\ .12 & \mathrm{U} & -.1 & \mathrm{U}\end{array}$

$<65$

U $V<E Q L ~ 1$

$u g / L \quad E X$

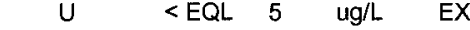

$\begin{array}{lllll}10300 & >4600 & 1 & \mathrm{ug} / \mathrm{L} & \mathrm{EX} \\ 5160 & >3000 & 2 & \mathrm{ug} / \mathrm{L} & \mathrm{EX}\end{array}$

Radium, total alpha-emitting

$\begin{array}{ll}1.43 & \\ 1.07 & \text { UI } \\ .14 & \text { UI }\end{array}$

$\begin{array}{ll} & -.24 \\ \text { UI } & .5 \\ \text { UI } & .12\end{array}$

$-$.

$\begin{array}{llllll}.176 & \mathrm{U} & <\mathrm{EQL} & 1 & \mathrm{pCi} / \mathrm{L} & \mathrm{GP} \\ .636 & \mathrm{U} & <\mathrm{EQL} & 1 & \mathrm{pCi} / \mathrm{L} & \mathrm{GP} \\ . .036 & \mathrm{U} & <\mathrm{EQL} & 1 & \mathrm{pCi} / \mathrm{L} & \mathrm{GP}\end{array}$

Notes: Concentrations in bold italics exceed the groundwater protection or monitoring constituent standards listed in Appendix A. Synchronous water levels are measured over a 3-5 day period or less. Dilution factors, Laboratory, and Filtered Data are for First Quarter 2000 data only

$+=$ exceeded the Groundwater Protection Standards or Monitoring Constituents listed in Appendix A for First Quarter 2000. 
Table D-7. Groundwater Monitoring Results for Background Wells, Met Lab HWMF WELL AMB 11D

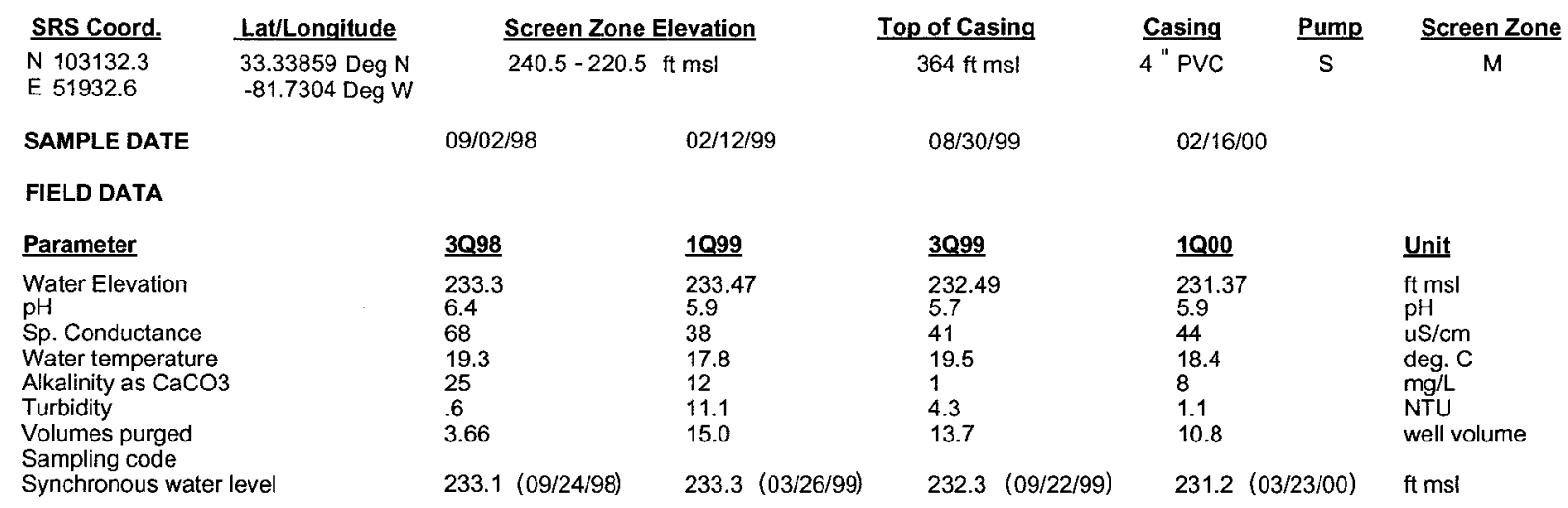

\section{ANALYTICAL DATA}

I. Groundwater Protection Standard

261 Appendix VIII/264 Appendix IX Hazardous Constituents

\begin{tabular}{|c|c|c|c|c|c|c|c|c|c|c|c|c|c|c|}
\hline$\underline{\mathrm{ST}}$ & Parameter & $\underline{3098}$ & CLPP EPA & 1099 & CLPP EPA & $\underline{3 Q 99}$ & CLPEPA & 1000 & CLP & EPA & Filt. & DF & Unit & Lab \\
\hline por & ganics & & & & & & & & & & & & & \\
\hline & Barium, total recoverable & 10.3 & & 4.7 & & 4.6 & & $<5.76$ & $\mathrm{U}$ & V & $<E Q L$ & 1 & $\mathrm{ug} / \mathrm{L}$ & EX \\
\hline & Cyanide & $<15.2$ & $\mathrm{U}$ & $<15.2$ & $\mathrm{U}$ & $<15.2$ & $\mathrm{U}$ & $<10$ & $\mathrm{U}$ & & $<E Q L$ & 1 & ug/L & EX \\
\hline & Lead, total recoverable & $<47$ & $\mathrm{U}$ & $<47$ & $U$ & $<47$ & $\mathrm{U}$ & $<10$ & $\mathrm{U}$ & & $<E Q L$ & 1 & $\mathrm{ug} / \mathrm{L}$ & $\mathrm{EX}$ \\
\hline & Nickel, total recoverable & $<26$ & $\mathrm{U}$ & $<26$ & $\mathrm{U}$ & $<26$ & $\mathrm{U}$ & $<9.87$ & JU & V & $<E Q L$ & 1 & $\mathrm{ug} / \mathrm{L}$ & EX \\
\hline & Selenium, total recoverable & $<66$ & $U$ & $<66$ & $\mathrm{U}$ & $<66$ & $\mathrm{U}$ & $<10$ & $\mathrm{U}$ & & $<E Q L$ & 1 & ug/L & EX \\
\hline rg & anics & & & & & & & & & & & & & \\
\hline & Chlorobenzene & $<5$ & $\mathrm{U}$ & $<5$ & $U$ & $<5$ & U & $<5$ & U & & $<E Q L$ & 1 & $\mathrm{ug} / \mathrm{L}$ & EX \\
\hline & 1,1-Dichloroethane & $<5$ & $\mathrm{U}$ & $<5$ & $\mathrm{U}$ & $<5$ & $U$ & $<5$ & $\mathrm{U}$ & & $<E Q L$ & 1 & $\mathrm{ug} / \mathrm{L}$ & EX \\
\hline & 1,1-Dichloroethylene & $<5$ & $\mathrm{U}$ & $<5$ & $U$ & $<5$ & $U$ & $<5$ & $\mathrm{U}$ & & $<\mathrm{EQL}$ & 1 & $\mathrm{ug} / \mathrm{L}$ & EX \\
\hline & $\begin{array}{l}\text { trans-1,2-Dichloroethylene } \\
\text { PCB } 1016\end{array}$ & $<5$ & $\mathrm{U}$ & $<5$ & $U$ & $<5$ & $\mathrm{U}$ & $<5$ & $\mathrm{U}$ & & $<\mathrm{EQL}$ & 1 & $\mathrm{ug} / \mathrm{L}$ & EX \\
\hline & PCB 1221 & & & & & & & & & & & & & \\
\hline & PCB 1232 & & & & & & & & & & & & & \\
\hline & PCB 1242 & & & & & & & & & & & & & \\
\hline & PCB 1248 & & & & & & & & & & & & & \\
\hline & РCB 1254 & & & & & & & & & & & & & \\
\hline & PCB 1260 & & & & & & & & & & & & & \\
\hline & 1,1,2,2-Tetrachloroethane & $<5$ & $U$ & $<5$ & $U$ & $<5$ & $U$ & $<5$ & $U$ & & $<\mathrm{EQL}$ & 1 & $\mathrm{ug} / \mathrm{L}$ & EX \\
\hline & Tetrachloroethylene & $<5$ & $U$ & $<5$ & $U$ & $<5$ & $U$ & $<5$ & $U$ & & $<\mathrm{EQL}$ & 1 & $\mathrm{ug} / \mathrm{L}$ & EX \\
\hline & 1,1,1-Trichloroethane & $<5$ & $U$ & $<5$ & $U$ & $<5$ & $\mathrm{U}$ & $<5$ & $\mathrm{U}$ & & $<E Q L$ & 1 & $\mathrm{ug} / \mathrm{L}$ & EX \\
\hline & Trichloroethylene & $<5$ & $u$ & $<5$ & $U$ & $<5$ & $\mathrm{U}$ & $<5$ & $U$ & & $<E Q L$ & 1 & ug/L & EX \\
\hline II. $\mathbf{M}$ & onitoring Constituents & & & & & & & & & & & & & \\
\hline$\underline{\mathbf{S T}}$ & Parameter & $\underline{3098}$ & CLPEPA & 1099 & CLP EPA & $\underline{3 Q 999}$ & CLPEPA & 1000 & CLP & EPA & Filt. & $\underline{\text { DF }}$ & Unit & $\underline{\text { Lab }}$ \\
\hline 0 & ganics & & & & & & & & & & & & & \\
\hline & Aluminum, total recoverable & 38.9 & $J$ & 118 & J I & 90.4 & $\mathrm{~J} \quad \mathrm{I}$ & 35.6 & $J$ & 1 & NDD & 1 & $\mathrm{ug} / \mathrm{L}$ & EX \\
\hline & Nitrate-nitrite as nitrogen & 186 & & 167 & & 225 & & $<500$ & $U$ & & $<\mathrm{EQL}$ & 5 & $\mathrm{ug} / \mathrm{L}$ & EX \\
\hline+ & Sodium, total recoverable & 6930 & & 6830 & & 6220 & & 8170 & & & $>4600$ & 1 & $\mathrm{ug} / \mathrm{L}$ & EX \\
\hline+ & Sulfate & 5310 & & 6540 & & 6180 & & 5650 & & & $>3000$ & 2 & $\mathrm{ug} / \mathrm{L}$ & EX \\
\hline 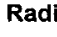 & onuclides & & & & & & & & & & & & & \\
\hline & Gross alpha & .34 & UIJ & .42 & $\mathrm{U}$ & .556 & $\mathrm{U}$ & .326 & U & & $<\mathrm{EQL}$ & 1 & $\mathrm{pCi} / \mathrm{L}$ & GP \\
\hline & Nonvolatile beta & .23 & UIJ & .16 & $\mathbf{U}$ & .574 & $U$ & .621 & $\mathrm{U}$ & & $<E Q L$ & 1 & $\mathrm{pCi} / \mathrm{L}$ & GP \\
\hline & Radium, total alpha-emitting & .15 & UI & .62 & U & -.1 & U & .371 & $\mathbf{J}$ & 1 & NDD & 1 & $\mathrm{pCi} / \mathrm{L}$ & GP \\
\hline
\end{tabular}

\footnotetext{
Notes: Concentrations in bold italics exceed the groundwater protection or monitoring constituent standards listed in Appendix A. Synchronous water levels are measured over a 3-5 day period or less. Dilution factors, Laboratory, and Filtered Data are for First Quarter 2000 data only.

$+=$ exceeded the Groundwater Protection Standards or Monitoring Constituents listed in Appendix A for First Quarter 2000.
} 
Table D-7. Groundwater Monitoring Results for Background Wells, Met Lab HWMF (Cont.) WELL AMB 12D

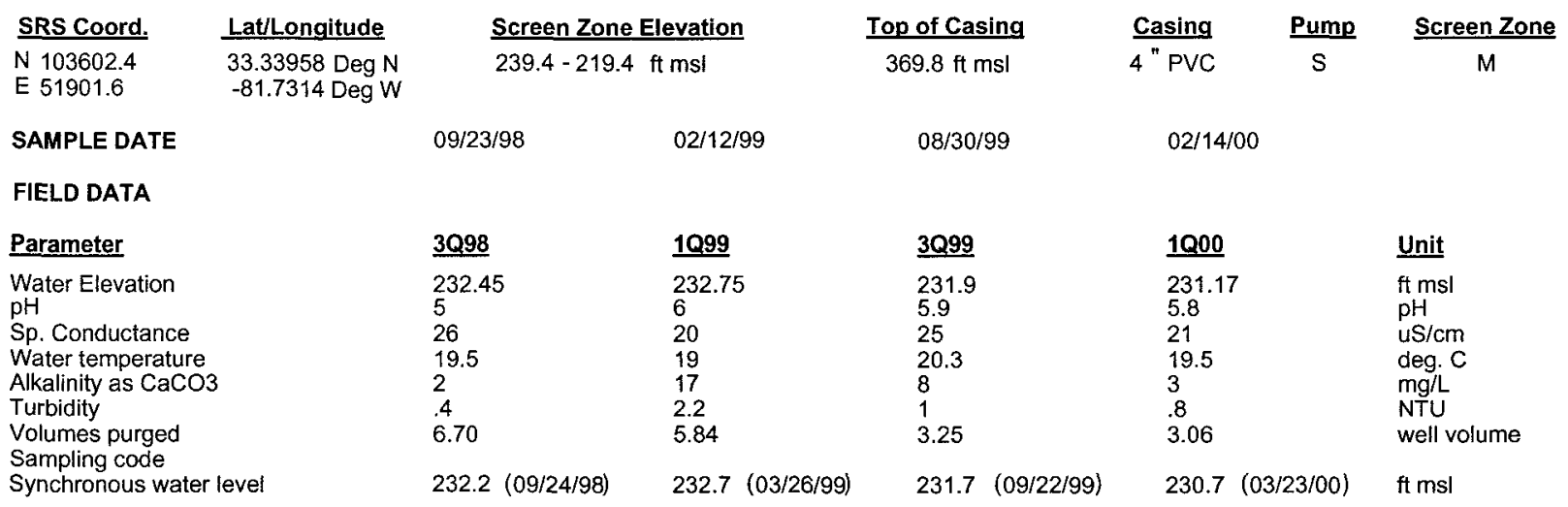

ANALYTICAL DATA

I. Groundwater Protection Standard

261 Appendix VIII/264 Appendix IX Hazardous Constituents

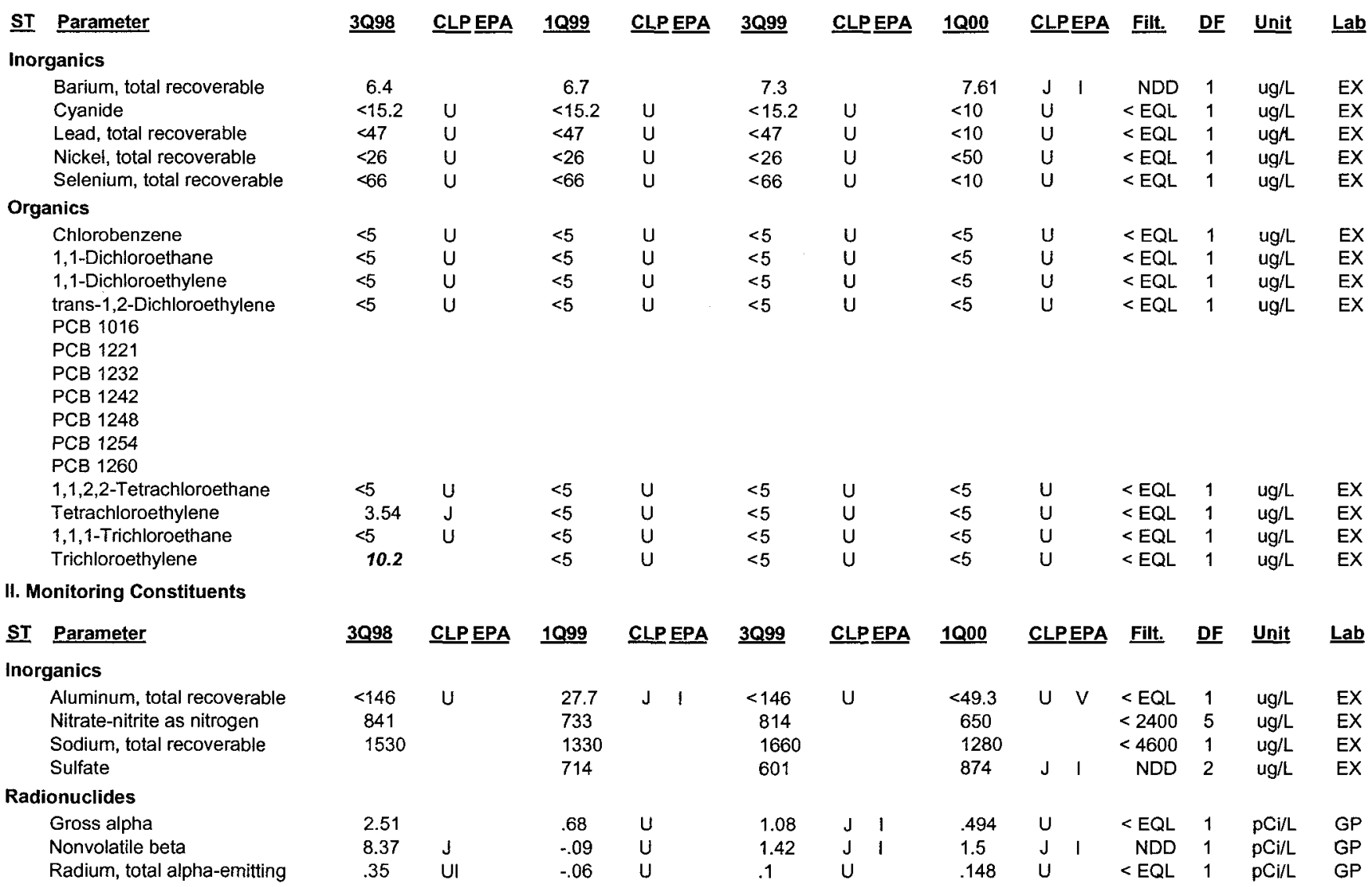

Notes: Concentrations in bold italics exceed the groundwater protection or monitoring constituent standards listed in Appendix A. Synchronous water levels are measured over a 3-5 day period or less. Dilution factors, Laboratory, and Filtered Data are for First Quarter 2000 data only.

$+=$ exceeded the Groundwater Protection Standards or Monitoring Constituents listed in Appendix A for First Quarter 2000. 
Table D-7. Groundwater Monitoring Results for Background Wells, Met Lab HWMF (Cont.) WELL MSB 29B

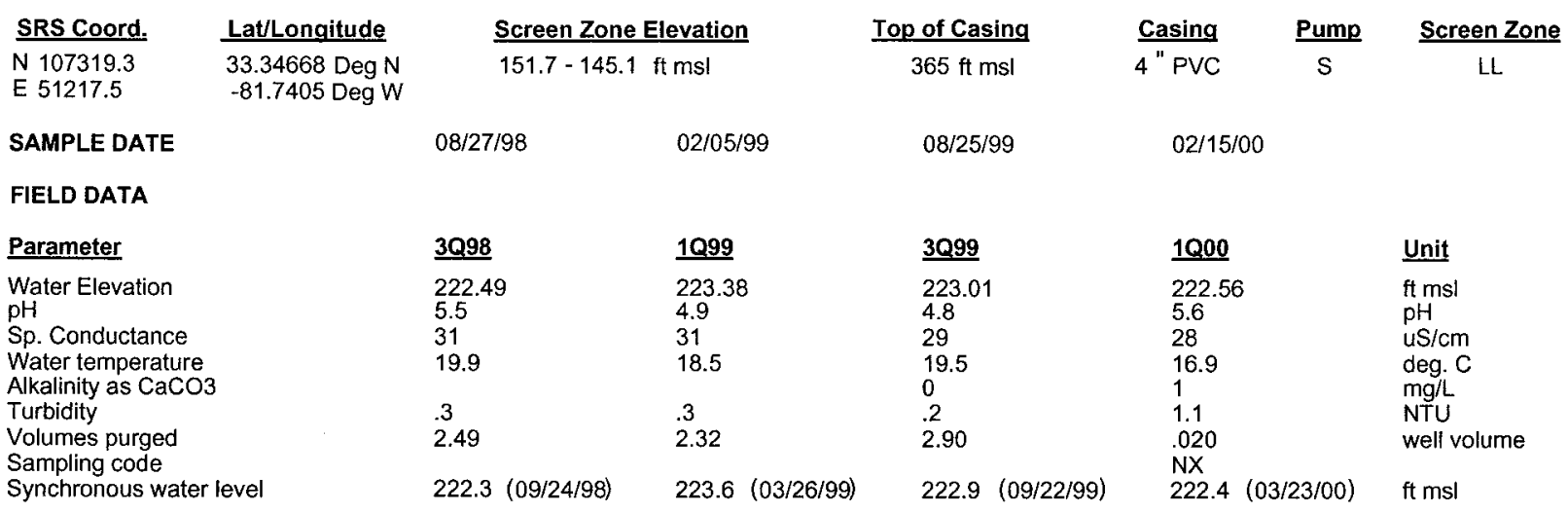

ANALYTICAL DATA

I. Groundwater Protection Standard

261 Appendix VIII/264 Appendix IX Hazardous Constituents

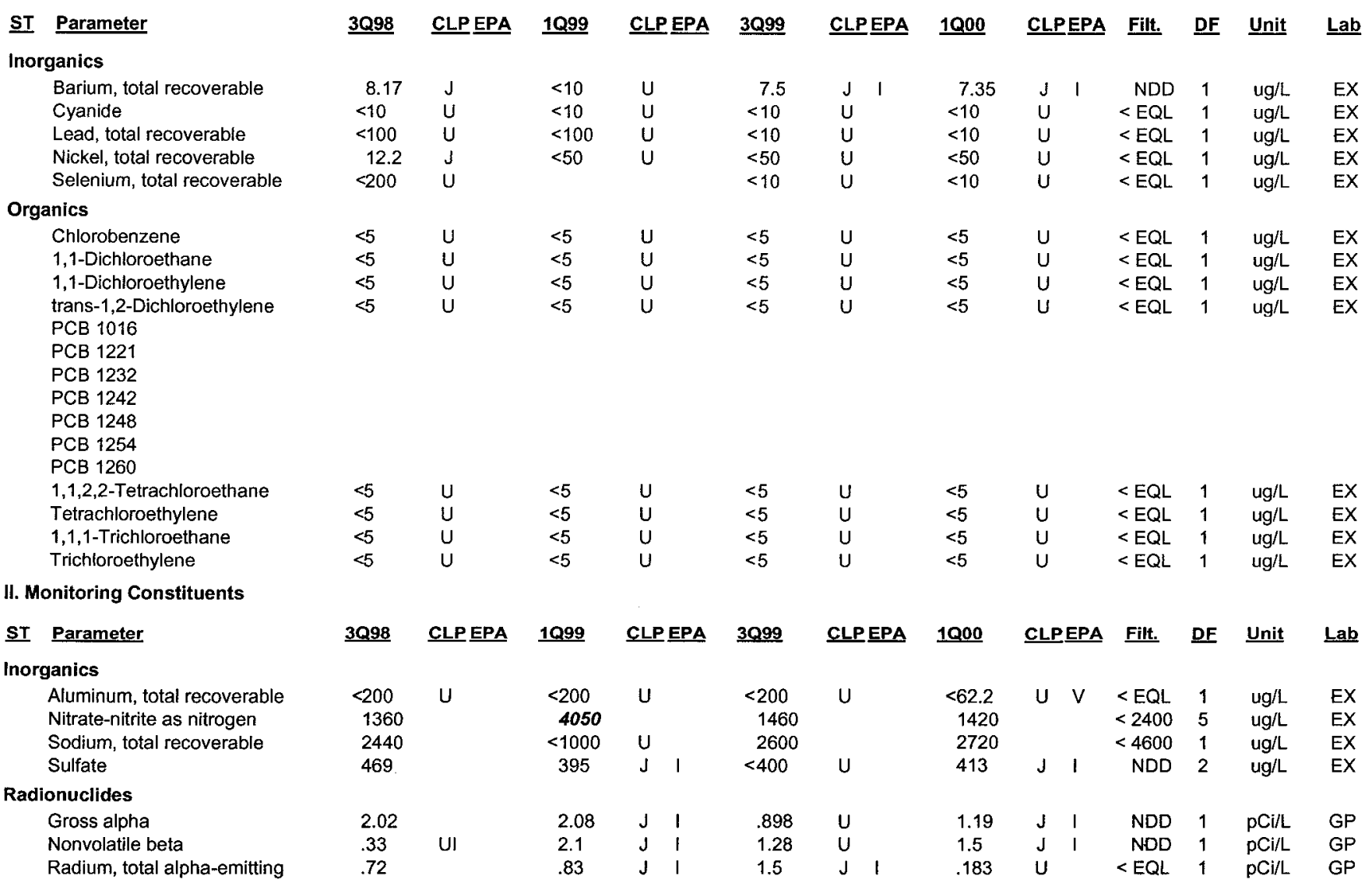

Notes: Concentrations in bold italics exceed the groundwater protection or monitoring constituent standards listed in Appendix A. Synchronous water levels are measured over a 3-5 day period or less. Dilution factors, Laboratory, and Filtered Data are for First Quarter 2000 data only.

$+=$ exceeded the Groundwater Protection Standards or Monitoring Constituents listed in Appendix A for First Quarter 2000. 
Table D-7. Groundwater Monitoring Results for Background Wells, Met Lab HWMF (Cont.) WELL MSB 29C

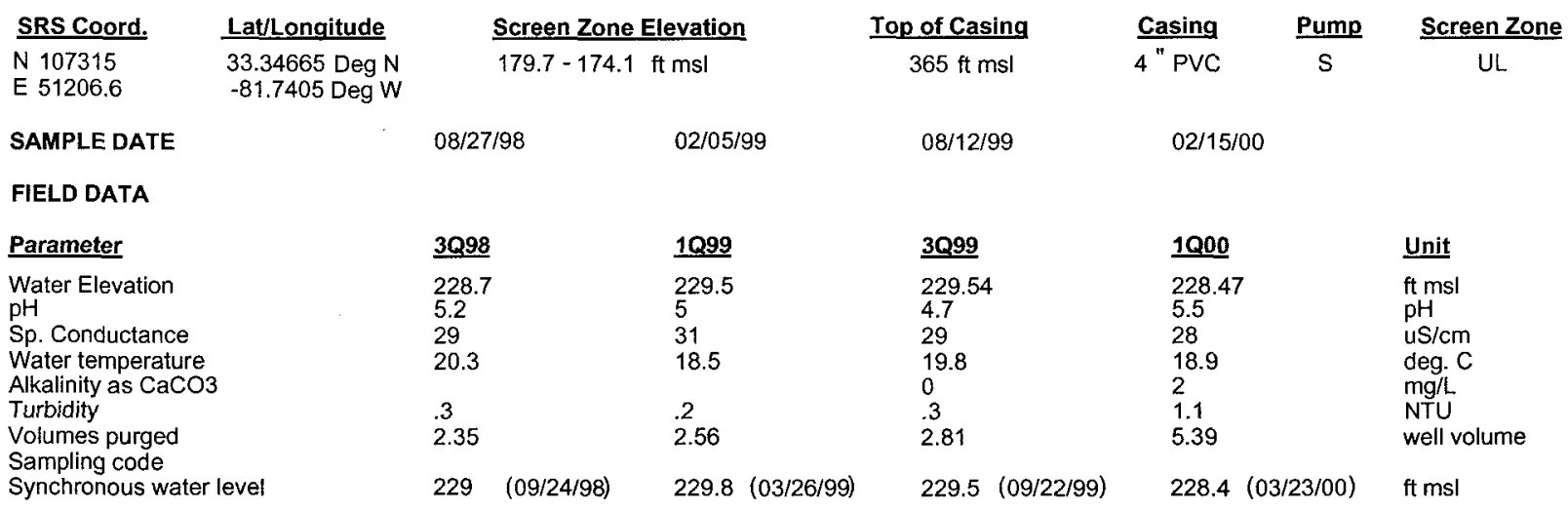

ANALYTICAL DATA

I. Groundwater Protection Standard

261 Appendix VIII/264 Appendix IX Hazardous Constituents

\begin{tabular}{|c|c|c|c|c|c|c|c|c|c|c|c|c|c|c|c|c|}
\hline \multirow{2}{*}{$\begin{array}{l}\underline{S T} \\
\text { Inorg }\end{array}$} & Parameter & $\underline{3 Q 98}$ & \multirow[t]{2}{*}{ CLP EPA } & \multirow[t]{2}{*}{$1 \mathrm{Q99}$} & \multicolumn{2}{|c|}{ CLP EPA } & \multirow[t]{2}{*}{ 3099 } & \multicolumn{2}{|c|}{ CLPPEPA } & \multirow[t]{2}{*}{1000} & \multicolumn{2}{|c|}{ CLPEPA } & \multirow[t]{2}{*}{ Filt. } & \multirow[t]{2}{*}{$\underline{\text { DF }}$} & \multirow[t]{2}{*}{ Unit } & \multirow[t]{2}{*}{ Lab } \\
\hline & ganics & & & & & & & & & & & & & & & \\
\hline & Barium, total recoverable & 6.13 & $J$ & $<10$ & $U$ & & 5.9 & J & 1 & 6 & 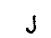 & I & NDD & 1 & $\mathrm{ug} / \mathrm{L}$ & EX \\
\hline & Cyanide & $<10$ & $u$ & $<10$ & $\mathrm{U}$ & & $<10$ & $\mathrm{U}$ & & $<10$ & U & & $<\mathrm{EQL}$ & 1 & $\mathrm{ug} / \mathrm{L}$ & EX \\
\hline & Lead, total recoverable & $<100$ & $U$ & $<100$ & $\mathrm{U}$ & & $<2.27$ & U & V & $<10$ & $U$ & & $<E Q L$ & 1 & $\mathrm{ug} / \mathrm{L}$ & EX \\
\hline & Nickel, total recoverable & 15.2 & J & $<50$ & $\mathrm{U}$ & & $<50$ & U & & $<50$ & $\mathrm{U}$ & & $<$ EQL & 1 & $\mathrm{ug} / \mathrm{L}$ & EX \\
\hline & Selenium, total recoverable & $<200$ & $\mathrm{U}$ & & & & $<2$ & $\mathrm{U}$ & & $<10$ & $\mathrm{U}$ & & $<E Q L$ & 1 & $u g / L$ & EX \\
\hline \multicolumn{17}{|c|}{ Organics } \\
\hline & Chlorobenzene & $<5$ & UJ & $<5$ & $\mathrm{U}$ & & $<5$ & $\mathrm{U}$ & & $<5$ & $\mathrm{U}$ & & $<\mathrm{EQL}$ & 1 & $u g / L$ & EX \\
\hline & 1,1-Dichloroethane & $<5$ & UJ & $<5$ & $U$ & & $<5$ & U & & $<5$ & $\mathrm{U}$ & & $<$ EQL & 1 & $u g / L$ & EX \\
\hline & 1,1-Dichloroethylene & $<5$ & UJ & $<5$ & U & & $<5$ & $u$ & & $<5$ & U & & $<E Q L$ & 1 & $u g / L$ & EX \\
\hline & trans-1,2-Dichloroethylene & $<5$ & UJ & $<5$ & $U$ & & $<5$ & $\mathrm{U}$ & & $<5$ & U & & $<E Q L$ & 1 & $\mathrm{ug} / \mathrm{L}$ & EX \\
\hline & PCB 1016 & & & & & & & & & & & & & & & \\
\hline & PCB 1221 & & & & & & & & & & & & & & & \\
\hline & PCB 1232 & & & & & & & & & & & & & & & \\
\hline & PCB 1242 & & & & & & & & & & & & & & & \\
\hline & PCB 1248 & & & & & & & & & & & & & & & \\
\hline & PCB 1254 & & & & & & & & & & & & & & & \\
\hline & PCB 1260 & & & & & & & & & & & & & & & \\
\hline & 1,1,2,2-Tetrachloroethane & $<5$ & UJ & $<5$ & $\mathrm{U}$ & & $<5$ & $\mathrm{U}$ & & $<5$ & $u$ & & $<\mathrm{EQL}$ & 1 & $\mathrm{ug} / \mathrm{L}$ & EX \\
\hline & Tetrachloroethylene & $<5$ & UJ & $<5$ & $\mathrm{U}$ & & $<5$ & U & & $<5$ & $\mathrm{U}$ & & $<\mathrm{EQL}$ & 1 & $\mathrm{ug} / \mathrm{L}$ & EX \\
\hline & 1,1,1-Trichloroethane & $<5$ & UJ & $<5$ & $U$ & & $<5$ & $\mathrm{U}$ & & $<5$ & $\mathrm{U}$ & & $<E Q L$ & 1 & $\mathrm{ug} / \mathrm{L}$ & EX \\
\hline & Trichloroethylene & $<5$ & UJ & $<5$ & $\mathrm{U}$ & & $<5$ & $\mathrm{U}$ & & $<5$ & $\mathrm{U}$ & & $<E Q L$ & 1 & $\mathrm{ug} / \mathrm{L}$ & $\mathrm{EX}$ \\
\hline \multicolumn{17}{|c|}{ II. Monitoring Constituents } \\
\hline$\underline{\mathbf{S T}}$ & Parameter & 3098 & CLP EPA & 1099 & $\underline{\text { CLP }}$ & EPA & $\underline{3099}$ & CLF & EPA & 1000 & $\underline{\text { CLF }}$ & EPA & Filt. & DF & $\underline{\text { Unit }}$ & Lab \\
\hline \multicolumn{17}{|c|}{ Inorganics } \\
\hline & Aluminum, total recoverable & $<200$ & $\mathrm{U}$ & $<200$ & $\mathrm{U}$ & & 77 & $J$ & 1 & $<59$ & $\mathrm{U}$ & v & $<\mathrm{EQL}$ & 1 & $\mathrm{ug} / \mathrm{L}$ & EX \\
\hline & Nitrate-nitrite as nitrogen & 1350 & & 1590 & & & 1440 & & & 1320 & & & $<2400$ & 5 & $\mathrm{ug} / \mathrm{L}$ & EX \\
\hline & Sodium, total recoverable & 2470 & & $<1000$ & $u$ & & 2500 & & & 2390 & & & $<4600$ & 1 & $\mathrm{ug} / \mathrm{L}$ & EX \\
\hline & Sulfate & 366 & J & 394 & $J$ & I & 535 & & & 591 & $J$ & 1 & NDD & 2 & $\mathrm{ug} / \mathrm{L}$ & EX \\
\hline \multicolumn{17}{|c|}{ Radionuclides } \\
\hline & Gross alpha & 3.73 & & 3.26 & & & 1.28 & $\jmath$ & IK & 2.45 & & & $<15$ & 1 & $\mathrm{pCi} / \mathrm{L}$ & GP \\
\hline & Nonvolatile beta & 3.85 & & 3.01 & $J$ & I & 2.58 & J & 1 & 2.56 & $J$ & 1 & NDD & 1 & $\mathrm{pCi} / \mathrm{L}$ & GP \\
\hline & Radium, total alpha-emitting & .34 & Ui & 1.06 & $J$ & 1 & .5 & $\jmath$ & 1 & .603 & J & $\mathrm{IL}$ & NDD & 1 & $\mathrm{pCi} / \mathrm{L}$ & GP \\
\hline
\end{tabular}

Notes: Concentrations in bold italics exceed the groundwater protection or monitoring constituent standards listed in Appendix A. Synchronous water levels are measured over a 3-5 day period or less. Dilution factors, Laboratory, and Filtered Data are for First Quarter 2000 data only.

+ = exceeded the Groundwater Protection Standards or Monitoring Constituents listed in Appendix A for First Quarter 2000. 
Table D-7. Groundwater Monitoring Results for Background Wells, Met Lab HWMF (Cont.) WELL MSB 29D

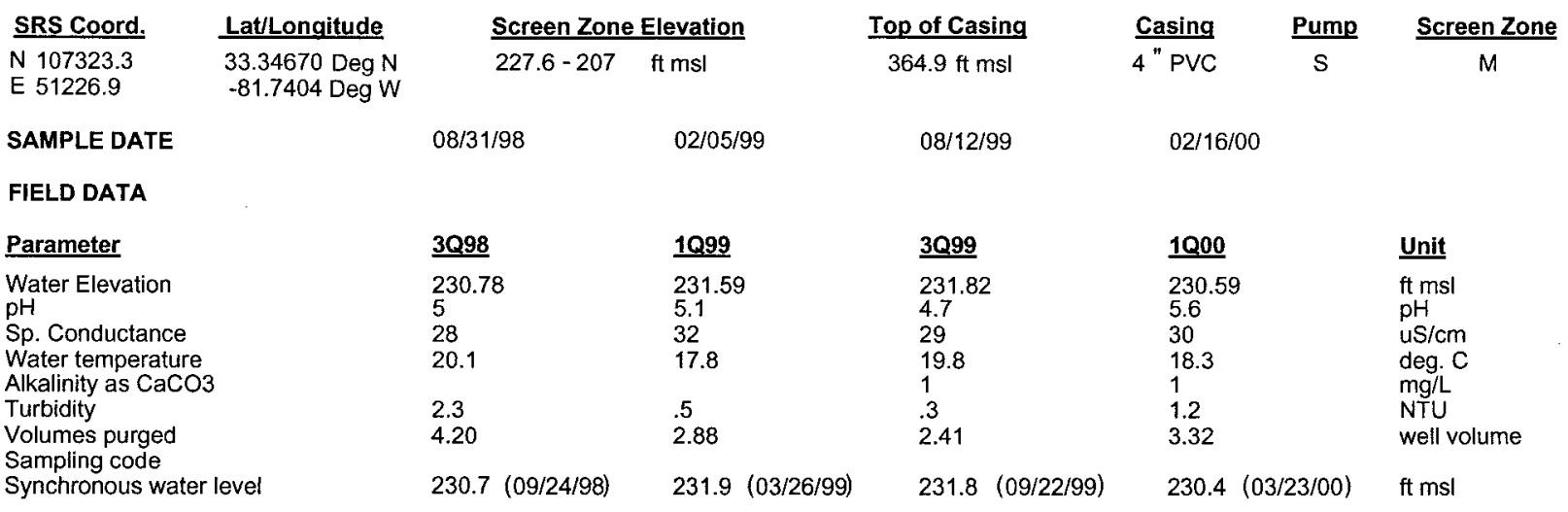

ANALYTICAL DATA

I. Groundwater Protection Standard

261 Appendix VIII/264 Appendix IX Hazardous Constituents

\begin{tabular}{|c|c|c|c|c|c|c|c|c|c|c|c|c|c|c|c|}
\hline$\underline{\text { ST }}$ & Parameter & $\underline{3 Q 98}$ & CLP EPA & 1099 & CLPEPA & $\underline{3099}$ & CLP & EPA & 1000 & CLF & EPA & Filt. & $\underline{\mathrm{DF}}$ & Unit & $\underline{\text { Lab }}$ \\
\hline tor & ganics & & & & & & & & & & & & & & \\
\hline & Barium, total recoverable & 8.84 & $J$ & $<10$ & $\mathrm{U}$ & 9.1 & $J$ & I & $<8.64$ & $\mathrm{U}$ & V & $<\mathrm{EQL}$ & 1 & $\mathrm{ug} / \mathrm{L}$ & EX \\
\hline & Cyanide & $<10$ & $\mathrm{U}$ & $<10$ & $\mathrm{U}$ & $<10$ & $\mathrm{U}$ & & $<10$ & $\mathrm{U}$ & & $<\mathrm{EQL}$ & 1 & $\mathrm{ug} / \mathrm{L}$ & EX \\
\hline & Lead, total recoverable & $<100$ & $\mathrm{U}$ & $<100$ & $u$ & $<4.14$ & $\mathrm{U}$ & v & 11.2 & & & $<15$ & 1 & $\mathrm{ug} / \mathrm{L}$ & EX \\
\hline & Nickel, total recoverable & $<50$ & $U$ & $<50$ & $U$ & $<9.5$ & JU & I & $<10.9$ & JU & V & $<E Q L$ & 1 & $\mathrm{ug} / \mathrm{L}$ & EX \\
\hline & Selenium, total recoverable & $<3.38$ & UJ & & & $<2$ & $\mathrm{U}$ & & $<10$ & $\mathrm{U}$ & & $<E Q L$ & 1 & $\mathrm{ug} / \mathrm{L}$ & EX \\
\hline Org & anics & & & & & & & & & & & & & & \\
\hline & Chlorobenzene & $<5$ & UJ & $<5$ & $\mathrm{U}$ & $<5$ & $\mathrm{u}$ & & $<5$ & $\mathrm{u}$ & & $<\mathrm{EQL}$ & 1 & $\mathrm{ug} / \mathrm{L}$ & EX \\
\hline & 1,1-Dichloroethane & $<5$ & UJ & $<5$ & $\mathrm{U}$ & $<5$ & $\mathrm{U}$ & & $<5$ & $u$ & & $<\mathrm{EQL}$ & 1 & $\mathrm{ug} / \mathrm{L}$ & EX \\
\hline & 1,1-Dichloroethylene & $<5$ & UJ & $<5$ & $U$ & $<5$ & $\mathrm{U}$ & & $<5$ & $\mathrm{U}$ & & $<E Q L$ & 1 & $\mathrm{ug} / \mathrm{L}$ & EX \\
\hline & $\begin{array}{l}\text { trans-1,2-Dichloroethylene } \\
\text { PCB } 1016\end{array}$ & $<5$ & UJ & $<5$ & $u$ & $<5$ & $\mathrm{U}$ & & $<5$ & $\mathrm{U}$ & & $<\mathrm{EQL}$ & 1 & $\mathrm{ug} / \mathrm{L}$ & EX \\
\hline & PCB 1221 & & & & & & & & & & & & & & \\
\hline & PCB 1232 & & & & & & & & & & & & & & \\
\hline & PCB 1242 & & & & & & & & & & & & & & \\
\hline & РСВ 1248 & & & & & & & & & & & & & & \\
\hline & PCB 1254 & & & & & & & & & & & & & & \\
\hline & PCB 1260 & & & & & & & & & & & & & & \\
\hline & $1,1,2,2$-Tetrachloroethane & $<5$ & UJ & $<5$ & $\mathrm{U}$ & $<5$ & 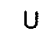 & & $<5$ & $\mathrm{u}$ & & $<$ EQL & 1 & ug/L & EX \\
\hline & Tetrachloroethylene & $<5$ & UJ & $<5$ & $\mathrm{U}$ & $<5$ & $U$ & & $<5$ & $U$ & & $<\mathrm{EQL}$ & 1 & $u g / L$ & EX \\
\hline & 1,1,1-Trichloroethane & $<5$ & UJ & $<5$ & $\mathrm{U}$ & $<5$ & $\mathrm{U}$ & & $<5$ & $\mathrm{U}$ & & $<\mathrm{EQL}$ & 1 & $\mathrm{ug} / \mathrm{L}$ & EX \\
\hline & Trichloroethylene & $<5$ & US & $<5$ & $U$ & $<5$ & U & & $<5$ & $\mathrm{U}$ & & $<\mathrm{EQL}$ & 1 & $\mathrm{ug} / \mathrm{L}$ & EX \\
\hline I. $\mathbf{M}$ & onitoring Constituents & & & & & & & & & & & & & & \\
\hline ST & Parameter & $\underline{3099}$ & CLPEPA & 1099 & CLPEPA & $\underline{3099}$ & CLP & EPA & 1000 & $\underline{\text { CLP }}$ & EPA & Filt. & $\underline{\mathrm{DF}}$ & Unit & $\underline{\text { Lab }}$ \\
\hline Inor & ganics & & & & & & & & & & & & & & \\
\hline & Aluminum, total recoverable & $<200$ & $\mathrm{U}$ & $<200$ & $\mathbf{U}$ & $<200$ & $U$ & & 59.7 & $J$ & 1 & NDD & 1 & ug/L & EX \\
\hline & Nitrate-nitrite as nitrogen & 867 & & 1870 & & 1400 & & & 1600 & & & $<2400$ & 5 & ug/L & EX \\
\hline & Sodium, total recoverable & 2700 & & $<1000$ & $\mathrm{U}$ & 3400 & & & 4120 & & & $<4600$ & 1 & $u g / L$ & EX \\
\hline & Sulfate & 356 & J & 284 & $\mathrm{~J} 1$ & 647 & & & 784 & $J$ & 1 & NDD & 2 & ug/L & EX \\
\hline Radi & ionuclides & & & & & & & & & & & & & & \\
\hline+ & Gross alpha & 28.5 & & 8.25 & & 12.4 & J & $\mathrm{K}$ & 18.1 & & & $>15$ & 1 & $\mathrm{pCi} / \mathrm{L}$ & GP \\
\hline & Nonvolatile beta & 19.3 & $\mathrm{~J}$ & 4.37 & & 4.93 & & & 6.01 & & & $<50$ & 1 & $\mathrm{pCi} / \mathrm{L}$ & GP \\
\hline+ & Radium, total alpha-emitting & 3.33 & & 2.22 & $\mathrm{~J} \quad \mathrm{I}$ & 3.7 & & & 6.27 & & & $>5$ & 1 & $\mathrm{pCi} / \mathrm{L}$ & GP \\
\hline
\end{tabular}


Table D-7. Groundwater Monitoring Results for Background Wells, Met Lab HWMF (Cont.) WELL MSB 43A

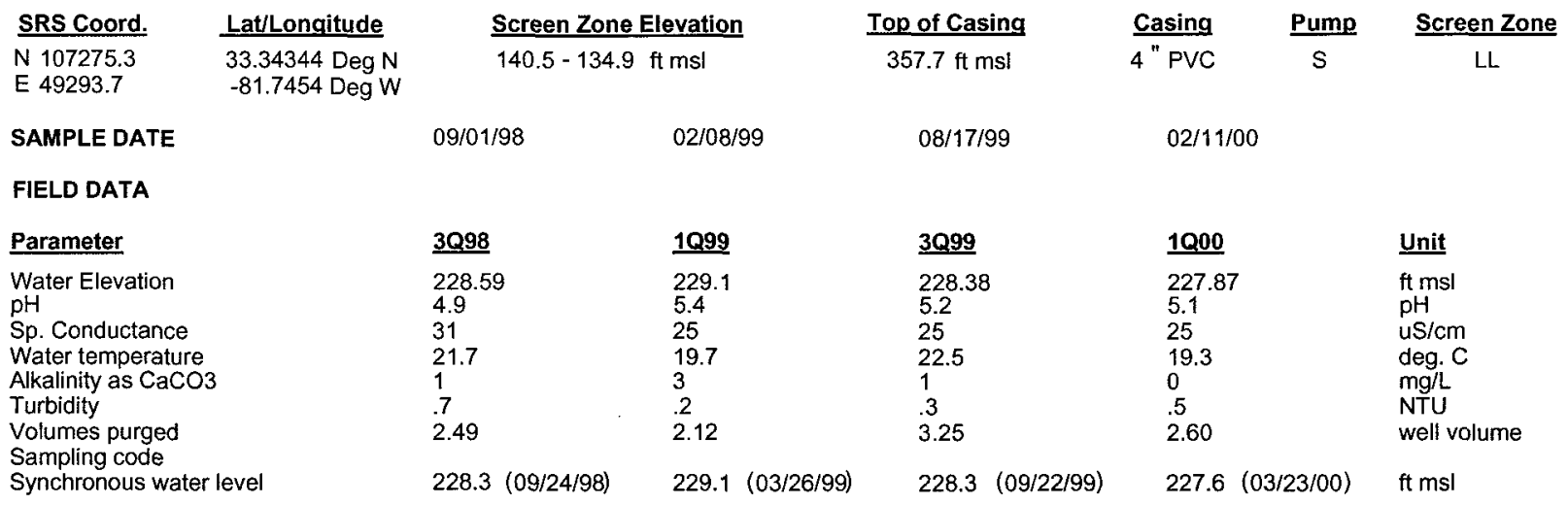

ANALYTICAL DATA

I. Groundwater Protection Standard

261 Appendix VIII/264 Appendix IX Hazardous Constituents

ST Parameter

Inorganics

Barium, total recoverable

Cyanide

Lead, total recoverable

Nickel, total recoverable

Selenium, total recoverable

Organics

Chlorobenzene

1,1-Dichloroethane

1,1-Dichloroethylene

trans-1,2-Dichloroethylene

PCB 1016

PCB 1221

PCB 1232

PCB 1242

PCB 1248

PCB 1254

PCB 1260

1,1,2,2-Tetrachloroethane

Tetrachloroethylene

1,1,1-Trichloroethane

Trichloroethylene

II. Monitoring Constituents

ST Param

Aluminum, total recoverable

Nitrate-nitrite as nitrogen

Sodium, total recoverable

Sulfate

Radionuclides

Gross aipha

Nonvolatile beta

Radium, total alpha-emitting

\section{$\underline{3 Q 98}$}

$\begin{array}{ll} & 14.1 \\ <10 & \\ <100 & U \\ <50 & U \\ <10 & U\end{array}$

$<10 \quad U$

$<5 \quad$ UJ

$<5 \quad$ UJ

$<5 \quad$ UJ

$\begin{array}{ll}<5 & \text { UJ } \\ <5 & \text { UJ } \\ <5 & \text { UJ } \\ <5 & \text { UJ }\end{array}$

$\begin{array}{ll}<5 & \mathrm{U} \\ <5 & \mathrm{U} \\ <5 & \mathrm{U} \\ <5 & \mathrm{U}\end{array}$

$\underline{3098}$

CLP EPA

1099 CLPEPA

3Q99

CLPEPA

$<200$
$\mathbf{3 8 7 0}$
1990
$<400$

u

$<200 \quad U$
1470
1700
284

$<400$

U

$\begin{array}{ll}.76 & \mathrm{UI} \\ 2.16 & \\ .72 & \mathrm{U}\end{array}$

$\begin{array}{ll}1.04 & \cup \\ .76 & U \\ .51 & U\end{array}$

$\begin{aligned} & 9.8 \\ < & 10 \\ < & 10 \\ < & 50 \\ < & 10 \\ & <5 \\ < & <5 \\ < & <5\end{aligned}$

\section{CLPEPA}

1000 CLPEPA Filt. DF Unit Lab

\begin{tabular}{|c|c|c|c|c|}
\hline 9.54 & $J$ & NDD & 1 & $u g / L$ \\
\hline$<10$ & $\mathrm{U}$ & $<E Q L$ & 1 & $\mathrm{ug} / \mathrm{L}$ \\
\hline$<1.93$ & JU & $<E Q L$ & 1 & $\mathrm{ug} / \mathrm{L}$ \\
\hline$<50$ & $\mathrm{U}$ & $<$ EQL & 1 & $\mathrm{ug} / \mathrm{L}$ \\
\hline$<10$ & $\mathrm{U}$ & $<E Q L$ & 1 & $\mathrm{ug} / \mathrm{L}$ \\
\hline$<5$ & U & $<E Q L$ & 1 & $\mathrm{ug} / \mathrm{L}$ \\
\hline$<5$ & $\mathrm{U}$ & $<\overline{E Q L}$ & 1 & $\mathrm{ug} / \mathrm{L}$ \\
\hline$<5$ & $\mathrm{U}$ & $<$ EQL & 1 & $\mathrm{ug} / \mathrm{L}$ \\
\hline$<5$ & $U$ & $<$ EQL & 1 & $\mathrm{ug} / \mathrm{L}$ \\
\hline
\end{tabular}

$\begin{array}{llllllll}<5 & U & <5 & U & <E Q L & 1 & u g / L & E X \\ <5 & U & <5 & U & <E Q L & 1 & u g / L & E X \\ <5 & U & <5 & U & <E Q L & 1 & u g / L & E X \\ <5 & U & <5 & U & <E Q L & 1 & u g / L & E X\end{array}$

levels are measured over a 3-5 day period or less. Dilution factors, Laboratory, and Filtered Data are for First Quarter 2000 data only.

$+=$ exceeded the Groundwater Protection Standards or Monitoring Constituents listed in Appendix A for First Quarter 2000. 
Table D-7. Groundwater Monitoring Results for Background Wells, Met Lab HWMF (Cont.) WELL MSB 43B

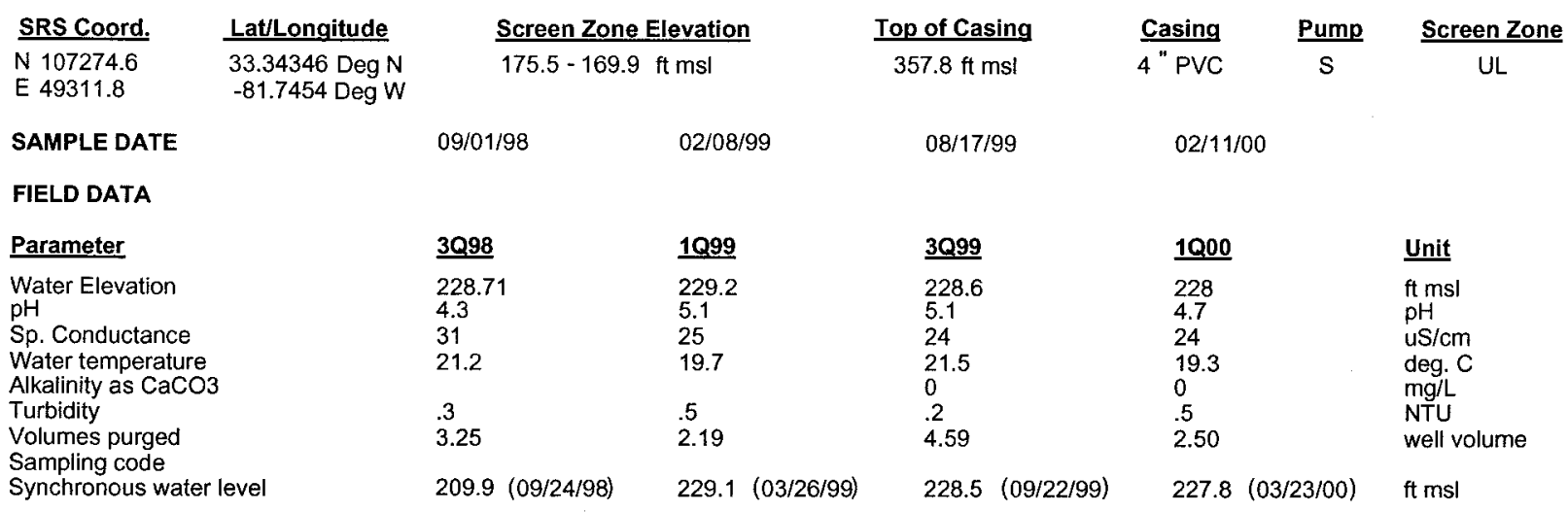

ANALYTICAL DATA

I. Groundwater Protection Standard

261 Appendix VIII/264 Appendix IX Hazardous Constituents

\begin{tabular}{|c|c|c|c|c|c|c|c|c|c|c|c|c|c|c|c|}
\hline \multirow{2}{*}{$\begin{array}{l}\text { ST Parameter } \\
\text { Inorganics }\end{array}$} & \multirow[t]{2}{*}{$\underline{3098}$} & \multirow[t]{2}{*}{ CLPEPA } & \multirow[t]{2}{*}{$1 \mathrm{Q99}$} & \multicolumn{2}{|c|}{ CLPEPA } & \multirow[t]{2}{*}{$\underline{3 Q 99}$} & \multicolumn{2}{|c|}{ CLPEPA } & \multirow[t]{2}{*}{1000} & \multicolumn{2}{|c|}{ CLPEPA } & \multirow[t]{2}{*}{ Filt. } & \multirow[t]{2}{*}{ DF } & \multirow[t]{2}{*}{ Unit } & \multirow[t]{2}{*}{$\underline{\mathrm{Lab}}$} \\
\hline & & & & & & & & & & & & & & & \\
\hline Barium, total recoverable & 2.89 & $J$ & 2.43 & J & 1 & 3.1 & $J$ & 1 & $<3.22$ & $u$ & $v$ & $<E Q L$ & 1 & $u g / L$ & EX \\
\hline Cyanide & $<10$ & $u$ & $<10$ & u & & $<10$ & $u$ & & $<10$ & $\mathrm{u}$ & & $<E Q L$ & 1 & $\mathrm{ug} / \mathrm{L}$ & EX \\
\hline Lead, total recoverable & $<100$ & $u$ & $<100$ & $u$ & & $<10$ & $u$ & & $<10$ & $u$ & & $<E Q L$ & 1 & $\mathrm{ug} / \mathrm{L}$ & EX \\
\hline Nickel, total recoverable & $<50$ & $u$ & $<50$ & $U$ & & $<6.7$ & JU & 1 & $<50$ & $u$ & & $<E Q L$ & 1 & $\mathrm{ug} / \mathrm{L}$ & EX \\
\hline Selenium, total recoverable & $<10$ & $u$ & $<200$ & $u$ & & $<10$ & $U$ & & $<10$ & $u$ & & $<E Q L$ & 1 & $\mathrm{ug} / \mathrm{L}$ & EX \\
\hline \multicolumn{16}{|l|}{ Organics } \\
\hline Chlorobenzene & $<5$ & UJ & $<5$ & $\mathrm{u}$ & & $<5$ & $u$ & & $<5$ & $u$ & & $<\mathrm{EQL}$ & 1 & ug/L & EX \\
\hline 1,1-Dichloroethane & $<5$ & UJ & $<5$ & $u$ & & $<5$ & $\mathrm{U}$ & & $<5$ & $\mathrm{U}$ & & $<E Q L$ & 1 & $\mathrm{ug} / \mathrm{L}$ & EX \\
\hline 1,1-Dichloroethylene & $<5$ & UJ & $<5$ & $u$ & & $<5$ & $u$ & & $<5$ & $u$ & & $<\mathrm{EQL}$ & 1 & $\mathrm{ug} / \mathrm{L}$ & $E x$ \\
\hline trans-1,2-Dichloroethylene & $<5$ & UJ & $<5$ & $U$ & & $<5$ & $U$ & & $<5$ & $\mathrm{u}$ & & $<\mathrm{EQL}$ & 1 & $\mathrm{ug} / \mathrm{L}$ & $E x$ \\
\hline $\begin{array}{l}\text { PCB } 1016 \\
\text { PCB } 1221\end{array}$ & & & & & & & & & & & & & & & \\
\hline PCB 1232 & & & & & & & & & & & & & & & \\
\hline PCB 1242 & & & & & & & & & & & & & & & \\
\hline PCB 1248 & & & & & & & & & & & & & & & \\
\hline PCB 1254 & & & & & & & & & & & & & & & \\
\hline PCB 1260 & & & & & & & & & & & & & & & \\
\hline $1,1,2,2$-Tetrachloroethane & $<5$ & UJ & $<5$ & $\mathrm{U}$ & & $<5$ & $\mathrm{U}$ & & $<5$ & u & & $<E Q L$ & 1 & $\mathrm{ug} / \mathrm{L}$ & EX \\
\hline Tetrachloroethylene & $<5$ & UJ & $<5$ & $u$ & & $<5$ & $\mathrm{u}$ & & $<5$ & $\mathrm{u}$ & & $<E Q L$ & 1 & $\mathrm{ug} / \mathrm{L}$ & EX \\
\hline 1,1,1-Trichloroethane & $<5$ & UJ & $<5$ & $\mathrm{u}$ & & $<5$ & $u$ & & $<5$ & $u$ & & $<E Q L$ & 1 & $\mathrm{ug} / \mathrm{L}$ & EX \\
\hline Trichloroethylene & $<5$ & UJ & $<5$ & $U$ & & $<5$ & $u$ & & $<5$ & $u$ & & $<E Q L$ & 1 & $u g / L$ & EX \\
\hline \multicolumn{16}{|l|}{ II. Monitoring Constituents } \\
\hline ST Parameter & $\underline{3098}$ & CLPEPA & 1099 & $\underline{\text { CLP }}$ & EPA & $\underline{3 Q 99}$ & $\underline{\text { CLP }}$ & EPA & 1000 & $\underline{\text { CLP }}$ & EPA & Filt. & $\underline{\text { DF }}$ & $\underline{\text { Unit }}$ & $\underline{\text { Lab }}$ \\
\hline \multicolumn{16}{|l|}{ Inorganics } \\
\hline Aluminum, total recoverable & $<200$ & $U$ & $<200$ & $U$ & & $<200$ & $U$ & & $<36.3$ & $U$ & V & $<E Q L$ & 1 & $u g / L$ & EX \\
\hline Nitrate-nitrite as nitrogen & 1410 & & 1550 & & & 1480 & & & 1430 & & & $<2400$ & 5 & $\mathrm{ug} / \mathrm{L}$ & EX \\
\hline Sodium, total recoverable & 2330 & & 2230 & & & 2400 & & & 2390 & & & $<4600$ & 1 & ug/L & EX \\
\hline Sulfate & 356 & $J$ & 521 & & & 374 & $J$ & 1 & $<1000$ & $u$ & & $<E Q L$ & 2 & $\mathrm{ug} / \mathrm{L}$ & EX \\
\hline \multicolumn{15}{|l|}{ Radionuclides } & \\
\hline Gross alpha & 3.76 & & 2.12 & $u$ & $\mathrm{~V}$ & 1.44 & $\mathrm{~J}$ & 1 & 2.57 & & & $<15$ & 1 & $\mathrm{pCi} / \mathrm{L}$ & GP \\
\hline Nonvolatile beta & 4.24 & & 1 & $\mathrm{U}$ & & 1.48 & $u$ & & 1.98 & $\mathrm{~J}$ & I & NDD & 1 & $\mathrm{pCi} / \mathrm{L}$ & GP \\
\hline Radium, total alpha-emitting & .99 & $u$ & 1.68 & $\mathrm{~J}$ & 1 & .5 & $u$ & v & .895 & $\mathrm{~J}$ & I & NDD & 1 & $\mathrm{pCi} / \mathrm{L}$ & $\mathrm{PP}$ \\
\hline
\end{tabular}


Table D-7. Groundwater Monitoring Results for Background Wells, Met Lab HWMF (Cont.) WELL MSB 43D

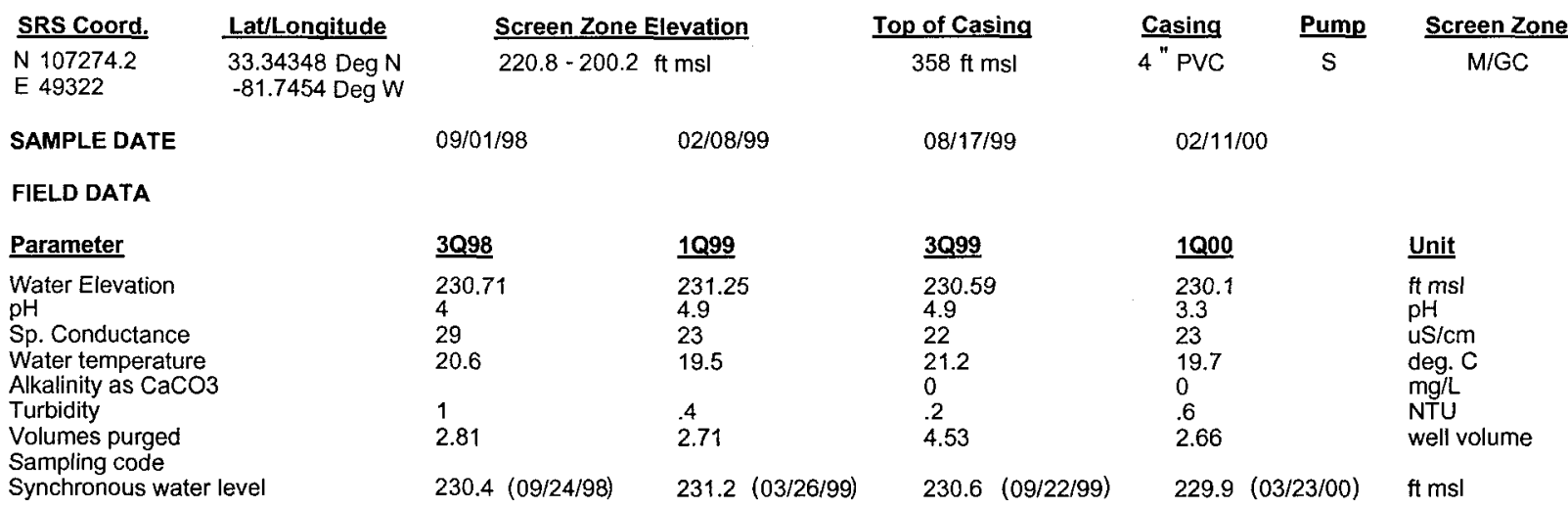

ANALYTICAL DATA

I. Groundwater Protection Standard

261 Appendix VIII/264 Appendix IX Hazardous Constituents

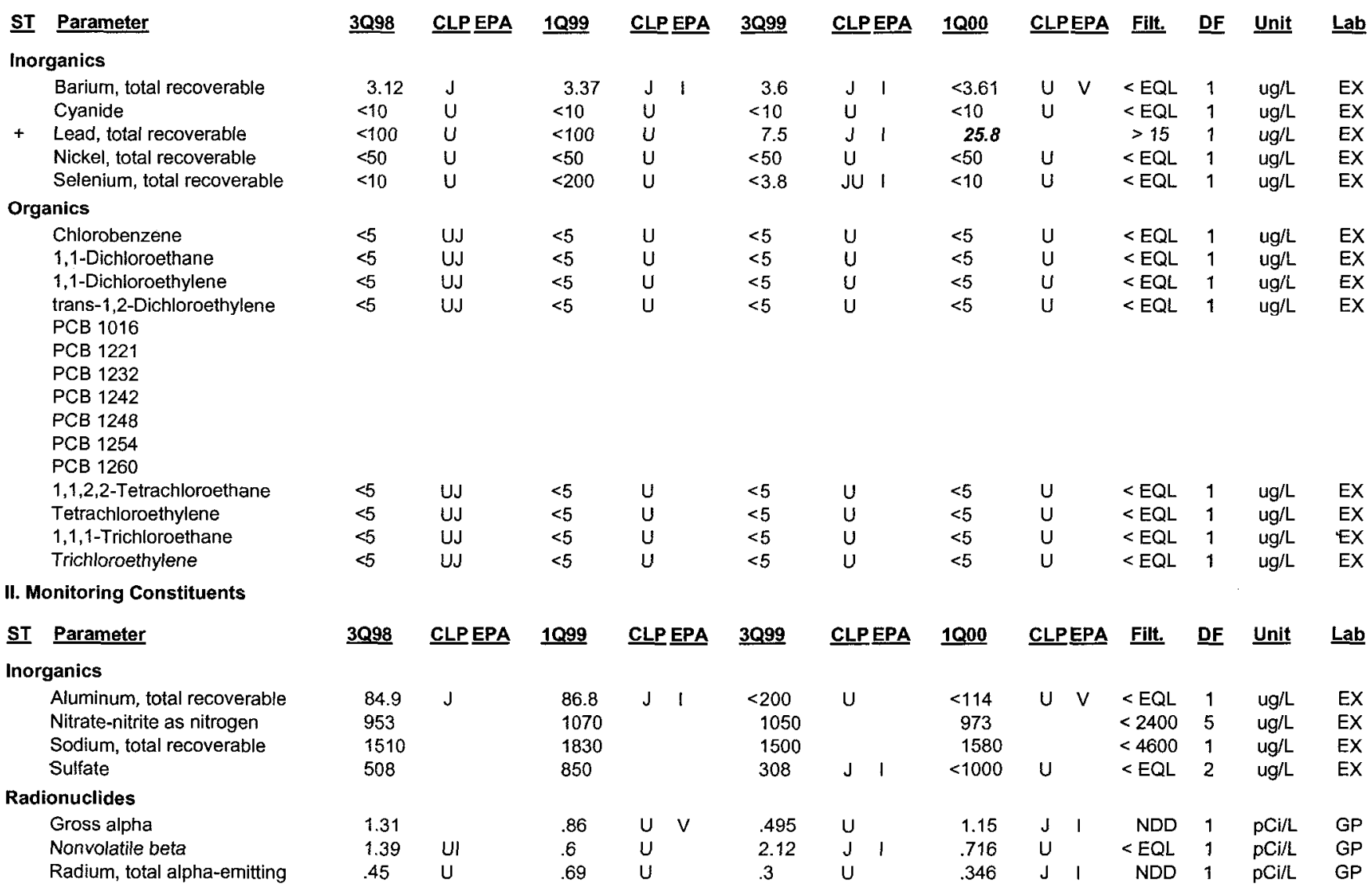

Notes: Concentrations in bold italics exceed the groundwater protection or monitoring constituent standards listed in Appendix A. Synchronous water levels are measured over a 3-5 day period or less. Dilution factors, Laboratory, and Filtered Data are for First Quarter 2000 data only.

+= exceeded the Groundwater Protection Standards or Monitoring Constituents listed in Appendix A for First Quarter 2000. 
Table D-8. Groundwater Monitoring Results for Plume Definition Wells, Met Lab HWMF

WELL AMB 4B

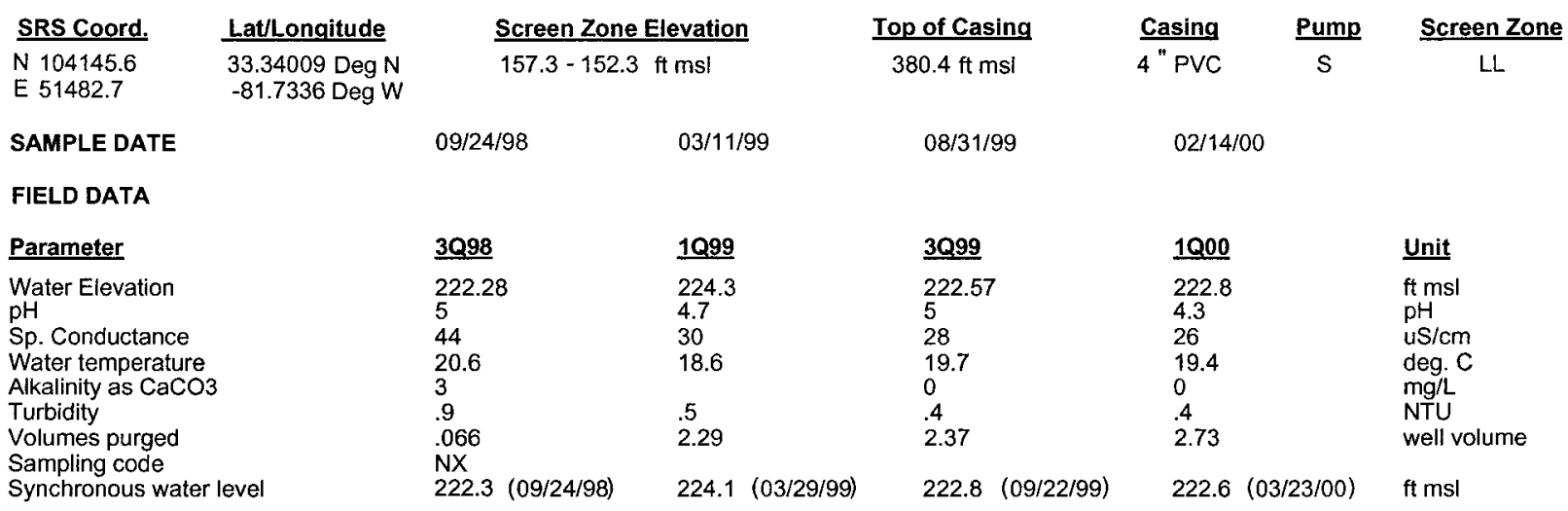

ANALYTICAL DATA

I. Groundwater Protection Standard

261 Appendix VIII/264 Appendix IX Hazardous Constituents

\begin{tabular}{|c|c|c|c|c|c|c|c|c|c|c|c|c|c|c|}
\hline \multirow{2}{*}{$\begin{array}{l}\text { ST Parameter } \\
\text { Inorganics }\end{array}$} & \multirow[t]{2}{*}{$\underline{3 Q 98}$} & \multirow[t]{2}{*}{ CLPEPA } & \multirow[t]{2}{*}{$\underline{1 Q 99}$} & \multicolumn{2}{|c|}{ CLP EPA } & \multirow[t]{2}{*}{$\underline{3099}$} & CLPEPA & \multirow[t]{2}{*}{$\underline{1000}$} & \multicolumn{2}{|c|}{ CLPEPA } & \multirow[t]{2}{*}{ Filt. } & \multirow[t]{2}{*}{ DF } & \multirow[t]{2}{*}{ Unit } & \multirow[t]{2}{*}{$\underline{L a b}$} \\
\hline & & & & & & & & & & & & & & \\
\hline Barium, total recoverable & 9.6 & $J$ & 5.1 & & & 6.1 & & 5.16 & J & I & NDD & 1 & $u g / L$ & EX \\
\hline Cyanide & $<15.2$ & $\mathrm{u}$ & $<15.2$ & $u$ & & $<15.2$ & $u$ & $<10$ & u & & $<E Q L$ & 1 & $\mathrm{ug} / \mathrm{L}$ & EX \\
\hline Lead, total recoverable & $<100$ & $u$ & $<47$ & $\mathrm{u}$ & & $<47$ & U & $<10$ & $u$ & & $<E Q L$ & 1 & $\mathrm{ug} / \mathrm{L}$ & EX \\
\hline Nickel, total recoverable & $<26$ & $u$ & $<26$ & u & & $<26$ & U & $<50$ & $u$ & & $<E Q L$ & 1 & $\mathrm{ug} / \mathrm{L}$ & EX \\
\hline Selenium, total recoverable & $<200$ & $u$ & $<66$ & $u$ & & $<66$ & $u$ & $<10$ & $\mathrm{U}$ & & $<E Q L$ & 1 & $\mathrm{ug} / \mathrm{L}$ & EX \\
\hline \multicolumn{15}{|l|}{ Organics } \\
\hline Chlorobenzene & $<5$ & $\mathrm{u}$ & $<5$ & $\mathrm{u}$ & & $<5$ & $u$ & $<5$ & $\mathrm{u}$ & & $<E Q L$ & 1 & $u g / L$ & EX \\
\hline 1,1-Dichloroethane & $<5$ & $u$ & $<5$ & u & & $<5$ & $\mathrm{u}$ & $<5$ & $\mathrm{u}$ & & $<E Q L$ & 1 & $\mathrm{ug} / \mathrm{L}$ & EX \\
\hline 1,1-Dichloroethylene & $<5$ & $\mathrm{u}$ & $<5$ & $u$ & & $<5$ & $u$ & $<5$ & $u$ & & $<E Q L$ & 1 & $\mathrm{ug} / \mathrm{L}$ & EX \\
\hline trans-1,2-Dichloroethylene & $<5$ & $\mathrm{u}$ & $<5$ & $u$ & & $<5$ & $u$ & $<5$ & $u$ & & $<E Q L$ & 1 & $\mathrm{ug} / \mathrm{L}$ & $\mathrm{EX}$ \\
\hline PCB 1016 & & & & & & & & & & & & & & \\
\hline PCB 1221 & & & & & & & & & & & & & & \\
\hline PCB 1232 & & & & & & & & & & & & & & \\
\hline PCB 1242 & & & & & & & & & & & & & & \\
\hline PCB 1248 & & & & & & & & & & & & & & \\
\hline PCB 1254 & & & & & & & & & & & & & & \\
\hline PCB 1260 & & & & & & & & & & & & & & \\
\hline 1,1,2,2-Tetrachloroethane & $<5$ & $\mathrm{u}$ & $<5$ & u & & $<5$ & $u$ & $<5$ & $\mathrm{u}$ & & $<E Q L$ & 1 & $\mathrm{ug} / \mathrm{L}$ & EX \\
\hline Tetrachloroethylene & $<5$ & $\mathrm{u}$ & $<5$ & $\mathrm{u}$ & & $<5$ & $u$ & $<5$ & $\mathrm{u}$ & & $<E Q L$ & 1 & $\mathrm{ug} / \mathrm{L}$ & EX \\
\hline $1,1,1$-Trichloroethane & $<5$ & u & $<5$ & u & & $<5$ & u & $<5$ & $u$ & & $<\mathrm{EQL}$ & 1 & $u g / L$ & EX \\
\hline Trichloroethylene & 3.56 & $\mathrm{~J}$ & 2.82 & $J$ & 1 & 3.26 & J 1 & 4.5 & $J$ & 1 & NDD & 1 & ug/L & EX \\
\hline \multicolumn{15}{|l|}{ II. Monitoring Constituents } \\
\hline ST Parameter & 3098 & CLPEPA & iQ999 & CLF & EPA & 3099 & CLPEPA & 1000 & $\underline{\text { CLP }}$ & EPA & Filt. & $\underline{\text { DF }}$ & Unit & $\underline{\text { Lab }}$ \\
\hline \multicolumn{15}{|l|}{ Inorganics } \\
\hline Aluminum, total recoverable & 292 & & 24.7 & $\mathrm{~J}$ & 1 & 29 & J 1 & $<49.7$ & $\mathrm{u}$ & $\mathrm{v}$ & $<E Q L$ & 1 & ug/L & EX \\
\hline Nitrate-nitrite as nitrogen & 440 & $\mathrm{~J}$ & 445 & & & 444 & & $<500$ & $\mathrm{u}$ & & $<E Q L$ & 5 & $\mathrm{ug} / \mathrm{L}$ & EX \\
\hline Sodium, total recoverable & 3210 & & 2870 & & & 2960 & & 2950 & & & $<4600$ & 1 & ug/L & EX \\
\hline Sulfate & 1180 & & 451 & & & 402 & & 797 & 子 & । & NDD & 2 & ug/L & EX \\
\hline \multicolumn{15}{|l|}{ Radionuclides } \\
\hline Gross alpha & 1.85 & & 2.84 & $\mathrm{~J}$ & 1 & .636 & $u$ & 1.75 & J & I & NDD & 1 & $\mathrm{pCi} / \mathrm{L}$ & GP \\
\hline Nonvolatile beta & 8.38 & UI & -1.59 & $\mathrm{u}$ & & .463 & u & 1.66 & j & $i$ & NDD & 1 & $\mathrm{pCi} / \mathrm{L}$ & GP \\
\hline Radium, total alpha-emitting & .74 & & 1.97 & $\mathrm{~J}$ & 1 & .3 & $\mathrm{U}$ & .355 & $\mathrm{~J}$ & 1 & NDD & 1 & $\mathrm{pCi} / \mathrm{L}$ & GP \\
\hline
\end{tabular}

\footnotetext{
Notes: Concentrations in bold italics exceed the groundwater protection or monitoring constituent standards listed in Appendix A. Synchronous water levels are measured over a 3-5 day period or less. Dilution factors, Laboratory, and Filtered Data are for First Quarter 2000 data only.

$+=$ exceeded the Groundwater Protection Standards or Monitoring Constituents listed in Appendix A for First Quarter 2000.
} 
Table D-8. Groundwater Monitoring Results for Plume Definition Wells, Met Lab HWMF (Cont.) WELL AMB 7

\begin{tabular}{|c|c|c|c|c|c|c|c|}
\hline SRS Coord. & Lat/Longitude & \multicolumn{2}{|c|}{ Screen Zone Elevation } & Top of Casing & Casing & Pump & Screen Zone \\
\hline $\begin{array}{l}\text { N } 103920 \\
E 51624.9\end{array}$ & $\begin{array}{l}33.33983 \operatorname{Deg} N \\
-81.7328 \operatorname{Deg} W\end{array}$ & \multicolumn{2}{|c|}{$242.1-222.1 \mathrm{ft} \mathrm{msl}$} & $369.9 \mathrm{ft} \mathrm{msl}$ & 4 "PVC & $S$ & \multirow[t]{3}{*}{$M$} \\
\hline SAMPLE DATE & & $09 / 02 / 98$ & $02 / 08 / 99$ & $08 / 28 / 99$ & \multicolumn{2}{|c|}{$02 / 15 / 00$} & \\
\hline \multicolumn{7}{|l|}{ FIELD DATA } & \\
\hline Parameter & & $\underline{3 Q 98}$ & $\underline{1099}$ & $\underline{3 Q 99}$ & \multicolumn{2}{|l|}{$\underline{1 Q 00}$} & Unit \\
\hline $\begin{array}{l}\text { Water Elevation } \\
\text { pH } \\
\text { Sp. Conductance } \\
\text { Water temperature } \\
\text { Alkalinity as CaCO3 } \\
\text { Turbidity } \\
\text { Volumes purged } \\
\text { Sampling code } \\
\text { Synchronous water le }\end{array}$ & level & $\begin{array}{l}231.66 \\
5.5 \\
67 \\
19.8 \\
16 \\
13 \\
163 \\
N X \\
231.4 \quad(09 / 24 / 98)\end{array}$ & $\begin{array}{l}232.04 \\
5.8 \\
51 \\
20.1 \\
16 \\
13.2 \\
.626 \\
\mathrm{NX} \\
232.1 \quad(03 / 26 / 99)\end{array}$ & $\begin{array}{l}231.6 \\
6.2 \\
54 \\
22.7 \\
19 \\
8.7 \\
.164 \\
\mathrm{NX} \\
231.3 \quad(09 / 22 / 99)\end{array}$ & \multicolumn{2}{|c|}{$\begin{array}{l}230.78 \\
6.5 \\
64 \\
17.5 \\
22 \\
11 \\
.539 \\
N X \\
230.7\end{array}$} & $\begin{array}{l}\mathrm{ft} \mathrm{msl} \\
\mathrm{pH} \\
\text { uS/cm } \\
\text { deg. C } \\
\mathrm{mg} / \mathrm{L} \\
\mathrm{NTU} \\
\text { well volume }\end{array}$ \\
\hline
\end{tabular}

ANALYTICAL DATA

I. Groundwater Protection Standard

261 Appendix VIII/264 Appendix IX Hazardous Constituents

ST Parameter

Inorganics

Barium, total recoverable

Cyanide

Lead, total recoverable

Nickel, total recoverable

Selenium, total recoverable

Organics

Chlorobenzene

1,1-Dichloroethane

1,1-Dichloroethylene

trans-1,2-Dichloroethylene

PCB 1016

PCB 1221

PCB 1232

PCB 1242

PCB 1248

PCB 1254

1,1,2,2-Tetrachloroethane

Tetrachloroethylene

1,1,1-Trichloroethane

Trichloroethylene

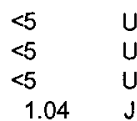

$\underline{309}$

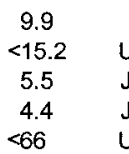

$<5 \quad U$

$<5 \quad U$

$<5 \quad U$

II. Monitoring Constituents

ST Parameter

Inorganics

Aluminum, total recoverable

Nitrate-nitrite as nitrogen

+ Sodium, total recoverable

+ Sulfate

Radionuclides

Gross alpha

Nonvolatile beta

Radium, total alpha-emitting

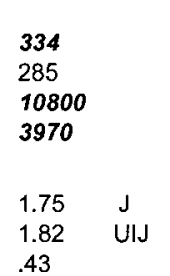

.43
CLPEPA 1 Q99

CLP EPA

$\underline{3099}$

\section{CLPEPA 1000 CLPEPA}

Filt. DF Unit Lab

$\begin{array}{cccc}6.8 & & & 10.1 \\ 2.41 & \mathrm{~J} & \mathrm{I} & <15.2 \\ 9.4 & \mathrm{~J} & \mathrm{I} & 6 \\ 3.8 & \mathrm{~J} & \mathrm{I} & 3.3 \\ <66 & \mathrm{U} & & <66 \\ & & & \\ <5 & \mathrm{JU} & \mathrm{Q} & <5 \\ <5 & \mathrm{JU} & \mathrm{Q} & <5 \\ <5 & \mathrm{JU} & \mathrm{Q} & <5 \\ <5 & \mathrm{JU} & \mathrm{Q} & <5\end{array}$

$\begin{array}{ll}U & \\ J & 1 \\ J & 1 \\ U & \\ U & \\ U & \\ U & \\ U & \end{array}$

10.1
$<10$
7.2
$<11.3$
$<10$

$<5$
$<5$
$<5$
$<5$

$\begin{array}{ll}\mathrm{U} & \\ \mathrm{U} & \mathrm{V}\end{array}$

$<2000$

$<$ EQL 1

NDD 1

EQL 1 ug/L EX

EQL 1 ug/L EX

$<$ EQL 11 ug/L EX

$<$ EQL 1 ug/L EX

$<$ EQL 1 ug/L EX

\begin{tabular}{|c|c|}
\hline$<5$ & JU \\
\hline$<5$ & JU \\
\hline$<5$ & JU \\
\hline$<5$ & JU \\
\hline
\end{tabular}

$\begin{array}{ll}<5 & U \\ <5 & U \\ <5 & U \\ <5 & U\end{array}$

$\begin{array}{ll}<5 & \mathrm{U} \\ <5 & \mathrm{U} \\ <5 & \mathrm{U} \\ <5 & \mathrm{U}\end{array}$

$\begin{array}{ll}<\mathrm{EQL} & 1 \\ <\mathrm{EQL} & 1 \\ <\mathrm{EQL} & 1\end{array}$

ug/L EX ug/L. EX

ug/L EX

$<$ EQL 1 ug/L EX

\section{CLPEPA}

\section{CLPEPA 1000 CLPEPA Filt. DF Unit Lab}

$\begin{array}{lllllllll}\mathbf{1 8 9} & \mathbf{3 4 2} & <367 & \mathrm{U} & <\mathrm{EQL} & 1 & \mathrm{ug} / \mathrm{L} & \mathrm{EX} \\ 357 & 575 & 379 & \mathrm{~J} & \mathrm{I} & \mathrm{NDD} & 5 & \mathrm{ug} / \mathrm{L} & \mathrm{EX} \\ \mathbf{9 2 3 0} & \mathbf{8 1 4 0} & \mathbf{1 2 4 0 0} & & >4600 & 1 & \mathrm{ug} / \mathrm{L} & \text { EX } \\ \mathbf{3 8 0 0} & \mathbf{4 0 1 0} & \mathbf{5 0 5 0} & & >3000 & 2 & \mathrm{ug} / \mathrm{L} & \mathrm{EX}\end{array}$

$\begin{array}{lllllllllrlll}1.57 & \mathrm{U} & \mathrm{V} & .55 & \mathrm{~J} & \mathrm{IK} & 1.25 & \mathrm{~J} & \mathrm{I} & \mathrm{NDD} & 1 & \mathrm{pCi} / \mathrm{L} & \mathrm{GP} \\ .71 & \mathrm{U} & & .93 & \mathrm{U} & & 1.16 & \mathrm{U} & & <\mathrm{EQL} & 1 & \mathrm{pCi} / \mathrm{L} & \mathrm{GP} \\ .13 & \mathrm{U} & & .5 & \mathrm{~J} & \mathrm{I} & .11 & \mathrm{U} & & <\mathrm{EQL} & 1 & \mathrm{pCi} / \mathrm{L} & \mathrm{GP}\end{array}$

\footnotetext{
Notes: Concentrations in bold italics exceed the groundwater protection or monitoring constituent standards listed in Appendix A. Synchronous water levels are measured over a 3-5 day period or less. Dilution factors, Laboratory, and Filtered Data are for First Quarter 2000 data only.
}

$+=$ exceeded the Groundwater Protection Standards or Monitoring Constituents listed in Appendix A for First Quarter 2000. 
Table D-8. Groundwater Monitoring Results for Plume Definition Wells, Met Lab HWMF (Cont.) WELL AMB 7A

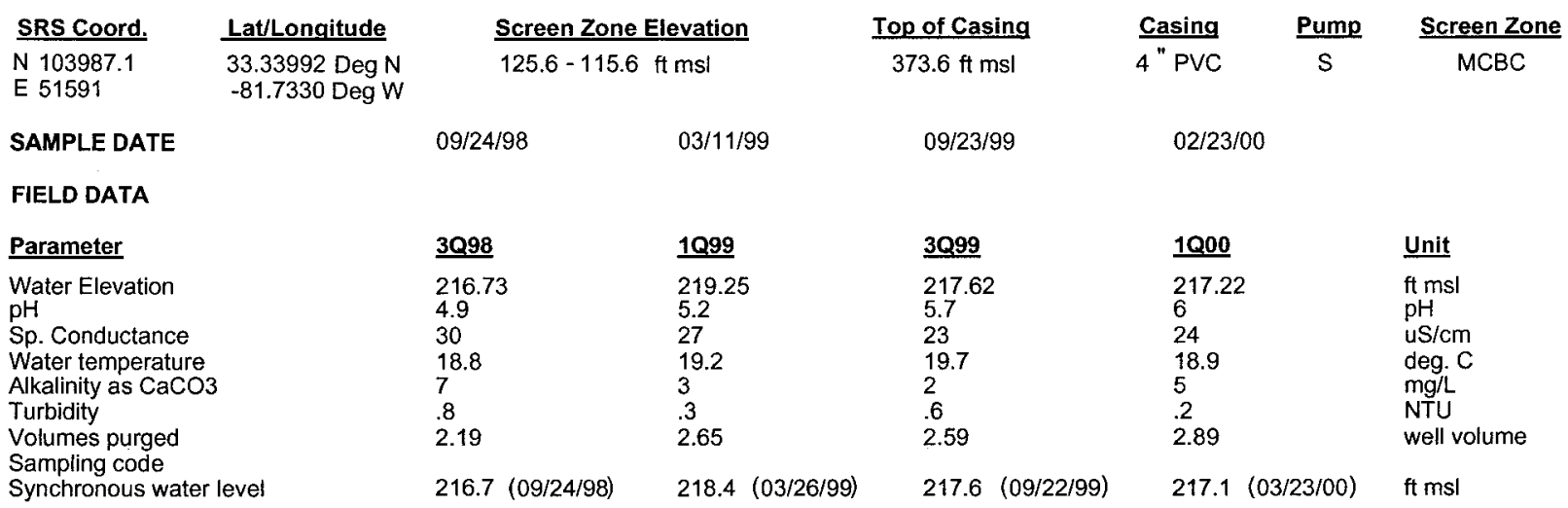

ANALYTICAL DATA

I. Groundwater Protection Standard

261 Appendix VIII/264 Appendix IX Hazardous Constituents

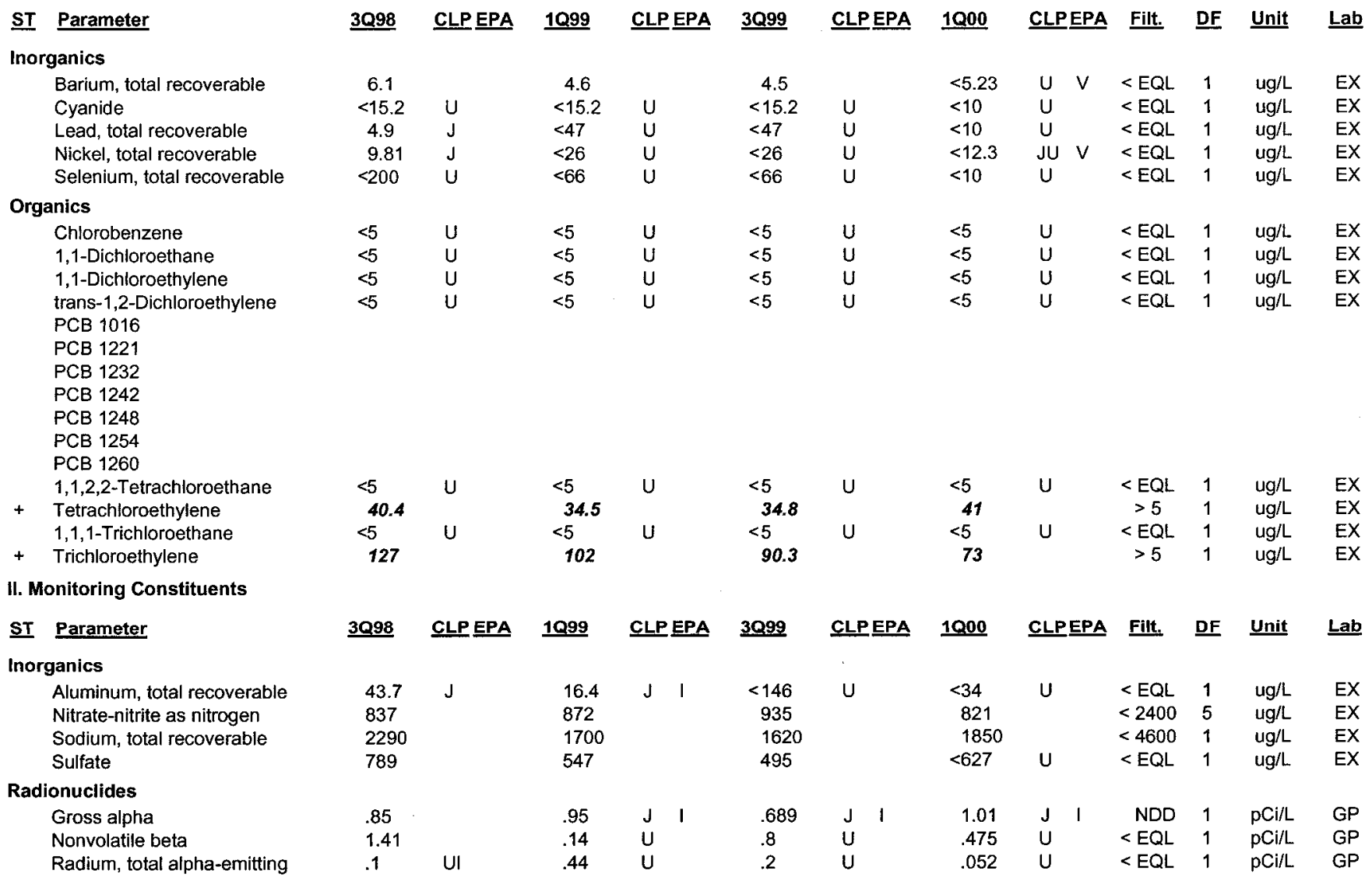

Notes: Concentrations in bold italics exceed the groundwater protection or monitoring constituent standards listed in Appendix A. Synchronous water levels are measured over a 3-5 day period or less. Dilution factors, Laboratory, and Filtered Data are for First Quarter 2000 data only.

$+=$ exceeded the Groundwater Protection Standards or Monitoring Constituents listed in Appendix A for First Quarter 2000. 
Table D-8. Groundwater Monitoring Results for Plume Definition Wells, Met Lab HWMF (Cont.) WELL AMB 7B

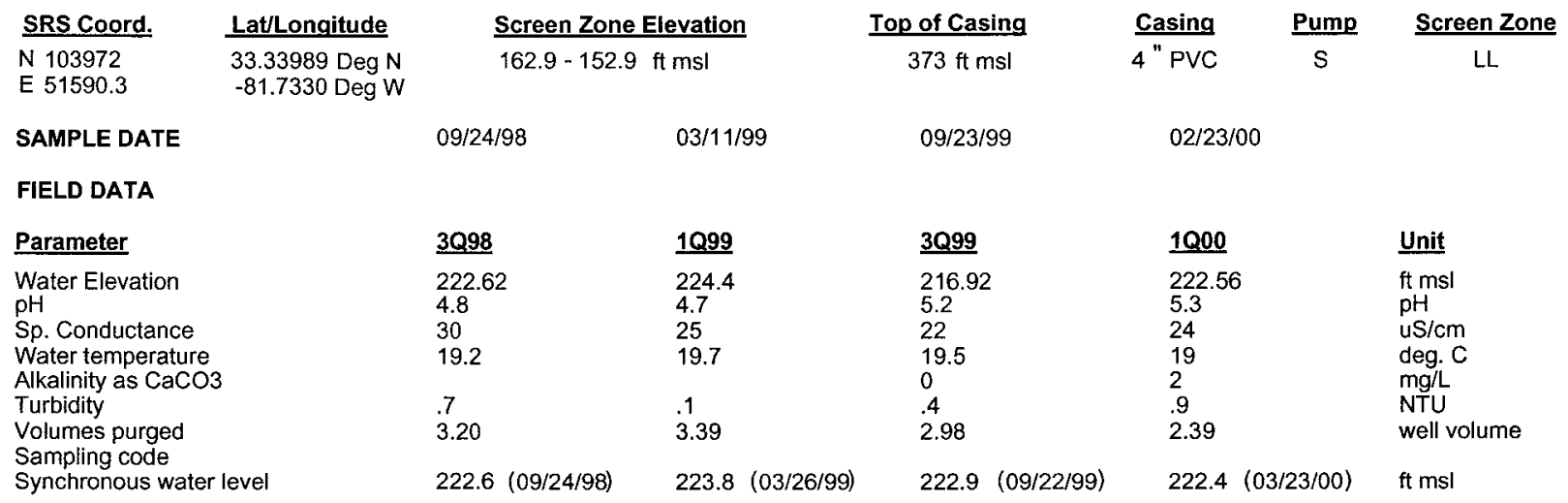

ANALYTICAL DATA

I. Groundwater Protection Standard

261 Appendix VIII/264 Appendix IX Hazardous Constituents

\begin{tabular}{|c|c|c|c|c|c|c|c|c|c|c|c|c|c|c|}
\hline$\underline{\text { ST }}$ & Parameter & $\underline{3 Q 98}$ & CLP EPA & 1099 & CLP EPA & $\underline{3 Q 99}$ & CLPEPA & $1 Q 00$ & $\underline{\text { CLP }}$ & EPA & Filt. & $\underline{D F}$ & $\underline{\text { Unit }}$ & $\underline{\text { Lab }}$ \\
\hline Inor & ganics & & & & & & & & & & & & & \\
\hline & 8arium, total recoverable & 2.5 & & 2.5 & & 2.8 & & $<2.88$ & $\mathrm{U}$ & V & $<E Q L$ & 1 & ug/L & EX \\
\hline & Cyanide & $<15.2$ & $U$ & $<15.2$ & $U$ & $<15.2$ & $\mathrm{U}$ & $<10$ & $\mathrm{U}$ & & $<\mathrm{EQL}$ & 1 & ug/L & EX \\
\hline & Lead, total recoverable & 18.6 & $\mathrm{~J}$ & $<100$ & $U$ & $<47$ & $\mathrm{U}$ & $<10$ & $\mathrm{U}$ & & $<\mathrm{EQL}$ & 1 & ug/L & EX \\
\hline & Nickel, total recoverable & $<26$ & $U$ & $<50$ & $U$ & $<26$ & $\mathrm{U}$ & $<5.95$ & JU & V & $<\mathrm{EQL}$ & 1 & ug/L & EX \\
\hline & Selenium, total recoverable & $<66$ & $U$ & $<200$ & $U$ & $<66$ & $\mathrm{U}$ & $<10$ & $U$ & & $<\mathrm{EQL}$ & 1 & $u g / L$ & EX \\
\hline Org & anics & & & & & & & & & & & & & \\
\hline & Chtorobenzene & $<5$ & $\mathrm{U}$ & $<5$ & $U$ & $<5$ & $u$ & $<5$ & $\mathrm{U}$ & & $<\mathrm{EQL}$ & 1 & ug/L & EX \\
\hline & 1,1-Dichloroethane & $<5$ & $\mathrm{U}$ & $<5$ & $\mathrm{U}$ & $<5$ & $\mathrm{U}$ & $<5$ & $\mathrm{U}$ & & $<\mathrm{EQL}$ & 1 & $\mathrm{ug} / \mathrm{L}$ & $x$ \\
\hline & 1,1-Dichloroethylene & $<5$ & $\mathrm{U}$ & $<5$ & $U$ & $<5$ & $\mathrm{U}$ & $<5$ & $\mathrm{U}$ & & $<\mathrm{EQL}$ & 1 & $\mathrm{ug} / \mathrm{L}$ & EX \\
\hline & trans-1,2-Dichloroethylene & $<5$ & $\mathrm{U}$ & $<5$ & $\mathrm{U}$ & $<5$ & $\mathrm{U}$ & $<5$ & $\mathrm{U}$ & & $<\mathrm{EQL}$ & 1 & $\mathrm{ug} / \mathrm{L}$ & EX \\
\hline & PCB 1016 & & & & & & & & & & & & & \\
\hline & PCB 1221 & & & & & & & & & & & & & \\
\hline & PCB 1232 & & & & & & & & & & & & & \\
\hline & PCB 1242 & & & & & & & & & & & & & \\
\hline & PCB 1248 & & & & & & & & & & & & & \\
\hline & PCB 1254 & & & & & & & & & & & & & \\
\hline & PCB 1260 & & & & & & & & & & & & & \\
\hline & 1,1,2,2-Tetrachloroethane & $<5$ & $\mathrm{U}$ & $<5$ & $\mathrm{U}$ & $<5$ & $U$ & $<5$ & $U$ & & $<\mathrm{EQL}$ & 1 & $\mathrm{ug} / \mathrm{L}$ & EX \\
\hline & Tetrachioroethylene & $<5$ & $\mathrm{U}$ & $<5$ & $\mathrm{U}$ & $<5$ & $\mathrm{U}$ & $<5$ & $\mathrm{U}$ & & $<\mathrm{EQL}$ & 1 & ug/L & $x$ \\
\hline & 1,1,1-Trichloroethane & $<5$ & $\mathrm{u}$ & $<5$ & $\mathrm{U}$ & $<5$ & $\mathrm{U}$ & $<5$ & $U$ & & $<\mathrm{EQL}$ & 1 & ug/L & $E x$ \\
\hline+ & Trichloroethylene & 6.06 & & 6.25 & & 5.26 & & 5.9 & & & $>5$ & 1 & $\mathrm{ug} / \mathrm{L}$ & \\
\hline II. M & onitoring Constituents & & & & & & & & & & & & & \\
\hline$\underline{\text { ST }}$ & Parameter & $\underline{3 Q 98}$ & $\underline{\text { CLP EPA }}$ & 1099 & CLP EPA & $\underline{3099}$ & CLPEPA & 1000 & CLP & EPA & Filt. & $\underline{\mathrm{DF}}$ & Unit & \\
\hline Inor & ganics & & & & & & & & & & & & & \\
\hline & Aluminum, total recoverable & 23.3 & J & 36.1 & $J 1$ & $<146$ & $U$ & 35.3 & J & 1 & NDD & 1 & ug/L & Ex \\
\hline & Nitrate-nitrite as nitrogen & 593 & & 776 & & 636 & & 647 & & & $<2400$ & 5 & $\mathrm{ug} / \mathrm{L}$ & $x$ \\
\hline & Sodium, total recoverable & 3300 & & 3890 & & 3130 & & 3750 & & & $<4600$ & 1 & $\mathrm{ug} / \mathrm{L}$ & EX \\
\hline & Sulfate & 836 & & 895 & & 821 & & $<1000$ & $U$ & & $<E Q L$ & 1 & ug/L & EX \\
\hline Rad & ionuclides & & & & & & & & & & & & & \\
\hline & Gross alpha & .7 & UI & 1.7 & $\mathrm{~J} \quad$ & .611 & $\mathrm{U}$ & .761 & $J$ & 1 & NDD & 1 & $\mathrm{pCi} / \mathrm{L}$ & GP \\
\hline & Nonvolatile beta & .4 & UIJ & .662 & $\mathrm{U}$ & .778 & $\mathrm{U}$ & .515 & $U$ & & $<E Q L$ & 1 & $\mathrm{pCi} / \mathrm{L}$ & GP \\
\hline & Radium, total alpha-emitting & .09 & Ui & .35 & $\mathrm{U}$ & .2 & $\mathrm{U}$ & .263 & $U$ & & $<E Q L$ & 1 & $\mathrm{pCi} / \mathrm{L}$ & \\
\hline
\end{tabular}

Notes: Concentrations in bold italics exceed the groundwater protection or monitoring constituent standards listed in Appendix A. Synchronous water levels are measured over a 3-5 day period or less. Dilution factors, Laboratory, and Filtered Data are for First Quarter 2000 data only.

$+=$ exceeded the Groundwater Protection Standards or Monitoring Constituents listed in Appendix A for First Quarter 2000. 
Table D-8. Groundwater Monitoring Results for Plume Definition Wells, Met Lab HWMF (Cont.) WELL AMB 11B

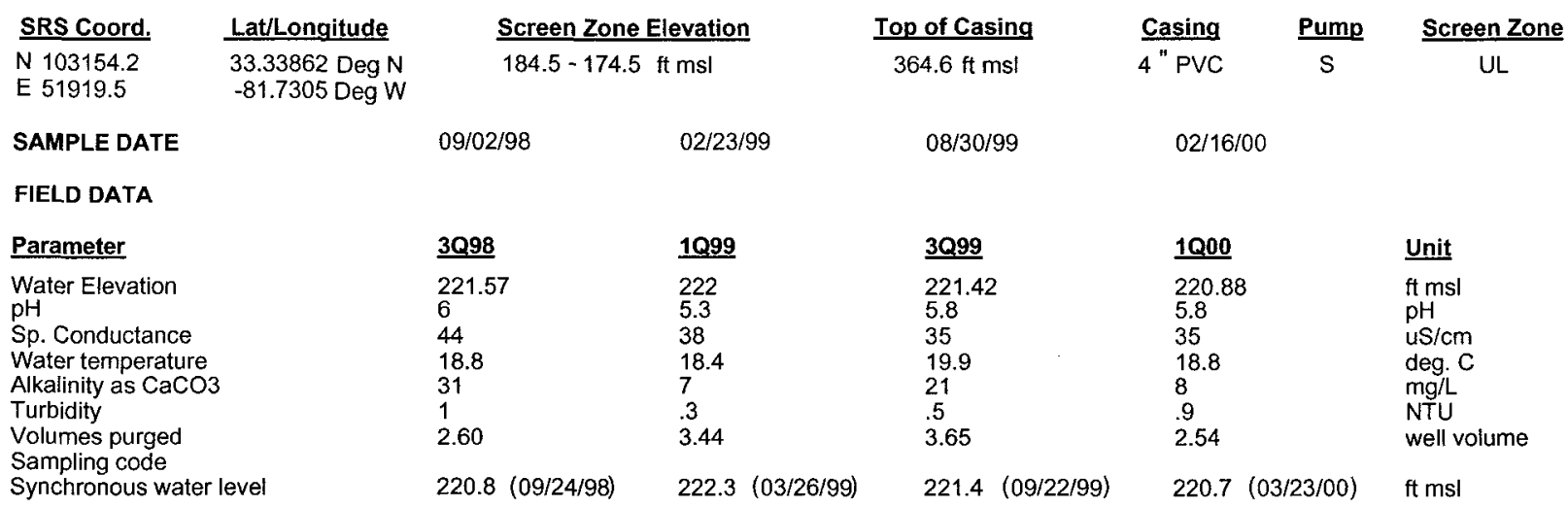

ANALYTICAL DATA

I. Groundwater Protection Standard

261 Appendix VIII/264 Appendix IX Hazardous Constituents

\begin{tabular}{|c|c|c|c|c|c|c|c|c|c|c|c|c|c|}
\hline \multirow{2}{*}{$\begin{array}{l}\text { ST Parameter } \\
\text { Inorganics }\end{array}$} & \multirow[t]{2}{*}{$\underline{3098}$} & \multirow[t]{2}{*}{ CLPEPA } & \multirow[t]{2}{*}{$\underline{1 Q 99}$} & CLPEPA & \multirow[t]{2}{*}{$\underline{30,99}$} & \multirow[t]{2}{*}{ CLPEPA } & \multirow[t]{2}{*}{1000} & \multicolumn{2}{|c|}{ CLPEPA } & \multirow[t]{2}{*}{ Filt. } & \multirow[t]{2}{*}{ DF } & \multirow[t]{2}{*}{$\underline{\text { Unit }}$} & $\underline{\underline{L}}$ \\
\hline & & & & & & & & & & & & & \\
\hline Barium, total recoverable & 24.9 & & 101 & & 19.5 & & 43.2 & & & $<2000$ & 1 & $\mathrm{ug} / \mathrm{h}$ & \\
\hline Cyanide & $<15.2$ & $u$ & $<15.2$ & $u$ & $<15.2$ & $u$ & $<10$ & $\mathrm{U}$ & & $<E Q L$ & 1 & $\mathrm{ug} / \mathrm{L}$ & \\
\hline Lead, total recoverable & $<47$ & $u$ & $<100$ & $\mathrm{u}$ & $<47$ & $u$ & $<10$ & $\mathrm{u}$ & & $<\mathrm{EQL}$ & 1 & ug/L & \\
\hline Nickel, total recoverable & $<26$ & $u$ & $<50$ & $u$ & $<26$ & $u$ & $<9.87$ & JU & $\mathrm{v}$ & $<\mathrm{EQL}$ & 1 & $\mathrm{ug} / \mathrm{L}$ & \\
\hline Selenium, total recoverable & $<66$ & $U$ & $<200$ & $u$ & $<66$ & $u$ & $<10$ & $U$ & & $<\mathrm{EQL}$ & 1 & $\mathrm{ug} / \mathrm{L}$ & \\
\hline \multicolumn{13}{|l|}{ Organics } & \\
\hline Chlorobenzene & $<5$ & $\mathrm{u}$ & $<5$ & $u$ & $<5$ & $u$ & $<5$ & $\mathrm{u}$ & & $<\mathrm{EQL}$ & 1 & $\mathrm{ug} / \mathrm{L}$ & \\
\hline 1,1-Dichloroethane & $<5$ & $\mathrm{u}$ & $<5$ & $u$ & $<5$ & $u$ & $<5$ & $u$ & & $<E Q L$ & 1 & $u g / L$ & \\
\hline 1,1-Dichloroethylene & $<5$ & $\mathrm{U}$ & $<5$ & $\mathrm{U}$ & $<5$ & $u$ & $<5$ & $u$ & & $<E Q L$ & 1 & $\mathrm{ug} / \mathrm{L}$ & \\
\hline $\begin{array}{l}\text { trans-1,2-Dichloroethylene } \\
\text { PCB } 1016\end{array}$ & $<5$ & $u$ & $<5$ & $u$ & $<5$ & $u$ & $<5$ & u & & $<E Q L$ & 1 & $\mathrm{ug} / \mathrm{L}$ & \\
\hline PCB 1221 & & & & & & & & & & & & & \\
\hline PCB 1232 & & & & & & & & & & & & & \\
\hline PCB 1242 & & & & & & & & & & & & & \\
\hline РCB 1248 & & & & & & & & & & & & & \\
\hline PCB 1254 & & & & & & & & & & & & & \\
\hline PCB 1260 & & & & & & & & & & & & & \\
\hline 1,1,2,2-Tetrachloroethane & $<5$ & $u$ & $<5$ & $\mathrm{u}$ & $<5$ & $\mathrm{u}$ & $<5$ & U & & $<E Q L$. & 1 & $\mathrm{ug} / \mathrm{L}$ & \\
\hline Tetrachioroethylene & $<5$ & $u$ & $<5$ & $u$ & $<5$ & $u$ & $<5$ & $\mathrm{u}$ & & $<E Q L$ & 1 & $\mathrm{ug} / \mathrm{L}$ & \\
\hline 1,1,1-Trichloroethane & $<5$ & $\mathrm{U}$ & $<5$ & $u$ & $<5$ & U & $<5$ & $U$ & & $<E Q L$ & 1 & $u g / L$ & \\
\hline Trichloroethylene & $<5$ & $u$ & $<5$ & $u$ & $<5$ & $u$ & $<5$ & $u$ & & $<E Q L$ & 1 & $\mathrm{ug} / \mathrm{L}$ & \\
\hline \multicolumn{14}{|l|}{ II. Monitoring Constituents } \\
\hline ST Parameter & $\underline{3098}$ & CLPEPA & 1099 & CLPEPA & $\underline{3099}$ & CLPEPA & $\underline{1000}$ & $\underline{\text { CLP }}$ & EPA & Filt. & $\underline{\text { DF }}$ & $\underline{\text { Unit }}$ & \\
\hline \multicolumn{14}{|l|}{ Inorganics } \\
\hline Aluminum, total recoverable & 34.7 & J & 29.8 & J 1 & $<146$ & $\mathrm{U}$ & 60.5 & $J$ & 1 & NDD & 1 & $\mathrm{ug} / \mathrm{L}$ & \\
\hline Nitrate-nitrite as nitrogen & 217 & & 216 & & 226 & & $<500$ & $u$ & & $<E Q L$ & 5 & ugh & \\
\hline Sodium, total recoverable & 2130 & & 2160 & & 1960 & & 2570 & & & $<4600$ & 1 & $u g / L$ & \\
\hline Sulfate & 465 & & 561 & & 463 & & 833 & $J$ & 1 & NDD & 2 & ug/L & \\
\hline \multicolumn{13}{|l|}{ Radionuclides } & \\
\hline Gross alpha & .97 & $\mathrm{~J}$ & 1.42 & J 1 & .425 & $u$ & .489 & $\mathrm{u}$ & & $<E Q L$ & 1 & $\mathrm{pCi} / \mathrm{L}$ & \\
\hline Nonvolatile beta & 6.67 & $J$ & .764 & U & .485 & U & .835 & u & & $<E Q L$ & 1 & $\mathrm{pCi} / \mathrm{L}$ & \\
\hline Radium, total al & .62 & & .61 & $u$ & & $\mathrm{U}$ & .584 & $\mathrm{~J}$ & & NDD & 1 & $\mathrm{pCl}$ & \\
\hline
\end{tabular}

Notes: Concentrations in bold italics exceed the groundwater protection or monitoring constituent standards listed in Appendix A. Synchronous water levels are measured over a 3-5 day period or less. Dilution factors, Laboratory, and Filtered Data are for First Quarter 2000 data only.

$+=$ exceeded the Groundwater Protection Standards or Monitoring Constituents listed in Appendix A for First Quarter 2000. 
Table D-8. Groundwater Monitoring Results for Plume Definition Wells, Met Lab HWMF (Cont.) WELL AMB 13AR

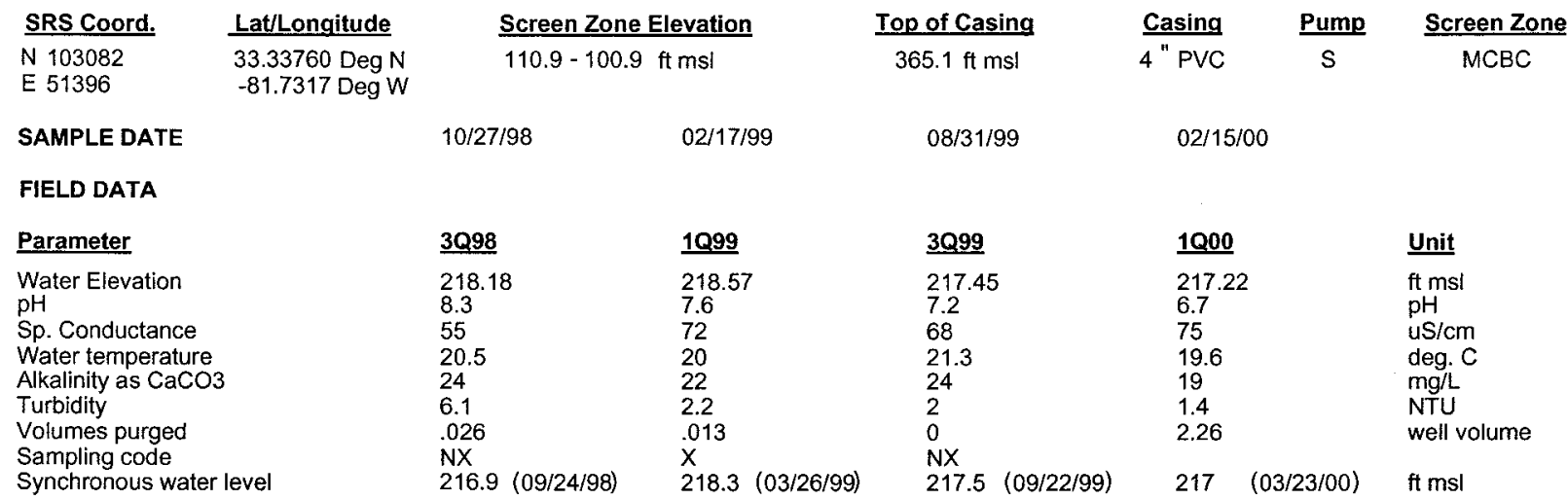

\section{ANALYTICAL DATA}

I. Groundwater Protection Standard

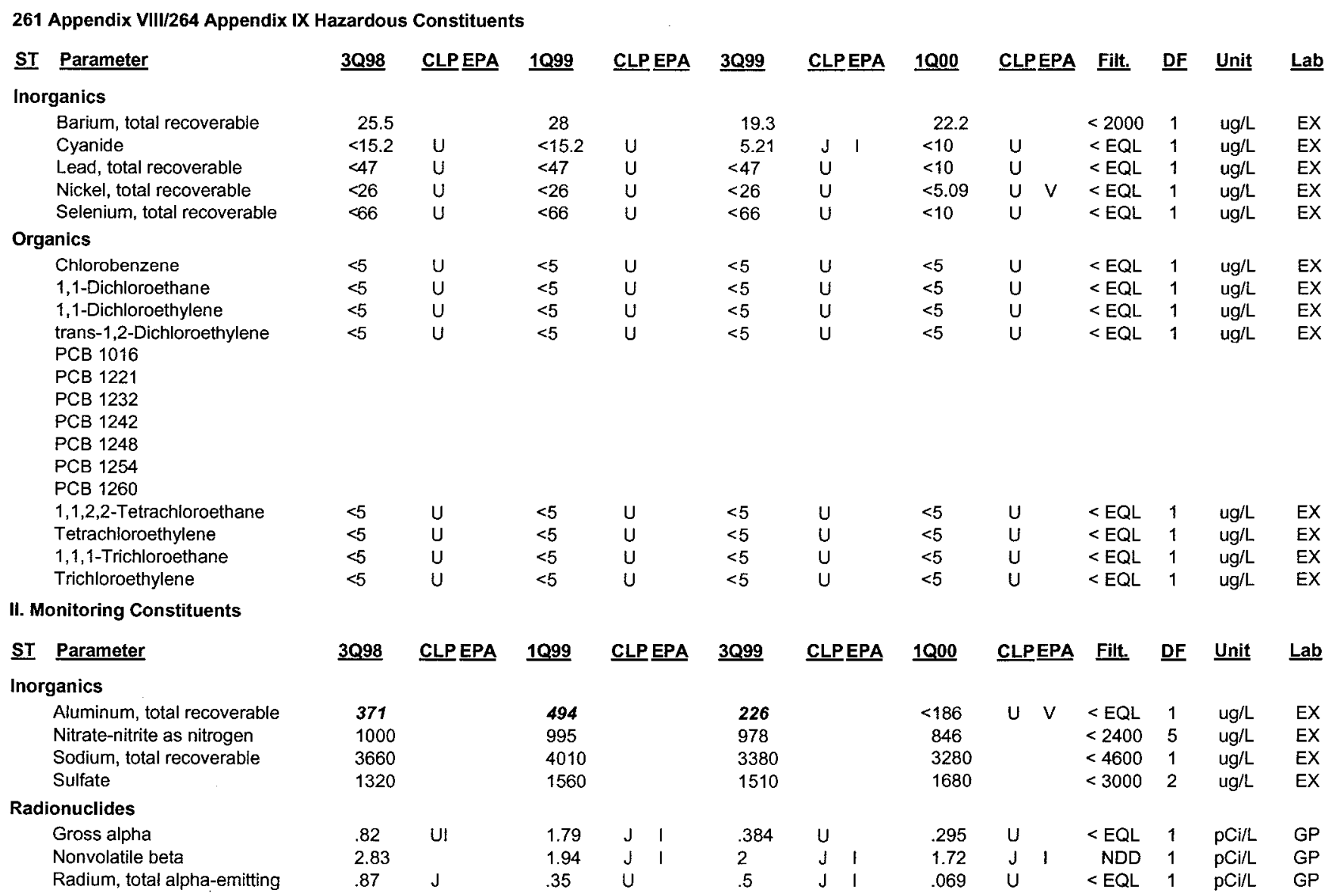

Notes: Concentrations in bold italics exceed the groundwater protection or monitoring constituent standards listed in Appendix A. Synchronous water levels are measured over a 3-5 day period or less. Dilution factors, Laboratory, and Filtered Data are for First Quarter 2000 data only.

$+=$ exceeded the Groundwater Protection Standards or Monitoring Constituents listed in Appendix A for First Quarter 2000. 
WSRC-TR- 2000-00242

Table D-8. Groundwater Monitoring Results for Plume Definition Wells, Met Lab HWMF (Cont.) WELL AMB 14D

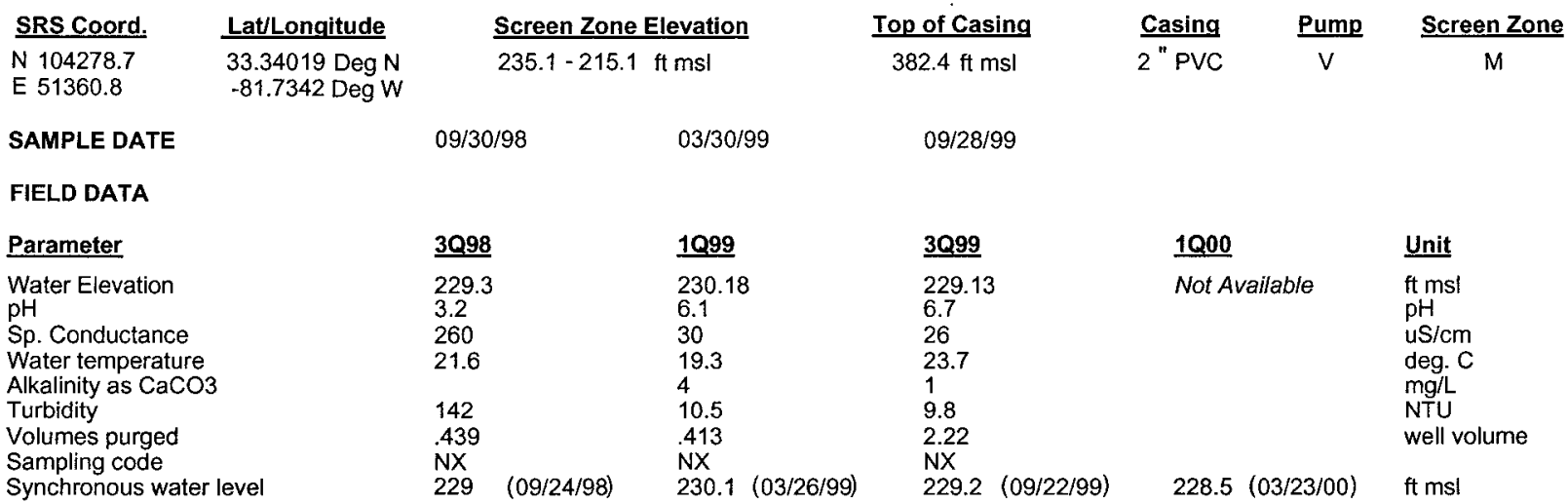

ANALYTICAL DATA

I. Groundwater Protection Standard

261 Appendix VIII/264 Appendix IX Hazardous Constituents

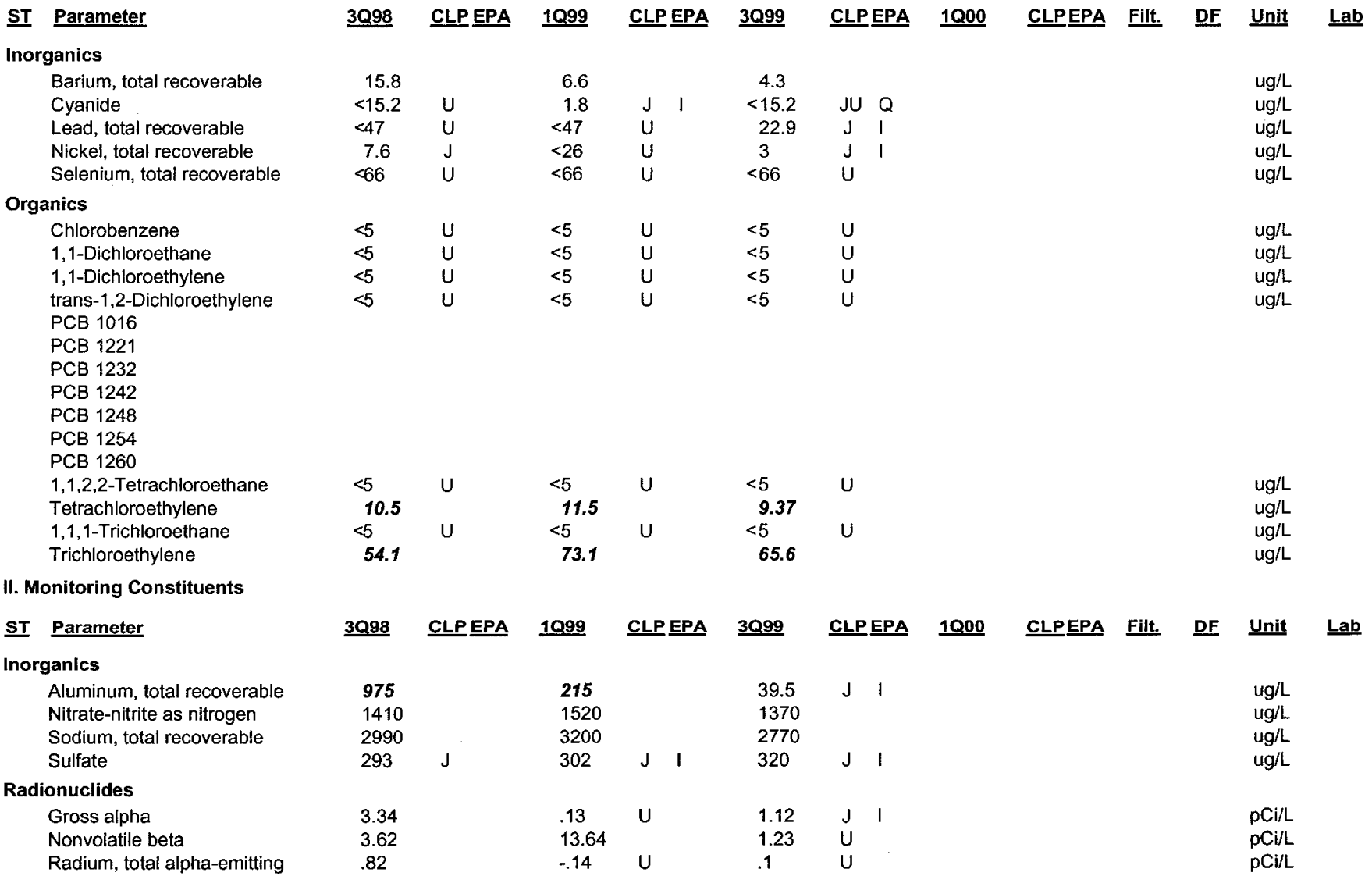

Notes: Concentrations in bold italics exceed the groundwater protection or monitoring constituent standards listed in Appendix A. Synchronous water levels are measured over a 3-5 day period or less. Dilution factors, Laboratory, and Filtered Data are for First Quarter 2000 data only.

$+=$ exceeded the Groundwater Protection Standards or Monitoring Constituents listed in Appendix A for First Quarter 2000. 
Table D-8. Groundwater Monitoring Results for Plume Definition Wells, Met Lab HWMF (Cont.) WELL AMB 15D

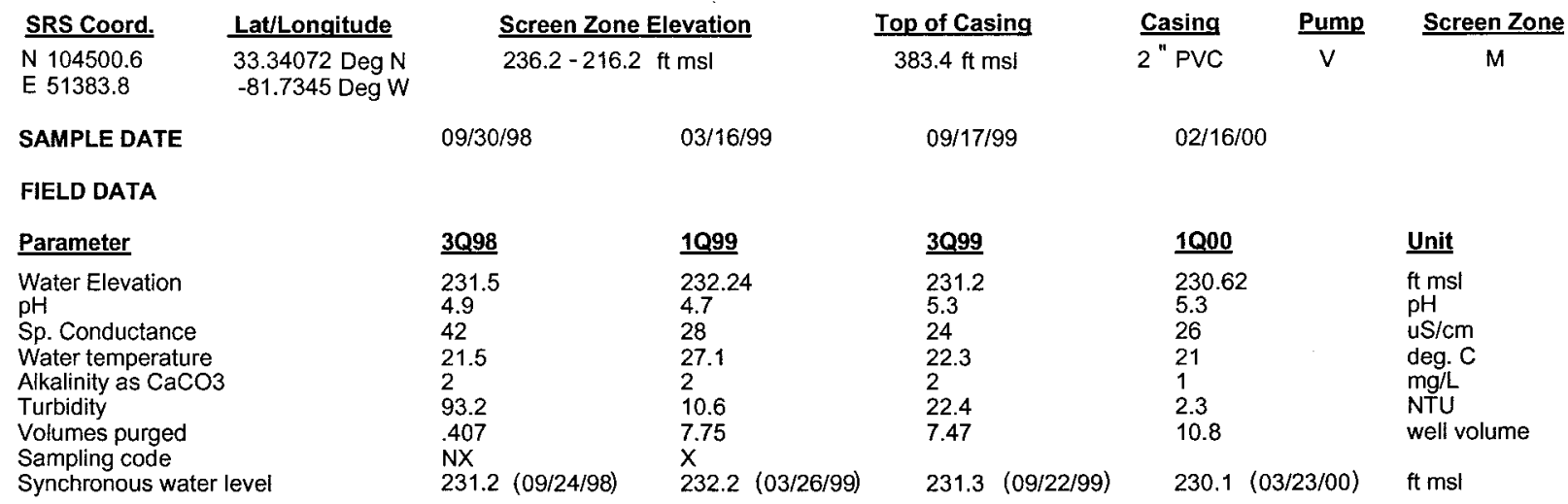

ANALYTICAL DATA

1. Groundwater Protection Standard

261 Appendix VIII/264 Appendix IX Hazardous Constituents

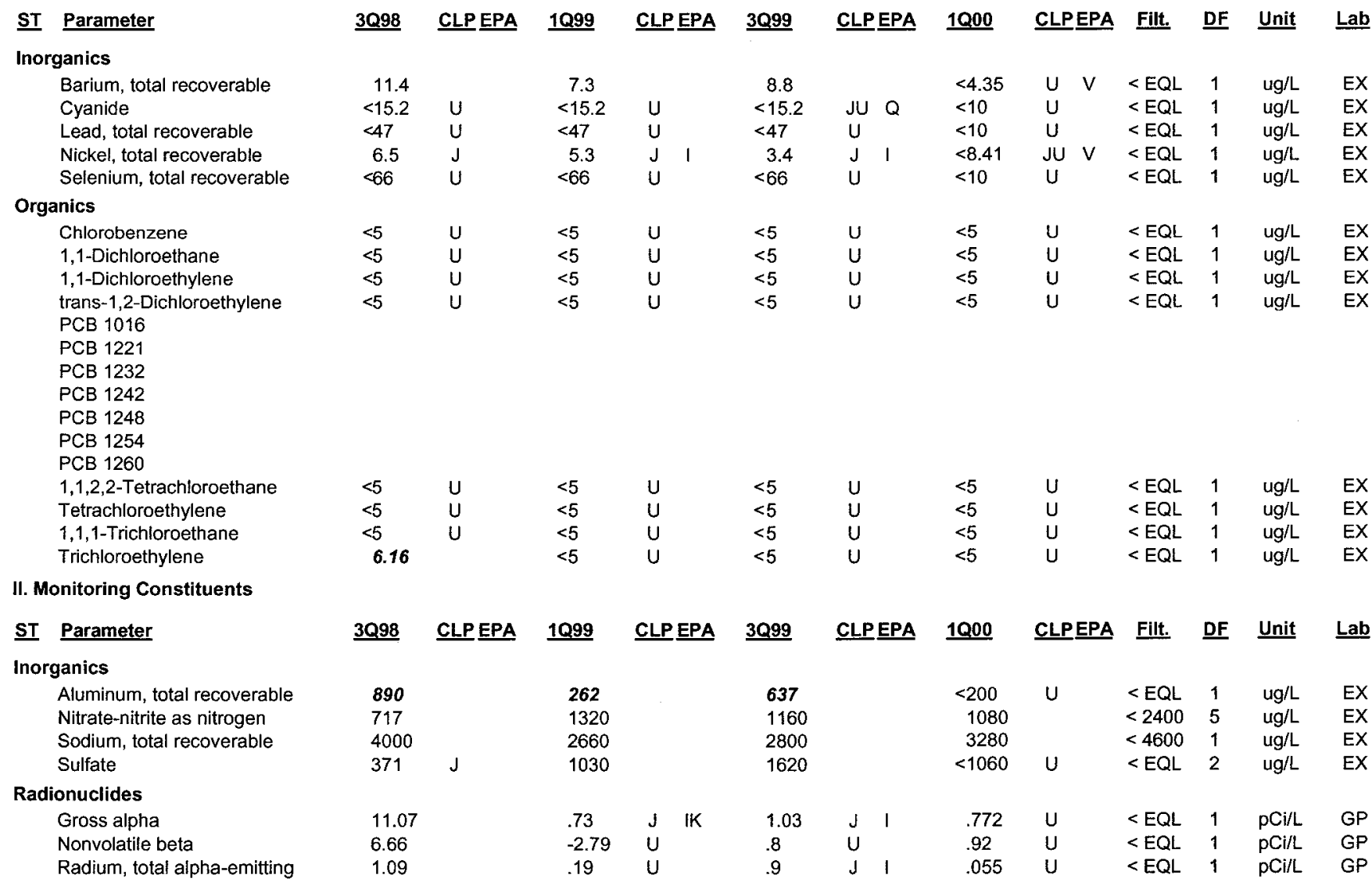

Notes: Concentrations in bold italics exceed the groundwater protection or monitoring constituent standards listed in Appendix A. Synchronous water levels are measured over a 3-5 day period or less. Dilution factors, Laboratory, and Filtered Data are for First Quarter 2000 data only.

+ = exceeded the Groundwater Protection Standards or Monitoring Constituents listed in Appendix A for First Quarter 2000. 
Table D-9A. Groundwater Monitoring Results for QA Wells, M-Area and Met Lab HWMF WELL AMB 7A

$\begin{array}{ll}\text { SRS Coord. } & \text { Lat/Longitude } \\ \text { N 103987.1 } & \text { 33.33992 Deg N } \\ \text { E 51591 } & -81.7330 \text { Deg W }\end{array}$

\section{SAMPLE DATE}

FIELD DATA

\section{Parameter}

Water Elevation

$\mathrm{pH}$

Sp. Conductance

Water temperature

Alkalinity as $\mathrm{CaCO} 3$

Turbidity

Volumes purged

Sampling code

Synchronous water level

\section{ANALYTICAL DATA}

ST Parameter

1,1,1-Trichloroethane

1,1,2,2-Tetrachloroethane

1,1-Dichloroethane

1,1-Dichloroethylene

1,2-Dichloroethane

2,4-Dichlorophenoxyacetic acid

Acetone

Aluminum, total recoverable

Arsenic, total recoverable

Barium, total recoverable

Carbon tetrachloride

Chloride

Chlorobenzene

Chloroethene (Vinyl chloride)

Chloroform

Chromium, total recoverable

Cyanide

Fluoride

Gross alpha

Iron, total recoverable

Lead, total recoverable

Lindane

Manganese, total recoverable

Mercury, total recoverable

Methyl methacrylate

Nickel, total recoverable

Nitrate-nitrite as nitrogen

Nonvolatile beta

Phenol

Radium, total alpha-emitting

Selenium, total recoverable

Silver, total recoverable

Sodium, total recoverable

Sulfate

Tetrachloroethylene

Total organic carbon

Total organic halogens

Total phosphates (as P)

Trichloroethylene

cis-1,2-Dichloroethylene

trans-1,2-Dichloroethylene

\begin{tabular}{l} 
Screen Zone Elevation \\
\hline $125.6-115.6 \mathrm{ft} \mathrm{msi}$ \\
$02 / 23 / 00$ \\
\\
$\underline{1000}$ \\
217.22 \\
6 \\
24 \\
18.9 \\
5 \\
.2 \\
2.89
\end{tabular}

\author{
Top of Casing \\ $373.6 \mathrm{ft} \mathrm{msl}$
}

Casing

Pump

Screen Zone

$\mathrm{MCBC}$

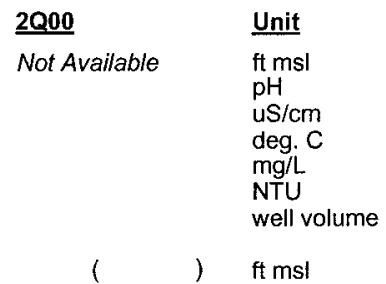

2Q00 CLPEPA Filt. DF Unit Lab

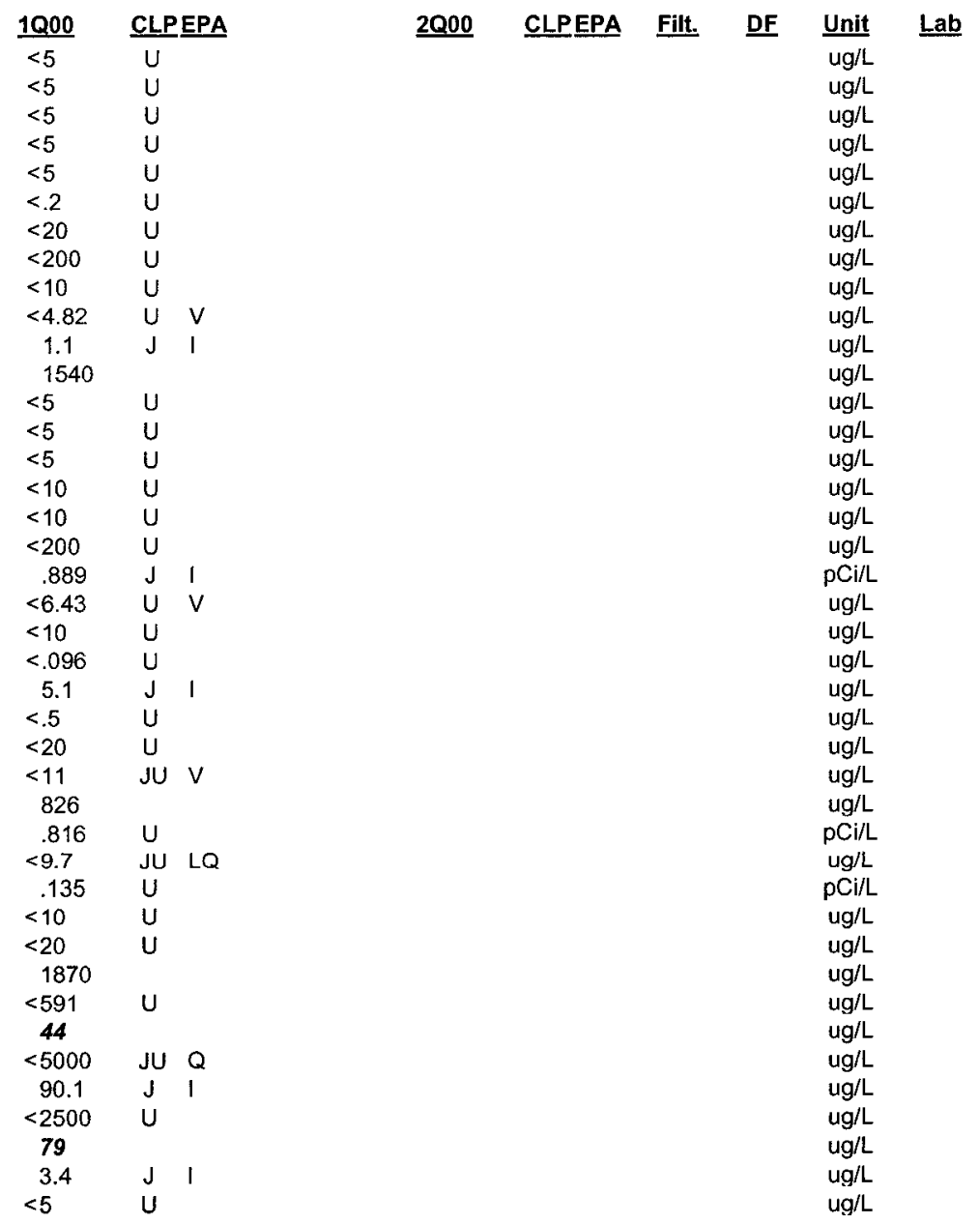

Notes: Concentrations in bold italics exceed the groundwater protection or monitoring constituent standards listed in Appendix A. Synchronous water levels are measured over a 3-5 day period or less. Dilution factors, Laboratory, and Filtered Data are for Second Quarter 2000 data only. $+=$ exceeded the groundwater protection or monitoring constituent standards listed in Appendix A for Second Quarter 2000. 
Table D-9A. Groundwater Monitoring Results for QA Wells, M-Area and Met Lab HWMF (Cont.) WELL ASB 5C

\begin{tabular}{|c|c|c|c|c|c|c|}
\hline SRS Coord. & Lat/Longitude & Screen Zone Elevation & Top of Casing & Casing & Pump & Screen Zone \\
\hline $\begin{array}{l}\text { N } 105884.8 \\
\text { E } 52837.8\end{array}$ & $\begin{array}{l}\text { 33.34615 Deg } N \\
\text {-81.7334 Deg } W\end{array}$ & $175.1-165.1 \mathrm{ft} \mathrm{msl}$ & $347.3 \mathrm{ft} \mathrm{msl}$ & 4 "PVC & $\mathrm{S}$ & LL \\
\hline SAMPLE DATE & & $03 / 23 / 00$ & & & & \\
\hline
\end{tabular}

FIELD DATA

Parameter

Water Elevation

$\mathrm{pH}$

Sp. Conductance

Water temperature

Alkalinity as $\mathrm{CaCO} 3$

Turbidity

Volumes purged

Sampling code

Synchronous water level

ANALYTICAL DATA

SI Parameter

1,1,1-Trichloroethane

1,1,2,2-Tetrachloroethane

1,1-Dichloroethane

1,1-Dichloroethylene

1,2-Dichloroethane

Acetone

Carbon tetrachloride

Chlorobenzene

Chloroethene (Vinyl chloride)

Chloroform

Tetrachloroethylene

Trichloroethylene

cis-1,2-Dichloroethylene

trans-1,2-Dichloroethylene

$\frac{1000}{220.28}$
6.1
45
18.9
11
1
2.77

2Q00

Not Available

Unit

$\mathrm{ft} \mathrm{ms}$

$\mathrm{pH}$

uS/cm

deg. $\mathrm{C}$

$\mathrm{mg} / \mathrm{L}$

NTU

well volume

$\mathrm{ft} \mathrm{msl}$

\begin{tabular}{|c|c|c|c|c|c|c|}
\hline 1000 & CLPEPA & 2000 & CLPEPA & Filt. & $\underline{\text { DF }}$ & Unit \\
\hline$<1$ & U & & & & & $\mathrm{ug} / \mathrm{L}$ \\
\hline$<1$ & U & & & & & $\mathrm{ug} / \mathrm{L}$ \\
\hline$<1$ & $U$ & & & & & $u g / L$ \\
\hline$<1$ & $\mathrm{U}$ & & & & & $u g / L$ \\
\hline$<1$ & U & & & & & $u g / L$ \\
\hline$<10$ & U & & & & & $u g / L$ \\
\hline$<1$ & $U$ & & & & & $u g / L$ \\
\hline$<1$ & $\mathrm{U}$ & & & & & $\mathrm{ug} / \mathrm{L}$ \\
\hline$<1$ & U & & & & & $\mathrm{ug} / \mathrm{L}$ \\
\hline$<1$ & $\mathrm{U}$ & & & & & $\mathrm{ug} / \mathrm{L}$ \\
\hline 55.3 & $J \quad K$ & & & & & $\mathrm{ug} / \mathrm{L}$ \\
\hline 182 & $J \quad K$ & & & & & $u g / L$ \\
\hline 3.51 & $J \quad K$ & & & & & $u g / L$ \\
\hline$<1$ & $\mathrm{U}$ & & & & & $\mathrm{ug} / \mathrm{L}$ \\
\hline
\end{tabular}

Notes: Concentrations in bold italics exceed the groundwater protection or monitoring constituent standards listed in Appendix A. Synchronous water levels are measured over a 3-5 day period or less. Dilution factors, Laboratory, and Filtered Data are for Second Quarter 2000 data only.

$+=$ exceeded the groundwater protection or monitoring constituent standards listed in Appendix A for Second Quarter 2000. 
Table D-9A. Groundwater Monitoring Results for QA Wells, M-Area and Met Lab HWMF (Cont.) WELL ASB 8TA

\begin{tabular}{|c|c|}
\hline SRS coord. & Lat/Longitude \\
\hline $\begin{array}{l}\text { N } 106375.8 \\
\text { E } 53124.7\end{array}$ & -8 \\
\hline
\end{tabular}

SAMPLE DATE

FIELD DATA

\section{Parameter}

Water Elevation

$\mathrm{pH}$

Sp. Conductance

Water temperature

Alkalinity as $\mathrm{CaCO} 3$

Turbidity

Volumes purged

Sampling code

Synchronous water level

\section{ANALYTICAL DATA}

ST Parameter

1,1,1-Trichloroethane

1,1,2,2-Tetrachloroethane

1,1-Dichloroethane

1,1-Dichloroethylene

1,2-Dichloroethane

Acetone

Carbon tetrachloride

Chlorobenzene

Chloroethene (Vinyl chloride)

Chloroform

Tetrachloroethylene

Trichloroethylene

cis-1,2-Dichloroethylene

trans-1,2-Dichloroethylene

\begin{tabular}{c} 
Screen Zone Elevation \\
\hline $24.6-19.4 \quad \mathrm{ft} \mathrm{msl}$ \\
$02 / 01 / 00$ \\
\\
$\frac{1000}{214.58}$ \\
4.9 \\
21 \\
18.5 \\
0 \\
1.1 \\
2.26
\end{tabular}

\author{
Top of Casing \\ $349.6 \mathrm{ft} \mathrm{msl}$
}

Casing

Pump

$S$

Screen Zone CBA

\begin{tabular}{|c|c|c|c|c|c|c|}
\hline 1000 & CLPEPA & $\underline{2 Q 00}$ & CLPEPA & Filt. & $\underline{\mathrm{DF}}$ & Unit \\
\hline$<1$ & JU & & & & & $u g / L$ \\
\hline$<1$ & JU & & & & & $\mathrm{ug} / \mathrm{L}$ \\
\hline$<1$ & JU & & & & & $\mathrm{ug} / \mathrm{L}$ \\
\hline$<1$ & JU & & & & & ug/h \\
\hline$<1$ & JU & & & & & ug/L \\
\hline$<10$ & $J$ & & & & & $\mathrm{ug} / \mathrm{t}$ \\
\hline$<1$ & JU & & & & & ug/L \\
\hline .28 & J I & & & & & ug $/ \mathrm{L}$ \\
\hline$<1$ & JU & & & & & $\mathrm{ug} / \mathrm{L}$ \\
\hline$<1$ & JU & & & & & ug/L \\
\hline$<1$ & JU & & & & & ug/L. \\
\hline$<1$ & JU & & & & & ug $/ \mathrm{L}$ \\
\hline$<1$ & JU & & & & & ug/L \\
\hline$<1$ & JU & & & & & $u g / L$ \\
\hline
\end{tabular}

Notes: Concentrations in bold italics exceed the groundwater protection or monitoring constituent standards listed in Appendix A. Synchronous water levels are measured over a 3-5 day period or less. Dilution factors, Laboratory, and Filtered Data are for Second Quarter 2000 data only. $+=$ exceeded the groundwater protection or monitoring constituent standards listed in Appendix A for Second Quarter 2000. 
Table D-9A. Groundwater Monitoring Results for QA Wells, M-Area and Met Lab HWMF (Cont.) WELL MSB 5B

\begin{tabular}{|c|c|c|c|c|c|c|}
\hline SRS Coord. & Lat/Longitude & Screen Zone Elevation & Top of Casing & Casing & Pump & Screen Zone \\
\hline $\begin{array}{l}N 101971.1 \\
E \text { 46983.6 }\end{array}$ & $\begin{array}{c}\text { 33.32794 Deg N } \\
-81.7412 \operatorname{Deg} W\end{array}$ & $136.1-131.4 \mathrm{ft} \mathrm{msl}$ & $345 \mathrm{ft} \mathrm{msl}$ & 4 " PVC & S & LL \\
\hline SAMPLE DATE & & $02 / 24 / 00$ & & & & \\
\hline
\end{tabular}

\section{FIELD DATA}

Parameter

Water Elevation

$\mathrm{pH}$

Sp. Conductance

Water temperature

Alkalinity as $\mathrm{CaCO} 3$

Turbidity

volumes purged

Sampling code

Synchronous water level

\section{ANALYTICAL DATA}

ST Parameter

1,1,1-Trichloroethane

1,1,2,2-Tetrachloroethane

1,1-Dichloroethane

1,1-Dichloroethylene

1,2-Dichloroethane

Acetone

Aluminum, total recoverable

Arsenic, total recoverable

Barium, total recoverable

Carbon tetrachloride

Chlorobenzene

Chloroethene (Vinyl chloride)

Chloroform

Chromium, total recoverable

Cobalt, total recoverable

Copper, total recoverable

Iron, total recoverable

Lead, total recoverable

Manganese, total recoverable

Mercury, total recoverable

Nickel, total recoverable

Selenium, total recoverable

Silver, total recoverable

Sodium, total recoverable

Tetrachloroethylene

Trichloroethylene

Zinc, total recoverable

cis-1,2-Dichloroethylene

trans-1,2-Dichloroethylene

1000
206.48
4.5
18
18.4
0
3
2.50

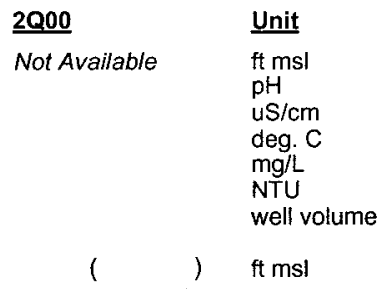

2000 CLPEPA Filt. DF Unit Lab

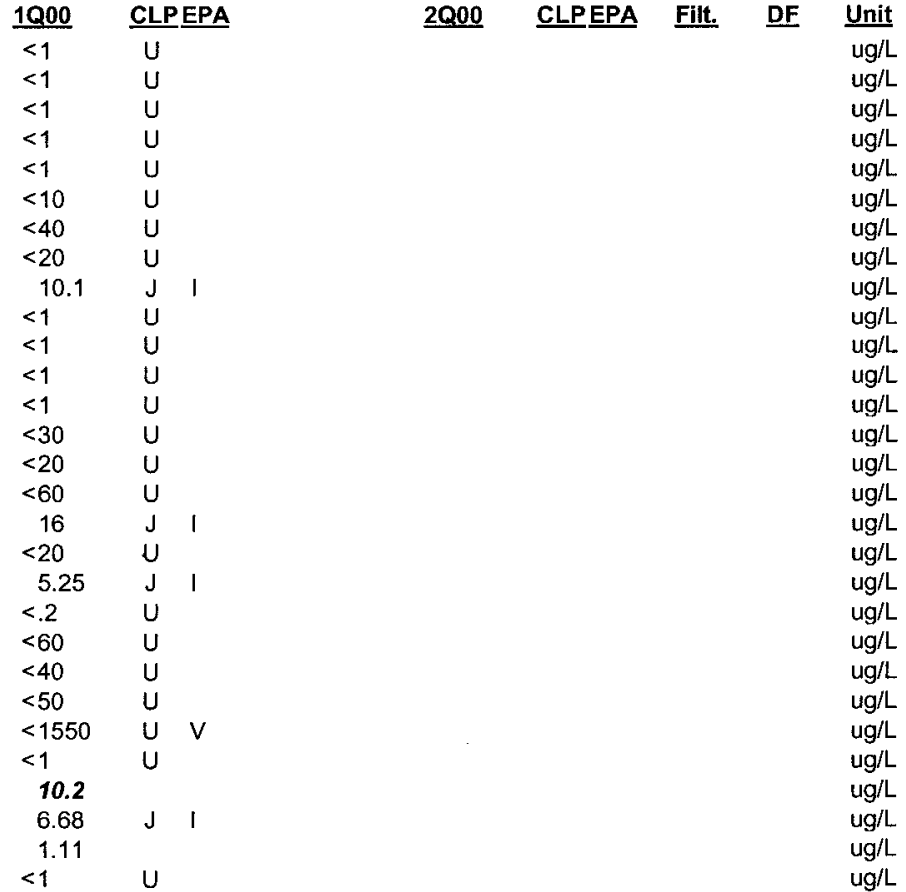

Notes: Concentrations in bold italics exceed the groundwater protection or monitoring constituent standards listed in Appendix A. Synchronous water levels are measured over a 3-5 day period or less. Dilution factors, Laboratory, and Filtered Data are for Second Quarter 2000 data only.

$+=$ exceeded the groundwater protection or monitoring constituent standards listed in Appendix A for Second Quarter 2000. 
Table D-9A. Groundwater Monitoring Results for QA Wells, M-Area and Met Lab HWMF (Cont.) WELL MSB 12B

$\begin{array}{ll}\text { SRS Coord. } & \text { Lat/Longitude } \\ \text { N 102272.76 } & \text { 33.32886 Deg N } \\ \text { E 47142.09 } & -81.7414 \text { Deg W }\end{array}$

\section{SAMPLE DATE}

FIELD DATA

Parameter

Water Elevation

$\mathrm{pH}$

Sp. Conductance

Water temperature

Alkalinity as $\mathrm{CaCO} 3$

Turbidity

Volumes purged

Sampling code

Synchronous water level

\section{ANALYTICAL DATA}

ST Parameter

1,1,1-Trichloroethane

1,1,2,2-Tetrachloroethane

1,1-Dichloroethane

1,1-Dichloroethylene

1,2-Dichloroethane

Acetone

Carbon tetrachloride

Chlorobenzene

Chloroethene (Vinyl chloride)

Chloroform

Tetrachloroethylene

Trichloroethylene

$\begin{array}{lllll}\text { Screen Zone Elevation } & \frac{\text { Top of Casing }}{350.3 \mathrm{ft} \mathrm{msl}} & \frac{\text { Casing }}{4 \text { " PVC }} & \frac{\text { Pump }}{\mathrm{S}} & \frac{\text { Screen Zone }}{\text { LLAZ }}\end{array}$

$02 / 29 / 00$

1Q00

216.68

5.1

88

18.4

2.2

2.50

$\begin{array}{ll}\text { 2Q00 } & \text { Unit } \\ \text { Not Available } & \mathrm{ft} \mathrm{msl} \\ & \mathrm{pH} \\ & \text { uS/cm } \\ & \mathrm{deg} . \mathrm{C} \\ & \mathrm{mg} / \mathrm{L} \\ & \mathrm{NTU} \\ & \text { well volume } \\ & \\ & \text { ft } \mathrm{msl}\end{array}$

2000 CLPEPA Filt. DF Unit Lab

$\mathrm{ug} / \mathrm{L}$

$u g / L$

$\mathrm{ug} / \mathrm{L}$

$\mathrm{ug} / \mathrm{L}$

$\mathrm{ug} / \mathrm{L}$

ug/L

$u g / L$

$\mathrm{ug} / \mathrm{L}$

ug/L

$\mathrm{ug} / \mathrm{L}$

ug/L

Notes: Concentrations in bold italics exceed the groundwater protection or monitoring constituent standards listed in Appendix A. Synchronous water levels are measured over a 3-5 day period or less. Dilution factors, Laboratory, and Filtered Data are for Second Quarter 2000 data only.

$+=$ exceeded the groundwater protection or monitoring constituent standards listed in Appendix A for Second Quarter 2000. 
Table D-9A. Groundwater Monitoring Results for QA Wells, M-Area and Met Lab HWMF (Cont.) WELL MSB 21B

\begin{tabular}{|c|c|c|c|c|c|c|c|c|c|c|}
\hline \multicolumn{2}{|c|}{ SRS Coord. } & Lat/Longitude & \multicolumn{2}{|c|}{ Screen Zone Elevation } & Top of Casing & Casing & \multicolumn{2}{|c|}{ Pump } & \multicolumn{2}{|c|}{ Screen Zone } \\
\hline \multicolumn{2}{|c|}{$\begin{array}{l}\text { N } 104000.1 \\
\text { E } 47271.8\end{array}$} & $\begin{array}{l}\text { 33.33289 Deg } N \\
-81.7444 \operatorname{Deg} W\end{array}$ & $147.2-142.5$ & & $355 \mathrm{ft} \mathrm{msl}$ & 4 "PVC & & $S$ & & $L$ \\
\hline \multicolumn{3}{|c|}{ SAMPLE DATE } & \multicolumn{2}{|c|}{$03 / 03 / 00$} & & & & & & \\
\hline \multicolumn{11}{|c|}{ FIELD DATA } \\
\hline \multicolumn{3}{|c|}{ Parameter } & \multicolumn{2}{|l|}{1000} & \multicolumn{2}{|l|}{$\underline{2 Q 00}$} & \multicolumn{2}{|l|}{ Unit } & & \\
\hline \multicolumn{3}{|c|}{$\begin{array}{l}\text { Water Elevation } \\
\text { pH } \\
\text { Sp. Conductance } \\
\text { Water temperature } \\
\text { Alkalinity as CaCO3 } \\
\text { Turbidity } \\
\text { Volumes purged } \\
\text { Sampling code }\end{array}$} & $\begin{array}{l}218.5 \\
5.9 \\
23 \\
19.1 \\
5 \\
.3 \\
2.85\end{array}$ & ) & Not Av & lable & $\begin{array}{l}\mathrm{ft} \mathrm{msl} \\
\mathrm{pH} \\
\text { uS/cm } \\
\mathrm{deg} . \mathrm{C} \\
\mathrm{mg} / \mathrm{L} \\
\mathrm{NTU} \\
\text { well vo } \\
\mathrm{ft} \mathrm{msl}\end{array}$ & tume & & \\
\hline \multicolumn{11}{|c|}{ ANALYTICAL DATA } \\
\hline \multirow[t]{13}{*}{$\underline{\text { ST }}$} & \multicolumn{2}{|c|}{ Parameter } & 1000 & CLPEPA & \multirow[t]{13}{*}{$\underline{2 Q 00}$} & \multirow[t]{13}{*}{ CLPEPA } & \multirow[t]{13}{*}{ Filt. } & \multirow[t]{13}{*}{$\underline{\mathbf{D F}}$} & Unit & \multirow[t]{13}{*}{$\underline{L a b}$} \\
\hline & $1,1,1-\mathrm{Tr}$ & ethane & $<1$ & $u$ & & & & & $\mathrm{ug} / \mathrm{L}$ & \\
\hline & $1,1,2,2-$ & hloroethane & $<1$ & $\mathrm{U}$ & & & & & $u g / L$ & \\
\hline & 1,1-Dich & hane & $<1$ & $\mathrm{U}$ & & & & & $\mathrm{ug} / \mathrm{L}$ & \\
\hline & 1,1-Dich & hylene & $<1$ & $\mathrm{U}$ & & & & & $u g / L$ & \\
\hline & 1,2-Dich & hane & $<1$ & $\mathrm{U}$ & & & & & $u g / L$ & \\
\hline & Acetone & & $<10$ & $\mathrm{U}$ & & & & & $\mathrm{ug} / \mathrm{L}$ & \\
\hline & Carbon & hloride & $<1$ & $\mathrm{U}$ & & & & & $\mathrm{ug} / \mathrm{L}$ & \\
\hline & Chlorob & & $<1$ & $U$ & & & & & $\mathrm{ug} / \mathrm{L}$ & \\
\hline & Chtoroe & (Vinyl chloride) & $<1$ & $U$ & & & & & $\mathrm{ug} / \mathrm{L}$ & \\
\hline & Chlorofo & . & $<1$ & $U$ & & & & & $\mathrm{ug} / \mathrm{L}$ & \\
\hline & Tetrachl & ylene & $<1$ & $U$ & & & & & $\mathrm{ug} / \mathrm{L}$ & \\
\hline & Trichlor & & 286 & & & & & & $\mathrm{ug} / \mathrm{L}$ & \\
\hline
\end{tabular}

Notes: Concentrations in bold italics exceed the groundwater protection or monitoring constituent standards listed in Appendix A. Synchronous water levels are measured over a 3-5 day period or less. Dilution factors, Laboratory, and Filtered Data are for Second Quarter 2000 data only.

$+=$ exceeded the groundwater protection or monitoring constituent standards listed in Appendix A for Second Quarter 2000. 
Table D-9A. Groundwater Monitoring Results for QA Wells, M-Area and Met Lab HWMF (Cont.) WELL MSB 30B

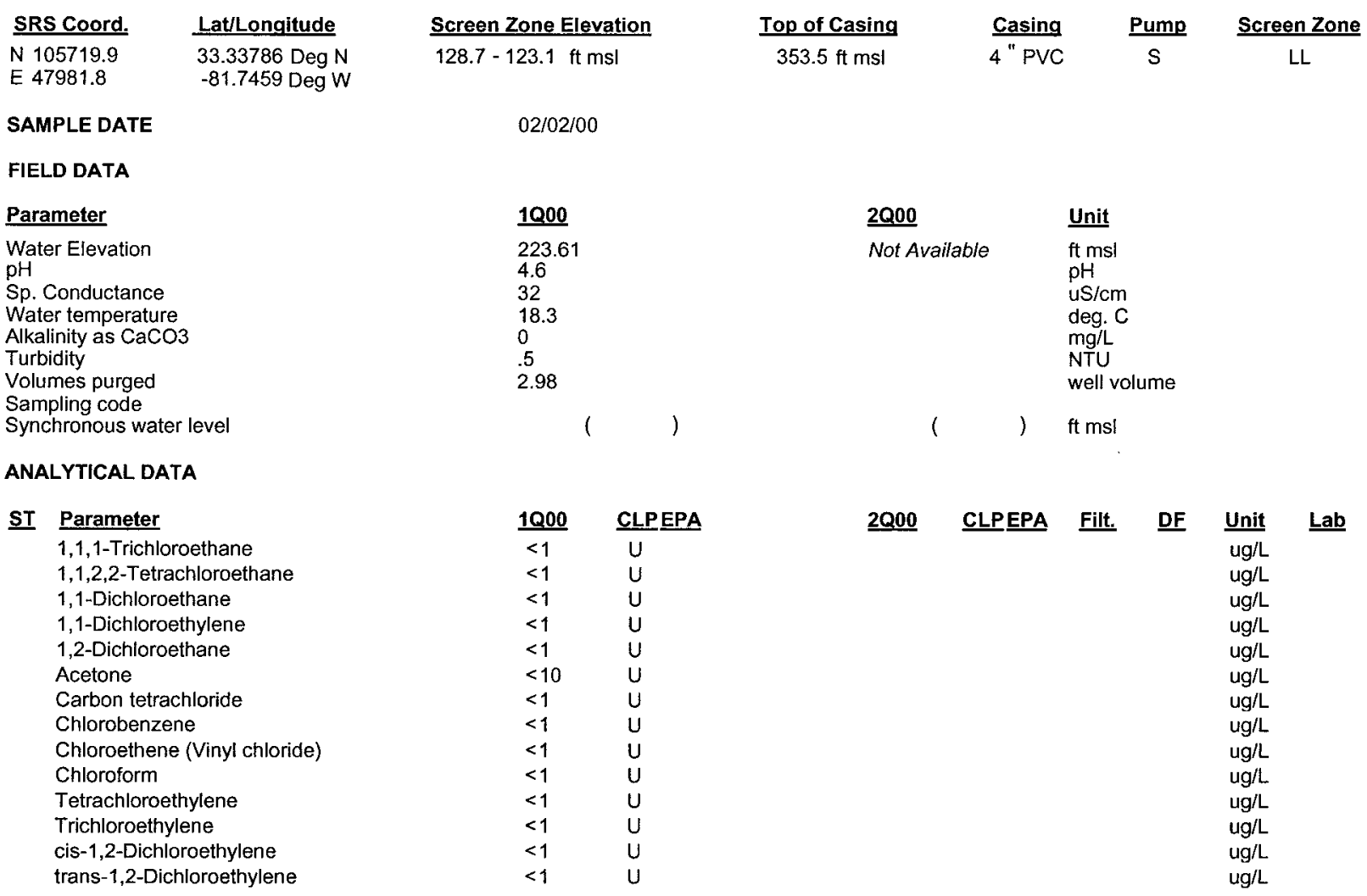

Notes: Concentrations in bold italics exceed the groundwater protection or monitoring constituent standards listed in Appendix A. Synchronous water levels are measured over a 3-5 day period or less. Dilution factors, Laboratory, and Filtered Data are for Second Quarter 2000 data only.

$+=$ exceeded the groundwater protection or monitoring constituent standards listed in Appendix A for Second Quarter 2000 . 
Table D-9A. Groundwater Monitoring Results for QA Wells, M-Area and Met Lab HWMF (Cont.) WELL MSB 41B

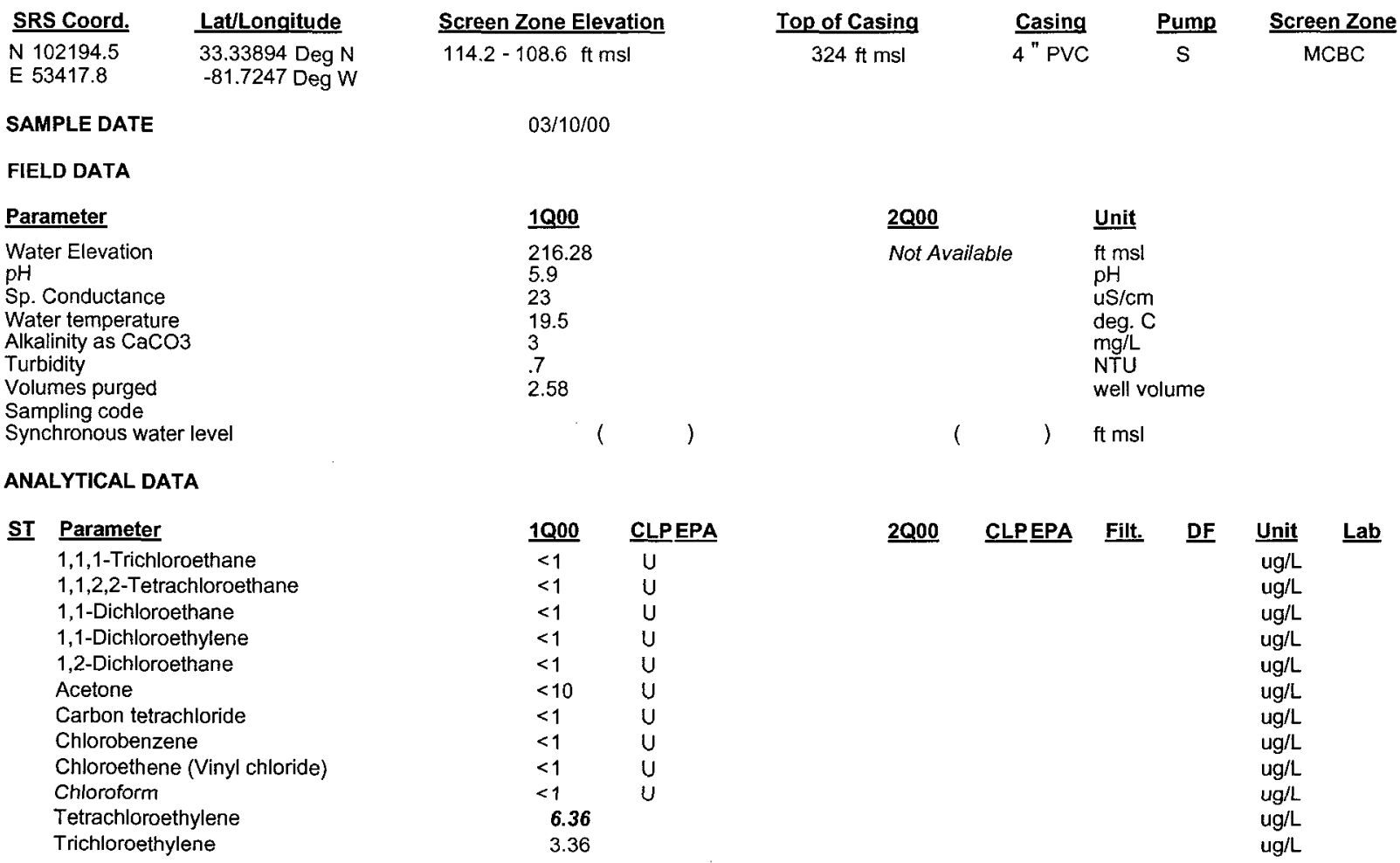


WSRC-TR- 2000-00242

Table D-9A. Groundwater Monitoring Results for QA Wells, M-Area and Met Lab HWMF (Cont.) WELL MSB 45A

$\begin{array}{ll}\text { SRS Coord. } & \text { Lat/Lonqitude } \\ \text { N } 103998.1 & \text { 33.33825 Deg N } \\ \text { E 50554.7 } & -81.7357 \text { Deg W }\end{array}$

SAMPLE DATE

FIELD DATA

\section{Parameter}

Water Elevation

$\mathrm{pH}$

Sp. Conductance

Water temperature

Alkalinity as $\mathrm{CaCO} 3$

Turbidity

Volumes purged

Sampling code

Synchronous water level

\section{ANALYTICAL DATA}

ST Parameter

1,1,1-Trichloroethane

1,1,2,2-Tetrachloroethane

1,1-Dichioroethane

1,1-Dichloroethylene

1,2-Dichloroethane

Acetone

Carbon tetrachloride

Chlorobenzene

Chloroethene (Vinyl chloride)

Chloroform

Tetrachloroethylene

Trichloroethylene

cis-1,2-Dichloroethylene

trans-1,2-Dichloroethylene

$\frac{\text { Screen Zone Elevation }}{139.2-129.2 \mathrm{ft} \mathrm{msl}}$

$03 / 13 / 00$

\section{0}

216.33

7.4

47

18.2

13

2.30

$\frac{\text { Top of Casing }}{380.8 \mathrm{ft} \mathrm{msl}} \quad \frac{\text { Casing }}{4 \text { " PVC }} \quad \frac{\text { Pump }}{\mathrm{S}} \quad \frac{\text { Screen Zone }}{\mathrm{LL}}$

2Q00

Not Available $\quad \mathrm{ft} \mathrm{msl}$

$\mathrm{pH}$

uS/cm

deg. C

$\mathrm{mg} / \mathrm{L}$

NTU

well volume

( ) ft msl

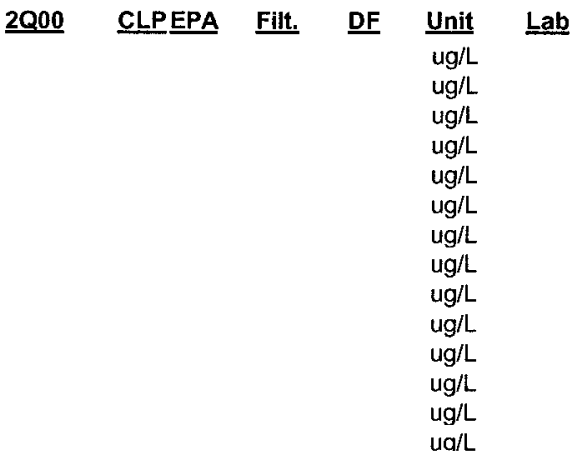

Notes: Concentrations in bold italics exceed the groundwater protection or monitoring constituent standards listed in Appendix A. Synchronous water levels are measured over a 3-5 day period or less. Dilution factors, Laboratory, and Filtered Data are for Second Quarter 2000 data only.

+ = exceeded the groundwater protection or monitoring constituent standards listed in Appendix A for Second Quarter 2000. 
WSRC-TR- 2000-00242

Table D-9A. Groundwater Monitoring Results for QA Wells, M-Area and Met Lab HWMF (Cont.) WELL MSB 64D

$\begin{array}{lcccccc}\text { SRS Coord. } & \text { Lat/Longitude } & \text { Screen Zone Elevation } & \text { Top of Casing } & \text { Casing } & \text { Pump } & \text { Screen Zone } \\ \begin{array}{l}\text { N 101854.8 } \\ \text { E 46598.5 }\end{array} & \begin{array}{l}33.32705 \mathrm{Deg} \mathrm{N} \\ 230.1-210.1 \mathrm{ft} \mathrm{msl}\end{array} & 348.6 \mathrm{ft} \mathrm{msl} & 4 \text { PVC } & \mathrm{S} & \mathrm{M} \\ \text { SAMPLE DATE } & 021.7420 \mathrm{Deg} \mathrm{W} & 02 / 15 / 00 & & & \end{array}$

FIELD DATA

Parameter
Water Elevation
pH
Sp. Conductance
Water temperature
Alkalinity as $\mathrm{CaCO} 3$
Turbidity
Volumes purged
Sampling code
Synchronous water level

\section{ANALYTICAL DATA}

ST Parameter

1,1,1-Trichloroethane $1,1,2,2$-Tetrachloroethane

1,1-Dichloroethane

1,1-Dichloroethylene

1,2-Dichloroethane

Acetone

Aluminum, total recoverable

Arsenic, total recoverable

Barium, total recoverable

Carbon tetrachloride

Chlorobenzene

Chloroethene (Vinyl chloride)

Chloroform

Chromium, total recoverable

Cobalt, total recoverable

Copper, total recoverable

Cyanide

Iron, total recoverable

Lead, total recoverable

Manganese, total recoverable

Mercury, total recoverable

Nickel, total recoverable

Selenium, total recoverable

Silver, total recoverable

Sodium, total recoverable

Tetrachloroethylene

Trichloroethylene

Zinc, total recoverable

cis-1,2-Dichloroethylene

trans-1,2-Dichloroethylene
1000

225.78

5.8

19.2

3.64

\begin{tabular}{|c|c|c|c|c|c|c|c|c|}
\hline 1000 & CLP & EPA & $2 \mathrm{QOOO}$ & CLPEPA & Filit. & $\underline{\text { DF }}$ & Unit & $\underline{\text { Lab }}$ \\
\hline$<1$ & $U$ & & & & & & ug/L & \\
\hline$<1$ & u & & & & & & $\mathrm{ug} / \mathrm{L}$ & \\
\hline$<1$ & U & & & & & & $\mathrm{ug} / \mathrm{L}$ & \\
\hline$<1$ & U & & & & & & $\mathrm{ug} / \mathrm{L}$ & \\
\hline$<1$ & U & & & & & & $\mathrm{ug} / \mathrm{L}$ & \\
\hline$<10$ & $U^{\prime}$ & & & & & & $\mathrm{ug} / \mathrm{L}$ & \\
\hline$<29.2$ & U & & & & & & $\mathrm{ug} / \mathrm{L}$ & \\
\hline$<20$ & U & & & & & & $\mathrm{ug} / \mathrm{L}$ & \\
\hline 12.6 & $J$ & I & & & & & ug/L & \\
\hline$<1$ & U & & & & & & $\mathrm{ug} / \mathrm{L}$ & \\
\hline$<1$ & U & & & & & & $\mathrm{ug} / \mathrm{L}$ & \\
\hline$<1$ & U & & & & & & $\mathrm{ug} / \mathrm{L}$ & \\
\hline$<1$ & U & & & & & & $\mathrm{ug} / \mathrm{L}$ & \\
\hline$<30$ & U & & & & & & $\mathrm{ug} / \mathrm{L}$ & \\
\hline$<20$ & $u$ & & & & & & $\mathrm{ug} / \mathrm{L}$ & \\
\hline$<60$ & U & & & & & & $\mathrm{ug} / \mathrm{L}$ & \\
\hline$<20$ & U & & & & & & $\mathrm{ug} / \mathrm{L}$ & \\
\hline 21.2 & J & 1 & & & & & $u g / L$ & \\
\hline$<20$ & U & & & & & & $u g / L$ & \\
\hline 7.96 & J & 1 & & & & & $\mathrm{ug} / \mathrm{L}$ & \\
\hline$<.128$ & JU & L & & & & & $u g / L$ & \\
\hline$<60$ & $u$ & & & & & & $u g / L$ & \\
\hline$<40$ & $u$ & & & & & & $\mathrm{ug} / \mathrm{L}$ & \\
\hline$<50$ & U & & & & & & $\mathrm{ug} / \mathrm{L}$ & \\
\hline 17000 & & & & & & & $\mathrm{ug} / \mathrm{L}$ & \\
\hline$<1$ & U & & & & & & $\mathrm{ug} / \mathrm{L}$ & \\
\hline$<1$ & U & & & & & & $\mathrm{ug} / \mathrm{L}$ & \\
\hline$<21.5$ & U & V & & & & & ug/L & \\
\hline$<1$ & U & & & & & & $\mathrm{ug} / \mathrm{L}$ & \\
\hline$<1$ & U & & & & & & $\mathrm{ug} / \mathrm{L}$ & \\
\hline
\end{tabular}

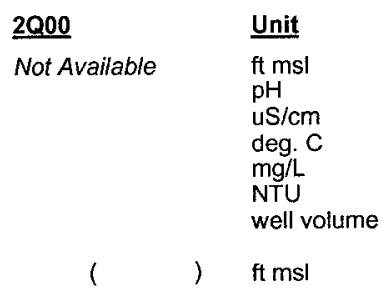

ab 
Table D-9A. Groundwater Monitoring Results for QA Wells, M-Area and Met Lab HWMF (Cont.) WELL MSB 68B

$\begin{array}{ll}\text { SRS Coord. } & \text { Lat/Longitude } \\ \text { N 106744.9 } & \text { 33.34719 Deg N } \\ \text { E 52308.5 } & -81.7365 \text { Deg W }\end{array}$

SAMPLE DATE

FIELD DATA

Parameter

Water Elevation

$\mathrm{pH}$

Sp. Conductance

Water temperature

Alkalinity as $\mathrm{CaCO} 3$

Turbidity

Volumes purged

Sampling code

Synchronous water level

\section{ANALYTICAL DATA}

ST Parameter

1,1,1-Trichloroethane

1,1,2,2-Tetrachloroethane

1,1-Dichłoroethane

1,1-Dichloroethylene

1,2-Dichloroethane

Acetone

Carbon tetrachloride

Chlorobenzene

Chloroethene (Vinyl chloride)

Chioroform

Tetrachloroethylene

Trichloroethylene

cis-1,2-Dichloroethylene

trans-1,2-Dichloroethylene

\begin{tabular}{l} 
Screen Zone Elevation \\
\hline $133-128.3 \mathrm{ft} \mathrm{msl}$ \\
$03 / 29 / 00$ \\
\\
$\mathbf{1 0 0 0}$ \\
216.82 \\
5.7 \\
25 \\
18.6 \\
2 \\
1.1 \\
3.51
\end{tabular}

Casing

$356.9 \mathrm{ft} \mathrm{msl}$

4 " PVC
Pump

$S$

Screen Zone

MCBC

\begin{tabular}{|c|c|c|c|c|c|c|}
\hline 1000 & CLPEPA & $\underline{2000}$ & CLPEPA & Filt. & $\underline{\mathrm{DF}}$ & Unit \\
\hline$<1$ & $u$ & & & & & $u g / L$ \\
\hline$<1$ & $u$ & & & & & $\mathrm{ug} / \mathrm{L}$ \\
\hline$<1$ & u & & & & & $\mathrm{ug} / \mathrm{L}$ \\
\hline$<1$ & u & & & & & $\mathrm{ug} / \mathrm{L}$ \\
\hline$<1$ & u & & & & & $u g / L$ \\
\hline$<10$ & $u$ & & & & & ug/L \\
\hline$<1$ & $u$ & & & & & ug/L \\
\hline$<1$ & $u$ & & & & & ug/L \\
\hline$<1$ & U & & & & & ug/L \\
\hline$<1$ & $U$ & & & & & ug/L \\
\hline 12.8 & & & & & & ug/L \\
\hline 199 & & & & & & $u g / L$ \\
\hline 5.88 & & & & & & $u g / L$ \\
\hline$<1$ & U & & & & & $\mathrm{ug} / \mathrm{L}$ \\
\hline
\end{tabular}

$\begin{array}{ll}\underline{2000} & \text { Unit } \\ \text { Not Available } & \mathrm{ft} \mathrm{msl} \\ & \mathrm{pH} \\ & \text { uS/cm } \\ \text { deg. C } \\ & \mathrm{mg} \mathrm{L} \\ & \mathrm{NTU} \\ & \text { well volume } \\ (, \quad \mathrm{ft} \mathrm{msl}\end{array}$

Notes: Concentrations in bold italics exceed the groundwater protection or monltoring constituent standards listed in Appendix A. Synchronous water levels are measured over a 3-5 day period or less. Dilution factors, Laboratory, and Filtered Data are for Second Quarter 2000 data only.

$+=$ exceeded the groundwater protection or monitoring constituent standards listed in Appendix A for Second Quarter 2000. 
Table D-9A. Groundwater Monitoring Results for QA Wells, M-Area and Met Lab HWMF (Cont.) WELL MSB 77C

\begin{tabular}{|c|c|}
\hline SRS Coord. & Lat/Longitude \\
\hline $\begin{array}{l}\text { N } 107078.3 \\
\text { E } 54225.9\end{array}$ & $\begin{array}{l}\text { 33.35106 Deg } \\
-81.7321 \text { Deg } V\end{array}$ \\
\hline
\end{tabular}

SAMPLE DATE

FIELD DATA

Parameter

Water Elevation

$\mathrm{pH}$

Sp. Conductance

Water temperature

Alkalinity as $\mathrm{CaCO} 3$

Turbidity

Volumes purged

Sampling code

Synchronous water level

ANALYTICAL DATA

ST Parameter

1,1,1-Trichloroethane

1,1,2,2-Tetrachloroethane

1,1 -Dichloroethane

1,1-Dichloroethylene

1,2-Dichloroethane

Acetone

Carbon tetrachloride

Chlorobenzene

Chloroethene (Vinyl chloride)

Chloroform

Tetrachloroethylene

Trichloroethylene

\section{Screen Zone Elevation}

$173.2-168.5 \mathrm{ft} \mathrm{ms}$

$03 / 11 / 00$

1000

222.26

6.2

27

18.9

13

.8
2.95

\begin{tabular}{|c|c|}
\hline$\underline{1000}$ & CLPEPA \\
\hline$<1$ & $U$ \\
\hline$<1$ & $U$ \\
\hline$<1$ & $\mathrm{U}$ \\
\hline$<1$ & U \\
\hline$<1$ & $\mathrm{U}$ \\
\hline$<10$ & $\mathrm{U}$ \\
\hline$<1$ & $\mathrm{U}$ \\
\hline$<1$ & $\mathrm{U}$ \\
\hline$<1$ & U \\
\hline$<1$ & $\mathrm{U}$ \\
\hline$<1$ & $U$ \\
\hline 15.9 & \\
\hline
\end{tabular}

$\begin{array}{llll}\text { Top of Casing } & \text { Casing } & \text { Pump } & \text { Screen Zone } \\ 357.2 \mathrm{ft} \mathrm{msl} & \text { "PVC } & \mathrm{SL}\end{array}$

UL

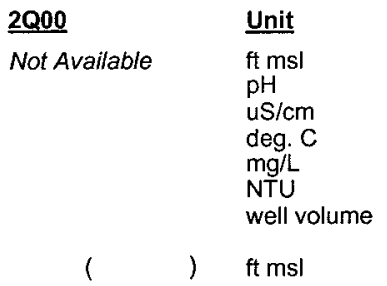

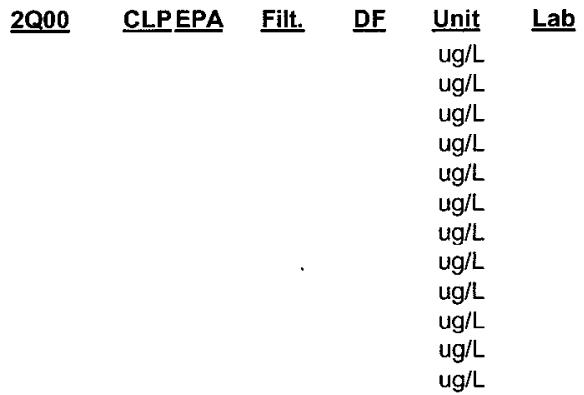

Notes: Concentrations in bold italics exceed the groundwater protection or monitoring constituent standards listed in Appendix A. Synchronous water levels are measured over a 3-5 day period or less. Dilution factors, Laboratory, and Filtered Data are for Second Quarter 2000 data only.

$+=$ exceeded the groundwater protection or monitoring constituent standards listed in Appendix A for Second Quarter 2000. 
Table D-9A. Groundwater Monitoring Results for QA Wells, M-Area and Met Lab HWMF (Cont.) WELL MSB 83B

$\begin{array}{ll}\text { SRS Coord. } & \text { Lat/Lonqitude } \\ \text { N } 108426.7 & \text { 33.35109 Deg N } \\ \text { E 52421.4 } & -81.7394 \text { Deg W }\end{array}$

SAMPLE DATE

FIELD DATA

Parameter

Water Elevation

pH

Sp. Conductance

Water temperature

Alkalinity as $\mathrm{CaCO} 3$

Turbidity

Volumes purged

Sampling code

Synchronous water level

ANALYTICAL DATA

ST Parameter

1,1,1-Trichloroethane

1,1,2,2-Tetrachloroethane

1,1-Dichloroethane

1,1-Dichloroethylene

1,2-Dichloroethane

Acetone

Carbon tetrachloride

Chlorobenzene

Chtoroethene (Vinyl chloride)

Chloroform

Tetrachloroethylene

Trichloroethylene

$\frac{\text { Screen Zone Elevation }}{146.3-141.6 \mathrm{ft} \mathrm{msl}} \quad \frac{\text { Top of Casing }}{371.8 \mathrm{ft} \mathrm{msl}} \quad \frac{\text { Casing }}{4^{\text {"PVC }}} \quad \frac{\text { Pump }}{\mathrm{S}} \quad \frac{\text { Screen Zone }}{\mathrm{MCBC}}$

$02 / 10 / 00$

1000

220.78

5.3

20

18.6

2

2.53

\begin{tabular}{|c|c|}
\hline $2 \mathrm{Q} 00$ & Unit \\
\hline Not Available & $\begin{array}{l}\text { ft msl } \\
\mathrm{pH} \\
\mathrm{uS} / \mathrm{cm} \\
\text { deg. C } \\
\mathrm{mg} / \mathrm{L} \\
\text { NTU } \\
\text { well volume }\end{array}$ \\
\hline( & $\mathrm{ft} \mathrm{msl}$ \\
\hline
\end{tabular}

2 CLPOEPA Filt. DF $\frac{\text { Unit Lab }}{\mathrm{ug} / \mathrm{L}} \quad \begin{gathered}\mathrm{ug} / \mathrm{L} \\ \mathrm{ug} / \mathrm{L} \\ \mathrm{ug} / \mathrm{L} \\ \mathrm{ug} / \mathrm{L} \\ \mathrm{ug} / \mathrm{L} \\ \mathrm{ug} / \mathrm{L} \\ \mathrm{ug} / \mathrm{L} \\ \mathrm{ug} / \mathrm{L} \\ \mathrm{ug} / \mathrm{L} \\ \mathrm{ug} / \mathrm{L} \\ \mathrm{ug} / \mathrm{L}\end{gathered}$

Notes: Concentrations in bold italics exceed the groundwater protection or monitoring constituent standards listed in Appendix A. Synchronous water levels are measured over a 3-5 day period or less. Dilution factors, Laboratory, and Filtered Data are for Second Quarter 2000 data only.

$+=$ exceeded the groundwater protection or monitoring constituent standards listed in Appendix A for Second Quarter 2000 . 
Table D-9A. Groundwater Monitoring Results for QA Wells, M-Area and Met Lab HWMF (Cont.) WELL RWM 2

\begin{tabular}{|c|c|c|c|c|c|c|}
\hline SRS Coord. & Lat/Longitude & Screen Zone Elevation & Top of Casing & $\underline{\text { Casing }}$ & Pump & Screen Zone \\
\hline $\begin{array}{l}\bar{N} 104434.1 \\
\text { E 49205.5 }\end{array}$ & $\begin{array}{l}\text { 33.33701 Deg N } \\
-81.7401 \text { Deg W }\end{array}$ & $\overline{148.3-138.3 \mathrm{ft} \mathrm{msl}}$ & $371.3 \mathrm{ft} \mathrm{msl}$ & $8 " \mathrm{CS}$ & $\mathrm{s}$ & UNK \\
\hline SAMPLE DAT & & & & & & \\
\hline
\end{tabular}

FIELD DATA

Parameter

Water Elevation

$\mathrm{pH}$

Sp. Conductance

Water temperature

Alkalinity as $\mathrm{CaCO} 3$

Turbidity

Volumes purged

Sampling code

Synchronous water level

ANALYTICAL DATA

ST Parameter

1,1,1-Trichloroethane

1,1,2,2-Tetrachloroethane

1,1-Dichloroethane

1,1-Dichloroethylene

1,2-Dichloroethane

Acetone

Carbon tetrachloride

Chlorobenzene

Chloroethene (Vinyl chloride)

Chloroform

Tetrachloroethylene

Trichloroethylene

cis-1,2-Dichloroethylene

trans-1,2-Dichloroethylene
1Q00

Not Available

1000 CLPEPA

\begin{tabular}{|c|c|}
\hline 20,00 & $\underline{\text { Unit }}$ \\
\hline $\begin{array}{l}\text { Not Available } \\
4.8\end{array}$ & $\begin{array}{l}\mathrm{ftmsl} \\
\mathrm{pH}\end{array}$ \\
\hline 81 & $\mathrm{uS} / \mathrm{cm}$ \\
\hline 22.9 & deg. C \\
\hline $\begin{array}{l}0 \\
.8\end{array}$ & $\mathrm{mg} / \mathrm{h}$ \\
\hline .8 & well volume \\
\hline C & \\
\hline
\end{tabular}

\begin{tabular}{ccc}
2000 & CLPEPA \\
\hline$<50$ & JU & L \\
$<50$ & JU & L \\
$<50$ & JU & L \\
$<50$ & JU & L \\
$<50$ & JU & L \\
$<500$ & JU & L \\
$<50$ & JU & L \\
$<50$ & JU & L \\
$<50$ & JU & L \\
$<50$ & JU & L \\
12900 & J & L \\
8820 & J & L \\
$<50$ & JU & L \\
$<50$ & JU & L
\end{tabular}

$\begin{array}{cc}\frac{\text { Filt. }}{\text { <EQL }} & \text { DF } \\ <\mathrm{EQL} & 50 \\ <\mathrm{EQL} & 50 \\ <\mathrm{EQL} & 50 \\ <\mathrm{EQL} & 50 \\ <\mathrm{EQL} & 50 \\ <\mathrm{EQL} & 50 \\ <\mathrm{EQL} & 50 \\ <\mathrm{EQL} & 50 \\ <\mathrm{EQL} & 50 \\ \mathrm{NDD} & 50 \\ \mathrm{NDD} & 50 \\ <\mathrm{EQL} & 50 \\ <\mathrm{EQL} & 50\end{array}$

\begin{tabular}{cc} 
Unit & Lab \\
\hline$u g / L$ & $M L$ \\
$u g / L$ & $M L$ \\
$u g / L$ & $M L$ \\
$u g / L$ & $M L$ \\
$u g / L$ & $M L$ \\
$u g / L$ & $M L$ \\
$u g / L$ & $M L$ \\
$u g / L$ & $M L$ \\
$u g / L$ & $M L$ \\
$u g / L$ & $M L$ \\
$u g / L$ & $M L$ \\
$u g / L$ & $M L$ \\
$u g / L$ & $M L$ \\
$u g / L$ & $M L$
\end{tabular}

Notes: Concentrations in bold italics exceed the groundwater protection or monitoring constituent standards listed in Appendix A. Synchronous water levels are measured over a 3-5 day period or less. Dilution factors, Laboratory, and Filtered Data are for Second Quarter 2000 data only.

+ = exceeded the groundwater protection or monitoring constituent standards listed in Appendix A for Second Quarter 2000. 
Table D-9A. Groundwater Monitoring Results for QA Wells, M-Area and Met Lab HWMF (Cont.) WELL RWM 4

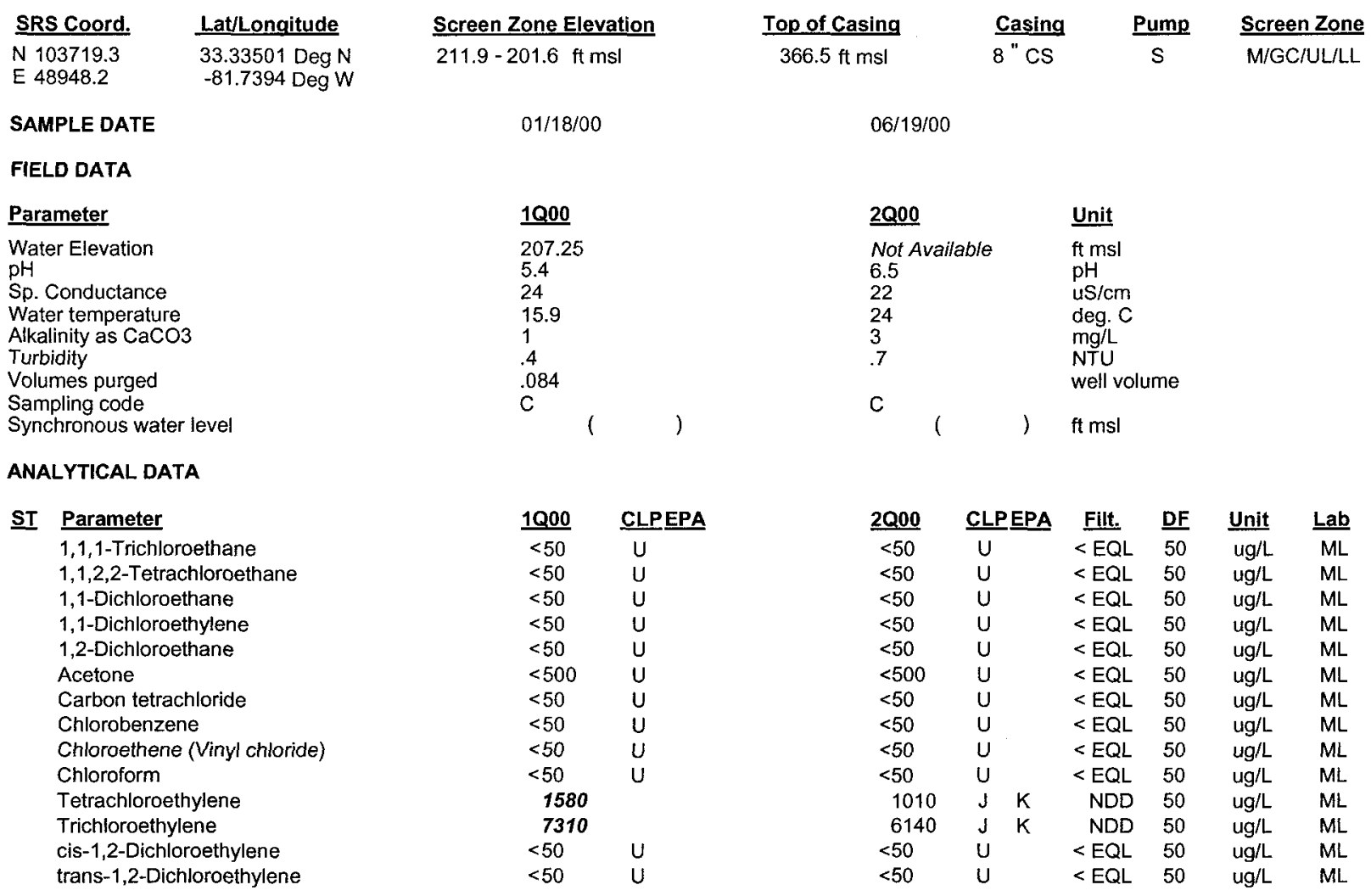

Notes: Concentrations in bold italics exceed the groundwater protection or monitoring constituent standards listed in Appendix A. Synchronous water levels are measured over a 3-5 day period or less. Dilution factors, Laboratory, and Filtered Data are for Second Quarter 2000 data only.

$+=$ exceeded the groundwater protection or monitoring constituent standards listed in Appendix A for Second Quarter 2000. 
Table D-9A. Groundwater Monitoring Results for QA Wells, M-Area and Met Lab HWMF (Cont.) WELL RWM 13B

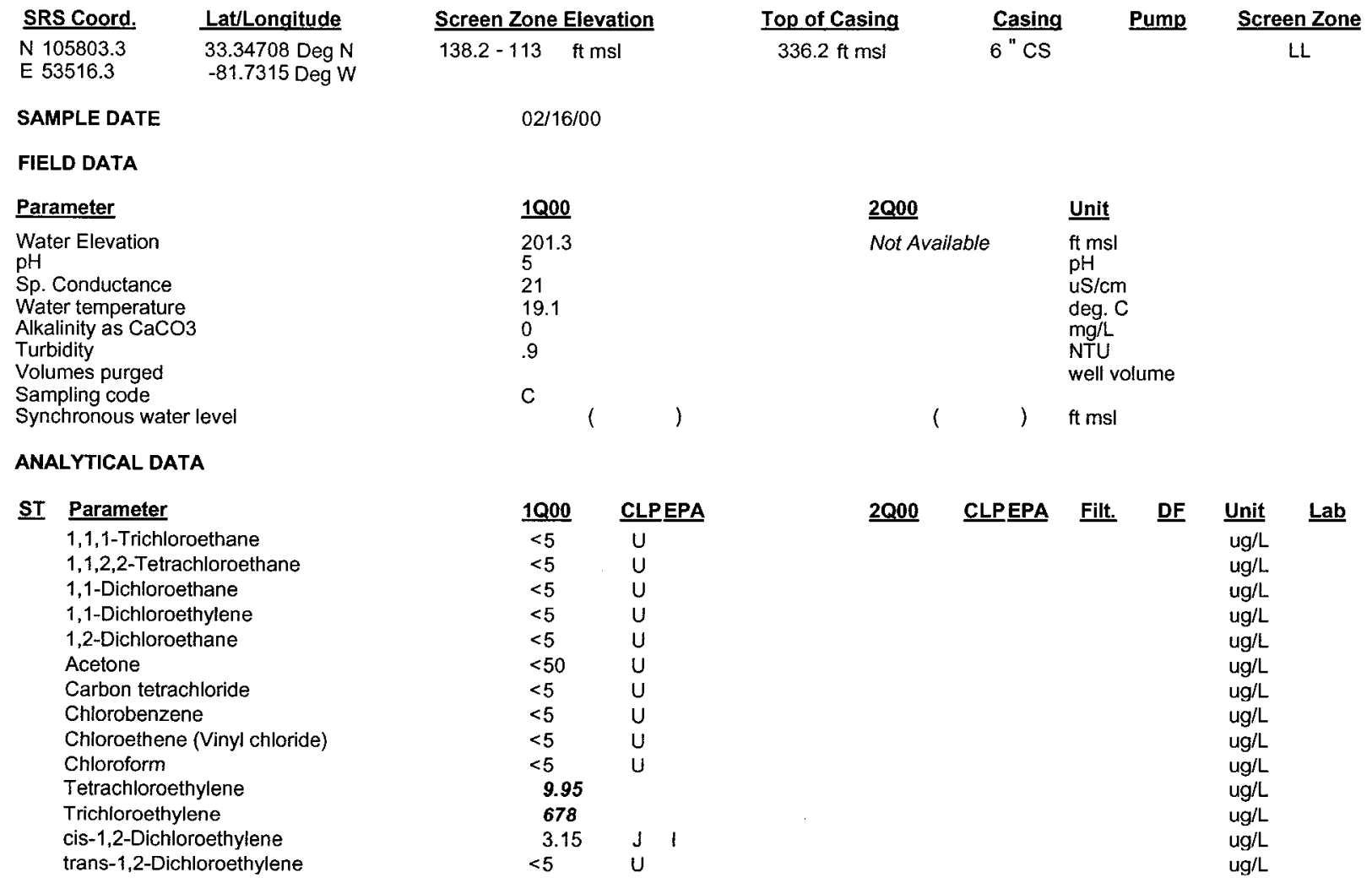

Notes: Concentrations in bold italics exceed the groundwater protection or monitoring constituent standards listed in Appendix A. Synchronous water levels are measured over a 3-5 day period or less. Dilution factors, Laboratory, and Filtered Data are for Second Quarter 2000 data only.

$+=$ exceeded the groundwater protection or monitoring constituent standards listed in Appendix A for Second Quarter 2000. 
Table D-9A. Groundwater Monitoring Results for QA Wells, M-Area and Met Lab HWMF (Cont.) WELL RWM 15B

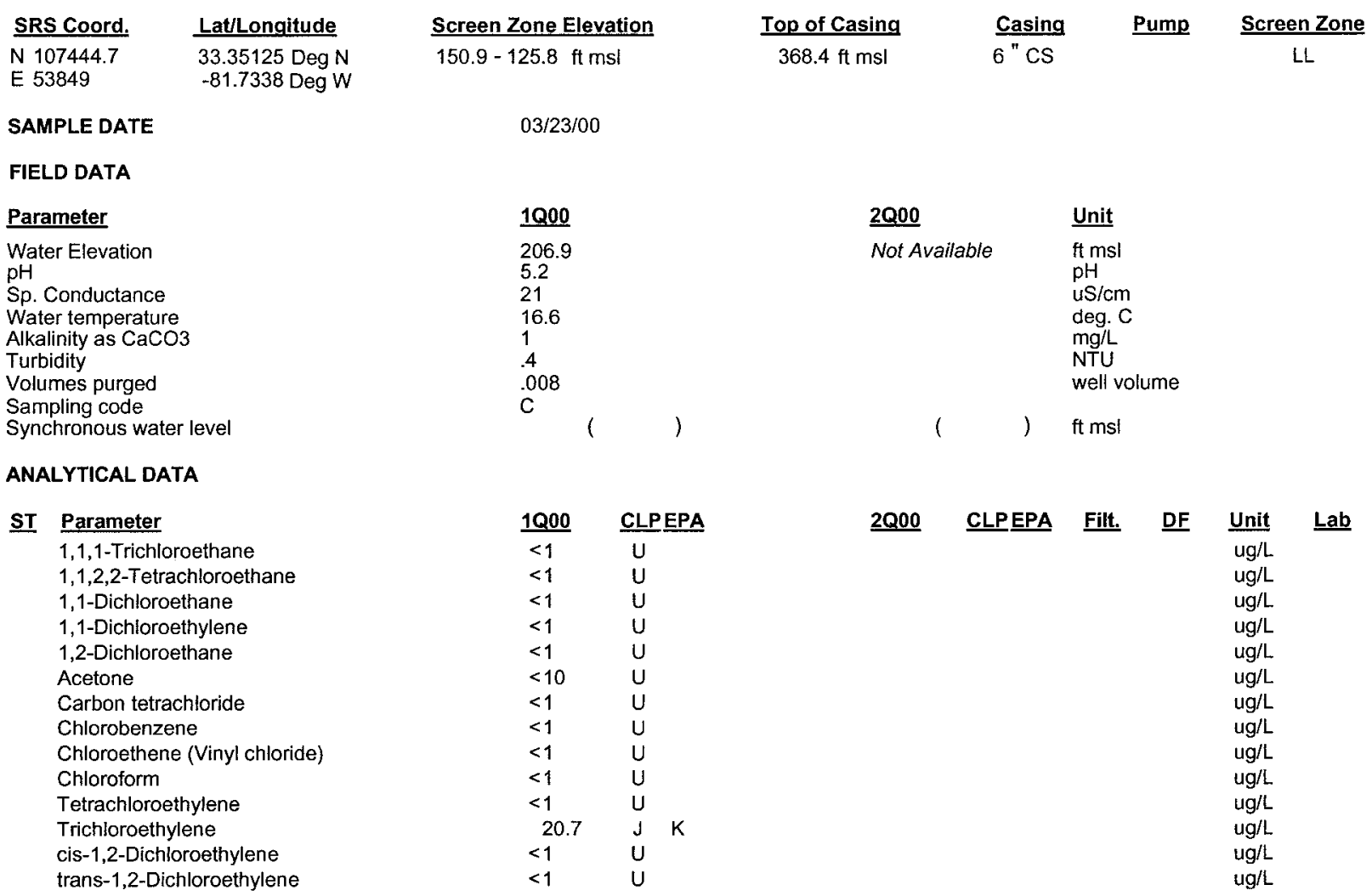

Notes: Concentrations in bold italics exceed the groundwater protection or monitoring constituent standards listed in Appendix A. Synchronous water levels are measured over a 3-5 day period or less. Dilution factors, Laboratory, and Filtered Data are for Second Quarter 2000 data only. 


\section{THIS PAGE LEFT BLANK INTENTIONALLY}


Table D-9B. Groundwater Monitoring Results for All Wells (Lab Duplicates), M-Area and Met Lab HWMF WELL AMB 4A

SAMPLE DATE

ANALYTICAL DATA

ST Parameter

Gross alpha

Nonvolatile beta

Radium, total alpha-emitting
$02 / 23 / 00$

\begin{tabular}{|c|c|c|c|c|c|c|}
\hline $1 Q 00$ & CLPEPA & $2 \mathrm{Q} 00$ & CLPEPA & Filt. & DF & Unit \\
\hline .238 & U & & & & & $\mathrm{pCi} / \mathrm{L}$ \\
\hline .409 & U & & & & & $\mathrm{pCi} / \mathrm{L}$ \\
\hline-.128 & U & & & & & $\mathrm{pCi} / \mathrm{L}$ \\
\hline
\end{tabular}

Notes: Concentrations in bold italics exceed the groundwater protection or monitoring constituent standards listed in Appendix A. Synchronous water levels are measured over a 3-5 day period or less. Dilution factors, Laboratory, and Filtered Data are for Second Quarter 2000 data only.

+ = exceeded the groundwater protection or monitoring constituent standards listed in Appendix A for Second Quarter 2000. 
WSRC-TR- 2000-00242

Unclassified

Table D-9B. Groundwater Monitoring Results for All Wells (Lab Duplicates), M-Area and Met Lab HWMF (Cont.) WELL AMB 7

SAMPLE DATE

ANALYTICAL DATA

ST Parameter
Chloride
Fluoride
Nitrate-nitrite as nitrogen
Sulfate
Total phosphates (as P)

ST Parameter

Fluoride

Total phosphates (as P)
$02 / 15 / 00$

\begin{tabular}{|c|c|c|c|c|c|c|}
\hline 1000 & CLPEPA & $\underline{2000}$ & CLPEPA & Filt. & $\underline{\mathrm{DF}}$ & Unit \\
\hline 1860 & & & & & & $u g / L$ \\
\hline$<200$ & $u$ & & & & & $\mathrm{ug} / \mathrm{L}$ \\
\hline 390 & J 1 & & & & & $\mathrm{ug} / \mathrm{L}$ \\
\hline 19700 & & & & & & $\mathrm{ug} / \mathrm{L}$ \\
\hline$<2500$ & $U$ & & & & & $\mathrm{ug} / \mathrm{L}$ \\
\hline
\end{tabular}

Notes: Concentrations in bold italics exceed the groundwater protection or monitoring constituent standards listed in Appendix A. Synchronous water levels are measured over a 3-5 day period or less. Dilution factors, Laboratory, and Filtered Data are for Second Quarter 2000 data only. $+=$ exceeded the groundwater protection or monitoring constituent standards listed in Appendix A for Second Quarter 2000. 
Table D-9B. Groundwater Monitoring Results for All Wells (Lab Duplicates), M-Area and Met Lab HWMF (Cont.) WELL AMB 7A

\section{SAMPLE DATE}

ANALYTICAL DATA

ST Parameter

2,4-Dichlorophenoxyacetic acid

Cyanide

Fluoride

Gross alpha

Lindane

Nitrate-nitrite as nitrogen

Nonvolatile beta

Phenol

Radium, total alpha-emitting
$02 / 23 / 00$

$\begin{array}{ccc}\frac{1 Q 00}{<2.08} & \text { CLPEPA } \\ & \text { U } & \\ <15.2 & \text { JU } & \text { LQ } \\ <19.3 & U & V \\ 1.05 & J & \text { I } \\ <.05 & J U & Q \\ 870 & J & Q \\ 1.48 & U & \\ <10 & J U & Q \\ 2.49 & U & V\end{array}$

2 CL00


Table D-9B. Groundwater Monitoring Results for All Wells (Lab Duplicates), M-Area and Met Lab HWMF (Cont.) WELL AMB 8D

SAMPLE DATE

ANALYTICAL DATA

ST Parameter

Total organic halogens
$02 / 14 / 00$

$\frac{1000}{15.5} \frac{\text { CLPEPA }}{\mathrm{J}} \quad \underline{2 \mathrm{Q} 00} \quad \underline{\text { CLPEPA }}$ Filt. $\quad \underline{\text { UF }} \frac{\text { Unit }}{\mathrm{ug} / \mathrm{L}}$

Notes: Concentrations in bold italics exceed the groundwater protection or monitoring constituent standards listed in Appendix A. Synchronous water levels are measured over a 3-5 day period or less. Dilution factors, Laboratory, and Filtered Data are for Second Quarter 2000 data only.

$+=$ exceeded the groundwater protection or monitoring constituent standards listed in Appendix A for Second Quarter 2000. 
Table D-9B. Groundwater Monitoring Results for All Wells (Lab Duplicates), M-Area and Met Lab HWMF (Cont.) WELL AMB 12D

\section{SAMPLE DATE}

$02 / 14 / 00$

ANALYTICAL DATA

ST Parameter
Chloride
Fluoride
Nitrate-nitrite as nitrogen
Sulfate
Total phosphates (as P)

$\begin{array}{lll}\frac{1 Q 00}{1320} & \text { CLPEPA } \\ <200 & \mathrm{U} \\ 620 & & \\ 904 & \mathrm{~J} & \mathrm{I} \\ <2500 & \mathrm{U} & \end{array}$

2000 CLPEPA Filt. DF Unit Lab

Notes: Concentrations in bold italics exceed the groundwater protection or monitoring constituent standards listed in Appendix A. Synchronous water levels are measured over a 3-5 day period or less. Dilution factors, Laboratory, and Filtered Data are for Second Quarter 2000 data only.

$+=$ exceeded the groundwater protection or monitoring constituent standards listed in Appendix A for Second Quarter 2000 . 
Table D-9B. Groundwater Monitoring Results for All Wells (Lab Duplicates), M-Area and Met Lab HWMF (Cont.) WELL MSB 29B

SAMPLE DATE

ANALYTICAL DATA

ST Parameter

Radium, total alpha-emitting
$02 / 15 / 00$

$\frac{1000}{.417} \frac{\text { CLPEPA }}{\mathrm{J}} \mathrm{I} \quad \underline{2000} \quad \underline{\text { CLPEPA }}$ Filt. $\underline{\text { DF }} \quad \underline{\mathrm{unit}} \underline{\mathrm{pCi} / \mathrm{L}}$


Table D-9B. Groundwater Monitoring Results for All Wells (Lab Duplicates), M-Area and Met Lab HWMF (Cont.) WELL MSB 29C

SAMPLE DATE

$02 / 15 / 00$

ANALYTICAL DATA

ST Parameter
$\begin{aligned} & \text { Gross alpha } \\ & \text { Nonvolatile beta }\end{aligned}$

\begin{tabular}{|c|c|}
\hline 1000 & CLPEP \\
\hline 2.17 & $J$ \\
\hline 2.29 & $J \quad 1$ \\
\hline
\end{tabular}

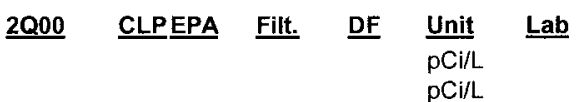

Notes: Concentrations in bold italics exceed the groundwater protection or monitoring constituent standards listed in Appendix A. Synchronous water levels are measured over a 3-5 day period or less. Dilution factors, Laboratory, and Filtered Data are for Second Quarter 2000 data only.

$+=$ exceeded the groundwater protection or monitoring constituent standards listed in Appendix A for Second Quarter 2000. 
WSRC-TR- 2000-00242

Unclassified

Table D-9B. Groundwater Monitoring Results for All Wells (Lab Duplicates), M-Area and Met Lab HWMF (Cont.) WELL MSB 29D

SAMPLE DATE

$02 / 16 / 00$

ANALYTICAL DATA

ST Parameter

Gross alpha

Nonvolatile beta

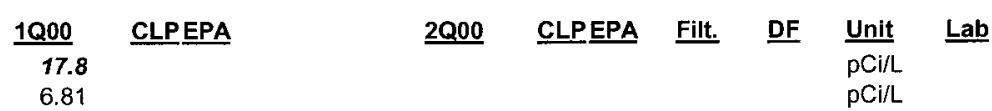

Notes: Concentrations in bold italics exceed the groundwater protection or monitoring constituent standards listed in Appendix A. Synchronous water levels are measured over a 3-5 day period or less. Dilution factors, Laboratory, and Filtered Data are for Second Quarter 2000 data only.

$+=$ exceeded the groundwater protection or monitoring constituent standards listed in Appendix A for Second Quarter 2000. 
Table D-10 Constituents Exceeding the Groundwater Protection or Monitoring Constituents Standard in Point of Compliance Wells, M Area HWMF

Well Name

Constituent

Unit

$1 Q 00$ CLP

M-Area Aquifer Zone

MSB 8A

MSB 62D

MSB 62D
Tetrachloroethylene

Tetrachloroethylene

Trichloroethylene $\mathrm{ug} / \mathrm{L} \quad 36.7$

$\mathrm{ug} / \mathrm{L} \quad 32.7$

$\mathrm{ug} / \mathrm{L} \quad 8.51$

Upper Lost Lake Aquifer Zone

MSB 6C

MSB 7C

MSB 7C

MSB 8C

MSB 8C

MSB 8C
Trichloroethylene

Tetrachloroethylene Trichloroethylene

1,1-Dichloroethylene Tetrachloroethylene Trichloroethylene
ug/L $\quad 11.3$

$\mathrm{ug} / \mathrm{L} \quad 62.1$

$\mathrm{ug} / \mathrm{L} \quad 20.9$

$u g / L \quad 7.96$

$\mathrm{ug} / \mathrm{L} \quad 68.1$

$\mathrm{ug} / \mathrm{L} \quad 43.4$

Lower Lost Lake Aquifer Zone

$\begin{array}{llll}\text { MSB 63B } & \text { Chlorobenzene } & \mathrm{ug} / \mathrm{L} & 6.5 \\ \text { MSB 63B } & \text { Tetrachloroethylene } & \mathrm{ug} / \mathrm{L} & 125\end{array}$ 
Table D-11 Constituents Exceeding the Groundwater Protection or Monitoring Constituents Standard in Point of Compliance Wells, Met Lab HWMF

\begin{tabular}{llll}
\hline Well Name & Constituent & Unit & 1000 \\
M-Area Aquifer Zone & & \\
& & & \\
AMB 6 & Sodium, total recoverable & $\mathrm{ug} / \mathrm{L}$ & 7030 \\
AMB 6 & Sulfate & $\mathrm{ug} / \mathrm{L}$ & 4010 \\
AMB 8D & Sodium, total recoverable & $\mathrm{ug} / \mathrm{L}$ & 6810 \\
AMB 9D & Sodium, total recoverable & $\mathrm{ug} / \mathrm{L}$ & 9760
\end{tabular}

\section{Upper Lost Lake Aquifer Zone}

$\begin{array}{llll}\text { AMB 18C } & \text { Lead, total recoverable } & \mathrm{ug} / \mathrm{L} & 15.7 \\ \text { AMB 19C } & \text { Lead, total recoverable } & \mathrm{ug} / \mathrm{L} & 98.8 \\ \text { AMB 19C } & \text { Sodium, total recoverable } & \mathrm{ug} / \mathrm{L} & 10300 \\ \text { AMB 19C } & \text { Sulfate } & \mathrm{ug} / \mathrm{L} & 5160\end{array}$

\section{Lower Lost Lake Aquifer Zone}

AMB IOB Sodium, total recoverable ug/L 4950

\section{Middle Sand Aquifer Zone-Crouch Branch Confining Unit}

AMB 17A

AMB 17A

AMB 17A

AMB 18A
Tetrachloroethylene

Total organic halogens

Trichloroethylene

Trichloroethylene $\mathrm{ug} / \mathrm{L} \quad 25$

ug/L $\quad 148$

$\mathrm{ug} / \mathrm{L} \quad 170$

$\mathrm{ug} / \mathrm{L} \quad 24$ 


\section{THIS PAGE LEFT BLANK INTENTIONALLY}




\section{Table D-12. Summary of Operating and Performance of the M-1 Air Stripper CY2000}

\begin{tabular}{|c|c|c|c|c|c|c|c|}
\hline Operation Parameter & $\underline{\text { Jan }}$ & Feb & $\underline{\text { Mar }}$ & Apr & May & $\underline{\text { Jun }}$ & $\frac{\text { Total / }}{\text { Average }}$ \\
\hline Average Operating Time (hrs) & 744 & 696 & 641 & 705.5 & 734 & 660 & $4,180.5$ \\
\hline Downtime (hrs) & 0 & 0 & 103 & 14.5 & 10 & 60 & 187.5 \\
\hline Throughput (gal) & $15,808,480$ & $14,515,776$ & $15,883,980$ & $19,192,422$ & $20,349,000$ & $18,074,150$ & $103,823,808$ \\
\hline Total Solvent Removed (Ibs) & 922.68 & 847.23 & 974.76 & 744.12 & $1,090.98$ & 863.52 & $5,443.29$ \\
\hline TCE Solvent Removed (Ibs) & 461.61 & 423.86 & 564.53 & 416.31 & 597.59 & 474.99 & $2,963.81$ \\
\hline PCE Solvent Removed (lbs) & 461.61 & 423.86 & 410.81 & 328.25 & 494.03 & 389.04 & 2546.59 \\
\hline \multicolumn{8}{|l|}{$\begin{array}{l}\text { Average Concentration }(\mu \mathrm{g} / \mathrm{L}) \\
\text { Stripper Influent }\end{array}$} \\
\hline PCE & 3500 & 3500 & 3100 & 2050 & 2910 & 2580 & 2,940 \\
\hline TCE & 3500 & 3500 & 4260 & 2600 & 3520 & 3150 & 3,422 \\
\hline Total & 7,000 & 7,000 & 7,360 & 4,650 & 6,430 & 5,730 & 6,362 \\
\hline \multicolumn{8}{|l|}{ Stripper Effluent } \\
\hline PCE & 0 & 0 & 0 & 0 & 0 & 0 & 0 \\
\hline TCE & 0 & 0 & 0 & 0 & 0 & 0 & 0 \\
\hline Total & 0 & 0 & 0 & 0 & 0 & 0 & 0 \\
\hline
\end{tabular}


THIS PAGE LEFT BLANK INTENTIONALLY 


\section{Table D-13. Summary of Operating and Performance of the A-2 Air Stripper CY2000}

\begin{tabular}{|c|c|c|c|c|c|c|c|}
\hline Operation Parameter & $\underline{\text { Jan }}$ & $\underline{\text { Feb }}$ & Mar & Apr & May & Jun & $\frac{\text { Total I }}{\text { Average }}$ \\
\hline Average Operating Time (hrs) & 738.5 & 696 & 727.5 & 720 & 741 & 720 & 4343 \\
\hline Downtime (hrs) & 5.5 & 0 & 16.5 & 0 & 3 & 0 & 25 \\
\hline Throughput (gal) & $13,177,815$ & $10,932,768$ & $10,934,325$ & $11,499,840$ & $12,742,236$ & $12,814,949$ & $72,101,933$ \\
\hline Total Solvent Removed (lbs.) & 158.2 & 129.4 & 125.8 & 108.1 & 113.6 & 124.0 & 759.3 \\
\hline PCE Removed (Pounds) & 1.10 & 0.90 & 0.91 & 2.18 & 1.02 & 1.14 & 7.30 \\
\hline TCE Removed (Pounds) & 157.22 & 128.61 & 124.98 & 106.02 & 112.69 & 122.95 & 754.43 \\
\hline
\end{tabular}

Average Concentration ( $\mu \mathrm{g} / \mathrm{L}$ )

Stripper Influent

PCE

TCE

\section{Stripper Effluent}

PCE

TCE

Total

$0 \quad 0$

0
1.6

0
1.4

0
1.5

0

1.5

1.6

1.4

1.5

1.2

0
1

0 


\section{THIS PAGE LEFT BLANK INTENTIONALLY}


Table D-14. Summary of Operation of the SVEU Vadose Unit 782-3M for CY2000

\begin{tabular}{|c|c|c|c|c|c|c|c|}
\hline $\begin{array}{l}\text { Operation } \\
\text { Parameter }\end{array}$ & $\underline{\text { Jan }}$ & $\underline{F e b}$ & Mar & Apr & May & $\underline{\text { Jun }}$ & $\frac{\text { Total / }}{\text { Average }}$ \\
\hline Operating Time (hours) & 744 & 678.5 & 741 & 720 & 737 & 716 & 4336.5 \\
\hline Downtime (hours) & 0 & 17.5 & 3 & 0 & 7 & 4 & 31.5 \\
\hline Total Solvent Removed (Ibs.) & 1516.56 & 1151.37 & 1118.31 & 1077.60 & 1219.95 & 1210.64 & 7294.43 \\
\hline $\begin{array}{l}\text { Average Vapor Flow Rate } \\
\text { (scfm) }\end{array}$ & 570.97 & 541.10 & 532.93 & 517.33 & 527.26 & 536.89 & 537.75 \\
\hline $\begin{array}{l}\text { Average Concentration } \\
\text { Influent (ppmv) }\end{array}$ & 141.26 & 125.04 & 113.01 & 114.94 & 124.70 & 125.29 & 124.04 \\
\hline
\end{tabular}




\section{THIS PAGE LEFT BLANK INTENTIONALLY}


Table D-15. Summary of Operation of the SVEU Vadose Unit 782-4M for CY2000

\begin{tabular}{|c|c|c|c|c|c|c|c|}
\hline $\begin{array}{l}\text { Operation } \\
\text { Parameter }\end{array}$ & $\underline{\text { Jan }}$ & $\underline{\text { Feb }}$ & Mar & Apr & May & $\underline{\text { Jun }}$ & $\frac{\text { Total / }}{\text { Average }}$ \\
\hline Operating Time (hours) & 744 & 690 & 736 & 720 & 744 & 718 & 4352 \\
\hline Downtime (hours) & 0 & 6 & 8 & 0 & 0 & 2 & 16 \\
\hline Total Solvent Removed (Ibs.) & 1520.16 & 1816.02 & 1549.60 & 1479.36 & 1800.24 & 1498.62 & 9664.00 \\
\hline $\begin{array}{l}\text { Average Vapor Flow Rate } \\
\text { (scfm) }\end{array}$ & 369.10 & 392.04 & 378.50 & 369.67 & 376.13 & 368.53 & 375.66 \\
\hline $\begin{array}{l}\text { Average Concentration } \\
\text { Influent (ppmv) }\end{array}$ & 226.06 & 272.11 & 225.82 & 225.62 & 260.35 & 228.54 & 239.75 \\
\hline
\end{tabular}




\section{THIS PAGE LEFT BLANK INTENTIONALLY}


Table D-16. Summary of Operation of the SVEU Vadose Unit 782-5M for CY2000

\begin{tabular}{|c|c|c|c|c|c|c|c|}
\hline $\begin{array}{l}\text { Operation } \\
\text { Parameter }\end{array}$ & $\underline{J a n}$ & Feb & Mar & Apr & May & Jun & $\frac{\text { Total / }}{\text { Average }}$ \\
\hline Operating Time (hours) & 0 & 0 & 0 & 0 & 0 & 0 & 0 \\
\hline Downtime (hours) & 744 & 696 & 744 & 720 & 744 & 720 & 4368 \\
\hline Total Solvent Removed (Ibs.) & 0 & 0 & 0 & 0 & 0 & 0 & 0 \\
\hline $\begin{array}{l}\text { Average Vapor Flow Rate } \\
\text { (scfm) }\end{array}$ & 0 & 0 & 0 & 0 & 0 & 0 & 0 \\
\hline $\begin{array}{l}\text { Average Concentration } \\
\text { Influent (ppmv) }\end{array}$ & 0 & 0 & 0 & 0 & 0 & 0 & 0 \\
\hline
\end{tabular}


WSRC-TR-2000-00242

Unclassified

\section{THIS PAGE LEFT BLANK INTENTIONALLY}

M-Area and Met Lab HWMFs

D16-2

First and Second Quarters 2000

$$
1
$$


Table D-17. Summary of Operation of the SVEU Vadose Unit 782-6M for CY2000

\begin{tabular}{|c|c|c|c|c|c|c|c|}
\hline$\frac{\text { Operation }}{\text { Parameter }}$ & $\underline{\text { Jan }}$ & Feb & Mar & Apr & May & Jun & $\frac{\text { Total I }}{\text { Average }}$ \\
\hline Operating Time (hours) & 742.5 & 695.5 & 156 & 0 & 0 & 0 & 1594 \\
\hline Downtime (hours) & 1.5 & 0.5 & 588 & 720 & 744 & 720 & 2774 \\
\hline Total Solvent Removed (lbs.) & 1892.64 & 2570.77 & 533.52 & 0 & 0 & 0 & 4996.93 \\
\hline $\begin{array}{l}\text { Average Vapor Flow Rate } \\
\text { (scfm) }\end{array}$ & 589.37 & 629.98 & 249.92 & 0 & 0 & 0 & 489.76 \\
\hline $\begin{array}{l}\text { Average Concentration } \\
\text { Influent (ppmv) }\end{array}$ & 193.36 & 234.66 & 545.00 & 0 & 0 & 0 & 324.34 \\
\hline
\end{tabular}


THIS PAGE LEFT BLANK INTENTIONALLY 
Table D-18. Summary of Operation of the SVEU Vadose Unit 782-7M for CY2000

\begin{tabular}{|c|c|c|c|c|c|c|c|}
\hline$\frac{\text { Operation }}{\text { Parameter }}$ & Jan & Eeb & Mar & Apr & May & Jun & $\frac{\text { Total } l}{\text { Average }}$ \\
\hline Operating Time (hours) & 742.5 & 694.5 & 744 & 720 & 741 & 720 & 4362 \\
\hline Downtime (hours) & 1.5 & 1.5 & 0 & 0 & 3 & 0 & 6 \\
\hline Total Solvent Removed (lbs.) & 270.63 & 227.33 & 203.52 & 184.92 & 156.55 & 130.56 & 1173.51 \\
\hline $\begin{array}{l}\text { Average Vapor Flow Rate } \\
(\mathrm{scfm})\end{array}$ & 325.00 & 325.00 & 325.00 & 325.00 & 325.00 & 325.00 & 325.00 \\
\hline $\begin{array}{l}\text { Average Concentration } \\
\text { Influent (ppmv) }\end{array}$ & 47.88 & 43.37 & 35.90 & 33.10 & 27.34 & 24.65 & 35.37 \\
\hline
\end{tabular}




\section{THIS PAGE LEFT BLANK INTENTIONALLY}


Table D-19. Summary of Operation of the SVEU Vadose Unit 782-8M for CY2000

\begin{tabular}{|c|c|c|c|c|c|c|c|}
\hline$\frac{\text { Operation }}{\text { Parameter }}$ & $\underline{\text { Jan }}$ & $\underline{\text { Feb }}$ & $\underline{\text { Mar }}$ & Apr & May & $\underline{\text { Jun }}$ & $\frac{\text { Total / }}{\text { Average }}$ \\
\hline Operating Time (hours) & 0 & 0 & 0 & 0 & 0 & 0 & 0 \\
\hline Downtime (hours) & 744 & 696 & 744 & 720 & 744 & 720 & 4368 \\
\hline Total Solvent Removed (lbs.) & 0 & 0 & 0 & 0 & 0 & 0 & 0 \\
\hline $\begin{array}{l}\text { Average Vapor Flow Rate } \\
\text { (scfm) }\end{array}$ & 0 & 0 & 0 & 0 & 0 & 0 & 0 \\
\hline $\begin{array}{l}\text { Average Concentration } \\
\text { Influent (ppmv) }\end{array}$ & 0 & 0 & 0 & 0 & 0 & 0 & 0 \\
\hline
\end{tabular}




\section{THIS PAGE LEFT BLANK INTENTIONALLY}


Table D-20. Summary of Volume Pumped form Recovery Wells 2000

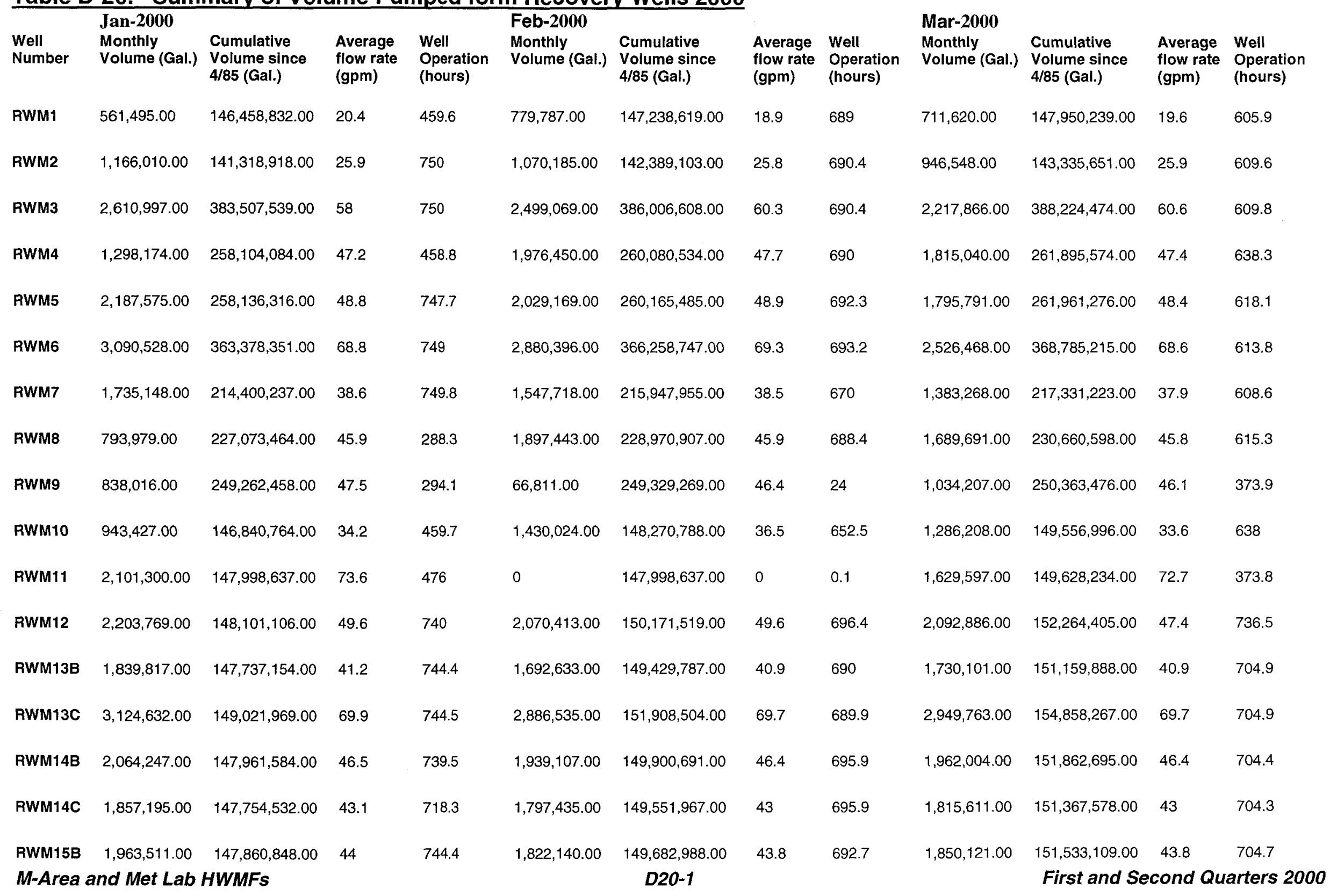


Table D-20. (cont.) Summary of Volume Pumped form Recovery Wells 2000

\begin{tabular}{|c|c|c|c|c|c|c|c|c|c|c|c|c|}
\hline & Apr-2000 & & & & May-2000 & & & & Jun-2000 & & & \\
\hline $\begin{array}{l}\text { Well } \\
\text { Number }\end{array}$ & $\begin{array}{l}\text { Monthly } \\
\text { Volume (Gal.) }\end{array}$ & $\begin{array}{l}\text { Cumulative } \\
\text { Volume since } \\
4 / 85 \text { (Gal.) }\end{array}$ & $\begin{array}{l}\text { Average } \\
\text { flow rate } \\
\text { (gpm) }\end{array}$ & $\begin{array}{l}\text { Well } \\
\text { Operation } \\
\text { (hours) }\end{array}$ & $\begin{array}{l}\text { Monthly } \\
\text { Volume (Gal.) }\end{array}$ & $\begin{array}{l}\text { Cumulative } \\
\text { Volume since } \\
4 / 85 \text { (Gal.) }\end{array}$ & $\begin{array}{l}\text { Average } \\
\text { flow rate } \\
\text { (gpm) }\end{array}$ & $\begin{array}{l}\text { Well } \\
\text { Operation } \\
\text { (hours) }\end{array}$ & $\begin{array}{l}\text { Monthly } \\
\text { Volume (Gal.) }\end{array}$ & $\begin{array}{l}\text { Cumulative } \\
\text { Volume since } \\
4 / 85 \text { (Gal.) }\end{array}$ & $\begin{array}{l}\text { Average } \\
\text { flow rate } \\
\text { (gpm) }\end{array}$ & $\begin{array}{l}\text { Well } \\
\text { Operation } \\
\text { (hours) }\end{array}$ \\
\hline RWM1 & $818,162.00$ & $148,768,401.00$ & 18.7 & 729.2 & $786,074.00$ & $149,554,475.00$ & 18.2 & 718.5 & $768,052.00$ & $150,322,527.00$ & 19.2 & 667.6 \\
\hline RWM2 & $1,112,977.00$ & $144,448,628.00$ & 25.4 & 730.3 & $1,093,240.00$ & $145,541,868.00$ & 25.3 & 721.3 & $406,865.00$ & $145,948,733.00$ & 25.1 & 270.4 \\
\hline RWM3 & $2,620,744.00$ & $390,845,218.00$ & 59.9 & 729.2 & $2,582,267.00$ & $393,427,485.00$ & 59.7 & 720.4 & $2,460,708.00$ & $395,888,193.00$ & 61.1 & 671.7 \\
\hline RWM4 & $1,990,917.00$ & $263,886,491.00$ & 47.1 & 704.5 & $2,044,407.00$ & $265,930,898.00$ & 47.2 & 721.7 & $1,909,471.00$ & $267,840,369.00$ & 47.4 & 672.1 \\
\hline RWM5 & $2,103,894,00$ & $264,065,170.00$ & 48.1 & 729 & $2,083,432.00$ & $266,148,602.00$ & 48.2 & 720.2 & $1,937,930.00$ & $268,086,532.00$ & 48.3 & 668.8 \\
\hline RWM6 & $2,982,888.00$ & $371,768,103.00$ & 68 & 731.1 & $2,950,797.00$ & $374,718,900.00$ & 67.8 & 725 & $2,746,254.00$ & $377,465,154.00$ & 68.8 & 665.2 \\
\hline RWM7 & $1,566,328.00$ & $218,897,551.00$ & 35.6 & 733.3 & $1,597,536.00$ & $220,495,087.00$ & 36.7 & 724.8 & $1,180,539.00$ & $221,675,626.00$ & 37.7 & 521.5 \\
\hline RWM8 & $1,990,374.00$ & $232,650,972.00$ & 48.1 & 689.7 & $2,005,456.00$ & $234,656,428.00$ & 43.9 & 761.8 & $1,855,207.00$ & $236,511,635.00$ & 46.2 & 668.8 \\
\hline RWM9 & $1,954,088.00$ & $252,317,564.00$ & 46.7 & 697.4 & $2,004,357.00$ & $254,321,921.00$ & 47.6 & 701.3 & $1,837,570$ & $256,159,491.00$ & 46.1 & 664.6 \\
\hline RWM10 & $1,412,072.00$ & $150,969,068.00$ & 32.2 & 729.9 & $1,353,150.00$ & $152,322,218.00$ & 32.3 & 697.6 & $1,181,812.00$ & $153,504,030.00$ & 32.6 & 604.7 \\
\hline RWM11 & $3,065,605.00$ & $152,693,839.00$ & 71.7 & 712.6 & $3,015,227.00$ & $155,709,066.00$ & 69.6 & 721.7 & $2,865,356.00$ & $158,574,422.00$ & 71.9 & 664.5 \\
\hline RWM12 & $2,204,706.00$ & $154,469,111.00$ & 51.9 & 708.1 & $2,211,600.00$ & $156,680,711.00$ & 49.5 & 744.5 & $2,059,078$ & $158,739,789.00$ & 49.3 & 696.2 \\
\hline RWM13B & $1,818,374.00$ & $152,978,262.00$ & 40.8 & 742.8 & $1,821,543.00$ & $154,799,805.00$ & 40.8 & 743.2 & $1,694,928.00$ & $156,494,733.00$ & 40.7 & 693.8 \\
\hline RWM13C & $3,106,389.00$ & $157,964,656.00$ & 69.7 & 742.8 & $3,085,561.00$ & $161,050,217.00$ & 70 & 740.1 & $2,886,276.00$ & $163,936,493.00$ & 69.4 & 693.6 \\
\hline RWM14B & $2,062,666.00$ & $153,925,361.00$ & 46.4 & 740.9 & $2,066,132.00$ & $155,991,493.00$ & 46.4 & 742.7 & $1,918,957.00$ & $157,910,450.00$ & 46.3 & 691.2 \\
\hline RWM14C & $1,907,334.00$ & $153,274,912.00$ & 42.9 & 741 & $1,866,112.00$ & $155,141,024.00$ & 42.9 & 724.6 & $1,793,459.00$ & $156,934,483.00$ & 42.9 & 696.1 \\
\hline RWM15B & $1,948,670.00$ & $153,481,779.00$ & 43.7 & 743.2 & $1,953,921.00$ & $155,435,700.00$ & 43.8 & 742.8 & $1,821,735.00$ & $157,257,435.00$ & 43.8 & 693.8 \\
\hline
\end{tabular}




\section{Table D-21 Summary of Average Tetrachloroethylene (PCE) \& Trichloroethylene (TCE) Concentrations in Recovery Wells}

\begin{tabular}{|c|c|c|c|c|c|c|c|c|c|}
\hline Well Number & \multicolumn{3}{|c|}{ Jan 2000} & \multicolumn{3}{|c|}{ Feb 2000} & \multicolumn{3}{|c|}{ Mar 2000} \\
\hline RWM 2 & 12,800 & 12,200 & 25,000 & 13,400 & 12,100 & 25,500 & 11,500 & 14,200 & 25,700 \\
\hline RWM 3 & 1,010 & 3,580 & 4,590 & 1,040 & 3,360 & 4,400 & 820 & 4,020 & 4,840 \\
\hline RWM 5 & 569 & 1,360 & 1,929 & 647 & 1,640 & 2,287 & 524 & 1,680 & 2,204 \\
\hline RWM 6 & 134 & 2,470 & 2,604 & 2,950 & 2,100 & 5,050 & 3,110 & 2,730 & 5,840 \\
\hline RWM 7 & 164 & 8,000 & 8,164 & 9,220 & 6,730 & 15,950 & 9,740 & 8,500 & 18,240 \\
\hline RWM 8 & 848 & 1,250 & 2,098 & 961 & 1,220 & 2,181 & 734 & 1,280 & 2,014 \\
\hline RWM 11 & 8 & 890 & 898 & 38 & 463 & 501 & 25 & 177 & 202 \\
\hline RWM 12 & 20 & 2,000 & 2,020 & 8 & 2,280 & 2,288 & 9 & 2,100 & 2,109 \\
\hline RWM 13B & 5 & 613 & 618 & 9 & 605 & 614 & 6 & 579 & 585 \\
\hline RWM 13C & 3 & 950 & 953 & 23 & 993 & 1,016 & 13 & 739 & 752 \\
\hline RWM 14B & 5 & 737 & 742 & 6 & 783 & 789 & 6 & 711 & 717 \\
\hline RWM 14C & 20 & 4,150 & 4,170 & 22 & 4,070 & 4,092 & 31 & 5,640 & 5,671 \\
\hline RWM 15B & 1 & 26 & 27 & 1 & 23 & 24 & 2 & 22 & 24 \\
\hline
\end{tabular}

NOTE: Numbers in bold are estimated because of missing data or equipment malfunction. Numbers in italics are averages of two or more samples. 
Table D-21 Summary of Average Tetrachloroethylene (PCE) \& Trichloroethylene (TCE) Concentrations in Recovery Wells (Cont.)

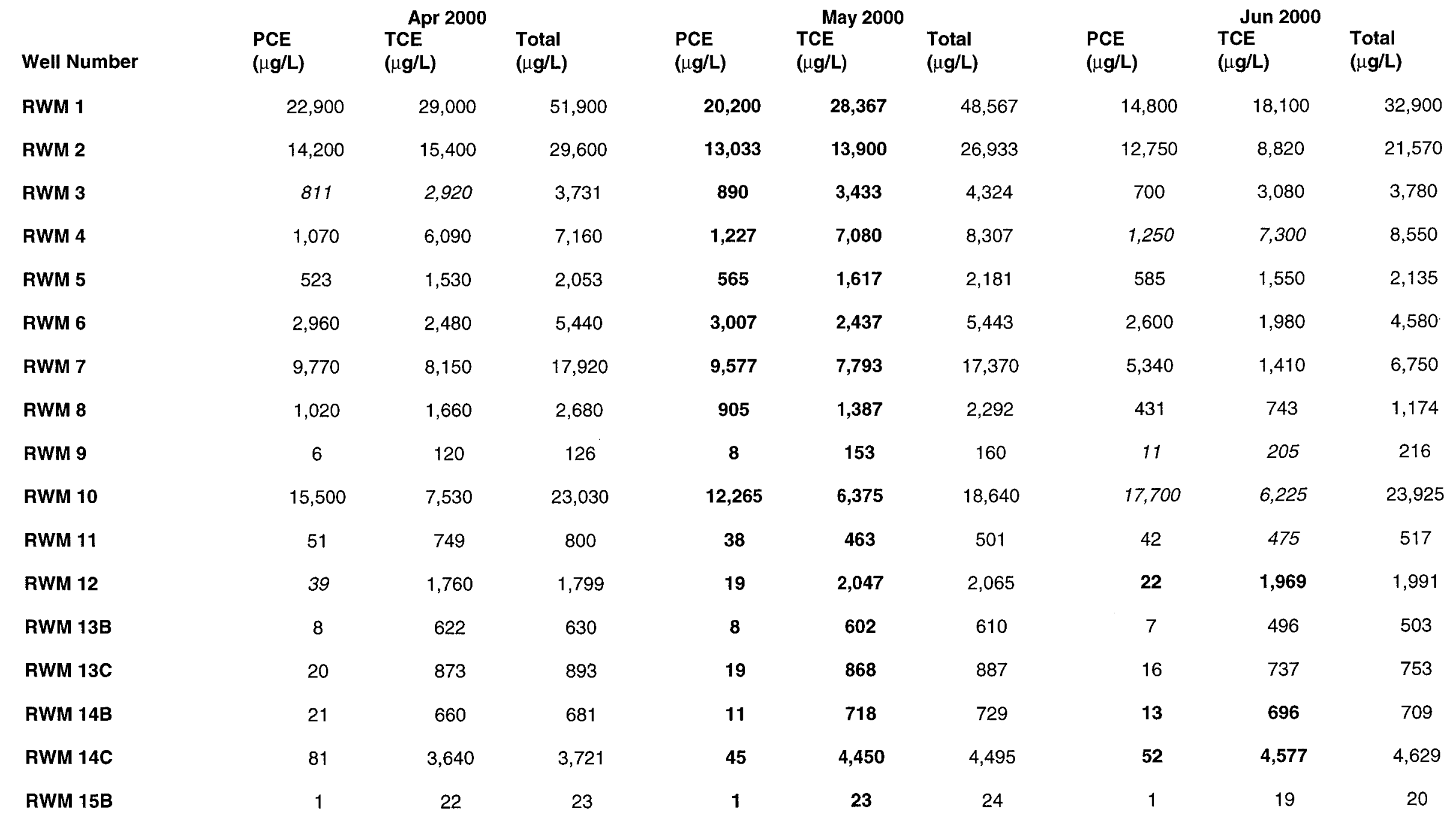

NOTE: Numbers in bold are estimated because of missing data or equipment malfunction. Numbers in italics are averages of two or more samples. 
Table D-22. Horizontal Gradients and Flow Rates in the Five Aquifer Zones

First Quarter 2000

\begin{tabular}{|c|c|c|c|c|}
\hline Aquifer Zone & Transect & Horizontal Gradient & $\begin{array}{l}\text { Flow Rate } \\
\text { (ft/day) }\end{array}$ & Flow Rate (ft/year) \\
\hline \multirow[t]{2}{*}{ M-Area } & $A-A^{, a}$ & 0.0073 & 0.99 & 361 \\
\hline & B-B' & 0.0026 & 0.35 & 128 \\
\hline Upper Lost Lake & $A-A^{\prime}$ & 0.0024 & 0.54 & 197 \\
\hline \multirow[t]{2}{*}{ Lower Lost Lake } & $A-A^{\prime}$ & 0.0039 & 0.61 & 222 \\
\hline & B-B' & 0.0025 & 0.92 & 337 \\
\hline \multirow{2}{*}{$\begin{array}{l}\text { Middle Sand of the } \\
\text { CBCU }\end{array}$} & $A-A^{\prime}$ & 0.0039 & 0.88 & 320 \\
\hline & B-B' & 0.0017 & 0.38 & 140 \\
\hline Crouch Branch & $A-A^{\prime}$ & 0.0034 & 0.68 & 248 \\
\hline
\end{tabular}

Second Quarter 2000

\begin{tabular}{lcccc} 
Aquifer Zone & Transect & Horizontal Gradient & $\begin{array}{c}\text { Flow Rate } \\
\text { (ft/day) }\end{array}$ & Flow Rate (ft/year) \\
\hline M-Area & A-A' & 0.0025 & 0.34 & 123 \\
Upper Lost Lake & B-B' $^{\prime \prime}$ & 0.0067 & 0.90 & 330 \\
Lower Lost Lake & A-A' & 0.0026 & 0.59 & 213 \\
& B-B' $^{\prime}$ & 0.0029 & 0.65 & 238 \\
Middle Sand of the & A-A' & 0.0025 & 0.56 & 205 \\
CBCU & B-B' & 0.0021 & 0.47 & 172 \\
& A-A' & 0.0040 & 0.90 & 329 \\
Crouch Branch & B-B' & 0.0015 & 0.34 & 123 \\
& A-A' & 0.0031 & 0.62 & 226
\end{tabular}

${ }^{a}$ Gradients were determined along transects oriented to represent the minimum and maximum gradient evident on the piezometric and potentiometric maps in Appendix $\mathrm{H}$ (Volume III) of this report. 
$-$

葛 
-<smiles>CCC</smiles> 


\section{Appendix E}

\section{Data Quality/Usability Assessment}


THIS PAGE LEFT BLANK INTENTIONALLY. 


\section{Data Quality/Usability Assessment}

Quality assurance/quality control (QA/QC) procedures relating to accuracy and precision of analyses performed on groundwater samples are followed in the field and laboratory, and QC data are reviewed prior to publication of results. The review by the Environmental Protection Department/ Environmental Monitoring Section (EPD/EMS) of the volume of analytical data acquired each quarter and presented in various reports is an ongoing process; EPD/EMS' review of the QC data cannot be completed in time to meet the deadlines for the groundwater monitoring reports required by the Resource Conservation and Recovery Act and associated regulations. Beginning first quarter 1996, the Quality Control Samples section of EMS' quarterly groundwater monitoring report contains detailed evaluation of the following indicators of data quality: precision, accuracy, representativeness, comparability, and completeness. Site and regulatory personnel can obtain further information on the data quality and usability in a variety of ways, including those described below.

\section{Data Qualification}

The contract laboratories continually assess their own accuracy and precision according to U.S. Environmental Protection Agency (EPA) guidelines. They submit sample- or batch-specific QC information either at the same time as analytical results or in quarterly summaries. Properly defined and used modifiers (also referred to as qualifiers) can be a key component in assessing data usability. Modifiers designed by EPA or EPD/EMS and used by the primary laboratories or EMS' data validators are presented in Appendix D.

\section{Assessment of Accuracy of the Data}

Accuracy, or the nearness of the reported result to the true concentration of a constituent in a sample, can be assessed in several ways.

A laboratory's general accuracy can be judged by evaluation of results obtained from known samples. The contract laboratories analyze commercial reference samples periodically at EPD/EMS' request. The results of these analyses from the non-radionuclide laboratories are presented in the EPD/EMS groundwater monitoring quarterly reports; the results from the radionuclide laboratories should be available on request from the laboratory subcontract representatives. The primary laboratories also seek or maintain state certification by participating periodically in performance studies. Reference samples and evaluation of results are provided by EPA. Results of these studies also are published in the EPD/EMS quarterly reports.

Analysis of blanks provides a tool for assessing the accuracy of both sampling and laboratory analysis. Results for all field blanks for the quarter can be found in the EPD/EMS quarterly reports. Any field or laboratory blanks that exceed established minimums are identified in the same reports, in tables associating them with groundwater samples analyzed in the same batches. In these regulatory reports, the modifier $V$ is assigned to every detected result that is run in the same batch as a laboratory blank with positive results for that analyte. A $U$ qualifier (reported as less than $[<]$ ) also is added if the analyte concentration is less than five times the concentration in the blank (or, for common laboratory contaminants, less than ten times).

Surrogates, organic compounds similar in chemical behavior to the compounds of interest but not normally found in environmental samples, are used to monitor the effect of the matrix on the accuracy of analyses for organic parameters. For example, for analyses of volatile organics by EPA Method 8240, three surrogate compounds are added to all samples and blanks in each analytical batch. In analyses of semivolatile organics by EPA Method 8270, three surrogates are required and an additional compound is advised for each fraction (acids and base/neutrals). Two surrogates are used in organochlorine pesticides analyses. Percent recoveries for surrogate analyses are calculated by laboratory personnel, reported to EPD/EMS, reviewed, and entered into the database. Beginning first quarter 1996, statistical summaries are published in the Quality Control Samples 
section of the EPD/EMS quarterly groundwater monitoring report. If recoveries are not within specified limits, the laboratory is expected to reanalyze the samples or attach qualifiers to the data identifying the anomalous results.

Sample-specific accuracy for both organic and inorganic parameters can be assessed by examination of matrix spike/matrix spike duplicate results. A portion of the sample is analyzed unspiked to determine a baseline set of values. A second portion of the sample is spiked with known concentrations of compounds appropriate to the analyses being performed, typically five volatile organic compounds for volatile organics analyses, eleven semivolatile compounds for semivolatiles, six pesticide compounds for pesticides, all metals for metals analyses by SW-846 methods (EPA, 1986), and a known quantity of cyanide for cyanide analysis. The percentage of the spike compound that is recovered (i.e., measured in excess of the value obtained for the unspiked sample) is a direct measure of analytical accuracy. EPA requires matrix spike/matrix spike duplicates to be run at least once per 20 samples of similar matrix.

Matrix spike/matrix spike duplicate results are reported to EPD/EMS. Beginning first quarter 1996, spike results and a statistical summary are published in the Quality Control Samples section of the EPD/EMS quarterly groundwater monitoring report. For organic compounds, according to EPA guidelines, no action is taken on the basis of matrix spike/matrix spike duplicate data alone (i.e., no result modifiers are assigned solely on the basis of matrix spike results); however, the results can indicate if a laboratory is having a systemic problem in the analysis of one or more analytes.

In the case of inorganic compounds, such as metals, the matrix spike sample analysis provides information about the effect of each sample matrix on the digestion and measurement methodology. Data qualifiers assigned by the laboratories on the basis of the percentage of spike recovery are reported in the EPD/EMS results tables.

\section{Assessment of Precision}

Precision of the analyses, or agreement of a set of replicate results among themselves, is assessed through the use of duplicates initiated by the laboratory and blind replicates provided by EPD/EMS. The results of duplicate and replicate analyses are presented in results tables that report only one quarter of data. Results tables that include more than one quarter of data present only the highest result for each analyte for each quarter of the year.

The laboratories assess precision by calculating the relative percent difference (RPD) for each pair of laboratory-initiated duplicate results. The EMS data validators apply a data qualifier to the results of entire preparation batches of metals analyses when the RPD for laboratory duplicates is greater than 20 percent and either the difference between the two samples is greater than the contractrequired detection limit (CRDL) or both sample results are greater than 5 times the CRDL. This qualifier is published in the EPD/EMS quarterly reports.

Additional statistical comparisons of laboratory duplicate and blind replicate results, both intra- and interlaboratory, are presented in the EPD/EMS quarterly reports. The calculation used for these reports is the mean relative difference (MRD), which is similar to EPA's RPD except that the MRD is the average of all the RPD values from one laboratory for each compound (intralaboratory MRD) 'or all the RPD values from all laboratories for each compound (interlaboratory MRD) during one quarter. Because detection limits may vary among samples, the MRD requires calculation of a reference detection limit, which is the detection limit at the 90th percentile of the array of limits in the population of all duplicate and replicate analyses for a given analyte during a particular quarter. The MRD is not method-specific.

\section{Method-Specific Accuracy and Precision}

The contract laboratories' EPA-approved laboratory procedures include QAVC requirements as an integral part of the methods. Thus, knowledge of the method used in obtaining data is an important component of determining data usability. EPA has conducted extensive research and development on the methods approved for the analysis of water and wastewater. Information on the accuracy and precision of a method is available from EPA publications, as is full information on required QAVC 
procedures. A listing of the methods used by the primary laboratories during fourth quarter 1995 is given below, along with the source for the method description. Many, if not all, of these sources include presentations of representative accuracy and precision results. The EPD/EMS quarterly reports provide the methods used by the laboratories each quarter.

Table E-1. Methods Used by the Contract Laboratories

\begin{tabular}{|c|c|c|}
\hline Method & Used to Analyze & Source \\
\hline EICHROMTC1MOD ${ }^{a}$ & Technetium isotopes & EiChrom Industrie \\
\hline EMLAM01MOD & Americium, curium isotopes & DOE, 1992 \\
\hline EMLPU02MOD & Neptunium, plutonium isotopes & DOE, 1992 \\
\hline EMLSR02MOD & Strontium isotopes & DOE, 1992 \\
\hline EMLTH01MOD & Thorium isotopes & DOE, 1992 \\
\hline EMLU02MOD & Uranium isotopes & DOE, 1992 \\
\hline ENICMOD & Carbon-14 & NA \\
\hline EPA120.1 & Specific conductance & EPA EMSL, 1983 \\
\hline EPA150.1 & $\mathrm{pH}$ & EPA EMSL, 1983 \\
\hline EPA160.1 & Total dissolved solids & EPA EMSL, 1983 \\
\hline EPA160.2 & Total dissolved solids, total suspended solids & EPA EMSL, 1983 \\
\hline EPA180.1 & Turbidity & EPA EMSL, 1983 \\
\hline EPA200.7 & Metals & EPA EMSL, 1983 \\
\hline EPA245.1 & Mercury & EPA EMSL, 1983 \\
\hline EPA300.0 & Chloride, nitrite, sulfate, fluoride & EPA EMSL, 1983 \\
\hline EPA310.1 & Alkalinity (as $\mathrm{CaCO}_{3}$ ) & EPA EMSL, 1983 \\
\hline EPA335.2 & Cyanide & EPA EMSL, 1983 \\
\hline EPA335.3 & Cyanide & EPA EMSL, 1983 \\
\hline EPA340.2 & Fluoride & EPA EMSL, 1983 \\
\hline EPA350.1 & Ammonia nitrogen & EPA EMSL, 1983 \\
\hline EPA350.3 & Ammonia & EPA EMSL, 1983 \\
\hline EPA353.1 & Nitrogen, nitrate-nitrite & EPA EMSL, 1983 \\
\hline EPA353.2 & Nitrogen, nitrate, nitrite, or combined & EPA EMSL, 1983 \\
\hline EPA365.2 & $\begin{array}{l}\text { Phosphorus, all forms (reported as total } \\
\text { phosphates) }\end{array}$ & EPA EMSL, 1983 \\
\hline EРАЗ65.3 & $\begin{array}{l}\text { Phosphorus, all forms (reported as total } \\
\text { phosphates) }\end{array}$ & EPA EMSL, 1983 \\
\hline EPA365.4 & $\begin{array}{l}\text { Phosphorus, all forms (reported as total } \\
\text { phosphates) }\end{array}$ & EPA EMSL, 1983 \\
\hline EPA405.1 & Five-day biochemiçal oxygen demand & EPA EMSL, 1983 \\
\hline EPA405.1/SM & Five-day biochemical oxygen demand ${ }^{\circ}$ & EPA EMSL, 1983 \\
\hline EPA410.4 & Chemical oxygen demand & EPA EMSL, 1983 \\
\hline EPA415.1 & $\begin{array}{l}\text { Dissolved organic carbon, total inorganic } \\
\text { carbon, total organic carbon }\end{array}$ & EPA EMSL, 1983 \\
\hline EPA418.1 & Total petroleum hydrocarbons & EPA EMSL, 1983 \\
\hline
\end{tabular}




\begin{tabular}{|c|c|c|}
\hline Method & Used to Analyze & Source \\
\hline EPA420.2 & Phenols & EPA EMSL, 1983 \\
\hline EPA450.1 & Total organic halogens & NA \\
\hline EPA900.0MOD & Gross alpha, nonvolatile beta & EPA EMSL, 1980 \\
\hline EPA901.1MOD & Gamma PHA, iodine isotopes & EPA EMSL, 1980 \\
\hline EPA903.0MOD & Total alpha-emitting radium, radium isotopes & EPA EMSL, 1980 \\
\hline EPA904.0MOD & Radium isotopes & EPA EMSL, 1980 \\
\hline EPA906.0MOD & Tritium & EPA EMSL, 1980 \\
\hline EPA6010 & Metals & EPA, 1986 \\
\hline EPA6010A & Metals & EPA, 1992 \\
\hline EPA7470 & Mercury & EPA, 1986 \\
\hline EPA8080 & Organochlorine pesticides and PCBs & EPA, 1986 \\
\hline EPA8150 & Chlorinated herbicides & EPA, 1986 \\
\hline EPA8240 & GCMS volatiles & EPA, 1986 \\
\hline EPA8260 & GCMS volatiles & EPA, 1986 \\
\hline EPA8270 & GCMS semivolatiles & EPA, 1986 \\
\hline EPA8280 & Dioxins and furans & EPA, 1986 \\
\hline EPA9020A & Total organic halogens & EPA, 1986 \\
\hline EPA9020B & Total organic halogens & EPA, 1994b \\
\hline EPA9060 & Total inorganic carbon & EPA, 1986 \\
\hline EPIA-001 & Gross alpha, nonvolatile beta & EP, 1996 \\
\hline EPIA- $002^{c}$ & Tritium & EP, 1996 \\
\hline $\mathrm{EPIA}-003^{\mathrm{C}}$ & Carbon-14 & $E P, 1996$ \\
\hline EPIA-004 ${ }^{c}$ & Strontium-90 & $E P, 1996$ \\
\hline EPIA-005 & Technetium-99 & $E P, 1996$ \\
\hline EPIA-006 ${ }^{c}$ & lodine-129 & $E P, 1996$ \\
\hline EPIA-008 ${ }^{\mathrm{C}}$ & Radium-226 & $E P, 1996$ \\
\hline EPIA-009 & Radium-228 & $E P, 1996$ \\
\hline EPIA-010 ${ }^{\mathrm{c}}$ & Radium, total alpha-emitting & EP, 1996 \\
\hline EPIA-011 ${ }^{\mathrm{c}}$ & Americium, curium, uranium isotopes & $E P, 1996$ \\
\hline EPIA-012 & Neptunium, plutonium, thorium isotopes & $E P, 1996$ \\
\hline$E P \mid A-013^{c}$ & Gamma PHA & EP, 1996 \\
\hline EPIA-022 $2^{c}$ & Nickel isotopes & EP, 1996 \\
\hline MMES16009MOD & Technetium-99 & NA \\
\hline $3 Q 1-6-1420$ & Total activity, tritium & NA \\
\hline
\end{tabular}

Method

EPA420.2

EPA900.0MOD

EPA901.1MOD

EPA906.0MOD

EPA6010

A6010A

EPA8080

EPA8240

8260

EPA9020A

EPA9020B

EPIA-003

EPIA-004

EPIA-008

EPIA-009

EPIA-010

EPIA-013

EPIA-022

MMES16009MOD
Source

EMSL, 1983

EPA EMSL, 1980

EPA EMSL, 1980

EPA EMSL, 1980

EPA, 1992

EPA, 1986

EPA, 1986

EPA, 1986

EPA, 1986

EPA, 1986

EPA, 1986

EP, 1996

1996

$E P, 1996$

996

$E P, 1996$

NA

NA 
Note: One of the labs reports the method for some metals determinations as CLP or CLP-MOD. This is presumed equivalent to Method 6010 or 6010A.

An example of method-specific QC information is that for EPA Method 8270 (EPA, 1986), which is used by both GE and Weston for analyses of semivolatile organics. The following table gives method-specific accuracy and precision as functions of concentration. Contract laboratories are expected to achieve or at least approach these limits.

Table E-2. Method Accuracy and Precision as Functions of Concentration for EPA Method 8270

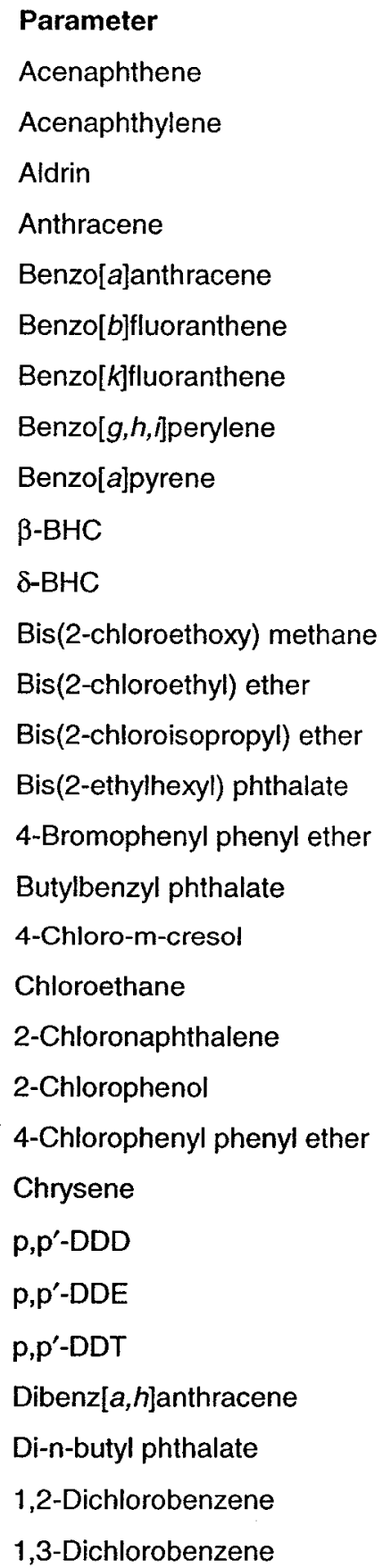

Accuracy, as recovery, $x^{\circ}$ ( $\left.\mu g / L\right)$

$0.96 \mathrm{C}+0.19$

$0.89 \mathrm{C}+0.74$

$0.78 \mathrm{C}+1.66$

$0.80 \mathrm{C}+0.68$

$0.88 \mathrm{C}-0.60$

$0.93 C-1.80$

$0.87 \mathrm{C}-1.56$

$0.98 \mathrm{C}-0.86$

0.90C-0.13

$0.87 \mathrm{C}-0.94$

$0.29 \mathrm{C}-1.09$

$1.12 \mathrm{C}-5.04$

$0.86 \mathrm{C}-1.54$

1.03C-2.31

$0.84 C-1.18$

$0.91 \mathrm{C}-1.34$

$0.66 \mathrm{C}-1.68$

$0.84 \mathrm{C}+0.35$

$0.99 \mathrm{C}-1.53$

$0.89 \mathrm{C}+0.01$

$0.78 C+0.29$

$0.91 \mathrm{C}+0.53$

$0.93 \mathrm{C}-1.00$

$0.56 \mathrm{C}-0.40$

$0.70 \mathrm{C}-0.54$

$0.79 \mathrm{C}-3.28$

$0.88 \mathrm{C}+4.72$

$0.59 \mathrm{C}+0.71$

$0.80 \mathrm{C}+0.28$

$0.86 \mathrm{C}-0.70$

\section{Single analyst} precision, $s_{r}^{\prime}(\mu g / L) \quad S^{\prime}(\mu g / L)$

$0.15 X-0.12$

$0.24 X-1.06$

$0.27 X-1.28$

$0.21 X-0.32$

$0.15 X+0.93$

$0.22 X+0.43$

$0.19 X+1.03$

$0.29 X+2.40$

$0.22 X+0.48$

$0.20 x-0.58$

$0.34 X+0.86$

$0.16 X+1.34$

$0.35 X-0.99$

$0.24 X+0.28$

$0.26 X+0.73$

$0.13 X+0.66$

$0.18 X+0.94$

$0.23 X+0.75$

$0.14 X-0.13$

$0.07 X+0.52$

$0.18 X+1.46$

$0.20 X-0.94$

$0.28 X+0.13$

$0.29 X-0.32$

$0.26 X-1.17$

$0.42 X+0.19$

$0.30 X+8.51$

$0.13 X+1.16$

$0.20 X+0.47$

$0.25 X+0.68$
$0.21 X-0.67$

$0.26 x-0.54$

$0.43 X+1.13$

$0.27 X-0.64$

$0.26 X-0.21$

$0.29 X+0.96$

$0.35 X+0.40$

$0.51 X-0.44$

$0.32 X+1.35$

$0.93 X+0.17$

$0.26 X+2.01$

$0.35 X+0.10$

$0.25 X+1.04$

$0.36 X+0.67$

$0.16 X+0.66$

$0.53 X+0.92$

$0.29 X+1.31$

$0.17 X-0.28$

$0.13 X+0.34$

$0.28 X+0.97$

$0.30 X-0.46$

$0.33 X-0.09$

$0.66 X-0.96$

$0.39 X-1.04$

$0.65 X-0.58$

$0.59 X+0.25$

$0.39 X+0.60$

$0.24 X+0.39$

$0.41 X+0.11$
$0.30 X+1.94$ 


\begin{tabular}{|c|c|c|c|}
\hline Parameter & $\begin{array}{l}\text { Accuracy, as } \\
\text { recovery, } x^{\prime}(\mu g / L)\end{array}$ & $\begin{array}{l}\text { Single analyst } \\
\text { precision, } s_{r}^{\prime}(\mu g / L)\end{array}$ & $\begin{array}{l}\text { Overall precision, } \\
S^{\prime}(\mu g / L)\end{array}$ \\
\hline 1,4-Dichlorobenzene & $0.73 C-1.47$ & $0.24 X+0.23$ & $0.29 X+0.36$ \\
\hline $3,3^{\prime}$-Dichlorobenzidine & $1.23 \mathrm{C}-12.65$ & $0.28 X+7.33$ & $0.47 X+3.45$ \\
\hline 2,4-Dichlorophenol & $0.87 C-0.13$ & $0.15 X+1.25$ & $0.21 X+1.28$ \\
\hline Dieldrin & $0.82 C-0.16$ & $0.20 x-0.16$ & $0.26 X-0.07$ \\
\hline Diethyl phthalate & $0.43 C+1.00$ & $0.28 X+1.44$ & $0.52 X+0.22$ \\
\hline 2,4-Dimethyl phenol & $0.71 \mathrm{C}+4.41$ & $0.16 X+1.21$ & $0.22 X+1.31$ \\
\hline Dimethyl phthalate & $0.20 \mathrm{C}+1.03$ & $0.54 X+0.19$ & $1.05 x-0.92$ \\
\hline 2,4-Dinitrophenol & $0.81 C-18.04$ & $0.38 X+2.36$ & $0.42 X+26.29$ \\
\hline 2,4-Dinitrotoluene & $0.92 C-4.81$ & $0.12 X+1.06$ & $0.21 X+1.50$ \\
\hline 2,6-Dinitrotoluene & $1.06 \mathrm{C}-3.60$ & $0.14 X+1.26$ & $0.19 X+0.35$ \\
\hline Di-n-octyl phthalate & $0.76 \mathrm{C}-0.79$ & $0.21 X+1.19$ & $0.37 X+1.19$ \\
\hline Endosulfan sulfate & $0.39 C+0.41$ & $0.12 X+2.47$ & $0.63 x-1.03$ \\
\hline Endrin aldehyde & $0.76 C-3.86$ & $0.18 X+3.91$ & $0.73 x-0.62$ \\
\hline Fluoranthene & $0.81 C+1.10$ & $0.22 x-0.73$ & $0.28 x-0.60$ \\
\hline Fluorene & $0.90 \mathrm{C}-0.00$ & $0.12 X+0.26$ & $0.13 X+0.61$ \\
\hline Heptachlor & $0.87 \mathrm{C}-2.97$ & $0.24 X-0.56$ & $0.50 x-0.23$ \\
\hline Heptachlor epoxide & $0.92 C-1.87$ & $0.33 x-0.46$ & $0.28 X+0.64$ \\
\hline Hexachlorobenzene & $0.74 C+0.66$ & $0.18 x-0.10$ & $0.43 x-0.52$ \\
\hline Hexachlorobutadiene & $0.71 C-1.01$ & $0.19 X+0.92$ & $0.26 X+0.49$ \\
\hline Hexachloroethane & $0.73 C-0.83$ & $0.17 X+0.67$ & $0.17 X+0.80$ \\
\hline Indeno[1,2,3-c, d]pyrene & $0.78 \mathrm{C}-3.10$ & $0.29 X+1.46$ & $0.50 x-0.44$ \\
\hline Isophorone & $1.12 \mathrm{C}+1.41$ & $0.27 X+0.77$ & $0.33 x+0.26$ \\
\hline 2-Methyl-4,6-dinitrophenol & $1.04 \mathrm{C}-28.04$ & $0.10 X+42.29$ & $0.26 X+23.10$ \\
\hline Naphthalene & $0.76 C+1.58$ & $0.21 x-0.41$ & $0.30 x-0.68$ \\
\hline Nitrobenzene & $1.09 \mathrm{C}-3.05$ & $0.19 X+0.92$ & $0.27 X+0.21$ \\
\hline 2-Nitrophenol & $0.07 C-1.15$ & $0.16 X+1.94$ & $0.27 X+2.60$ \\
\hline 4-Nitrophenol & $0.61 C-1.22$ & $0.38 X+2.57$ & $0.44 X+3.24$ \\
\hline N-Nitrosodi-n-propylamine & $1.12 \mathrm{C}-6.22$ & $0.27 X+0.68$ & $0.44 X+0.47$ \\
\hline PCB 1260 & $0.81 C-10.86$ & $0.35 X+3.61$ & $0.43 X+1.82$ \\
\hline Pentachlorophenol & $0.93 C+1.99$ & $0.24 X+3.03$ & $0.30 X+4.33$ \\
\hline Phenanthrene & $0.87 \mathrm{C}+0.06$ & $0.12 X+0.57$ & $0.15 X+0.25$ \\
\hline Phenol & $0.43 C+1.26$ & $0.26 X+0.73$ & $0.35 X+0.58$ \\
\hline Pyrene & $0.84 C-0.16$ & $0.16 X+0.06$ & $0.15 X+0.31$ \\
\hline 1,2,4-Trichlorobenzene & $0.94 C-0.79$ & $0.15 X+0.85$ & $0.21 X+0.39$ \\
\hline 2,4,6-Trichlorophenol & $0.91 C-0.18$ & $0.16 X+2.22$ & $0.22 X+1.81$ \\
\hline
\end{tabular}

$x^{\prime}=$ Expected recovery for one or more measurements of a sample containing a concentration of $C$, in $\mu g / L$. 
$\mathbf{S}_{r}{ }^{\prime}=$ Expected single analyst standard deviation of measurements at an average concentration of $X$, in $\mu \mathrm{g} / \mathrm{L}$.

$S^{\prime}=$ Expected interlaboratory standard deviation of measurements at an average concentration of $X$, in $\mu \mathrm{g} / \mathrm{L}$.

$\mathrm{C}=$ True value for the concentration, in $\mu \mathrm{g} / \mathrm{L}$.

$X=$ Average recovery found for measurements of samples containing a concentration of $C$, in $\mu \mathrm{g} / \mathrm{L}$. 
THIS PAGE LEFT BLANK INTENTIONALLY. 


\section{Appendix $\mathrm{F}$}

\section{Time Series Plots}


THIS PAGE LEFT BLANK INTENTIONALLY. 


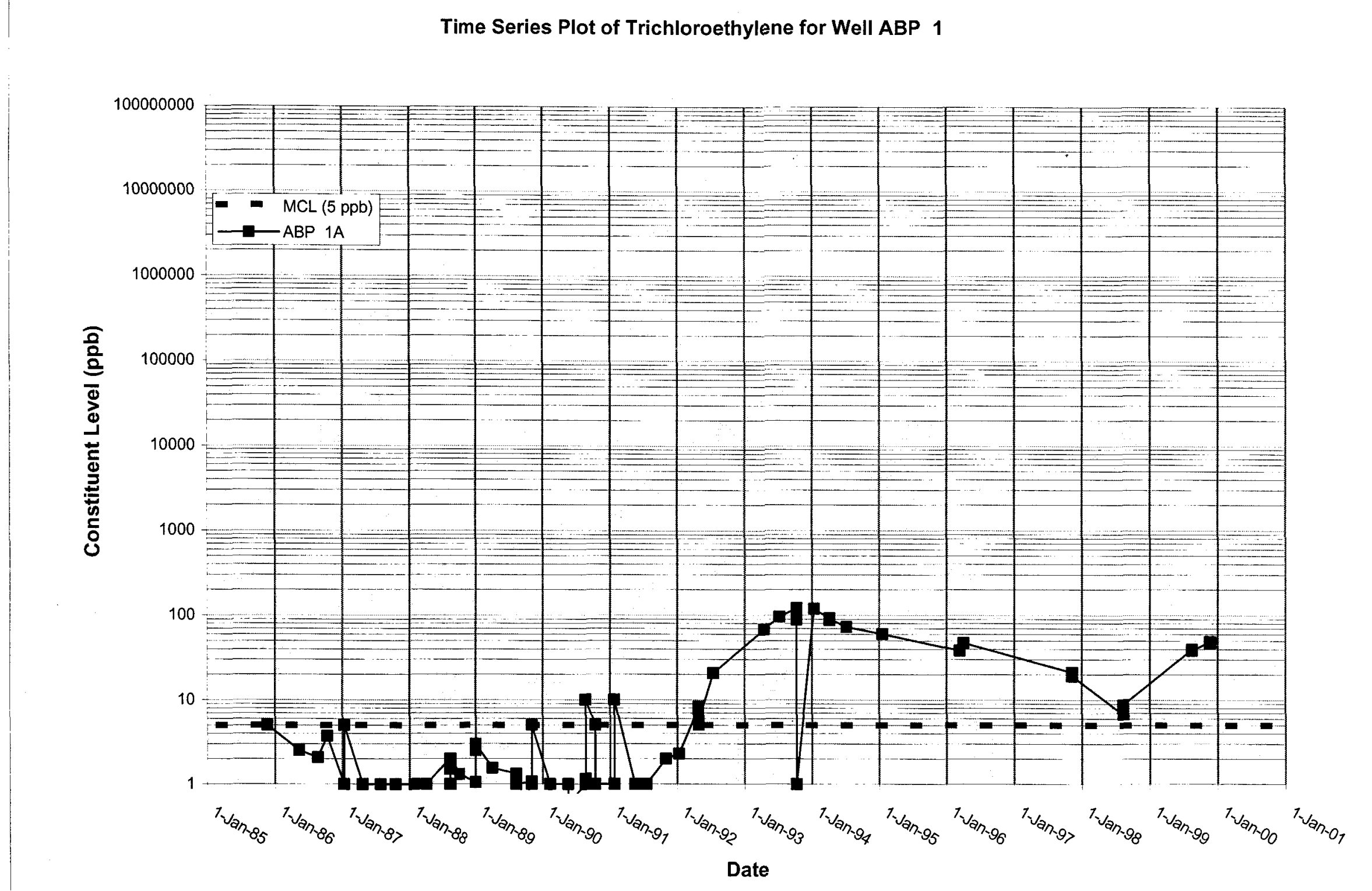




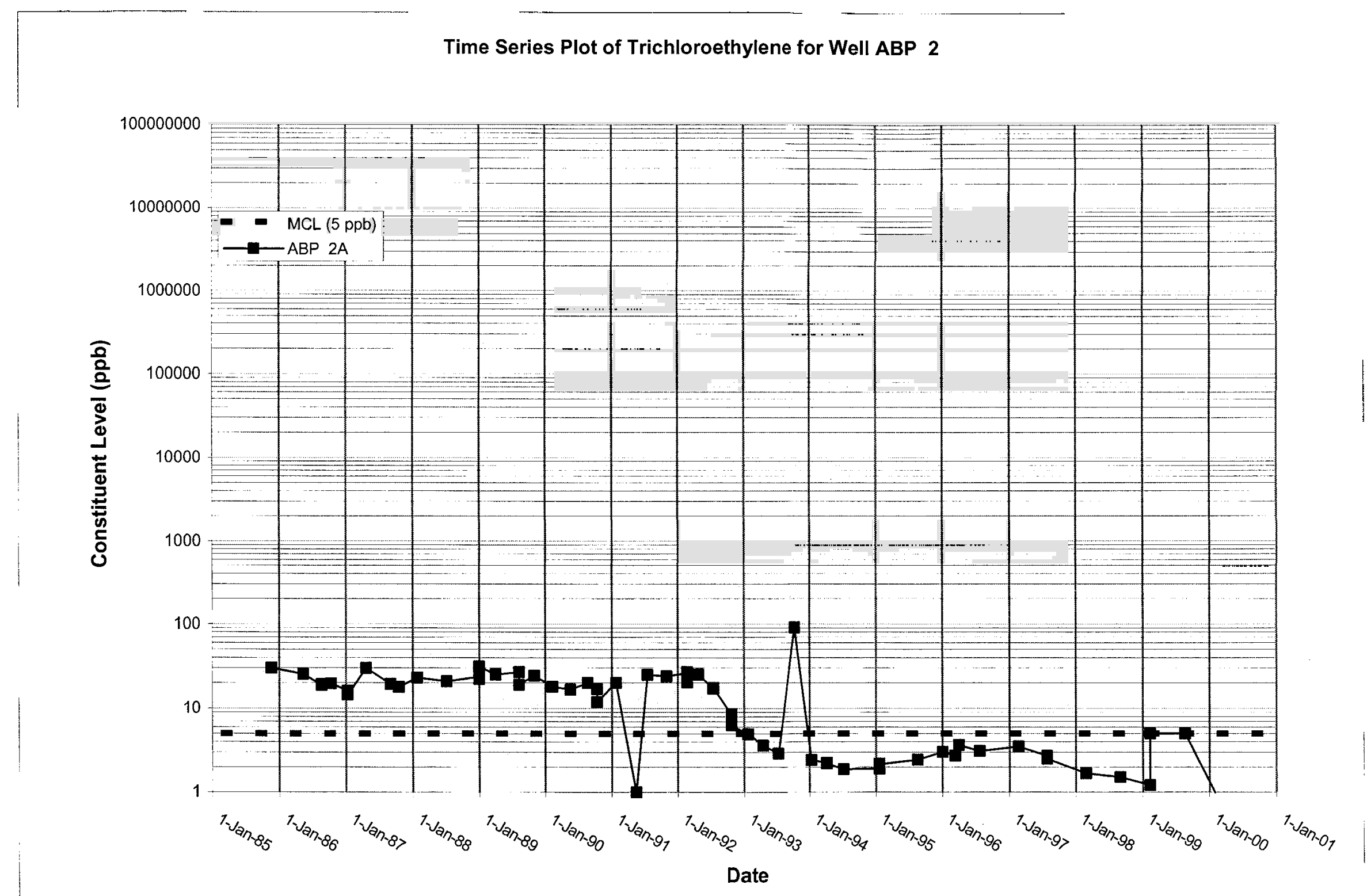




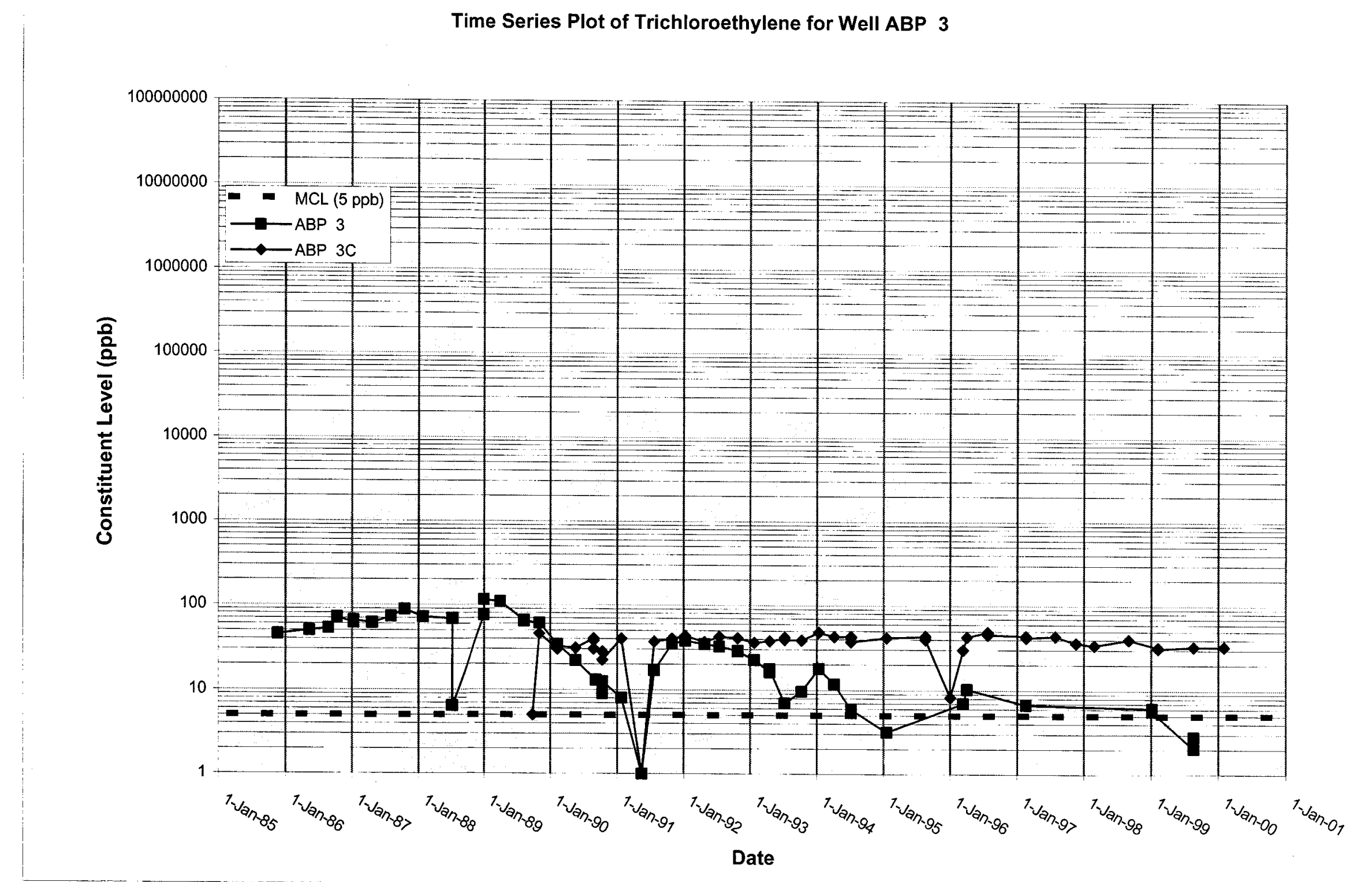


WSRC-TR-2000-00242 UNCLASSIFIED

\section{Time Series Plot of Trichloroethylene for Well ABP 4}

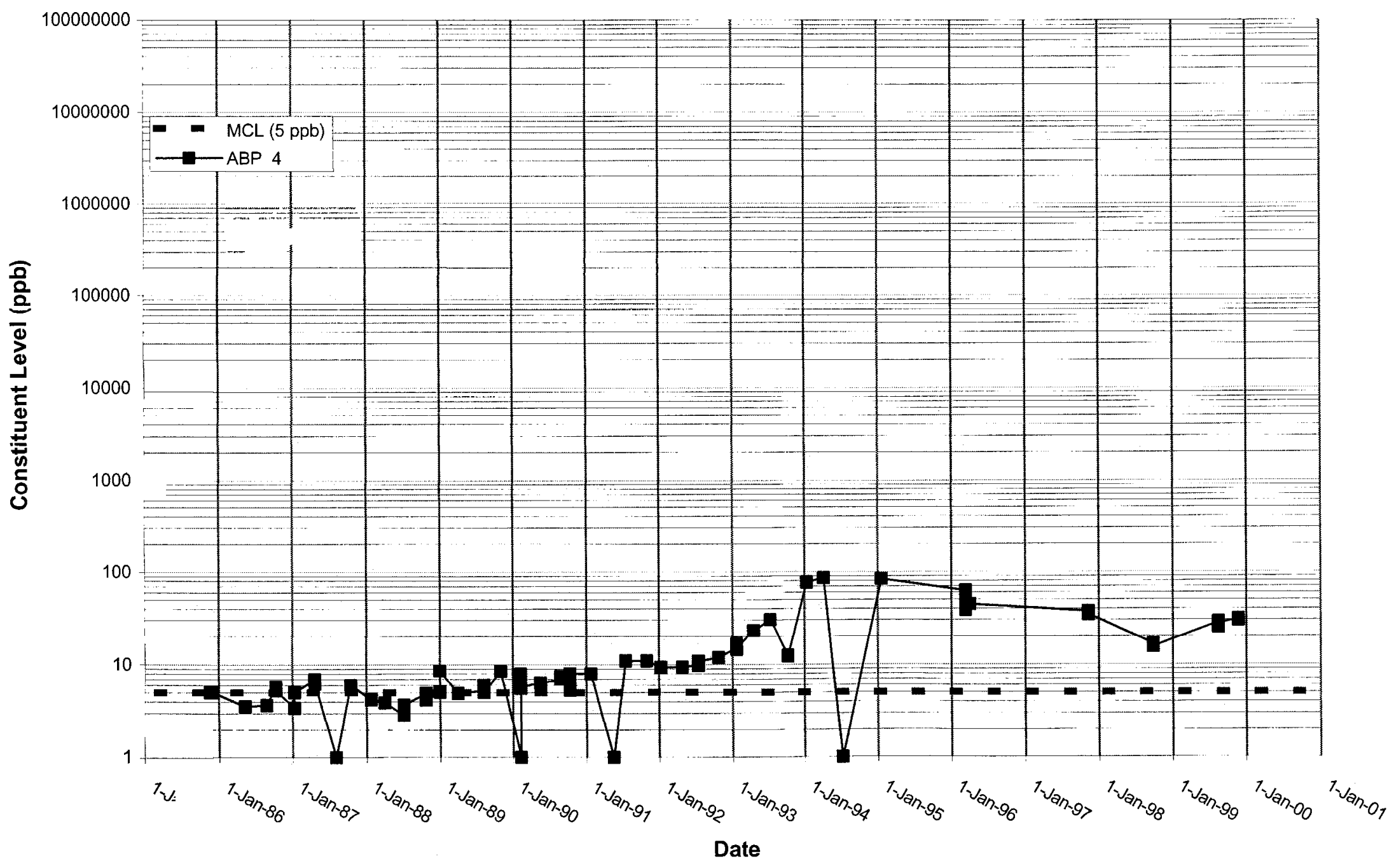




\section{Time Series Plot of Trichloroethylene for Well ABP 8}

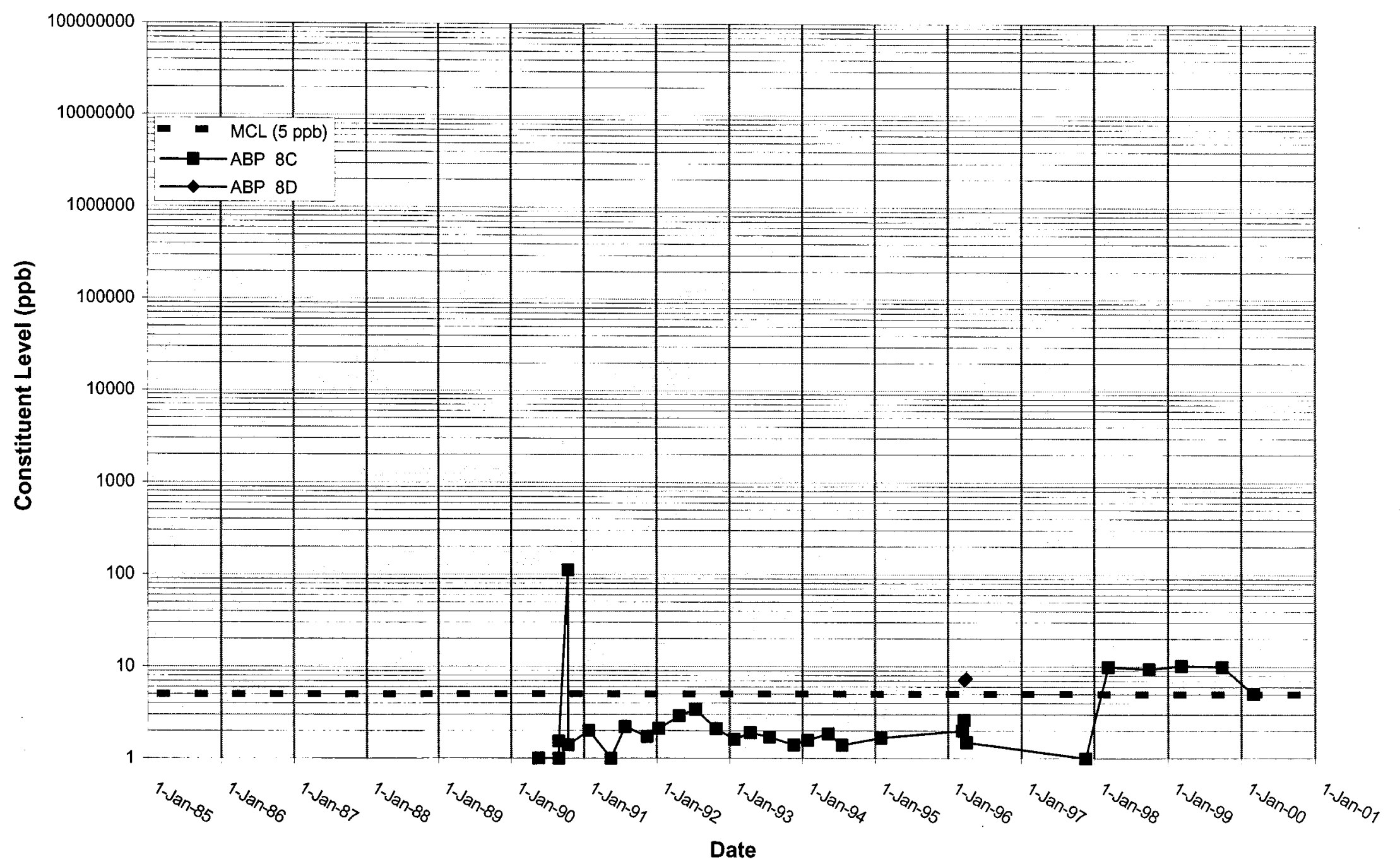


WSRC-TR-2000-00242 UNCL $\Lambda$ SSIFIED

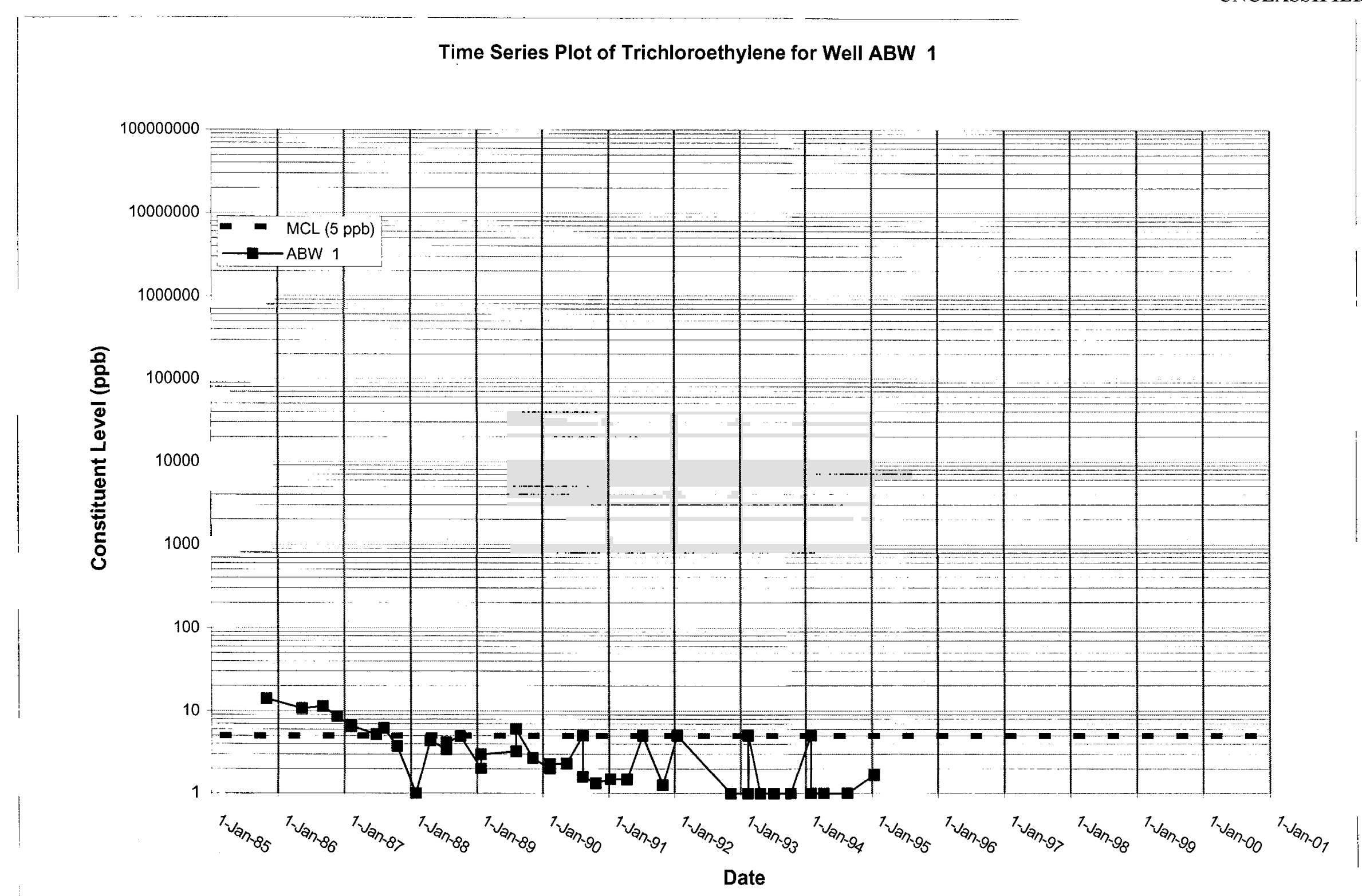




\section{Time Series Plot of Trichloroethylene for Well AC 1}

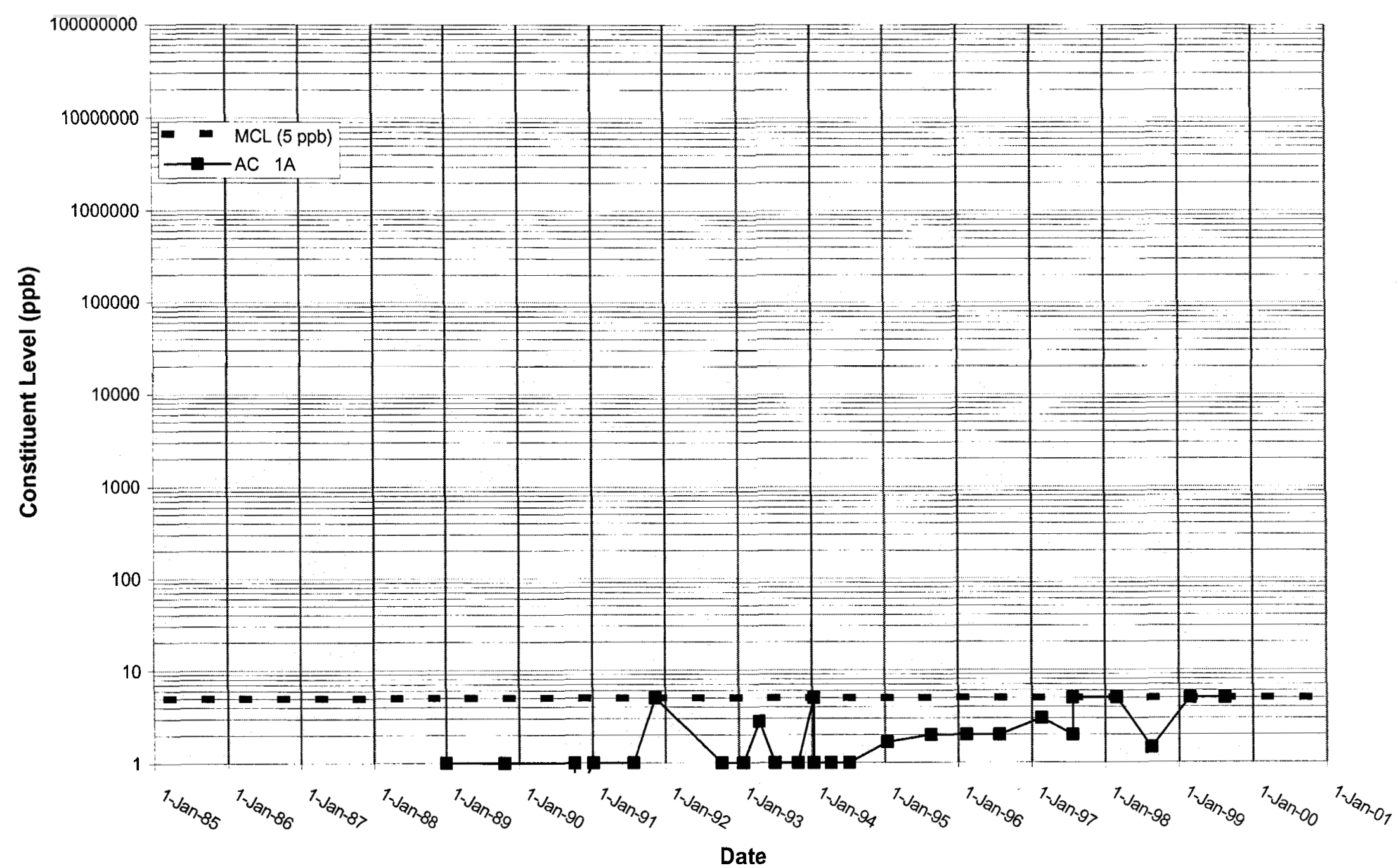




\section{Time Series Plot of Trichloroethylene for Well AC 2}

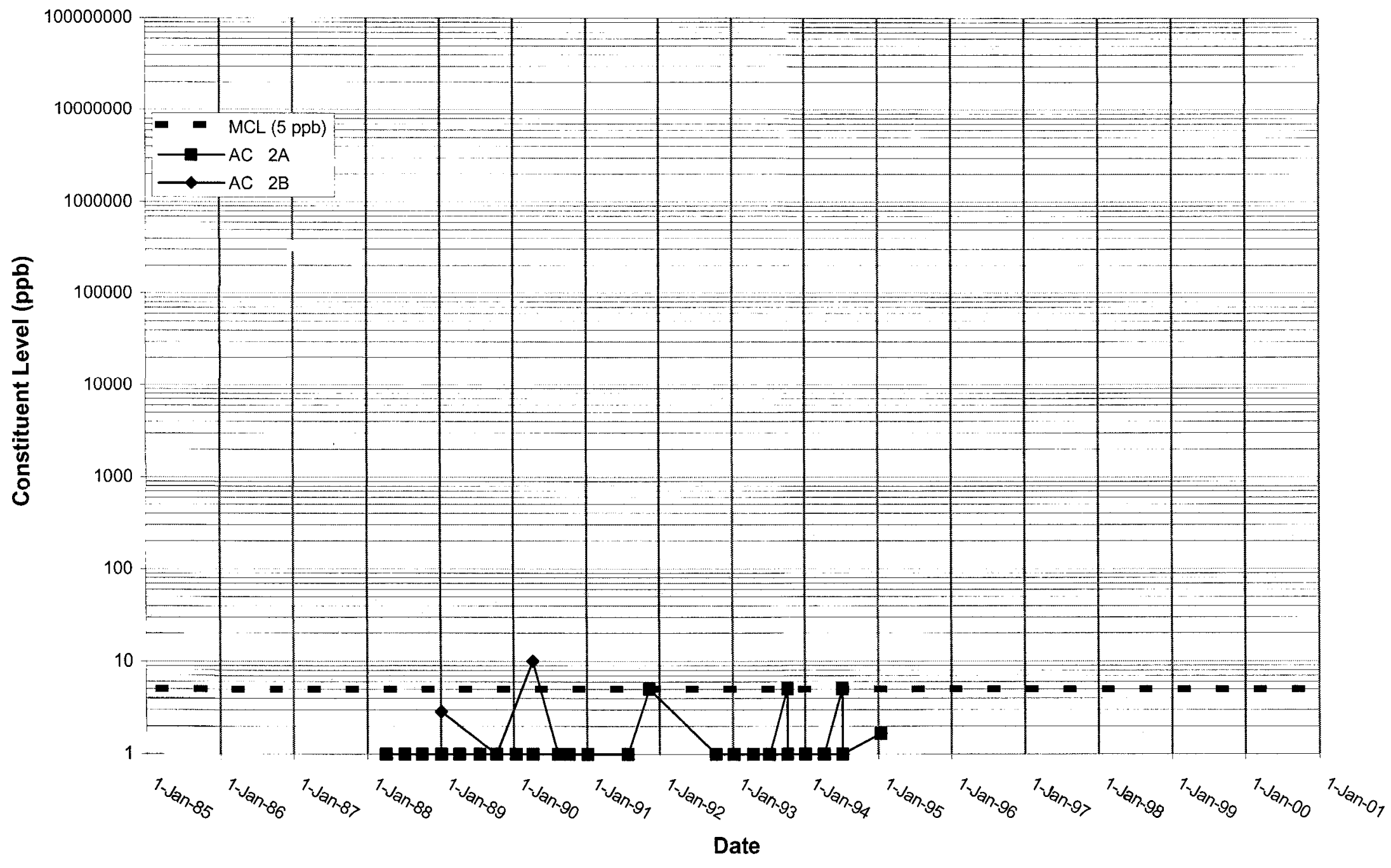




\section{Time Series Plot of Trichloroethylene for Well AC 3}

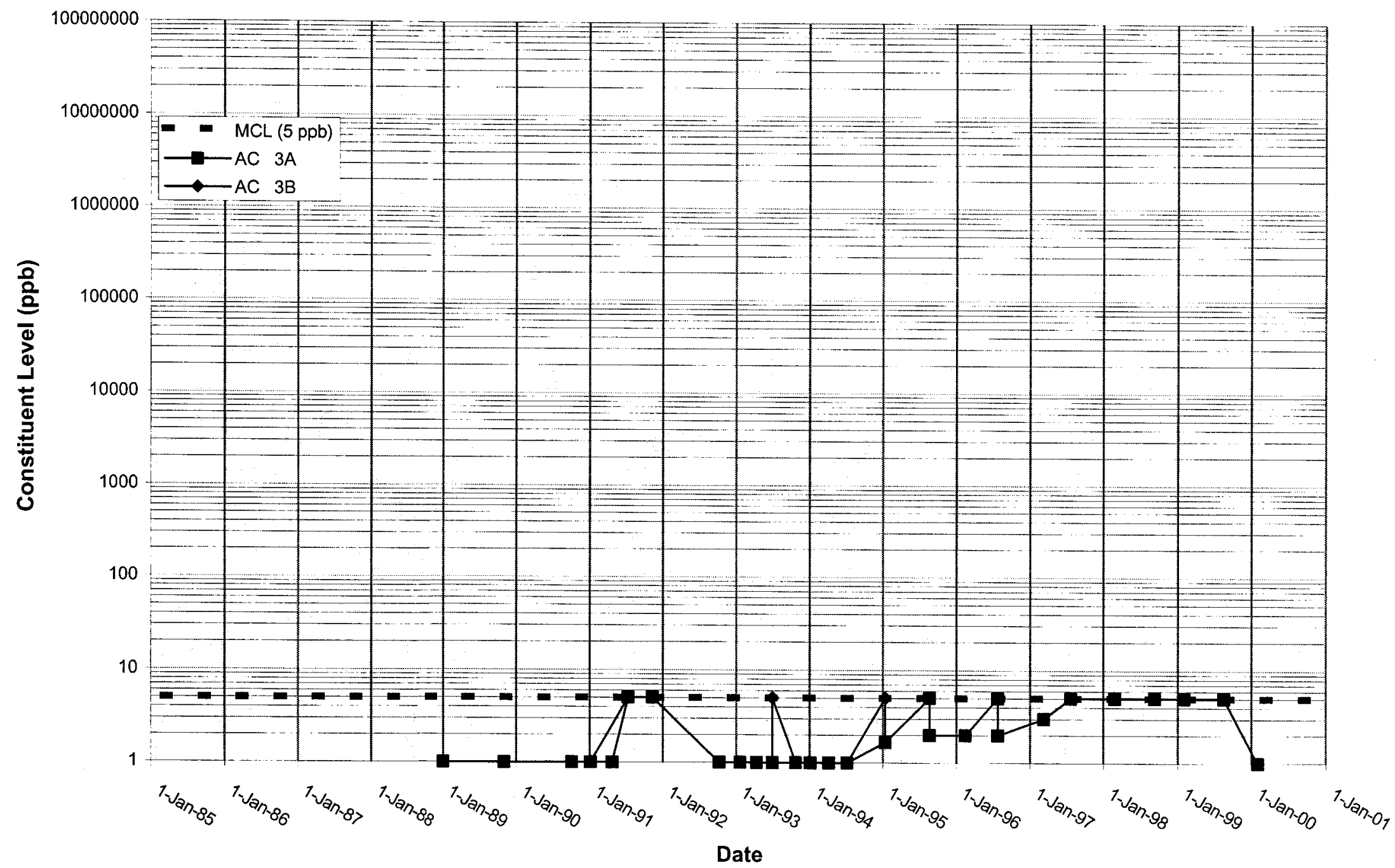


WSRC-TR-2000-00242

UNCLASSIFIED

Time Series Plot of Trichloroethylene for Well ACB 1

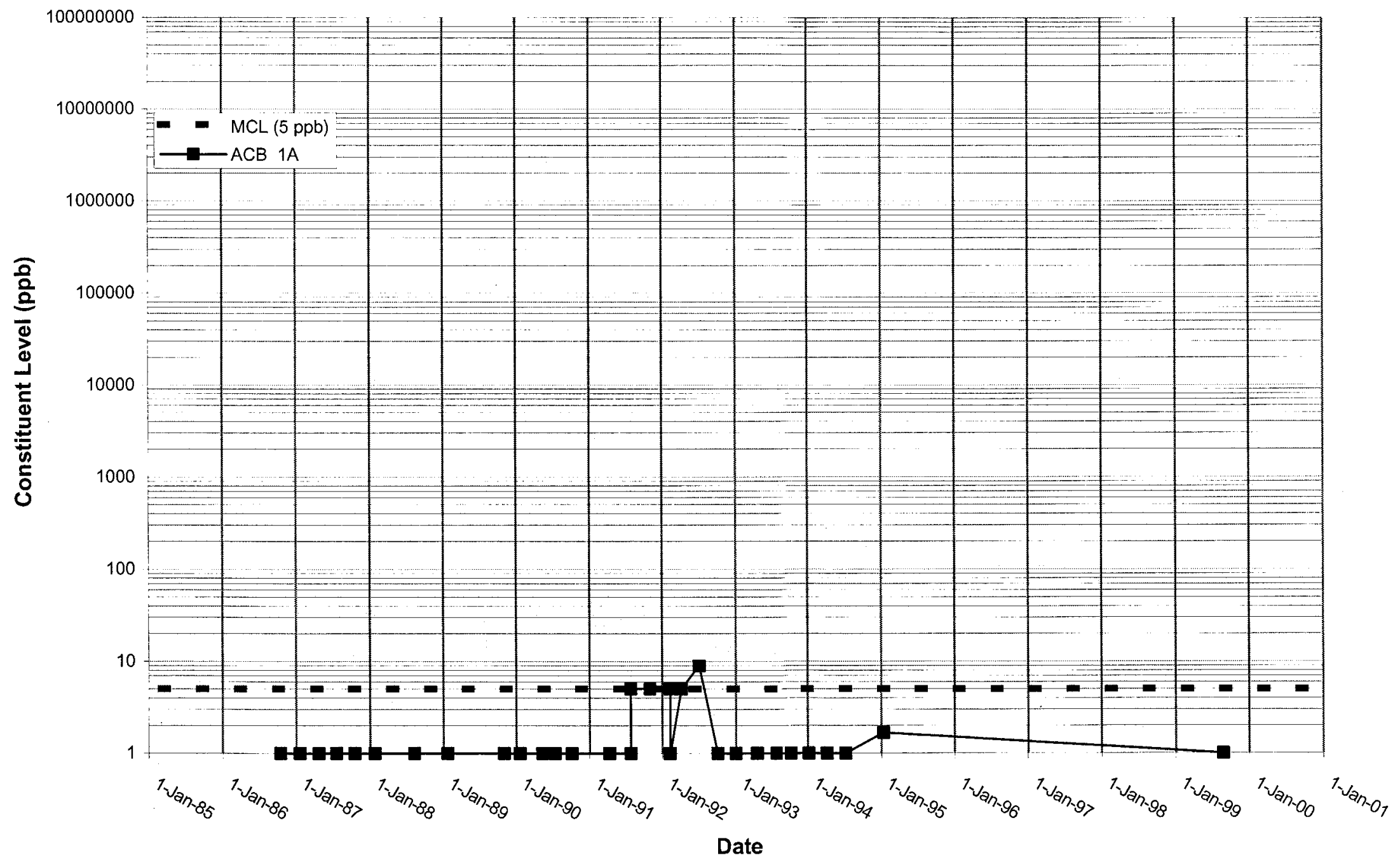


Time Series Plot of Trichloroethylene for Well ACB 2

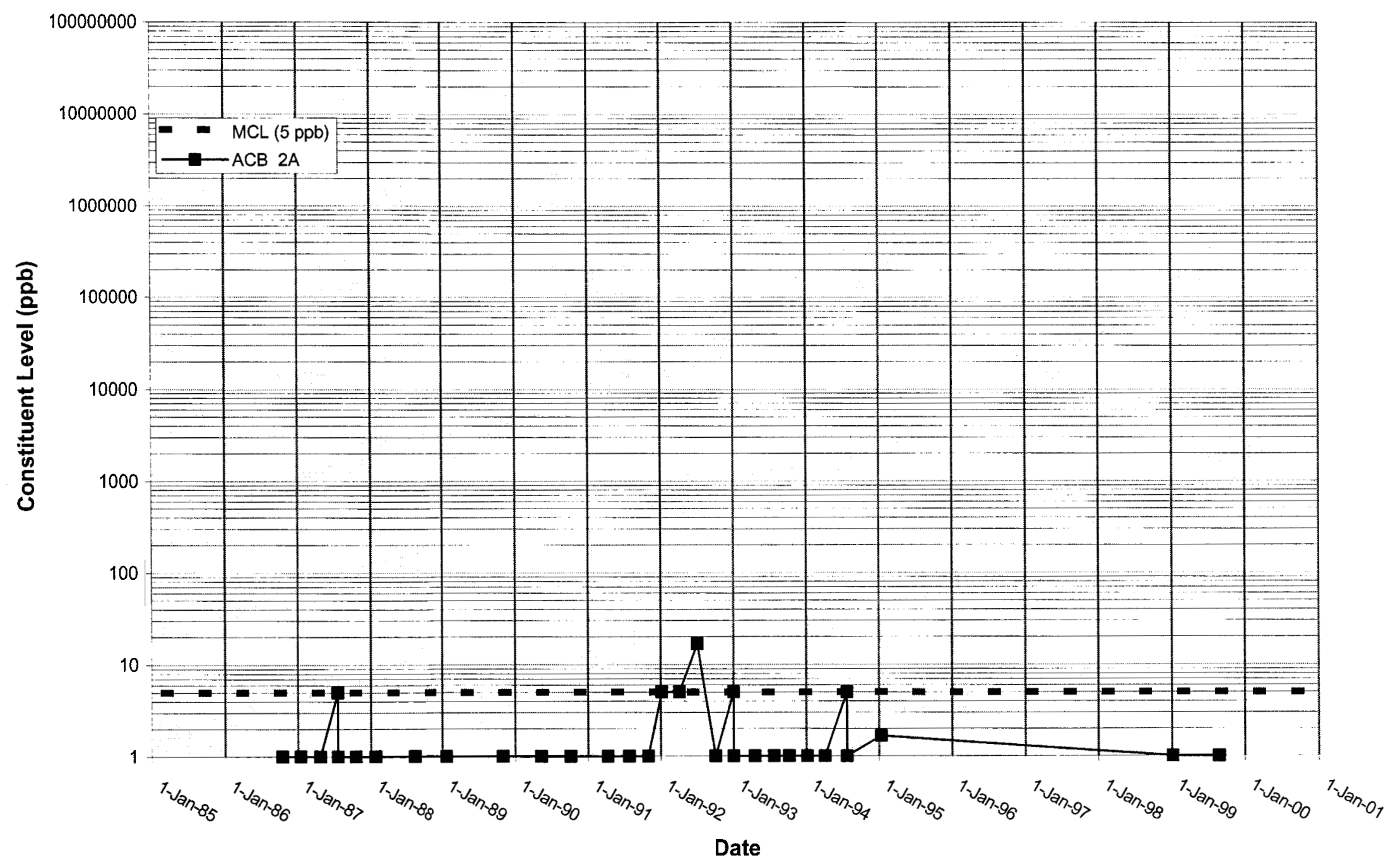




\section{Time Series Plot of Trichloroethylene for Well ACB 3}

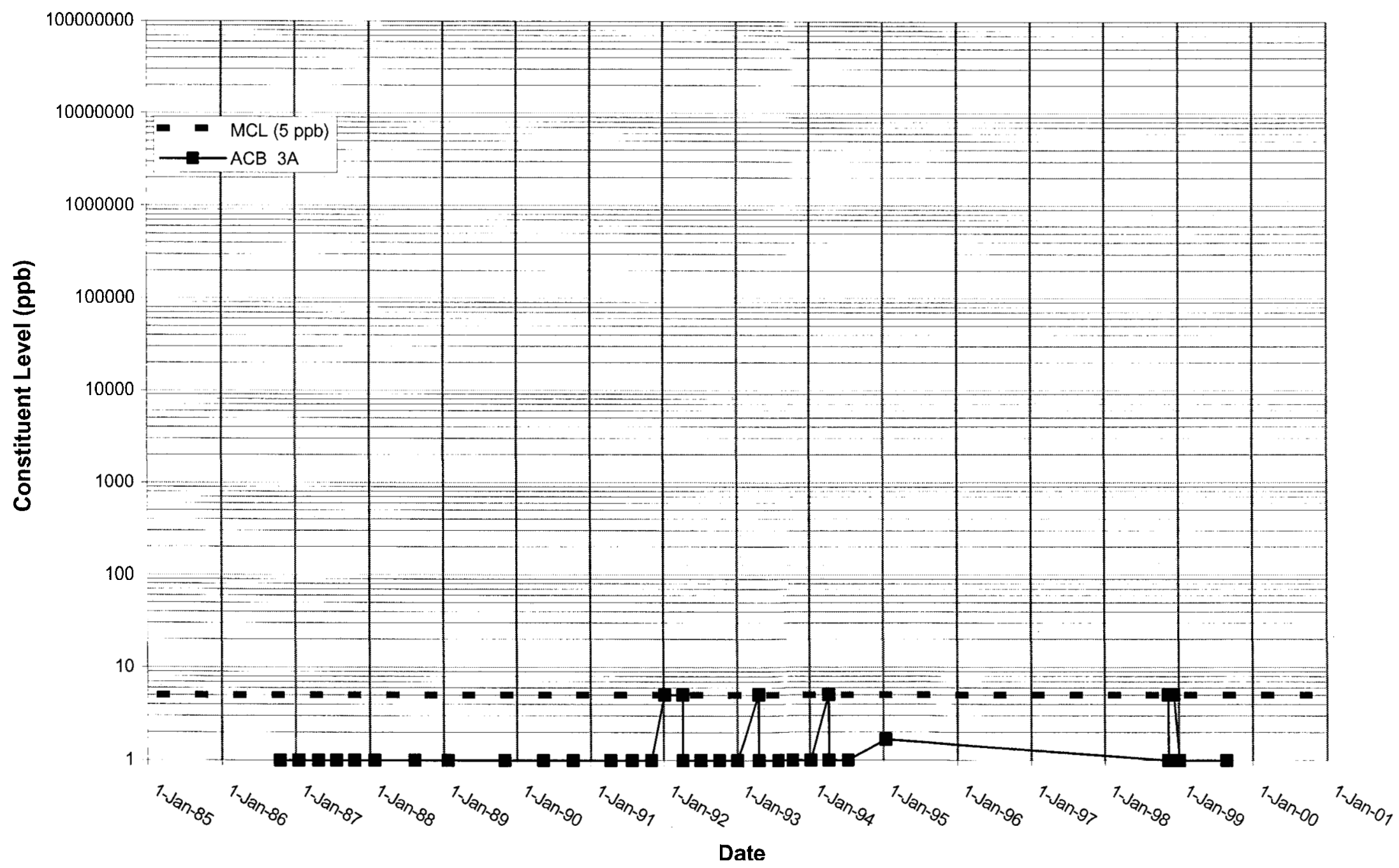


Time Series Plot of Trichloroethylene for Well ACB 4

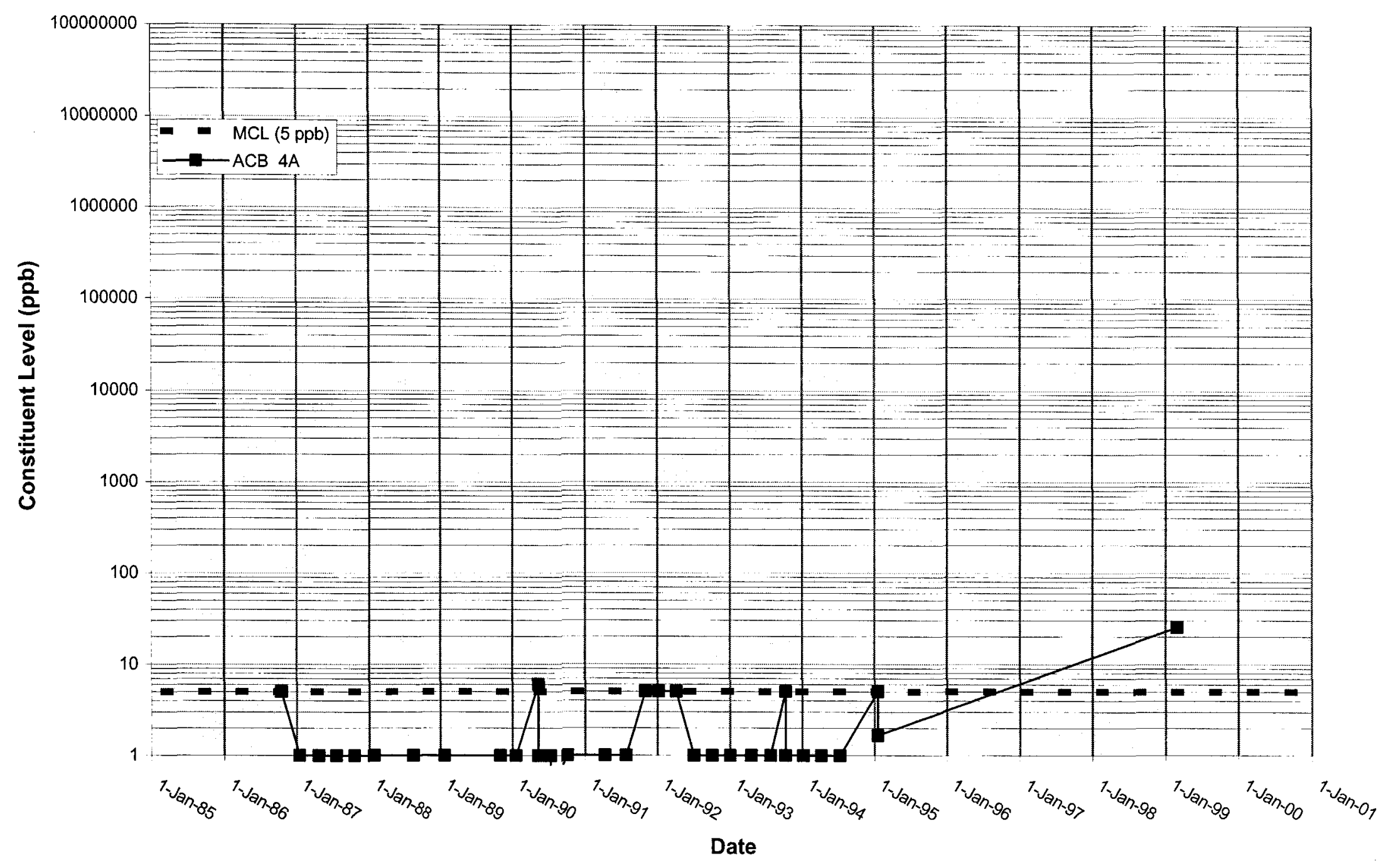


WSRC-TR-2000-00242 UNCLASSIFIED

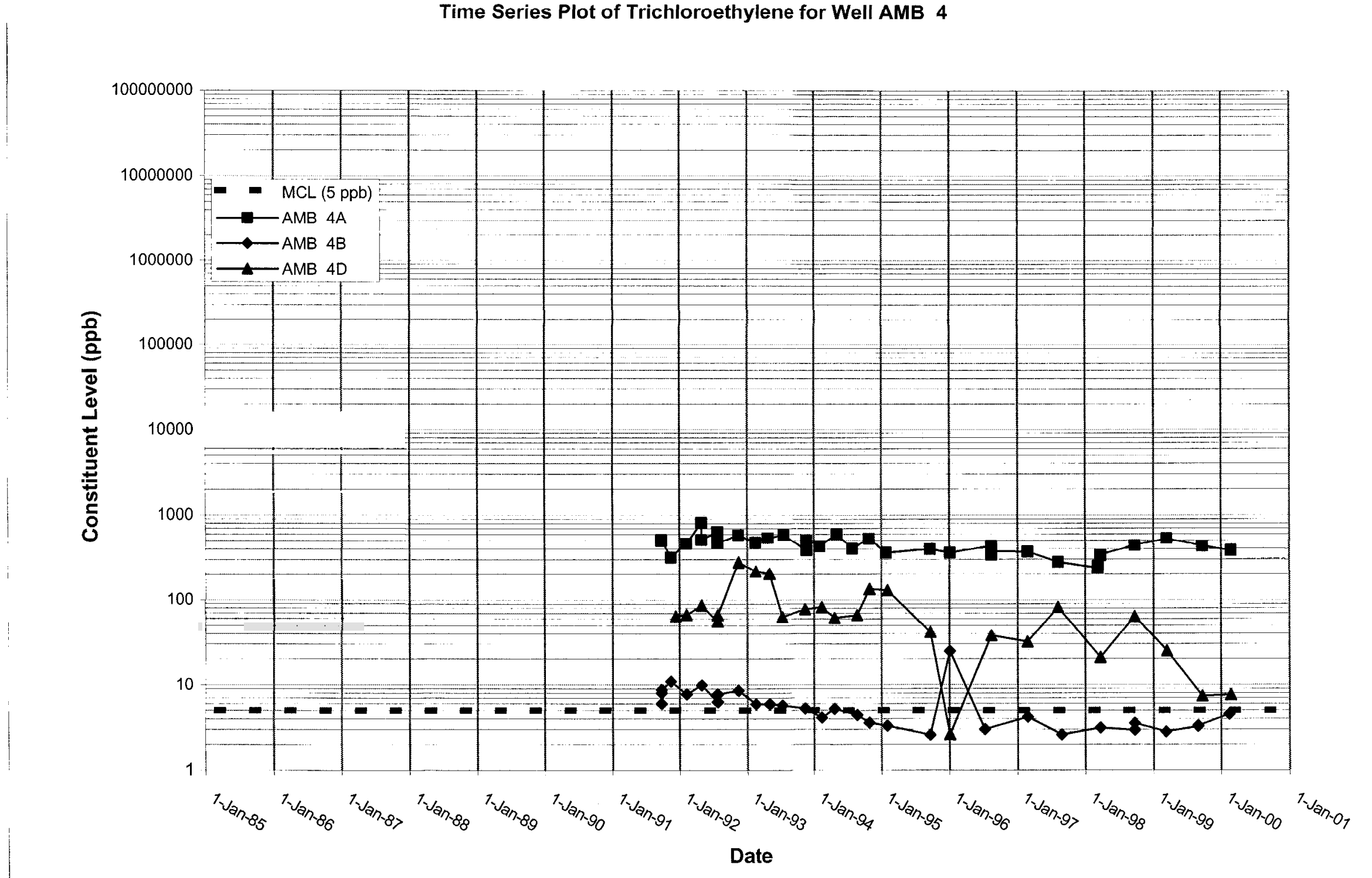




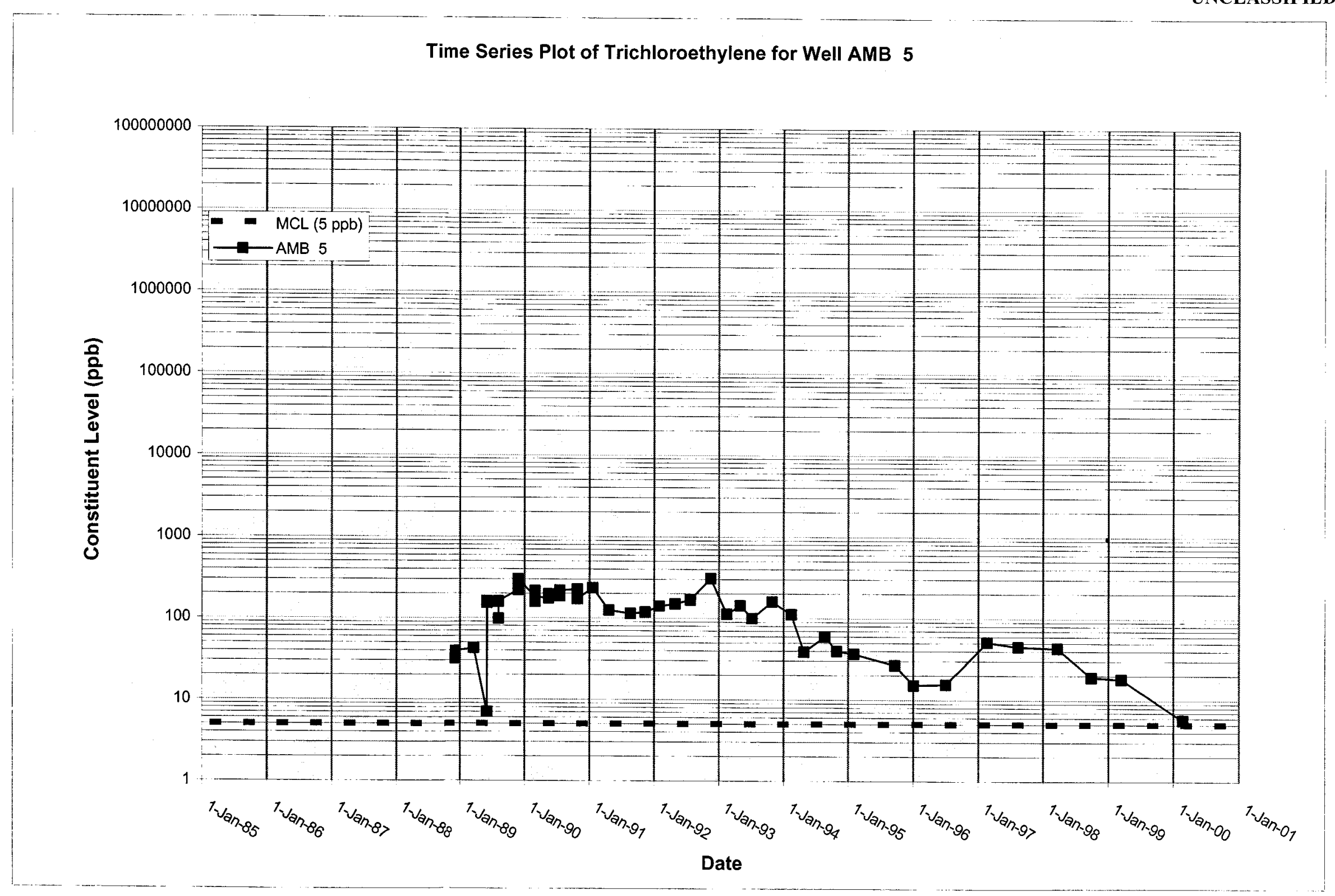


WSRC-TR-2000-00242 UNCLASSIFIED

\section{Time Series Plot of Trichloroethylene for Well AMB 6}

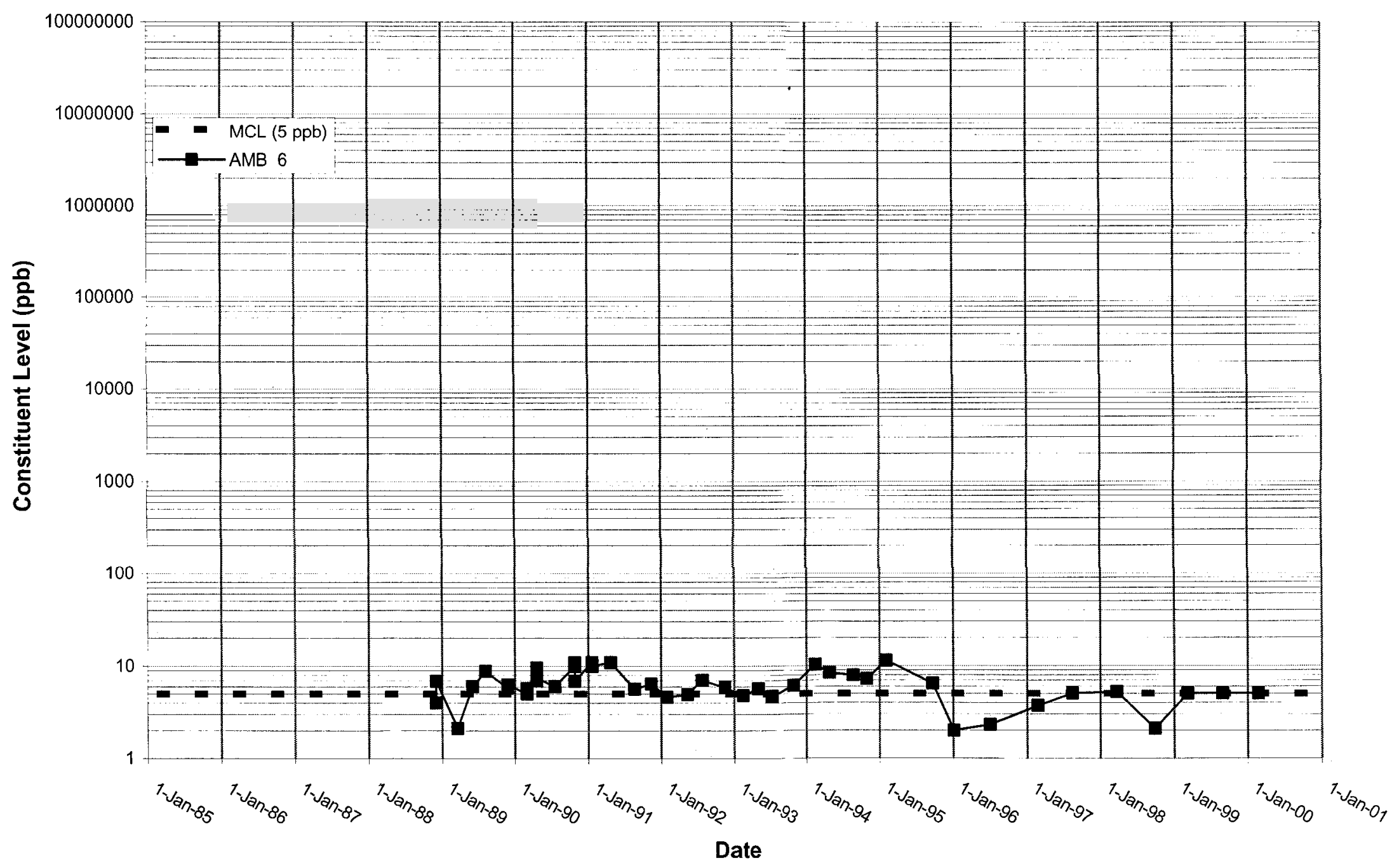




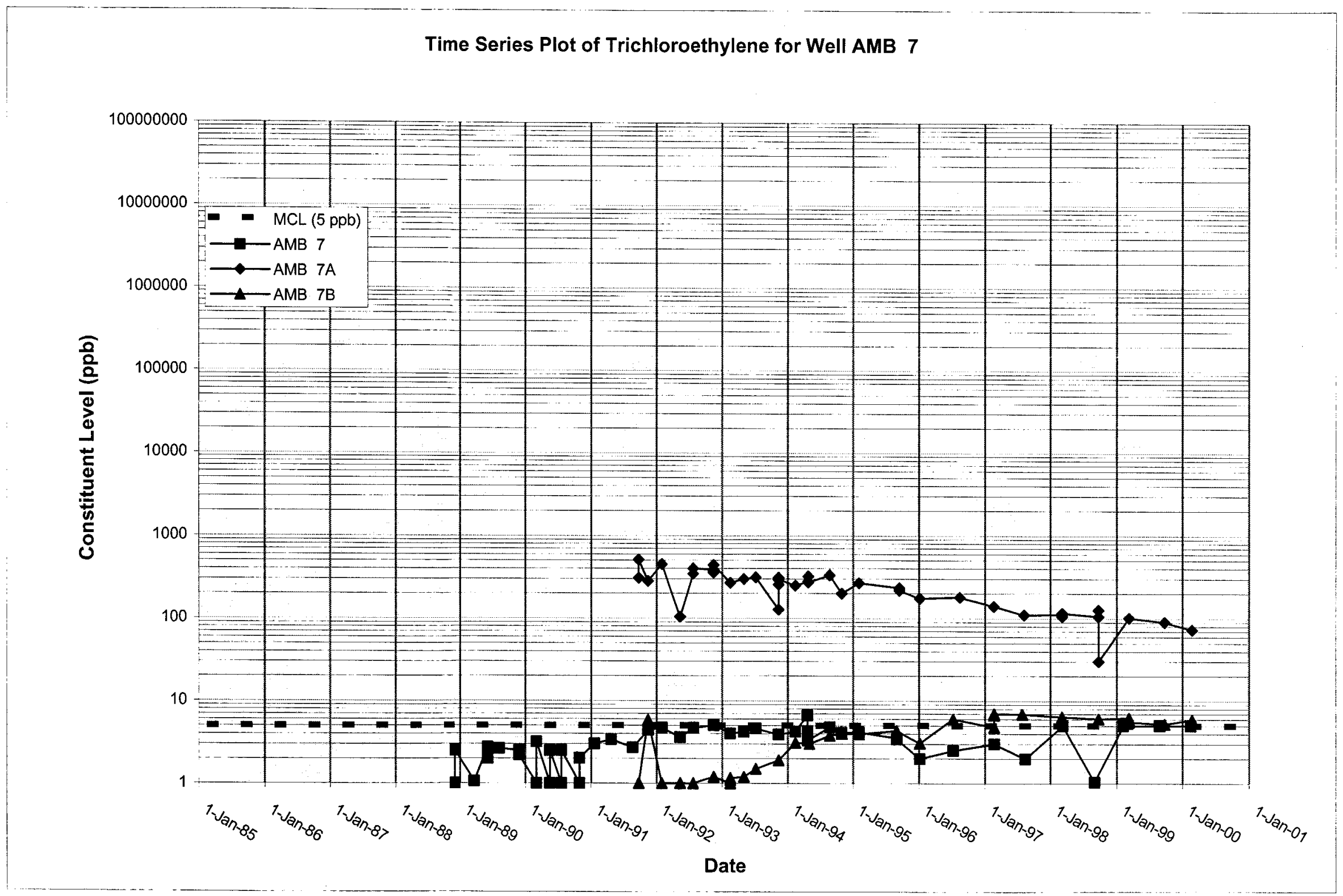


WSRC-TR-2000-00242 UNCLASSIFIED

Time Series Plot of Trichloroethylene for Well AMB 8

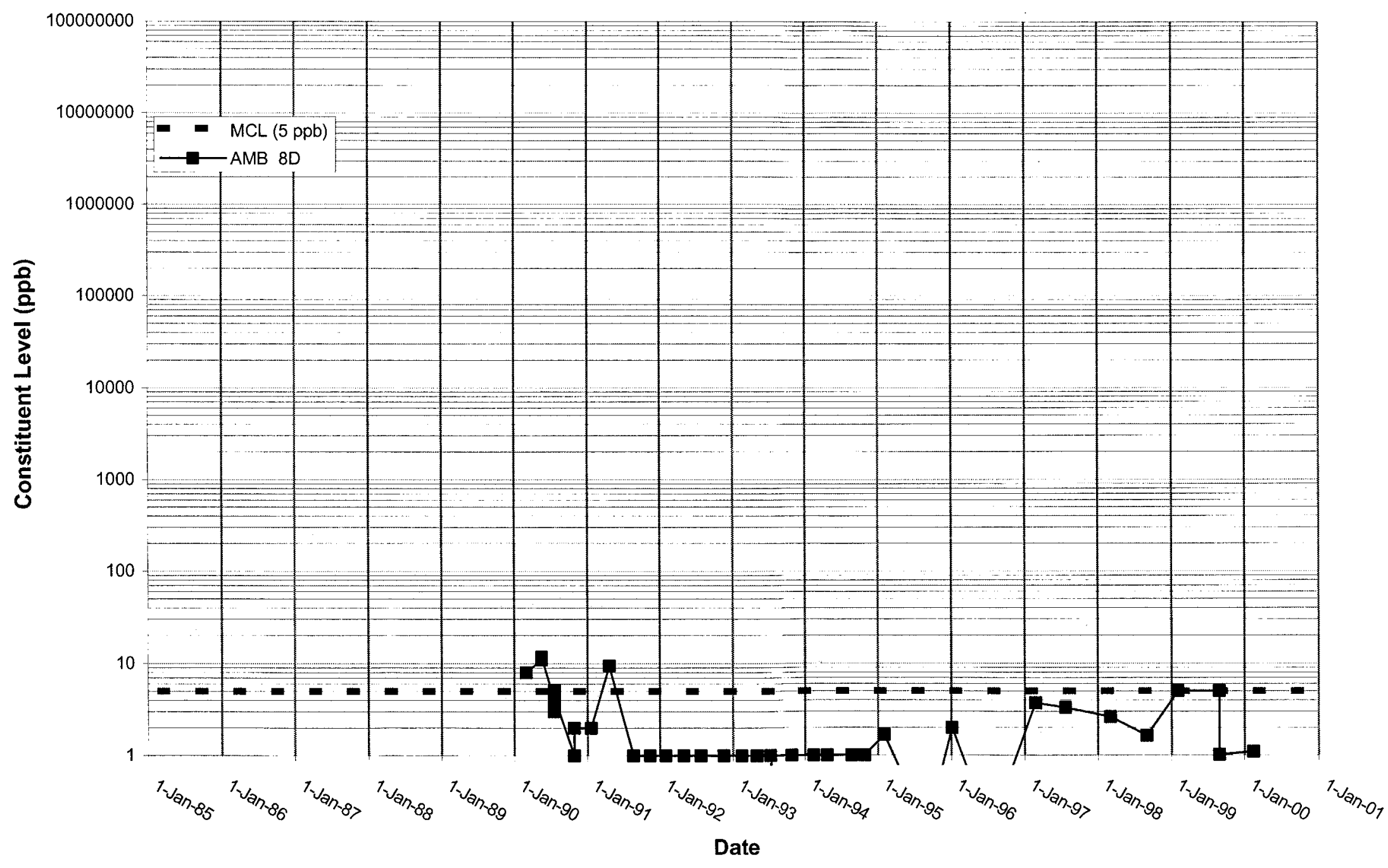


Time Series Plot of Trichloroethylene for Well AMB 9

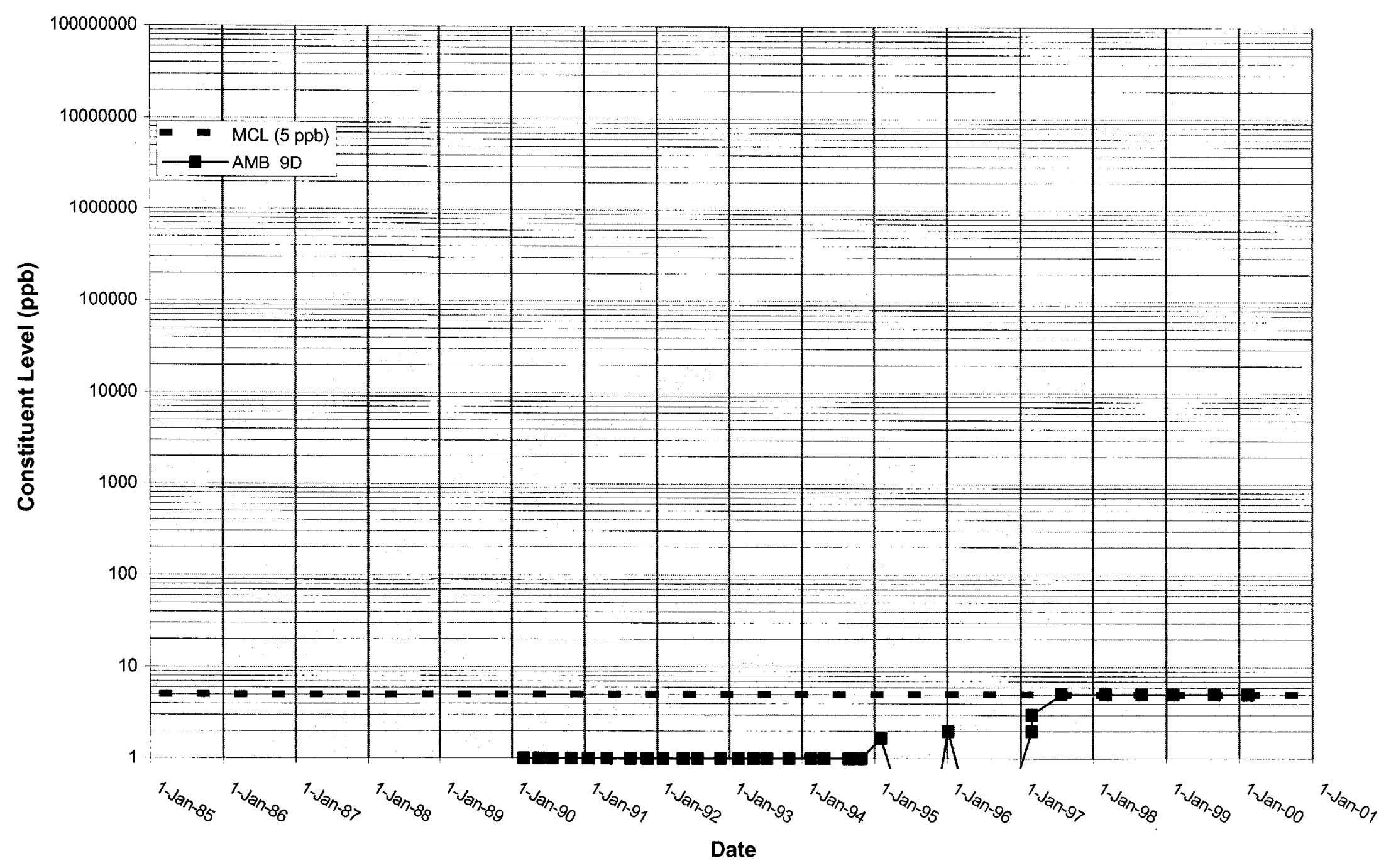


WSRC-TR-2000-00242 UNCLASSIFIED

Time Series Plot of Trichloroethylene for Well AMB 10

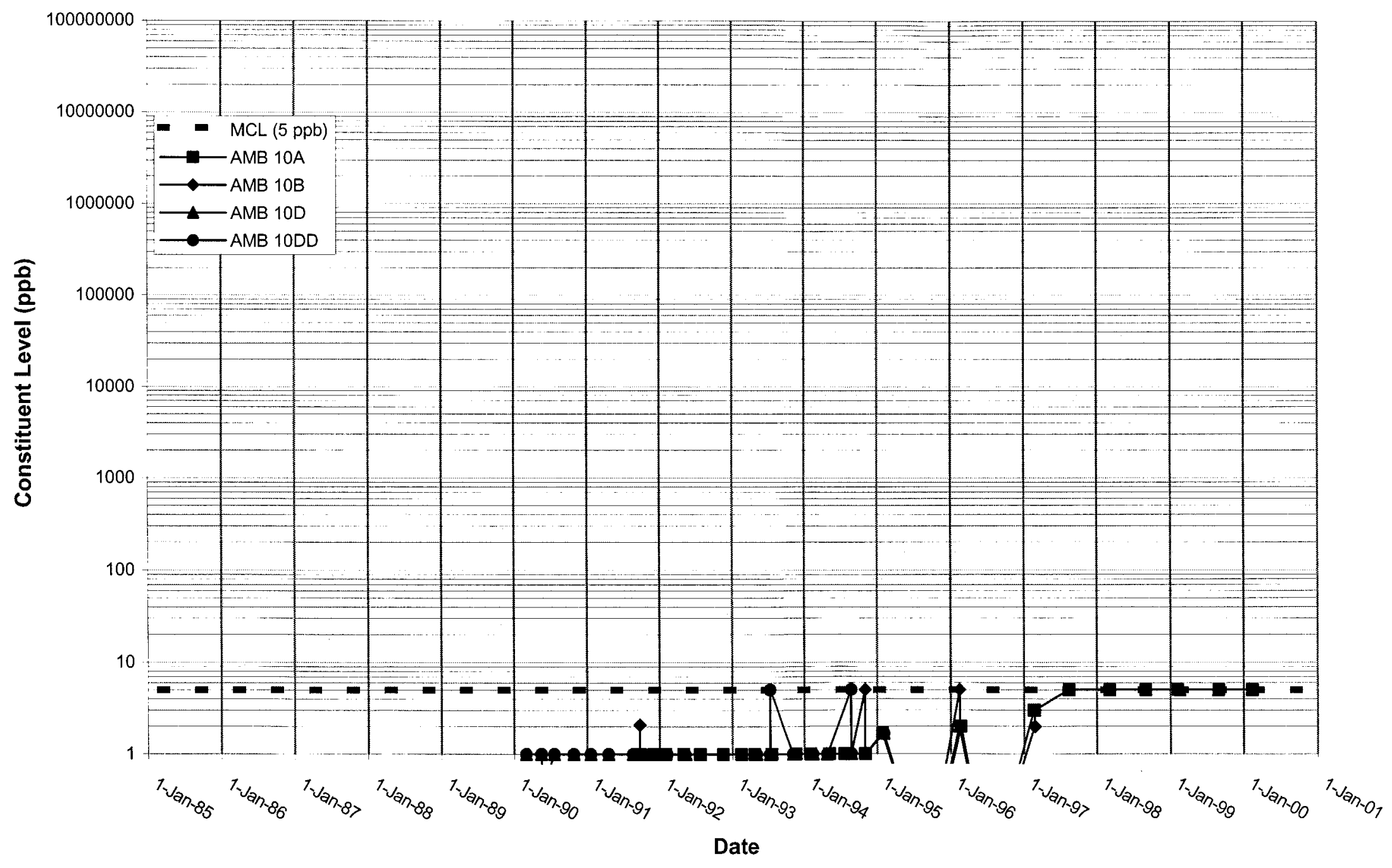


Time Series Plot of Trichloroethylene for Well AMB 11

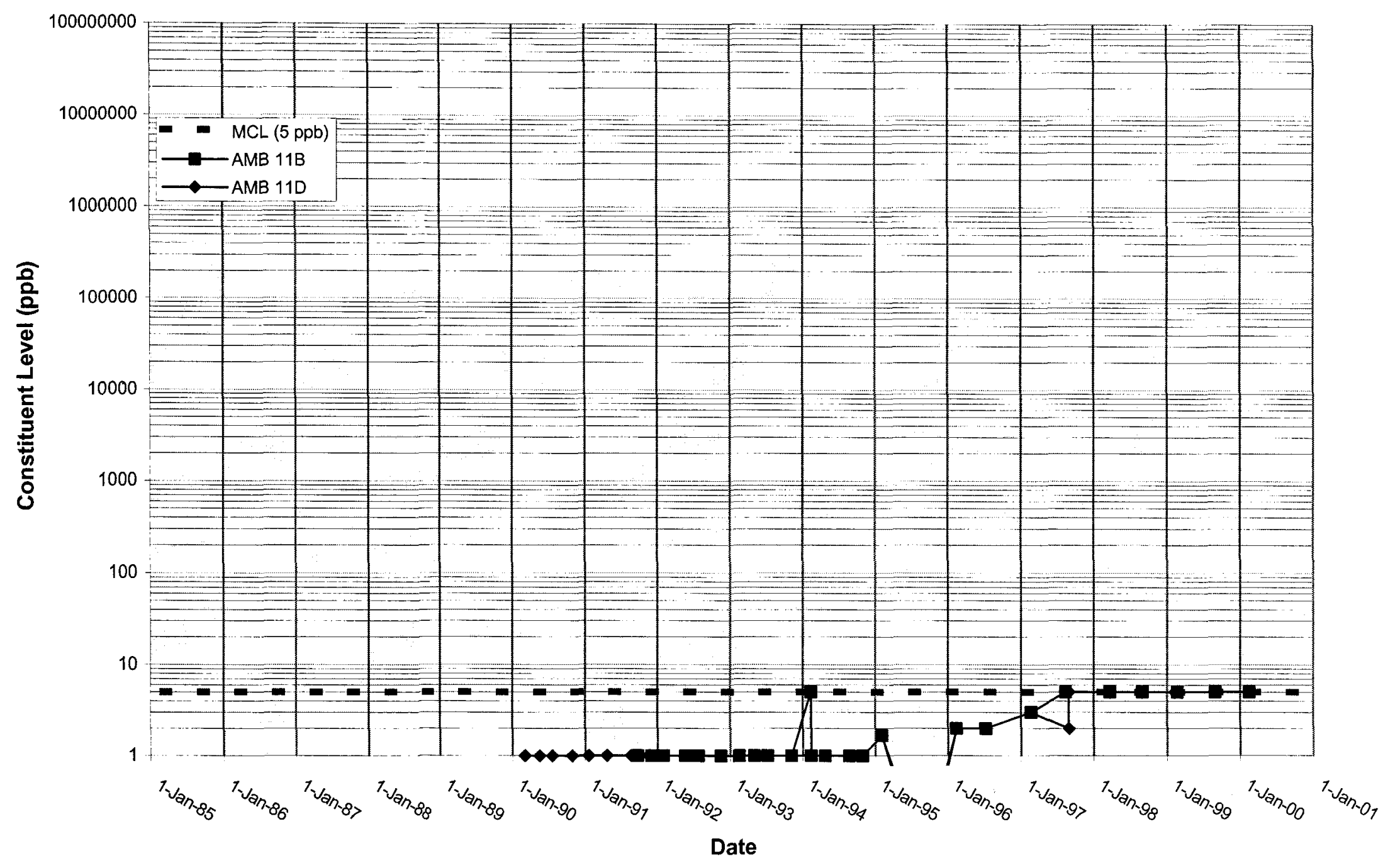


WSRC-TR-2000-00242

UNCLASSIFIED

\section{Time Series Plot of Trichloroethylene for Well AMB 12}

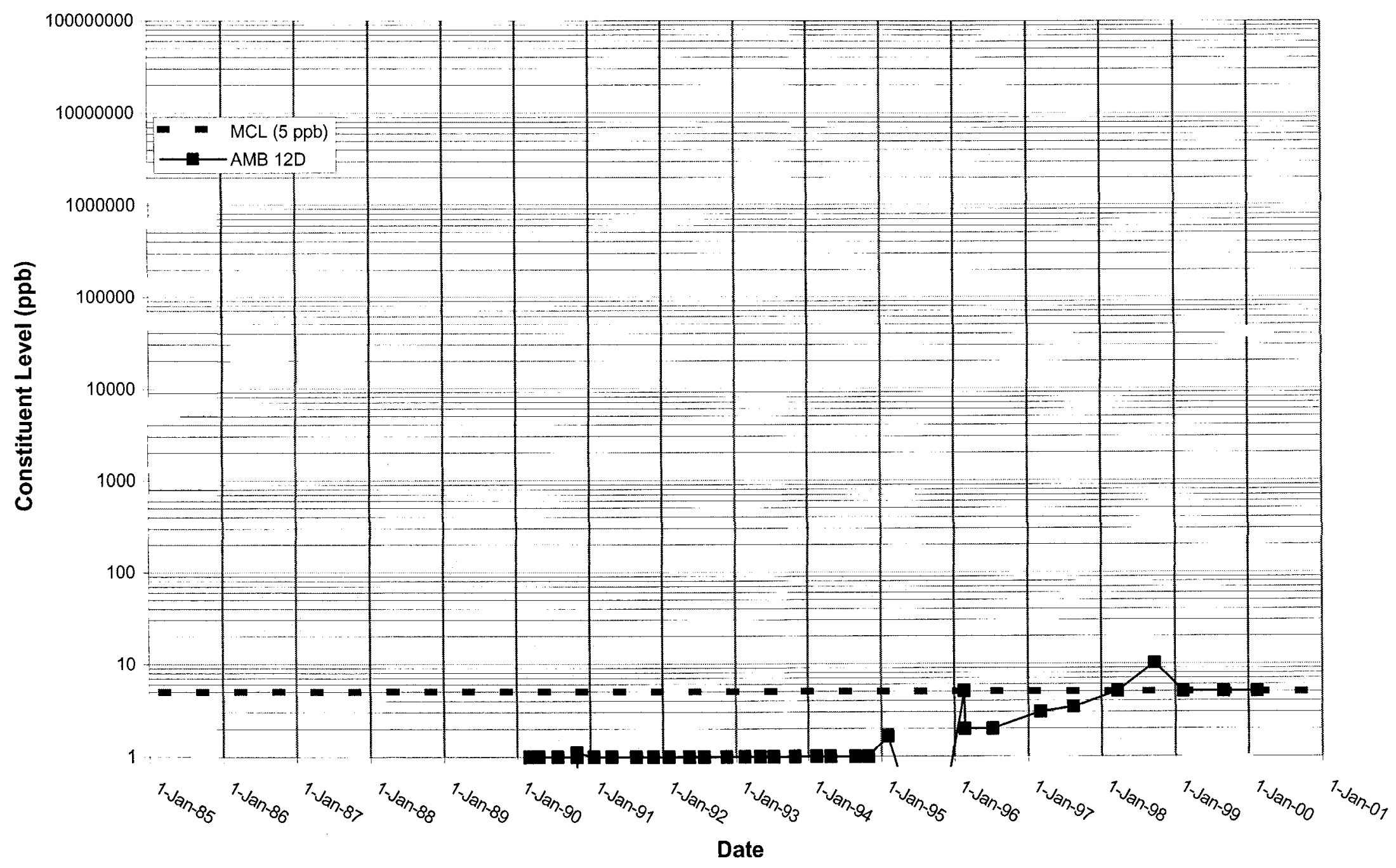


Time Series Plot of Trichloroethylene for Well AMB 13

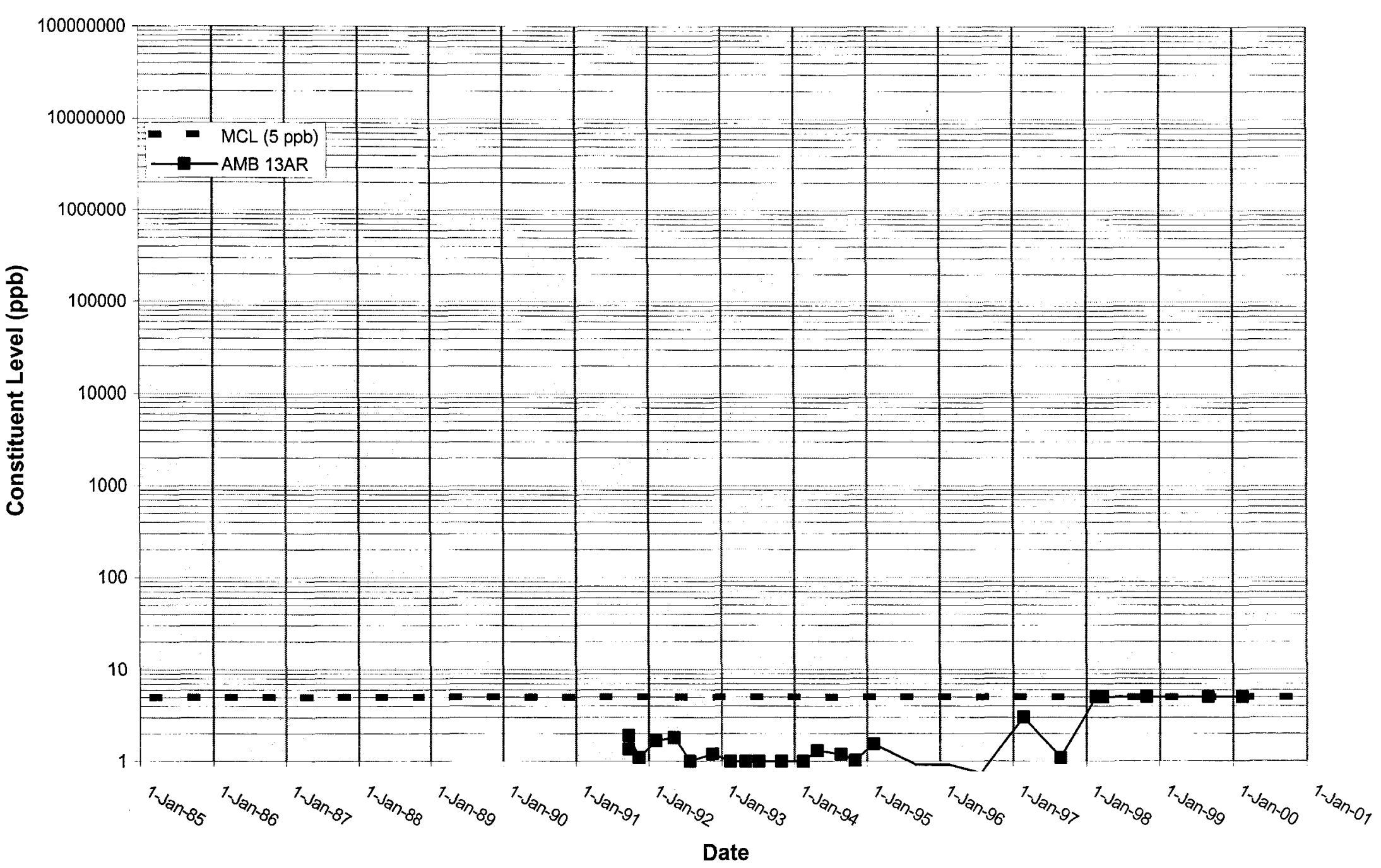




\section{Time Series Plot of Trichloroethylene for Well AMB 14}

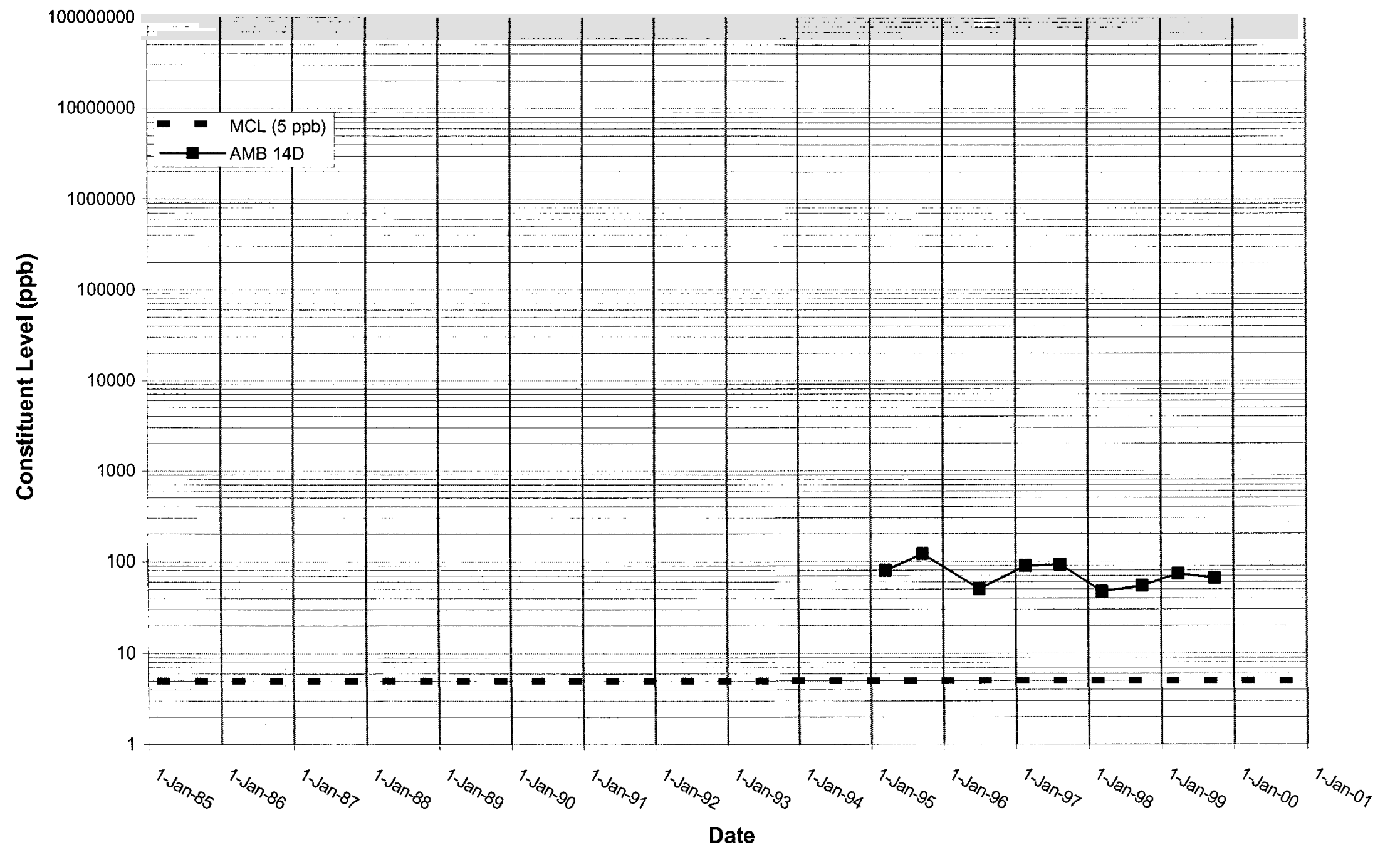




\section{Time Series Plot of Trichloroethylene for Well AMB 15}

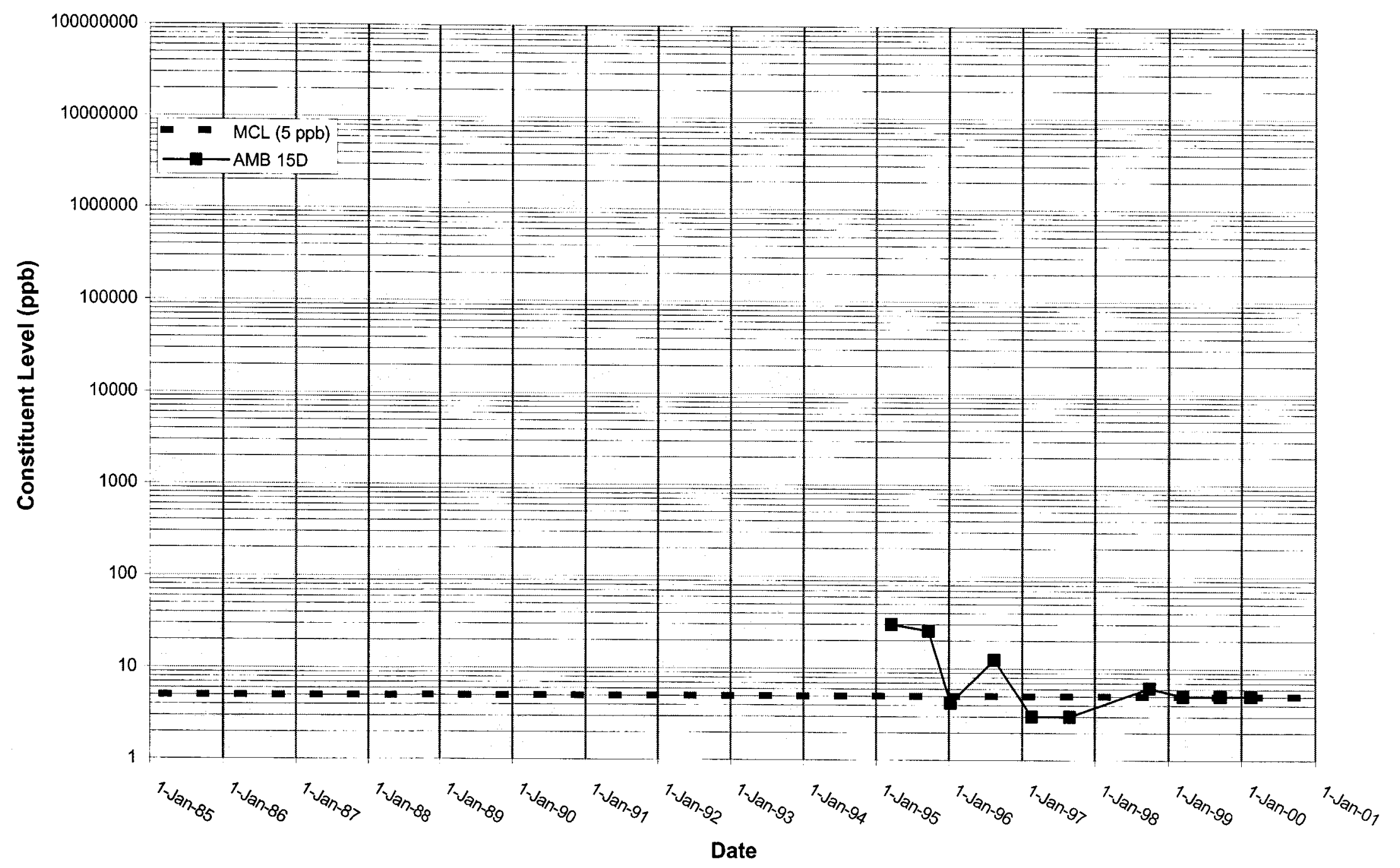


WSRC-TR-2000-00242 UNCLASSIFIED

\section{Time Series Plot of Trichloroethylene for Well AMB 16}

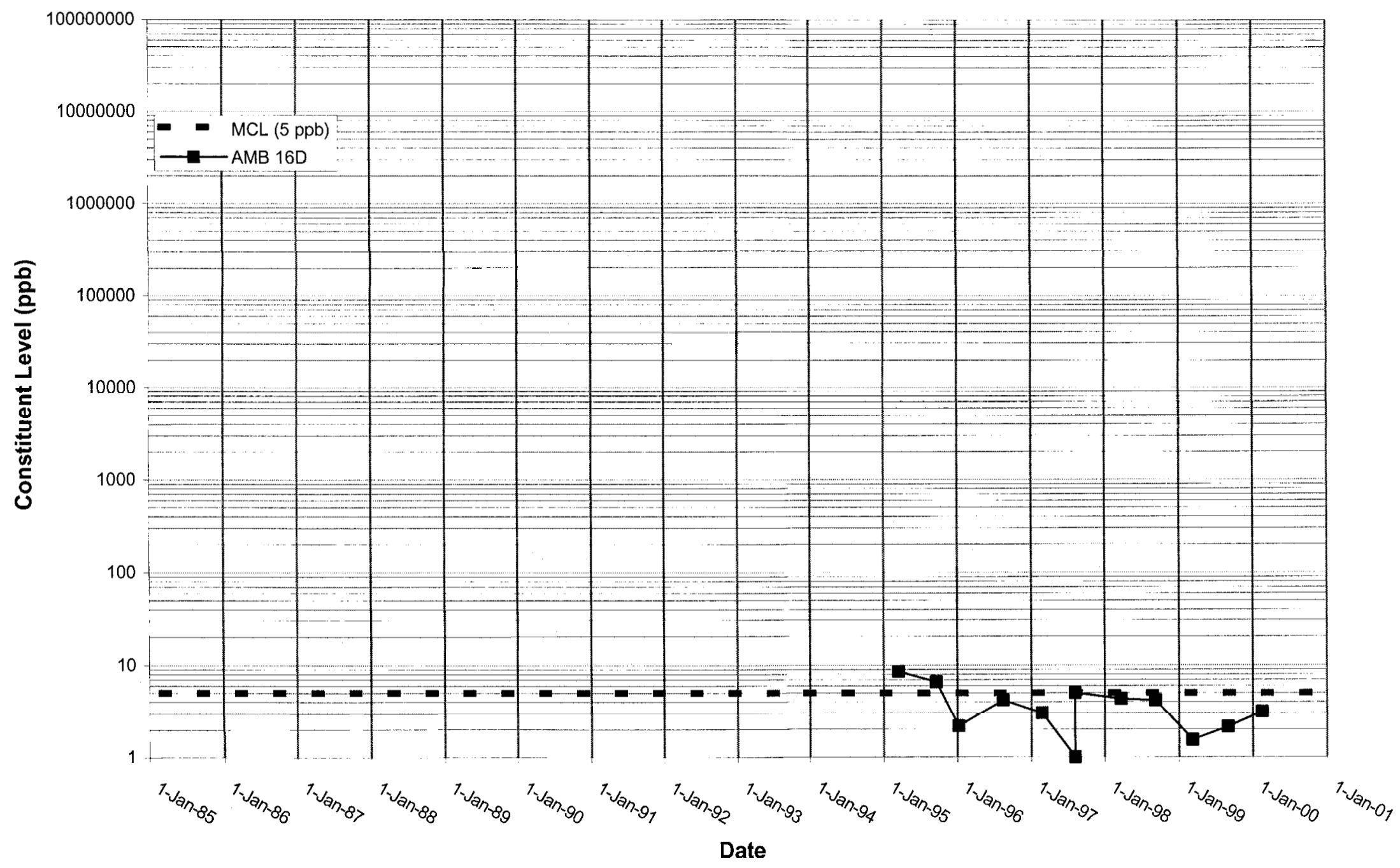




\section{Time Series Plot of Trichloroethylene for Well AMB 17}

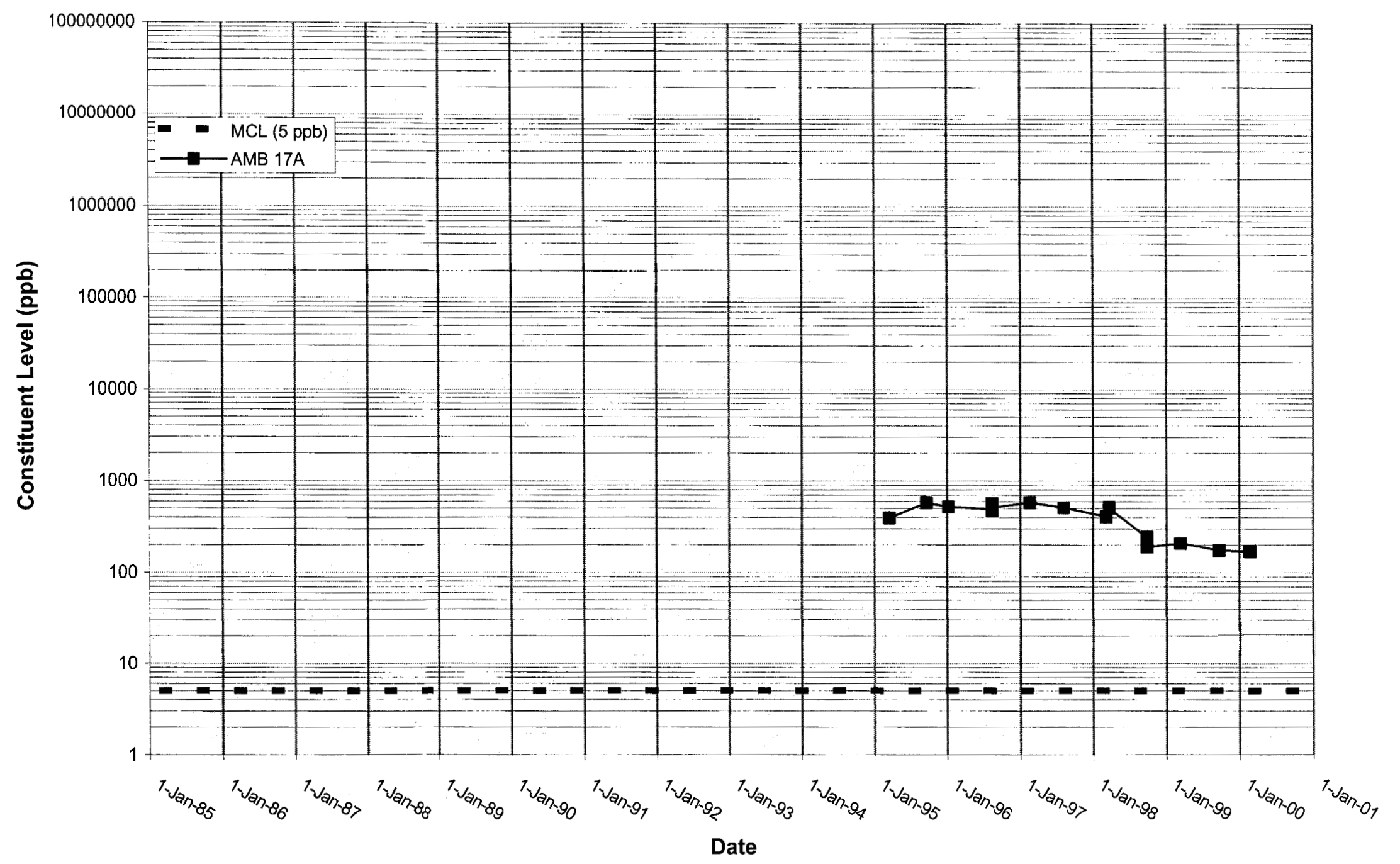


WSRC-TR-2000-00242 UNCLASSIFIED

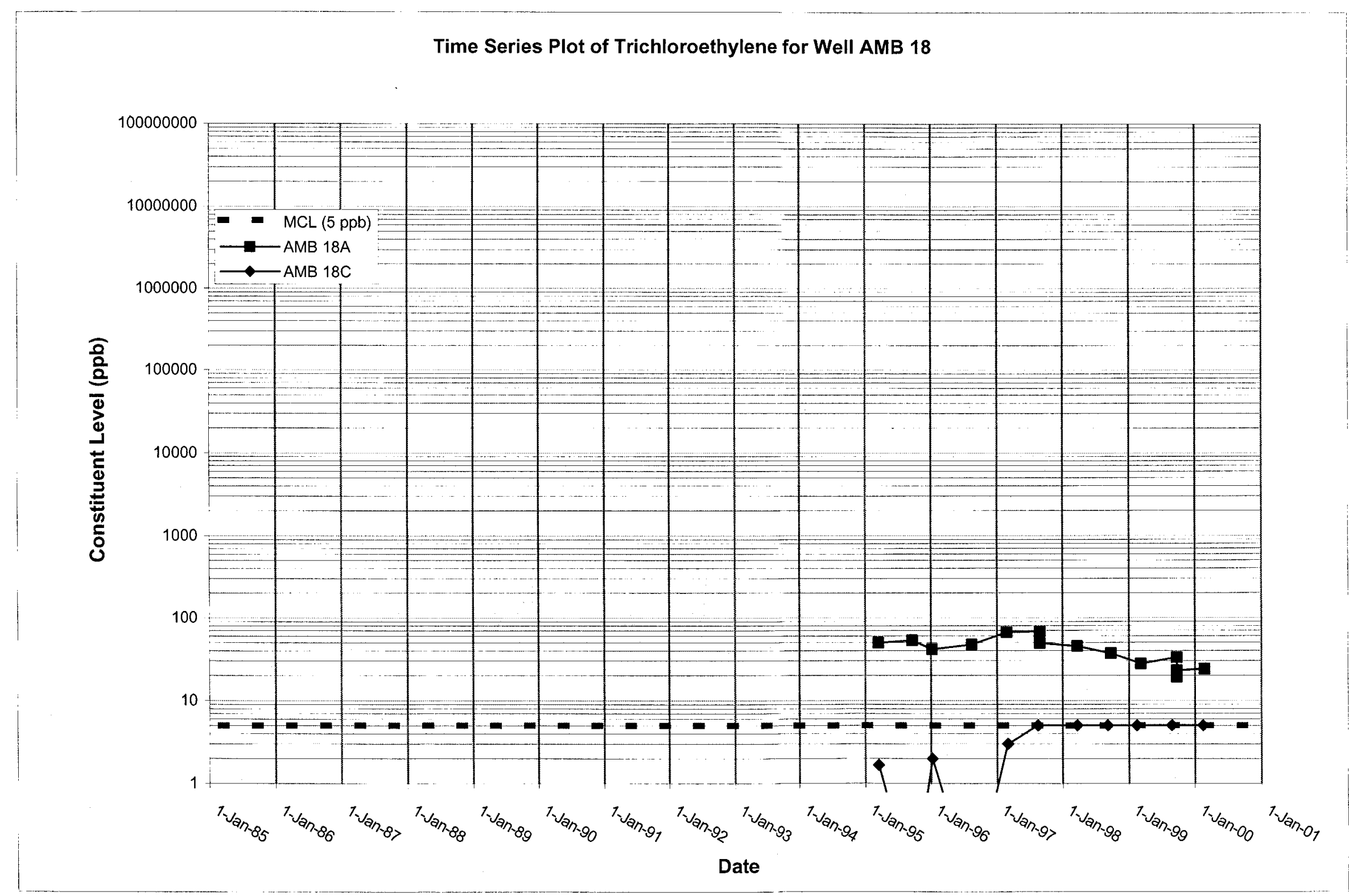


WSRC-TR-2000-00242 UNCLASSIFIED

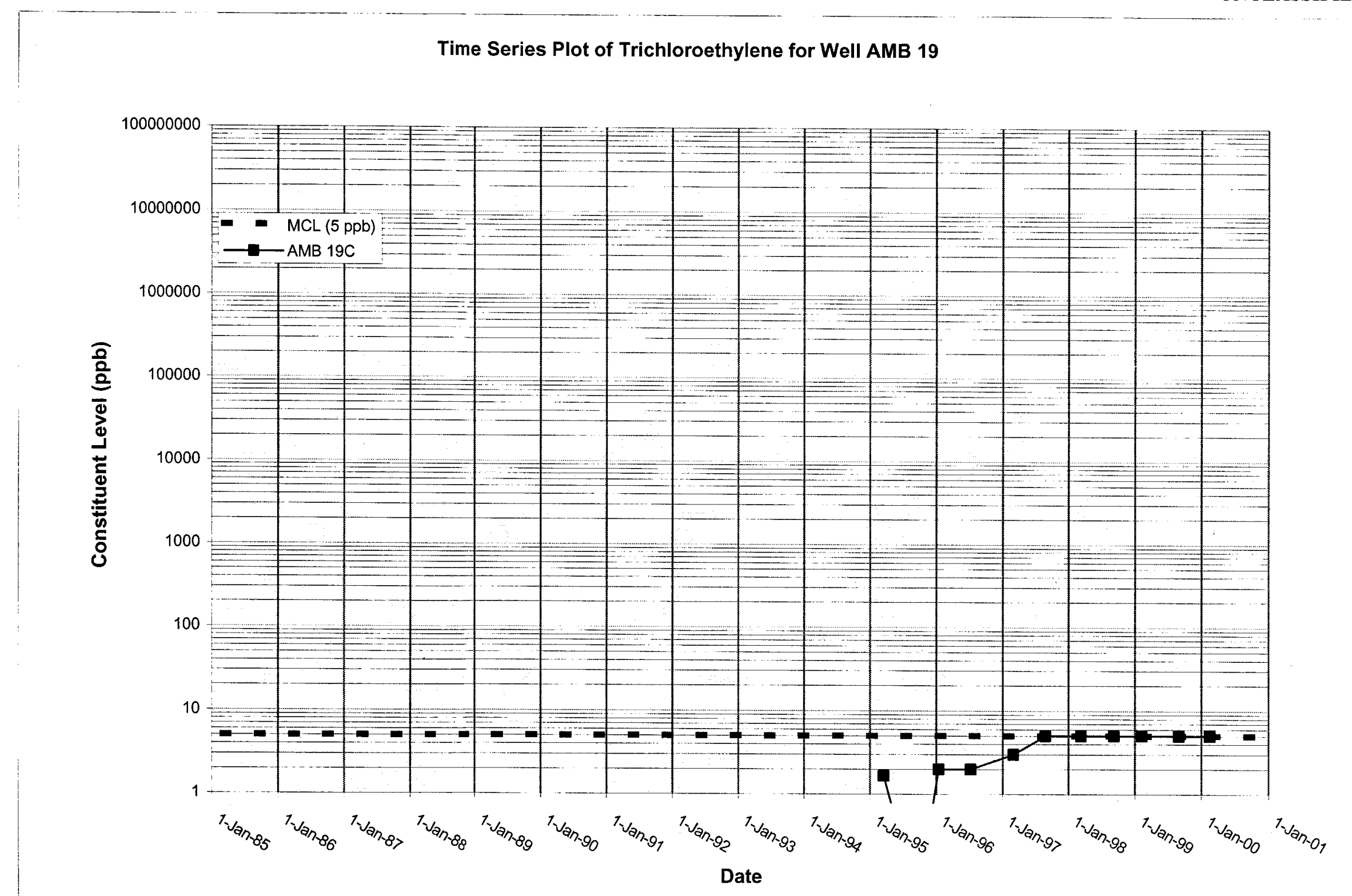


WSRC-TR-2000-00242 UNCLASSIFIED

Time Series Plot of Trichloroethylene for Well AOB 1

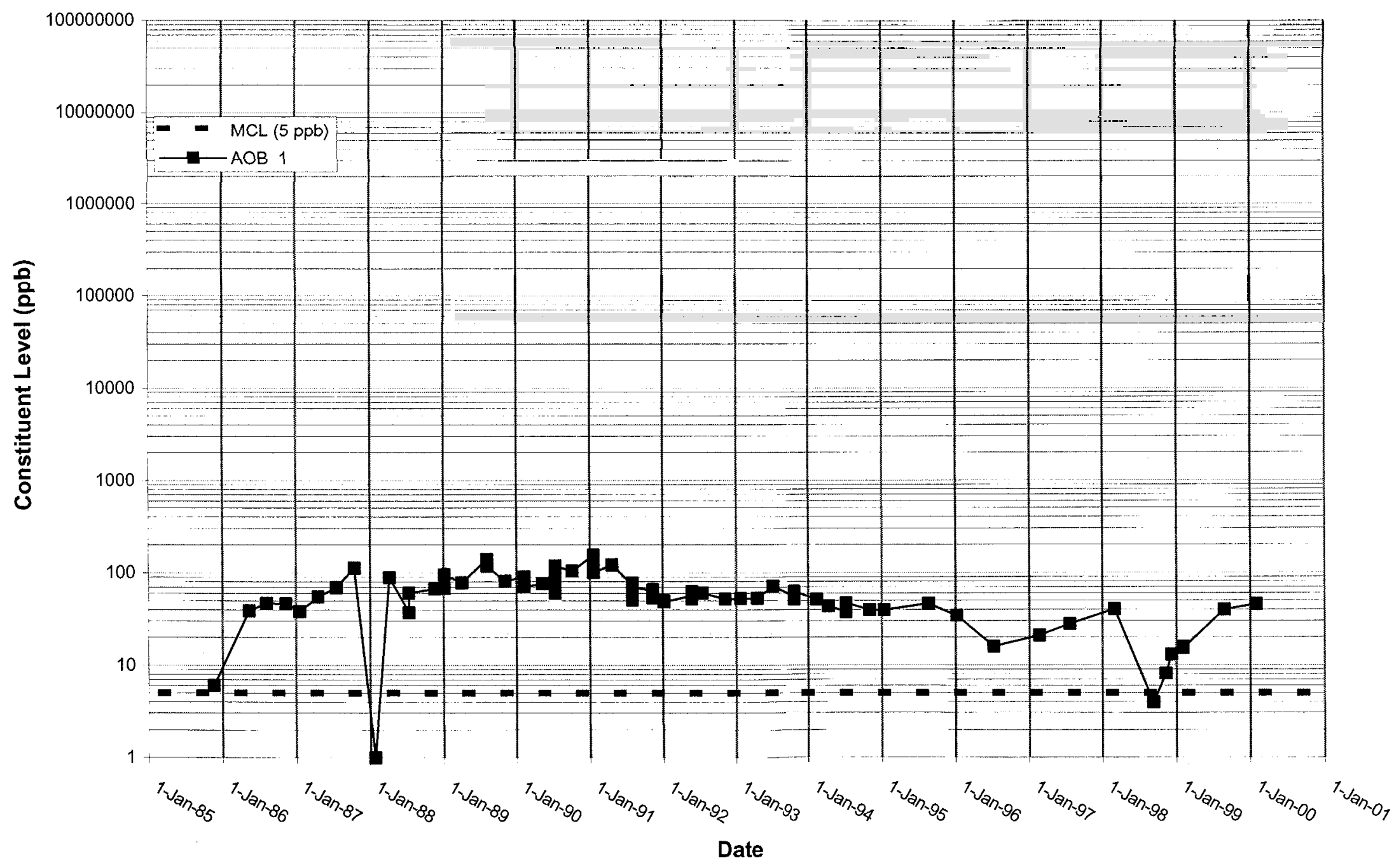


WSRC-TR-2000-00242 UNCLASSIFIED

Time Series Plot of Trichloroethylene for Well AOB 2

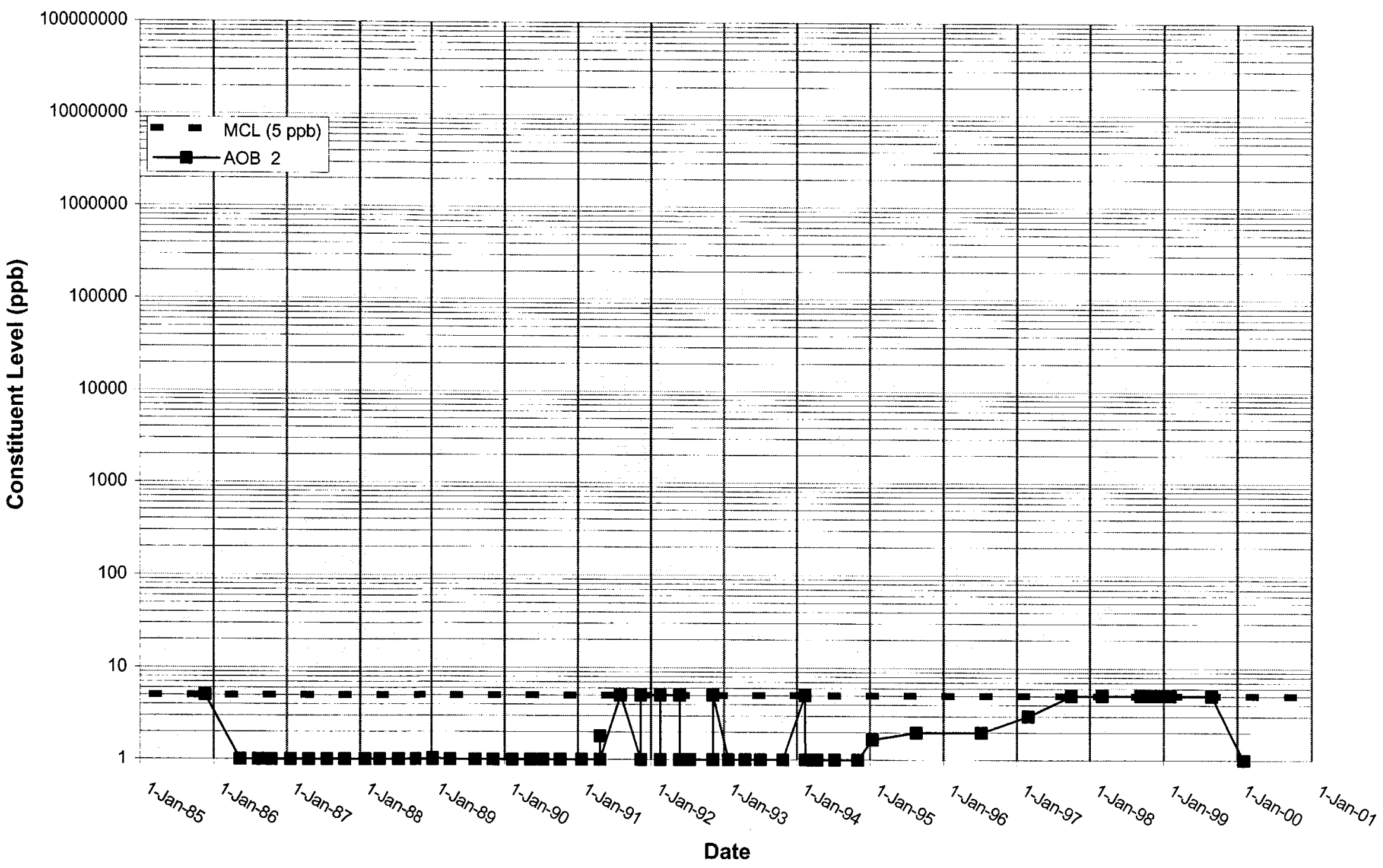




\section{Time Series Plot of Trichloroethylene for Well AOB 3}

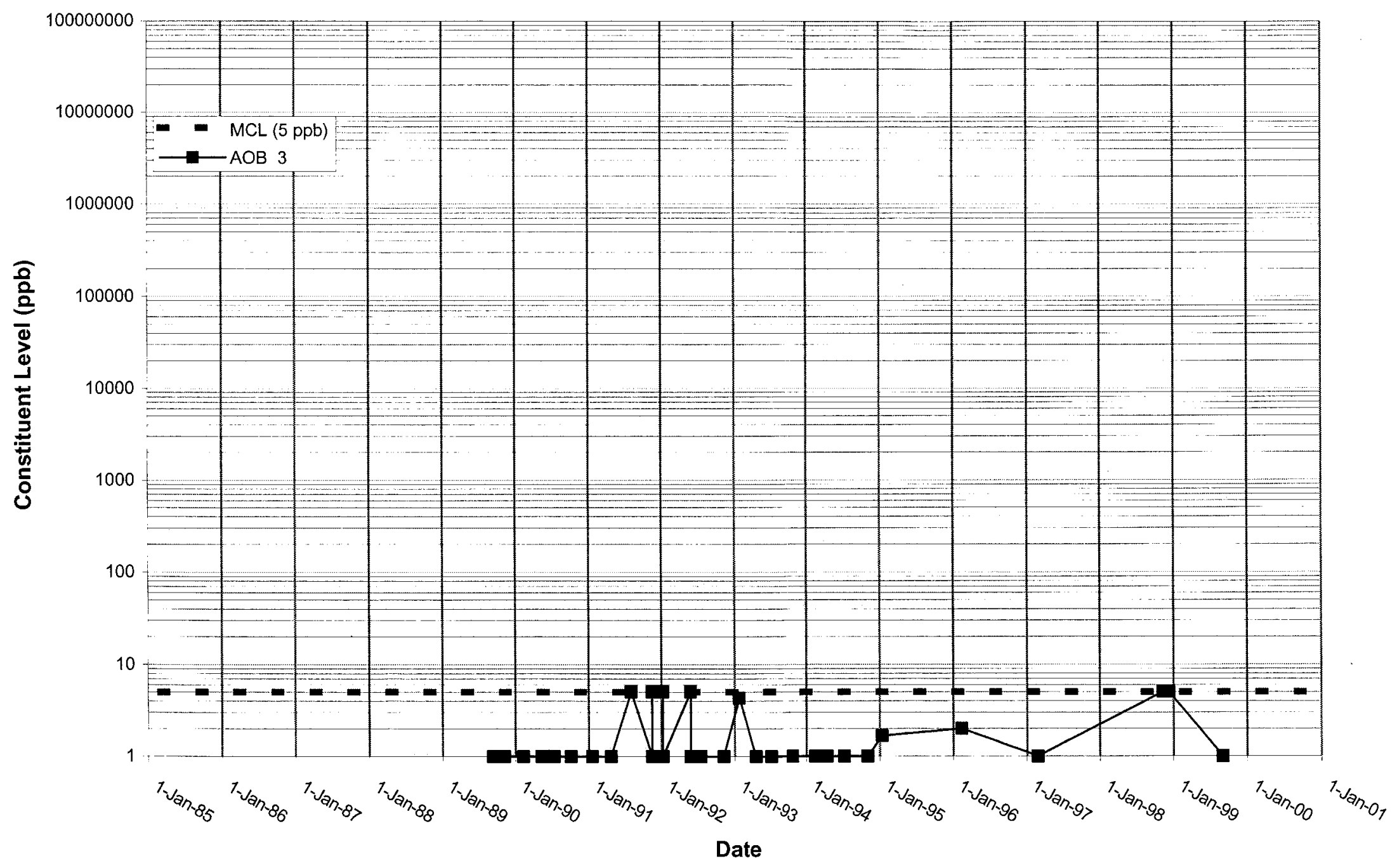


Time Series Plot of Trichloroethylene for Well ARP 1

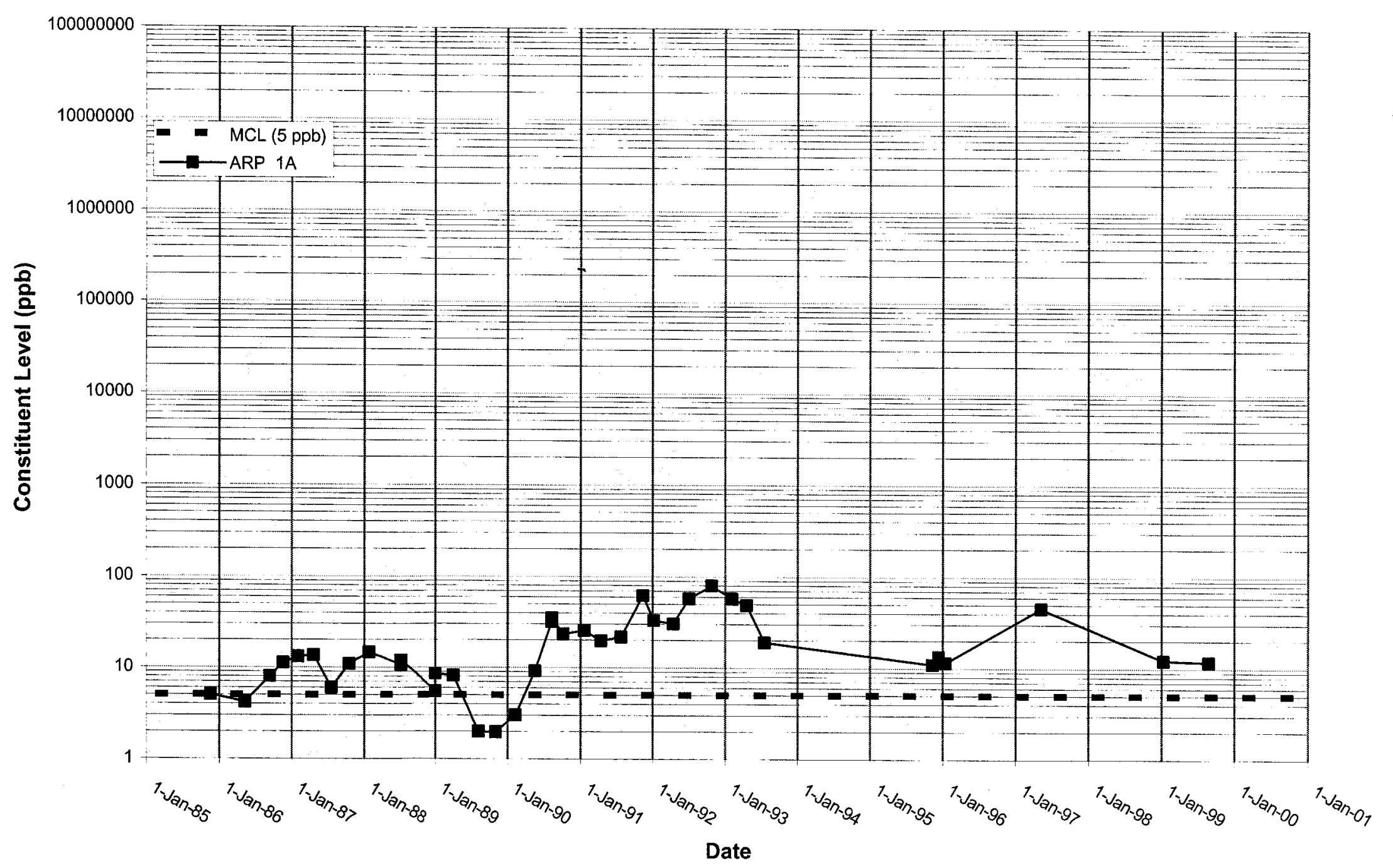


Time Series Plot of Trichloroethylene for Well ARP 2

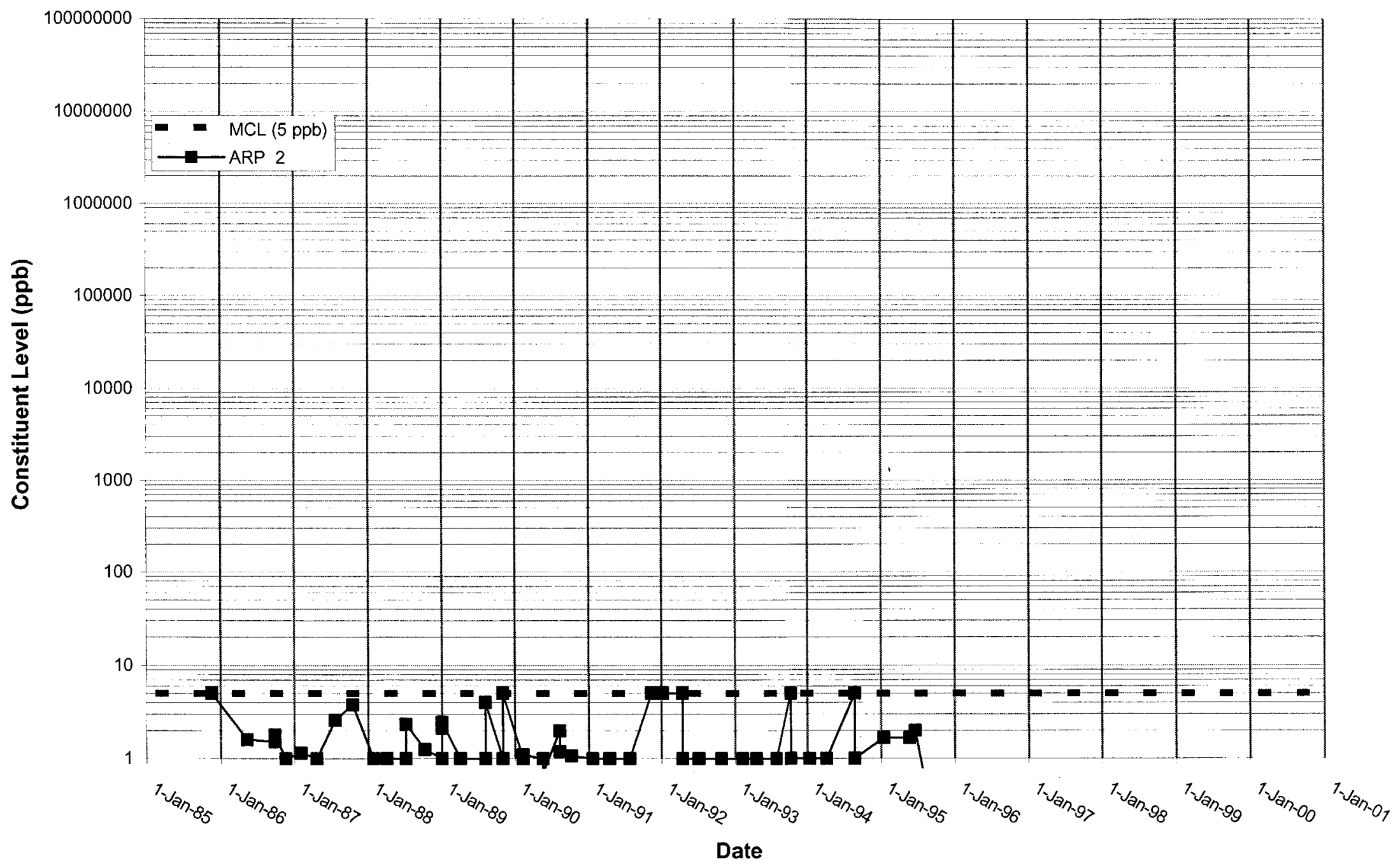




\section{Time Series Plot of Trichloroethylene for Well ARP 3}

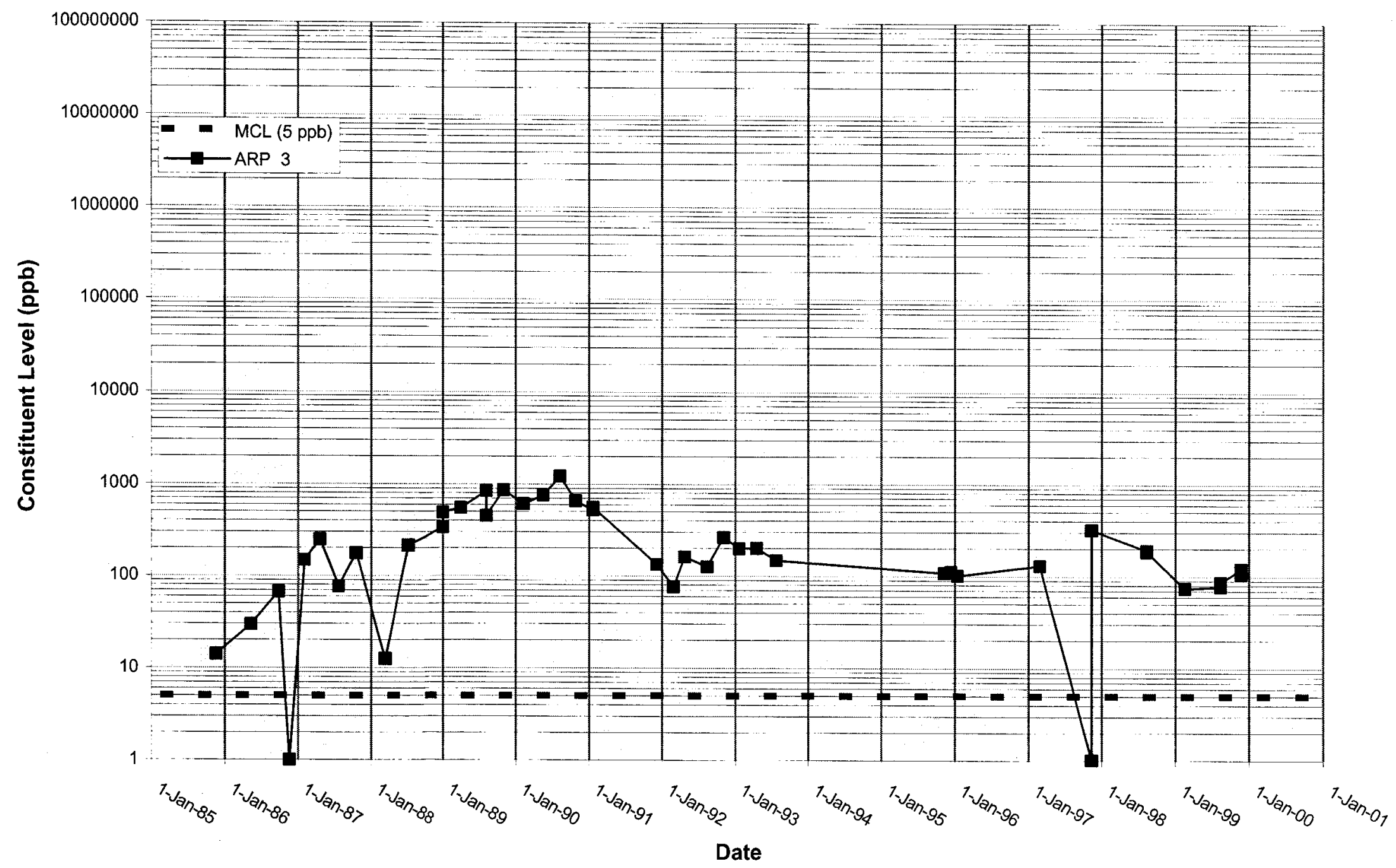


Time Series Plot of Trichloroethylene for Well ARP 4

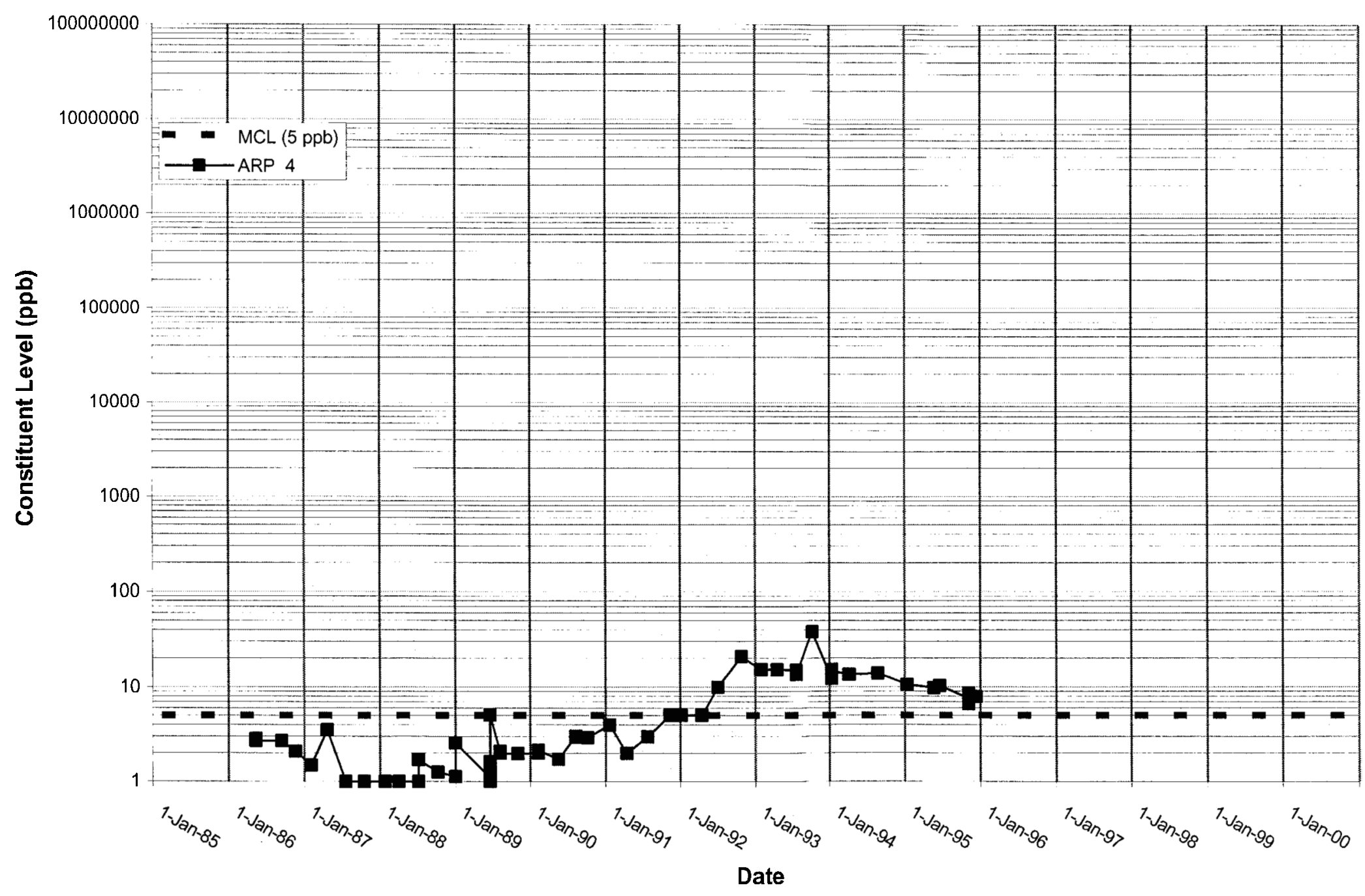


Time Series Plot of Trichloroethylene for Well ASB 1

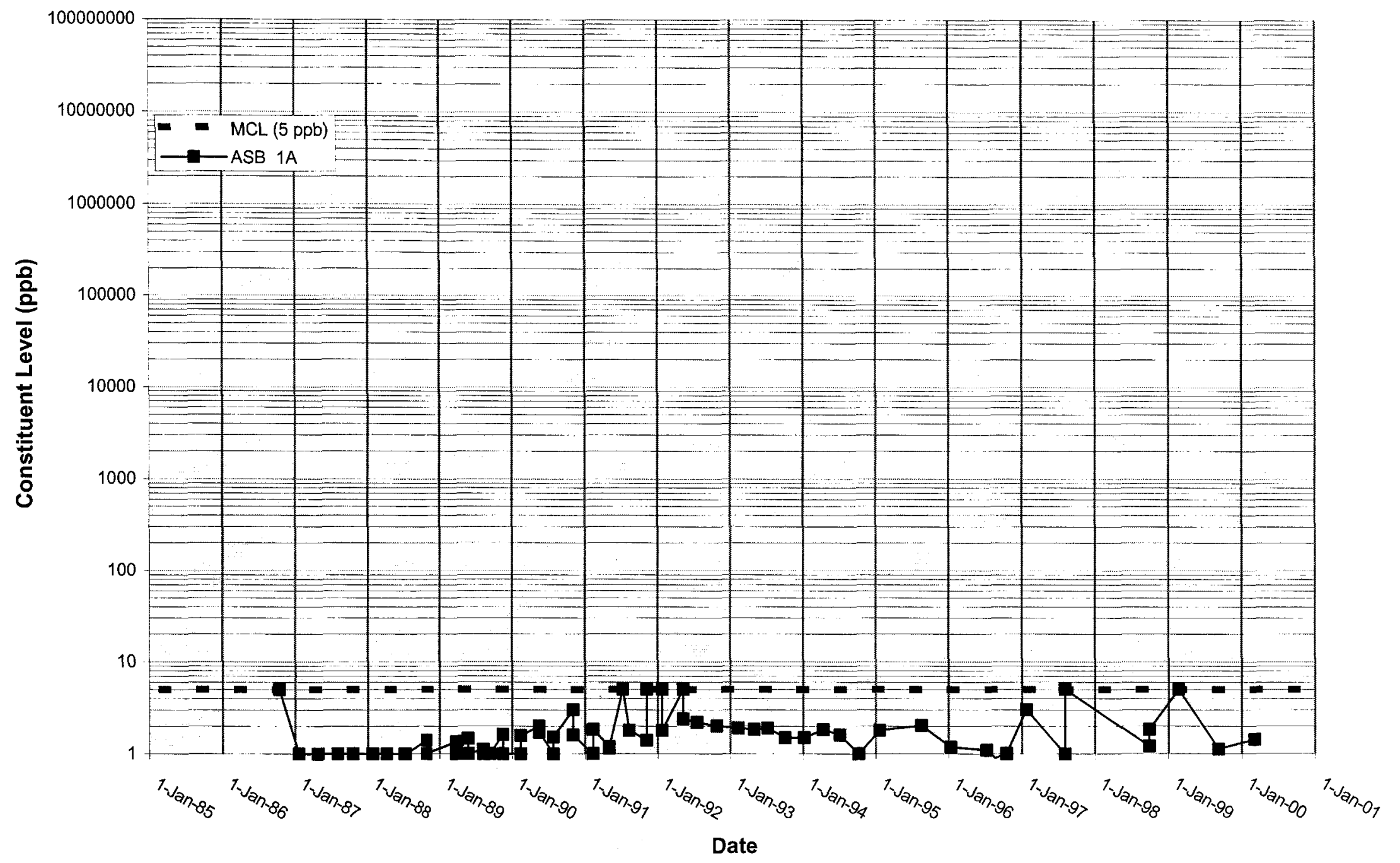


WSRC-TR-2000-00242 UNCLASSIFIED

\section{Time Series Plot of Trichloroethylene for Well ASB 2}

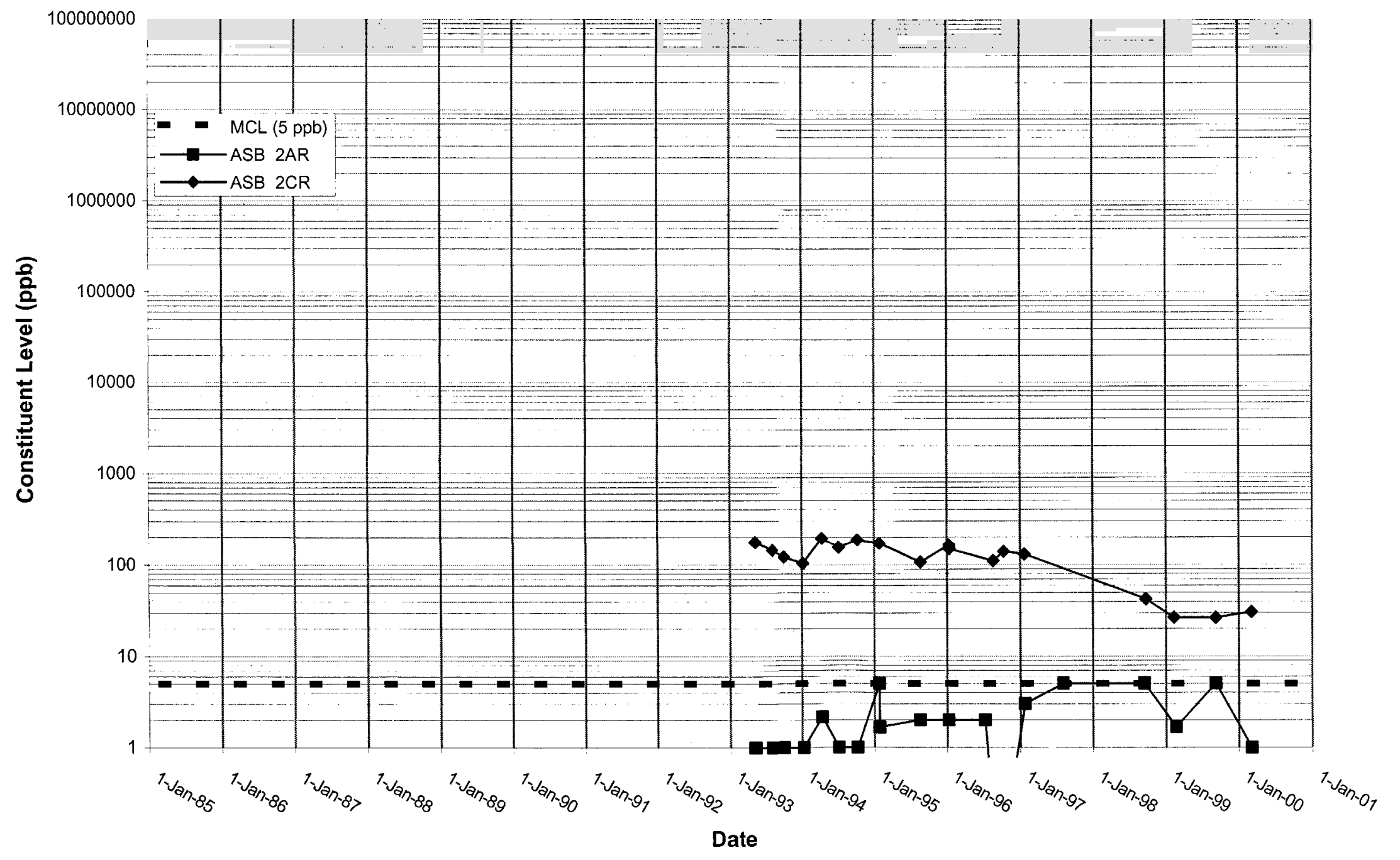


WSRC-TR-2000-00242 UNCLASSIFIED

Time Series Plot of Trichloroethylene for Well ASB 3

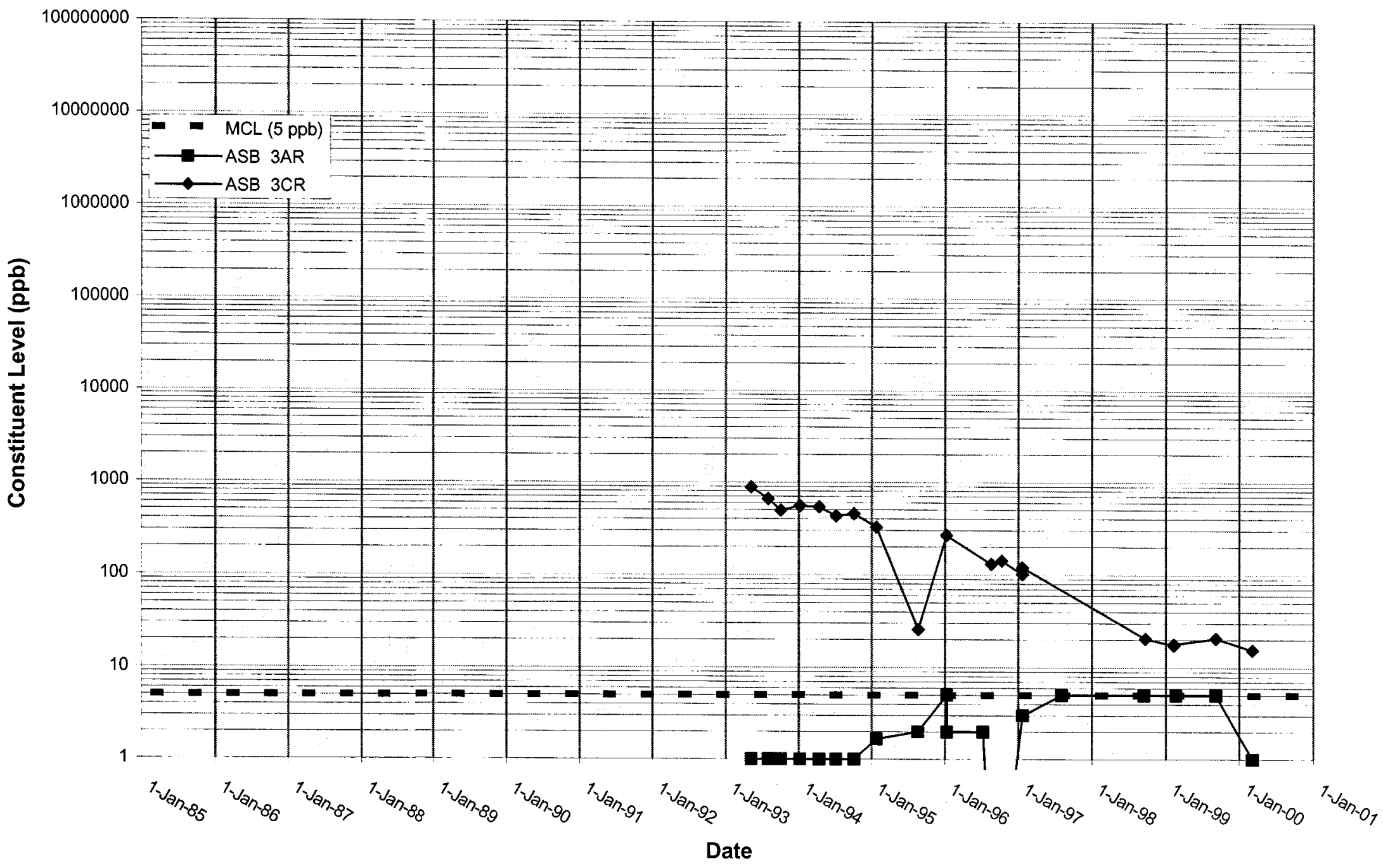


WSRC-TR-2000-00242 UNCLASSIFIED

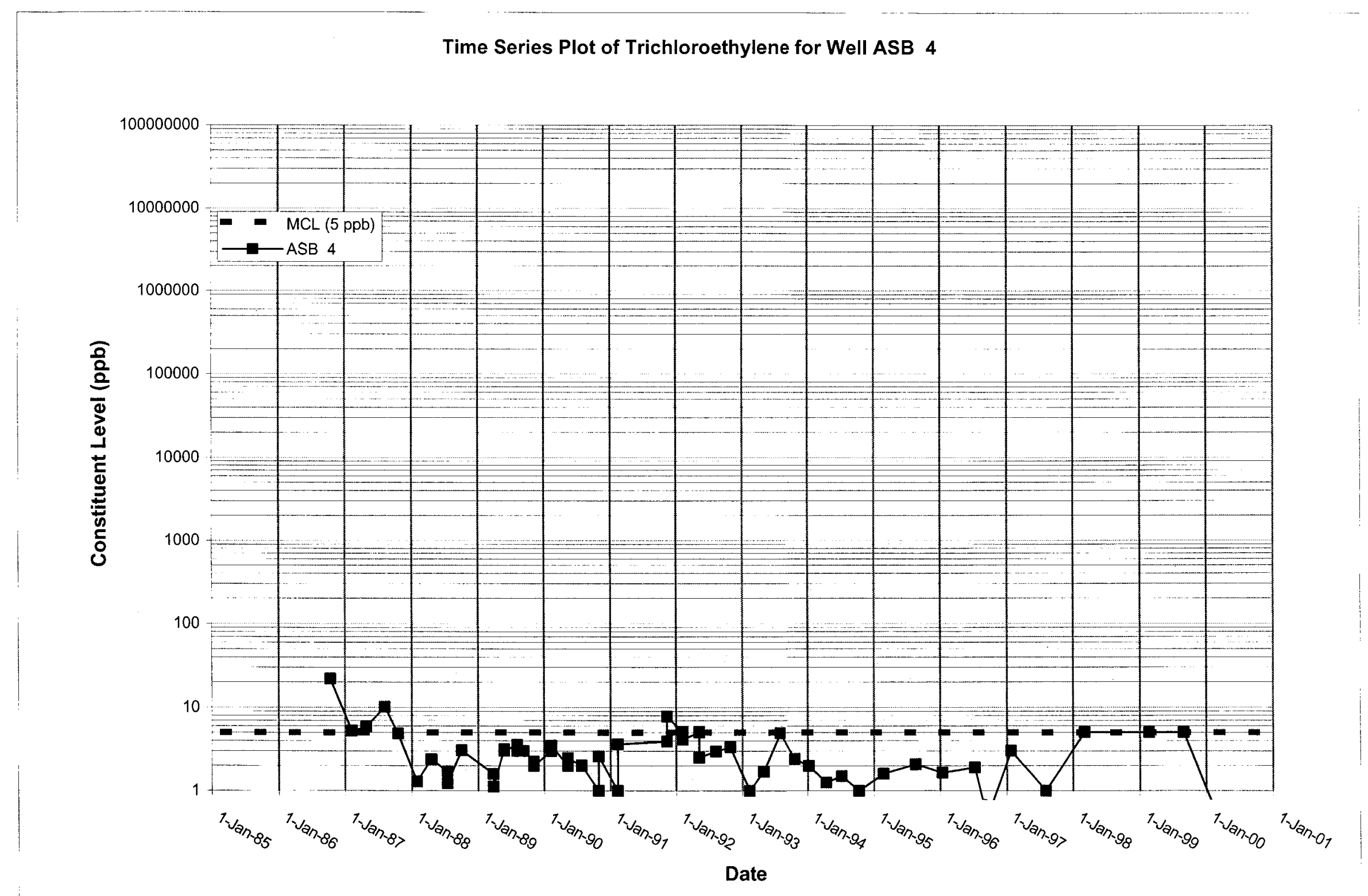


WSRC-TR-2000-00242

Time Series Plot of Trichloroethylene for Well ASB 5

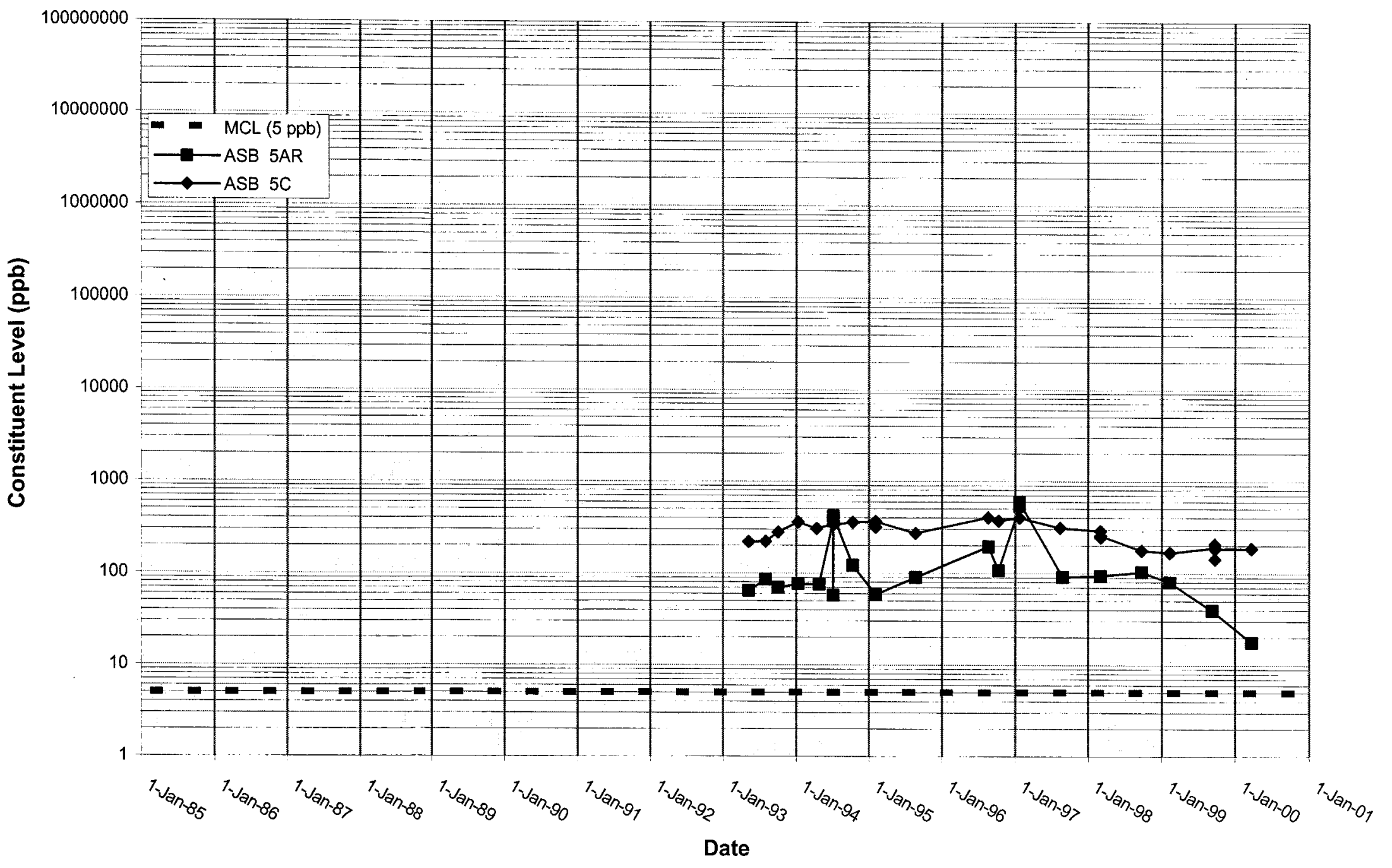


Time Series Plot of Trichloroethylene for Well ASB 6

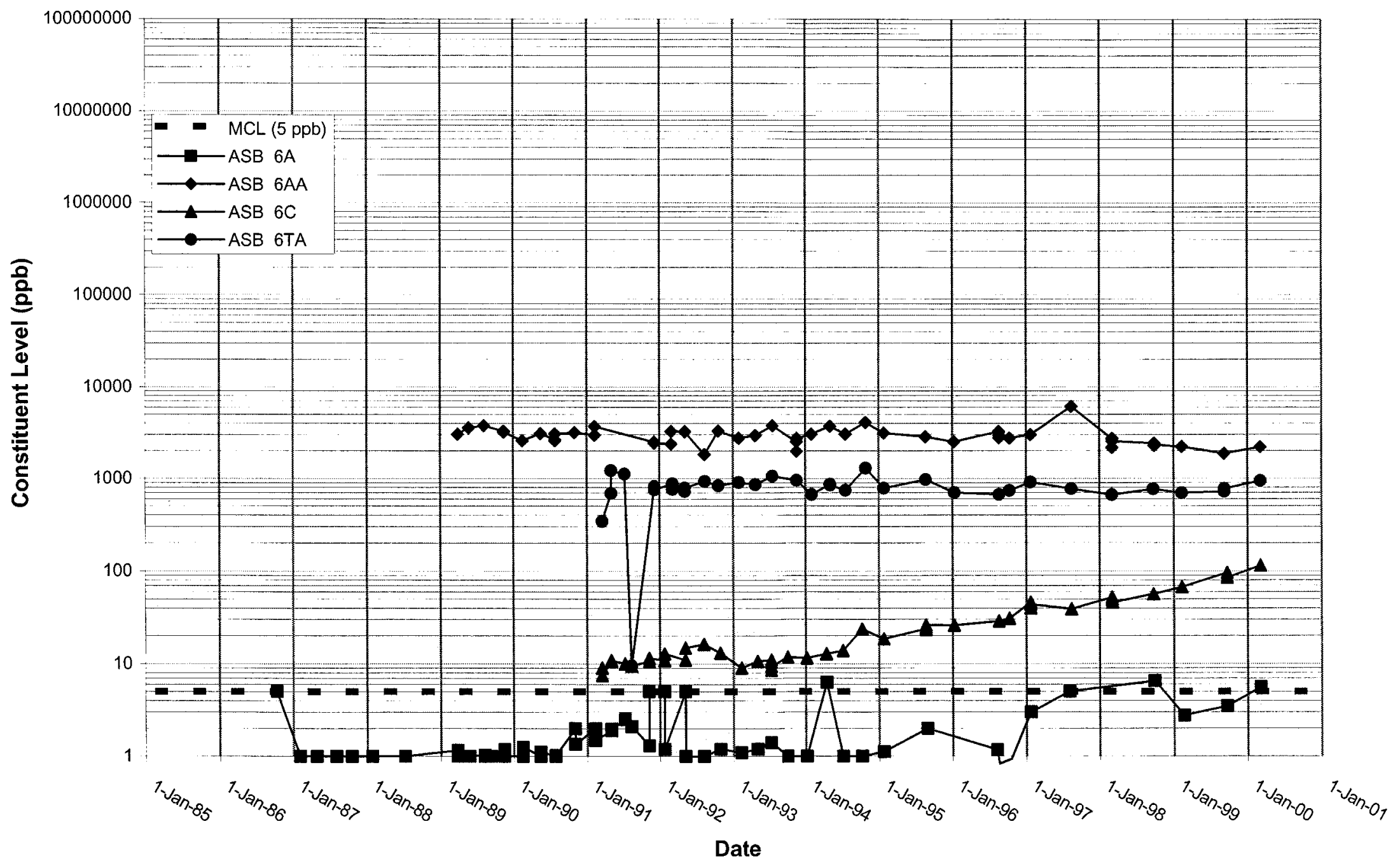


Time Series Plot of Trichloroethylene for Well ASB 8

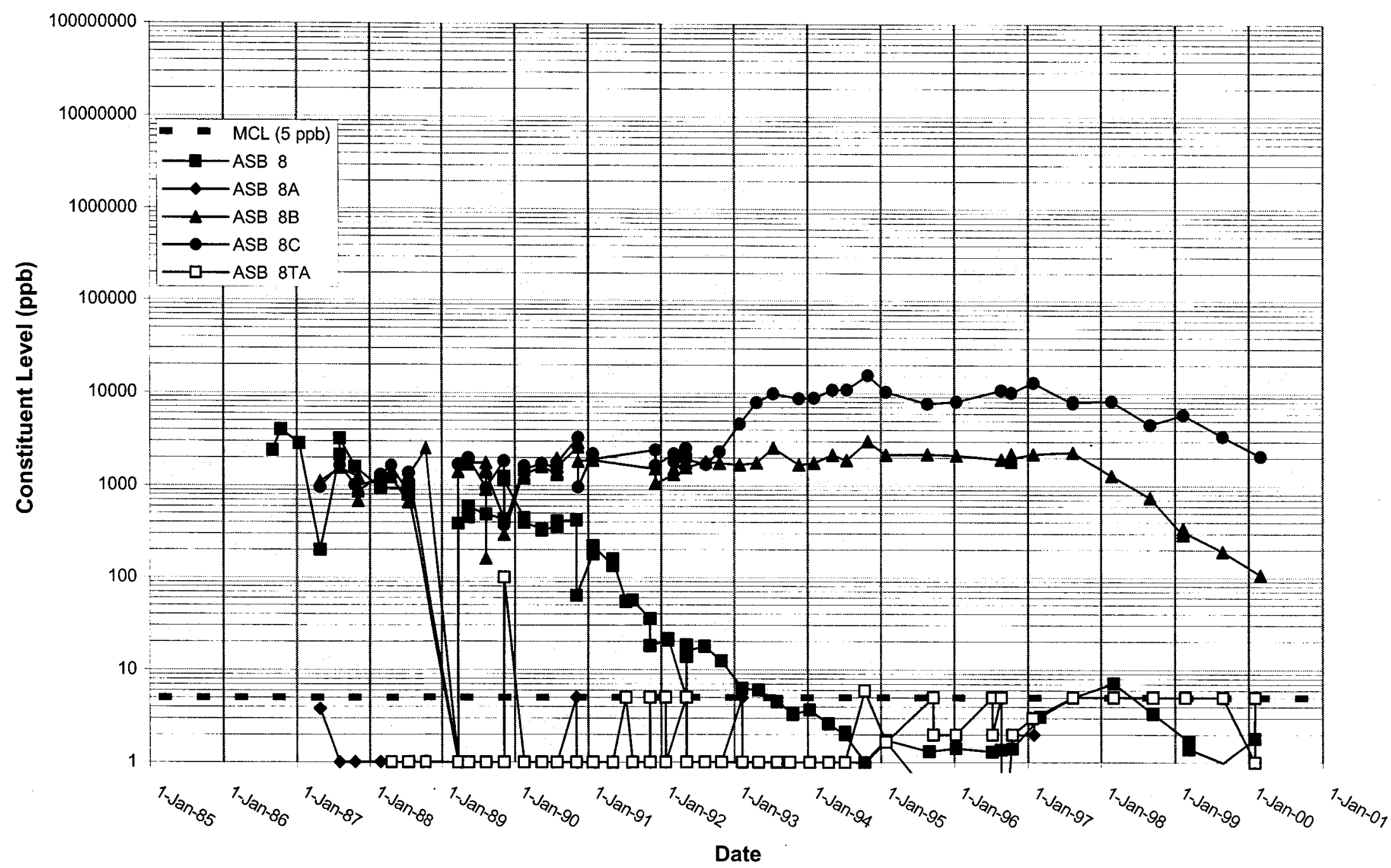


Time Series Plot of Trichloroethylene for Well ASB 9

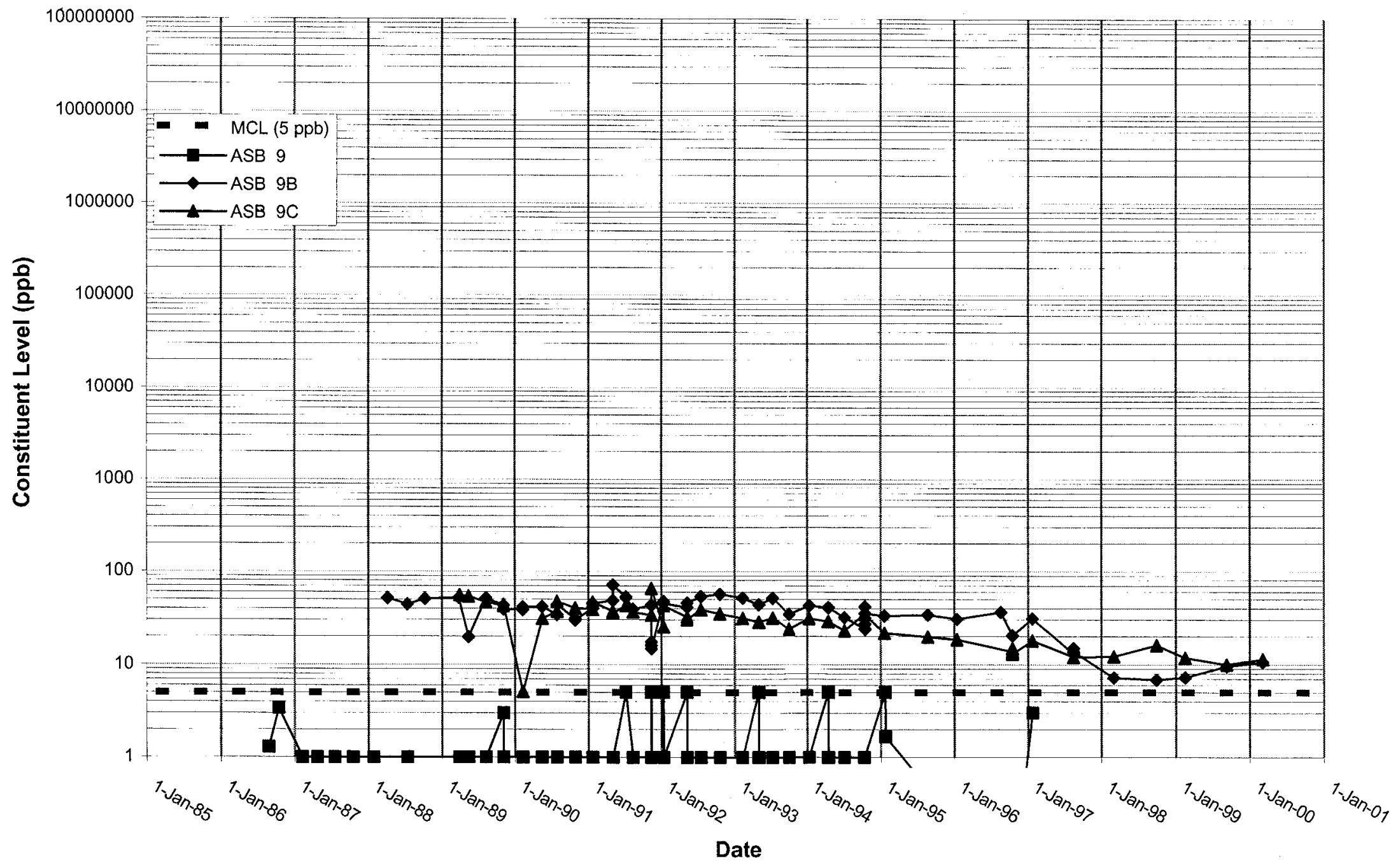


WSRC-TR-2000-00242 UNCLASSIFIED

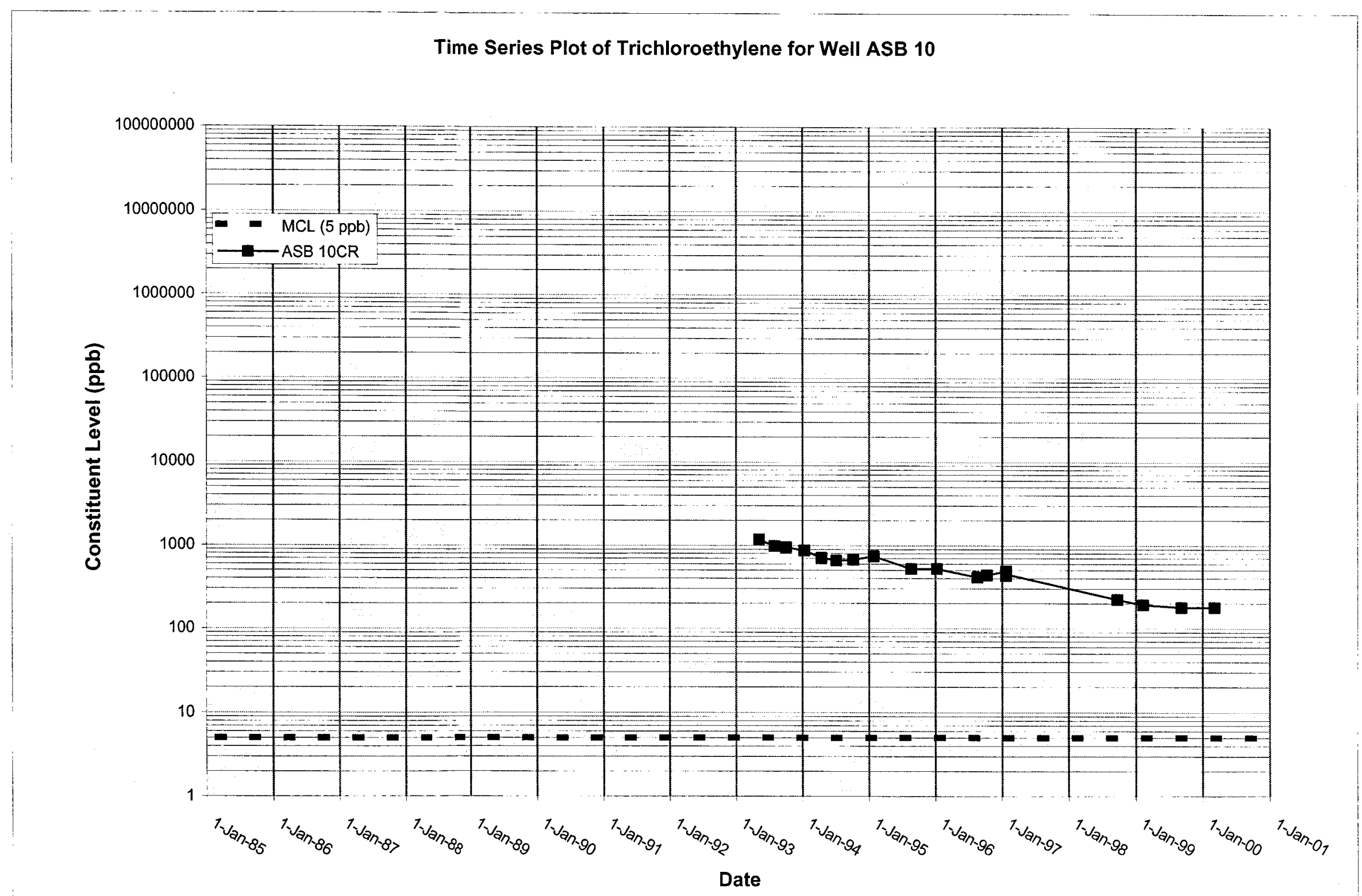


WSRC-TR-2000-00242 UNCLASSIFIED

Time Series Plot of Trichloroethylene for Well MCB 2

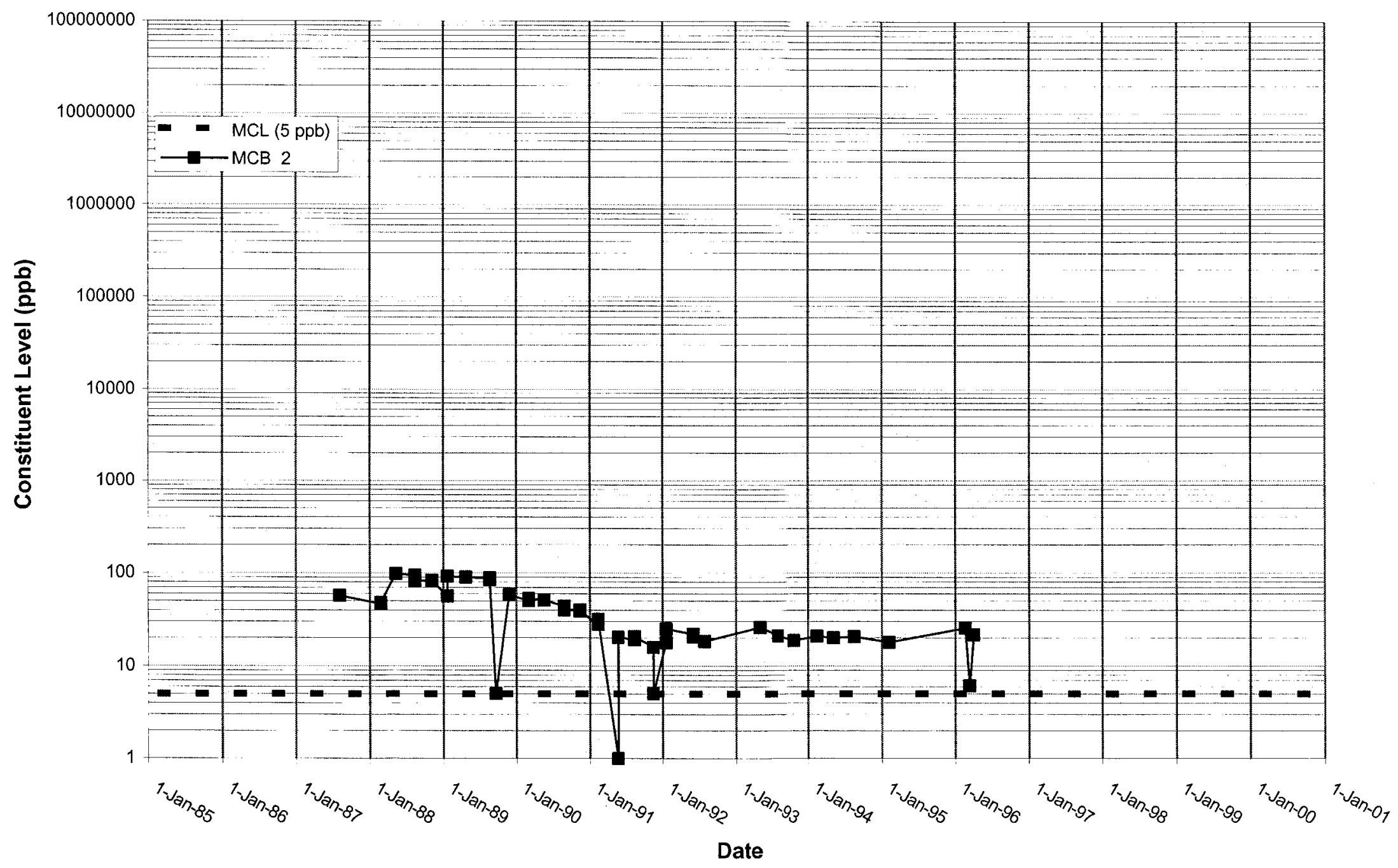


WSRC-TR-2000-00242 UNCLASSIFIED

Time Series Plot of Trichloroethylene for Well MCB 4

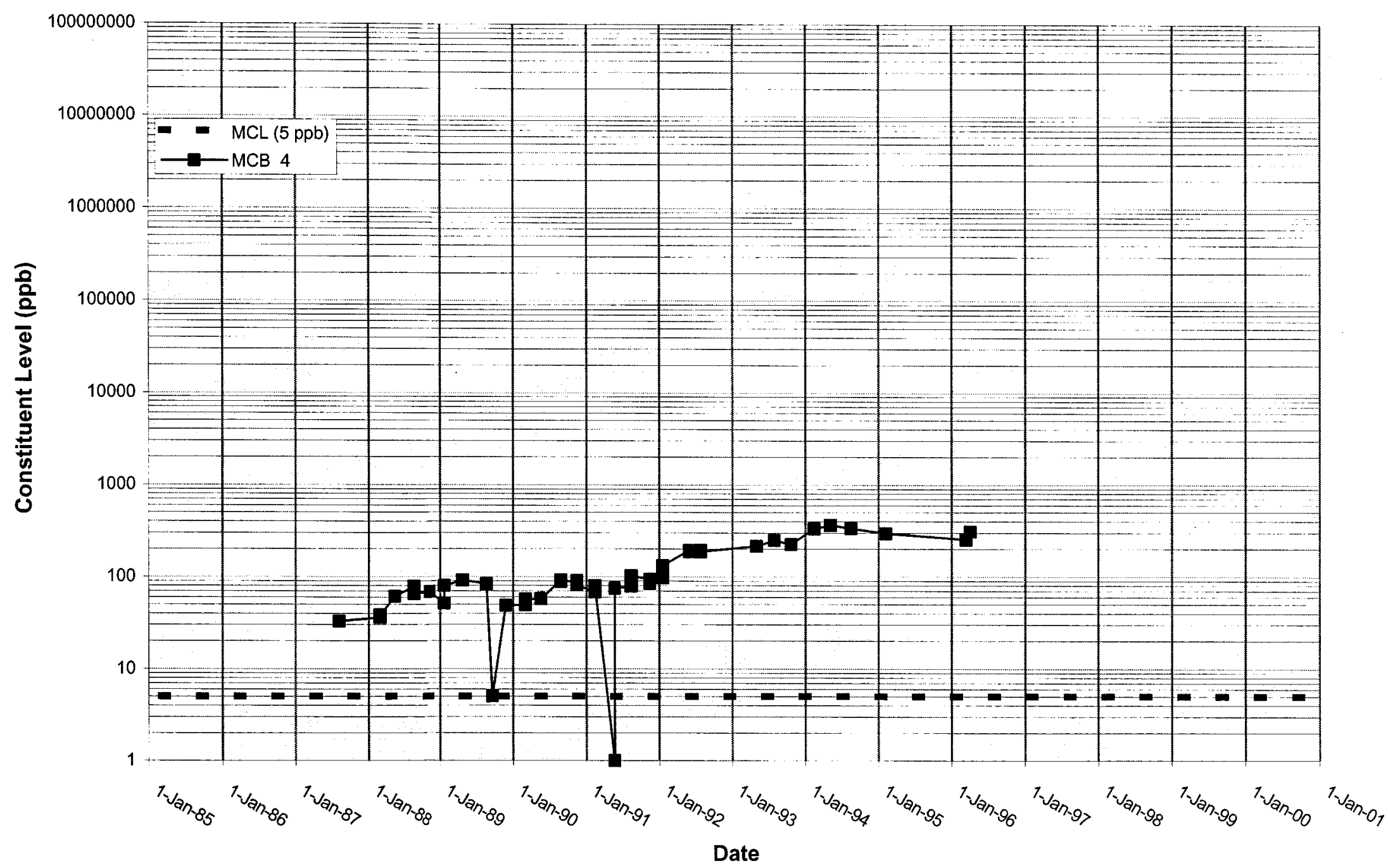




\section{Time Series Plot of Trichloroethylene for Well MCB 5}

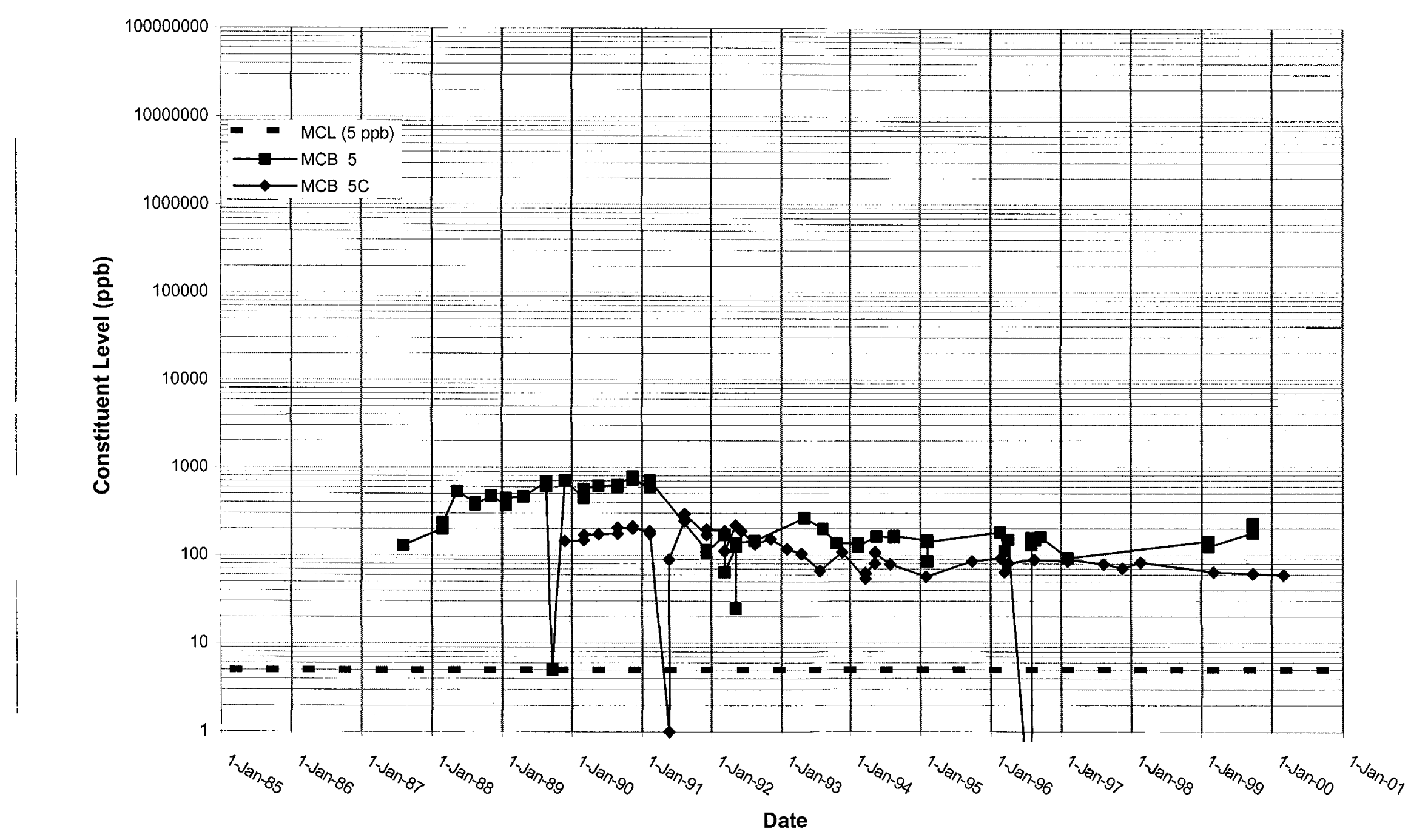




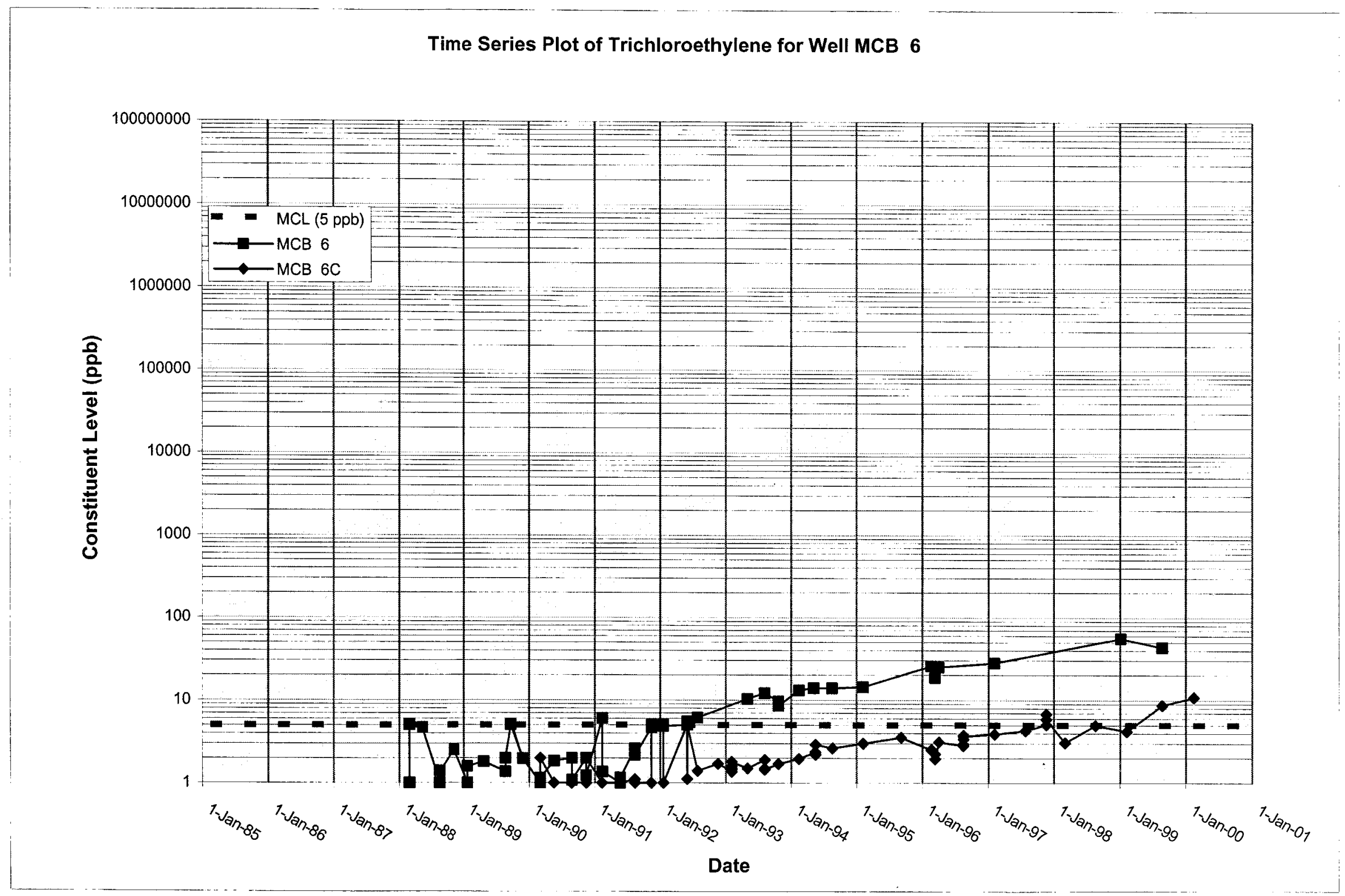




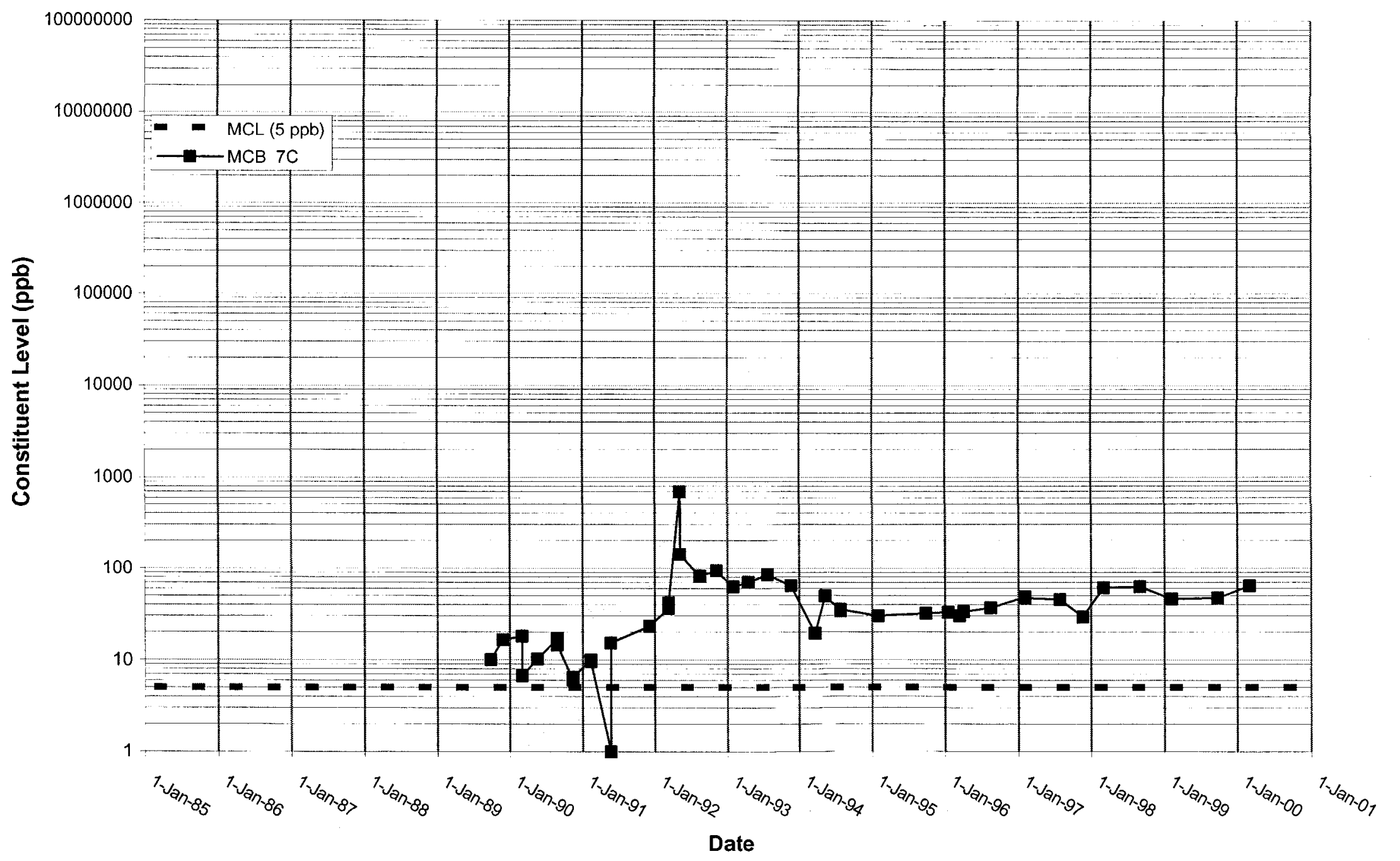




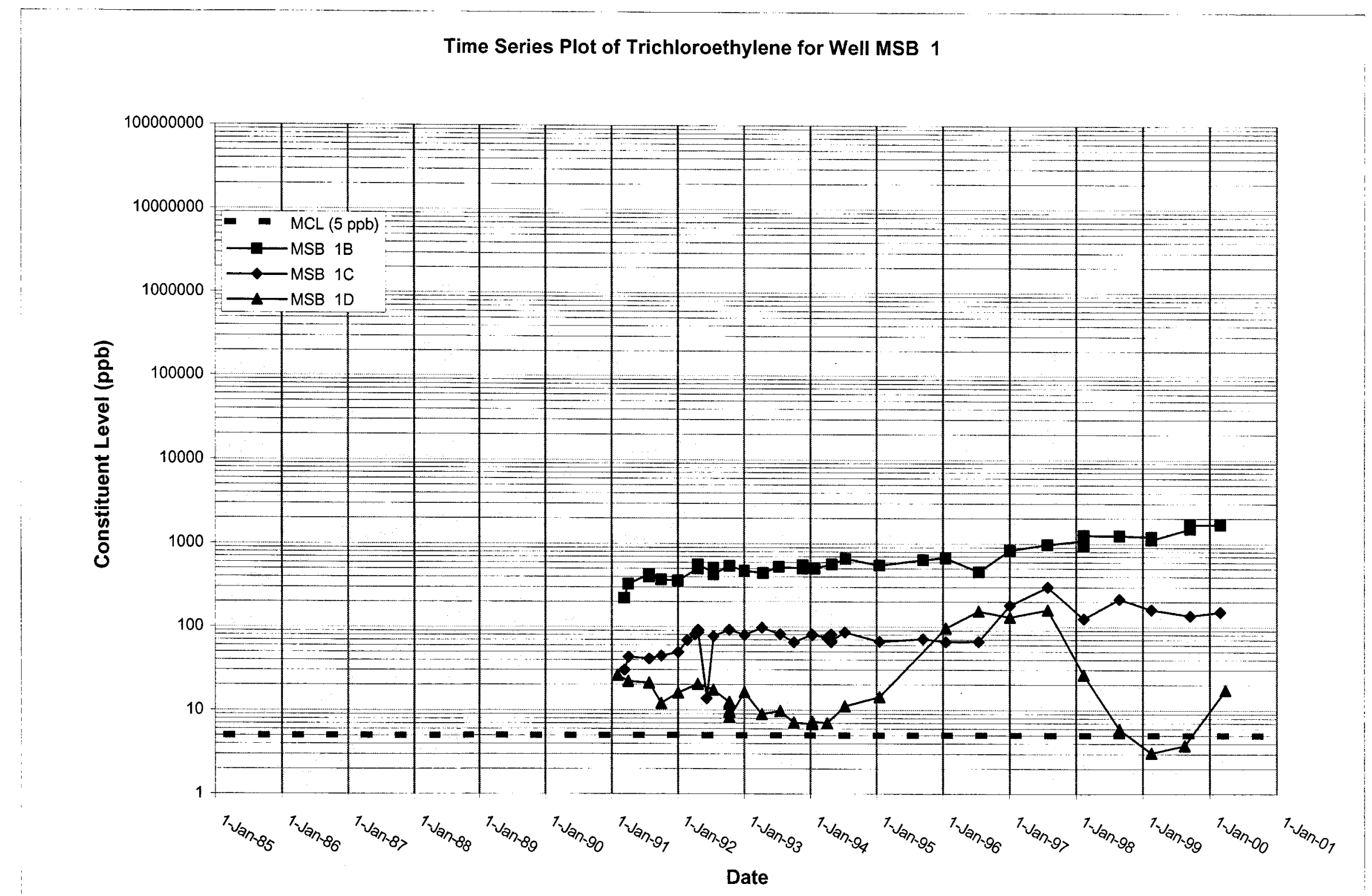


Time Series Plot of Trichloroethylene for Well MSB 2

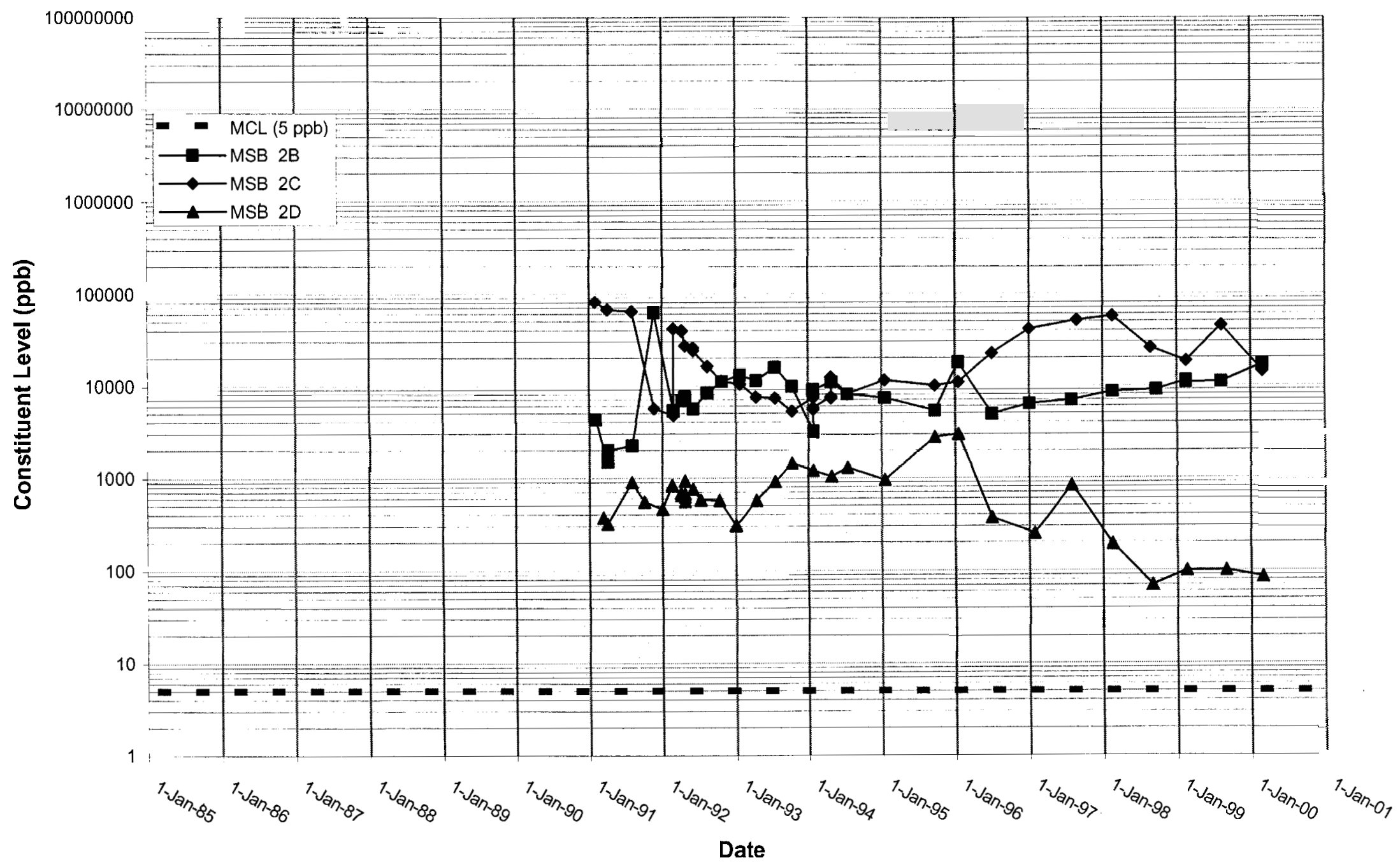


WSRC-TR-2000-00242 UNCLASSIFIED

Time Series Plot of Trichloroethylene for Well MSB 3

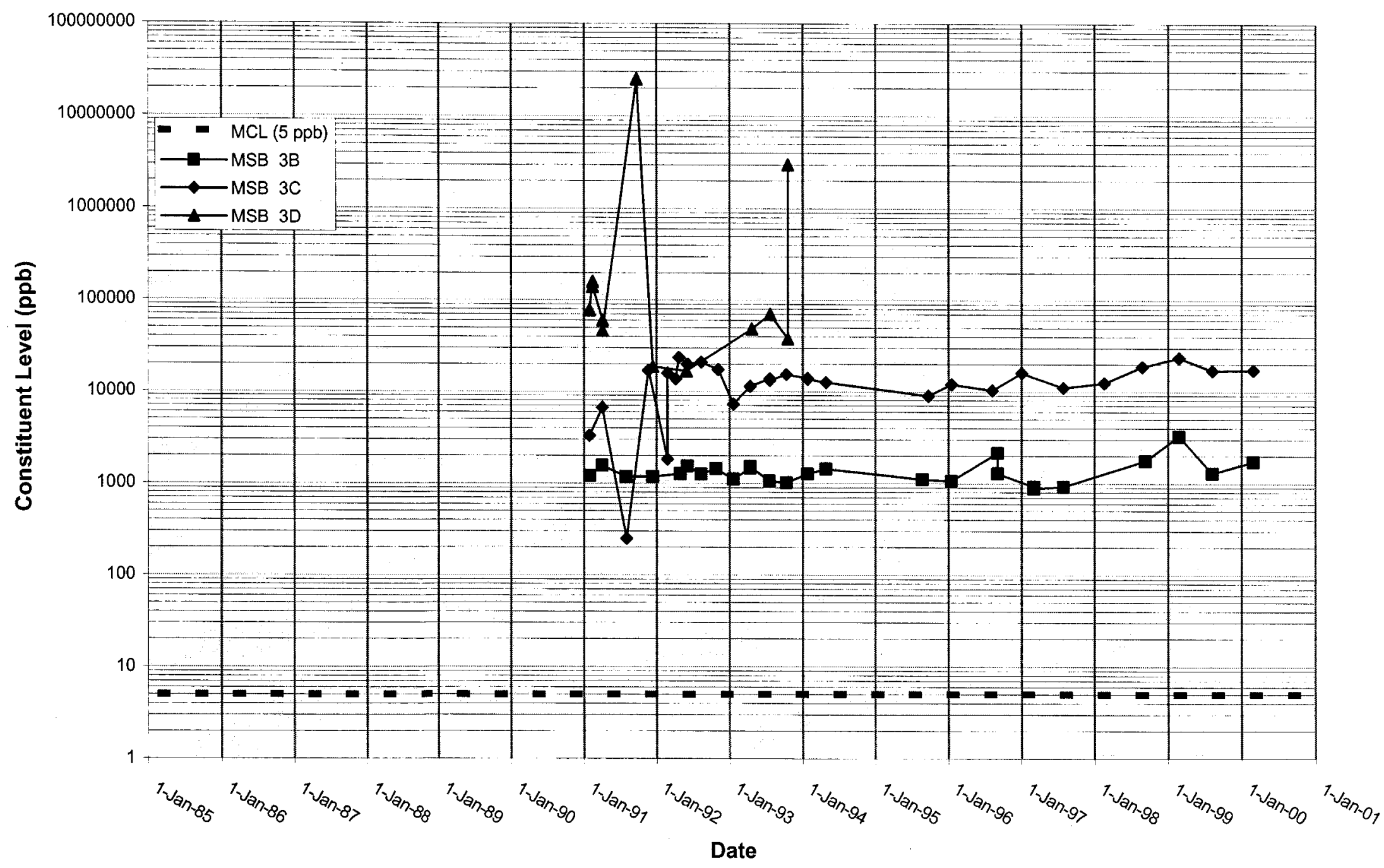




\section{Time Series Plot of Trichloroethylene for Well MSB 4}

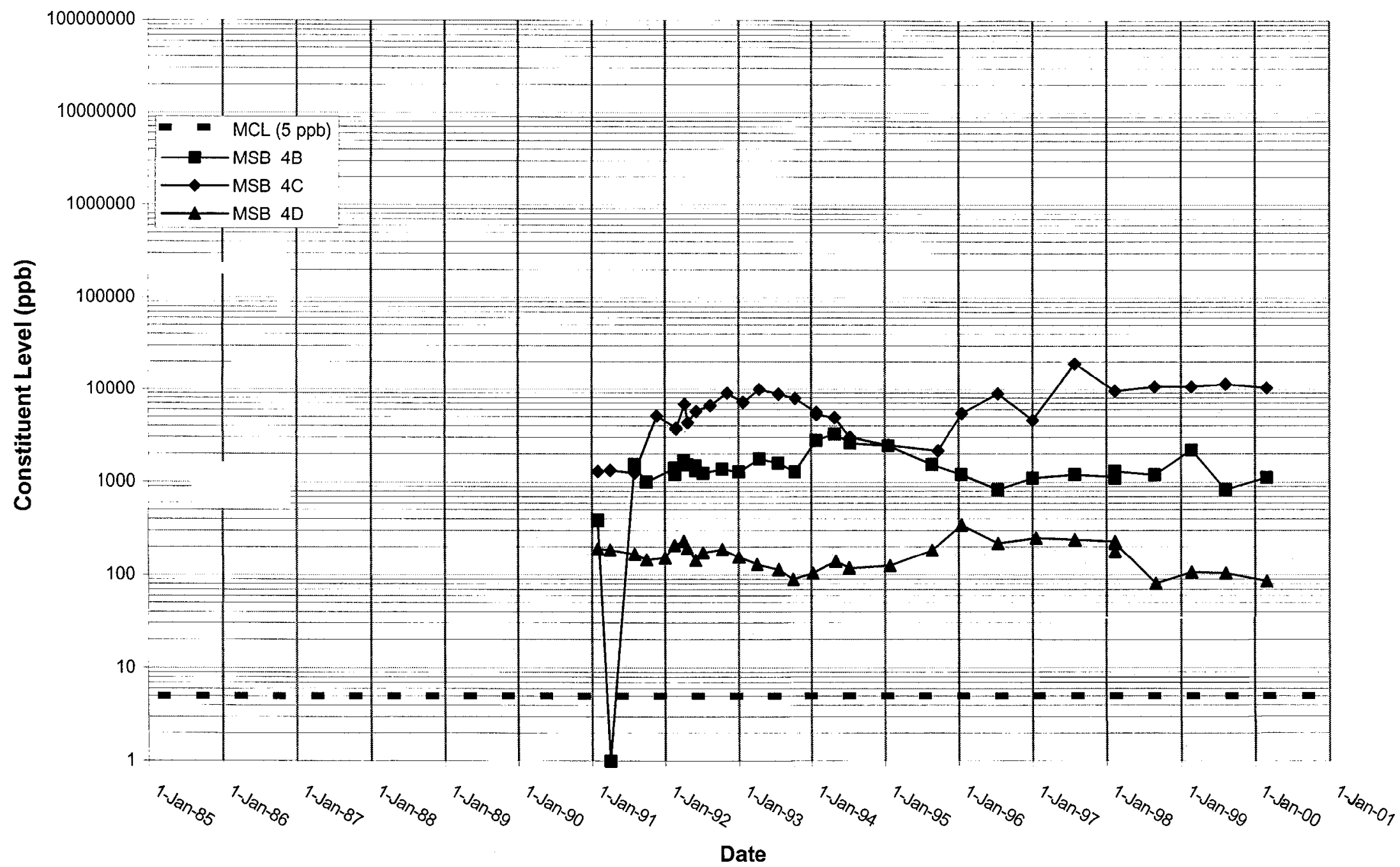


WSRC-TR-2000-00242 UNCLASSIFIED

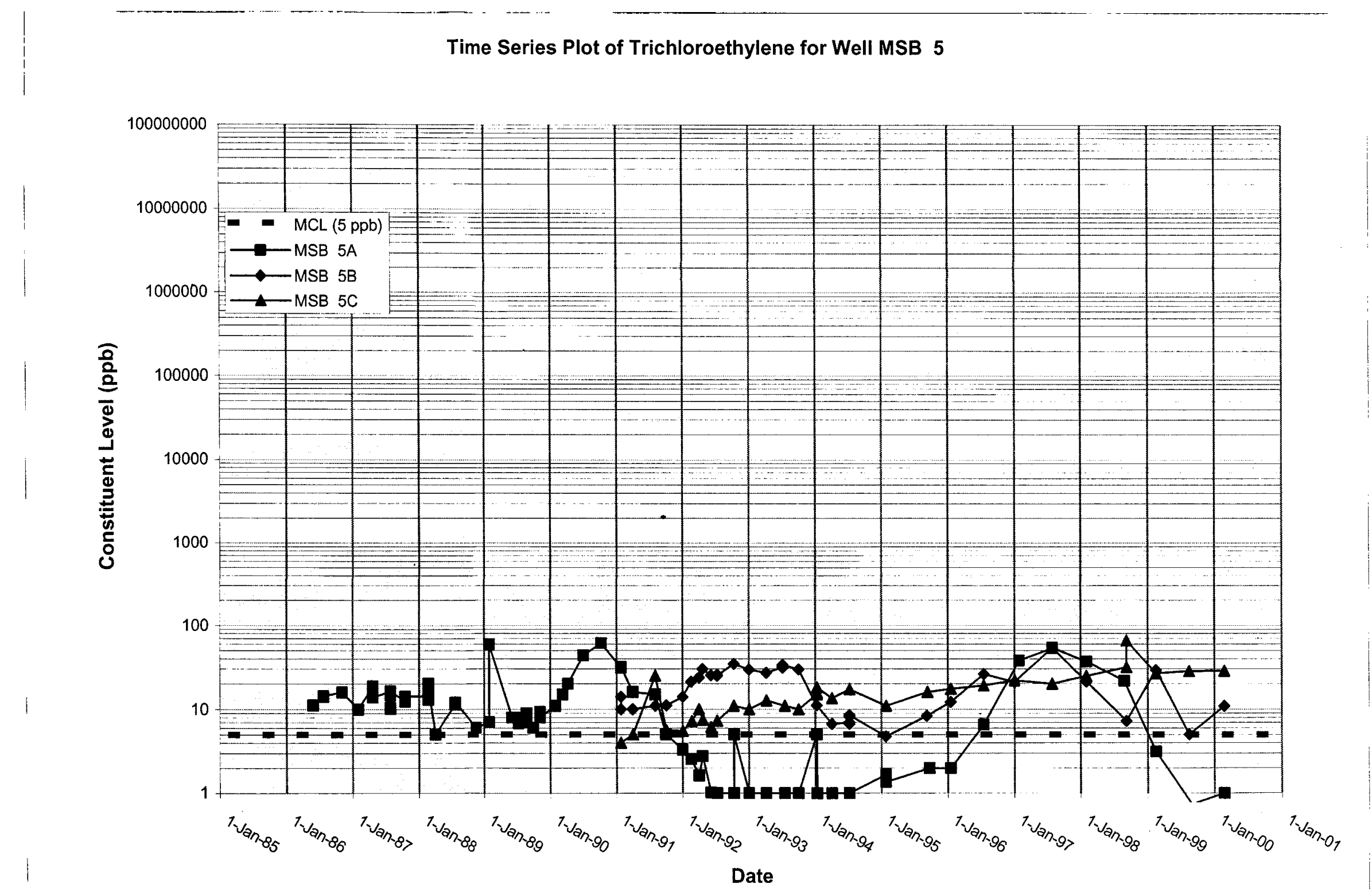




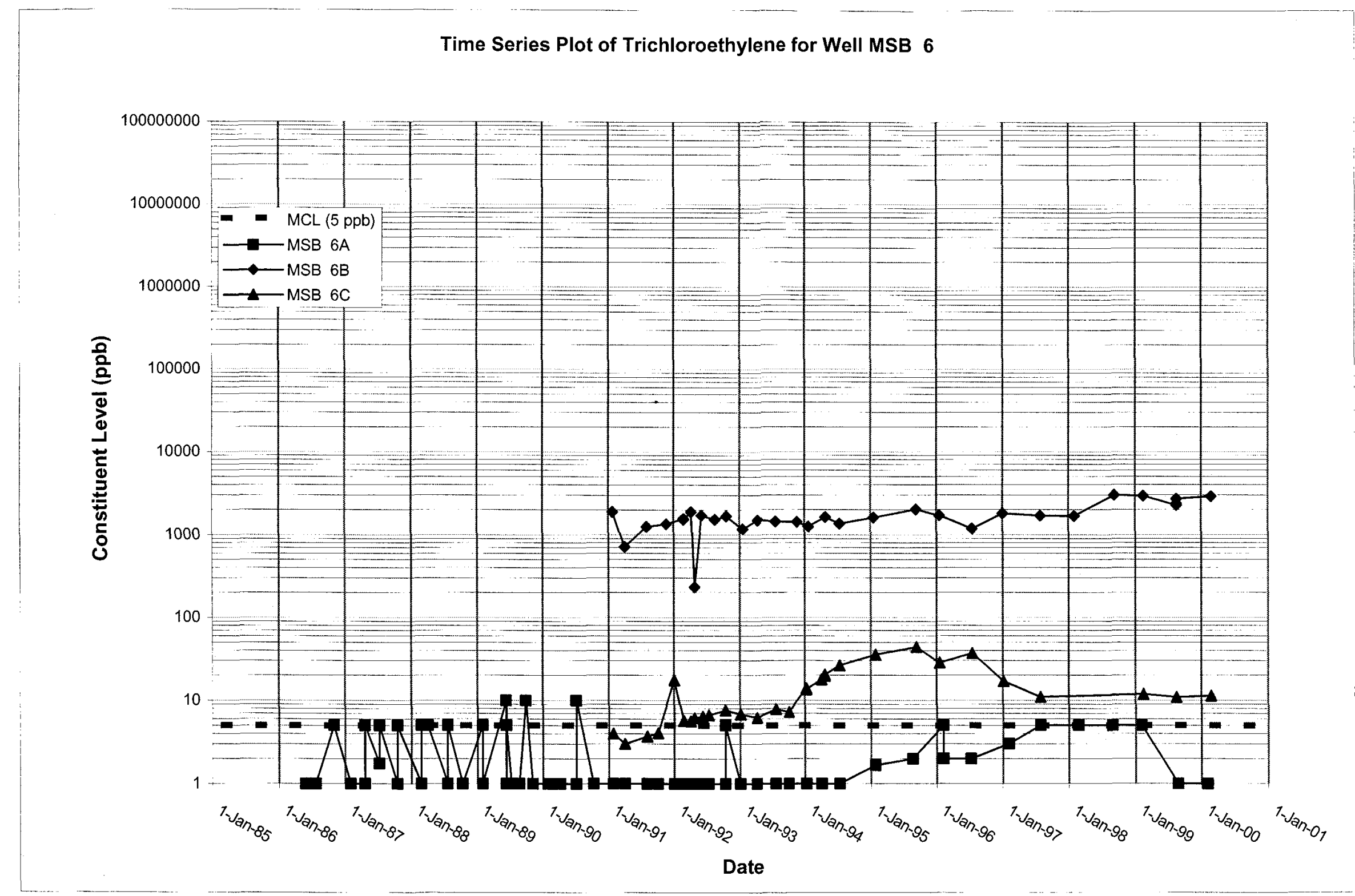




\section{Time Series Plot of Trichloroethylene for Well MSB 7}

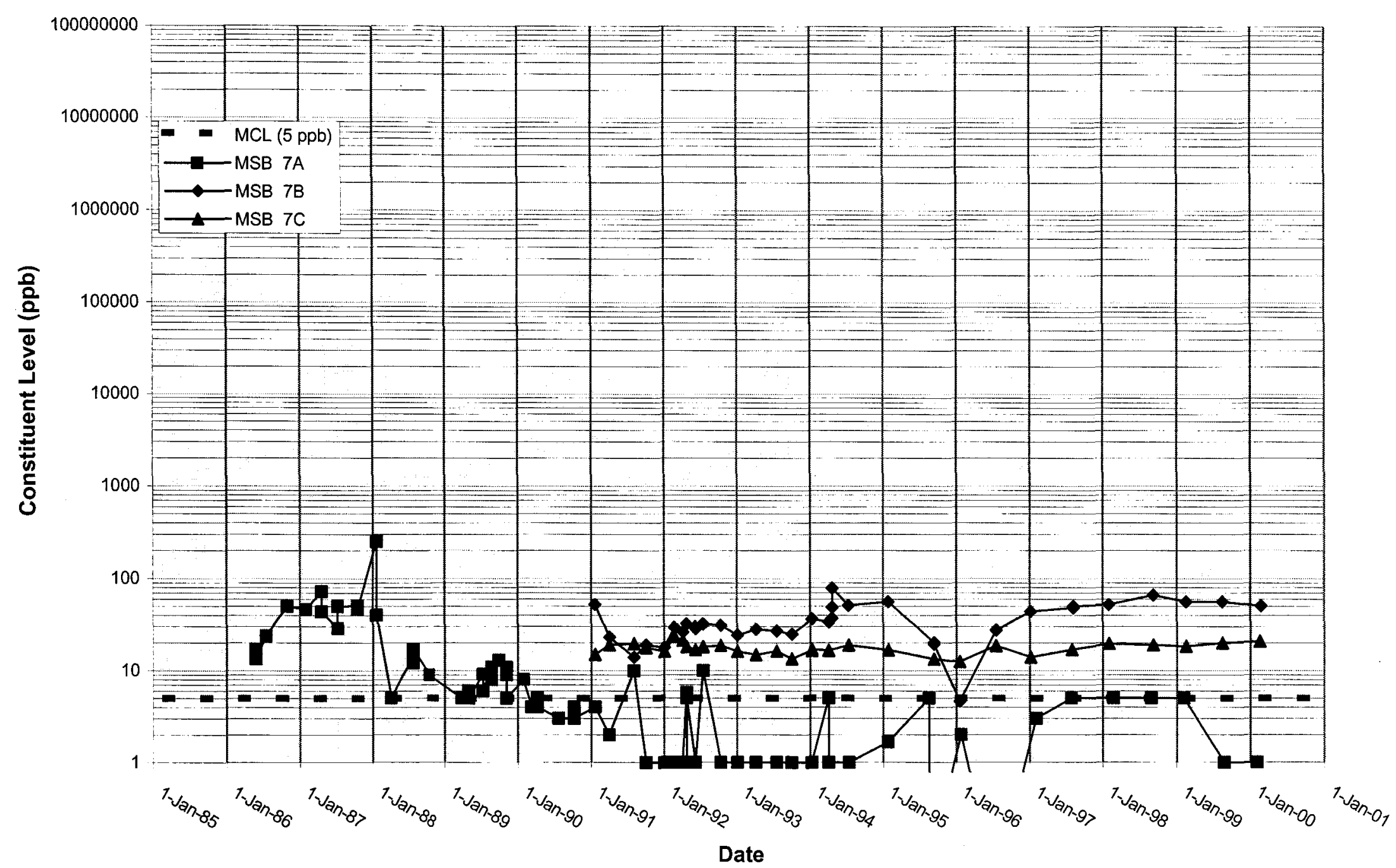


WSRC-TR-2000-00242

UNCLASSIFIED

Time Series Plot of Trichloroethylene for Well MSB 8

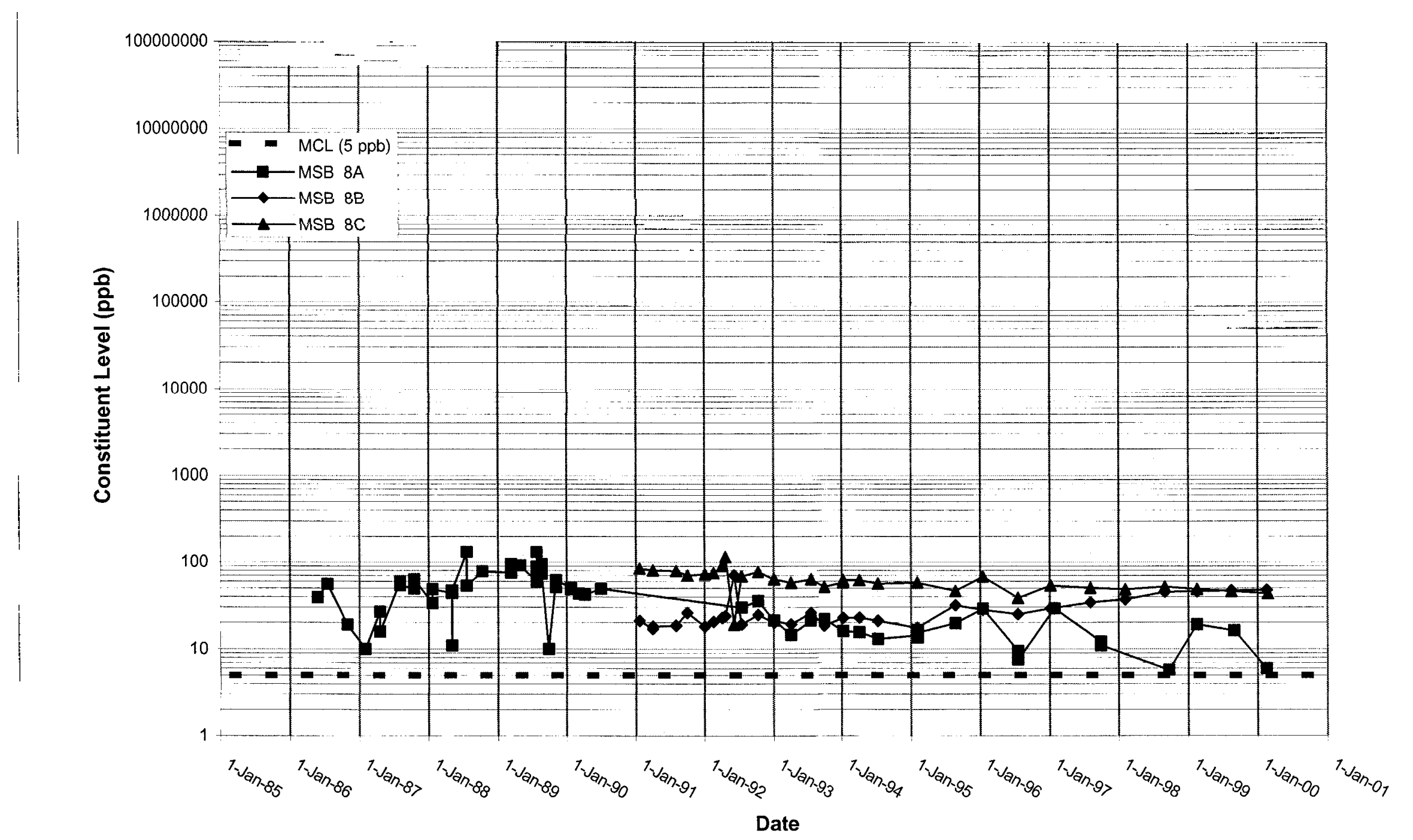




\section{Time Series Plot of Trichloroethylene for Well MSB 9}

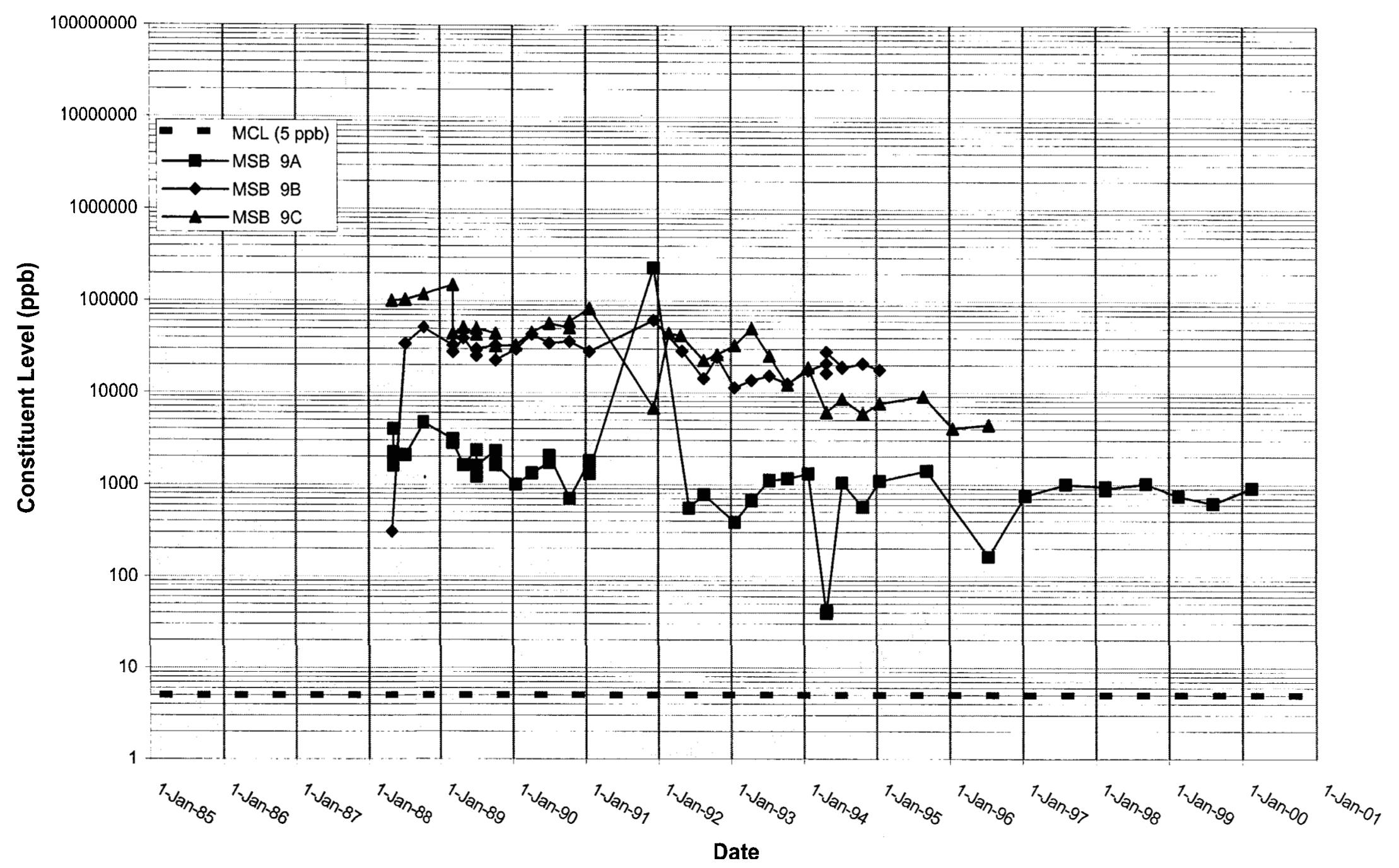


WSRC-TR-2000-00242 UNCLASSIFIED

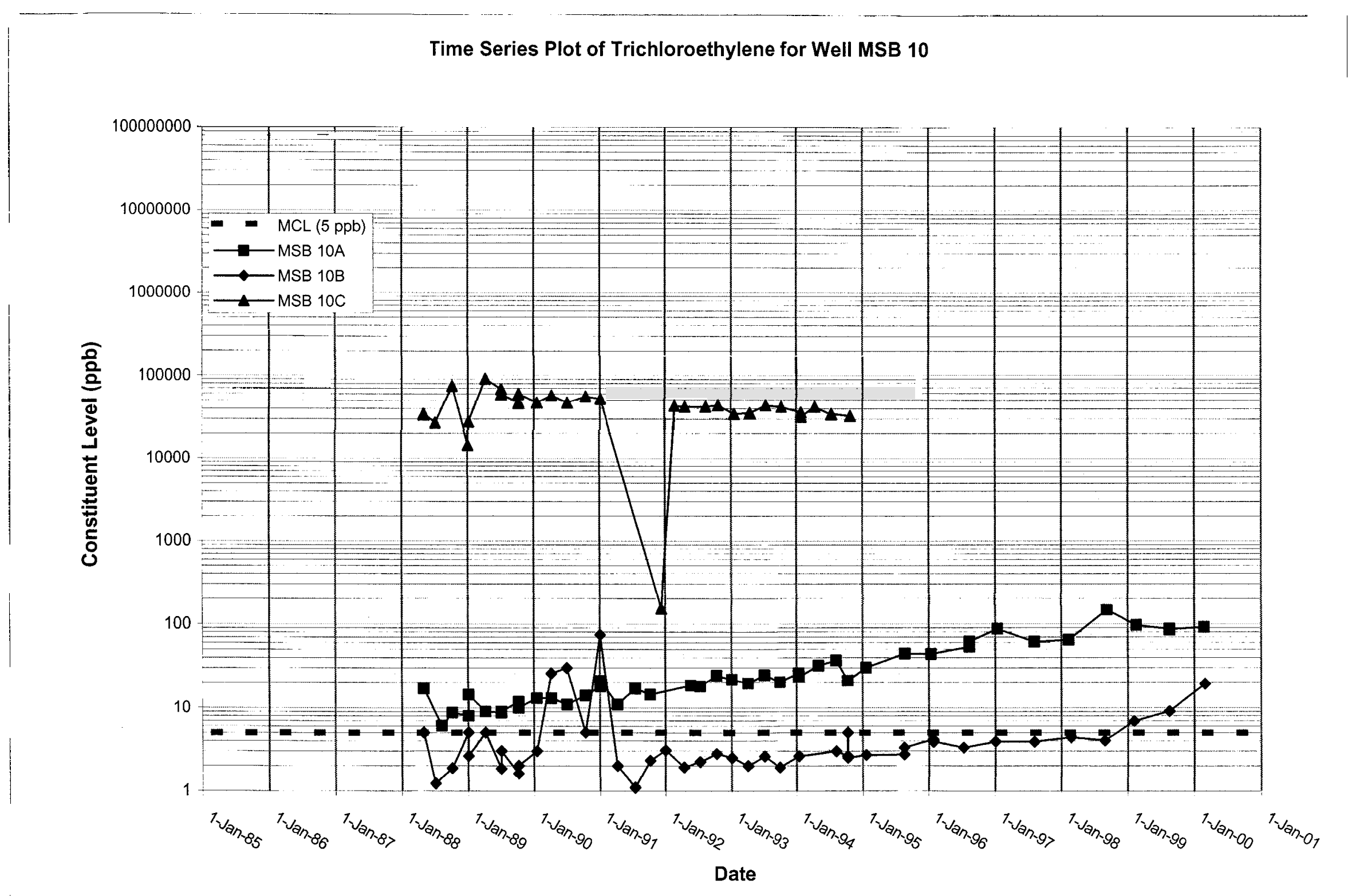




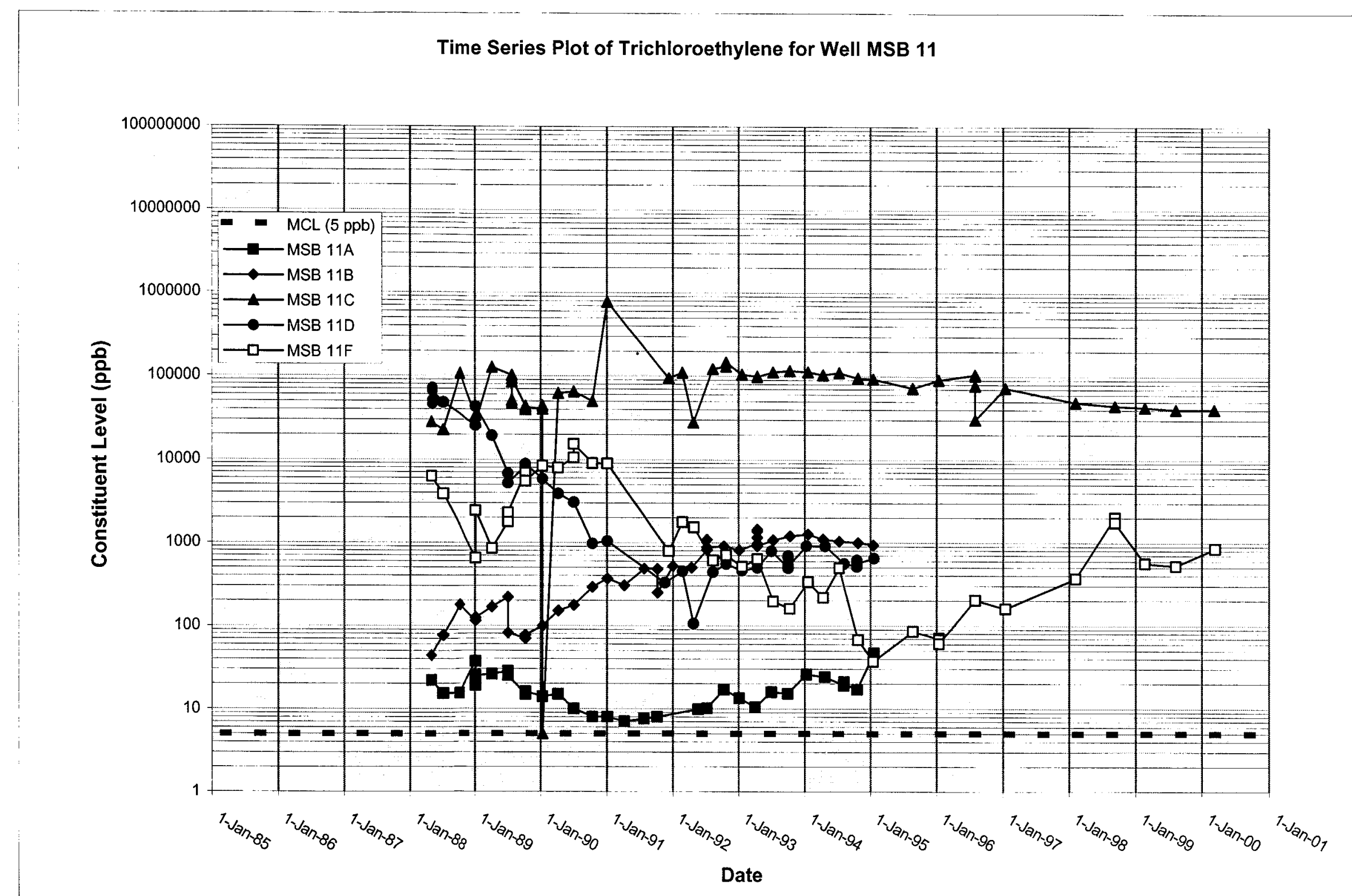




\section{Time Series Plot of Trichloroethylene for Well MSB 12}

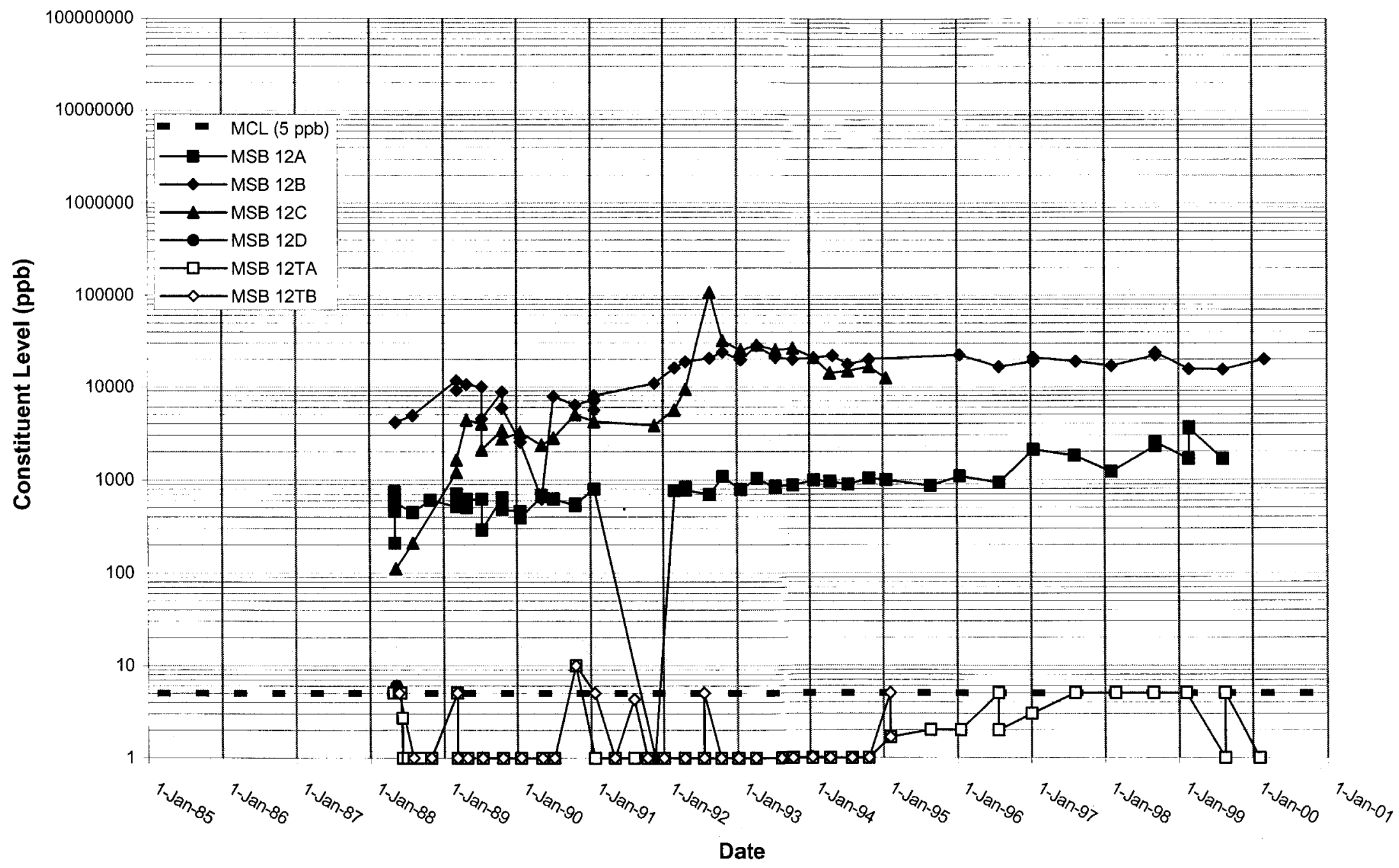




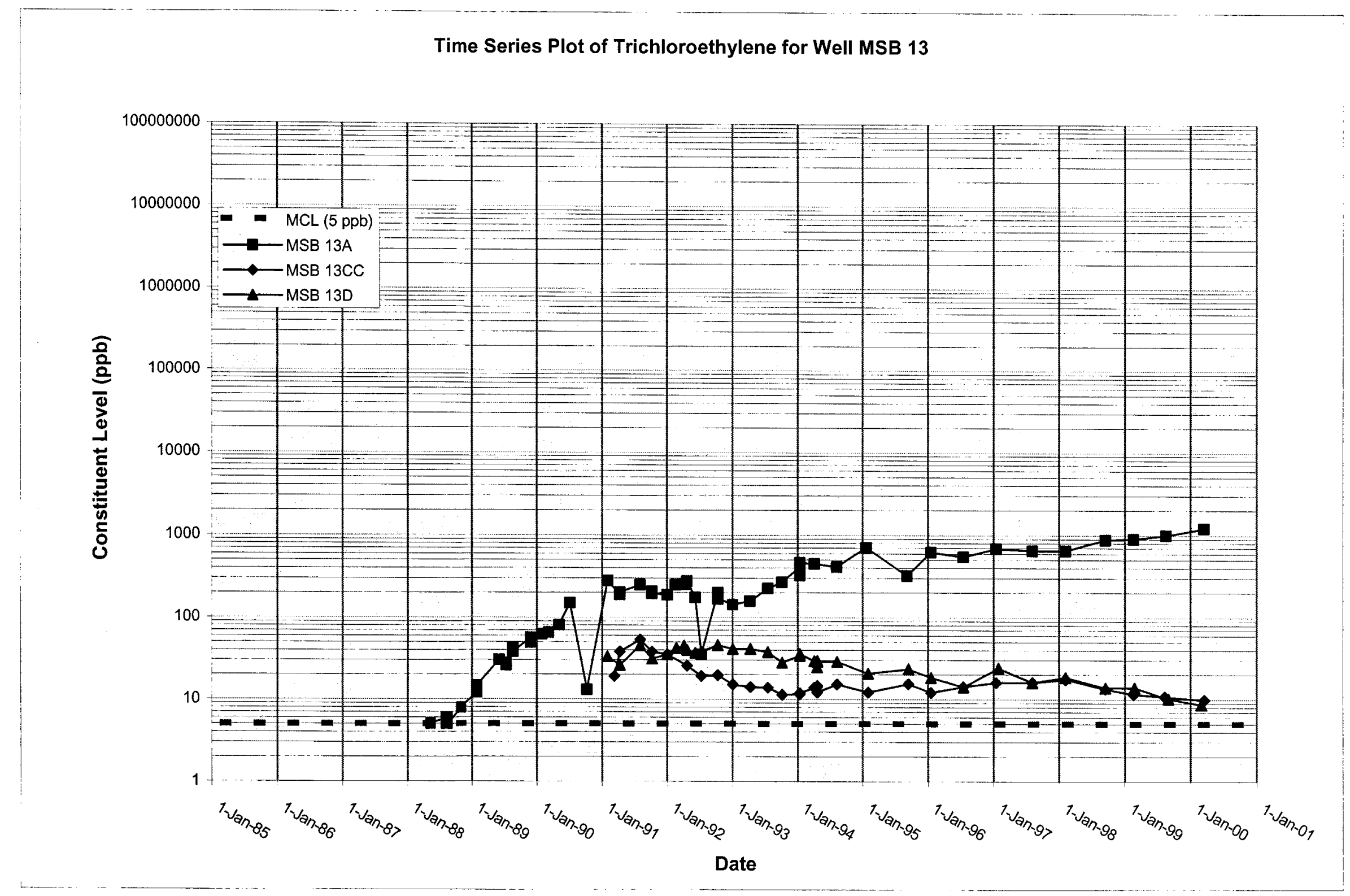




\section{Time Series Plot of Trichloroethylene for Well MSB 14}

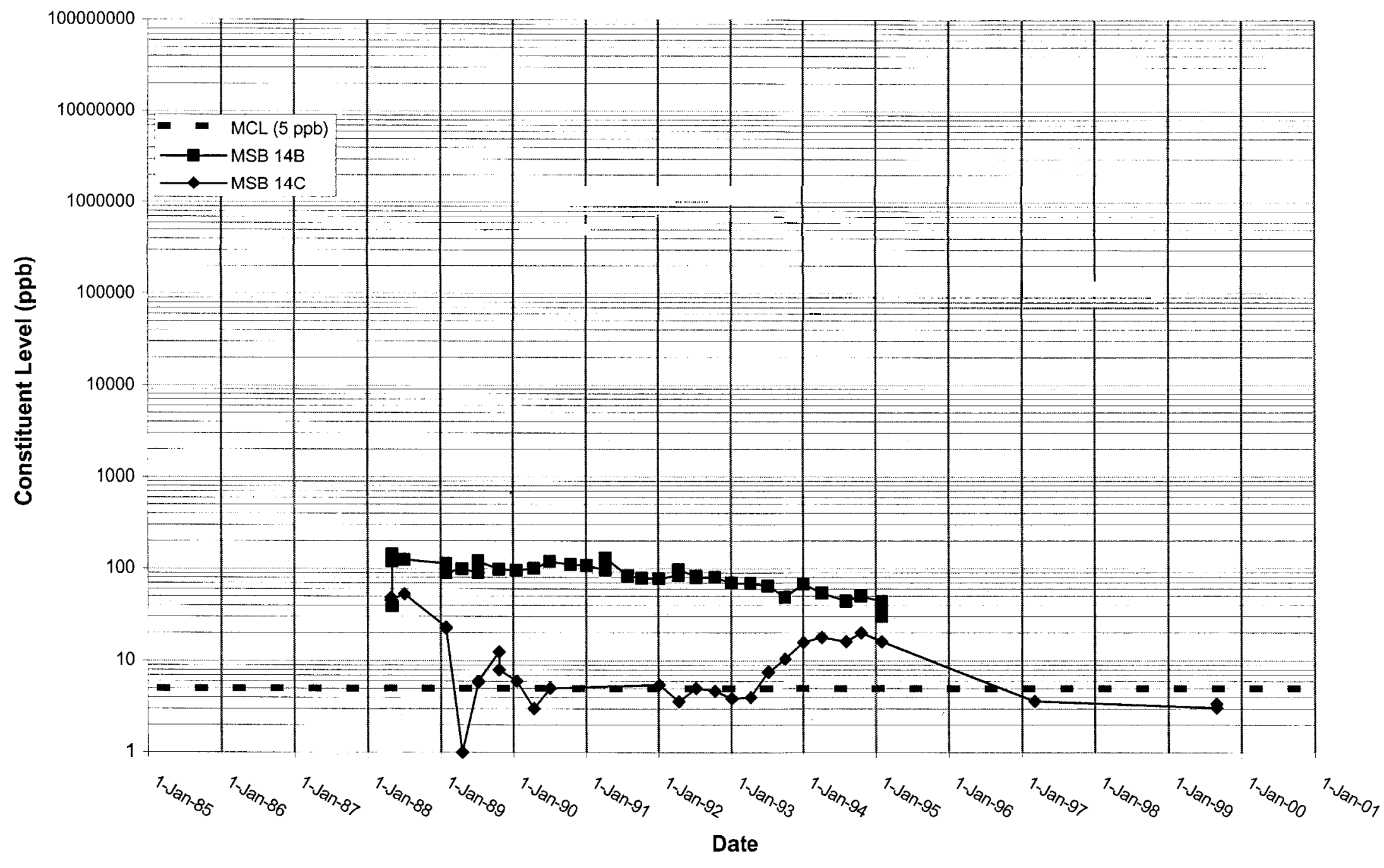


WSRC-TR-2000-00242 UNCLASSIFIED

Time Series Plot of Trichloroethylene for Well MSB 15

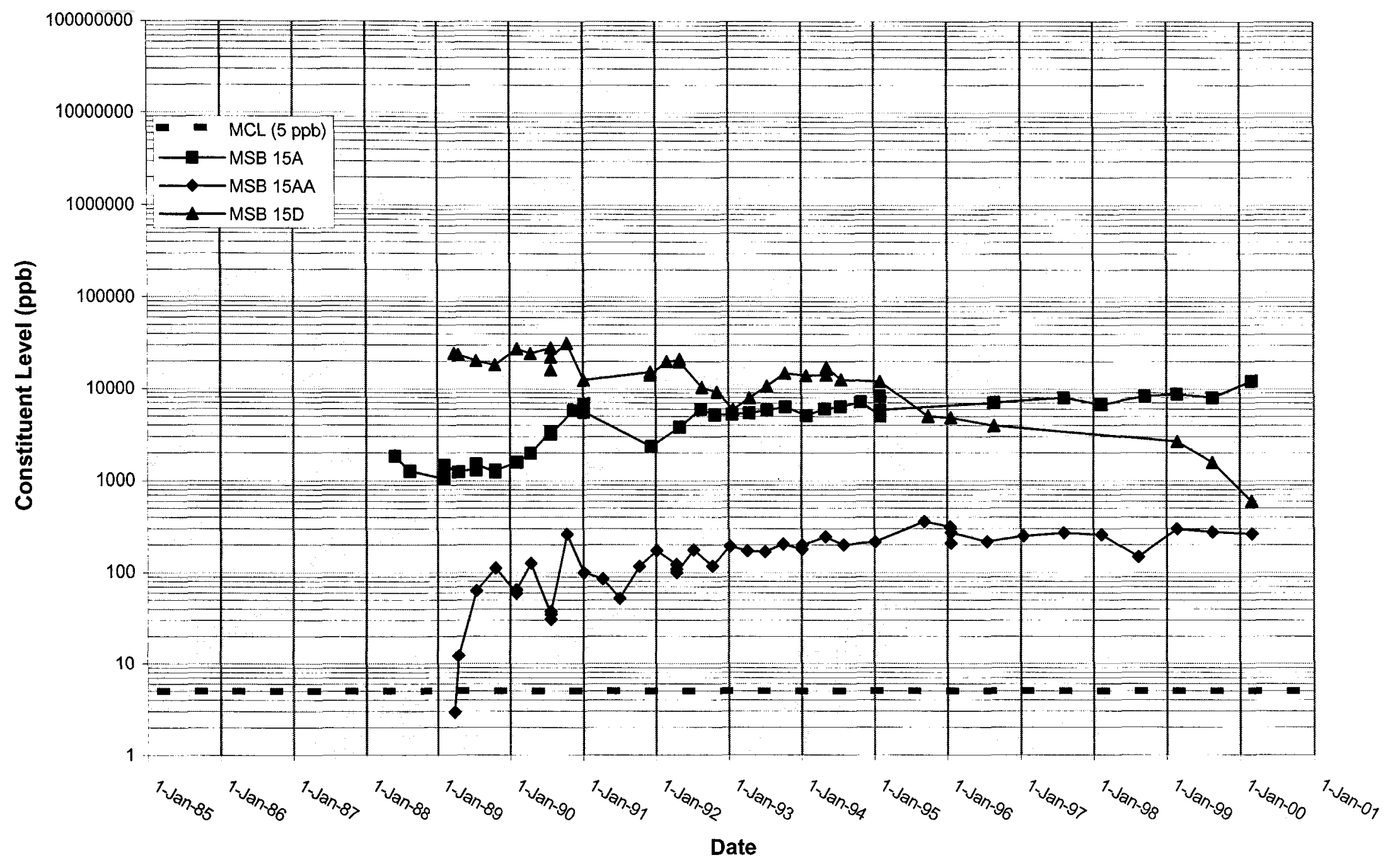




\section{Time Series Plot of Trichloroethylene for Well MSB 16}

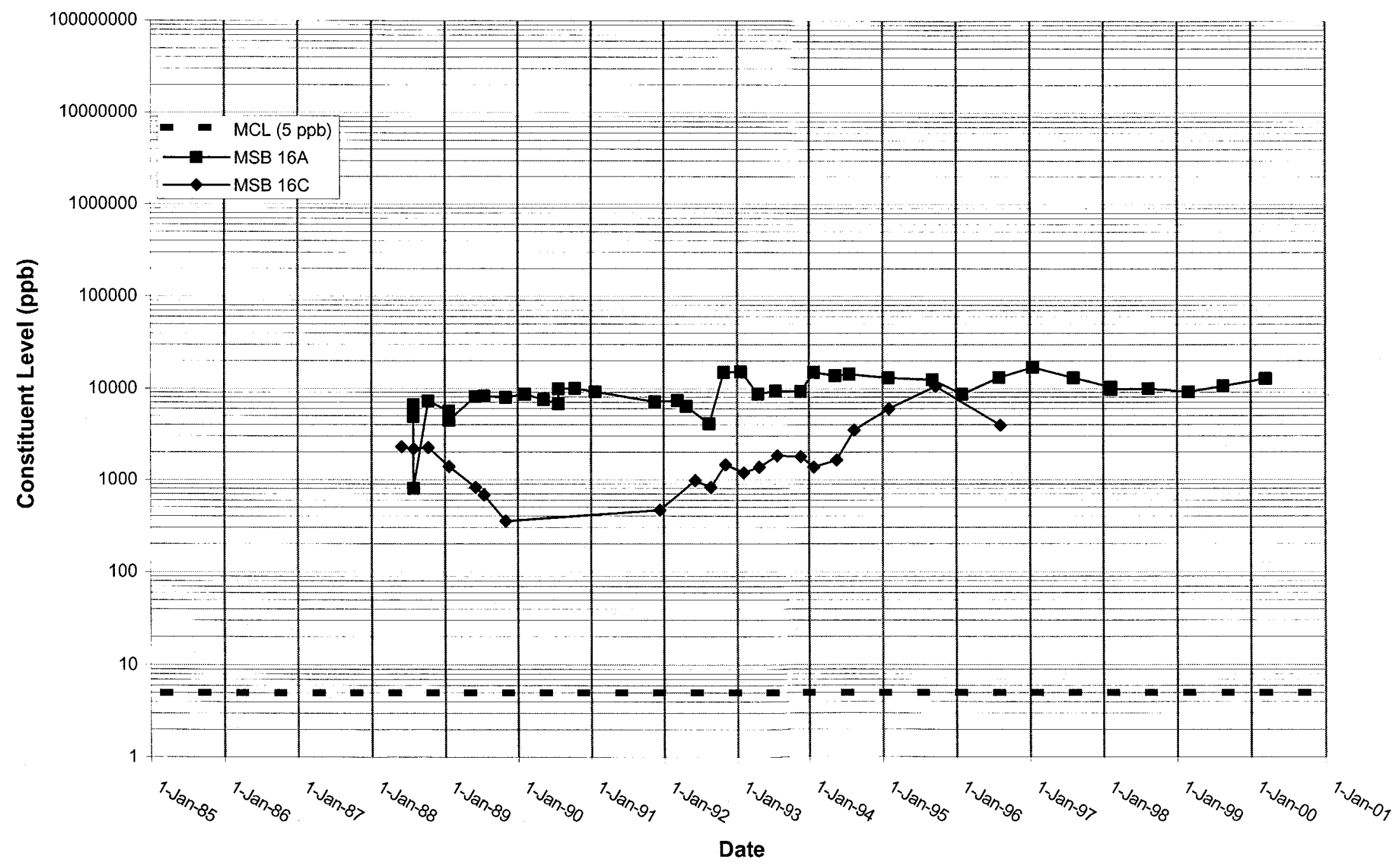


Time Series Plot of Trichloroethylene for Well MSB 17

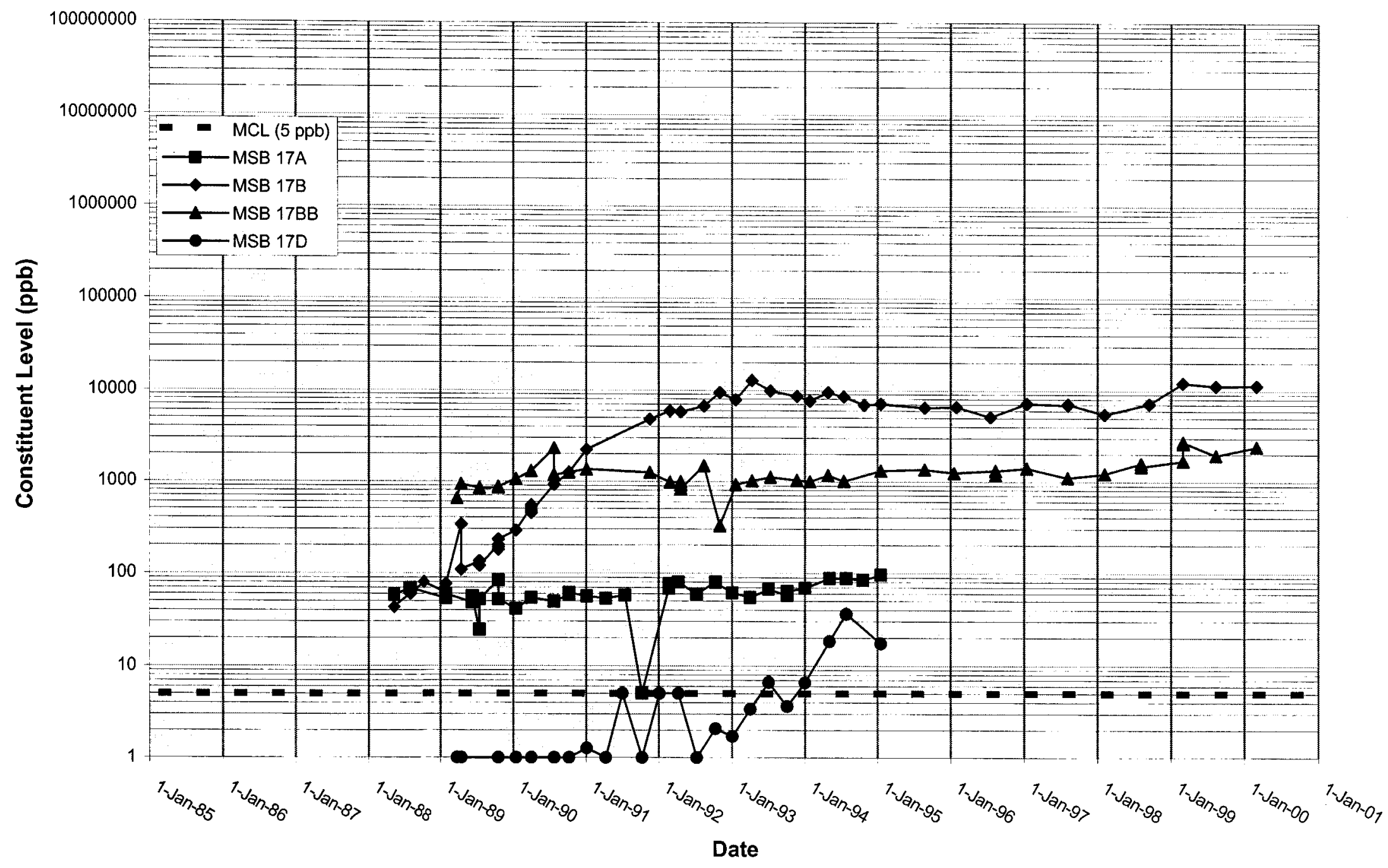


WSRC-TR-2000-00242 UNCLASSIFIED

Time Series Plot of Trichloroethylene for Well MSB 18

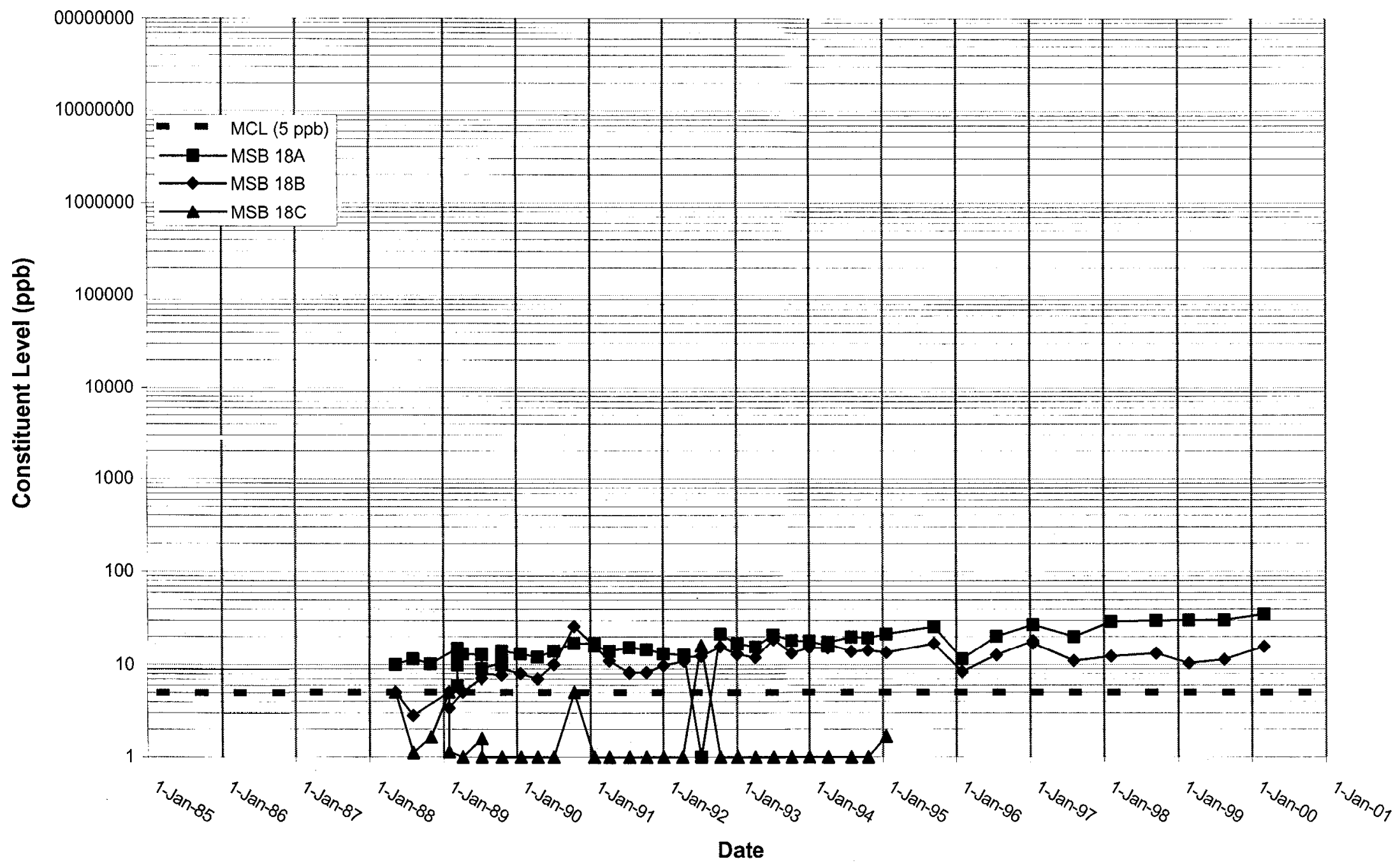


Time Series Plot of Trichloroethylene for Well MSB 19

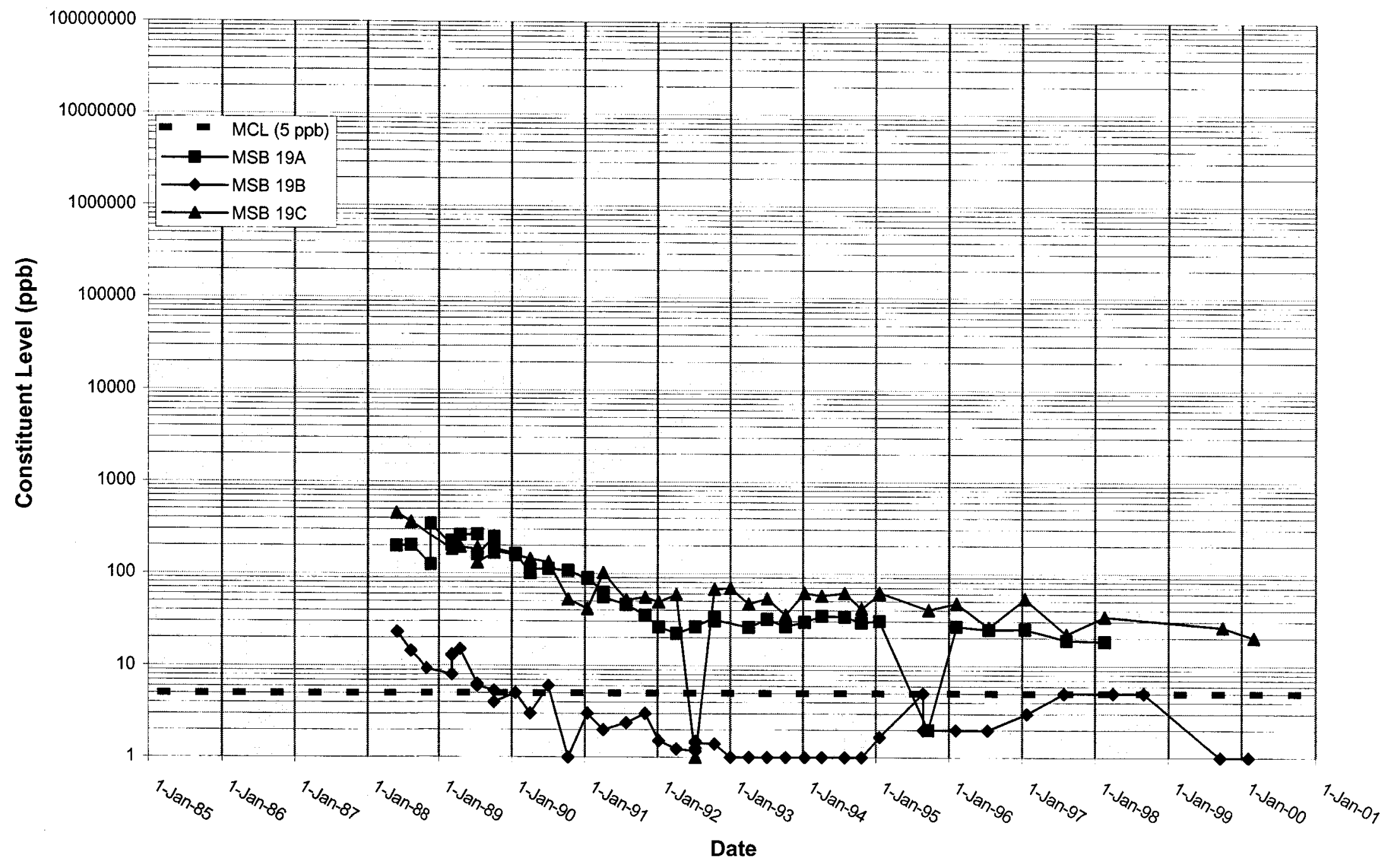


WSRC-TR-2000-00242 UNCLASSIFIED

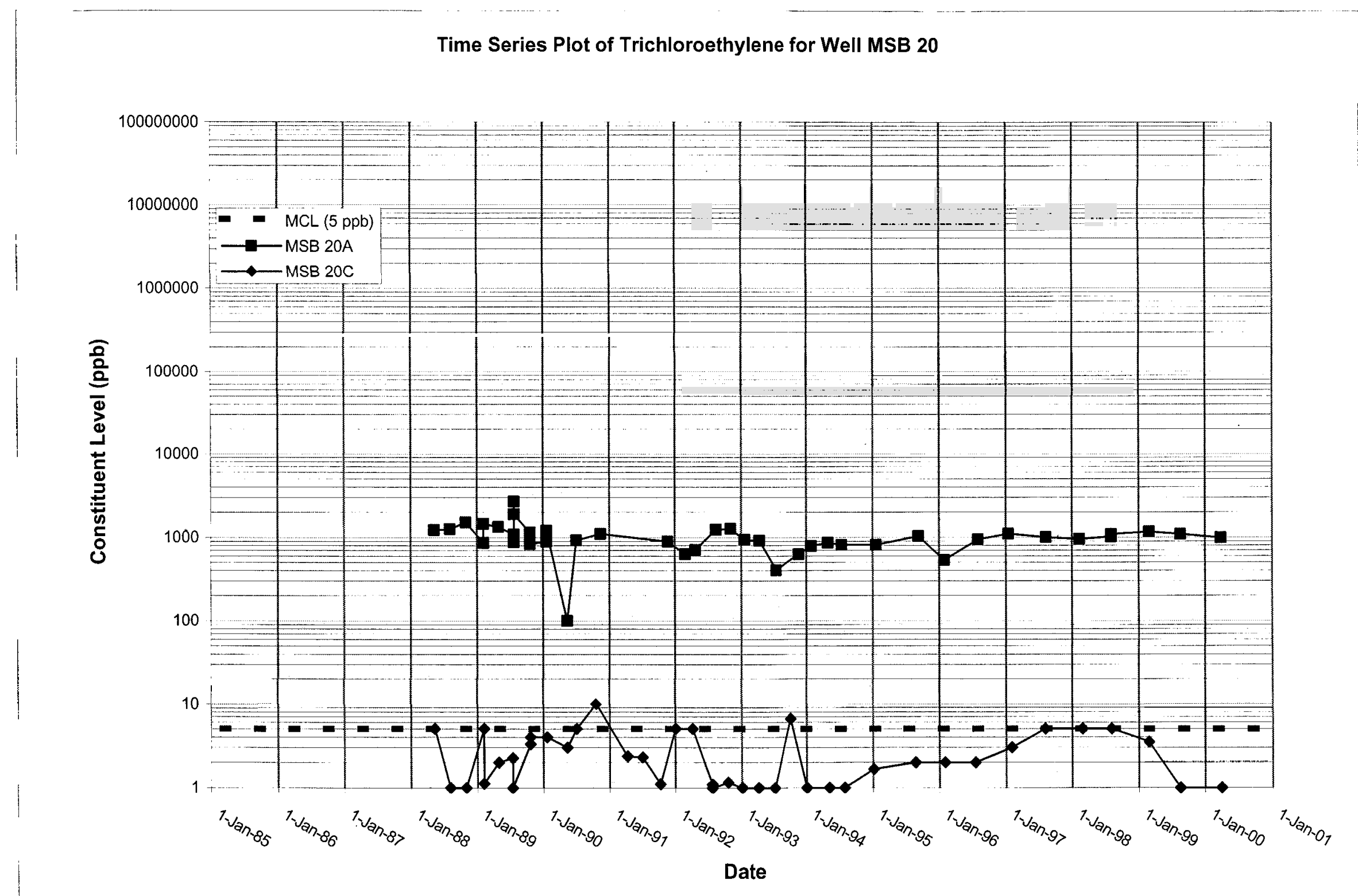




\section{Time Series Plot of Trichloroethylene for Well MSB 21}

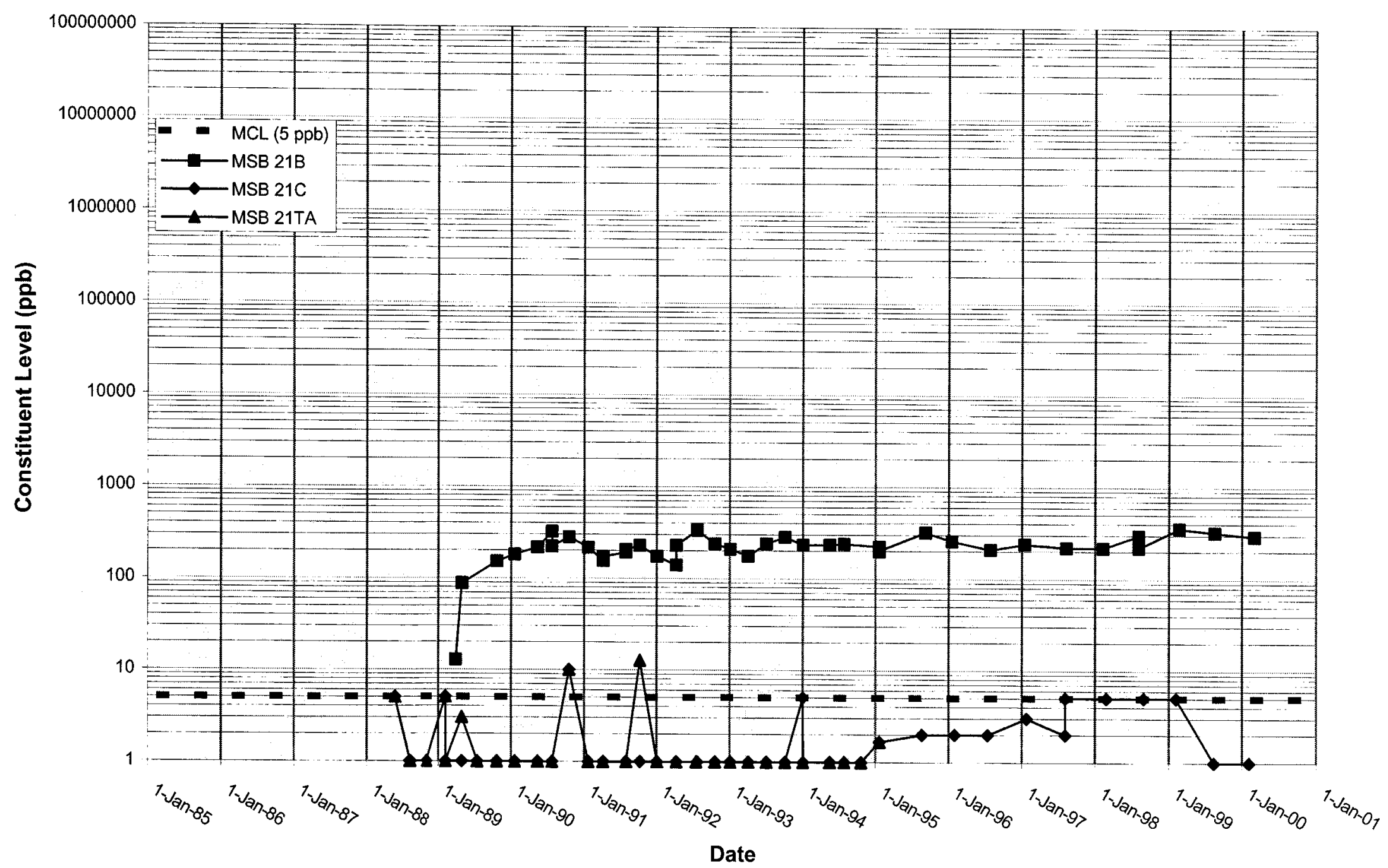


WSRC-TR-2000-00242 UNCLASSIFIED

\section{Time Series Plot of Trichloroethylene for Well MSB 22}

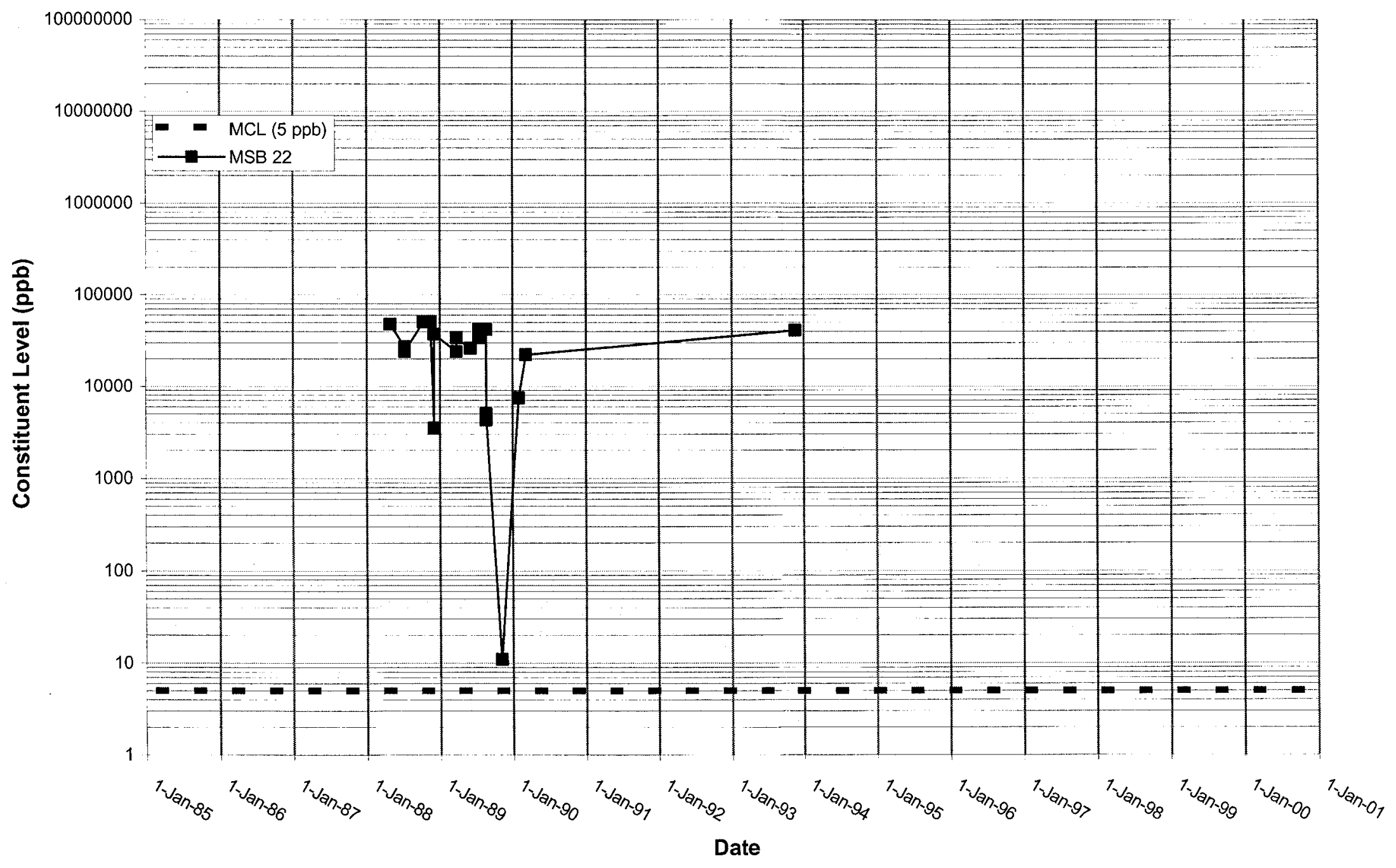


Time Series Plot of Trichloroethylene for Well MSB 23

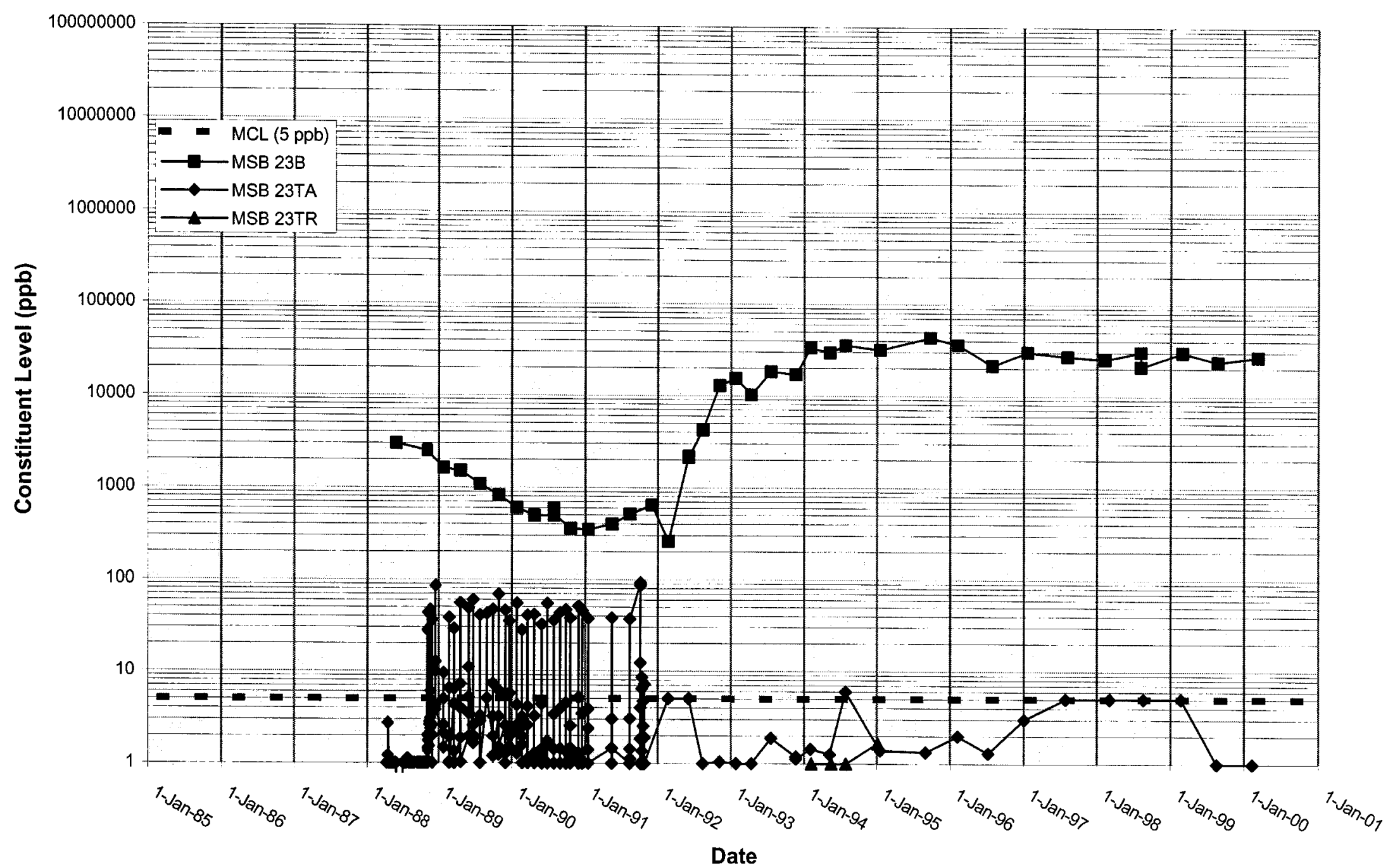


WSRC-TR-2000-00242 UNCLASSIFIED

Time Series Plot of Trichloroethylene for Well MSB 24

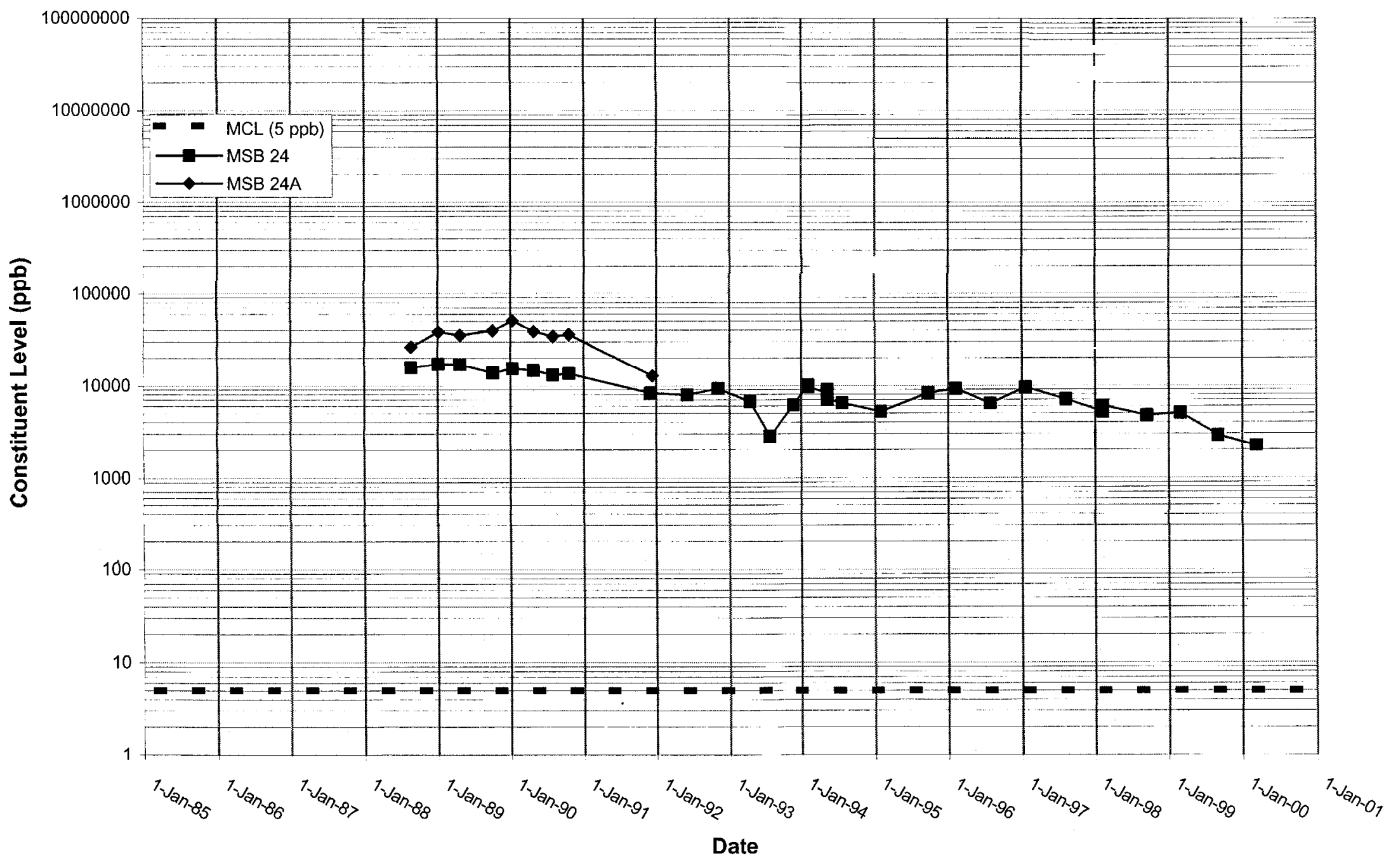


Time Series Plot of Trichloroethylene for Well MSB 25

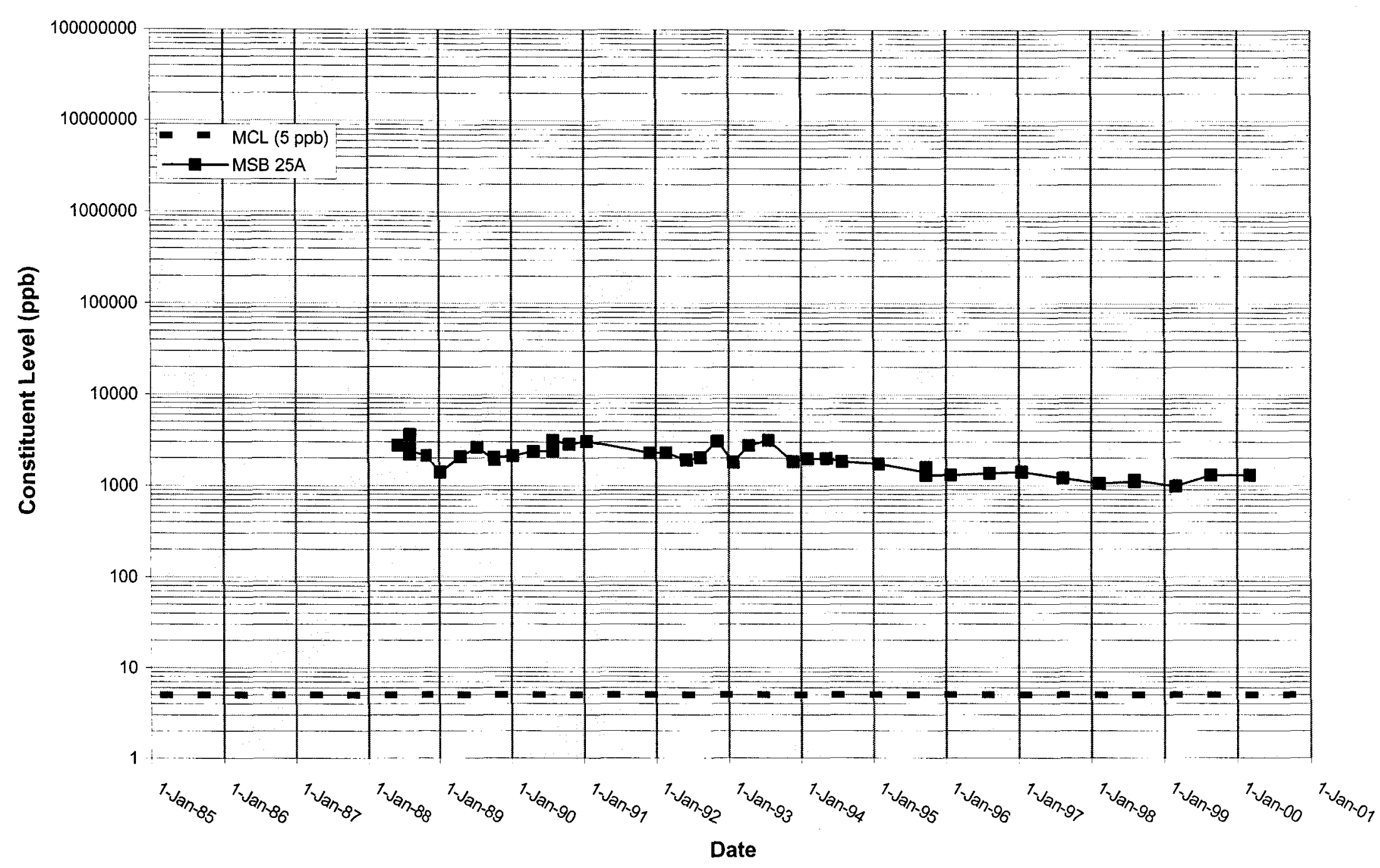


WSRC-TR-2000-00242 UNCLASSIFIED

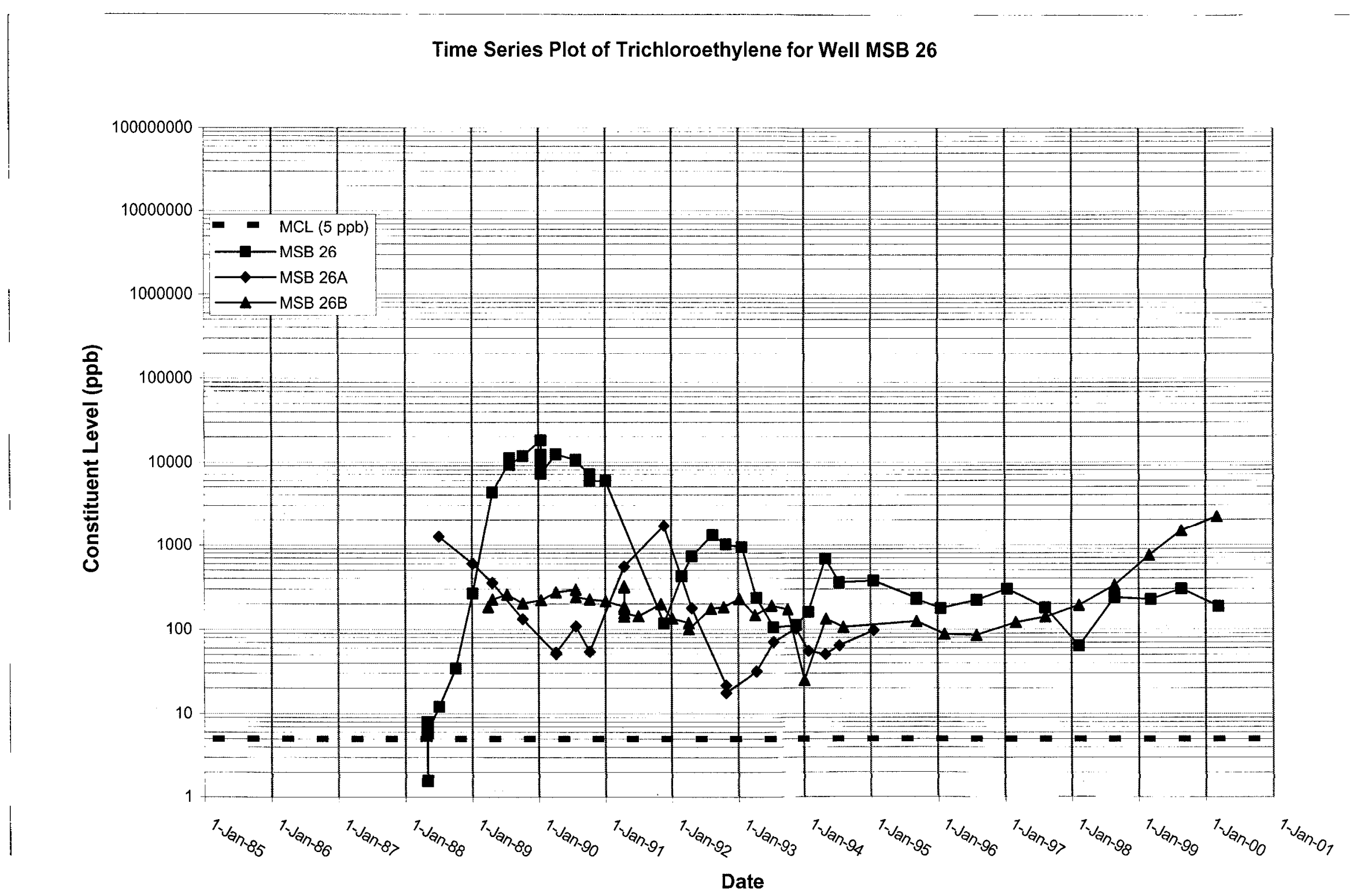


Time Series Plot of Trichloroethylene for Well MSB 27

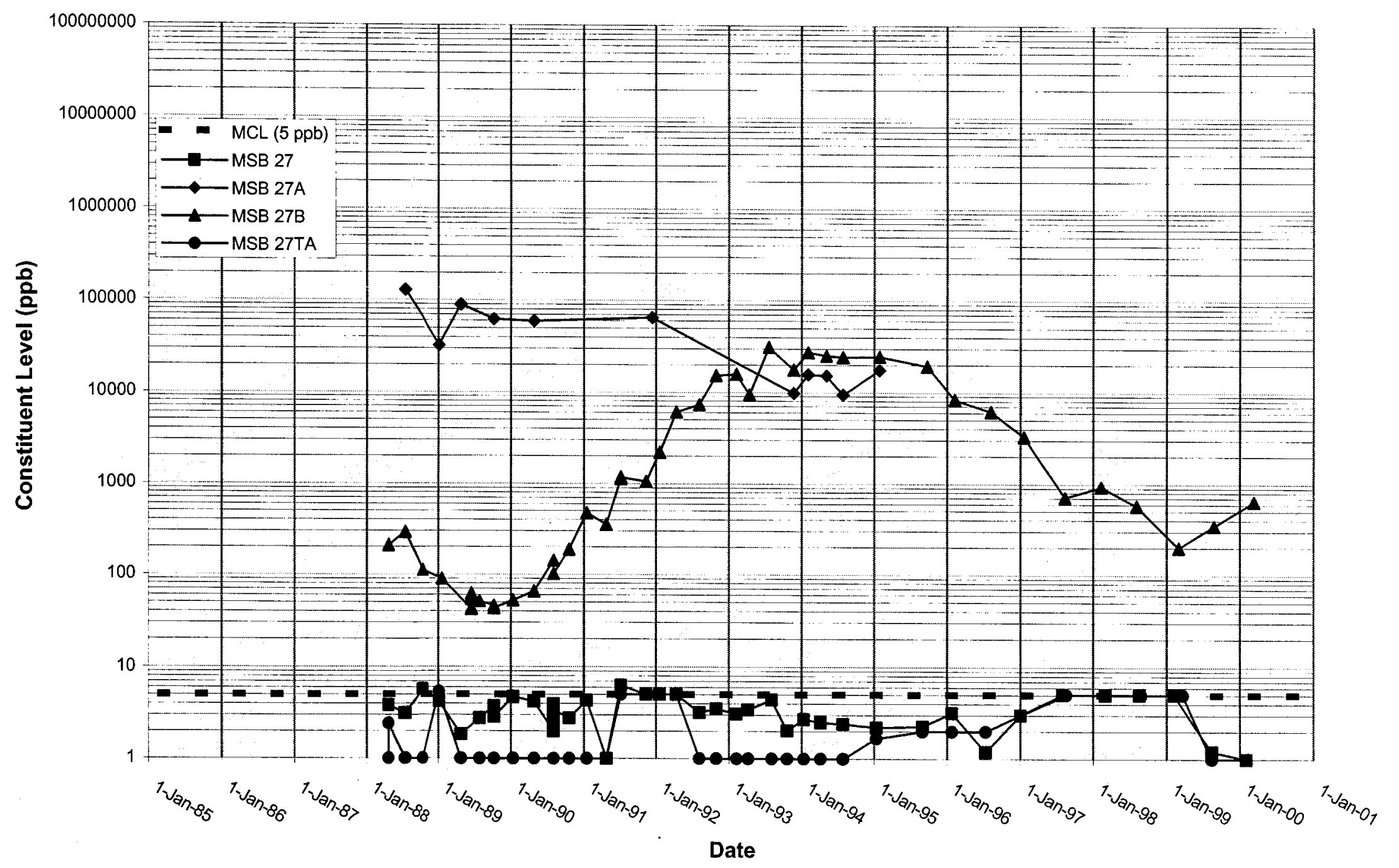


Time Series Plot of Trichloroethylene for Well MSB 28

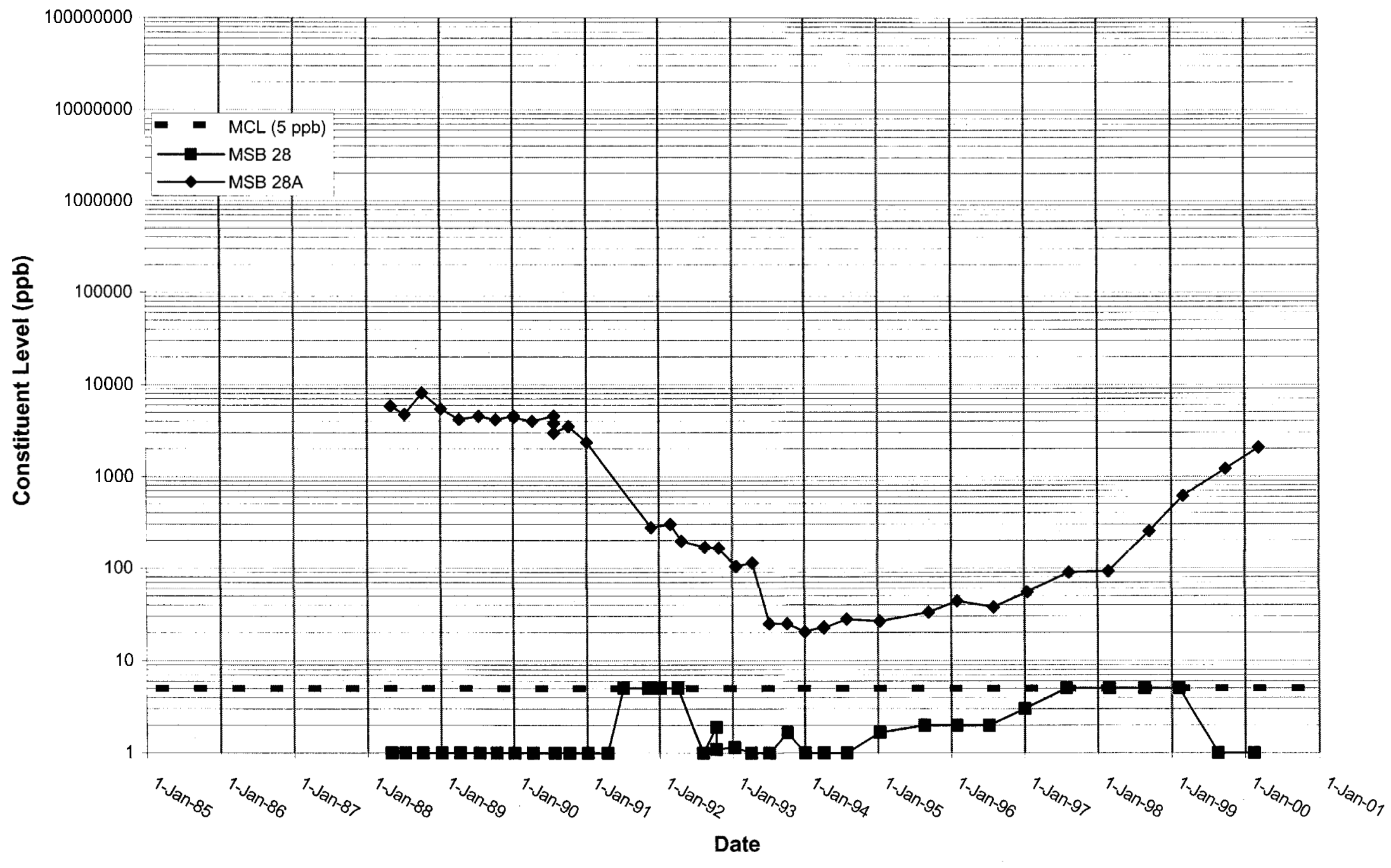




\section{Time Series Plot of Trichloroethylene for Well MSB 29}

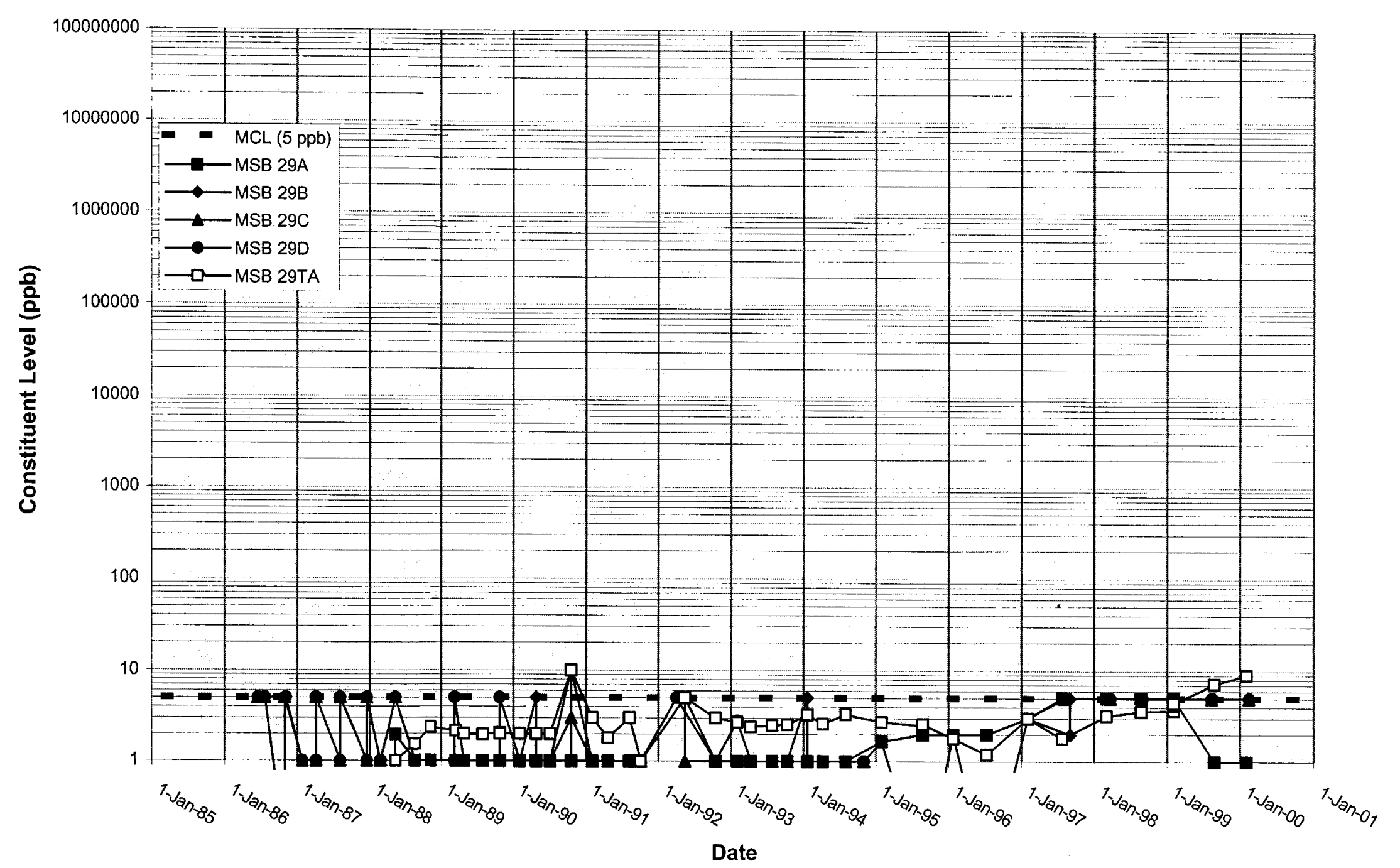


WSRC-TR-2000-00242 UNCLASSIFIED

Time Series Plot of Trichloroethylene for Well MSB 30

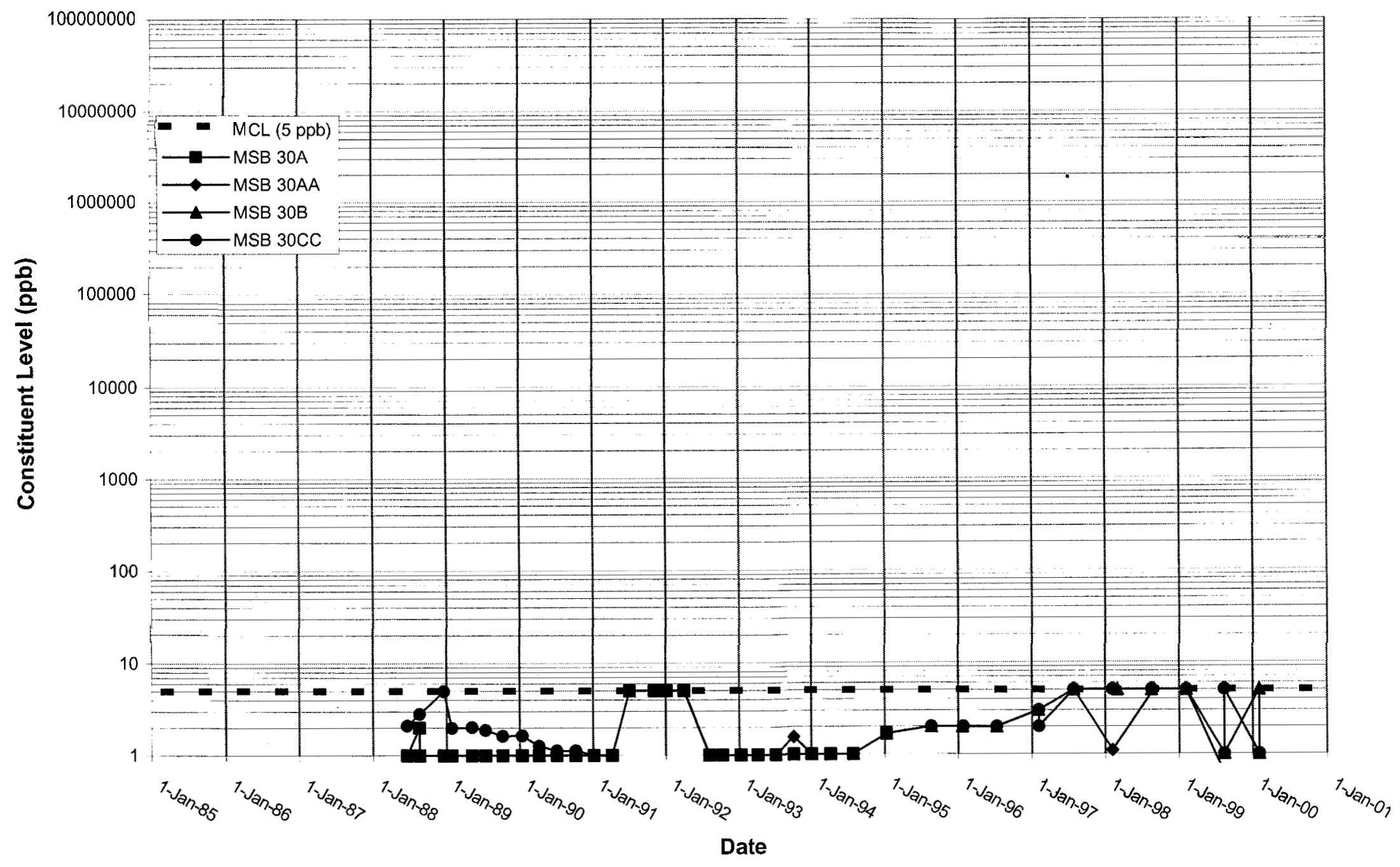


WSRC-TR-2000-00242 UNCLASSIFIED

Time Series Plot of Trichloroethylene for Well MSB 31

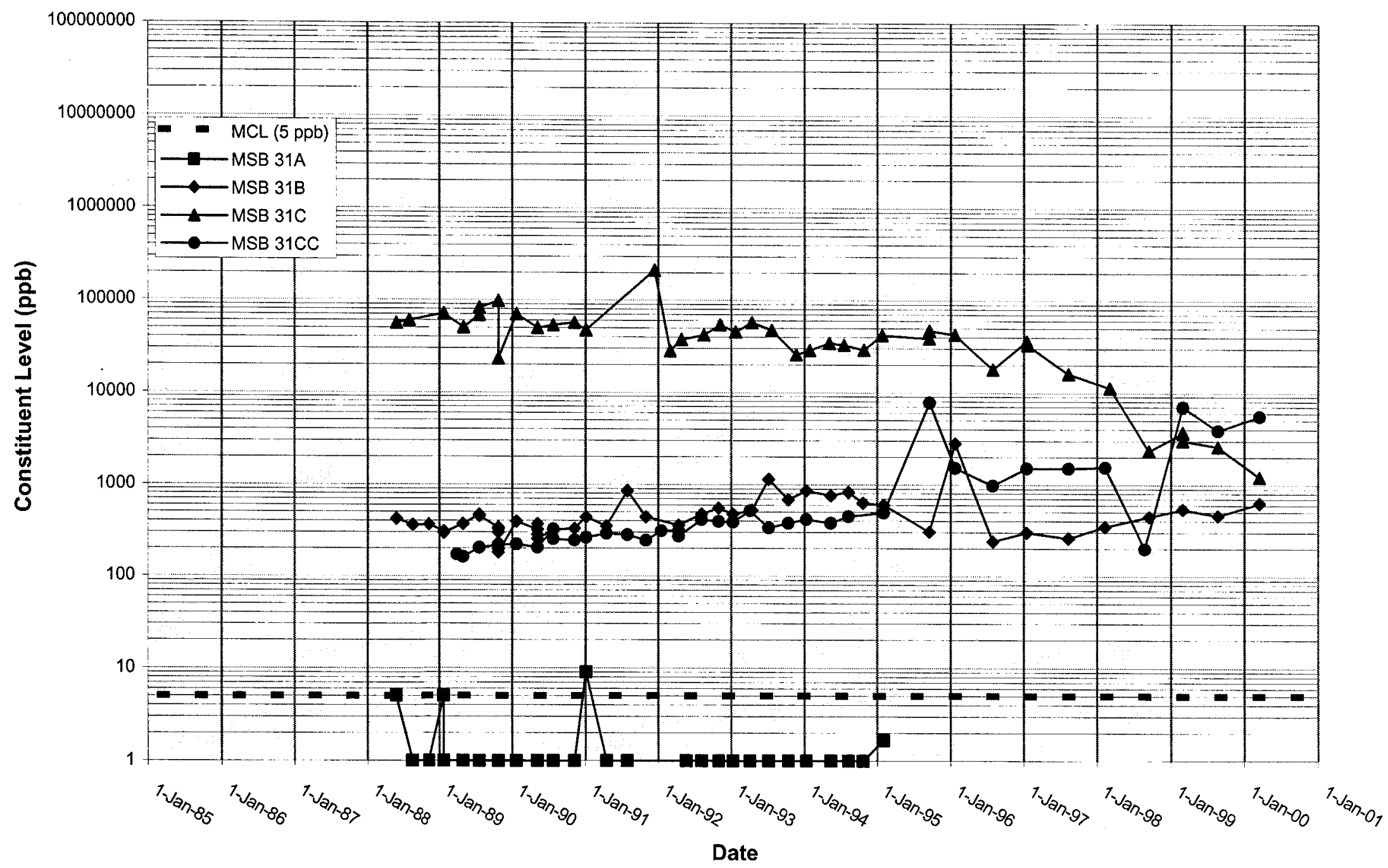


WSRC-TR-2000-00242 UNCLASSIFIED

Time Series Plot of Trichloroethylene for Well MSB 32

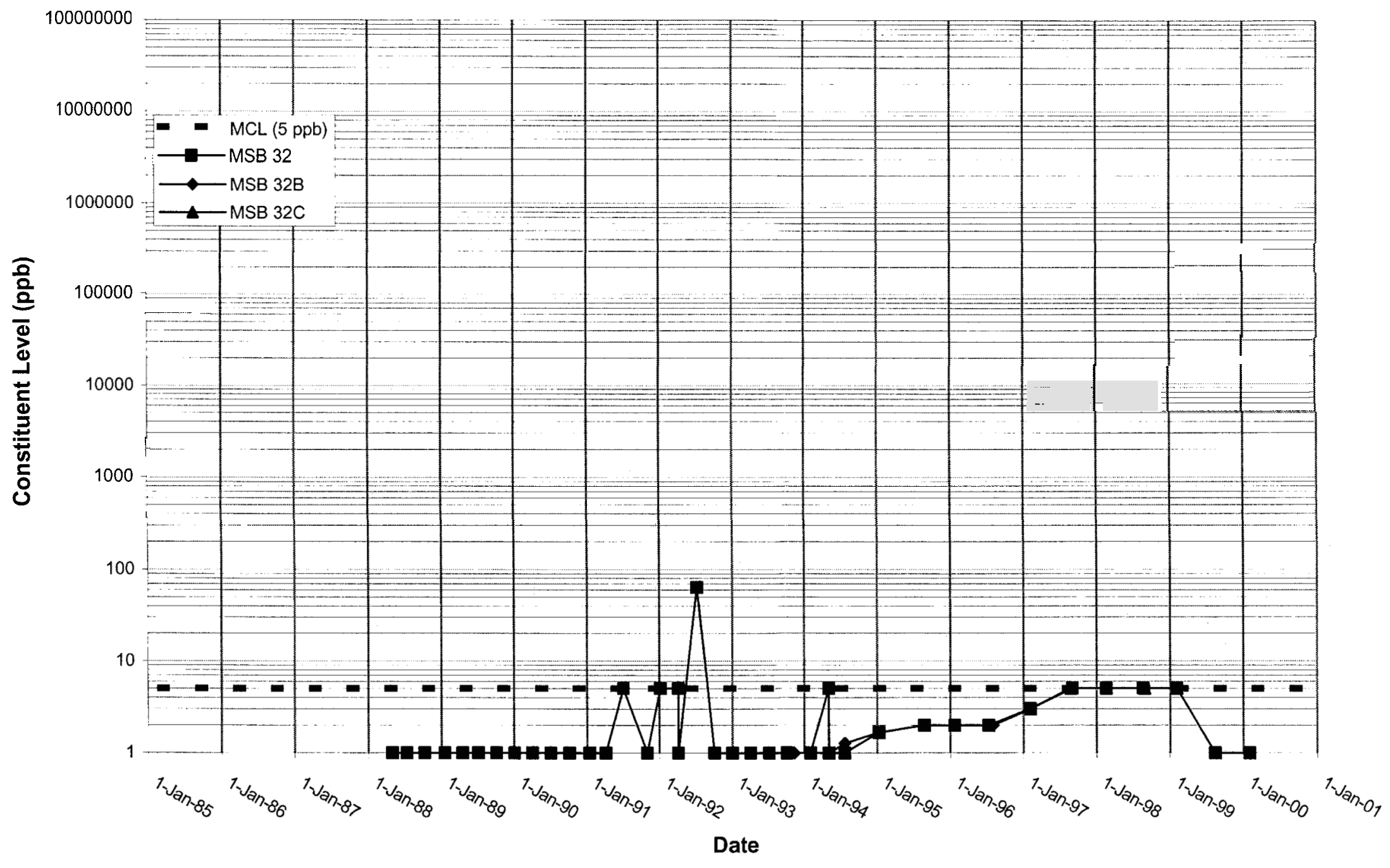


Time Series Plot of Trichloroethylene for Well MSB 33

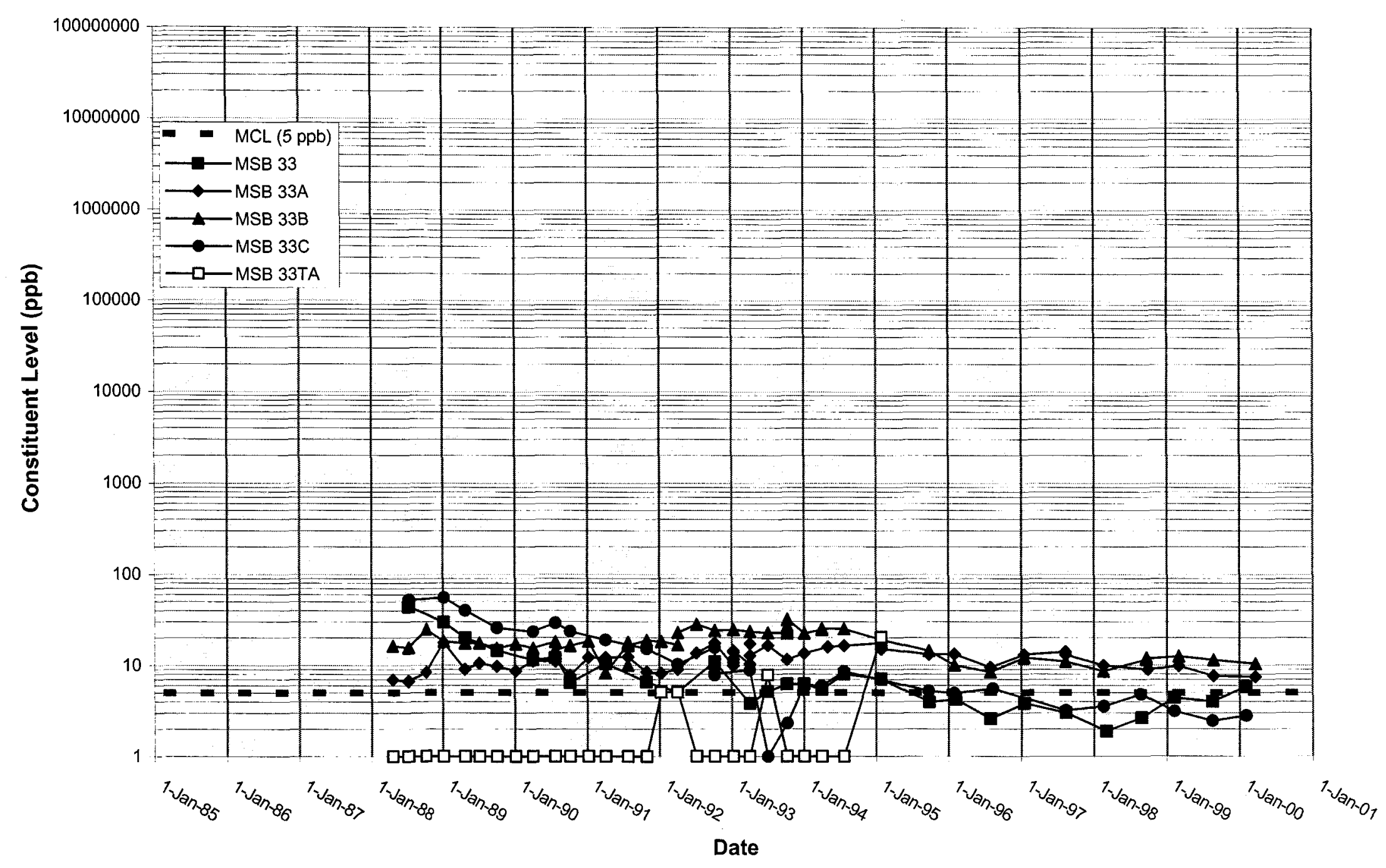


WSRC-TR-2000-00242 UNCLASSIFIED

Time Series Plot of Trichloroethylene for Well MSB 34

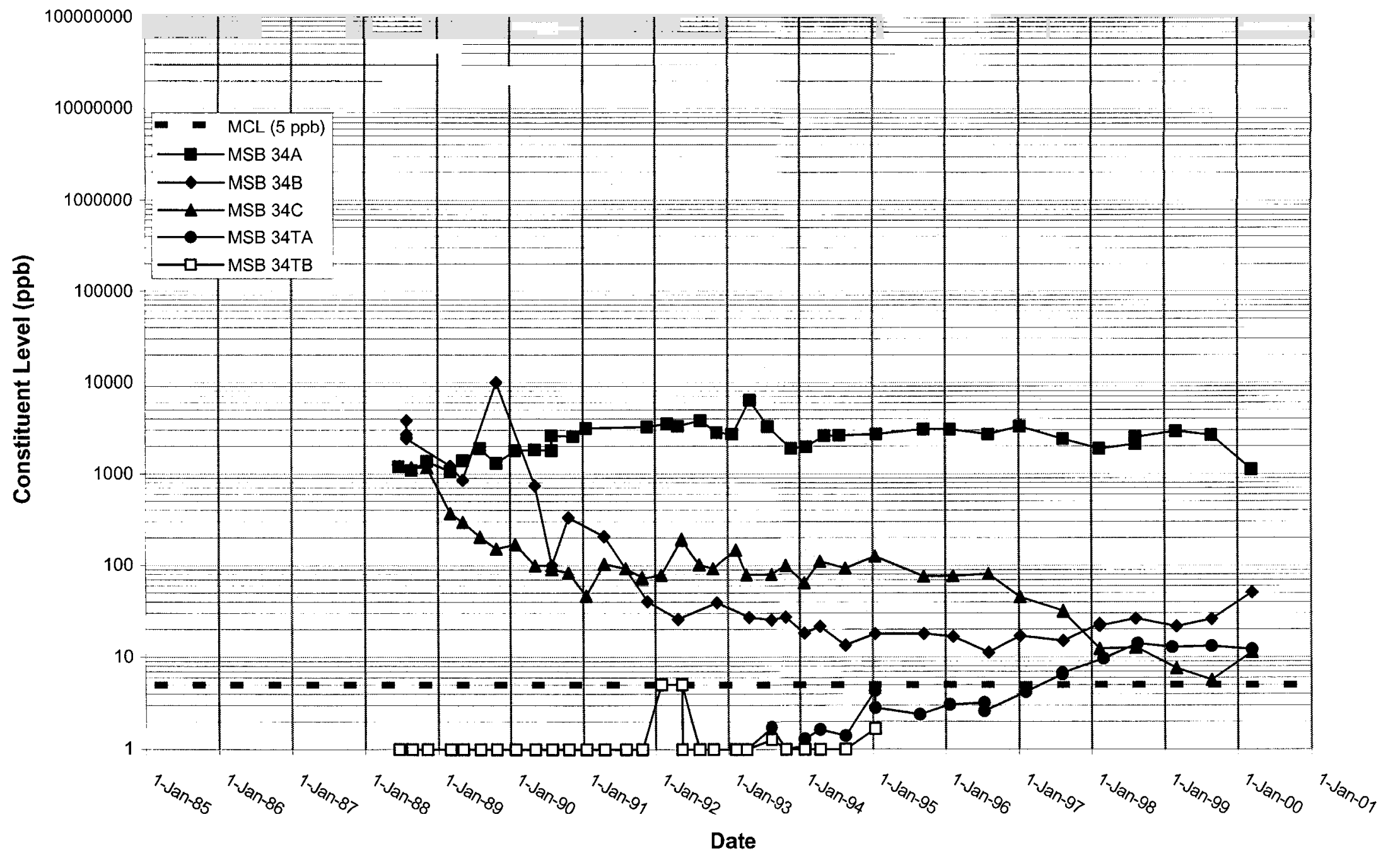


WSRC-TR-2000-00242 UNCLASSIFIED

Time Series Plot of Trichloroethylene for Well MSB 35

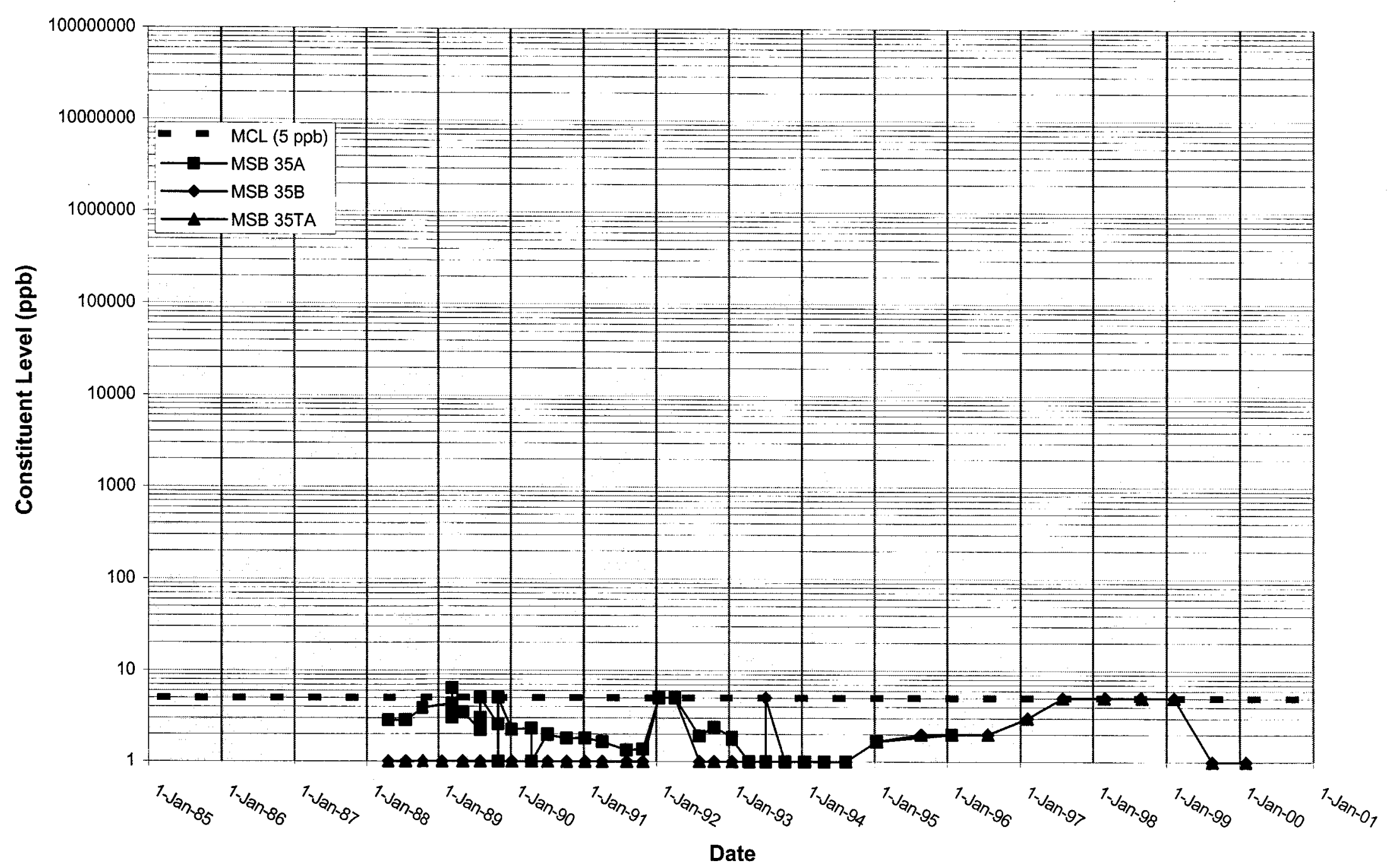




\section{Time Series Plot of Trichloroethylene for Well MSB 36}

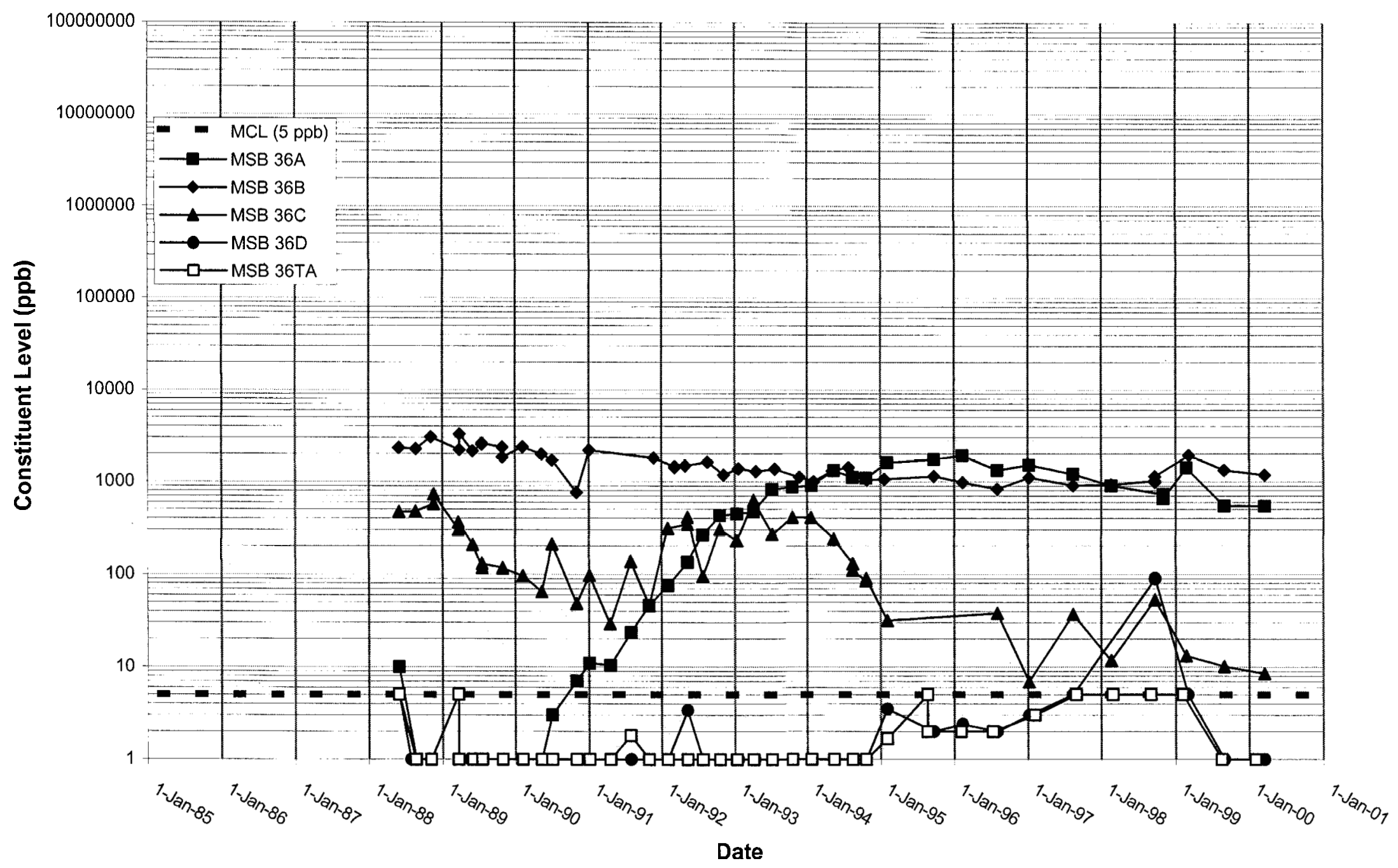


Time Series Plot of Trichloroethylene for Well MSB 37

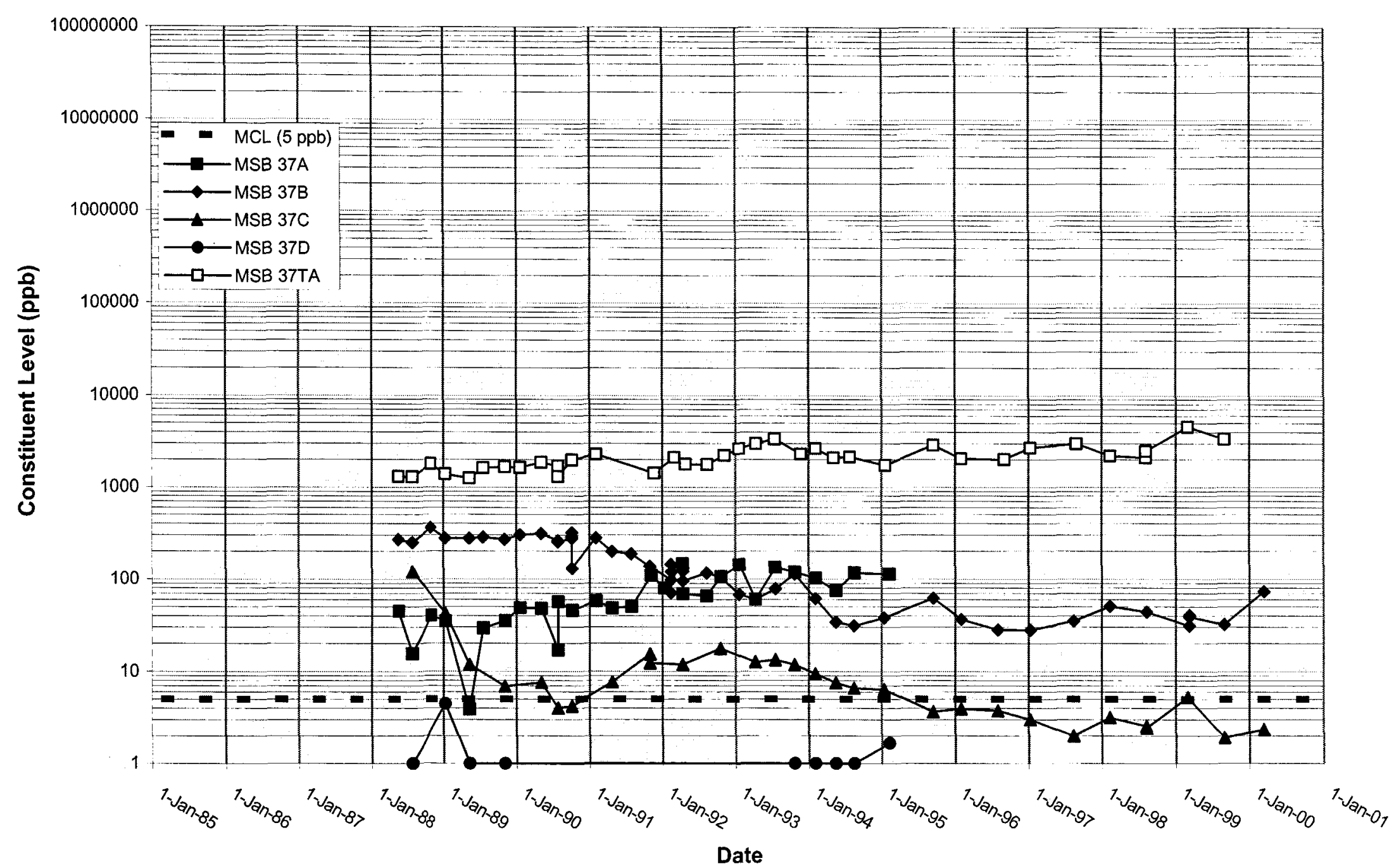




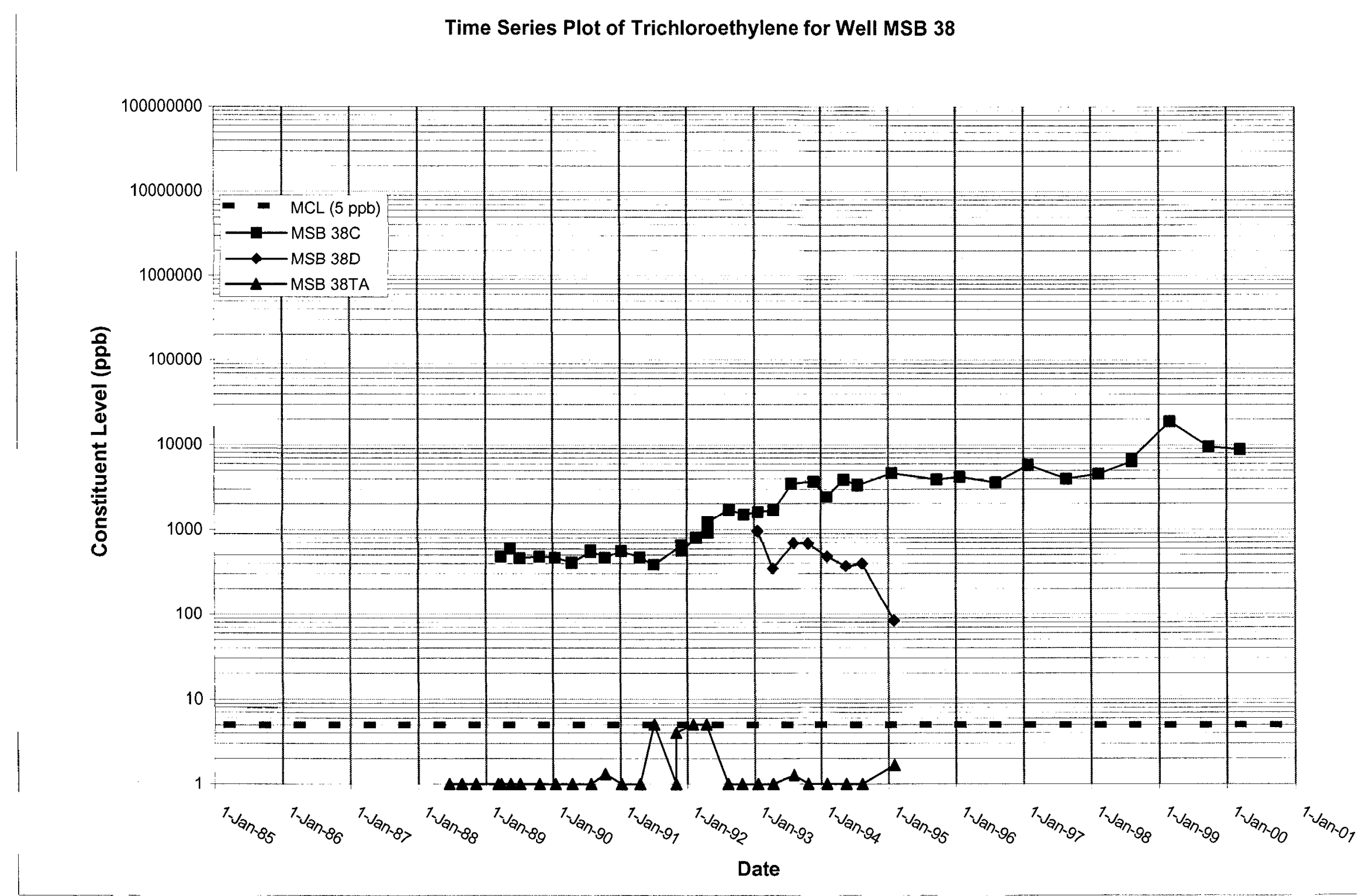




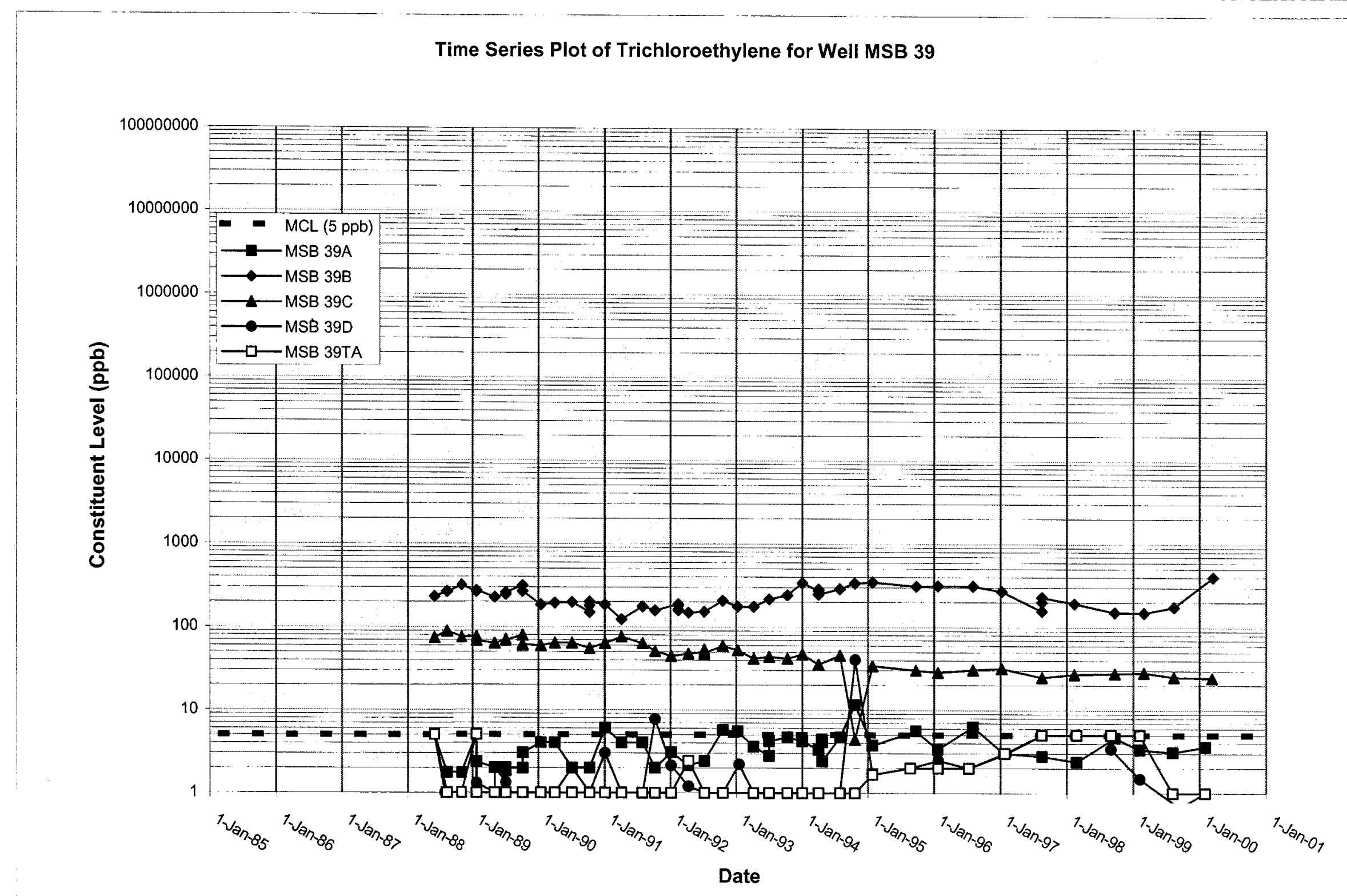




\section{Time Series Plot of Trichloroethylene for Well MSB 40}

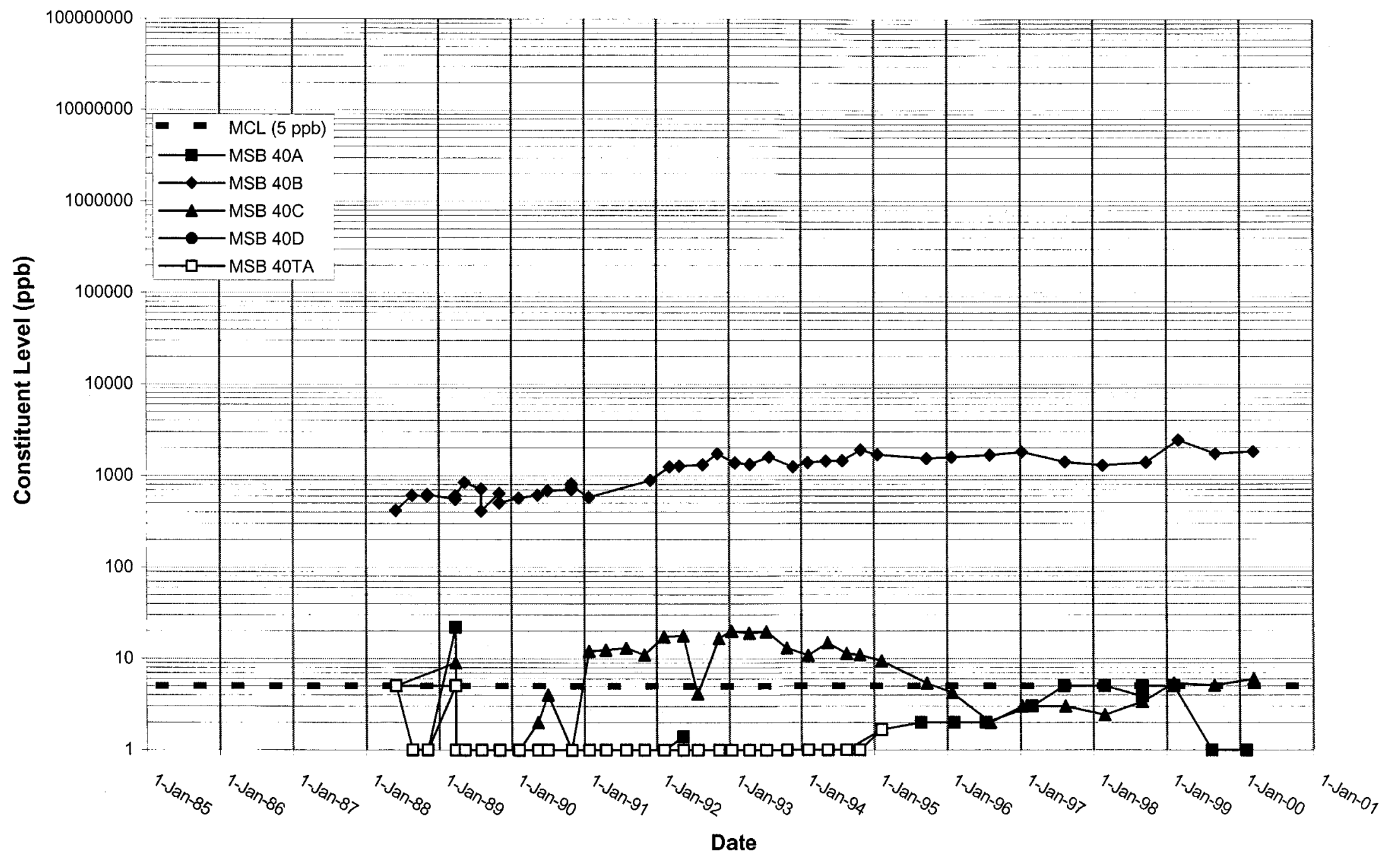




\section{Time Series Plot of Trichloroethylene for Well MSB 41}

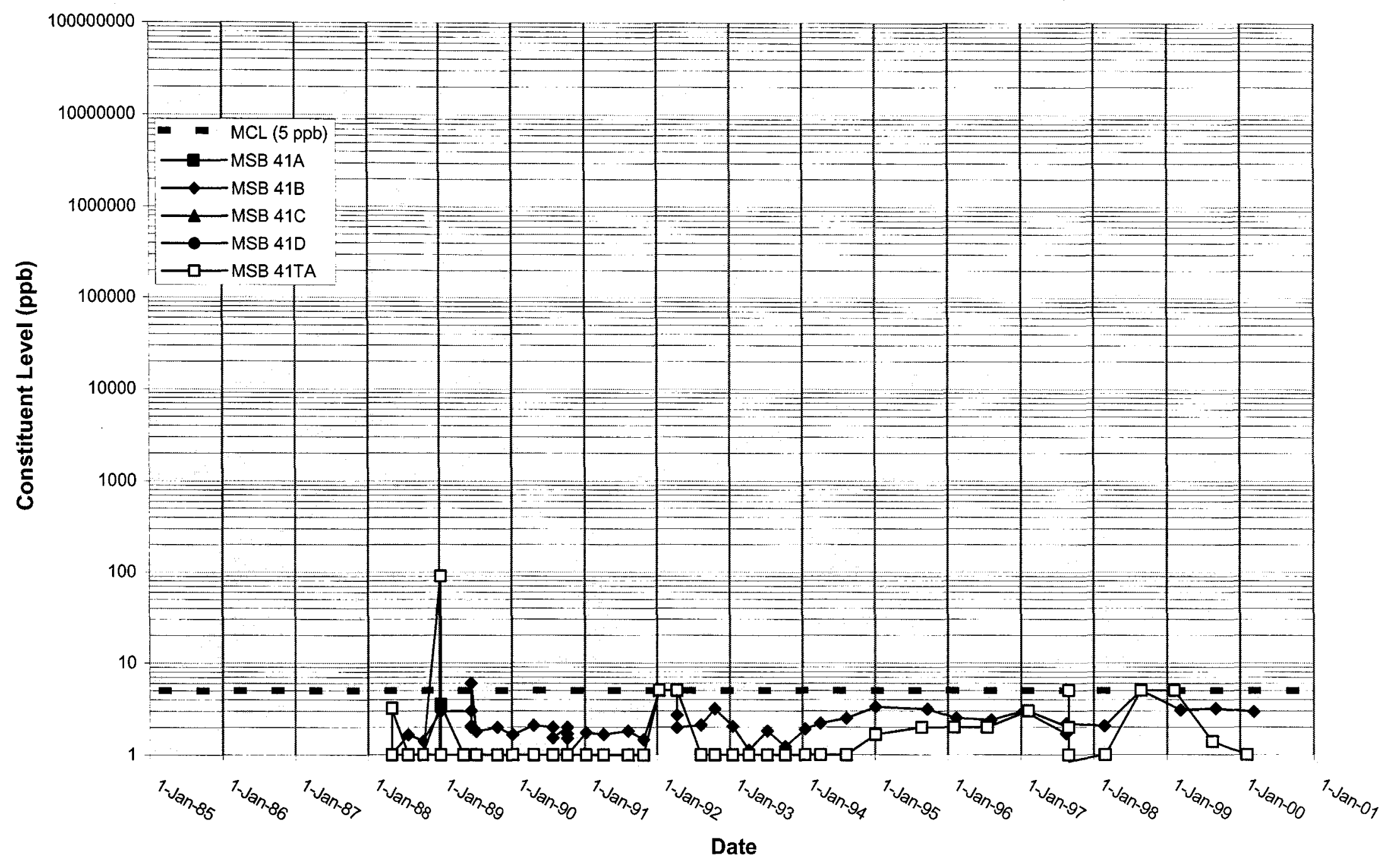




\section{Time Series Plot of Trichloroethylene for Well MSB 42}

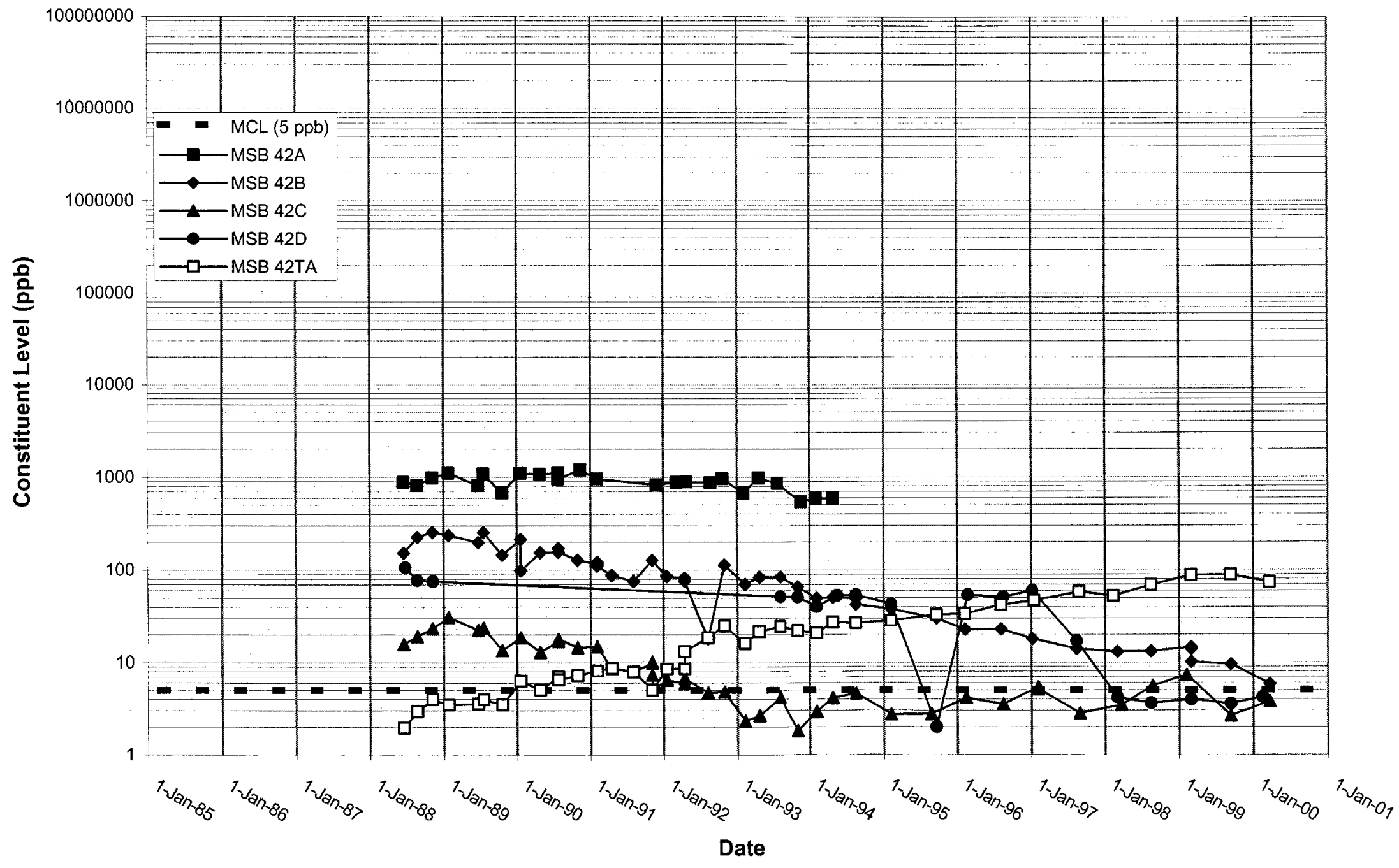


Time Series Plot of Trichloroethylene for Well MSB 43

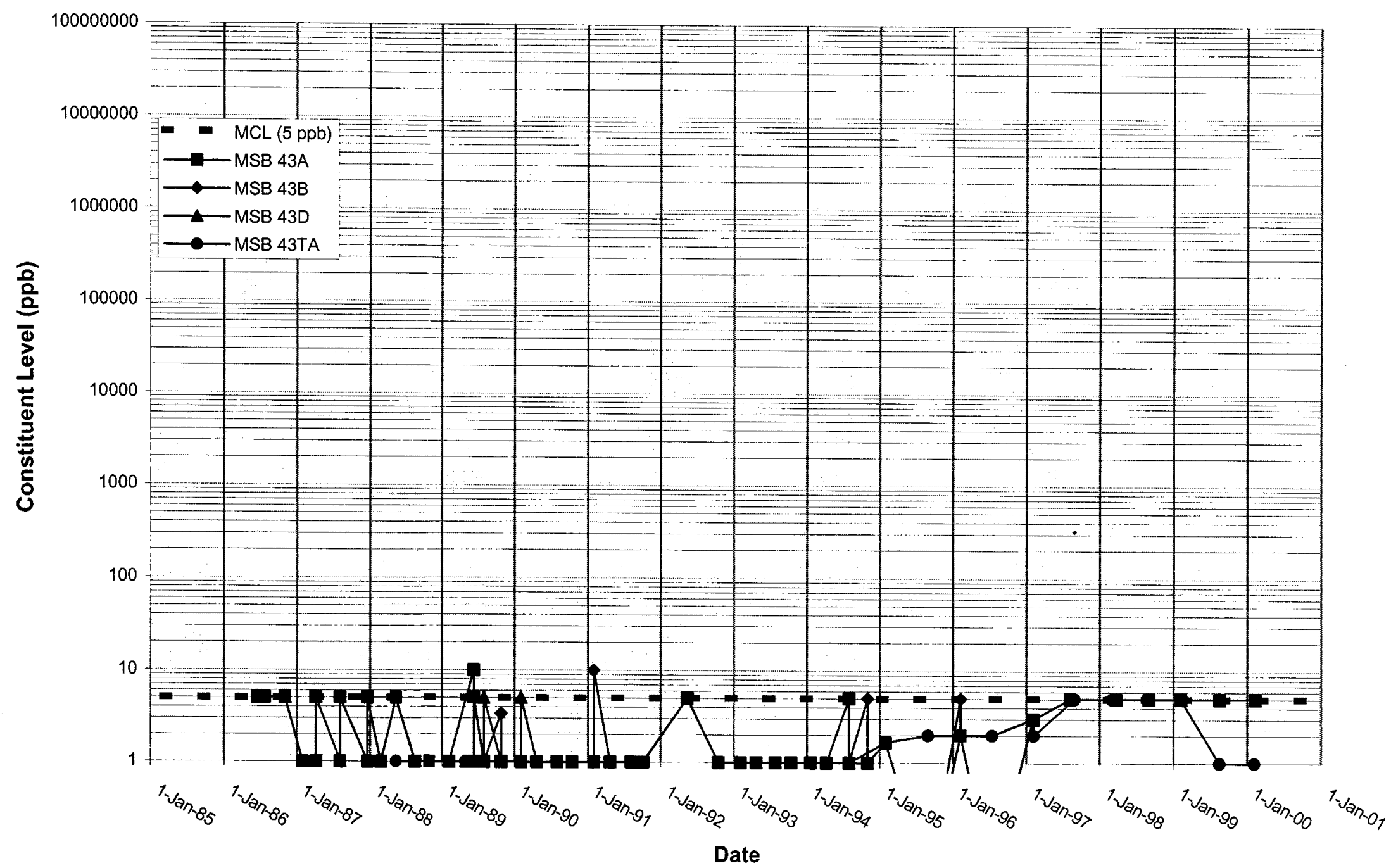




\section{Time Series Plot of Trichloroethylene for Well MSB 44}

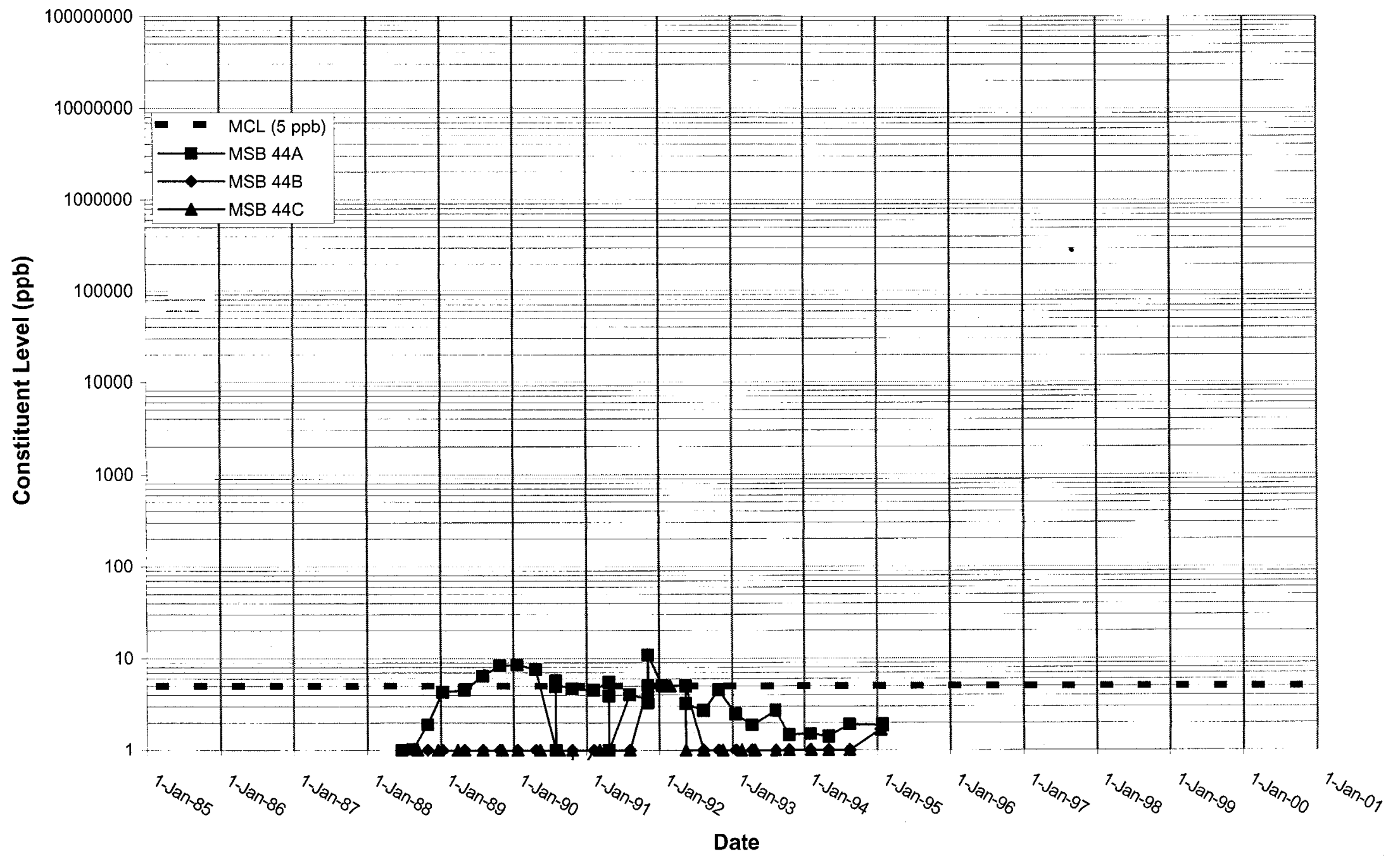




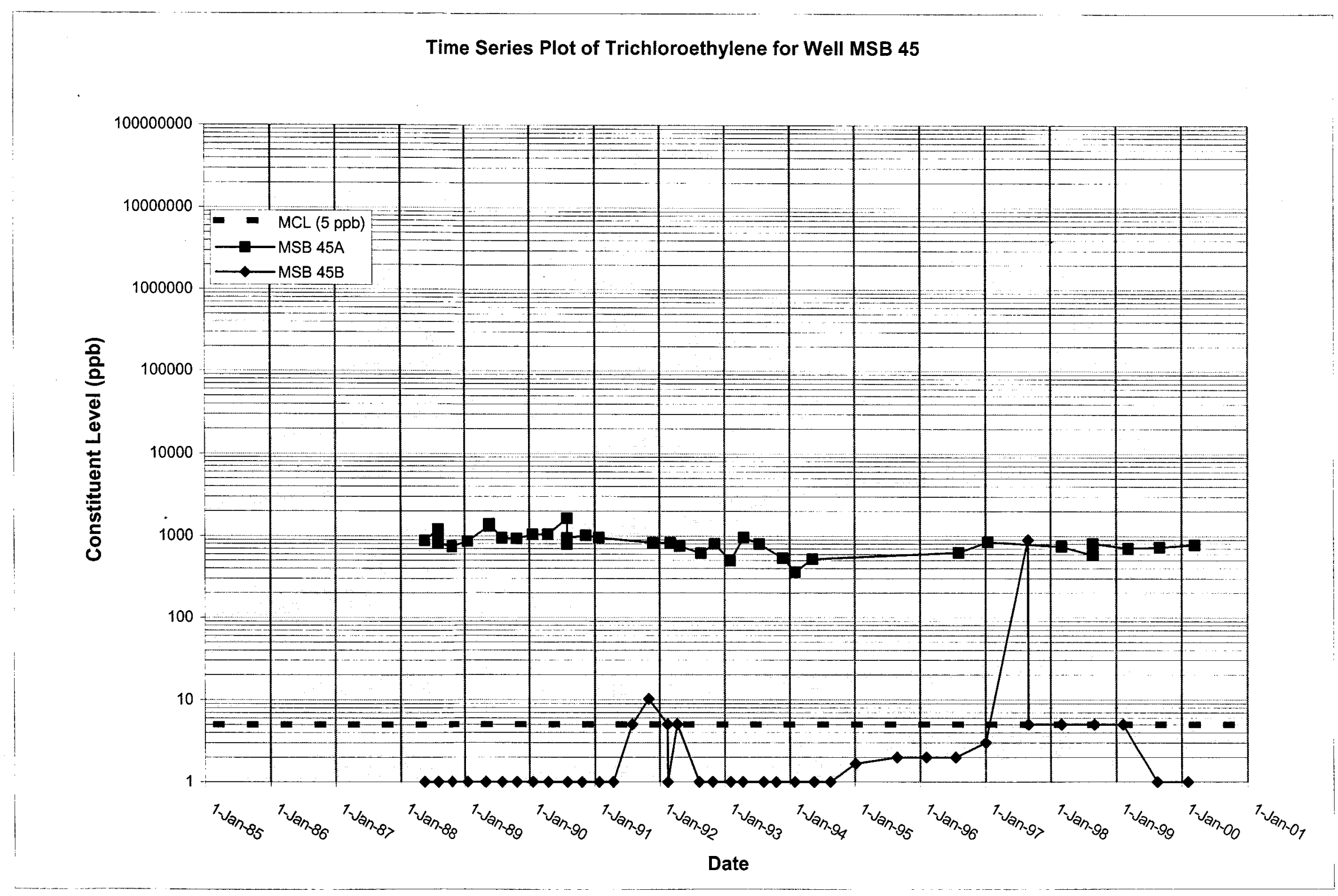


WSRC-TR-2000-00242 UNCLASSIFIED

Time Series Plot of Trichloroethylene for Well MSB 46

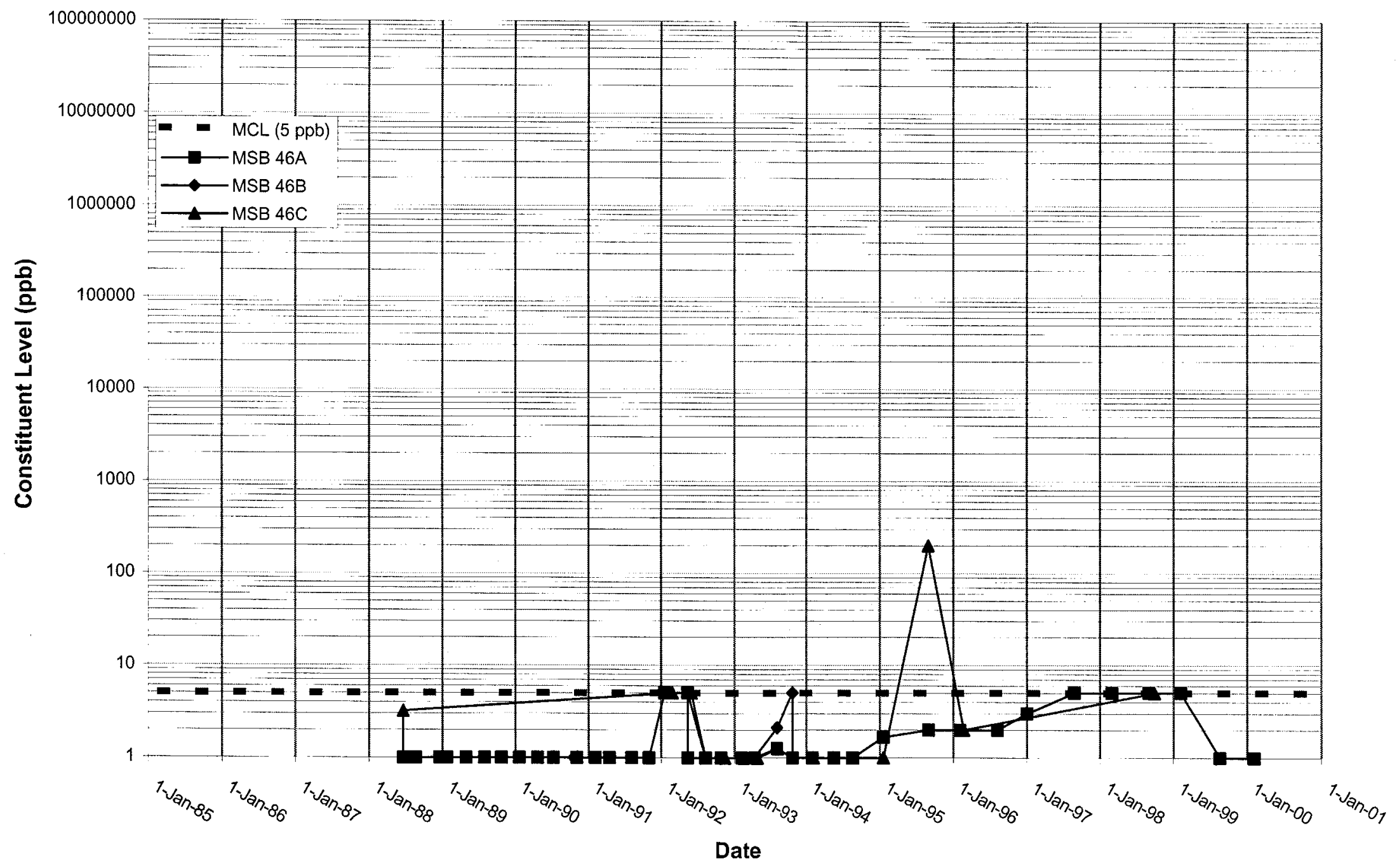




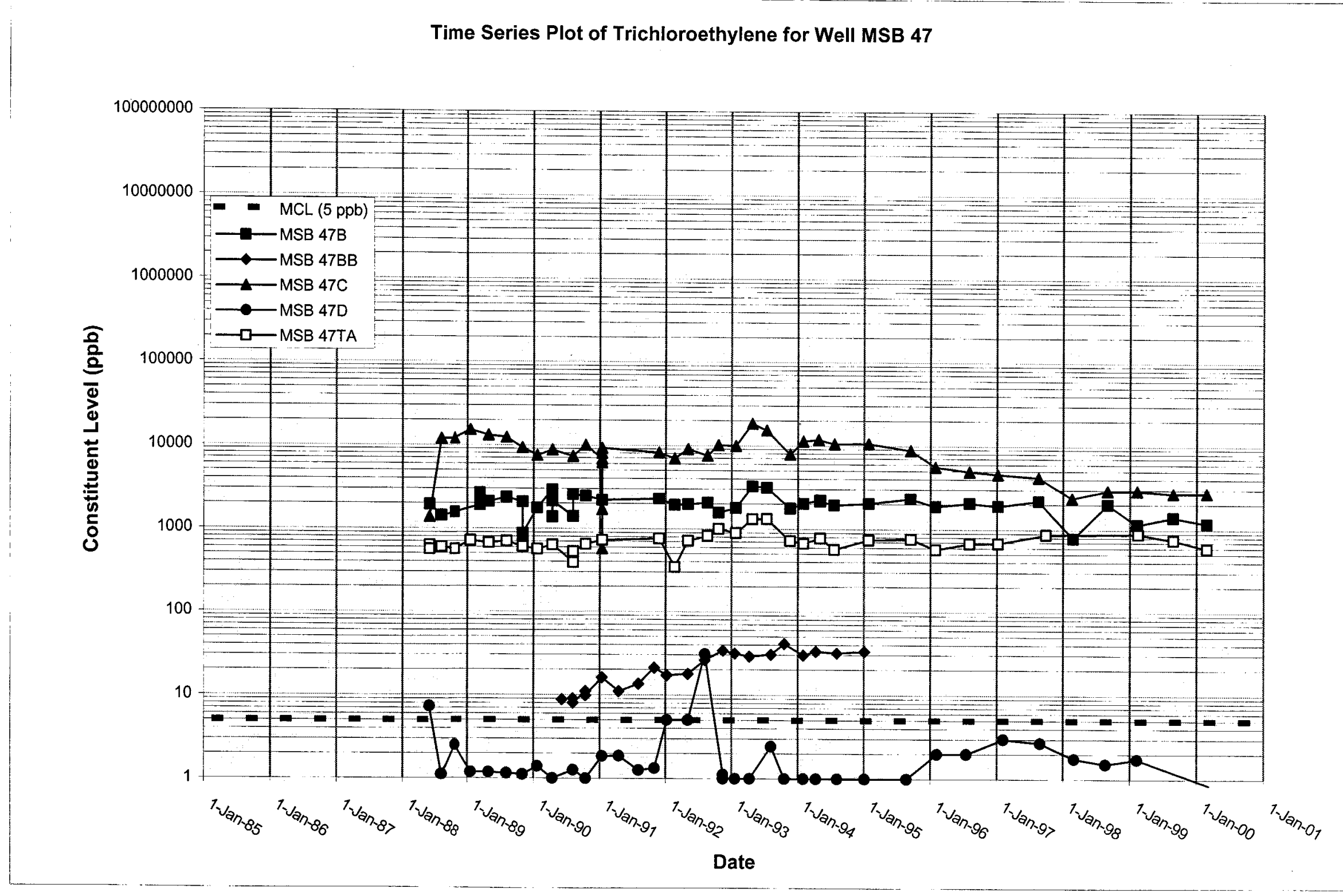


WSRC-TR-2000-00242 UNCLASSIFIED

Time Series Plot of Trichloroethylene for Well MSB 48

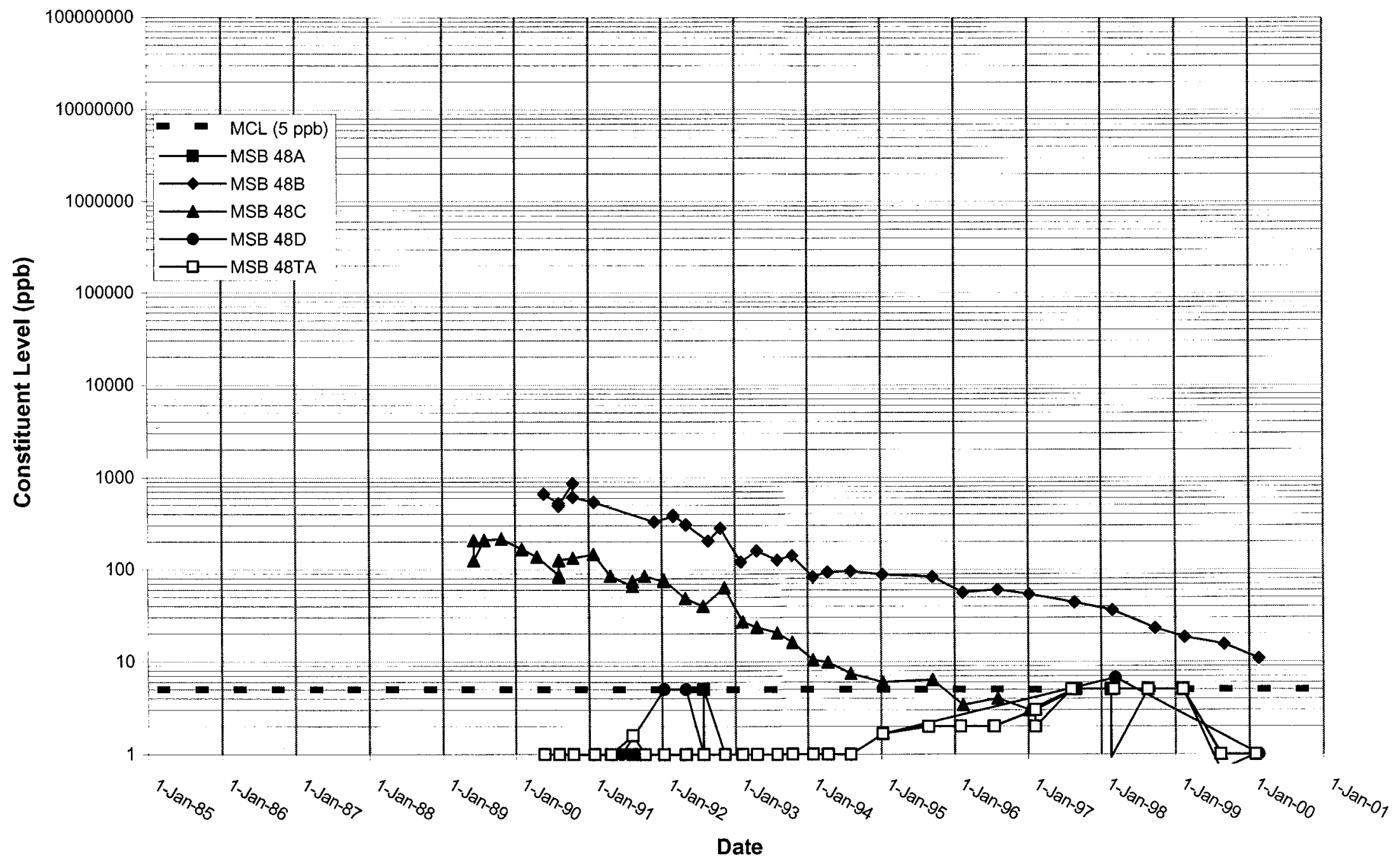




\section{Time Series Plot of Trichloroethylene for Well MSB 49}

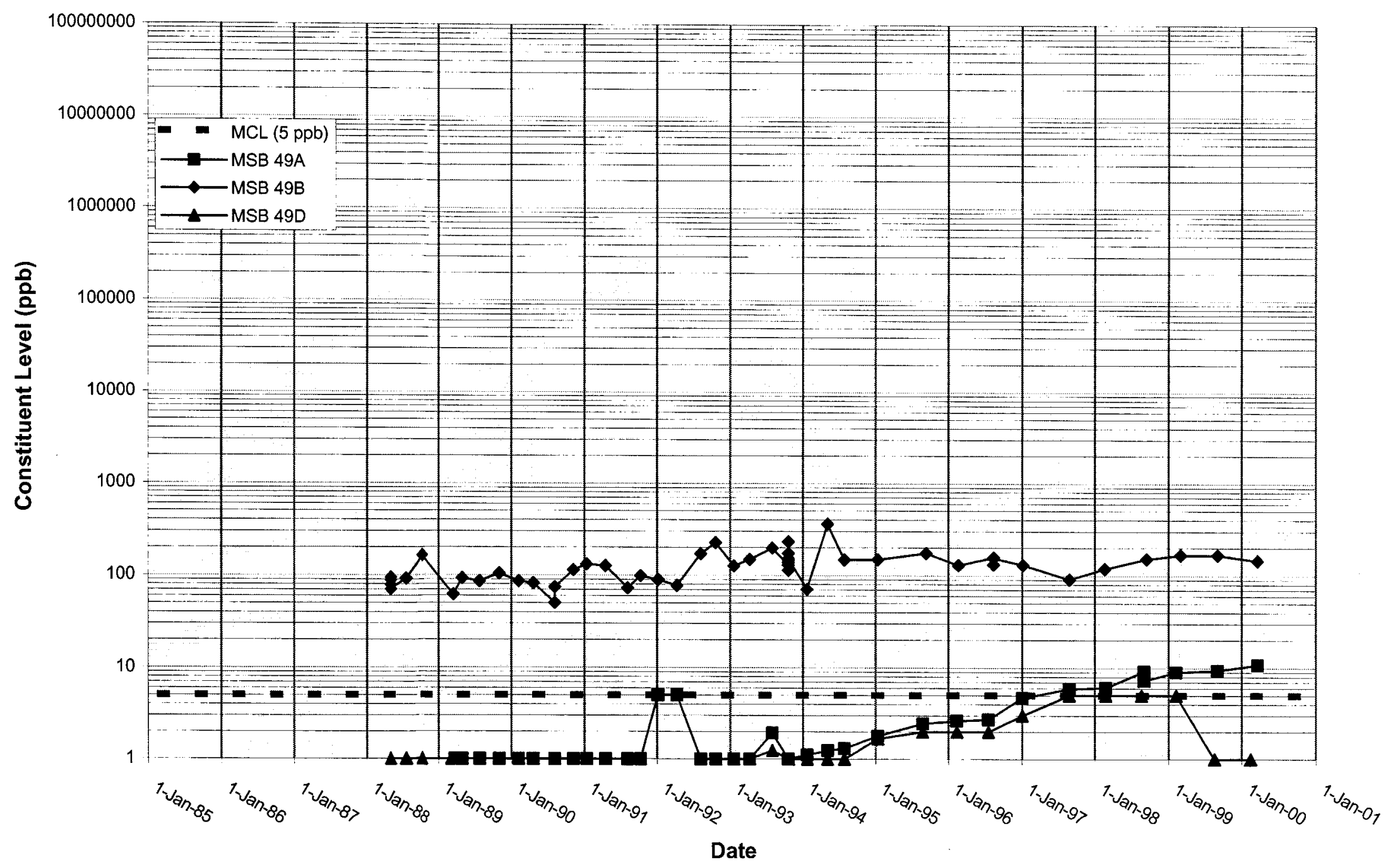


Time Series Plot of Trichloroethylene for Well MSB 50

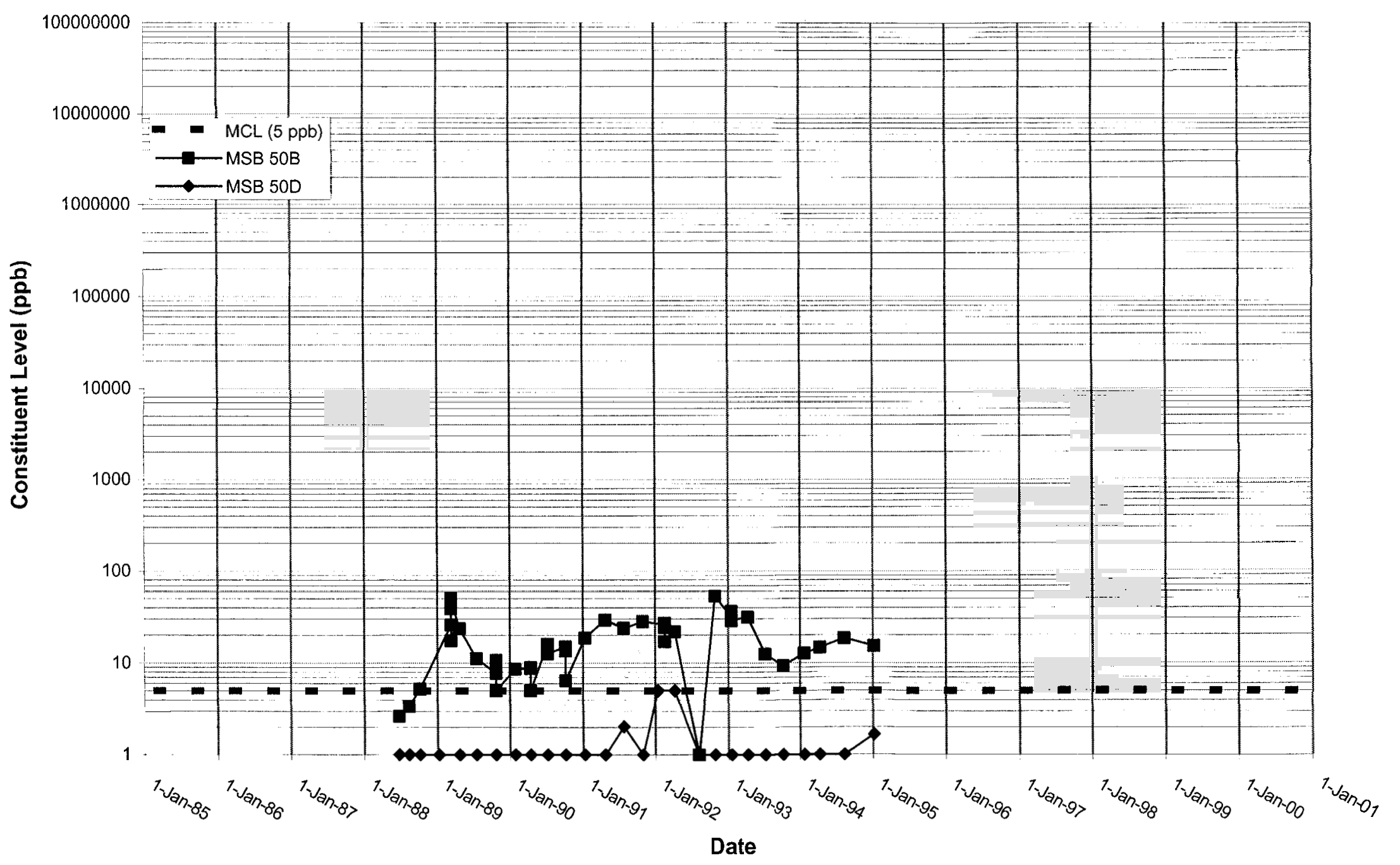


WSRC-TR-2000-00242 UNCLASSIFIED

\section{Time Series Plot of Trichloroethylene for Well MSB 51}

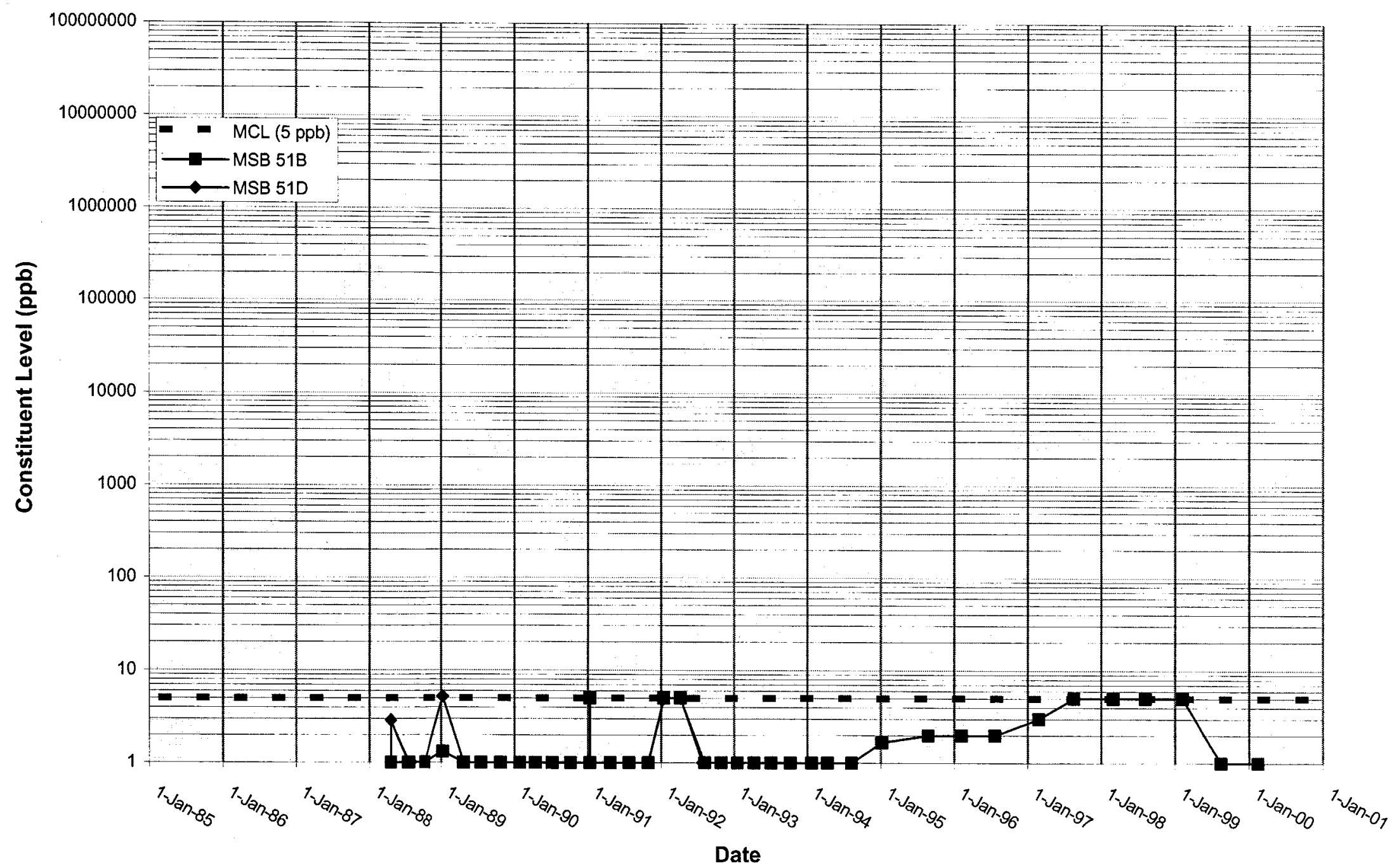




\section{Time Series Plot of Trichloroethylene for Well MSB 52}

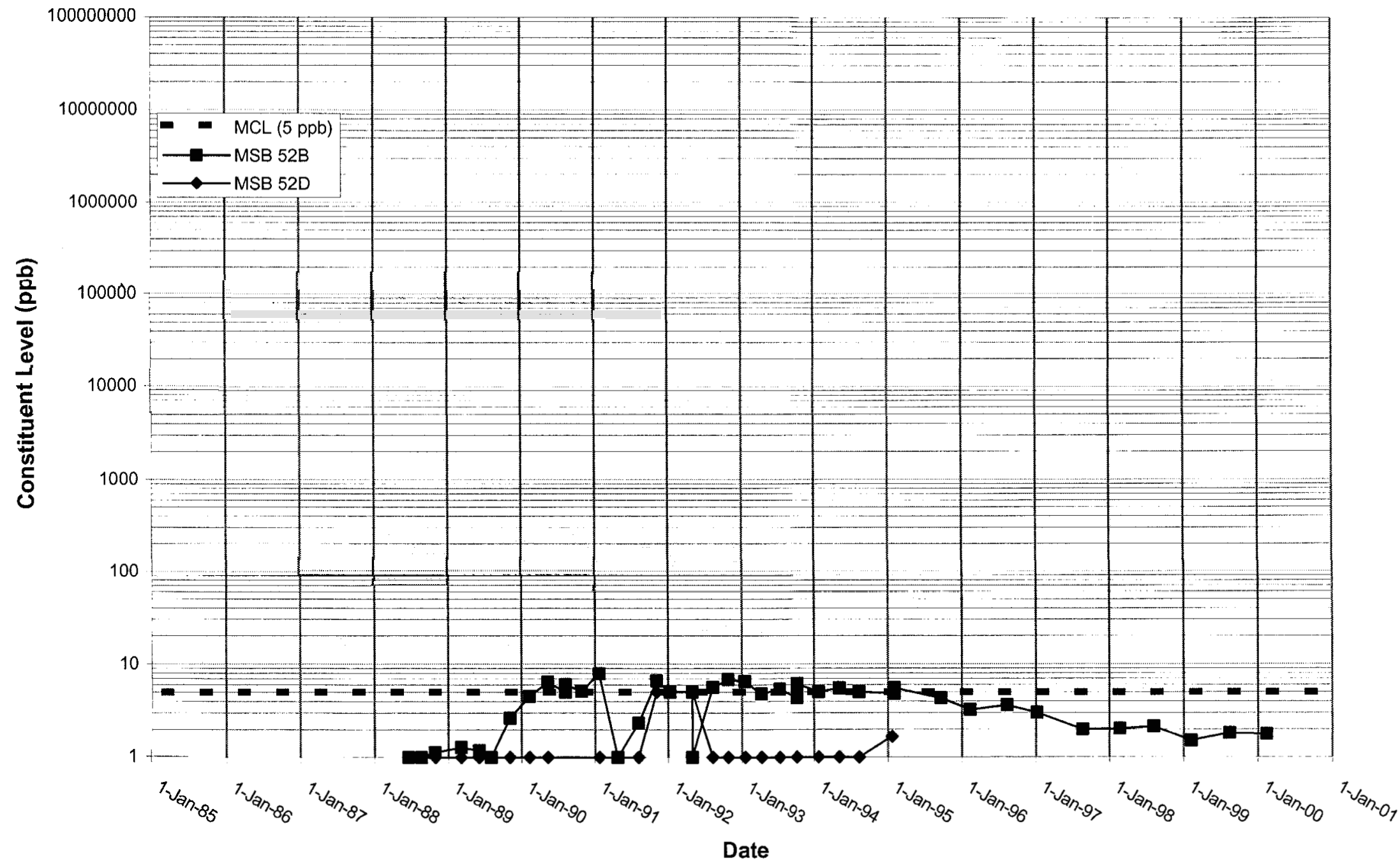


Time Series Plot of Trichloroethylene for Well MSB 53

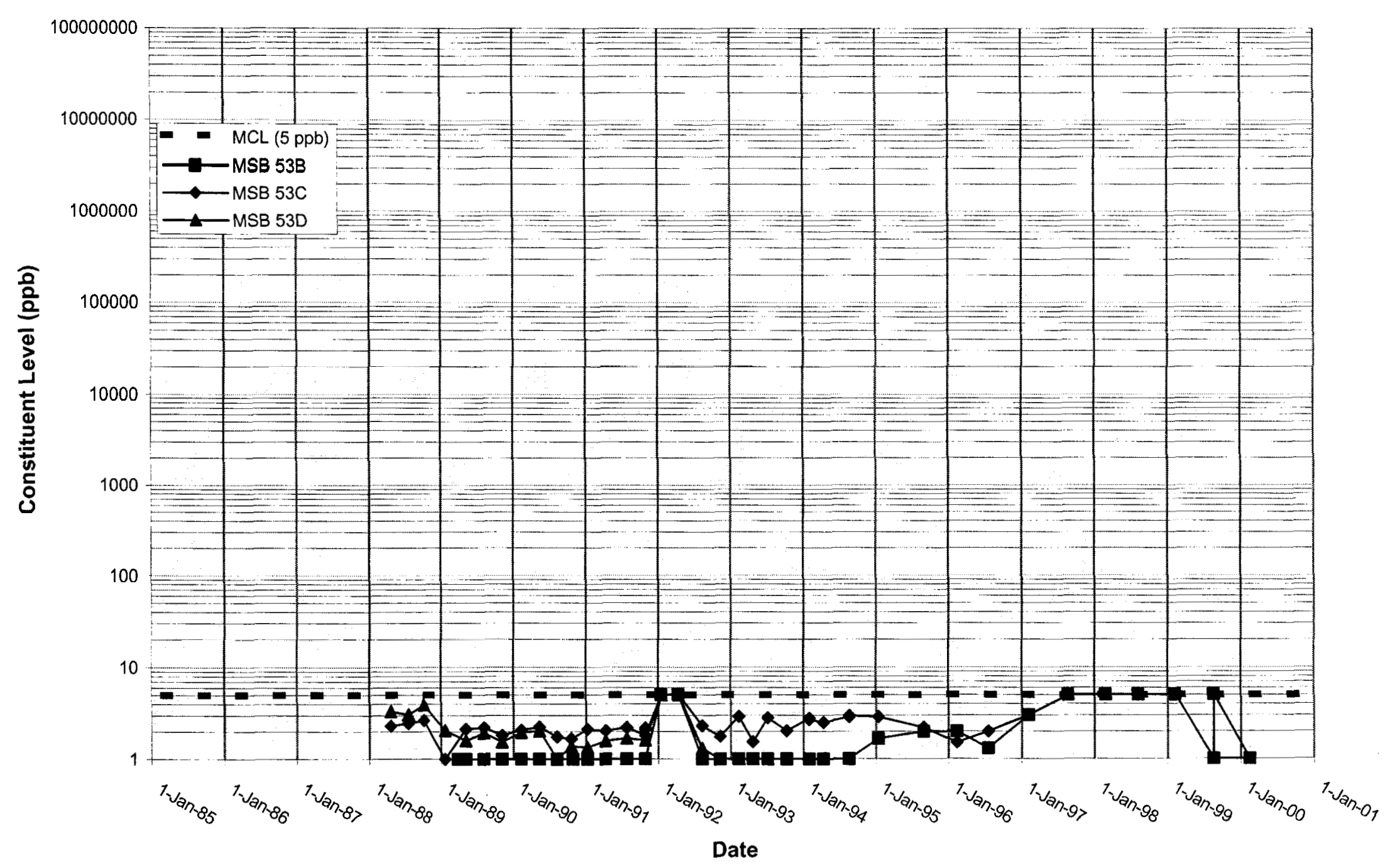


WSRC-TR-2000-00242 UNCLASSIFIED

Time Series Plot of Trichloroethylene for Well MSB 54

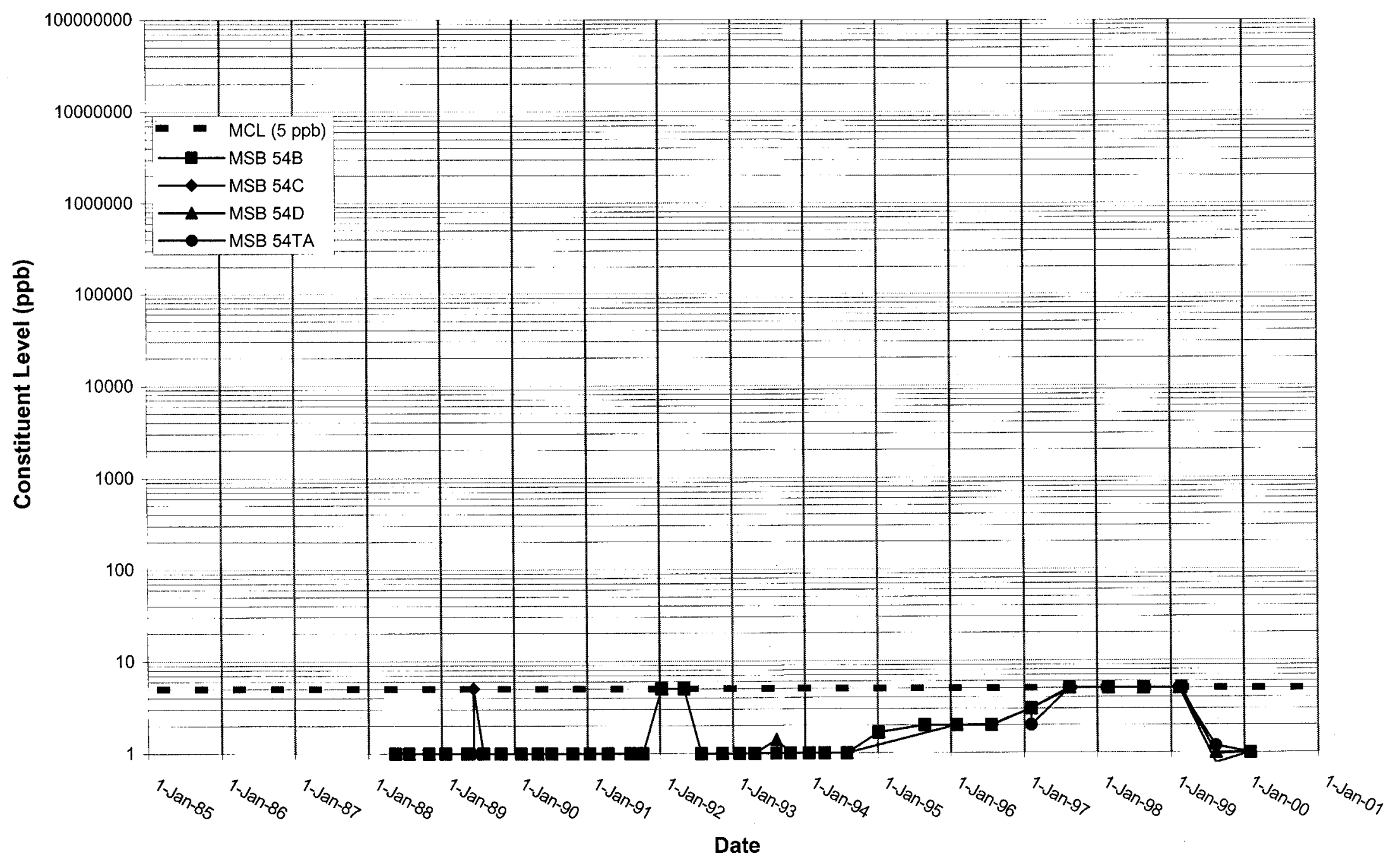


Time Series Plot of Trichloroethylene for Well MSB 55

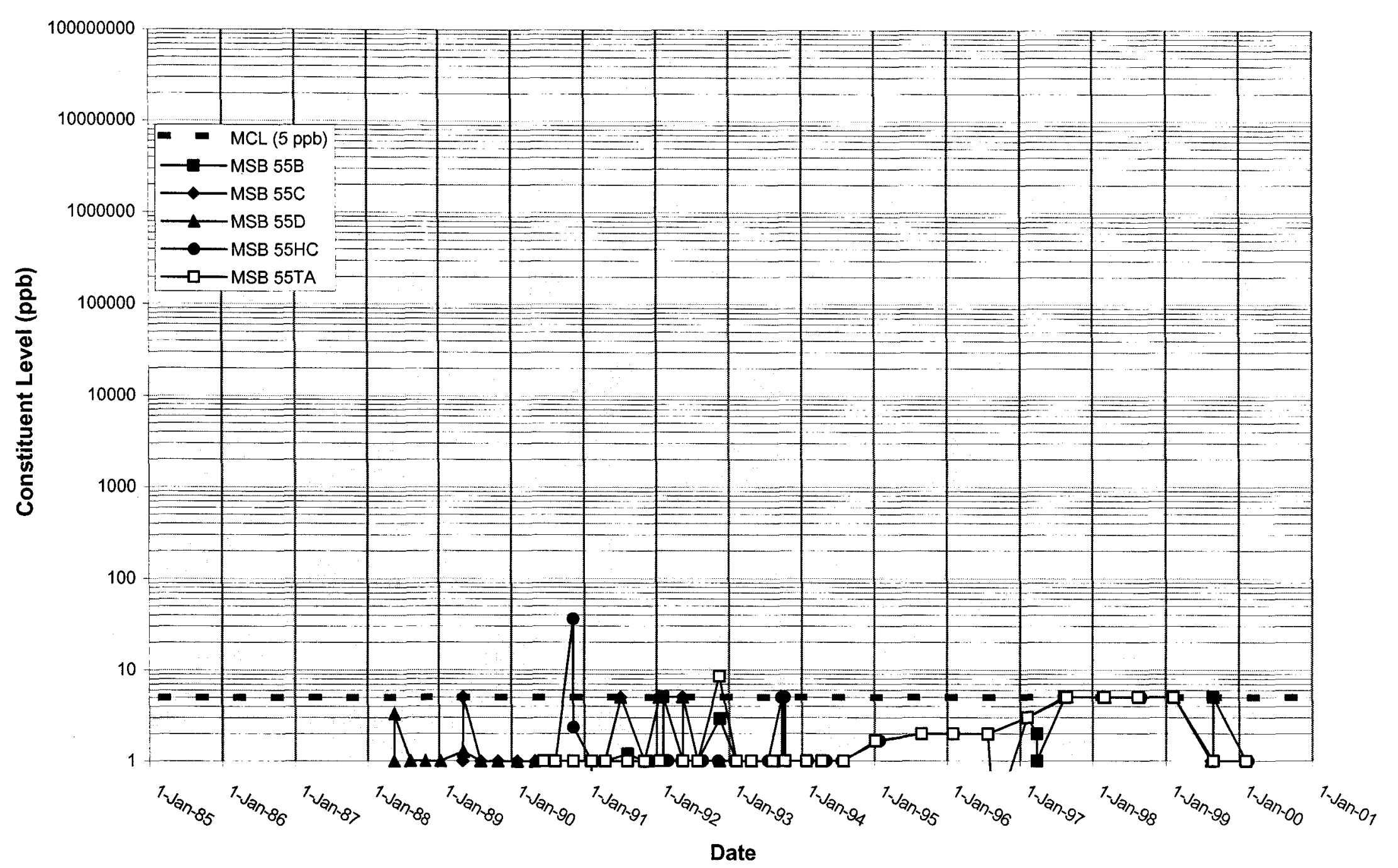


WSRC-TR-2000-00242 UNCLASSIFIED

Time Series Plot of Trichloroethylene for Well MSB 56

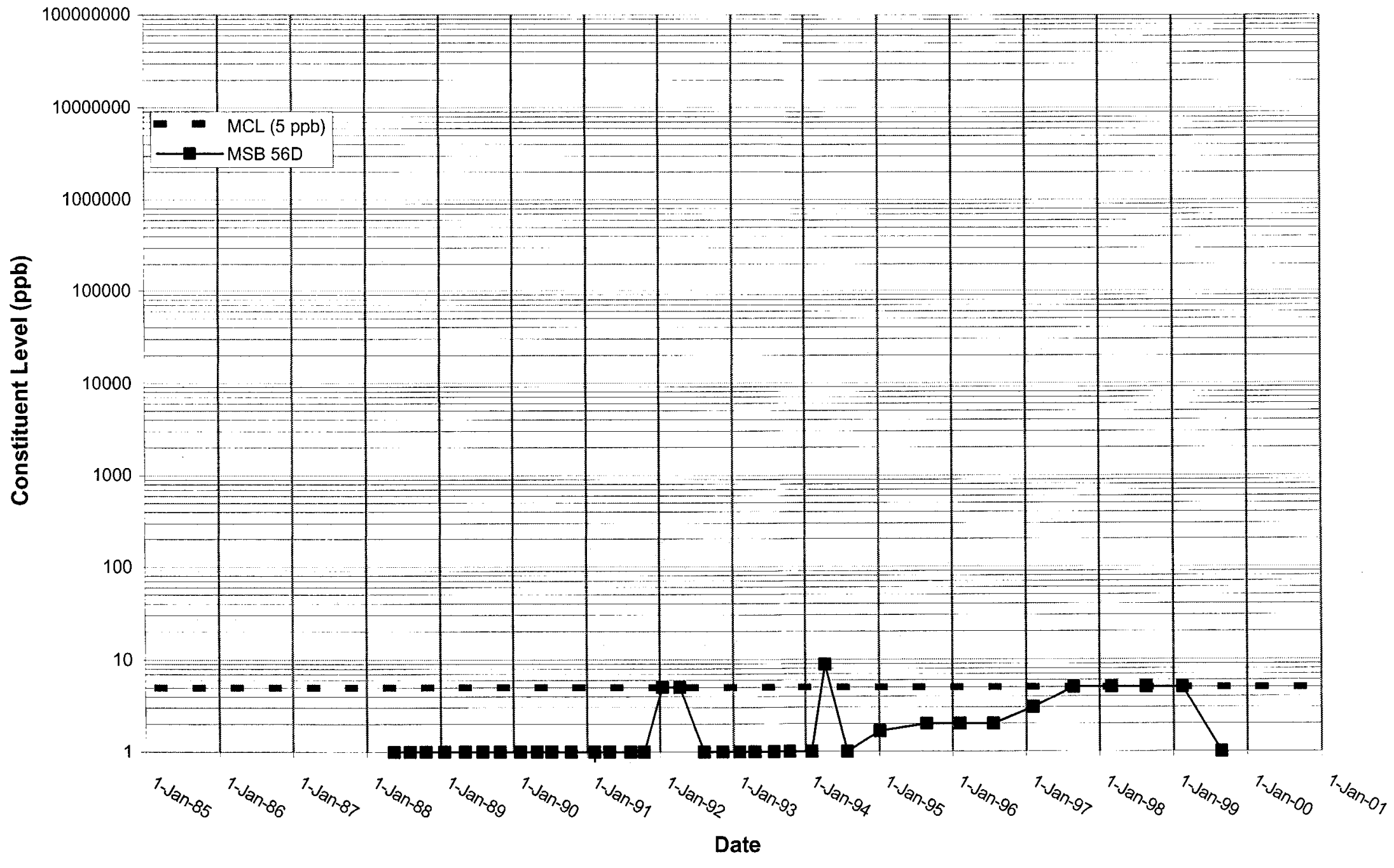


Time Series Plot of Trichloroethylene for Well MSB 57

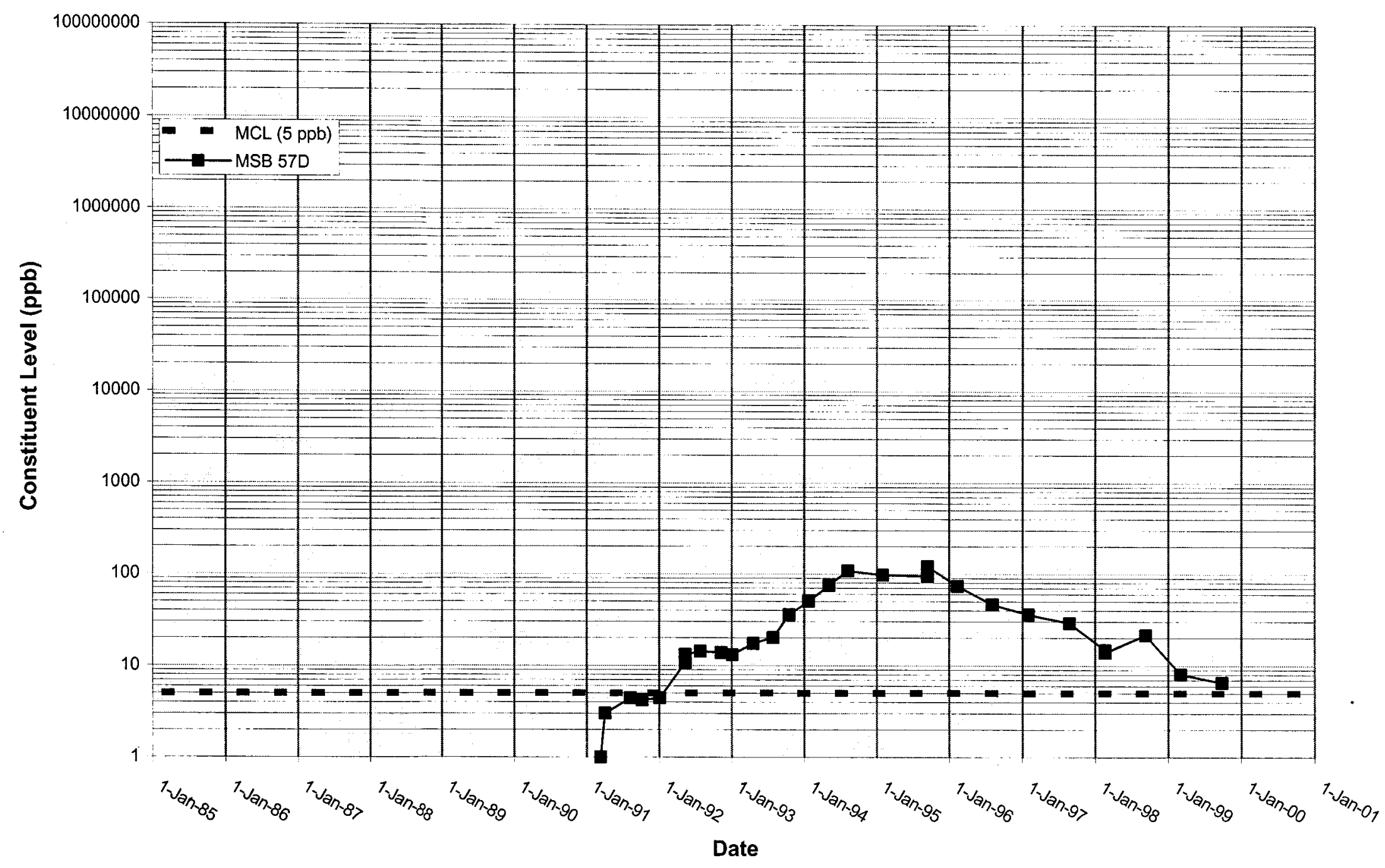


WSRC-TR-2000-00242 UNCLASSIFIED

Time Series Plot of Trichloroethylene for Well MSB 58

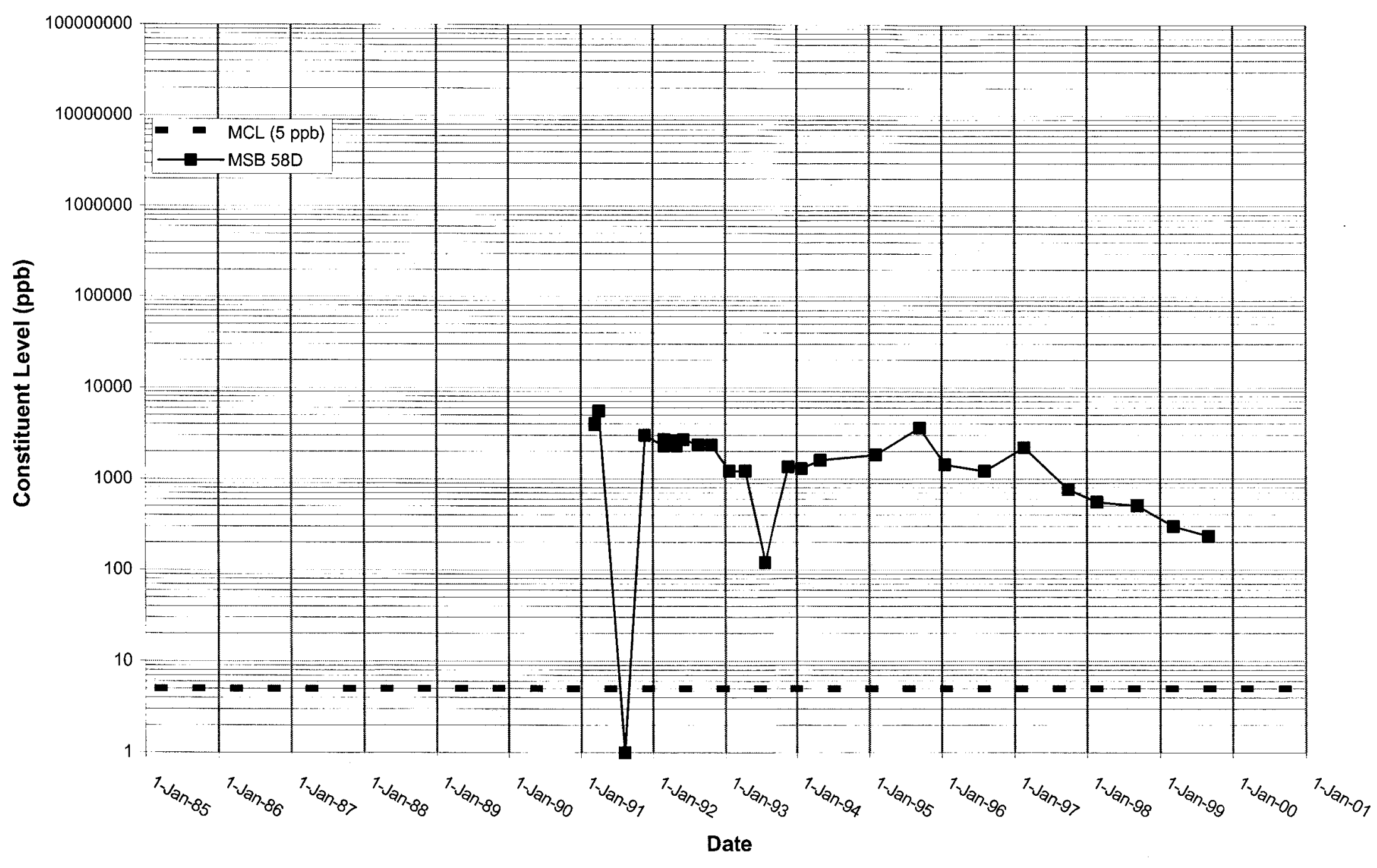


WSRC-TR-2000-00242 UNCLASSIFIED

\section{Time Series Plot of Trichloroethylene for Well MSB 59}

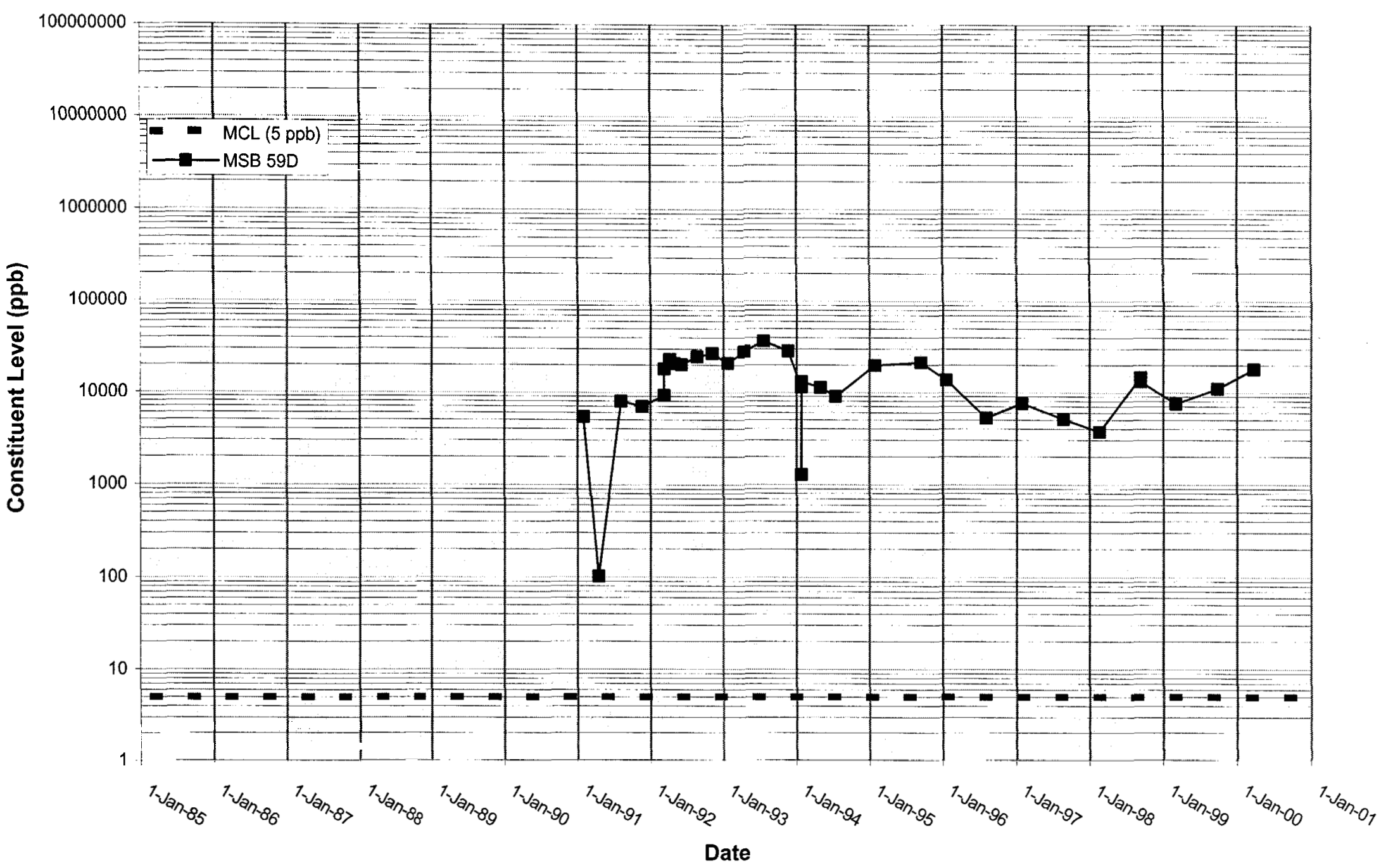




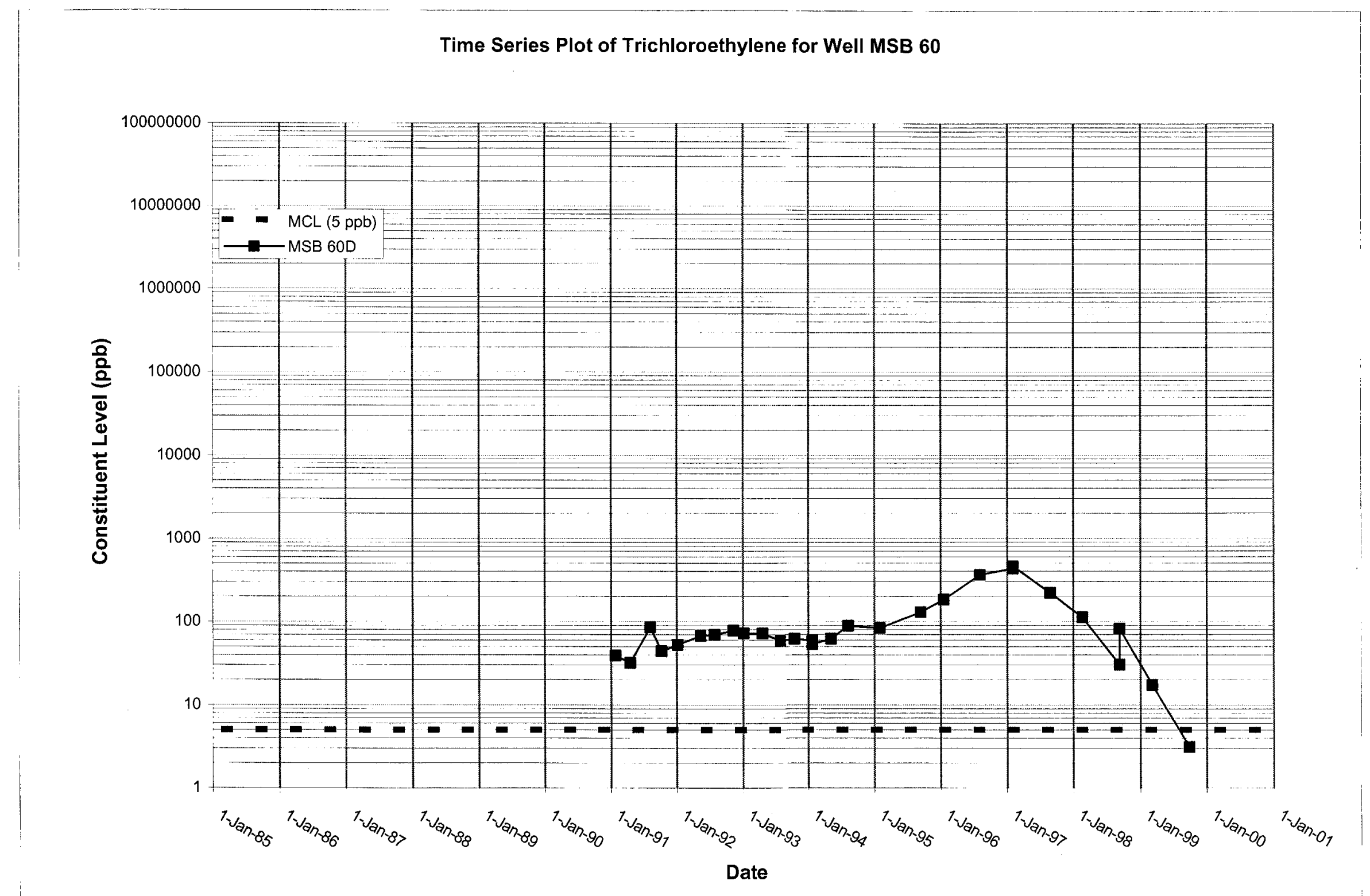




\section{Time Series Plot of Trichloroethylene for Well MSB 61}

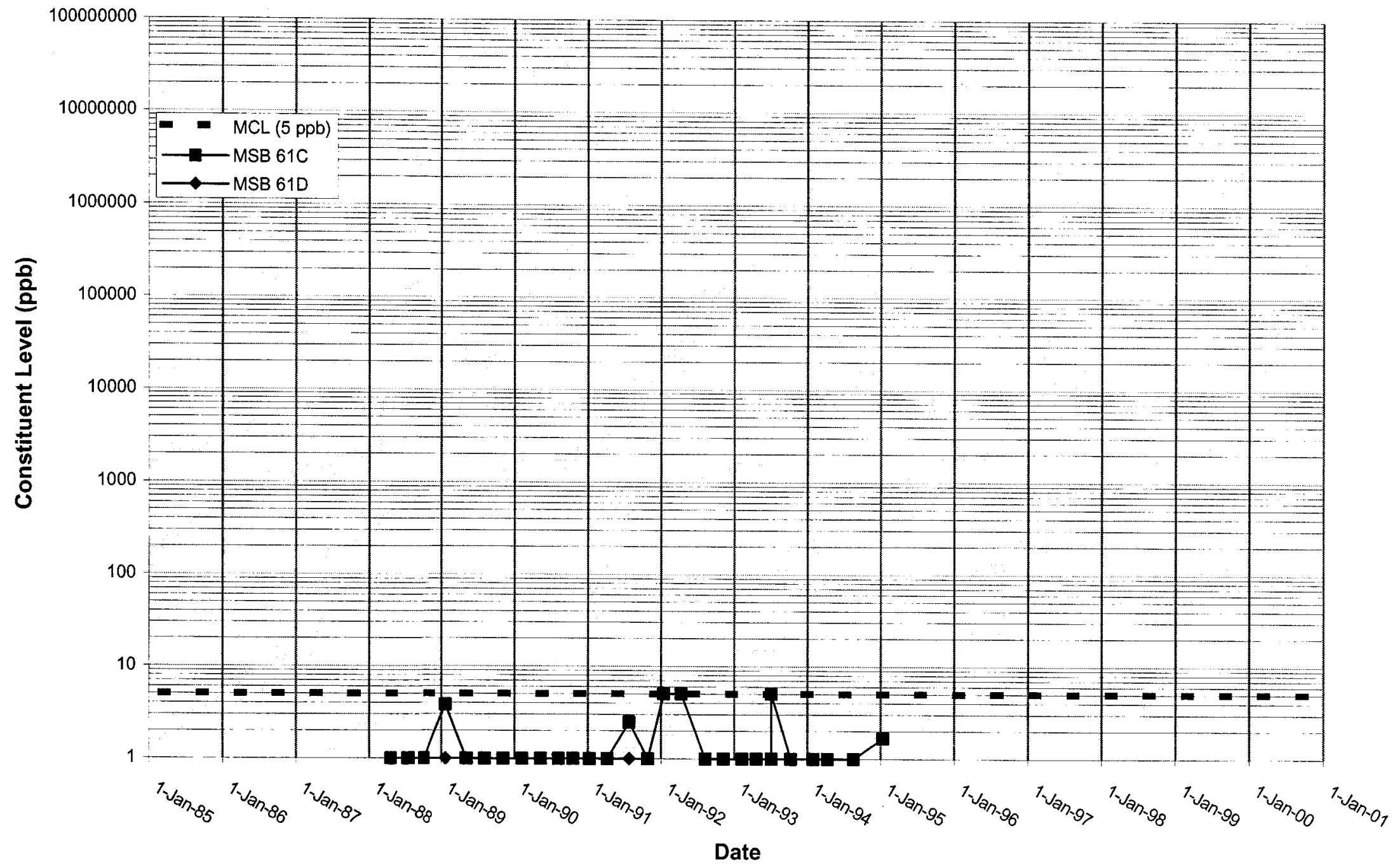


WSRC-TR-2000-00242 UNCLASSIFIED

Time Series Plot of Trichloroethylene for Well MSB 62

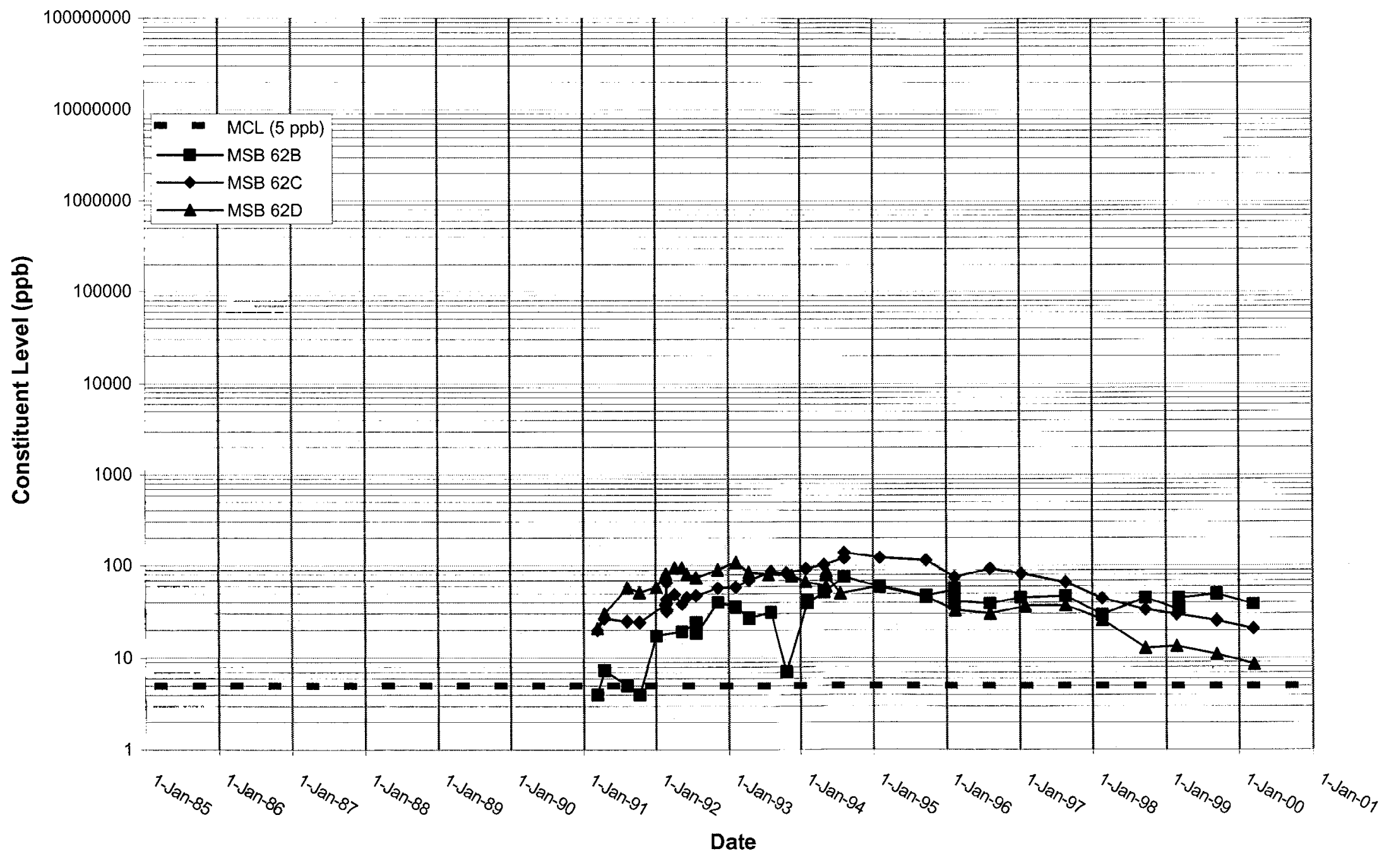




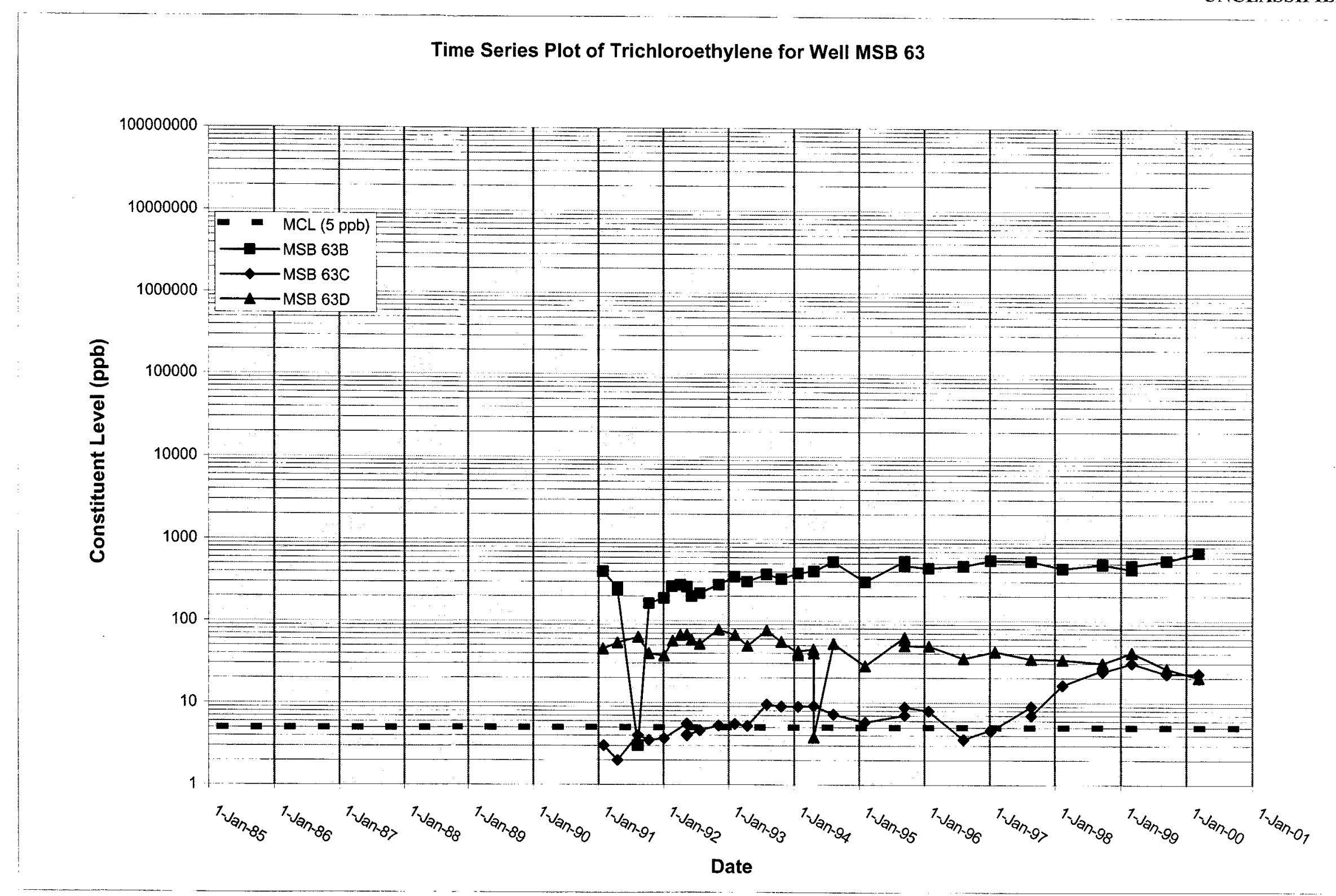


WSRC-TR-2000-00242 UNCLASSIFIED

Time Series Plot of Trichloroethylene for Well MSB 64

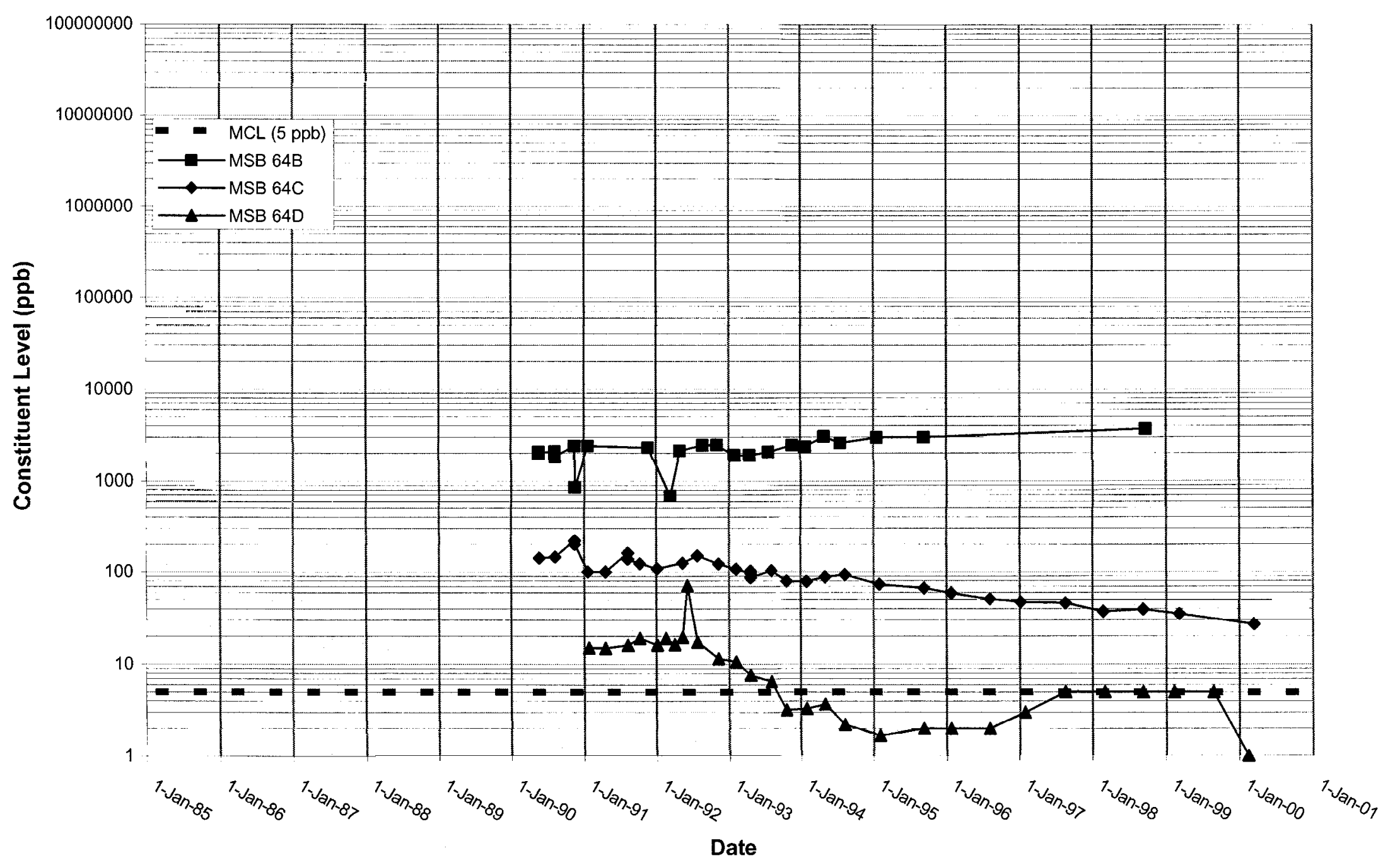


WSRC-TR-2000-00242

UNCLASSIFIED

Time Series Plot of Trichloroethylene for Well MSB 65

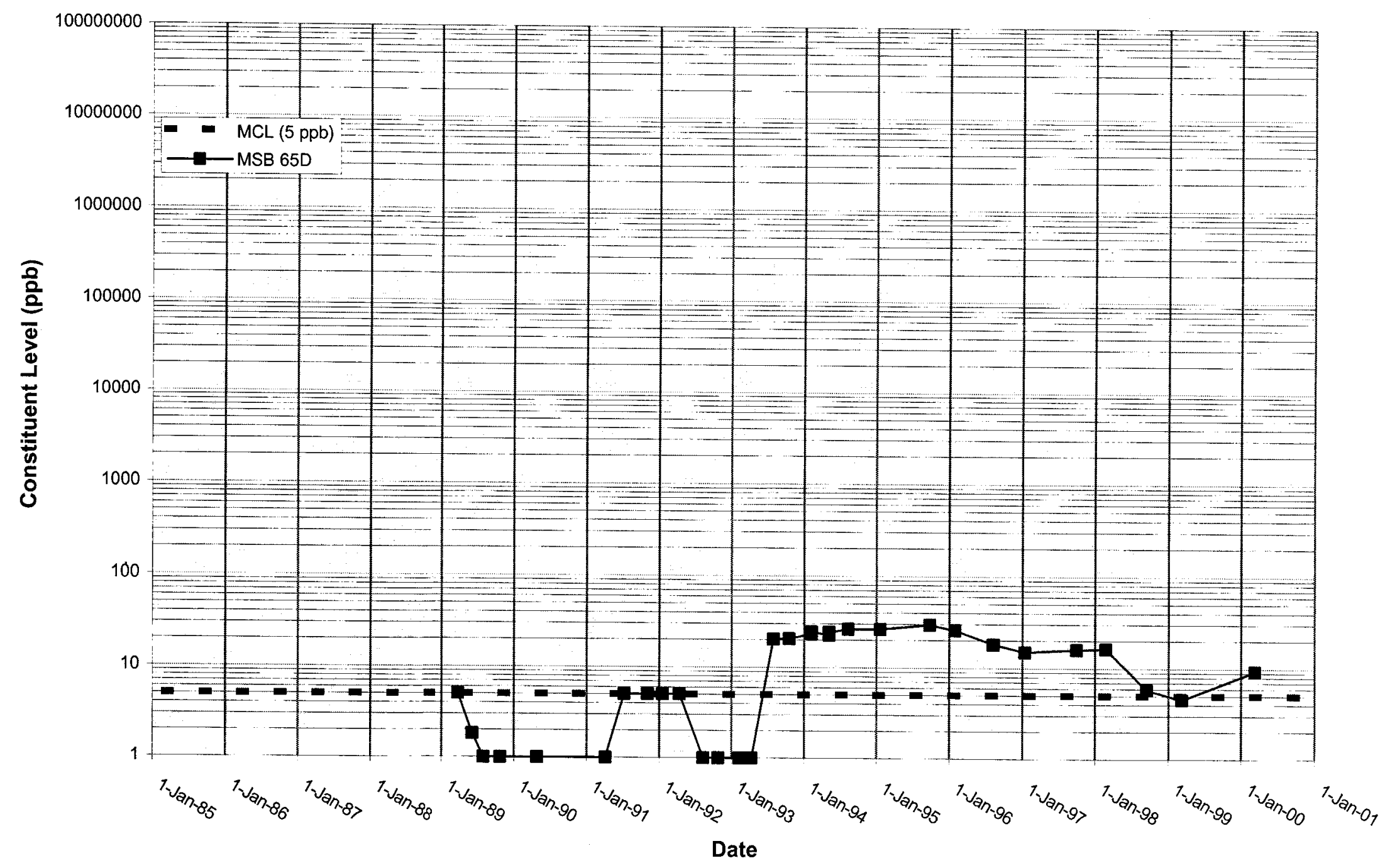




\section{Time Series Plot of Trichloroethylene for Well MSB 66}

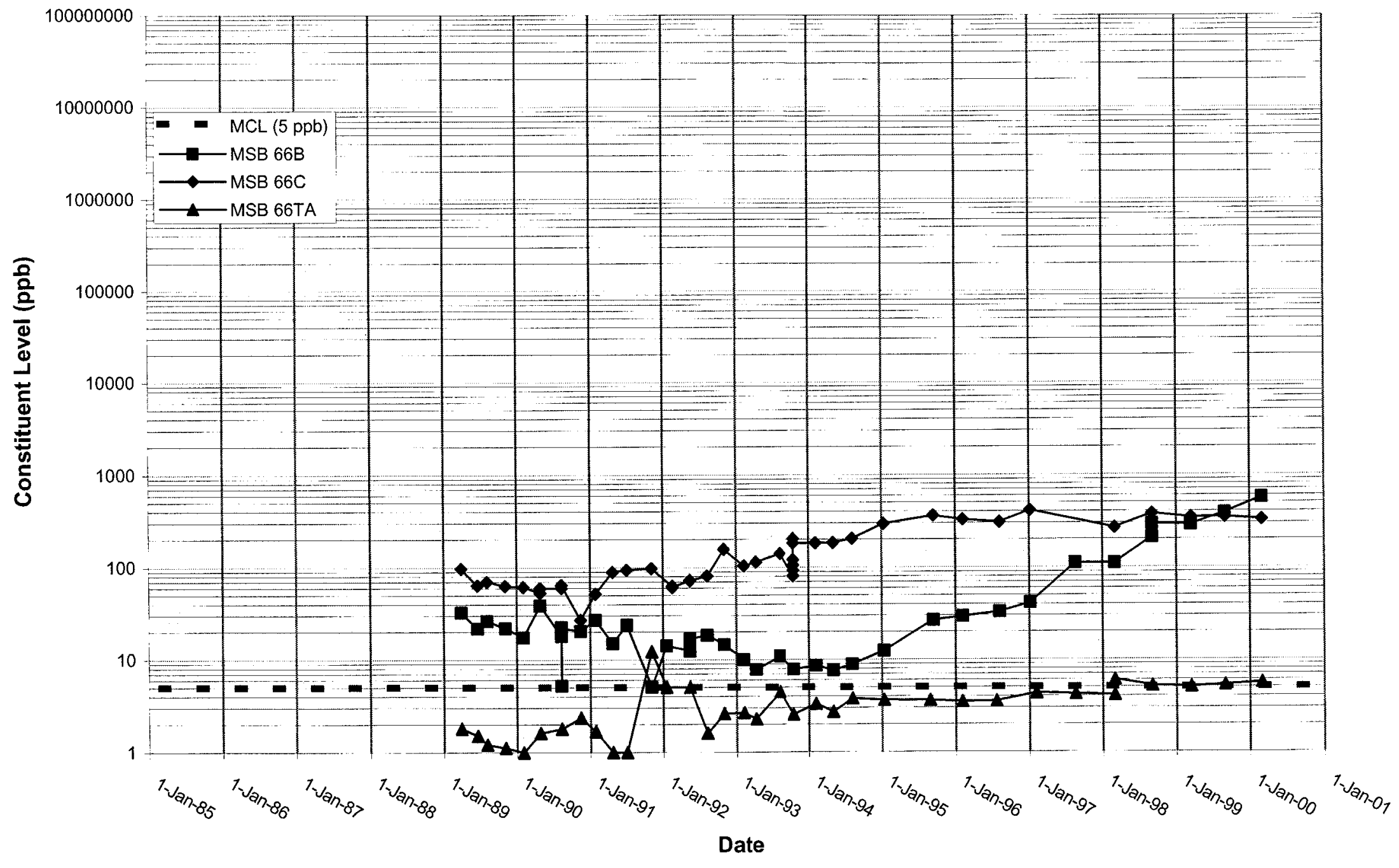




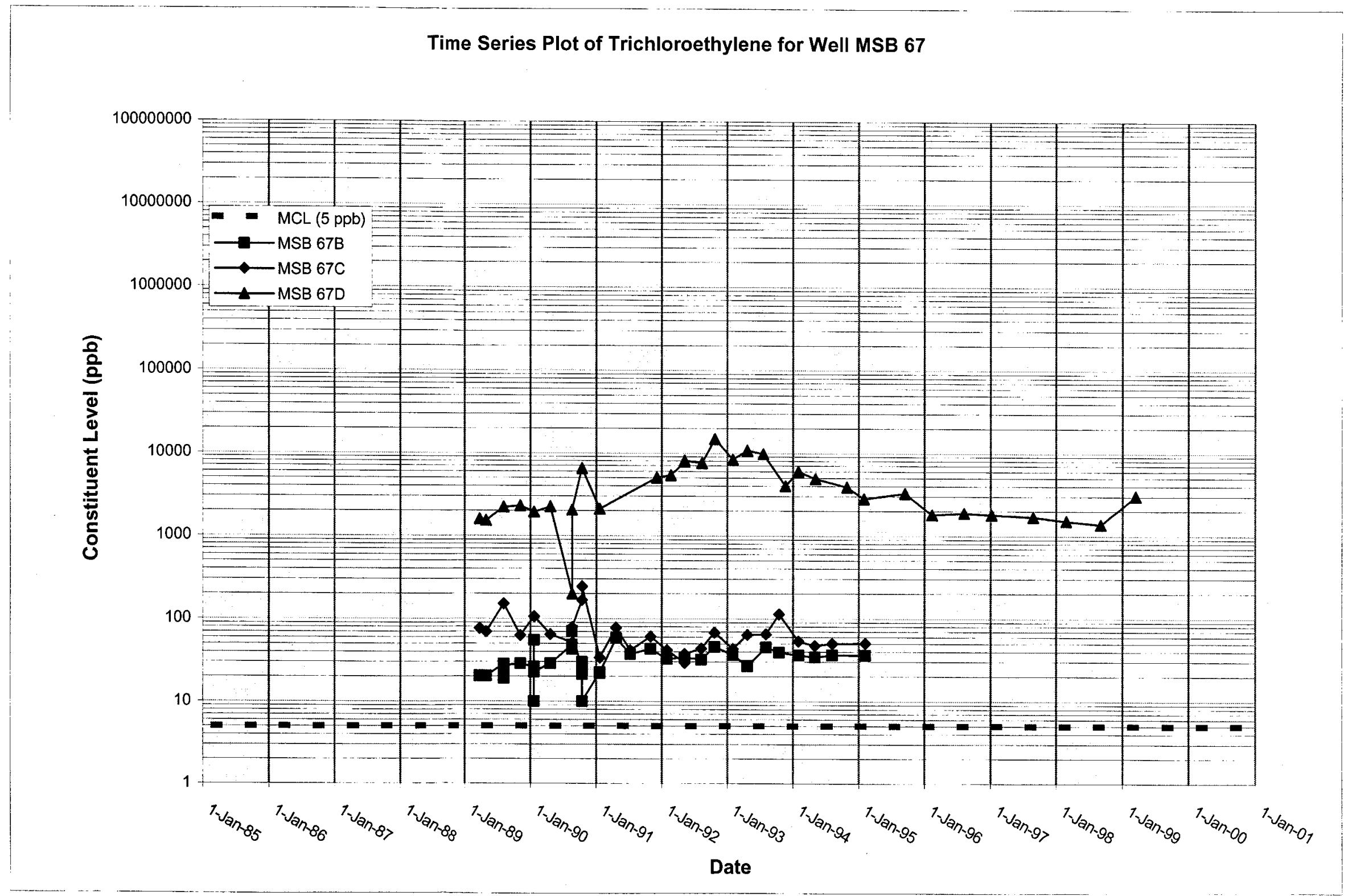




\section{Time Series Plot of Trichloroethylene for Well MSB 68}

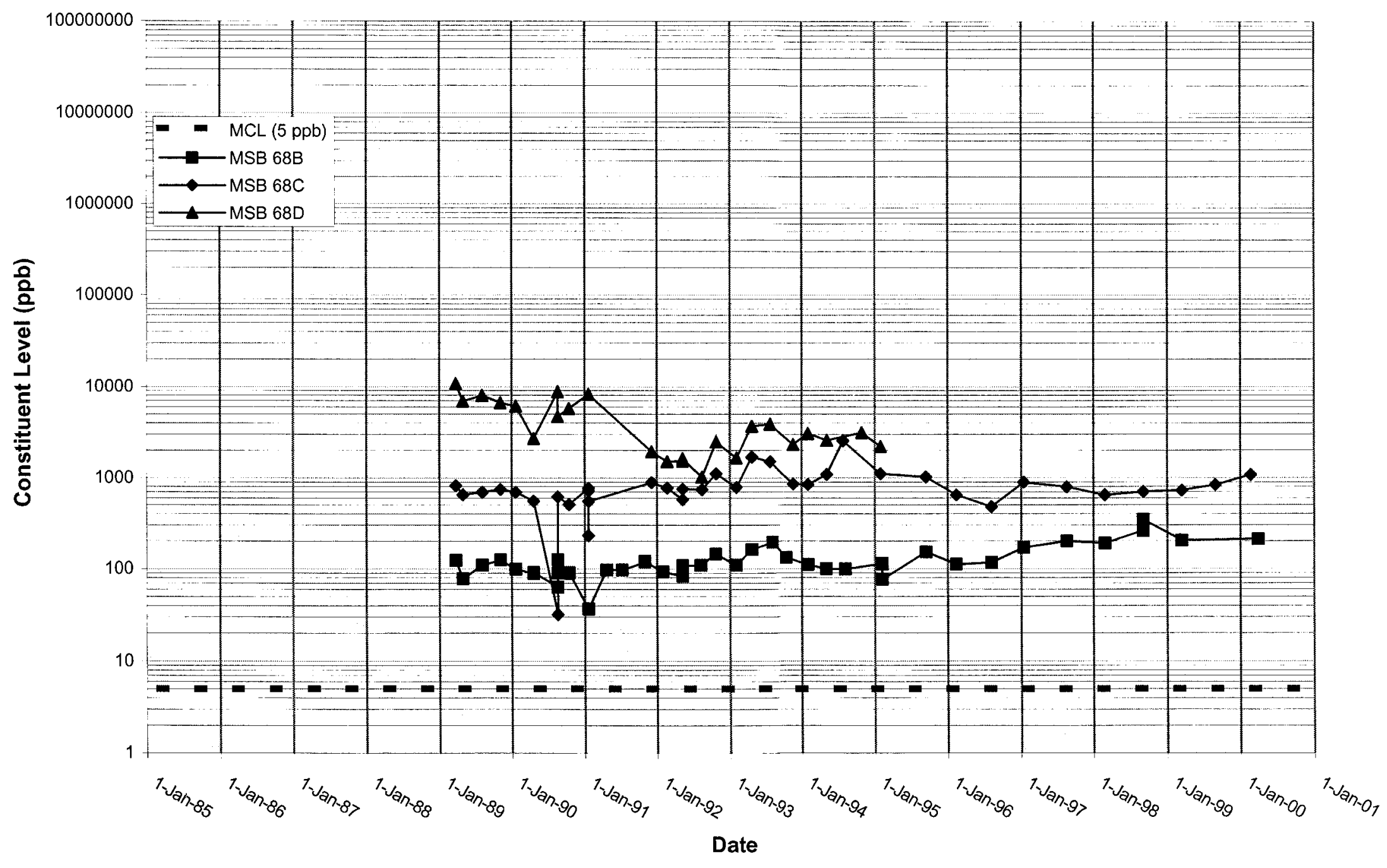




\section{Time Series Plot of Trichloroethylene for Well MSB 69}

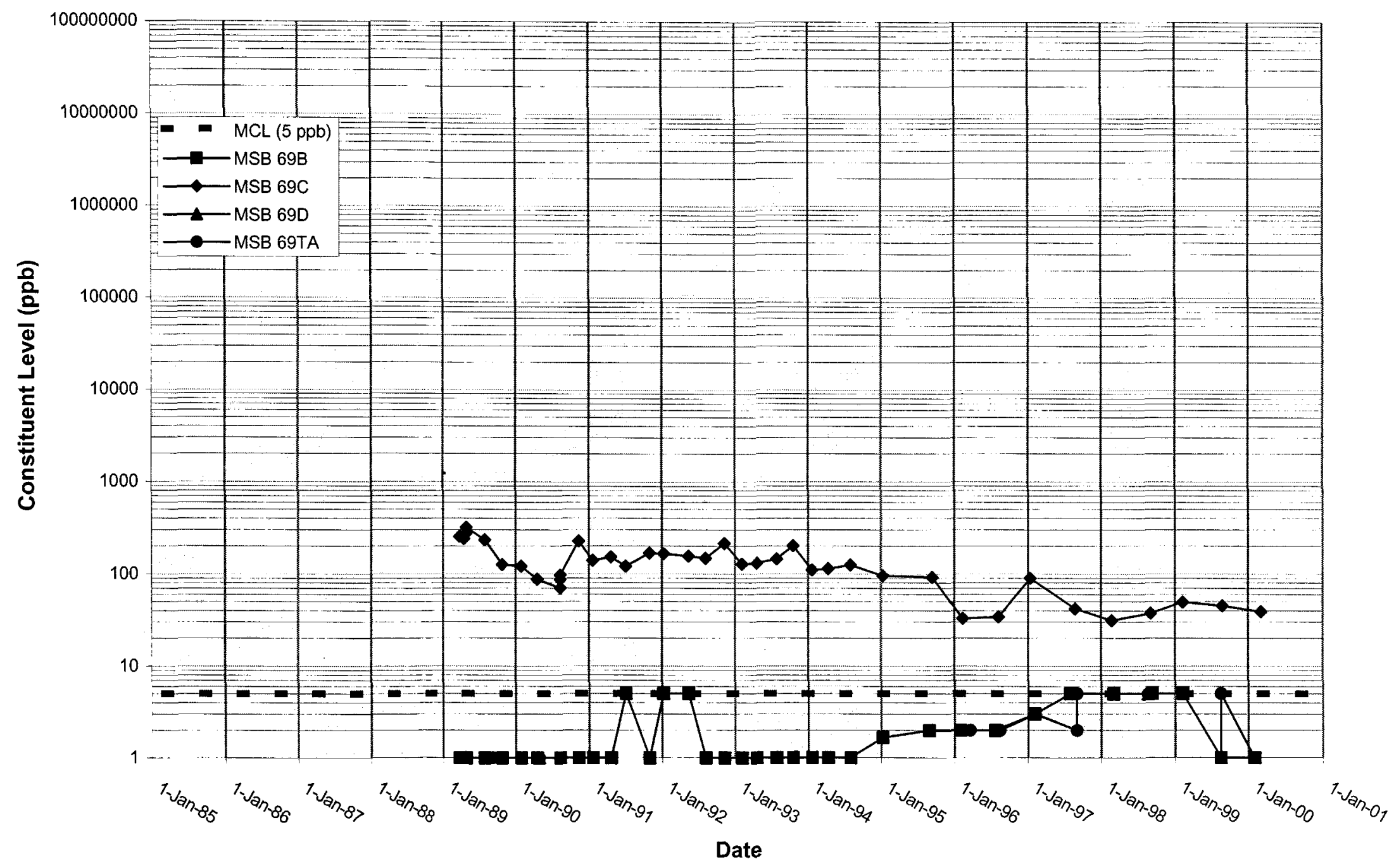


WSRC-TR-2000-00242 UNCLASSIFIED

Time Series Plot of Trichloroethylene for Well MSB 70

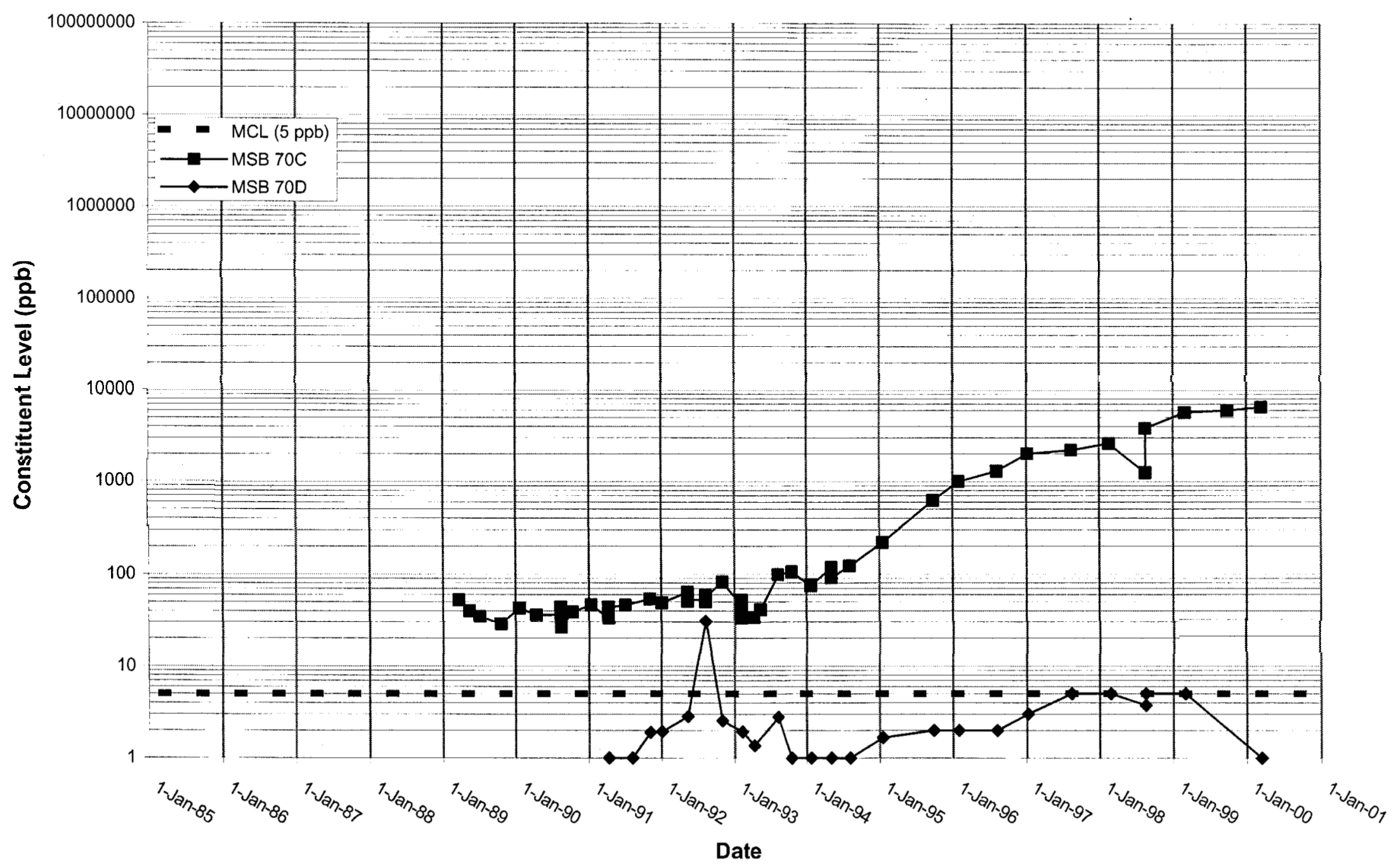


WSRC-TR-2000-00242 UNCLASSIFIED

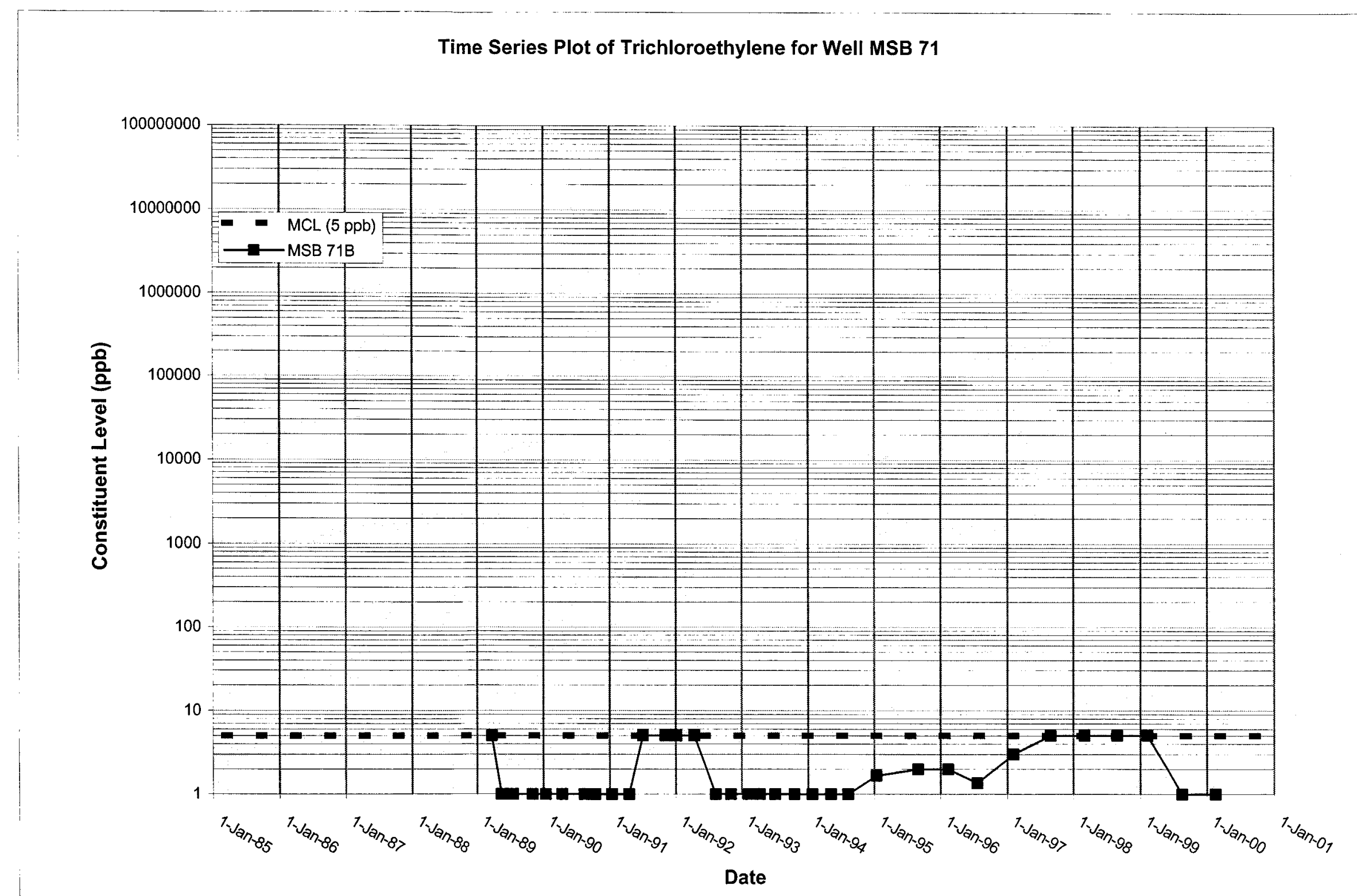


Time Series Plot of Trichloroethylene for Well MSB 72

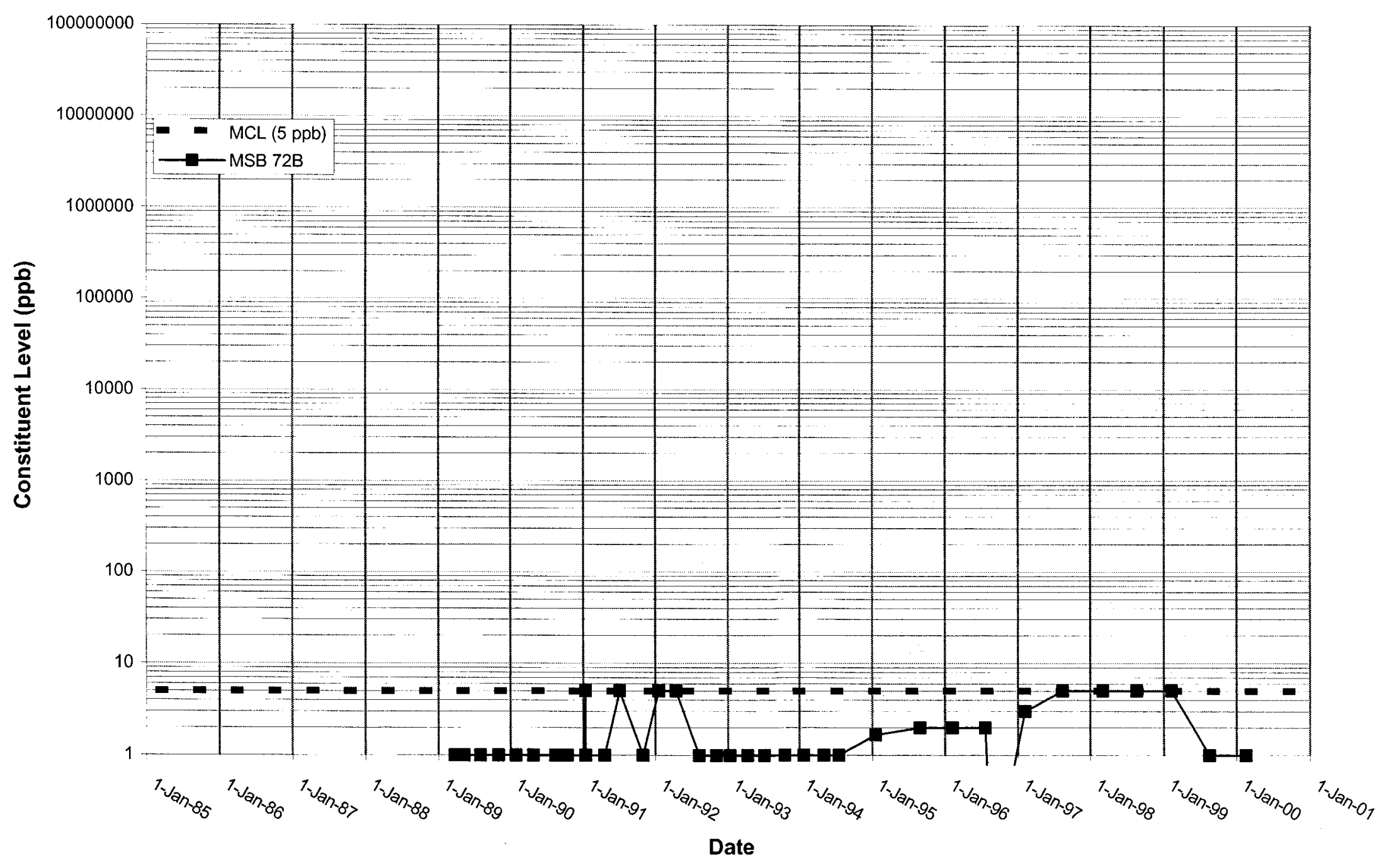


Tìme Series Plot of Trichloroethylene for Well MSB 73

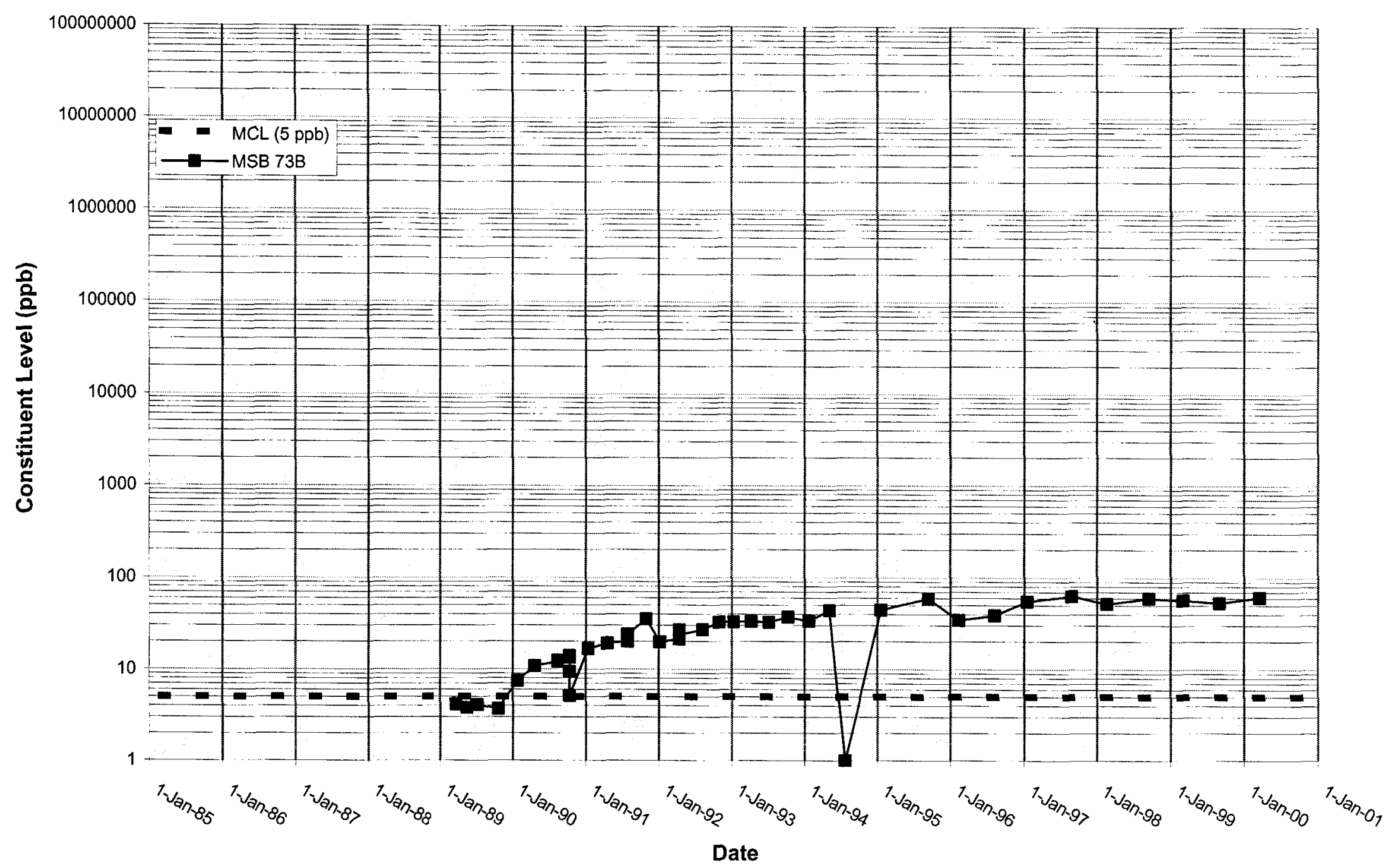




\section{Time Series Plot of Trichloroethylene for Well MSB 74}

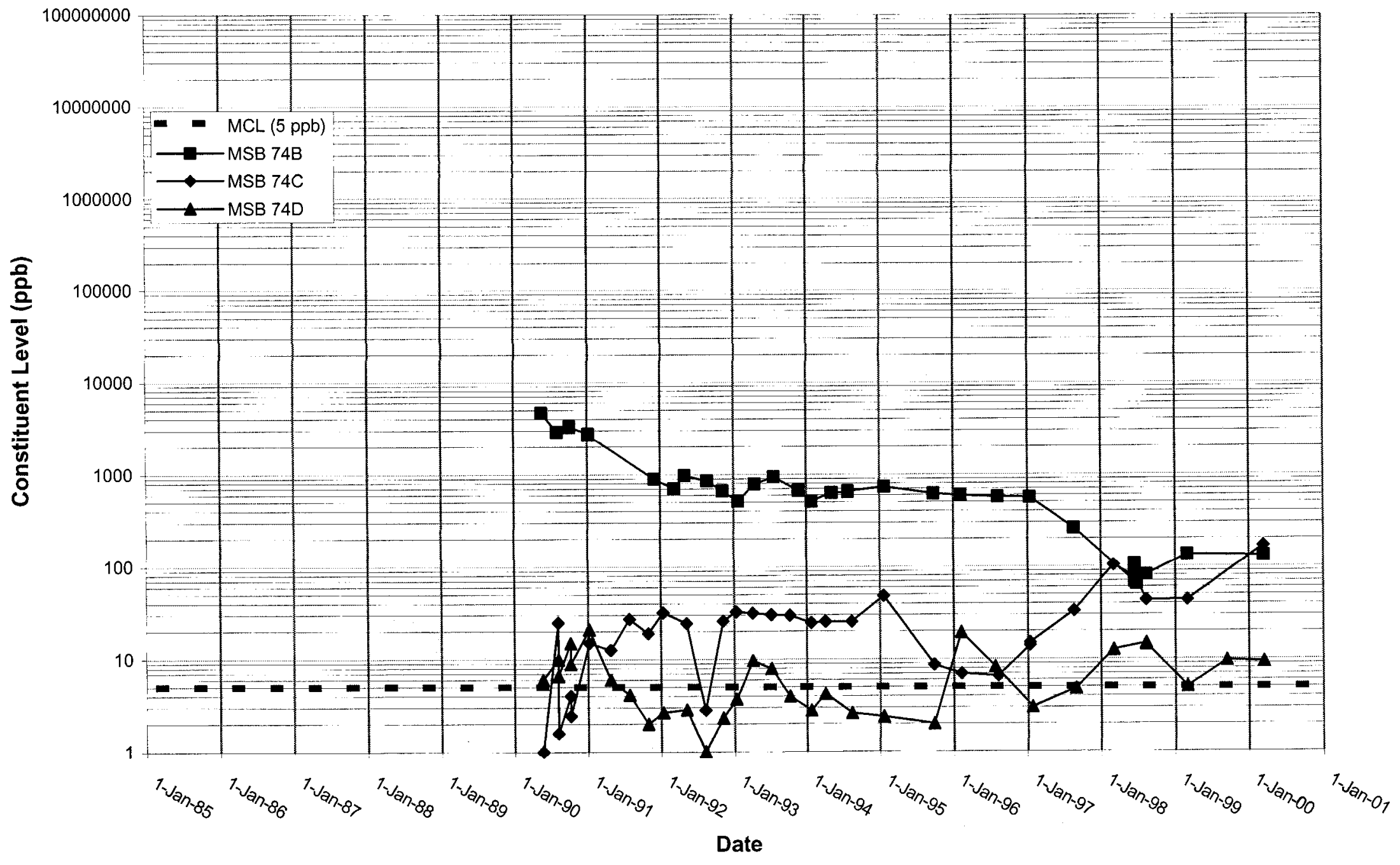


WSRC-TR-2000-00242 UNCLASSIFIED

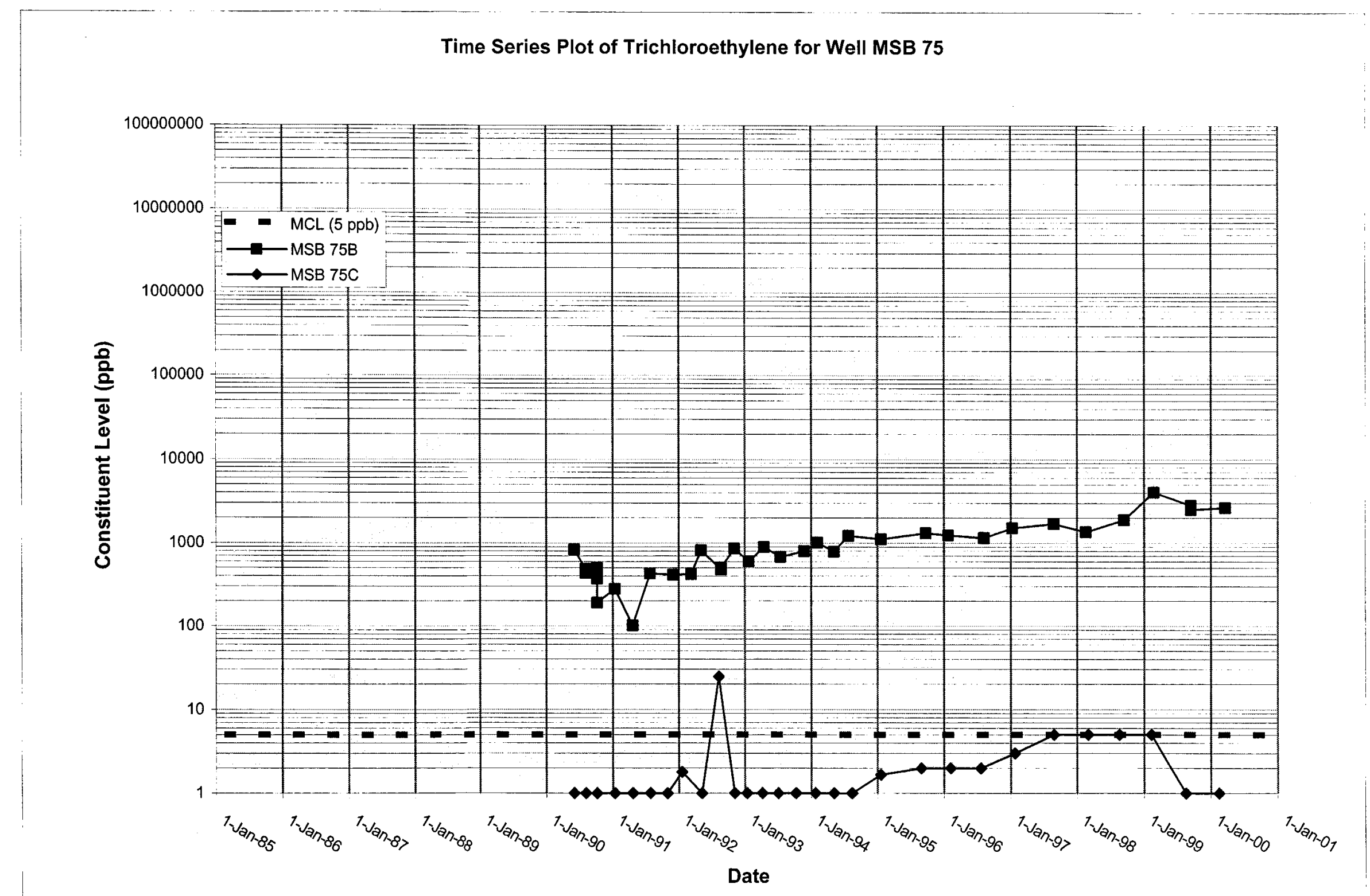




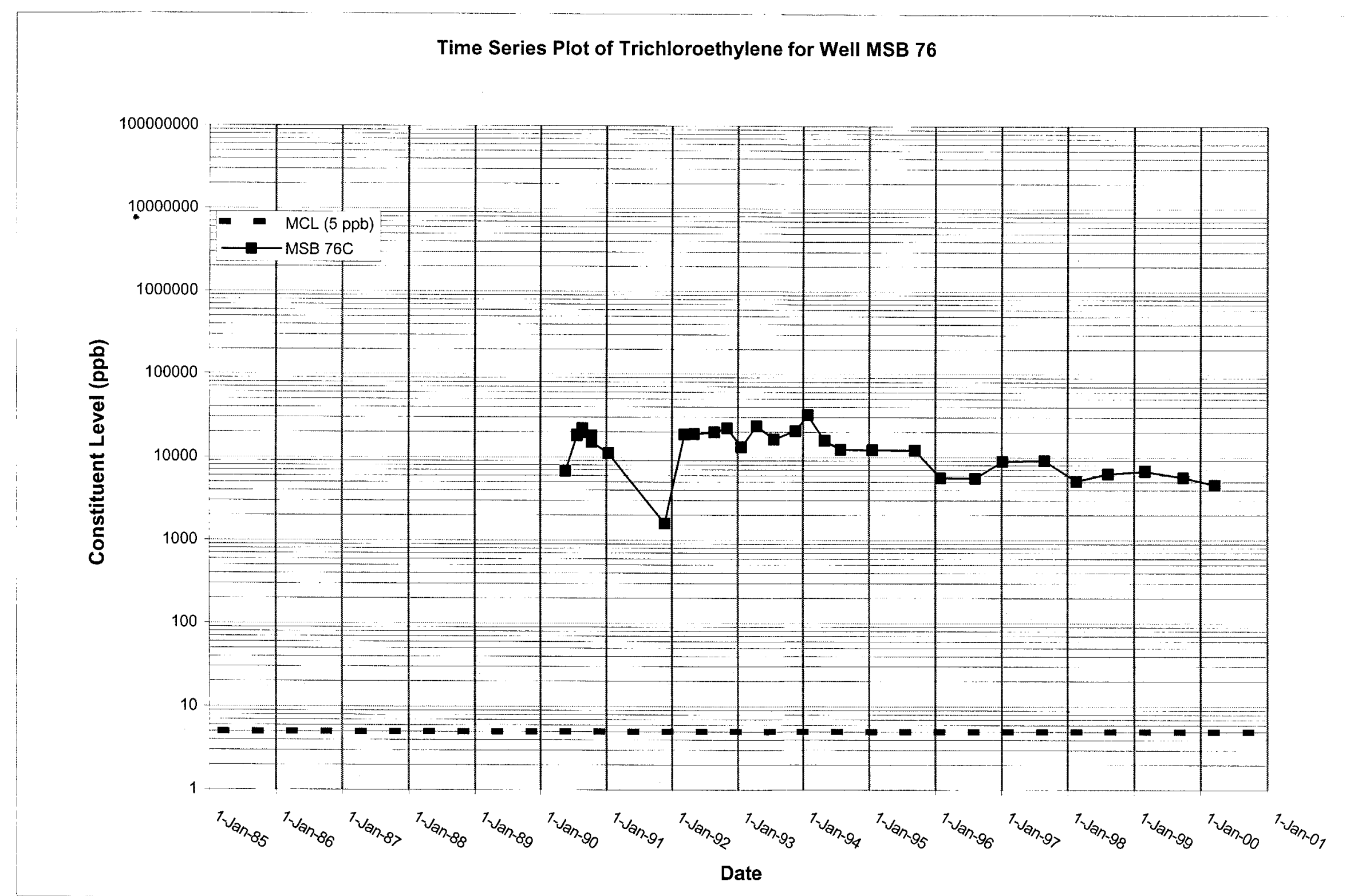


WSRC-TR-2000-00242 UNCLASSIFIED

Time Series Plot of Trichloroethylene for Well MSB 77

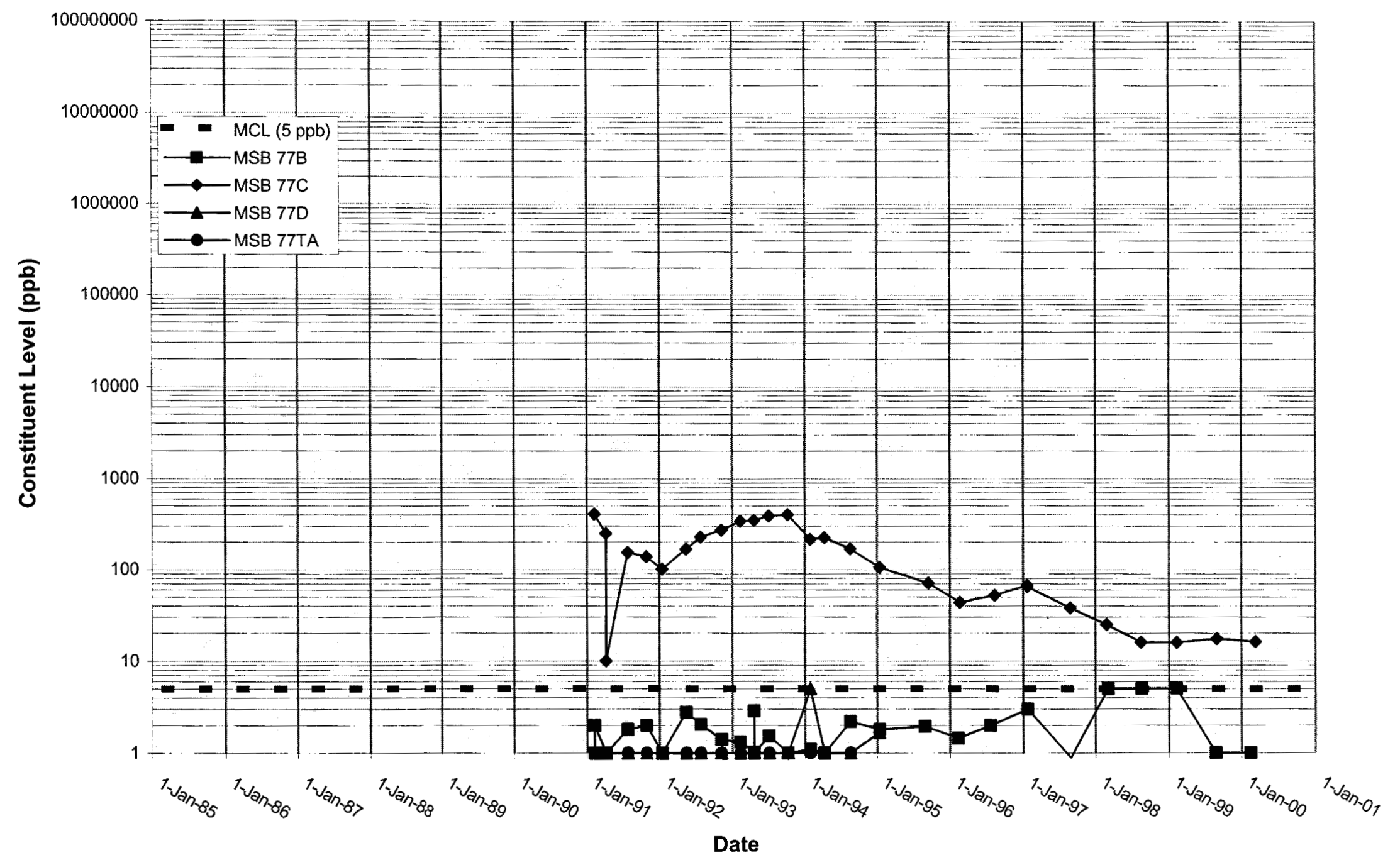




\section{Time Series Plot of Trichloroethylene for Well MSB 78}

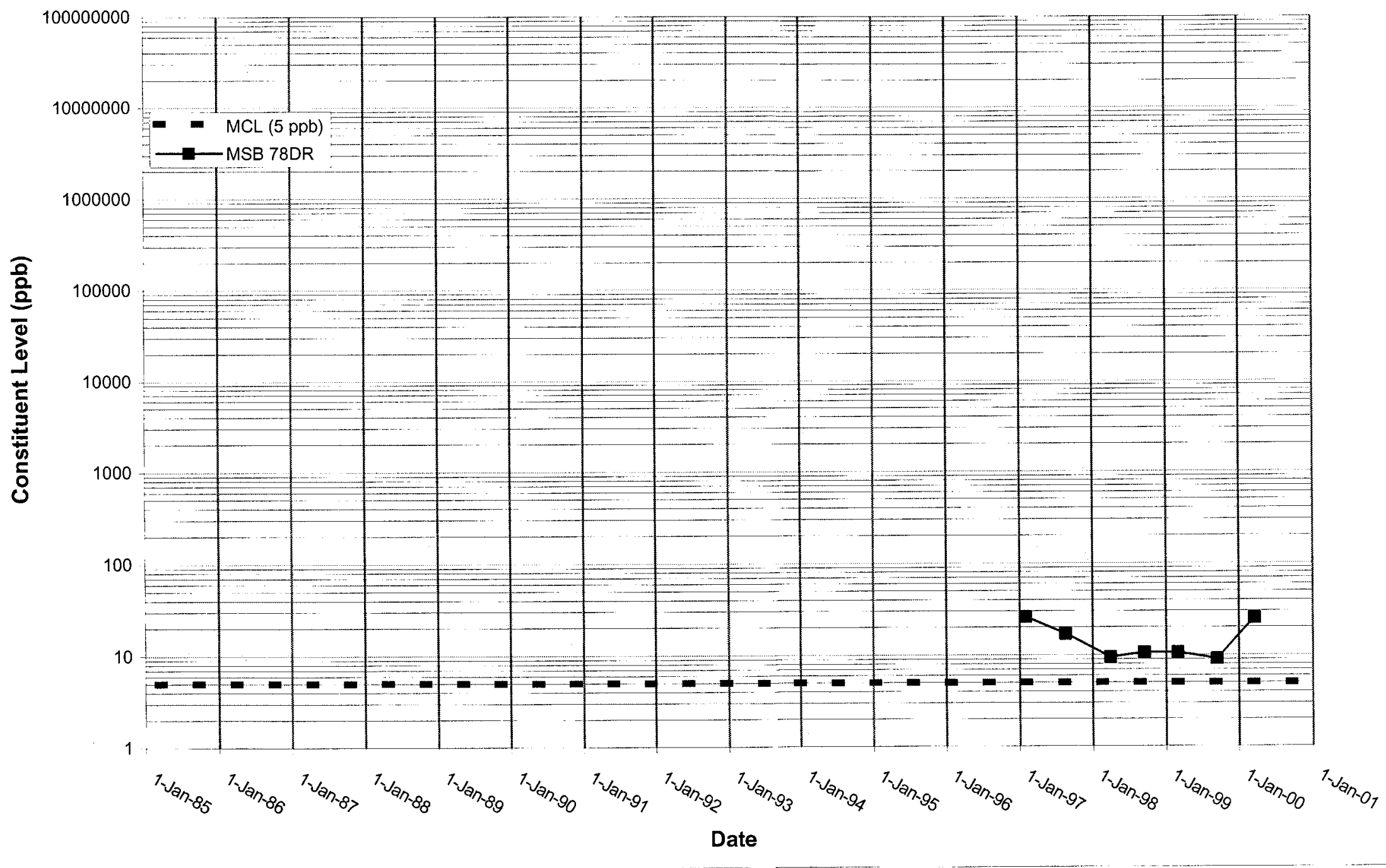




\section{1}

WSRC-TR-2000-00242

UNCLASSIFIED

Time Series Plot of Trichloroethylene for Well MSB 79

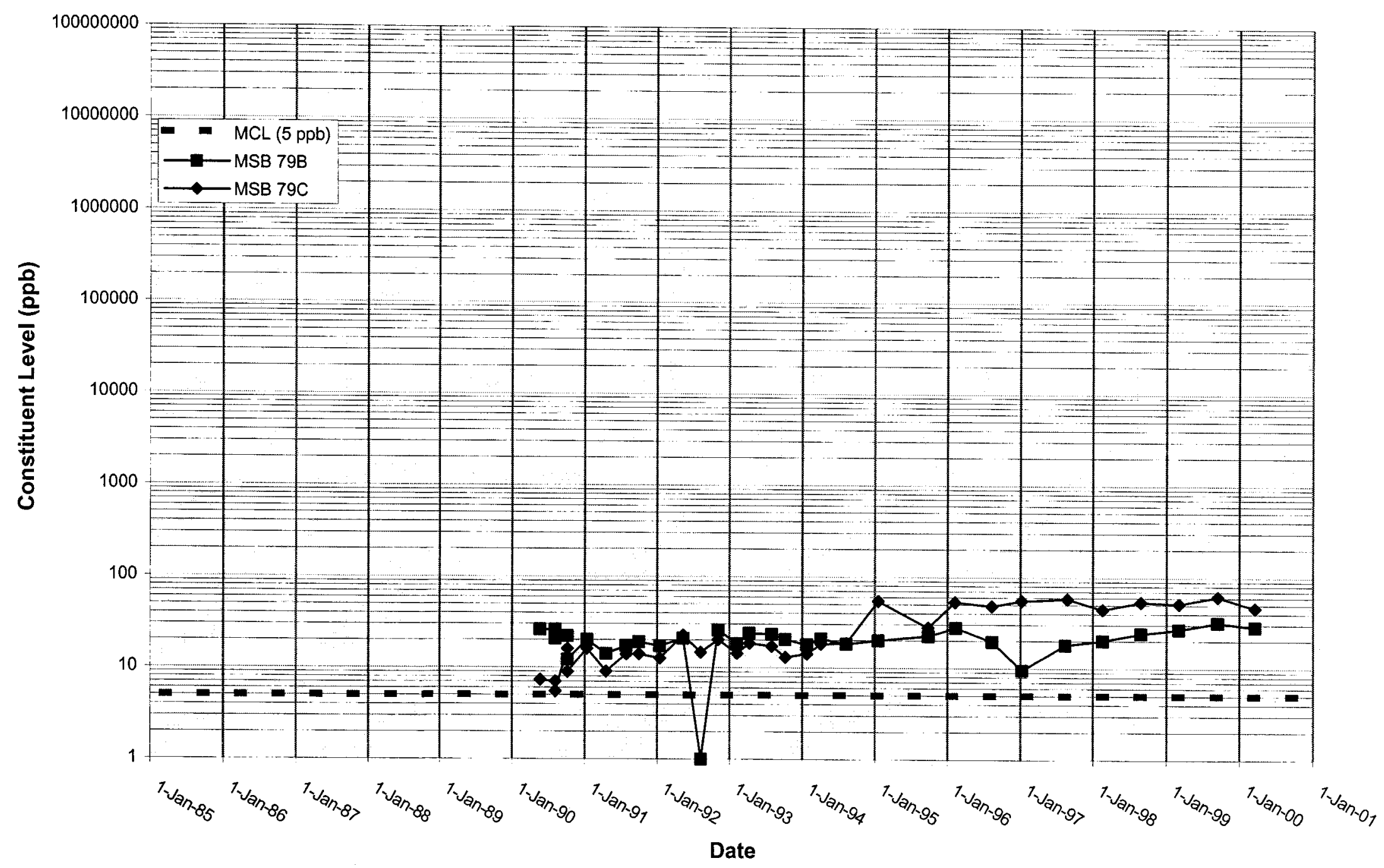




\section{Time Series Plot of Trichloroethylene for Well MSB 81}

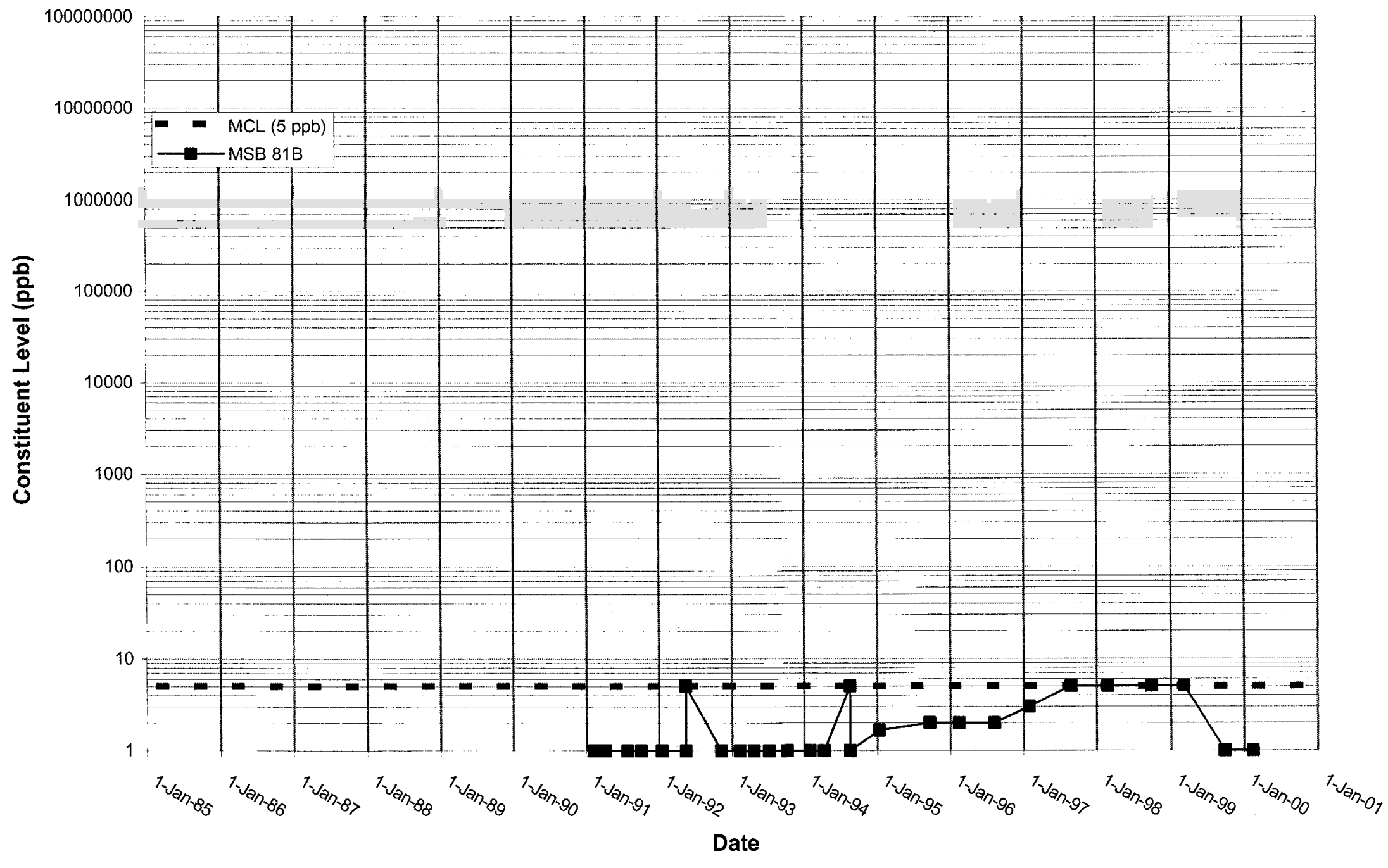


Time Series Plot of Trichloroethylene for Well MSB 82

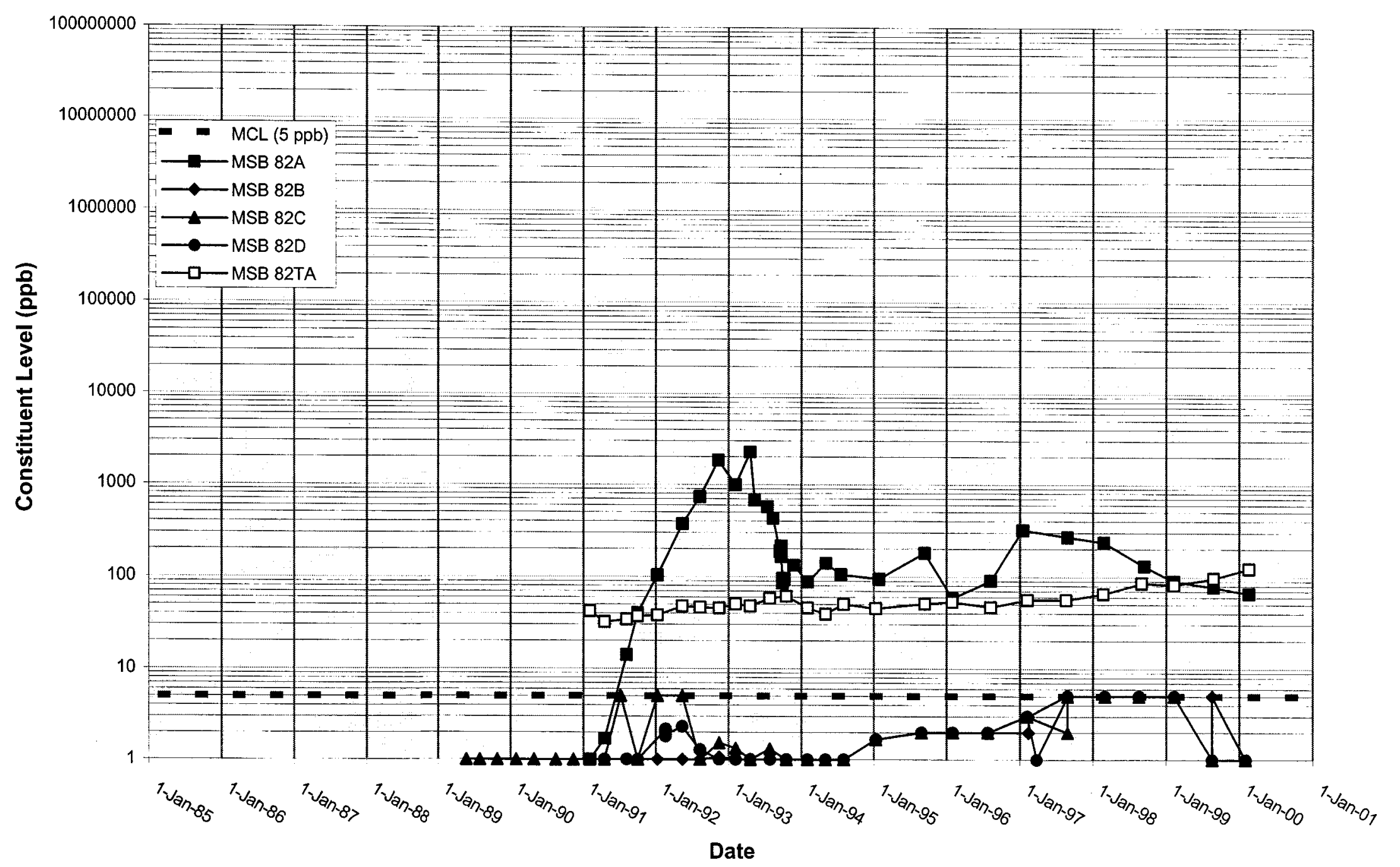




\section{Time Series Plot of Trichloroethylene for Well MSB 83}

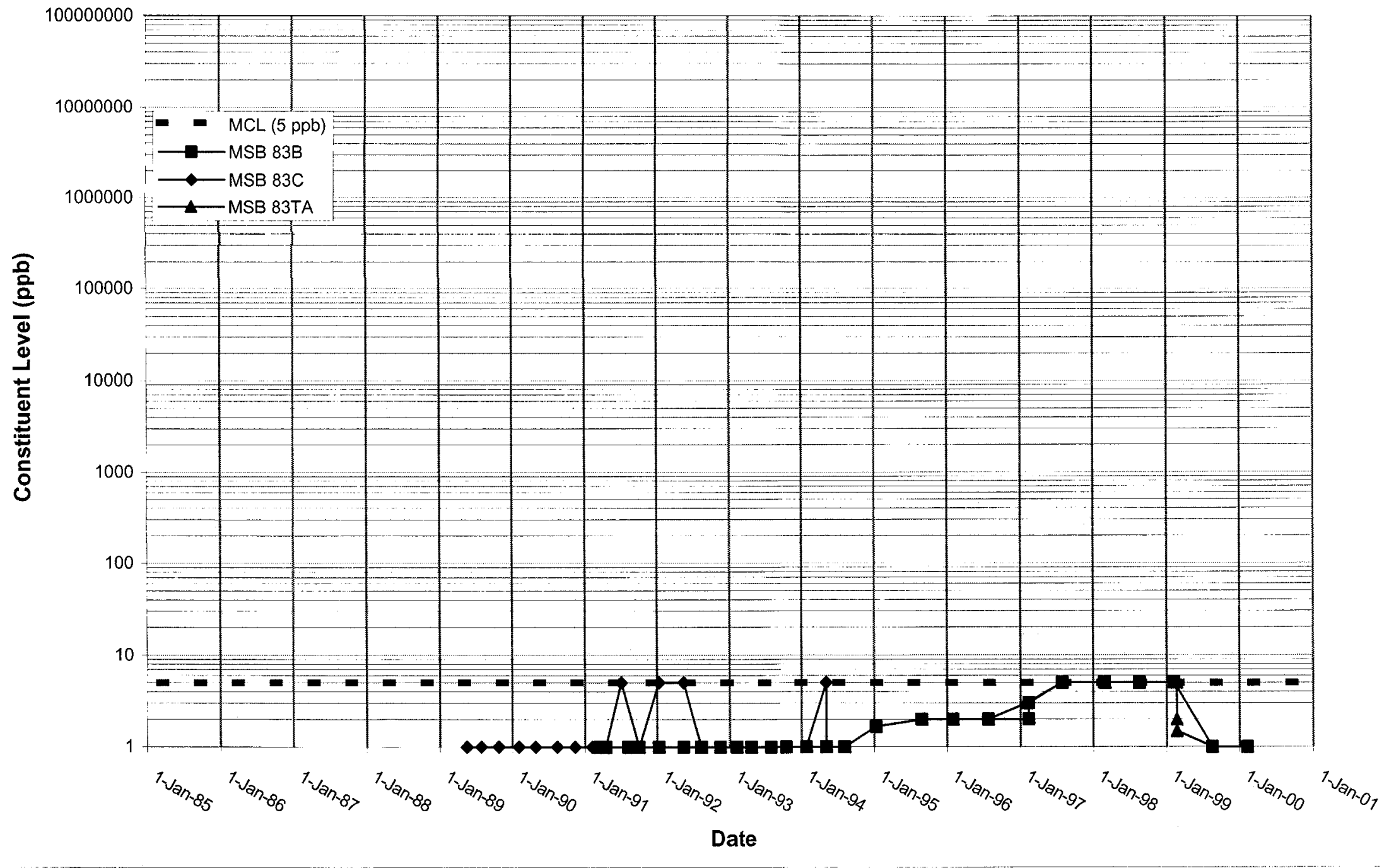




\section{Time Series Plot of Trichloroethylene for Well MSB 84}

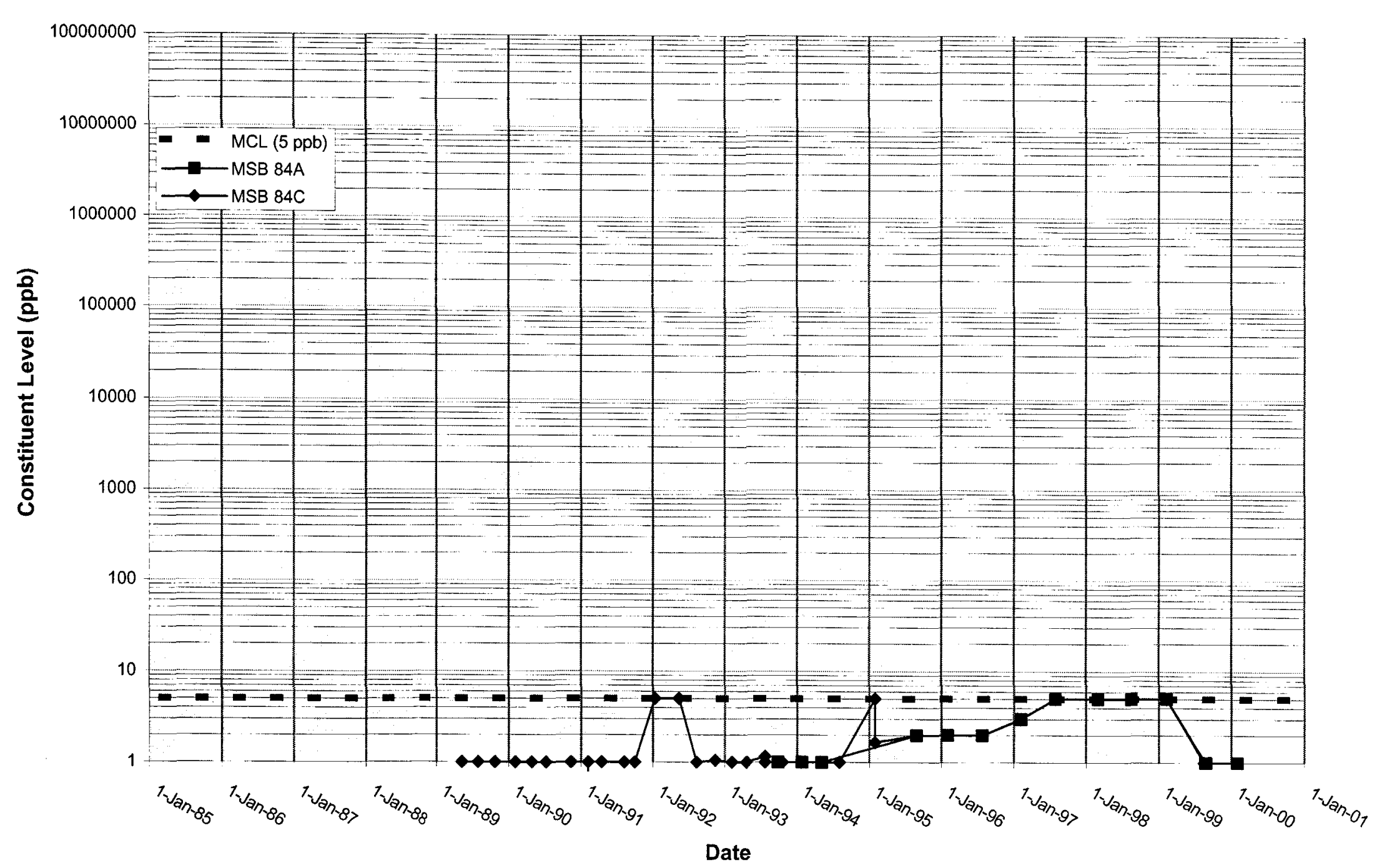


Time Series Plot of Trichloroethylene for Well MSB 85

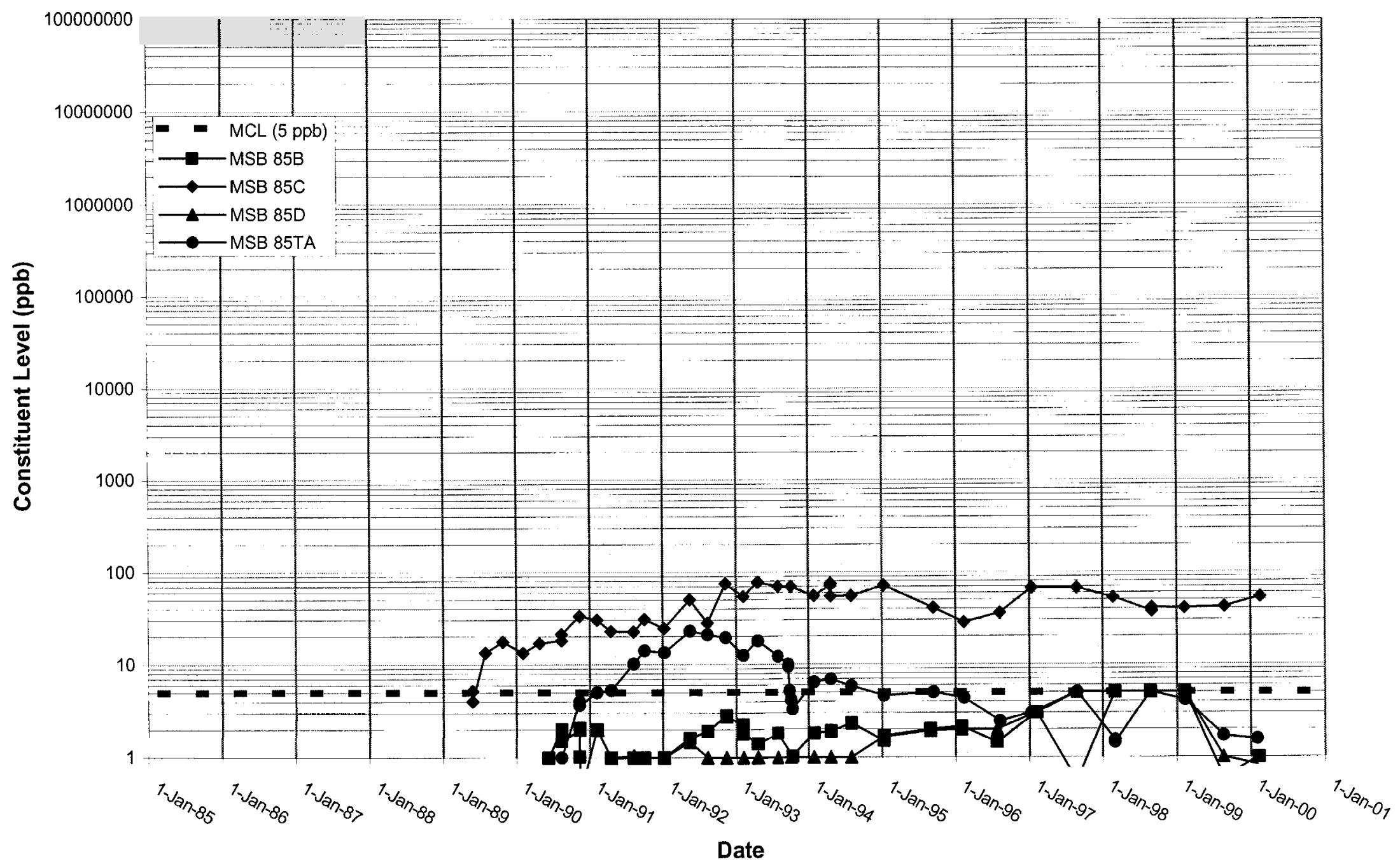




\section{Time Series Plot of Trichloroethylene for Well MSB 86}

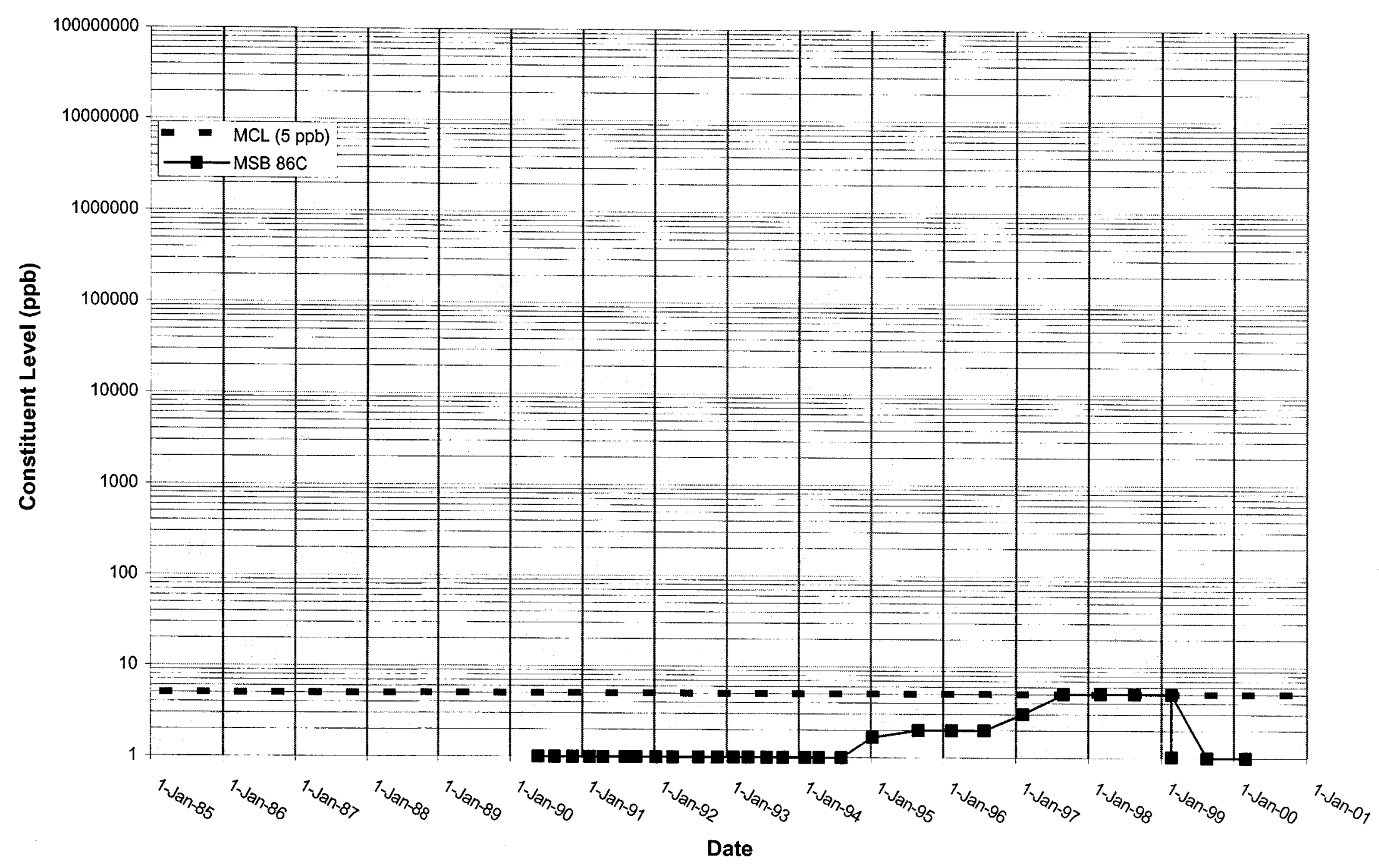


WSRC-TR-2000-00242 UNCLASSIFIED

\section{Time Series Plot of Trichloroethylene for Well MSB 87}

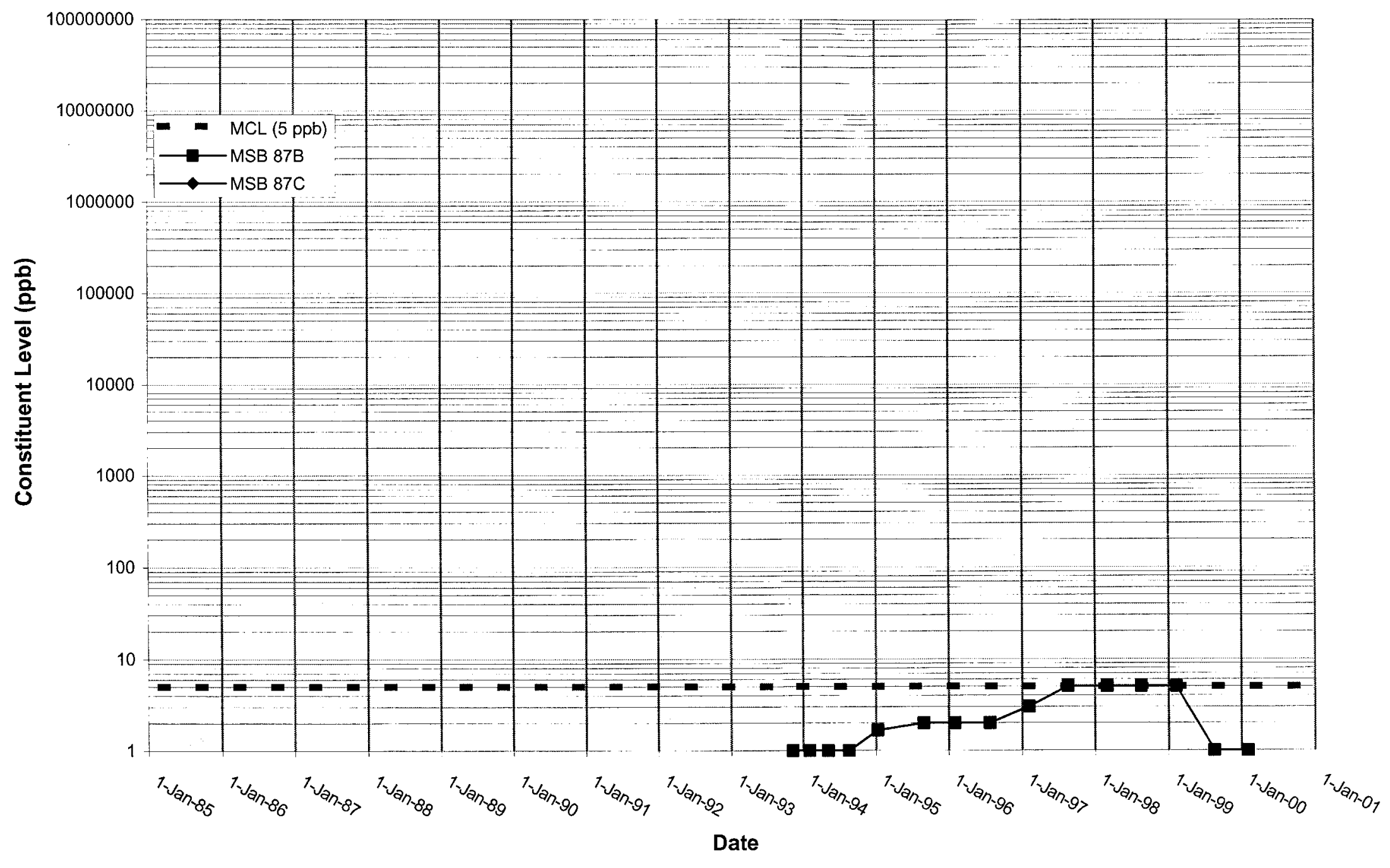




\section{Time Series Plot of Trichloroethylene for Well MSB 88}

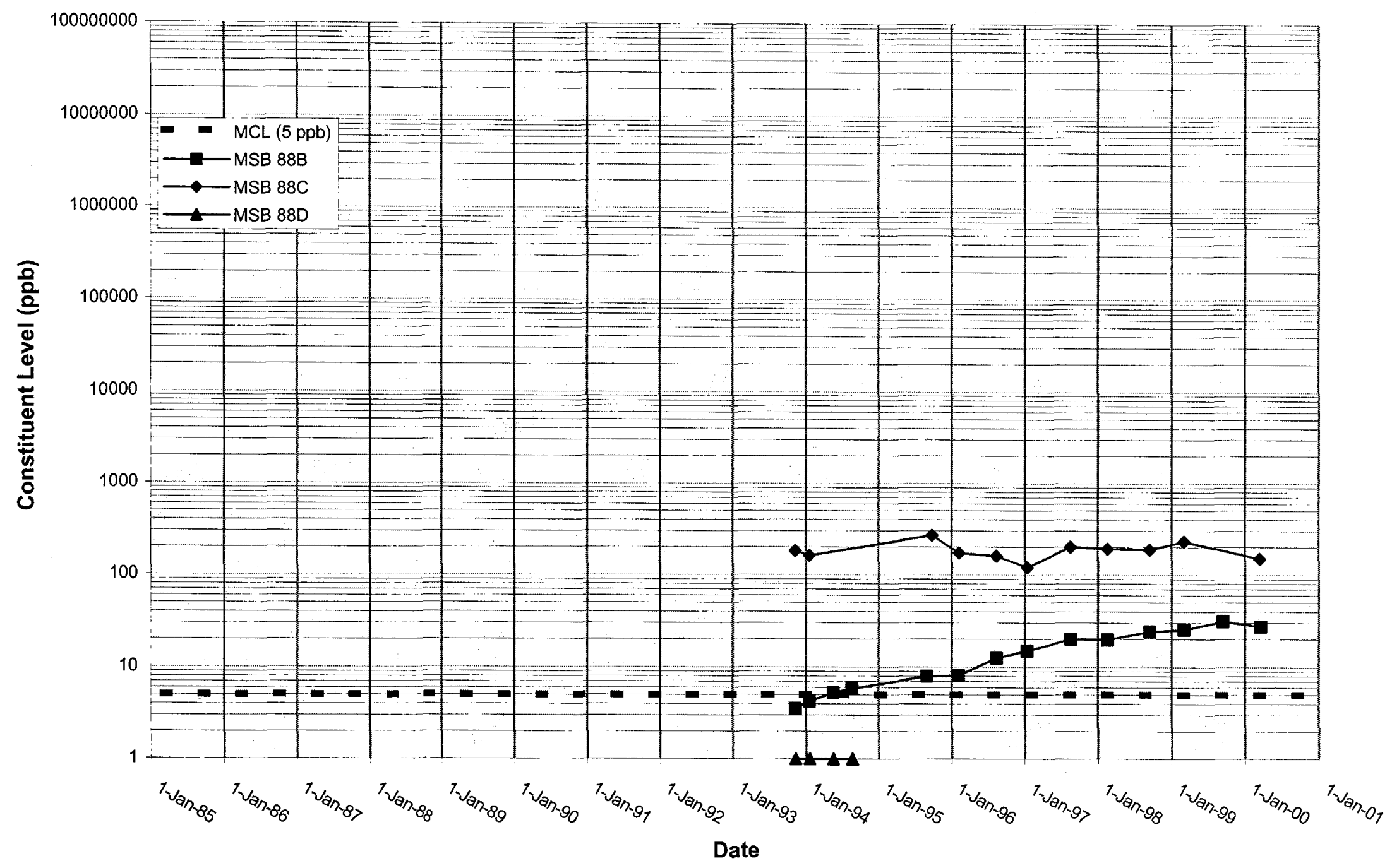




\section{Time Series Plot of Trichloroethylene for Well MSB 89}

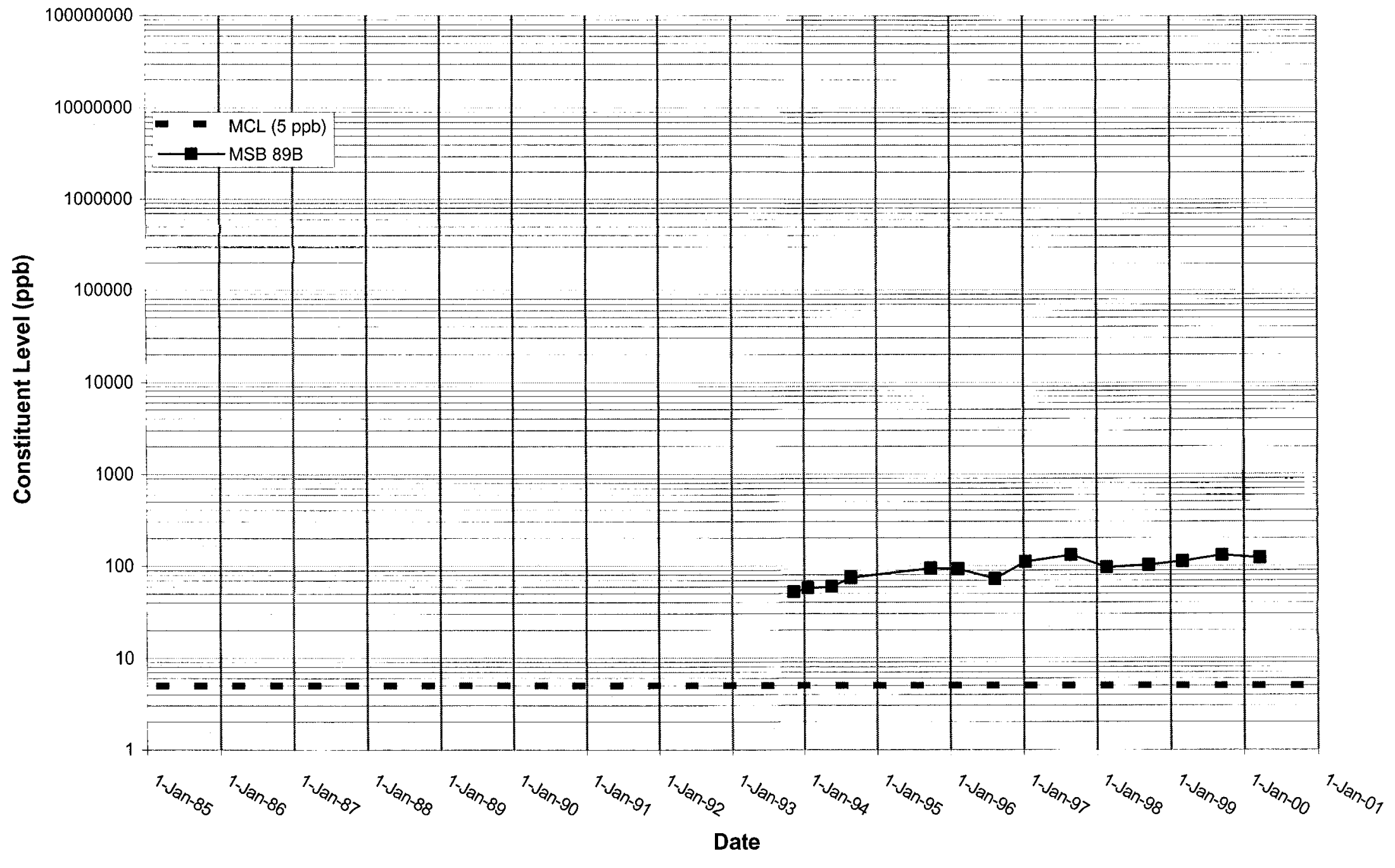


Time Series Plot of Trichloroethylene for Well RWM 1

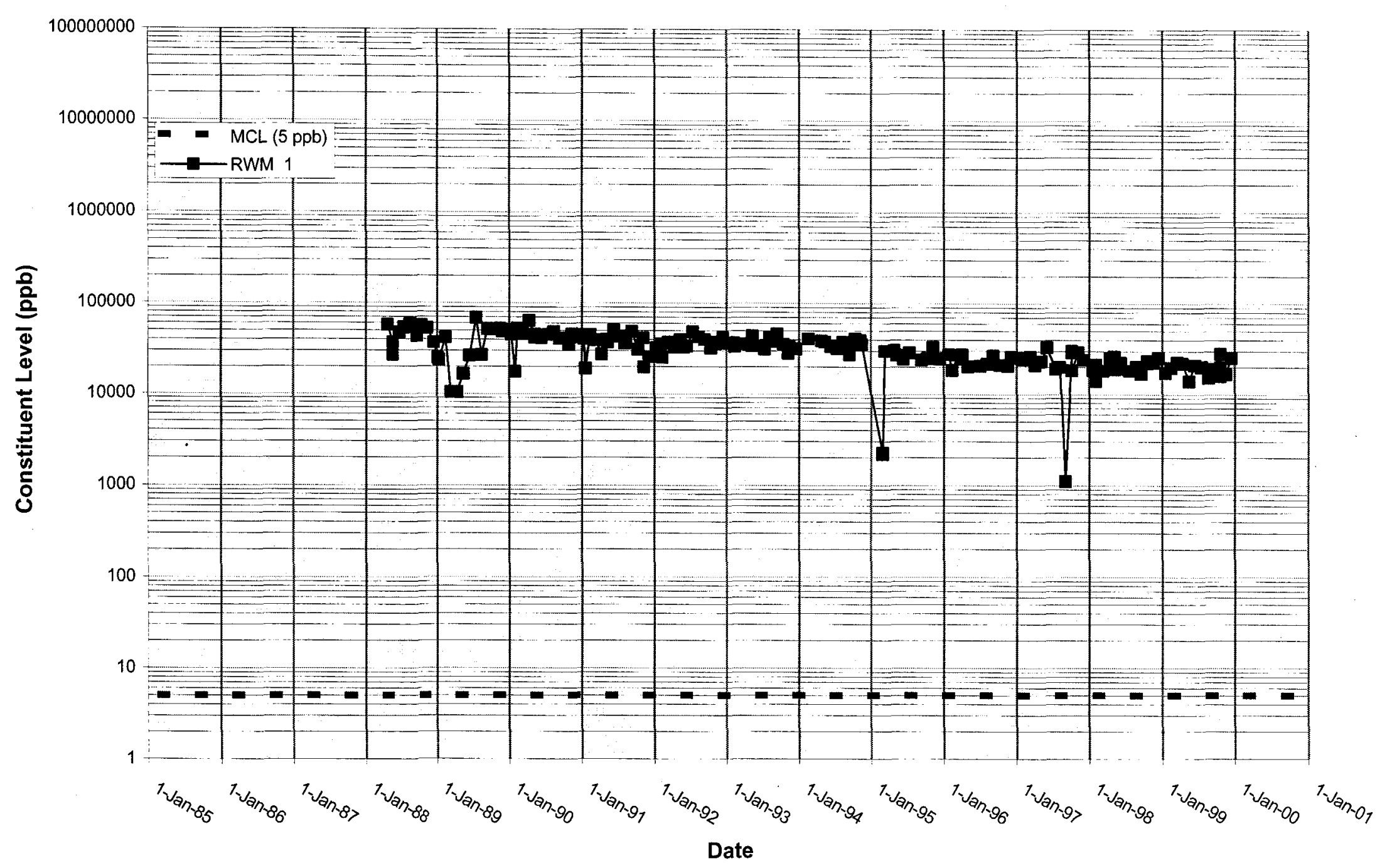




\section{Time Series Plot of Trichloroethylene for Well RWM 2}

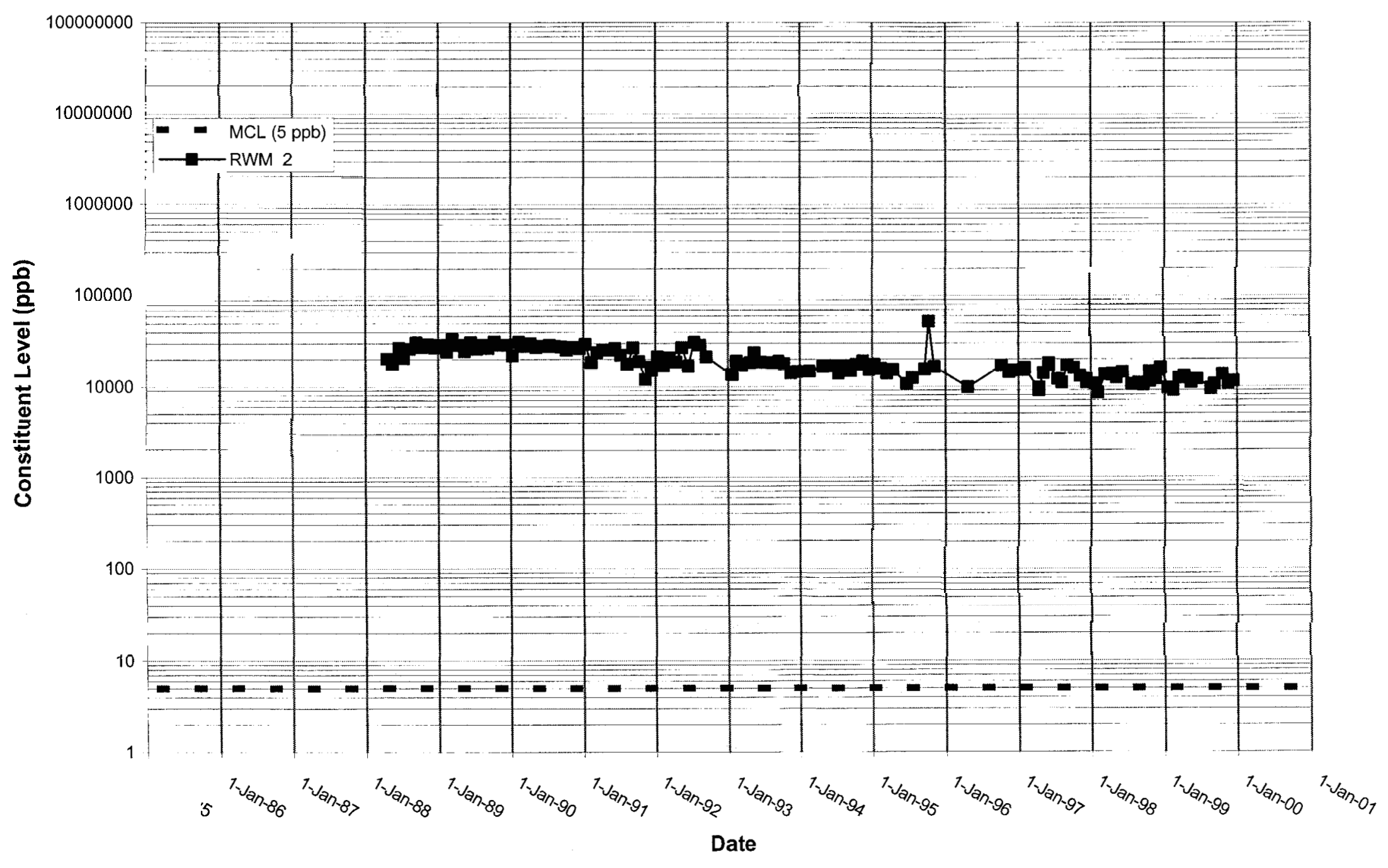


Time Series Plot of Trichloroethylene for Well RWM 3

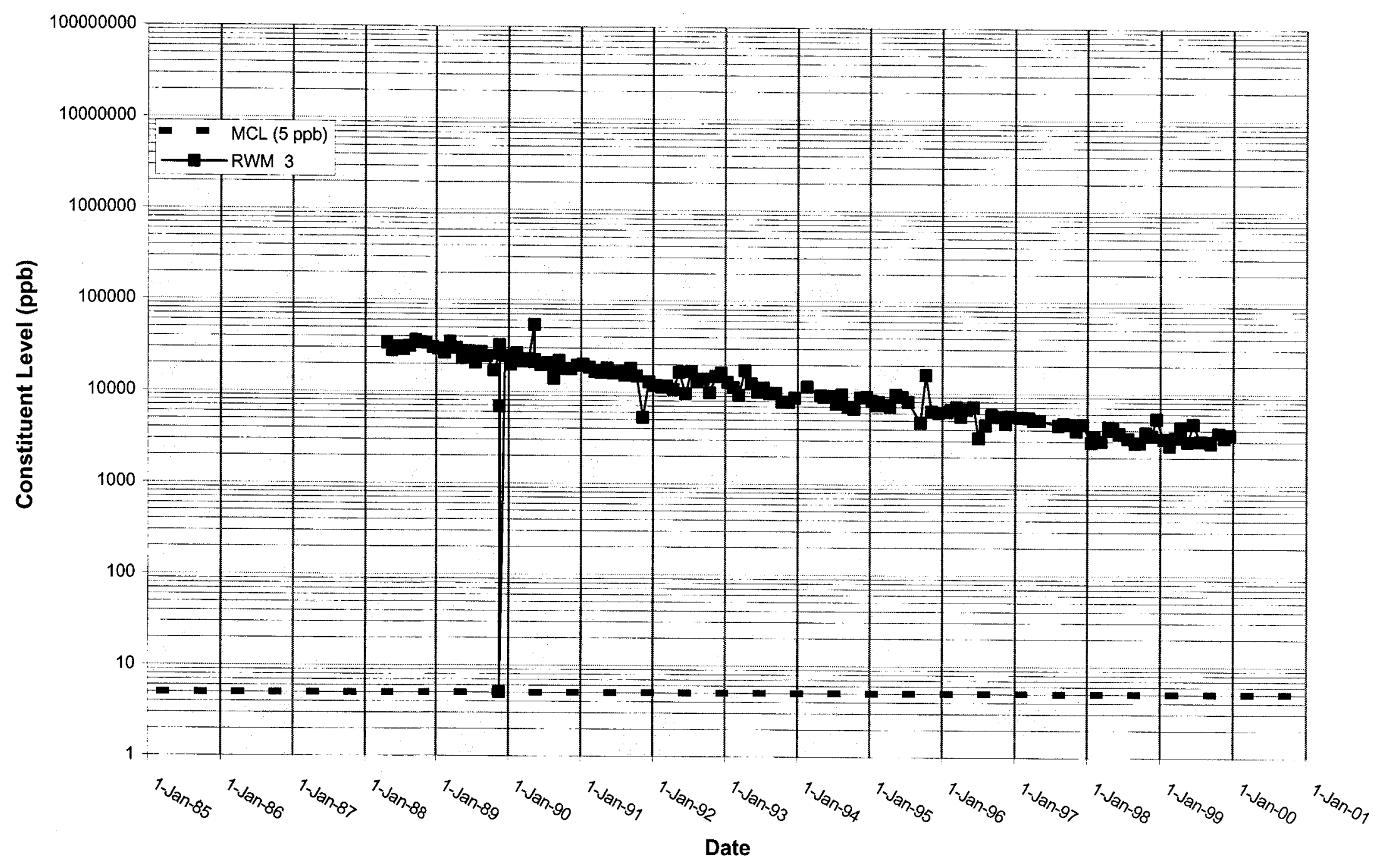


Time Series Plot of Trichloroethylene for Well RWM 4

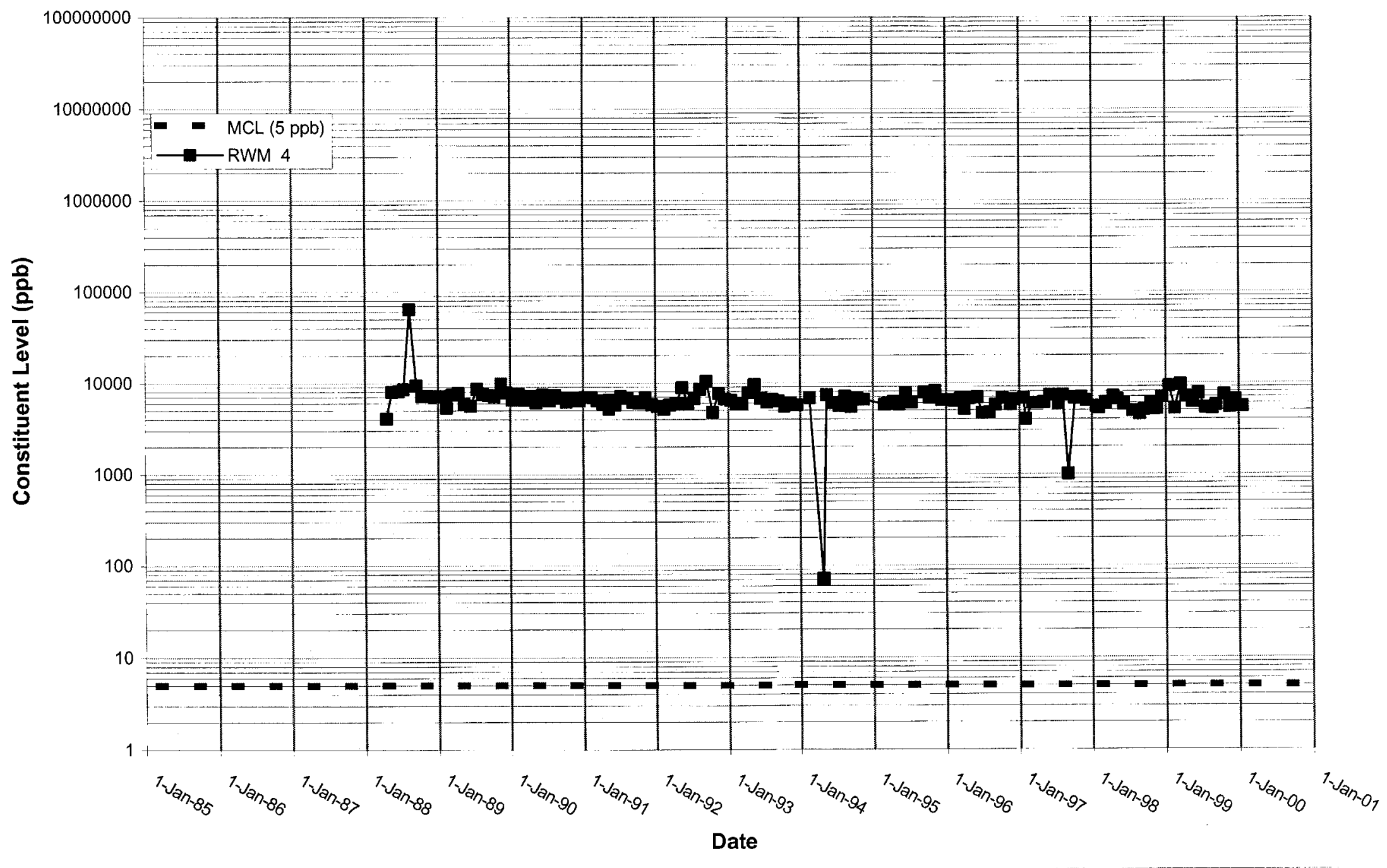


Time Series Plot of Trichloroethylene for Well RWM 5

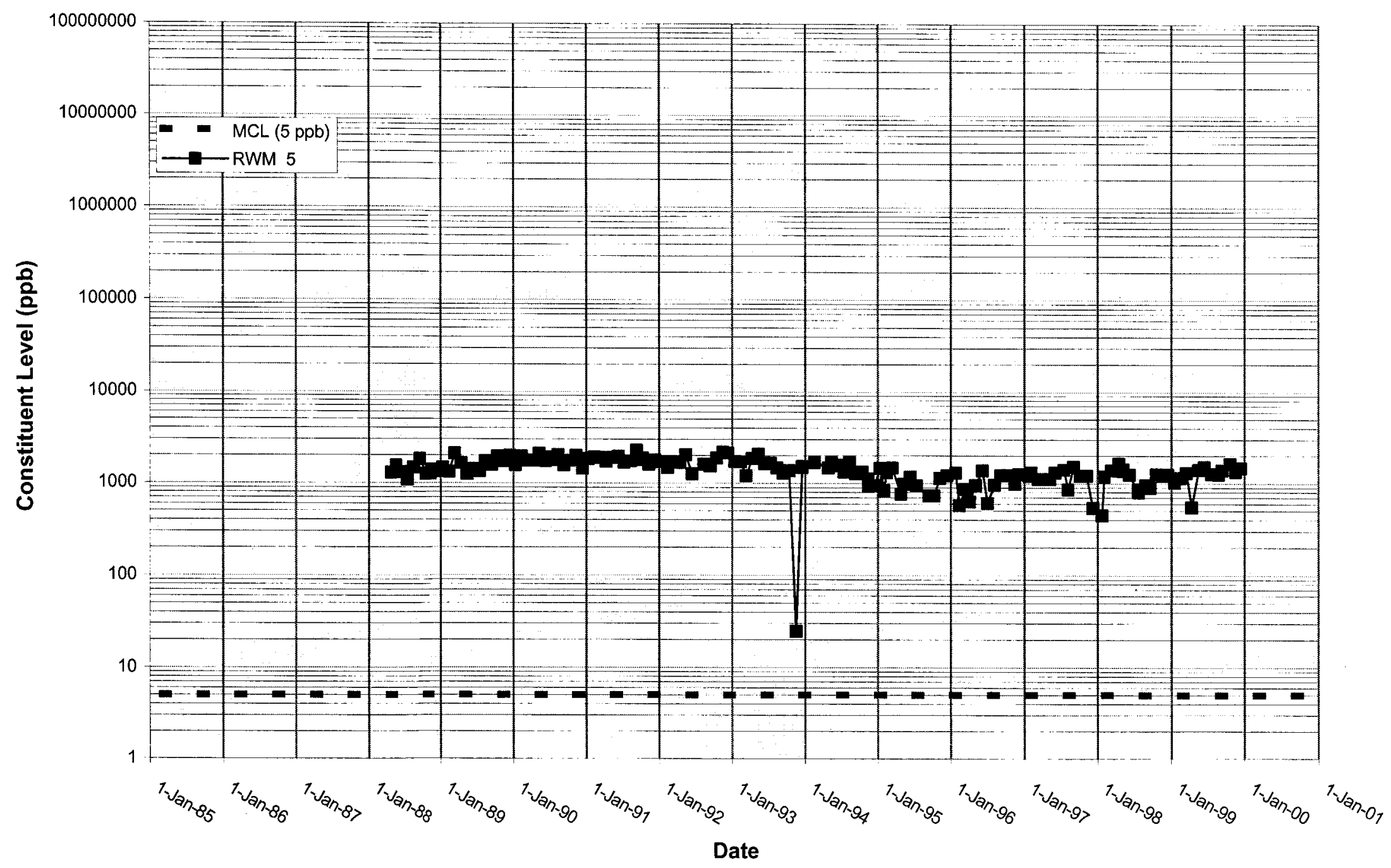


WSRC-TR-2000-00242 UNCLASSIFIED

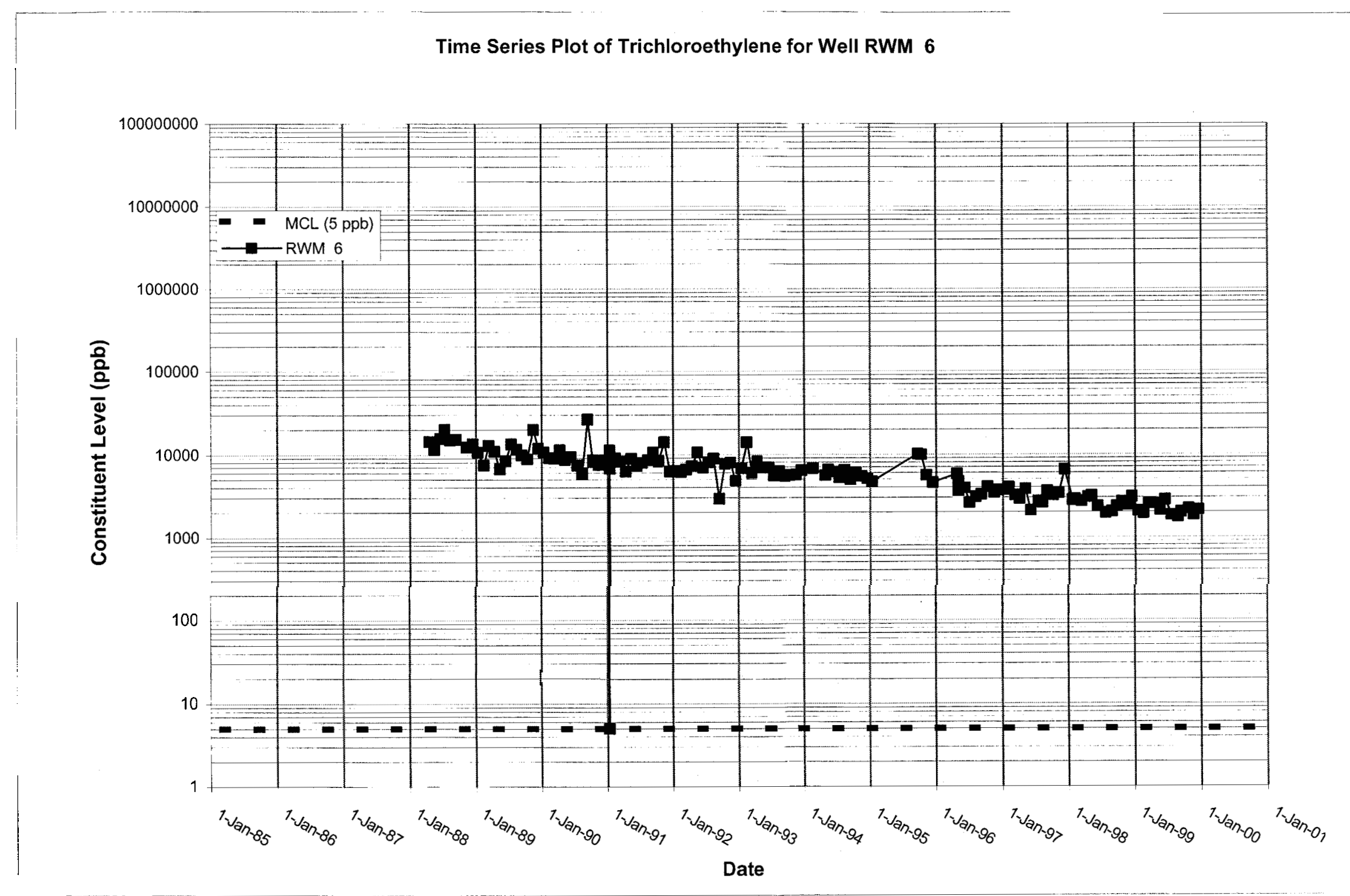




\section{Time Series Plot of Trichloroethylene for Well RWM 7}

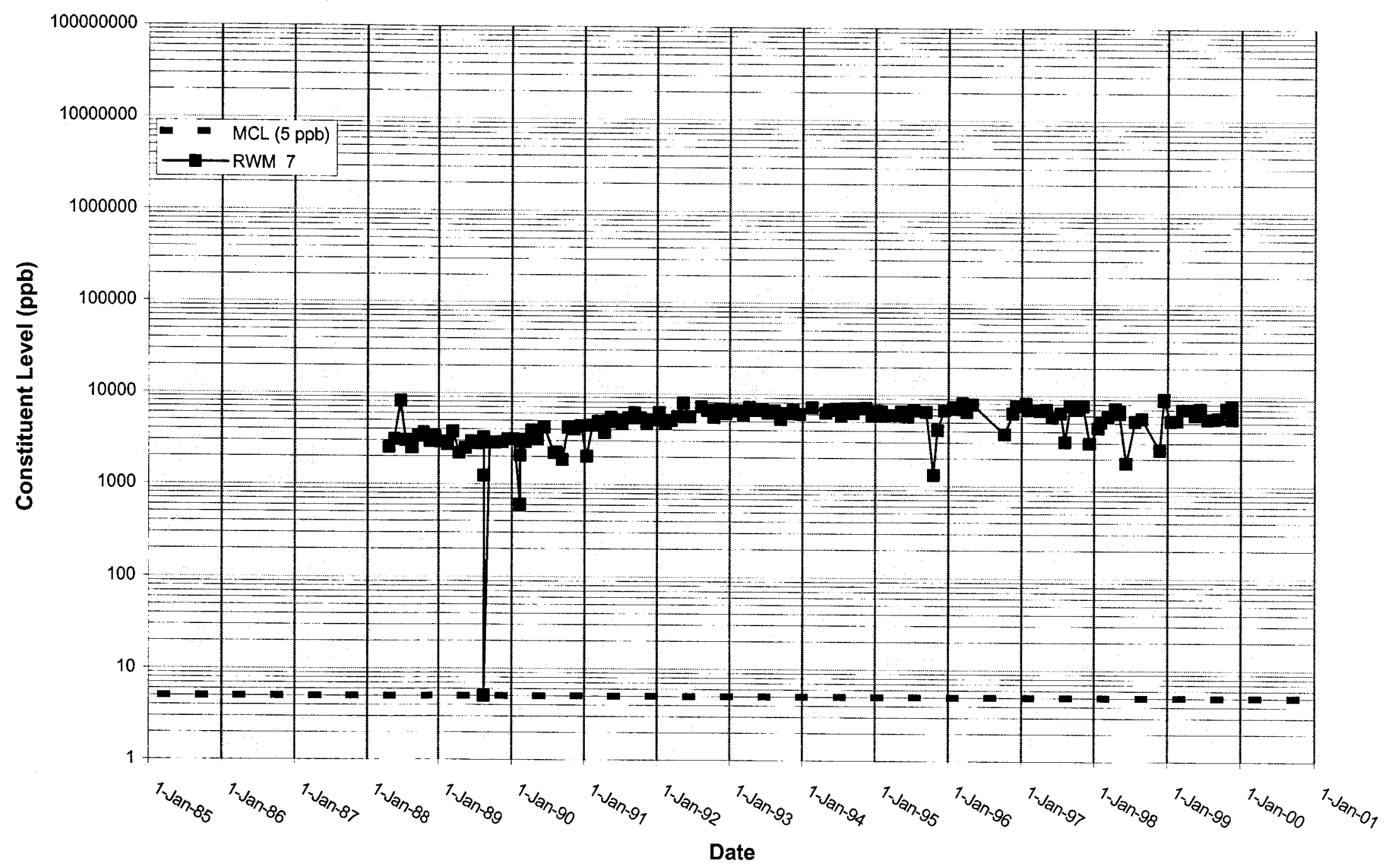


WSRC-TR-2000-00242 UNCLASSIFIED

Time Series Plot of Trichloroethylene for Well RWM 8

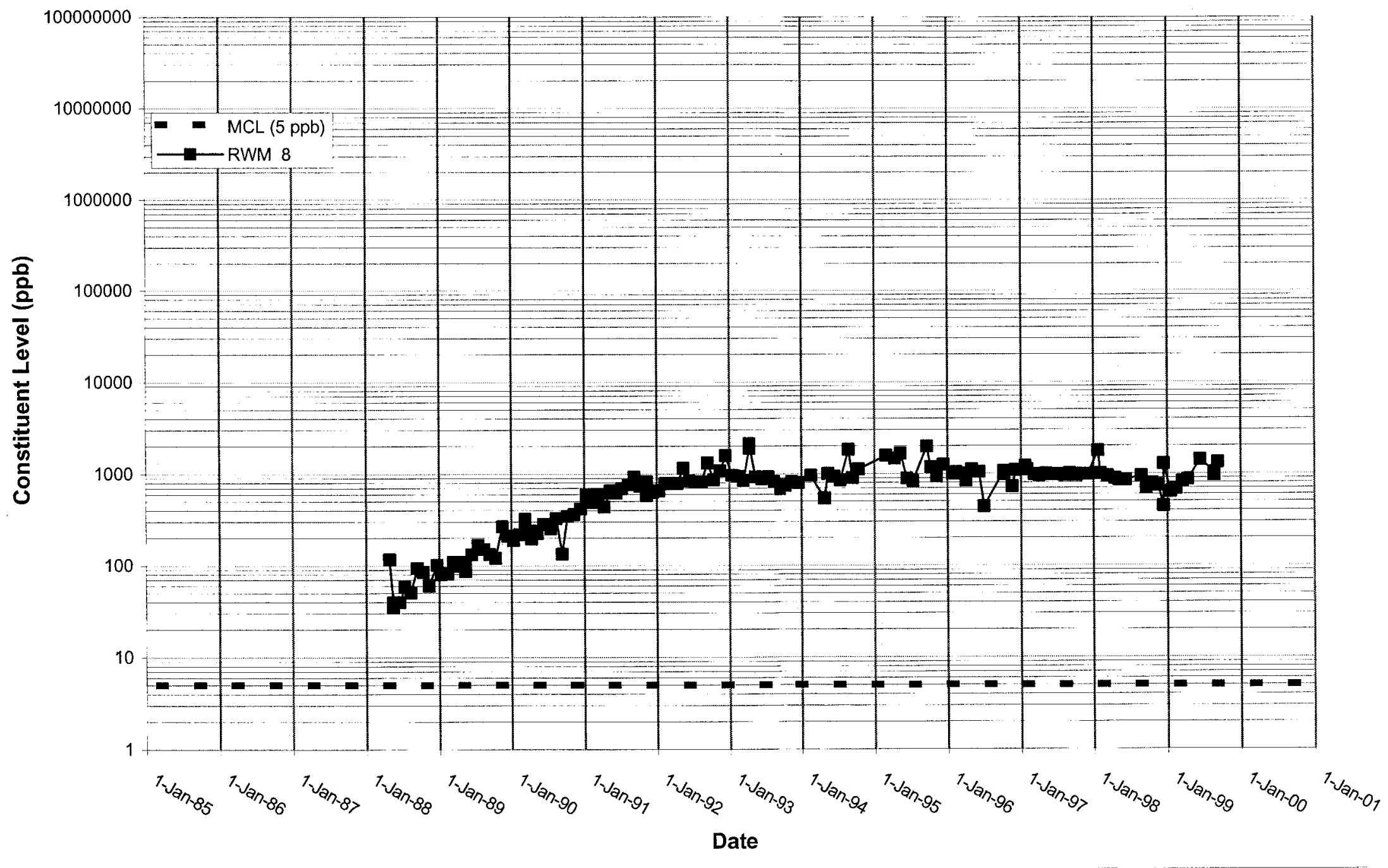




\section{Time Series Plot of Trichloroethylene for Well RWM 9}

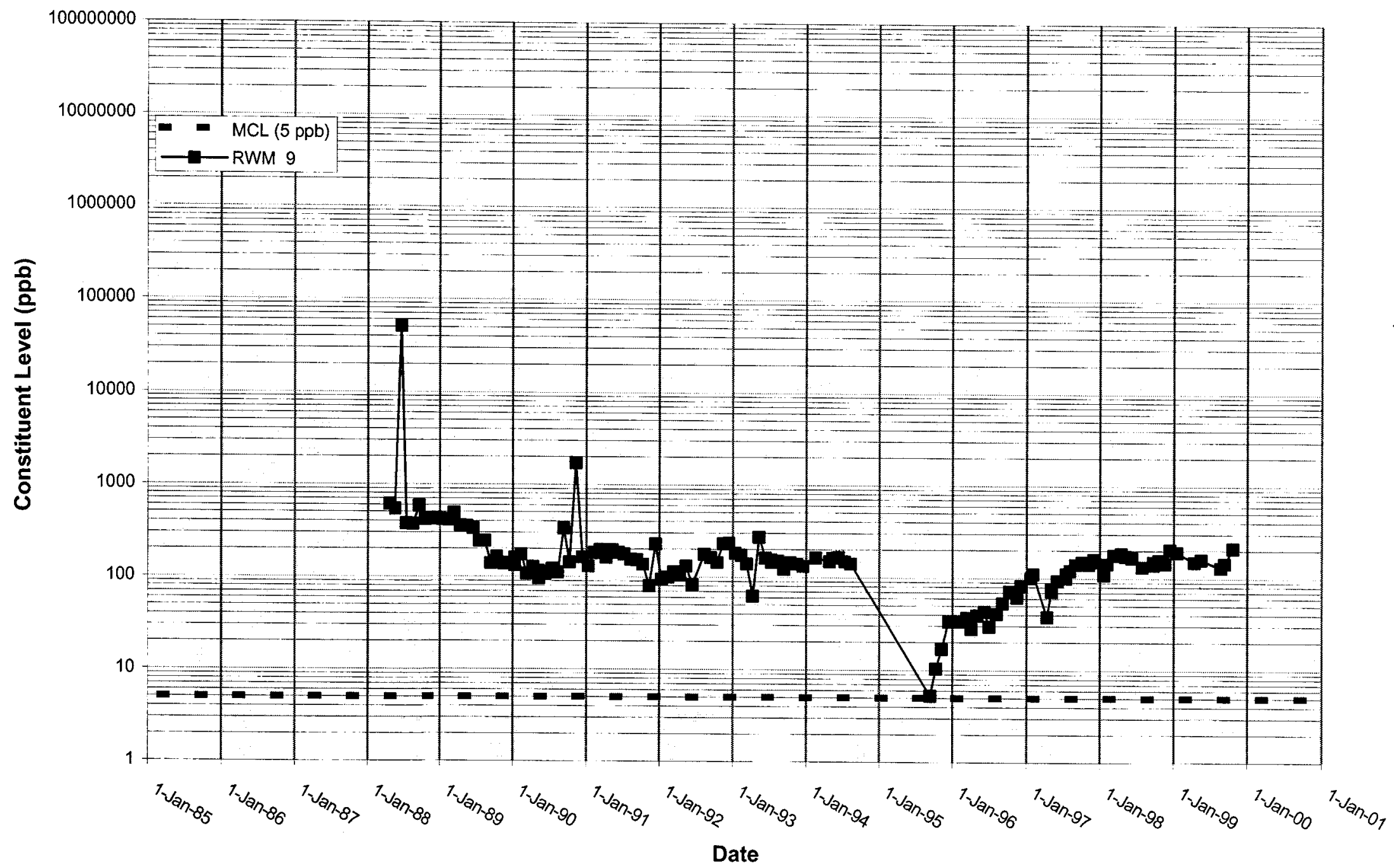


WSRC-TR-2000-00242 UNCLASSIFIED

Time Series Plot of Trichloroethylene for Well RWM 10

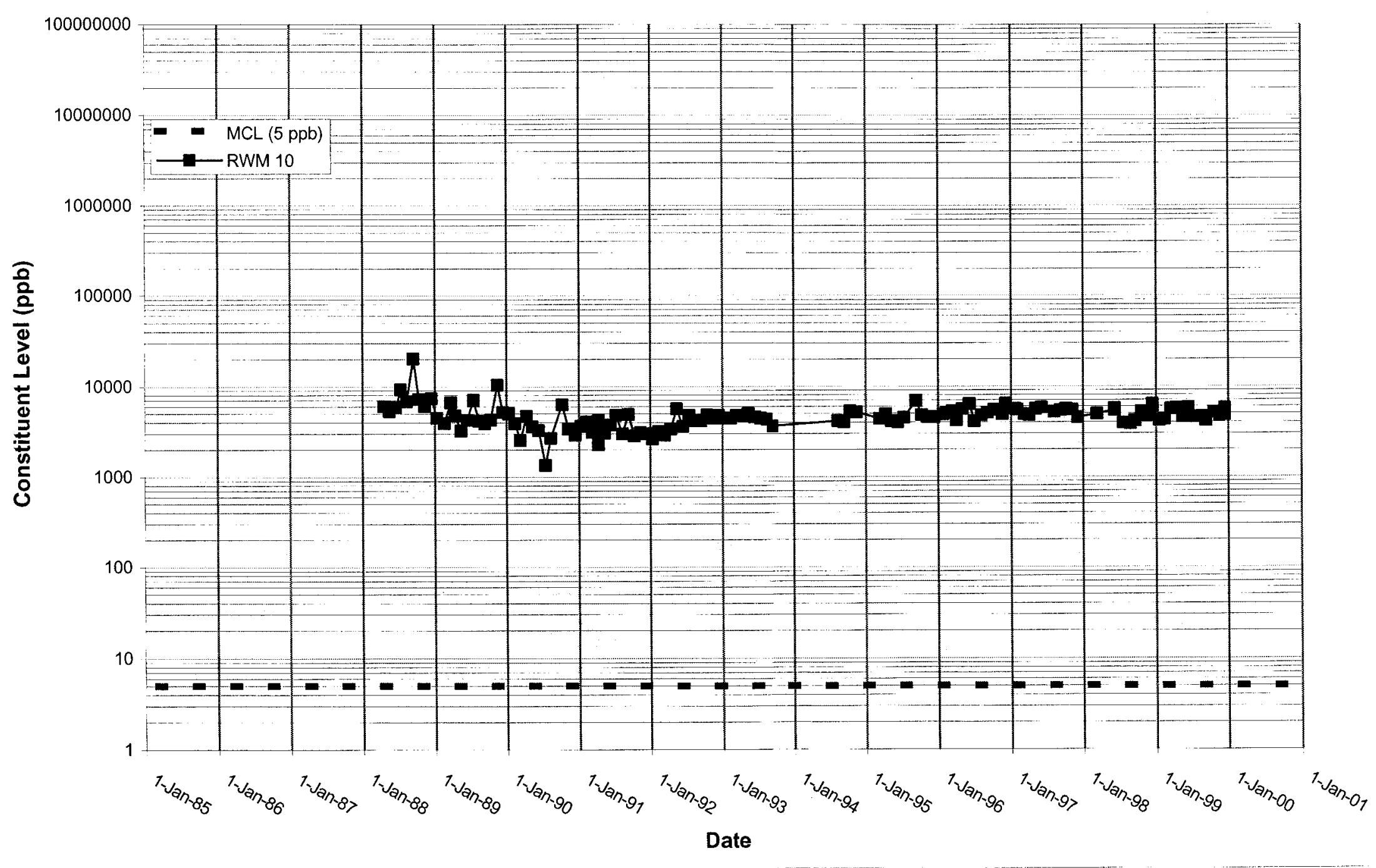




\section{Time Series Plot of Trichloroethylene for Well RWM 11}

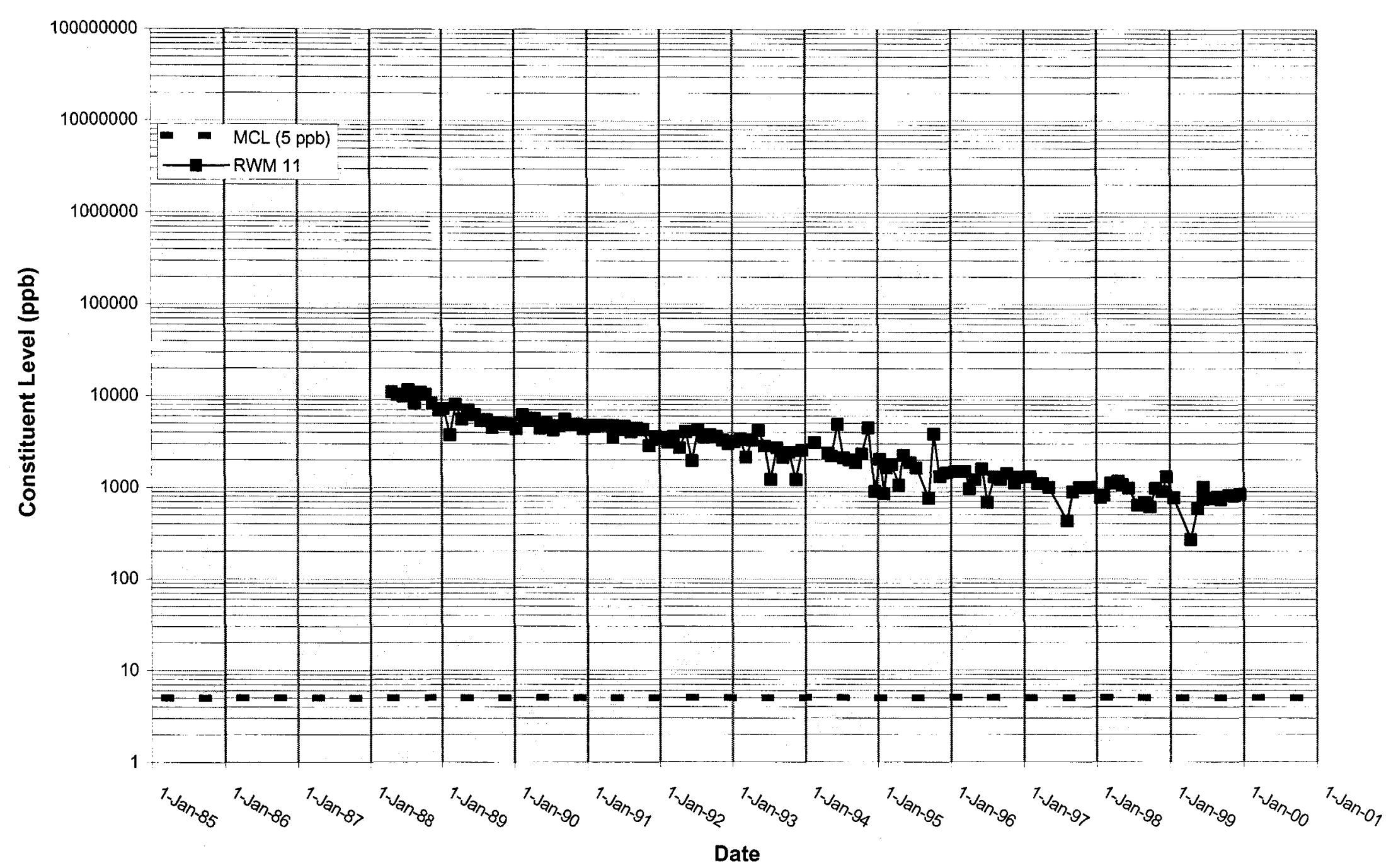


WSRC-TR-2000-00242 UNCLASSIFIED

\section{Time Series Plot of Trichloroethylene for Well RWM 12}

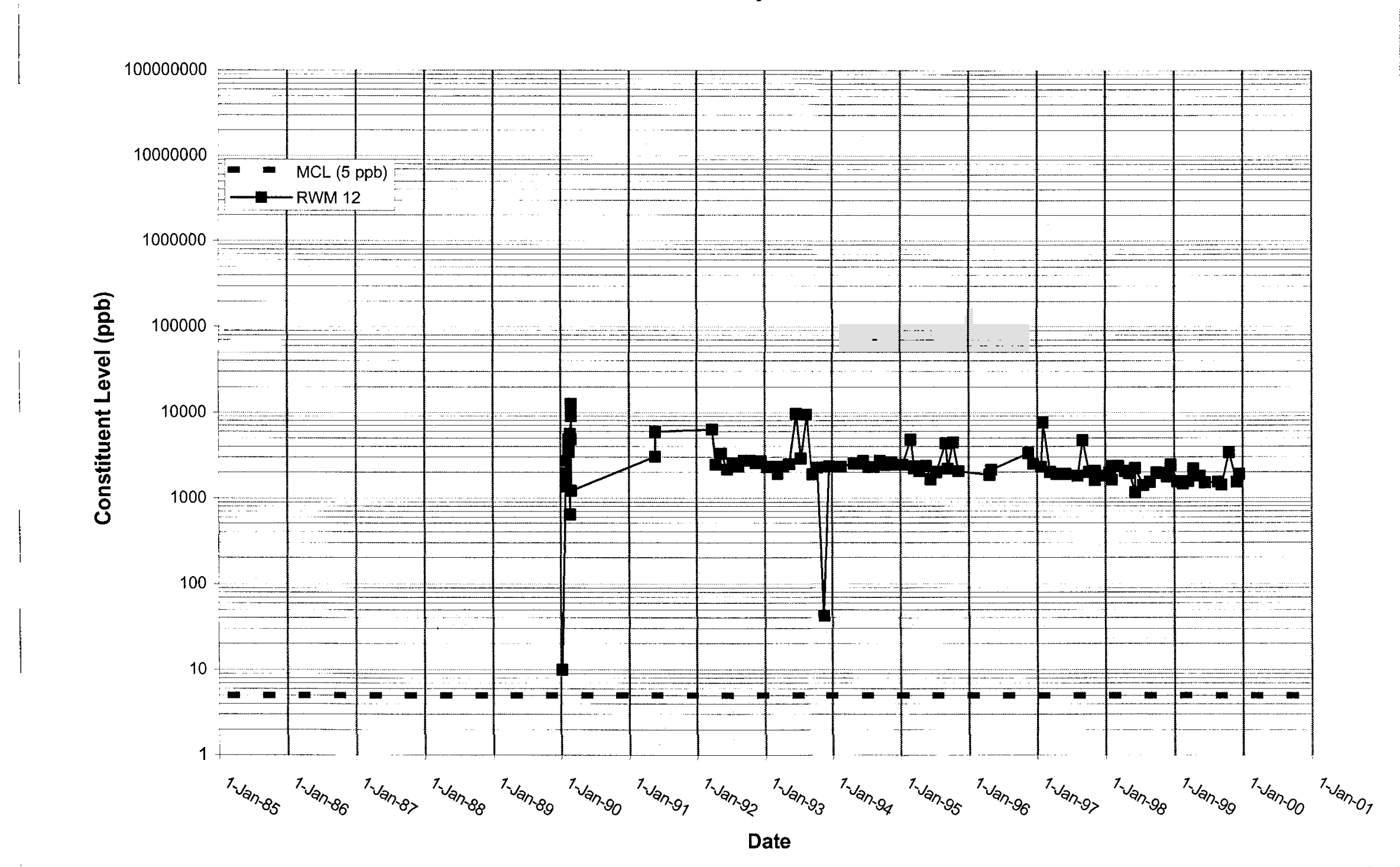


WSRC-TR-2000-00242 UNCLASSIFIED

\section{Time Series Plot of Trichloroethylene for Well RWM 13}

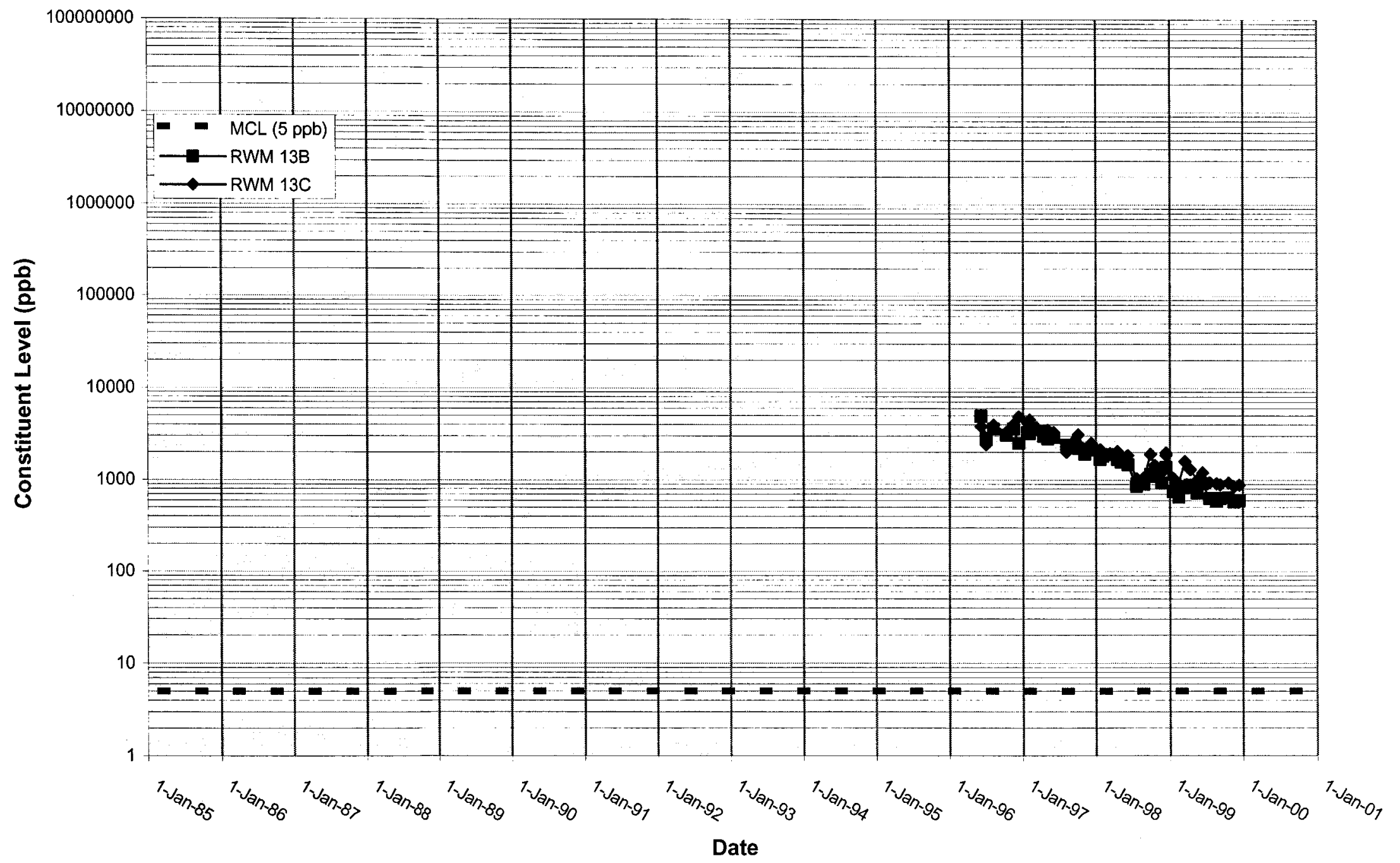


WSRC-TR-2000-00242 UNCLASSIFIED

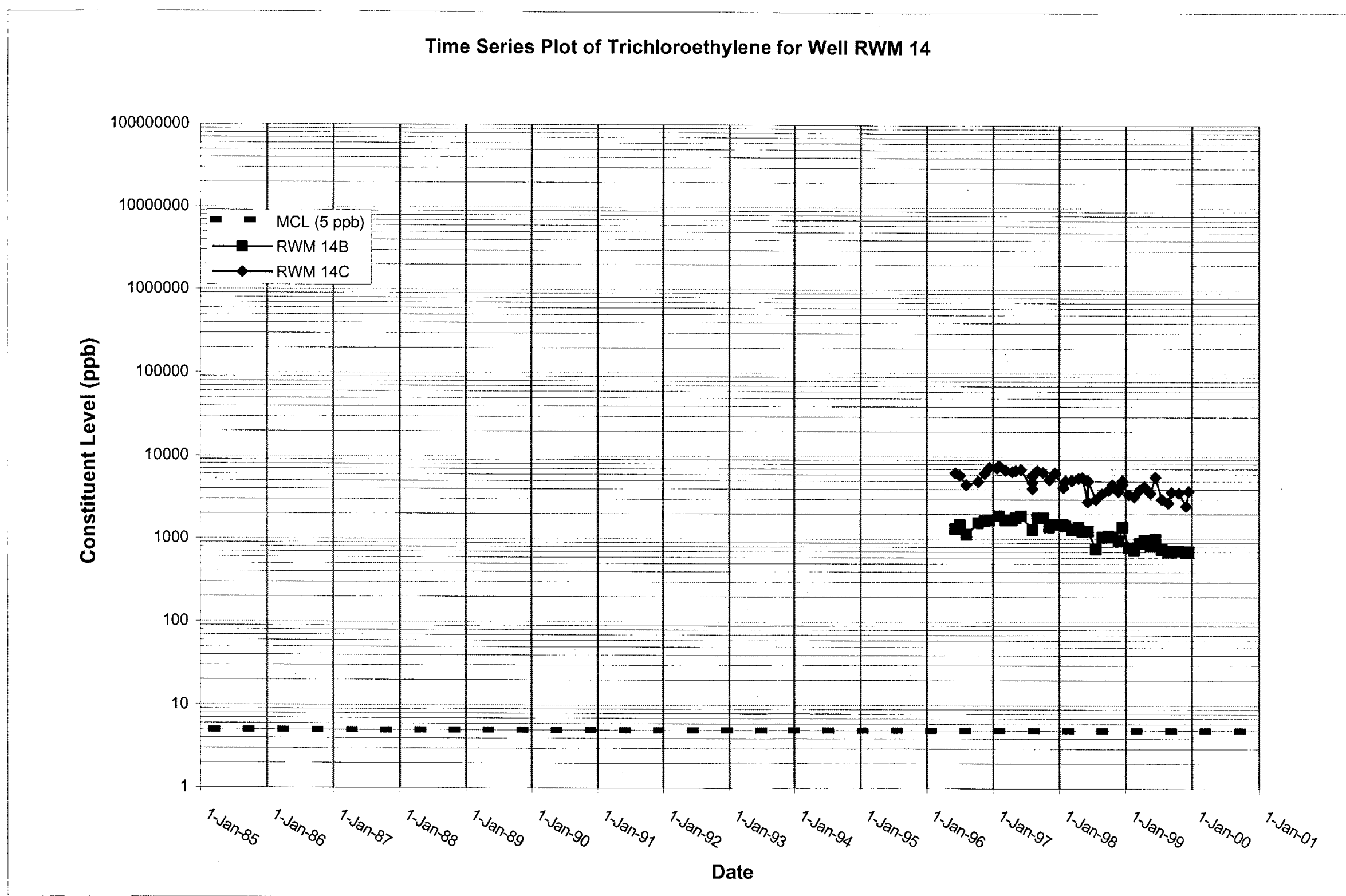




\section{Time Series Plot of Trichloroethylene for Well RWM 15}

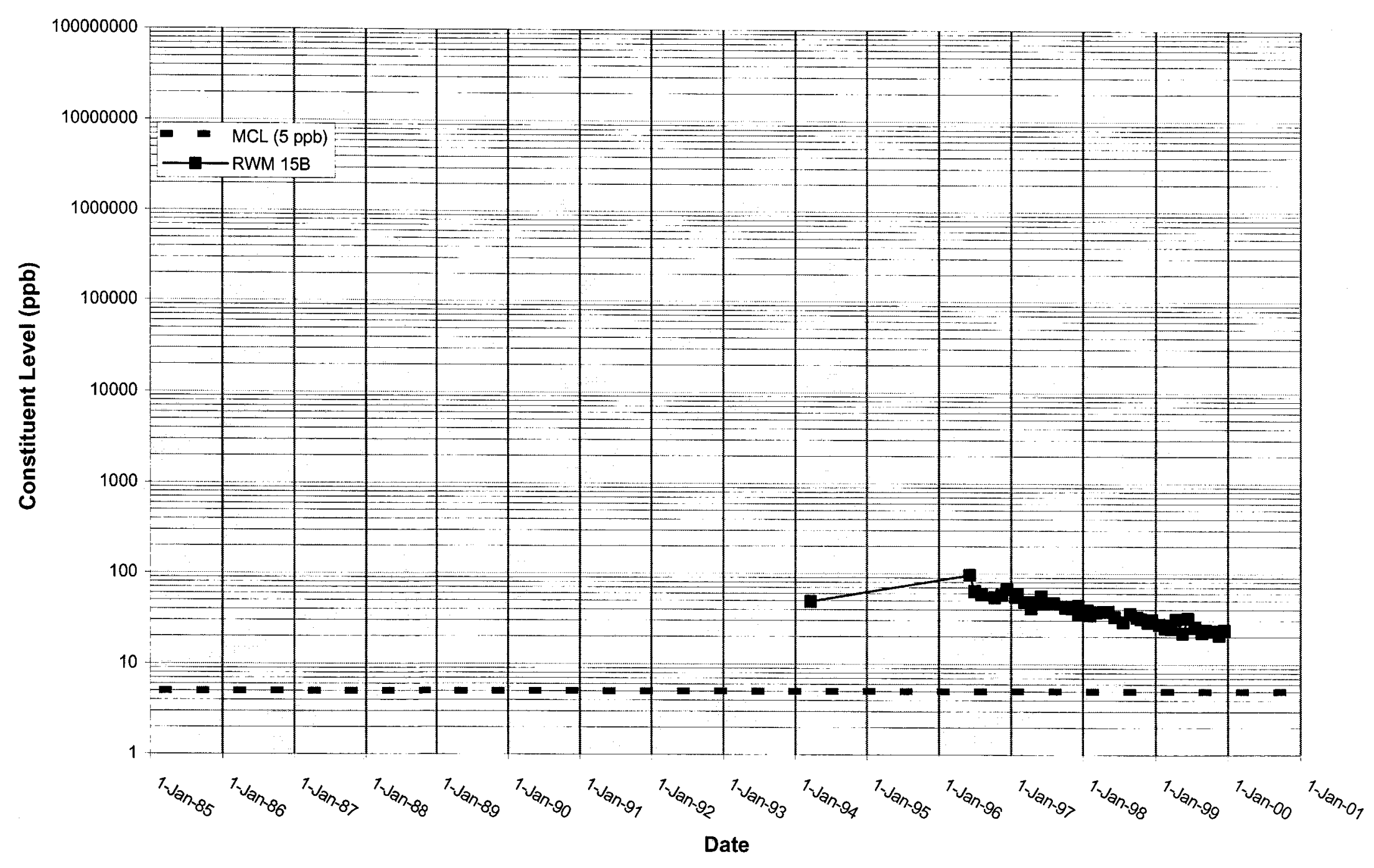


WSRC-TR-2000-00242 UNCLASSIFIED

Time Series Plot of Trichloroethylene for Well SRW 1

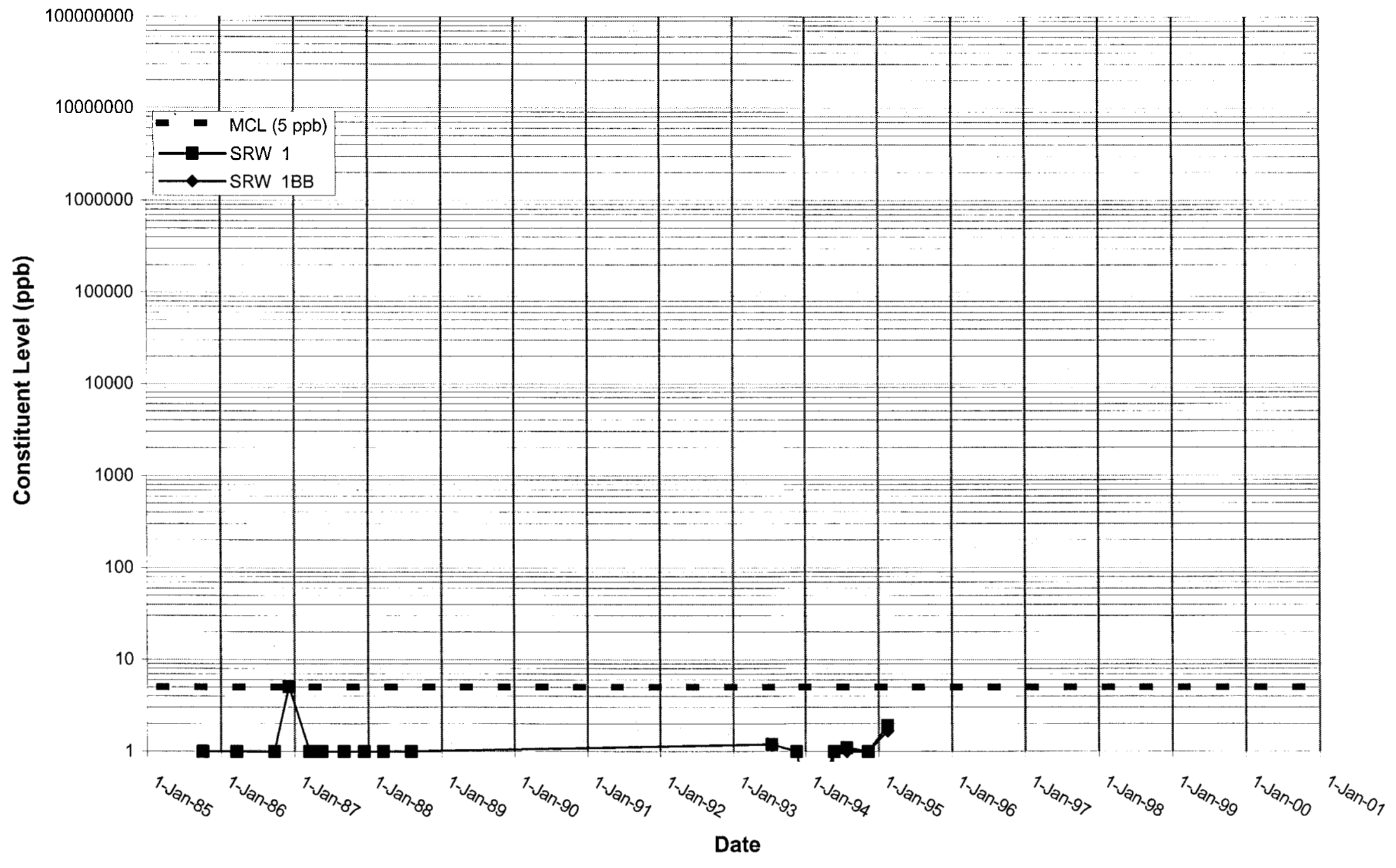


WSRC-TR-2000-00242 UNCLASSIFIED

Time Series Plot of Trichloroethylene for Well SRW 2

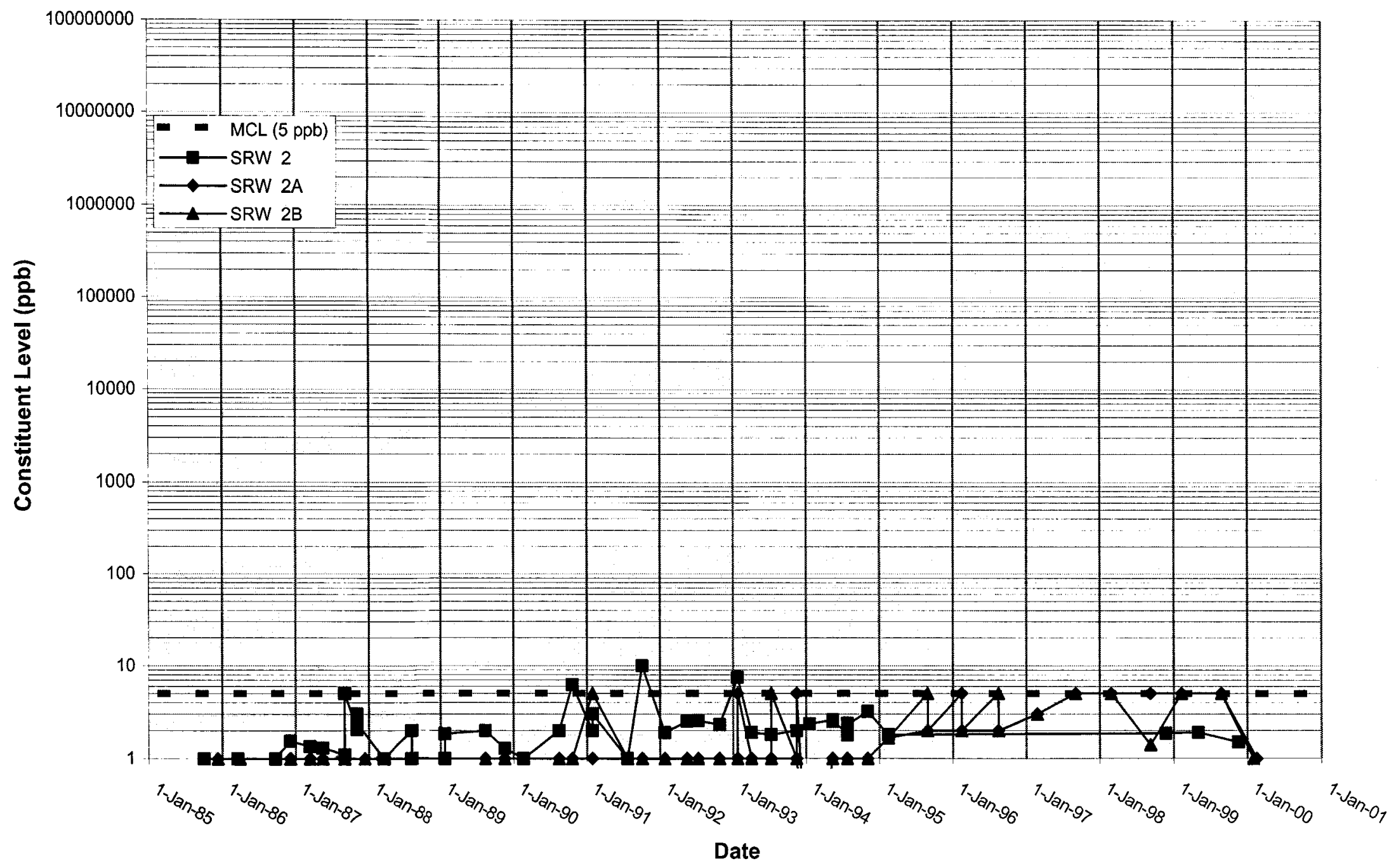


Time Series Plot of Trichloroethylene for Well SRW 3

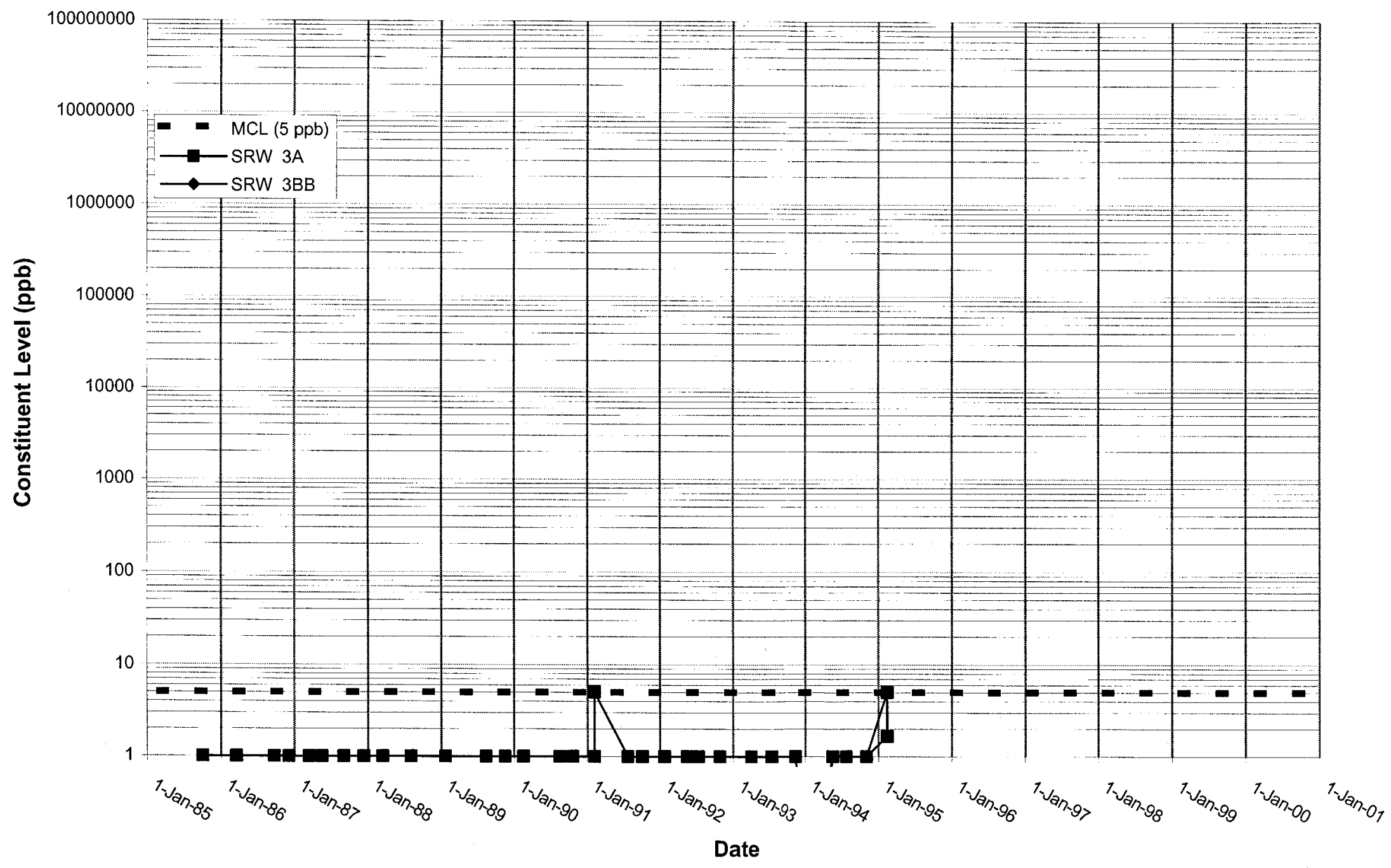




\section{1}

Time Series Plot of Trichloroethylene for Well SRW 4

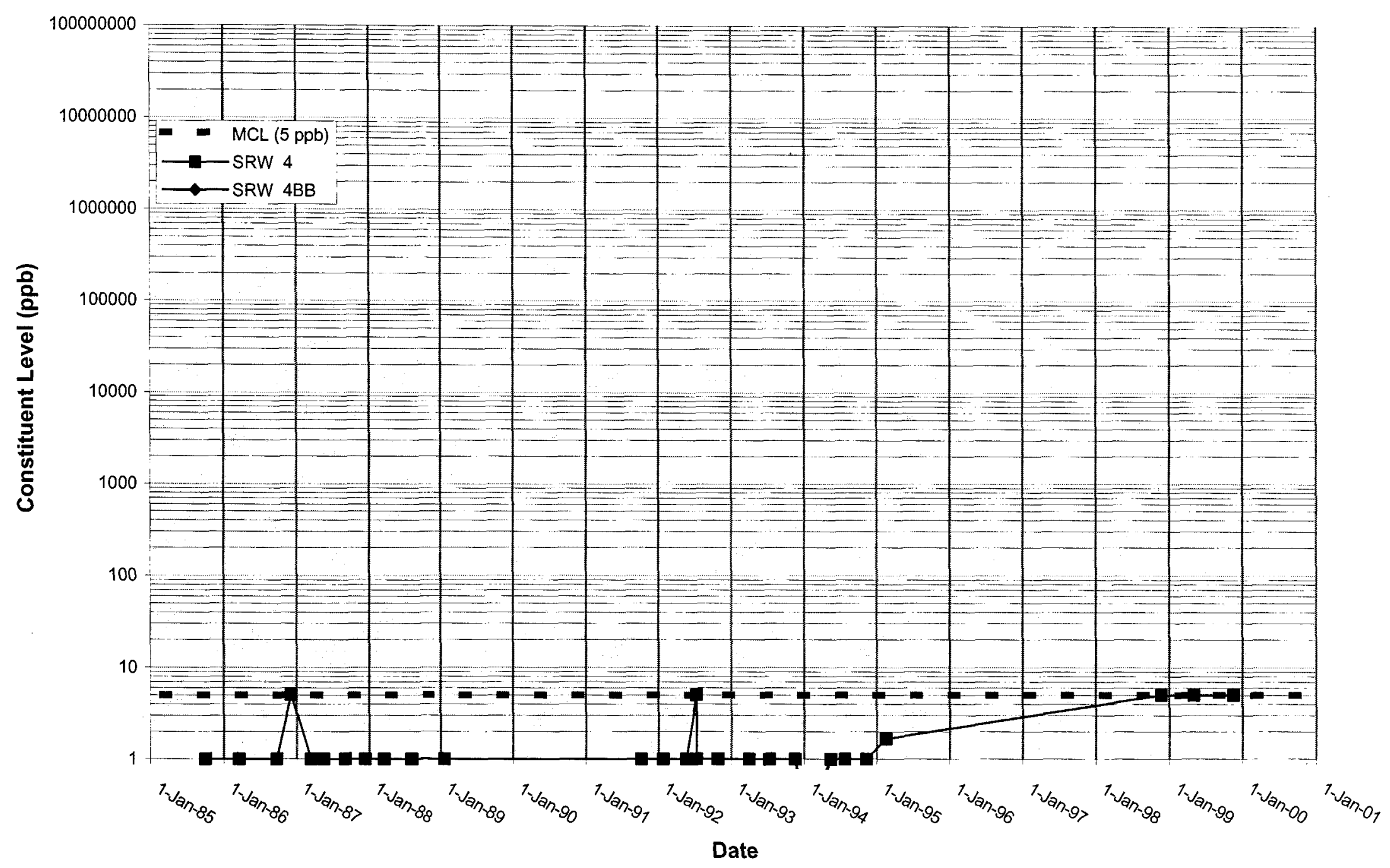




\section{Time Series Plot of Trichloroethylene for Well SRW 5}

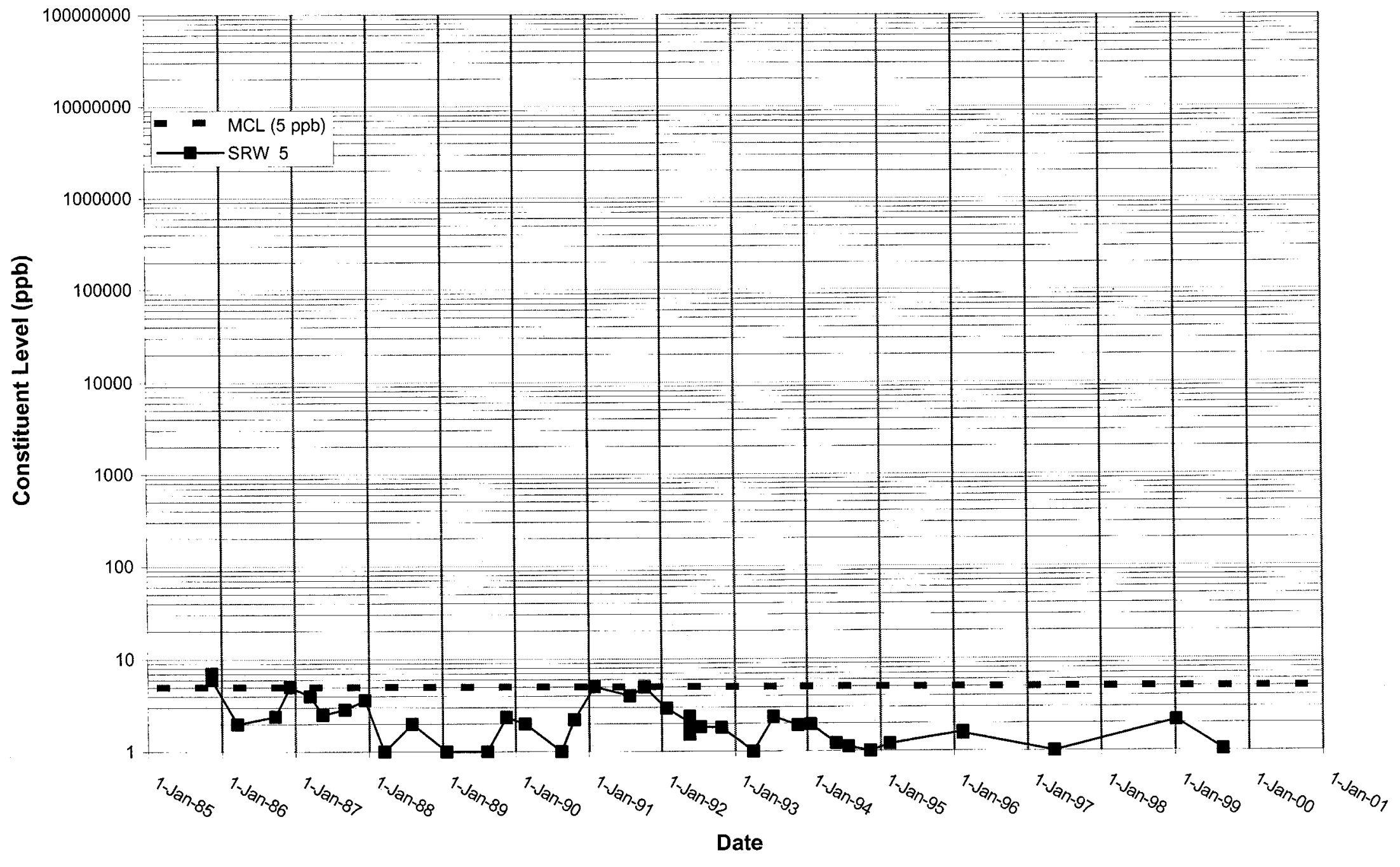


WSRC-TR-2000-00242 UNCLASSIFIED

Time Series Plot of Trichloroethylene for Well SRW 6

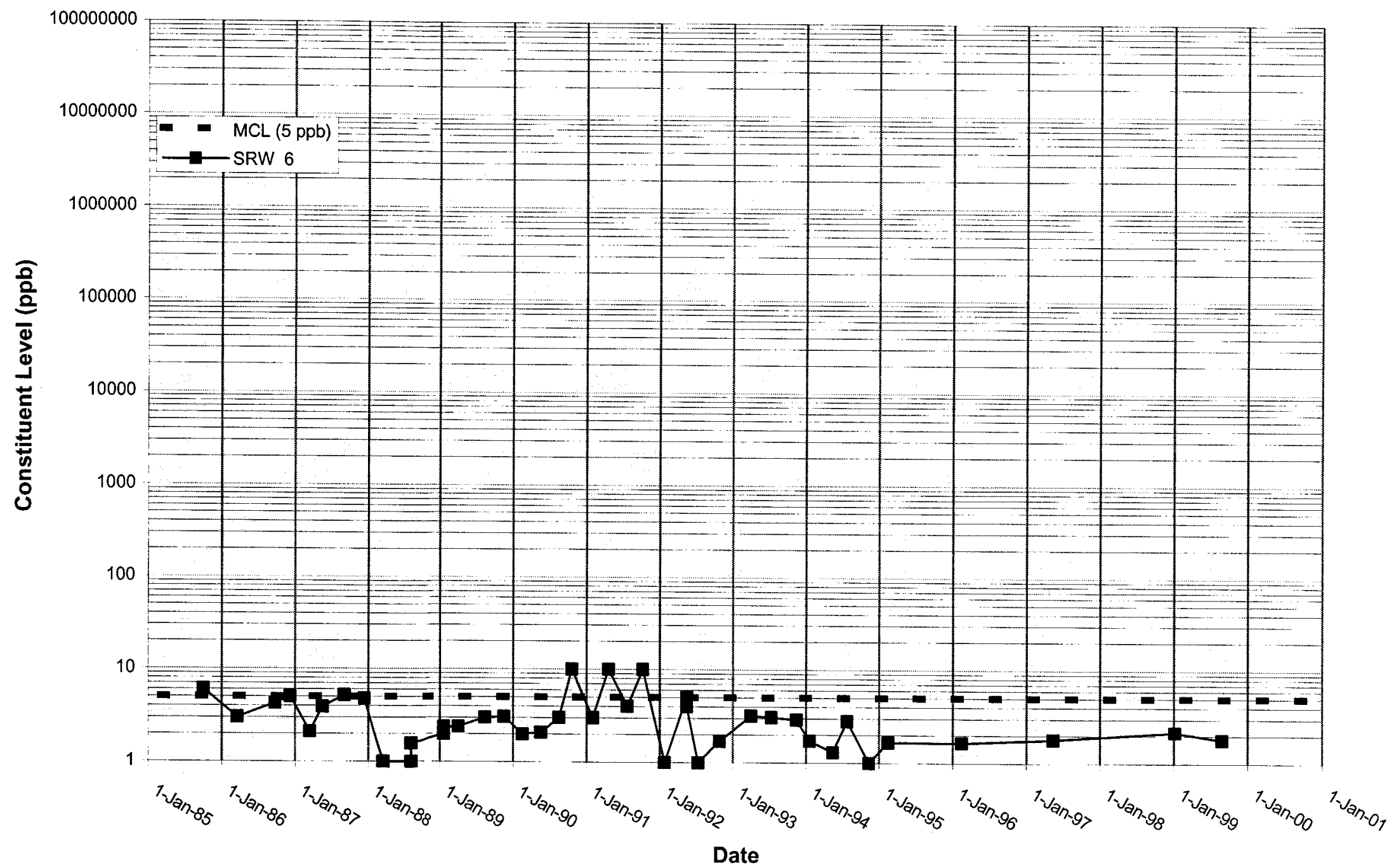




\section{Time Series Plot of Trichloroethylene for Well SRW 7}

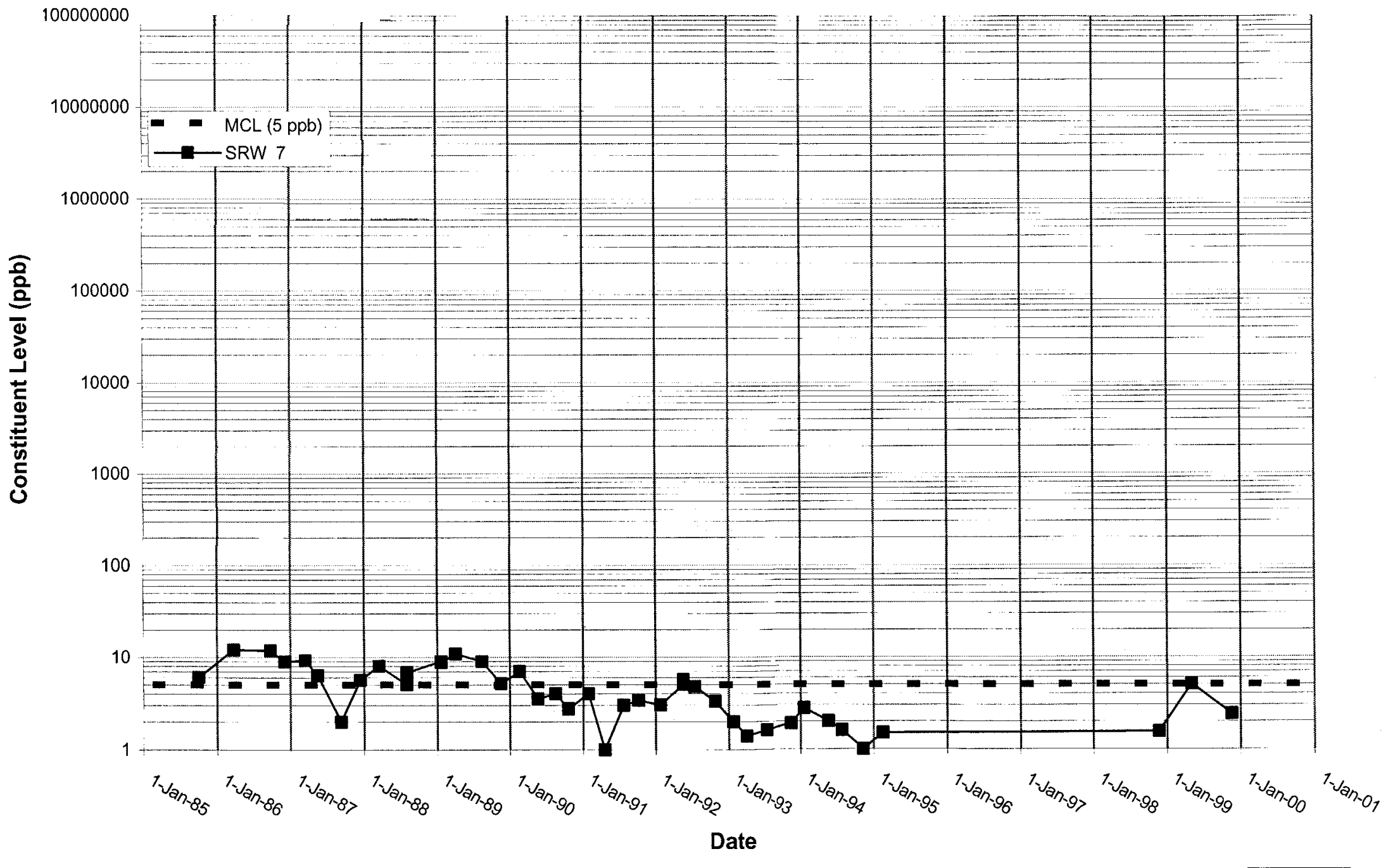


WSRC-TR-2000-00242 UNCLASSIFIED

Time Series Plot of Trichloroethylene for Well SRW 8

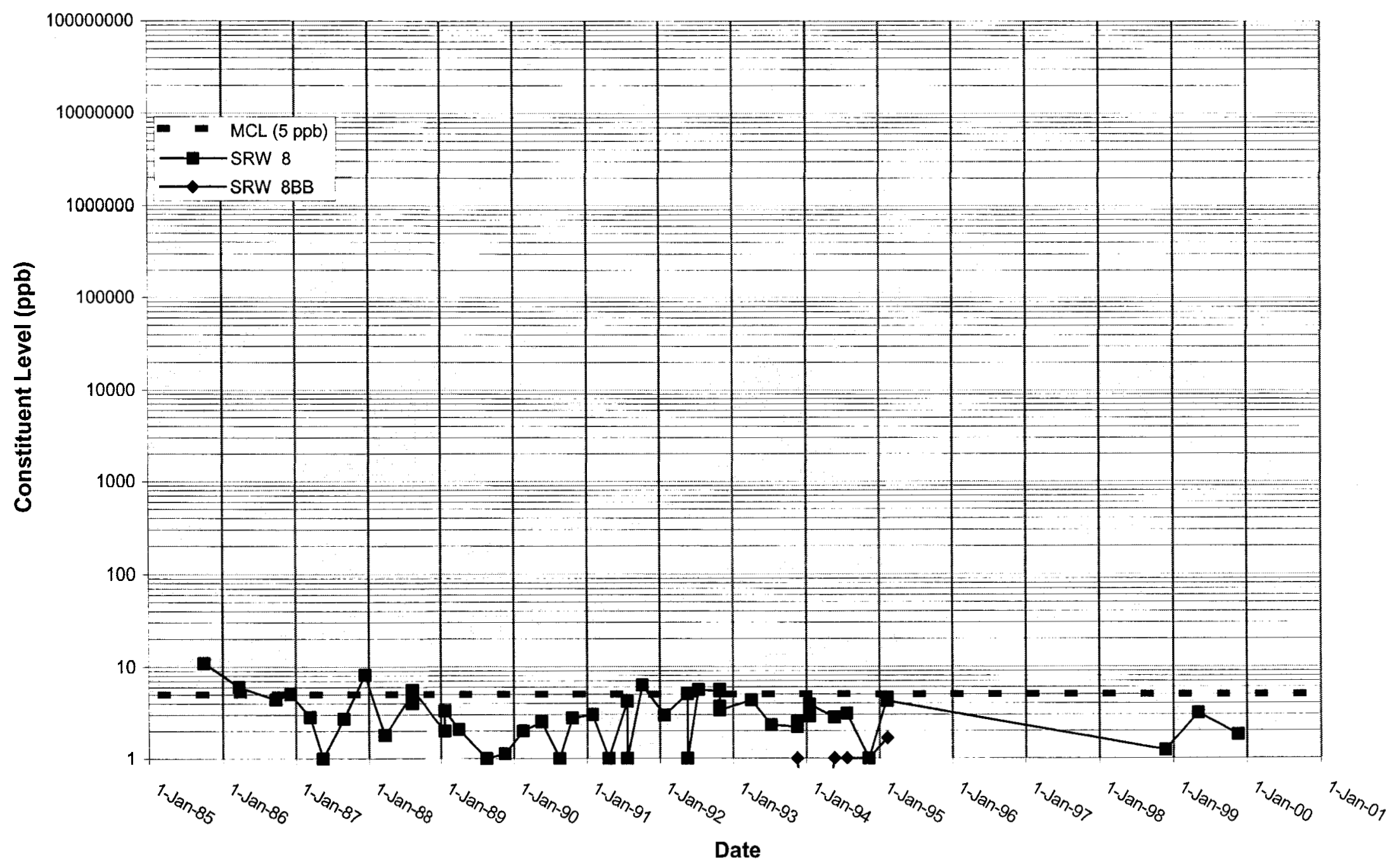


Time Series Plot of Trichloroethylene for Well SRW 9

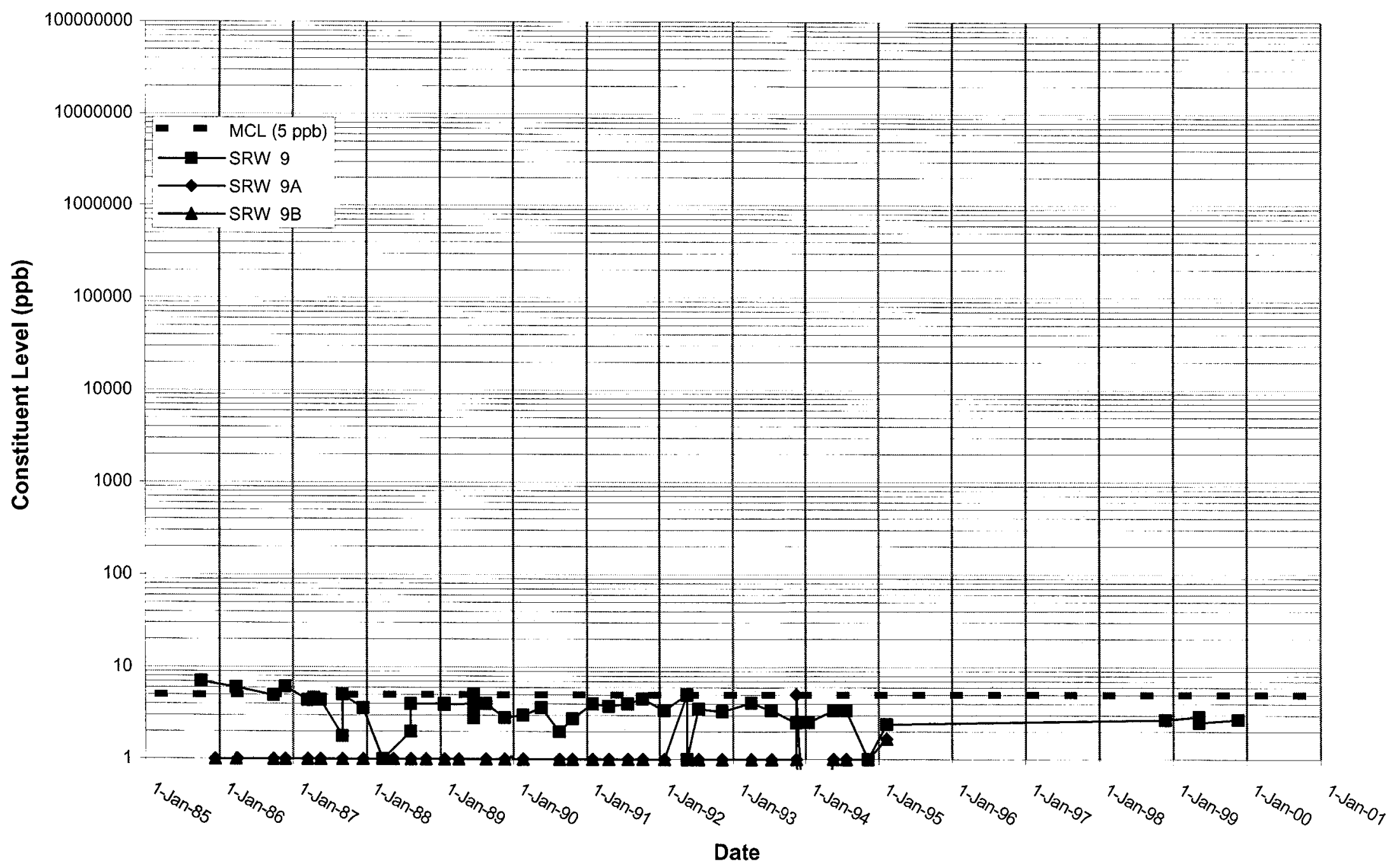


Time Series Plot of Trichloroethylene for Well SRW 10

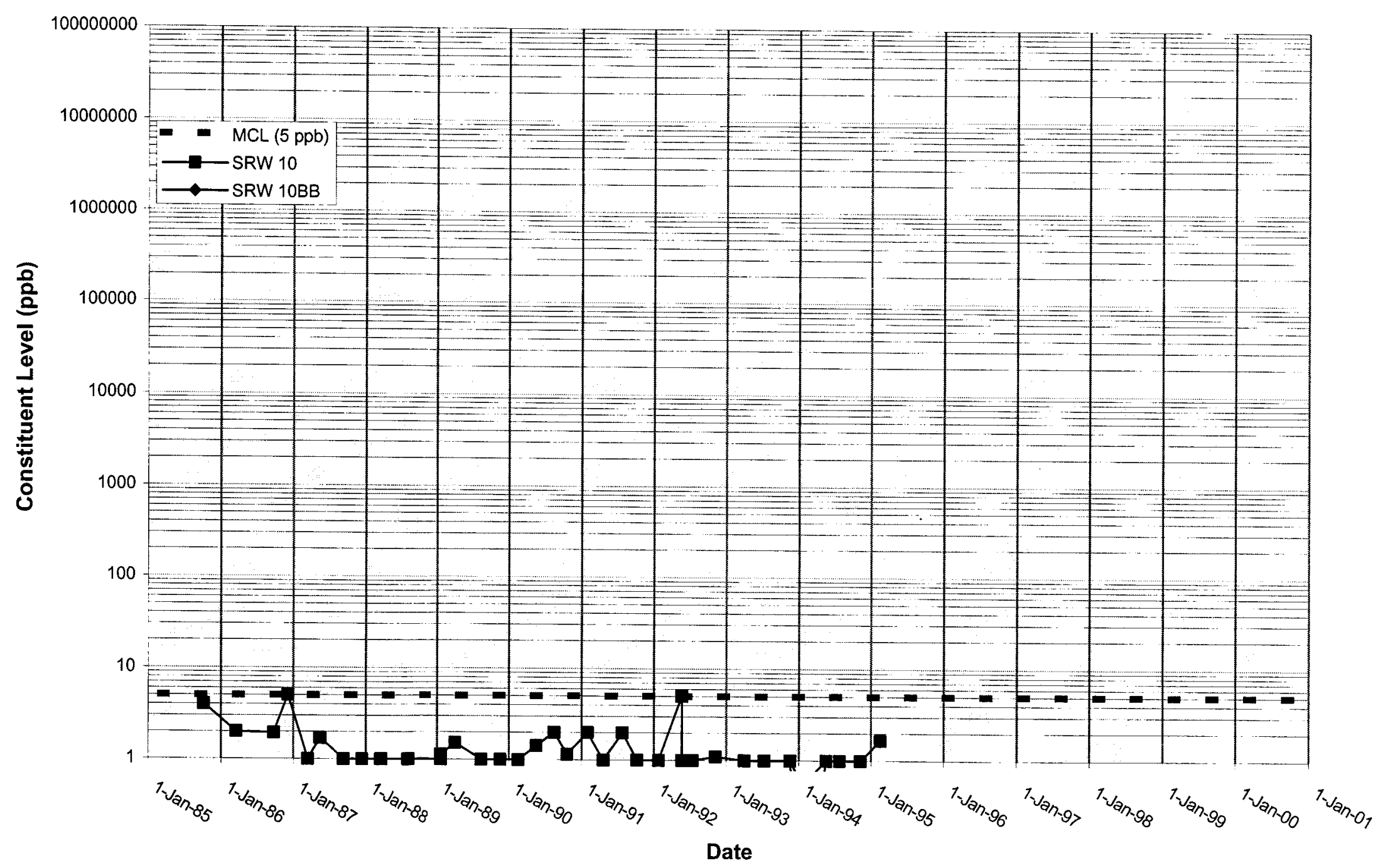


WSRC-TR-2000-00242 UNCLASSIFIED

Time Series Plot of Trichloroethylene for Well SRW 11

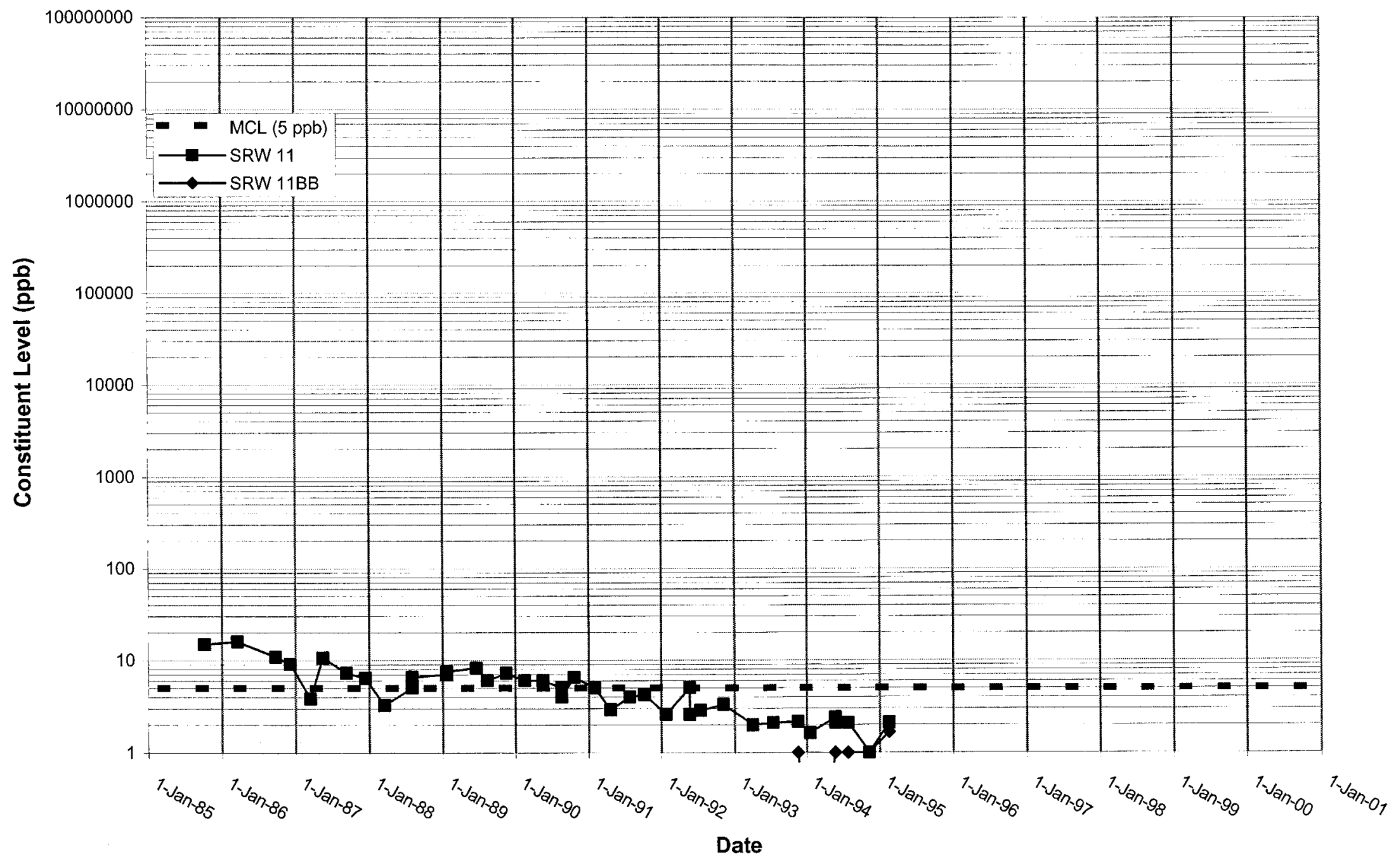


Time Series Plot of Trichloroethylene for Well SRW 12

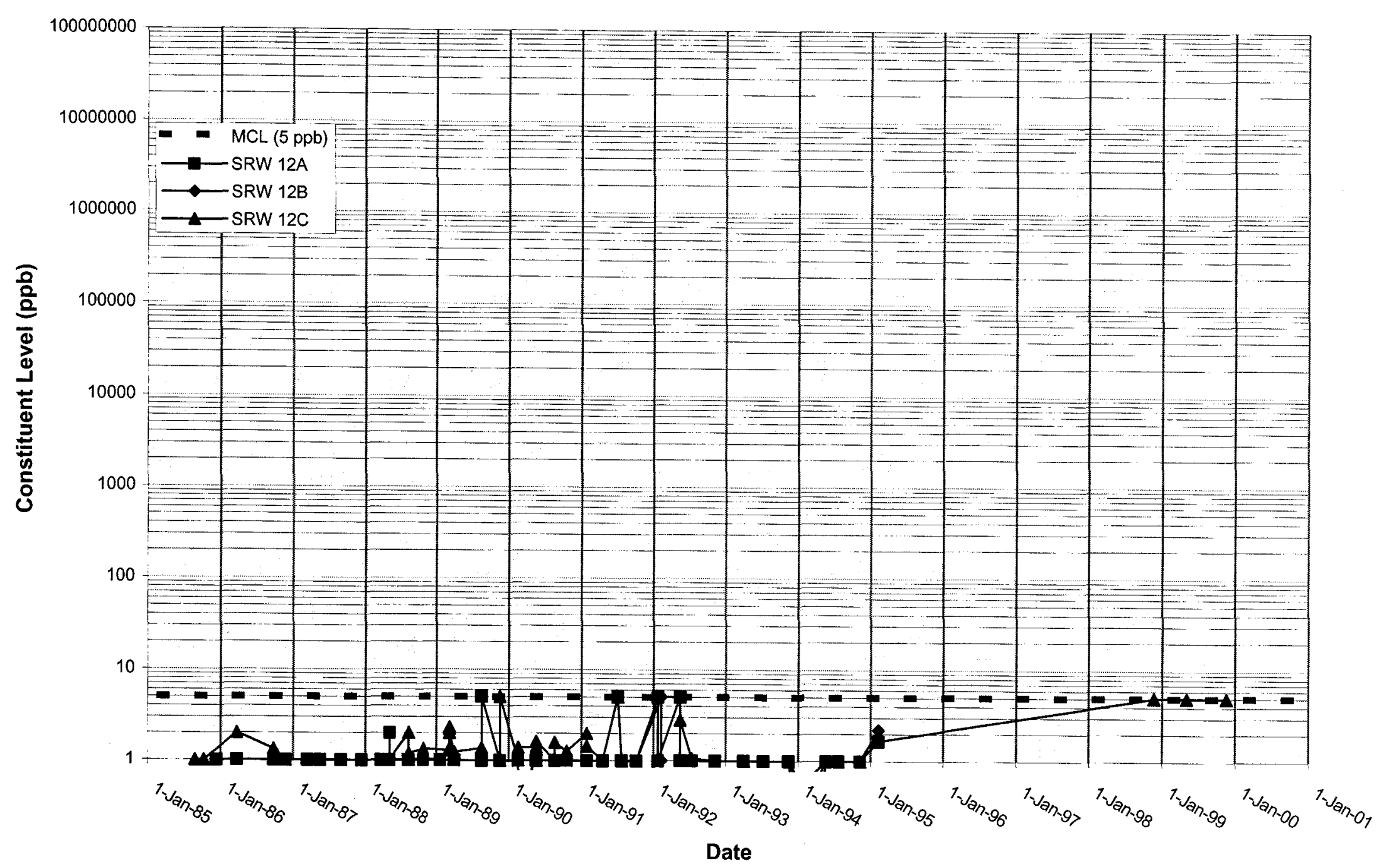


WSRC-TR-2000-00242 UNCLASSIFIED

Time Series Plot of Trichloroethylene for Well SRW 13

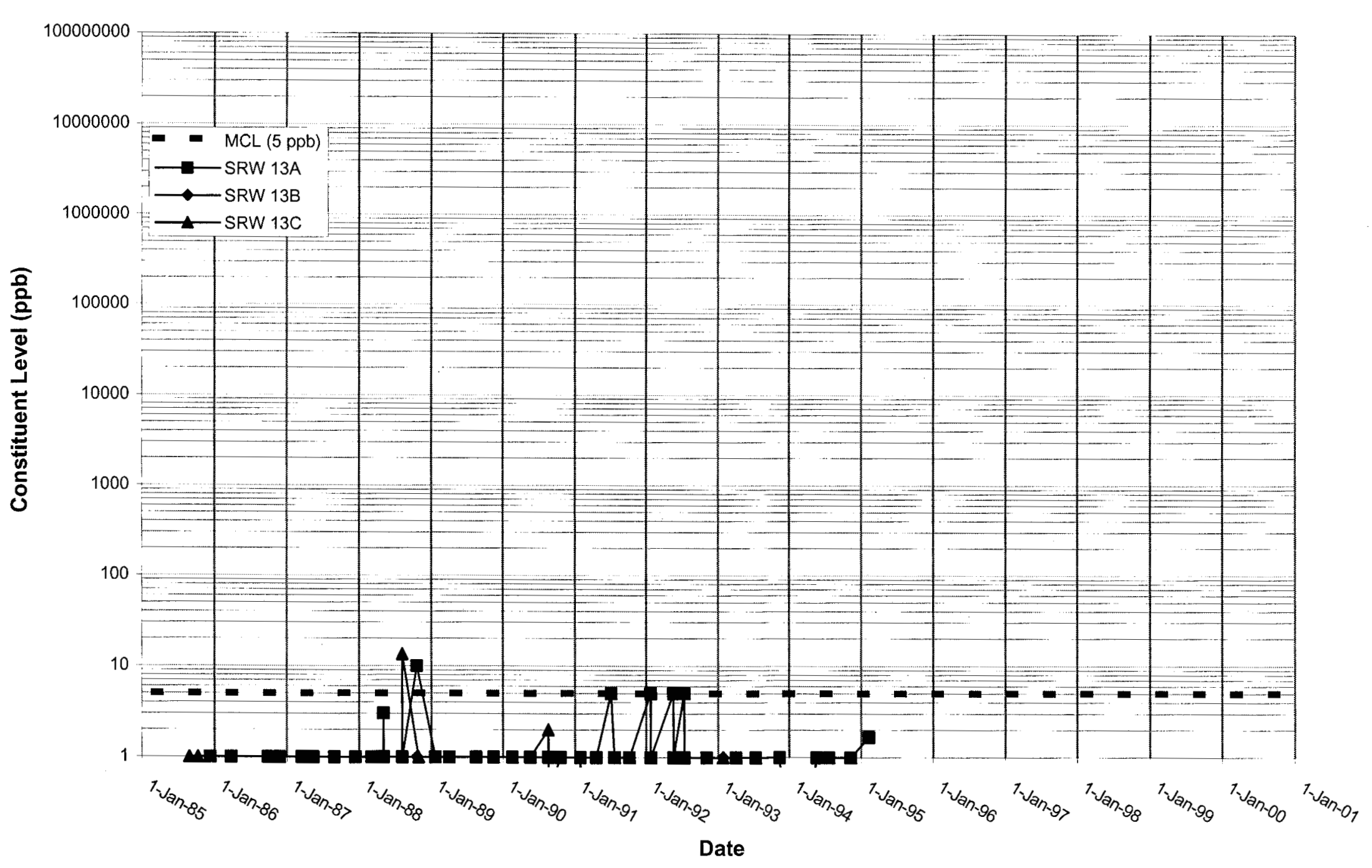


WSRC-TR-2000-00242 UNCLASSIFIED

Time Series Plot of Trichloroethylene for Well SRW 14

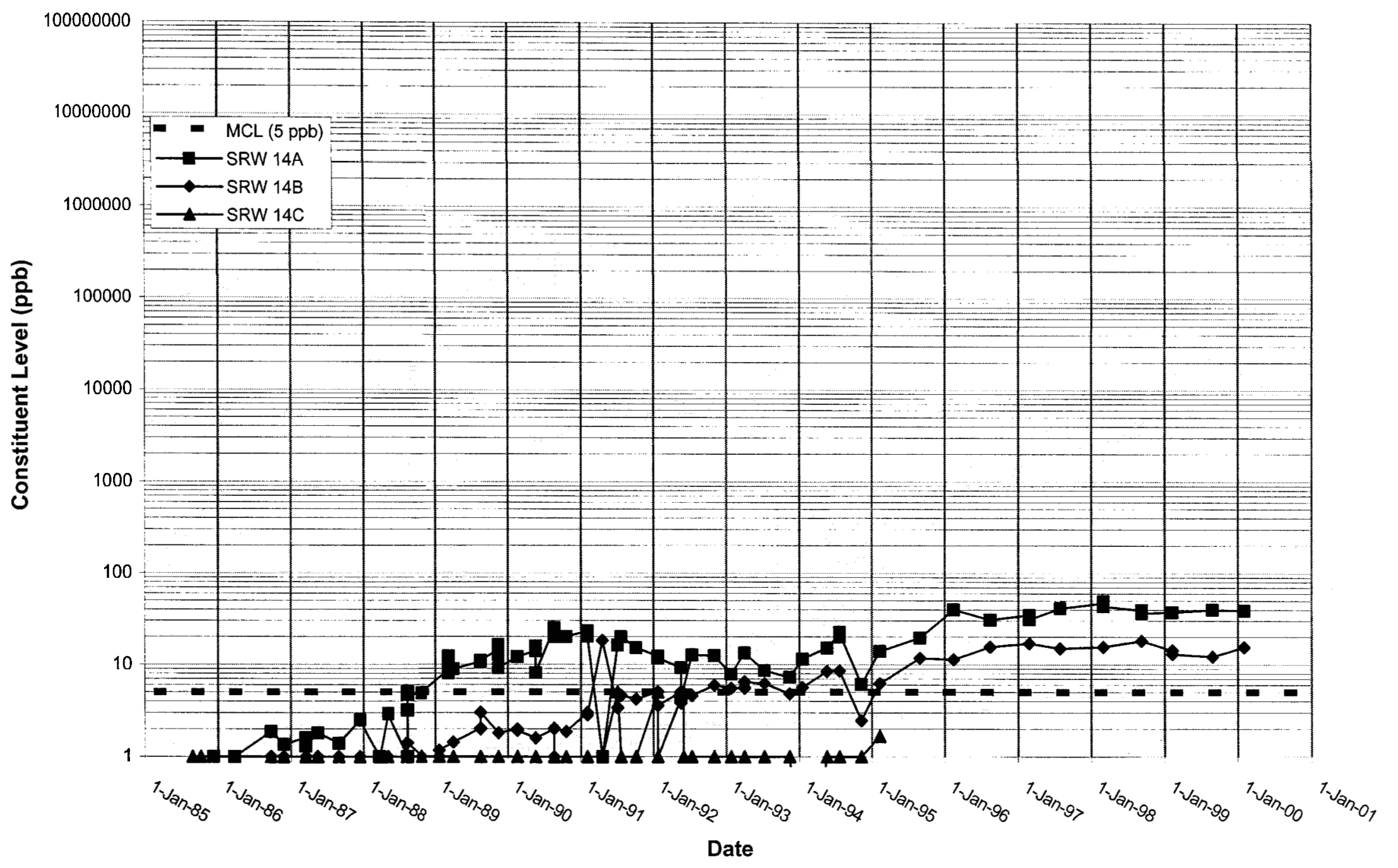




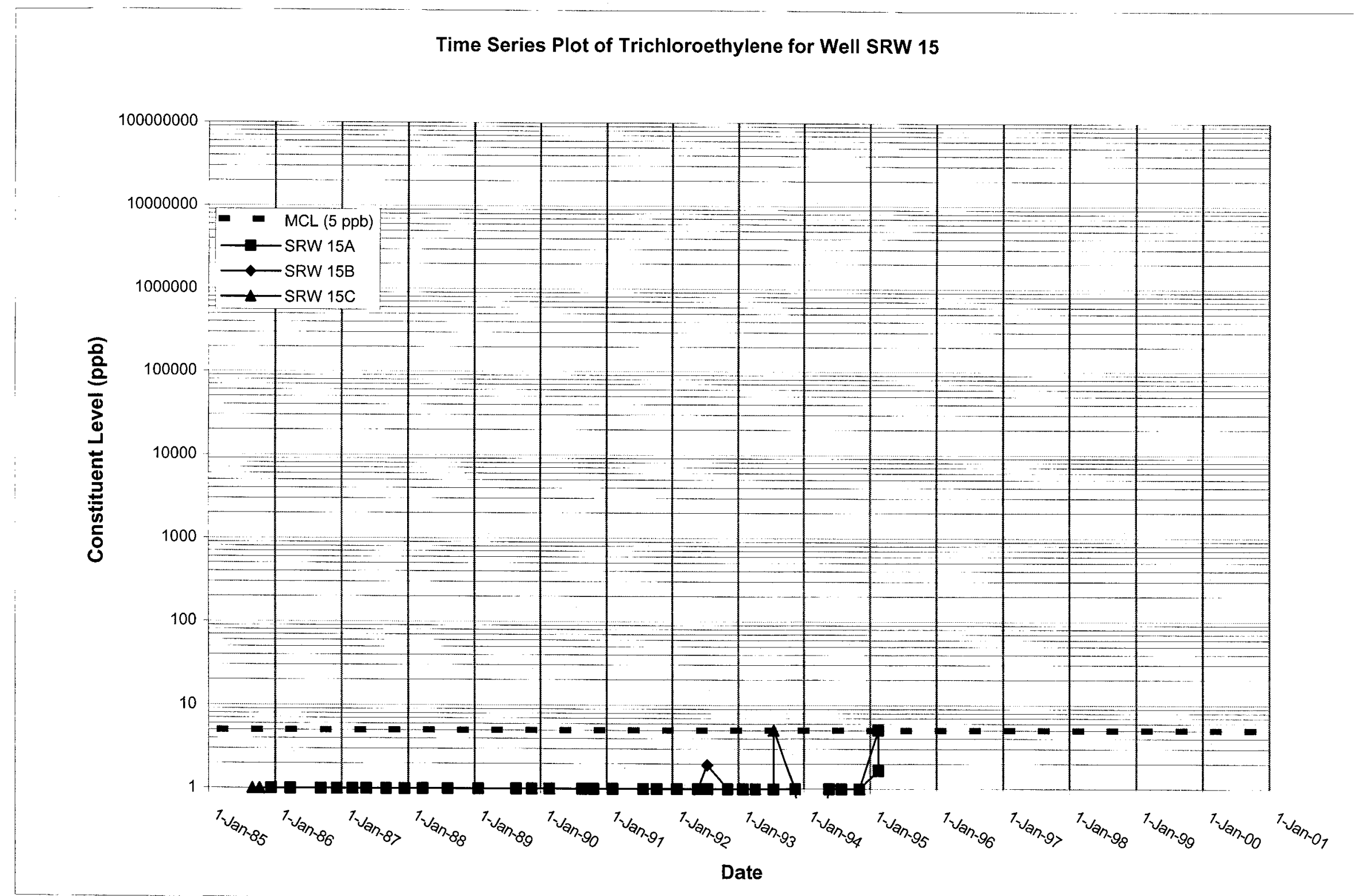


Time Series Plot of Trichloroethylene for Well SRW 16

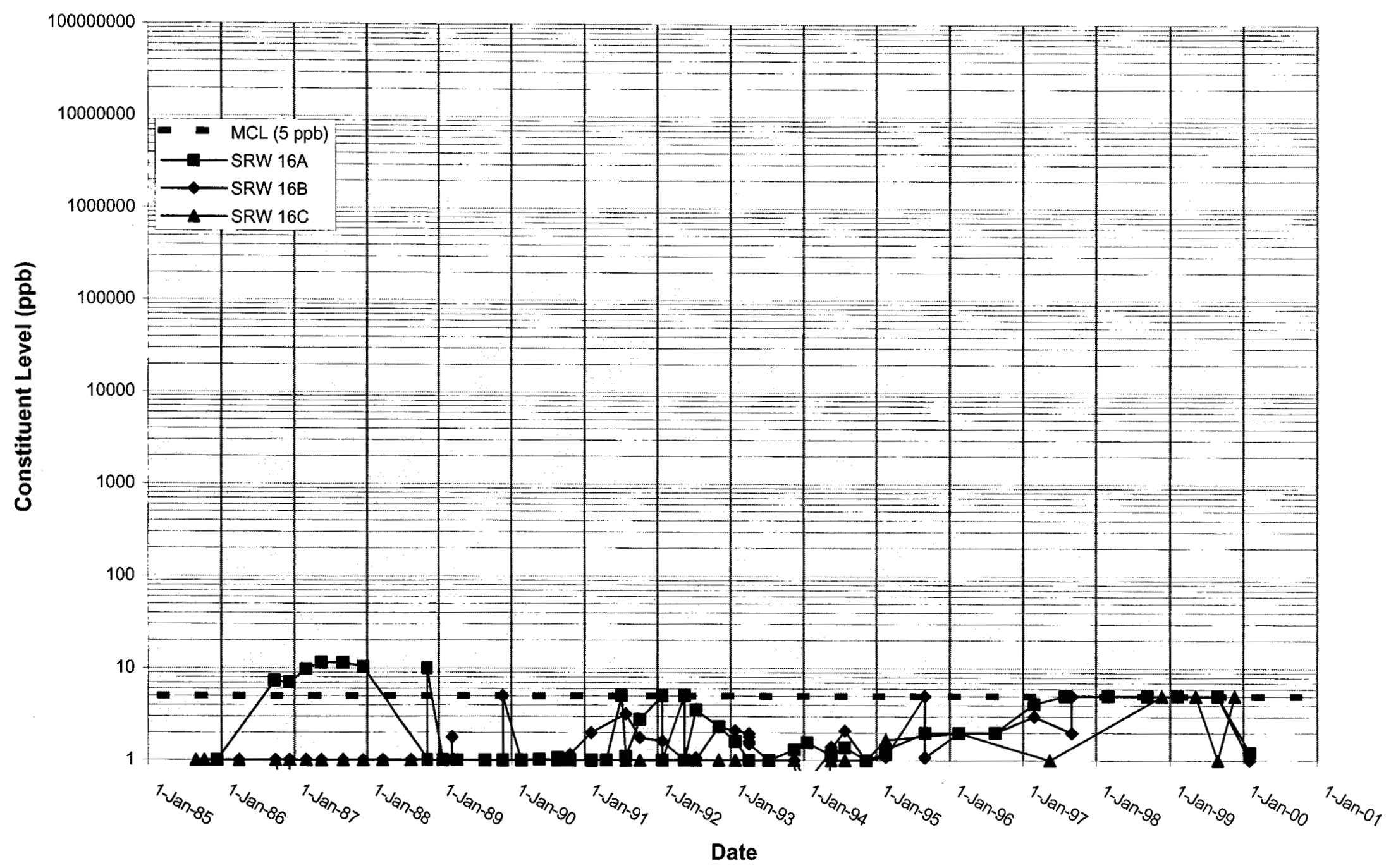


Time Series Plot of Trichloroethylene for Well SRW 17

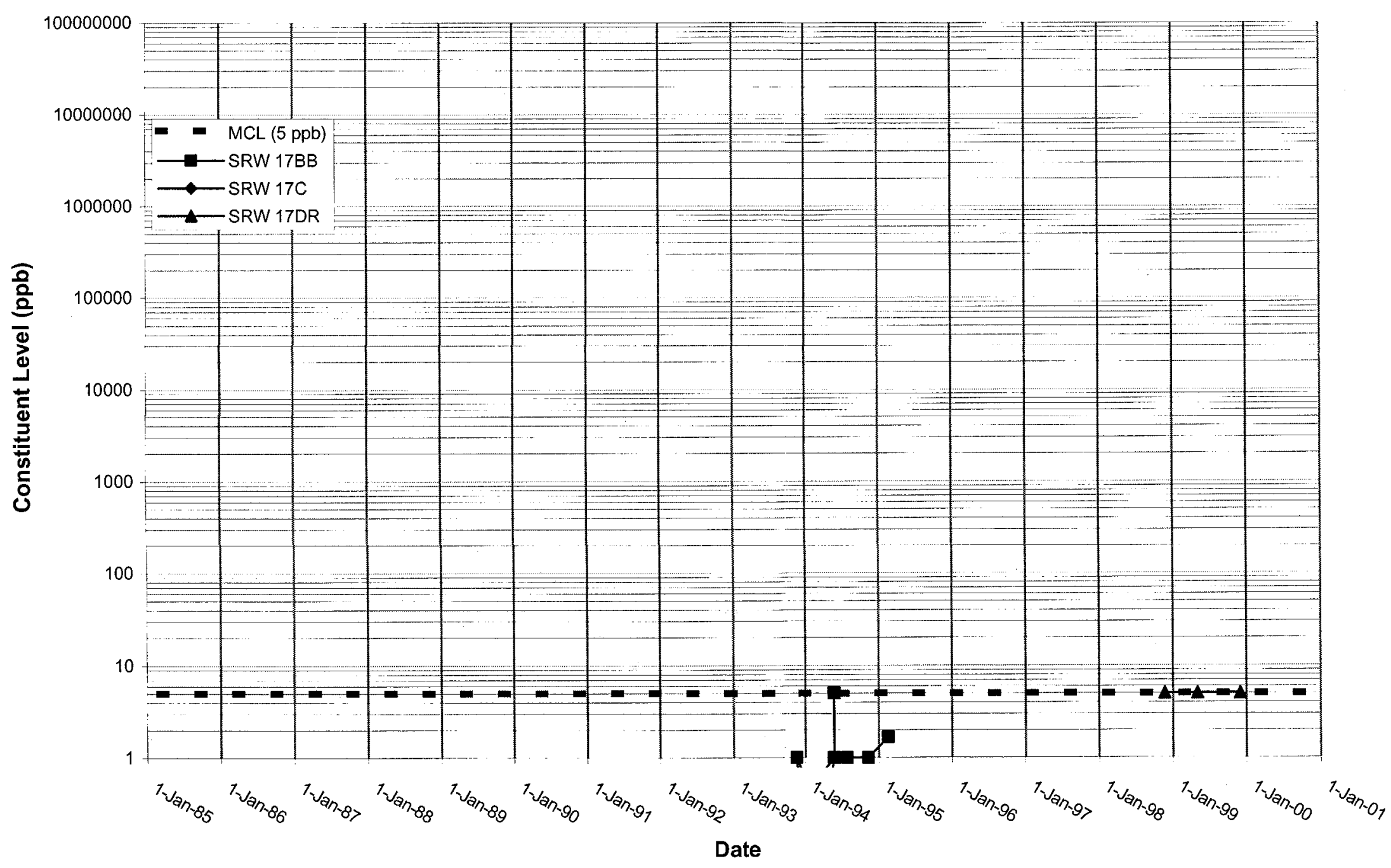


WSRC-TR-2000-00242 UNCLASSIFIED

Time Series Plot of Trichloroethylene for Well SRW 18

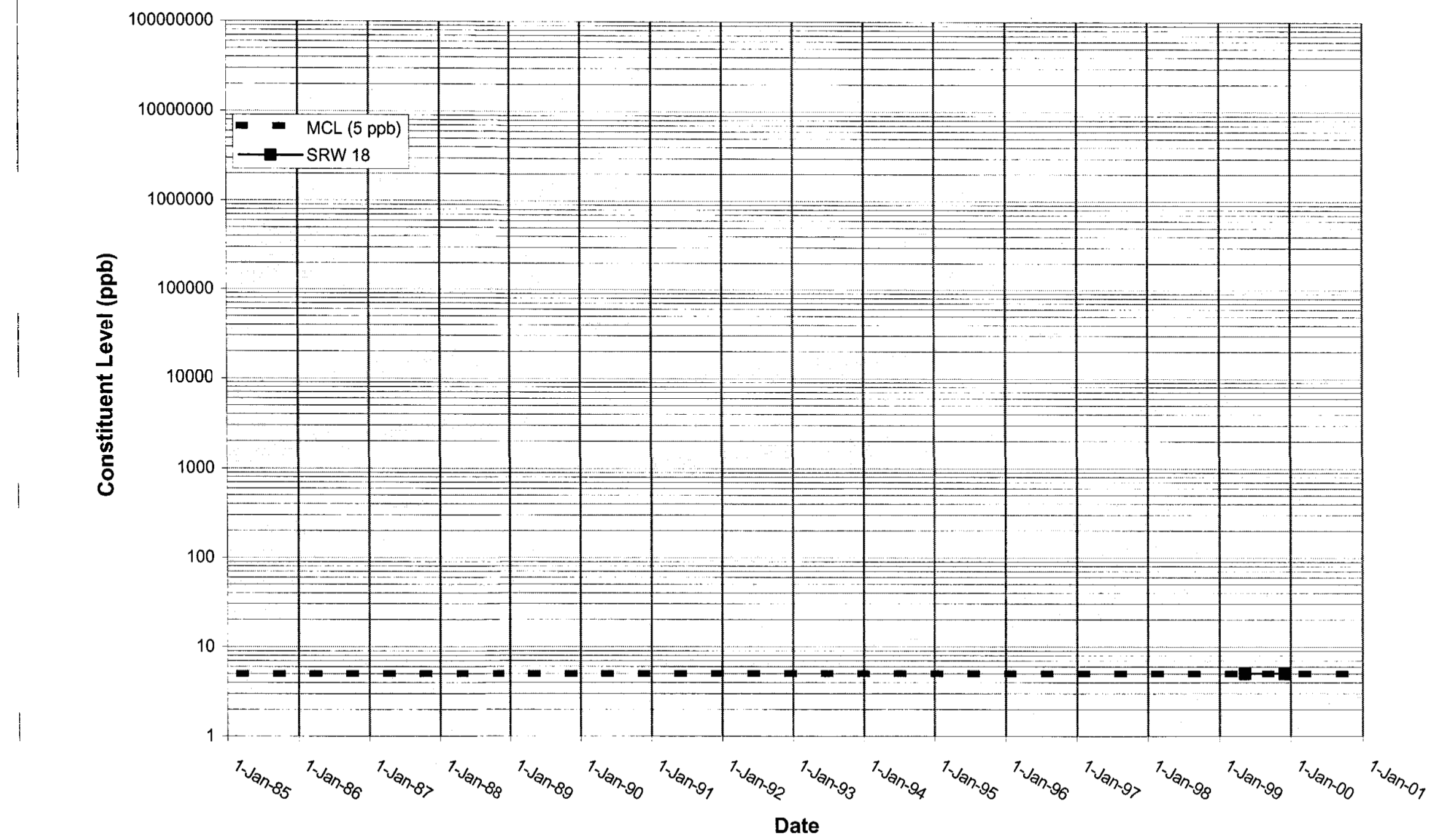




\section{Time Series Plot of Trichloroethylene for Well SRW 19}

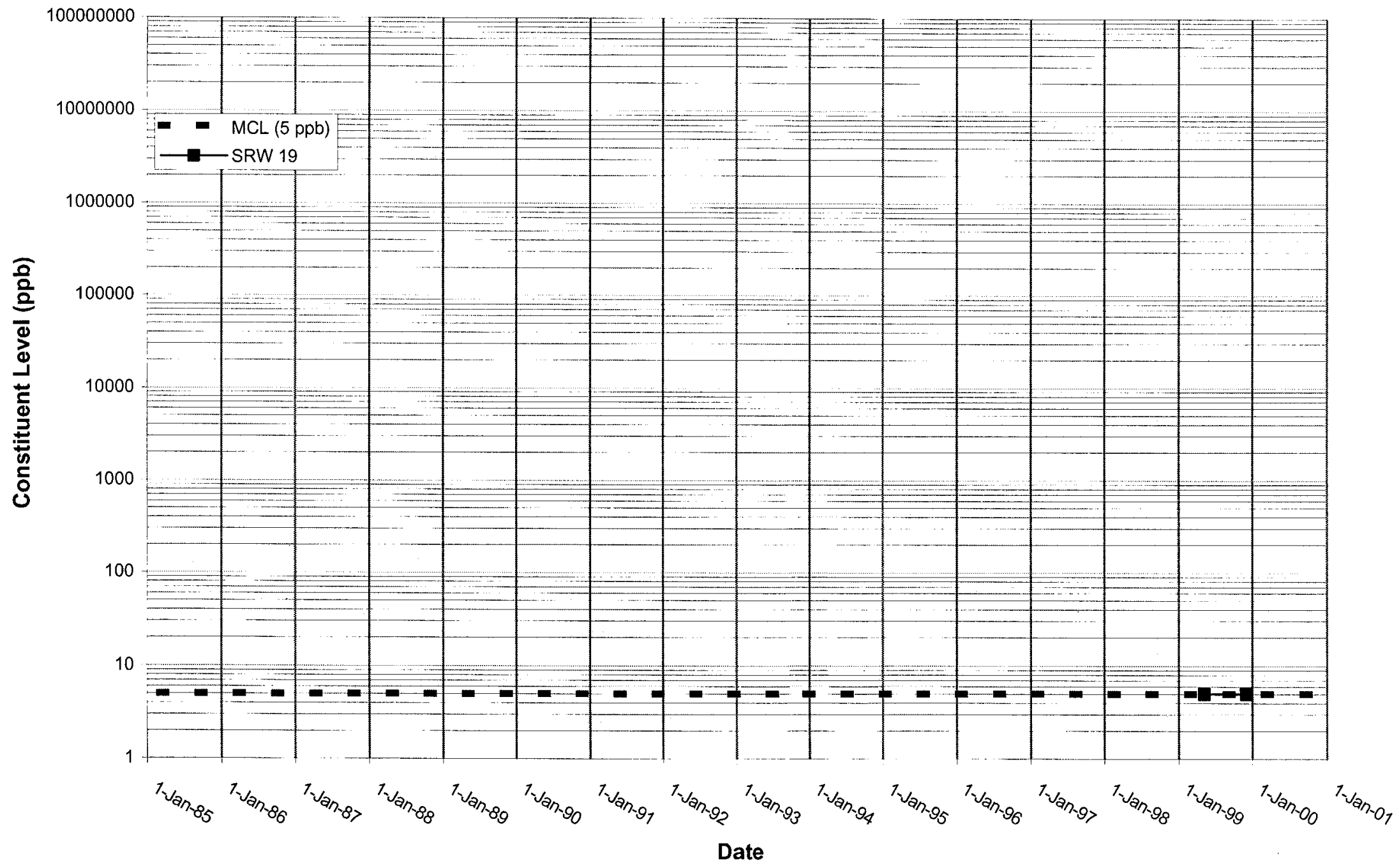


WSRC-TR-2000-00242

\section{Time Series Plot of Tetrachloroethylene for Well ABP 1}

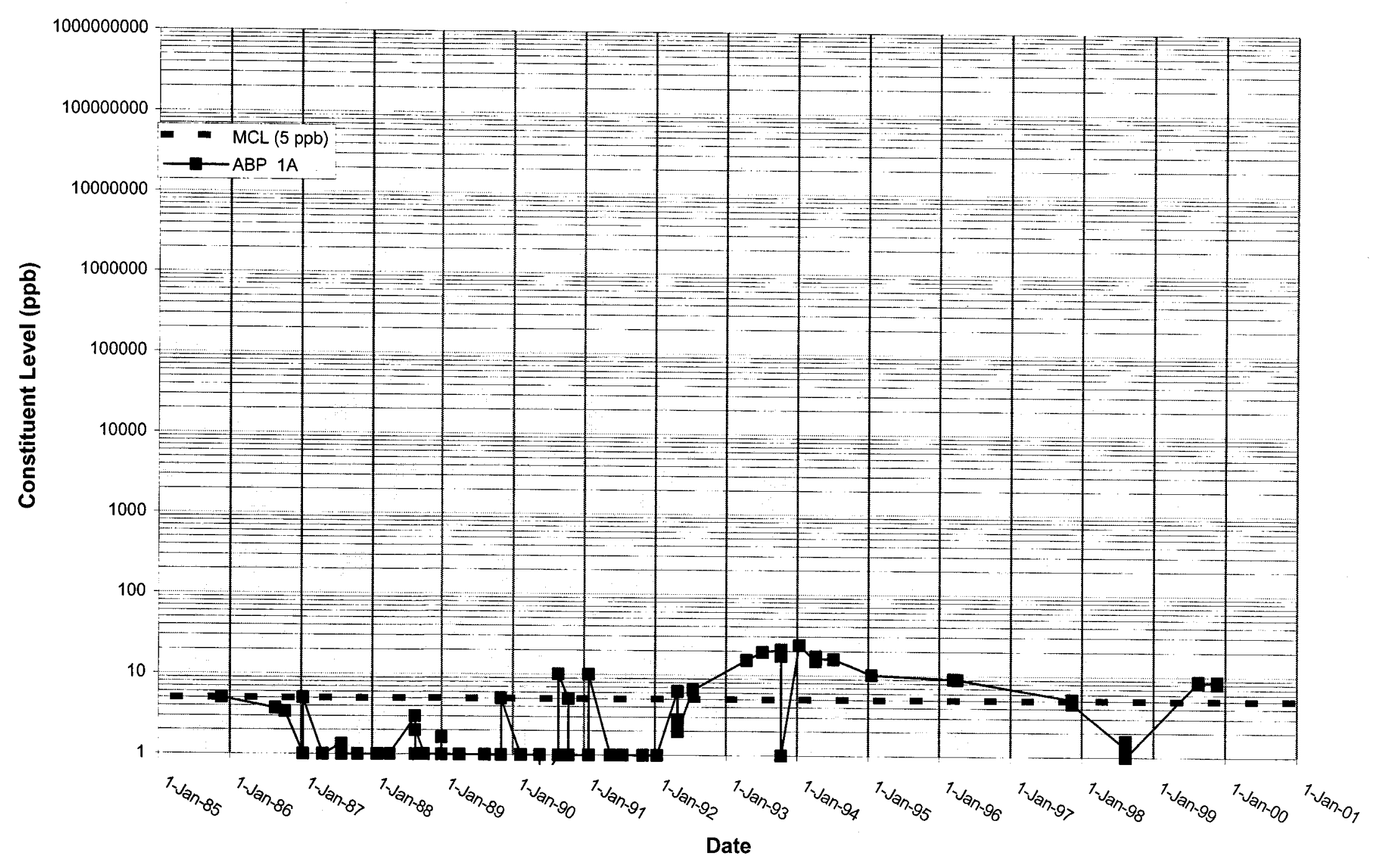


Time Series Plot of Tetrachloroethylene for Well ABP 2

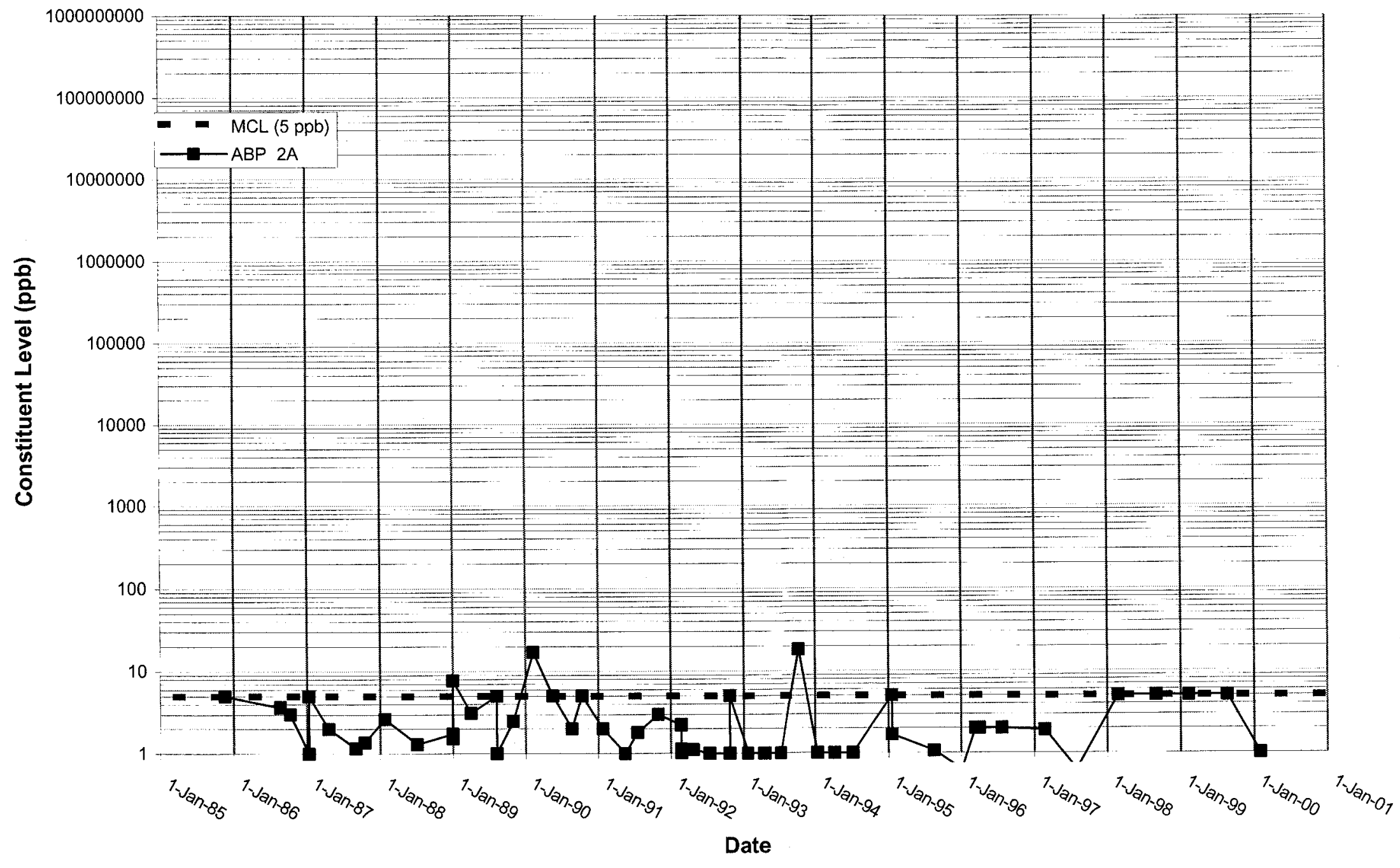


WSRC-TR-2000-00242 UNCLASSIFIED

Time Series Plot of Tetrachloroethylene for Well ABP 3

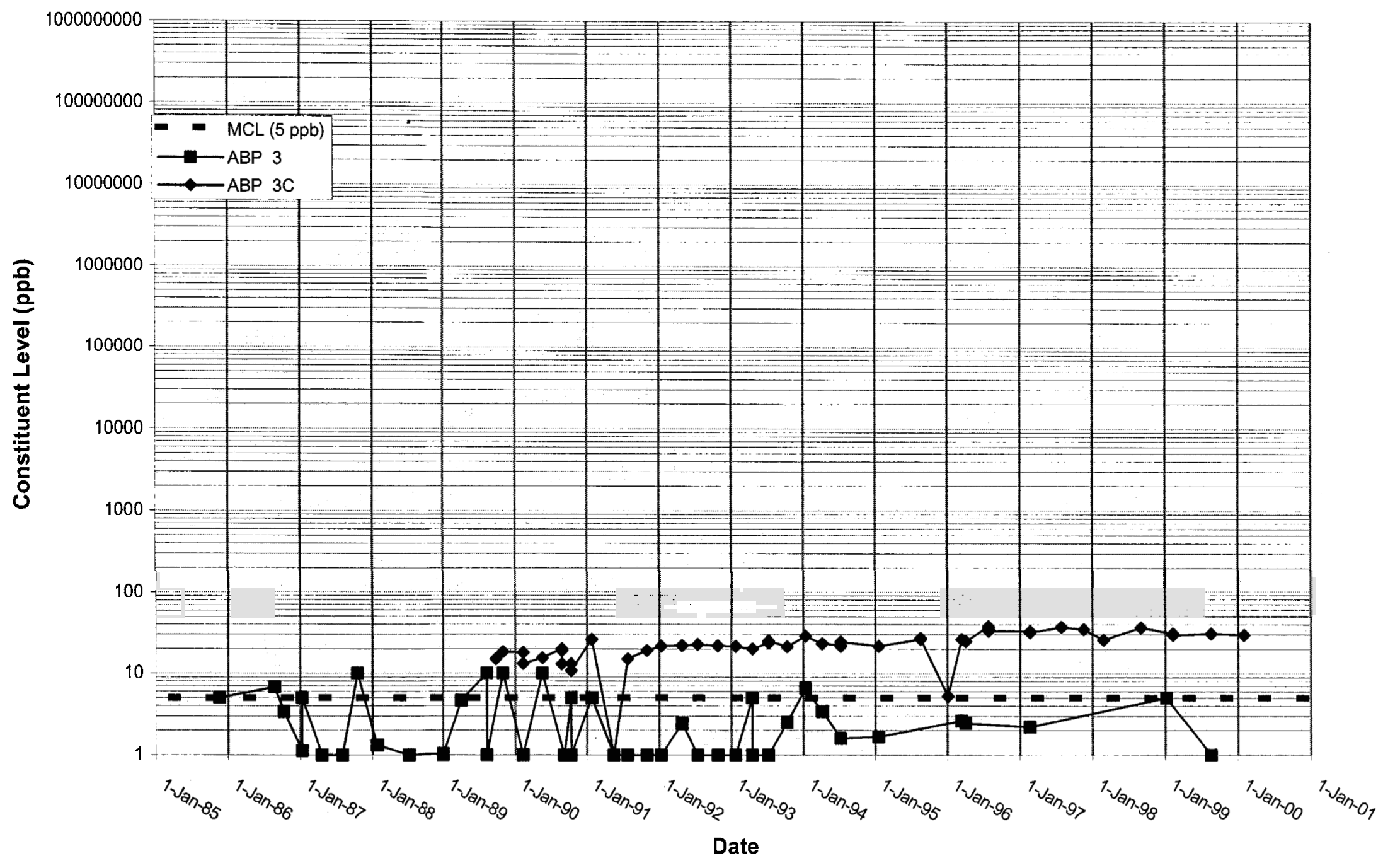




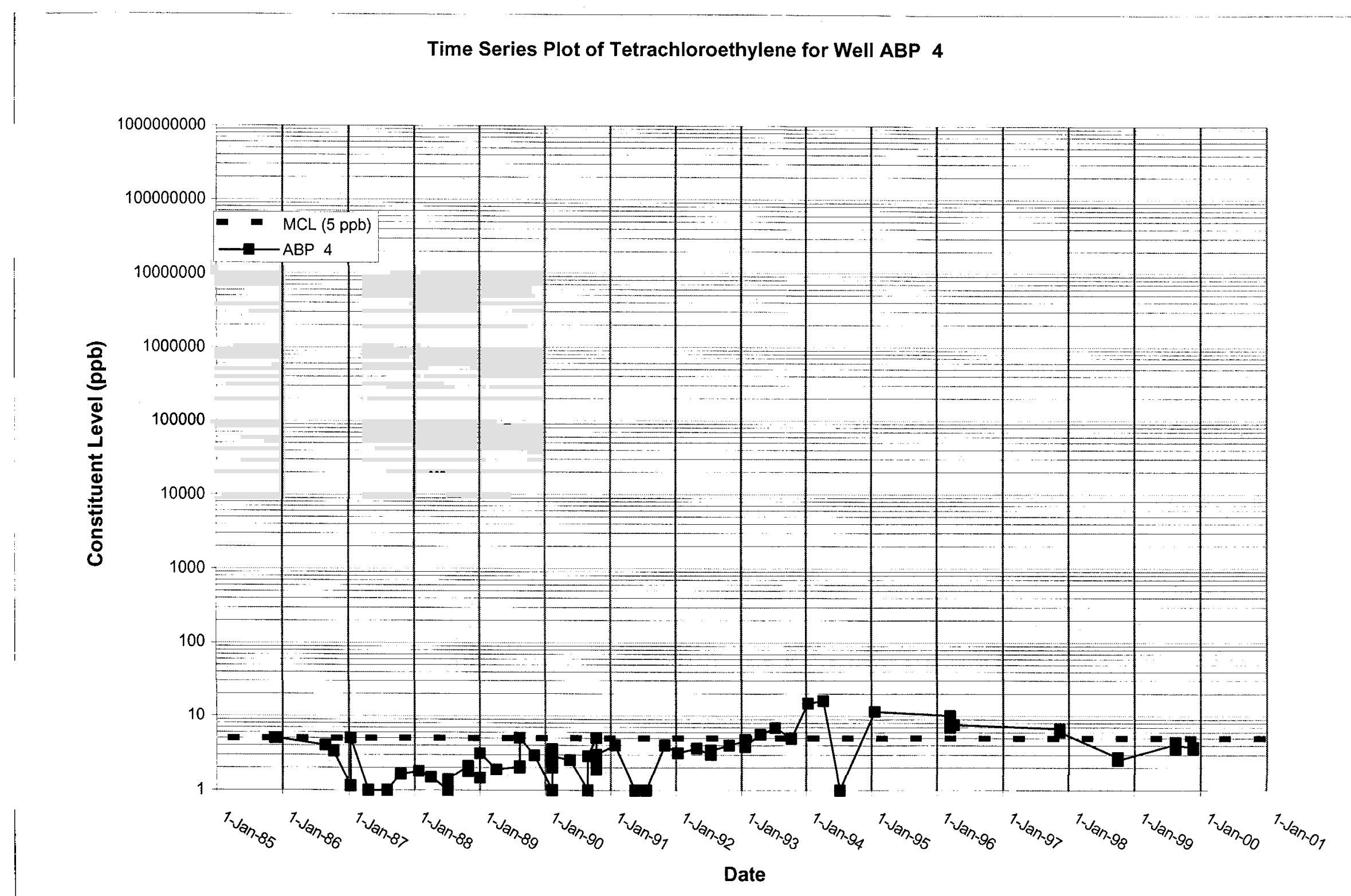


Time Series Plot of Tetrachloroethylene for Well ABP 8

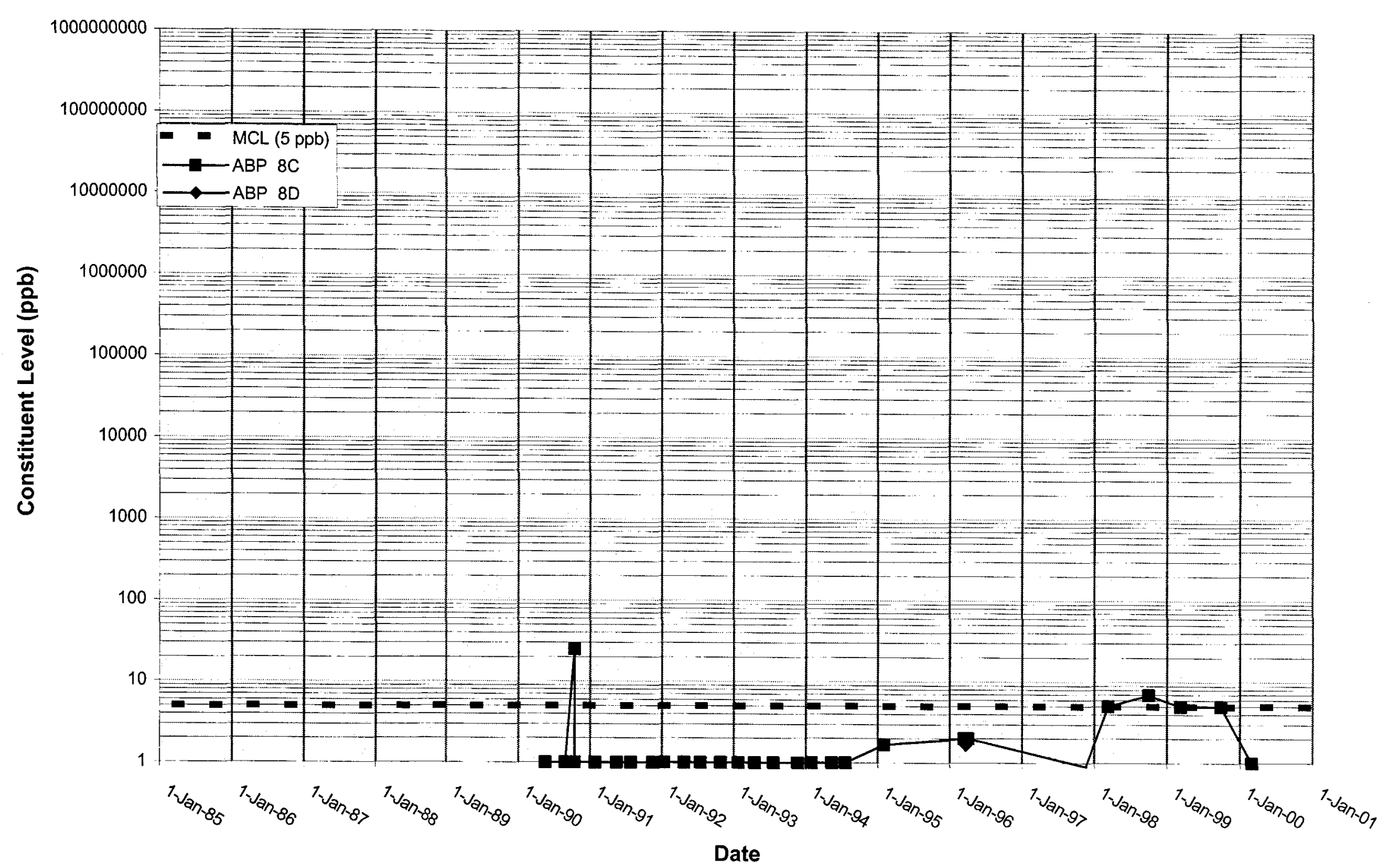


Time Series Plot of Tetrachloroethylene for Well ABW 1

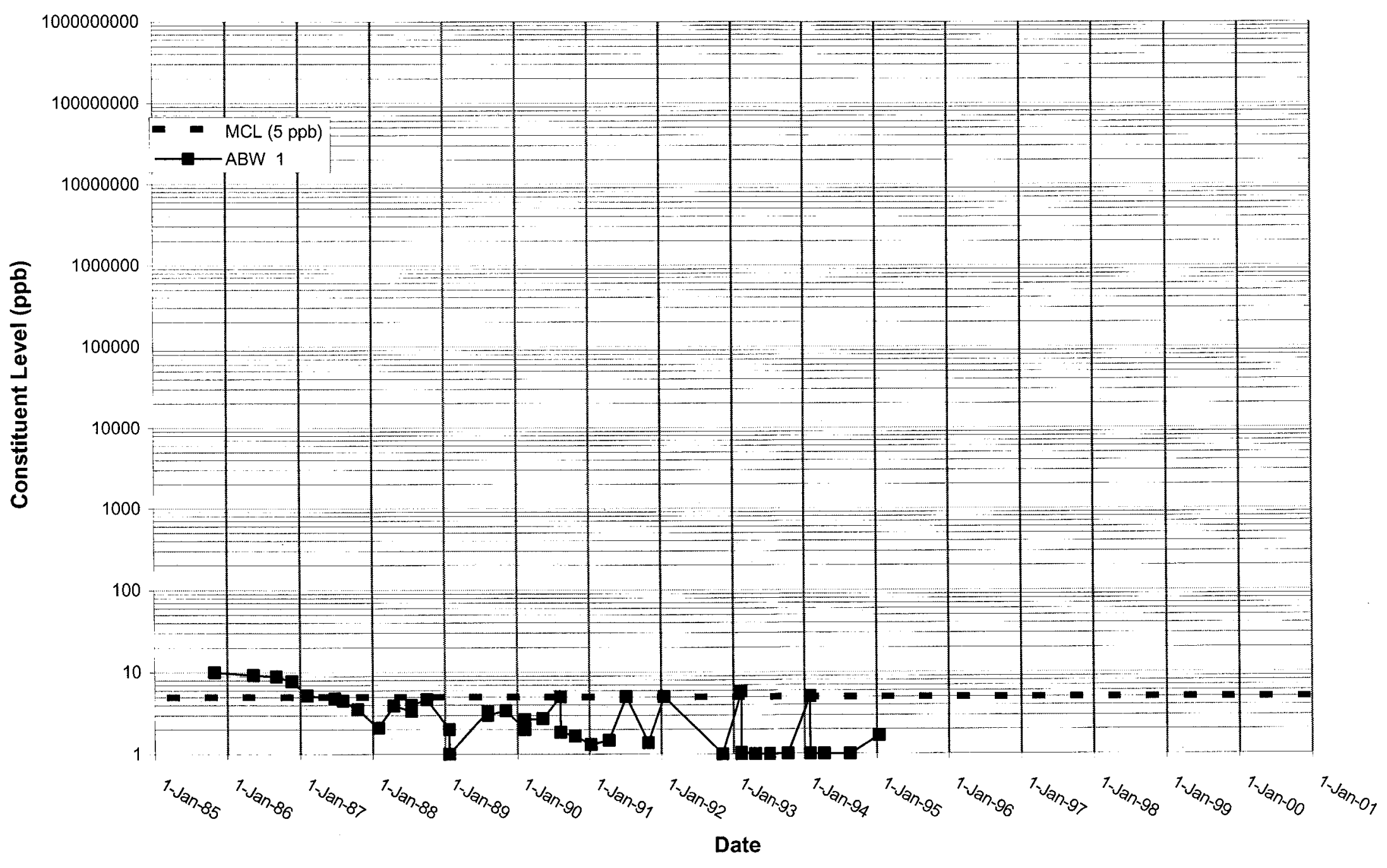


Time Series Plot of Tetrachloroethylene for Well AC 1

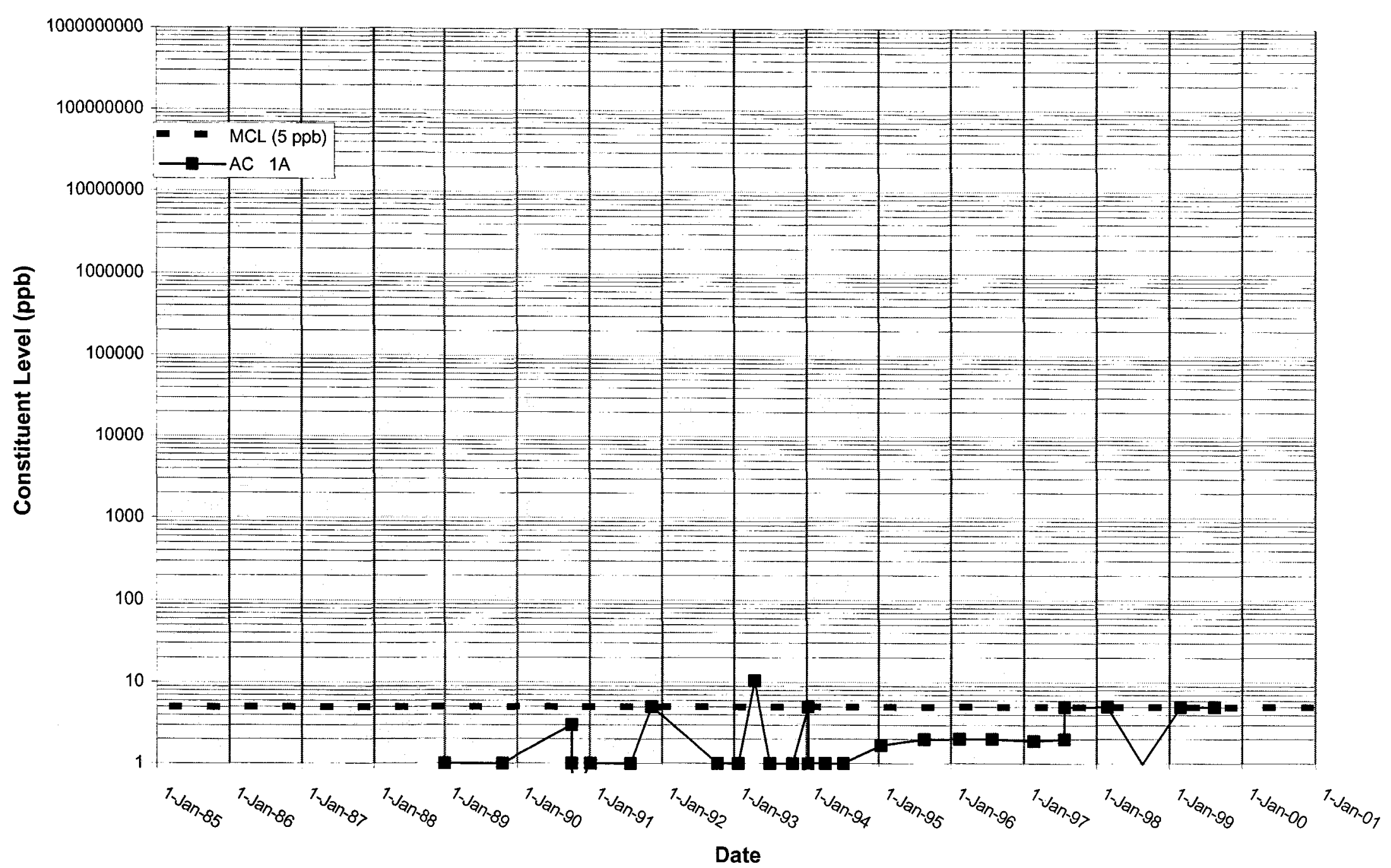


Time Series Plot of Tetrachloroethylene for Well AC 2

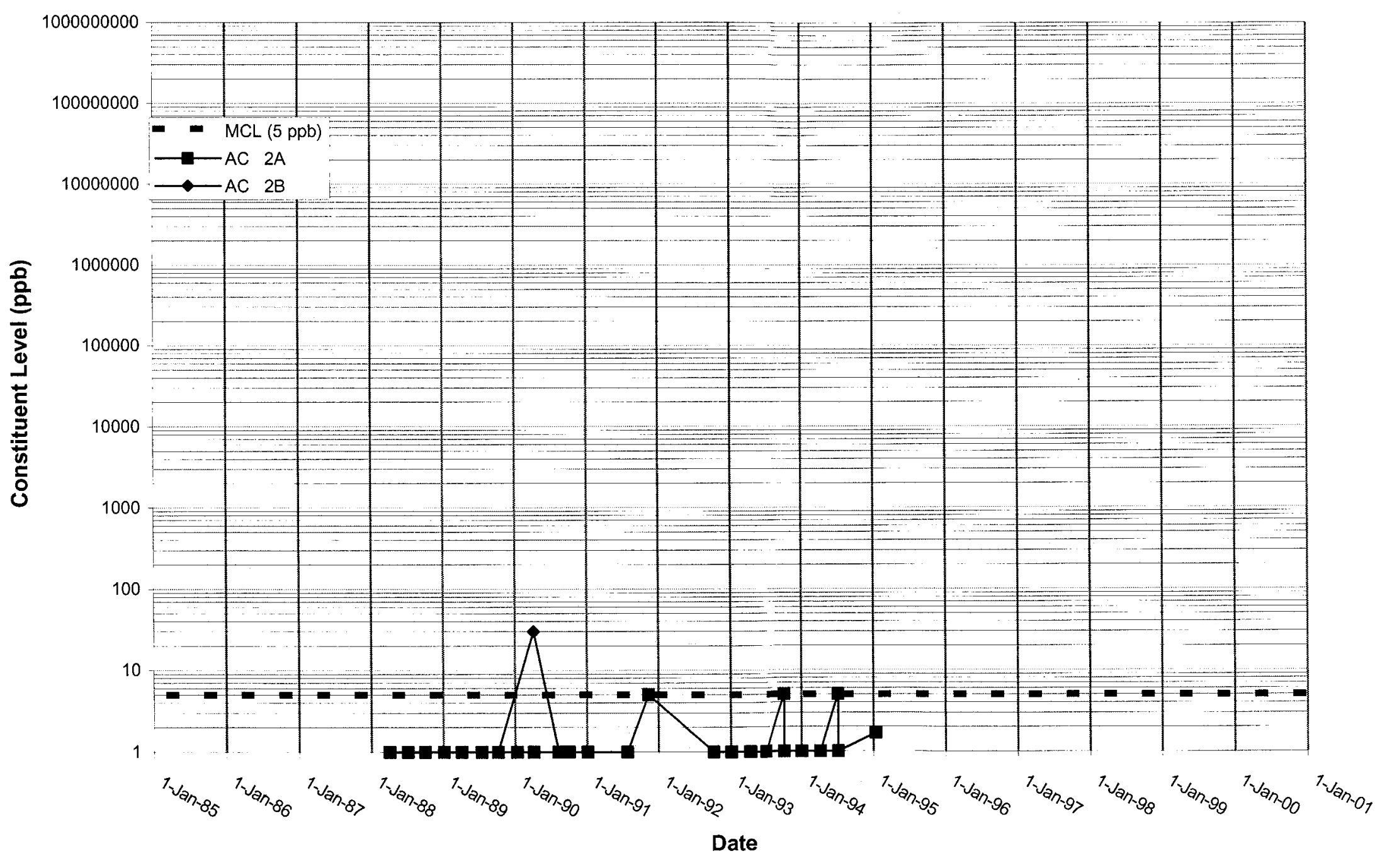


WSRC-TR-2000-00242 UNCLASSIFIED

Time Series Plot of Tetrachloroethylene for Well AC 3

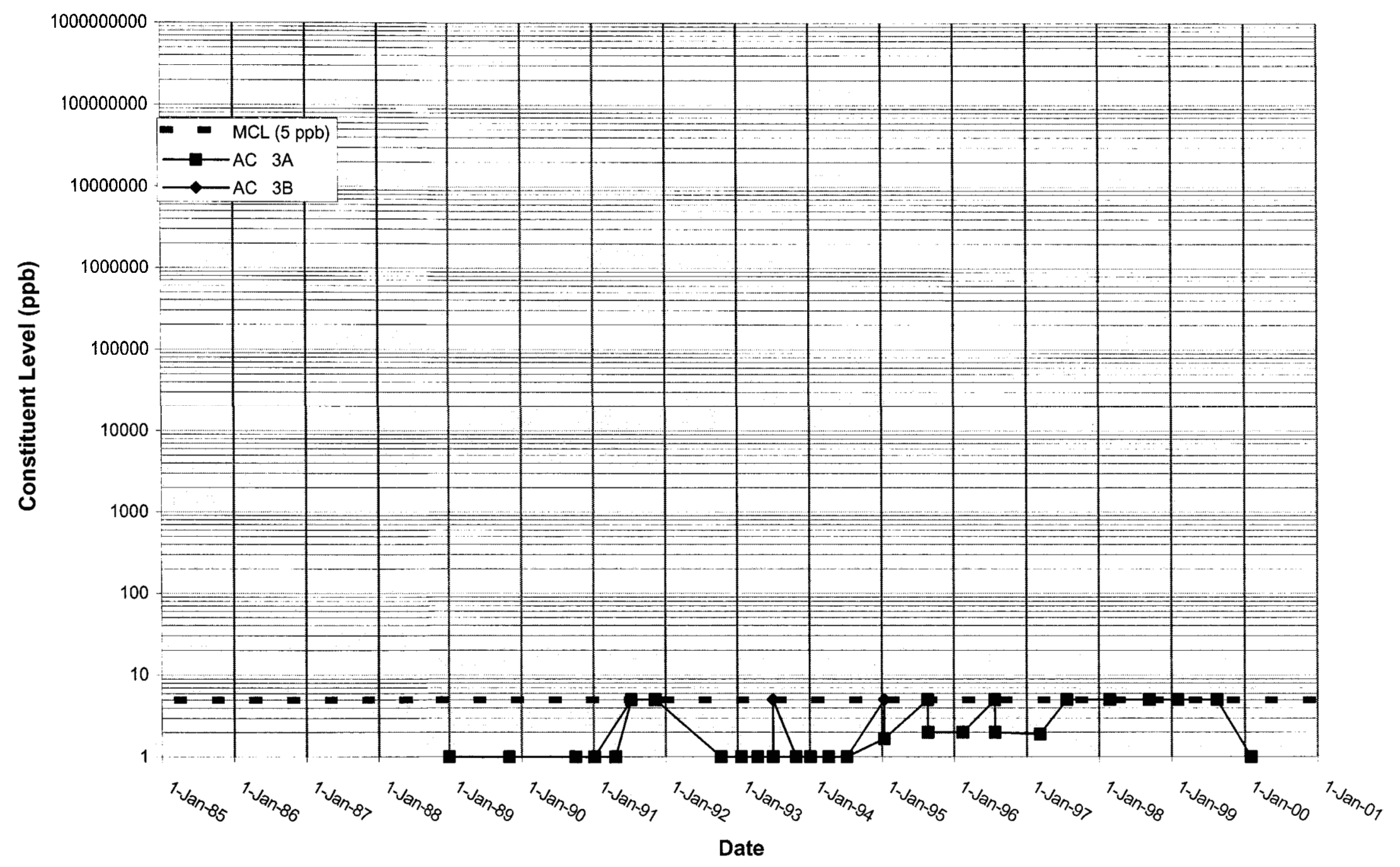


Time Series Plot of Tetrachloroethylene for Well ACB 1

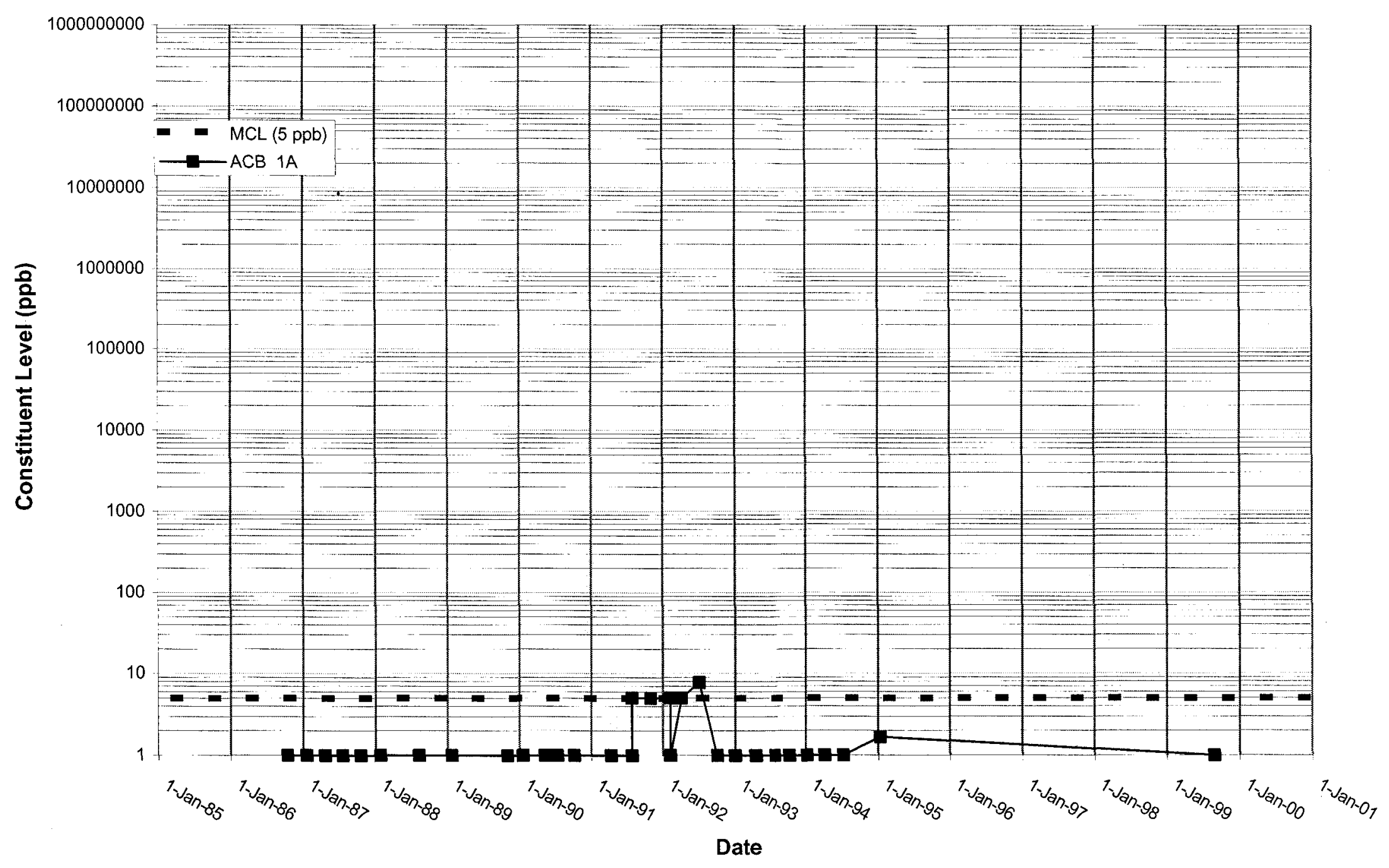


Time Series Plot of Tetrachloroethylene for Well ACB 2

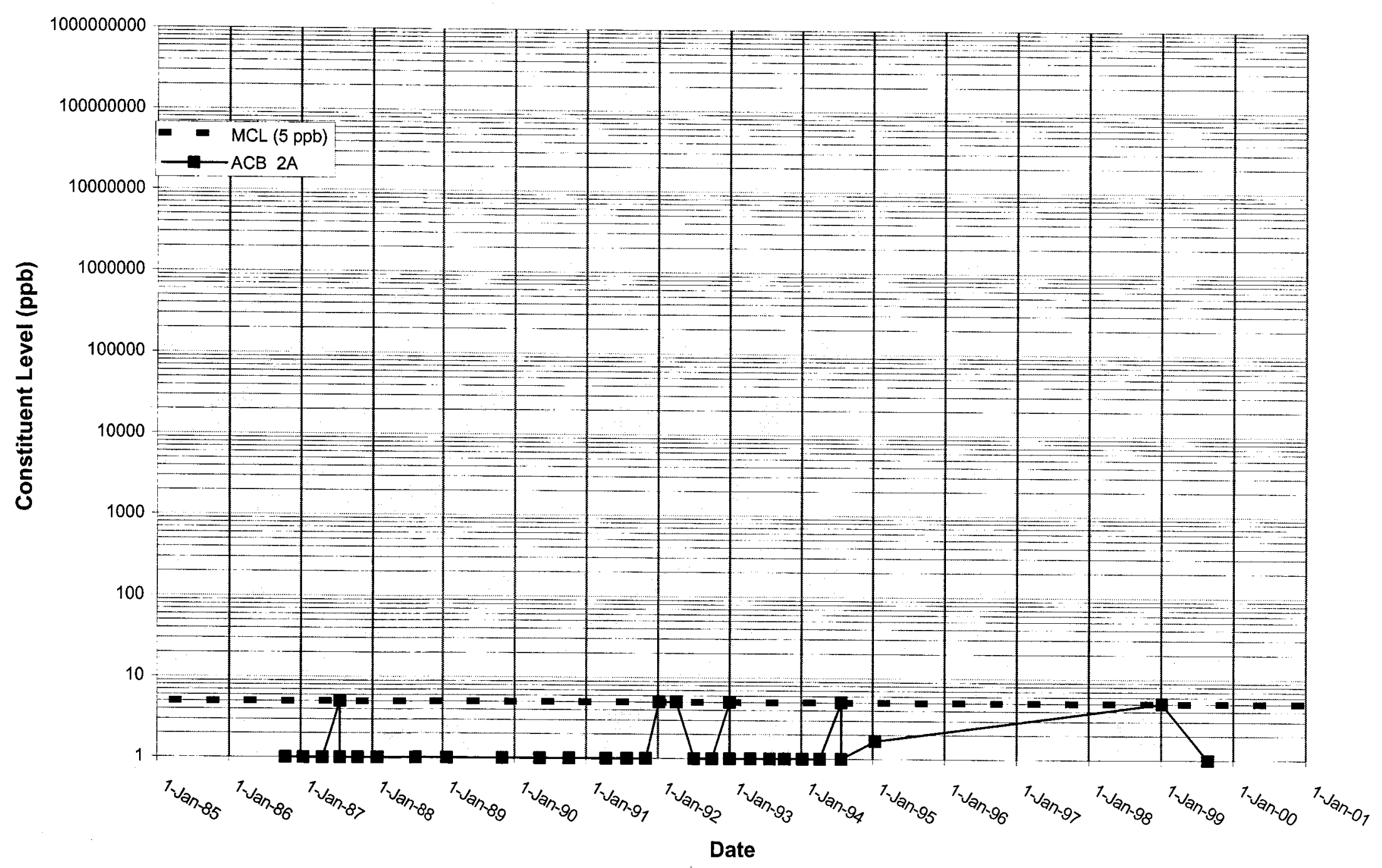


Time Series Plot of Tetrachloroethylene for Well ACB 3

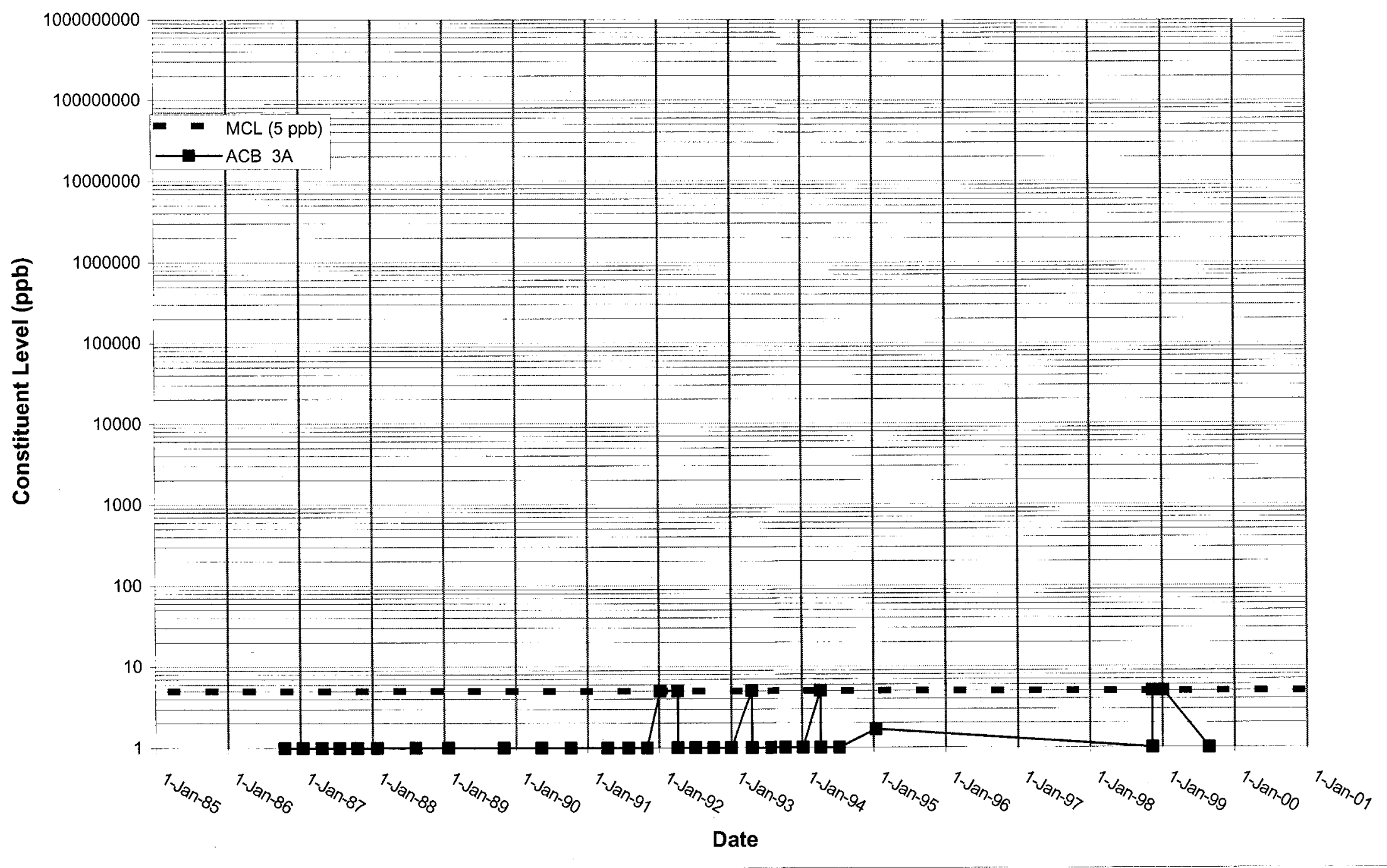


WSRC-TR-2000-00242 UNCLASSIFIED

Time Series Plot of Tetrachloroethylene for Well ACB 4

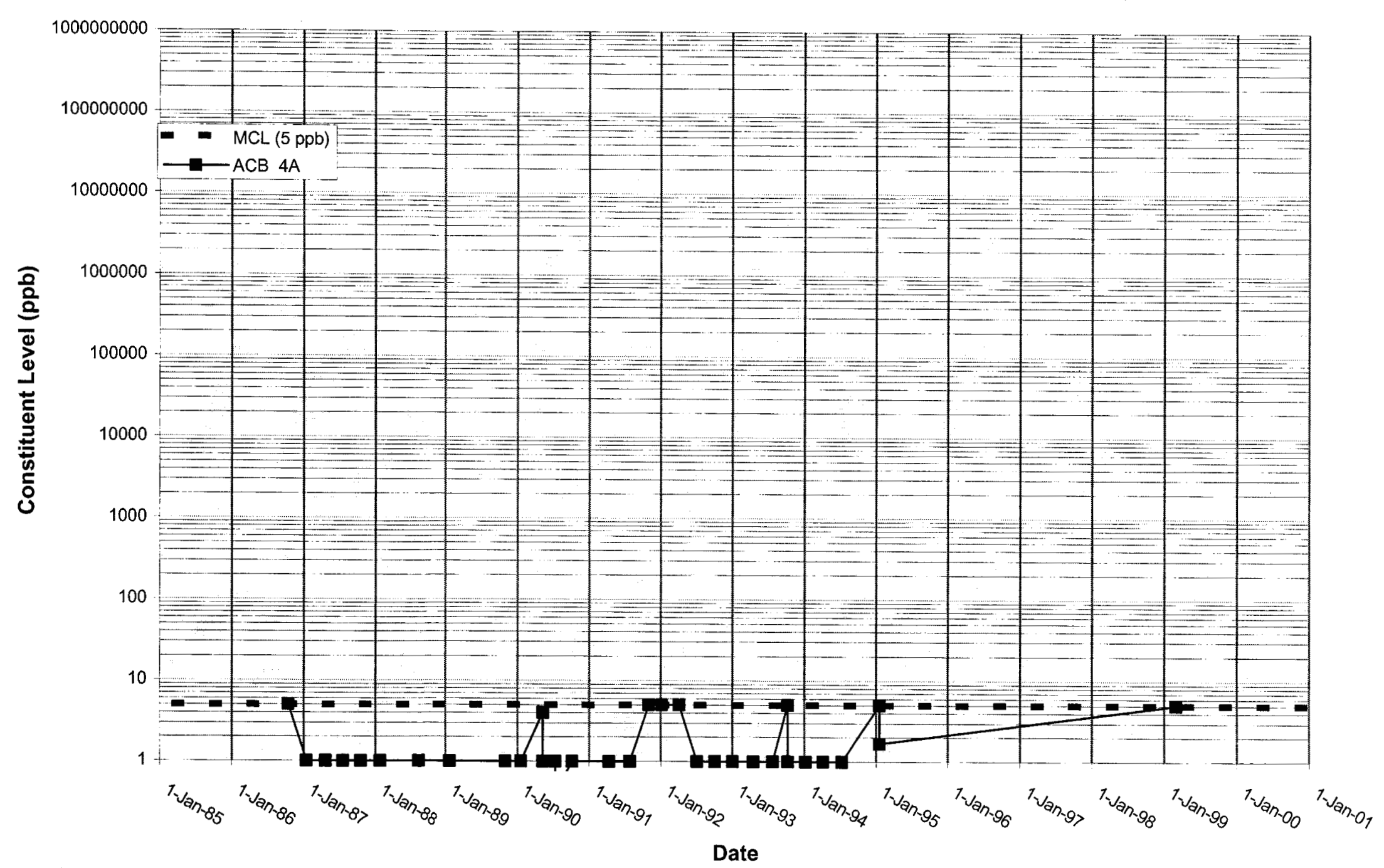


Time Series Plot of Tetrachloroethylene for Well AMB 4

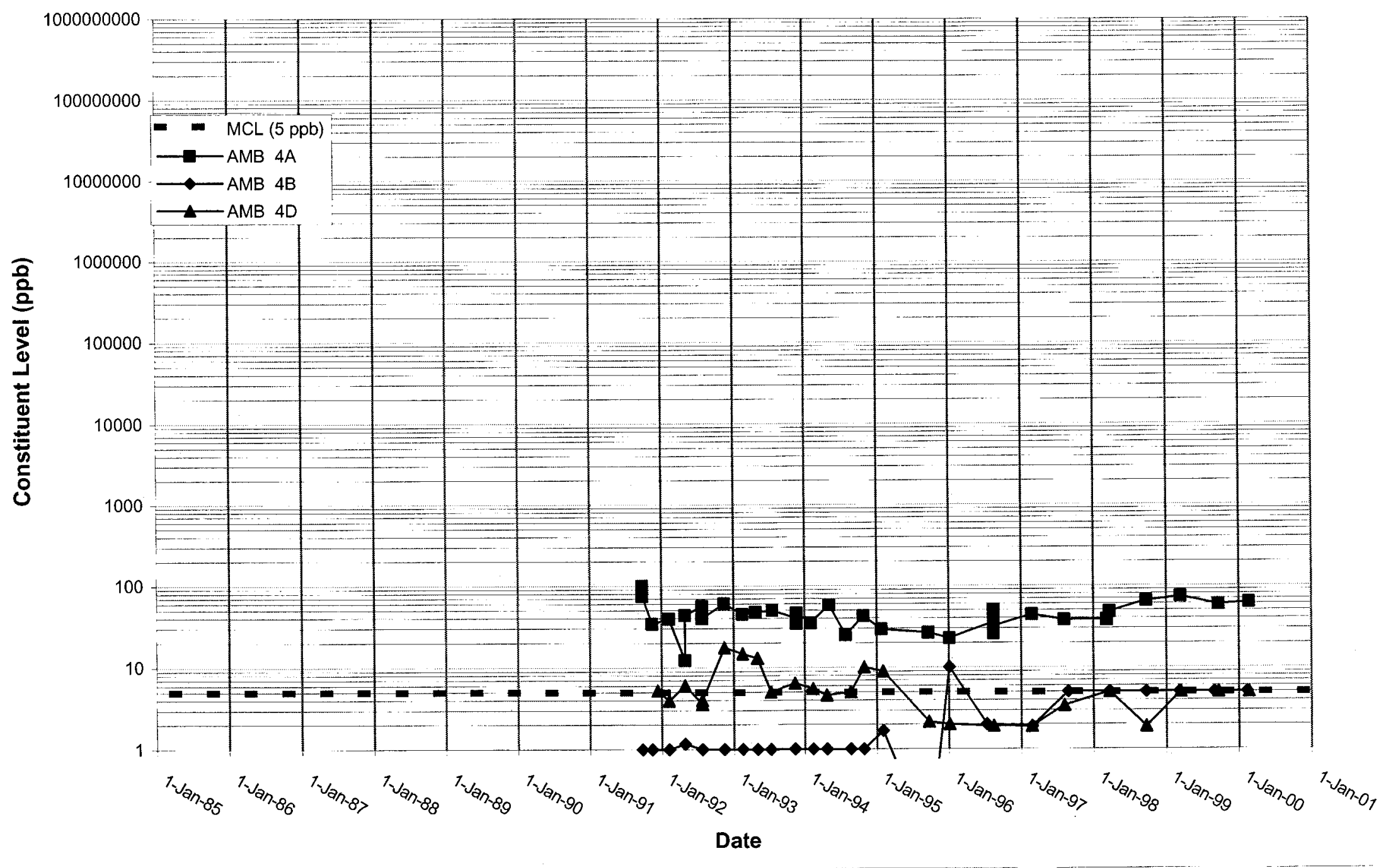


WSRC-TR-2000-00242 UNCLASSIFIED

Time Series Plot of Tetrachloroethylene for Well AMB 5

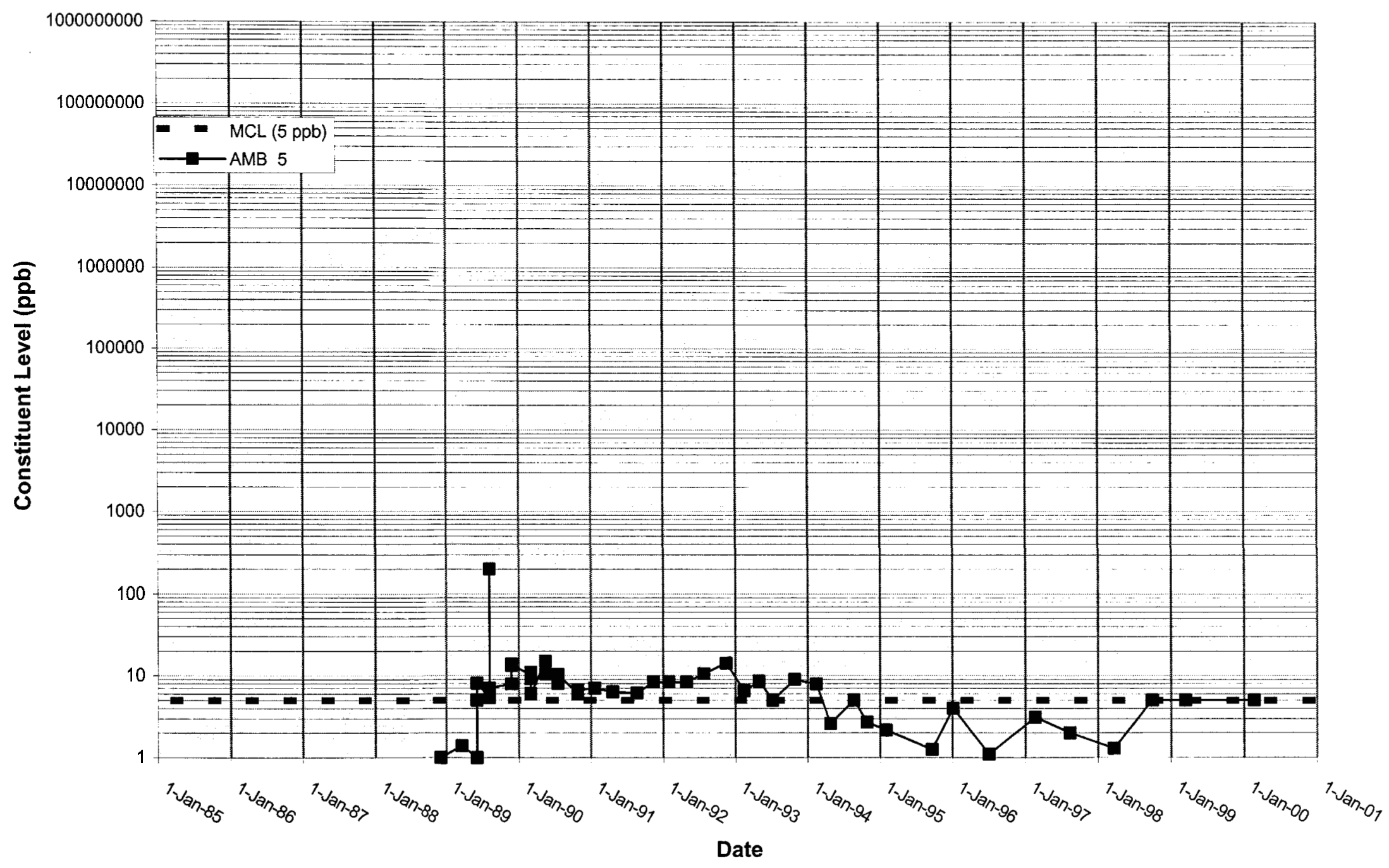




\section{Time Series Plot of Tetrachloroethylene for Well AMB 6}

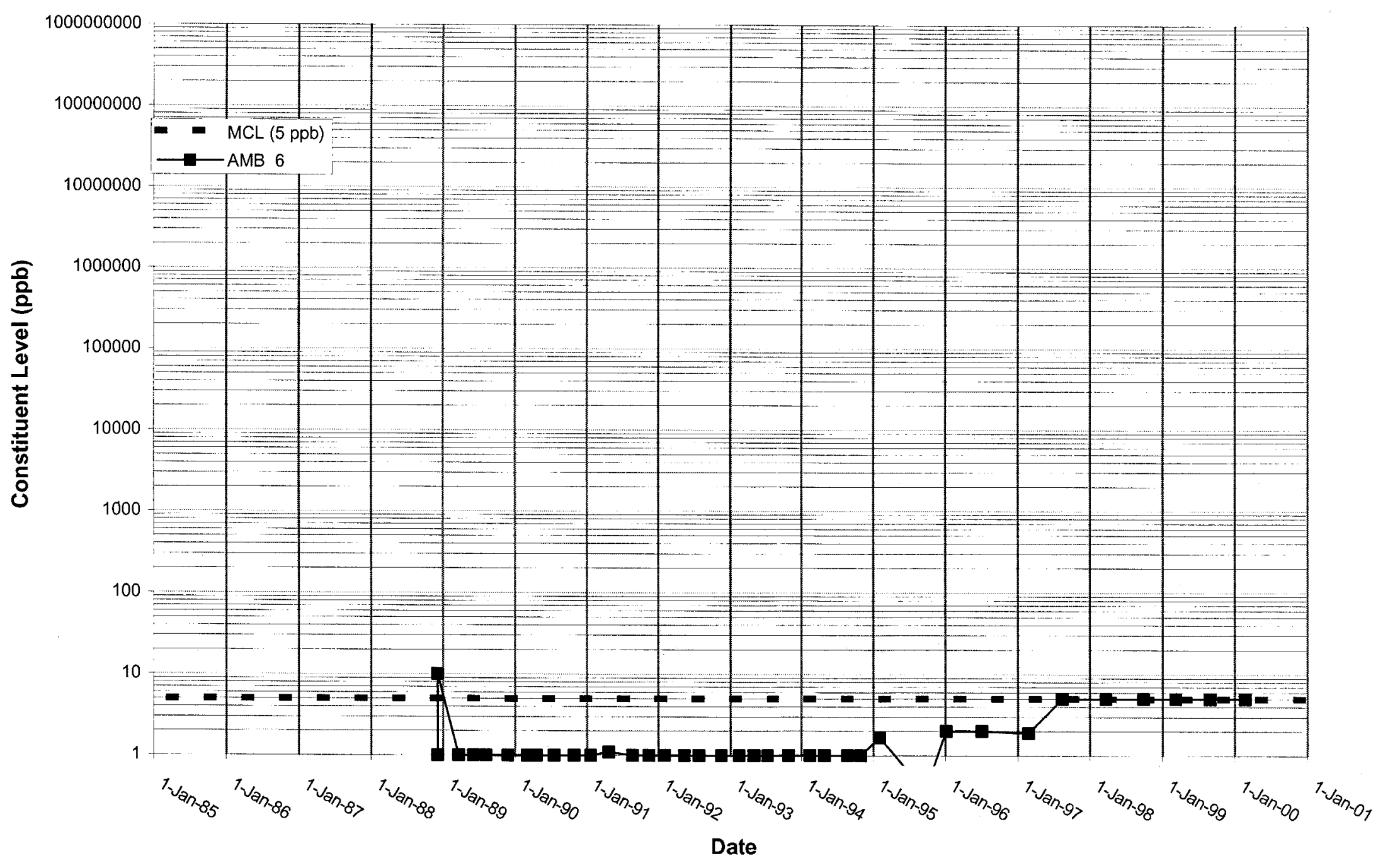


Time Series Plot of Tetrachloroethylene for Well AMB 7

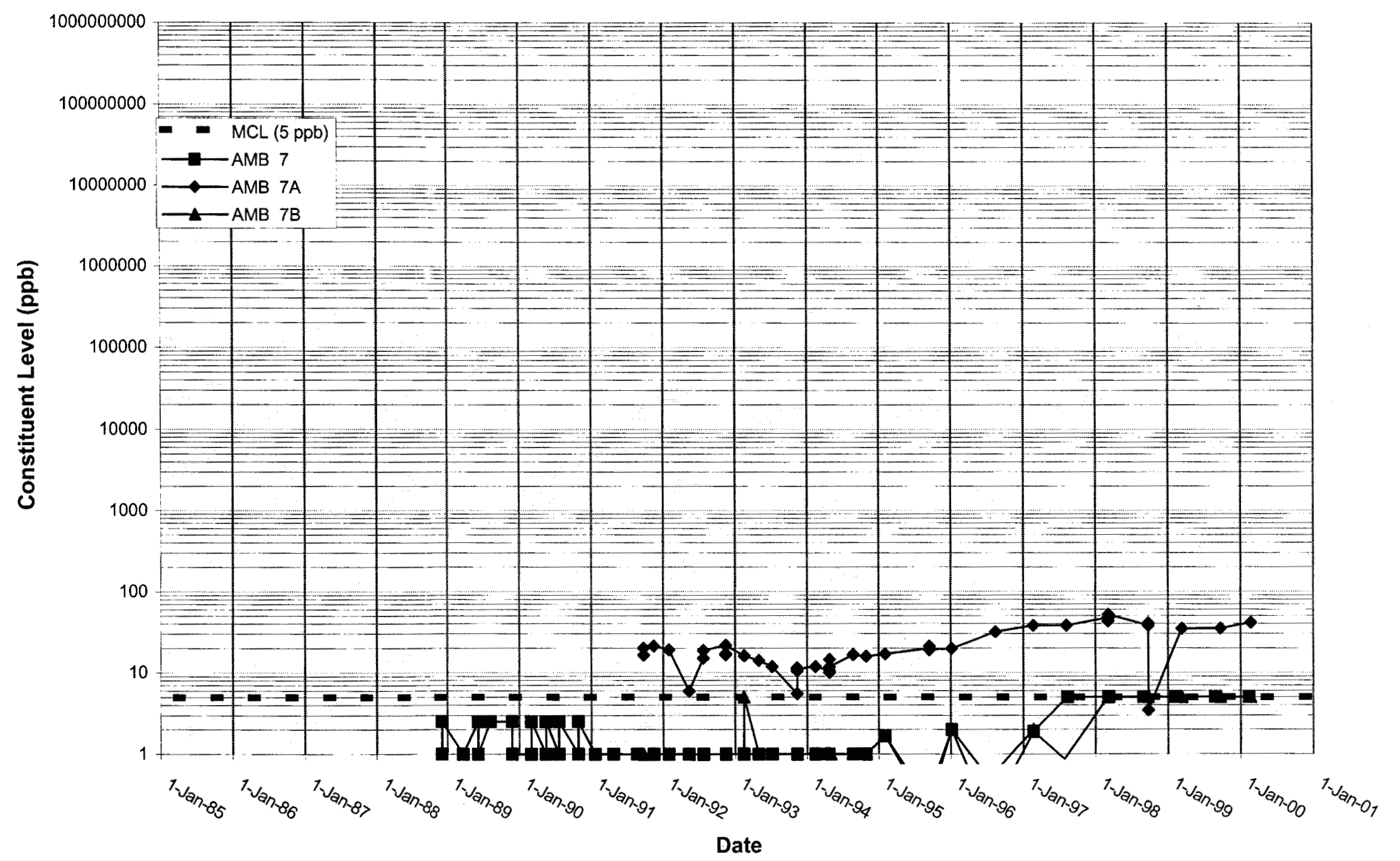


Time Series Plot of Tetrachloroethylene for Well AMB 8

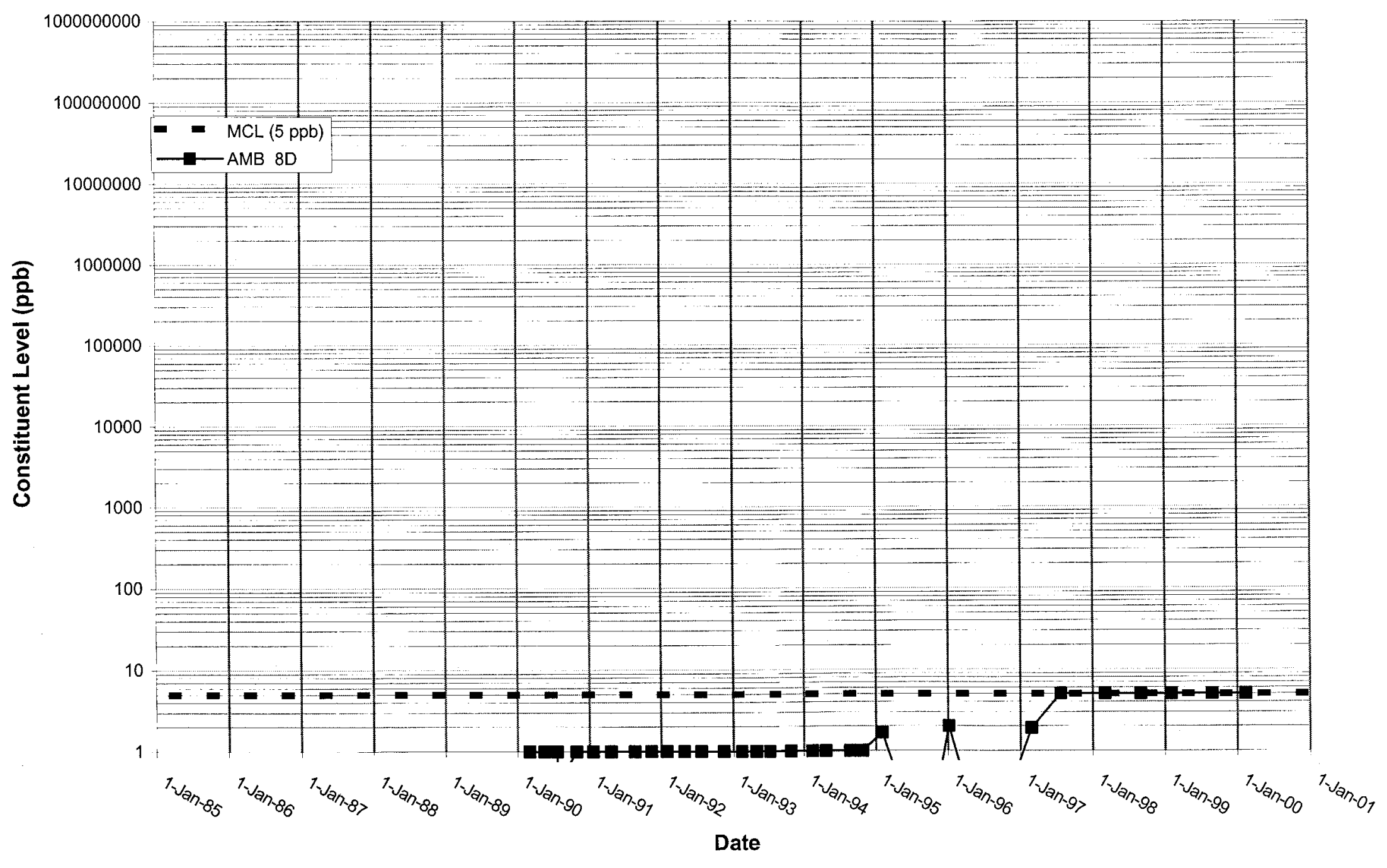


WSRC-TR-2000-00242 UNCLASSIFIED

\section{Time Series Plot of Tetrachloroethylene for Well AMB 9}

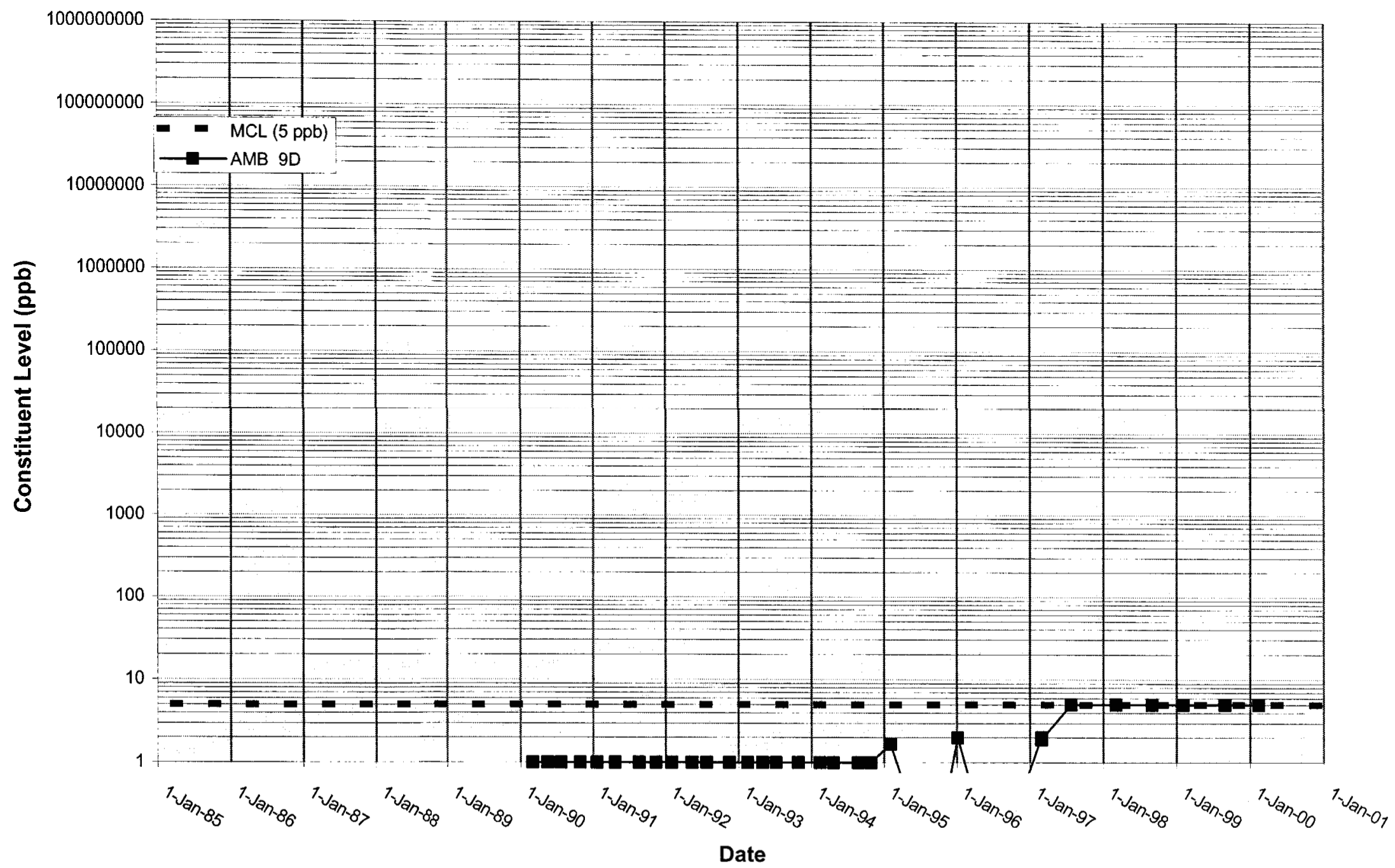




\section{Time Series Plot of Tetrachloroethylene for Well AMB 10}

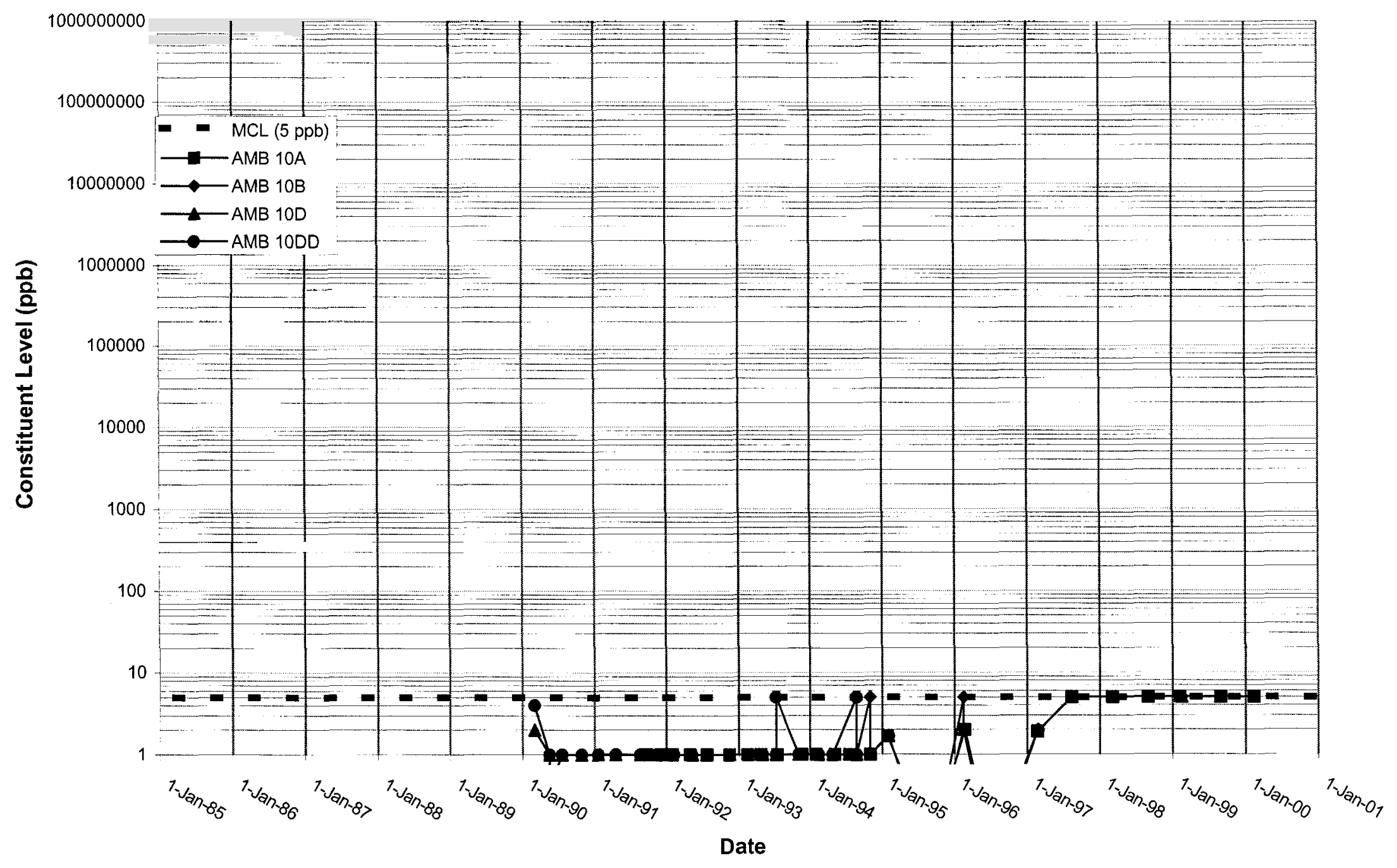




\section{Time Series Plot of Tetrachloroethylene for Well AMB 11}

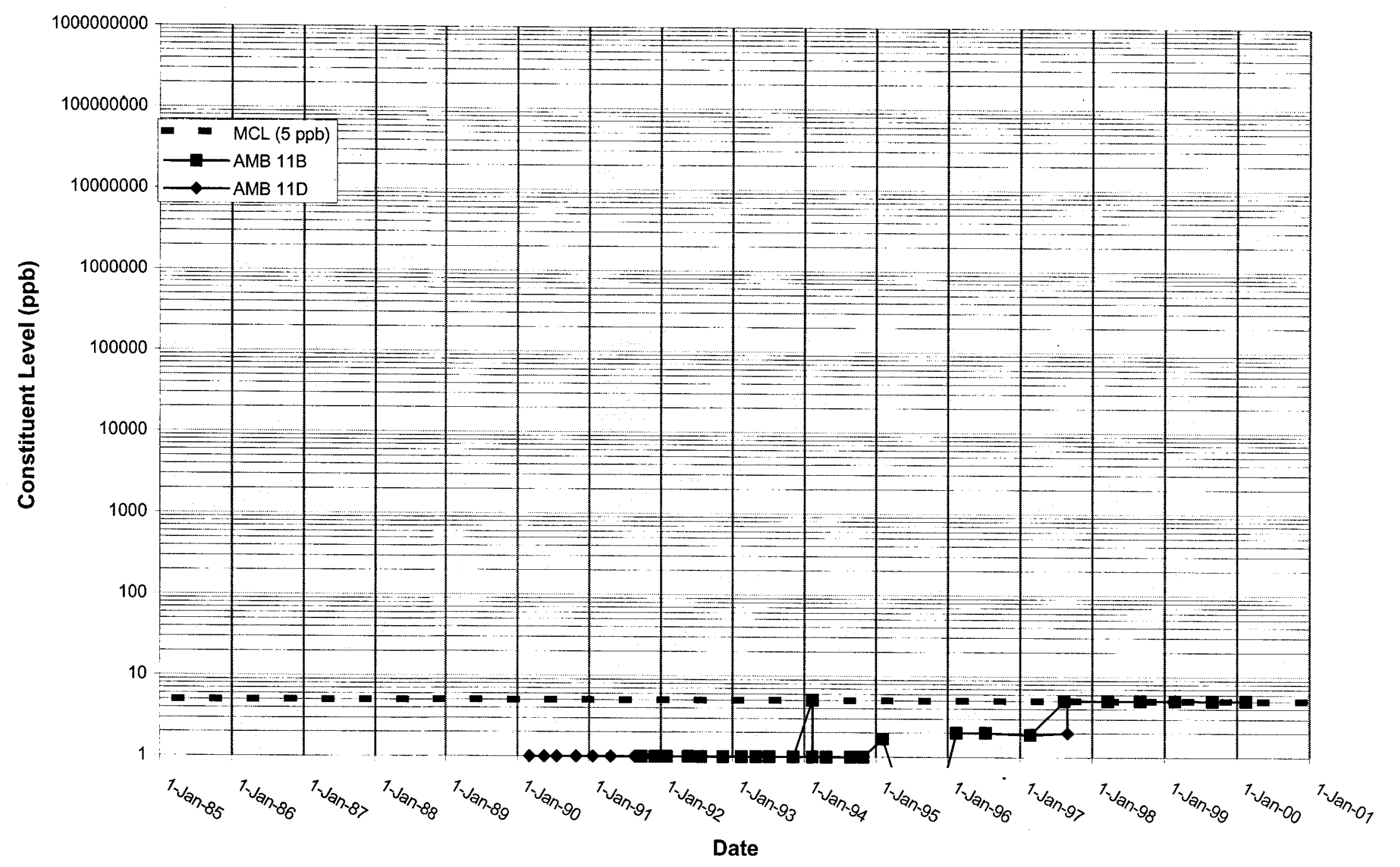


WSRC-TR-2000-00242 UNCLASSIFIED

Time Series Plot of Tetrachloroethylene for Well AMB 12

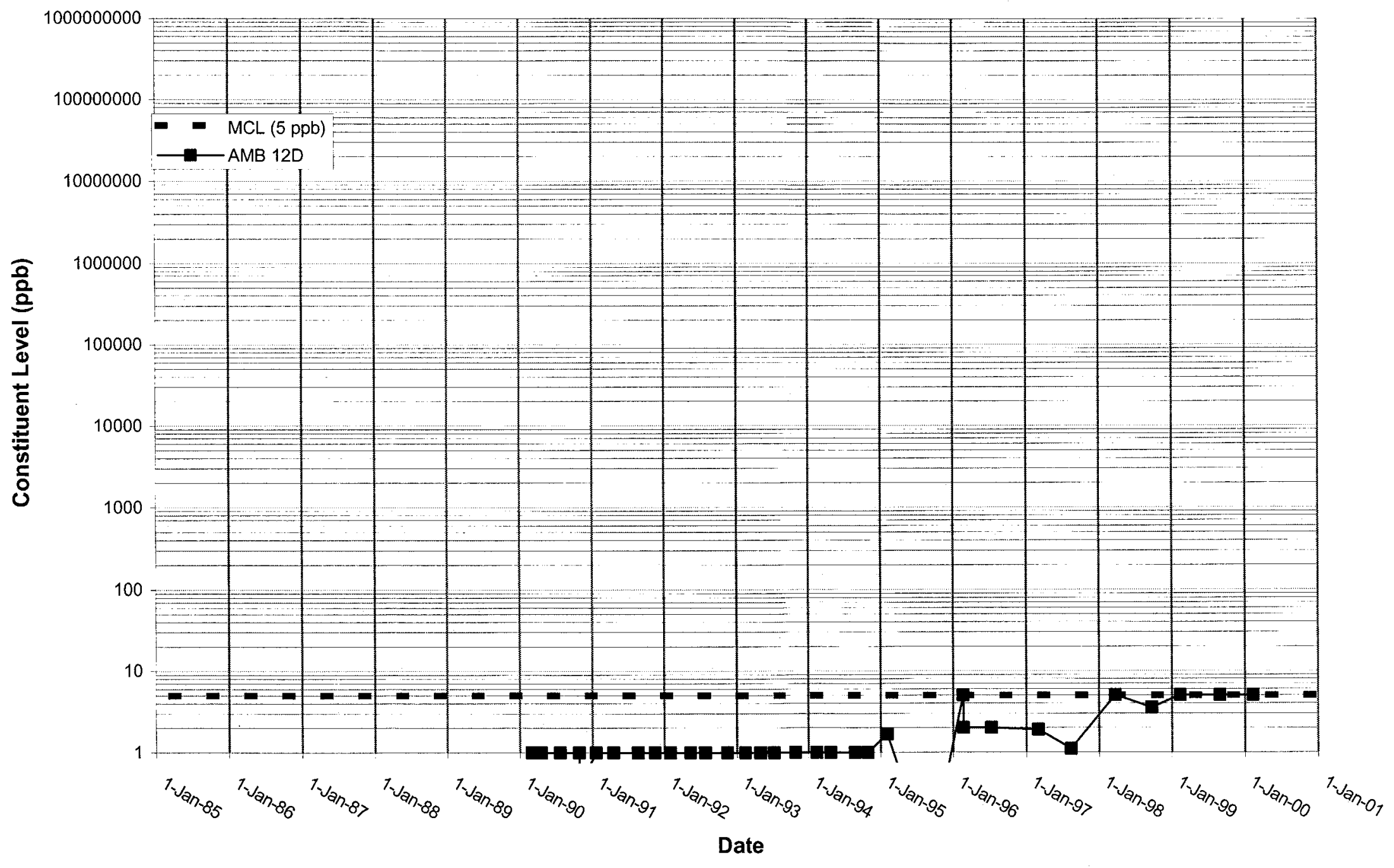




\section{Time Series Plot of Tetrachloroethylene for Well AMB 13}

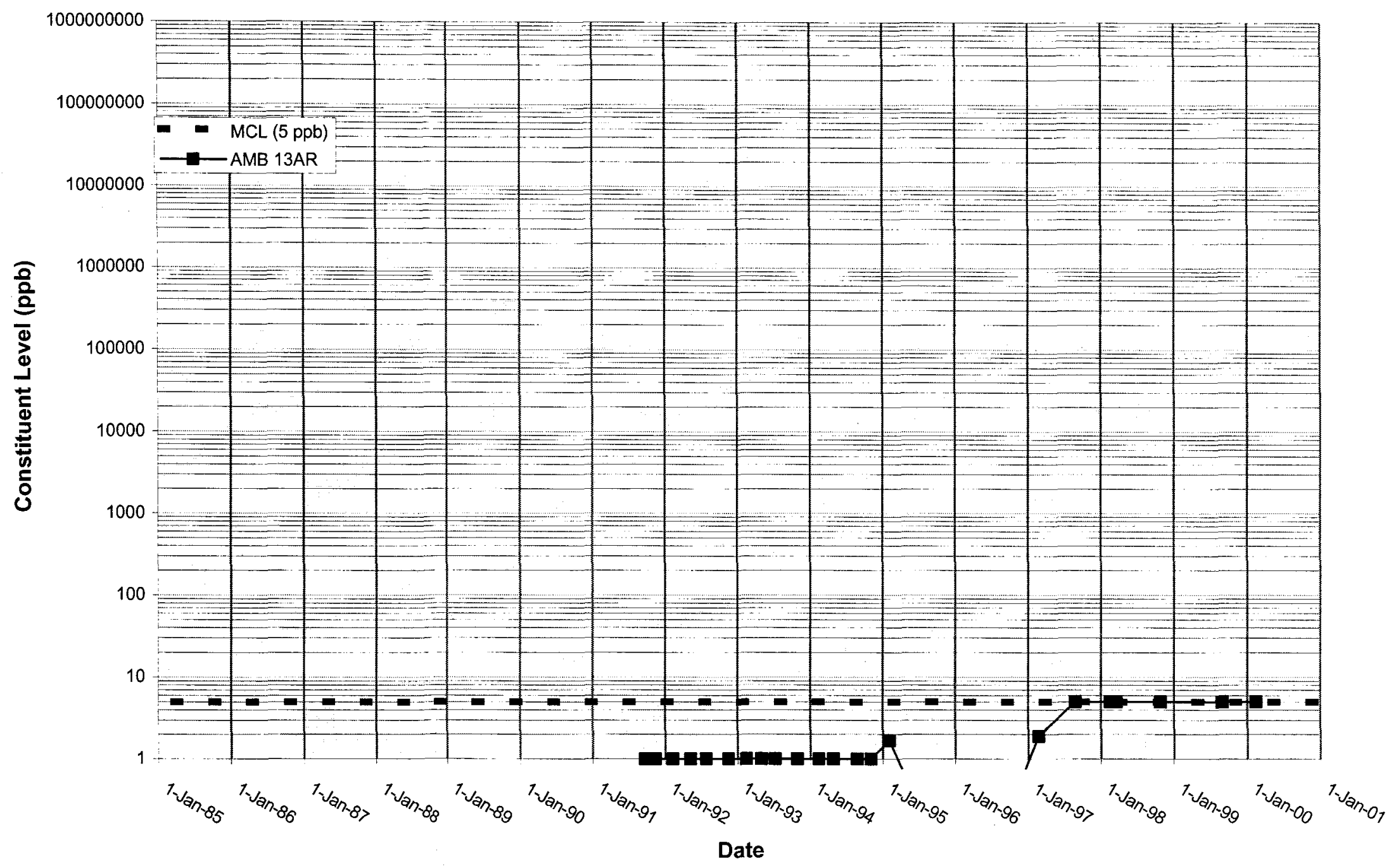


WSRC-TR-2000-00242 UNCLASSIFIED

\section{Time Series Plot of Tetrachloroethylene for Well AMB 14}

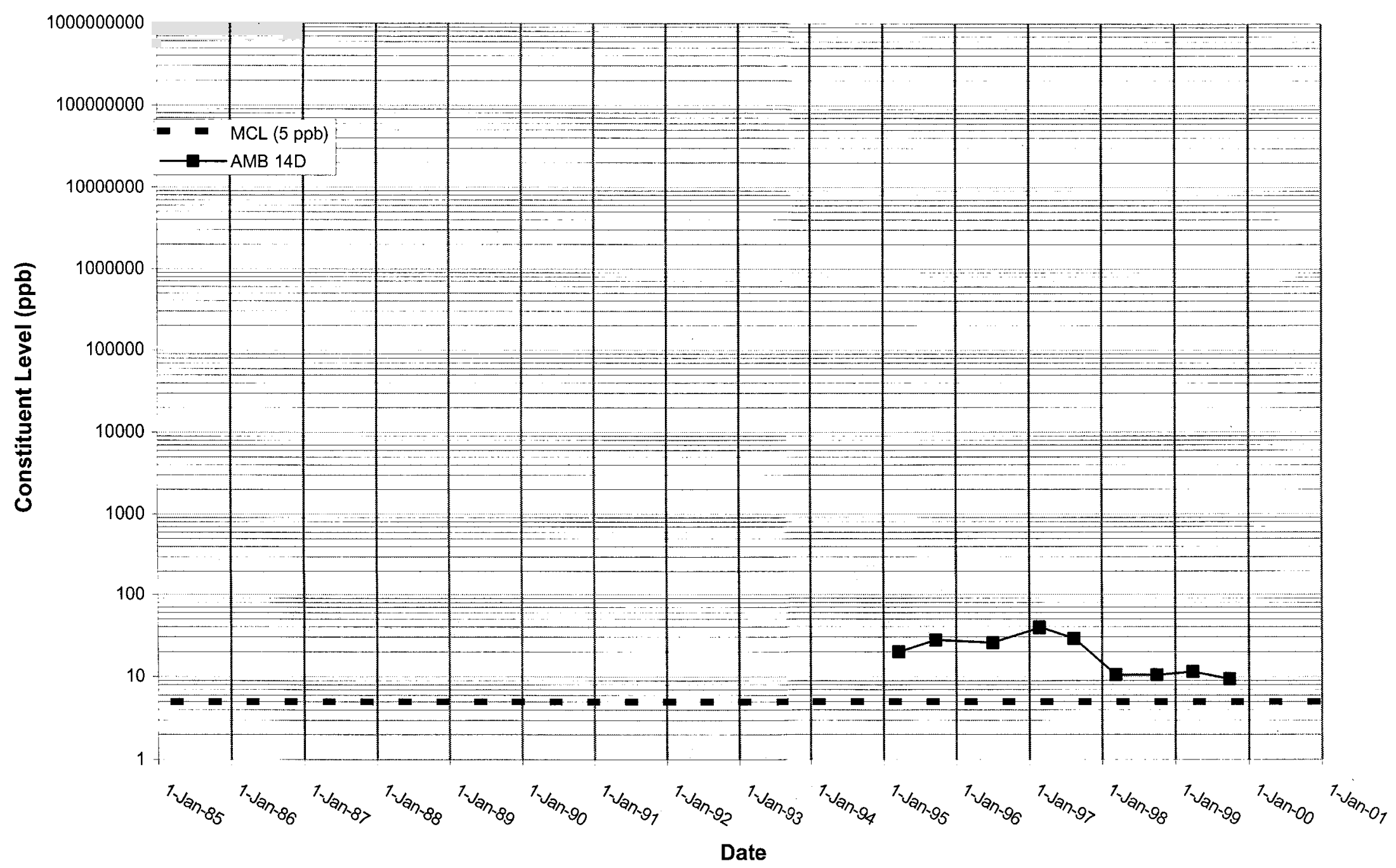


WSRC-TR-2000-00242

Time Series Plot of Tetrachloroethylene for Well AMB 15

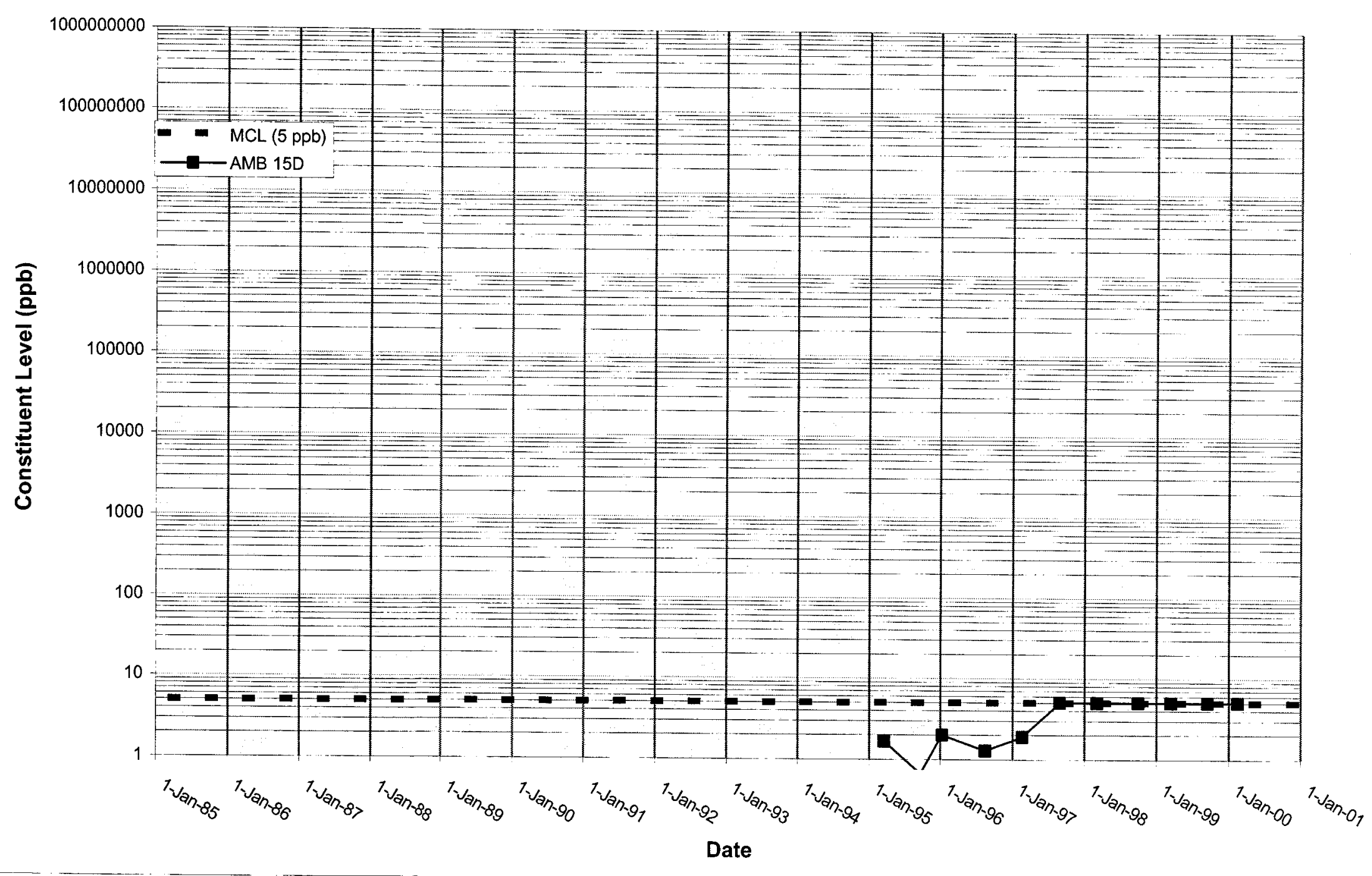




\section{Time Series Plot of Tetrachloroethylene for Well AMB 16}

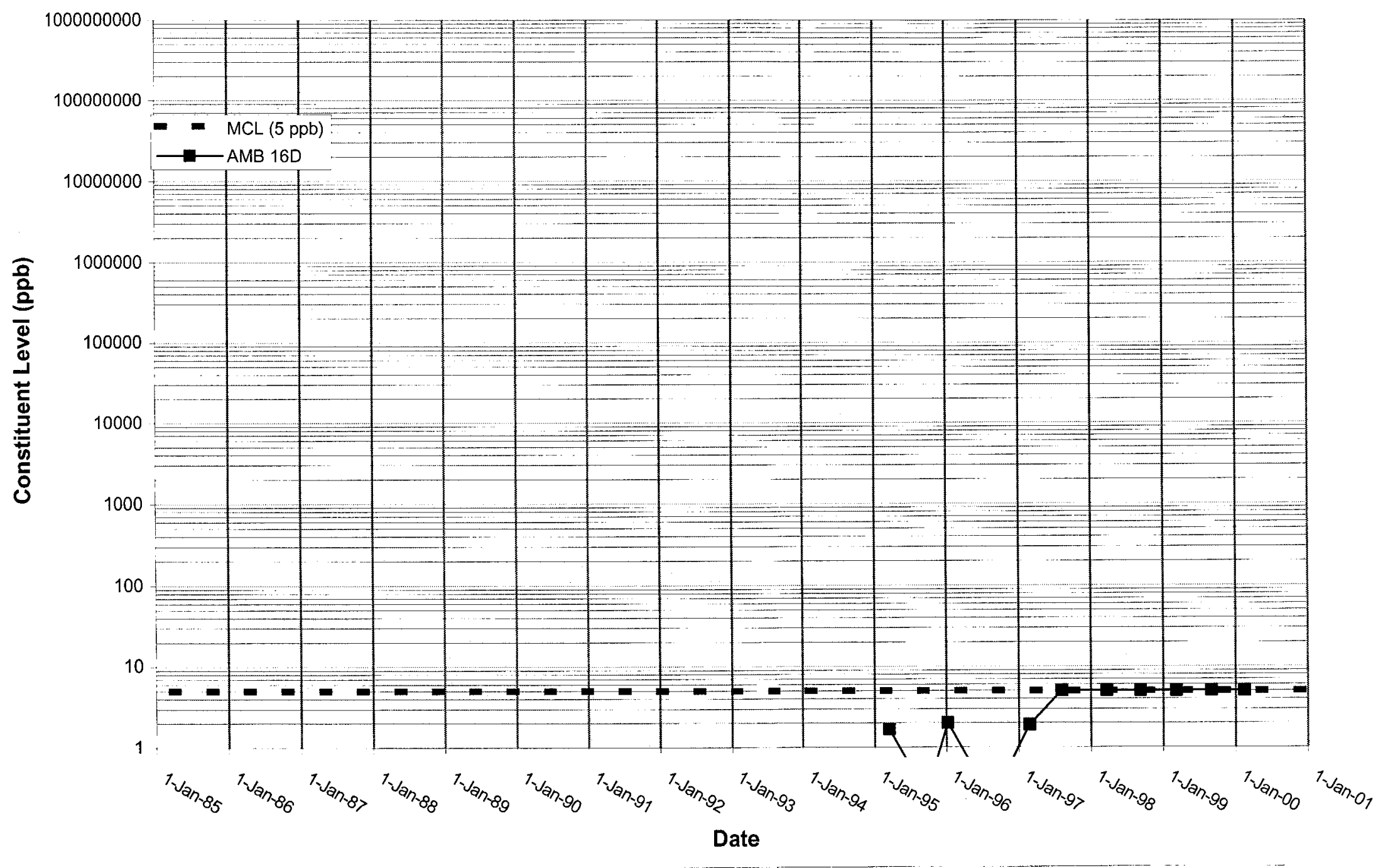


WSRC-TR-2000-00242 UNCLASSIFIED

Time Series Plot of Tetrachloroethylene for Well AMB 17

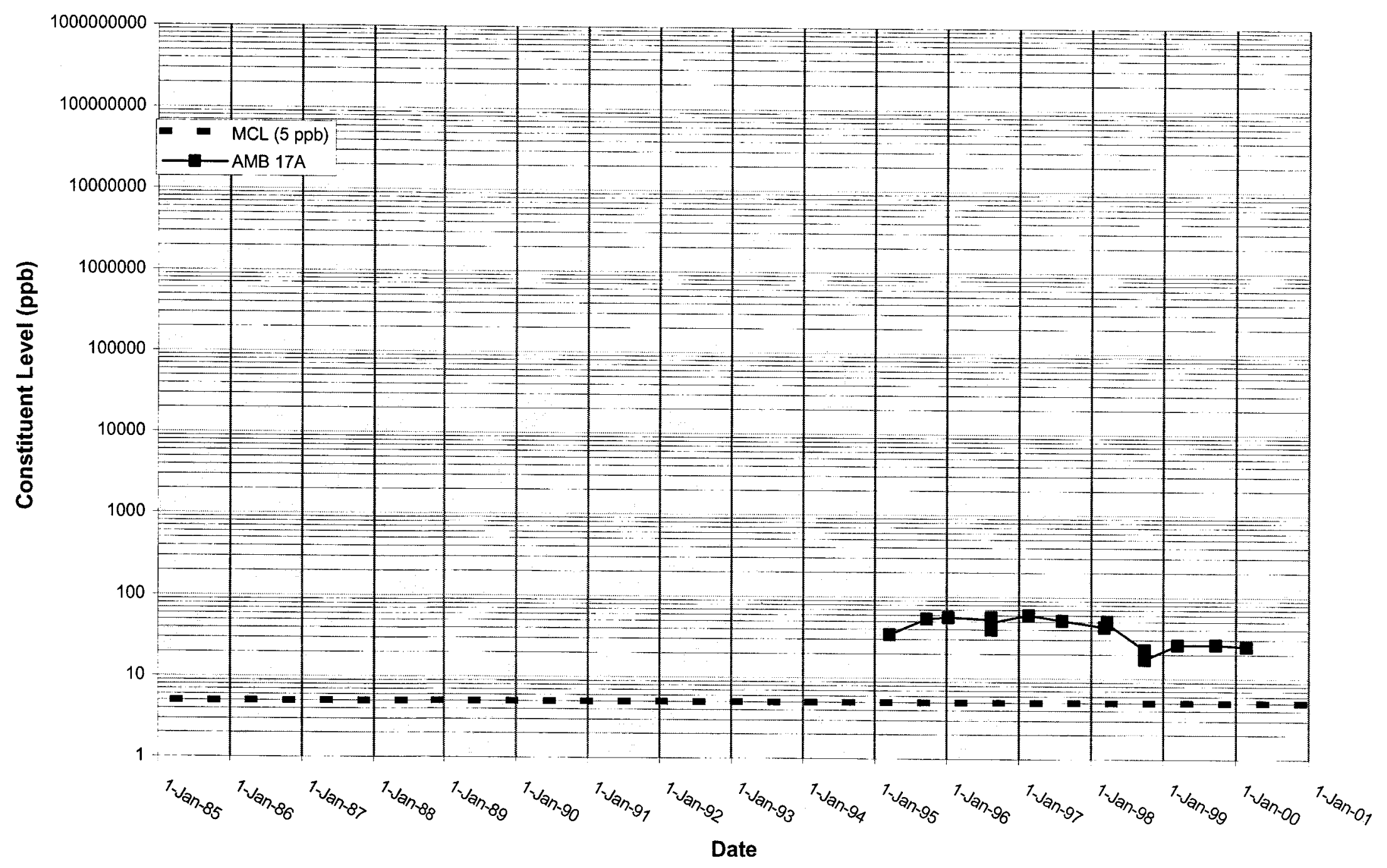


WSRC-TR-2000-00242

UNCLASSIFIED

\section{Time Series Plot of Tetrachloroethylene for Well AMB 18}

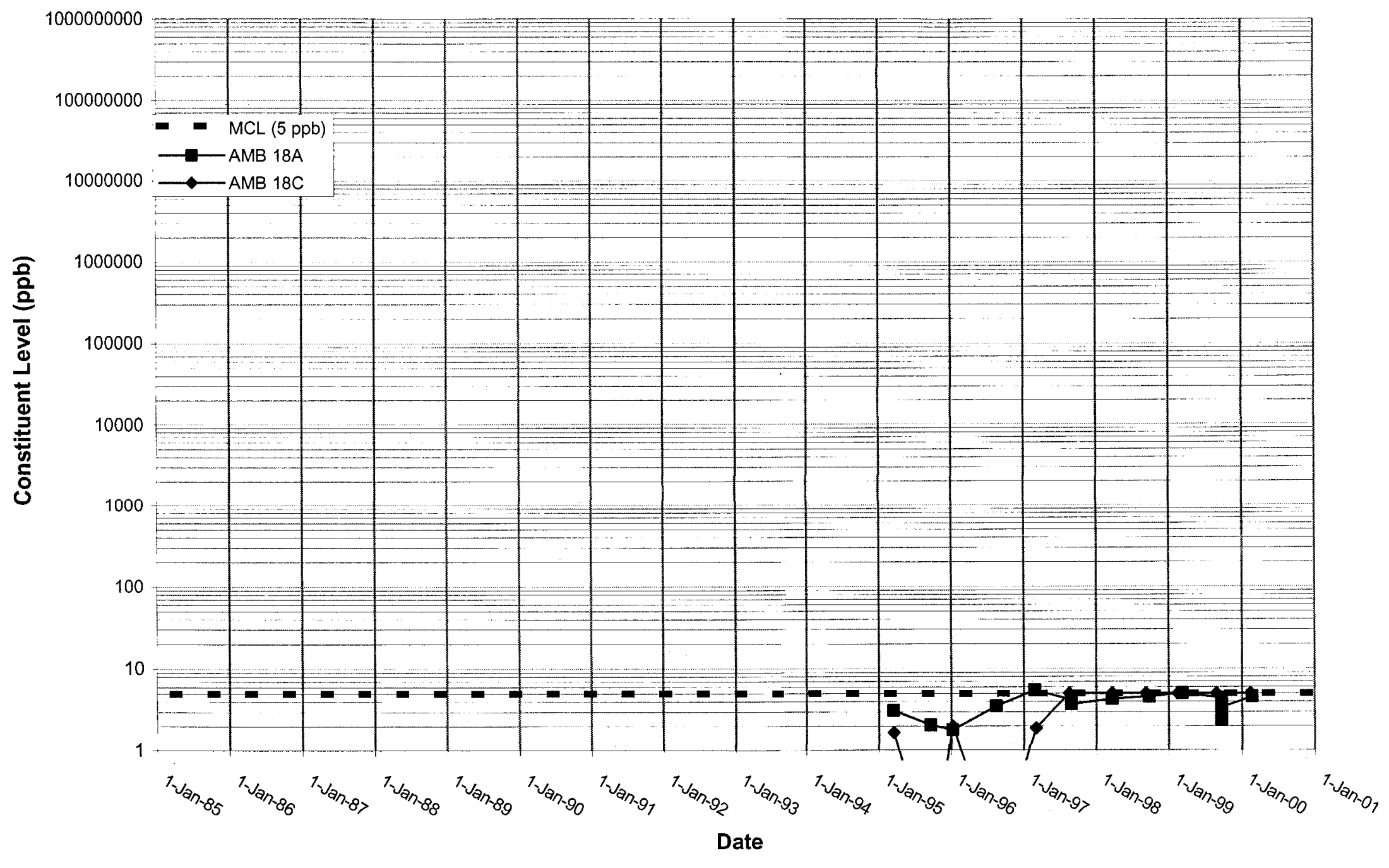




\section{Time Series Plot of Tetrachloroethylene for Well AMB 19}

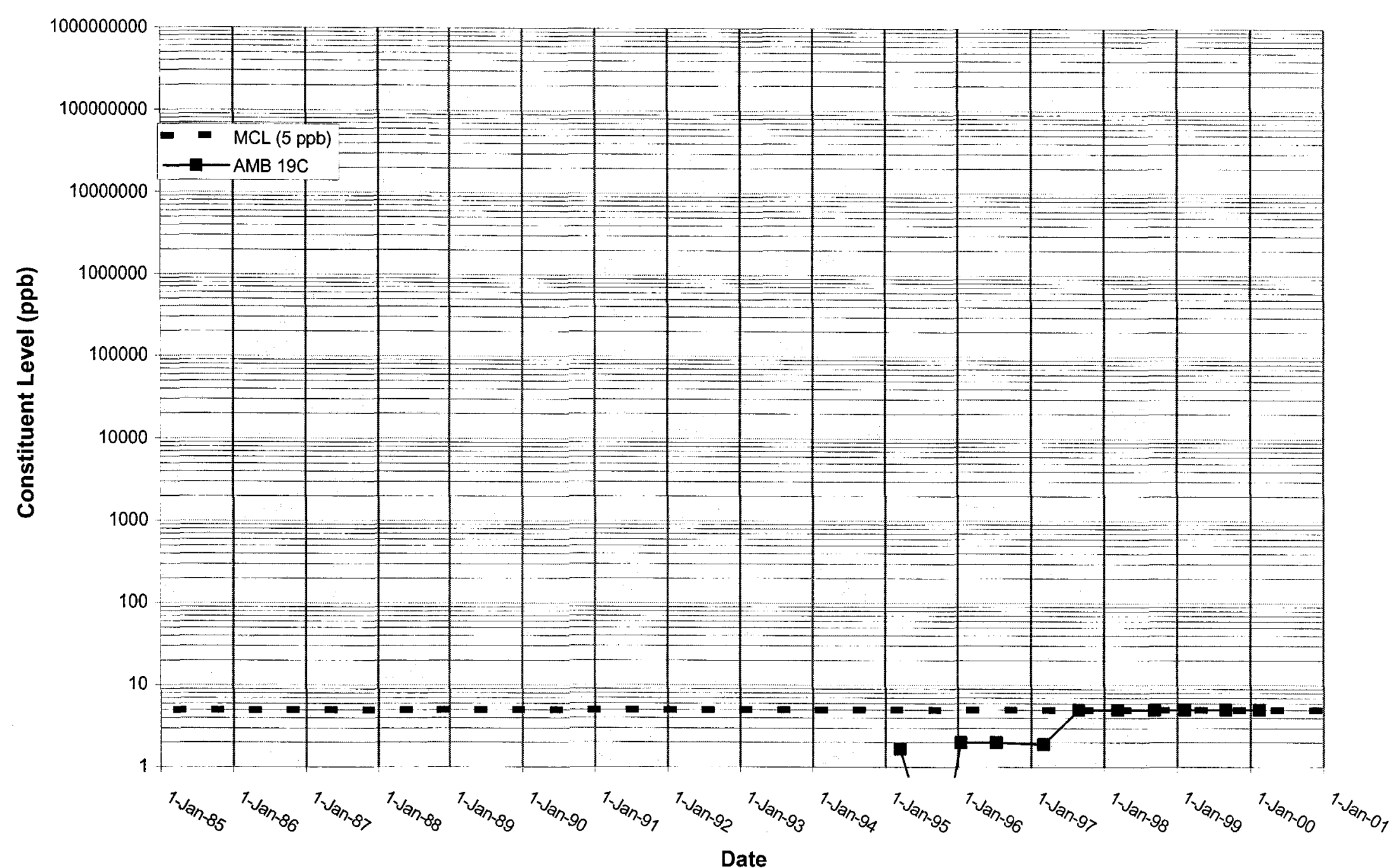


Time Series Plot of Tetrachloroethylene for Well AOB 1

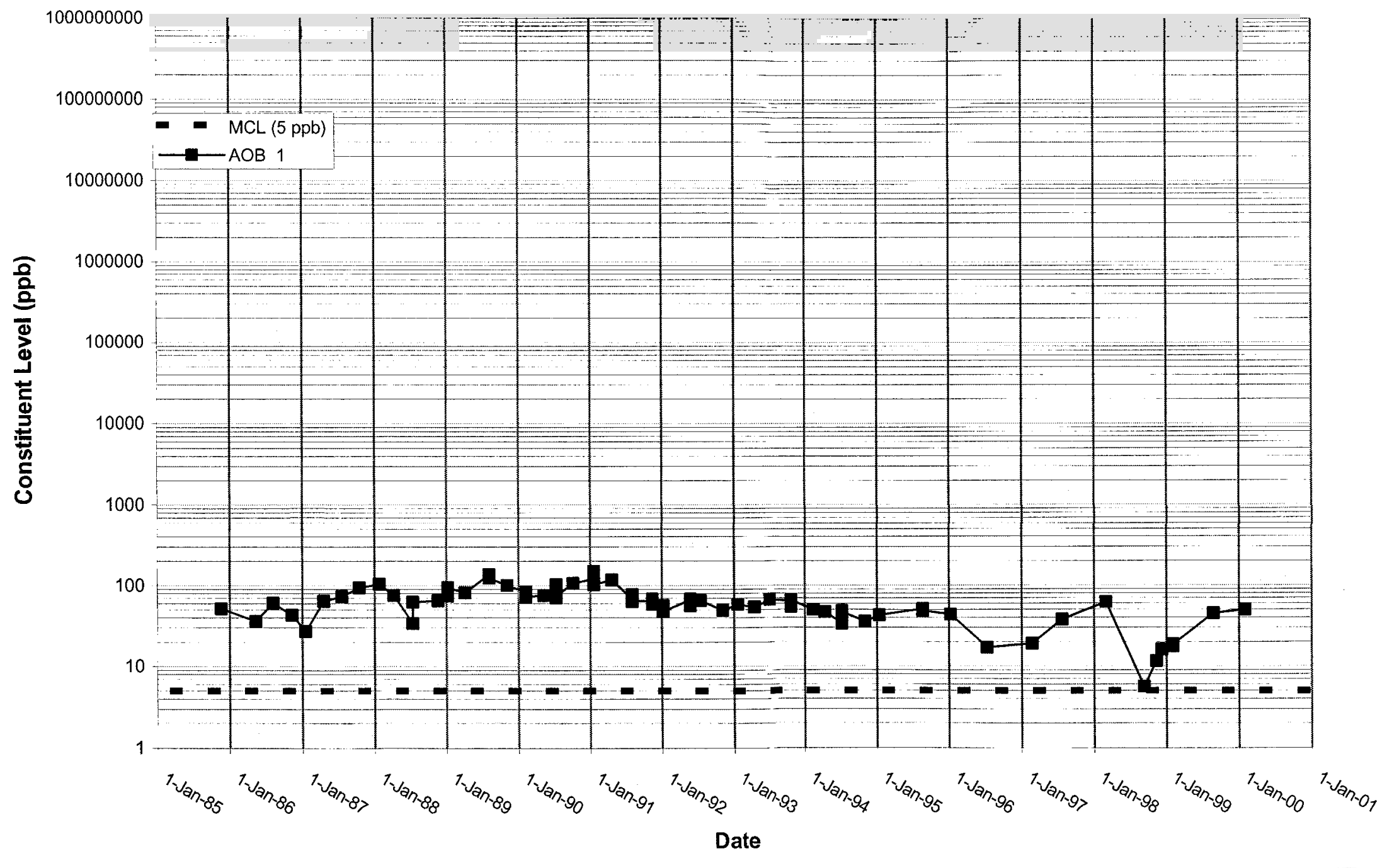


WSRC-TR-2000-00242 UNCLASSIFIED

Time Series Plot of Tetrachloroethylene for Well AOB 2

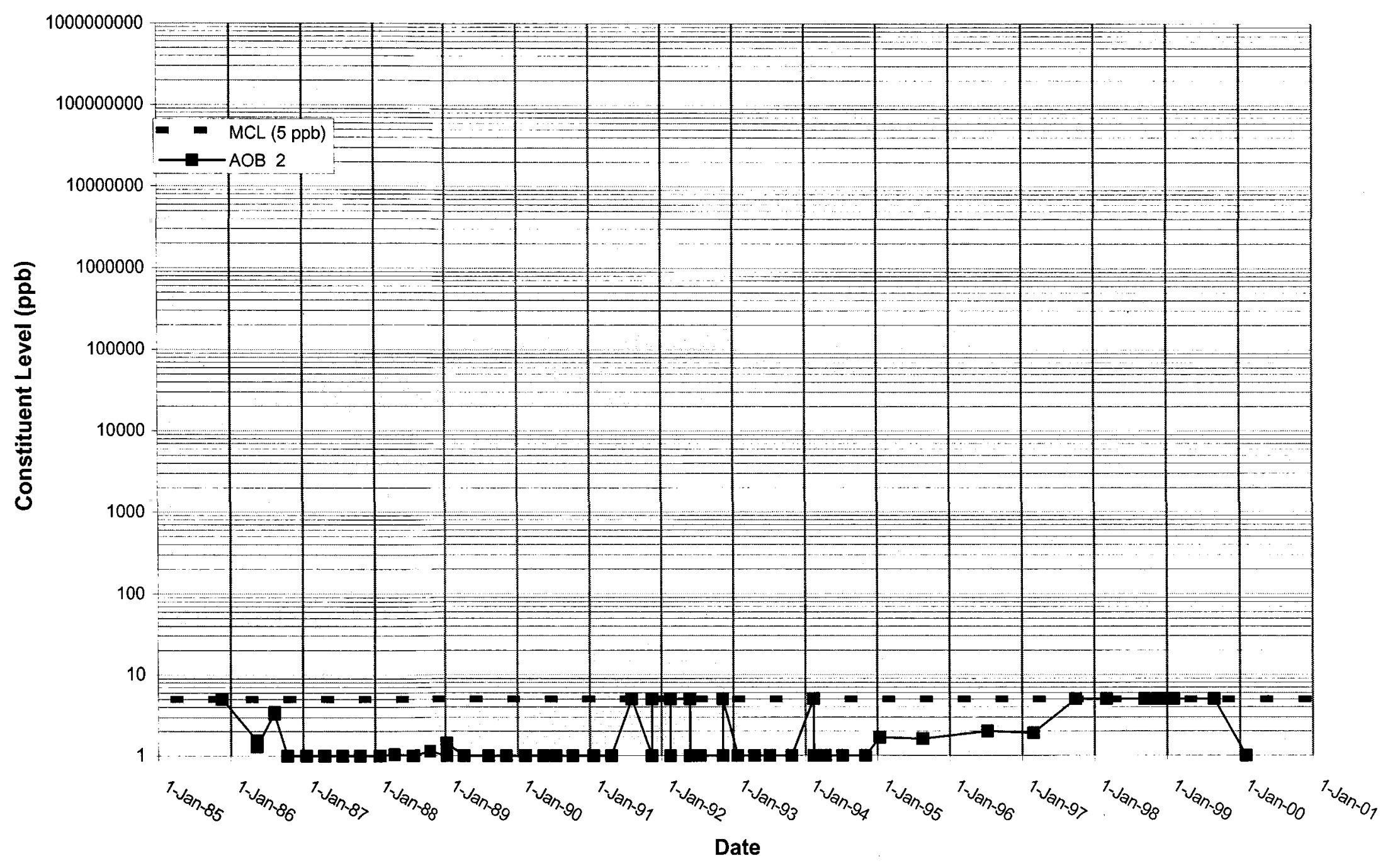


WSRC-TR-2000-00242 UNCLASSIFIED

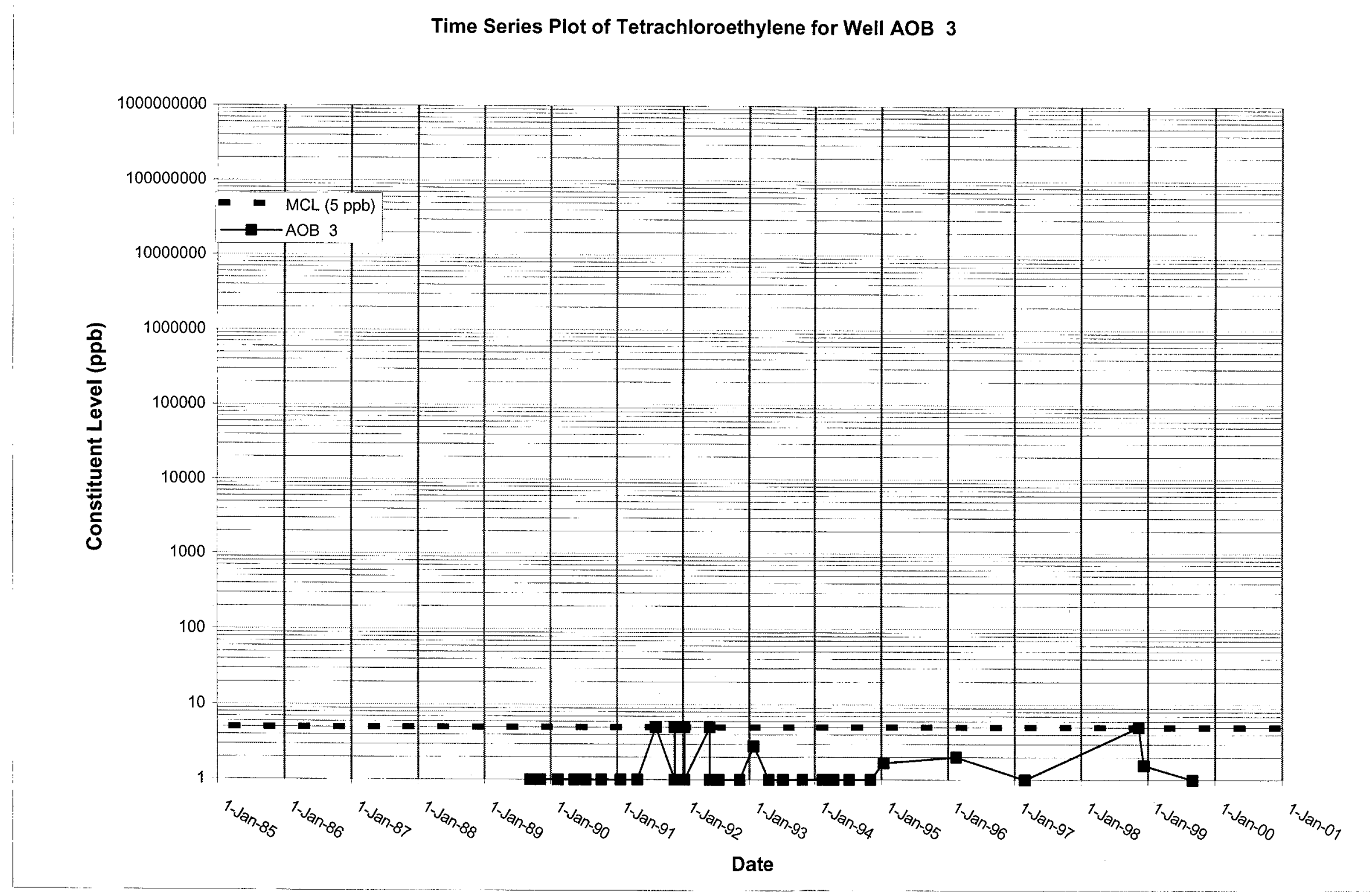




\section{Time Series Plot of Tetrachloroethylene for Well ARP 1}

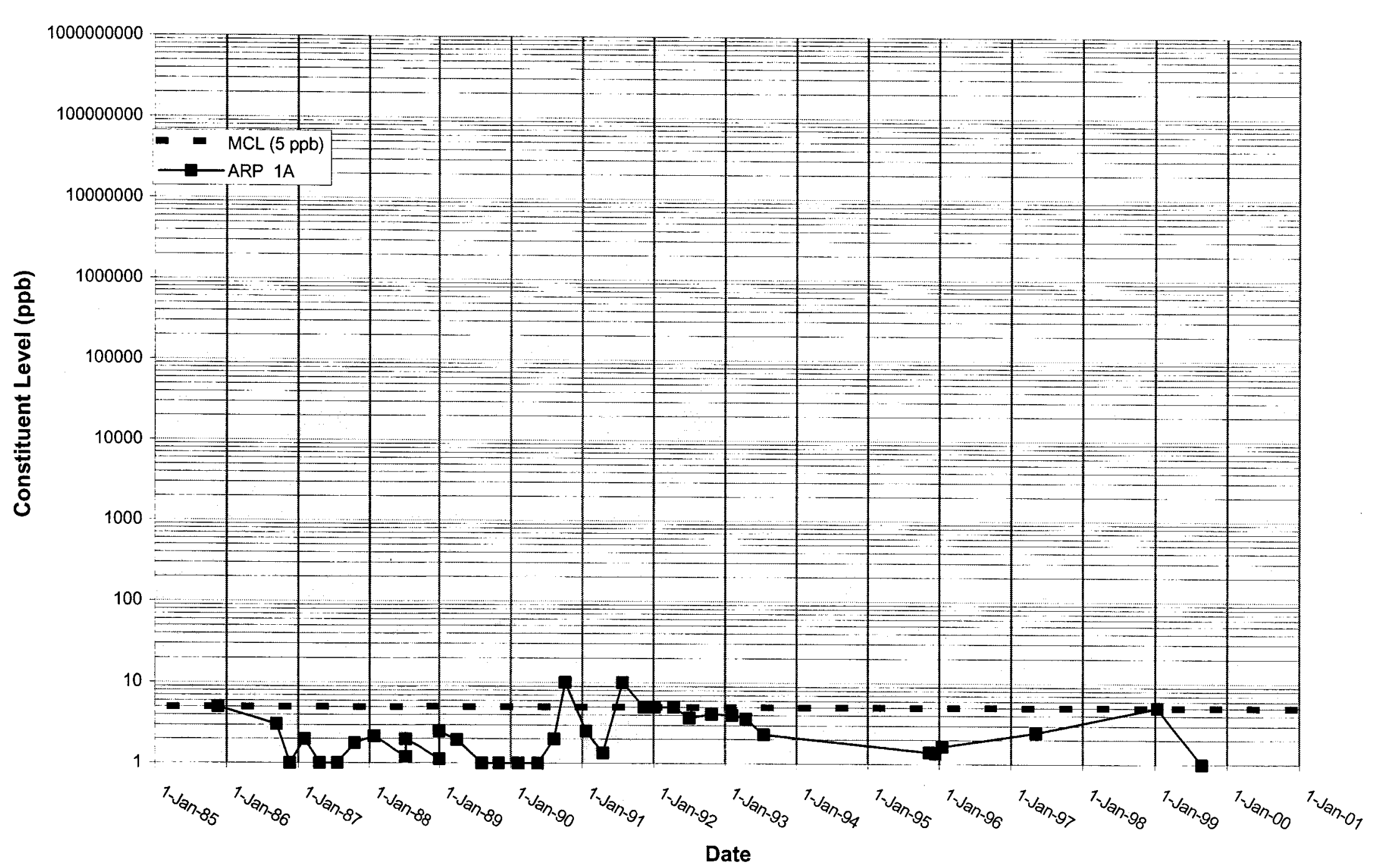




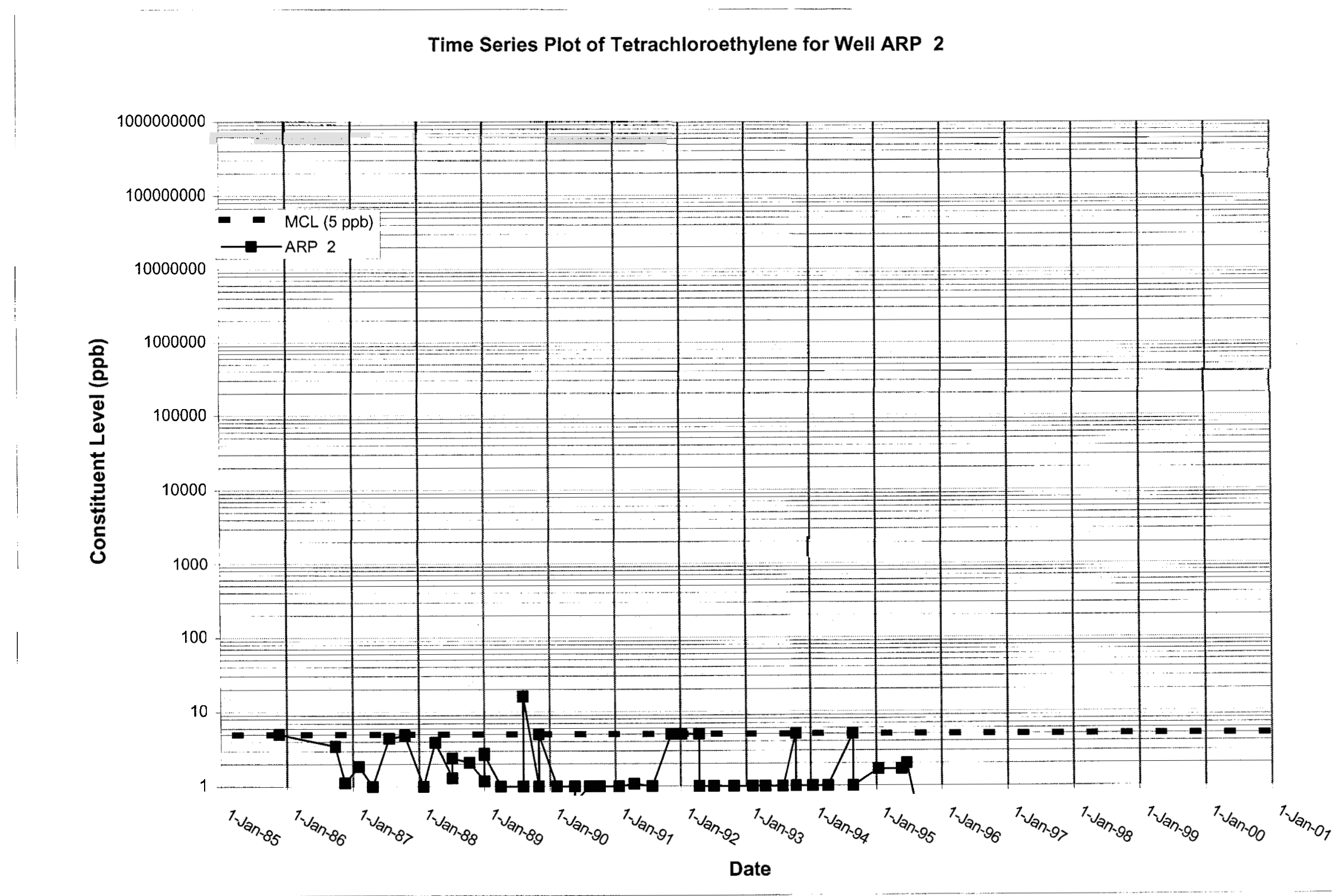




\section{Time Series Plot of Tetrachloroethylene for Well ARP 3}

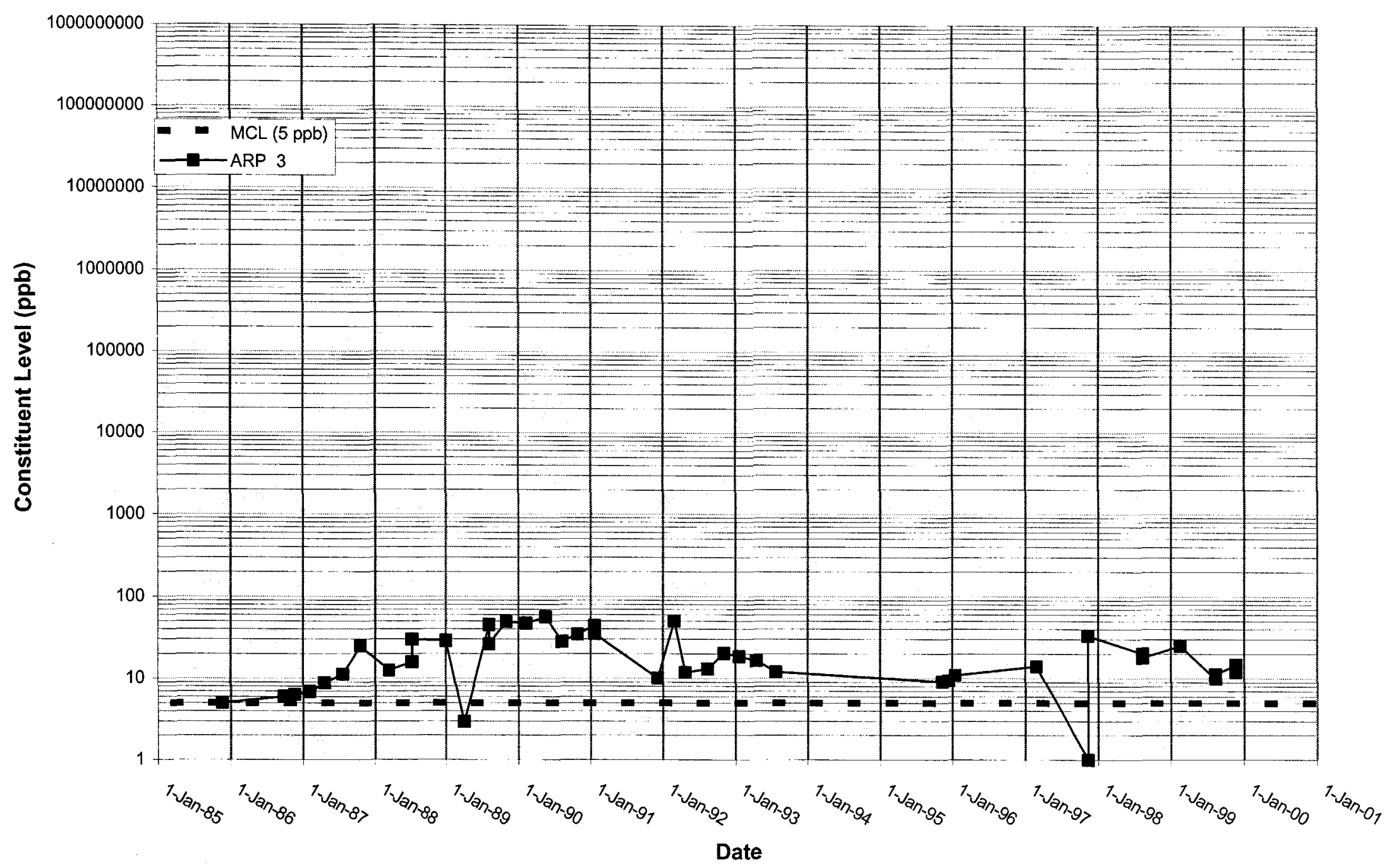


WSRC-TR-2000-00242 UNCLASSIFIED

Time Series Plot of Tetrachloroethylene for Well ARP 4

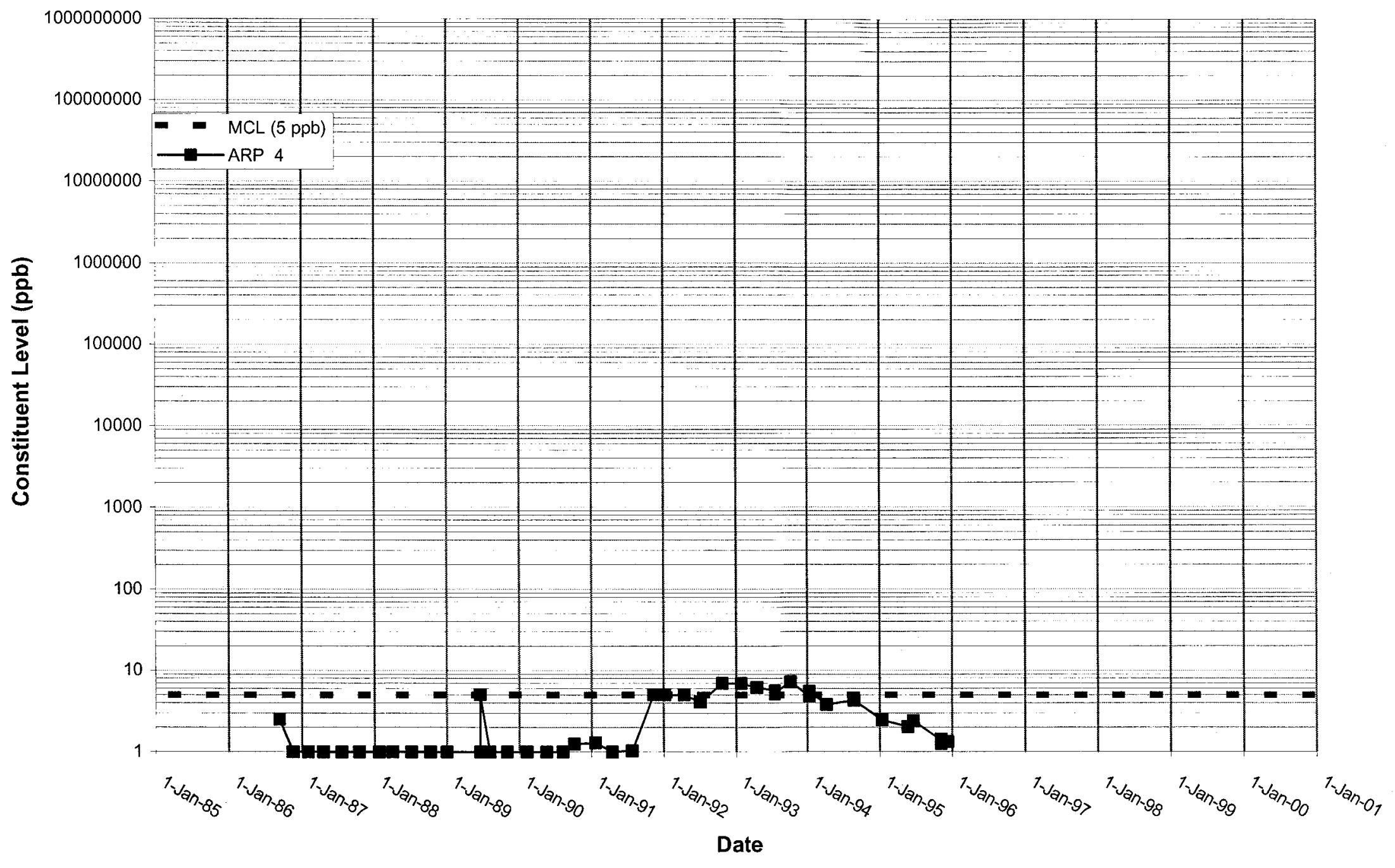


Time Series Plot of Tetrachloroethylene for Well ASB 1

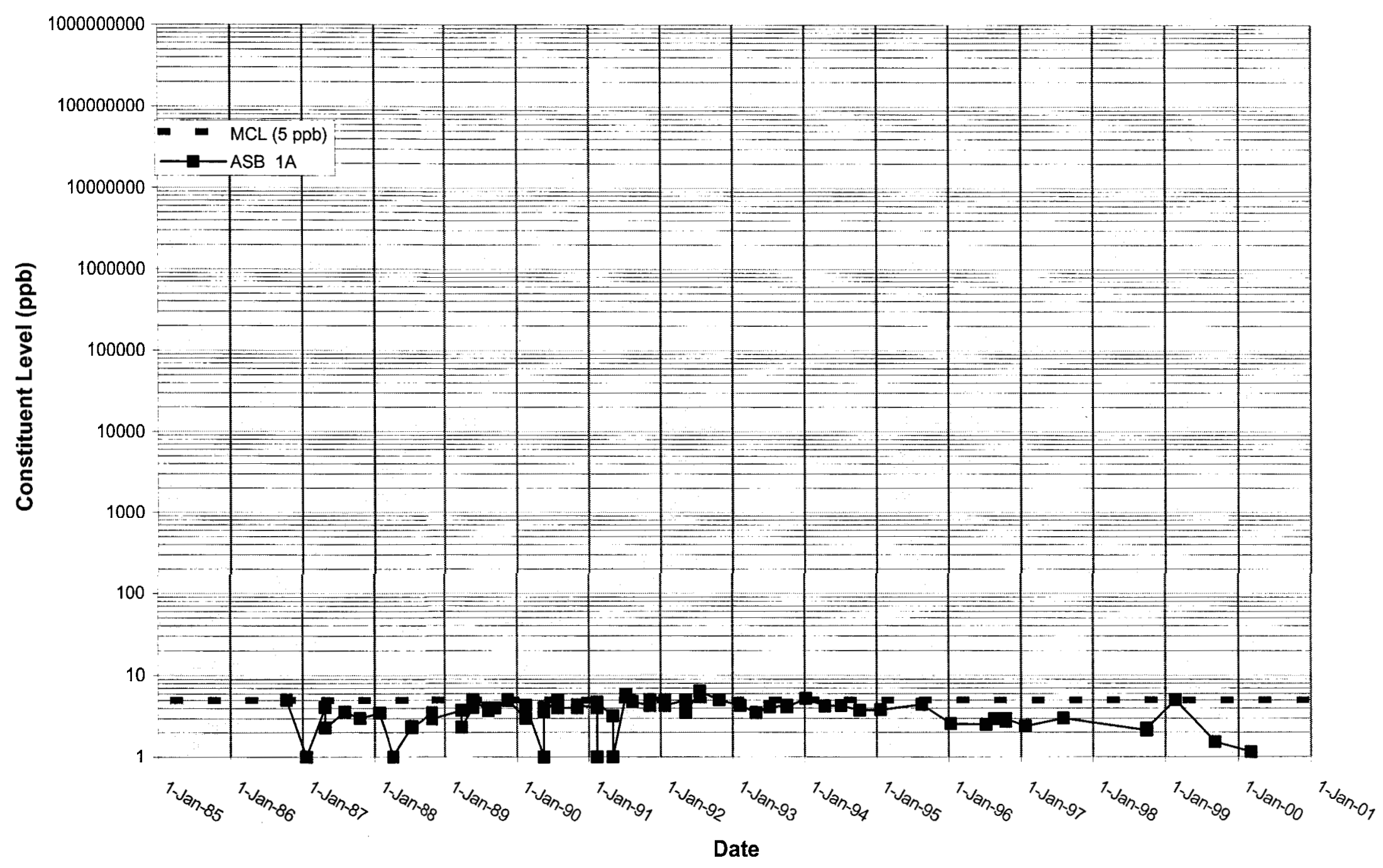


WSRC-TR-2000-00242 UNCLASSIFIED

\section{Time Series Plot of Tetrachloroethylene for Well ASB 2}

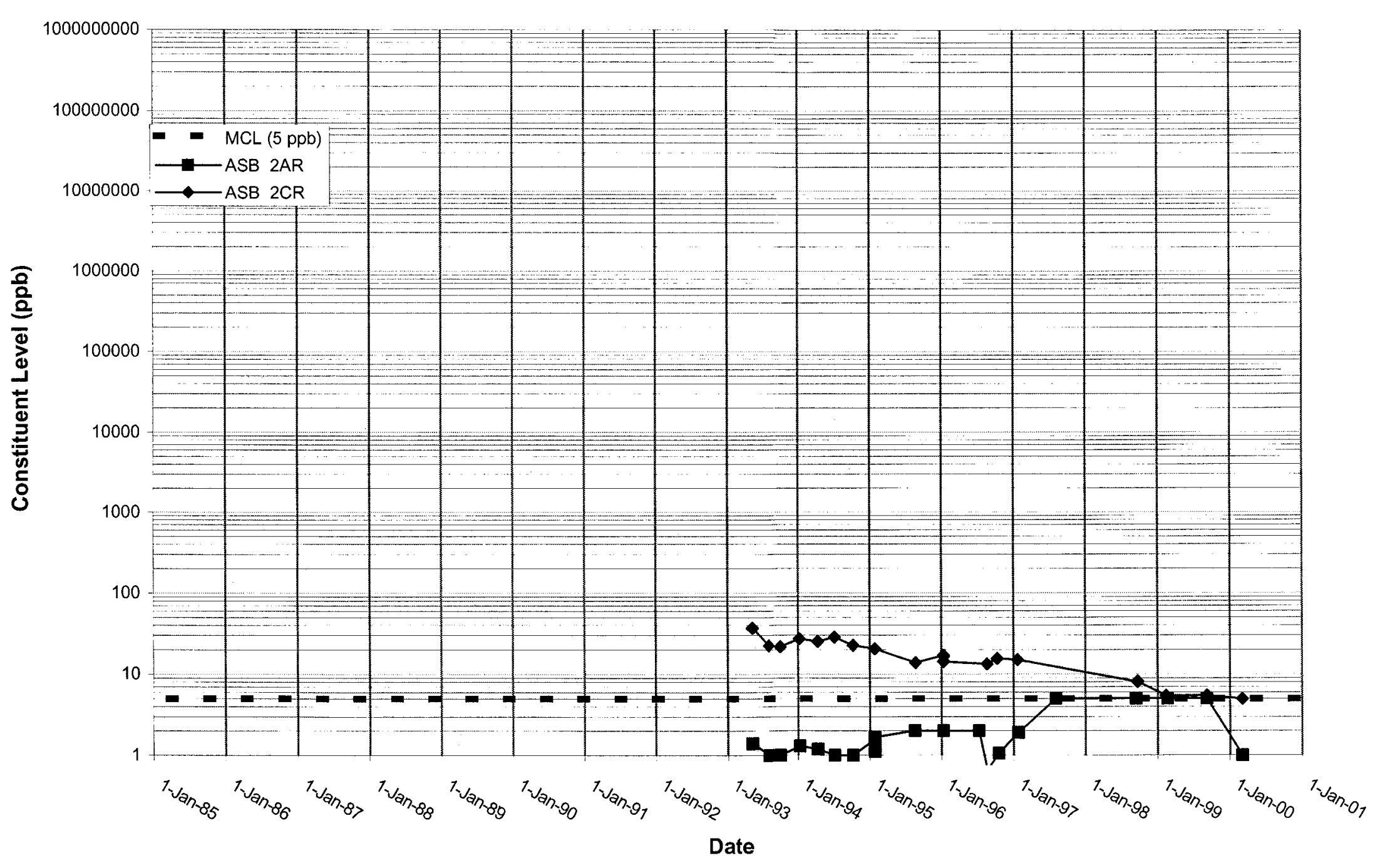


WSRC-TR-2000-00242 UNCLASSIFIED

Time Series Plot of Tetrachloroethylene for Well ASB 3

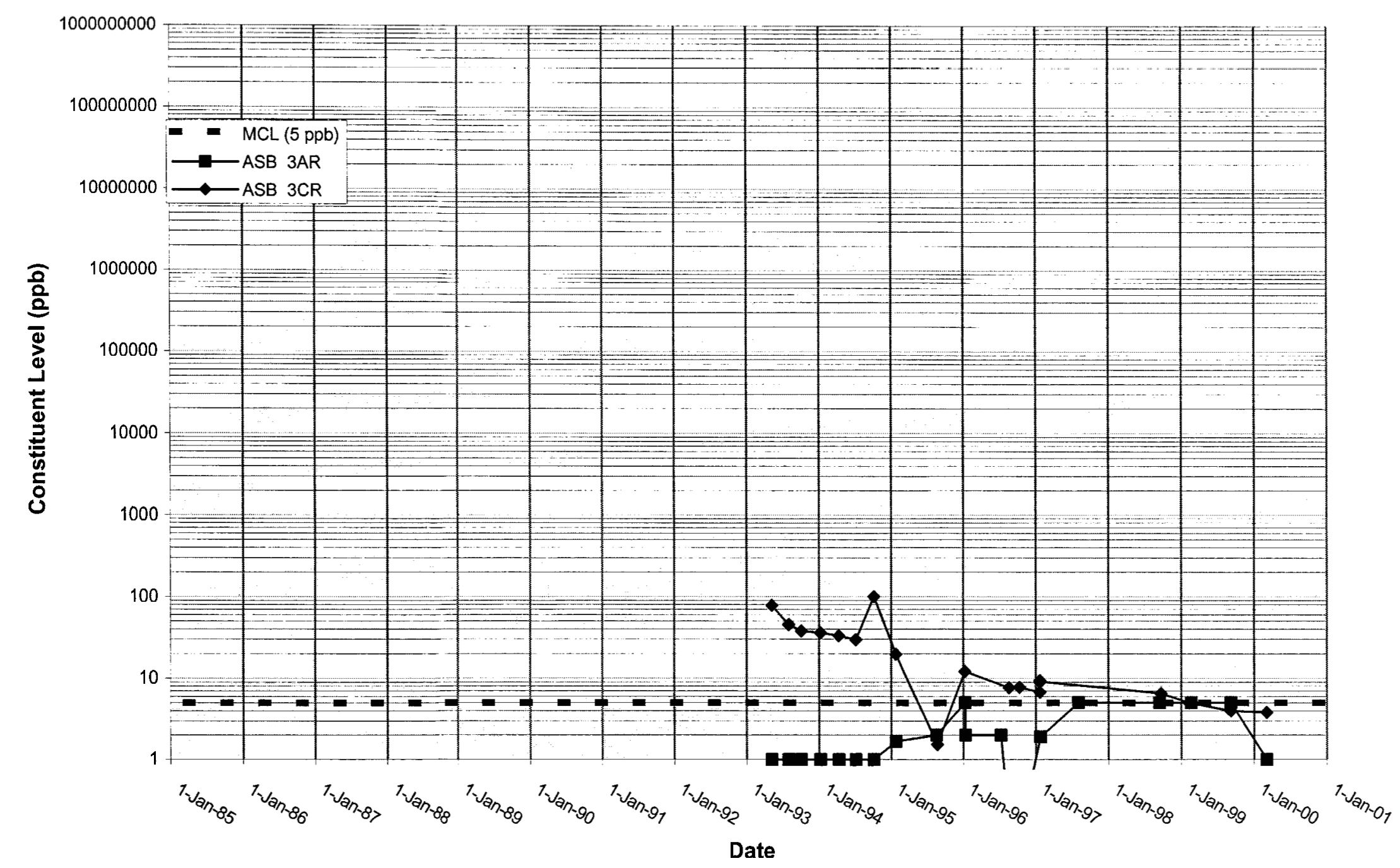


WSRC-TR-2000-00242

UNCLASSIFIED

Time Series Plot of Tetrachloroethylene for Well ASB 4

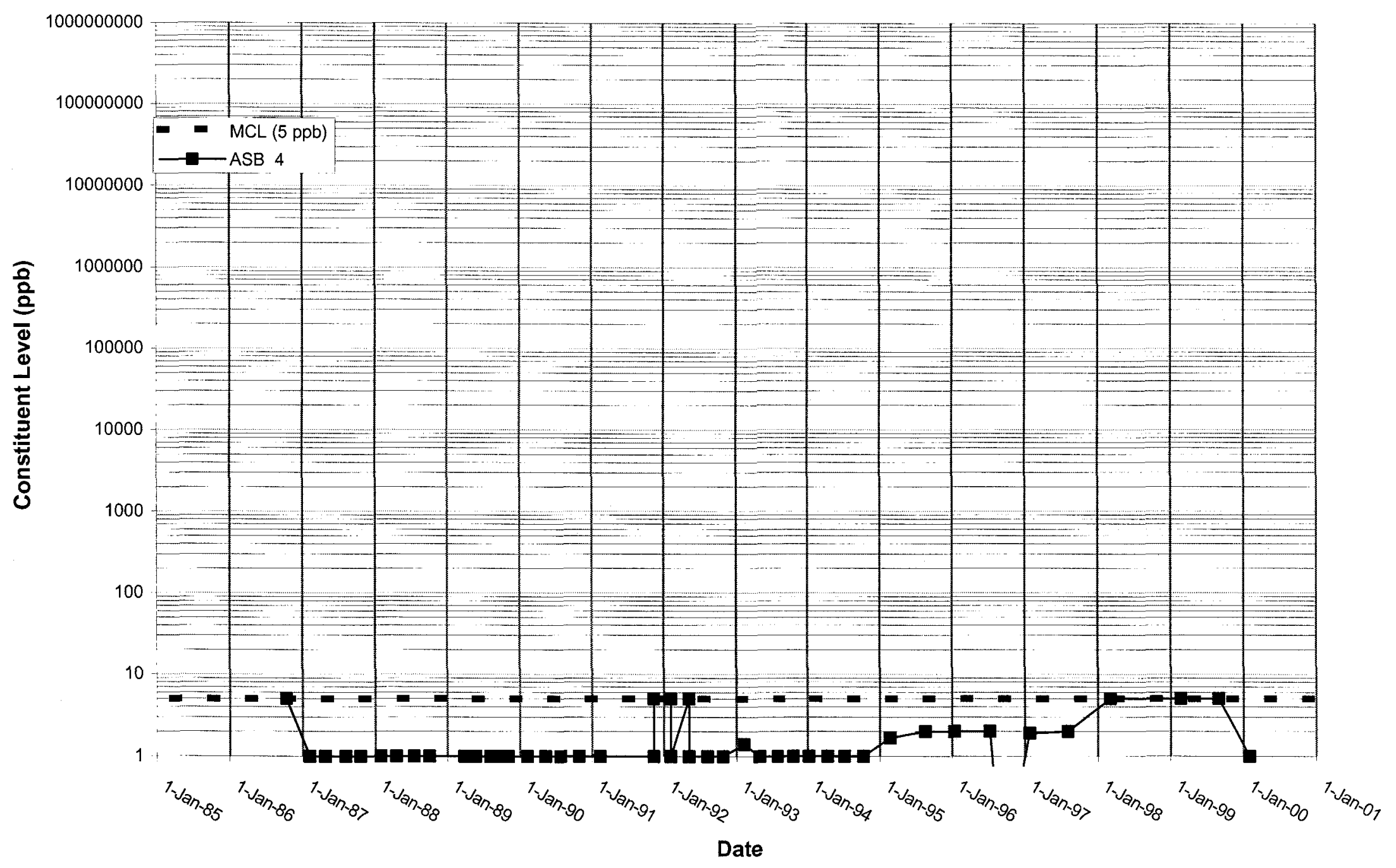


Time Series Plot of Tetrachloroethylene for Well ASB 5

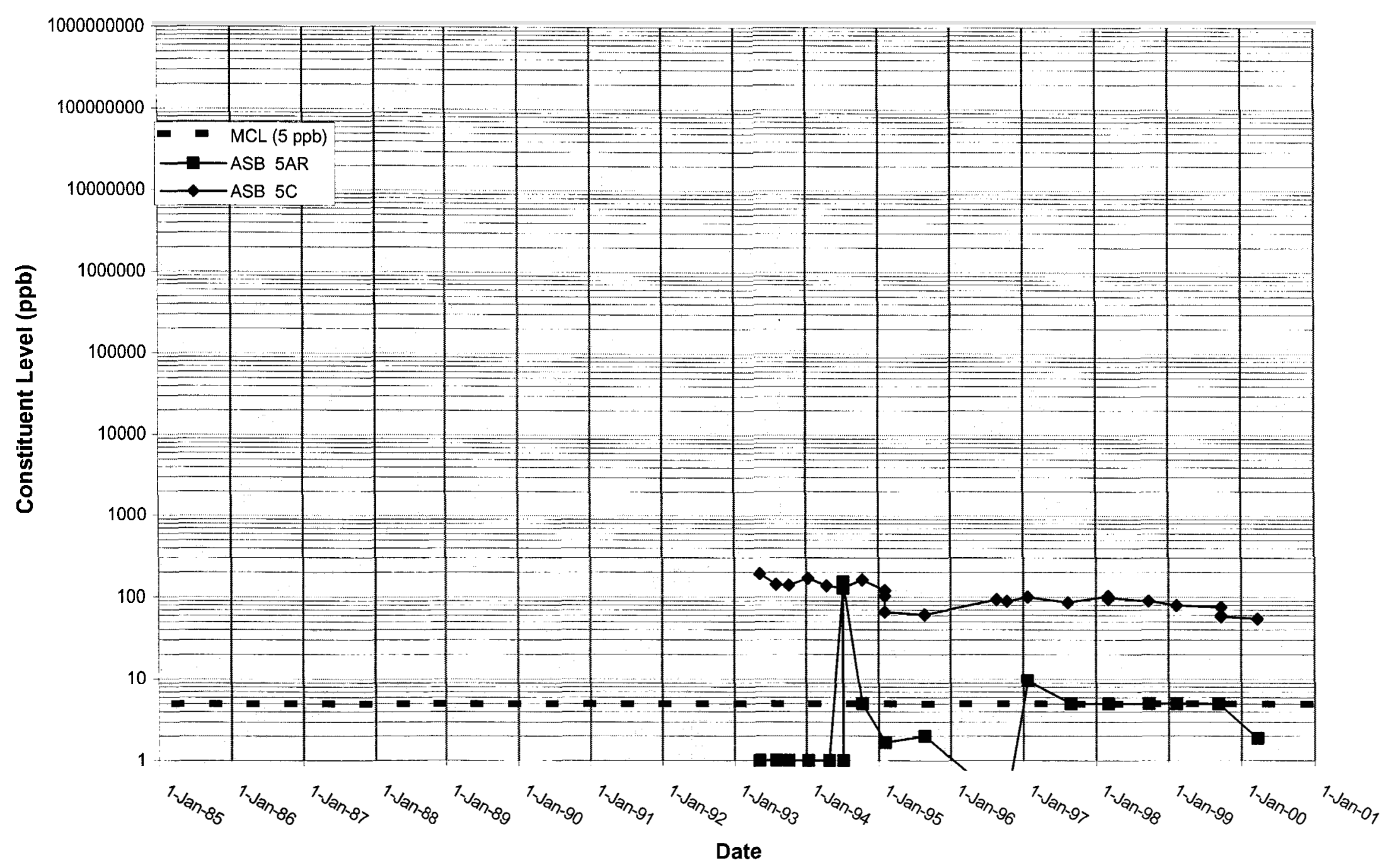


Time Series Plot of Tetrachloroethylene for Well ASB 6

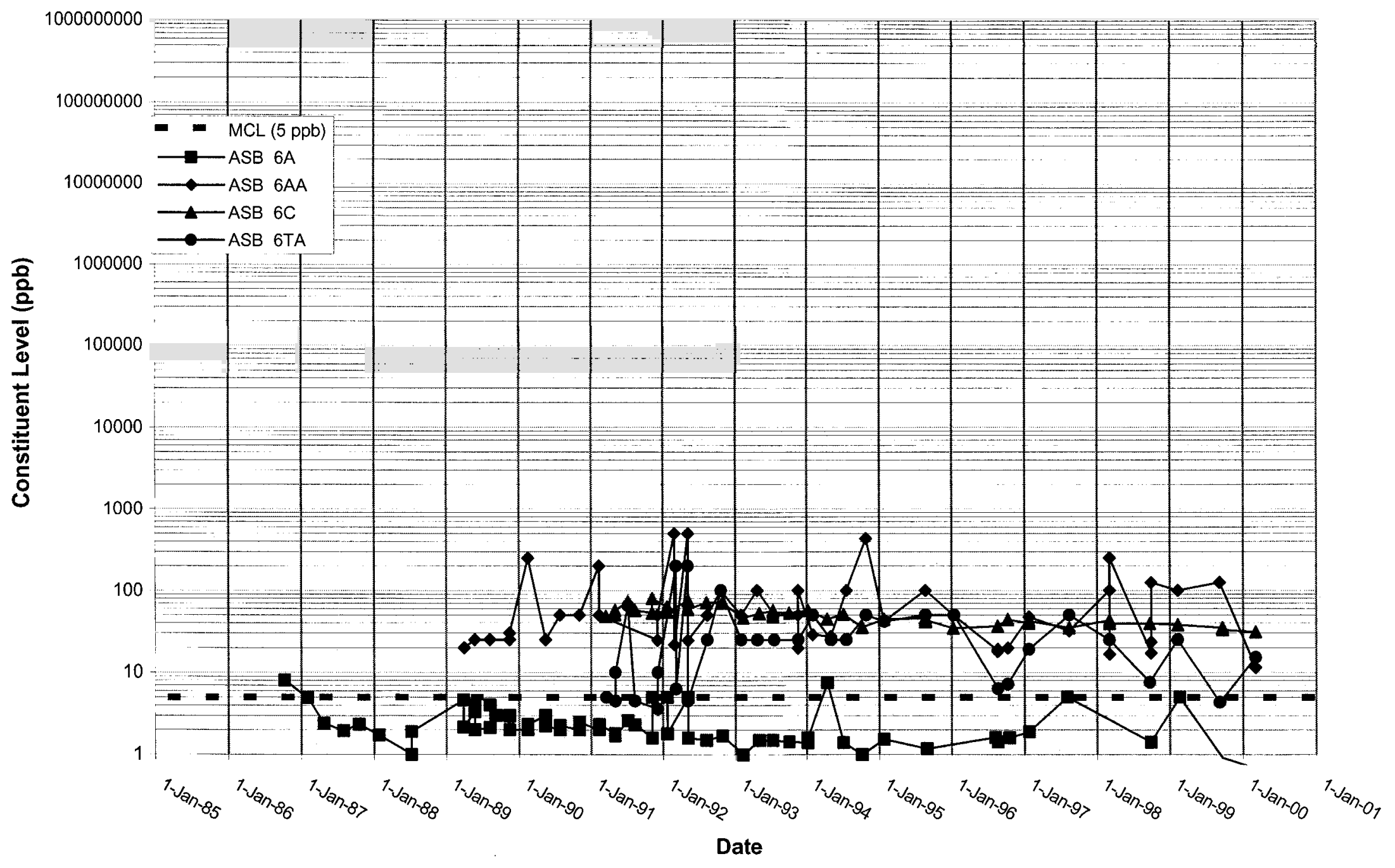


Time Series Plot of Tetrachloroethylene for Well ASB 8

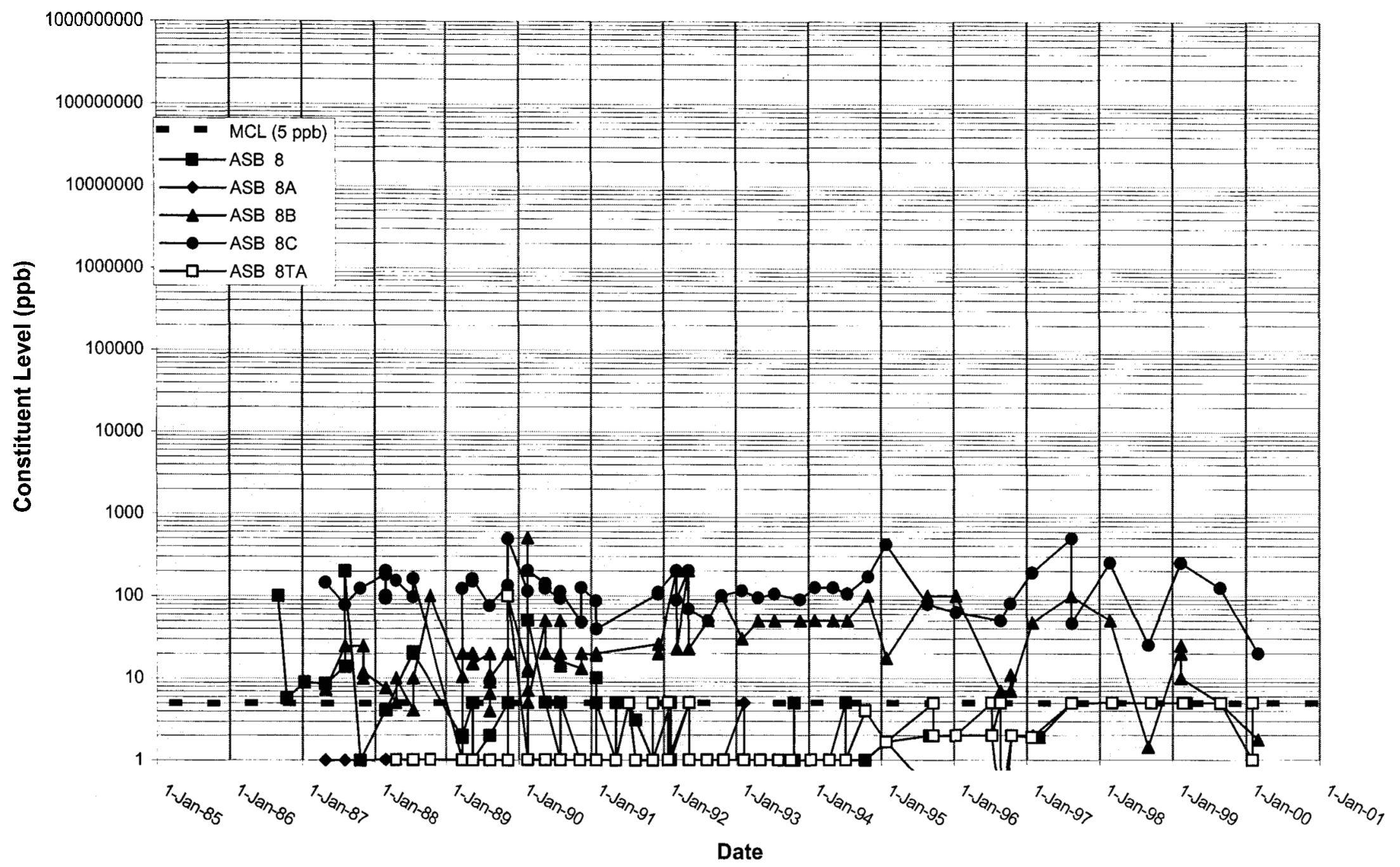


Time Series Plot of Tetrachloroethylene for Well ASB 9

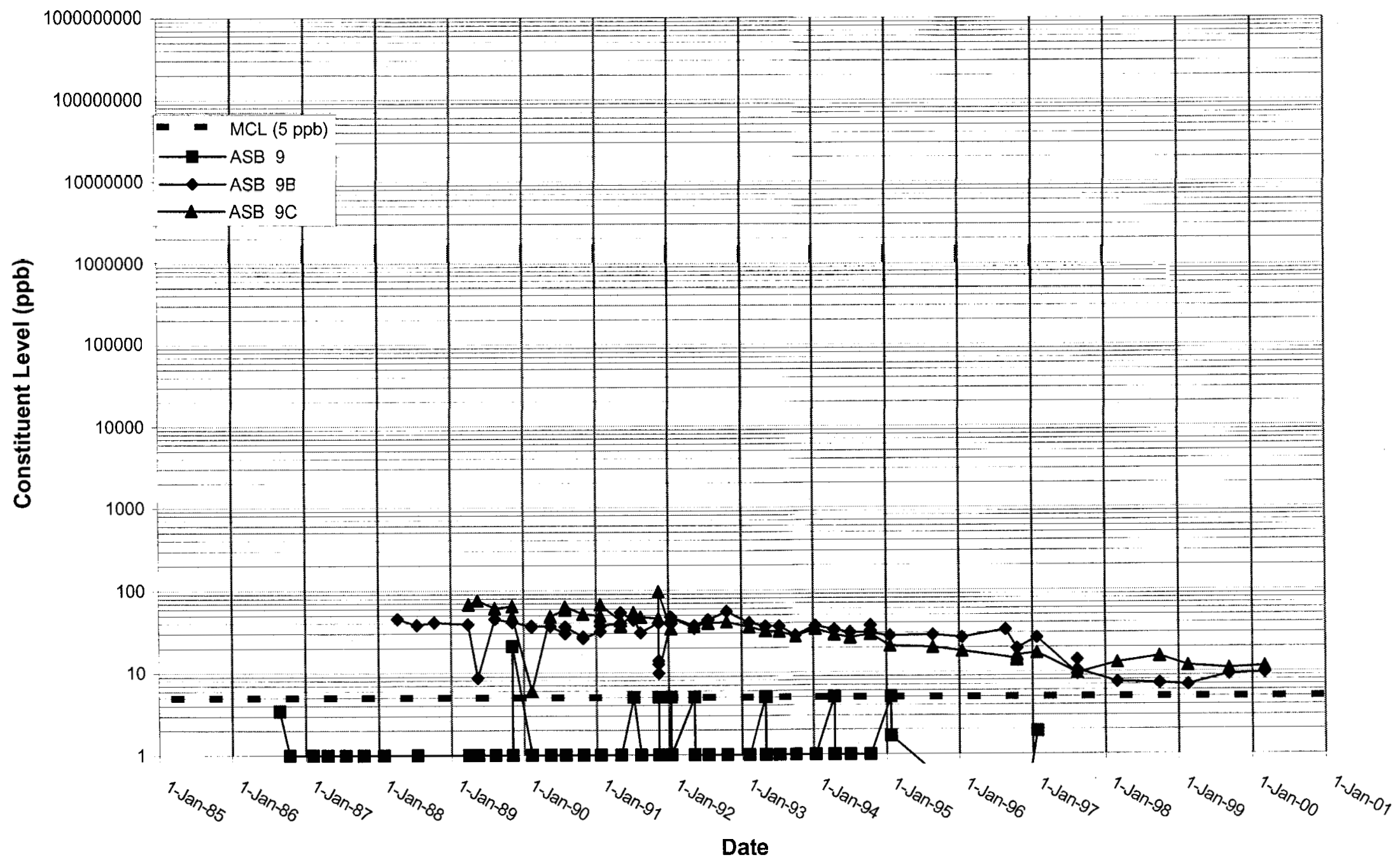




\section{Time Series Plot of Tetrachloroethylene for Well ASB 10}

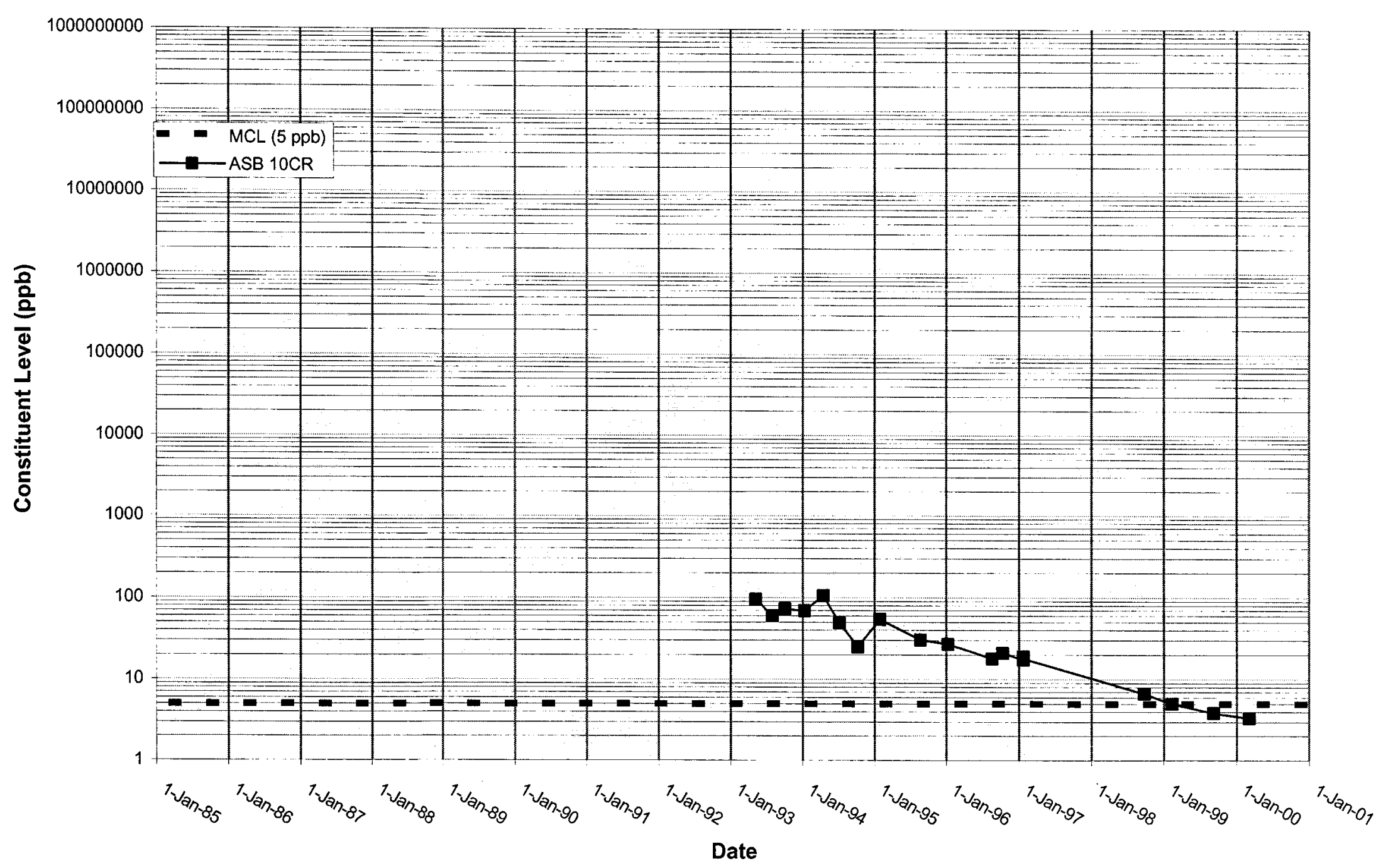


WSRC-TR-2000-00242 UNCLASSIFIED

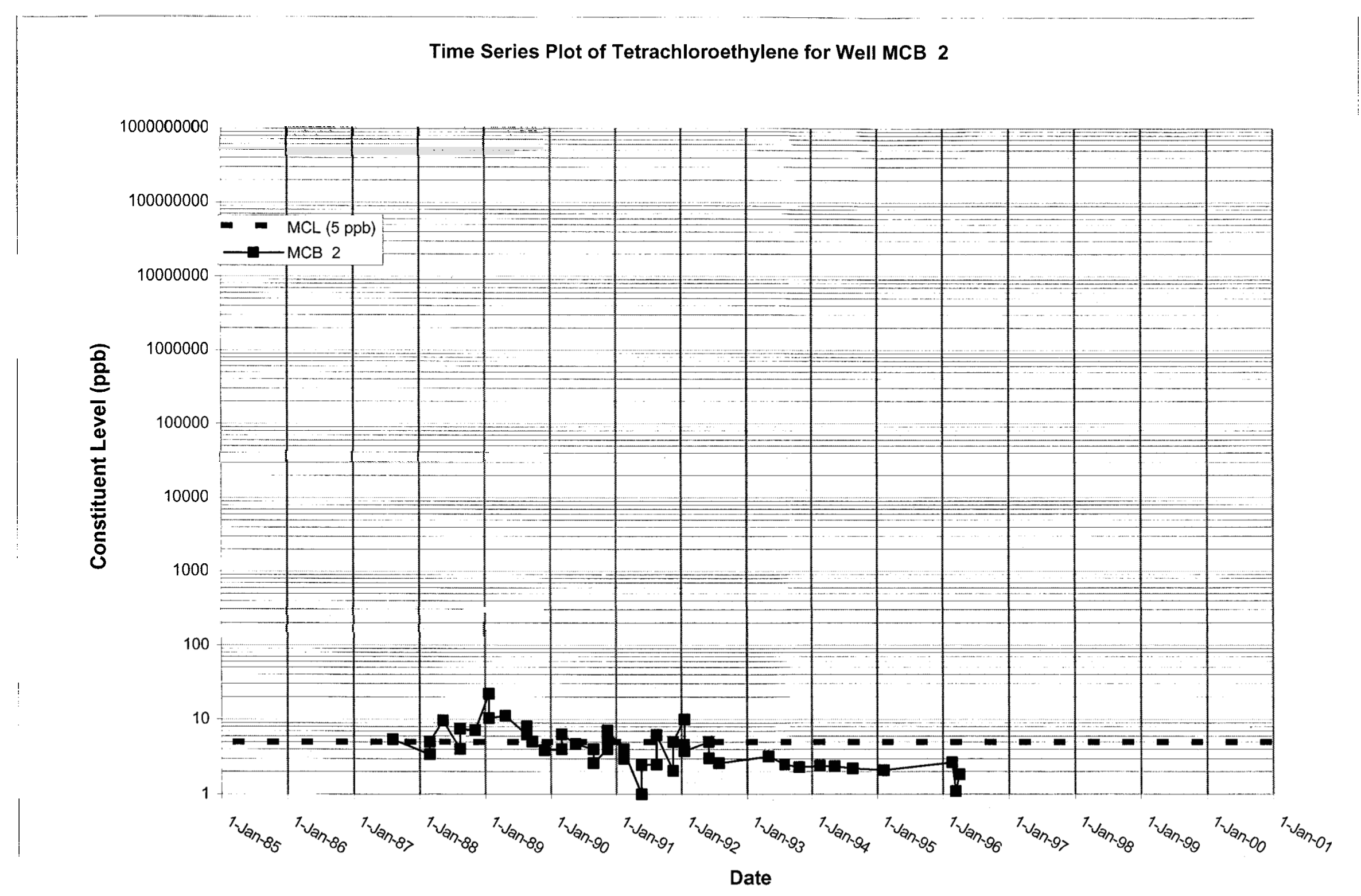


Time Series Plot of Tetrachloroethylene for Well MCB 4

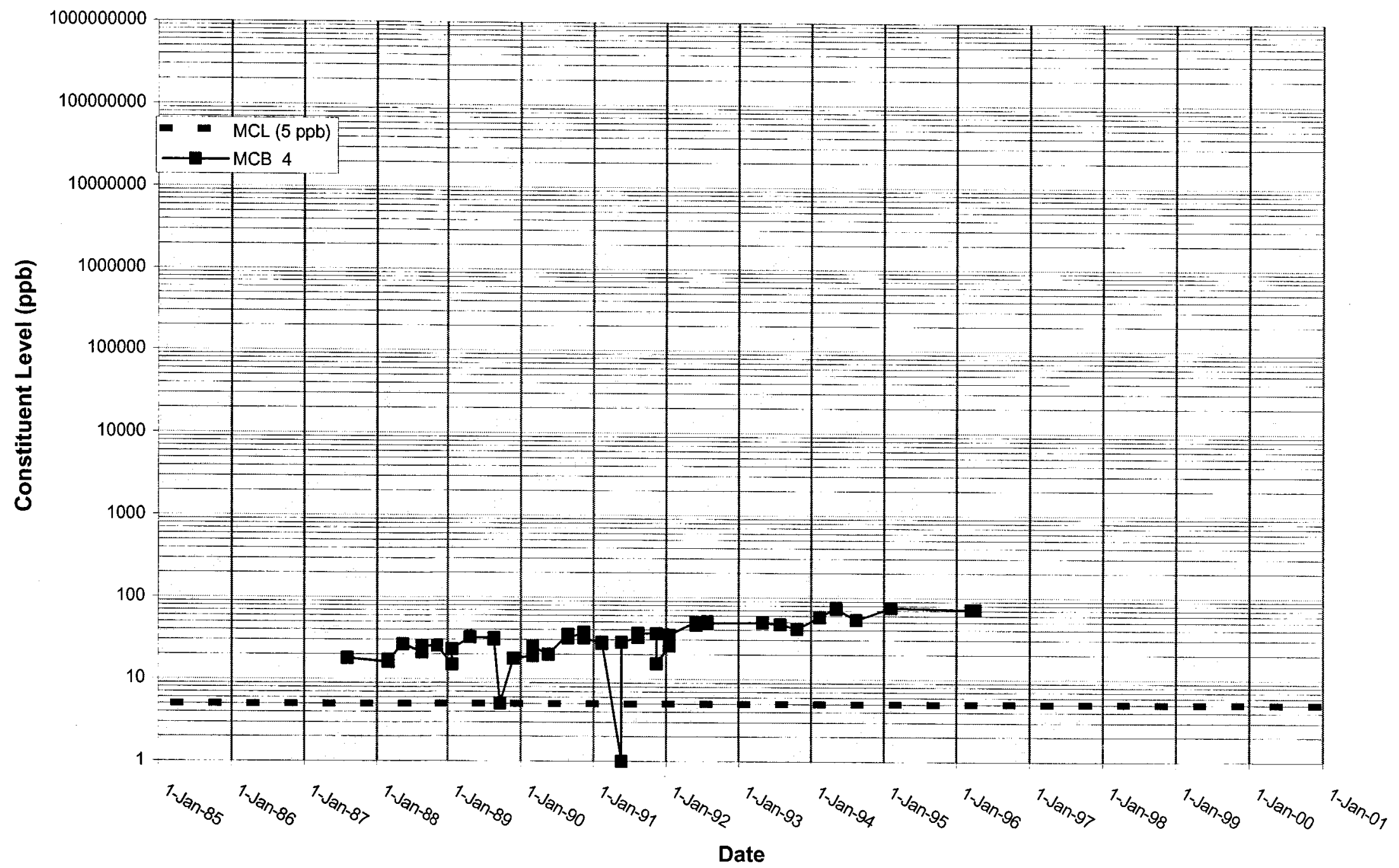


Time Series Plot of Tetrachloroethylene for Well MCB 5

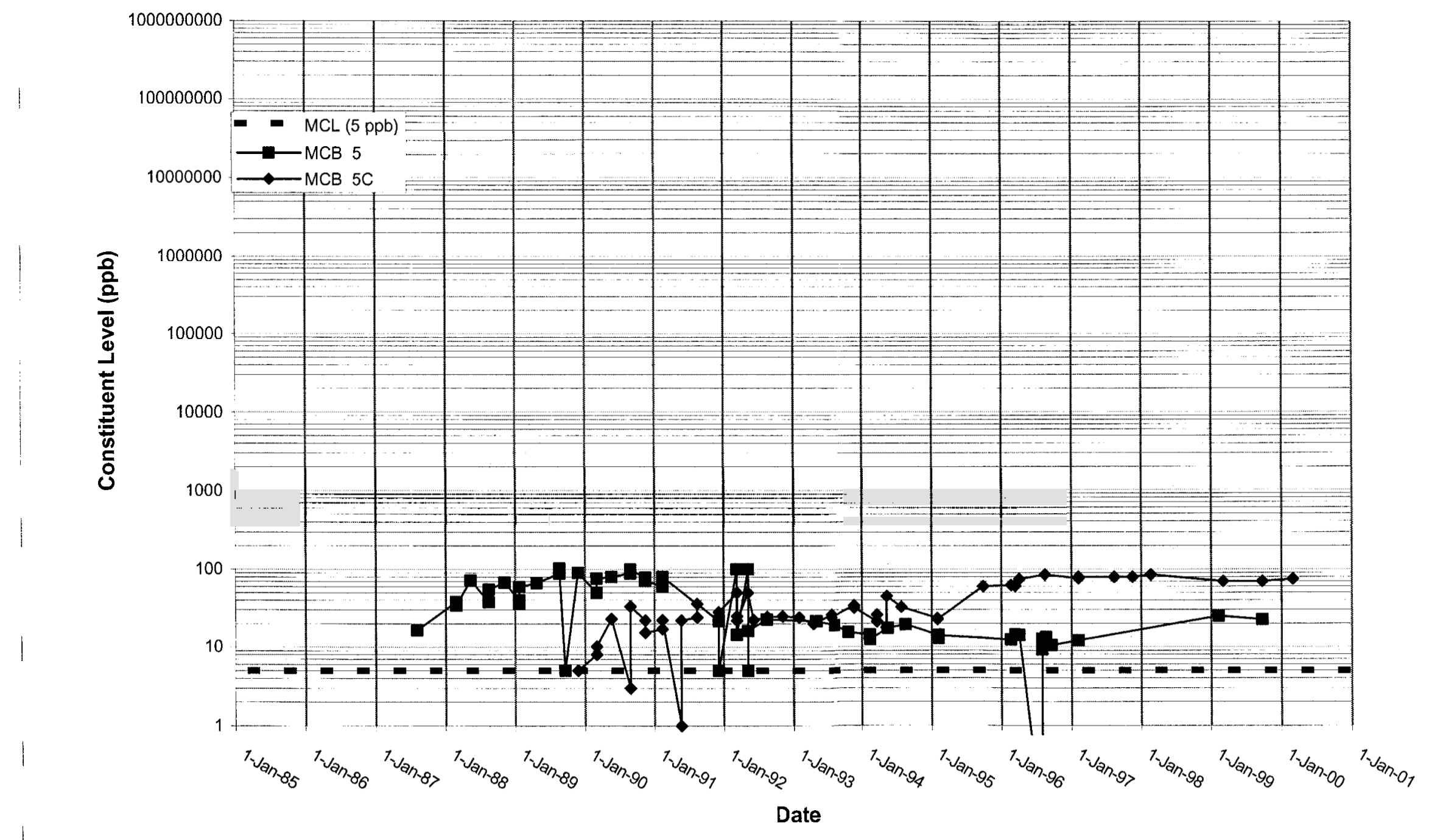




\section{Time Series Plot of Tetrachloroethylene for Well MCB 6}

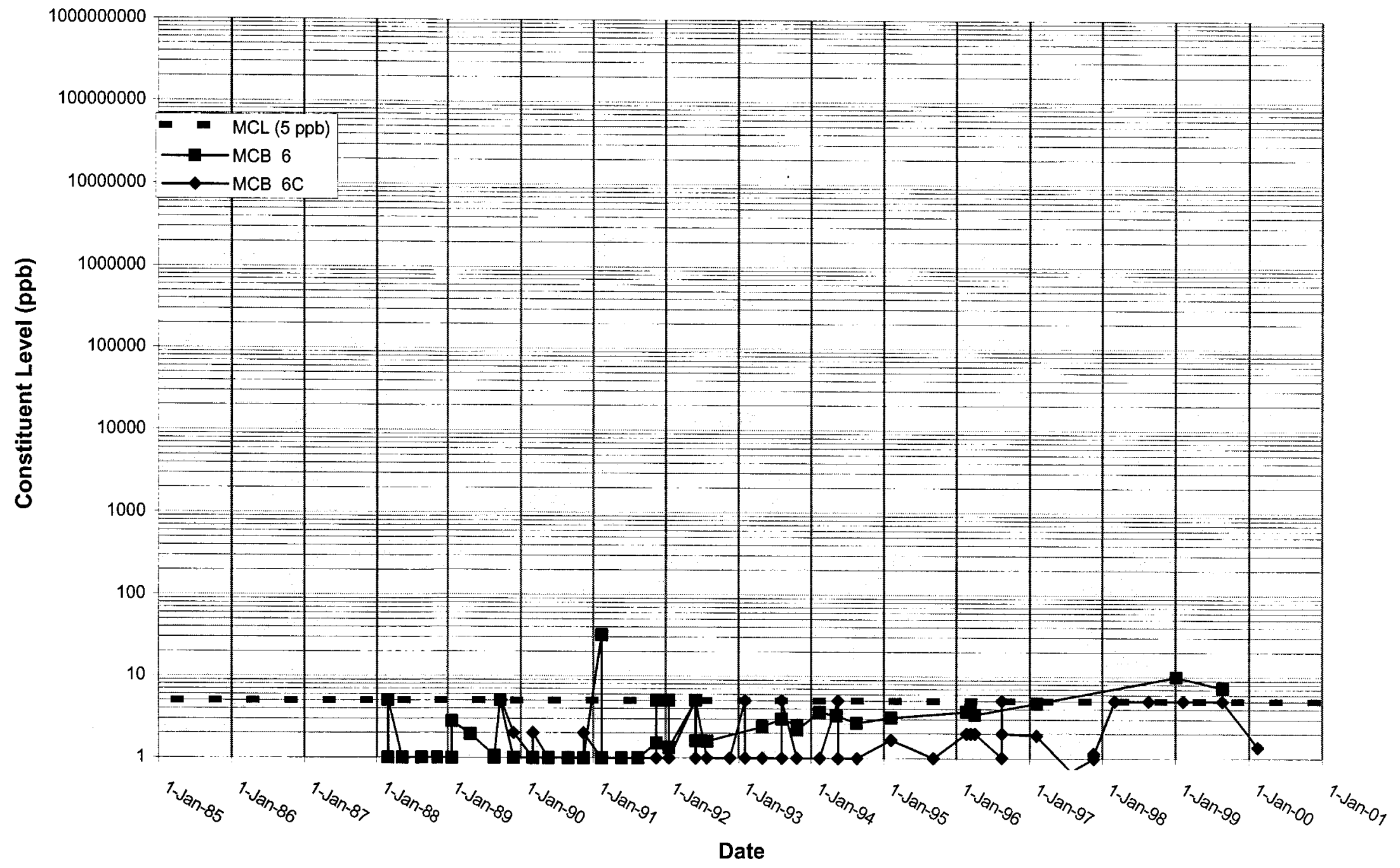


WSRC-TR-2000-00242 UNCLASSIFIED

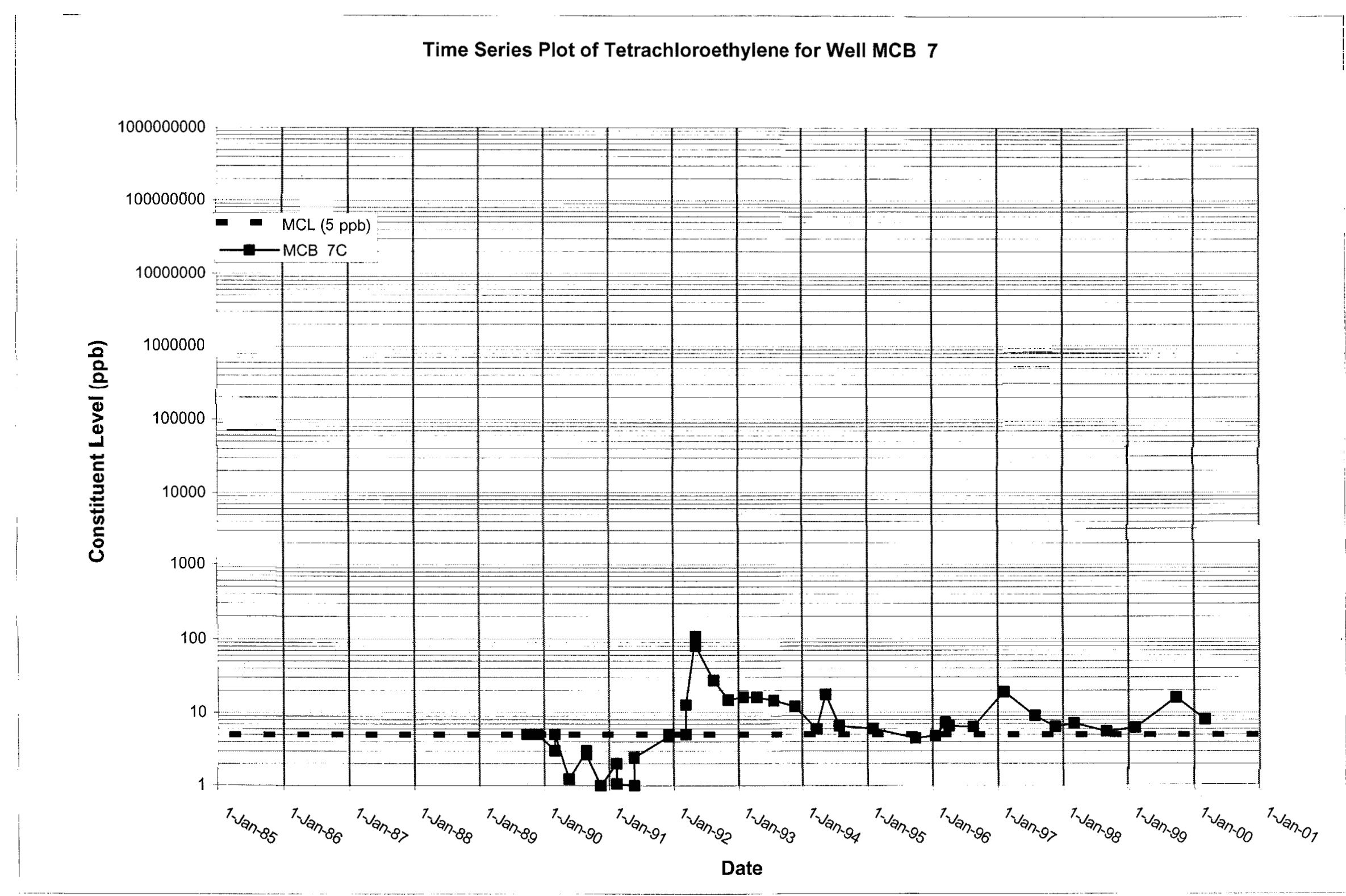


Time Series Plot of Tetrachloroethylene for Well MSB 1

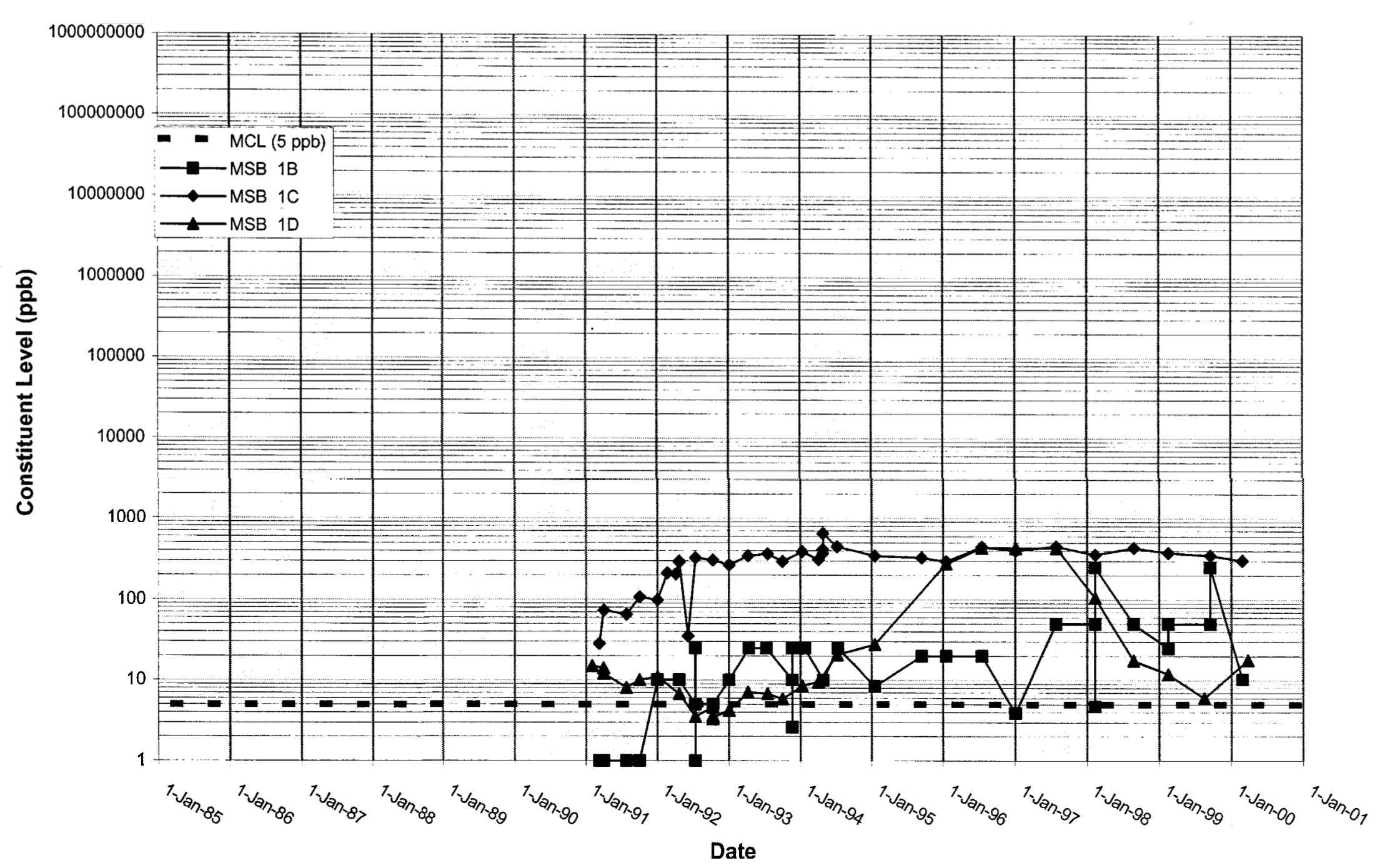


WSRC-TR-2000-00242 UNCLASSIFIED

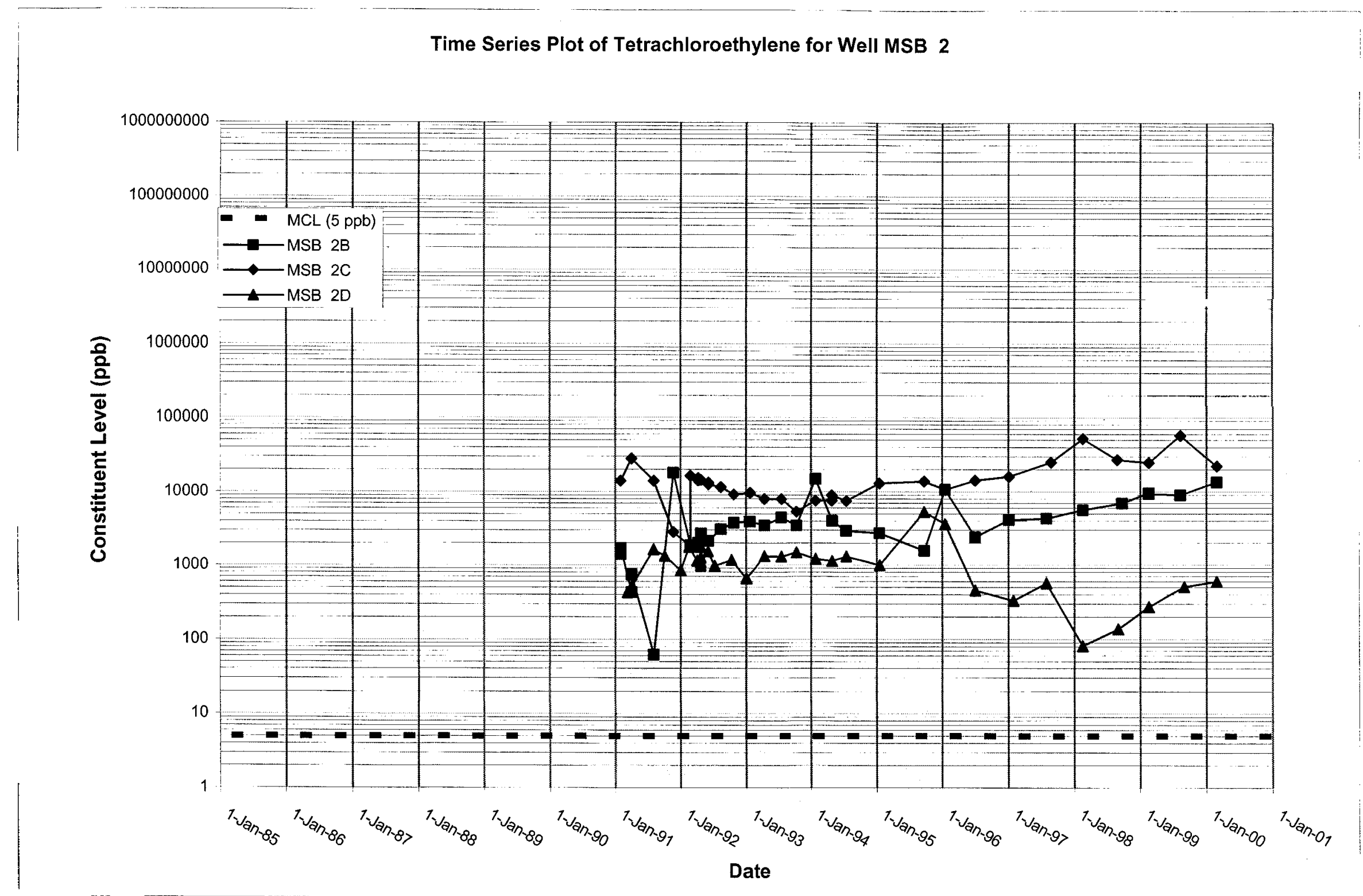


Time Series Plot of Tetrachloroethylene for Well MSB 3

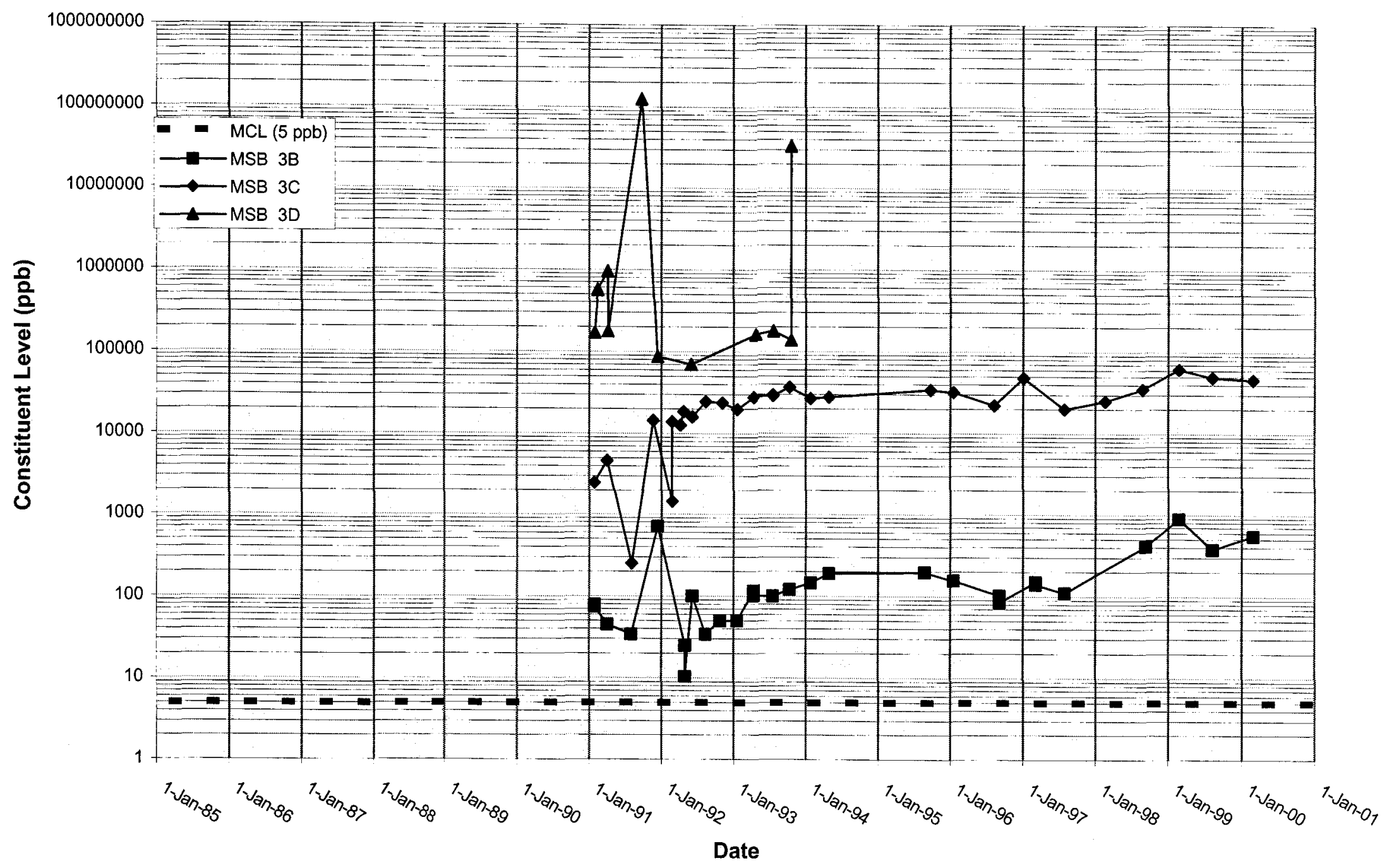


Time Series Plot of Tetrachloroethylene for Well MSB 4

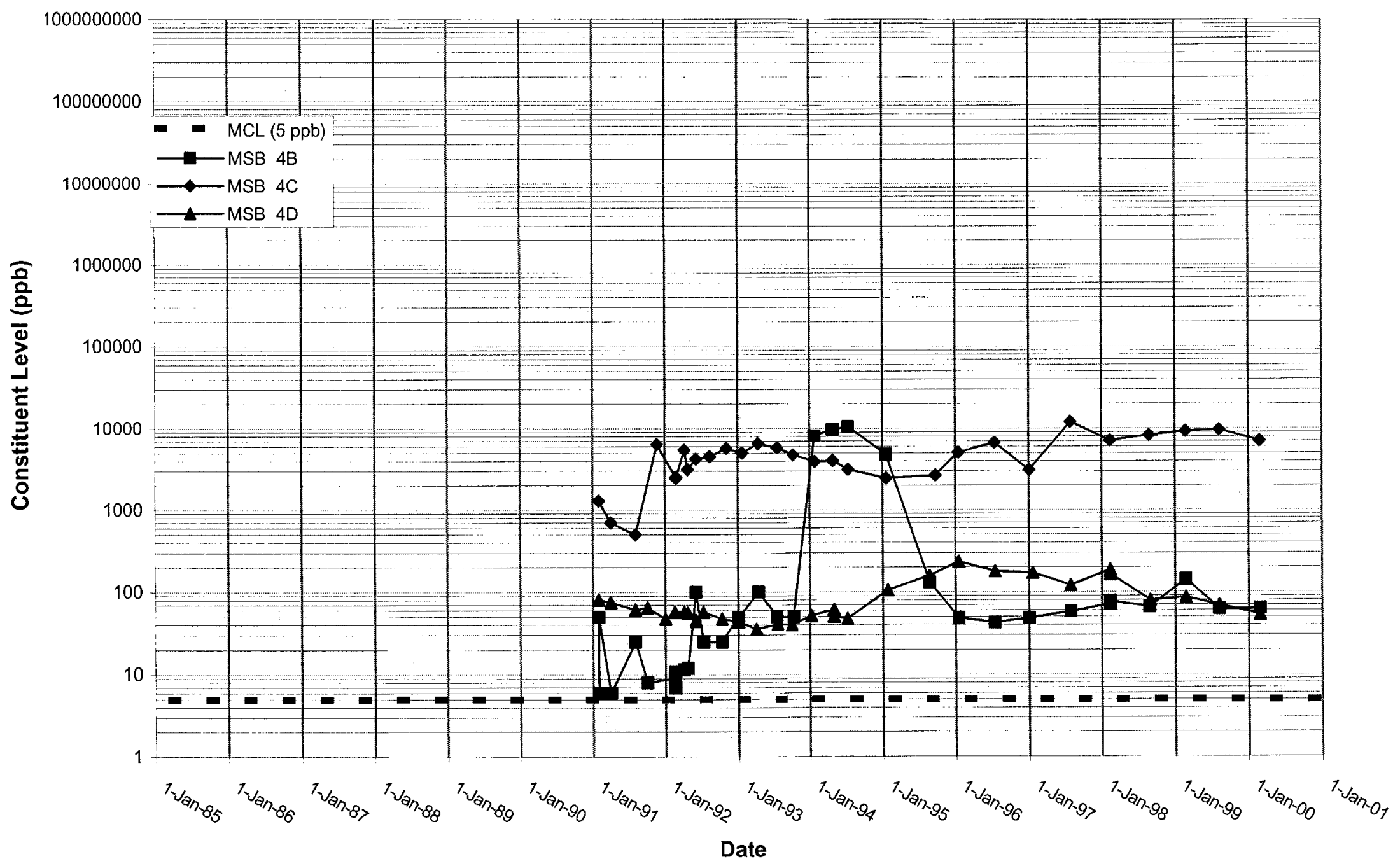


Time Series Plot of Tetrachloroethylene for Well MSB 5

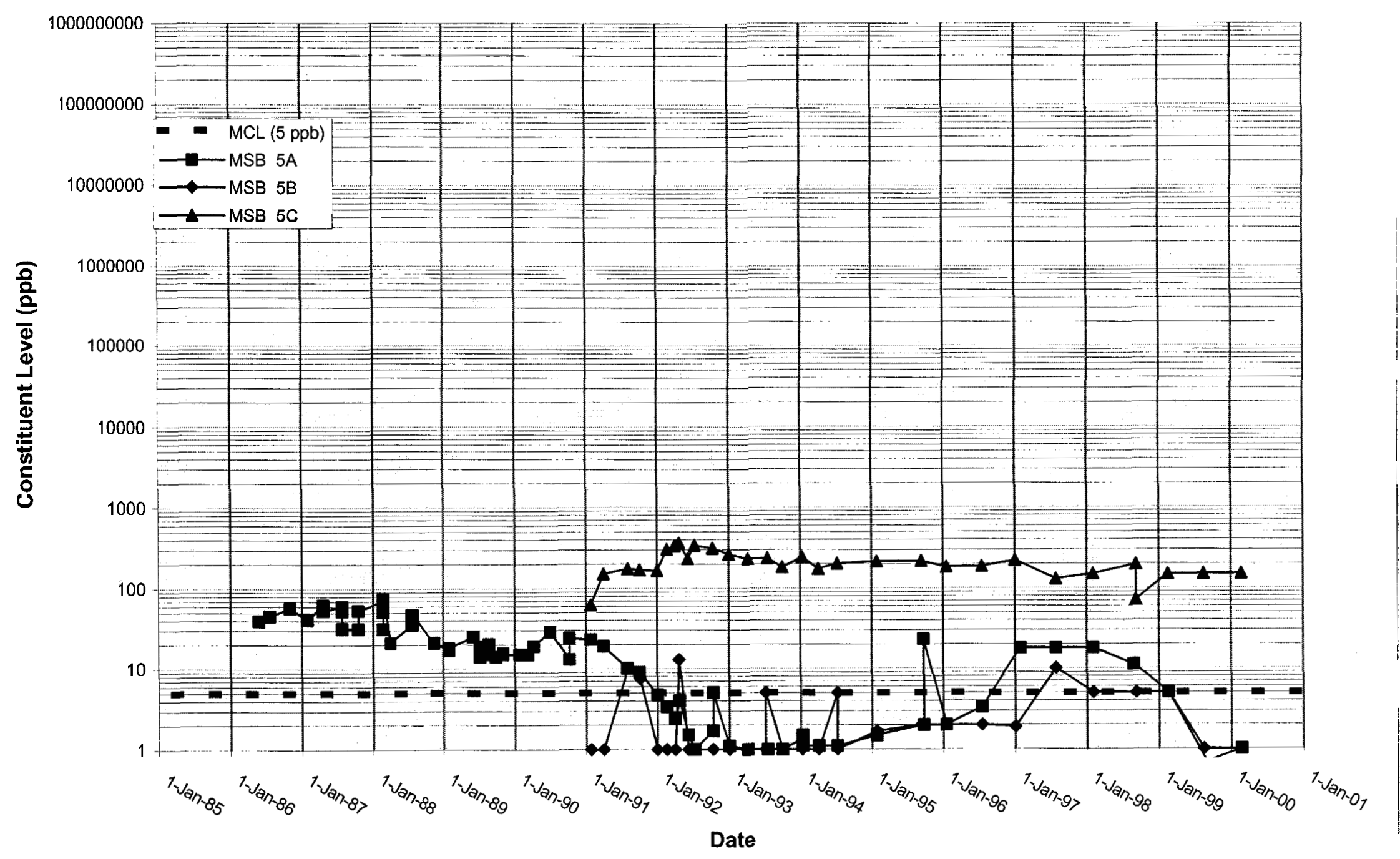


Time Series Plot of Tetrachloroethylene for Well MSB 6

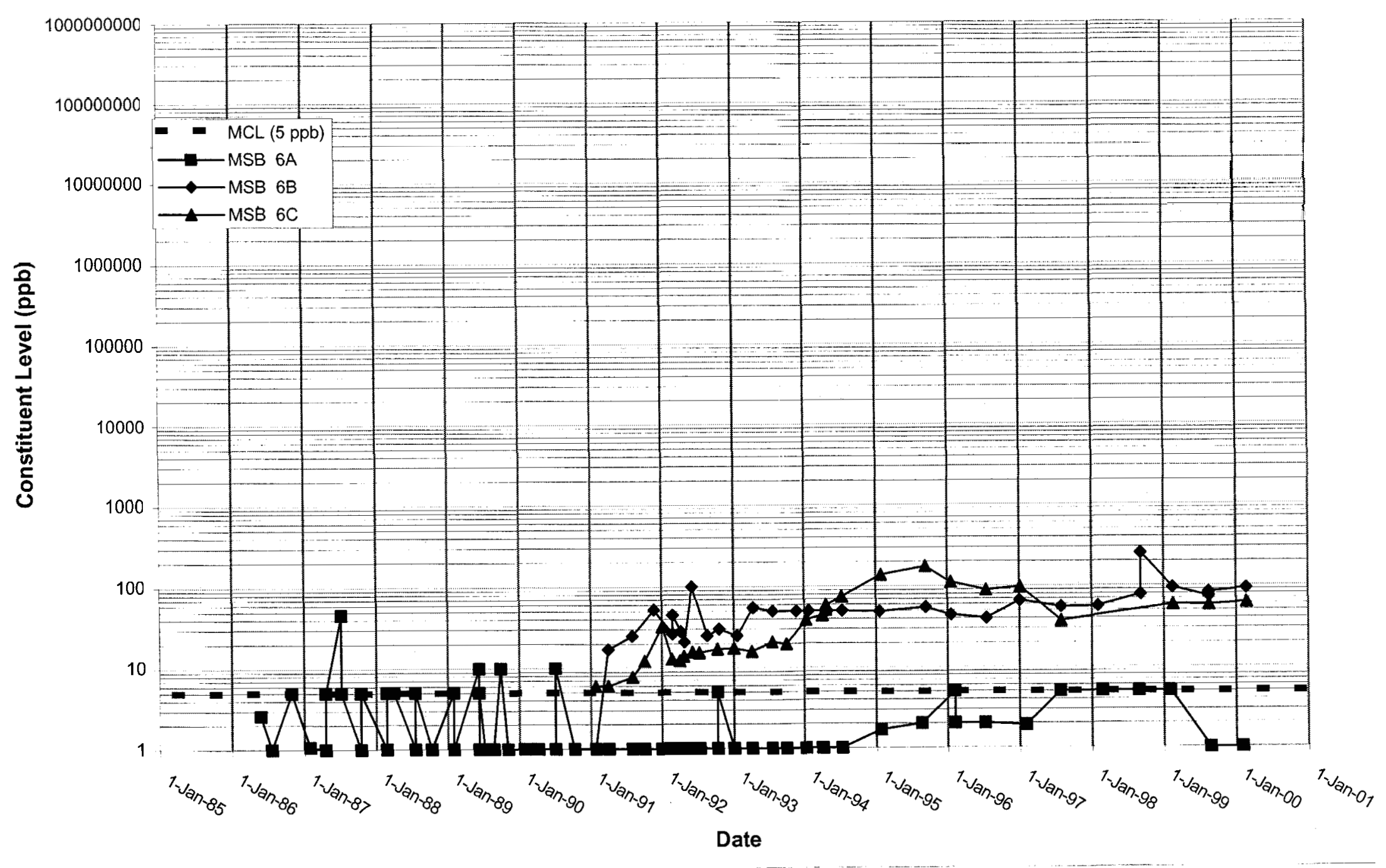


Time Series Plot of Tetrachloroethylene for Well MSB 7

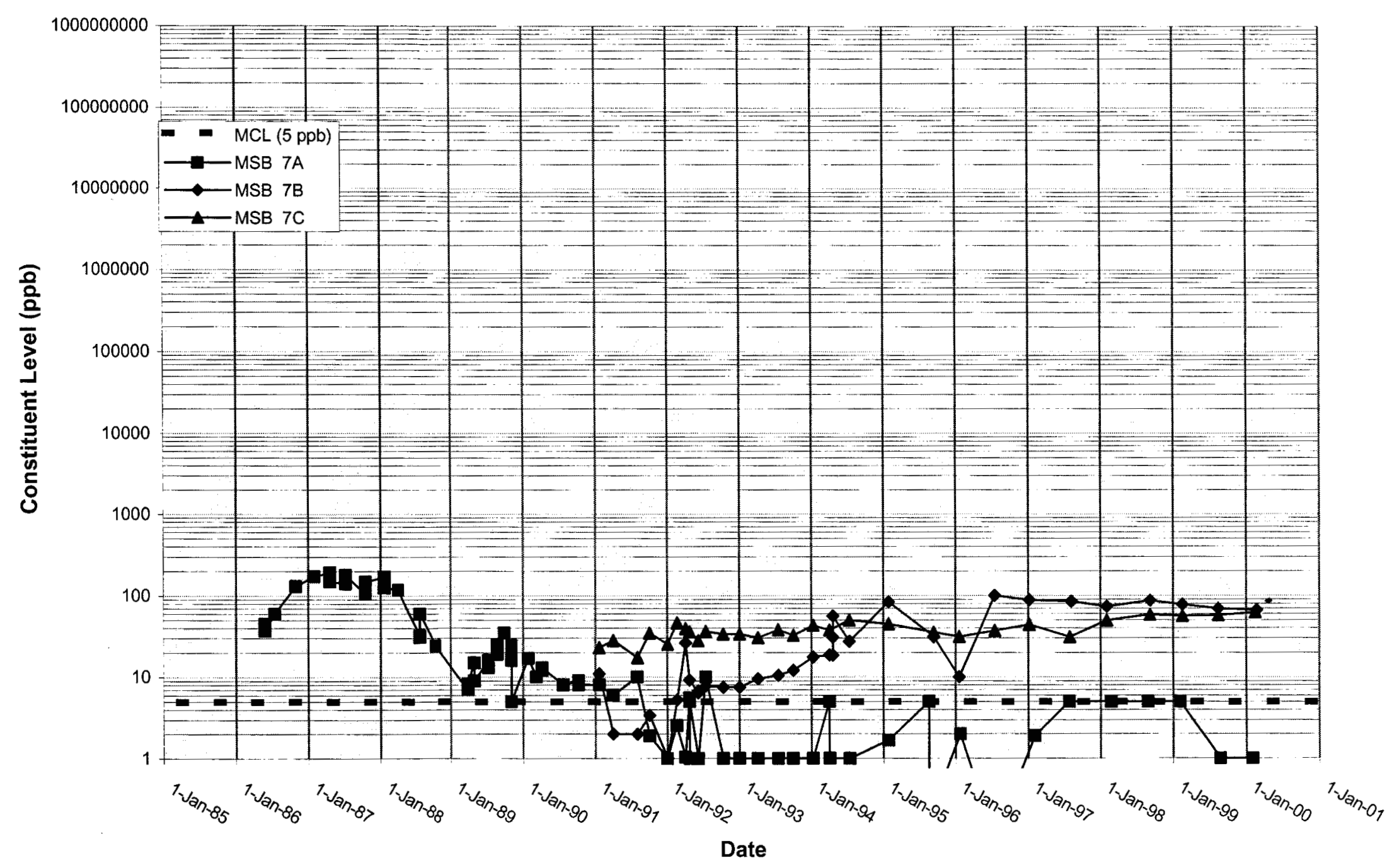




\section{Time Series Plot of Tetrachloroethylene for Well MSB 8}

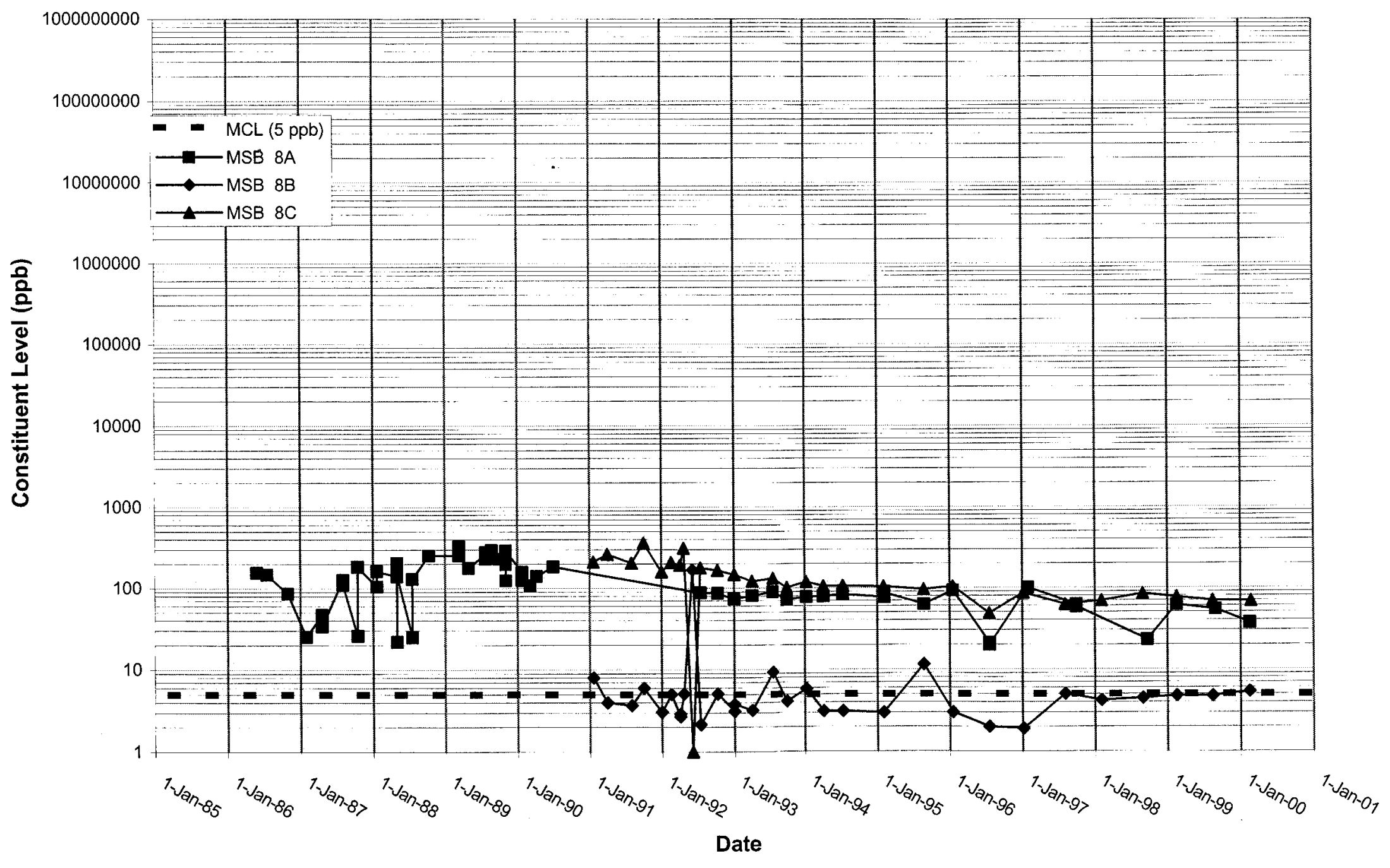


Time Series Plot of Tetrachloroethylene for Well MSB 9

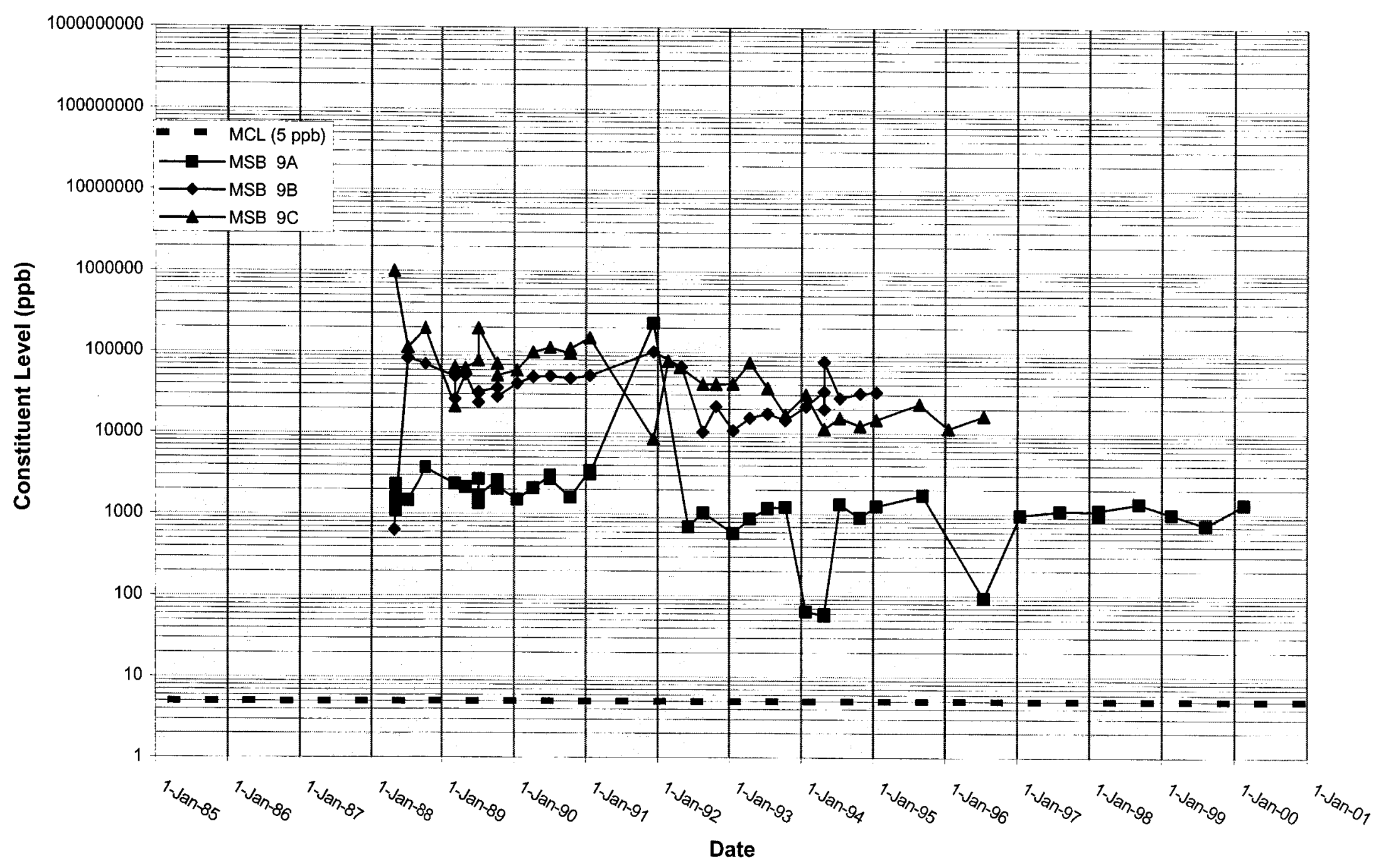


Time Series Plot of Tetrachloroethylene for Well MSB 10

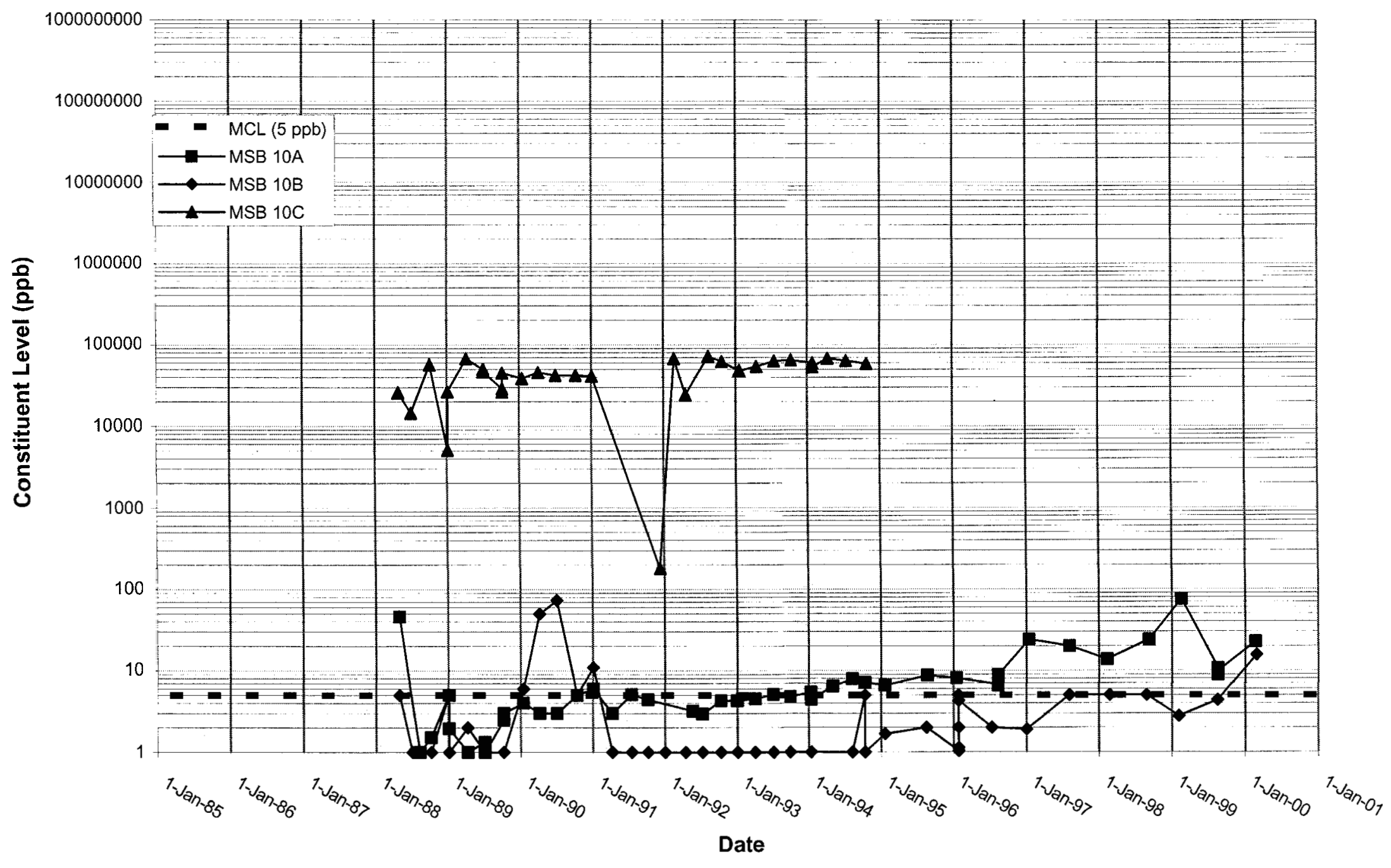


Time Series Plot of Tetrachloroethylene for Well MSB 11

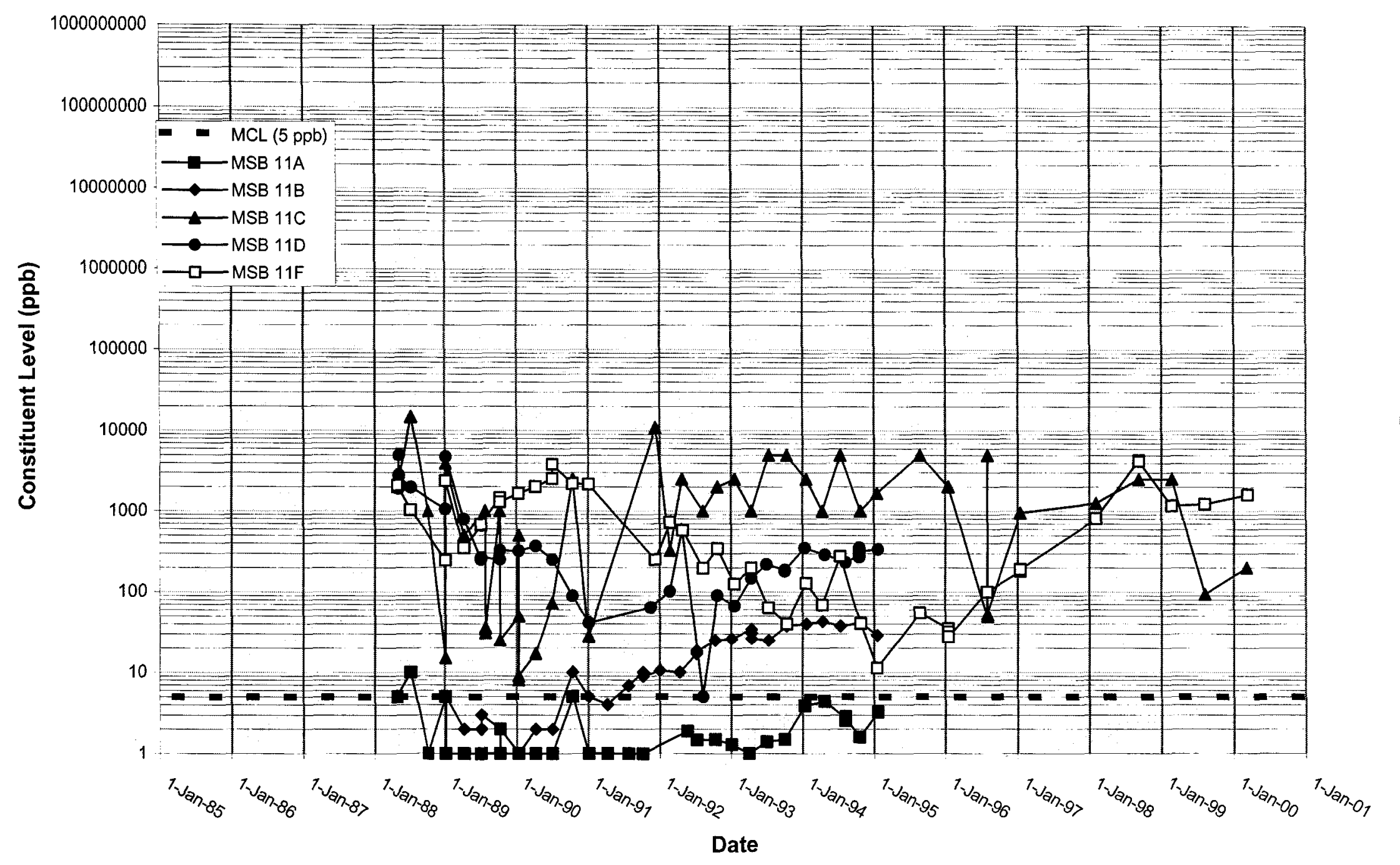




\section{Time Series Plot of Tetrachloroethylene for Well MSB 12}

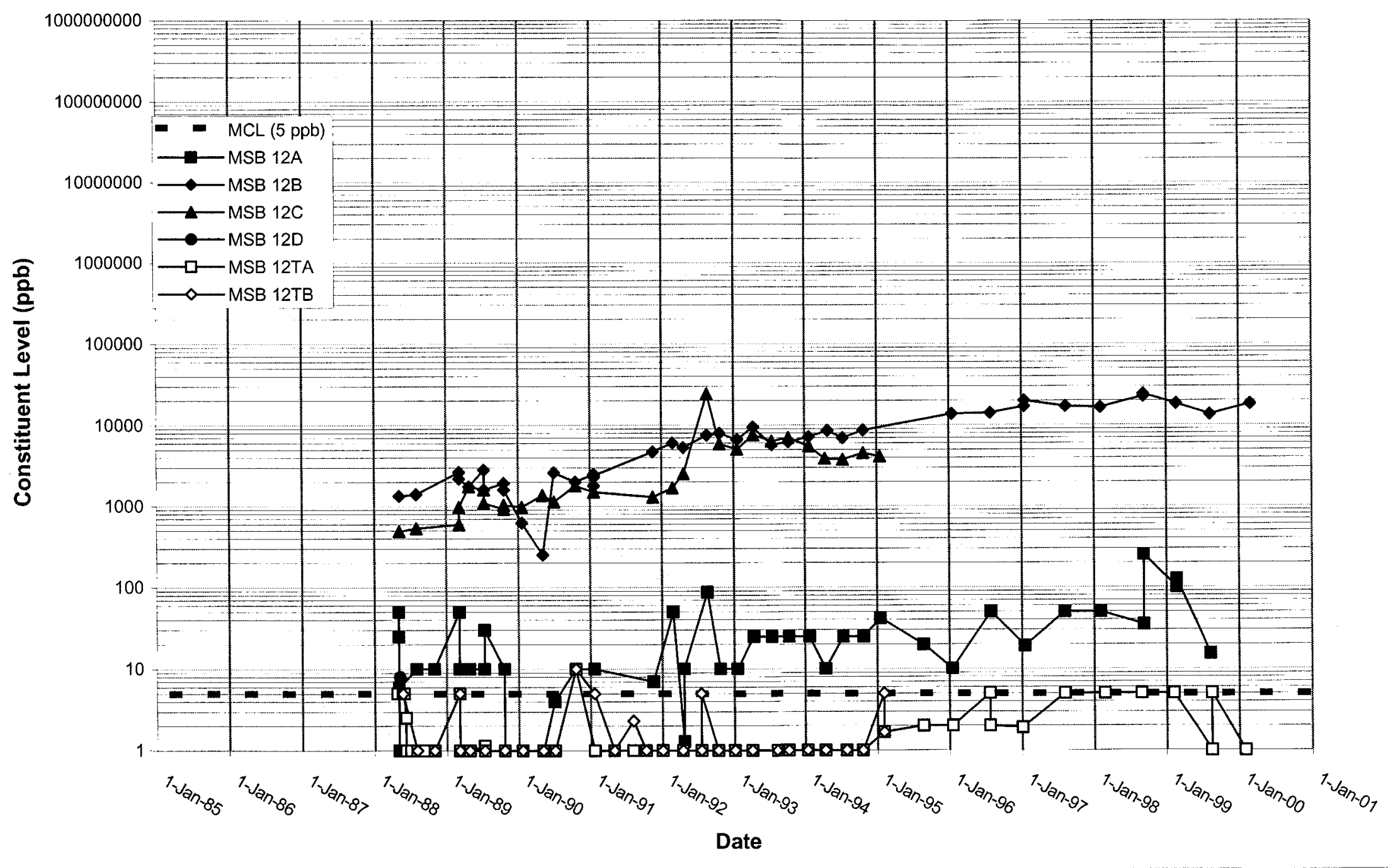


WSRC-TR-2000-00242 UNCLASSIFIED

\section{Time Series Plot of Tetrachloroethylene for Well MSB 13}

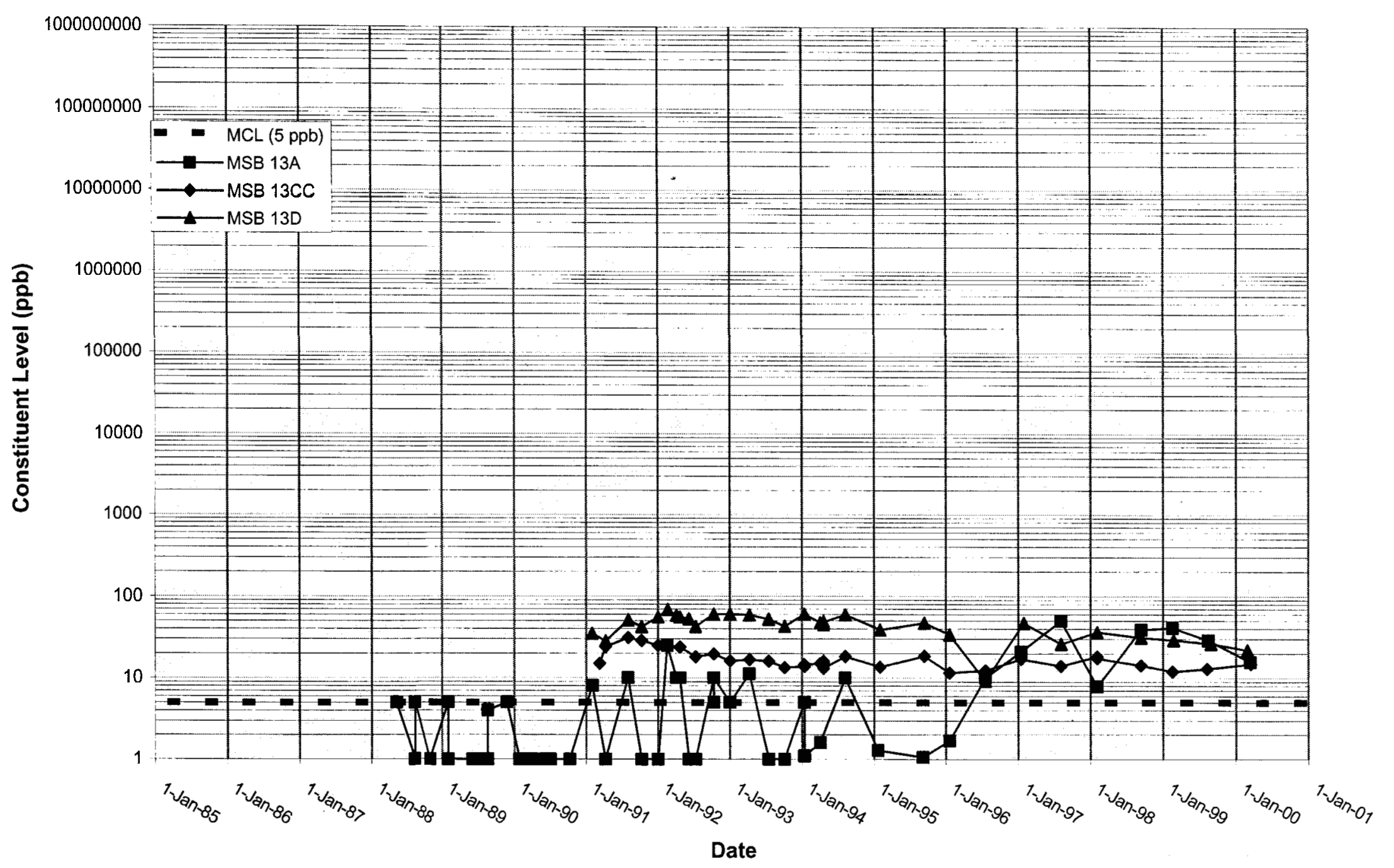




\section{Time Series Plot of Tetrachloroethylene for Well MSB 14}

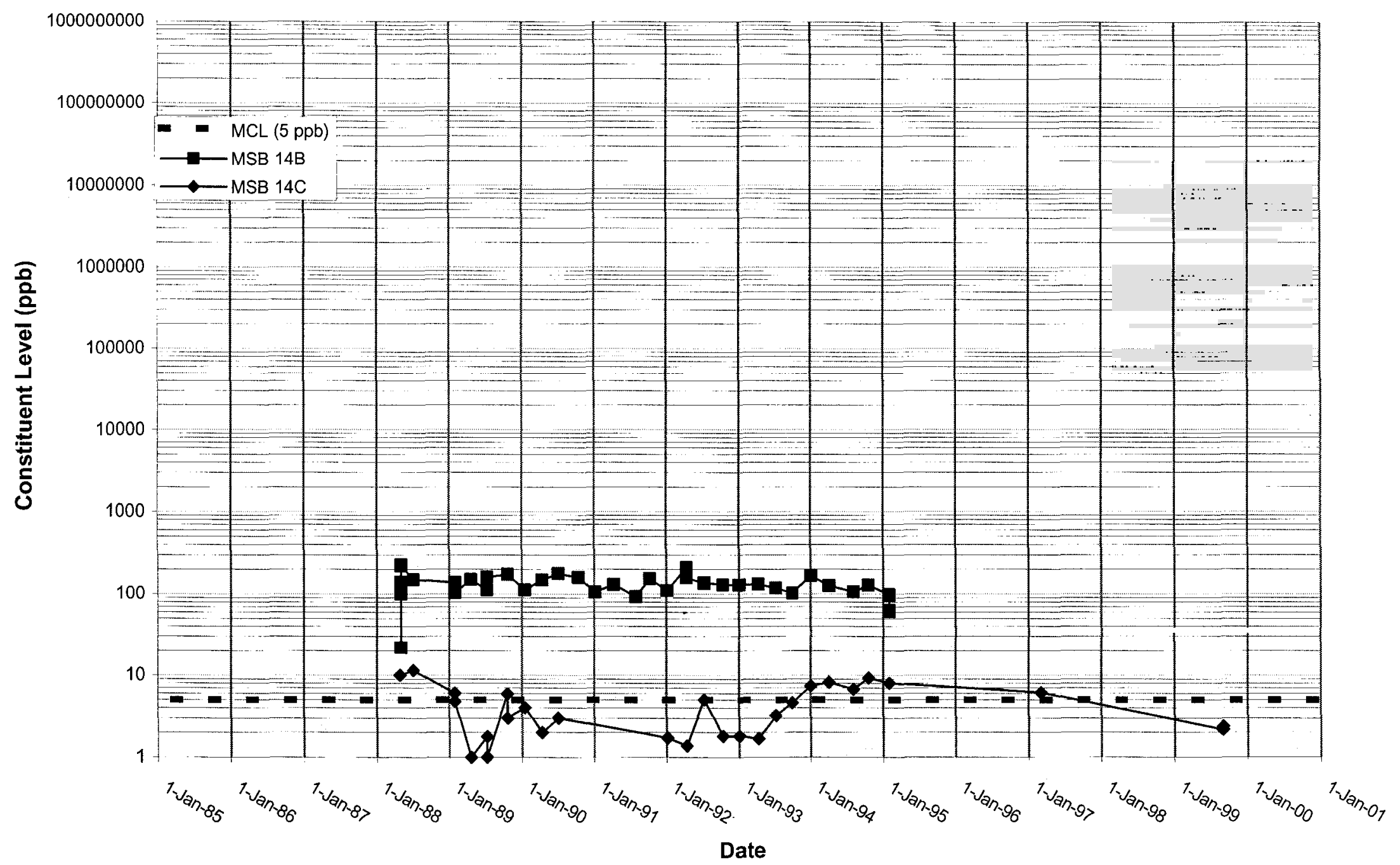


Time Series Plot of Tetrachloroethylene for Well MSB 15

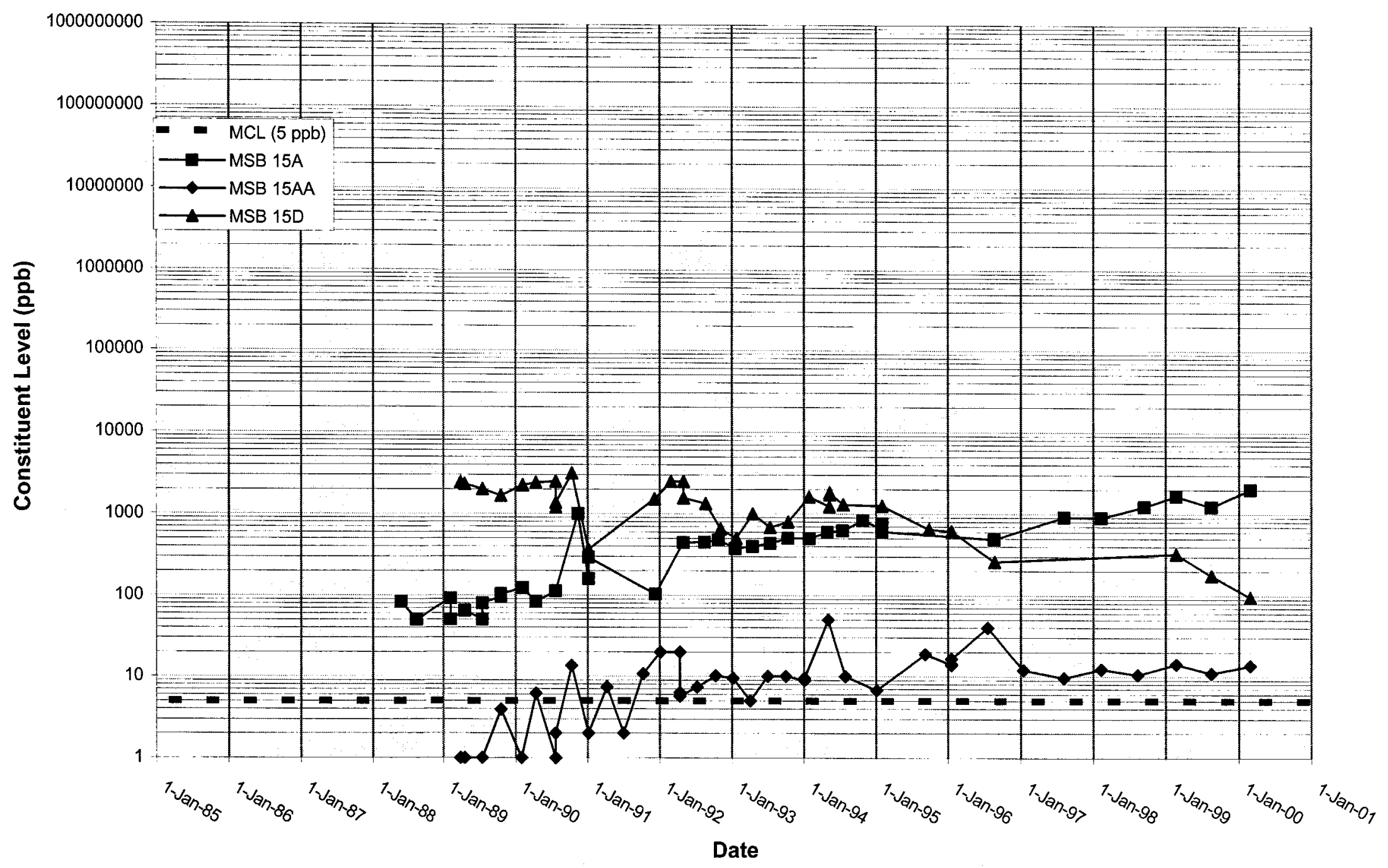


WSRC-TR-2000-00242

UNCLASSIFIED

Time Series Plot of Tetrachloroethylene for Well MSB 16

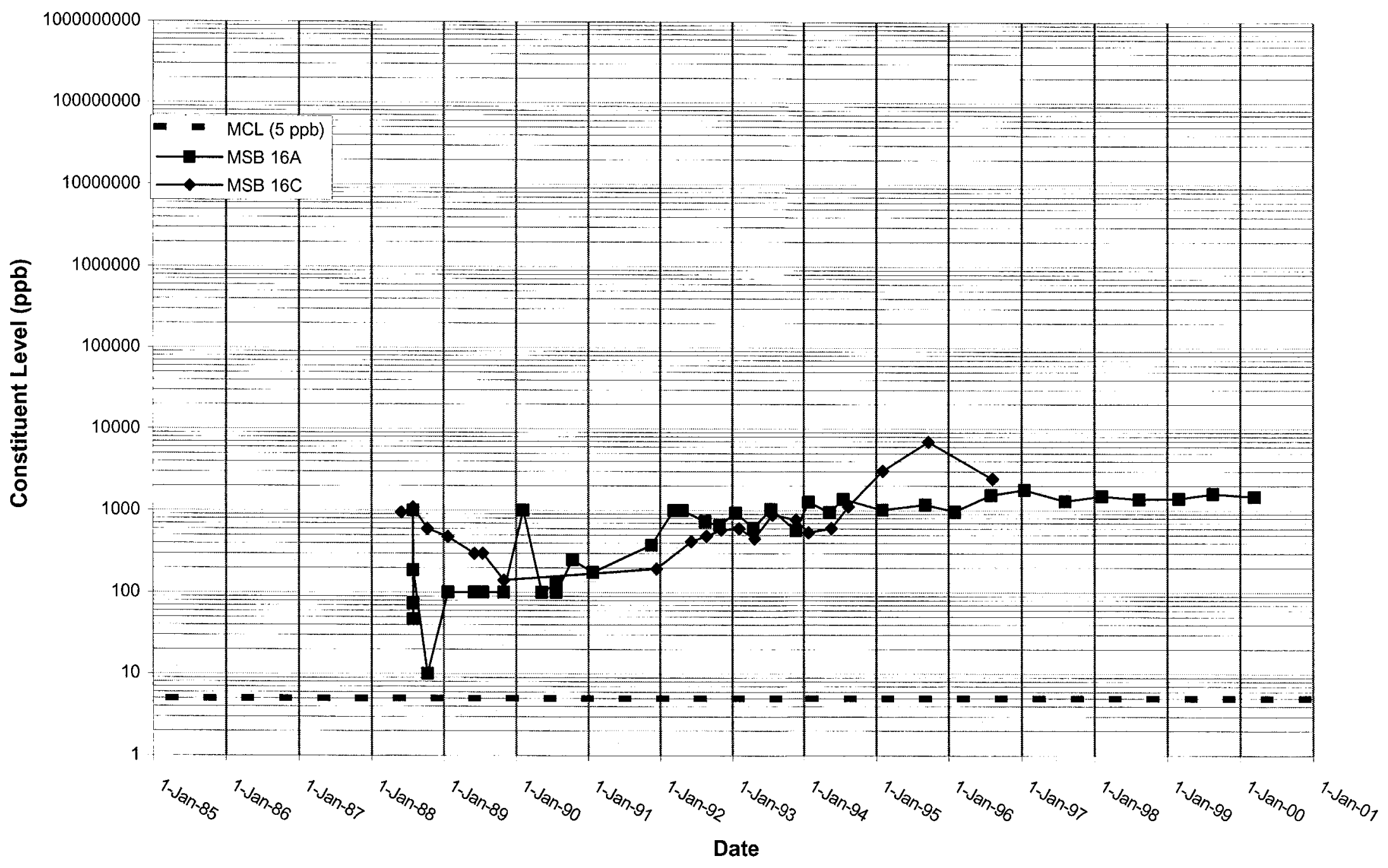




\section{Time Series Plot of Tetrachloroethylene for Well MSB 17}

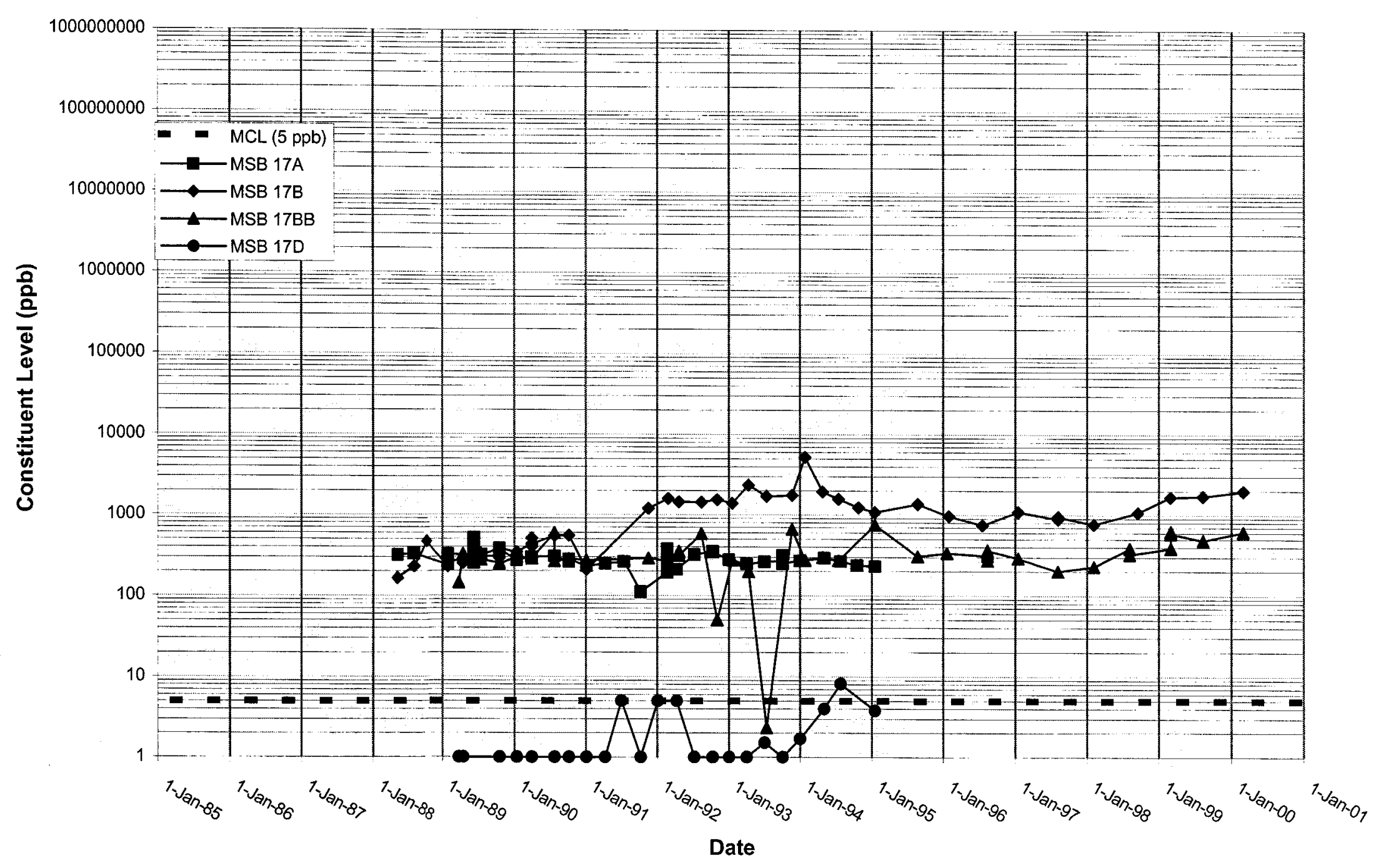


WSRC-TR-2000-00242 UNCLASSIFIED

Time Series Plot of Tetrachloroethylene for Well MSB 18

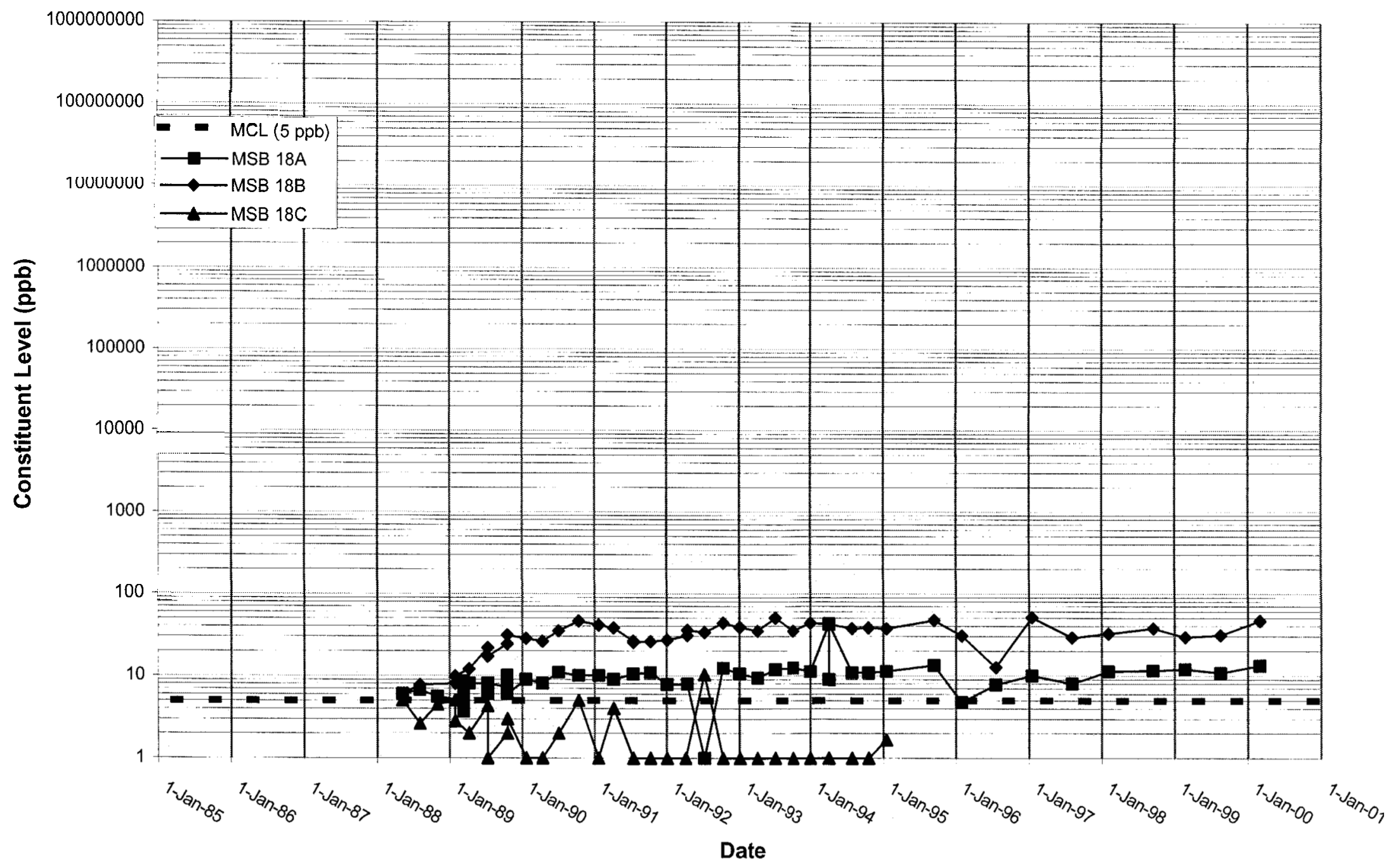


WSRC-TR-2000-00242 UNCLASSIFIED

Time Series Plot of Tetrachloroethylene for Well MSB 19

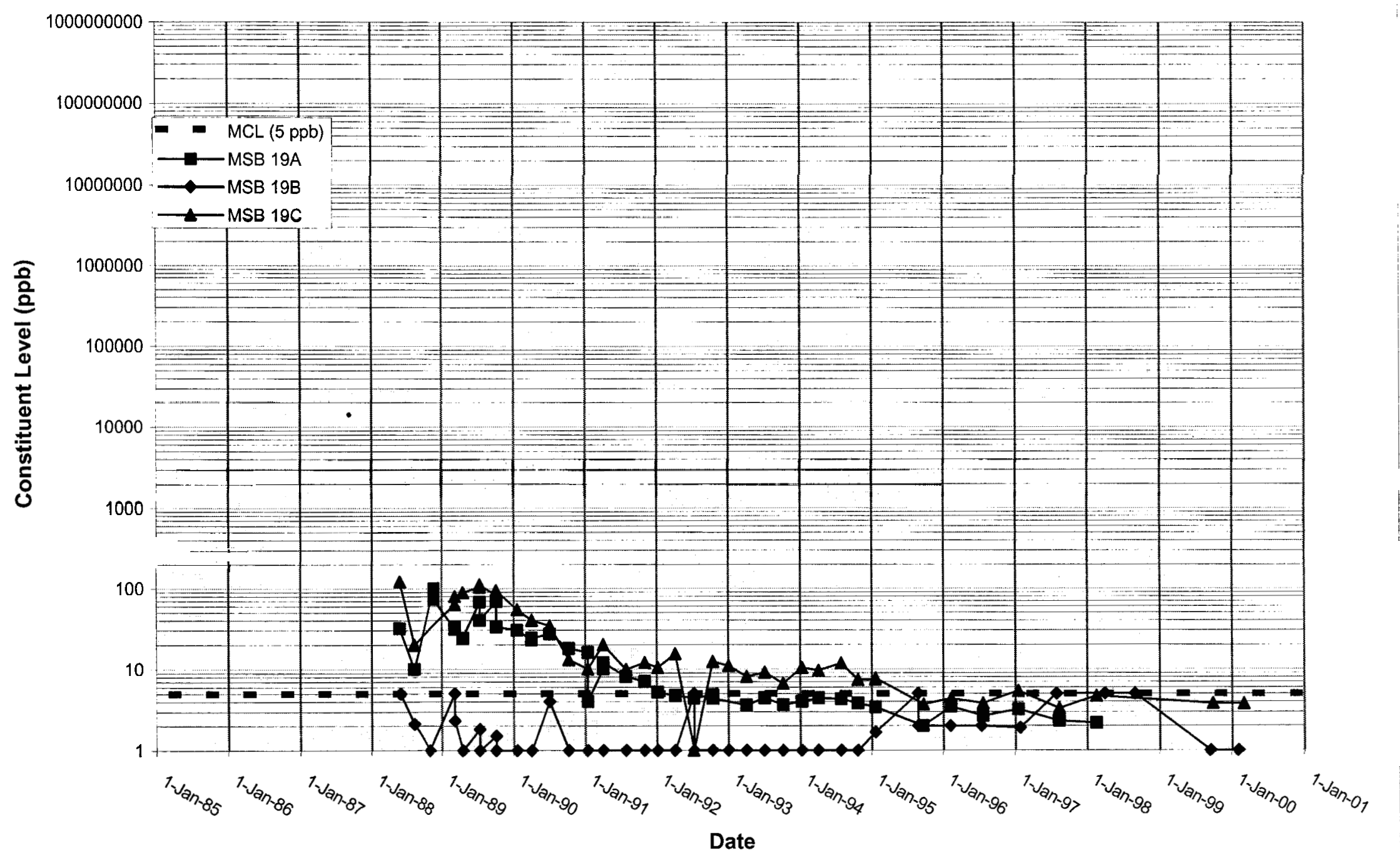




\section{Time Series Plot of Tetrachloroethylene for Well MSB 20}

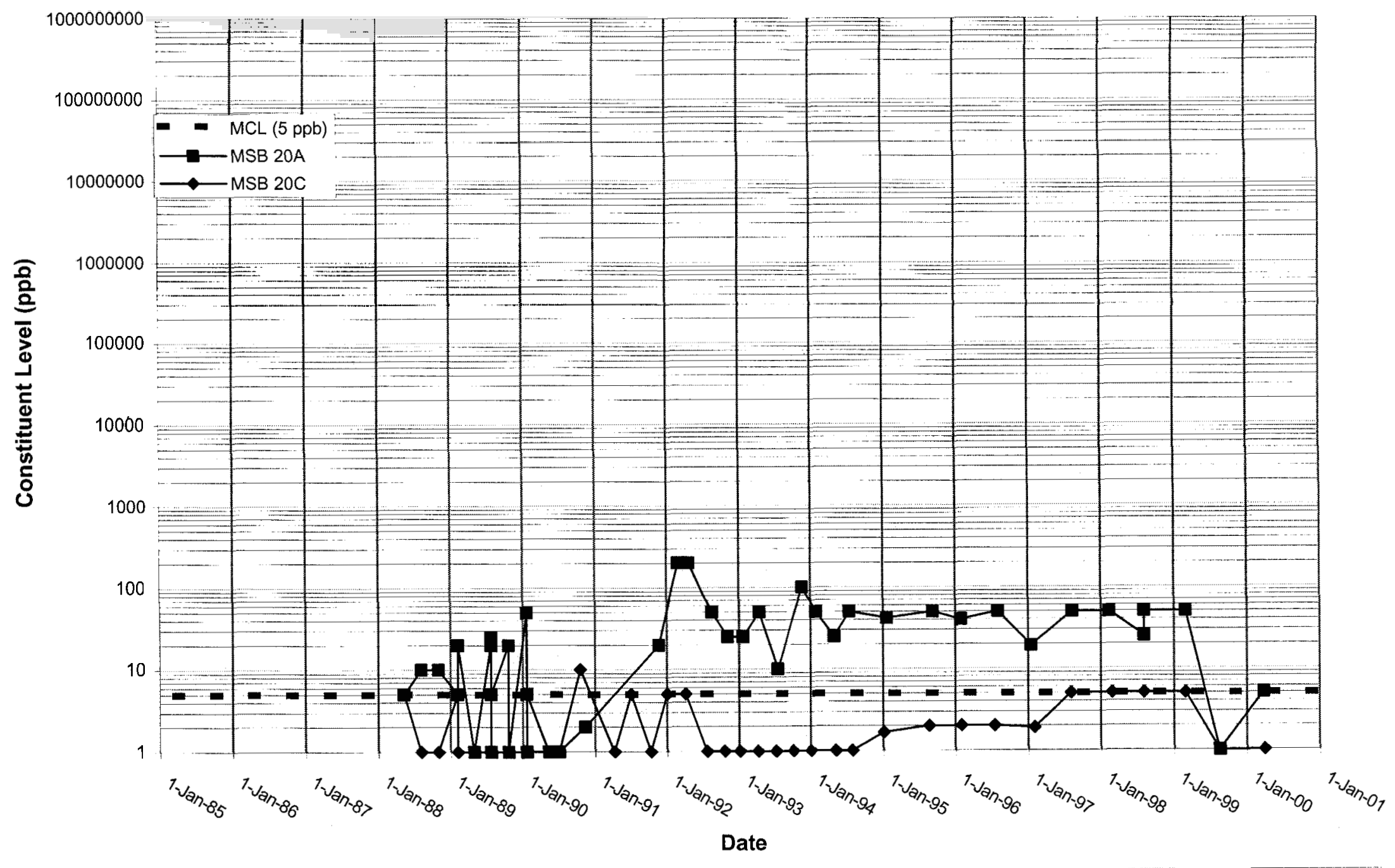




\section{Time Series Plot of Tetrachloroethylene for Well MSB 21}

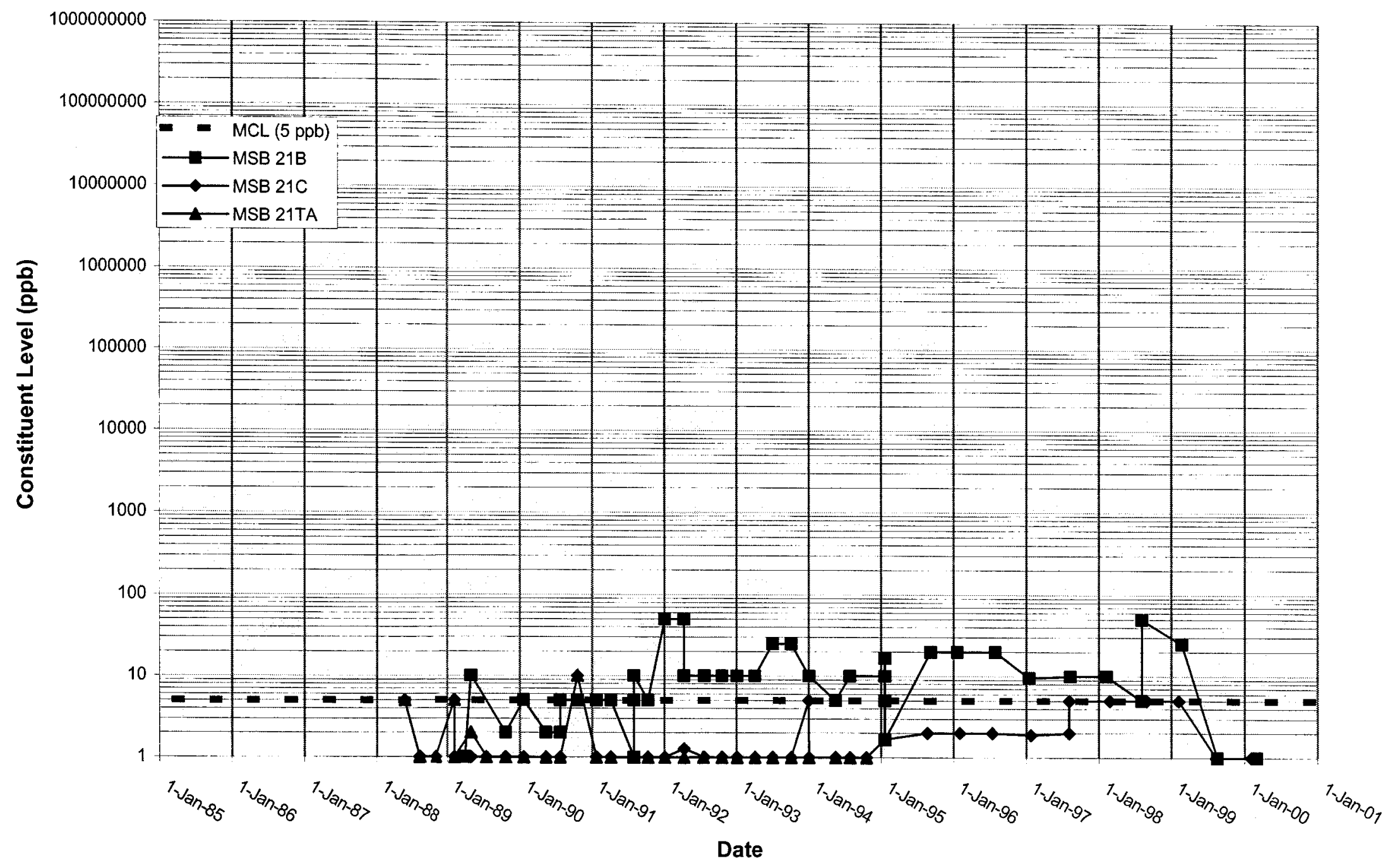


WSRC-TR-2000-00242 UNCLASSIFIED

\section{Time Series Plot of Tetrachloroethylene for Well MSB 22}

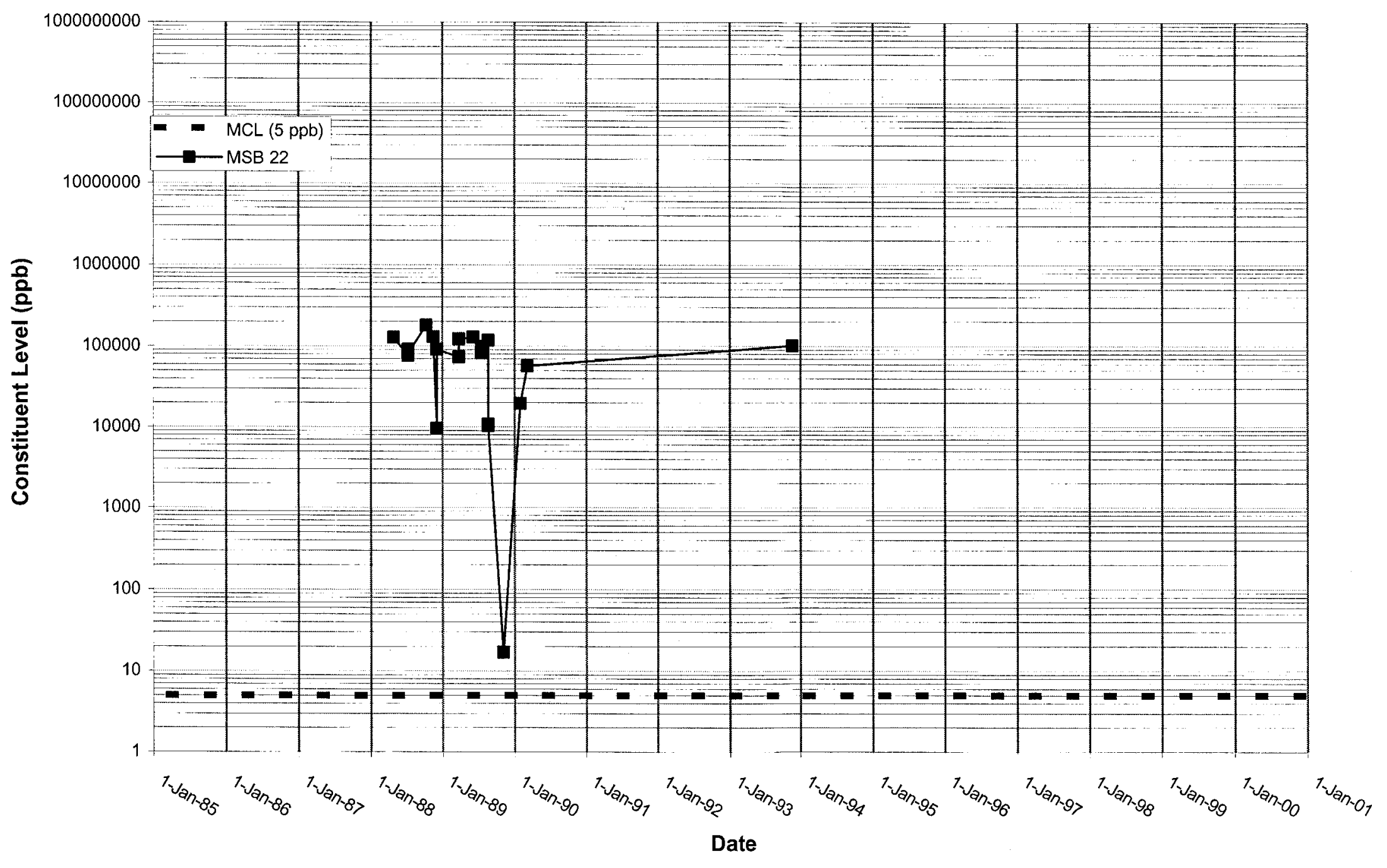




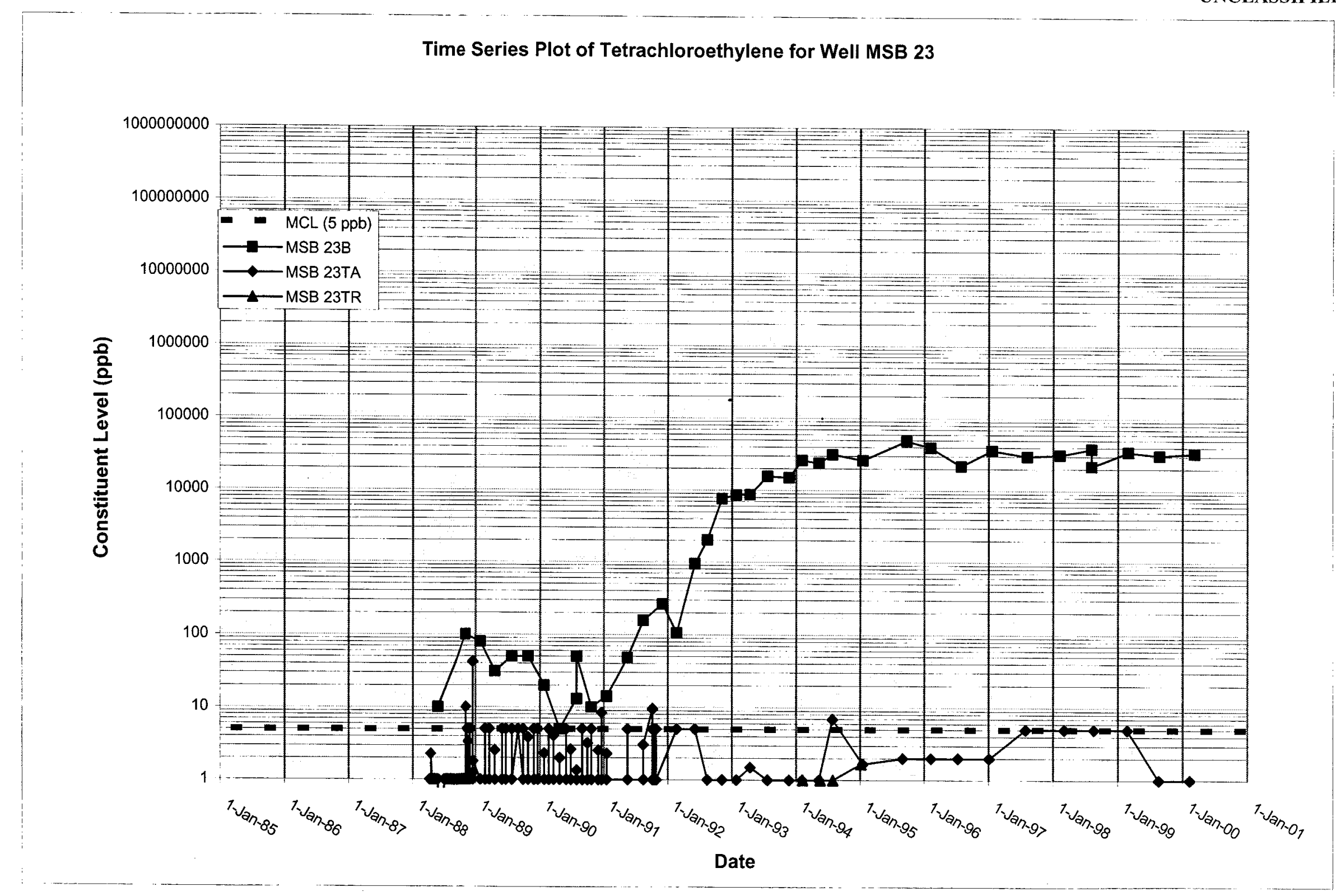


WSRC-TR-2000-00242 UNCLASSIFIED

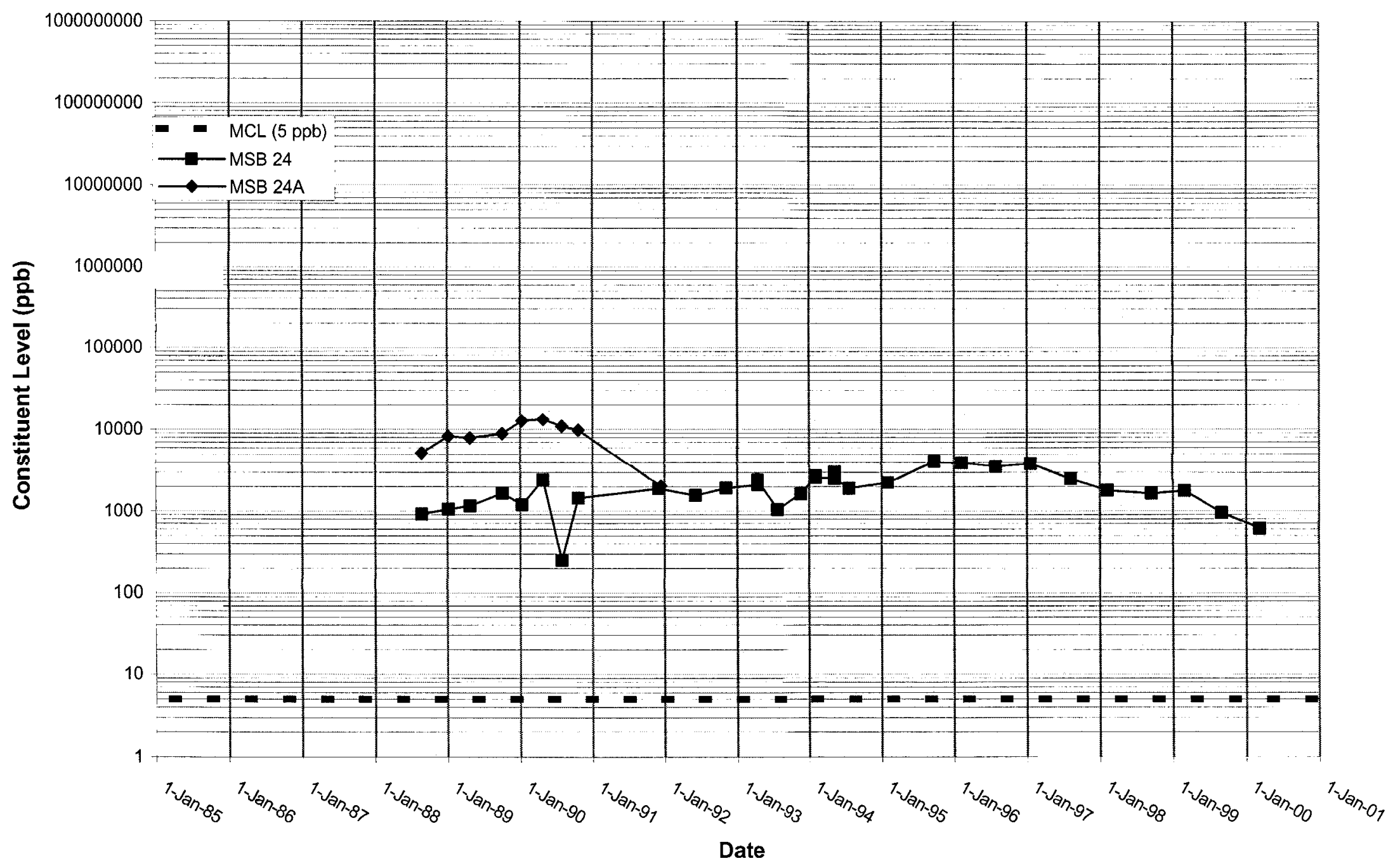




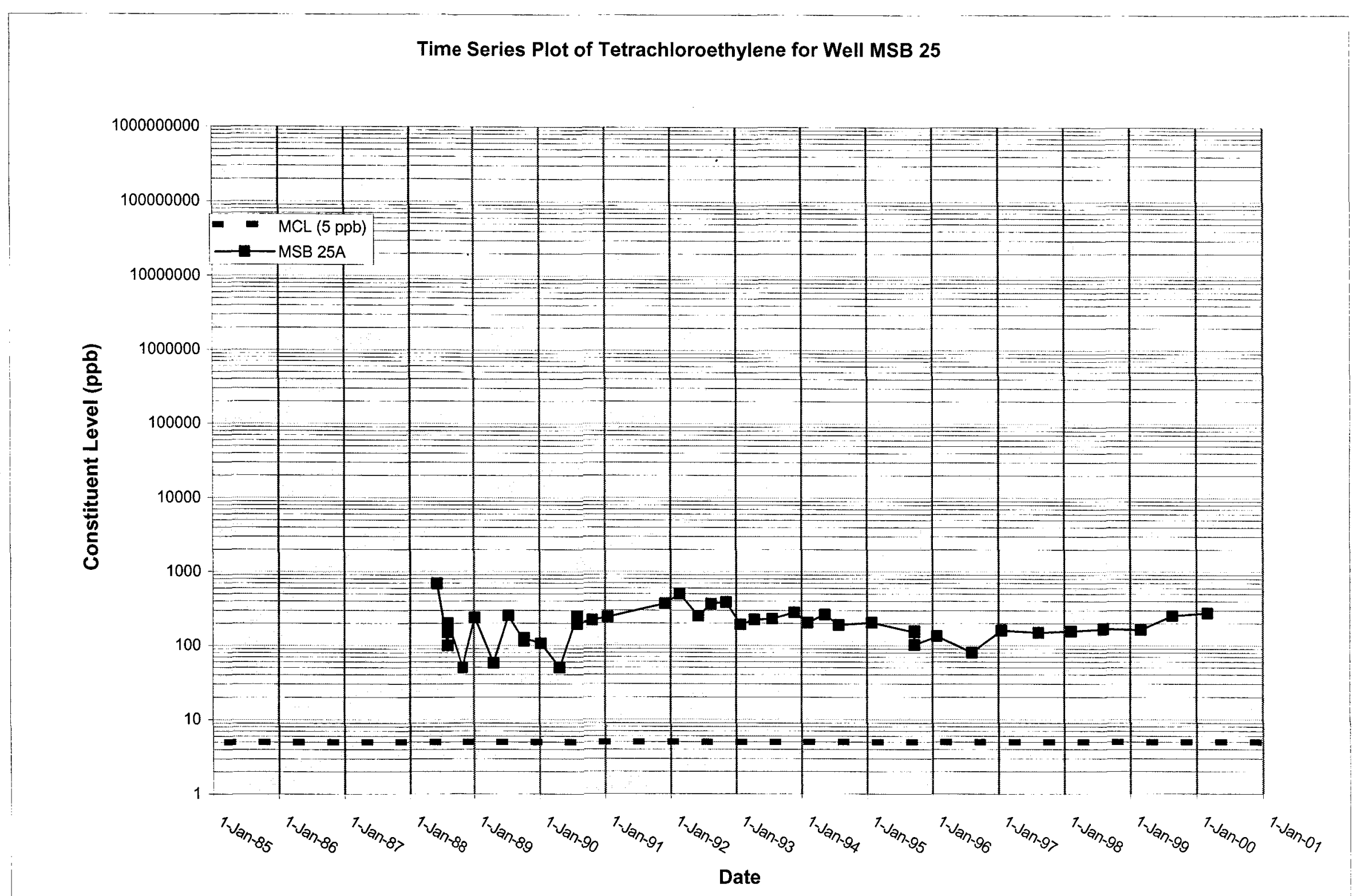


WSRC-TR-2000-00242 UNCLASSIFIED

Time Series Plot of Tetrachloroethylene for Well MSB 26

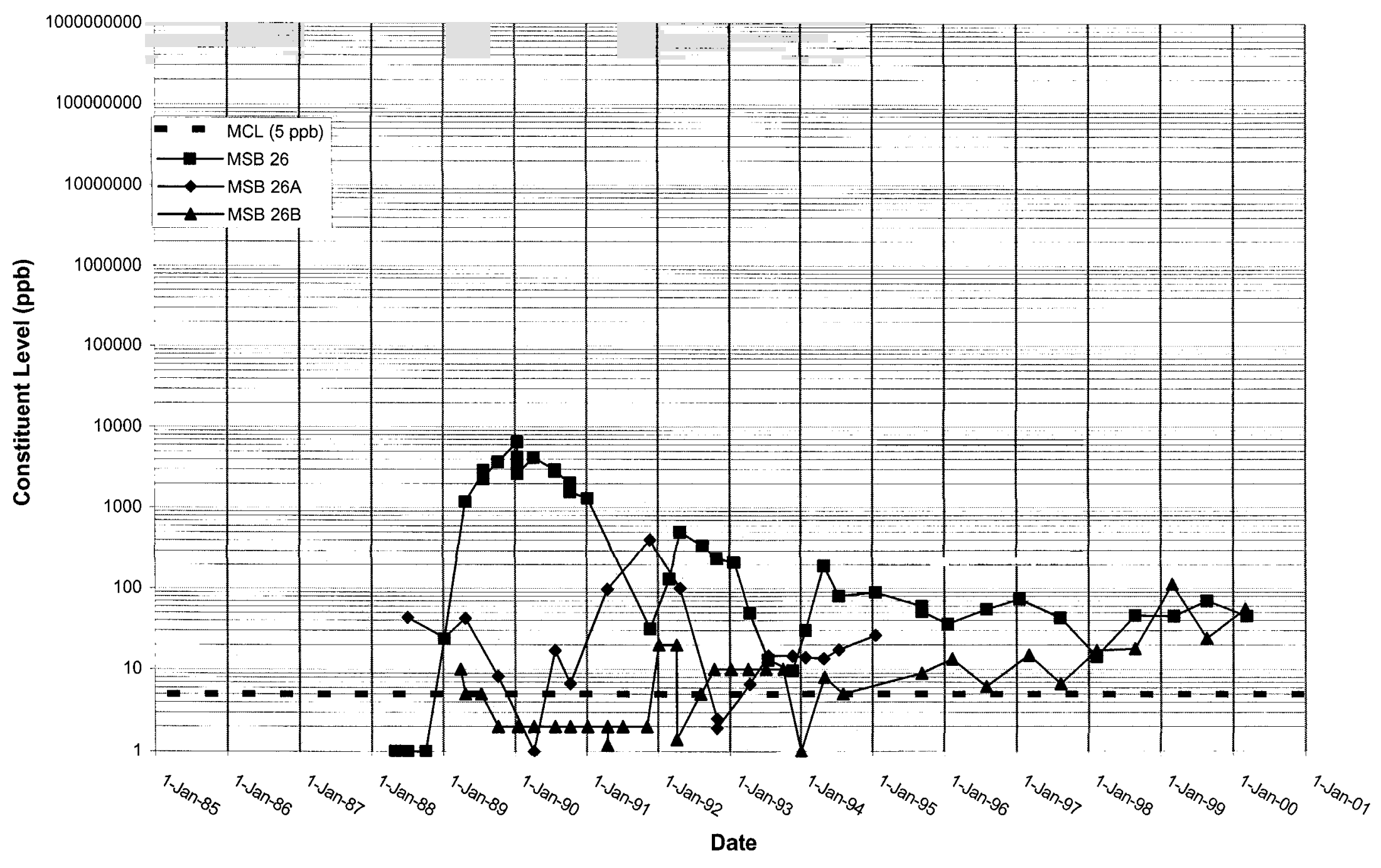


Time Series Plot of Tetrachloroethylene for Well MSB 27

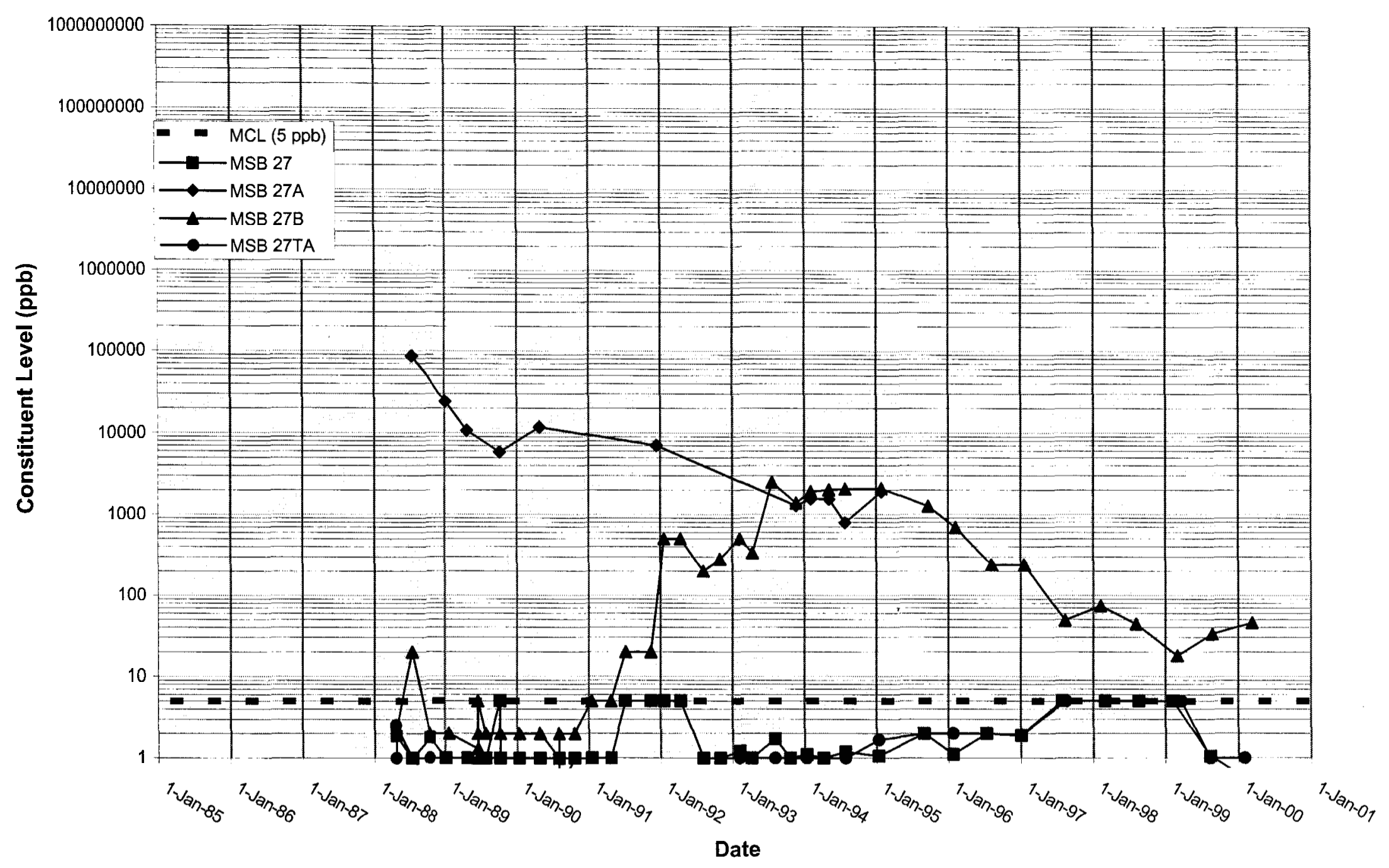


WSRC-TR-2000-00242 UNCLASSIFIED

Time Series Plot of Tetrachloroethylene for Well MSB 28

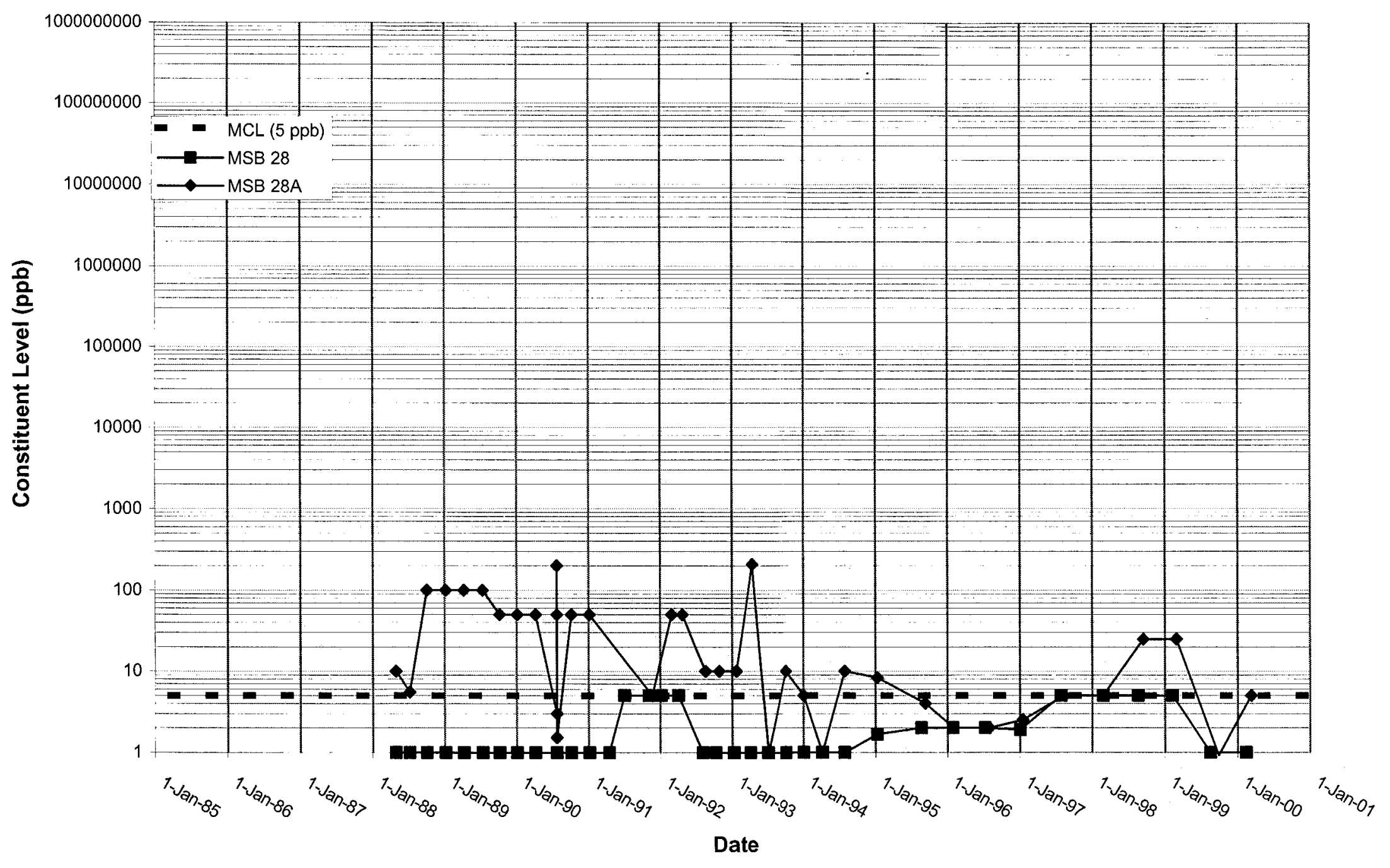


Time Series Plot of Tetrachloroethylene for Well MSB 29

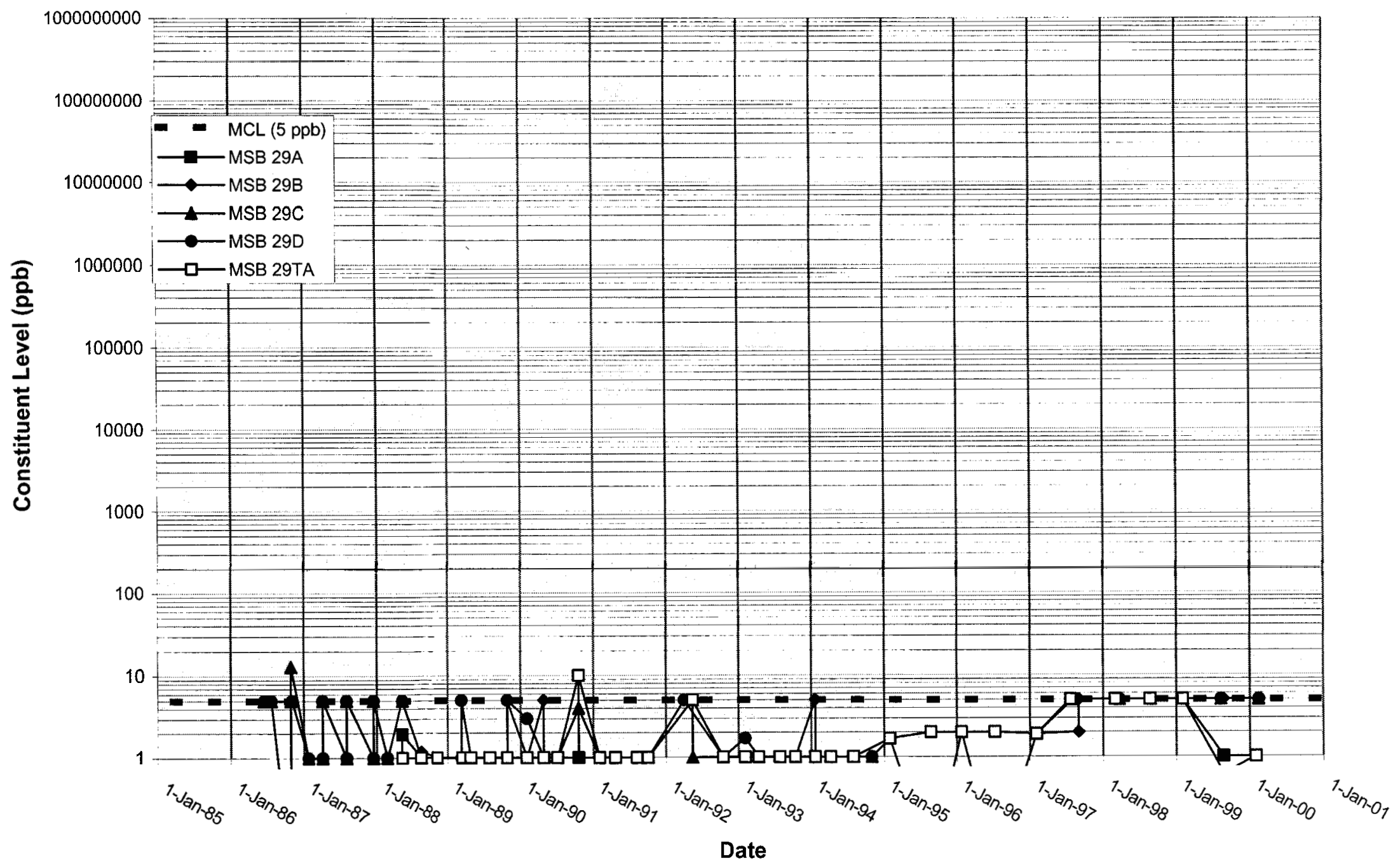


WSRC-TR-2000-00242 UNCLASSIFIED

Time Series Plot of Tetrachloroethylene for Well MSB $\mathbf{3 0}$

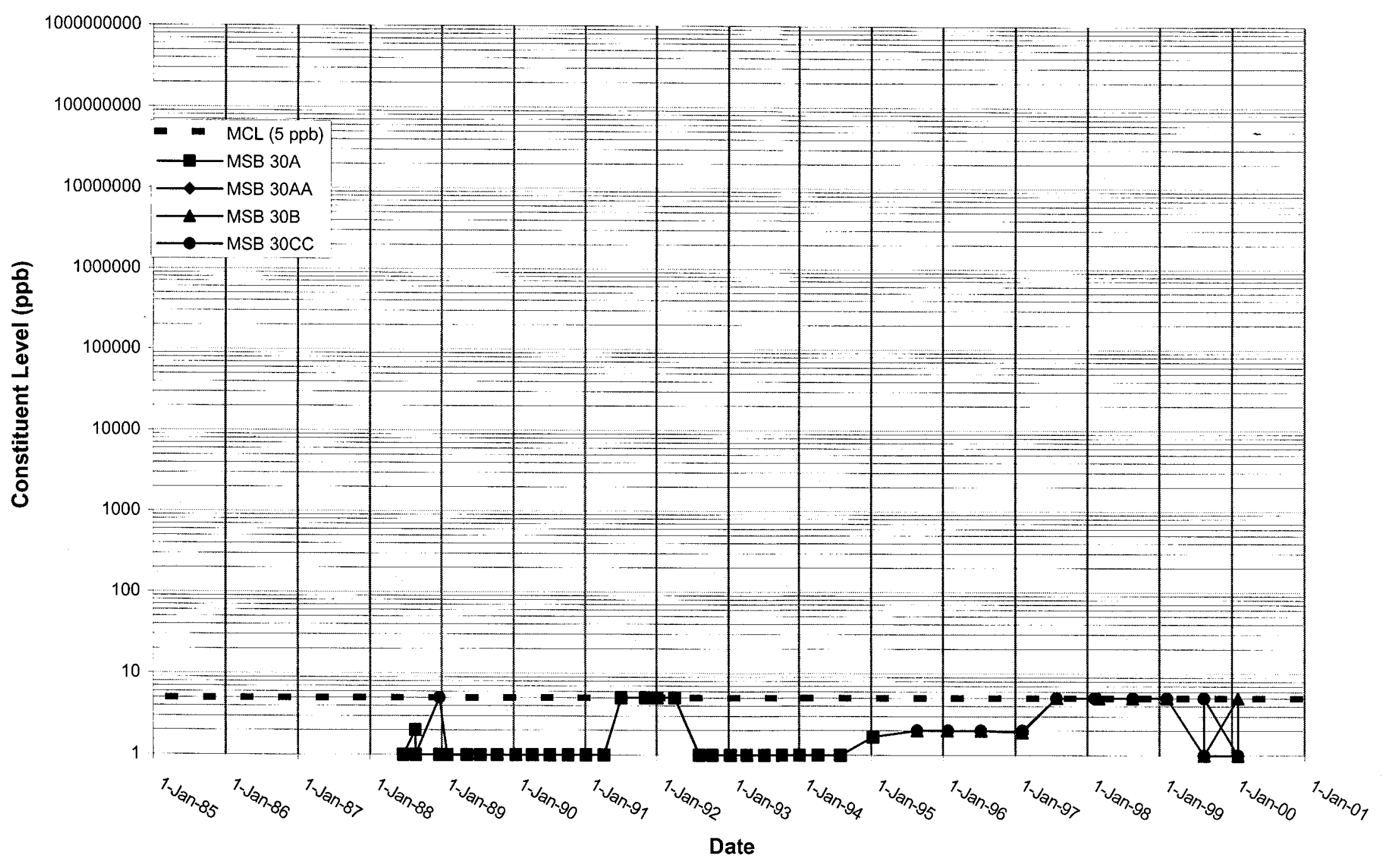


Time Series Plot of Tetrachloroethylene for Well MSB 31

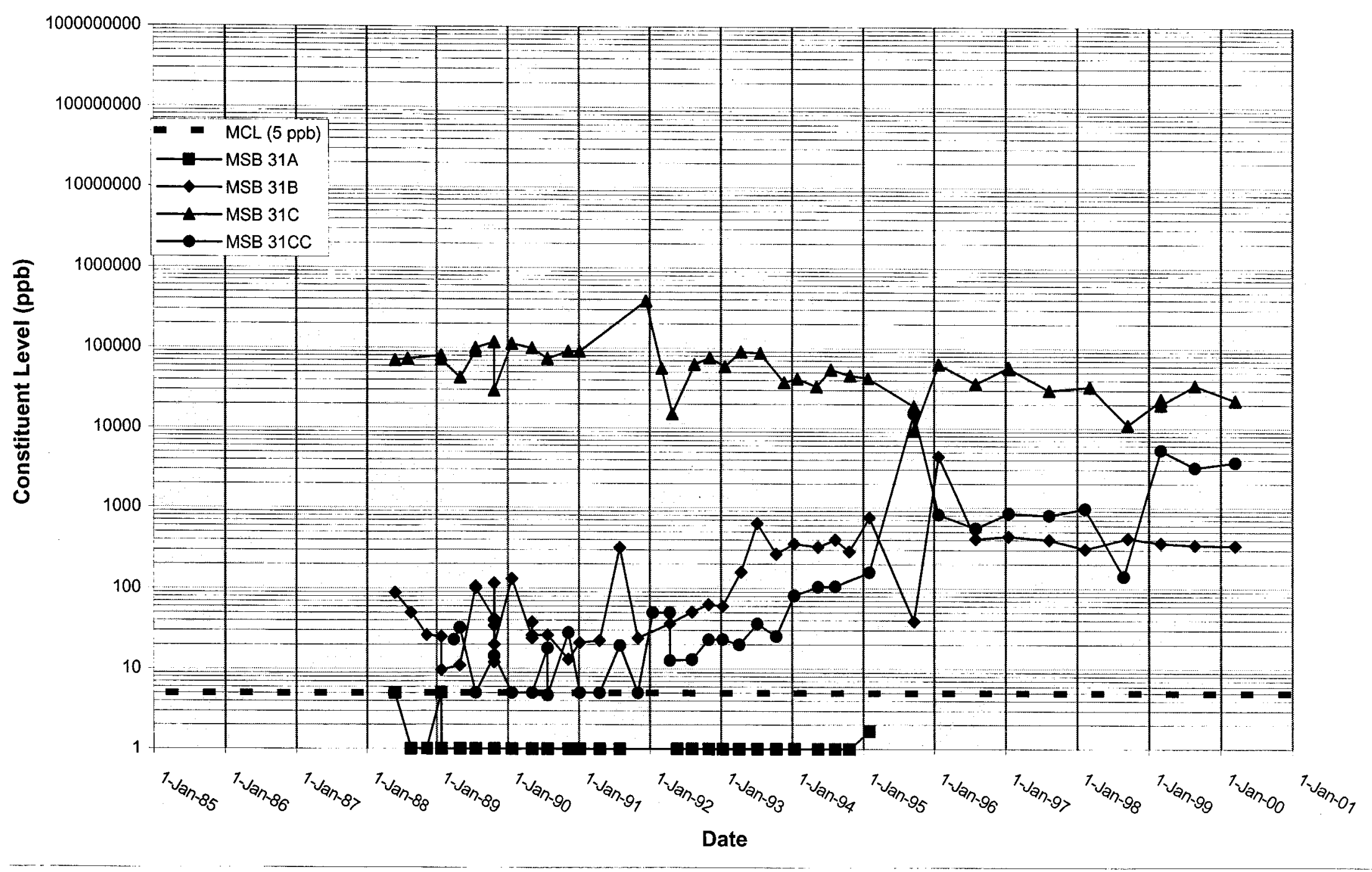


WSRC-TR-2000-00242 UNCLASSIFIED

Time Series Plot of Tetrachloroethylene for Well MSB 32

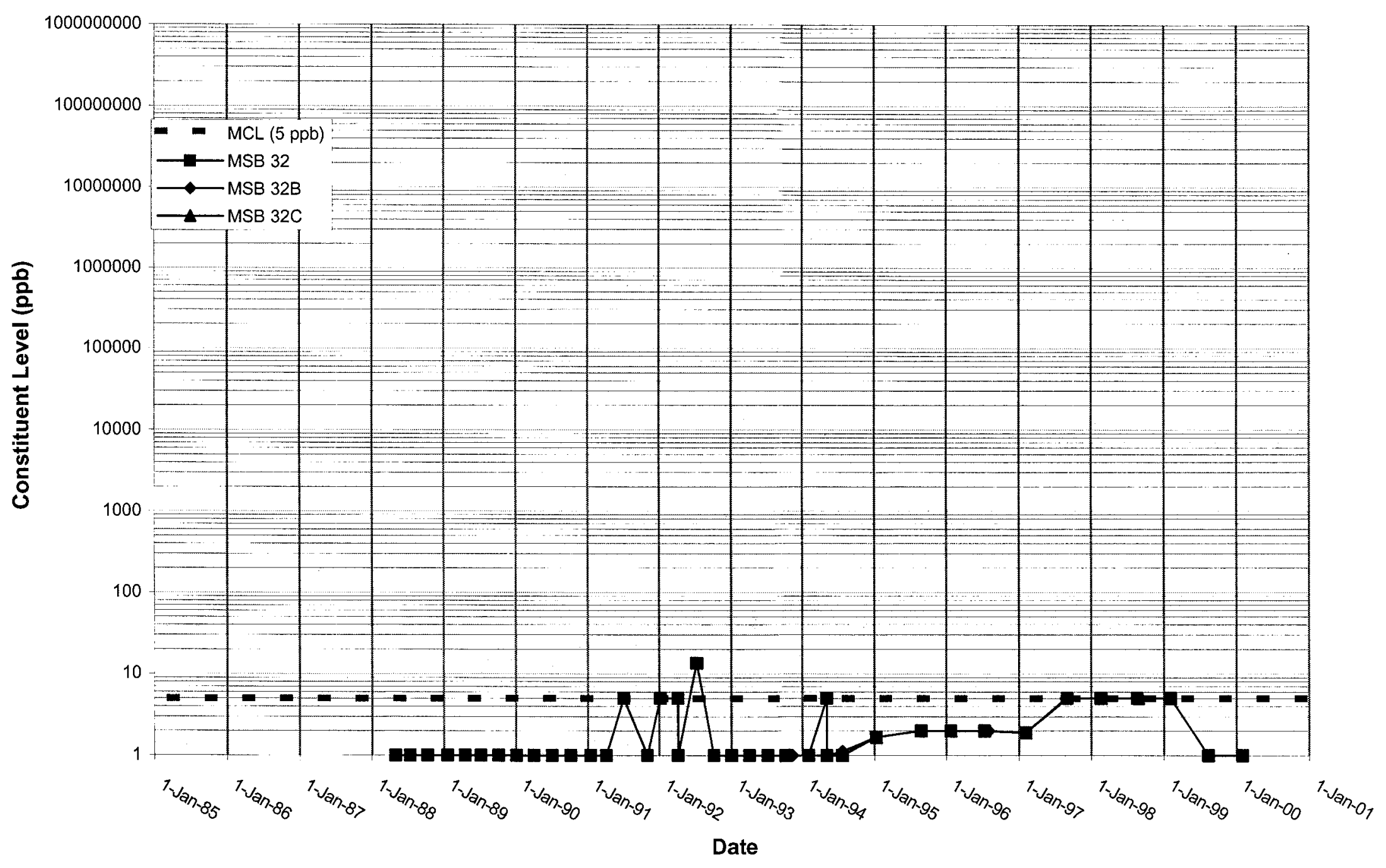


WSRC-TR-2000-00242 UNCLASSIFIED

Time Series Plot of Tetrachloroethylene for Well MSB 33

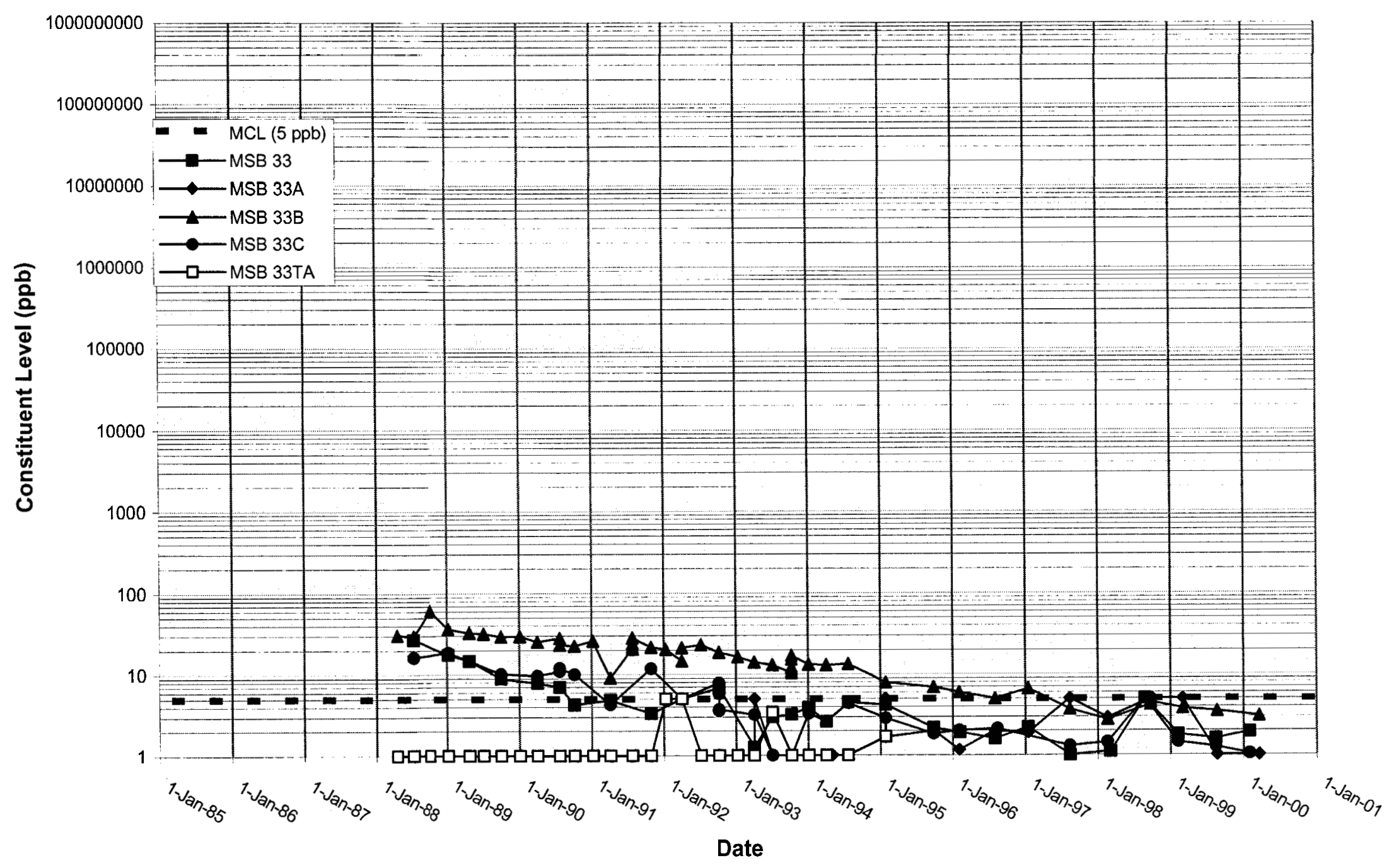


Time Series Plot of Tetrachloroethylene for Well MSB 34

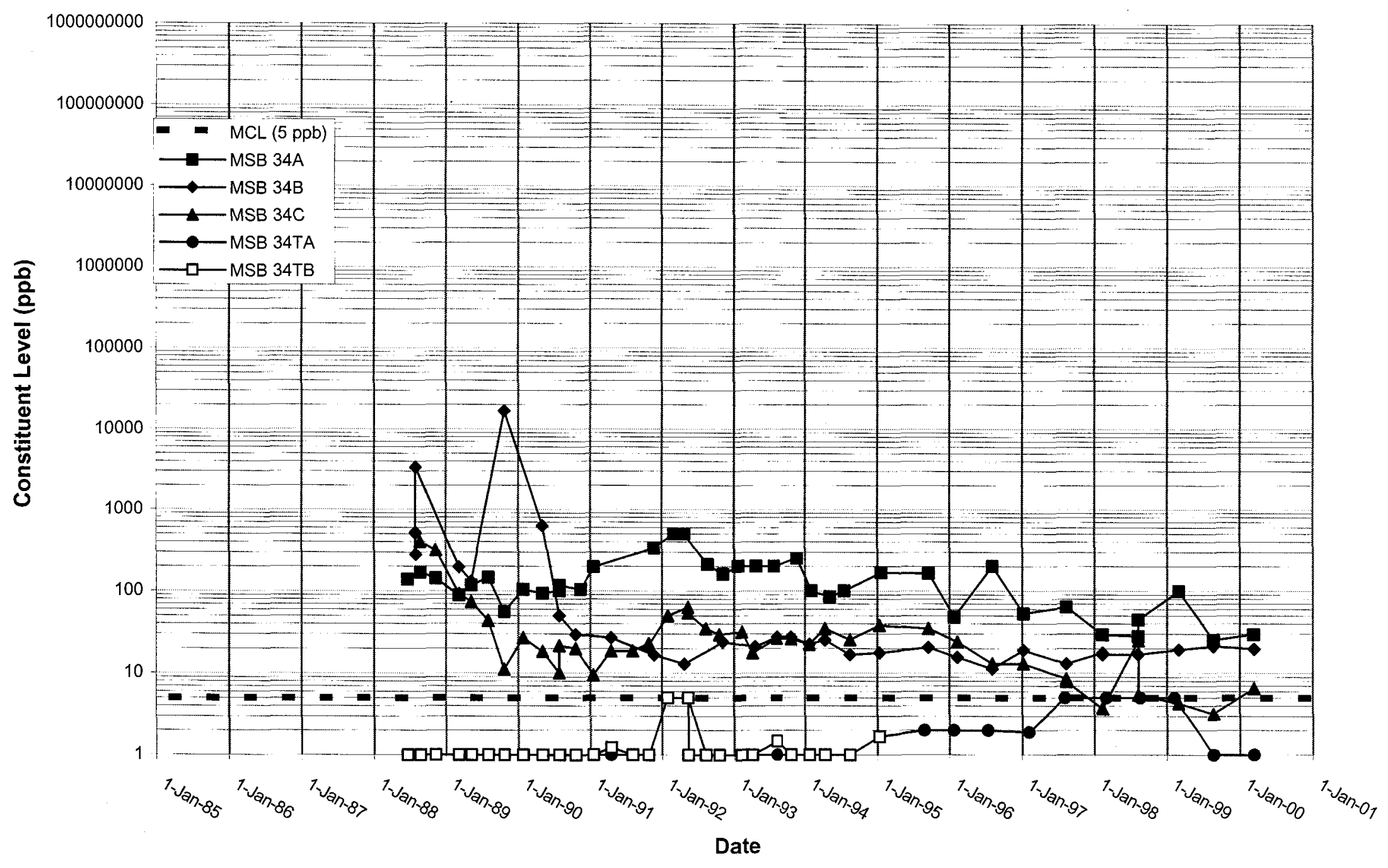


Time Series Plot of Tetrachloroethylene for Well MSB 35

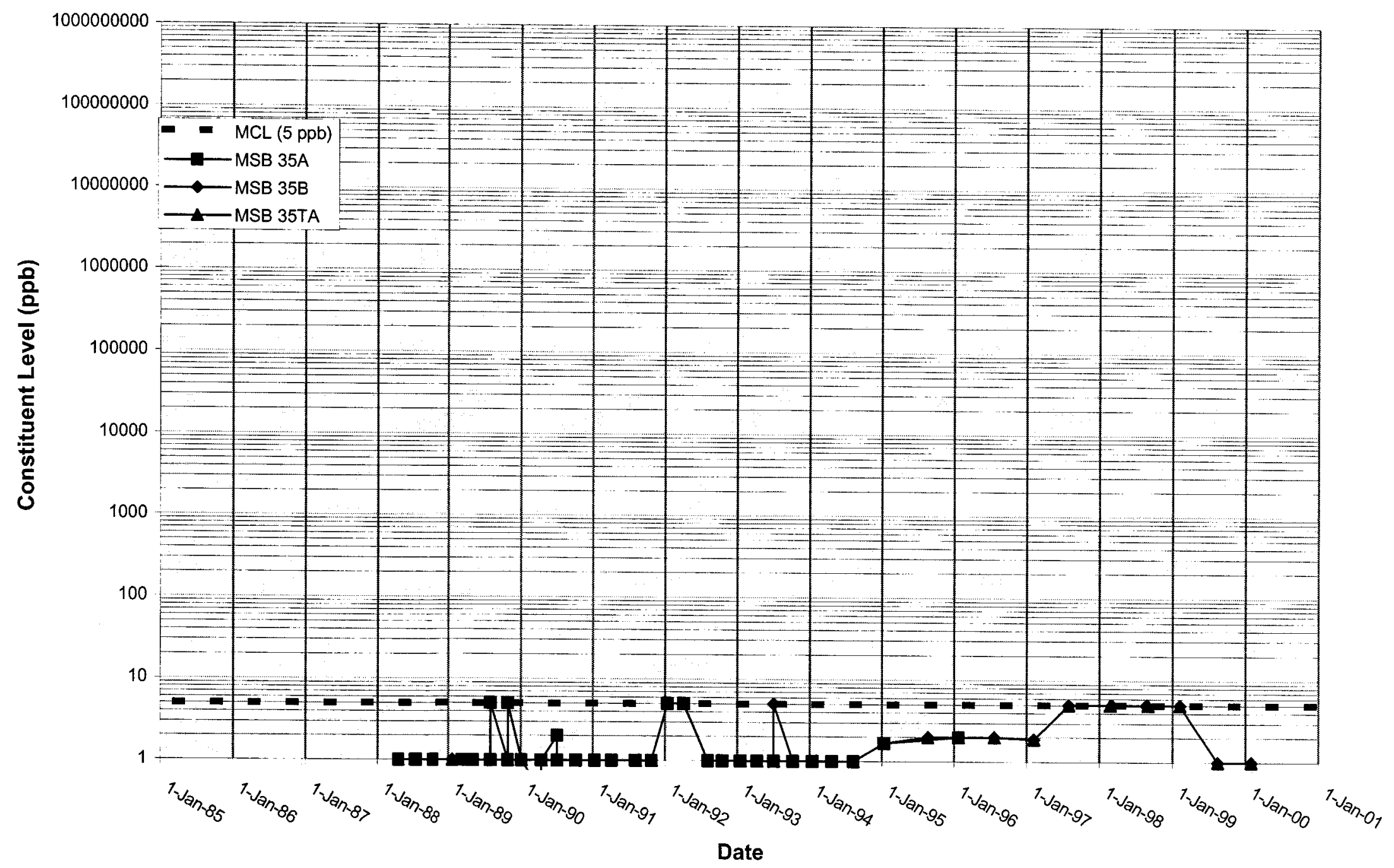




\section{Time Series Plot of Tetrachloroethylene for Well MSB 36}

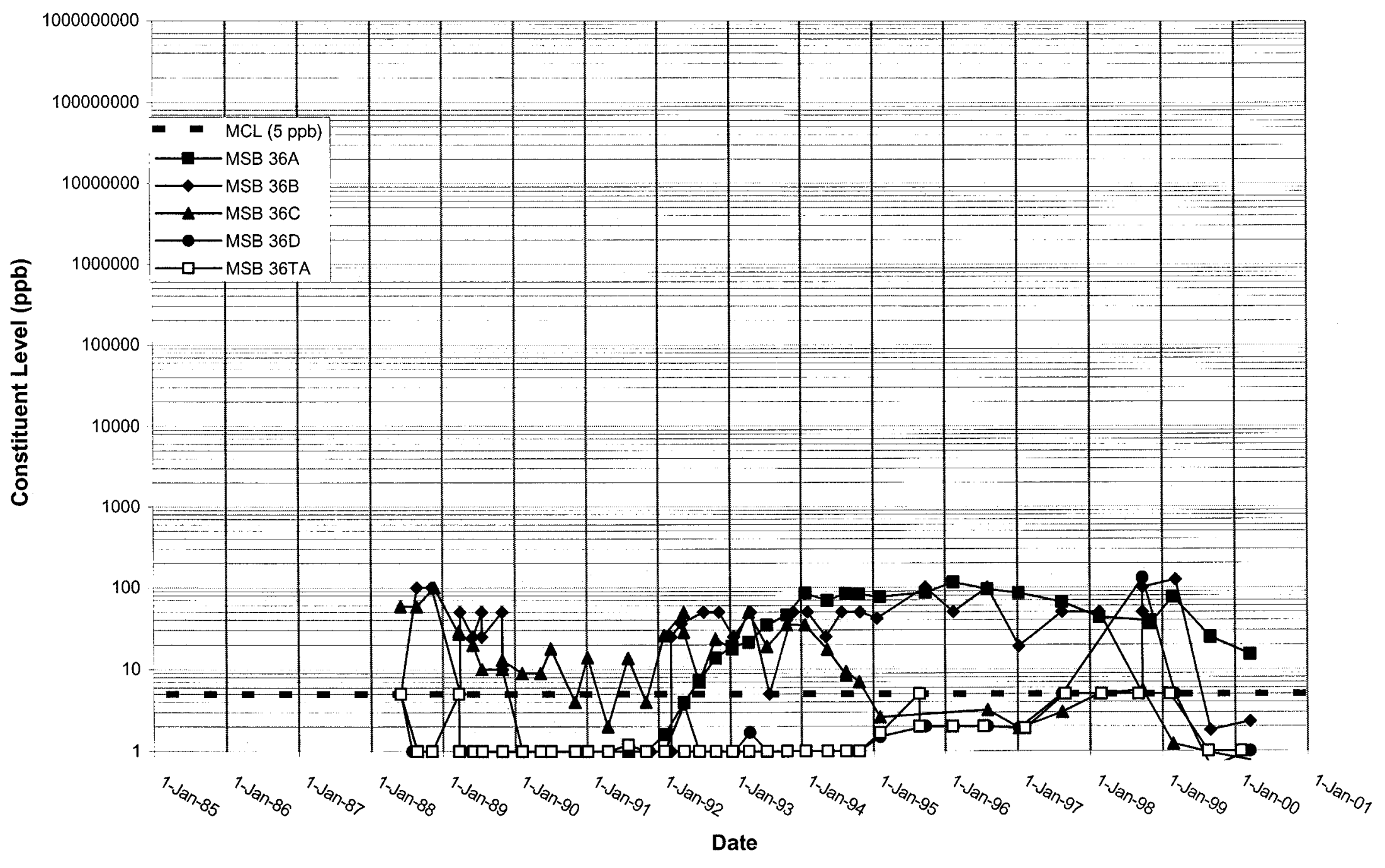


WSRC-TR-2000-00242

UNCLASSIFIED

Time Series Plot of Tetrachloroethylene for Well MSB 37

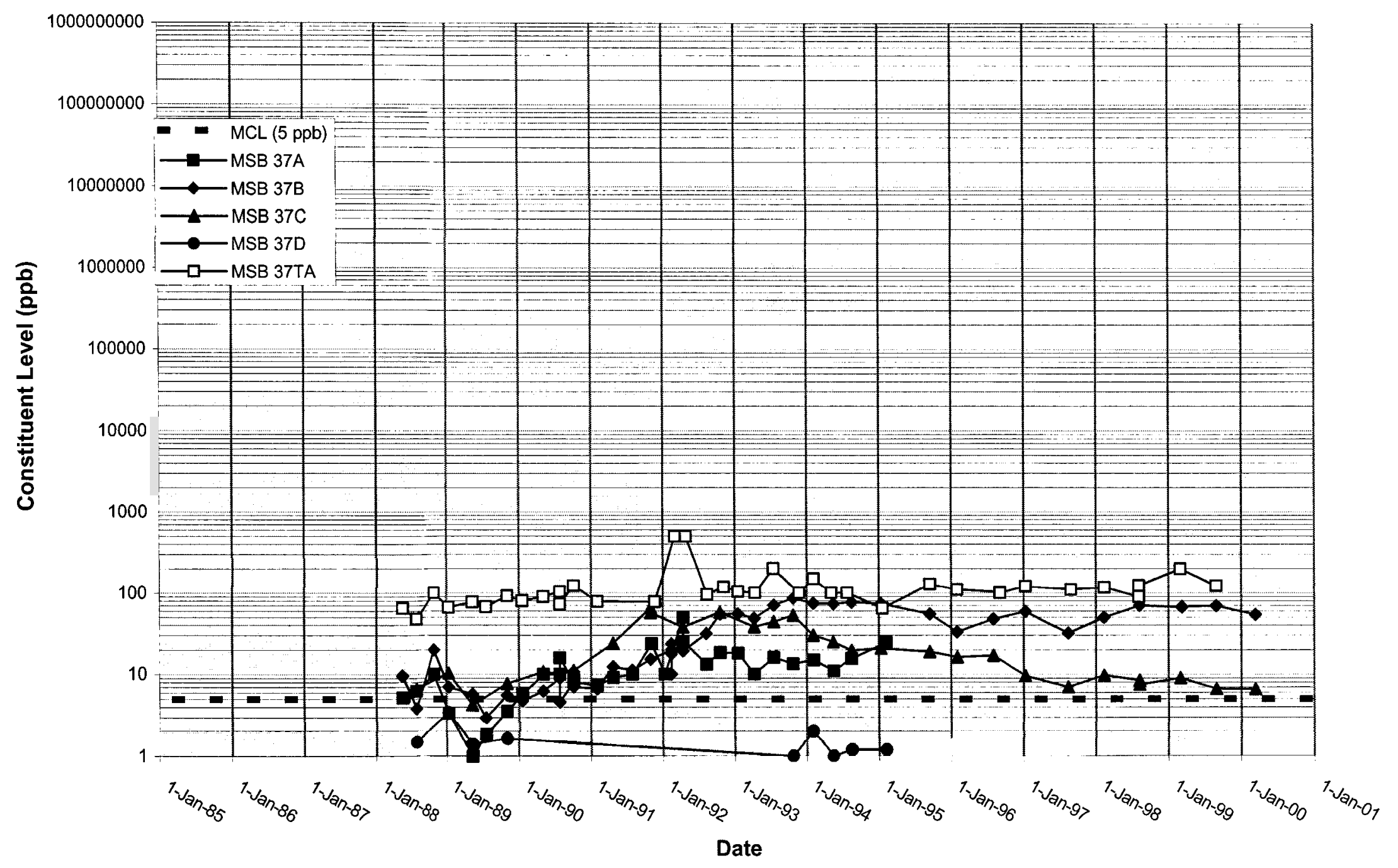


Time Series Plot of Tetrachloroethylene for Well MSB 38

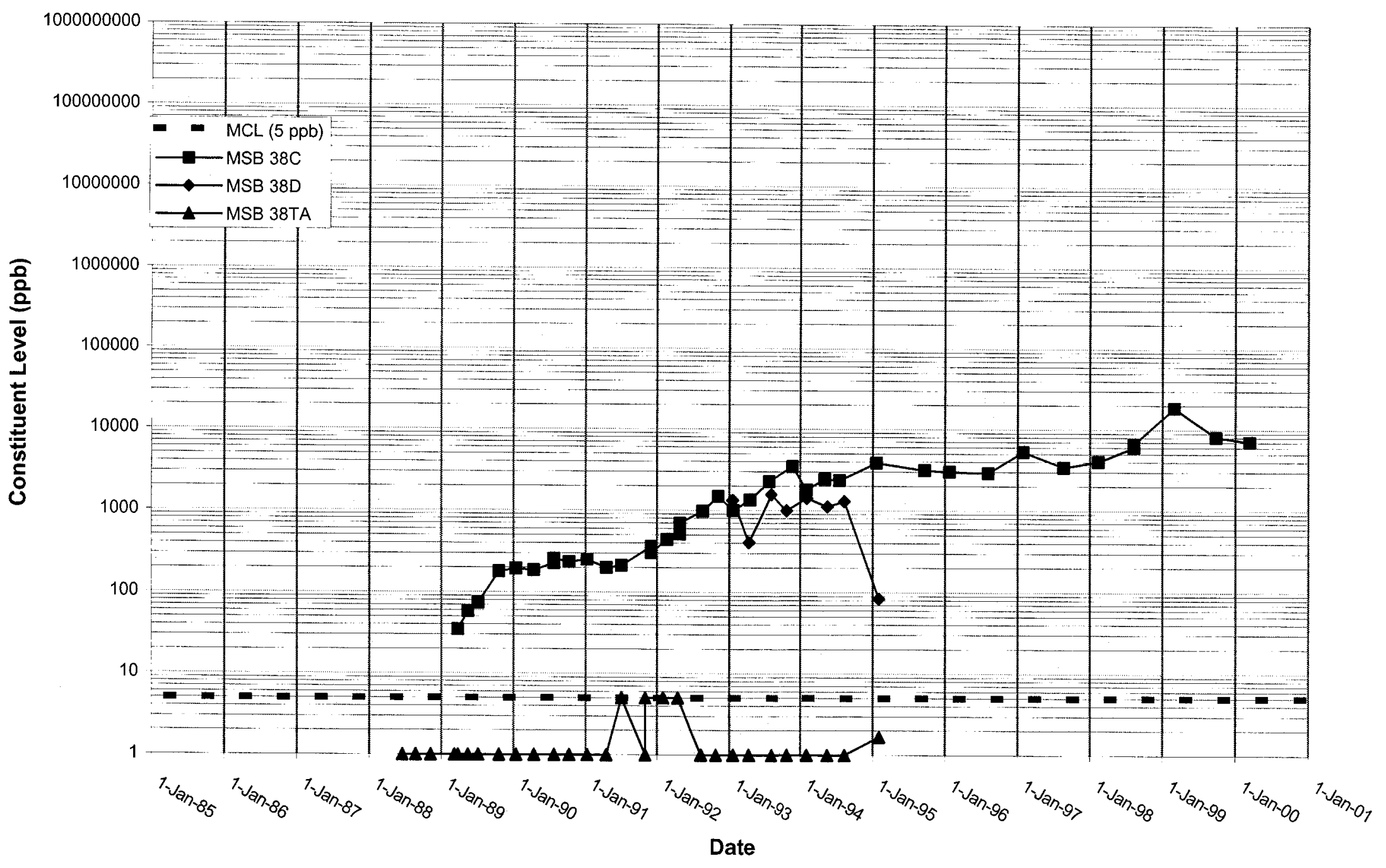




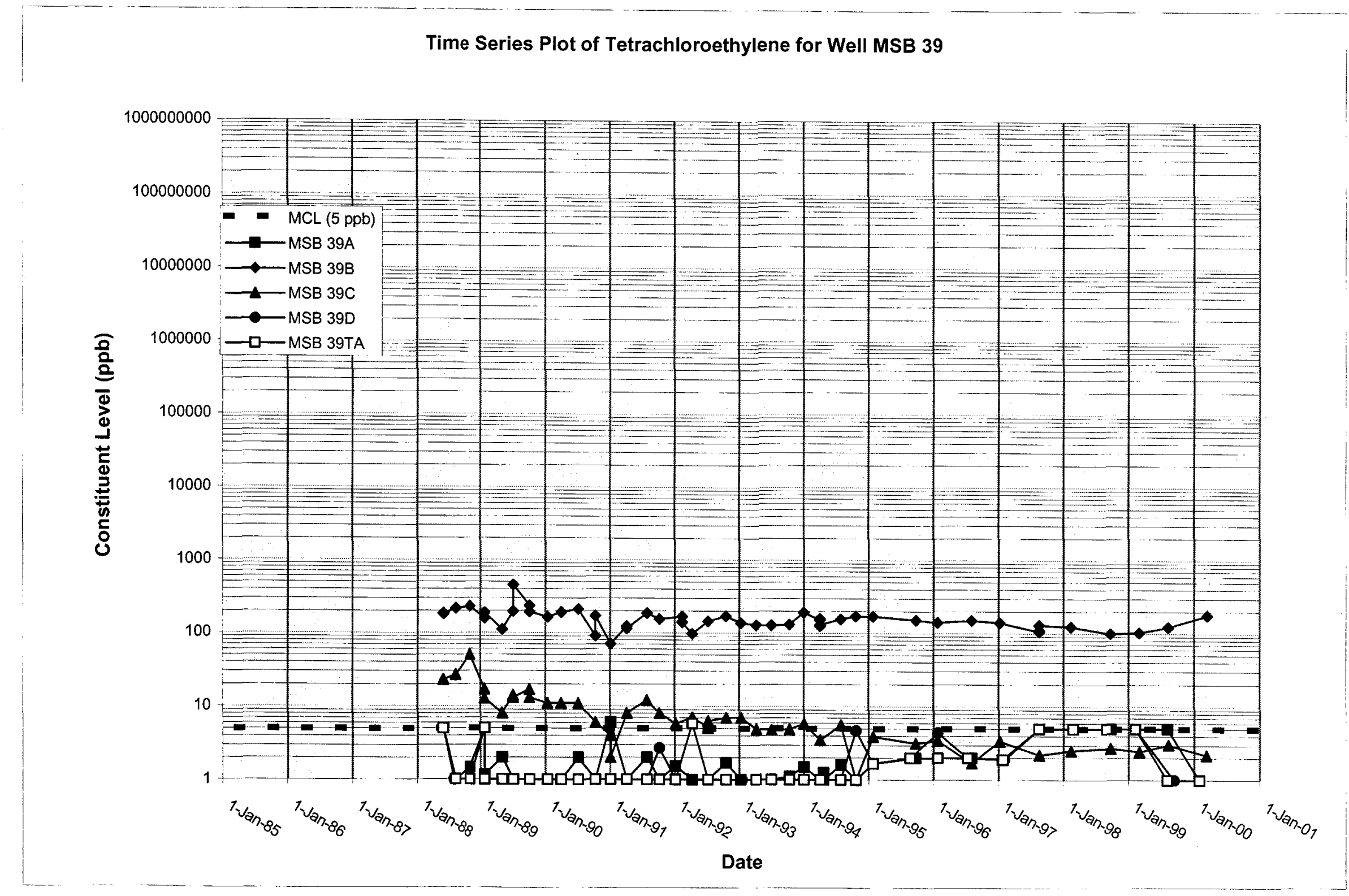


WSRC-TR-2000-00242 UNCLASSIFIED

Time Series Plot of Tetrachloroethylene for Well MSB 40

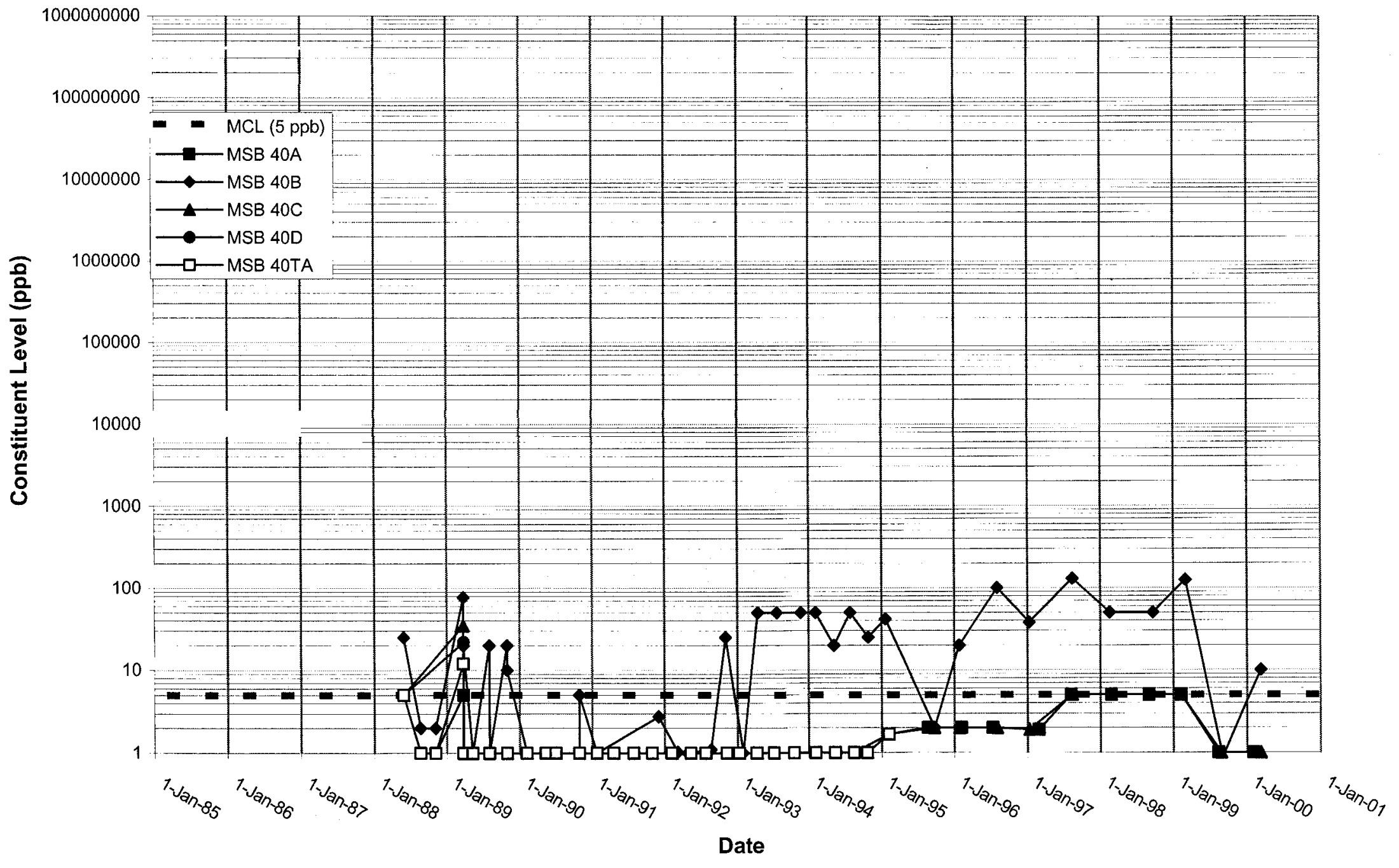


WSRC-TR-2000-00242

UNCLASSIFIED

Time Series Plot of Tetrachloroethylene for Well MSB 41

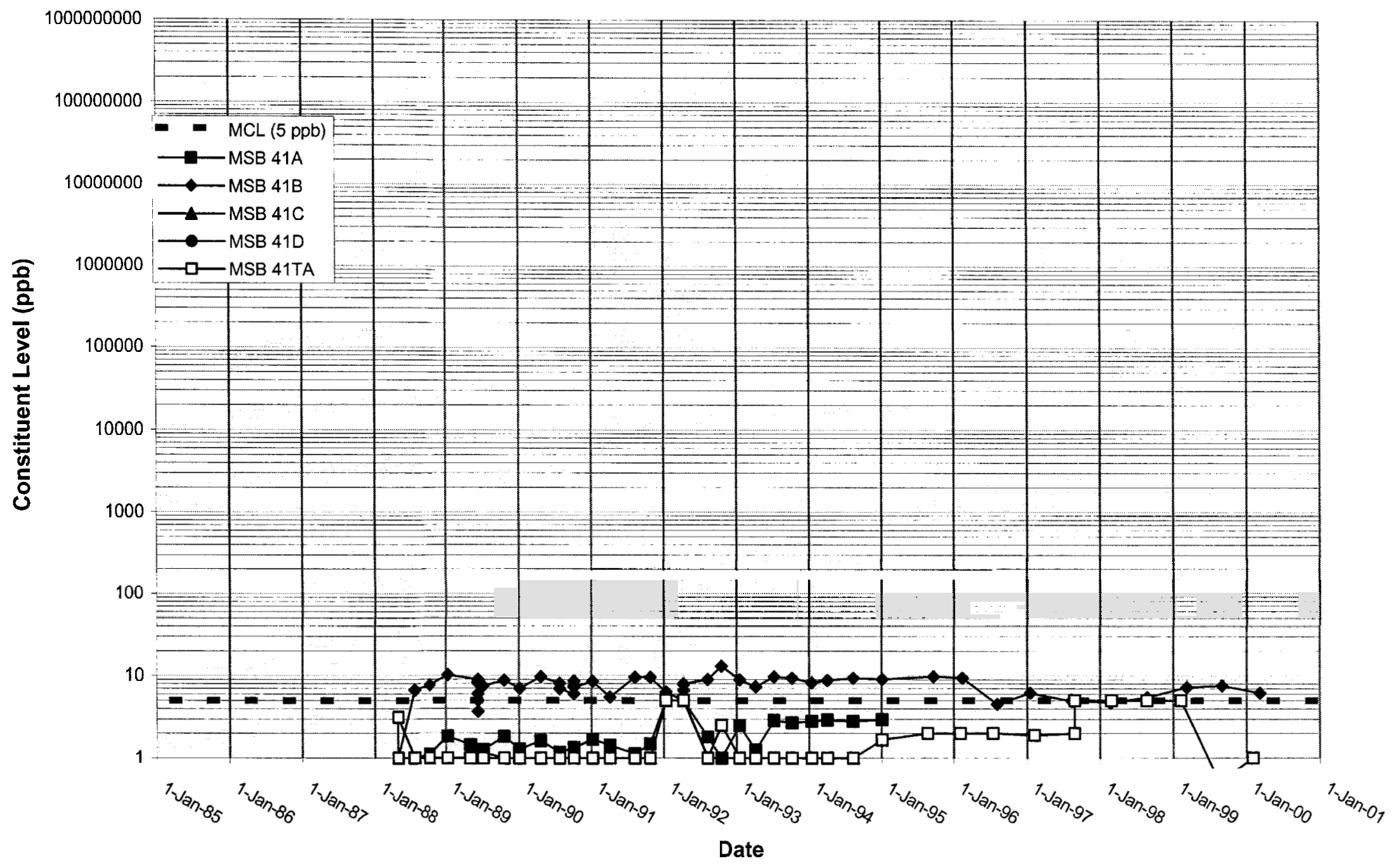




\section{Time Series Plot of Tetrachloroethylene for Well MSB 42}

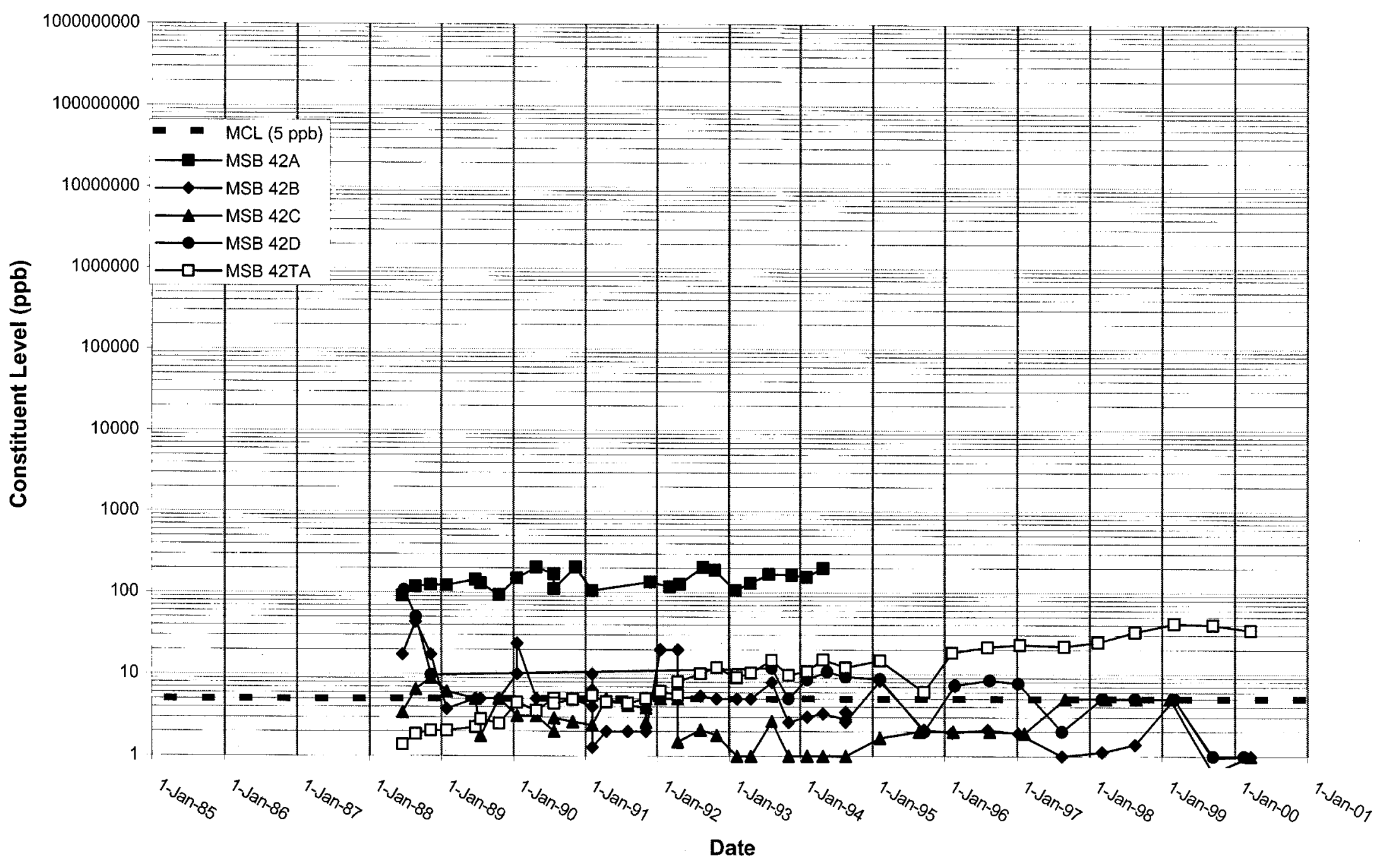




\section{Time Series Plot of Tetrachloroethylene for Well MSB 43}

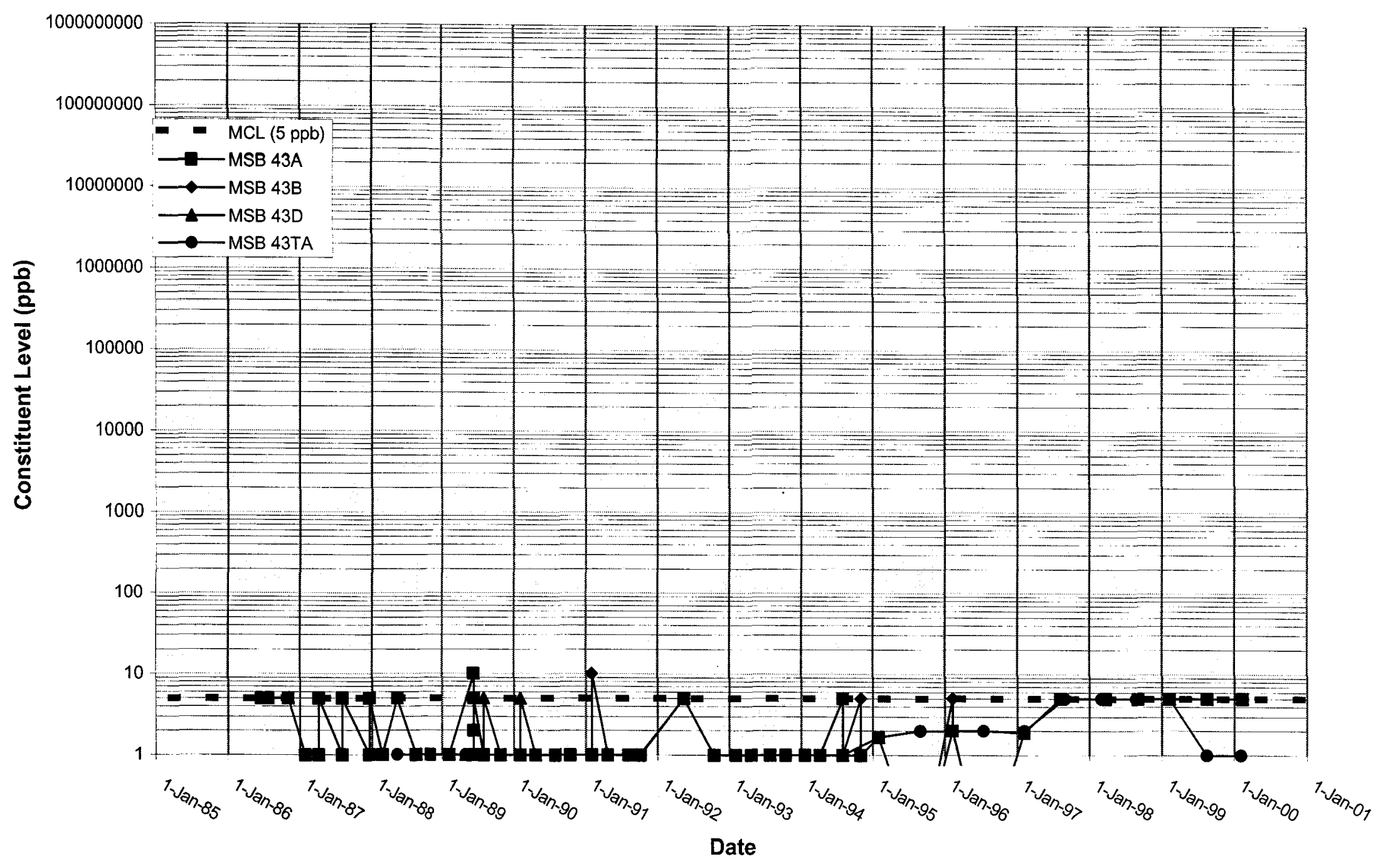


WSRC-TR-2000-00242 UNCLASSIFIED

\section{Time Series Plot of Tetrachloroethylene for Well MSB 44}

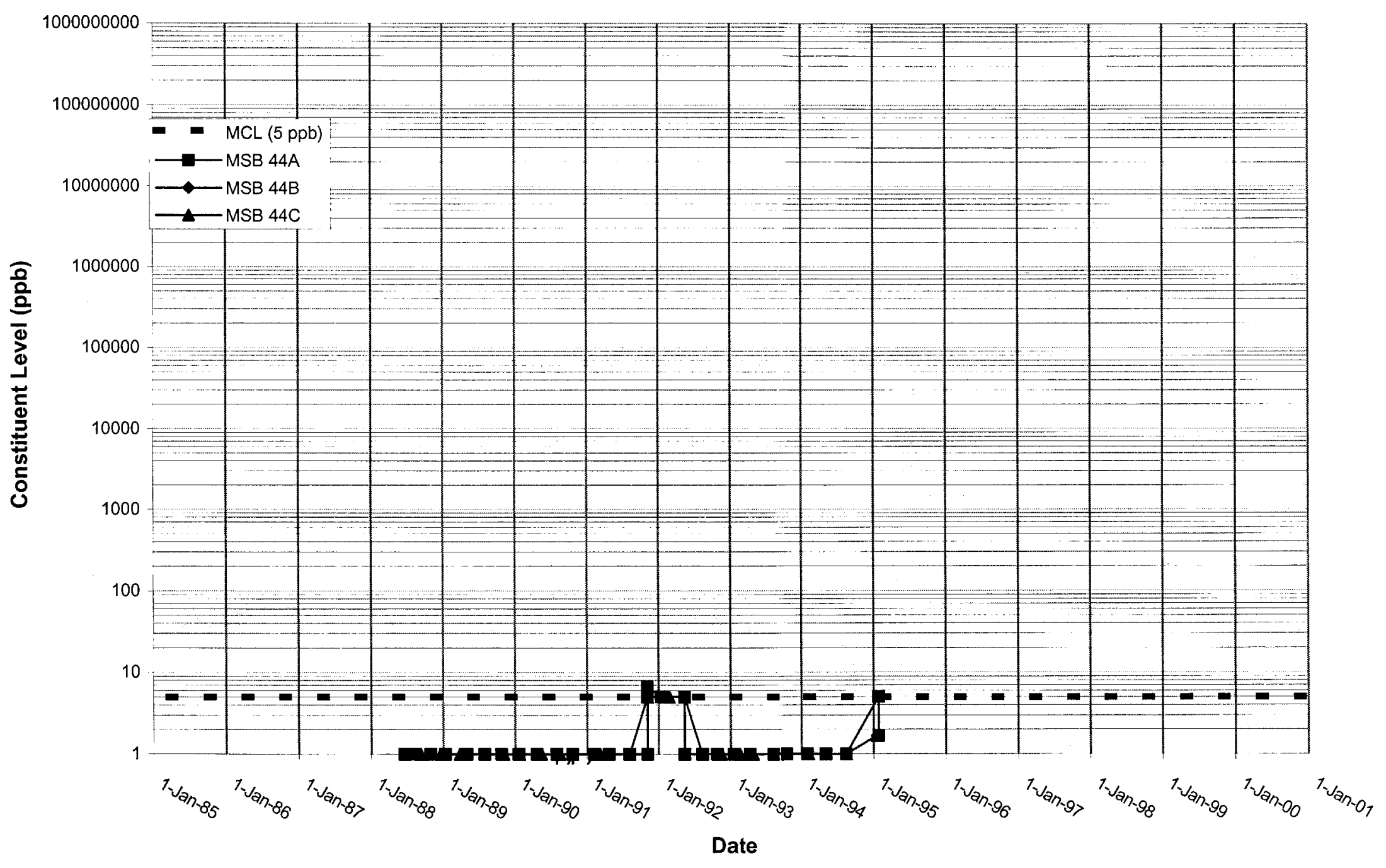


WSRC-TR-2000-00242 UNCLASSIFIED

\section{Time Series Plot of Tetrachloroethylene for Well MSB 45}

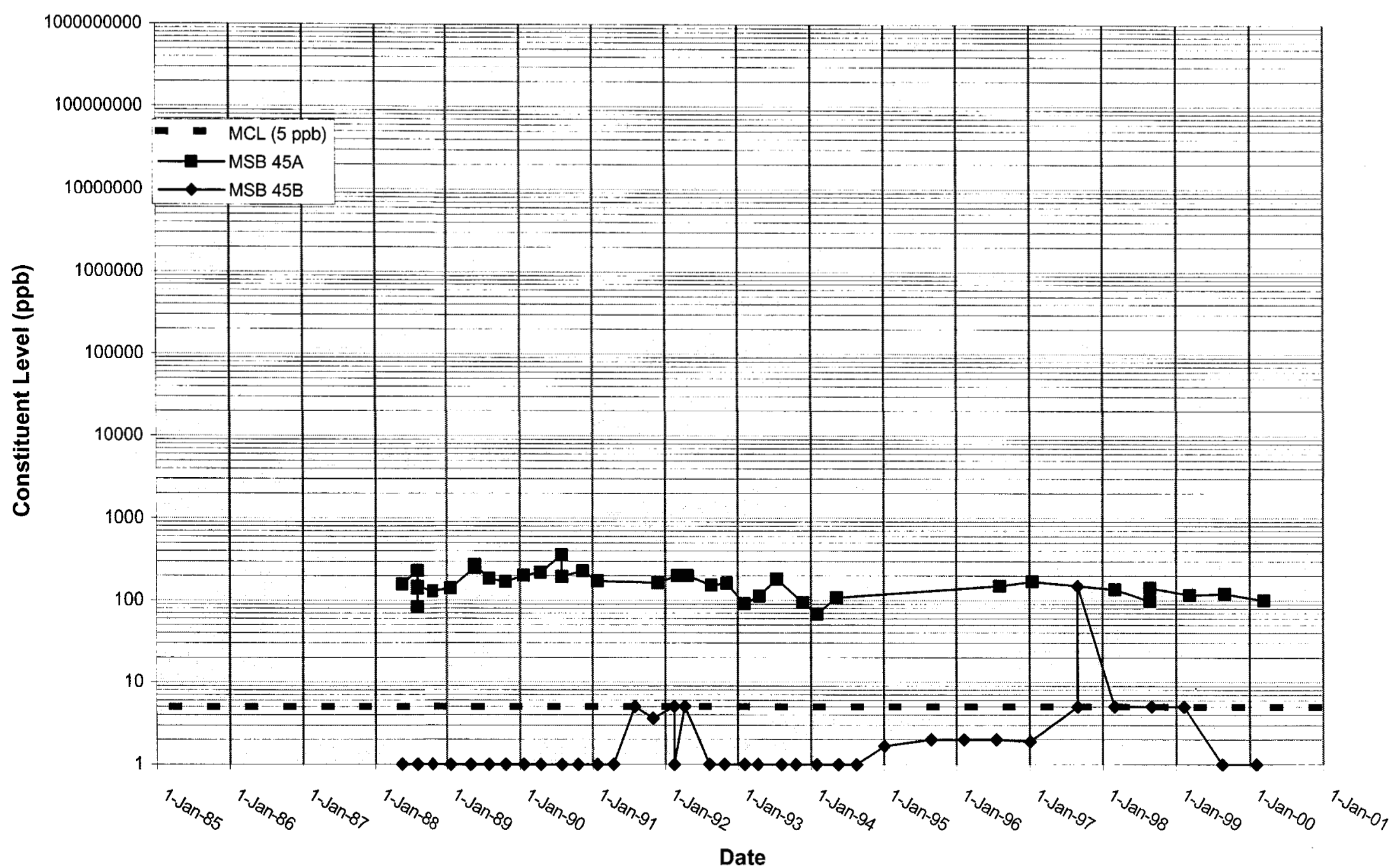


Time Series Plot of Tetrachloroethylene for Well MSB 46

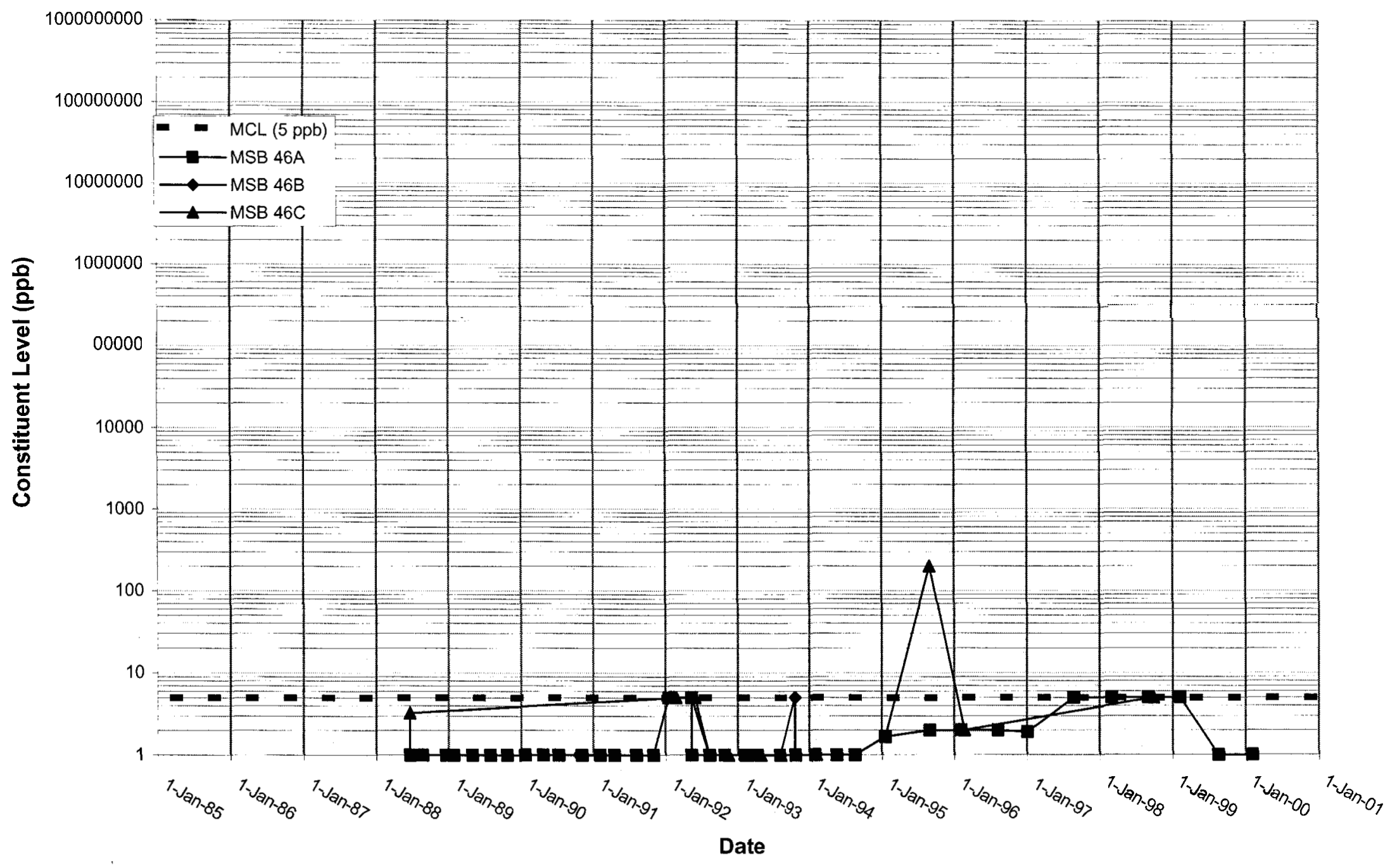


Time Series Plot of Tetrachloroethylene for Well MSB 47

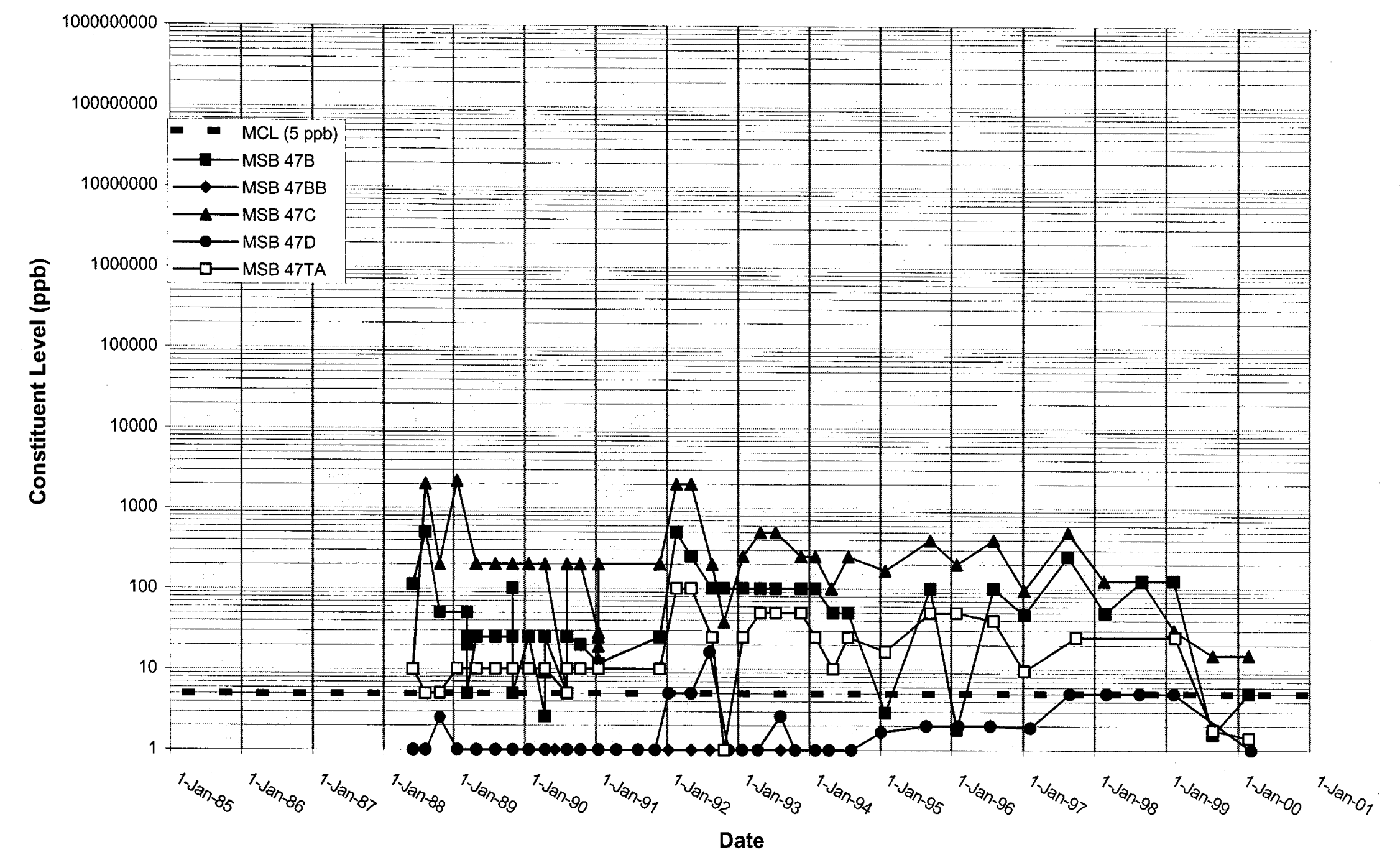


WSRC-TR-2000-00242 UNCLASSIFIED

Time Series Plot of Tetrachloroethylene for Well MSB 48

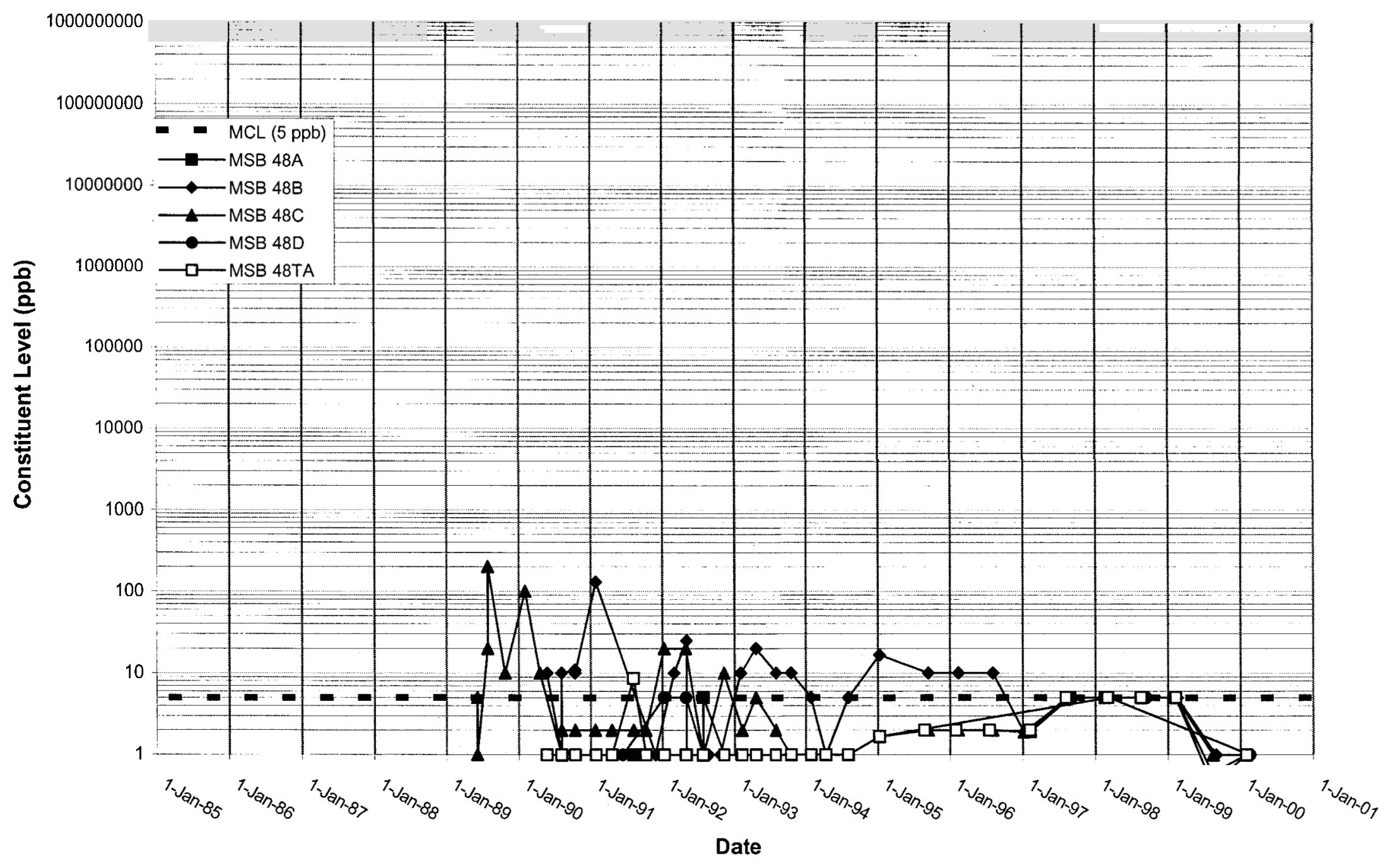


WSRC-TR-2000-00242 UNCLASSIFIED

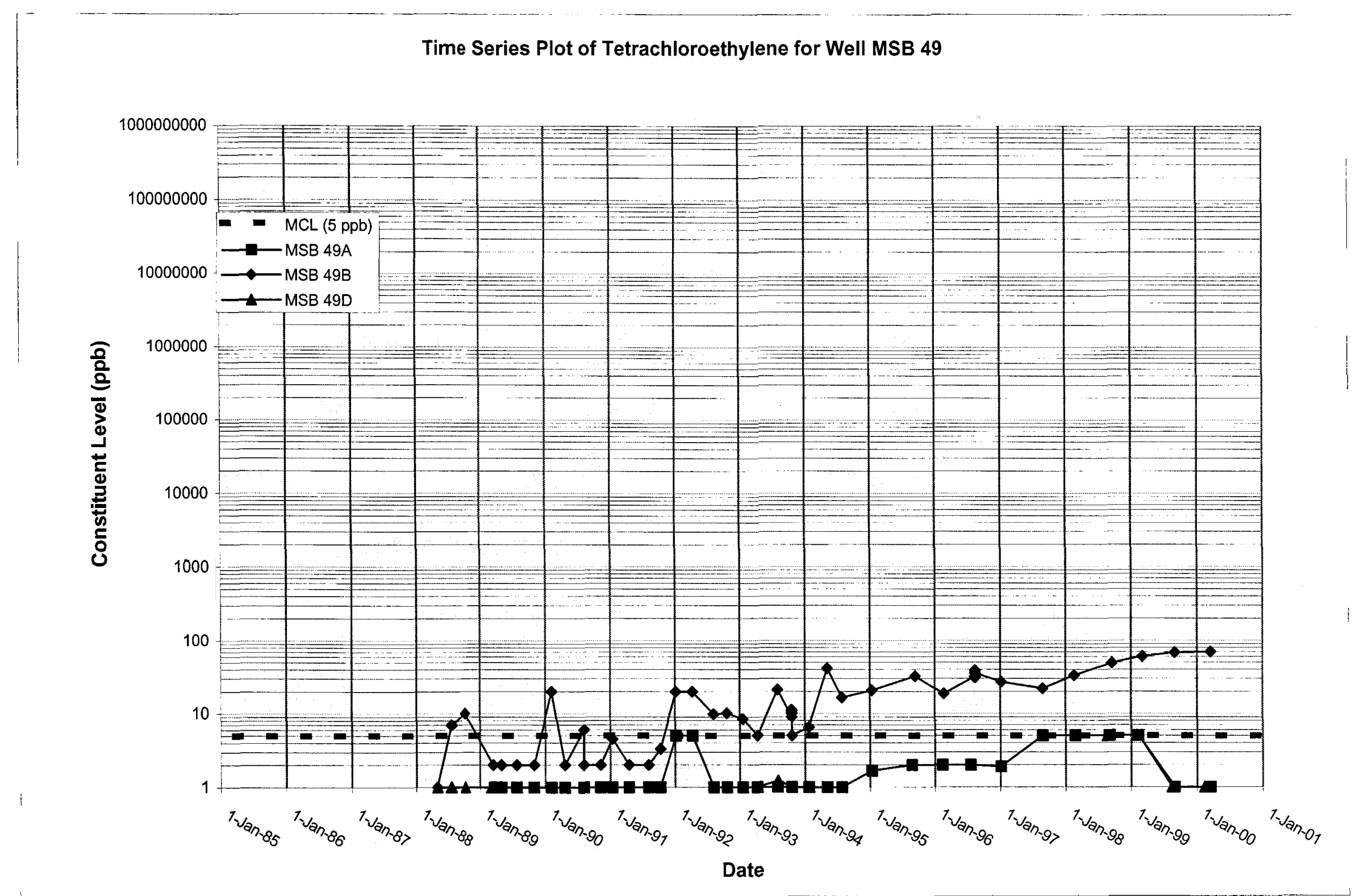




\section{Time Series Plot of Tetrachloroethylene for Well MSB 50}

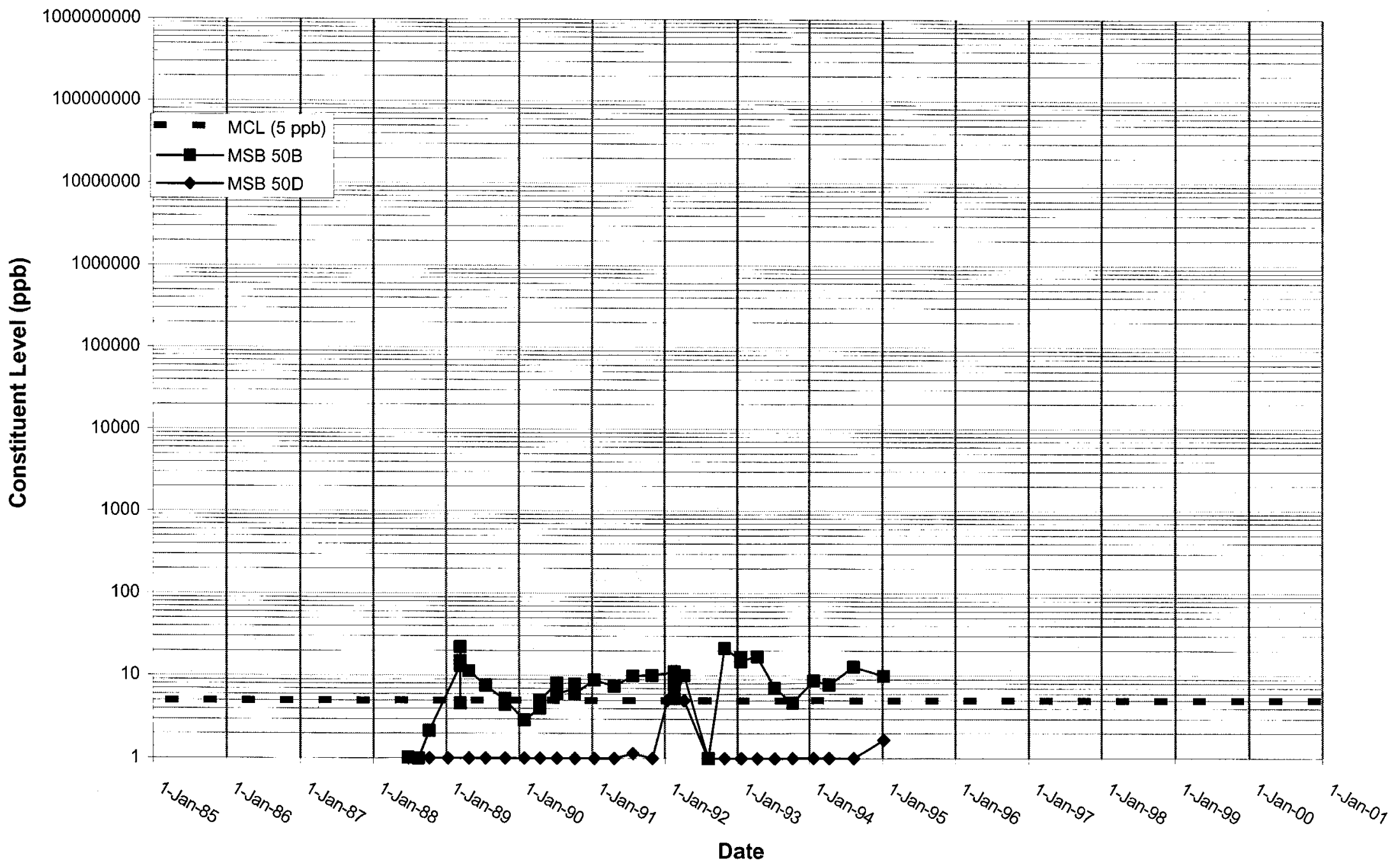




\section{Time Series Plot of Tetrachloroethylene for Well MSB 51}

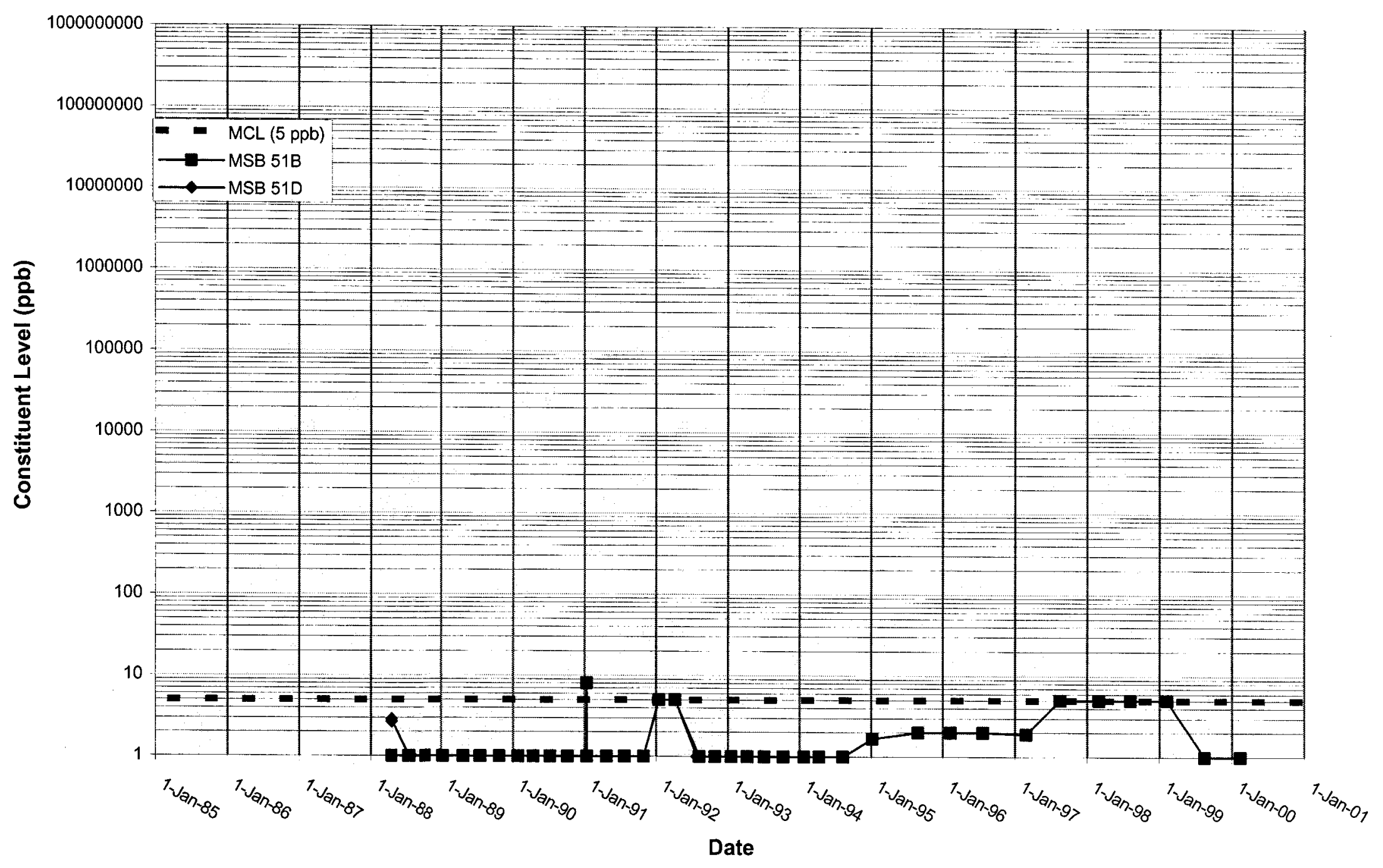


WSRC-TR-2000-00242 UNCLASSIFIED

Time Series Plot of Tetrachloroethylene for Well MSB 52

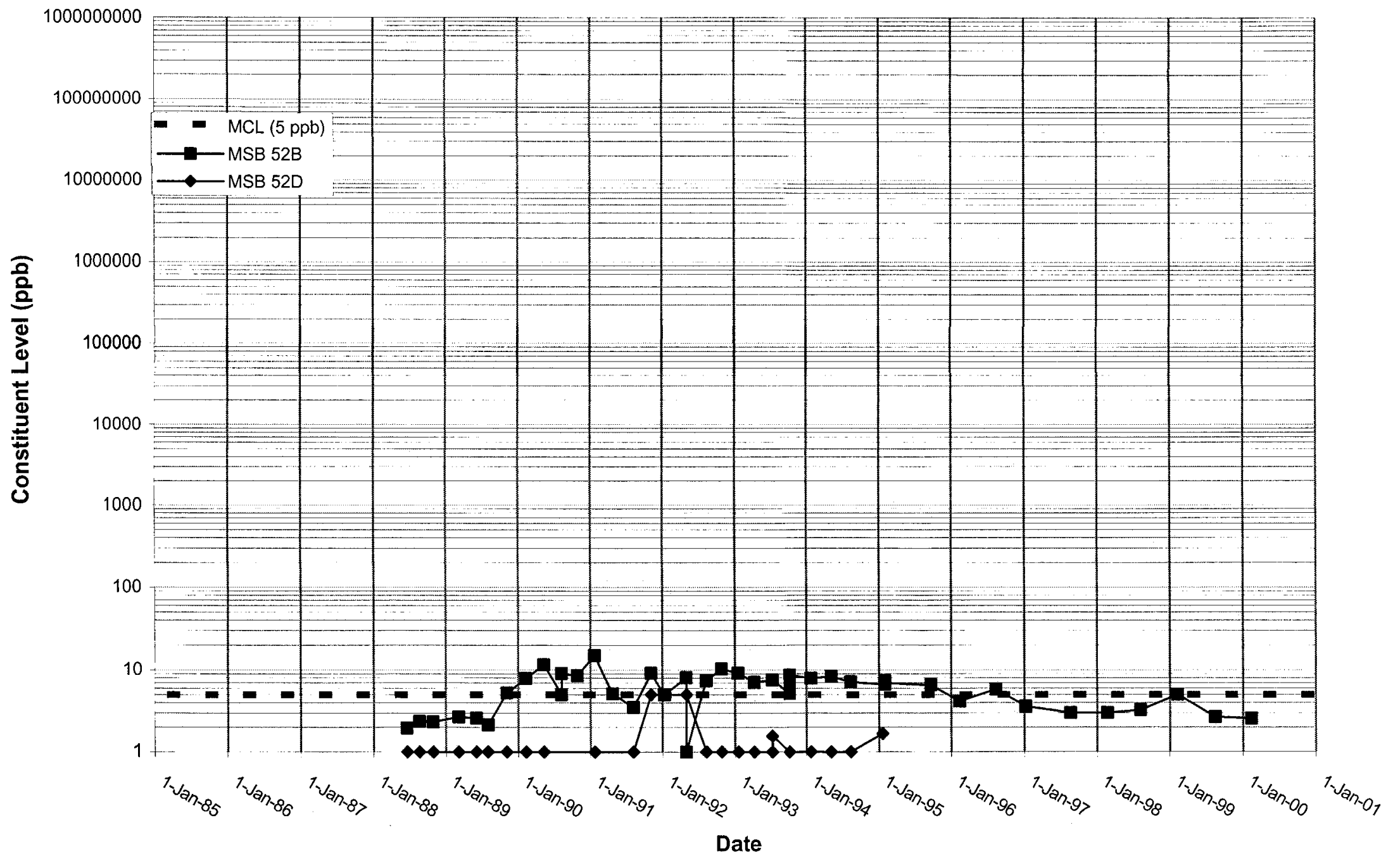




\section{Time Series Plot of Tetrachloroethylene for Well MSB 53}

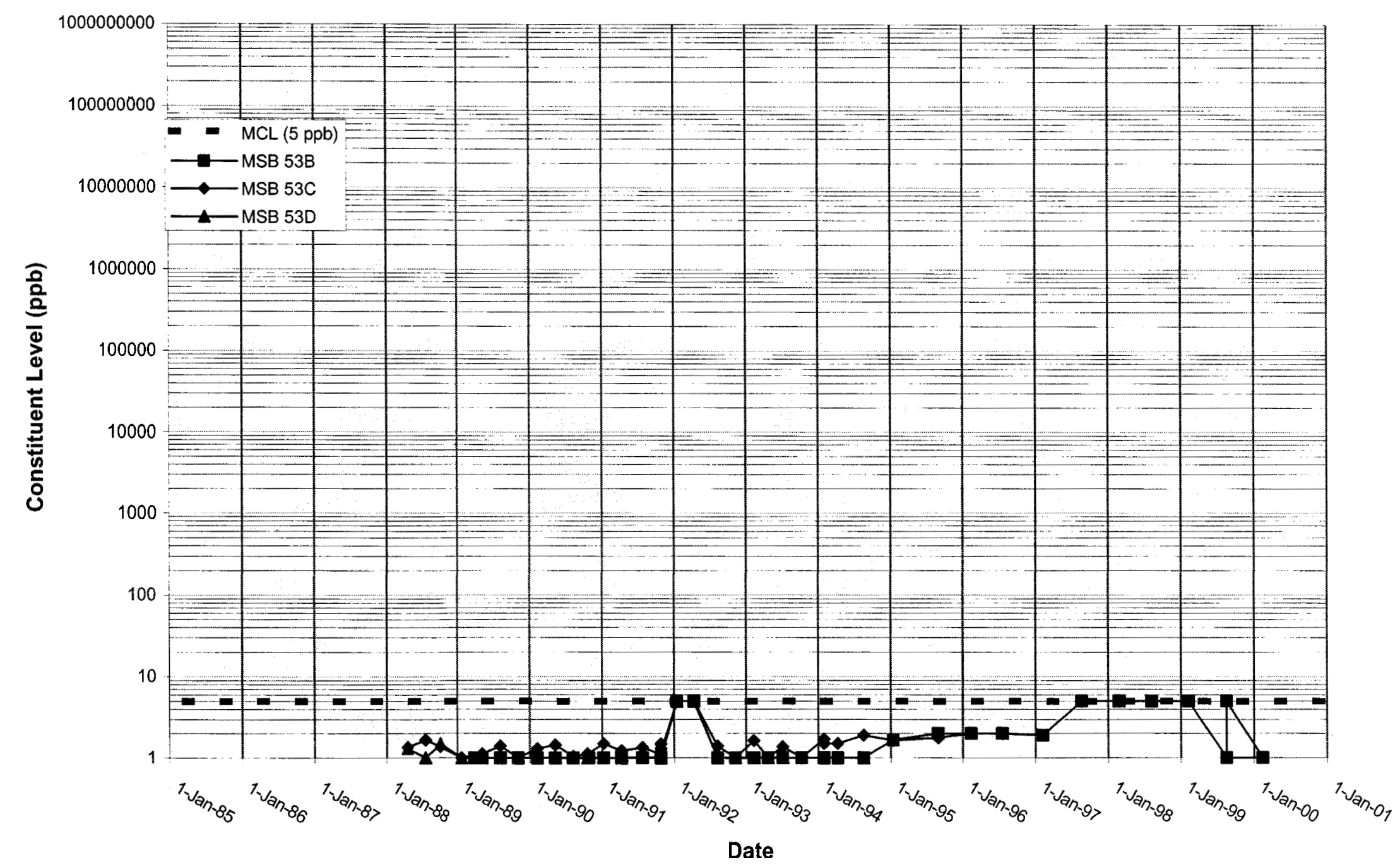


WSRC-TR-2000-00242 UNCLASSIFIED

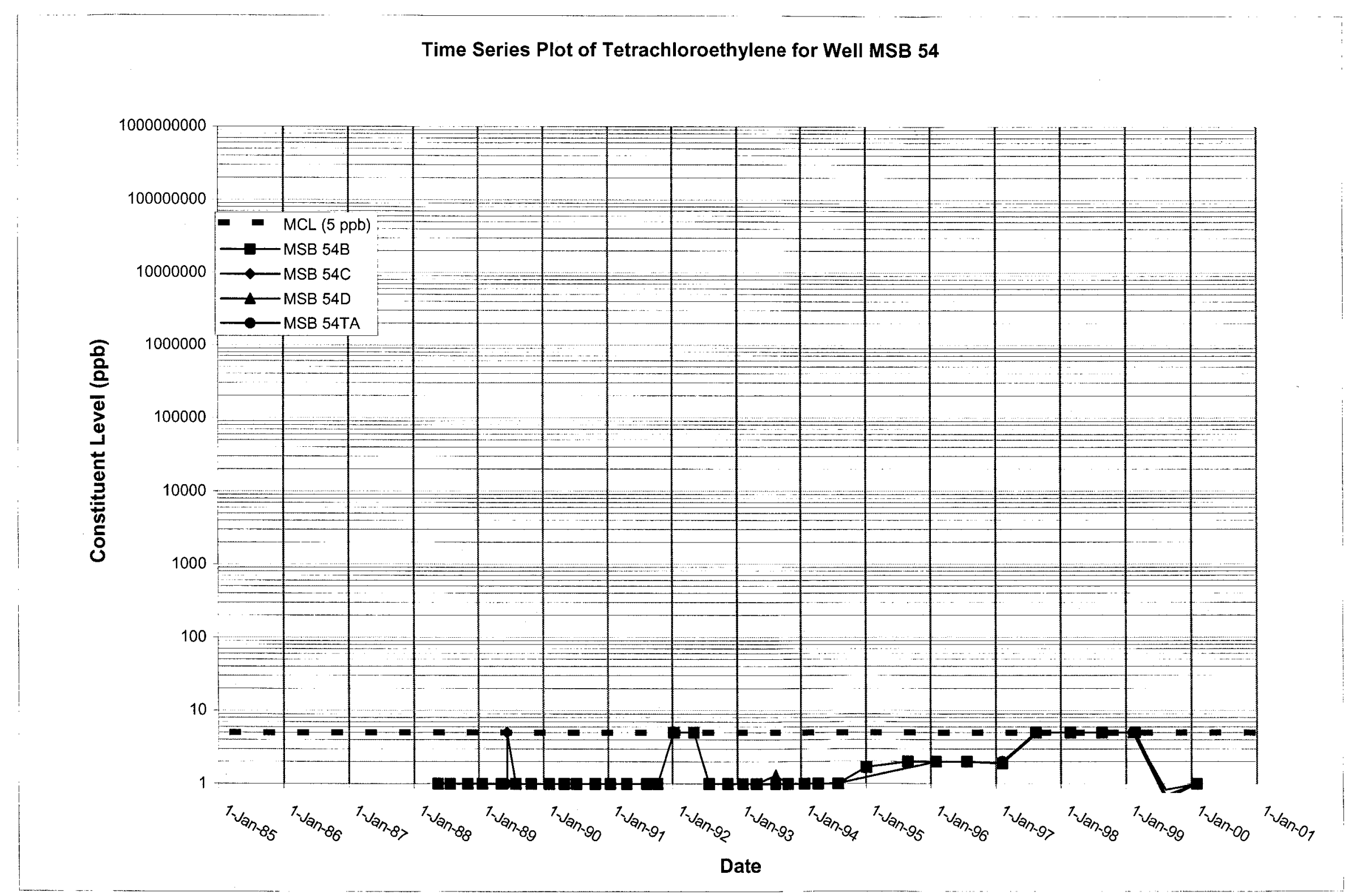




\section{Time Series Plot of Tetrachloroethylene for Well MSB 55}

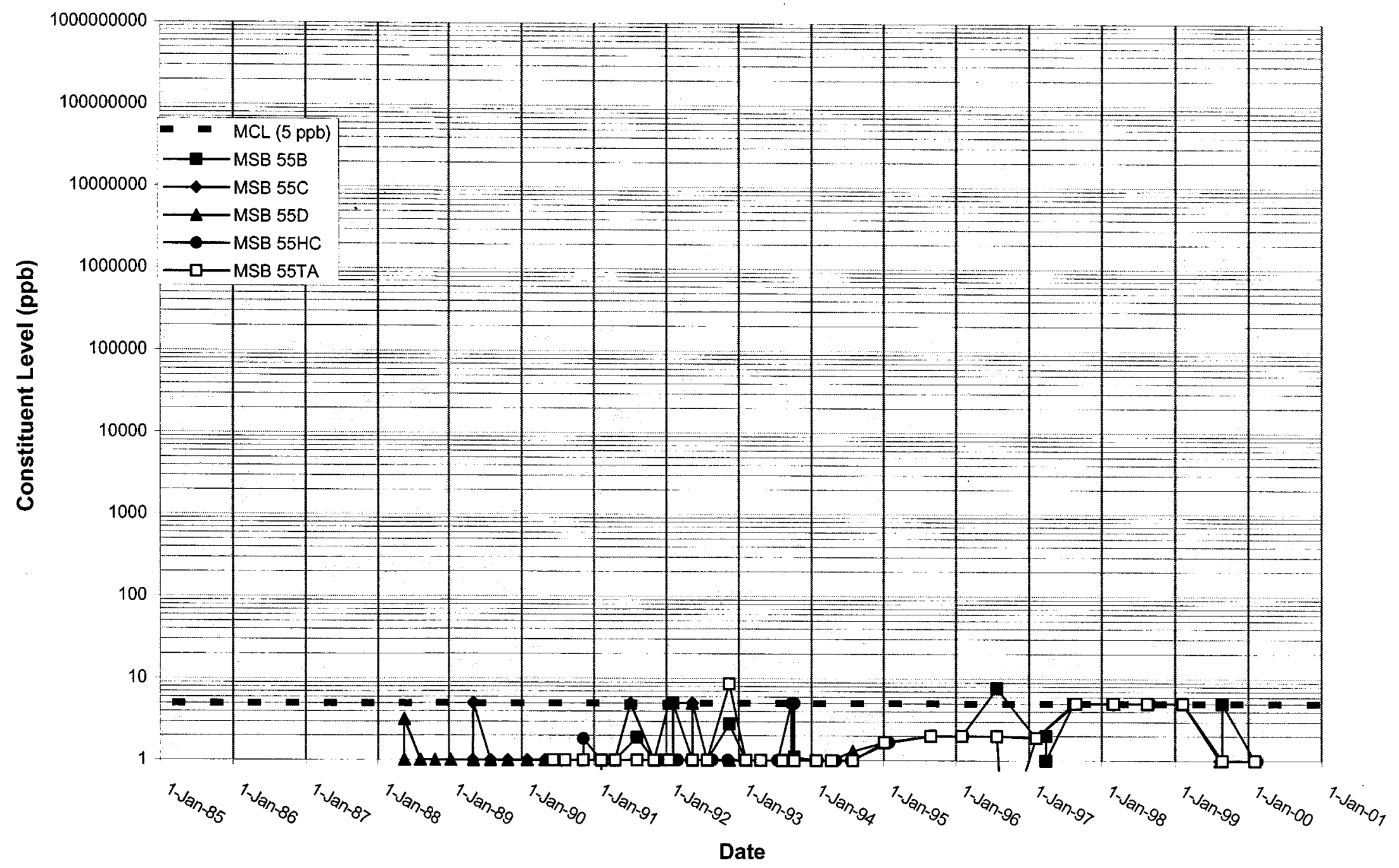


WSRC-TR-2000-00242 UNCLASSIFIED

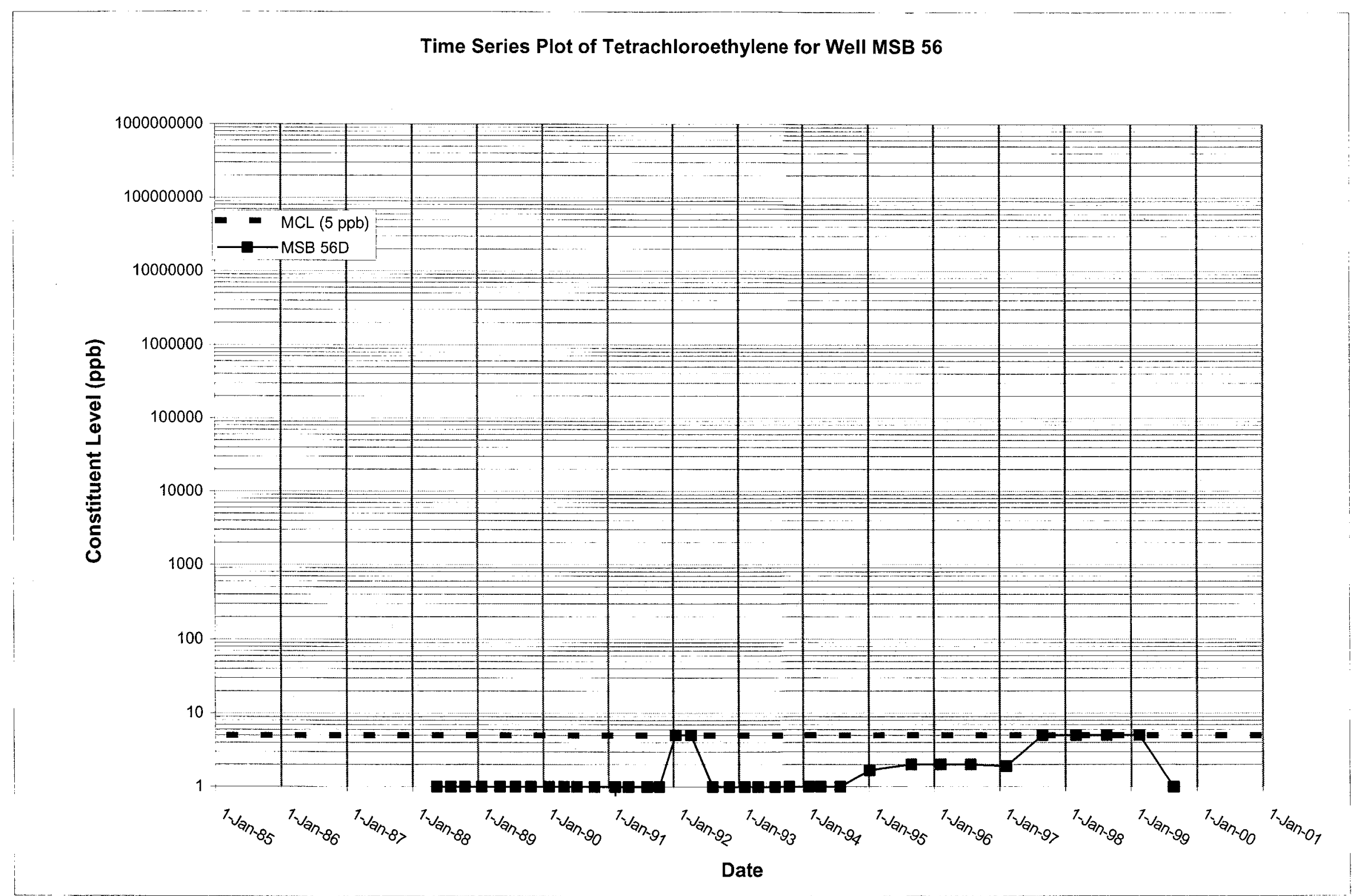


Time Series Plot of Tetrachloroethylene for Well MSB 57

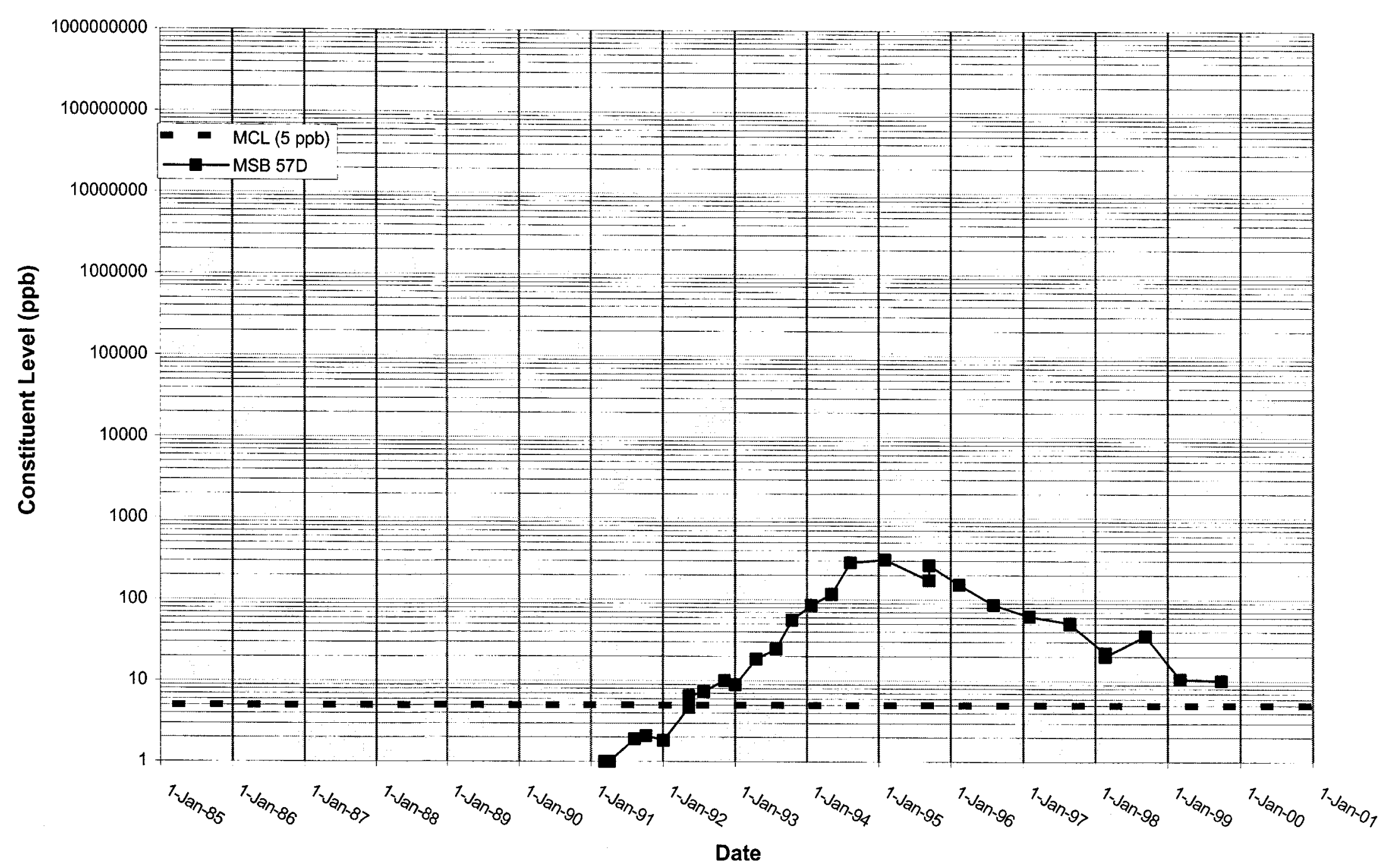


WSRC-TR-2000-00242 UNCLASSIFIED

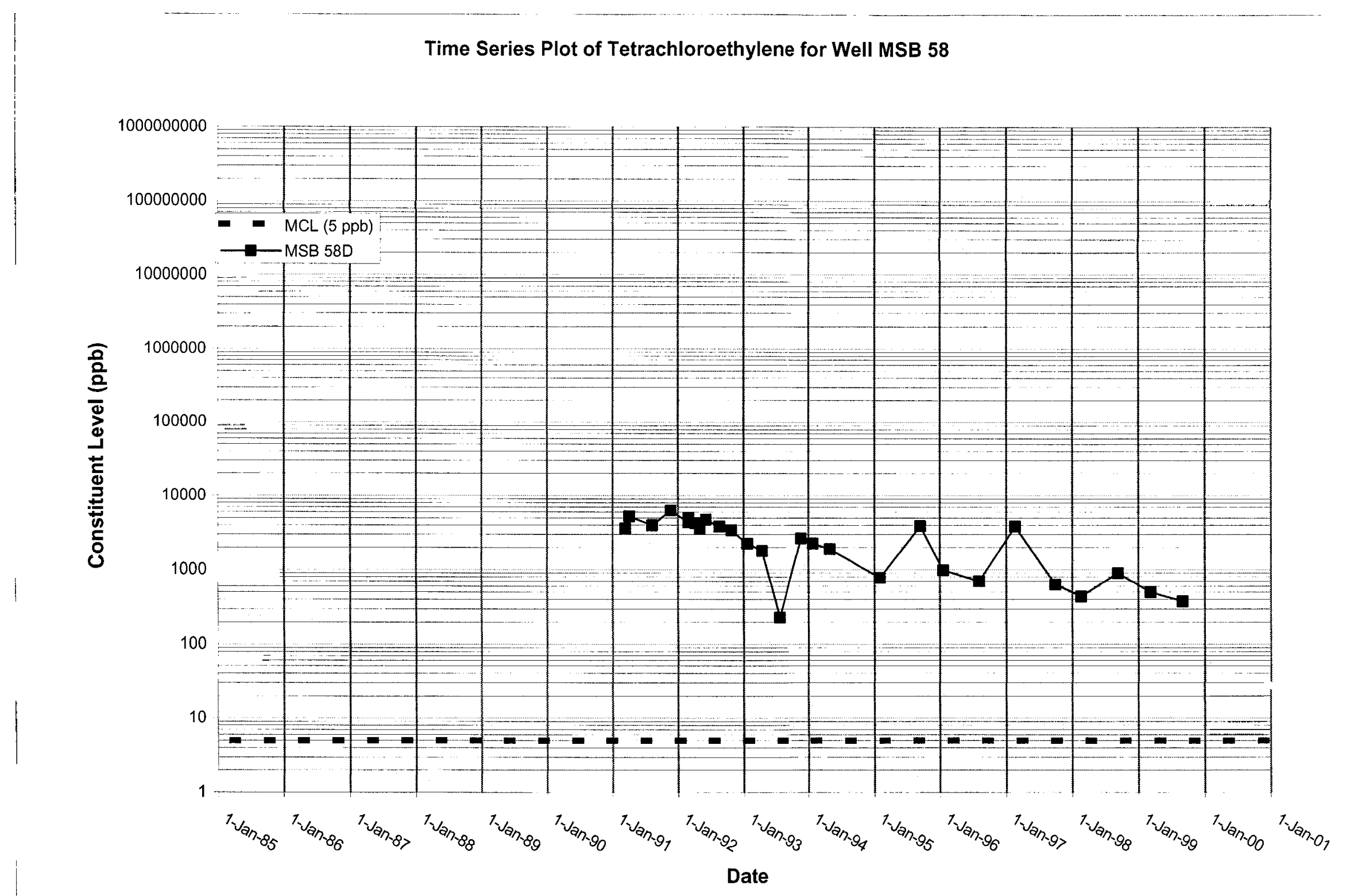




\section{Time Series Plot of Tetrachloroethylene for Well MSB 59}

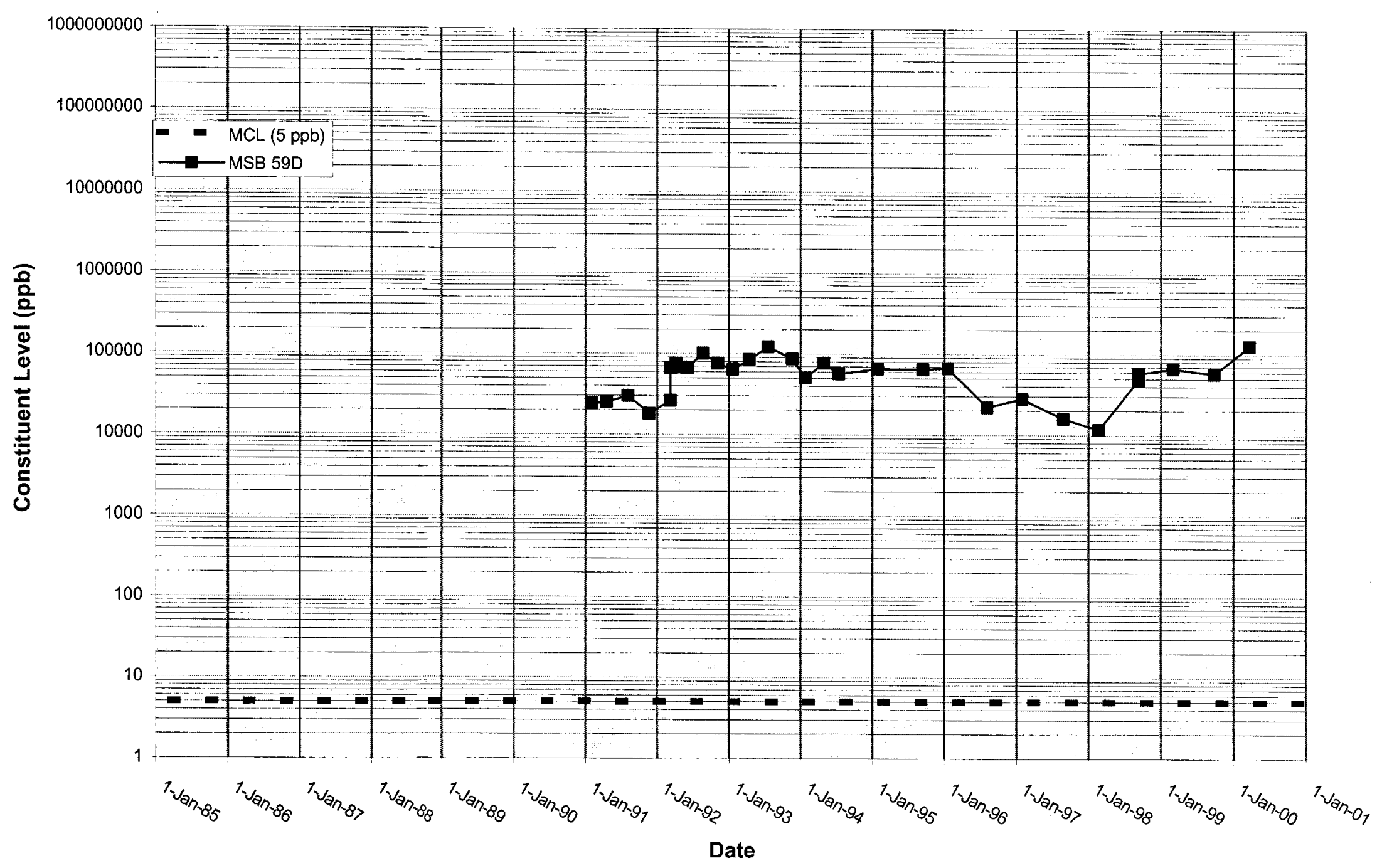


WSRC-TR-2000-00242 UNCLASSIFIED

Time Series Plot of Tetrachloroethylene for Well MSB 60

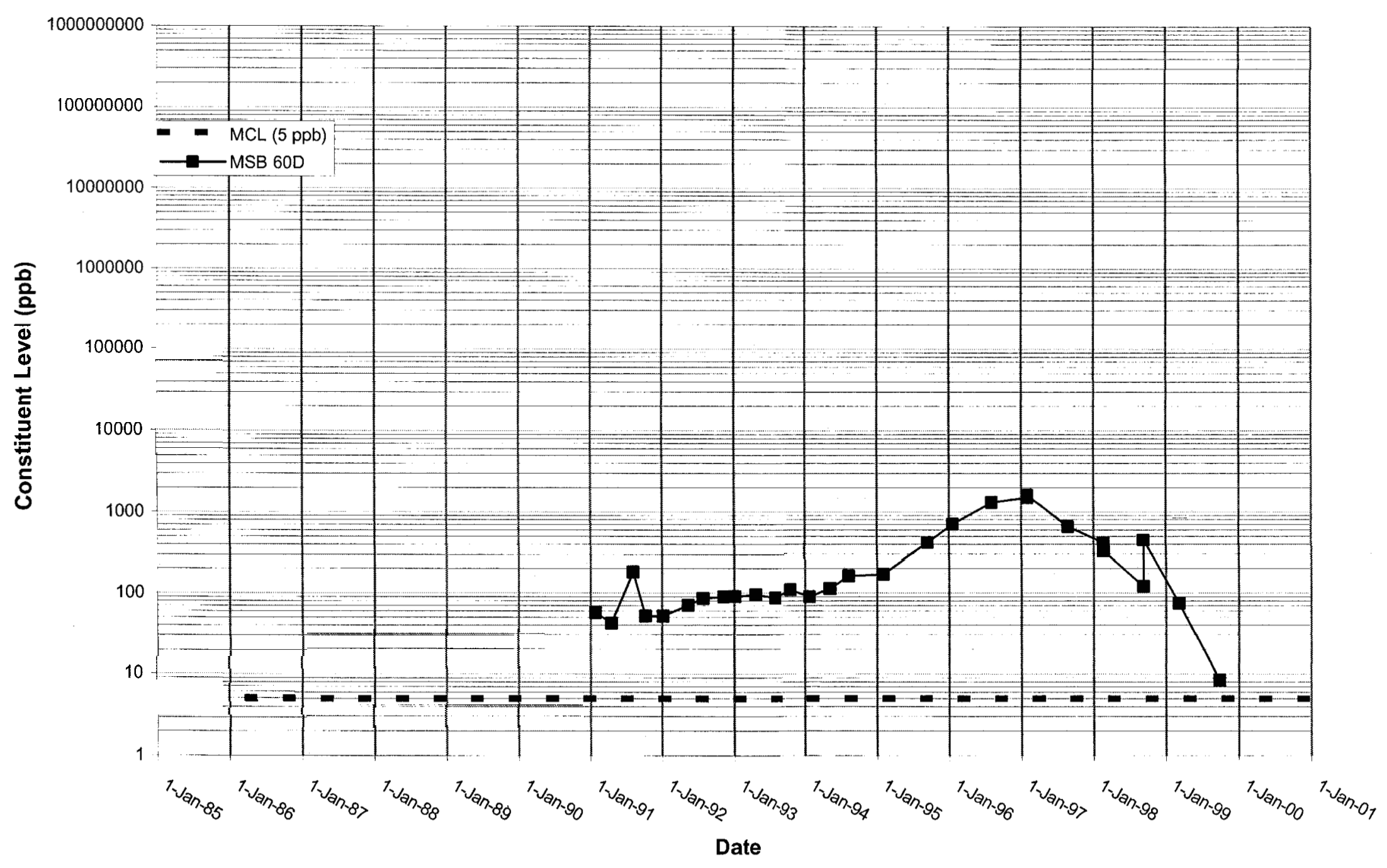


Time Series Plot of Tetrachloroethylene for Well MSB 61

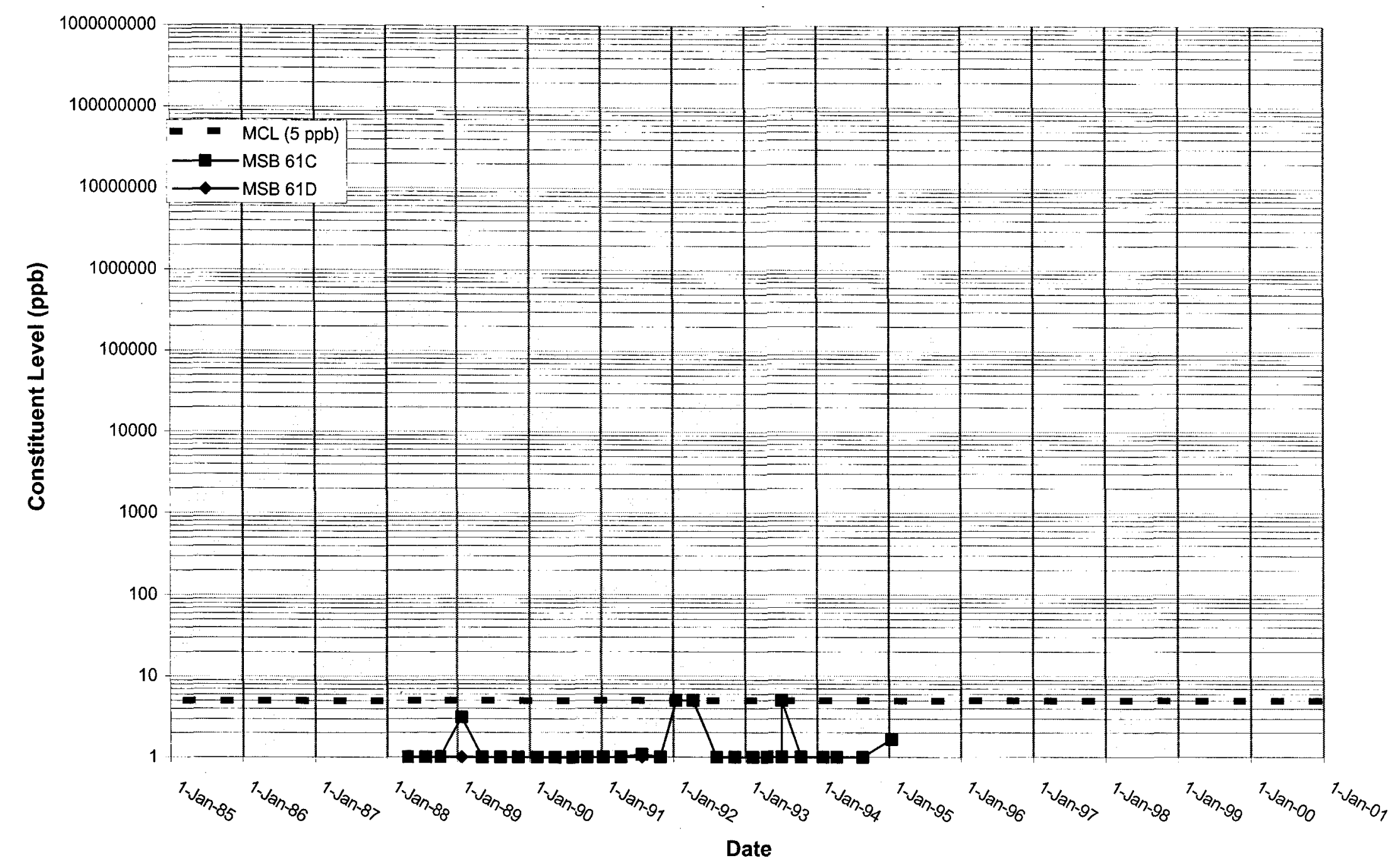


WSRC-TR-2000-00242 UNCLASSIFIED

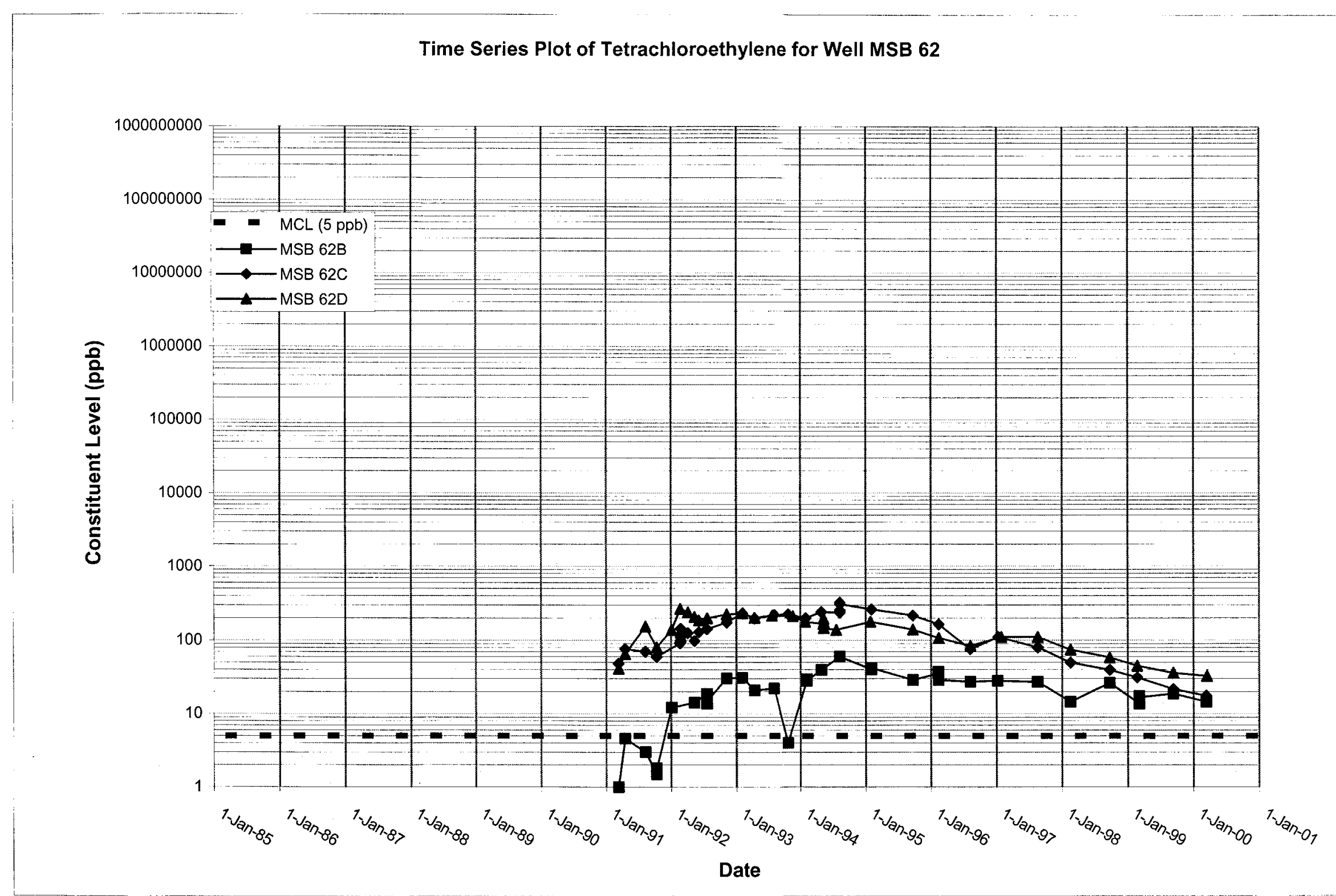


Time Series Plot of Tetrachloroethylene for Well MSB 63

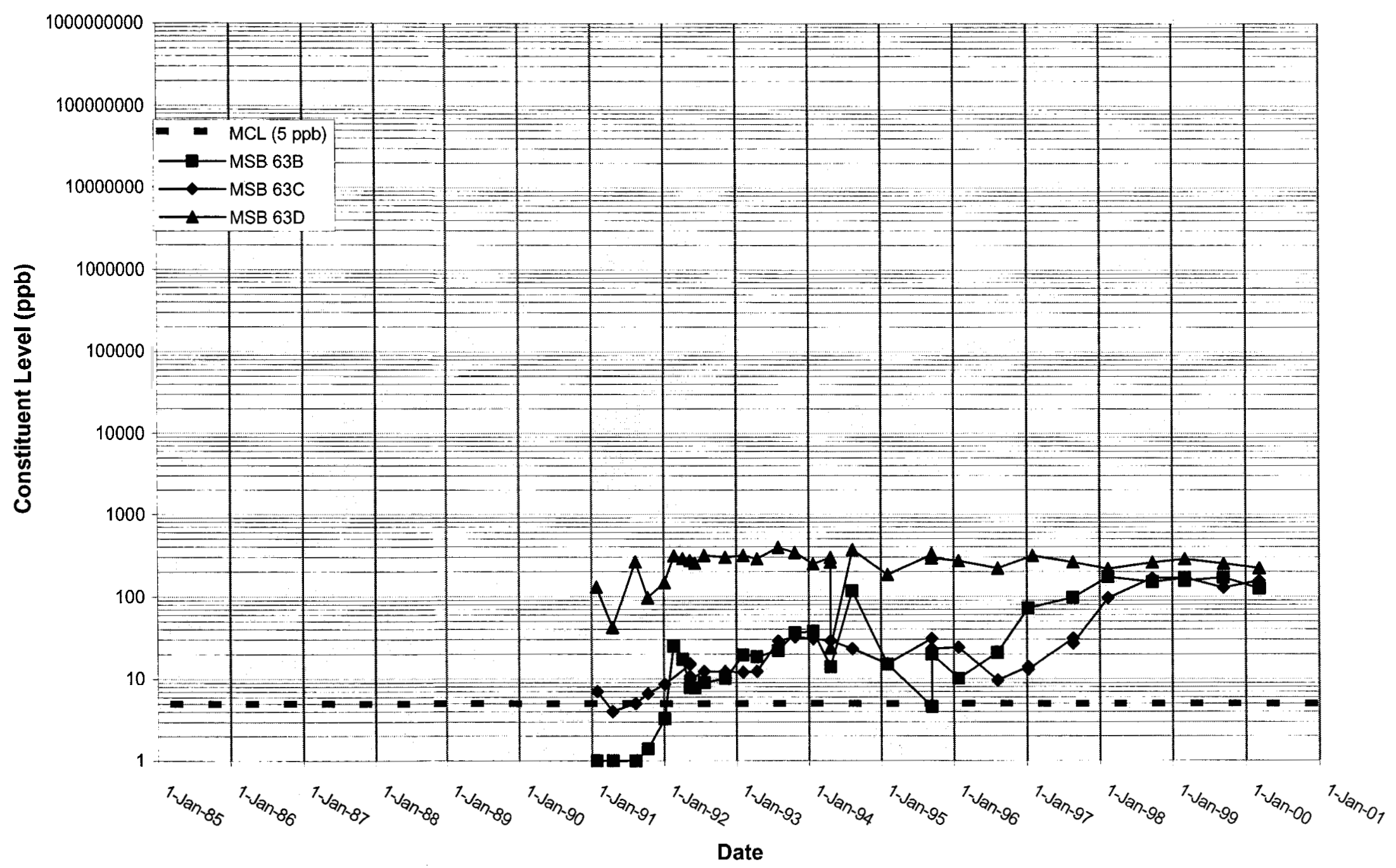




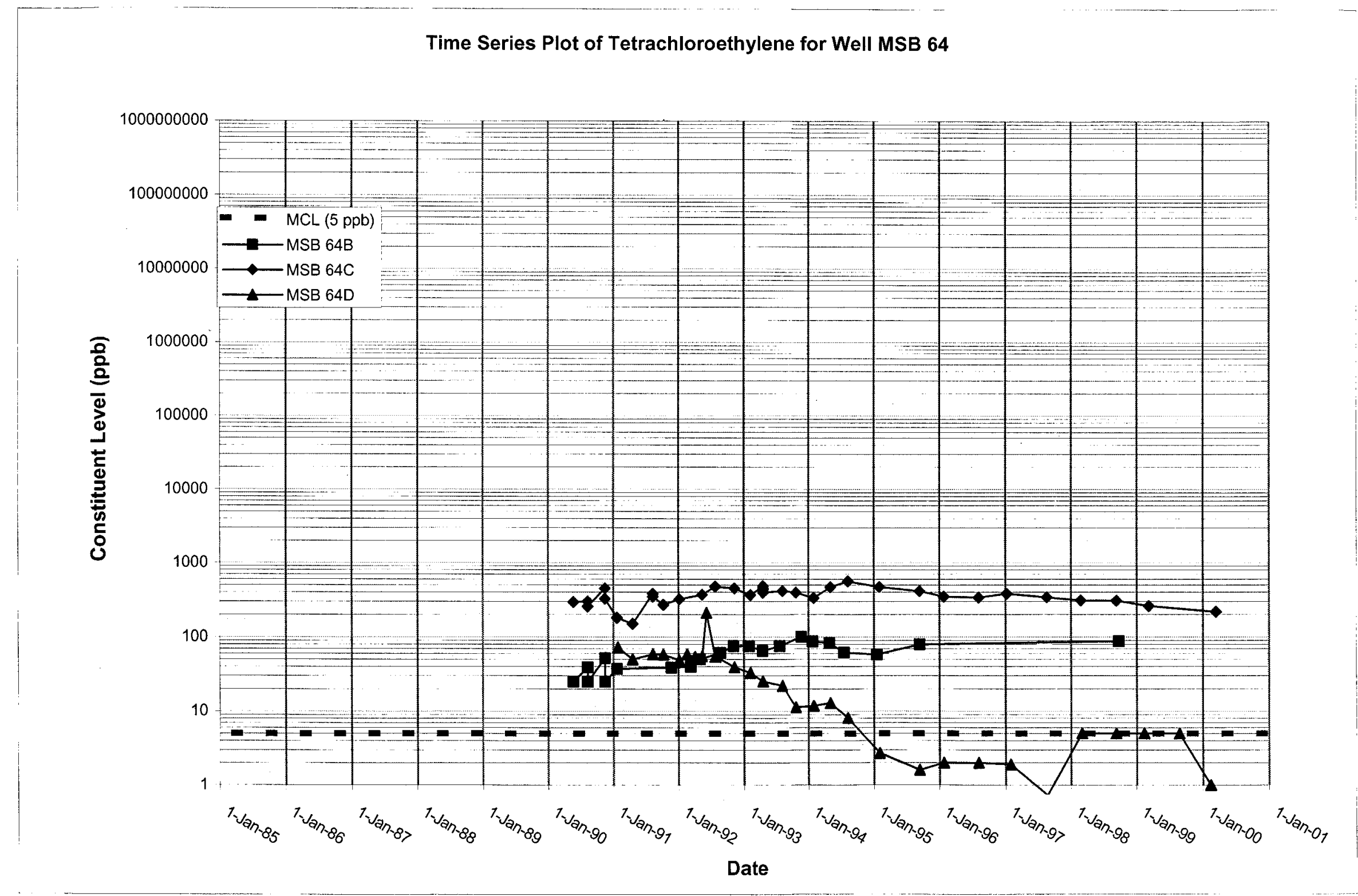




\section{Time Series Plot of Tetrachloroethylene for Well MSB 65}

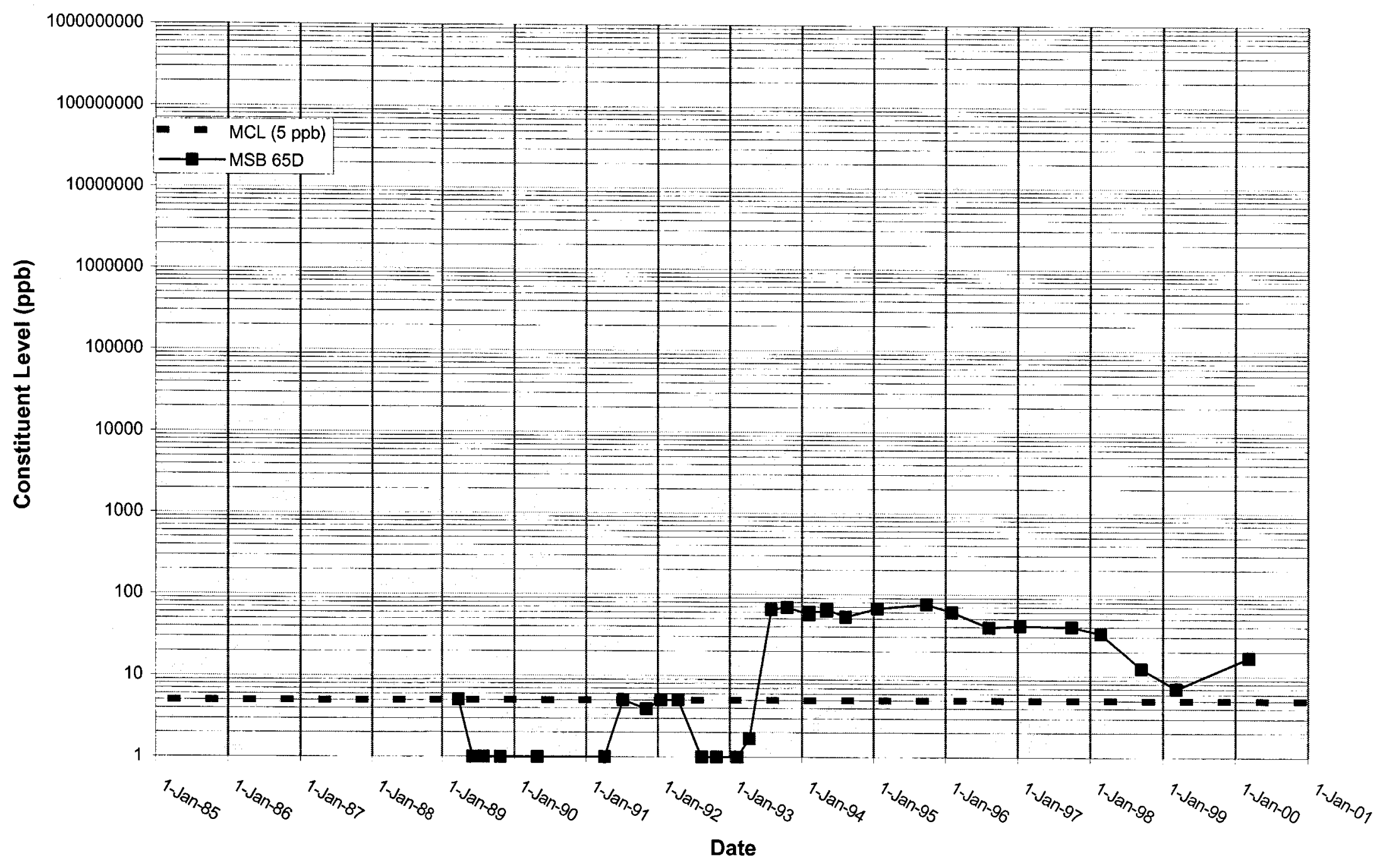


WSRC-TR-2000-00242 UNCLASSIFIED

Time Series Plot of Tetrachloroethylene for Well MSB 66

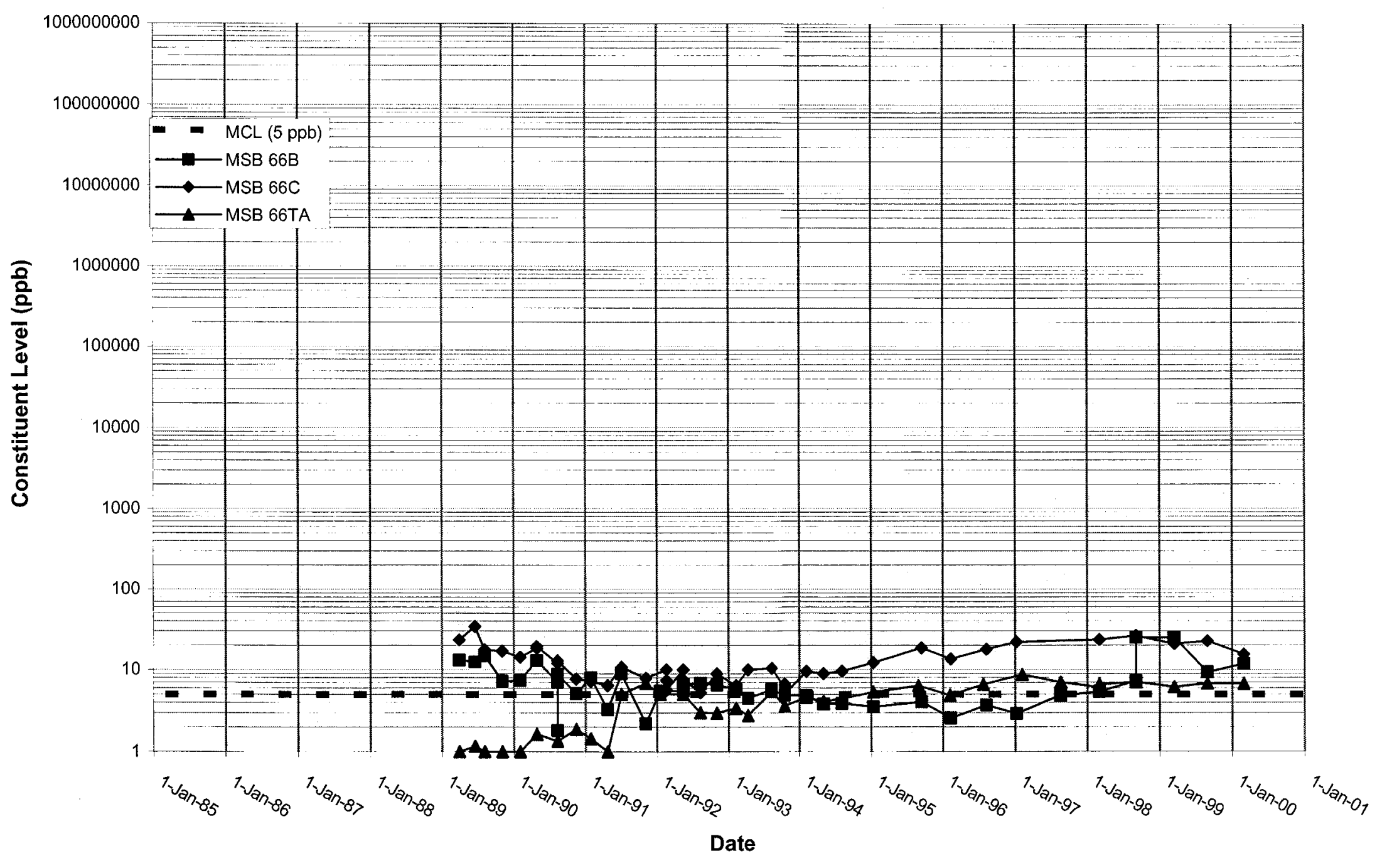




\section{Time Series Plot of Tetrachloroethylene for Well MSB 67}

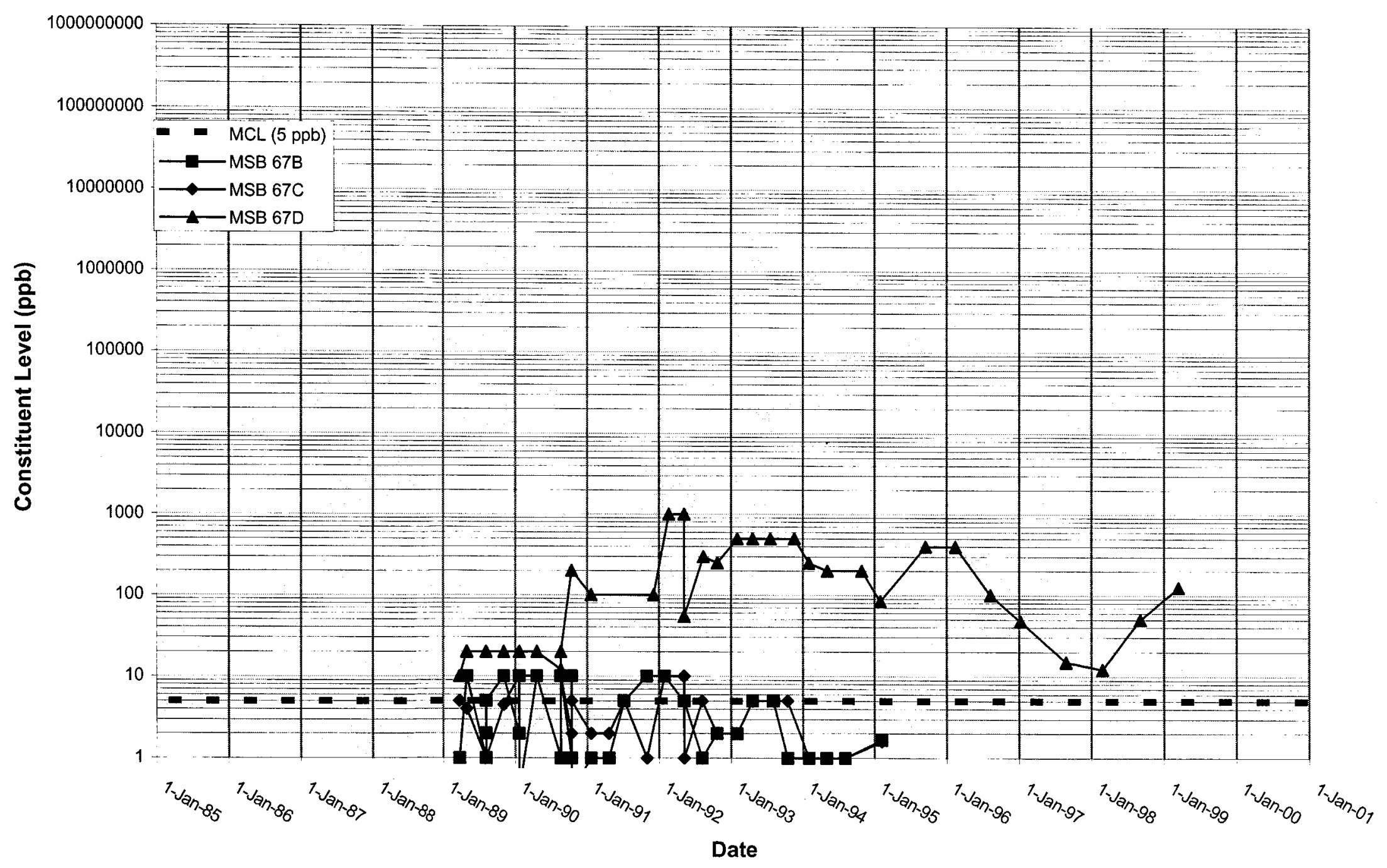


WSRC-TR-2000-00242 UNCLASSIFIED

\section{Time Series Plot of Tetrachloroethylene for Well MSB 68}

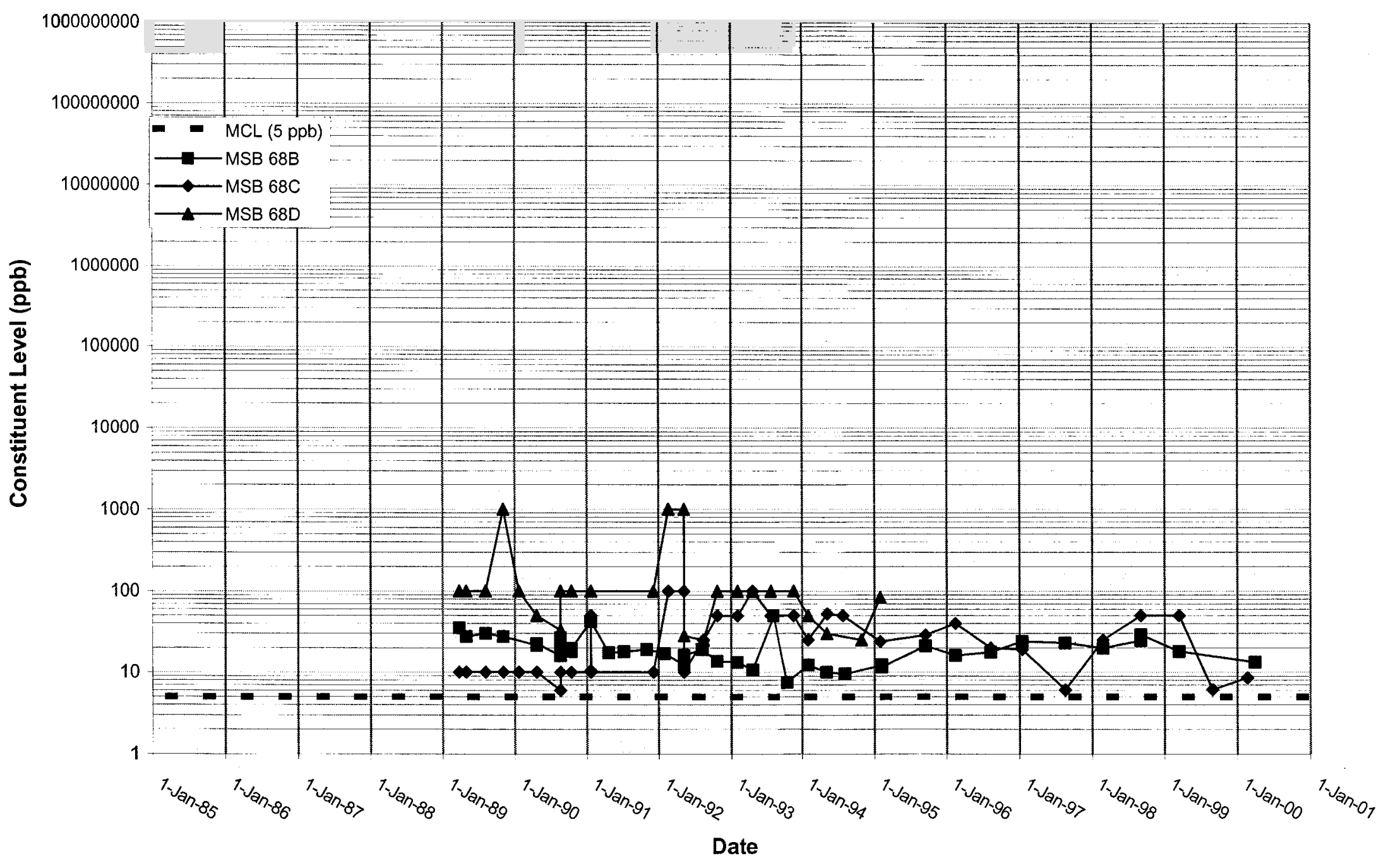


Time Series Plot of Tetrachloroethylene for Well MSB 69

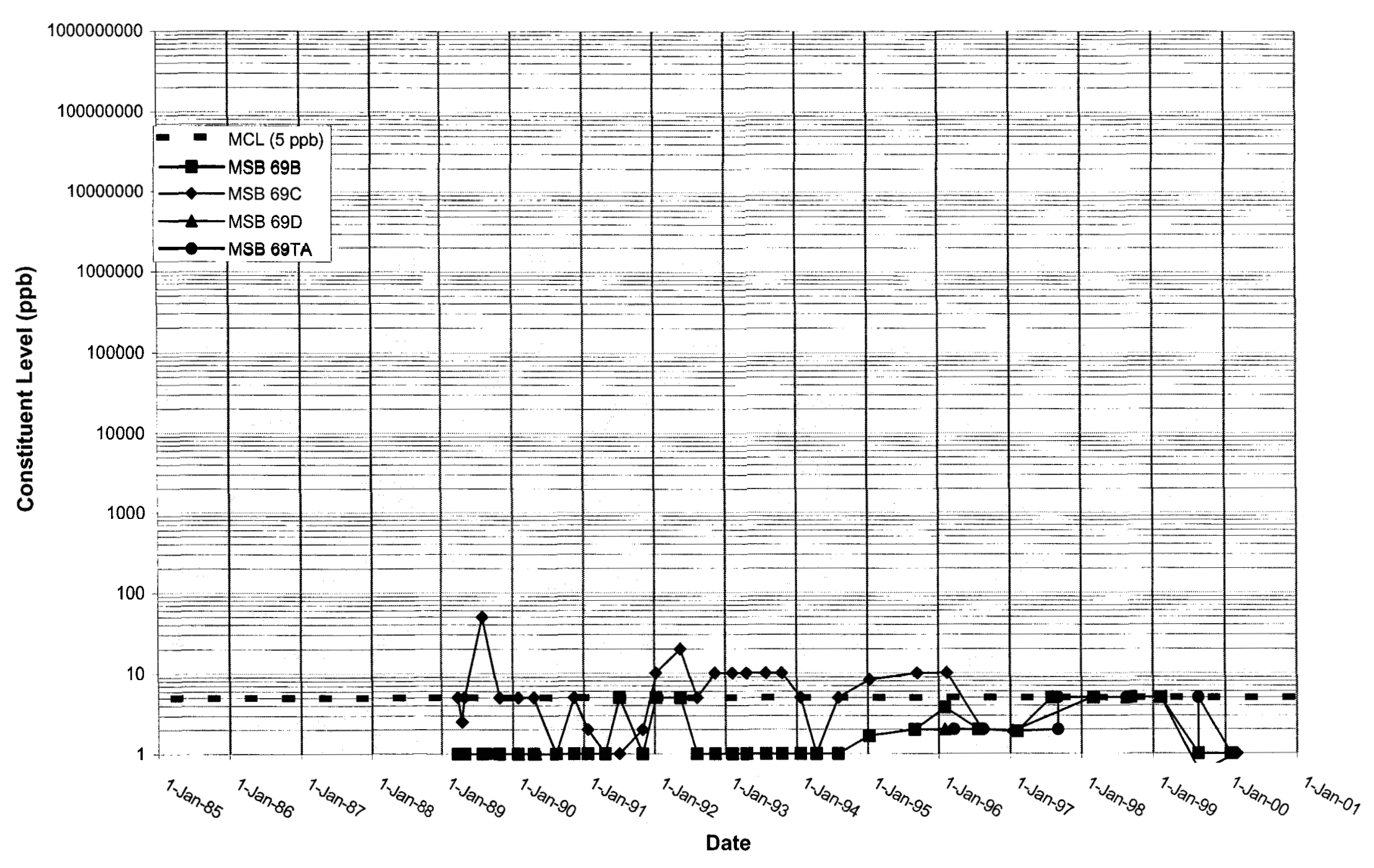


WSRC-TR-2000-00242 UNCLASSIFIED

\section{Time Series Plot of Tetrachloroethylene for Well MSB 70}

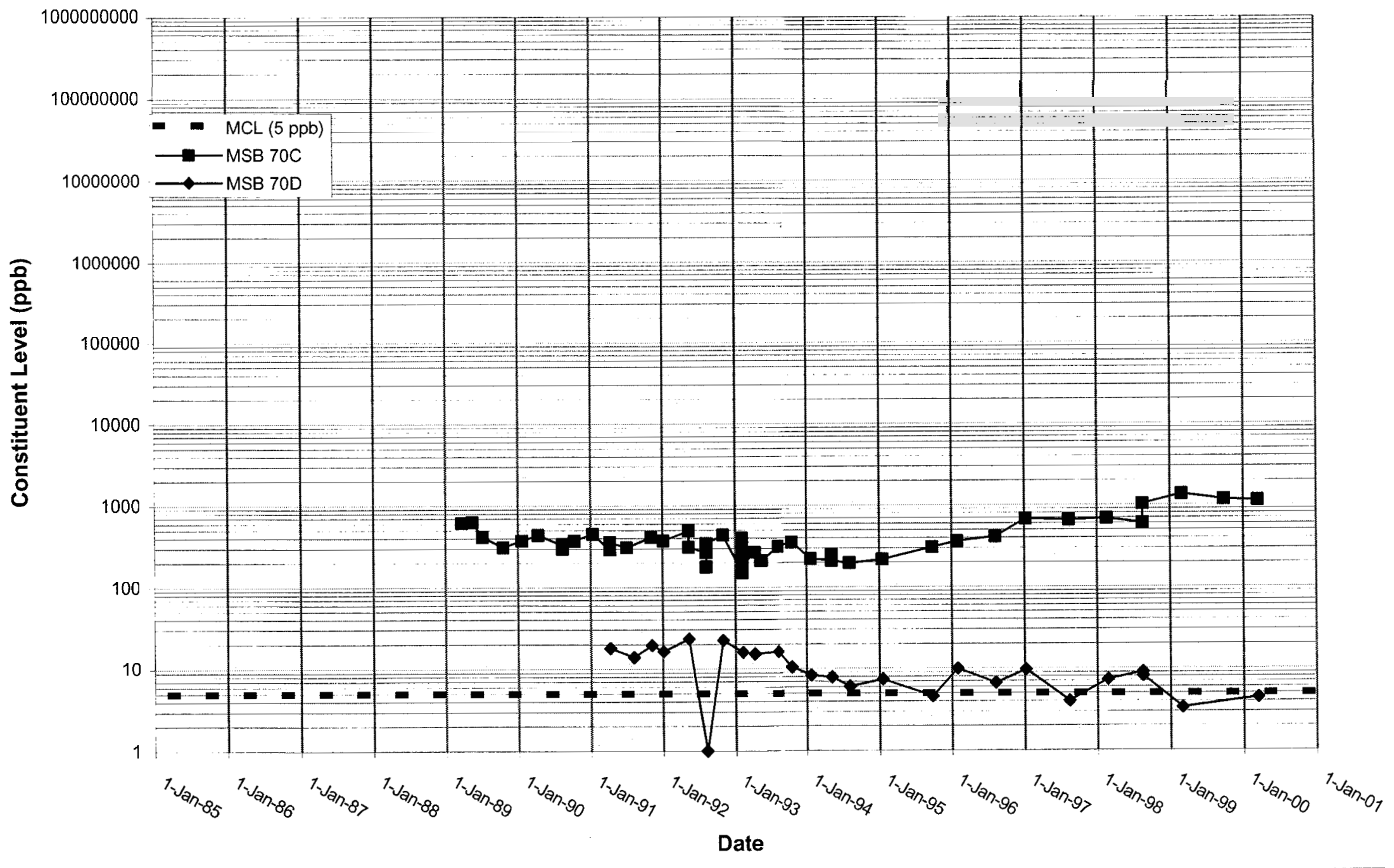




\section{Time Series Plot of Tetrachloroethylene for Well MSB 71}

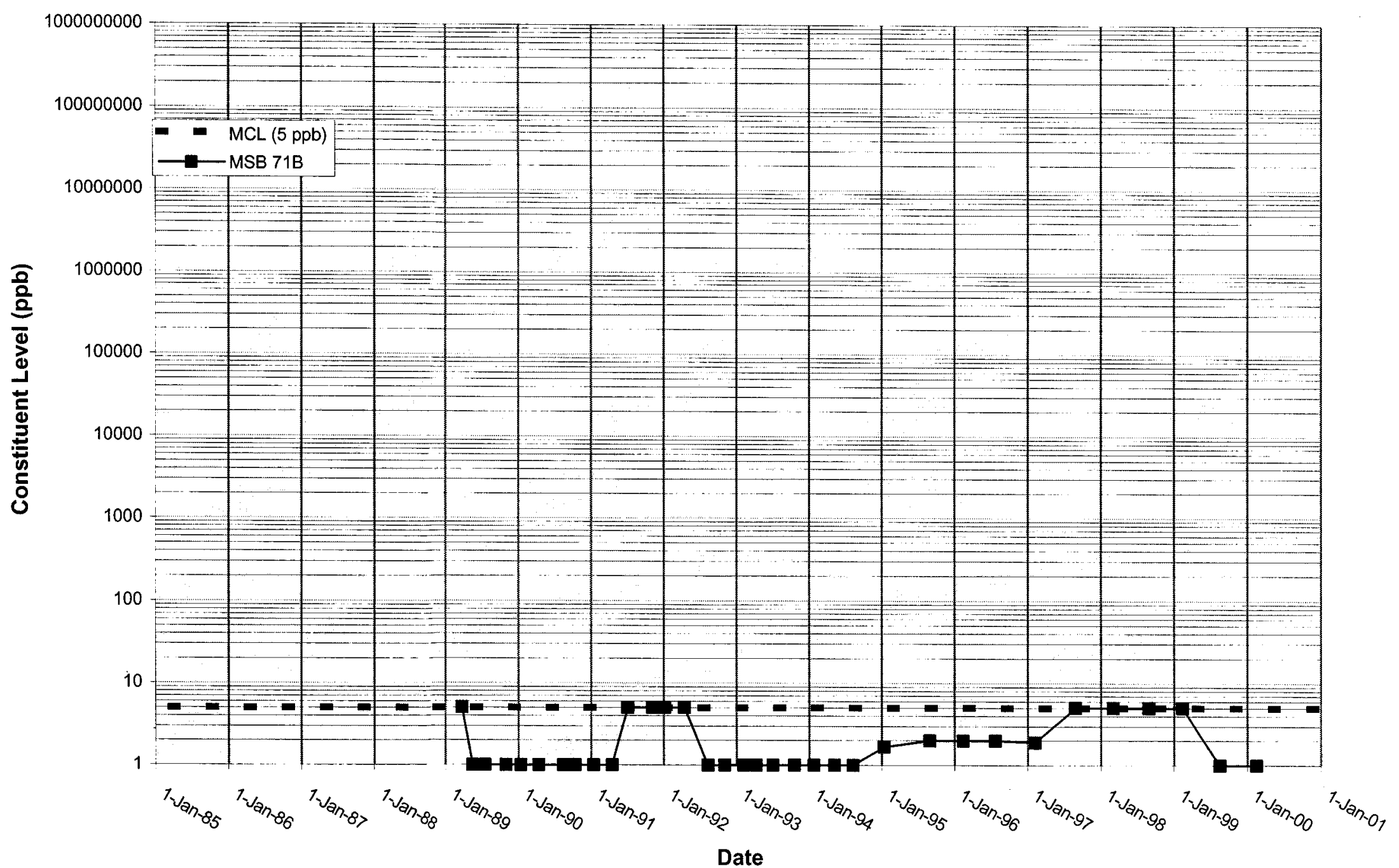


WSRC-TR-2000-00242 UNCLASSIFIED

Time Series Plot of Tetrachloroethylene for Well MSB 72

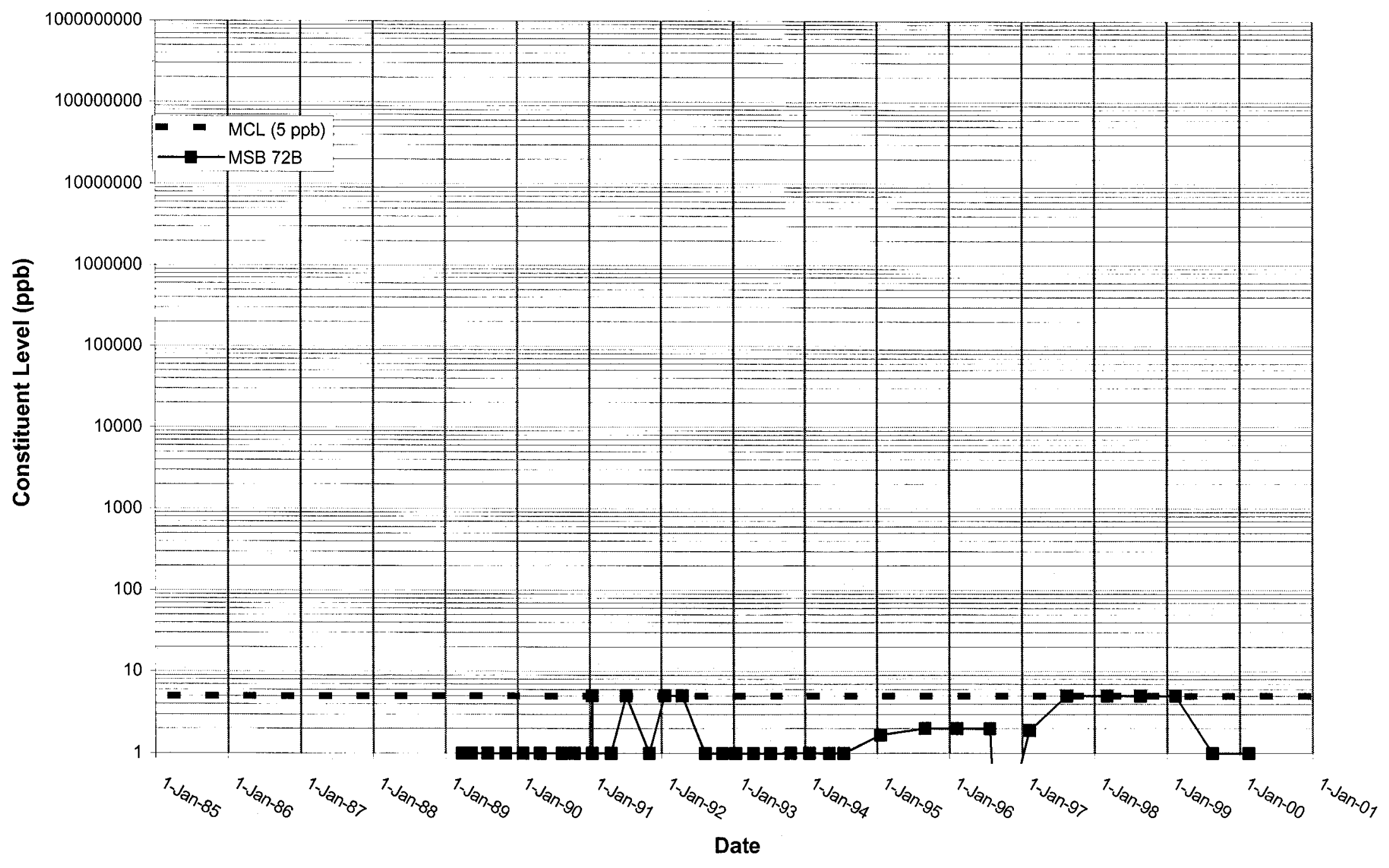




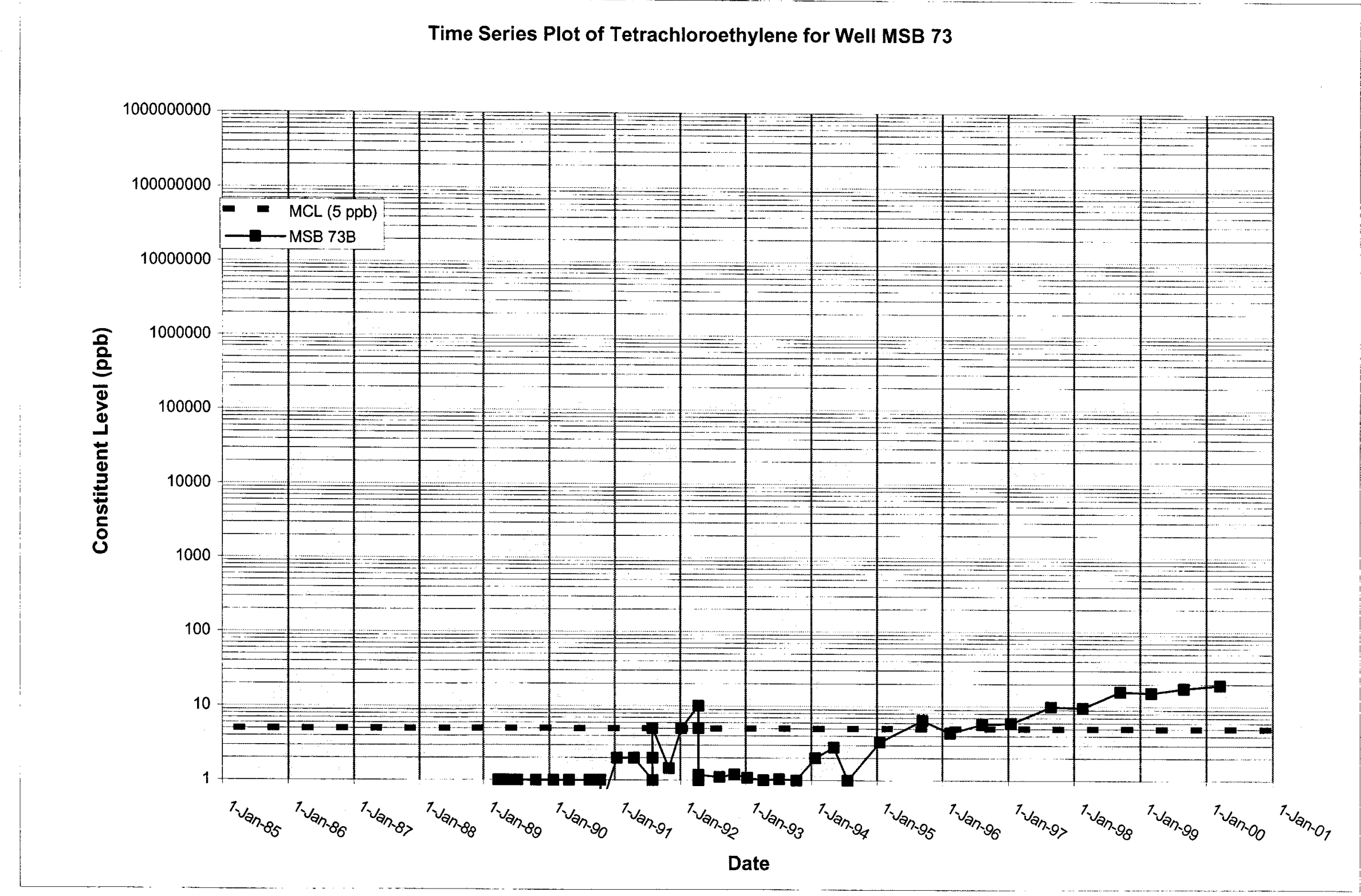


WSRC-TR-2000-00242 UNCLASSIFIED

Time Series Plot of Tetrachloroethylene for Well MSB 74

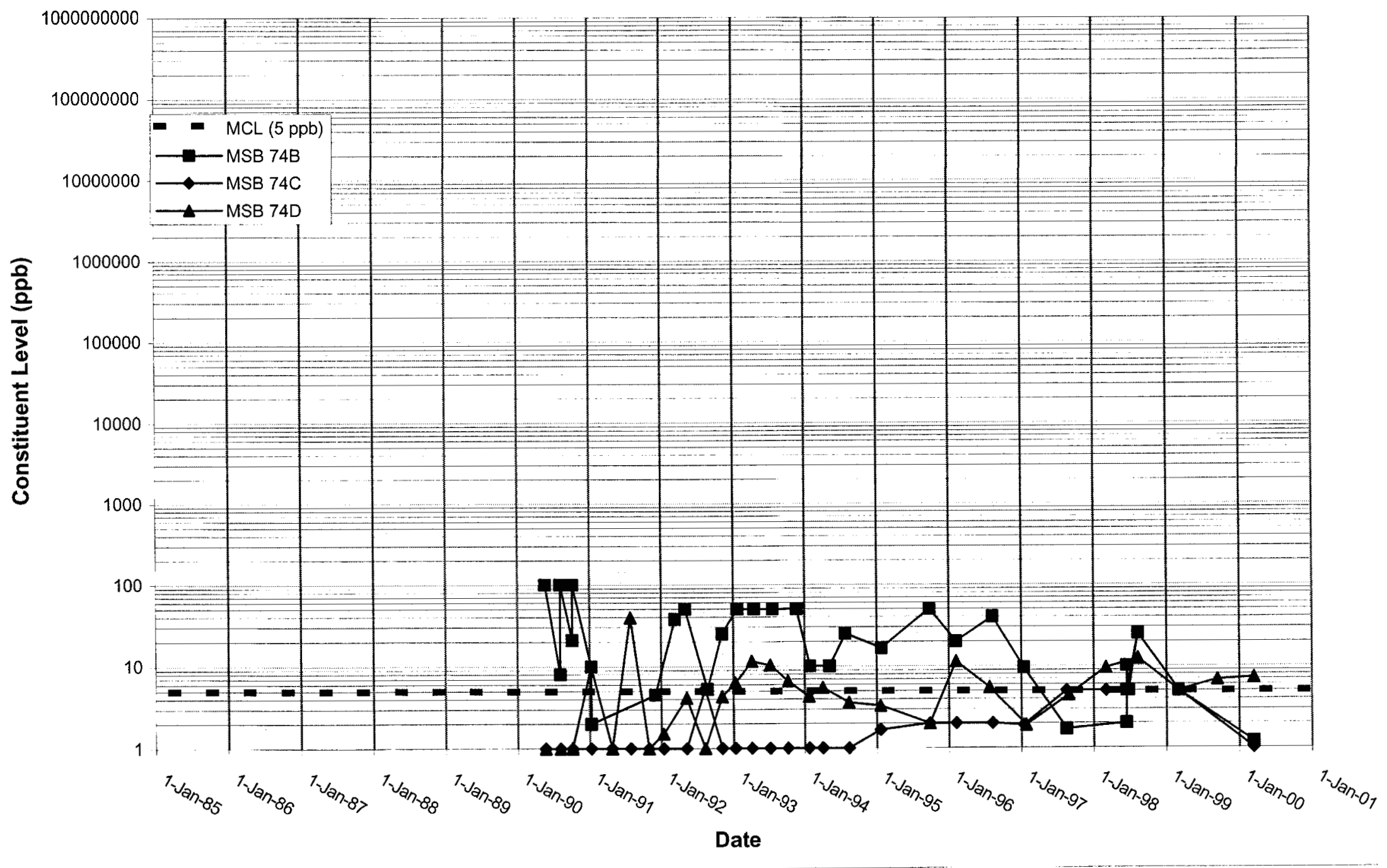




\section{Time Series Plot of Tetrachloroethylene for Well MSB 75}

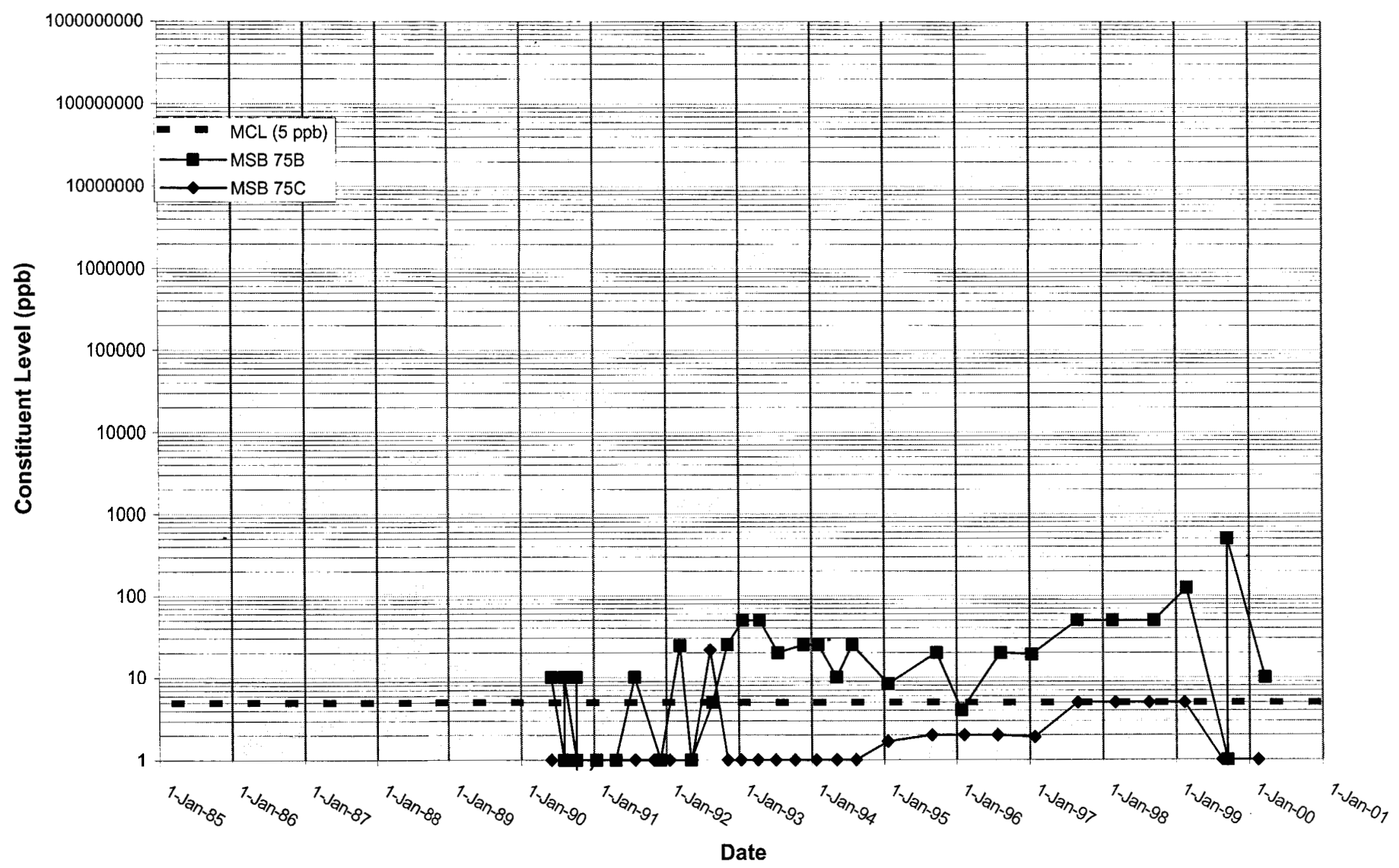


WSRC-TR-2000-00242 UNCLASSIFIED

Time Series Plot of Tetrachloroethylene for Well MSB 76

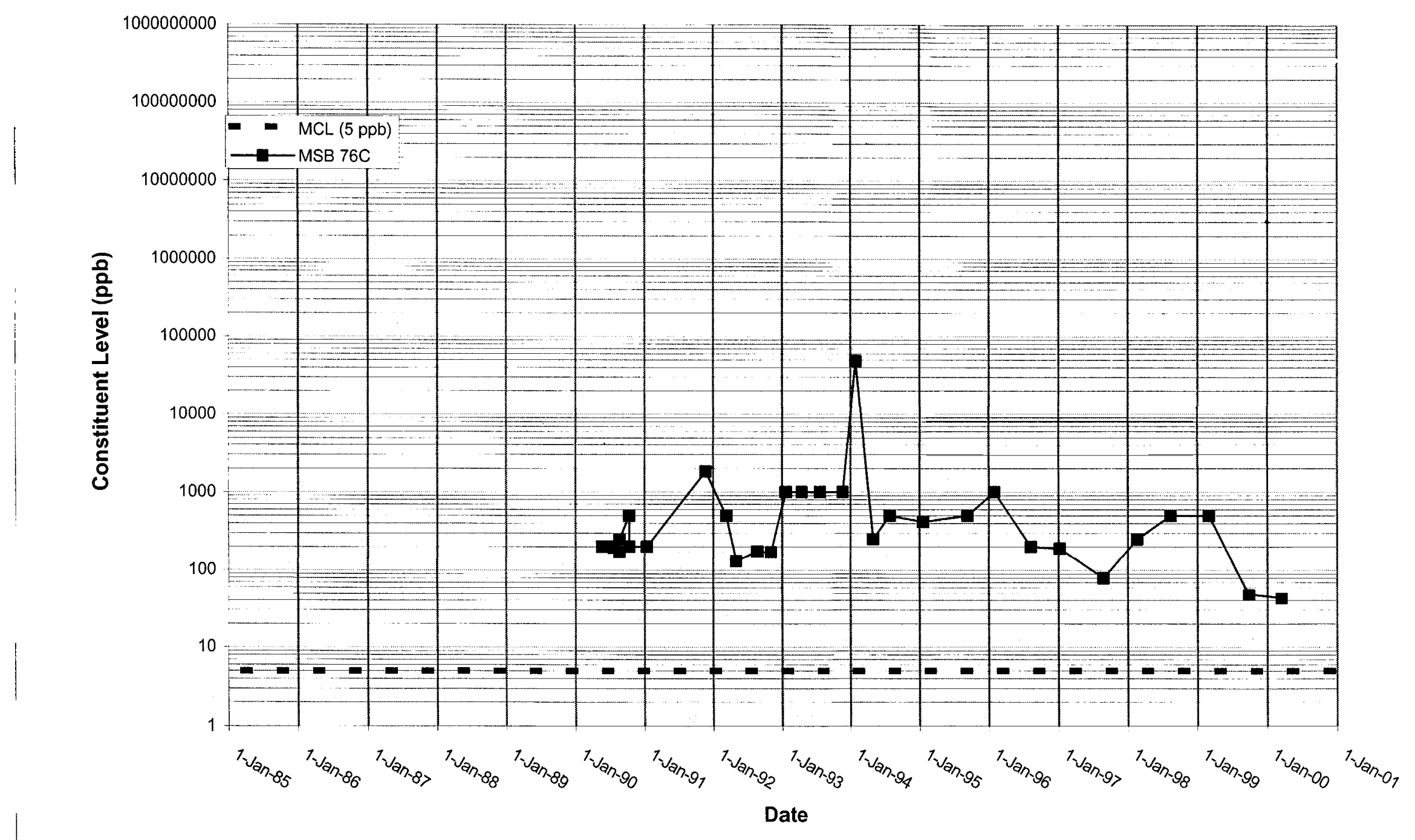




\section{1}

Time Series Plot of Tetrachloroethylene for Well MSB 77

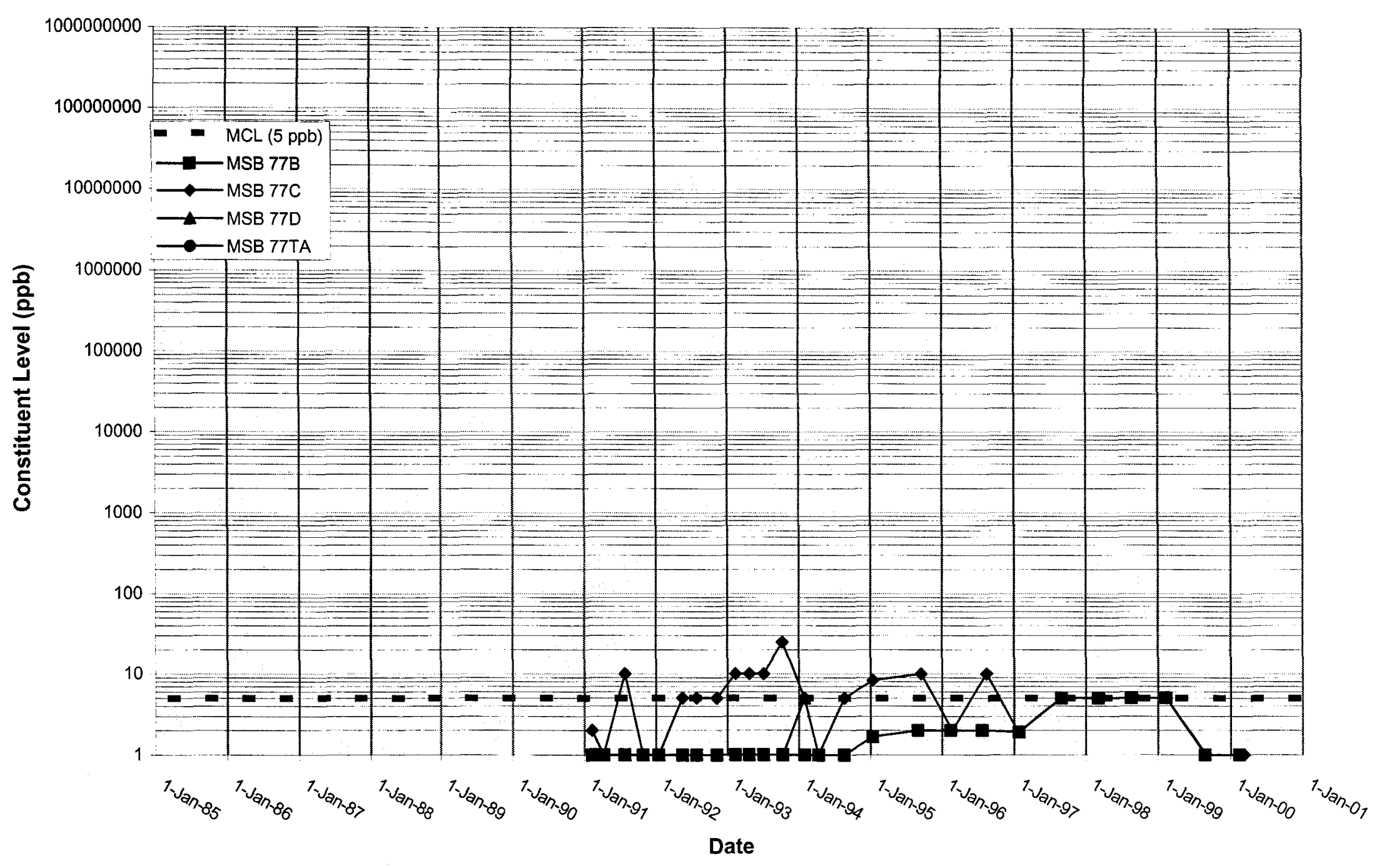


WSRC-TR-2000-00242 UNCLASSIFIED

\section{Time Series Plot of Tetrachloroethylene for Well MSB 78}

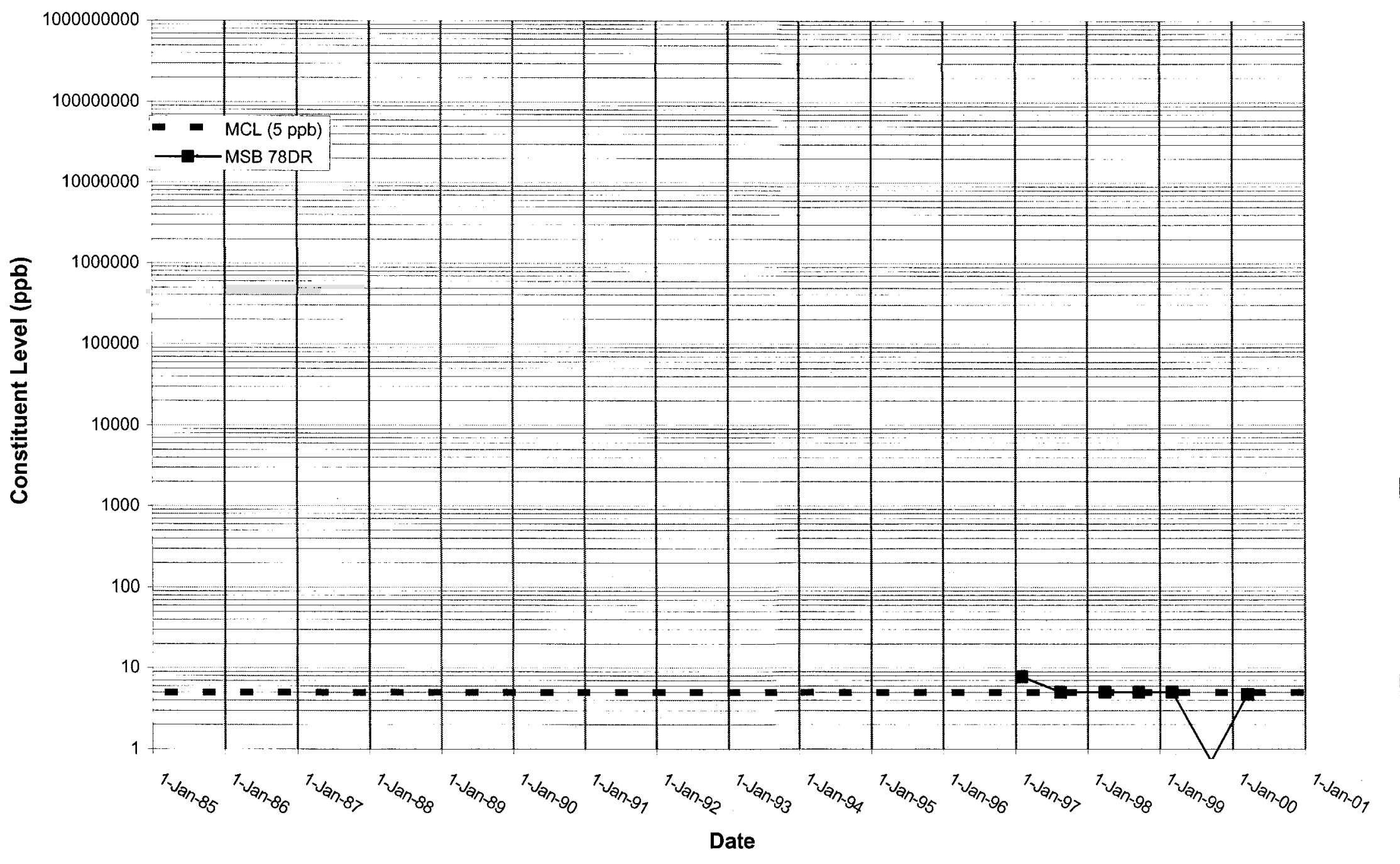




\section{Time Series Plot of Tetrachloroethylene for Well MSB 79}

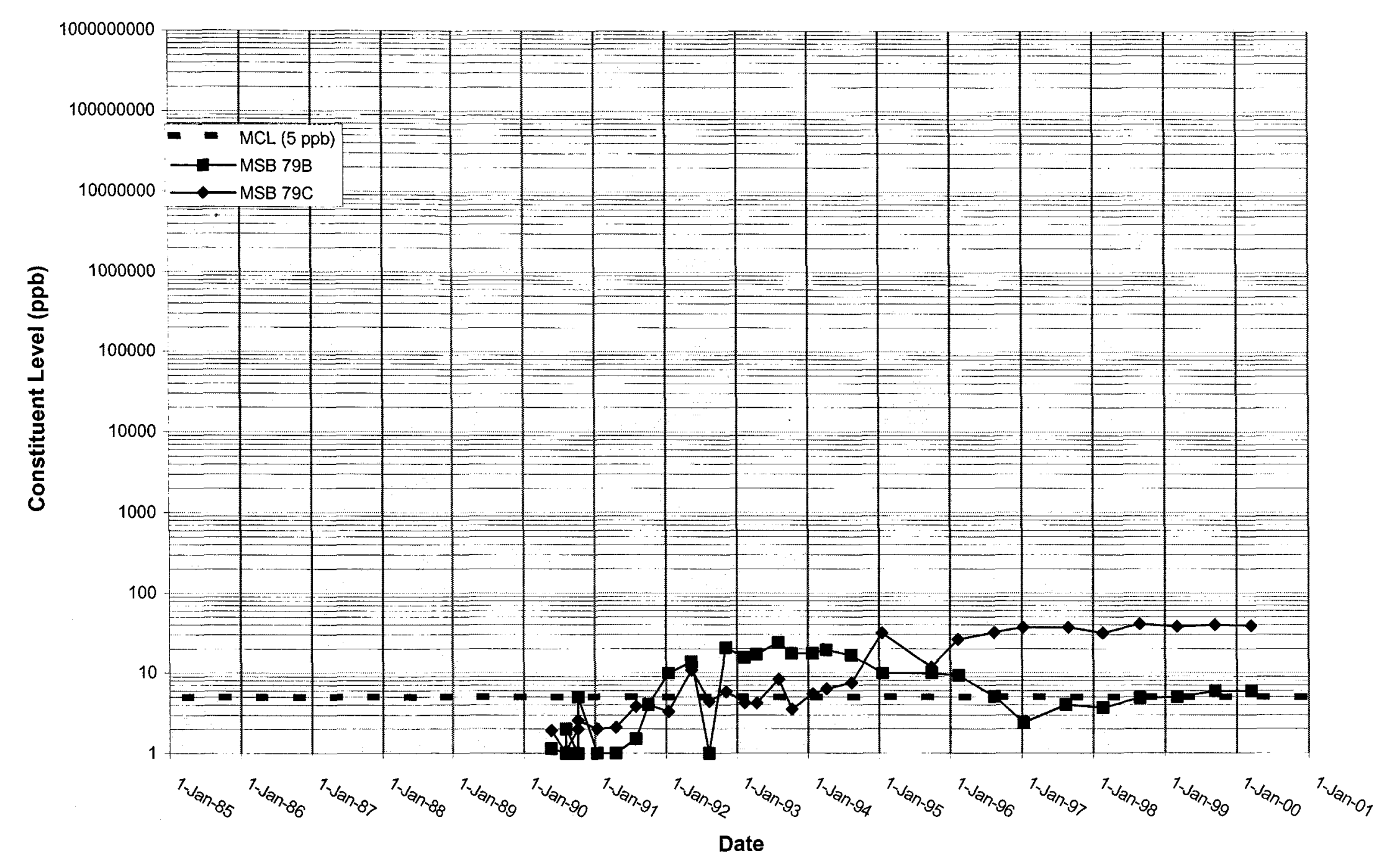


WSRC-TR-2000-00242 UNCLASSIFIED

\section{Time Series Plot of Tetrachloroethylene for Well MSB 81}

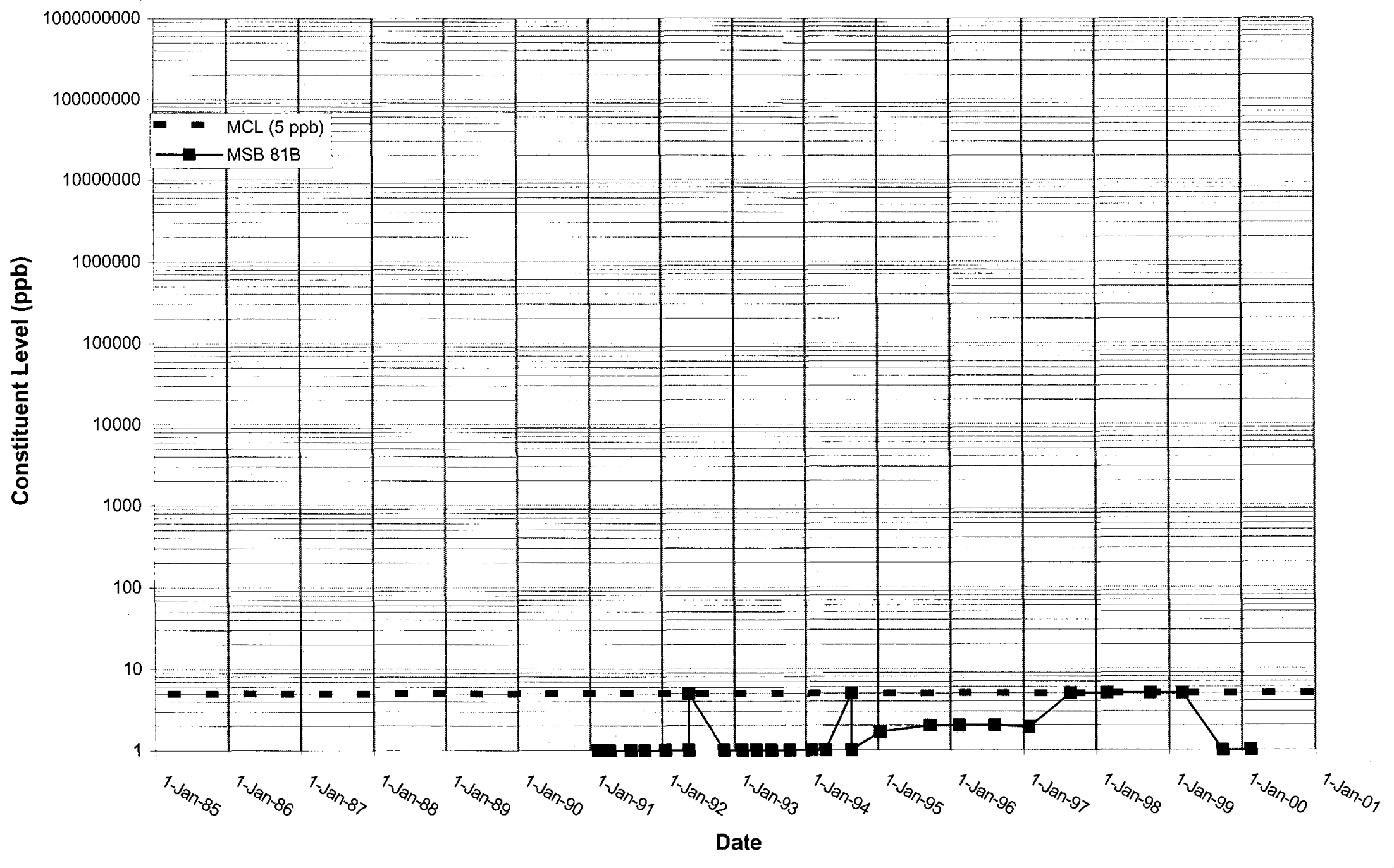




\section{Time Series Plot of Tetrachloroethylene for Well MSB 82}

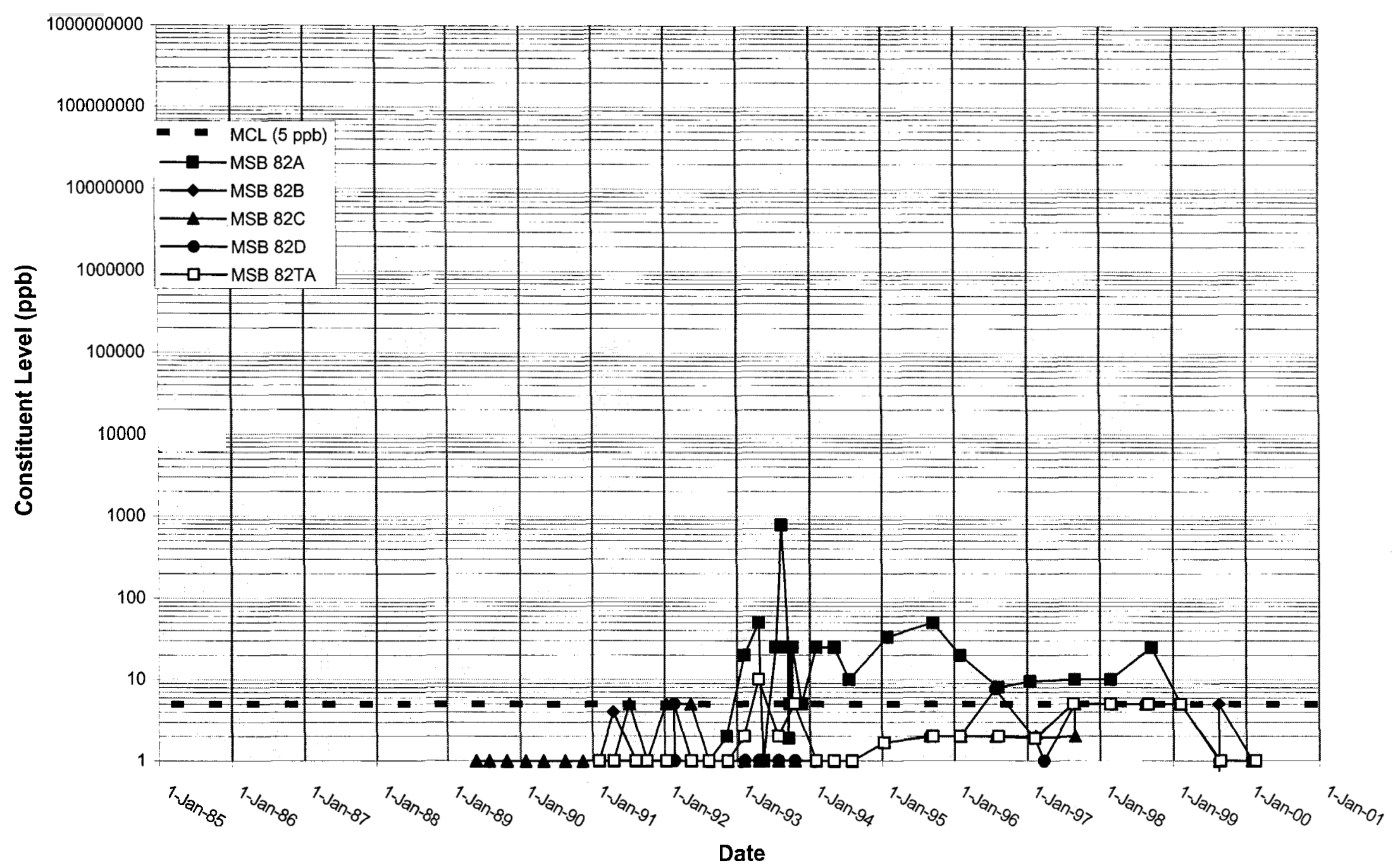


WSRC-TR-2000-00242 UNCLASSIFIED

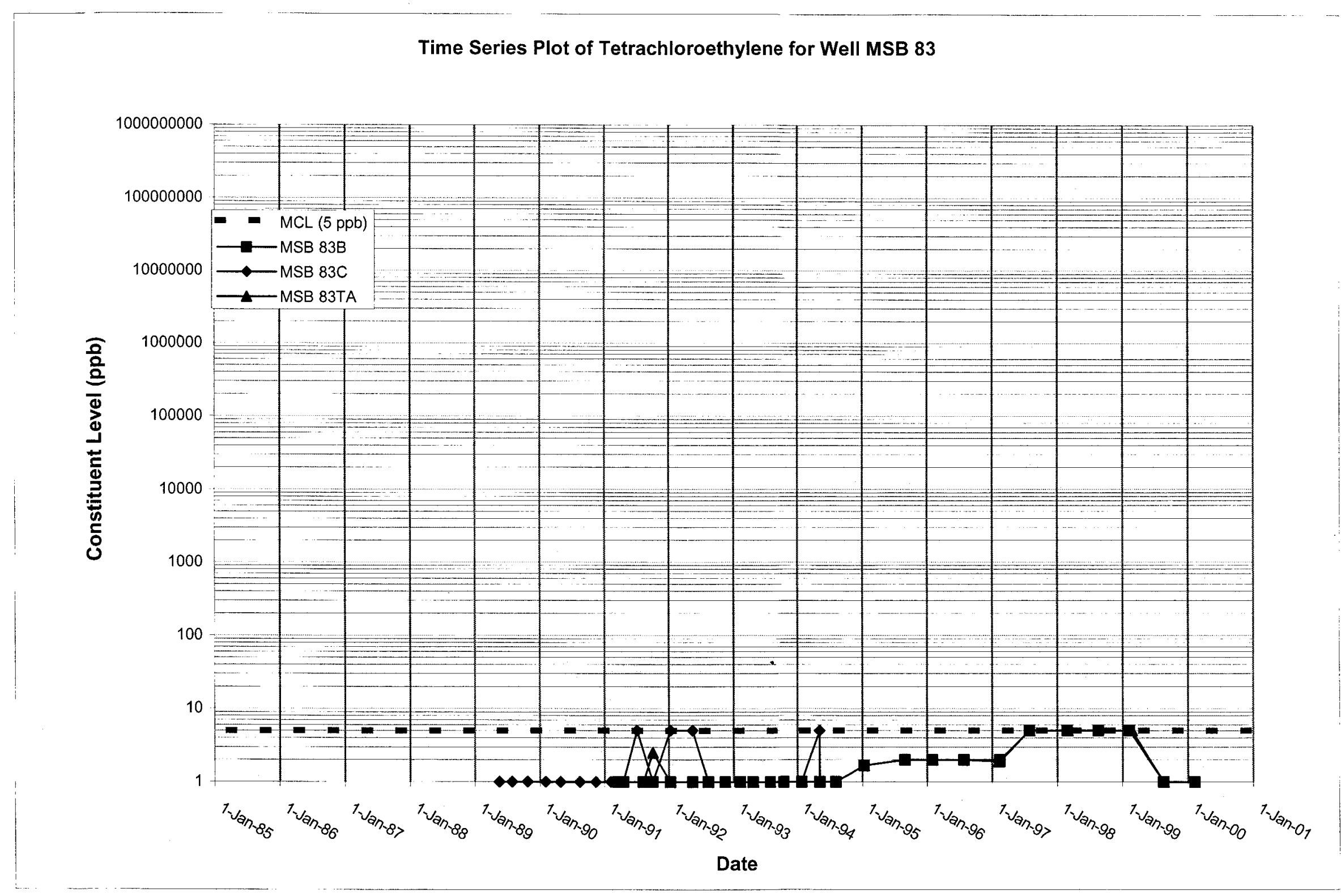




\section{Time Series Plot of Tetrachloroethylene for Well MSB 84}

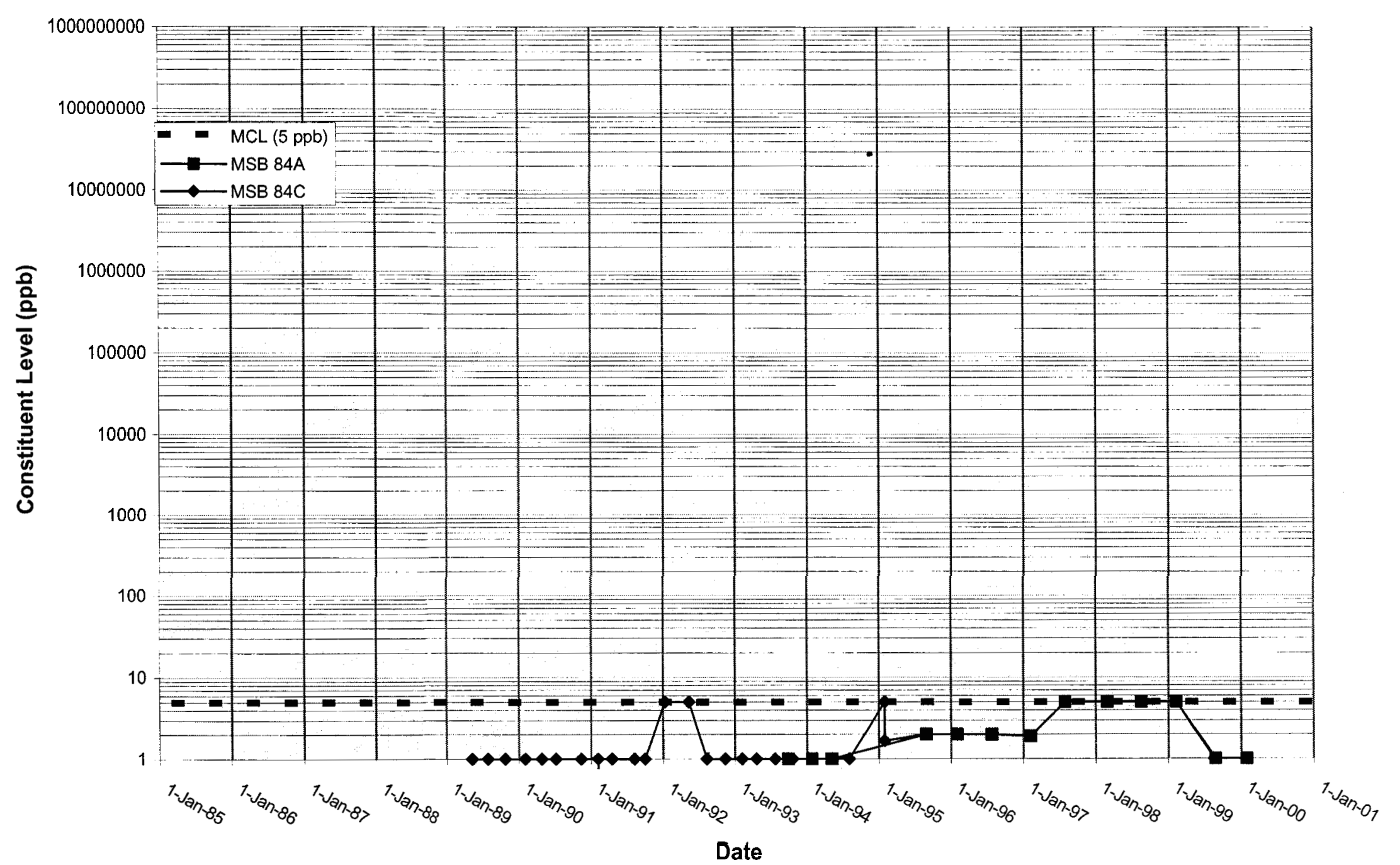


WSRC-TR-2000-00242 UNCLASSIFIED

Time Series Plot of Tetrachloroethylene for Well MSB 85

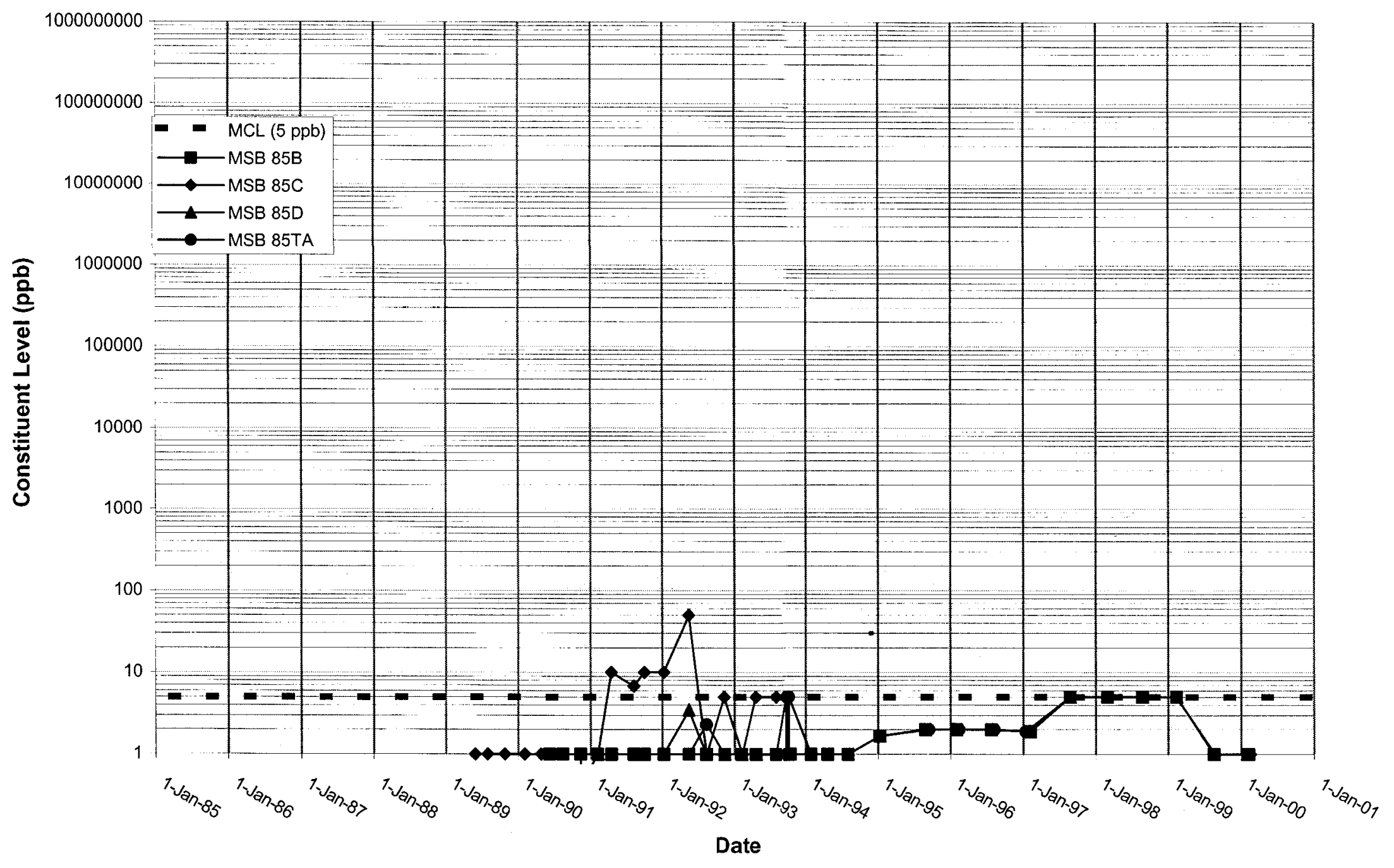




\section{Time Series Plot of Tetrachloroethylene for Well MSB 86}

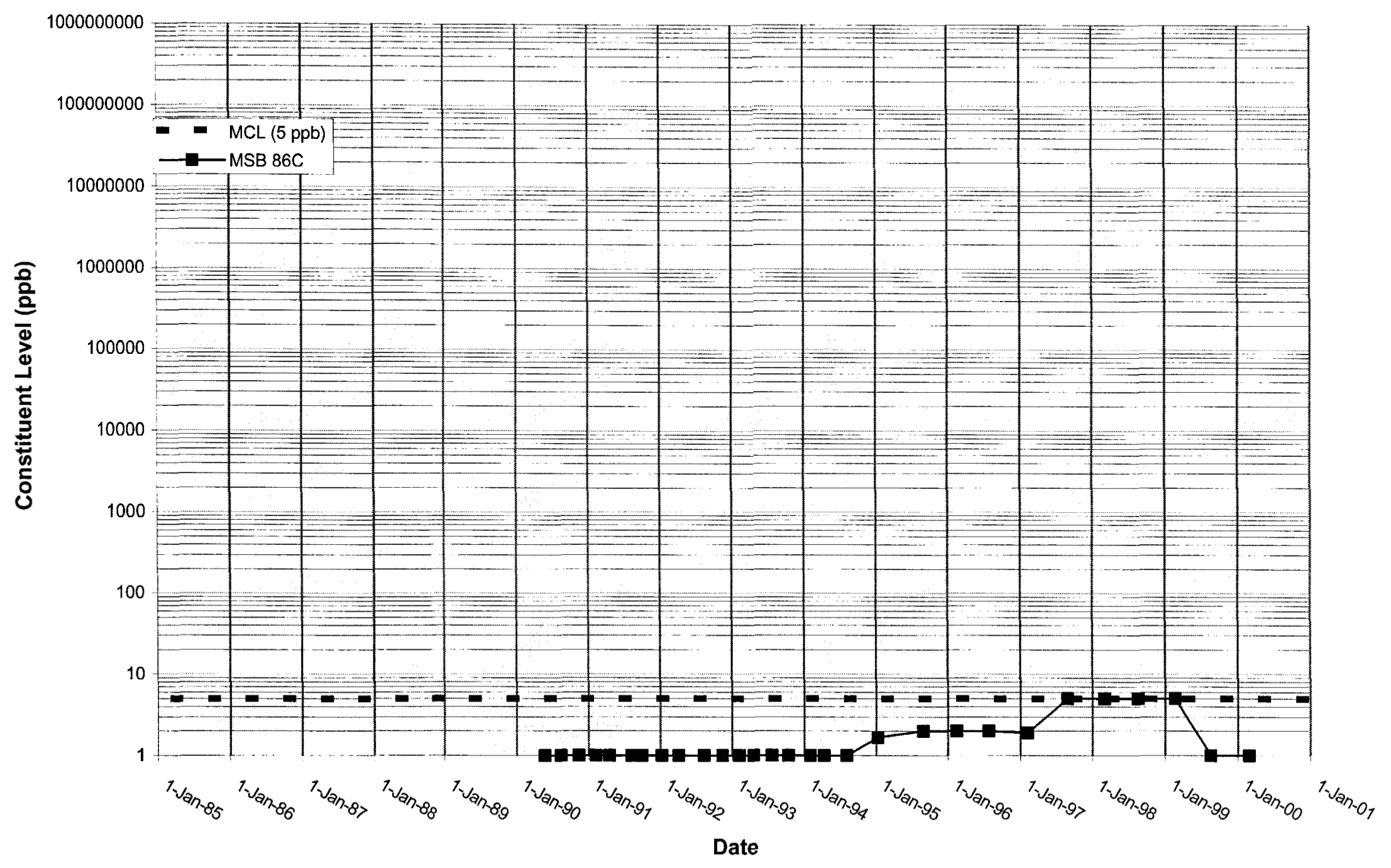


WSRC-TR-2000-00242 UNCLASSIFIED

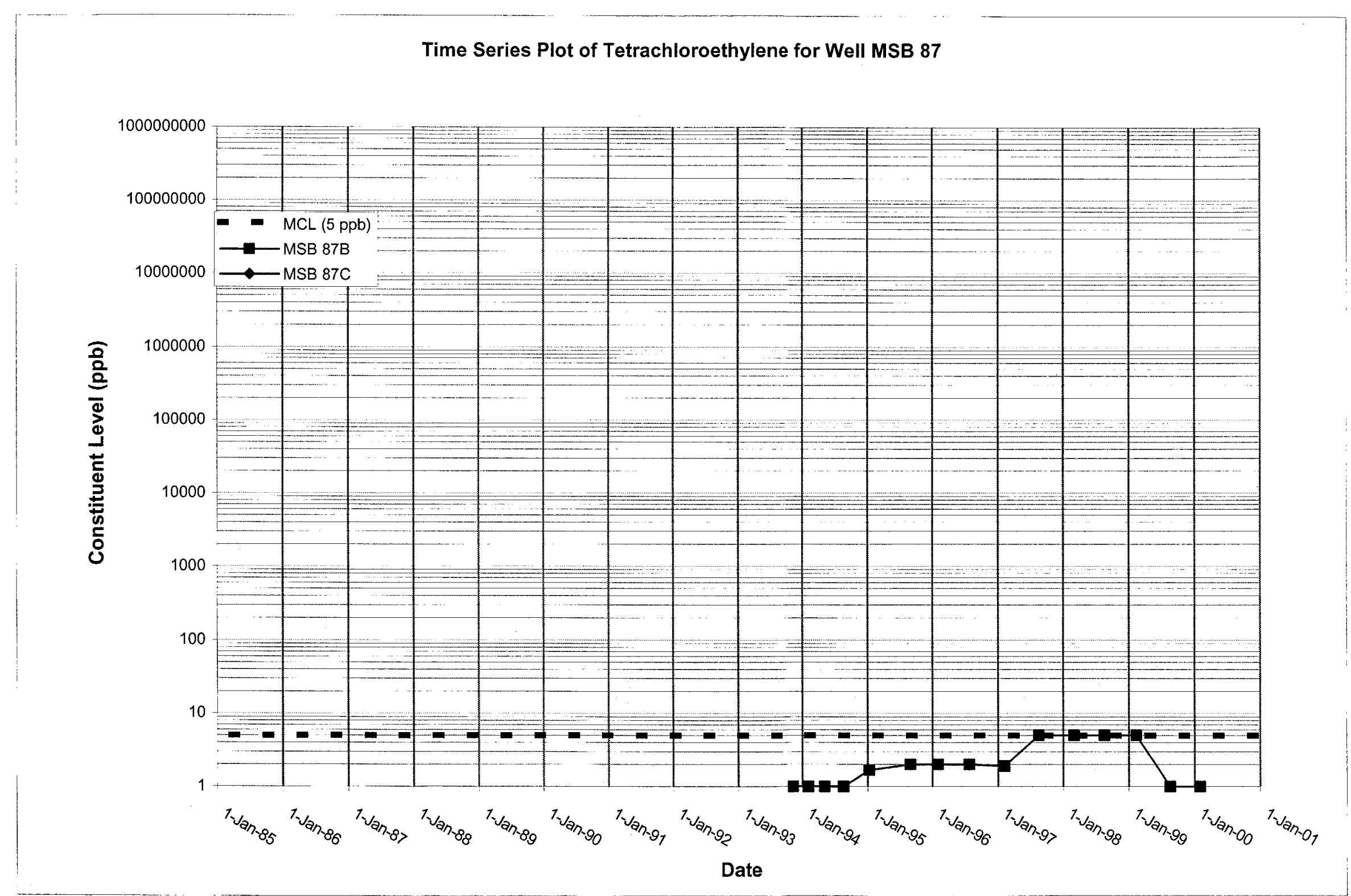




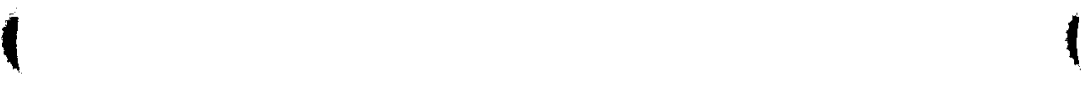

WSRC-TR-2000-00242

UNCLASSIFIED

\section{Time Series Plot of Tetrachloroethylene for Well MSB 88}

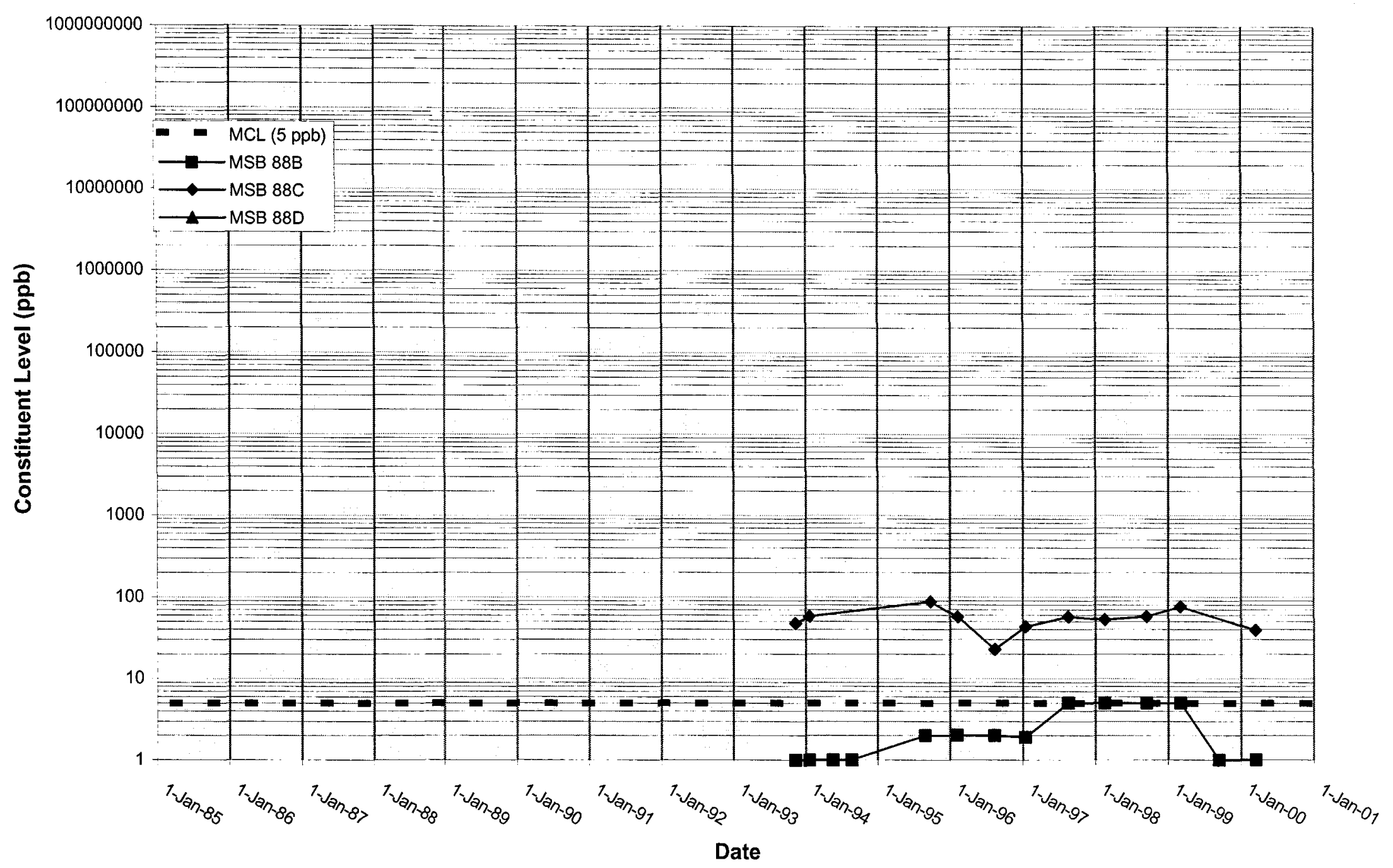


WSRC-TR-2000-00242 UNCLASSIFIED

Time Series Plot of Tetrachloroethylene for Well MSB 89

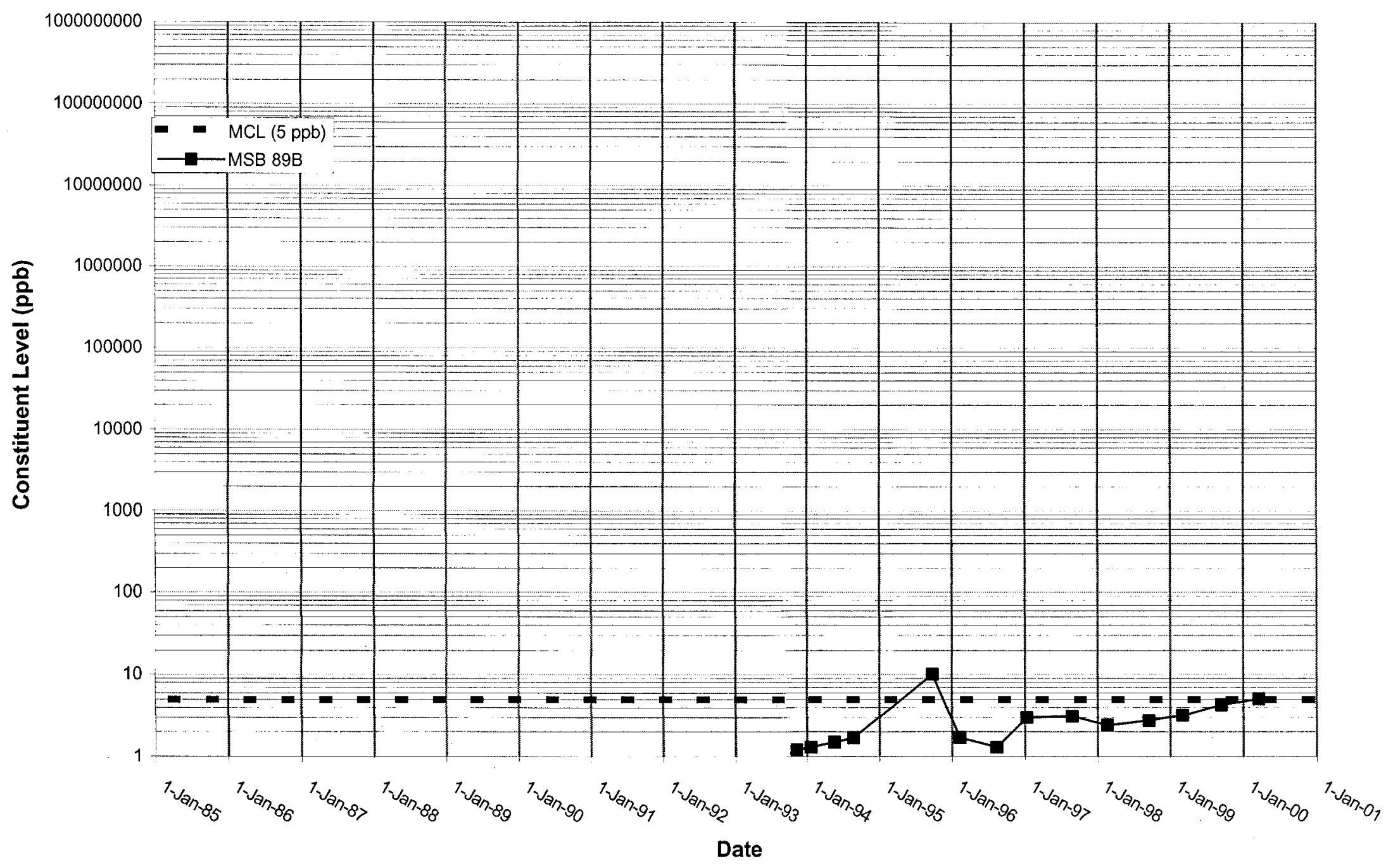




\section{Time Series Plot of Tetrachloroethylene for Well RWM 1}

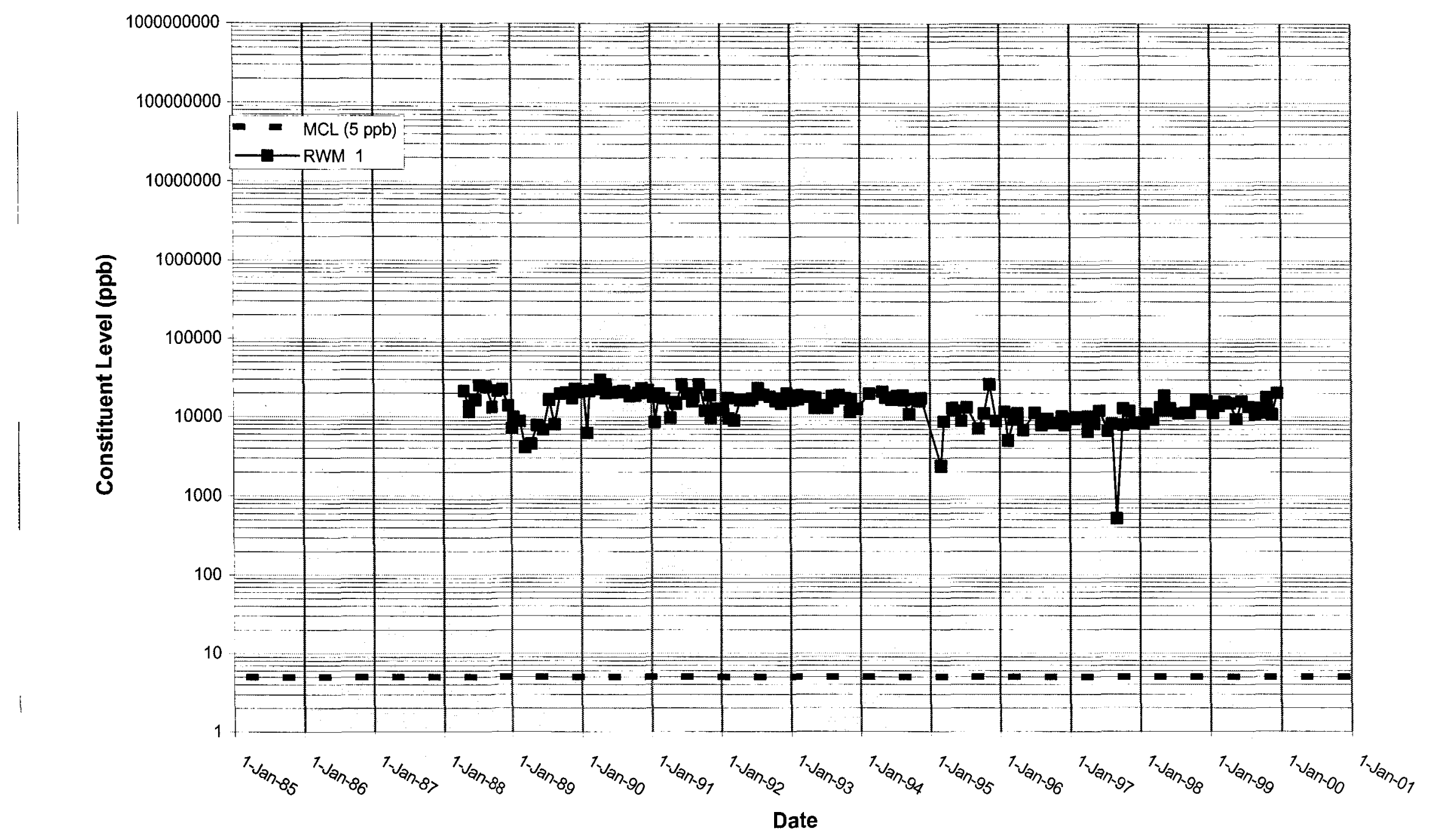


WSRC-TR-2000-00242 UNCLASSIFIED

Time Series Plot of Tetrachloroethylene for Well RWM 2

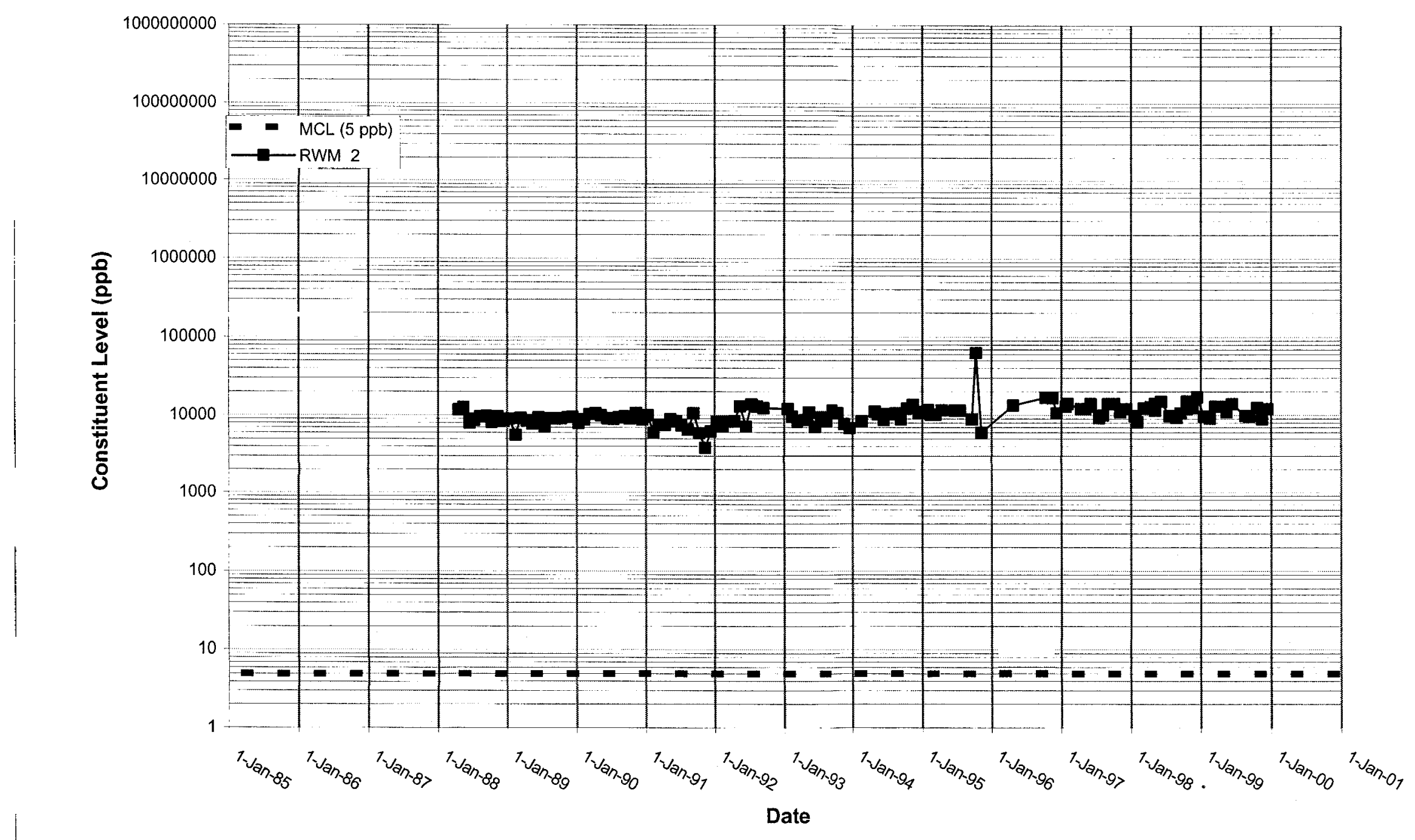




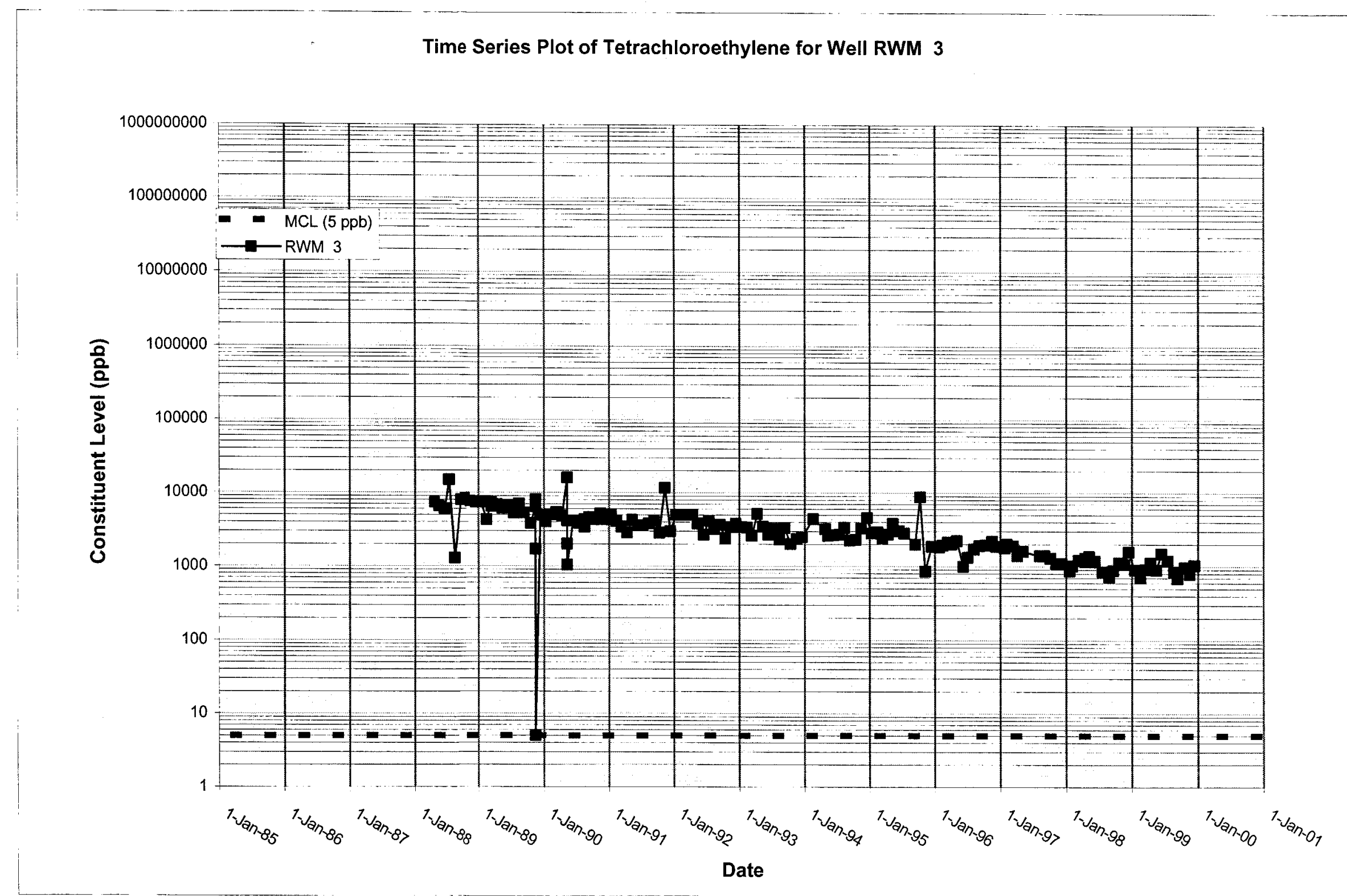


WSRC-TR-2000-00242 UNCLASSIFIED

Time Series Plot of Tetrachloroethylene for Well RWM 4

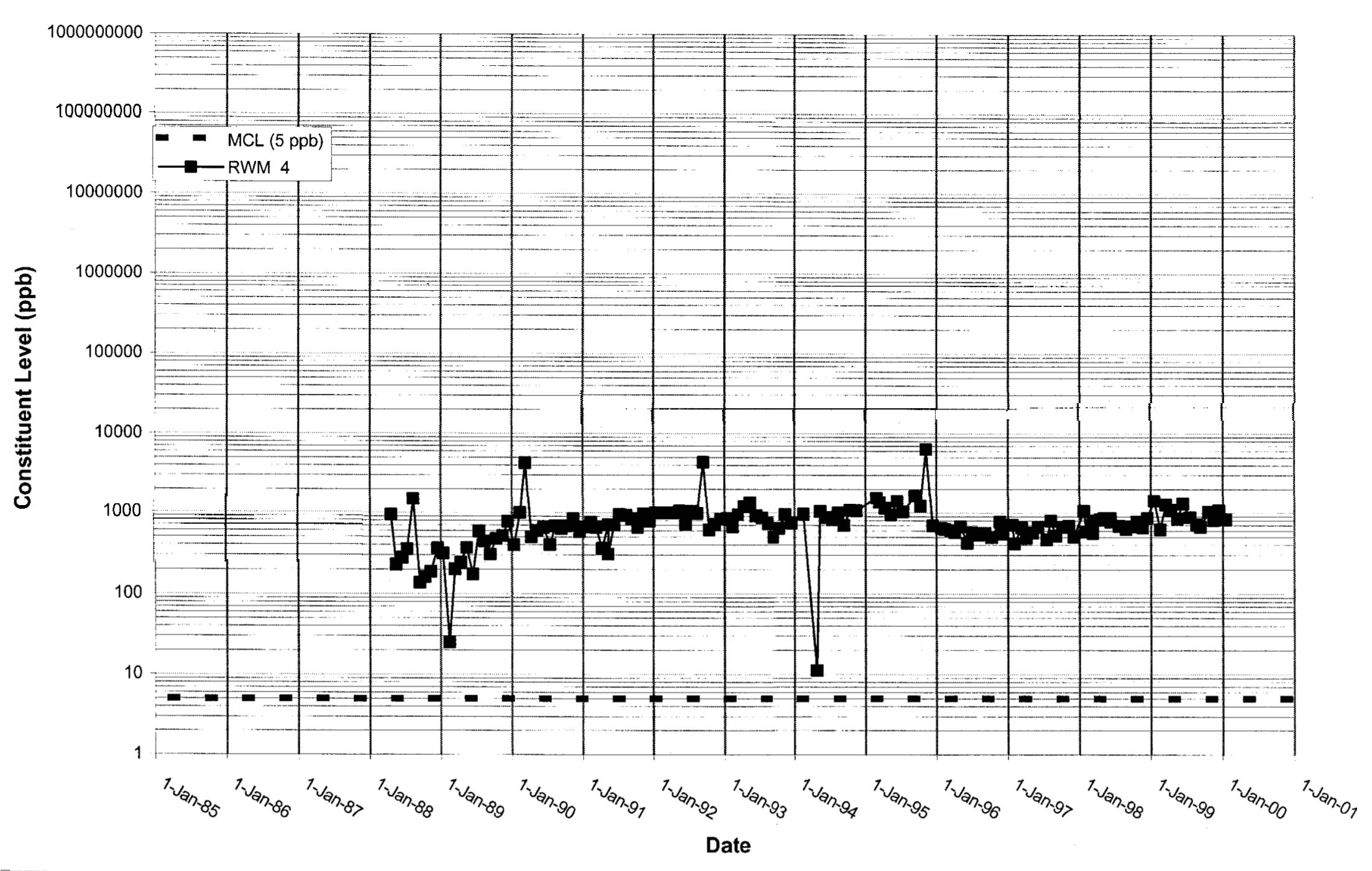


Time Series Plot of Tetrachloroethylene for Well RWM 5

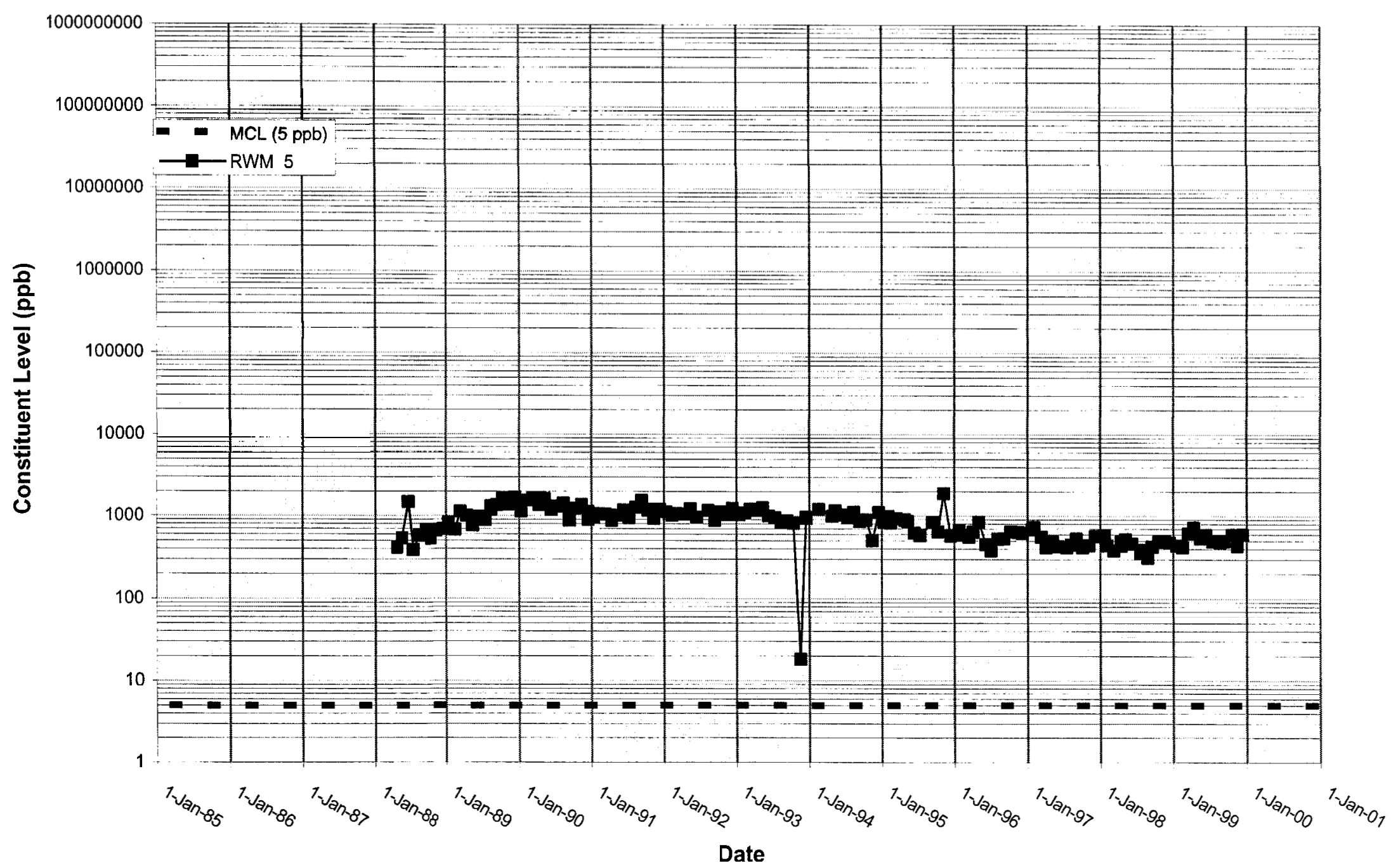


Time Series Plot of Tetrachloroethylene for Well RWM 6

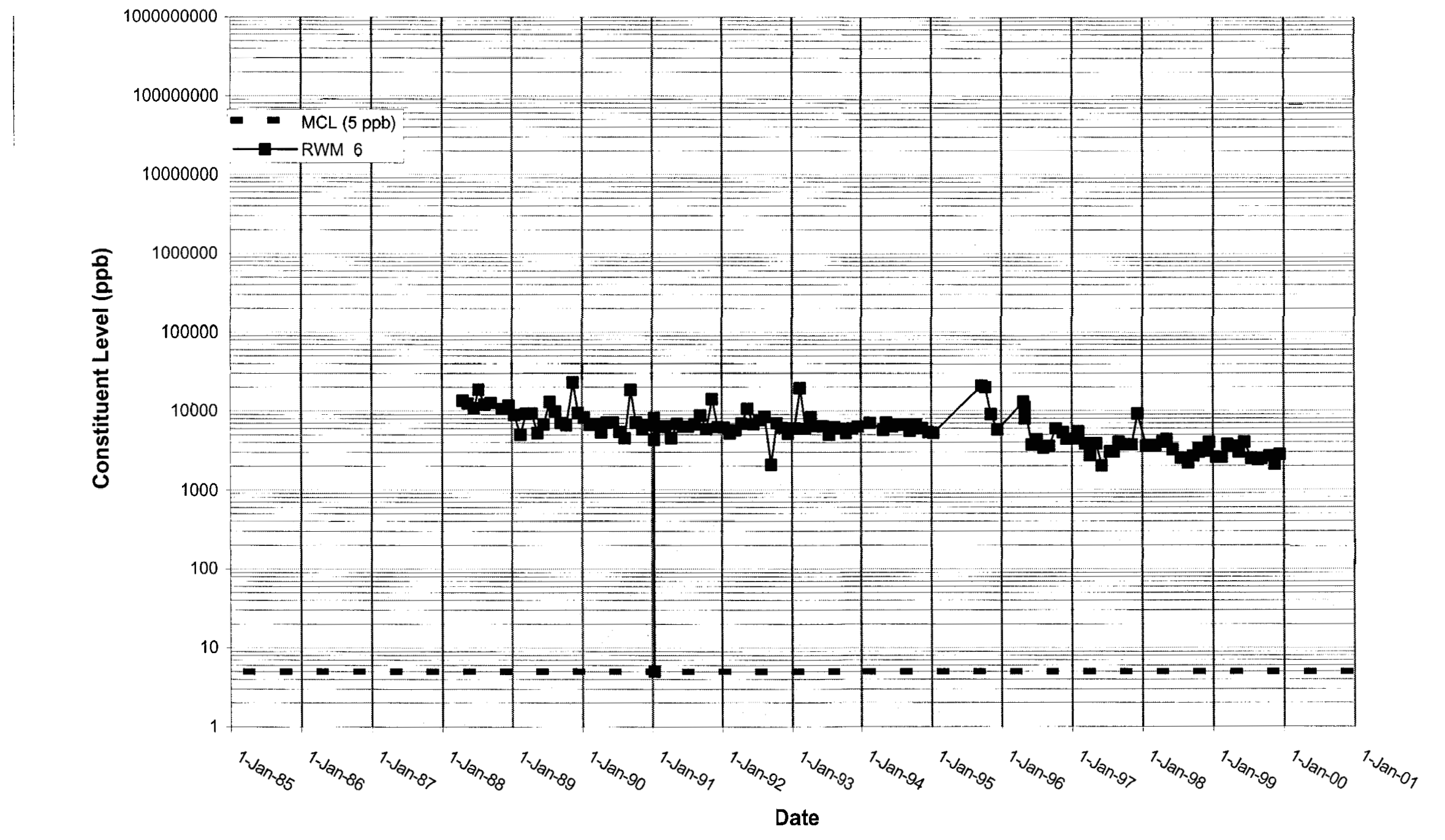




\section{Time Series Plot of Tetrachloroethylene for Well RWM 7}

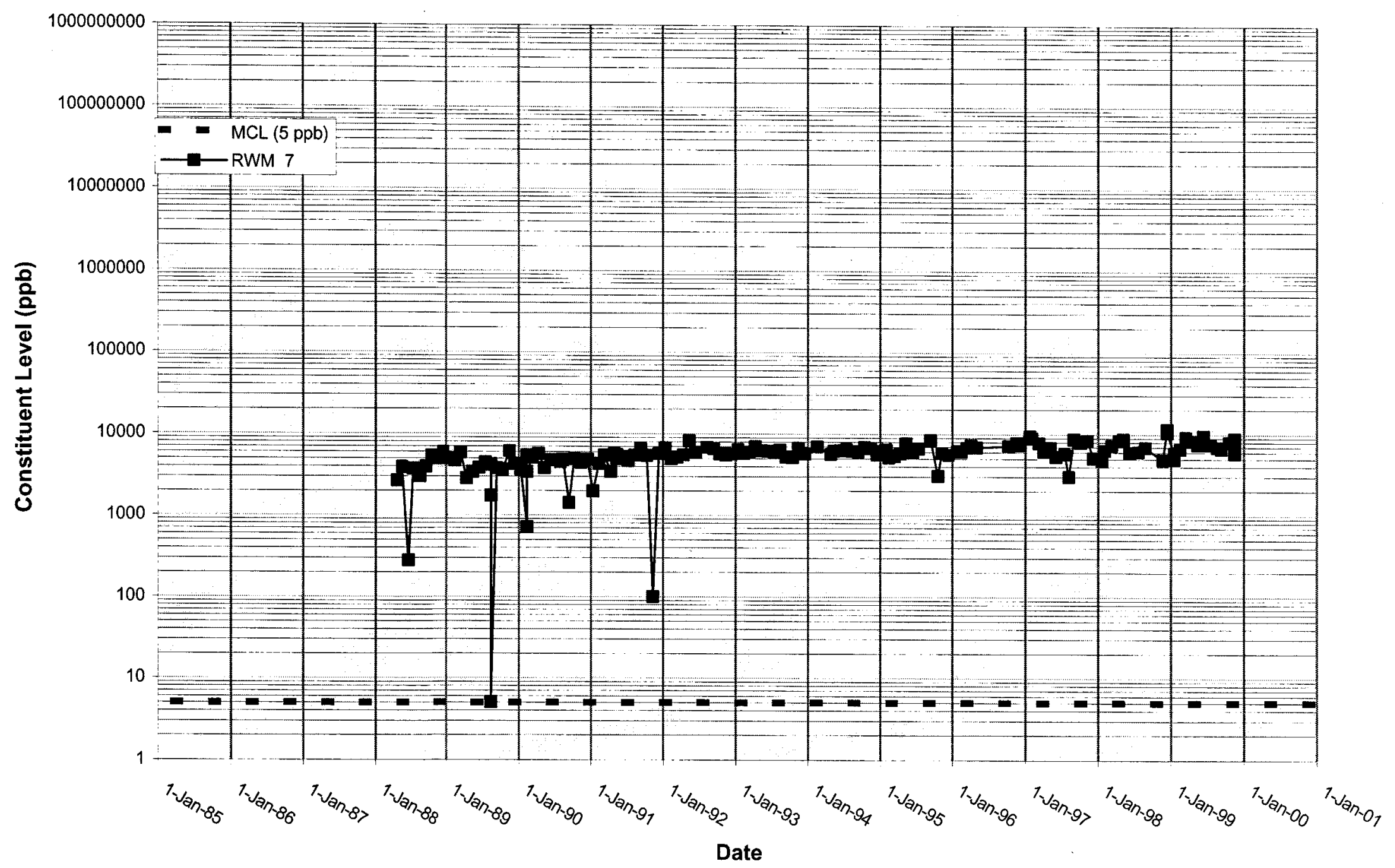


WSRC-TR-2000-00242 UNCLASSIFIED

\section{Time Series Plot of Tetrachloroethylene for Well RWM 8}

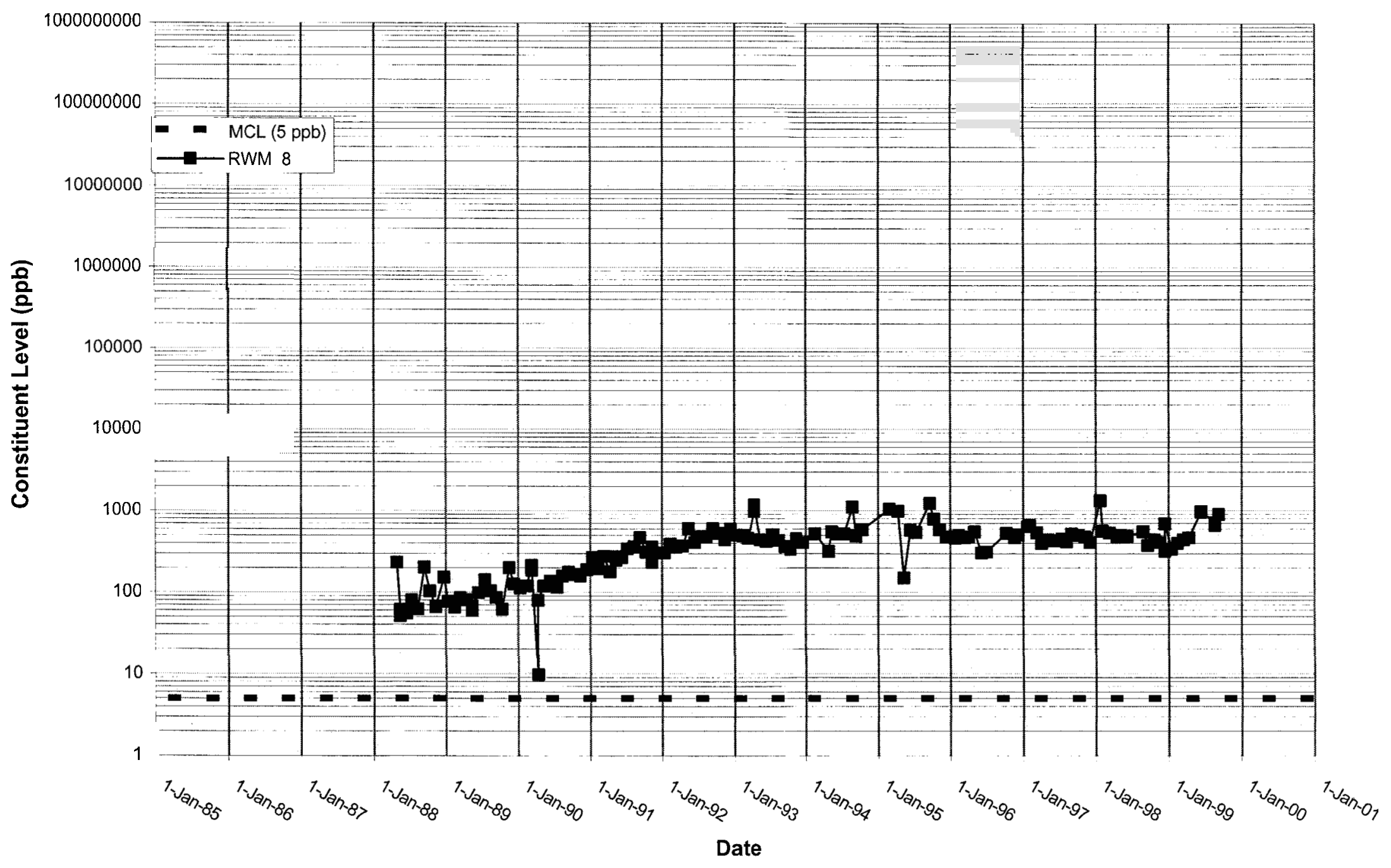


Time Series Plot of Tetrachloroethylene for Well RWM 9

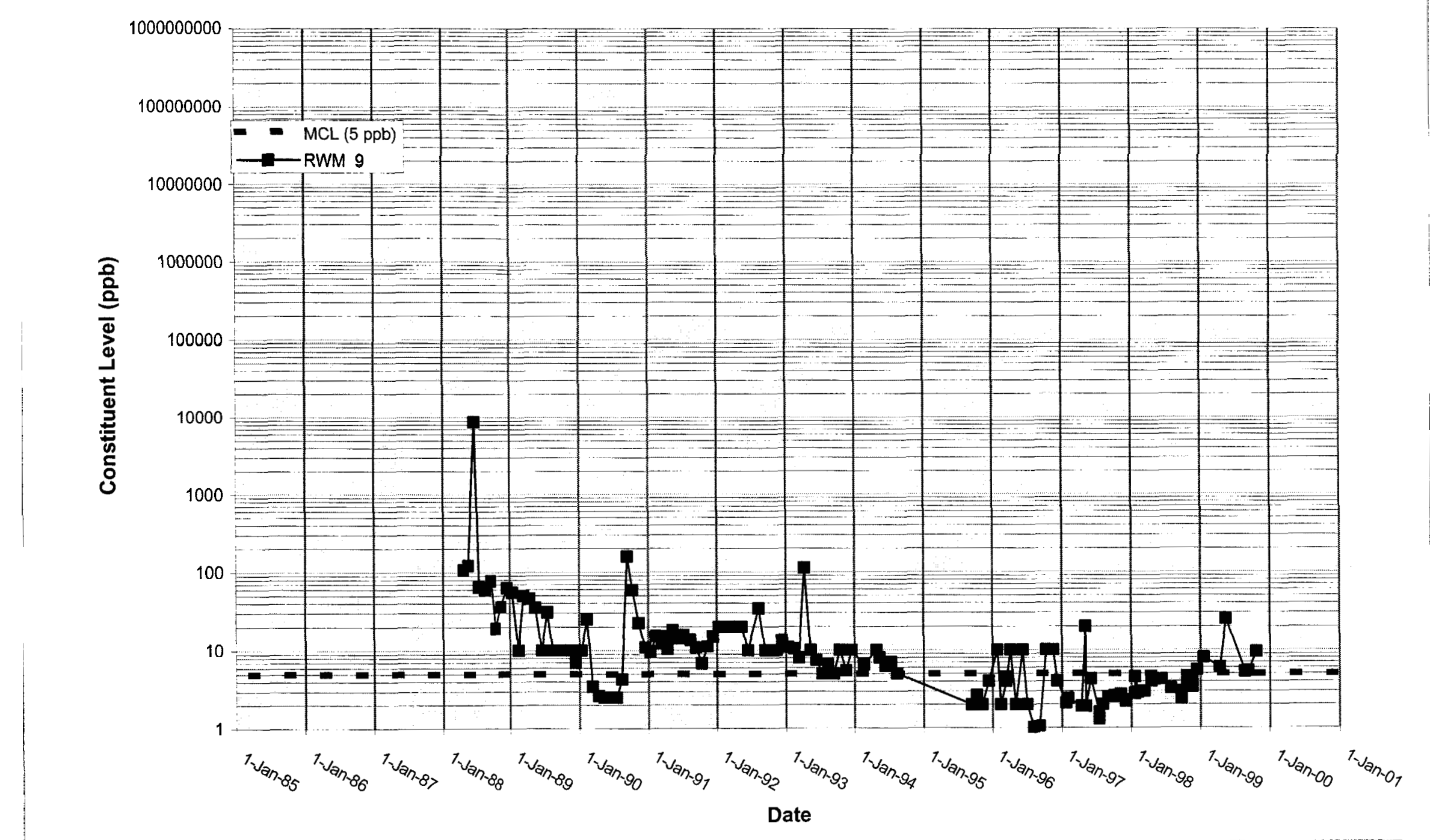


WSRC-TR-2000-00242 UNCLASSIFIED

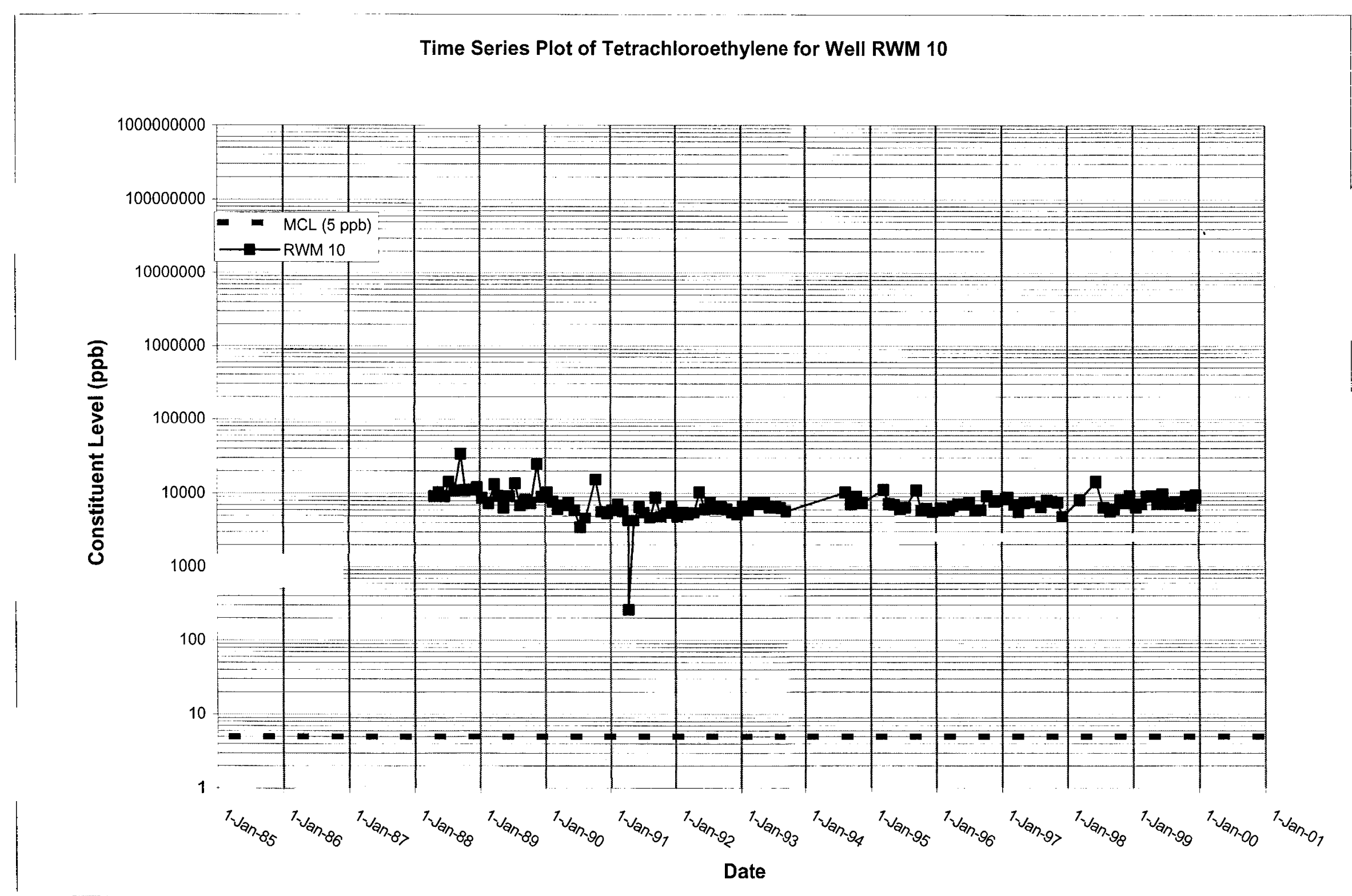




\section{Time Series Plot of Tetrachloroethylene for Well RWM 11}

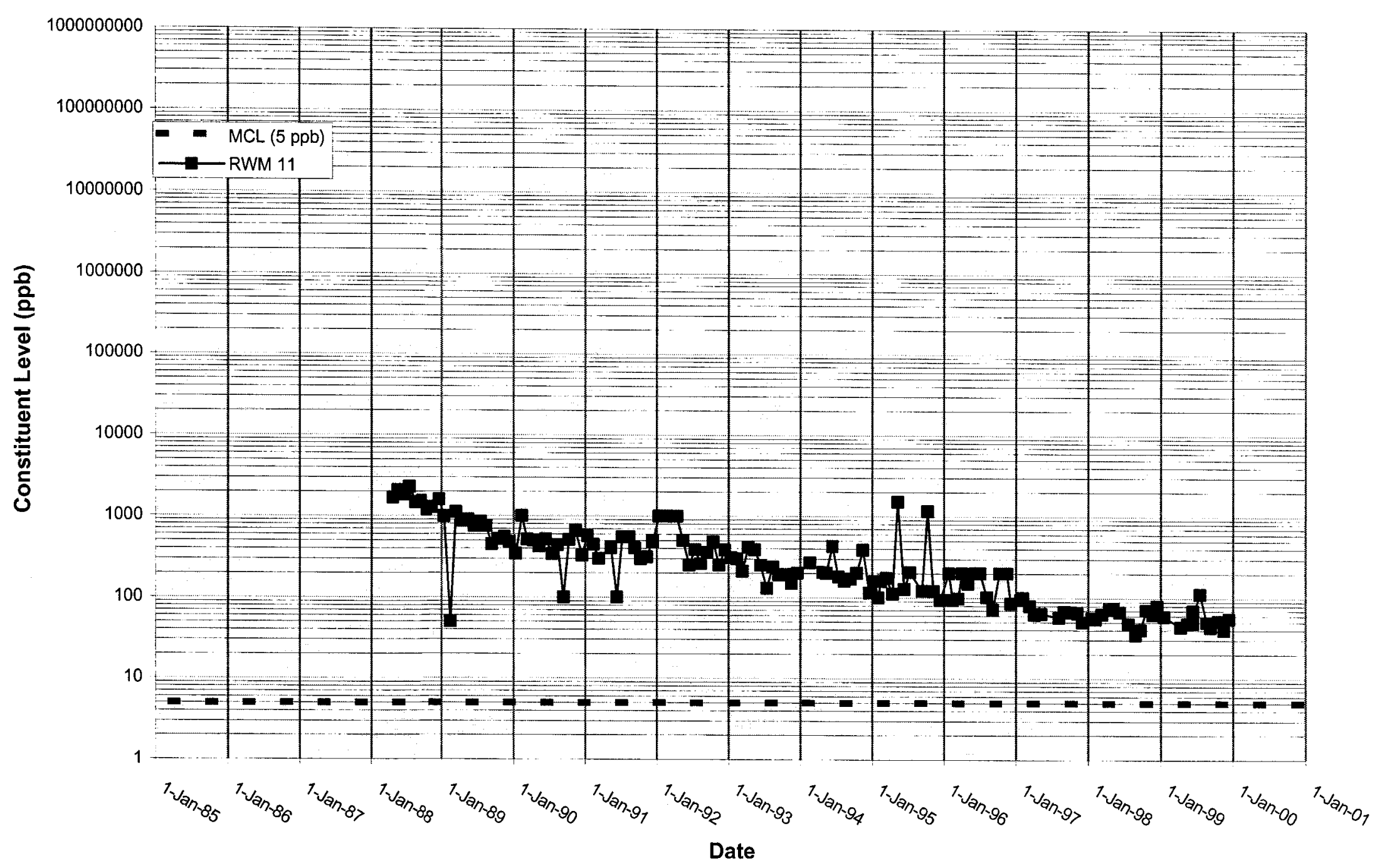


WSRC-TR-2000-00242 UNCLASSIFIED

Time Series Plot of Tetrachloroethylene for Well RWM 12

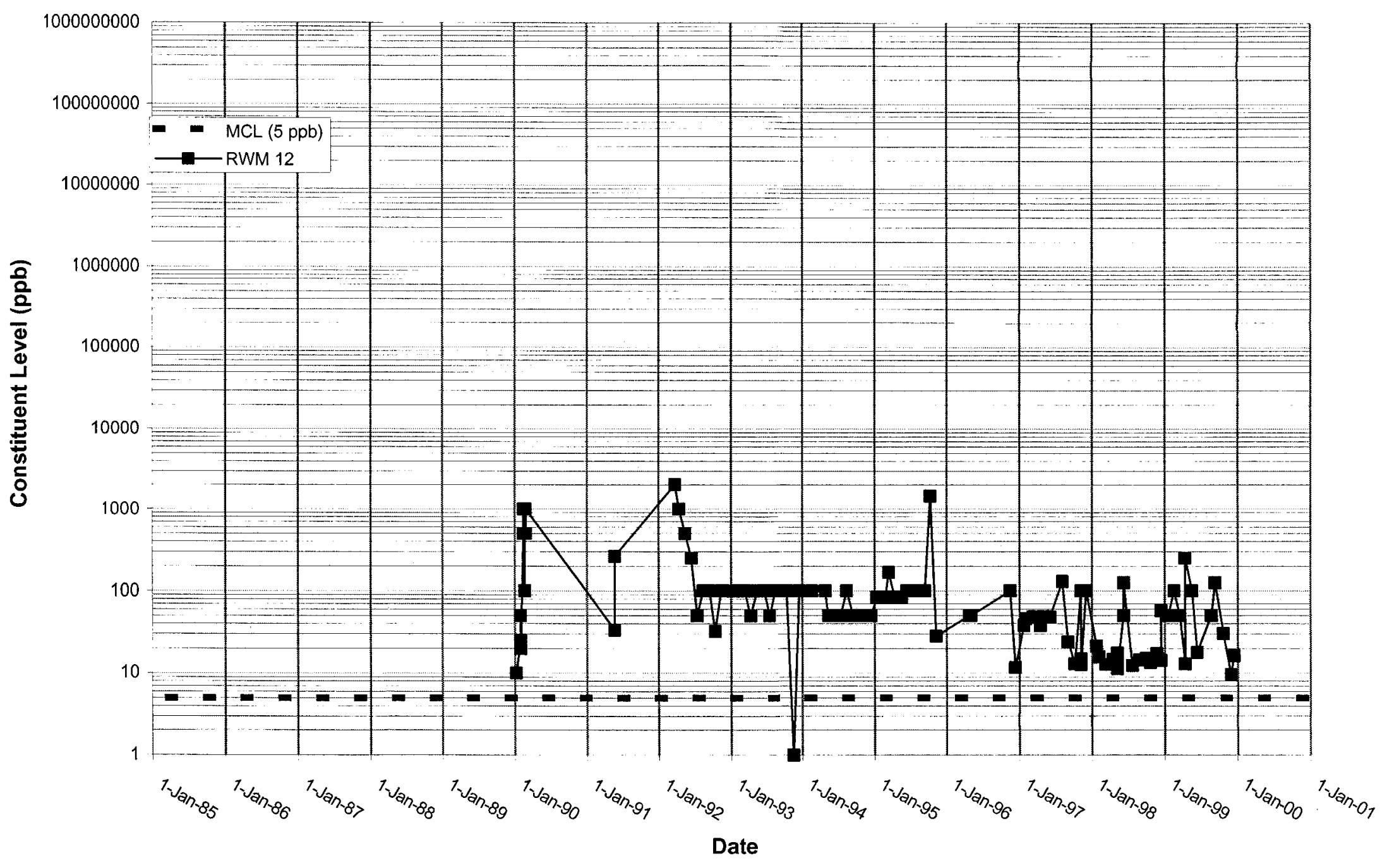




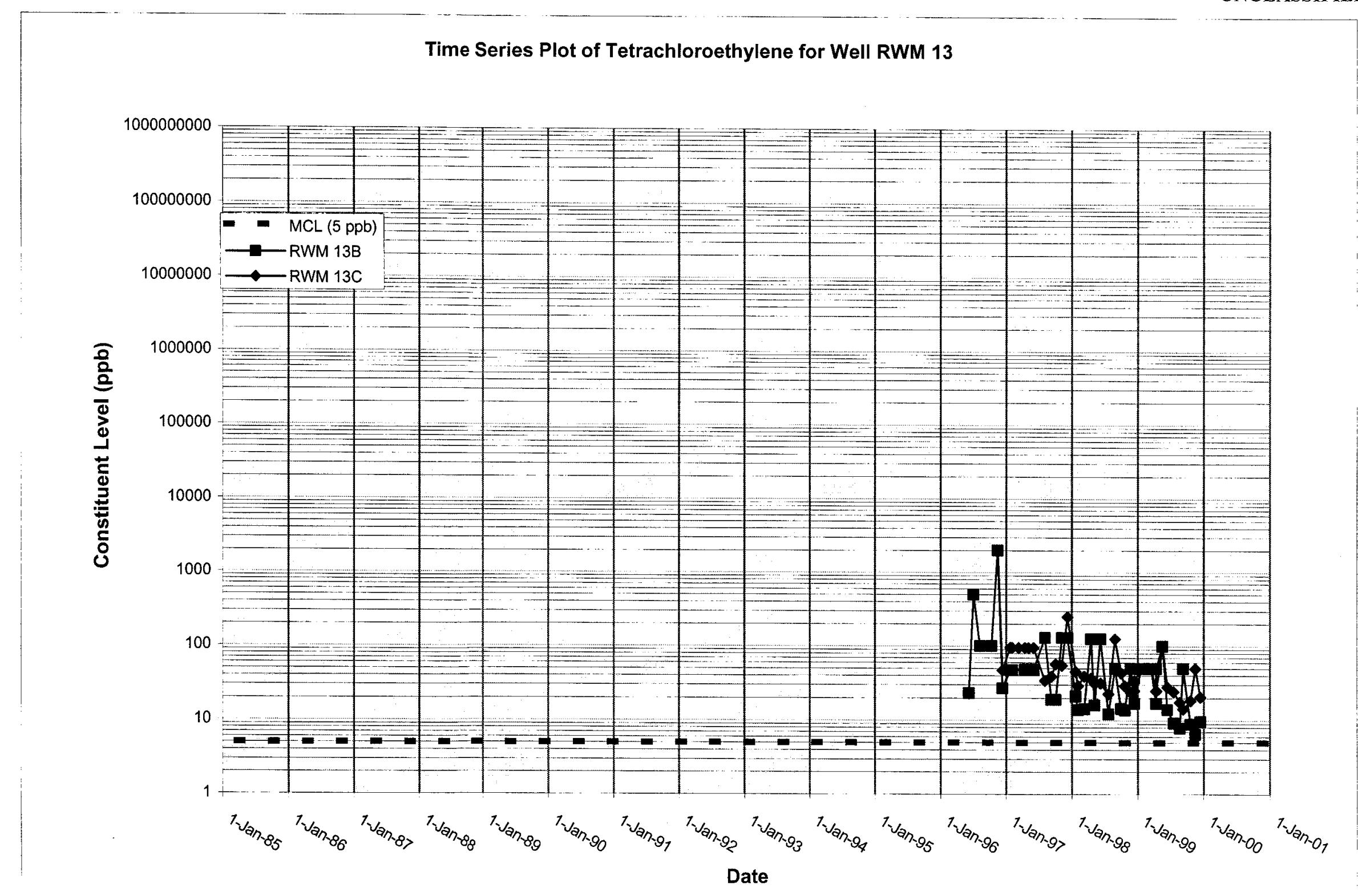


WSRC-TR-2000-00242

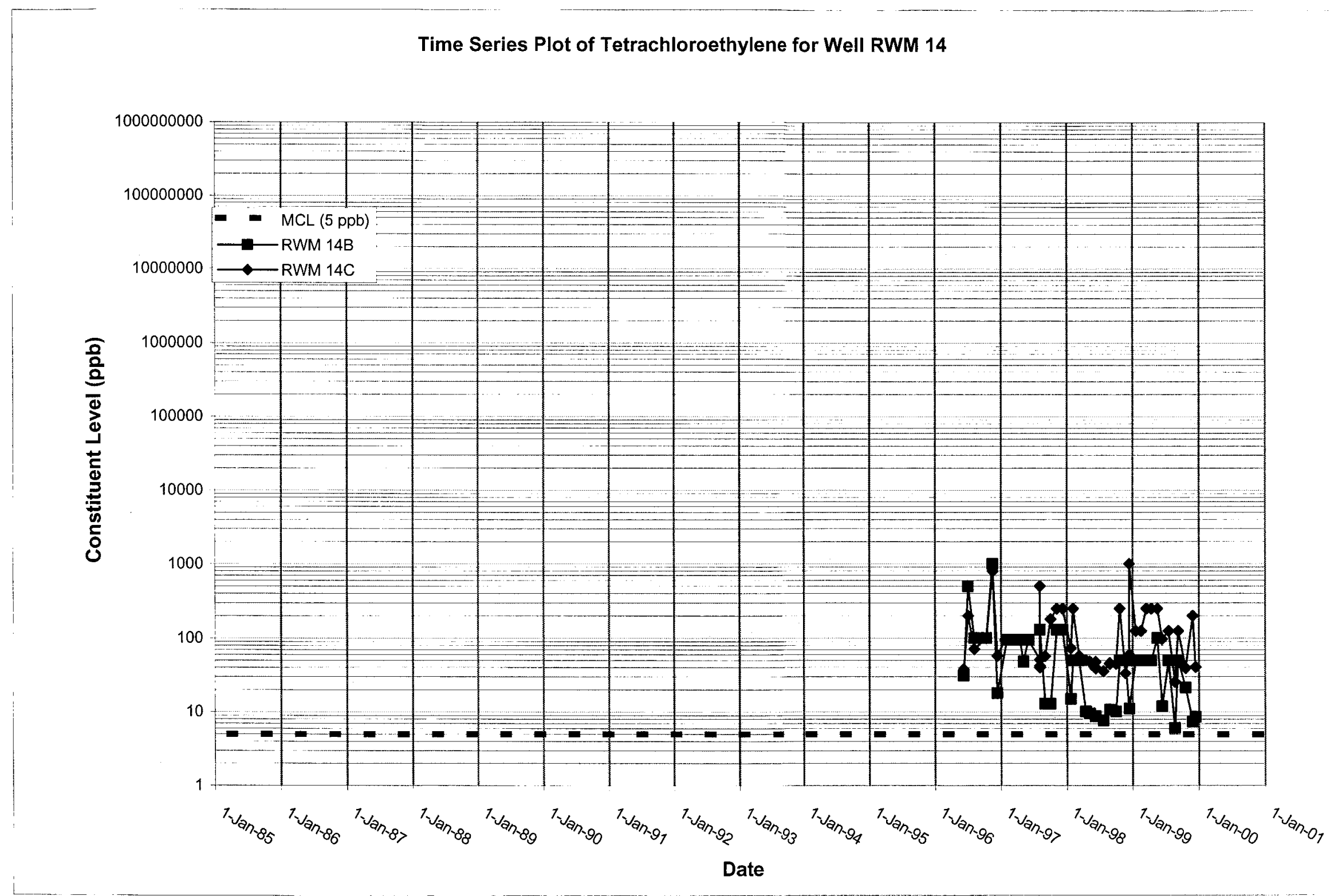




\section{Time Series Plot of Tetrachloroethylene for Well RWM 15}

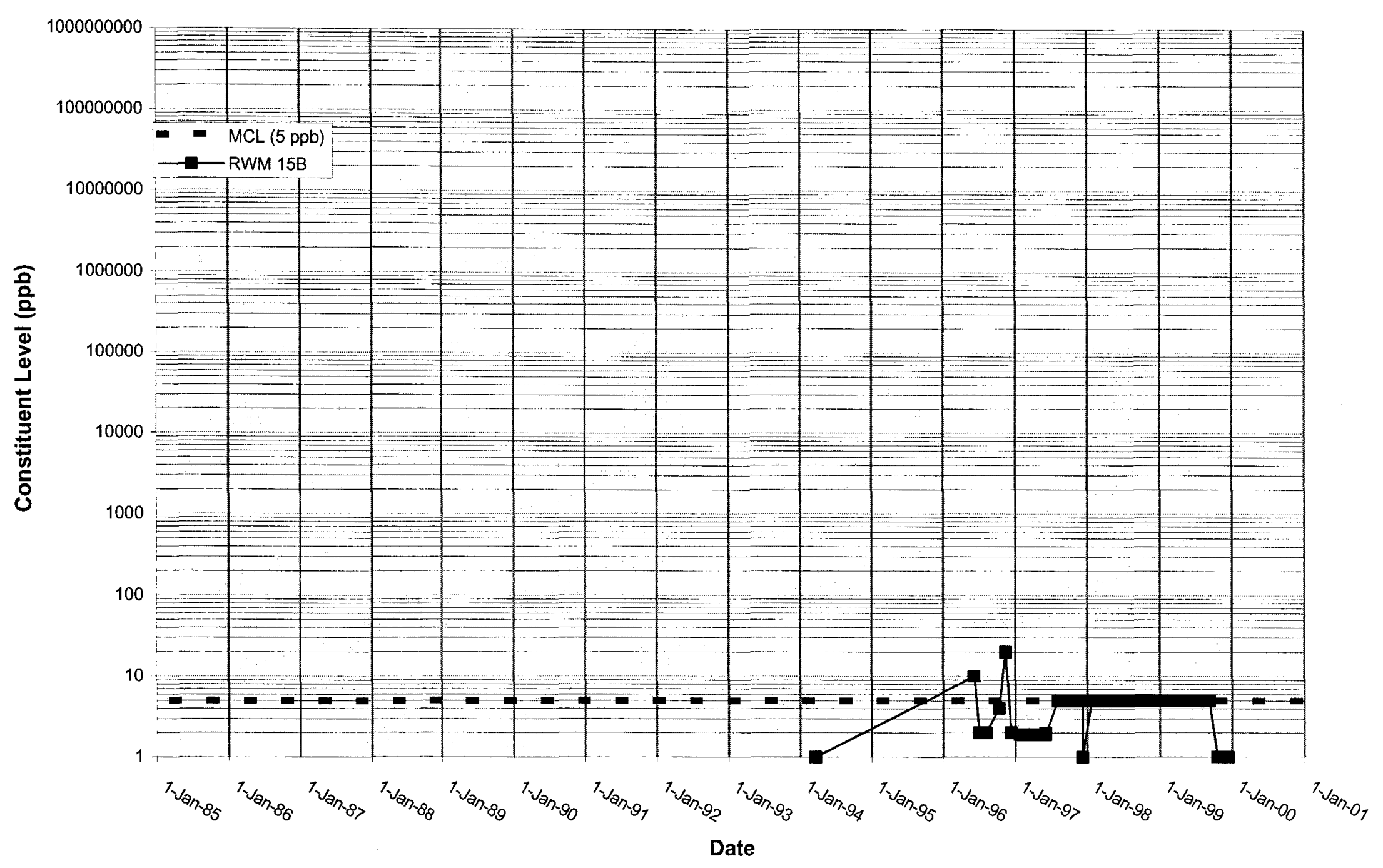


WSRC-TR-2000-00242 UNCLASSIFIED

\section{Time Series Plot of Tetrachloroethylene for Well SRW 1}

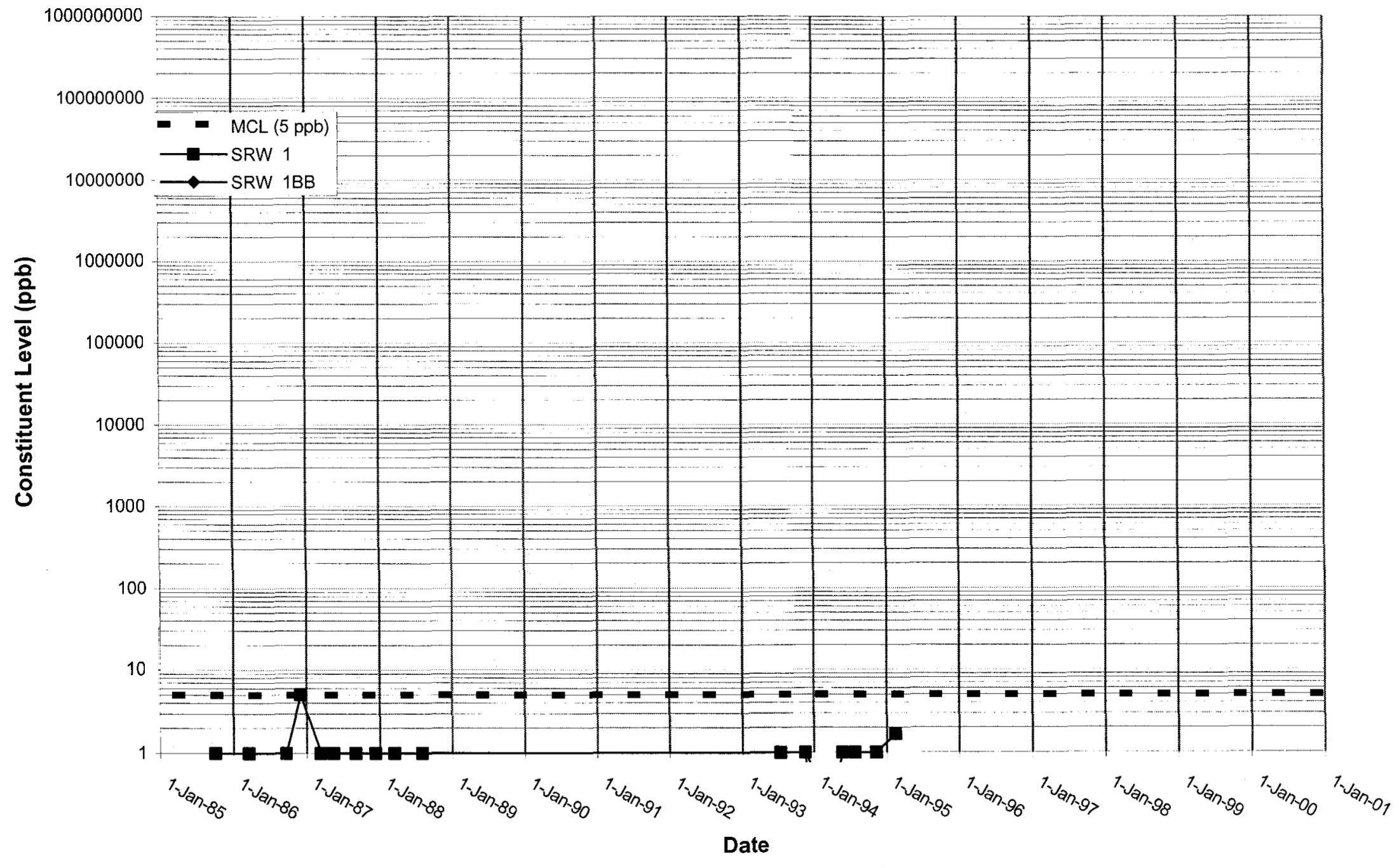


Time Series Plot of Tetrachloroethylene for Well SRW 2

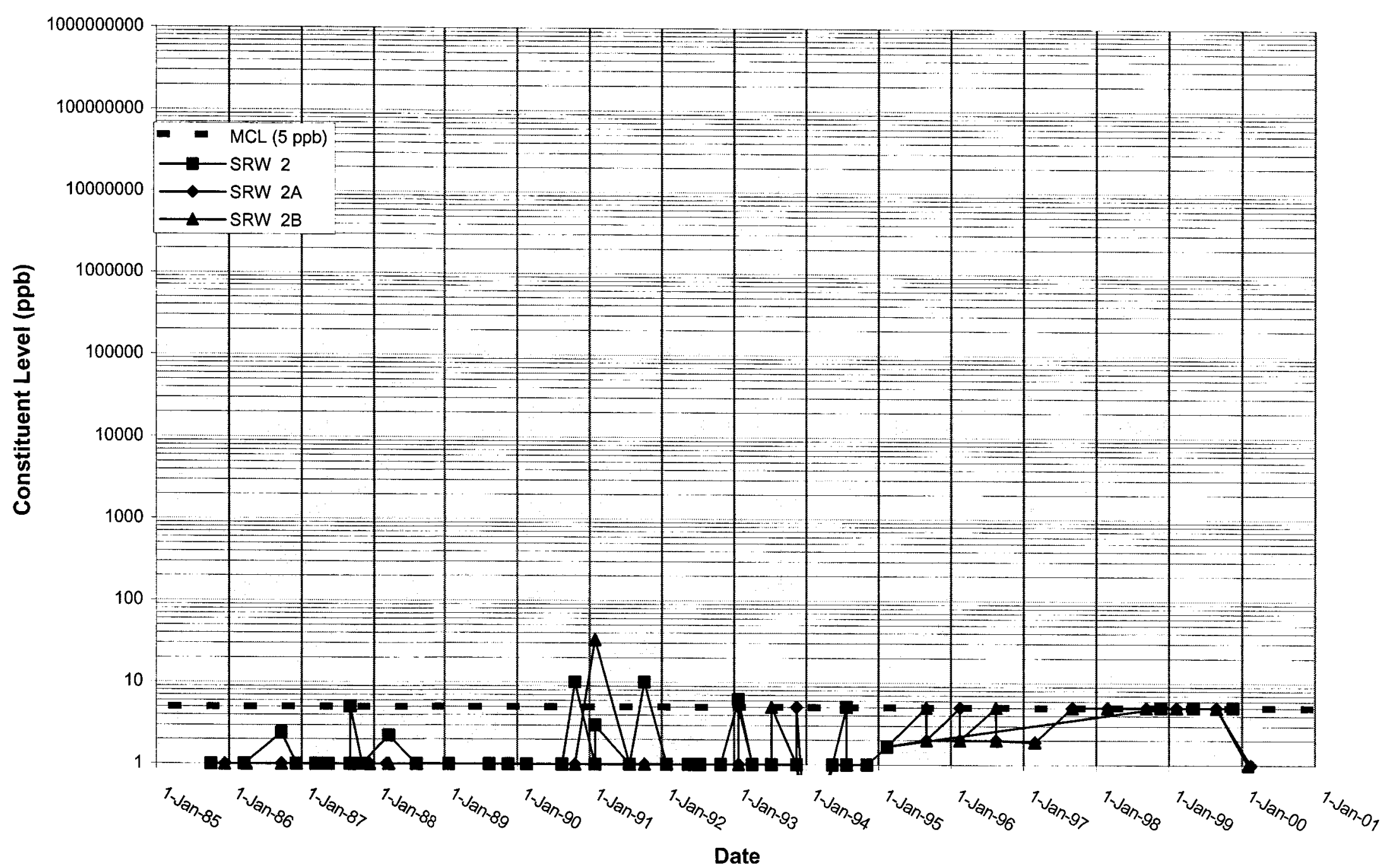


WSRC-TR-2000-00242 UNCLASSIFIED

\section{Time Series Plot of Tetrachloroethylene for Well SRW 3}

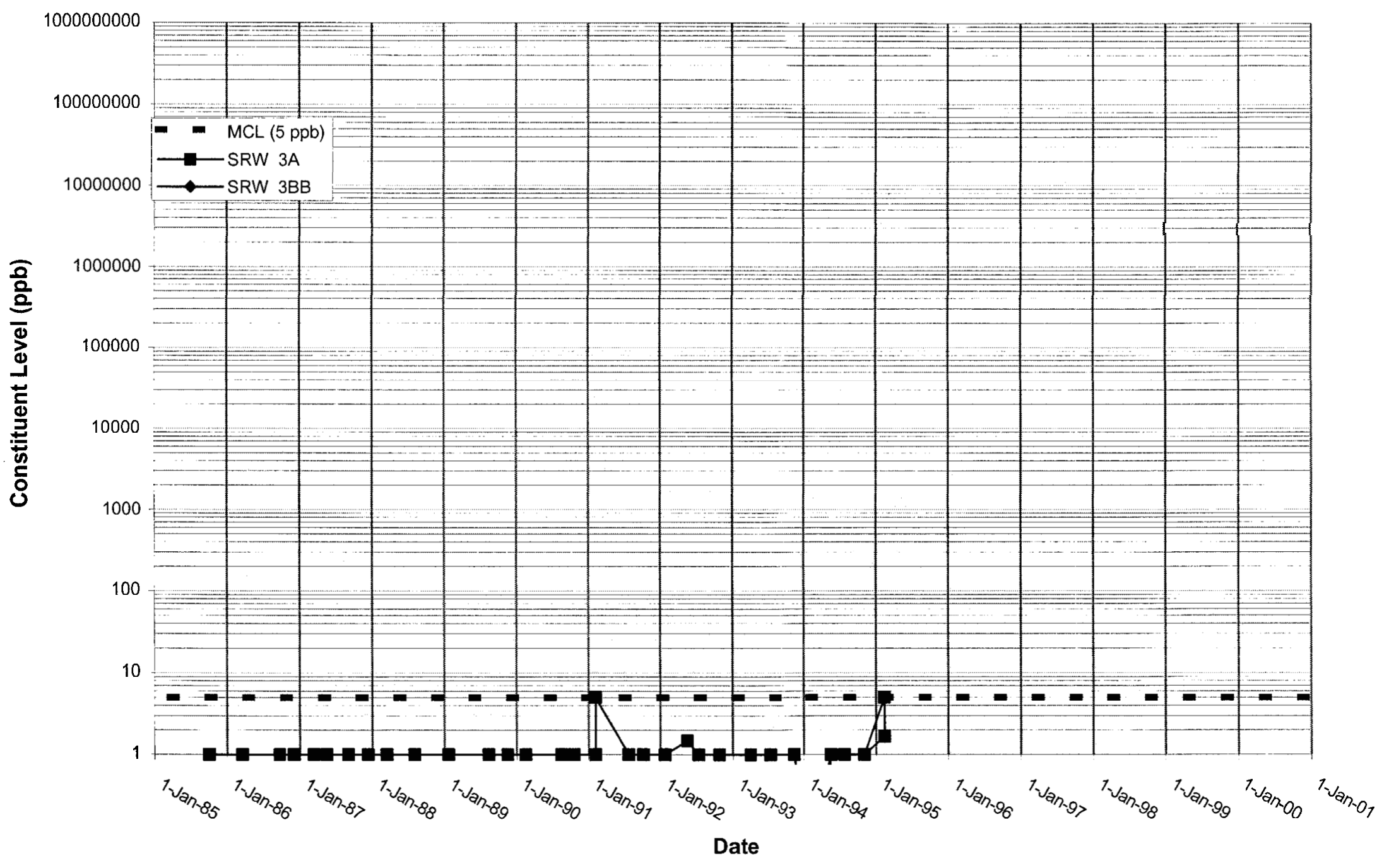


Time Series Plot of Tetrachloroethylene for Well SRW 4

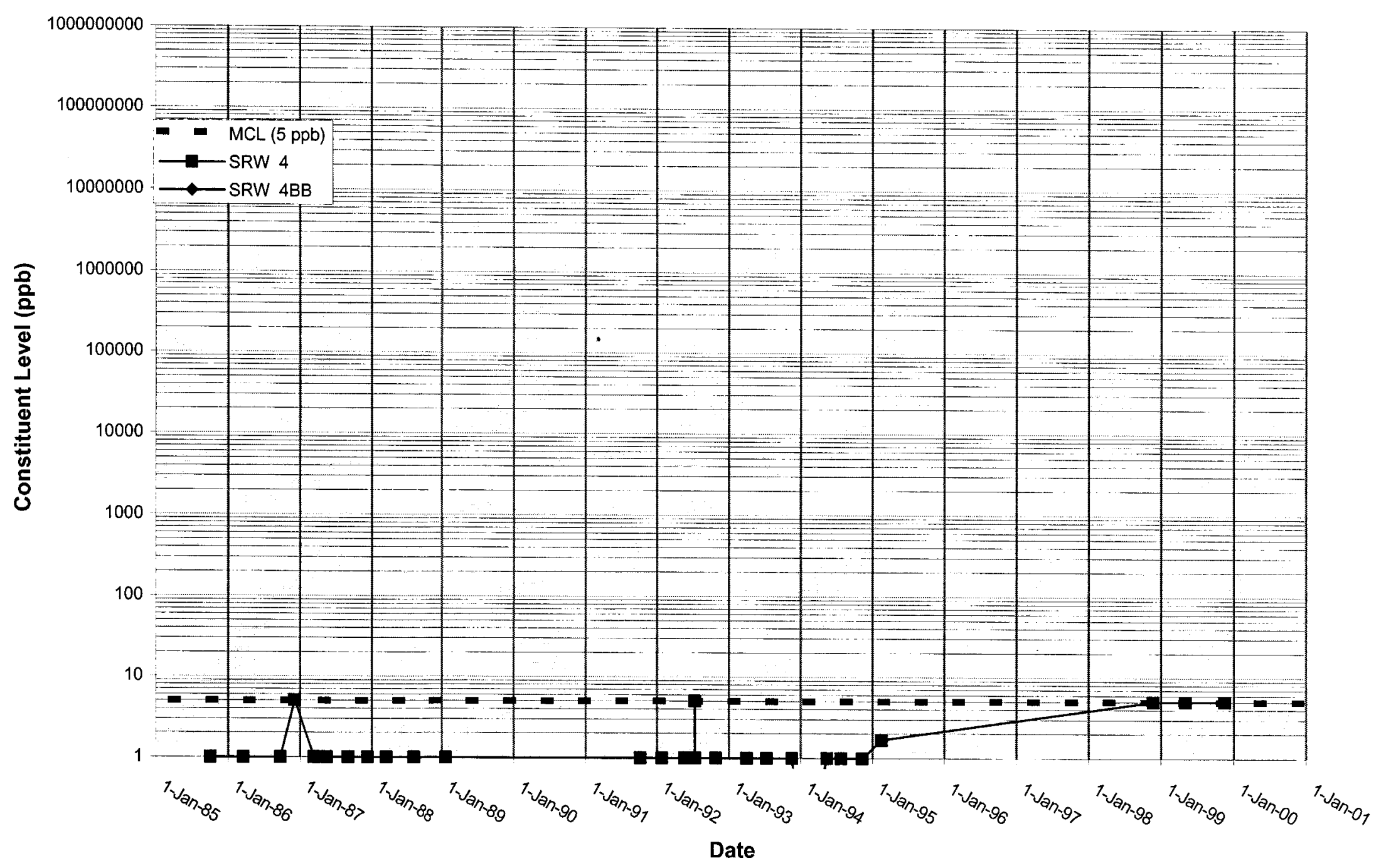


WSRC-TR-2000-00242 UNCLASSIFIED

Time Series Plot of Tetrachloroethylene for Well SRW 5

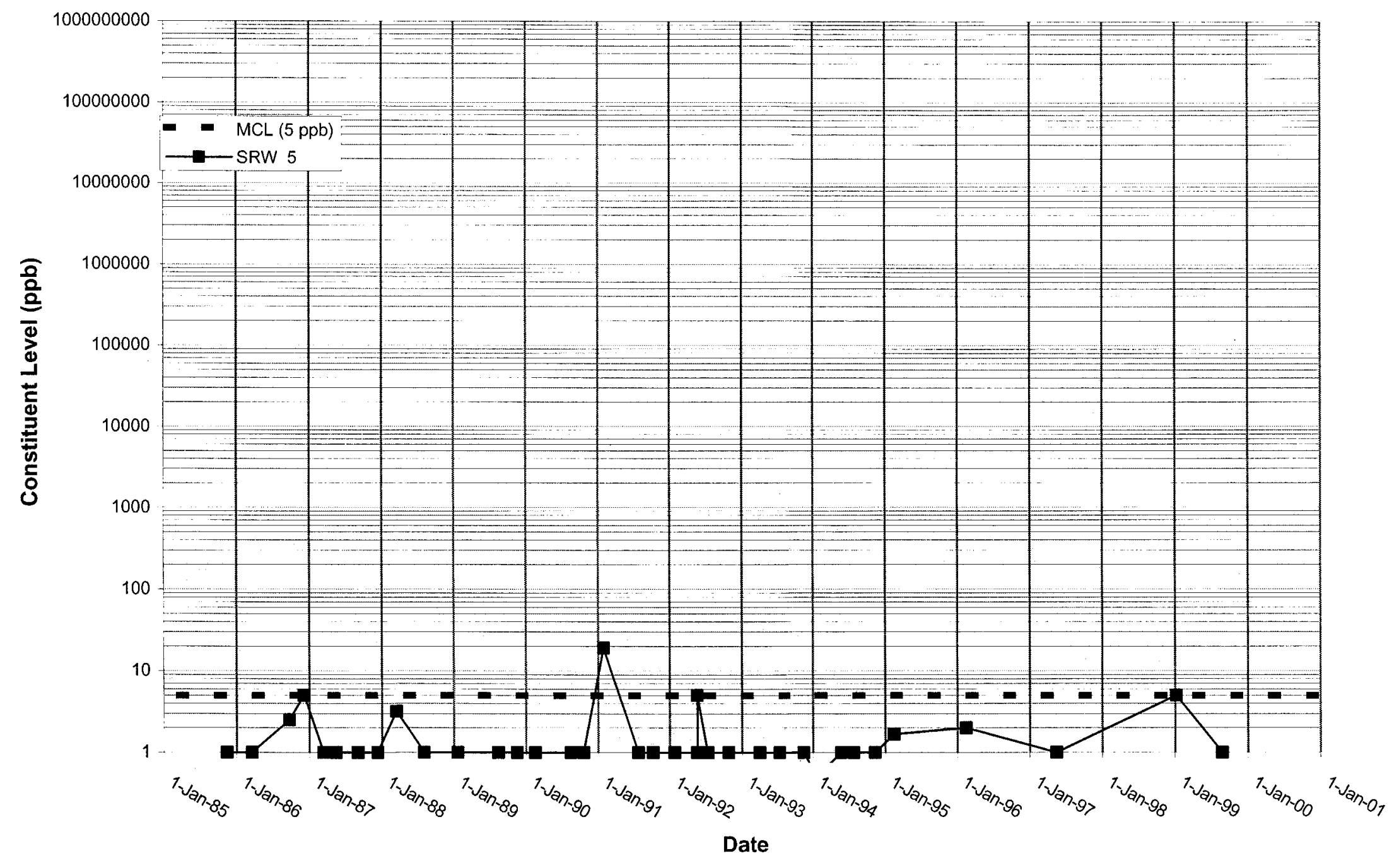


WSRC-TR-2000-00242

UNCLASSIFIED

\section{Time Series Plot of Tetrachloroethylene for Well SRW 6}

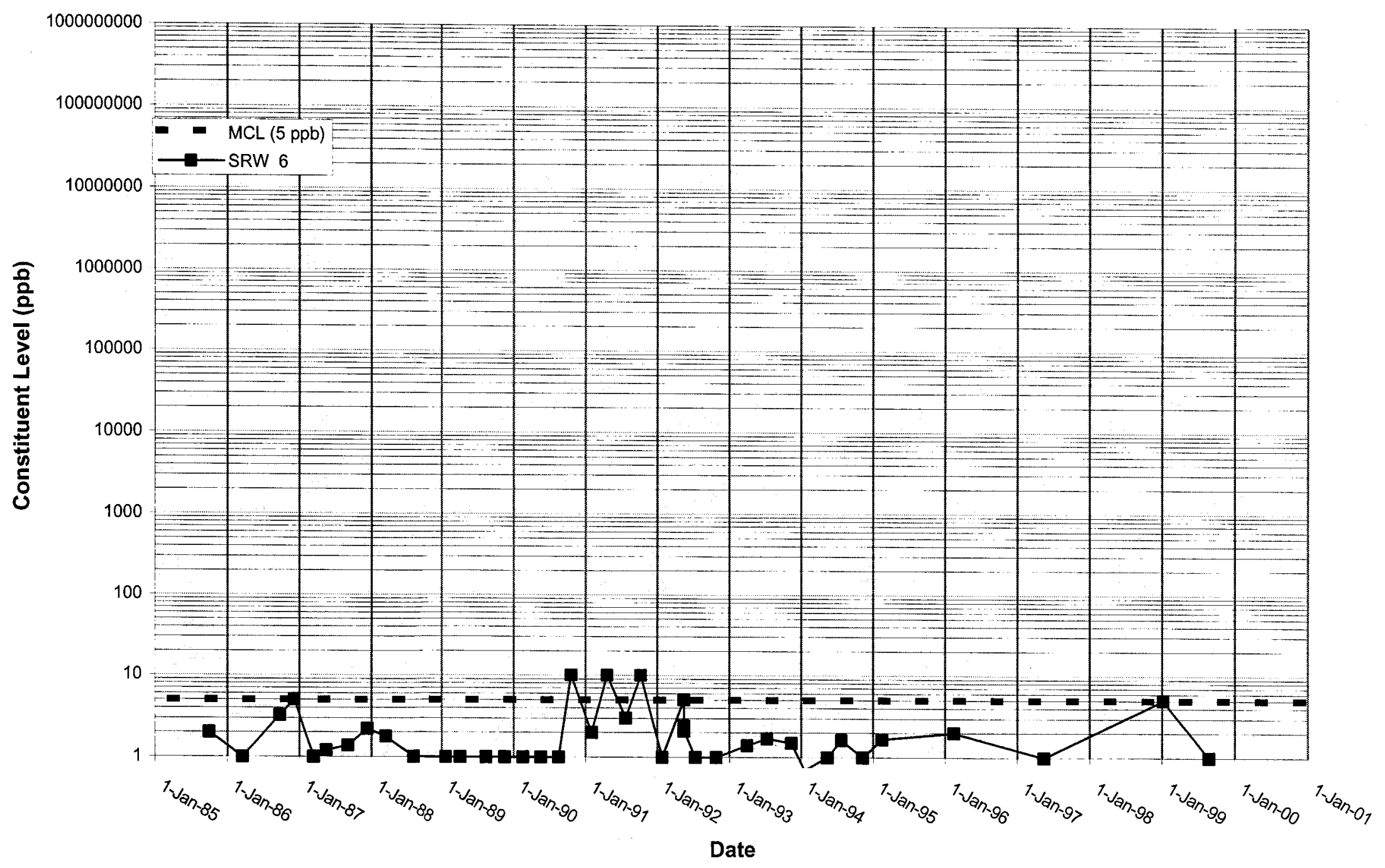


WSRC-TR-2000-00242 UNCLASSIFIED

\section{Time Series Plot of Tetrachloroethylene for Well SRW 7}

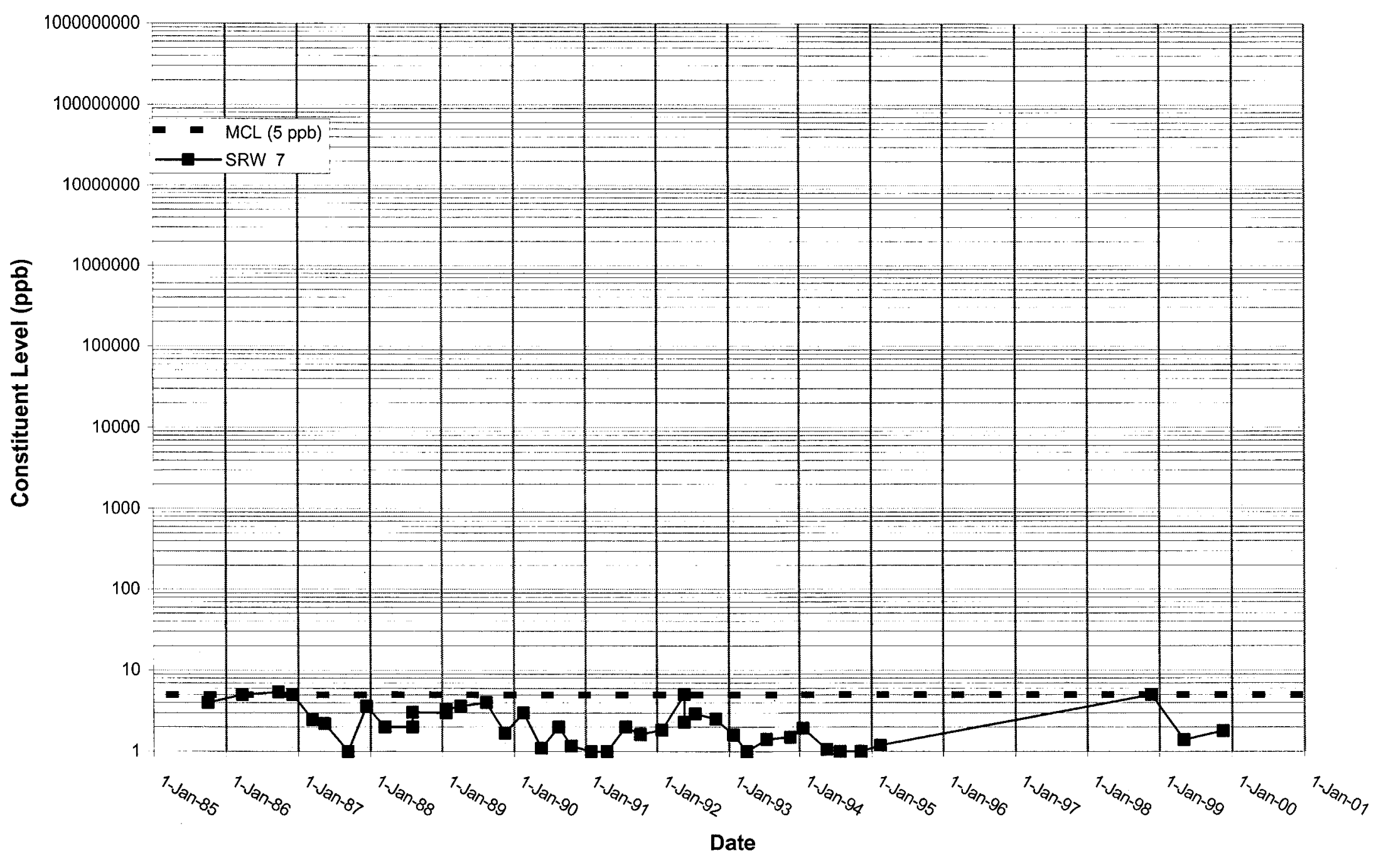


Time Series Plot of Tetrachloroethylene for Well SRW 8

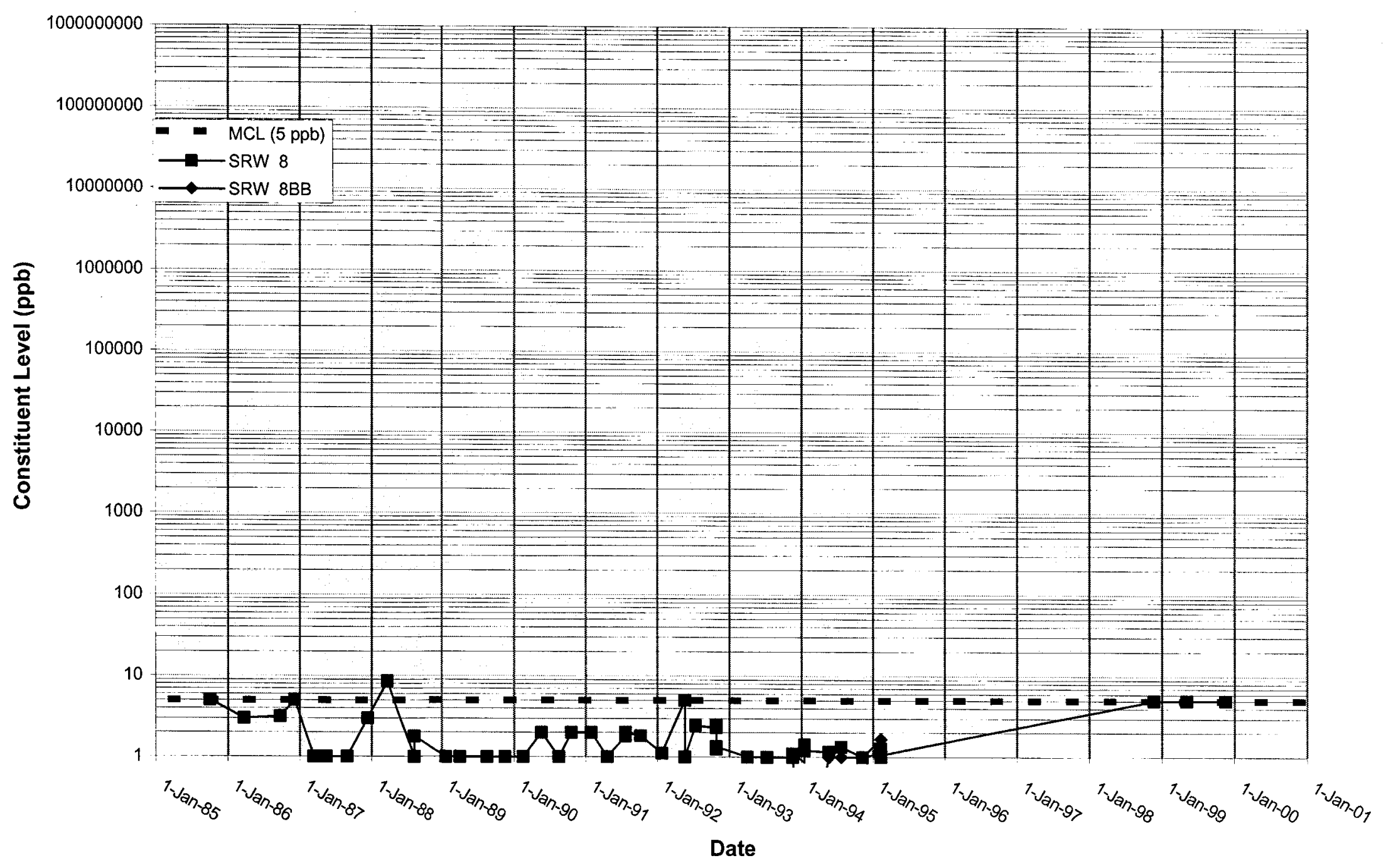


WSRC-TR-2000-00242 UNCLASSIFIED

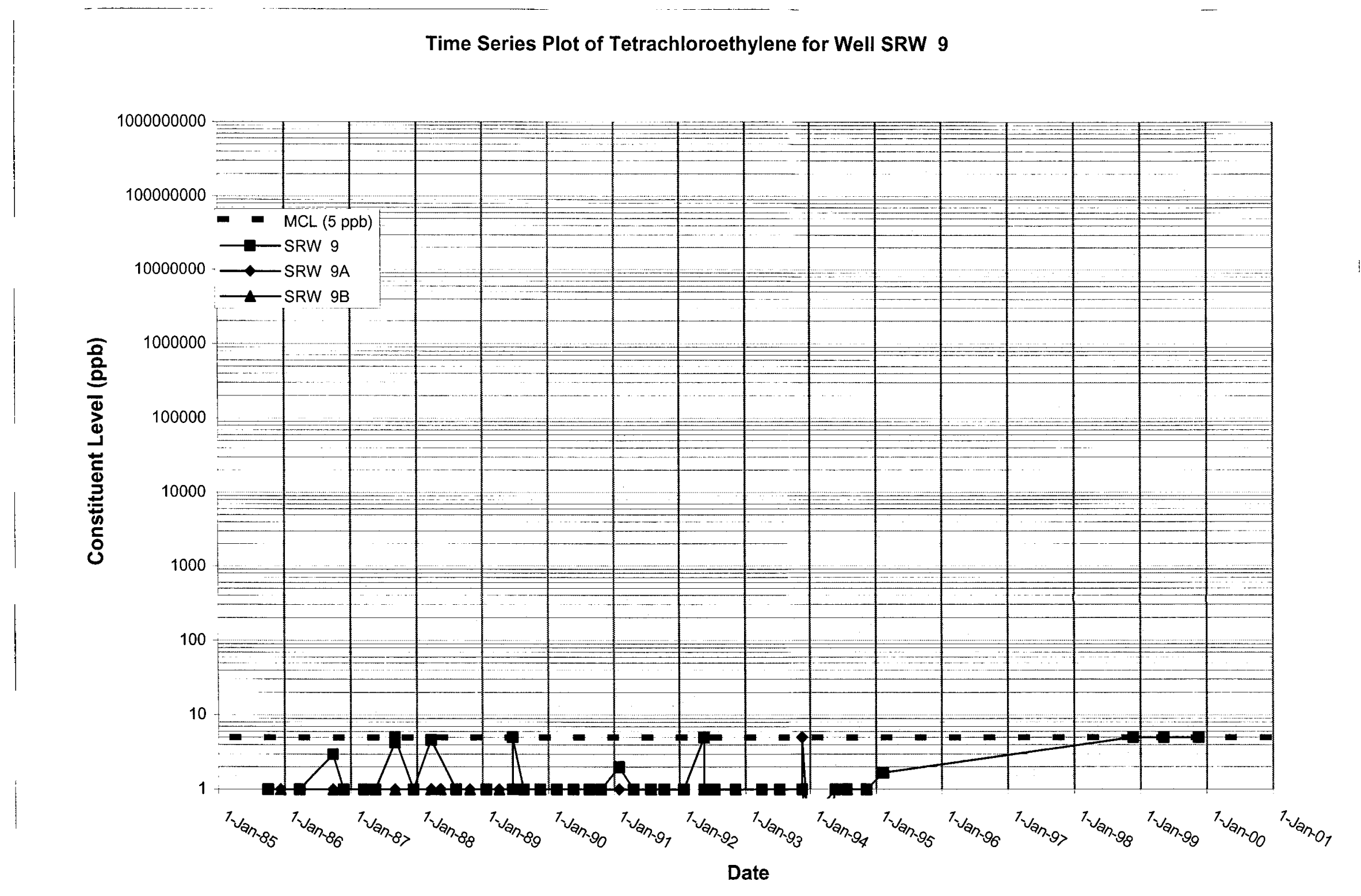


WSRC-TR-2000-00242

UNCLASSIFIED

Time Series Plot of Tetrachloroethylene for Well SRW 10

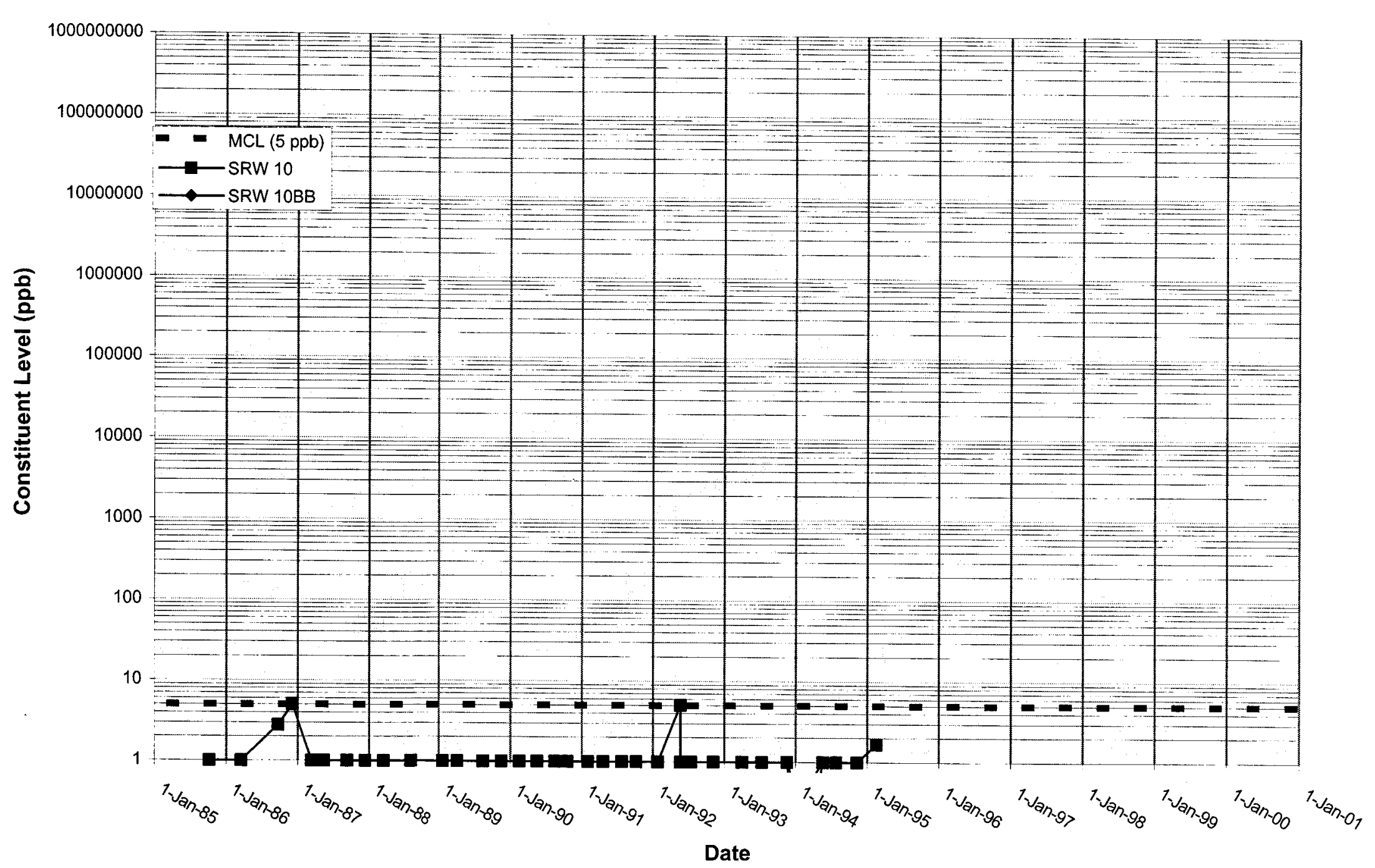


WSRC-TR-2000-00242 UNCLASSIFIED

Time Series Plot of Tetrachloroethylene for Well SRW 11

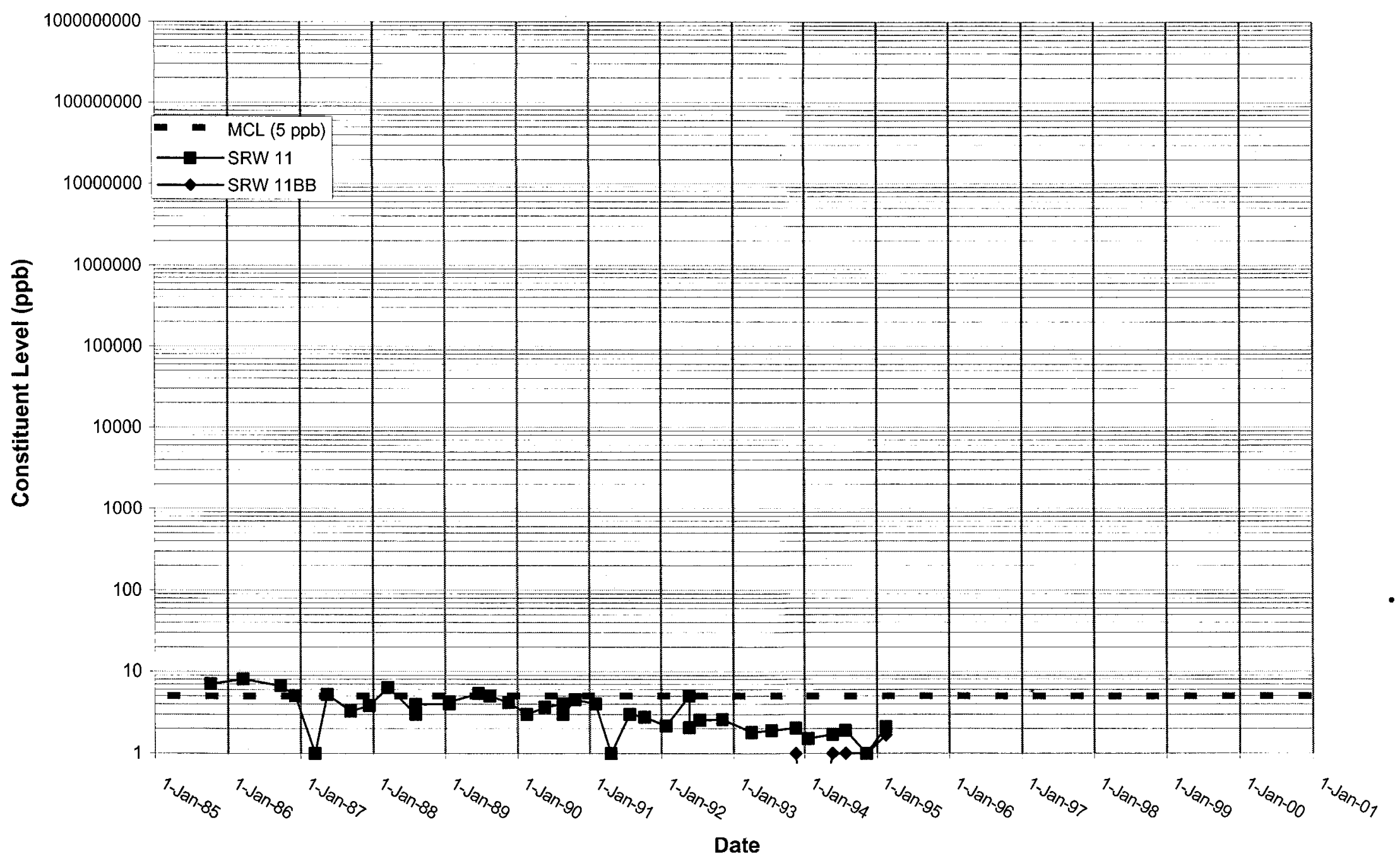




\section{Time Series Plot of Tetrachloroethylene for Well SRW 12}

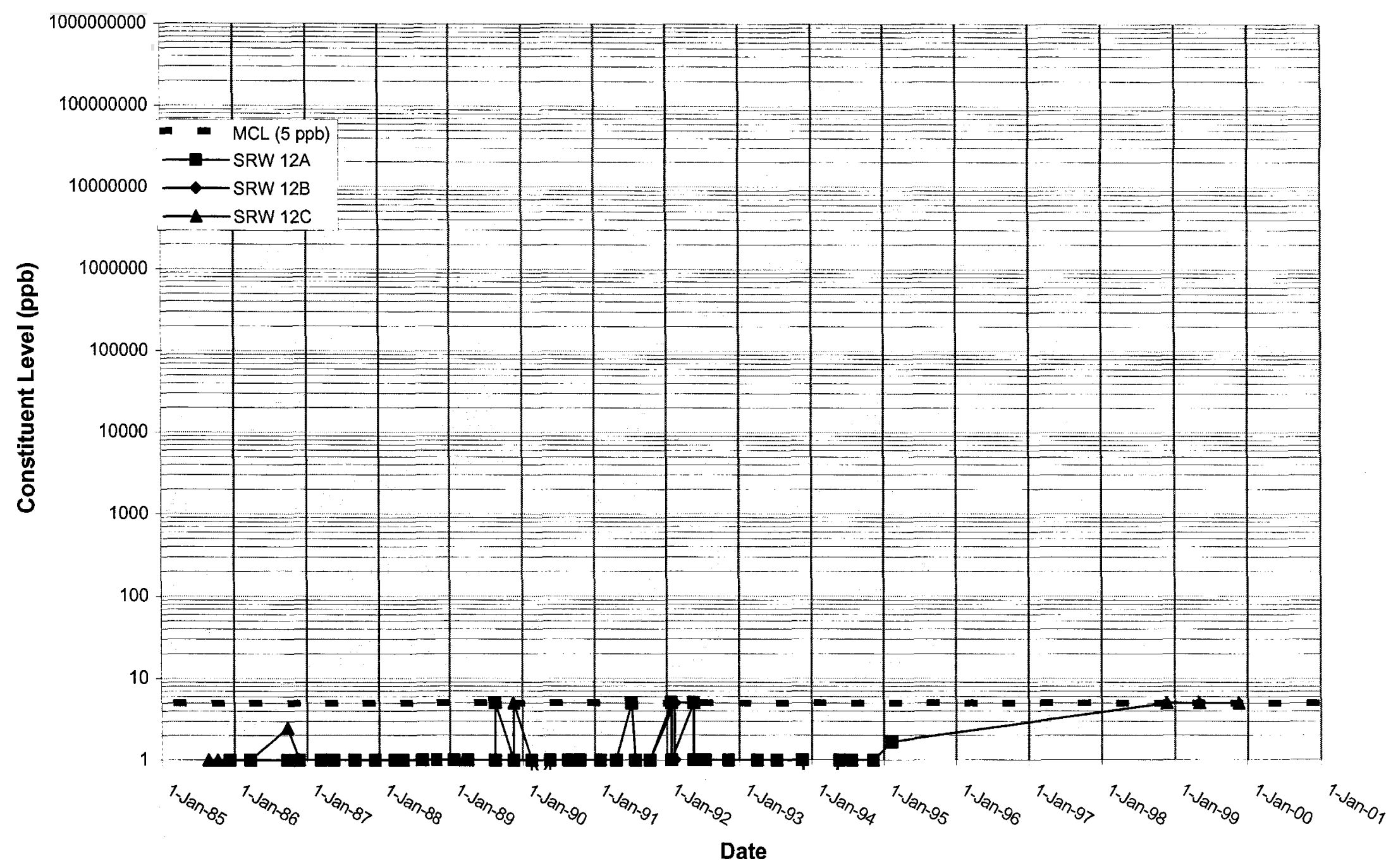


WSRC-TR-2000-00242 UNCLASSIFIED

Time Series Plot of Tetrachloroethylene for Well SRW 13

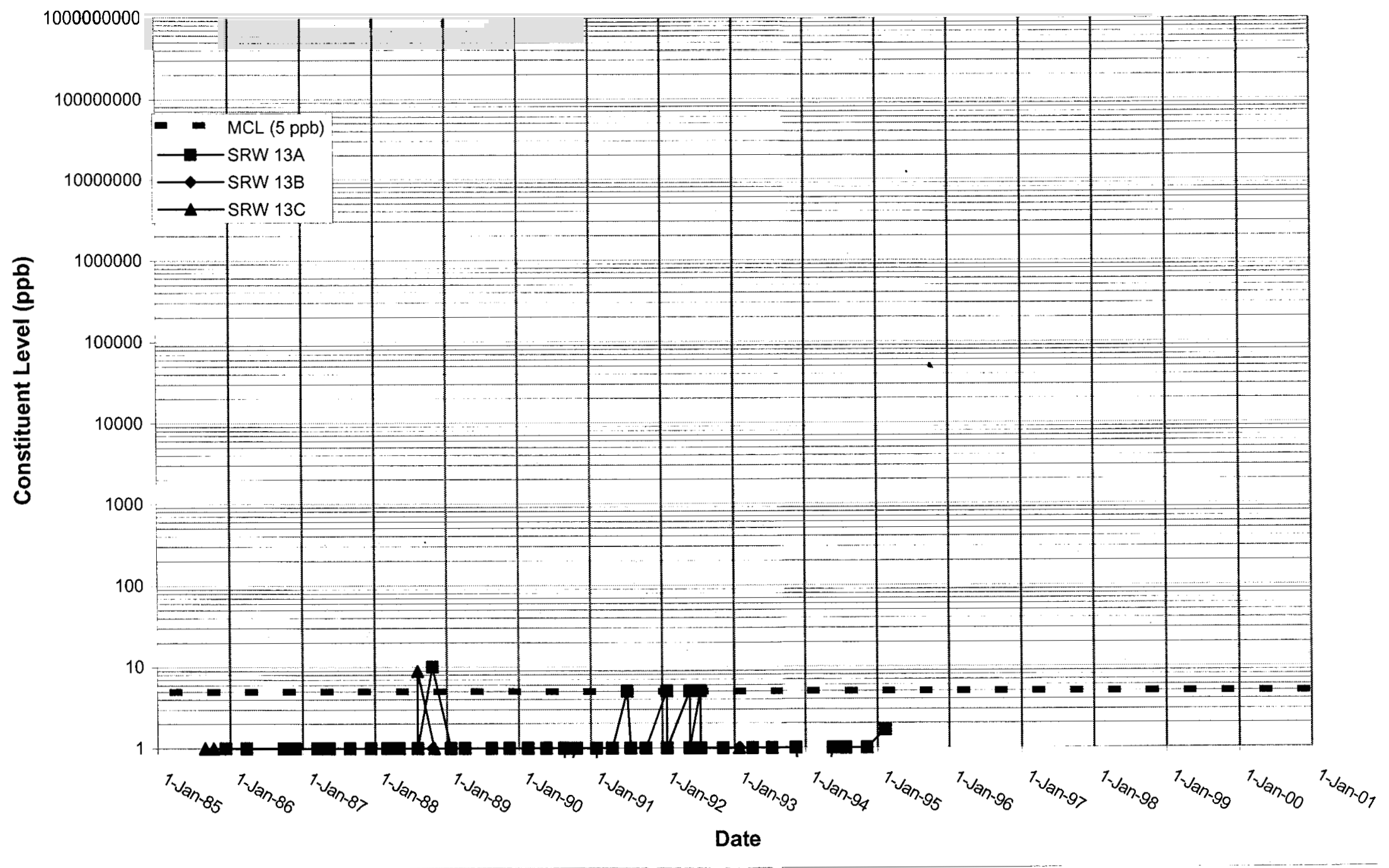




\section{Time Series Plot of Tetrachloroethylene for Well SRW 14}

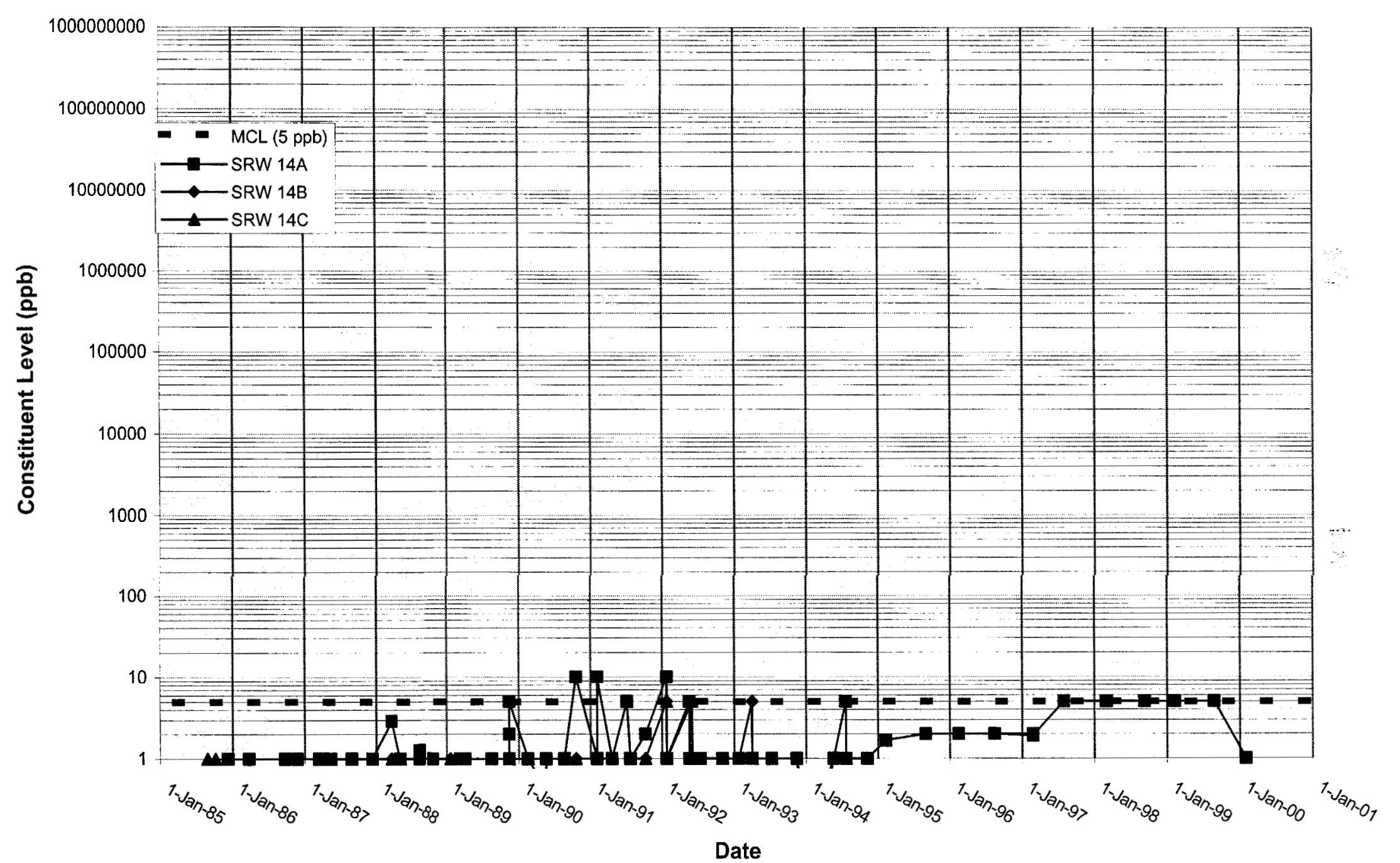


WSRC-TR-2000-00242 UNCLASSIFIED

Time Series Plot of Tetrachloroethylene for Well SRW 15

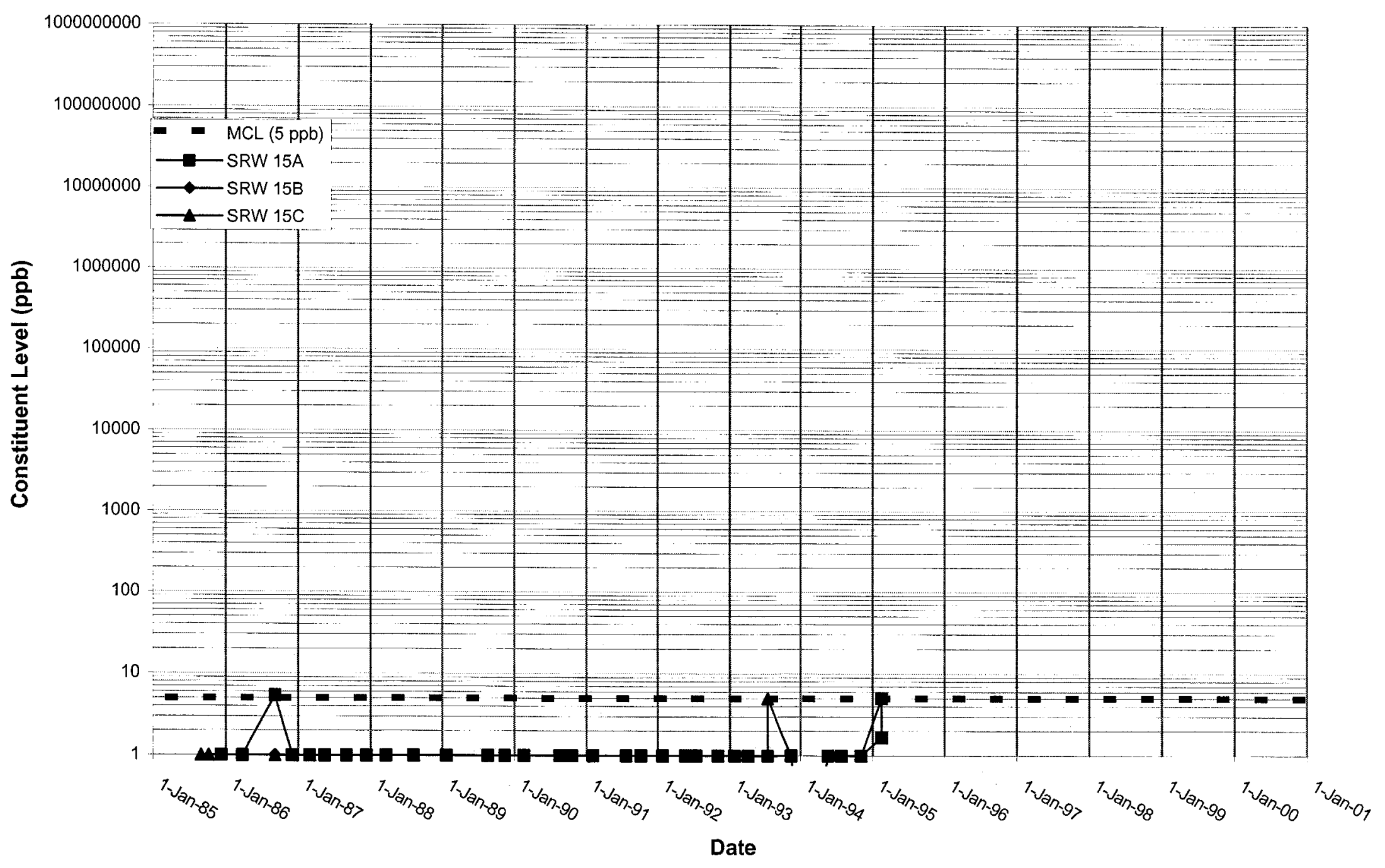


Time Series Plot of Tetrachloroethylene for Well SRW 16

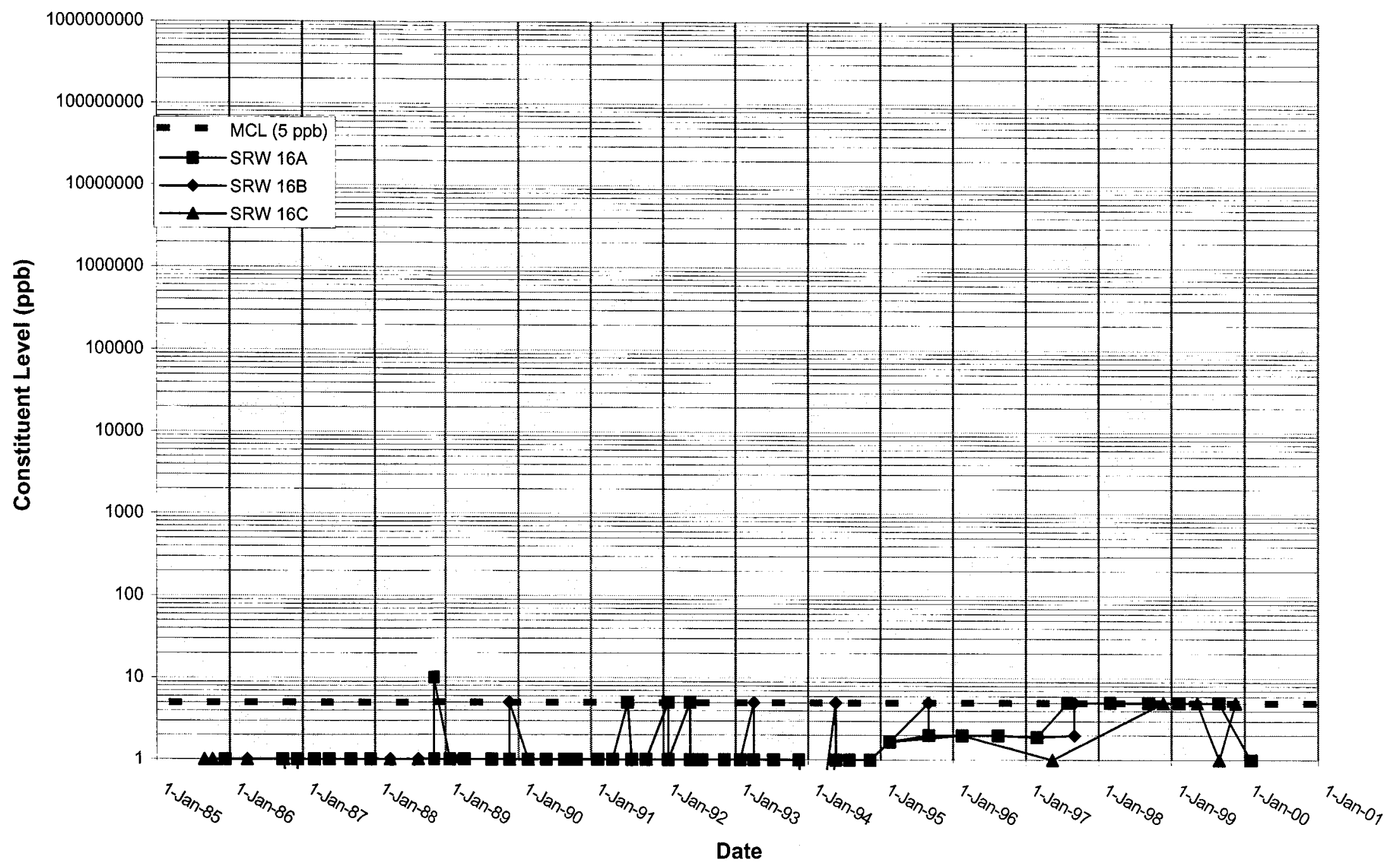


WSRC-TR-2000-00242 UNCLASSIFIED

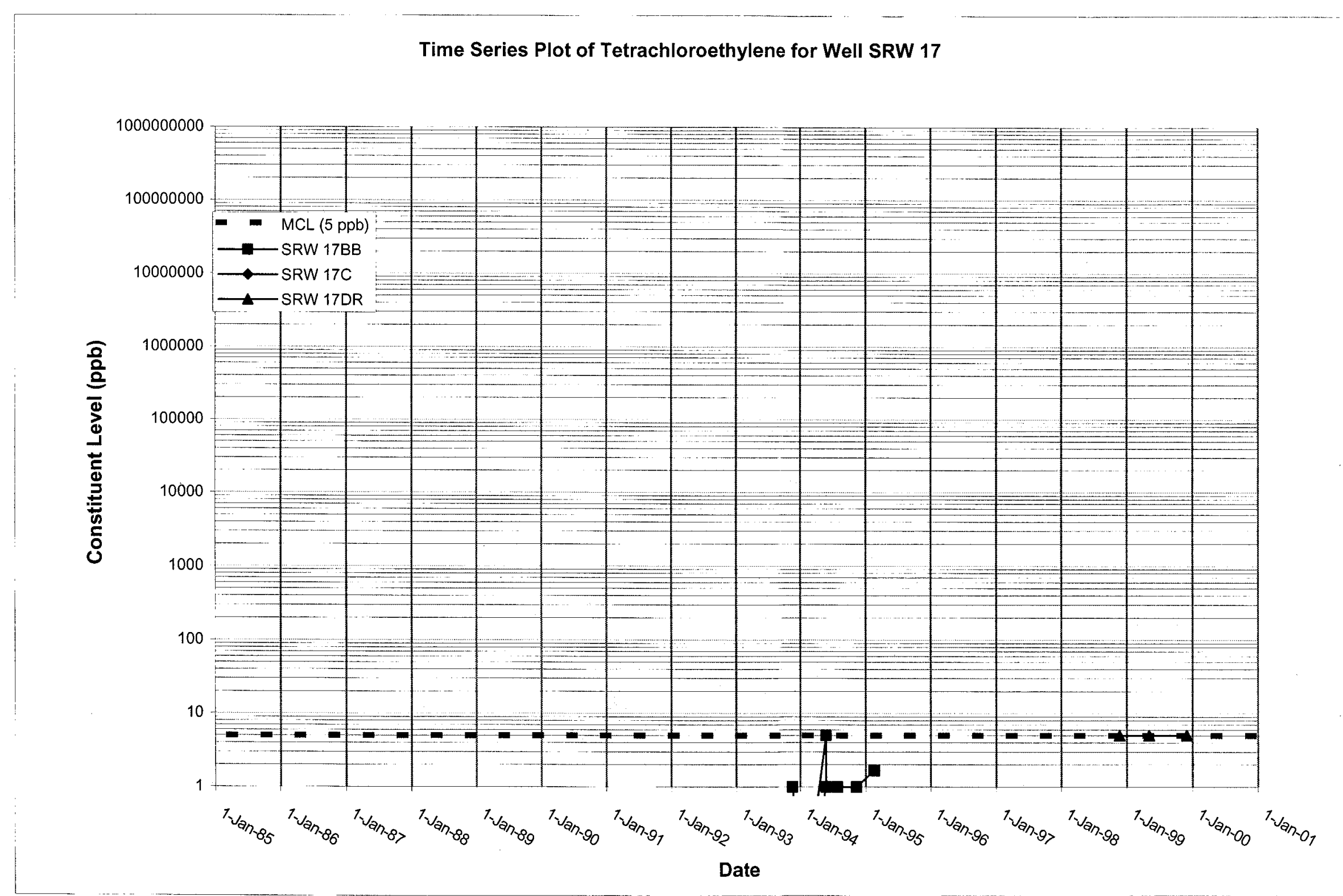




\section{Time Series Plot of Tetrachloroethylene for Well SRW 18}

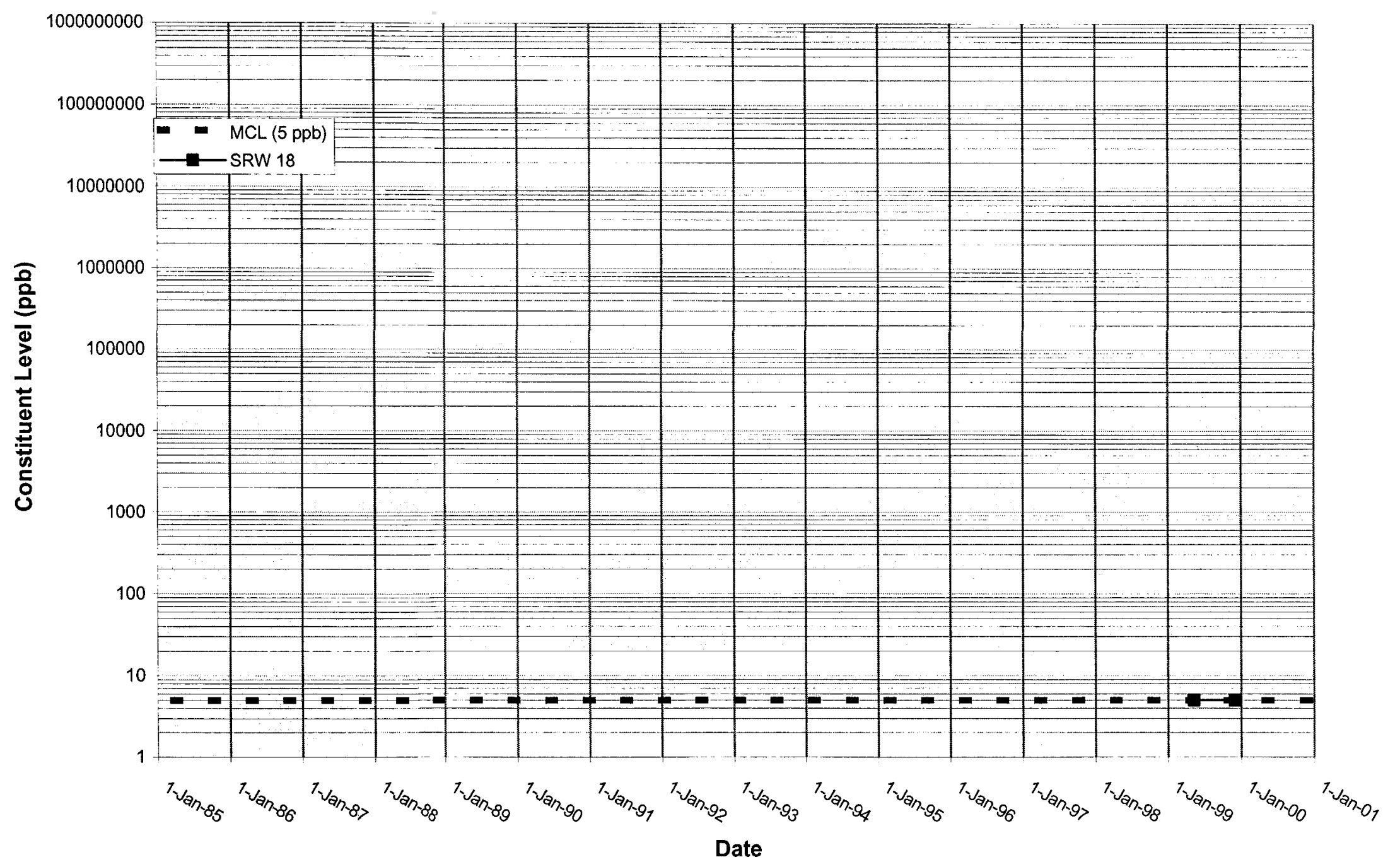


WSRC-TR-2000-00242 UNCLASSIFIED

Time Series Plot of Tetrachloroethylene for Well SRW 19

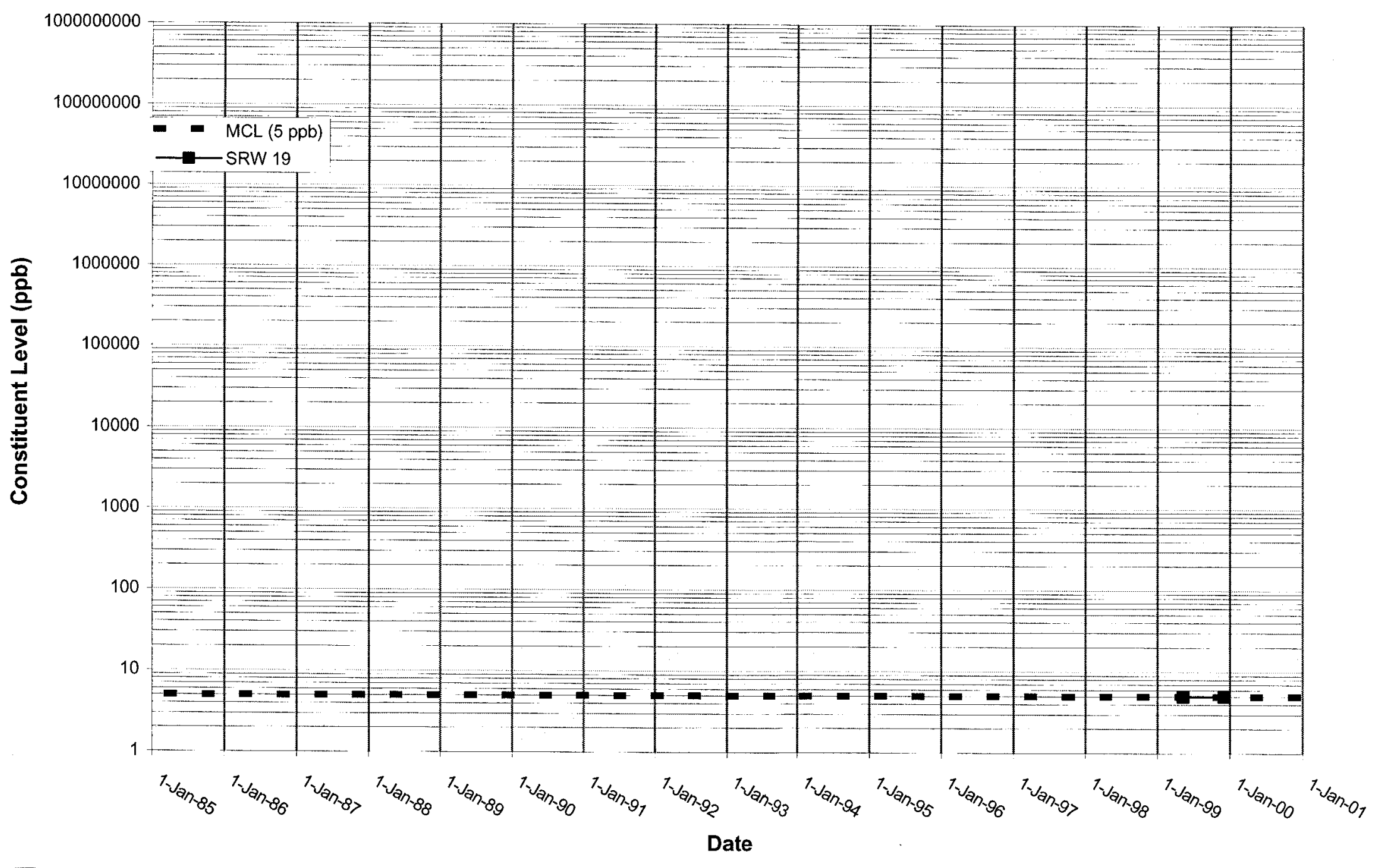


WSRC-TR-2000-00242

Unclassified

\section{Appendix G}

\section{Hydrographs}


WSRC-TR-2000-00242

Unclassified

THIS PAGE LEFT BLANK INTENTIONALLY. 


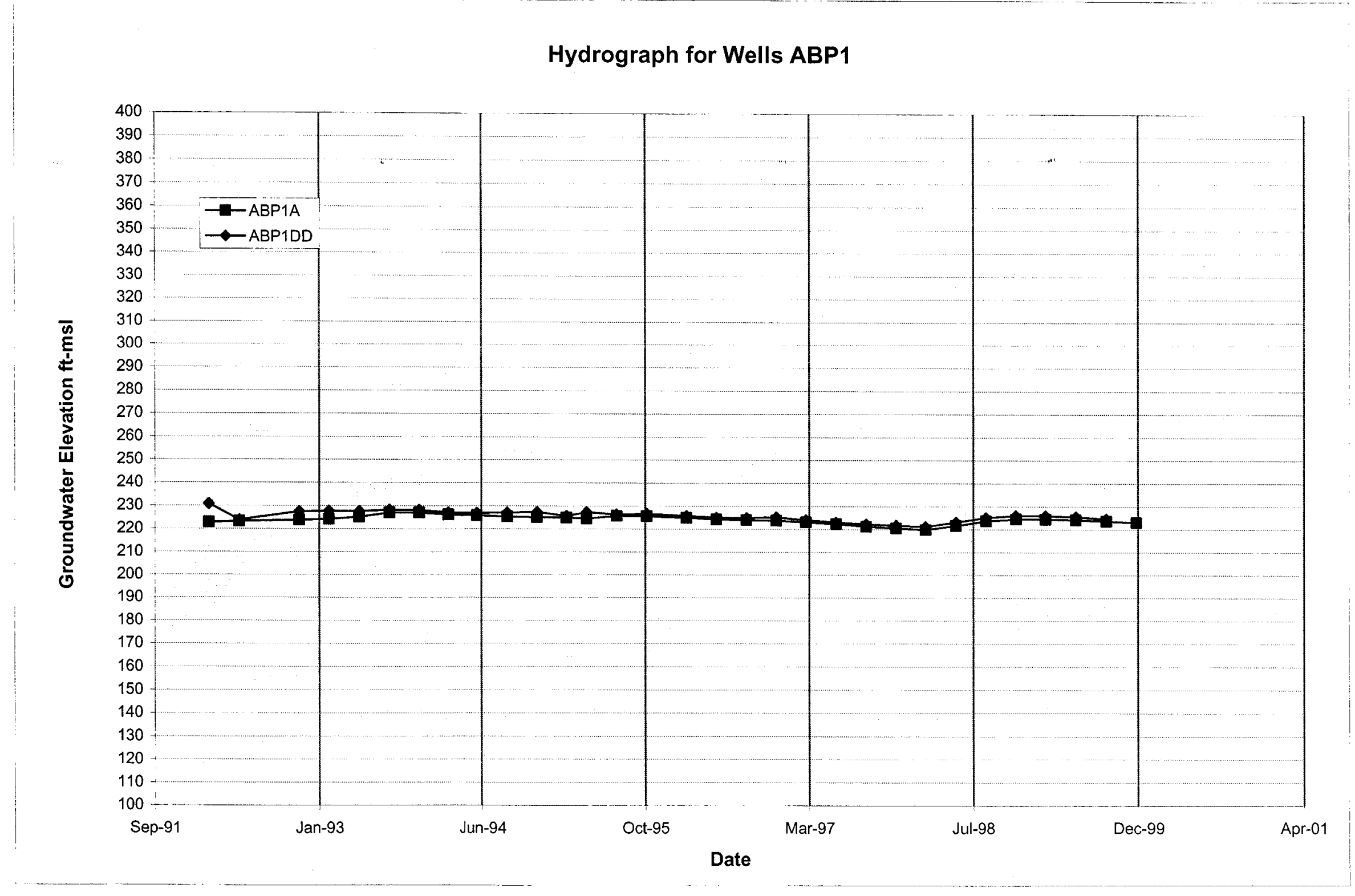


WSRC-TR-2000-00242 UNCLASSIFIED

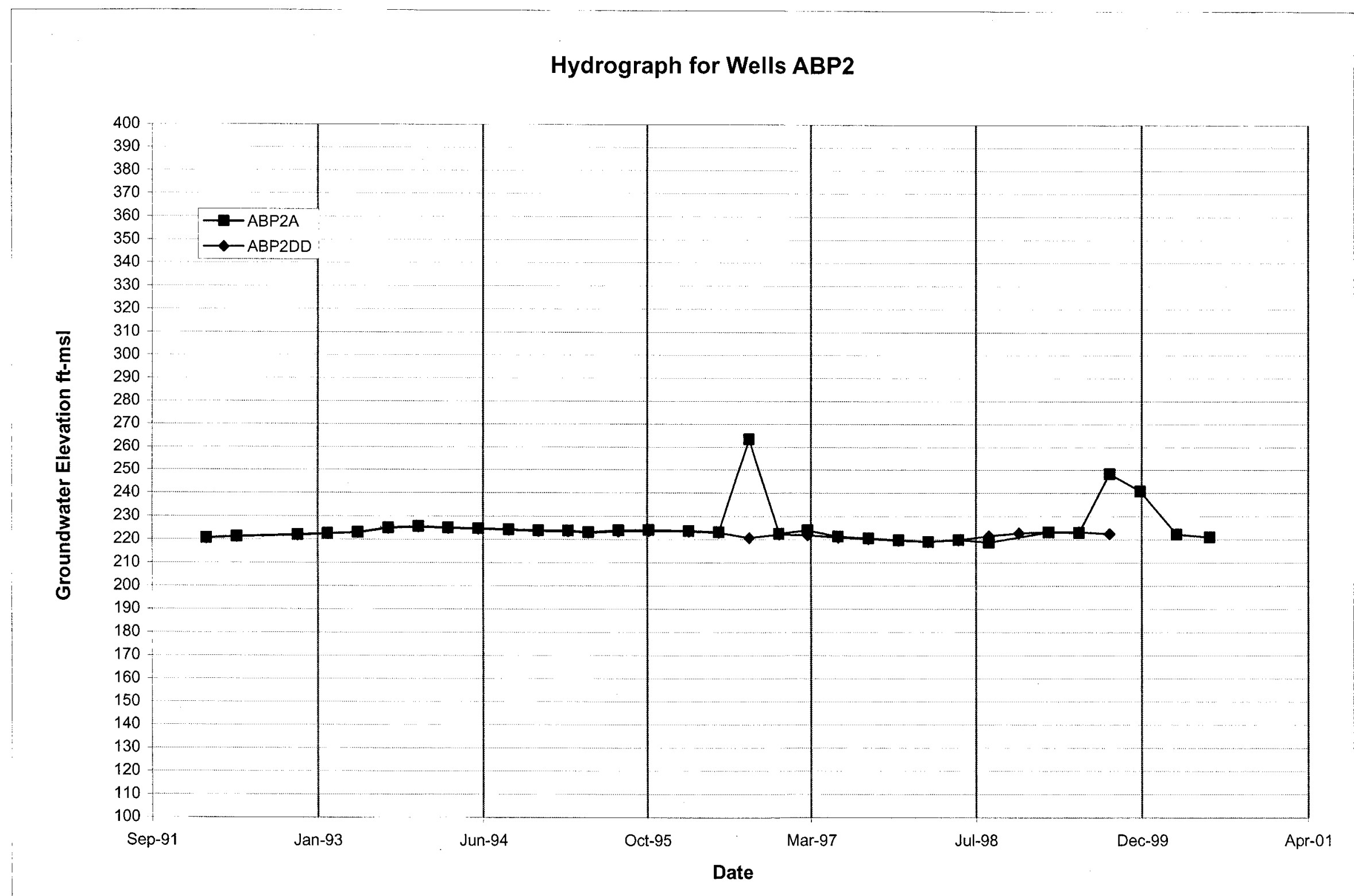




\section{Hydrograph for Wells ABP3}

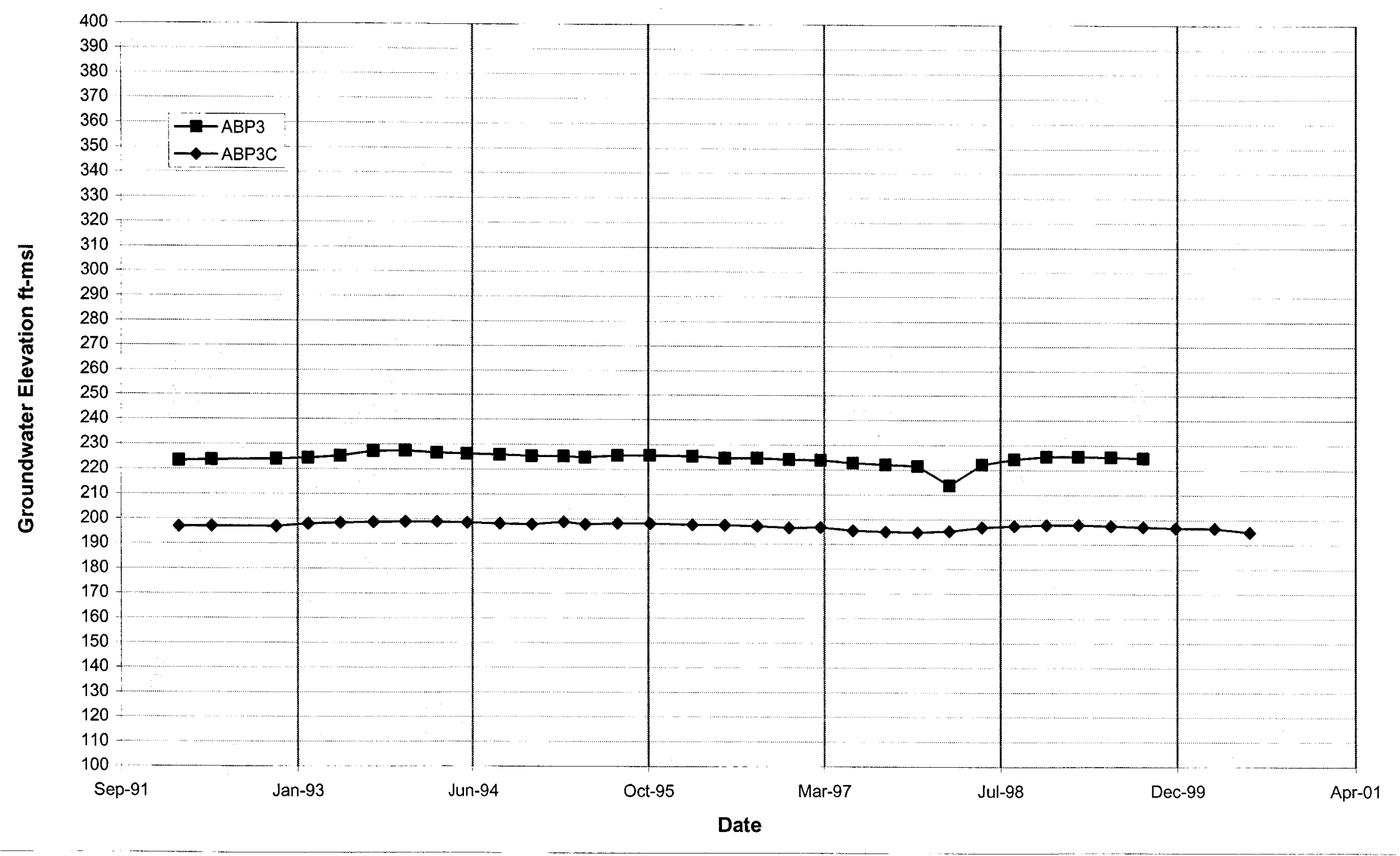


WSRC-TR-2000-00242 UNCLASSIFIED

Hydrograph for Wells ABP4

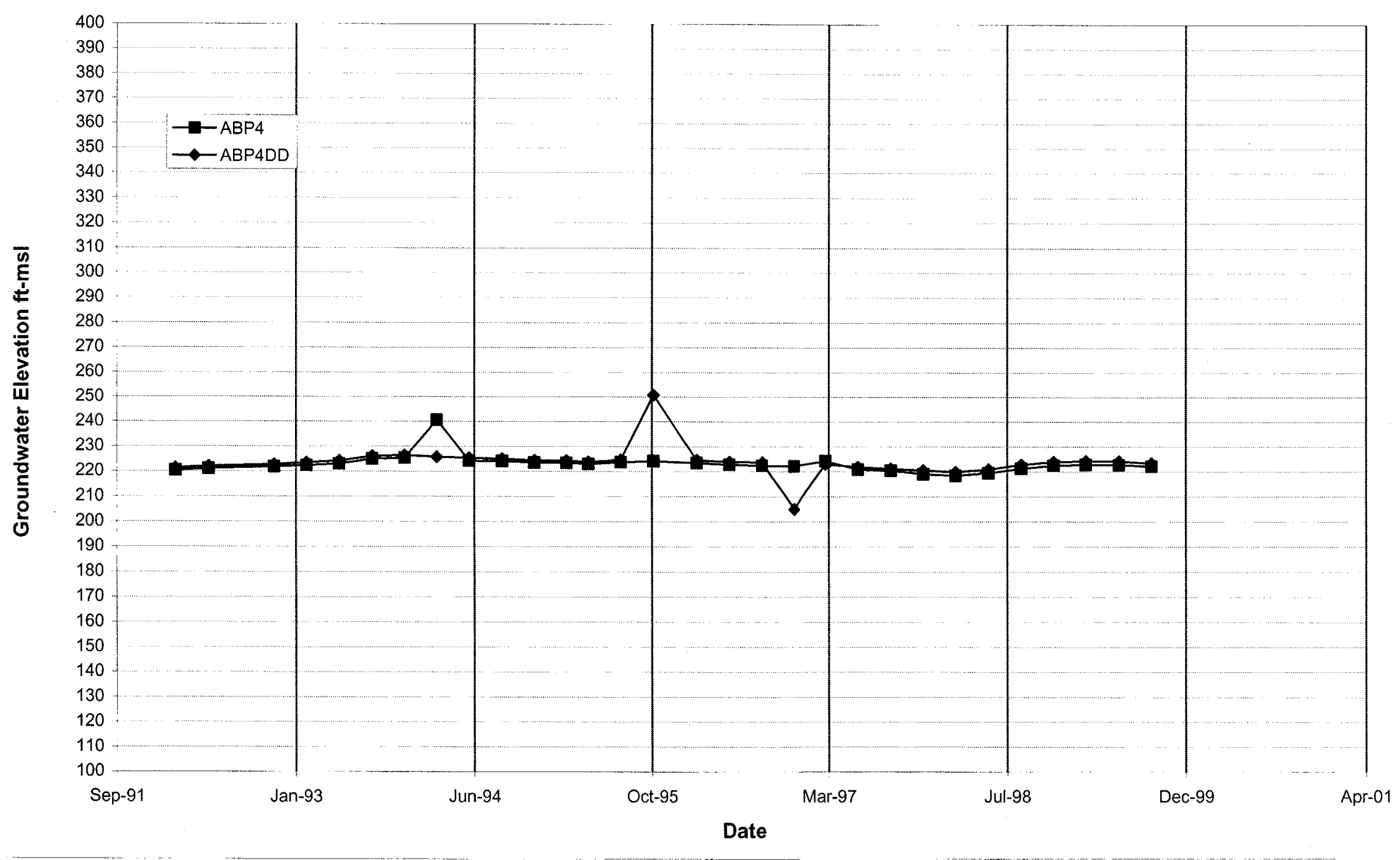


WSRC-TR-2000-00242 UNCLASSIFIED

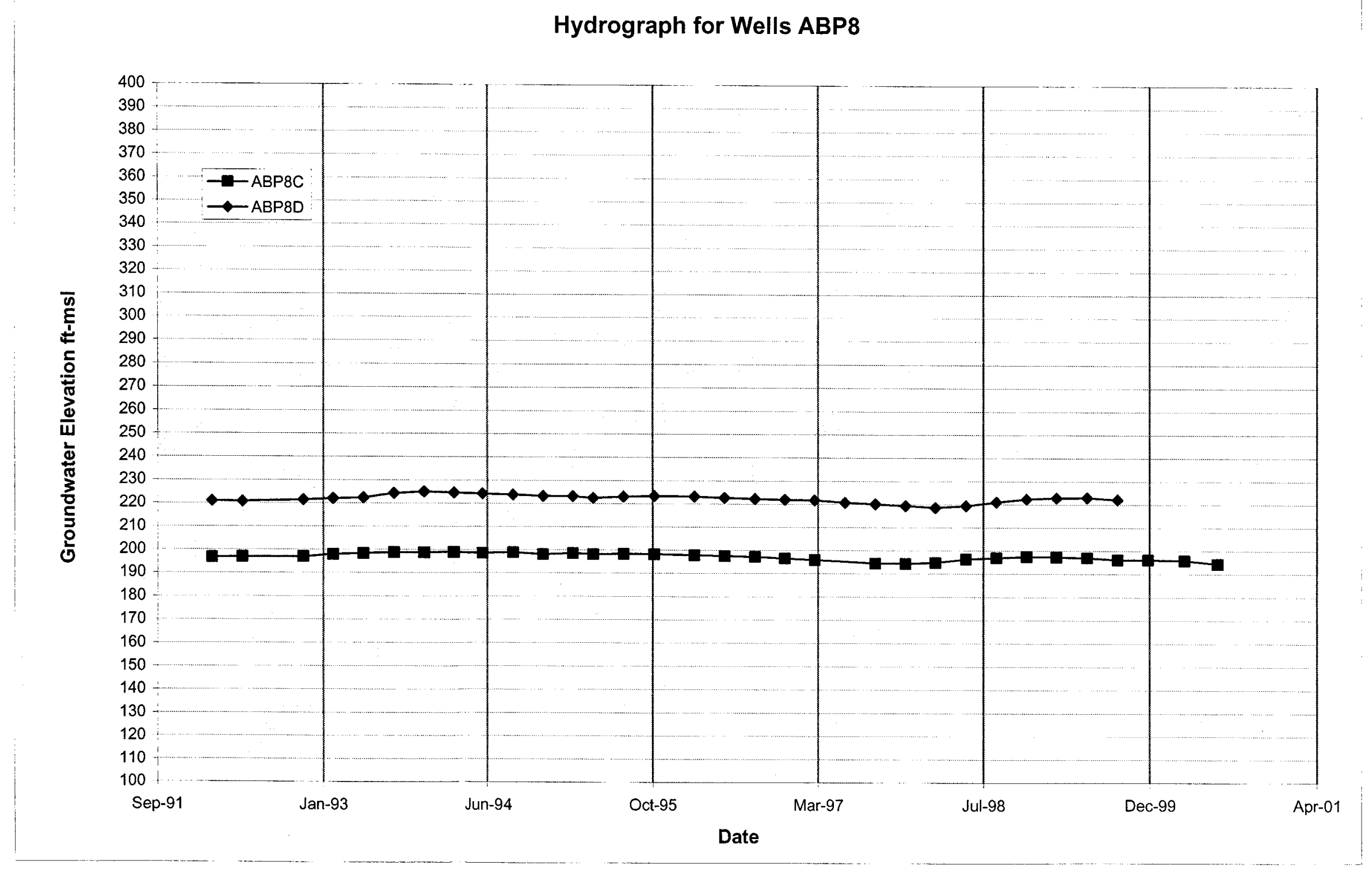


WSRC-TR-2000-00242 UNCLASSIFIED

\section{Hydrograph for Wells ABW1}

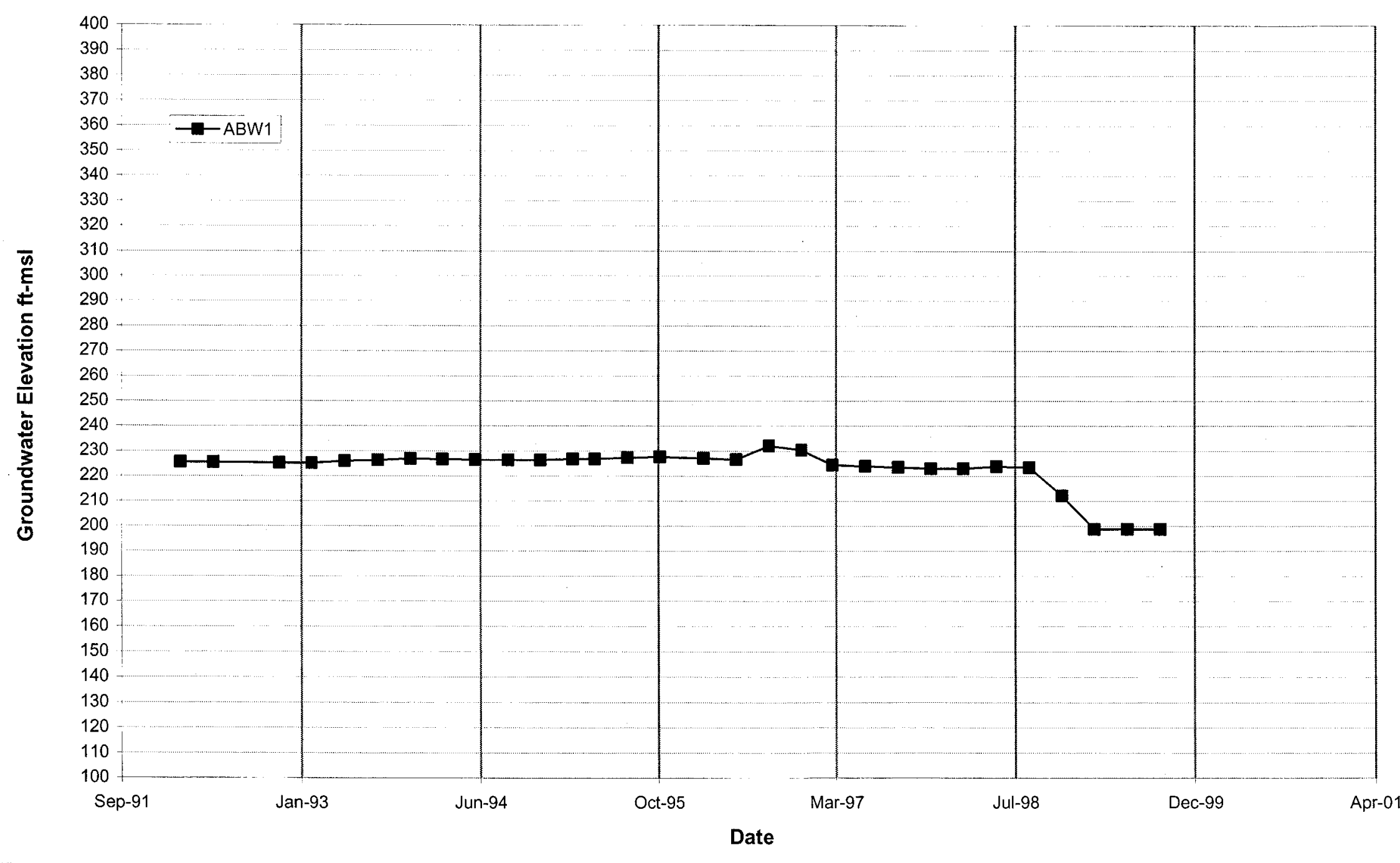




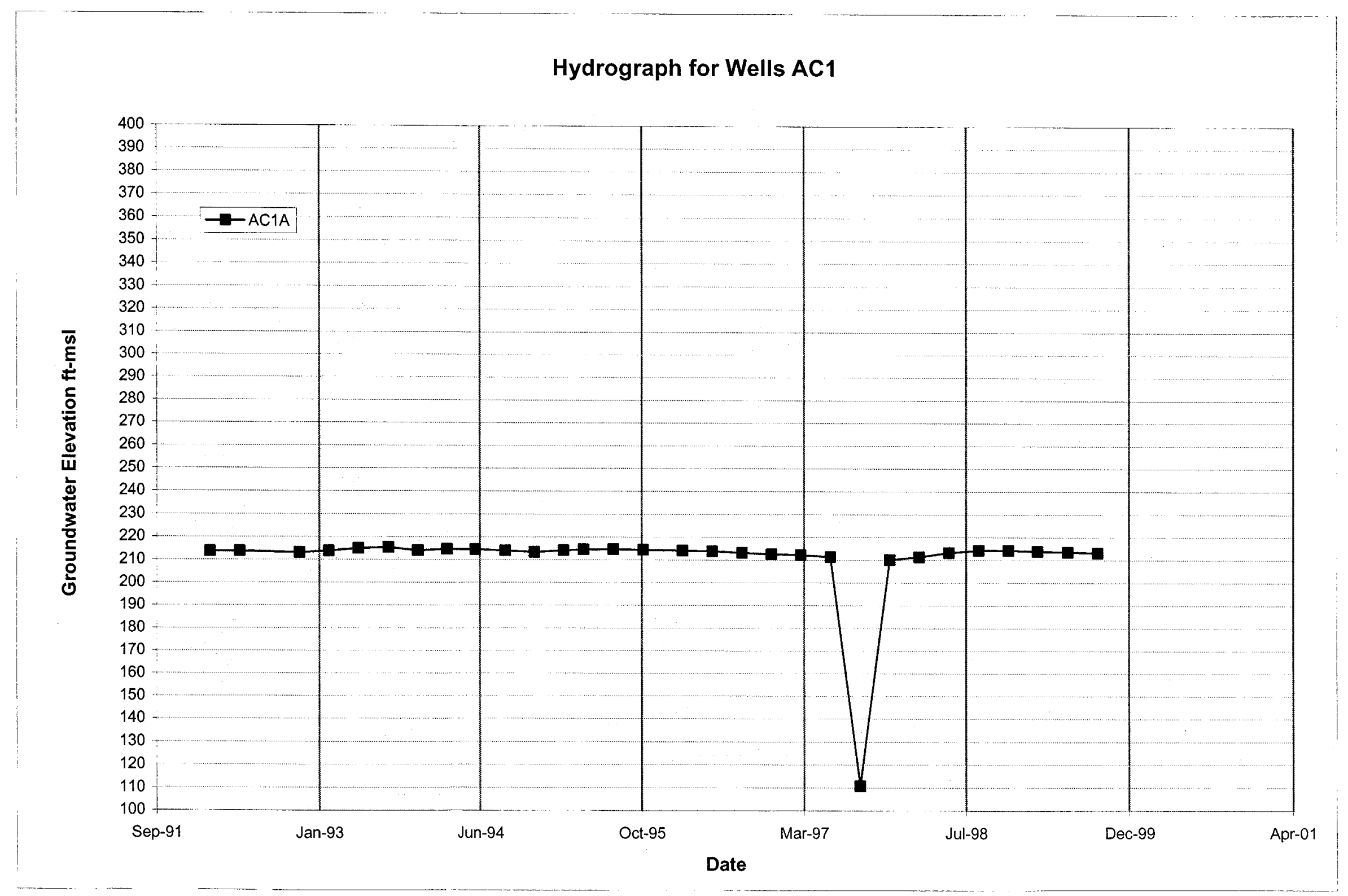


WSRC-TR-2000-00242 UNCLASSIFIED

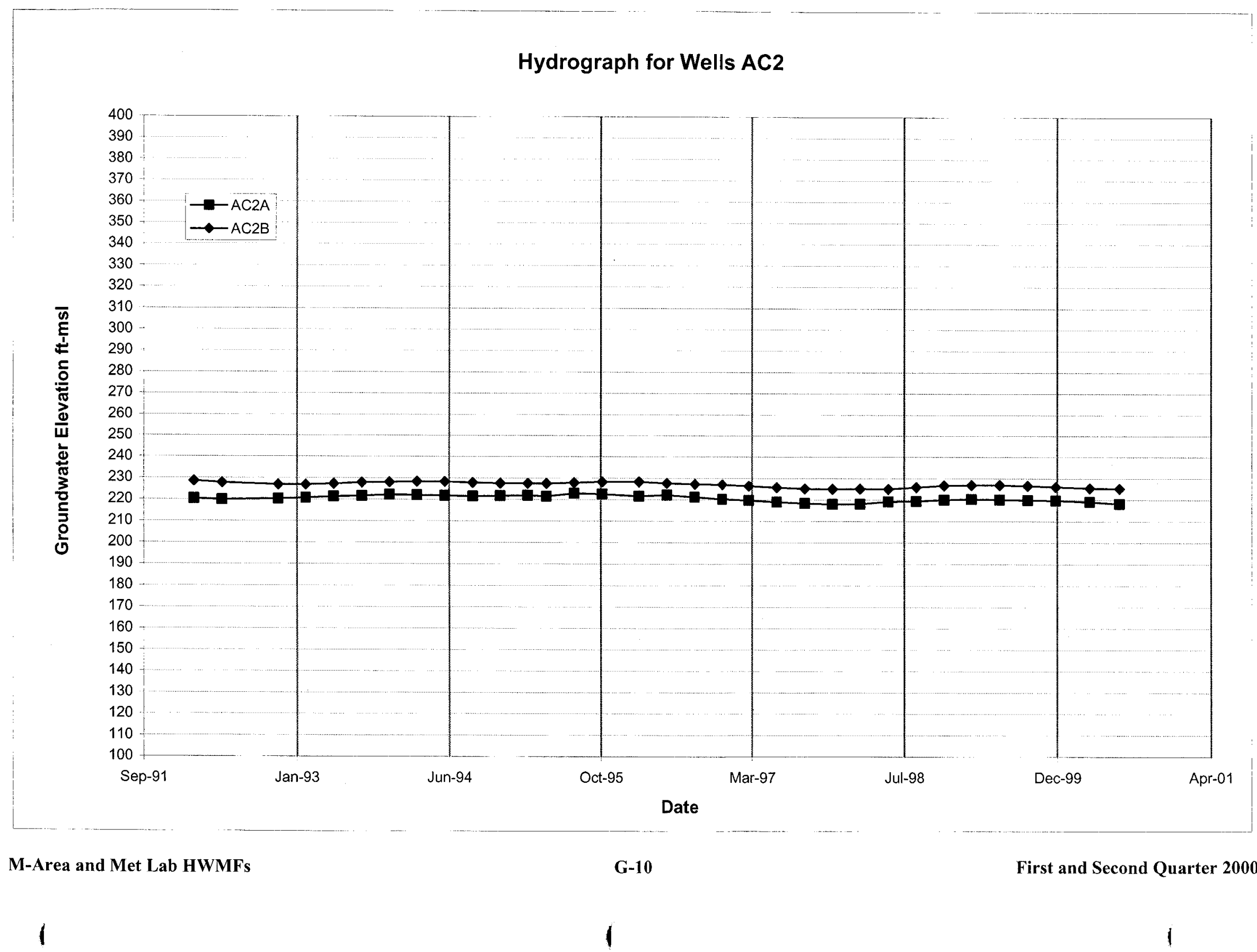


WSRC-TR-2000-00242 UNCLASSIFIED

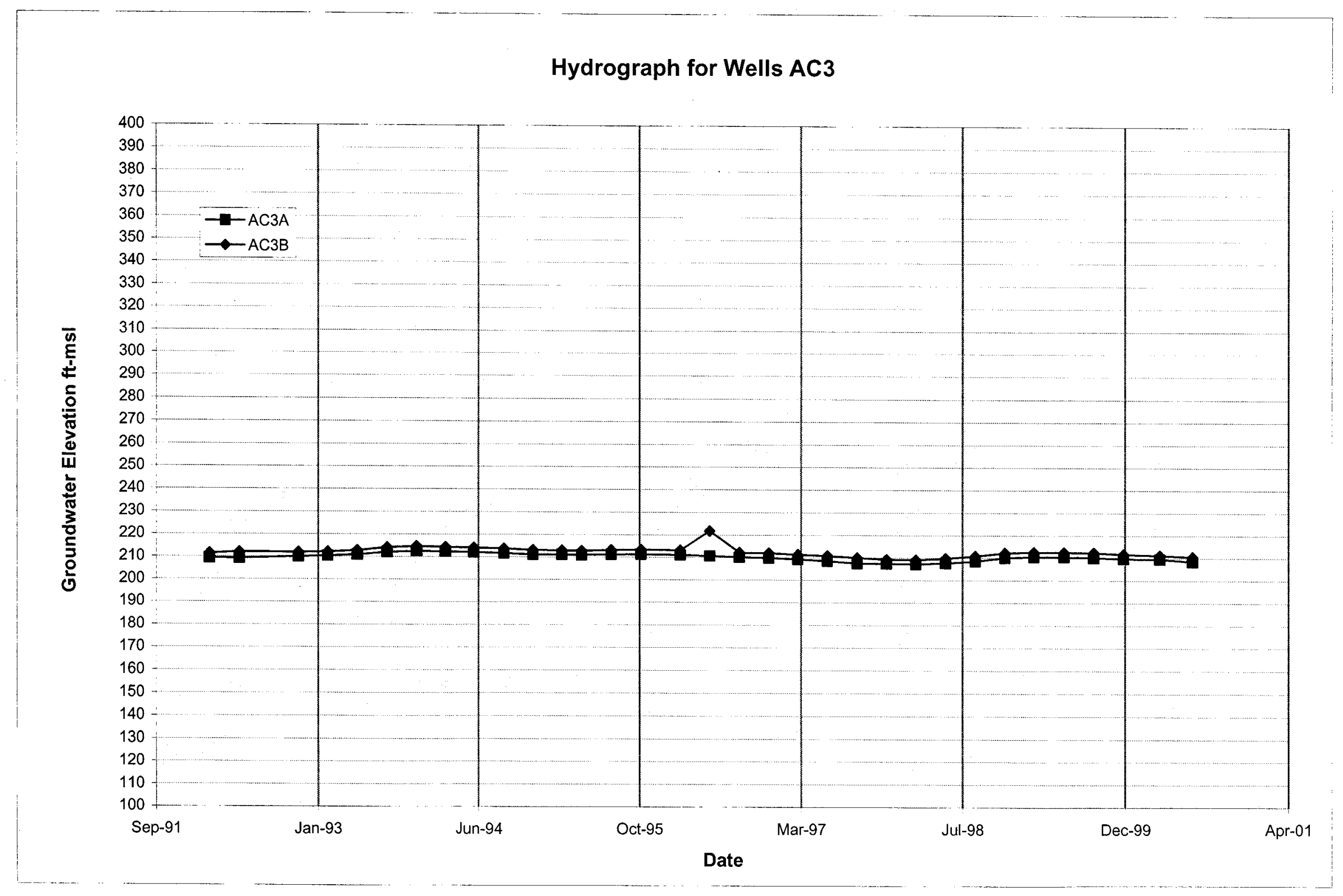


WSRC-TR-2000-00242 UNCLASSIFIED

\section{Hydrograph for Wells ACB2}

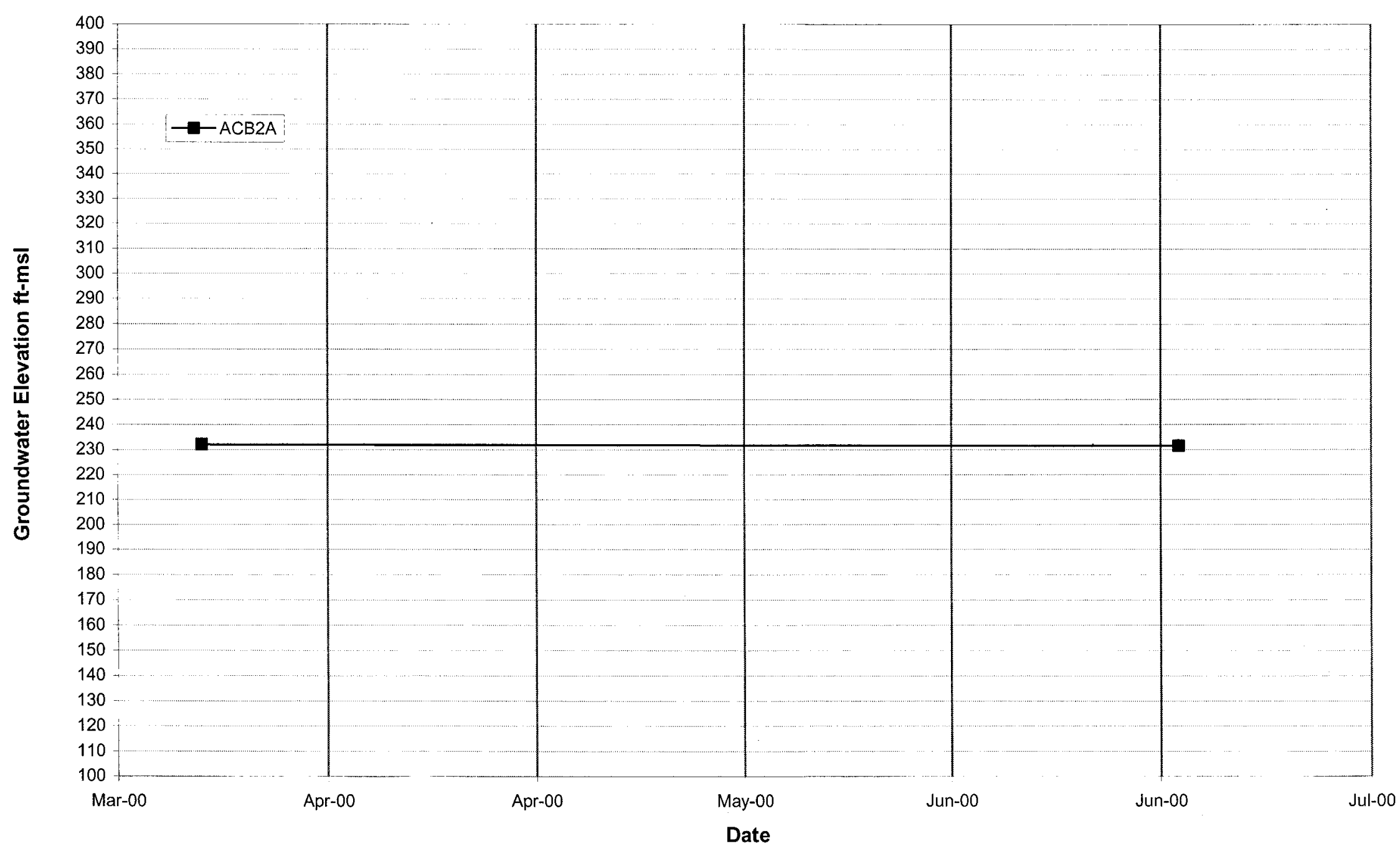


Hydrograph for Wells ACB4

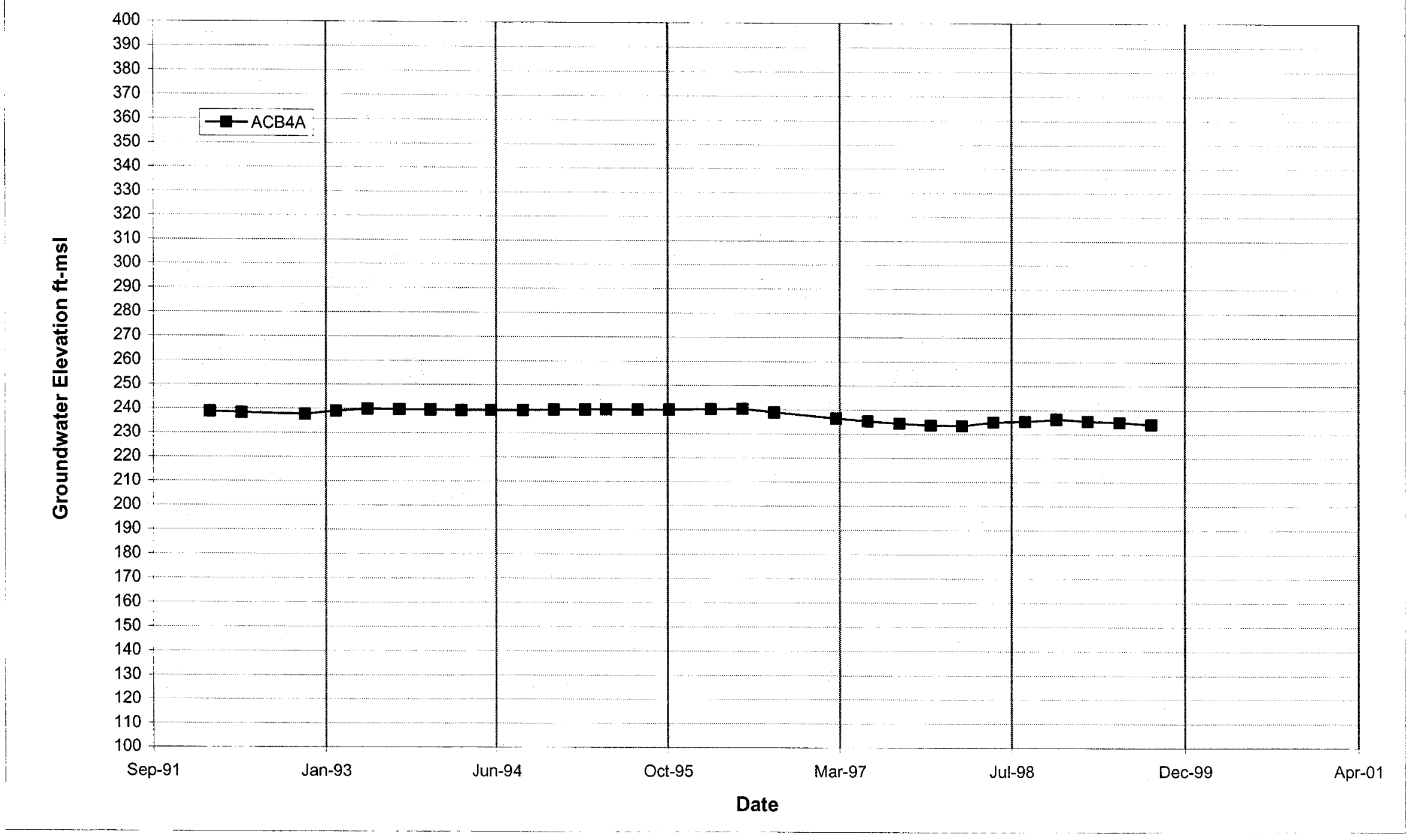


WSRC-TR-2000-00242 UNCLASSIFIED

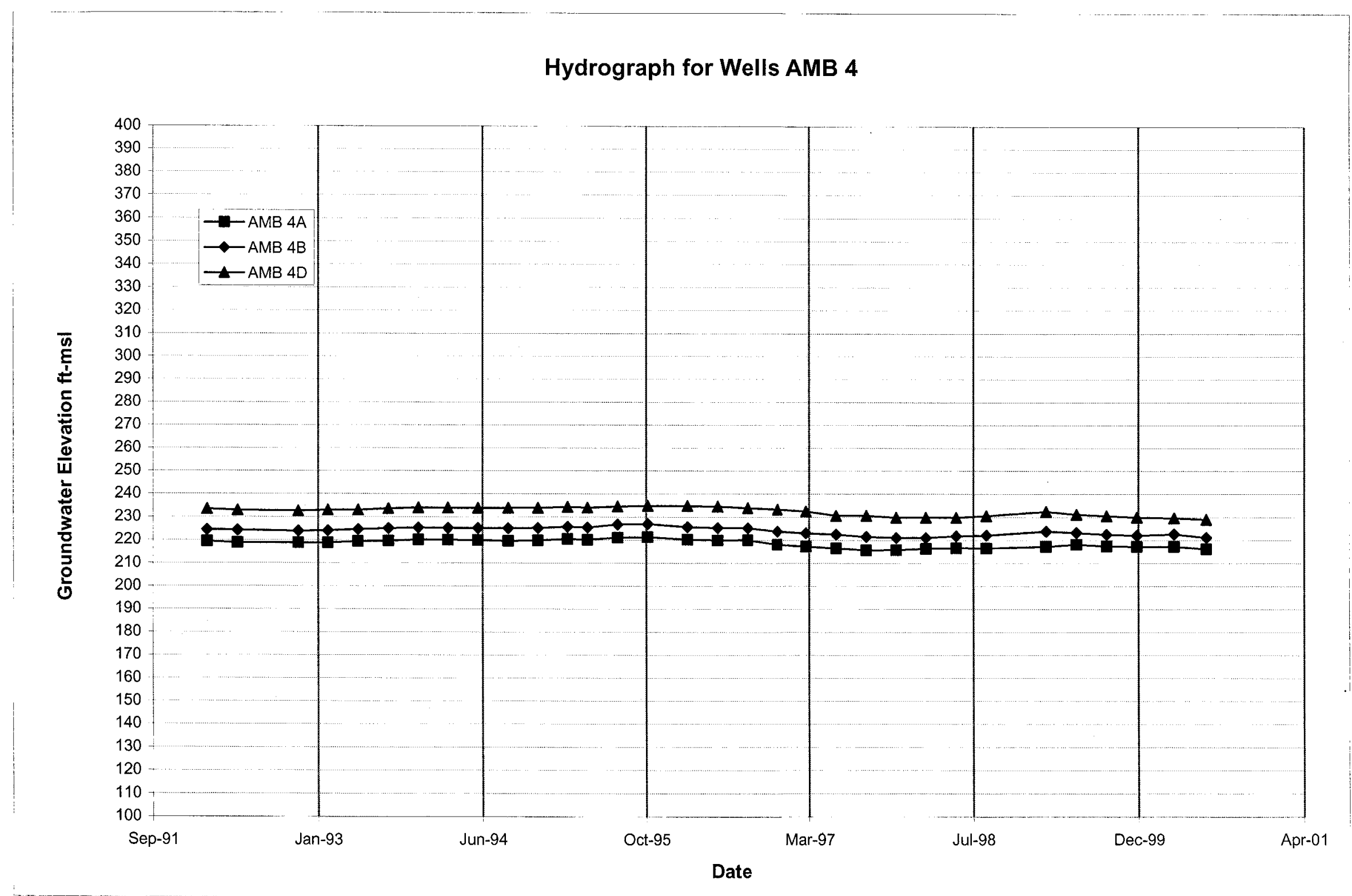




\section{Hydrograph for Wells AMB 5}

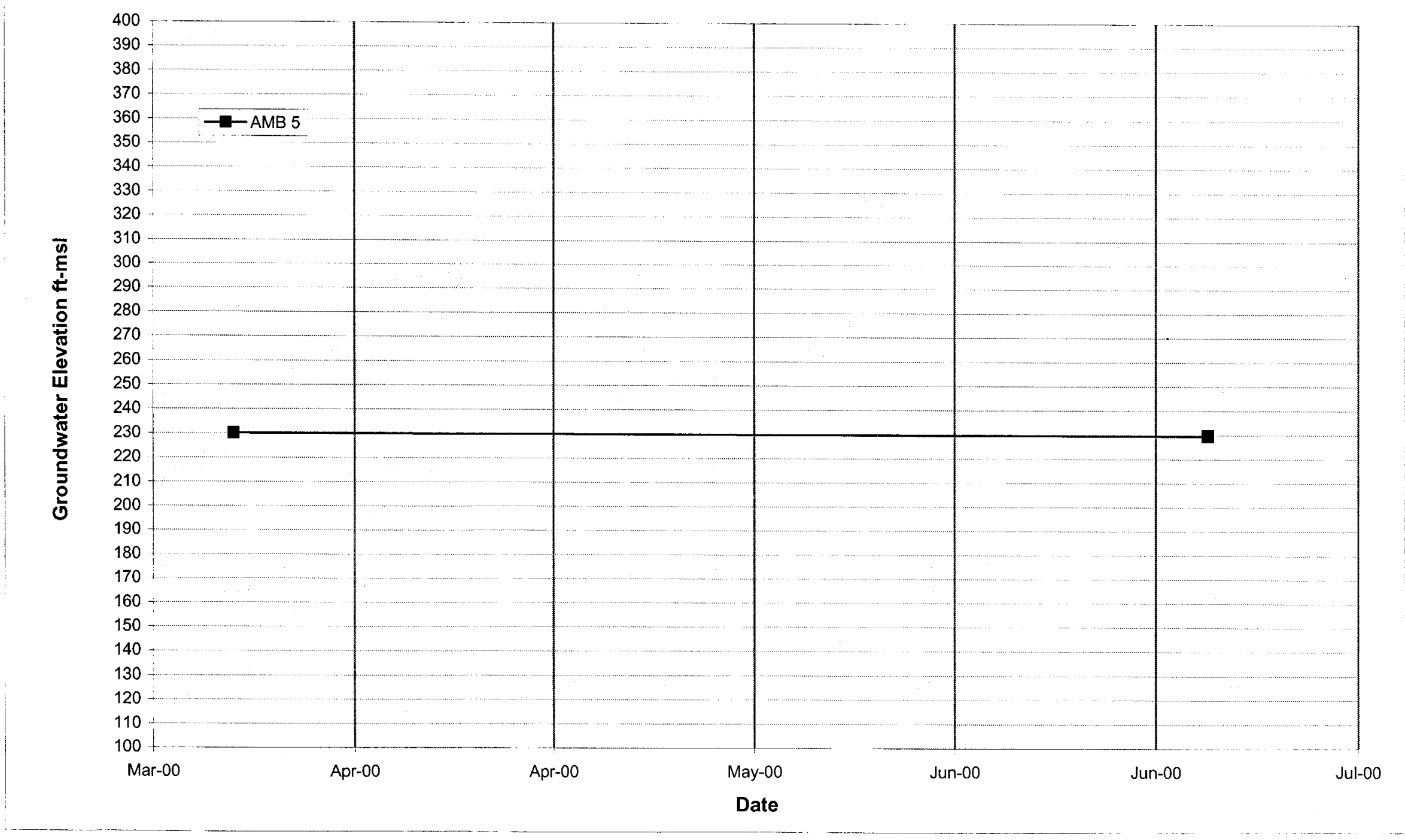




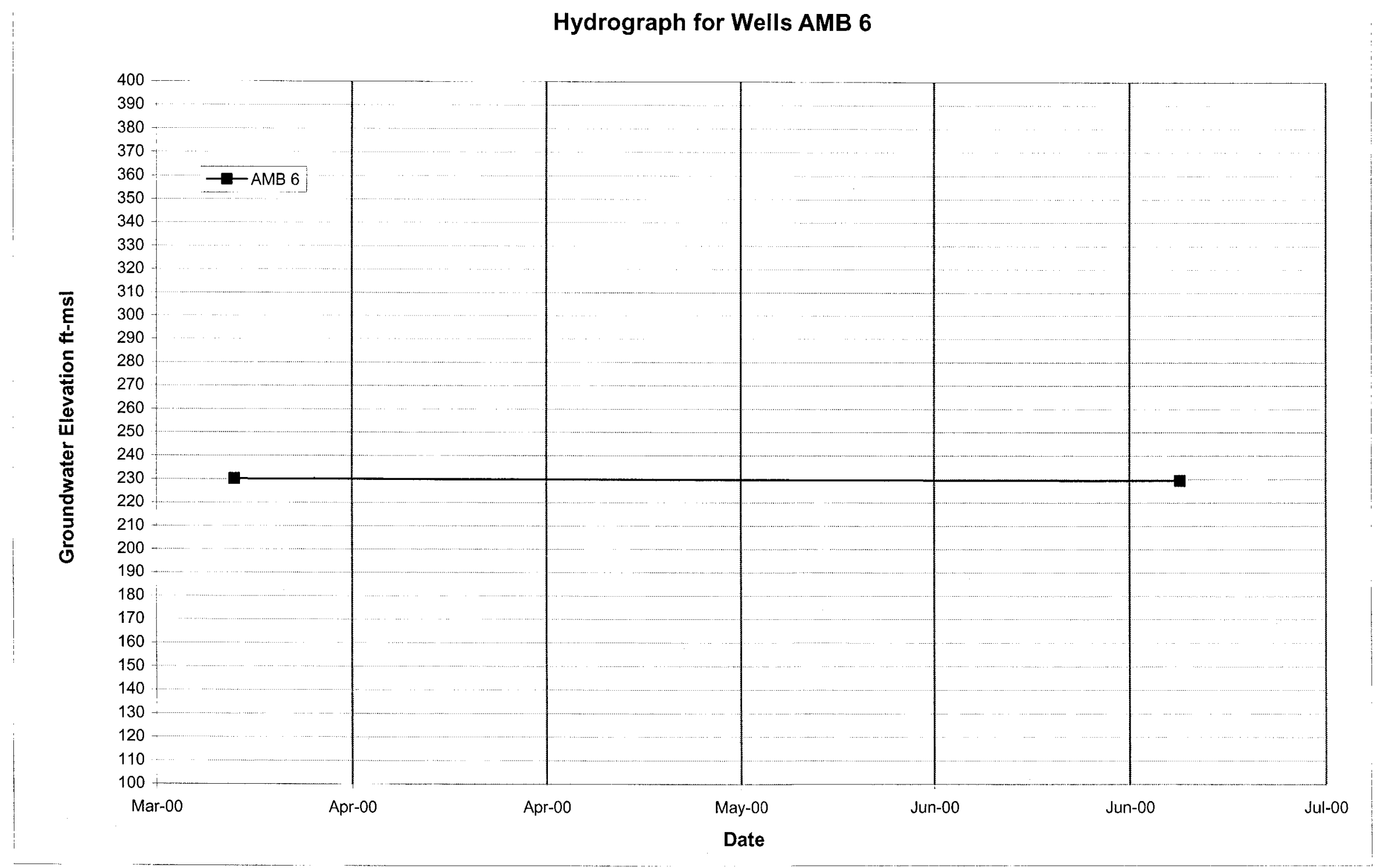


WSRC-TR-2000-00242 UNCLASSIFIED

\section{Hydrograph for Wells AMB 7}

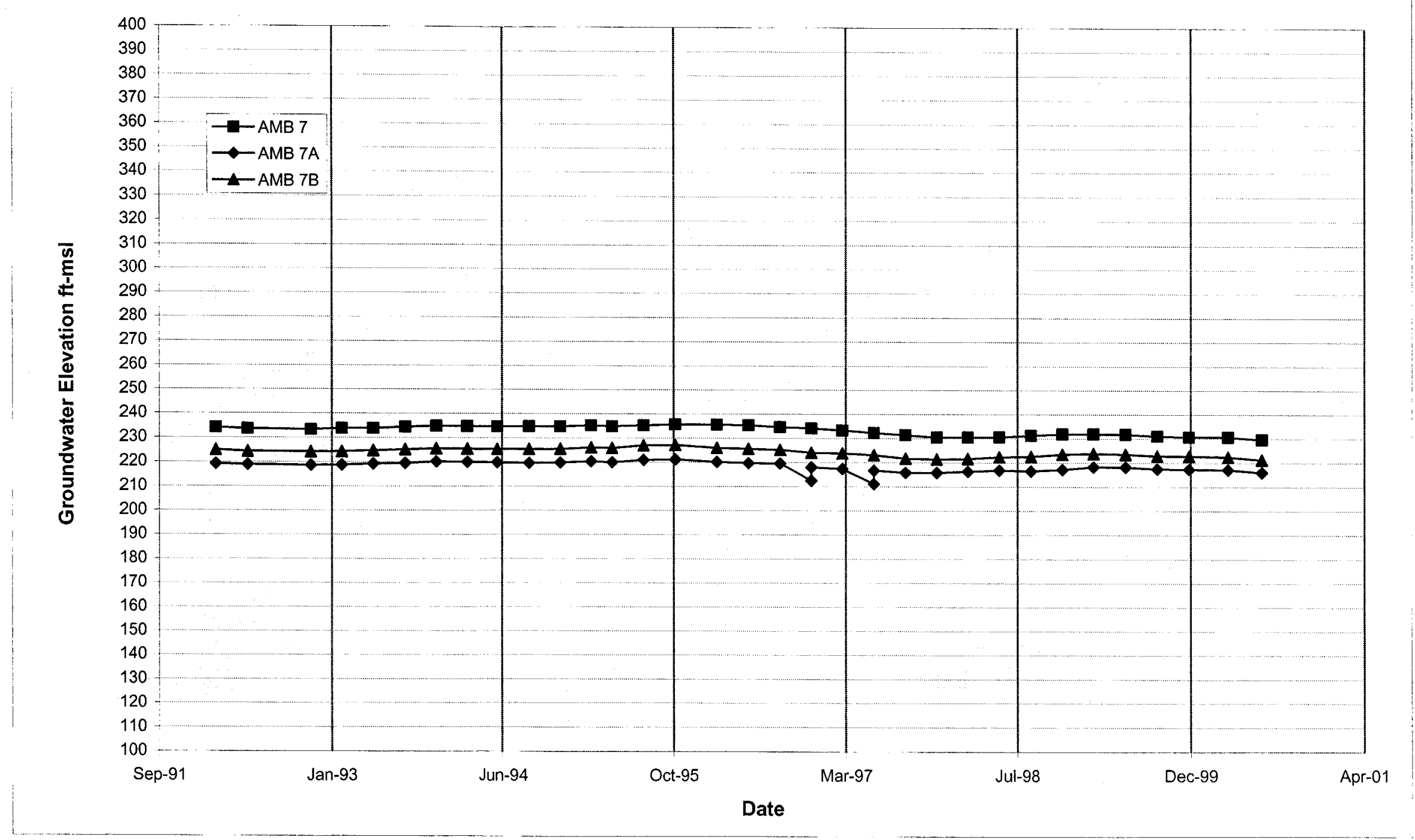


WSRC-TR-2000-00242

UNCLASSIFIED

Hydrograph for Wells AMB 8

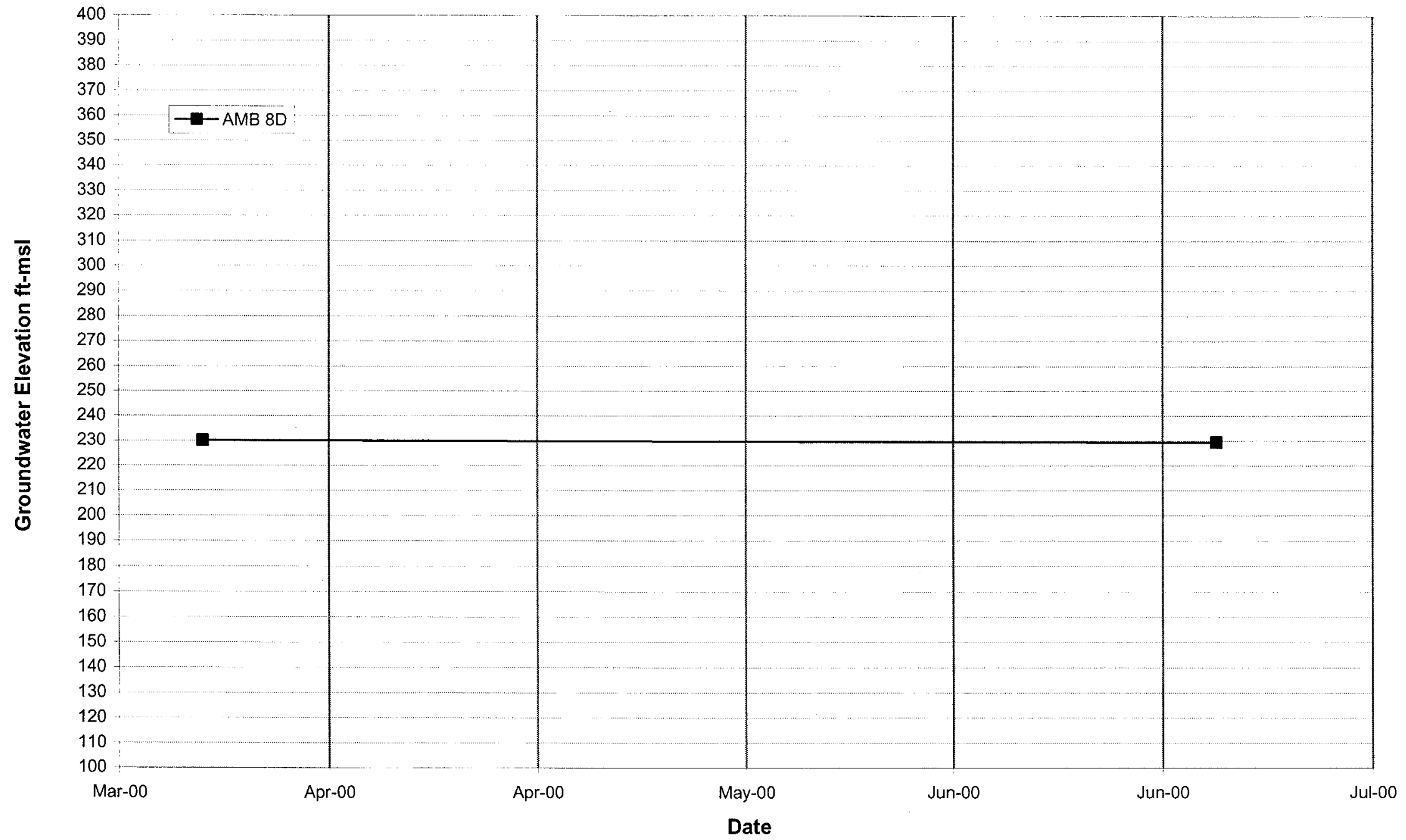

M-Area and Met Lab HWMFs

G-18

First and Second Quarter 2000 


\section{Hydrograph for Wells AMB 9}

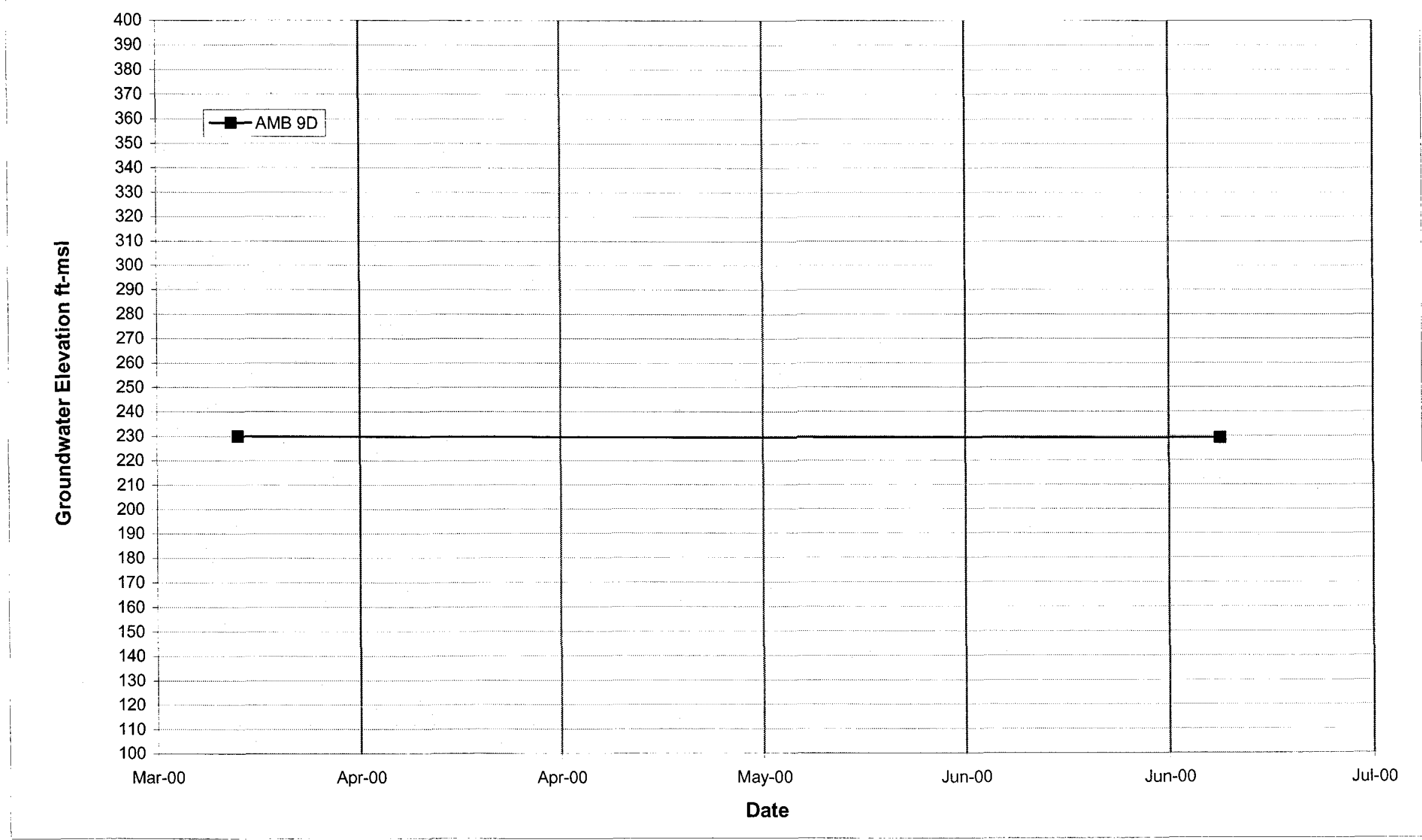


WSRC-TR-2000-00242 UNCLASSIFIED

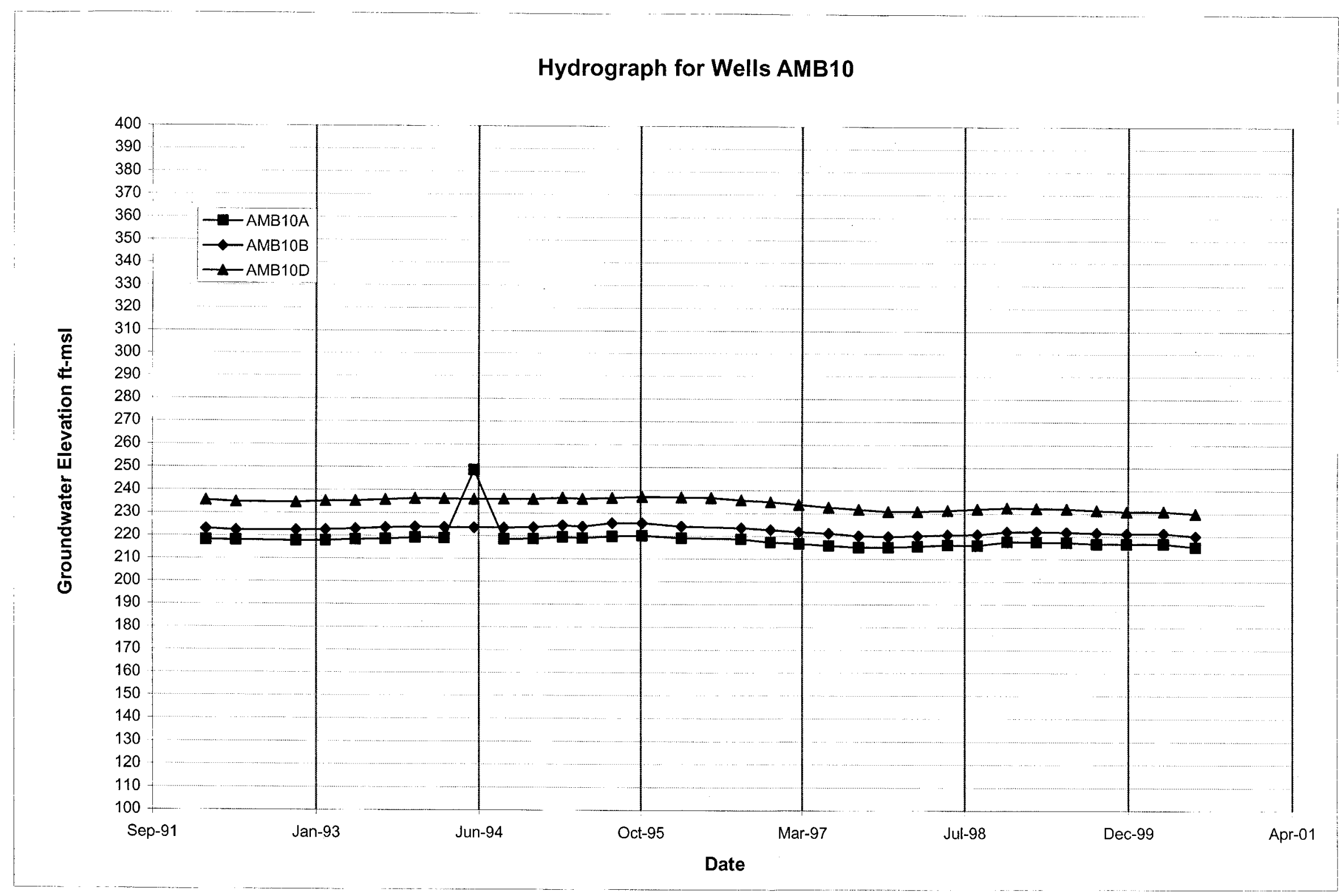




\section{Hydrograph for Wells AMB11}

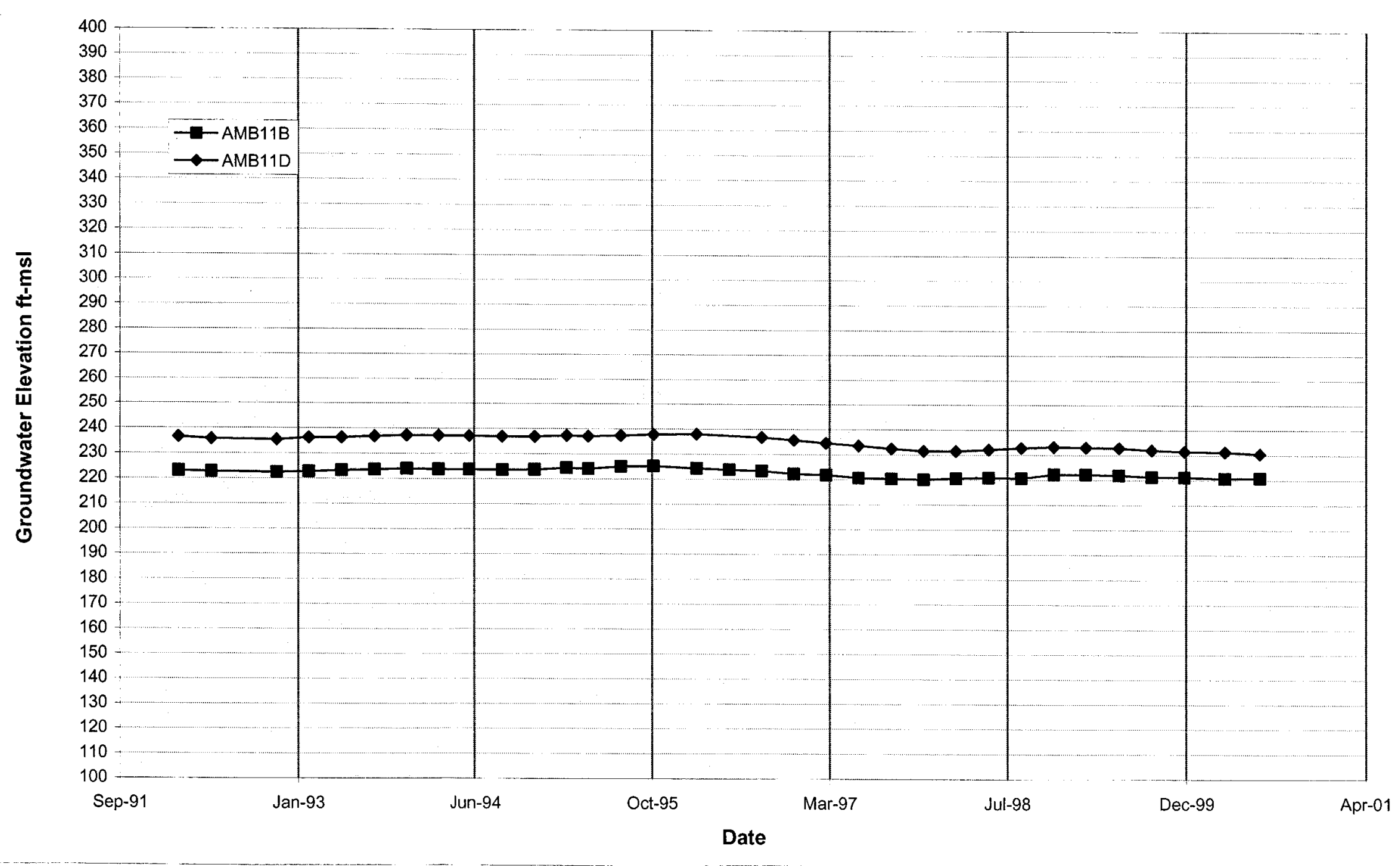


WSRC-TR-2000-00242 UNCLASSIFIED

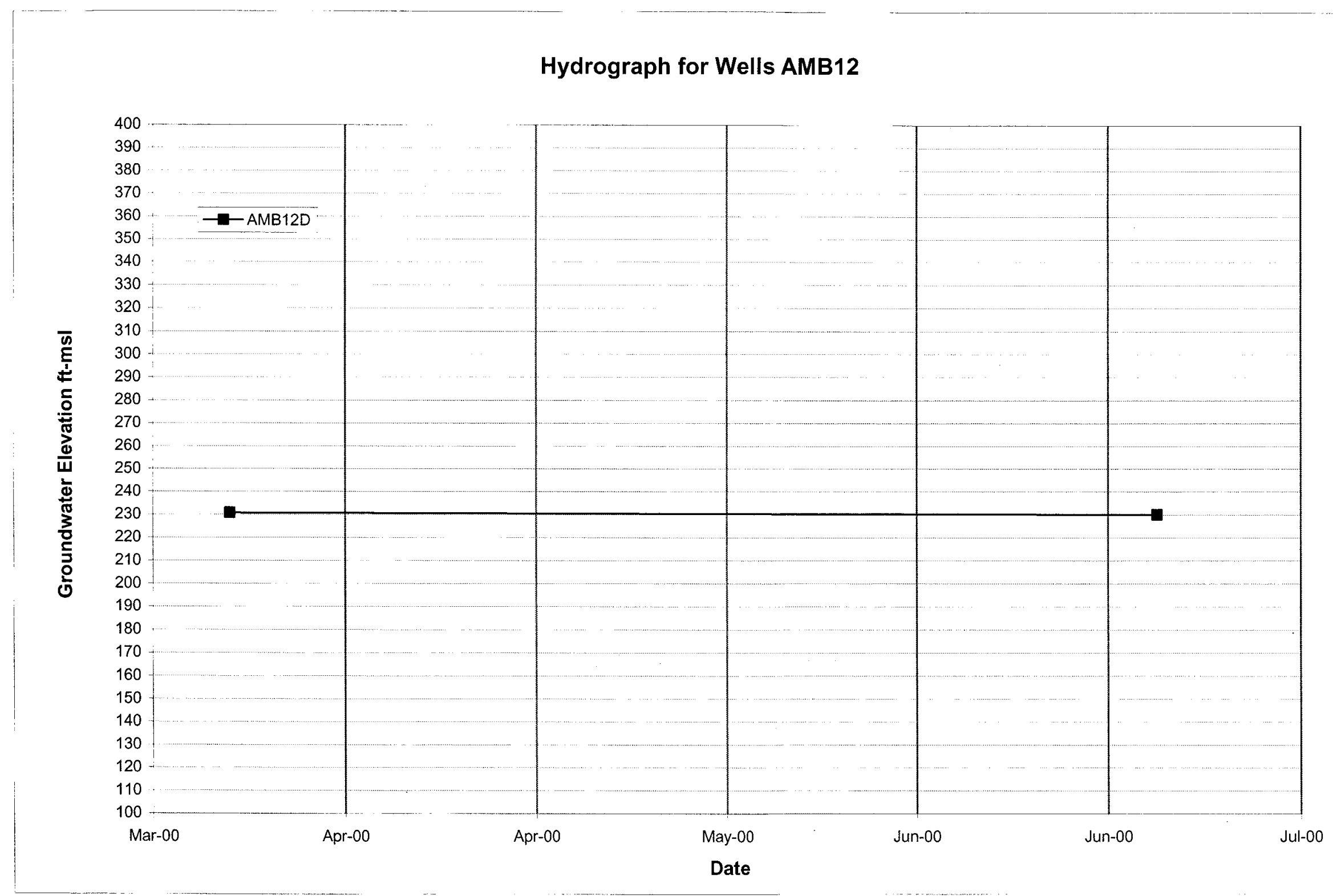


WSRC-TR-2000-00242 UNCLASSIFIED

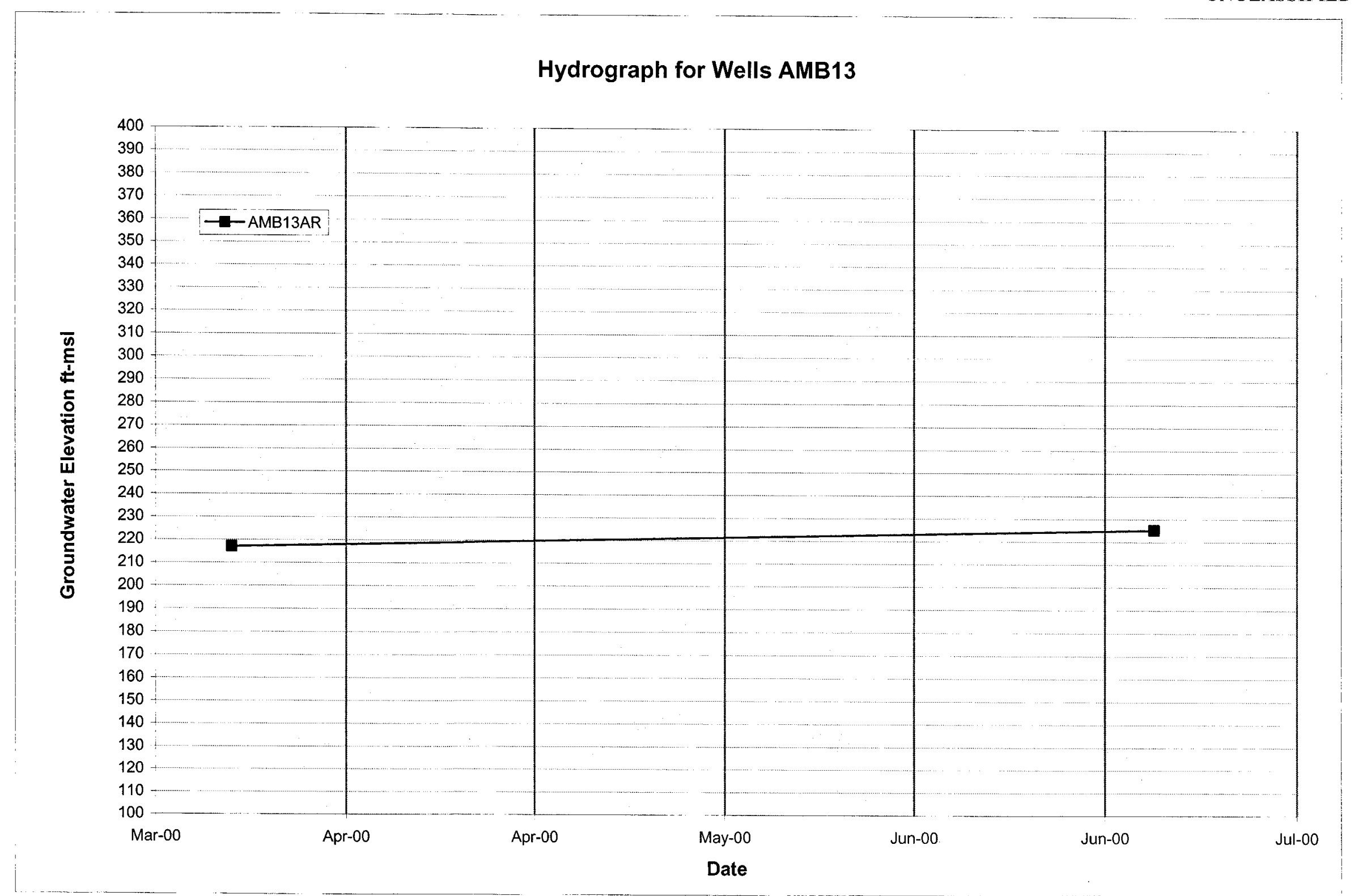


WSRC-TR-2000-00242 UNCLASSIFIED

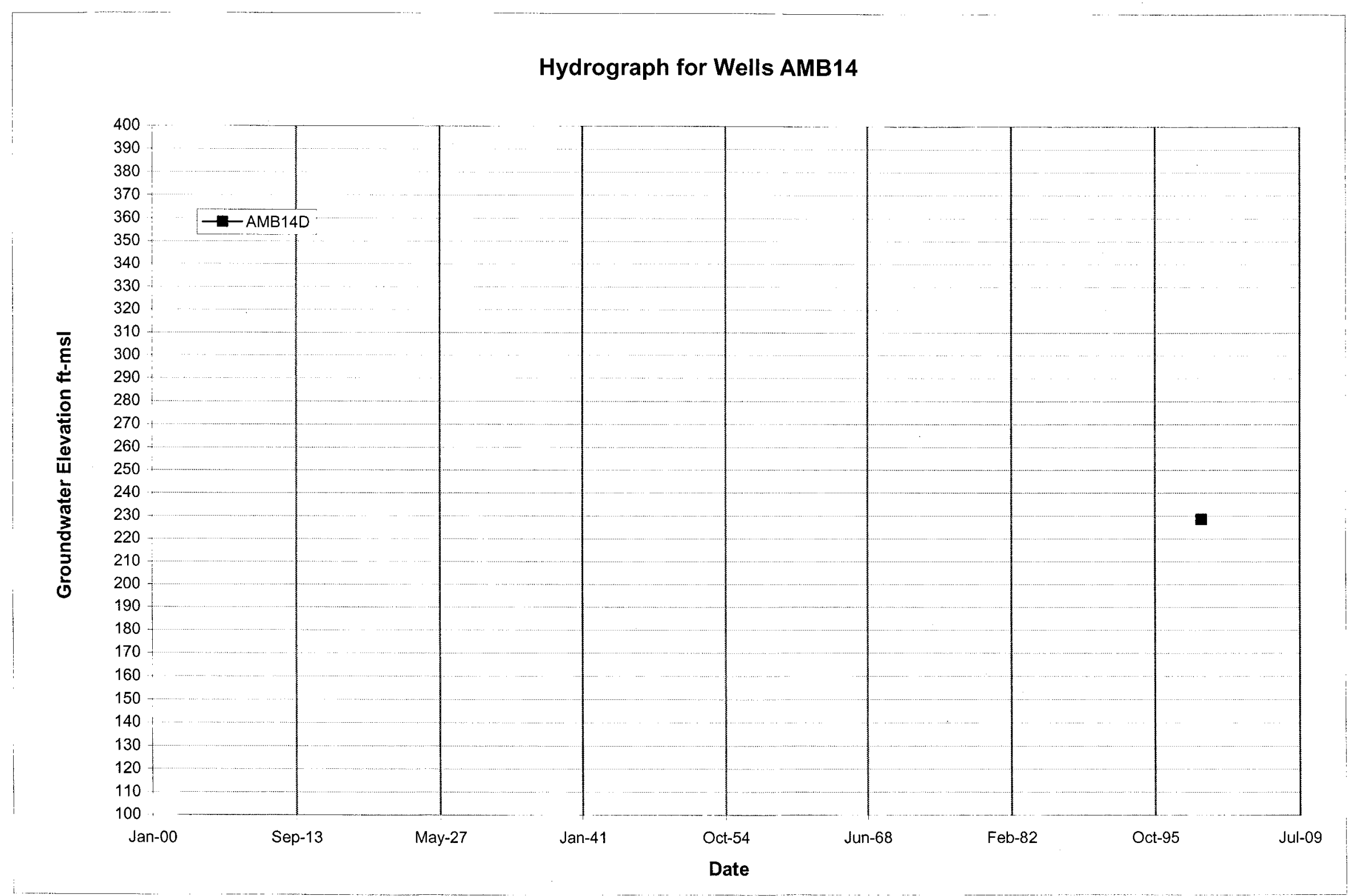




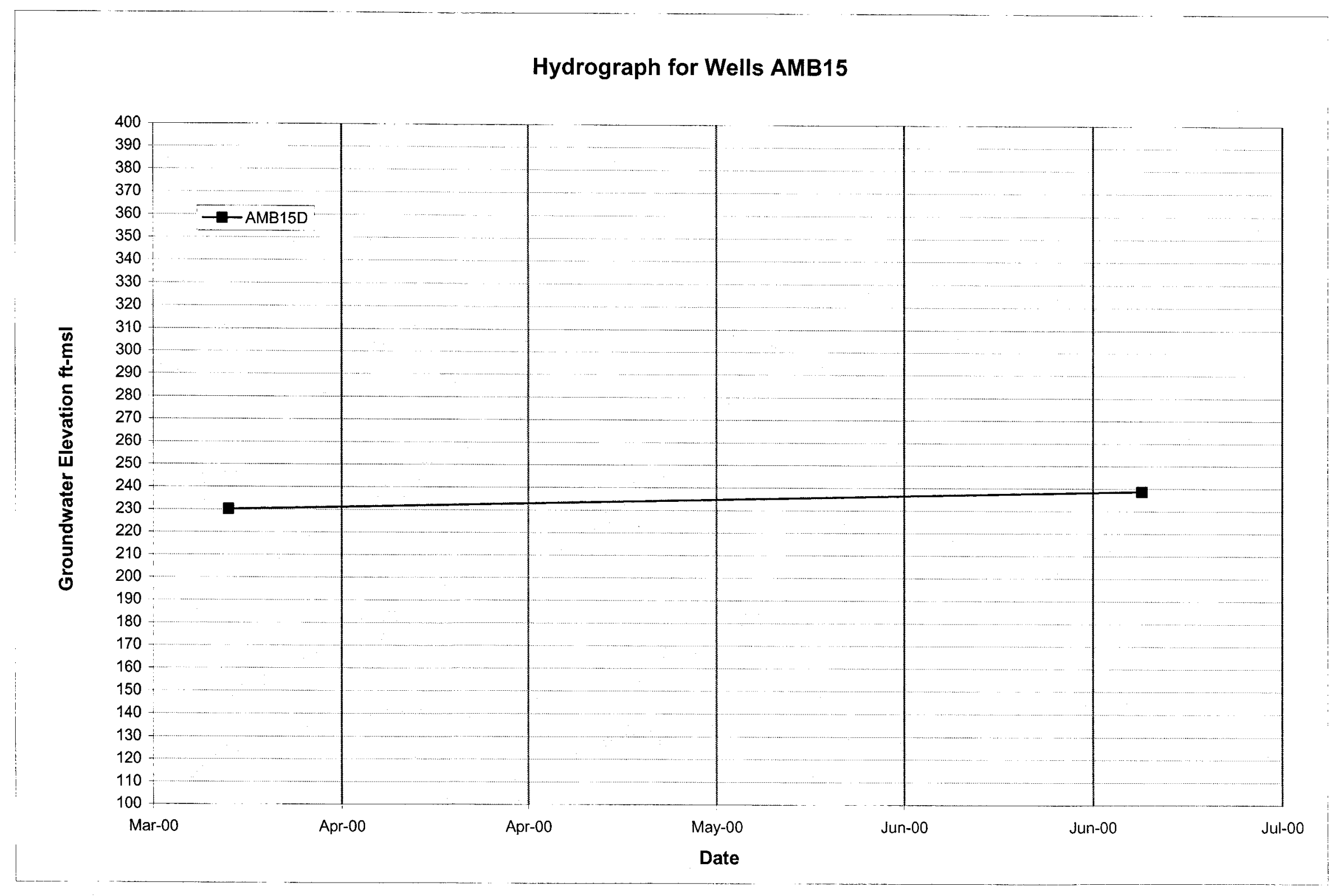


WSRC-TR-2000-00242 UNCLASSIFIED

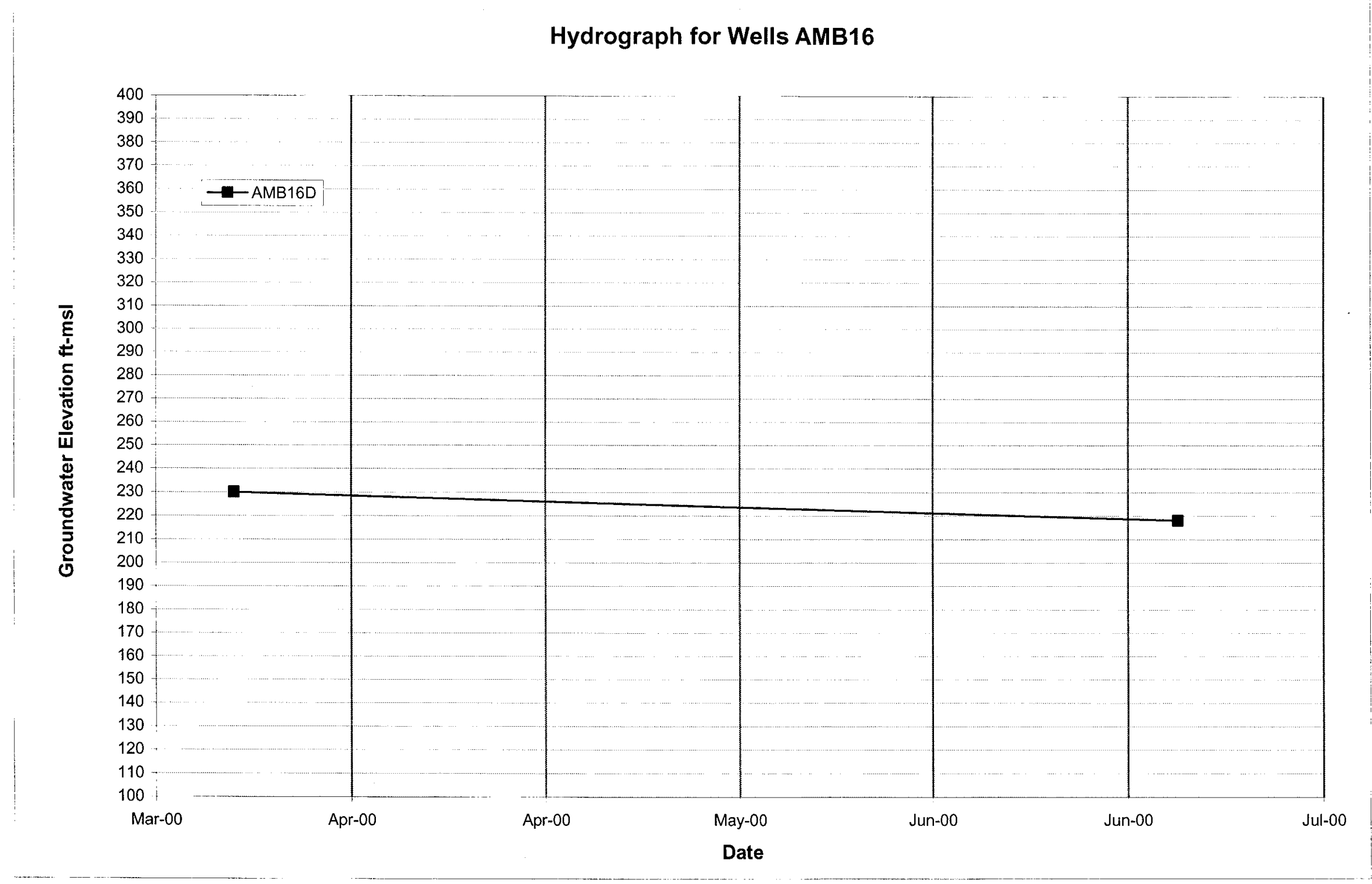


WSRC-TR-2000-00242 UNCLASSIFIED

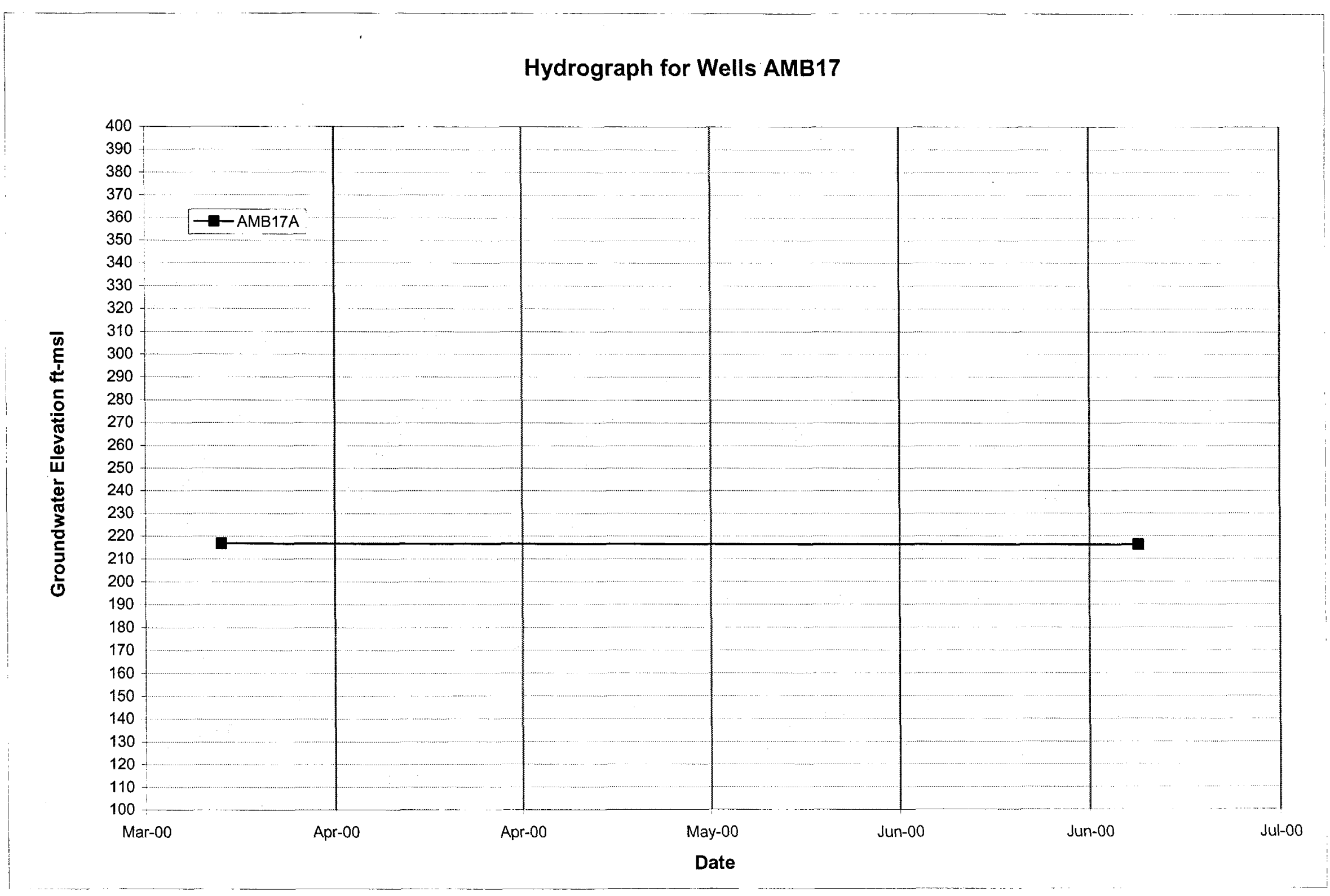


WSRC-TR-2000-00242 UNCLASSIFIED

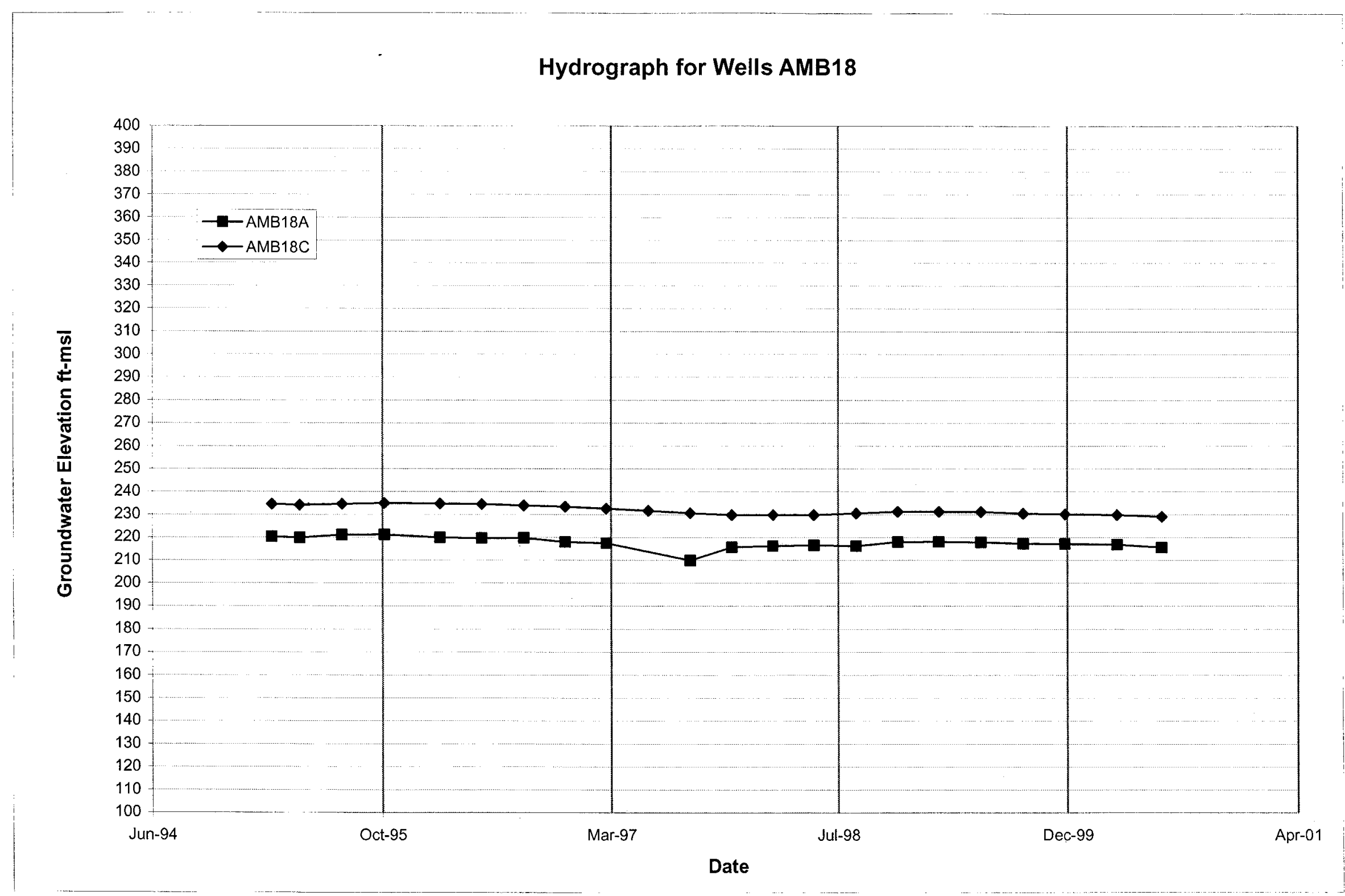


WSRC-TR-2000-00242 UNCLASSIFIED

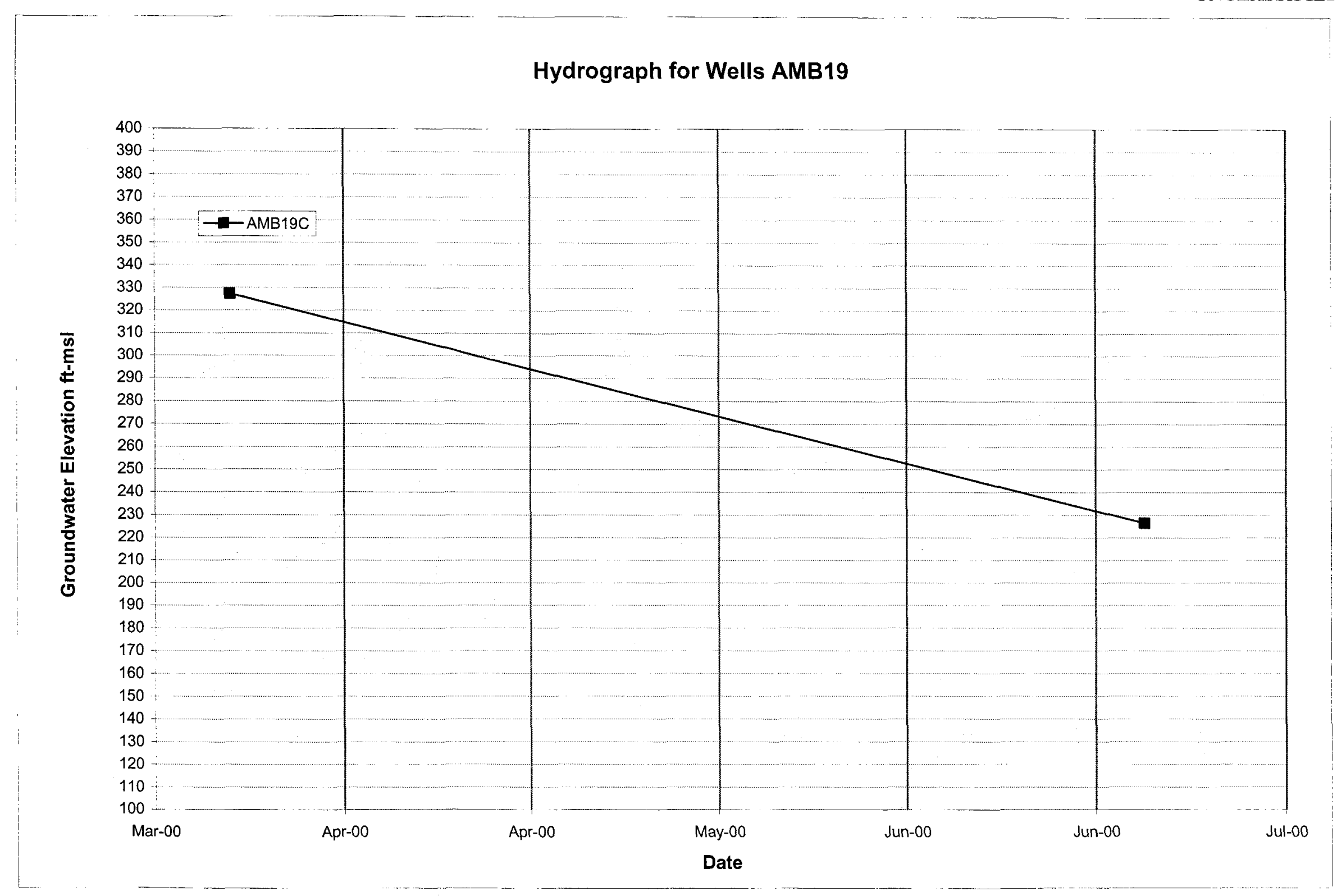


WSRC-TR-2000-00242 UNCLASSIFIED

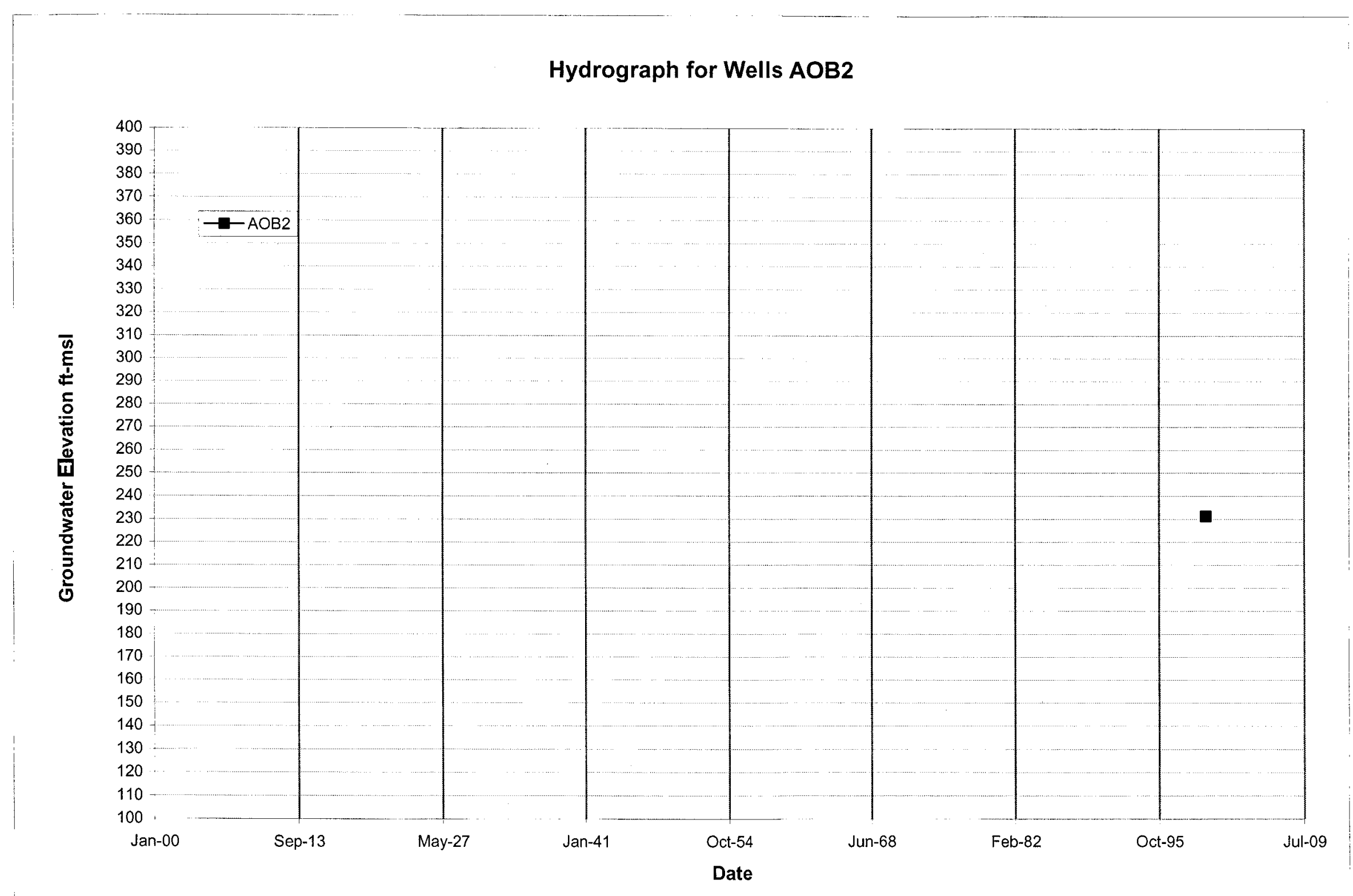




\section{Hydrograph for Wells ARP2}

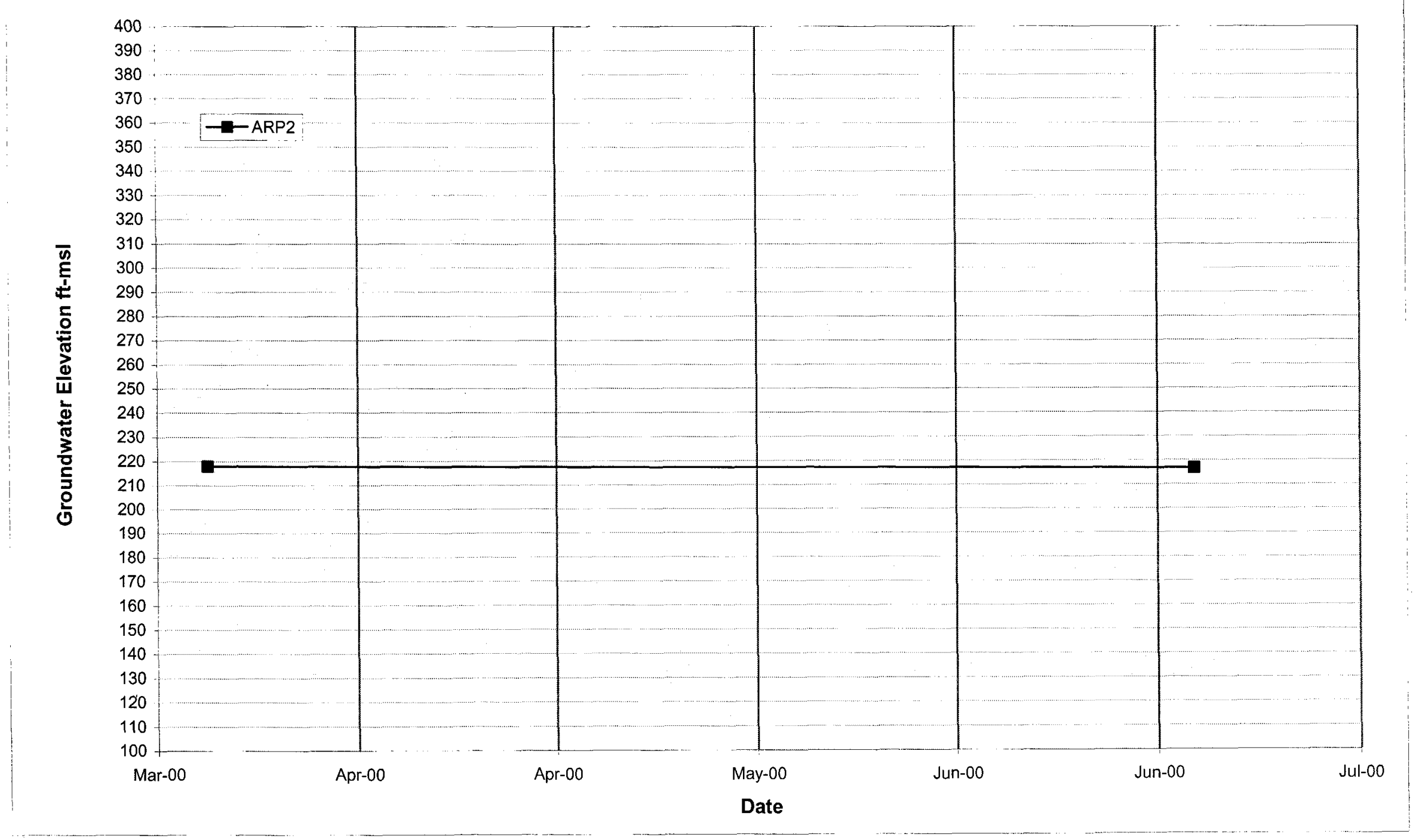


WSRC-TR-2000-00242 UNCLASSIFIED

Hydrograph for Wells ARP3

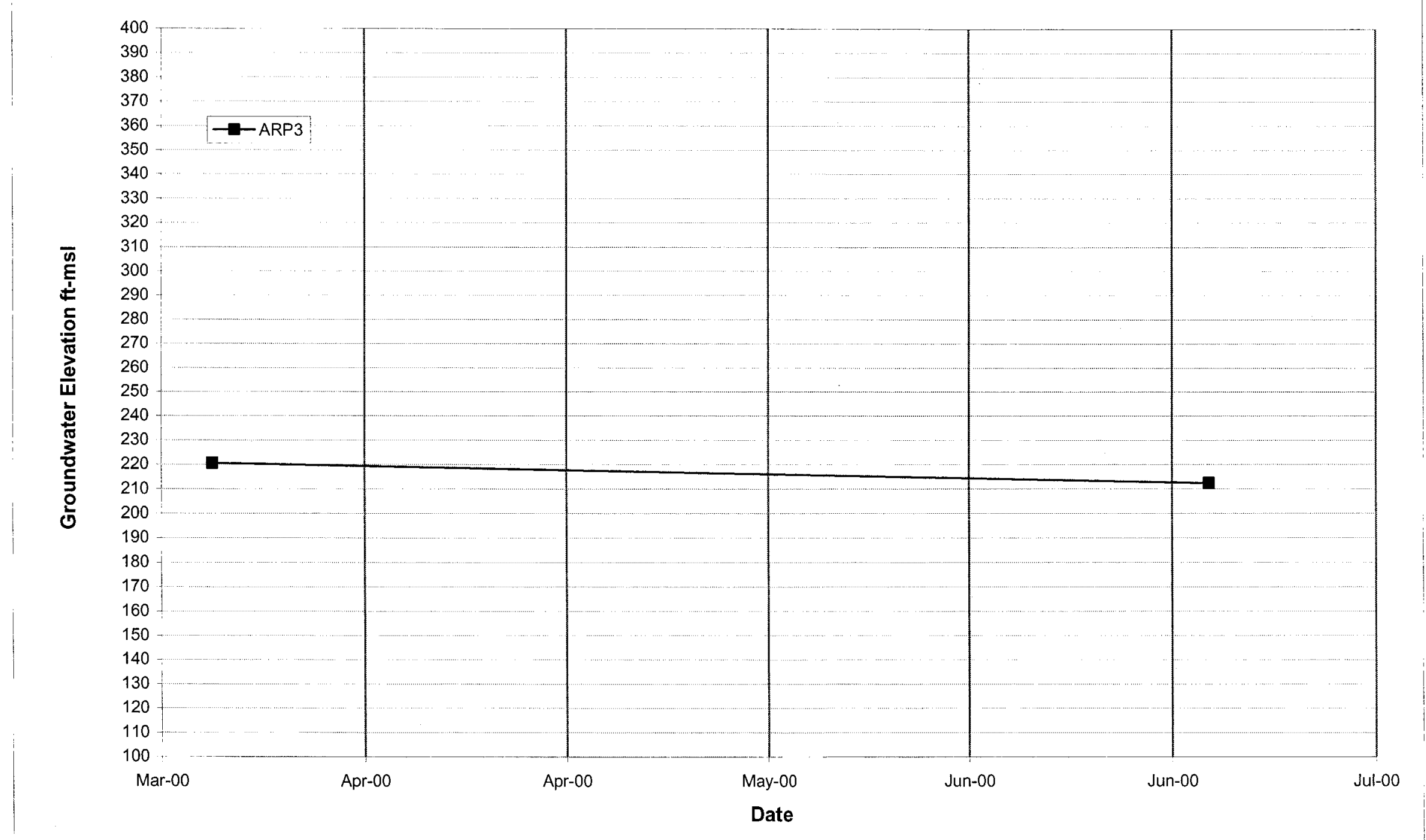




\section{Hydrograph for Wells ARP4}

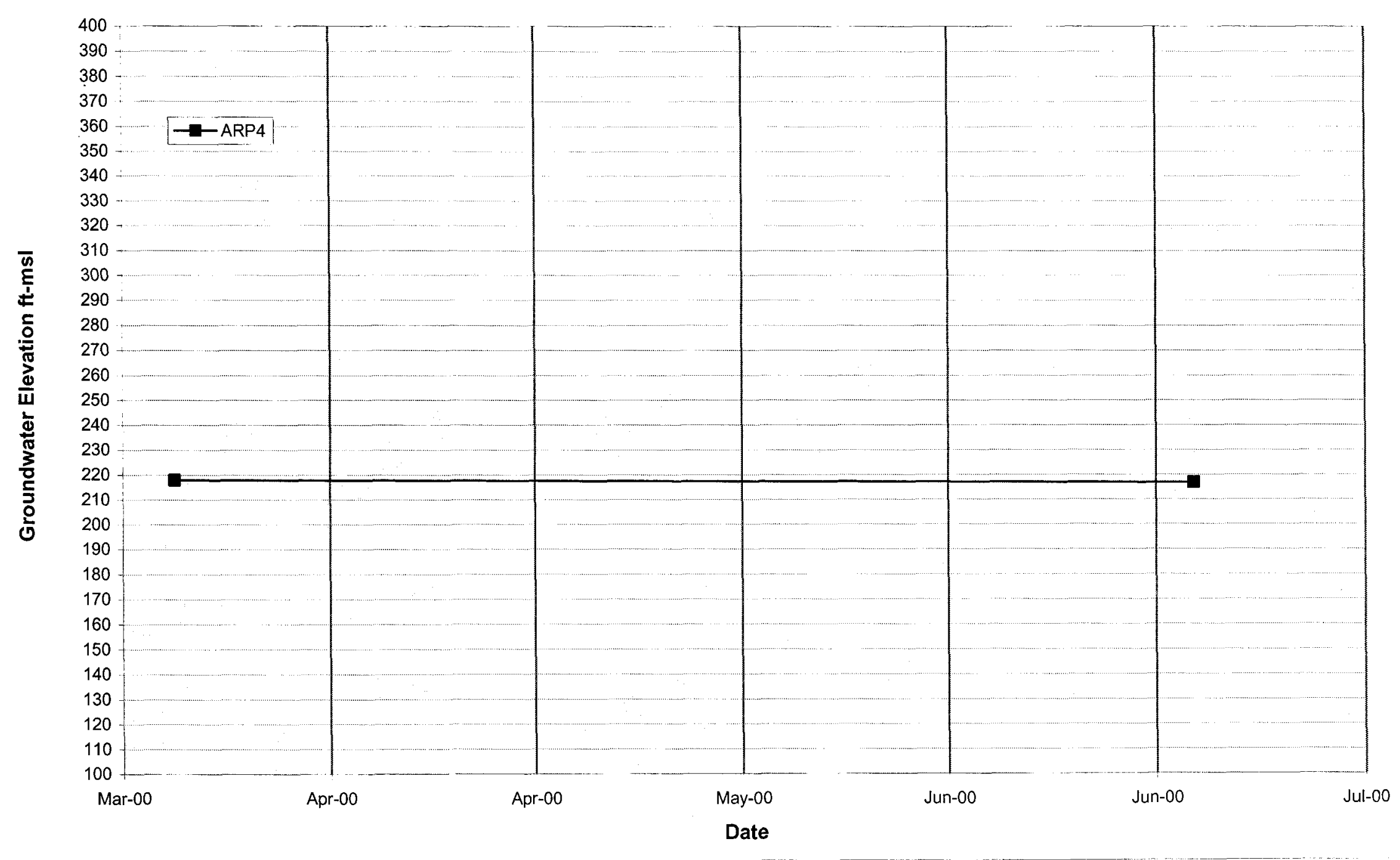


WSRC-TR-2000-00242 UNCLASSIFIED

\section{Hydrograph for Wells ASB 1}

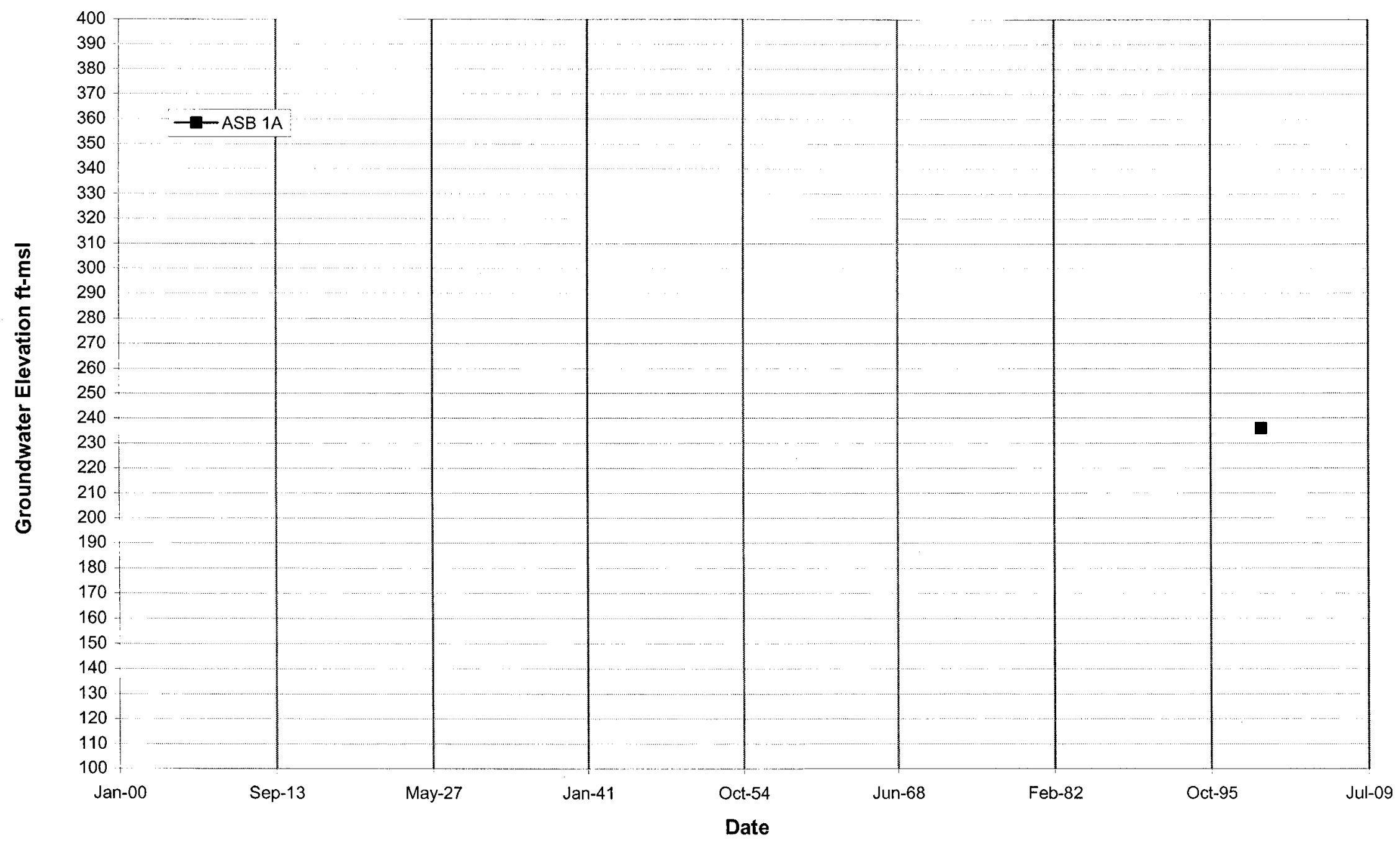




\section{Hydrograph for Wells ASB 2}

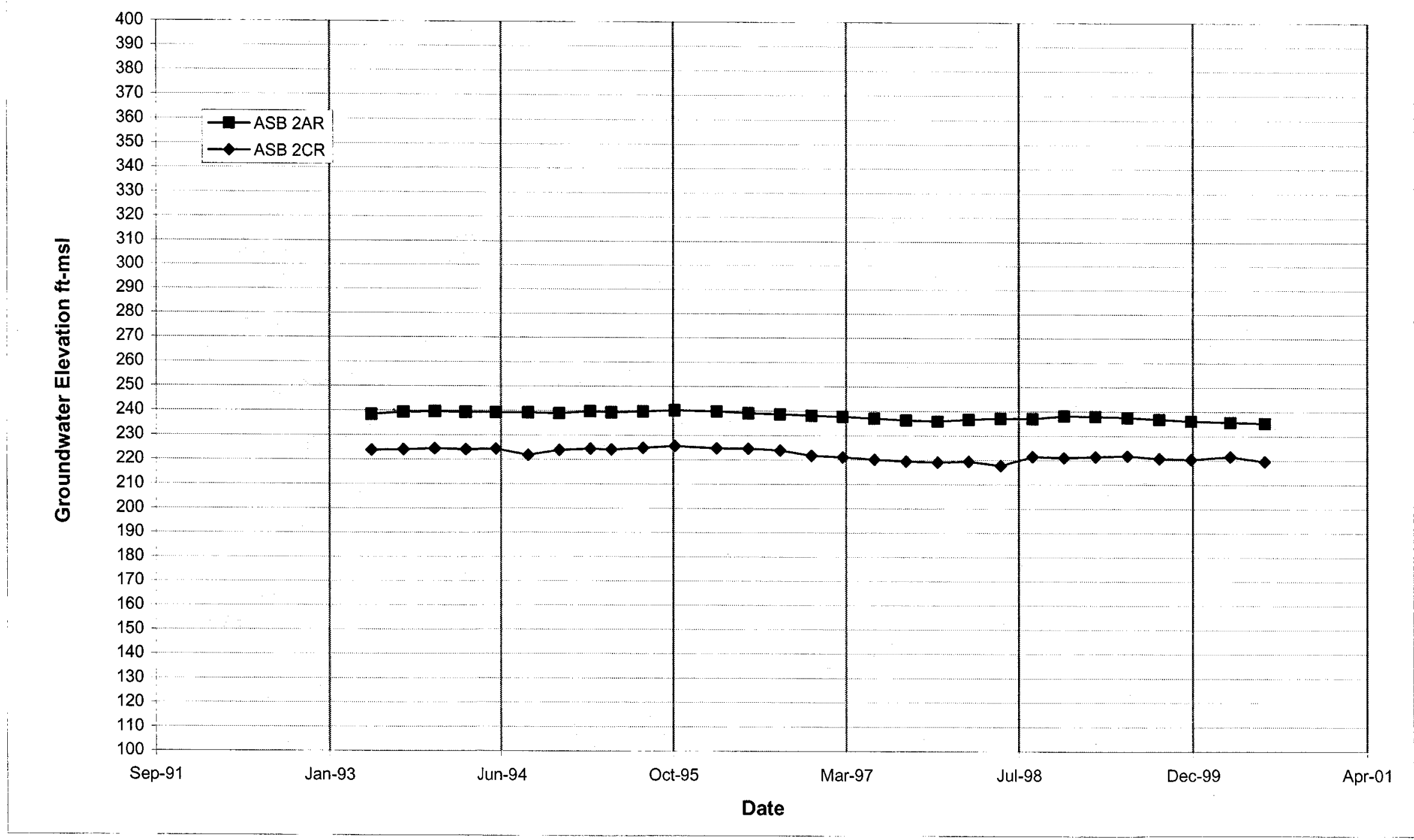


WSRC-TR-2000-00242 UNCLASSIFIED

Hydrograph for Wells ASB 3

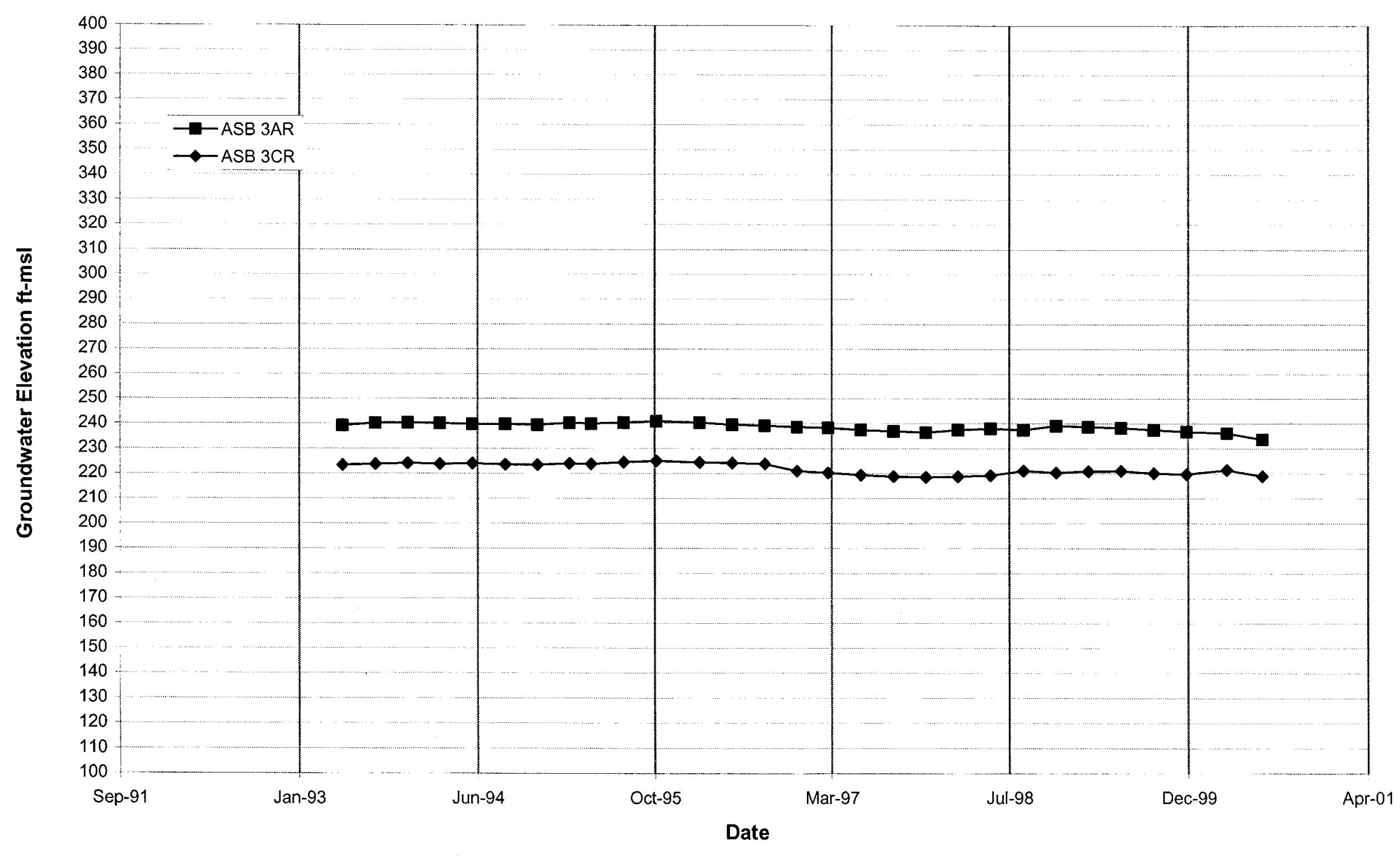


WSRC-TR-2000-00242

UNCLASSIFIED

Hydrograph for Wells ASB 4

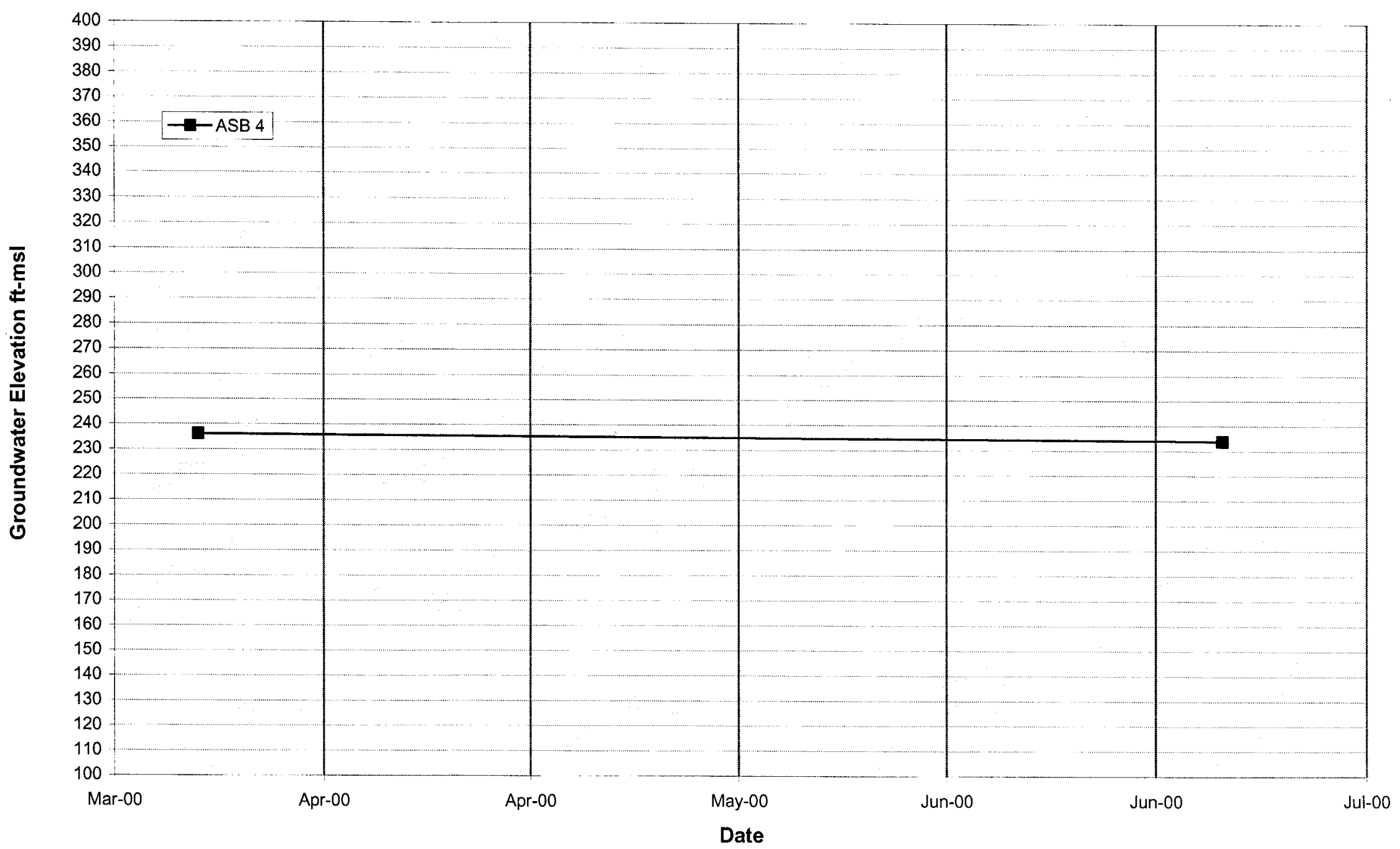

M-Area and Met Lab HWMFs

G-37

First and Second Quarter 2000 
WSRC-TR-2000-00242 UNCLASSIFIED

\section{Hydrograph for Wells ASB 5}

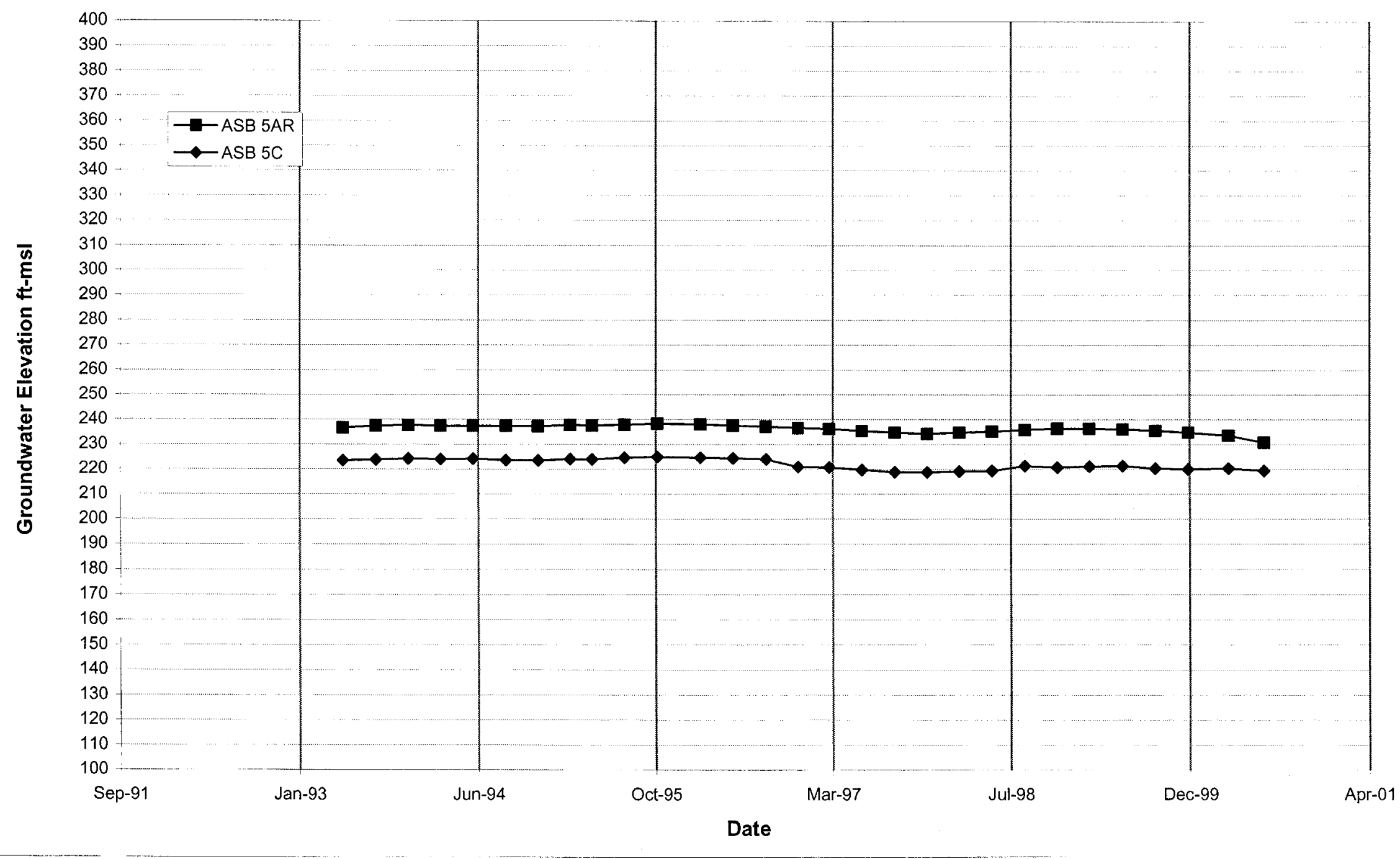


WSRC-TR-2000-00242 UNCLASSIFIED

Hydrograph for Wells ASB 6

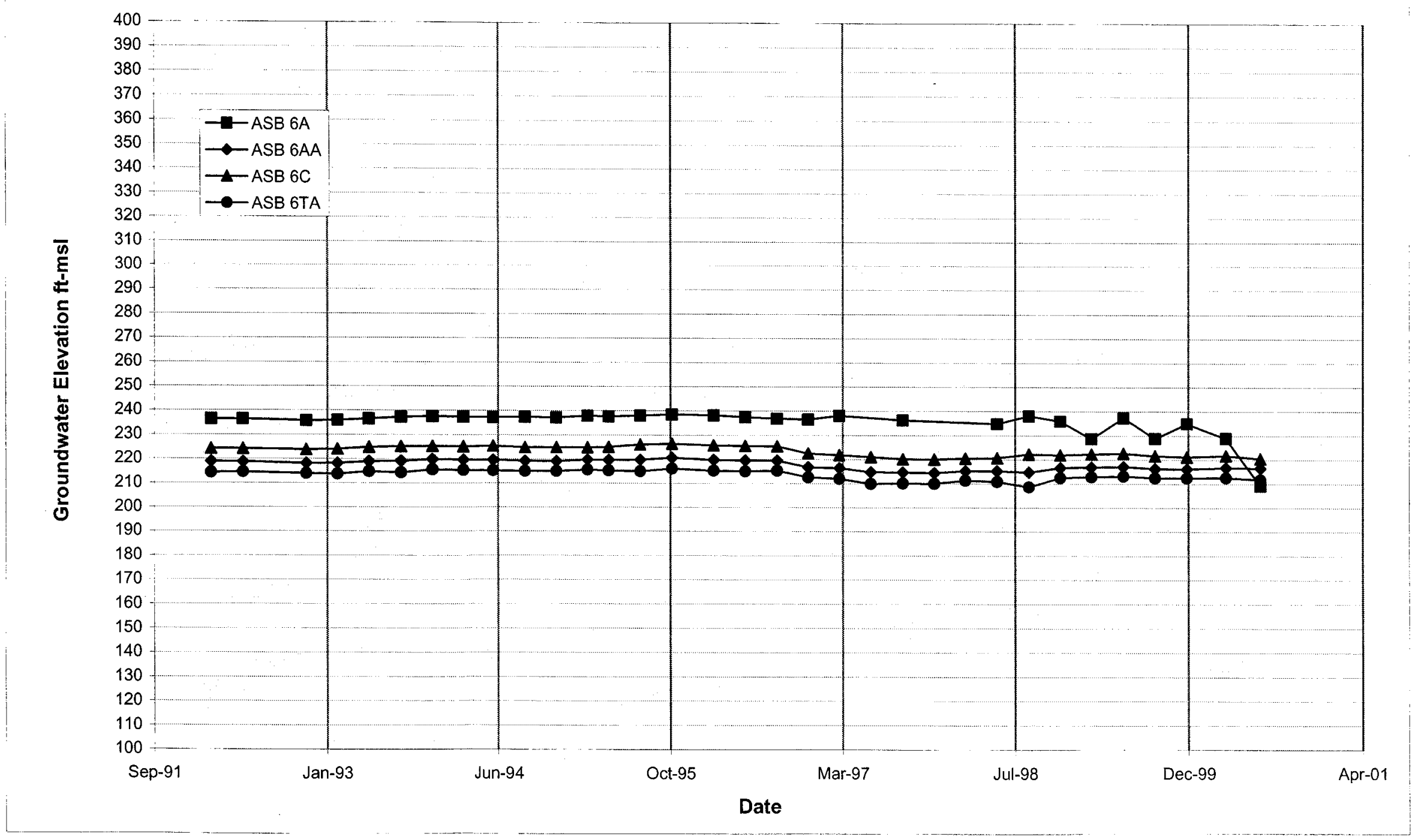


WSRC-TR-2000-00242 UNCLASSIFIED

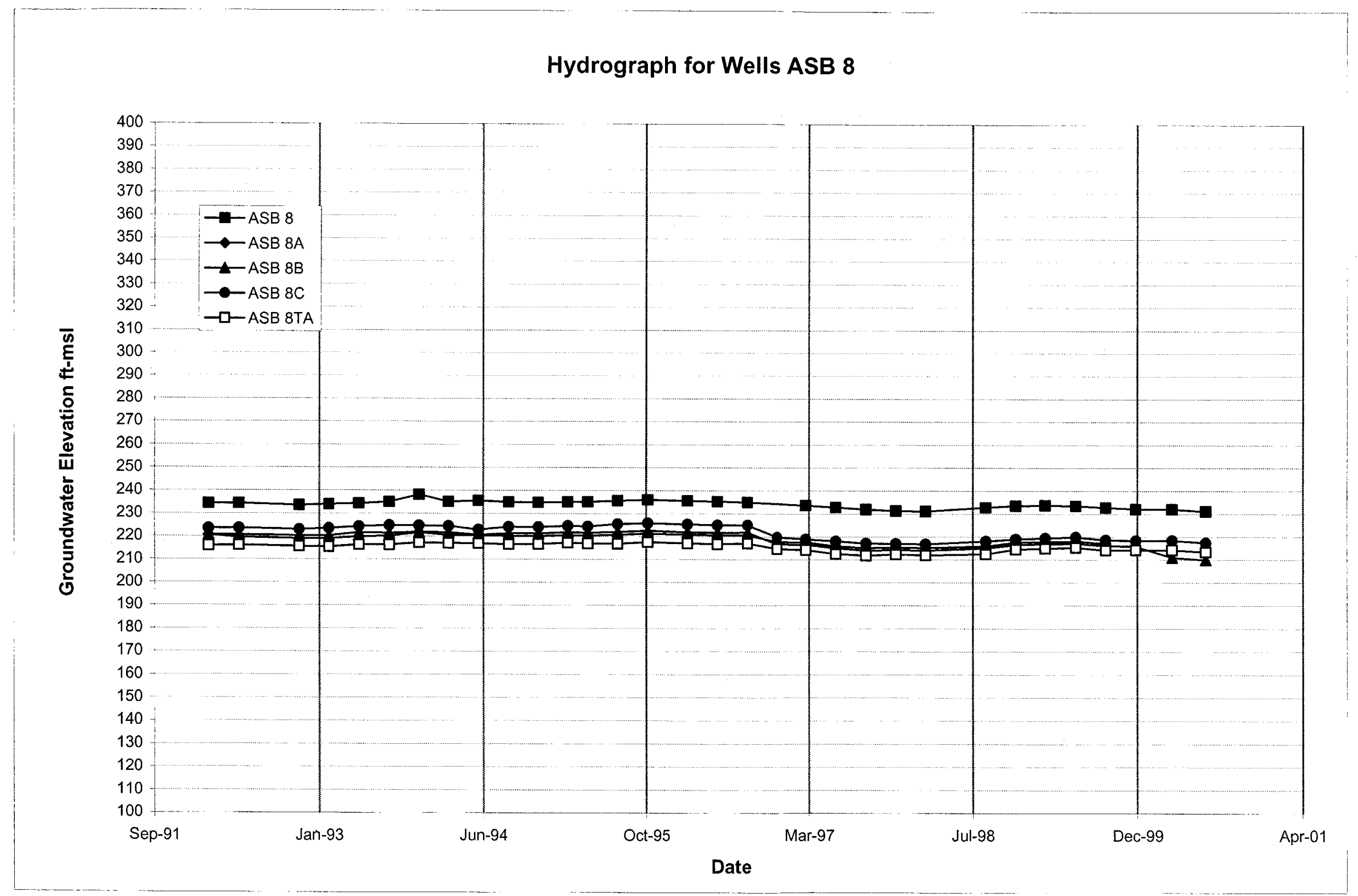




\section{Hydrograph for Wells ASB 9}

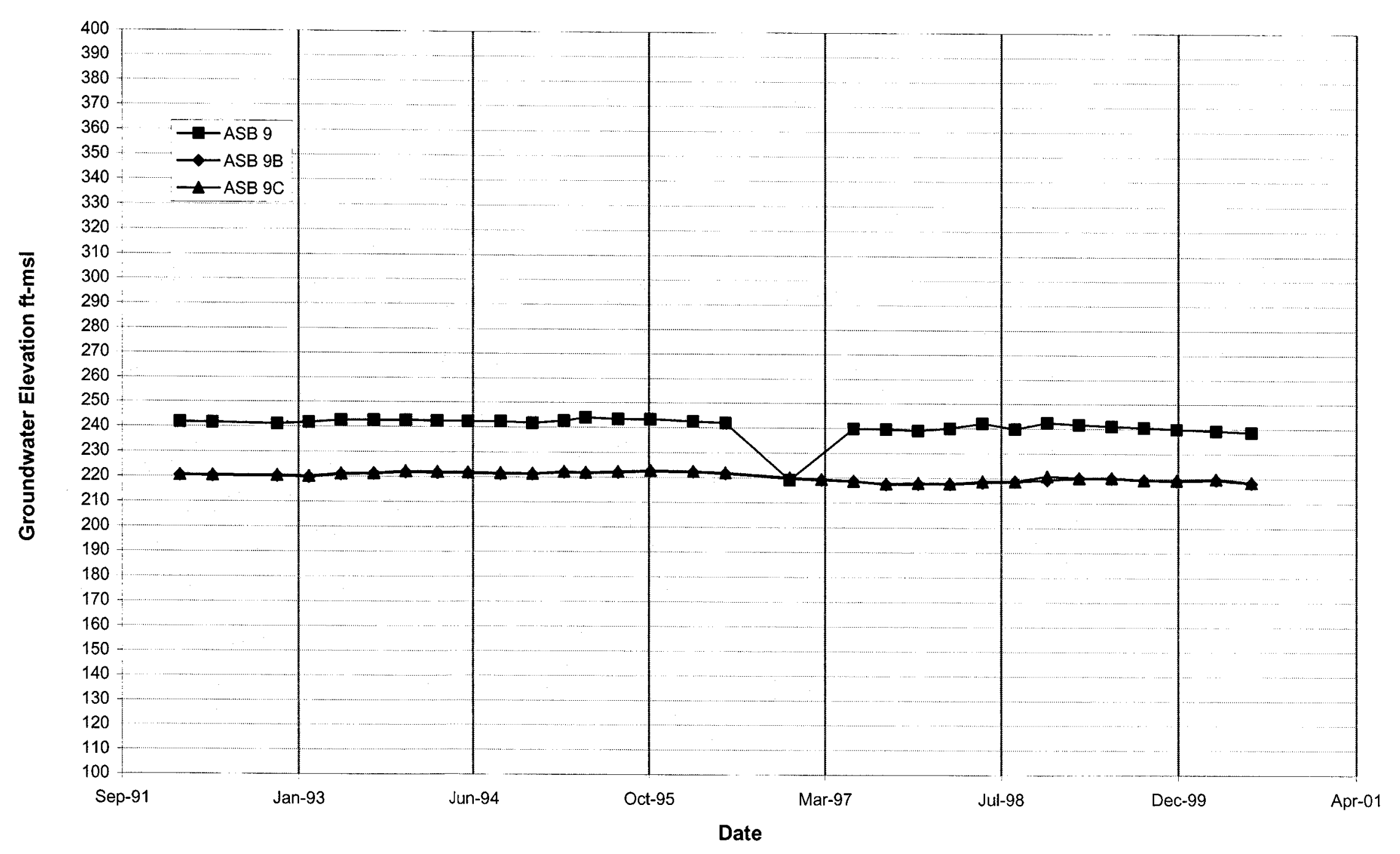


WSRC-TR-2000-00242

UNCLASSIFIED

Hydrograph for Wells ASB10

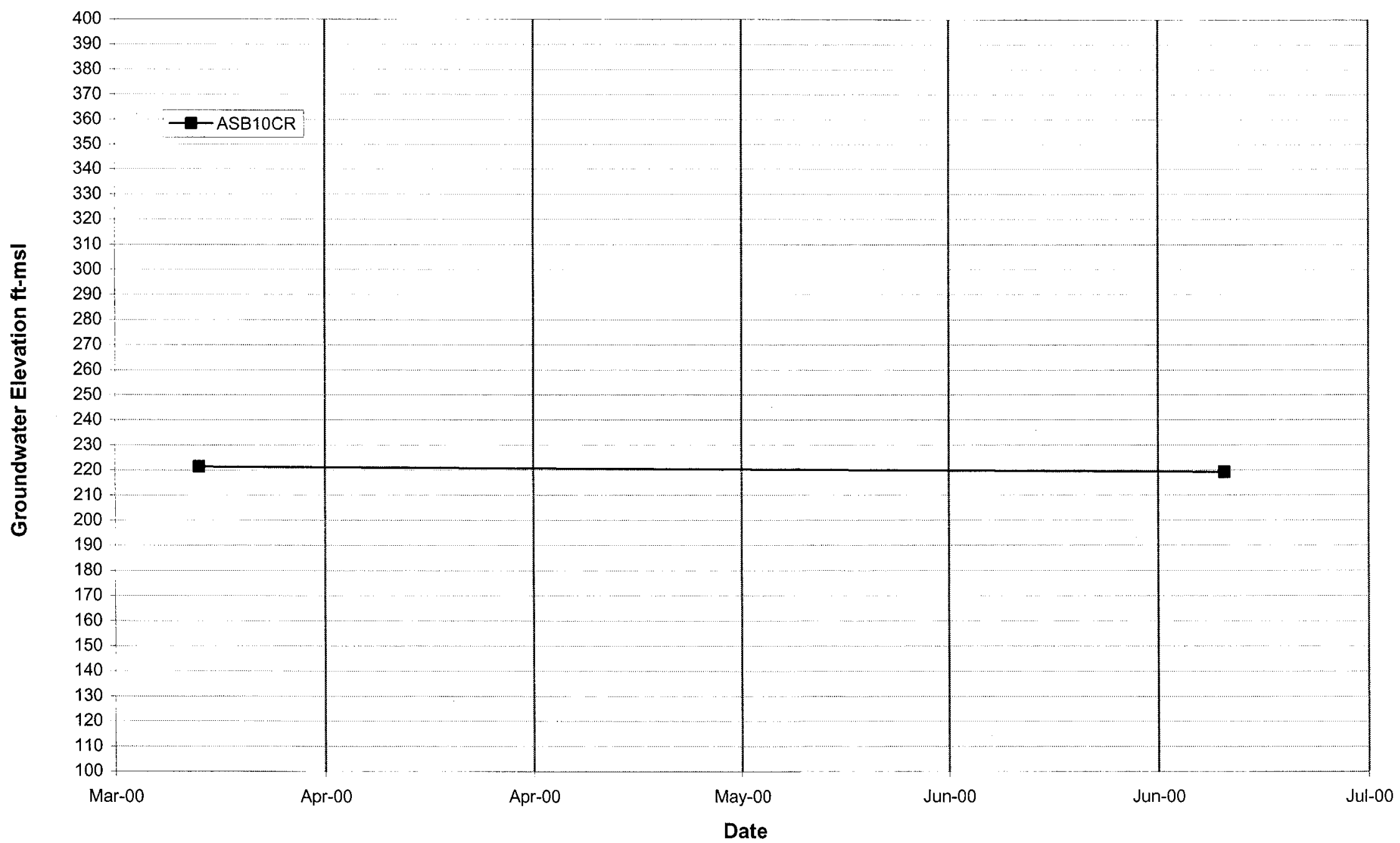

M-Area and Met Lab HWMFs

G-42

First and Second Quarter 2000 
WSRC-TR-2000-00242 UNCLASSIFIED

\section{Hydrograph for Wells MCB 2}

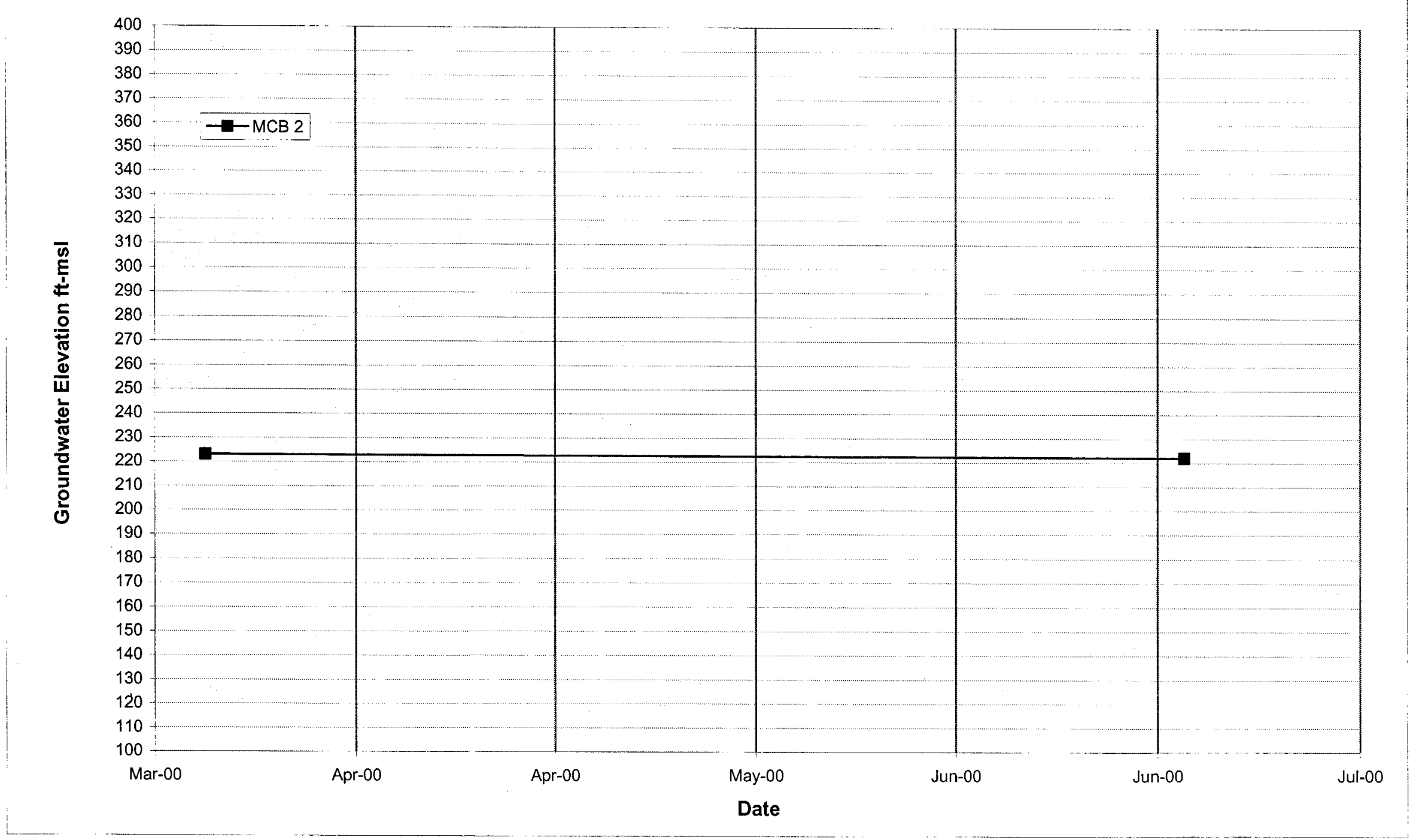


WSRC-TR-2000-00242 UNCLASSIFIED

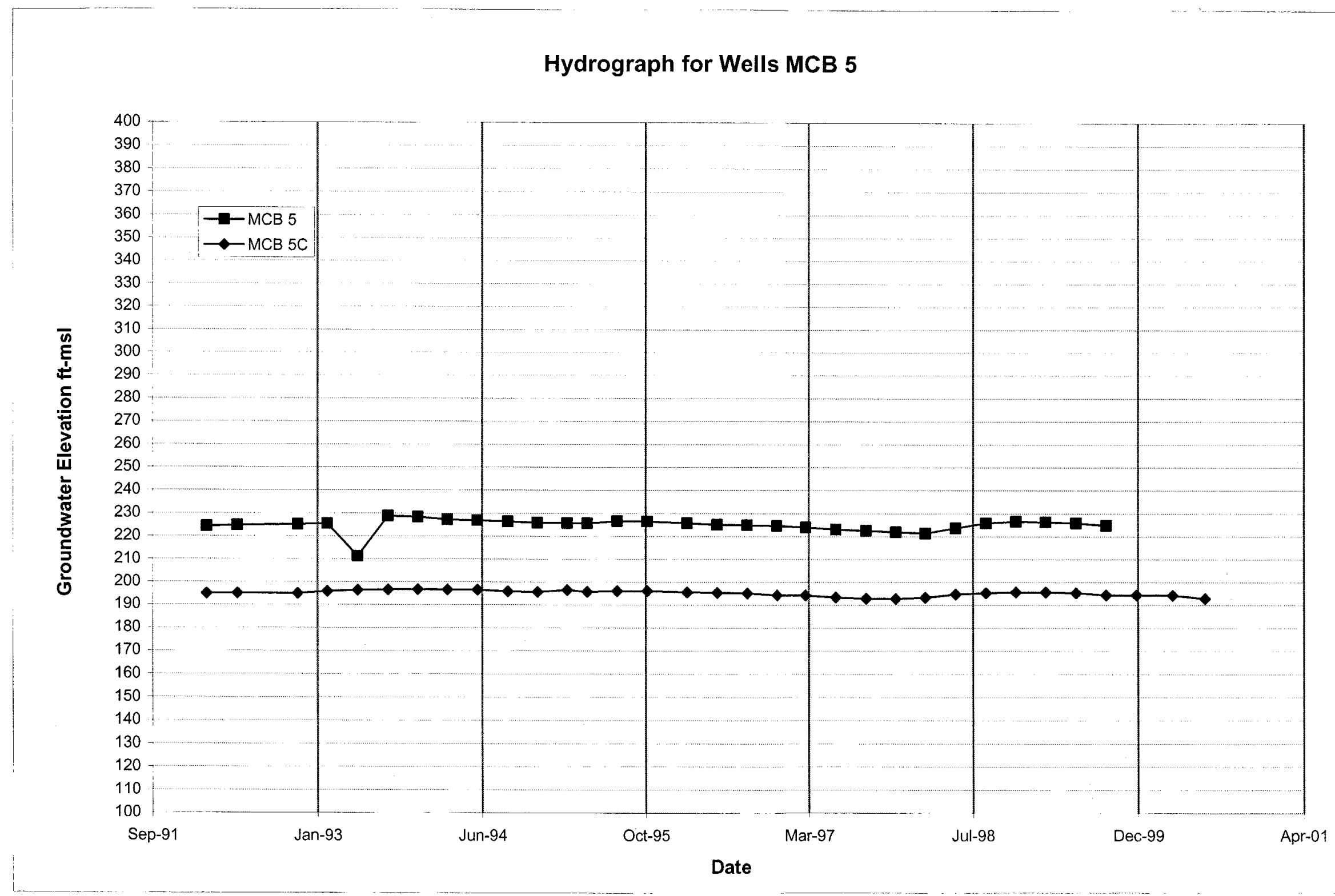


Hydrograph for Wells MCB 6

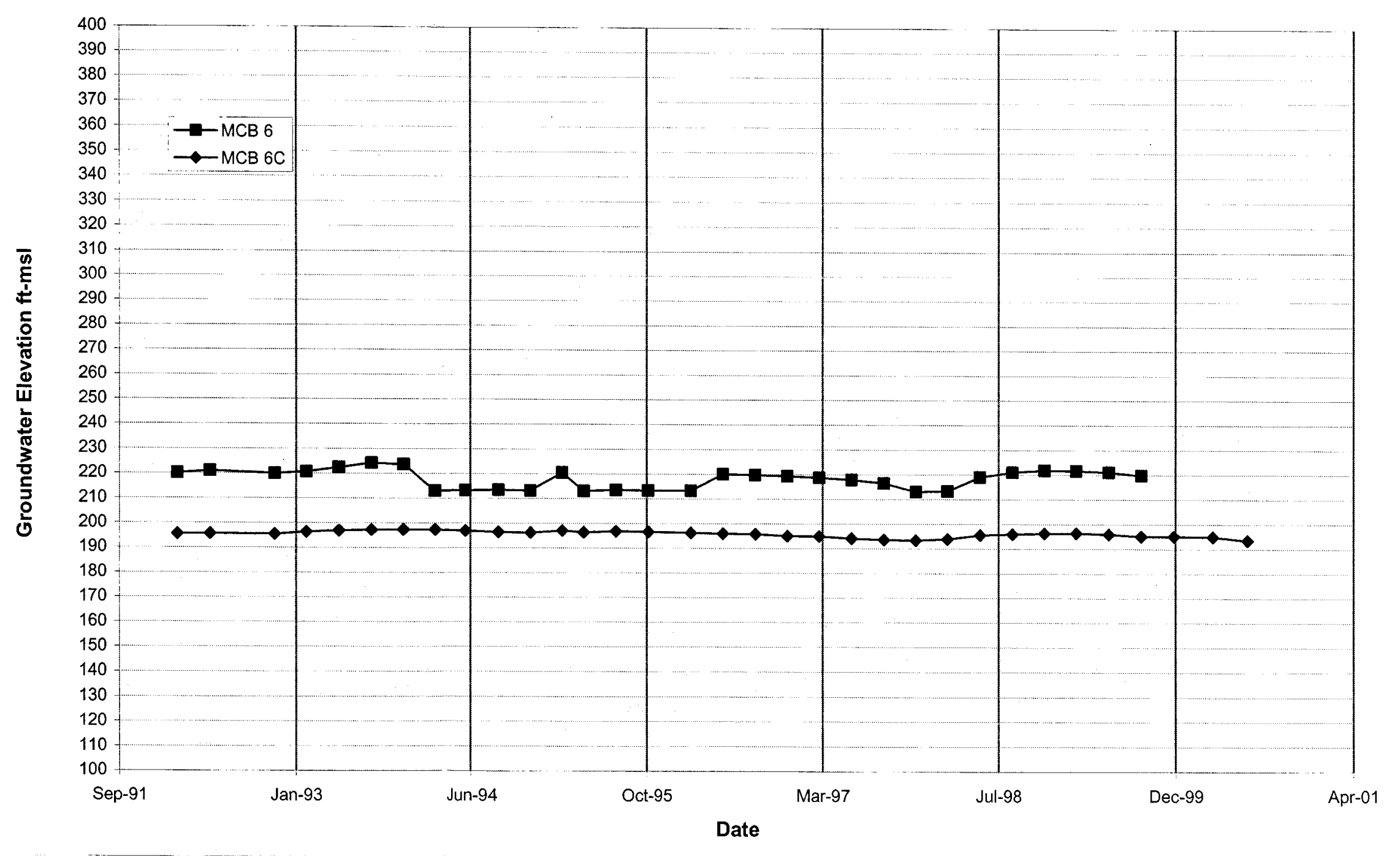


WSRC-TR-2000-00242 UNCLASSIFIED

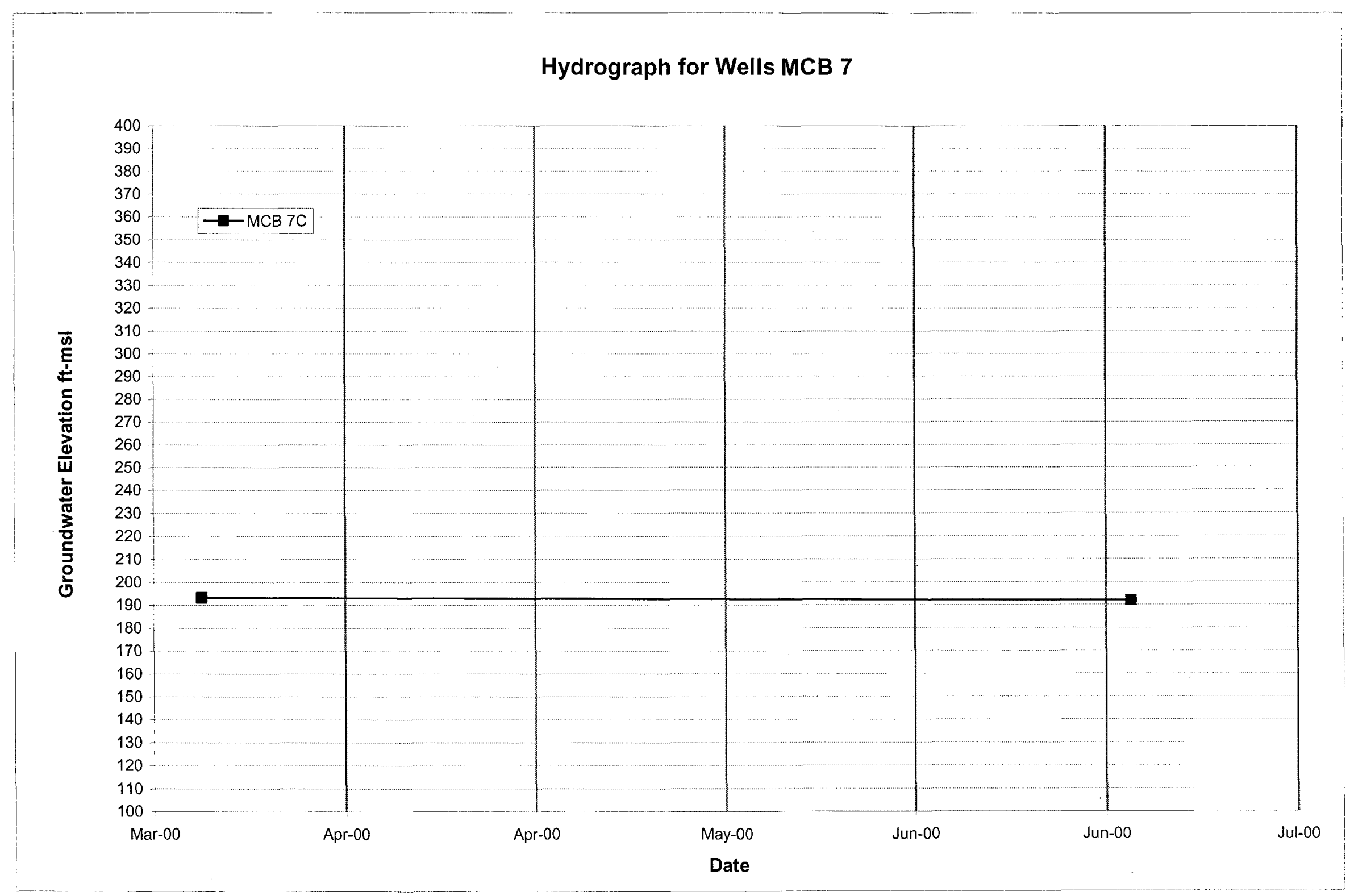




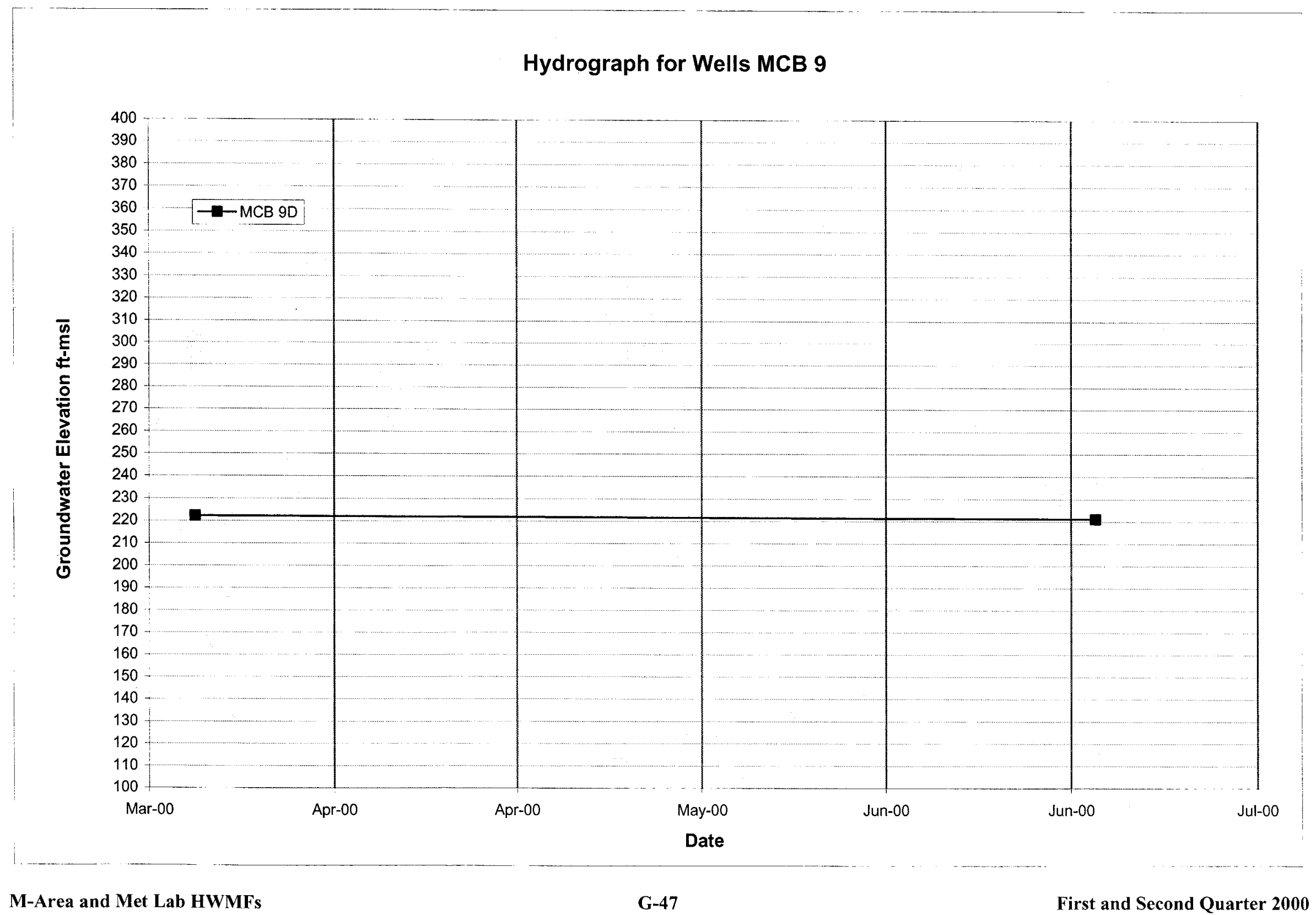


WSRC-TR-2000-00242 UNCLASSIFIED

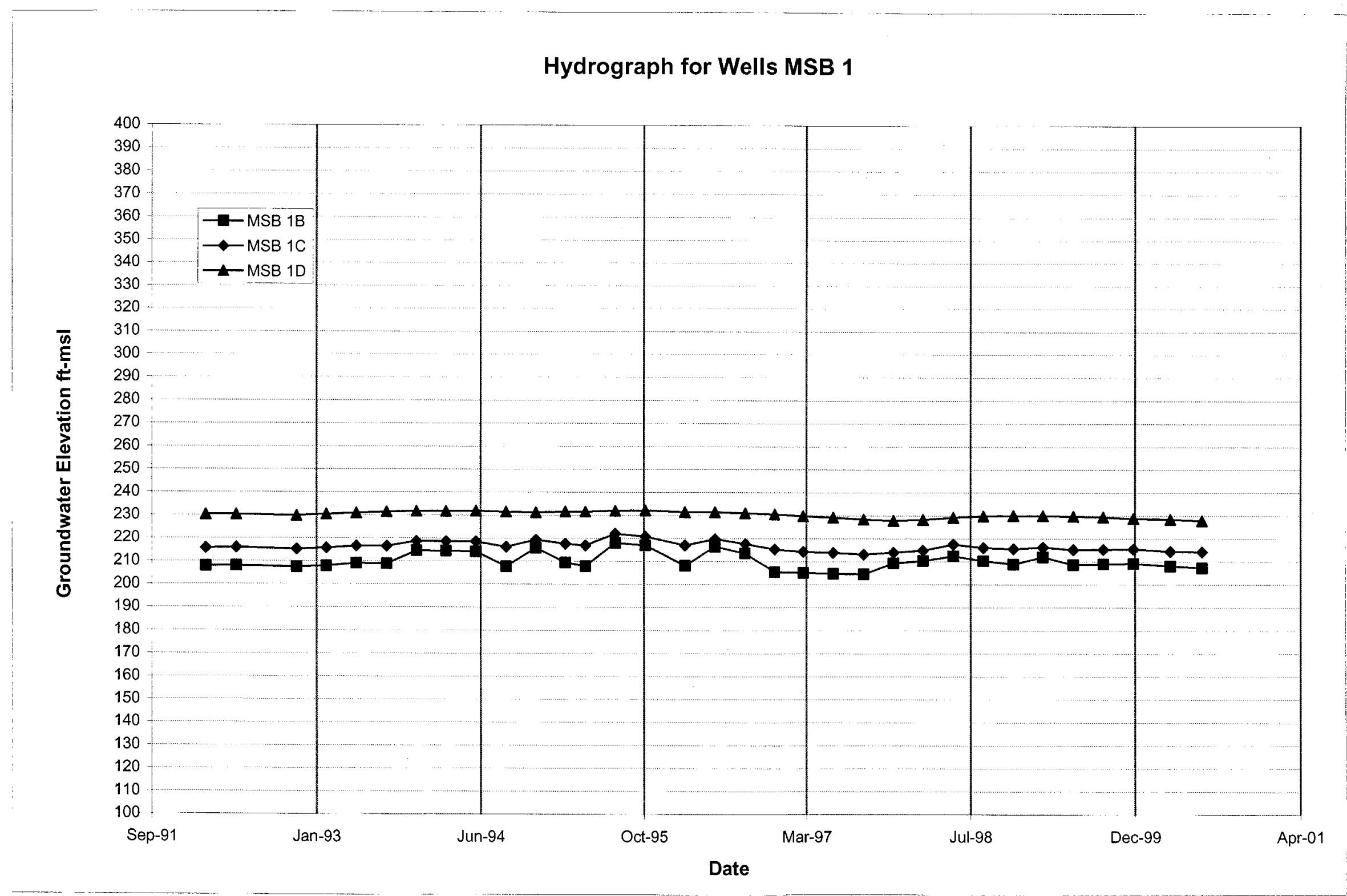


WSRC-TR-2000-00242 UNCLASSIFIED

Hydrograph for Wells MSB 2

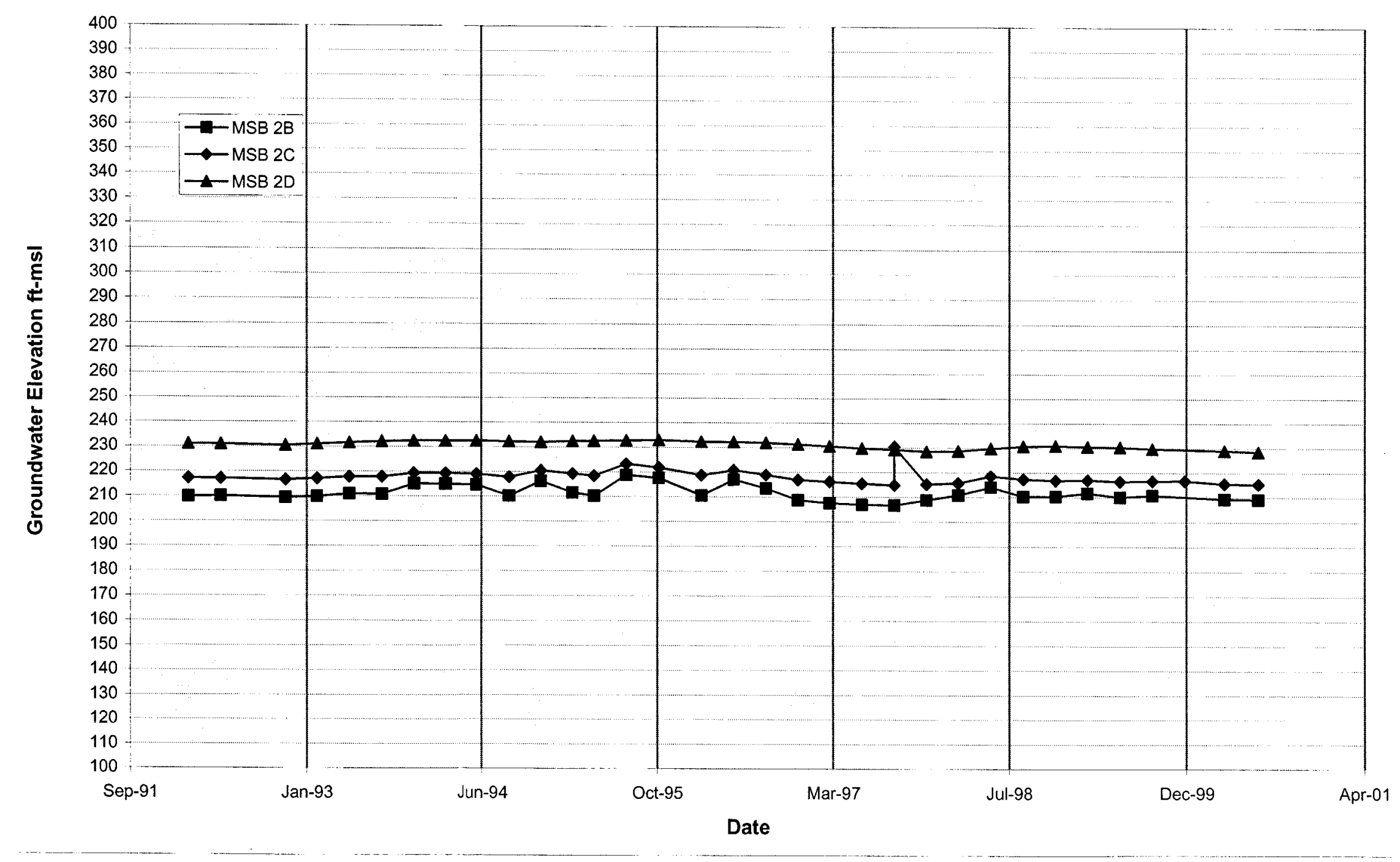


WSRC-TR-2000-00242 UNCLASSIFIED

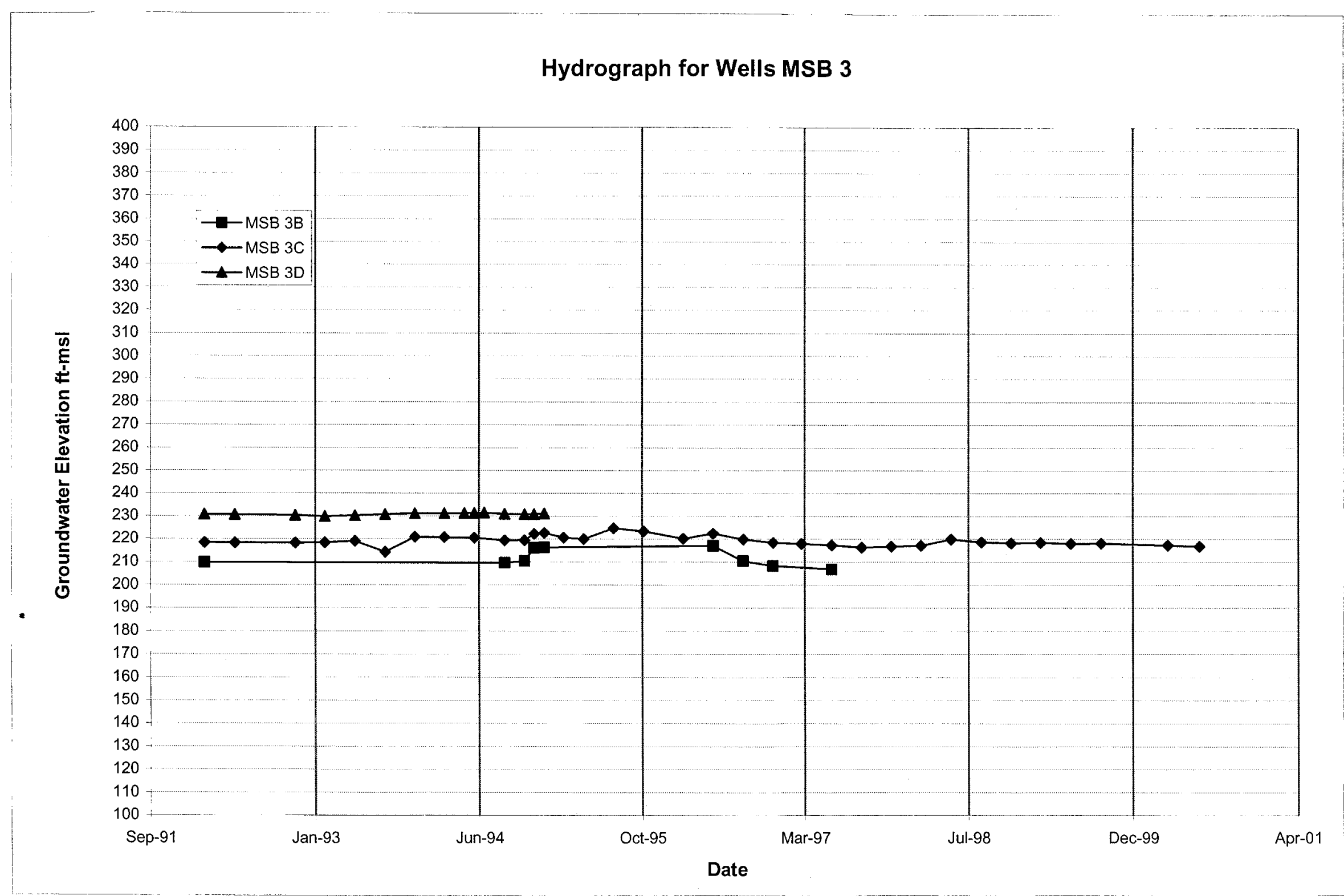


WSRC-TR-2000-00242 UNCLASSIFIED

\section{Hydrograph for Wells MSB 4}

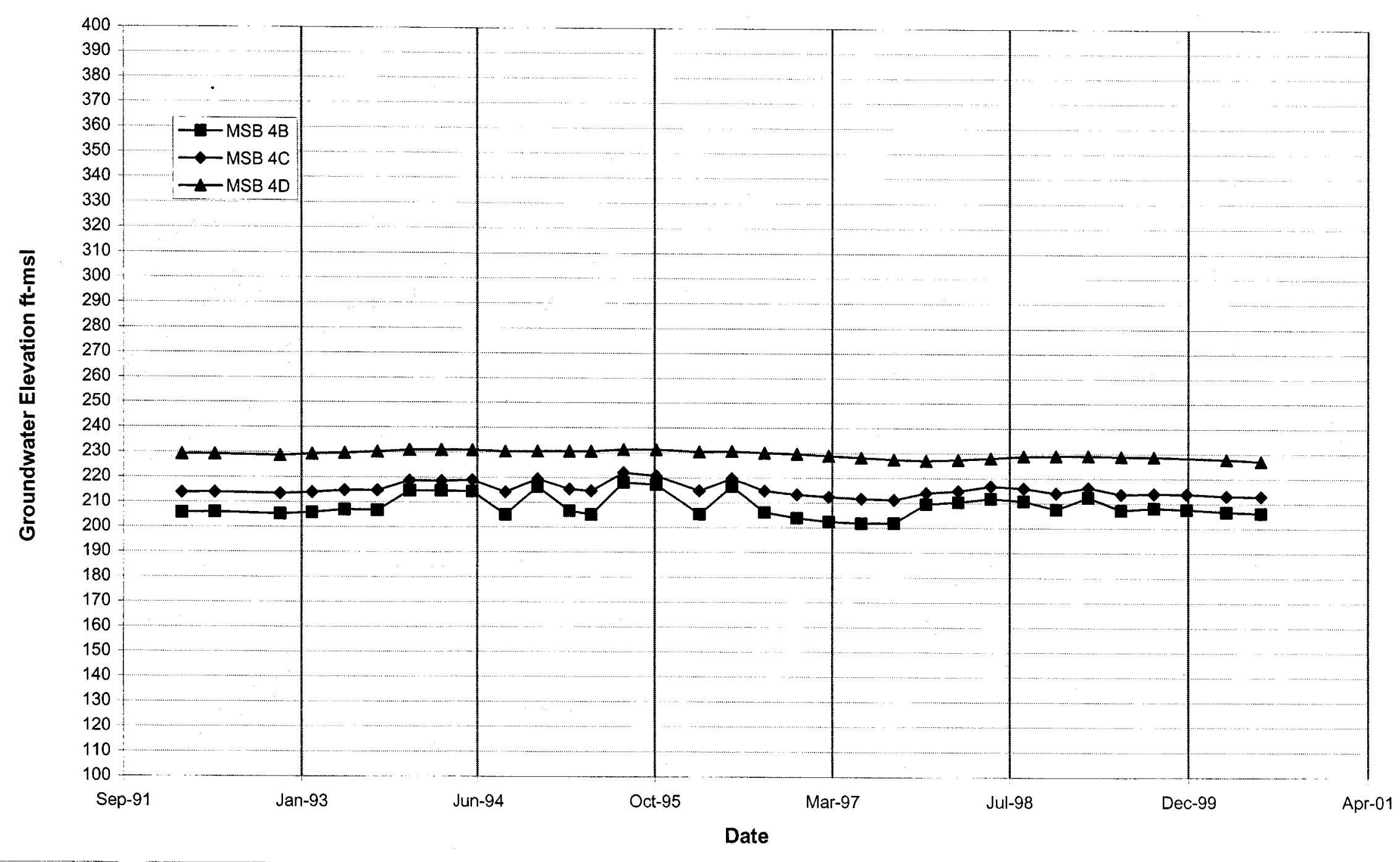




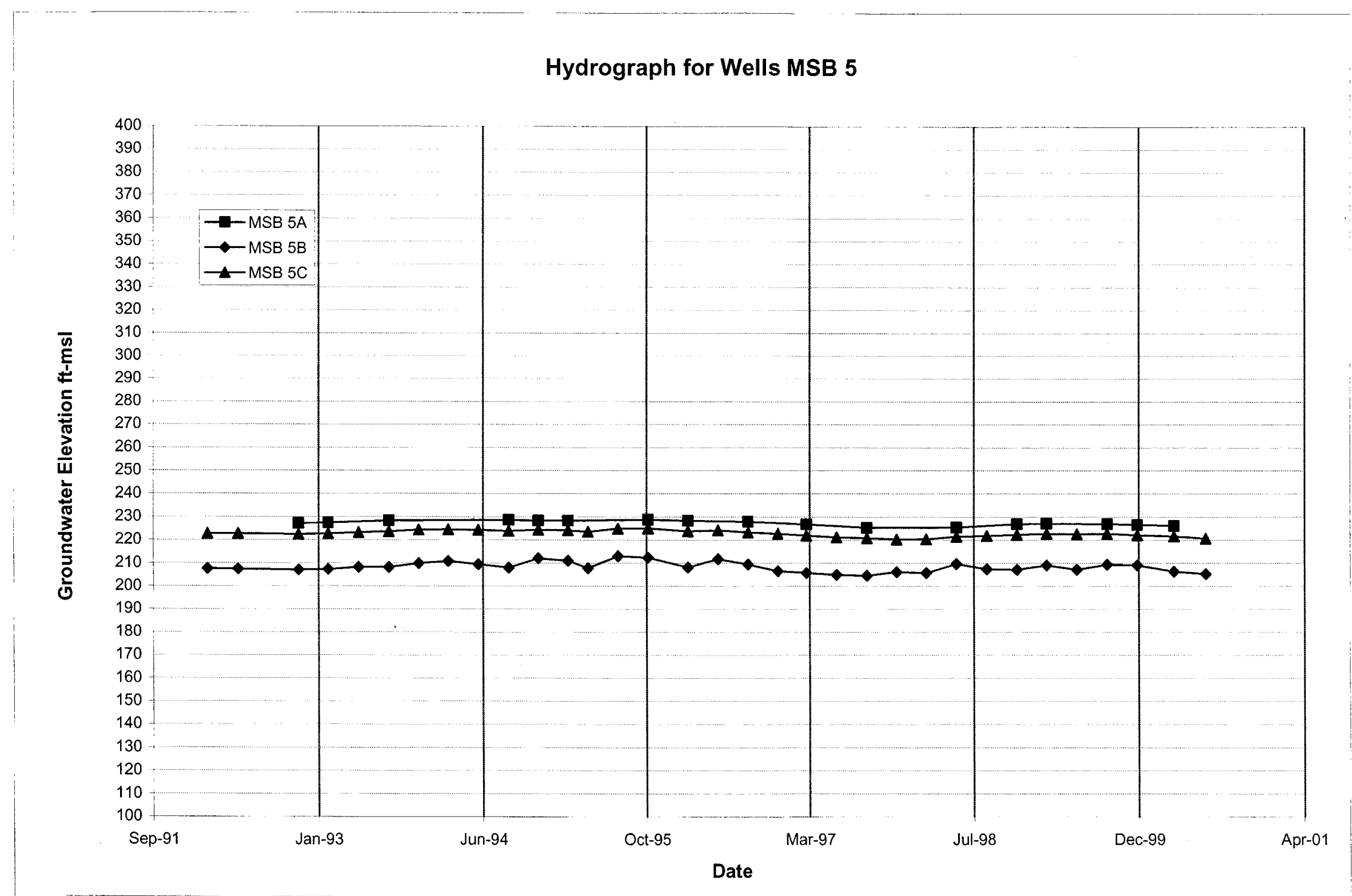


Hydrograph for Wells MSB 6

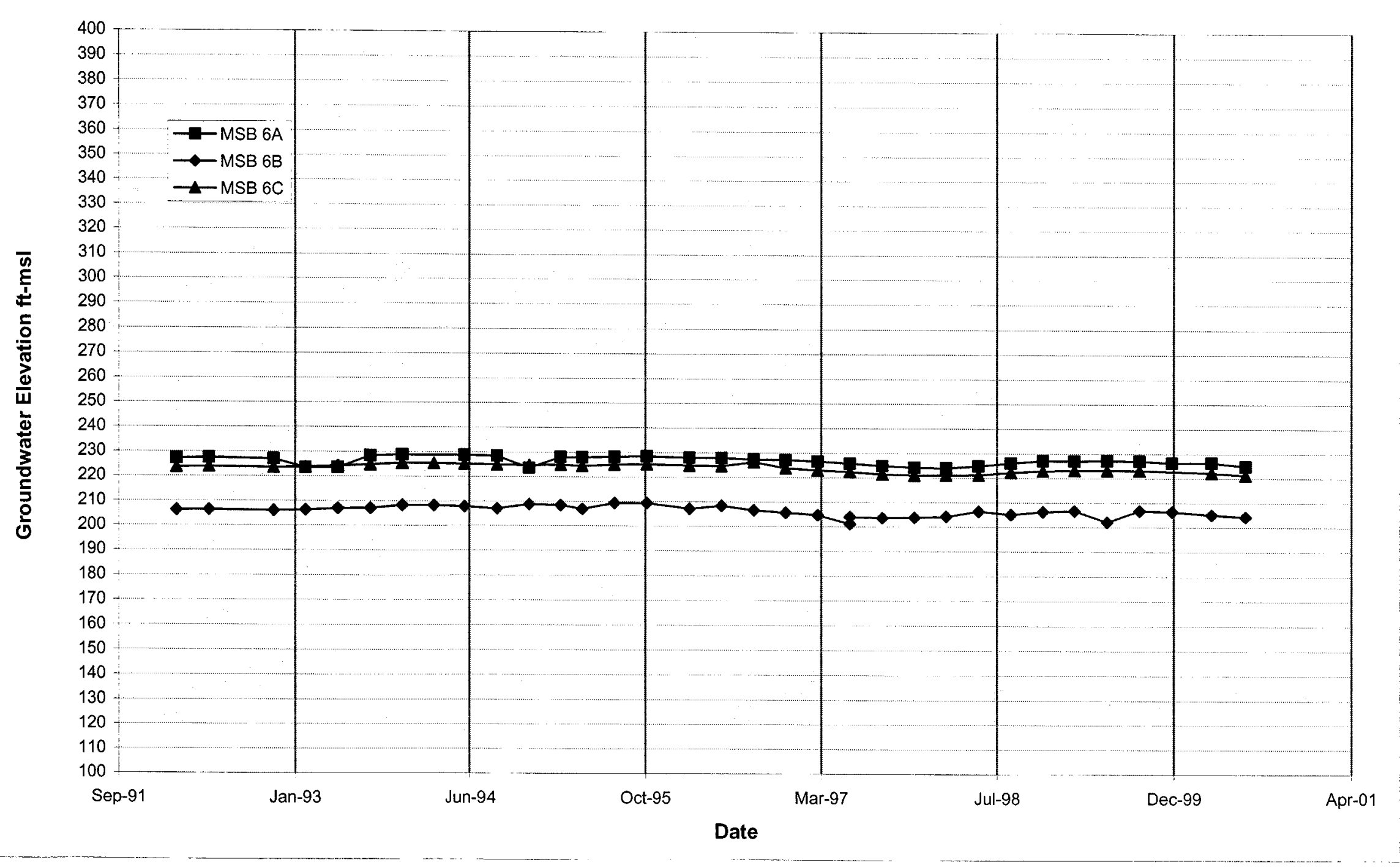


WSRC-TR-2000-00242 UNCLASSIFIED

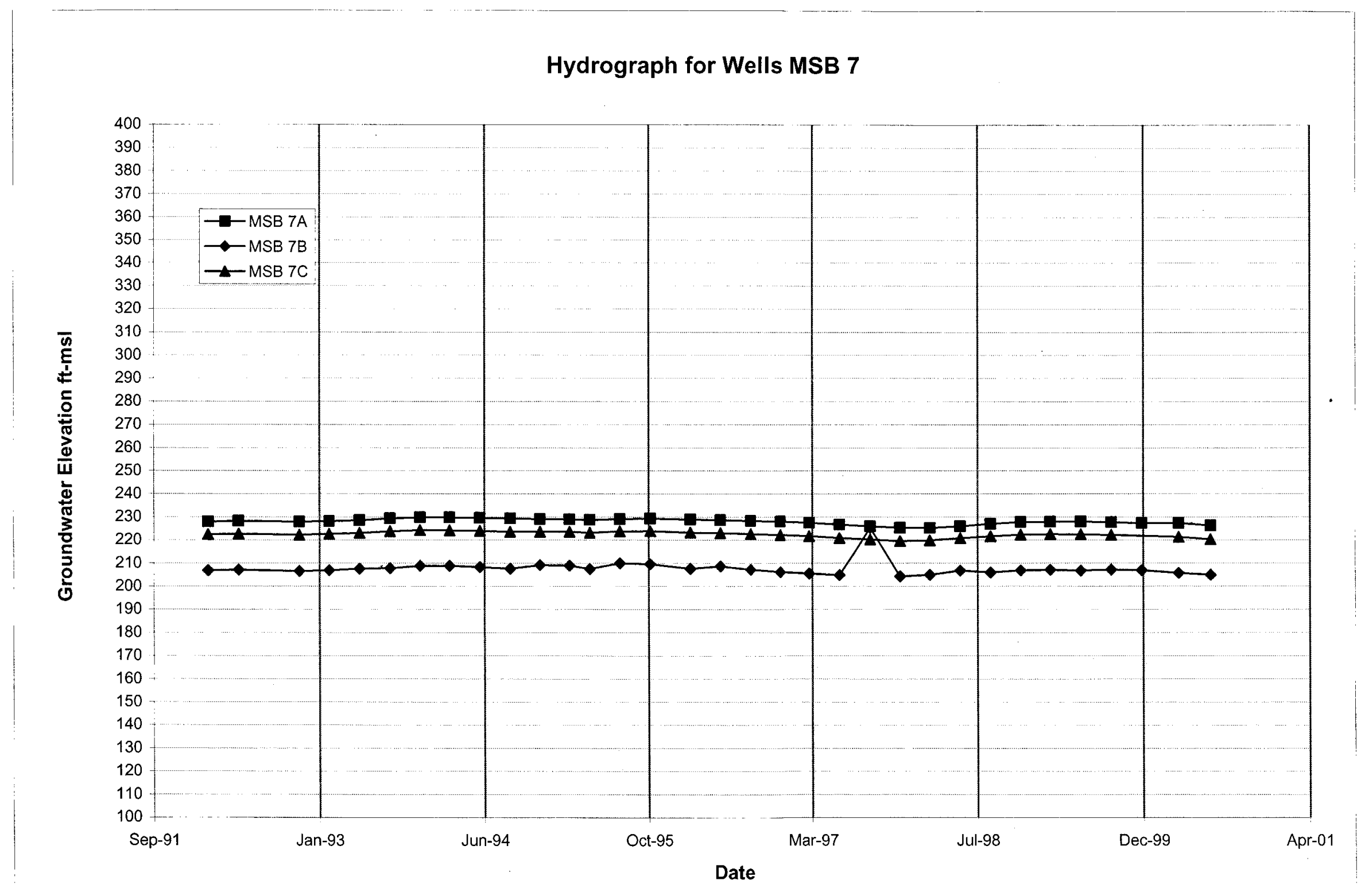


WSRC-TR-2000-00242 UNCLASSIFIED

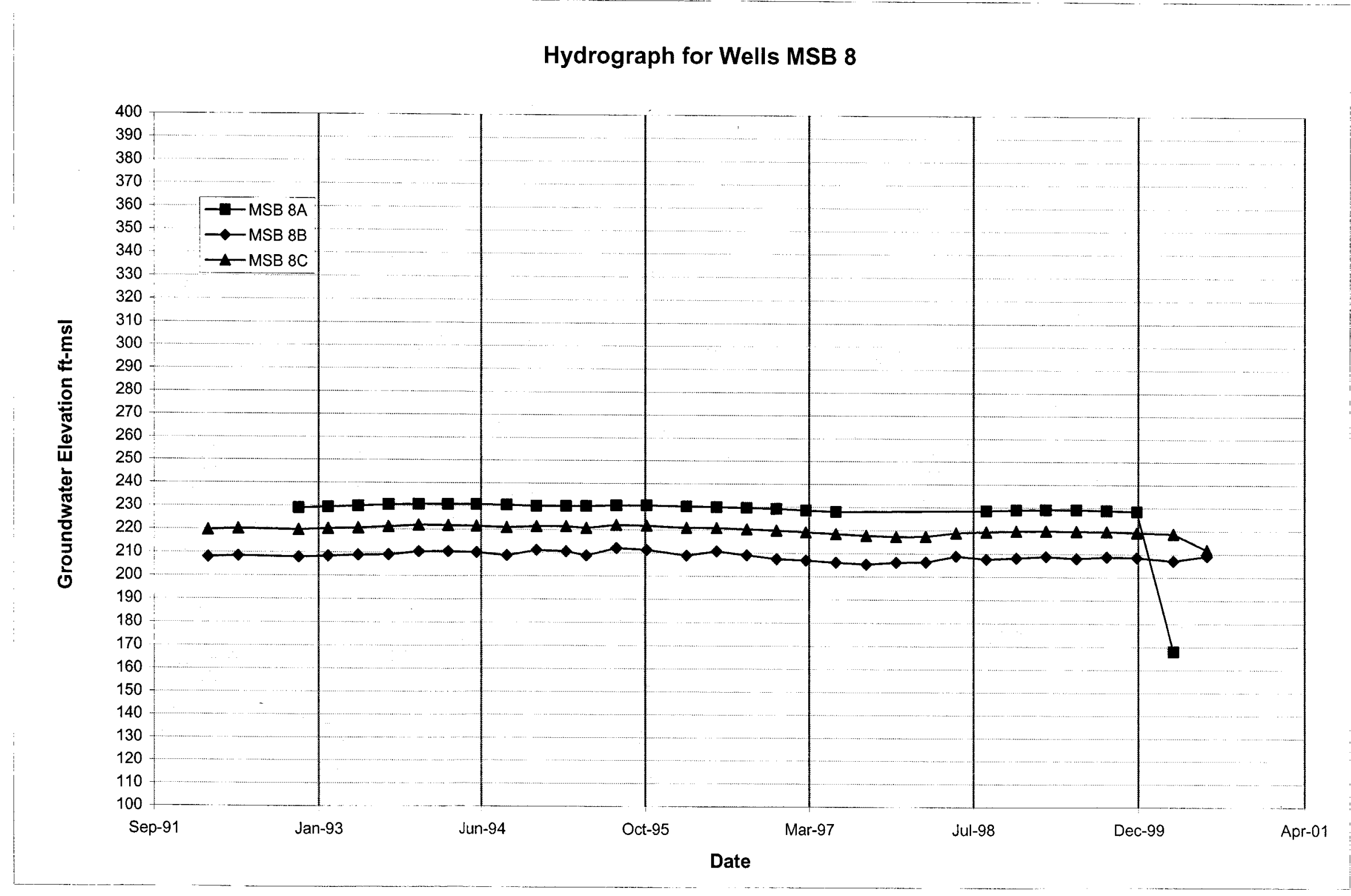


WSRC-TR-2000-00242 UNCLASSIFIED

\section{Hydrograph for Wells MSB 9}

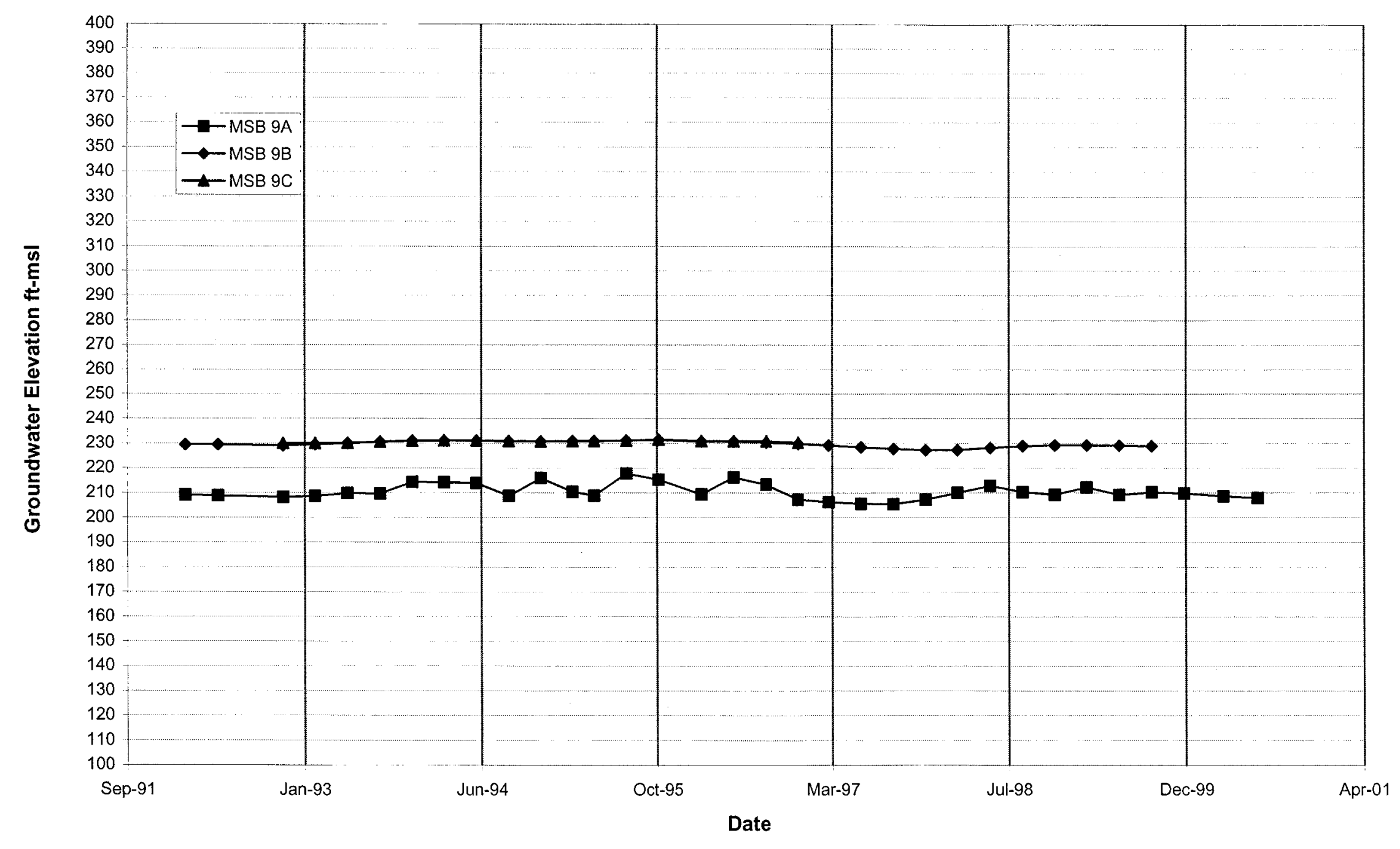


Hydrograph for Wells MSB10

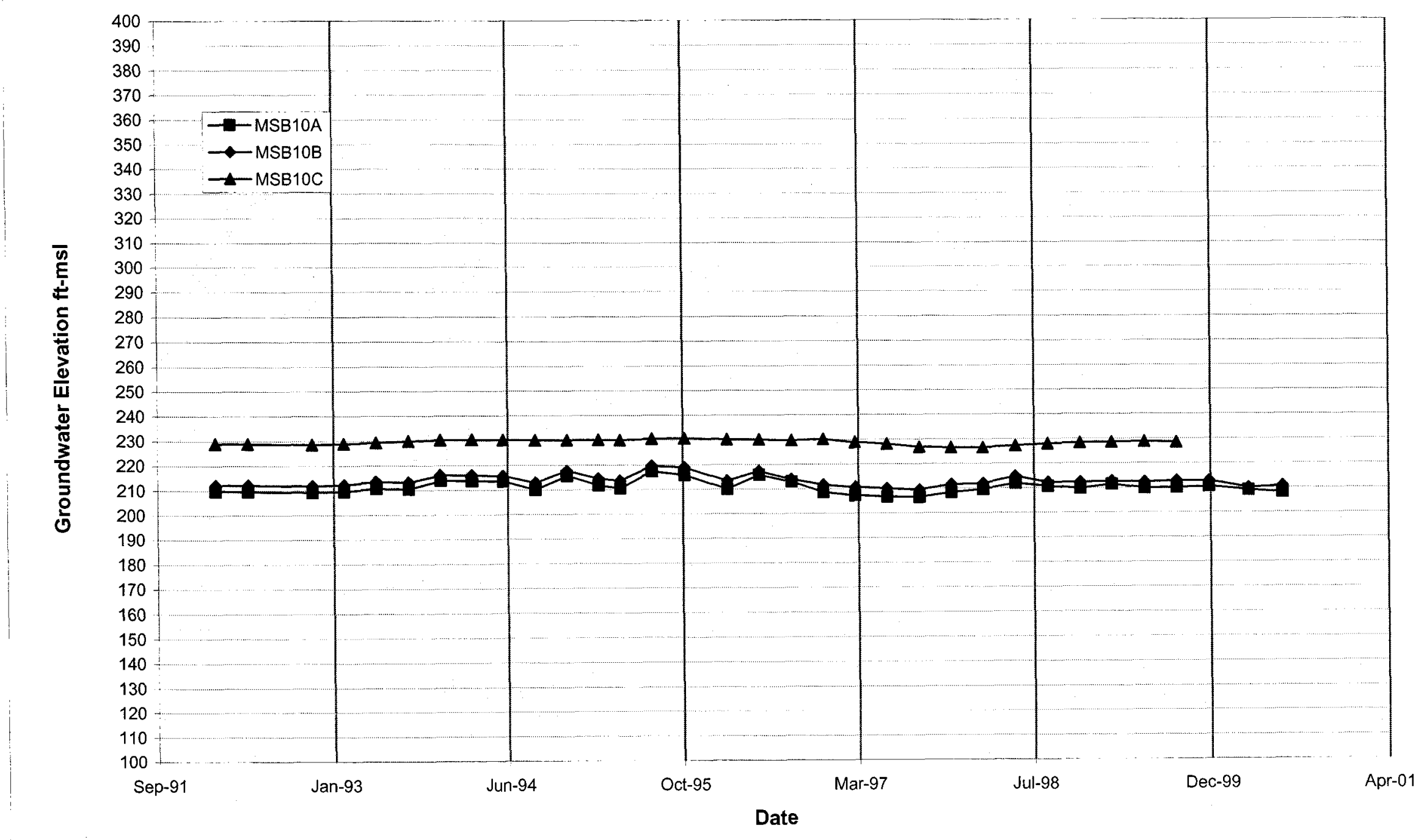


WSRC-TR-2000-00242 UNCLASSIFIED

\section{Hydrograph for Wells MSB11}

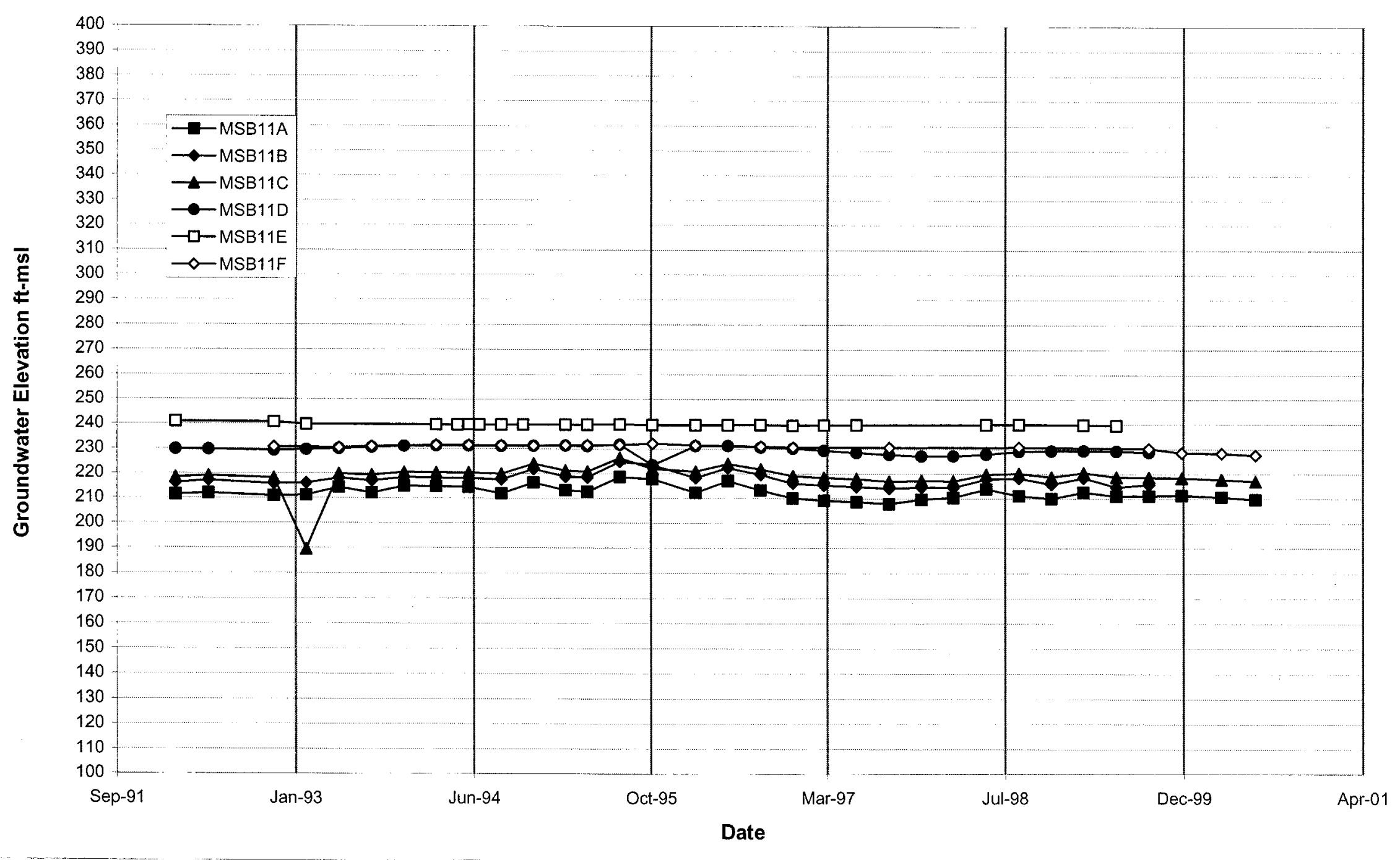


WSRC-TR-2000-00242 UNCLASSIFIED

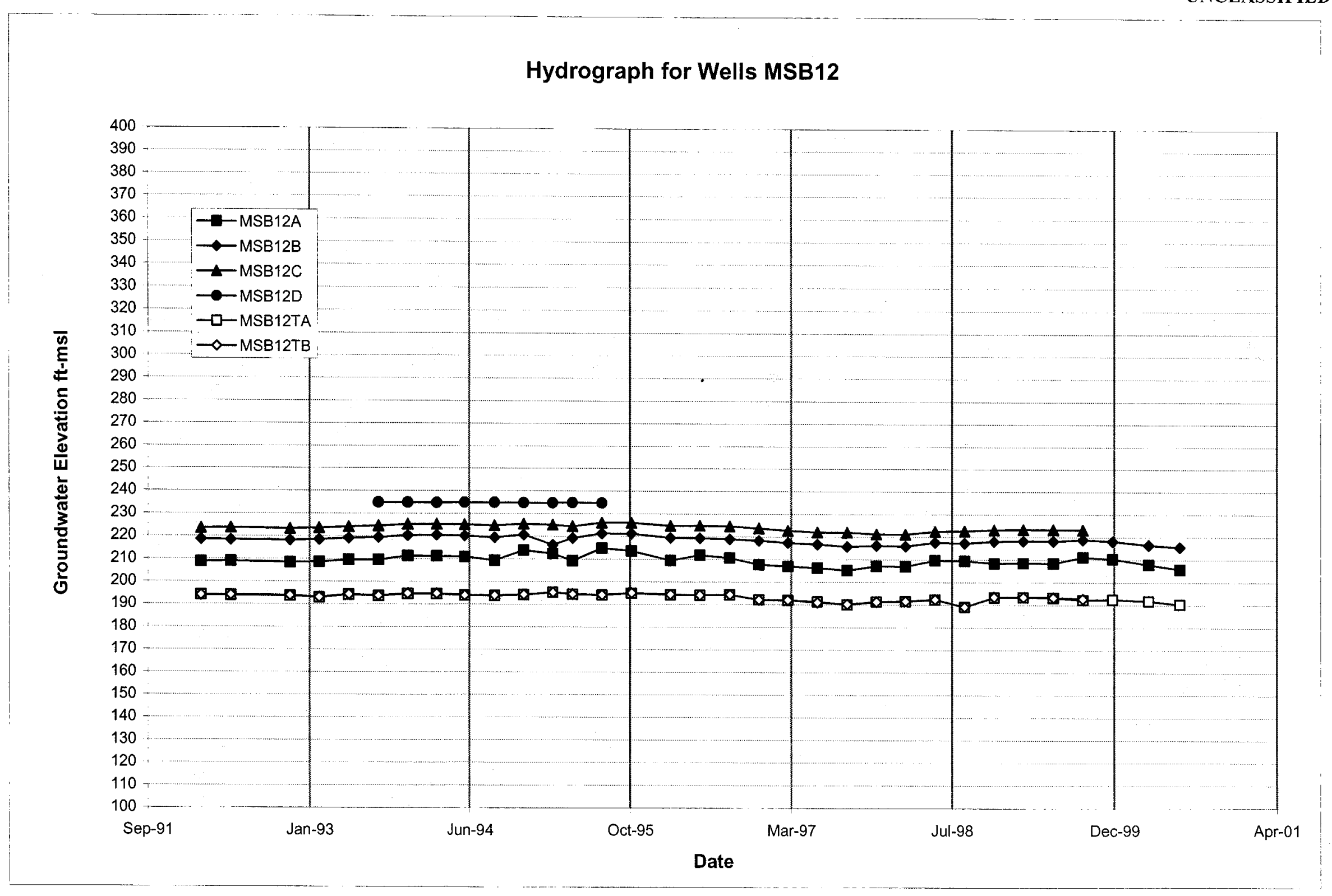


WSRC-TR-2000-00242 UNCLASSIFIED

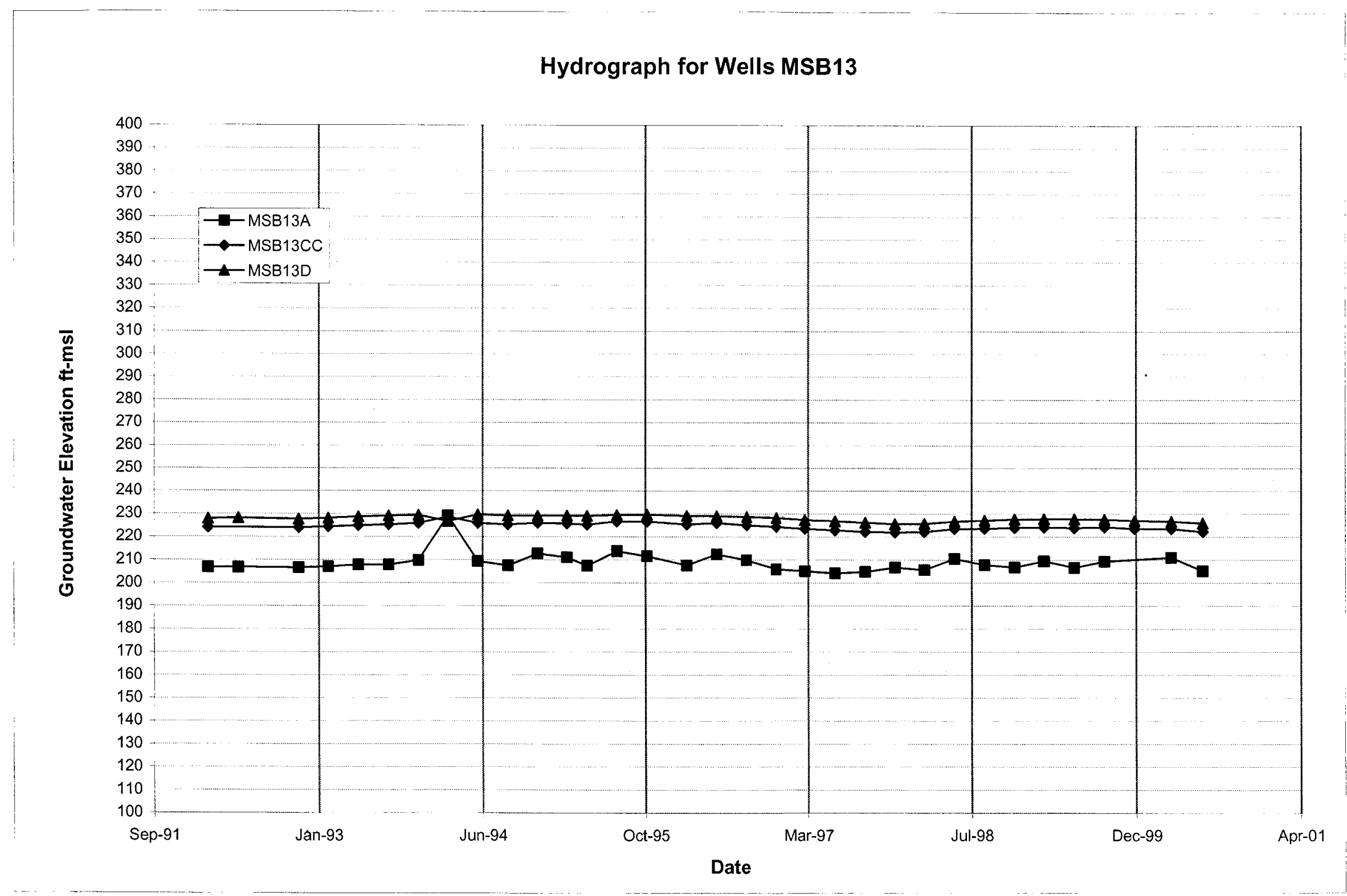




\section{Hydrograph for Wells MSB14}

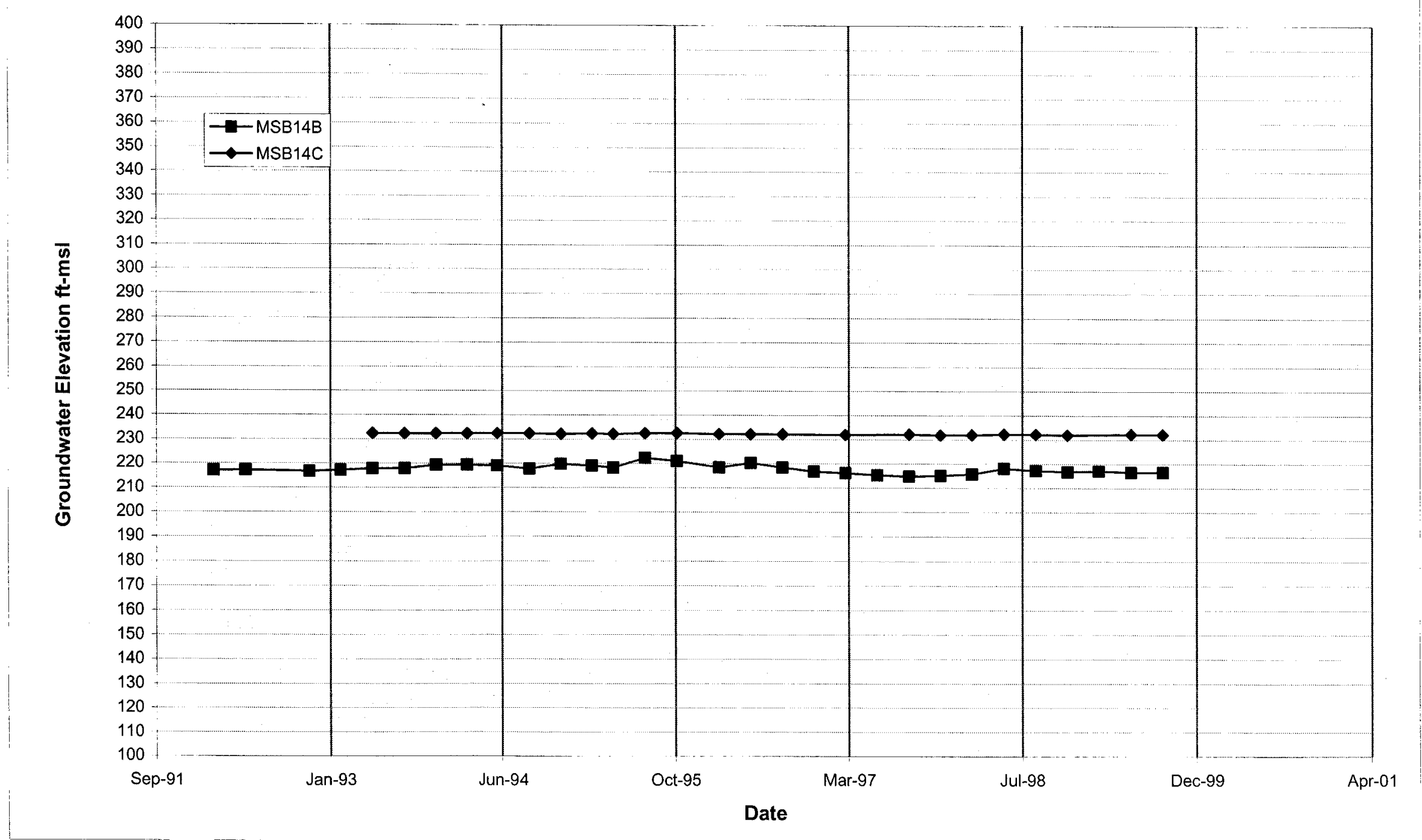


Hydrograph for Wells MSB15

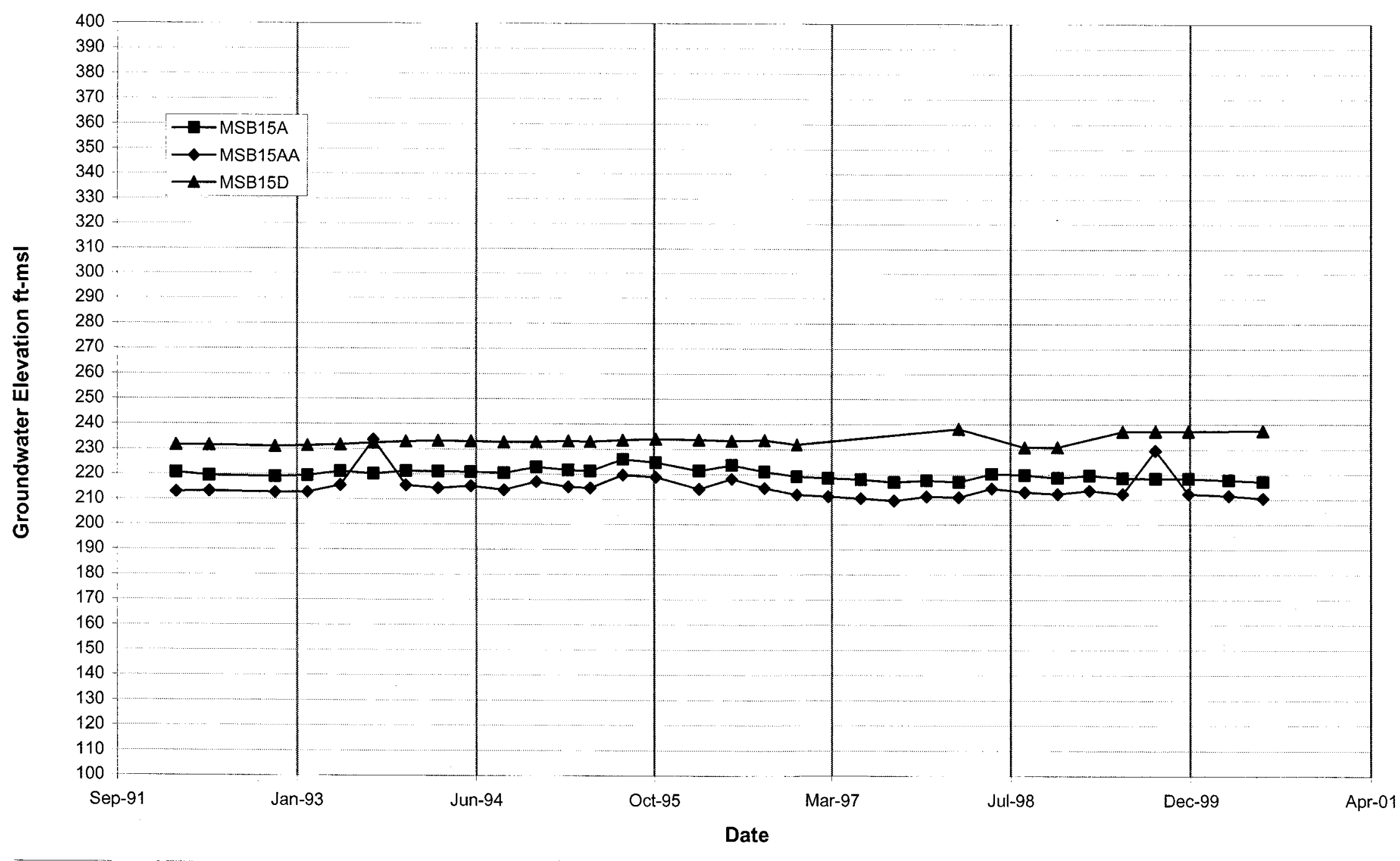




\section{Hydrograph for Wells MSB16}

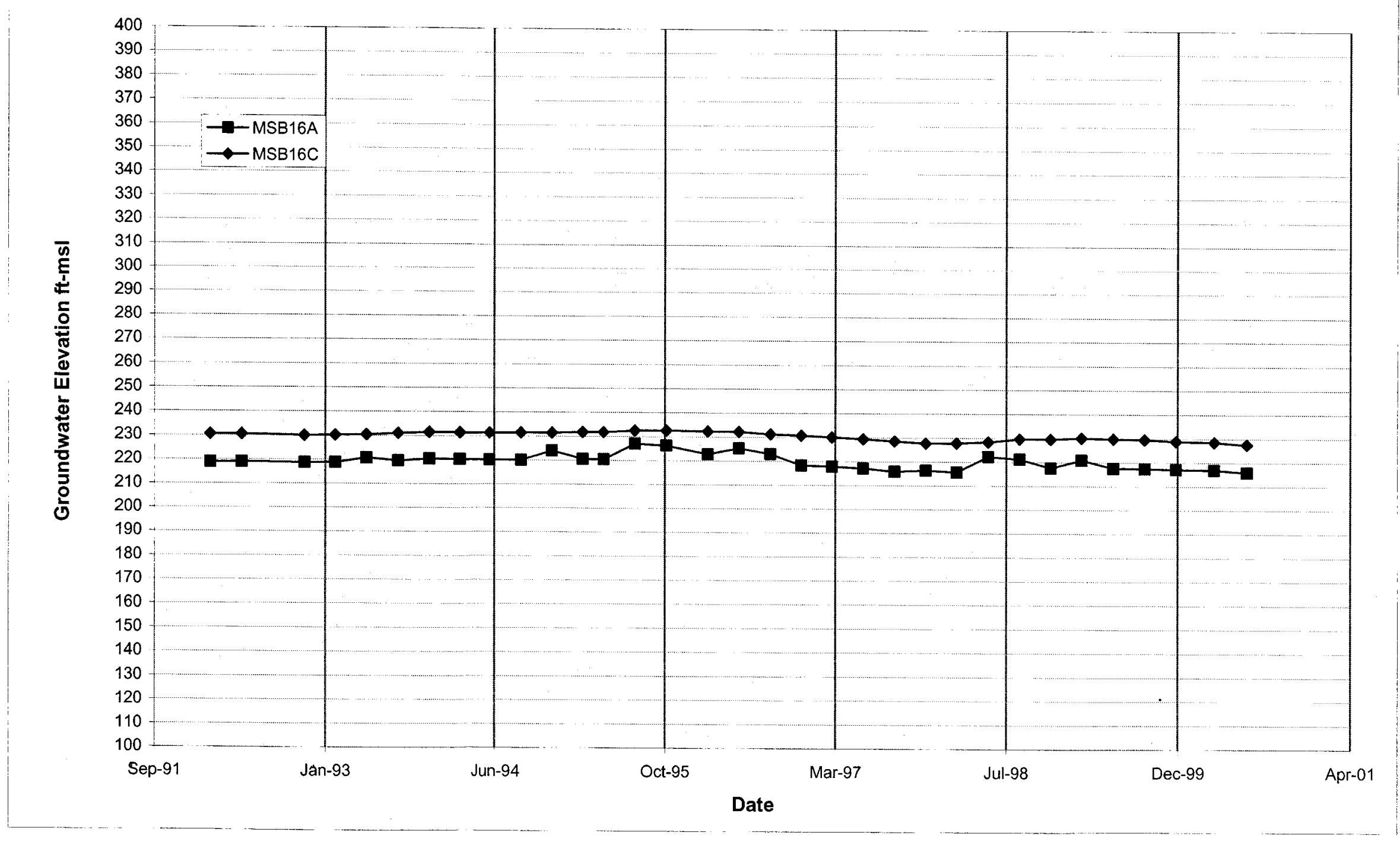


WSRC-TR-2000-00242

UNCLASSIFIED

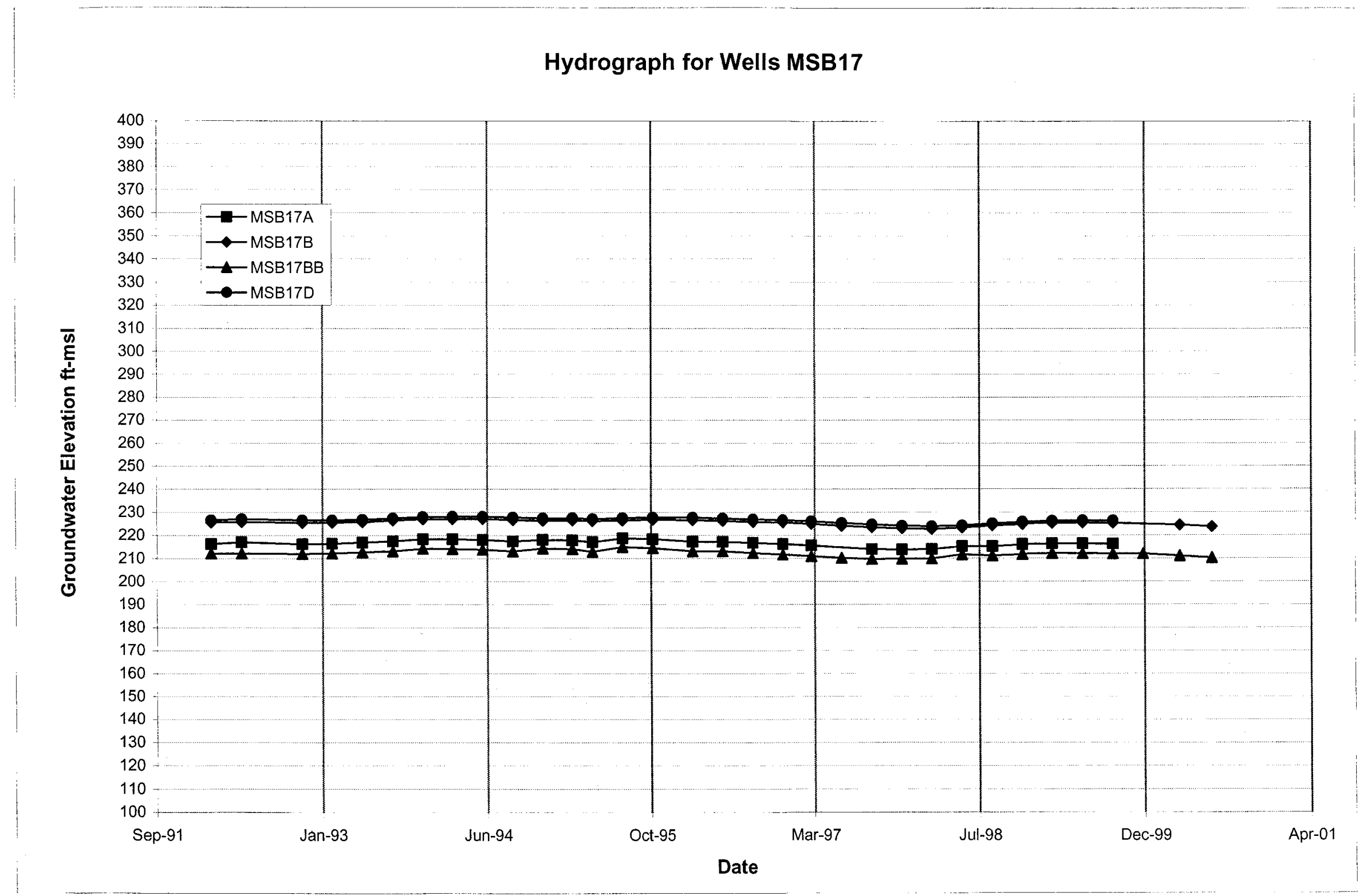




\section{Hydrograph for Wells MSB18}

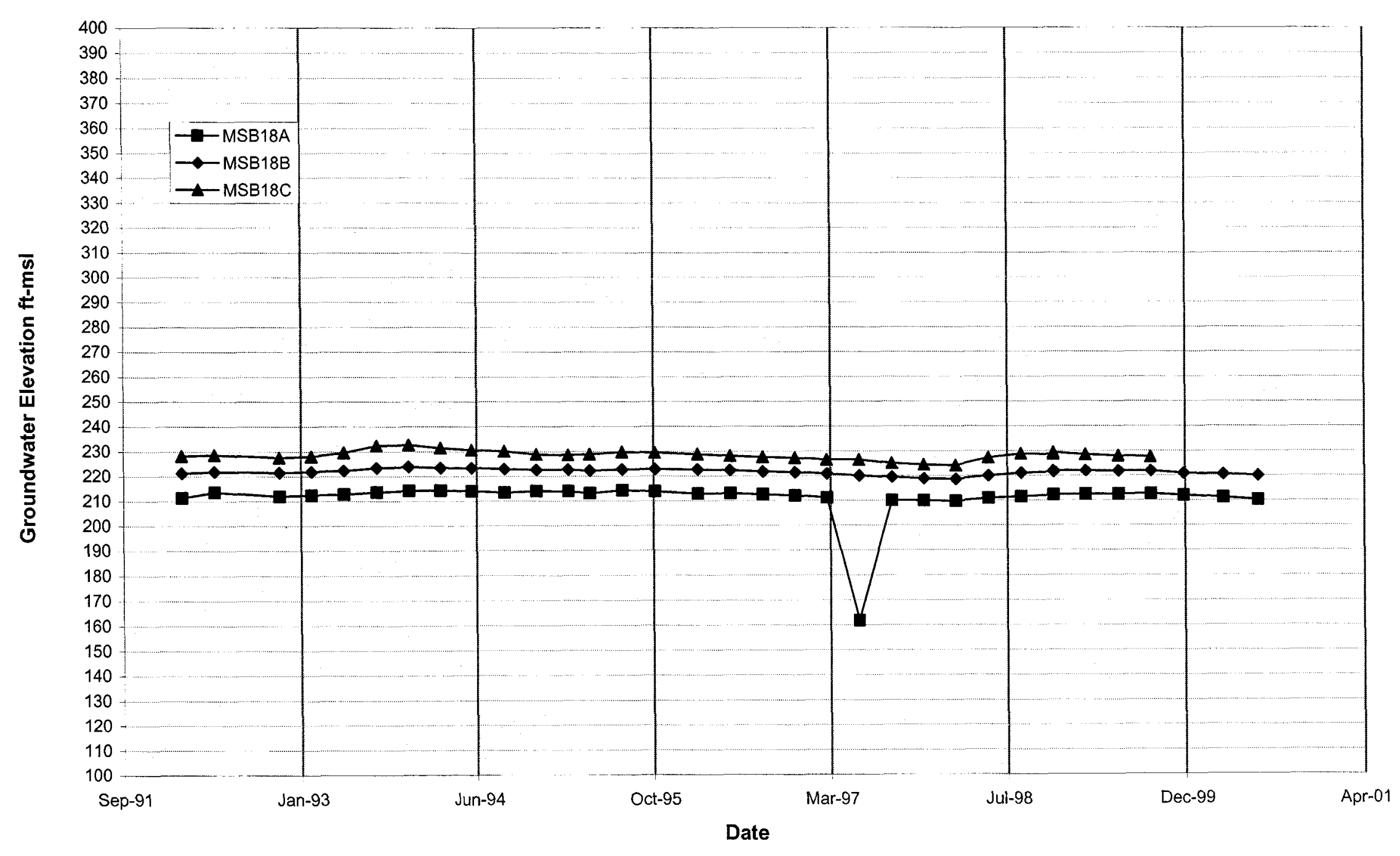


WSRC-TR-2000-00242 UNCLASSIFIED

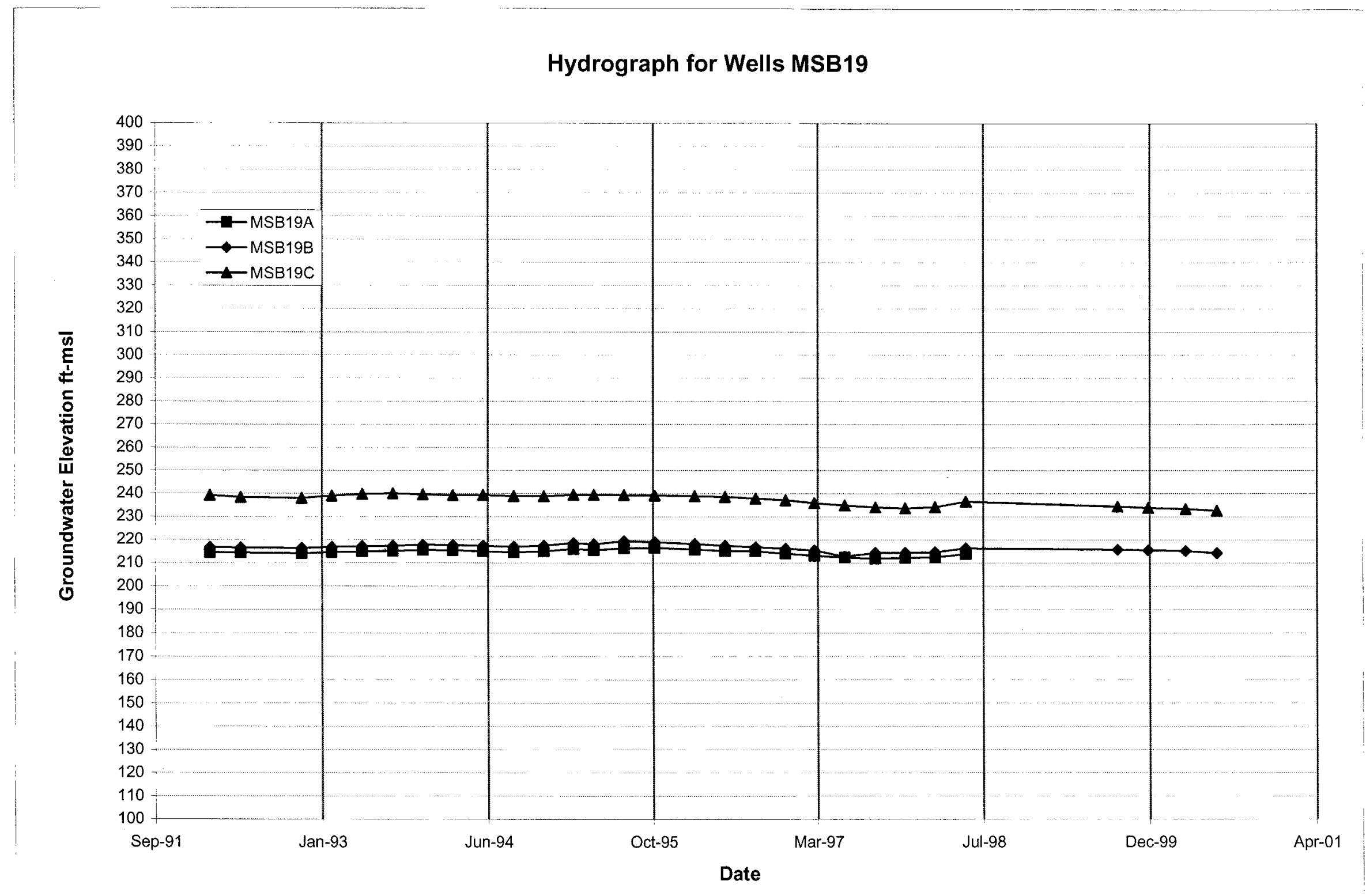




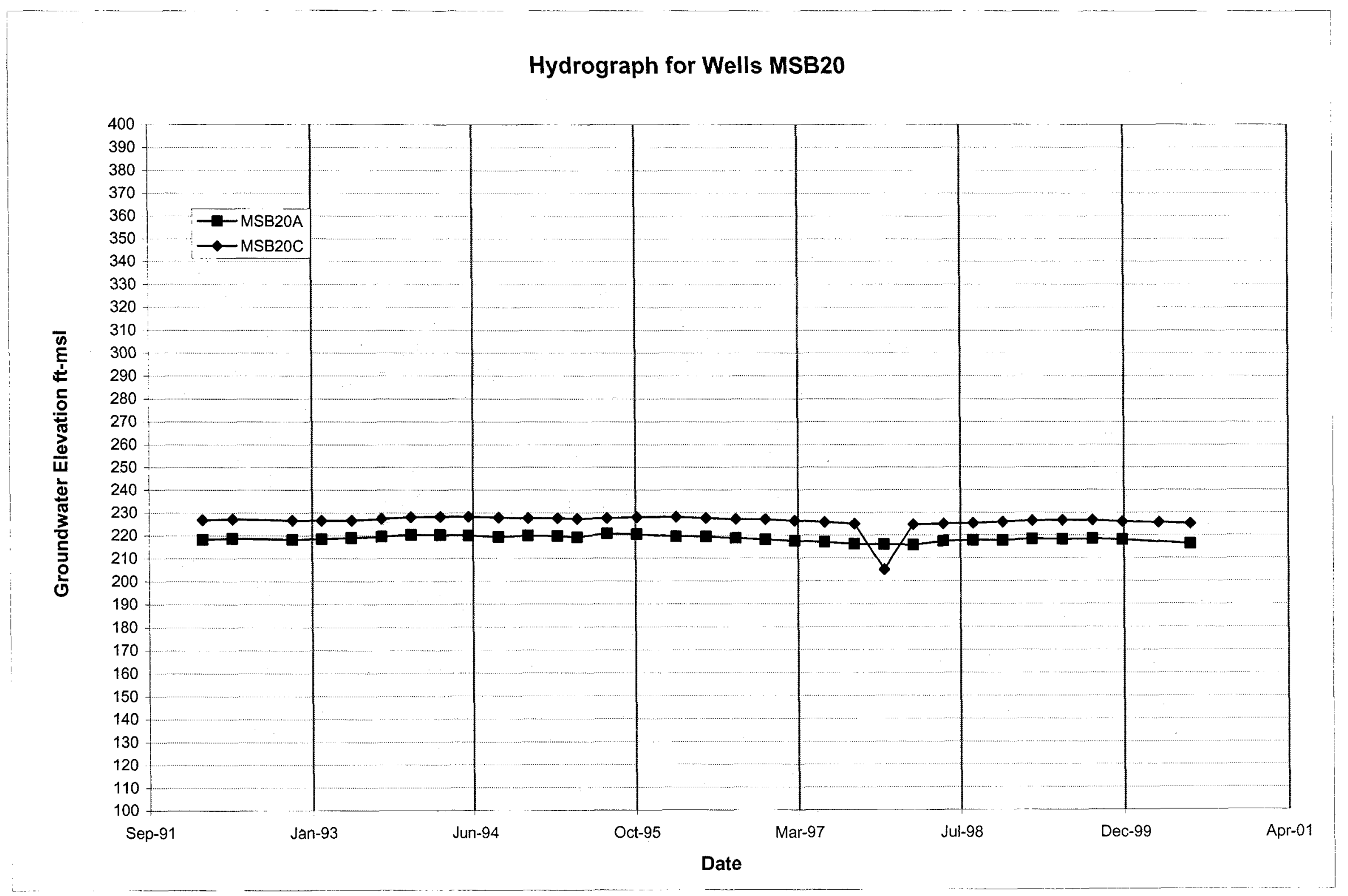




\section{Hydrograph for Wells MSB21}

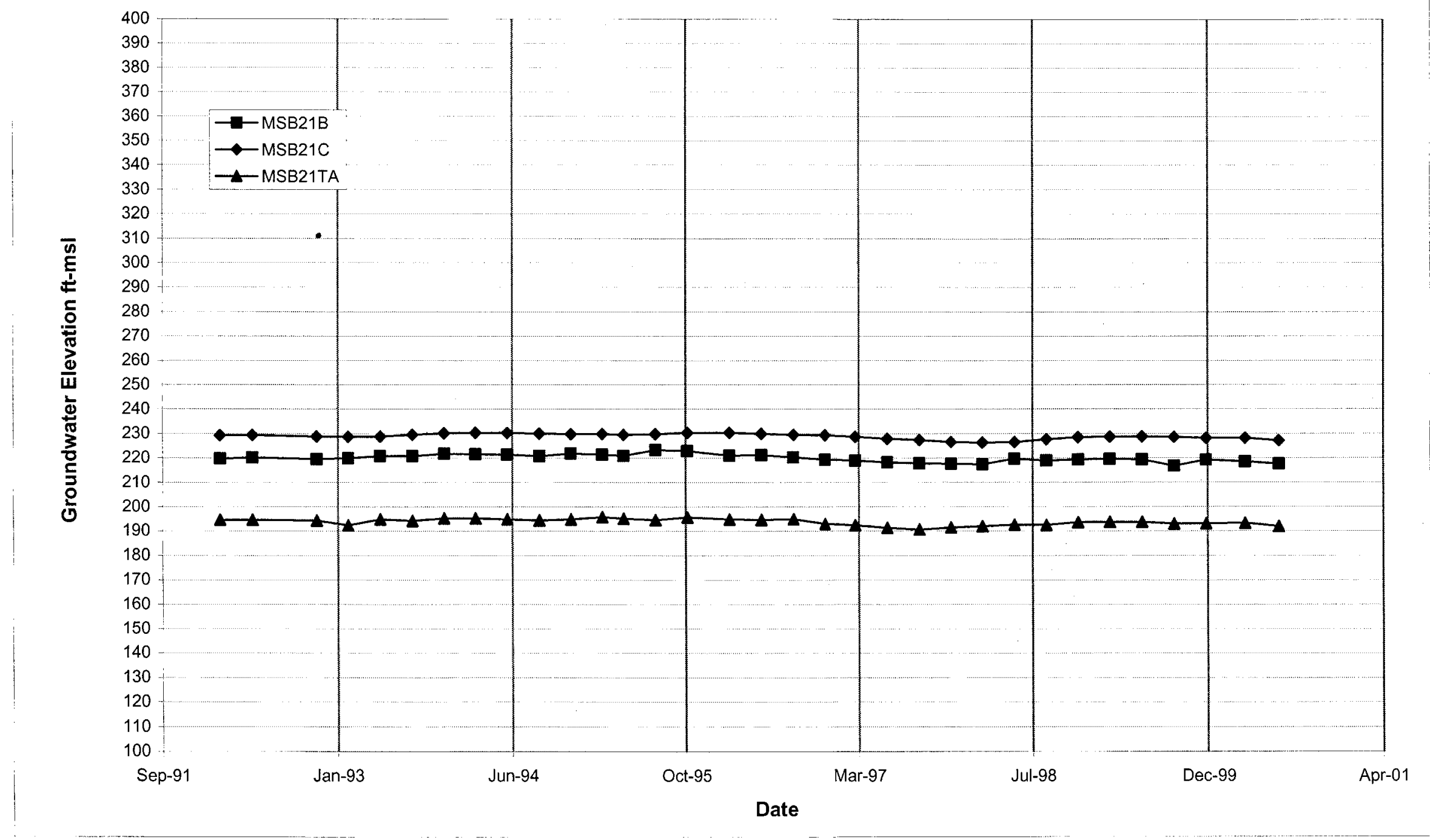


WSRC-TR-2000-00242 UNCLASSIFIED

Hydrograph for Wells MSB23

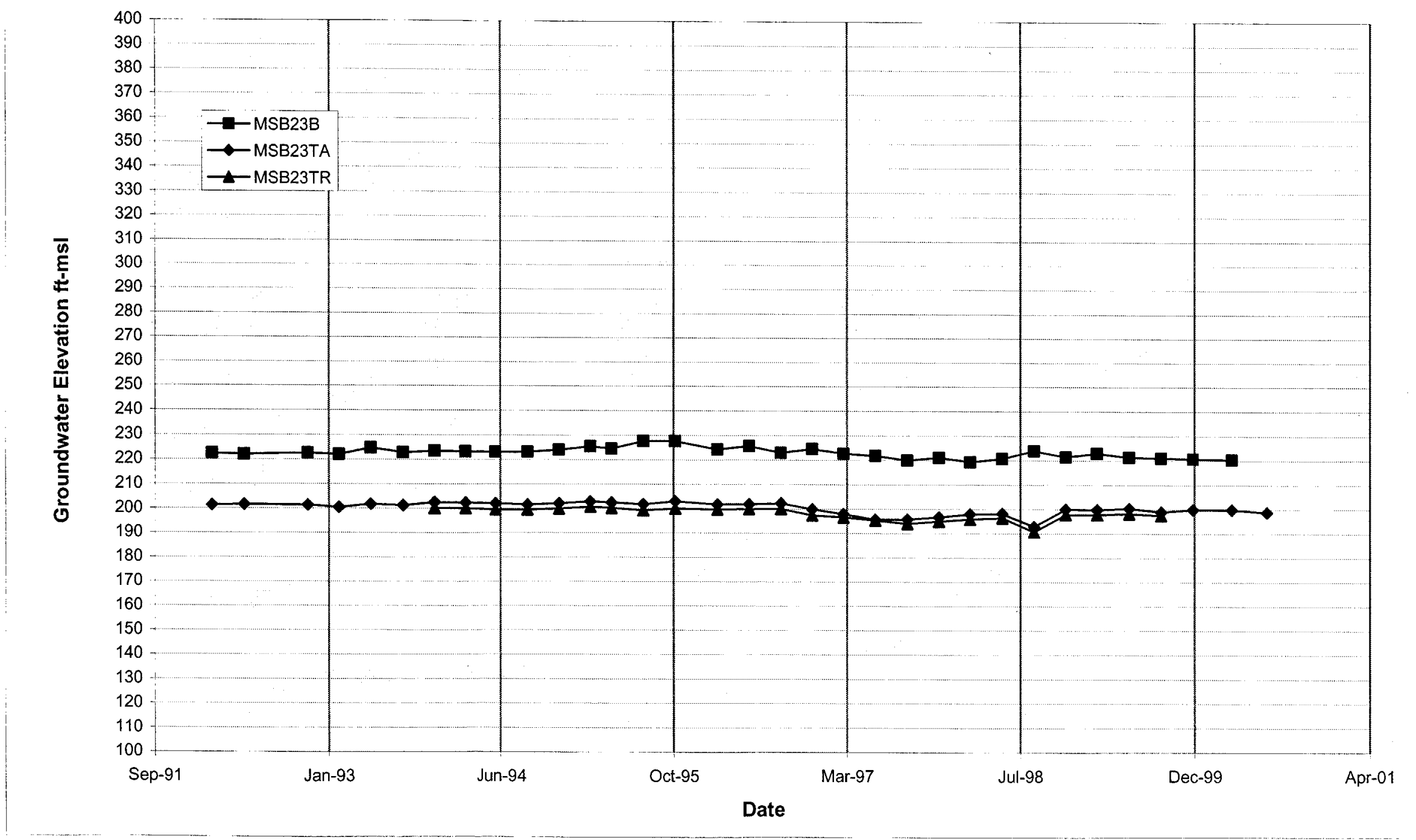




\section{Hydrograph for Wells MSB24}

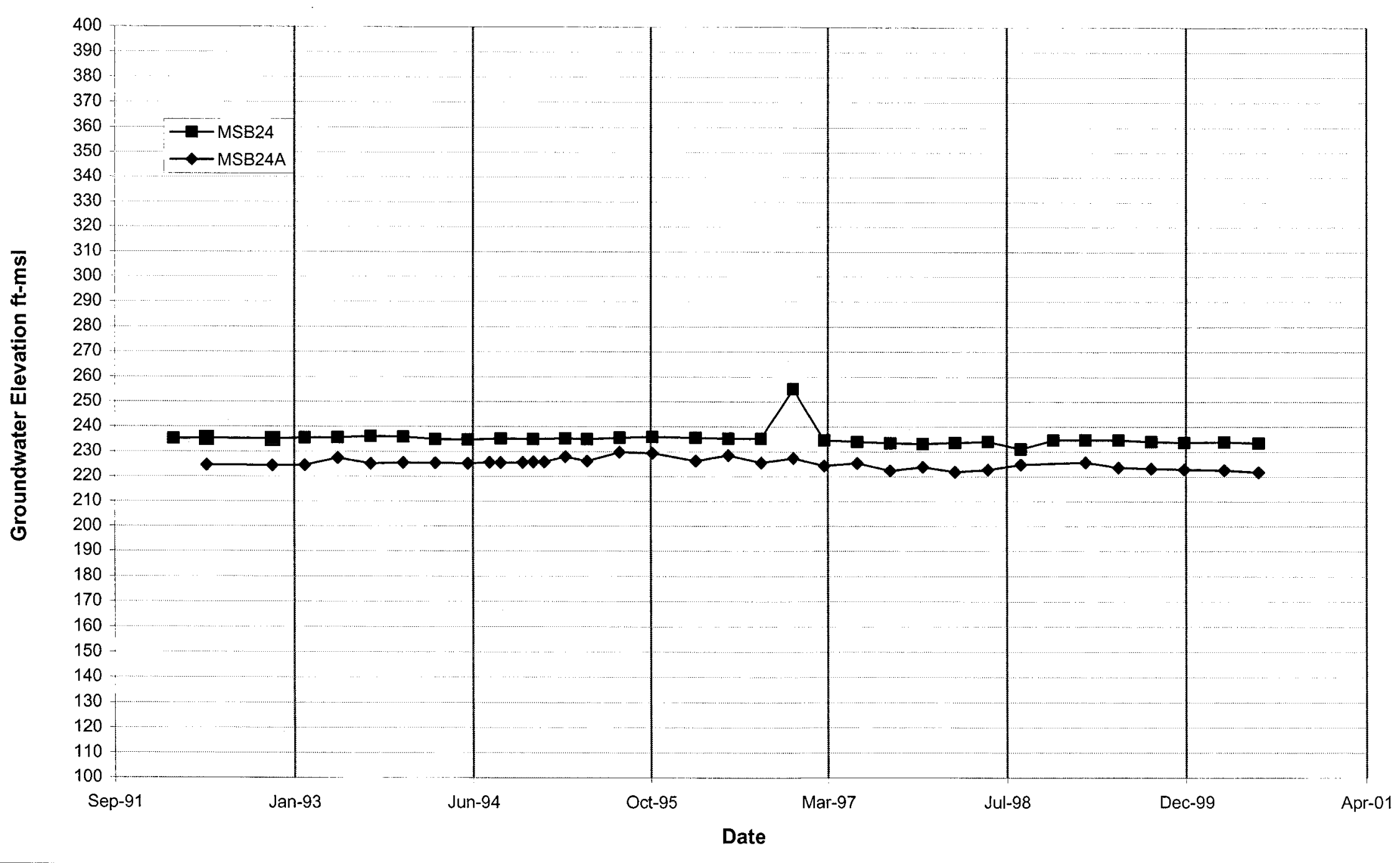




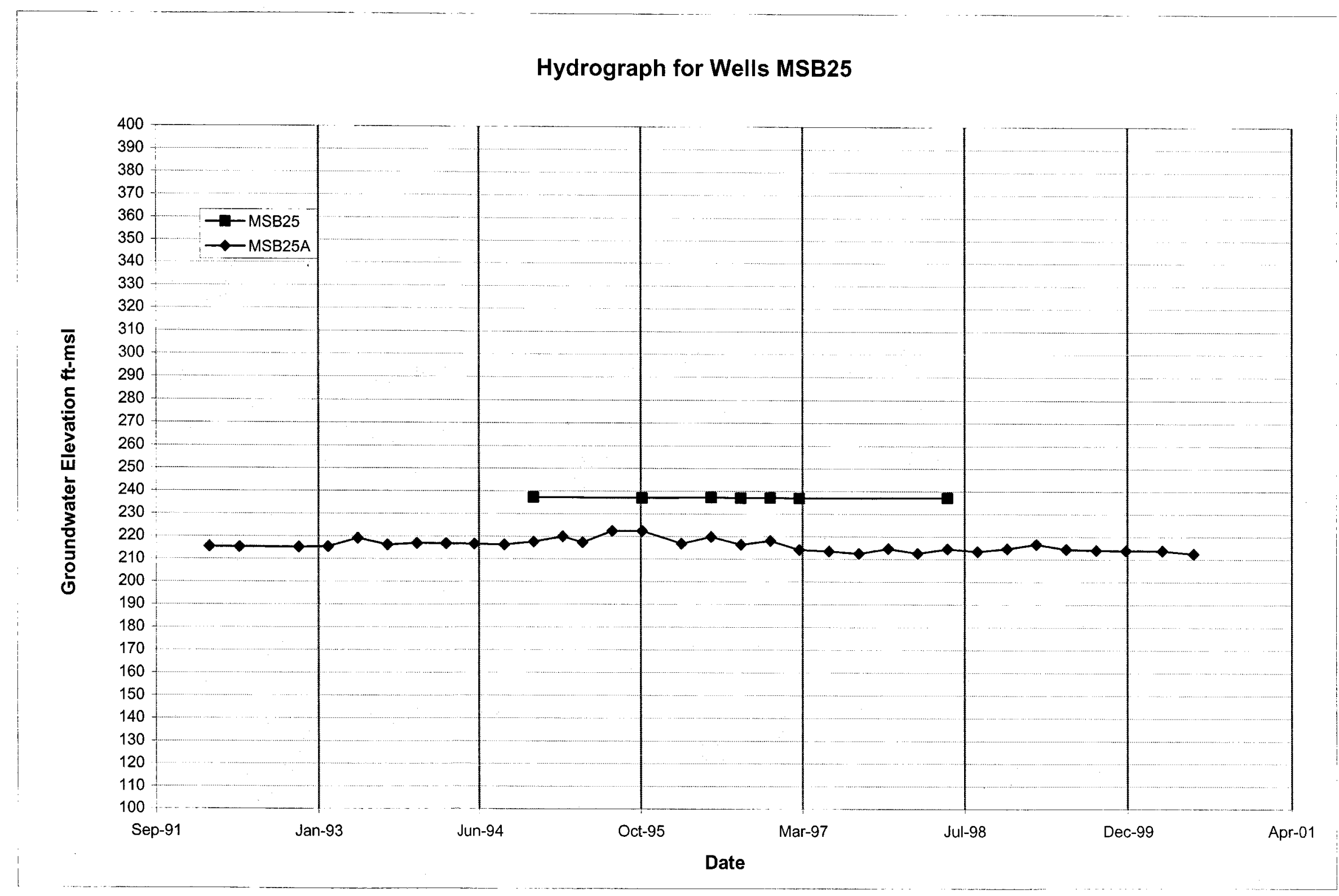




\section{Hydrograph for Wells MSB26}

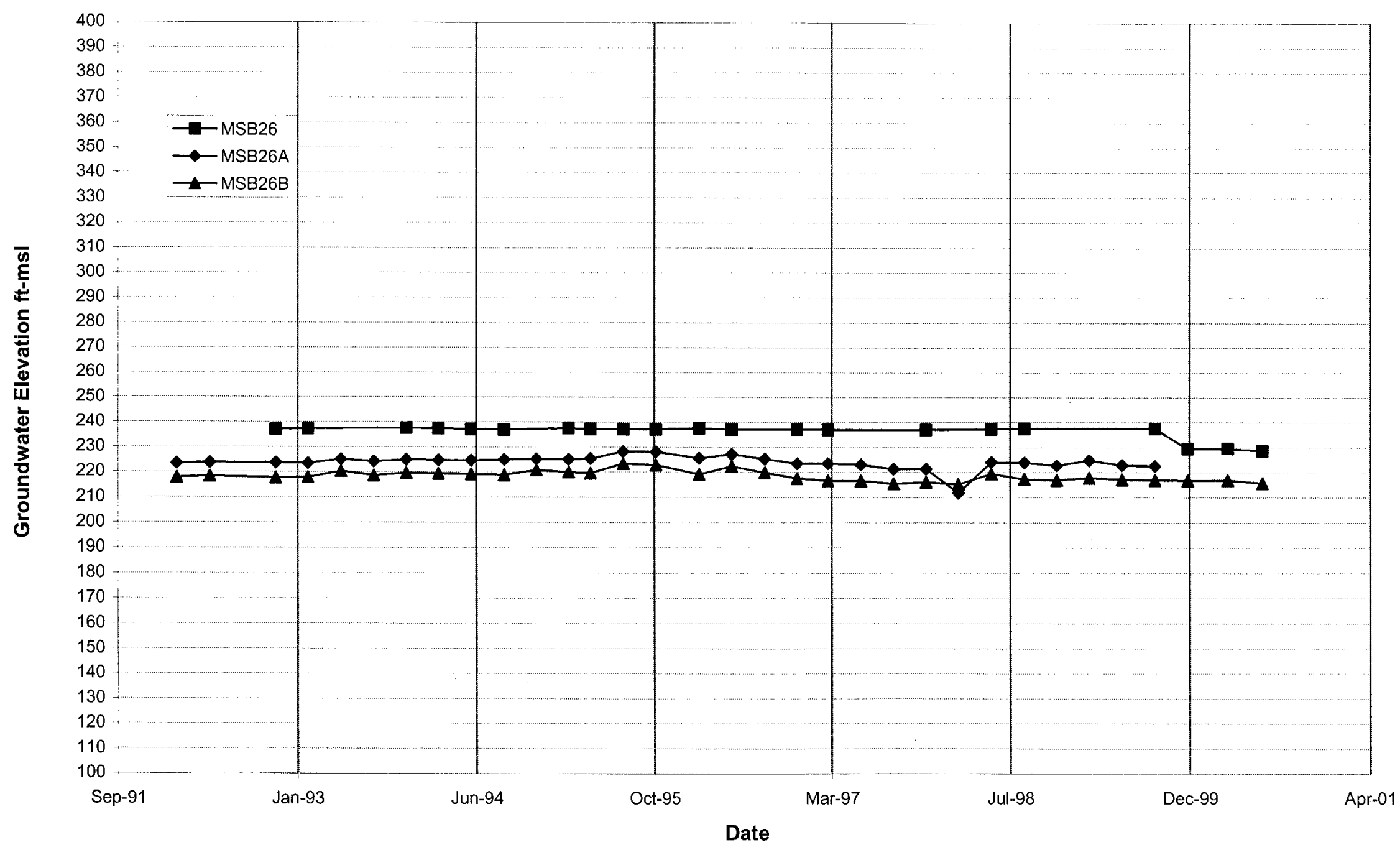




\section{Hydrograph for Wells MSB27}

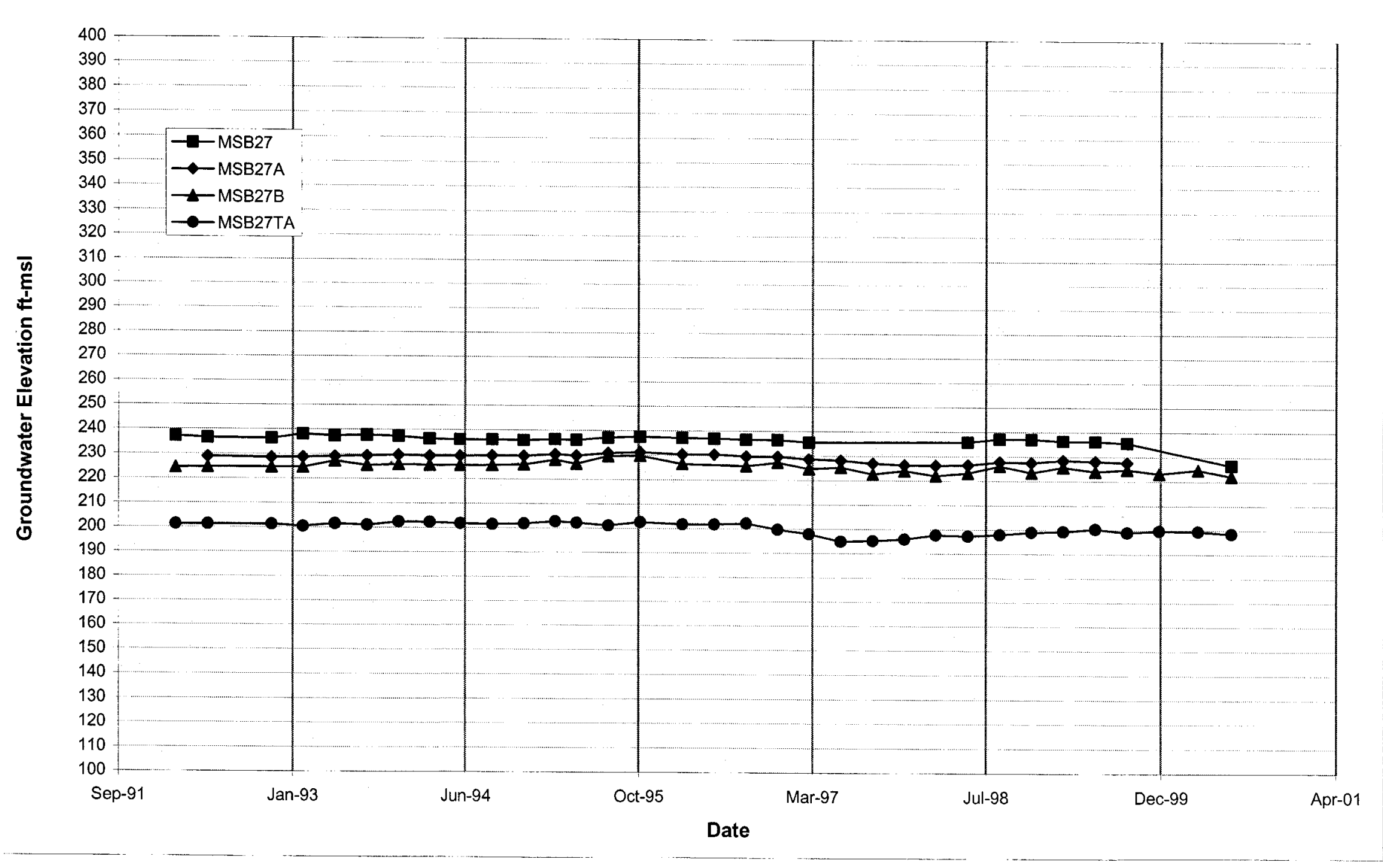


WSRC-TR-2000-00242 UNCLASSIFIED

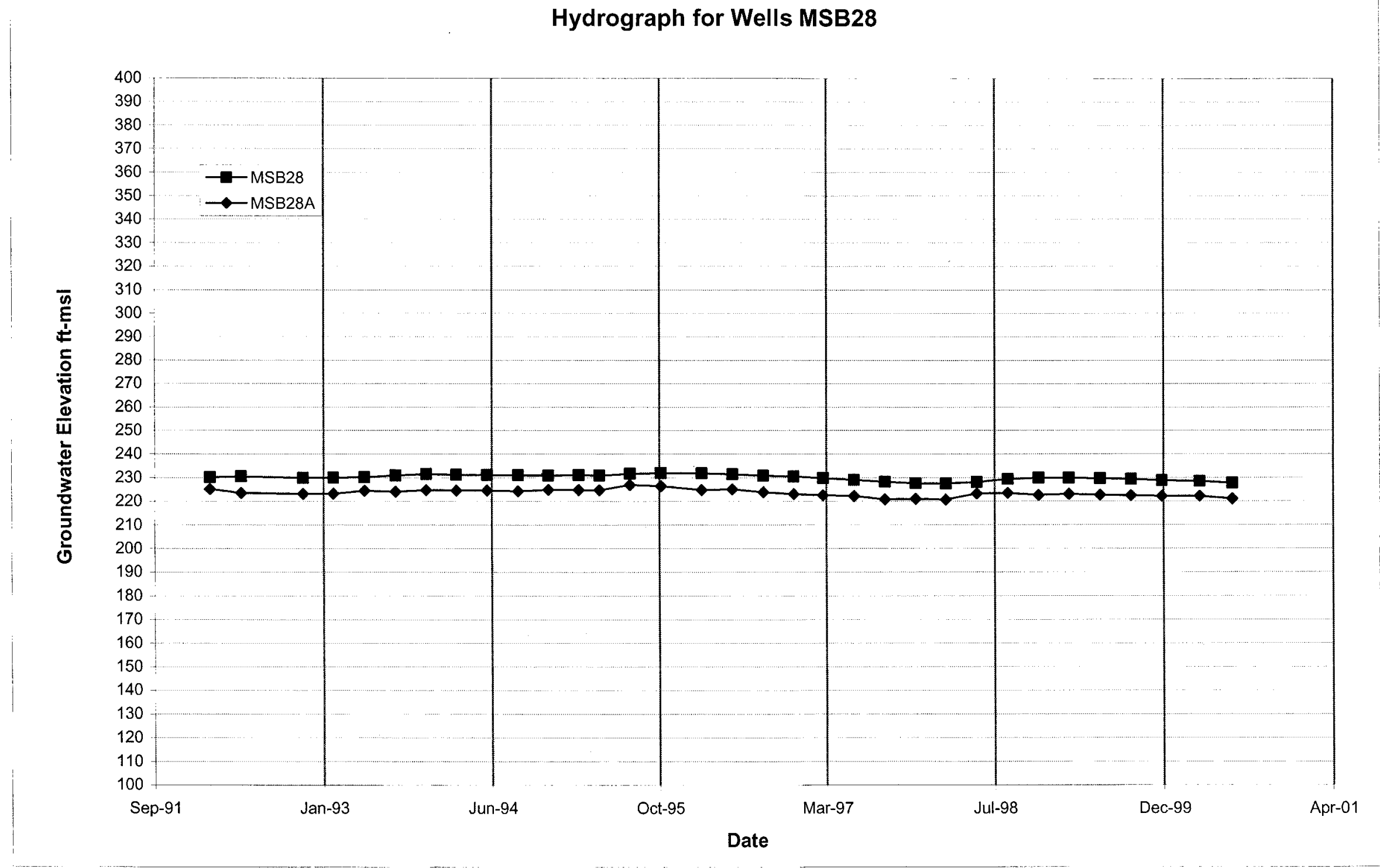




\section{Hydrograph for Wells MSB29}

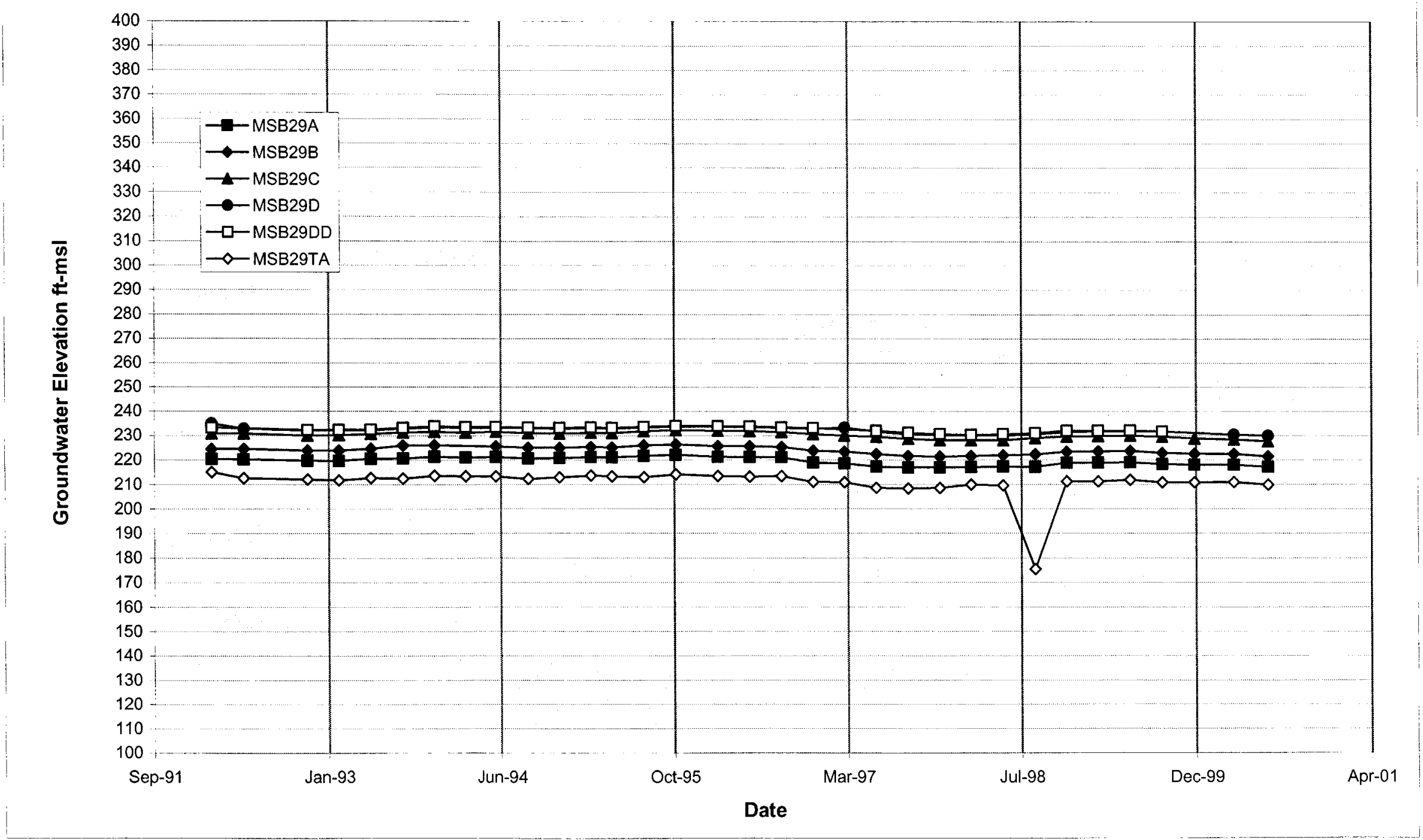




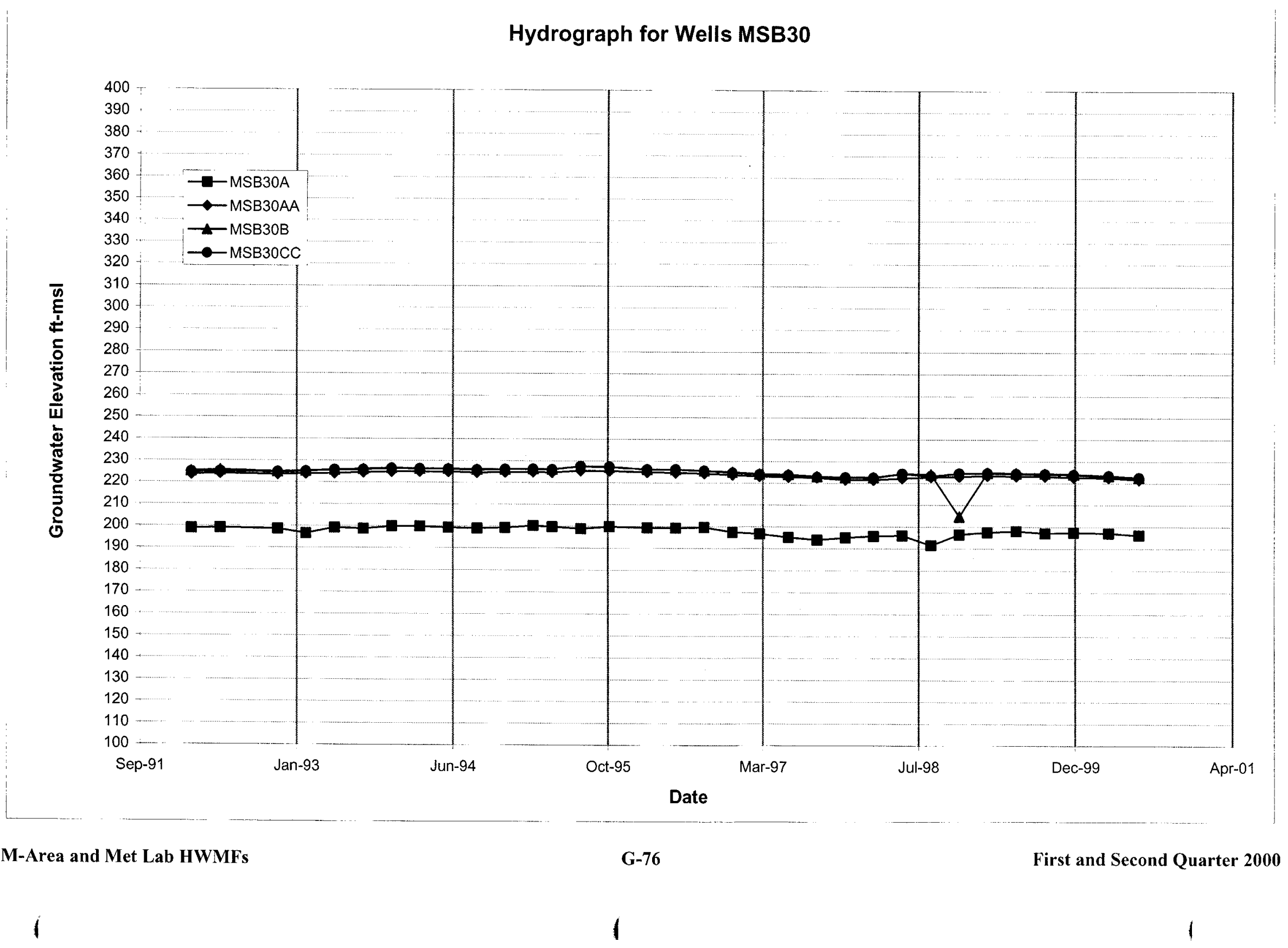




\section{Hydrograph for Wells MSB31}

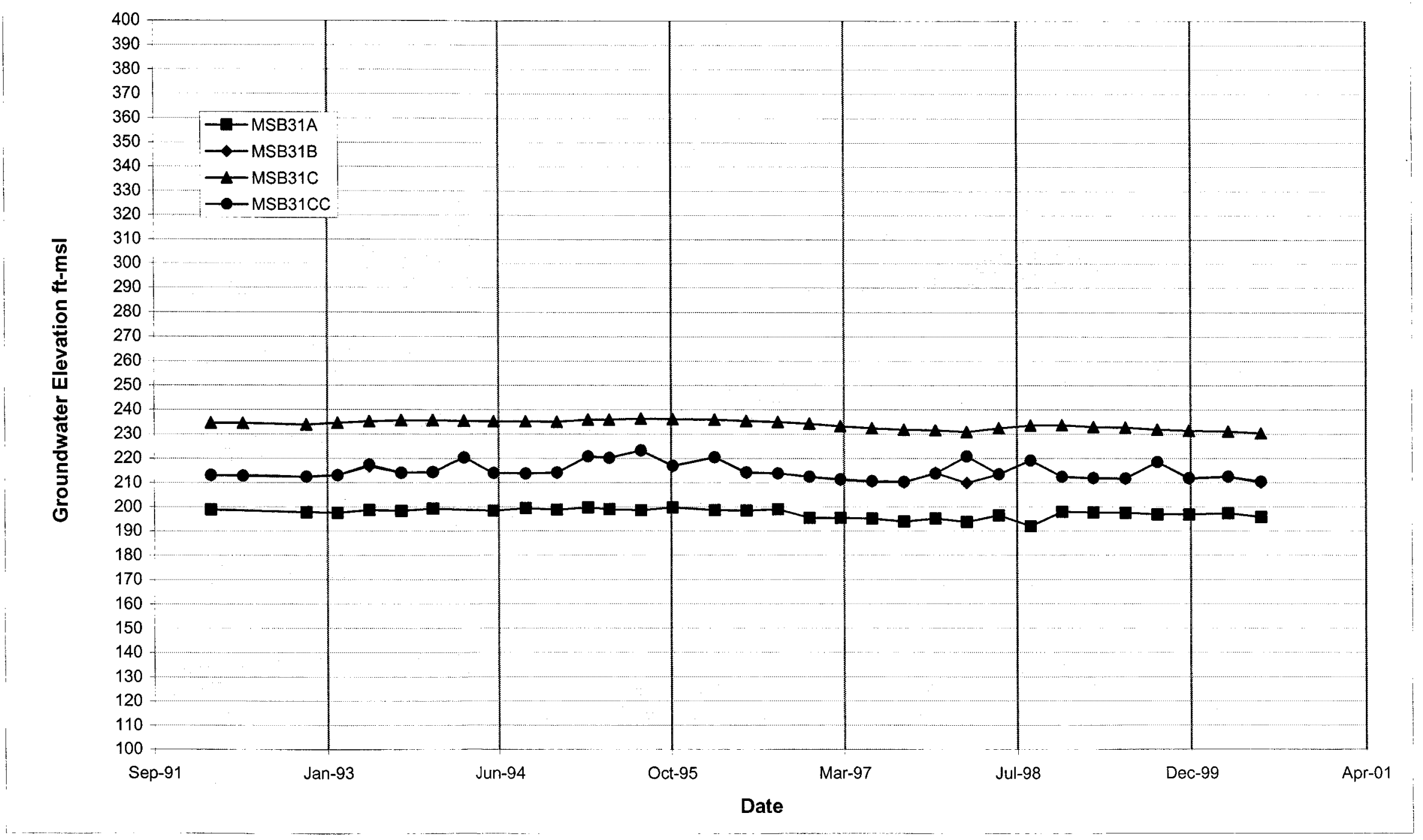


WSRC-TR-2000-00242 UNCLASSIFIED

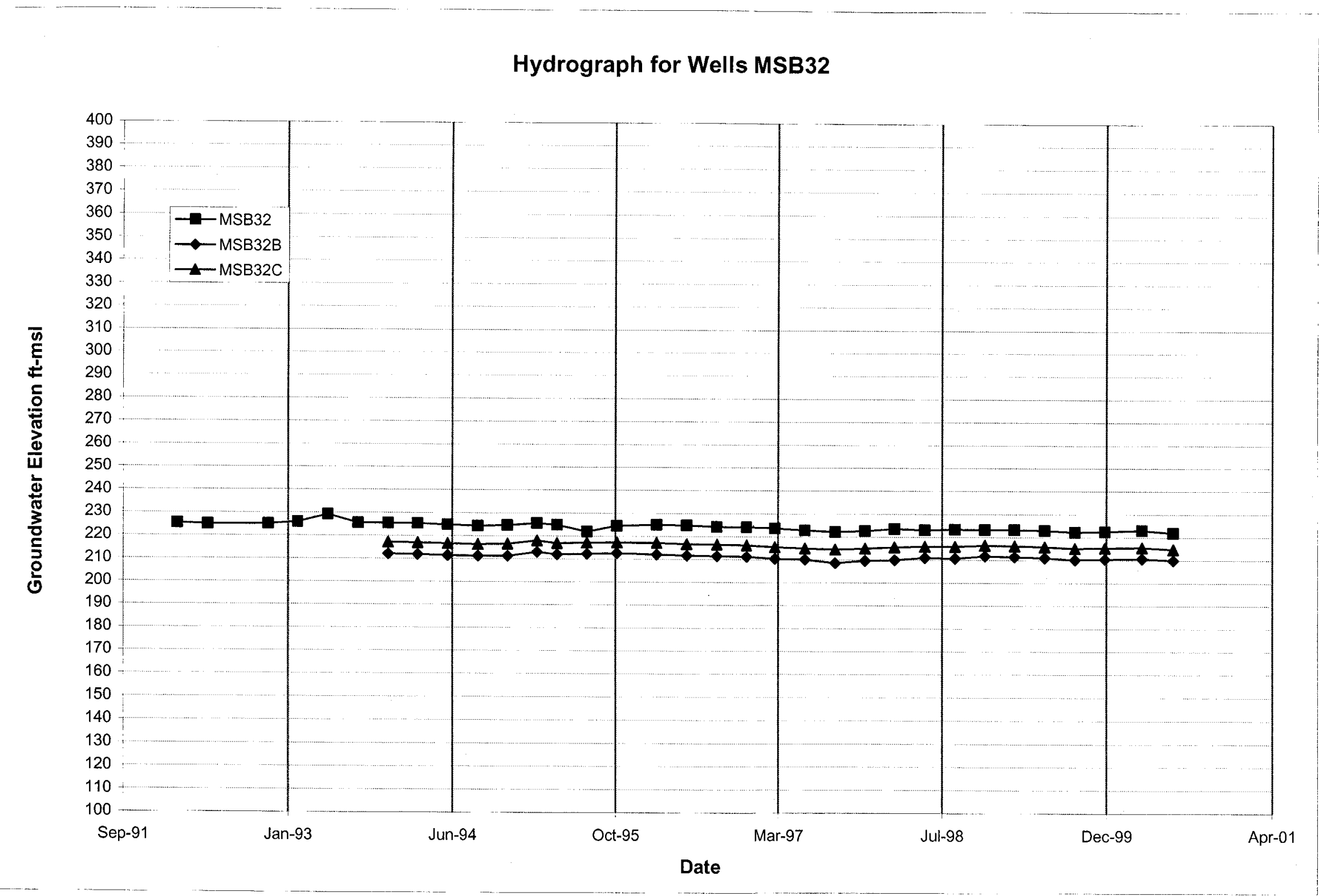


WSRC-TR-2000-00242 UNCLASSIFIED

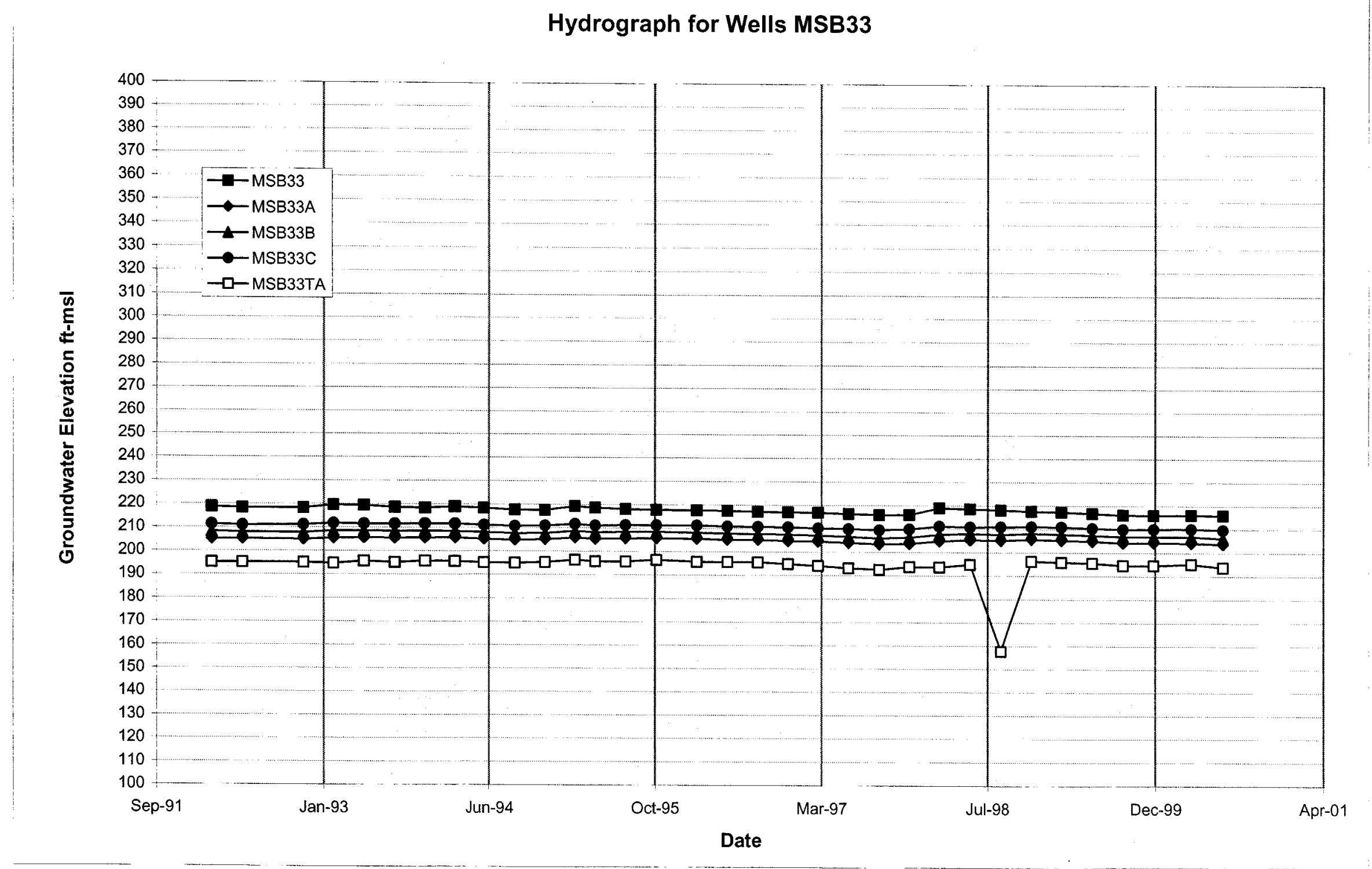




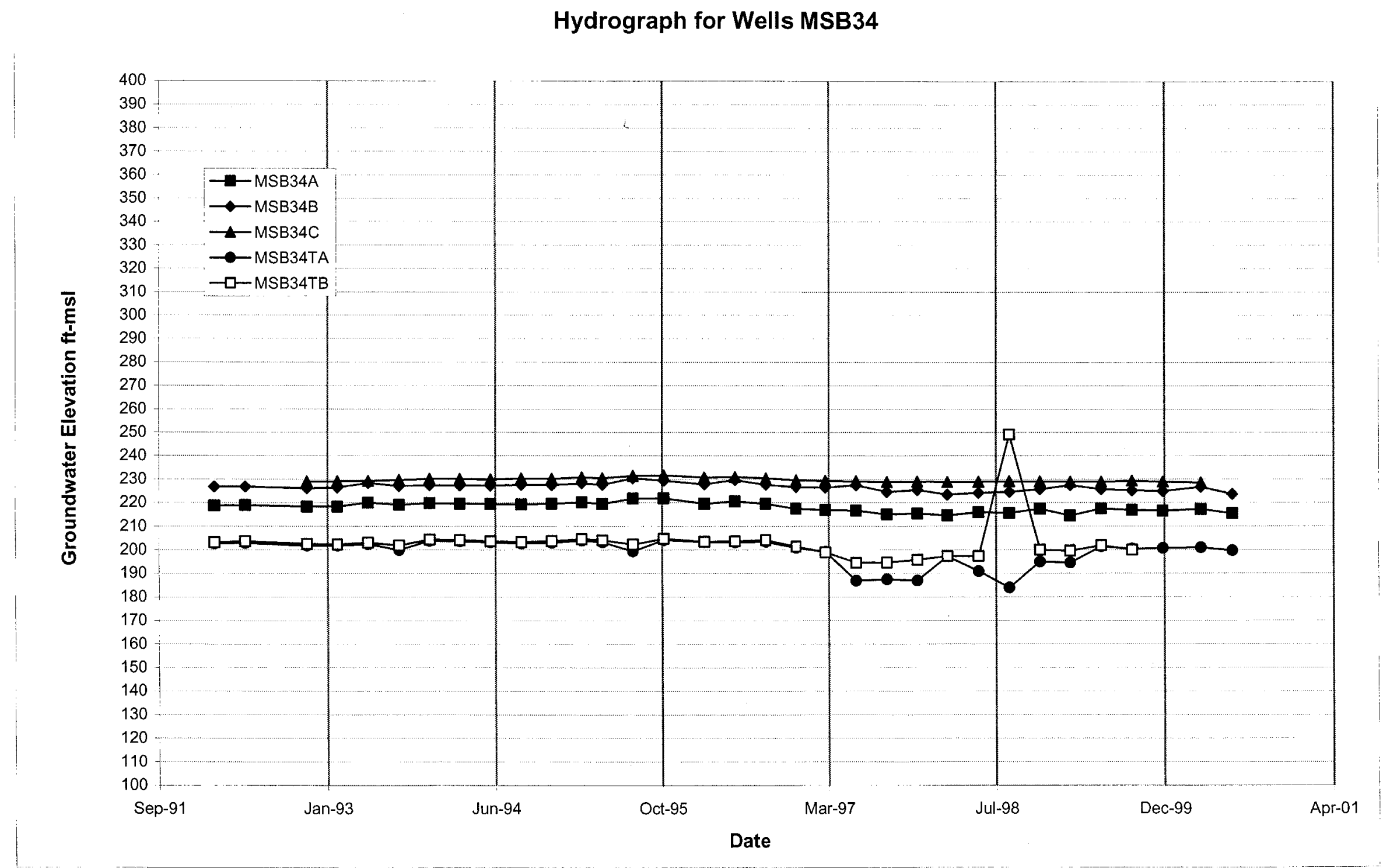


WSRC-TR-2000-00242 UNCLASSIFIED

Hydrograph for Wells MSB35

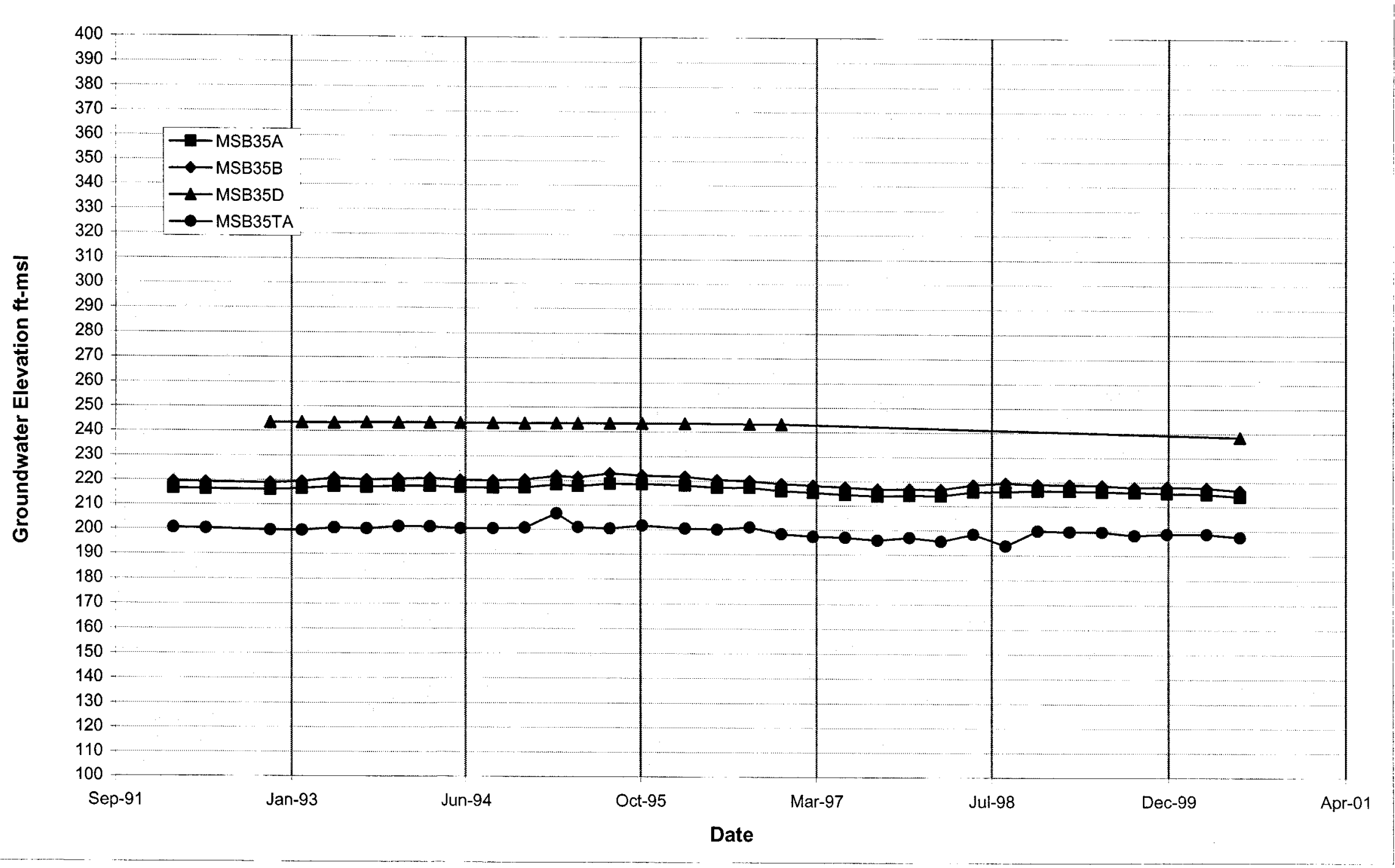




\section{Hydrograph for Wells MSB36}

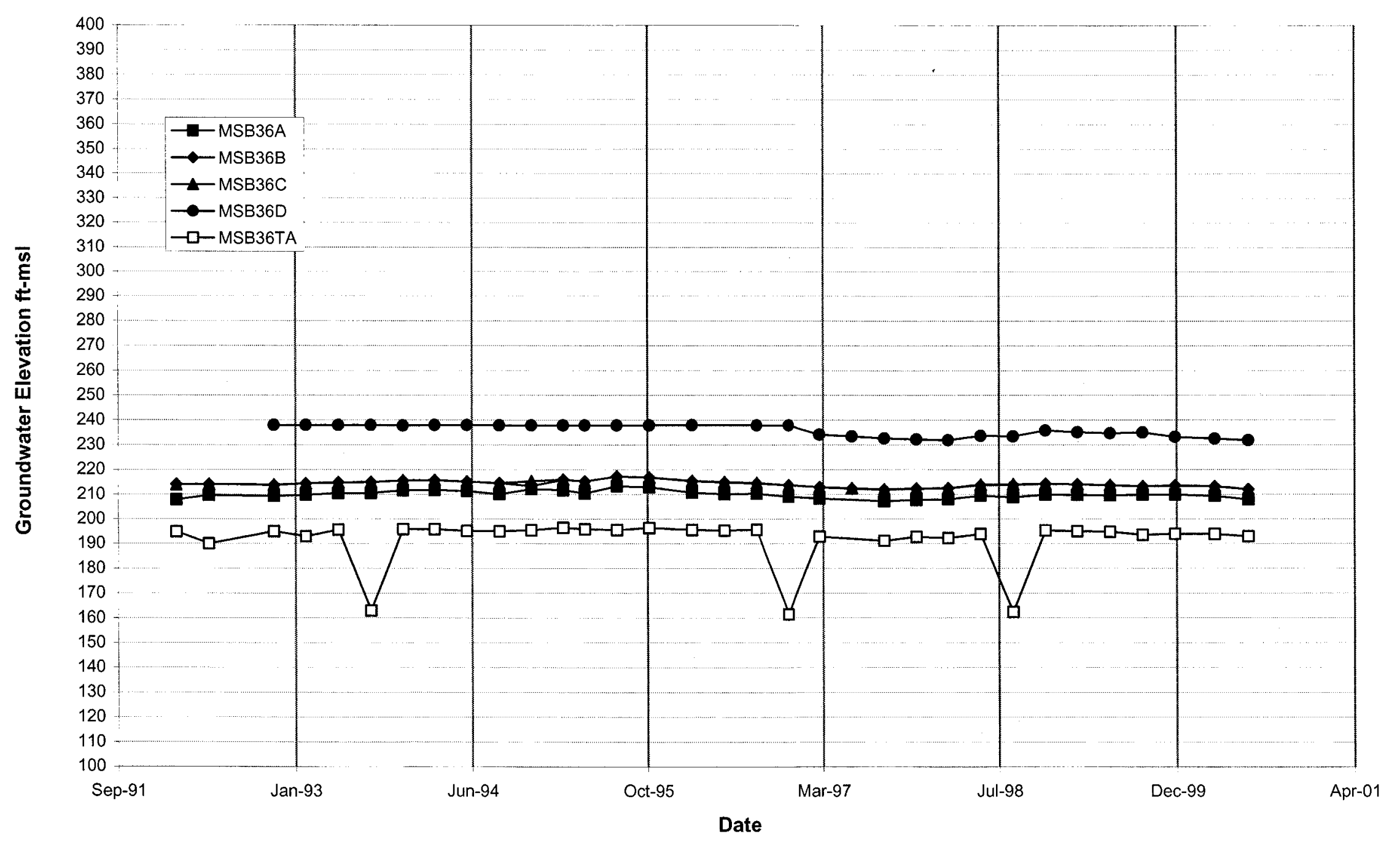




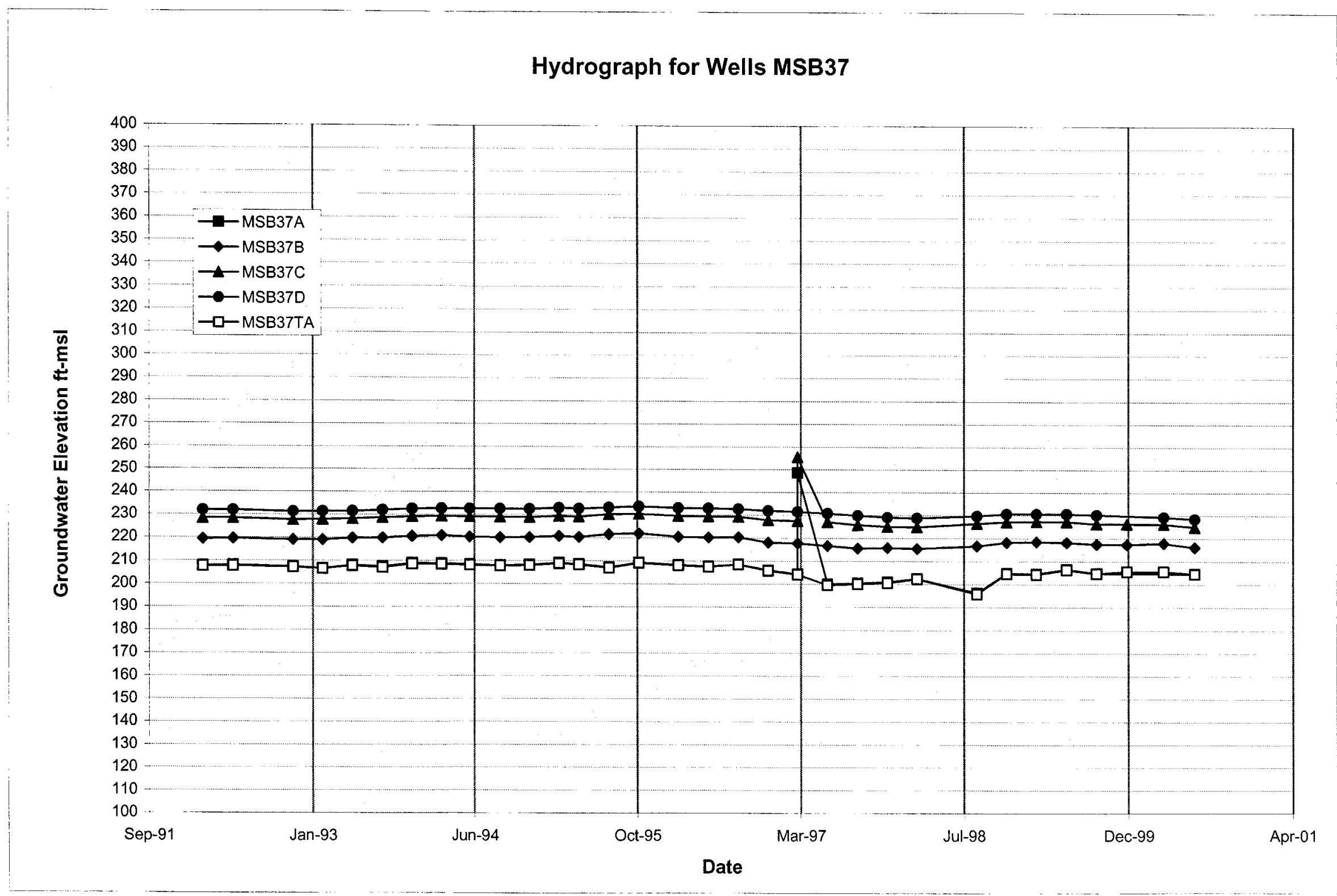




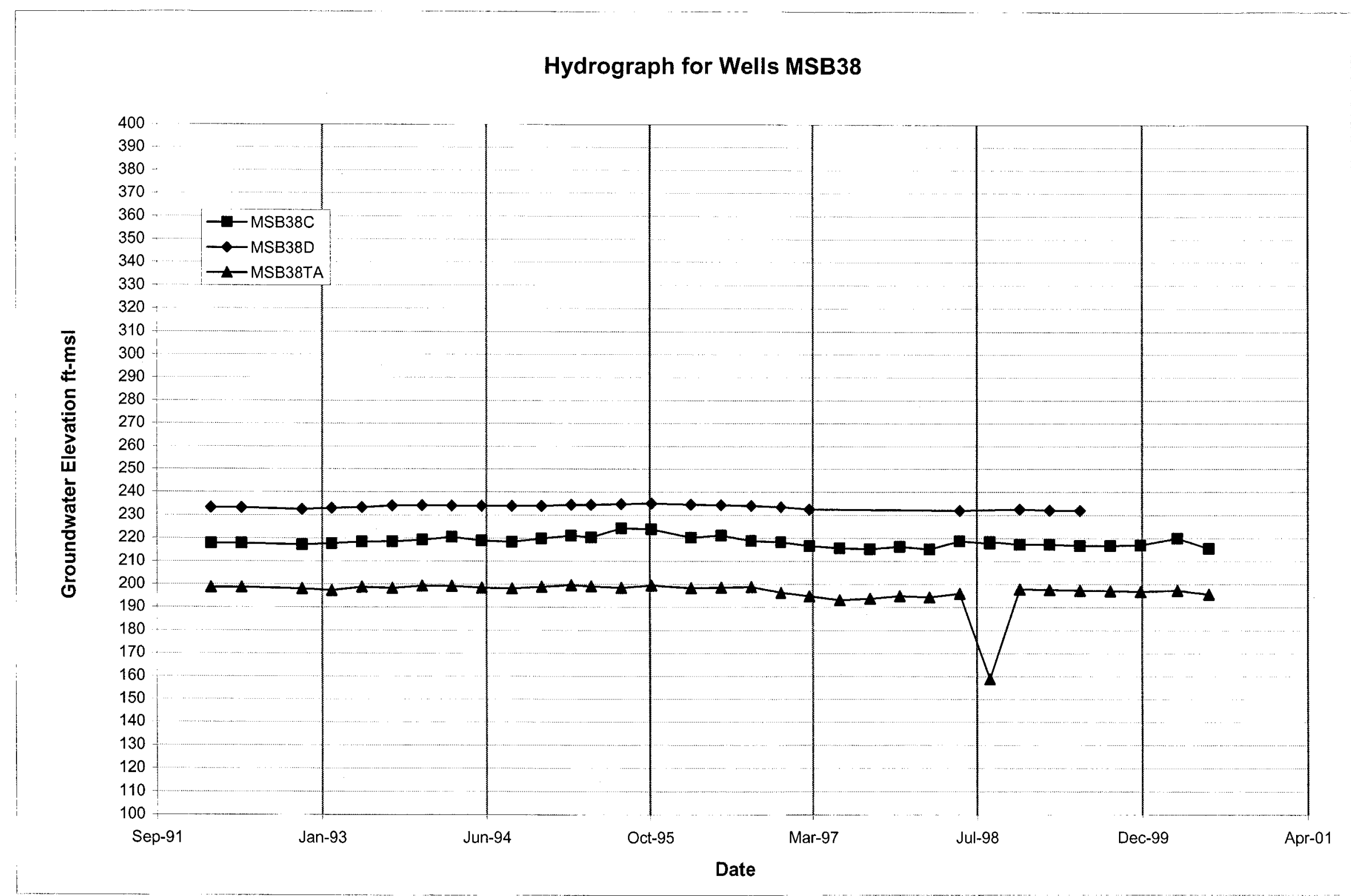




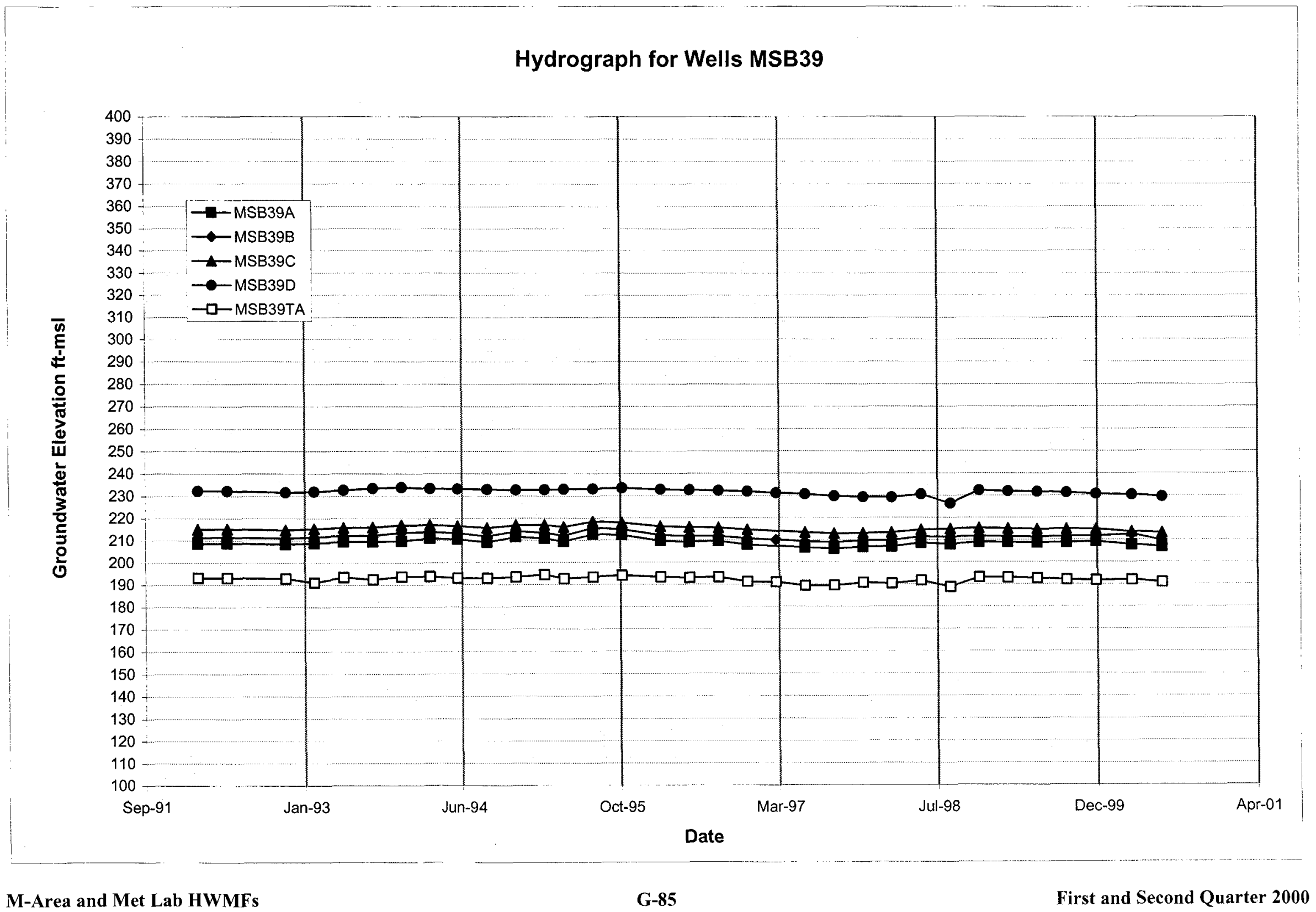




\section{Hydrograph for Wells MSB40}

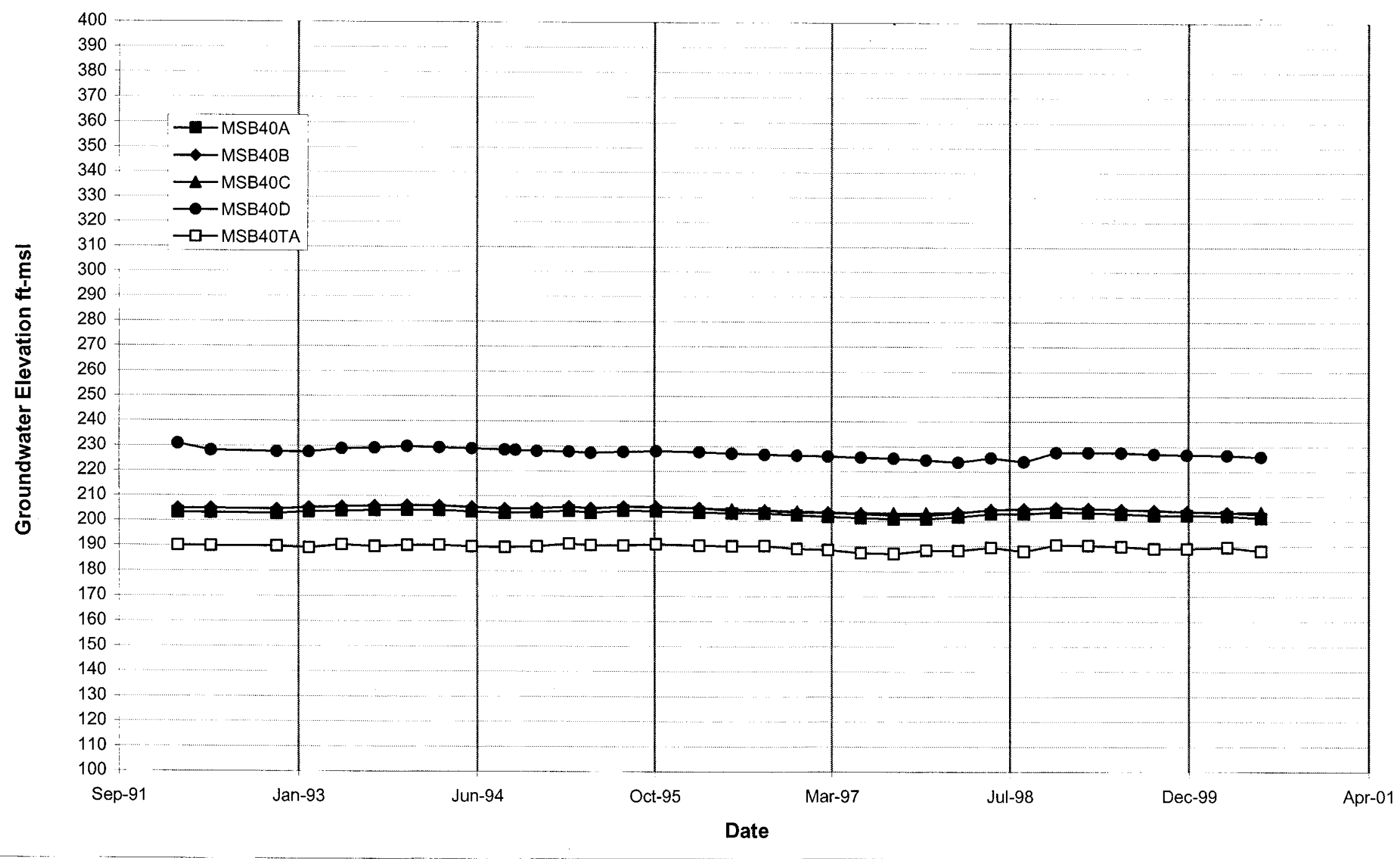




\section{Hydrograph for Wells MSB41}

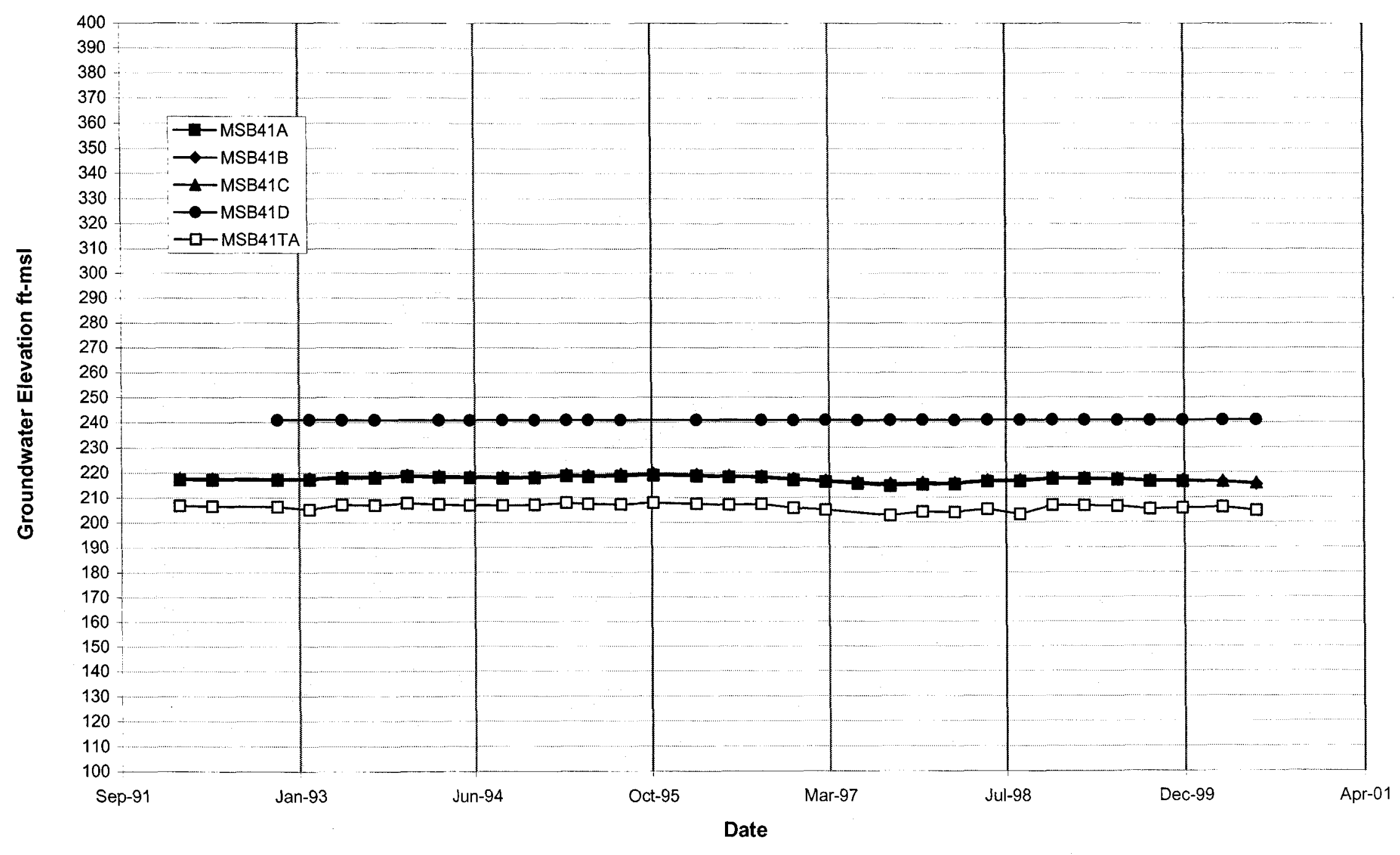




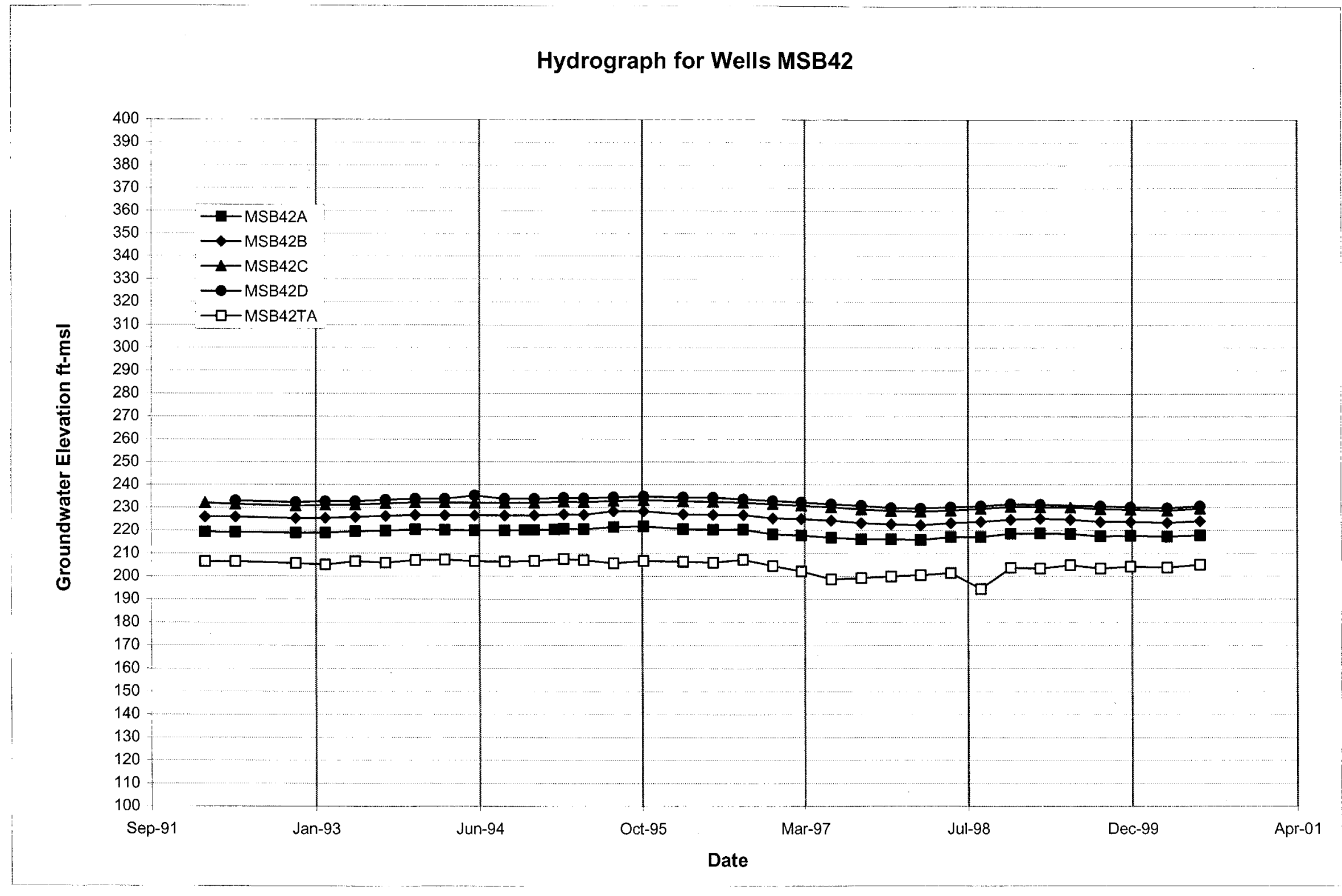




\section{Hydrograph for Wells MSB43}

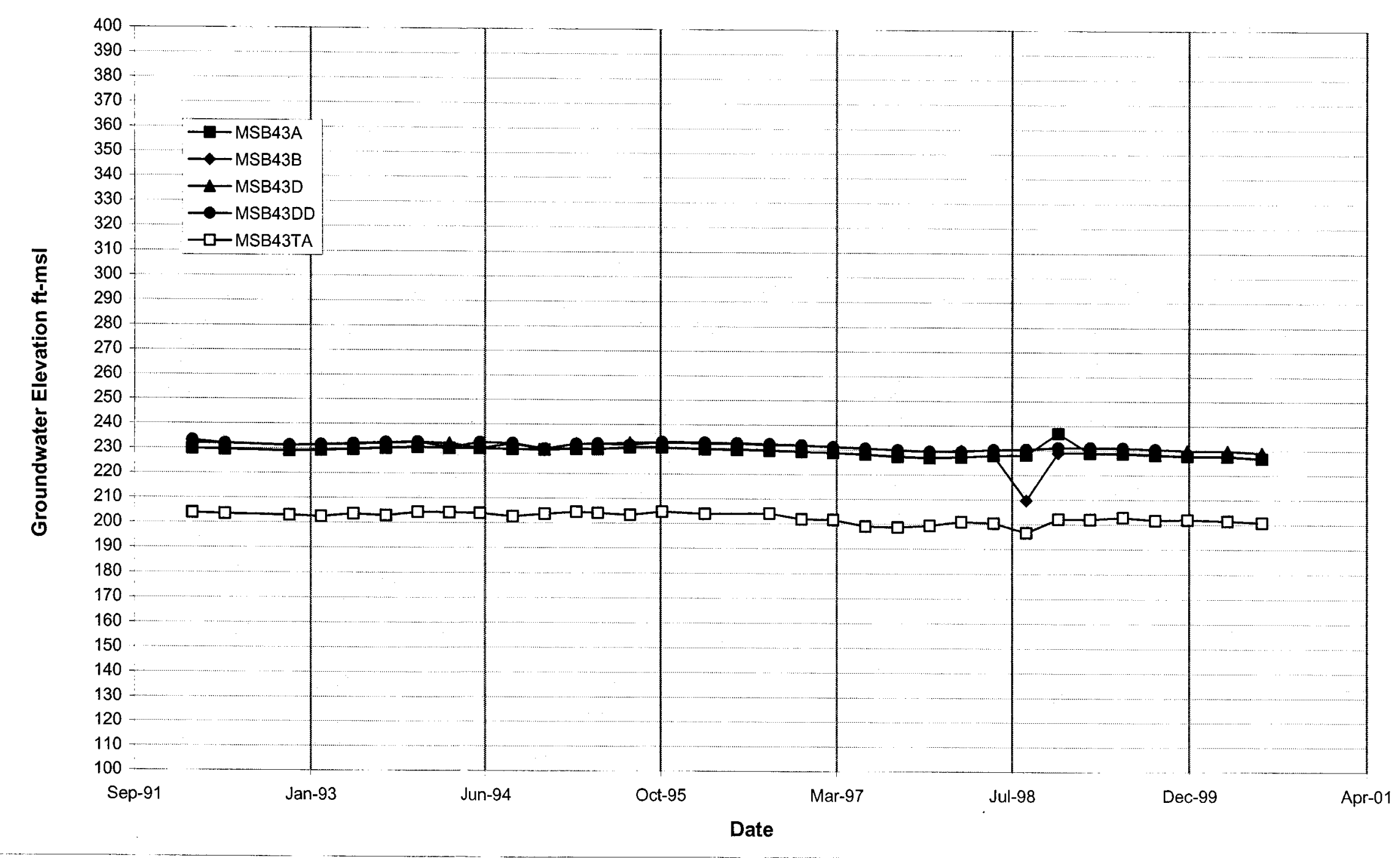


WSRC-TR-2000-00242 UNCLASSIFIED

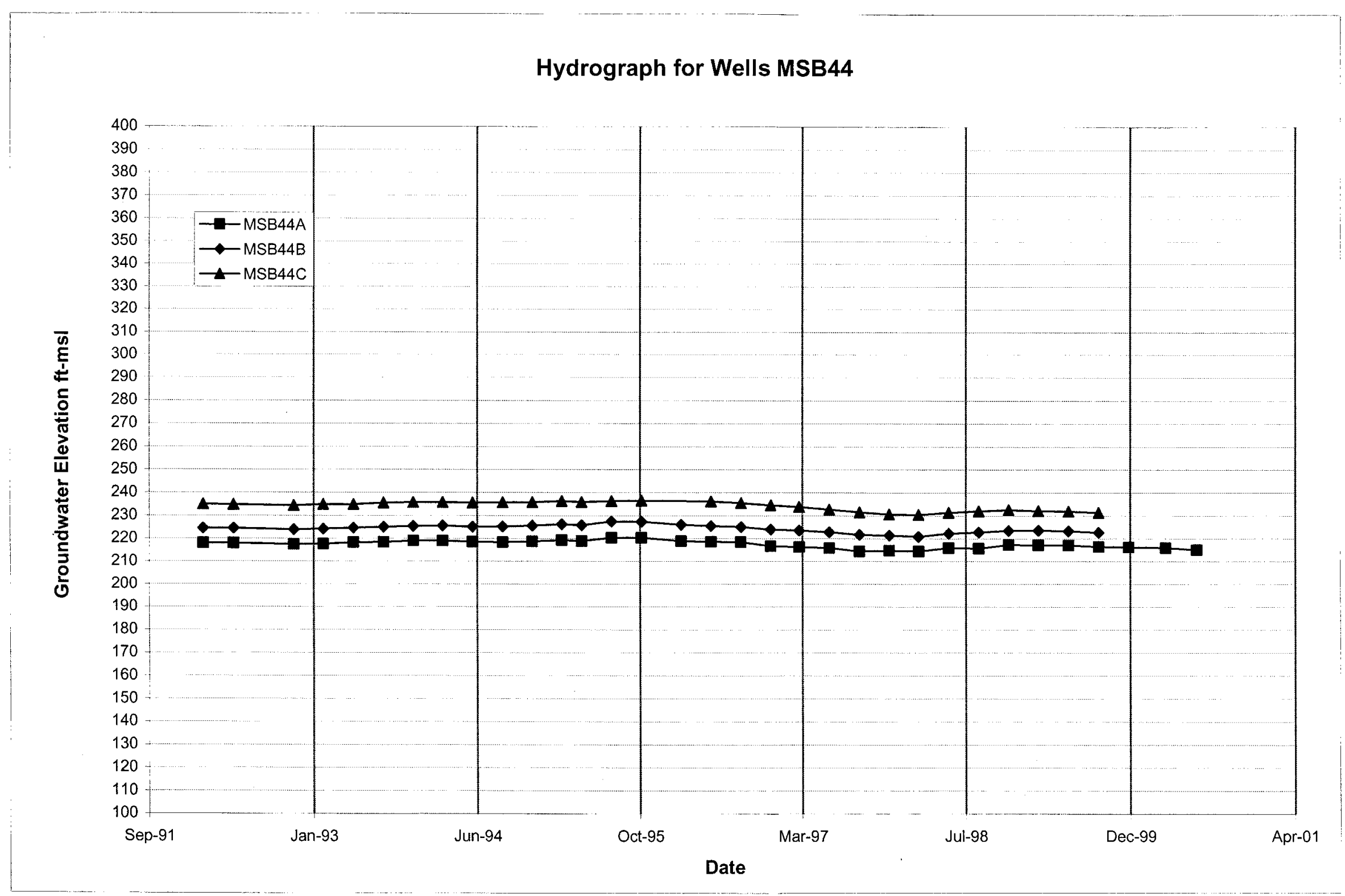




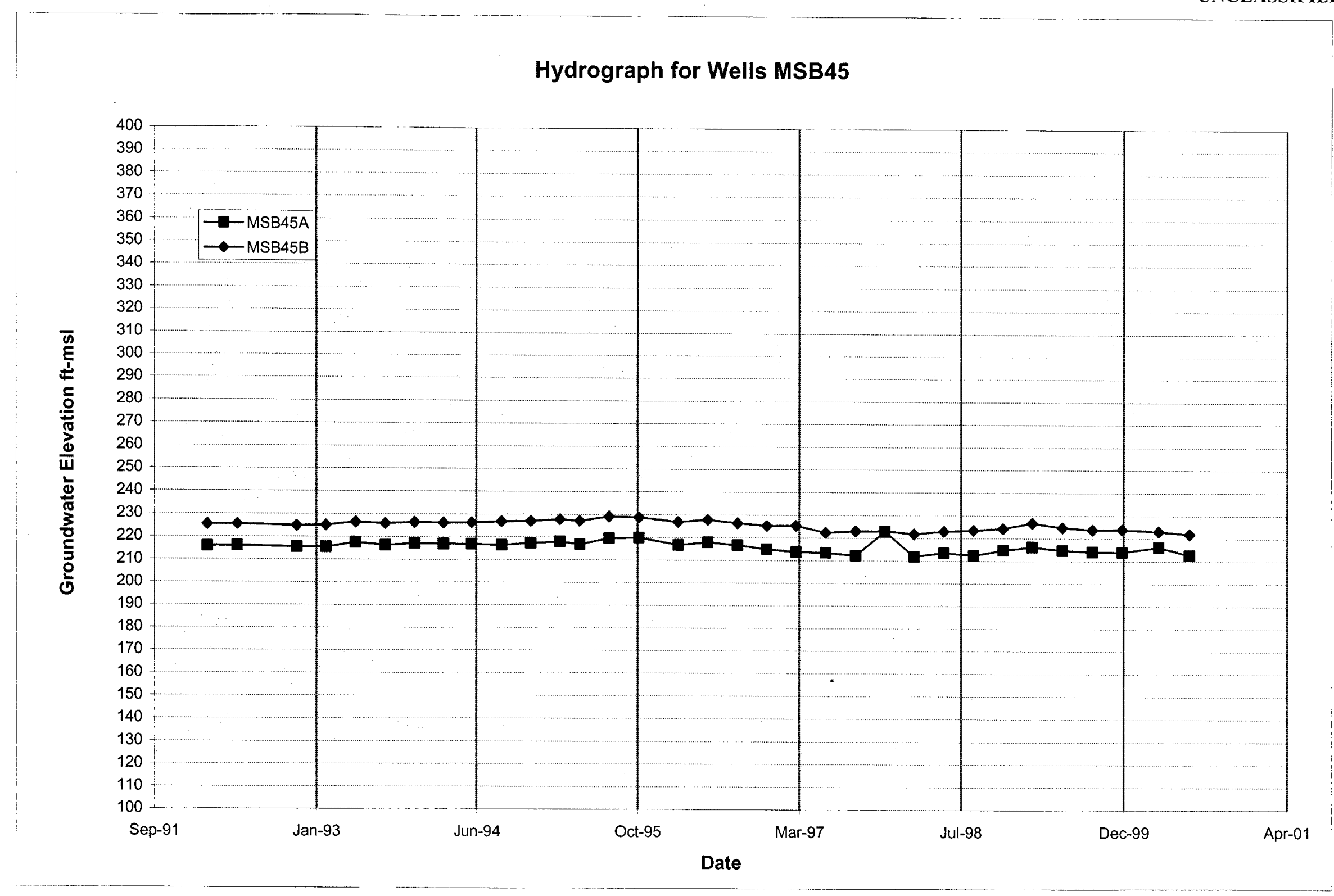


WSRC-TR-2000-00242 UNCLASSIFIED

Hydrograph for Wells MSB46

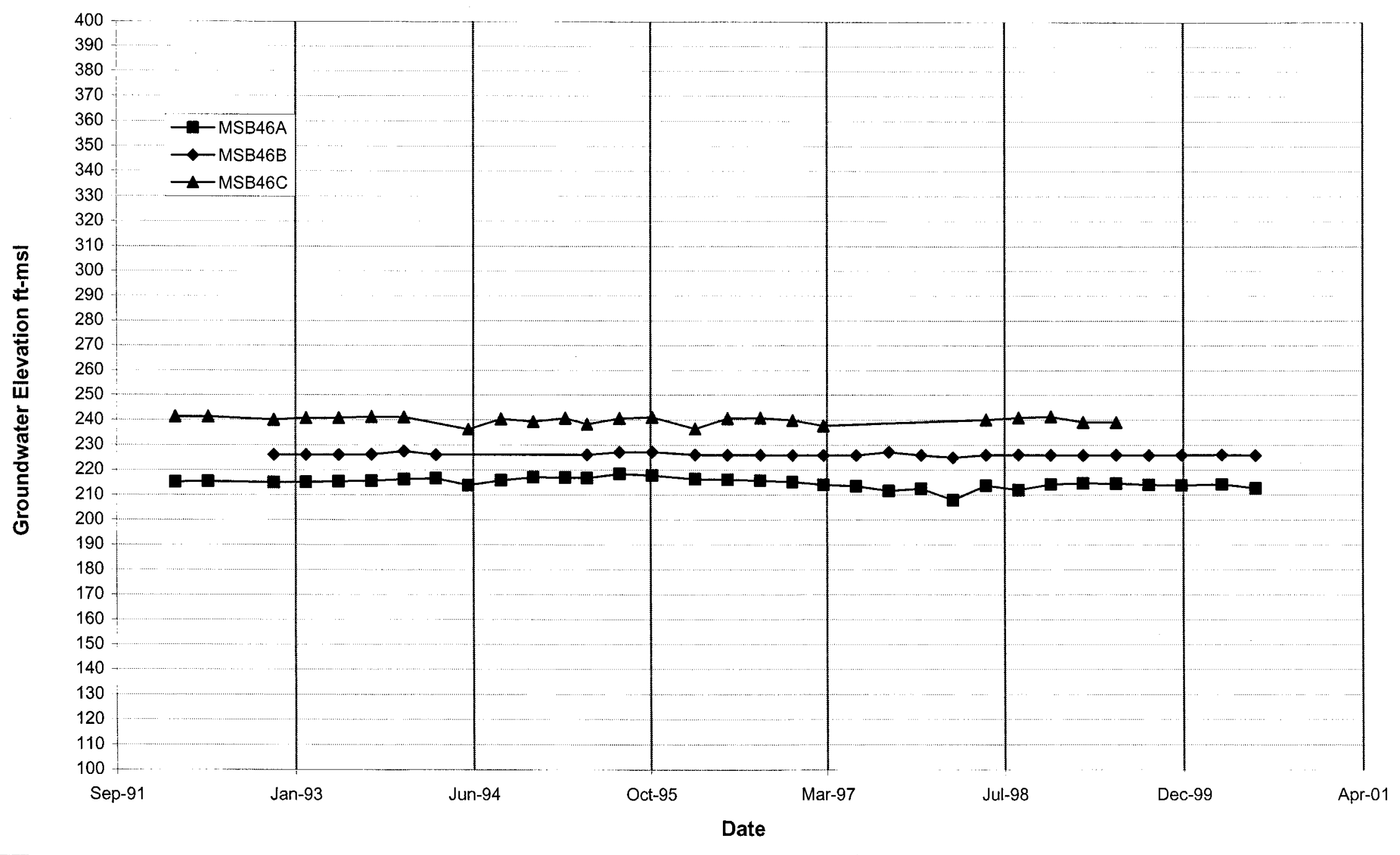




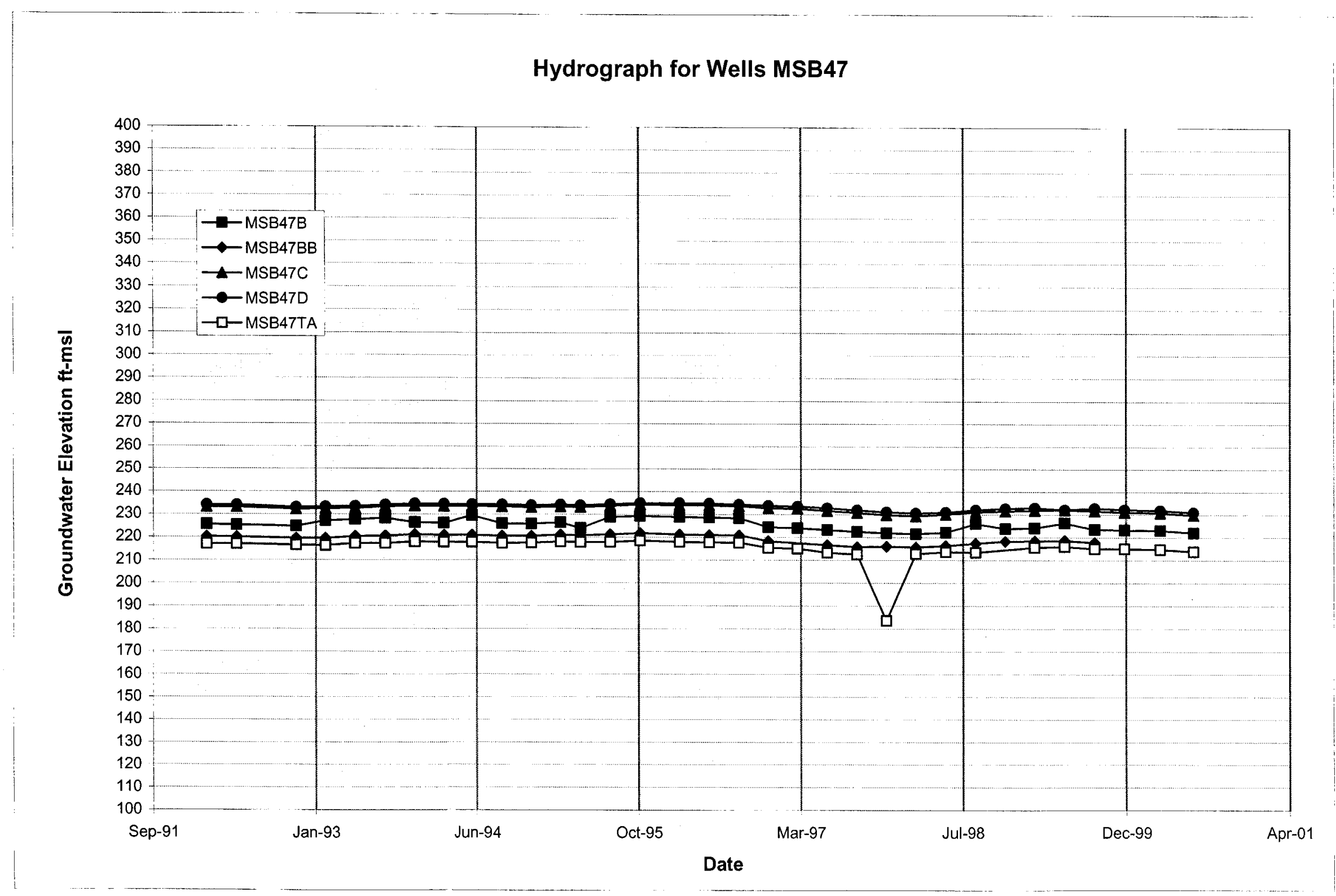




\section{Hydrograph for Wells MSB48}

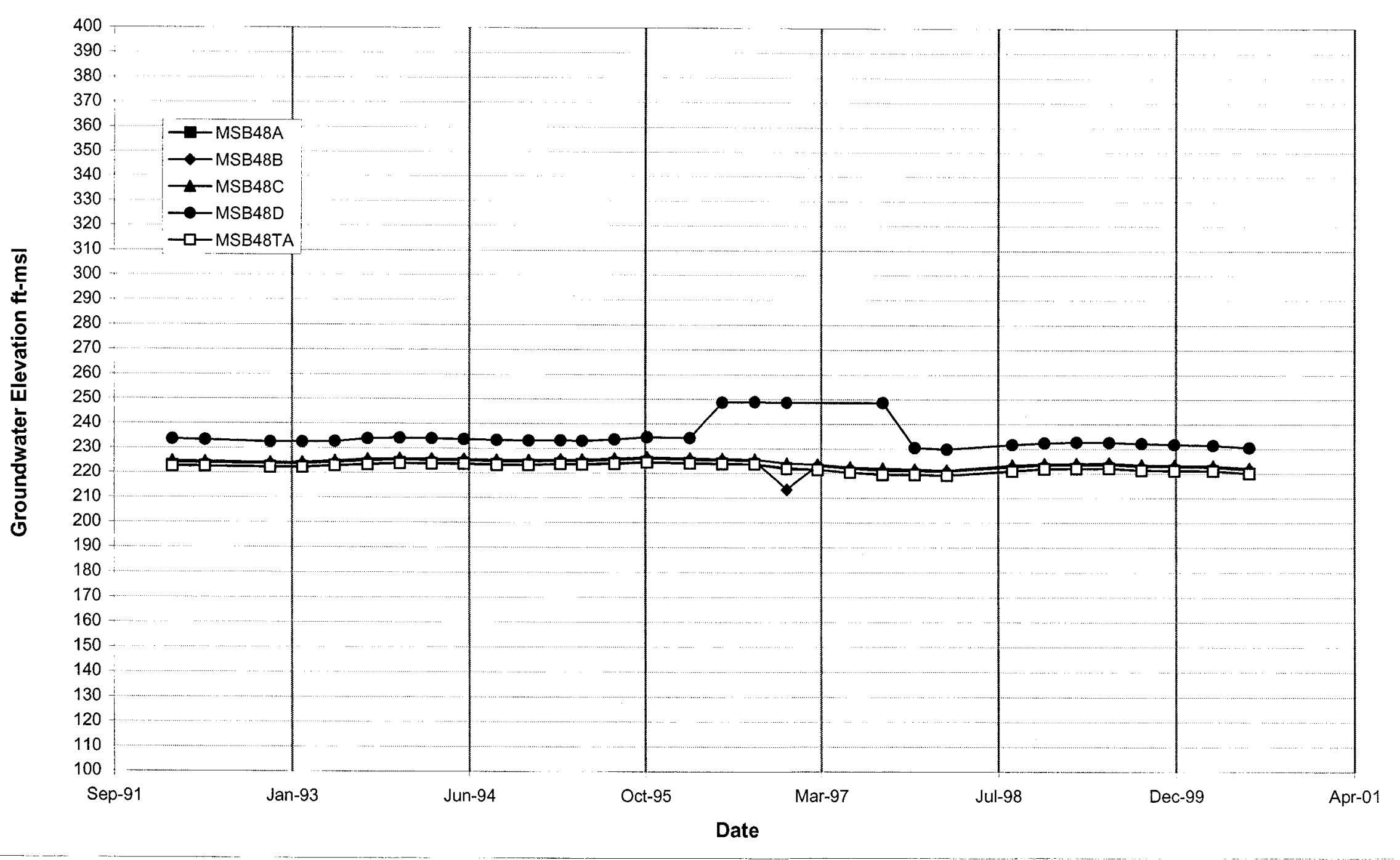




\section{Hydrograph for Wells MSB49}

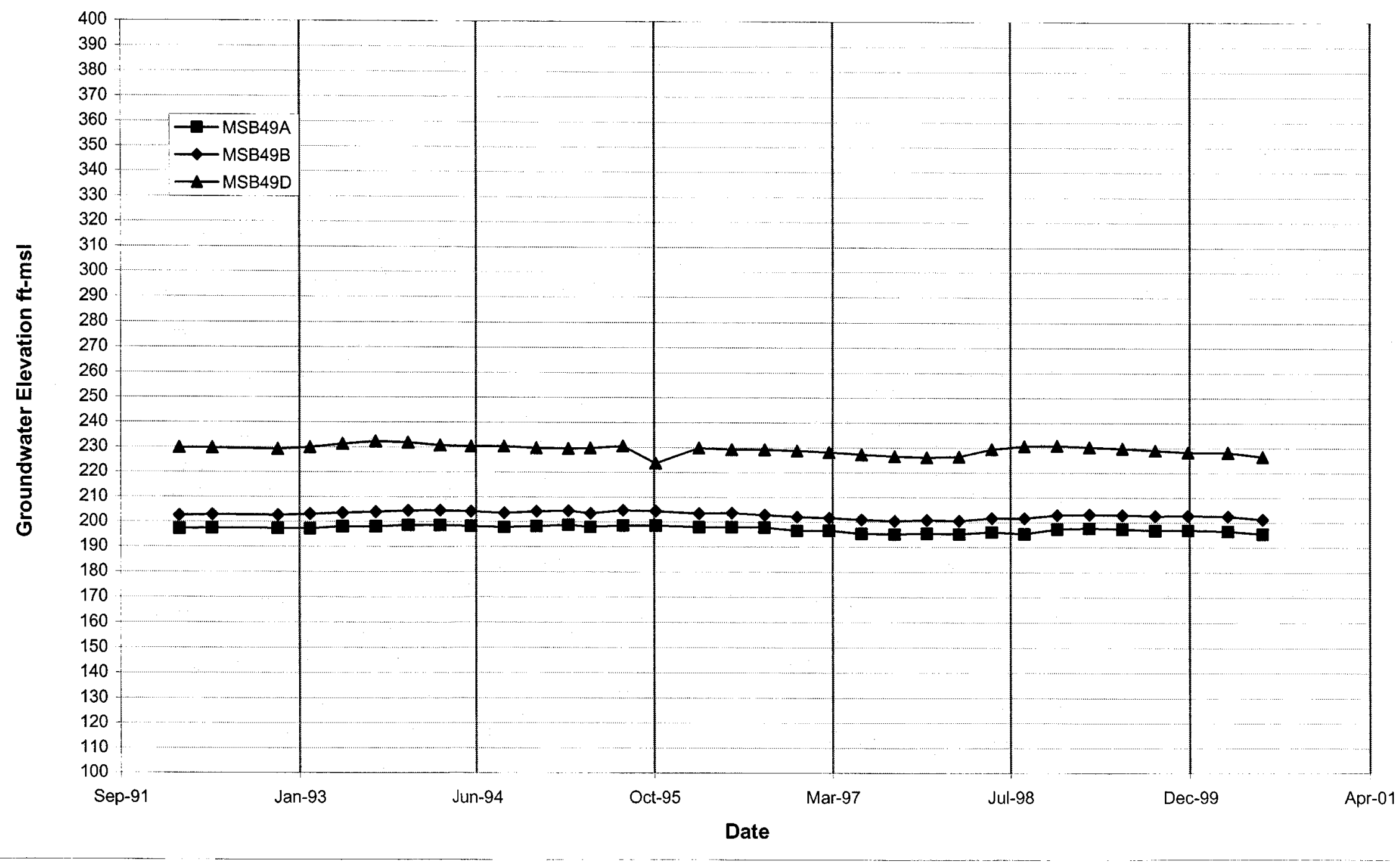


WSRC-TR-2000-00242

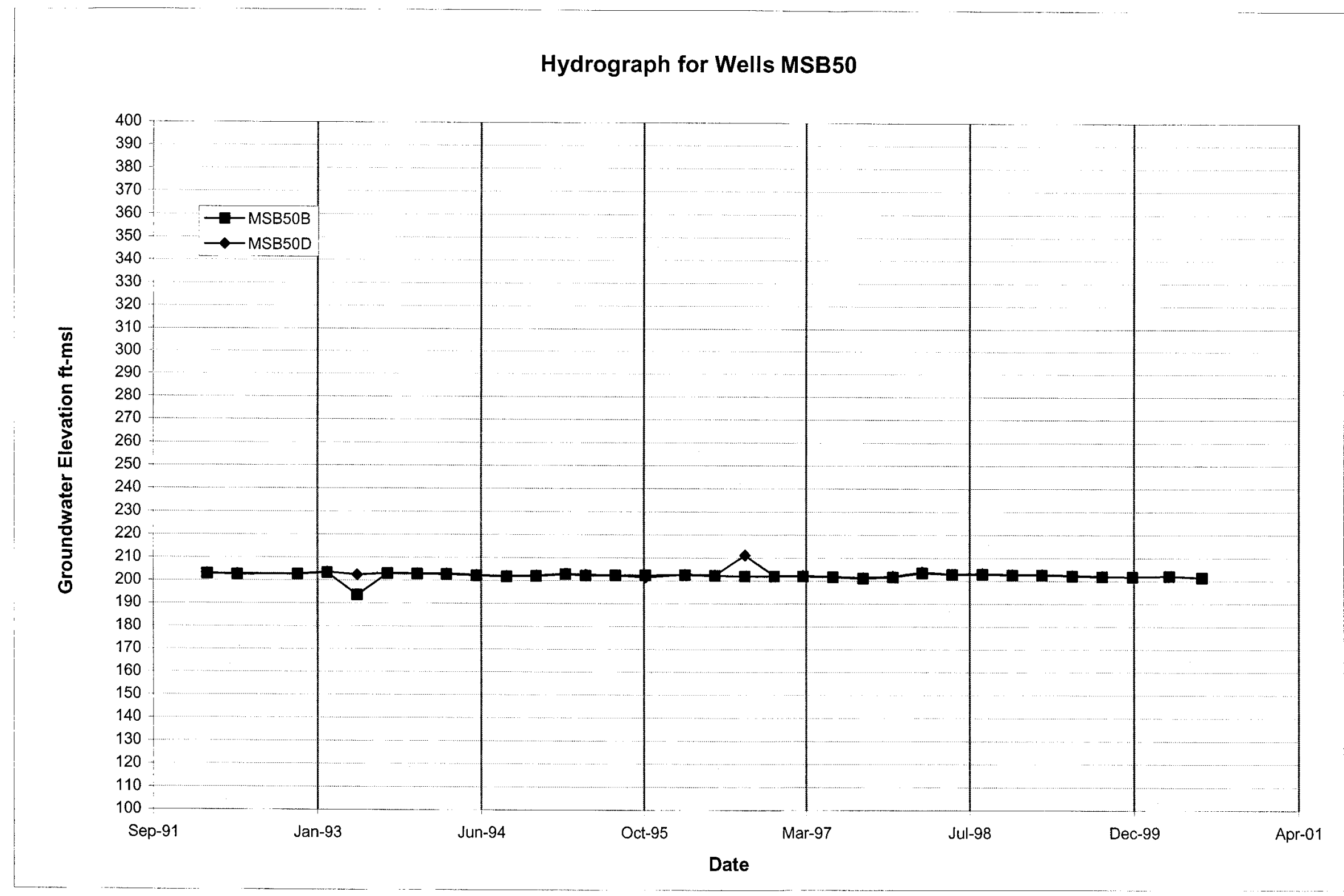




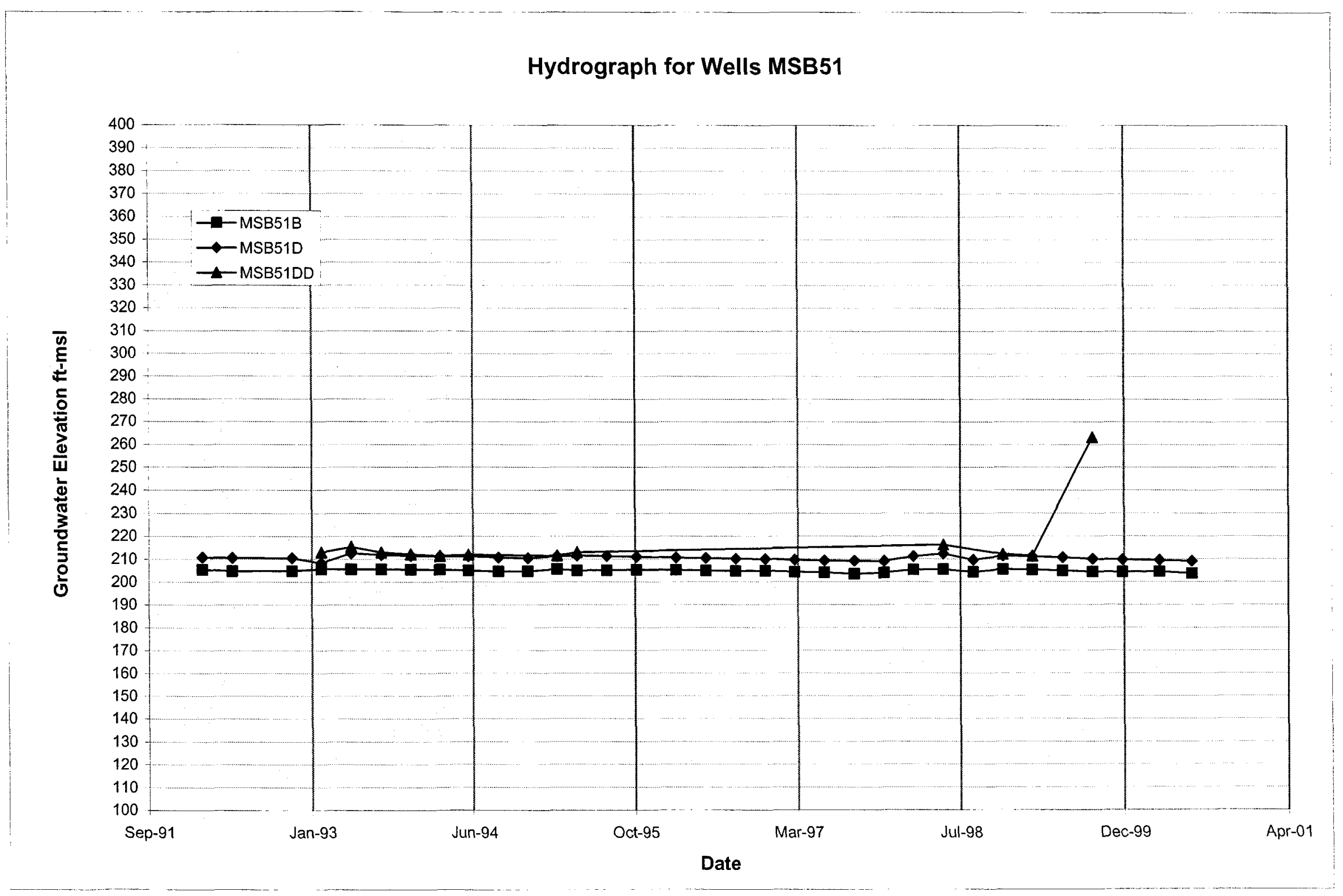




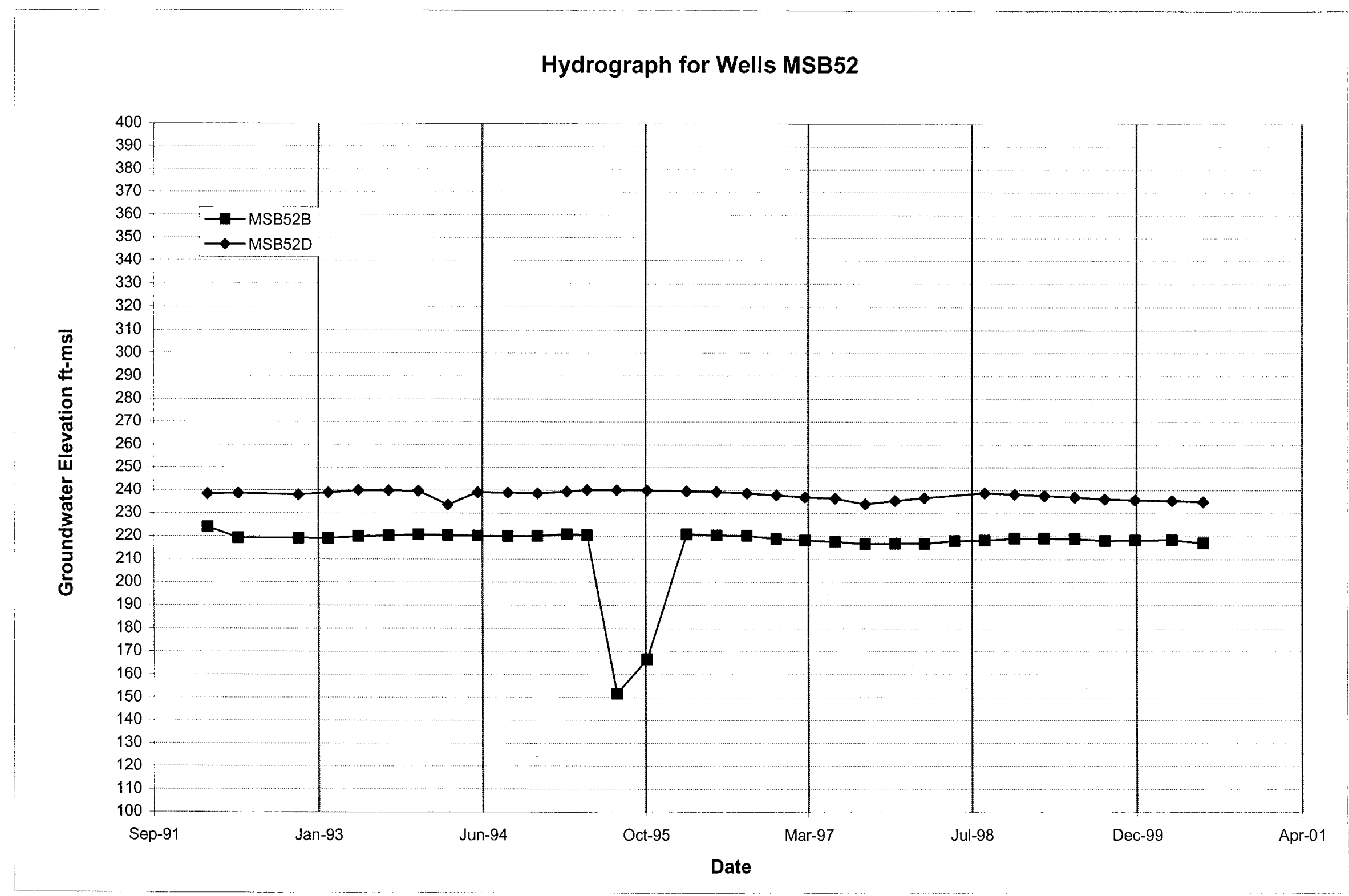


WSRC-TR-2000-00242

UNCLASSIFIED

Hydrograph for Wells MSB53

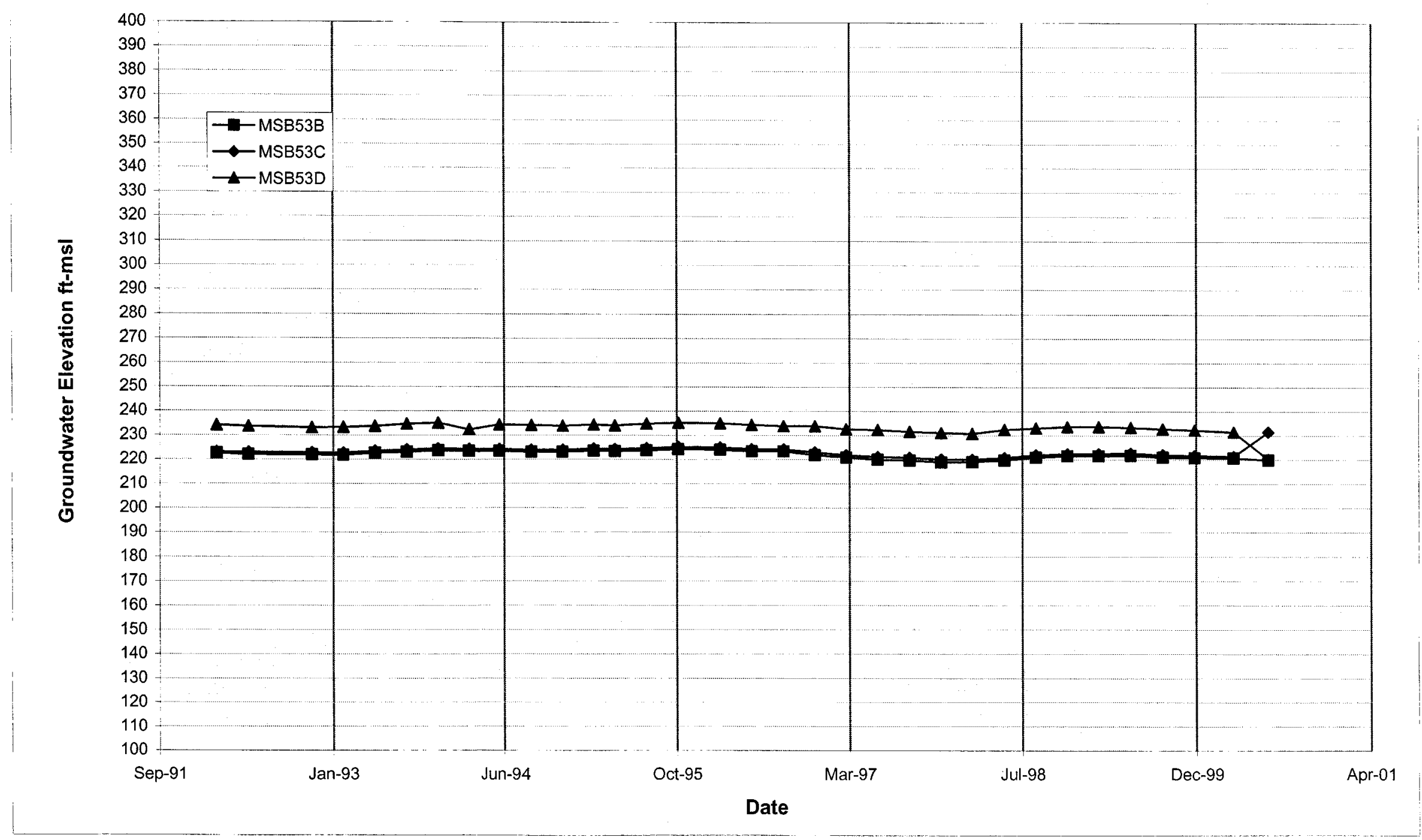




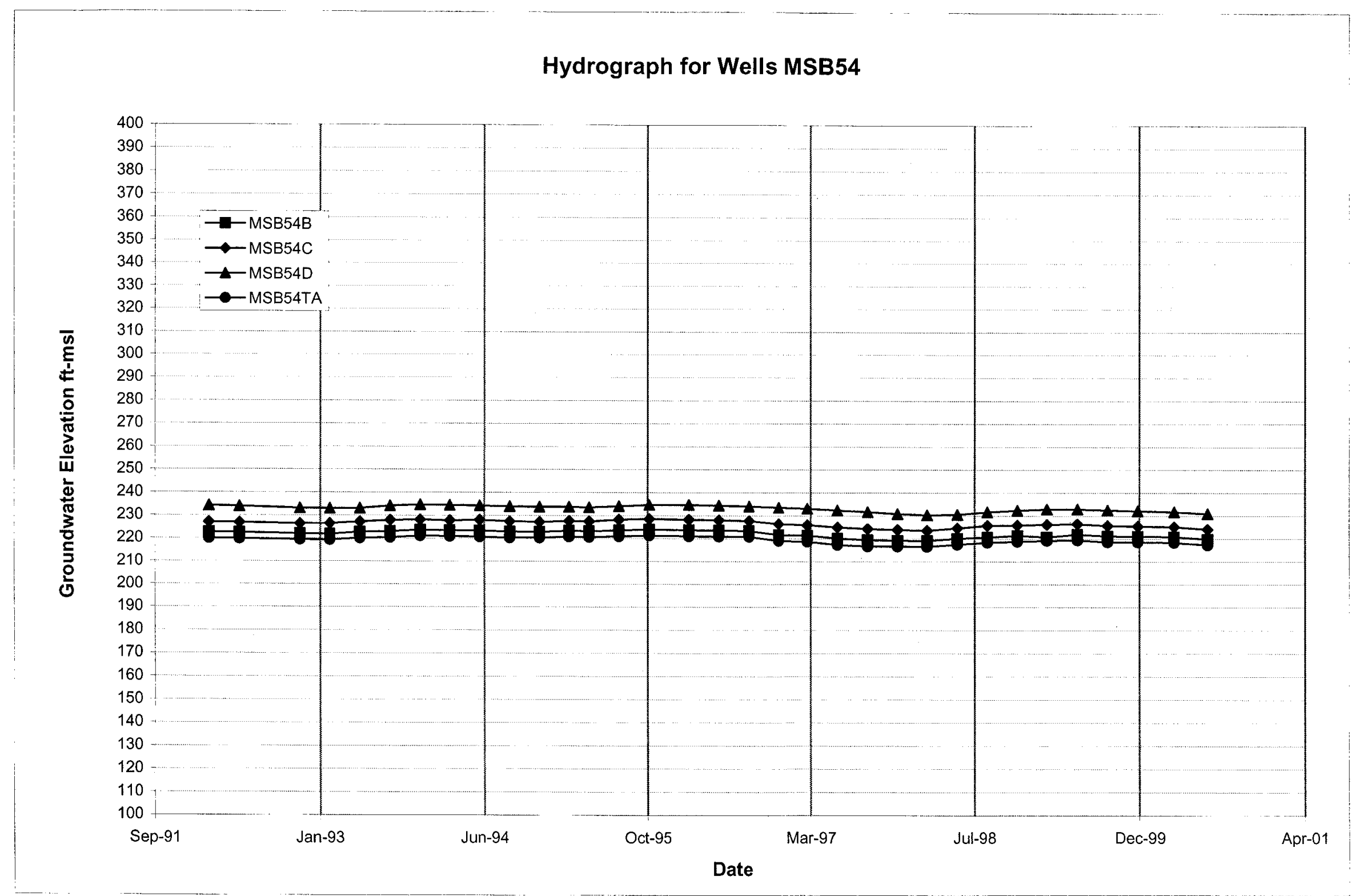




\section{Hydrograph for Wells MSB55}

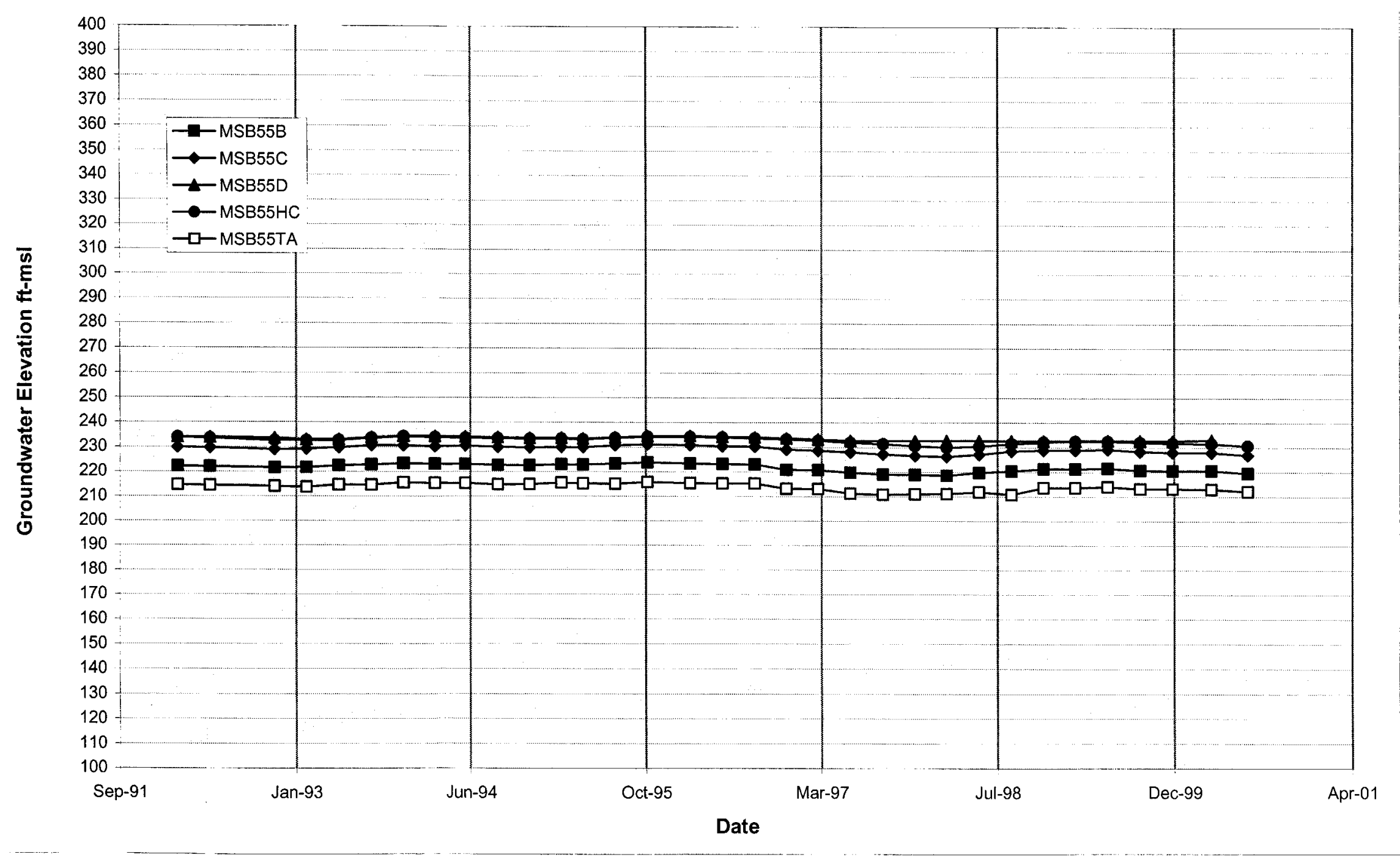


WSRC-TR-2000-00242 UNCLASSIFIED

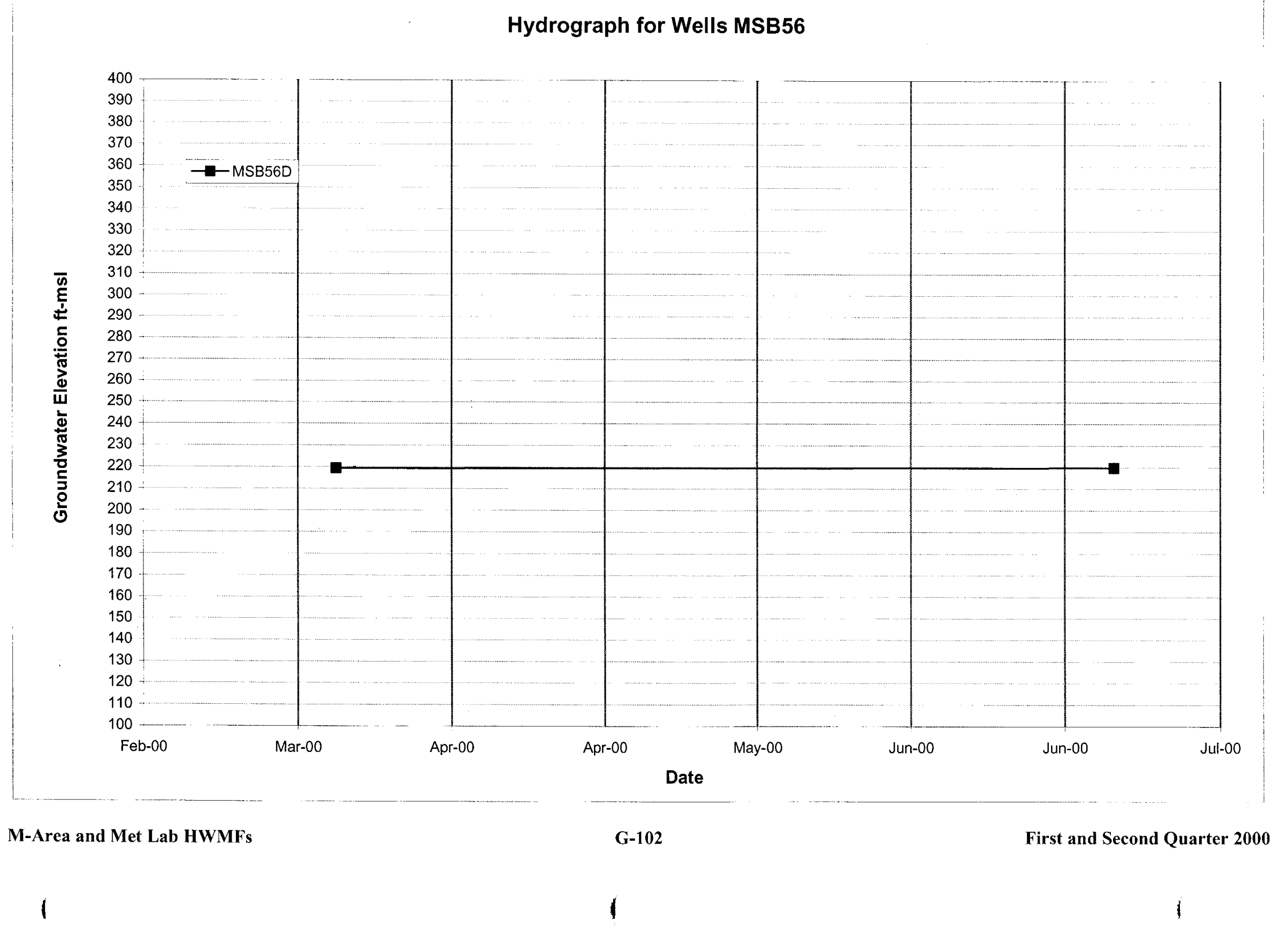




\section{Hydrograph for Wells MSB59}

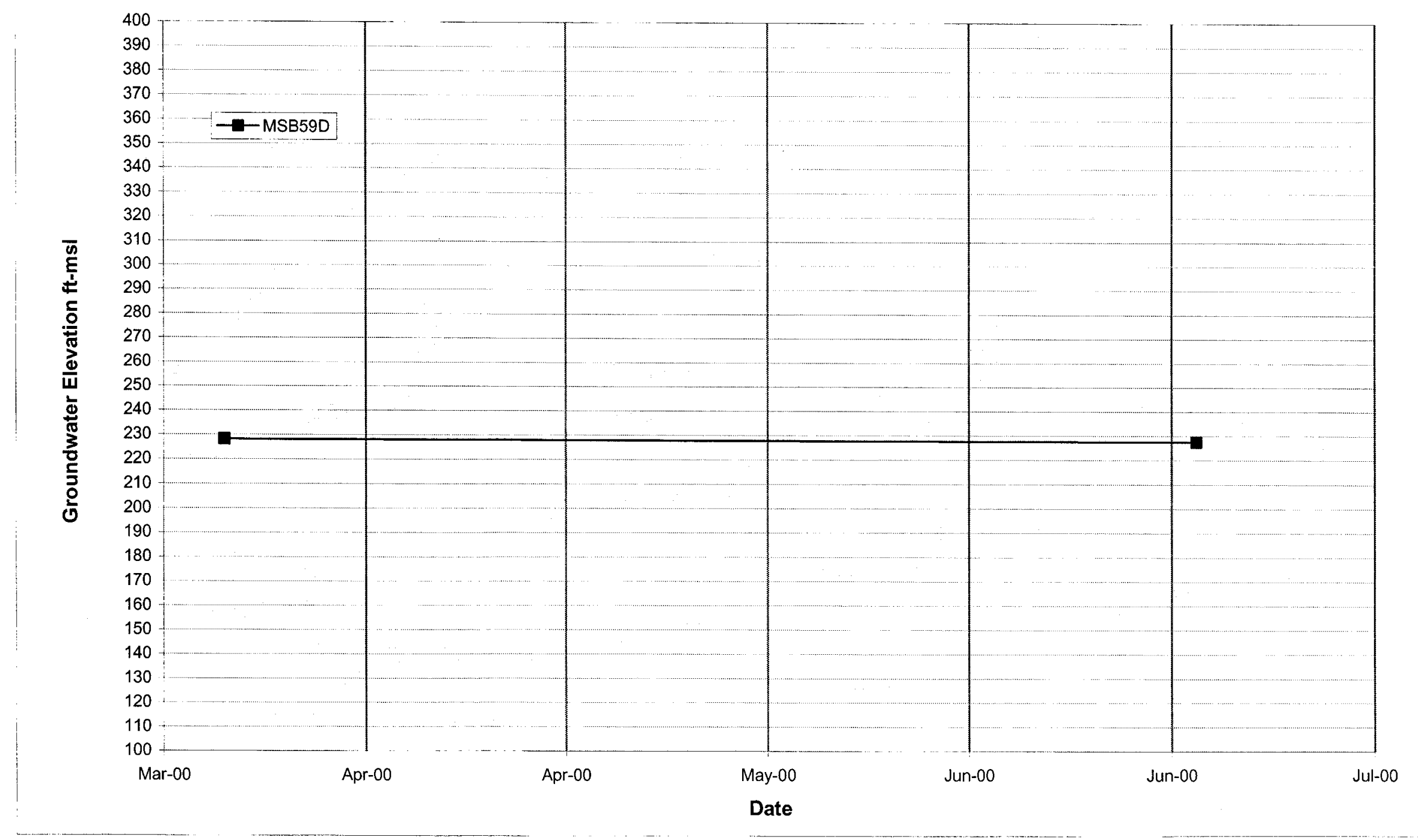



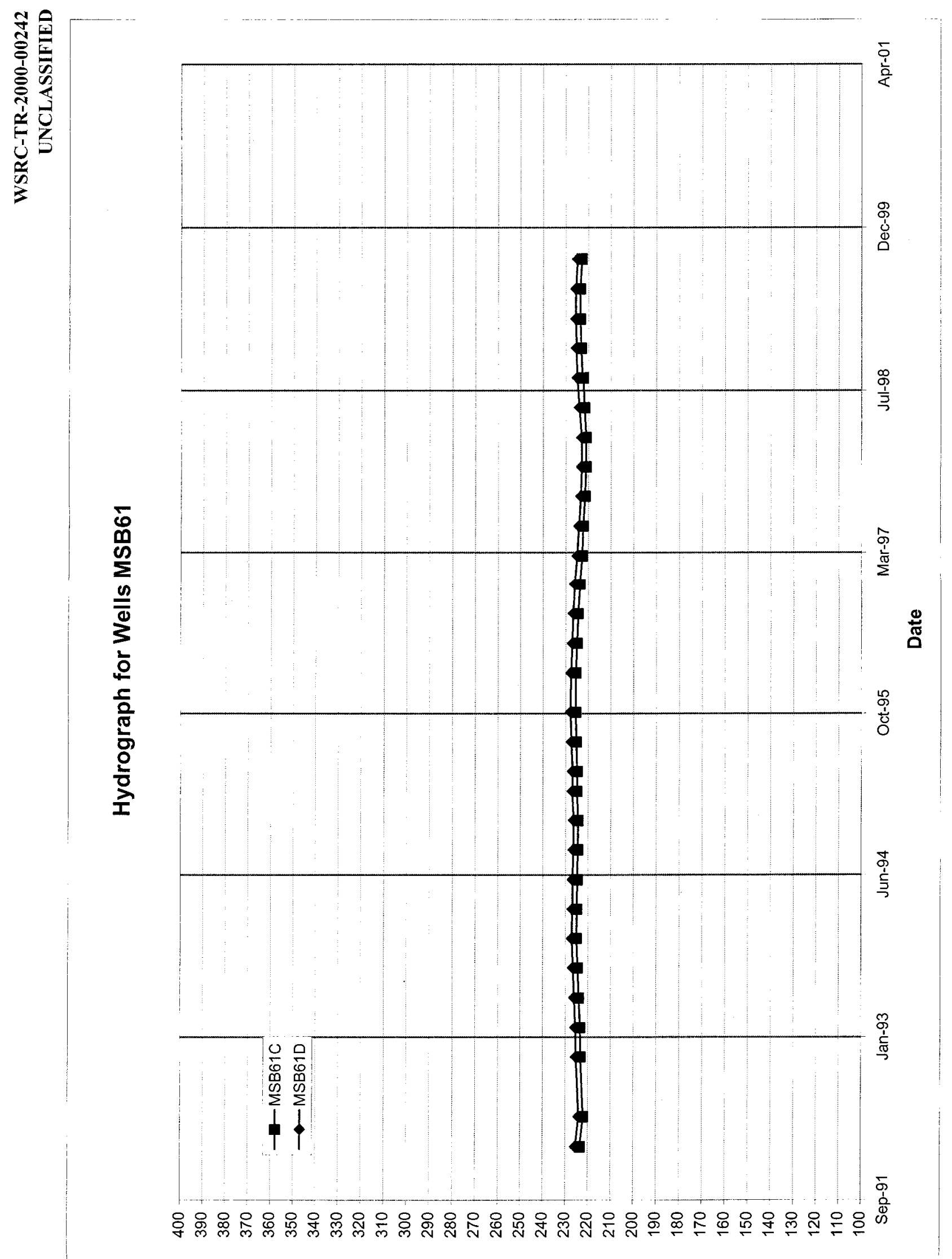

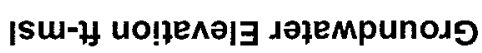
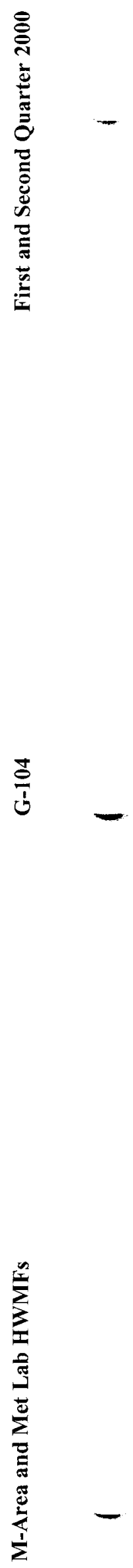


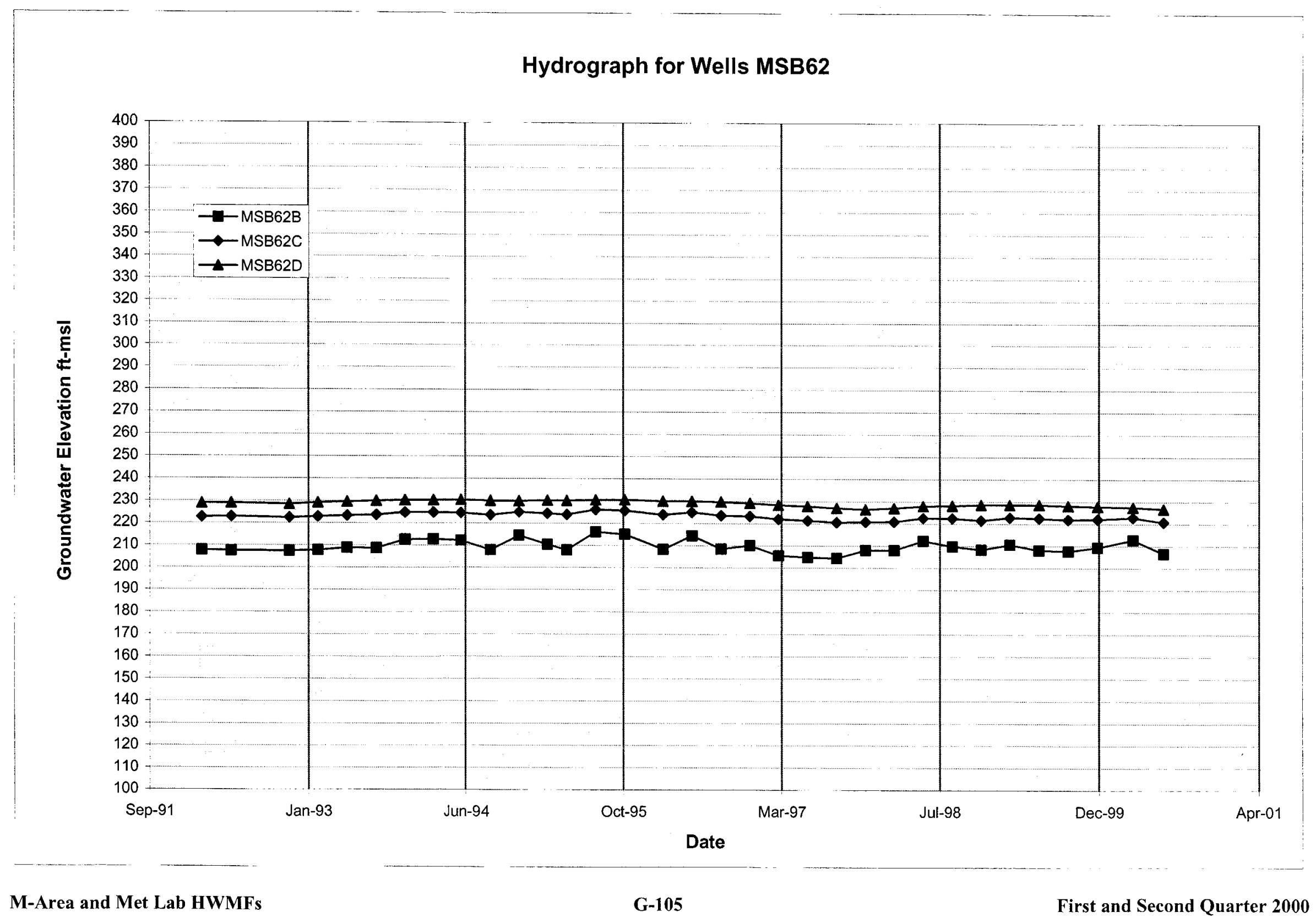


WSRC-TR-2000-00242 UNCLASSIFIED

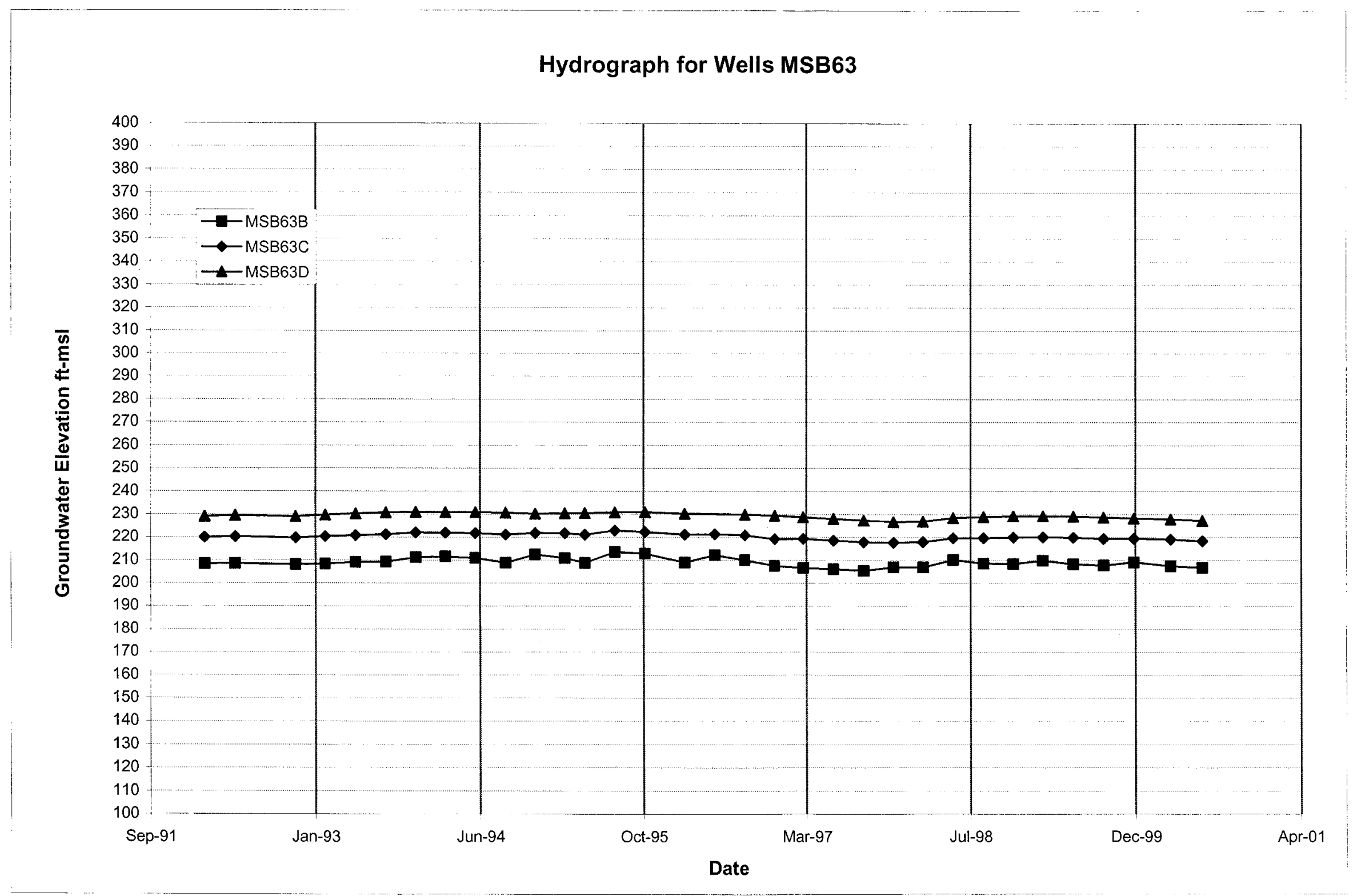


WSRC-TR-2000-00242

UNCLASSIFIED

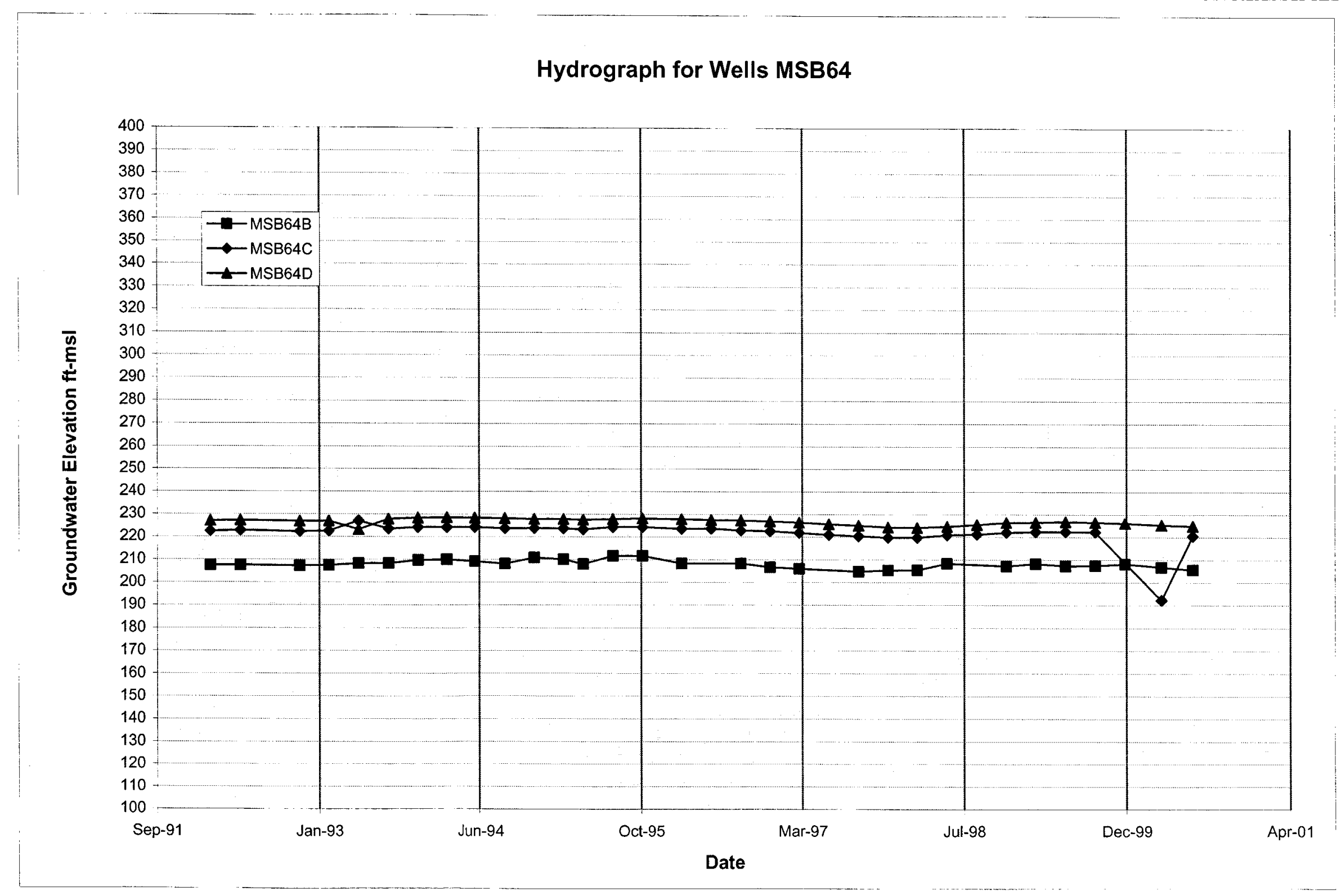


WSRC-TR-2000-00242 UNCLASSIFIED

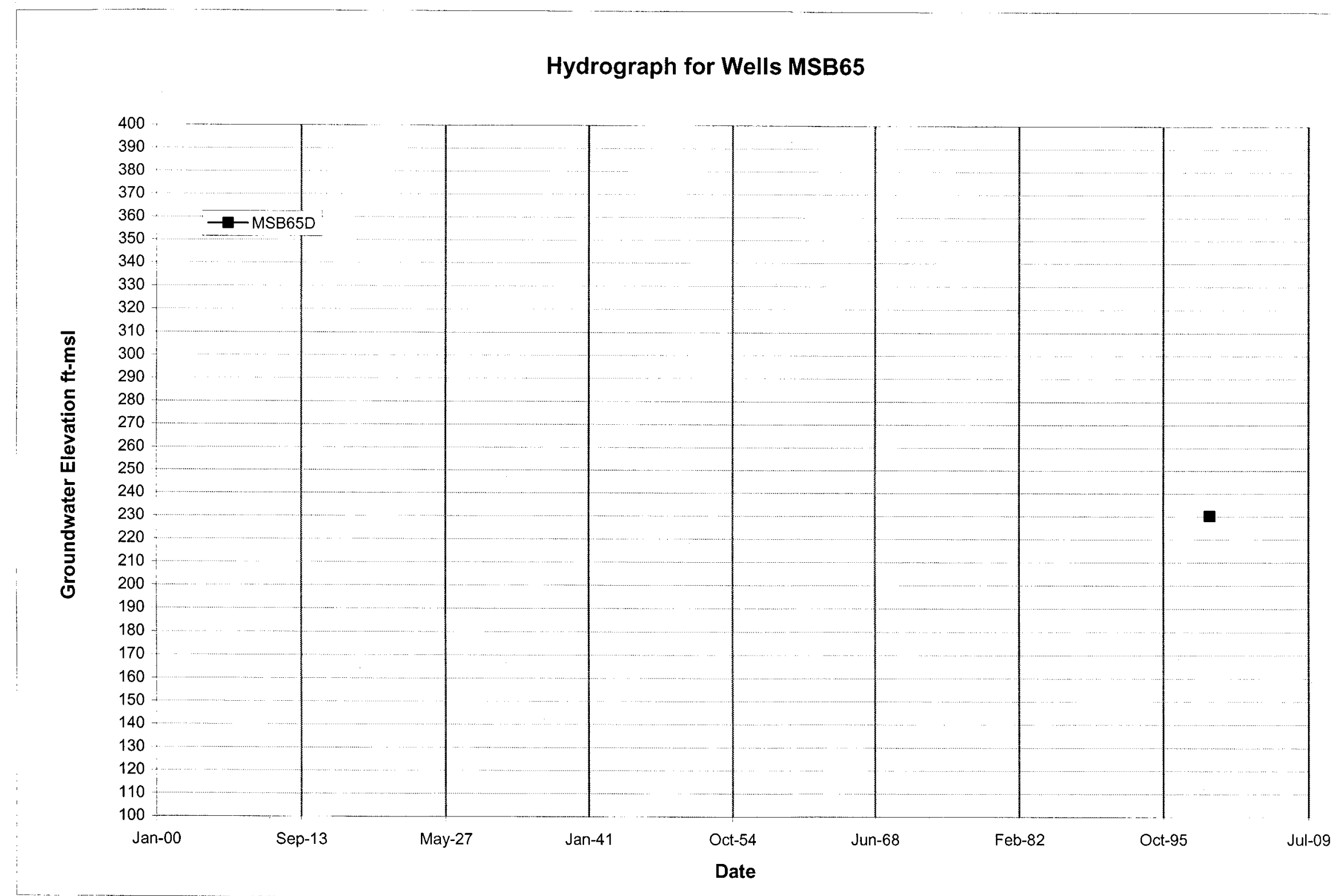




\section{Hydrograph for Wells MSB66}

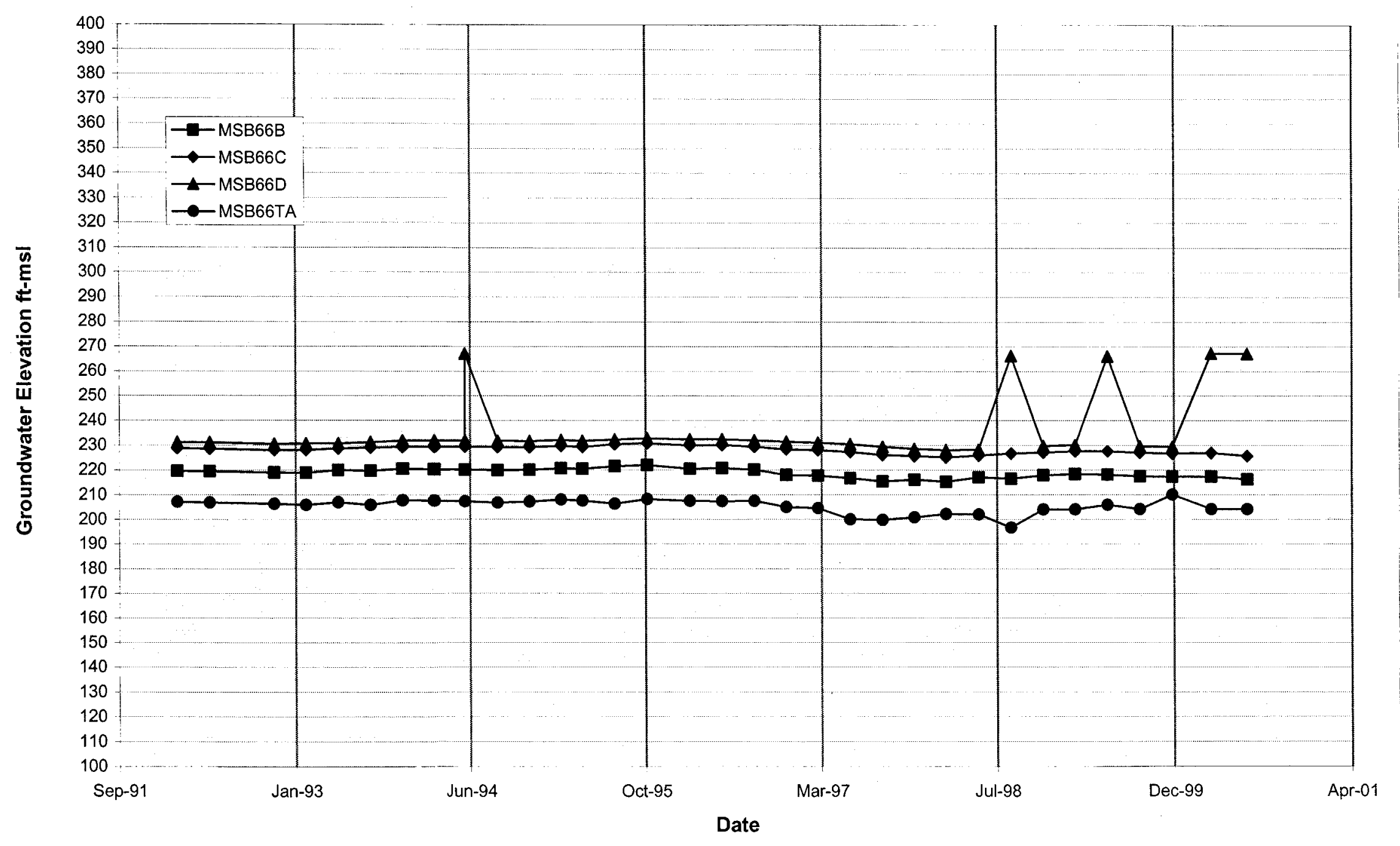


WSRC-TR-2000-00242 UNCLASSIFIED

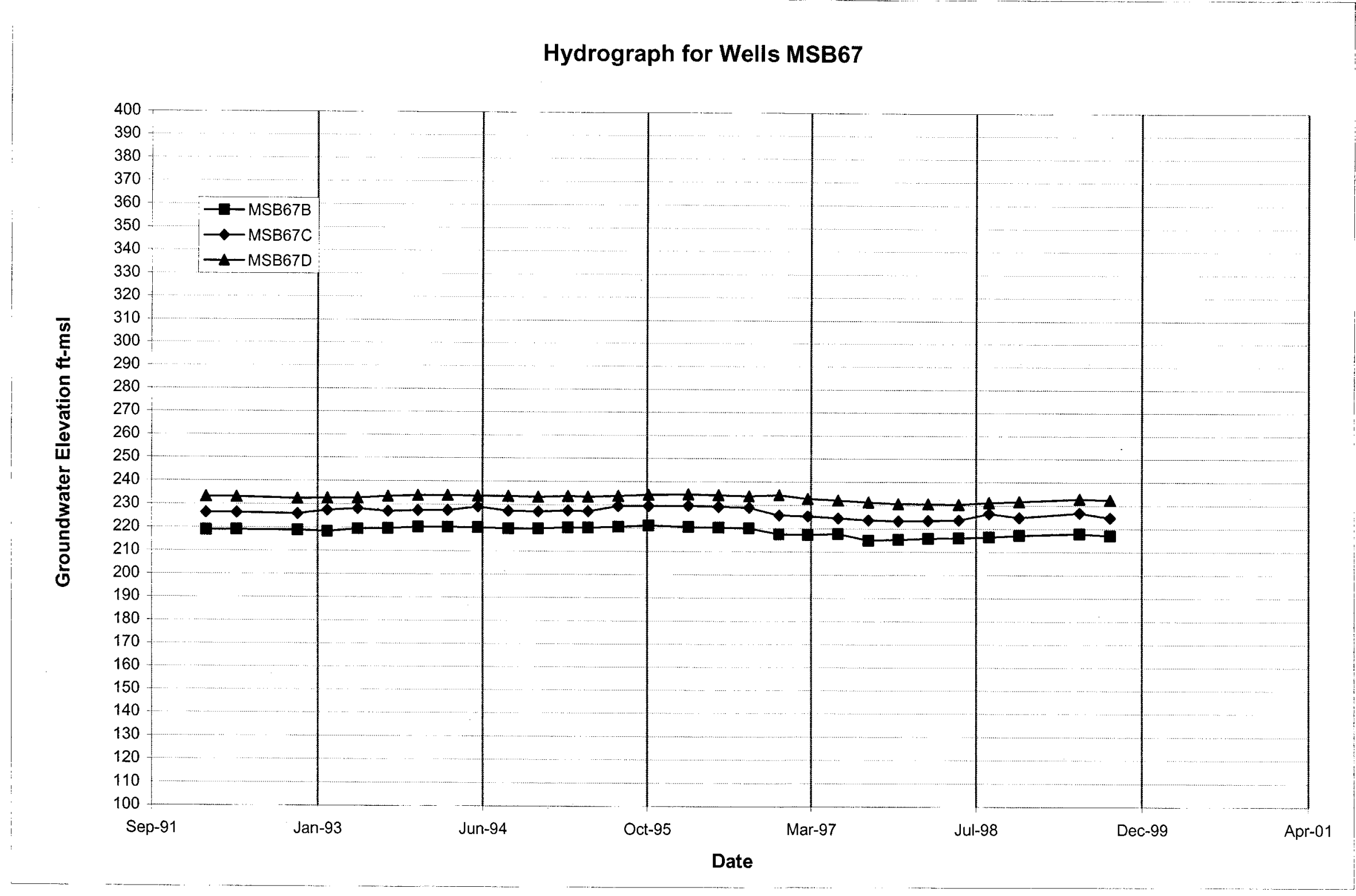




\section{Hydrograph for Wells MSB68}

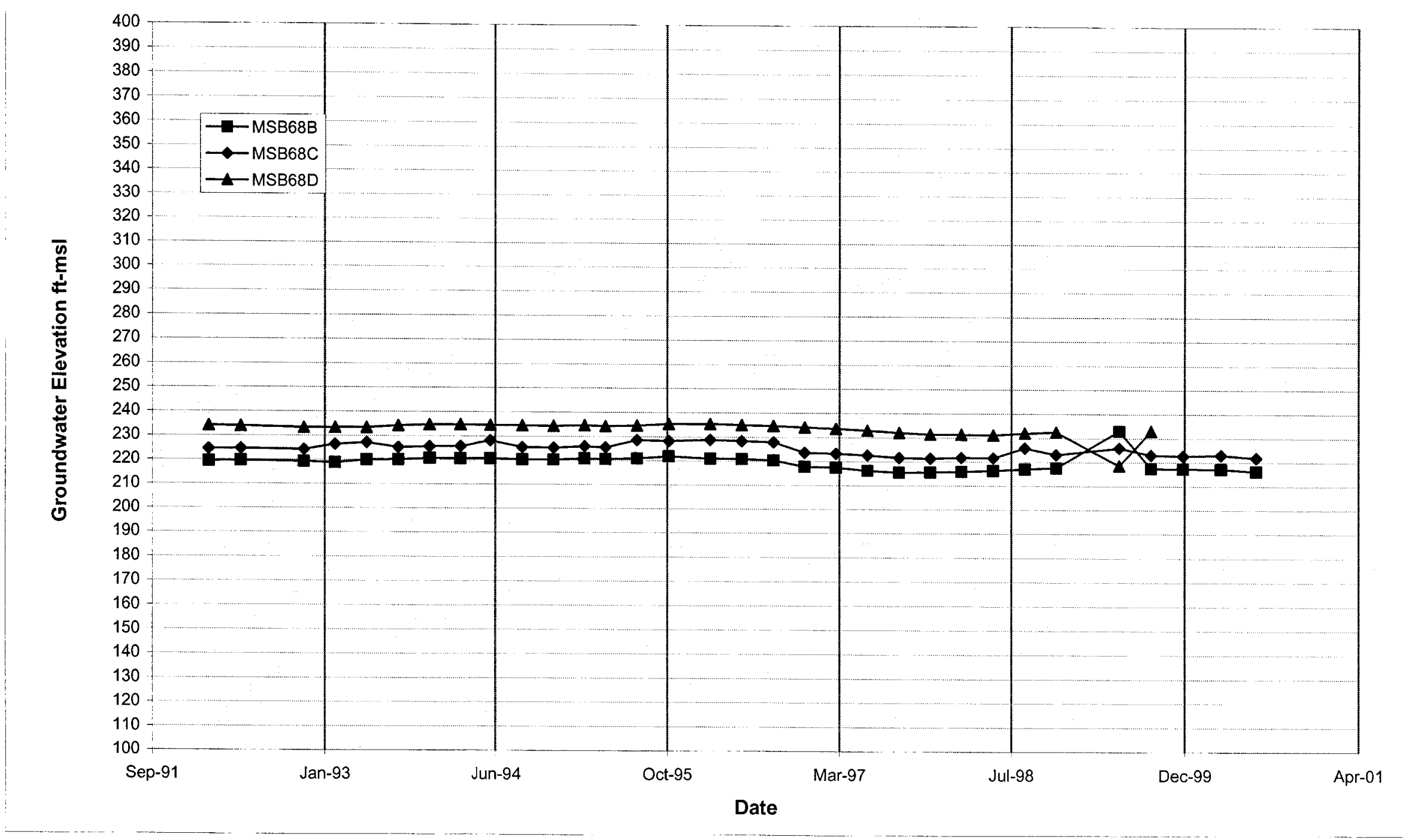


WSRC-TR-2000-00242 UNCLASSIFIED

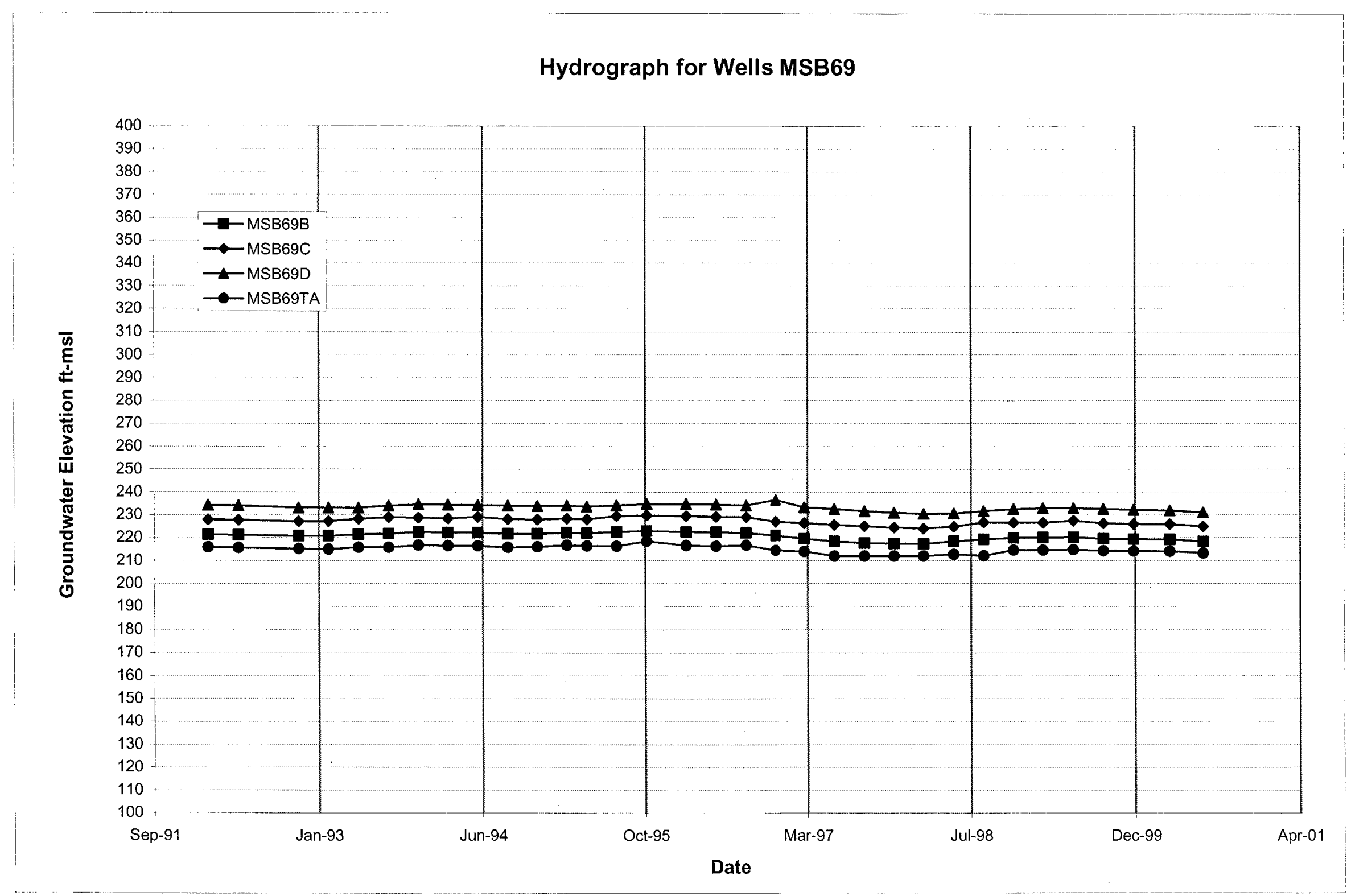




\section{Hydrograph for Wells MSB70}

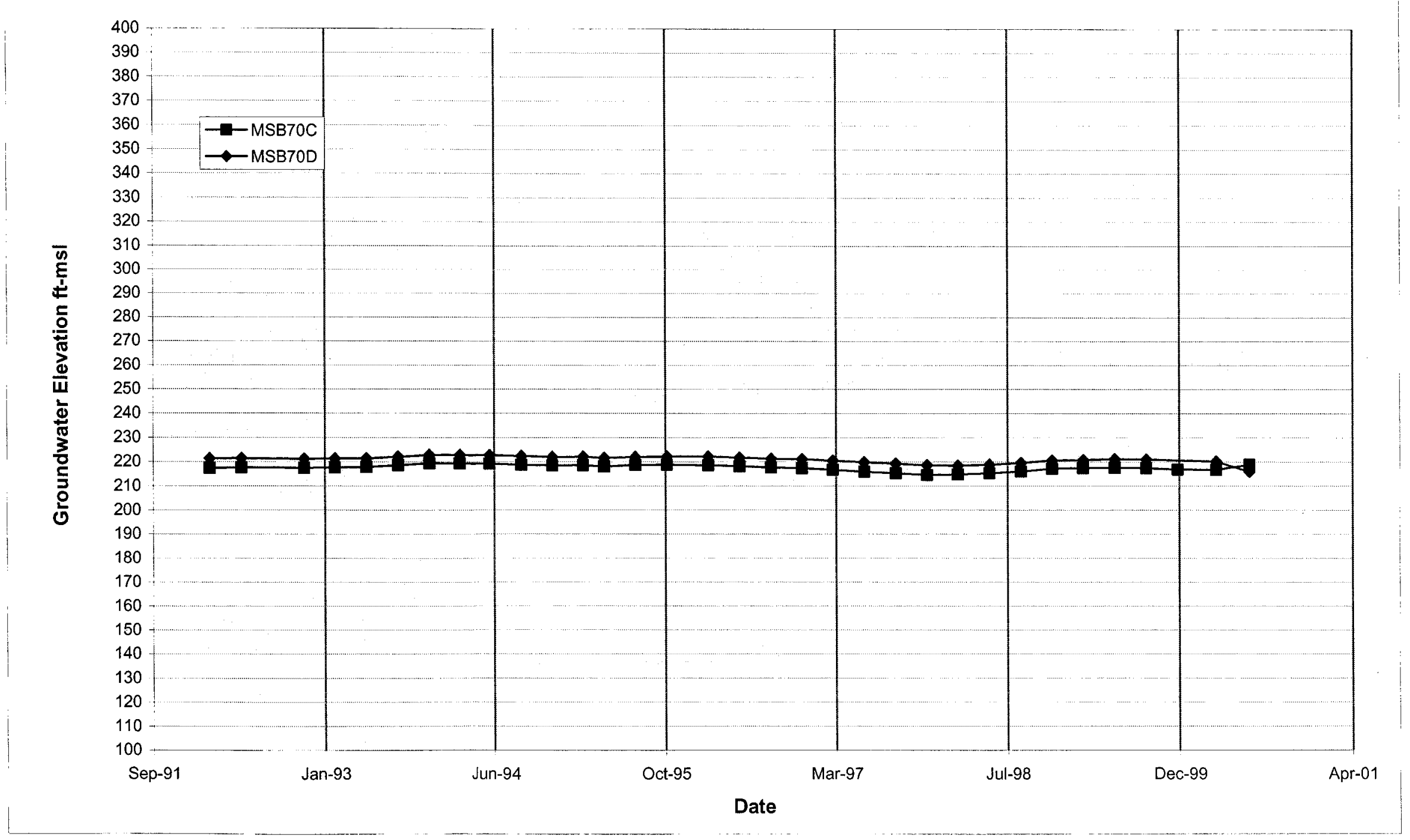


WSRC-TR-2000-00242 UNCLASSIFIED

\section{Hydrograph for Wells MSB71}

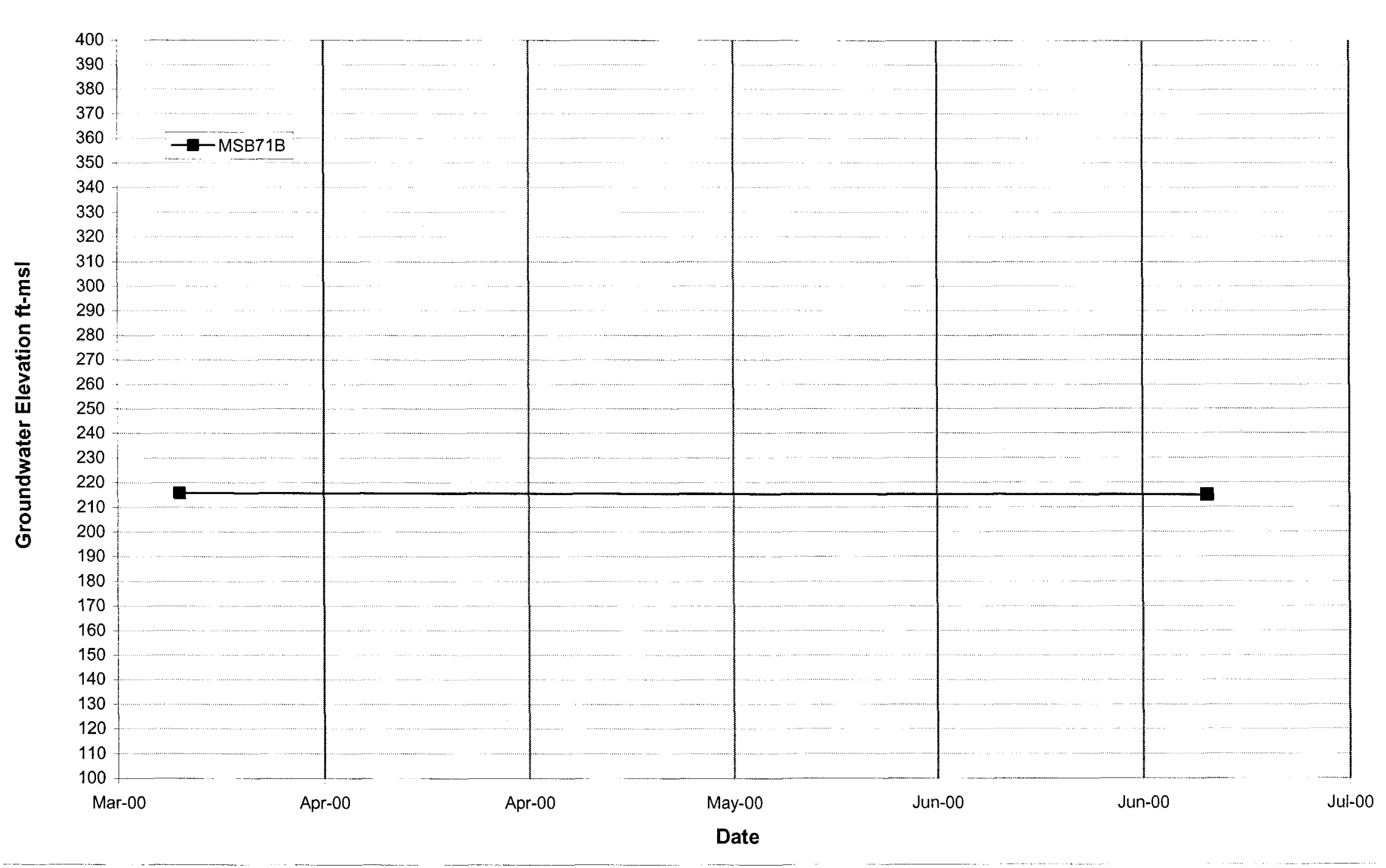




\section{Hydrograph for Wells MSB72}

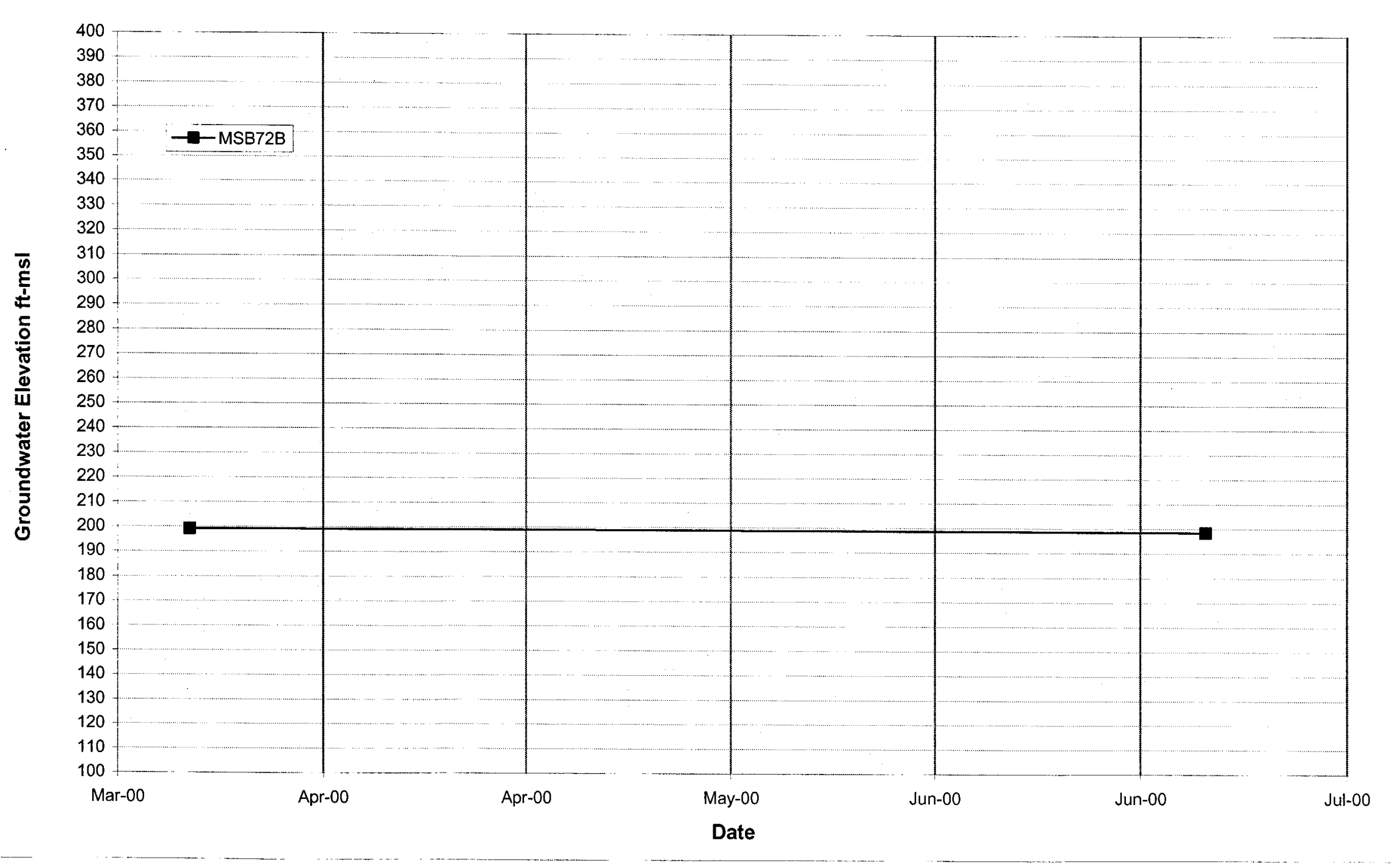


WSRC-TR-2000-00242 UNCLASSIFIED

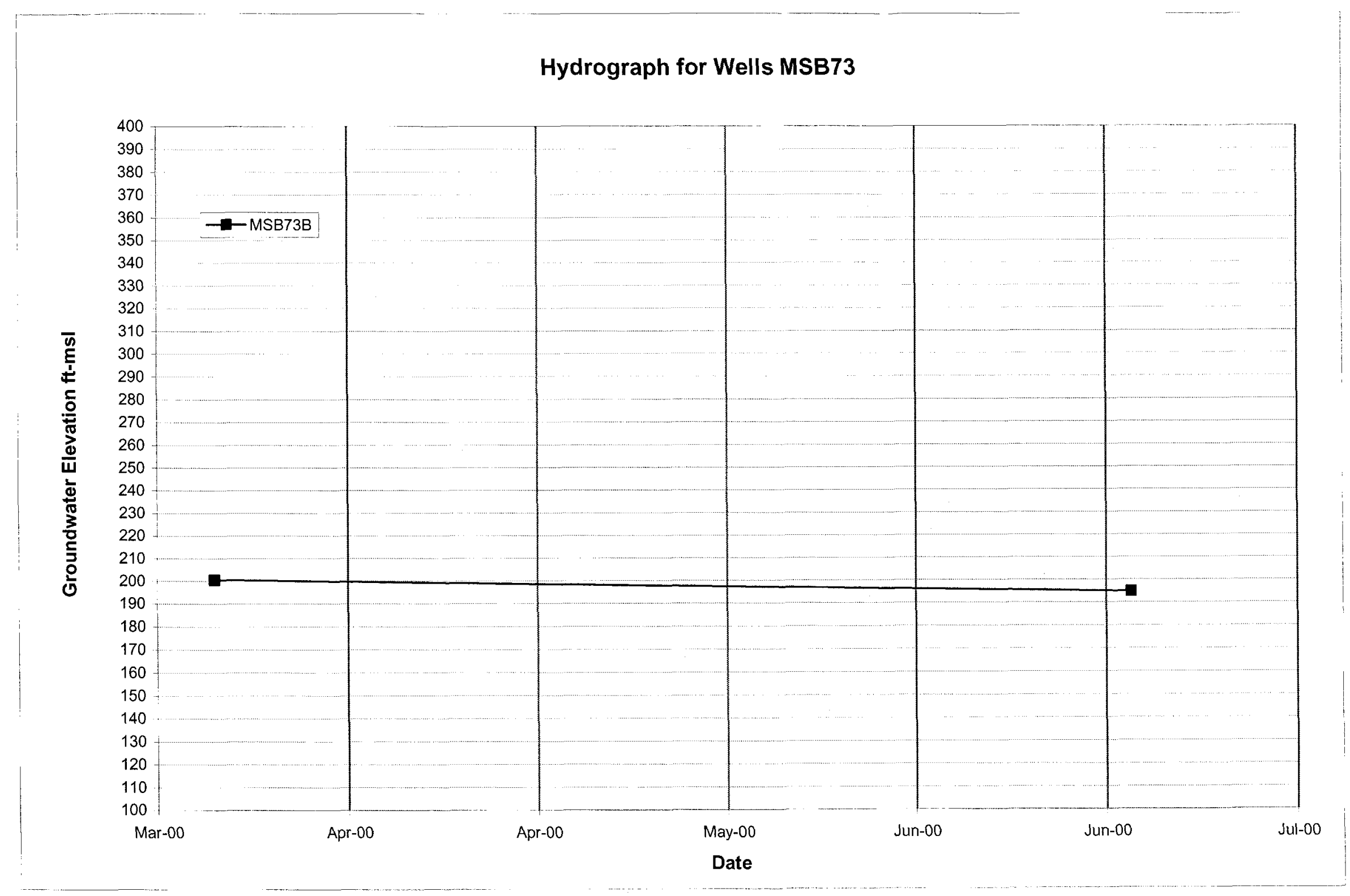


WSRC-TR-2000-00242

UNCLASSIFIED

\section{Hydrograph for Wells MSB74}

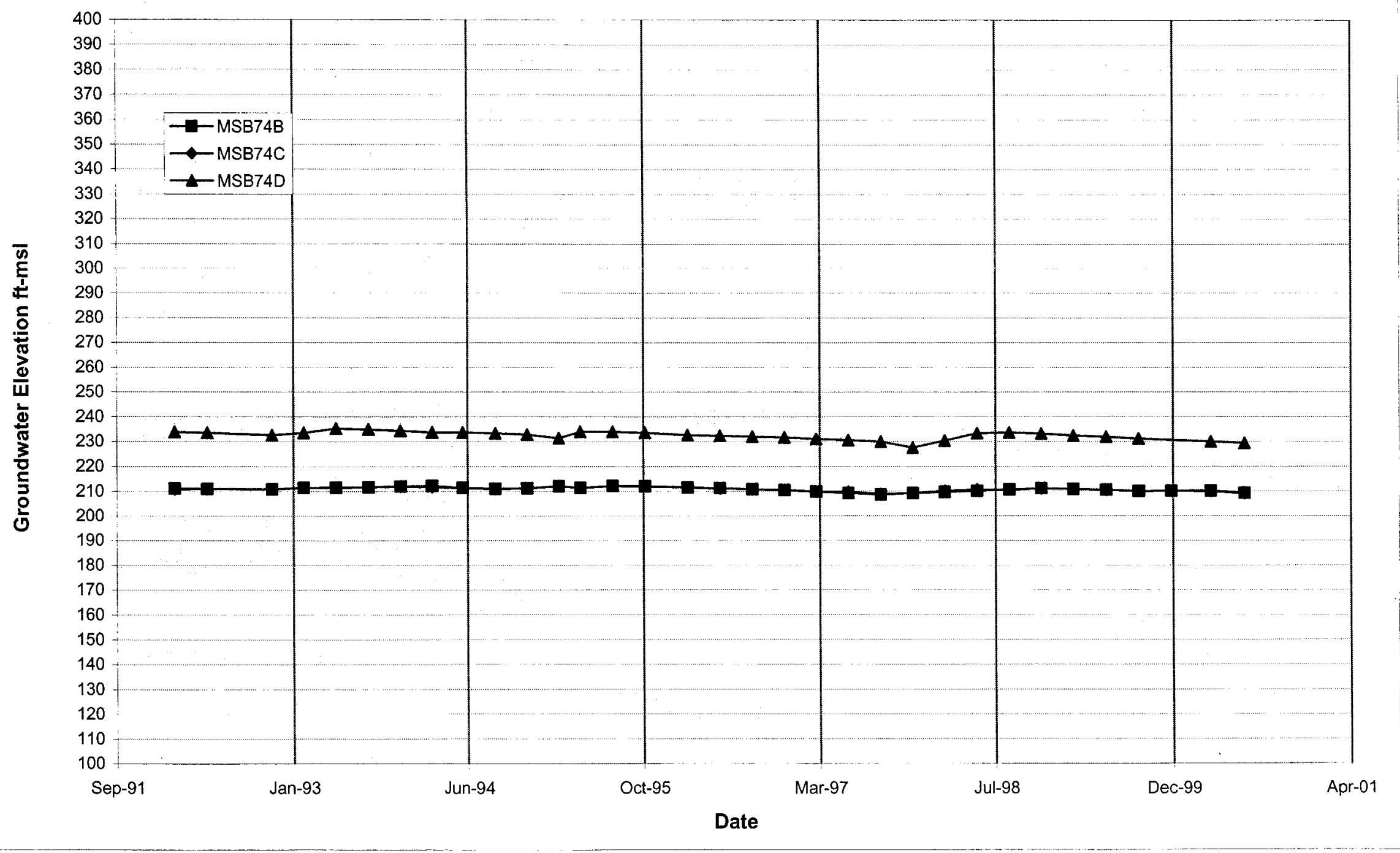




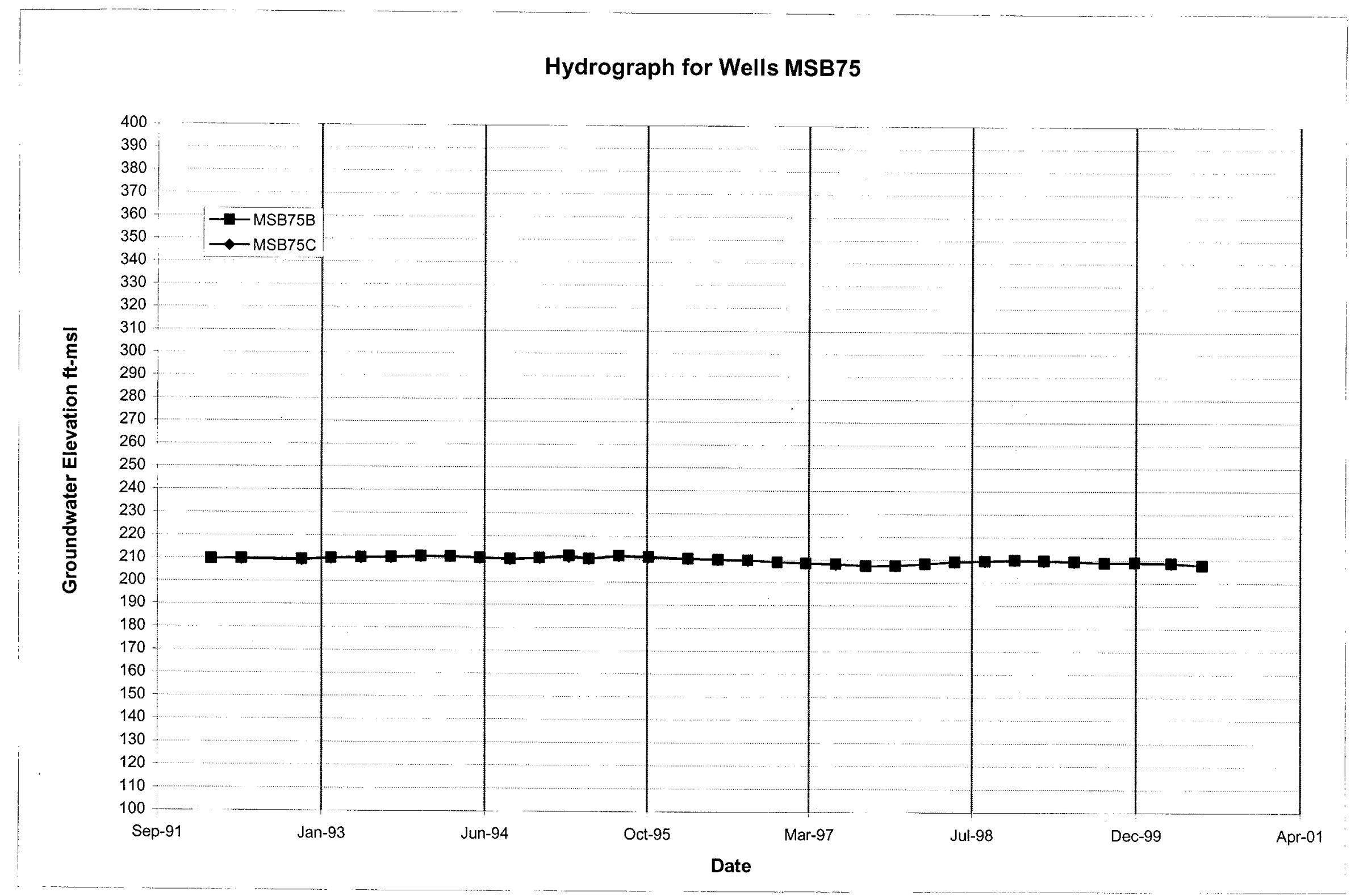


WSRC-TR-2000-00242 UNCLASSIFIED

\section{Hydrograph for Wells MSB76}

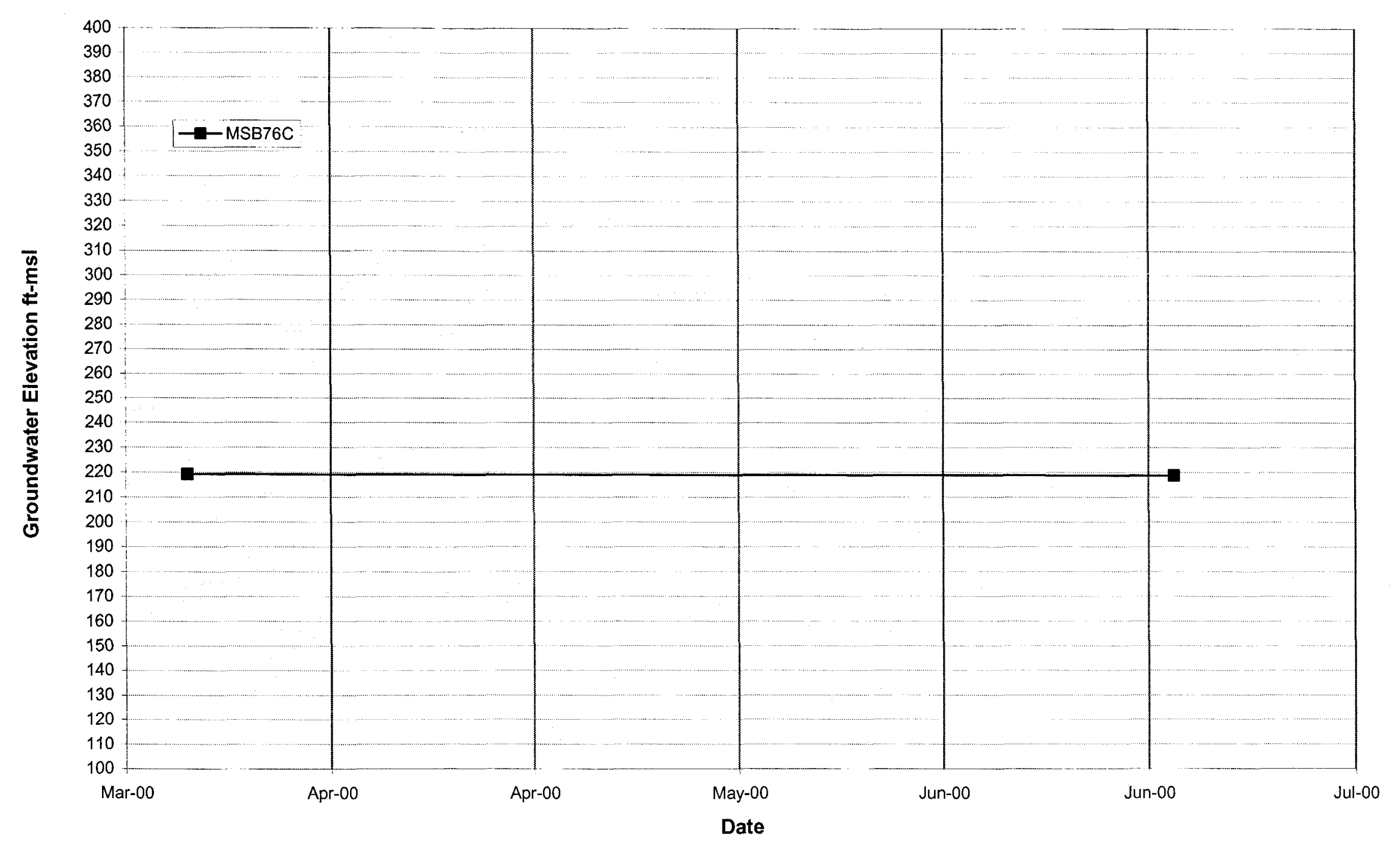


WSRC-TR-2000-00242 UNCLASSIFIED

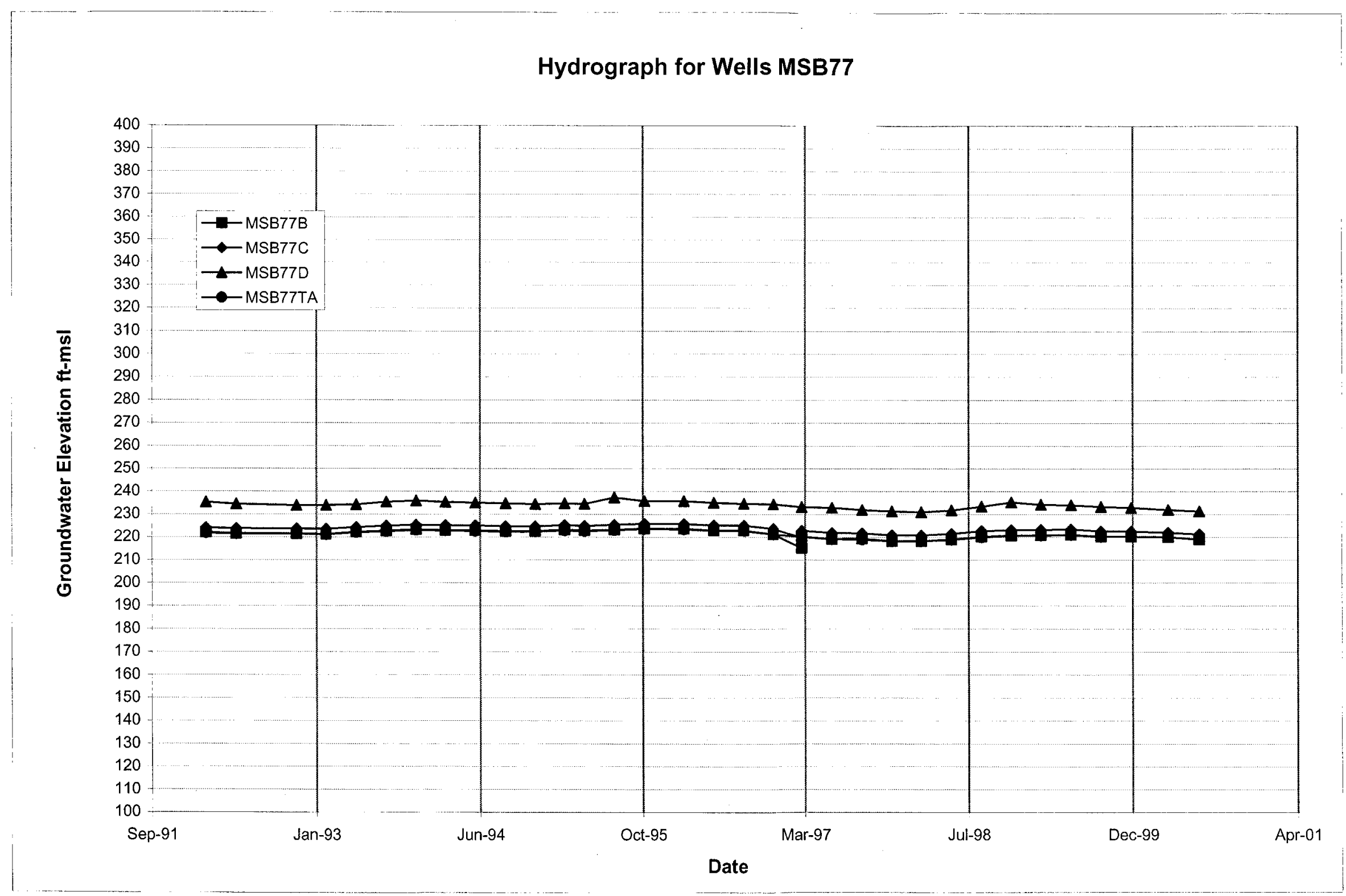




\section{Hydrograph for Wells MSB78}

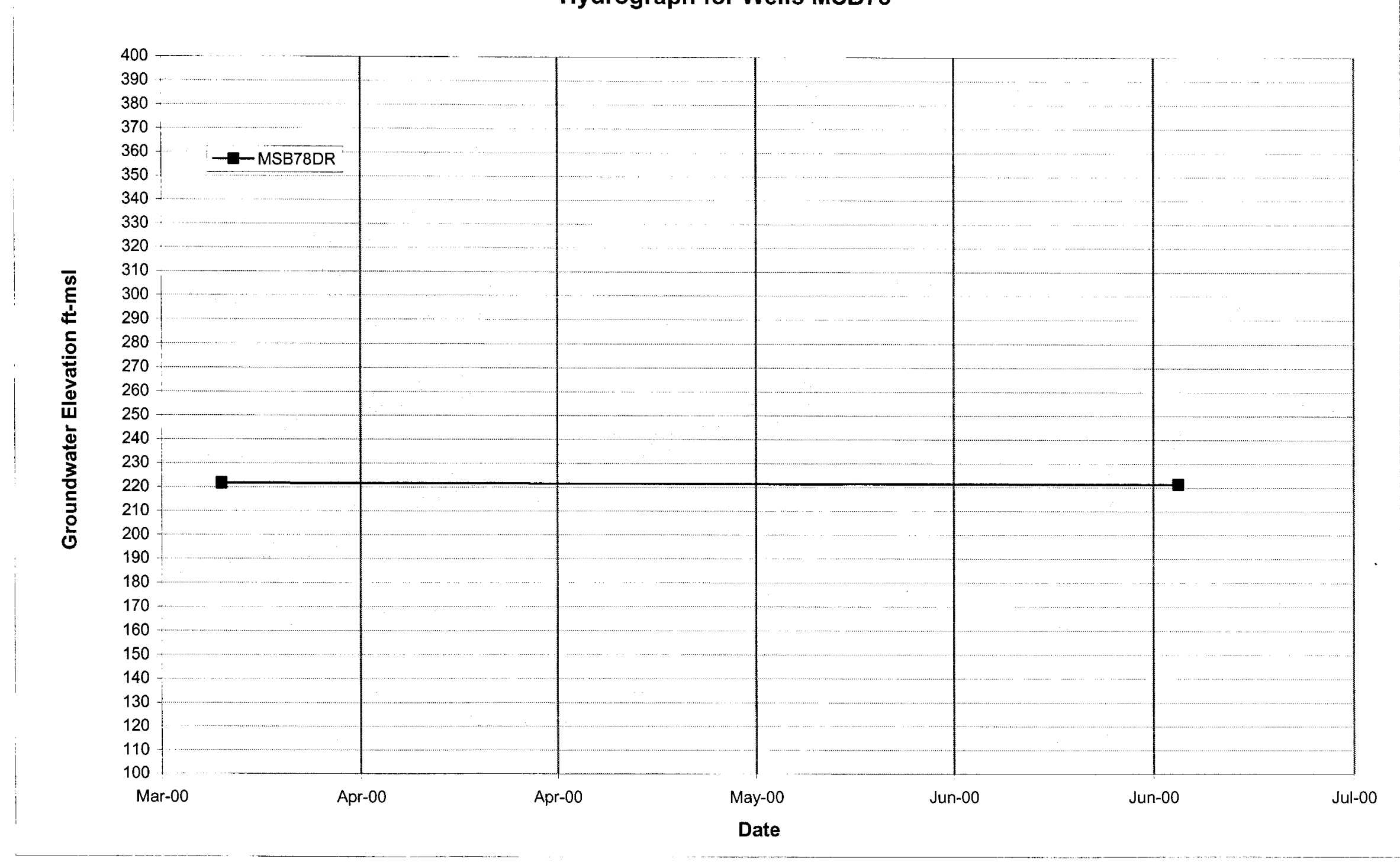


WSRC-TR-2000-00242 UNCLASSIFIED

Hydrograph for Wells MSB79

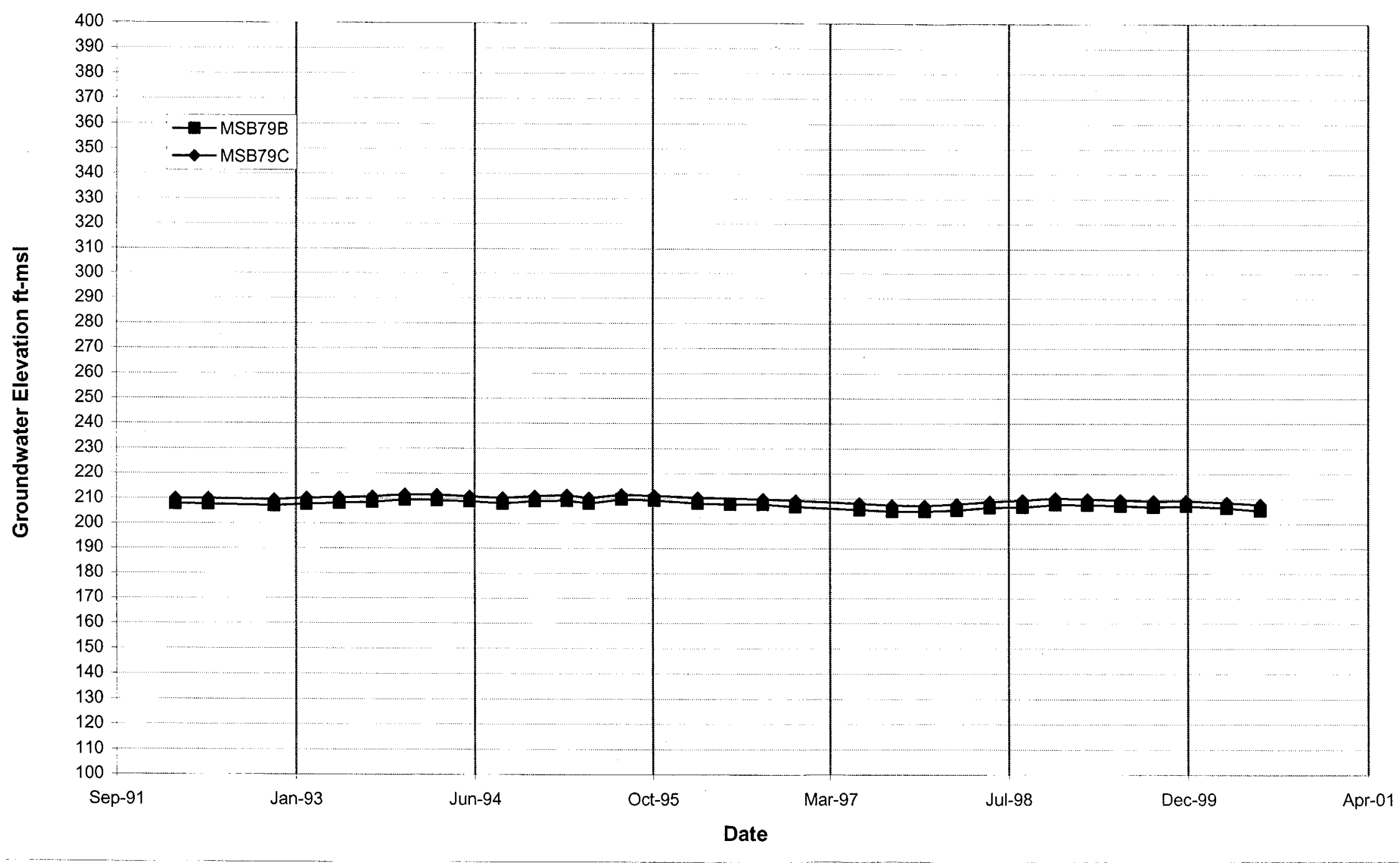




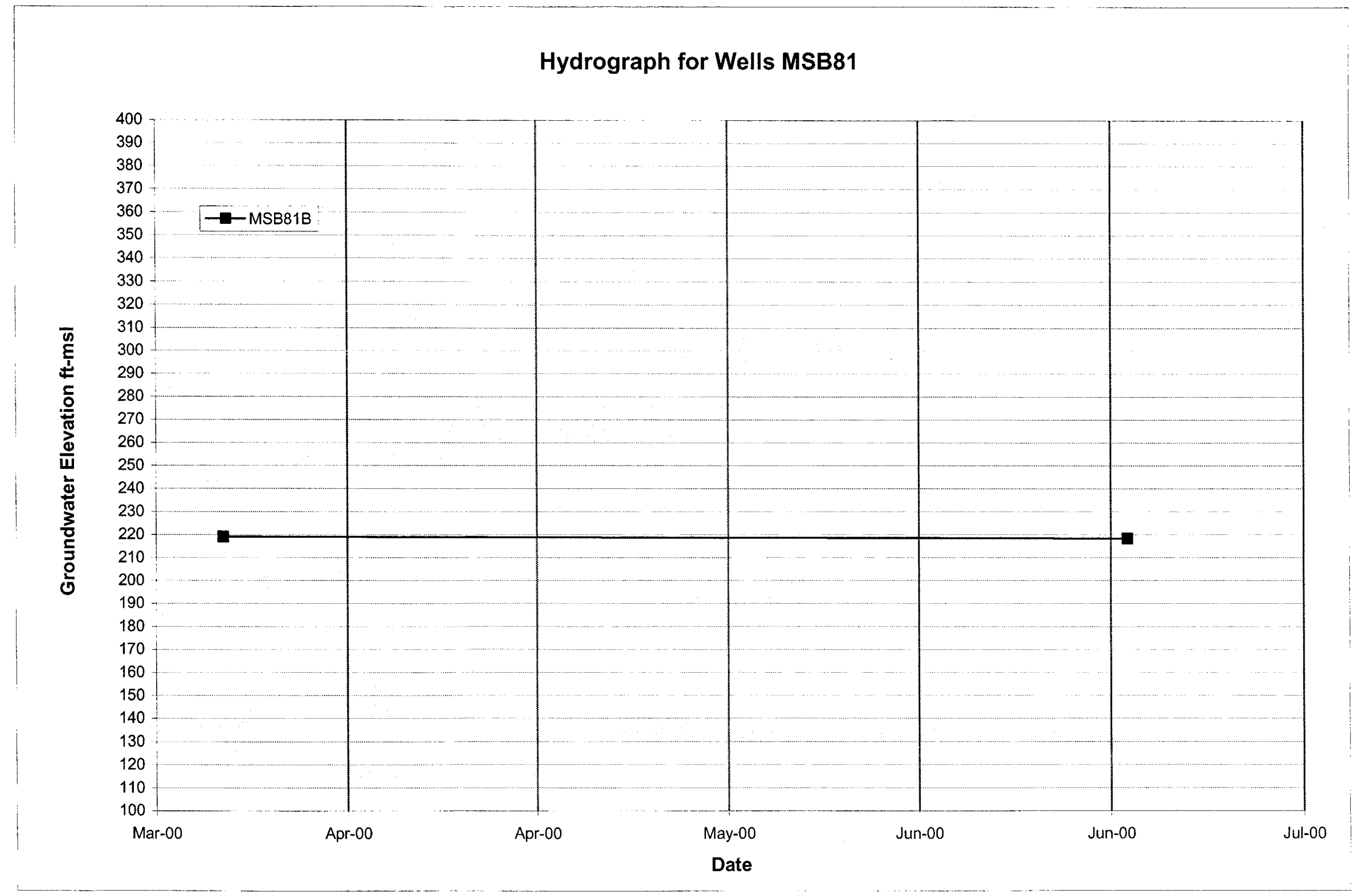


Hydrograph for Wells MSB82

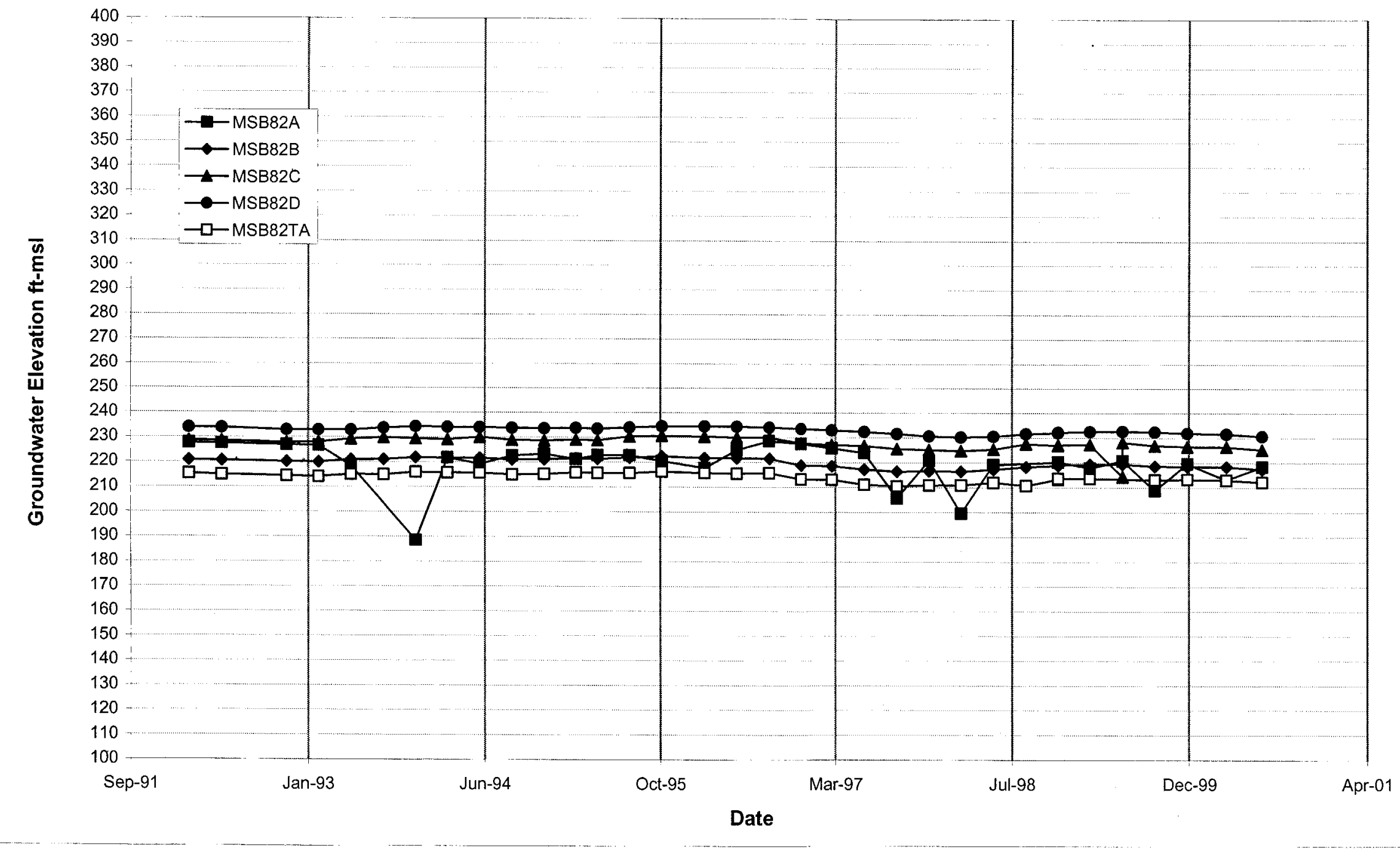


Hydrograph for Wells MSB83

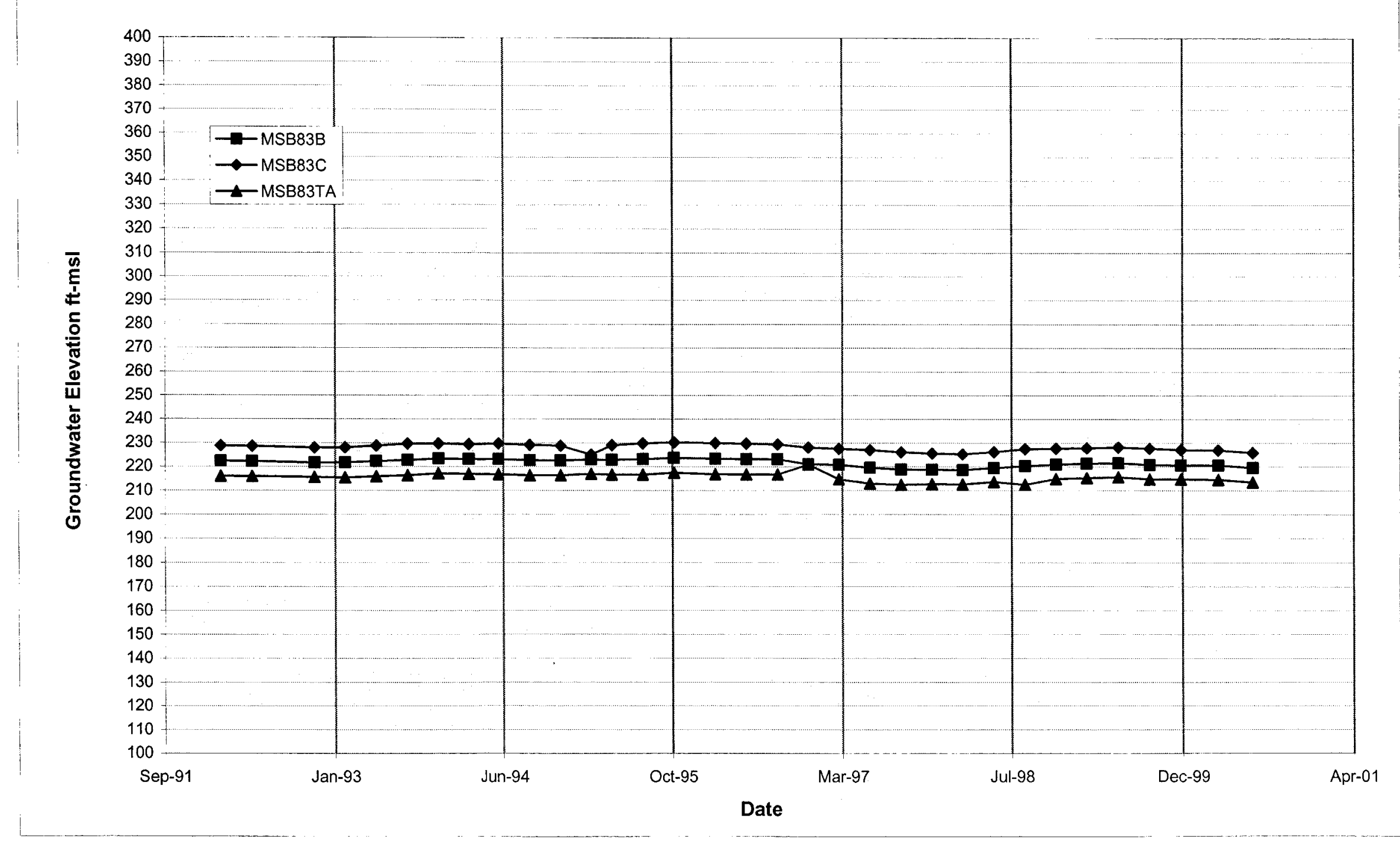




\section{Hydrograph for Wells MSB84}

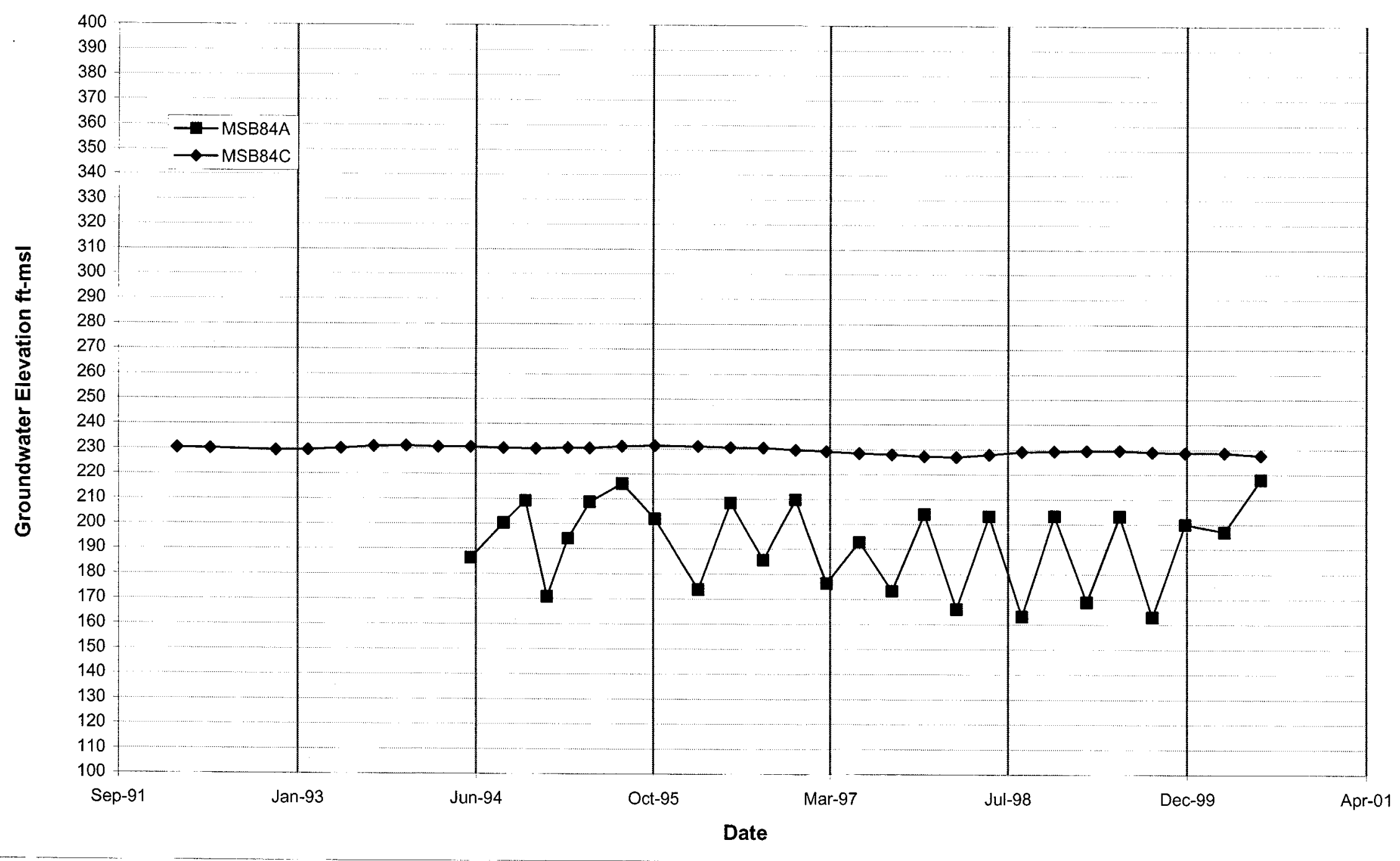




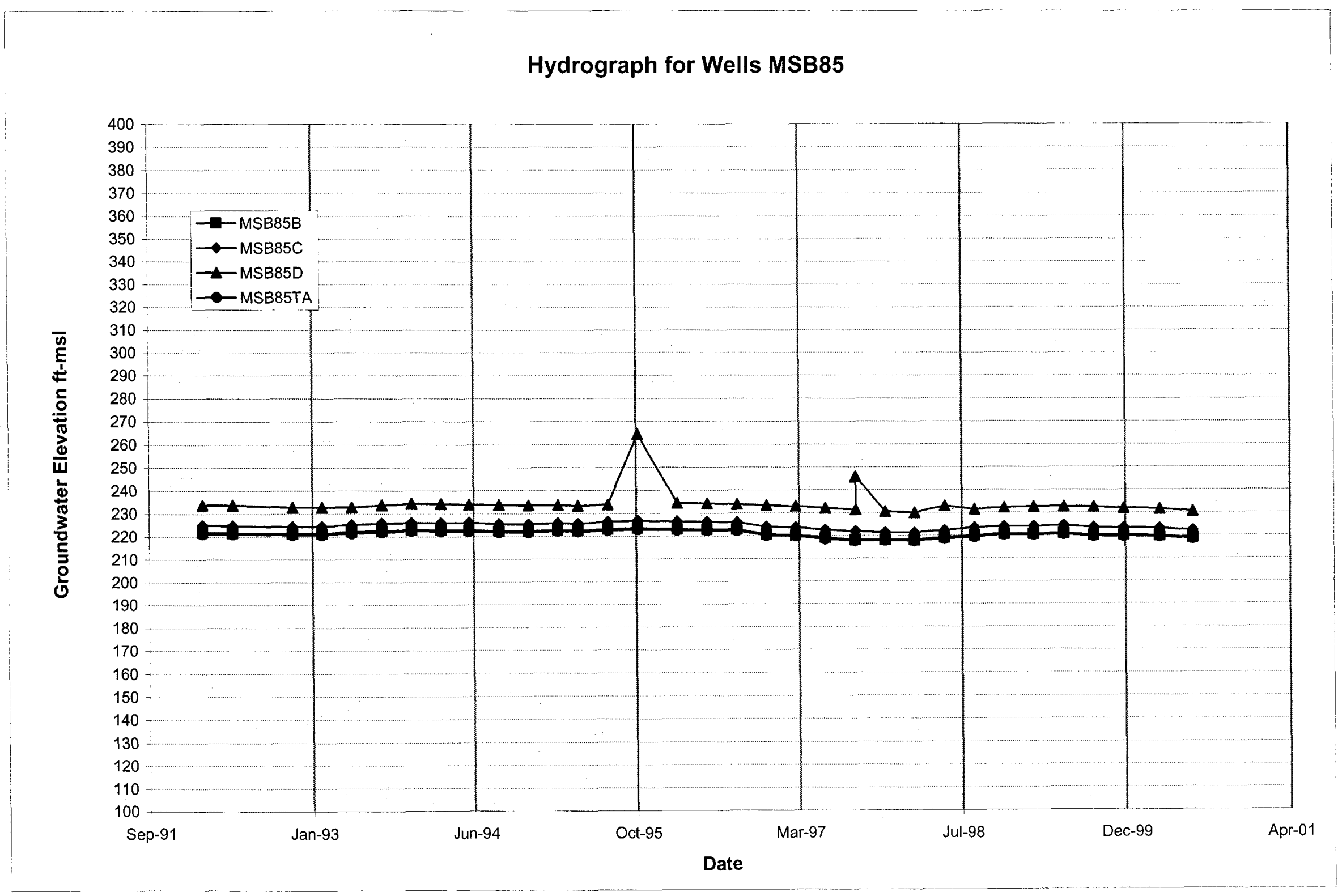


WSRC-TR-2000-00242 UNCLASSIFIED

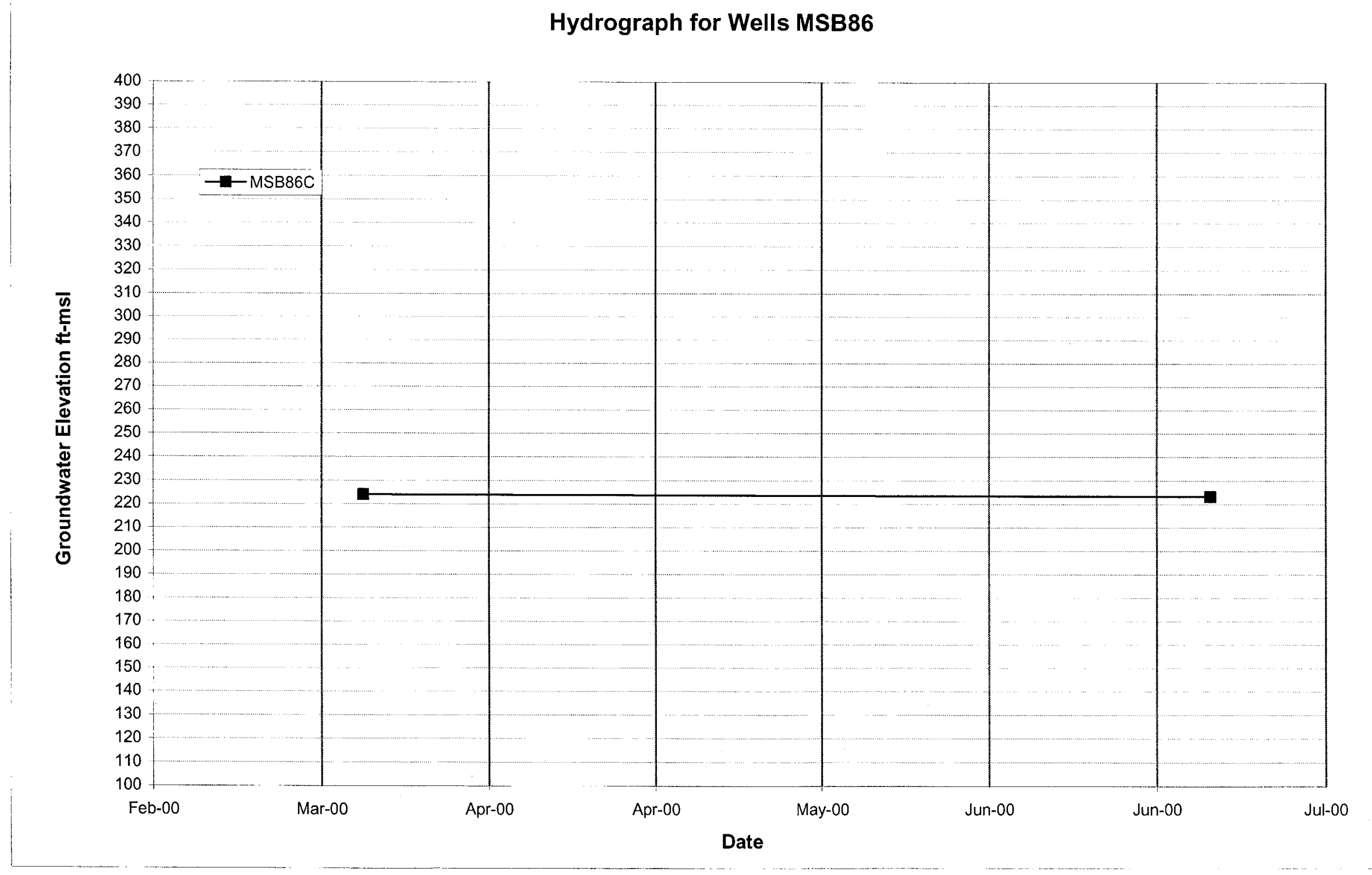




\section{Hydrograph for Wells MSB87}

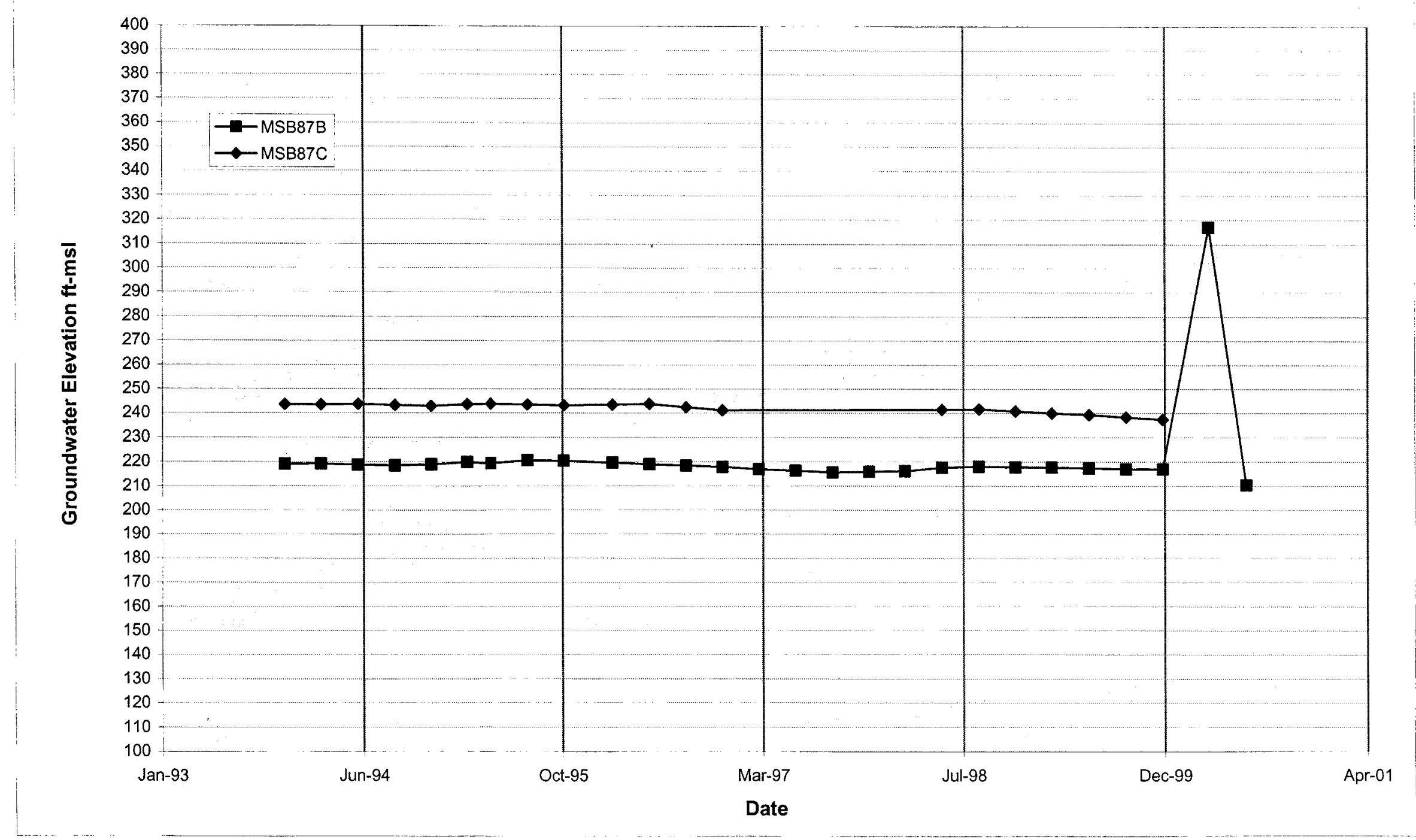


WSRC-TR-2000-00242

UNCLASSIFIED

Hydrograph for Wells MSB88

|5

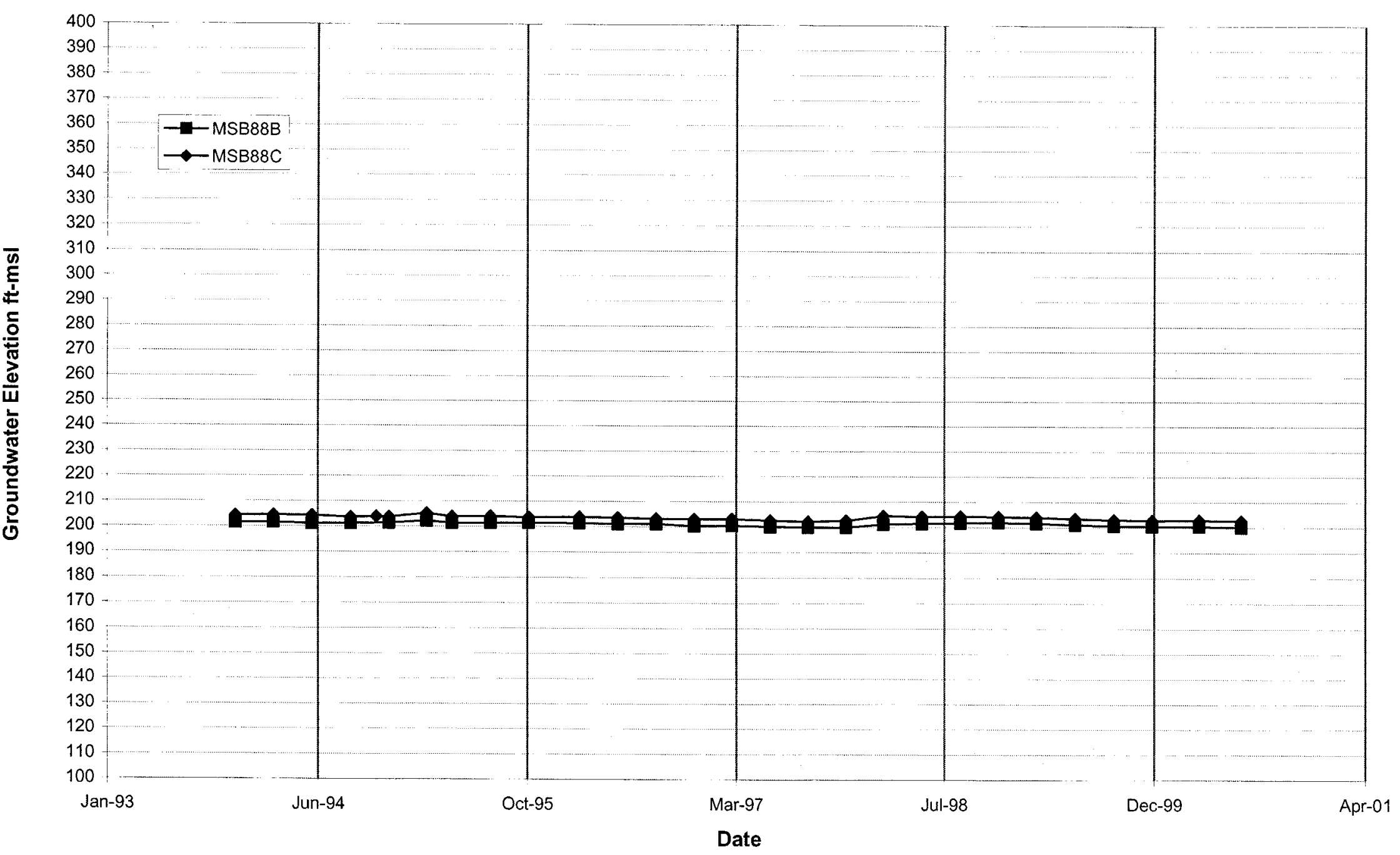

M-Area and Met Lab HWMFs

G-130

First and Second Quarter 2000 
WSRC-TR-2000-00242 UNCLASSIFIED

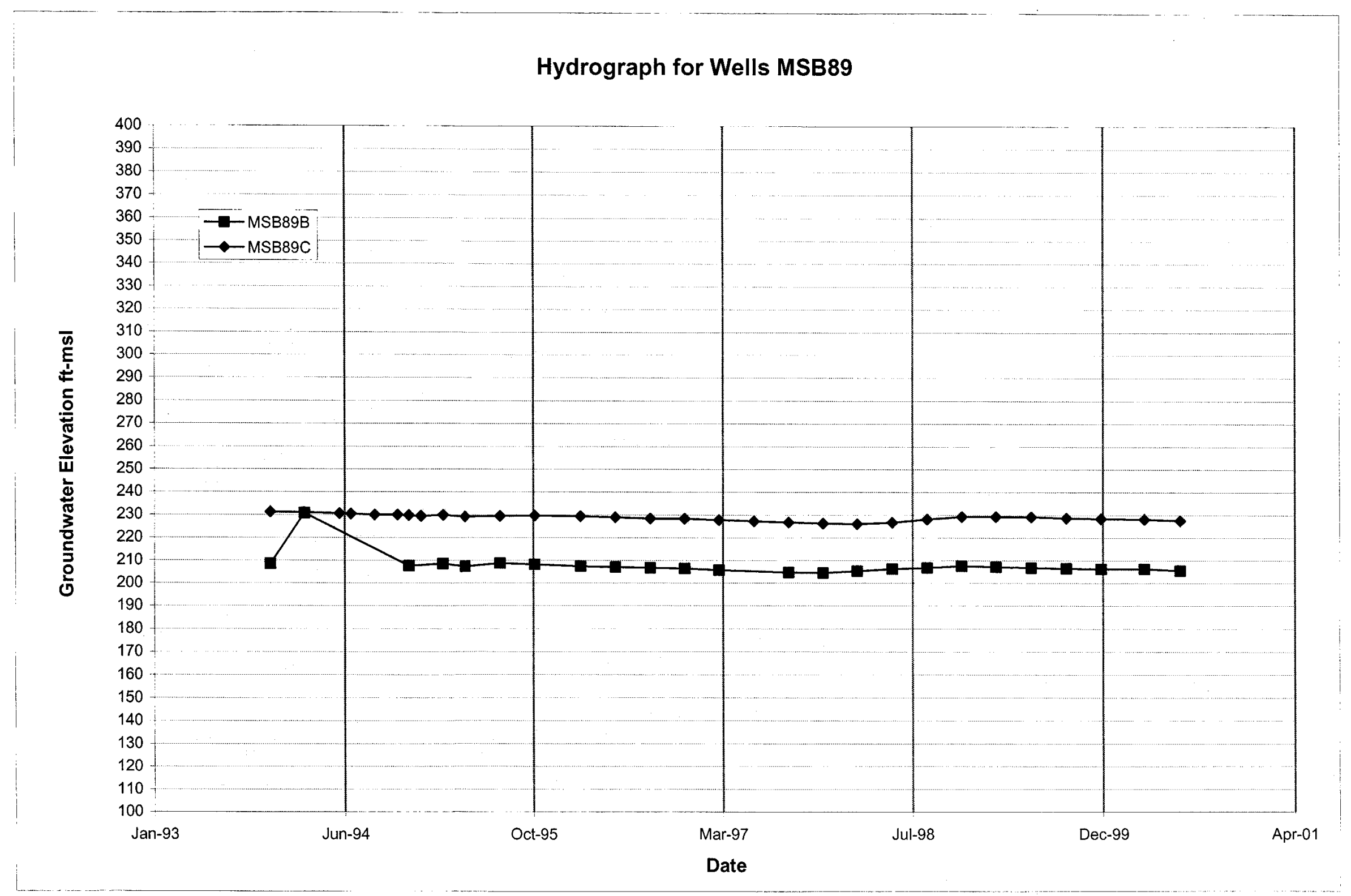


WSRC-TR-2000-00242 UNCLASSIFIED

\section{Hydrograph for Wells RWM13}

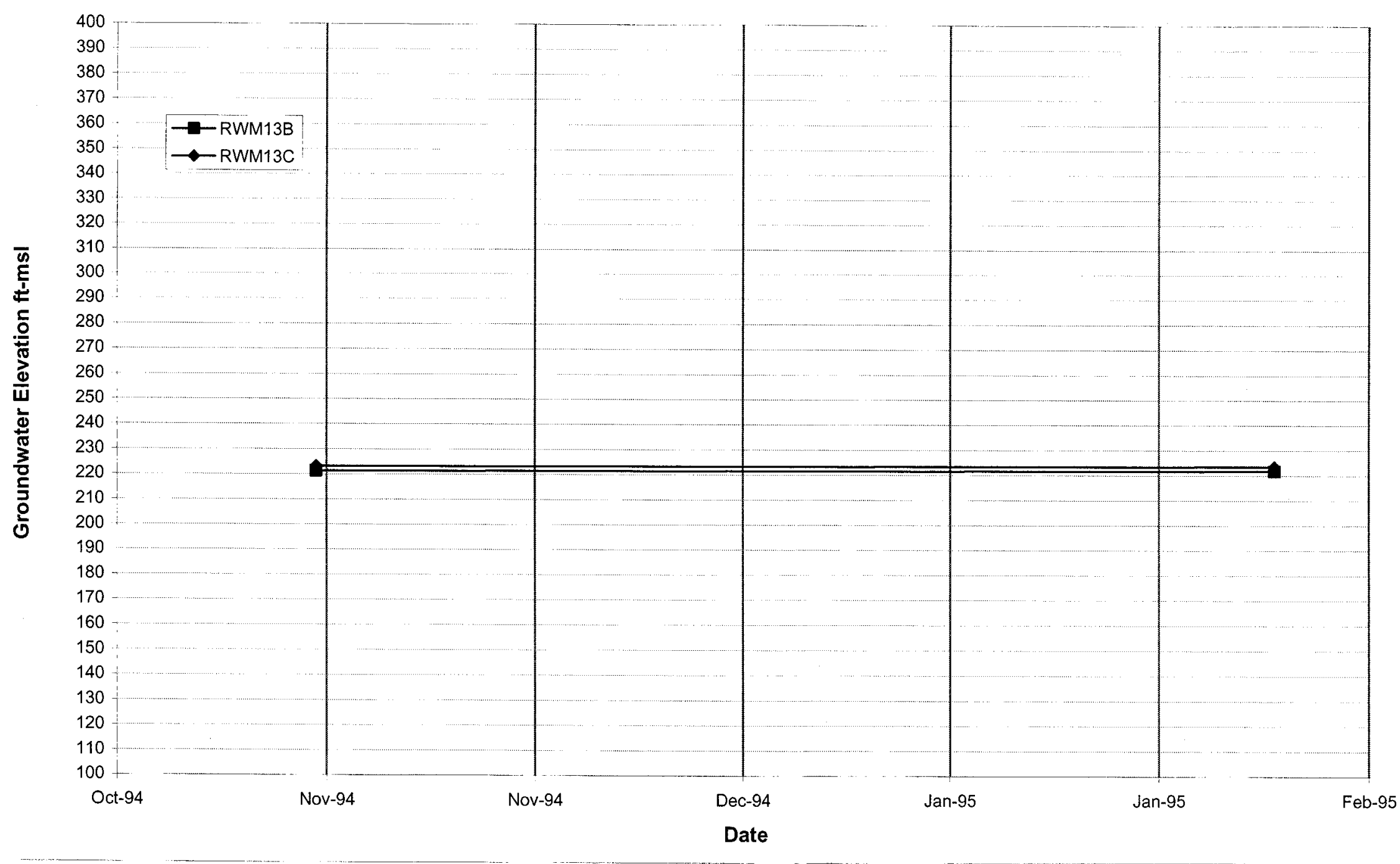




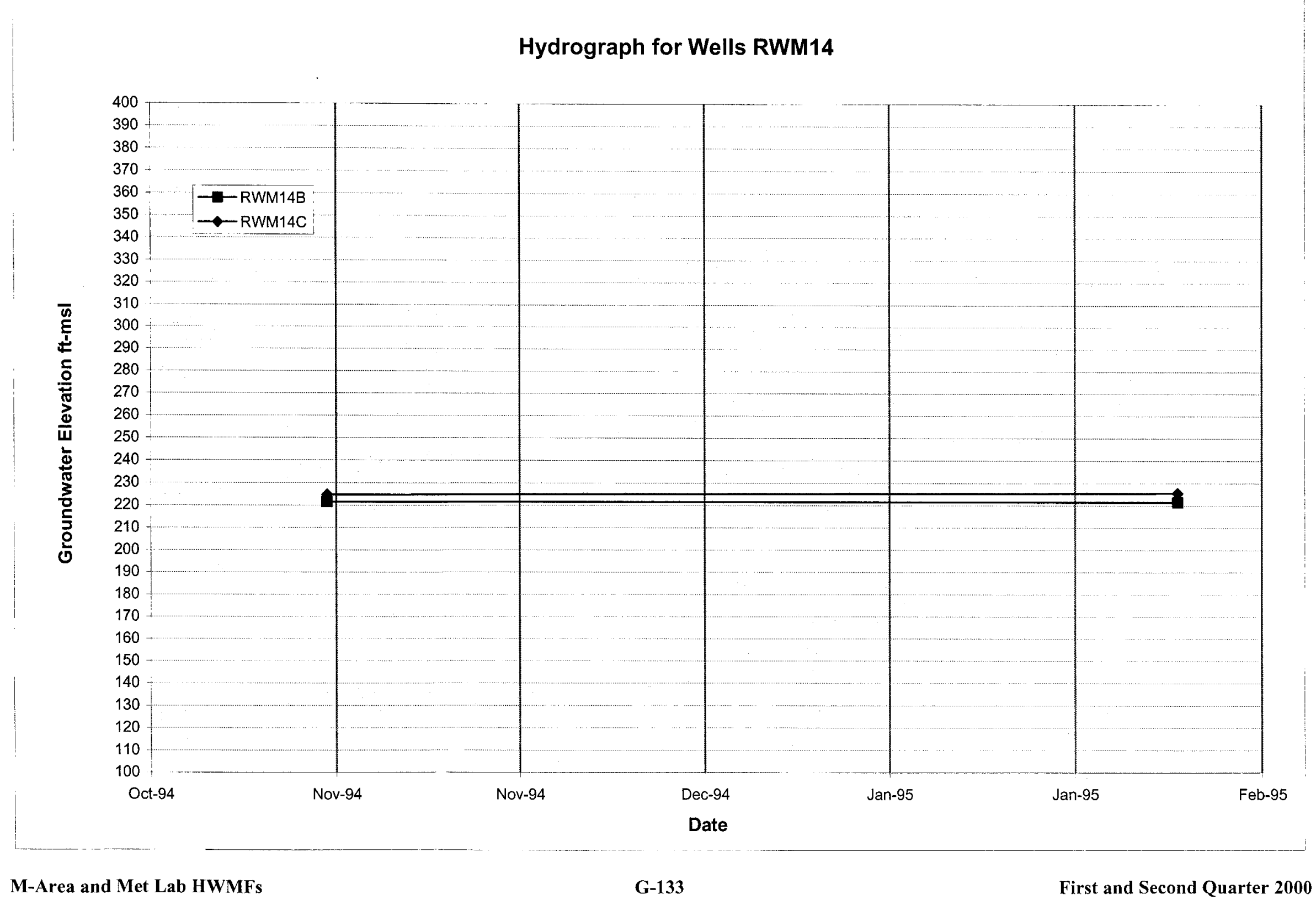


WSRC-TR-2000-00242

UNCLASSIFIED

Hydrograph for Wells RWM15

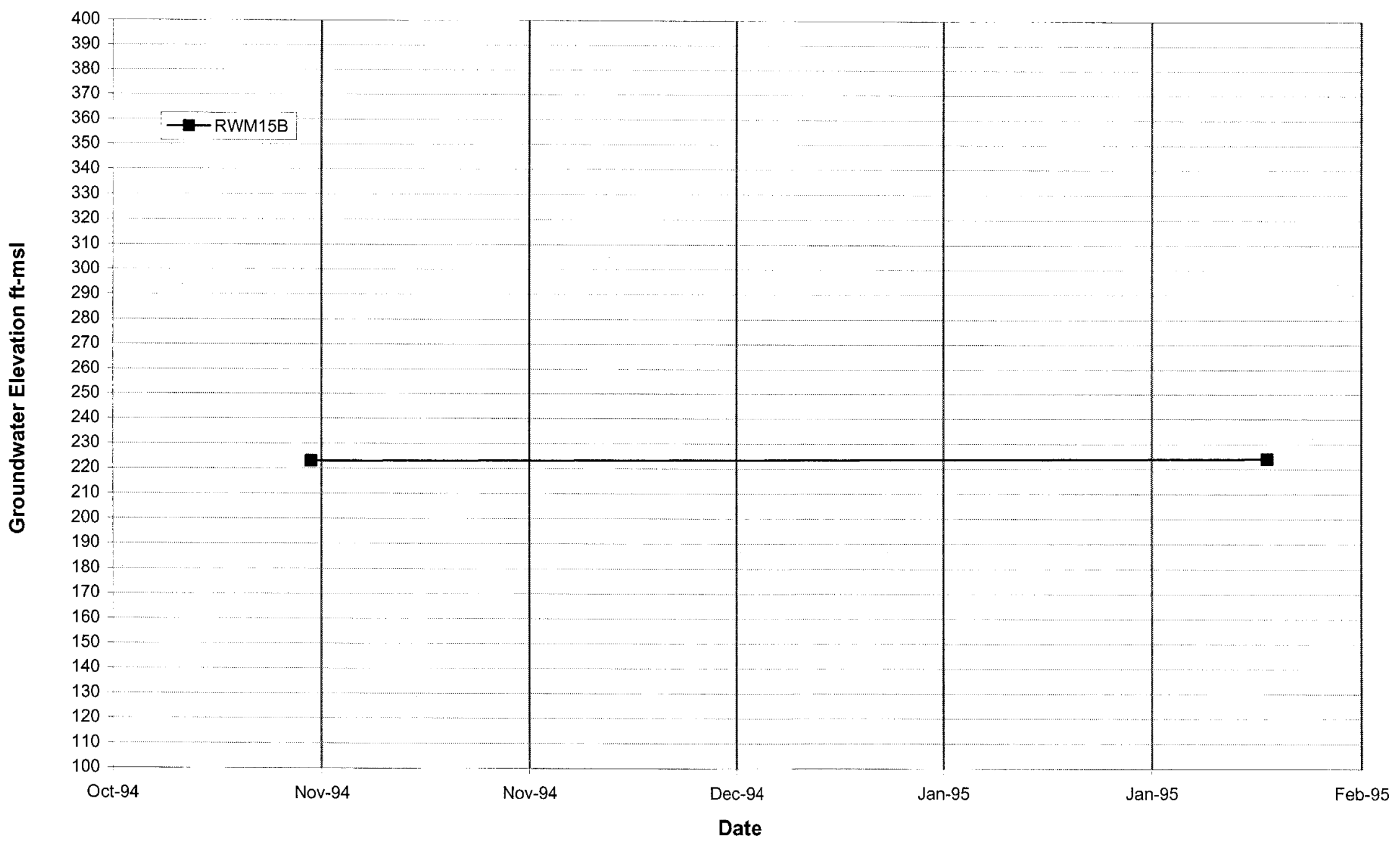

M-Area and Met Lab HWMFs

G-134

First and Second Quarter 2000 
WSRC-TR-2000-00242 UNCLASSIFIED

Hydrograph for Wells SRW 1

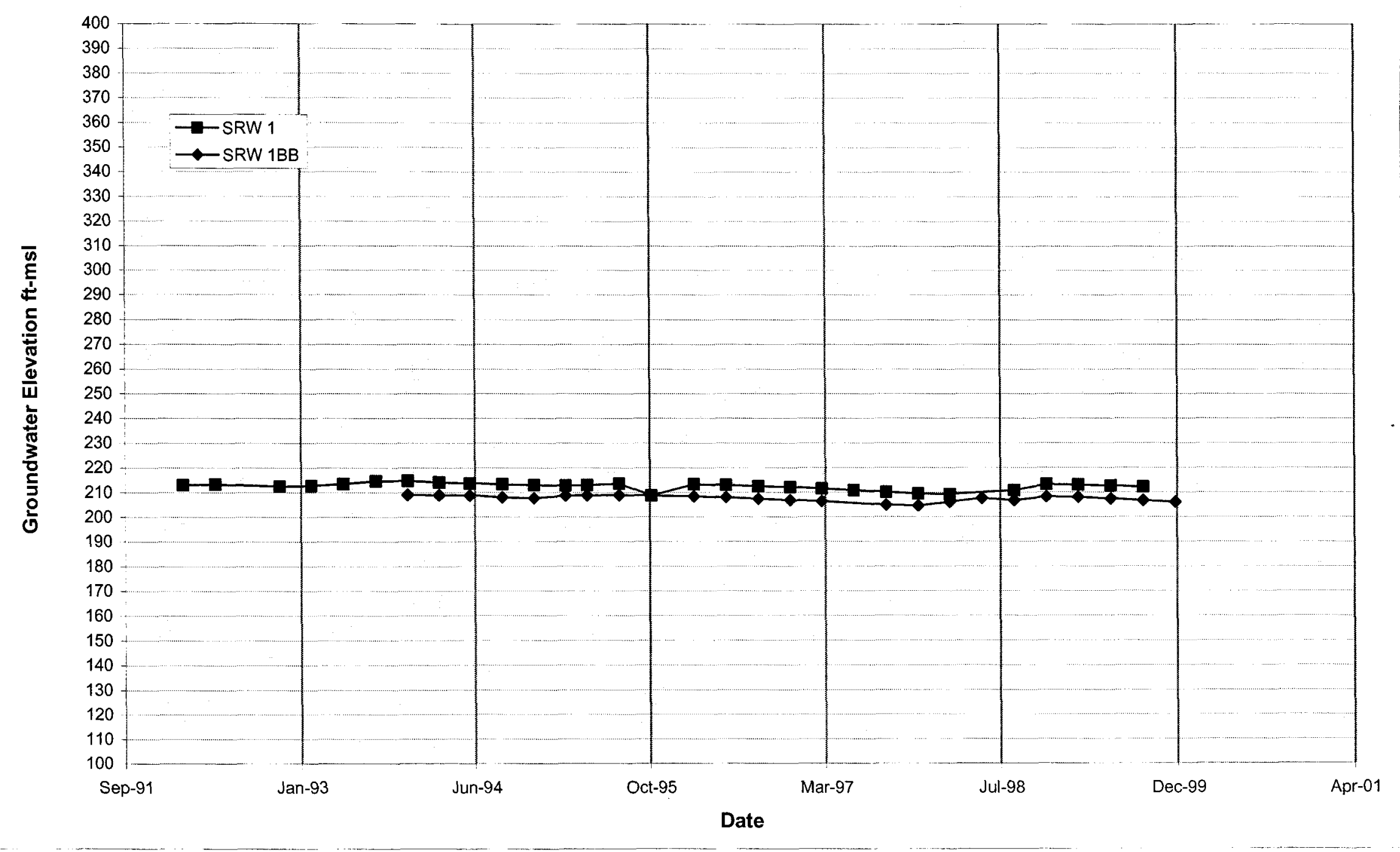


WSRC-TR-2000-00242 UNCLASSIFIED

\section{Hydrograph for Wells SRW 2}

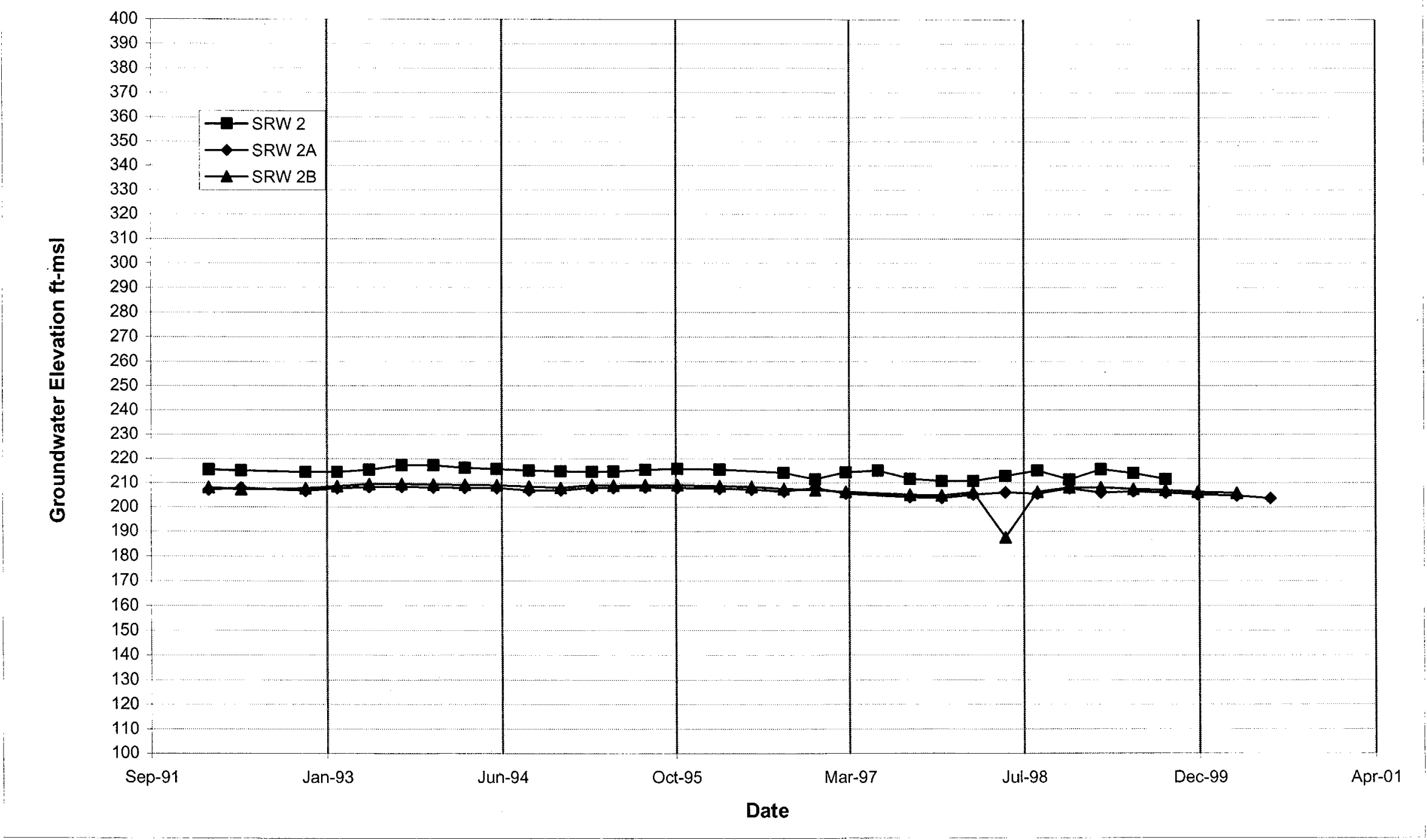




\section{Hydrograph for Wells SRW 3}

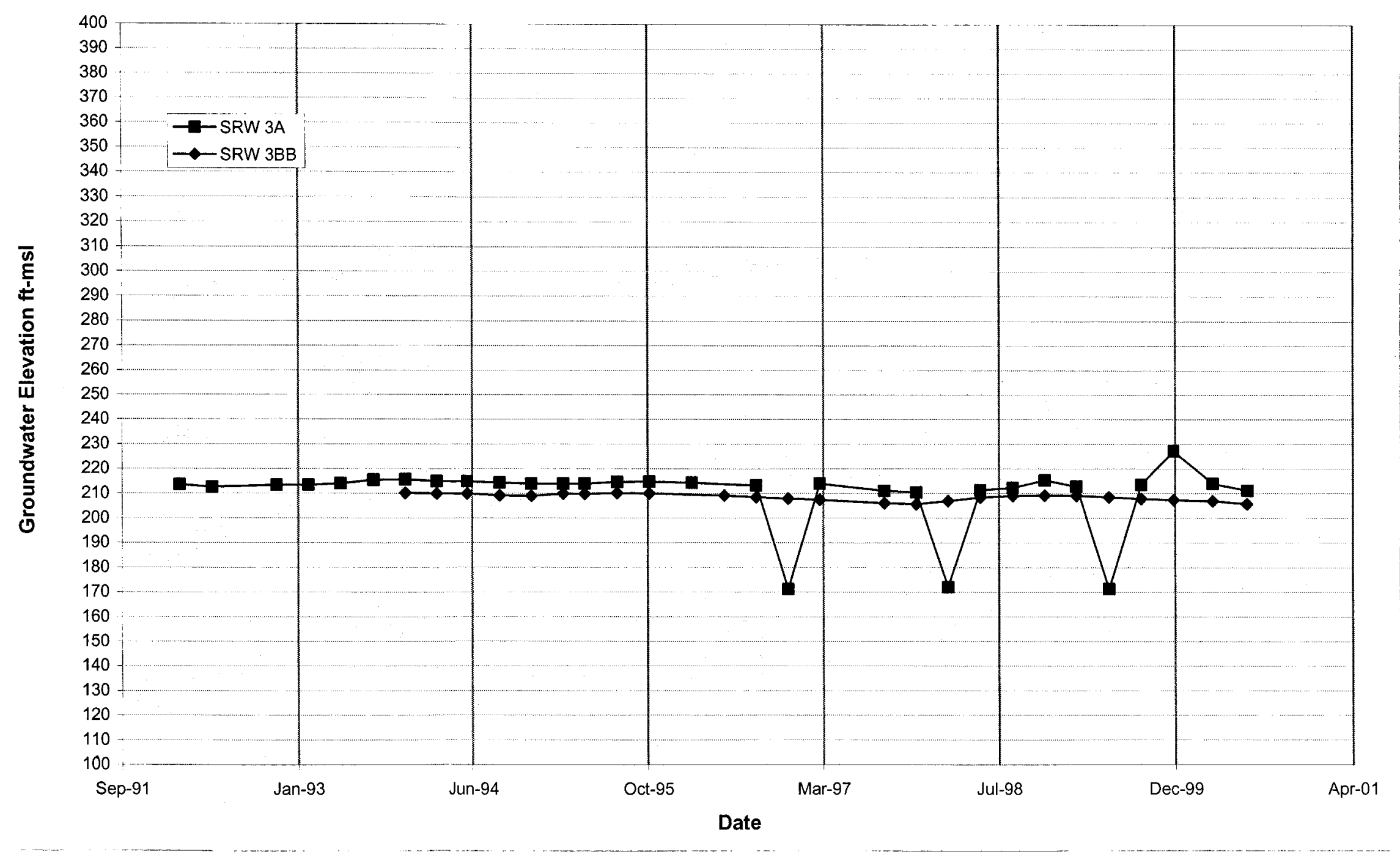


WSRC-TR-2000-00242 UNCLASSIFIED

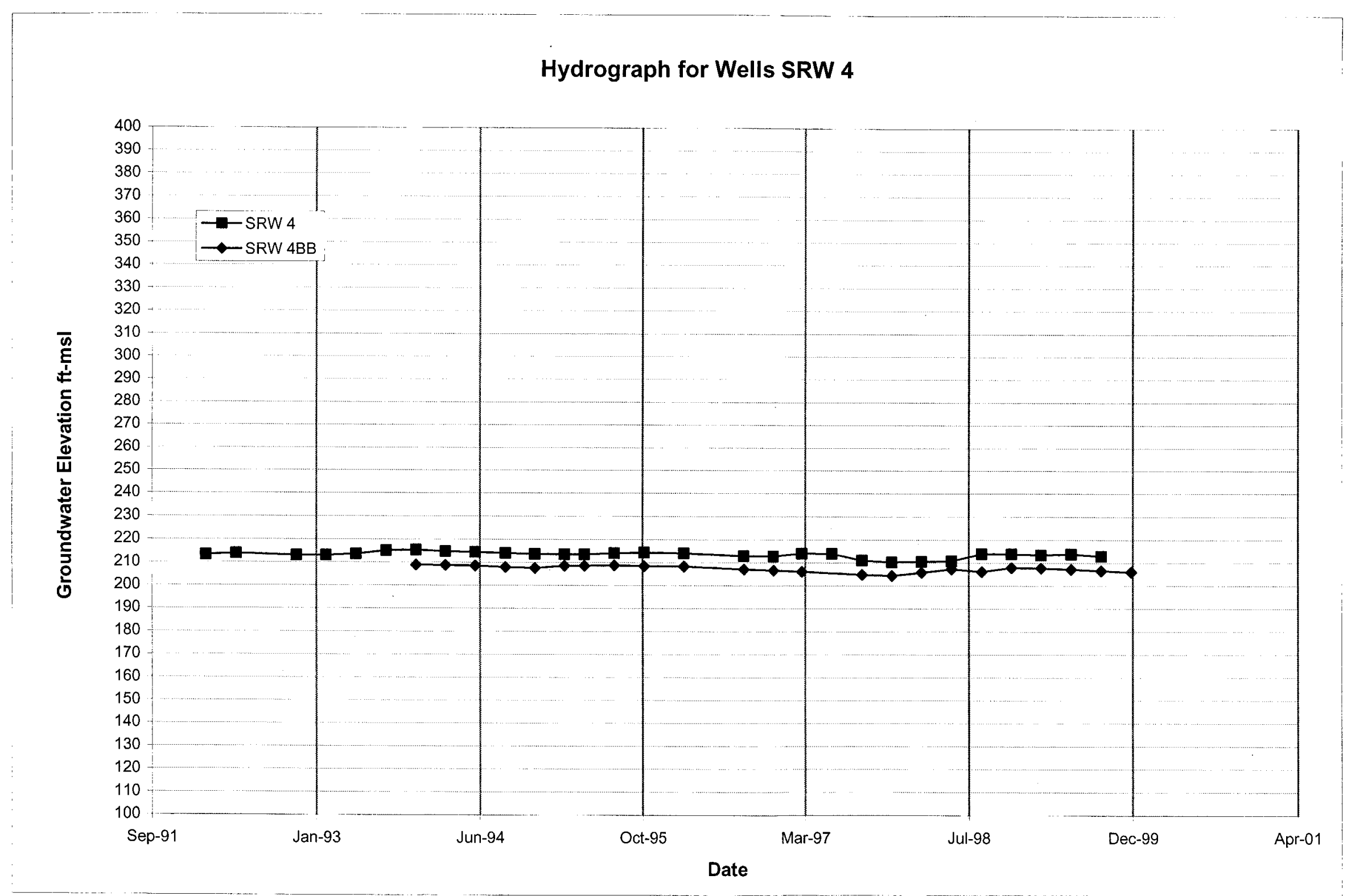


WSRC-TR-2000-00242 UNCLASSIFIED

\section{Hydrograph for Wells SRW 8}

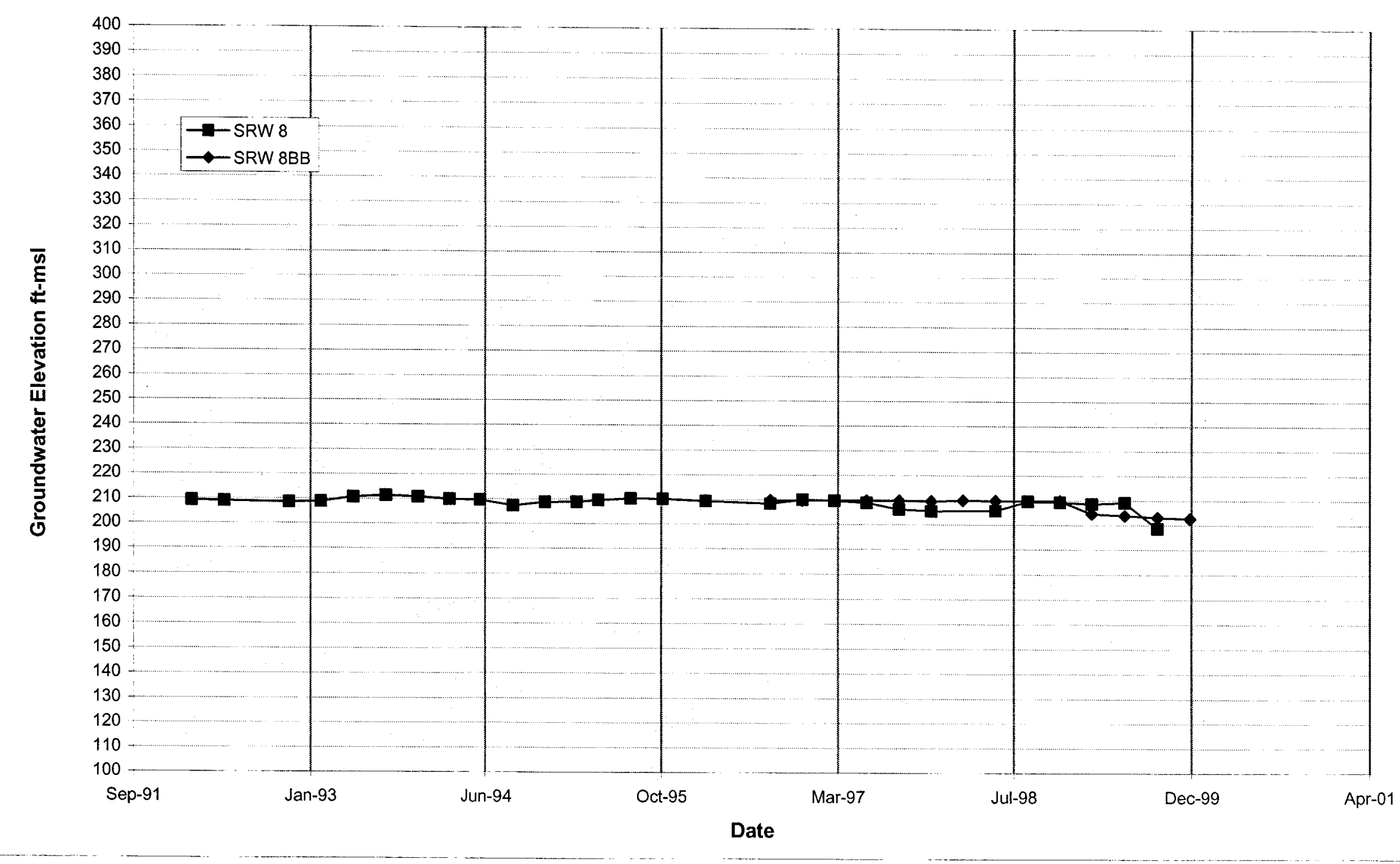


WSRC-TR-2000-00242 UNCLASSIFIED

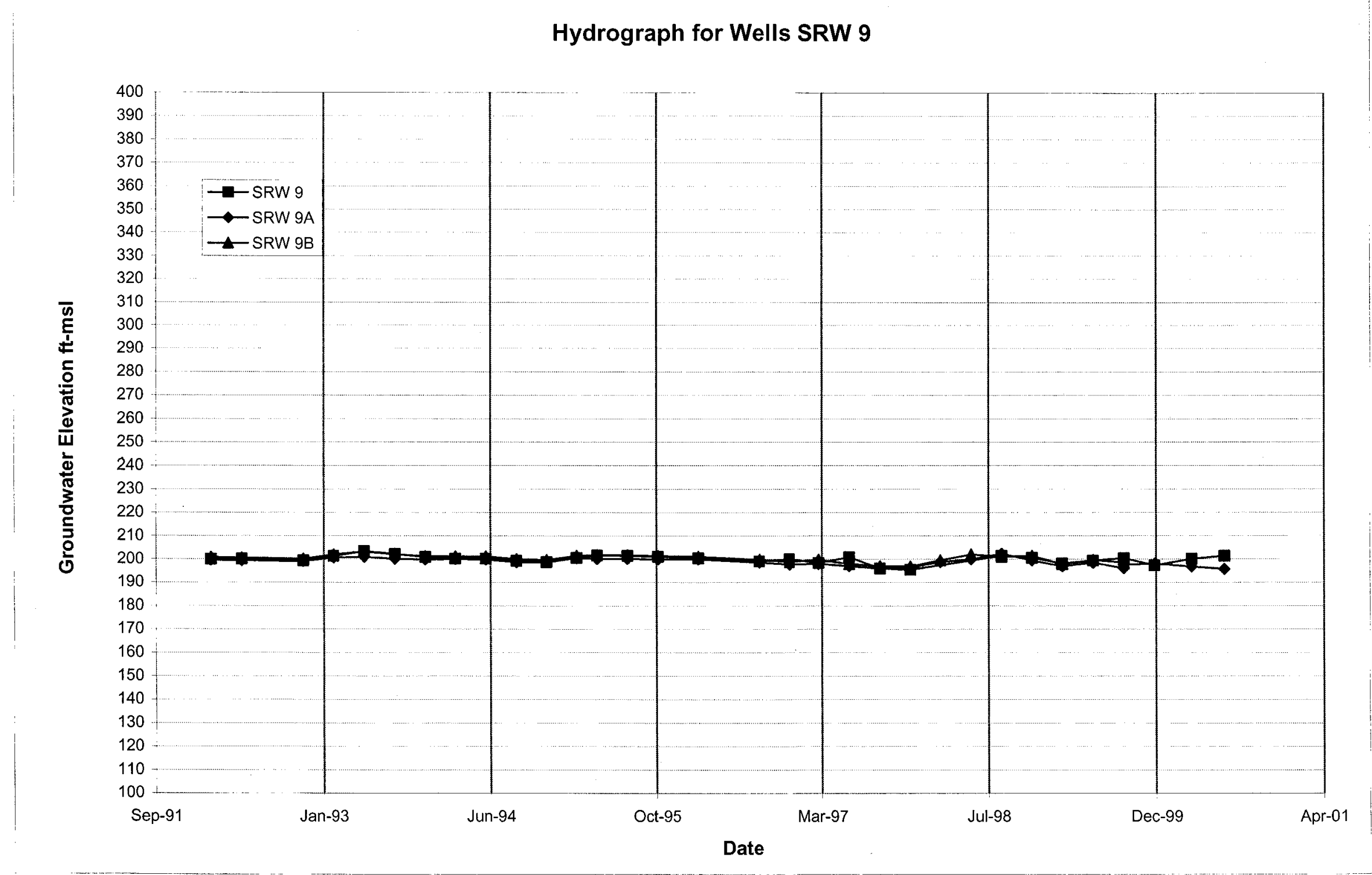




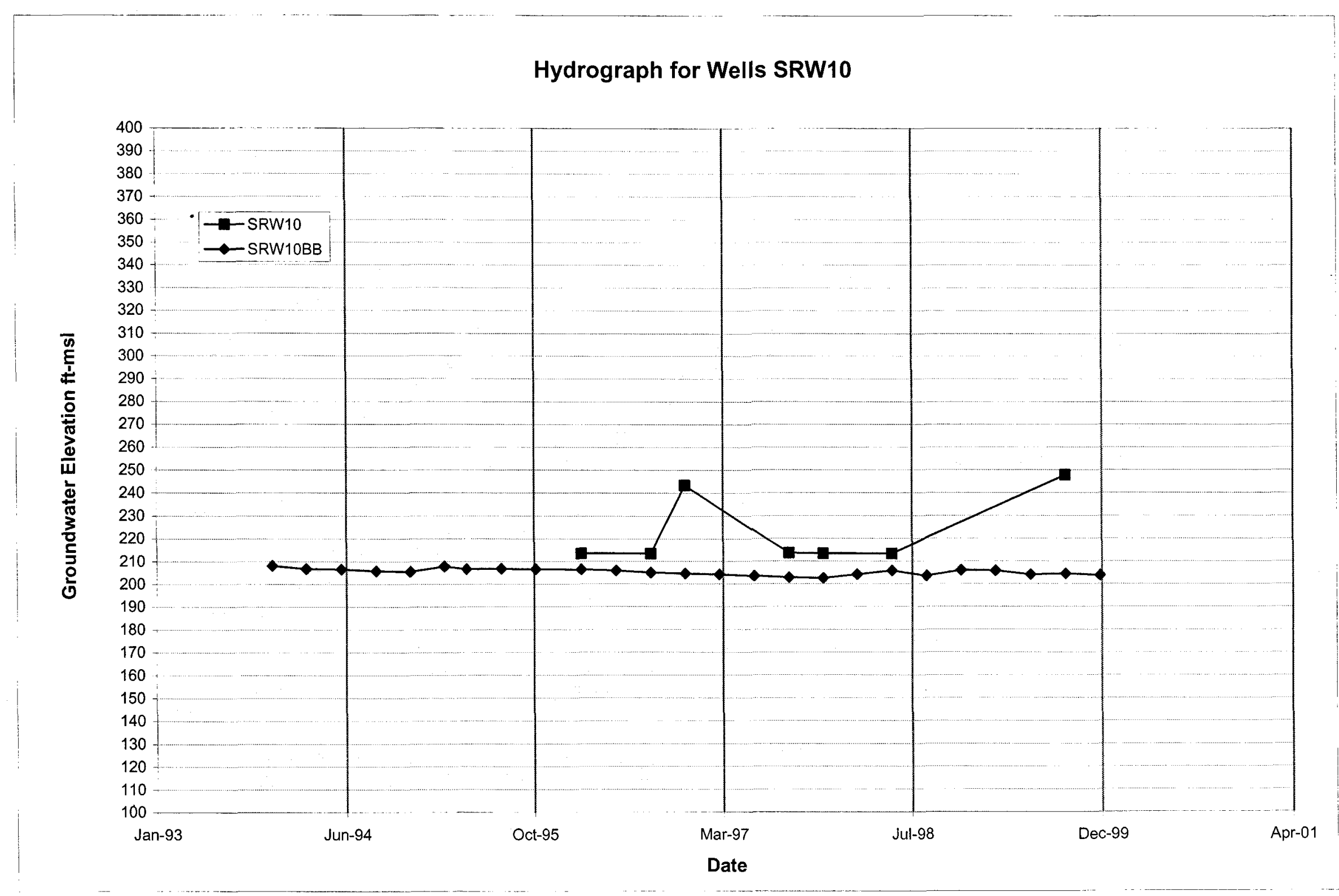


WSRC-TR-2000-00242 UNCLASSIFIED

Hydrograph for Wells SRW11

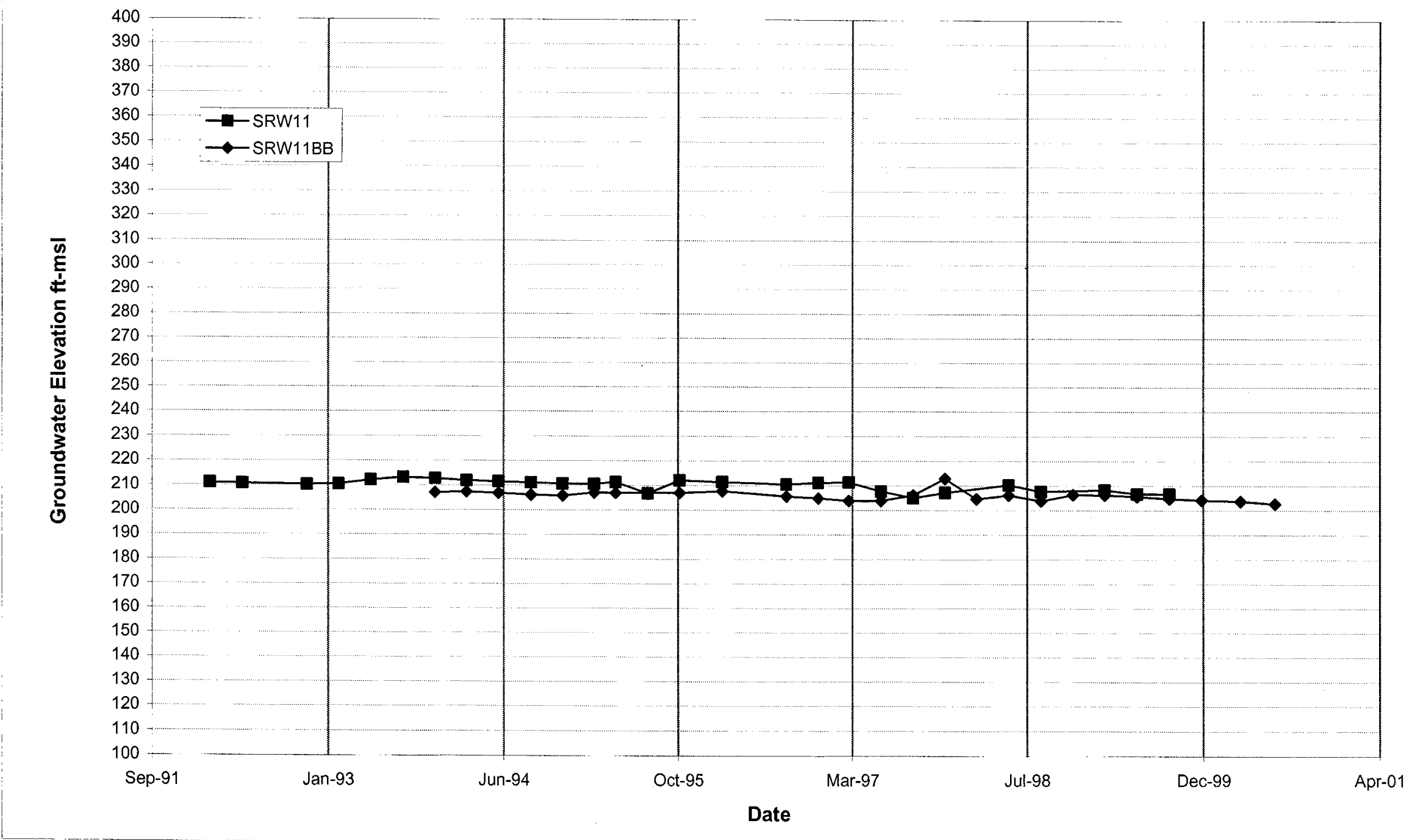




\section{Hydrograph for Wells SRW12}

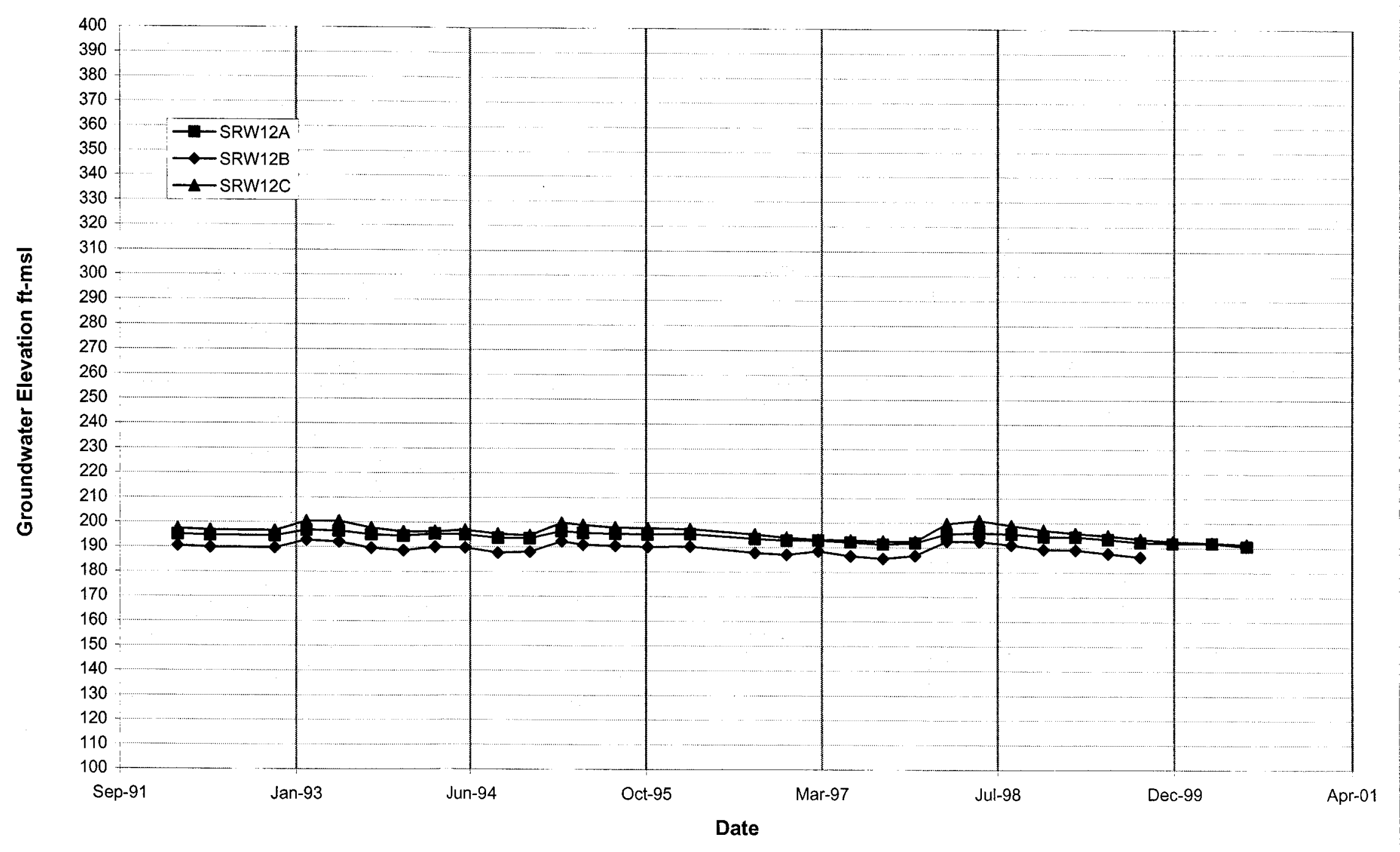


WSRC-TR-2000-00242 UNCLASSIFIED

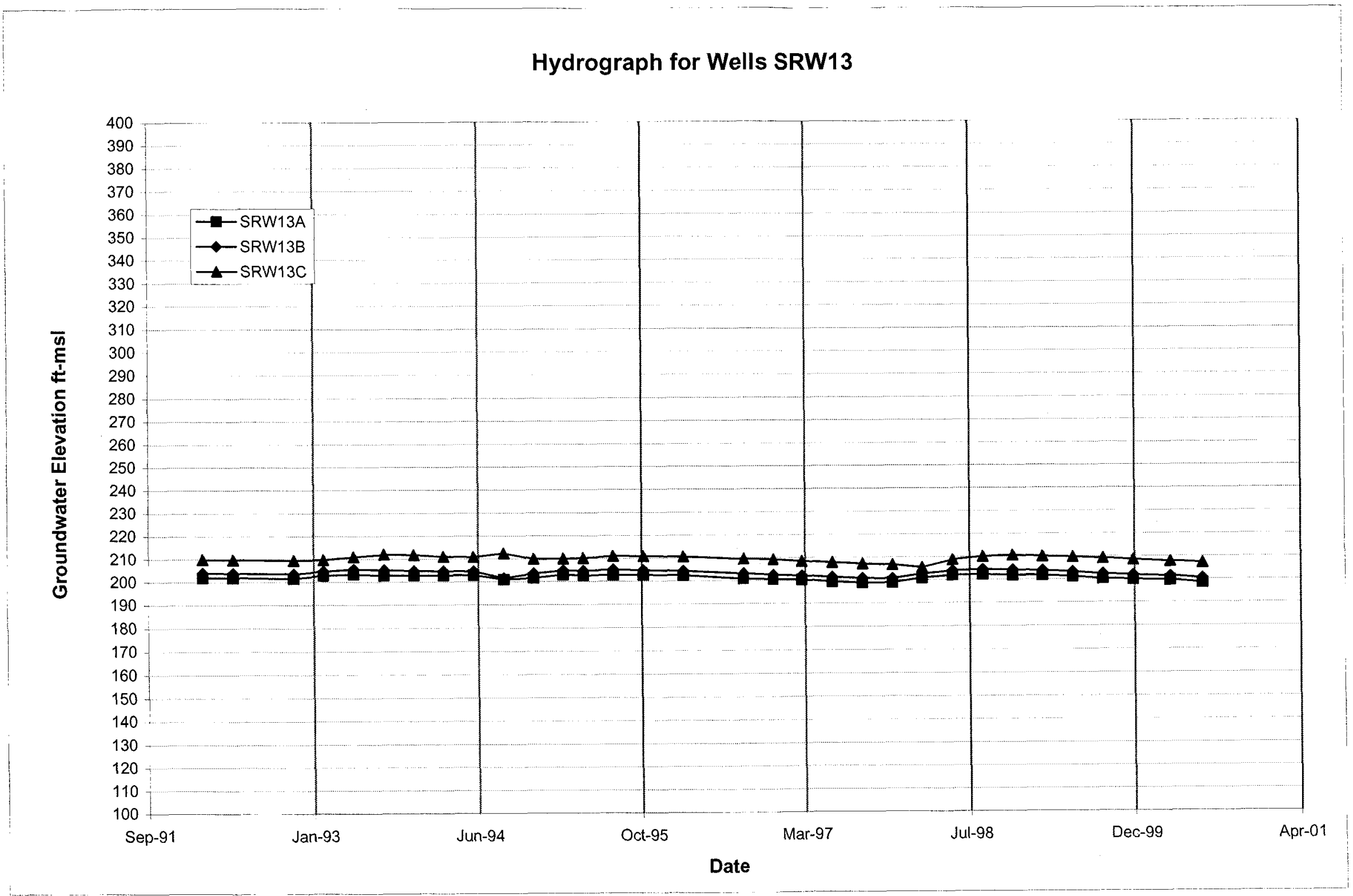




\section{Hydrograph for Wells SRW14}

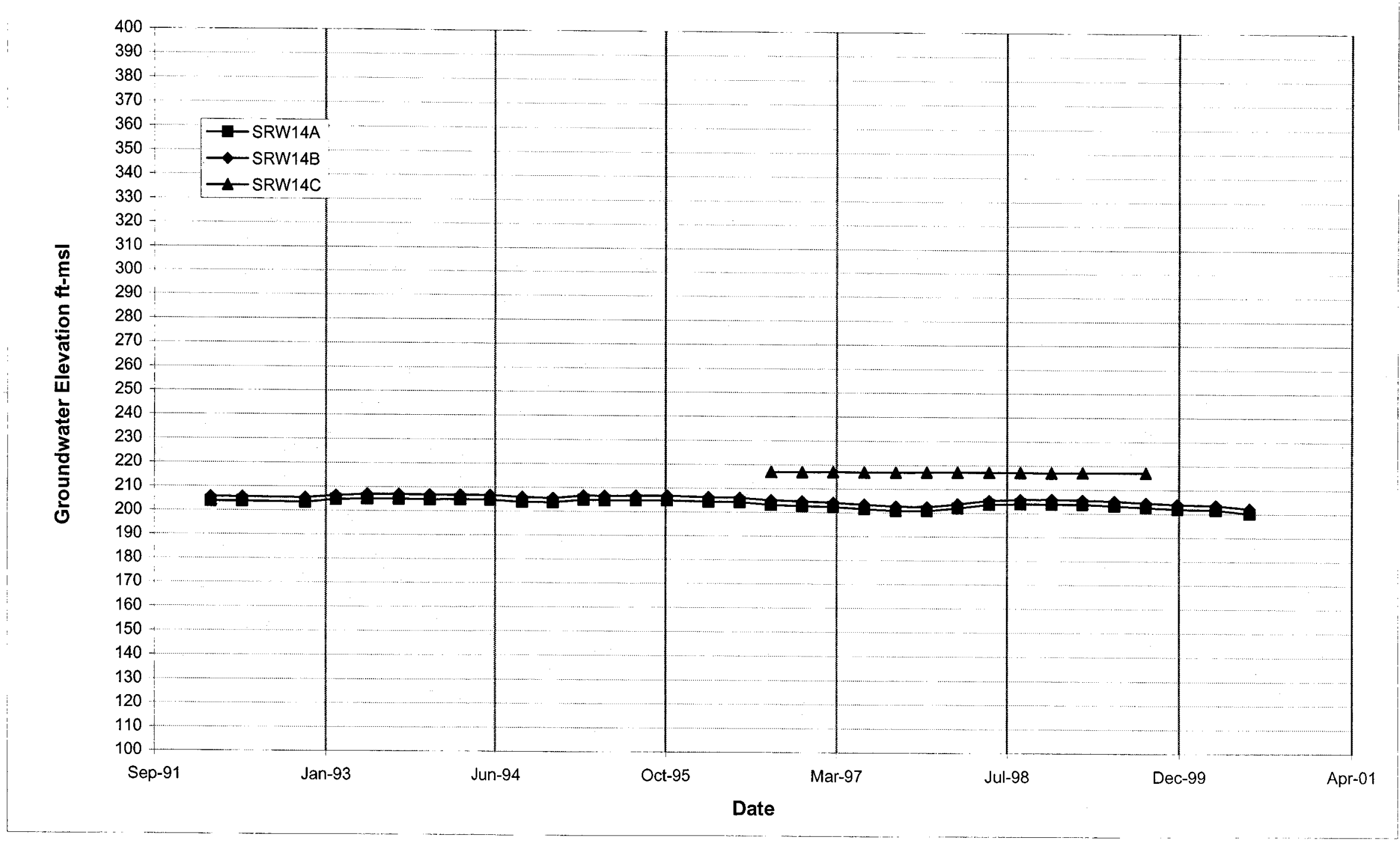




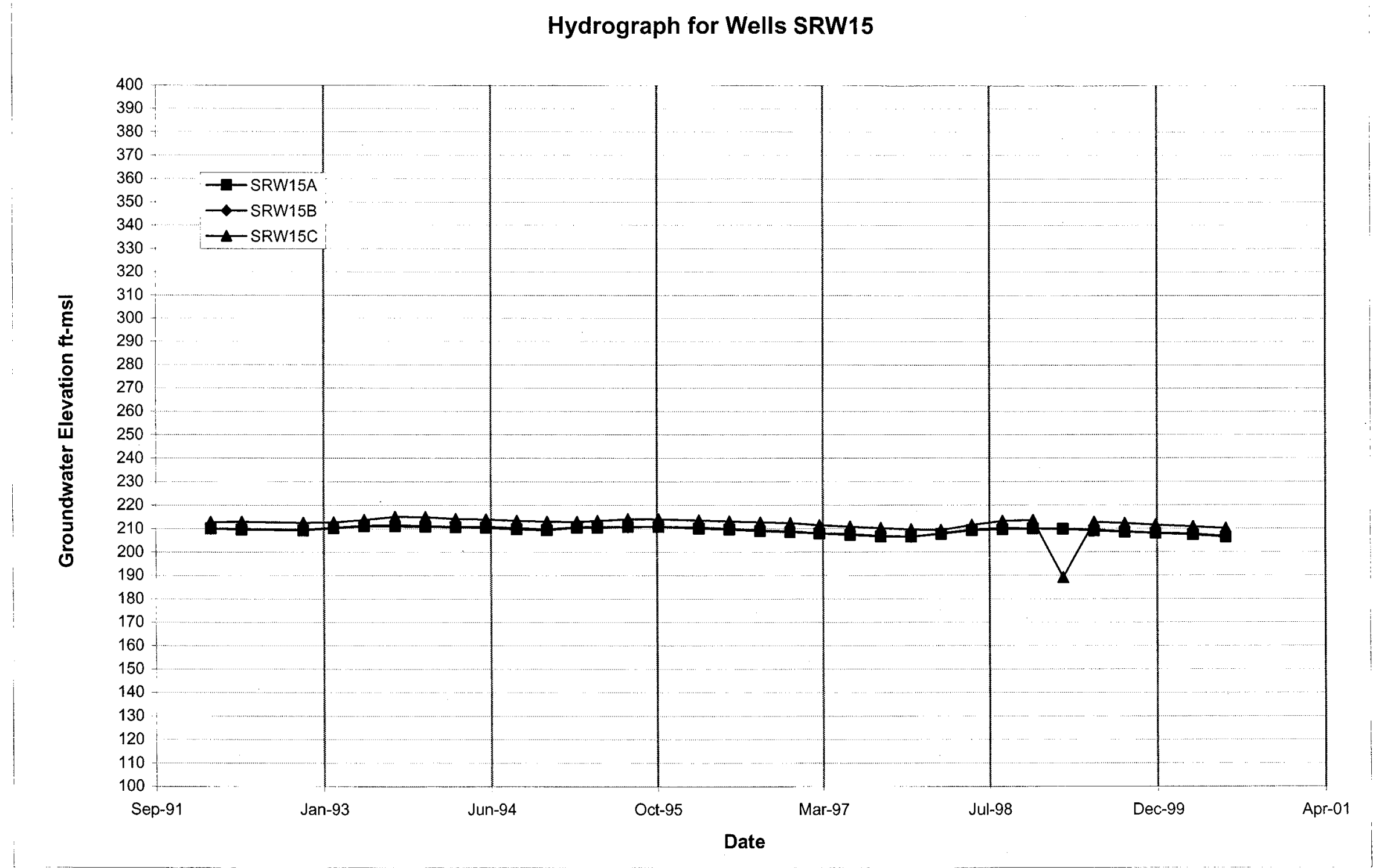




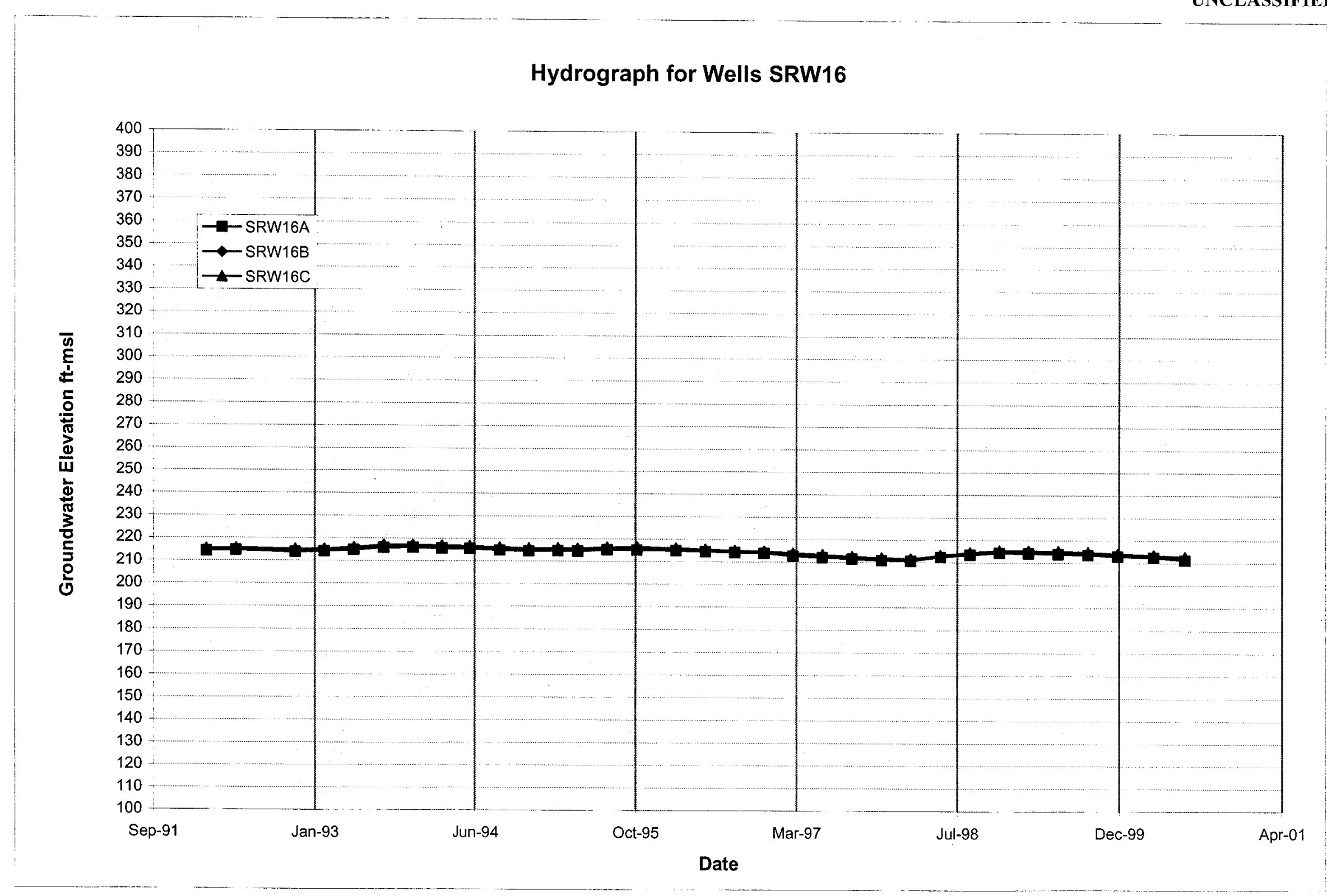


WSRC-TR-2000-00242 UNCLASSIFIED

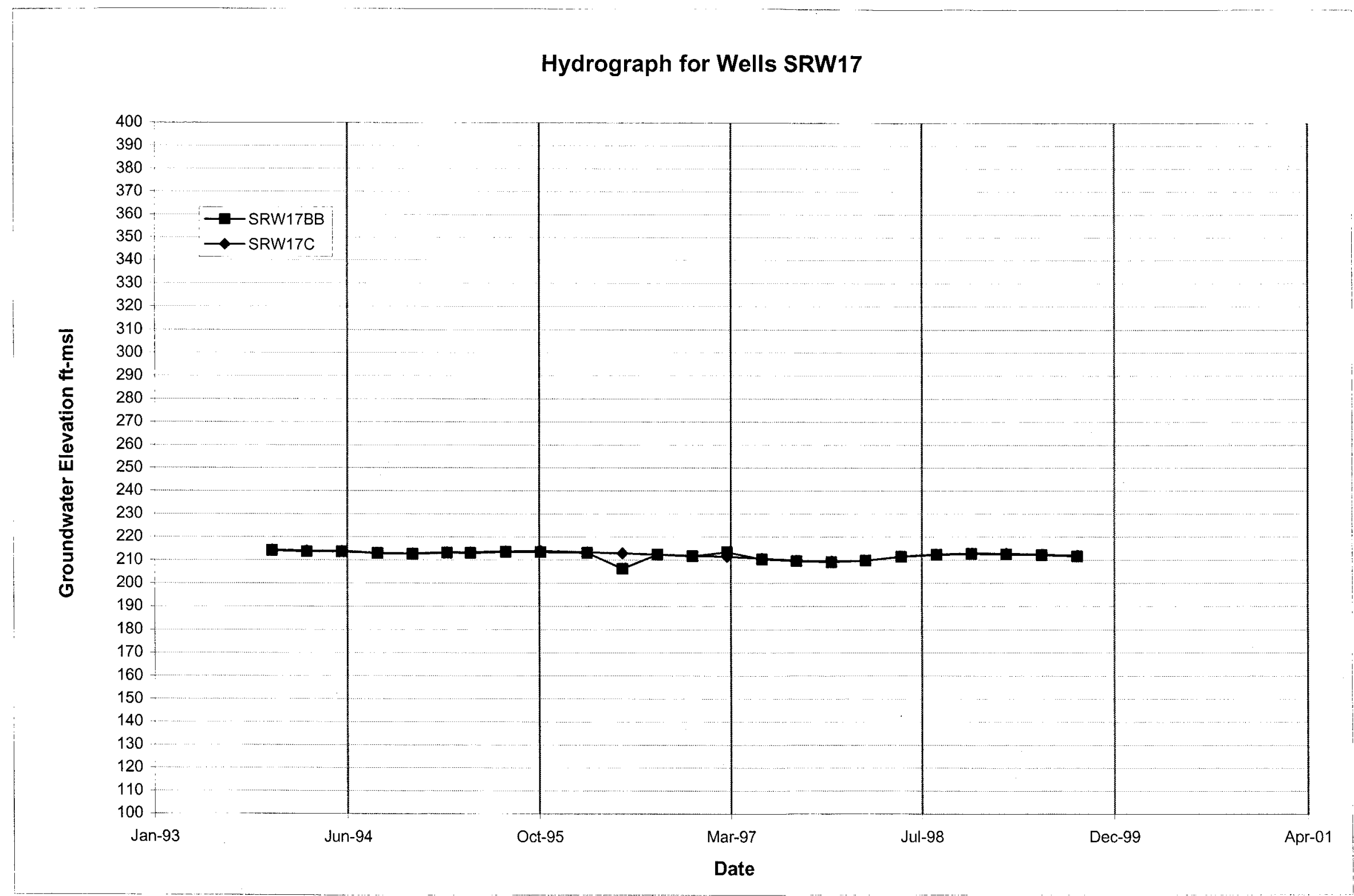




\section{Q/2Q00 M-Area and Metallurgical Laboratory Hazardous Waste Management Facilities Groundwater Monitoring and Corrective-Action Report - First and Second Quarters 2000 - Volumes I, II, and II}

by

J. Chase

Westinghouse Savannah River Company

Savannah River Site

Aiken, South Carolina 29808

This paper was prepared in connection with work done under the above contract number with the U.S. Department of Energy. By acceptance of this paper, the publisher and/or recipient acknowledges the U. S. Government's right to retain a nonexclusive, royalty-free license in and to any copyright covering this paper, along with the right to reproduce and to authorize others to reproduce all or part of the copyrighted paper. 

WSRC-TR-2000-00242

Unclassified

\section{Q/2Q00 M-AREA AND METALLURGICAL LABORATORY HAZARDOUS WASTE : MANAGEMENT FACILITIES GROUNDWATER MONITORING AND CORRECTIVE-ACTION REPORT (U)}

FIRST AND SECOND QUARTERS 2000

VOLUME III

Publication Date: September 2000

Authorized Derivative Classifier and Reviewing Official:

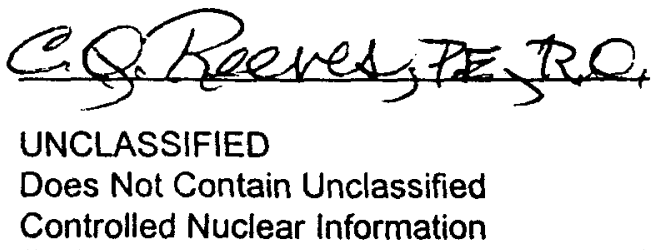

Westinghouse Savannah River Company Savannah River Site

Aiken, SC 29808

Prepared for the U.S. Department of Energy under Control Contract No. DE-AC09-89SR18035 

WSRC-TR-2000-00242

Unclassified

\section{Q/2Q00 M-AREA AND METALLURGICAL LABORATORY HAZARDOUS WASTE MANAGEMENT FACILITIES GROUNDWATER MONITORING AND CORRECTIVE-ACTION REPORT (U)}

FIRST AND SECOND QUARTERS 2000

VOLUME III

Publication Date: September 2000

Authorized Derivative Classifier and Reviewing Official:

UNCLASSIFIED

Does Not Contain Unclassified

Controlled Nuclear Information

Westinghouse Savannah River Company

Savannah River Site

Aiken, SC 29808

Prepared for the U.S. Department of Energy under Control Contract No. DE-AC09-89SR18035 
WSRC-TR-2000-00242

Unclassified

\section{DISCLAIMER}

This report was prepared as an account of work sponsored by an agency of the United States Government. Neither the United States Government nor any agency thereof, nor any of their employees, makes any warranty, express or implied, or assumes any legal liability or responsibility for the accuracy, completeness, or usefulness of any information, apparatus, product or process disclosed, or represents that its use would not infringe privately owned rights. Reference herein to any specific commercial product, process or service by trade name, trademark, manufacturer, or otherwise does not necessarily constitute or imply its endorsement, recommendation, or favoring by the United States Government or any agency thereof. The views and opinions of authors expressed herein do not necessarily state or reflect those of the United States Government or any agency thereof.

This report has been reproduced directly from the best available copy.

Available for sale to the public, in paper, from: U.S. Department of Commerce, National Technical Information Service, 5285 Port Royal Road, Springfield, VA 22161, phone: (800) 553-6847

fax: (703) 605-6900

email: orders@ntis.fedworld.gov

online ordering: http://www.ntis.gov/ordering.htm

Available electronically at http://www.doe.gov/bridge

Available for a processing fee to U.S. Department of Energy and its contractors, in paper, from: U.S. Department of Energy, Office of Scientific and Technical Information, P.O. Box 62, Oak Ridge, TN 37831-0062, phone: (865) 576-8401

fax: (865) 576-5728

email: reports@adonis.osti.gov 


\section{Notice}

Page(s) size did not permit electronic reproduction. Information may be purchased by the general public from the National Technical Information Service, U.S. Department of Commerce, Springfield, VA 22161 (1800-553-6847). DOE and DOE contractors may purchase information by contacting DOE's Office of Scientific and Technical Information, P.O. Box 62, Oak Ridge, TN 37831-0062, Attn: Information Services (1865-576-8401). 